\section{Pacific Northwest}

National Laboratory

Operated by Battelle for the

U.S. Department of Energy

\title{
Database and Interim Glass Property Models for Hanford HLW and LAW Glasses
}

\author{
J. Vienna \\ D. Kim \\ P. Hrma
}

September 2002

Prepared for the U.S. Department of Energy under Contract DE-AC06-76RL01830 


\title{
DISCLAIMER
}

This report was prepared as an account of work sponsored by an agency of the United States Government. Neither the United States Government nor any agency thereof, nor Battelle Memorial Institute, nor any of their employees, makes any warranty, express or implied, or assumes any legal liability or responsibility for the accuracy, completeness, or usefulness of any information, apparatus, product, or process disclosed, or represents that its use would not infringe privately owned rights. Reference herein to any specific commercial product, process, or service by trade name, trademark, manufacturer, or otherwise does not necessarily constitute or imply its endorsement, recommendation, or favoring by the United States Government or any agency thereof, or Battelle Memorial Institute. The views and opinions of authors expressed herein do not necessarily state or reflect those of the United States Government or any agency thereof.

\author{
PACIFIC NORTHWEST NATIONAL LABORATORY \\ operated by \\ BATTELLE \\ for the \\ UNITED STATES DEPARTMENT OF ENERGY \\ under Contract DE-ACO6-76RLO183O
}

Printed in the United States of America

Available to DOE and DOE contractors from the Office of Scientific and Technical Information,

P.O. Box 62, Oak Ridge, TN 37831-0062;

ph: (865) 576-8401

fax: (865) 576-5728

email: reports@adonis.osti.gov

Available to the public from the National Technical Information Service,

U.S. Department of Commerce, 5285 Port Royal Rd., Springfield, VA 22161

ph: (800) 553-6847

fax: (703) 605-6900

email: orders@ntis.fedworld.gov

online ordering: http://www.ntis.gov/ordering.htm

This document was printed on recycled paper.

$(8 / 00)$ 


\title{
Database and Interim Glass Property Models for Hanford HLW and LAW Glasses
}

\author{
J. D. Vienna \\ D.-S. Kim \\ P. Hrma
}

September 2002

Prepared for the U.S. Department of Energy under Contract DE-AC06-76RL01830

Pacific Northwest National Laboratory

Richland, Washington 99352 


\begin{abstract}
This report discusses a methodology for increasing the efficiency and decreasing the cost of vitrifying nuclear waste by optimizing waste-glass formulation. This methodology involves collecting and generating a property-composition database for glass properties that determine waste-glass processability and acceptability and relating these properties to glass composition via property-composition models. The report explains how the property-composition models are developed, fitted to data and evaluated, validated with additional data, used for glass-formulation optimization, and continuously updated in response to changes in waste-composition estimates and processing technologies. Further, the report describes a waste-glass property-composition database compiled from literature sources and presents the results from a critical evaluation and screening of the data for applicability to Hanford waste glasses.

Finally, the report provides interim property-composition models for melt viscosity, liquidus temperature (with spinel and zircon primary crystalline phases), and Product Consistency Test normalized releases of $\mathrm{B}, \mathrm{Na}$, and Li. Models were fitted to a subset of the database deemed most relevant for the anticipated Hanford waste-glass composition region.
\end{abstract}




\section{Summary}

Efforts are being made to increase the efficiency and decrease the cost of vitrifying radioactive waste stored in tanks at U.S. Department of Energy waste sites. The compositions of acceptable and processable high-level waste and low-activity waste glasses need to be optimized to minimize the wasteform volume and, hence, to save cost. A database of properties and associated compositions for simulated waste glasses was collected at Pacific Northwest National Laboratory for developing propertycomposition models. The database includes waste-glass compositions and properties, such as Product Consistency Test (PCT) response, viscosity $(\eta)$, Toxicity Characteristic Leach Procedure response, density $(\rho)$, and liquidus temperature $\left(T_{L}\right)$, that are important for processability and product performance. Data from Pacific Northwest National Laboratory, West Valley Demonstration Project, Savannah River Technology Center, Vitreous State Laboratory at Catholic University of America, Idaho National Engineering and Environmental Laboratory, and several other institutions were reviewed and compiled into a single, easy-to-use database. This database, although not comprehensive, represents a large fraction of data on waste-glass compositions and properties that were available at the time of this report. Because of the size of the database, two versions of this report were printed, one version with the database attached as Appendix A and one version without the database attached.

Glass property-composition models were fit to subsets of the database for several key glass properties. Models were generated for normalized boron, sodium, and lithium release in the PCT, $r_{B}, r_{N a}$, and $r_{L i}$, respectively; $T_{L}$ in the spinel ([Fe,Ni,Mn] $\left.[\mathrm{Fe}, \mathrm{Cr}]_{2} \mathrm{O}_{4}\right)$ and zircon $\left(\mathrm{ZrSiO}_{4}\right)$ primary phase fields; $\eta$ at $1150^{\circ} \mathrm{C}\left(\eta_{1150}\right)$; Arrhenius $\eta$ coefficients $(A$ and $B)$; and molar volume $(\mathrm{V})$. Tables $\mathrm{S} 1$ through S4 summarize these models and the composition regions over which they are valid.

These models were developed from available data in a limited period of time to meet a client deadline. Selected statistical model development, evaluation, and validation methodologies were applied, but there was insufficient time for a complete evaluation and validation. Ordinary "good practices" quality assurance (QA) practices were followed, but the QA was not compliant with RW-0333P (DOERW 2002) or Nuclear Quality Assurance Requirements and Descriptions requirements (PNNL 2000). Future updates of models could be developed to tighter QA requirements when required.

These models should be considered for interim use in calculating properties of Hanford waste glasses. The models presented here represent updates of the models developed by Hrma et al. (2001) and appear to perform as well or better than those models; they cover broader composition regions with comparable or

higher $\mathrm{R}^{2}$ values, especially for validation datasets. However, as we discuss below, the models should be updated when the database is updated.

Table S1. Partial Molar Coefficients for Normalized B, Li, and Na Releases in the PCT 


\begin{tabular}{|c|c|c|c|}
\hline Component & $\overline{b_{B, i}}$ & $\overline{b_{L i, i}}$ & $\overline{b_{N a, i}}$ \\
\hline $\mathrm{Al}_{2} \mathrm{O}_{3}$ & -28.483 & -28.866 & -27.370 \\
\hline $\mathrm{B}_{2} \mathrm{O}_{3}$ & 13.749 & 11.087 & 8.981 \\
\hline $\mathrm{CaO}$ & -11.537 & -7.367 & -4.040 \\
\hline $\mathrm{F}$ & 15.957 & 24.631 & 11.990 \\
\hline $\mathrm{Fe}_{2} \mathrm{O}_{3}$ & -9.692 & -10.013 & -11.597 \\
\hline $\mathrm{K}_{2} \mathrm{O}$ & 8.055 & 2.931 & 11.807 \\
\hline $\mathrm{Li}_{2} \mathrm{O}$ & 9.061 & 8.996 & 7.175 \\
\hline $\mathrm{MgO}$ & 7.031 & 5.323 & 9.058 \\
\hline $\mathrm{MnO}$ & -17.752 & -17.695 & -11.371 \\
\hline $\mathrm{Na}_{2} \mathrm{O}$ & 11.508 & 12.616 & 15.189 \\
\hline $\mathrm{NiO}$ & 34.479 & 28.840 & 39.979 \\
\hline $\mathrm{P}_{2} \mathrm{O}_{5}$ & -24.522 & -8.028 & -19.351 \\
\hline $\mathrm{SiO}_{2}$ & -4.352 & -3.938 & -4.669 \\
\hline $\mathrm{SO}_{3}$ & 75.518 & -- & 49.951 \\
\hline $\mathrm{SrO}$ & -9.824 & -20.210 & 17.360 \\
\hline $\mathrm{ThO}_{2}$ & -17.107 & -- & -- \\
\hline $\mathrm{TiO}_{2}$ & -27.156 & -20.204 & -30.906 \\
\hline $\mathrm{ZnO}$ & -3.511 & 14.493 & -21.494 \\
\hline $\mathrm{ZrO}_{2}$ & -19.611 & -18.661 & -17.045 \\
\hline Others & 1.909 & 0.640 & -1.987 \\
\hline $\mathrm{R}^{2}$ & 0.749 & 0.769 & 0.772 \\
\hline $\mathrm{R}_{\text {adj }}^{2}$ & 0.739 & 0.757 & 0.762 \\
\hline $\mathrm{R}_{\text {pred }}^{2}$ & 0.724 & 0.739 & 0.747 \\
\hline $\mathrm{s}$ & 0.547 & 0.485 & 0.446 \\
\hline min response $\left(\ln \left[\mathrm{r}_{\mathrm{i}}\right]\right)$ & -2.72 & -1.635 & -2.749 \\
\hline mean response $\left(\ln \left[\mathrm{r}_{\mathrm{i}}\right]\right)$ & -0.658 & -0.457 & -0.71 \\
\hline $\max$ response $\left(\ln \left[\mathrm{r}_{\mathrm{i}}\right]\right)$ & 3.22 & 2.09 & 2.64 \\
\hline \# observations & 516 & 371 & 445 \\
\hline
\end{tabular}

Note: Normalized B, Li, and Na releases in the PCT are calculated with the formula:

$$
r_{j}=\exp \left(\sum_{i=1}^{N} r_{j i} x_{i}\right)
$$

where:

$j=\mathrm{B}, \mathrm{Li}, \mathrm{Na}$ is the element released

$i=$ oxide component

$N=$ number of components

$x_{i}=i$-th component mole fraction where $\sum_{i=1}^{N} x_{i}=1$

$r_{j i}=$ coefficient listed in Table S1.

Note: Melt viscosity at $1150^{\circ} \mathrm{C}$ is calculated with the formula: 


$$
\eta_{1150}=\exp \sum_{i=1}^{N} h_{i} x_{i}
$$

Melt viscosity at a given temperature $\mathrm{T}$ is calculated with the formula:

$$
\eta=\exp \sum_{i=1}^{N}\left[\left(A_{i}+B_{i} / T\right) x_{i}\right]
$$

where:

$$
\begin{aligned}
i & =\text { oxide component } \\
N & =\text { number of components } \\
x_{i} & =i \text {-th component mole fraction where } \sum_{i=1}^{N} x_{i}=1
\end{aligned}
$$

$h_{i}, A_{i}$ and $B_{i}=$ coefficients listed in Table S2

Table S2. Partial Molar Coefficients for Viscosity

\begin{tabular}{||l|c|c|c||}
\hline Component & \multicolumn{1}{|c|}{$\boldsymbol{h}_{\boldsymbol{i}}$} & $\boldsymbol{A}_{\boldsymbol{i}}$ & $\boldsymbol{B}_{\boldsymbol{i}}$ \\
\hline $\mathrm{Al}_{2} \mathrm{O}_{3}$ & 19.791 & 2.554 & 23392.8 \\
\hline $\mathrm{B}_{2} \mathrm{O}_{3}$ & -9.52 & -23.120 & 19322.4 \\
\hline $\mathrm{CaO}$ & -5.286 & -11.840 & 9158.0 \\
\hline $\mathrm{Fe}_{2} \mathrm{O}_{3}$ & -3.433 & -14.951 & 15620.9 \\
\hline $\mathrm{K}_{2} \mathrm{O}$ & -8.678 & -8.380 & 1270.8 \\
\hline $\mathrm{Li}_{2} \mathrm{O}$ & -16.25 & -7.886 & -11261.2 \\
\hline $\mathrm{MgO} \mathrm{O}$ & -1.393 & -18.479 & 24230.9 \\
\hline $\mathrm{Na}_{2} \mathrm{O}$ & -10.53 & -11.008 & 837.1 \\
\hline $\mathrm{P}_{2} \mathrm{O}_{5}$ & 14.747 & -18.382 & 43075.7 \\
\hline $\mathrm{SiO}_{2}$ & 9.028 & -8.839 & 25425.3 \\
\hline $\mathrm{ZrO}_{2}$ & 13.246 & -53.976 & 94861.2 \\
\hline $\mathrm{LN}_{2} \mathrm{O}_{3}$ & -11.53 & -51.517 & 52422.2 \\
\hline $\mathrm{BaO}^{\mathrm{F}}$ & -8.507 & 27.367 & -50506.4 \\
\hline $\mathrm{MnO}$ & -7.921 & -13.866 & 9173.6 \\
\hline $\mathrm{SrO}$ & -7.578 & -2.611 & -6619.8 \\
\hline $\mathrm{ThO}_{2}$ & -4.64 & -3.084 & -176.8 \\
\hline $\mathrm{TiO}$ & 13.443 & --- & --- \\
\hline $\mathrm{V}_{2} \mathrm{O}_{5}$ & -6.516 & -5.508 & 1826.5 \\
\hline $\mathrm{ZnO}$ & -9.038 & -45.533 & 50114.4 \\
\hline $\mathrm{Others}$ & --- & -49.368 & 71022.4 \\
\hline $\mathrm{R}^{2}$ & 0.502 & -22.152 & 33384.5 \\
\hline $\mathrm{R}_{\mathrm{adj}}^{2}$ & 0.9707 & & 0.9787 \\
\hline $\mathrm{s}$ & 0.9691 & & 0.9784 \\
\hline \hline
\end{tabular}

Note: $T_{L}$ is calculated with the formula: 


$$
T_{L}=\sum_{N i, C r, M n} T_{i} x_{i}+\sum_{A l k, A l k E}\left(t_{i o n}+\Theta_{i o n} P_{i}\right) x_{i}+\sum_{\text {remaining }}\left(t_{\mathrm{cov}}+\Theta_{\mathrm{cov}} P_{i}\right) x_{i}
$$

where:

$$
\begin{aligned}
i & =\text { electropositive-element component } \\
x_{i} & =i \text {-th element mole fraction where } \sum_{i} x_{i}=1
\end{aligned}
$$

$P_{i}=Z_{i} / r_{i}$, where $Z_{i}$ is the valance of the ion and $r_{i}$ is the crystal radius of the ion given by Shannon (1976)

$\begin{gathered}T_{i}, t_{i o n}, \Theta_{i o n}, \\ t_{c o v}, \text { and } \Theta_{c o v}\end{gathered}=$ coefficients listed in Table S3.

In Equation S.4, components are broken into three groups represented by the three terms in Equation S.4. The first group includes the major spinel components minus Fe, the second group includes the alkali and alkaline-earth components, and the last group includes all components not in the first two groups.

Table S3. Partial Molar Coefficients for Liquidus

Temperature with Spinel Primary Phase

\begin{tabular}{||l|c||}
\hline Item & Value \\
\hline$t_{\text {ion }}$ & $-1,942$ \\
\hline$\Theta_{\text {ion }}$ & 1,996 \\
\hline$t_{c o v}$ & 6,038 \\
\hline$\Theta_{c o v}$ & -500 \\
\hline$T_{C r}$ & 33,271 \\
\hline$T_{M n}$ & 1,316 \\
\hline$T_{N i}$ & 13,675 \\
\hline$\#$ Observations & 320 \\
\hline Min $\mathrm{T}_{\mathrm{L}}\left({ }^{\circ} \mathrm{C}\right)$ & 859 \\
\hline Max $\mathrm{T}_{\mathrm{L}}\left({ }^{\circ} \mathrm{C}\right)$ & 1,310 \\
\hline $\mathrm{Mean}_{\mathrm{L}}\left({ }^{\circ} \mathrm{C}\right)$ & 1,063 \\
\hline $\mathrm{R}^{2}$ & 0.897 \\
\hline $\mathrm{R}^{2}$ adj & 0.895 \\
\hline $\mathrm{s}\left({ }^{\circ} \mathrm{C}\right)$ & 32.77 \\
\hline Max residual $\left({ }^{\circ} \mathrm{C}\right)$ & 78 \\
\hline \hline
\end{tabular}


Note: $T_{L}$ is calculated with the formula:

$$
T_{L}=T_{Z r} x_{Z r}+\sum_{\text {Alk, }, \text { AlkE }}\left(t_{\text {ion }}+\Theta_{i o n} P_{i}\right) x_{i}+\sum_{\text {remaining }}\left(t_{\mathrm{cov}}+\Theta_{\mathrm{cov}} P_{i}\right) x_{i}
$$

where:

$$
\begin{aligned}
i & =\text { electropositive-element component } \\
x_{i} & =i \text {-th element mole fraction where } \sum_{i} x_{i}=1 \\
P_{i} & =Z_{i} / r_{i}, \text { where } Z_{i} \text { is the valance of the ion and } r_{i} \text { is the crystal radius of the ion given by }
\end{aligned}
$$
Shannon (1976)

$T_{Z r}, t_{i o n}, \Theta_{i o n},=$ coefficients listed in Table S4.
$t_{c o v}$, and $\Theta_{c o v}$

In this equation, components are broken into three groups represented by the three terms in Equation S.5. The first group includes only $\mathrm{Zr}$, the second group includes the alkali and alkaline-earth components, and the last group includes all components not in the first two groups.

Table S4. Partial Molar Coefficients for Liquidus

Temperature with Zircon Primary Phase

\begin{tabular}{||l|c|}
\hline \hline Parameter & Value \\
\hline$t_{i o n}$ & $-3,979$ \\
\hline$\Theta_{i o n}$ & 3,524 \\
\hline$t_{c o v}$ & 2,505 \\
\hline$\Theta_{c o v}$ & -146 \\
\hline$T_{Z r}$ & 12,254 \\
\hline $\mathrm{R}^{2}$ & 0.868 \\
\hline $\mathrm{R}_{\text {adj }}^{2}$ & 0.859 \\
\hline $\mathrm{s}\left({ }^{\circ} \mathrm{C}\right)$ & 29.9 \\
\hline$\#$ Observations & 64 \\
\hline
\end{tabular}

Note: Molar volume $(V)$ is calculated with the formula:

$$
V=\sum_{i=1}^{N} V_{i} x_{i}
$$

where:

$i=$ the component

$N=$ number of components

$x_{i}=i$-th component mole fraction where $\sum_{i} x_{i}=1$

$V_{i}=$ the partial molar volume of $i$-th component listed in Table S5. 
The density $(\rho)$ is calculated as follows:

$$
\rho=\frac{\sum_{i=1}^{N} M_{i} x_{i}}{V}
$$

where $M_{i}$ is the molecular weight of $i$-th component.

Table S5. Partial Molar Volume for Density

\begin{tabular}{|c|c|c|c|c|c|}
\hline Comnonent & 18-Camn $V$ & 56-Comn $V$ : & Fommonent & 18-Comn $V$ : & 56-Comn $V$ \\
\hline $\mathrm{Al}_{2} \mathrm{O}_{3}$ & 46.149 & 46.149 & $\mathrm{Ag}_{2} \mathrm{O}$ & & 20.000 \\
\hline $\mathrm{B}_{2} \mathrm{O}_{3}$ & 30.048 & 30.048 & $\mathrm{As}_{2} \mathrm{O}_{5}$ & & 59.810 \\
\hline $\mathrm{BaO}$ & 18.866 & 18.866 & $\mathrm{Ce}_{2} \mathrm{O}_{3}$ & & 45.341 \\
\hline $\mathrm{CaO}$ & 15.214 & 15.214 & $\mathrm{Cr}_{2} \mathrm{O}_{3}$ & & 38.262 \\
\hline $\mathrm{F}$ & 7.526 & 7.526 & $\mathrm{CuO}$ & & 13.960 \\
\hline $\mathrm{Fe}_{2} \mathrm{O}_{3}$ & 39.158 & 39.158 & $\mathrm{Eu}_{2} \mathrm{O}_{3}$ & & 46.855 \\
\hline $\mathrm{K}_{2} \mathrm{O}$ & 37.741 & 37.741 & $\mathrm{Gd}_{2} \mathrm{O}_{3}$ & & 46.491 \\
\hline $\mathrm{Li}_{2} \mathrm{O}$ & 9.943 & 9.943 & $\mathrm{MoO}_{3}$ & & 36.731 \\
\hline $\mathrm{MgO}$ & 13.028 & 13.028 & $\mathrm{Nd}_{2} \mathrm{O}_{3}$ & & 48.117 \\
\hline $\mathrm{MnO}_{\mathrm{x}}$ & 13.175 & 13.175 & $\mathrm{P}_{2} \mathrm{O}_{5}$ & & 59.307 \\
\hline $\mathrm{Na}_{2} \mathrm{O}$ & 19.834 & 19.834 & $\mathrm{PdO}$ & & 15.069 \\
\hline $\mathrm{NiO}$ & 12.668 & 12.668 & $\mathrm{Pr}_{2} \mathrm{O}_{3}$ & & 48.640 \\
\hline $\mathrm{SiO}_{2}$ & 25.316 & 25.316 & $\mathrm{Rh}_{2} \mathrm{O}_{3}$ & & 38.855 \\
\hline $\mathrm{SrO}$ & 17.611 & 17.611 & $\mathrm{RuO}_{2}$ & & 25.070 \\
\hline $\mathrm{TiO}_{2}$ & 17.964 & 17.964 & $\mathrm{SeO}_{3}$ & & 35.709 \\
\hline $\mathrm{ZrO}_{2}$ & 27.081 & 27.081 & $\mathrm{Sm}_{2} \mathrm{O}_{3}$ & & 47.227 \\
\hline $\mathrm{ZnO}$ & 15.069 & 15.069 & $\mathrm{SO}_{3}$ & & 35.526 \\
\hline $\operatorname{Re} \cap$ & & 7800 & Ten。 & & 25307 \\
\hline $\mathrm{Bi}_{2} \mathrm{O}_{3}$ & & 45.000 & $\mathrm{UO}_{3}$ & & 37.606 \\
\hline $\mathrm{CdO}$ & & 17.600 & $\mathrm{~V}_{2} \mathrm{O}_{5}$ & & 61.155 \\
\hline $\mathrm{CoO}$ & & 14.500 & $\mathrm{WO}_{3}$ & & 36.784 \\
\hline $\mathrm{Cs}_{2} \mathrm{O}$ & & 47.000 & Others & 42.812 & 33.479 \\
\hline $\mathrm{Ga} a_{2} \mathrm{O}$, & & 4) 500 & Numher of Glasses & 365 & 365 \\
\hline $\mathrm{HfO}_{2}$ & & 27.500 & $\mathrm{R}^{2}$, molar volume base & 0.949 & 0.946 \\
\hline $\mathrm{La}_{2} \mathrm{O}_{3}$ & & 40.000 & $\mathrm{R}^{2}$ (Adjusted), molar volume base & 0.946 & 0.937 \\
\hline $\mathrm{Nb}_{2} \mathrm{O}_{5}$ & & 56.000 & $\mathrm{R}^{2}$, density base & 0.921 & 0.917 \\
\hline $\mathrm{PbO}$ & & 22.250 & $\mathrm{R}^{2}$ (Adjusted), density base & 0.918 & 0.902 \\
\hline $\mathrm{Rh} \cap \mathrm{O}$ & & $430 n n$ & & & \\
\hline $\mathrm{Sb}_{2} \mathrm{O}_{5}$ & & 47.000 & & & \\
\hline $\mathrm{Sc}_{2} \mathrm{O}_{3}$ & & 28.000 & & & \\
\hline $\mathrm{SnO}_{2}$ & & 28.800 & & & \\
\hline $\mathrm{Ta}_{2} \mathrm{O}_{3}$ & & 52.000 & & & \\
\hline $\mathrm{ThO}_{2}$ & & 31.700 & & & \\
\hline $\mathrm{Y}_{2} \mathrm{O}_{3}$ & & 35.000 & & & \\
\hline
\end{tabular}




\section{References}

DOE-RW. 2002. Quality Assurance Requirements and Description (QARD), DOE/RW-0333P, Rev. 12, U.S. Department of Energy, Office of Civilian Radioactive Waste Management, Washington, D.C.

Hrma P, GF Piepel, JD Vienna, SK Cooley, DS Kim, RL Russell. 2001. Database and Interim Glass Property Models for Hanford HLW Glasses, PNNL-13573, Pacific Northwest National Laboratory, Richland, WA.

PNNL. 2000. Nuclear Quality Assurance Requirements and Description (NQARD) QA Program Manual, Rev. 1, PNNL-13427, Pacific Northwest National Laboratory, Richland, WA.

Shannon RD. 1976. "Revised Effective Ionic Radii and Systematic Study of Interatomic Distances in Halides and Chalcogenides," Acta Cryst. A32:751-767. 


\section{Abbreviations}

ASTM American Society for Testing and Materials

BNFL British Nuclear Fuels, Limited

BNI Bechtel National, Inc.

CCC canister centerline cooled

CUA Catholic University of America

CVS composition variation study

DOE U.S. Department of Energy

DWPF Defense Waste Processing Facility

EA environmental assessment

EDS energy dispersive spectroscopy

HAW high-activity waste

HLW high-level waste

HWVP Hanford Waste Vitrification Plant

HT heat treated

HTWOS Hanford Tank Waste Optimization Simulator

INEEL Idaho National Engineering and Environmental Laboratory

INTEC Idaho Nuclear Technology and Engineering Center

IP ion potential

ISV in situ vitrification

LAW low-activity waste

LM light microscopy

$\mathrm{LN}_{2} \mathrm{O}_{3} \quad$ mixed lanthanide oxide

$\left(\mathrm{LN}_{2} \mathrm{O}_{3}=\mathrm{Ce}_{2} \mathrm{O}_{3}+\mathrm{Eu}_{2} \mathrm{O}_{3}+\mathrm{Gd}_{2} \mathrm{O}_{3}+\mathrm{La}_{2} \mathrm{O}_{3}+\mathrm{Nd}_{2} \mathrm{O}_{3}+\mathrm{Pr}_{2} \mathrm{O}_{3}+\mathrm{Sm}_{2} \mathrm{O}_{3}+\mathrm{Y}_{2} \mathrm{O}_{3}\right)$

MCC Materials Characterization Center

MS melter study

NCAW neutralized current acid waste

NQARD Nuclear Quality Assurance Requirements and Descriptions

OM optical microscopy

ORP Office of River Protection

PCT product consistency test

PNNL Pacific Northwest National Laboratory

PVTD PNNL Vitrification Technology Development 


$\begin{array}{ll}\text { Q } & \text { quenched (glasses) } \\ \text { QA } & \text { quality assurance } \\ \text { RPP } & \text { River Protection Project } \\ \text { RFETS } & \text { Rocky Flats Environmental Technology Site } \\ \text { RPP-WTP } & \text { River Protection Project-Waste Treatment Plant } \\ \text { RMSE } & \text { root mean squared error } \\ \text { SBW } & \text { sodium-bearing waste } \\ \text { SEM } & \text { scanning electron microscopy } \\ \text { SG } & \text { Savannah River Glass } \\ \text { SP } & \text { spinel (study) } \\ \text { SRS } & \text { Savannah River Site } \\ \text { SRTC } & \text { Savannah River Technology Center } \\ \text { TCLP } & \text { toxicity characteristic leaching procedure } \\ \text { TEM } & \text { transmission electron microscopy } \\ \text { THERMO } & \text { thermodynamic hydration energy reaction model } \\ \text { TRU } & \text { Transuranic } \\ \text { TWRS } & \text { Tank Waste Remediation System } \\ \text { VHT } & \text { vapor hydration test } \\ \text { VSL } & \text { Vitreous State Laboratory } \\ \text { VFT } & \text { Vogel -Fulcher-Tamman } \\ \text { WAPS } & \text { Waste Acceptance Product Specifications } \\ \text { WQR } & \text { Waste Form Qualification Report } \\ \text { WTP } & \text { Waste Treatment Plant } \\ \text { WVDP } & \text { West Valley Demonstration Project } \\ \text { WVNS } & \text { West Valley Nuclear Services } \\ \text { WVST } & \text { West Valley Support Task } \\ \text { XRD } & \text { X-ray dion } \\ & \end{array}$




\section{List of Symbols}

\begin{tabular}{|c|c|c|}
\hline Symbol & Definition & Equation \\
\hline $\mathrm{a}$ & constant in molar volume equation & $(6.3)$ \\
\hline$a^{\prime}$ & constant in molar volume equation $=\operatorname{ar}_{0}^{3} / 2$ & $(6.4)$ \\
\hline $\mathrm{A}, \mathrm{A}_{\mathrm{VFT}}$ & composition-dependent coefficients & $(1.9),(1.10)$ \\
\hline$A_{i}$ & i-th component coefficient in Arrhenius viscosity equation & $(1.12),(3.2)$ \\
\hline $\mathrm{A}_{\mathrm{VFT}, \mathrm{i}}$ & i-th component coefficient in VFT viscosity equation & $(1.11)$ \\
\hline $\mathrm{b}$ & constant in molar volume equation & $(6.3),(6.4)$ \\
\hline $\mathrm{B}, \mathrm{B}_{\mathrm{VFT}}$ & composition-dependent coefficients & $(1.9),(1.10)$ \\
\hline $\mathrm{B}_{\mathrm{i}}$ & i-th component coefficient in viscosity equation & $(1.12),(3.2)$ \\
\hline $\mathrm{B}_{\mathrm{VFT}, \mathrm{i}}$ & i-th component coefficient in viscosity equation & $(1.11)$ \\
\hline $\mathrm{b}_{\alpha, \mathrm{i}}$ & i-th component coefficient for $\alpha$-th property & $(1.3),(1.4)$ \\
\hline $\mathrm{c}_{\alpha}$ & $\alpha$-th property constraint constant & $(8.1)$ \\
\hline $\mathrm{f}_{\alpha}$ & $\alpha$-th property-composition function & $(1.1),(1.3)$ \\
\hline $\mathbf{F}_{\mathrm{W}}$ & composition vector function & \\
\hline $\mathrm{g}_{\alpha}$ & $\alpha$-th property-composition function (inverse) & $(1.4)$ \\
\hline $\mathrm{h}_{\mathrm{i}}$ & i-th component coefficient for $\ln \left[\eta_{1150}\right]$ & $(3.1)$ \\
\hline $\mathrm{K}$ & number of constraints & \\
\hline $\mathrm{L}_{\mathrm{i}}$ & i-th component atomic/thermodynamic characteristic & $(1.6),(1.7)$ \\
\hline $\mathrm{m}_{\alpha}$ & $\alpha$-th property empirical coefficient & $(1.6),(1.7)$ \\
\hline $\mathrm{M}_{\mathrm{i}}$ & i-th element molecular weight & $(6.1)$ \\
\hline $\mathrm{n}_{\alpha}$ & $\alpha$-th property empirical coefficient & $(1.6),(1.7)$ \\
\hline $\mathrm{N}$ & number of components in glass & (1.2), etc. \\
\hline $\mathbf{p}$ & property vector & \\
\hline $\mathrm{p}_{\alpha}$ & $\alpha$-th property & $(1.1),(1.3)$ \\
\hline $\mathrm{p}_{\alpha}^{\prime}$ & $\alpha$-th property of the corrected glass & $(8.4)$ \\
\hline $\mathrm{P}_{\mathrm{i}}$ & $\mathrm{i}$-th ion potential $=\mathrm{Z}_{\mathrm{i}} / \mathrm{r}_{\mathrm{i}}$ & $(4.1)$, etc. \\
\hline $\mathrm{Q}_{0}, \mathrm{Q}_{\mathrm{i}}$ & i-th component constraint constants & $(8.2)$ \\
\hline$r_{i}$ & i-th component ionic radius & $(6.3),(6.4)$ \\
\hline$r_{j}$ & $\mathrm{j}$-th element normalized PCT release from quenched glass & $(5.1)$ \\
\hline$r_{j, i}$ & $\mathrm{i}$-th component coefficient for $\mathrm{j}$-th element (PCT release) & $(5.1)$ \\
\hline $\mathrm{t}_{\mathrm{cov}}$ & $\begin{array}{l}\text { intercept of the line relating the effect of other components on } T_{L} \text { with their } \\
\text { ion potential }\end{array}$ & (4.1), etc. \\
\hline$t_{\text {ion }}$ & $\begin{array}{l}\text { intercept of the line relating the effect of ionic components on } T_{L} \text { with their } \\
\text { ion potential }\end{array}$ & (4.1), etc. \\
\hline $\mathrm{T}$ & temperature (on absolute scale unless otherwise specified) & (1.8), etc. \\
\hline $\mathrm{T}_{0}$ & composition-dependent coefficient & $(1.9)$ \\
\hline $\mathrm{T}_{0, \mathrm{i}}$ & i-th component coefficient in viscosity equation & $(1.11)$ \\
\hline
\end{tabular}




$\begin{array}{lll}\mathrm{T}_{\mathrm{i}} & \text { liquidus temperature i-th component coefficient } & (4.1), \text { etc. } \\ T_{L} & \text { liquidus temperature } & (4.1),(4.4) \\ \mathbf{G} & \text { glass composition region with acceptable properties } & (8.3) \\ \mathrm{V}_{\mathrm{i}} & \text { i-th component apparent partial molar volume } & (6.3),(6.4) \\ \mathrm{V} & \text { molar volume } & (6.1),(6.2) \\ \mathrm{V}_{\mathrm{i}} & \text { i-th component partial molar volume } & (6.2) \\ \mathrm{W} & \text { waste loading } & (8.3) \\ \mathbf{x} & \text { composition vector } & (1.1),(1.8) \\ \mathrm{x}_{\mathrm{i}} & \text { i-th component mole fraction } & (1.2), \text { etc. } \\ \mathrm{x}_{\mathrm{i}} & \text { i-th component mole fraction in corrected glass } & (8.4) \\ \mathbf{x}_{\mathbf{0}} & \text { optimized glass composition vector } & (6.3),(6.4) \\ \mathrm{Z}_{\mathrm{i}} & \text { i-th component charge (valence) } & \\ \varepsilon & \text { melt electrical conductivity } & (1.9), \text { etc. } \\ \eta & \text { melt viscosity } & (6.1),(6.2) \\ \rho & \text { glass density } & (6.2) \\ \rho_{\mathrm{i}} & \text { i-th component coefficient for glass viscosity } & (4.1), \text { etc. } \\ \theta_{\text {cov }} & \text { slope of the line relating the effect of other components on } \mathrm{T}_{\mathrm{L}} \text { with their ion } & \text { potential } \\ \theta_{\text {ion }} & \text { slope of the line relating the effect of ionic components on } \mathrm{T}_{\mathrm{L}} \text { with their ion } & (4.1), \text { etc. }\end{array}$




\section{Acknowledgments}

The authors are grateful to Bill Holtzscheiter (SRTC) for management and guidance, Greg Piepel (PNNL) and Steve Lambert (NHC) for helpful discussions and useful suggestions, Wayne Cosby (PNNL) for careful editing, Carol Jantzen (SRTC) for supplying Savannah River Technology Center data, Ian Pegg (VSL) for supplying Vitreous State Laboratory data, and David Peeler (SRCT), Denis Strachan (PNNL), Greg Piepel (PNNL), and Steve Lambert (NHC) for careful review of the text and helpful comments. This work was performed at the Pacific Northwest National Laboratory, which is operated for the U.S. Department of Energy (DOE) by Battelle under Contract DE-AC06-76RL01830. The DOE Office of Environmental Management funded this work through the Tanks Focus Area Immobilization Program.

\section{Quality Assurance}

Ordinary "good practices" quality assurance (QA) was performed in developing the database and models. Formal QA documentation, such as would be required for Nuclear Quality Assurance Requirements and Descriptions, was not performed because of the interim nature of the work. 


\section{Contents}

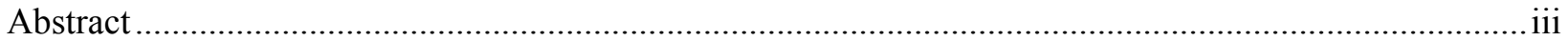

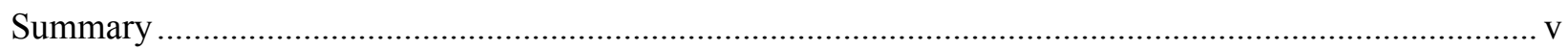

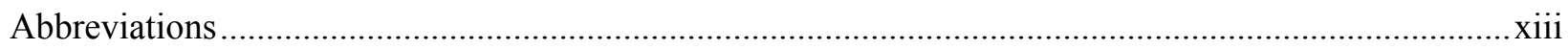

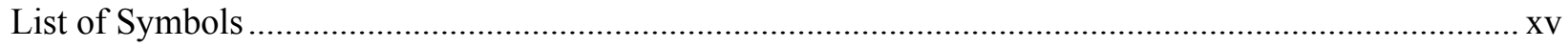

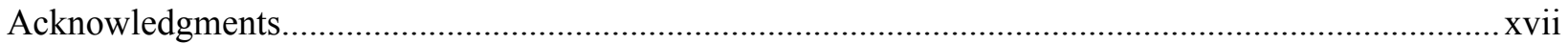

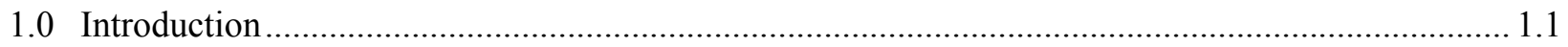

1.1 The Role of Property-Composition Models ...................................................................... 1.2

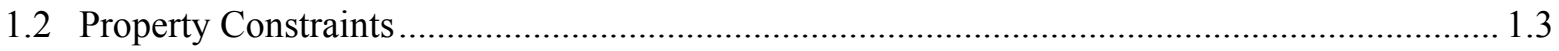

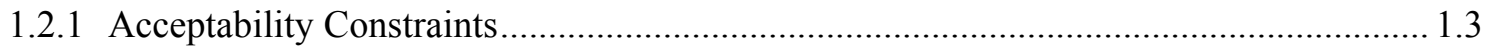

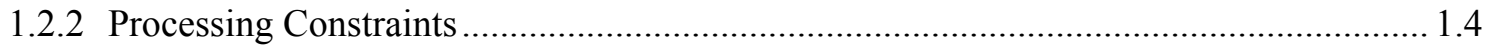

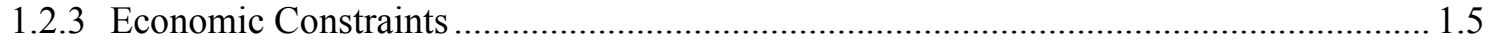

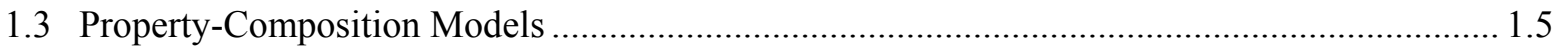

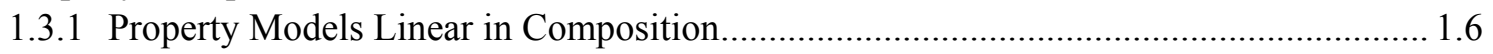

1.3.2 Property Models Linear in Composition and Nonlinear in Temperature ....................... 1.8

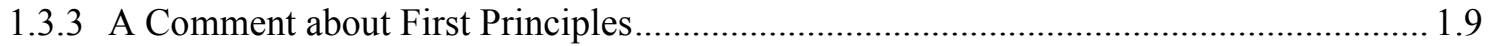

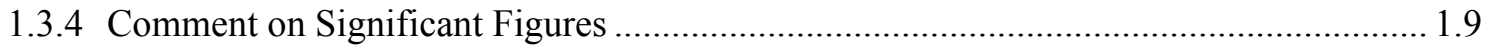

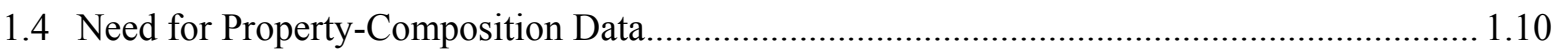

1.5 Data Reduction: Partial Specific/Molar Coefficients ...................................................... 1.11

1.6 Existing Property Composition Models for Hanford HLW Glass ...................................... 1.13

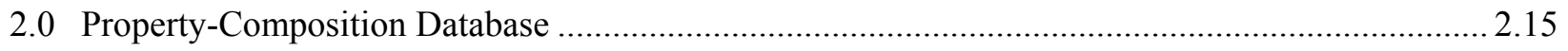

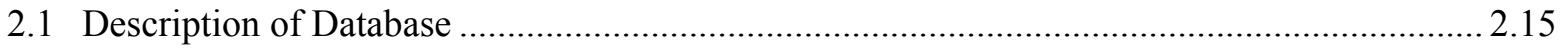

2.2 Discussion of Data Sets Used in Model Development....................................................... 2.16

2.2.1 Idaho National Engineering and Environmental Laboratory Composition Variation

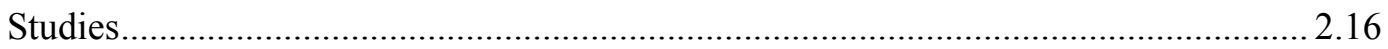

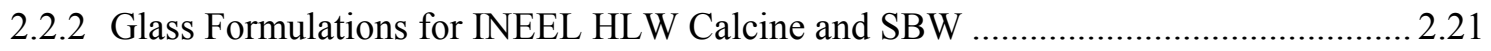

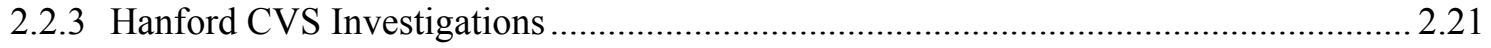

2.2.4 HLW Glass Formulation for Hanford Wastes at the Vitreous State Laboratory ........... 2.22

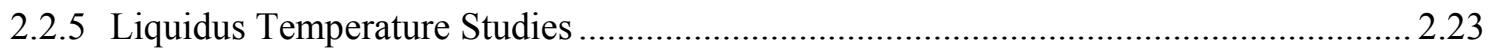

2.2.6 Other Property-Composition Relation Studies ...................................................... 2.24

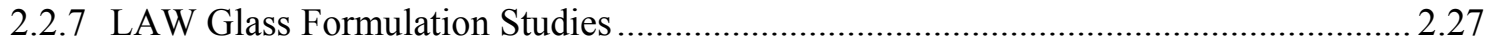




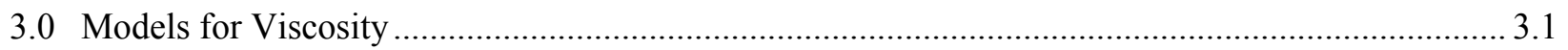

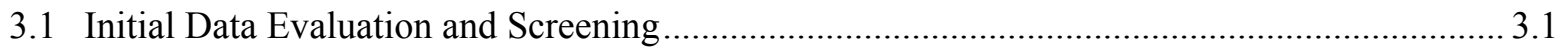

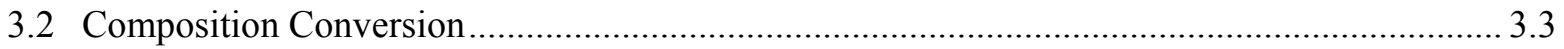

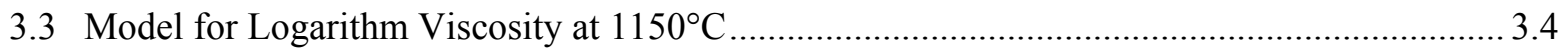

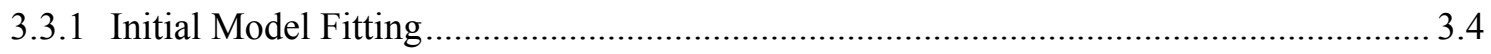

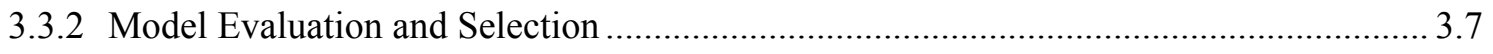

3.4 Model for Viscosity as a Function of Temperature ....................................................... 3.11

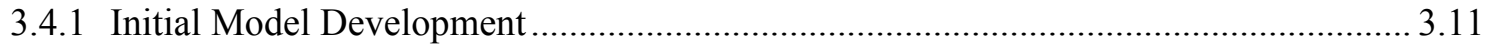

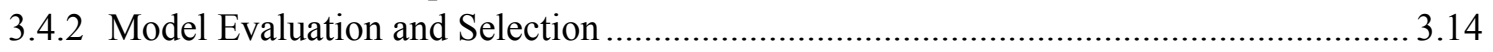

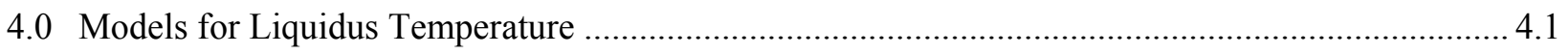

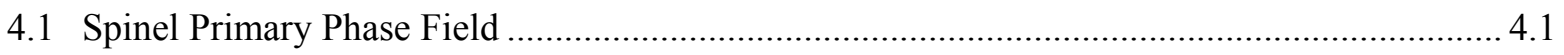

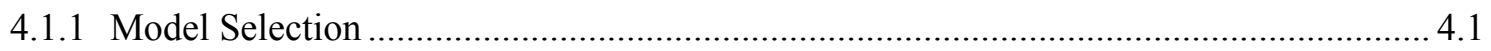

4.1.2 Composition Conversion and Initial Data Screening .................................................... 4.1

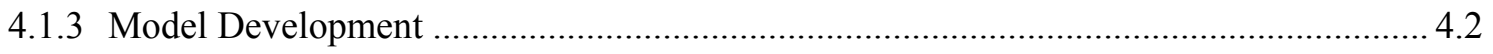

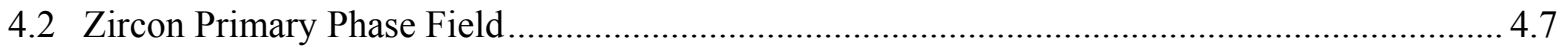

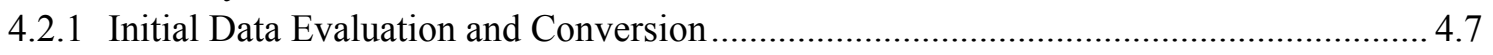

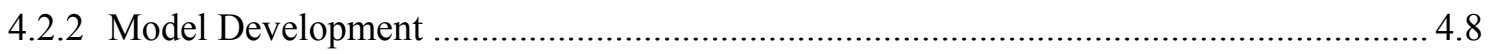

5.0 Models for Product Consistency Test Response …...................................................................... 5.1

5.1 Initial Data Evaluation, Screening, and Conversion …................................................... 5.1

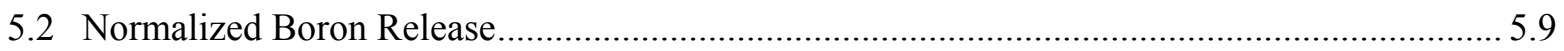

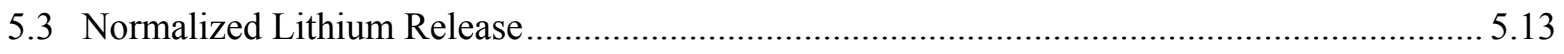

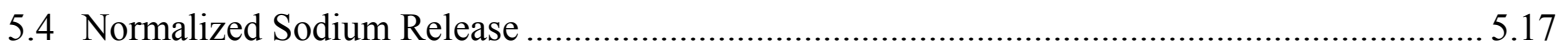

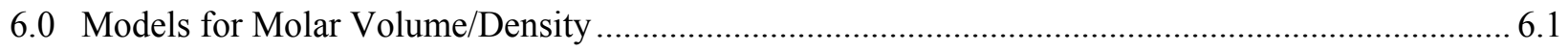

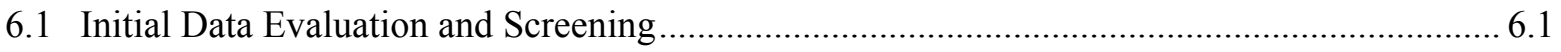

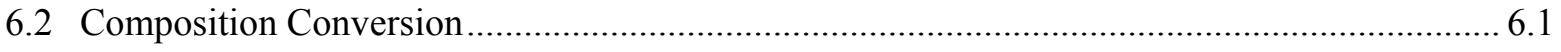

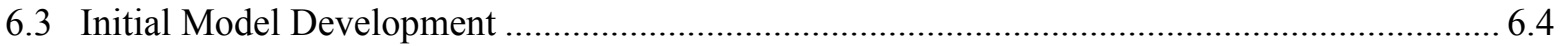

6.3.1 General Forms of the Density and Molar Volume Models ............................................ 6.4

6.3.2 Selection of Model Forms for Density Modeling ......................................................... 6.5

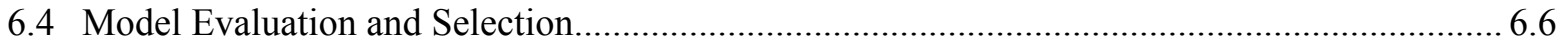

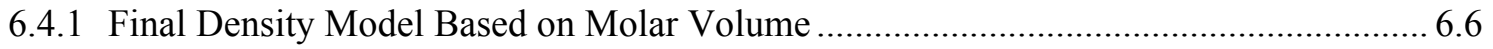

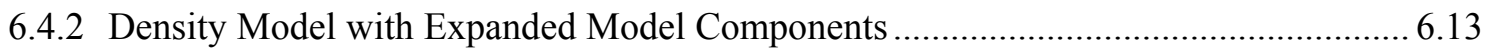




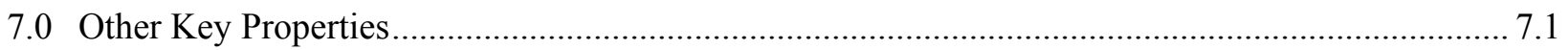

7.1 Toxicity Characteristic Leaching Procedure Response ..................................................... 7.1

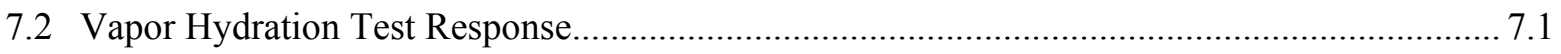

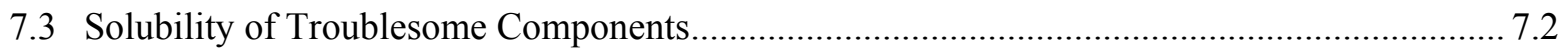

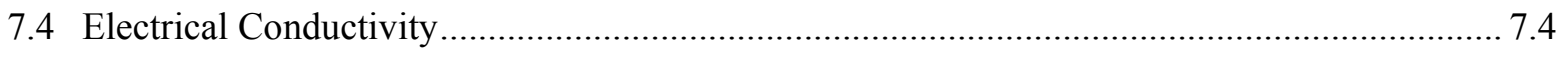

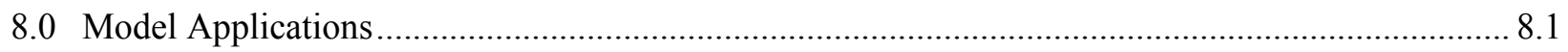

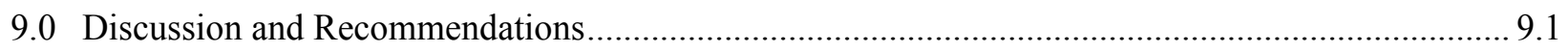

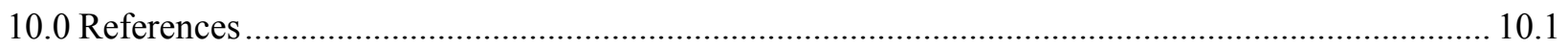

\section{Figures}

Figure 3-1. Comparison of Predicted and Measured $\ln \left[\eta_{1150}(\mathrm{~Pa} \cdot \mathrm{s})\right]$ by the Initial Model with Two Data Points (shown as solid triangles) Removed ........................................................................... 3.5

Figure 3-2. Comparison of Predicted and Measured $\ln [\eta]$ Values for Validation Glasses (not used in

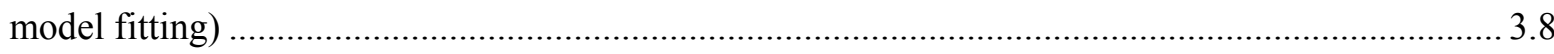

Figure 3-3. Scatter Plot Matrix for $\ln \left[\eta_{1150}\right]$ Model Data Component Concentrations .......................... 3.10

Figure 3-4. Comparison of Measured and Predicted $\ln [\eta(\mathrm{Pa} \cdot \mathrm{s})]$ from Initial 24-Component Arrhenius

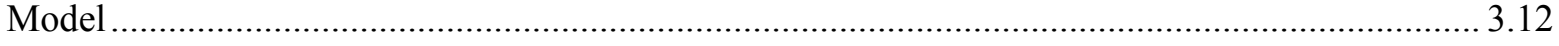

Figure 3-5. Comparison of Calculated and Measured $\ln [\eta]$ for the Complete Validation Data Set ....... 3.15

Figure 3-6. Comparison of Calculated and Measured $\ln [\eta]$ for the Reduced Validation Data Set........ 3.15

Figure 3-7. Comparison of $a_{i}$ Values from Different Models (Hrma represents Hrma et al. 2001, and

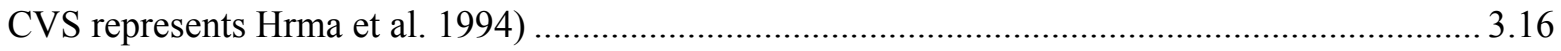

Figure 3-8. Comparison of $b_{i}$ Values from Different Models (Hrma represents Hrma et al. 2001, and CVS represents Hrma et al. 1994) ….................................................................................. 3.17

Figure 4-1. Comparison of Calculated and Measured $\mathrm{T}_{\mathrm{L}}$ Values .................................................... 4.4

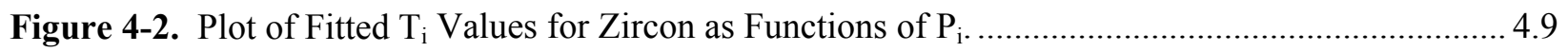

Figure 4-3. Plot Comparing Measured and Calculated Zircon $T_{L}$ Values from IP Model................... 4.10

Figure 5-1. Scatter Plot Matrix for $\ln \left[\mathrm{r}_{\mathrm{B}}\right]$ Model Data Component Concentrations ............................ 5.11 
Figure 5-2. Scatter Plot Matrix for $\ln \left[\mathrm{r}_{\mathrm{Li}}\right]$ Model Data Component Concentrations......

Figure 5-1. Scatter Plot Matrix for $\ln \left[\mathrm{r}_{\mathrm{Na}}\right]$ Model Data Component Concentrations

Figure 6-1. Plot of Appen's Molar Volume Coefficients Versus Model Fit Coefficients from the 21Component Linear Mixture Model (the bars on selected points cover range of reported values with mean values used as data points)

Figure 6-2. Plot of Appen's Molar Volume Coefficients versus Model Fit Coefficients from the 18Component Linear Mixture Model (error bars cover range of reported values with mean values used as data points)

Figure 6-3. Plot of Calculated Versus Measured Density Values for Data Used to Fit the 18-Component Molar Volume Model

Figure 6-4. Plot of Calculated Versus Measured Density Values for the Glasses Deleted and Not Used to Develop the 18-Component Linear Mixture Model

Figure 6-5. Plot of Appen's Molar Volume Coefficients Versus Model Fit Coefficients from 18Component Model

\section{Tables}

Table 1-1. Advantages and Disadvantages of Two Approaches for Exploring a Glass Composition Region of Interest.

Table 2-1. Summary of Number of Glasses Tested and Properties Measured in Each Data Set 2.18

Table 3-1. List of Glasses Excluded from Viscosity Model Development.

Table 3-2. Mole Fraction Range for Components in Viscosity Model Data Glasses

Table 3-3. Summary of Initial Model for $\ln \left[\eta_{1150}\right]$

Table 3-4. Summary of $\ln \left[\eta_{1150}\right]$ Models.......

Table 3-5. Comparison of Component Coefficients for the New Model with Previous, Hanford CVS Model

Table 3-6. Component Concentration Ranges (in mass fractions) for Accurate Prediction of $\ln \left[\eta_{1150}\right] 3.11$

Table 3-7. Summary of Arrhenius Viscosity Models.

Table 4-1. Glasses with Extreme Component Concentrations or $T_{L}$ Values. 


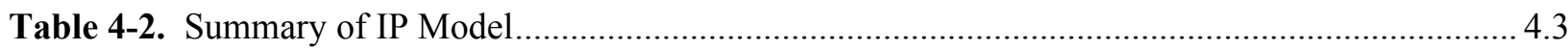

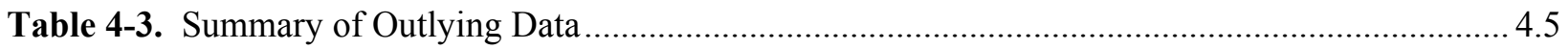

Table 4-4. IP Model Validity Concentration Ranges (in mass\% of oxides and F) .............................. 4.6

Table 4-5. $\mathrm{Z}_{\mathrm{i}}, \mathrm{r}_{\mathrm{i}}, \mathrm{P}_{\mathrm{i}}$, and $\mathrm{T}_{\mathrm{i}}$ Values for Typical HLW Components (i)................................................... 4.7

Table 4-6. $\mathrm{T}_{\mathrm{i}}$ Values for the First-Order Model Calculated from the Zircon Data Set ......................... 4.8

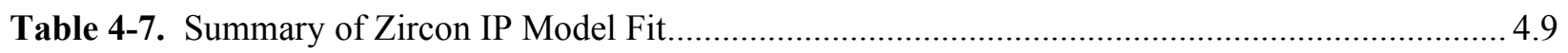

Table 4-8. $\mathrm{Z}_{\mathrm{i}}, \mathrm{r}_{\mathrm{i}}, \mathrm{P}_{\mathrm{i}}$, and $\mathrm{T}_{\mathrm{i}}$ Values for Typical HLW Components (i).............................................11

Table 4-9. Concentration Ranges and Outlier Composition (in mass\%) for the Data that Were Used to Fit the $\mathrm{T}_{\mathrm{L}}$ Model in the Zircon Primary Phase Field .................................................................... 4.12

Table 5-1. Ranges of Selected Glass Components (mass\% oxide or halogen) ................................... 5.2

Table 5-2. Glasses Excluded from Model Development Because Their Compositions are Outside the Region of Expected Hanford Waste Glasses (concentrations of components are in mass\% of the

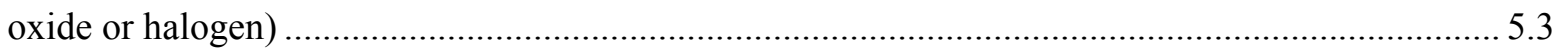

Table 5-3. List of Glasses Excluded Due to Data Problems ............................................................... 5.6

Table 5-4. Glasses with Extreme Compositions Excluded from PCT Model Development Data Set (with components that were in extreme concentrations listed) ......................................................... 5.7

Table 5-5. Glasses Selected for Validation of PCT Release Models ............................................... 5.8

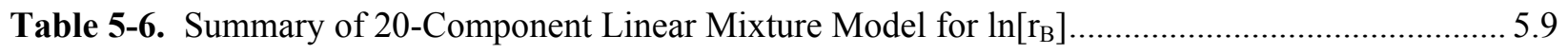

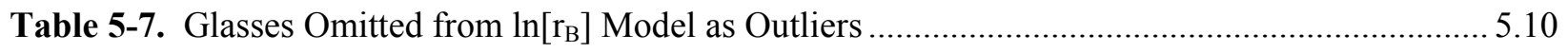

Table 5-8. Component Concentration Ranges for Data Used to Fit $\ln \left[\mathrm{r}_{\mathrm{B}}\right]$ Model (mole\%) ................. 5.10

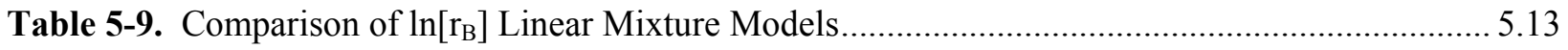

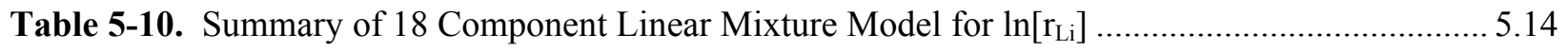

Table 5-11. Glasses Omitted from $r_{\mathrm{Li}}$ Model as Outliers ............................................................. 5.14

Table 5-8. Component Concentration Ranges for Data Used to Fit $\ln \left[\mathrm{r}_{\mathrm{Li}}\right]$ Model (mole\%) ................. 5.14

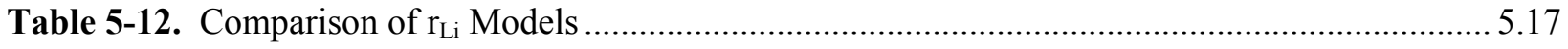

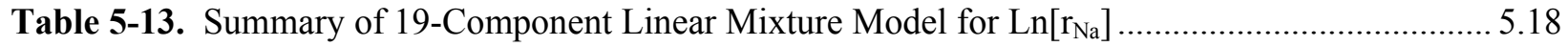

Table 5-14. Glasses Omitted from $\mathrm{r}_{\mathrm{Na}}$ Model as Outliers................................................................ 5.18 
Table 5-8. Component Concentration Ranges for Data Used to Fit $\ln \left[\mathrm{r}_{\mathrm{Na}}\right]$ Model (mole\%)

Table 5-15. Comparison of $r_{\mathrm{Na}}$ Models

Table 6-1. Glasses Deleted during Screening for Density Model Development (composition expressed in mole fraction)

Table 6-2. Mole Fraction Range for Components in Density Model Data Glasses .

Table 6-3. Mass Fraction Range for Components in Density Model Data Glasses .....

Table 6-4. Comparison of Model Statistics for Different Density Model Forms from the Reduced Data Set, where $20 \%$ of Data were Not Included in the Model Development But Were Used for Model Validation

Table 6-5. Comparison of Model Statistics for Different Model Forms for Different Data Sets; all $\mathrm{R}^{2}$ Statistics are Calculated Based on Density

Table 6-6. Summary Statistics for Molar-Volume-Based Models for Density Modeling. 6.7

Table 6-7. Partial Molar Volumes for Glass Components Published by Appen (1970) 6.8

Table 6-8. Model Coefficients and Standard Errors for the Final 18-Component Linear Mixture Model for Molar Volume 6.11

Table 6-9. Constants in Equation 6.4

Table 6-10. Comparison of Partial Molar Volumes and Fit Statistics Obtained from Linear-Mixture Model Fit, Appen, and Equation 6.4 and Those Obtained by Combining the Three Models 6.17

Table 7-1. Single- and Multi-Component Concentration Constraints in Mass\% 7.2 


\subsection{Introduction}

This report discusses interim property-composition models developed for Hanford high-level waste (HLW) and low-activity waste (LAW) glasses. This effort is aimed at improving the understanding of composition effects on glass properties over the broad composition space of expected Hanford waste glasses. With this increased understanding, the ability to more accurately estimate glass volumes, cleanup cost and schedule, and the impacts of changing property constraints and flowsheet options will be possible.

The interim models were developed from a glass property-composition database compiled for this purpose. Because the compiled database covers a large fraction of the regions of HLW and LAW glass compositions considered potentially of interest for Hanford, the resulting interim property-composition models are considered global models (Piepel, Hrma, and Vienna 1998). Global models are useful when it is necessary to make property predictions over a wide HLW glass-composition region. Global models are likely to yield less-accurate property predictions (compared to local models near specific waste glass compositions), but still can be useful. Eventually, when target compositions and composition-variation regions around target compositions for specific waste types ${ }^{(a)}$ are better defined, it may be advisable to develop local models to predict HLW glass properties over the composition-variation region corresponding to a target composition for a given waste type. Local models, because they cover smaller composition regions centered near the glass composition of interest, are typically more accurate.

The glass property-composition models presented in this report are referred to as interim models for several reasons. The most obvious reason is that property-composition models will continue to be developed as more property-composition data become available, and better information about HLW compositions $^{(\mathrm{b})}$ and corresponding waste-glass compositions become available. However, the models should also be considered interim due to limitations in the data used to develop them. Ideally, to provide good support for developing property-composition models (per Piepel, Hrma, and Vienna 1998), a wasteglass-composition region of interest should be defined and covered with property-composition data derived with the use of statistical experimental design methods.

The interim models presented in this report were developed from a compilation of existing data. The data were screened so that only relevant data (i.e., data in the appropriate composition region) were used. However, the coverage of the waste glass-composition region(s) of interest was not as extensive as desired. Hence, the quality of the property-composition models discussed in this report may be affected by the limitations in the available data upon which they were based. Further, the time to develop, evaluate, and validate the interim-property models was limited. The available time did not permit the use of many statistical regression diagnostic methods (Draper and Smith 1998; Montgomery and Peck 1992) to identify outlying or influential data points and to identify the subset of waste-glass components that should be included in the interim model for a given property. Statistical model-validation methods (Montgomery and Peck 1992, Chapter 10) were not applied. However, informal model-validation

(a) A waste type is defined in the Waste Acceptance Product Specifications (WAPS) (DOE 1996) as "the waste material fed to each vitrification plant, the composition and properties of which will remain relatively constant over an extended period of time during waste-form production."

(b) It is expected that waste feed compositions will be updated as tank characterization, retrieval/blending scenarios, and definition of chemical impacts from separations and pretreatment processes are improved. 
methods were applied to identify validation points for which model predictions are not close to measured values. In summary, although the interim property-composition models presented in this report have certain limitations, they should be useful for the intended purpose. These models are expected to give improved predictions of glass properties over those previously developed for Hanford HLW waste glasses.

The following subsections in this introduction address the role of property-composition models, property constraints implemented through models, the need for property-composition data to develop models, the forms of property-composition models, model fitting and reduction, and previous work to develop property models for Hanford HLW glasses. Section 2.0 presents a property-composition database compiled from previous studies to provide a basis for developing HLW property-composition models. Sections 3.0 through 7.0 discusses the interim property-composition models developed for Hanford HLW glasses, the data used to develop the models, and the glass-composition regions over which the models are valid for predicting glass properties. Section 8.0 discusses the use of propertycomposition models and mathematically constrained optimization methods to develop glass formulations and estimate waste-glass volumes. Section 9.0 summarizes a number of suggestions for future studies to complete the objectives of this research.

\subsection{The Role of Property-Composition Models}

The processability and acceptability of a waste glass is specified in terms of its properties. To make waste glass, the waste must be mixed with glass-forming additives in proportions to obtain a target glass composition that must have properties within prescribed limits. High-level waste and LAW glass compositions vary as a result of the changing composition of waste. The economic aspect of vitrification requires that waste-glass composition should minimize the expense.

It is practically impossible to develop an optimized waste glass for each waste composition on a purely experimental basis. Mathematical, statistical, and optimization methods are extremely useful in developing optimal glass compositions. Therefore, the task of developing optimum glass compositions must be addressed within a mathematical framework. An indispensable element of such a framework is a set of property-composition models. These models should be developed for waste glasses covering both the glass composition region of interest and a sufficiently broad range of values for glass properties of interest.

Property-composition models applicable to Hanford waste glasses have been developed at Pacific Northwest National Laboratory (PNNL) for over a decade. Initially, only one HLW waste stream was considered that would be processed in a certain type of melter without any attempt for optimization (Hrma et al. 1994). However, the need for property-composition models broadened over time because the waste-composition estimates changed, new waste streams were taken into consideration, new types of melters were tested, and economic considerations became more important. Thus, it became clear that the composition region of waste glass for the development of property-composition models was too narrow, and the ranges of existing property-composition models were not broad enough for practical applications.

The composition region of waste glass is determined by compositions and concentrations of waste and additives. Which are, in turn, determined by the range of glass properties that define acceptability for waste form storage, transport, and disposal; the processing technology related constraints; and the 
applicable economic considerations. These factors are not fixed; all are frequently changing, and some are interdependent. Estimated waste compositions change with new samples and chemical analyses, pretreatment methods, retrieval strategies, and blending options. The acceptability conditions for HLW glass have been relatively stable for the last decade. ${ }^{(c)}$ However, opinions vary as to how much crystallinity should be allowed in the glass and whether the glass can include immiscible amorphous phase-separation. New LAW glass-acceptability constraints have been recently developed (BNI 2001). It is conceivable that additional acceptability conditions may be imposed in the future as additional concerns regarding safety are raised. Finally, different melter types have been considered in the past or are considered for the future, e.g., Joule-heated melter, stirred melter, high-temperature melter, and coldwalled induction-heated melter. Different melters require different sets of glass-property values for processability. Economic considerations are often assessed in terms of waste loading in glass.

Maximizing waste loading generally minimizes the costs of producing and disposing of the waste glass if produced at a fixed rate.

With each change, whether in

- the estimated composition of waste in the tanks currently considered for vitrification

- the group of tanks being considered for processing

- the key glass components resulting from pretreatment or

- the melter type being considered for vitrification,

the set of property-composition models needs to be updated. Extrapolation beyond the composition region on which the models are established can lead to misleading results. There is another reason for periodic updating of property-composition models. As more glasses are being tested to support various programs, more and more glass-property data accumulate. These data can be used to validate the existing property-composition models and ultimately to update these models. The development of propertycomposition models must respond to continuous changes. Consequently, developing propertycomposition models for waste glass is a continuous process.

\subsection{Property Constraints}

Three kinds of constraints on waste-glass properties exist: acceptability constraints, processing constraints, and economic constraints. Each of these kinds of constraints is discussed below.

\subsubsection{Acceptability Constraints}

Acceptability constraints are concerned with the acceptability of the final product. Roughly, waste glass should have sufficiently high chemical durability ${ }^{(d)}$ and should retain this durability over thousands of years. Although the engineered barriers in the repository and the geology of the repository itself are designed to prevent the spreading of radioactive elements into the environment, the glass itself should

(c) The acceptability of HLW glass is described in the Waste Acceptance Product Specifications (WAPS) (DOE 1996).

(d) Chemical durability is typically defined as resistance to degredation caused by chemical attack. In the case of waste glasses, chemical durability can be considered the ability of the glass to withstand attack from an aqueous medium without the release of hazardous or radioactive components. 
have good resistance against corrosion by water. The benchmark test for U. S. HLW glass is the Product Consistency Test (PCT) (ASTM 1998). A rough statement of an applicable PCT constraint is that no acceptable HLW glass should have a higher release of boron, sodium, or lithium than the environmental assessment (EA) glass after normalization for their fractions in glass (DOE 1996). As our knowledge of glass behavior increases, glasses are routinely formulated that surpass the EA constraint by nearly an order of magnitude. However, to implement this constraint, various uncertainties must be accounted for in PCT measurement, release predictions from models that are functions of glass composition, and glass compositions used in predicting PCT release.

Under the current River Protection Project-Waste Treatment Plant (RPP-WTP) contract (BNI 2001), the LAW glass at Hanford must also be tested with the PCT and yield normalized boron and sodium releases of less than $2 \mathrm{~g} \cdot \mathrm{m}^{-2}$ after 7 days. In addition, the rate of alteration as measured by a vapor hydration test (VHT) at $200^{\circ} \mathrm{C}$ should be less than $50 \mathrm{~g} \cdot \mathrm{m}^{-2} \cdot \mathrm{d}^{-1}$.

High-level waste glass properties may strongly depend on the temperature history of the glass. Slowly cooled glass is prone to amorphous phase separation and crystallization. Crystals are usually durable, which means that they remove components from the glass phase that endow glass with durability. Thus, the crystallization of certain minerals, such as nepheline, may produce non-durable glass. Therefore, PCT performance should be studied and modeled for glass that is quickly cooled, such as quenched glass, and glass with the slowest rate of cooling, such as canister-centerline-cooled; see Marra and Jantzen (1993) and Riley, Rosario, and Hrma (2001). Although the primary consideration in waste form acceptance is its performance, the formation of additional phases impacts the ability to predict the performance, such as PCT release. This imposes a further restriction on glass composition by increasing the error margin in PCT release for which an account must be given.

\subsubsection{Processing Constraints}

The second type of constraint is added to assure the processability of glass in the melter. The acceptable ranges for processability-related glass properties differ for different melter types. Generally, glass is processable when its viscosity $(\eta)$ is lower than 10 or $15 \mathrm{~Pa} \cdot \mathrm{s}$ - the lower the viscosity, the higher the rate of processing. However, when $\eta$ is below 1 or $2 \mathrm{~Pa} \cdot \mathrm{s}$, glass becomes more corrosive to those materials that are in contact with the melt (e.g., refractories, electrodes, and bubblers) and steam excursions $^{(\mathrm{e})}$ become more likely. Therefore, between 1 and $2 \mathrm{~Pa} \cdot \mathrm{s}$ is generally considered the lower limit for melt viscosity, although lower viscosities may be acceptable.

An important property of HLW glass is its liquidus temperature $\left(T_{L}\right)$, the highest temperature at which a solid phase can exist in the melt at equilibrium. ${ }^{(\mathrm{f})}$ With increasing waste loading, the $T_{L}$ increases until it reaches and exceeds the melter operating temperature. When this happens, solids can precipitate in the

(e) Steam excursions may occur when water from the melter feed is intimately mixed with a hot glass or salt melt. The heat from the melt is quickly transferred to the water which abruptly transforms the liquid into a steam. This is only likely if the water and hot melt are allowed to quickly mix as might be the case with an exceedingly low viscosity melt.

(f) Frequently the presence of melt insolubles from the waste, such as noble metals and their oxides, are ignored. This is consistent with the definition of $\mathrm{T}_{\mathrm{L}}$ since these components are not considered part of the melt (or thermodynamic system) and therefore are more like the crucible the melt is held in. However, for practical purposes, the presence of melt insolubles is an important consideration in glass processing. 
melter. If these solids are not removed from the melter as the glass is poured, they accumulate and can eventually obstruct melter operation. To prevent a premature shutdown of these melters, the $T_{L}$ is required to be lower than a certain temperature appropriate for the melter. Typically, a $100^{\circ} \mathrm{C}$ or more safety margin is used so that $T_{L} \leq T_{M}-100^{\circ} \mathrm{C}$, where $T_{M}$ is the nominal melter operating temperature.

Processability constraints may be modified in the future. In particular, the $T_{L}$ constraint currently limits waste loading in most of Hanford and Savannah River HLW glasses and, thus, has a huge economic impact. Hence, the $T_{L}$ constraint is likely to be scrutinized and made less restrictive without putting solid-phase-sensitive melters at risk. For example, it may be acceptable to allow some smallvolume fraction of certain crystals in the melter as long as they do not segregate to the melter bottom, cause other processing problems, or affect the acceptability of the HLW glass. Other constraints may be added to the existing list. For example, constraints could be developed that would prevent the segregation of molten salts in the melter or prevent an unacceptably low melting rate or high corrosion rate.

Another property that has been required to be within certain limits is electrical conductivity $(\varepsilon)$ of the melt. Because glass is heated by an electric current passing through it, the glass conductivity must be substantially higher than that of the melter walls and sufficiently low to prevent electrode damage or thermal instability. The permitted range of $\varepsilon$ of molten glass is wide, usually 10 to $100 \mathrm{~S} / \mathrm{m}$. With this wide range, $\varepsilon$ is unlikely to affect the formulation of HLW glasses that meet viscosity and PCT constraints. Once the power system for the melter has been built, the acceptable range for $\varepsilon$ may be narrowed to meet the specific power output of the system. Because of the broad range of acceptable $\varepsilon$, this property rarely limits the waste loading of HLW glasses and therefore was not modeled in this study.

\subsubsection{Economic Constraints}

The cost of producing HLW glass depends on the total volume of glass to be produced and the required processing time. By increasing the waste loading of HLW glass, i.e., the fraction of HLW incorporated in the glass, the volume of HLW glass will be reduced. Thus, the cost will be reduced by decreasing the time required to process the waste at the same rate and by decreasing the space and handling required for the canistered waste. Hence, constraints may be placed on the waste loading of HLW glass to control the cost of producing the glass. For example, it is often desirable to optimize glass composition to have the maximum waste loading while meeting other product quality and processability constraints. To some extent, the cost of LAW glass production is also determined by loading of waste in the glass along with production rate.

Acceptability constraints and processing constraints are typically placed on waste glass properties, whereas waste loading (economic) constraints are placed directly on the waste glass composition itself. For glass development and qualification work, property-composition models provide a way to implement property constraints as functions of glass composition.

\subsection{Property-Composition Models}

Property-composition models or constitutive equations are functions of the form

$$
p_{\alpha}=\mathrm{f}_{\alpha}(\mathbf{x})
$$


where $p_{\alpha}$ is the $\alpha$-th property, $\mathbf{x}$ is the composition vector, and $\mathrm{f}_{\alpha}$ denotes the functional form of the model. The composition is defined as $\mathbf{x}=\left(x_{1}, x_{2}, \ldots, x_{N-1}\right)$, where $x_{i}$ is the $i$-th component mass or mole fraction, and $N$ is the number of components. Only $N-1$ components are independent because the mass and mole fractions must sum to 1 :

$$
\sum_{i=1}^{N} x_{i}=1
$$

Typically, the functional form $\mathrm{f}_{\alpha}$ involves parameters or coefficients that are independent of state variables, such as $\mathbf{x}$. Values of these coefficients must be determined by measurement. Models in which all coefficients are estimated from data are called empirical models. Models in which some coefficients are derived from fundamental principles of physics and chemistry, while other coefficients are estimated from data, are called semi-empirical models. Models that are developed from the fundamental laws of physics, e.g., quantum and statistical mechanics, without the use of any experimental data are called firstprinciple models. First-principle models ${ }^{(\mathrm{g})}$ are not applicable to the prediction of multi-component wasteglass properties but may be useful in understanding fundamental relationships that guide model development.

The following two subsections discuss property models linear in composition, and property models linear in composition and nonlinear in temperature. The final subsection briefly discusses the concept of first principles.

\subsubsection{Property Models Linear in Composition}

A simple but very useful property-composition model form is given by

$$
p_{\alpha}=\mathrm{f}_{\alpha}\left(\sum_{i=1}^{N} b_{\alpha i} x_{i}\right)
$$

or, more conveniently,

$$
\mathrm{g}_{\alpha}\left(p_{\alpha}\right)=\sum_{i=1}^{N} b_{\alpha i} x_{i}
$$

where $b_{\alpha i}$ is the $i$-th component coefficient for $\alpha$-th property and $g_{\alpha}$ is the inverse of $\mathrm{f}_{\alpha}$. Note that the mathematical transformation, $g_{\alpha}$, could be the identity transformation, i.e., no transformation. Also note that the sum runs from 1 to $N$, and thus not all $x_{i}$ are independent. This form enabled us to write Equation 1.4 without a constant, i.e., an intercept term, because, by Equation 1.2,

(g) A good working definition of first-prinicple's was given by Cohen (1994) "In a first principles approach, one uses fundamental quantum physics to obtain energetics and the static and dynamic properties of a system, without fitting any experimental data." 


$$
x_{N}=1-\sum_{i=1}^{N} x_{i}
$$

Because $\mathrm{g}_{\alpha}\left(p_{\alpha}\right)$ in Equation 1.4 is also a property, we can identify the $b_{\alpha i}$ coefficients as partialspecific or partial-molar properties. Models of the form (1.4) are often referred to as first-order, i.e., linear, mixture experiment models (Cornell 2002) because the functional form is a first-order, i.e., linear, polynomial. Note that in classical thermodynamics, Equation 1.4 is used for non-ideal mixtures. In these mixtures, partial specific or molar properties are functions of composition. Glass is generally a non-ideal mixture. If individual components in waste glasses are confined to sufficiently narrow ranges of concentrations, non-linear functions of composition may be approximated as linear with acceptable error. As components vary over wider ranges, linear functions of composition may not adequately approximate the underlying nonlinear relationship. In such cases, nonlinear approximating functions may be required.

Glass composition (the $\mathbf{x}$ vector) can be expressed in three basic ways.

1) Glass composition is traditionally expressed as a mixture of single metal oxides such as $\mathrm{SiO}_{2}$, $\mathrm{Fe}_{2} \mathrm{O}_{3}, \mathrm{SO}_{3}, \mathrm{Na}_{2} \mathrm{O}$, etc. and halogens, e.g., $\mathrm{F}$. Multivalent oxides are all represented with the most prevalent oxidation state. Though the oxygen content in the glass is not counted accurately, the composition is uniquely defined and is probably the most suitable for technological and engineering applications, especially when mass fractions are used. Accounting for the true oxidation-reduction states of all components is not practicable because this state depends on glass-making conditions such as selection of raw materials, temperature, and atmosphere during glass making. However, models have been previously developed that account for variations in oxygen fugacity by including separate terms for $\mathrm{FeO}$ and $\mathrm{Fe}_{2} \mathrm{O}_{3}$.

2) Glass can be viewed as a mixture of electronegative elements, such as $\mathrm{O}$ and $\mathrm{F}$, and electropositive elements, such as $\mathrm{Si}, \mathrm{B}, \mathrm{Fe}, \mathrm{Na}$, etc. The problem with the redox state, e.g., $\mathrm{Fe}(\mathrm{II})$ and $\mathrm{Fe}(\mathrm{III})$, and the fraction of oxygen does not arise if only electropositive constituents are considered. This may be advantageous for semi-empirical models that include fundamental properties of ionic species, such as the size or charge of the ions.

3) Glass composition can be resolved into simple silicates and borates (e.g., see Jantzen 1992). This representation is not unique and can be accomplished in different ways for different properties, dependent on which structural units are associated with different transport properties, hydration reactions, or crystallization behavior. This may be advantageous for modeling the effects of elements that fill multiple structural roles in glass, depending on composition. This method has not been applied to models in this report. However, this method may be useful in developing future models.

Approach 1 has been used in developing $\eta$ and PCT release models in this study. Approach 2 has been used in the $T_{L}$ and $V$ models in this report. Approach 3 has been applied in the hydration energy model (Jantzen et al. 1995).

The disadvantage of an empirical model, such as Equation 1.4, is that a large number of coefficients must be estimated from data. Waste glass can have up to 80 elements, and some of these elements can be in multiple oxidation states. It would be difficult to cover a large composition region with enough 
compositions to estimate 80 or more coefficients. However, in practice, most glass properties are only significantly affected by "major" glass components, e.g., those appearing at mass or mole fractions greater than 0.005 . Hence, the number of components for which $b_{a i}$ coefficients in Equation 1.4 must be estimated is usually much smaller than the total number of glass components.

The problem of empirically estimating a large number of component coefficients in Equation 1.4 can be resolved if the coefficients are related to some basic atomic or thermodynamic characteristic of the components. If such relationships are simple, the number of empirical coefficients can drastically decrease. Suppose that

$$
b_{\alpha i}=n_{\alpha}+m_{\alpha} L_{i}
$$

where $L_{i}$ is the atomic or thermodynamic characteristic of the $i$-th component, and $n_{\alpha}$ and $m_{\alpha}$ are empirical coefficients for $\alpha$-th property. Combining Equations 1.4 and 1.6, we obtain a semi-empirical relationship

$$
\mathrm{g}_{\alpha}\left(p_{\alpha}\right)=n_{\alpha}+m_{\alpha} \sum_{i=1}^{N} L_{i} x_{i}
$$

Because Equation 1.7 has only two empirical coefficients, it will generally yield less accurate property predictions than the fully empirical Equation 1.4. However, Equation 1.7 may yield better results if $b_{\alpha i}$ coefficients are not known for some influential components. A semi-empirical relationship such as Equation 1.7 is easier to develop and use, and $N$ can be larger than for an empirical relationship represented by Equation 1.4. Comparing empirical and semi-empirical $b_{\alpha i}$ values can identify shortcomings in the assumptions inherent in Equation 1.7; see, for example, Piepel, Redgate, and Masuga (1996) and Piepel, Redgate, and Masuga (1997).

\subsubsection{Property Models Linear in Composition and Nonlinear in Temperature}

Some glass properties, such as $\eta$ and $\varepsilon$, are functions of temperature $(T)$ as well as composition. For such properties, models are of the form

$$
p_{\alpha}=\mathrm{f}_{\alpha}(\mathbf{x}, T)
$$

For a given waste glass, the temperature dependence of a property, such as viscosity, is often approximated by the Vogel-Fulcher-Tammann equation ${ }^{(\mathrm{h})}$

$$
\eta=\exp \left(A_{V F T}+\frac{B_{V F T}}{T-T_{0}}\right)
$$

or, in a narrow temperature interval, by the Arrhenius equation

(h) See H. Vogel, Phys. Z. 22, 645-646 (1921), G. S. Fulcher, J. Am. Ceram. Soc. 8, 339-366 (1925), G. S. Fulcher, J. Am. Ceram. Soc. 8, 789-794 (1925), and G. Tammann and W. Hesse, Z. Anorg. Allg. Chem., 156, $245-257$ (1926). 


$$
\eta=\exp \left(A+\frac{B}{T}\right)
$$

where $A_{V F T}, B_{V F T}, T_{0}, A$, and $B$ are temperature-independent coefficients.

In any of these equations, the parameters $A_{V F T}, B_{V F T}, T_{0}, A$, and $B$ can be expressed as functions of composition to also capture the dependence of the property on composition. Expanding the parameters in Equations 1.9 and 1.10 as linear functions of composition. i.e., linear mixture models, yields

$$
\ln (\eta)=\sum_{i=1}^{N} A_{V F T, i} x_{i}+\frac{\sum_{i=1}^{N} B_{V F T, i} x_{i}}{T-\sum_{i=1}^{N} T_{0, i} x_{i}}
$$

and

$$
\ln (\eta)=\sum_{i=1}^{N} A_{i} x_{i}+\frac{\sum_{i=1}^{N} B_{i} x_{i}}{T}
$$

Coefficients $A_{V F T}, B_{V F T}, T_{0}, A$, and $B$ can be treated as composition-dependent properties, and thus they may be reparameterized as in Equation 1.7.

\subsubsection{A Comment about First Principles}

In classical field theory, material parameters or properties are mathematically established as constitutive equations that relate these parameters to state variables, such as temperature, pressure, and composition. These relationships are distinct from the first-principles that postulate the basic laws of physics, such as the balance of energy. The constitutive equations themselves are not first-principles. They just define material objects whose behavior is subject to the basic laws, i.e., the first-principles.

Can constitutive equations themselves be based on first-principles? Yes, if these equations were based on quantum mechanics, as in the ab initio calculations (see Cohen 1994). In such models, no empirical coefficients would be needed. They would be based solely on the first-principles or basic laws. Such an approach is not realistic at present. For example, properties of even a single-component glass $\left(\mathrm{SiO}_{2}\right)$ are much easier to measure than to accurately calculate from first-principles. The term "firstprinciple" is occasionally erroneously used to describe semi-empirical models.

\subsubsection{Co m ment on Significant Figures}

Throughout this document a number of models coefficients and other values are reported a with higher number of figures than are significant. Past experience has suggested that prediction of property models is slightly better with more figures than significant. However, a detailed evaluation of the 
appropriate number of figures to report and use was not performed. We therefore suggest use of all reported figures for consistency.

\subsection{Need for Property-Composition Data}

Partial properties in Equation 1.4 or empirical coefficients in Equation 1.7 can only be measured or estimated from data. The temperature dependence of a property, such as $\eta$, can be determined by: 1) measuring the property at a series of constant temperatures, or 2) continuously measuring the property while the temperature is gradually increasing or decreasing. To establish a $\eta-T$ relationship in analytical terms, an empirical model is fitted to measured data. Such a relationship or model ${ }^{(i)}$ is ready for use in applications, for example, in the Navier-Stokes equation for fluid flow (see Fung 1994 for example) with a variable temperature. It can also be used for computing viscosity at a given temperature if such a number is needed for understanding a more complex behavior, such as the settling of solid particles. With empirical models, it is important to be aware of uncertainties, such as the uncertainty of measured data, the uncertainty of the empirical model form, i.e., how closely it represents or approximates the true behavior, and the uncertainty of model input values, e.g., the composition of the glass or the degree of uniformity of the melter feed.

To determine the empirical coefficients in property-composition models, a set of propertycomposition data that adequately covers the composition region of interest is needed. Historical data sets can be compiled for this purpose, or a test matrix of compositions can be designed and the properties measured. Unlike temperature, which is expressed by one number, composition is expressed as a vector with $N$-1 dimensions. The goal in compiling property-composition data to develop property-composition models is to adequately cover the $(N-1)$-dimensional glass-composition region with a manageable number of compositions.

Several approaches can be used to select a test matrix to explore an $(N-1)$-dimensional composition region. Three common approaches are to 1) vary each component one-at-a-time, i.e., adding or removing a single component to or from a baseline composition with the remaining components adjusting for the change while maintaining constant proportions, or replacing one component with another, and 2) changing the fractions of several (up to $N-1$ ) components at a time. Table 1-1 summarizes some advantages and disadvantages of these three approaches.

(i) The terms "model" and "relationship" are interchangeable in this report, though their connotations are different in materials science and statistics. 
Table 1-1. Advantages and Disadvantages of Two Approaches for Exploring a Glass Composition Region of Interest

\begin{tabular}{|c|c|c|}
\hline Approach & Advantages & Disadvantages \\
\hline $\begin{array}{l}\text { One-at-a-time } \\
\text { variations from } \\
\text { baseline } \\
\text { composition or } \\
\text { replacing one } \\
\text { component with } \\
\text { another. }\end{array}$ & $\begin{array}{l}\text { 1. Component effects can be } \\
\text { graphically visualized. } \\
\text { 2. The linearity or nonlinearity of } \\
\text { component effects is immediately } \\
\text { apparent. } \\
\text { 3. Does not require sophisticated } \\
\text { software for design or evaluation. } \\
\text { 4. Component replacement is useful if } \\
\text { one component makes up the } \\
\text { majority of the glass. }\end{array}$ & $\begin{array}{l}\text { 1. Results may depend on the baseline } \\
\text { composition. } \\
\text { 2. Does not provide information about } \\
\text { non-linear blending, i.e., "interaction," } \\
\text { effects of the components. } \\
\text { 3. Inefficient way to cover composition } \\
\text { space and generate data for property- } \\
\text { composition models. }\end{array}$ \\
\hline $\begin{array}{l}\text { Many-at-a-time } \\
\text { variations within } \\
\text { a defined glass } \\
\text { composition } \\
\text { region. }\end{array}$ & $\begin{array}{l}\text { 1. Provides information about nonlinear } \\
\text { blending, i.e., "interaction," effects } \\
\text { of the components. } \\
\text { 2. Provides information about linear } \\
\text { effects of components over the } \\
\text { region. } \\
\text { 3. Provides for best coverage of the } \\
\text { composition region. }\end{array}$ & $\begin{array}{l}\text { 1. Requires statistical optimal } \\
\text { experimental design methods and } \\
\text { software to implement. } \\
\text { 2. Requires models to assess whether } \\
\text { components have linear or non-linear, } \\
\text { i.e., curvilinear or interaction, } \\
\text { blending effects. }\end{array}$ \\
\hline
\end{tabular}

The large number of components in Hanford HLW glass means that there are seldom enough data to develop highly accurate constitutive equations over the entire glass-composition region in question. Moreover, the dependence of waste-glass properties on glass composition is fundamentally nonlinear, and thus the linear representation in Equations 1.4 or 1.7 has limited validity. Fortunately, the "true," but unknown, property-composition relationships are generally expected to be smooth or piecewise smooth as functions of composition, such as $T_{L}$. For smooth functions of composition, linear functions provide satisfactory approximations over sufficiently small portions of the composition space. Luckily, in manycomponent HLW glasses, the range of interest for each component is likely to be sufficiently narrow so that a linear function provides a reasonable approximation to the "true" property-composition relationship.

Linear mixture models such as (1.4) can be expanded to include nonlinear blending terms in cases where a linear approximation is not adequate, and there are sufficient data to estimate the coefficients of linear and nonlinear blending terms (Cornell 2002; Piepel, Szychowski, and Loeppky 2002). If glass components are likely to have significant nonlinear blending effects for a given waste-glass composition region, more property-composition data points covering the composition space will be needed to support fitting models with nonlinear blending terms.

\subsection{Data Reduction: Partial Specific/Molar Coefficients}

Databases alone are insufficient for developing an adequate HLW glass formulation because too many constraints need to be satisfied, and the waste-glass volume should be as small as possible, i.e., the waste loading in the glass should be as high as possible (assuming constant processing rate). Therefore, 
data reduction is necessary. As discussed in Section 1.1, the goal of data reduction for formulation purposes is to develop property-composition models that adequately approximate the "true," unknown property-composition relationships. Such models can then be used to predict waste-glass property values as functions of waste-glass composition and temperature, where applicable, anywhere within the composition region of validity for the models.

In this report, we focus attention on models with linear-composition dependence, such as in Equations $1.4,1.11$, and 1.12. Such models can be fit to an appropriate property-composition data set, thereby reducing the data to partial-specific or molar properties. Composition in the models can be expressed in one of the three possible divisions of the mixture to constituents discussed in Section 1.3. Partial properties have been well established in thermodynamics, including their physical meaning and methods of evaluation from data. They can themselves be functions of composition. Hence, Equation 1.4 is useful, even when the composition relationship is nonlinear, that is, $b_{\alpha i}=\mathrm{f}_{\alpha i}(\mathbf{x})$. Attempts have been made to use this approach to develop higher-order models, but, for some properties, these attempts were discontinued for the lack of sufficient data or because of the limited time available to develop models for inclusion in this report.

After fitting property-composition data to a linear mixture model-Equation 1.4-and thus estimating partial specific or molar properties for the components in the model, the next step is to assess the adequacy of the model. As noted previously, a model linear in composition may or may not adequately approximate the "true," unknown property-composition relationship over the composition region covered by the available data. Provided the data set includes sufficient replicate data points, or adequate estimates of experimental and property-measurement uncertainties exist, statistical methods for assessing model lack-of-fit (Draper and Smith 1998; Montgomery and Peck 1992) can be applied. If a model does not have a statistically significant lack-of-fit, then it should be validated with data not used to develop it or cross-validated with the data used to develop it (Montgomery and Peck 1992, Chapter 10). However, empirical models with statistically significant lack-of-fits or less than ideal validation or cross-validation performance can still be useful in earlier stages of glass development and formulation studies, provided the models provide reasonable fits and predictive ability. Global models linear in composition may fall into this category, but would still be useful in supporting early waste-retrieval, waste-blending, and glassdevelopment activities. As waste composition and variation information improve, local models can be developed to more accurately predict glass properties over smaller glass-composition regions corresponding to specific waste types. The uncertainty of these local models can be significantly lower than the uncertainties of the global models, approaching the uncertainties in property measurements and compositions in the data used in their development.

If a model linear in composition-Equations 1.4, 1.7, and 1.12-for a given property provides an adequate fit to the data, the next step in the data reduction is to focus more attention on the estimated partial-specific or molar properties obtained from the model fit. For example, the partial properties can be further analyzed to find correlations between them or relationships with more fundamental characteristics of the constituents, such as the electric charge and radius of ions or the free enthalpy of simple silicates and borates (Jantzen 1992; Vienna et al. 2001a). Such a semi-empirical approach to developing propertycomposition models reduces property-composition data to the maximum practicable degree. 


\subsection{Existing Property Composition Models for Hanford HLW Glass}

Historically, simple relations between properties and composition have been developed for commercial glasses for nearly a century. The book by Scholtze (1990) provides a good review of property-composition relationships for simple glasses. A similar approach became a necessity for HLW glass to deal with the large composition region of Hanford HLWs. Before the Hanford experience, a semi-empirical equation, originally developed by Paul for assessing glass durability (Paul 1977), was applied to HLW at Savannah River (Jantzen 1992, p.153-217).

The Hanford HLW composition region is not fully known, but reasonable estimates of its boundaries have been made based on HLW sample analyses and process inventories. For this estimated HLW composition region, a glass-composition region for neutralized current acid waste (NCAW) was assessed and represented by more than 100 compositions, for which several properties were measured (Hrma et al. 1994; Piepel, Redgate, and Hrma 1995). Property-composition models were then developed from these data (Hrma et al. 1994, 1995a, 1995b).

The basic processing properties measured and modeled (Hrma et al. 1995b, Vienna et al. 1996b) were $\eta, \varepsilon$, and $T_{L}$. Other properties were also characterized and modeled: glass-transition temperature, thermalexpansion coefficients of solid and molten glass, and density (Hrma et al. 1994). Considerable attention focused on PCT response (Hrma 1995a; Vienna et al. 1996a; Kot and Pegg 2001), and other studies for Hanford HLW glass have focused some attention on Toxicity Characteristic Leach Procedure (TCLP) (EPA) to a lesser extent (Vienna et al. 1998, Kot and Pegg 2001, Jantzen et al. 2000). These outcomes of specific tests are, strictly speaking, not properties, but can be treated as such because they are reproducible results of well-defined experimental procedures. For Idaho HLW glass-composition regions, preliminary property-composition models were developed for $\eta$ and PCT (Edwards et al. 2000; Piepel et al. 2002).

The form of the function $\mathrm{g}_{\alpha}$ in Equations 1.4 and 1.7 is the natural logarithmic function for $\eta$, $\varepsilon$, and normalized PCT elemental releases. For other properties, $\mathrm{g}_{\alpha}$ is the identity function. The transport properties, $\eta$ and $\varepsilon$, are also functions of temperature, as discussed in Section 1.3. For $\eta$, Hrma et al. (1995a) developed property-composition-temperature models of the form (1.11) and (1.12) as presented in Section 1.3. For $\varepsilon$, they developed models of the form (1.12). Models for $\eta$ and $\varepsilon$ at a constant temperature were also developed. Hrma et al. (1995b) also used a model of the form (1.7) for PCT normalized elemental releases. A semi-empirical model based on the hydration energies of silicate, borate, and oxide components of glass (Jantzen et al. 1995) did not work well for Hanford HLW glass (Piepel, Redgate, and Masuga 1996 and 1997). Feng, Saad, and Pegg (1990) and Feng and Metzger (1996) developed a semi-empirical model for viscosity and PCT release based on the heat of formation of oxide components in glass. Jantzen (1991) developed a model for $T_{L}$ based on the free energies of formation of three mineral phases. Jantzen (1991) also developed a model for viscosity based on a calculation of the number of non-bridging oxygen atoms.

Hrma et al. (2001) developed a database of glass compositions and properties from literature. This database was used to fit interim glass-property models for use in predicting the properties of projected Hanford HLW glasses. However, several shortcomings of the database used to develop the models were identified: 1) the data developed in support of the Office of River Protection's (ORP's) Waste Treatment 
Plant (WTP) project were not included in the database due to the timing of data release, 2) there was insufficient coverage of the expected composition regions for many properties, primarily $T_{L}, 3$ ) several datasets were inputted to the database, but were not verified and tested for consistency with other data and therefore could not be used in model development, and 4) compositions of interest to Hanford LAW glasses were not considered during model development. In the study reported here, we take the next logical step in the series of required studies to develop and refine the models for Hanford waste glass properties. 


\subsection{Property-Composition Database}

This section describes the waste-glass property-composition database that was compiled to support developing property-composition models. Section 2.1 briefly describes the structure and contents of the database. Section 2.2 lists the major sources of data incorporated in the database and used for model development in Sections 3.0 to 7.0.

The database certainly does not contain all property values of HLW glasses ever measured. Some available data sets were not included because of incomplete documentation to confirm the accuracy of the data. More data will be added as necessary after checking for accuracy or when new reports become available after this report is issued to validate and improve property-composition models.

\subsection{Description of Database}

The current database was compiled in an Excel spreadsheet with IDs of glasses in rows and compositions and properties in columns as described below.

- Study, Data Source, and Glass ID: Each set of data is distinguished by a study name, i.e., the names for a group or groups of data, and the source of the data, i.e., a reference. The same Glass IDs are used as given in the original Data Source.

- Glass Composition (mass fraction): Target (-t) and analyzed (-a) compositions are entered in separate columns in alphabetical order for 61 components. The Others component is used only when there is no information available to separate Others into individual component concentrations, and the sum of oxide components in the target composition did not equal one.

- Melting Temperature $\left(T_{M}\right.$ in $\left.{ }^{\circ} \mathrm{C}\right)$ : The melting temperatures are the actual temperatures used to fabricate the glass. When a glass was melted two or three times, only the final melting temperature was entered.

- Liquidus Temperature $\left(T_{L}\right.$ in $\left.{ }^{\circ} \mathrm{C}\right)$ : The $T_{L} \mathrm{~s}$ measured by a gradient-furnace method and by a uniformtemperature method are given in separate columns. The primary phase was also recorded in a column.

- Crystallinity and Homogeneity: The information on the crystalline and amorphous phase separation was given for quenched, canister centerline cooled (CCC), and heat-treated glasses. The results from visual/light microscopic (LM) observation, electron microscopic, i.e., scanning electron microscopy/electron-dispersive spectroscopy (SEM/EDS) or transmission electron microscopy (TEM), and X-ray diffraction (XRD) were recorded in separate columns.

- Density $\left(\rho\right.$ in $\left.\mathrm{g} / \mathrm{cm}^{3}\right)$ : Density data are available for a limited number of glasses.

- Viscosity (n inPa $s$ ): The coefficients for Vogel-Fulcher-Tamann and Arrhenius equations; the calculated viscosity at $1150^{\circ} \mathrm{C}$; and temperatures at 2,5 , and $10 \mathrm{~Pa} \cdot \mathrm{s}$, based on these coefficients, are 
entered as provided in the references. The $T_{n}$ and $V_{n}(\mathrm{n} \equiv$ data point number $=1$ to 14$)$ columns are for the viscosity-at-temperature data.

- PCT Normalized Releases $\left(r_{j}\right.$ in $\left.g / \mathrm{m}^{2}\right)$ : Normalized elemental releases of $\mathrm{j}=\mathrm{B}, \mathrm{Li}, \mathrm{Na}$, and $\mathrm{Si}$ and final $\mathrm{pH}$ values from standard 7-day $\mathrm{PCT}$ at $90^{\circ} \mathrm{C}$ and target surface area-to-volume ratio $(\mathrm{S} / \mathrm{V})$ of $2000 \mathrm{~m}^{-1}$ are given for quenched (Q) and CCC glasses. The releases are all normalized to $\mathrm{S} / \mathrm{V}$ in addition to the element concentration in glass, giving $r_{j}$ values in $\mathrm{g} / \mathrm{m}^{2}$. Some studies measured PCT at $20^{\circ} \mathrm{C}$; these results are in separate columns.

- TCLP Response (in ppm): The TCLP data are available for a limited number of glasses.

The database compiled in this report contains glasses designed for the vitrification of HLW and LAW that were used for the model development.

\subsection{Discussion of Data Sets Used in Model Development}

This subsection provides a list of studies represented in the database with brief explanations of each study. Table 2-1 summarizes these studies in terms of the number of glasses tested, their compositions, and properties provided. The figures in parentheses in the "property" column indicate the number of glasses for which the property data are provided.

\subsubsection{Idaho National Engineering and Environmental Laboratory Composition Variation Studies}

This series of studies includes the composition variation study (CVS) to develop glass compositions for immobilizing the HLW calcine and the sodium-bearing waste (SBW) stored at the Idaho Nuclear Technology and Engineering Center (INTEC) conducted at the Idaho National Engineering and Environmental Laboratory (INEEL).

\section{INEEL CVS Phase 1 (Staples et al. 1999)}

The first phase of the CVS addressed waste compositions based on the high-activity waste (HAW) fraction from the initial separations flowsheet. This study investigated how glass properties depend on composition within a region compatible with the expected range of INEEL INTEC HAW. Given the range of the HAW compositions, statistical design techniques were applied to derive a formulation matrix for the first phase of the CVS (Piepel, Vienna, and Hrma 1999). Formulations selected from this matrix were characterized with respect to the crystallinity of both quenched and CCC glasses, PCT releases of quenched glasses, $\eta$, and $T_{L}$. The $\mathrm{T}_{\mathrm{M}}$ data are provided for all 44 glasses. Because of batching errors that were discovered after the report was issued, the original report contained some incorrect target compositions and PCT releases that were calculated based on the target compositions. The correct compositions and PCT releases were recalculated later from the original batch sheets and entered in the database. The corrected compositions are documented in a paper by Piepel, Szychowski, and Loeppky (2002). Analyzed compositions were not given in the original report; the analyzed compositions entered in this database were those obtained after the Staples et al. (1999) report was issued.

INEEL CVS Phase 2a (Edwards et al. 2000) 
This set of glasses, which was designated as Phase 2a, was tested as a preliminary study before designing the test matrix of 37 CVS Phase 2 glasses, referred to as Phase $2 b$ to distinguish them from the preliminary test glasses (Staples et al. 2000). This report presented the glass-composition experimental region and the methodology used to develop a statistically designed Phase $2 \mathrm{~b}$ test matrix. Viscosity at $1150^{\circ} \mathrm{C}$ and the $\mathrm{PCT}$ of quenched glasses were entered in the database.

\section{INEEL CVS Phase 2 (Staples et al. 2000)}

A test matrix of 37 glasses was developed (Edwards et al. 2000) and tested in Phase 2 to investigate property-composition relationships within the alkali borosilicate glass-composition region compatible with estimates of INTEC calcined compositions with a direct vitrification flowsheet and of HAW fractions proposed to be separated from dissolved calcine. A secondary objective of the Phase $2 \mathrm{~b}$ test matrix was to investigate the composition boundaries of $\mathrm{P}_{2} \mathrm{O}_{5}, \mathrm{~F}, \mathrm{CaO}$, and $\mathrm{Al}_{2} \mathrm{O}_{3}$. These glasses were characterized with respect to the crystallinity of quenched and CCC glasses, PCT releases of quenched glasses, $\eta, T_{L}$, and density. $T_{M}$ data are provided.

\section{INEEL CVS Phase 3 (Scholes, Peeler, and Vienna 2000)}

Phase 3 of the INEEL CVS focused on glass compositions that may be appropriate for either the INEEL separations/pretreatment scenario or the direct vitrification of calcined HLW. Through application of statistical techniques, a test matrix was defined to augment Phase 1 and 2 data with additional data points (Piepel et al. 2002). These glasses were characterized for crystallinity of quenched and CCC glasses, PCT releases of quenched glasses, $\eta, T_{L}$, and density. $T_{M}$ data are provided for all 30 glasses.

\section{$\underline{\text { SBW CVS Phase } 1 \text { (Scholes et al. 2002) }}$}

This report presented the results of the first phase of a composition variation study conducted for the development of glass compositions for the vitrification of SBW located at the INEEL. The purpose of this study was to investigate and model the effects of glass composition on key properties within the expected region of immobilizing the liquid SBW stored in tanks at the INEEL. The $T_{M}, T_{L}$, crystallinity of quenched and CCC treated glasses, $\rho, \eta$ at $T$, and PCT of quenched glasses were entered in the database. 
Table 2-1. Summary of Number of Glasses Tested and Properties Measured in Each Data Set

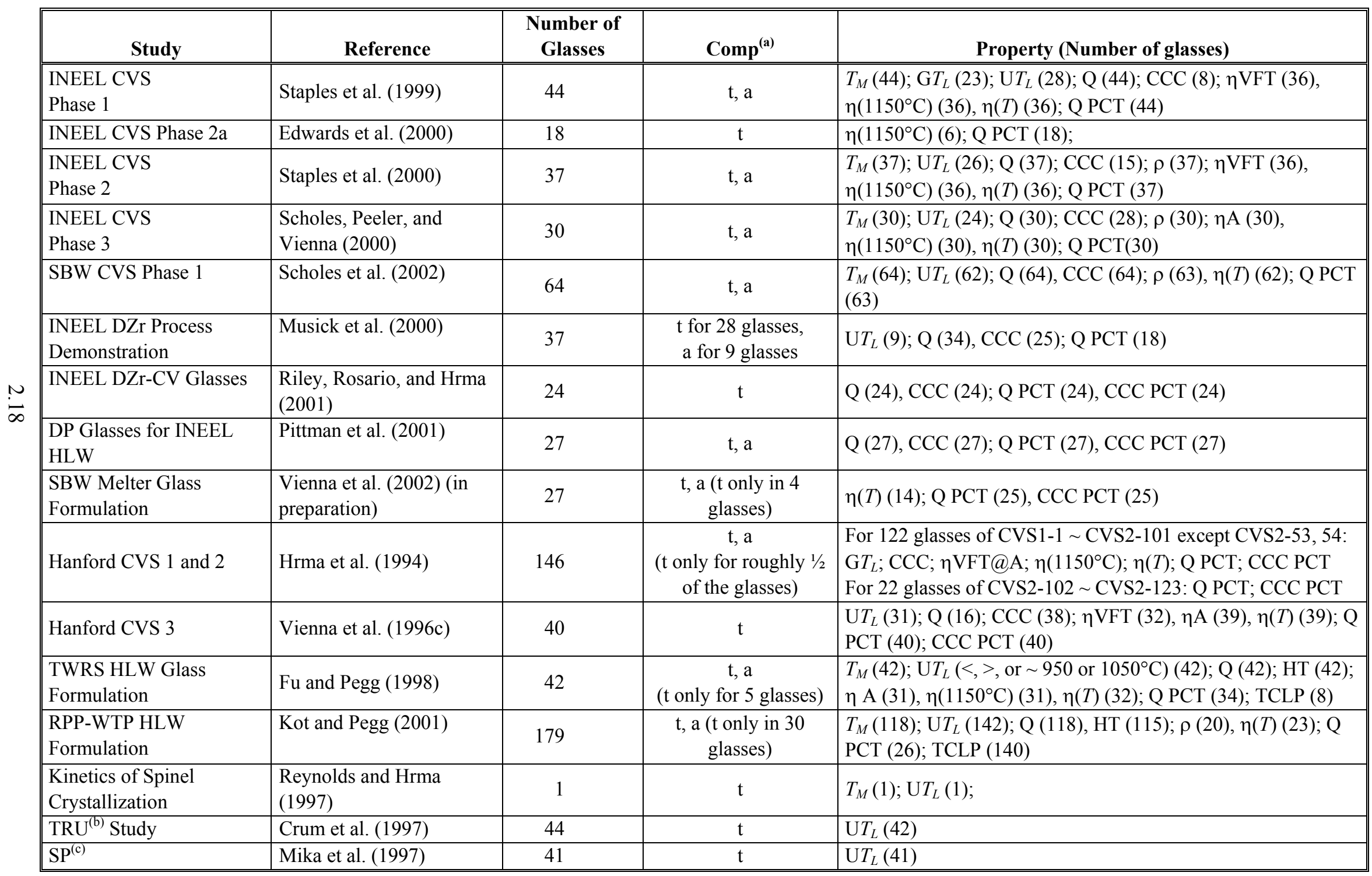


Table 2.1 (Contd)

\begin{tabular}{|c|c|c|c|c|}
\hline Study & Reference & $\begin{array}{c}\text { Number of } \\
\text { Glasses }\end{array}$ & $\operatorname{Comp}^{(\mathbf{a})}$ & Property (Number of glasses) \\
\hline MS & $\begin{array}{l}\text { Hrma (1999) and Wilson } \\
\text { et al. (2001) }\end{array}$ & 28 & $\mathrm{t}$ & $\mathrm{U} T_{L}(28)$ \\
\hline SG & Hrma et al. (1999) & 63 & $\mathrm{t}$ & $T_{M}(63) ; \mathrm{U} T_{L}(63)$ \\
\hline SP3, SP $\times 4$, Misc. & Vienna et al. (2001a) & 50 & $\mathrm{t}$ & $\mathrm{U} T_{L}(50)$ \\
\hline $\begin{array}{l}\text { Comp. Vs Properties } \\
\text { Study }\end{array}$ & Chick et al. (1981) & 102 & a & $\left.T_{M}(102) ; \mathrm{Q}(77) ; \mathrm{CCC}(44) ; \eta\right\rceil 1250^{\circ} \mathrm{C}(102)$ \\
\hline ISV Glass & $\begin{array}{l}\text { Carter, Koegler, and Bates } \\
(1988)\end{array}$ & 4 & a & $T(10 \mathrm{~Pa} \cdot \mathrm{s})(4)$ \\
\hline $\begin{array}{l}\text { WV HLW Formulation } \\
\text { Study }\end{array}$ & Chick et al. (1984) & 103 & $\mathrm{t}, \mathrm{a}$ & $\begin{array}{l}\text { Q (103); CCC (103); HT (103); T @ } 10 \text { Pa·s (88); T @ } 3 \text { Pa·s } \\
\text { (96) }\end{array}$ \\
\hline HWVP 85 & Bates (1985) & 1 & $\mathrm{t}$ & $\eta(T) ; \rho$ \\
\hline $\begin{array}{l}\text { WV Glasses by VSL and } \\
\text { PNL }\end{array}$ & $\begin{array}{l}\text { Johnston, Piepel, and } \\
\text { Pulsipher }(1990)^{(\mathrm{j})}\end{array}$ & 128 & & $\begin{array}{l}\text { Q PCT (128) - } 98 \text { glasses } r_{B} \text { only, } 30 \text { glasses } r_{B}, r_{L i}, r_{N a}, r_{S i} \text { and } \\
\text { pH }\end{array}$ \\
\hline $\begin{array}{l}\text { Glass Dissolution } \\
\text { Chemistry }\end{array}$ & Ramsey (1995) & 30 & $\mathrm{t}, \mathrm{a}$ & Q PCT (30) \\
\hline West Valley WQR ${ }^{(\mathrm{e})}$ & WVNS (1995) & 48 & $\mathrm{t}$ & $\eta\left(1150^{\circ} \mathrm{C}\right)(10), \eta(T)(10) ;$ Q PCT (48) \\
\hline DWPF $^{(\mathrm{f})}$ PCT Model & Jantzen et al. (1995) & 177 & $\mathrm{t}, \mathrm{a}$ & Q PCT (177) \\
\hline Plutonium Vitrification & $\begin{array}{l}\begin{array}{l}\text { Bulkley and Vienna } \\
(1997)\end{array} \\
\end{array}$ & 45 & $\mathrm{t}$ & $T_{M}(41) ; \eta \mathrm{A}(40), \eta\left(1150^{\circ} \mathrm{C}\right)(36), \eta(T)(40) ;$ Q PCT (39) \\
\hline $\begin{array}{l}\text { SRS M-Area Mixed } \\
\text { Waste Glass }\end{array}$ & Fu et al. (1997) & 6 & $\mathrm{t}, \mathrm{a}$ & $T_{M}(6) ; \operatorname{TCLP}(6)$ \\
\hline $\begin{array}{l}\text { TWRS Envelope D HLW } \\
\text { Glass }\end{array}$ & Crawford et al. (1998) & 1 & $\mathrm{t}, \mathrm{a}$ & Q PCT (1) \\
\hline $\begin{array}{l}\text { Hanford LLW Glass } \\
\text { Formulation }\end{array}$ & Feng et al. (1996) & 79 & $\begin{array}{l}\text { t, a (t only in } 49 \\
\text { glasses) }\end{array}$ & $\eta(T)$ (51); Q PCT (78) \\
\hline
\end{tabular}

(j) J. W. Johnston, G. F. Piepel, and B. A. Pulsipher. 1990. "Evaluation of Empirical Models for Glass Durability,” Letter Report Prepared for West Valley Nuclear Services, Pacific Northwest National Laboratory. 
Table 2.1 (Contd)

\begin{tabular}{|c|c|c|c|c|}
\hline Study & Reference & $\begin{array}{c}\text { Number of } \\
\text { Glasses }\end{array}$ & Comp $^{(a)}$ & Property (Number of glasses) \\
\hline $\begin{array}{l}\text { TWRS LAW } \\
\text { Formulation }^{(\mathrm{g})}\end{array}$ & Muller and Pegg (1998) & 51 & $\mathrm{t}, \mathrm{a}$ & $\mathrm{U} T_{L}(46) ; \rho(4) ; \eta(T)(46) ; \mathrm{Q}$ PCT @ $20^{\circ} \mathrm{C}(51) ; \mathrm{TCLP}(4)$ \\
\hline $\begin{array}{l}\text { TWRS LAW Formulation } \\
2^{(\mathrm{g})}\end{array}$ & Ferrara et al. (1998) & 3 & $\mathrm{t}, \mathrm{a}$ & Q PCT @ 20ㄷ (3); TCLP (3) \\
\hline $\begin{array}{l}\text { RPP-WTP LAW } \\
\text { Formulation }^{(\mathrm{g})}\end{array}$ & $\begin{array}{l}\text { Muller, Buechele, and } \\
\text { Pegg (2001) }\end{array}$ & 107 & $\begin{array}{l}\mathrm{t}, \mathrm{a} \text { (t only in } 42 \\
\text { glasses) }\end{array}$ & 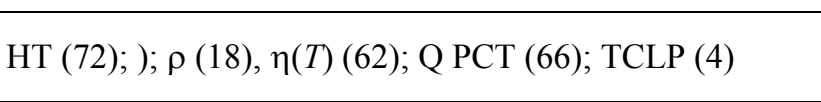 \\
\hline HLP glasses $^{(\mathrm{g})}$ & Vienna et al. (2001b) & 78 & $t, a$ & $\rho(63) ;$ Q PCT (75) \\
\hline
\end{tabular}

(a) Compositions given in the database; $\mathrm{t}=$ target and $\mathrm{a}=$ analyzed.

(b) TRU $=$ transuranic

(c) SP = spinel study

(d) WVDP $=$ West Valley Demonstration Project

(e) $\mathrm{WQR}=$ Waste Form Qualification Report

(f) DWPF $=$ Defense Waste Processing Facility

(g) LAW glasses

Other abbreviations: A and VFT $=$ Arrhenius and Vogel-Fulcher-Tammann viscosity coefficients, respectively, $\mathrm{CCC}$ and $\mathrm{Q}=$ crystallinity of CCC and quenched

glasses, respectively, CCC PCT and Q PCT $=r_{i}(i=\mathrm{B}, \mathrm{Na}, \mathrm{Li})$ from CCC and glasses, respectively, $\mathrm{G} T_{L}$ and $\mathrm{U} T_{L}=T_{L}$ measured with gradient temperature and

uniform temperature furnace methods, respectively, $\mathrm{HT}=$ heat-treated. 


\subsubsection{Glass Formulations for INEEL HLW Calcine and SB W}

\section{INEEL DZr Process Demonstration (Musick et al. 2000)}

This report summarized the results of glass formulations that meet the applicable waste acceptance and processing requirements and pilot-scale melter tests to demonstrate vitrification feasibility for INEEL HLW calcines. The major focus of the glass-development efforts was to achieve a high solubility of calcium fluoride to maximize the waste loading. The $T_{L}$, crystallinity on quenched and CCC treated glasses, and PCT releases of quenched glasses were entered in the database.

\section{INEEL DZr-CV Glasses (Riley, Rosario, and Hrma 2001)}

This report collected and discussed existing data on the PCT releases of boron and sodium from glasses that precipitated crystalline phases upon CCC or isothermal heat treatment. The results of the glass-formulation study for INEEL Run 78 calcine, i.e., glasses designated as DZr-CV, were included in this report when the data became available to assess the effect of crystallization on PCT releases. An initial evaluation of the results was treated in a separate report (Crum et al. 2002), which did not include the detailed data on glass composition and crystallinity. The crystallinity and PCT of quenched and CCC treated glasses were entered in the database.

\section{DP Glasses for INEEL HLW (Pittman et al. 2001)}

The objective of this study was to investigate the specific property-composition relationships of glasses over a compositional envelope compatible with INEEL calcine compositions. An important element of this objective was to determine the influence of CCC on the promotion of crystallization and its ultimate impact on durability. This report presents the development of the glass-composition experimental region and the experimental results of specific glass properties as a function of composition. The crystallinity and PCT of quenched and CCC treated glasses were entered in the database.

\section{$\underline{\text { SBW Melter Glass Formulation (Vienna et al. 2002) }}$}

This report summarizes the results of glass formulation and testing to develop glasses that can incorporate the maximum concentration of sulfate for the vitrification of sodium-bearing waste at INEEL. The $\eta$ at $T$ and PCT releases of quenched and CCC treated glasses were entered in the database.

\subsubsection{Hanford CVS Investigations}

This series includes the three stages of CVS performed within the Hanford Waste Vitrification Plant (HWVP) and PNNL Vitrification Technology Development (PVTD) programs in support of a future HLW vitrification plant at the Hanford site.

\section{Hanford CVS 1 and 2 (Hrma et al. 1994)}

The report presents two CVS data sets, CVS-1 and -2. These studies were performed at PNNL for the Hanford HLW program. Property-composition relationships were obtained for 146 glasses in five statistically designed experimental phases. The properties measured include $\eta, \varepsilon$, glass-transition temperature $\left(T_{g}\right)$, thermal-expansion coefficients of solid glass and molten glass, crystallinity (quenched and CCC glasses), $T_{L}$, and durability based on normalized elemental releases from the Materials Characterization Center-1 28-day dissolution test (MCC-1) (quenched glasses) and the standard PCT (quenched and CCC glasses). Amorphous phase separation was also evaluated with TEM for selected 
glasses. The statistical experimental design strategy used in the CVS involved defining a glasscomposition region expected to contain glasses that might be made from NCAW and expected to be processed by the previously planned HWVP_-scoping tests considering some additional waste types were also included - and then selecting specific compositions for study so as to appropriately cover this region. Ten glass components, $\mathrm{SiO}_{2}, \mathrm{~B}_{2} \mathrm{O}_{3}, \mathrm{Na}_{2} \mathrm{O}, \mathrm{Li}_{2} \mathrm{O}, \mathrm{CaO}, \mathrm{MgO}, \mathrm{Fe}_{2} \mathrm{O}_{3}, \mathrm{Al}_{2} \mathrm{O}_{3}, \mathrm{ZrO}_{2}$, and Others, i.e., the remaining waste constituents, were systematically varied in the study. The individual component concentrations contained in the Others component were separated and entered in the database. The compositions in the database are the target mass-fraction values with analyzed compositions for roughly half of the glasses.

\section{Hanford CVS 3 (Vienna et al. 1996c)}

This data set, CVS-3, covers a different glass composition region than CVS- 1 and -2 to allow for higher-temperature-melting glasses with higher waste loadings to be produced. The properties measured include the viscosity-temperature relationship, $T_{g}, \varepsilon, T_{L}, \mathrm{CCC}$ crystallinity, and PCT from quenched and $\mathrm{CCC}$ glasses. This study varied glass components one-at-a-time from a boron-free alkali-aluminosilicate baseline glass while maintaining constant ratios of all other components. The components varied were $\mathrm{SiO}_{2}, \mathrm{~B}_{2} \mathrm{O}_{3}, \mathrm{Na}_{2} \mathrm{O}, \mathrm{Li}_{2} \mathrm{O}, \mathrm{Fe}_{2} \mathrm{O}_{3}, \mathrm{Al}_{2} \mathrm{O}_{3}, \mathrm{ZrO}_{2}, \mathrm{Bi}_{2} \mathrm{O}_{3}, \mathrm{UO}_{2}, \mathrm{P}_{2} \mathrm{O}_{5}$, and Others. The compositions in the database are the target mass-fraction values.

\subsubsection{HL W Glass Formulation for Hanford Wastes at the Vitreous State Laboratory}

This series includes two studies on the glass-formulation development for Hanford HLW conducted at the Vitreous State Laboratory (VSL) of the Catholic University of America.

\section{TWRS HLW Glass Formulation (Fu and Pegg 1998)}

This report presented the results of glass-formulation development with Tank Waste Remediation System (TWRS) HLW simulants during TWRS Phase I, Part A. Glasses were formulated based on the HLW Envelope D composition specifications provided by the TWRS contract. After specific glassformulation design parameters were identified, glasses were formulated on the basis of previous experience with the objective of meeting the constraints imposed on those parameters and characterized with respect to $T_{L}, \eta$, and PCT and TCLP releases. The $T_{M}, T_{L}, \eta$ at $T$, PCT releases of quenched glasses and TCLP results were entered in the database. The $T_{L}$ data were given as "greater or less than a reference temperature" without information on the primary phase.

\section{RPP-WTP HLW Formulation (Kot and Pegg 2001)}

This report presented the results from HLW glass formulation work that was conducted at the VSL during Part B1 of the RPP-WTP Project, formally known as TWRS-P, in support of the privatization contractor, British Nuclear Fuels, Inc. (BNFL, Inc.). This work was built on the results obtained during TWRS Part A (Fu and Pegg 1998), which was directed towards an assessment of the viability of the contractually specified waste envelope and product requirements and identification of the risks associated with complying with those specifications. Two approaches were adopted for glass formulation. In the first approach, glasses were formulated on the basis of existing knowledge and information, and in the second approach, a matrix of glass compositions was designed with a statistical method. Samples of these glasses were melted using simulants and characterized with respect to the properties of interest. The resulting set of glass compositions and properties data were the primary subject of this report. The $T_{M}, T_{L}$ 
(without primary phases), crystallinity of quenched and heat treated samples, $\eta$ at $T$, PCT of quenched glasses, and TCLP results were entered in the database.

\subsubsection{Iiquidus Temperature Studies}

These studies are specifically concentrated on the effect of glass compositions on the $T_{L}$ of important crystalline phases, such as spinel and zirconium silicate, that can occur in the HLW glass melter during operation. The $T_{L}$ models described in Section 3.4 are primarily based on the data from these studies.

\section{Kinetics of Spinel Crystallization (Reynolds and Hrma 1997)}

This study investigated the kinetics of spinel crystallization under isothermal conditions from a molten high-iron simulated HLW glass designed to maximize the waste loading for a blend of Hanford site wastes. The $T_{M}$ and $T_{L}$ for this glass were recorded in the database.

\section{Transuranic (TRU) Study (Crum et al. 1997)}

This study of glasses was based on a simulated TRU waste with high concentrations of $\mathrm{ZrO}_{2}$ and $\mathrm{Bi}_{2} \mathrm{O}_{3}$ to determine the composition dependence of primary crystalline phase and $T_{L}$. Starting from a baseline composition, glasses were formulated by changing one-at-a-time the mass fractions of $\mathrm{Al}_{2} \mathrm{O}_{3}$, $\mathrm{B}_{2} \mathrm{O}_{3}, \mathrm{Bi}_{2} \mathrm{O}_{3}, \mathrm{CeO}_{2}, \mathrm{Li}_{2} \mathrm{O}, \mathrm{Na}_{2} \mathrm{O}, \mathrm{P}_{2} \mathrm{O}_{5}, \mathrm{SiO}_{2}$, and $\mathrm{ZrO}_{2}$, while keeping the remaining components in the same relative proportions as in the baseline glass. The $T_{L}$ values are given along with the corresponding primary crystalline phases, which were mostly zircon $\left(\mathrm{ZrSiO}_{4}\right)$ with some baddeleyite $\left(\mathrm{ZrO}_{2}\right)$ and cerium oxide.

\section{$\underline{\text { SP (Mika et al. 1997) }}$}

This study was performed to measure the $T_{L}$ of melts in the spinel primary phase field as a function of glass composition. The test glasses were based on high-iron Hanford tank wastes. A test matrix was designed containing 33 glasses with 10 components: $\mathrm{Al}_{2} \mathrm{O}_{3}, \mathrm{Cr}_{2} \mathrm{O}_{3}, \mathrm{Fe}_{2} \mathrm{O}_{3}, \mathrm{MgO}, \mathrm{MnO}, \mathrm{Na}_{2} \mathrm{O}, \mathrm{NiO}, \mathrm{SiO}_{2}$, $\mathrm{B}_{2} \mathrm{O}_{3}$, and $\mathrm{Li}_{2} \mathrm{O}$ and Others. The test matrix was derived from the baseline glass containing $47 \mathrm{mass} \%$ water-washed Hanford nominal waste blend. The baseline composition was altered, one-component-at-atime, while maintaining the same relative proportions of the remaining components. All glasses tested had spinel as the primary phase.

\section{Zr Study (Vienna et al. 1999)}

The objective of this study was to develop preliminary glass formulations for the HAW fraction of INEEL zirconia calcine and then to recommend a waste loading and frit composition for use in an INEEL scaled melter test. This study focused on the development of glass compositions with a high concentration of $\mathrm{ZrO}_{2}$ because the estimated waste composition contained $92.58 \mathrm{wt} \% \mathrm{ZrO}_{2}$. In 29 glass compositions tested, the concentrations of $\mathrm{ZrO}_{2}, \mathrm{Al}_{2} \mathrm{O}_{3}, \mathrm{~B}_{2} \mathrm{O}_{3}$, and $\mathrm{Li}_{2} \mathrm{O}$ were varied independently, and then the concentrations of $\mathrm{SiO}_{2}$ and $\mathrm{Na}_{2} \mathrm{O}$ were adjusted to maintain a calculated viscosity of $6 \mathrm{~Pa} \cdot \mathrm{s}$ at $1150^{\circ} \mathrm{C}$. The PCT releases and $T_{L}$ values with the corresponding primary crystalline phases are given. The primary phases identified in this study were mostly zircon $\left(\mathrm{ZrSiO}_{4}\right)$, baddeleyite $\left(\mathrm{ZrO}_{2}\right)$, and parakeldyshite $\left(\mathrm{Na}_{2} \mathrm{ZrSi}_{2} \mathrm{O}_{7}\right)$.

\section{Melter Settling (MS) (Hrma 1999; Wilson et al. 2001)}

The goal of the study by Hrma (1999) was to develop a basic understanding of the dynamics of spinel formation and motion in velocity, temperature, and redox fields that are characteristic for the glass- 
melting process. Nine glasses with different compositions, MS-1 to MS-9, were formulated and measured for $T_{L}$. In a subsequent study (Wilson et al. 2001), the glasses were formulated by increasing or decreasing concentrations of $\mathrm{Al}_{2} \mathrm{O}_{3}, \mathrm{Cr}_{2} \mathrm{O}_{3}, \mathrm{Fe}_{2} \mathrm{O}_{3}, \mathrm{Li}_{2} \mathrm{O}, \mathrm{MgO}, \mathrm{Na}_{2} \mathrm{O}$, or NiO, one-at-a-time, from a baseline composition (MS-7). The $T_{L}$ data from this study were also included in this data set.

\section{Savannah River Glass (SG) (Hrma et al. 1999)}

A $T_{L}$ versus composition study was performed for the DWPF. The main objective of the study was to decrease the uncertainty in $T_{L}$ model predictions of glasses produced at SRS by developing a database for glass-composition effects on $T_{L}$. A series of 53 glass compositions was statistically designed to cover the DWPF composition region by means of a layered design approach (Edwards 1997). The test matrix contained glass compositions within and just outside the DWPF composition region. The components that varied in mass fractions in parentheses were $\mathrm{SiO}_{2}(0.43$ to 0.59$), \mathrm{B}_{2} \mathrm{O}_{3}(0.05$ to 0.10$), \mathrm{Na}_{2} \mathrm{O}(0.06$ to 0.11), $\mathrm{Li}_{2} \mathrm{O}$ (0.03 to 0.06), $\mathrm{Fe}_{2} \mathrm{O}_{3}$ (0.06 to .015), $\mathrm{Al}_{2} \mathrm{O}_{3}$ (0.025 to 0.08), $\mathrm{CaO}$ (0.003 to 0.02), $\mathrm{MgO}(0.005$ to0.025), $\mathrm{K}_{2} \mathrm{O}$ (0.015 to 0.038$), \mathrm{U}_{3} \mathrm{O}_{8}$ (0 to 0.055$), \mathrm{MnO}$ (0.01 to 0.03$), \mathrm{NiO}(0.001$ to 0.02$), \mathrm{TiO}_{2}(0.002$ to 0.006 ), and $\mathrm{Cr}_{2} \mathrm{O}_{3}$ (0.001 to 0.003$)$. The properties measured and reported included $T_{L}$ with primary phases. Spinel was the primary phase for most of glasses while some glasses had a clinopyroxene primary phase. The compositions of the glasses were confirmed by chemical analyses after the report was issued. Those analyzed compositions, although not currently included in the database, did not show any significant deviation from target values.

\section{$\underline{S P-3, S P \times 4, \text { Misc. (Vienna et al. 2001a) }}$}

This study compiled all the glasses in the spinel primary phase field, including MS, SG, and SP studies previously described, to develop a new model that can accurately predict the $T_{L}$ of spinel. This reference includes the results of further studies on the spinel crystallization, designated as SP-3 and SP $\times 4$. The SP-3 study expanded the SP series by five components, $\mathrm{CaO}, \mathrm{K}_{2} \mathrm{O}, \mathrm{RuO}_{2}, \mathrm{TiO}_{2}$, and $\mathrm{ZrO}_{2}$, and the range of variation for $\mathrm{SiO}_{2}, \mathrm{Li}_{2} \mathrm{O}$, and $\mathrm{Fe}_{2} \mathrm{O}_{3}$ was increased. Another study was also performed to determine $T_{L}$ in the spinel primary crystalline phase field. This study, designated as $\mathrm{SP} \times 4$, was based on the original baseline glass SP-1 but varied four components-at-a-time while maintaining the concentrations of all other components in constant relative proportions. The four components varied were $\mathrm{Al}_{2} \mathrm{O}_{3}, \mathrm{Cr}_{2} \mathrm{O}_{3}, \mathrm{Na}_{2} \mathrm{O}$, and $\mathrm{NiO}$, which were found to have the strongest impact on the $T_{L}$ of those that were varied in the original study. A number of glasses that had been fabricated for different purposes or studies were grouped into the "Misc." study and were used in $T_{L}$ model validation.

\subsubsection{Other Property-Composition Relation Studies}

\section{Composition vs. Properties Study (Chick et al. 1981)}

The purpose of this study was to evaluate and refine the statistical methods used to produce the empirical property models. Ninety simplified waste-glass compositions - 102 glasses including replicates - within an 11-component oxide composition matrix were tested for crystallinity, $\eta$, and MCC- 1 releases. The $\eta$ at $1250^{\circ} \mathrm{C}$ data and the results of crystallinity observations on quenched and $\mathrm{CCC}$ treated glasses were entered in the database.

\section{WV HLW Formulation Study (Chick et al. 1984)}

This report describes the statistically designed study performed at PNNL to develop the glass composition recommended for the vitrification of HLW stored at West Valley, New York. This study assessed the effects of seven oxide components: $\mathrm{SiO}_{2}, \mathrm{~B}_{2} \mathrm{O}_{3}, \mathrm{CaO}, \mathrm{Fe}_{2} \mathrm{O}_{3}, \mathrm{Al}_{2} \mathrm{O}_{3}, \mathrm{Na}_{2} \mathrm{O}$, and waste mix on 
glass properties. Over 100 melts combining the seven components into a wide variety of compositions were tested for $\eta, \varepsilon$, thermal expansion, crystallinity, and MCC-1 releases. The estimated temperatures at $10 \mathrm{~Pa} \cdot \mathrm{s}$ and $3 \mathrm{~Pa} \cdot \mathrm{s}$ and the results of crystallinity observation on quenched, $\mathrm{CCC}$ treated, and heat-treated glasses were recorded in the database.

\section{HWVP 85 (Bates 1985)}

This report describes the result of development of a reference-glass composition for the HWVP. The reference-glass composition developed from this study was designated HW39 and characterized for melt $\eta, \varepsilon$, and density.

\section{SRL 165 Glasses (Pye 1985)}

This report presented the results of the measurements of various physical and thermal properties of simulated nuclear waste glasses and their melts. Three SRL 165 glasses, designated as high iron, TDS, and high alumina, were provided by the Savannah River Laboratory. The properties measured included thermal diffusivity, thermal expansion, Young's modulus, shear modulus, modulus of rupture, Poisson's ratio, density, and viscosity. Density and viscosity at $1150^{\circ} \mathrm{C}$ were entered in the database.

\section{ISV Glass (Carter, Koegler, and Bates 1988)}

This report summarized the results of laboratory and pilot-scale tests of the in situ vitrification (ISV) process developed at PNNL that used actual Oak Ridge National Laboratory soil and limestone. The $T$ data at $10 \mathrm{~Pa} \cdot \mathrm{s}$ for vitrified ISV products were recorded in the database.

\section{WV Glasses by VSL and PNL (Johnston, Piepel, and Pulsipher 1990) ${ }^{(k)}$}

This report describes the development of a database of PCT leach test results. The compositional and leach data pertinent to West Valley waste forms from the VSL at the Catholic University of America (CUA) and PNNL were gathered to compare the free-energy-of-hydration model with several empirical models that predict boron releases from chemical composition. The data from PNNL were included in another data set (Reimus et al. 1988) and so were excluded in this data set. The VSL PCT data, which were entered in the database, were those transferred to PNNL via diskettes at the time of this study.

\section{West Valley Demonstration Project (WVDP) Support (Olson 1993 and 1994)}

These data are from two studies conducted as part of glass-composition variability testing under the PNNL West Valley Support Program to support the establishment of a glass-composition control strategy by West Valley Nuclear Services (WVNS). The results of PCT releases for 20 glasses (PNL series, Alkali series) were reported in Olson (1993) while the results of viscosity measurement for the same 20 glasses and 10 more glasses of the Ratio series are reported in Olson (1994). The PCT releases and $\eta$ at $T$ data from this data set were entered in the database. The viscosity data for 10 glasses of the Ratio series were entered as part of another data set, "West Valley WQR" (WVNS 1995), which contained also the PCT data along with other West Valley waste-form qualification glasses.

\section{West Valley CVS Glasses PCT (Olson et al. 1994)}

To assist WVDP, the PCT method was used to evaluate 44 West Valley glasses. These glasses were fabricated as sets of CVS glasses for studies performed by the West Valley Support Task (WVST) at

(k) The Johnston et al. 1990 report is the only (incomplete) source available to us for this particular VSL data set. 
PNNL and were initially tested with a modified MCC-3 test method. The glasses were retested with the PCT method after WAPS included the PCT in an acceptance specification.

\section{Glass Dissolution Chemistry (Ramsey 1995)}

This data set includes glasses from a statistically designed study of the following 6-component glasscomposition region, which was intended to include alkali borosilicate glasses that successfully encapsulate the high-level defense wastes, $\mathrm{SiO}_{2}, \mathrm{Al}_{2} \mathrm{O}_{3}, \mathrm{~B}_{2} \mathrm{O}_{3}, \mathrm{Fe}_{2} \mathrm{O}_{3}, \mathrm{CaO}$, and $\mathrm{Na}_{2} \mathrm{O}$. The analyzed compositions in the database contain the concentrations of iron oxides in both $\mathrm{FeO}$ and $\mathrm{Fe}_{2} \mathrm{O}_{3}$, which were calculated from the result of glass redox analysis. PCT releases in elemental leachate concentrations given in the reference were converted to normalized releases and entered in the database. Oksoy et al. (1994) used this data set to perform a study on the canonical correlation of glass compositions and PCT releases.

\section{West Valley WQR (WVNS 1995)}

WVNS developed a data set consisting of 58 glass compositions. These data were used to develop PCT release models for use in WVDP, a high-level nuclear waste glass facility. The 58 glasses were selected to evenly cover a glass-composition region centered on the WVDP target glass composition with the boundary taken to be three times the expected process variation. Only nine components known to influence PCT releases were varied in these glasses, and the composition data were provided based on these nine components. The full composition data entered in the database were those found in Piepel, Redgate, and Masuga (1996). Out of 58 glasses given in this report, 10 glasses of the alkali series (Alkali1 to Ref6Qtr2) were already included in data set of "WVDP Support" (Olson 1993 and 1994) and were not included in this data set. The viscosity data for 10 glasses of the Ratio series (Ratio2 to PNL190) were from Olson (1994).

\section{DWPF PCT Model (Jantzen et al. 1995)}

This study was conducted to develop the Thermodynamic Hydration Energy Reaction Model (THERMO) to predict glass PCT releases from a glass composition based on the estimated glasshydration free energy. The THERMO is being used in DWPF to assess product consistency and quality. The result of this study was registered as a U.S. patent (Jantzen et al. 1998). The glasses examined in this study were fabricated under a variety of laboratory and pilot-scale conditions by various researchers and vendors. The target compositions and PCT data entered in the database were those supplied by the Savannah River Technology Center (SRTC) to PNNL in electronic data form. The analyzed compositions are from the SRTC report (Jantzen et al. 1995). PCT releases were converted to $\mathrm{g} / \mathrm{m}^{2}$ from $\mathrm{g} / \mathrm{L}$ reported.

\section{Plutonium Vitrification (Bulkley and Vienna 1997)}

The objective of this study was to examine how variations in the waste-stream compositions would affect the key properties of glasses for immobilization of plutonium-bearing materials at Rocky Flats Environmental Technology Site (RFETS), Hanford, and other DOE sites. A one-component-at-a-time change matrix was designed, keeping the remaining components in the same relative proportions as in a baseline composition. The glasses were tested for viscosity and PCT releases. The published reference (Bulkley and Vienna 1997) contains only a part of the data, the temperature at $5 \mathrm{~Pa} \cdot \mathrm{s}$, and PCT releases for $\mathrm{B}$ and $\mathrm{Na}$, whereas the database contains all the data obtained during this study, including viscosity-attemperature, Arrhenius coefficients for viscosity, PCT releases for Li and Si, and final pH from PCT. 
The database also contains nine more glasses with different or replicate compositions that were not included in the reference.

Savannah River Site (SRS) M-Area Mixed Waste Glass (Fu et al. 1997)

This study was a third phase of glass-formulation studies to determine the feasibility of vitrifying Savannah River M-Area mixed wastes. This study reported the effect of varying the boron-to-total-alkali ratio and the effect of substitutions such as $\mathrm{ZrO}_{2}$ for waste and $\mathrm{TiO}_{2}$ for $\mathrm{SiO}_{2}$ on the chemical durability and processability of M-Area waste glasses. The requirement for safe disposal of this waste stream is to pass the TCLP. The $T_{M}$ and TCLP results were recorded in the database. The glass compositions given in $\mathrm{mole} \%$ were converted to mass fraction for inclusion in the database.

\section{TWRS Envelope D HLW Glass (Crawford et al. 1998)}

This study was a radioactive demonstration of production and characterization of HLW glass, performed at SRTC, using the actual Hanford radioactive waste that consisted of HLW sludge slurry (Envelope D), elute waste streams containing high levels of ${ }^{137} \mathrm{Cs}$ and ${ }^{99} \mathrm{Tc}$, and solids containing ${ }^{90} \mathrm{Sr}$ and TRU. The PCT of one quenched radioactive glass was recorded in the database.

\subsubsection{LA W Glass Formulation Studies}

\section{Hanford LLW Glass Formulation (Feng et al. 1996)}

This report presented the results of the glass formulation work for Hanford low-level waste to develop optimized compositions containing the maximum fraction of waste, acceptable processing characteristics, and adequate flexibility to handle waste variations. In addition, this report included an evaluation of the impacts of minor components, characterization of melter vendor glasses, and the determination of $T_{L}$ and crystallization kinetics. This report also summarized relevant work on high-iron glasses for Hanford tank wastes conducted through the Mixed Waste Integrated Program and work at SRTC to optimize glass formulations with a Plackett-Burnam experimental design. The $\eta$ at $T$ and PCT releases of quenched glasses were entered in the database.

\section{TWRS LAW Formulation (Muller and Pegg 1998)}

This report presented the results of glass-formulation development with TWRS LAW simulants during TWRS Phase I, Part A. Glasses were formulated based on the LAW Envelope A, B, and C composition specifications provided by the TWRS contract. After specific glass-formulation design parameters were identified, glasses were formulated on the basis of previous experience with the objective of meeting the constraints imposed on those parameters and characterized with respect to $T_{L}, \eta$, $\varepsilon$, and PCT releases. It should be noted that PCT releases were measured at $20^{\circ} \mathrm{C}$ instead of the standard $90^{\circ} \mathrm{C}$ used in HLW glasses. The $T_{L}, \eta$ at $T, \rho, 20^{\circ} \mathrm{C}$ PCT releases of quenched glasses, and TCLP results were entered in the database. The $T_{L}$ data were given as "greater or less than a reference temperature" without information on the primary phase.

\section{TWRS LAW Formulation 2 (Ferrara et al. 1998)}

This study characterized three LAW glasses produced from Hanford radioactive waste samples as part of a demonstration for BNFL. The three LAW glasses were produced from radioactive supernate samples that had been treated to remove most of the radionuclides. The TCLP results and PCT releases of quenched glasses are recorded in the database. 
RPP-WTP LAW Formulation (Muller, Buechele, and Pegg 2001)

This report presented the results from LAW glass formulation development and testing activities performed at the VSL in support of Part B1 of the RPP-WTP privatization project led by BNFL, Inc. The selection of new glass formulation was guided by extensive past experience and the glass-property models and database developed and continuously updated at VSL. The development of glass formulations was an iterative process in that prospective glasses were formulated and characterized to obtain propertycomposition information. The results were then analyzed, and revised compositions were generated to optimize the properties of the glass to the imposed constraints. Therefore, the formulations were referred to as "actively designed" rather than "statistically designed." The resulting set of glass-compositionproperty data was summarized in this report. The crystallinity of heat-treated glasses, $\rho, \eta$ at $T$, PCT of quenched glasses, and TCLP results were entered in the database.

\section{HLP Glasses (Vienna et al. 2001b)}

This report provided the results of a study to help determine the composition range of LAW glasses that will meet performance expectations of the Hanford site burial facility. This study was a continuation of the Hanford Immobilized Low Activity Waste Product Acceptance: Tanks Focus Area Testing Data Package II (Schulz et al. 2000). An original matrix of 55 glasses (Phase I, HLP-01 through -55) was developed to identify the impact of glass composition on long-term corrosion behavior and to develop an acceptable composition region for Hanford LAW glasses. A second matrix consisting of 20 glasses (Phase II, HLP-58 through -77) was developed to help determine the influence on the test responses of key glass components that were not varied systematically in Phase I. The $\rho$ and PCT of quenched glasses were entered in the database. 


\subsection{Models for Viscosity}

This section discusses the interim viscosity-composition models developed for Hanford HLW and LAW glasses and the portions of the database, discussed in Section 2.0, used to develop the models.

\subsection{Initial Data Evaluation and Screening}

The initial database available for developing the property-composition models (see Section 2.0) contained data from 2001 glass compositions expressed in terms of 71 components. Those glasses with viscosity $(\eta)$-temperature (T) data were selected for initial model development. A total of 623 glasses had at least $2 \eta-T$ data points. These 623 glasses were taken as the starting data set. For those glasses with both target and analyzed compositions, the compositions were compared. Any glass with more than 20\% difference in target and analyzed concentrations of more than one major component, i.e., a component with more than 1 mass $\%$ in glass, were excluded from model fit. This removed 110 glass compositions, listed in Table 3.1. Of the remaining 513 glasses, 10 were reported with only analyzed compositions (no target composition was available), and one glass had a target composition that summed to less than $99 \%$ (LAWB42S); these 11 compositions (see Table 3-1) were removed from the data set, and target compositions were exclusively used in model development. Of the remaining 502 glasses, 55 were reported in their source documentation to be phase separated, either crystalline or amorphous, and were removed (also listed in Table 3-1). Of the 447 remaining glasses, 79 were found to be extreme in either $\eta$ at $1150^{\circ} \mathrm{C}(1<\eta>100 \mathrm{~Pa} \cdot \mathrm{s})$ and marked either $\eta<1$ or $\eta>100$ in Table 3-1; or extreme concentrations of single components were marked with either "value $<$ component" or "value $>$ component," where the values are in mass $\%$ of the oxide in glass. These restrictions were added to remove the influence of extreme compositions and/or property values on the property model. The component ranges for the remaining data can be used as an initial assessment of the model-validity region. Two additional glasses were removed (not in Table 3-1) because of large data scatter in the $\eta$-T relationship - CVS1-18 and CVS3-13. This left a total of 366 glasses for use in $\eta$-composition modeling. Some of these data were removed as outliers during the model fitting as described in the following sections. 
Table 3-1. List of Glasses Excluded from Viscosity Model Development

\begin{tabular}{|c|c|c|c|c|c|c|c|}
\hline \multicolumn{8}{|c|}{ "Glasses excluded because of differences in target and analyzed composition } \\
\hline LAWB18 & CVS2-7 & CVS2-38 & LAWA16 & LAWC4 & LAWB34 & LAWA19 & HLWD1-01 \\
\hline IG2-04 & CVS2-19 & CVS2-39 & LAWA17 & LAWC5 & LDM-2 & LAWPC2 & HLWD1-04 \\
\hline IG2-15 & CVS2-20 & CVS2-40 & LAWA20 & LAWPC7 & LDM-3 & LAWC3 & HLWD1-14 \\
\hline IG2-23 & CVS2-21 & CVS2-41 & LAWA21 & LAWPC8 & LDM-4 & IG3-06 & HLW98-03 \\
\hline IG2-26 & CVS2-22 & CVS2-43 & LAWA24 & LAWPC9 & LDM-5412 & IG3-10b & IG3-05 \\
\hline IG2-37 & CVS2-25 & CVS2-44 & LAWB12 & LAWPC10 & LDMS-1 & LAWA25H & IG1-08 \\
\hline IG3-08b & CVS2-28 & CVS2-46 & LAWB13 & HLWD1-03 & LRM-4 & LAWA26H & IG2-10 \\
\hline IG3-15 & CVS2-29 & CVS2-49 & LAWB14 & HLWD3-01 & LRMS-1 & IG2-36 & IG3-13 \\
\hline IG3-16 & CVS2-30 & CVS2-50 & LAWB15 & HLWD2-02 & SSHTM-3 & IG3-01 & HLWD1-13 \\
\hline IG3-19 & CVS2-31 & CVS2-51 & LAWB17 & HLWD2-03 & SBW1-42 & IG3-20 & HLWD1-17 \\
\hline IG3-26 & CVS2-32 & CVS2-58 & LAWB20 & HLW98-14 & SBW1-43 & LAWA15 & IG3-09 \\
\hline IG3-29 & CVS2-35 & CVS2-97 & LAWB28 & HLW98-44 & HLWD1-19 & LAWC11 for AN107 & CVS2-69 \\
\hline CVS1-15 & CVS2-36 & Alkali4 & LAWB29 & HLW98-61 & HLWD2-04 & LAWC12 for AN107 & \\
\hline CVS1-23 & CVS2-37 & Alkali9 & LAWC2 & LAWA98S & CVS2-52 & LAWB16 & \\
\hline \multicolumn{8}{|c|}{ Glasses excluded because target glass compositions weren't available (or in the case of LAWB42S, were incomplete) } \\
\hline Ratio2 & Ratio5 & LoTh4 & $\mathrm{HiFe} 2$ & $\mathrm{HiFe} 4$ & LAWB42S & & \\
\hline Ratio4 & LoTh2 & LoTh5 & HiFe3 & PNL190 & & & \\
\hline \multicolumn{8}{|c|}{ Glasses excluded because they were reported as phase separated } \\
\hline CVS3-16 & CVS3-5 & HLWD1-07 & IG1-01 & IG1-23 & IG1-32 & IG2-01 & IG2-22 \\
\hline CVS3-17 & HLW98-06 & HLWD1-11 & IG1-02 & IG1-24 & IG1-34 & IG2-05 & IG2-27 \\
\hline CVS3-20 & HLW98-07 & HLWD1-21 & IG1-09 & IG1-25 & IG1-36 & IG2-06 & IG2-34 \\
\hline CVS3-38 & HLW98-08 & HLWD1-23 & IG1-14 & IG1-26 & IG1-40 & IG2-07 & IG3-03 \\
\hline CVS3-39 & HLW98-12 & HLWD1-27 & IG1-17 & IG1-27 & IG1-41 & IG2-09 & IG3-04 \\
\hline CVS3-4 & HLW98-21 & HLWD2-05 & IG1-19 & IG1-28 & IG1-42 & IG2-17 & IG3-07 \\
\hline CVS3-40 & HLWD1-05 & HLWD3-04 & IG1-21 & IG1-31 & IG1-44 & IG2-18 & \\
\hline \multicolumn{8}{|c|}{ Glasses excluded because they were extreme in composition or viscosity at $1150^{\circ} \mathrm{C}$} \\
\hline Extreme & Glass ID & Extreme & Glass ID & Extreme & Glass ID & Extreme & Glass ID \\
\hline$>1 \mathrm{Y}_{2} \mathrm{O}_{3}$ & LAWA47 & $>2 \mathrm{TiO}_{2}$ & LAWA42 & $\eta<1$ & CVS1-11 & $<25 \mathrm{SiO}_{2}$ & HLWD1-18 \\
\hline$>1 \mathrm{Y}_{2} \mathrm{O}_{3}$ & LAWPC3 & $>2 \mathrm{TiO}_{2}$ & LAWA82 & $\eta<1$ & CVS1-12 & $<4.5 \mathrm{Na}_{2} \mathrm{O}$ & HLWD1-10 \\
\hline$>25 \mathrm{Na}_{2} \mathrm{O}$ & L7-30 & $>2 \mathrm{TiO}_{2}$ & LAWA89 & $\eta<1$ & CVS2-81 & $<4.5 \mathrm{Na}_{2} \mathrm{O}$ & SRC-Na-1 \\
\hline$>25 \mathrm{Na}_{2} \mathrm{O}$ & L7-35 & $>2 \mathrm{TiO}_{2}$ & LAWABP1 & $\eta<1$ & CVS2-83 & $<4.5 \mathrm{Na}_{2} \mathrm{O},>10 \mathrm{MnO},>20 \mathrm{SrO}, \mathrm{\eta}<1$ & HLWMS-08 \\
\hline$>2 \mathrm{CdO}$ & HLWD1-09 & $>2 \mathrm{TiO}_{2}$ & SRC-Ti-2 & $\eta<1$ & CVS2-92 & $<4.5 \mathrm{Na}_{2} \mathrm{O},>10 \mathrm{MnO},>20 \mathrm{SrO}, \eta<1$ & HLWMS-11 \\
\hline$>2 \mathrm{CdO}$ & HLWD3-03 & $>3 \mathrm{Bi}_{2} \mathrm{O}_{3}$ & CVS3-28 & $\eta<1$ & CVS2-93 & $<4.5 \mathrm{Na}_{2} \mathrm{O},>20 \mathrm{SrO}$ & HLWMS-15 \\
\hline$>2 \mathrm{CdO}, \eta<1$ & HLW99-15 & $>3 \mathrm{Bi}_{2} \mathrm{O}_{3}$ & CVS3-29 & $\eta<1$ & CVS2-94 & $<4.5 \mathrm{Na}_{2} \mathrm{O},>2 \mathrm{CdO}$ & HLWD1-08 \\
\hline$>2 \mathrm{CdO}, \eta<1$ & HLWD3-06 & $>3 \mathrm{Bi}_{2} \mathrm{O}_{3}$ & CVS3-30 & $\eta<1$ & PNL 1 & $>10 \mathrm{U}_{3} \mathrm{O}_{8}$ & CVS3-35 \\
\hline$>2 \mathrm{~F}$ & IG2-29 & $>3 \mathrm{Bi}_{2} \mathrm{O}_{3}$ & CVS3-31 & $\eta<1$ & PNL 3 & $>10 \mathrm{U}_{3} \mathrm{O}_{8}$ & CVS3-36 \\
\hline$>2 \mathrm{~F}$ & IG2-32 & $>4 \mathrm{P}_{2} \mathrm{O}_{5}$ & IG1-35 & $\eta>100$ & CVS3-12 & $>1 \mathrm{CoO}$ & LAWA18 \\
\hline$>2 \mathrm{~F}$ & IG2-33 & $>4 \mathrm{P}_{2} \mathrm{O}_{5}$ & LAWA84 & $\eta>100$ & CVS3-2 & $>1 \mathrm{Cr}_{2} \mathrm{O}_{3}$ & CVS2-66 \\
\hline$>2 \mathrm{~F}$ & IG2-35 & $>4 \mathrm{P}_{2} \mathrm{O}_{5}$ & LAWA96 & $\eta>100$ & CVS3-9 & $>1 \mathrm{Cr}_{2} \mathrm{O}_{3}$ & CVS2-68 \\
\hline$>2 \mathrm{~F}$ & IG3-14 & $>5 \mathrm{Ce}_{2} \mathrm{O}_{3}$ & SRC-Ce-3 & $\eta>100$ & IG2-14 & $>1 \mathrm{Cr}_{2} \mathrm{O}_{3}$ & CVS2-70 \\
\hline$>2 \mathrm{~F}$ & IG3-23 & $>5 \mathrm{Ce}_{2} \mathrm{O}_{3}$ & SRC-Ce-4 & $\eta>100$ & L4-615 & $>1 \mathrm{Cr}_{2} \mathrm{O}_{3}$ & HW39 \\
\hline$>2 \mathrm{~F}$ & IG3-24 & $>5 \mathrm{~F}$ & IG2-12 & $\eta>100$ & L6-3312 & $>1 \mathrm{CuO},>1 \mathrm{Y}_{2} \mathrm{O}_{3}$ & LAWPC5 \\
\hline$>2 \mathrm{~F}$ & IG3-25 & $>5 \mathrm{Gd}_{2} \mathrm{O}_{3}$ & SRC-Gd-3 & $\eta>100$ & L7-15 & $>1 \mathrm{Eu}_{2} \mathrm{O}_{3}$ & SRC-Eu-1 \\
\hline$>2 \mathrm{~F}$ & IG3-27 & $>5 \mathrm{Gd}_{2} \mathrm{O}_{3}$ & SRC-Gd-4 & $\eta>100$ & L8-1 & $>1 \mathrm{Ga}_{2} \mathrm{O}_{3}$ & LAWA46 \\
\hline$>2 \mathrm{~F}$ & IG3-28 & $>5 \mathrm{SrO}$ & HLW98-23 & $\eta>100$ & L8-2 & $>1 \mathrm{SnO}_{2}$ & SRC-Sn-1 \\
\hline$>2 \mathrm{PbO}$ & SRC-Pb-2 & $>5 \mathrm{SrO}$ & HLW98-34 & $\eta>100$ & L8-3 & $>1 \mathrm{SnO}_{2}$ & SRC-Sn-2 \\
\hline$>2 \mathrm{PbO}$ & SRC-Pb-3 & $>5 \mathrm{SrO}$ & LAWA64 & $\eta>100$ & LRM-3 & & \\
\hline
\end{tabular}




\subsection{Composition Conversion}

The glass compositions used in viscosity modeling are listed in Appendix A on a targeted mass fractions $\left(g_{i}\right)$ of oxides and halogens basis. These compositions were converted into mole fractions $\left(x_{i}\right)$ of oxides and halogens by standard methods. Several components were listed in the database with multiple oxidation states. ${ }^{(1)}$ These components were combined into groups of like metal oxides according to: ${ }^{(\mathrm{m})}$

$$
\begin{aligned}
\mathrm{Ce}_{2} \mathrm{O}_{\mathrm{x}} & =\mathrm{Ce}_{2} \mathrm{O}_{3}+0.5 \mathrm{CeO}_{2} \\
\mathrm{CoO}_{\mathrm{x}} & =\mathrm{CoO}+2 \mathrm{Co}_{2} \mathrm{O}_{3} \\
\mathrm{Fe}_{2} \mathrm{O}_{\mathrm{x}} & =\mathrm{Fe}_{2} \mathrm{O}_{3}+0.5 \mathrm{FeO} \\
\mathrm{MnO}_{\mathrm{x}} & =\mathrm{MnO}+\mathrm{MnO}_{2} \\
\mathrm{MoO}_{\mathrm{x}} & =\mathrm{MoO}_{3}+\mathrm{MoO} \\
\mathrm{PdO}_{\mathrm{x}} & =\mathrm{PdO}+\mathrm{PdO}_{2} \\
\mathrm{Pr}_{2} \mathrm{O}_{\mathrm{x}} & =\mathrm{Pr}_{2} \mathrm{O}_{3}+3 \mathrm{Pr}_{6} \mathrm{O}_{11} \\
\mathrm{ReO}_{\mathrm{x}} & =\mathrm{ReO}_{2}+2 \mathrm{Re}_{2} \mathrm{O}_{7}+\mathrm{ReO} \\
\mathrm{Rh}_{2} \mathrm{O}_{\mathrm{x}} & =\mathrm{Rh}_{2} \mathrm{O}_{3}+0.5 \mathrm{RhO}_{2} \\
\mathrm{Sb}_{2} \mathrm{O}_{\mathrm{x}} & =\mathrm{Sb}_{2} \mathrm{O}_{3}+\mathrm{Sb}_{2} \mathrm{O}_{5} \\
\mathrm{SnO}_{\mathrm{x}} & =\mathrm{SnO}_{2}+\mathrm{SnO} \\
\mathrm{Tl}_{2} \mathrm{O}_{\mathrm{x}} & =\mathrm{Tl}_{2} \mathrm{O}_{3}+\mathrm{Tl}_{2} \mathrm{O} \\
\mathrm{UO}_{\mathrm{x}} & =\mathrm{UO}_{3}+\mathrm{UO}_{2}+3 \mathrm{U}_{3} \mathrm{O}_{8}
\end{aligned}
$$

When only one oxide form had at least one glass with non-zero concentration, this oxide form was used instead of the combined form, e.g., $\mathrm{MnO}$ was used instead of $\mathrm{MnO}_{\mathrm{x}}$ if no glass had $\mathrm{MnO}_{2}$. The resultant compositions were then normalized to 1 . In addition, the lanthanide oxides and $\mathrm{Y}_{2} \mathrm{O}_{3}$ were combined to form a single component, $\mathrm{LN}_{2} \mathrm{O}_{3}$, given by

$$
\mathrm{LN}_{2} \mathrm{O}_{3}=\mathrm{Ce}_{2} \mathrm{O}_{3}+\mathrm{Eu}_{2} \mathrm{O}_{3}+\mathrm{Gd}_{2} \mathrm{O}_{3}+\mathrm{La}_{2} \mathrm{O}_{3}+\mathrm{Nd}_{2} \mathrm{O}_{3}+\mathrm{Pr}_{2} \mathrm{O}_{3}+\mathrm{Sm}_{2} \mathrm{O}_{3}+\mathrm{Y}_{2} \mathrm{O}_{3} .
$$

This combined component was formed because the separate components had insufficient concentrations and insufficient variations in most glasses to justify fitting separate coefficients for them. However, combined they had sufficient concentration and showed sufficient variation to support a combined coefficient. Generally, their effects on glass properties are similar and vary only with ionic radius; this variation with radius was not accounted for in this study.

After forming the combined components, the 366 glass compositions were expressed as normalized mole fractions of 49 components. The mole-fraction ranges of these 49 components are listed in Table 3-2. Those components with concentrations greater than $1.5 \mathrm{~mole} \%$ in at least one glass were considered

(1) It is assumed that all glasses were equilibrated with air during melting or heat-treatment, and thus the proportions of valence states of multivalent oxides are uniquely determined for each glass by the temperature at which the glass has been equilibrated with the partial pressure of oxygen of $2.13 \times 10^{4} \mathrm{~Pa}$. However, this proportion is rarely determined experimentally. The oxides are listed in databases as a certain nominal valency that may $\left(\mathrm{Fe}_{2} \mathrm{O}_{3}\right)$ or may not $\left(\mathrm{MnO}_{2}\right)$ represent the dominant valence state of that oxide in glass

(m) These equations should not be interpreted as balanced equations, only actual steps performed to reduce the number of waste composition variables. Following these steps, the composition is renormalized. 
as possible model components-leaving the 23 components between $\mathrm{SiO}_{2}$ and $\mathrm{ThO}_{2}$ in $\mathrm{Table}^{3-2}$. This left a minimum sum of included components of $97.7 \mathrm{~mole} \%$ for all glasses.

Table 3-2. Mole Fraction Range for Components in Viscosity Model Data Glasses

\begin{tabular}{||l|l|l||l|l|l||l|l|l||}
\hline Component & Min & Max & Comp & Min & Max & Comp & Min & Max \\
\hline $\mathrm{SiO}_{2}$ & 0.3596 & 0.6589 & $\mathrm{~V}_{2} \mathrm{O}_{5}$ & 0.0000 & 0.0213 & $\mathrm{SeO}_{2}$ & 0.0000 & 0.0012 \\
\hline $\mathrm{Na}_{2} \mathrm{O}$ & 0.0484 & 0.2700 & $\mathrm{NiO}$ & 0.0000 & 0.0195 & $\mathrm{TeO}_{2}$ & 0.0000 & 0.0009 \\
\hline $\mathrm{B}_{2} \mathrm{O}_{3}$ & 0.0000 & 0.1953 & $\mathrm{P}_{2} \mathrm{O}_{5}$ & 0.0000 & 0.0179 & $\mathrm{I}$ & 0.0000 & 0.0008 \\
\hline $\mathrm{Li}_{2} \mathrm{O}$ & 0.0000 & 0.1821 & $\mathrm{TiO}_{2}$ & 0.0000 & 0.0174 & $\mathrm{Ag}_{2} \mathrm{O}$ & 0.0000 & 0.0007 \\
\hline $\mathrm{CaO}$ & 0.0000 & 0.1445 & $\mathrm{Cl}$ & 0.0000 & 0.0168 & $\mathrm{Sb}_{2} \mathrm{O}_{\mathrm{x}}$ & 0.0000 & 0.0007 \\
\hline $\mathrm{MgO}$ & 0.0000 & 0.1343 & $\mathrm{ThO}_{2}$ & 0.0000 & 0.0154 & $\mathrm{As}_{2} \mathrm{O}_{5}$ & 0.0000 & 0.0007 \\
\hline $\mathrm{Al}_{2} \mathrm{O}_{3}$ & 0.0000 & 0.1326 & $\mathrm{SO}_{3}$ & 0.0000 & 0.0124 & $\mathrm{Br}$ & 0.0000 & 0.0006 \\
\hline $\mathrm{Fe}_{2} \mathrm{O}_{\mathrm{x}}$ & 0.0000 & 0.1040 & $\mathrm{CdO}$ & 0.0000 & 0.0090 & $\mathrm{WO}_{3}$ & 0.0000 & 0.0006 \\
\hline $\mathrm{ZrO}_{2}$ & 0.0000 & 0.0802 & $\mathrm{MoO}_{\mathrm{x}}$ & 0.0000 & 0.0061 & $\mathrm{Tl}_{2} \mathrm{O}_{\mathrm{x}}$ & 0.0000 & 0.0005 \\
\hline $\mathrm{K}_{2} \mathrm{O}$ & 0.0000 & 0.0750 & $\mathrm{Cr}_{2} \mathrm{O}_{3}$ & 0.0000 & 0.0038 & $\mathrm{PdO}$ & 0.0000 & 0.0005 \\
\hline $\mathrm{F}$ & 0.0000 & 0.0414 & $\mathrm{PbO}_{\mathrm{x}}$ & 0.0000 & 0.0034 & $\mathrm{SnO}_{\mathrm{x}}$ & 0.0000 & 0.0004 \\
\hline $\mathrm{MnO} \mathrm{x}_{\mathrm{x}}$ & 0.0000 & 0.0343 & $\mathrm{Bi}_{2} \mathrm{O}_{3}$ & 0.0000 & 0.0031 & $\mathrm{Rb}_{2} \mathrm{O}$ & 0.0000 & 0.0003 \\
\hline $\mathrm{ZnO}$ & 0.0000 & 0.0326 & $\mathrm{Cs}_{2} \mathrm{O}$ & 0.0000 & 0.0027 & $\mathrm{ReO}_{\mathrm{x}}$ & 0.0000 & 0.0003 \\
\hline $\mathrm{SrO}$ & 0.0000 & 0.0293 & $\mathrm{CuO} \mathrm{CuO}_{2}$ & 0.0000 & 0.0023 & $\mathrm{Rh}_{2} \mathrm{O}_{\mathrm{x}}$ & 0.0000 & 0.0002 \\
\hline $\mathrm{LN} \mathrm{O}_{3}$ & 0.0000 & 0.0245 & $\mathrm{Ga}_{2} \mathrm{O}_{3}$ & 0.0000 & 0.0019 & $\mathrm{Nb}_{2} \mathrm{O}_{5}$ & 0.0000 & 0.0001 \\
\hline $\mathrm{UO} \mathrm{I}_{\mathrm{x}}$ & 0.0000 & 0.0237 & $\mathrm{CoO}_{\mathrm{x}}$ & 0.0000 & 0.0019 & & & \\
\hline $\mathrm{BaO}$ & 0.0000 & 0.0213 & $\mathrm{RuO}_{2}$ & 0.0000 & 0.0014 & & & \\
\hline \hline
\end{tabular}

\subsection{Model for Logarithm Viscosity at $1150^{\circ} \mathrm{C}$}

\subsubsection{Initial Model Fitting}

The natural logarithm of viscosity at $1150^{\circ} \mathrm{C}$ was modeled as a linear combination of composition according to:

$$
\ln \left[\eta_{1150}(P a \cdot s)\right]=\sum_{i=1}^{N} h_{i} x_{i}
$$

where $\mathrm{h}_{\mathrm{i}}$ and $\mathrm{x}_{\mathrm{i}}$ are the $\mathrm{i}^{\text {th }}$ component coefficient and mole fraction in glass, respectively, and $\mathrm{N}$ is the number of components in the glass - initially 24, including 23 components listed above plus Others. Two glasses, CVS2-79 and CVS2-80, were found to have residuals $>1$ in $\ln \left[\eta_{1150}(\mathrm{~Pa} \cdot \mathrm{s})\right]$ and were removed from the fit (Figure 3-1). ${ }^{(n)}$ The resulting model is summarized in Table 3-3 along with $R^{2}$ and $s$

(n) Although it is preferable to determine whether glasses are "well-predicted" by a model using standardized residuals (residual/standard deviation), it is easier to use residuals, and for the purposes of this modeling effort, it was deemed the appropriate level of effort. However, final models developed for plant operation or wasteform qualification should use the full, more rigorous methods. 
statistics. ${ }^{(o)}$ Five of the components, $\mathrm{Cl}, \mathrm{NiO}$, Others, $\mathrm{ZnO}$, and $\mathrm{UO}_{\mathrm{x}}$, had coefficients close to response values (the range of coefficient \pm one standard error overlapped with the range of responses). An additional two components, $\mathrm{SrO}$ and $\mathrm{ThO}_{2}$, had coefficients, slightly further from the response values (the range of coefficient \pm two standard errors overlapped the property response range). ${ }^{(p)}$ The effects of these components on $\ln \left[\eta_{1150}\right]$ are less significant than other components. Models were fitted to these two subsets of components; the 24 original components minus $\mathrm{Cl}, \mathrm{NiO}, \mathrm{ZnO}$, and $\mathrm{UO}_{\mathrm{x}}$ to make a 20component model and the 20 components minus $\mathrm{SrO}$ and $\mathrm{ThO}_{2}$ to make an 18-component model. ${ }^{(\mathrm{q})}$ In these latter two models, only Others was found to have a coefficient within one standard error of the range of property responses, and $\mathrm{SrO}, \mathrm{ThO}_{2}, \mathrm{TiO}_{2}$, and $\mathrm{MgO}$ were found to have coefficients within two standard errors of the range of property responses. Table 3-4 summarizes these two models. Further reduction in components, e.g., the removal of $\mathrm{TiO}_{2}$ and $\mathrm{MgO}$, was not deemed necessary for the purpose of this modeling effort. These models, as stated in the Introduction, are aimed at supplying reasonable estimates of viscosity over the relatively broad range of Hanford waste-glass composition regions.

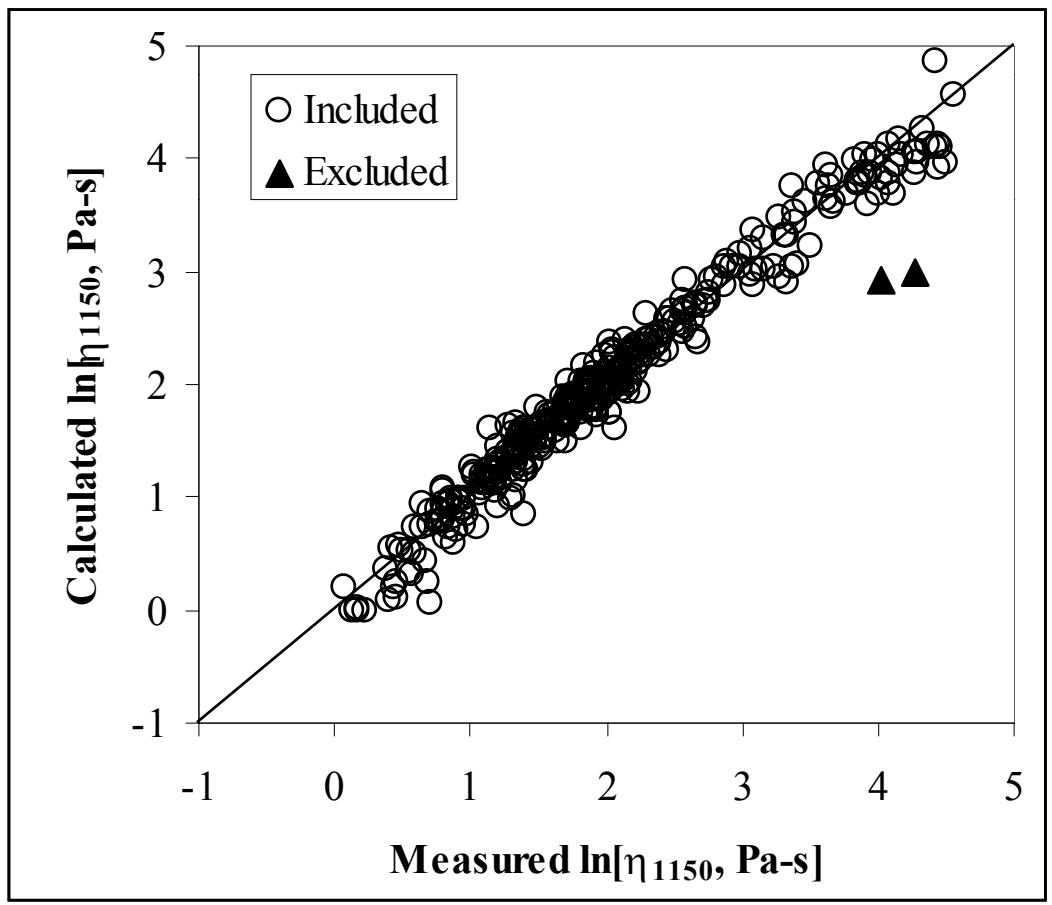

Figure 3-1. Comparison of Predicted and Measured $\ln \left[\eta_{1150}(\mathrm{~Pa} \cdot \mathrm{s})\right]$ by the Initial Model with Two Data Points (shown as solid triangles) Removed

(o) The $\mathrm{R}^{2}$ statistic is a measure of the fraction of the variation in response accounted for by the model and $\mathrm{s}$ (or root mean square error) is a measure of the prediction uncertainty and is compared to measurement uncertainty (standard deviation, $\sigma$ ). Other methods are required to determine if there is a "statistical lack of fit".

(p) For detailed discussion of the rigorous methods used to reduce terms in a statistical model see Piepel and Redgate (1997). The less rigorous methods described here were deemed sufficient for the purposes of this effort.

(q) In all modeling efforts, the component Others is redefined to include the concentrations of all components other than those with coefficients. 
Table 3-3. Summary of Initial Model for $\ln \left[\eta_{1150}\right]$

\begin{tabular}{|c|c|c|c|c|}
\hline Component & $\mathbf{h}_{\mathbf{i}}$ & std. err. & Statisitic & Value \\
\hline $\mathrm{Al}_{2} \mathrm{O}_{3}$ & 19.832652 & 0.43823 & $\mathrm{R}^{2}$ & 0.9710 \\
\hline $\mathrm{B}_{2} \mathrm{O}_{3}$ & -9.447488 & 0.254518 & $\mathrm{R}_{\text {adi }}^{2}$ & 0.9690 \\
\hline $\mathrm{CaO}$ & -5.212173 & 0.31748 & $\mathrm{R}_{\text {pred }}^{2}$ & 0.9654 \\
\hline $\mathrm{Fe}_{2} \mathrm{O}_{\mathrm{x}}$ & -3.598369 & 0.629876 & $\mathrm{~S}$ & 0.176 \\
\hline $\mathrm{K}_{2} \mathrm{O}$ & -8.77593 & 0.815398 & Min & 0.08 \\
\hline $\mathrm{Li}_{2} \mathrm{O}$ & -16.28959 & 0.255676 & Mean & 2.01 \\
\hline $\mathrm{MgO}$ & -1.304641 & 0.418957 & Max & 4.55 \\
\hline $\mathrm{Na}_{2} \mathrm{O}$ & -10.6205 & 0.252595 & $\mathrm{~N}$ & 364 \\
\hline $\mathrm{P}_{2} \mathrm{O}_{5}$ & 14.779068 & 3.128487 & & \\
\hline $\mathrm{SiO}_{2}$ & 9.0365457 & 0.099332 & & \\
\hline $\mathrm{ZrO}_{2}$ & 13.266995 & 0.713292 & & \\
\hline $\mathrm{LN}_{2} \mathrm{O}_{3}$ & -15.78739 & 3.400612 & & \\
\hline $\mathrm{BaO}$ & -7.564176 & 3.022988 & & \\
\hline $\mathrm{Cl}$ & 5.7657005 & 3.94834 & & \\
\hline $\mathrm{F}$ & -9.069534 & 1.491206 & & \\
\hline $\mathrm{MnO}$ & -7.457774 & 2.878181 & & \\
\hline $\mathrm{NiO}$ & 9.0815729 & 5.315329 & & \\
\hline $\mathrm{SrO}$ & -4.150929 & 2.706841 & & \\
\hline $\mathrm{ThO}_{2}$ & 13.632387 & 5.527846 & & \\
\hline $\mathrm{TiO}_{2}$ & -7.075945 & 3.26553 & & \\
\hline $\mathrm{UO}_{\mathrm{x}}$ & -1.625638 & 5.771282 & & \\
\hline $\mathrm{V}_{2} \mathrm{O}_{5}$ & -8.70675 & 3.211984 & & \\
\hline $\mathrm{ZnO}$ & -0.614569 & 1.425769 & & \\
\hline Others & -0.39505 & 3.1501 & & \\
\hline
\end{tabular}

Table 3-4. Summary of $\ln \left[\eta_{1150}\right]$ Models

\begin{tabular}{||l|r|l|l|l|l|l||}
\hline Comp. & 20 comp. $\mathbf{h}_{\mathbf{i}}$ & $\begin{array}{c}\text { 20 comp. } \\
\text { std. error }\end{array}$ & $\mathbf{1 8}$ comp. $\mathbf{h}_{\mathbf{i}}$ & $\begin{array}{c}\text { 18 comp. } \\
\text { std. Error }\end{array}$ & \multicolumn{1}{|c||}{ Statisitic } & Value \\
\hline $\mathrm{Al}_{2} \mathrm{O}_{3}$ & 19.7913912 & 1.089093 & 19.7257004 & 1.045584 & 20 component & \\
\hline $\mathrm{B}_{2} \mathrm{O}_{3}$ & -9.5197338 & 1.027234 & -9.5341696 & 0.981596 & $\mathrm{R}^{2}$ & 0.9707 \\
\hline $\mathrm{CaO}$ & -5.2862988 & 1.096003 & -5.4241256 & 1.056964 & $\mathrm{R}_{\text {adj }}^{2}$ & 0.9691 \\
\hline $\mathrm{Fe}_{2} \mathrm{O}_{3}$ & -3.4331938 & 1.266525 & -3.3040116 & 1.239384 & $\mathrm{R}_{\text {pred }}$ & 0.9661 \\
\hline $\mathrm{K}_{2} \mathrm{O}$ & -8.6783098 & 1.180412 & -7.9009786 & 1.082242 & $s$ & 0.175 \\
\hline $\mathrm{Li}_{2} \mathrm{O}$ & -16.2455438 & 1.036035 & -16.2528056 & 0.990857 & & \\
\hline $\mathrm{MgO}$ & -1.3932898 & 1.157175 & -1.3225766 & 1.093959 & 18 component & \\
\hline $\mathrm{Na}_{2} \mathrm{O}$ & -10.5253238 & 1.037976 & -10.6596156 & 0.993271 & $\mathrm{R}^{2}$ & 0.9700 \\
\hline $\mathrm{P}_{2} \mathrm{O}_{5}$ & 14.7468362 & 3.361107 & 16.3538324 & 3.310263 & $\mathrm{R}_{\text {adj }}^{2}$ & 0.9685 \\
\hline $\mathrm{SiO}_{2}$ & 9.0280829 & 0.995036 & 9.0741542 & 0.923224 & $\mathrm{R}_{\text {pred }}^{2}$ & 0.9658 \\
\hline $\mathrm{ZrO}_{2}$ & 13.2464352 & 1.267737 & 12.9501184 & 1.233309 & $\mathrm{~s}$ & 0.177 \\
\hline $\mathrm{LN}_{2} \mathrm{O}_{3}$ & -11.5263138 & 2.272914 & -12.1995456 & 2.240728 & & \\
\hline $\mathrm{BaO}^{2}-8.5073418$ & 2.95368 & -9.5766916 & 2.862491 & Data & \\
\hline $\mathrm{F}$ & -7.9212428 & 1.895651 & -8.2422436 & 1.788564 & min & 0.08 \\
\hline $\mathrm{MnO}$ & -7.5777358 & 2.974306 & -6.4337356 & 2.880346 & mean & 2.01 \\
\hline $\mathrm{SrO}$ & -4.6401028 & 2.849546 & & & max & 4.55 \\
\hline $\mathrm{ThO}_{2}$ & 13.4430212 & 5.407617 & & & $n$ & 364 \\
\hline $\mathrm{TiO}_{2}$ & -6.5164958 & 3.621772 & -4.5216196 & 3.461289 & & \\
\hline $\mathrm{V}_{2} \mathrm{O}_{5}$ & -9.0378278 & 3.361092 & -8.9763446 & 3.325748 & & \\
\hline $\mathrm{Others}$ & 0.5019462 & 0.994674 & 0.0253444 & 0.934159 & & \\
\hline \hline
\end{tabular}




\subsubsection{Model Evaluation and Selection}

The value of a model is in its capability to predict the properties of glasses not used in model development. As all of the prescreened data were used in model development, we must rely on other bases to validate the models. One option is a leave-one-out crossvalidation method, wherein model predictions are made for each data point without using that point to fit the model. Special formulas implemented in software perform the calculations without actually having to refit the model leaving each data point out. The $\mathrm{R}_{\text {pred }}^{2}$ shown in Tables 3.3 and 3.4 summarize the leave-one-out crossvalidation

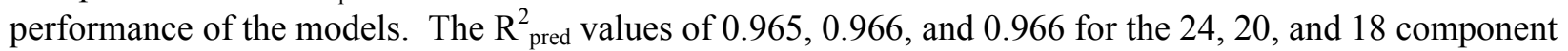
models, respectively, are not much smaller than the $\mathrm{R}^{2}$ or $\mathrm{R}_{\text {adj }}^{2}$ values for those models. Hence, the models have good crossvalidation performance.

Another option for model validation is to use the data that were removed during the screening process. However, model predictions for many of these points will be extrapolations beyond the region of validity for the models, which should be kept in mind when assessing the results. The 250 screened-out data points with $\eta_{1150}$ values reported were used to validate the models. Figure 3-2 shows a comparison of the measured and predicted $\ln \left[\eta_{1150}\right]$ values from the validation data set, using each of the three models labeled 24, 20, and 18 for the number of components in each model. The resulting validation $\mathrm{R}^{2}\left(\mathrm{R}_{\text {val }}^{2}\right)$ values were $0.858,0.866$, and 0.860 for the 24,20 , and 18 component models, respectively. As can be seen in Figure 3-2, there are two data points with higher residuals by all three models than any other points-IG2-07 (near 4.5 measured and 1.5 predicted) and HLWD1-19 (near -1.1 measured and -2.9

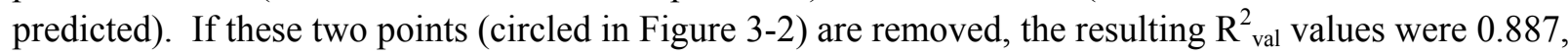
0.893 , and 0.886 for the 24,20 , and 18 component models, respectively.

Figure 3.2 indicates a tendency for all three models on average to underpredict $\ln \left[\eta_{1150}\right]$, especially for smaller and larger values. This tendency was not observed in Figure 3.1, so it may be due to the extrapolative predictions for compositions removed from the modeling process.

Considering the $\mathrm{R}_{\text {pred }}^{2}$ and $\mathrm{R}^{2}$ val values, there is very little difference in the predictive performances of the 24-, 20-, and 18-component models. Because of its slight advantage, the 20-component model is recommended for use in predicting $\ln \left[\eta_{1150}\right]$ of Hanford LAW and HLW glasses. 


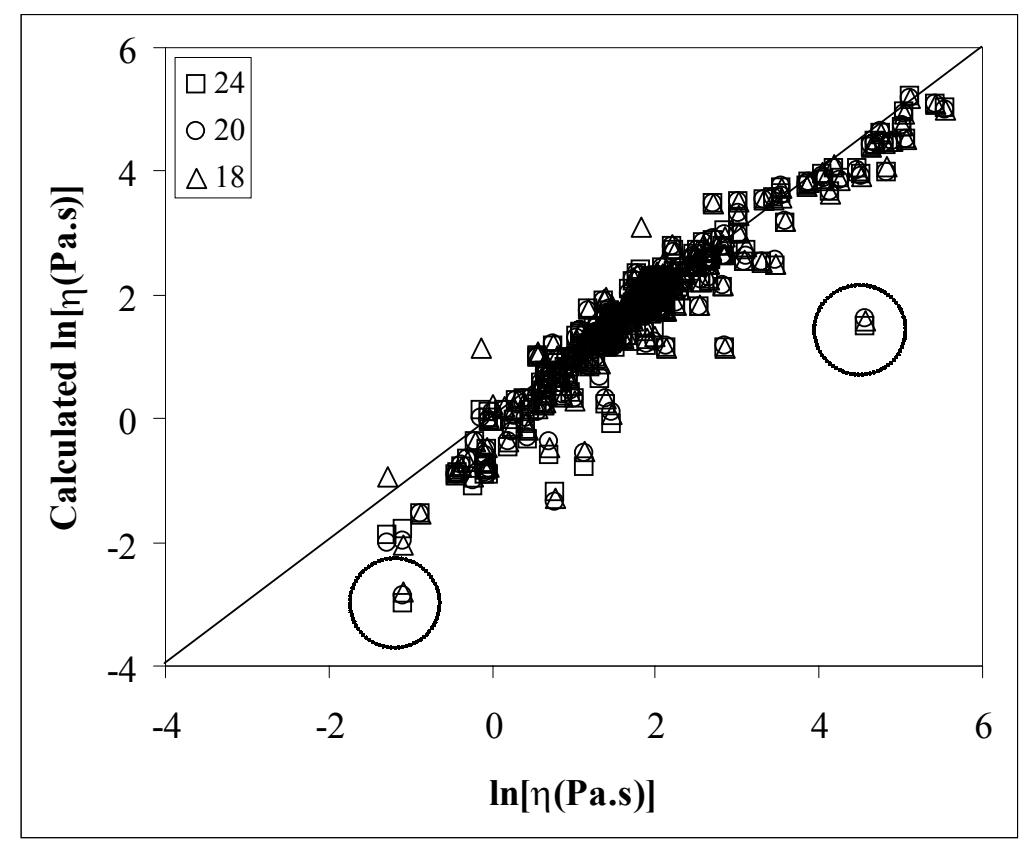

Figure 3-2. Comparison of Predicted and Measured $\ln [\eta]$ Values for Validation Glasses (not used in model fitting)

Table 3-5 compares the model coefficients of the new, 20 component, model with the previous model for $\ln \left[\eta_{1150}\right]$, the CVS model (Hrma et al. 1994). The percent difference shows that all new coefficients are within $40 \%$ of the old ones except the coefficient for the Others component, showing good general agreement. The Others coefficient should ideally be within the range of property responses (near the mean response value), which would indicate that it has a negligible effect on $\ln \left[\eta_{1150}\right]$. That is the case for the new model, but not the old model. The new model also accounts for the effects of several components that the old one did not $-\mathrm{K}_{2} \mathrm{O}, \mathrm{P}_{2} \mathrm{O}_{5}, \mathrm{LN}_{2} \mathrm{O}_{3}, \mathrm{BaO}, \mathrm{F}, \mathrm{MnO}, \mathrm{SrO}, \mathrm{ThO}_{2}, \mathrm{TiO}_{2}$, and $\mathrm{V}_{2} \mathrm{O}_{5}-$ all of which are possibly significant components in Hanford HLW or LAW glasses.

Table 3-5. Comparison of Component Coefficients for the New Model with Previous, Hanford CVS Model

\begin{tabular}{||l|c|c|c||l|l|l|l||}
\hline Component & $\mathbf{2 0}$ & CVS & \% diff & Component & 20 & CVS & \% diff \\
\hline $\mathrm{Al}_{2} \mathrm{O}_{3}$ & 19.791 & 17.088 & $16 \%$ & $\mathrm{BaO}$ & -8.507 & & \\
\hline $\mathrm{B}_{2} \mathrm{O}_{3}$ & -9.520 & -6.842 & $39 \%$ & $\mathrm{~F}$ & -7.921 & & \\
\hline $\mathrm{CaO}$ & -5.286 & -6.279 & $-16 \%$ & $\mathrm{MnO}$ & -7.578 & & \\
\hline $\mathrm{Fe}_{2} \mathrm{O}_{3}$ & -3.433 & -2.525 & $36 \%$ & $\mathrm{SrO}$ & -4.640 & & \\
\hline $\mathrm{K}_{2} \mathrm{O}$ & -8.678 & & & $\mathrm{ThO}_{2}$ & 13.443 & & \\
\hline $\mathrm{Li}_{2} \mathrm{O}$ & -16.246 & -15.030 & $8 \%$ & $\mathrm{TiO}_{2}$ & -6.516 & & \\
\hline $\mathrm{MgO}$ & -1.393 & -1.198 & $16 \%$ & $\mathrm{~V}_{2} \mathrm{O}_{5}$ & -9.038 & & \\
\hline $\mathrm{Na}_{2} \mathrm{O}$ & -10.525 & -11.017 & $-4 \%$ & Others & 0.502 & -3.561 & \\
\hline $\mathrm{P}_{2} \mathrm{O}_{5}$ & 14.747 & & & $\mathrm{R}^{2}$ & 0.9707 & 0.9447 & \\
\hline $\mathrm{SiO}_{2}$ & 9.028 & 8.498 & $6 \%$ & $\mathrm{R}_{\text {adj }}^{2}$ & 0.9691 & 0.9404 & \\
\hline $\mathrm{ZrO}_{2}$ & 13.246 & 12.811 & $3 \%$ & $\mathrm{R}_{\text {pred }}^{2}$ & 0.9661 & 0.9324 & \\
\hline $\mathrm{LN}_{2} \mathrm{O}_{3}$ & -11.526 & & & \multicolumn{7}{|l|}{} \\
\hline
\end{tabular}


From the model coefficients of all models, but specifically the recommended 20-component model, we can evaluate component effects on the viscosity of waste glasses. Those coefficients with values higher than the range of $\ln \left[\eta_{1150}\right]$ will increase $\eta_{1150}$, while those with values below the range will decrease $\eta_{1150}$. Those coefficients within the range of response generally have a small, but possibly statistically significant, effect on viscosity. The ranges of response in the model data set and validation data sets are $0.08 \leq \ln \left[\eta_{1150}\right] \leq 4.55$ and $-1.28 \leq \ln \left[\eta_{1150}\right] \leq 5.55$, respectively. Therefore, the following components decrease viscosity - in order of magnitude:

$$
\mathrm{Li}_{2} \mathrm{O}>\mathrm{LN}_{2} \mathrm{O}_{3}>\mathrm{Na}_{2} \mathrm{O}>\mathrm{B}_{2} \mathrm{O}_{3}>\mathrm{V}_{2} \mathrm{O}_{5}>\mathrm{K}_{2} \mathrm{O}>\mathrm{BaO}>\mathrm{F}>\mathrm{MnO}>\mathrm{TiO}_{2}>\mathrm{CaO}>\mathrm{SrO}>\mathrm{Fe}_{2} \mathrm{O}_{3}>\mathrm{MgO}
$$

and the following components increase viscosity — in order of magnitude:

$$
\mathrm{Al}_{2} \mathrm{O}_{3}>\mathrm{P}_{2} \mathrm{O}_{5}>\mathrm{ThO}_{2}>\mathrm{ZrO}_{2}>\mathrm{SiO}_{2}
$$

All other components of the 23 major components considered in this modeling effort, i.e., $\mathrm{Cl}, \mathrm{NiO}, \mathrm{ZnO}$, and $\mathrm{UO}_{3}$, have little impact on viscosity. These groupings are not surprising from our understanding of glass chemistry. However, the order of components within each category is of interest and suggests something about the chemical and physical processes responsible for viscous flow in waste-glass melts. Further evaluation of these chemical and physical processes is beyond the scope of this work.

Since it is not straight forward to directly convert the model's valid component concentration ranges from mole fractions to mass fractions, we have chosen to examine the composition region in mass fractions of oxides over which this model was found to predict well. The first step taken was to estimate the $\eta_{1150}$ of all 623 glasses with $\eta-T$ data. We then compared predicted and measured values of $\ln \left[\eta_{1150}\right]$ for each glass. Glasses with residuals, i.e., absolute value of predicted minus measured $\ln \left[\eta_{1150}(\mathrm{~Pa} \cdot \mathrm{s})\right]$, greater than $2 \mathrm{~s}(\geq 0.35)$ were marked as not well predicted - accounting for 92 data points. The data were then sorted by mass fractions of individual oxide components (corresponding to the 20 components with coefficients in the model) to determine the concentration ranges that separate those glasses with residuals $\geq 0.35$ from those with residuals $<0.35$. By removing an additional 75 glasses with residuals $<0.35$, one could separate the removed glasses from the remaining glasses simply by limiting the mass fractions of single-component oxides to those levels listed in Table 3-6. Although this is not to be interpreted as the model validity range, which is given in mole fraction of oxides in Section 3.2, it can be used to estimate whether $\ln \left[\eta_{1150}\right]$ of glasses are likely to be well predicted by this model. It is interesting to note that the concentration ranges listed for $\mathrm{Fe}_{2} \mathrm{O}_{3}, \mathrm{Al}_{2} \mathrm{O}_{3}, \mathrm{Na}_{2} \mathrm{O}, \mathrm{SiO}_{2}, \mathrm{MnO}$, and $\mathrm{SrO}$ are narrower than in the glass compositions expected to be produced at Hanford.

Pair-wise distributions of single component concentrations of the glasses used in model fitting are shown in Figure 3-3. There are no correlation coefficients higher than $|0.3|$ except for the $\mathrm{Li}_{2} \mathrm{O}: \mathrm{Na}_{2} \mathrm{O}$ which has a correlation coefficient of -0.666 . There is a good general distribution of the first 11 components and reasonable coverage of all components. 


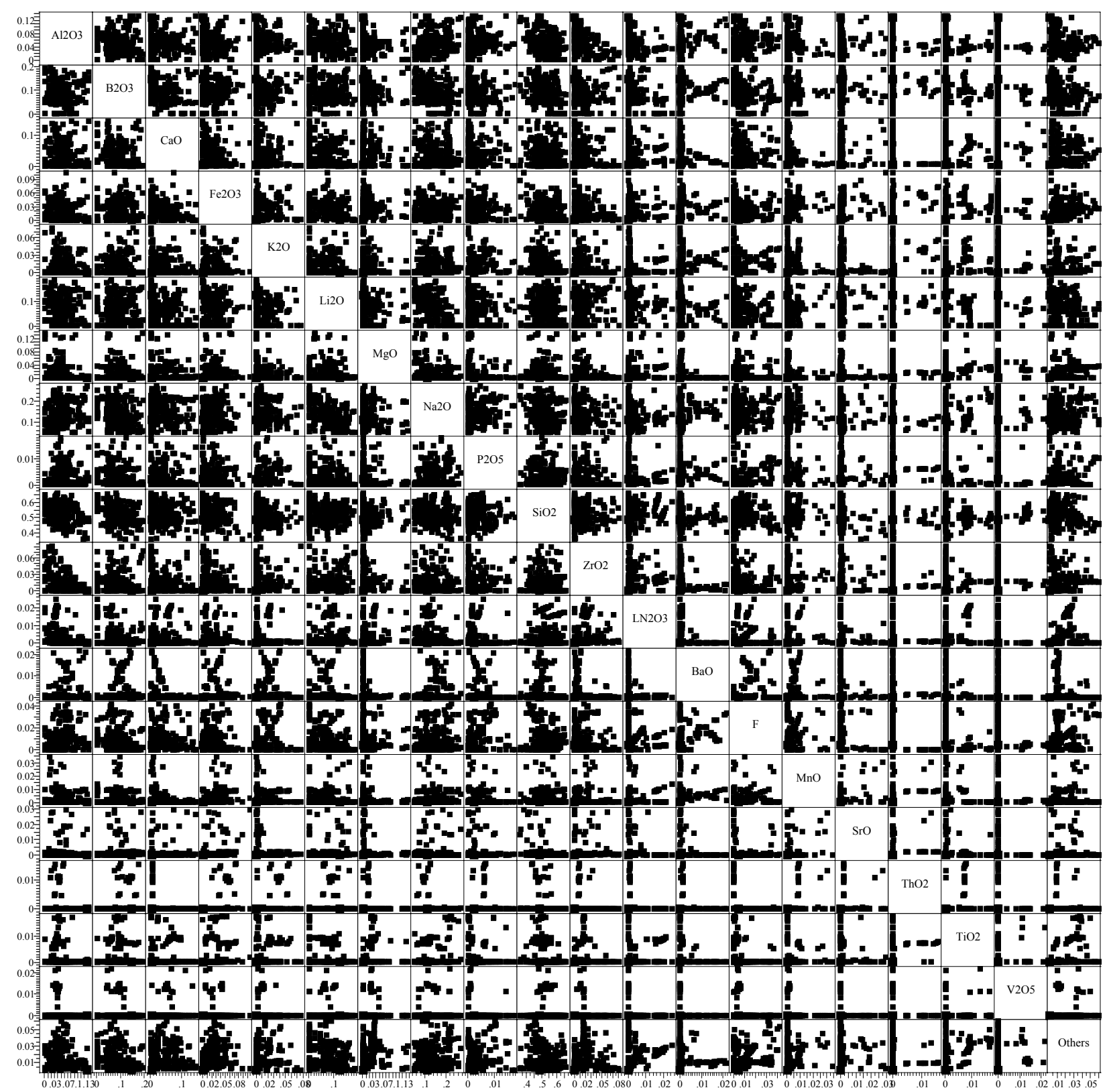

Figure 3-3. Scatter Plot Matrix for $\ln \left[\eta_{1150}\right]$ Model Data Component Concentrations 
Table 3-6. Component Concentration Ranges

(in mass fractions) for Accurate Prediction of $\ln \left[\eta_{1150}\right]$

\begin{tabular}{||l|c|c||l|c|c||}
\hline \hline Component & Min & Max & Component & Min & Max \\
\hline $\mathrm{Al}_{2} \mathrm{O}_{3}$ & 0.0000 & 0.1821 & $\mathrm{ZrO}_{2}$ & 0.0000 & 0.1167 \\
\hline $\mathrm{B}_{2} \mathrm{O}_{3}$ & 0.0000 & 0.2000 & $\mathrm{LN}_{2} \mathrm{O}_{3}$ & 0.0000 & 0.0356 \\
\hline $\mathrm{CaO}$ & 0.0000 & 0.1200 & $\mathrm{BaO}$ & 0.0000 & 0.0387 \\
\hline $\mathrm{Fe}_{2} \mathrm{O}_{3}$ & 0.0000 & 0.1550 & $\mathrm{~F}$ & 0.0000 & 0.0250 \\
\hline $\mathrm{K}_{2} \mathrm{O}$ & 0.0000 & 0.1000 & $\mathrm{MnO}$ & 0.0000 & 0.0310 \\
\hline $\mathrm{Li}_{2} \mathrm{O}$ & 0.0000 & 0.0846 & $\mathrm{SrO}$ & 0.0000 & 0.0917 \\
\hline $\mathrm{MgO}$ & 0.0000 & 0.0800 & $\mathrm{ThO}_{2}$ & 0.0000 & 0.0534 \\
\hline $\mathrm{Na}_{2} \mathrm{O}$ & 0.0500 & 0.2500 & $\mathrm{TiO}_{2}$ & 0.0000 & 0.0501 \\
\hline $\mathrm{P}_{2} \mathrm{O}_{5}$ & 0.0000 & 0.0402 & $\mathrm{~V}_{2} \mathrm{O}_{5}$ & 0.0000 & 0.0598 \\
\hline $\mathrm{SiO}_{2}$ & 0.3500 & 0.6278 & Others & 0.0000 & 0.1502 \\
\hline \hline
\end{tabular}

\subsection{Model for Viscosity as a Function of Temperature}

Although $1150^{\circ} \mathrm{C}$ is the current estimate of nominal Hanford waste-glass melter operation, there is value in predicting the melt viscosity as a function of temperature for several reasons, e.g., evaluation of melter idling conditions. It has been well established that the Vogel-Tamman-Fulcher (VTF) model can be used to estimate the effect of temperature on viscosity over nearly 20 orders of magnitude for silicatebased glasses (see Varsneya 1994, for example). However, fitting empirical composition models to the VTF model was found to require a great number of fit parameters that may not be justified for the purposes of this model, e.g., 30 parameters in the work by Hrma et al. (1994) on glasses with only 10 varied components. Over relatively narrow ranges of viscosity, e.g., 2 orders of magnitude, 1 to $100 \mathrm{~Pa} \cdot \mathrm{s}$, the Arrhenius model can adequately approximate the $\eta-T$ relationship, requires less fit parameters, and has the added advantage of being linear with inverse temperature (Hrma et al. 1994; Hrma et al. 2001). Therefore, we have chosen to use the Arrhenius model as the basis of our $\eta-T-x_{i}$ model in this work.

\subsubsection{Initial Model Development}

The natural logarithm of viscosity as a function of temperature was modeled by means of the Arrhenius model with its parameters expanded as linear mixture models:

$$
\ln \left[\eta_{1150}(P a \cdot s)\right]=\sum_{i=1}^{N} A_{i} x_{i}+\frac{\sum_{i=1}^{N} B_{i} x_{i}}{T}
$$

where $a_{i}$ and $b_{i}$ are the $i^{\text {th }}$ component Arrhenius model coefficients, $\mathrm{T}$ is absolute temperature, and $\mathrm{N}$ is the number of components in the glass. We started with the normalized mole fractions of 24 components (23 components plus Others) and 366 glass compositions as discussed in Section 3.2. As with the $\ln \left[\eta_{1150}\right]$ model, outliers were identified (IG2-02, CVS1-09, CVS2-79, CVS2-80, and HLWD3-08), and the model 
was refit. The resulting data set includes 361 glasses, $2868 \eta$ - $T$ data points with $\eta$ and $T$ ranges of $0.713 \leq \ln [\eta(\mathrm{Pa} \cdot \mathrm{s})] \leq 6.26$ and $1130 \leq \mathrm{T}(\mathrm{K}) \leq 1769$, respectively. The mean values for $\ln [\eta]$ and $\mathrm{T}$ of the model data set were 2.208 and $1413 \mathrm{~K}$, which translate to $9.1 \mathrm{~Pa} \cdot \mathrm{s}$ and $1140^{\circ} \mathrm{C}$, respectively.

Table 3-7 summarizes the new 24-component Arrhenius viscosity model. Figure 3-4 shows the predicted and measured $\ln [\eta(\mathrm{Pa} \cdot \mathrm{s})]$ for model glasses, with the excluded data points shown as squares. This model represents the data well with an $\mathrm{R}^{2}$ value of 0.980 . An examination of the coefficient values and their standard deviations suggests that the model may be improved by removing $\mathrm{NiO}$ and $\mathrm{Cl}$ as components, which led to the 22-component model summarized in Table 3-7. This model also represents the data well with an $\mathrm{R}^{2}$ value of 0.979 . The coefficients for $\mathrm{ThO}_{2}$ and $\mathrm{UO}_{3}$, both $\mathrm{a}_{\mathrm{i}}$ and $\mathrm{b}_{\mathrm{i}}$, were found to have the values inconsistent with our current understanding of those components effects on properties and were removed. This left the 20-component model. In this latter model, only the $\mathrm{BaO}$ coefficient standard errors were found to be significantly higher than those for other components. The coefficients for $\mathrm{BaO}$ were removed, resulting in the 19-component model described in Table 3-7. Again, more rigorous methods for selection of model components were reported by Piepel and Redgate (1997). The methods used here were determined sufficient for the purposes of this study. There is a clear decrease in $\mathrm{s}$ and increase in both $\mathrm{R}^{2}$ and $\mathrm{R}_{\text {adj }}^{2}$ values with increasing $\mathrm{N}$. These models will be evaluated in Section 3.4.2.

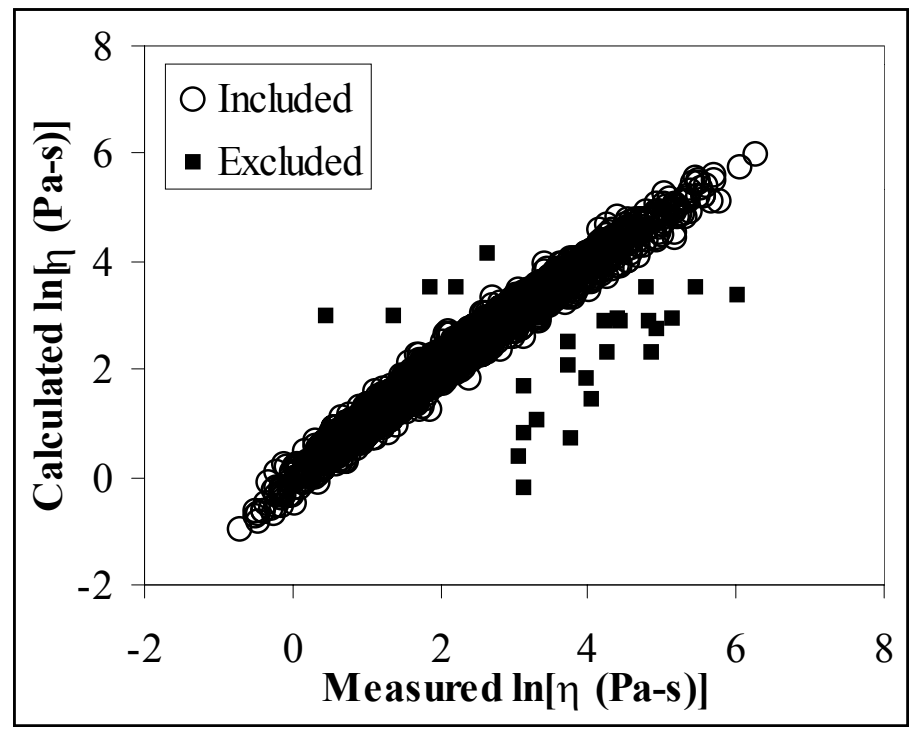

Figure 3-4. Comparison of Measured and Predicted $\ln [\eta(\mathrm{Pa} \cdot \mathrm{s})]$ from Initial 24-Component Arrhenius Model

Table 3-7. Summary of Arrhenius Viscosity Models

\begin{tabular}{|c|c|c|c|c|c|c|c|c|}
\hline & \multicolumn{2}{|c|}{24 Comn Model } & \multicolumn{2}{|c|}{22 Comn. Model } & \multicolumn{2}{|c|}{20 Comn. Model } & \multicolumn{2}{|c|}{19 Comn. Model } \\
\hline Comnonent & a: & std. err. & a: & std err. & a: & std. err. & a: & Std. err. \\
\hline $\mathrm{Al}_{2} \mathrm{O}_{3}$ & 0.588065 & 1.9 & 2.0466138 & 1.9 & 2.5543153 & 1.9 & 3.5835442 & 2.0 \\
\hline $\mathrm{B}_{2} \mathrm{O}_{3}$ & -24.38013 & 1.1 & -23.51997 & 1.1 & -23.12003 & 1.1 & -23.08111 & 1.1 \\
\hline $\mathrm{CaO}$ & -12.39029 & 1.3 & -12.10363 & 1.3 & -11.83956 & 1.3 & -12.58415 & 1.3 \\
\hline $\mathrm{Fe}_{2} \mathrm{O}_{3}$ & -14.58128 & 2.9 & -16.30602 & 2.9 & -14.95076 & 2.9 & -16.3428 & 2.9 \\
\hline $\mathrm{K}_{2} \mathrm{O}$ & -16.22202 & 3.8 & -12.49379 & 3.7 & -8.379555 & 3.5 & -6.828983 & 3.5 \\
\hline $\mathrm{Li}_{2} \mathrm{O}$ & -5.703848 & 1.1 & -7.953139 & 1.1 & -7.886365 & 1.1 & -8.340798 & 1.1 \\
\hline
\end{tabular}




\begin{tabular}{|c|c|c|c|c|c|c|c|c|}
\hline & \multicolumn{2}{|c|}{24 Comn Model } & \multicolumn{2}{|c|}{22 Comn. Model } & \multicolumn{2}{|c|}{20 Comn. Model } & \multicolumn{2}{|c|}{19 Comn. Model } \\
\hline$\overline{~ C o m n o n e n t ~}$ & $\mathbf{a}:$ & $\begin{array}{l}\text { std. err. } \\
\end{array}$ & a: & \begin{tabular}{|l|} 
std err. \\
\end{tabular} & a: & std. err. & a: & Std. err. \\
\hline $\mathrm{MgO}$ & -20.24774 & 2.1 & -18.63367 & 2.1 & -18.47942 & 2.1 & -19.68245 & 2.1 \\
\hline $\mathrm{Na}_{2} \mathrm{O}$ & -9.885539 & 1.2 & -10.68107 & 1.2 & -11.0084 & 1.1 & -11.15226 & 1.2 \\
\hline $\mathrm{P}_{2} \mathrm{O}_{5}$ & -11.68989 & 12.3 & -26.71842 & 11.9 & -18.38244 & 11.7 & -24.19039 & 11.9 \\
\hline $\mathrm{SiO}_{2}$ & -8.993515 & 0.4 & -8.836743 & 0.4 & -8.838998 & 0.4 & -8.778731 & 0.4 \\
\hline $\mathrm{ZrO}_{2}$ & -52.86406 & 3.1 & -52.76029 & 3.1 & -53.97564 & 3.1 & -54.78701 & 3.1 \\
\hline $\mathrm{LN}_{2} \mathrm{O}_{3}$ & -26.83475 & 15.0 & -54.45905 & 13.6 & -51.51733 & 10.7 & -69.37717 & 10.5 \\
\hline $\mathrm{BaO}$ & 18.51618 & 15.4 & 38.454578 & 15.1 & 27.367476 & 14.9 & & \\
\hline $\mathrm{Cl}$ & 8.129332 & 16.4 & & & & & & \\
\hline F & -10.68322 & 6.9 & -12.05659 & 6.8 & -13.86623 & 6.3 & -13.95969 & 6.4 \\
\hline $\mathrm{MnO}$ & 4.092693 & 14.4 & -15.48788 & 13.9 & -2.611432 & 13.0 & 0.9254979 & 13.1 \\
\hline $\mathrm{NiO}$ & -131.8808 & 22.1 & & & & & & \\
\hline $\mathrm{SrO}$ & \begin{tabular}{|l|}
-8.467963 \\
\end{tabular} & 13.7 & -6.60889 & 13.9 & -3.083549 & 13.8 & -6.377174 & 14.0 \\
\hline $\mathrm{ThO}_{2}$ & 85.9681 & 30.1 & 82.000602 & 30.4 & & & & \\
\hline $\mathrm{TiO}_{2}$ & -24.09365 & 14.3 & -13.43509 & 13.5 & -5.5078 & 13.4 & -13.93102 & 13.6 \\
\hline $\mathrm{UO}_{3}$ & 59.1457 & 28.1 & 37.547609 & 27.9 & & & & \\
\hline $\mathrm{V}_{2} \mathrm{O}_{5}$ & -47.79726 & 17.7 & -44.6834 & 17.6 & -45.53306 & 17.7 & -43.20154 & 17.9 \\
\hline $\mathrm{ZnO}$ & -54.02427 & 6.6 & -47.77983 & 6.6 & -49.36786 & 6.4 & -50.15485 & 6.5 \\
\hline \multirow[t]{2}{*}{ Others } & 6.587927 & 14.1 & -15.03723 & 10.4 & -22.15227 & 7.6 & -4.963217 & 7.0 \\
\hline & $\mathbf{b}_{\mathrm{i}}$ & std. err. & $b_{i}$ & std. err. & $b_{i}$ & std. err. & $\mathbf{b}_{\mathrm{i}}$ & Std. err. \\
\hline $\mathrm{Al}_{2} \mathrm{O}_{3}$ & 26234.92 & 2681 & 24090.916 & 2690 & 23392.834 & 2710 & 21719.847 & 2736 \\
\hline $\mathrm{B}_{2} \mathrm{O}_{3}$ & 21190.53 & 1586 & 19792.599 & 1546 & 19322.371 & 1536 & 19196.345 & 1536 \\
\hline $\mathrm{CaO}$ & 9971.479 & 1775 & 9561.6416 & 1765 & 9157.9755 & 1766 & 10349.85 & 1787 \\
\hline $\mathrm{Fe}_{2} \mathrm{O}_{3}$ & 14827.42 & 4097 & 17120.619 & 4093 & 15620.93 & 4030 & 17834.262 & 4085 \\
\hline $\mathrm{K}_{2} \mathrm{O}$ & 11162.71 & 5227 & 6310.3968 & 5083 & 1270.8166 & 4825 & -1250.997 & 4840 \\
\hline $\mathrm{Li}_{2} \mathrm{O}$ & -14470.56 & 1599 & -11228.19 & 1554 & -11261.19 & 1559 & -10547.26 & 1581 \\
\hline $\mathrm{MgO}$ & 26791.39 & 2913 & 24401.612 & 2901 & 24230.913 & 2921 & 26147.971 & 2955 \\
\hline $\mathrm{Na}_{2} \mathrm{O}$ & -877.4744 & 1652 & 416.81426 & 1606 & 837.06103 & 1576 & 1030.831 & 1599 \\
\hline $\mathrm{P}_{2} \mathrm{O}_{5}$ & 31856.38 & 17031 & 53131.386 & 16553 & 43075.714 & 16346 & 51974.792 & 16559 \\
\hline $\mathrm{SiO}_{2}$ & 25673.29 & 623 & 25429.115 & 625 & 25425.28 & 623 & 25354.665 & 626 \\
\hline $\mathrm{ZrO}_{2}$ & 93724.64 & 4283 & 93497.131 & 4331 & 94861.187 & 4324 & 96153.204 & 4375 \\
\hline $\mathrm{LN}_{2} \mathrm{O}_{3}$ & 16960.1 & 20882 & 56108.16 & 19078 & 52422.177 & 15453 & 81198.69 & 15090 \\
\hline $\mathrm{BaO}$ & -34830.48 & 21190 & -64629.16 & 20803 & -50506.39 & 20525 & & \\
\hline $\mathrm{Cl}$ & -1624.732 & 22542 & & & & & & \\
\hline F & 4421.361 & 9487 & 6930.2503 & 9404 & 9173.5771 & 8694 & 9754.377 & 8826 \\
\hline $\mathrm{MnO}$ & -14695.05 & 19871 & 11516.455 & 19297 & -6619.81 & 18034 & -12285.33 & 18188 \\
\hline $\mathrm{NiO}$ & 198454.4 & 30716 & & & & & & \\
\hline $\mathrm{SrO}$ & 7303.827 & 18973 & 4807.0424 & 19145 & -176.7816 & 19081 & 5392.7199 & 19270 \\
\hline $\mathrm{ThO}_{2}$ & -97982.25 & 41427 & -91772.52 & 41895 & & & & \\
\hline $\mathrm{TiO}_{2}$ & 23127.85 & 19529 & 11669.5 & 18356 & 1826.5471 & 18341 & 14973.294 & 18520 \\
\hline $\mathrm{UO}_{3}$ & -99217.04 & 42594 & -69099.76 & 42327 & & & & \\
\hline $\mathrm{V}_{2} \mathrm{O}_{5}$ & 55467.67 & 24335 & 48929.976 & 24190 & 50114.369 & 24331 & 47875.034 & 24702 \\
\hline $\mathrm{ZnO}$ & 78241.33 & 9126 & 69075.629 & 9048 & 71022.386 & 8853 & 73083.664 & 8925 \\
\hline Others & -12650.47 & 19628 & 24548.528 & 14294 & 33384.492 & 10669 & 5218.009 & 9762 \\
\hline \multicolumn{9}{|c|}{ Regression Statistics } \\
\hline$\overline{\mathrm{R}^{2}}$ & \begin{tabular}{ll|}
0.9797 \\
\end{tabular} & & 0.9792 & & 0.9787 & & 0.9781 & \\
\hline $\mathrm{R}_{\text {adj }}^{2}$ & 0.9793 & & 0.9788 & & 0.9784 & & 0.9778 & \\
\hline $\mathrm{s}$ & 0.166 & & 0.168 & & 0.170 & & 0.173 & \\
\hline
\end{tabular}




\subsubsection{Model Evaluation and Selection}

Four models were developed from the viscosity model set as described in Section 3.4.1. These models all fit the experimental data well with $\mathrm{R}^{2}$ values ranging from 0.9781 to 0.9797 - suggesting that roughly $98 \%$ of the variation in $\ln [\eta]$ is described by these models. Unfortunately, many of the model coefficients for the same components differ significantly from model to model. For example, the $\mathrm{a}_{\mathrm{MnO}}$ varies from -15.5 in the 22 -component model to +4.1 in the 20 -component model. In the same manner as the $\ln \left[\eta_{1150}\right]$ model selection (see Section 3.3.2), we turned to the data set excluded from model fitting to determine the most appropriate model. The four models were each used to calculate the viscosity of glasses excluded from model fitting (listed in Table 3-1). Figure 3-5 shows a comparison between the calculated and measured $\ln [\eta]$ values for the validation data set. It is clear from this figure that all four models predict fairly well the $\ln [\eta]$ of glasses not used in fitting them. The $\mathrm{R}_{\text {val }}^{2}$ values were 0.869 , $0.874,0.879$, and 0.878 for the 24-, 22-, 20-, and 19-component models, respectively. It is also clear that a handful of data are not predicted as well as most. These data-glasses HLWD2-05, IG2-07, CVS3-05, CVS3-20, LAWB42S, LAWPC7, HLWD1-19, and IG2-10 - were removed, ${ }^{(\mathrm{r})}$ and the goodness of prediction was recalculated. The measured and calculated $\ln [\eta]$ values for this reduced validation data set are compared in Figure 3-6. The $\mathrm{R}_{\text {val }}^{2}$ values for the reduced data set were $0.916,0.919,0.925$, and 0.923 for the 24-, 22-, 20-, and 19-component models, respectively. It is interesting to note that significantly more of the validation data were over predicted than under predicted. This tendency to over predict data outside the model validity region should be considered when attempting to expand the composition region of validity and may require adding interaction terms.

(r) Despite the differences in data used to model the $\ln [\eta 1150]$ and this $\eta-T-\mathbf{x}$ model, the model validity single component concentration ranges are the same. 


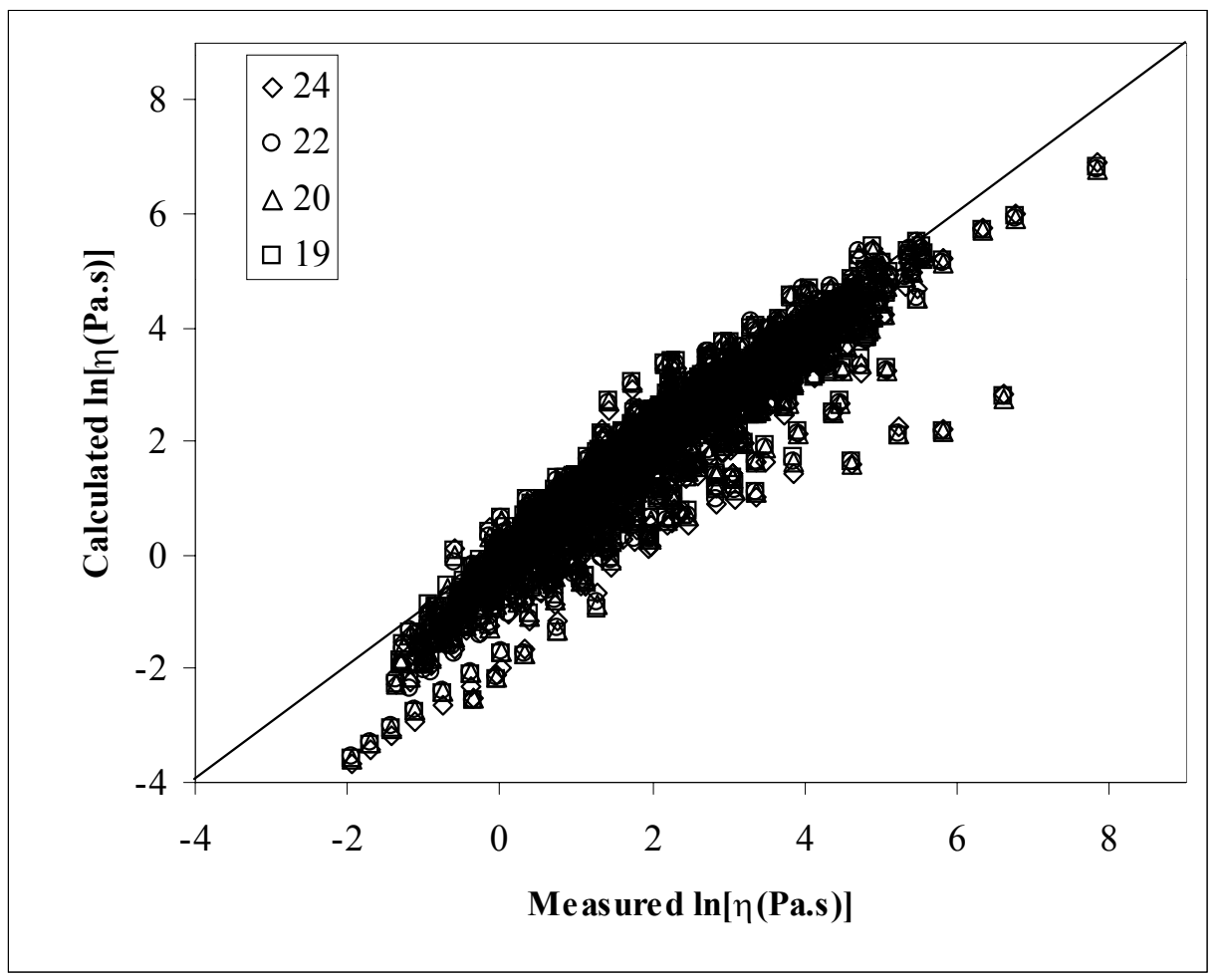

Figure 3-5. Comparison of Calculated and Measured $\ln [\eta]$ for the Complete Validation Data Set

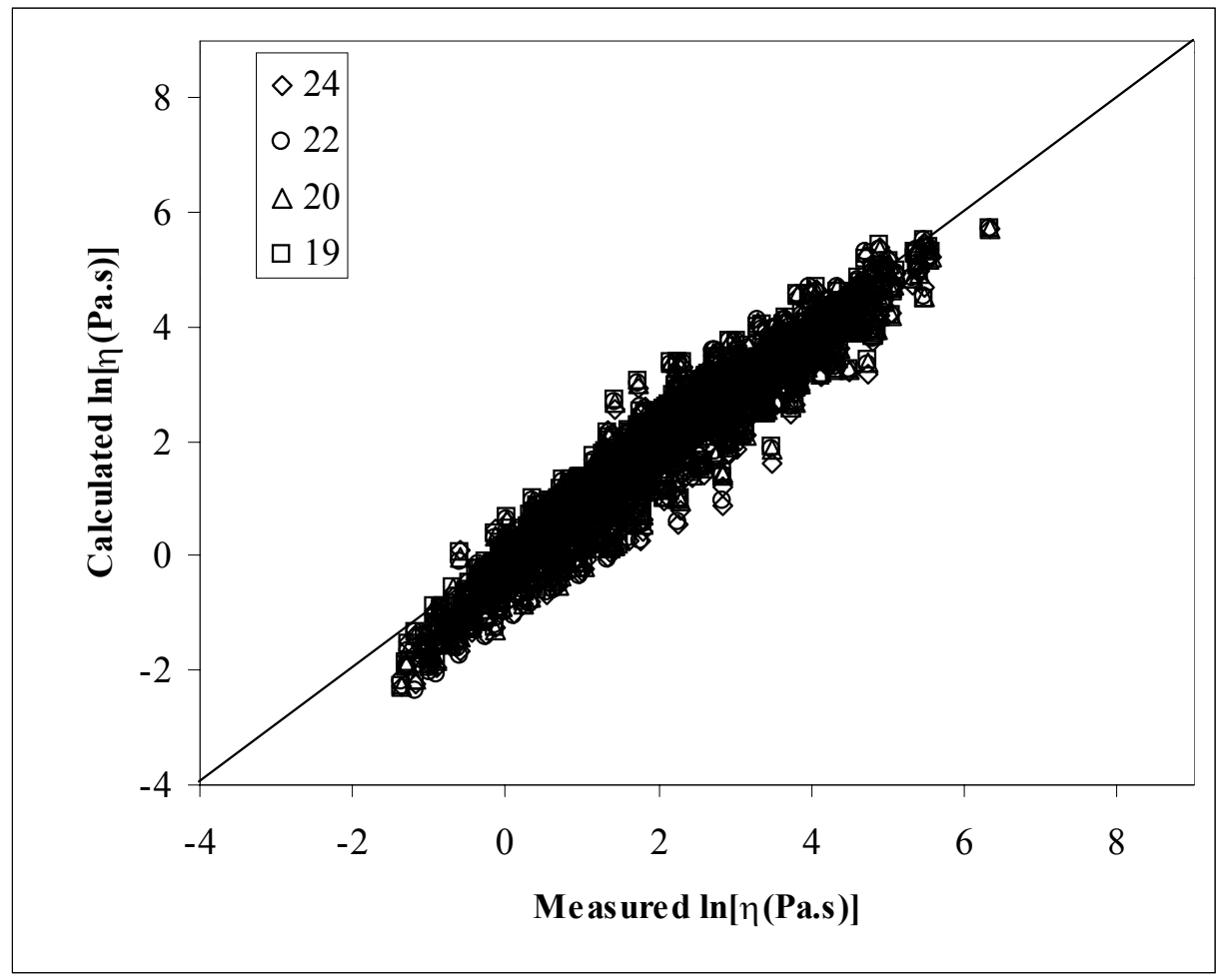

Figure 3-6. Comparison of Calculated and Measured $\ln [\eta]$ for the Reduced Validation Data Set 
The coefficients for each model, along with those reported by Hrma et al. (1994) and Hrma et al. (2001), are compared in Figure 3-7 and Figure 3-8. It is clear from these figures that there is considerable overlap between the coefficients for each component with the exception of the $\mathrm{LN}_{2} \mathrm{O}_{3}$ and $\mathrm{F}$ coefficients from Hrma et al. (2001). The NiO coefficients from the 24-component model also appear to be outlying. Generally, the coefficient standard errors are higher for the Hrma et al. (2001) and Hrma et al. (1994) models than those developed in this study. The 20-component model had model coefficients with the lowest standard errors. The lower standard errors combined with the slightly improved $\mathrm{R}_{\text {val values lead }}^{2}$ us to recommend the use of the 20-component model for predicting $\ln [\eta]$ as functions of composition and temperature. However, for the prediction of $\ln \left[\eta_{1150}\right]$, we recommend the model selected in Section 3.3.2.

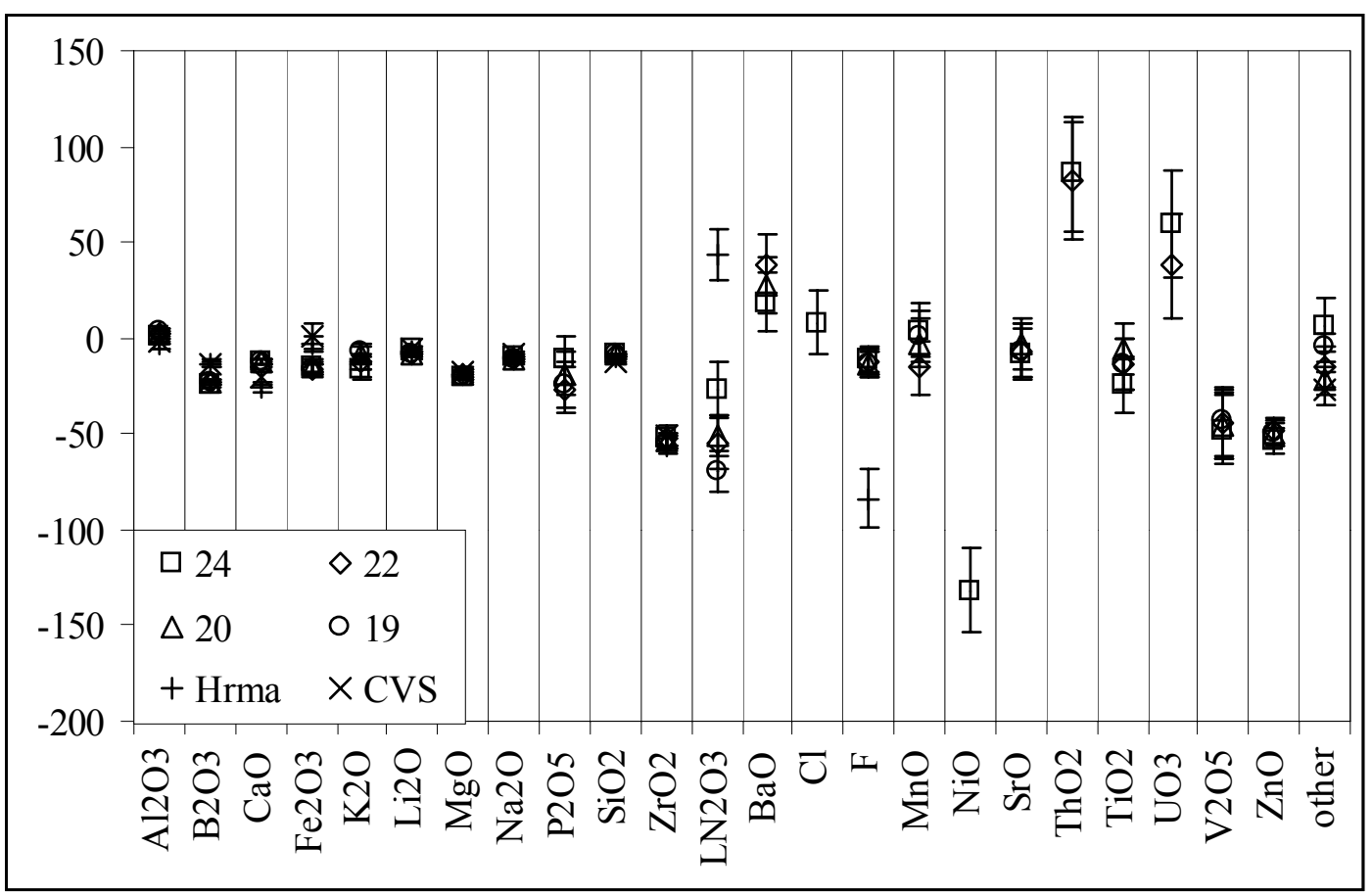

Figure 3-7. Comparison of $a_{i}$ Values from Different Models (Hrma represents Hrma et al. 2001, and CVS represents Hrma et al. 1994) 


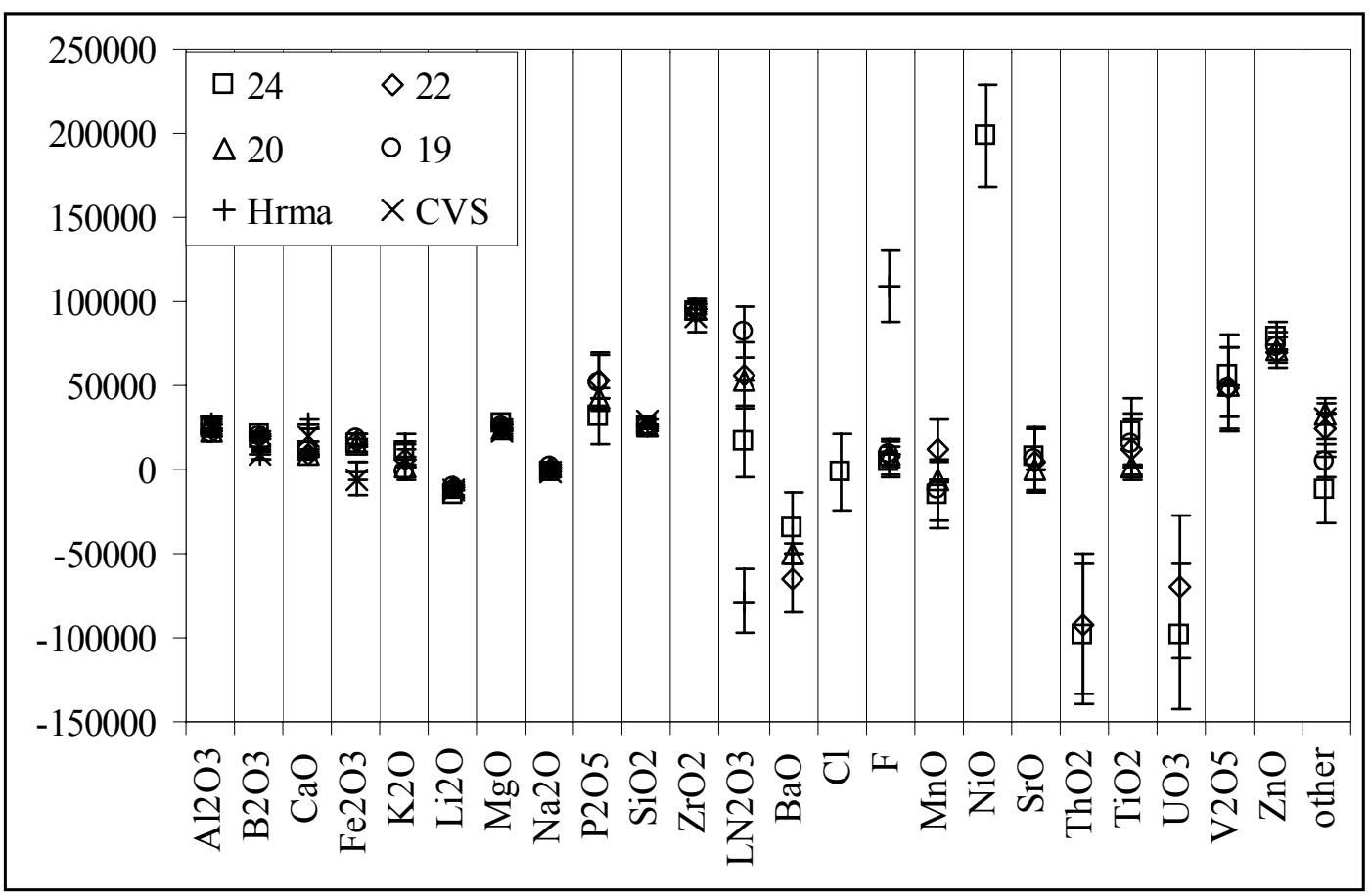

Figure 3-8. Comparison of $b_{i}$ Values from Different Models (Hrma represents Hrma et al. 2001, and CVS represents Hrma et al. 1994) 


\subsection{Models for Liquidus Temperature}

This section describes the development of models to predict the $T_{L}$ of glass melts in the spinel and zircon primary phase fields.

\subsection{Spinel Primary Phase Field}

\subsubsection{Model Selection}

The ion potential (IP) model (Vienna et al. 2001) was selected as the basis for $T_{L}$ model-development efforts in this work. Vienna (2002) compared this model form with other forms available at the time of this work. He concluded that only a purely empirical model could predict $\mathrm{T}_{\mathrm{L}}$ data better than the IP model and only for some of the data sets. The $s$, root-mean squared error (RMSE), values for some of the validation data sets were lower by the IP model than by an empirical model and vise-versa, with only small differences overall. The IP model was selected since 1) only a small difference between the predictability of the empirical model and the IP model was found, 2) the IP model has the advantage that it estimates the effects of all electropositive components on the $T_{L}$ of HLW glasses, and 3) the IP model has less than half the fitted parameters of the empirical model.

\subsubsection{Composition Conversion and Initial Data Screening}

The initial database available for developing property-composition models (see Section 2.0) contained data from 2001 glass compositions expressed in terms of 71 components. Those glasses with $\mathrm{T}_{\mathrm{L}}$ data within the spinel primary phase field were selected for model development - yielding a total of 389 glasses. Of the 389 glasses, 43 glasses were reported with only analyzed compositions, 222 glasses were reported with only target compositions, and 124 were reported with both target and analyzed compositions. The glasses with both target and analyzed composition were evaluated for consistency between composition estimates. Only five glasses - CVS2-22, -24, -30, -46, and -49-showed more than $30 \%$ relative difference in analyzed and target concentrations of the major components. ${ }^{(\mathrm{s})}$ These glasses were retained in the initial $T_{L}$ modeling data set but will be discussed later.

As discussed above, the IP model form (Vienna et al. 2001a; Vienna 2002) was assumed. In this model, the composition is expressed in mole fractions of electropositive components, i.e., cations or oxides expressed in the form of single metals, e.g., $\mathrm{AlO}_{3 / 2}$. For those components that were reported in more than one oxidation state, the most likely or prevalent oxidation state was chosen, e.g., $\mathrm{Mn}^{2+}, \mathrm{Fe}^{3+}$, and $\mathrm{U}^{6+}$. The compositions of the 389 glasses were converted to this basis according to normal procedures. Of the 389 glasses, 42 were found to be extreme in either $T_{L}$ values or single-component concentrations; e.g., when plotted on a histogram of all data, these compositions were significantly separated from most of the data. Table 4-1 lists the glasses and the extremes to which they correspond. These glasses were excluded from model fitting as they would likely be too influential in determining component effects. ${ }^{(t)}$ This left a total of 347 glasses for use in modeling. Some of these data were removed as outliers during the model fitting, as described in the following sections.

s) Major components were defined as those with $\geq 1$ mass $\%$ in glass.

(t) One exception to the data-exclusion method was for $\mathrm{ThO}_{2}$. There were only four glasses with $\mathrm{ThO}_{2}$ that were left in the data set because the effect of $\mathrm{ThO}_{2}$ on $\mathrm{T}_{\mathrm{L}}$ is an important parameter-SPA- $41,-42,-44$, and -45 . 
Table 4-1. Glasses with Extreme Component Concentrations or $\mathrm{T}_{\mathrm{L}}$ Values

\begin{tabular}{||c|c||}
\hline Glasses & Mole\% element \\
\hline CVS1-17, SP-MC-8 and SP-MC-2 & $<2 \mathrm{Al}$ \\
\hline SP-B-5 & $>25 \mathrm{~B}$ \\
\hline SP-B-1, SP-MC-8, CVS3-1 to -39 (minus-7 and -26) & $<1 \mathrm{~B}$ \\
\hline SPA-02 and SPA-04 & $>5 \mathrm{~K}$ \\
\hline SP-Li-6 & $>20 \mathrm{Li}$ \\
\hline CVS2-22 and SP-Mg-3 & $>4 \mathrm{Mg}$ \\
\hline SP-MC-9 & $<8.5 \mathrm{Na}$ \\
\hline SP-Si-4 and SPA-07 & $<23 \mathrm{Si}$ \\
\hline SPA-04 & $>1 \mathrm{Sr}$ \\
\hline SP-MC-2 & $>2.5 \mathrm{Zr}$ \\
\hline SG29 and CVS2-24 & $\mathrm{T}_{\mathrm{L}}<850^{\circ} \mathrm{C}$ \\
\hline SPA-02, SPA-25, Sp-HLHH, and SP-Na-1 & $\mathrm{T}_{\mathrm{L}}>1350^{\circ} \mathrm{C}$ \\
\hline
\end{tabular}

\subsubsection{Model Development}

The IP model (Vienna 2002; Vienna et al. 2001a), has the form:

$$
T_{L}=\sum_{N i, C r, M n} T_{i} x_{i}+\sum_{\text {Alk, AlkE }}\left(t_{\text {ion }}+\Theta_{\text {ion }} P_{i}\right) x_{i}+\sum_{\text {remaining }}\left(t_{\mathrm{cov}}+\Theta_{\mathrm{cov}} P_{i}\right) x_{i}
$$

where:

$\mathrm{x}_{\mathrm{i}}=\mathrm{i}^{\text {th }}$ electropositive component mole fraction in glass on a single metal oxide basis, e.g., $\mathrm{AlO}_{3 / 2}$

$P_{i}=Z_{i} / r_{i}$, where $Z_{i}$ is formal valence and $r_{i}$ is ionic radius of the $i^{\text {th }}$ component, respectively

$\mathrm{T}_{\mathrm{i}}=\mathrm{i}^{\text {th }}$ component coefficient $(\mathrm{i}=\mathrm{Ni}, \mathrm{Cr}$, and $\mathrm{Mn})$

$t_{\text {ion }}$ and $\Theta_{\text {ion }}=$ intercept and slope of the line relating the effect of alkali and alkaline earth components on $\mathrm{T}_{\mathrm{L}}$ with their ion potential

$t_{\text {cov }}$ and $\Theta_{\text {cov }}=$ intercept and slope of the line relating the effect of all other components on $T_{L}$ with their $\mathrm{P}_{\mathrm{i}}$, respectively

$\mathrm{T}_{\mathrm{L}}=$ liquidus temperature in ${ }^{\circ} \mathrm{C}$.

This model was fit directly to the experimental data from the 347 glasses described in Section 4.1.1 by means of standard, i.e., unweighted least squares, regression techniques (minimizing $\mathrm{s}=\mathrm{RMSE}$ values). The initial fit to experimental data resulted in several data points being outliers defined by high residuals, i.e., the absolute value of measured $\mathrm{T}_{\mathrm{L}}$ minus calculated $\mathrm{T}_{\mathrm{L}}$. The glass with the highest residual was removed, and the model was refitted. This process was repeated until the residuals from model fit data were all less than $80^{\circ} \mathrm{C}$. ${ }^{\text {u })}$ The excluded data, generally, can be used to define the composition

(u) Occasionally, when the glass with the highest residual remaining in the fit data set was removed, a glass previously removed had a lower residual than the newly fitted model. In those cases, the glass was returned to the model fit data set, and the model was refit. 
region of model validity. However, as discussed in Section 3.3, it is better to use standardized residuals. These excluded data will be discussed below.

Table 4-2 summarizes the model-fit results. Generally, the model-fit statistics and parameter estimates are similar to those reported by Vienna (2002) and reflect a more accurate estimate of Mn effects than those reported by Vienna et al. (2001a). Figure 4-1 compares the measured and calculated $\mathrm{T}_{\mathrm{L}}$ values by this model. There were 320 glasses in the final model fit. The remaining 27 glasses, $7.8 \%$ of the data set, were defined as outliers. The 27 outliers are listed in Table 4-3. All of these outlying data were found to be either at extremes in composition, based on two component concentrations, or showed peculiar results for another reason, except SPA-13 and -24.

Table 4-2. Summary of IP Model

\begin{tabular}{||l|c||}
\hline Item & Value \\
\hline $\mathrm{t}_{\text {ion }}$ & $-1,942$ \\
\hline$\Theta_{\text {ion }}$ & 1,996 \\
\hline $\mathrm{t}_{\text {cov }}$ & 6,038 \\
\hline$\Theta_{\text {cov }}$ & -500 \\
\hline $\mathrm{T}_{\mathrm{Cr}}$ & 33,271 \\
\hline $\mathrm{T}_{\mathrm{Mn}}$ & 1,316 \\
\hline $\mathrm{T}_{\mathrm{Ni}}$ & 13,675 \\
\hline Count & 320 \\
\hline Min $\mathrm{T}_{\mathrm{L}}\left({ }^{\circ} \mathrm{C}\right)$ & 859 \\
\hline Max $\mathrm{T}_{\mathrm{L}}\left({ }^{\circ} \mathrm{C}\right)$ & 1,310 \\
\hline Mean $\mathrm{T}_{\mathrm{L}}\left({ }^{\circ} \mathrm{C}\right)$ & 1,063 \\
\hline $\mathrm{R}^{2}$ & 0.897 \\
\hline $\mathrm{R}^{2}{ }_{\text {Adj }}$ & 0.895 \\
\hline $\mathrm{s}\left({ }^{\circ} \mathrm{C}\right)$ & 32.77 \\
\hline Max residual $\left({ }^{\circ} \mathrm{C}\right)$ & 78 \\
\hline
\end{tabular}




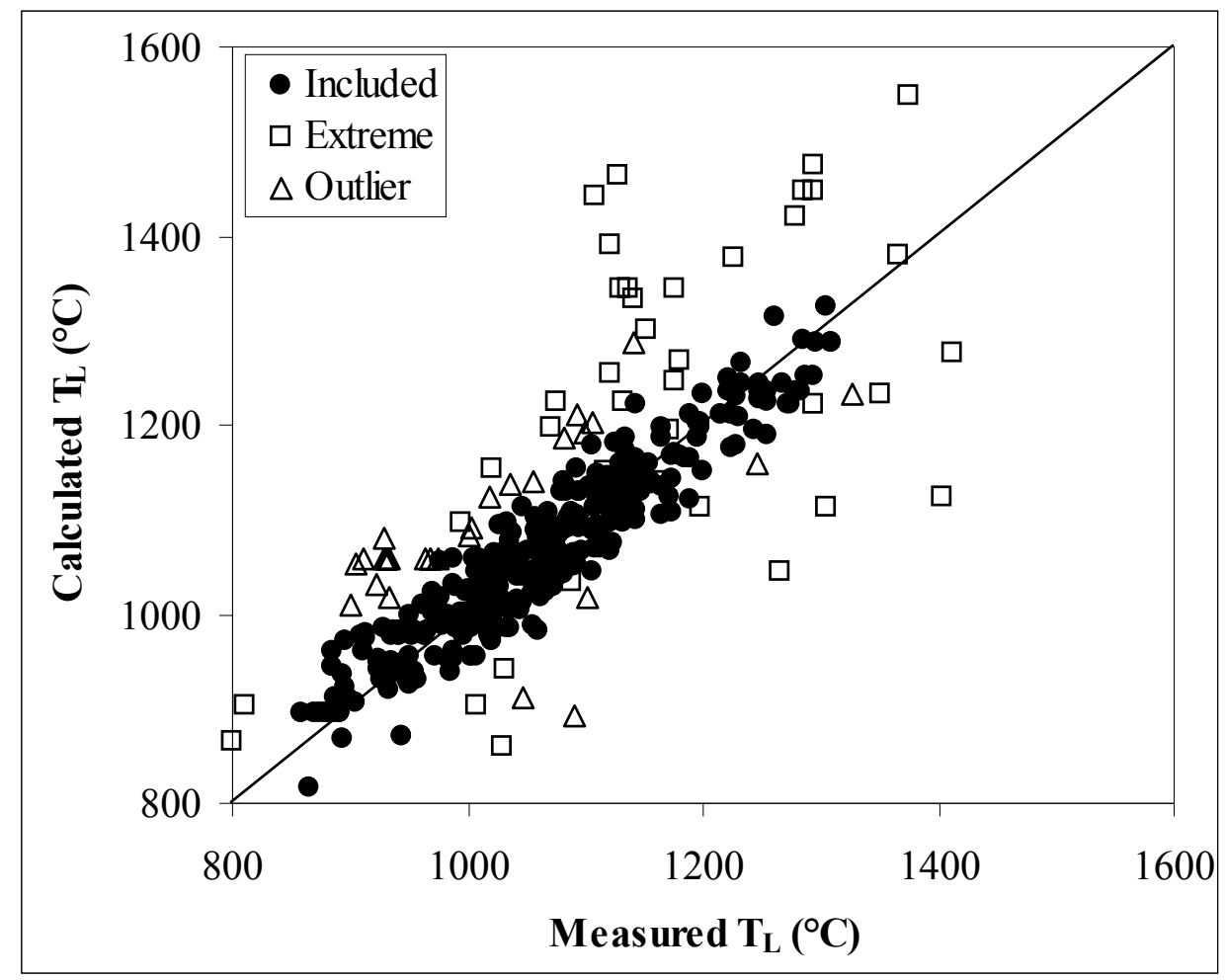

Figure 4-1. Comparison of Calculated and Measured $T_{L}$ Values

These excluded points were used in combination with the extreme compositions discussed in Table 4-1 to define a model validity range. The model validity ranges were defined in terms of mass $\%$ of oxides as the glasses excluded were found to be at the edge of the composition region on both mole and mass fraction bases between the excluded glasses by both composition bases. These composition ranges of model validity are listed in Table 4-4. Also listed in the table are comments on those components with model-validity concentration ranges thought to be inadequate for the purpose of this global model for all Hanford HLW glasses. The adequacy of the concentration ranges was determined from three sources: 1) the study performed by Perez et al. (2001), 2) the current WTP contract specifications (BNI 2001), and 3 ) current knowledge of pretreatment options. Expansion of the model-validity concentration ranges will require additional data and modeling efforts. Based on these comments, future studies should aim at expanding the concentration ranges of $\mathrm{SrO}, \mathrm{ThO}_{2}, \mathrm{Cr}_{2} \mathrm{O}_{3}, \mathrm{MnO}, \mathrm{K}_{2} \mathrm{O}, \mathrm{U}_{3} \mathrm{O}_{8}$, and $\mathrm{CdO}$. As mentioned earlier, this model is aimed at providing relatively low precision $\mathrm{T}_{\mathrm{L}}$ estimates over relatively broad (global) composition regions. Additional modeling, and possibly data collection, is required to develop more precise models that cover smaller (local) composition regions. 
Table 4-3. Summary of Outlying Data

\begin{tabular}{|c|c|c|c|c|}
\hline Glass ID & Study & $\mathrm{T}_{\mathrm{L}}\left({ }^{\circ} \mathrm{C}\right)$ & "calc $\mathbf{T}_{\mathrm{L}}\left({ }^{\circ} \mathrm{C}\right)$ & Comment \\
\hline AH $165 \mathrm{AL}$ & DWPF & 933 & 1017 & $\mathrm{GF}^{(\mathrm{a})}$ \\
\hline AH $165 \mathrm{AL}$ & DWPF & 905 & 1052 & GF \\
\hline AH $165 \mathrm{AV}$ & DWPF & 923 & 1031 & GF \\
\hline AH 165FE-RED & DWPF & 1100 & 1191 & reduced glass, GF \\
\hline AH 200AL & DWPF & 900 & 1010 & GF \\
\hline AH 202AL & DWPF & 930 & 1081 & GF \\
\hline AH 202FE-RED & DWPF & 1093 & 1210 & reduced glass, GF \\
\hline c106a-2 & Misc & 1004 & 1091 & high $\mathrm{Mg}$ and low $\mathrm{Li}$, high $\mathrm{Mg}$ and high $\mathrm{Na}$ \\
\hline CVS1-11 & CVS & 1035 & 1138 & $\mathrm{GF}, \mathrm{CaO}>6$ \\
\hline CVS1-17 & CVS & 1000 & 1083 & $\mathrm{GF}, \mathrm{Al}_{2} \mathrm{O}_{3}<1 \%, \mathrm{CaO}>6 \%$ \\
\hline CVS2-30 & CVS & 1090 & 894 & $\mathrm{GF}, \mathrm{B}_{2} \mathrm{O}_{3}>18.6 \%$ \\
\hline CVS3-26 & CVS & 1047 & 912 & GF \\
\hline LSi-Cr-008 & LSi & 1107 & 1203 & high $\mathrm{Cr}$ and $\mathrm{Cd}$, high $\mathrm{Cr}$ and high $\mathrm{Na}$ \\
\hline LSi-Cr-012 & LSi & 1140 & 1286 & high $\mathrm{Cr}$ and $\mathrm{Cd}$, high $\mathrm{Cr}$ and high $\mathrm{Na}$ \\
\hline SG06a & SG & 911 & 1060 & $\mathrm{SG}^{(\mathrm{b})}$ \\
\hline SG06b & SG & 931 & 1060 & SG06 \\
\hline SG06c & SG & 929 & 1059 & SG06 \\
\hline SG1-07(=SG06s1) & SG-1 & 965 & 1059 & SG06 \\
\hline SG1-08(=SG06s2) & \begin{tabular}{|l|} 
SG-1 \\
\end{tabular} & 968 & 1059 & SG06 \\
\hline SG1-14(=SG06) & SG-1 & 934 & 1059 & SG06 \\
\hline SG1-15(=SG06p1) & SG-1 & 974 & 1059 & SG06 \\
\hline SPA-11 & SPA & 1326 & 1232 & $\mathrm{~T}_{\mathrm{L}}>1310^{\circ} \mathrm{C}, \mathrm{OM} / \mathrm{XRD}^{(\mathrm{c})}$ \\
\hline SPA-13 & SPA & 1246 & 1158 & \\
\hline SPA-16 & SPA & 1081 & 1187 & high $\mathrm{Si}$ and high $\mathrm{Sr}$ \\
\hline SPA-24 & SPA & 1055 & 1140 & \\
\hline SPA-42 & SPA & 1018 & 1123 & high Th and high $\mathrm{Sr}$ \\
\hline Sp-LHLH(b) & SPx4 & 1102 & 1019 & different from Sp-LHLH with $\mathrm{T}_{\mathrm{L}}=1063^{\circ} \mathrm{C}$ \\
\hline \multicolumn{5}{|c|}{$\begin{array}{l}\text { (a) GF-Measured by gradient furnace method with uncertainties as high as } \pm 200^{\circ} \mathrm{C} \text { (Plodinec 1999) } \\
\text { (b) SG06-The SG06 glass composition has been measured a number of times and repeatedly been } \\
\text { found to be an outlier by a number of model forms. Of all the measurements, the last measurement } \\
\text { was performed with significantly longer heat treatments to ensure redox equilibrium was obtained } \\
\text { and this data point matches models the closest of any data points }\left(988^{\circ} \mathrm{C} \text { for SG1-20=SG06p2) }\right. \\
\text { (c) OM/XRD-One hundred of the } 347 \text { glasses were measured by both the optical microscopy (OM) } \\
\text { method and the X-ray diffraction (XRD) method. Of these } 100 \text { glasses, only six had a } \mathrm{T}_{\mathrm{L}} \text { difference } \\
\text { of more than } 50^{\circ} \mathrm{C} \text { between the two methods, three of them with } \mathrm{T}_{\mathrm{L}} \text { by XRD below } \mathrm{T}_{\mathrm{L}} \text { by OM- } \\
\text { SPA-25, SPA-11, and SP-Na- } 1 \text {. In that case, it is likely that the OM measurement was strongly } \\
\text { affected by volatile losses. }\end{array}$} \\
\hline
\end{tabular}


Table 4-4. IP Model Validity Concentration Ranges (in mass\% of oxides and F)

\begin{tabular}{|c|c|c|c|}
\hline Component & $\min$ & $\max$ & Comments \\
\hline $\mathrm{Al}_{2} \mathrm{O}_{3}$ & 1.0 & 16.1 & may need higher $\mathrm{Al}_{2} \mathrm{O}_{3}$ concentrations \\
\hline $\mathrm{B}_{2} \mathrm{O}_{3}$ & 1.0 & 18.6 & adequate $^{(\mathrm{a})}$ \\
\hline $\mathrm{Bi}_{2} \mathrm{O}_{3}$ & 0.0 & 7.0 & adequate \\
\hline $\mathrm{CaO}$ & 0.0 & 6.0 & adequate \\
\hline $\mathrm{CdO}$ & 0.0 & 2.0 & $\mathrm{CS}^{(\mathrm{b})}=3$ \\
\hline $\mathrm{Cr}_{2} \mathrm{O}_{3}$ & 0.0 & 0.8 & need overlap with eskolaite phase field \\
\hline $\mathrm{F}$ & 0.0 & 2.0 & adequate \\
\hline $\mathrm{Fe}_{2} \mathrm{O}_{3}$ & 2.0 & 23.0 & adequate \\
\hline $\mathrm{K}_{2} \mathrm{O}$ & 0.0 & 4.5 & $\begin{array}{l}\text { most effective component at reducing } \mathrm{T}_{\mathrm{L}} \text { (and } \\
\text { may be added at higher concentrations to } \\
\text { optimize glass composition) }\end{array}$ \\
\hline $\mathrm{Li}_{2} \mathrm{O}$ & 0.0 & 7.0 & adequate \\
\hline $\mathrm{MgO}$ & 0.0 & 5.0 & adequate \\
\hline $\mathrm{MnO}$ & 0.0 & 6.0 & $\mathrm{Sr} / \mathrm{TRU} \mathrm{ppt}^{(\mathrm{c})}$ \\
\hline $\mathrm{Na}_{2} \mathrm{O}$ & 5.8 & 25.0 & adequate \\
\hline $\mathrm{Nd}_{2} \mathrm{O}_{3}$ & 0.0 & 2.0 & adequate \\
\hline $\mathrm{NiO}$ & 0.0 & 3.1 & adequate \\
\hline $\mathrm{P}_{2} \mathrm{O}_{5}$ & 0.0 & 2.5 & adequate \\
\hline $\mathrm{SiO}_{2}$ & 30.0 & 60.0 & adequate \\
\hline $\mathrm{SrO}$ & 0.0 & 2.0 & Sr/TRU ppt. \\
\hline $\mathrm{ThO}_{2}$ & 0.0 & 2.0 & $\mathrm{CS}=4$, only 4 data points \\
\hline $\mathrm{TiO}_{2}$ & 0.0 & 5.0 & adequate \\
\hline $\mathrm{U}_{3} \mathrm{O}_{8}$ & 0.0 & 5.5 & $\mathrm{CS}=8$ as $\mathrm{UO}_{2}$ \\
\hline $\mathrm{ZnO}$ & 0.0 & 2.0 & adequate \\
\hline $\mathrm{ZrO}_{2}$ & 0.0 & 6.2 & adequate \\
\hline Others & $\overline{0.0}$ & 3.5 & concentration of all other components combined \\
\hline $\mathrm{T}_{\mathrm{L}}{ }^{\circ} \mathrm{C}$ & 859 & 1310 & adequate \\
\hline \multicolumn{4}{|c|}{$\begin{array}{l}\text { (a) adequate-Based on current estimates, the concentration ranges are } \\
\text { adequate to represent expected Hanford HLW glasses. } \\
\text { (b) } \mathrm{CS} \text { - Current WTP contract specifications (for defining minimum waste } \\
\text { loading) include higher concentrations than the current maximum model } \\
\text { validity concentration. } \\
\text { (c) } \mathrm{Sr} / \mathrm{TRU} \text { ppt.-SrO and MnO concentrations may be very high in HLW } \\
\text { glasses due to chemical additives for Sr and TRU precipitation (ppt) from } \\
\text { LAW. The concentration of MnO may be further increased if permanganate } \\
\text { oxidative leaching is applied to HLW. }\end{array}$} \\
\hline
\end{tabular}

Table 4-5 lists the $Z_{i}, r_{i}, P_{i}$, and $T_{i}$ (where $T_{i}=T_{i}$ for $i=M n, N i$, and $C r, T_{i}=t_{i o n}+\Theta_{i o n} P_{i}$ for $i=$ alkali and alkaline earth components, and $\mathrm{T}_{\mathrm{i}}=\mathrm{t}_{\text {cov }}+\Theta_{\text {cov }} \mathrm{P}_{\mathrm{i}}$ for $\mathrm{i}=$ all other components) for typical glass components. 
Table 4-5. $\mathrm{Z}_{\mathrm{i}}, \mathrm{r}_{\mathrm{i}}, \mathrm{P}_{\mathrm{i}}$, and $\mathrm{T}_{\mathrm{i}}$ Values for Typical HLW Components (i)

\begin{tabular}{|c|c|c|c|c|c|c|c|}
\hline Element (i) & $\overline{Z_{i}^{(a)}}$ & $\mathbf{r}_{i}^{(\mathbf{b})}(\AA)$ & $\overline{T_{\mathbf{i}}\left({ }^{\circ} \mathbf{C}\right)}$ & Element (i) & $\overline{\overline{Z_{i}}}$ & $\overline{\mathbf{r}_{\mathbf{i}}(\AA)}$ & $\overline{T_{i}\left({ }^{\circ} \mathbf{C}\right)}$ \\
\hline$\overline{\mathrm{Ag}}$ & 1 & 1.080 & 5575.7733 & $\mathrm{P}$ & 5 & 0.310 & -2021.2207 \\
\hline $\mathrm{Al}$ & 3 & 0.530 & 3209.9674 & $\mathrm{~Pb}$ & 2 & 1.430 & 5339.5773 \\
\hline As & 5 & 0.475 & 778.4574 & $\mathrm{Pd}$ & 2 & 1.000 & 5039.0584 \\
\hline $\mathrm{B}$ & 3 & 0.250 & 42.0572 & $\operatorname{Pr}$ & 3 & 1.266 & 4854.3351 \\
\hline $\mathrm{Ba}$ & 2 & 1.560 & 616.3770 & $\mathrm{Rb}$ & 1 & 1.750 & -801.8076 \\
\hline $\mathrm{Bi}$ & 3 & 1.170 & 4757.1763 & $\mathrm{Rh}$ & 3 & 0.805 & 4176.2222 \\
\hline $\mathrm{Ca}$ & 2 & 1.260 & 1225.5628 & $\mathrm{Ru}$ & 4 & 0.760 & 3408.4580 \\
\hline $\mathrm{Cd}$ & 2 & 1.090 & 5121.5777 & $\mathrm{~S}$ & 6 & 0.260 & -5493.0826 \\
\hline $\mathrm{Ce}$ & 4 & 1.010 & 4059.4483 & $\mathrm{Sb}$ & 5 & 0.740 & 2662.1065 \\
\hline $\mathrm{Co}$ & 2 & 0.720 & 4650.4027 & $\mathrm{Se}$ & 6 & 0.420 & -1100.1145 \\
\hline $\mathrm{Cr}$ & 3 & 0.755 & 33271.4288 & $\mathrm{Si}$ & 4 & 0.400 & 1041.4574 \\
\hline $\mathrm{Cs}$ & 1 & 1.880 & -880.6648 & $\mathrm{Sm}$ & 3 & 1.219 & 4808.6798 \\
\hline $\mathrm{Cu}$ & 2 & 0.870 & 4889.7227 & $\mathrm{Sr}$ & 2 & 1.400 & 908.7862 \\
\hline $\mathrm{Fe}$ & 3 & 0.630 & 3658.9343 & $\mathrm{Te}$ & 4 & 0.800 & 3539.9580 \\
\hline $\mathrm{K}$ & 1 & 1.650 & -732.6927 & $\mathrm{Th}$ & 4 & 1.080 & 4187.7175 \\
\hline $\mathrm{La}$ & 3 & 1.300 & 4885.3045 & $\mathrm{Ti}$ & 4 & 0.560 & 2469.1721 \\
\hline $\mathrm{Li}$ & 1 & 0.900 & 275.2329 & $\mathrm{U}$ & 6 & 0.870 & 2592.2509 \\
\hline $\mathrm{Mg}$ & 2 & 0.860 & 2698.9425 & $\mathrm{~V}$ & 5 & 0.680 & 2364.1931 \\
\hline $\mathrm{Mn}$ & 2 & 0.970 & 1316.4376 & $\mathrm{~W}$ & 6 & 0.740 & 1986.8360 \\
\hline Mo & 6 & 0.730 & 1931.3344 & $\mathrm{Y}$ & 3 & 1.159 & 4745.0157 \\
\hline $\mathrm{Na}$ & 1 & 1.160 & -221.7787 & $\mathrm{Zn}$ & 2 & 0.740 & 4687.9178 \\
\hline $\mathrm{Nd}$ & 3 & 1.249 & 4838.2182 & $\mathrm{Zr}$ & 4 & 0.860 & 3714.2720 \\
\hline $\mathrm{Ni}$ & 2 & 0.830 & 13674.8724 & & & & \\
\hline \multicolumn{8}{|c|}{$\begin{array}{l}\text { (a) For multivalent components, the most abundant valence states were assumed. } \\
\text { (b) The crystal radii from Shannon (1976) were used assuming the most abundant } \\
\text { coordination environment. }\end{array}$} \\
\hline
\end{tabular}

\subsection{Zircon Primary Phase Field}

\subsubsection{Initial Data Evaluation and Conversion}

A total of 72 glasses was reported with zircon or an unknown zirconia-containing phase on the primary phase field. Of the 72 glasses in this data set, two, Sp-MC-7 and Sp-Zr-2, were reported to have an unknown zirconia-containing primary phase, four, CVS1-07, CVS1-08, CVS2-14, and CVS2-80, were reported as inequalities (e.g., $\mathrm{T}_{\mathrm{L}}>1117^{\circ} \mathrm{C}$ ), and one, CVS3-21, was from an alkali-aluminosilicate glass family. The 65 remaining glasses were used in initial model development.

To determine if the IP model form may be applicable to the zircon primary phase field, compositions were first converted to the basis of that model, e.g., mole fractions of single metal oxides, e.g., $\mathrm{AlO}_{3 / 2}$, as was done in Section 4.1.1. 


\subsubsection{Model Development}

A first-order empirical fit of the zircon $\mathrm{T}_{\mathrm{L}}$ data was made. Table 4-6 summarizes this empirical fit. In this fit, one glass, $\mathrm{Zr}-27$, was found to be an outlier with a residual of $130^{\circ} \mathrm{C}$. It is not clear why this glass is not accurately predicted by the first-order approximation, but it was excluded from the fit. Figure 4-2 shows the correlation between $P_{i}$ and $T_{i}$ for the expansion of zircon $T_{L}$ in composition. Missing from this figure is $\mathrm{T}_{\mathrm{Zr}}$, which is off scale for the plot and a major component of zircon $\left(\mathrm{ZrSiO}_{4}\right)$ and $\mathrm{T}_{\text {Others. }}$ "Others" represents the sum of other components besides the nine used in the fit and therefore does not have a distinct $P_{i}$ value. It is clear from the plot that the relationship between $T_{i}$ and $P_{i}$ remains valid in the zircon primary phase field-yielding two straight lines with the equations:

$$
\mathrm{T}_{\mathrm{i}}=\mathrm{t}_{\text {ion }}+\Theta_{\text {ion }} \mathrm{P}_{\mathrm{i}}
$$

where $\mathrm{t}_{\text {ion }}=-3834, \Theta_{\text {ion }}=3247$, and $\mathrm{i}=\mathrm{Ca}, \mathrm{Li}, \mathrm{Mg}, \mathrm{Na}$ and

$$
\mathrm{T}_{\mathrm{i}}=\mathrm{t}_{\mathrm{cov}}+\Theta_{\mathrm{cov}} \mathrm{P}_{\mathrm{i}}
$$

where $\mathrm{t}_{\mathrm{cov}}=3865, \Theta_{\mathrm{cov}}=-270$, and $\mathrm{i}=\mathrm{Al}, \mathrm{B}, \mathrm{Fe}, \mathrm{Si}$. This relationship leads directly to an IP model of the form:

$$
T_{L}=T_{Z r} x_{Z r}+\sum_{\text {Alk, AlkE }}\left(t_{\text {ion }}+\Theta_{\text {ion }} P_{i}\right) x_{i}+\sum_{\text {remaining }}\left(t_{\text {cov }}+\Theta_{\text {cov }} P_{i}\right) x_{i}
$$

which was fitted to the 64 data points used in the empirical expansion. Table 4-7 summarizes the results of the IP model fit to the zircon data, and Figure 4-3 compares the predicted and measured values. The goodness of fit, as judged by $\mathrm{R}^{2}, \mathrm{R}_{\text {adj }}^{2}$, and $\mathrm{s}$, are only slightly better for the empirical fit with 10 fitted parameters than for the IP model with only 5 fitted parameters.

Table 4-6. $\mathrm{T}_{\mathrm{i}}$ Values for the First-Order Model Calculated from the Zircon Data Set

\begin{tabular}{|l|r|l|l|c||}
\hline Element & \multicolumn{1}{c|}{$\mathbf{T}_{\mathbf{i}}$} & Std. Error & \multicolumn{1}{c|}{ Statistic } & Value \\
\hline $\mathrm{Al}$ & $2,240.85$ & 281.12 & $\mathrm{R}^{2}$ & 0.926 \\
\hline $\mathrm{B}$ & 717.98 & 235.62 & $\mathrm{R}^{2}$ adi & 0.914 \\
\hline $\mathrm{Fe}$ & $2,692.88$ & 546.07 & $\mathrm{~s}\left({ }^{\circ} \mathrm{C}\right)$ & 23.31 \\
\hline $\mathrm{Si}$ & $1,044.30$ & 217.21 & min response $\left({ }^{\circ} \mathrm{C}\right)$ & 897 \\
\hline $\mathrm{Ca}$ & $1,054.86$ & 327.31 & mean response $\left({ }^{\circ} \mathrm{C}\right)$ & 1068 \\
\hline $\mathrm{Li}$ & 129.36 & 214.33 & max response $\left({ }^{\circ} \mathrm{C}\right)$ & 1350 \\
\hline $\mathrm{Mg}$ & $3,788.52$ & 325.78 & Observations & 64 \\
\hline $\mathrm{Na}$ & $-1,195.28$ & 260.23 & & \\
\hline $\mathrm{Zr}$ & $11,465.94$ & 633.87 & & \\
\hline Others & $1,850.97$ & 207.28 & & \\
\hline
\end{tabular}




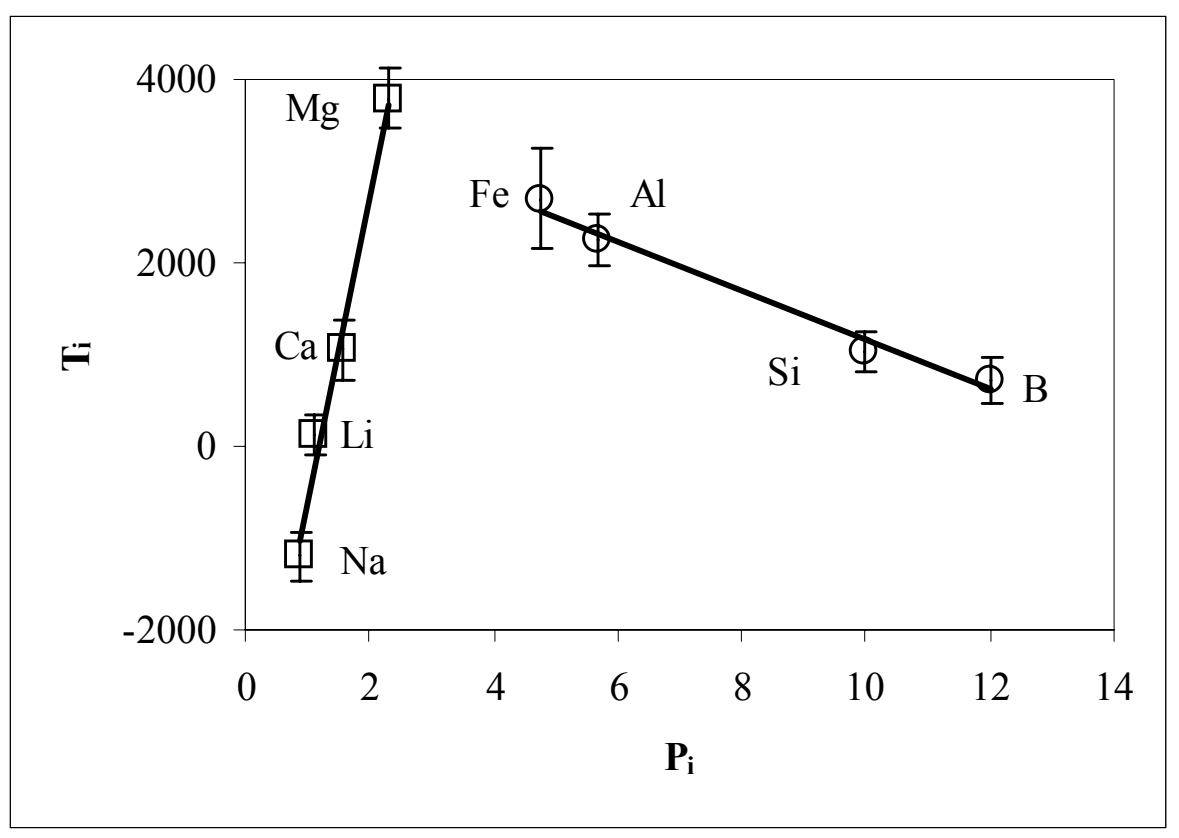

Figure 4-2. Plot of Fitted $T_{i}$ Values for Zircon as Functions of $P_{i}$.

Table 4-7. Summary of Zircon IP Model Fit

\begin{tabular}{||l|c||}
\hline Parameter & Value \\
\hline $\mathrm{t}_{\text {ion }}$ & $-3,979$ \\
\hline$\Theta_{\text {ion }}$ & 3,524 \\
\hline $\mathrm{t}_{\mathrm{cov}}$ & 2,505 \\
\hline$\Theta_{\mathrm{cov}}$ & -146 \\
\hline $\mathrm{T}_{\mathrm{Zr}}$ & 12,254 \\
\hline $\mathrm{R}^{2}$ & 0.868 \\
\hline $\mathrm{R}_{\text {adj }}{ }$ & 0.859 \\
\hline $\mathrm{s}\left({ }^{\circ} \mathrm{C}\right)$ & 29.9 \\
\hline Observations & 64 \\
\hline
\end{tabular}




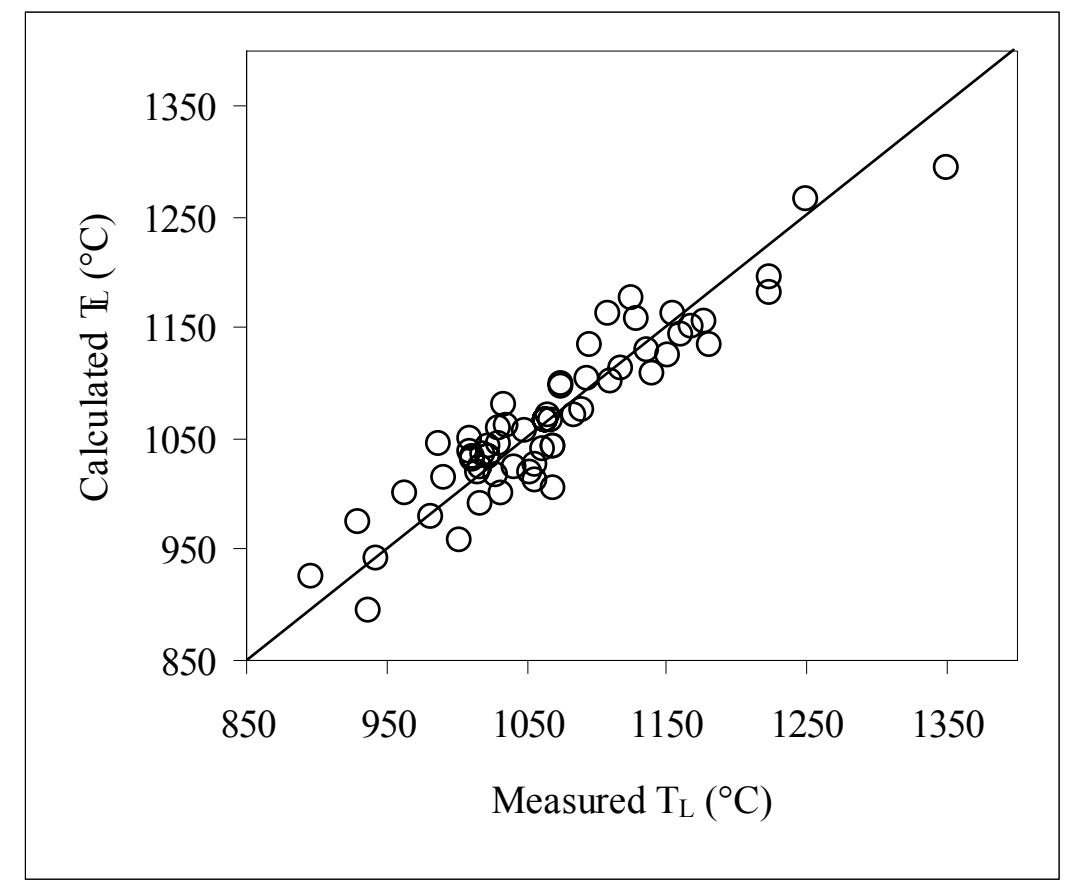

Figure 4-3. Plot Comparing Measured and Calculated Zircon $T_{L}$ Values from IP Model

Table 4-8 lists the $Z_{i}, r_{i}, P_{i}$, and $T_{i}$ (where $T_{i}=T_{i}$ for $i=M n, N i$, and Cr, $T_{i}=t_{\text {ion }}+\Theta_{\text {ion }} P_{i}$ for $i=$ alkali and alkaline earth components, and $\mathrm{T}_{\mathrm{i}}=\mathrm{t}_{\mathrm{cov}}+\Theta_{\mathrm{cov}} \mathrm{P}_{\mathrm{i}}$ for $\mathrm{i}=$ all other components) for typical glass components. 
Table 4-8. $\mathrm{Z}_{\mathrm{i}}, \mathrm{r}_{\mathrm{i}}, \mathrm{P}_{\mathrm{i}}$, and $\mathrm{T}_{\mathrm{i}}$ Values for Typical HLW Components (i)

\begin{tabular}{||l|c|c|c||l|c|c|c||}
\hline Element (i) & $\mathbf{Z}_{\mathbf{i}}$ & $\mathbf{r}_{\mathbf{i}}(\mathbf{\AA})$ & $\mathbf{T}_{\mathbf{i}}\left({ }^{\circ} \mathbf{C}\right)$ & $\mathbf{E l e m e n t}(\mathbf{i})$ & $\mathbf{Z}_{\mathbf{i}}$ & $\mathbf{r}_{\mathbf{i}}(\mathbf{\AA})$ & $\mathbf{T}_{\mathbf{i}}\left({ }^{\circ} \mathbf{C}\right)$ \\
\hline $\mathrm{Ag}$ & 1 & 1.08 & 2369.8763 & $\mathrm{Nd}$ & 3 & 1.25 & 2154.0937 \\
\hline $\mathrm{Al}$ & 3 & 0.53 & 1677.725 & $\mathrm{Ni}$ & 2 & 0.83 & 2152.9655 \\
\hline $\mathrm{B}$ & 3 & 0.25 & 750.90633 & $\mathrm{P}$ & 5 & 0.31 & 147.26404 \\
\hline $\mathrm{Ba}$ & 2 & 1.56 & 538.88459 & $\mathrm{~Pb}$ & 2 & 1.43 & 2300.7737 \\
\hline $\mathrm{Bi}$ & 3 & 1.17 & 2130.3837 & $\mathrm{Pr}$ & 3 & 1.13 & 2117.1144 \\
\hline $\mathrm{Ca}$ & 2 & 1.26 & 1614.6523 & $\mathrm{Rb}$ & 1 & 1.75 & -1965.503 \\
\hline $\mathrm{Cd}$ & 2 & 1.09 & 2236.9947 & $\mathrm{Re}$ & 4 & 0.77 & 1745.7892 \\
\hline $\mathrm{Ce}$ & 4 & 1.01 & 1926.2531 & $\mathrm{Rh}$ & 3 & 0.81 & 1960.4171 \\
\hline $\mathrm{Co}$ & 2 & 0.72 & 2099.1456 & $\mathrm{Ru}$ & 4 & 0.76 & 1735.7964 \\
\hline $\mathrm{Cr}$ & 3 & 0.76 & 1924.336 & $\mathrm{Sb}$ & 5 & 0.74 & 1517.4403 \\
\hline $\mathrm{Cs}$ & 1 & 1.88 & -2104.757 & $\mathrm{Se}$ & 6 & 0.42 & 416.74721 \\
\hline $\mathrm{Cu}$ & 2 & 0.87 & 2169.1621 & $\mathrm{Si}$ & 4 & 0.40 & 1043.2956 \\
\hline $\mathrm{Fe}$ & 3 & 0.63 & 1809.0769 & $\mathrm{Sm}$ & 3 & 1.22 & 2145.4519 \\
\hline $\mathrm{Gd}$ & 3 & 1.19 & 2137.6107 & $\mathrm{Sn}$ & 4 & 0.83 & 1800.6894 \\
\hline $\mathrm{K}$ & 1 & 1.65 & -1843.452 & $\mathrm{~S}$ & 6 & 0.26 & -868.4802 \\
\hline $\mathrm{La}$ & 3 & 1.30 & 2167.8695 & $\mathrm{Sr}$ & 2 & 1.40 & 1055.2531 \\
\hline $\mathrm{Li}$ & 1 & 0.90 & -63.54531 & $\mathrm{Te}$ & 4 & 0.80 & 1774.2686 \\
\hline $\mathrm{Mg}$ & 2 & 0.86 & 4216.509 & $\mathrm{Ti}$ & 4 & 0.56 & 1460.9945 \\
\hline $\mathrm{Mn}$ & 2 & 0.97 & 2203.8095 & $\mathrm{~W}$ & 6 & 0.74 & 1319.88 \\
\hline $\mathrm{Mo}$ & 6 & 0.73 & 1303.6421 & $\mathrm{Y}$ & 3 & 1.16 & 2126.826 \\
\hline $\mathrm{Na}$ & 1 & 1.16 & -941.2234 & $\mathrm{Zn}$ & 2 & 0.74 & 2110.1211 \\
\hline $\mathrm{Nb}$ & 5 & 0.78 & 1568.0967 & $\mathrm{Zr}$ & 4 & 0.86 & 12253.597 \\
\hline \hline
\end{tabular}

The ranges of model-fit data were used to determine the model-validity ranges, which are reported in terms of mass \% of oxides in Table 4-9. Also listed in the table is the composition of the outlier glass, $\mathrm{Zr}-27$, which appears to be at the upper edge of $\mathrm{Li}_{2} \mathrm{O}$ and $\mathrm{SiO}_{2}$ concentrations and at the lower edge of the concentration ranges for a number of components. The concentration ranges and model in general are not yet adequate for the intended purposes. However, to date, it appears to be the best predicting model for $\mathrm{T}_{\mathrm{L}}$ in the zircon primary phase field and should be used until further data and models are available. As mentioned earlier, this is a global model aimed at providing lower-precision $T_{L}$ estimates over broader composition regions (relative to local models). Additional modeling, and possibly data collection, is required to develop more precise local models that cover smaller composition regions. 
Table 4-9. Concentration Ranges and Outlier Composition (in mass\%) for the Data that Were Used to Fit the $\mathrm{T}_{\mathrm{L}}$ Model in the Zircon Primary Phase Field

\begin{tabular}{||l|r|r|c||}
\hline Oxide & min & max & Zr-27 \\
\hline $\mathrm{Al}_{2} \mathrm{O}_{3}$ & 0.0 & 10.3 & 3.0 \\
\hline $\mathrm{B}_{2} \mathrm{O}_{3}$ & 2.0 & 20.0 & 5.0 \\
\hline $\mathrm{Bi}_{2} \mathrm{O}_{3}$ & 0.0 & 10.0 & 0.0 \\
\hline $\mathrm{CaO}$ & 0.0 & 8.0 & 0.1 \\
\hline $\mathrm{CdO}$ & 0.0 & 1.3 & 0.0 \\
\hline $\mathrm{CeO}_{2}$ & 0.0 & 3.0 & 0.0 \\
\hline $\mathrm{Fe}_{2} \mathrm{O}_{3}$ & 0.0 & 7.4 & 0.0 \\
\hline $\mathrm{La}_{2} \mathrm{O}_{3}$ & 0.0 & 1.2 & 0.0 \\
\hline $\mathrm{Li}_{2} \mathrm{O}$ & 1.0 & 9.0 & 9.0 \\
\hline $\mathrm{MgO} \mathrm{O}$ & 0.0 & 8.0 & 0.0 \\
\hline $\mathrm{MnO}_{2} \mathrm{Na}_{2} \mathrm{O}$ & 4.0 & 1.9 & 0.0 \\
\hline $\mathrm{Na}_{2} \mathrm{O}$ & 0.0 & 2.2 & 0.0 \\
\hline $\mathrm{Nd}_{2} \mathrm{O}_{3}$ & 0.0 & 1.0 & 0.0 \\
\hline $\mathrm{NiO}^{2} \mathrm{P}_{2} \mathrm{O}_{5}$ & 0.0 & 5.0 & 0.2 \\
\hline $\mathrm{SiO}_{2}$ & 41.0 & 59.6 & 57.2 \\
\hline $\mathrm{ZrO}_{2}$ & 3.7 & 16.5 & 13.5 \\
\hline \hline
\end{tabular}




\subsection{Models for Product Consistency Test Response}

This section discusses initial data evaluation, screening/conversion, and modeling of normalized boron, lithium, and sodium releases during PCT.

\subsection{Initial Data Evaluation, Screening, and Conversion}

Of the 2001 data in the database, 1252 data points (glasses) report normalized releases of either B, $\mathrm{Na}$, or Li by the product consistency test (PCT) (ASTM 1998), denoted $\mathrm{r}_{\mathrm{B}}, \mathrm{r}_{\mathrm{Na}}, \mathrm{r}_{\mathrm{Li}}$, respectively. Only PCT release values for quenched glasses are considered. Of the 1252 glasses, there are $1229,887,1152$, and 1050 that have $\mathrm{r}_{\mathrm{B}}, \mathrm{r}_{\mathrm{Li}}, \mathrm{r}_{\mathrm{Na}}$, and $\mathrm{r}_{\mathrm{Si}}$ values, respectively. To determine the impacts of CCC on PCT release, the reader should turn to other documents, e.g., Riley et al. (2001). Analyzed compositions were available for 881 of the glasses while target compositions were available for 1053 of the 1252 glasses.

Preliminary calculations and previous experience suggested that fitting PCT release models with all the experimental data would not yield acceptable results. So initial glass screening was performed. The data were evaluated to select a subset of data that was appropriate for model fitting according to the following procedure (in order of operation).

- Results from the WTP glass formulation activities (Muller, Buechele, and Pegg 2001; Kot and Pegg 2001), the Tanks Focus Area melter study (Perez et al. 2001), and glass-formulation experience were used to narrow the likely composition region for Hanford waste glasses. Table 5-1 lists the concentration ranges of components used to restrict the database to those compositions thought to be of interest to Hanford. This process removed 424 glasses from the model fit data set listed in Table $5-2$.

- The target and measured compositions of test glasses were compared for those glasses with both types of compositions. Any glass with more than two major components, i.e., $>1$ mass $\%$ in target concentration, with more than $20 \%$ difference between analyzed and target concentrations was removed. This procedure resulted in the removal of 34 glasses.

- Those glasses with compositions that summed to less than 95 mass $\%$ for analyzed or 99 mass \% for target were excluded. This procedure resulted in the removal of 5 additional glasses.

- Those glasses reported to be multi-phased were removed from the data set (26 additional glasses).

- Those glasses that were highly reduced- $\mathrm{Fe}^{2+} /\left(\mathrm{Fe}^{2+}+\mathrm{Fe}^{3+}\right)>5 \%$-were removed so that $\mathrm{FeO}$ and $\mathrm{Fe}_{2} \mathrm{O}_{3}$ could be combined into a single component. This procedure removed an additional 25 glasses.

- Finally, one glass was reported to have foamed over and out of the crucible during fabrication.

This screening resulted in the removal of 515 glasses, leaving 737 in the preliminary data set for model fitting. Table 5-2 (for composition ranges) and Table 5-3 (for other purposes) list the glasses removed from the initial model-fit data set. It should be noted that a number of excluded glasses may have fit in more than one of the categories described above, but each glass is marked with only one category determined by the order of exclusion. 
Table 5-1. Ranges of Selected Glass Components (mass\% oxide or halogen)

\begin{tabular}{||l|c|c||l|c|c||l|c|c||}
\hline \hline Component & Min & Max & Component & Min & Max & Component & Min & Max \\
\hline $\mathrm{Al}_{2} \mathrm{O}_{3}$ & & 20 & $\mathrm{Ga}_{2} \mathrm{O}_{3}$ & & 0.5 & $\mathrm{SiO}_{2}$ & 30 & 60 \\
\hline $\mathrm{B}_{2} \mathrm{O}_{3}$ & 4 & 20 & $\mathrm{Gd}_{2} \mathrm{O}_{3}$ & & 2 & $\mathrm{Sm}_{2} \mathrm{O}_{3}$ & & 1 \\
\hline $\mathrm{CaO}$ & & 10 & $\mathrm{~K}_{2} \mathrm{O}$ & & 10 & $\mathrm{SnO}_{2}$ & & 1 \\
\hline $\mathrm{Ce}_{2} \mathrm{O}_{3}$ & & 2 & $\mathrm{MoO}_{3}$ & & 1 & $\mathrm{TiO}_{2}$ & & 5 \\
\hline $\mathrm{Cr}_{2} \mathrm{O}_{3}$ & & 2 & $\mathrm{Na}_{2} \mathrm{O}$ & 5 & 20 & $\mathrm{Y}_{2} \mathrm{O}_{3}$ & & 2 \\
\hline $\mathrm{Eu}_{2} \mathrm{O}_{3}$ & & 2 & $\mathrm{Nd}_{2} \mathrm{O}_{3}$ & & 5 & $\mathrm{ZrO}_{2}$ & & 15 \\
\hline $\mathrm{F}$ & & 2 & $\mathrm{NiO}$ & & 3 & \multicolumn{4}{|l||}{} \\
\cline { 1 - 4 } $\mathrm{Fe}_{2} \mathrm{O}_{3}$ & & 25 & $\mathrm{P}_{2} \mathrm{O}_{5}$ & & 3 & &
\end{tabular}


Table 5-2. Glasses Excluded from Model Development Because Their Compositions are Outside the Region of Expected Hanford Waste Glasses (concentrations of components are in mass $\%$ of the oxide or halogen)

\begin{tabular}{|c|c|c|c|c|c|c|c|c|c|}
\hline Glass ID & Conc. & Glass ID & Conc. & Glass ID & Conc. & Glass ID & Conc. & Glass ID & Conc. \\
\hline 4 & $\mathrm{Na}>20$ & CVS3-6 & $\mathrm{Nd}>5$ & HLP-15 & $\mathrm{Ti}>5$ & IG2-22 & $\mathrm{K}>10, \mathrm{Na}<5$ & $\mathrm{P} 2-3 \mathrm{Ca}-0 \mathrm{~F}$ & $\mathrm{Si}>60$ \\
\hline 7 & $\mathrm{Na}>20$ & CVS3-7 & $\mathrm{Nd}>5$ & HLP-16 & $\mathrm{Na}>20$ & IG2-27 & $\mathrm{F}>2$ & $\mathrm{P} 2-3 \mathrm{Ca}-4 \mathrm{~F}$ & $\mathrm{~F}>2$ \\
\hline 9 & $\mathrm{Na}>20$ & CVS3-8 & $\mathrm{B}<4, \mathrm{Na}>20, \mathrm{Nd}>5$ & HLP-18 & $\mathrm{Na}>20$ & IG2-29 & $\mathrm{F}>2$ & $\mathrm{P} 2-3 \mathrm{Ca}-5 \mathrm{~F}$ & $\mathrm{~F}>2$ \\
\hline 10 & $\mathrm{Na}>20$ & CVS3-9 & $\mathrm{B}<4, \mathrm{Nd}>5$ & HLP-20 & $\mathrm{Na}>20$ & IG2-32 & $\mathrm{F}>2$ & P2-9Ca-3F & $\mathrm{F}>2$ \\
\hline 13 & $\mathrm{Na}>20$ & DP-1 & $\mathrm{Ca}>10, \mathrm{~F}>2$ & HLP-23 & $\mathrm{Na}>20$ & IG2-33 & $\mathrm{F}>2$ & P2-9Ca-4F & $\mathrm{F}>2$ \\
\hline 15 & $\mathrm{Na}>20$ & DP-10 & $\mathrm{F}>2$ & HLP-27 & $\mathrm{Na}>20$ & IG2-34 & $\mathrm{F}>2, \mathrm{Mo}>1$ & P2-9Ca-5F & $\mathrm{F}>2$ \\
\hline 16 & $\mathrm{Na}>20$ & DP-11 & $\mathrm{F}>2$ & HLP-29 & $\mathrm{Na}>20$ & IG2-35 & $\mathrm{F}>2$ & PBG3-.5C & $\mathrm{Ce}>2, \mathrm{Gd}>2$ \\
\hline 18 & $\mathrm{Na}>20$ & DP-12 & $\mathrm{Ca}>10, \mathrm{~F}>2$ & HLP-31 & $\mathrm{Na}>20$ & IG2-36 & $\mathrm{F}>2$ & PEI & $B<4$ \\
\hline 19 & $\mathrm{Na}>20$ & DP-13 & $\mathrm{Ca}>10, \mathrm{~F}>2$ & HLP-33 & $\mathrm{Na}>20$ & IG2-37 & $\mathrm{F}>2$ & SBW1-20 & $\mathrm{Na}>20$ \\
\hline 20 & $\mathrm{Na}>20$ & DP-14 & $\mathrm{Ca}>10, \mathrm{~F}>2$ & HLP-35 & $\mathrm{Na}>20$ & IG3-01 & $\mathrm{F}>2$ & SBW1-30 & $\mathrm{Na}>20$ \\
\hline 30 & $\mathrm{Na}>20$ & DP-15 & $\mathrm{Ca}>10, \mathrm{~F}>2$ & HLP-37 & $\mathrm{Na}>20, \mathrm{Ti}>5$ & IG3-02b & $\mathrm{Ca}>10, \mathrm{Mo}>1$ & SBW1-32 & $\mathrm{Si}>60$ \\
\hline 31 & $\mathrm{Na}>20$ & DP-16 & $\mathrm{F}>2$ & HLP-38 & $\mathrm{Ti}>5$ & IG3-03 & $\mathrm{Mo}>1$ & SBW1-36 & $\mathrm{Na}>20$ \\
\hline ARM-1-7 & $\mathrm{Ce}>2, \mathrm{Mo}>1, \mathrm{Nd}>5$ & DP-17 & $\mathrm{F}>2$ & HLP-39 & $\mathrm{Na}>20, \mathrm{Ti}>5$ & IG3-05 & $\mathrm{Ca}>10, \mathrm{~F}>2, \mathrm{Mo}>1$ & SBW1-37 & $\mathrm{Na}>20$ \\
\hline ARM-1-7 (10/91) & $\mathrm{Ce}>2, \mathrm{Mo}>1, \mathrm{Nd}>5$ & DP-18 & $\mathrm{F}>2$ & HLP-40Q & $\mathrm{Ti}>5$ & IG3-06 & $\mathrm{Ca}>10, \mathrm{~F}>2$ & SBW1-41 & $\mathrm{Na}>20$ \\
\hline ARM-1-7 (12/90) & $\mathrm{Ce}>2, \mathrm{Mo}>1, \mathrm{Nd}>5$ & DP-19 & $\mathrm{F}>2$ & HLP-42Q & $\mathrm{Ti}>5, \mathrm{Zn}>4$ & IG3-07 & $\mathrm{Mo}>1$ & SF10 & $\mathrm{Si}>60, \mathrm{Na}<5, \mathrm{P}>3$ \\
\hline ARM-1-7 (4/88) & $\mathrm{Ce}>2, \mathrm{Mo}>1, \mathrm{Nd}>5$ & DP-2 & $\mathrm{Ca}>10, \mathrm{~F}>2$ & HLP-48 & $\mathrm{Zn}>4$ & IG3-08b & $\mathrm{Mo}>1$ & SRC-Al-1 & $\mathrm{Ce}>2, \mathrm{Gd}>2$ \\
\hline ARM-1-7 (4/93) & $\mathrm{Ce}>2, \mathrm{Mo}>1, \mathrm{Nd}>5$ & DP-20 & $\mathrm{F}>2$ & HLP-52 & $\mathrm{B}<4, \mathrm{Na}>20$ & IG3-09 & $\mathrm{F}>2, \mathrm{Mo}>1$ & SRC-Al-2 & $\mathrm{Ce}>2, \mathrm{Gd}>2$ \\
\hline ARM-1-7 (5/89) & $\mathrm{Ce}>2, \mathrm{Mo}>1, \mathrm{Nd}>5$ & DP-21 & $\mathrm{Ca}>10, \mathrm{~F}>2$ & HLP-61 & $\mathrm{Ti}>5, \mathrm{Zn}>4$ & IG3-10b & $\mathrm{F}>2$ & SRC-B-1 & $\mathrm{B}<4, \mathrm{Ce}>2, \mathrm{Gd}>2$ \\
\hline ARM-1-7 (5/91) & $\mathrm{Ce}>2, \mathrm{Mo}>1, \mathrm{Nd}>5$ & DP-22 & $\mathrm{F}>2$ & HLP-62 & $\mathrm{Ti}>5$ & IG3-11 & $\mathrm{Ca}>10$ & SRC-B-2 & $\mathrm{Ce}>2, \mathrm{Gd}>2$ \\
\hline ARM-1-7 (7/90) & $\mathrm{Ce}>2, \mathrm{Mo}>1, \mathrm{Nd}>5$ & DP-23 & $\mathrm{Ca}>10, \mathrm{~F}>2$ & HLP-63 & $\mathrm{Na}>20, \mathrm{Ti}>5$ & IG3-13 & $\mathrm{F}>2$ & SRC-B-3 & $\mathrm{Ce}>2, \mathrm{Gd}>2$ \\
\hline ARM-1-7 (8/93) & $\mathrm{Ce}>2, \mathrm{Mo}>1, \mathrm{Nd}>5$ & DP-24 & $\mathrm{F}>2$ & HLP-64 & $\mathrm{Zn}>4$ & IG3-14 & $\mathrm{F}>2$ & SRC-Ca-1 & $\mathrm{Ce}>2, \mathrm{Gd}>2$ \\
\hline ARM-1-7 (10/92) & $\mathrm{Ce}>2, \mathrm{Mo}>1, \mathrm{Nd}>5$ & DP-3 & $\mathrm{F}>2$ & HLP-66 & $\mathrm{Na}>20$ & IG3-15 & $\mathrm{F}>2$ & SRC-Ca-2 & $\mathrm{Ce}>2, \mathrm{Gd}>2$ \\
\hline ARM-1-7 (6/93) & $\mathrm{Ce}>2, \mathrm{Mo}>1, \mathrm{Nd}>5$ & DP-4 & $\mathrm{F}>2$ & HLP-67 & $\mathrm{Na}>20, \mathrm{Zn}>4$ & IG3-19 & $\mathrm{F}>2$ & SRC-Ca-3 & $\mathrm{Ce}>2, \mathrm{Gd}>2$ \\
\hline $\begin{array}{l}\text { BATCH 1 STUDY- } \\
\text { 10B-7 }\end{array}$ & $\mathrm{Si}>60$ & DP-5 & $F>2$ & HLP-69 & $\mathrm{Ti}>5, \mathrm{Zn}>4$ & IG3-20 & $\mathrm{F}>2$ & SRC-Ce-1 & $\mathrm{Gd}>2$ \\
\hline $\begin{array}{l}\text { BATCH 1 STUDY- } \\
15-7\end{array}$ & $\mathrm{Si}>60$ & DP-6 & $\mathrm{F}>2$ & HLW98-12 & $\mathrm{Na}<5$ & IG3-23 & $\mathrm{F}>2$ & SRC-Ce-2 & $\mathrm{Ce}>2, \mathrm{Gd}>2$ \\
\hline CU37 & $\mathrm{Ti}>5$ & DP-7 & $F>2$ & HLW98-32A & $\mathrm{Na}<5$ & IG3-24 & $\mathrm{F}>2$ & SRC-Ce-3 & $\mathrm{Ce}>2, \mathrm{Gd}>2$ \\
\hline CU38 & $\mathrm{Cr}>2$ & DP-8 & $\mathrm{F}>2$ & HLWD1-08 & $\mathrm{Na}<5$ & IG3-25 & $\mathrm{F}>2$ & SRC-Ce-4 & $\mathrm{Ce}>2, \mathrm{Gd}>2$ \\
\hline CU39 & $\mathrm{Cr}>2$ & DP-9 & $\mathrm{F}>2$ & HLWD1-10 & $\mathrm{Na}<5$ & IG3-27 & $\mathrm{F}>2$ & SRC-Eu-1 & $\mathrm{Ce}>2, \mathrm{Eu}>4$ \\
\hline
\end{tabular}


Table 5.2 (Contd)

\begin{tabular}{|c|c|c|c|c|c|c|c|c|c|}
\hline Glass ID & Conc. & Glass ID & Conc. & Glass ID & Conc. & Glass ID & Conc. & Glass ID & Conc. \\
\hline CU40 & $\mathrm{Cr}>2$ & DP-BL1 & $\mathrm{Ca}>10, \mathrm{~F}>2$ & HLWD1-11 & $S \mathrm{Si}<30, \mathrm{Fe}>25$ & IG3-28 & $\mathrm{F}>2$ & SRC-Gd-1 & $\mathrm{Ce}>2$ \\
\hline CU44 & $\mathrm{Cu}>1$ & DP-centroid & $\mathrm{Ca}>10, \mathrm{~F}>2$ & HLWD1-17 & $\mathrm{Si}<30$ & L1-15 & $\mathrm{Si}>60, \mathrm{~B}<4$ & SRC-Gd-3 & $\mathrm{Ce}>2, \mathrm{Gd}>2$ \\
\hline CU49 & $\mathrm{Ni}>3$ & DZr-10-78-38 & $\mathrm{Ca}>10, \mathrm{~F}>2$ & HLWD1-19 & $\mathrm{Si}<30, \mathrm{~F}>2$ & L4-69 & $\mathrm{Si}>60$ & SRC-K-1 & $\mathrm{Ce}>2, \mathrm{Gd}>2$ \\
\hline CU53 & $\mathrm{Na}<5, \mathrm{P}>3$ & DZr-10-78-40 & $\mathrm{Ca}>10, \mathrm{~F}>2$ & HLWD1-20 & $\mathrm{Si}<30, \mathrm{~F}>2$ & L4-96 & $\mathrm{Si}>60$ & SRC-K-2 & $\mathrm{Ce}>2, \mathrm{Gd}>2$ \\
\hline CU54 & $\mathrm{Na}<5, \mathrm{P}>3$ & DZr-9-78-38 & $\mathrm{Ca}>10, \mathrm{~F}>2$ & HLWD2-03 & $\mathrm{Si}<30$ & L5-1212 & $\mathrm{B}<4, \mathrm{Ca}>10$ & SRC-Li-1 & $\mathrm{Ce}>2, \mathrm{Gd}>2$ \\
\hline CU57 & $\mathrm{Na}<5, \mathrm{P}>3$ & DZr-CV-10 & $\mathrm{Ca}>10, \mathrm{~F}>2$ & HLWD3-03 & $\mathrm{Si}<30, \mathrm{P}>3$ & L5-612 & $B<4$ & SRC-Mg-1 & $\mathrm{Ce}>2, \mathrm{Gd}>2$ \\
\hline CU58 & $\mathrm{Si}>60, \mathrm{Na}<5, \mathrm{P}>3$ & DZr-CV-11 & $\mathrm{Ca}>10, \mathrm{~F}>2$ & HLWD3-04 & $\mathrm{Si}<30, \mathrm{~F}>2$ & L5-615 & $B<4$ & SRC-Mg-2 & $\mathrm{Ce}>2, \mathrm{Gd}>2$ \\
\hline CUOD & $\mathrm{Na}<5, \mathrm{P}>3$ & DZr-CV-12 & $\mathrm{Ca}>10, \mathrm{~F}>2$ & HLWD3-06 & $\mathrm{Si}<30, \mathrm{P}>3$ & L5-69 & $\mathrm{Si}>60, \mathrm{~B}<4$ & SRC-Na-1 & $\mathrm{Ce}>2, \mathrm{Gd}>2, \mathrm{Na}<5$ \\
\hline CUOI & $\mathrm{Na}<5, \mathrm{P}>3$ & DZr-CV-13 & $\mathrm{Ca}>10, \mathrm{~F}>2$ & HLWD3-07 & $\mathrm{Si}<30$ & L5-912 & $B<4$ & SRC-Na-2 & $\mathrm{Ce}>2, \mathrm{Gd}>2$ \\
\hline CUOJ & $\mathrm{Si}>60, \mathrm{Na}<5, \mathrm{P}>3$ & DZr-CV-14 & $\mathrm{Ca}>10, \mathrm{~F}>2$ & HLWD3-08 & $\mathrm{Si}<30, \mathrm{Na}>20$ & L5-915 & $B<4$ & SRC-Pb-1 & $\mathrm{Ce}>2, \mathrm{Gd}>2$ \\
\hline CUOK & $\mathrm{Na}<5, \mathrm{P}>3$ & DZr-CV-15 & $\mathrm{Ca}>10, \mathrm{~F}>2$ & HLWMS-08 & $\mathrm{Na}<5$ & L5-96 & $\mathrm{Si}>60, \mathrm{~B}<4$ & SRC-Pb-2 & $\mathrm{Ce}>2, \mathrm{Gd}>2$ \\
\hline CVS2-67 & $\mathrm{Al}>20$ & DZr-CV-2 & $\mathrm{Ca}>10, \mathrm{~F}>2$ & IG1-03 & $\mathrm{Na}>20, \mathrm{P}>3$ & L7-25 & $\mathrm{Na}>20$ & SRC-Sn-1 & $\mathrm{Ce}>2, \mathrm{Gd}>2, \mathrm{Sn}>1$ \\
\hline CVS2-68 & $\mathrm{Cr}>2$ & DZr-CV-20 & $\mathrm{Ca}>10, \mathrm{~F}>2$ & IG1-04 & $\mathrm{P}>3$ & L7-30 & $\mathrm{Na}>20$ & SRC-Sn-2 & $\mathrm{Ce}>2, \mathrm{Gd}>2, \mathrm{Sn}>1$ \\
\hline \begin{tabular}{|l|} 
CVS2-69 \\
\end{tabular} & $\mathrm{Cr}>2, \mathrm{P}>3$ & DZr-CV-21 & $\mathrm{Ca}>10, \mathrm{~F}>2$ & IG1-05 & $\mathrm{Na}>20, \mathrm{P}>3$ & L7-35 & $\mathrm{B}<4, \mathrm{Na}>20$ & SRC-Ti-1 & $\mathrm{Ce}>2, \mathrm{Gd}>2$ \\
\hline CVS3-1 & $\mathrm{B}<4, \mathrm{Nd}>5$ & DZr-CV-22 & $\mathrm{Ca}>10, \mathrm{~F}>2$ & IG1-08 & $\mathrm{P}>3$ & L8-2 & $B<4$ & SRC-Ti-2 & $\mathrm{Ce}>2, \mathrm{Gd}>2, \mathrm{Ti}>5$ \\
\hline CVS3-10 & $\mathrm{B}<4, \mathrm{Nd}>5$ & DZr-CV-23 & $\mathrm{Ca}>10, \mathrm{~F}>2$ & IG1-09 & $\mathrm{Na}>20, \mathrm{P}>3$ & L8-3 & $B<4$ & SRC-Zr-1 & $\mathrm{Ce}>2, \mathrm{Gd}>2$ \\
\hline CVS3-11 & $\mathrm{B}<4, \mathrm{Nd}>5$ & DZr-CV-24 & $\mathrm{Ca}>10, \mathrm{~F}>2$ & IG1-14 & $\mathrm{P}>3$ & L8-5 & $B<4$ & SRC-Zr-2 & $\mathrm{Ce}>2, \mathrm{Gd}>2$ \\
\hline CVS3-12 & $\mathrm{B}<4, \mathrm{Nd}>5$ & DZr-CV-3 & $\mathrm{Ca}>10, \mathrm{~F}>2$ & IG1-15 & $\mathrm{P}>3$ & L8-6 & $B<4$ & SS-ARM-1 & $\mathrm{Ce}>2, \mathrm{Mo}>1, \mathrm{Nd}>5$ \\
\hline CVS3-13 & $\mathrm{B}<4, \mathrm{Nd}>5$ & DZr-CV-4 & $\mathrm{Ca}>10, \mathrm{~F}>2$ & IG1-16 & $\mathrm{P}>3$ & LAWA104 & $\mathrm{Na}>20$ & SS-ARM-1 & $\mathrm{Ce}>2, \mathrm{Mo}>1, \mathrm{Nd}>5$ \\
\hline CVS3-14 & $\mathrm{B}<4, \mathrm{Nd}>5$ & DZr-CV-5 & $F>2$ & IG1-17 & $\mathrm{P}>3$ & LAWA105 & $\mathrm{Na}>20$ & T-ARM-1 & $\mathrm{Ce}>2, \mathrm{Mo}>1, \mathrm{Nd}>5$ \\
\hline CVS3-15 & $\mathrm{B}<4, \mathrm{Nd}>5$ & DZr-CV-6 & $\mathrm{Ca}>10, \mathrm{~F}>2$ & IG1-18 & $\mathrm{P}>3$ & LAWA46 & $\mathrm{Ga}>0$ & TC27 & $\mathrm{Na}<5$ \\
\hline$\overline{\text { CVS3-16 }}$ & $\mathrm{B}<4, \mathrm{Al}>20$ & DZr-CV-7 & $\mathrm{Ca}>10, \mathrm{~F}>2$ & IG1-19 & $\mathrm{P}>3$ & LAWA47 & $\mathrm{Y}>2$ & WV205 & $\mathrm{Si}>60, \mathrm{Na}<5, \mathrm{P}>3$ \\
\hline $\begin{array}{l}\text { CVS3-17 } \\
\end{array}$ & $\mathrm{B}<4, \mathrm{Nd}>5$ & DZr-CV-8 & $\mathrm{Ca}>10, \mathrm{~F}>2$ & IG1-20 & $\mathrm{P}>3$ & LAWA48 & $\mathrm{Ga}>0$ & WVCM57 & $\mathrm{Na}<5, \mathrm{P}>3$ \\
\hline
\end{tabular}


Table 5.2 (Contd)

\begin{tabular}{|c|c|c|c|c|c|c|c|c|c|}
\hline Glass ID & Conc. & Glass ID & Conc. & Glass ID & Conc. & Glass ID & Conc. & Glass ID & Conc. \\
\hline CVS3-2 & $\mathrm{Si}>60, \mathrm{~B}<4, \mathrm{Nd}>5$ & $\begin{array}{l}\text { FRIT-202- } \\
\text { CLEAR } \\
\end{array}$ & $\mathrm{Si}>60$ & IG1-23 & $\mathrm{P}>3$ & LAWA51 & $\mathrm{Ga}>0$ & WVDG-21 & $\mathrm{P}>3$ \\
\hline CVS3-20 & $\mathrm{B}<4, \mathrm{Nd}>5, \mathrm{Zr}>15$ & FRIT-202-INT & $\mathrm{Si}>60$ & IG1-24 & $\mathrm{P}>3$ & LAWA84 & $\mathrm{P}>3$ & WVDG-23 & $\mathrm{P}>3$ \\
\hline CVS3-21 & $\mathrm{B}<4, \mathrm{Nd}>5$ & \begin{tabular}{|l|} 
FRIT-202- \\
WHITE \\
\end{tabular} & $\mathrm{Si}>60$ & IG1-25 & $\mathrm{P}>3$ & LAWA86 & $\mathrm{P}>3$ & WVDG-25 & $\mathrm{P}>3$ \\
\hline CVS3-22 & $\mathrm{B}<4, \mathrm{Nd}>5$ & Frit-5-78-30 & $\mathrm{Ca}>10, \mathrm{~F}>2$ & IG1-26 & $\mathrm{P}>3$ & LAWA96 & $\mathrm{P}>3$ & WVDG-27 & $\mathrm{P}>3$ \\
\hline CVS3-23 & $\mathrm{B}<4, \mathrm{Nd}>5$ & Frit-5-78-35 & $\mathrm{Ca}>10, \mathrm{~F}>2$ & IG1-27 & $\mathrm{P}>3$ & LAWB30 & $\mathrm{Zn}>4$ & WVDG-29 & $\mathrm{P}>3$ \\
\hline CVS3-24 & $\mathrm{B}<4, \mathrm{Ce}>2, \mathrm{Nd}>5$ & Frit-5-78-37 & $\mathrm{Ca}>10, \mathrm{~F}>2$ & IG1-28 & $\mathrm{P}>3$ & LAWB33 & $\mathrm{P}>3$ & WVDG-39 & $\mathrm{P}>3$ \\
\hline CVS3-25 & $\mathrm{B}<4$ & Frit-5-78-40 & $\mathrm{Ca}>10, \mathrm{~F}>2$ & IG1-32 & $\mathrm{P}>3$ & LAWB37 & $\mathrm{P}>3$ & WVDG-41 & $\mathrm{P}>3$ \\
\hline CVS3-27 & $\mathrm{B}<4, \mathrm{Nd}>5$ & GLA 78-10-14 & $\mathrm{F}>2, \mathrm{P}>3$ & IG1-34 & $\mathrm{P}>3$ & LAWB38 & $\mathrm{P}>3$ & WVDG-42 & $\mathrm{P}>3$ \\
\hline CVS3-28 & $\mathrm{B}<4, \mathrm{Nd}>5$ & GLA 78-10-15 & $\mathrm{F}>2, \mathrm{P}>3$ & IG1-35 & $\mathrm{P}>3$ & LAWC12 & $\mathrm{Zn}>4$ & WVDG-45 & $\mathrm{P}>3$ \\
\hline CVS3-29 & $\mathrm{B}<4, \mathrm{Nd}>5$ & GLA 78-10-16 & $\mathrm{F}>2, \mathrm{P}>3$ & IG1-40 & $\mathrm{P}>3$ & LD5-912 & $B<4$ & WVDG-47 & $\mathrm{P}>3$ \\
\hline CVS3-3 & $\mathrm{B}<4, \mathrm{Nd}>5$ & GLA 78-21 & $\mathrm{Ca}>10, \mathrm{~F}>2$ & IG1-41 & $\mathrm{P}>3$ & LDM-1 & $\mathrm{B}<4$ & WVDG-48 & $\mathrm{Ce}>2, \mathrm{P}>3$ \\
\hline CVS3-30 & $\mathrm{B}<4, \mathrm{Nd}>5$ & GLA 78-22 & $\mathrm{Ca}>10, \mathrm{~F}>2$ & IG1-42 & $\mathrm{P}>3$ & LDM-2 & $\mathrm{B}<4$ & WVDG-6 & $\mathrm{P}>3$ \\
\hline CVS3-31 & $\mathrm{B}<4, \mathrm{Nd}>5$ & GLA 78-23 & $\mathrm{Ca}>10, \mathrm{~F}>2, \mathrm{P}>3$ & IG1-43 & $\mathrm{P}>3$ & LDM-912 & $\mathrm{B}<4$ & WVUTH31 & $\mathrm{P}>3$ \\
\hline CVS3-32 & $B<4$ & GLA 78-9-11 & $\mathrm{Ca}>10, \mathrm{~F}>2, \mathrm{P}>3$ & IG1-44 & $\mathrm{P}>3$ & LDMS-1 & $\mathrm{B}<4$ & Zr-12 & $\mathrm{Na}<5, \mathrm{Zr}>15$ \\
\hline CVS3-33 & $B<4$ & GLA 78-9-15 & $\mathrm{Ca}>10, \mathrm{~F}>2$ & IG2-01 & $\mathrm{F}>2$ & LRM-1 & $\mathrm{B}<4$ & Zr-13 & $\mathrm{Zr}>15$ \\
\hline CVS3-34 & $B<4$ & GLA 78-9-18 & $\mathrm{Ca}>10, \mathrm{~F}>2, \mathrm{P}>3$ & IG2-02 & $\mathrm{Ca}>10$ & LRM-2 & $\mathrm{B}<4$ & $\mathrm{Zr}-15$ & $\mathrm{Zr}>15$ \\
\hline CVS3-35 & $B<4$ & HLP-02 & $\mathrm{Na}>20$ & IG2-03 & $\mathrm{F}>2$ & LRM-912 & $\mathrm{B}<4$ & $\mathrm{Zr}-17$ & $\mathrm{Na}<5, \mathrm{Zr}>15$ \\
\hline CVS3-36 & $B<4$ & HLP-04 & $\mathrm{Na}>20$ & IG2-05 & $\mathrm{Ca}>10$ & LRMS-1 & $\mathrm{B}<4$ & $\mathrm{Zr}-18$ & $\mathrm{Zr}>15$ \\
\hline CVS3-37 & $\mathrm{B}<4, \mathrm{Nd}>5$ & HLP-05 & $\mathrm{Na}>20$ & IG2-07 & $\mathrm{Ca}>10, \mathrm{~F}>2$ & MG 18-7 & $\mathrm{Na}>20$ & Zr-19 & $\mathrm{Zr}>15$ \\
\hline CVS3-38 & $\mathrm{B}<4, \mathrm{Nd}>5, \mathrm{P}>3$ & HLP-08 & $\mathrm{Na}>20$ & IG2-08 & $\mathrm{Ca}>10$ & MG 9-7 & $\mathrm{Na}>20$ & Zr-20 & $\mathrm{Zr}>15$ \\
\hline CVS3-39 & $\mathrm{B}<4, \mathrm{Nd}>5, \mathrm{P}>3$ & HLP-10 & $\mathrm{Na}>20$ & IG2-09 & $\mathrm{Ca}>10, \mathrm{~F}>2$ & NBS SRM 623-7 & $\mathrm{Si}>60$ & Zr-21 & $\mathrm{Na}<5$ \\
\hline CVS3-4 & $\mathrm{B}<4, \mathrm{Ce}>2, \mathrm{Nd}>5$ & HLP-11 & $\mathrm{Na}>20$ & IG2-10 & $\mathrm{F}>2$ & $\mathrm{P} 2-0 \mathrm{Ca}-0 \mathrm{~F}$ & $\mathrm{Si}>60$ & Zr-25 & $\mathrm{Na}<5$ \\
\hline CVS3-40 & $\mathrm{B}<4, \mathrm{Nd}>5, \mathrm{P}>3$ & HLP-13 & $\mathrm{Na}>20$ & IG2-12 & $\mathrm{F}>2$ & P2-0Ca-4F & $\mathrm{F}>2$ & Zr-3 & $\mathrm{Zr}>15$ \\
\hline CVS3-5 & $\mathrm{B}<4, \mathrm{Ce}>2, \mathrm{Nd}>5$ & HLP-14 & $\mathrm{Na}>20$ & IG2-17 & $\mathrm{F}>2$ & P2-0Ca-5F & $\mathrm{F}>2$ & & \\
\hline
\end{tabular}


Table 5-3. List of Glasses Excluded Due to Data Problems

\begin{tabular}{|c|c|c|c|c|c|c|c|}
\hline Glass ID & Type $^{(\mathbf{A})}$ & Glass ID & Type & Glass ID & Type & "Glass ID & Type \\
\hline SBW1-38B & foam & HLW99-52 & $\mathrm{h}$ & T-EA & red & Alkali3 & $\mathrm{t}-\mathrm{a}$ \\
\hline IG1-31 & $\mathrm{h}$ & SBW1-24 & $\mathrm{h}$ & SS-EA-19 & red & 22 & $\mathrm{t}-\mathrm{a}$ \\
\hline IG1-36 & $\mathrm{h}$ & SBW1-25 & $\mathrm{h}$ & SS-EA-15 & red & 23 & $\mathrm{t}-\mathrm{a}$ \\
\hline IG2-06 & $\mathrm{h}$ & SBW1-33A & $\mathrm{h}$ & SS-EA-1-7 & red & 29 & $\mathrm{t}-\mathrm{a}$ \\
\hline IG2-18 & $\mathrm{h}$ & WVUTH8 & lcomp & SS-EA-2-7 & red & 32 & $\mathrm{t}-\mathrm{a}$ \\
\hline IG3-04 & $\mathrm{h}$ & WVUTH38 & lcomp & SRS-SEA-A-7 & red & 33 & $\mathrm{t}-\mathrm{a}$ \\
\hline Envelope D & $\mathrm{h}$ & WVUTH49 & lcomp & SRS-SEA-B-7 & red & HLWD2-02 & $\mathrm{t}-\mathrm{a}$ \\
\hline HLWD1-02 & $\mathrm{h}$ & WVUTH81 & lcomp & CUASEA-A-7 & red & HLW98-61 & $\mathrm{t}-\mathrm{a}$ \\
\hline HLWD1-03 & $\mathrm{h}$ & HLWD1-25 & lcomp, h & CUASEA-B-7 & red & LAWB34 & $\mathrm{t}-\mathrm{a}$ \\
\hline HLWD1-04 & $\mathrm{h}$ & HG-3-2-7 & red & MG 25-7 & red & HLP-22 & $\mathrm{t}-\mathrm{a}$ \\
\hline HLWD1-05 & $\mathrm{h}$ & HG-3-3-7 & red & MG 28-7 & red & SSHTM-3 & $\mathrm{t}-\mathrm{a}$ \\
\hline HLWD1-07 & $\mathrm{h}$ & AH-165 FE-7 & red & IG1-33 & $\mathrm{t}-\mathrm{a}$ & B1G9-0IIC4 & $\mathrm{t}-\mathrm{a}$ \\
\hline HLWD1-13 & $\mathrm{h}$ & AH-131 AV-7 & red & IG3-16 & $\mathrm{t}-\mathrm{a}$ & B1G9-013C5 & $\mathrm{t}-\mathrm{a}$ \\
\hline HLWD1-21 & $\mathrm{h}$ & AH-131 FE-7 & red & IG3-26 & $\mathrm{t}-\mathrm{a}$ & B1G9-014C & $\mathrm{t}-\mathrm{a}$ \\
\hline HLWD1-23 & $\mathrm{h}$ & AH-168 Al-7 & red & IG3-29 & $\mathrm{t}-\mathrm{a}$ & D1G4-022P2 & $\mathrm{t}-\mathrm{a}$ \\
\hline HLWD1-27 & $\mathrm{h}$ & AH-168 AV-7 & red & CVS2-5 & $\mathrm{t}-\mathrm{a}$ & M1G1-008P & $\mathrm{t}-\mathrm{a}$ \\
\hline HLWD2-01 & $\mathrm{h}$ & AH-168 FE-7 & red & CVS2-14 & $\mathrm{t}-\mathrm{a}$ & M1G1-011P & $\mathrm{t}-\mathrm{a}$ \\
\hline HLW98-21 & $\mathrm{h}$ & AH-200 FE-7 & red & CVS2-19 & $\mathrm{t}-\mathrm{a}$ & V1M2 632040 P2 & $\mathrm{t}-\mathrm{a}$ \\
\hline HLW98-53A & $\mathrm{h}$ & AH-202 FE-7 & red & CVS2-28 & $\mathrm{t}-\mathrm{a}$ & V1M3 632059 P1 & $\mathrm{t}-\mathrm{a}$ \\
\hline HLW98-59 & $\mathrm{h}$ & EA-1-7 & red & CVS2-46 & $\mathrm{t}-\mathrm{a}$ & V1M3 632075 P2 & $\mathrm{t}-\mathrm{a}$ \\
\hline HLW98-60 & $\mathrm{h}$ & EA-1-7 & red & CVS2-51 & $\mathrm{t}-\mathrm{a}$ & SBW-16-18.5 & $\mathrm{t}-\mathrm{a}$ \\
\hline HLW99-15 & $\mathrm{h}$ & EA-2-7 & red & CVS2-97 & $\mathrm{t}-\mathrm{a}$ & HLWD1-01 & $\mathrm{t}-\mathrm{a}, \mathrm{h}$ \\
\hline HLW99-27 & $\mathrm{h}$ & EA-7 & red & BATCH 1 STUDY-6-7 & $\mathrm{t}-\mathrm{a}$ & & \\
\hline \multicolumn{8}{|c|}{$\begin{array}{l}\text { (a) The type of exclusion indicates whether the glasses were excluded due to foaming out of the crucible during } \\
\text { fabrication (foam), contained multiple phases (h), had component concentrations that summed to less than } 99 \% \\
\text { for target or } 95 \% \text { for analyzed (lcomp), were excessively reduced (red), or had significant differences between } \\
\text { target and analyzed compositions (t-a). }\end{array}$} \\
\hline
\end{tabular}

The compositions of the resulting 737 glasses were converted into mole fractions of oxides and (with a single oxide representing each multivalent element) by standard techniques. The concentration ranges of oxide components were evaluated to assure that coverage of the composition region over which models will be fit was adequate. Extreme compositions were excluded from model fitting by plotting the component concentrations in a histogram and removing data that were far from the bulk of the compositions. This was performed by evaluating each component in alphabetical order, excluding any outliers, before moving to the next component. By this method, the glasses with extreme concentrations of a component may be different before and after the removal of other data. Therefore, the procedure was repeated iteratively until the data covered the resulting composition region with a more or less normal distribution. Fifty-eight glasses were removed by this procedure (listed in Table 5-4), resulting in 679 glasses for model development. 
Table 5-4. Glasses with Extreme Compositions Excluded from PCT Model Development Data Set (with components that were in extreme concentrations listed)

\begin{tabular}{|c|c|c|c|c|c|c|c|}
\hline Glass ID & "Comp & "Glass ID & "Comp & Glass ID & "Comp & Glass ID & Comp \\
\hline IG2-04 & $\mathrm{Al}_{2} \mathrm{O}_{3}$ & P2-3Ca-2F & $\mathrm{F}$ & IG2-11 & $\mathrm{Li}_{2} \mathrm{O}$ & HLW98-51R & $\mathrm{SrO}$ \\
\hline IG2-14 & $\mathrm{Al}_{2} \mathrm{O}_{3}$ & P2-3Ca-3F & $\mathrm{F}$ & IG2-13 & $\mathrm{Li}_{2} \mathrm{O}$ & LAWA64 & $\mathrm{SrO}$ \\
\hline CVS2-63 & $\mathrm{Al}_{2} \mathrm{O}_{3}$ & P2-9Ca-2F & $\mathrm{F}$ & IG2-30 & $\mathrm{Li}_{2} \mathrm{O}$ & CU36 & $\mathrm{TiO}_{2}$ \\
\hline WSTC & $\mathrm{Al}_{2} \mathrm{O}_{3}$ & 6 & $\mathrm{Fe}_{2} \mathrm{O}_{3}$ & $\mathrm{Zr}-11$ & $\mathrm{Li}_{2} \mathrm{O}$ & LAWA82 & $\mathrm{TiO}_{2}$ \\
\hline SBW1-35 & $\mathrm{Al}_{2} \mathrm{O}_{3}$ & 8 & $\mathrm{Fe}_{2} \mathrm{O}_{3}$ & Zr-23 & $\mathrm{Li}_{2} \mathrm{O}$ & LAWA89 & $\mathrm{TiO}_{2}$ \\
\hline SBW1-40 & $\mathrm{BaO}$ & 17 & $\mathrm{Fe}_{2} \mathrm{O}_{3}$ & Zr-27 & $\mathrm{Li}_{2} \mathrm{O}$ & HLP-32 & $\mathrm{TiO}_{2}$ \\
\hline SBW1-45 & $\mathrm{BaO}$ & EnvDSR1 & $\mathrm{Fe}_{2} \mathrm{O}_{3}$ & SBW1-63 & $\mathrm{Li}_{2} \mathrm{O}$ & HLP-34 & $\mathrm{TiO}_{2}$ \\
\hline HLWD1-09 & $\mathrm{CdO}$ & IG1-06 & $\mathrm{K}_{2} \mathrm{O}$ & HLW98-34 & $\mathrm{MnO}$ & HLP-75 & $\mathrm{TiO}_{2}$ \\
\hline HLP-47 & $\mathrm{Cl}$ & IG1-12 & $\mathrm{K}_{2} \mathrm{O}$ & HLWD3-01 & $\mathrm{Na}_{2} \mathrm{O}$ & HLWD3-02 & $\mathrm{U}_{3} \mathrm{O}_{8}$ \\
\hline HLP-76 & $\mathrm{Cl}$ & IG1-13 & $\mathrm{K}_{2} \mathrm{O}$ & CU47 & $\mathrm{NiO}$ & HLW98-50 & $\mathrm{U}_{3} \mathrm{O}_{8}$ \\
\hline HLP-77 & $\mathrm{Cl}$ & IG3-12 & $\mathrm{La}_{2} \mathrm{O}_{3}$ & LAWA76 & $\mathrm{SO}_{3}$ & LAWC14 & $\mathrm{V}_{2} \mathrm{O}_{5}$ \\
\hline 202P w/o Mn-7 & $\mathrm{Cu}$ & IG3-21 & $\mathrm{La}_{2} \mathrm{O}_{3}$ & SBW-11-30 & $\mathrm{SO}_{3}$ & CU42 & $\mathrm{ZnO}$ \\
\hline IG2-23 & $\mathrm{F}$ & CU52 & $\mathrm{La}_{2} \mathrm{O}_{3}$ & HLW98-22 & $\mathrm{SrO}$ & LAWA42 & $\mathrm{ZnO}$ \\
\hline P2-0Ca-2F & $\mathrm{F}$ & IG1-07 & $\mathrm{Li}_{2} \mathrm{O}$ & HLW98-23 & $\mathrm{SrO}$ & HLP-17 & $\mathrm{ZnO}$ \\
\hline P2-0Ca-3F & $F$ & IG1-10 & $\mathrm{Li}_{2} \mathrm{O}$ & & & & \\
\hline
\end{tabular}

The value of a model lies in its capability to predict the properties of glasses not used in model development. Therefore, a subset of the 679 glass data set was removed for use in model evaluation and validation. To select the glasses to be removed, the data were sorted by $\ln \left[\mathrm{r}_{\mathrm{B}}\right]$ values, and every fifth data point was chosen as a validation data point. This resulted in roughly $20 \%$ of the data being used to validate models. Table 5-5 lists the glasses selected for use in the validation data set. The remaining 534 glasses were used in model development. 
Table 5-5. Glasses Selected for Validation of PCT Release Models

\begin{tabular}{|c|c|c|c|c|c|c|c|}
\hline Glass ID & Glass ID & \begin{tabular}{|l|} 
Glass ID \\
\end{tabular} & \begin{tabular}{|l} 
Glass ID \\
\end{tabular} & \begin{tabular}{|l|} 
Glass ID \\
\end{tabular} & \begin{tabular}{|l} 
Glass ID \\
\end{tabular} & \begin{tabular}{|l} 
Glass ID \\
\end{tabular} & Glass ID \\
\hline WVCM42 & WVUTH103 & IG2-19 & CVS2-49 & HG-2-2-7 & BLEND 2-3611 & HLW98-31 & LD6-5510 \\
\hline WVCM45 & WVDG-3 & IG2-21 & CVS2-58 & HG-2-3-7 & HM 1-3824 & HLW98-62 & LDM-4 \\
\hline WVCM47 & WVDG-7 & IG2-24 & CVS2-66 & AH-165 Al-7 & HM-2-2 (4099A) & LAWA87 & SBW1-02 \\
\hline WVUTH7 & WVDG-14R & IG3-17 & CVS2-75 & AH-2-7 & HM-3-3 (4357) & LAWA88 & SBW1-19 \\
\hline WVUTH19 & WVDG-18 & IG3-30 & CVS2-77 & AH-5-7 & HM-4-2 (5641) & LAWB39 & SBW1-28 \\
\hline WVUTH22 & WVDG-26 & CVS1-4 & CVS2-80 & AH-7-7 & PX 2-2 (4509) & LAWB41 & SBW1-46 \\
\hline WVUTH28 & $\mathrm{HiFe} 3$ & CVS1-16 & CVS2-86 & AH-13-7 & PX 3-2 (5818) & LAWC13 & SBW1-50 \\
\hline WVUTH41 & HiFe4 & CVS1-17 & CVS2-88 & SFRIT1 & PX 5-10 (6972) & LAWC21S & SBW1-52 \\
\hline WVUTH42 & FY93\#3 & CVS1-20 & CVS2-95 & SFRIT2 & H-GLAS-0112 & PNLREF (LD6-5412) & SBW1-57 \\
\hline WVUTH51 & FY93\#4 & CVS2-21 & CVS2-99 & 202G w/o Mn-7 & H-GLAS-0293 & TFA-BASE (HLP-01) & SBW1-59 \\
\hline WVUTH53 & FY93\#5 & CVS2-24 & CVS2-100 & 200R-7 & H-GLAS-0308 & HLP-19 & SBW1-60 \\
\hline WVUTH66 & FY93\#9 & CVS2-25 & CVS2-101 & 131-TDS-3A-SOPER-7 & H-GLAS-0421 & HLP-24 & SBW-13-18.5 \\
\hline WVUTH67 & FY94\#3 & CVS2-26 & CVS2-102 & BLEND 1.6-7 & CUOE & \begin{tabular}{|l|} 
HLP-28 \\
\end{tabular} & SBW-14-18.5 \\
\hline WVUTH68 & Sigma1 & CVS2-37 & CVS2-109 & BATCH 2-1.6 & $\mathrm{CUOO}$ & HLP-46 & SBW-17-18.5 \\
\hline WVUTH73 & Sigma3 & CVS2-38 & CVS2-117 & BATCH 4-7 (2) & CU34 & \begin{tabular}{|l} 
HLP-55 \\
\end{tabular} & SBW-18-18.5 \\
\hline WVUTH94 & IG1-11 & CVS2-39 & HG-1-1-7 & BLEND 1-3457 & \begin{tabular}{|l|} 
Alkali5 \\
\end{tabular} & L4-915 & SBW-22-18.5 \\
\hline WVUTH98 & IG1-38 & CVS2-42 & HG-2-1-7 & BLEND 1-3526 & HLW98-04 & L8-1 & SBW-25-25 \\
\hline
\end{tabular}




\subsection{Normalized Boron Release}

The data set for modeling $\ln \left[\mathrm{r}_{\mathrm{B}}\right]$ was developed as described in Sections 2.0 and 5.1. A histogram of the $\ln \left[r_{B}\right]$ values was plotted and found to be roughly normal without significant outliers. For initial model fitting, we assumed that $\ln \left[\mathrm{r}_{\mathrm{B}}\right]$ was linear in composition, e.g.,

$$
\ln \left[r_{j}\right]=\sum_{i=1}^{N} r_{j, i} x_{i}
$$

where, $r_{j}$ is the normalized release of the $j^{\text {th }}$ component from glass (in $\left.g / \mathrm{m}^{2}\right), r_{j, i}$ and $x_{i}$ are the $i^{\text {th }}$ component $r_{j}$ coefficient and mole fraction in glass, respectively, and $\mathrm{N}$ is the number of model components. We also assumed that required model components would be the set of components with at least one mole $\%$ in at least one glass. All other components were combined into a pseudo-component called Others.

Through an iterative process, several data points were determined to be outliers, and several model components were determined to have insignificant effects on $\ln \left[\mathrm{r}_{\mathrm{B}}\right]$. The outlying data were determined by a histogram plot of the residuals after each preliminary model fit to the data. Those data with residuals that were outside of a roughly normal distribution were considered outliers for the purpose of this model development. The resulting model is summarized in Table 5-6. Eighteen glasses - listed in Table 5-7were excluded from the model fit as outliers.

Table 5-6. Summary of 20-Component Linear Mixture Model for $\ln \left[\mathrm{r}_{\mathrm{B}}\right]$

\begin{tabular}{|c|c|c|c|c|c|c|c|}
\hline Comp & $\mathbf{r}_{B, i}$ & std. err. & Comp & $\mathbf{r}_{\mathrm{B}, \mathbf{i}}$ & std. err. & Statistic & value \\
\hline$\overline{\mathrm{Al}_{2} \mathrm{O}_{3}}$ & -28.4828 & 6.08 & $\mathrm{NiO}$ & 34.4786 & 14.95 & $\overline{\mathrm{R}^{2}}$ & 0.749 \\
\hline $\mathrm{B}_{2} \mathrm{O}_{3}$ & 13.7490 & 6.10 & $\mathrm{P}_{2} \mathrm{O}_{5}$ & -24.5220 & 8.96 & $\mathrm{R}_{\text {adj }}^{2}$ & 0.739 \\
\hline $\mathrm{CaO}$ & -11.5370 & 5.91 & $\mathrm{SiO}_{2}$ & -4.3515 & 6.01 & $\mathrm{R}_{\text {pred }}^{2}$ & 0.724 \\
\hline$F$ & 15.9574 & 9.10 & $\mathrm{SO}_{3}$ & 75.5175 & 19.79 & $\mathrm{~s}$ & 0.547 \\
\hline $\mathrm{Fe}_{2} \mathrm{O}_{3}$ & -9.6922 & 6.45 & $\mathrm{SrO}$ & -9.8242 & 15.48 & min response & -2.72 \\
\hline $\mathrm{K}_{2} \mathrm{O}$ & 8.0550 & 6.27 & $\mathrm{ThO}_{2}$ & -17.1074 & 11.13 & mean response & -0.658 \\
\hline $\mathrm{Li}_{2} \mathrm{O}$ & 9.0610 & 6.10 & $\mathrm{TiO}_{2}$ & -27.1564 & 8.26 & max response & 3.22 \\
\hline $\mathrm{MgO}$ & 7.0305 & 6.24 & $\mathrm{ZnO}$ & -3.5114 & 8.18 & Observations & 525 \\
\hline $\mathrm{MnO}$ & -17.7517 & 7.21 & $\mathrm{ZrO}_{2}$ & -19.6106 & 6.14 & & \\
\hline $\mathrm{Na}_{2} \mathrm{O}$ & 11.5079 & 6.17 & Others & 1.9089 & 6.00 & & \\
\hline
\end{tabular}


Table 5-7. Glasses Omitted from $\ln \left[\mathrm{r}_{\mathrm{B}}\right]$ Model as Outliers

\begin{tabular}{||l|l|l||}
\hline 2 & CVS2-82 & PX 5-5 (6839) \\
\hline PX 5-8 (6884) & CVS2-84 & PX 5-4 (6820) \\
\hline IG1-30 & CVS2-30 & CVS2-76 \\
\hline PX 6-1 (7340) & Zr-29 & CVS1-7 \\
\hline Ratio5 & PX 5-6 (6862) & CVS2-32 \\
\hline PX 5-7 (6871) & PX 5-3 (6812) & IG1-37 \\
\hline
\end{tabular}

The single component concentration ranges of $\ln \left[\mathrm{r}_{B}\right]$ model data are listed in Table 5-8 and pair-wise component concentrations are shown in Figure 5-1. Generally, the correlations between components are small and the coverage of pair-wise component concentrations is good. Seven of the component pairs have correlation coefficients $>|0.50|--\mathrm{MnO}: \mathrm{Fe}_{2} \mathrm{O}_{3}(0.55), \mathrm{Na}_{2} \mathrm{O}: \mathrm{Li}_{2} \mathrm{O}(-0.64), \mathrm{NiO}: \mathrm{MnO}(0.56)$, $\mathrm{ThO}_{2}: \mathrm{Fe}_{2} \mathrm{O}_{3}(0.54), \mathrm{P}_{2} \mathrm{O}_{5}: \mathrm{ThO}_{2}$ (0.64), F:Others (0.53), S:Others (0.51).

Table 5-8. Component Concentration Ranges for Data Used to Fit $\ln \left[\mathrm{r}_{\mathrm{B}}\right]$ Model (mole\%)

\begin{tabular}{||l|r|r|l|r|r||}
\hline Component & Min & Max & Component & Min & Max \\
\hline $\mathrm{Al}_{2} \mathrm{O}_{3}$ & 0.00 & 11.29 & $\mathrm{NiO}$ & 0.00 & 1.47 \\
\hline $\mathrm{B}_{2} \mathrm{O}_{3}$ & 3.88 & 19.53 & $\mathrm{P}_{2} \mathrm{O}_{5}$ & 0.00 & 1.37 \\
\hline $\mathrm{CaO}$ & 0.00 & 12.07 & $\mathrm{SiO}_{2}$ & 39.28 & 64.19 \\
\hline $\mathrm{F}$ & 0.00 & 3.41 & $\mathrm{SO}_{3}$ & 0.00 & 0.91 \\
\hline $\mathrm{Fe}_{2} \mathrm{O}_{3}$ & 0.00 & 8.08 & $\mathrm{SrO}$ & 0.00 & 1.92 \\
\hline $\mathrm{K}_{2} \mathrm{O}$ & 0.00 & 5.87 & $\mathrm{ThO}_{2}$ & 0.00 & 1.67 \\
\hline $\mathrm{Li}_{2} \mathrm{O}$ & 0.00 & 15.82 & $\mathrm{TiO}_{2}$ & 0.00 & 2.59 \\
\hline $\mathrm{MgO}$ & 0.00 & 13.43 & $\mathrm{ZnO}_{2}$ & 0.00 & 2.54 \\
\hline $\mathrm{MnO}$ & 0.00 & 3.43 & $\mathrm{ZrO}_{2}$ & 0.00 & 7.75 \\
\hline $\mathrm{Na} \mathrm{Na}_{2} \mathrm{O}$ & 4.82 & 22.40 & Others & 0.00 & 2.49 \\
\hline
\end{tabular}




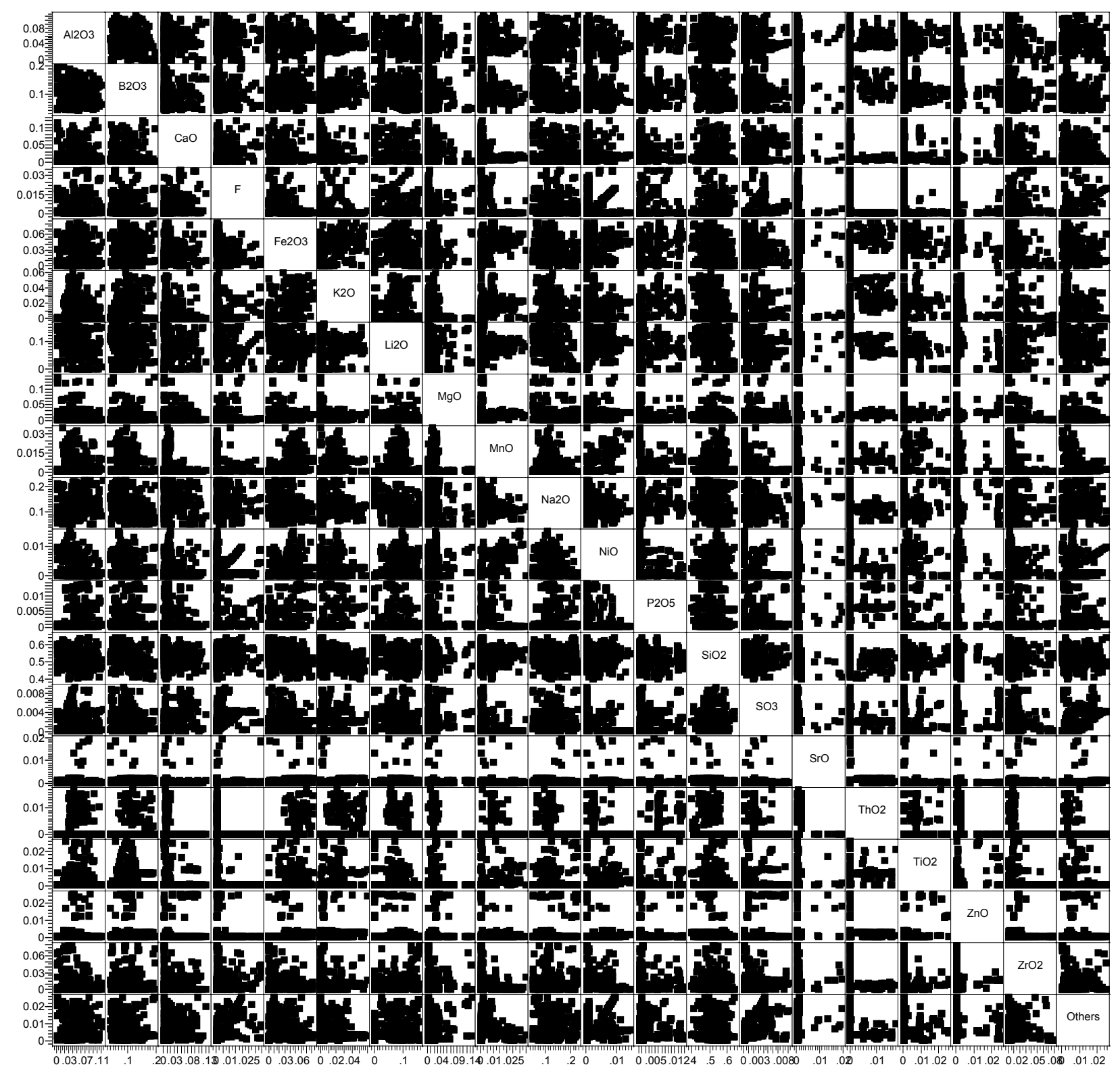

Figure 5-1. Scatter Plot Matrix for $\ln \left[\mathrm{r}_{\mathrm{B}}\right]$ Model Data Component Concentrations

Table 5-9 compares this model with two of the previously reported models. The previously reported models were those reported by Hrma et al. (1994) (CVS) and Hrma et al. (2001) (Interim). It should be noted that all three models were fit on a mole fraction of oxide component basis, and the Interim model was based on a glass composition normalized to only the model components. However, the other two, current and CVS, lumped additional components into Others. We first consider the differences in goodness of fit as judged by $\mathrm{R}^{2}, \mathrm{R}^{2}$ adj, and s values. The current model reports a lower $\mathrm{R}^{2}$ value than the previous two models. However, the $\mathrm{R}^{2}$ values are better for both the model data set (all 534 data, before removal of outliers) and for the validation data set. This discrepancy is due to the use of a significantly broader data set for the current study than those used in either the CVS or the Interim model development. As the purpose of these models is to give reasonably accurate predictions of properties from glasses over a very broad composition region, we conclude that the current model is superior to the other two. Second, 
we look at the number of model coefficients. The current model uses 20 coefficients while the previous two models used 10 and 12 coefficients. An attempt was made to further reduce the number of coefficients in the model by either removing coefficients for components with relatively small concentrations in glass $\left(\mathrm{SO}_{3}, \mathrm{NiO}, \mathrm{ThO}_{2}\right)$ or removing components with relatively uncertain coefficients (those coefficients that would be within the range of the property responses if the standard error were added or subtracted to the coefficient $-\mathrm{K}_{2} \mathrm{O}, \mathrm{MgO}, \mathrm{Li}_{2} \mathrm{O}, \mathrm{SiO}_{2}$ ) or removing both. However, each attempt at removing one or more of the components resulted in a marked increase in $\mathrm{s}$ or in the number of outliers. Therefore, the number of coefficients stayed at 20. In earlier iterations, coefficients for $\mathrm{BaO}$, $\mathrm{V}_{2} \mathrm{O}_{5}, \mathrm{U}_{3} \mathrm{O}_{8}$, and $\mathrm{Cl}$ were removed.

We also consider the coefficient values themselves. There is a reasonable match between the current coefficients, those reported earlier, and our expectations based on knowledge of the physical and chemical processes responsible for PCT release for $\mathrm{Al}_{2} \mathrm{O}_{3}, \mathrm{~B}_{2} \mathrm{O}_{3}, \mathrm{CaO}, \mathrm{Fe}_{2} \mathrm{O}_{3}, \mathrm{Li}_{2} \mathrm{O}, \mathrm{SiO}_{2}, \mathrm{ZrO}_{2}$, and Others. The coefficient for $\mathrm{F}, 15.957$, suggests that additions of $\mathrm{F}$ strongly increase $\mathrm{r}_{\mathrm{B}}$, which matches our expectations well. The coefficient for $\mathrm{K}_{2} \mathrm{O}, 8.055$, suggests that additions of $\mathrm{K}_{2} \mathrm{O}$ increase $\mathrm{r}_{\mathrm{B}}$, which matches our expectations well, but is different from that from the interim model, -1.547, which suggests little effect of $\mathrm{K}_{2} \mathrm{O}$ on $\mathrm{r}_{\mathrm{B}}$. The coefficients for $\mathrm{MgO}$ from this study, 7.031, and the CVS, 7.044, match closely with our expectations that $\mathrm{MgO}$ increases $\mathrm{r}_{\mathrm{B}}$; however, that for the Interim model, 3.513, suggests that $\mathrm{MgO}$ has little effect on $\mathrm{r}_{\mathrm{B}}$. The coefficients for $\mathrm{MnO}$ in the current study and the interim model, -17.752 and -21.220 , match fairly closely, but are difficult to explain from our current knowledge of the physical and chemical processes that control PCT release. More work will be required to further understand this result. The $\mathrm{Na}_{2} \mathrm{O}$ coefficient in this study, 11.508 , is significantly lower than those from the CVS and Interim studies, 17.258 and 17.013. While all three suggest that $r_{B}$ is strongly increased by adding $\mathrm{Na}_{2} \mathrm{O}$, the current study suggests that the effect is not as strong as previously measured. There is no explanation for this change. The NiO coefficient, 34.479, is very high, suggesting that the addition of $\mathrm{NiO}$ strongly increases $\mathrm{r}_{\mathrm{B}}$. This is difficult to explain from our current knowledge of the physical and chemical processes that control PCT release. More work will be required to further understand this result. There is a significant difference in $\mathrm{P}_{2} \mathrm{O}_{5}$ coefficients between this study, -24.522 , and the Interim model, -41.830. Both suggest a decrease in $r_{B}$, as would be expected, but this study suggests that the decrease would not be as extreme as in the Interim model. The coefficient from this study is a more moderate and likely more accurate assessment of the average effect of $\mathrm{P}_{2} \mathrm{O}_{5}$ over a broader composition region. The coefficient for $\mathrm{SO}_{3}, 75.517$, determined only in this study, suggests that sulfur increases $\mathrm{r}_{\mathrm{B}}$ more strongly than any other component. This is hard to justify with our current understanding of the physical and chemical processes responsible for PCT release and will require further study to verify. The coefficients for $\mathrm{SrO}, \mathrm{ThO}_{2}, \mathrm{TiO}_{2}$, and $\mathrm{ZnO}$ all match expectations but were not reported previously. To summarize, most coefficients seem appropriate and reproducible between studies. The exceptions that require further study include $\mathrm{SO}_{3}, \mathrm{NiO}, \mathrm{MnO}$, and possibly $\mathrm{Na}_{2} \mathrm{O}$. This study should be the focus of further research.

As stated repeatedly, the purpose of this model is to supply a tool for the prediction of $r_{B}$ over the entire composition region expected of Hanford waste glasses. Further research, i.e., model development and possibly data generation, is required to generate models with higher precision over narrower composition regions. In addition, the exploration of other model types and forms should be considered for future study. 
Table 5-9. Comparison of $\ln \left[\mathrm{r}_{\mathrm{B}}\right]$ Linear Mixture Models

\begin{tabular}{|c|c|c|c|c|c|c|c|}
\hline Comp & Current & CVS & Interim & Statistic & Current & CVS & Interim \\
\hline $\mathrm{Al}_{2} \mathrm{O}_{3}$ & -28.483 & -41.077 & -33.419 & \multicolumn{4}{|c|}{ As Reported } \\
\hline $\mathrm{B}_{2} \mathrm{O}_{3}$ & 13.749 & 13.009 & 13.403 & $\mathrm{R}^{2}$ & 0.749 & 0.806 & 0.814 \\
\hline $\mathrm{CaO}$ & -11.537 & -7.473 & -9.183 & $\mathrm{R}_{\text {adj }}^{2}$ & 0.739 & 0.790 & 0.808 \\
\hline $\mathrm{F}$ & 15.957 & & & $\mathrm{~s}$ & 0.547 & & 0.531 \\
\hline $\mathrm{Fe}_{2} \mathrm{O}_{3}$ & -9.692 & -9.027 & -11.947 & \# of glasses & 525 & 123 & 383 \\
\hline $\mathrm{K}_{2} \mathrm{O}$ & 8.055 & & -1.547 & \multicolumn{4}{|c|}{ Model Data Set (534 glasses) } \\
\hline $\mathrm{Li}_{2} \mathrm{O}$ & 9.061 & 10.431 & 9.382 & $\mathrm{R}_{\text {(val) }}^{2}$ & 0.705 & 0.496 & 0.561 \\
\hline $\mathrm{MgO}$ & 7.031 & 7.044 & 3.513 & $\mathrm{R}_{\text {adj, (val) }}^{2}$ & 0.694 & 0.488 & 0.552 \\
\hline $\mathrm{MnO}$ & -17.752 & & -21.220 & $\mathrm{~S}_{(\mathrm{val})}$ & 0.647 & 0.837 & 0.783 \\
\hline $\mathrm{Na}_{2} \mathrm{O}$ & 11.508 & 17.258 & 17.013 & \multicolumn{4}{|c|}{ Validation Data Set (136 glasses) } \\
\hline $\mathrm{NiO}$ & 34.479 & & & $\mathrm{R}_{\text {val }}^{2}$ & 0.702 & 0.498 & 0.586 \\
\hline $\mathrm{P}_{2} \mathrm{O}_{5}$ & -24.522 & & -41.830 & $\mathrm{R}_{\text {adj,val }}^{2}$ & 0.653 & 0.462 & 0.549 \\
\hline $\mathrm{SiO}_{2}$ & -4.351 & -3.917 & -4.106 & $\mathrm{~S}_{\mathrm{val}}$ & 0.685 & 0.852 & 0.780 \\
\hline $\mathrm{SO}_{3}$ & 75.517 & & & & & & \\
\hline $\mathrm{SrO}$ & -9.824 & & & & & & \\
\hline $\mathrm{ThO}_{2}$ & -17.107 & & & & & & \\
\hline $\mathrm{TiO}_{2}$ & -27.156 & & & & & & \\
\hline $\mathrm{ZnO}$ & -3.511 & & & & & & \\
\hline $\mathrm{ZrO}_{2}$ & -19.611 & -21.246 & -14.458 & & & & \\
\hline Others & 1.909 & -1.067 & & & & & \\
\hline
\end{tabular}

\subsection{Normalized Lithium Release}

The data set for modeling $\ln \left[\mathrm{r}_{\mathrm{Li}}\right]$ was developed as described in Sections 2.0 and 5.1. Of the 534glass model data set, only 394 reported $\mathrm{r}_{\mathrm{Li}}$ values. A histogram of the $\ln \left[\mathrm{r}_{\mathrm{Li}}\right]$ values was plotted, and three glasses were found to be outliers (WVDG-30 and IG1-37 with $\ln \left[\mathrm{r}_{\mathrm{Li}}\right]<-2.5$ and Frit-131-7 with $\ln \left[\mathrm{r}_{\mathrm{Li}}\right]$ $>3$ ), leaving 391 glasses for initial model fitting. For initial model fitting, we assumed that $\ln \left[\mathrm{r}_{\mathrm{Li}}\right]$ was linear in composition as done for $r_{B}$ models in Section 5.2. We also assumed that required model components would be the set of components with at least one mole $\%$ in at least one glass. All other components were combined into a pseudo-component called Others.

Through an iterative process, several data points were determined to be outliers, and several model components were determined to have insignificant effects on $\ln \left[\mathrm{r}_{\mathrm{Li}}\right]$. The outlying data were determined by a histogram plot of the residuals after each preliminary model fit to the data. Those data with residuals that were outside of a roughly normal distribution, estimated by gross visual interpretation, were considered outliers for the purpose of this model development. The resulting model is summarized in Table 5-10. Twenty glasses - listed in Table 5-11—were excluded from the model fit as outliers. 
Table 5-10. Summary of 18 Component Linear Mixture Model for $\ln \left[\mathrm{r}_{\mathrm{Li}}\right]$

\begin{tabular}{|c|c|c|c|c|}
\hline Comp. & $\overline{\mathbf{r}_{\mathrm{Li}, \mathrm{i}}}$ & std. err. & Statistic & value \\
\hline $\mathrm{Al}_{2} \mathrm{O}_{3}$ & -28.8662 & 4.42 & $\mathrm{R}^{2}$ & 0.769 \\
\hline $\mathrm{B}_{2} \mathrm{O}_{3}$ & 11.0871 & 4.37 & $\mathrm{R}_{\text {adj }}^{2}$ & 0.757 \\
\hline $\mathrm{CaO}$ & -7.3671 & 4.28 & $\mathrm{R}_{\text {pred }}^{2}$ & 0.739 \\
\hline $\mathrm{F}$ & 24.6314 & 7.64 & $\mathrm{~S}$ & 0.485 \\
\hline $\mathrm{Fe}_{2} \mathrm{O}_{3}$ & -10.0128 & 4.80 & min response & -1.635 \\
\hline $\mathrm{K}_{2} \mathrm{O}$ & 2.9308 & 4.96 & mean response & -0.457 \\
\hline $\mathrm{Li}_{2} \mathrm{O}$ & 8.9964 & 4.25 & max response & 2.09 \\
\hline $\mathrm{MgO}$ & 5.3232 & 4.50 & observations & 371 \\
\hline $\mathrm{MnO}$ & -17.6948 & 5.41 & & \\
\hline $\mathrm{Na}_{2} \mathrm{O}$ & 12.6156 & 4.34 & & \\
\hline $\mathrm{NiO}$ & 28.8398 & 12.10 & & \\
\hline $\mathrm{P}_{2} \mathrm{O}_{5}$ & -8.0281 & 10.95 & & \\
\hline $\mathrm{SiO}_{2}$ & -3.9379 & 4.30 & & \\
\hline $\mathrm{SrO}$ & -20.2103 & 17.11 & & \\
\hline $\mathrm{TiO}_{2}$ & -20.2039 & 11.00 & & \\
\hline $\mathrm{ZnO}$ & 14.4925 & 17.43 & & \\
\hline $\mathrm{ZrO}_{2}$ & -18.6611 & 4.18 & & \\
\hline Others & 0.6400 & 4.22 & & \\
\hline
\end{tabular}

Table 5-11. Glasses Omitted from $r_{L i}$ Model as Outliers

\begin{tabular}{||l|ll|l|l||}
\hline \hline PX 5-6 (6862) & CVS1-21 & AH-202 Al-7 & CVS2-30 \\
\hline P2-3Ca-1F & PX 5-5 & $(6839)$ & CVS2-32 & SBW-23-25 \\
\hline CVS1-2 & PX 5-4 & $(6820)$ & WVDG-22 & SBW-23-20 \\
\hline CVS2-33 & Ratio5 & & CVS1-7 & SBW-23-18.5 \\
\hline PX 6-1 (7340) & PX 5-3 & $(6812)$ & P2-0Ca-1F & SBW-23-15 \\
\hline
\end{tabular}

The single component concentration ranges of $\ln \left[\mathrm{r}_{\mathrm{Li}}\right]$ model data are listed in Table 5-12 and pairwise component concentrations are shown in Figure 5-2. Generally, the correlations between components are small and the coverage of pair-wise component concentrations is good. Four of the component pairs have correlation coefficients $>|0.50|--\mathrm{MnO}: \mathrm{Fe}_{2} \mathrm{O}_{3}(0.51), \mathrm{NiO}: \mathrm{MnO}(0.56), \mathrm{Fe}_{2} \mathrm{O}_{3}: \mathrm{TiO}_{2}(0.57)$, $\mathrm{TiO}_{2}: \mathrm{K}_{2} \mathrm{O}(0.56)$, and $\mathrm{ZnO}: \mathrm{SrO}(0.54)$.

Table 5-12. Component Concentration Ranges for Data Used to Fit $\ln \left[\mathrm{r}_{\mathrm{Li}}\right]$ Model (mole\%)

\begin{tabular}{|l|r|r|l|r|r|l|r|r||}
\hline \hline Component & Min & Max & Component & Min & Max & Component & Min & Max \\
\hline $\mathrm{Al}_{2} \mathrm{O}_{3}$ & 0.00 & 11.29 & $\mathrm{Li}_{2} \mathrm{O}$ & 0.63 & 15.82 & $\mathrm{SiO}_{2}$ & 39.28 & 64.19 \\
\hline $\mathrm{B}_{2} \mathrm{O}_{3}$ & 3.88 & 19.53 & $\mathrm{MgO}$ & 0.00 & 13.43 & $\mathrm{SrO}$ & 0.00 & 1.92 \\
\hline $\mathrm{CaO}$ & 0.00 & 12.07 & $\mathrm{MnO}$ & 0.00 & 3.43 & $\mathrm{TiO}_{2}$ & 0.00 & 2.59 \\
\hline $\mathrm{F}$ & 0.00 & 3.41 & $\mathrm{Na}_{2} \mathrm{O}$ & 4.82 & 22.40 & $\mathrm{ZnO}$ & 0.00 & 1.81 \\
\hline $\mathrm{Fe}_{2} \mathrm{O}_{3}$ & 0.00 & 8.08 & $\mathrm{NiO}$ & 0.00 & 1.47 & $\mathrm{ZrO}_{2}$ & 0.00 & 7.75 \\
\hline $\mathrm{K}_{2} \mathrm{O}$ & 0.00 & 5.56 & $\mathrm{P}_{2} \mathrm{O}_{5}$ & 0.00 & 1.36 & Others & 0.00 & 3.21 \\
\hline \hline
\end{tabular}




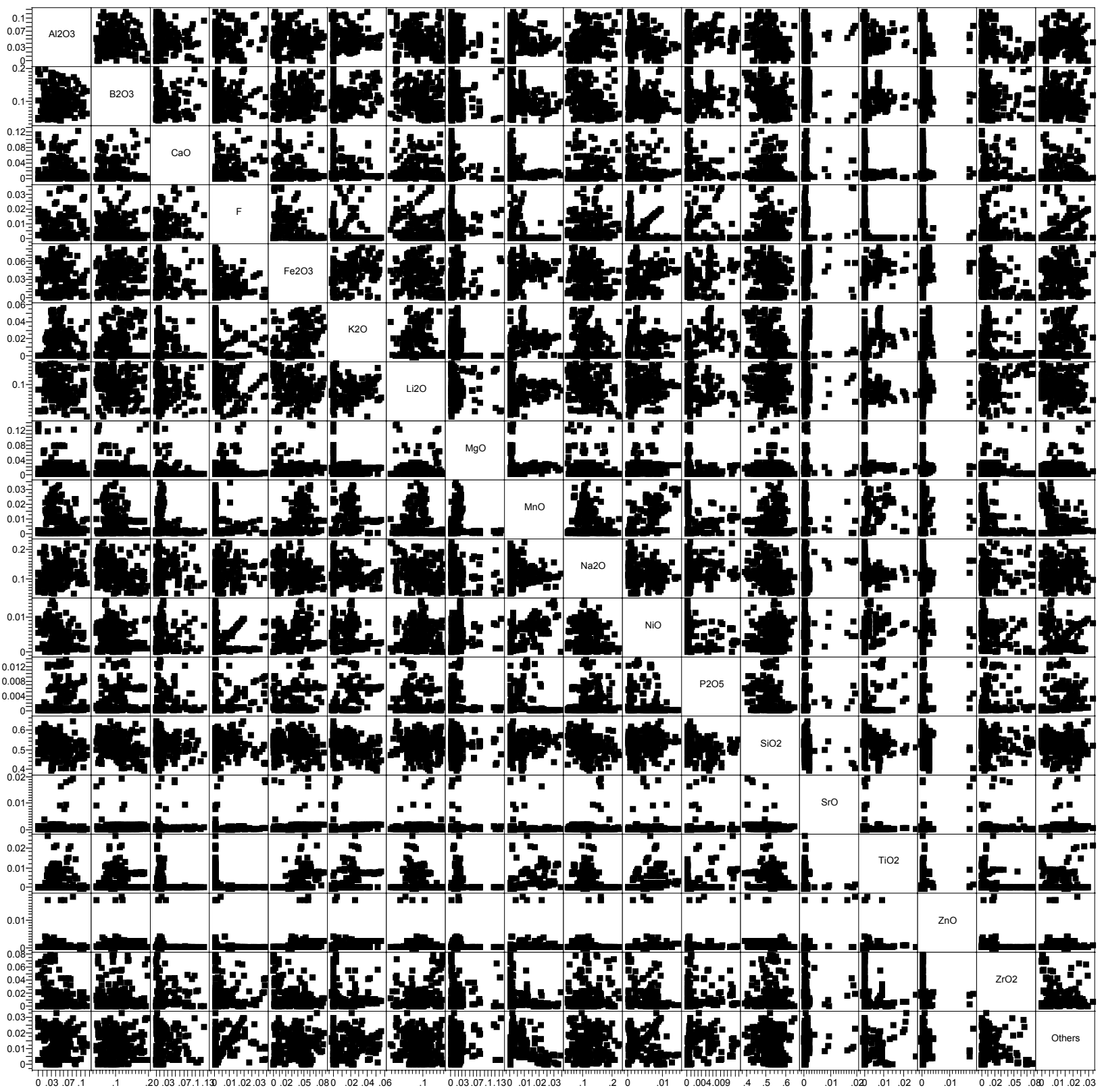

Figure 5-2. Scatter Plot Matrix for $\ln \left[\mathrm{r}_{\mathrm{Li}}\right]$ Model Data Component Concentrations

This model was compared with two of the previously reported models in Table 5-13. The previously reported models were those reported by Hrma et al. (1994) (CVS) and Hrma et al. (2001) (Interim). It should be noted that all three models were fit on a mole fraction of oxide component basis, and the Interim model was based on a glass composition normalized to only the model components. However, the other two, i.e., current and CVS, lumped additional components into Others. We first consider the differences in goodness of fit as judged by $\mathrm{R}^{2}, \mathrm{R}_{\text {adj }}^{2}$, and $\mathrm{s}$ values. The current model reports a lower $\mathrm{R}^{2}$ value than the previous two models. However, the $\mathrm{R}^{2}$ values are better for both the model data set - all 394 data, before removal of outliers or extreme release glasses - and for the validation data set. This discrepancy is due to the use of a significantly broader data set for the current study than those used in 
either the CVS or the Interim model development. Interestingly, the $\mathrm{R}^{2}$ for the validation data set is higher than that for the model data set. This result is due to the influence of the 20 outliers removed during the model fit. As the purpose of these models is to give reasonably accurate predictions of properties from glasses over a very broad composition region, we conclude that the current model is superior to the other two.

Second, we look at the number of model coefficients. The current model uses 18 coefficients while the previous two models used 10 and 12 coefficients. An attempt was made to further reduce the number of coefficients in the model by either removing coefficients for components with relatively small concentrations in glass $(\mathrm{NiO})$ and/or components with relatively uncertain coefficients, e.g., those coefficients that would be within the range of the property responses if the standard error were added or subtracted to the coefficient $-\mathrm{K}_{2} \mathrm{O}, \mathrm{MgO}, \mathrm{SiO}_{2}$. However, each attempt at removing one or more of the components resulted in a marked increase in $\mathrm{s}$ or in the number of outliers. Therefore, the number of coefficients stayed at 18. In earlier iterations, coefficients for $\mathrm{SO}_{3}, \mathrm{ThO}_{2}, \mathrm{BaO}, \mathrm{V}_{2} \mathrm{O}_{5}, \mathrm{U}_{3} \mathrm{O}_{8}$, and $\mathrm{Cl}$ were removed.

We also consider the coefficient values themselves. There is a reasonable match between the current coefficients, those reported earlier, and our expectations based on knowledge of the physical and chemical processes responsible for PCT release for $\mathrm{Al}_{2} \mathrm{O}_{3}, \mathrm{~B}_{2} \mathrm{O}_{3}, \mathrm{CaO}, \mathrm{Fe}_{2} \mathrm{O}_{3}, \mathrm{Li}_{2} \mathrm{O}, \mathrm{Na}_{2} \mathrm{O}, \mathrm{SiO}_{2}, \mathrm{ZrO}_{2}$ and Others. The coefficient for $\mathrm{F}, 24.631$, suggests that additions of $\mathrm{F}$ strongly increase $\mathrm{r}_{\mathrm{Li}}$, which matches our expectations. The coefficient for $\mathrm{K}_{2} \mathrm{O}, 2.931$, suggests that additions of $\mathrm{K}_{2} \mathrm{O}$ increase $\mathrm{r}_{\mathrm{Li}}$, which matches our expectations well, but is different from that from the interim model, -0.814 , which suggests little effect of $\mathrm{K}_{2} \mathrm{O}$ on $\mathrm{r}_{\mathrm{Li}}$. The coefficients for $\mathrm{MgO}$ from this study, 5.323, and the CVS, 4.592, match closely with our expectations that $\mathrm{MgO}$ increases $\mathrm{r}_{\mathrm{Li}}$; however, that for the Interim model, 1.719, suggests that $\mathrm{MgO}$ has no effect on $\mathrm{r}_{\mathrm{Li}}$. The coefficients for $\mathrm{MnO}$ in the current study and the interim model, -17.695 and -15.880 , match fairly closely, but are difficult to explain from our current knowledge of the physical and chemical processes that control PCT release. More work will be required to further understand this result. The NiO coefficient, 28.840, is very high, suggesting that adding NiO strongly increases $\mathrm{r}_{\mathrm{Li}}$. This is difficult to explain from our current knowledge of the physical and chemical processes that control PCT release. More work will be required to further understand this result. There is a significant difference in $\mathrm{P}_{2} \mathrm{O}_{5}$ coefficients between this study, -8.028, and the Interim model, -31.893. Both suggest a decrease in $\mathrm{r}_{\mathrm{Li}}$, as would be expected, but this study suggests that the decrease would not be as extreme as that of the Interim model. The coefficient from this study is a more moderate and likely more accurate assessment of the average effect of $\mathrm{P}_{2} \mathrm{O}_{5}$ over a broader composition region. The coefficient for $\mathrm{TiO}_{2}$ matches expectations but was not reported previously. The coefficients for $\mathrm{ZnO}$ and $\mathrm{SrO}$ appear to be high and low relative to expectations, 14.493 and -20.210 , and have high standard errors, suggesting that more data are required to more accurately predict their effects. Interestingly, they are opposite the trend as seen in the $\mathrm{r}_{\mathrm{Na}}$ model. To summarize, most coefficients seem appropriate and reproducible between studies. The exceptions that require further study include $\mathrm{NiO}, \mathrm{MnO}, \mathrm{SrO}$, and $\mathrm{ZnO}$. This study should be the focus of further research.

As stated repeatedly, the purpose of this model is to supply a tool to predict $\mathrm{r}_{\mathrm{Li}}$ over the entire composition region expected of Hanford waste glasses. Further research, i.e., model development and possibly data generation, is required to generate models with higher precision over narrower composition regions. In addition, the exploration of other model types and forms should be considered for future study. 
Table 5-13. Comparison of $\mathrm{r}_{\mathrm{Li}}$ Models

\begin{tabular}{|c|c|c|c|c|c|c|c|}
\hline & Current & CVS & Interim & Statistic & Current & CVS & Interim \\
\hline $\mathrm{Al}_{2} \mathrm{O}_{3}$ & -28.866 & -36.078 & -29.999 & \multicolumn{4}{|c|}{ As Reported } \\
\hline $\mathrm{B}_{2} \mathrm{O}_{3}$ & 11.087 & 11.044 & 11.238 & $\mathrm{R}^{2}$ & 0.769 & 0.785 & 0.797 \\
\hline $\mathrm{CaO}$ & -7.367 & -4.629 & -6.144 & $\mathrm{R}_{\text {adj }}^{2}$ & 0.757 & 0.768 & 0.791 \\
\hline $\mathrm{F}$ & 24.631 & & & $\mathrm{~s}$ & 0.485 & & 0.468 \\
\hline $\mathrm{Fe}_{2} \mathrm{O}_{3}$ & -10.013 & -12.051 & -13.028 & \multicolumn{4}{|c|}{ Model Data Set (394 glasses) } \\
\hline $\mathrm{K}_{2} \mathrm{O}$ & 2.931 & & -0.814 & $\mathrm{R}^{2}$ & 0.692 & 0.603 & 0.643 \\
\hline $\mathrm{Li}_{2} \mathrm{O}$ & 8.996 & 8.487 & 9.279 & $\mathrm{R}_{\text {adj }}^{2}$ & 0.678 & 0.593 & 0.633 \\
\hline $\mathrm{MgO}$ & 5.323 & 4.592 & 1.719 & $\mathrm{~S}$ & 0.620 & 0.697 & 0.662 \\
\hline $\mathrm{MnO}$ & -17.695 & & -15.880 & \multicolumn{4}{|c|}{ Validation Data Set (100 glasses) } \\
\hline $\mathrm{Na}_{2} \mathrm{O}$ & 12.616 & 13.719 & 13.404 & $\mathrm{R}^{2}$ & 0.727 & 0.599 & 0.667 \\
\hline $\mathrm{NiO}$ & 28.840 & & & $\mathrm{R}_{\text {adj }}^{2}$ & 0.670 & 0.559 & 0.625 \\
\hline $\mathrm{P}_{2} \mathrm{O}_{5}$ & -8.028 & & -31.893 & $\mathrm{~s}$ & 0.591 & 0.683 & 0.630 \\
\hline $\mathrm{SiO}_{2}$ & -3.938 & -2.904 & -3.431 & & & & \\
\hline $\mathrm{SrO}$ & -20.210 & & & & & & \\
\hline $\mathrm{TiO}_{2}$ & -20.204 & & & & & & \\
\hline $\mathrm{ZnO}$ & 14.493 & & & & & & \\
\hline $\mathrm{ZrO}_{2}$ & -18.661 & -20.152 & -12.906 & & & & \\
\hline Others & 0.640 & 0.247 & & & & & \\
\hline
\end{tabular}

\subsection{Normalized Sodium Release}

The data set for modeling $\ln \left[\mathrm{r}_{\mathrm{Na}}\right]$ was developed as described in Sections 2.0 and 5.1. Of the 534glass model data set, only 484 reported $\mathrm{r}_{\mathrm{Na}}$ values. A histogram of the $\ln \left[\mathrm{r}_{\mathrm{Na}}\right]$ values was plotted, and three glasses were found to be outliers (Frit-131-7, CVS2-82, and CVS2-83 with $\ln \left[\mathrm{r}_{\mathrm{Na}}\right]>3$ ), leaving 481 glasses for initial model fitting. For initial model fitting, we assumed that $\ln \left[\mathrm{r}_{\mathrm{Na}}\right]$ was linear in composition as done for $r_{B}$ and $r_{L i}$ models in Section 5.2. We also assumed that required model components would be the set of components with at least one mole $\%$ in at least one glass. All other components were combined into a pseudo-component called Others.

Through an iterative process, several data points were determined to be outliers, and several model components were insignificant. The outlying data were determined by a histogram plot of the residuals after each preliminary model fit to the data. Those data with residuals that were outside of a roughly normal distribution, estimated by gross visual interpretation, were considered outliers for the purpose of this model development. The resulting model is summarized in Table 5-14. Thirty-six glasses were excluded from the model fit as outliers-listed in Table 5-15. 


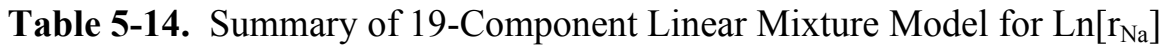

\begin{tabular}{||l|c|l|l|l|c|l|c||}
\hline \hline Comp & \multicolumn{1}{|c|}{$\mathbf{r}_{\mathbf{N a}, \mathbf{i}}$} & std. err. & $\mathbf{C o m p}$ & \multicolumn{1}{c|}{$\mathbf{r}_{\mathbf{N a}, \mathbf{i}}$} & std. err. & \multicolumn{1}{c|}{ Statistic } & Value \\
\hline $\mathrm{Al}_{2} \mathrm{O}_{3}$ & -27.3698 & 4.64 & $\mathrm{NiO}$ & 39.9792 & 11.93 & $\mathrm{R}^{2}$ & 0.772 \\
\hline $\mathrm{B}_{2} \mathrm{O}_{3}$ & 8.9811 & 4.61 & $\mathrm{P}_{2} \mathrm{O}_{5}$ & -19.3508 & 8.18 & $\mathrm{R}_{\text {adj }}$ & 0.762 \\
\hline $\mathrm{CaO}$ & -4.0404 & 4.39 & $\mathrm{SiO}_{2}$ & -4.6689 & 4.48 & $\mathrm{R}_{\text {pred }}$ & 0.747 \\
\hline $\mathrm{F}$ & 11.9897 & 6.58 & $\mathrm{SrO}$ & 17.3596 & 13.18 & $\mathrm{~s}$ & 0.446 \\
\hline $\mathrm{Fe}_{2} \mathrm{O}_{3}$ & -11.5968 & 4.98 & $\mathrm{TiO}_{2}$ & -30.9056 & 7.20 & min response & -2.749 \\
\hline $\mathrm{K}_{2} \mathrm{O}$ & 11.8066 & 5.14 & $\mathrm{ZnO}^{2}$ & -21.4935 & 6.03 & mean response & -0.710 \\
\hline $\mathrm{Li}_{2} \mathrm{O}$ & 7.1754 & 4.55 & $\mathrm{ZrO}_{2}$ & -17.0450 & 4.68 & max response & 2.64 \\
\hline $\mathrm{MgO}$ & 9.0581 & 4.71 & $\mathrm{SO}_{3}$ & 49.9505 & 18.07 & observations & 445 \\
\hline $\mathrm{MnO}$ & -11.3707 & 5.42 & Others & -1.9870 & 4.47 & & \\
\hline $\mathrm{Na}_{2} \mathrm{O}$ & 15.1889 & 4.55 & & & & & \\
\hline
\end{tabular}

Table 5-15. Glasses Omitted from $r_{\mathrm{Na}}$ Model as Outliers

\begin{tabular}{||c|c|c|c|c|c||}
\hline \hline CVS2-114 & Zr-29 & CVS1-21 & CVS2-90 & SBW-23-25 & PX 5-3 (6812) \\
\hline Ratio5 & CVS2-1 & PX 5-8 (6884) & SBW-23-15 & SBW-23-20 & PX 5-4 (6820) \\
\hline 24 & PX 5-2 (6795) & V1M2 6 32 011 P1 & CVS2-32 & SBW-23-18.5 & CVS2-33 \\
\hline CVS2-115 & CVS2-53 & V1M4 6 32 088 P1 & HLP-30 & CVS2-76 & CVS2-30 \\
\hline CU41 & V1M4 6 32 096 P2 & 5 & PX 5-7 (6871) & PX 5-5 (6839) & PX 6-1 (7340) \\
\hline CVS2-84 & CVS2-6 & CVS1-7 & 2 & PX 5-6 (6862) & IG1-37 \\
\hline
\end{tabular}

The single component concentration ranges of $\ln \left[\mathrm{r}_{\mathrm{Na}}\right]$ model data are listed in Table 5-16 and pairwise component concentrations are shown in Figure 5-3. Generally, the correlations between components are small and the coverage of pair-wise component concentrations is good. Three of the component pairs have correlation coefficients $>|0.50|--\mathrm{MnO}: \mathrm{Fe}_{2} \mathrm{O}_{3}(0.54), \mathrm{Na}_{2} \mathrm{O}: \mathrm{Li}_{2} \mathrm{O}(-0.64)$, and $\mathrm{NiO}: \mathrm{MnO}(0.65)$.

Table 5-16. Component Concentration Ranges for Data Used to Fit $\ln \left[\mathrm{r}_{\mathrm{Na}}\right]$ Model (mole\%)

\begin{tabular}{||l|r|r|l|r|r||}
\hline Component & Min & Max & Component & Min & Max \\
\hline $\mathrm{Al}_{2} \mathrm{O}_{3}$ & 0.00 & 11.29 & $\mathrm{NiO}$ & 0.00 & 1.47 \\
\hline $\mathrm{B}_{2} \mathrm{O}_{3}$ & 3.88 & 19.53 & $\mathrm{P}_{2} \mathrm{O}_{5}$ & 0.00 & 1.36 \\
\hline $\mathrm{CaO}$ & 0.00 & 12.07 & $\mathrm{SiO}_{2}$ & 39.28 & 64.19 \\
\hline $\mathrm{F}$ & 0.00 & 3.41 & $\mathrm{SO}_{3}$ & 0.00 & 0.88 \\
\hline $\mathrm{Fe}_{2} \mathrm{O}_{3}$ & 0.00 & 8.08 & $\mathrm{SrO}$ & 0.00 & 1.92 \\
\hline $\mathrm{K}_{2} \mathrm{O}$ & 0.00 & 5.87 & $\mathrm{TiO}_{2}$ & 0.00 & 2.59 \\
\hline $\mathrm{Li}_{2} \mathrm{O}$ & 0.00 & 15.82 & $\mathrm{ZnO}$ & 0.00 & 2.54 \\
\hline $\mathrm{MgO}$ & 0.00 & 13.43 & $\mathrm{ZrO}_{2}$ & 0.00 & 7.75 \\
\hline $\mathrm{MnO}$ & 0.00 & 3.43 & Other & 0.00 & 2.64 \\
\hline $\mathrm{Na}_{2} \mathrm{O}$ & 4.82 & 22.40 & & & \\
\hline
\end{tabular}




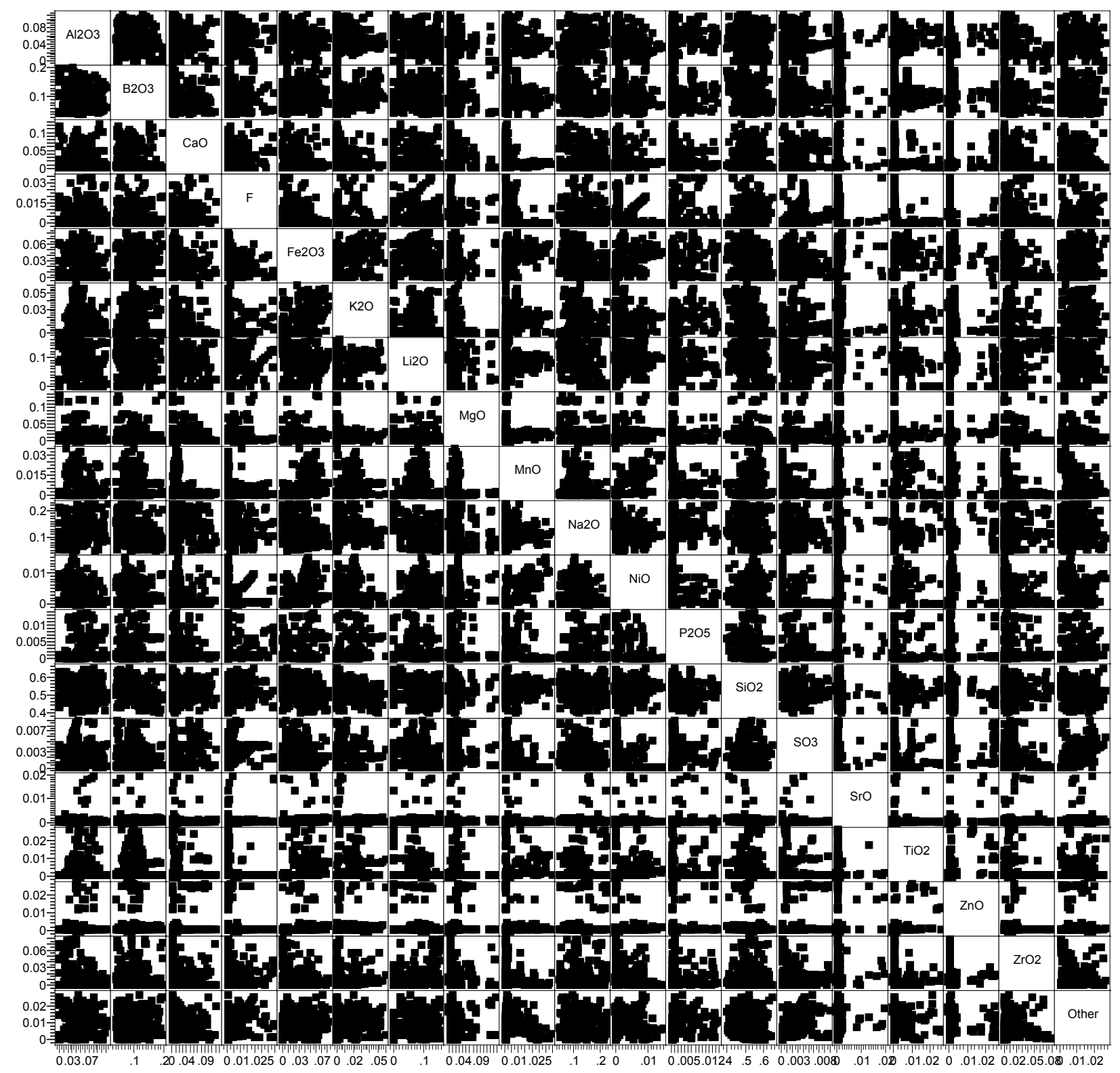

Figure 5-3. Scatter Plot Matrix for $\ln \left[\mathrm{r}_{\mathrm{Na}}\right]$ Model Data Component Concentrations

This model was compared with two of the previously reported models in Table 5-17. The previously reported models were those reported by Hrma et al. (1994) (CVS) and Hrma et al. (2001) (Interim). It should be noted that all three models were fit on a mole fraction of oxide component basis, and the Interim model was based on a glass composition normalized to only the model components, while the other two, current and CVS, lumped additional components into Others. We first consider the differences in goodness of fit as judged by $\mathrm{R}^{2}, \mathrm{R}_{\text {adj }}^{2}$, and $\mathrm{s}$ values. The current model reports a lower $\mathrm{R}^{2}$ value than the previous two models. However, the $\mathrm{R}^{2}$ values are better for both the model data set, all 484 data, before removal of outliers or extreme release glasses, and for the validation data set. This discrepancy is due to the use of a significantly broader data set for the current study than those used in either the CVS or 
the Interim model development. Interestingly, the $\mathrm{R}^{2}$ for the validation data set is higher than that for the model data set. This result is due to the influence of the 36 outliers removed during the model fit. As the purpose of these models is to give reasonably accurate predictions of properties from glasses over a very broad composition region, we conclude that the current model is superior to the other two. Second, we look at the number of model coefficients. The current model uses 19 coefficients while the previous two models used 10 and 12 coefficients. An attempt was made to further reduce the number of coefficients in the model by either removing coefficients for components with relatively small concentrations in glass $\left(\mathrm{NiO}, \mathrm{SO}_{3}\right)$ or removing components with relatively uncertain coefficients, e.g., those coefficients that would be within the range of the property responses if the standard error were added or subtracted to the coefficient $\left(\mathrm{CaO}, \mathrm{Li}_{2} \mathrm{O}, \mathrm{SiO}_{2}, \mathrm{SrO}\right)$ or removing both. However, each attempt at removing one or more of the components resulted in a marked increase in $\mathrm{s}$ or in the number of outliers. Therefore, the number of coefficients stayed at 19. In earlier iterations, coefficients for $\mathrm{ThO}_{2}, \mathrm{BaO}, \mathrm{V}_{2} \mathrm{O}_{5}, \mathrm{U}_{3} \mathrm{O}_{8}$, and $\mathrm{Cl}$ were removed.

We also consider the coefficient values themselves. There is a reasonable match between the current coefficients, those reported earlier, and our expectations based on knowledge of the physical and chemical processes responsible for PCT release for $\mathrm{Al}_{2} \mathrm{O}_{3}, \mathrm{~B}_{2} \mathrm{O}_{3}, \mathrm{Fe}_{2} \mathrm{O}_{3}, \mathrm{Li}_{2} \mathrm{O}, \mathrm{Na}_{2} \mathrm{O}, \mathrm{SiO}_{2}, \mathrm{ZrO}_{2}$ and Others. The coefficient for $\mathrm{F}, 11.990$, suggests that additions of $\mathrm{F}$ strongly increase $\mathrm{r}_{\mathrm{Na}}$, which matches our expectations. The coefficient for $\mathrm{K}_{2} \mathrm{O}, 11.807$, suggests that additions of $\mathrm{K}_{2} \mathrm{O}$ increase $\mathrm{r}_{\mathrm{Na}}$, which matches our expectations well, but is different from that from the interim model, 2.363, which suggests little effect of $\mathrm{K}_{2} \mathrm{O}$ on $\mathrm{r}_{\mathrm{Na}}$. The coefficients for $\mathrm{MgO}$ from this study, 9.058, and the CVS, 7.483, match closely with our expectations that $\mathrm{MgO}$ increases $\mathrm{r}_{\mathrm{Na}}$; however, that for the Interim model, 3.549, suggests that $\mathrm{MgO}$ has little effect on $\mathrm{r}_{\mathrm{Na}}$. The coefficients for $\mathrm{MnO}$ in the current study and the interim model, -11.371 and 16.419, match fairly closely, but are difficult to explain from our current knowledge of the physical and chemical processes that control PCT release. More work will be required to further understand this result. The $\mathrm{NiO}$ coefficient, 39.979, is very high, suggesting that adding $\mathrm{NiO}$ strongly increases $\mathrm{r}_{\mathrm{Na}}$. This is difficult to explain from our current knowledge of the physical and chemical processes that control PCT release. More work will be required to further understand this result. There is a significant difference in $\mathrm{P}_{2} \mathrm{O}_{5}$ coefficients between this study, -19.351, and the Interim model, -39.952. Both suggest a decrease in $\mathrm{r}_{\mathrm{Na}}$, as would be expected, but this study suggests that the decrease would not be as extreme as in the Interim model. The coefficient from this study is a more moderate and likely more accurate assessment of the average effect of $\mathrm{P}_{2} \mathrm{O}_{5}$ over a broader composition region. The coefficient for $\mathrm{SO}_{3}, 49.951$, determined only in this study, suggests that sulfur increases $r_{\mathrm{Na}}$ more strongly than any other component. This is hard to justify with our current understanding of the physical and chemical processes responsible for PCT release and will require further study to verify. The coefficients for $\mathrm{SrO}$ and $\mathrm{ZnO}$ appear to be high and low relative to expectations, 17.360 and -21.493, and to have high standard errors, suggesting that more data are required to more accurately predict their effects. Interestingly, they are opposite the trend as seen in the $\mathrm{r}_{\mathrm{Li}}$ model. The coefficient for $\mathrm{TiO}_{2}$ matches expectations but was not reported previously. To summarize, most coefficients seem appropriate and reproducible between studies. The exceptions that require further study include $\mathrm{SO}_{3}, \mathrm{NiO}, \mathrm{ZnO}, \mathrm{SrO}$, and $\mathrm{MnO}$. This study should be the focus of further research.

As stated repeatedly, the purpose of this model is to supply a tool to predict $\mathbf{r}_{\mathrm{Na}}$ over the entire composition region expected of Hanford waste glasses. Further research (model development and possibly data generation) is required to generate models with higher precision over narrower composition 
regions. In addition, the exploration of other model types and forms should be considered for future study.

Table 5-17. Comparison of $r_{\mathrm{Na}}$ Models

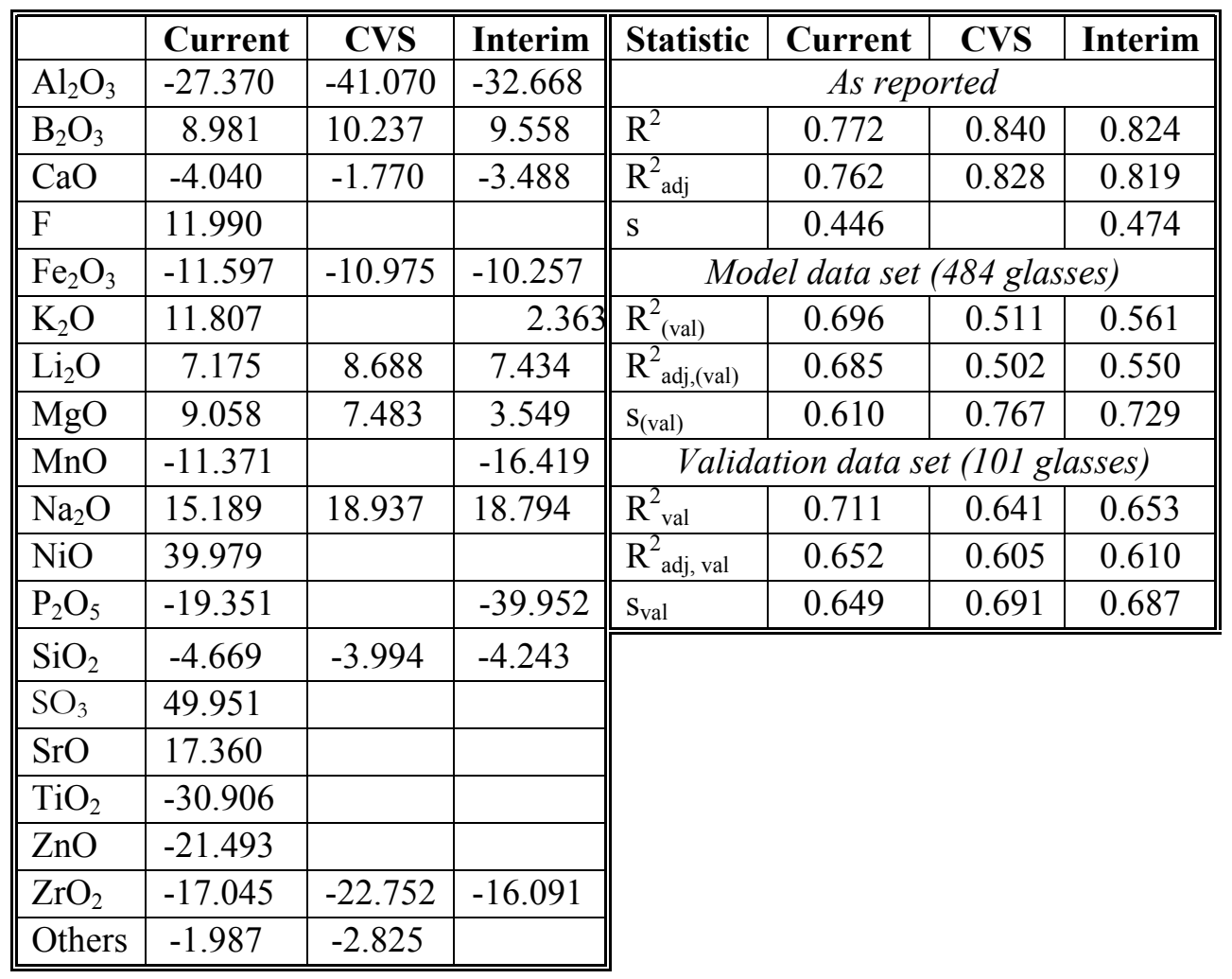




\subsection{Models for Molar Volume/Density}

This section discusses initial data evaluation and screening for molar volume/density as well as processes for converting the composition of samples, initially developing models, and evaluating and selecting models.

\subsection{Initial Data Evaluation and Screening}

Out of 2001 glasses in the database, 392 had density data. Before modeling density, some glasses were removed from the data set because of the characteristics that are considered undesirable for density modeling. These characteristics include glasses a) identified in the database as multi-phase, i.e., crystallized or amorphous phase separated, b) with extreme density values, and c) with extreme component concentrations. Justifications for removing these glasses are a) the density of glasses with crystalline phases can be significantly different from those of single-phase glasses, b) glasses with high density value could degrade predictive performance of models, and c) glasses with extreme component concentration values can be very influential in developing property-composition models. Glasses with measured densities $\geq 3.15 \mathrm{~g} / \mathrm{cm}^{3}$ were not used for modeling; the remaining glasses have densities $<3$ $\mathrm{g} / \mathrm{cm}^{3}$. A summary of the glasses deleted from the density data set during the above data screening is in Table 6-1. This left a total of 368 glasses for use in the density-composition modeling. Table 6-1 also contains three glasses deleted later as model outliers, which will be explained in Section 6.3.2.

Regarding multi-phase glasses, it should be noted that most of the glasses in the database did not have information indicating whether they were single-phase or multi-phase. Hence, some multi-phase glasses could remain in the subsets of glasses used to develop density models. On the other hand, the glasses identified as multi-phase do not have information on the amount of crystalline phases. Hence, some multi-phase glasses could have a very small fraction of crystals so that the effect on density may be virtually negligible.

\subsection{Composition Conversion}

The same procedure as in Section 3.2 to convert mass fractions to mole fractions and to combine like oxides into groups was applied to the density data set. After forming the combined components, the 368 glass compositions were expressed as normalized mole fractions of 48 components. The mole fractions ranges of these 48 components are listed Table 6-2. Those components with concentrations greater than $1.0 \mathrm{~mole} \%$ in at least one glass were first considered as possible model components. However, components without a sufficient number of glasses with reasonable distribution, i.e., $\mathrm{Bi}_{2} \mathrm{O}_{3}, \mathrm{Cl}, \mathrm{Cr}_{2} \mathrm{O}_{3}$, and $\mathrm{V}_{2} \mathrm{O}_{5}$, were excluded from the model components-leaving the 22 components from $\mathrm{Al}_{2} \mathrm{O}_{3}$ to $\mathrm{UO}_{\mathrm{x}}$ in Table 6-2. 
Table 6-1. Glasses Deleted during Screening for Density Model Development (composition expressed in mole fraction)

\begin{tabular}{|c|c|c|}
\hline Glass & Study & Reason \\
\hline IG2-01 & INEEL CVS Phase 2 & Multi-phase \\
\hline IG2-03 & INEEL CVS Phase 2 & Multi-phase \\
\hline IG2-05 & INEEL CVS Phase 2 & Multi-phase \\
\hline IG2-06 & INEEL CVS Phase 2 & Multi-phase \\
\hline IG2-07 & INEEL CVS Phase 2 & Multi-phase \\
\hline IG2-09 & INEEL CVS Phase 2 & Multi-phase \\
\hline IG2-10 & INEEL CVS Phase 2 & Multi-phase \\
\hline IG2-14 & INEEL CVS Phase 2 & Multi-phase \\
\hline IG2-17 & INEEL CVS Phase 2 & Multi-phase \\
\hline IG2-18 & INEEL CVS Phase 2 & Multi-phase \\
\hline IG2-22 & INEEL CVS Phase 2 & Multi-phase \\
\hline IG2-27 & INEEL CVS Phase 2 & Multi-phase \\
\hline IG2-34 & INEEL CVS Phase 2 & Multi-phase \\
\hline IG3-03 & INEEL CVS Phase 3 & Multi-phase \\
\hline IG3-04 & INEEL CVS Phase 3 & Multi-phase \\
\hline IG3-05 & INEEL CVS Phase 3 & Multi-phase \\
\hline IG3-07 & INEEL CVS Phase 3 & Multi-phase \\
\hline IG3-09 & INEEL CVS Phase 3 & Multi-phase \\
\hline IG3-13 & INEEL CVS Phase 3 & Multi-phase \\
\hline HLWMS-11 & RPP-WTP HLW Formulation & $\rho>3 \mathrm{~g} / \mathrm{cm}^{3}(3.37)$ \\
\hline HLW99-05 & RPP-WTP HLW Formulation & $\rho>3 \mathrm{~g} / \mathrm{cm}^{3}(3.15)$ \\
\hline IG2-12 & INEEL CVS Phase 2 & High $\mathrm{F}=0.1828$, for all other glasses $\mathrm{F} \leq 0.1137$ \\
\hline HLW98-34 & RPP-WTP HLW Formulation & High $\mathrm{SrO}=0.0495$, for all others $\mathrm{SrO} \leq 0.0278$ \\
\hline HLP-52 & HLP glasses & $\mathrm{B}_{2} \mathrm{O}_{3}=0$, for all others $\mathrm{B}_{2} \mathrm{O}_{3} \geq 0.0274$ \\
\hline HLW99-08 & RPP-WTP HLW Formulation & Outlier \\
\hline HLW99-13 & RPP-WTP HLW Formulation & Outlier \\
\hline SPA-43 & SPA Glasses & Outlier \\
\hline
\end{tabular}


Table 6-2. Mole Fraction Range for Components in Density Model Data Glasses

\begin{tabular}{|c|c|c|c|c|c|c|c|c|}
\hline Component & Min & Max & Component & Min & Max & Component & Min & Max \\
\hline $\mathrm{Al}_{2} \mathrm{O}_{3}$ & 0.0000 & 0.1325 & $\mathrm{ZnO}$ & 0.0000 & 0.0369 & I & 0.0000 & 0.0007 \\
\hline $\mathrm{B}_{2} \mathrm{O}_{3}$ & 0.0274 & 0.2113 & $\mathrm{BaO}$ & 0.0000 & 0.0213 & $\mathrm{MoO}_{3}$ & 0.0000 & 0.0062 \\
\hline $\mathrm{CaO}$ & 0.0000 & 0.1445 & $\mathrm{CdO}$ & 0.0000 & 0.0170 & $\mathrm{PbO}$ & 0.0000 & 0.0033 \\
\hline $\mathrm{Fe}_{2} \mathrm{O}_{3}$ & 0.0000 & \begin{tabular}{|l|}
0.0737 \\
\end{tabular} & $\mathrm{LN}_{2} \mathrm{O}_{3}$ & 0.0000 & 0.0111 & $\mathrm{PdO}$ & 0.0000 & 0.0007 \\
\hline $\mathrm{K}_{2} \mathrm{O}$ & 0.0000 & 0.0682 & \begin{tabular}{|l|}
$\mathrm{ThO}_{2}$ \\
\end{tabular} & 0.0000 & 0.0106 & $\mathrm{Rb}_{2} \mathrm{O}$ & 0.0000 & 0.0003 \\
\hline $\mathrm{Li}_{2} \mathrm{O}$ & 0.0000 & 0.1922 & $\mathrm{UO}_{\mathrm{x}}$ & 0.0000 & 0.0220 & $\mathrm{ReO}_{\mathrm{x}}$ & 0.0000 & 0.0003 \\
\hline $\mathrm{MgO}$ & 0.0000 & 0.1342 & $\mathrm{Ag}_{2} \mathrm{O}$ & 0.0000 & 0.0008 & $\mathrm{Rh}_{2} \mathrm{O}_{3}$ & 0.0000 & 0.0002 \\
\hline $\mathrm{Na}_{2} \mathrm{O}$ & 0.0451 & 0.2830 & $\mathrm{As}_{2} \mathrm{O}_{3}$ & 0.0000 & 0.0007 & $\mathrm{RuO}_{2}$ & 0.0000 & 0.0014 \\
\hline $\mathrm{NiO}$ & 0.0000 & 0.0292 & $\mathrm{Bi}_{2} \mathrm{O}_{3}$ & 0.0000 & 0.0115 & $\mathrm{Sb}_{2} \mathrm{O}_{\mathrm{x}}$ & 0.0000 & 0.0007 \\
\hline $\mathrm{P}_{2} \mathrm{O}_{5}$ & 0.0000 & \begin{tabular}{|l|l|}
0.0189 \\
\end{tabular} & $\mathrm{Br}$ & 0.0000 & 0.0006 & $\mathrm{SeO}_{2}$ & 0.0000 & 0.0020 \\
\hline $\mathrm{SiO}_{2}$ & 0.3276 & 0.6417 & $\mathrm{Cl}$ & 0.0000 & 0.0166 & $\mathrm{SnO}_{2}$ & 0.0000 & 0.0007 \\
\hline $\mathrm{ZrO}_{2}$ & 0.0000 & 0.0792 & $\mathrm{CoO}$ & 0.0000 & 0.0020 & $\mathrm{SO}_{3}$ & 0.0000 & 0.0085 \\
\hline $\mathrm{F}$ & 0.0000 & 0.1137 & $\mathrm{Cr}_{2} \mathrm{O}_{3}$ & 0.0000 & 0.0121 & $\mathrm{TeO}_{2}$ & 0.0000 & 0.0009 \\
\hline $\mathrm{MnO}_{\mathrm{x}}$ & 0.0000 & 0.0621 & $\mathrm{Cs}_{2} \mathrm{O}$ & 0.0000 & 0.0007 & $\mathrm{Tl}_{2} \mathrm{O}_{3}$ & 0.0000 & 0.0003 \\
\hline $\mathrm{SrO}$ & 0.0000 & 0.0278 & $\mathrm{CuO}$ & 0.0000 & 0.0023 & $\mathrm{~V}_{2} \mathrm{O}_{5}$ & 0.0000 & 0.0213 \\
\hline $\mathrm{TiO}_{2}$ & 0.0000 & 0.0739 & $\mathrm{Ga}_{2} \mathrm{O}_{3}$ & 0.0000 & 0.0018 & $\mathrm{WO}_{3}$ & 0.0000 & 0.0006 \\
\hline
\end{tabular}

Table 6-3 shows the composition range of 368 glasses in mass fraction. The components included in $\mathrm{LN}_{2} \mathrm{O}_{3}$ were separately shown in Table 6-3.

Table 6-3. Mass Fraction Range for Components in Density Model Data Glasses

\begin{tabular}{|c|c|c|c|c|c|c|c|c|}
\hline Component & Min & Max & Component & Min & Max & Component & Min & Max \\
\hline $\mathrm{Al}_{2} \mathrm{O}_{3}$ & 0.0000 & 0.2043 & $\mathrm{CdO}$ & 0.0000 & 0.0300 & $\mathrm{CuO}$ & 0.0000 & 0.0027 \\
\hline $\mathrm{B}_{2} \mathrm{O}_{3}$ & 0.0300 & 0.2001 & $\mathrm{ThO}_{2}$ & 0.0000 & 0.0410 & $\mathrm{Ga}_{2} \mathrm{O}_{3}$ & 0.0050 & 0.0050 \\
\hline $\mathrm{CaO}$ & 0.0000 & 0.1200 & $\mathrm{UO}_{3}$ & 0.0000 & 0.0847 & I & 0.0000 & 0.0013 \\
\hline $\mathrm{Fe}_{2} \mathrm{O}_{3}$ & 0.0000 & \begin{tabular}{|l|}
0.1577 \\
\end{tabular} & $\mathrm{Ce}_{2} \mathrm{O}_{3}$ & 0.0000 & 0.0044 & $\mathrm{MoO}_{3}$ & 0.0000 & 0.0135 \\
\hline $\mathrm{K}_{2} \mathrm{O}$ & 0.0000 & 0.0993 & $\mathrm{Gd}_{2} \mathrm{O}_{3}$ & 0.0000 & 0.0007 & $\mathrm{PbO}$ & 0.0000 & 0.0100 \\
\hline $\mathrm{Li}_{2} \mathrm{O}$ & 0.0000 & 0.0894 & $\mathrm{La}_{2} \mathrm{O}_{3}$ & 0.0000 & 0.0500 & $\mathrm{PdO}$ & 0.0000 & 0.0014 \\
\hline $\mathrm{MgO}$ & 0.0000 & 0.0800 & $\mathrm{Nd}_{2} \mathrm{O}_{3}$ & 0.0000 & 0.0513 & $\mathrm{Rb}_{2} \mathrm{O}$ & 0.0000 & 0.0009 \\
\hline $\mathrm{Na}_{2} \mathrm{O}$ & 0.0450 & 0.2514 & $\operatorname{Pr}_{6} \mathrm{O}_{11}$ & 0.0000 & 0.0019 & $\mathrm{ReO}_{2}$ & 0.0000 & 0.0011 \\
\hline $\mathrm{NiO}$ & 0.0000 & 0.0300 & $\mathrm{Sm}_{2} \mathrm{O}_{3}$ & 0.0000 & 0.0009 & $\mathrm{Rh}_{2} \mathrm{O}_{3}$ & 0.0000 & 0.0009 \\
\hline $\mathrm{P}_{2} \mathrm{O}_{5}$ & 0.0000 & 0.0402 & $\mathrm{Y}_{2} \mathrm{O}_{3}$ & 0.0000 & 0.0009 & $\mathrm{RuO}_{2}$ & 0.0000 & 0.0027 \\
\hline $\mathrm{SiO}_{2}$ & 0.2801 & 0.6044 & $\mathrm{Ag}_{2} \mathrm{O}$ & 0.0000 & 0.0025 & $\mathrm{Sb}_{2} \mathrm{O}_{3}$ & 0.0000 & 0.0032 \\
\hline $\mathrm{ZrO}_{2}$ & 0.0000 & 0.1548 & $\mathrm{As}_{2} \mathrm{O}_{3}$ & 0.0000 & 0.0020 & $\mathrm{SeO}_{2}$ & 0.0000 & 0.0036 \\
\hline $\mathrm{F}$ & 0.0000 & 0.0334 & $\mathrm{Bi}_{2} \mathrm{O}_{3}$ & 0.0000 & 0.0700 & $\mathrm{SnO}_{2}$ & 0.0000 & 0.0016 \\
\hline $\mathrm{MnO}$ & 0.0000 & 0.0600 & $\mathrm{Br}$ & 0.0005 & 0.0008 & $\mathrm{SO}_{3}$ & 0.0000 & 0.0110 \\
\hline $\mathrm{SrO}$ & 0.0000 & 0.0405 & $\mathrm{Cl}$ & 0.0000 & 0.0085 & $\mathrm{TeO}_{2}$ & 0.0000 & 0.0020 \\
\hline $\mathrm{TiO}_{2}$ & 0.0000 & 0.0859 & $\mathrm{CoO}$ & 0.0000 & 0.0020 & $\mathrm{Tl}_{2} \mathrm{O}_{3}$ & 0.0000 & 0.0020 \\
\hline $\mathrm{ZnO}$ & 0.0000 & \begin{tabular}{|l|}
0.0430 \\
\end{tabular} & $\mathrm{Cr}_{2} \mathrm{O}_{3}$ & 0.0000 & 0.0297 & $\mathrm{~V}_{2} \mathrm{O}_{5}$ & 0.0000 & 0.0570 \\
\hline $\mathrm{BaO}$ & 0.0000 & \begin{tabular}{|l|}
0.0470 \\
\end{tabular} & $\mathrm{Cs}_{2} \mathrm{O}$ & 0.0000 & 0.0027 & $\mathrm{WO}_{3}$ & 0.0000 & 0.0020 \\
\hline
\end{tabular}




\subsection{Initial Model Development}

\subsubsection{General Forms of the Density and Molar Volume Models}

The composition model for density can have different forms, depending on which properties are used for modeling, i.e., density, molar volume, or specific volume. Molar volume $(V)$ is expressed as

$$
V=\frac{\sum_{i=1}^{N} M_{i} x_{i}}{\rho}
$$

where

$$
\begin{aligned}
M_{i} & =\text { molecular weight of } i \text {-th oxide component } \\
x_{i} & =\text { mole fraction of } i \text {-th component } \\
N & =\text { number of oxide component in glass } \\
\rho & =\text { density of glass. }
\end{aligned}
$$

When the mass fraction is used for glass composition, it is common to use the specific volume $(1 / \rho)$.

The general form of the composition models for $\rho$ or $V$ can be written as

$$
\rho=\sum_{i=1}^{N} \rho_{i} x_{i}, \text { or } V=\sum_{i=1}^{N} V_{i} x_{i}
$$

where $\rho_{i}$ and $V_{i}$ are the ${ }_{\mathrm{i}}$-th component partial molar density and partial molar volume. When the specific volume is used, $x_{i}$ is replaced by the mass fraction, $g_{i}$, in Equation 6.2. These models are linear-mixture models of the form (1.4) presented in Section 1.3.

Only a limited number of components can be included in modeling, although the present glass composition-property database is based on 71 glass components, mainly because some components are present only in a very small number of glasses. For components not included in the model, two different approaches can be applied: 1) use an Others component as a sum of all the components not included in the model and 2) use compositions normalized to sum to one after deleting the components not included in the model. Therefore, the density models of four different forms are considered: 1) $\rho$-based with the Others component, 2) $V$-based with the Others component, 3) $\rho$-based with normalized composition, and 4) $V$-based with normalized composition.

The advantage of using density is its simplicity compared to using molar volume, which requires a conversion from density for model prediction. However, using molar volume has an advantage in that it can lead to a better understanding of the relation between the model coefficients and the effect of each component on glass structure. This can lead to estimation of the component coefficients that are not available from the model fit or in the literature to make the model more applicable to wider composition regions. 


\subsubsection{Selection of Model Forms for Density Modeling}

To make a fair comparison between models, the 368 glasses in the density data set after the first screening were sorted in the order of density (then glass ID) and were divided into two subsets by reserving every fifth glass for use in validating models. This process resulted in 73 validation glasses. The other 295 glasses were used for model development, i.e., model glasses. The following 15 components selected as model components in preliminary modeling studies ${ }^{(\mathrm{v})}$ were used to evaluate the different model forms: $\mathrm{Al}_{2} \mathrm{O}_{3}, \mathrm{~B}_{2} \mathrm{O}_{3}, \mathrm{CaO}, \mathrm{Fe}_{2} \mathrm{O}_{3}, \mathrm{~K}_{2} \mathrm{O}, \mathrm{Li}_{2} \mathrm{O}, \mathrm{MgO}, \mathrm{Na}_{2} \mathrm{O}, \mathrm{SiO}_{2}, \mathrm{ZrO}_{2}, \mathrm{~F}, \mathrm{MnO}_{\mathrm{x}}, \mathrm{SrO}$, $\mathrm{TiO}_{2}$, and $\mathrm{ZnO}$.

The results of the model statistics for four different model forms are summarized in Table 6-4. The $\mathrm{R}_{\text {val }}^{2}$ is calculated for glasses that were not used in the model development but reserved for validation. When comparing the models calculated based on different properties, it is necessary to compare the $\mathrm{R}^{2}$ statistics that were calculated based on the same property, in this case, the density. For models based on molar volume, the $\mathrm{R}^{2}$ statistics calculated based on density are also included in Table $6-4$. The $\mathrm{R}^{2}$ statistics that were calculated based on density are more important because the main objective of the modeling efforts is to be able to predict the density.

The models using an Others component as the sum of all the components not included in the model have better $\mathrm{R}^{2}$ statistics than the models using compositions normalized to sum to one after deleting the components not included in the model. This suggests that one or more components included in Others have an effect on density. Models for density and molar volume when an Others component is used have $\mathrm{R}^{2}$ values almost the same, but the molar-volume model had a better $\mathrm{R}_{\text {val }}^{2}$ value. In summary, the molarvolume-based model using an Others component performed best.

Table 6-4. Comparison of Model Statistics for Different Density Model Forms from the Reduced Data Set, where $20 \%$ of Data were Not Included in the Model Development But Were Used for Model Validation

\begin{tabular}{||l|c|c|c|c||}
\cline { 2 - 5 } \multicolumn{1}{c|}{} & \multicolumn{4}{c||}{ Model } \\
\cline { 2 - 5 } \multicolumn{1}{c|}{} & Using Others Component & \multicolumn{2}{c||}{ Using Normalized Composition } \\
\hline \hline \multicolumn{1}{|c|}{ Model Statistics } & Density & Molar Volume & Density & Molar Volume \\
\hline \hline Number of glasses & 295 & 295 & 295 & 295 \\
\hline $\mathrm{R}^{2}$ & 0.900 & 0.930 & 0.765 & 0.924 \\
\hline $\mathrm{R}_{\text {adj }}^{2}$ & 0.895 & 0.927 & 0.752 & 0.920 \\
\hline $\mathrm{R}_{\text {val }}^{2}$ & 0.876 & 0.941 & 0.731 & 0.930 \\
\hline \hline \multicolumn{5}{|c|}{$R^{2}$ Statistics Calculated Based on Density } \\
\hline $\mathrm{R}^{2}$ & 0.900 & 0.893 & 0.765 & 0.883 \\
\hline $\mathrm{R}_{\text {adj }}^{2}$ & 0.895 & 0.888 & 0.752 & 0.876 \\
\hline $\mathrm{R}_{\text {val }}^{2}$ & 0.876 & 0.916 & 0.731 & 0.900 \\
\hline \hline
\end{tabular}

(a) The data set used in preliminary modeling studies had 248 glasses and contained the glasses of which density was re-measured later because some of their data were suspect (Hanford CVS 1 and 2 glasses). 
One thing to note in Table 6-4 is that in molar-volume models, the $\mathrm{R}_{\text {val }}^{2}$ is larger than the model $\mathrm{R}^{2}$ values. This rather unusual result seems to be caused by a few outlying data points included in model glasses. Table 6-5 shows the $\mathrm{R}^{2}$ comparisons when all 368 the glasses were used for model calculation. There was little difference between density-based and $V$-based models. In this model, however, it was found that the three glasses that had been poorly predicted had a strong influence on the model in $V$-based models. So the model calculations were also compared for this reduced data set after removing these three glasses. By removing the three glasses, the $\mathrm{R}^{2}$ increased slightly. For the final density modeling, these three glasses were deleted (as listed in Table 6-1). In conclusion, the models with an Others component are better than the models with normalized compositions. The $V$-based model is at least equal to or better than the density-based model.

Table 6-5. Comparison of Model Statistics for Different Model Forms for Different Data Sets; all $\mathrm{R}^{2}$ Statistics are Calculated Based on Density

\begin{tabular}{|c|c|c|c|c|}
\hline & \multicolumn{4}{|c|}{ Model } \\
\hline & \multicolumn{2}{|c|}{ Using Others Component } & \multicolumn{2}{|c|}{ Using Normalized Composition } \\
\hline Model Statistics & Density & Molar Volume & Density & Molar Volume \\
\hline \multicolumn{5}{|c|}{ Using all 368 glasses } \\
\hline Number of glasses & 368 & 368 & 368 & 368 \\
\hline $\mathrm{R}^{2}$ & 0.899 & 0.900 & 0.765 & 0.889 \\
\hline $\mathrm{R}_{\text {adj }}^{2}$ & 0.894 & 0.895 & 0.752 & 0.883 \\
\hline \multicolumn{5}{|c|}{ Using 365 glasses after deleting three outliers } \\
\hline Number of glasses & 365 & 365 & 365 & 365 \\
\hline $\mathrm{R}^{2}$ & 0.894 & 0.910 & 0.778 & 0.897 \\
\hline $\mathrm{R}_{\text {adj }}^{2}$ & 0.890 & 0.906 & 0.766 & 0.893 \\
\hline
\end{tabular}

In selecting the proper model forms, the simplicity of the density-based model is appealing from a practical-application point of view. However, the better performance and other benefits discussed in Section 6.3.1 can justify the use of a molar-volume-based model. Hence, the molar-volume-based model with an Others component was selected for density modeling. The 365 glasses after deleting three outliers were used for model development in the following section.

\subsection{Model Evaluation and Selection}

\subsubsection{Final Density Model Based on Molar Volume}

The molar-volume model that used 365 glasses was initially developed with 23 components (22 components selected in Section 6.2 plus Others), which were identified as the maximum number of components that have reasonable ranges of mole fractions and distributions of mol-fraction values within the range to consider including them in the density models. Then the next models were calculated based on the reduced number of components, removing the component(s) of higher uncertainty or of less importance. As mentioned earlier, see Piepel and Redgate (1997) for description of more rigorous method for component removal from model. Table 6-6 shows which components were included in the 23-, 21-, 20-, 18-, 17-, and 16-component molar-volume-based models by providing model coefficient values, i.e., 
partial molar volumes. Summary statistics from the regression analysis for each of these models based on molar volume and $\mathrm{R}^{2}$ statistics calculated based on density are also included in Table 6-6.

Table 6-6. Summary Statistics for Molar-Volume-Based Models for Density Modeling

\begin{tabular}{|c|c|c|c|c|c|c|}
\hline \multirow[b]{2}{*}{ Component } & \multicolumn{6}{|c|}{ Model } \\
\hline & 23-Comp. & 21-Comp. & 20-Comp. & 18-Comp. & 17-Comp. & 16-Comp. \\
\hline $\mathrm{Al}_{2} \mathrm{O}_{3}$ & 46.169 & 46.084 & 46.076 & 46.149 & 45.551 & 46.148 \\
\hline $\mathrm{B}_{2} \mathrm{O}_{3}$ & 30.268 & 30.200 & 30.068 & 30.048 & 30.095 & 29.899 \\
\hline $\mathrm{CaO}$ & 14.848 & 14.891 & 15.231 & 15.214 & 16.211 & 15.727 \\
\hline $\mathrm{Fe}_{2} \mathrm{O}_{3}$ & 40.287 & 39.833 & 39.215 & 39.158 & 37.110 & 38.706 \\
\hline $\mathrm{K}_{2} \mathrm{O}$ & 35.166 & 35.879 & 36.855 & 37.741 & 35.147 & 36.126 \\
\hline $\mathrm{Li}_{2} \mathrm{O}$ & 10.018 & 9.926 & 9.946 & 9.943 & 9.871 & 9.581 \\
\hline $\mathrm{MgO}$ & 13.076 & 12.967 & 13.105 & 13.028 & 13.376 & 12.863 \\
\hline $\mathrm{Na}_{2} \mathrm{O}$ & 19.849 & 19.954 & 19.797 & 19.834 & 20.033 & 19.825 \\
\hline $\mathrm{SiO}_{2}$ & 25.264 & 25.319 & 25.341 & 25.316 & 25.509 & 25.489 \\
\hline $\mathrm{ZrO}_{2}$ & 26.494 & 26.647 & 27.275 & 27.081 & 26.861 & 27.042 \\
\hline $\mathrm{F}$ & 5.704 & 6.634 & 7.788 & 7.526 & 9.262 & 7.422 \\
\hline $\mathrm{MnO}_{\mathrm{x}}$ & 10.611 & 11.164 & 12.566 & 13.175 & & 13.142 \\
\hline $\mathrm{SrO}$ & 14.703 & 17.745 & 18.167 & 17.611 & 10.733 & 16.744 \\
\hline $\mathrm{TiO}_{2}$ & 18.458 & 18.213 & 17.913 & 17.964 & 16.456 & 17.170 \\
\hline $\mathrm{ZnO}$ & 14.059 & 15.545 & 13.884 & 15.069 & 19.312 & 18.398 \\
\hline $\mathrm{NiO}$ & 30.007 & 20.500 & 13.773 & 12.668 & 21.202 & \\
\hline $\mathrm{P}_{2} \mathrm{O}_{5}$ & 64.435 & 63.405 & & & & \\
\hline $\mathrm{BaO}$ & 22.767 & 20.115 & 17.309 & 18.866 & 24.713 & \\
\hline $\mathrm{CdO}$ & 3.757 & & & & & \\
\hline $\mathrm{Ln}_{2} \mathrm{O}_{3}$ & 50.709 & 43.808 & 29.078 & & & \\
\hline $\mathrm{ThO}_{2}$ & 82.812 & & & & & \\
\hline $\mathrm{UO}_{\mathrm{x}}$ & 32.308 & 39.944 & 42.121 & & & \\
\hline Others & 41.782 & 34.392 & 44.749 & 42.812 & 29.431 & 32.725 \\
\hline \multicolumn{7}{|c|}{ Model Statistics } \\
\hline Number of Glasses & 365 & 365 & 365 & 365 & 365 & 365 \\
\hline $\mathrm{R}^{2}$ & 0.954 & 0.952 & 0.949 & 0.949 & 0.934 & 0.942 \\
\hline $\mathrm{R}_{\text {adj }}^{2}$ & 0.951 & 0.949 & 0.946 & 0.946 & 0.931 & 0.939 \\
\hline $\mathrm{R}_{\text {pred }}^{2}$ & 0.942 & 0.944 & 0.942 & 0.942 & 0.924 & 0.935 \\
\hline s (RMSE) & 0.2453 & 0.2507 & 0.2574 & 0.2577 & 0.2921 & 0.2729 \\
\hline \multicolumn{7}{|c|}{$R^{2}$ Statistics Based on Density } \\
\hline $\mathrm{R}^{2}$ & 0.930 & 0.926 & 0.922 & 0.921 & 0.896 & 0.910 \\
\hline $\mathrm{R}_{\text {adj }}^{2}$ & 0.925 & 0.922 & 0.918 & 0.918 & 0.892 & 0.906 \\
\hline
\end{tabular}


When selecting the components to remove for the next model with a reduced number of components, the following were considered: 1 ) the ratio of a component coefficient to its standard deviation, ${ }^{(\mathrm{w})} 2$ ) the comparison of component coefficients with the partial molar volume data found in literature, and 3) the relative importance of the components to the application for Hanford waste. For example, the partial molar volume for $\mathrm{CdO}$ and $\mathrm{ThO}_{2}$ in the 23-component model in Table 6-6 were unreasonably low or high compared to the literature values (Table 6-7 below), indicating that these components may not be appropriate for inclusion in the model component, which was also supported by the low ratio of the component coefficient to its standard deviation - not shown in Table 6-6.

In selecting the components for the final model, consideration was given that it would be useful to have as many components as possible with reasonably low uncertainty, i.e., reliability. Before making final selection of the model components, the model coefficients obtained in this study were compared with literature data by Appen (1970). The partial molar volumes for 34 components were published, which are reproduced in Table 6-7. For $\mathrm{B}_{2} \mathrm{O}_{3}, \mathrm{SiO}_{2}, \mathrm{TiO}_{2}, \mathrm{CdO}$, and $\mathrm{PbO}$, the coefficients are given as a range of coefficients, which vary with the range of specified component compositions.

Table 6-7. Partial Molar Volumes for Glass Components Published by Appen (1970)

\begin{tabular}{||l|c||l|c||}
\hline Component & Coefficient & Component & Coefficient \\
\hline $\mathrm{Al}_{2} \mathrm{O}_{3}$ & 40.4 & $\mathrm{Na}_{2} \mathrm{O}$ & 20.2 \\
\hline $\mathrm{B}_{2} \mathrm{O}_{3}$ & $18.5-38.0$ & $\mathrm{Nb}_{2} \mathrm{O}_{5}$ & 56 \\
\hline $\mathrm{BaO}$ & 22 & $\mathrm{NiO}$ & 13 \\
\hline $\mathrm{BeO}$ & 7.8 & $\mathrm{PbO}$ & $21.0-23.5$ \\
\hline $\mathrm{Bi}_{2} \mathrm{O}_{3}$ & 45 & $\mathrm{Rb}_{2} \mathrm{O}$ & 43 \\
\hline $\mathrm{CaO}$ & 14.4 & $\mathrm{Sb}_{2} \mathrm{O}_{3}$ & 47 \\
\hline $\mathrm{CdO}$ & $17.0-18.2$ & $\mathrm{Sc}_{2} \mathrm{O}_{3}$ & 28 \\
\hline $\mathrm{CoO}$ & 14.5 & $\mathrm{SiO}_{2}$ & $26.1-27.25$ \\
\hline $\mathrm{Cs} \mathrm{S}_{2} \mathrm{O}$ & 47 & $\mathrm{SnO}_{2}$ & 28.8 \\
\hline $\mathrm{FeO}$ & 16.5 & $\mathrm{SrO}$ & 17.5 \\
\hline $\mathrm{Ga}_{2} \mathrm{O}_{3}$ & 42.5 & $\mathrm{Ta}_{2} \mathrm{O}_{3}$ & 52 \\
\hline $\mathrm{HfO} \mathrm{H}_{2}$ & 27.5 & $\mathrm{ThO}_{2}$ & 31.7 \\
\hline $\mathrm{K}_{2} \mathrm{O}$ & 34.1 & $\mathrm{TiO}_{2}$ & $19.0-22.5$ \\
\hline $\mathrm{Li}_{2} \mathrm{O}$ & 11 & $\mathrm{Tl}_{2} \mathrm{O}$ & 63 \\
\hline $\mathrm{La}_{2} \mathrm{O}_{3}$ & 40 & $\mathrm{Y}_{2} \mathrm{O}_{3}$ & 35 \\
\hline $\mathrm{MgO}$ & 12.5 & $\mathrm{ZnO}_{3}$ & 14.5 \\
$\mathrm{MnO}^{\mathrm{MnO} O}$ & 17.2 & $\mathrm{ZrO}_{2}$ & 23 \\
\hline
\end{tabular}

The coefficients obtained from the 21-component model are compared with Appen's coefficient in Figure 6-1. For those components with the range of coefficient values, i.e., $\mathrm{CdO}, \mathrm{PbO}, \mathrm{SiO}_{2}$, and $\mathrm{TiO}_{2}$, center values were used in Figure 6-1 with the error bars indicating the range of coefficients. The coefficient of $\mathrm{La}_{2} \mathrm{O}_{3}$ in Appen (1970) was used to compare with that of $\mathrm{LN}_{2} \mathrm{O}_{3}$ in the model fit, and the

(w) The ratio of a coefficient to its standard error forms a t-statistic, which is used to assess whether the coefficient is statistically different from zero. In a linear-mixture model, this hypothesis is not directly related to the significance of the effect of the component coefficient. However, by considering several other things, this information can be considered in reducing the number of components in a model. 
coefficient of $\mathrm{FeO}$ was multiplied by two to compare with that of $\mathrm{Fe}_{2} \mathrm{O}_{3}$ in the model fit. Also included in Figure 6-1 are two lines. One is the 45-degree line representing the perfect agreement between Appen's and linear mixture model coefficients. The other is the least-squares fit between the two sets of coefficients, with equation $y=0.7982 x+3.6581$ and $R^{2}=0.9216$. In the least-squares fit for the components with coefficient ranges, the center value was used.

As can be seen in Figure 6-1, the agreement between two coefficient sets is reasonable except for two components, $\mathrm{MnO}_{\mathrm{x}}$ and $\mathrm{NiO}$. This may indicate that the reliability of the model coefficients for $\mathrm{MnO}_{\mathrm{x}}$ and $\mathrm{NiO}$ obtained in the 21-component model may not be good enough because of the lack of a sufficient data range. The similar comparison for the 18-component model is shown in Figure 6-2, with the fitted line given by $\mathrm{y}=0.7802 \mathrm{x}+4.2796$ with $\mathrm{R}^{2}=0.9548$. Comparing Figure 6-2 with Figure 6-1 shows better agreement between the 18-component model and Appen coefficients compared to the 21component model, which is also supported by a slighltly larger $\mathrm{R}^{2}$ value. The discrepancy in $\mathrm{MnO}_{\mathrm{x}}$ and $\mathrm{NiO}$ coefficients is decreased.

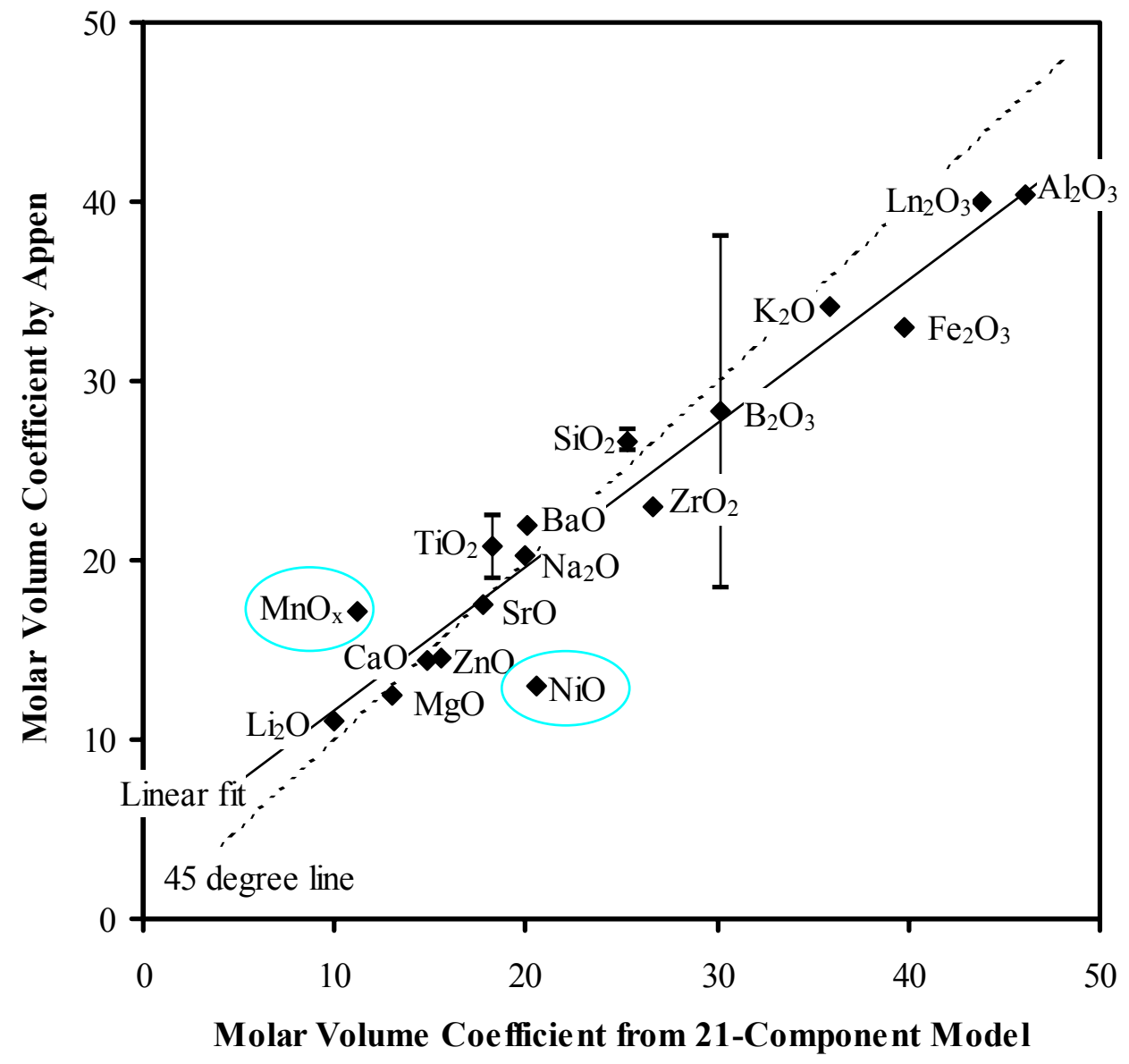

Figure 6-1. Plot of Appen's Molar Volume Coefficients Versus Model Fit Coefficients from the 21Component Linear Mixture Model (the bars on selected points cover range of reported values with mean values used as data points) 


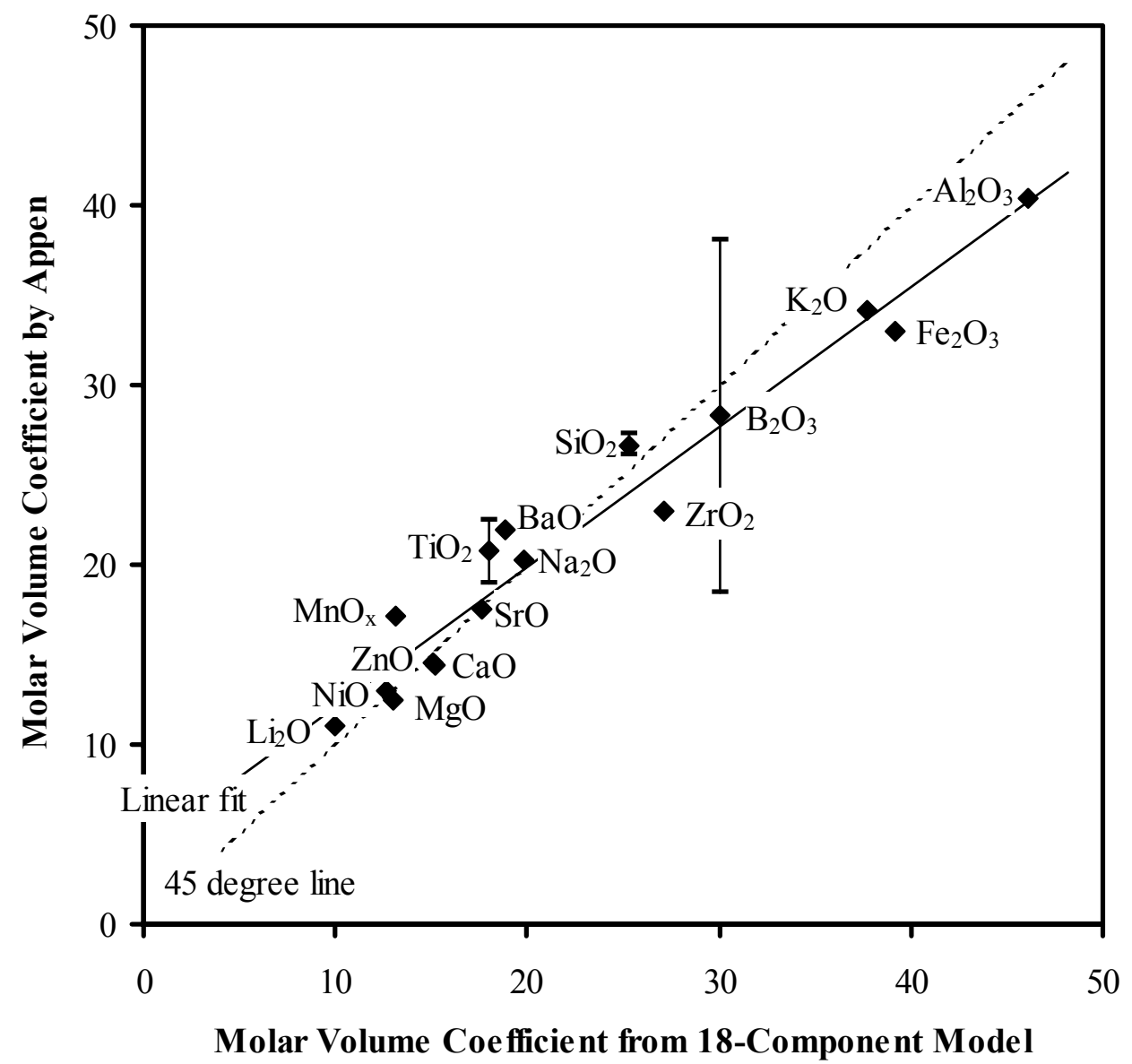

Figure 6-2. Plot of Appen's Molar Volume Coefficients versus Model Fit Coefficients from the 18Component Linear Mixture Model (error bars cover range of reported values with mean values used as data points)

In both Figure 6-1 and Figure 6-2, the linear-fit line deviates from 45-degree line, indicating that there are differences in the partial molar volumes determined by fitting a linear-mixture model as compared to Appen's values. The linear-mixture model coefficients are significant extrapolations of the partial molar volumes because the data used to fit the linear-mixture models are for a constrained subspace of the full mixture space. The overall agreement is good considering that Appen's coefficients were mainly obtained from 3 to 5 component glasses, which may imply that the coefficients for those components can be extrapolated from rather simple glasses to the multi-component HLW glasses.

Based on the above analysis, the 18-component model was selected as the final model. The model coefficients, standard errors, and the regression statistics are included in Table 6-8. 
Table 6-8. Model Coefficients and Standard Errors for the Final 18-Component Linear Mixture Model for Molar Volume

\begin{tabular}{|c|c|c|}
\hline & \multicolumn{2}{|c|}{ Final Molar Volume Model } \\
\hline Component & Coefficient & Std. Error \\
\hline $\mathrm{Al}_{2} \mathrm{O}_{3}$ & 46.149 & 0.577 \\
\hline $\mathrm{B}_{2} \mathrm{O}_{3}$ & 30.048 & 0.342 \\
\hline $\mathrm{CaO}$ & 15.214 & 0.456 \\
\hline $\mathrm{Fe}_{2} \mathrm{O}_{3}$ & 39.158 & 0.947 \\
\hline $\mathrm{K}_{2} \mathrm{O}$ & 37.741 & 1.381 \\
\hline $\mathrm{Li}_{2} \mathrm{O}$ & 9.943 & 0.347 \\
\hline $\mathrm{MgO}$ & 13.028 & 0.507 \\
\hline $\mathrm{Na}_{2} \mathrm{O}$ & 19.834 & 0.308 \\
\hline $\mathrm{SiO}_{2}$ & 25.316 & 0.133 \\
\hline $\mathrm{ZrO}_{2}$ & 27.081 & 0.993 \\
\hline $\mathrm{F}$ & 7.526 & 0.995 \\
\hline $\mathrm{MnO}_{\mathrm{x}}$ & 13.175 & 2.127 \\
\hline $\mathrm{SrO}$ & 17.611 & 3.656 \\
\hline $\mathrm{TiO}_{2}$ & 17.964 & 1.568 \\
\hline $\mathrm{ZnO}$ & 15.069 & 2.075 \\
\hline $\mathrm{NiO}$ & 12.668 & 4.350 \\
\hline $\mathrm{BaO}$ & 18.866 & 3.709 \\
\hline Others & 42.812 & 1.917 \\
\hline \multicolumn{3}{|c|}{ Model Statistics } \\
\hline Number of Glasses & \multicolumn{2}{|c|}{365} \\
\hline $\mathrm{R}^{2}$ & \multicolumn{2}{|c|}{0.949} \\
\hline $\mathrm{R}_{\text {adj }}^{2}$ & \multicolumn{2}{|c|}{0.946} \\
\hline $\mathrm{R}_{\text {pred }}^{2}$ & \multicolumn{2}{|c|}{0.942} \\
\hline s (RMSE) & \multicolumn{2}{|c|}{0.2577} \\
\hline \multicolumn{3}{|c|}{$R^{2}$ Statistics Based on Density } \\
\hline $\mathrm{R}^{2}$ & \multicolumn{2}{|c|}{0.921} \\
\hline $\mathrm{R}_{\text {adj }}^{2}$ & \multicolumn{2}{|c|}{0.918} \\
\hline
\end{tabular}

Figure 6-3 is the calculated-versus-measured plot for the data points used to fit the density model. Figure 6-4 shows the calculated-versus-measured plot for the glasses that were deleted from the screening process as discussed in Section 6.1. Surprisingly, the glasses deleted because of extreme density and concentration values fit the model very well. For those glasses deleted because they were crystallized, only two glasses showed the noticeable deviation from the model, which may suggest that the crystallinity in rest of the glasses was sufficiently low so that the density was not affected. Based on the observations in Figure 6-4 and also considering the high $\mathrm{R}_{\text {val }}^{2}$ values discussed in Section 6.3.2, the present density model may be extrapolated outside the composition region used to develop the model with reasonably high confidence. 
The two glasses with noticeable deviation from the model (Figure 6-4) had unusually low density $\left(\sim 2.3 \mathrm{~g} / \mathrm{cm}^{3}\right)$ below the expected limit for typical multicomponent glasses, considering that the density of pure crystalline $\mathrm{SiO}_{2}$ (cristobalite) is $2.3 \mathrm{~g} / \mathrm{cm}^{3}$ and $\mathrm{SiO}_{2}$ glass is $2.2 \mathrm{~g} / \mathrm{cm}^{3}$. The observed lower bound of density for the glasses used in the model was $2.4 \mathrm{~g} / \mathrm{cm}^{3}$ as seen in Figure 6-3, which seems reasonable.

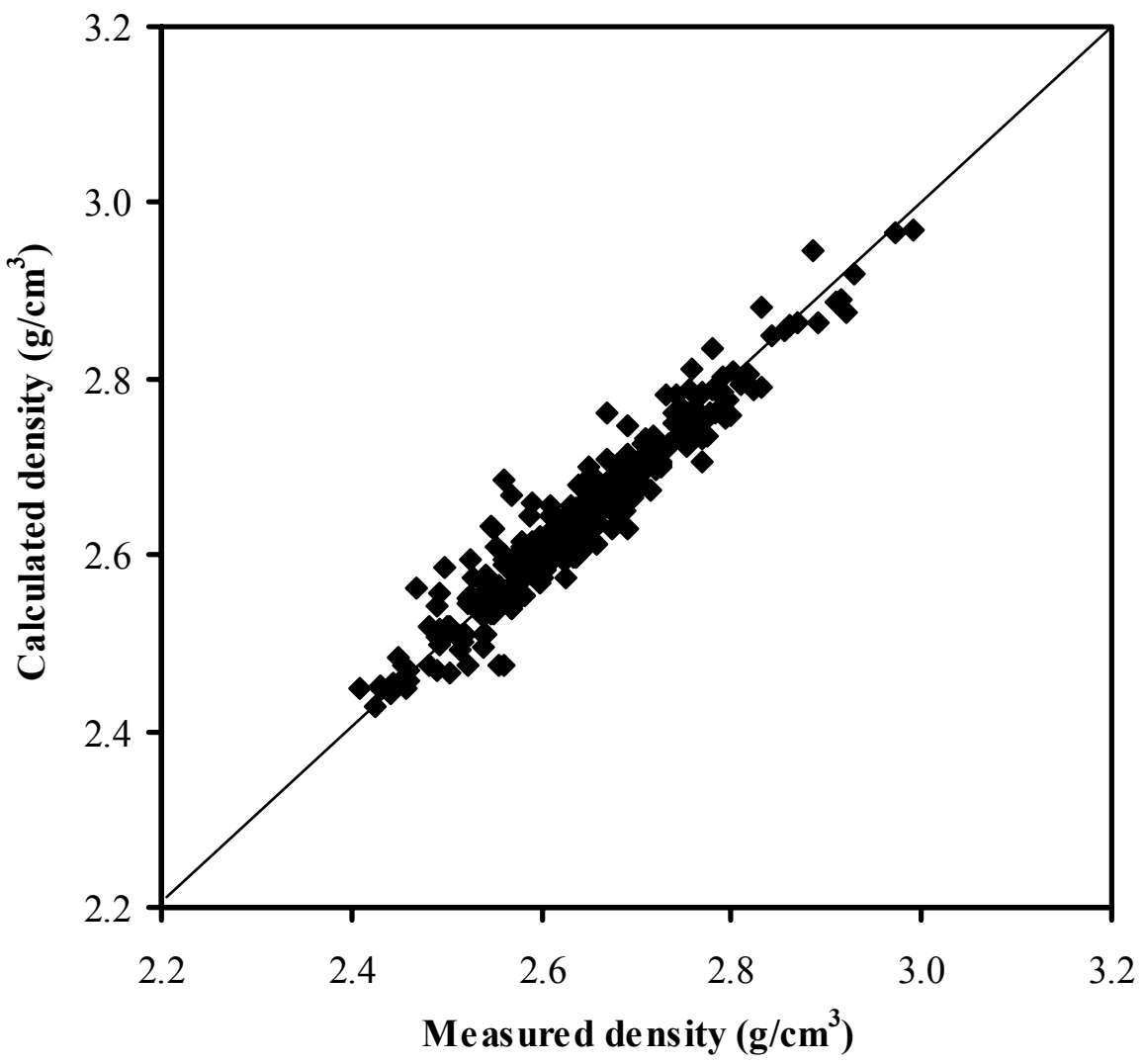

Figure 6-3. Plot of Calculated Versus Measured Density Values for Data Used to Fit the 18-Component Molar Volume Model 


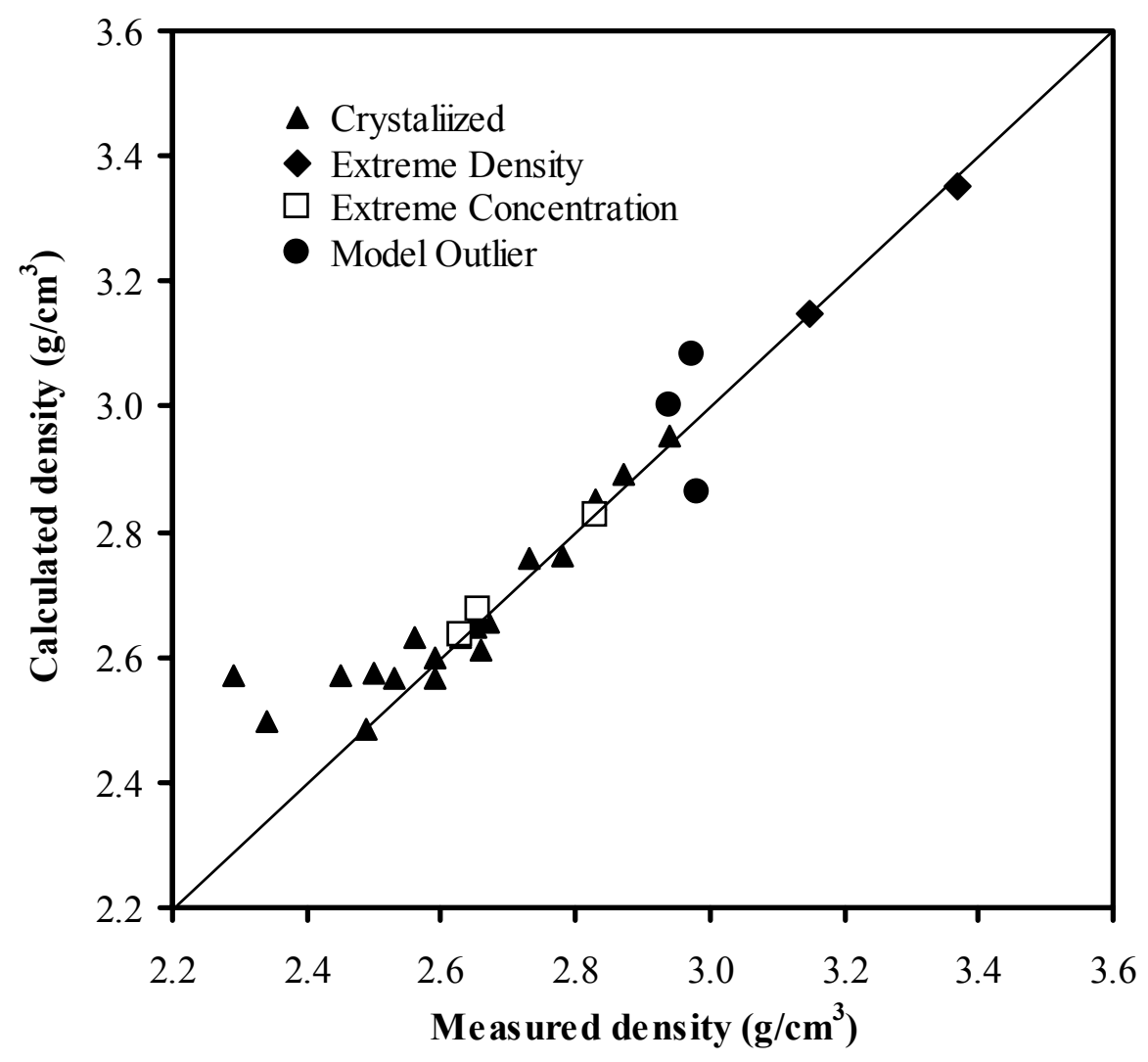

Figure 6-4. Plot of Calculated Versus Measured Density Values for the Glasses Deleted and Not Used to Develop the 18-Component Linear Mixture Model

\subsubsection{Density Model with Expanded Model Components}

As discussed in Section 6.4.1, the reasonable agreement between model coefficients and Appen's coefficients suggest that Appen's coefficients can be used for those components that could not be included in the model because of lack of data. Although Appen's coefficients can cover the majority of components of interest to HLW glass formulation, there are still some important components that are not included in Appen, like $\mathrm{UO}_{3}, \mathrm{P}_{2} \mathrm{O}_{5}$, etc.

Considering that the molar volume of glass will be proportional to the volume of cation and oxygen ions, the partial molar volume per cation structural unit (not oxide) can be expressed as a sum of the contributions by cation and oxygen ions as indicated below:

$$
v_{i}=a r_{O}^{3} \frac{Z_{i}}{2}+b r_{i}^{3}
$$

where 


$$
\begin{aligned}
Z_{i} & =\text { valence of cation in component } i \\
v_{i} & =\text { "apparent" partial molar volume per cation }\left(v_{i}=V_{i} \text { if } Z_{i}=2,4,6 ; v_{i}=1 / 2 V_{i} \text { if } Z_{i}=1,3,5\right) \\
r_{i} & =\text { ionic radius of cation } \\
r_{O} & =\text { radius of oxygen ion } \\
a \text { and } b & =\text { constants. }
\end{aligned}
$$

The expression, "apparent," was used because $v_{i}$ is not exactly the partial molar volume per cation obtained from the condition that satisfies $\Sigma y_{i}=1$, where $y_{i}$ is the mole fraction of cation in glass normalized to total cations. Here the term, $r_{O}{ }^{3} Z_{i} / 2$, represents the volume of oxygen ions per cation, while $r_{i}^{3}$ represents the volume of the cation. If the molar volume depends only on the total volume of anions and cations, then $a$ equals $b$ in Equation 6.3. Then, $v_{i}$ in Equation 6.3 will be inversely proportional to the overall packing fraction. However, Equation 6.3 assumes that the contribution to the packing fraction by oxygen would be different from that by cations, depending on the structure of glass to obtain two empirical constants, $a$ and $b$. Rewriting Equation 6.3 leads to

$$
\frac{v_{i}}{Z_{i}}=a^{\prime}+b \frac{r_{i}^{3}}{Z_{i}}
$$

where $a^{\prime}=a r_{O}^{3} / 2$.

For the purpose of applying Equation 6.4, it would be more appropriate to calculate the model coefficients based on the concentration of cations instead of oxides. However, since the Appen coefficients are given as oxide coefficients, the models were fit based on oxides to make direct comparison with Appen and to obtain combined coefficients with Equation 6.4.

The 18-component model coefficients, 17 glass components plus Others, from Table 6-8 and Appen's coefficients were fitted to the Equation 6.4 by plotting the $v_{i} / Z_{i}$ as a function of $r_{i}{ }^{3} / Z_{i}$, as shown in Figure 6-5, to obtain the constants $a$ ' and $b$. Table 6-9 summarizes constants $a$ ' and $b$ for three different cases of model fit, Appen, and both. 


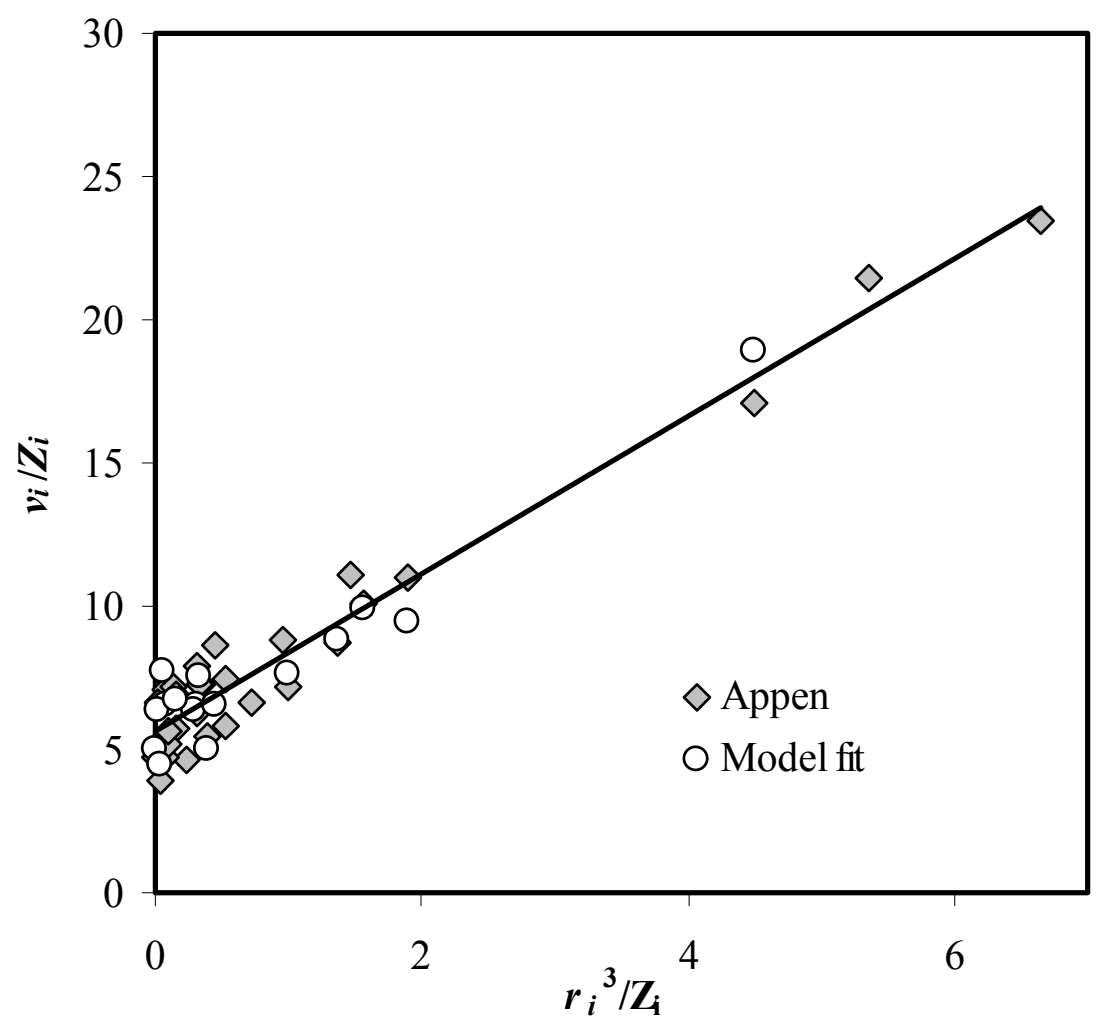

Figure 6-5. Plot of Appen's Molar Volume Coefficients Versus Model Fit Coefficients from 18-Component Model

Table 6-9. Constants in Equation 6.4

\begin{tabular}{||l|c|c|c||}
\hline \hline & Model Fit & Appen & Model Fit and Appen \\
\hline \# of data points & 16 & 33 & 49 \\
\hline$a^{\prime}$ & 5.56 & 5.59 & 5.58 \\
\hline$B$ & 2.76 & 2.76 & 2.76 \\
\hline $\mathrm{R}^{2}$ & 0.9119 & 0.9508 & 0.9428 \\
\hline
\end{tabular}

The values $a$ ' and $b$ are the proportionality constants for the change of molar volume as the number of oxygens per cation, i.e., valence of cation, and the volume of the cation change. Applying the $r_{O}=1.28 \AA$, then $a \sim a$ '. So the fit results, $a \sim 5.6$ and $\mathrm{b} \sim 2.8$, imply that the molar-volume change is almost twice by the added number of anions (oxygen) from higher valence cations than the increase of cation size. This is what would be expected if the packing fraction of ions were about 0.5 . However, the packing fraction cannot be estimated from these constants because the $v_{i}$ in this study is not true partial molar volume per cation.

Based on above discussion, the model coefficients and Appen data fit Equation 6.4 well, which may imply that Equation 6.4 can be extrapolated to the components not covered by Model and Appen data. The second and the third columns in Table 6-10 show the fit statistics obtained when Appen coefficients and Equation 6.4 are used to calculate the molar volume and density. The 18-component model results are also shown for comparison, and the combined final expanded model is given in the last column. In 
the Appen column, only the two fit parameters, $\mathrm{F}$ and Others coefficients, are calculated with the least squares method. The parameters $a$ and $b$ are additional fit parameters in Equation 6.4. The component $\mathrm{Ln}_{2} \mathrm{O}_{3}$ is no longer used in the models with expanded components.

The Appen coefficients and Equation 6.4 predict the multi-component HLW glasses reasonably well, although they are subject to many inevitable errors. The Appen coefficients were obtained from rather simple glasses. Also, some of them, such as $\mathrm{B}_{2} \mathrm{O}_{3}$ given as concentration range, were dependent on the glass composition, which was not considered in this study. The use of Equation 6.4 also involves several inherent errors because 1) only one valence state of the multivalent cations was assumed, 2) the composition dependence of coordination number of cations was not considered, and 3) the ionic radii of cations and oxygen in crystals were used.

In summary, the 18-component linear mixture model is recommended for glasses with a small total fraction of Others component, and the combined model in Table 6-10 is recommend for glasses with a relatively high concentration of those components included in Others. 
Table 6-10. Comparison of Partial Molar Volumes and Fit Statistics Obtained from Linear-Mixture Model Fit, Appen, and Equation 6.4 and Those Obtained by Combining the Three Models

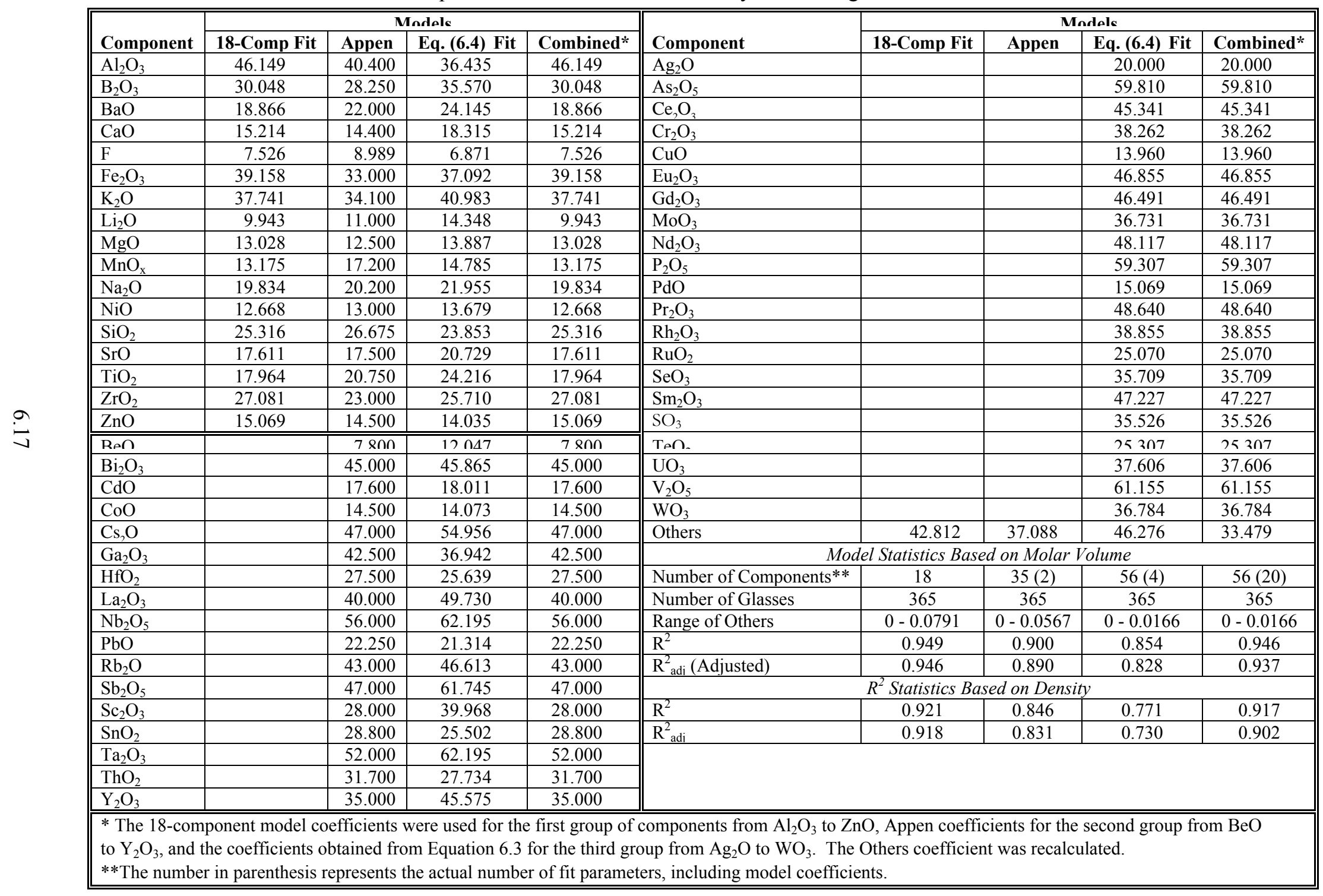




\subsection{Other Key Properties}

Sections 3.0 through 6.0 discuss the development of models for viscosity, $\mathrm{T}_{\mathrm{L}}$, PCT release, and density of waste glasses. However, several more glass properties are important for the successful immobilization of waste glasses at Hanford. The status of modeling these properties is described below.

\subsection{Toxicity Characteristic Leaching Procedure Response}

Most Hanford tank waste is listed as being toxic, primarily due to organic content. Current plans include the delisting of the glass products made from these wastes to dispose of them as radioactive, not mixed, waste. One of the criteria typically applied to determine whether a material is toxic is to examine the release of toxic metals from the material exposed to an aqueous solution under the conditions of the TCLP (EPA).

An evaluation of the TCLP data currently included in the database suggested that an effective TCLP response model could not be developed. It is strongly recommended that a TCLP response-composition model be eventually developed for Hanford waste glasses. The TCLP of preliminary glass compositions developed for the WTP suggest that the TCLP release of Cd may limit the composition of glasses produced from at least some of the initial Hanford HLWs (Kot and Pegg 2001). Preliminary models based on limited data were developed to predict TCLP based on glass composition (Vienna et al. 1998; Fu and Pegg 2000; Gan and Pegg 2002). However, the predictive ability of these models is not expected to be high, and the validity regions are relatively narrow.

It is recommended that the model reported by Gan and Pegg (2002) be used as an indication of TCLP response until sufficient additional data are available, and an improved model is developed. However, it is recommended that due to the high uncertainty of this model, the glass composition should not be restricted by its application. In other words, it is recommended that the model be employed to give property indications but not used to calculate glass compositions or waste loadings.

\subsection{Vapor Hydration Test Response}

One of the current contract specifications for WTP LAW glass performance is an alteration rate of less than $50 \mathrm{~g} \cdot \mathrm{m}^{-2} \cdot \mathrm{d}^{-1}$ by the VHT method conducted at $200^{\circ} \mathrm{C}$ (BNI 2001). However, as a relatively new technique in waste-glass characterization, there are currently insufficient data to accurately model composition effects on the VHT response. Attempts were made to develop preliminary models for VHT (Vienna et al. 2001b; Lu et al. 2002). These attempts clearly showed that a model linear-in-composition is unlikely to successfully model VHT response. This means that significantly more data are required to develop nonlinear-in-composition models. Until sufficient data are available to accurately model VHT response as a function of composition, it is recommended that the second-order model reported by Vienna et al. (2001b) can be used as a gross indicator of VHT response but not used to limit waste loading or glass composition. The model of Lu et al. (2002) may also be used; however, the difficulty of specifying initial glass thickness that is required to obtain their model variable makes its application problematic. 


\subsection{Solubility of Troublesome Components}

The solubilities of components in glass melts are generally strong functions of both temperature and composition. If solid phases precipitate when the concentration of a sparsely soluble component is higher than some critical value, the appropriate method for addressing this solubility is through $\mathrm{T}_{\mathrm{L}}$ modeling. If a molten salt segregates when the concentration of a sparsely soluble component is higher than some critical value, then both solubility, as functions of temperature and composition, and segregation kinetics need to be considered. There are insufficient data and understanding of the temperature and composition effects on the maximum allowable concentration of many sparsely soluble components, e.g., S, P, Cr, ... We, therefore, cannot model these effects. However, we expect many of these to limit the loading of some Hanford wastes in glass. As an interim solution to this dilemma, we suggest using some single- and multi-component concentration constraints to give a rough assessment of the impacts of the concentrations of these sparsely soluble components. If during the evaluation of glass-volume estimates by Hanford Tank Waste Optimization Simulator (HTWOS) (or other exercises), it is determined that one or more of these component constraints is highly influential, then it is recommended that further study be performed to put their concentration limits on a sounder technical basis. The recommended single- and multi-component constraints are listed in Table 7-1.

Clearly, developing the appropriate data and models to predict the acceptable concentration of these components is critical to developing optimized glass compositions and more immediately to accurately estimate the volume of glass likely to be produced from Hanford's tank wastes.

Table 7-1. Single- and Multi-Component Concentration Constraints in Mass\%

\begin{tabular}{|c|c|c|}
\hline Constraint & Limit & Explanation \\
\hline $\mathrm{Cr}_{2} \mathrm{O}_{3}$ & $\leq 1$ & $\begin{array}{l}\text { Eskolaite }\left(\boldsymbol{C r}_{2} \mathrm{O}_{3}\right) \boldsymbol{T}_{\boldsymbol{L}} \text { and Spinel } \boldsymbol{T}_{\boldsymbol{L}} \\
\text { At a concentration above its solubility, } \mathrm{Cr}_{2} \mathrm{O}_{3} \text { will either crystallize as } \\
\text { eskolaite or incorporate into spinel crystals. The effect of composition on } \mathrm{T}_{\mathrm{L}} \\
\text { in the eskolaite primary phase field is unknown, and the model to predict the } \\
\mathrm{T}_{\mathrm{L}} \text { in the spinel primary phase field is typically valid at }<1 \mathrm{wt} \% \mathrm{Cr}_{2} \mathrm{O}_{3} \text {. } \\
\text { Recent study on the } \mathrm{Cr}_{2} \mathrm{O}_{3} \text { solubility in a HLW glass suggests that glass } \\
\text { compositions in the eskolaite primary phase field and optimized for } \mathrm{Cr}_{2} \mathrm{O}_{3} \\
\text { solubility should be capable of achieving at least } 1 \text { mass\%. }\end{array}$ \\
\hline $\mathrm{P}_{2} \mathrm{O}_{5}$ & $\leq 2.5$ & $\begin{array}{l}\text { Immiscibility } \\
\text { Jantzen et al. (2000) showed that generally, borosilicate waste-glass melts } \\
\text { with higher than } 2.6 \text { mass } \% \text { of } \mathrm{P}_{2} \mathrm{O}_{5} \text { tended to separate into two or more } \\
\text { immiscible liquid phases. Therefore, } 2.5 \text { mass } \% \text { of } \mathrm{P}_{2} \mathrm{O}_{5} \text { was adopted as a } \\
\text { limit to help assure single-phase glass because the impact of the separated } \\
\text { liquid phases on glass durability is not well known; e.g., if separate phases } \\
\text { form, the durability may or may not be adequate, and the durability models } \\
\text { will not be accurate. }\end{array}$ \\
\hline $\mathrm{F}$ & $\leq 2$ & $\begin{array}{l}\text { Immiscibility } \\
\text { The solubility of fluoride, or the concentration of fluorine that a melt can } \\
\text { contain without the formation of a fluorine-containing phase, such as } \mathrm{CaF}_{2} \text {, } \\
\text { at melt temperature or during cooling, can vary from roughly } 2 \text { to } 8 \mathrm{mass}^{2} \text {. }\end{array}$ \\
\hline
\end{tabular}


Table 7.1 (Contd)

\begin{tabular}{|c|c|c|}
\hline Constraint & Limit & Explanation \\
\hline & & $\begin{array}{l}\text { As most of the Hanford wastes are relatively low in fluorine, we adopt the } \\
\text { conservative limit of } 2 \text { mass } \% \text { fluorine. However, if this limit were found to } \\
\text { influence the waste loading significantly, detailed studies would be required } \\
\text { to determine temperature and composition effects on solubility. }\end{array}$ \\
\hline $\mathrm{ThO}_{2}$ & $\leq 4$ & $\begin{array}{l}\text { Solubility } \\
\text { Glass is difficult to melt if } \mathrm{ThO}_{2}>4 \text { mass\%, especially when present } \\
\text { together with } \mathrm{ZrO}_{2} \text {. The } 4 \text { mass } \% \text { limit from the current WTP contract (BNI } \\
\text { 2001) should be used until more detailed information becomes available. If } \\
\text { it is found that the } \mathrm{ThO}_{2} \text { limit is restricting the loading of a significant } \\
\text { fraction of the Hanford } \mathrm{HLW} \text { in glass, then additional data on } \mathrm{ThO}_{2} \\
\text { solubility should be generated and modeled. }\end{array}$ \\
\hline $\mathrm{SO}_{3}$ & $\leq 0.8$ & $\begin{array}{l}\text { Salt Formation } \\
\text { A number of studies found that immiscible salt phases form and segregate } \\
\text { from melts with } \mathrm{SO}_{3} \text { concentrations higher than roughly } 0.6 \text { to } 1.2 \text { mass } \% \text {. } \\
\text { We adopt a limit of } 0.8 \text { mass } \% \mathrm{SO}_{3} \text { to avoid salt segregation in the melter. }\end{array}$ \\
\hline $\mathrm{RuO}_{2}+\mathrm{Rh}_{2} \mathrm{O}_{3}$ & $\leq 0.25$ & $\begin{array}{l}\text { Accumulation in the Melter } \\
\text { The combined concentrations of } \mathrm{RuO}_{2} \text { and } \mathrm{Rh}_{2} \mathrm{O}_{3} \text { of } 0.10 \text { mass\% limit is } \\
\text { used to avoid noble metals accumulation in the melter. Other noble metals } \\
\text { are either not expected to be a problem in the melter at the concentrations } \\
\text { currently thought to be in Hanford wastes, e.g., } \mathrm{Au}_{2} \mathrm{O} \text {, or are not actively } \\
\text { tracked in tank-waste inventories. It is well understood that } \mathrm{Ag}_{2} \mathrm{O} \\
\text { concentrations in the melter can exceed those of } \mathrm{RuO}_{2} \text { and/or } \mathrm{Rh}_{2} \mathrm{O}_{3} \text { without } \\
\text { the detrimental effects that they pose. Therefore, the WTP contract limit of } \\
0.25 \text { mass } \% \mathrm{Ag}_{2} \mathrm{O} \text { should be adopted until it is shown to either restrict } \\
\text { loading of significant amounts of Hanford HLW in glass or be an insufficient } \\
\text { limit to assure that processing-related problems do not occur. }\end{array}$ \\
\hline $\begin{array}{l}\mathrm{SiO}_{2} /\left(\mathrm{SiO}_{2}+\right. \\
\left.\mathrm{Na}_{2} \mathrm{O}+\mathrm{Al}_{2} \mathrm{O}_{3}\right)\end{array}$ & $\leq 0.62$ & $\begin{array}{l}\text { Nepheline Formation on Cooling - Durability } \\
\text { This constraint is a composition rule designed to help avoid the formation of } \\
\text { nepheline, } \mathrm{NaAlSiO}_{4} \text {. Li et al. (1997) showed that alumino-borosilicate } \\
\text { glasses with } \mathrm{Na}_{2} \mathrm{O} \cdot \mathrm{Al}_{2} \mathrm{O}_{3} \cdot \mathrm{SiO}_{2} \text { sub-mixtures within the nepheline primary } \\
\text { phase field in that ternary mixture are susceptible to nepheline formation, } \\
\text { which can significantly decrease the chemical durability of partly crystallized } \\
\text { glasses. For practical purposes, glasses with } \mathrm{SiO}_{2} /\left(\mathrm{SiO}_{2}+\mathrm{Na}_{2} \mathrm{O}+\mathrm{Al}_{2} \mathrm{O}_{3}\right) \\
\geq 0.62 \text { are less susceptible to nepheline formation. }\end{array}$ \\
\hline $\begin{array}{l}\left(\mathrm{Na}_{2} \mathrm{O}+\mathrm{Li}_{2} \mathrm{O}\right) / \\
\left(\mathrm{Na}_{2} \mathrm{O}+\mathrm{Li}_{2} \mathrm{O}+\right. \\
\left.\mathrm{B}_{2} \mathrm{O}_{3}+\mathrm{SiO}_{2}\right)\end{array}$ & $\geq 0.12$ & $\begin{array}{l}\text { Liquid-Liquid Phase Separation } \\
\text { This constraint is a composition rule designed to help avoid a silica-rich } \\
\text { immiscible melt on cooling of the glass. Glasses with }\left(\mathrm{Na}_{2} \mathrm{O}+\mathrm{Li}_{2} \mathrm{O}\right) /\left(\mathrm{Na}_{2} \mathrm{O}\right. \\
\left.+\mathrm{Li}_{2} \mathrm{O}+\mathrm{B}_{2} \mathrm{O}_{3}+\mathrm{SiO}_{2}\right) \geq 0.12 \text { are less susceptible to silica-rich immiscible } \\
\text { liquid formation (Peeler and Hrma 1998). }\end{array}$ \\
\hline
\end{tabular}




\subsection{Electrical Conductivity}

Although an important processing-related property, electrical conductivity $(\varepsilon)$ has rarely been found to restrict the composition or waste loading of glasses melted in Joule-heated ceramic melters operating below $1250^{\circ} \mathrm{C}$. Therefore, little effort was invested in updating $\varepsilon$ models from those previously reported (Hrma et al. 1994). It is recommended that the $\varepsilon$ model reported by Hrma et al. (1994) be used to estimate the conductivity of glasses, but not restrict their composition or waste loading.

Additional data, developed since the work of Hrma et al. (1994), are currently available for the development of an updated $\varepsilon$ model. If it is determined that $\varepsilon$ is a more important parameter for processing Hanford HLW glasses, then an updated model should be developed. In addition, if glasses with significant concentrations of $\mathrm{K}_{2} \mathrm{O}$ or other $\varepsilon$ influencing components, e.g., $\mathrm{F}$, are used, then further data collection and model development would be required. 


\subsection{Model Applications}

As mentioned in Section 1.2, waste glasses must meet property and composition constraints. The task is to determine, for a given waste stream, the composition, or composition region, of glass that has admissible properties and the minimum possible, or acceptably small, volume. Thus, we first need to solve the reverse problem to developing property-composition relationships. That is, we need to find the relationship $\mathbf{x}=\mathrm{F}_{\mathbf{w}}[\mathbf{p}]$, where $\mathbf{p} \equiv\left[p_{1}, p_{2}, \ldots p_{K}\right]$ is the property vector, and the parameter $\mathbf{w}$ stands for waste composition. The next step is to identify the composition region, $\mathbf{G}$, of glasses with acceptable properties, i.e., the region of glasses made from the given waste composition and meeting all acceptability and processability constraints. Finally, we need to find on $\mathbf{G}$ a glass $\mathbf{x}_{\mathbf{0}} \in \mathbf{G}$ with maximum waste loading or a minimum allowable waste loading with maximum allowable variation in $\mathbf{w}$.

Note that $\mathbf{x}_{\mathbf{0}}$ is subject to Equation 1.2. Property constraints have a form $\pm p_{\alpha} \leq c_{\alpha}$, where $p_{\alpha}$ is the $\alpha$-th property, and $c_{\alpha}$ is a constant; the \pm sign depends on whether the constraint is an upper or a lower limit. By Equation 1.4, a property constraint assumes the form ${ }^{(\mathrm{x})}$

$$
\pm \sum_{i=1}^{N} b_{\alpha i} x_{i}-c_{\alpha} \geq 0
$$

for each $\alpha=1,2, \ldots, K$.

Not all properties that limit glass acceptability and processability have models or other relationships as functions of glass composition. For example, insoluble noble metals, chromium, sulfates, fluorides, and phosphates, may negatively impact glass processing, or the crystallization of nepheline and cristobalite may negatively impact glass acceptability. These limitations are expressed as composition constraints, some of which are provisory and will be used until the nature of the constraining property is mathematically formulated, and the property is measured and evaluated as a function of glass composition. Composition constraints are of a general form

$$
\sum_{i=1}^{N} Q_{i} x_{i}+Q_{0} \geq 0
$$

Here the $Q_{j}$ values may be 0 for some or most components. Lower and upper bounds on single wasteglass components, on linear combinations of waste-glass components, or on ratios of linear combinations of waste-glass components can all be represented in the form of Equation 8.2. Similarly, lower and upper bounds on waste-glass properties, implemented through property-composition models of the form (1.4), (1.7), (1.12), etc., can be represented in the form (8.1).

(x) Inequalities (8.1) and (8.2) follow the convention, common in many statistical software packages, that a nonnegative value means inside (pass) the constraint, while a negative value means outside (fail) the constraint. Thus, their form (in vector notation) is $\pm \mathbf{b . x}-\mathbf{c} \geq 0$. 
The maximum waste-loading requirement can be expressed as

$$
\boldsymbol{W}=\max \text { on } \mathbf{G}
$$

where $W$ is the waste loading.

The solution of the constrained optimization problem of finding $\mathbf{x}_{\mathbf{0}} \in \mathbf{G}$ has been outlined several times in the past; see, for example, Hrma and Robertus (1993) and Hrma (1994). This approach to developing waste-glass formulations has been widely applied at Hanford and Idaho (Vienna et al. 2000). By making use of this approach, glasses are being formulated for experimental melter runs.

Of course, glass for costly melter runs, not to mention vitrification plants, cannot be formulated on computers and paper only. Because of uncertainties in measured data and the property-composition models, the real-property values associated with the "optimized" glass composition are subject to uncertainty and may differ from the model-predicted property values, depending on the accuracy and precision of the property-composition models. Consequently, experimental verification of the acceptability of mathematically optimized glass formulations is absolutely necessary. Models are then used again to assure that acceptable glass will be made from the full range of expected waste-composition variation.

To verify a mathematically optimized glass formulation, the glass is made in the laboratory, its properties are measured, and the measured and model-predicted property values are compared. If the measured and predicted property values differ beyond an acceptable tolerance, e.g., as determined by applicable statistical model-validation methods, the candidate glass formulation must be corrected. One correction approach is to start with the candidate formulation as a baseline. If the corrected glass composition is $\mathbf{x}^{\prime}$, the property value $\left(p_{\alpha}{ }^{\prime}\right)$ of the corrected glass can be expressed as

$$
g_{\alpha}\left(p_{\alpha}^{\prime}\right)=g_{\alpha}\left(p_{\alpha}\right)+\sum_{i=1}^{N} b_{\alpha i}\left(x_{i}^{\prime}-x_{i}\right)
$$

where $p_{\alpha}$ is the measured property value of the baseline glass. Equation 8.4 is a consequence of Equation 1.4 .

However, Equation 8.4 is insufficient to make the property-composition model appropriate for local sub-regions of glass composition space because local $b_{\alpha i}$ values may be different from the global ones, i.e., those determined for a large composition region. Therefore, we need to check the corrected glass and make as many corrections as needed. However, the process of checking and correcting may be unnecessary or shorter if local glass composition regions and models are the basis for the glass optimization.

Only a glass whose properties have been confirmed by property measurement can be used for a melter test run or waste-glass production. Such a run may reveal that laboratory testing did not exactly match the glass produced in the melter. For example, glass redox can have a different value because the glass from a laboratory crucible is close to redox equilibrium with the furnace atmosphere, whereas the redox state of glass from the melter is governed by the feed-melting reactions. A difference in the redox state might have an impact on some properties, such as $T_{L}$. Any such discrepancies must be resolved 
before the glass is committed to large-scale production. Resolutions may include modifying propertycomposition models to account for redox effects so that such effects are accounted for in future efforts to optimize formulations with the mathematical approach.

Generating a large number of data within a sufficiently close neighborhood of the baseline composition will make the measurement-prediction iterative process described above more efficient. A local property-composition model developed from the data would adequately respond to small composition variations within a relatively small neighborhood of glass compositions, but could not be extrapolated beyond this close neighborhood.

Whether the "iterations" or "local response" approach is chosen depends on its effectiveness with respect to the intended application. For processing a large HLW batch of a known average composition with specified variations, a set of local property-composition models might be preferred. In this situation, the detailed knowledge of the local neighborhood is needed to show that the expected composition variations, which are unavoidable in the real process, will yield acceptable glasses. For a short-term medium-scale melter run, one or two composition iterations followed by calculations by making use of Equation 8.4 may be enough.

Additionally, fully radioactive glasses, made from tank sludge treated according to the appropriate flowsheet unit operations, should be fabricated and tested to confirm that the chemical simulation of the waste adequately represented the real waste from a key glass-property perspective.

There are several benefits to developing waste glasses by the process of 1) generating propertycomposition data to cover a waste-glass-composition region of interest, 2) developing propertycomposition models that adequately fit the data, 3) making use of mathematically constrained optimization methods to develop candidate optimized glass formulations, and 4) verifying or improving the candidate glass formulation, either via local property-composition models or measurement-calculation iterations:

- The process is much faster and cheaper than developing a glass from scratch for each new waste composition to be vitrified at Hanford or when a property constraint is changed.

- Step 4) of the process provides strong evidence that the resulting glass composition is optimal for given constraints. Stopping after Step 3) would always leave open to question whether a better solution had been missed. As has been repeatedly shown in practice (Musick et al. 2000; Peeler et al. 2001; Vienna et al. 2000), Step 4) does provide for obtaining improved glass formulations over the result after Step 3).

- Additional data are generated in Step 4) that can be used for validating and/or improving the existing property-composition models.

Planning Hanford's large-scale vitrification program will require considering a large number of parameters and alternatives. To help in making important decisions, optimization programs such as the HTWOS have been developed that allow working with compositions of all Hanford waste streams simultaneously. These programs can assess the effects of influential parameters on important global criteria, such as the total number of HLW glass canisters to be produced, and compare alternatives in equipment, schedules, pretreatments, etc. 
A useful tool in trying to develop optimal glass formulations is to develop charts that plot glass volume, i.e., the number of canisters, or cleanup-cost estimates as functions of property constraints. The following hypothetical situations illustrate the usefulness of such investigations:

- Suppose a change in a certain property constraint (say, the lower limit for $T_{L}$ ) has a strong impact on the total number of canisters, and thus on the overall cost. This information would provide an incentive for either changing this constraint if the risk associated with it proves exaggerated or developing a technology that is not sensitive to the influential property.

- Suppose a certain constraint (say, the upper limit for viscosity) can be changed without a significant impact on the number of canisters, but a change in this constraint would substantially increase melter output, i.e., the production rate. In this case, large potential savings could be achieved by formulating glass with a revised constraint, e.g., lower viscosity.

- Suppose a more durable glass (when a less durable product is acceptable) can be produced at the expense of an insignificant increase of the total volume. A decision might be made to produce more durable glass. Such a decision would increase the safety margin for protection of the environment that might be much appreciated by the general public.

These and similar studies can be performed only when dependable property-composition models are available, and if these models are regularly updated to meet changing demands and changes in input data. Though data generation, development of property-composition models, and development of sophisticated optimization algorithms are complex endeavors, the output can be presented in a form that is easily comprehended and convincing. This would help in adopting unbiased views and informed decisions.

Models of glass durability are also required to assure the regulators and the public that Hanford LAW and HLW can be safely produced and disposed. 


\subsection{Discussion and Recomm endations}

The preceding sections of this report describe the utility and status of continued development of models for waste-glass properties in support of Hanford's tank waste immobilization efforts. It is recommended that the models described in this report be used to estimate the properties of Hanford waste glasses. Once adequate (and final) glass-property models are developed by the WTP, those models should be used for the initial few waste tanks and compositions similar to them, and the more global models developed in this study or their successors should be used in predicting glass properties for Hanford's balance-of-mission glasses.

The models described in this report were shown to more accurately represent the larger data set than our previously published models and provide reasonable estimates of various properties over the composition regions of overlap between expected Hanford waste glasses and available data. However, they are far from adequate for the purpose of predicting properties of glasses to be produced from all expected Hanford tank wastes with sufficient accuracy and precision. In that sense, this report represents a work in progress. Additional efforts should be focused on those areas that give the highest level of uncertainty in overall program cost and schedule. Some of these more important efforts, described in detail in the previous sections, are summarized below:

- Development of a $T_{L}$ model in the eskolaite primary phase field - Initial calculations (Perez et al. 2001) suggest that the single most important factor in determining the volume of HLW glass to be produced at Hanford is chromium. There are many aspects to the $\mathrm{Cr}$ uncertainties. First, there is uncertainty in the quantity and distribution of $\mathrm{Cr}$ in the waste tanks. Second, there is high uncertainty in the amount of $\mathrm{Cr}$ that can be efficiently removed from HLW during pretreatment. Third, there is high uncertainty in the solubility of $\mathrm{Cr}$ in glass melts that are in the eskolaite primary phase field. Finally, there is uncertainty in the boundary between the eskolaite and spinel primary phase fields. Studies aimed at lowering these uncertainties would make considerable progress toward reducing the uncertainty in glass volumes to be produced from Hanford HLWs.

- Development of sub-space models or overlapping local models for $T_{L}$ in the spinel primary phase field - Most HLW batches are currently expected to be within the spinel primary phase field. However, the $T_{L}$ model presented in this report has validity over a limited composition region and has lower than acceptable precision. These problems can only be resolved by developing a non-linear model or developing linear models that are valid over smaller composition regions. An initial evaluation of these two options suggests that developing linear models that are valid over limited composition regions that overlap each other is likely to give prediction uncertainties as low as measurement uncertainty. This approach should allow $\mathrm{T}_{\mathrm{L}}$ to be calculated over as broad a composition region as necessary to support the Hanford mission and one that is supported by the current database.

- Determination of acceptable sulfur content in LAW melter feed - The current WTP technical basis for LAW loading in glass is that the concentrations of $\mathrm{Na}_{2} \mathrm{O}$ and $\mathrm{SO}_{3}$ in mass $\%$ targeted in final glass must be below 5 when multiplied, e.g., the rule-of-five: $\left[\mathrm{Na}_{2} \mathrm{O}\right]\left[\mathrm{SO}_{3}\right] \leq 5$. Calculations suggest that this will double the amount of glass required to immobilize all of Hanford LAW as compared with the volume of glass that would be required if loading were 20 mass $\% \mathrm{Na}_{2} \mathrm{O}$. Results from several tests, including some performed in support of the WTP, have suggested that $\left[\mathrm{SO}_{3}\right]$ as high as 1 mass \% in glass should be processable in joule-heated ceramic-melters without an undesirable segregated salt 
layer accumulating. However, the salt layer often forms at $\left[\mathrm{SO}_{3}\right]$ below the solubility limit, and the acceptable $\left[\mathrm{SO}_{3}\right]$ is a complex function of a number of physical and chemical parameters. Initial studies suggest that the appropriate level should be between 0.6 and 1.0 mass $\% \mathrm{SO}_{3}$. However, more research is required to accurately assess the limit of $\left[\mathrm{SO}_{3}\right]$ in glass and the values of key chemical and physical parameters that are required to successfully process high sulfur wastes in the currently designed plant.

- Development of initial models for VHT and TCLP - Toxicity Characteristic Leach Procedure and VHT have both been used to show that there are composition and/or waste loading limits for Hanford waste glasses. The VSL has developed preliminary data sets and models for VHT and TCLP (Muller, Buechele, and Pegg 2001; Kot and Pegg 2001; Lu et al. 2002; Gan and Pegg 2002). However, those data sets and models are very preliminary and are not yet adequate for predicting TCLP and VHT responses. Additional VHT and TCLP data covering the glass-composition regions of interest to the WTP must be developed. These data should be used with existing data to develop, evaluate, and validate property-composition models.

- Improve estimates of Hanford waste glasses and then update models - Because of uncertainty about the compositions of wastes in Hanford tanks and further uncertainties about pretreatment and retrieval scenarios, the composition region of glasses expected to be produced from Hanford wastes changes dramatically at times. The models reported here should be applied to estimate glass-composition regions expected to be produced from future Hanford HLWs, which may require ignoring model validity ranges. Then, additional glass-property-composition data should be developed, and the models should be refit to acceptably predict glass properties within the appropriate composition region(s). This process should be performed iteratively until adequate glass-property models are available.

- Models should be developed for other key glass properties - Many properties, such as melt corrosivity (to glass-contact materials), melting rate, electrical conductivity, crystal formation on cooling, impact of slow cooling on durability, thermal conductivity, etc., are important for waste immobilization at Hanford. Data collection and model development for these properties should be performed to allow for the optimization of acceptable glass compositions and the determination of glass volumes to be produced at Hanford.

Performing these tasks is necessary for many purposes, including 1) accurate estimation of glass volumes to be produced at Hanford, 2) optimization of glass compositions for specific wastes, 3) detailed planning required to conduct the River Protection Program scope, 4) ability to estimate the impacts of different process or product changes on cleanup cost and schedule, 5) evaluation of the impacts of new technologies and strategies, and 6) a host of other tasks. 


\subsection{References}

American Society for Testing and Materials (ASTM). 1998. Standard Test Methods for Determining Chemical Durability of Nuclear Waste Glasses: Product Consistency Test (PCT), ASTM-C-1285-97, 1998 Annual Book of ASTM Standards, West Conshohecken, PA.

Appen, A. A. 1970. Chemistry of Glass, Publishing House "Khimia”, Leningrad Department. (in Russian).

Bates, S. O. 1985. HWVP Baseline Milestone 020202B: Issue a Recommended HWVP Glass

Composition for the NCAW-CRW Stream for Future Work, Pacific Northwest National Laboratory, Richland, WA.

Bechtel National, Inc. (BNI). 2001. "Design, Construction, and Commissioning of the Hanford Tank Waste Treatment and Immobilization Plant," Contract Number: DE-AC27-01RV14136, US Department of Energy, Office of River Protection, Richland WA.

BNI, see Bechtel National, Inc.

Bulkley, S. A., and J. D. Vienna. 1997. "Composition Effects on Viscosity and Chemical Durability of Simulated Plutonium Residue Glasses," Mat. Res. Soc. Symp. Proc., 465:1243-50.

Carter, J. G., S. S. Koegler, and S. O. Bates. 1988. Process Performance of the Pilot Scale ISV of a Simulated Waste Disposal Site at ORN, PNL-6530, Pacific Northwest National Laboratory, Richland, WA.

Chick, L. A., G. F. Piepel, G. B. Mellinger, R. P. May, W. J. Gray, and C. Q. Buckwalter. 1981. The Effects of Composition on Properties in an 11-Component Nuclear Waste Glass System, PNL-3188, Pacific Northwest National Laboratory, Richland, WA.

Chick, L. A., W. M. Bowen, R. O. Lokken, J. W. Wald, L. R. Bunnell, and D. M. Strachan. 1984. West Valley High-Level Nuclear Waste Glass Development: A Statistically Designed Mixture Study, PNL-4992, Pacific Northwest National Laboratory, Richland, WA.

Cohen, R. E. 1994. "First-Principles Theory of Crystalline $\mathrm{SiO}_{2}$," in Reviews in Mineralogy, Vol. 29,

Silica: Physical Behavior, Geochemistry, and Materials Applications, Mineralogical Society of America, Chelsea, MI.

Cornell, J. A. 2002. Experiments With Mixtures: Designs, Models, and the Analysis of Mixture Data, Third Edition, John Wiley \& Sons, NY.

Crawford, C. L., D. M. Ferrara, B. C. Ha, and N. E. Bibler. 1998. "Production of a High-Level Waste Glass from Hanford Waste Samples." In: Proceedings of the International Conference on Decommissioning and Decontamination and on Nuclear Hazardous Waste Management, Volume 1, American Nuclear Society, La Grange Park, IL.

Crum, J. V., M. J. Schweiger, P. Hrma, and J. D. Vienna. 1997. "Liquidus Temperature Model for Hanford High-Level Waste Glasses with High Concentrations of Zirconia," Mat. Res. Soc. Symp. Proc., 465:79-85. 
Crum, J. V., J. D. Vienna, D. K. Peeler, I. A. Reamer, and D. J. Pittman. 2002. "The Effect of Glass Composition on Crystallinity and Durability for INEEL Run 78 Calcine Waste Simulant," in Ceramic Transactions 132, pp. 267-278, American Ceramic Society, Westerville, OH.

DOE, see U.S. Department of Energy

Draper, N. R., and H. Smith. 1998. Applied Regression Analysis, Third Edition, John Wiley \& Sons, NY.

Edwards, T. B. 1997. A Statistically Designed Sampling Plan for Investigating Liquidus Temperature Versus Glass Composition, SRT-SCS-97-0022, Westinghouse Savannah River Company, Aiken, SC.

Edwards, T. B., D. K. Peeler, I. A. Reamer, G. F. Piepel, J. D. Vienna, and H. Li. 2000. Phase $2 b$ Experimental Design For the INEEL Glass Composition Variation Study, WSRC-TR-99-00224, Rev. 0, Westinghouse Savannah River Company, Aiken, SC.

EPA, see U. S. Environmental Protection Agency

Feng, X., E. E. Saad, and I. L. Pegg. 1990. "A Model for the Viscosity of Multicomponent Glass Melts," Ceram. Trans. 9:457-468.

Feng, X., and T. B. Metzger. 1996. “A Structural Bond Strength Model for Glass Durability,” Ceram. Trans. 72:51-60.

Feng, X., P. R. Hrma, J. H. Westsik, N. R. Brown, M. J. Schweiger, H. Li, J. D. Vienna, G. Chen, G. F. Piepel, D. E. Smith, B. P. McGrail, S. E. Palmer, D. S. Kim, Y. Peng, W. K. Hahn, A. J. Bakel, W. L. Ebert, D. K. Peeler, and C. Chang. 1996. Glass Optimization for Vitrification of Hanford Site Low-Level Tank Waste, PNNL-10918, Pacific Northwest National Laboratory, Richland, WA.

Ferrara, D. M., C. L. Crawford, B. C. Ha, and N. E. Bibler. 1998. "Vitrification of Three Low-Activity Radioactive Waste Streams from Hanford." In: Proceedings of the International Conference on Decommissioning and Decontamination and on Nuclear and Hazardous Waste Management, Vol. 1, 706-713.

Fu, S. S., H. Gan, I. S. Muller, I. L. Pegg, and P. B. Macedo. 1997. “Optimization of Savannah River MArea Mixed Waste for Vitrification.” In: MRS Symposium Proceedings, Vol. 465:139-146.

Fu, S. S., and I. L. Pegg. 1998. Glass Formulation and Testing with TWRS HLW Simulants, VSL Final Report. January 18, 1998, Vitreous State Laboratory, The Catholic University of America, Washington D. C.

Fu, S. S., and I. L. Pegg. 2000. "The Effect of Composition on TCLP Leach Rates for Glasses with Similar Melt Viscosities," Ceram. Trans. 107:261-269.

Fung, Y. C. 1994. A First Course in Continuum Mechanics, for Physical and Biological Engineers and Scientists, $3^{\text {rd }}$ Ed., Pentice Hall, Englewood Cliffs, NJ.

Gan H., and I. L. Pegg. 2002. "Effect of Glass Composition on the Leaching Behavior of HLW Glasses Under TCLP Conditions," Ceram. Trans. 132:335-344. 
Hrma, P., and R. J. Robertus. 1993. "Waste Glass Design Based on Property Composition Functions," Ceram. Eng. Sci. Proc. 14 (11-12):187-203.

Hrma, P., G. F. Piepel, M. J. Schweiger, D. E. Smith, D.-S. Kim, P. E. Redgate, J. D. Vienna, C. A. LoPresti, D. B. Simpson, D. K. Peeler, and M. H. Langowski. 1994. Property/Composition Relationships for Hanford High-Level Waste Glasses Melting at $1150^{\circ} \mathrm{C}$, PNL-10359, Vol. 1 and 2, Pacific Northwest Laboratory, Richland, WA.

Hrma, P. 1994. "Toward Optimization of Nuclear Waste Glasses: Constraints, Property Models, and Waste Loading," Ceram. Trans. 45:391-401.

Hrma, P., G. F. Piepel, J. D. Vienna, P. E. Redgate, M. J. Schweiger, and D. E. Smith. 1995a. "Prediction of Nuclear Waste Glass Dissolution as a Function of Composition," Ceram. Trans. 61:497504.

Hrma, P., G. F. Piepel, P. E. Redgate, D. E. Smith, M. J. Schweiger, J. D. Vienna, and D.-S. Kim. 1995 b. "Prediction of Processing Properties for Nuclear Waste Glasses," Ceram. Trans. 61:505-513.

Hrma, P. 1999. "Modeling of Spinel Settling in Waste Glass Melter." In: Science to Support DOE Site Cleanup, PNNL-12208, Pacific Northwest National Laboratory, Richland, WA.

Hrma, P., J. D. Vienna, M. Mika, J. V. Crum, and G. F. Piepel. 1999. Liquidus Temperature Data for DWPF Glass, PNNL-11790, Pacific Northwest National Laboratory, Richland, WA.

Hrma, P., G. F. Piepel, J. D. Vienna, S. K. Cooley, D. S. Kim, R. L. Russell. 2001. Database and Interim Glass Property Models for Hanford HLW Glasses, PNNL-13573, Pacific Northwest National Laboratory, Richland, WA.

Jantzen, C. M. 1991. "Relationship of Glass Composition to Glass Viscosity, Resistivity, Liquidus Temperature, and Durability: First-Principle Process Product Models for Vitrification of Nuclear Waste," Ceram. Trans. 23:37-51.

Jantzen, C. M. 1992. “Thermodynamic Approach to Glass Corrosion.” In: Corrosion of Glass, Ceramics and Ceramic Superconductors. D. E. Clark and K. Ziotos (Eds.), Noyes, Park Ridge, NJ.

Jantzen, C. M., J. B. Pickett, K. G. Brown, T. B. Edwards, and D. C. Beam. 1995. Process/Product Models for the Defense Waste Processing Facility (DWPF): Part I. Predicting Glass Durability from Composition Using a Thermodynamic Hydration Energy Reaction MOdel (THERMO), US DOE Report WSRC-TR-93-0672, Westinghouse Savannah River Company, Aiken, SC.

Jantzen, C. M., J. B. Pickett, K. G. Brown, and T. B. Edwards. 1998. "Method of Determining Glass Durability," US Patent 5846278, Westinghouse Savannah River Company, Aiken, SC.

Jantzen, C. M., K. G. Brown, J. B. Pickett, and G. L. Ritzhaupt. 2000. Crystalline Phase Separation in Phosphate Containing Waste Glasses: Relevancy to Vitrification of Idaho National Engineering and Environmental Laboratory (I NEEL) High Activity Waste (U), WSRC-TR-2000-00339, Westinghouse Savannah River Company, Aiken, SC.

Jantzen, C. M., J. B. Pickett, and I. Joseph. 2000. "Toxic Characteristic leaching Procedure (TCLP) Testing of Waste Glass and K-3 Refractory: Revisited," in Ceramic Transactions, vol. 107, 271-280, American Ceramic Society, Westerville, OH. 
Johnston, J. W., G. F. Piepel, and B. A. Pulsipher. 1990. "Evaluation of Empirical Models for Glass Durability," Letter Report Prepared for West Valley Nuclear Services, Pacific Northwest National Laboratory, Richland, WA.

Kot, W. K., and I. L. Pegg. 2001. Glass Formulation and Testing with RPP-WTP HLW Simulants - Final Report, VSL-01R2540-2, Vitreous State Laboratory, The Catholic University of America, Washington D.C.

Li, H., J. D. Vienna, P. Hrma, D. E. Smith, and M. J. Schweiger. 1997. "Nepheline Precipitation in High-Level Waste Glasses: Compositional Effects and Impact on the Waste Form Acceptability." In Scientific Basis for Nuclear Waste Management XX, pp. 261-268, Materials Research Society, Pittsburgh, PA.

Lu, X., F. Perez-Cardenas, H. Gan, A. C. Buechele, and I. L. Pegg. 2002. "Kinetics of Alteration in Vapor Phase Hydration Tests on High Sodium Waste Glass," Ceram. Trans. 132:311-322.

Marra, S. L., and C. M. Jantzen. 1993. Characterization of Projected DWPF Glasses Heat Treated to Simulate Canister Centerline Cooling (U), WSRC-TR-92-142, Rev. 1. Westinghouse Savannah River Company, Aiken, SC.

Mika, M., M. J. Schweiger, J. D. Vienna, and P. Hrma. 1997. "Liquidus Temperature of Spinel Precipitating High-Level Waste Glasses," Mat. Res. Soc. Symp. Proc., 465:71-8.

Montgomery, D. C., and E. A. Peck. 1992. Introduction to Linear Regression Analysis, Second Edition, John Wiley \& Sons, NY.

Muller, I. S., and I. L. Pegg. 1998. Glass Formulation and Testing with TWRS LAW Simulants, Final Report for GTS Durateck Inc. and BNFL Inc., Catholic University of America, Washington D.C.

Muller, I. S., A. C. Buechele, and I. L. Pegg. 2001. Glass Formulation and Testing with RPP-WTP LAW Simulants - Final Report, VSL-01R3560-2, Vitreous State Laboratory, The Catholic University of America, Washington D. C.

Musick, C. A., B. A. Scholes, R. D. Tillotson, D. M. Bennert, J. D. Vienna, J. V. Crum, D. K. Peeler, I. A. Reamer, D. F. Bickford, J. C. Marra, and N. L. Waldo. 2000. Technical Status Report: Vitrification Technology Development Using INEEL Run 78 Pilot Plant Calcine, INEELIEXT-2000-00110, Idaho National Engineering and Environmental Laboratory, Idaho Falls, ID.

Oksoy, D. L., D. Pye, D. F. Bickford, and W. G. Ramsey. 1994. "Canonical Correlation of Waste Glass Compositions and Durability, Including pH," Ceram. Trans. 39:365-380.

Olson, K. M. 1993. Fabrication and Leaching of West Valley Demonstration Project Glasses: Ten Quarter 2 and Ten Quarter 3 Glasses, Pacific Northwest National Laboratory, Richland, WA.

Olson, K. M. 1994. Viscosity Testing of 30 WVDP Glasses, WVSP 94-16, Pacific Northwest National Laboratory, Richland, WA.

Olson, K. M., G. F. Piepel, S. C. Marschman, and G. K. Whiting. 1994. Product Consistency Testing of West Valley Compositional Variation Glasses, PNL-10191, Pacific Northwest National Laboratory, Richland, WA. 
Paul, A. 1977. "Chemical Durability of Glasses a Thermodynamic Approach,” J. Mat. Sci. 12, pp 22462268.

Piepel, G. F., and P. E. Redgate. 1997. "Mixture Experiment Techniques for Reducing the Number of Components Applied to Modeling Waste Glass Sodium Release," J. Am. Ceram. Soc., 80:3038-3044.

Peeler, D. K., and P. Hrma. 1998. "Predicting Liquid Immiscibility in Multicomponent Nuclear Waste Glasses," Ceram. Trans. 45:219-229.

Peeler, D. K., T. H. Lorier, D. F. Bickford, D. C. Witt, T. B. Edwards, K. G. Brown, I. A. Reamer, R. J. Workman, and J. D. Vienna. 2001. Melt Rate Improvement For DWPF MB3: Frit Development and Model Assessment (U), WSRC-TR-2001-00131, Westinghouse Savannah River Company, Aiken, SC.

Perez, J. M., D. F. Bickford, D. E. Day, D. S. Kim, S. L. Lambert, S. L. Marra, D. K. Peeler, D. M. Strachan, M. B. Triplett, J. D. Vienna, R. S. Wittman. 2001. High-Level Waste Melter Study Report, PNNL-13582, Pacific Northwest National Laboratory, Richland, WA.

Piepel, G. F., P. E. Redgate, and P. Hrma. 1995. "Statistical Experimental Design of a Waste Glass Study," Ceram. Trans. 61:489-496.

Piepel, G. F., P. E. Redgate, and P. Masuga. 1996. Mixture Models Versus Free Energy of Hydration Models for Waste Glass Durability, PNL-10823, Pacific Northwest National Laboratory, Richland, WA.

Piepel, G. F., P. E. Redgate, and P. Masuga. 1997. "Comparison of Mixture Models and Free Energy of Hydration Models for Waste Glass Releases," Glass Tech. 38:210-215.

Piepel, G. F., P. Hrma, and J. D. Vienna. 1998. “Glass Chemistry Development Strategy For Hanford High Level Waste (HLW).” In: Science and Technology for Disposal of Radioactive Tank Wastes, pp. 393-402, Plenum Press, NY.

Piepel, G. F., J. D. Vienna, and P. Hrma. 1999. Phase 1 Experimental Design for the INEEL HLW Glass Composition Variation Study, PNNL-SA-29594, Rev. 2, Pacific Northwest National Laboratory, Richland, WA.

Piepel, G. F., J. M. Szychowski, and J. L. Loeppky. 2002. “Augmenting Scheffé Linear Mixture Models with Squared and/or Crossproduct Terms," J. Quality Tech., 34:297-314.

Piepel, G. F., S. K. Cooley, D. K. Peeler, J. D. Vienna, and T. B. Edwards. 2002. “Augmenting a Waste Glass Mixture Experiment Study with Additional Glass Components and Experimental Runs," Quality Eng., 15:91-111.

Pittman, D. J., I. A. Reamer, D. K. Peeler, and T. B. Edwards. 2001. Property-Composition Relationships for the DP Glasses: Effect of Crystallization on Durability (U), WSRC-TR-2001-00166, Westinghouse Savannah River Company, Aiken, SC.

Plodinec, M. J. 1999. "Solubility Approach for Modeling Waste Glass Liquidus.” Mat. Res. Soc. Symp. Proc. 556:223-230.

Pye, L. D. 1985. The Physical and Thermal Properties of Simulated Nuclear Waste Glasses and Their Melts, DPST-85-397, New York State College of Ceramics, Alfred University, Alfred, NY. 
Ramsey, W. G. 1995. Glass Dissolution Chemistry of the System $\mathrm{Na}_{2} \mathrm{O} \cdot \mathrm{B}_{2} \mathrm{O}_{3} \cdot \mathrm{SiO}_{2} \cdot \mathrm{Al}_{2} \mathrm{O}_{3} \cdot \mathrm{Fe}_{2} \mathrm{O}_{3} \cdot \mathrm{CaO}$, $\mathrm{PhD}$ Thesis, Clemson University, Clemson, SC.

Reimus, M. A. H., G. B. Mellinger, G. F. Piepel, and L. R. Bunnell. 1988. West Valley Glass Product Qualification Durability Studies, FY 1987-1988: Effects of Composition, Redox State, Thermal History, and Groundwater. PNL-6723. Pacific Northwest Laboratory, Richland, WA.

Reynolds, J. G., and P. Hrma. 1997. "The Kinetics of Spinel Crystallization from a High-Level Waste Glass.” In: MRS Symposium Proceedings, Vol. 465:65-69.

Riley, B. J., J. A. Rosario, and P. Hrma. 2001. Impact of HLW Glass Crystallinity on the PCT Response. PNNL-13491. Pacific Northwest Laboratory, Richland, WA.

Scholes, B. A., D. K. Peeler, and J. D. Vienna. 2000. The Preparation and Characterization of INTEC Phase 3 Composition Variation Study Glasses, INEEL/EXT-2000-01566, Idaho National Engineering and Environmental Laboratory, Idaho Falls, ID.

Scholes, B. A., J. D. Vienna, D. K. Peeler, and T. B. Edwards. 2002. The preparation and Characterization of INTEC Sodium Bearing Waste Phase 1 Composition Variation Study Glasses, INEEL/EXT-02-00386, Idaho National Engineering and Environmental Laboratory, Idaho Falls, ID.

Scholtze, H. 1990. Glass Nature, Structure, and Properties, Springer, NY.

Schulz, R. L., T. H. Lorier, D. K. Peeler, K. G. Brown, I. A. Reamer, J. D. Vienna, A. Jiricka, B. M. Jorgensen, D. E. Smith. 2000. Hanford Immobilized LAW Product Acceptance: Tanks Focus Area Testing Data Package II, PNNL-13344, Pacific Northwest National Laboratory, Richland, WA.

Shannon, R. D. 1976. "Revised Effective Ionic Radii and Systematic Study of Interatomic Distances in Halides and Chalcogenides," Acta Cryst. A32:751-767.

Staples, B. A., D. K. Peeler, J. D. Vienna, B. A. Scholes, and C. A. Musick. 1999. The Preparation and Characterization of INTEC HAW Phase 1 Composition Variation Study Glasses, Idaho National Engineering and Environmental Laboratory, INEEL/EXT-98-00970, Idaho Falls, ID.

Staples, B. A., B. A. Scholes, D. K. Peeler, L. L. Torres, J. D. Vienna, C. A. Musick, and B. R. Boyle. 2000. The Preparation and Characterization of INTEC Phase $2 b$ Composition Variation Study Glasses, INEEL/EXT-99-01322, Idaho National Engineering and Environmental Laboratory, Idaho Falls, ID.

U. S. Department of Energy (DOE). 1996. Waste Acceptance Product Specifications for Vitrified HighLevel Waste Forms, EM-WAPS Rev. 02, Office of Environmental Management, Washington, D. C.

U. S. Environmental Protection Agency. SW-846, Test Methods for Evaluating Solid Waste, Physical/Chemical Methods. U.S. Environmental Protection Agency, Office of Solid Waste and Emergency Response, Washington, D.C.

Varshneya, A. K. 1994. Fundamentals of Inorganic Glasses, Academic Press, San Diego, CA.

Vienna, J. D., P. Hrma, M. J. Schweiger, and M. H. Langowski. 1996a. "Compositional Dependence of Elemental Release from HLW Glasses by the Product Consistency Test: One Component at a Time Study," Ceram. Trans. 72:307-316. 
Vienna, J. D., P. Hrma, D.-S. Kim, M. J. Schweiger, and D. E. Smith. 1996b. “Compositional Dependence of Viscosity, Electrical Conductivity, and Liquidus Temperature of Multicomponent Borosilicate Glasses," Ceram. Trans. 72:427-436.

Vienna, J. D., P. R. Hrma, M. J. Schweiger, M. H. Langowski, P. E. Redgate, D.-S. Kim, G. F. Piepel, D. E. Smith, C. Y. Chang, D. E. Rinehart, S. E. Palmer, and H. Li. 1996c. Effect of Composition and Temperature on the Properties of High-Level Waste (HLW) Glass Melting Above $1200^{\circ}$ C, PNNL-10987, Pacific Northwest National Laboratory, Richland, WA.

Vienna, J. D., R. P. Thimke, G. F. Piepel, J. V. Crum, M. L. Elliott, R. K. Nakaoka, and G. W. Veazey. 1998. Glass Development for Treatment of Evaporator Bottoms Waste, PNNL-11865, Pacific Northwest National Laboratory, Richland, WA.

Vienna, J. D., D. K. Peeler, R. L. Plaisted, T. J. Plaisted, I. A. Reamer, R. D. Tillotson, J. V. Crum, C. A. Musick, and T. L. James. 1999. Glass Formulation for Idaho National Engineering and Environmental Laboratory Zirconia Calcine High-Activity Waste, PNNL-12202, Pacific Northwest National Laboratory, Richland, WA.

Vienna, J. D., T. J. Plaisted, R. L. Plaisted, J. V. Crum, D. K. Peeler, and I. A. Reamer. 2000. "Glass Formulation for Idaho Engineering Environmental Laboratory Zirconia High-Activity Waste," Ceram. Trans. 107:451-459.

Vienna, J. D., P. Hrma, J. V. Crum, and M. Mika. 2001a. "Liquidus Temperature-Composition Model for Multi-Component Glasses in the Fe, Cr, Ni, and Mn Spinel Primary Phase Field," J. Non-Cryst. Solids 292:1-24.

Vienna, J. D., P. Hrma, A. Jiricka, D. E. Smith, T. H. Lorier, I. A. Reamer, and R. L. Schulz. 2001 b. Hanford Immobilized LAW Product Acceptance Testing: Tanks Focus Area Results, PNNL-13744, Pacific Northwest National Laboratory, Richland, WA.

Vienna, J. D. 2002. The Effects of Temperature and Composition on the Solubility of Chromium in Multi-component Alkali-borosilicate Glass Melts, Ph.D. Dissertation, Washington State University, Pullman, WA.

Vienna, J. D., W. C. Buchmiller, J. V. Crum, D. D. Graham, D. S. Kim, B. D. MacIsaac, M. J. Schweiger, D. K. Peeler, T. B. Edwards, I. A. Reamer, R. J. Workman. 2002. Glass Formulation Development for INEEL Sodium-Bearing Waste, PNNL-14050, Pacific Northwest National Laboratory, Richland, WA.

West Valley Nuclear Services (WVNS). 1995. West Valley Demonstration Project Waste Form Qualification Report - Waste Form Specifications, WVDP-186, Section 1.3, Rev. 1, West Valley Nuclear Services Company Inc., West Valley, NY.

Wilson, B. K., T. J. Plaisted, J. Alton, and P. Hrma. 2001. "The Effect of Composition on Spinel Equilibrium and Crystal Size in High-Level Waste Glass," submitted for publication. 
INEEL CVS Phase 1 (Staples et al. 1999)

\begin{tabular}{|c|c|c|c|c|c|c|c|c|c|c|c|c|c|c|c|c|c|c|c|c|c|}
\hline Glass ID & $\begin{array}{c}\mathrm{A} 12 \mathrm{O} 3 \\
-\mathrm{t}\end{array}$ & $\begin{array}{c}\mathrm{B} 2 \mathrm{O} 3 \\
-\mathrm{t}\end{array}$ & $\begin{array}{c}\mathrm{CaO} \\
-\mathrm{t}\end{array}$ & $\begin{array}{c}\mathrm{Fe} 2 \mathrm{O} 3 \\
-\mathrm{t}\end{array}$ & $\begin{array}{c}\mathrm{FeO} \\
-\mathrm{t}\end{array}$ & $\begin{array}{c}\mathrm{K} 2 \mathrm{O} \\
-\mathrm{t}\end{array}$ & $\begin{array}{c}\mathrm{Li} 2 \mathrm{O} \\
-\mathrm{t}\end{array}$ & $\begin{array}{c}\mathrm{MgO} \\
-\mathrm{t}\end{array}$ & $\begin{array}{c}\mathrm{Na} 2 \mathrm{O} \\
-\mathrm{t}\end{array}$ & $\begin{array}{c}\mathrm{NiO} \\
-\mathrm{t}\end{array}$ & $\begin{array}{c}\mathrm{P} 2 \mathrm{O} 5 \\
-\mathrm{t}\end{array}$ & $\begin{array}{c}\mathrm{SiO} 2 \\
-\mathrm{t}\end{array}$ & $\begin{array}{c}\mathrm{ZrO} 2 \\
-\mathrm{t}\end{array}$ & $\begin{array}{c}\mathrm{Ag} 2 \mathrm{O} \\
-\mathrm{t}\end{array}$ & $\begin{array}{c}\mathrm{As} 2 \mathrm{O} 3 \\
-\mathrm{t}\end{array}$ & $\begin{array}{c}\mathrm{BaO} \\
-\mathrm{t}\end{array}$ & $\begin{array}{c}\mathrm{Bi} 2 \mathrm{O} 3 \\
-\mathrm{t}\end{array}$ & $\begin{array}{c}\mathrm{Br} \\
-\mathrm{t}\end{array}$ & $\begin{array}{c}\mathrm{CdO} \\
-\mathrm{t}\end{array}$ & $\begin{array}{c}\mathrm{Ce} 2 \mathrm{O} 3 \\
-\mathrm{t}\end{array}$ & $\begin{array}{c}\mathrm{CeO} 2 \\
-\mathrm{t}\end{array}$ \\
\hline IG1-01 & \begin{tabular}{|l|}
0.0552 \\
\end{tabular} & 0.0947 & 0.0007 & 0.0005 & & 0.0425 & 0.0546 & 0.0000 & 0.1432 & & 0.0703 & 0.4706 & 0.0649 & & & 0.0001 & & & 0.0000 & & 0.0006 \\
\hline IG1-02 & 0.0656 & 0.1312 & 0.0007 & 0.0005 & & 0.0874 & 0.0714 & 0.0000 & 0.0819 & & 0.1312 & 0.4273 & 0.0000 & & & 0.0001 & & & 0.0000 & & 0.0006 \\
\hline IG1-03 & 0.0000 & 0.0438 & 0.0007 & 0.0005 & & 0.0875 & 0.0010 & 0.0000 & 0.2131 & & 0.1312 & 0.3971 & 0.1224 & & & 0.0001 & & & 0.0000 & & 0.0006 \\
\hline IG1-04 & 0.0000 & 0.0437 & 0.0007 & 0.0005 & & 0.0000 & 0.0787 & 0.0000 & 0.0953 & & 0.1310 & 0.5248 & 0.1225 & & & 0.0001 & & & 0.0000 & & 0.0006 \\
\hline IG1-05 & 0.0657 & 0.1313 & 0.0007 & 0.0005 & & 0.0000 & 0.0014 & 0.0000 & 0.2131 & & 0.1310 & 0.4537 & 0.0000 & & & 0.0001 & & & 0.0000 & & 0.0006 \\
\hline IG1-06 & 0.0000 & 0.1501 & 0.0008 & 0.0005 & & 0.1000 & 0.0482 & 0.0000 & 0.0500 & & 0.0000 & 0.5722 & 0.0751 & & & 0.0001 & & & 0.0000 & & 0.0007 \\
\hline IG1-07 & 0.1501 & 0.1501 & 0.0008 & 0.0005 & & 0.0000 & 0.0845 & 0.0000 & 0.0500 & & 0.0000 & 0.5308 & 0.0301 & & & 0.0001 & & & 0.0000 & & 0.0007 \\
\hline IG1-08 & 0.1312 & 0.0437 & 0.0007 & 0.0005 & & 0.0875 & 0.0722 & 0.0000 & 0.0819 & & 0.1312 & 0.4221 & 0.0262 & & & 0.0001 & & & 0.0000 & & 0.0006 \\
\hline IG1-09 & 0.0360 & 0.1312 & 0.0007 & 0.0005 & & 0.0000 & 0.0333 & 0.0000 & 0.2019 & & 0.1312 & 0.3411 & 0.1215 & & & 0.0001 & & & 0.0000 & & 0.0006 \\
\hline IG1-10 & 0.0000 & 0.0654 & 0.0008 & 0.0005 & & 0.0000 & 0.0900 & 0.0000 & 0.1560 & & 0.0000 & 0.5441 & 0.1401 & & & 0.0001 & & & 0.0000 & & 0.0007 \\
\hline IG1-11 & 0.1464 & 0.0500 & 0.0008 & 0.0006 & & 0.0000 & 0.0511 & 0.0000 & 0.2000 & & 0.0000 & 0.5479 & 0.0000 & & & 0.0001 & & & 0.0000 & & 0.0007 \\
\hline IG1-12 & 0.0317 & 0.1501 & 0.0008 & 0.0005 & & 0.1001 & 0.0000 & 0.0000 & 0.1530 & & 0.0000 & 0.4206 & 0.1401 & & & 0.0001 & & & 0.0000 & & 0.0007 \\
\hline IG1-13 & 0.0759 & 0.0501 & 0.0008 & 0.0006 & & 0.1001 & 0.0709 & 0.0000 & 0.1720 & & 0.0000 & 0.5266 & 0.0000 & & & 0.0001 & & & 0.0000 & & 0.0007 \\
\hline IG1-14 & 0.1087 & 0.0724 & 0.0007 & 0.0006 & & 0.0242 & 0.0507 & 0.0000 & 0.1674 & & 0.0362 & 0.5023 & 0.0338 & & & 0.0001 & & & 0.0000 & & 0.0006 \\
\hline IG1-15 & 0.0644 & 0.0677 & 0.0007 & 0.0005 & & 0.0677 & 0.0600 & 0.0000 & 0.1086 & & 0.1016 & 0.4943 & 0.0316 & & & 0.0001 & & & 0.0000 & & 0.0006 \\
\hline IG1-16 & 0.0725 & 0.0725 & 0.0007 & 0.0006 & & 0.0242 & 0.0464 & 0.0000 & 0.1674 & & 0.0362 & 0.4752 & 0.1014 & & & 0.0001 & & & 0.0000 & & 0.0007 \\
\hline IG1-17 & 0.0677 & 0.0678 & 0.0007 & 0.0005 & & 0.0226 & 0.0386 & 0.0000 & 0.1763 & & 0.1016 & 0.4266 & 0.0948 & & & 0.0001 & & & 0.0000 & & 0.0006 \\
\hline IG1-18 & 0.0684 & 0.1129 & 0.0007 & 0.0005 & & 0.0226 & 0.0563 & 0.0000 & 0.1086 & & 0.1016 & 0.4941 & 0.0316 & & & 0.0001 & & & 0.0000 & & 0.0006 \\
\hline IG1-19 & 0.0683 & 0.1126 & 0.0007 & 0.0005 & & 0.0226 & 0.0564 & 0.0000 & 0.1086 & & 0.1016 & 0.4944 & 0.0316 & & & 0.0001 & & & 0.0000 & & 0.0006 \\
\hline IG1-20 & 0.0449 & 0.1129 & 0.0007 & 0.0005 & & 0.0226 & 0.0528 & 0.0000 & 0.1086 & & 0.1016 & 0.4578 & 0.0948 & & & 0.0001 & & & 0.0000 & & 0.0006 \\
\hline IG1-21 & 0.0890 & 0.1207 & 0.0007 & 0.0005 & & 0.0724 & 0.0652 & 0.0000 & 0.0950 & & 0.0362 & 0.4323 & 0.0848 & & & 0.0001 & & & 0.0000 & & 0.0006 \\
\hline IG1-22 & 0.1013 & 0.1118 & 0.0007 & 0.0005 & & 0.0226 & 0.0610 & 0.0000 & 0.1316 & & 0.1017 & 0.4045 & 0.0615 & & & 0.0001 & & & 0.0000 & & 0.0006 \\
\hline IG1-23 & 0.0979 & 0.0677 & 0.0007 & 0.0005 & & 0.0663 & 0.0610 & 0.0000 & 0.1086 & & 0.1016 & 0.4282 & 0.0647 & & & 0.0001 & & & 0.0000 & & 0.0006 \\
\hline IG1-24 & 0.0863 & 0.1207 & 0.0007 & 0.0006 & & 0.0724 & 0.0652 & 0.0000 & 0.0971 & & 0.0362 & 0.4302 & 0.0876 & & & 0.0001 & & & 0.0000 & & 0.0006 \\
\hline IG1-25 & 0.0724 & 0.0724 & 0.0007 & 0.0006 & & 0.0724 & 0.0492 & 0.0000 & 0.1334 & & 0.0362 & 0.4580 & 0.1014 & & & 0.0001 & & & 0.0000 & & 0.0006 \\
\hline IG1-26 & 0.0552 & 0.0947 & 0.0007 & 0.0005 & & 0.0425 & 0.0546 & 0.0000 & 0.1432 & & 0.0703 & 0.4706 & 0.0649 & & & 0.0001 & & & 0.0000 & & 0.0006 \\
\hline IG1-27 & 0.1312 & 0.0437 & 0.0007 & 0.0005 & & 0.0875 & 0.0721 & 0.0000 & 0.0819 & & 0.1312 & 0.4221 & 0.0263 & & & 0.0001 & & & 0.0000 & & 0.0006 \\
\hline IG1-28 & 0.1312 & 0.0437 & 0.0007 & 0.0005 & & 0.0875 & 0.0722 & 0.0000 & 0.0819 & & 0.1312 & 0.4221 & 0.0263 & & & 0.0001 & & & 0.0000 & & 0.0006 \\
\hline IG1-29 & 0.1400 & 0.0839 & 0.0000 & 0.0200 & & 0.0000 & 0.0700 & 0.0000 & 0.1063 & 0.0011 & 0.0006 & 0.5700 & 0.0000 & & & 0.0013 & & & 0.0000 & & 0.0003 \\
\hline IG1-30 & 0.0203 & 0.0607 & 0.0042 & 0.0051 & & 0.0000 & 0.0707 & 0.0012 & 0.1833 & 0.0094 & 0.0049 & 0.4598 & 0.1112 & & & 0.0115 & & & 0.0000 & & 0.0025 \\
\hline IG1-31 & 0.1500 & 0.1500 & 0.0009 & 0.0005 & & 0.0000 & 0.0900 & 0.0000 & 0.1187 & 0.0000 & 0.0000 & 0.4565 & 0.0300 & & & 0.0000 & & & 0.0000 & & 0.0007 \\
\hline IG1-32 & 0.1500 & 0.0500 & 0.0009 & 0.0005 & & 0.1000 & 0.0836 & 0.0000 & 0.0500 & 0.0000 & 0.0500 & 0.5116 & 0.0000 & & & 0.0000 & & & 0.0000 & & 0.0007 \\
\hline IG1-33 & 0.0000 & 0.0500 & 0.0009 & 0.0005 & & 0.1000 & 0.0116 & 0.0000 & 0.2000 & 0.0000 & 0.0000 & 0.4936 & 0.1400 & & & 0.0000 & & & 0.0000 & & 0.0007 \\
\hline IG1-34 & 0.0000 & 0.0701 & 0.0009 & 0.0005 & & 0.0000 & 0.0900 & 0.0000 & 0.0500 & 0.0000 & 0.0500 & 0.5951 & 0.1400 & & & 0.0000 & & & 0.0000 & & 0.0007 \\
\hline IG1-35 & 0.0750 & 0.1500 & 0.0009 & 0.0005 & & 0.0000 & 0.0016 & 0.0000 & 0.2000 & 0.0000 & 0.0500 & 0.5186 & 0.0000 & & & 0.0000 & & & 0.0000 & & 0.0007 \\
\hline IG1-36 & 0.0750 & 0.0500 & 0.0009 & 0.0005 & & 0.1000 & 0.0900 & 0.0000 & 0.1174 & 0.0000 & 0.0000 & 0.5628 & 0.0000 & & & 0.0000 & & & 0.0000 & & 0.0007 \\
\hline IG1-37 & 0.0762 & 0.1013 & 0.0009 & 0.0005 & & 0.0497 & 0.0568 & 0.0000 & 0.1191 & 0.0000 & 0.0260 & 0.4902 & 0.0759 & & & 0.0000 & & & 0.0000 & & 0.0007 \\
\hline IG1-38 & 0.0375 & 0.1250 & 0.0009 & 0.0005 & & 0.0250 & 0.0631 & 0.0000 & 0.0875 & 0.0000 & 0.0125 & 0.5396 & 0.1050 & & & 0.0000 & & & 0.0000 & & 0.0007 \\
\hline IG1-39 & 0.0751 & 0.1250 & 0.0009 & 0.0005 & & 0.0750 & 0.0675 & 0.0000 & 0.0883 & 0.0000 & 0.0125 & 0.4469 & 0.1049 & & & 0.0000 & & & 0.0000 & & 0.0007 \\
\hline IG1-40 & 0.0546 & 0.1022 & 0.0009 & 0.0005 & & 0.0750 & 0.0559 & 0.0000 & 0.0875 & 0.0000 & 0.0375 & 0.5475 & 0.0350 & & & 0.0000 & & & 0.0000 & & 0.0007 \\
\hline IG1-41 & 0.0513 & 0.0750 & 0.0009 & 0.0005 & & 0.0750 & 0.0471 & 0.0000 & 0.1268 & 0.0000 & 0.0375 & 0.5475 & 0.0350 & & & 0.0000 & & & 0.0000 & & 0.0007 \\
\hline IG1-42 & 0.1084 & 0.0750 & 0.0009 & 0.0005 & & 0.0734 & 0.0675 & 0.0000 & 0.0875 & 0.0000 & 0.0375 & 0.4743 & 0.0716 & & & 0.0000 & & & 0.0000 & & 0.0007 \\
\hline IG1-43 & 0.0704 & 0.1250 & 0.0009 & 0.0005 & & 0.0250 & 0.0249 & 0.0000 & 0.1625 & 0.0000 & 0.0375 & 0.4449 & 0.1050 & & & 0.0000 & & & 0.0000 & & 0.0007 \\
\hline IG1-44 & 0.0000 & 0.0701 & 0.0009 & 0.0005 & & 0.0000 & 0.0900 & 0.0000 & 0.0500 & 0.0000 & 0.0500 & 0.5951 & 0.1400 & & & 0.0000 & & & 0.0000 & & 0.0007 \\
\hline
\end{tabular}


INEEL CVS Phase 1 (Staples et al. 1999)

\begin{tabular}{|c|c|c|c|c|c|c|c|c|c|c|c|c|c|c|c|c|c|c|c|c|c|}
\hline Glass ID & $\begin{array}{l}\mathrm{Cl} \\
-\mathrm{t}\end{array}$ & $\begin{array}{c}\mathrm{CoO} \\
-\mathrm{t}\end{array}$ & $\begin{array}{c}\mathrm{Co} 2 \mathrm{O} 3 \\
-\mathrm{t}\end{array}$ & $\begin{array}{c}\mathrm{Cr} 2 \mathrm{O} 3 \\
-\mathrm{t}\end{array}$ & $\begin{array}{c}\mathrm{Cs} 2 \mathrm{O} \\
-\mathrm{t}\end{array}$ & $\begin{array}{c}\mathrm{CuO} \\
-\mathrm{t}\end{array}$ & $\begin{array}{c}\mathrm{Eu} 2 \mathrm{O} 3 \\
-\mathrm{t}\end{array}$ & $\begin{array}{l}F \\
-t\end{array}$ & $\begin{array}{c}\mathrm{Ga} 2 \mathrm{O} 3 \\
-\mathrm{t}\end{array}$ & $\begin{array}{c}\mathrm{Gd} 2 \mathrm{O} 3 \\
-\mathrm{t}\end{array}$ & $\begin{array}{c}\mathrm{HgO} \\
-\mathrm{t}\end{array}$ & $\begin{array}{l}I \\
-t\end{array}$ & $\begin{array}{c}\mathrm{La} 2 \mathrm{O} 3 \\
-\mathrm{t}\end{array}$ & $\begin{array}{c}\mathrm{MnO} 2 \\
-\mathrm{t}\end{array}$ & $\begin{array}{c}\mathrm{MnO} \\
-\mathrm{t}\end{array}$ & $\begin{array}{c}\mathrm{MoO} \\
-\mathrm{t}\end{array}$ & $\begin{array}{c}\mathrm{MoO} 3 \\
-\mathrm{t}\end{array}$ & $\begin{array}{c}\mathrm{Nb} 2 \mathrm{O} 5 \\
-\mathrm{t}\end{array}$ & $\begin{array}{c}\mathrm{Nd} 2 \mathrm{O} 3 \\
-\mathrm{t}\end{array}$ & $\begin{array}{c}\mathrm{PbO} \\
-\mathrm{t}\end{array}$ & $\begin{array}{c}\mathrm{PdO} 2 \\
-\mathrm{t}\end{array}$ \\
\hline IG1-01 & 0.0000 & & & 0.0000 & 0.0006 & 0.0007 & & & & 0.0007 & & 0.0000 & 0.0000 & 0.0000 & 0.0000 & & 0.0001 & 0.0000 & 0.0000 & 0.0000 & \\
\hline IG1-02 & 0.0000 & & & 0.0000 & 0.0006 & 0.0006 & & & & 0.0007 & & 0.0000 & 0.0000 & 0.0000 & 0.0000 & & 0.0001 & 0.0000 & 0.0000 & 0.0000 & \\
\hline IG1-03 & 0.0000 & & & 0.0000 & 0.0005 & 0.0006 & & & & 0.0007 & & 0.0000 & 0.0000 & 0.0000 & 0.0000 & & 0.0001 & 0.0000 & 0.0000 & 0.0000 & \\
\hline IG1-04 & 0.0000 & & & 0.0000 & 0.0005 & 0.0007 & & & & 0.0007 & & 0.0000 & 0.0000 & 0.0000 & 0.0000 & & 0.0001 & 0.0000 & 0.0000 & 0.0000 & \\
\hline IG1-05 & 0.0000 & & & 0.0000 & 0.0005 & 0.0007 & & & & 0.0006 & & 0.0000 & 0.0000 & 0.0000 & 0.0000 & & 0.0001 & 0.0000 & 0.0000 & 0.0000 & \\
\hline IG1-06 & 0.0000 & & & 0.0000 & 0.0006 & 0.0007 & & & & 0.0008 & & 0.0000 & 0.0000 & 0.0000 & 0.0000 & & 0.0001 & 0.0000 & 0.0000 & 0.0000 & \\
\hline IG1-07 & 0.0000 & & & 0.0000 & 0.0006 & 0.0007 & & & & 0.0008 & & 0.0000 & 0.0000 & 0.0000 & 0.0000 & & 0.0001 & 0.0000 & 0.0000 & 0.0000 & \\
\hline IG1-08 & 0.0000 & & & 0.0000 & 0.0006 & 0.0006 & & & & 0.0007 & & 0.0000 & 0.0000 & 0.0000 & 0.0000 & & 0.0001 & 0.0000 & 0.0000 & 0.0000 & \\
\hline IG1-09 & 0.0000 & & & 0.0000 & 0.0005 & 0.0006 & & & & 0.0007 & & 0.0000 & 0.0000 & 0.0000 & 0.0000 & & 0.0001 & 0.0000 & 0.0000 & 0.0000 & \\
\hline IG1-10 & 0.0000 & & & 0.0000 & 0.0006 & 0.0007 & & & & 0.0007 & & 0.0000 & 0.0000 & 0.0000 & 0.0000 & & 0.0001 & 0.0000 & 0.0000 & 0.0000 & \\
\hline IG1-11 & 0.0000 & & & 0.0000 & 0.0006 & 0.0008 & & & & 0.0008 & & 0.0000 & 0.0000 & 0.0000 & 0.0000 & & 0.0001 & 0.0000 & 0.0000 & 0.0000 & \\
\hline IG1-12 & 0.0000 & & & 0.0000 & 0.0006 & 0.0007 & & & & 0.0008 & & 0.0000 & 0.0000 & 0.0000 & 0.0000 & & 0.0001 & 0.0000 & 0.0000 & 0.0000 & \\
\hline IG1-13 & 0.0000 & & & 0.0000 & 0.0006 & 0.0007 & & & & 0.0008 & & 0.0000 & 0.0000 & 0.0000 & 0.0000 & & 0.0001 & 0.0000 & 0.0000 & 0.0000 & \\
\hline IG1-14 & 0.0000 & & & 0.0000 & 0.0006 & 0.0007 & & & & 0.0007 & & 0.0000 & 0.0000 & 0.0000 & 0.0000 & & 0.0001 & 0.0000 & 0.0000 & 0.0000 & \\
\hline IG1-15 & 0.0000 & & & 0.0000 & 0.0005 & 0.0007 & & & & 0.0007 & & 0.0000 & 0.0000 & 0.0000 & 0.0000 & & 0.0001 & 0.0000 & 0.0000 & 0.0000 & \\
\hline IG1-16 & 0.0000 & & & 0.0000 & 0.0006 & 0.0007 & & & & 0.0007 & & 0.0000 & 0.0000 & 0.0000 & 0.0000 & & 0.0001 & 0.0000 & 0.0000 & 0.0000 & \\
\hline IG1-17 & 0.0000 & & & 0.0000 & 0.0005 & 0.0007 & & & & 0.0007 & & 0.0000 & 0.0000 & 0.0000 & 0.0000 & & 0.0001 & 0.0000 & 0.0000 & 0.0000 & \\
\hline IG1-18 & 0.0000 & & & 0.0000 & 0.0005 & 0.0007 & & & & 0.0007 & & 0.0000 & 0.0000 & 0.0000 & 0.0000 & & 0.0001 & 0.0000 & 0.0000 & 0.0000 & \\
\hline IG1-19 & 0.0000 & & & 0.0000 & 0.0005 & 0.0007 & & & & 0.0007 & & 0.0000 & 0.0000 & 0.0000 & 0.0000 & & 0.0001 & 0.0000 & 0.0000 & 0.0000 & \\
\hline IG1-20 & 0.0000 & & & 0.0000 & 0.0005 & 0.0007 & & & & 0.0007 & & 0.0000 & 0.0000 & 0.0000 & 0.0000 & & 0.0001 & 0.0000 & 0.0000 & 0.0000 & \\
\hline IG1-21 & 0.0000 & & & 0.0000 & 0.0006 & 0.0007 & & & & 0.0007 & & 0.0000 & 0.0000 & 0.0000 & 0.0000 & & 0.0001 & 0.0000 & 0.0000 & 0.0000 & \\
\hline IG1-22 & 0.0000 & & & 0.0000 & 0.0006 & 0.0007 & & & & 0.0007 & & 0.0000 & 0.0000 & 0.0000 & 0.0000 & & 0.0001 & 0.0000 & 0.0000 & 0.0000 & \\
\hline IG1-23 & 0.0000 & & & 0.0000 & 0.0006 & 0.0007 & & & & 0.0007 & & 0.0000 & 0.0000 & 0.0000 & 0.0000 & & 0.0001 & 0.0000 & 0.0000 & 0.0000 & \\
\hline IG1-24 & 0.0000 & & & 0.0000 & 0.0006 & 0.0007 & & & & 0.0007 & & 0.0000 & 0.0000 & 0.0000 & 0.0000 & & 0.0001 & 0.0000 & 0.0000 & 0.0000 & \\
\hline IG1-25 & 0.0000 & & & 0.0000 & 0.0006 & 0.0007 & & & & 0.0007 & & 0.0000 & 0.0000 & 0.0000 & 0.0000 & & 0.0001 & 0.0000 & 0.0000 & 0.0000 & \\
\hline IG1-26 & 0.0000 & & & 0.0000 & 0.0006 & 0.0007 & & & & 0.0007 & & 0.0000 & 0.0000 & 0.0000 & 0.0000 & & 0.0001 & 0.0000 & 0.0000 & 0.0000 & \\
\hline IG1-27 & 0.0000 & & & 0.0000 & 0.0006 & 0.0006 & & & & 0.0007 & & 0.0000 & 0.0000 & 0.0000 & 0.0000 & & 0.0001 & 0.0000 & 0.0000 & 0.0000 & \\
\hline IG1-28 & 0.0000 & & & 0.0000 & 0.0006 & 0.0006 & & & & 0.0007 & & 0.0000 & 0.0000 & 0.0000 & 0.0000 & & 0.0001 & 0.0000 & 0.0000 & 0.0000 & \\
\hline IG1-29 & 0.0000 & & & 0.0003 & 0.0002 & 0.0003 & & & & 0.0000 & & 0.0000 & 0.0011 & 0.0000 & 0.0003 & & 0.0005 & 0.0000 & 0.0022 & 0.0000 & \\
\hline IG1-30 & 0.0000 & & & 0.0021 & 0.0015 & 0.0025 & & & & 0.0000 & & 0.0000 & 0.0102 & 0.0000 & 0.0020 & & 0.0049 & 0.0000 & 0.0190 & 0.0000 & \\
\hline IG1-31 & 0.0000 & & & 0.0000 & 0.0010 & 0.0007 & & & & 0.0007 & & 0.0000 & 0.0000 & 0.0000 & 0.0000 & & 0.0000 & 0.0000 & 0.0000 & 0.0000 & \\
\hline IG1-32 & 0.0000 & & & 0.0000 & 0.0010 & 0.0007 & & & & 0.0007 & & 0.0000 & 0.0000 & 0.0000 & 0.0000 & & 0.0000 & 0.0000 & 0.0000 & 0.0000 & \\
\hline IG1-33 & 0.0000 & & & 0.0000 & 0.0010 & 0.0007 & & & & 0.0007 & & 0.0000 & 0.0000 & 0.0000 & 0.0000 & & 0.0000 & 0.0000 & 0.0000 & 0.0000 & \\
\hline IG1-34 & 0.0000 & & & 0.0000 & 0.0010 & 0.0007 & & & & 0.0007 & & 0.0000 & 0.0000 & 0.0000 & 0.0000 & & 0.0000 & 0.0000 & 0.0000 & 0.0000 & \\
\hline IG1-35 & 0.0000 & & & 0.0000 & 0.0010 & 0.0007 & & & & 0.0007 & & 0.0000 & 0.0000 & 0.0000 & 0.0000 & & 0.0000 & 0.0000 & 0.0000 & 0.0000 & \\
\hline IG1-36 & 0.0000 & & & 0.0000 & 0.0010 & 0.0007 & & & & 0.0007 & & 0.0000 & 0.0000 & 0.0000 & 0.0000 & & 0.0000 & 0.0000 & 0.0000 & 0.0000 & \\
\hline IG1-37 & 0.0000 & & & 0.0000 & 0.0010 & 0.0007 & & & & 0.0007 & & 0.0000 & 0.0000 & 0.0000 & 0.0000 & & 0.0000 & 0.0000 & 0.0000 & 0.0000 & \\
\hline IG1-38 & 0.0000 & & & 0.0000 & 0.0010 & 0.0007 & & & & 0.0007 & & 0.0000 & 0.0000 & 0.0000 & 0.0000 & & 0.0000 & 0.0000 & 0.0000 & 0.0000 & \\
\hline IG1-39 & 0.0000 & & & 0.0000 & 0.0010 & 0.0007 & & & & 0.0007 & & 0.0000 & 0.0000 & 0.0000 & 0.0000 & & 0.0000 & 0.0000 & 0.0000 & 0.0000 & \\
\hline IG1-40 & 0.0000 & & & 0.0000 & 0.0010 & 0.0007 & & & & 0.0007 & & 0.0000 & 0.0000 & 0.0000 & 0.0000 & & 0.0000 & 0.0000 & 0.0000 & 0.0000 & \\
\hline IG1-41 & 0.0000 & & & 0.0000 & 0.0010 & 0.0007 & & & & 0.0007 & & 0.0000 & 0.0000 & 0.0000 & 0.0000 & & 0.0000 & 0.0000 & 0.0000 & 0.0000 & \\
\hline IG1-42 & 0.0000 & & & 0.0000 & 0.0010 & 0.0007 & & & & 0.0007 & & 0.0000 & 0.0000 & 0.0000 & 0.0000 & & 0.0000 & 0.0000 & 0.0000 & 0.0000 & \\
\hline IG1-43 & 0.0000 & & & 0.0000 & 0.0010 & 0.0007 & & & & 0.0007 & & 0.0000 & 0.0000 & 0.0000 & 0.0000 & & 0.0000 & 0.0000 & 0.0000 & 0.0000 & \\
\hline IG1-44 & 0.0000 & & & 0.0000 & 0.0010 & 0.0007 & & & & 0.0007 & & 0.0000 & 0.0000 & 0.0000 & 0.0000 & & 0.0000 & 0.0000 & 0.0000 & 0.0000 & \\
\hline
\end{tabular}


INEEL CVS Phase 1 (Staples et al. 1999)

\begin{tabular}{|c|c|c|c|c|c|c|c|c|c|c|c|c|c|c|c|c|c|c|c|c|c|}
\hline Glass ID & $\begin{array}{c}\mathrm{PdO} \\
-\mathrm{t}\end{array}$ & $\begin{array}{c}\mathrm{Pr} 2 \mathrm{O} 3 \\
-\mathrm{t}\end{array}$ & $\begin{array}{c}\text { Pr6O11 } \\
-\mathrm{t}\end{array}$ & $\begin{array}{c}\mathrm{Rb} 2 \mathrm{O} \\
-\mathrm{t}\end{array}$ & $\begin{array}{c}\mathrm{ReO} \\
-\mathrm{t}\end{array}$ & $\begin{array}{c}\mathrm{ReO} 2 \\
-\mathrm{t}\end{array}$ & $\begin{array}{c}\mathrm{Rh} 2 \mathrm{O} 3 \\
-\mathrm{t}\end{array}$ & $\begin{array}{c}\mathrm{RhO} 2 \\
-\mathrm{t}\end{array}$ & $\begin{array}{c}\mathrm{RuO}_{2} \\
-\mathrm{t}\end{array}$ & $\begin{array}{c}\mathrm{Sb} 2 \mathrm{O} 3 \\
-\mathrm{t}\end{array}$ & $\begin{array}{c}\mathrm{Sb} 2 \mathrm{O} 5 \\
-\mathrm{t}\end{array}$ & $\begin{array}{c}\mathrm{SeO} 2 \\
-\mathrm{t}\end{array}$ & $\begin{array}{c}\mathrm{Sm} 2 \mathrm{O} 3 \\
-\mathrm{t}\end{array}$ & $\begin{array}{c}\mathrm{SnO} \\
-\mathrm{t}\end{array}$ & $\begin{array}{c}\mathrm{SnO} 2 \\
-\mathrm{t}\end{array}$ & $\begin{array}{c}\mathrm{SO} 3 \\
-\mathrm{t}\end{array}$ & $\begin{array}{c}\mathrm{SrO} \\
-\mathrm{t}\end{array}$ & $\begin{array}{c}\mathrm{Tc} 2 \mathrm{O} 7 \\
-\mathrm{t}\end{array}$ & $\begin{array}{c}\mathrm{TeO} 2 \\
-\mathrm{t}\end{array}$ & $\begin{array}{c}\mathrm{ThO} 2 \\
-\mathrm{t}\end{array}$ & $\begin{array}{c}\mathrm{TiO} 2 \\
-\mathrm{t}\end{array}$ \\
\hline IG1-01 & 0.0000 & 0.0000 & 0.0000 & 0.0000 & & 0.0000 & 0.0000 & & 0.0000 & & & & 0.0000 & & 0.0000 & 0.0000 & 0.0001 & & 0.0000 & & \\
\hline IG1-02 & 0.0000 & 0.0000 & 0.0000 & 0.0000 & & 0.0000 & 0.0000 & & 0.0000 & & & & 0.0000 & & 0.0000 & 0.0000 & 0.0001 & & 0.0000 & & \\
\hline IG1-03 & 0.0000 & 0.0000 & 0.0000 & 0.0000 & & 0.0000 & 0.0000 & & 0.0000 & & & & 0.0000 & & 0.0000 & 0.0000 & 0.0001 & & 0.0000 & & \\
\hline IG1-04 & 0.0000 & 0.0000 & 0.0000 & 0.0000 & & 0.0000 & 0.0000 & & 0.0000 & & & & 0.0000 & & 0.0000 & 0.0000 & 0.0001 & & 0.0000 & & \\
\hline IG1-05 & 0.0000 & 0.0000 & 0.0000 & 0.0000 & & 0.0000 & 0.0000 & & 0.0000 & & & & 0.0000 & & 0.0000 & 0.0000 & 0.0001 & & 0.0000 & & \\
\hline IG1-06 & 0.0000 & 0.0000 & 0.0000 & 0.0000 & & 0.0000 & 0.0000 & & 0.0000 & & & & 0.0000 & & 0.0000 & 0.0000 & 0.0001 & & 0.0000 & & \\
\hline IG1-07 & 0.0000 & 0.0000 & 0.0000 & 0.0000 & & 0.0000 & 0.0000 & & 0.0000 & & & & 0.0000 & & 0.0000 & 0.0000 & 0.0001 & & 0.0000 & & \\
\hline IG1-08 & 0.0000 & 0.0000 & 0.0000 & 0.0000 & & 0.0000 & 0.0000 & & 0.0000 & & & & 0.0000 & & 0.0000 & 0.0000 & 0.0001 & & 0.0000 & & \\
\hline IG1-09 & 0.0000 & 0.0000 & 0.0000 & 0.0000 & & 0.0000 & 0.0000 & & 0.0000 & & & & 0.0000 & & 0.0000 & 0.0000 & 0.0001 & & 0.0000 & & \\
\hline IG1-10 & 0.0000 & 0.0000 & 0.0000 & 0.0000 & & 0.0000 & 0.0000 & & 0.0000 & & & & 0.0000 & & 0.0000 & 0.0000 & 0.0001 & & 0.0000 & & \\
\hline IG1-11 & 0.0000 & 0.0000 & 0.0000 & 0.0000 & & 0.0000 & 0.0000 & & 0.0000 & & & & 0.0000 & & 0.0000 & 0.0000 & 0.0001 & & 0.0000 & & \\
\hline IG1-12 & 0.0000 & 0.0000 & 0.0000 & 0.0000 & & 0.0000 & 0.0000 & & 0.0000 & & & & 0.0000 & & 0.0000 & 0.0000 & 0.0001 & & 0.0000 & & \\
\hline IG1-13 & 0.0000 & 0.0000 & 0.0000 & 0.0000 & & 0.0000 & 0.0000 & & 0.0000 & & & & 0.0000 & & 0.0000 & 0.0000 & 0.0001 & & 0.0000 & & \\
\hline IG1-14 & 0.0000 & 0.0000 & 0.0000 & 0.0000 & & 0.0000 & 0.0000 & & 0.0000 & & & & 0.0000 & & 0.0000 & 0.0000 & 0.0001 & & 0.0000 & & \\
\hline IG1-15 & 0.0000 & 0.0000 & 0.0000 & 0.0000 & & 0.0000 & 0.0000 & & 0.0000 & & & & 0.0000 & & 0.0000 & 0.0000 & 0.0001 & & 0.0000 & & \\
\hline IG1-16 & 0.0000 & 0.0000 & 0.0000 & 0.0000 & & 0.0000 & 0.0000 & & 0.0000 & & & & 0.0000 & & 0.0000 & 0.0000 & 0.0001 & & 0.0000 & & \\
\hline IG1-17 & 0.0000 & 0.0000 & 0.0000 & 0.0000 & & 0.0000 & 0.0000 & & 0.0000 & & & & 0.0000 & & 0.0000 & 0.0000 & 0.0001 & & 0.0000 & & \\
\hline IG1-18 & 0.0000 & 0.0000 & 0.0000 & 0.0000 & & 0.0000 & 0.0000 & & 0.0000 & & & & 0.0000 & & 0.0000 & 0.0000 & 0.0001 & & 0.0000 & & \\
\hline IG1-19 & 0.0000 & 0.0000 & 0.0000 & 0.0000 & & 0.0000 & 0.0000 & & 0.0000 & & & & 0.0000 & & 0.0000 & 0.0000 & 0.0001 & & 0.0000 & & \\
\hline IG1-20 & 0.0000 & 0.0000 & 0.0000 & 0.0000 & & 0.0000 & 0.0000 & & 0.0000 & & & & 0.0000 & & 0.0000 & 0.0000 & 0.0001 & & 0.0000 & & \\
\hline IG1-21 & 0.0000 & 0.0000 & 0.0000 & 0.0000 & & 0.0000 & 0.0000 & & 0.0000 & & & & 0.0000 & & 0.0000 & 0.0000 & 0.0001 & & 0.0000 & & \\
\hline IG1-22 & 0.0000 & 0.0000 & 0.0000 & 0.0000 & & 0.0000 & 0.0000 & & 0.0000 & & & & 0.0000 & & 0.0000 & 0.0000 & 0.0001 & & 0.0000 & & \\
\hline IG1-23 & 0.0000 & 0.0000 & 0.0000 & 0.0000 & & 0.0000 & 0.0000 & & 0.0000 & & & & 0.0000 & & 0.0000 & 0.0000 & 0.0001 & & 0.0000 & & \\
\hline IG1-24 & 0.0000 & 0.0000 & 0.0000 & 0.0000 & & 0.0000 & 0.0000 & & 0.0000 & & & & 0.0000 & & 0.0000 & 0.0000 & 0.0001 & & 0.0000 & & \\
\hline IG1-25 & 0.0000 & 0.0000 & 0.0000 & 0.0000 & & 0.0000 & 0.0000 & & 0.0000 & & & & 0.0000 & & 0.0000 & 0.0000 & 0.0001 & & 0.0000 & & \\
\hline IG1-26 & 0.0000 & 0.0000 & 0.0000 & 0.0000 & & 0.0000 & 0.0000 & & 0.0000 & & & & 0.0000 & & 0.0000 & 0.0000 & 0.0001 & & 0.0000 & & \\
\hline IG1-27 & 0.0000 & 0.0000 & 0.0000 & 0.0000 & & 0.0000 & 0.0000 & & 0.0000 & & & & 0.0000 & & 0.0000 & 0.0000 & 0.0001 & & 0.0000 & & \\
\hline IG1-28 & 0.0000 & 0.0000 & 0.0000 & 0.0000 & & 0.0000 & 0.0000 & & 0.0000 & & & & 0.0000 & & 0.0000 & 0.0000 & 0.0001 & & 0.0000 & & \\
\hline IG1-29 & 0.0001 & 0.0000 & 0.0002 & 0.0000 & & 0.0001 & 0.0000 & & 0.0003 & & & & 0.0001 & & 0.0000 & 0.0000 & 0.0007 & & 0.0000 & & \\
\hline IG1-30 & 0.0008 & 0.0000 & 0.0017 & 0.0000 & & 0.0008 & 0.0000 & & 0.0025 & & & & 0.0008 & & 0.0000 & 0.0000 & 0.0059 & & 0.0000 & & \\
\hline IG1-31 & 0.0000 & 0.0000 & 0.0000 & 0.0000 & & 0.0000 & 0.0000 & & 0.0000 & & & & 0.0000 & & 0.0000 & 0.0000 & 0.0002 & & 0.0000 & & \\
\hline IG1-32 & 0.0000 & 0.0000 & 0.0000 & 0.0000 & & 0.0000 & 0.0000 & & 0.0000 & & & & 0.0000 & & 0.0000 & 0.0000 & 0.0002 & & 0.0000 & & \\
\hline IG1-33 & 0.0000 & 0.0000 & 0.0000 & 0.0000 & & 0.0000 & 0.0000 & & 0.0000 & & & & 0.0000 & & 0.0000 & 0.0000 & 0.0002 & & 0.0000 & & \\
\hline IG1-34 & 0.0000 & 0.0000 & 0.0000 & 0.0000 & & 0.0000 & 0.0000 & & 0.0000 & & & & 0.0000 & & 0.0000 & 0.0000 & 0.0002 & & 0.0000 & & \\
\hline IG1-35 & 0.0000 & 0.0000 & 0.0000 & 0.0000 & & 0.0000 & 0.0000 & & 0.0000 & & & & 0.0000 & & 0.0000 & 0.0000 & 0.0002 & & 0.0000 & & \\
\hline IG1-36 & 0.0000 & 0.0000 & 0.0000 & 0.0000 & & 0.0000 & 0.0000 & & 0.0000 & & & & 0.0000 & & 0.0000 & 0.0000 & 0.0002 & & 0.0000 & & \\
\hline IG1-37 & 0.0000 & 0.0000 & 0.0000 & 0.0000 & & 0.0000 & 0.0000 & & 0.0000 & & & & 0.0000 & & 0.0000 & 0.0000 & 0.0002 & & 0.0000 & & \\
\hline IG1-38 & 0.0000 & 0.0000 & 0.0000 & 0.0000 & & 0.0000 & 0.0000 & & 0.0000 & & & & 0.0000 & & 0.0000 & 0.0000 & 0.0002 & & 0.0000 & & \\
\hline IG1-39 & 0.0000 & 0.0000 & 0.0000 & 0.0000 & & 0.0000 & 0.0000 & & 0.0000 & & & & 0.0000 & & 0.0000 & 0.0000 & 0.0002 & & 0.0000 & & \\
\hline IG1-40 & 0.0000 & 0.0000 & 0.0000 & 0.0000 & & 0.0000 & 0.0000 & & 0.0000 & & & & 0.0000 & & 0.0000 & 0.0000 & 0.0002 & & 0.0000 & & \\
\hline IG1-41 & 0.0000 & 0.0000 & 0.0000 & 0.0000 & & 0.0000 & 0.0000 & & 0.0000 & & & & 0.0000 & & 0.0000 & 0.0000 & 0.0002 & & 0.0000 & & \\
\hline IG1-42 & 0.0000 & 0.0000 & 0.0000 & 0.0000 & & 0.0000 & 0.0000 & & 0.0000 & & & & 0.0000 & & 0.0000 & 0.0000 & 0.0002 & & 0.0000 & & \\
\hline IG1-43 & 0.0000 & 0.0000 & 0.0000 & 0.0000 & & 0.0000 & 0.0000 & & 0.0000 & & & & 0.0000 & & 0.0000 & 0.0000 & 0.0002 & & 0.0000 & & \\
\hline IG1-44 & 0.0000 & 0.0000 & 0.0000 & 0.0000 & & 0.0000 & 0.0000 & & 0.0000 & & & & 0.0000 & & 0.0000 & 0.0000 & 0.0002 & & 0.0000 & & \\
\hline
\end{tabular}


INEEL CVS Phase 1 (Staples et al. 1999)

\begin{tabular}{|c|c|c|c|c|c|c|c|c|c|c|c|c|c|c|c|c|c|c|c|c|c|}
\hline Glass ID & $\begin{array}{c}\mathrm{T} 12 \mathrm{O} 3 \\
-\mathrm{t}\end{array}$ & $\begin{array}{c}\mathrm{U} 3 \mathrm{O} 8 \\
-\mathrm{t}\end{array}$ & $\begin{array}{c}\mathrm{UO} 2 \\
-\mathrm{t}\end{array}$ & $\begin{array}{c}\mathrm{UO} 3 \\
-\mathrm{t}\end{array}$ & $\begin{array}{c}\mathrm{V} 2 \mathrm{O} 5 \\
-\mathrm{t}\end{array}$ & $\begin{array}{c}\text { WO3 } \\
-t\end{array}$ & $\begin{array}{c}\mathrm{Y} 2 \mathrm{O} 3 \\
-\mathrm{t}\end{array}$ & $\begin{array}{c}\mathrm{ZnO} \\
-\mathrm{t}\end{array}$ & $\begin{array}{c}\text { Others } \\
-t\end{array}$ & $\begin{array}{c}\text { Sum } \\
-t\end{array}$ & $\begin{array}{c}\mathrm{A} 12 \mathrm{O} 3 \\
-\mathrm{a}\end{array}$ & \begin{tabular}{|c}
$\mathrm{B} 2 \mathrm{O} 3$ \\
$-\mathrm{a}$
\end{tabular} & $\begin{array}{c}\mathrm{CaO} \\
-\mathrm{a}\end{array}$ & $\begin{array}{c}\mathrm{Fe} 2 \mathrm{O} 3 \\
-\mathrm{a}\end{array}$ & $\begin{array}{c}\mathrm{FeO} \\
-\mathrm{a}\end{array}$ & $\begin{array}{c}\mathrm{K} 2 \mathrm{O} \\
-\mathrm{a}\end{array}$ & $\begin{array}{c}\mathrm{Li} 2 \mathrm{O} \\
-\mathrm{a}\end{array}$ & $\begin{array}{c}\mathrm{MgO} \\
-\mathrm{a}\end{array}$ & $\begin{array}{c}\mathrm{Na} 2 \mathrm{O} \\
-\mathrm{a}\end{array}$ & $\begin{array}{c}\mathrm{NiO} \\
-\mathrm{a}\end{array}$ & $\begin{array}{c}\mathrm{P} 2 \mathrm{O} 5 \\
-\mathrm{a}\end{array}$ \\
\hline IG1-01 & & & & & & & 0.0000 & & & 1.0000 & 0.0548 & 0.0857 & 0.0009 & & & 0.0440 & 0.0507 & & 0.1436 & & 0.0663 \\
\hline IG1-02 & & & & & & & 0.0000 & & & 1.0000 & & & & & & & & & & & \\
\hline IG1-03 & & & & & & & 0.0000 & & & 1.0000 & & & & & & & & & & & \\
\hline IG1-04 & & & & & & & 0.0000 & & & 1.0000 & 0.0013 & 0.0369 & 0.0009 & & & 0.0009 & 0.0693 & & 0.0921 & & 0.1170 \\
\hline IG1-05 & & & & & & & 0.0000 & & & 1.0000 & 0.0629 & 0.1265 & 0.0009 & & & 0.0009 & 0.0015 & & 0.2187 & & 0.1255 \\
\hline IG1-06 & & & & & & & 0.0000 & & & 1.0000 & 0.0008 & 0.1403 & 0.0009 & & & 0.0916 & 0.0458 & & 0.0491 & & 0.0007 \\
\hline IG1-07 & & & & & & & 0.0000 & & & 1.0000 & 0.1397 & 0.1410 & 0.0010 & & & 0.0009 & 0.0792 & & 0.0498 & & 0.0007 \\
\hline IG1-08 & & & & & & & 0.0000 & & & 1.0000 & 0.1269 & 0.0350 & 0.0009 & & & 0.0901 & 0.0669 & & 0.0826 & & 0.1237 \\
\hline IG1-09 & & & & & & & 0.0000 & & & 1.0000 & 0.0343 & 0.1278 & 0.0010 & & & 0.0009 & 0.0332 & & 0.2100 & & 0.1285 \\
\hline IG1-10 & & & & & & & 0.0000 & & & 1.0000 & 0.0011 & 0.0575 & 0.0009 & & & 0.0009 & 0.0805 & & 0.1483 & & 0.0007 \\
\hline IG1-11 & & & & & & & 0.0000 & & & 1.0000 & 0.1361 & 0.0473 & 0.0011 & & & 0.0009 & 0.0475 & & 0.1897 & & 0.0007 \\
\hline IG1-12 & & & & & & & 0.0000 & & & 1.0000 & 0.0307 & 0.1440 & 0.0009 & & & 0.1014 & 0.0001 & & 0.1547 & & 0.0007 \\
\hline IG1-13 & & & & & & & 0.0000 & & & 1.0000 & 0.0709 & 0.0443 & 0.0011 & & & 0.1028 & 0.0701 & & 0.1786 & & 0.0081 \\
\hline IG1-14 & & & & & & & 0.0000 & & & 1.0000 & 0.0989 & 0.0643 & 0.0010 & & & 0.0239 & 0.0454 & & 0.1574 & & 0.0320 \\
\hline IG1-15 & & & & & & & 0.0000 & & & 1.0000 & & & & & & & & & & & \\
\hline IG1-16 & & & & & & & 0.0000 & & & 1.0000 & 0.0686 & 0.0730 & 0.0009 & & & 0.0246 & 0.0438 & & 0.1641 & & 0.0357 \\
\hline IG1-17 & & & & & & & 0.0000 & & & 1.0000 & & & & & & & & & & & \\
\hline IG1-18 & & & & & & & 0.0000 & & & 1.0000 & 0.0644 & 0.1060 & 0.0010 & & & 0.0228 & 0.0536 & & 0.1076 & & 0.0981 \\
\hline IG1-19 & & & & & & & 0.0000 & & & 1.0000 & & & & & & & & & & & \\
\hline IG1-20 & & & & & & & 0.0000 & & & 1.0000 & 0.0435 & 0.1067 & 0.0009 & & & 0.0230 & 0.0497 & & 0.1076 & & 0.0979 \\
\hline IG1-21 & & & & & & & 0.0000 & & & 1.0000 & 0.0855 & 0.1131 & 0.0009 & & & 0.0734 & 0.0603 & & 0.0944 & & 0.0340 \\
\hline IG1-22 & & & & & & & 0.0000 & & & 1.0000 & & & & & & & & & & & \\
\hline IG1-23 & & & & & & & 0.0000 & & & 1.0000 & & & & & & & & & & & \\
\hline IG1-24 & & & & & & & 0.0000 & & & 1.0000 & 0.0798 & 0.1141 & 0.0009 & & & 0.0725 & 0.0594 & & 0.0933 & & 0.0324 \\
\hline IG1-25 & & & & & & & 0.0000 & & & 1.0000 & 0.0693 & 0.0689 & 0.0010 & & & 0.0696 & 0.0470 & & 0.1290 & & 0.0348 \\
\hline IG1-26 & & & & & & & 0.0000 & & & 1.0000 & 0.0538 & 0.0819 & 0.0009 & & & 0.0414 & 0.0521 & & 0.1412 & & 0.0687 \\
\hline IG1-27 & & & & & & & 0.0000 & & & 1.0000 & & & & & & & & & & & \\
\hline IG1-28 & & & & & & & 0.0000 & & & 1.0000 & & & & & & & & & & & \\
\hline IG1-29 & & & & & & & 0.0001 & & & 1.0000 & 0.1313 & 0.0814 & 0.0003 & & & 0.0009 & 0.0659 & & 0.1053 & & 0.0007 \\
\hline IG1-30 & & & & & & & 0.0008 & & & 1.0000 & 0.0208 & 0.0561 & 0.0040 & & & 0.0009 & 0.0664 & & 0.1823 & & 0.0050 \\
\hline IG1-31 & & & & & & & 0.0000 & & & 1.0000 & 0.1412 & 0.1367 & 0.0011 & & & 0.0008 & 0.0833 & & 0.1145 & & 0.0013 \\
\hline IG1-32 & & & & & & & 0.0000 & & & 1.0000 & 0.1413 & 0.0537 & 0.0011 & & & 0.0884 & 0.0789 & & 0.0510 & & 0.0476 \\
\hline IG1-33 & & & & & & & 0.0000 & & & 1.0000 & 0.0018 & 0.0340 & 0.0012 & & & 0.0765 & 0.0117 & & 0.2099 & & 0.0015 \\
\hline IG1-34 & & & & & & & 0.0000 & & & 1.0000 & 0.0026 & 0.0742 & 0.0011 & & & 0.0008 & 0.0848 & & 0.0511 & & 0.0476 \\
\hline IG1-35 & & & & & & & 0.0000 & & & 1.0000 & 0.0720 & 0.1496 & 0.0010 & & & 0.0005 & 0.0017 & & 0.2013 & & 0.0500 \\
\hline IG1-36 & & & & & & & 0.0000 & & & 1.0000 & 0.0727 & 0.0563 & 0.0011 & & & 0.0914 & 0.0867 & & 0.1179 & & 0.0014 \\
\hline IG1-37 & & & & & & & 0.0000 & & & 1.0000 & 0.0739 & 0.0963 & 0.0011 & & & 0.0445 & 0.0545 & & 0.1202 & & 0.0255 \\
\hline IG1-38 & & & & & & & 0.0000 & & & 1.0000 & 0.0371 & 0.1207 & 0.0011 & & & 0.0229 & 0.0601 & & 0.0883 & & 0.0129 \\
\hline IG1-39 & & & & & & & 0.0000 & & & 1.0000 & 0.0719 & 0.1110 & 0.0009 & & & 0.0645 & 0.0629 & & 0.0884 & & 0.0123 \\
\hline IG1-40 & & & & & & & 0.0000 & & & 1.0000 & 0.0532 & 0.1065 & 0.0010 & & & 0.0658 & 0.0544 & & 0.0924 & & 0.0390 \\
\hline IG1-41 & & & & & & & 0.0000 & & & 1.0000 & 0.0501 & 0.0670 & 0.0011 & & & 0.0669 & 0.0463 & & 0.1313 & & 0.0388 \\
\hline IG1-42 & & & & & & & 0.0000 & & & 1.0000 & 0.1038 & 0.0871 & 0.0011 & & & 0.0651 & 0.0658 & & 0.0932 & & 0.0388 \\
\hline IG1-43 & & & & & & & 0.0000 & & & 1.0000 & 0.0672 & 0.1164 & 0.0010 & & & 0.0227 & 0.0242 & & 0.1654 & & 0.0395 \\
\hline IG1-44 & & & & & & & 0.0000 & & & 1.0000 & 0.0025 & 0.0646 & 0.0010 & & & 0.0006 & 0.0868 & & 0.0517 & & 0.0501 \\
\hline
\end{tabular}


Appendix A. Database - mass fraction

INEEL CVS Phase 1 (Staples et al. 1999)

\begin{tabular}{|c|c|c|c|c|c|c|c|c|c|c|c|c|c|c|c|c|c|c|c|c|c|}
\hline Glass ID & $\begin{array}{c}\mathrm{SiO} 2 \\
-\mathrm{a} \\
\end{array}$ & $\begin{array}{c}\mathrm{ZrO} 2 \\
-\mathrm{a} \\
\end{array}$ & $\begin{array}{c}\mathrm{Ag} 2 \mathrm{O} \\
-\mathrm{a}\end{array}$ & $\begin{array}{c}\mathrm{As} 2 \mathrm{O} 3 \\
-\mathrm{a} \\
\end{array}$ & $\begin{array}{c}\mathrm{BaO} \\
-\mathrm{a}\end{array}$ & $\begin{array}{c}\mathrm{Bi} 2 \mathrm{O} 3 \\
-\mathrm{a}\end{array}$ & $\begin{array}{l}\mathrm{Br} \\
-\mathrm{a}\end{array}$ & $\begin{array}{c}\mathrm{CdO} \\
-\mathrm{a}\end{array}$ & $\begin{array}{c}\mathrm{Ce} 2 \mathrm{O} 3 \\
-\mathrm{a}\end{array}$ & $\begin{array}{c}\mathrm{CeO} 2 \\
-\mathrm{a}\end{array}$ & $\begin{array}{l}\mathrm{Cl} \\
-\mathrm{a}\end{array}$ & $\begin{array}{c}\mathrm{CoO} \\
-\mathrm{a}\end{array}$ & $\begin{array}{c}\mathrm{Co} 2 \mathrm{O} 3 \\
-\mathrm{a}\end{array}$ & $\begin{array}{c}\mathrm{Cr} 2 \mathrm{O} 3 \\
-\mathrm{a}\end{array}$ & $\begin{array}{c}\mathrm{Cs} 2 \mathrm{O} \\
-\mathrm{a}\end{array}$ & $\begin{array}{c}\mathrm{CuO} \\
-\mathrm{a}\end{array}$ & $\begin{array}{c}\mathrm{Eu} 2 \mathrm{O} 3 \\
-\mathrm{a}\end{array}$ & $\begin{array}{c}\mathrm{F} \\
-\mathrm{a}\end{array}$ & $\begin{array}{c}\mathrm{Ga} 2 \mathrm{O} 3 \\
-\mathrm{a}\end{array}$ & $\begin{array}{c}\mathrm{Gd} 2 \mathrm{O} 3 \\
-\mathrm{a}\end{array}$ & $\begin{array}{c}\mathrm{HgO} \\
-\mathrm{a}\end{array}$ \\
\hline IG1-01 & 0.4503 & 0.0667 & & & & & & & & & & & & & & & & & & & \\
\hline \multicolumn{22}{|l|}{ IG1-02 } \\
\hline \multicolumn{22}{|l|}{ IG1-03 } \\
\hline IG1-04 & 0.4936 & 0.1116 & & & & & & & & & & & & & & & & & & & \\
\hline IG1-05 & 0.4540 & 0.0001 & & & & & & & & & & & & & & & & & & & \\
\hline IG1-06 & 0.5717 & 0.0724 & & & & & & & & & & & & & & & & & & & \\
\hline IG1-07 & 0.5311 & 0.0290 & & & & & & & & & & & & & & & & & & & \\
\hline IG1-08 & 0.3968 & 0.0271 & & & & & & & & & & & & & & & & & & & \\
\hline IG1-09 & 0.3471 & 0.1133 & & & & & & & & & & & & & & & & & & & \\
\hline IG1-10 & 0.5273 & 0.1405 & & & & & & & & & & & & & & & & & & & \\
\hline IG1-11 & 0.5247 & 0.0004 & & & & & & & & & & & & & & & & & & & \\
\hline IG1-12 & 0.3947 & 0.1348 & & & & & & & & & & & & & & & & & & & \\
\hline IG1-13 & 0.5343 & 0.0074 & & & & & & & & & & & & & & & & & & & \\
\hline IG1-14 & 0.4819 & 0.0305 & & & & & & & & & & & & & & & & & & & \\
\hline \multicolumn{22}{|l|}{ IG1-15 } \\
\hline IG1-16 & 0.4680 & 0.0981 & & & & & & & & & & & & & & & & & & & \\
\hline \multicolumn{22}{|l|}{ IG1-17 } \\
\hline IG1-18 & 0.4872 & 0.0297 & & & & & & & & & & & & & & & & & & & \\
\hline \multicolumn{22}{|l|}{ IG1-19 } \\
\hline IG1-20 & 0.4690 & 0.0952 & & & & & & & & & & & & & & & & & & & \\
\hline IG1-21 & 0.4300 & 0.0847 & & & & & & & & & & & & & & & & & & & \\
\hline \multicolumn{22}{|l|}{ IG1-22 } \\
\hline \multicolumn{22}{|l|}{ IG1-23 } \\
\hline IG1-24 & 0.4300 & 0.0855 & & & & & & & & & & & & & & & & & & & \\
\hline IG1-25 & 0.4690 & 0.0967 & & & & & & & & & & & & & & & & & & & \\
\hline IG1-26 & 0.4514 & 0.0633 & & & & & & & & & & & & & & & & & & & \\
\hline \multicolumn{22}{|l|}{ IG1-27 } \\
\hline \multicolumn{22}{|l|}{ IG1-28 } \\
\hline IG1-29 & 0.5445 & 0.0002 & & & & & & & & & & & & & & & & & & & \\
\hline IG1-30 & 0.4412 & 0.1046 & & & & & & & & & & & & & & & & & & & \\
\hline IG1-31 & 0.4626 & 0.0293 & & & & & & & & & & & & & & & & & & & \\
\hline IG1-32 & 0.5022 & 0.0002 & & & & & & & & & & & & & & & & & & & \\
\hline IG1-33 & 0.5150 & 0.1393 & & & & & & & & & & & & & & & & & & & \\
\hline IG1-34 & 0.5680 & 0.1353 & & & & & & & & & & & & & & & & & & & \\
\hline IG1-35 & 0.4930 & 0.0003 & & & & & & & & & & & & & & & & & & & \\
\hline IG1-36 & 0.5370 & 0.0002 & & & & & & & & & & & & & & & & & & & \\
\hline IG1-37 & 0.4840 & 0.0747 & & & & & & & & & & & & & & & & & & & \\
\hline IG1-38 & 0.5250 & 0.1022 & & & & & & & & & & & & & & & & & & & \\
\hline IG1-39 & 0.4270 & 0.1024 & & & & & & & & & & & & & & & & & & & \\
\hline IG1-40 & 0.5289 & 0.0367 & & & & & & & & & & & & & & & & & & & \\
\hline IG1-41 & 0.5359 & 0.0345 & & & & & & & & & & & & & & & & & & & \\
\hline IG1-42 & 0.4590 & 0.0723 & & & & & & & & & & & & & & & & & & & \\
\hline IG1-43 & 0.4490 & 0.1079 & & & & & & & & & & & & & & & & & & & \\
\hline IG1-44 & 0.5835 & 0.1384 & & & & & & & & & & & & & & & & & & & \\
\hline
\end{tabular}


Appendix A. Database - mass fraction

INEEL CVS Phase 1 (Staples et al. 1999)

\begin{tabular}{|c|c|c|c|c|c|c|c|c|c|c|c|c|c|c|c|c|c|c|c|c|c|}
\hline Glass ID & $\begin{array}{c}\mathrm{I} \\
-\mathrm{a}\end{array}$ & $\begin{array}{c}\mathrm{La} 2 \mathrm{O} 3 \\
-\mathrm{a}\end{array}$ & $\begin{array}{c}\mathrm{MnO} 2 \\
-\mathrm{a}\end{array}$ & $\begin{array}{c}\mathrm{MnO} \\
-\mathrm{a}\end{array}$ & $\begin{array}{c}\mathrm{MoO} \\
-\mathrm{a}\end{array}$ & $\begin{array}{c}\mathrm{MoO} 3 \\
-\mathrm{a}\end{array}$ & $\begin{array}{c}\mathrm{Nb} 2 \mathrm{O} 5 \\
-\mathrm{a}\end{array}$ & $\begin{array}{c}\mathrm{Nd} 2 \mathrm{O} 3 \\
-\mathrm{a}\end{array}$ & $\begin{array}{c}\mathrm{PbO} \\
-\mathrm{a}\end{array}$ & $\begin{array}{c}\mathrm{PdO} 2 \\
-\mathrm{a}\end{array}$ & $\begin{array}{c}\mathrm{PdO} \\
-\mathrm{a}\end{array}$ & $\begin{array}{c}\mathrm{Pr} 2 \mathrm{O} 3 \\
-\mathrm{a}\end{array}$ & $\begin{array}{c}\text { Pr6O11 } \\
-\mathrm{a}\end{array}$ & $\begin{array}{c}\mathrm{Rb} 2 \mathrm{O} \\
-\mathrm{a}\end{array}$ & $\begin{array}{c}\mathrm{ReO} \\
-\mathrm{a}\end{array}$ & $\begin{array}{c}\mathrm{ReO} 2 \\
-\mathrm{a}\end{array}$ & $\begin{array}{c}\mathrm{Rh} 2 \mathrm{O} 3 \\
-\mathrm{a}\end{array}$ & $\begin{array}{c}\mathrm{RhO}_{2} \\
-\mathrm{a}\end{array}$ & $\begin{array}{c}\mathrm{RuO} 2 \\
-\mathrm{a}\end{array}$ & $\begin{array}{c}\mathrm{Sb} 2 \mathrm{O} 3 \\
-\mathrm{a}\end{array}$ & $\begin{array}{c}\mathrm{Sb} 2 \mathrm{O} 5 \\
-\mathrm{a}\end{array}$ \\
\hline IG1-01 & & & & & & & & & & & & & & & & & & & & & \\
\hline IG1-02 & & & & & & & & & & & & & & & & & & & & & \\
\hline IG1-03 & & & & & & & & & & & & & & & & & & & & & \\
\hline IG1-04 & & & & & & & & & & & & & & & & & & & & & \\
\hline IG1-05 & & & & & & & & & & & & & & & & & & & & & \\
\hline IG1-06 & & & & & & & & & & & & & & & & & & & & & \\
\hline IG1-07 & & & & & & & & & & & & & & & & & & & & & \\
\hline IG1-08 & & & & & & & & & & & & & & & & & & & & & \\
\hline IG1-09 & & & & & & & & & & & & & & & & & & & & & \\
\hline IG1-10 & & & & & & & & & & & & & & & & & & & & & \\
\hline IG1-11 & & & & & & & & & & & & & & & & & & & & & \\
\hline IG1-12 & & & & & & & & & & & & & & & & & & & & & \\
\hline IG1-13 & & & & & & & & & & & & & & & & & & & & & \\
\hline IG1-14 & & & & & & & & & & & & & & & & & & & & & \\
\hline IG1-15 & & & & & & & & & & & & & & & & & & & & & \\
\hline IG1-16 & & & & & & & & & & & & & & & & & & & & & \\
\hline IG1-17 & & & & & & & & & & & & & & & & & & & & & \\
\hline IG1-18 & & & & & & & & & & & & & & & & & & & & & \\
\hline IG1-19 & & & & & & & & & & & & & & & & & & & & & \\
\hline IG1-20 & & & & & & & & & & & & & & & & & & & & & \\
\hline IG1-21 & & & & & & & & & & & & & & & & & & & & & \\
\hline IG1-22 & & & & & & & & & & & & & & & & & & & & & \\
\hline IG1-23 & & & & & & & & & & & & & & & & & & & & & \\
\hline IG1-24 & & & & & & & & & & & & & & & & & & & & & \\
\hline IG1-25 & & & & & & & & & & & & & & & & & & & & & \\
\hline IG1-26 & & & & & & & & & & & & & & & & & & & & & \\
\hline IG1-27 & & & & & & & & & & & & & & & & & & & & & \\
\hline IG1-28 & & & & & & & & & & & & & & & & & & & & & \\
\hline IG1-29 & & & & & & & & & & & & & & & & & & & & & \\
\hline IG1-30 & & & & & & & & & & & & & & & & & & & & & \\
\hline IG1-31 & & & & & & & & & & & & & & & & & & & & & \\
\hline IG1-32 & & & & & & & & & & & & & & & & & & & & & \\
\hline IG1-33 & & & & & & & & & & & & & & & & & & & & & \\
\hline IG1-34 & & & & & & & & & & & & & & & & & & & & & \\
\hline IG1-35 & & & & & & & & & & & & & & & & & & & & & \\
\hline IG1-36 & & & & & & & & & & & & & & & & & & & & & \\
\hline IG1-37 & & & & & & & & & & & & & & & & & & & & & \\
\hline IG1-38 & & & & & & & & & & & & & & & & & & & & & \\
\hline IG1-39 & & & & & & & & & & & & & & & & & & & & & \\
\hline IG1-40 & & & & & & & & & & & & & & & & & & & & & \\
\hline IG1-41 & & & & & & & & & & & & & & & & & & & & & \\
\hline IG1-42 & & & & & & & & & & & & & & & & & & & & & \\
\hline IG1-43 & & & & & & & & & & & & & & & & & & & & & \\
\hline IG1-44 & & & & & & & & & & & & & & & & & & & & & \\
\hline
\end{tabular}


Appendix A. Database - mass fraction

INEEL CVS Phase 1 (Staples et al. 1999)

\begin{tabular}{|c|c|c|c|c|c|c|c|c|c|c|c|c|c|c|c|c|c|c|c|c|}
\hline Glass ID & $\begin{array}{c}\mathrm{SeO} 2 \\
-\mathrm{a}\end{array}$ & $\begin{array}{c}\mathrm{Sm} 2 \mathrm{O} 3 \\
-\mathrm{a}\end{array}$ & $\begin{array}{c}\mathrm{SnO} \\
-\mathrm{a} \\
\end{array}$ & $\begin{array}{c}\mathrm{SnO} 2 \\
-\mathrm{a}\end{array}$ & $\begin{array}{c}\mathrm{SO} 3 \\
-\mathrm{a}\end{array}$ & $\begin{array}{c}\mathrm{SrO} \\
-\mathrm{a}\end{array}$ & $\begin{array}{c}\mathrm{Tc} 2 \mathrm{O} 7 \\
-\mathrm{a}\end{array}$ & $\begin{array}{c}\mathrm{TeO} 2 \\
-\mathrm{a}\end{array}$ & $\begin{array}{c}\mathrm{ThO} 2 \\
-\mathrm{a}\end{array}$ & $\begin{array}{c}\mathrm{TiO} 2 \\
-\mathrm{a}\end{array}$ & $\begin{array}{c}\mathrm{T} 12 \mathrm{O} 3 \\
-\mathrm{a}\end{array}$ & $\begin{array}{c}\mathrm{U} 3 \mathrm{O} 8 \\
-\mathrm{a}\end{array}$ & $\begin{array}{c}\mathrm{UO} 2 \\
-\mathrm{a}\end{array}$ & $\begin{array}{c}\mathrm{UO3} \\
-\mathrm{a}\end{array}$ & $\begin{array}{c}\mathrm{V} 2 \mathrm{O} 5 \\
-\mathrm{a}\end{array}$ & $\begin{array}{c}\text { WO3 } \\
-\mathrm{a}\end{array}$ & $\begin{array}{c}\mathrm{Y} 2 \mathrm{O} 3 \\
-\mathrm{a}\end{array}$ & $\begin{array}{c}\mathrm{ZnO} \\
-\mathrm{a}\end{array}$ & $\begin{array}{c}\text { Others } \\
-\mathrm{a}\end{array}$ & $\begin{array}{c}\text { Sum } \\
-\mathrm{a}\end{array}$ \\
\hline IG1-01 & & & & & & & & & & & & & & & & & & & & 0.9630 \\
\hline IG1-02 & & & & & & & & & & & & & & & & & & & & \\
\hline IG1-03 & & & & & & & & & & & & & & & & & & & & \\
\hline IG1-04 & & & & & & & & & & & & & & & & & & & & 0.9236 \\
\hline IG1-05 & & & & & & & & & & & & & & & & & & & & 0.9910 \\
\hline IG1-06 & & & & & & & & & & & & & & & & & & & & 0.9733 \\
\hline IG1-07 & & & & & & & & & & & & & & & & & & & & 0.9724 \\
\hline IG1-08 & & & & & & & & & & & & & & & & & & & & 0.9500 \\
\hline IG1-09 & & & & & & & & & & & & & & & & & & & & 0.9961 \\
\hline IG1-10 & & & & & & & & & & & & & & & & & & & & 0.9577 \\
\hline IG1-11 & & & & & & & & & & & & & & & & & & & & 0.9484 \\
\hline IG1-12 & & & & & & & & & & & & & & & & & & & & 0.9620 \\
\hline IG1-13 & & & & & & & & & & & & & & & & & & & & 1.0176 \\
\hline IG1-14 & & & & & & & & & & & & & & & & & & & & 0.9353 \\
\hline IG1-15 & & & & & & & & & & & & & & & & & & & & \\
\hline IG1-16 & & & & & & & & & & & & & & & & & & & & 0.9768 \\
\hline IG1-17 & & & & & & & & & & & & & & & & & & & & \\
\hline IG1-18 & & & & & & & & & & & & & & & & & & & & 0.9704 \\
\hline IG1-19 & & & & & & & & & & & & & & & & & & & & \\
\hline IG1-20 & & & & & & & & & & & & & & & & & & & & 0.9935 \\
\hline IG1-21 & & & & & & & & & & & & & & & & & & & & 0.9763 \\
\hline IG1-22 & & & & & & & & & & & & & & & & & & & & \\
\hline IG1-23 & & & & & & & & & & & & & & & & & & & & \\
\hline IG1-24 & & & & & & & & & & & & & & & & & & & & 0.9679 \\
\hline IG1-25 & & & & & & & & & & & & & & & & & & & & 0.9853 \\
\hline IG1-26 & & & & & & & & & & & & & & & & & & & & 0.9547 \\
\hline IG1-27 & & & & & & & & & & & & & & & & & & & & \\
\hline IG1-28 & & & & & & & & & & & & & & & & & & & & \\
\hline IG1-29 & & & & & & & & & & & & & & & & & & & & 0.9305 \\
\hline IG1-30 & & & & & & & & & & & & & & & & & & & & 0.8813 \\
\hline IG1-31 & & & & & & & & & & & & & & & & & & & & 0.9708 \\
\hline IG1-32 & & & & & & & & & & & & & & & & & & & & 0.9644 \\
\hline IG1-33 & & & & & & & & & & & & & & & & & & & & 0.9909 \\
\hline IG1-34 & & & & & & & & & & & & & & & & & & & & 0.9655 \\
\hline IG1-35 & & & & & & & & & & & & & & & & & & & & 0.9694 \\
\hline IG1-36 & & & & & & & & & & & & & & & & & & & & 0.9647 \\
\hline IG1-37 & & & & & & & & & & & & & & & & & & & & 0.9747 \\
\hline IG1-38 & & & & & & & & & & & & & & & & & & & & 0.9703 \\
\hline IG1-39 & & & & & & & & & & & & & & & & & & & & 0.9413 \\
\hline IG1-40 & & & & & & & & & & & & & & & & & & & & 0.9779 \\
\hline IG1-41 & & & & & & & & & & & & & & & & & & & & 0.9719 \\
\hline IG1-42 & & & & & & & & & & & & & & & & & & & & 0.9862 \\
\hline IG1-43 & & & & & & & & & & & & & & & & & & & & 0.9933 \\
\hline IG1-44 & & & & & & & & & & & & & & & & & & & & 0.9792 \\
\hline
\end{tabular}


Appendix A. Database - mass fraction

INEEL CVS Phase 1 (Staples et al. 1999)

\begin{tabular}{|c|c|c|c|c|c|c|c|c|}
\hline Glass ID & $\begin{array}{l}\mathrm{TM} \\
\left({ }^{\circ} \mathrm{C}\right)\end{array}$ & $\begin{array}{l}\text { Gradient } \\
\text { TL }\left({ }^{\circ} \mathrm{C}\right)\end{array}$ & $\begin{array}{l}\text { Uniform } \\
\text { TL }\left({ }^{\circ} \mathrm{C}\right)\end{array}$ & Primary Phase & $\begin{array}{c}\text { Quenched } \\
\text { Visual/OM }\end{array}$ & $\begin{array}{c}\text { Quenched } \\
\text { SEM/EDS or TEM }\end{array}$ & $\begin{array}{l}\text { Quenched } \\
\text { XRD }\end{array}$ & $\begin{array}{c}\text { Quenched } \\
\text { Homogeneous? }\end{array}$ \\
\hline IG1-01 & 1150 & 897 & 902 & Li3PO4 & Homogeneous & Phase separated & Li3PO4 & no \\
\hline IG1-02 & 1150 & & & $\mathrm{Li3PO4}$ & Phase Separated & & $\mathrm{Li3PO4}$ & no \\
\hline IG1-03 & 1150 & & & $\mathrm{Na} 3 \mathrm{PO} 4$ & Phase Separated & & $\mathrm{Na} 3 \mathrm{PO} 4$ & no \\
\hline IG1-04 & 1150 & & & & Phase Separated & Phase Separated & & no \\
\hline IG1-05 & 1150 & & & & Phase Separated & Phase Separated & & no \\
\hline IG1-06 & 1150 & $883-855$ & 887 & $\mathrm{SiO} 2, \mathrm{LiB} 2 \mathrm{O} 4$ & Homogeneous & & Amorphous & yes \\
\hline IG1-07 & 1150 & $915-897$ & 885 & LiAlSi3O8 & Homogeneous & & Amorphous & yes \\
\hline IG1-08 & 1150 & & & & Phase Separated & Phase Separated & & no \\
\hline IG1-09 & 1150 & & 861 & $\mathrm{Na} 2 \mathrm{Si} 3 \mathrm{O} 7$, & Homogeneous & Phase separated & $\mathrm{Li} 2 \mathrm{NaPO} 4$ & no \\
\hline IG1-10 & 1150 & $985-965$ & 981 & $\mathrm{Na} 2 \mathrm{ZrSi} 2 \mathrm{O} 7$, & Homogeneous & & Amorphous & yes \\
\hline IG1-11 & 1150 & $848-743$ & 827 & $\mathrm{NaAlSiO} 4, \mathrm{Li} 2 \mathrm{SiO} 3$ & Homogeneous & & Amorphous & yes \\
\hline IG1-12 & 1150 & & 1075 & $\mathrm{~K} 2 \mathrm{ZrSi} 3 \mathrm{O} 9$ & Homogeneous & Homogeneous & Amorphous & yes \\
\hline IG1-13 & 1150 & $775-770$ & 765 & $\mathrm{Li} 2 \mathrm{SiO} 3$ & Homogeneous & Homogeneous & Amorphous & yes \\
\hline IG1-14 & 1150 & $850-840$ & 862 & Li3PO4 & Homogeneous & Phase separated & Li3PO4 & no \\
\hline IG1-15 & 1150 & & & & Phase Separated & & & no \\
\hline IG1-16 & 1150 & $908-882$ & 917 & $\mathrm{Li} 3 \mathrm{PO} 4, \mathrm{Zr} 2 \mathrm{P} 2 \mathrm{O} 7$ & Homogeneous & Homogeneous & Amorphous & yes \\
\hline IG1-17 & 1150 & $930-925$ & 928 & $\begin{array}{l}(\mathrm{Li}, \mathrm{Na}) 3 \mathrm{PO} 4, \\
\mathrm{Li} 2 \mathrm{NaPO} 4\end{array}$ & Homogeneous & Phase separated & $\mathrm{Li} 2 \mathrm{NaPO} 4$ & no \\
\hline IG1-18 & 1150 & & & & Phase separated & & & no \\
\hline IG1-19 & 1150 & & & & Phase separated & & & no \\
\hline IG1-20 & 1150 & & & & Phase separated & Phase separated & & no \\
\hline IG1-21 & 1150 & $918-898$ & 927 & $\mathrm{Li} 3 \mathrm{PO} 4$ & Homogeneous & Phase separated & $\mathrm{Li3PO4}$ & no \\
\hline IG1-22 & 1150 & & & & Phase separated & & & no \\
\hline IG1-23 & 1150 & & & & Phase separated & & & no \\
\hline IG1-24 & 1150 & 918 & 905 & $\mathrm{Li3PO4}$ & Homogeneous & Phase separated & Li3PO4, Na2ZrSiO5 & no \\
\hline IG1-25 & 1150 & $990-980$ & 991 & K2ZrSi3O9, Li3PO4 & Homogeneous & Questionable & Li3PO4 & no \\
\hline IG1-26 & 1150 & 888 & 875 & $\mathrm{Li3PO4}$ & Homogeneous & Phase separated & Li3PO4 & no \\
\hline IG1-27 & 1150 & & & & Phase separated & & & no \\
\hline IG1-28 & 1150 & & & & Phase separated & & & no \\
\hline IG1-29 & 1150 & $1090-1080$ & & & Homogeneous & & & yes \\
\hline IG1-30 & 1150 & 1135-1125 & & & Homogeneous & & & yes \\
\hline IG1-31 & 1150 & 785 & 791 & $\mathrm{Li} 2 \mathrm{SiO} 3, \mathrm{ZrO} 2$ & Homogeneous & & $\mathrm{Li} 2 \mathrm{SiO} 3, \mathrm{ZrO} 2$ & no \\
\hline IG1-32 & 1150 & & & & Phase Separated & & Li3PO4 & no \\
\hline IG1-33 & 1450 & & 1310 & $\mathrm{Na} 2 \mathrm{ZrSi} 2 \mathrm{O} 7$ & Homogeneous & & Amorphous & yes \\
\hline IG1-34 & 1150 & & 1142 & $\begin{array}{l}\text { LiNaZrSi6O15, } \\
\text { Li3PO4 }\end{array}$ & Homogeneous & Phase separated & Li3PO4 & no \\
\hline IG1-35 & 1150 & 863 & 850 & $\mathrm{Na3PO} 4$ & Homogeneous & Homogeneous & Amorphous & yes \\
\hline IG1-36 & 1150 & 865 & 855 & $\mathrm{Li} 2 \mathrm{SiO}_{3}$ & Homogeneous & & $\mathrm{Li} 2 \mathrm{SiO} 3$ & no \\
\hline IG1-37 & 1150 & 871 & 887 & Li3PO4, ZrO2 & Homogeneous & Homogeneous & Amorphous & yes \\
\hline IG1-38 & 1150 & $900-885$ & 922 & LiNaZrSi6O15 & Homogeneous & Homogeneous & Amorphous & yes \\
\hline IG1-39 & 1150 & $875-840$ & 855 & $\mathrm{~K} 2 \mathrm{ZrSi} 3 \mathrm{O} 9$ & Homogeneous & Homogeneous & Amorphous & yes \\
\hline IG1-40 & 1150 & 927 & 927 & $\mathrm{Li} 3 \mathrm{PO} 4$ & Homogeneous & Homogeneous & $\mathrm{Li3PO4}$ & no \\
\hline IG1-41 & 1150 & $835-827$ & 847 & Li3PO4 & Homogeneous & Questionable & Li3PO4 & no \\
\hline IG1-42 & 1150 & $960-953$ & 954 & Li3PO4 & Homogeneous & Phase separated & Li3PO4 & no \\
\hline IG1-43 & 1150 & 815 & 811 & Li3PO4 & Homogeneous & Homogeneous & Amorphous & yes \\
\hline IG1-44 & 1150 & & 1142 & $\begin{array}{l}\text { LiNaZrSi6O15, } \\
\text { Li3PO4 }\end{array}$ & Homogeneous & Phase separated & Li3PO4 & no \\
\hline
\end{tabular}


Appendix A. Database - mass fraction

\section{INEEL CVS Phase 1 (Staples et al. 1999)}

\begin{tabular}{|c|c|c|c|c|c|}
\hline Glass ID & $\begin{array}{c}\mathrm{CCC} \\
\text { Visual/OM }\end{array}$ & $\begin{array}{c}\text { CCC } \\
\text { SEM/EDS or TEM }\end{array}$ & $\begin{array}{l}\text { CCC } \\
\text { XRD }\end{array}$ & $\begin{array}{l}\text { Heat Treated } \\
\text { Visual/OM }\end{array}$ & $\begin{array}{c}\text { Heat Treated } \\
\text { SEM/EDS or TEM }\end{array}$ \\
\hline \multicolumn{6}{|l|}{ IG1-01 } \\
\hline \multicolumn{6}{|l|}{ IG1-02 } \\
\hline \multicolumn{6}{|l|}{ IG1-03 } \\
\hline \multicolumn{6}{|l|}{ IG1-04 } \\
\hline \multicolumn{6}{|l|}{ IG1-05 } \\
\hline \multicolumn{6}{|l|}{ IG1-06 } \\
\hline \multicolumn{6}{|l|}{ IG1-07 } \\
\hline \multicolumn{6}{|l|}{ IG1-08 } \\
\hline \multicolumn{6}{|l|}{ IG1-09 } \\
\hline \multicolumn{6}{|l|}{ IG1-10 } \\
\hline \multicolumn{6}{|l|}{ IG1-11 } \\
\hline \multicolumn{6}{|l|}{ IG1-12 } \\
\hline \multicolumn{6}{|l|}{ IG1-13 } \\
\hline IG1-14 & Phase separated & & $\mathrm{Li3PO4}$ & & \\
\hline \multicolumn{6}{|l|}{ IG1-15 } \\
\hline IG1-16 & homogenous & & Li3PO4 & & \\
\hline \multicolumn{6}{|l|}{ IG1-17 } \\
\hline \multicolumn{6}{|l|}{ IG1-18 } \\
\hline \multicolumn{6}{|l|}{ IG1-19 } \\
\hline \multicolumn{6}{|l|}{ IG1-20 } \\
\hline \multicolumn{6}{|l|}{ IG1-21 } \\
\hline \multicolumn{6}{|l|}{ IG1-22 } \\
\hline \multicolumn{6}{|l|}{ IG1-23 } \\
\hline \multicolumn{6}{|l|}{ IG1-24 } \\
\hline \multicolumn{6}{|l|}{ IG1-25 } \\
\hline \multicolumn{6}{|l|}{ IG1-26 } \\
\hline IG1-27 & & & & & \\
\hline IG1-28 & & & & & \\
\hline IG1-29 & & & & & \\
\hline IG1-30 & & & & & \\
\hline IG1-31 & & & & & \\
\hline IG1-32 & & & & & \\
\hline IG1-33 & & & & & \\
\hline IG1-34 & & & & & \\
\hline IG1-35 & homogenous & & $\mathrm{Na} 3 \mathrm{PO} 4$ & & \\
\hline IG1-36 & & & & & \\
\hline IG1-37 & homogenous & & Li3PO4 & & \\
\hline IG1-38 & homogenous & & none & & \\
\hline IG1-39 & homogenous & & none & & \\
\hline IG1-40 & & & & & \\
\hline IG1-41 & Phase separated & & $\mathrm{Li3PO} 4$ & & \\
\hline IG1-42 & & & & & \\
\hline IG1-43 & homogenous & & Li3PO4 & & \\
\hline IG1-44 & & & & & \\
\hline
\end{tabular}


INEEL CVS Phase 1 (Staples et al. 1999)

\begin{tabular}{|c|c|c|c|c|c|c|c|c|c|c|c|c|c|c|c|}
\hline Glass ID & $\begin{array}{c}\text { Heat Treated } \\
\text { XRD } \\
\end{array}$ & $\begin{array}{l}\text { Density } \\
\left(\mathrm{g} / \mathrm{cm}^{3}\right)\end{array}$ & $\begin{array}{c}\text { Fulc Visc } \\
\mathrm{A}\end{array}$ & $\begin{array}{c}\text { Fulc Visc } \\
\text { B } \\
\end{array}$ & \begin{tabular}{|c} 
Fulc Visc \\
To \\
\end{tabular} & $\begin{array}{c}\mathrm{FV} 1150^{\circ} \mathrm{C} \\
(\mathrm{Pa} \cdot \mathrm{s})\end{array}$ & $\begin{array}{c}\text { Arrh Visc } \\
\text { A }\end{array}$ & $\begin{array}{c}\text { Arrh Visc } \\
\text { B }\end{array}$ & $\begin{array}{c}1150^{\circ} \mathrm{C} \\
\left(\mathrm{D}_{0}, \mathrm{C}\right)\end{array}$ & \begin{tabular}{|c|}
$\mathrm{T}\left({ }^{\circ} \mathrm{C}\right)$ at \\
$2 \mathrm{~Pa} \cdot \mathrm{s}$ \\
\end{tabular} & $\begin{array}{c}\mathrm{T}\left({ }^{\circ} \mathrm{C}\right) \text { at } \\
5 \mathrm{~Pa} \cdot \mathrm{s} \\
\end{array}$ & \begin{tabular}{|c|}
$\mathrm{T}\left({ }^{\circ} \mathrm{C}\right)$ at \\
$10 \mathrm{~Pa} \cdot \mathrm{s}$ \\
\end{tabular} & $\begin{array}{c}\mathrm{T} 1 \\
\left({ }^{\circ} \mathrm{C}\right) \\
\end{array}$ & $\begin{array}{c}\mathrm{V} 1 \\
(\mathrm{~Pa} \cdot \mathrm{s}) \\
\end{array}$ & $\begin{array}{c}\mathrm{T} 2 \\
\left({ }^{\circ} \mathrm{C}\right) \\
\end{array}$ \\
\hline IG1-01 & & & -5.8791 & 6252.6 & 296.52 & 4.25 & -11.624 & 18626.3 & 4.33 & & & & 1160 & 3.8 & 1105.8 \\
\hline IG1-02 & & & -3.5888 & 2400.8 & 584.24 & 1.92 & -10.903 & 16498.7 & 2.00 & & & & 1162.2 & 1.7 & 1109.3 \\
\hline \multicolumn{16}{|l|}{ IG1-03 } \\
\hline \multicolumn{16}{|l|}{ IG1-04 } \\
\hline \multicolumn{16}{|l|}{ IG1-05 } \\
\hline IG1-06 & & & -5.9344 & 6419 & 337.53 & 7.14 & -12.931 & 21236.6 & 7.33 & & & & 1156.1 & 6.66 & 1105.9 \\
\hline IG1-07 & & & -5.061 & 6470.2 & 264.42 & 9.44 & -10.218 & 17762.1 & 9.62 & & & & 1159.5 & 8.76 & 1106.6 \\
\hline IG1-08 & & & -1.1538 & 1153.3 & 849.2 & 14.59 & -13.273 & 22753.9 & 15.14 & & & & 1159 & 13.18 & 1107.9 \\
\hline IG1-09 & & & -7.0245 & 6030 & 353.72 & 1.73 & -14.109 & 20894.0 & 1.78 & & & & 1155.9 & 1.64 & 1105.5 \\
\hline IG1-10 & & & -7.3343 & 7607.1 & 242.89 & 2.86 & -12.854 & 19823.4 & 2.94 & & & & 1159.1 & 2.68 & 1106.4 \\
\hline IG1-11 & & & -4.8207 & 6807 & 216.48 & 11.83 & -9.233 & 16682.8 & 12.07 & & & & 1158.6 & 11.19 & 1105.4 \\
\hline IG1-12 & & & -9.6209 & 10796 & 209.54 & 6.41 & -16.359 & 25957.7 & 6.57 & & & & 1158.7 & 5.67 & 1108 \\
\hline IG1-13 & & & -5.5947 & 6261.9 & 183.91 & 2.43 & -9.104 & 14234.8 & 2.46 & & & & 1156 & 2.33 & 1105.1 \\
\hline IG1-14 & & & -5.3455 & 6601.5 & 255.8 & 7.67 & -10.419 & 17754.0 & 7.83 & & & & 1157.6 & 7.18 & 1106.6 \\
\hline \multicolumn{16}{|l|}{ IG1-15 } \\
\hline IG1-16 & & & -5.9717 & 6781.3 & 305.43 & 7.83 & -12.405 & 20626.9 & 8.09 & & & & 1162.1 & 7.05 & 1109 \\
\hline IG1-17 & & & -6.0772 & 6626.8 & 338.08 & 8.04 & -13.320 & 21964.5 & 8.29 & & & & 1159.9 & 7.25 & 1107.4 \\
\hline \multicolumn{16}{|l|}{ IG1-18 } \\
\hline IG1-19 & & & -5.4745 & 6233.2 & 325.58 & 8.05 & -10.958 & 18584.3 & 8.18 & & & & 1160.8 & 7.23 & 1108.5 \\
\hline \multicolumn{16}{|l|}{ IG1-20 } \\
\hline IG1-21 & & & -6.1136 & 6360 & 285.07 & 3.45 & -11.656 & 18383.0 & 3.53 & & & & 1158 & 3.24 & 1105.8 \\
\hline \multicolumn{16}{|l|}{ IG1-22 } \\
\hline IG1-23 & & & -5.8418 & 6380.9 & 337.04 & 7.44 & -11.773 & 19632.8 & 7.57 & & & & 1158 & 6.88 & 1107 \\
\hline IG1-24 & & & -5.7323 & 5834.4 & 311.32 & 3.40 & -11.364 & 17948.6 & 3.49 & & & & 1162.4 & 3.06 & 1109.8 \\
\hline IG1-25 & & & -6.1325 & 6734.2 & 307.96 & 6.46 & -12.618 & 20654.5 & 6.67 & & & & 1159.6 & 5.92 & 1107.2 \\
\hline IG1-26 & & & -5.9698 & 6285.1 & 297.72 & 4.07 & -11.742 & 18738.4 & 4.16 & & & & 1158 & 3.78 & 1107 \\
\hline IG1-27 & & & -1.2374 & 1166.9 & 848.61 & 13.93 & -13.892 & 23590.1 & 14.67 & & & & 1159.9 & 12.38 & 1109 \\
\hline IG1-28 & & & -1.8747 & 1522.3 & 811.08 & 13.69 & -14.667 & 24669.0 & 14.42 & & & & 1158.1 & 12.17 & 1107 \\
\hline IG1-29 & & & -4.6669 & 6594.3 & 238.87 & 13.07 & -9.329 & 16959.1 & 13.31 & & & & 1163.2 & 11.74 & 1110.3 \\
\hline IG1-30 & & & -5.0111 & 4049.1 & 425.74 & 1.79 & -11.533 & 17273.8 & 1.83 & & & & 1155.3 & 1.71 & 1104.1 \\
\hline IG1-31 & & & -5.816 & 6034.3 & 220.07 & 1.96 & -9.786 & 14901.3 & 1.99 & & & & 1157.3 & 1.87 & 1106.6 \\
\hline IG1-32 & & & -4.9198 & 6656.4 & 273.09 & 14.45 & -10.040 & 18112.8 & 14.71 & & & & 1157.1 & 13.75 & 1106.1 \\
\hline \multicolumn{16}{|l|}{ IG1-33 } \\
\hline IG1-34 & & & -5.8934 & 6799.9 & 373.14 & 17.46 & -13.818 & 23769.5 & 17.92 & & & & 1158 & 15.95 & 1106.9 \\
\hline IG1-35 & & & -5.1402 & 5750.5 & 366.4 & 9.01 & -12.244 & 20609.6 & 9.39 & & & & 1158.5 & 8.25 & 1107.4 \\
\hline IG1-36 & & & -5.5219 & 6460.8 & 189.34 & 3.33 & -9.230 & 14871.2 & 3.39 & & & & 1155.9 & 3.25 & 1106 \\
\hline IG1-37 & & & -5.849 & 6571.6 & 286.65 & 5.83 & -11.618 & 19076.5 & 5.98 & & & & 1157.5 & 5.45 & 1106.8 \\
\hline IG1-38 & & & -5.9294 & 6569.3 & 319.64 & 7.26 & -12.583 & 20764.4 & 7.46 & & & & 1157.5 & 6.74 & 1106.7 \\
\hline IG1-39 & & & -6.3307 & 6346.2 & 288.7 & 2.82 & -11.949 & 18514.0 & 2.89 & & & & 1159.2 & 2.62 & 1107.7 \\
\hline IG1-40 & & & -5.2199 & 6054.7 & 313.58 & 7.53 & -11.169 & 18797.4 & 7.70 & & & & 1157.9 & 6.92 & 1106.7 \\
\hline IG1-41 & & & -5.3757 & 6490.9 & 274.82 & 7.70 & -10.784 & 18279.6 & 7.86 & & & & 1158 & 7.16 & 1106.9 \\
\hline IG1-42 & & & -5.7084 & 6720.2 & 289.77 & 8.20 & -11.691 & 19668.3 & 8.42 & & & & 1158.6 & 7.6 & 1107.2 \\
\hline IG1-43 & & & -6.0533 & 6513.7 & 343.86 & 7.59 & -13.339 & 21906.5 & 7.81 & & & & 1159.7 & 6.82 & 1107.2 \\
\hline IG1-44 & & & -5.9383 & 6830.5 & 371.37 & 17.02 & -13.833 & 23754.6 & 17.47 & & & & 1158.9 & 15.43 & 1107.5 \\
\hline
\end{tabular}




\section{INEEL CVS Phase 1 (Staples et al. 1999)}

\begin{tabular}{|c|c|c|c|c|c|c|c|c|c|c|c|c|c|c|c|c|c|c|c|c|c|}
\hline Glass ID & $\begin{array}{c}\mathrm{V} 2 \\
(\mathrm{~Pa} \cdot \mathrm{s})\end{array}$ & $\begin{array}{c}\mathrm{T} 3 \\
\left({ }^{\circ} \mathrm{C}\right)\end{array}$ & $\begin{array}{c}\mathrm{V} 3 \\
(\mathrm{~Pa} \cdot \mathrm{s})\end{array}$ & $\begin{array}{l}\mathrm{T} 4 \\
\left({ }^{\circ} \mathrm{C}\right)\end{array}$ & $\begin{array}{c}\mathrm{V} 4 \\
(\mathrm{~Pa} \cdot \mathrm{s})\end{array}$ & $\begin{array}{l}\text { T5 } \\
\left({ }^{\circ} \mathrm{C}\right)\end{array}$ & $\begin{array}{c}\mathrm{V} 5 \\
(\mathrm{~Pa} \cdot \mathrm{s})\end{array}$ & $\begin{array}{l}\mathrm{T} 6 \\
\left({ }^{\circ} \mathrm{C}\right)\end{array}$ & $\begin{array}{c}\mathrm{V} 6 \\
(\mathrm{~Pa} \cdot \mathrm{s})\end{array}$ & $\begin{array}{l}\mathrm{T} 7 \\
\left({ }^{\circ} \mathrm{C}\right)\end{array}$ & $\begin{array}{c}\mathrm{V} 7 \\
(\mathrm{~Pa} \cdot \mathrm{s})\end{array}$ & $\begin{array}{l}\mathrm{T} 8 \\
\left({ }^{\circ} \mathrm{C}\right)\end{array}$ & $\begin{array}{c}\mathrm{V} 8 \\
(\mathrm{~Pa} \cdot \mathrm{s})\end{array}$ & $\begin{array}{l}\mathrm{T} 9 \\
\left({ }^{\circ} \mathrm{C}\right)\end{array}$ & $\begin{array}{c}\mathrm{V} 9 \\
(\mathrm{~Pa} \cdot \mathrm{s})\end{array}$ & $\begin{array}{l}\mathrm{T} 10 \\
\left({ }^{\circ} \mathrm{C}\right)\end{array}$ & $\begin{array}{c}\mathrm{V} 10 \\
(\mathrm{~Pa} \cdot \mathrm{s})\end{array}$ & $\begin{array}{l}\mathrm{T} 11 \\
\left({ }^{\circ} \mathrm{C}\right)\end{array}$ & $\begin{array}{c}\mathrm{V} 11 \\
(\mathrm{~Pa} \cdot \mathrm{s})\end{array}$ & $\begin{array}{l}\mathrm{T} 12 \\
\left({ }^{\circ} \mathrm{C}\right)\end{array}$ & $\begin{array}{c}\mathrm{V} 12 \\
(\mathrm{~Pa} \cdot \mathrm{s})\end{array}$ \\
\hline IG1-01 & 6.22 & 1055.1 & 10.32 & 1108 & 6.42 & 1158.7 & 3.99 & 1206.5 & 2.69 & 1255.2 & 1.87 & 1153.4 & 4.23 & 1005.5 & 19.2 & 956 & 36.53 & & & & \\
\hline IG1-02 & 2.6 & 1058.4 & 4.23 & 1109.5 & 2.68 & 1158.7 & 1.85 & 1207.9 & 1.32 & 1255.9 & 0.96 & 1156.3 & 1.92 & 1008 & 8.1 & & & & & & \\
\hline \multicolumn{22}{|l|}{ IG1-03 } \\
\hline \multicolumn{22}{|l|}{ IG1-04 } \\
\hline \multicolumn{22}{|l|}{ IG1-05 } \\
\hline IG1-06 & 11.21 & 1056 & 20 & 1107.9 & 10.93 & 1157.5 & 6.6 & 1206.1 & 4.27 & 1255.3 & 2.89 & 1155.2 & 6.98 & 1006.7 & 39.19 & 957.2 & 83.12 & & & & \\
\hline IG1-07 & 13.83 & 1055.6 & 22.59 & 1107.6 & 13.61 & 1158 & 8.86 & 1206.1 & 6.1 & 1254.9 & 4.31 & 1154.4 & 9.22 & 1005.9 & 39.25 & 955.9 & 72.99 & & & & \\
\hline IG1-08 & 27.35 & 1158.6 & 12.48 & 1207.2 & 8.14 & 1256 & 5.31 & 1156.2 & 13.76 & & & & & & & & & & & & \\
\hline IG1-09 & 2.7 & 1055.6 & 4.77 & 1105.9 & 2.71 & 1155.2 & 1.65 & 1204.1 & 1.06 & 1252.9 & 0.72 & 1154.6 & 1.69 & 1006 & 9.31 & 956.9 & 19.38 & & & & \\
\hline IG1-10 & 4.47 & 1055.8 & 7.7 & 1107.3 & 4.38 & 1157 & 2.71 & 1206.1 & 1.75 & 1256 & 1.17 & 1155 & 2.77 & 1006.1 & 14.07 & 956.8 & 27.35 & & & & \\
\hline IG1-11 & 17.35 & 1055.4 & 27.25 & 1107.2 & 16.99 & 1156.6 & 11.34 & 1205.8 & 7.83 & 1254.7 & 5.54 & 1154 & 11.8 & 1006.1 & 45.23 & 956.6 & 78.54 & & & & \\
\hline IG1-12 & 10.8 & 1057.9 & 21.25 & 1109.5 & 10.59 & 1158.8 & 5.77 & 1207.6 & 3.29 & 1255.8 & 1.97 & 1156.2 & 6.58 & 1007.9 & 50.7 & 958.6 & 120.84 & & & & \\
\hline IG1-13 & 3.35 & 1055 & 4.94 & 1105.3 & 3.36 & 1154.8 & 2.37 & 1204 & 1.72 & 1253.1 & 1.27 & 1154.1 & 2.42 & 1005.2 & 7.69 & 956.1 & 12.12 & & & & \\
\hline IG1-14 & 11.3 & 1056.3 & 18.29 & 1106.7 & 11.3 & 1156.2 & 7.34 & 1205.2 & 4.99 & 1254.2 & 3.49 & 1155.4 & 7.51 & 1006.4 & 31.97 & 957 & 57.77 & & & & \\
\hline \multicolumn{22}{|l|}{ IG1-15 } \\
\hline IG1-16 & 12.03 & 1058.4 & 21.33 & 1109.5 & 11.97 & 1158.8 & 7.28 & 1207.7 & 4.67 & 1256.4 & 3.14 & 1156.6 & 7.48 & 1008.1 & 40.4 & 958.9 & 80.74 & & & & \\
\hline IG1-17 & 12.77 & 1056.5 & 23.31 & 1107.6 & 12.61 & 1157.1 & 7.63 & 1206.2 & 4.75 & 1255.2 & 3.1 & 1155.4 & 7.76 & 1006.5 & 47.37 & 957.2 & 100.39 & & & & \\
\hline \multicolumn{22}{|l|}{ IG1-18 } \\
\hline IG1-19 & 11.95 & 1057.6 & 21.06 & 1108.9 & 11.94 & 1158.3 & 7.42 & 1207.1 & 4.93 & 1255.8 & 3.43 & 1156.1 & 7.74 & & & & & & & & \\
\hline \multicolumn{22}{|l|}{ IG1-20 } \\
\hline IG1-21 & 5.2 & 1055.4 & 8.62 & 1107.9 & 5.06 & 1157.3 & 3.26 & 1206.1 & 2.2 & 1255 & 1.54 & 1154.8 & 3.37 & 1005.9 & 15.16 & 956.7 & 28.39 & & & & \\
\hline \multicolumn{22}{|l|}{ IG1-22 } \\
\hline IG1-23 & 11.53 & 1056.8 & 20.36 & 1107.2 & 11.55 & 1156.5 & 7.01 & 1205.5 & 4.52 & 1254.4 & 3 & 1155.7 & 7.19 & & & & & & & & \\
\hline IG1-24 & 4.78 & 1059 & 7.79 & 1110.2 & 4.76 & 1159.5 & 3.14 & 1208.3 & 2.17 & 1256.9 & 1.57 & 1157.3 & 3.29 & 1008.7 & 14.23 & 959.6 & 26.37 & & & & \\
\hline IG1-25 & 10.09 & 1056.5 & 17.8 & 1107.9 & 10 & 1157.4 & 6.12 & 1206.3 & 3.92 & 1255.1 & 2.6 & 1155.3 & 6.33 & 1006.5 & 34.28 & 957.3 & 68.32 & & & & \\
\hline IG1-26 & 6.03 & 1057.1 & 10.01 & 1107.1 & 6.06 & 1156.9 & 3.86 & 1206 & 2.59 & 1255 & 1.79 & 1156.2 & 3.94 & 1007 & 18.25 & 957.6 & 34.67 & & & & \\
\hline IG1-27 & 25.34 & 1158.5 & 12.54 & 1107.6 & 27 & 1158.8 & 11.91 & 1207.5 & 7.6 & 1256.2 & 5.11 & 1156.3 & 13.36 & & & & & & & & \\
\hline IG1-28 & 26.29 & 1106.2 & 27.59 & 1156.4 & 12.02 & 1205.4 & 7.27 & 1254.3 & 4.92 & 1155.5 & 12.76 & & & & & & & & & & \\
\hline IG1-29 & 18.22 & 1059.5 & 29.02 & 1110.9 & 18.24 & 1160 & 12.13 & 1208.9 & 8.38 & 1258 & 6.02 & 1158.1 & 12.45 & 1009.4 & 49.44 & 960.2 & 86.88 & & & & \\
\hline IG1-30 & 2.55 & 1055.8 & 4.05 & 1107.4 & 2.53 & 1157 & 1.72 & 1206.1 & 1.2 & 1255.2 & 0.87 & 1155.6 & 1.73 & 1006.9 & 7.25 & 957.1 & 13.49 & & & & \\
\hline IG1-31 & 2.7 & 1056.2 & 4.05 & 1106.7 & 2.71 & 1156.1 & 1.89 & 1205.4 & 1.36 & 1254.2 & 1 & 1155.4 & 1.9 & 1006.2 & 6.43 & 956.9 & 10.65 & & & & \\
\hline IG1-32 & 21.69 & 1055.9 & 35.77 & 1106.4 & 21.65 & 1155.8 & 13.83 & 1204.8 & 9.23 & 1253.8 & 6.35 & 1155 & 14.05 & 1006.1 & 63.75 & & & & & & \\
\hline \multicolumn{22}{|l|}{ IG1-33 } \\
\hline IG1-34 & 29.34 & 1056.5 & 57.34 & 1106.7 & 29.46 & 1156.1 & 16.34 & 1205.1 & 9.7 & 1254.1 & 6.06 & 1155.2 & 16.67 & 1006.1 & 124.1 & & & & & & \\
\hline IG1-35 & 13.59 & 1057.1 & 23.86 & 1107.4 & 13.66 & 1156.9 & 8.54 & 1205.8 & 5.66 & 1254.7 & 3.94 & 1155.8 & 8.86 & 1006.8 & 48.16 & 957.5 & 101.63 & & & & \\
\hline IG1-36 & 4.66 & 1056.1 & 6.98 & 1106.7 & 4.64 & 1156.1 & 3.23 & 1205.3 & 2.31 & 1254.2 & 1.68 & 1155.4 & 3.27 & 1006.4 & 10.99 & 957.1 & 17.74 & & & & \\
\hline IG1-37 & 8.81 & 1056.7 & 14.75 & 1107 & 8.8 & 1156.5 & 5.58 & 1205.5 & 3.68 & 1254.4 & 2.51 & 1155.6 & 5.7 & 1006.7 & 26.9 & 957.6 & 51.17 & & & & \\
\hline IG1-38 & 11.31 & 1056.4 & 20.03 & 1106.6 & 11.34 & 1156.1 & 6.89 & 1205.2 & 4.42 & 1254.2 & 2.96 & 1155.3 & 7.03 & 1006.3 & 38.39 & 956.9 & 78.91 & & & & \\
\hline IG1-39 & 4.17 & 1057.1 & 6.92 & 1107.4 & 4.2 & 1156.7 & 2.7 & 1205.6 & 1.81 & 1254.4 & 1.25 & 1155.5 & 2.73 & 1006.6 & 12.48 & 957.3 & 23.35 & & & & \\
\hline IG1-40 & 11.09 & 1056.5 & 18.48 & 1107 & 11.12 & 1156.5 & 7.17 & 1205.5 & 4.83 & 1254.4 & 3.36 & 1155.6 & 7.31 & 1006.8 & 34.26 & 957.4 & 65.23 & & & & \\
\hline IG1-41 & 11.32 & 1056.5 & 18.64 & 1106.9 & 11.39 & 1156.2 & 7.34 & 1205.3 & 4.95 & 1254.1 & 3.45 & 1155.2 & 7.57 & 1006.3 & 33.42 & 956.9 & 62.31 & & & & \\
\hline IG1-42 & 12.51 & 1056.7 & 21.47 & 1107.2 & 12.57 & 1156.6 & 7.8 & 1205.5 & 5.11 & 1254.5 & 3.45 & 1155.5 & 7.97 & 1006.7 & 39.82 & 957.5 & 76.91 & & & & \\
\hline IG1-43 & 11.98 & 1057.1 & 21.86 & 1107.8 & 11.89 & 1157.7 & 7.08 & 1206.2 & 4.47 & 1255.2 & 2.94 & 1155.5 & 7.39 & 1006.3 & 43.86 & 958.8 & 92.76 & & & & \\
\hline IG1-44 & 28.44 & 1057 & 55.43 & 1107.3 & 28.61 & 1156.6 & 15.92 & 1205.5 & 9.42 & 1254.4 & 5.88 & 1155.5 & 16.16 & 1006.5 & 119.96 & & & & & & \\
\hline
\end{tabular}


Appendix A. Database - mass fraction

INEEL CVS Phase 1 (Staples et al. 1999)

\begin{tabular}{|c|c|c|c|c|c|c|c|c|c|c|c|c|c|c|c|c|}
\hline Glass ID & $\begin{array}{l}\mathrm{T} 13 \\
\left({ }^{\circ} \mathrm{C}\right)\end{array}$ & $\begin{array}{c}\mathrm{V} 13 \\
(\mathrm{~Pa} \cdot \mathrm{s}) \\
\end{array}$ & $\begin{array}{l}\mathrm{T} 14 \\
\left({ }^{\circ} \mathrm{C}\right)\end{array}$ & $\begin{array}{c}\mathrm{V} 14 \\
(\mathrm{~Pa} \cdot \mathrm{s})\end{array}$ & $\begin{array}{c}\text { Q PCT } \\
\mathrm{B}\left(\mathrm{g} / \mathrm{m}^{2}\right)\end{array}$ & $\begin{array}{c}\text { Q PCT } \\
\mathrm{Li}\left(\mathrm{g} / \mathrm{m}^{2}\right)\end{array}$ & $\begin{array}{c}\text { Q PCT } \\
\mathrm{Na}\left(\mathrm{g} / \mathrm{m}^{2}\right)\end{array}$ & $\begin{array}{c}\text { Q PCT } \\
\mathrm{Si}\left(\mathrm{g} / \mathrm{m}^{2}\right)\end{array}$ & $\begin{array}{c}\text { Q PCT } \\
\text { pH }\end{array}$ & $\begin{array}{c}\text { CCC PCT } \\
\mathrm{B}\left(\mathrm{g} / \mathrm{m}^{2}\right)\end{array}$ & $\begin{array}{l}\text { CCC PCT } \\
\mathrm{Li}\left(\mathrm{g} / \mathrm{m}^{2}\right)\end{array}$ & $\begin{array}{l}\mathrm{CCC} \text { PCT } \\
\mathrm{Na}\left(\mathrm{g} / \mathrm{m}^{2}\right)\end{array}$ & $\begin{array}{l}\text { CCC PCT } \\
\mathrm{Si}\left(\mathrm{g} / \mathrm{m}^{2}\right)\end{array}$ & $\begin{array}{c}\text { CCC PCT } \\
\mathrm{pH}\end{array}$ & $\begin{array}{l}\text { Q PCT B at } \\
20^{\circ} \mathrm{C}\left(\mathrm{g} / \mathrm{m}^{2}\right)\end{array}$ & $\begin{array}{l}\text { Q PCT Li at } \\
20^{\circ} \mathrm{C}\left(\mathrm{g} / \mathrm{m}^{2}\right)\end{array}$ \\
\hline IG1-01 & & & & & 2.790 & 1.636 & 2.279 & 0.670 & & & & & & & & \\
\hline IG1-02 & & & & & 3.546 & 0.877 & 2.984 & 0.4225 & & & & & & & & \\
\hline IG1-03 & & & & & 21.404 & 6.168 & 16.491 & 2.569 & & & & & & & & \\
\hline IG1-04 & & & & & 0.384 & 1.686 & 0.599 & 0.200 & & & & & & & & \\
\hline IG1-05 & & & & & 4.061 & 0.129 & 2.330 & 0.401 & & & & & & & & \\
\hline IG1-06 & & & & & 9.634 & 9.516 & 8.923 & 1.583 & & & & & & & & \\
\hline IG1-07 & & & & & 0.282 & 0.362 & 0.063 & 0.2575 & & & & & & & & \\
\hline IG1-08 & & & & & 0.183 & 0.283 & 0.339 & 0.1265 & & & & & & & & \\
\hline IG1-09 & & & & & 2.865 & 1.115 & 2.292 & 0.2495 & & & & & & & & \\
\hline IG1-10 & & & & & 14.658 & 10.665 & 9.512 & 5.6345 & & & & & & & & \\
\hline IG1-11 & & & & & 0.291 & 0.380 & 0.938 & 0.2825 & & & & & & & & \\
\hline IG1-12 & & & & & 4.605 & & 3.999 & 0.155 & & & & & & & & \\
\hline IG1-13 & & & & & 30.051 & 23.368 & 29.596 & 20.071 & & & & & & & & \\
\hline IG1-14 & & & & & 0.498 & 0.285 & 0.650 & 0.1945 & & & & & & & & \\
\hline IG1-15 & & & & & 0.493 & 0.395 & 0.584 & 0.203 & & & & & & & & \\
\hline IG1-16 & & & & & 0.353 & 0.216 & 0.594 & 0.1565 & & & & & & & & \\
\hline IG1-17 & & & & & 0.202 & 0.066 & 0.335 & 0.1185 & & & & & & & & \\
\hline IG1-18 & & & & & 0.318 & 0.290 & 0.353 & 0.1355 & & & & & & & & \\
\hline IG1-19 & & & & & 0.279 & 0.251 & 0.323 & 0.1245 & & & & & & & & \\
\hline IG1-20 & & & & & 0.254 & 0.219 & 0.282 & 0.1095 & & & & & & & & \\
\hline IG1-21 & & & & & 0.905 & 0.736 & 0.738 & 0.1295 & & & & & & & & \\
\hline IG1-22 & & & & & 0.396 & 0.223 & 0.396 & 0.1285 & & & & & & & & \\
\hline IG1-23 & & & & & 0.176 & 0.207 & 0.328 & 0.112 & & & & & & & & \\
\hline IG1-24 & & & & & 1.042 & 0.725 & 0.750 & 0.133 & & & & & & & & \\
\hline IG1-25 & & & & & 0.450 & 0.401 & 0.632 & 0.1445 & & & & & & & & \\
\hline IG1-26 & & & & & 3.143 & 1.812 & 2.495 & 0.7185 & & & & & & & & \\
\hline IG1-27 & & & & & 0.151 & 0.293 & 0.336 & 0.118 & & & & & & & & \\
\hline IG1-28 & & & & & 0.181 & 0.315 & 0.357 & 0.1295 & & & & & & & & \\
\hline IG1-29 & & & & & 0.227 & 0.357 & 0.175 & 0.205 & & & & & & & & \\
\hline IG1-30 & & & & & 12.142 & 8.152 & 8.975 & 3.4275 & & & & & & & & \\
\hline IG1-31 & & & & & 1.512 & 1.270 & 0.783 & 0.2645 & & & & & & & & \\
\hline IG1-32 & & & & & 0.194 & 0.289 & 0.268 & 0.150 & & & & & & & & \\
\hline IG1-33 & & & & & 5.822 & 9.658 & 10.615 & 4.285 & & & & & & & & \\
\hline IG1-34 & & & & & 0.543 & 0.541 & 0.119 & 0.257 & & & & & & & & \\
\hline IG1-35 & & & & & 0.440 & 18.427 & 0.358 & 0.1515 & & & & & & & & \\
\hline IG1-36 & & & & & 8.858 & 8.973 & 8.924 & 4.634 & & & & & & & & \\
\hline IG1-37 & & & & & 7.792 & 0.057 & 6.358 & 0.4835 & & & & & & & & \\
\hline IG1-38 & & & & & 1.225 & 1.108 & 0.737 & 0.3145 & & & & & & & & \\
\hline IG1-39 & & & & & 1.694 & 1.396 & 1.080 & 0.1695 & & & & & & & & \\
\hline IG1-40 & & & & & 4.115 & 2.835 & 3.623 & 1.0755 & & & & & & & & \\
\hline IG1-41 & & & & & 20.179 & 8.174 & 16.802 & 6.3355 & & & & & & & & \\
\hline IG1-42 & & & & & 0.202 & 0.264 & 0.307 & 0.126 & & & & & & & & \\
\hline IG1-43 & & & & & 0.725 & 0.355 & 0.552 & 0.1195 & & & & & & & & \\
\hline IG1-44 & & & & & 0.580 & 0.573 & 0.125 & 0.2745 & & & & & & & & \\
\hline
\end{tabular}


Appendix A. Database - mass fraction

INEEL CVS Phase 1 (Staples et al. 1999)

\begin{tabular}{|c|c|c|c|c|c|c|c|c|c|c|c|c|}
\hline Glass ID & $\begin{array}{l}\text { Q PCT Na at } \\
20^{\circ} \mathrm{C}\left(\mathrm{g} / \mathrm{m}^{2}\right)\end{array}$ & $\begin{array}{l}\text { Q PCT Si at } \\
20^{\circ} \mathrm{C}\left(\mathrm{g} / \mathrm{m}^{2}\right)\end{array}$ & $\begin{array}{c}\mathrm{QpH} \\
\text { at } 20^{\circ} \mathrm{C}\end{array}$ & $\begin{array}{c}\text { TCLP Ag } \\
(\mathrm{ppm})\end{array}$ & $\begin{array}{c}\text { TCLP As } \\
(\mathrm{ppm})\end{array}$ & $\begin{array}{c}\text { TCLP Ba } \\
(\mathrm{ppm})\end{array}$ & $\begin{array}{c}\text { TCLP Cd } \\
(\mathrm{ppm})\end{array}$ & $\begin{array}{c}\text { TCLP Cr } \\
(\mathrm{ppm})\end{array}$ & $\begin{array}{c}\text { TCLP Ni } \\
(\mathrm{ppm})\end{array}$ & $\begin{array}{c}\text { TCLP Pb } \\
(\mathrm{ppm})\end{array}$ & $\begin{array}{l}\text { TCLP Se } \\
(\mathrm{ppm})\end{array}$ & $\begin{array}{c}\text { TCLP Zn } \\
(\mathrm{ppm})\end{array}$ \\
\hline \multicolumn{13}{|c|}{ 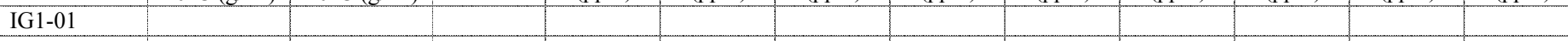 } \\
\hline \multicolumn{13}{|l|}{ IG1-02 } \\
\hline \multicolumn{13}{|l|}{ IG1-03 } \\
\hline \multicolumn{13}{|l|}{ IG1-04 } \\
\hline \multicolumn{13}{|l|}{ IG1-05 } \\
\hline \multicolumn{13}{|l|}{ IG1-06 } \\
\hline \multicolumn{13}{|l|}{ IG1-07 } \\
\hline \multicolumn{13}{|l|}{ IG1-08 } \\
\hline \multicolumn{13}{|l|}{ IG1-09 } \\
\hline \multicolumn{13}{|l|}{ IG1-10 } \\
\hline \multicolumn{13}{|l|}{ IG1-11 } \\
\hline \multicolumn{13}{|l|}{ IG1-12 } \\
\hline \multicolumn{13}{|l|}{ IG1-13 } \\
\hline \multicolumn{13}{|l|}{ IG1-14 } \\
\hline \multicolumn{13}{|l|}{ IG1-15 } \\
\hline \multicolumn{13}{|l|}{ IG1-16 } \\
\hline \multicolumn{13}{|l|}{ IG1-17 } \\
\hline \multicolumn{13}{|l|}{ IG1-18 } \\
\hline \multicolumn{13}{|l|}{ IG1-19 } \\
\hline \multicolumn{13}{|l|}{ IG1-20 } \\
\hline IG1-21 & & & & & & & & & & & & \\
\hline IG1-22 & & & & & & & & & & & & \\
\hline IG1-23 & & & & & & & & & & & & \\
\hline IG1-24 & & & & & & & & & & & & \\
\hline IG1-25 & & & & & & & & & & & & \\
\hline IG1-26 & & & & & & & & & & & & \\
\hline IG1-27 & & & & & & & & & & & & \\
\hline IG1-28 & & & & & & & & & & & & \\
\hline IG1-29 & & & & & & & & & & & & \\
\hline IG1-30 & & & & & & & & & & & & \\
\hline IG1-31 & & & & & & & & & & & & \\
\hline IG1-32 & & & & & & & & & & & & \\
\hline IG1-33 & & & & & & & & & & & & \\
\hline IG1-34 & & & & & & & & & & & & \\
\hline IG1-35 & & & & & & & & & & & & \\
\hline IG1-36 & & & & & & & & & & & & \\
\hline IG1-37 & & & & & & & & & & & & \\
\hline IG1-38 & & & & & & & & & & & & \\
\hline IG1-39 & & & & & & & & & & & & \\
\hline IG1-40 & & & & & & & & & & & & \\
\hline IG1-41 & & & & & & & & & & & & \\
\hline IG1-42 & & & & & & & & & & & & \\
\hline IG1-43 & & & & & & & & & & & & \\
\hline IG1-44 & & & & & & & & & & & & \\
\hline
\end{tabular}


INEEL CVS Phase 2 (Staples et al. 2000)

\begin{tabular}{|c|c|c|c|c|c|c|c|c|c|c|c|c|c|c|c|c|c|c|c|c|c|}
\hline Glass ID & $\begin{array}{c}\mathrm{A} 12 \mathrm{O} 3 \\
-\mathrm{t}\end{array}$ & $\begin{array}{c}\mathrm{B} 2 \mathrm{O} 3 \\
-\mathrm{t}\end{array}$ & $\begin{array}{c}\mathrm{CaO} \\
-\mathrm{t}\end{array}$ & $\begin{array}{c}\mathrm{Fe} 2 \mathrm{O} 3 \\
-\mathrm{t}\end{array}$ & $\begin{array}{c}\mathrm{FeO} \\
-\mathrm{t}\end{array}$ & $\begin{array}{c}\mathrm{K} 2 \mathrm{O} \\
-\mathrm{t}\end{array}$ & $\begin{array}{c}\mathrm{Li} 2 \mathrm{O} \\
-\mathrm{t}\end{array}$ & $\begin{array}{c}\mathrm{MgO} \\
-\mathrm{t}\end{array}$ & $\mathrm{Na} 2 \mathrm{O}$ & $\begin{array}{c}\mathrm{NiO} \\
-\mathrm{t}\end{array}$ & $\begin{array}{c}\mathrm{P} 2 \mathrm{O} 5 \\
-\mathrm{t}\end{array}$ & $\begin{array}{c}\mathrm{SiO} 2 \\
-\mathrm{t}\end{array}$ & $\begin{array}{c}\mathrm{ZrO} 2 \\
-\mathrm{t}\end{array}$ & $\begin{array}{c}\mathrm{Ag} 2 \mathrm{O} \\
-\mathrm{t}\end{array}$ & $\begin{array}{c}\mathrm{As} 2 \mathrm{O} 3 \\
-\mathrm{t}\end{array}$ & $\begin{array}{c}\mathrm{BaO} \\
-\mathrm{t}\end{array}$ & $\begin{array}{c}\mathrm{Bi} 2 \mathrm{O} 3 \\
-\mathrm{t}\end{array}$ & $\begin{array}{c}\mathrm{Br} \\
-\mathrm{t}\end{array}$ & $\begin{array}{c}\mathrm{CdO} \\
-\mathrm{t}\end{array}$ & $\begin{array}{c}\mathrm{Ce} 2 \mathrm{O} 3 \\
-\mathrm{t}\end{array}$ & $\begin{array}{c}\mathrm{CeO} 2 \\
-\mathrm{t}\end{array}$ \\
\hline IG2-01 & 0.1986 & 0.0496 & 0.0000 & 0.0000 & & 0.0000 & 0.0000 & 0.0021 & 0.1986 & 0.0149 & 0.0000 & 0.4715 & 0.0000 & & & 0.0000 & & & 0.0000 & & 0.0000 \\
\hline IG2-02 & 0.1986 & 0.1787 & 0.1191 & 0.0000 & & 0.0000 & 0.0000 & 0.0021 & 0.0496 & 0.0000 & 0.0298 & 0.4170 & 0.0000 & & & 0.0000 & & & 0.0000 & & 0.0000 \\
\hline IG2-03 & 0.0348 & 0.1790 & 0.0000 & 0.0796 & & 0.0000 & 0.0000 & 0.0021 & 0.0497 & 0.0149 & 0.0000 & 0.5969 & 0.0000 & & & 0.0000 & & & 0.0000 & & 0.0001 \\
\hline IG2-04 & 0.1986 & 0.1787 & 0.0000 & 0.0000 & & 0.0993 & 0.0894 & 0.0021 & 0.0496 & 0.0149 & 0.0000 & 0.3474 & 0.0149 & & & 0.0000 & & & 0.0000 & & 0.0000 \\
\hline IG2-05 & 0.0347 & 0.0496 & 0.1191 & 0.0794 & & 0.0099 & 0.0000 & 0.0021 & 0.1986 & 0.0149 & 0.0000 & 0.3476 & 0.1390 & & & 0.0000 & & & 0.0000 & & 0.0000 \\
\hline IG2-06 & 0.0645 & 0.1787 & 0.0000 & 0.0794 & & 0.0000 & 0.0894 & 0.0021 & 0.0496 & 0.0149 & 0.0298 & 0.3475 & 0.1390 & & & 0.0000 & & & 0.0000 & & 0.0000 \\
\hline IG2-07 & 0.0794 & 0.0496 & 0.1191 & 0.0000 & & 0.0993 & 0.0000 & 0.0021 & 0.0496 & 0.0149 & 0.0298 & 0.3525 & 0.1390 & & & 0.0000 & & & 0.0000 & & 0.0000 \\
\hline IG2-08 & 0.0347 & 0.1787 & 0.1191 & 0.0000 & & 0.0993 & 0.0000 & 0.0021 & 0.1268 & 0.0000 & 0.0000 & 0.4342 & 0.0000 & & & 0.0000 & & & 0.0000 & & 0.0000 \\
\hline IG2-09 & 0.1986 & 0.0496 & 0.1191 & 0.0794 & & 0.0000 & 0.0736 & 0.0021 & 0.0496 & 0.0000 & 0.0000 & 0.3633 & 0.0000 & & & 0.0000 & & & 0.0000 & & 0.0000 \\
\hline IG2-10 & 0.0347 & 0.0496 & 0.0000 & 0.0794 & & 0.0993 & 0.0834 & 0.0021 & 0.0496 & 0.0000 & 0.0298 & 0.5074 & 0.0000 & & & 0.0000 & & & 0.0000 & & 0.0000 \\
\hline IG2-11 & 0.0347 & 0.0496 & 0.0746 & 0.0000 & & 0.0000 & 0.0894 & 0.0021 & 0.1588 & 0.0149 & 0.0298 & 0.5410 & 0.0000 & & & 0.0000 & & & 0.0000 & & 0.0000 \\
\hline IG2-12 & 0.0347 & 0.1787 & 0.0000 & 0.0000 & & 0.0000 & 0.0052 & 0.0021 & 0.1986 & 0.0000 & 0.0000 & 0.4044 & 0.1116 & & & 0.0000 & & & 0.0000 & & 0.0000 \\
\hline IG2-13 & 0.0485 & 0.0706 & 0.0000 & 0.0000 & & 0.0000 & 0.0894 & 0.0021 & 0.0496 & 0.0000 & 0.0000 & 0.5957 & 0.1390 & & & 0.0000 & & & 0.0000 & & 0.0000 \\
\hline IG2-14 & 0.1986 & 0.0613 & 0.0000 & 0.0794 & & 0.0993 & 0.0000 & 0.0021 & 0.1489 & 0.0000 & 0.0298 & 0.3556 & 0.0199 & & & 0.0000 & & & 0.0000 & & 0.0000 \\
\hline IG2-15 & 0.0794 & 0.0596 & 0.0397 & 0.0050 & & 0.0298 & 0.0596 & 0.0021 & 0.1489 & 0.0050 & 0.0199 & 0.4963 & 0.0397 & & & 0.0000 & & & 0.0000 & & 0.0000 \\
\hline IG2-16 & 0.1092 & 0.1191 & 0.0397 & 0.0050 & & 0.0298 & 0.0298 & 0.0021 & 0.1489 & 0.0050 & 0.0199 & 0.3971 & 0.0794 & & & 0.0000 & & & 0.0000 & & 0.0000 \\
\hline IG2-17 & 0.0794 & 0.0596 & 0.0794 & 0.0050 & & 0.0298 & 0.0298 & 0.0021 & 0.0993 & 0.0050 & 0.0199 & 0.4814 & 0.0794 & & & 0.0000 & & & 0.0000 & & 0.0000 \\
\hline IG2-18 & 0.1191 & 0.0596 & 0.0397 & 0.0298 & & 0.0596 & 0.0298 & 0.0021 & 0.1489 & 0.0050 & 0.0099 & 0.4021 & 0.0794 & & & 0.0000 & & & 0.0000 & & 0.0000 \\
\hline IG2-19 & 0.0794 & 0.0596 & 0.0397 & 0.0050 & & 0.0298 & 0.0596 & 0.0021 & 0.1489 & 0.0099 & 0.0199 & 0.4914 & 0.0397 & & & 0.0000 & & & 0.0000 & & 0.0000 \\
\hline IG2-20 & 0.0794 & 0.0596 & 0.0397 & 0.0050 & & 0.0596 & 0.0298 & 0.0021 & 0.1489 & 0.0099 & 0.0199 & 0.4914 & 0.0397 & & & 0.0000 & & & 0.0000 & & 0.0000 \\
\hline IG2-21 & 0.0794 & 0.0596 & 0.0794 & 0.0199 & & 0.0596 & 0.0298 & 0.0021 & 0.0993 & 0.0099 & 0.0099 & 0.4964 & 0.0397 & & & 0.0000 & & & 0.0000 & & 0.0000 \\
\hline IG2-22 & 0.1134 & 0.0651 & 0.0375 & 0.0048 & & 0.2098 & 0.0611 & 0.0021 & 0.0140 & 0.0043 & 0.0168 & 0.3825 & 0.0747 & & & 0.0000 & & & 0.0000 & & 0.0000 \\
\hline IG2-23 & 0.1050 & 0.1191 & 0.0397 & 0.0050 & & 0.0298 & 0.0298 & 0.0021 & 0.1489 & 0.0050 & 0.0199 & 0.3971 & 0.0794 & & & 0.0000 & & & 0.0000 & & 0.0000 \\
\hline IG2-24 & 0.1191 & 0.0596 & 0.0794 & 0.0050 & & 0.0596 & 0.0564 & 0.0021 & 0.0993 & 0.0099 & 0.0099 & 0.4053 & 0.0794 & & & 0.0000 & & & 0.0000 & & 0.0000 \\
\hline IG2-25 & 0.1191 & 0.0596 & 0.0794 & 0.0050 & & 0.0596 & 0.0576 & 0.0021 & 0.0993 & 0.0050 & 0.0099 & 0.4090 & 0.0794 & & & 0.0000 & & & 0.0000 & & 0.0000 \\
\hline IG2-26 & 0.0817 & 0.0611 & 0.0257 & 0.0051 & & 0.0611 & 0.0505 & 0.0022 & 0.1018 & 0.0051 & 0.0102 & 0.5088 & 0.0814 & & & 0.0000 & & & 0.0000 & & 0.0001 \\
\hline IG2-27 & 0.0981 & 0.0596 & 0.0794 & 0.0050 & & 0.0298 & 0.0310 & 0.0021 & 0.0993 & 0.0099 & 0.0199 & 0.4963 & 0.0397 & & & 0.0000 & & & 0.0000 & & 0.0000 \\
\hline IG2-28 & 0.0794 & 0.0596 & 0.0735 & 0.0298 & & 0.0596 & 0.0298 & 0.0021 & 0.0993 & 0.0099 & 0.0099 & 0.4924 & 0.0397 & & & 0.0000 & & & 0.0000 & & 0.0000 \\
\hline IG2-29 & 0.0987 & 0.0915 & 0.0489 & 0.0256 & & 0.0440 & 0.0392 & 0.0000 & 0.1154 & 0.0074 & 0.0144 & 0.4361 & 0.0579 & & & 0.0000 & & & 0.0000 & & 0.0000 \\
\hline IG2-30 & 0.1503 & 0.1503 & 0.0008 & 0.0005 & & 0.0000 & 0.0846 & 0.0000 & 0.0501 & 0.0000 & 0.0000 & 0.5314 & 0.0301 & & & 0.0000 & & & 0.0000 & & 0.0006 \\
\hline IG2-31 & 0.0376 & 0.1252 & 0.0009 & 0.0005 & & 0.0250 & 0.0632 & 0.0000 & 0.0876 & 0.0000 & 0.0125 & 0.5403 & 0.1051 & & & 0.0000 & & & 0.0000 & & 0.0007 \\
\hline IG2-32 & 0.0980 & 0.0908 & 0.0485 & 0.0254 & & 0.0437 & 0.0389 & 0.0021 & 0.1146 & 0.0073 & 0.0143 & 0.4331 & 0.0575 & & & 0.0000 & & & 0.0000 & & 0.0000 \\
\hline IG2-33 & 0.0980 & 0.0908 & 0.0485 & 0.0254 & & 0.0437 & 0.0389 & 0.0021 & 0.1146 & 0.0073 & 0.0143 & 0.4331 & 0.0575 & & & 0.0000 & & & 0.0000 & & 0.0000 \\
\hline IG2-34 & 0.0960 & 0.0890 & 0.0476 & 0.0249 & & 0.0428 & 0.0381 & 0.0021 & 0.1123 & 0.0072 & 0.0140 & 0.4243 & 0.0563 & & & 0.0000 & & & 0.0000 & & 0.0000 \\
\hline IG2-35 & 0.0975 & 0.0904 & 0.0483 & 0.0252 & & 0.0435 & 0.0387 & 0.0022 & 0.1139 & 0.0073 & 0.0142 & 0.4304 & 0.0572 & & & 0.0000 & & & 0.0000 & & 0.0001 \\
\hline IG2-36 & 0.0960 & 0.0890 & 0.0476 & 0.0249 & & 0.0428 & 0.0381 & 0.0021 & 0.1123 & 0.0072 & 0.0140 & 0.4243 & 0.0563 & & & 0.0000 & & & 0.0000 & & 0.0000 \\
\hline IG2-37 & 0.0940 & 0.0872 & 0.0466 & 0.0244 & & 0.0419 & 0.0373 & 0.0021 & 0.1100 & 0.0071 & 0.0137 & 0.4155 & 0.0552 & & & 0.0000 & & & 0.0000 & & 0.0000 \\
\hline
\end{tabular}

\section{INEEL CVS Phase 3 (Scholes et al. 2000)}

\begin{tabular}{|c|c|c|c|c|c|c|c|c|c|c|c|c|c|c|}
\hline IG3-01 & 0.0850 & 0.0925 & 0.0487 & 0.0228 & 0.0088 & 0.0396 & 0.0024 & 0.1246 & 0.0073 & 0.0107 & 0.4207 & 0.0622 & 0.0000 & 0.0001 \\
\hline IG3-02b & 0.0450 & 0.1625 & 0.1057 & 0.0046 & 0.0018 & 0.0079 & 0.0024 & 0.0652 & 0.0015 & 0.0021 & 0.5158 & 0.0124 & 0.0000 & 0.0001 \\
\hline IG3-03 & 0.0400 & 0.0500 & 0.0000 & 0.0000 & 0.0200 & 0.0078 & 0.0024 & 0.2000 & 0.0150 & 0.0250 & 0.4272 & 0.1400 & 0.0000 & 0.0001 \\
\hline IG3-04 & 0.0350 & 0.1800 & 0.0000 & 0.0300 & 0.0000 & 0.0268 & 0.0024 & 0.0500 & 0.0150 & 0.0250 & 0.5432 & 0.0000 & 0.0000 & 0.0001 \\
\hline
\end{tabular}


INEEL CVS Phase 2 (Staples et al. 2000)

\begin{tabular}{|c|c|c|c|c|c|c|c|c|c|c|c|c|c|c|c|c|c|c|c|c|c|}
\hline Glass ID & $\begin{array}{l}\mathrm{Cl} \\
-\mathrm{t}\end{array}$ & $\begin{array}{c}\mathrm{CoO} \\
-\mathrm{t}\end{array}$ & $\begin{array}{c}\mathrm{Co} 2 \mathrm{O} 3 \\
-\mathrm{t}\end{array}$ & $\begin{array}{c}\mathrm{Cr} 2 \mathrm{O} 3 \\
-\mathrm{t}\end{array}$ & $\begin{array}{c}\mathrm{Cs} 2 \mathrm{O} \\
-\mathrm{t}\end{array}$ & $\begin{array}{c}\mathrm{CuO} \\
-\mathrm{t}\end{array}$ & $\begin{array}{c}\mathrm{Eu} 2 \mathrm{O} 3 \\
-\mathrm{t}\end{array}$ & $\begin{array}{l}F \\
-t\end{array}$ & $\begin{array}{c}\mathrm{Ga} 2 \mathrm{O} 3 \\
-\mathrm{t}\end{array}$ & {$\left[\begin{array}{c}\mathrm{Gd} 2 \mathrm{O} 3 \\
-\mathrm{t}\end{array}\right.$} & $\begin{array}{c}\mathrm{HgO} \\
-\mathrm{t}\end{array}$ & $\begin{array}{l}I \\
-t\end{array}$ & $\begin{array}{c}\mathrm{La} 2 \mathrm{O} 3 \\
-\mathrm{t}\end{array}$ & $\begin{array}{c}\mathrm{MnO} 2 \\
-\mathrm{t}\end{array}$ & $\begin{array}{c}\mathrm{MnO} \\
-\mathrm{t}\end{array}$ & $\begin{array}{c}\mathrm{MoO} \\
-\mathrm{t}\end{array}$ & $\begin{array}{c}\mathrm{MoO} 3 \\
-\mathrm{t}\end{array}$ & $\begin{array}{c}\mathrm{Nb} 2 \mathrm{O} 5 \\
-\mathrm{t}\end{array}$ & $\begin{array}{c}\mathrm{Nd} 2 \mathrm{O} 3 \\
-\mathrm{t}\end{array}$ & $\begin{array}{c}\mathrm{PbO} \\
-\mathrm{t}\end{array}$ & $\begin{array}{c}\mathrm{PdO} 2 \\
-\mathrm{t}\end{array}$ \\
\hline IG2-01 & 0.0003 & & & 0.0006 & 0.0000 & 0.0000 & & 0.0596 & & 0.0000 & & 0.0000 & 0.0000 & 0.0000 & 0.0001 & & 0.0001 & 0.0002 & 0.0001 & 0.0000 & \\
\hline IG2-02 & 0.0003 & & & 0.0006 & 0.0000 & 0.0000 & & 0.0000 & & 0.0000 & & 0.0000 & 0.0000 & 0.0000 & 0.0001 & & 0.0001 & 0.0002 & 0.0001 & 0.0000 & \\
\hline IG2-03 & 0.0003 & & & 0.0007 & 0.0000 & 0.0000 & & 0.0378 & & 0.0000 & & 0.0000 & 0.0000 & 0.0000 & 0.0001 & & 0.0001 & 0.0002 & 0.0001 & 0.0000 & \\
\hline IG2-04 & 0.0003 & & & 0.0006 & 0.0000 & 0.0000 & & 0.0000 & & 0.0000 & & 0.0000 & 0.0000 & 0.0000 & 0.0001 & & 0.0001 & 0.0002 & 0.0001 & 0.0000 & \\
\hline IG2-05 & 0.0003 & & & 0.0006 & 0.0000 & 0.0000 & & 0.0000 & & 0.0000 & & 0.0000 & 0.0000 & 0.0000 & 0.0001 & & 0.0001 & 0.0002 & 0.0001 & 0.0000 & \\
\hline IG2-06 & 0.0003 & & & 0.0006 & 0.0000 & 0.0000 & & 0.0000 & & 0.0000 & & 0.0000 & 0.0000 & 0.0000 & 0.0001 & & 0.0001 & 0.0002 & 0.0001 & 0.0000 & \\
\hline IG2-07 & 0.0003 & & & 0.0006 & 0.0000 & 0.0000 & & 0.0596 & & 0.0000 & & 0.0000 & 0.0000 & 0.0000 & 0.0001 & & 0.0001 & 0.0002 & 0.0001 & 0.0000 & \\
\hline IG2-08 & 0.0003 & & & 0.0006 & 0.0000 & 0.0000 & & 0.0000 & & 0.0000 & & 0.0000 & 0.0000 & 0.0000 & 0.0001 & & 0.0001 & 0.0002 & 0.0001 & 0.0000 & \\
\hline IG2-09 & 0.0003 & & & 0.0006 & 0.0000 & 0.0000 & & 0.0596 & & 0.0000 & & 0.0000 & 0.0000 & 0.0000 & 0.0001 & & 0.0001 & 0.0002 & 0.0001 & 0.0000 & \\
\hline IG2-10 & 0.0003 & & & 0.0006 & 0.0000 & 0.0000 & & 0.0596 & & 0.0000 & & 0.0000 & 0.0000 & 0.0000 & 0.0001 & & 0.0001 & 0.0002 & 0.0001 & 0.0000 & \\
\hline IG2-11 & 0.0003 & & & 0.0006 & 0.0000 & 0.0000 & & 0.0000 & & 0.0000 & & 0.0000 & 0.0000 & 0.0000 & 0.0001 & & 0.0001 & 0.0002 & 0.0001 & 0.0000 & \\
\hline IG2-12 & 0.0003 & & & 0.0006 & 0.0000 & 0.0000 & & 0.0596 & & 0.0000 & & 0.0000 & 0.0000 & 0.0000 & 0.0001 & & 0.0001 & 0.0002 & 0.0001 & 0.0000 & \\
\hline IG2-13 & 0.0003 & & & 0.0006 & 0.0000 & 0.0000 & & 0.0000 & & 0.0000 & & 0.0000 & 0.0000 & 0.0000 & 0.0001 & & 0.0001 & 0.0002 & 0.0001 & 0.0000 & \\
\hline IG2-14 & 0.0003 & & & 0.0006 & 0.0000 & 0.0000 & & 0.0000 & & 0.0000 & & 0.0000 & 0.0000 & 0.0000 & 0.0001 & & 0.0001 & 0.0002 & 0.0001 & 0.0000 & \\
\hline IG2-15 & 0.0003 & & & 0.0006 & 0.0000 & 0.0000 & & 0.0099 & & 0.0000 & & 0.0000 & 0.0000 & 0.0000 & 0.0001 & & 0.0001 & 0.0002 & 0.0001 & 0.0000 & \\
\hline IG2-16 & 0.0003 & & & 0.0006 & 0.0000 & 0.0000 & & 0.0099 & & 0.0000 & & 0.0000 & 0.0000 & 0.0000 & 0.0001 & & 0.0001 & 0.0002 & 0.0001 & 0.0000 & \\
\hline IG2-17 & 0.0003 & & & 0.0006 & 0.0000 & 0.0000 & & 0.0248 & & 0.0000 & & 0.0000 & 0.0000 & 0.0000 & 0.0001 & & 0.0001 & 0.0002 & 0.0001 & 0.0000 & \\
\hline IG2-18 & 0.0003 & & & 0.0006 & 0.0000 & 0.0000 & & 0.0099 & & 0.0000 & & 0.0000 & 0.0000 & 0.0000 & 0.0001 & & 0.0001 & 0.0002 & 0.0001 & 0.0000 & \\
\hline IG2-19 & 0.0003 & & & 0.0006 & 0.0000 & 0.0000 & & 0.0099 & & 0.0000 & & 0.0000 & 0.0000 & 0.0000 & 0.0001 & & 0.0001 & 0.0002 & 0.0001 & 0.0000 & \\
\hline IG2-20 & 0.0003 & & & 0.0006 & 0.0000 & 0.0000 & & 0.0099 & & 0.0000 & & 0.0000 & 0.0000 & 0.0000 & 0.0001 & & 0.0001 & 0.0002 & 0.0001 & 0.0000 & \\
\hline IG2-21 & 0.0003 & & & 0.0006 & 0.0000 & 0.0000 & & 0.0099 & & 0.0000 & & 0.0000 & 0.0000 & 0.0000 & 0.0001 & & 0.0001 & 0.0002 & 0.0001 & 0.0000 & \\
\hline IG2-22 & 0.0003 & & & 0.0006 & 0.0000 & 0.0000 & & 0.0091 & & 0.0000 & & 0.0000 & 0.0000 & 0.0000 & 0.0001 & & 0.0000 & 0.0002 & 0.0001 & 0.0000 & \\
\hline IG2-23 & 0.0003 & & & 0.0006 & 0.0000 & 0.0000 & & 0.0141 & & 0.0000 & & 0.0000 & 0.0000 & 0.0000 & 0.0001 & & 0.0001 & 0.0002 & 0.0001 & 0.0000 & \\
\hline IG2-24 & 0.0003 & & & 0.0006 & 0.0000 & 0.0000 & & 0.0099 & & 0.0000 & & 0.0000 & 0.0000 & 0.0000 & 0.0001 & & 0.0001 & 0.0002 & 0.0001 & 0.0000 & \\
\hline IG2-25 & 0.0003 & & & 0.0006 & 0.0000 & 0.0000 & & 0.0099 & & 0.0000 & & 0.0000 & 0.0000 & 0.0000 & 0.0001 & & 0.0001 & 0.0002 & 0.0001 & 0.0000 & \\
\hline IG2-26 & 0.0003 & & & 0.0007 & 0.0000 & 0.0000 & & 0.0000 & & 0.0000 & & 0.0000 & 0.0000 & 0.0000 & 0.0001 & & 0.0001 & 0.0002 & 0.0001 & 0.0000 & \\
\hline IG2-27 & 0.0003 & & & 0.0006 & 0.0000 & 0.0000 & & 0.0248 & & 0.0000 & & 0.0000 & 0.0000 & 0.0000 & 0.0001 & & 0.0001 & 0.0002 & 0.0001 & 0.0000 & \\
\hline IG2-28 & 0.0003 & & & 0.0006 & 0.0000 & 0.0000 & & 0.0099 & & 0.0000 & & 0.0000 & 0.0000 & 0.0000 & 0.0001 & & 0.0001 & 0.0002 & 0.0001 & 0.0000 & \\
\hline IG2-29 & 0.0000 & & & 0.0000 & 0.0000 & 0.0000 & & 0.0209 & & 0.0000 & & 0.0000 & 0.0000 & 0.0000 & 0.0000 & & 0.0000 & 0.0000 & 0.0000 & 0.0000 & \\
\hline IG2-30 & 0.0000 & & & 0.0000 & 0.0009 & 0.0000 & & 0.0000 & & 0.0000 & & 0.0000 & 0.0000 & 0.0000 & 0.0000 & & 0.0001 & 0.0000 & 0.0000 & 0.0000 & \\
\hline IG2-31 & 0.0000 & & & 0.0000 & 0.0010 & 0.0000 & & 0.0000 & & 0.0000 & & 0.0000 & 0.0000 & 0.0000 & 0.0000 & & 0.0001 & 0.0000 & 0.0000 & 0.0000 & \\
\hline IG2-32 & 0.0003 & & & 0.0006 & 0.0000 & 0.0000 & & 0.0207 & & 0.0000 & & 0.0000 & 0.0000 & 0.0000 & 0.0001 & & 0.0001 & 0.0002 & 0.0001 & 0.0000 & \\
\hline IG2-33 & 0.0003 & & & 0.0006 & 0.0000 & 0.0000 & & 0.0207 & & 0.0000 & & 0.0000 & 0.0000 & 0.0000 & 0.0001 & & 0.0001 & 0.0002 & 0.0001 & 0.0000 & \\
\hline IG2-34 & 0.0003 & & & 0.0006 & 0.0000 & 0.0000 & & 0.0203 & & 0.0000 & & 0.0000 & 0.0000 & 0.0000 & 0.0001 & & 0.0201 & 0.0002 & 0.0001 & 0.0000 & \\
\hline IG2-35 & 0.0003 & & & 0.0007 & 0.0000 & 0.0000 & & 0.0206 & & 0.0000 & & 0.0000 & 0.0000 & 0.0000 & 0.0001 & & 0.0053 & 0.0002 & 0.0001 & 0.0000 & \\
\hline IG2-36 & 0.0003 & & & 0.0006 & 0.0000 & 0.0000 & & 0.0203 & & 0.0000 & & 0.0000 & 0.0000 & 0.0000 & 0.0001 & & 0.0001 & 0.0002 & 0.0001 & 0.0000 & \\
\hline IG2-37 & 0.0003 & & & 0.0006 & 0.0000 & 0.0000 & & 0.0199 & & 0.0000 & & 0.0000 & 0.0000 & 0.0000 & 0.0001 & & 0.0001 & 0.0002 & 0.0001 & 0.0000 & \\
\hline
\end{tabular}

\section{INEEL CVS Phase 3 (Scholes et al. 2000)}

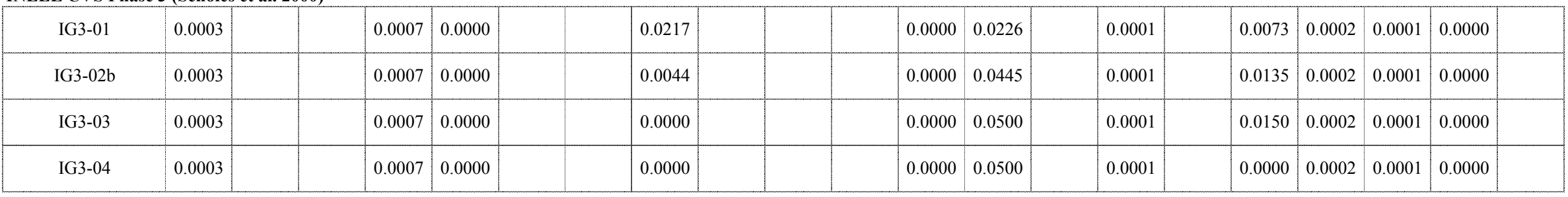


INEEL CVS Phase 2 (Staples et al. 2000)

\begin{tabular}{|c|c|c|c|c|c|c|c|c|c|c|c|c|c|c|c|c|c|c|c|c|c|}
\hline Glass ID & $\begin{array}{c}\mathrm{PdO} \\
-\mathrm{t}\end{array}$ & $\begin{array}{c}\mathrm{Pr} 2 \mathrm{O} 3 \\
-\mathrm{t}\end{array}$ & $\begin{array}{c}\text { Pr6O11 } \\
-t\end{array}$ & $\begin{array}{c}\mathrm{Rb} 2 \mathrm{O} \\
-\mathrm{t}\end{array}$ & $\begin{array}{c}\mathrm{ReO} \\
-\mathrm{t}\end{array}$ & $\begin{array}{c}\mathrm{ReO} 2 \\
-\mathrm{t}\end{array}$ & $\begin{array}{c}\mathrm{Rh} 2 \mathrm{O} 3 \\
-\mathrm{t}\end{array}$ & $\begin{array}{c}\mathrm{RhO} 2 \\
-\mathrm{t}\end{array}$ & $\begin{array}{c}\mathrm{RuO} 2 \\
-\mathrm{t}\end{array}$ & $\begin{array}{c}\mathrm{Sb} 2 \mathrm{O} 3 \\
-\mathrm{t}\end{array}$ & $\begin{array}{c}\mathrm{Sb} 2 \mathrm{O} 5 \\
-\mathrm{t}\end{array}$ & $\begin{array}{c}\mathrm{SeO} 2 \\
-\mathrm{t}\end{array}$ & $\begin{array}{c}\mathrm{Sm} 2 \mathrm{O} 3 \\
-\mathrm{t}\end{array}$ & $\begin{array}{c}\mathrm{SnO} \\
-\mathrm{t}\end{array}$ & $\begin{array}{c}\mathrm{SnO} 2 \\
-\mathrm{t}\end{array}$ & $\begin{array}{c}\mathrm{SO} 3 \\
-\mathrm{t}\end{array}$ & $\begin{array}{c}\mathrm{SrO} \\
-\mathrm{t}\end{array}$ & $\begin{array}{c}\mathrm{Tc} 2 \mathrm{O} 7 \\
-\mathrm{t}\end{array}$ & $\begin{array}{c}\mathrm{TeO} 2 \\
-\mathrm{t}\end{array}$ & $\begin{array}{c}\mathrm{ThO} 2 \\
-\mathrm{t}\end{array}$ & $\begin{array}{c}\mathrm{TiO} 2 \\
-\mathrm{t}\end{array}$ \\
\hline IG2-01 & 0.0000 & 0.0000 & 0.0000 & 0.0000 & & 0.0000 & 0.0000 & & 0.0000 & & & & 0.0000 & & 0.0005 & 0.0022 & 0.0005 & & 0.0000 & & 0.0000 \\
\hline IG2-02 & 0.0000 & 0.0000 & 0.0000 & 0.0000 & & 0.0000 & 0.0000 & & 0.0000 & & & & 0.0000 & & 0.0005 & 0.0022 & 0.0005 & & 0.0000 & & 0.0000 \\
\hline IG2-03 & 0.0000 & 0.0000 & 0.0000 & 0.0000 & & 0.0000 & 0.0000 & & 0.0000 & & & & 0.0000 & & 0.0005 & 0.0022 & 0.0006 & & 0.0000 & & 0.0000 \\
\hline IG2-04 & 0.0000 & 0.0000 & 0.0000 & 0.0000 & & 0.0000 & 0.0000 & & 0.0000 & & & & 0.0000 & & 0.0005 & 0.0022 & 0.0005 & & 0.0000 & & 0.0000 \\
\hline IG2-05 & 0.0000 & 0.0000 & 0.0000 & 0.0000 & & 0.0000 & 0.0000 & & 0.0000 & & & & 0.0000 & & 0.0005 & 0.0022 & 0.0005 & & 0.0000 & & 0.0000 \\
\hline IG2-06 & 0.0000 & 0.0000 & 0.0000 & 0.0000 & & 0.0000 & 0.0000 & & 0.0000 & & & & 0.0000 & & 0.0005 & 0.0022 & 0.0005 & & 0.0000 & & 0.0000 \\
\hline IG2-07 & 0.0000 & 0.0000 & 0.0000 & 0.0000 & & 0.0000 & 0.0000 & & 0.0000 & & & & 0.0000 & & 0.0005 & 0.0022 & 0.0005 & & 0.0000 & & 0.0000 \\
\hline IG2-08 & 0.0000 & 0.0000 & 0.0000 & 0.0000 & & 0.0000 & 0.0000 & & 0.0000 & & & & 0.0000 & & 0.0005 & 0.0022 & 0.0005 & & 0.0000 & & 0.0000 \\
\hline IG2-09 & 0.0000 & 0.0000 & 0.0000 & 0.0000 & & 0.0000 & 0.0000 & & 0.0000 & & & & 0.0000 & & 0.0005 & 0.0022 & 0.0005 & & 0.0000 & & 0.0000 \\
\hline IG2-10 & 0.0000 & 0.0000 & 0.0000 & 0.0000 & & 0.0000 & 0.0000 & & 0.0000 & & & & 0.0000 & & 0.0005 & 0.0022 & 0.0005 & & 0.0000 & & 0.0000 \\
\hline IG2-11 & 0.0000 & 0.0000 & 0.0000 & 0.0000 & & 0.0000 & 0.0000 & & 0.0000 & & & & 0.0000 & & 0.0005 & 0.0022 & 0.0005 & & 0.0000 & & 0.0000 \\
\hline IG2-12 & 0.0000 & 0.0000 & 0.0000 & 0.0000 & & 0.0000 & 0.0000 & & 0.0000 & & & & 0.0000 & & 0.0005 & 0.0022 & 0.0005 & & 0.0000 & & 0.0000 \\
\hline IG2-13 & 0.0000 & 0.0000 & 0.0000 & 0.0000 & & 0.0000 & 0.0000 & & 0.0000 & & & & 0.0000 & & 0.0005 & 0.0022 & 0.0005 & & 0.0000 & & 0.0000 \\
\hline IG2-14 & 0.0000 & 0.0000 & 0.0000 & 0.0000 & & 0.0000 & 0.0000 & & 0.0000 & & & & 0.0000 & & 0.0005 & 0.0022 & 0.0005 & & 0.0000 & & 0.0000 \\
\hline IG2-15 & 0.0000 & 0.0000 & 0.0000 & 0.0000 & & 0.0000 & 0.0000 & & 0.0000 & & & & 0.0000 & & 0.0005 & 0.0022 & 0.0005 & & 0.0000 & & 0.0000 \\
\hline IG2-16 & 0.0000 & 0.0000 & 0.0000 & 0.0000 & & 0.0000 & 0.0000 & & 0.0000 & & & & 0.0000 & & 0.0005 & 0.0022 & 0.0005 & & 0.0000 & & 0.0000 \\
\hline IG2-17 & 0.0000 & 0.0000 & 0.0000 & 0.0000 & & 0.0000 & 0.0000 & & 0.0000 & & & & 0.0000 & & 0.0005 & 0.0022 & 0.0005 & & 0.0000 & & 0.0000 \\
\hline IG2-18 & 0.0000 & 0.0000 & 0.0000 & 0.0000 & & 0.0000 & 0.0000 & & 0.0000 & & & & 0.0000 & & 0.0005 & 0.0022 & 0.0005 & & 0.0000 & & 0.0000 \\
\hline IG2-19 & 0.0000 & 0.0000 & 0.0000 & 0.0000 & & 0.0000 & 0.0000 & & 0.0000 & & & & 0.0000 & & 0.0005 & 0.0022 & 0.0005 & & 0.0000 & & 0.0000 \\
\hline IG2-20 & 0.0000 & 0.0000 & 0.0000 & 0.0000 & & 0.0000 & 0.0000 & & 0.0000 & & & & 0.0000 & & 0.0005 & 0.0022 & 0.0005 & & 0.0000 & & 0.0000 \\
\hline IG2-21 & 0.0000 & 0.0000 & 0.0000 & 0.0000 & & 0.0000 & 0.0000 & & 0.0000 & & & & 0.0000 & & 0.0005 & 0.0022 & 0.0005 & & 0.0000 & & 0.0000 \\
\hline IG2-22 & 0.0000 & 0.0000 & 0.0000 & 0.0000 & & 0.0000 & 0.0000 & & 0.0000 & & & & 0.0000 & & 0.0005 & 0.0022 & 0.0003 & & 0.0000 & & 0.0000 \\
\hline IG2-23 & 0.0000 & 0.0000 & 0.0000 & 0.0000 & & 0.0000 & 0.0000 & & 0.0000 & & & & 0.0000 & & 0.0005 & 0.0022 & 0.0005 & & 0.0000 & & 0.0000 \\
\hline IG2-24 & 0.0000 & 0.0000 & 0.0000 & 0.0000 & & 0.0000 & 0.0000 & & 0.0000 & & & & 0.0000 & & 0.0005 & 0.0022 & 0.0005 & & 0.0000 & & 0.0000 \\
\hline IG2-25 & 0.0000 & 0.0000 & 0.0000 & 0.0000 & & 0.0000 & 0.0000 & & 0.0000 & & & & 0.0000 & & 0.0005 & 0.0022 & 0.0005 & & 0.0000 & & 0.0000 \\
\hline IG2-26 & 0.0000 & 0.0000 & 0.0000 & 0.0000 & & 0.0000 & 0.0000 & & 0.0000 & & & & 0.0000 & & 0.0005 & 0.0022 & 0.0006 & & 0.0000 & & 0.0000 \\
\hline IG2-27 & 0.0000 & 0.0000 & 0.0000 & 0.0000 & & 0.0000 & 0.0000 & & 0.0000 & & & & 0.0000 & & 0.0005 & 0.0022 & 0.0005 & & 0.0000 & & 0.0000 \\
\hline IG2-28 & 0.0000 & 0.0000 & 0.0000 & 0.0000 & & 0.0000 & 0.0000 & & 0.0000 & & & & 0.0000 & & 0.0005 & 0.0022 & 0.0005 & & 0.0000 & & 0.0000 \\
\hline IG2-29 & 0.0000 & 0.0000 & 0.0000 & 0.0000 & & 0.0000 & 0.0000 & & 0.0000 & & & & 0.0000 & & 0.0000 & 0.0000 & 0.0000 & & 0.0000 & & 0.0000 \\
\hline IG2-30 & 0.0000 & 0.0000 & 0.0000 & 0.0000 & & 0.0000 & 0.0000 & & 0.0000 & & & & 0.0000 & & 0.0000 & 0.0000 & 0.0002 & & 0.0000 & & 0.0000 \\
\hline IG2-31 & 0.0000 & 0.0000 & 0.0000 & 0.0000 & & 0.0000 & 0.0000 & & 0.0000 & & & & 0.0000 & & 0.0000 & 0.0000 & 0.0002 & & 0.0000 & & 0.0000 \\
\hline IG2-32 & 0.0000 & 0.0000 & 0.0000 & 0.0000 & & 0.0000 & 0.0000 & & 0.0000 & & & & 0.0000 & & 0.0005 & 0.0022 & 0.0005 & & 0.0000 & & 0.0000 \\
\hline IG2-33 & 0.0000 & 0.0000 & 0.0000 & 0.0000 & & 0.0000 & 0.0000 & & 0.0000 & & & & 0.0000 & & 0.0005 & 0.0022 & 0.0005 & & 0.0000 & & 0.0000 \\
\hline IG2-34 & 0.0000 & 0.0000 & 0.0000 & 0.0000 & & 0.0000 & 0.0000 & & 0.0000 & & & & 0.0000 & & 0.0005 & 0.0022 & 0.0005 & & 0.0000 & & 0.0000 \\
\hline IG2-35 & 0.0000 & 0.0000 & 0.0000 & 0.0000 & & 0.0000 & 0.0000 & & 0.0000 & & & & 0.0000 & & 0.0005 & 0.0023 & 0.0006 & & 0.0000 & & 0.0000 \\
\hline IG2-36 & 0.0000 & 0.0000 & 0.0000 & 0.0000 & & 0.0000 & 0.0000 & & 0.0000 & & & & 0.0000 & & 0.0005 & 0.0022 & 0.0206 & & 0.0000 & & 0.0000 \\
\hline IG2-37 & 0.0000 & 0.0000 & 0.0000 & 0.0000 & & 0.0000 & 0.0000 & & 0.0000 & & & & 0.0000 & & 0.0005 & 0.0022 & 0.0405 & & 0.0000 & & 0.0000 \\
\hline
\end{tabular}

\section{INEEL CVS Phase 3 (Scholes et al. 2000)}

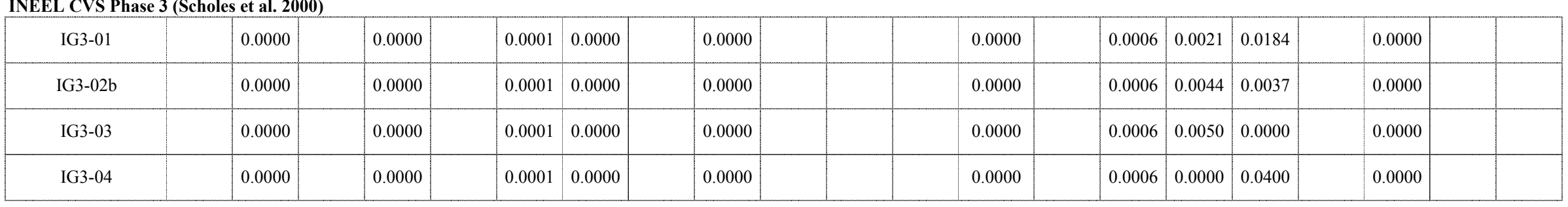


INEEL CVS Phase 2 (Staples et al. 2000)

\begin{tabular}{|c|c|c|c|c|c|c|c|c|c|c|c|c|c|c|c|c|c|c|c|c|c|}
\hline Glass ID & $\begin{array}{c}\mathrm{T} 12 \mathrm{O} 3 \\
-\mathrm{t}\end{array}$ & $\begin{array}{c}\text { U3O8 } \\
-t\end{array}$ & $\begin{array}{c}\mathrm{UO} 2 \\
-\mathrm{t}\end{array}$ & $\begin{array}{c}\text { UO3 } \\
-t\end{array}$ & $\begin{array}{c}\mathrm{V} 2 \mathrm{O} 5 \\
-\mathrm{t}\end{array}$ & $\begin{array}{c}\text { WO3 } \\
-t\end{array}$ & $\begin{array}{c}\mathrm{Y} 2 \mathrm{O} 3 \\
-\mathrm{t}\end{array}$ & $\begin{array}{c}\mathrm{ZnO} \\
-\mathrm{t}\end{array}$ & $\begin{array}{c}\text { Others } \\
-t\end{array}$ & $\begin{array}{c}\text { Sum } \\
-t\end{array}$ & $\begin{array}{c}\mathrm{A} 12 \mathrm{O} 3 \\
-\mathrm{a}\end{array}$ & $\begin{array}{c}\mathrm{B} 2 \mathrm{O} 3 \\
-\mathrm{a}\end{array}$ & $\begin{array}{c}\mathrm{CaO} \\
-\mathrm{a}\end{array}$ & $\begin{array}{c}\mathrm{Fe} 2 \mathrm{O} 3 \\
-\mathrm{a}\end{array}$ & $\begin{array}{c}\mathrm{FeO} \\
-\mathrm{a}\end{array}$ & $\begin{array}{c}\mathrm{K} 2 \mathrm{O} \\
-\mathrm{a}\end{array}$ & $\begin{array}{c}\mathrm{Li} 2 \mathrm{O} \\
-\mathrm{a}\end{array}$ & $\begin{array}{c}\mathrm{MgO} \\
-\mathrm{a}\end{array}$ & $\begin{array}{c}\mathrm{Na} 2 \mathrm{O} \\
-\mathrm{a}\end{array}$ & $\begin{array}{c}\mathrm{NiO} \\
-\mathrm{a}\end{array}$ & $\begin{array}{c}\mathrm{P} 2 \mathrm{O} 5 \\
-\mathrm{a}\end{array}$ \\
\hline IG2-01 & & & & & & & 0.0000 & & & 1.0000 & 0.2070 & 0.0522 & 0.0004 & 0.0002 & & 0.0000 & 0.0000 & & 0.2000 & 0.0155 & 0.0000 \\
\hline IG2-02 & & & & & & & 0.0000 & & & 1.0000 & 0.1990 & 0.1900 & 0.1130 & 0.0003 & & 0.0000 & 0.0000 & & 0.0541 & 0.0000 & 0.0286 \\
\hline IG2-03 & & & & & & & 0.0000 & & & 1.0000 & 0.0364 & 0.1810 & 0.0004 & 0.0820 & & 0.0000 & 0.0000 & & 0.0508 & 0.0153 & 0.0005 \\
\hline IG2-04 & & & & & & & 0.0000 & & & 1.0000 & 0.1990 & 0.2010 & 0.0002 & 0.0003 & & 0.1300 & 0.0931 & & 0.0563 & 0.0148 & 0.0000 \\
\hline IG2-05 & & & & & & & 0.0000 & & & 1.0000 & 0.0368 & 0.0549 & 0.1170 & 0.0781 & & 0.0118 & 0.0000 & & 0.1990 & 0.0148 & 0.0000 \\
\hline IG2-06 & & & & & & & 0.0000 & & & 1.0000 & 0.0649 & 0.2040 & 0.0003 & 0.0766 & & 0.0000 & 0.1067 & & 0.0512 & 0.0142 & 0.0288 \\
\hline IG2-07 & & & & & & & 0.0000 & & & 1.0000 & 0.0848 & 0.0573 & 0.1040 & 0.0002 & & 0.1190 & 0.0000 & & 0.0523 & 0.0146 & 0.0280 \\
\hline IG2-08 & & & & & & & 0.0000 & & & 1.0000 & 0.0356 & 0.1880 & 0.1150 & 0.0002 & & 0.1110 & 0.0000 & & 0.1470 & 0.0000 & 0.0000 \\
\hline IG2-09 & & & & & & & 0.0000 & & & 1.0000 & 0.2000 & 0.0543 & 0.1140 & 0.0756 & & 0.0000 & 0.0756 & & 0.0581 & 0.0000 & 0.0000 \\
\hline IG2-10 & & & & & & & 0.0000 & & & 1.0000 & 0.0379 & 0.0661 & 0.0006 & 0.0701 & & 0.0868 & 0.0921 & & 0.0669 & 0.0000 & 0.0306 \\
\hline IG2-11 & & & & & & & 0.0000 & & & 1.0000 & 0.0379 & 0.0543 & 0.0729 & 0.0002 & & 0.0000 & 0.0911 & & 0.1790 & 0.0145 & 0.0281 \\
\hline IG2-12 & & & & & & & 0.0000 & & & 1.0000 & 0.0385 & 0.2007 & 0.0002 & 0.0002 & & 0.0000 & 0.0060 & & 0.2240 & 0.0000 & 0.0000 \\
\hline IG2-13 & & & & & & & 0.0000 & & & 1.0000 & 0.0494 & 0.0784 & 0.0007 & 0.0004 & & 0.0000 & 0.0964 & & 0.0554 & 0.0000 & 0.0000 \\
\hline IG2-14 & & & & & & & 0.0000 & & & 1.0000 & 0.1940 & 0.0659 & 0.0003 & 0.0761 & & 0.1090 & 0.0000 & & 0.1570 & 0.0000 & 0.0275 \\
\hline IG2-15 & & & & & & & 0.0000 & & & 1.0000 & 0.0802 & 0.0833 & 0.0400 & 0.0050 & & 0.0325 & 0.0579 & & 0.1590 & 0.0047 & 0.0181 \\
\hline IG2-16 & & & & & & & 0.0000 & & & 1.0000 & 0.1070 & 0.1270 & 0.0389 & 0.0050 & & 0.0313 & 0.0294 & & 0.1420 & 0.0046 & 0.0192 \\
\hline IG2-17 & & & & & & & 0.0000 & & & 1.0000 & 0.0721 & 0.0641 & 0.0675 & 0.0047 & & 0.0320 & 0.0306 & & 0.1040 & 0.0049 & 0.0176 \\
\hline IG2-18 & & & & & & & 0.0000 & & & 1.0000 & 0.1150 & 0.0630 & 0.0397 & 0.0277 & & 0.0607 & 0.0309 & & 0.1660 & 0.0046 & 0.0093 \\
\hline IG2-19 & & & & & & & 0.0000 & & & 1.0000 & 0.0768 & 0.0647 & 0.0383 & 0.0051 & & 0.0311 & 0.0631 & & 0.1660 & 0.0092 & 0.0172 \\
\hline IG2-20 & & & & & & & 0.0000 & & & 1.0000 & 0.0793 & 0.0620 & 0.0396 & 0.0048 & & 0.0612 & 0.0290 & & 0.1700 & 0.0093 & 0.0181 \\
\hline IG2-21 & & & & & & & 0.0000 & & & 1.0000 & 0.0791 & 0.0605 & 0.0759 & 0.0166 & & 0.0697 & 0.0326 & & 0.0980 & 0.0093 & 0.0087 \\
\hline IG2-22 & & & & & & & 0.0000 & & & 1.0000 & 0.1130 & 0.0648 & 0.0374 & 0.0048 & & 0.2090 & 0.0609 & & 0.0139 & 0.0043 & 0.0167 \\
\hline IG2-23 & & & & & & & 0.0000 & & & 1.0000 & 0.1010 & 0.1180 & 0.0382 & 0.0047 & & 0.0359 & 0.0299 & & 0.1600 & 0.0047 & 0.0187 \\
\hline IG2-24 & & & & & & & 0.0000 & & & 1.0000 & 0.1050 & 0.0582 & 0.0662 & 0.0059 & & 0.0662 & 0.0589 & & 0.0975 & 0.0094 & 0.0090 \\
\hline IG2-25 & & & & & & & 0.0000 & & & 1.0000 & 0.1060 & 0.0573 & 0.0640 & 0.0051 & & 0.0616 & 0.0536 & & 0.0951 & 0.0046 & 0.0086 \\
\hline IG2-26 & & & & & & & 0.0000 & & & 1.0000 & 0.0772 & 0.0538 & 0.0251 & 0.0224 & & 0.0609 & 0.0554 & & 0.1050 & 0.0046 & 0.0089 \\
\hline IG2-27 & & & & & & & 0.0000 & & & 1.0000 & 0.0992 & 0.0557 & 0.0765 & 0.0048 & & 0.0327 & 0.0338 & & 0.0969 & 0.0092 & 0.0173 \\
\hline IG2-28 & & & & & & & 0.0000 & & & 1.0000 & 0.0761 & 0.0564 & 0.0700 & 0.0268 & & 0.0696 & 0.0273 & & 0.1060 & 0.0090 & 0.0089 \\
\hline IG2-29 & & & & & & & 0.0000 & & & 1.0000 & 0.0962 & 0.1070 & 0.0466 & 0.0261 & & 0.0480 & 0.0390 & & 0.1280 & 0.0070 & 0.0132 \\
\hline IG2-30 & & & & & & & 0.0000 & & & 1.0000 & 0.1480 & 0.1470 & 0.0012 & 0.0014 & & 0.0000 & 0.0970 & & 0.0513 & 0.0000 & 0.0000 \\
\hline IG2-31 & & & & & & & 0.0000 & & & 1.0000 & 0.0415 & 0.1300 & 0.0022 & 0.0000 & & 0.0262 & 0.0599 & & 0.0877 & 0.0000 & 0.0123 \\
\hline IG2-32 & & & & & & & 0.0000 & & & 1.0000 & 0.1000 & 0.0966 & 0.0476 & 0.0259 & & 0.0466 & 0.0425 & & 0.1120 & 0.0071 & 0.0143 \\
\hline IG2-33 & & & & & & & 0.0000 & & & 1.0000 & 0.0968 & 0.0924 & 0.0489 & 0.0251 & & 0.0439 & 0.0424 & & 0.1150 & 0.0074 & 0.0139 \\
\hline IG2-34 & & & & & & & 0.0000 & & & 1.0000 & 0.0992 & 0.0992 & 0.0481 & 0.0248 & & 0.0431 & 0.0370 & & 0.1080 & 0.0070 & 0.0137 \\
\hline IG2-35 & & & & & & & 0.0000 & & & 1.0000 & 0.1000 & 0.0970 & 0.0485 & 0.0253 & & 0.0489 & 0.0360 & & 0.1220 & 0.0074 & 0.0142 \\
\hline IG2-36 & & & & & & & 0.0000 & & & 1.0000 & 0.0980 & 0.0909 & 0.0486 & 0.0244 & & 0.0447 & 0.0367 & & 0.1100 & 0.0073 & 0.0140 \\
\hline IG2-37 & & & & & & & 0.0000 & & & 1.0000 & 0.0970 & 0.0829 & 0.0478 & 0.0244 & & 0.0461 & 0.0357 & & 0.1020 & 0.0073 & 0.0130 \\
\hline
\end{tabular}

\section{INEEL CVS Phase 3 (Scholes et al. 2000)}

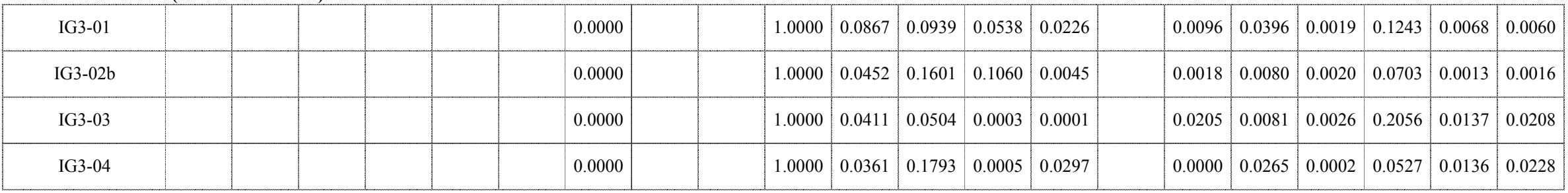


INEEL CVS Phase 2 (Staples et al. 2000)

\begin{tabular}{|c|c|c|c|c|c|c|c|c|c|c|c|c|c|c|c|c|c|c|c|c|c|}
\hline Glass ID & $\begin{array}{c}\mathrm{SiO} 2 \\
-\mathrm{a}\end{array}$ & $\begin{array}{c}\mathrm{ZrO} 2 \\
-\mathrm{a}\end{array}$ & $\begin{array}{c}\mathrm{Ag} 2 \mathrm{O} \\
-\mathrm{a}\end{array}$ & $\begin{array}{c}\mathrm{As} 2 \mathrm{O} 3 \\
-\mathrm{a}\end{array}$ & $\begin{array}{c}\mathrm{BaO} \\
-\mathrm{a}\end{array}$ & $\begin{array}{c}\mathrm{Bi} 2 \mathrm{O} 3 \\
-\mathrm{a}\end{array}$ & $\begin{array}{l}\mathrm{Br} \\
-\mathrm{a}\end{array}$ & $\begin{array}{c}\mathrm{CdO} \\
-\mathrm{a}\end{array}$ & $\begin{array}{c}\mathrm{Ce} 2 \mathrm{O} 3 \\
-\mathrm{a}\end{array}$ & $\begin{array}{c}\mathrm{CeO} 2 \\
-\mathrm{a}\end{array}$ & $\begin{array}{l}\mathrm{Cl} \\
-\mathrm{a}\end{array}$ & $\begin{array}{c}\mathrm{CoO} \\
-\mathrm{a}\end{array}$ & $\begin{array}{c}\mathrm{Co} 2 \mathrm{O} 3 \\
-\mathrm{a}\end{array}$ & $\begin{array}{c}\mathrm{Cr} 2 \mathrm{O} 3 \\
-\mathrm{a}\end{array}$ & $\begin{array}{c}\mathrm{Cs} 2 \mathrm{O} \\
-\mathrm{a}\end{array}$ & $\begin{array}{c}\mathrm{CuO} \\
-\mathrm{a}\end{array}$ & $\begin{array}{c}\mathrm{Eu} 2 \mathrm{O} 3 \\
-\mathrm{a}\end{array}$ & $\begin{array}{c}F \\
-a\end{array}$ & $\begin{array}{c}\mathrm{Ga} 2 \mathrm{O} 3 \\
-\mathrm{a}\end{array}$ & {$\left[\begin{array}{c}\mathrm{Gd} 2 \mathrm{O} 3 \\
-\mathrm{a}\end{array}\right.$} & $\begin{array}{c}\mathrm{HgO} \\
-\mathrm{a}\end{array}$ \\
\hline IG2-01 & 0.4780 & 0.0000 & & & & & & & & & & & & & & & & 0.0607 & & & \\
\hline IG2-02 & 0.4250 & 0.0000 & & & & & & & & & & & & & & & & 0.0000 & & & \\
\hline IG2-03 & 0.5930 & 0.0000 & & & & & & & & & & & & & & & & 0.0138 & & & \\
\hline IG2-04 & 0.3410 & 0.0147 & & & & & & & & & & & & & & & & 0.0000 & & & \\
\hline IG2-05 & 0.3650 & 0.1410 & & & & & & & & & & & & & & & & 0.0000 & & & \\
\hline IG2-06 & 0.3630 & 0.1340 & & & & & & & & & & & & & & & & 0.0000 & & & \\
\hline IG2-07 & 0.3780 & 0.1280 & & & & & & & & & & & & & & & & 0.0548 & & & \\
\hline IG2-08 & 0.4450 & 0.0000 & & & & & & & & & & & & & & & & 0.0000 & & & \\
\hline IG2-09 & 0.3840 & 0.0000 & & & & & & & & & & & & & & & & 0.0591 & & & \\
\hline IG2-10 & 0.4910 & 0.0000 & & & & & & & & & & & & & & & & 0.0565 & & & \\
\hline IG2-11 & 0.5450 & 0.0000 & & & & & & & & & & & & & & & & 0.0000 & & & \\
\hline IG2-12 & 0.3940 & 0.1160 & & & & & & & & & & & & & & & & 0.0581 & & & \\
\hline IG2-13 & 0.5970 & 0.1360 & & & & & & & & & & & & & & & & 0.0000 & & & \\
\hline IG2-14 & 0.3450 & 0.0195 & & & & & & & & & & & & & & & & 0.0000 & & & \\
\hline IG2-15 & 0.5050 & 0.0385 & & & & & & & & & & & & & & & & 0.0095 & & & \\
\hline IG2-16 & 0.3910 & 0.0799 & & & & & & & & & & & & & & & & 0.0095 & & & \\
\hline IG2-17 & 0.4880 & 0.0768 & & & & & & & & & & & & & & & & 0.0209 & & & \\
\hline IG2-18 & 0.4220 & 0.0791 & & & & & & & & & & & & & & & & 0.0083 & & & \\
\hline IG2-19 & 0.5130 & 0.0399 & & & & & & & & & & & & & & & & 0.0089 & & & \\
\hline IG2-20 & 0.4820 & 0.0382 & & & & & & & & & & & & & & & & 0.0084 & & & \\
\hline IG2-21 & 0.4890 & 0.0380 & & & & & & & & & & & & & & & & 0.0091 & & & \\
\hline IG2-22 & 0.3810 & 0.0744 & & & & & & & & & & & & & & & & 0.0091 & & & \\
\hline IG2-23 & 0.3800 & 0.0777 & & & & & & & & & & & & & & & & 0.0132 & & & \\
\hline IG2-24 & 0.4080 & 0.0779 & & & & & & & & & & & & & & & & 0.0091 & & & \\
\hline IG2-25 & 0.3950 & 0.0740 & & & & & & & & & & & & & & & & 0.0092 & & & \\
\hline IG2-26 & 0.4730 & 0.0778 & & & & & & & & & & & & & & & & 0.0000 & & & \\
\hline IG2-27 & 0.4840 & 0.0391 & & & & & & & & & & & & & & & & 0.0231 & & & \\
\hline IG2-28 & 0.4820 & 0.0382 & & & & & & & & & & & & & & & & 0.0097 & & & \\
\hline IG2-29 & 0.4250 & 0.0556 & & & & & & & & & & & & & & & & 0.0203 & & & \\
\hline IG2-30 & 0.4960 & 0.0294 & & & & & & & & & & & & & & & & 0.0000 & & & \\
\hline IG2-31 & 0.5230 & 0.1060 & & & & & & & & & & & & & & & & 0.0000 & & & \\
\hline IG2-32 & 0.4490 & 0.0573 & & & & & & & & & & & & & & & & 0.0195 & & & \\
\hline IG2-33 & 0.4430 & 0.0557 & & & & & & & & & & & & & & & & 0.0193 & & & \\
\hline IG2-34 & 0.4510 & 0.0543 & & & & & & & & & & & & & & & & 0.0177 & & & \\
\hline IG2-35 & 0.4330 & 0.0546 & & & & & & & & & & & & & & & & 0.0199 & & & \\
\hline IG2-36 & 0.3900 & 0.0554 & & & & & & & & & & & & & & & & 0.0195 & & & \\
\hline IG2-37 & 0.3970 & 0.0526 & & & & & & & & & & & & & & & & 0.0195 & & & \\
\hline
\end{tabular}

\section{INEEL CVS Phase 3 (Scholes et al. 2000)}

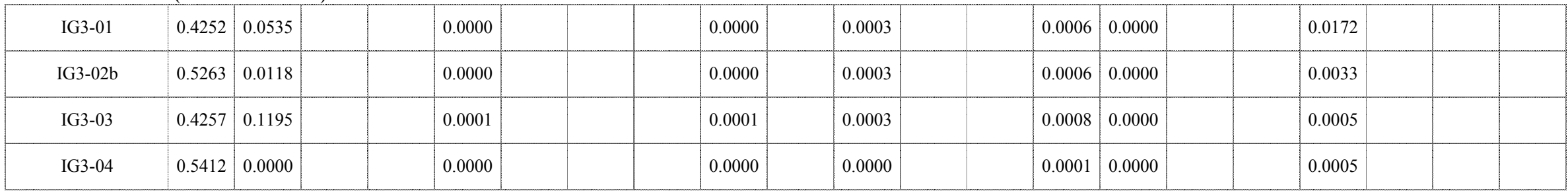


INEEL CVS Phase 2 (Staples et al. 2000)

\begin{tabular}{|c|c|c|c|c|c|c|c|c|c|c|c|c|c|c|c|c|c|c|c|c|c|}
\hline Glass ID & $\begin{array}{c}\mathrm{I} \\
-\mathrm{a} \\
\end{array}$ & $\begin{array}{c}\mathrm{La} 2 \mathrm{O} 3 \\
-\mathrm{a} \\
\end{array}$ & $\begin{array}{c}\mathrm{MnO} 2 \\
\text {-a }\end{array}$ & $\begin{array}{c}\mathrm{MnO} \\
-\mathrm{a} \\
\end{array}$ & $\begin{array}{c}\mathrm{MoO} \\
-\mathrm{a}\end{array}$ & $\begin{array}{c}\mathrm{MoO} 3 \\
-\mathrm{a} \\
\end{array}$ & $\begin{array}{c}\mathrm{Nb} 2 \mathrm{O} 5 \\
-\mathrm{a}\end{array}$ & $\begin{array}{c}\mathrm{Nd} 2 \mathrm{O} 3 \\
-\mathrm{a} \\
\end{array}$ & $\begin{array}{c}\mathrm{PbO} \\
-\mathrm{a}\end{array}$ & $\begin{array}{c}\mathrm{PdO} 2 \\
-\mathrm{a}\end{array}$ & $\begin{array}{c}\mathrm{PdO} \\
-\mathrm{a} \\
\end{array}$ & $\begin{array}{c}\mathrm{Pr} 2 \mathrm{O} 3 \\
-\mathrm{a}\end{array}$ & $\begin{array}{c}\text { Pr6O11 } \\
-\mathrm{a}\end{array}$ & $\begin{array}{c}\mathrm{Rb} 2 \mathrm{O} \\
-\mathrm{a}\end{array}$ & $\begin{array}{c}\mathrm{ReO} \\
-\mathrm{a}\end{array}$ & $\begin{array}{c}\mathrm{ReO} 2 \\
-\mathrm{a} \\
\end{array}$ & $\begin{array}{c}\mathrm{Rh} 2 \mathrm{O} 3 \\
-\mathrm{a}\end{array}$ & $\begin{array}{c}\mathrm{RhO}_{2} \\
-\mathrm{a}\end{array}$ & $\begin{array}{c}\mathrm{RuO} 2 \\
-\mathrm{a}\end{array}$ & \begin{tabular}{|c}
$\mathrm{Sb} 2 \mathrm{O} 3$ \\
$-\mathrm{a}$ \\
\end{tabular} & $\begin{array}{c}\mathrm{Sb} 2 \mathrm{O} 5 \\
-\mathrm{a}\end{array}$ \\
\hline IG2-01 & & & & & & 0.0000 & & & & & & & & & & & & & & & \\
\hline IG2-02 & & & & & & 0.0000 & & & & & & & & & & & & & & & \\
\hline IG2-03 & & & & & & 0.0000 & & & & & & & & & & & & & & & \\
\hline IG2-04 & & & & & & 0.0000 & & & & & & & & & & & & & & & \\
\hline IG2-05 & & & & & & 0.0000 & & & & & & & & & & & & & & & \\
\hline IG2-06 & & & & & & 0.0000 & & & & & & & & & & & & & & & \\
\hline IG2-07 & & & & & & 0.0000 & & & & & & & & & & & & & & & \\
\hline IG2-08 & & & & & & 0.0000 & & & & & & & & & & & & & & & \\
\hline IG2-09 & & & & & & 0.0000 & & & & & & & & & & & & & & & \\
\hline IG2-10 & & & & & & 0.0000 & & & & & & & & & & & & & & & \\
\hline IG2-11 & & & & & & 0.0000 & & & & & & & & & & & & & & & \\
\hline IG2-12 & & & & & & 0.0000 & & & & & & & & & & & & & & & \\
\hline IG2-13 & & & & & & 0.0000 & & & & & & & & & & & & & & & \\
\hline IG2-14 & & & & & & 0.0000 & & & & & & & & & & & & & & & \\
\hline IG2-15 & & & & & & 0.0000 & & & & & & & & & & & & & & & \\
\hline IG2-16 & & & & & & 0.0000 & & & & & & & & & & & & & & & \\
\hline IG2-17 & & & & & & 0.0000 & & & & & & & & & & & & & & & \\
\hline IG2-18 & & & & & & 0.0000 & & & & & & & & & & & & & & & \\
\hline IG2-19 & & & & & & 0.0000 & & & & & & & & & & & & & & & \\
\hline IG2-20 & & & & & & 0.0000 & & & & & & & & & & & & & & & \\
\hline IG2-21 & & & & & & 0.0000 & & & & & & & & & & & & & & & \\
\hline IG2-22 & & & & & & 0.0000 & & & & & & & & & & & & & & & \\
\hline IG2-23 & & & & & & 0.0000 & & & & & & & & & & & & & & & \\
\hline IG2-24 & & & & & & 0.0000 & & & & & & & & & & & & & & & \\
\hline IG2-25 & & & & & & 0.0000 & & & & & & & & & & & & & & & \\
\hline IG2-26 & & & & & & 0.0000 & & & & & & & & & & & & & & & \\
\hline IG2-27 & & & & & & 0.0000 & & & & & & & & & & & & & & & \\
\hline IG2-28 & & & & & & 0.0000 & & & & & & & & & & & & & & & \\
\hline IG2-29 & & & & & & 0.0000 & & & & & & & & & & & & & & & \\
\hline IG2-30 & & & & & & 0.0000 & & & & & & & & & & & & & & & \\
\hline IG2-31 & & & & & & 0.0000 & & & & & & & & & & & & & & & \\
\hline IG2-32 & & & & & & 0.0000 & & & & & & & & & & & & & & & \\
\hline IG2-33 & & & & & & 0.0000 & & & & & & & & & & & & & & & \\
\hline IG2-34 & & & & & & 0.0191 & & & & & & & & & & & & & & & \\
\hline IG2-35 & & & & & & 0.0050 & & & & & & & & & & & & & & & \\
\hline IG2-36 & & & & & & 0.0000 & & & & & & & & & & & & & & & \\
\hline IG2-37 & & & & & & 0.0000 & & & & & & & & & & & & & & & \\
\hline
\end{tabular}

\section{INEEL CVS Phase 3 (Scholes et al. 2000)}

\begin{tabular}{|c|c|c|c|c|c|c|c|c|c|c|c|c|}
\hline IG3-01 & 0.0000 & 0.0211 & 0.0001 & 0.0069 & 0.0002 & 0.0001 & 0.0000 & 0.0000 & 0.0000 & 0.0000 & 0.0000 & 0.0000 \\
\hline IG3-02b & 0.0000 & 0.0410 & 0.0001 & 0.0130 & 0.0002 & 0.0001 & 0.0000 & 0.0000 & 0.0000 & 0.0000 & 0.0000 & 0.0000 \\
\hline IG3-03 & 0.0000 & 0.0462 & 0.0001 & 0.0142 & 0.0002 & 0.0001 & 0.0000 & 0.0000 & 0.0000 & 0.0001 & 0.0000 & 0.0000 \\
\hline IG3-04 & 0.0000 & 0.0447 & 0.0000 & 0.0002 & 0.0000 & 0.0000 & 0.0000 & 0.0000 & 0.0000 & 0.0000 & 0.0000 & 0.0000 \\
\hline
\end{tabular}


Appendix A. Database - mass fraction

INEEL CVS Phase 2 (Staples et al. 2000)

\begin{tabular}{|c|c|c|c|c|c|c|c|c|c|c|c|c|c|c|c|c|c|c|c|c|}
\hline Glass ID & $\begin{array}{c}\mathrm{SeO} 2 \\
-\mathrm{a} \\
\end{array}$ & $\begin{array}{c}\mathrm{Sm} 2 \mathrm{O} 3 \\
-\mathrm{a} \\
\end{array}$ & $\begin{array}{c}\mathrm{SnO} \\
-\mathrm{a} \\
\end{array}$ & $\begin{array}{c}\mathrm{SnO} 2 \\
-\mathrm{a} \\
\end{array}$ & $\begin{array}{c}\mathrm{SO} 3 \\
-\mathrm{a} \\
\end{array}$ & $\begin{array}{c}\mathrm{SrO} \\
-\mathrm{a} \\
\end{array}$ & $\begin{array}{c}\mathrm{Tc} 2 \mathrm{O} 7 \\
-\mathrm{a}\end{array}$ & $\begin{array}{c}\mathrm{TeO} 2 \\
-\mathrm{a}\end{array}$ & $\begin{array}{c}\mathrm{ThO} 2 \\
-\mathrm{a}\end{array}$ & $\begin{array}{c}\mathrm{TiO} 2 \\
-\mathrm{a} \\
\end{array}$ & $\begin{array}{c}\mathrm{T} 12 \mathrm{O} 3 \\
-\mathrm{a} \\
\end{array}$ & $\begin{array}{c}\mathrm{U} 3 \mathrm{O} 8 \\
-\mathrm{a} \\
\end{array}$ & $\begin{array}{c}\mathrm{UO} 2 \\
-\mathrm{a}\end{array}$ & $\begin{array}{c}\mathrm{UO} 3 \\
-\mathrm{a} \\
\end{array}$ & $\begin{array}{c}\mathrm{V} 2 \mathrm{O} 5 \\
-\mathrm{a} \\
\end{array}$ & $\begin{array}{c}\text { WO3 } \\
-\mathrm{a} \\
\end{array}$ & $\begin{array}{c}\mathrm{Y} 2 \mathrm{O} 3 \\
-\mathrm{a}\end{array}$ & $\begin{array}{c}\mathrm{ZnO} \\
-\mathrm{a} \\
\end{array}$ & $\begin{array}{c}\text { Others } \\
-\mathrm{a}\end{array}$ & $\begin{array}{l}\text { Sum } \\
-\mathrm{a}\end{array}$ \\
\hline IG2-01 & & & & & & 0.0003 & & & & & & & & & & & & & & 1.0143 \\
\hline IG2-02 & & & & & & 0.0003 & & & & & & & & & & & & & & 1.0103 \\
\hline IG2-03 & & & & & & 0.0004 & & & & & & & & & & & & & & 0.9736 \\
\hline IG2-04 & & & & & & 0.0003 & & & & & & & & & & & & & & 1.0507 \\
\hline IG2-05 & & & & & & 0.0004 & & & & & & & & & & & & & & 1.0188 \\
\hline IG2-06 & & & & & & 0.0003 & & & & & & & & & & & & & & 1.0440 \\
\hline IG2-07 & & & & & & 0.0003 & & & & & & & & & & & & & & 1.0213 \\
\hline IG2-08 & & & & & & 0.0004 & & & & & & & & & & & & & & 1.0422 \\
\hline IG2-09 & & & & & & 0.0002 & & & & & & & & & & & & & & 1.0209 \\
\hline IG2-10 & & & & & & 0.0003 & & & & & & & & & & & & & & 0.9989 \\
\hline IG2-11 & & & & & & 0.0004 & & & & & & & & & & & & & & 1.0234 \\
\hline IG2-12 & & & & & & 0.0003 & & & & & & & & & & & & & & 1.0380 \\
\hline IG2-13 & & & & & & 0.0003 & & & & & & & & & & & & & & 1.0140 \\
\hline IG2-14 & & & & & & 0.0003 & & & & & & & & & & & & & & 0.9946 \\
\hline IG2-15 & & & & & & 0.0003 & & & & & & & & & & & & & & 1.0340 \\
\hline IG2-16 & & & & & & 0.0004 & & & & & & & & & & & & & & 0.9852 \\
\hline IG2-17 & & & & & & 0.0003 & & & & & & & & & & & & & & 0.9835 \\
\hline IG2-18 & & & & & & 0.0003 & & & & & & & & & & & & & & 1.0266 \\
\hline IG2-19 & & & & & & 0.0003 & & & & & & & & & & & & & & 1.0336 \\
\hline IG2-20 & & & & & & 0.0003 & & & & & & & & & & & & & & 1.0022 \\
\hline IG2-21 & & & & & & 0.0003 & & & & & & & & & & & & & & 0.9868 \\
\hline IG2-22 & & & & & & 0.0003 & & & & & & & & & & & & & & 0.9896 \\
\hline IG2-23 & & & & & & 0.0003 & & & & & & & & & & & & & & 0.9823 \\
\hline IG2-24 & & & & & & 0.0003 & & & & & & & & & & & & & & 0.9716 \\
\hline IG2-25 & & & & & & 0.0003 & & & & & & & & & & & & & & 0.9344 \\
\hline IG2-26 & & & & & & 0.0003 & & & & & & & & & & & & & & 0.9644 \\
\hline IG2-27 & & & & & & 0.0003 & & & & & & & & & & & & & & 0.9726 \\
\hline IG2-28 & & & & & & 0.0003 & & & & & & & & & & & & & & 0.9803 \\
\hline IG2-29 & & & & & & 0.0000 & & & & & & & & & & & & & & 1.0120 \\
\hline IG2-30 & & & & & & 0.0000 & & & & & & & & & & & & & & 0.9713 \\
\hline IG2-31 & & & & & & 0.0000 & & & & & & & & & & & & & & 0.9888 \\
\hline IG2-32 & & & & & & 0.0003 & & & & & & & & & & & & & & 1.0187 \\
\hline IG2-33 & & & & & & 0.0003 & & & & & & & & & & & & & & 1.0041 \\
\hline IG2-34 & & & & & & 0.0003 & & & & & & & & & & & & & & 1.0225 \\
\hline IG2-35 & & & & & & 0.0003 & & & & & & & & & & & & & & 1.0121 \\
\hline IG2-36 & & & & & & 0.0148 & & & & & & & & & & & & & & 0.9543 \\
\hline IG2-37 & & & & & & 0.0300 & & & & & & & & & & & & & & 0.9553 \\
\hline
\end{tabular}

\section{INEEL CVS Phase 3 (Scholes et al. 2000)}

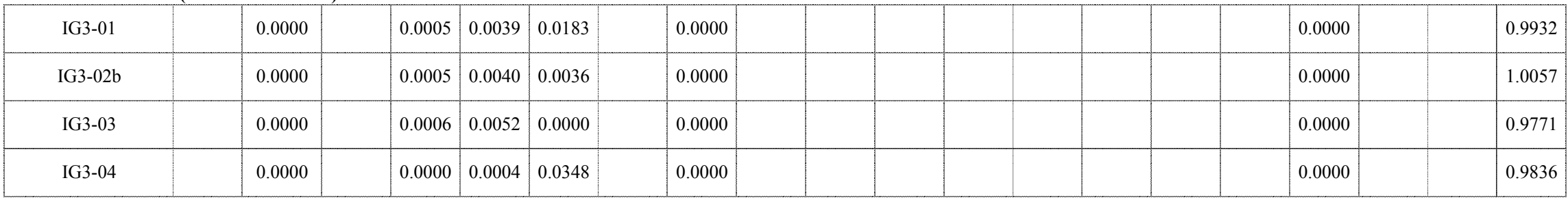


INEEL CVS Phase 2 (Staples et al. 2000)

\begin{tabular}{|c|c|c|c|c|c|c|c|c|}
\hline Glass ID & $\begin{array}{l}\mathrm{TM} \\
\left({ }^{\circ} \mathrm{C}\right)\end{array}$ & $\begin{array}{l}\text { Gradient } \\
\text { TL }\left({ }^{\circ} \mathrm{C}\right)\end{array}$ & $\begin{array}{l}\text { Uniform } \\
\text { TL }\left({ }^{\circ} \mathrm{C}\right)\end{array}$ & Primary Phase & $\begin{array}{c}\text { Quenched } \\
\text { Visual/OM }\end{array}$ & $\begin{array}{c}\text { Quenched } \\
\text { SEM/EDS or TEM }\end{array}$ & $\begin{array}{l}\text { Quenched } \\
\text { XRD }\end{array}$ & $\begin{array}{c}\text { Quenched } \\
\text { Homogeneous? }\end{array}$ \\
\hline IG2-01 & 1250 & & & & Inhomogeneous & & Na3AlF6 & no \\
\hline IG2-02 & 1250 & & 1133 & $\mathrm{Ca} 5(\mathrm{PO} 4) 3(\mathrm{OH})$ & Homogeneous & & Amorphous & yes \\
\hline IG2-03 & 1550 & & & & Inhomogeneous & & $\mathrm{Fe} 2 \mathrm{O} 3, \mathrm{NaA1O} 2$ & no \\
\hline IG2-04 & 1150 & & 913 & $\mathrm{NiO}, \mathrm{Na} 2 \mathrm{ZrO} 3$ & Homogeneous & & Amorphous & yes \\
\hline IG2-05 & 1250 & & & & Inhomogeneous & & $\mathrm{ZrO} 2$ & no \\
\hline IG2-06 & 1250 & & & & Inhomogeneous & & $\mathrm{ZrSiO} 4, \mathrm{ZrO} 2$ & no \\
\hline IG2-07 & 1250 & & & & Inhomogeneous & & $\mathrm{ZrO} 2, \mathrm{CaF} 2$ & no \\
\hline IG2-08 & 1150 & & 861 & $\mathrm{CaSiO} 3$ & Homogeneous & & Amorphous & yes \\
\hline IG2-09 & 1150 & & & & Inhomogeneous & & Amorphous & no \\
\hline IG2-10 & 1150 & & & & Inhomogeneous & & Unidentified Phase & no \\
\hline IG2-11 & 1150 & & 773 & $\mathrm{Li} 2 \mathrm{SiO} 3$ & Homogeneous & & Amorphous & yes \\
\hline IG2-12 & 1150 & & 823 & unknown phase & Homogeneous & & Amorphous & yes \\
\hline IG2-13 & 1250 & & 1223 & $\mathrm{ZrSiO} 4$ & Homogeneous & & Amorphous & yes \\
\hline IG2-14 & 1250 & & 1133 & $\mathrm{NaAlSiO} 4$ & Homogeneous & & Amorphous & yes \\
\hline IG2-15 & 1150 & & 823 & $\mathrm{Ca} 5(\mathrm{PO} 4) 3 \mathrm{OH}$ & Homogeneous & & Amorphous & yes \\
\hline IG2-16 & 1150 & & 948 & $\mathrm{Ca} 5(\mathrm{PO} 4) 3 \mathrm{~F}$ & Homogeneous & & Amorphous & yes \\
\hline IG2-17 & 1250 & & & & Homogeneous & & $\mathrm{Ca} 5(\mathrm{PO} 4) 3 \mathrm{~F}$ & no \\
\hline IG2-18 & 1250 & & 1407 & $\mathrm{ZrO} 2$ & Homogeneous & & $\mathrm{ZrO} 2$ & no \\
\hline IG2-19 & 1150 & & 841 & Li2SiO3, & Homogeneous & & Amorphous & yes \\
\hline IG2-20 & 1150 & & 843 & $\mathrm{Ca} 5(\mathrm{PO} 4) 3 \mathrm{~F}$ & Homogeneous & & Amorphous & yes \\
\hline IG2-21 & 1150 & & 961 & $\mathrm{Ca} 5(\mathrm{PO} 4) 3 \mathrm{~F}$ & Homogeneous & & Amorphous & yes \\
\hline IG2-22 & 1250 & & & & Homogeneous & & $\mathrm{SiO} 2, \mathrm{SiP} 2 \mathrm{O} 7, \mathrm{AlPO} 4$ & no \\
\hline IG2-23 & 1150 & & 923 & $\mathrm{Ca} 5(\mathrm{PO} 4) 3 \mathrm{~F}$ & Homogeneous & & Amorphous & yes \\
\hline IG2-24 & 1250 & & 1382 & $\mathrm{ZrO} 2$ & Homogeneous & & Amorphous & yes \\
\hline IG2-25 & 1250 & & 1362 & $\mathrm{ZrO} 2$ & Homogeneous & & Amorphous & yes \\
\hline IG2-26 & 1150 & & 966 & $\mathrm{Li} 2 \mathrm{SiO} 3$ & Homogeneous & & Amorphous & yes \\
\hline IG2-27 & 1250 & & & & Inhomogeneous & & Ca5(PO4)3F & no \\
\hline IG2-28 & 1150 & & 938 & $\mathrm{Ca} 5(\mathrm{PO} 4) 3 \mathrm{~F}$ & Homogeneous & & Amorphous & yes \\
\hline IG2-29 & 1150 & & 923 & $\mathrm{Ca} 5(\mathrm{PO} 4) 3 \mathrm{~F}$ & Homogeneous & & Amorphous & yes \\
\hline IG2-30 & 1150 & & 948 & LiAlSi3O8 & Homogeneous & & Amorphous & yes \\
\hline IG2-31 & 1150 & & 883 & $\begin{array}{l}\text { LiNaZrSi6O15, } \\
\text { LiPO4 }\end{array}$ & Homogeneous & & Amorphous & yes \\
\hline IG2-32 & 1150 & & 933 & Ca5(PO4)3F & Homogeneous & & Amorphous & yes \\
\hline IG2-33 & 1150 & & 913 & $\mathrm{Ca} 5(\mathrm{PO} 4) 3 \mathrm{~F}$ & Homogeneous & & Amorphous & yes \\
\hline IG2-34 & 1250 & & & & Inhomogeneous & & $\mathrm{Ca} 5(\mathrm{PO} 4) 3 \mathrm{~F}, \mathrm{KAlSiO} 4$ & no \\
\hline IG2-35 & 1150 & & 931 & $\mathrm{Ca} 5(\mathrm{PO} 4) 3 \mathrm{~F}$ & Homogeneous & & Amorphous & yes \\
\hline IG2-36 & 1150 & & 923 & $\mathrm{Ca} 5(\mathrm{PO} 4) 3 \mathrm{~F}$ & Homogeneous & & Amorphous & yes \\
\hline IG2-37 & 1150 & & 938 & $\mathrm{Ca} 5(\mathrm{PO} 4) 3 \mathrm{~F}$ & Homogeneous & & Amorphous & yes \\
\hline
\end{tabular}

\section{INEEL CVS Phase 3 (Scholes et al. 2000)}

\begin{tabular}{|c|c|c|c|c|c|}
\hline IG3-01 & 1150 & 873 & $\begin{array}{l}\mathrm{Ca} 10(\mathrm{SiO} 4) 3(\mathrm{SO} 4) 3 \mathrm{~F} \\
2\end{array}$ & amorphous & yes \\
\hline IG3-02b & 1250 & 963 & single-phase & amorphous & yes \\
\hline IG3-03 & 1350 & & multi-phase & $\begin{array}{l}\mathrm{Na} 2 \mathrm{MoO} 4 \\
\mathrm{Na}(\mathrm{A} 1 \mathrm{Si} 3 \mathrm{O} 8)\end{array}$ & no \\
\hline IG3-04 & 1250 & & multi-phase & $\begin{array}{l}\text { LaPO4, Na5P3O10, } \\
\text { SiP2O7 }\end{array}$ & no \\
\hline
\end{tabular}


Appendix A. Database - mass fraction

INEEL CVS Phase 2 (Staples et al. 2000)

\begin{tabular}{|c|c|c|c|c|c|}
\hline Glass ID & $\begin{array}{c}\mathrm{CCC} \\
\text { Visual/OM } \\
\end{array}$ & $\begin{array}{c}\text { CCC } \\
\text { SEM/EDS or TEM }\end{array}$ & $\begin{array}{l}\mathrm{CCC} \\
\mathrm{XRD}\end{array}$ & $\begin{array}{c}\text { Heat Treated } \\
\text { Visual/OM }\end{array}$ & $\begin{array}{c}\text { Heat Treated } \\
\text { SEM/EDS or TEM }\end{array}$ \\
\hline \multicolumn{6}{|l|}{ IG2-01 } \\
\hline \multicolumn{6}{|l|}{ IG2-02 } \\
\hline \multicolumn{6}{|l|}{ IG2-03 } \\
\hline \multicolumn{6}{|l|}{ IG2-04 } \\
\hline \multicolumn{6}{|l|}{ IG2-05 } \\
\hline \multicolumn{6}{|l|}{ IG2-06 } \\
\hline \multicolumn{6}{|l|}{ IG2-07 } \\
\hline \multicolumn{6}{|l|}{ IG2-08 } \\
\hline IG2-09 & Inhomogenous & & $\mathrm{LiAlSiO} 4$ and $\mathrm{NaAlSiO} 4$ & & \\
\hline IG2-10 & Inhomogenous & & $\mathrm{Li} 3 \mathrm{PO} 4$ and $\mathrm{LiF}$ & & \\
\hline IG2-11 & homogenous & & Amorphous & & \\
\hline IG2-12 & Inhomogenous & & $\mathrm{NaF}, \mathrm{LiAlOCl} 2$, and $\mathrm{NaAl} 2 \mathrm{Si} 2 \mathrm{O} 9$ & & \\
\hline \multicolumn{6}{|l|}{ IG2-13 } \\
\hline \multicolumn{6}{|l|}{ IG2-14 } \\
\hline IG2-15 & homogenous & & Amorphous & & \\
\hline \multicolumn{6}{|l|}{ IG2-16 } \\
\hline \multicolumn{6}{|l|}{ IG2-17 } \\
\hline \multicolumn{6}{|l|}{ IG2-18 } \\
\hline IG2-19 & homogenous & & Amorphous & & \\
\hline IG2-20 & homogenous & & Amorphous & & \\
\hline \multicolumn{6}{|l|}{ IG2-21 } \\
\hline \multicolumn{6}{|l|}{ IG2-22 } \\
\hline IG2-23 & Inhomogenous & & $\mathrm{Ca} 5(\mathrm{PO} 4) 3 \mathrm{~F}$ & & \\
\hline \multicolumn{6}{|l|}{ IG2-24 } \\
\hline \multicolumn{6}{|l|}{ IG2-25 } \\
\hline IG2-26 & homogenous & & Amorphous & & \\
\hline \multicolumn{6}{|l|}{ IG2-27 } \\
\hline IG2-28 & homogenous & & Amorphous & & \\
\hline IG2-29 & Inhomogenous & & $\mathrm{Ca} 5(\mathrm{PO} 4) 3 \mathrm{~F}$ & & \\
\hline \multicolumn{6}{|l|}{ IG2-30 } \\
\hline \multicolumn{6}{|l|}{ IG2-31 } \\
\hline \multicolumn{6}{|l|}{ IG2-32 } \\
\hline IG2-33 & Inhomogenous & & $\mathrm{Ca} 5(\mathrm{PO} 4) 3 \mathrm{~F}$ & & \\
\hline \multicolumn{6}{|l|}{ IG2-34 } \\
\hline IG2-35 & Inhomogenous & & $\mathrm{Ca} 5(\mathrm{PO} 4) 3 \mathrm{~F}$ & & \\
\hline IG2-36 & Inhomogenous & & $\mathrm{Ca} 5(\mathrm{PO} 4) 3 \mathrm{~F}$ & & \\
\hline IG2-37 & & & & & \\
\hline
\end{tabular}

\section{INEEL CVS Phase 3 (Scholes et al. 2000)}

IG3-01 brown colored glass is multi-phase and transparent with

small fraction of large clear crystal clusters throughout

IG3-02b opaque colored glass is multi-phase and devitrified with large

clear crystals

dark reddish brown glass is multi-phase with very small clear

granular crystals making the glass appear cloudy

opaque-brown glass with a lighter brown surface is multi-

phased and devitrified with fern type crystals

amorphous
amorphous
$\mathrm{La} 2 \mathrm{Mo} 2 \mathrm{O} 9$ and KAlP2O7
$\mathrm{La}(\mathrm{PO} 4)$ and $\mathrm{La} 2(\mathrm{MoO} 4) 3$


INEEL CVS Phase 2 (Staples et al. 2000)

\begin{tabular}{|c|c|c|c|c|c|c|c|c|c|c|c|c|c|c|c|}
\hline Glass ID & $\begin{array}{l}\text { Heat Treated } \\
\text { XRD }\end{array}$ & $\begin{array}{l}\text { Density } \\
\left(\mathrm{g} / \mathrm{cm}^{3}\right)\end{array}$ & \begin{tabular}{|c|} 
Fulc Visc \\
A
\end{tabular} & \begin{tabular}{|c|} 
Fulc Visc \\
B
\end{tabular} & \begin{tabular}{|c|} 
Fulc Visc \\
To \\
\end{tabular} & $\begin{array}{c}\mathrm{FV} 1150^{\circ} \mathrm{C} \\
(\mathrm{Pa} \cdot \mathrm{s})\end{array}$ & $\begin{array}{c}\text { Arrh Visc } \\
\text { A }\end{array}$ & $\begin{array}{c}\text { Arrh Visc } \\
\text { B }\end{array}$ & $1150^{\circ} \mathrm{C}$ & $\begin{array}{c}\mathrm{T}\left({ }^{\circ} \mathrm{C}\right) \text { at } \\
2 \mathrm{~Pa} \cdot \mathrm{s} \\
\end{array}$ & $\begin{array}{c}\mathrm{T}\left({ }^{\circ} \mathrm{C}\right) \text { at } \\
5 \mathrm{~Pa} \cdot \mathrm{s}\end{array}$ & $\begin{array}{c}\mathrm{T}\left({ }^{\circ} \mathrm{C}\right) \text { at } \\
10 \mathrm{~Pa} \cdot \mathrm{s}\end{array}$ & $\begin{array}{l}\mathrm{T} 1 \\
\left({ }^{\circ} \mathrm{C}\right)\end{array}$ & $\begin{array}{c}\mathrm{V} 1 \\
(\mathrm{~Pa} \cdot \mathrm{s})\end{array}$ & $\begin{array}{l}\mathrm{T} 2 \\
\left({ }^{\circ} \mathrm{C}\right)\end{array}$ \\
\hline IG2-01 & & 2.49 & -3.6815 & 6380.2 & 259.2 & 32.49 & -8.170 & 16656.0 & 34.29 & 1606 & & 1317 & 1156.7 & 29.94 & 1105.7 \\
\hline IG2-02 & & 2.45 & -4.6429 & 5769.9 & 516.35 & 86.74 & -14.185 & 26612.9 & 91.56 & 1516 & & 1341 & 1254.9 & 23.36 & 1205 \\
\hline IG2-03 & & 2.34 & & & & & & & & & & & & & \\
\hline IG2-04 & & 2.45 & -5.7656 & 5989.6 & 201.68 & 1.73 & -9.374 & 14141.8 & 1.76 & 1132 & & 938 & 1157.1 & 1.64 & 1106.2 \\
\hline IG2-05 & & 2.94 & -16.402 & 27111 & -385.51 & 3.50 & -15.192 & 23401.5 & 3.50 & 1200 & & 1065 & 1256.3 & 1.17 & 1205.8 \\
\hline IG2-06 & & 2.73 & -10.533 & 13589 & -144.05 & 0.97 & -11.671 & 16572.6 & 0.98 & 1067 & & 913 & 1007.7 & 3.84 & 958 \\
\hline IG2-07 & & 2.78 & -33.64 & 63084 & -501.3 & 95.84 & $(-28.623)$ & 47202.154 & $\left(94.43^{\prime}\right)$ & 1337 & & 1253 & 1107.2 & 338.1 & 1057.2 \\
\hline IG2-08 & & 2.56 & -5.4958 & 4279.5 & 422.59 & 1.47 & -12.232 & 18004.0 & 1.52 & 1120 & & 966 & 1157.8 & 1.37 & 1107.4 \\
\hline IG2-09 & & 2.66 & -5.2587 & 3936.8 & 454.74 & 1.50 & -12.400 & 18270.4 & 1.55 & 1122 & & 970 & 1159.4 & 1.34 & 1108.1 \\
\hline IG2-10 & & 2.45 & -5.5952 & 5698.8 & 301.94 & 3.08 & -11.321 & 17729.3 & 3.12 & 1203 & & 1028 & 1059.1 & 6.92 & 1007.7 \\
\hline IG2-11 & & 2.55 & -5.8924 & 5992.5 & 206.39 & 1.58 & -10.113 & 15046.1 & 1.58 & 1119 & & 939 & 1059.4 & 3.05 & 1007.4 \\
\hline IG2-12 & & 2.63 & -6.4399 & 5817.5 & 336 & 2.03 & -13.871 & 20743.0 & 2.03 & 1151 & & 1010 & 1061.8 & 4.34 & 957.6 \\
\hline IG2-13 & & 2.59 & -5.8504 & 7017.6 & 364.12 & 21.74 & -12.412 & 22087.8 & 22.42 & 1413 & & 1228 & 1254.8 & 7.54 & 1204.7 \\
\hline IG2-14 & & 2.29 & -27.698 & 96203 & -1806.8 & 126.24 & -12.483 & 24733.0 & 134.01 & 1604 & & 1400 & 1255.5 & 44.31 & 1204.9 \\
\hline IG2-15 & & 2.56 & -5.5756 & 6249.9 & 256.64 & 4.14 & -10.366 & 16800.0 & 4.22 & 1246 & & 1053 & 1157.1 & 3.86 & 1106.4 \\
\hline IG2-16 & & 2.61 & -5.9936 & 6386.2 & 302.7 & 4.68 & -11.982 & 19284.0 & 4.80 & 1248 & & 1077 & 1156.1 & 4.37 & 1105.3 \\
\hline IG2-17 & & 2.65 & -5.7477 & 6537.2 & 363.42 & 12.98 & -12.163 & 20998.0 & 13.37 & 1360 & & 1178 & 1254.3 & 4.74 & 1204.1 \\
\hline IG2-18 & & 2.67 & -7.1023 & 8434.5 & 222.83 & 7.35 & -12.183 & 20260.0 & 7.80 & 1300 & & 1125 & 1254.9 & 2.86 & 1205.1 \\
\hline IG2-19 & & 2.58 & -5.7004 & 6278.3 & 258.55 & 3.83 & -10.540 & 16939.0 & 3.91 & 1235 & & 1046 & 1156.1 & 3.57 & 1105.6 \\
\hline IG2-20 & & 2.58 & -5.6886 & 6772.7 & 267.91 & 7.31 & -11.146 & 18736.0 & 7.54 & 1309 & & 1120 & 1158.7 & 6.65 & 1107.3 \\
\hline IG2-21 & & 2.61 & -5.5949 & 6870.3 & 297.01 & 11.70 & -11.831 & 20386.0 & 12.12 & 1355 & & 1169 & 1158.7 & 10.79 & 1108 \\
\hline IG2-22 & & 2.59 & -7.2824 & 8396 & 234.93 & 6.64 & -13.137 & 21426.0 & 6.82 & 1276 & & 1115 & 1158.1 & 6 & 1116.7 \\
\hline IG2-23 & & 2.62 & -6.2059 & 6387.6 & 303.18 & 3.81 & -12.176 & 19270.0 & 3.92 & 1224 & & 1058 & 1158.4 & 3.51 & 1107.5 \\
\hline IG2-24 & & 2.66 & -7.5443 & 8590.7 & 183.59 & 3.84 & -12.327 & 19490.0 & 3.93 & 1224 & & 1059 & 1159.2 & 3.56 & 1108.9 \\
\hline IG2-25 & & 2.66 & -7.4458 & 8626.8 & 174.48 & 4.05 & -12.059 & 19182.0 & 4.14 & 1231 & & 1062 & 1158.9 & 3.73 & 1108.3 \\
\hline IG2-26 & & 2.6 & -5.5274 & 6772.5 & 302.42 & 11.74 & -11.848 & 20409.0 & 12.11 & 1354 & & 1169 & 1156.7 & 11 & 1106.6 \\
\hline IG2-27 & & 2.59 & -5.4888 & 6358.8 & 369.17 & 14.22 & -12.222 & 21200.0 & 14.53 & 1368 & & 1186 & 1157.2 & 12.94 & 1106.8 \\
\hline IG2-28 & & 2.62 & -5.7848 & 6668.3 & 299.18 & 7.79 & -11.922 & 19929.0 & 8.03 & 1307 & & 1128 & 1158.5 & 7.38 & 1107.5 \\
\hline IG2-29 & & 2.62 & -5.8416 & 6430.1 & 274.81 & 4.51 & -11.180 & 18085.0 & 4.61 & 1250 & & 1068 & 1158.5 & 4.11 & 1107.2 \\
\hline IG2-30 & & 2.41 & -5.0017 & 6565.9 & 261.49 & 10.89 & -10.119 & 17829.0 & 11.14 & 1376 & & 1162 & 1157.7 & 10.22 & 1107.7 \\
\hline IG2-31 & & 2.56 & -5.7592 & 6542.4 & 320.54 & 8.40 & -12.345 & 20636.0 & 8.64 & 1310 & & 1136 & 1158.3 & 7.75 & 1107.1 \\
\hline IG2-32 & & 2.61 & -5.8462 & 6466.9 & 283.55 & 5.04 & -11.396 & 18555.0 & 5.17 & 1262 & & 1081 & 1160.2 & 4.54 & 1108.7 \\
\hline IG2-33 & & 2.62 & -6.0101 & 6340 & 288.38 & 3.85 & -11.584 & 18437.0 & 3.94 & 1229 & & 1055 & 1157.6 & 3.53 & 1106.4 \\
\hline IG2-34 & & 2.63 & -5.8429 & 6386.1 & 292.35 & 4.97 & -11.549 & 18751.0 & 5.09 & 1259 & & 1081 & 1157.3 & 4.58 & 1106.8 \\
\hline IG2-35 & & 2.61 & -5.8817 & 6398.6 & 282.21 & 4.44 & -11.353 & 18313.0 & 4.56 & 1247 & & 1068 & 1156.7 & 4.16 & 1106.7 \\
\hline IG2-36 & & 2.64 & -5.7538 & 6211.9 & 289.88 & 4.34 & -11.228 & 18100.0 & 4.44 & 1245 & & 1065 & 1158.4 & 3.94 & 1107.4 \\
\hline IG2-37 & & 2.67 & -5.8505 & 6358.3 & 287.47 & 4.58 & -11.400 & 18422.0 & 4.69 & 1250 & & 1071 & 1157.1 & 4.25 & 1107.3 \\
\hline
\end{tabular}

\section{INEEL CVS Phase 3 (Scholes et al. 2000)}

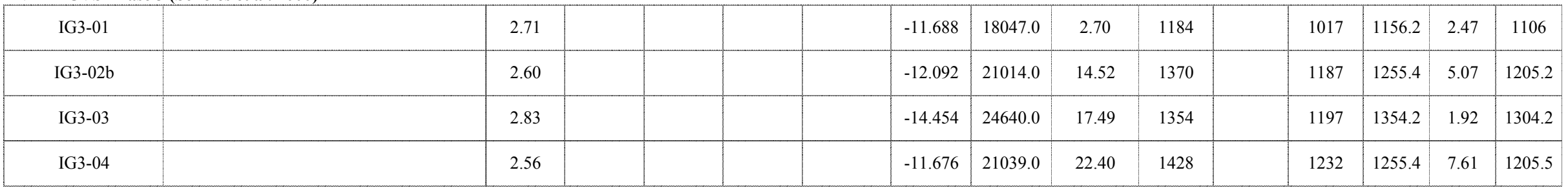




\section{INEEL CVS Phase 2 (Staples et al. 2000)}

\begin{tabular}{|c|c|c|c|c|c|c|c|c|c|c|c|c|c|c|c|c|c|c|c|c|c|}
\hline Glass ID & $\begin{array}{c}\mathrm{V} 2 \\
(\mathrm{~Pa} \cdot \mathrm{s})\end{array}$ & $\begin{array}{l}\mathrm{T} 3 \\
\left({ }^{\circ} \mathrm{C}\right)\end{array}$ & $\begin{array}{c}\mathrm{V} 3 \\
(\mathrm{~Pa} \cdot \mathrm{s})\end{array}$ & $\begin{array}{l}\mathrm{T} 4 \\
\left({ }^{\circ} \mathrm{C}\right)\end{array}$ & $\begin{array}{c}\mathrm{V} 4 \\
(\mathrm{~Pa} \cdot \mathrm{s})\end{array}$ & $\begin{array}{l}\text { T5 } \\
\left({ }^{\circ} \mathrm{C}\right)\end{array}$ & $\begin{array}{c}\text { V5 } \\
(\mathrm{Pa} \cdot \mathrm{s})\end{array}$ & $\begin{array}{l}\mathrm{T} 6 \\
\left({ }^{\circ} \mathrm{C}\right)\end{array}$ & $\begin{array}{c}\mathrm{V} 6 \\
(\mathrm{~Pa} \cdot \mathrm{s})\end{array}$ & $\begin{array}{l}\mathrm{T} 7 \\
\left({ }^{\circ} \mathrm{C}\right)\end{array}$ & $\begin{array}{c}\text { V7 } \\
(\mathrm{Pa} \cdot \mathrm{s})\end{array}$ & $\begin{array}{c}\text { T8 } \\
\left({ }^{\circ} \mathrm{C}\right)\end{array}$ & $\begin{array}{c}\mathrm{V} 8 \\
(\mathrm{~Pa} \cdot \mathrm{s})\end{array}$ & $\begin{array}{c}\text { T9 } \\
\left({ }^{\circ} \mathrm{C}\right)\end{array}$ & $\begin{array}{c}\mathrm{V} 9 \\
(\mathrm{~Pa} \cdot \mathrm{s})\end{array}$ & $\begin{array}{l}\mathrm{T} 10 \\
\left({ }^{\circ} \mathrm{C}\right)\end{array}$ & $\begin{array}{c}\mathrm{V} 10 \\
(\mathrm{~Pa} \cdot \mathrm{s})\end{array}$ & $\begin{array}{l}\text { T11 } \\
\left({ }^{\circ} \mathrm{C}\right)\end{array}$ & $\begin{array}{c}\mathrm{V} 11 \\
(\mathrm{~Pa} \cdot \mathrm{s})\end{array}$ & $\begin{array}{l}\mathrm{T} 12 \\
\left({ }^{\circ} \mathrm{C}\right)\end{array}$ & $\begin{array}{c}\mathrm{V} 12 \\
(\mathrm{~Pa} \cdot \mathrm{s})\end{array}$ \\
\hline IG2-01 & 46.49 & 1055.4 & 76.18 & 1006.5 & 130.91 & 956.6 & 237.94 & 1155.6 & 31.02 & 1204.6 & 21.84 & 1254.5 & 15.76 & 1303.8 & 11.31 & 1353.8 & 8.56 & & & & \\
\hline IG2-02 & 42.21 & 1155.5 & 81 & 1105.8 & 170.14 & 1056.1 & 415.42 & 1156.6 & 84.56 & 1205.9 & 42.01 & 1255 & 23.46 & 1304.5 & 13.93 & 1354.7 & 9.39 & 1405.5 & 6.62 & 1255.6 & 22.74 \\
\hline IG2-03 & & & & & & & & & & & & & & & & & & & & & \\
\hline IG2-04 & 2.35 & 1056.1 & 3.47 & 1006.4 & 5.35 & 956.8 & 8.64 & 1156.5 & 1.66 & 1205.6 & 1.22 & 1255.7 & 0.91 & 1156.9 & 1.7 & & & & & & \\
\hline IG2-05 & 1.98 & 1156.1 & 3.24 & 1106.7 & 5.83 & 1254.7 & 1.02 & 1304.2 & 0.71 & 1254.6 & 1.15 & & & & & & & & & & \\
\hline IG2-06 & 5.77 & 1157.4 & 0.85 & 1206.7 & 0.63 & 1256.1 & 0.45 & 1157.1 & 0.92 & & & & & & & & & & & & \\
\hline IG2-07 & 749.13 & 1157.4 & 99.78 & 1206.4 & 29.18 & 1255.2 & 7.22 & 1304.5 & 2.94 & 1354 & 1.91 & 1255.8 & 8.86 & & & & & & & & \\
\hline IG2-08 & 2.11 & 1057.3 & 3.46 & 1007.5 & 6.21 & 957.7 & 12.36 & 1058.7 & 3.41 & 1157.9 & 1.39 & 1206.7 & 0.97 & 1255.6 & 0.7 & 1156.8 & 1.43 & & & & \\
\hline IG2-09 & 2.1 & 1057.7 & 3.4 & 1007.9 & 5.97 & 958.3 & 13.25 & 1059.1 & 3.93 & 1158.2 & 1.42 & 1207.4 & 0.98 & 1256.6 & 0.7 & 1157.6 & 1.46 & & & & \\
\hline IG2-10 & 11.73 & 956.8 & 21.69 & 906.8 & 46.76 & 1057.3 & 7.28 & 1106.8 & 4.25 & 1156.2 & 2.86 & 1205.5 & 2.04 & 1254.8 & 1.45 & 1155.7 & 3.04 & 1056.3 & 7.13 & 1156.7 & 3.02 \\
\hline IG2-11 & 4.88 & 956.9 & 8.06 & 906.9 & 14.19 & 857.1 & 27.45 & 957.5 & 8.02 & 1057.1 & 3.17 & 1106.7 & 2.15 & 1156.3 & 1.53 & 1205.7 & 1.1 & 1255.2 & 0.82 & 1156 & 1.54 \\
\hline IG2-12 & 18.06 & 907.3 & 42 & 857.3 & 112.57 & 958.1 & 18.82 & 1007.3 & 9.33 & 1057.4 & 4.82 & 1106.9 & 2.85 & 1156.3 & 1.84 & 1205.5 & 1.25 & 1254.8 & 0.88 & 1155.7 & 2.06 \\
\hline IG2-13 & 12.19 & 1155.2 & 20.65 & 1105.6 & 36.93 & 1055.7 & 71.19 & 1156.1 & 20.72 & 1205.5 & 12.36 & 1254.9 & 7.7 & 1304.4 & 4.96 & 1354.3 & 3.37 & 1255.1 & 7.7 & & \\
\hline IG2-14 & 68.02 & 1155.4 & 120.9 & 1205.1 & 66.89 & 1254.3 & 39.05 & 1303.8 & 24.58 & 1403 & 9.21 & 1452.9 & 6.09 & 1353.7 & 15.54 & 1254.7 & 44.17 & 1353.8 & 15.97 & 1452.8 & 6.31 \\
\hline IG2-15 & 5.9 & 1056.1 & 9.48 & 956.4 & 28.44 & 1057.4 & 9.44 & 1156.6 & 3.93 & 1205.7 & 2.76 & 1254.9 & 1.97 & 1155.6 & 4.01 & & & & & & \\
\hline IG2-16 & 7.14 & 1054.8 & 12.25 & 1004.9 & 22.29 & 955.2 & 44.05 & 1055.7 & 12.2 & 1155.3 & 4.45 & 1204.7 & 2.95 & 1254.2 & 2.03 & 1155.4 & 4.64 & & & & \\
\hline IG2-17 & 7.51 & 1154.4 & 12.4 & 1104.7 & 21.58 & 1054.8 & 40.11 & 1155.2 & 12.55 & 1204.5 & 7.61 & 1253.9 & 4.89 & 1303.5 & 3.33 & 1253.9 & 5.07 & & & & \\
\hline IG2-18 & 4.42 & 1155.6 & 7.1 & 1106.1 & 11.88 & 1056.3 & 20.72 & 1006.6 & 39 & 956.9 & 79.49 & 1057.8 & 20.41 & 1157 & 6.89 & 1255.1 & 2.89 & 1304.4 & 1.99 & 1354 & 1.42 \\
\hline IG2-19 & 5.52 & 1055.5 & 8.84 & 1007 & 14.81 & 957.3 & 26.43 & 1058.2 & 8.72 & 1157.3 & 3.64 & 1206.5 & 2.53 & 1255.7 & 1.79 & 1156.6 & 3.74 & & & & \\
\hline IG2-20 & 10.93 & 1056.9 & 18.44 & 1006.9 & 32.56 & 957.2 & 62 & 1058.2 & 18.51 & 1157.6 & 6.96 & 1206.6 & 4.64 & 1255.8 & 3.14 & 1156.6 & 7.27 & & & & \\
\hline IG2-21 & 17.95 & 1057.7 & 31.62 & 1007.5 & 59.57 & 957.6 & 120.82 & 1058.8 & 31.53 & 1158.1 & 11.11 & 1207.1 & 7.17 & 1256.1 & 4.69 & & & & & & \\
\hline IG2-22 & 9.29 & 1058.4 & 18.49 & 1007.6 & 35.81 & 957.9 & 75.12 & 1059.3 & 19.21 & 1158.4 & 6.4 & 1207.2 & 3.83 & 1256.2 & 2.52 & 1157 & 6.3 & & & & \\
\hline IG2-23 & 5.68 & 1057.2 & 9.75 & 1007.4 & 17.65 & 957.7 & 34.49 & 1058.8 & 9.73 & 1157.9 & 3.56 & 1206.9 & 2.37 & 1256 & 1.63 & 1156.8 & 3.64 & & & & \\
\hline IG2-24 & 5.85 & 1058.7 & 9.95 & 1008.9 & 17.85 & 959.3 & 33.4 & 1060.1 & 9.7 & 1158.5 & 3.59 & 1207.1 & 2.33 & 1256.2 & 1.56 & 1157 & 3.66 & & & & \\
\hline IG2-25 & 6.15 & 1058.2 & 10.46 & 1008.3 & 18.65 & 958.6 & 34.14 & 1059.7 & 10.04 & 1158.8 & 3.79 & 1207.8 & 2.46 & 1256.6 & 1.65 & 1157.3 & 3.87 & & & & \\
\hline IG2-26 & 18.27 & 1056.6 & 32.31 & 1006.8 & 60.42 & 957.1 & 121.48 & 1057.9 & 31.7 & 1157.3 & 10.98 & 1206.5 & 7.16 & 1255.7 & 4.79 & 1156.3 & 11.15 & & & & \\
\hline IG2-27 & 22.34 & 1056.7 & 43.41 & 1157.2 & 13.54 & 1206.3 & 8.33 & 1255.4 & 5.37 & 1156.2 & 13.23 & & & & & & & & & & \\
\hline IG2-28 & 12.07 & 1056.7 & 20.88 & 1006.8 & 38.38 & 957 & 76.51 & 1057.7 & 20.63 & 1157 & 7.16 & 1206.2 & 4.72 & 1255.3 & 3.29 & 1156.2 & 7.42 & & & & \\
\hline IG2-29 & 6.56 & 1056.3 & 11.04 & 1006.4 & 19.21 & 956.5 & 35.85 & 1057.4 & 10.91 & 1156.6 & 4.3 & 1205.6 & 2.9 & 1254.8 & 2.03 & 1155.7 & 4.48 & & & & \\
\hline IG2-30 & 15.8 & 1057.6 & 25.8 & 1007.9 & 44.42 & 958.2 & 82.84 & 1059.3 & 25.46 & 1158.4 & 10.26 & 1207 & 6.96 & 1255.9 & 4.89 & 1156.8 & 10.45 & & & & \\
\hline IG2-31 & 12.9 & 1056.9 & 22.8 & 1008.2 & 43.08 & 958.5 & 88.52 & 1059.6 & 22.54 & 1156.9 & 7.89 & 1205.7 & 5.1 & 1254.5 & 3.45 & 1155.5 & 8.08 & & & & \\
\hline IG2-32 & 7.31 & 1058 & 12.29 & 1008 & 21.89 & 958.2 & 41.66 & 1059.3 & 12.37 & 1158.4 & 4.72 & 1207.2 & 3.18 & 1256.3 & 2.19 & 1157.1 & 4.94 & & & & \\
\hline IG2-33 & 5.69 & 1056.1 & 9.54 & 1006.3 & 16.86 & 956.6 & 32.02 & 1057.4 & 9.59 & 1156.9 & 3.62 & 1206 & 2.46 & 1255.2 & 1.72 & 1155.8 & 3.79 & & & & \\
\hline IG2-34 & 7.38 & 1056.6 & 12.47 & 1006.7 & 22.31 & 956.9 & 42.8 & 1057.8 & 12.26 & 1157.1 & 4.67 & 1206.1 & 3.14 & 1255.3 & 2.18 & 1156 & 4.9 & & & & \\
\hline IG2-35 & 6.56 & 1056.8 & 10.91 & 1006.9 & 19.21 & 957.2 & 36.18 & 1058.3 & 10.69 & 1157.5 & 4.19 & 1206.6 & 2.83 & 1255.7 & 1.96 & 1156.4 & 4.35 & & & & \\
\hline IG2-36 & 6.26 & 1057.1 & 10.41 & 1007.2 & 18.35 & 957.4 & 34.72 & 1058.3 & 10.35 & 1157.6 & 4.1 & 1206.7 & 2.78 & 1256 & 1.95 & 1156.8 & 4.26 & & & & \\
\hline IG2-37 & 6.69 & 1057.4 & 11.19 & 1007.6 & 19.76 & 957.8 & 37.61 & 1058.8 & 11.03 & 1158 & 4.29 & 1207.3 & 2.88 & 1256.5 & 2.01 & 1157.4 & 4.49 & & & & \\
\hline
\end{tabular}

\section{INEEL CVS Phase 3 (Scholes et al. 2000)}

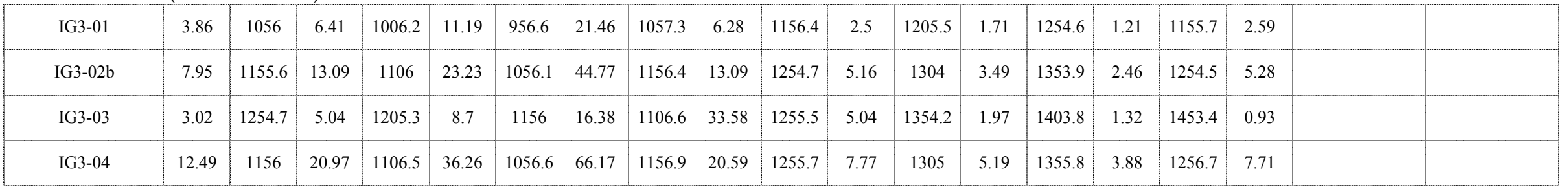


Appendix A. Database - mass fraction

INEEL CVS Phase 2 (Staples et al. 2000)

\begin{tabular}{|c|c|c|c|c|c|c|c|c|c|c|c|c|c|c|c|c|}
\hline Glass ID & $\begin{array}{l}\mathrm{T} 13 \\
\left({ }^{\circ} \mathrm{C}\right) \\
\end{array}$ & $\begin{array}{c}\mathrm{V} 13 \\
(\mathrm{~Pa} \cdot \mathrm{s})\end{array}$ & $\begin{array}{l}\mathrm{T} 14 \\
\left({ }^{\circ} \mathrm{C}\right) \\
\end{array}$ & $\begin{array}{c}\mathrm{V} 14 \\
(\mathrm{~Pa} \cdot \mathrm{s})\end{array}$ & $\begin{array}{c}\text { Q PCT } \\
\text { B }\left(\mathrm{g} / \mathrm{m}^{2}\right)\end{array}$ & $\begin{array}{c}\text { Q PCT } \\
\mathrm{Li}\left(\mathrm{g} / \mathrm{m}^{2}\right)\end{array}$ & $\begin{array}{c}\text { Q PCT } \\
\mathrm{Na}\left(\mathrm{g} / \mathrm{m}^{2}\right)\end{array}$ & $\begin{array}{c}\text { Q PCT } \\
\mathrm{Si}\left(\mathrm{g} / \mathrm{m}^{2}\right)\end{array}$ & $\begin{array}{c}\text { Q PCT } \\
\mathrm{pH}\end{array}$ & $\begin{array}{l}\text { CCC PCT } \\
\mathrm{B}\left(\mathrm{g} / \mathrm{m}^{2}\right)\end{array}$ & $\begin{array}{l}\text { CCC PCT } \\
\mathrm{Li}\left(\mathrm{g} / \mathrm{m}^{2}\right) \\
\end{array}$ & \begin{tabular}{|l|}
$\mathrm{CCC}$ PCT \\
$\mathrm{Na}\left(\mathrm{g} / \mathrm{m}^{2}\right)$ \\
\end{tabular} & $\begin{array}{c}\text { CCC PCT } \\
\mathrm{Si}\left(\mathrm{g} / \mathrm{m}^{2}\right) \\
\end{array}$ & $\begin{array}{c}\begin{array}{c}\text { CCC PCT } \\
\mathrm{pH}\end{array} \\
\end{array}$ & $\begin{array}{l}\text { Q PCT B at } \\
20^{\circ} \mathrm{C}\left(\mathrm{g} / \mathrm{m}^{2}\right)\end{array}$ & $\begin{array}{l}\text { Q PCT Li at } \\
20^{\circ} \mathrm{C}\left(\mathrm{g} / \mathrm{m}^{2}\right)\end{array}$ \\
\hline IG2-01 & & & & & 0.226 & & 0.213 & 0.131 & 8.50 & & & & & & & \\
\hline IG2-02 & & & & & 0.090 & & 0.107 & 0.041 & 7.68 & & & & & & & \\
\hline IG2-03 & & & & & 12.404 & & 5.449 & 0.214 & 6.79 & & & & & & & \\
\hline IG2-04 & & & & & 2.884 & 2.601 & 1.597 & 0.250 & 10.88 & & & & & & & \\
\hline IG2-05 & & & & & 0.490 & & 1.429 & 0.163 & 11.27 & & & & & & & \\
\hline IG2-06 & & & & & 2.067 & 1.661 & 0.525 & 0.184 & 9.50 & & & & & & & \\
\hline IG2-07 & & & & & 0.307 & & 0.351 & 0.072 & 9.34 & & & & & & & \\
\hline IG2-08 & & & & & 0.440 & & 0.694 & 0.208 & 10.11 & & & & & & & \\
\hline IG2-09 & & & & & 0.100 & 0.223 & 0.087 & 0.059 & 9.67 & & & & & & & \\
\hline IG2-10 & & & & & 1.397 & 1.377 & 1.427 & 0.246 & 10.44 & & & & & & & \\
\hline IG2-11 & 1056.3 & 3.28 & 1156.6 & 1.53 & 1.981 & 2.506 & 2.606 & 1.376 & 11.72 & & & & & & & \\
\hline IG2-12 & 1056.2 & 5.96 & 1156.7 & 2.08 & 11.673 & 10.471 & 7.379 & 0.123 & 8.60 & & & & & & & \\
\hline IG2-13 & & & & & 0.289 & 0.533 & 0.023 & 0.210 & 10.19 & & & & & & & \\
\hline IG2-14 & & & & & 0.425 & & 0.593 & 0.185 & 10.44 & & & & & & & \\
\hline IG2-15 & & & & & 0.739 & 1.016 & 1.139 & 0.269 & 11.12 & & & & & & & \\
\hline IG2-16 & & & & & 0.766 & 0.639 & 0.546 & 0.086 & 10.01 & & & & & & & \\
\hline IG2-17 & & & & & 0.213 & 0.378 & 0.335 & 0.086 & 9.90 & & & & & & & \\
\hline IG2-18 & 1255 & 3.04 & & & 0.391 & 0.478 & 0.783 & 0.156 & 10.78 & & & & & & & \\
\hline IG2-19 & & & & & 0.729 & 0.959 & 1.102 & 0.261 & 11.23 & & & & & & & \\
\hline IG2-20 & & & & & 0.622 & 0.846 & 0.946 & 0.233 & 11.13 & & & & & & & \\
\hline IG2-21 & & & & & 0.253 & 0.526 & 0.428 & 0.103 & 10.26 & & & & & & & \\
\hline IG2-22 & & & & & 0.542 & 1.161 & 0.756 & 0.186 & 11.30 & & & & & & & \\
\hline IG2-23 & & & & & 0.911 & 0.735 & 0.786 & 0.115 & 10.23 & & & & & & & \\
\hline IG2-24 & & & & & 0.379 & 0.702 & 0.563 & 0.095 & 10.68 & & & & & & & \\
\hline IG2-25 & & & & & 0.431 & 0.816 & 0.619 & 0.050 & 10.83 & & & & & & & \\
\hline IG2-26 & & & & & 0.303 & 0.533 & 0.481 & 0.144 & 10.54 & & & & & & & \\
\hline IG2-27 & & & & & 0.287 & 0.500 & 0.371 & 0.106 & 10.05 & & & & & & & \\
\hline IG2-28 & & & & & 0.380 & 0.730 & 0.727 & 0.110 & 10.54 & & & & & & & \\
\hline IG2-29 & & & & & 0.481 & 0.503 & 0.549 & 0.121 & 10.27 & & & & & & & \\
\hline IG2-30 & & & & & 0.558 & 0.647 & 0.040 & 0.360 & 9.23 & & & & & & & \\
\hline IG2-31 & & & & & 1.617 & 1.506 & 0.942 & 0.320 & 9.99 & & & & & & & \\
\hline IG2-32 & & & & & 0.566 & 0.681 & 0.653 & 0.136 & 10.11 & & & & & & & \\
\hline IG2-33 & & & & & 0.501 & 0.547 & 0.581 & 0.131 & 10.26 & & & & & & & \\
\hline IG2-34 & & & & & 0.536 & 0.667 & 0.574 & 0.135 & 10.22 & & & & & & & \\
\hline IG2-35 & & & & & 0.408 & 0.470 & 0.493 & 0.122 & 10.13 & & & & & & & \\
\hline IG2-36 & & & & & 0.378 & 0.461 & 0.466 & 0.119 & 10.14 & & & & & & & \\
\hline IG2-37 & & & & & 0.449 & 0.590 & 0.473 & 0.125 & 10.18 & & & & & & & \\
\hline
\end{tabular}

\section{INEEL CVS Phase 3 (Scholes et al. 2000)}

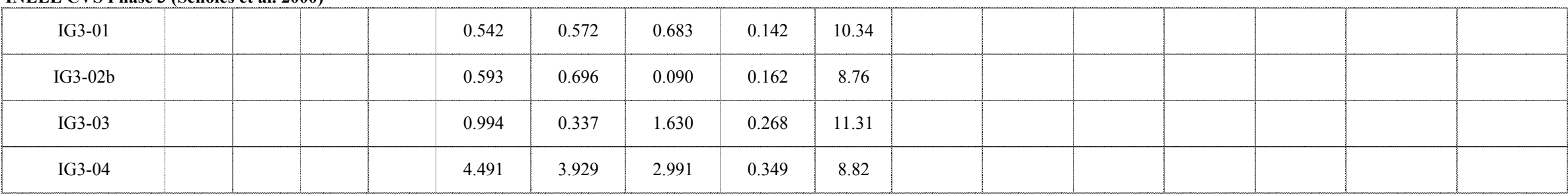


Appendix A. Database - mass fraction

INEEL CVS Phase 2 (Staples et al. 2000)

\begin{tabular}{|c|c|c|c|c|c|c|c|c|c|c|c|c|}
\hline Glass ID & $\begin{array}{l}\text { Q PCT Na at } \\
20^{\circ} \mathrm{C}\left(\mathrm{g} / \mathrm{m}^{2}\right)\end{array}$ & $\begin{array}{l}\text { Q PCT Si at } \\
20^{\circ} \mathrm{C}\left(\mathrm{g} / \mathrm{m}^{2}\right)\end{array}$ & $\begin{array}{c}\mathrm{QpH} \\
\text { at } 20^{\circ} \mathrm{C}\end{array}$ & $\begin{array}{c}\text { TCLP Ag } \\
(\mathrm{ppm})\end{array}$ & $\begin{array}{c}\text { TCLP As } \\
(\mathrm{ppm})\end{array}$ & $\begin{array}{c}\text { TCLP Ba } \\
(\mathrm{ppm})\end{array}$ & $\begin{array}{c}\text { TCLP Cd } \\
(\mathrm{ppm})\end{array}$ & $\begin{array}{c}\text { TCLP Cr } \\
(\mathrm{ppm})\end{array}$ & $\begin{array}{c}\text { TCLP Ni } \\
(\mathrm{ppm})\end{array}$ & $\begin{array}{c}\text { TCLP Pb } \\
(\mathrm{ppm})\end{array}$ & $\begin{array}{c}\text { TCLP Se } \\
(\mathrm{ppm})\end{array}$ & $\begin{array}{c}\text { TCLP Zn } \\
(\mathrm{ppm})\end{array}$ \\
\hline \multicolumn{13}{|l|}{ IG2-01 } \\
\hline \multirow{2}{*}{\multicolumn{13}{|c|}{ IG2-02 }} \\
\hline \multirow{2}{*}{\multicolumn{13}{|c|}{$\frac{\text { IG2-03 }}{\text { IG2-04 }}$}} \\
\hline & & & & & & & & & & & & \\
\hline \multicolumn{13}{|l|}{ IG2-05 } \\
\hline \multicolumn{13}{|l|}{ IG2-06 } \\
\hline \multicolumn{13}{|l|}{ IG2-07 } \\
\hline \multirow{2}{*}{\multicolumn{13}{|c|}{ IG2-08 }} \\
\hline \multirow{2}{*}{\multicolumn{8}{|c|}{$\begin{array}{l}\text { IG2-09 } \\
\text { IG2-10 }\end{array}$}} & & & & & \\
\hline & & & & & & & & & & & & \\
\hline \multicolumn{13}{|l|}{ IG2-11 } \\
\hline \multicolumn{13}{|l|}{ IG2-12 } \\
\hline \multicolumn{13}{|l|}{ IG2-13 } \\
\hline \multicolumn{13}{|l|}{ IG2-14 } \\
\hline \multirow{2}{*}{\multicolumn{13}{|c|}{$\begin{array}{l}\text { IG2-15 } \\
\text { IG2-16 }\end{array}$}} \\
\hline & & & & & & & & & & & & \\
\hline \multicolumn{13}{|l|}{ IG2-17 } \\
\hline \multicolumn{13}{|l|}{ IG2-18 } \\
\hline \multicolumn{13}{|l|}{ IG2-19 } \\
\hline \multicolumn{13}{|l|}{ IG2-20 } \\
\hline \multicolumn{13}{|l|}{ IG2-21 } \\
\hline \multicolumn{13}{|l|}{ IG2-22 } \\
\hline \multicolumn{13}{|l|}{ IG2-23 } \\
\hline \multicolumn{13}{|l|}{ IG2-24 } \\
\hline \multicolumn{13}{|l|}{ IG2-25 } \\
\hline \multicolumn{13}{|l|}{ IG2-26 } \\
\hline \multicolumn{13}{|l|}{ IG2-27 } \\
\hline IG2-28 & & & & & & & & & & & & \\
\hline IG2-29 & & & & & & & & & & & & \\
\hline IG2-30 & & & & & & & & & & & & \\
\hline IG2-31 & & & & & & & & & & & & \\
\hline IG2-32 & & & & & & & & & & & & \\
\hline IG2-33 & & & & & & & & & & & & \\
\hline IG2-34 & & & & & & & & & & & & \\
\hline IG2-35 & & & & & & & & & & & & \\
\hline IG2-36 & & & & & & & & & & & & \\
\hline IG2-37 & & & & & & & & & & & & \\
\hline
\end{tabular}

\section{INEEL CVS Phase 3 (Scholes et al. 2000)}

\begin{tabular}{|c|c|c|c|c|c|c|c|c|c|c|c|c|}
\hline IG3-01 & & & & & & & & & \\
\hline IG3-02b & & & & & & & & & \\
\hline IG3-03 & & & & & & & & \\
\hline IG3-04 & & & & & & & & \\
\hline
\end{tabular}


Appendix A. Database - mass fraction

INEEL CVS Phase 3 (Scholes et al. 2000)

\begin{tabular}{|c|c|c|c|c|c|c|c|c|c|c|c|c|c|c|c|c|c|c|c|c|c|}
\hline Glass ID & $\begin{array}{c}\mathrm{A} 12 \mathrm{O} 3 \\
-\mathrm{t}\end{array}$ & $\begin{array}{c}\mathrm{B} 2 \mathrm{O} 3 \\
-\mathrm{t}\end{array}$ & $\begin{array}{c}\mathrm{CaO} \\
-\mathrm{t} \\
\end{array}$ & $\begin{array}{c}\mathrm{Fe} 2 \mathrm{O} 3 \\
-\mathrm{t} \\
\end{array}$ & $\begin{array}{c}\mathrm{FeO} \\
-\mathrm{t}\end{array}$ & $\begin{array}{c}\mathrm{K} 2 \mathrm{O} \\
-\mathrm{t}\end{array}$ & $\begin{array}{c}\mathrm{Li} 2 \mathrm{O} \\
-\mathrm{t} \\
\end{array}$ & $\begin{array}{c}\mathrm{MgO} \\
-\mathrm{t}\end{array}$ & $\begin{array}{c}\mathrm{Na} 2 \mathrm{O} \\
-\mathrm{t}\end{array}$ & $\begin{array}{c}\mathrm{NiO} \\
-\mathrm{t}\end{array}$ & $\begin{array}{c}\mathrm{P} 2 \mathrm{O} 5 \\
-\mathrm{t}\end{array}$ & $\begin{array}{c}\mathrm{SiO} 2 \\
-\mathrm{t} \\
\end{array}$ & $\begin{array}{c}\mathrm{ZrO} 2 \\
-\mathrm{t}\end{array}$ & $\begin{array}{c}\mathrm{Ag} 2 \mathrm{O} \\
-\mathrm{t}\end{array}$ & $\begin{array}{c}\mathrm{As} 2 \mathrm{O} 3 \\
-\mathrm{t}\end{array}$ & $\begin{array}{c}\mathrm{BaO} \\
-\mathrm{t} \\
\end{array}$ & $\begin{array}{c}\mathrm{Bi} 2 \mathrm{O} 3 \\
-\mathrm{t}\end{array}$ & $\begin{array}{l}\mathrm{Br} \\
-\mathrm{t}\end{array}$ & $\begin{array}{c}\mathrm{CdO} \\
-\mathrm{t} \\
\end{array}$ & $\begin{array}{c}\mathrm{Ce} 2 \mathrm{O} 3 \\
-\mathrm{t}\end{array}$ & $\begin{array}{c}\mathrm{CeO} 2 \\
-\mathrm{t} \\
\end{array}$ \\
\hline IG3-05 & 0.0350 & 0.0500 & 0.1200 & 0.0000 & & 0.0200 & 0.0000 & 0.0024 & 0.1201 & 0.0150 & 0.0000 & 0.5332 & 0.0000 & & & 0.0000 & & & & 0.0001 & \\
\hline IG3-06 & 0.0400 & 0.0500 & 0.1200 & 0.0000 & & 0.0200 & 0.0302 & 0.0024 & 0.0500 & 0.0150 & 0.0250 & 0.4214 & 0.1400 & & & 0.0000 & & & & 0.0001 & \\
\hline IG3-07 & 0.1690 & 0.1800 & 0.0000 & 0.0800 & & 0.0000 & 0.0000 & 0.0024 & 0.1860 & 0.0150 & 0.0000 & 0.3500 & 0.0000 & & & 0.0000 & & & & 0.0001 & \\
\hline IG3-08b & 0.0535 & 0.1538 & 0.0146 & 0.0628 & & 0.0026 & 0.0119 & 0.0024 & 0.1190 & 0.0127 & 0.0032 & 0.4086 & 0.1167 & & & 0.0000 & & & & 0.0001 & \\
\hline IG3-09 & 0.0350 & 0.0500 & 0.0000 & 0.0800 & & 0.0200 & 0.0900 & 0.0024 & 0.0568 & 0.0000 & 0.0250 & 0.5626 & 0.0000 & & & 0.0000 & & & & 0.0001 & \\
\hline IG3-10b & 0.0490 & 0.1625 & 0.0097 & 0.0457 & & 0.0178 & 0.0390 & 0.0024 & 0.0649 & 0.0135 & 0.0021 & 0.3641 & 0.1244 & & & 0.0000 & & & & 0.0001 & \\
\hline IG3-11 & 0.1205 & 0.0500 & 0.1200 & 0.0800 & & 0.0000 & 0.0146 & 0.0024 & 0.2000 & 0.0000 & 0.0000 & 0.3649 & 0.0000 & & & 0.0000 & & & & 0.0001 & \\
\hline IG3-12 & 0.0350 & 0.1800 & 0.0899 & 0.0300 & & 0.0000 & 0.0000 & 0.0024 & 0.1151 & 0.0000 & 0.0000 & 0.3500 & 0.1000 & & & 0.0000 & & & & 0.0001 & \\
\hline IG3-13 & 0.0400 & 0.0754 & 0.0000 & 0.0800 & & 0.0000 & 0.0467 & 0.0024 & 0.1335 & 0.0150 & 0.0144 & 0.3500 & 0.1400 & & & 0.0000 & & & & 0.0001 & \\
\hline IG3-14 & 0.0800 & 0.0600 & 0.0400 & 0.0300 & & 0.0100 & 0.0600 & 0.0024 & 0.1000 & 0.0050 & 0.0150 & 0.4925 & 0.0400 & & & 0.0000 & & & & 0.0001 & \\
\hline IG3-15 & 0.1000 & 0.0600 & 0.0800 & 0.0050 & & 0.0100 & 0.0425 & 0.0024 & 0.1000 & 0.0100 & 0.0050 & 0.4000 & 0.0800 & & & 0.0000 & & & & 0.0001 & \\
\hline IG3-16 & 0.0989 & 0.0751 & 0.0800 & 0.0050 & & 0.0100 & 0.0600 & 0.0024 & 0.1000 & 0.0050 & 0.0150 & 0.4000 & 0.0800 & & & 0.0000 & & & & 0.0001 & \\
\hline IG3-17 & 0.0893 & 0.0600 & 0.0800 & 0.0300 & & 0.0097 & 0.0600 & 0.0024 & 0.1000 & 0.0050 & 0.0150 & 0.4000 & 0.0800 & & & 0.0000 & & & & 0.0001 & \\
\hline IG3-18 & 0.1200 & 0.0600 & 0.0400 & 0.0050 & & 0.0100 & 0.0580 & 0.0024 & 0.1500 & 0.0100 & 0.0145 & 0.4000 & 0.0600 & & & 0.0000 & & & & 0.0001 & \\
\hline IG3-19 & 0.1200 & 0.0600 & 0.0800 & 0.0150 & & 0.0100 & 0.0600 & 0.0024 & 0.1051 & 0.0050 & 0.0150 & 0.4000 & 0.0439 & & & 0.0000 & & & & 0.0001 & \\
\hline IG3-20 & 0.0800 & 0.1200 & 0.0800 & 0.0300 & & 0.0050 & 0.0305 & 0.0024 & 0.1000 & 0.0050 & 0.0050 & 0.4120 & 0.0400 & & & 0.0000 & & & & 0.0001 & \\
\hline IG3-21 & 0.1000 & 0.1115 & 0.0400 & 0.0150 & & 0.0050 & 0.0600 & 0.0024 & 0.1000 & 0.0050 & 0.0050 & 0.4075 & 0.0800 & & & 0.0000 & & & & 0.0001 & \\
\hline IG3-22 & 0.1000 & 0.0600 & 0.0400 & 0.0300 & & 0.0050 & 0.0538 & 0.0024 & 0.1500 & 0.0050 & 0.0050 & 0.4000 & 0.0800 & & & 0.0000 & & & & 0.0001 & \\
\hline
\end{tabular}


Appendix A. Database - mass fraction

INEEL CVS Phase 3 (Scholes et al. 2000)

\begin{tabular}{|c|c|c|c|c|c|c|c|c|c|c|c|c|c|c|c|c|c|c|c|c|c|}
\hline Glass ID & $\begin{array}{l}\mathrm{Cl} \\
-\mathrm{t} \\
\end{array}$ & $\begin{array}{c}\mathrm{CoO} \\
-\mathrm{t} \\
\end{array}$ & $\begin{array}{c}\mathrm{Co} 2 \mathrm{O} 3 \\
-\mathrm{t}\end{array}$ & $\begin{array}{c}\mathrm{Cr} 2 \mathrm{O} 3 \\
-\mathrm{t}\end{array}$ & $\begin{array}{c}\mathrm{Cs} 2 \mathrm{O} \\
-\mathrm{t}\end{array}$ & $\begin{array}{c}\mathrm{CuO} \\
-\mathrm{t} \\
\end{array}$ & $\begin{array}{c}\mathrm{Eu} 2 \mathrm{O} 3 \\
-\mathrm{t}\end{array}$ & $\begin{array}{l}F \\
-t\end{array}$ & $\begin{array}{c}\mathrm{Ga} 2 \mathrm{O} 3 \\
-\mathrm{t}\end{array}$ & $\begin{array}{c}\mathrm{Gd} 2 \mathrm{O} 3 \\
-\mathrm{t}\end{array}$ & $\begin{array}{c}\mathrm{HgO} \\
-\mathrm{t} \\
\end{array}$ & $\begin{array}{l}I \\
-t\end{array}$ & $\begin{array}{c}\mathrm{La} 2 \mathrm{O} 3 \\
-\mathrm{t}\end{array}$ & $\begin{array}{c}\mathrm{MnO} 2 \\
-\mathrm{t}\end{array}$ & $\begin{array}{c}\mathrm{MnO} \\
-\mathrm{t}\end{array}$ & $\begin{array}{c}\mathrm{MoO} \\
-\mathrm{t} \\
\end{array}$ & $\begin{array}{c}\mathrm{MoO} 3 \\
-\mathrm{t}\end{array}$ & $\begin{array}{c}\mathrm{Nb} 2 \mathrm{O} 5 \\
-\mathrm{t}\end{array}$ & $\begin{array}{c}\mathrm{Nd} 2 \mathrm{O} 3 \\
-\mathrm{t}\end{array}$ & $\begin{array}{c}\mathrm{PbO} \\
-\mathrm{t}\end{array}$ & $\begin{array}{c}\mathrm{PdO} 2 \\
-\mathrm{t}\end{array}$ \\
\hline IG3-05 & 0.0003 & & & 0.0007 & 0.0000 & & & 0.0467 & & & & 0.0000 & 0.0000 & & 0.0001 & & 0.0150 & 0.0002 & 0.0001 & 0.0000 & \\
\hline IG3-06 & 0.0003 & & & 0.0007 & 0.0000 & & & 0.0334 & & & & 0.0000 & 0.0500 & & 0.0001 & & 0.0000 & 0.0002 & 0.0001 & 0.0000 & \\
\hline IG3-07 & 0.0003 & & & 0.0007 & 0.0000 & & & 0.0000 & & & & 0.0000 & 0.0000 & & 0.0001 & & 0.0150 & 0.0002 & 0.0001 & 0.0000 & \\
\hline IG3-08b & 0.0003 & & & 0.0007 & 0.0000 & & & 0.0065 & & & & 0.0000 & 0.0068 & & 0.0001 & & 0.0127 & 0.0002 & 0.0001 & 0.0000 & \\
\hline IG3-09 & 0.0003 & & & 0.0007 & 0.0000 & & & 0.0556 & & & & 0.0000 & 0.0000 & & 0.0001 & & 0.0150 & 0.0002 & 0.0001 & 0.0000 & \\
\hline IG3-10b & 0.0003 & & & 0.0007 & 0.0000 & & & 0.0333 & & & & 0.0000 & 0.0274 & & 0.0001 & & 0.0015 & 0.0002 & 0.0001 & 0.0000 & \\
\hline IG3-11 & 0.0003 & & & 0.0007 & 0.0000 & & & 0.0000 & & & & 0.0000 & 0.0000 & & 0.0001 & & 0.0000 & 0.0002 & 0.0001 & 0.0000 & \\
\hline IG3-12 & 0.0003 & & & 0.0007 & 0.0000 & & & 0.0000 & & & & 0.0000 & 0.0500 & & 0.0001 & & 0.0000 & 0.0002 & 0.0001 & 0.0000 & \\
\hline IG3-13 & 0.0003 & & & 0.0007 & 0.0000 & & & 0.0600 & & & & 0.0000 & 0.0000 & & 0.0001 & & 0.0000 & 0.0002 & 0.0001 & 0.0000 & \\
\hline IG3-14 & 0.0003 & & & 0.0007 & 0.0000 & & & 0.0250 & & & & 0.0000 & 0.0150 & & 0.0001 & & 0.0100 & 0.0002 & 0.0001 & 0.0000 & \\
\hline IG3-15 & 0.0003 & & & 0.0007 & 0.0000 & & & 0.0250 & & & & 0.0000 & 0.0350 & & 0.0001 & & 0.0100 & 0.0002 & 0.0001 & 0.0000 & \\
\hline IG3-16 & 0.0003 & & & 0.0007 & 0.0000 & & & 0.0100 & & & & 0.0000 & 0.0150 & & 0.0001 & & 0.0100 & 0.0002 & 0.0001 & 0.0000 & \\
\hline IG3-17 & 0.0003 & & & 0.0007 & 0.0000 & & & 0.0100 & & & & 0.0000 & 0.0150 & & 0.0001 & & 0.0100 & 0.0002 & 0.0001 & 0.0000 & \\
\hline IG3-18 & 0.0003 & & & 0.0007 & 0.0000 & & & 0.0100 & & & & 0.0000 & 0.0150 & & 0.0001 & & 0.0100 & 0.0002 & 0.0001 & 0.0000 & \\
\hline IG3-19 & 0.0003 & & & 0.0007 & 0.0000 & & & 0.0250 & & & & 0.0000 & 0.0350 & & 0.0001 & & 0.0100 & 0.0002 & 0.0001 & 0.0000 & \\
\hline IG3-20 & 0.0003 & & & 0.0007 & 0.0000 & & & 0.0250 & & & & 0.0000 & 0.0200 & & 0.0001 & & 0.0100 & 0.0002 & 0.0001 & 0.0000 & \\
\hline IG3-21 & 0.0003 & & & 0.0007 & 0.0000 & & & 0.0100 & & & & 0.0000 & 0.0350 & & 0.0001 & & 0.0100 & 0.0002 & 0.0001 & 0.0000 & \\
\hline IG3-22 & 0.0003 & & & 0.0007 & 0.0000 & & & 0.0100 & & & & 0.0000 & 0.0200 & & 0.0001 & & 0.0050 & 0.0002 & 0.0001 & 0.0000 & \\
\hline
\end{tabular}


Appendix A. Database - mass fraction

INEEL CVS Phase 3 (Scholes et al. 2000)

\begin{tabular}{|c|c|c|c|c|c|c|c|c|c|c|c|c|c|c|c|c|c|c|c|c|c|}
\hline Glass ID & $\begin{array}{c}\mathrm{PdO} \\
-\mathrm{t}\end{array}$ & $\begin{array}{c}\mathrm{Pr} 2 \mathrm{O} 3 \\
-\mathrm{t}\end{array}$ & $\begin{array}{c}\text { Pr6O11 } \\
-t\end{array}$ & $\begin{array}{c}\mathrm{Rb} 2 \mathrm{O} \\
-\mathrm{t}\end{array}$ & $\begin{array}{c}\mathrm{ReO} \\
-\mathrm{t}\end{array}$ & $\begin{array}{c}\mathrm{ReO} 2 \\
-\mathrm{t}\end{array}$ & $\begin{array}{c}\mathrm{Rh} 2 \mathrm{O} 3 \\
-\mathrm{t}\end{array}$ & $\begin{array}{c}\mathrm{RhO} 2 \\
-\mathrm{t}\end{array}$ & $\begin{array}{c}\mathrm{RuO}^{2} \\
-\mathrm{t}\end{array}$ & $\begin{array}{c}\mathrm{Sb} 2 \mathrm{O} 3 \\
-\mathrm{t} \\
\end{array}$ & $\begin{array}{c}\mathrm{Sb} 2 \mathrm{O} 5 \\
-\mathrm{t}\end{array}$ & $\begin{array}{c}\mathrm{SeO} 2 \\
-\mathrm{t} \\
\end{array}$ & $\begin{array}{c}\mathrm{Sm} 2 \mathrm{O} 3 \\
-\mathrm{t}\end{array}$ & $\begin{array}{c}\mathrm{SnO} \\
-\mathrm{t}\end{array}$ & $\begin{array}{c}\mathrm{SnO} 2 \\
-\mathrm{t}\end{array}$ & $\begin{array}{c}\mathrm{SO} 3 \\
-\mathrm{t}\end{array}$ & $\begin{array}{c}\mathrm{SrO} \\
-\mathrm{t}\end{array}$ & $\begin{array}{c}\mathrm{Tc} 2 \mathrm{O} 7 \\
-\mathrm{t}\end{array}$ & $\begin{array}{c}\mathrm{TeO} 2 \\
-\mathrm{t}\end{array}$ & $\begin{array}{c}\mathrm{ThO} 2 \\
-\mathrm{t}\end{array}$ & $\begin{array}{c}\mathrm{TiO} 2 \\
-\mathrm{t} \\
\end{array}$ \\
\hline IG3-05 & & 0.0000 & & 0.0000 & & 0.0001 & 0.0000 & & 0.0000 & & & & 0.0000 & & 0.0006 & 0.0000 & 0.0400 & & 0.0000 & & \\
\hline IG3-06 & & 0.0000 & & 0.0000 & & 0.0001 & 0.0000 & & 0.0000 & & & & 0.0000 & & 0.0006 & 0.0000 & 0.0000 & & 0.0000 & & \\
\hline IG3-07 & & 0.0000 & & 0.0000 & & 0.0001 & 0.0000 & & 0.0000 & & & & 0.0000 & & 0.0006 & 0.0000 & 0.0000 & & 0.0000 & & \\
\hline IG3-08b & & 0.0000 & & 0.0000 & & 0.0001 & 0.0000 & & 0.0000 & & & & 0.0000 & & 0.0006 & 0.0041 & 0.0055 & & 0.0000 & & \\
\hline IG3-09 & & 0.0000 & & 0.0000 & & 0.0001 & 0.0000 & & 0.0000 & & & & 0.0000 & & 0.0006 & 0.0050 & 0.0000 & & 0.0000 & & \\
\hline IG3-10b & & 0.0000 & & 0.0000 & & 0.0001 & 0.0000 & & 0.0000 & & & & 0.0000 & & 0.0006 & 0.0044 & 0.0357 & & 0.0000 & & \\
\hline IG3-11 & & 0.0000 & & 0.0000 & & 0.0001 & 0.0000 & & 0.0000 & & & & 0.0000 & & 0.0006 & 0.0050 & 0.0400 & & 0.0000 & & \\
\hline IG3-12 & & 0.0000 & & 0.0000 & & 0.0001 & 0.0000 & & 0.0000 & & & & 0.0000 & & 0.0006 & 0.0050 & 0.0400 & & 0.0000 & & \\
\hline IG3-13 & & 0.0000 & & 0.0000 & & 0.0001 & 0.0000 & & 0.0000 & & & & 0.0000 & & 0.0006 & 0.0000 & 0.0400 & & 0.0000 & & \\
\hline IG3-14 & & 0.0000 & & 0.0000 & & 0.0001 & 0.0000 & & 0.0000 & & & & 0.0000 & & 0.0006 & 0.0025 & 0.0100 & & 0.0000 & & \\
\hline IG3-15 & & 0.0000 & & 0.0000 & & 0.0001 & 0.0000 & & 0.0000 & & & & 0.0000 & & 0.0006 & 0.0025 & 0.0300 & & 0.0000 & & \\
\hline IG3-16 & & 0.0000 & & 0.0000 & & 0.0001 & 0.0000 & & 0.0000 & & & & 0.0000 & & 0.0006 & 0.0010 & 0.0300 & & 0.0000 & & \\
\hline IG3-17 & & 0.0000 & & 0.0000 & & 0.0001 & 0.0000 & & 0.0000 & & & & 0.0000 & & 0.0006 & 0.0010 & 0.0300 & & 0.0000 & & \\
\hline IG3-18 & & 0.0000 & & 0.0000 & & 0.0001 & 0.0000 & & 0.0000 & & & & 0.0000 & & 0.0006 & 0.0025 & 0.0300 & & 0.0000 & & \\
\hline IG3-19 & & 0.0000 & & 0.0000 & & 0.0001 & 0.0000 & & 0.0000 & & & & 0.0000 & & 0.0006 & 0.0010 & 0.0100 & & 0.0000 & & \\
\hline IG3-20 & & 0.0000 & & 0.0000 & & 0.0001 & 0.0000 & & 0.0000 & & & & 0.0000 & & 0.0006 & 0.0025 & 0.0300 & & 0.0000 & & \\
\hline IG3-21 & & 0.0000 & & 0.0000 & & 0.0001 & 0.0000 & & 0.0000 & & & & 0.0000 & & 0.0006 & 0.0010 & 0.0100 & & 0.0000 & & \\
\hline IG3-22 & & 0.0000 & & 0.0000 & & 0.0001 & 0.0000 & & 0.0000 & & & & 0.0000 & & 0.0006 & 0.0025 & 0.0287 & & 0.0000 & & \\
\hline
\end{tabular}


Appendix A. Database - mass fraction

INEEL CVS Phase 3 (Scholes et al. 2000)

\begin{tabular}{|c|c|c|c|c|c|c|c|c|c|c|c|c|c|c|c|c|c|c|c|c|c|}
\hline Glass ID & $\begin{array}{c}\mathrm{T} 12 \mathrm{O} 3 \\
-\mathrm{t}\end{array}$ & $\begin{array}{c}\mathrm{U} 3 \mathrm{O} 8 \\
-\mathrm{t}\end{array}$ & $\begin{array}{c}\mathrm{UO} 2 \\
-\mathrm{t}\end{array}$ & $\begin{array}{c}\text { UO3 } \\
-t\end{array}$ & $\begin{array}{c}\mathrm{V} 2 \mathrm{O} 5 \\
-\mathrm{t}\end{array}$ & $\begin{array}{c}\text { WO3 } \\
-t\end{array}$ & $\begin{array}{c}\mathrm{Y} 2 \mathrm{O} 3 \\
-\mathrm{t}\end{array}$ & $\begin{array}{c}\mathrm{ZnO} \\
-\mathrm{t} \\
\end{array}$ & $\begin{array}{c}\text { Others } \\
-t\end{array}$ & $\begin{array}{c}\text { Sum } \\
-t\end{array}$ & $\begin{array}{c}\mathrm{A} 12 \mathrm{O} 3 \\
-\mathrm{a}\end{array}$ & $\begin{array}{c}\mathrm{B} 2 \mathrm{O} 3 \\
-\mathrm{a}\end{array}$ & $\begin{array}{c}\mathrm{CaO} \\
-\mathrm{a}\end{array}$ & $\begin{array}{c}\mathrm{Fe} 2 \mathrm{O} 3 \\
-\mathrm{a} \\
\end{array}$ & $\begin{array}{c}\mathrm{FeO} \\
-\mathrm{a} \\
\end{array}$ & $\begin{array}{c}\mathrm{K} 2 \mathrm{O} \\
-\mathrm{a}\end{array}$ & $\begin{array}{c}\mathrm{Li} 2 \mathrm{O} \\
-\mathrm{a} \\
\end{array}$ & $\begin{array}{c}\mathrm{MgO} \\
-\mathrm{a}\end{array}$ & $\begin{array}{c}\mathrm{Na} 2 \mathrm{O} \\
-\mathrm{a}\end{array}$ & $\begin{array}{c}\mathrm{NiO} \\
-\mathrm{a}\end{array}$ & $\begin{array}{c}\mathrm{P} 2 \mathrm{O} 5 \\
-\mathrm{a}\end{array}$ \\
\hline IG3-05 & & & & & & & 0.0000 & & & 1.0000 & 0.0366 & 0.0506 & 0.1200 & 0.0001 & & 0.0204 & 0.0007 & 0.0002 & 0.1245 & 0.0142 & 0.0002 \\
\hline IG3-06 & & & & & & & 0.0000 & & & 1.0000 & 0.0420 & 0.0499 & 0.1210 & 0.0006 & & 0.0211 & 0.0295 & 0.0005 & 0.0508 & 0.0135 & 0.0093 \\
\hline IG3-07 & & & & & & & 0.0000 & & & 1.0000 & 0.1687 & 0.1790 & 0.0001 & 0.0805 & & 0.0000 & 0.0008 & 0.0004 & 0.1894 & 0.0137 & 0.0002 \\
\hline IG3-08b & & & & & & & 0.0000 & & & 1.0000 & 0.0555 & 0.1569 & 0.0173 & 0.0650 & & 0.0027 & 0.0122 & 0.0002 & 0.1432 & 0.0116 & 0.0021 \\
\hline IG3-09 & & & & & & & 0.0000 & & & 1.0000 & 0.0268 & 0.0522 & 0.0001 & 0.0821 & & 0.0225 & 0.0930 & 0.0025 & 0.0650 & 0.0001 & 0.0246 \\
\hline IG3-10b & & & & & & & 0.0000 & & & 1.0000 & 0.0404 & 0.1685 & 0.0122 & 0.0471 & & 0.0190 & 0.0404 & 0.0023 & 0.0683 & 0.0127 & 0.0013 \\
\hline IG3-11 & & & & & & & 0.0000 & & & 1.0000 & 0.1195 & 0.0503 & 0.1199 & 0.0796 & & 0.0000 & 0.0141 & 0.0042 & 0.2032 & 0.0001 & 0.0002 \\
\hline IG3-12 & & & & & & & 0.0000 & & & 1.0000 & 0.0371 & 0.1793 & 0.0908 & 0.0295 & & 0.0000 & 0.0010 & 0.0025 & 0.1139 & 0.0001 & 0.0002 \\
\hline IG3-13 & & & & & & & 0.0000 & & & 1.0000 & 0.0418 & 0.0767 & 0.0007 & 0.0795 & & 0.0000 & 0.0471 & 0.0003 & 0.1422 & 0.0143 & 0.0054 \\
\hline IG3-14 & & & & & & & 0.0000 & & & 1.0000 & 0.0805 & 0.0602 & 0.0434 & 0.0298 & & 0.0110 & 0.0595 & 0.0015 & 0.1058 & 0.0046 & 0.0075 \\
\hline IG3-15 & & & & & & & 0.0000 & & & 1.0000 & 0.1028 & 0.0623 & 0.0842 & 0.0051 & & 0.0111 & 0.0439 & 0.0025 & 0.1042 & 0.0025 & 0.0041 \\
\hline IG3-16 & & & & & & & 0.0000 & & & 1.0000 & 0.0970 & 0.0745 & 0.0828 & 0.0049 & & 0.0089 & 0.0583 & 0.0007 & 0.1031 & 0.0050 & 0.0062 \\
\hline IG3-17 & & & & & & & 0.0000 & & & 1.0000 & 0.0886 & 0.0597 & 0.0833 & 0.0300 & & 0.0108 & 0.0588 & 0.0009 & 0.1049 & 0.0045 & 0.0092 \\
\hline IG3-18 & & & & & & & 0.0000 & & & 1.0000 & 0.1206 & 0.0616 & 0.0440 & 0.0047 & & 0.0091 & 0.0582 & 0.0015 & 0.1510 & 0.0049 & 0.0081 \\
\hline IG3-19 & & & & & & & 0.0000 & & & 1.0000 & 0.1227 & 0.0640 & 0.0857 & 0.0153 & & 0.0112 & 0.0616 & 0.0007 & 0.1067 & 0.0046 & 0.0059 \\
\hline IG3-20 & & & & & & & 0.0000 & & & 1.0000 & 0.0823 & 0.1218 & 0.0821 & 0.0310 & & 0.0042 & 0.0321 & 0.0014 & 0.1032 & 0.0045 & 0.0041 \\
\hline IG3-21 & & & & & & & 0.0000 & & & 1.0000 & 0.0996 & 0.1137 & 0.0438 & 0.0151 & & 0.0056 & 0.0597 & 0.0008 & 0.1021 & 0.0044 & 0.0028 \\
\hline IG3-22 & & & & & & & 0.0000 & & & 1.0000 & 0.1006 & 0.0622 & 0.0445 & 0.0333 & & 0.0056 & 0.0544 & 0.0017 & 0.1591 & 0.0044 & 0.0028 \\
\hline
\end{tabular}


Appendix A. Database - mass fraction

INEEL CVS Phase 3 (Scholes et al. 2000)

\begin{tabular}{|c|c|c|c|c|c|c|c|c|c|c|c|c|c|c|c|c|c|c|c|c|c|}
\hline Glass ID & $\begin{array}{c}\mathrm{SiO} 2 \\
-\mathrm{a}\end{array}$ & $\begin{array}{c}\mathrm{ZrO} 2 \\
-\mathrm{a}\end{array}$ & $\begin{array}{c}\mathrm{Ag} 2 \mathrm{O} \\
-\mathrm{a}\end{array}$ & $\begin{array}{c}\mathrm{As} 2 \mathrm{O} 3 \\
-\mathrm{a} \\
\end{array}$ & $\begin{array}{c}\mathrm{BaO} \\
-\mathrm{a}\end{array}$ & $\begin{array}{c}\mathrm{Bi} 2 \mathrm{O} 3 \\
-\mathrm{a}\end{array}$ & $\begin{array}{l}\mathrm{Br} \\
-\mathrm{a}\end{array}$ & $\begin{array}{c}\mathrm{CdO} \\
-\mathrm{a}\end{array}$ & $\begin{array}{c}\mathrm{Ce} 2 \mathrm{O} 3 \\
-\mathrm{a}\end{array}$ & $\begin{array}{c}\mathrm{CeO} 2 \\
-\mathrm{a}\end{array}$ & $\begin{array}{l}\mathrm{Cl} \\
-\mathrm{a}\end{array}$ & $\begin{array}{c}\mathrm{CoO} \\
-\mathrm{a}\end{array}$ & $\begin{array}{c}\mathrm{Co} 2 \mathrm{O} 3 \\
-\mathrm{a}\end{array}$ & $\begin{array}{c}\mathrm{Cr} 2 \mathrm{O} 3 \\
-\mathrm{a}\end{array}$ & $\begin{array}{c}\mathrm{Cs} 2 \mathrm{O} \\
-\mathrm{a} \\
\end{array}$ & $\begin{array}{c}\mathrm{CuO} \\
-\mathrm{a}\end{array}$ & $\begin{array}{c}\mathrm{Eu} 2 \mathrm{O} 3 \\
-\mathrm{a}\end{array}$ & $\begin{array}{c}\mathrm{F} \\
-\mathrm{a}\end{array}$ & $\begin{array}{c}\mathrm{Ga} 2 \mathrm{O} 3 \\
-\mathrm{a}\end{array}$ & $\begin{array}{c}\mathrm{Gd} 2 \mathrm{O} 3 \\
-\mathrm{a}\end{array}$ & $\begin{array}{c}\mathrm{HgO} \\
-\mathrm{a}\end{array}$ \\
\hline IG3-05 & 0.5348 & 0.0003 & & & 0.0000 & & & & 0.0000 & & 0.0000 & & & 0.0001 & 0.0000 & & & 0.0368 & & & \\
\hline IG3-06 & 0.4295 & 0.1061 & & & 0.0000 & & & & 0.0000 & & 0.0001 & & & 0.0001 & 0.0000 & & & 0.0261 & & & \\
\hline IG3-07 & 0.3530 & 0.0002 & & & 0.0000 & & & & 0.0000 & & 0.0001 & & & 0.0001 & 0.0000 & & & 0.0005 & & & \\
\hline IG3-08b & 0.4156 & 0.1025 & & & 0.0000 & & & & 0.0000 & & 0.0000 & & & 0.0001 & 0.0000 & & & 0.0005 & & & \\
\hline IG3-09 & 0.6001 & 0.0000 & & & 0.0001 & & & & 0.0001 & & 0.0003 & & & 0.0008 & 0.0000 & & & 0.0347 & & & \\
\hline IG3-10b & 0.3904 & 0.1146 & & & 0.0000 & & & & 0.0001 & & 0.0003 & & & 0.0007 & 0.0000 & & & 0.0145 & & & \\
\hline IG3-11 & 0.3674 & 0.0000 & & & 0.0001 & & & & 0.0001 & & 0.0006 & & & 0.0013 & 0.0001 & & & 0.0005 & & & \\
\hline IG3-12 & 0.3562 & 0.0905 & & & 0.0000 & & & & 0.0001 & & 0.0003 & & & 0.0007 & 0.0000 & & & 0.0005 & & & \\
\hline IG3-13 & 0.3658 & 0.1224 & & & 0.0000 & & & & 0.0000 & & 0.0000 & & & 0.0001 & 0.0000 & & & 0.0426 & & & \\
\hline IG3-14 & 0.5081 & 0.0325 & & & 0.0000 & & & & 0.0000 & & 0.0002 & & & 0.0004 & 0.0000 & & & 0.0213 & & & \\
\hline IG3-15 & 0.4134 & 0.0742 & & & 0.0000 & & & & 0.0001 & & 0.0003 & & & 0.0007 & 0.0000 & & & 0.0193 & & & \\
\hline IG3-16 & 0.4027 & 0.0630 & & & 0.0000 & & & & 0.0000 & & 0.0001 & & & 0.0002 & 0.0000 & & & 0.0073 & & & \\
\hline IG3-17 & 0.4001 & 0.0659 & & & 0.0000 & & & & 0.0000 & & 0.0001 & & & 0.0003 & 0.0000 & & & 0.0076 & & & \\
\hline IG3-18 & 0.4022 & 0.0482 & & & 0.0000 & & & & 0.0000 & & 0.0002 & & & 0.0005 & 0.0000 & & & 0.0075 & & & \\
\hline IG3-19 & 0.4161 & 0.0347 & & & 0.0000 & & & & 0.0000 & & 0.0001 & & & 0.0002 & 0.0000 & & & 0.0198 & & & \\
\hline IG3-20 & 0.4140 & 0.0376 & & & 0.0000 & & & & 0.0000 & & 0.0002 & & & 0.0004 & 0.0000 & & & 0.0193 & & & \\
\hline IG3-21 & 0.4193 & 0.0708 & & & 0.0000 & & & & 0.0000 & & 0.0001 & & & 0.0003 & 0.0000 & & & 0.0077 & & & \\
\hline IG3-22 & 0.4209 & 0.0715 & & & 0.0000 & & & & 0.0000 & & 0.0002 & & & 0.0005 & 0.0000 & & & 0.0080 & & & \\
\hline
\end{tabular}


Appendix A. Database - mass fraction

INEEL CVS Phase 3 (Scholes et al. 2000)

\begin{tabular}{|c|c|c|c|c|c|c|c|c|c|c|c|c|c|c|c|c|c|c|c|c|c|}
\hline Glass ID & $\begin{array}{c}\mathrm{I} \\
-\mathrm{a} \\
\end{array}$ & $\begin{array}{c}\mathrm{La} 2 \mathrm{O} 3 \\
-\mathrm{a}\end{array}$ & $\begin{array}{c}\mathrm{MnO} 2 \\
-\mathrm{a}\end{array}$ & $\begin{array}{c}\mathrm{MnO} \\
-\mathrm{a} \\
\end{array}$ & $\begin{array}{c}\mathrm{MoO} \\
-\mathrm{a} \\
\end{array}$ & $\begin{array}{c}\mathrm{MoO} 3 \\
-\mathrm{a}\end{array}$ & $\begin{array}{c}\mathrm{Nb} 2 \mathrm{O} 5 \\
-\mathrm{a}\end{array}$ & $\begin{array}{c}\mathrm{Nd} 2 \mathrm{O} 3 \\
-\mathrm{a} \\
\end{array}$ & $\begin{array}{c}\mathrm{PbO} \\
-\mathrm{a} \\
\end{array}$ & $\begin{array}{c}\mathrm{PdO} 2 \\
-\mathrm{a}\end{array}$ & $\begin{array}{c}\mathrm{PdO} \\
-\mathrm{a} \\
\end{array}$ & $\begin{array}{c}\mathrm{Pr} 2 \mathrm{O} 3 \\
-\mathrm{a}\end{array}$ & $\begin{array}{c}\text { Pr6O11 } \\
-\mathrm{a}\end{array}$ & $\begin{array}{c}\mathrm{Rb} 2 \mathrm{O} \\
-\mathrm{a}\end{array}$ & $\begin{array}{c}\mathrm{ReO} \\
-\mathrm{a} \\
\end{array}$ & $\begin{array}{c}\mathrm{ReO} 2 \\
-\mathrm{a}\end{array}$ & $\begin{array}{c}\mathrm{Rh} 2 \mathrm{O} 3 \\
-\mathrm{a}\end{array}$ & $\begin{array}{c}\mathrm{RhO}_{2} \\
-\mathrm{a}\end{array}$ & $\begin{array}{c}\mathrm{RuO2} \\
-\mathrm{a}\end{array}$ & $\begin{array}{c}\mathrm{Sb} 2 \mathrm{O} 3 \\
-\mathrm{a} \\
\end{array}$ & $\begin{array}{c}\mathrm{Sb} 2 \mathrm{O} 5 \\
-\mathrm{a}\end{array}$ \\
\hline IG3-05 & 0.0000 & 0.0001 & & 0.0000 & & 0.0149 & 0.0000 & 0.0000 & 0.0000 & & & 0.0000 & & 0.0000 & & 0.0000 & 0.0000 & & 0.0000 & & \\
\hline IG3-06 & 0.0000 & 0.0473 & & 0.0000 & & 0.0002 & 0.0000 & 0.0000 & 0.0000 & & & 0.0000 & & 0.0000 & & 0.0000 & 0.0000 & & 0.0000 & & \\
\hline IG3-07 & 0.0000 & 0.0001 & & 0.0000 & & 0.0145 & 0.0000 & 0.0000 & 0.0000 & & & 0.0000 & & 0.0000 & & 0.0000 & 0.0000 & & 0.0000 & & \\
\hline IG3-08b & 0.0000 & 0.0064 & & 0.0000 & & 0.0121 & 0.0000 & 0.0000 & 0.0000 & & & 0.0000 & & 0.0000 & & 0.0000 & 0.0000 & & 0.0000 & & \\
\hline IG3-09 & 0.0000 & 0.0001 & & 0.0001 & & 0.0149 & 0.0002 & 0.0001 & 0.0000 & & & 0.0000 & & 0.0000 & & 0.0001 & 0.0000 & & 0.0000 & & \\
\hline IG3-10b & 0.0000 & 0.0272 & & 0.0001 & & 0.0013 & 0.0002 & 0.0001 & 0.0000 & & & 0.0000 & & 0.0000 & & 0.0000 & 0.0000 & & 0.0000 & & \\
\hline IG3-11 & 0.0000 & 0.0001 & & 0.0002 & & 0.0002 & 0.0004 & 0.0002 & 0.0000 & & & 0.0000 & & 0.0000 & & 0.0001 & 0.0000 & & 0.0001 & & \\
\hline IG3-12 & 0.0000 & 0.0462 & & 0.0001 & & 0.0002 & 0.0002 & 0.0001 & 0.0000 & & & 0.0000 & & 0.0000 & & 0.0001 & 0.0000 & & 0.0000 & & \\
\hline IG3-13 & 0.0000 & 0.0001 & & 0.0000 & & 0.0002 & 0.0000 & 0.0000 & 0.0000 & & & 0.0000 & & 0.0000 & & 0.0000 & 0.0000 & & 0.0000 & & \\
\hline IG3-14 & 0.0000 & 0.0142 & & 0.0001 & & 0.0097 & 0.0001 & 0.0001 & 0.0000 & & & 0.0000 & & 0.0000 & & 0.0000 & 0.0000 & & 0.0000 & & \\
\hline IG3-15 & 0.0000 & 0.0326 & & 0.0001 & & 0.0096 & 0.0002 & 0.0001 & 0.0000 & & & 0.0000 & & 0.0000 & & 0.0001 & 0.0000 & & 0.0000 & & \\
\hline IG3-16 & 0.0000 & 0.0141 & & 0.0000 & & 0.0097 & 0.0001 & 0.0000 & 0.0000 & & & 0.0000 & & 0.0000 & & 0.0000 & 0.0000 & & 0.0000 & & \\
\hline IG3-17 & 0.0000 & 0.0139 & & 0.0000 & & 0.0094 & 0.0001 & 0.0000 & 0.0000 & & & 0.0000 & & 0.0000 & & 0.0000 & 0.0000 & & 0.0000 & & \\
\hline IG3-18 & 0.0000 & 0.0138 & & 0.0001 & & 0.0093 & 0.0001 & 0.0001 & 0.0000 & & & 0.0000 & & 0.0000 & & 0.0000 & 0.0000 & & 0.0000 & & \\
\hline IG3-19 & 0.0000 & 0.0325 & & 0.0000 & & 0.0096 & 0.0001 & 0.0000 & 0.0000 & & & 0.0000 & & 0.0000 & & 0.0000 & 0.0000 & & 0.0000 & & \\
\hline IG3-20 & 0.0000 & 0.0184 & & 0.0001 & & 0.0094 & 0.0001 & 0.0001 & 0.0000 & & & 0.0000 & & 0.0000 & & 0.0000 & 0.0000 & & 0.0000 & & \\
\hline IG3-21 & 0.0000 & 0.0322 & & 0.0000 & & 0.0095 & 0.0001 & 0.0000 & 0.0000 & & & 0.0000 & & 0.0000 & & 0.0000 & 0.0000 & & 0.0000 & & \\
\hline IG3-22 & 0.0000 & 0.0187 & & 0.0001 & & 0.0047 & 0.0002 & 0.0001 & 0.0000 & & & 0.0000 & & 0.0000 & & 0.0000 & 0.0000 & & 0.0000 & & \\
\hline
\end{tabular}


Appendix A. Database - mass fraction

INEEL CVS Phase 3 (Scholes et al. 2000)

\begin{tabular}{|c|c|c|c|c|c|c|c|c|c|c|c|c|c|c|c|c|c|c|c|c|}
\hline Glass ID & $\begin{array}{c}\mathrm{SeO} 2 \\
-\mathrm{a}\end{array}$ & $\begin{array}{c}\mathrm{Sm} 2 \mathrm{O} 3 \\
-\mathrm{a}\end{array}$ & $\begin{array}{c}\mathrm{SnO} \\
-\mathrm{a} \\
\end{array}$ & $\begin{array}{c}\mathrm{SnO} 2 \\
-\mathrm{a}\end{array}$ & $\begin{array}{c}\mathrm{SO} 3 \\
-\mathrm{a} \\
\end{array}$ & $\begin{array}{c}\mathrm{SrO} \\
-\mathrm{a} \\
\end{array}$ & $\begin{array}{c}\mathrm{Tc} 2 \mathrm{O} 7 \\
-\mathrm{a}\end{array}$ & $\begin{array}{c}\mathrm{TeO} 2 \\
-\mathrm{a}\end{array}$ & $\begin{array}{c}\mathrm{ThO} 2 \\
-\mathrm{a}\end{array}$ & $\begin{array}{c}\mathrm{TiO} 2 \\
-\mathrm{a} \\
\end{array}$ & $\begin{array}{c}\mathrm{T} 12 \mathrm{O} 3 \\
-\mathrm{a} \\
\end{array}$ & $\begin{array}{c}\mathrm{U} 3 \mathrm{O} 8 \\
-\mathrm{a}\end{array}$ & $\begin{array}{c}\mathrm{UO} 2 \\
-\mathrm{a}\end{array}$ & $\begin{array}{c}\text { UO3 } \\
-\mathrm{a}\end{array}$ & $\begin{array}{c}\mathrm{V} 2 \mathrm{O} 5 \\
-\mathrm{a} \\
\end{array}$ & $\begin{array}{c}\text { WO3 } \\
-\mathrm{a}\end{array}$ & $\begin{array}{c}\mathrm{Y} 2 \mathrm{O} 3 \\
-\mathrm{a}\end{array}$ & $\begin{array}{c}\mathrm{ZnO} \\
-\mathrm{a}\end{array}$ & $\begin{array}{c}\text { Others } \\
-\mathrm{a}\end{array}$ & $\begin{array}{c}\text { Sum } \\
-\mathrm{a}\end{array}$ \\
\hline IG3-05 & & 0.0000 & & 0.0000 & 0.0004 & 0.0359 & & 0.0000 & & & & & & & & & 0.0000 & & & 0.9910 \\
\hline IG3-06 & & 0.0000 & & 0.0001 & 0.0010 & 0.0000 & & 0.0000 & & & & & & & & & 0.0000 & & & 0.9486 \\
\hline IG3-07 & & 0.0000 & & 0.0001 & 0.0008 & 0.0000 & & 0.0000 & & & & & & & & & 0.0000 & & & 1.0023 \\
\hline IG3-08b & & 0.0000 & & 0.0000 & 0.0004 & 0.0056 & & 0.0000 & & & & & & & & & 0.0000 & & & 1.0098 \\
\hline IG3-09 & & 0.0000 & & 0.0006 & 0.0052 & 0.0000 & & 0.0000 & & & & & & & & & 0.0000 & & & 1.0265 \\
\hline IG3-10b & & 0.0000 & & 0.0006 & 0.0048 & 0.0333 & & 0.0000 & & & & & & & & & 0.0000 & & & 1.0009 \\
\hline IG3-11 & & 0.0001 & & 0.0010 & 0.0087 & 0.0343 & & 0.0000 & & & & & & & & & 0.0000 & & & 1.0067 \\
\hline IG3-12 & & 0.0000 & & 0.0006 & 0.0051 & 0.0353 & & 0.0000 & & & & & & & & & 0.0000 & & & 0.9907 \\
\hline IG3-13 & & 0.0000 & & 0.0001 & 0.0006 & 0.0365 & & 0.0000 & & & & & & & & & 0.0000 & & & 0.9767 \\
\hline IG3-14 & & 0.0000 & & 0.0004 & 0.0030 & 0.0103 & & 0.0000 & & & & & & & & & 0.0000 & & & 1.0043 \\
\hline IG3-15 & & 0.0000 & & 0.0006 & 0.0050 & 0.0284 & & 0.0000 & & & & & & & & & 0.0000 & & & 1.0077 \\
\hline IG3-16 & & 0.0000 & & 0.0002 & 0.0014 & 0.0278 & & 0.0000 & & & & & & & & & 0.0000 & & & 0.9680 \\
\hline IG3-17 & & 0.0000 & & 0.0002 & 0.0018 & 0.0274 & & 0.0000 & & & & & & & & & 0.0000 & & & 0.9775 \\
\hline IG3-18 & & 0.0000 & & 0.0004 & 0.0031 & 0.0279 & & 0.0000 & & & & & & & & & 0.0000 & & & 0.9773 \\
\hline IG3-19 & & 0.0000 & & 0.0002 & 0.0014 & 0.0101 & & 0.0000 & & & & & & & & & 0.0000 & & & 1.0033 \\
\hline IG3-20 & & 0.0000 & & 0.0003 & 0.0029 & 0.0277 & & 0.0000 & & & & & & & & & 0.0000 & & & 0.9972 \\
\hline IG3-21 & & 0.0000 & & 0.0002 & 0.0017 & 0.0103 & & 0.0000 & & & & & & & & & 0.0000 & & & 0.9999 \\
\hline IG3-22 & & 0.0000 & & 0.0004 & 0.0034 & 0.0271 & & 0.0000 & & & & & & & & & 0.0000 & & & 1.0246 \\
\hline
\end{tabular}


Appendix A. Database - mass fraction

INEEL CVS Phase 3 (Scholes et al. 2000)

\begin{tabular}{|c|c|c|c|c|c|c|c|c|}
\hline Glass ID & $\begin{array}{l}\mathrm{TM} \\
\left({ }^{\circ} \mathrm{C}\right)\end{array}$ & $\begin{array}{l}\text { Gradient } \\
\text { TL }\left({ }^{\circ} \mathrm{C}\right)\end{array}$ & $\begin{array}{l}\text { Uniform } \\
\text { TL }\left({ }^{\circ} \mathrm{C}\right)\end{array}$ & Primary Phase & $\begin{array}{r}\text { Quenched } \\
\text { Visual/OM }\end{array}$ & $\begin{array}{c}\text { Quenched } \\
\text { SEM/EDS or TEM }\end{array}$ & $\begin{array}{l}\text { Quenched } \\
\text { XRD }\end{array}$ & $\begin{array}{c}\text { Quenched } \\
\text { Homogeneous? }\end{array}$ \\
\hline IG3-05 & 1150 & & & & multi-phase & & amorphous & no \\
\hline IG3-06 & 1350 & & $>1500$ & $\mathrm{ZrO} 2$ & single-phase & & amorphous & yes \\
\hline IG3-07 & 1250 & & & & multi-phase & & amorphous & no \\
\hline IG3-08b & 1275 & & $>1350$ & $\mathrm{ZrO} 2$ & single-phase & & amorphous & yes \\
\hline IG3-09 & 1150 & & & & multi-phase & & $\begin{array}{l}\text { LiF, Li3PO4, LiFeO2, } \\
\text { Fe7S8 }\end{array}$ & no \\
\hline IG3-10b & 1250 & & $>1500$ & $\mathrm{ZrO} 2$ & single-phase & & amorphous & yes \\
\hline IG3-11 & 1150 & & 1013 & Na7Al6Si6O24S3 & single-phase & & amorphous & yes \\
\hline IG3-12 & 1150 & & 973 & $\mathrm{CaAl} 2(\mathrm{SiO} 4) 2$ & single-phase & & amorphous & yes \\
\hline IG3-13 & 1150 & & & & multi-phase & & $\begin{array}{l}\mathrm{LiF}, \mathrm{Fe} 2 \mathrm{O} 3, \mathrm{ZrO} 2 \\
\mathrm{Na} \text {, }\end{array}$ & no \\
\hline IG3-14 & 1150 & & 943 & $(\mathrm{Ca} 9 \mathrm{Sr})(\mathrm{PO} 4) 6 \mathrm{~F} 2$ & single-phase & & amorphous & yes \\
\hline IG3-15 & 1200 & & 1203 & $\mathrm{ZrO} 2$ & single-phase & & amorphous & yes \\
\hline IG3-16 & 1150 & & 1133 & $\mathrm{ZrO} 2$ & single-phase & & amorphous & yes \\
\hline IG3-17 & 1150 & & 1173 & $\mathrm{ZrO} 2$ & single-phase & & amorphous & yes \\
\hline IG3-18 & 1150 & & 1023 & $\begin{array}{l}\mathrm{Na} 6 \mathrm{Ca} 2(\mathrm{Al} 6 \mathrm{Si} 6 \mathrm{O} 24)( \\
\mathrm{SO} 4) 2\end{array}$ & single-phase & & amorphous & yes \\
\hline IG3-19 & 1150 & & 893 & $\mathrm{La} 2 \mathrm{NiO} 4$ & single-phase & & amorphous & yes \\
\hline IG3-20 & 1150 & & 893 & $\begin{array}{l}\mathrm{Ca} 10(\mathrm{SiO} 4) 3(\mathrm{SO} 4) 3 \mathrm{~F} \\
2\end{array}$ & single-phase & & amorphous & yes \\
\hline IG3-21 & 1150 & & 1013 & $\mathrm{ZrO} 2$ & single-phase & & amorphous & yes \\
\hline IG3-22 & 1200 & & 1163 & $\mathrm{ZrO} 2$ & single-phase & & amorphous & yes \\
\hline
\end{tabular}


Appendix A. Database - mass fraction

\begin{tabular}{|c|c|c|c|c|c|}
\hline Glass ID & $\begin{array}{c}\mathrm{CCC} \\
\text { Visual/OM }\end{array}$ & $\begin{array}{c}\text { CCC } \\
\text { SEM/EDS or TEM }\end{array}$ & $\begin{array}{l}\text { CCC } \\
\text { XRD }\end{array}$ & $\begin{array}{l}\text { Heat Treated } \\
\text { Visual/OM }\end{array}$ & $\begin{array}{c}\text { Heat Treated } \\
\text { SEM/EDS or TEM }\end{array}$ \\
\hline IG3-05 & $\begin{array}{l}\text { brown colored glass is multiphase and devitrified with large } \\
\text { clear crystals throughout, the top surface is semi-transparent, } \\
\text { but with a rough scale like appearance }\end{array}$ & & $\mathrm{CaF} 2, \mathrm{FeO}$, and $\mathrm{LiAlSi} 2 \mathrm{O} 6$ & & \\
\hline IG3-06 & $\begin{array}{l}\text { dark brown colored glass is multi-phase and translucent, not } \\
\text { devitrified }\end{array}$ & & $\mathrm{Ca} 8 \mathrm{La} 2(\mathrm{PO} 4) 5 \mathrm{O} 2$ and $(\mathrm{CaLa}) \mathrm{Si} 2$ & & \\
\hline IG3-07 & $\begin{array}{l}\text { dark reddish brown colored glass completely devitrified with } \\
\text { dark snowflake type crystals throughout the glass }\end{array}$ & & (FeNi2)o2(BO3) & & \\
\hline IG3-08b & $\begin{array}{l}\text { separated into regions of either transparent glass or opaque } \\
\text { phase-separated glass with well-defined boundaries, but no } \\
\text { signs of devitrification }\end{array}$ & & amorphous & & \\
\hline IG3-09 & $\begin{array}{l}\text { green-opaque colored glass appears to be completely } \\
\text { devitrified with very small clear granular crystals throughout } \\
\text { with some red colored ones at the top and outer surfaces }\end{array}$ & & $\begin{array}{l}\text { LiF, Li3PO4, Li2SiO3, LiKSO4, and } \\
\text { AlPS4 }\end{array}$ & & \\
\hline IG3-10b & $\begin{array}{l}\text { brown colored glass is single-phase and transparent, not } \\
\text { devitrified }\end{array}$ & & amorphous & & \\
\hline IG3-11 & $\begin{array}{l}\text { dark brown colored glass appears crumbly and about half is } \\
\text { transparent with the rest devitrified with solid brown groups of } \\
\text { crystals }\end{array}$ & & $\begin{array}{l}\text { Na4Ca4(Si6O18)NaAlSiO4, and } \\
\text { CaSrO }\end{array}$ & & \\
\hline IG3-12 & $\begin{array}{l}\text { dark green colored glass is single-phase and transparent with } \\
\text { lots of small air bubbles }\end{array}$ & & amorphous & & \\
\hline IG3-13 & $\begin{array}{l}\text { solid reddish brown colored glass is multi-phase with very } \\
\text { small reddish granular crystals throughout the glass and on to } \\
\text { are clear granular crystals with reddish star-type crystals }\end{array}$ & & $\begin{array}{l}\mathrm{Sr} 9 \mathrm{NaBO} 2(\mathrm{PO} 4) 6, \mathrm{LiF}, \mathrm{Li} 2 \mathrm{NiF} 4 \text {, and } \\
\mathrm{Fe} 2 \mathrm{O} 3\end{array}$ & & \\
\hline IG3-14 & $\begin{array}{l}\text { brownish green colored glass is multi-phase and devitrified } \\
\text { with clear clusters of crystals throughout }\end{array}$ & & $\begin{array}{l}\mathrm{CaF} 2, \mathrm{LiAlSi} 2 \mathrm{O} 6, \mathrm{Sr} 2 \mathrm{FeO} 4 \text {, and } \\
\mathrm{La} 2 \mathrm{O} 2 \mathrm{~S}\end{array}$ & & \\
\hline IG3-15 & green colored glass appears to be single-phase and transparent & & amorphous & & \\
\hline IG3-16 & $\begin{array}{l}\text { dark green colored glass is mostly translucent with lots of } \\
\text { small air bubbles, very small percent of clear cubic crystals ar } \\
\text { present throughout the glass }\end{array}$ & & amorphous & & \\
\hline IG3-17 & $\begin{array}{l}\text { brown colored glass is multi-phase and transparent, but } \\
\text { devitrified with small white-flaky and rod-type crystals } \\
\text { throughout the glass }\end{array}$ & & amorphous & & \\
\hline IG3-18 & $\begin{array}{l}\text { dark brown glass is multi-phased with areas of opaque green } \\
\text { near the bottom and devitrified with a small percent of } \\
\text { snowflake type crystals throughout }\end{array}$ & & amorphous & & \\
\hline IG3-19 & $\begin{array}{l}\text { brown colored glass is transparent, but devitrified with white } \\
\text { fern-like crystals forming on the bottom and sides }\end{array}$ & & La2NiO4 & & \\
\hline IG3-20 & $\begin{array}{l}\text { dark brown colored glass appears to be transparent with lots o } \\
\text { small air bubbles, but contains a small percent of clear crystal } \\
\text { clusters thoughout }\end{array}$ & & amorphous & & \\
\hline IG3-21 & $\begin{array}{l}\text { brown colored glass is single-phase and transparent, not } \\
\text { devitrified }\end{array}$ & & amorphous & & \\
\hline IG3-22 & $\begin{array}{l}\text { reddish brown colored glass is single-phase and transparent } \\
\text { with some air bubbles, not devitrified }\end{array}$ & & amorphous & & \\
\hline
\end{tabular}


Appendix A. Database - mass fraction

INEEL CVS Phase 3 (Scholes et al. 2000)

\begin{tabular}{|c|c|c|c|c|c|c|c|c|c|c|c|c|c|c|c|}
\hline Glass ID & $\begin{array}{c}\text { Heat Treated } \\
\text { XRD } \\
\end{array}$ & $\begin{array}{l}\text { Density } \\
\left(\mathrm{g} / \mathrm{cm}^{3}\right)\end{array}$ & \begin{tabular}{|c|} 
Fulc Visc \\
$\mathrm{A}$
\end{tabular} & \begin{tabular}{|c|c|} 
Fulc Visc \\
B \\
\end{tabular} & \begin{tabular}{|c|} 
Fulc Visc \\
To \\
\end{tabular} & $\begin{array}{c}\mathrm{FV} 1150^{\circ} \mathrm{C} \\
(\mathrm{Pa} \cdot \mathrm{s})\end{array}$ & $\begin{array}{c}\text { Arrh Visc } \\
\mathrm{A}\end{array}$ & $\begin{array}{c}\text { Arrh Visc } \\
\text { B } \\
\end{array}$ & $\begin{array}{c}\mathrm{Ar} \\
1150^{\circ} \mathrm{C} \\
\left(\mathrm{D}_{0.0+}\right)\end{array}$ & \begin{tabular}{|c|}
$\mathrm{T}\left({ }^{\circ} \mathrm{C}\right)$ at \\
$2 \mathrm{~Pa} \cdot \mathrm{s}$ \\
\end{tabular} & \begin{tabular}{|c|}
$\mathrm{T}\left({ }^{\circ} \mathrm{C}\right)$ at \\
$5 \mathrm{~Pa} \cdot \mathrm{s}$ \\
\end{tabular} & $\begin{array}{c}\mathrm{T}\left({ }^{\circ} \mathrm{C}\right) \text { at } \\
10 \mathrm{~Pa} \cdot \mathrm{s} \\
\end{array}$ & $\begin{array}{l}\mathrm{T} 1 \\
\left({ }^{\circ} \mathrm{C}\right) \\
\end{array}$ & $\begin{array}{c}\mathrm{V} 1 \\
(\mathrm{~Pa} \cdot \mathrm{s}) \\
\end{array}$ & $\begin{array}{c}\mathrm{T} 2 \\
\left({ }^{\circ} \mathrm{C}\right) \\
\end{array}$ \\
\hline IG3-05 & & 2.65 & & & & & -12.817 & 20915.0 & 6.56 & 1275 & & 1110 & 1158.7 & 5.66 & 1107.6 \\
\hline IG3-06 & & 2.91 & & & & & -15.390 & 24906.0 & 8.27 & 1275 & & 1135 & 1256.2 & 2.34 & 1206.3 \\
\hline IG3-07 & & 2.53 & & & & & -10.088 & 17358.0 & 8.25 & 1337 & & 1128 & 1256.8 & 3.46 & 1206.4 \\
\hline IG3-08b & & 2.71 & & & & & -12.949 & 21483.0 & 8.57 & 1302 & & 1135 & 1256.3 & 2.86 & 1206.4 \\
\hline IG3-09 & & 2.50 & & & & & -10.851 & 17520.0 & 4.31 & 1245 & & 1059 & 1158 & 3.86 & 1106.8 \\
\hline IG3-10b & & 2.81 & & & & & -13.478 & 20120.0 & 1.94 & 1147 & & 1002 & 1159 & 1.79 & 1107.7 \\
\hline IG3-11 & & 2.74 & & & & & -11.935 & 18283.0 & 2.49 & 1175 & & 1011 & 1158.8 & 2.24 & 1107.6 \\
\hline IG3-12 & & 2.87 & & & & & -16.362 & 24601.0 & 2.52 & 1169 & & 1045 & 1158.8 & 2.17 & 1107.8 \\
\hline IG3-13 & & 2.87 & & & & & -12.111 & 17109.0 & 0.92 & 1063 & & 914 & 1160.1 & 0.82 & 1108.8 \\
\hline IG3-14 & & 2.63 & & & & & -11.133 & 17804.0 & 3.97 & 1232 & & 1052 & 1158.5 & 3.59 & 1107.3 \\
\hline IG3-15 & & 2.79 & & & & & -12.660 & 19591.0 & 3.03 & 1194 & & 1036 & 1158.6 & 2.68 & 1107.5 \\
\hline IG3-16 & & 2.74 & & & & & -12.427 & 18809.0 & 2.21 & 1160 & & 1004 & 1159 & 1.98 & 1107.7 \\
\hline IG3-17 & & 2.77 & & & & & -12.267 & 18380.0 & 1.91 & 1145 & & 988 & 1157.1 & 1.76 & 1106.2 \\
\hline IG3-18 & & 2.69 & & & & & -11.520 & 17694.0 & 2.50 & 1176 & & 1007 & 1157 & 2.29 & 1106.2 \\
\hline IG3-19 & & 2.71 & & & & & -11.594 & 17476.0 & 1.99 & 1149 & & 984 & 1160.8 & 1.76 & 1108.9 \\
\hline IG3-20 & & 2.72 & & & & & -11.911 & 18117.0 & 2.27 & 1164 & & 1001 & 1160.4 & 1.99 & 1108.9 \\
\hline IG3-21 & & 2.71 & & & & & -11.878 & 18053.0 & 2.24 & 1163 & & 1000 & 1159.5 & 2.02 & 1107.6 \\
\hline IG3-22 & & 2.75 & & & & & -12.222 & 18589.0 & 2.32 & 1166 & & 1007 & 1156.2 & 2.12 & 1106.1 \\
\hline
\end{tabular}


Appendix A. Database - mass fraction

\begin{tabular}{|c|c|c|c|c|c|c|c|c|c|c|c|c|c|c|c|c|c|c|c|c|c|}
\hline Glass ID & $\begin{array}{c}\mathrm{V} 2 \\
(\mathrm{~Pa} \cdot \mathrm{s})\end{array}$ & $\begin{array}{l}\mathrm{T} 3 \\
\left({ }^{\circ} \mathrm{C}\right)\end{array}$ & $\begin{array}{c}\mathrm{V} 3 \\
(\mathrm{~Pa} \cdot \mathrm{s})\end{array}$ & $\begin{array}{l}\mathrm{T} 4 \\
\left({ }^{\circ} \mathrm{C}\right)\end{array}$ & $\begin{array}{c}\mathrm{V} 4 \\
(\mathrm{~Pa} \cdot \mathrm{s})\end{array}$ & $\begin{array}{c}\mathrm{T} 5 \\
\left({ }^{\circ} \mathrm{C}\right) \\
\end{array}$ & $\begin{array}{c}\text { V5 } \\
(\mathrm{Pa} \cdot \mathrm{s})\end{array}$ & $\begin{array}{c}\text { T6 } \\
\left({ }^{\circ} \mathrm{C}\right) \\
\end{array}$ & $\begin{array}{c}\mathrm{V} 6 \\
(\mathrm{~Pa} \cdot \mathrm{s})\end{array}$ & $\begin{array}{c}\mathrm{T} 7 \\
\left({ }^{\circ} \mathrm{C}\right) \\
\end{array}$ & $\begin{array}{c}\mathrm{V} 7 \\
(\mathrm{~Pa} \cdot \mathrm{s})\end{array}$ & $\begin{array}{c}\mathrm{T} 8 \\
\left({ }^{\circ} \mathrm{C}\right) \\
\end{array}$ & $\begin{array}{c}\mathrm{V} 8 \\
(\mathrm{~Pa} \cdot \mathrm{s})\end{array}$ & $\begin{array}{c}\mathrm{T} 9 \\
\left({ }^{\circ} \mathrm{C}\right) \\
\end{array}$ & $\begin{array}{c}\mathrm{V} 9 \\
(\mathrm{~Pa} \cdot \mathrm{s}) \\
\end{array}$ & $\begin{array}{l}\mathrm{T} 10 \\
\left({ }^{\circ} \mathrm{C}\right)\end{array}$ & $\begin{array}{c}\mathrm{V} 10 \\
(\mathrm{~Pa} \cdot \mathrm{s})\end{array}$ & $\begin{array}{l}\mathrm{T} 11 \\
\left({ }^{\circ} \mathrm{C}\right)\end{array}$ & $\begin{array}{c}\mathrm{V} 11 \\
(\mathrm{~Pa} \cdot \mathrm{s})\end{array}$ & $\begin{array}{l}\mathrm{T} 12 \\
\left({ }^{\circ} \mathrm{C}\right)\end{array}$ & $\begin{array}{c}\mathrm{V} 12 \\
(\mathrm{~Pa} \cdot \mathrm{s})\end{array}$ \\
\hline IG3-05 & 9.71 & 1057 & 17.5 & 1007.1 & 33.83 & 957.5 & 71.8 & 1058.3 & 17.33 & 1157.6 & 5.76 & 1206.5 & 3.8 & 1256 & 2.63 & 1157 & 6.28 & & & & \\
\hline IG3-06 & 3.91 & 1156.8 & 8.07 & 1157.8 & 7.79 & 1256 & 2.27 & 1305.5 & 1.49 & 1354.8 & 0.97 & 1255.4 & 2.55 & & & & & & & & \\
\hline IG3-07 & 5.07 & 1156.7 & 7.67 & 1107.1 & 11.98 & 1057.2 & 19.88 & 1157.6 & 7.66 & 1256 & 3.53 & 1305.3 & 2.51 & 1354.8 & 1.82 & 1255.7 & 3.56 & & & & \\
\hline IG3-08b & 4.53 & 1156.9 & 7.66 & 1107.3 & 13.7 & 1057.2 & 26.1 & 1157.7 & 7.87 & 1256.3 & 2.98 & 1305.5 & 1.97 & 1354.8 & 1.38 & 1255.5 & 2.97 & & & & \\
\hline IG3-09 & 5.99 & 1056.4 & 9.68 & 1006.3 & 16.81 & 956.4 & 32.59 & 1156.9 & 4.02 & 1205.9 & 2.78 & 1255.1 & 1.98 & 1155.7 & 4.11 & & & & & & \\
\hline IG3-10b & 2.94 & 1057 & 5.22 & 1006.9 & 9.58 & 957.1 & 18.7 & 1057.9 & 4.75 & 1157 & 1.72 & 1206.1 & 1.13 & 1255.4 & 0.78 & 1156 & 1.78 & & & & \\
\hline IG3-11 & 3.52 & 1056.9 & 5.9 & 1006.9 & 10.5 & 957.1 & 20.22 & 1058 & 5.86 & 1157.2 & 2.27 & 1206.2 & 1.54 & 1255.5 & 1.13 & 1156.3 & 2.3 & & & & \\
\hline IG3-12 & 3.93 & 1057.5 & 7.7 & 1007.5 & 17.16 & 957.8 & 44.46 & 1058.5 & 7.57 & 1157.7 & 2.2 & 1206.7 & 1.36 & 1255.9 & 0.89 & 1156.4 & 2.26 & & & & \\
\hline IG3-13 & 1.3 & 1058.2 & 2.11 & 1008.2 & 3.47 & 958.6 & 5.93 & 1059.1 & 2.06 & 1158.2 & 0.86 & 1207.3 & 0.58 & 1256.3 & 0.4 & 1157 & 0.88 & & & & \\
\hline IG3-14 & 5.67 & 1056.9 & 9.33 & 1007.2 & 16 & 957.6 & 29.38 & 1058.5 & 9.21 & 1157.8 & 3.6 & 1206.8 & 2.47 & 1255.9 & 1.77 & 1156.6 & 3.76 & & & & \\
\hline IG3-15 & 4.46 & 1057.1 & 7.73 & 1007.1 & 14.06 & 957.3 & 27.74 & 1057.9 & 7.54 & 1157 & 2.78 & 1206.2 & 1.82 & 1255.4 & 1.24 & 1155.9 & 2.9 & & & & \\
\hline IG3-16 & 3.16 & 1057 & 5.35 & 1006.8 & 9.64 & 957 & 18.79 & 1057.8 & 5.35 & 1157.2 & 2.05 & 1206.3 & 1.36 & 1255.4 & 0.94 & 1156 & 2.07 & & & & \\
\hline IG3-17 & 2.79 & 1055.7 & 4.69 & 1005.8 & 8.26 & 956.1 & 15.4 & 1056.4 & 4.57 & 1155.8 & 1.79 & 1205.3 & 1.19 & 1255.1 & 0.82 & 1155.6 & 1.83 & & & & \\
\hline IG3-18 & 3.56 & 1055.7 & 5.85 & 1005.8 & 10.16 & 956 & 18.75 & 1056.4 & 5.81 & 1155.9 & 2.34 & 1205.3 & 1.6 & 1254.7 & 1.12 & 1155.2 & 2.39 & & & & \\
\hline IG3-19 & 2.73 & 1058.1 & 4.49 & 1008.2 & 7.71 & 958.3 & 14.39 & 1059.5 & 4.42 & 1158.6 & 1.82 & 1207.4 & 1.26 & 1256.5 & 0.9 & 1157.1 & 1.87 & & & & \\
\hline IG3-20 & 3.13 & 1058.1 & 5.23 & 1008.1 & 9.36 & 958.3 & 17.79 & 1059.5 & 5.21 & 1158.6 & 2.07 & 1207.5 & 1.42 & 1256.6 & 1 & 1157.1 & 2.14 & & & & \\
\hline IG3-21 & 3.18 & 1056.8 & 5.27 & 1006.8 & 9.29 & 957 & 17.5 & 1058 & 5.23 & 1157.1 & 2.08 & 1206.1 & 1.41 & 1255.2 & 0.99 & 1155.7 & 2.11 & & & & \\
\hline IG3-22 & 3.36 & 1056.1 & 5.67 & 1006.2 & 10.07 & 956.5 & 19.13 & 1057.7 & 5.62 & 1157 & 2.18 & 1205.8 & 1.44 & 1254.9 & 1 & 1155.5 & 2.18 & & & & \\
\hline
\end{tabular}


Appendix A. Database - mass fraction

INEEL CVS Phase 3 (Scholes et al. 2000)

\begin{tabular}{|c|c|c|c|c|c|c|c|c|c|c|c|c|c|c|c|c|}
\hline Glass ID & $\begin{array}{l}\mathrm{T} 13 \\
\left({ }^{\circ} \mathrm{C}\right) \\
\end{array}$ & $\begin{array}{l}\text { V13 } \\
(\mathrm{Pa} \cdot \mathrm{s}) \\
\end{array}$ & $\begin{array}{l}\mathrm{T} 14 \\
\left({ }^{\circ} \mathrm{C}\right) \\
\end{array}$ & $\begin{array}{c}\text { V14 } \\
(\mathrm{Pa} \cdot \mathrm{s}) \\
\end{array}$ & $\begin{array}{c}\text { Q PCT } \\
\mathrm{B}\left(\mathrm{g} / \mathrm{m}^{2}\right)\end{array}$ & $\begin{array}{c}\text { Q PCT } \\
\mathrm{Li}\left(\mathrm{g} / \mathrm{m}^{2}\right)\end{array}$ & $\begin{array}{c}\text { Q PCT } \\
\mathrm{Na}\left(\mathrm{g} / \mathrm{m}^{2}\right)\end{array}$ & $\begin{array}{c}\text { Q PCT } \\
\mathrm{Si}\left(\mathrm{g} / \mathrm{m}^{2}\right)\end{array}$ & $\begin{array}{c}\text { Q PCT } \\
\mathrm{pH}\end{array}$ & $\begin{array}{c}\text { CCC PCT } \\
\mathrm{B}\left(\mathrm{g} / \mathrm{m}^{2}\right)\end{array}$ & $\begin{array}{l}\text { CCC PCT } \\
\mathrm{Li}\left(\mathrm{g} / \mathrm{m}^{2}\right)\end{array}$ & $\begin{array}{l}\text { CCC PCT } \\
\mathrm{Na}\left(\mathrm{g} / \mathrm{m}^{2}\right)\end{array}$ & $\begin{array}{l}\text { CCC PCT } \\
\mathrm{Si}\left(\mathrm{g} / \mathrm{m}^{2}\right)\end{array}$ & $\begin{array}{c}\text { CCC PCT } \\
\mathrm{pH} \\
\end{array}$ & $\begin{array}{l}\text { Q PCT B at } \\
20^{\circ} \mathrm{C}\left(\mathrm{g} / \mathrm{m}^{2}\right)\end{array}$ & $\begin{array}{l}\text { Q PCT Li at } \\
20^{\circ} \mathrm{C}\left(\mathrm{g} / \mathrm{m}^{2}\right)\end{array}$ \\
\hline IG3-05 & & & & & 0.200 & & 0.568 & 0.135 & 10.23 & & & & & & & \\
\hline IG3-06 & & & & & 0.194 & 0.386 & 0.270 & 0.055 & 9.87 & & & & & & & \\
\hline IG3-07 & & & & & 4.859 & & 2.617 & 0.104 & 9.00 & & & & & & & \\
\hline IG3-08b & & & & & 0.792 & 0.801 & 0.545 & 0.103 & 8.83 & & & & & & & \\
\hline IG3-09 & & & & & 0.681 & 0.965 & 0.684 & 0.346 & 9.88 & & & & & & & \\
\hline IG3-10b & & & & & 1.581 & 1.451 & 1.124 & 0.097 & 8.96 & & & & & & & \\
\hline IG3-11 & & & & & 0.313 & 0.985 & 1.825 & 0.171 & 11.56 & & & & & & & \\
\hline IG3-12 & & & & & 0.581 & & 0.699 & 0.073 & 9.16 & & & & & & & \\
\hline IG3-13 & & & & & 0.410 & 0.458 & 0.658 & 0.099 & 10.11 & & & & & & & \\
\hline IG3-14 & & & & & 0.401 & 0.540 & 0.513 & 0.175 & 10.44 & & & & & & & \\
\hline IG3-15 & & & & & 0.227 & 0.428 & 0.448 & 0.083 & 10.68 & & & & & & & \\
\hline IG3-16 & & & & & 0.444 & 0.709 & 0.713 & 0.161 & 11.13 & & & & & & & \\
\hline IG3-17 & & & & & 0.493 & 0.727 & 0.736 & 0.163 & 11.09 & & & & & & & \\
\hline IG3-18 & & & & & 0.642 & 0.735 & 1.214 & 0.243 & 11.33 & & & & & & & \\
\hline IG3-19 & & & & & 0.377 & 0.583 & 0.641 & 0.150 & 11.01 & & & & & & & \\
\hline IG3-20 & & & & & 0.315 & 0.424 & 0.421 & 0.083 & 9.95 & & & & & & & \\
\hline IG3-21 & & & & & 0.498 & 0.606 & 0.517 & 0.143 & 10.66 & & & & & & & \\
\hline IG3-22 & & & & & 0.553 & 0.613 & 1.006 & 0.185 & 11.34 & & & & & & & \\
\hline
\end{tabular}


Appendix A. Database - mass fraction

INEEL CVS Phase 3 (Scholes et al. 2000)

\begin{tabular}{|c|c|c|c|c|c|c|c|c|c|c|c|c|}
\hline Glass ID & $\begin{array}{l}\text { Q PCT Na at } \\
20^{\circ} \mathrm{C}\left(\mathrm{g} / \mathrm{m}^{2}\right)\end{array}$ & $\begin{array}{l}\text { Q PCT Si at } \\
20^{\circ} \mathrm{C}\left(\mathrm{g} / \mathrm{m}^{2}\right)\end{array}$ & $\begin{array}{c}\mathrm{QpH} \\
\text { at } 20^{\circ} \mathrm{C}\end{array}$ & $\begin{array}{c}\text { TCLP Ag } \\
(\mathrm{ppm})\end{array}$ & $\begin{array}{c}\text { TCLP As } \\
(\mathrm{ppm})\end{array}$ & $\begin{array}{c}\text { TCLP Ba } \\
(\mathrm{ppm})\end{array}$ & $\begin{array}{c}\text { TCLP Cd } \\
(\mathrm{ppm})\end{array}$ & $\begin{array}{c}\text { TCLP Cr } \\
(\mathrm{ppm})\end{array}$ & $\begin{array}{c}\text { TCLP Ni } \\
(\mathrm{ppm})\end{array}$ & $\begin{array}{c}\text { TCLP Pb } \\
(\mathrm{ppm})\end{array}$ & $\begin{array}{c}\text { TCLP Se } \\
(\mathrm{ppm})\end{array}$ & $\begin{array}{c}\text { TCLP Zn } \\
(\mathrm{ppm})\end{array}$ \\
\hline IG3-05 & & & & & & & & & & & & \\
\hline IG3-06 & & & & & & & & & & & & \\
\hline IG3-07 & & & & & & & & & & & & \\
\hline IG3-08b & & & & & & & & & & & & \\
\hline IG3-09 & & & & & & & & & & & & \\
\hline IG3-10b & & & & & & & & & & & & \\
\hline IG3-11 & & & & & & & & & & & & \\
\hline IG3-12 & & & & & & & & & & & & \\
\hline IG3-13 & & & & & & & & & & & & \\
\hline IG3-14 & & & & & & & & & & & & \\
\hline IG3-15 & & & & & & & & & & & & \\
\hline IG3-16 & & & & & & & & & & & & \\
\hline IG3-17 & & & & & & & & & & & & \\
\hline IG3-18 & & & & & & & & & & & & \\
\hline IG3-19 & & & & & & & & & & & & \\
\hline IG3-20 & & & & & & & & & & & & \\
\hline IG3-21 & & & & & & & & & & & & \\
\hline IG3-22 & & & & & & & & & & & & \\
\hline
\end{tabular}


INEEL CVS Phase 3 (Scholes et al. 2000)

\begin{tabular}{|c|c|c|c|c|c|c|c|c|c|c|c|c|c|c|c|c|c|c|c|c|c|}
\hline Glass ID & $\begin{array}{c}\mathrm{A} 12 \mathrm{O} 3 \\
-\mathrm{t}\end{array}$ & $\begin{array}{c}\mathrm{B} 2 \mathrm{O} 3 \\
-\mathrm{t}\end{array}$ & $\begin{array}{c}\mathrm{CaO} \\
-\mathrm{t}\end{array}$ & $\begin{array}{c}\mathrm{Fe} 2 \mathrm{O} 3 \\
-\mathrm{t}\end{array}$ & $\begin{array}{c}\mathrm{FeO} \\
-\mathrm{t}\end{array}$ & $\begin{array}{c}\mathrm{K} 2 \mathrm{O} \\
-\mathrm{t}\end{array}$ & $\begin{array}{c}\mathrm{Li} 2 \mathrm{O} \\
-\mathrm{t}\end{array}$ & $\begin{array}{c}\mathrm{MgO} \\
-\mathrm{t}\end{array}$ & $\begin{array}{c}\mathrm{Na} 2 \mathrm{O} \\
-\mathrm{t}\end{array}$ & $\begin{array}{c}\mathrm{NiO} \\
-\mathrm{t}\end{array}$ & $\begin{array}{c}\mathrm{P} 2 \mathrm{O} 5 \\
-\mathrm{t}\end{array}$ & $\begin{array}{c}\mathrm{SiO} 2 \\
-\mathrm{t}\end{array}$ & $\begin{array}{c}\mathrm{ZrO} 2 \\
-\mathrm{t}\end{array}$ & $\begin{array}{c}\mathrm{Ag} 2 \mathrm{O} \\
-\mathrm{t}\end{array}$ & $\begin{array}{c}\mathrm{As} 2 \mathrm{O} 3 \\
-\mathrm{t}\end{array}$ & $\begin{array}{c}\mathrm{BaO} \\
-\mathrm{t}\end{array}$ & $\begin{array}{c}\mathrm{Bi} 2 \mathrm{O} 3 \\
-\mathrm{t}\end{array}$ & $\begin{array}{l}\mathrm{Br} \\
-\mathrm{t}\end{array}$ & $\begin{array}{c}\mathrm{CdO} \\
-\mathrm{t}\end{array}$ & $\begin{array}{c}\mathrm{Ce} 2 \mathrm{O} 3 \\
-\mathrm{t}\end{array}$ & $\begin{array}{c}\mathrm{CeO} 2 \\
-\mathrm{t}\end{array}$ \\
\hline IG3-23 & 0.1200 & 0.0600 & 0.0400 & 0.0050 & & 0.0050 & 0.0300 & 0.0024 & 0.1497 & 0.0100 & 0.0050 & 0.4478 & 0.0400 & & & 0.0000 & & & & 0.0001 & \\
\hline IG3-24 & 0.1200 & 0.0600 & 0.0400 & 0.0050 & & 0.0050 & 0.0319 & 0.0024 & 0.1500 & 0.0100 & 0.0050 & 0.4506 & 0.0400 & & & 0.0000 & & & & 0.0001 & \\
\hline IG3-25 & 0.1200 & 0.0600 & 0.0400 & 0.0050 & & 0.0050 & 0.0300 & 0.0024 & 0.1441 & 0.0050 & 0.0050 & 0.4434 & 0.0400 & & & 0.0000 & & & & 0.0001 & \\
\hline IG3-26 & 0.1200 & 0.0843 & 0.0800 & 0.0150 & & 0.0050 & 0.0300 & 0.0024 & 0.1000 & 0.0050 & 0.0150 & 0.4147 & 0.0600 & & & 0.0000 & & & & 0.0001 & \\
\hline IG3-27 & 0.0843 & 0.0917 & 0.0483 & 0.0226 & & 0.0087 & 0.0393 & 0.0024 & 0.1237 & 0.0072 & 0.0106 & 0.4173 & 0.0617 & & & 0.0000 & & & & 0.0001 & \\
\hline IG3-28 & 0.0850 & 0.0925 & 0.0487 & 0.0228 & & 0.0088 & 0.0396 & 0.0024 & 0.1246 & 0.0073 & 0.0107 & 0.4207 & 0.0622 & & & 0.0000 & & & & 0.0001 & \\
\hline IG3-29 & 0.1501 & 0.1501 & 0.0008 & 0.0005 & & 0.0000 & 0.0845 & 0.0014 & 0.0500 & 0.0000 & 0.0000 & 0.5308 & 0.0301 & & & 0.0000 & & & & 0.0000 & \\
\hline IG3-30 & 0.0375 & 0.1250 & 0.0009 & 0.0005 & & 0.0250 & 0.0631 & 0.0016 & 0.0875 & 0.0000 & 0.0125 & 0.5396 & 0.1050 & & & 0.0000 & & & & 0.0000 & \\
\hline
\end{tabular}

\section{Hanford CVS 1 (Hrma et al. 1994)}

\begin{tabular}{|c|c|c|c|c|c|c|c|c|c|c|c|c|c|c|c|c|c|}
\hline CVS1-1 & 0.0636 & 0.1142 & 0.0275 & 0.0568 & 0.0000 & 0.0376 & 0.0363 & 0.1003 & 0.0042 & 0.0007 & 0.4802 & 0.0429 & 0.0000 & 0.0007 & 0.0000 & 0.0055 & 0.0011 \\
\hline CVS1-2 & 0.1500 & 0.0500 & 0.1000 & 0.0200 & 0.0000 & 0.0700 & 0.0000 & 0.0500 & 0.0010 & 0.0002 & 0.5500 & 0.0000 & 0.0000 & 0.0002 & 0.0000 & 0.0013 & 0.0003 \\
\hline CVS1-3 & 0.1400 & 0.2000 & 0.0000 & 0.0200 & 0.0000 & 0.0700 & 0.0800 & 0.0500 & 0.0010 & 0.0002 & 0.4200 & 0.0100 & 0.0000 & 0.0002 & 0.0000 & 0.0013 & 0.0003 \\
\hline CVS1-4 & 0.0000 & 0.2000 & 0.0200 & 0.0200 & 0.0000 & 0.0100 & 0.0800 & 0.0900 & 0.0010 & 0.0002 & 0.5700 & 0.0000 & 0.0000 & 0.0002 & 0.0000 & 0.0013 & 0.0003 \\
\hline CVS1-5 & 0.0800 & 0.0500 & 0.0000 & 0.1500 & 0.0000 & 0.0700 & 0.0000 & 0.0700 & 0.0010 & 0.0002 & 0.5700 & 0.0000 & 0.0000 & 0.0002 & 0.0000 & 0.0013 & 0.0003 \\
\hline CVS1-6 & 0.0000 & 0.2001 & 0.0000 & 0.0200 & 0.0000 & 0.0700 & 0.0000 & 0.0500 & 0.0103 & 0.0018 & 0.4402 & 0.1200 & 0.0000 & 0.0018 & 0.0000 & 0.0134 & 0.0027 \\
\hline CVS1-7 & 0.0000 & 0.0500 & 0.1000 & 0.0336 & 0.0000 & 0.0100 & 0.0000 & 0.0964 & 0.0010 & 0.0002 & 0.5700 & 0.1300 & 0.0000 & 0.0002 & 0.0000 & 0.0013 & 0.0003 \\
\hline CVS1-8 & 0.0000 & 0.0500 & 0.0000 & 0.1500 & 0.0000 & 0.0100 & 0.0800 & 0.0837 & 0.0010 & 0.0002 & 0.5363 & 0.0800 & 0.0000 & 0.0002 & 0.0000 & 0.0013 & 0.0003 \\
\hline CVS1-9 & 0.0000 & 0.1963 & 0.0000 & 0.1401 & 0.0000 & 0.0100 & 0.0800 & 0.0538 & 0.0103 & 0.0018 & 0.4202 & 0.0000 & 0.0000 & 0.0018 & 0.0000 & 0.0134 & 0.0027 \\
\hline CVS1-10 & 0.1201 & 0.0851 & 0.0000 & 0.0200 & 0.0000 & 0.0100 & 0.0000 & 0.0949 & 0.0103 & 0.0018 & 0.5703 & 0.0000 & 0.0000 & 0.0018 & 0.0000 & 0.0134 & 0.0027 \\
\hline CVS1-11 & 0.1401 & 0.1550 & 0.1000 & 0.0200 & 0.0000 & 0.0100 & 0.0000 & 0.0751 & 0.0082 & 0.0014 & 0.4202 & 0.0000 & 0.0000 & 0.0014 & 0.0000 & 0.0107 & 0.0022 \\
\hline CVS1-12 & 0.0000 & 0.1764 & 0.1000 & 0.1500 & 0.0000 & 0.0700 & 0.0000 & 0.0736 & 0.0010 & 0.0002 & 0.4200 & 0.0000 & 0.0000 & 0.0002 & 0.0000 & 0.0013 & 0.0003 \\
\hline CVS1-13 & 0.0038 & 0.2000 & 0.0000 & 0.0200 & 0.0000 & 0.0100 & 0.0000 & 0.1862 & 0.0010 & 0.0002 & 0.5700 & 0.0000 & 0.0000 & 0.0002 & 0.0000 & 0.0013 & 0.0003 \\
\hline CVS1-14 & 0.0238 & 0.2000 & 0.0000 & 0.0200 & 0.0000 & 0.0100 & 0.0000 & 0.1862 & 0.0010 & 0.0002 & 0.4200 & 0.1300 & 0.0000 & 0.0002 & 0.0000 & 0.0013 & 0.0003 \\
\hline CVS1-15 & 0.0000 & 0.0500 & 0.0000 & 0.0200 & 0.0000 & 0.0700 & 0.0800 & 0.1212 & 0.0103 & 0.0018 & 0.5592 & 0.0000 & 0.0000 & 0.0018 & 0.0000 & 0.0134 & 0.0027 \\
\hline
\end{tabular}


Appendix A. Database - mass fraction

INEEL CVS Phase 3 (Scholes et al. 2000)

\begin{tabular}{|c|c|c|c|c|c|c|c|c|c|c|c|c|c|c|c|c|c|c|c|c|c|}
\hline Glass ID & $\begin{array}{l}\mathrm{Cl} \\
-\mathrm{t}\end{array}$ & $\begin{array}{c}\mathrm{CoO} \\
-\mathrm{t}\end{array}$ & $\begin{array}{c}\mathrm{Co} 2 \mathrm{O} 3 \\
-\mathrm{t}\end{array}$ & $\begin{array}{c}\mathrm{Cr} 2 \mathrm{O} 3 \\
-\mathrm{t}\end{array}$ & $\begin{array}{c}\mathrm{Cs} 2 \mathrm{O} \\
-\mathrm{t}\end{array}$ & $\begin{array}{c}\mathrm{CuO} \\
-\mathrm{t}\end{array}$ & $\begin{array}{c}\mathrm{Eu} 2 \mathrm{O} 3 \\
-\mathrm{t}\end{array}$ & $\begin{array}{l}F \\
-t\end{array}$ & $\begin{array}{c}\mathrm{Ga} 2 \mathrm{O} 3 \\
-\mathrm{t}\end{array}$ & $\begin{array}{c}\mathrm{Gd} 2 \mathrm{O} 3 \\
-\mathrm{t}\end{array}$ & $\begin{array}{c}\mathrm{HgO} \\
-\mathrm{t}\end{array}$ & $\begin{array}{l}I \\
-t\end{array}$ & $\begin{array}{c}\mathrm{La} 2 \mathrm{O} 3 \\
-\mathrm{t}\end{array}$ & $\begin{array}{c}\mathrm{MnO} 2 \\
-\mathrm{t}\end{array}$ & $\begin{array}{c}\mathrm{MnO} \\
-\mathrm{t}\end{array}$ & $\begin{array}{c}\mathrm{MoO} \\
-\mathrm{t}\end{array}$ & $\begin{array}{c}\mathrm{MoO} 3 \\
-\mathrm{t}\end{array}$ & $\begin{array}{c}\mathrm{Nb} 2 \mathrm{O} 5 \\
-\mathrm{t}\end{array}$ & $\begin{array}{c}\mathrm{Nd} 2 \mathrm{O} 3 \\
-\mathrm{t}\end{array}$ & $\begin{array}{c}\mathrm{PbO} \\
-\mathrm{t}\end{array}$ & $\begin{array}{c}\mathrm{PdO} 2 \\
-\mathrm{t}\end{array}$ \\
\hline IG3-23 & 0.0003 & & & 0.0007 & 0.0000 & & & 0.0250 & & & & 0.0000 & 0.0150 & & 0.0001 & & 0.0100 & 0.0002 & 0.0001 & 0.0000 & \\
\hline IG3-24 & 0.0003 & & & 0.0007 & 0.0000 & & & 0.0250 & & & & 0.0000 & 0.0350 & & 0.0001 & & 0.0050 & 0.0002 & 0.0001 & 0.0000 & \\
\hline IG3-25 & 0.0003 & & & 0.0007 & 0.0000 & & & 0.0250 & & & & 0.0000 & 0.0350 & & 0.0001 & & 0.0050 & 0.0002 & 0.0001 & 0.0000 & \\
\hline IG3-26 & 0.0003 & & & 0.0007 & 0.0000 & & & 0.0100 & & & & 0.0000 & 0.0350 & & 0.0001 & & 0.0100 & 0.0002 & 0.0001 & 0.0000 & \\
\hline IG3-27 & 0.0003 & & & 0.0007 & 0.0000 & & & 0.0216 & & & & 0.0000 & 0.0225 & & 0.0001 & & 0.0072 & 0.0002 & 0.0001 & 0.0000 & \\
\hline IG3-28 & 0.0003 & & & 0.0007 & 0.0000 & & & 0.0217 & & & & 0.0000 & 0.0226 & & 0.0001 & & 0.0073 & 0.0002 & 0.0001 & 0.0000 & \\
\hline IG3-29 & 0.0002 & & & 0.0004 & 0.0000 & & & 0.0000 & & & & 0.0000 & 0.0000 & & 0.0001 & & 0.0001 & 0.0001 & 0.0001 & 0.0000 & \\
\hline IG3-30 & 0.0002 & & & 0.0005 & 0.0000 & & & 0.0000 & & & & 0.0000 & 0.0000 & & 0.0001 & & 0.0000 & 0.0002 & 0.0001 & 0.0000 & \\
\hline
\end{tabular}

\section{Hanford CVS 1 (Hrma et al. 1994)}

\begin{tabular}{|c|c|c|c|c|c|c|c|c|c|c|}
\hline CVS1-1 & 0.0000 & 0.0009 & 0.0011 & 0.0011 & 0.0022 & 0.0046 & 0.0009 & 0.0022 & 0.0090 & 0.0000 \\
\hline CVS1-2 & 0.0000 & 0.0002 & 0.0003 & 0.0003 & 0.0005 & 0.0011 & 0.0002 & 0.0005 & 0.0022 & 0.0000 \\
\hline CVS1-3 & 0.0000 & 0.0002 & 0.0003 & 0.0003 & 0.0005 & 0.0011 & 0.0002 & 0.0005 & 0.0022 & 0.0000 \\
\hline CVS1-4 & 0.0000 & 0.0002 & 0.0003 & 0.0003 & 0.0005 & 0.0011 & 0.0002 & 0.0005 & 0.0022 & 0.0000 \\
\hline CVS1-5 & 0.0000 & 0.0002 & 0.0003 & 0.0003 & 0.0005 & 0.0011 & 0.0002 & 0.0005 & 0.0022 & 0.0000 \\
\hline CVS1-6 & 0.0000 & 0.0022 & 0.0027 & 0.0027 & 0.0054 & 0.0112 & 0.0022 & 0.0054 & 0.0221 & 0.0000 \\
\hline CVS1-7 & 0.0000 & 0.0002 & 0.0003 & 0.0003 & 0.0005 & 0.0011 & 0.0002 & 0.0005 & 0.0022 & 0.0000 \\
\hline CVS1-8 & 0.0000 & 0.0002 & 0.0003 & 0.0003 & 0.0005 & 0.0011 & 0.0002 & 0.0005 & 0.0022 & 0.0000 \\
\hline CVS1-9 & 0.0000 & 0.0022 & 0.0027 & 0.0027 & 0.0054 & 0.0112 & 0.0022 & 0.0054 & 0.0221 & 0.0000 \\
\hline CVS1-10 & 0.0000 & 0.0022 & 0.0027 & 0.0027 & 0.0054 & 0.0112 & 0.0022 & 0.0054 & 0.0221 & 0.0000 \\
\hline CVS1-11 & 0.0000 & 0.0018 & 0.0022 & 0.0022 & 0.0043 & 0.0090 & 0.0018 & 0.0043 & 0.0177 & 0.0000 \\
\hline CVS1-12 & 0.0000 & 0.0002 & 0.0003 & 0.0003 & 0.0005 & 0.0011 & 0.0002 & 0.0005 & 0.0022 & 0.0000 \\
\hline CVS1-13 & 0.0000 & 0.0002 & 0.0003 & 0.0003 & 0.0005 & 0.0011 & 0.0002 & 0.0005 & 0.0022 & 0.0000 \\
\hline CVS1-14 & 0.0000 & 0.0002 & 0.0003 & 0.0003 & 0.0005 & 0.0011 & 0.0002 & 0.0005 & 0.0022 & 0.0000 \\
\hline CVS1-15 & 0.0000 & 0.0022 & 0.0027 & 0.0027 & 0.0054 & 0.0112 & 0.0022 & 0.0054 & 0.0221 & 0.0000 \\
\hline
\end{tabular}


INEEL CVS Phase 3 (Scholes et al. 2000)

\begin{tabular}{|c|c|c|c|c|c|c|c|c|c|c|c|c|c|c|c|c|c|c|c|c|c|}
\hline Glass ID & $\begin{array}{c}\mathrm{PdO} \\
-\mathrm{t}\end{array}$ & $\begin{array}{c}\mathrm{Pr} 2 \mathrm{O} 3 \\
-\mathrm{t}\end{array}$ & $\begin{array}{c}\mathrm{Pr} 6 \mathrm{O} 11 \\
-\mathrm{t}\end{array}$ & $\begin{array}{c}\mathrm{Rb} 2 \mathrm{O} \\
-\mathrm{t}\end{array}$ & $\begin{array}{c}\mathrm{ReO} \\
-\mathrm{t}\end{array}$ & $\begin{array}{c}\mathrm{ReO} 2 \\
-\mathrm{t}\end{array}$ & $\begin{array}{c}\mathrm{Rh} 2 \mathrm{O} 3 \\
-\mathrm{t}\end{array}$ & $\begin{array}{c}\mathrm{RhO} 2 \\
-\mathrm{t}\end{array}$ & $\begin{array}{c}\mathrm{RuO}_{2} \\
-\mathrm{t}\end{array}$ & $\begin{array}{c}\mathrm{Sb} 2 \mathrm{O} 3 \\
-\mathrm{t}\end{array}$ & $\begin{array}{c}\mathrm{Sb} 2 \mathrm{O} 5 \\
-\mathrm{t}\end{array}$ & $\begin{array}{c}\mathrm{SeO} 2 \\
-\mathrm{t} \\
\end{array}$ & $\begin{array}{c}\mathrm{Sm} 2 \mathrm{O} 3 \\
-\mathrm{t}\end{array}$ & $\begin{array}{c}\mathrm{SnO} \\
-\mathrm{t}\end{array}$ & $\begin{array}{c}\mathrm{SnO} 2 \\
-\mathrm{t}\end{array}$ & $\begin{array}{c}\mathrm{SO} 3 \\
-\mathrm{t}\end{array}$ & $\begin{array}{c}\mathrm{SrO} \\
-\mathrm{t} \\
\end{array}$ & $\begin{array}{c}\mathrm{Tc} 2 \mathrm{O} 7 \\
-\mathrm{t}\end{array}$ & $\begin{array}{c}\mathrm{TeO} 2 \\
-\mathrm{t}\end{array}$ & $\begin{array}{c}\mathrm{ThO} 2 \\
-\mathrm{t}\end{array}$ & $\begin{array}{c}\mathrm{TiO} 2 \\
-\mathrm{t} \\
\end{array}$ \\
\hline IG3-23 & & 0.0000 & & 0.0000 & & 0.0001 & 0.0000 & & 0.0000 & & & & 0.0000 & & 0.0006 & 0.0025 & 0.0300 & & 0.0000 & & \\
\hline IG3-24 & & 0.0000 & & 0.0000 & & 0.0001 & 0.0000 & & 0.0000 & & & & 0.0000 & & 0.0006 & 0.0025 & 0.0100 & & 0.0000 & & \\
\hline IG3-25 & & 0.0000 & & 0.0000 & & 0.0001 & 0.0000 & & 0.0000 & & & & 0.0000 & & 0.0006 & 0.0025 & 0.0300 & & 0.0000 & & \\
\hline IG3-26 & & 0.0000 & & 0.0000 & & 0.0001 & 0.0000 & & 0.0000 & & & & 0.0000 & & 0.0006 & 0.0010 & 0.0100 & & 0.0000 & & \\
\hline IG3-27 & & 0.0000 & & 0.0000 & & 0.0001 & 0.0000 & & 0.0000 & & & & 0.0000 & & 0.0006 & 0.0100 & 0.0183 & & 0.0000 & & \\
\hline IG3-28 & & 0.0000 & & 0.0000 & & 0.0001 & 0.0000 & & 0.0000 & & & & 0.0000 & & 0.0006 & 0.0021 & 0.0184 & & 0.0000 & & \\
\hline IG3-29 & & 0.0000 & & 0.0000 & & 0.0000 & 0.0000 & & 0.0000 & & & & 0.0000 & & 0.0003 & 0.0000 & 0.0001 & & 0.0000 & & \\
\hline IG3-30 & & 0.0000 & & 0.0000 & & 0.0000 & 0.0000 & & 0.0000 & & & & 0.0000 & & 0.0004 & 0.0000 & 0.0002 & & 0.0000 & & \\
\hline
\end{tabular}

\section{Hanford CVS 1 (Hrma et al. 1994)}

\begin{tabular}{|c|c|c|c|c|c|c|c|c|c|c|}
\hline CVS1-1 & 0.0004 & 0.0007 & 0.0004 & 0.0004 & 0.0011 & 0.0004 & 0.0020 & 0.0007 & 0.0000 & 0.0000 \\
\hline CVS1-2 & 0.0001 & 0.0002 & 0.0001 & 0.0001 & 0.0003 & 0.0001 & 0.0005 & 0.0002 & 0.0000 & 0.0000 \\
\hline CVS1-3 & 0.0001 & 0.0002 & 0.0001 & 0.0001 & 0.0003 & 0.0001 & 0.0005 & 0.0002 & 0.0000 & 0.0000 \\
\hline CVS1-4 & 0.0001 & 0.0002 & 0.0001 & 0.0001 & 0.0003 & 0.0001 & 0.0005 & 0.0002 & 0.0000 & 0.0000 \\
\hline CVS1-5 & 0.0001 & 0.0002 & 0.0001 & 0.0001 & 0.0003 & 0.0001 & 0.0005 & 0.0002 & 0.0000 & 0.0000 \\
\hline CVS1-6 & 0.0009 & 0.0018 & 0.0009 & 0.0009 & 0.0027 & 0.0009 & 0.0049 & 0.0018 & 0.0000 & 0.0000 \\
\hline CVS1-7 & 0.0001 & 0.0002 & 0.0001 & 0.0001 & 0.0003 & 0.0001 & 0.0005 & 0.0002 & 0.0000 & 0.0000 \\
\hline CVS1-8 & 0.0001 & 0.0002 & 0.0001 & 0.0001 & 0.0003 & 0.0001 & 0.0005 & 0.0002 & 0.0000 & 0.0000 \\
\hline CVS1-9 & 0.0009 & 0.0018 & 0.0009 & 0.0009 & 0.0027 & 0.0009 & 0.0049 & 0.0018 & 0.0000 & 0.0000 \\
\hline CVS1-10 & 0.0009 & 0.0018 & 0.0009 & 0.0009 & 0.0027 & 0.0009 & 0.0049 & 0.0018 & 0.0000 & 0.0000 \\
\hline CVS1-11 & 0.0007 & 0.0014 & 0.0007 & 0.0007 & 0.0022 & 0.0007 & 0.0039 & 0.0014 & 0.0000 & 0.0000 \\
\hline CVS1-12 & 0.0001 & 0.0002 & 0.0001 & 0.0001 & 0.0003 & 0.0001 & 0.0005 & 0.0002 & 0.0000 & 0.0000 \\
\hline CVS1-13 & 0.0001 & 0.0002 & 0.0001 & 0.0001 & 0.0003 & 0.0001 & 0.0005 & 0.0002 & 0.0000 & 0.0000 \\
\hline CVS1-14 & 0.0001 & 0.0002 & 0.0001 & 0.0001 & 0.0003 & 0.0001 & 0.0005 & 0.0002 & 0.0000 & 0.0000 \\
\hline CVS1-15 & 0.0009 & 0.0018 & 0.0009 & 0.0009 & 0.0027 & 0.0009 & 0.0049 & 0.0018 & 0.0000 & 0.0000 \\
\hline
\end{tabular}




\section{INEEL CVS Phase 3 (Scholes et al. 2000)}

\begin{tabular}{|c|c|c|c|c|c|c|c|c|c|c|c|c|c|c|c|c|c|c|c|c|c|}
\hline Glass ID & $\begin{array}{c}\mathrm{T} 12 \mathrm{O} 3 \\
-\mathrm{t}\end{array}$ & $\begin{array}{c}\mathrm{U} 3 \mathrm{O} 8 \\
-\mathrm{t}\end{array}$ & $\begin{array}{c}\mathrm{UO} 2 \\
-\mathrm{t}\end{array}$ & $\begin{array}{c}\mathrm{UO} 3 \\
-\mathrm{t}\end{array}$ & $\begin{array}{c}\mathrm{V} 2 \mathrm{O} 5 \\
-\mathrm{t}\end{array}$ & $\begin{array}{c}\mathrm{WO} 3 \\
-\mathrm{t}\end{array}$ & $\begin{array}{c}\mathrm{Y} 2 \mathrm{O} 3 \\
-\mathrm{t}\end{array}$ & $\begin{array}{c}\mathrm{ZnO} \\
-\mathrm{t}\end{array}$ & $\begin{array}{c}\text { Others } \\
-t\end{array}$ & $\begin{array}{c}\text { Sum } \\
-t\end{array}$ & $\begin{array}{c}\mathrm{A} 12 \mathrm{O} 3 \\
-\mathrm{a}\end{array}$ & $\begin{array}{c}\mathrm{B} 2 \mathrm{O} 3 \\
-\mathrm{a} \\
\end{array}$ & $\begin{array}{c}\mathrm{CaO} \\
-\mathrm{a}\end{array}$ & $\begin{array}{c}\mathrm{Fe} 2 \mathrm{O} 3 \\
-\mathrm{a} \\
\end{array}$ & $\begin{array}{c}\mathrm{FeO} \\
-\mathrm{a} \\
\end{array}$ & $\begin{array}{c}\mathrm{K} 2 \mathrm{O} \\
-\mathrm{a}\end{array}$ & $\begin{array}{c}\mathrm{Li} 2 \mathrm{O} \\
-\mathrm{a} \\
\end{array}$ & $\begin{array}{c}\mathrm{MgO} \\
-\mathrm{a}\end{array}$ & $\begin{array}{c}\mathrm{Na} 2 \mathrm{O} \\
-\mathrm{a} \\
\end{array}$ & $\begin{array}{c}\mathrm{NiO} \\
-\mathrm{a} \\
\end{array}$ & $\begin{array}{c}\mathrm{P} 2 \mathrm{O} 5 \\
-\mathrm{a}\end{array}$ \\
\hline IG3-23 & & & & & & & 0.0000 & & & 1.0000 & 0.1225 & 0.0617 & 0.0442 & 0.0055 & & 0.0047 & 0.0308 & 0.0016 & 0.1513 & 0.0088 & 0.0041 \\
\hline IG3-24 & & & & & & & 0.0000 & & & 1.0000 & 0.1204 & 0.0605 & 0.0441 & 0.0050 & & 0.0054 & 0.0314 & 0.0014 & 0.1557 & 0.0093 & 0.0044 \\
\hline IG3-25 & & & & & & & 0.0000 & & & 1.0000 & 0.1241 & 0.0626 & 0.0448 & 0.0049 & & 0.0046 & 0.0316 & 0.0017 & 0.1496 & 0.0046 & 0.0046 \\
\hline IG3-26 & & & & & & & 0.0000 & & & 1.0000 & 0.1222 & 0.0866 & 0.0819 & 0.0145 & & 0.0045 & 0.0312 & 0.0006 & 0.1017 & 0.0045 & 0.0074 \\
\hline IG3-27 & & & & & & & 0.0000 & & & 1.0000 & 0.0862 & 0.0961 & 0.0542 & 0.0231 & & 0.0081 & 0.0402 & 0.0054 & 0.1302 & 0.0001 & 0.0055 \\
\hline IG3-28 & & & & & & & 0.0000 & & & 1.0000 & 0.0874 & 0.0963 & 0.0510 & 0.0248 & & 0.0097 & 0.0409 & 0.0013 & 0.1289 & 0.0068 & 0.0081 \\
\hline IG3-29 & & & & & & & 0.0000 & & & 1.0000 & 0.1213 & 0.0872 & 0.0834 & 0.0148 & & 0.0045 & 0.0308 & 0.0007 & 0.0999 & 0.0043 & 0.0087 \\
\hline IG3-30 & & & & & & & 0.0000 & & & 1.0000 & 0.0384 & 0.1257 & 0.0016 & 0.0001 & & 0.0258 & 0.0634 & 0.0002 & 0.0888 & 0.0001 & 0.0097 \\
\hline
\end{tabular}

\section{Hanford CVS 1 (Hrma et al. 1994)}

\begin{tabular}{|c|c|c|c|c|c|c|c|c|c|c|c|c|c|c|c|}
\hline CVS1-1 & 0.0000 & 0.0000 & 0.0004 & 0.0000 & 0.9999 & 0.0642 & 0.1160 & 0.0277 & 0.0566 & 0.0080 & 0.0340 & 0.0359 & 0.0891 & 0.0044 & 0.0007 \\
\hline CVS1-2 & 0.0000 & 0.0000 & 0.0001 & 0.0000 & 1.0000 & 0.1500 & 0.0511 & 0.1020 & 0.0203 & 0.0040 & 0.0615 & 0.0000 & 0.0504 & 0.0013 & 0.0002 \\
\hline CVS1-3 & 0.0000 & 0.0000 & 0.0001 & 0.0000 & 1.0000 & 0.1380 & 0.2010 & 0.0004 & 0.0202 & 0.0090 & 0.0601 & 0.0776 & 0.0511 & 0.0014 & 0.0002 \\
\hline CVS1-4 & 0.0000 & 0.0000 & 0.0001 & 0.0000 & 1.0000 & 0.0000 & 0.2000 & 0.0204 & 0.0210 & 0.0050 & 0.0086 & 0.0791 & 0.0893 & 0.0014 & 0.0002 \\
\hline CVS1-5 & 0.0000 & 0.0000 & 0.0001 & 0.0000 & 1.0000 & 0.0803 & 0.0517 & 0.0001 & 0.1490 & 0.0090 & 0.0620 & 0.0000 & 0.0641 & 0.0014 & 0.0002 \\
\hline CVS1-6 & 0.0000 & 0.0000 & 0.0009 & 0.0000 & 0.9999 & 0.0009 & 0.1920 & 0.0012 & 0.0190 & 0.0070 & 0.0616 & 0.0000 & 0.0537 & 0.0094 & 0.0018 \\
\hline CVS1-7 & 0.0000 & 0.0000 & 0.0001 & 0.0000 & 1.0000 & 0.0010 & 0.0507 & 0.1000 & 0.0334 & 0.0070 & 0.0087 & 0.0000 & 0.0946 & 0.0014 & 0.0002 \\
\hline CVS1-8 & 0.0000 & 0.0000 & 0.0001 & 0.0000 & 1.0000 & 0.0009 & 0.0527 & 0.0003 & 0.1520 & 0.0060 & 0.0092 & 0.0807 & 0.0780 & 0.0015 & 0.0002 \\
\hline CVS1-9 & 0.0000 & 0.0000 & 0.0009 & 0.0000 & 1.0000 & 0.0000 & 0.2020 & 0.0004 & 0.1400 & 0.0000 & 0.0091 & 0.0816 & 0.0562 & 0.0106 & 0.0018 \\
\hline CVS1-10 & 0.0000 & 0.0000 & 0.0009 & 0.0000 & 1.0000 & 0.1210 & 0.0848 & 0.0005 & 0.0206 & 0.0050 & 0.0087 & 0.0000 & 0.0924 & 0.0105 & 0.0018 \\
\hline CVS1-11 & 0.0000 & 0.0000 & 0.0007 & 0.0000 & 1.0001 & 0.1450 & 0.1600 & 0.1030 & 0.0206 & 0.0000 & 0.0098 & 0.0000 & 0.0730 & 0.0084 & 0.0014 \\
\hline CVS1-12 & 0.0000 & 0.0000 & 0.0001 & 0.0000 & 1.0000 & 0.0000 & 0.1810 & 0.1020 & 0.1490 & 0.0000 & 0.0703 & 0.0000 & 0.0761 & 0.0014 & 0.0002 \\
\hline CVS1-13 & 0.0000 & 0.0000 & 0.0001 & 0.0000 & 1.0000 & 0.0043 & 0.2030 & 0.0003 & 0.0205 & 0.0000 & 0.0086 & 0.0000 & 0.1740 & 0.0013 & 0.0002 \\
\hline CVS1-14 & 0.0000 & 0.0000 & 0.0001 & 0.0000 & 1.0000 & 0.0240 & 0.1960 & 0.0002 & 0.0194 & 0.0060 & 0.0093 & 0.0000 & 0.1850 & 0.0013 & 0.0002 \\
\hline CVS1-15 & 0.0000 & 0.0000 & 0.0009 & 0.0000 & 1.0000 & 0.0000 & 0.0507 & 0.0005 & 0.0201 & 0.0100 & 0.0568 & 0.0760 & 0.1120 & 0.0101 & 0.0018 \\
\hline
\end{tabular}


Appendix A. Database - mass fraction

INEEL CVS Phase 3 (Scholes et al. 2000)

\begin{tabular}{|c|c|c|c|c|c|c|c|c|c|c|c|c|c|c|c|c|c|c|c|c|c|}
\hline Glass ID & $\begin{array}{c}\mathrm{SiO} 2 \\
-\mathrm{a}\end{array}$ & $\begin{array}{c}\mathrm{ZrO} 2 \\
-\mathrm{a}\end{array}$ & $\begin{array}{c}\mathrm{Ag} 2 \mathrm{O} \\
-\mathrm{a}\end{array}$ & $\begin{array}{c}\mathrm{As} 2 \mathrm{O} 3 \\
-\mathrm{a} \\
\end{array}$ & $\begin{array}{c}\mathrm{BaO} \\
-\mathrm{a}\end{array}$ & $\begin{array}{c}\mathrm{Bi} 2 \mathrm{O} 3 \\
-\mathrm{a}\end{array}$ & $\begin{array}{l}\mathrm{Br} \\
-\mathrm{a}\end{array}$ & $\begin{array}{c}\mathrm{CdO} \\
-\mathrm{a}\end{array}$ & $\begin{array}{c}\mathrm{Ce} 2 \mathrm{O} 3 \\
-\mathrm{a}\end{array}$ & $\begin{array}{c}\mathrm{CeO} 2 \\
-\mathrm{a}\end{array}$ & $\begin{array}{l}\mathrm{Cl} \\
-\mathrm{a}\end{array}$ & $\begin{array}{c}\mathrm{CoO} \\
-\mathrm{a} \\
\end{array}$ & $\begin{array}{c}\mathrm{Co} 2 \mathrm{O} 3 \\
-\mathrm{a}\end{array}$ & $\begin{array}{c}\mathrm{Cr} 2 \mathrm{O} 3 \\
-\mathrm{a}\end{array}$ & $\begin{array}{c}\mathrm{Cs} 2 \mathrm{O} \\
-\mathrm{a} \\
\end{array}$ & $\begin{array}{c}\mathrm{CuO} \\
-\mathrm{a}\end{array}$ & $\begin{array}{c}\mathrm{Eu} 2 \mathrm{O} 3 \\
-\mathrm{a}\end{array}$ & $\begin{array}{l}\mathrm{F} \\
-\mathrm{a}\end{array}$ & $\begin{array}{c}\mathrm{Ga} 2 \mathrm{O} 3 \\
-\mathrm{a}\end{array}$ & $\begin{array}{c}\mathrm{Gd} 2 \mathrm{O} 3 \\
-\mathrm{a}\end{array}$ & $\begin{array}{c}\mathrm{HgO} \\
-\mathrm{a}\end{array}$ \\
\hline IG3-23 & 0.4658 & 0.0373 & & & 0.0000 & & & & 0.0000 & & 0.0002 & & & 0.0005 & 0.0000 & & & 0.0209 & & & \\
\hline IG3-24 & 0.4573 & 0.0382 & & & 0.0000 & & & & 0.0000 & & 0.0002 & & & 0.0004 & 0.0000 & & & 0.0205 & & & \\
\hline IG3-25 & 0.4482 & 0.0384 & & & 0.0000 & & & & 0.0000 & & 0.0002 & & & 0.0005 & 0.0000 & & & 0.0202 & & & \\
\hline IG3-26 & 0.4241 & 0.0471 & & & 0.0000 & & & & 0.0000 & & 0.0001 & & & 0.0002 & 0.0000 & & & 0.0072 & & & \\
\hline IG3-27 & 0.4268 & 0.0532 & & & 0.0001 & & & & 0.0001 & & 0.0007 & & & 0.0016 & 0.0001 & & & 0.0182 & & & \\
\hline IG3-28 & 0.4236 & 0.0559 & & & 0.0000 & & & & 0.0000 & & 0.0002 & & & 0.0004 & 0.0000 & & & 0.0180 & & & \\
\hline IG3-29 & 0.4156 & 0.0487 & & & 0.0000 & & & & 0.0000 & & 0.0001 & & & 0.0002 & 0.0000 & & & 0.0074 & & & \\
\hline IG3-30 & 0.5477 & 0.0910 & & & 0.0000 & & & & 0.0000 & & 0.0000 & & & 0.0001 & 0.0000 & & & 0.0005 & & & \\
\hline
\end{tabular}

Hanford CVS 1 (Hrma et al. 1994)

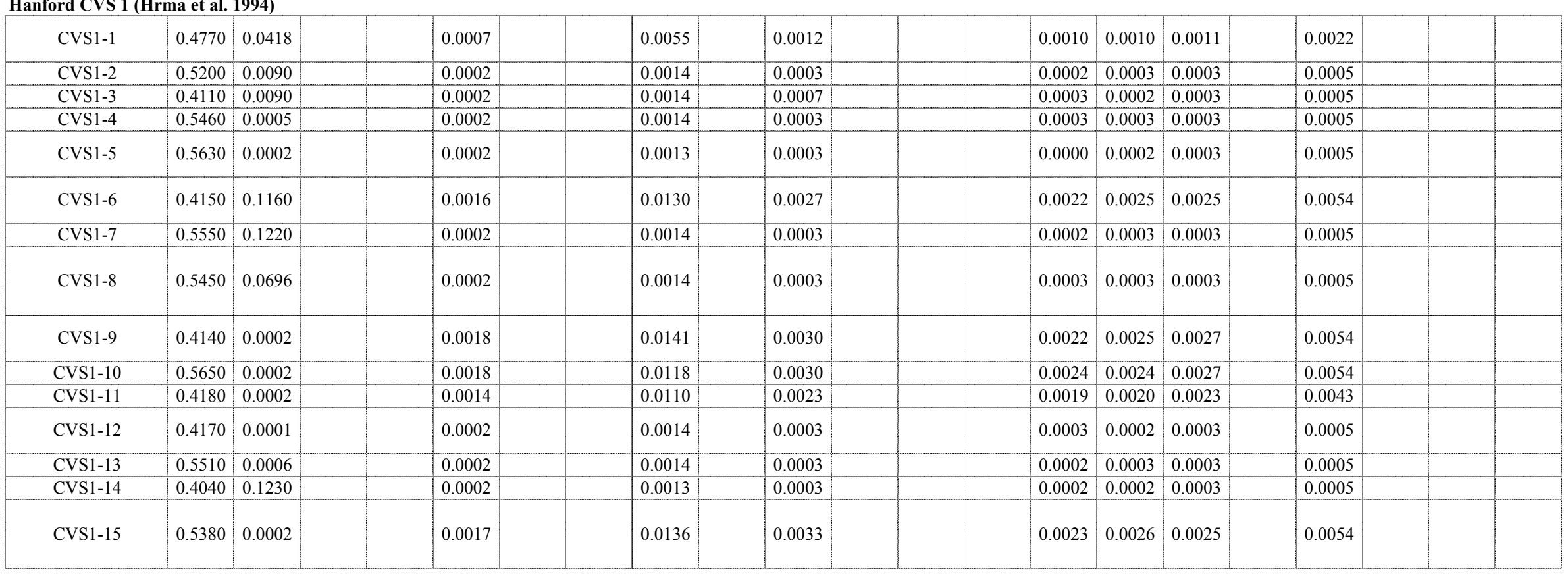


INEEL CVS Phase 3 (Scholes et al. 2000)

\begin{tabular}{|c|c|c|c|c|c|c|c|c|c|c|c|c|c|c|c|c|c|c|c|c|c|}
\hline Glass ID & $\begin{array}{c}\mathrm{I} \\
-\mathrm{a} \\
\end{array}$ & $\begin{array}{c}\mathrm{La} 2 \mathrm{O} 3 \\
-\mathrm{a}\end{array}$ & $\begin{array}{c}\mathrm{MnO} 2 \\
-\mathrm{a}\end{array}$ & $\begin{array}{c}\mathrm{MnO} \\
-\mathrm{a}\end{array}$ & $\begin{array}{c}\mathrm{MoO} \\
-\mathrm{a}\end{array}$ & $\begin{array}{c}\mathrm{MoO} 3 \\
-\mathrm{a} \\
\end{array}$ & $\begin{array}{c}\mathrm{Nb} 2 \mathrm{O} 5 \\
-\mathrm{a}\end{array}$ & $\begin{array}{c}\mathrm{Nd} 2 \mathrm{O} 3 \\
-\mathrm{a}\end{array}$ & $\begin{array}{c}\mathrm{PbO} \\
-\mathrm{a}\end{array}$ & $\begin{array}{c}\mathrm{PdO} 2 \\
-\mathrm{a}\end{array}$ & $\begin{array}{c}\mathrm{PdO} \\
-\mathrm{a}\end{array}$ & $\begin{array}{c}\mathrm{Pr} 2 \mathrm{O} 3 \\
-\mathrm{a}\end{array}$ & $\begin{array}{c}\text { Pr6O11 } \\
-\mathrm{a}\end{array}$ & $\begin{array}{c}\mathrm{Rb} 2 \mathrm{O} \\
-\mathrm{a}\end{array}$ & $\begin{array}{c}\mathrm{ReO} \\
-\mathrm{a}\end{array}$ & $\begin{array}{c}\mathrm{ReO} 2 \\
-\mathrm{a}\end{array}$ & $\begin{array}{c}\mathrm{Rh} 2 \mathrm{O} 3 \\
-\mathrm{a}\end{array}$ & $\begin{array}{c}\mathrm{RhO} 2 \\
-\mathrm{a}\end{array}$ & $\begin{array}{c}\mathrm{RuO} 2 \\
-\mathrm{a}\end{array}$ & $\begin{array}{c}\mathrm{Sb} 2 \mathrm{O} 3 \\
-\mathrm{a}\end{array}$ & $\begin{array}{c}\mathrm{Sb} 2 \mathrm{O} 5 \\
-\mathrm{a}\end{array}$ \\
\hline IG3-23 & 0.0000 & 0.0140 & & 0.0001 & & 0.0095 & 0.0002 & 0.0001 & 0.0000 & & & 0.0000 & & 0.0000 & & 0.0000 & 0.0000 & & 0.0000 & & \\
\hline IG3-24 & 0.0000 & 0.0326 & & 0.0001 & & 0.0048 & 0.0001 & 0.0001 & 0.0000 & & & 0.0000 & & 0.0000 & & 0.0000 & 0.0000 & & 0.0000 & & \\
\hline IG3-25 & 0.0000 & 0.0328 & & 0.0001 & & 0.0048 & 0.0002 & 0.0001 & 0.0000 & & & 0.0000 & & 0.0000 & & 0.0000 & 0.0000 & & 0.0000 & & \\
\hline IG3-26 & 0.0000 & 0.0325 & & 0.0000 & & 0.0097 & 0.0001 & 0.0000 & 0.0000 & & & 0.0000 & & 0.0000 & & 0.0000 & 0.0000 & & 0.0000 & & \\
\hline IG3-27 & 0.0001 & 0.0213 & & 0.0003 & & 0.0069 & 0.0005 & 0.0002 & 0.0000 & & & 0.0001 & & 0.0001 & & 0.0001 & 0.0001 & & 0.0001 & & \\
\hline IG3-28 & 0.0000 & 0.0212 & & 0.0001 & & 0.0070 & 0.0001 & 0.0000 & 0.0000 & & & 0.0000 & & 0.0000 & & 0.0000 & 0.0000 & & 0.0000 & & \\
\hline IG3-29 & 0.0000 & 0.0315 & & 0.0000 & & 0.0094 & 0.0001 & 0.0000 & 0.0000 & & & 0.0000 & & 0.0000 & & 0.0000 & 0.0000 & & 0.0000 & & \\
\hline IG3-30 & 0.0000 & 0.0001 & & 0.0000 & & 0.0002 & 0.0000 & 0.0000 & 0.0000 & & & 0.0000 & & 0.0000 & & 0.0000 & 0.0000 & & 0.0000 & & \\
\hline
\end{tabular}

Hanford CVS 1 (Hrma et al. 1994)

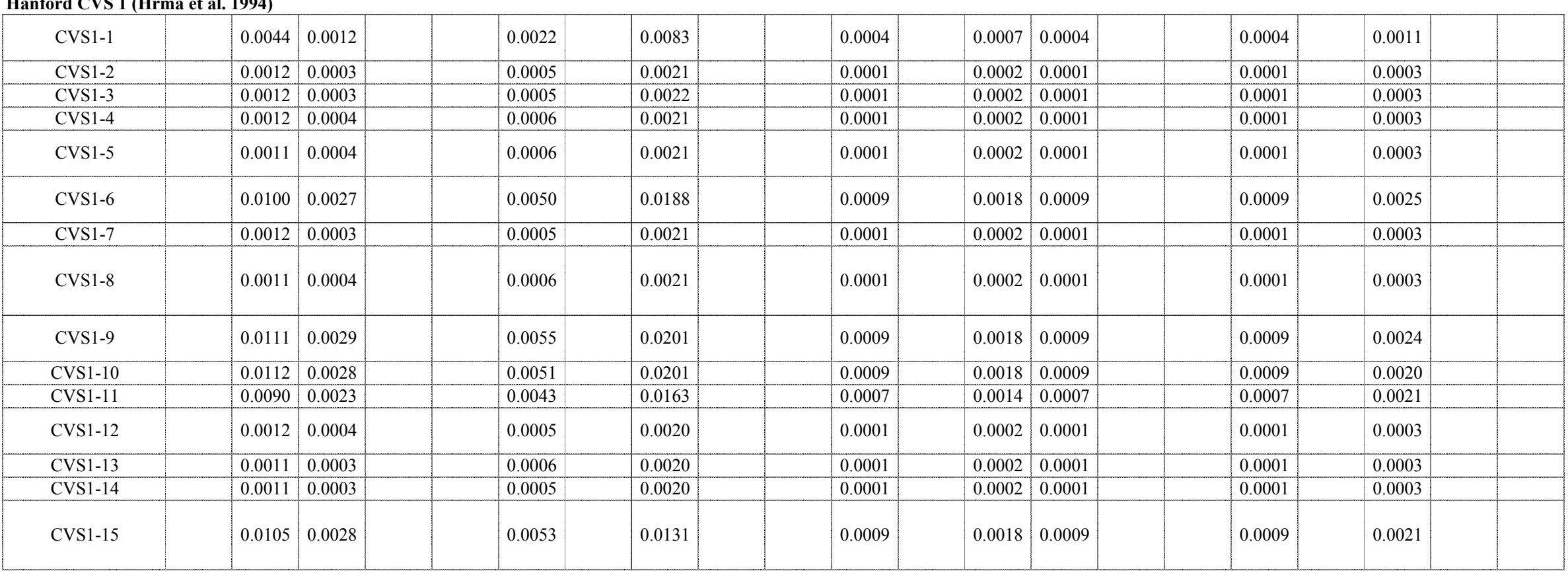


Appendix A. Database - mass fraction

INEEL CVS Phase 3 (Scholes et al. 2000)

\begin{tabular}{|c|c|c|c|c|c|c|c|c|c|c|c|c|c|c|c|c|c|c|c|c|}
\hline Glass ID & $\begin{array}{c}\mathrm{SeO} 2 \\
-\mathrm{a}\end{array}$ & $\begin{array}{c}\mathrm{Sm} 2 \mathrm{O} 3 \\
-\mathrm{a}\end{array}$ & $\begin{array}{c}\mathrm{SnO} \\
-\mathrm{a} \\
\end{array}$ & $\begin{array}{c}\mathrm{SnO} 2 \\
-\mathrm{a}\end{array}$ & $\begin{array}{c}\mathrm{SO} 3 \\
-\mathrm{a}\end{array}$ & $\begin{array}{c}\mathrm{SrO} \\
-\mathrm{a} \\
\end{array}$ & $\begin{array}{c}\mathrm{Tc} 2 \mathrm{O} 7 \\
-\mathrm{a}\end{array}$ & $\begin{array}{c}\mathrm{TeO} 2 \\
-\mathrm{a}\end{array}$ & $\begin{array}{c}\mathrm{ThO} 2 \\
-\mathrm{a}\end{array}$ & $\begin{array}{c}\mathrm{TiO} 2 \\
-\mathrm{a}\end{array}$ & $\begin{array}{c}\mathrm{T} 12 \mathrm{O} 3 \\
-\mathrm{a} \\
\end{array}$ & $\begin{array}{c}\mathrm{U} 3 \mathrm{O} 8 \\
-\mathrm{a}\end{array}$ & $\begin{array}{c}\mathrm{UO} 2 \\
-\mathrm{a}\end{array}$ & $\begin{array}{c}\mathrm{UO3} \\
-\mathrm{a} \\
\end{array}$ & $\begin{array}{c}\mathrm{V} 2 \mathrm{O} 5 \\
-\mathrm{a}\end{array}$ & $\begin{array}{c}\text { WO3 } \\
-\mathrm{a}\end{array}$ & $\begin{array}{c}\mathrm{Y} 2 \mathrm{O} 3 \\
-\mathrm{a}\end{array}$ & $\begin{array}{c}\mathrm{ZnO} \\
-\mathrm{a}\end{array}$ & $\begin{array}{c}\text { Others } \\
-\mathrm{a}\end{array}$ & $\begin{array}{c}\text { Sum } \\
-\mathrm{a}\end{array}$ \\
\hline IG3-23 & & 0.0000 & & 0.0004 & 0.0033 & 0.0278 & & 0.0000 & & & & & & & & & 0.0000 & & & 1.0154 \\
\hline IG3-24 & & 0.0000 & & 0.0004 & 0.0030 & 0.0102 & & 0.0000 & & & & & & & & & 0.0000 & & & 1.0057 \\
\hline IG3-25 & & 0.0000 & & 0.0004 & 0.0034 & 0.0284 & & 0.0000 & & & & & & & & & 0.0000 & & & 1.0110 \\
\hline IG3-26 & & 0.0000 & & 0.0002 & 0.0013 & 0.0104 & & 0.0000 & & & & & & & & & 0.0000 & & & 0.9881 \\
\hline IG3-27 & & 0.0001 & & 0.0013 & 0.0111 & 0.0180 & & 0.0000 & & & & & & & & & 0.0000 & & & 1.0102 \\
\hline IG3-28 & & 0.0000 & & 0.0003 & 0.0026 & 0.0179 & & 0.0000 & & & & & & & & & 0.0000 & & & 1.0027 \\
\hline IG3-29 & & 0.0000 & & 0.0002 & 0.0015 & 0.0101 & & 0.0000 & & & & & & & & & 0.0000 & & & 0.9804 \\
\hline IG3-30 & & 0.0000 & & 0.0000 & 0.0004 & 0.0000 & & 0.0000 & & & & & & & & & 0.0000 & & & 0.9939 \\
\hline
\end{tabular}

\section{Hanford CVS 1 (Hrma et al. 1994)}

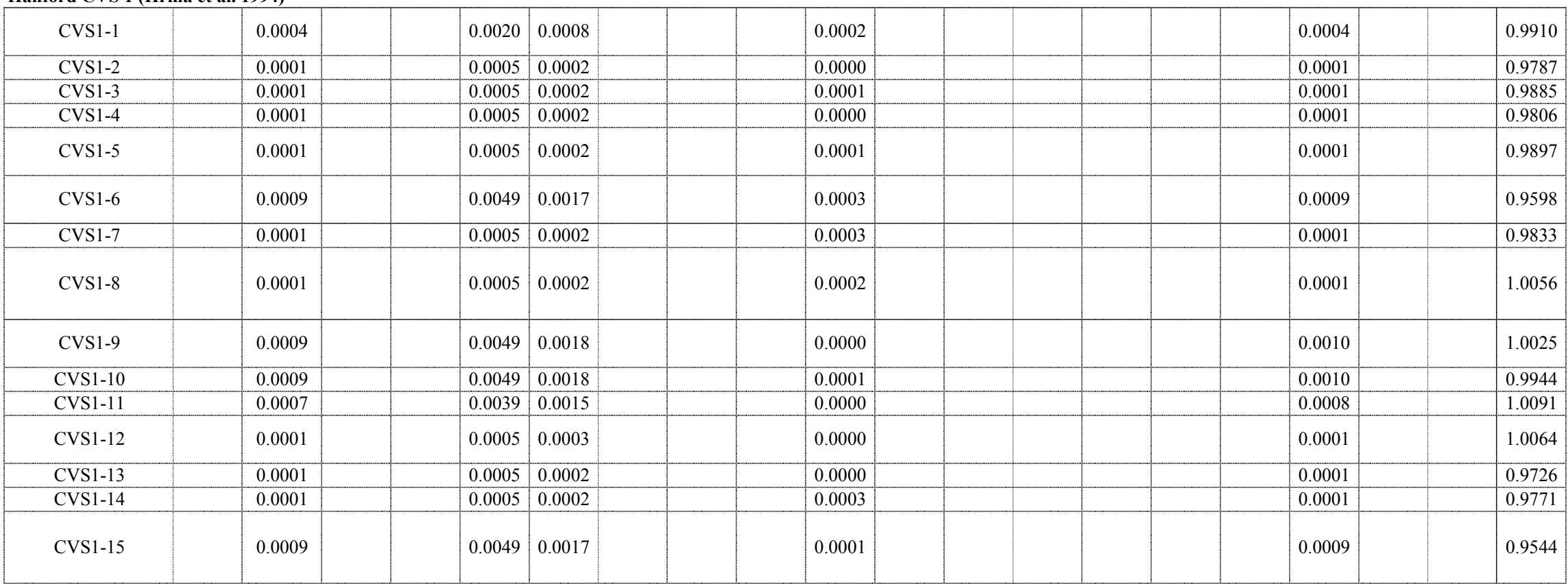


Appendix A. Database - mass fraction

INEEL CVS Phase 3 (Scholes et al. 2000)

\begin{tabular}{|c|c|c|c|c|c|c|c|c|}
\hline Glass ID & $\begin{array}{l}\mathrm{TM} \\
\left({ }^{\circ} \mathrm{C}\right)\end{array}$ & $\begin{array}{l}\text { Gradient } \\
\text { TL }\left({ }^{\circ} \mathrm{C}\right)\end{array}$ & $\begin{array}{l}\text { Uniform } \\
\text { TL }\left({ }^{\circ} \mathrm{C}\right)\end{array}$ & Primary Phase & $\begin{array}{r}\text { Quenched } \\
\text { Visual/OM }\end{array}$ & $\begin{array}{c}\text { Quenched } \\
\text { SEM/EDS or TEM }\end{array}$ & $\begin{array}{l}\text { Quenched } \\
\text { XRD }\end{array}$ & $\begin{array}{c}\text { Quenched } \\
\text { Homogeneous? }\end{array}$ \\
\hline IG3-23 & 1150 & & 903 & $\begin{array}{l}\mathrm{Na} 6 \mathrm{Ca} 2(\mathrm{Al} 16 \mathrm{Si} 6 \mathrm{O} 24)( \\
\mathrm{SO} 4) 2\end{array}$ & single-phase & & amorphous & yes \\
\hline IG3-24 & 1150 & & 883 & $\mathrm{La} 8(\mathrm{SiO} 4) 6 \mathrm{O} 2$ & single-phase & & amorphous & yes \\
\hline IG3-25 & 1150 & & 903 & $\mathrm{La} 2 \mathrm{NiO} 4$ & single-phase & & amorphous & yes \\
\hline IG3-26 & 1150 & & 1063 & $\begin{array}{l}\mathrm{Ca} 10(\mathrm{SiO} 4) 3(\mathrm{SO} 4) 3 \mathrm{~F} \\
2\end{array}$ & single-phase & & amorphous & yes \\
\hline IG3-27 & 1150 & & 953 & $\begin{array}{l}\mathrm{Ca} 10(\mathrm{SiO} 4) 3(\mathrm{SO} 4) 3 \mathrm{~F} \\
2\end{array}$ & single-phase & & amorphous & yes \\
\hline IG3-28 & 1150 & & 873 & $\begin{array}{l}\mathrm{Ca} 10(\mathrm{SiO} 4) 3(\mathrm{SO} 4) 3 \mathrm{~F} \\
2\end{array}$ & single-phase & & amorphous & yes \\
\hline IG3-29 & 1150 & & 873 & LiAlSi3O8 & single-phase & & amorphous & yes \\
\hline IG3-30 & 1150 & & 883 & $\mathrm{NaLiZrSi6O15}$ & single-phase & & amorphous & yes \\
\hline
\end{tabular}

\section{Hanford CVS 1 (Hrma et al. 1994)}

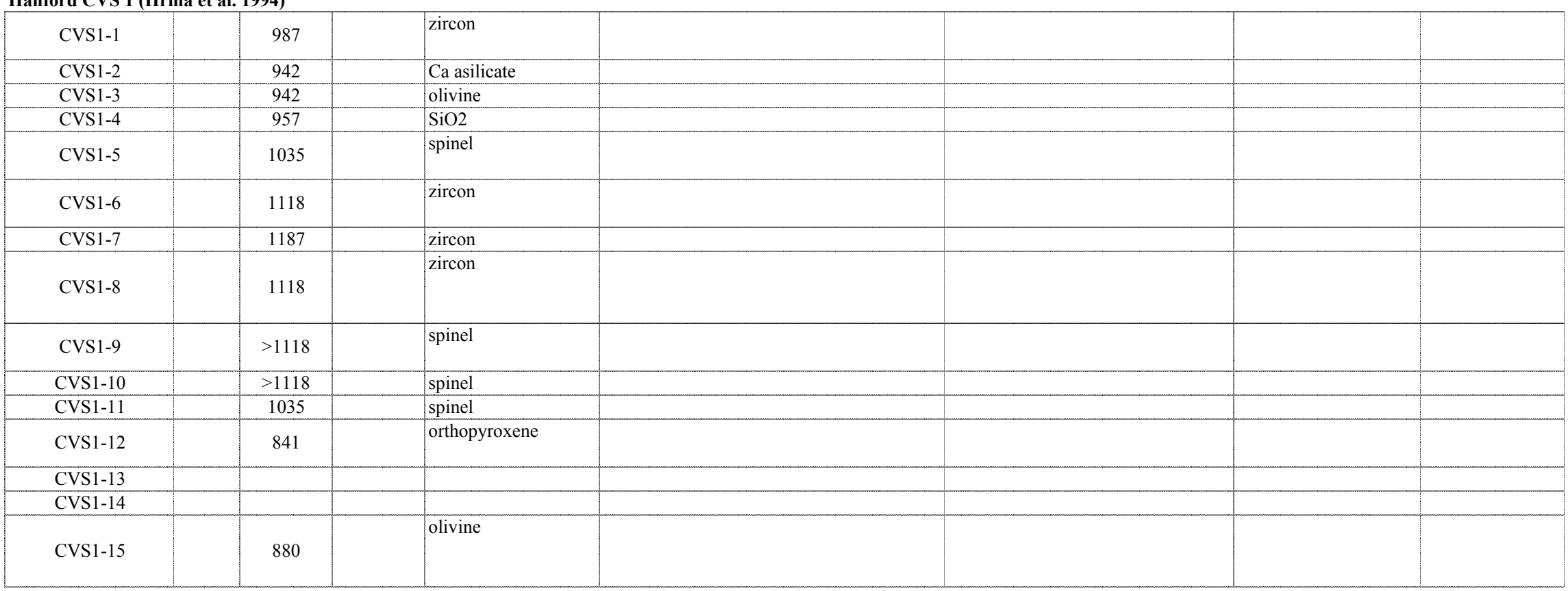


Appendix A. Database - mass fraction

INEEL CVS Phase 3 (Scholes et al. 2000)

\begin{tabular}{|c|c|c|c|c|c|}
\hline Glass ID & $\begin{array}{c}\mathrm{CCC} \\
\text { Visual/OM } \\
\end{array}$ & $\begin{array}{c}\text { CCC } \\
\text { SEM/EDS or TEM }\end{array}$ & $\begin{array}{l}\text { CCC } \\
\text { XRD }\end{array}$ & $\begin{array}{l}\text { Heat Treated } \\
\text { Visual/OM }\end{array}$ & $\begin{array}{c}\text { Heat Treated } \\
\text { SEM/EDS or TEM }\end{array}$ \\
\hline IG3-23 & $\begin{array}{l}\text { brown colored glass is multi-phased with very small granular } \\
\text { crystals and lots of small air bubbles making the glass appear } \\
\text { translucent. The top surface has a thin layer of transparent } \\
\text { glass with some small white flaky crystals just forming }\end{array}$ & & SiF4 & & \\
\hline IG3-24 & $\begin{array}{l}\text { brown colored glass is multi-phased with very small chain } \\
\text { forming granular or long flaky crystals making the glass } \\
\text { appear translucent, the top surface has a thin layer of } \\
\text { transparent glass }\end{array}$ & & amorphous & & \\
\hline IG3-25 & $\begin{array}{l}\text { green colored glass is multi-phase with very small chain- } \\
\text { forming granular or long flaky crystals and small air bubbles } \\
\text { making the glass appear translucent }\end{array}$ & & amorphous & & \\
\hline IG3-26 & $\begin{array}{l}\text { brown colored glass is completely devitrified with lighter } \\
\text { green round or rectangular snowflake-type crystals }\end{array}$ & & $(\mathrm{Ca}, \mathrm{Na}) 5(\mathrm{SiO} 4, \mathrm{PO} 4) 3 \mathrm{~F}$ & & \\
\hline IG3-27 & $\begin{array}{l}\text { green colored glass is multi-phased with very small clear } \\
\text { granular crystals and thin sheet-type crystals on the surface }\end{array}$ & & SiF4 and CaSrSi4 & & \\
\hline IG3-28 & $\begin{array}{l}\text { brown colored glass is multi-phased and transparent, with a } \\
\text { small fraction of large clear crystal clusters throughout }\end{array}$ & & amorphous & & \\
\hline IG3-29 & & & & & \\
\hline IG3-30 & & & & & \\
\hline
\end{tabular}

\section{Hanford CVS 1 (Hrma et al. 1994)}

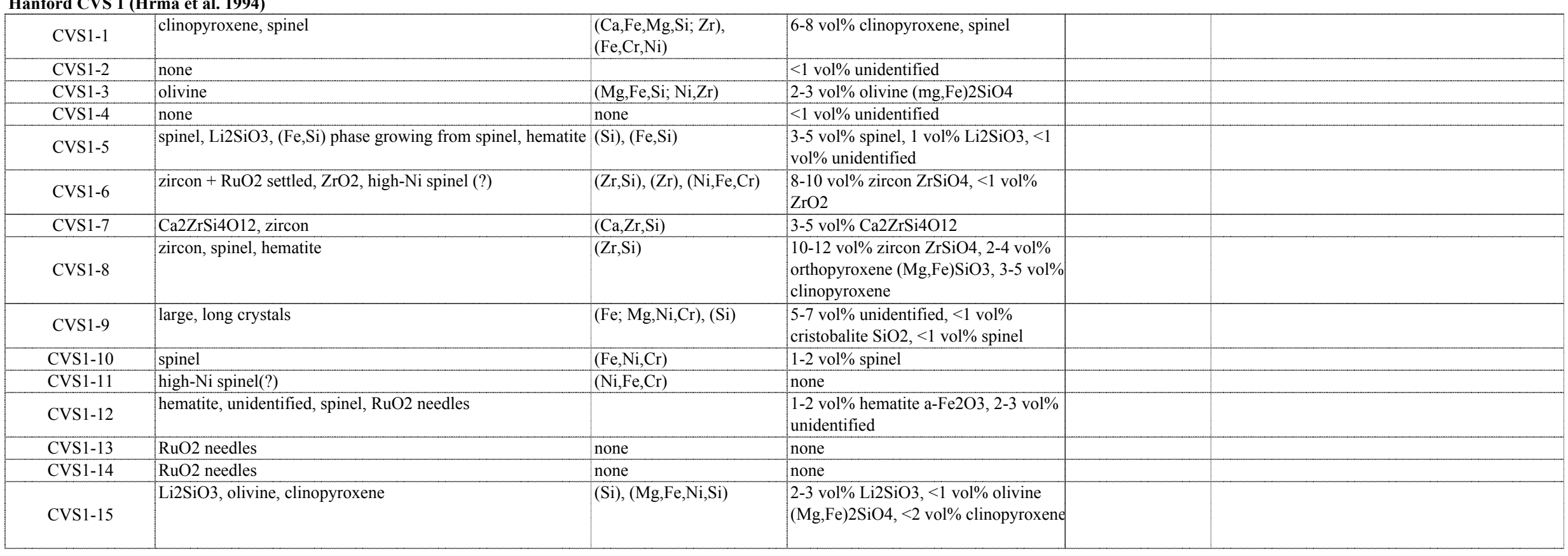


INEEL CVS Phase 3 (Scholes et al. 2000)

\begin{tabular}{|c|c|c|c|c|c|c|c|c|c|c|c|c|c|c|c|}
\hline Glass ID & $\begin{array}{c}\text { Heat Treated } \\
\text { XRD } \\
\end{array}$ & $\begin{array}{l}\text { Density } \\
\left(\mathrm{g} / \mathrm{cm}^{3}\right)\end{array}$ & $\begin{array}{c}\text { Fulc Visc } \\
\mathrm{A}\end{array}$ & $\begin{array}{c}\text { Fulc Visc } \\
\text { B } \\
\end{array}$ & $\begin{array}{c}\text { Fulc Visc } \\
\text { To } \\
\end{array}$ & $\begin{array}{c}\mathrm{FV} 1150^{\circ} \mathrm{C} \\
(\mathrm{Pa} \cdot \mathrm{s})\end{array}$ & $\begin{array}{c}\text { Arrh Visc } \\
\mathrm{A}\end{array}$ & $\begin{array}{c}\text { Arrh Visc } \\
\text { B } \\
\end{array}$ & $\begin{array}{r}1150^{\circ} \mathrm{C} \\
\text { (Dawa) }\end{array}$ & $\begin{array}{c}\mathrm{T}\left({ }^{\circ} \mathrm{C}\right) \text { at } \\
2 \mathrm{~Pa} \cdot \mathrm{s}\end{array}$ & $\begin{array}{c}\mathrm{T}\left({ }^{\circ} \mathrm{C}\right) \text { at } \\
5 \mathrm{~Pa} \cdot \mathrm{s}\end{array}$ & $\begin{array}{l}\mathrm{T}\left({ }^{\circ} \mathrm{C}\right) \text { at } \\
10 \mathrm{~Pa} \cdot \mathrm{s}\end{array}$ & $\begin{array}{l}\mathrm{T} 1 \\
\left({ }^{\circ} \mathrm{C}\right) \\
\end{array}$ & $\begin{array}{c}\mathrm{V} 1 \\
(\mathrm{~Pa} \cdot \mathrm{s}) \\
\end{array}$ & $\begin{array}{c}\mathrm{T} 2 \\
\left({ }^{\circ} \mathrm{C}\right) \\
\end{array}$ \\
\hline IG3-23 & & 2.67 & & & & & -11.539 & 19014.0 & 6.19 & 1281 & & 1101 & 1159 & 5.52 & 1107.8 \\
\hline IG3-24 & & 2.66 & & & & & -11.534 & 19016.0 & 6.23 & 1282 & & 1101 & 1158.7 & 5.56 & 1107.5 \\
\hline IG3-25 & & 2.69 & & & & & -11.723 & 19251.0 & 6.08 & 1277 & & 1099 & 1160.3 & 5.3 & 1109.1 \\
\hline IG3-26 & & 2.72 & & & & & -13.543 & 22053.0 & 7.06 & 1276 & & 1119 & 1160.6 & 6.07 & 1109.1 \\
\hline IG3-27 & & 2.70 & & & & & -11.712 & 18175.0 & 2.89 & 1192 & & 1024 & 1159 & 2.58 & 1107.9 \\
\hline IG3-28 & & 2.69 & & & & & -11.761 & 18154.0 & 2.71 & 1185 & & 1018 & 1160.1 & 2.41 & 1108.7 \\
\hline IG3-29 & & 2.43 & & & & & -10.103 & 17535.0 & 9.20 & 1351 & & 1140 & 1160 & 8.3 & 1108.7 \\
\hline IG3-30 & & 2.58 & & & & & -12.223 & 20261.0 & 7.50 & 1296 & & 1122 & 1159.3 & 6.79 & 1108.1 \\
\hline
\end{tabular}

\section{Hanford CVS 1 (Hrma et al. 1994)}

\begin{tabular}{|c|c|c|c|c|c|c|c|c|c|c|c|}
\hline CVS1-1 & 2.6839 & -5.31 & 5390.83 & 387.05 & 5.78 & -12.744 & 20714.4 & 6.13 & 1250 & 2.54 & 1150 \\
\hline CVS1-2 & 2.5501 & -5.14 & 6367.00 & 326.47 & 13.29 & -11.854 & 20617.5 & 13.94 & 1248 & 5.81 & 1149 \\
\hline CVS1-3 & 2.5131 & -6.47 & 6286.50 & 294.28 & 2.39 & -12.805 & 19435.3 & 2.35 & 1149 & 2.39 & 1050 \\
\hline CVS1-4 & 2.4818 & -7.11 & 7998.68 & 287.57 & 8.70 & -15.012 & 24398.4 & 8.45 & 1149 & 8.79 & 1051 \\
\hline CVS1-5 & 2.6185 & -5.72 & 7937.47 & 194.09 & 13.24 & -10.031 & 18036.5 & 14.07 & 1348 & 3.19 & 1250 \\
\hline CVS1-6 & 2.7439 & -6.30 & 5392.86 & 379.94 & 2.01 & & & & 1052 & 5.59 & 952 \\
\hline CVS1-7 & 2.7826 & -6.86 & 8127.95 & 420.63 & 72.88 & -15.257 & 27789.3 & 71.64 & 1348 & 6.75 & 1248 \\
\hline CVS1-8 & 2.7973 & -5.67 & 7000.00 & 375.91 & 29.26 & & & & 1350 & 4.57 & 1252 \\
\hline CVS1-9 & 2.7447 & -7.18 & 7000.00 & 333.99 & 4.06 & & & & 1251 & 1.58 & 1152 \\
\hline CVS1-10 & 2.5425 & -2.56 & 4100.51 & 563.65 & 83.83 & -10.189 & 20778.3 & 82.50 & $(1449)$ & (7.98) & 1350 \\
\hline CVS1-11 & 2.6253 & & & & & & & & 1251 & 5.68 & 1152 \\
\hline CVS1-12 & 2.7537 & -5.18 & 2995.90 & 454.08 & 0.42 & -11.558 & 15162.3 & 0.41 & 1148 & 0.42 & 1049 \\
\hline CVS1-13 & 2.541 & -4.48 & 3586.72 & 518.39 & 3.31 & -13.672 & 21274.1 & 3.59 & 1247 & 1.55 & 1148 \\
\hline CVS1-14 & 2.6836 & -5.99 & 5055.15 & 449.86 & 3.42 & -15.257 & 23573.7 & 3.70 & 1249 & 1.41 & 1150 \\
\hline CVS1-15 & 2.6913 & -5.47 & 5493.19 & 293.09 & 2.55 & -10.472 & 16285.9 & 2.65 & 1251 & 1.3 & 1151 \\
\hline
\end{tabular}


Appendix A. Database - mass fraction

INEEL CVS Phase 3 (Scholes et al. 2000)

\begin{tabular}{|c|c|c|c|c|c|c|c|c|c|c|c|c|c|c|c|c|c|c|c|c|c|}
\hline Glass ID & $\begin{array}{c}\mathrm{V} 2 \\
(\mathrm{~Pa} \cdot \mathrm{s})\end{array}$ & $\begin{array}{c}\mathrm{T} 3 \\
\left({ }^{\circ} \mathrm{C}\right) \\
\end{array}$ & $\begin{array}{c}\mathrm{V} 3 \\
(\mathrm{~Pa} \cdot \mathrm{s})\end{array}$ & $\begin{array}{c}\mathrm{T} 4 \\
\left({ }^{\circ} \mathrm{C}\right) \\
\end{array}$ & $\begin{array}{c}\mathrm{V} 4 \\
(\mathrm{~Pa} \cdot \mathrm{s})\end{array}$ & $\begin{array}{c}\mathrm{T} 5 \\
\left({ }^{\circ} \mathrm{C}\right) \\
\end{array}$ & $\begin{array}{c}\mathrm{V} 5 \\
(\mathrm{~Pa} \cdot \mathrm{s})\end{array}$ & $\begin{array}{c}\mathrm{T} 6 \\
\left({ }^{\circ} \mathrm{C}\right) \\
\end{array}$ & $\begin{array}{c}\mathrm{V} 6 \\
(\mathrm{~Pa} \cdot \mathrm{s})\end{array}$ & $\begin{array}{c}\mathrm{T} 7 \\
\left({ }^{\circ} \mathrm{C}\right) \\
\end{array}$ & $\begin{array}{c}\mathrm{V} 7 \\
(\mathrm{~Pa} \cdot \mathrm{s})\end{array}$ & $\begin{array}{c}\mathrm{T} 8 \\
\left({ }^{\circ} \mathrm{C}\right) \\
\end{array}$ & $\begin{array}{c}\mathrm{V} 8 \\
(\mathrm{~Pa} \cdot \mathrm{s})\end{array}$ & $\begin{array}{c}\text { T9 } \\
\left({ }^{\circ} \mathrm{C}\right)\end{array}$ & $\begin{array}{c}\mathrm{V} 9 \\
(\mathrm{~Pa} \cdot \mathrm{s})\end{array}$ & $\begin{array}{l}\mathrm{T} 10 \\
\left({ }^{\circ} \mathrm{C}\right) \\
\end{array}$ & $\begin{array}{c}\mathrm{V} 10 \\
(\mathrm{~Pa} \cdot \mathrm{s})\end{array}$ & $\begin{array}{l}\mathrm{T} 11 \\
\left({ }^{\circ} \mathrm{C}\right) \\
\end{array}$ & $\begin{array}{c}\mathrm{V} 11 \\
(\mathrm{~Pa} \cdot \mathrm{s})\end{array}$ & $\begin{array}{l}\mathrm{T} 12 \\
\left({ }^{\circ} \mathrm{C}\right) \\
\end{array}$ & $\begin{array}{c}\mathrm{V} 12 \\
(\mathrm{~Pa} \cdot \mathrm{s})\end{array}$ \\
\hline IG3-23 & 8.87 & 1057.4 & 15.3 & 1007.3 & 27.24 & 957.5 & 52.48 & 1058.6 & 15.56 & 1157.6 & 5.62 & 1206.3 & 3.74 & 1255.3 & 2.62 & 1156 & 5.9 & & & & \\
\hline IG3-24 & 8.99 & 1056.7 & 15.26 & 1006.7 & 27.46 & 956.9 & 54.17 & 1058 & 15.54 & 1157.1 & 5.68 & 1205.8 & 3.8 & 1254.9 & 2.65 & 1155.6 & 6.01 & & & & \\
\hline IG3-25 & 8.66 & 1058.6 & 14.75 & 1008.6 & 26.87 & 958.9 & 52.51 & 1059.8 & 15.23 & 1158.8 & 5.44 & 1207.5 & 3.62 & 1256.5 & 2.53 & 1157.3 & 5.78 & & & & \\
\hline IG3-26 & 10.56 & 1058.3 & 19.53 & 1008.3 & 38.58 & 958.6 & 84.87 & 1059.7 & 20.06 & 1158.7 & 6.32 & 1207.3 & 3.94 & 1256 & 2.61 & 1156.9 & 6.44 & & & & \\
\hline IG3-27 & 4.07 & 1057.6 & 6.76 & 1007.6 & 11.97 & 958 & 22.42 & 1058.8 & 6.76 & 1157.7 & 2.66 & 1206.6 & 1.81 & 1255.5 & 1.27 & 1156.3 & 2.72 & & & & \\
\hline IG3-28 & 3.77 & 1058 & 6.31 & 1007.9 & 11.08 & 958.1 & 21.37 & 1059.1 & 6.22 & 1158.1 & 2.48 & 1207 & 1.69 & 1256 & 1.19 & 1156.9 & 2.56 & & & & \\
\hline IG3-29 & 12.88 & 1058.1 & 20.89 & 1008.1 & 35.81 & 958.4 & 65.94 & 1059.5 & 20.78 & 1158.3 & 8.45 & 1207 & 5.8 & 1255.6 & 4.11 & 1156.6 & 8.67 & & & & \\
\hline IG3-30 & 11.14 & 1057.8 & 19.33 & 1008 & 36.12 & 958.4 & 73.65 & 1059.4 & 19.09 & 1158.3 & 6.76 & 1207 & 4.39 & 1255.7 & 2.97 & 1156.5 & 6.99 & & & & \\
\hline
\end{tabular}

\section{Hanford CVS 1 (Hrma et al. 1994)}

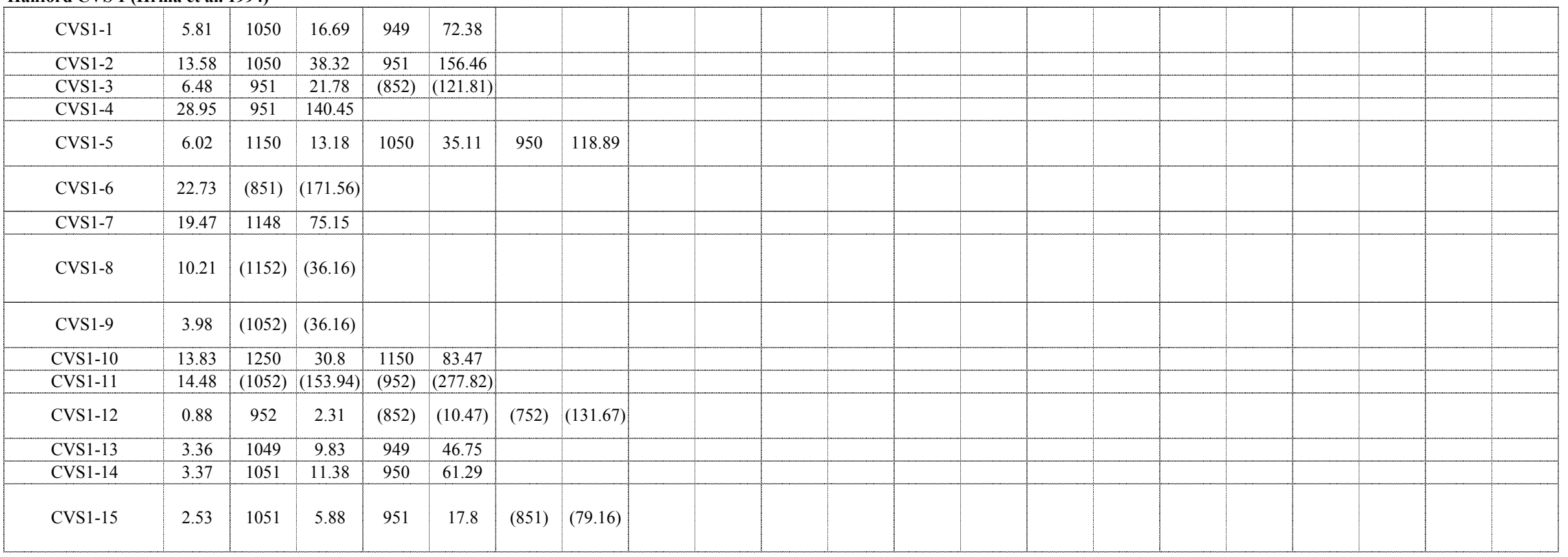


Appendix A. Database - mass fraction

INEEL CVS Phase 3 (Scholes et al. 2000)

\begin{tabular}{|c|c|c|c|c|c|c|c|c|c|c|c|c|c|c|c|c|}
\hline Glass ID & $\begin{array}{l}\mathrm{T} 13 \\
\left({ }^{\circ} \mathrm{C}\right) \\
\end{array}$ & $\begin{array}{c}\mathrm{V} 13 \\
(\mathrm{~Pa} \cdot \mathrm{s}) \\
\end{array}$ & $\begin{array}{l}\mathrm{T} 14 \\
\left({ }^{\circ} \mathrm{C}\right) \\
\end{array}$ & $\begin{array}{c}\mathrm{V} 14 \\
(\mathrm{~Pa} \cdot \mathrm{s}) \\
\end{array}$ & $\begin{array}{c}\text { Q PCT } \\
\text { B }\left(\mathrm{g} / \mathrm{m}^{2}\right)\end{array}$ & $\begin{array}{c}\text { Q PCT } \\
\mathrm{Li}\left(\mathrm{g} / \mathrm{m}^{2}\right)\end{array}$ & $\begin{array}{c}\text { Q PCT } \\
\mathrm{Na}\left(\mathrm{g} / \mathrm{m}^{2}\right) \\
\end{array}$ & $\begin{array}{c}\text { Q PCT } \\
\mathrm{Si}\left(\mathrm{g} / \mathrm{m}^{2}\right)\end{array}$ & $\begin{array}{c}\text { Q PCT } \\
\text { pH }\end{array}$ & $\begin{array}{c}\text { CCC PCT } \\
\mathrm{B}\left(\mathrm{g} / \mathrm{m}^{2}\right) \\
\end{array}$ & $\begin{array}{l}\text { CCC PCT } \\
\mathrm{Li}\left(\mathrm{g} / \mathrm{m}^{2}\right) \\
\end{array}$ & $\begin{array}{l}\text { CCC PCT } \\
\mathrm{Na}\left(\mathrm{g} / \mathrm{m}^{2}\right) \\
\end{array}$ & $\begin{array}{c}\text { CCC PCT } \\
\mathrm{Si}\left(\mathrm{g} / \mathrm{m}^{2}\right) \\
\end{array}$ & $\begin{array}{c}\text { CCC PCT } \\
\mathrm{pH}\end{array}$ & $\begin{array}{l}\text { Q PCT B at } \\
20^{\circ} \mathrm{C}\left(\mathrm{g} / \mathrm{m}^{2}\right)\end{array}$ & $\begin{array}{l}\text { Q PCT Li at } \\
20^{\circ} \mathrm{C}\left(\mathrm{g} / \mathrm{m}^{2}\right)\end{array}$ \\
\hline IG3-23 & & & & & 0.261 & 0.291 & 0.492 & 0.116 & 10.78 & & & & & & & \\
\hline IG3-24 & & & & & 0.300 & 0.325 & 0.565 & 0.135 & 10.78 & & & & & & & \\
\hline IG3-25 & & & & & 0.256 & 0.321 & 0.539 & 0.114 & 10.73 & & & & & & & \\
\hline IG3-26 & & & & & 0.164 & 0.333 & 0.288 & 0.067 & 10.42 & & & & & & & \\
\hline IG3-27 & & & & & 0.396 & 0.453 & 0.507 & 0.117 & 10.61 & & & & & & & \\
\hline IG3-28 & & & & & 0.433 & 0.460 & 0.542 & 0.126 & 10.62 & & & & & & & \\
\hline IG3-29 & & & & & 0.607 & 0.676 & 0.065 & 0.394 & 9.59 & & & & & & & \\
\hline IG3-30 & & & & & 2.129 & 1.779 & 1.154 & 0.378 & 10.29 & & & & & & & \\
\hline
\end{tabular}

\section{Hanford CVS 1 (Hrma et al. 1994)}

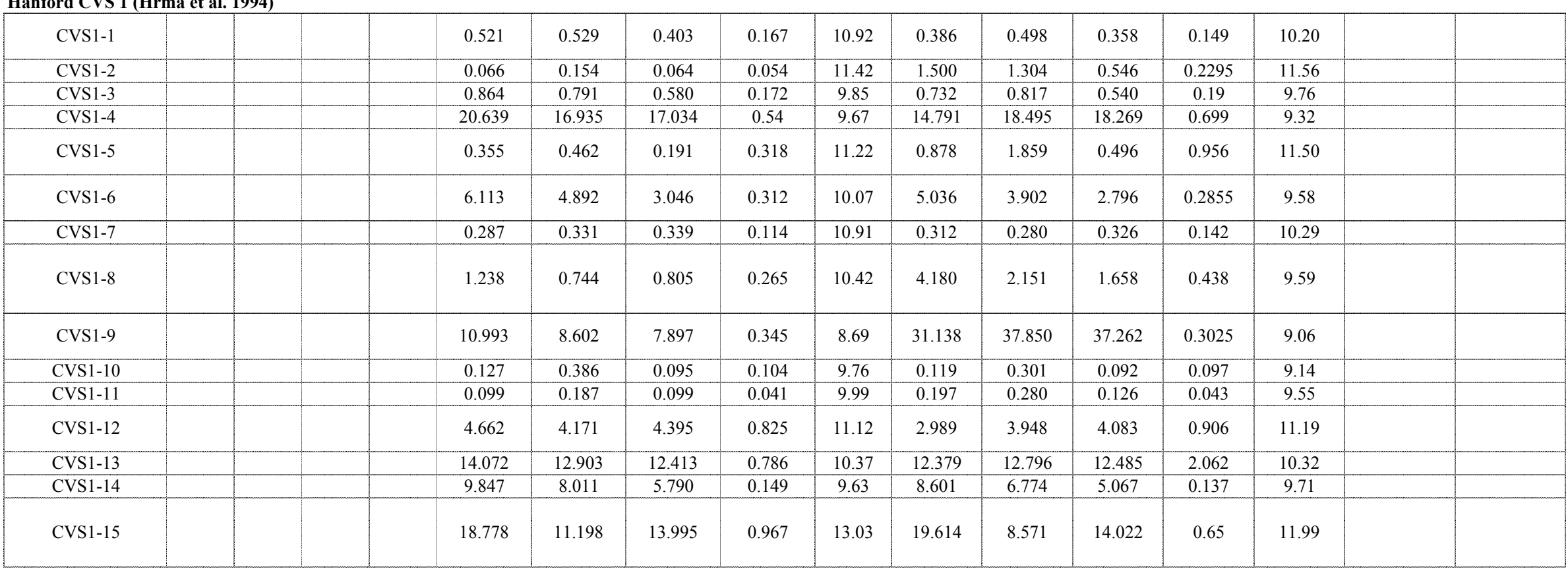


Appendix A. Database - mass fraction

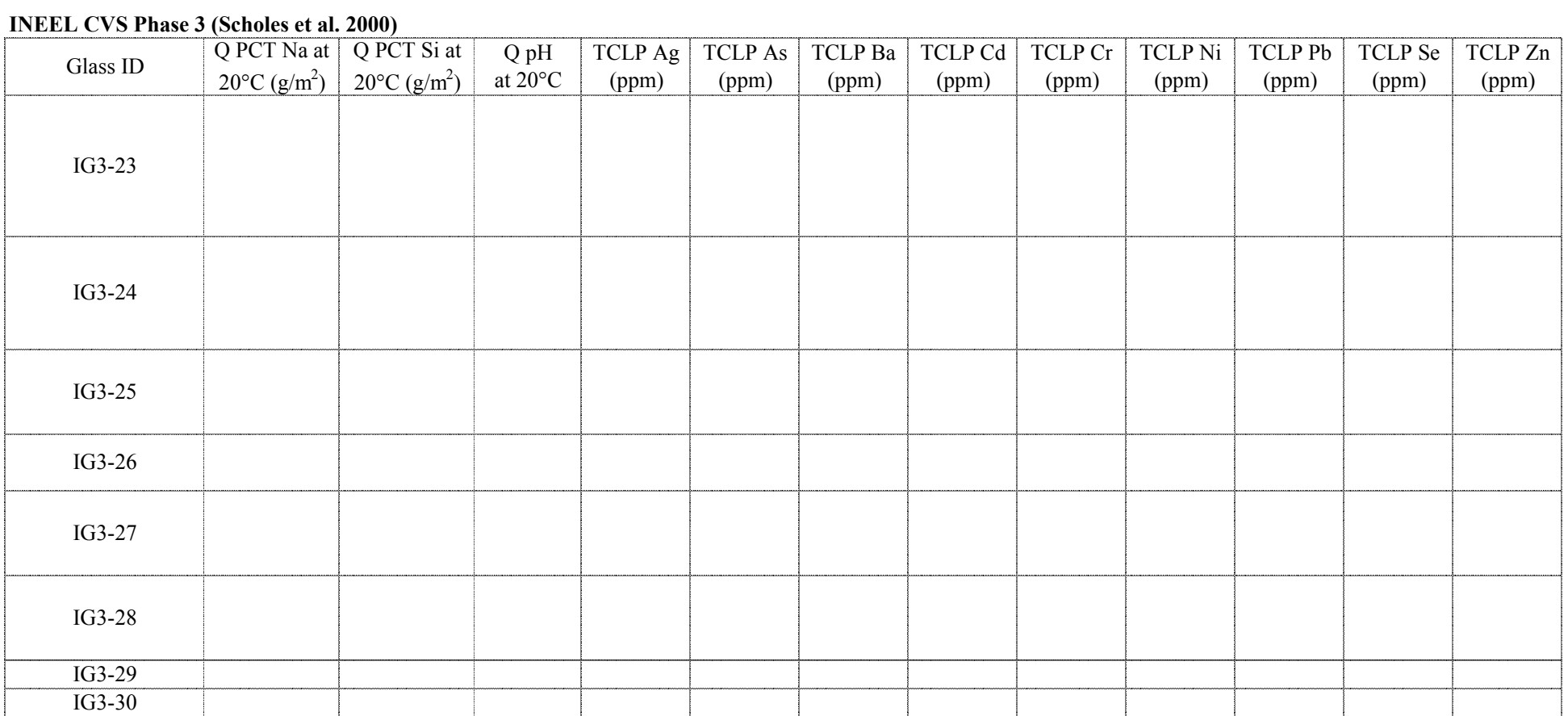

\section{Hanford CVS 1 (Hrma et al 1994)}

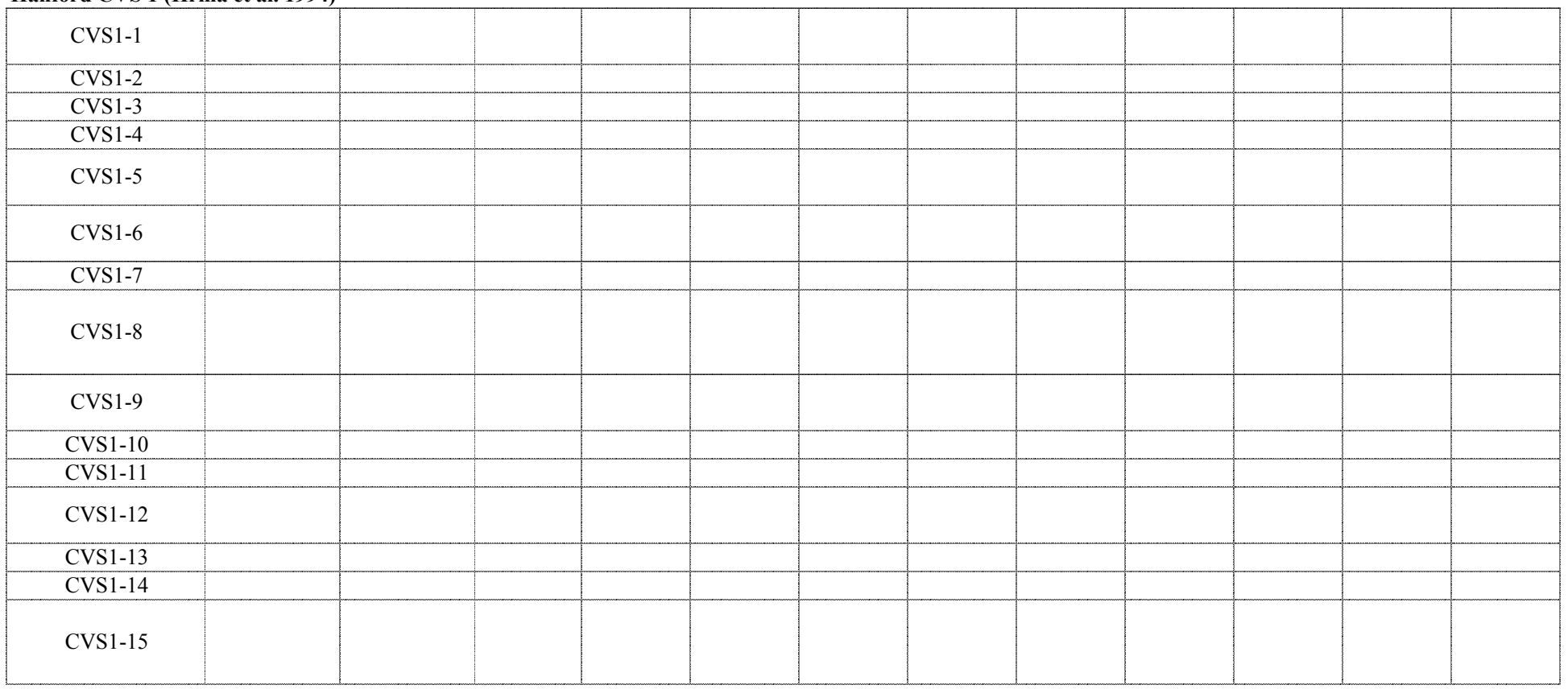


Hanford CVS 1 (Hrma et al. 1994)

\begin{tabular}{|c|c|c|c|c|c|c|c|c|c|c|c|c|c|c|c|c|c|c|c|c|c|}
\hline Glass ID & $\begin{array}{c}\mathrm{A} 12 \mathrm{O} 3 \\
-\mathrm{t}\end{array}$ & $\begin{array}{c}\mathrm{B} 2 \mathrm{O} 3 \\
-\mathrm{t}\end{array}$ & $\begin{array}{c}\mathrm{CaO} \\
-\mathrm{t} \\
\end{array}$ & $\begin{array}{c}\mathrm{Fe} 2 \mathrm{O} 3 \\
-\mathrm{t}\end{array}$ & $\begin{array}{c}\mathrm{FeO} \\
-\mathrm{t}\end{array}$ & $\begin{array}{c}\mathrm{K} 2 \mathrm{O} \\
-\mathrm{t}\end{array}$ & $\begin{array}{c}\mathrm{Li} 2 \mathrm{O} \\
-\mathrm{t}\end{array}$ & $\begin{array}{c}\mathrm{MgO} \\
-\mathrm{t}\end{array}$ & $\begin{array}{c}\mathrm{Na} 2 \mathrm{O} \\
-\mathrm{t}\end{array}$ & $\begin{array}{c}\mathrm{NiO} \\
-\mathrm{t}\end{array}$ & $\begin{array}{c}\mathrm{P} 2 \mathrm{O} 5 \\
-\mathrm{t}\end{array}$ & $\begin{array}{c}\mathrm{SiO} 2 \\
-\mathrm{t}\end{array}$ & $\begin{array}{c}\mathrm{ZrO} 2 \\
-\mathrm{t}\end{array}$ & $\begin{array}{c}\mathrm{Ag} 2 \mathrm{O} \\
-\mathrm{t}\end{array}$ & $\begin{array}{c}\mathrm{As} 2 \mathrm{O} 3 \\
-\mathrm{t}\end{array}$ & $\begin{array}{c}\mathrm{BaO} \\
-\mathrm{t}\end{array}$ & $\begin{array}{c}\mathrm{Bi} 2 \mathrm{O} 3 \\
-\mathrm{t}\end{array}$ & $\begin{array}{l}\mathrm{Br} \\
-\mathrm{t}\end{array}$ & $\begin{array}{c}\mathrm{CdO} \\
-\mathrm{t}\end{array}$ & $\begin{array}{c}\mathrm{Ce} 2 \mathrm{O} 3 \\
-\mathrm{t}\end{array}$ & $\begin{array}{c}\mathrm{CeO} 2 \\
-\mathrm{t}\end{array}$ \\
\hline CVS1-16 & 0.1442 & 0.0500 & 0.0000 & 0.0858 & & 0.0000 & 0.0100 & 0.0800 & 0.1873 & 0.0010 & 0.0002 & 0.4327 & 0.0000 & 0.0000 & & 0.0002 & 0.0000 & & 0.0013 & & 0.0003 \\
\hline CVS1-17 & 0.0000 & 0.0500 & 0.1000 & 0.1401 & & 0.0000 & 0.0100 & 0.0000 & 0.1456 & 0.0103 & 0.0018 & 0.4547 & 0.0000 & 0.0000 & & 0.0018 & 0.0000 & & 0.0134 & & 0.0027 \\
\hline CVS1-18 & 0.0000 & 0.0500 & 0.0200 & 0.0200 & & 0.0000 & 0.0700 & 0.0800 & 0.1186 & 0.0093 & 0.0016 & 0.4216 & 0.1301 & 0.0000 & & 0.0016 & 0.0000 & & 0.0121 & & 0.0024 \\
\hline CVS1-19 & 0.0636 & 0.1142 & 0.0275 & 0.0568 & & 0.0000 & 0.0376 & 0.0363 & 0.1003 & 0.0042 & 0.0007 & 0.4802 & 0.0429 & 0.0000 & & 0.0007 & 0.0000 & & 0.0055 & & 0.0011 \\
\hline CVS1-20 & 0.0636 & 0.1142 & 0.0275 & 0.0568 & & 0.0000 & 0.0376 & 0.0363 & 0.1003 & 0.0042 & 0.0007 & 0.4802 & 0.0429 & 0.0000 & & 0.0007 & 0.0000 & & 0.0055 & & 0.0011 \\
\hline CVS1-21 & 0.0000 & 0.2000 & 0.0200 & 0.0200 & & 0.0000 & 0.0100 & 0.0800 & 0.0900 & 0.0010 & 0.0002 & 0.5700 & 0.0000 & 0.0000 & & 0.0002 & 0.0000 & & 0.0013 & & 0.0003 \\
\hline CVS1-22 & 0.0000 & 0.0500 & 0.0000 & 0.1500 & & 0.0000 & 0.0100 & 0.0800 & 0.0837 & 0.0010 & 0.0002 & 0.5363 & 0.0800 & 0.0000 & & 0.0002 & 0.0000 & & 0.0013 & & 0.0003 \\
\hline CVS1-23 & 0.0456 & 0.0956 & 0.0289 & 0.1179 & & 0.0000 & 0.0375 & 0.0084 & 0.1052 & 0.0040 & 0.0007 & 0.5154 & 0.0063 & 0.0000 & & 0.0007 & 0.0000 & & 0.0053 & & 0.0011 \\
\hline
\end{tabular}

\section{Hanford CVS 2 (Hrma et al. 1994)}

\begin{tabular}{|c|c|c|c|c|c|c|c|c|c|c|c|c|c|c|c|c|c|}
\hline CVS2-1 & 0.0800 & 0.0874 & 0.0000 & 0.0400 & 0.0000 & 0.0600 & 0.0500 & 0.0700 & 0.0082 & 0.0014 & 0.5228 & 0.0100 & 0.0000 & 0.0014 & 0.0000 & 0.0107 & 0.0022 \\
\hline CVS2-2 & 0.1100 & 0.0700 & 0.0700 & 0.0450 & 0.0000 & 0.0600 & 0.0000 & 0.0883 & 0.0026 & 0.0004 & 0.5018 & 0.0300 & 0.0000 & 0.0004 & 0.0000 & 0.0034 & 0.0007 \\
\hline CVS2-3 & 0.1032 & 0.1320 & 0.0700 & 0.0450 & 0.0000 & 0.0435 & 0.0100 & 0.0700 & 0.0026 & 0.0004 & 0.4645 & 0.0368 & 0.0000 & 0.0004 & 0.0000 & 0.0034 & 0.0007 \\
\hline CVS2-4 & 0.0619 & 0.1095 & 0.0700 & 0.0400 & 0.0000 & 0.0536 & 0.0000 & 0.0700 & 0.0026 & 0.0004 & 0.5600 & 0.0100 & 0.0000 & 0.0004 & 0.0000 & 0.0034 & 0.0007 \\
\hline CVS2-5 & 0.0800 & 0.1591 & 0.0348 & 0.0400 & 0.0000 & 0.0200 & 0.0000 & 0.1010 & 0.0082 & 0.0014 & 0.4752 & 0.0100 & 0.0000 & 0.0014 & 0.0000 & 0.0107 & 0.0022 \\
\hline CVS2-6 & 0.0159 & 0.0700 & 0.0700 & 0.1200 & 0.0000 & 0.0382 & 0.0046 & 0.0700 & 0.0066 & 0.0011 & 0.5374 & 0.0100 & 0.0000 & 0.0011 & 0.0000 & 0.0086 & 0.0017 \\
\hline CVS2-7 & 0.0953 & 0.1701 & 0.0094 & 0.0400 & 0.0000 & 0.0591 & 0.0000 & 0.0700 & 0.0067 & 0.0012 & 0.4815 & 0.0100 & 0.0000 & 0.0012 & 0.0000 & 0.0087 & 0.0017 \\
\hline CVS2-8 & 0.0610 & 0.0700 & 0.0000 & 0.1140 & 0.0000 & 0.0600 & 0.0500 & 0.0985 & 0.0026 & 0.0004 & 0.5116 & 0.0100 & 0.0000 & 0.0004 & 0.0000 & 0.0034 & 0.0007 \\
\hline CVS2-9 & 0.0138 & 0.0944 & 0.0000 & 0.0712 & 0.0000 & 0.0600 & 0.0000 & 0.0924 & 0.0026 & 0.0004 & 0.5432 & 0.1000 & 0.0000 & 0.0004 & 0.0000 & 0.0034 & 0.0007 \\
\hline CVS2-10 & 0.1043 & 0.1700 & 0.0000 & 0.0669 & 0.0000 & 0.0200 & 0.0000 & 0.1306 & 0.0030 & 0.0005 & 0.4695 & 0.0100 & 0.0000 & 0.0005 & 0.0000 & 0.0039 & 0.0008 \\
\hline CVS2-11 & 0.0100 & 0.0751 & 0.0700 & 0.0400 & 0.0000 & 0.0600 & 0.0100 & 0.0833 & 0.0069 & 0.0012 & 0.4918 & 0.0935 & 0.0000 & 0.0012 & 0.0000 & 0.0089 & 0.0018 \\
\hline CVS2-12 & 0.0901 & 0.1700 & 0.0700 & 0.0400 & 0.0000 & 0.0466 & 0.0100 & 0.0700 & 0.0026 & 0.0004 & 0.4684 & 0.0100 & 0.0000 & 0.0004 & 0.0000 & 0.0034 & 0.0007 \\
\hline CVS2-13 & 0.0896 & 0.0700 & 0.0300 & 0.0400 & 0.0000 & 0.0225 & 0.0500 & 0.1692 & 0.0026 & 0.0004 & 0.4937 & 0.0100 & 0.0000 & 0.0004 & 0.0000 & 0.0034 & 0.0007 \\
\hline CVS2-14 & 0.0243 & 0.1312 & 0.0500 & 0.0400 & 0.0000 & 0.0486 & 0.0200 & 0.0802 & 0.0047 & 0.0008 & 0.4601 & 0.1000 & 0.0000 & 0.0008 & 0.0000 & 0.0061 & 0.0012 \\
\hline CVS2-15 & 0.0756 & 0.0700 & 0.0601 & 0.0400 & 0.0000 & 0.0214 & 0.0000 & 0.1701 & 0.0082 & 0.0014 & 0.4731 & 0.0100 & 0.0000 & 0.0014 & 0.0000 & 0.0107 & 0.0022 \\
\hline CVS2-16 & 0.0231 & 0.1053 & 0.0083 & 0.0719 & 0.0000 & 0.0375 & 0.0084 & 0.1125 & 0.0061 & 0.0011 & 0.5354 & 0.0385 & 0.0000 & 0.0011 & 0.0000 & 0.0080 & 0.0016 \\
\hline CVS2-17 & 0.0636 & 0.1142 & 0.0275 & 0.0568 & 0.0000 & 0.0376 & 0.0363 & 0.1003 & 0.0042 & 0.0007 & 0.4802 & 0.0429 & 0.0000 & 0.0007 & 0.0000 & 0.0055 & 0.0011 \\
\hline CVS2-18 & 0.0231 & 0.1053 & 0.0083 & 0.0719 & 0.0000 & 0.0375 & 0.0084 & 0.1125 & 0.0061 & 0.0011 & 0.5354 & 0.0385 & 0.0000 & 0.0011 & 0.0000 & 0.0080 & 0.0016 \\
\hline CVS2-19 & 0.0235 & 0.1048 & 0.0082 & 0.0733 & 0.0000 & 0.0373 & 0.0084 & 0.1129 & 0.0060 & 0.0010 & 0.5330 & 0.0392 & 0.0000 & 0.0010 & 0.0000 & 0.0065 & 0.0002 \\
\hline CVS2-20 & 0.0100 & 0.0500 & 0.0000 & 0.0600 & 0.0000 & 0.0669 & 0.0000 & 0.1031 & 0.0010 & 0.0002 & 0.5700 & 0.1300 & 0.0000 & 0.0002 & 0.0000 & 0.0013 & 0.0003 \\
\hline CVS2-21 & 0.0686 & 0.1314 & 0.0000 & 0.0200 & 0.0000 & 0.0700 & 0.0800 & 0.0500 & 0.0010 & 0.0002 & 0.5700 & 0.0000 & 0.0000 & 0.0002 & 0.0000 & 0.0013 & 0.0003 \\
\hline CVS2-22 & 0.0365 & 0.0500 & 0.0000 & 0.0200 & 0.0000 & 0.0700 & 0.0800 & 0.0735 & 0.0103 & 0.0018 & 0.5703 & 0.0000 & 0.0000 & 0.0018 & 0.0000 & 0.0134 & 0.0027 \\
\hline CVS2-23 & 0.0578 & 0.0522 & 0.0800 & 0.0200 & 0.0000 & 0.0100 & 0.0000 & 0.2000 & 0.0010 & 0.0002 & 0.5700 & 0.0000 & 0.0000 & 0.0002 & 0.0000 & 0.0013 & 0.0003 \\
\hline CVS2-24 & 0.0961 & 0.2001 & 0.0000 & 0.0200 & 0.0000 & 0.0700 & 0.0000 & 0.0736 & 0.0097 & 0.0017 & 0.4466 & 0.0000 & 0.0000 & 0.0017 & 0.0000 & 0.0126 & 0.0025 \\
\hline CVS2-25 & 0.0033 & 0.0500 & 0.0800 & 0.1500 & 0.0000 & 0.0700 & 0.0000 & 0.0841 & 0.0058 & 0.0010 & 0.5060 & 0.0000 & 0.0000 & 0.0010 & 0.0000 & 0.0076 & 0.0015 \\
\hline CVS2-26 & 0.0257 & 0.2000 & 0.0800 & 0.0200 & 0.0000 & 0.0700 & 0.0000 & 0.0512 & 0.0010 & 0.0002 & 0.4431 & 0.1000 & 0.0000 & 0.0002 & 0.0000 & 0.0013 & 0.0003 \\
\hline
\end{tabular}


Hanford CVS 1 (Hrma et al. 1994)

\begin{tabular}{|c|c|c|c|c|c|c|c|c|c|c|c|c|c|c|c|c|c|c|c|c|c|}
\hline Glass ID & $\begin{array}{l}\mathrm{Cl} \\
-\mathrm{t}\end{array}$ & $\begin{array}{c}\mathrm{CoO} \\
-\mathrm{t} \\
\end{array}$ & $\begin{array}{c}\mathrm{Co} 2 \mathrm{O} 3 \\
-\mathrm{t}\end{array}$ & $\begin{array}{c}\mathrm{Cr} 2 \mathrm{O} 3 \\
-\mathrm{t}\end{array}$ & $\begin{array}{c}\mathrm{Cs} 2 \mathrm{O} \\
-\mathrm{t}\end{array}$ & $\begin{array}{c}\mathrm{CuO} \\
-\mathrm{t}\end{array}$ & $\begin{array}{c}\mathrm{Eu} 2 \mathrm{O} 3 \\
-\mathrm{t}\end{array}$ & $\begin{array}{l}F \\
-t\end{array}$ & $\begin{array}{c}\mathrm{Ga} 2 \mathrm{O} 3 \\
-\mathrm{t}\end{array}$ & $\begin{array}{c}\mathrm{Gd} 2 \mathrm{O} 3 \\
-\mathrm{t}\end{array}$ & $\begin{array}{c}\mathrm{HgO} \\
-\mathrm{t}\end{array}$ & $\begin{array}{l}I \\
-t\end{array}$ & $\begin{array}{c}\mathrm{La} 2 \mathrm{O} 3 \\
-\mathrm{t}\end{array}$ & $\begin{array}{c}\mathrm{MnO} 2 \\
-\mathrm{t}\end{array}$ & $\begin{array}{c}\mathrm{MnO} \\
-\mathrm{t}\end{array}$ & $\begin{array}{c}\mathrm{MoO} \\
-\mathrm{t}\end{array}$ & $\begin{array}{c}\mathrm{MoO} 3 \\
-\mathrm{t}\end{array}$ & $\begin{array}{c}\mathrm{Nb} 2 \mathrm{O} 5 \\
-\mathrm{t}\end{array}$ & $\begin{array}{c}\mathrm{Nd} 2 \mathrm{O} 3 \\
-\mathrm{t}\end{array}$ & $\begin{array}{c}\mathrm{PbO} \\
-\mathrm{t}\end{array}$ & $\begin{array}{c}\mathrm{PdO} 2 \\
-\mathrm{t}\end{array}$ \\
\hline CVS1-16 & & & 0.0000 & 0.0002 & 0.0003 & 0.0003 & & 0.0005 & & & & & 0.0011 & 0.0002 & & & 0.0005 & & 0.0022 & 0.0000 & \\
\hline CVS1-17 & & & 0.0000 & 0.0022 & 0.0027 & 0.0027 & & 0.0054 & & & & & 0.0112 & 0.0022 & & & 0.0054 & & 0.0221 & 0.0000 & \\
\hline CVS1-18 & & & 0.0000 & 0.0020 & 0.0024 & 0.0024 & & 0.0048 & & & & & 0.0101 & 0.0020 & & & 0.0048 & & 0.0199 & 0.0000 & \\
\hline CVS1-19 & & & 0.0000 & 0.0009 & 0.0011 & 0.0011 & & 0.0022 & & & & & 0.0046 & 0.0009 & & & 0.0022 & & 0.0090 & 0.0000 & \\
\hline CVS1-20 & & & 0.0000 & 0.0009 & 0.0011 & 0.0011 & & 0.0022 & & & & & 0.0046 & 0.0009 & & & 0.0022 & & 0.0090 & 0.0000 & \\
\hline CVS1-21 & & & 0.0000 & 0.0002 & 0.0003 & 0.0003 & & 0.0005 & & & & & 0.0011 & 0.0002 & & & 0.0005 & & 0.0022 & 0.0000 & \\
\hline CVS1-22 & & & 0.0000 & 0.0002 & 0.0003 & 0.0003 & & 0.0005 & & & & & 0.0011 & 0.0002 & & & 0.0005 & & 0.0022 & 0.0000 & \\
\hline CVS1-23 & & & 0.0000 & 0.0009 & 0.0011 & 0.0011 & & 0.0021 & & & & & 0.0044 & 0.0009 & & & 0.0021 & & 0.0087 & 0.0000 & \\
\hline
\end{tabular}

\section{Hanford CVS 2 (Hrma et al. 1994)}

\begin{tabular}{|c|c|c|c|c|c|c|c|c|c|c|}
\hline CVS2-1 & 0.0000 & 0.0018 & 0.0022 & 0.0022 & 0.0043 & 0.0090 & 0.0018 & 0.0043 & 0.0177 & 0.0000 \\
\hline CVS2-2 & 0.0000 & 0.0006 & 0.0007 & 0.0007 & 0.0013 & 0.0028 & 0.0005 & 0.0013 & 0.0055 & 0.0000 \\
\hline CVS2-3 & 0.0000 & 0.0006 & 0.0007 & 0.0007 & 0.0013 & 0.0028 & 0.0005 & 0.0013 & 0.0055 & 0.0000 \\
\hline CVS2-4 & 0.0000 & 0.0006 & 0.0007 & 0.0007 & 0.0013 & 0.0028 & 0.0005 & 0.0013 & 0.0055 & 0.0000 \\
\hline CVS2-5 & 0.0000 & 0.0018 & 0.0022 & 0.0022 & 0.0043 & 0.0090 & 0.0018 & 0.0043 & 0.0177 & 0.0000 \\
\hline CVS2-6 & 0.0000 & 0.0014 & 0.0017 & 0.0017 & 0.0034 & 0.0072 & 0.0014 & 0.0034 & 0.0142 & 0.0000 \\
\hline CVS2-7 & 0.0000 & 0.0015 & 0.0017 & 0.0017 & 0.0035 & 0.0073 & 0.0014 & 0.0035 & 0.0143 & 0.0000 \\
\hline CVS2-8 & 0.0000 & 0.0006 & 0.0007 & 0.0007 & 0.0013 & 0.0028 & 0.0005 & 0.0013 & 0.0055 & 0.0000 \\
\hline CVS2-9 & 0.0000 & 0.0006 & 0.0007 & 0.0007 & 0.0013 & 0.0028 & 0.0005 & 0.0013 & 0.0055 & 0.0000 \\
\hline CVS2-10 & 0.0000 & 0.0006 & 0.0008 & 0.0008 & 0.0015 & 0.0032 & 0.0006 & 0.0015 & 0.0064 & 0.0000 \\
\hline CVS2-11 & 0.0000 & 0.0015 & 0.0018 & 0.0018 & 0.0036 & 0.0074 & 0.0015 & 0.0036 & 0.0147 & 0.0000 \\
\hline CVS2-12 & 0.0000 & 0.0006 & 0.0007 & 0.0007 & 0.0013 & 0.0028 & 0.0005 & 0.0013 & 0.0055 & 0.0000 \\
\hline CVS2-13 & 0.0000 & 0.0006 & 0.0007 & 0.0007 & 0.0013 & 0.0028 & 0.0005 & 0.0013 & 0.0055 & 0.0000 \\
\hline CVS2-14 & 0.0000 & 0.0010 & 0.0012 & 0.0012 & 0.0025 & 0.0051 & 0.0010 & 0.0025 & 0.0101 & 0.0000 \\
\hline CVS2-15 & 0.0000 & 0.0018 & 0.0022 & 0.0022 & 0.0043 & 0.0090 & 0.0018 & 0.0043 & 0.0177 & 0.0000 \\
\hline CVS2-16 & 0.0000 & 0.0013 & 0.0016 & 0.0016 & 0.0032 & 0.0066 & 0.0013 & 0.0032 & 0.0131 & 0.0000 \\
\hline CVS2-17 & 0.0000 & 0.0009 & 0.0011 & 0.0011 & 0.0022 & 0.0046 & 0.0009 & 0.0022 & 0.0090 & 0.0000 \\
\hline CVS2-18 & 0.0000 & 0.0013 & 0.0016 & 0.0016 & 0.0032 & 0.0066 & 0.0013 & 0.0032 & 0.0131 & 0.0000 \\
\hline CVS2-19 & 0.0000 & 0.0013 & 0.0015 & 0.0016 & 0.0019 & 0.0155 & 0.0013 & 0.0031 & 0.0057 & 0.0000 \\
\hline CVS2-20 & 0.0000 & 0.0002 & 0.0003 & 0.0003 & 0.0005 & 0.0011 & 0.0002 & 0.0005 & 0.0022 & 0.0000 \\
\hline CVS2-21 & 0.0000 & 0.0002 & 0.0003 & 0.0003 & 0.0005 & 0.0011 & 0.0002 & 0.0005 & 0.0022 & 0.0000 \\
\hline CVS2-22 & 0.0000 & 0.0022 & 0.0027 & 0.0027 & 0.0054 & 0.0112 & 0.0022 & 0.0054 & 0.0221 & 0.0000 \\
\hline CVS2-23 & 0.0000 & 0.0002 & 0.0003 & 0.0003 & 0.0005 & 0.0011 & 0.0002 & 0.0005 & 0.0022 & 0.0000 \\
\hline CVS2-24 & 0.0000 & 0.0021 & 0.0025 & 0.0025 & 0.0050 & 0.0105 & 0.0021 & 0.0050 & 0.0208 & 0.0000 \\
\hline CVS2-25 & 0.0000 & 0.0013 & 0.0015 & 0.0015 & 0.0030 & 0.0063 & 0.0012 & 0.0030 & 0.0125 & 0.0000 \\
\hline CVS2-26 & 0.0000 & 0.0002 & 0.0003 & 0.0003 & 0.0005 & 0.0011 & 0.0002 & 0.0005 & 0.0022 & 0.0000 \\
\hline
\end{tabular}


Hanford CVS 1 (Hrma et al. 1994)

\begin{tabular}{|c|c|c|c|c|c|c|c|c|c|c|c|c|c|c|c|c|c|c|c|c|c|}
\hline Glass ID & $\begin{array}{c}\mathrm{PdO} \\
-\mathrm{t}\end{array}$ & $\begin{array}{c}\mathrm{Pr} 2 \mathrm{O} 3 \\
-\mathrm{t}\end{array}$ & $\begin{array}{c}\text { Pr6O11 } \\
-t\end{array}$ & $\begin{array}{c}\mathrm{Rb} 2 \mathrm{O} \\
-\mathrm{t} \\
\end{array}$ & $\begin{array}{c}\mathrm{ReO} \\
-\mathrm{t}\end{array}$ & $\begin{array}{c}\mathrm{ReO} 2 \\
-\mathrm{t}\end{array}$ & $\begin{array}{c}\mathrm{Rh} 2 \mathrm{O} 3 \\
-\mathrm{t}\end{array}$ & $\begin{array}{c}\mathrm{RhO}^{2} \\
-\mathrm{t}\end{array}$ & $\begin{array}{c}\mathrm{RuO} 2 \\
-\mathrm{t}\end{array}$ & $\begin{array}{c}\mathrm{Sb} 2 \mathrm{O} 3 \\
-\mathrm{t}\end{array}$ & $\begin{array}{c}\mathrm{Sb} 2 \mathrm{O} 5 \\
-\mathrm{t}\end{array}$ & $\begin{array}{c}\mathrm{SeO} 2 \\
-\mathrm{t} \\
\end{array}$ & $\begin{array}{c}\mathrm{Sm} 2 \mathrm{O} 3 \\
-\mathrm{t}\end{array}$ & $\begin{array}{c}\mathrm{SnO} \\
-\mathrm{t} \\
\end{array}$ & $\begin{array}{c}\mathrm{SnO} 2 \\
-\mathrm{t}\end{array}$ & $\begin{array}{c}\mathrm{SO} 3 \\
-\mathrm{t}\end{array}$ & $\begin{array}{c}\mathrm{SrO} \\
-\mathrm{t}\end{array}$ & $\begin{array}{c}\mathrm{Tc} 2 \mathrm{O} 7 \\
-\mathrm{t}\end{array}$ & $\begin{array}{c}\mathrm{TeO} 2 \\
-\mathrm{t}\end{array}$ & $\begin{array}{c}\mathrm{ThO}_{2} \\
-\mathrm{t}\end{array}$ & $\begin{array}{c}\mathrm{TiO} 2 \\
-\mathrm{t} \\
\end{array}$ \\
\hline CVS1-16 & 0.0001 & & 0.0002 & 0.0001 & & & 0.0001 & & 0.0003 & & & & 0.0001 & & & 0.0005 & 0.0002 & & 0.0000 & & 0.0000 \\
\hline CVS1-17 & 0.0009 & & 0.0018 & 0.0009 & & & 0.0009 & & 0.0027 & & & & 0.0009 & & & 0.0049 & 0.0018 & & 0.0000 & & 0.0000 \\
\hline CVS1-18 & 0.0008 & & 0.0016 & 0.0008 & & & 0.0008 & & 0.0024 & & & & 0.0008 & & & 0.0044 & 0.0016 & & 0.0000 & & 0.0000 \\
\hline CVS1-19 & 0.0004 & & 0.0007 & 0.0004 & & & 0.0004 & & 0.0011 & & & & 0.0004 & & & 0.0020 & 0.0007 & & 0.0000 & & 0.0000 \\
\hline CVS1-20 & 0.0004 & & 0.0007 & 0.0004 & & & 0.0004 & & 0.0011 & & & & 0.0004 & & & 0.0020 & 0.0007 & & 0.0000 & & 0.0000 \\
\hline CVS1-21 & 0.0001 & & 0.0002 & 0.0001 & & & 0.0001 & & 0.0003 & & & & 0.0001 & & & 0.0005 & 0.0002 & & 0.0000 & & 0.0000 \\
\hline CVS1-22 & 0.0001 & & 0.0002 & 0.0001 & & & 0.0001 & & 0.0003 & & & & 0.0001 & & & 0.0005 & 0.0002 & & 0.0000 & & 0.0000 \\
\hline CVS1-23 & 0.0004 & & 0.0007 & 0.0004 & & & 0.0004 & & 0.0011 & & & & 0.0004 & & & 0.0019 & 0.0007 & & 0.0000 & & 0.0000 \\
\hline
\end{tabular}

\section{Hanford CVS 2 (Hrma et al. 1994)}

\begin{tabular}{|c|c|c|c|c|c|c|c|c|c|c|}
\hline CVS2-1 & 0.0007 & 0.0014 & 0.0007 & 0.0007 & 0.0022 & 0.0007 & 0.0039 & 0.0014 & 0.0000 & 0.0000 \\
\hline CVS2-2 & 0.0002 & 0.0004 & 0.0002 & 0.0002 & 0.0007 & 0.0002 & 0.0012 & 0.0004 & 0.0000 & 0.0000 \\
\hline CVS2-3 & 0.0002 & 0.0004 & 0.0002 & 0.0002 & 0.0007 & 0.0002 & 0.0012 & 0.0004 & 0.0000 & 0.0000 \\
\hline CVS2-4 & 0.0002 & 0.0004 & 0.0002 & 0.0002 & 0.0007 & 0.0002 & 0.0012 & 0.0004 & 0.0000 & 0.0000 \\
\hline CVS2-5 & 0.0007 & 0.0014 & 0.0007 & 0.0007 & 0.0022 & 0.0007 & 0.0039 & 0.0014 & 0.0000 & 0.0000 \\
\hline CVS2-6 & 0.0006 & 0.0011 & 0.0006 & 0.0006 & 0.0017 & 0.0006 & 0.0032 & 0.0011 & 0.0000 & 0.0000 \\
\hline CVS2-7 & 0.0006 & 0.0012 & 0.0006 & 0.0006 & 0.0017 & 0.0006 & 0.0032 & 0.0012 & 0.0000 & 0.0000 \\
\hline CVS2-8 & 0.0002 & 0.0004 & 0.0002 & 0.0002 & 0.0007 & 0.0002 & 0.0012 & 0.0004 & 0.0000 & 0.0000 \\
\hline CVS2-9 & 0.0002 & 0.0004 & 0.0002 & 0.0002 & 0.0007 & 0.0002 & 0.0012 & 0.0004 & 0.0000 & 0.0000 \\
\hline CVS2-10 & 0.0003 & 0.0005 & 0.0003 & 0.0003 & 0.0008 & 0.0003 & 0.0014 & 0.0005 & 0.0000 & 0.0000 \\
\hline CVS2-11 & 0.0006 & 0.0012 & 0.0006 & 0.0006 & 0.0018 & 0.0006 & 0.0033 & 0.0012 & 0.0000 & 0.0000 \\
\hline CVS2-12 & 0.0002 & 0.0004 & 0.0002 & 0.0002 & 0.0007 & 0.0002 & 0.0012 & 0.0004 & 0.0000 & 0.0000 \\
\hline CVS2-13 & 0.0002 & 0.0004 & 0.0002 & 0.0002 & 0.0007 & 0.0002 & 0.0012 & 0.0004 & 0.0000 & 0.0000 \\
\hline CVS2-14 & 0.0004 & 0.0008 & 0.0004 & 0.0004 & 0.0012 & 0.0004 & 0.0023 & 0.0008 & 0.0000 & 0.0000 \\
\hline CVS2-15 & 0.0007 & 0.0014 & 0.0007 & 0.0007 & 0.0022 & 0.0007 & 0.0039 & 0.0014 & 0.0000 & 0.0000 \\
\hline CVS2-16 & 0.0005 & 0.0011 & 0.0005 & 0.0005 & 0.0016 & 0.0005 & 0.0029 & 0.0011 & 0.0000 & 0.0000 \\
\hline CVS2-17 & 0.0004 & 0.0007 & 0.0004 & 0.0004 & 0.0011 & 0.0004 & 0.0020 & 0.0007 & 0.0000 & 0.0000 \\
\hline CVS2-18 & 0.0005 & 0.0011 & 0.0005 & 0.0005 & 0.0016 & 0.0005 & 0.0029 & 0.0011 & 0.0000 & 0.0000 \\
\hline CVS2-19 & 0.0000 & 0.0019 & 0.0000 & 0.0000 & 0.0015 & 0.0000 & 0.0044 & 0.0000 & 0.0000 & 0.0000 \\
\hline CVS2-20 & 0.0001 & 0.0002 & 0.0001 & 0.0001 & 0.0003 & 0.0001 & 0.0005 & 0.0002 & 0.0000 & 0.0000 \\
\hline CVS2-21 & 0.0001 & 0.0002 & 0.0001 & 0.0001 & 0.0003 & 0.0001 & 0.0005 & 0.0002 & 0.0000 & 0.0000 \\
\hline CVS2-22 & 0.0009 & 0.0018 & 0.0009 & 0.0009 & 0.0027 & 0.0009 & 0.0049 & 0.0018 & 0.0000 & 0.0000 \\
\hline CVS2-23 & 0.0001 & 0.0002 & 0.0001 & 0.0001 & 0.0003 & 0.0001 & 0.0005 & 0.0002 & 0.0000 & 0.0000 \\
\hline CVS2-24 & 0.0008 & 0.0017 & 0.0008 & 0.0008 & 0.0025 & 0.0008 & 0.0046 & 0.0017 & 0.0000 & 0.0000 \\
\hline CVS2-25 & 0.0005 & 0.0010 & 0.0005 & 0.0005 & 0.0015 & 0.0005 & 0.0028 & 0.0010 & 0.0000 & 0.0000 \\
\hline CVS2-26 & 0.0001 & 0.0002 & 0.0001 & 0.0001 & 0.0003 & 0.0001 & 0.0005 & 0.0002 & 0.0000 & 0.0000 \\
\hline
\end{tabular}


Hanford CVS 1 (Hrma et al. 1994)

\begin{tabular}{|c|c|c|c|c|c|c|c|c|c|c|c|c|c|c|c|c|c|c|c|c|c|}
\hline Glass ID & $\begin{array}{c}\mathrm{T} 12 \mathrm{O} 3 \\
-\mathrm{t}\end{array}$ & $\begin{array}{c}\mathrm{U} 3 \mathrm{O} 8 \\
-\mathrm{t}\end{array}$ & $\begin{array}{c}\mathrm{UO} 2 \\
-\mathrm{t}\end{array}$ & $\begin{array}{c}\text { UO3 } \\
-t\end{array}$ & $\begin{array}{c}\mathrm{V} 2 \mathrm{O} 5 \\
-\mathrm{t}\end{array}$ & $\begin{array}{c}\text { WO3 } \\
-t\end{array}$ & $\begin{array}{c}\mathrm{Y} 2 \mathrm{O} 3 \\
-\mathrm{t}\end{array}$ & $\begin{array}{c}\mathrm{ZnO} \\
-\mathrm{t}\end{array}$ & $\begin{array}{c}\text { Others } \\
-t\end{array}$ & $\begin{array}{c}\text { Sum } \\
-t\end{array}$ & $\begin{array}{c}\mathrm{A} 12 \mathrm{O} 3 \\
-\mathrm{a}\end{array}$ & $\begin{array}{c}\mathrm{B} 2 \mathrm{O} 3 \\
-\mathrm{a}\end{array}$ & $\begin{array}{c}\mathrm{CaO} \\
-\mathrm{a} \\
\end{array}$ & $\begin{array}{c}\mathrm{Fe} 2 \mathrm{O} 3 \\
-\mathrm{a}\end{array}$ & $\begin{array}{c}\mathrm{FeO} \\
-\mathrm{a}\end{array}$ & $\begin{array}{c}\mathrm{K} 2 \mathrm{O} \\
-\mathrm{a}\end{array}$ & $\begin{array}{c}\mathrm{Li} 2 \mathrm{O} \\
-\mathrm{a}\end{array}$ & $\begin{array}{c}\mathrm{MgO} \\
-\mathrm{a}\end{array}$ & $\begin{array}{c}\mathrm{Na} 2 \mathrm{O} \\
-\mathrm{a}\end{array}$ & $\begin{array}{c}\mathrm{NiO} \\
-\mathrm{a}\end{array}$ & $\begin{array}{c}\mathrm{P} 2 \mathrm{O} 5 \\
-\mathrm{a} \\
\end{array}$ \\
\hline CVS1-16 & & 0.0000 & & & & 0.0000 & 0.0001 & 0.0000 & & 1.0000 & 0.1430 & 0.0509 & 0.0004 & 0.0851 & & 0.0070 & 0.0085 & 0.0771 & 0.1740 & 0.0014 & 0.0002 \\
\hline CVS1-17 & & 0.0000 & & & & 0.0000 & 0.0009 & 0.0000 & & 1.0000 & 0.0000 & 0.0513 & 0.0995 & 0.1370 & & 0.0040 & 0.0091 & 0.0000 & 0.1540 & 0.0104 & 0.0018 \\
\hline CVS1-18 & & 0.0000 & & & & 0.0000 & 0.0008 & 0.0000 & & 0.9999 & 0.0009 & 0.0516 & 0.0220 & 0.0202 & & 0.0130 & 0.0616 & 0.0754 & 0.1150 & 0.0093 & 0.0016 \\
\hline CVS1-19 & & 0.0000 & & & & 0.0000 & 0.0004 & 0.0000 & & 0.9999 & 0.0636 & 0.1150 & 0.0289 & 0.0559 & & 0.0080 & 0.0345 & 0.0355 & 0.0964 & 0.0043 & 0.0007 \\
\hline CVS1-20 & & 0.0000 & & & & 0.0000 & 0.0004 & 0.0000 & & 0.9999 & 0.0637 & 0.1160 & 0.0270 & 0.0555 & & 0.0140 & 0.0350 & 0.0344 & 0.1100 & 0.0044 & 0.0007 \\
\hline CVS1-21 & & 0.0000 & & & & 0.0000 & 0.0001 & 0.0000 & & 1.0000 & 0.0074 & 0.2010 & 0.0214 & 0.0207 & & 0.0000 & 0.0086 & 0.0795 & 0.0855 & 0.0014 & 0.0002 \\
\hline CVS1-22 & & 0.0000 & & & & 0.0000 & 0.0001 & 0.0000 & & 1.0000 & 0.0008 & 0.0512 & 0.0009 & 0.1480 & & 0.0070 & 0.0096 & 0.0754 & 0.0836 & 0.0018 & 0.0002 \\
\hline CVS1-23 & & 0.0000 & & & & 0.0000 & 0.0004 & 0.0000 & & 0.9999 & 0.0434 & 0.0945 & 0.0294 & 0.1140 & & 0.0090 & 0.0296 & 0.0078 & 0.0943 & 0.0040 & 0.0007 \\
\hline
\end{tabular}

\section{Hanford CVS 2 (Hrma et al. 1994)}

\begin{tabular}{|c|c|c|c|c|c|c|c|c|c|c|c|c|c|c|c|}
\hline CVS2-1 & 0.0000 & 0.0000 & 0.0007 & 0.0000 & 0.9998 & 0.0795 & 0.0880 & 0.0008 & 0.0400 & 0.0330 & 0.0580 & 0.0465 & 0.0700 & 0.0088 & 0.0014 \\
\hline CVS2-2 & 0.0000 & 0.0000 & 0.0002 & 0.0000 & 1.0000 & 0.1065 & 0.0705 & 0.0690 & 0.0425 & 0.0150 & 0.0530 & 0.0000 & 0.0780 & 0.0031 & 0.0005 \\
\hline CVS2-3 & 0.0000 & 0.0000 & 0.0002 & 0.0000 & 0.9999 & 0.0995 & 0.1305 & 0.0690 & 0.0435 & 0.0340 & 0.0405 & 0.0097 & 0.0650 & 0.0033 & 0.0005 \\
\hline CVS2-4 & 0.0000 & 0.0000 & 0.0002 & 0.0000 & 0.9999 & 0.0595 & 0.1090 & 0.0700 & 0.0385 & 0.0067 & 0.0465 & 0.0000 & 0.0630 & 0.0030 & 0.0005 \\
\hline CVS2-5 & 0.0000 & 0.0000 & 0.0007 & 0.0000 & 0.9997 & 0.0690 & 0.1545 & 0.0260 & 0.0345 & 0.0170 & 0.0170 & 0.0000 & 0.0890 & 0.0083 & 0.0014 \\
\hline CVS2-6 & 0.0000 & 0.0000 & 0.0006 & 0.0000 & 0.9999 & 0.0155 & 0.0690 & 0.0680 & 0.1125 & 0.0100 & 0.0350 & 0.0047 & 0.0630 & 0.0068 & 0.0012 \\
\hline CVS2-7 & 0.0000 & 0.0000 & 0.0006 & 0.0000 & 0.9999 & 0.0895 & 0.1570 & 0.0170 & 0.0385 & 0.0083 & 0.0495 & 0.0000 & 0.0770 & 0.0066 & 0.0012 \\
\hline CVS2-8 & 0.0000 & 0.0000 & 0.0002 & 0.0000 & 1.0000 & 0.0580 & 0.0695 & 0.0014 & 0.1065 & 0.0190 & 0.0540 & 0.0445 & 0.0900 & 0.0078 & 0.0005 \\
\hline CVS2-9 & 0.0000 & 0.0000 & 0.0002 & 0.0000 & 0.9999 & 0.0140 & 0.0950 & 0.0006 & 0.0700 & 0.0150 & 0.0545 & 0.0000 & 0.0860 & 0.0030 & 0.0005 \\
\hline CVS2-10 & 0.0000 & 0.0000 & 0.0003 & 0.0000 & 1.0000 & 0.1005 & 0.1690 & 0.0013 & 0.0635 & 0.0110 & 0.0019 & 0.0000 & 0.1180 & 0.0033 & 0.0005 \\
\hline CVS2-11 & 0.0000 & 0.0000 & 0.0006 & 0.0000 & 1.0000 & 0.0104 & 0.0745 & 0.0680 & 0.0395 & 0.0045 & 0.0550 & 0.0111 & 0.0910 & 0.0069 & 0.0012 \\
\hline CVS2-12 & 0.0000 & 0.0000 & 0.0002 & 0.0000 & 1.0000 & 0.0900 & 0.1745 & 0.0760 & 0.0400 & 0.0130 & 0.0450 & 0.0100 & 0.0690 & 0.0030 & 0.0005 \\
\hline CVS2-13 & 0.0000 & 0.0000 & 0.0002 & 0.0000 & 0.9999 & 0.0895 & 0.0710 & 0.0320 & 0.0405 & 0.0370 & 0.0215 & 0.0480 & 0.1620 & 0.0031 & 0.0005 \\
\hline CVS2-14 & 0.0000 & 0.0000 & 0.0004 & 0.0000 & 0.9999 & 0.0245 & 0.1320 & 0.0310 & 0.0395 & 0.0170 & 0.0340 & 0.0450 & 0.0700 & 0.0050 & 0.0008 \\
\hline CVS2-15 & 0.0000 & 0.0000 & 0.0007 & 0.0000 & 0.9999 & 0.0745 & 0.0775 & 0.0550 & 0.0380 & 0.0079 & 0.0205 & 0.0000 & 0.1440 & 0.0082 & 0.0014 \\
\hline CVS2-16 & 0.0000 & 0.0000 & 0.0005 & 0.0000 & 0.9998 & 0.0230 & 0.1040 & 0.0092 & 0.0700 & 0.0310 & 0.0360 & 0.0086 & 0.1050 & 0.0065 & 0.0011 \\
\hline CVS2-17 & 0.0000 & 0.0000 & 0.0004 & 0.0000 & 0.9999 & 0.0615 & 0.1135 & 0.0300 & 0.0550 & 0.0180 & 0.0330 & 0.0330 & 0.0890 & 0.0045 & 0.0007 \\
\hline CVS2-18 & 0.0000 & 0.0000 & 0.0005 & 0.0000 & 0.9998 & 0.0235 & 0.1060 & 0.0093 & 0.0705 & 0.0280 & 0.0365 & 0.0086 & 0.1090 & 0.0066 & 0.0011 \\
\hline CVS2-19 & 0.0000 & 0.0000 & 0.0000 & 0.0049 & 0.9999 & 0.0300 & 0.1010 & 0.0108 & 0.0610 & 0.0000 & 0.0344 & 0.0809 & 0.1160 & 0.0063 & 0.0010 \\
\hline CVS2-20 & 0.0000 & 0.0000 & 0.0001 & 0.0000 & 0.9999 & 0.0096 & 0.0486 & 0.0004 & 0.0564 & 0.0000 & 0.0628 & 0.0004 & 0.1455 & 0.0010 & 0.0008 \\
\hline CVS2-21 & 0.0000 & 0.0000 & 0.0001 & 0.0000 & 1.0000 & 0.0669 & 0.1290 & 0.0005 & 0.0190 & 0.0000 & 0.0646 & 0.0705 & 0.0847 & 0.0010 & 0.0004 \\
\hline CVS2-22 & 0.0000 & 0.0000 & 0.0009 & 0.0000 & 0.9999 & 0.0366 & 0.0502 & 0.0006 & 0.0203 & 0.0020 & 0.0703 & 0.0731 & 0.1355 & 0.0093 & 0.0020 \\
\hline CVS2-23 & 0.0000 & 0.0000 & 0.0001 & 0.0000 & 0.9999 & 0.0561 & 0.0508 & 0.0760 & 0.0188 & 0.0000 & 0.0081 & 0.0004 & 0.2265 & 0.0010 & 0.0002 \\
\hline CVS2-24 & 0.0000 & 0.0000 & 0.0008 & 0.0000 & 0.9999 & 0.0940 & 0.2008 & 0.0007 & 0.0185 & 0.0200 & 0.0673 & 0.0002 & 0.1290 & 0.0087 & 0.0020 \\
\hline CVS2-25 & 0.0000 & 0.0000 & 0.0005 & 0.0000 & 0.9998 & 0.0040 & 0.0496 & 0.0765 & 0.1415 & 0.0000 & 0.0663 & 0.0007 & 0.1295 & 0.0054 & 0.0010 \\
\hline CVS2-26 & 0.0000 & 0.0000 & 0.0001 & 0.0000 & 0.9999 & 0.0250 & 0.2000 & 0.0770 & 0.0190 & 0.0000 & 0.0680 & 0.0010 & 0.0520 & 0.0009 & 0.0004 \\
\hline
\end{tabular}


Appendix A. Database - mass fraction

Hanford CVS 1 (Hrma et al. 1994)

\begin{tabular}{|c|c|c|c|c|c|c|c|c|c|c|c|c|c|c|c|c|c|c|c|c|c|}
\hline Glass ID & $\begin{array}{c}\mathrm{SiO} 2 \\
-\mathrm{a} \\
\end{array}$ & $\begin{array}{c}\mathrm{ZrO} 2 \\
-\mathrm{a}\end{array}$ & $\begin{array}{c}\mathrm{Ag} 2 \mathrm{O} \\
-\mathrm{a}\end{array}$ & $\begin{array}{c}\mathrm{As} 2 \mathrm{O} 3 \\
-\mathrm{a} \\
\end{array}$ & $\begin{array}{c}\mathrm{BaO} \\
-\mathrm{a}\end{array}$ & $\begin{array}{c}\mathrm{Bi} 2 \mathrm{O} 3 \\
-\mathrm{a} \\
\end{array}$ & $\begin{array}{l}\mathrm{Br} \\
-\mathrm{a}\end{array}$ & $\begin{array}{c}\mathrm{CdO} \\
-\mathrm{a}\end{array}$ & $\begin{array}{c}\mathrm{Ce} 2 \mathrm{O} 3 \\
-\mathrm{a}\end{array}$ & $\begin{array}{c}\mathrm{CeO} 2 \\
-\mathrm{a}\end{array}$ & $\begin{array}{l}\mathrm{Cl} \\
-\mathrm{a}\end{array}$ & $\begin{array}{c}\mathrm{CoO} \\
-\mathrm{a} \\
\end{array}$ & $\begin{array}{c}\mathrm{Co} 2 \mathrm{O} 3 \\
-\mathrm{a}\end{array}$ & $\begin{array}{c}\mathrm{Cr} 2 \mathrm{O} 3 \\
-\mathrm{a}\end{array}$ & $\begin{array}{c}\mathrm{Cs} 2 \mathrm{O} \\
-\mathrm{a}\end{array}$ & $\begin{array}{c}\mathrm{CuO} \\
-\mathrm{a}\end{array}$ & $\begin{array}{c}\mathrm{Eu} 2 \mathrm{O} 3 \\
-\mathrm{a}\end{array}$ & $\begin{array}{c}\mathrm{F} \\
-\mathrm{a} \\
\end{array}$ & $\begin{array}{c}\mathrm{Ga} 2 \mathrm{O} 3 \\
-\mathrm{a}\end{array}$ & $\begin{array}{c}\mathrm{Gd} 2 \mathrm{O} 3 \\
-\mathrm{a}\end{array}$ & $\begin{array}{c}\mathrm{HgO} \\
-\mathrm{a} \\
\end{array}$ \\
\hline CVS1-16 & 0.4230 & 0.0002 & & & 0.0002 & & & 0.0014 & & 0.0003 & & & & 0.0002 & 0.0003 & 0.0003 & & 0.0005 & & & \\
\hline CVS1-17 & 0.4380 & 0.0004 & & & 0.0017 & & & 0.0136 & & 0.0029 & & & & 0.0023 & 0.0026 & 0.0026 & & 0.0054 & & & \\
\hline CVS1-18 & 0.4190 & 0.0975 & & & 0.0016 & & & 0.0126 & & 0.0026 & & & & 0.0022 & 0.0022 & 0.0024 & & 0.0048 & & & \\
\hline CVS1-19 & 0.4760 & 0.0400 & & & 0.0007 & & & 0.0057 & & 0.0014 & & & & 0.0010 & 0.0010 & 0.0011 & & 0.0022 & & & \\
\hline CVS1-20 & 0.4750 & 0.0411 & & & 0.0007 & & & 0.0055 & & 0.0012 & & & & 0.0010 & 0.0011 & 0.0011 & & 0.0022 & & & \\
\hline CVS1-21 & 0.5540 & 0.0002 & & & 0.0002 & & & 0.0014 & & 0.0003 & & & & 0.0004 & 0.0003 & 0.0003 & & 0.0005 & & & \\
\hline CVS1-22 & 0.5340 & 0.0703 & & & 0.0002 & & & 0.0014 & & 0.0003 & & & & 0.0002 & 0.0003 & 0.0006 & & 0.0005 & & & \\
\hline CVS1-23 & 0.5140 & 0.0064 & & & 0.0007 & & & 0.0052 & & 0.0011 & & & & 0.0009 & 0.0010 & 0.0010 & & 0.0021 & & & \\
\hline
\end{tabular}

\section{Hanford CVS 2 (Hrma et al. 1994)}

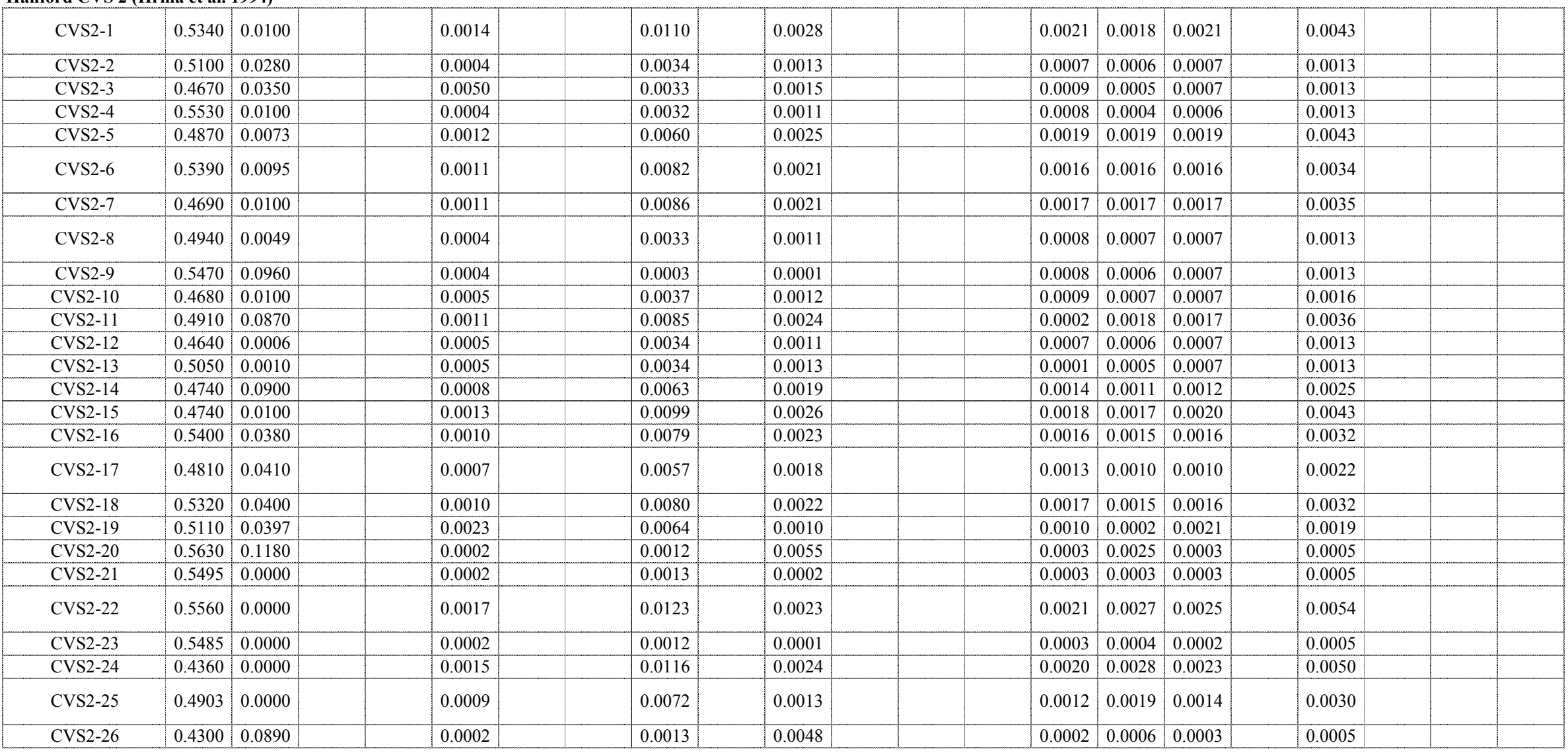


Hanford CVS 1 (Hrma et al. 1994)

\begin{tabular}{|c|c|c|c|c|c|c|c|c|c|c|c|c|c|c|c|c|c|c|c|c|c|}
\hline Glass ID & $\begin{array}{c}\mathrm{I} \\
-\mathrm{a}\end{array}$ & $\begin{array}{c}\mathrm{La} 2 \mathrm{O} 3 \\
-\mathrm{a}\end{array}$ & $\begin{array}{c}\mathrm{MnO} 2 \\
-\mathrm{a}\end{array}$ & $\begin{array}{c}\mathrm{MnO} \\
-\mathrm{a}\end{array}$ & $\begin{array}{c}\mathrm{MoO} \\
-\mathrm{a}\end{array}$ & $\begin{array}{c}\mathrm{MoO} 3 \\
-\mathrm{a}\end{array}$ & $\begin{array}{c}\mathrm{Nb} 2 \mathrm{O} 5 \\
-\mathrm{a}\end{array}$ & $\begin{array}{c}\mathrm{Nd} 2 \mathrm{O} 3 \\
-\mathrm{a}\end{array}$ & $\begin{array}{c}\mathrm{PbO} \\
-\mathrm{a}\end{array}$ & $\begin{array}{c}\mathrm{PdO} 2 \\
-\mathrm{a}\end{array}$ & $\begin{array}{c}\mathrm{PdO} \\
-\mathrm{a}\end{array}$ & $\begin{array}{c}\mathrm{Pr} 2 \mathrm{O} 3 \\
-\mathrm{a}\end{array}$ & $\begin{array}{c}\text { Pr6O11 } \\
-\mathrm{a}\end{array}$ & $\begin{array}{c}\mathrm{Rb} 2 \mathrm{O} \\
-\mathrm{a}\end{array}$ & $\begin{array}{c}\mathrm{ReO} \\
-\mathrm{a}\end{array}$ & $\begin{array}{c}\mathrm{ReO} 2 \\
-\mathrm{a}\end{array}$ & $\begin{array}{c}\mathrm{Rh} 2 \mathrm{O} 3 \\
-\mathrm{a}\end{array}$ & $\begin{array}{c}\mathrm{RhO} 2 \\
-\mathrm{a}\end{array}$ & $\begin{array}{c}\mathrm{RuO} 2 \\
-\mathrm{a}\end{array}$ & $\begin{array}{c}\mathrm{Sb} 2 \mathrm{O} 3 \\
-\mathrm{a}\end{array}$ & $\begin{array}{c}\mathrm{Sb} 2 \mathrm{O} 5 \\
-\mathrm{a}\end{array}$ \\
\hline CVS1-16 & & 0.0011 & 0.0004 & & & 0.0005 & & 0.0021 & & & 0.0001 & & 0.0002 & 0.0001 & & & 0.0001 & & 0.0003 & & \\
\hline CVS1-17 & & 0.0108 & 0.0029 & & & 0.0053 & & 0.0195 & & & 0.0009 & & 0.0018 & 0.0009 & & & 0.0009 & & 0.0021 & & \\
\hline CVS1-18 & & 0.0097 & 0.0025 & & & 0.0049 & & 0.0179 & & & 0.0008 & & 0.0016 & 0.0008 & & & 0.0008 & & 0.0021 & & \\
\hline CVS1-19 & & 0.0044 & 0.0012 & & & 0.0022 & & 0.0081 & & & 0.0004 & & 0.0007 & 0.0004 & & & 0.0004 & & 0.0016 & & \\
\hline CVS1-20 & & 0.0043 & 0.0011 & & & 0.0022 & & 0.0081 & & & 0.0004 & & 0.0007 & 0.0004 & & & 0.0004 & & 0.0013 & & \\
\hline CVS1-21 & & 0.0013 & 0.0004 & & & 0.0006 & & 0.0021 & & & 0.0001 & & 0.0002 & 0.0001 & & & 0.0001 & & 0.0003 & & \\
\hline CVS1-22 & & 0.0011 & 0.0003 & & & 0.0005 & & 0.0020 & & & 0.0001 & & 0.0002 & 0.0001 & & & 0.0001 & & 0.0003 & & \\
\hline CVS1-23 & & 0.0041 & 0.0012 & & & 0.0021 & & 0.0076 & & & 0.0004 & & 0.0007 & 0.0004 & & & 0.0004 & & 0.0011 & & \\
\hline
\end{tabular}

\section{Hanford CVS 2 (Hrma et al. 1994)}

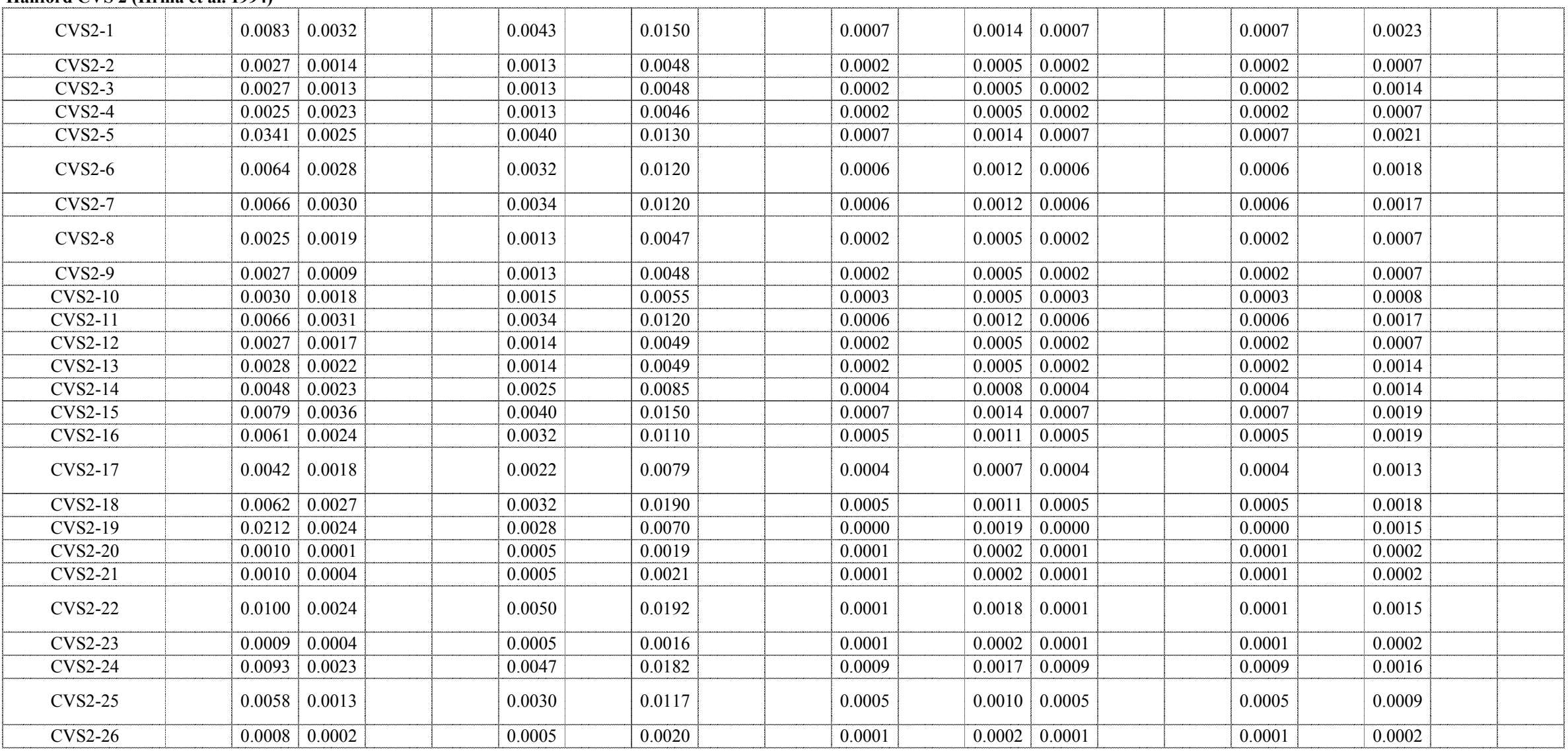


Appendix A. Database - mass fraction

Hanford CVS 1 (Hrma et al. 1994)

\begin{tabular}{|c|c|c|c|c|c|c|c|c|c|c|c|c|c|c|c|c|c|c|c|c|}
\hline Glass ID & $\begin{array}{c}\mathrm{SeO} 2 \\
-\mathrm{a}\end{array}$ & $\begin{array}{c}\mathrm{Sm} 2 \mathrm{O} 3 \\
-\mathrm{a}\end{array}$ & $\begin{array}{c}\mathrm{SnO} \\
-\mathrm{a}\end{array}$ & $\begin{array}{c}\mathrm{SnO} 2 \\
-\mathrm{a}\end{array}$ & $\begin{array}{c}\mathrm{SO} 3 \\
-\mathrm{a}\end{array}$ & $\begin{array}{c}\mathrm{SrO} \\
-\mathrm{a}\end{array}$ & $\begin{array}{c}\mathrm{Tc} 2 \mathrm{O} 7 \\
-\mathrm{a}\end{array}$ & $\begin{array}{c}\mathrm{TeO} 2 \\
-\mathrm{a}\end{array}$ & $\begin{array}{c}\mathrm{ThO} 2 \\
-\mathrm{a}\end{array}$ & $\begin{array}{c}\mathrm{TiO} 2 \\
-\mathrm{a}\end{array}$ & $\begin{array}{c}\mathrm{T} 12 \mathrm{O} 3 \\
-\mathrm{a}\end{array}$ & $\begin{array}{c}\mathrm{U} 3 \mathrm{O} 8 \\
-\mathrm{a}\end{array}$ & $\begin{array}{c}\mathrm{UO} 2 \\
-\mathrm{a}\end{array}$ & $\begin{array}{c}\mathrm{UO3} \\
-\mathrm{a}\end{array}$ & $\begin{array}{c}\mathrm{V} 2 \mathrm{O} 5 \\
-\mathrm{a}\end{array}$ & $\begin{array}{c}\text { WO3 } \\
-\mathrm{a}\end{array}$ & $\begin{array}{c}\mathrm{Y} 2 \mathrm{O} 3 \\
-\mathrm{a}\end{array}$ & $\begin{array}{c}\mathrm{ZnO} \\
-\mathrm{a}\end{array}$ & $\begin{array}{c}\text { Others } \\
-\mathrm{a}\end{array}$ & $\begin{array}{c}\text { Sum } \\
-\mathrm{a}\end{array}$ \\
\hline CVS1-16 & & 0.0001 & & & 0.0005 & 0.0002 & & & & 0.0000 & & & & & & & 0.0001 & & & 0.9797 \\
\hline CVS1-17 & & 0.0009 & & & 0.0049 & 0.0018 & & & & 0.0000 & & & & & & & 0.0009 & & & 0.9901 \\
\hline CVS1-18 & & 0.0008 & & & 0.0044 & 0.0016 & & & & 0.0003 & & & & & & & 0.0008 & & & 0.9645 \\
\hline CVS1-19 & & 0.0004 & & & 0.0020 & 0.0007 & & & & 0.0002 & & & & & & & 0.0004 & & & 0.9949 \\
\hline CVS1-20 & & 0.0004 & & & 0.0020 & 0.0007 & & & & 0.0002 & & & & & & & 0.0004 & & & 1.0122 \\
\hline CVS1-21 & & 0.0001 & & & 0.0005 & 0.0002 & & & & 0.0000 & & & & & & & 0.0001 & & & 0.9892 \\
\hline CVS1-22 & & 0.0001 & & & 0.0005 & 0.0002 & & & & 0.0002 & & & & & & & 0.0001 & & & 0.9920 \\
\hline CVS1-23 & & 0.0004 & & & 0.0019 & 0.0007 & & & & 0.0001 & & & & & & & 0.0004 & & & 0.9805 \\
\hline
\end{tabular}

\section{Hanford CVS 2 (Hrma et al. 1994)}

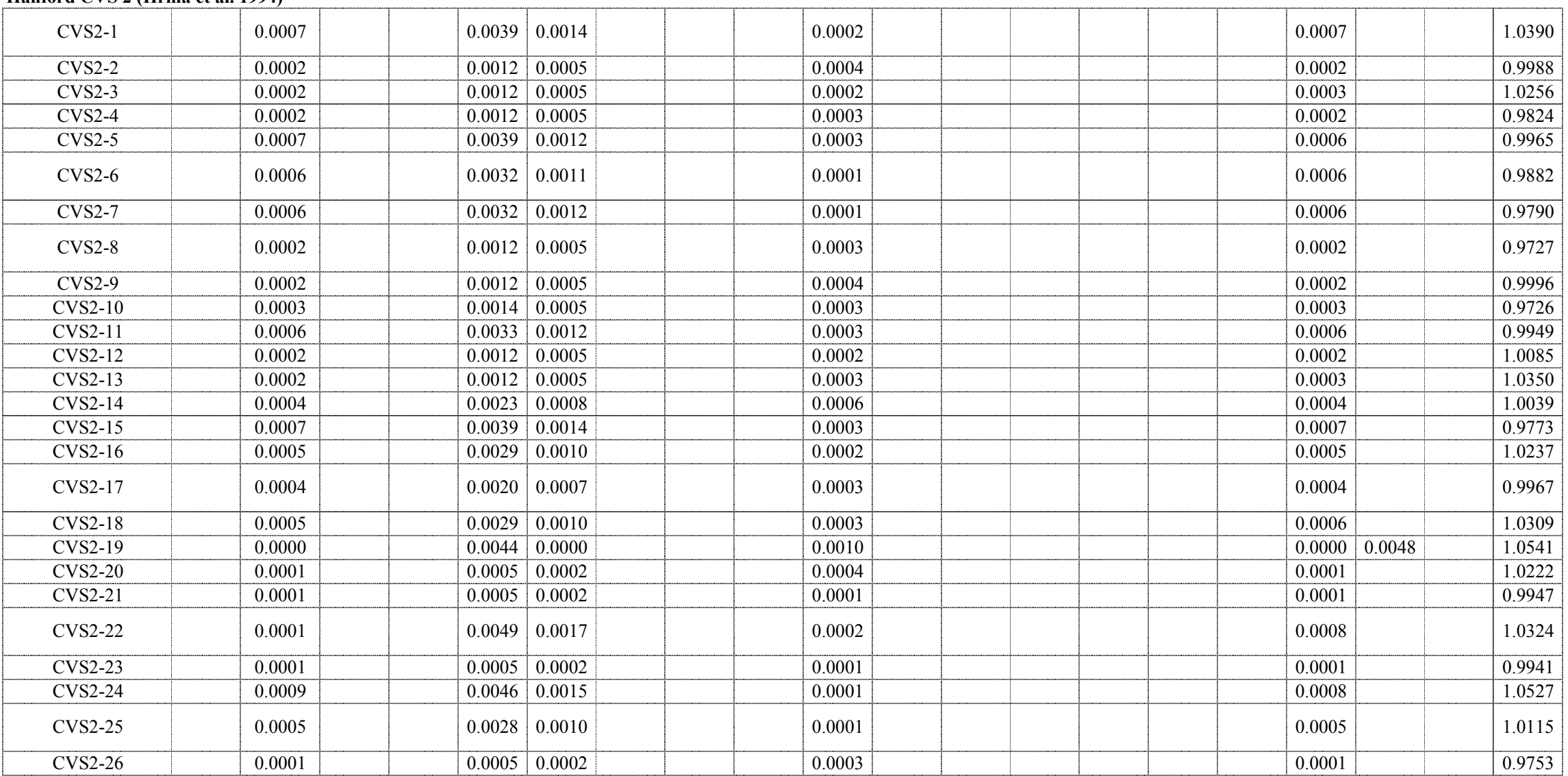


Appendix A. Database - mass fraction

Hanford CVS 1 (Hrma et al. 1994)

\begin{tabular}{|c|c|c|c|c|c|c|c|c|}
\hline Glass ID & $\begin{array}{l}\mathrm{TM} \\
\left({ }^{\circ} \mathrm{C}\right)\end{array}$ & $\begin{array}{l}\text { Gradient } \\
\text { TL }\left({ }^{\circ} \mathrm{C}\right)\end{array}$ & $\begin{array}{l}\text { Uniform } \\
\text { TL }\left({ }^{\circ} \mathrm{C}\right)\end{array}$ & Primary Phase & $\begin{array}{l}\text { Quenched } \\
\text { Visual/OM }\end{array}$ & $\begin{array}{c}\text { Quenched } \\
\text { SEM/EDS or TEM }\end{array}$ & $\begin{array}{l}\text { Quenched } \\
\text { XRD }\end{array}$ & $\begin{array}{c}\text { Quenched } \\
\text { Homogeneous? }\end{array}$ \\
\hline CVS1-16 & & $>1118$ & & spinel & & & & \\
\hline CVS1-17 & & 1000 & & spinel & & & & \\
\hline CVS1-18 & & $>1118$ & & $\mathrm{ZrO} 2$ & & & & \\
\hline CVS1-19 & & 961 & & spinel & & & & \\
\hline CVS1-20 & & 966 & & spinel & & & & \\
\hline CVS1-21 & & 957 & & $\mathrm{SiO} 2$ & & & & \\
\hline CVS1-22 & & $>1118$ & & spinel & & & & \\
\hline CVS1-23 & & 980 & & spinel & & & & \\
\hline
\end{tabular}

Hanford CVS 2 (Hrma et al. 1994)

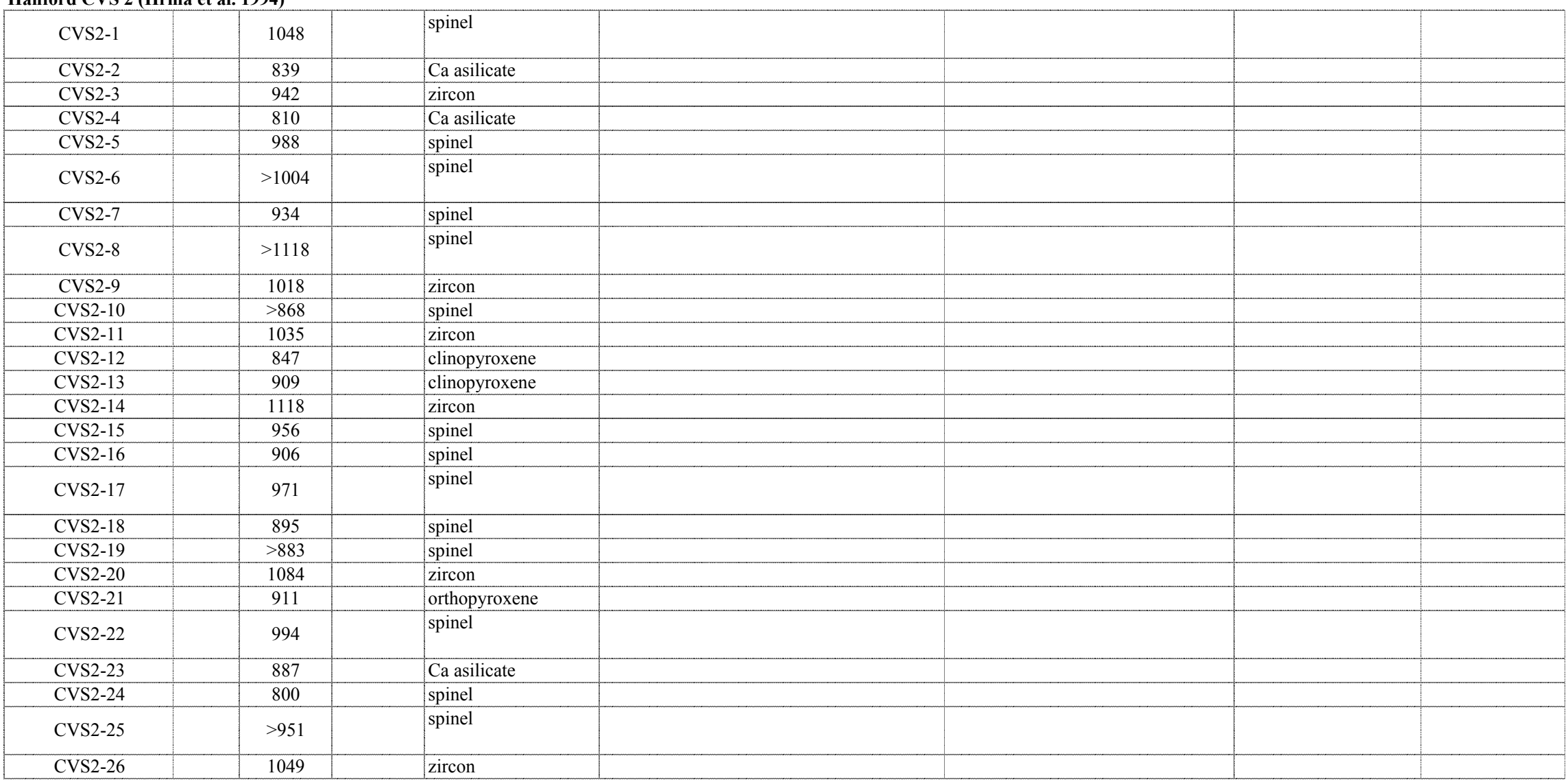


Appendix A. Database - mass fraction

\begin{tabular}{|c|c|c|c|c|c|}
\hline \multicolumn{6}{|c|}{ Hanford CVS 1 (Hrma et al. 1994) } \\
\hline Glass ID & $\begin{array}{c}\text { CCC } \\
\text { Visual/OM }\end{array}$ & $\begin{array}{c}\text { CCC } \\
\text { SEM/EDS or TEM }\end{array}$ & $\begin{array}{l}\mathrm{CCC} \\
\mathrm{XRD}\end{array}$ & $\begin{array}{c}\text { Heat Treated } \\
\text { Visual/OM }\end{array}$ & $\begin{array}{c}\text { Heat Treated } \\
\text { SEM/EDS or TEM }\end{array}$ \\
\hline CVS1-16 & nepheline, spinel & $(\mathrm{Fe} ; \mathrm{Ni}, \mathrm{Cr})$ & $\begin{array}{l}\text { 15-18 vol\% nepheline NaAlSiO4, 1-2 } \\
\text { vol\% spinel }\end{array}$ & & \\
\hline CVS1-17 & spinel, clinopyroxene & $(\mathrm{Fe}, \mathrm{Ni}, \mathrm{Cr}),(\mathrm{Ca}, \mathrm{Fe}, \mathrm{Si})$ & $1-2$ vol $\%$ maghemite g-Fe2O3 & & \\
\hline CVS1-18 & ZrO2, Li2SiO3, clinopyroxene & $\begin{array}{l}(\mathrm{zr}),(\mathrm{Si}),(\mathrm{Ca}, \mathrm{Mg}, \mathrm{Si} \\
\mathrm{Fe}, \mathrm{Ni})\end{array}$ & 12-13 vol\% $\mathrm{ZrO} 2,2-3$ vol\% $\mathrm{Li} 2 \mathrm{SiO} 3$ & & \\
\hline CVS1-19 & clinopyroxene, spinel & $\begin{array}{l}(\mathrm{Ca}, \mathrm{Fe}, \mathrm{Mg}, \mathrm{Si} ; \mathrm{Zr}) \\
(\mathrm{Fe}, \mathrm{Ni}, \mathrm{Cr})\end{array}$ & $8-10 \mathrm{vol} \%$ clinopyroxene & & \\
\hline CVS1-20 & clinopyroxene, spinel & & 8-10 vol\% clinopyroxene & & \\
\hline CVS1-21 & none & none & $<1$ vol $\%$ unidentified & & \\
\hline CVS1-22 & zircon, orthopyroxene, clinopyroxene, spinel, hematite & $(\mathrm{Zr}, \mathrm{Si}),(\mathrm{Mg}, \mathrm{Fe}, \mathrm{Si}),(\mathrm{Fe}, \mathrm{Si})$ & $\begin{array}{l}10-12 \text { vol\% zircon } \mathrm{ZrSiO} 4,2-4 \mathrm{vol} \% \\
\text { orthopyroxene }(\mathrm{Mg}, \mathrm{Fe}) \mathrm{SiO}, 2-3 \mathrm{vol} \% \\
\text { clinopyroxene }\end{array}$ & & \\
\hline CVS1-23 & clinopyroxene, spinel & $\begin{array}{l}(\mathrm{Ca}, \mathrm{Fe}, \mathrm{Si} ; \mathrm{Na}, \mathrm{Al}) \\
(\mathrm{Fe}, \mathrm{Ni}, \mathrm{Cr})\end{array}$ & $2-3$ vol\% clinopyroxene & & \\
\hline
\end{tabular}

\section{Hanford CVS 2 (Hrma et al, 1994)}

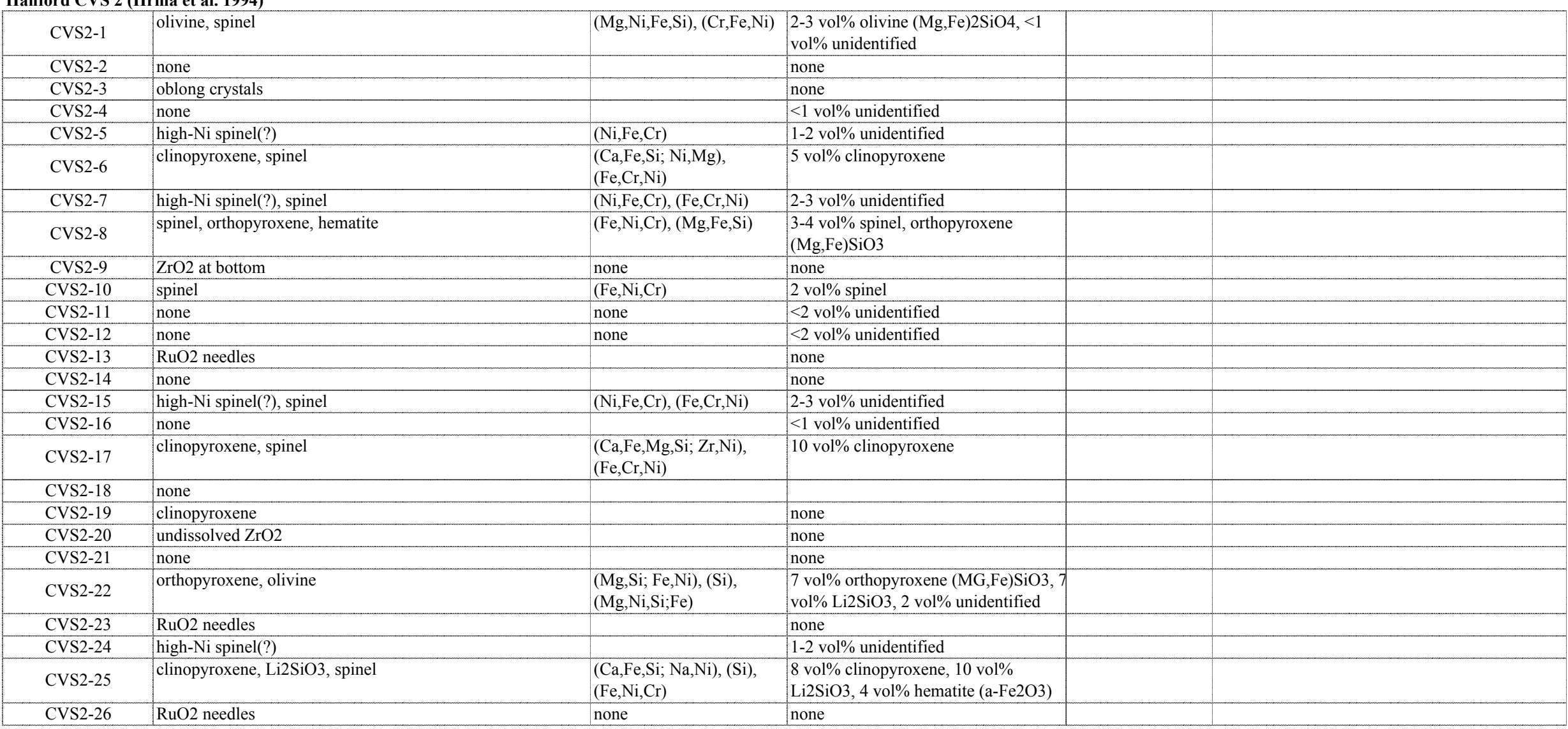


Hanford CVS 1 (Hrma et al. 1994)

\begin{tabular}{|c|c|c|c|c|c|c|c|c|c|c|c|c|c|c|c|}
\hline Glass ID & $\begin{array}{c}\text { Heat Treated } \\
\text { XRD }\end{array}$ & $\begin{array}{l}\text { Density } \\
\left(\mathrm{g} / \mathrm{cm}^{3}\right)\end{array}$ & $\begin{array}{c}\text { Fulc Visc } \\
\text { A }\end{array}$ & \begin{tabular}{|c|} 
Fulc Visc \\
B \\
\end{tabular} & \begin{tabular}{|c|} 
Fulc Visc \\
To \\
\end{tabular} & $\begin{array}{c}\mathrm{FV} 1150^{\circ} \mathrm{C} \\
(\mathrm{Pa} \cdot \mathrm{s})\end{array}$ & $\begin{array}{c}\text { Arrh Visc } \\
\text { A }\end{array}$ & $\begin{array}{c}\text { Arrh Visc } \\
\text { B }\end{array}$ & $\begin{array}{l}1150^{\circ} \mathrm{C} \\
\left(\mathrm{D}_{\mathrm{a}}, \mathrm{C}\right)\end{array}$ & $\begin{array}{c}\mathrm{T}\left({ }^{\circ} \mathrm{C}\right) \text { at } \\
2 \mathrm{~Pa} \cdot \mathrm{s}\end{array}$ & $\begin{array}{c}\mathrm{T}\left({ }^{\circ} \mathrm{C}\right) \text { at } \\
5 \mathrm{~Pa} \cdot \mathrm{s}\end{array}$ & $\begin{array}{c}\mathrm{T}\left({ }^{\circ} \mathrm{C}\right) \text { at } \\
10 \mathrm{~Pa} \cdot \mathrm{s}\end{array}$ & $\begin{array}{l}\mathrm{T} 1 \\
\left({ }^{\circ} \mathrm{C}\right) \\
\end{array}$ & $\begin{array}{c}\mathrm{V} 1 \\
(\mathrm{~Pa} \cdot \mathrm{s}) \\
\end{array}$ & $\begin{array}{c}\mathrm{T} 2 \\
\left({ }^{\circ} \mathrm{C}\right) \\
\end{array}$ \\
\hline CVS1-16 & & 2.5984 & -6.15 & 7080.86 & 365.44 & 17.81 & -12.047 & 21223.4 & 17.59 & & & & 1348 & 2.89 & 1249 \\
\hline CVS1-17 & & 2.9205 & -5.14 & 4146.93 & 451.94 & 2.23 & -12.736 & 19360.4 & 2.39 & & & & 1252 & 1.04 & 1152 \\
\hline CVS1-18 & & 2.9163 & -34.31 & 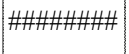 & -2730.03 & 1.87 & & & & & & & (1251) & $(0.77)$ & (1052) \\
\hline CVS1-19 & & 2.6705 & -6.45 & 7170.87 & 275.46 & 5.76 & -12.484 & 20317.4 & 6.01 & & & & 1251 & 2.46 & 1151 \\
\hline CVS1-20 & & 2.6709 & -6.53 & 7629.94 & 228.06 & 5.71 & -11.740 & 19235.1 & 5.91 & & & & 1252 & 2.52 & 1152 \\
\hline CVS1-21 & & 2.502 & -3.90 & 4025.10 & 494.15 & 9.36 & -13.194 & 22133.7 & 10.59 & & & & 1249 & 4.14 & 1150 \\
\hline CVS1-22 & & 2.7923 & -6.16 & 7000.00 & 435.70 & 38.11 & -13.629 & 24521.0 & 36.71 & & & & 1351 & 4.43 & 1252 \\
\hline CVS1-23 & & 2.6919 & -6.00 & 7237.84 & 214.12 & 5.69 & -10.642 & 17661.9 & 5.87 & & & & 1252 & 2.66 & 1153 \\
\hline
\end{tabular}

\section{Hanford CVS 2 (Hrma et al. 1994)}

\begin{tabular}{|c|c|c|c|c|c|c|c|c|c|c|c|}
\hline CVS2-1 & 2.6447 & -7.66 & 10547.27 & 63.07 & 7.74 & -11.860 & 19788.6 & 7.74 & 1199 & 5.11 & 1123 \\
\hline CVS2-2 & 2.6513 & -5.83 & 6776.14 & 265.76 & 6.26 & -11.352 & 18785.1 & 6.35 & 1249 & 2.9 & 1199 \\
\hline CVS2-3 & 2.6312 & -5.47 & 5748.80 & 349.90 & 5.56 & -12.226 & 19866.7 & 5.67 & 1249 & 2.56 & 1198 \\
\hline CVS2-4 & 2.6008 & -5.63 & 6567.10 & 272.32 & 6.37 & -11.428 & 18906.0 & 6.41 & 1198 & 4.37 & 1124 \\
\hline CVS2-5 & 2.6127 & -4.67 & 5076.07 & 400.58 & 8.18 & -12.139 & 20299.4 & 8.38 & 1250 & 3.72 & 1200 \\
\hline CVS2-6 & 2.7703 & -4.77 & 4954.98 & 398.91 & 6.19 & -12.001 & 19705.3 & 6.34 & 1250 & 2.87 & 1199 \\
\hline CVS2-7 & 2.554 & -4.67 & 4761.86 & 371.35 & 4.26 & -10.811 & 17473.7 & 4.34 & 1250 & 2.17 & 1199 \\
\hline CVS2-8 & 2.6705 & -5.83 & 6627.35 & 242.42 & 4.36 & -10.480 & 17050.6 & 4.49 & 1299 & 1.57 & 1250 \\
\hline CVS2-9 & 2.7096 & -6.50 & 7389.73 & 278.00 & 7.23 & -13.167 & 21579.4 & 7.37 & 1250 & 3.04 & 1200 \\
\hline CVS2-10 & 2.5353 & -4.02 & 4618.29 & 406.77 & 8.99 & -10.994 & 18801.1 & 9.19 & 1250 & 4.39 & 1200 \\
\hline CVS2-11 & 2.8228 & -6.37 & 5985.39 & 351.41 & 3.07 & -13.876 & 21352.4 & 3.09 & 1198 & 2.01 & 1123 \\
\hline CVS2-12 & 2.5975 & -4.24 & 3704.99 & 470.67 & 3.38 & -12.361 & 19332.4 & 3.40 & 1198 & 2.37 & 1123 \\
\hline CVS2-13 & 2.6198 & -6.36 & 7751.57 & 221.29 & 7.27 & -11.573 & 19310.5 & 7.37 & 1248 & 3.32 & 1199 \\
\hline CVS2-14 & 2.7735 & -5.65 & 4912.78 & 420.85 & 2.97 & -13.573 & 20898.7 & 3.04 & 1249 & 1.33 & 1199 \\
\hline CVS2-15 & 2.5615 & -5.34 & 5529.18 & 341.84 & 4.47 & -11.615 & 18686.6 & 4.56 & 1249 & 2.16 & 1199 \\
\hline CVS2-16 & 2.6786 & -6.70 & 8140.32 & 202.92 & 6.61 & -11.864 & 19585.6 & 6.68 & 1248 & 2.93 & 1199 \\
\hline CVS2-17 & 2.6744 & -5.44 & 5744.01 & 343.47 & 5.37 & -11.995 & 19490.2 & 5.48 & 1250 & 2.46 & 1199 \\
\hline CVS2-18 & 2.6868 & -6.12 & 7093.77 & 260.62 & 6.41 & -11.780 & 19427.9 & 6.51 & 1248 & 2.89 & 1199 \\
\hline CVS2-19 & 2.6987 & -5.69 & 6319.75 & 319.03 & 6.76 & -12.176 & 20074.3 & 6.90 & 1249 & 2.95 & 1200 \\
\hline CVS2-20 & 2.7333 & -6.53 & 7702.25 & 298.49 & 12.31 & -13.843 & 23295.3 & 12.52 & 1245 & 5.07 & 1194 \\
\hline CVS2-21 & 2.492 & -5.82 & 6876.21 & 246.69 & 6.01 & -11.021 & 18250.7 & 6.08 & 1244 & 2.93 & 1195 \\
\hline CVS2-22 & 2.6611 & -5.57 & 6443.11 & 273.26 & 5.92 & -11.012 & 18221.9 & 6.01 & 1248 & 2.79 & 1198 \\
\hline CVS2-23 & 2.591 & -5.27 & 6320.83 & 313.98 & 9.91 & -11.693 & 19924.7 & 10.06 & 1243 & 4.63 & 1194 \\
\hline CVS2-24 & 2.5796 & -6.84 & 7662.12 & 131.74 & 1.99 & -10.294 & 15638.9 & 2.01 & 1247 & 1.03 & 1199 \\
\hline CVS2-25 & 2.8316 & -5.15 & 4096.93 & 398.47 & 1.35 & -11.162 & 16332.5 & 1.37 & 1246 & 0.72 & 1197 \\
\hline CVS2-26 & 2.6967 & -5.99 & 4592.64 & 411.51 & 1.26 & -13.167 & 19093.2 & 1.28 & 1244 & 0.63 & 1194 \\
\hline
\end{tabular}




\section{Hanford CVS 1 (Hrma et al. 1994)}

\begin{tabular}{|c|c|c|c|c|c|c|c|c|c|c|c|c|c|c|c|c|c|c|c|c|c|}
\hline Glass ID & $\begin{array}{c}\mathrm{V} 2 \\
(\mathrm{~Pa} \cdot \mathrm{s}) \\
\end{array}$ & $\begin{array}{c}\mathrm{T} 3 \\
\left({ }^{\circ} \mathrm{C}\right) \\
\end{array}$ & $\begin{array}{c}\mathrm{V} 3 \\
(\mathrm{~Pa} \cdot \mathrm{s})\end{array}$ & $\begin{array}{c}\mathrm{T} 4 \\
\left({ }^{\circ} \mathrm{C}\right) \\
\end{array}$ & $\begin{array}{c}\mathrm{V} 4 \\
(\mathrm{~Pa} \cdot \mathrm{s})\end{array}$ & $\begin{array}{c}\mathrm{T} 5 \\
\left({ }^{\circ} \mathrm{C}\right) \\
\end{array}$ & $\begin{array}{c}\text { V5 } \\
(\mathrm{Pa} \cdot \mathrm{s}) \\
\end{array}$ & $\begin{array}{c}\mathrm{T} 6 \\
\left({ }^{\circ} \mathrm{C}\right) \\
\end{array}$ & $\begin{array}{c}\mathrm{V} 6 \\
(\mathrm{~Pa} \cdot \mathrm{s})\end{array}$ & $\begin{array}{l}\mathrm{T} 7 \\
\left({ }^{\circ} \mathrm{C}\right) \\
\end{array}$ & $\begin{array}{c}\mathrm{V} 7 \\
(\mathrm{~Pa} \cdot \mathrm{s}) \\
\end{array}$ & $\begin{array}{c}\mathrm{T} 8 \\
\left({ }^{\circ} \mathrm{C}\right) \\
\end{array}$ & $\begin{array}{c}\mathrm{V} 8 \\
(\mathrm{~Pa} \cdot \mathrm{s})\end{array}$ & $\begin{array}{c}\mathrm{T} 9 \\
\left({ }^{\circ} \mathrm{C}\right) \\
\end{array}$ & $\begin{array}{c}\mathrm{V} 9 \\
(\mathrm{~Pa} \cdot \mathrm{s})\end{array}$ & $\begin{array}{l}\mathrm{T} 10 \\
\left({ }^{\circ} \mathrm{C}\right) \\
\end{array}$ & $\begin{array}{c}\text { V10 } \\
(\mathrm{Pa} \cdot \mathrm{s})\end{array}$ & $\begin{array}{l}\mathrm{T} 11 \\
\left({ }^{\circ} \mathrm{C}\right) \\
\end{array}$ & $\begin{array}{c}\mathrm{V} 11 \\
(\mathrm{~Pa} \cdot \mathrm{s})\end{array}$ & $\begin{array}{l}\mathrm{T} 12 \\
\left({ }^{\circ} \mathrm{C}\right) \\
\end{array}$ & $\begin{array}{c}\mathrm{V} 12 \\
(\mathrm{~Pa} \cdot \mathrm{s}) \\
\end{array}$ \\
\hline CVS1-16 & 6.48 & 1149 & 18.02 & (1049) & (113.81) & & & & & & & & & & & & & & & & \\
\hline CVS1-17 & 2.22 & 1052 & 5.83 & 953 & 23.1 & & & & & & & & & & & & & & & & \\
\hline CVS1-18 & (4.62) & (952) & $(12.23)$ & & & & & & & & & & & & & & & & & & \\
\hline CVS1-19 & 5.73 & 1051 & 16.35 & 951 & 64.53 & & & & & & & & & & & & & & & & \\
\hline CVS1-20 & 5.51 & 1053 & 15.31 & 953 & 53.9 & & & & & & & & & & & & & & & & \\
\hline CVS1-21 & 9.58 & (1049) & $(28.12)$ & 950 & 138.75 & & & & & & & & & & & & & & & & \\
\hline CVS1-22 & 11.2 & 1153 & 35.97 & & & & & & & & & & & & & & & & & & \\
\hline CVS1-23 & 5.55 & 1053 & 13.91 & 953 & 44.72 & & & & & & & & & & & & & & & & \\
\hline
\end{tabular}

\section{Hanford CVS 2 (Hrma et al. 1994)}

\begin{tabular}{|c|c|c|c|c|c|c|c|c|c|c|c|c|c|c|c|c|c|c|c|c|c|}
\hline CVS2-1 & 9.81 & 1048 & 21.29 & 958 & 70.77 & & & & & & & & & & & & & & & & \\
\hline CVS2-2 & 4.24 & 1150 & 6.14 & 1149 & 6.21 & 1147 & 6.47 & 1100 & 9.72 & 1097 & 10.44 & 1047 & 17.22 & 999 & 30.68 & 948 & 60.06 & & & & \\
\hline CVS2-3 & 3.58 & 1150 & 5.54 & 1148 & 5.6 & 1147 & 5.86 & 1100 & 8.82 & 1096 & 9.65 & 1048 & 15.67 & 998 & 30.09 & 949 & 61.85 & & & & \\
\hline CVS2-4 & 7.81 & 1048 & 17.36 & 948 & 59.47 & & & & & & & & & & & & & & & & \\
\hline CVS2-5 & 5.41 & 1149 & 7.89 & 1150 & 8.07 & 1147 & 8.53 & 1097 & 13.6 & 1100 & 13.65 & 1047 & 23.82 & 999 & 46.32 & 948 & 98.42 & & & & \\
\hline CVS2-6 & 4.1 & 1150 & 6.16 & 1149 & 6.18 & 1148 & 6.44 & 1100 & 9.96 & 1098 & 10.01 & 1048 & 17.25 & 999 & 33.3 & 949 & 68.39 & & & & \\
\hline CVS2-7 & 2.85 & 1150 & 4.23 & 1148 & 4.35 & 1149 & 4.37 & 1099 & 6.54 & 1098 & 6.57 & 1048 & 10.67 & 998 & 19.03 & 949 & 35.5 & & & & \\
\hline CVS2-8 & 2.13 & 1200 & 2.93 & 1150 & 4.27 & 1148 & 4.35 & 1145 & 4.64 & 1100 & 6.59 & 1097 & 7.08 & 1046 & 11.22 & 995 & 19.86 & 947 & 35.44 & & \\
\hline CVS2-9 & 4.52 & 1198 & 4.68 & 1150 & 7.23 & 1149 & 7.33 & 1148 & 7.38 & 1123 & 9.57 & 1100 & 12.01 & 1099 & 12.43 & 1048 & 22.09 & 1048 & 23.07 & 999 & 42.76 \\
\hline CVS2-10 & 6.07 & 1149 & 8.67 & 1150 & 8.7 & 1147 & 9.43 & 1100 & 14.24 & 1098 & 14.54 & 1048 & 24.11 & 999 & 44.78 & 949 & 88.75 & & & & \\
\hline CVS2-11 & 3.98 & 1048 & 9.22 & 948 & 38.83 & & & & & & & & & & & & & & & & \\
\hline CVS2-12 & 4.18 & 1048 & 8.93 & 945 & 35.62 & & & & & & & & & & & & & & & & \\
\hline CVS2-13 & 4.85 & 1149 & 7.02 & 1148 & 7.17 & 1146 & 7.67 & 1099 & 11.77 & 1096 & 12.5 & 1046 & 20.52 & 997 & 39.31 & 948 & 72.4 & & & & \\
\hline CVS2-14 & 2 & 1149 & 2.88 & 1150 & 2.88 & 1148 & 2.99 & 1100 & 4.99 & 1099 & 5.03 & 1048 & 8.86 & 998 & 17.54 & 949 & 38.36 & & & & \\
\hline CVS2-15 & 2.94 & 1149 & 4.4 & 1150 & 4.44 & 1148 & 4.74 & 1100 & 6.95 & 1099 & 7.18 & 1048 & 11.71 & 998 & 22.69 & 949 & 42.4 & & & & \\
\hline CVS2-16 & 4.27 & 1198 & 4.42 & 1148 & 6.68 & 1149 & 6.71 & 1147 & 6.91 & 1123 & 8.38 & 1099 & 10.59 & 1097 & 11.24 & 1047 & 18.61 & 1048 & 18.88 & 998 & 34.49 \\
\hline CVS2-17 & 3.45 & 1149 & 5.37 & 1149 & 5.57 & 1147 & 5.69 & 1100 & 8.53 & 1098 & 8.85 & 1048 & 14.9 & 999 & 27.35 & 949 & 57.68 & & & & \\
\hline CVS2-18 & 4.2 & 1148 & 6.46 & 1150 & 6.47 & 1145 & 6.84 & 1099 & 10.51 & 1097 & 10.53 & 1047 & 17.78 & 997 & 33.96 & 948 & 66.75 & & & & \\
\hline CVS2-19 & 4.44 & 1149 & 6.76 & 1150 & 6.76 & 1148 & 7.14 & 1100 & 11.02 & 1097 & 11.51 & 1048 & 18.73 & 999 & 36.93 & 949 & 77.01 & & & & \\
\hline CVS2-20 & 7.8 & 1144 & 12.77 & 1144 & 13.02 & 1144 & 13.12 & 1094 & 23.47 & 1094 & 23.52 & 1044 & 44.72 & 994 & 94.47 & 944 & 218.42 & & & & \\
\hline CVS2-21 & 4.16 & 1145 & 6.17 & 1146 & 6.23 & 1145 & 6.4 & 1096 & 9.66 & 1096 & 9.85 & 1046 & 16.2 & 995 & 28.76 & 946 & 55.62 & & & & \\
\hline CVS2-22 & 4.1 & 1148 & 5.97 & 1148 & 6.05 & 1148 & 6.17 & 1098 & 9.31 & 1098 & 9.36 & 1047 & 15.63 & 997 & 28.16 & 947 & 54.27 & & & & \\
\hline CVS2-23 & 6.76 & 1145 & 10.16 & 1145 & 10.45 & 1144 & 10.64 & 1095 & 16.96 & 1096 & 16.96 & 1045 & 29.41 & 995 & 53.72 & 945 & 117.22 & & & & \\
\hline CVS2-24 & 1.41 & 1144 & 1.99 & 1148 & 2.02 & 1147 & 2.11 & 1098 & 2.99 & 1097 & 3.06 & 1047 & 4.66 & 997 & 7.33 & 947 & 13.11 & & & & \\
\hline CVS2-25 & 0.97 & 1148 & 1.37 & 1147 & 1.38 & 1148 & 1.44 & 1098 & 1.98 & 1097 & 2 & 1048 & 3.14 & 998 & 5.45 & 947 & 10.11 & & & & \\
\hline CVS2-26 & 0.88 & 1146 & 1.27 & 1145 & 1.3 & 1147 & 1.3 & 1097 & 2.09 & 1095 & 2.13 & 1046 & 3.48 & 996 & 6.34 & 946 & 13.65 & & & & \\
\hline
\end{tabular}


Appendix A. Database - mass fraction

Hanford CVS 1 (Hrma et al. 1994)

\begin{tabular}{|c|c|c|c|c|c|c|c|c|c|c|c|c|c|c|c|c|}
\hline Glass ID & $\begin{array}{l}\mathrm{T} 13 \\
\left({ }^{\circ} \mathrm{C}\right)\end{array}$ & $\begin{array}{c}\mathrm{V} 13 \\
(\mathrm{~Pa} \cdot \mathrm{s}) \\
\end{array}$ & $\begin{array}{l}\mathrm{T} 14 \\
\left({ }^{\circ} \mathrm{C}\right)\end{array}$ & $\begin{array}{c}\mathrm{V} 14 \\
(\mathrm{~Pa} \cdot \mathrm{s}) \\
\end{array}$ & $\begin{array}{c}\text { Q PCT } \\
\text { B }\left(\mathrm{g} / \mathrm{m}^{2}\right)\end{array}$ & $\begin{array}{c}\text { Q PCT } \\
\mathrm{Li}\left(\mathrm{g} / \mathrm{m}^{2}\right)\end{array}$ & $\begin{array}{c}\text { Q PCT } \\
\mathrm{Na}\left(\mathrm{g} / \mathrm{m}^{2}\right)\end{array}$ & $\begin{array}{c}\text { Q PCT } \\
\mathrm{Si}\left(\mathrm{g} / \mathrm{m}^{2}\right)\end{array}$ & $\begin{array}{c}\text { Q PCT } \\
\mathrm{pH}\end{array}$ & $\begin{array}{c}\text { CCC PCT } \\
\mathrm{B}\left(\mathrm{g} / \mathrm{m}^{2}\right)\end{array}$ & $\begin{array}{l}\text { CCC PCT } \\
\mathrm{Li}\left(\mathrm{g} / \mathrm{m}^{2}\right) \\
\end{array}$ & $\begin{array}{l}\text { CCC PCT } \\
\mathrm{Na}\left(\mathrm{g} / \mathrm{m}^{2}\right)\end{array}$ & $\begin{array}{c}\text { CCC PCT } \\
\mathrm{Si}\left(\mathrm{g} / \mathrm{m}^{2}\right)\end{array}$ & $\begin{array}{c}\text { CCC PCT } \\
\mathrm{pH}\end{array}$ & $\begin{array}{l}\text { Q PCT B at } \\
20^{\circ} \mathrm{C}\left(\mathrm{g} / \mathrm{m}^{2}\right)\end{array}$ & $\begin{array}{l}\text { Q PCT Li at } \\
20^{\circ} \mathrm{C}\left(\mathrm{g} / \mathrm{m}^{2}\right)\end{array}$ \\
\hline CVS1-16 & & & & & 0.523 & 0.266 & 0.540 & 0.241 & 12.01 & 21.688 & 10.075 & 8.058 & 1.717 & 12.28 & & \\
\hline CVS1-17 & & & & & 2.235 & 2.075 & 2.304 & 0.663 & 11.57 & 1.122 & 1.200 & 1.220 & 0.4335 & 11.45 & & \\
\hline CVS1-18 & & & & & 11.238 & 8.065 & 8.040 & 1.531 & 12.25 & 22.186 & 5.714 & 12.216 & 1.699 & 12.30 & & \\
\hline CVS1-19 & & & & & 0.523 & 0.533 & 0.433 & 0.166 & 10.29 & 0.410 & 0.503 & 0.363 & 0.138 & 10.11 & & \\
\hline CVS1-20 & & & & & 0.455 & 0.468 & 0.396 & 0.156 & 10.16 & 0.443 & 0.515 & 0.364 & 0.149 & 10.21 & & \\
\hline CVS1-21 & & & & & 18.850 & 15.591 & 15.536 & 0.459 & 9.68 & 16.881 & 19.355 & 18.718 & 0.694 & 9.32 & & \\
\hline CVS1-22 & & & & & 1.119 & 0.704 & 0.760 & 0.259 & 9.81 & 3.859 & 2.000 & 1.538 & 0.458 & 9.62 & & \\
\hline CVS1-23 & & & & & 0.525 & 0.535 & 0.487 & 0.229 & 10.29 & 0.370 & 0.373 & 0.339 & 0.193 & 9.87 & & \\
\hline
\end{tabular}

\section{Hanford CVS 2 (Hrma et al. 1994)}

\begin{tabular}{|c|c|c|c|c|c|c|c|c|c|c|c|c|c|c|c|}
\hline CVS2-1 & & & & & 0.312 & 0.369 & 0.208 & 0.17 & 10.21 & 0.386 & 0.305 & 0.248 & 0.237 & 10.30 & \\
\hline CVS2-2 & & & & & 0.128 & 0.276 & 0.188 & 0.075 & 10.92 & 0.136 & 0.215 & 0.160 & 0.077 & 10.77 & \\
\hline CVS2-3 & & & & & 0.137 & 0.222 & 0.125 & 0.064 & 10.08 & 0.134 & 0.198 & 0.116 & 0.06 & 10.14 & \\
\hline CVS2-4 & & & & & 0.158 & 0.300 & 0.182 & 0.101 & 10.40 & 0.147 & 0.241 & 0.154 & 0.09 & 10.30 & \\
\hline CVS2-5 & & & & & 0.284 & 0.382 & 0.284 & 0.106 & 9.58 & 0.263 & 0.285 & 0.264 & 0.146 & 9.61 & \\
\hline CVS2-6 & & & & & 1.185 & 1.303 & 1.284 & 0.421 & 10.48 & 1.240 & 1.064 & 0.934 & 0.418 & 10.26 & \\
\hline CVS2-7 & & & & & 0.740 & 0.812 & 0.343 & 0.164 & 9.89 & 0.653 & 0.666 & 0.337 & 0.189 & 9.82 & \\
\hline CVS2-8 & & & & & 0.484 & 0.523 & 0.445 & 0.263 & 10.75 & 0.850 & 1.183 & 0.561 & 0.522 & 11.34 & \\
\hline CVS2-9 & 948 & 93.07 & 943 & 111.05 & 0.560 & 0.620 & 0.374 & 0.241 & 10.34 & 0.545 & 0.484 & 0.314 & 0.236 & 10.24 & \\
\hline CVS2-10 & & & & & 1.332 & 1.322 & 0.828 & 0.115 & 9.46 & 0.965 & 0.968 & 0.645 & 0.162 & 9.51 & \\
\hline CVS2-11 & & & & & 1.587 & 1.564 & 1.370 & 0.51 & 11.46 & 1.019 & 1.108 & 1.092 & 0.426 & 11.33 & \\
\hline CVS2-12 & & & & & 0.194 & 0.262 & 0.184 & 0.075 & 9.90 & 0.136 & 0.213 & 0.156 & 0.082 & 9.90 & \\
\hline CVS2-13 & & & & & 0.360 & 0.362 & 0.478 & 0.182 & 11.14 & 0.345 & 0.335 & 0.390 & 0.177 & 10.94 & \\
\hline CVS2-14 & & & & & 1.656 & 1.468 & 1.268 & 0.261 & 10.46 & 1.580 & 1.254 & 1.210 & 0.258 & 10.50 & \\
\hline CVS2-15 & & & & & 0.331 & 0.451 & 0.608 & 0.18 & 11.30 & 1.493 & 1.558 & 0.163 & 0.208 & 9.87 & \\
\hline CVS2-16 & 948 & 65.71 & 948 & 70.62 & 2.937 & 2.349 & 2.182 & 0.558 & 10.36 & 2.634 & 1.878 & 1.722 & 0.535 & 10.30 & \\
\hline CVS2-17 & & & & & 0.495 & 0.571 & 0.474 & 0.167 & 10.27 & 0.676 & 0.629 & 0.423 & 0.156 & 10.30 & \\
\hline CVS2-18 & & & & & 2.578 & 2.094 & 1.886 & 0.546 & 10.34 & 2.443 & 1.778 & 1.617 & 0.559 & 10.23 & \\
\hline CVS2-19 & & & & & 1.990 & 1.654 & 1.481 & 0.45 & 10.35 & 1.358 & 1.095 & 1.045 & 0.377 & 10.26 & \\
\hline CVS2-20 & & & & & 0.347 & 0.386 & 0.279 & 0.187 & 10.89 & 0.322 & 0.402 & 0.268 & 0.206 & 10.90 & \\
\hline CVS2-21 & & & & & 3.854 & 2.534 & 2.089 & 0.506 & 10.26 & 3.181 & 2.458 & 1.752 & 0.487 & 10.34 & \\
\hline CVS2-22 & & & & & 9.646 & 5.453 & 6.097 & 2.249 & 11.72 & 5.932 & 3.978 & 4.497 & 2.166 & 11.48 & \\
\hline CVS2-23 & & & & & 0.173 & 0.604 & 0.809 & 0.144 & 11.81 & 0.203 & 0.559 & 0.708 & 0.14 & 11.76 & \\
\hline CVS2-24 & & & & & 4.522 & 3.226 & 2.060 & 0.179 & 9.80 & 4.019 & 3.226 & 1.831 & 0.187 & 9.91 & \\
\hline CVS2-25 & & & & & 4.662 & 2.765 & 3.526 & 1.214 & 11.91 & 1.775 & 4.255 & 1.202 & 1.345 & 12.04 & \\
\hline CVS2-26 & & & & & 1.628 & 1.436 & 1.349 & 0.275 & 10.11 & 1.326 & 1.567 & 1.448 & 0.328 & 10.17 & \\
\hline
\end{tabular}


Appendix A. Database - mass fraction

\begin{tabular}{|c|c|c|c|c|c|c|c|c|c|c|c|c|}
\hline & & & & & & & & & & & & \\
\hline Glass ID & $\begin{array}{l}\text { Q PCT Na at } \\
20^{\circ} \mathrm{C}\left(\mathrm{g} / \mathrm{m}^{2}\right)\end{array}$ & $\begin{array}{l}\text { Q PCT Si at } \\
20^{\circ} \mathrm{C}\left(\mathrm{g} / \mathrm{m}^{2}\right)\end{array}$ & $\begin{array}{c}\mathrm{QpH} \\
\text { at } 20^{\circ} \mathrm{C}\end{array}$ & $\begin{array}{c}\text { TCLP Ag } \\
(\mathrm{ppm})\end{array}$ & $\begin{array}{c}\text { TCLP As } \\
(\mathrm{ppm})\end{array}$ & $\begin{array}{c}\text { TCLP Ba } \\
(\mathrm{ppm})\end{array}$ & $\begin{array}{c}\text { TCLP Cd } \\
(\mathrm{ppm})\end{array}$ & $\begin{array}{c}\text { TCLP Cr } \\
(\mathrm{ppm})\end{array}$ & $\begin{array}{c}\text { TCLP Ni } \\
(\mathrm{ppm})\end{array}$ & $\begin{array}{c}\text { TCLP Pb } \\
(\mathrm{ppm})\end{array}$ & $\begin{array}{c}\text { TCLP Se } \\
(\mathrm{ppm})\end{array}$ & $\begin{array}{c}\text { TCLP Zn } \\
(\mathrm{ppm})\end{array}$ \\
\hline CVS1-16 & & & & & & & & & & & & \\
\hline CVS1-17 & & & & & & & & & & & & \\
\hline CVS1-18 & & & & & & & & & & & & \\
\hline CVS1-19 & & & & & & & & & & & & \\
\hline CVS1-20 & & & & & & & & & & & & \\
\hline CVS1-21 & & & & & & & & & & & & \\
\hline CVS1-22 & & & & & & & & & & & & \\
\hline CVS1-23 & & & & & & & & & & & & \\
\hline
\end{tabular}

\section{Hanford CVS 2 (Hrma et al. 1994)}

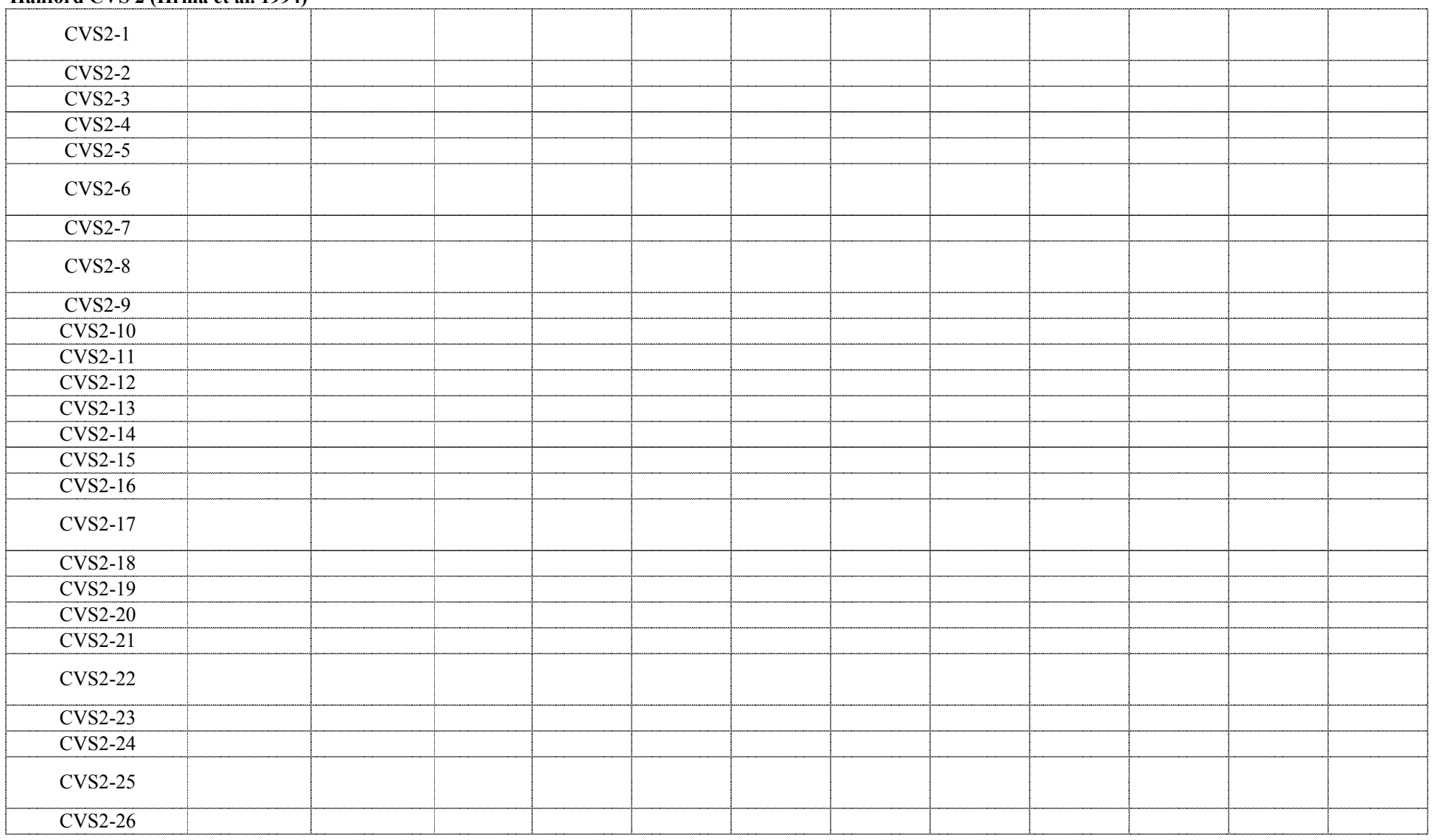




\section{Hanford CVS 2 (Hrma et al. 1994)}

\begin{tabular}{|c|c|c|c|c|c|c|c|c|c|c|c|c|c|c|c|c|c|c|c|c|c|}
\hline \multirow[b]{2}{*}{ Glass ID } & & & & & & & & & & & & & & & & & & & & & \\
\hline & $\begin{array}{c}\mathrm{A} 12 \mathrm{O} 3 \\
-\mathrm{t}\end{array}$ & $\begin{array}{c}\mathrm{B} 2 \mathrm{O} 3 \\
-\mathrm{t}\end{array}$ & $\begin{array}{c}\mathrm{CaO} \\
-\mathrm{t}\end{array}$ & $\begin{array}{c}\mathrm{Fe} 2 \mathrm{O} 3 \\
-\mathrm{t}\end{array}$ & $\begin{array}{c}\mathrm{FeO} \\
-\mathrm{t}\end{array}$ & $\begin{array}{c}\mathrm{K} 2 \mathrm{O} \\
-\mathrm{t}\end{array}$ & $\begin{array}{c}\mathrm{Li} 2 \mathrm{O} \\
-\mathrm{t}\end{array}$ & $\begin{array}{c}\mathrm{MgO} \\
-\mathrm{t}\end{array}$ & $\begin{array}{c}\mathrm{Na} 2 \mathrm{O} \\
-\mathrm{t}\end{array}$ & $\begin{array}{c}\mathrm{NiO} \\
-\mathrm{t}\end{array}$ & $\begin{array}{c}\mathrm{P} 2 \mathrm{O} 5 \\
-\mathrm{t}\end{array}$ & $\begin{array}{c}\mathrm{SiO}^{2} \\
-\mathrm{t}\end{array}$ & $\begin{array}{c}\mathrm{ZrO} 2 \\
-\mathrm{t}\end{array}$ & $\begin{array}{c}\mathrm{Ag} 2 \mathrm{O} \\
-\mathrm{t}\end{array}$ & $\begin{array}{c}\mathrm{As} 2 \mathrm{O} 3 \\
-\mathrm{t}\end{array}$ & $\begin{array}{c}\mathrm{BaO} \\
-\mathrm{t}\end{array}$ & $\begin{array}{c}\mathrm{Bi} 2 \mathrm{O} 3 \\
-\mathrm{t}\end{array}$ & $\begin{array}{c}\mathrm{Br} \\
-\mathrm{t}\end{array}$ & $\begin{array}{c}\mathrm{CdO} \\
-\mathrm{t}\end{array}$ & $\begin{array}{c}\mathrm{Ce} 2 \mathrm{O} 3 \\
-\mathrm{t}\end{array}$ & $\begin{array}{c}\mathrm{CeO} 2 \\
-\mathrm{t}\end{array}$ \\
\hline CVS2-27 & 0.0782 & 0.0500 & 0.0000 & 0.0200 & & 0.0000 & 0.0155 & 0.0800 & 0.2000 & 0.0010 & 0.0002 & 0.5463 & 0.0000 & 0.0000 & & 0.0002 & 0.0000 & & 0.0013 & & 0.0003 \\
\hline CVS2-28 & 0.0555 & 0.0500 & 0.0000 & 0.0200 & & 0.0000 & 0.0126 & 0.0000 & 0.2000 & 0.0103 & 0.0018 & 0.5622 & 0.0000 & 0.0000 & & 0.0018 & 0.0000 & & 0.0134 & & 0.0027 \\
\hline CVS2-29 & 0.0000 & 0.2001 & 0.0800 & 0.0200 & & 0.0000 & 0.0100 & 0.0000 & 0.0675 & 0.0103 & 0.0018 & 0.4393 & 0.0834 & 0.0000 & & 0.0018 & 0.0000 & & 0.0134 & & 0.0027 \\
\hline CVS2-30 & 0.0458 & 0.2000 & 0.0000 & 0.1320 & & 0.0000 & 0.0100 & 0.0000 & 0.0832 & 0.0010 & 0.0002 & 0.5190 & 0.0000 & 0.0000 & & 0.0002 & 0.0000 & & 0.0013 & & 0.0003 \\
\hline CVS2-31 & 0526 & 0.1843 & 0.0800 & 0.0200 & & 0.0000 & 0.0331 & 0.0000 & 0.0500 & 0.0010 & 0.0002 & 0.5700 & 0.0000 & 0.0000 & & 0.0002 & 0.0000 & & 0.0013 & & 0.0003 \\
\hline CVS2-32 & 0.0027 & 0.0500 & 0.0000 & 0.0200 & & 0.0000 & 0.0428 & 0.0000 & 0.2000 & 0.0010 & 0.0002 & 0.5445 & 0.1300 & 0.0000 & & 0.0002 & 0.0000 & & 0.0013 & & 0.0003 \\
\hline CVS2-33 & 0.0892 & 0.0544 & 0.0000 & 0.0200 & & 0.0000 & 0.0364 & 0.0800 & 0.2001 & 0.0103 & 0.0018 & 0.4202 & 0.0000 & 0.0000 & & 0.0018 & 0.0000 & & 0.0134 & & 0.0027 \\
\hline CVS2-34 & 0.1388 & 0.1743 & 0.0000 & 0.0200 & & 0.0000 & 0.0369 & 0.0000 & 0.2000 & 0.0010 & 0.0002 & 0.4200 & 0.0000 & 0.0000 & & 0.0002 & 0.0000 & & 0.0013 & & 0.0003 \\
\hline CVS2-35 & 0.1340 & 0.0500 & 0.0800 & 0.0632 & & 0.0000 & 0.0428 & 0.0000 & 0.2000 & 0.0010 & 0.0002 & 0.4200 & 0.0000 & 0.0000 & & 0.0002 & 0.0000 & & 0.0013 & & 0.0003 \\
\hline CVS2-36 & 0.0088 & 0.0500 & 0.0800 & 0.1500 & & 0.0000 & 0.0700 & 0.0000 & 0.0891 & 0.0010 & 0.0002 & 0.5421 & 0.0000 & 0.0000 & & 0.0002 & 0.0000 & & 0.0013 & & 0.0003 \\
\hline CVS2-37 & 0.1400 & 0.0839 & 0.0000 & 0.0200 & & 0.0000 & 0.0700 & 0.0000 & 1061 & 0.0010 & 0.0002 & 0.5700 & 0.0000 & 0.0000 & & 0.0002 & 0.0000 & & 0.0013 & & 0.0003 \\
\hline CVS2-38 & 0.0272 & 0.1109 & 0.0000 & 0.1428 & & 0.0000 & 0.0100 & 0.0800 & 0.1044 & 0.0010 & 0.0002 & 0.5147 & 0.0000 & 0.0000 & & 0.0002 & 0.0000 & & 0.0013 & & 0.0003 \\
\hline CVS2-39 & 0.0258 & 0.0500 & 0.0000 & 0.0742 & & 0000 & 0.0700 & 0.0800 & 0.1362 & 0.0010 & 0.0002 & 0.4838 & 0.0700 & 0.0000 & & 0.0002 & 0.0000 & & 0.0013 & & 0.0003 \\
\hline CVS2-40 & 0.1000 & 0.0639 & 0.0200 & 0.0200 & & 0.0000 & 0.0421 & 0.0500 & 0.1500 & 0.0031 & 0.0005 & 0.5040 & 0.0200 & 0.0000 & & 0.0005 & 0.0000 & & 0.0040 & & 0.0008 \\
\hline CVS2-41 & 0.1000 & 0.0694 & 0.0500 & 0.0300 & & 0.0000 & 0.0700 & 0.0200 & 0.0781 & 0.0031 & 0.0005 & 0.5325 & 0.0200 & 0.0000 & & 0.0005 & 0.0000 & & 0.0040 & & 0.0008 \\
\hline CVS2-42 & 0.0300 & 0.0500 & 0.0320 & 0.1000 & & 0.0000 & 0.0700 & 0.0380 & 0.0625 & 0.0031 & 0.0005 & 0.5675 & 0.0200 & 0.0000 & & 0.0005 & 0.0000 & & 0.0040 & & 0.0008 \\
\hline CVS2-43 & 0.0500 & 0.1477 & 0.0200 & 0.0300 & & 0000 & 0.0653 & 0.0300 & 0.0500 & 0.0031 & 0.0005 & 0.5070 & 0.0700 & 0.0000 & & 0.0005 & 0.0000 & & 0.0040 & & 0.0008 \\
\hline CVS2-44 & 0.0623 & 0.1078 & 0.0500 & 0.0200 & & 0.0000 & 0.0699 & 0.0200 & 0.0500 & 0.0031 & 0.0005 & 0.5700 & 0.0200 & 0.0000 & & 0.0005 & 0.0000 & & 0.0040 & & 0.0008 \\
\hline CVS2-45 & 0.0592 & 0.1106 & 0.0200 & 0.0308 & & 0.0000 & 0.0595 & 0.0500 & 0.0500 & 0.0072 & 0.0013 & 0.5301 & 0.0200 & 0.0000 & & 0.0013 & 0.0000 & & 0.0094 & & 0.0019 \\
\hline CVS2-46 & 0.0746 & 0.1259 & 0.0200 & 0.0200 & & 0000 & 0.0700 & 0.0200 & 0.0577 & 0.0067 & 0.0012 & 0.5266 & 0.0200 & 0.0000 & & 0.0012 & 0.0000 & & 0.0088 & & 0.0018 \\
\hline CVS2-47 & 0.0400 & 0.0500 & 0.0500 & 0.0200 & & 0.0000 & 0.0429 & 0.0200 & 0.1277 & 0.0072 & 0.0013 & 0.5296 & 0.0500 & 0.0000 & & 0.0013 & 0.0000 & & 0.0094 & & 0.0019 \\
\hline CVS2-48 & 0.0854 & 0.1442 & 0.0500 & 0.0200 & & 0.0000 & 0.0390 & 0.0200 & 0.0968 & 0.0056 & 0.0010 & 0.4701 & 0.0200 & 0.0000 & & 0.0010 & 0.0000 & & 0.0073 & & 0.0015 \\
\hline CVS2-49 & 0.0785 & 0.1357 & 0.0200 & 0.0515 & & 0.0000 & 0.0413 & 0.0200 & 0.0957 & 0.0031 & 0.0005 & 0.5073 & 0.0200 & 0.0000 & & 0.0005 & 0.0000 & & 0.0040 & & 0.0008 \\
\hline CVS2-50 & 0.0636 & 0.1142 & 0.0275 & 0.0568 & & 0.0000 & 0.0376 & 0.0363 & 0.1003 & 0.0042 & 0.0007 & 0.4802 & 0.0429 & 0.0000 & & 0.0007 & 0.0000 & & 0.0055 & & 0.0011 \\
\hline CVS2-51 & 0.0235 & 0.1048 & 0.0082 & 0.0733 & & 0.0000 & 0.0373 & 0.0084 & 0.1129 & 0.0060 & 0.0010 & 0.5330 & 0.0392 & 0.0000 & & 0.0010 & 0.0000 & & 0.0065 & & 0.0002 \\
\hline CVS2-52 & 0.0233 & 0.0817 & 0.0008 & 0.0720 & & 0.0000 & 0.0788 & 0.0009 & 0.0450 & 0.0061 & 0.0011 & 0.6002 & 0.0385 & 0.0000 & & 0.0011 & 0.0000 & & 0.0079 & & 0.0016 \\
\hline CVS2-53 & 0.0800 & 0.0874 & 0.0000 & 0.0400 & & 0.0000 & 0.0600 & 0.0500 & 0.0700 & 0.0082 & 0.0014 & 0.5226 & 0.0100 & 0.0000 & & 0.0014 & 0.0000 & & 0.0107 & & 0.0022 \\
\hline CVS2-54 & 0.0235 & 0.1048 & 0.0082 & 0.0733 & & 0.0000 & 0.0373 & 0.0084 & 0.1129 & 0.0061 & 0.0010 & 0.5328 & 0.0392 & 0.0000 & & 0.0010 & 0.0000 & & 0.0065 & & 0.0002 \\
\hline CVS2-55 & 0.0235 & 0.1048 & 0.0082 & 0.0733 & & 0.0000 & 0.0373 & 0.0084 & 0.1129 & 0.0000 & 0.0000 & 0.5328 & 0.0392 & 0.0000 & 0.0000 & 0.0000 & 0.0000 & & 0.0000 & & 0.0000 \\
\hline CVS2-56 & 0.0235 & 0.1048 & 0.0082 & 0.0733 & & 0.0000 & 0.0373 & 0.0084 & 0.1129 & 0.0000 & 0.0000 & 0.5328 & 0.0392 & 0.0000 & 0.0000 & 0.0000 & 0.0000 & & 0.0000 & & 0.0000 \\
\hline CVS2-57 & 0.0235 & 0.1048 & 0.0082 & 0.0733 & & 0.0000 & 0.0373 & 0.0084 & 0.1129 & 0.0000 & 0.0000 & 0.5328 & 0.0392 & 0.0000 & & 0.0000 & 0.0000 & & 0.0000 & & 0.0000 \\
\hline CVS2-58 & 0.1500 & 0.2000 & 0.0200 & 0.0200 & & 0.0000 & 0.0700 & 0.0800 & 0.0500 & 0.0010 & 0.0002 & 0.3900 & 0.0100 & 0.0000 & & 0.0002 & 0.0000 & & 0.0013 & & 0.0003 \\
\hline CVS2-59 & 0.1150 & 0.1718 & 0.0375 & 0.0200 & & 0.0000 & 0.0727 & 0.0005 & 0.1268 & 0.0011 & 0.0002 & 0.4380 & 0.0075 & 0.0000 & & 0.0002 & 0.0000 & & 0.0014 & & 0.0003 \\
\hline CVS2-60 & 0.0925 & 0.0876 & 0.0063 & 0.0200 & & 0.0000 & 0.0743 & 0.0005 & 0.1725 & 0.0011 & 0.0002 & 0.5281 & 0.0075 & 0.0000 & & 0.0002 & 0.0000 & & 0.0014 & & 0.0003 \\
\hline CVS2-61 & 0.1625 & 0.0664 & 0.0000 & 0.0200 & & 0.0000 & 0.0730 & 0.0000 & 0.1200 & 0.0013 & 0.0002 & 0.5281 & 0.0175 & 0.0000 & & 0.0002 & 0.0000 & & 0.0017 & & 0.0003 \\
\hline CVS2-62 & \begin{tabular}{|l|}
0.0500 \\
\end{tabular} & 0.1765 & 0.0500 & 0.0200 & & 0.0000 & 0.0156 & 0.0005 & 0.1125 & 0.0010 & 0.0002 & 0.5579 & 0.0075 & 0.0000 & & 0.0002 & 0.0000 & & 0.0013 & & 0.0003 \\
\hline CVS2-63 & 0.1800 & 0.1717 & 0.1000 & 0.0200 & & 0.0000 & 0.0051 & 0.0000 & 0.1900 & 0.0010 & 0.0002 & 0.3232 & 0.0000 & 0.0000 & & 0.0002 & 0.0000 & & 0.0013 & & 0.0003 \\
\hline CVS2-64 & 0.0288 & 0.0509 & 0.0025 & 0.0812 & & 0.0009 & 0.0642 & 0.0008 & 0.0925 & 0.0098 & 0.0037 & 0.5697 & 0.0431 & 0.0000 & & 0.0000 & 0.0000 & & 0.0129 & & 0.0026 \\
\hline CVS2-65 & 0.0196 & 0.1128 & 0.0007 & 0.0013 & & 0.0087 & 0.0697 & 0.0004 & 0.0860 & 0.0000 & 0.0000 & 0.5344 & 0.1548 & 0.0000 & & 0.0000 & 0.0000 & & 0.0000 & & 0.0000 \\
\hline
\end{tabular}


Hanford CVS 2 (Hrma et al. 1994)

\begin{tabular}{|c|c|c|c|c|c|c|c|c|c|c|c|c|c|c|c|c|c|c|c|c|c|}
\hline Glass ID & $\begin{array}{l}\mathrm{Cl} \\
-\mathrm{t}\end{array}$ & $\begin{array}{c}\mathrm{CoO} \\
-\mathrm{t}\end{array}$ & $\begin{array}{c}\mathrm{Co} 2 \mathrm{O} 3 \\
-\mathrm{t}\end{array}$ & $\begin{array}{c}\mathrm{Cr} 2 \mathrm{O} 3 \\
-\mathrm{t}\end{array}$ & $\begin{array}{c}\mathrm{Cs} 2 \mathrm{O} \\
-\mathrm{t}\end{array}$ & $\begin{array}{c}\mathrm{CuO} \\
-\mathrm{t}\end{array}$ & $\begin{array}{c}\mathrm{Eu} 2 \mathrm{O} 3 \\
-\mathrm{t}\end{array}$ & $\begin{array}{l}F \\
-t\end{array}$ & $\begin{array}{c}\mathrm{Ga} 2 \mathrm{O} 3 \\
-\mathrm{t}\end{array}$ & $\begin{array}{c}\mathrm{Gd} 2 \mathrm{O} 3 \\
-\mathrm{t}\end{array}$ & $\begin{array}{c}\mathrm{HgO} \\
-\mathrm{t}\end{array}$ & $\begin{array}{l}I \\
-t\end{array}$ & $\begin{array}{c}\mathrm{La} 2 \mathrm{O} 3 \\
-\mathrm{t}\end{array}$ & $\begin{array}{c}\mathrm{MnO} 2 \\
-\mathrm{t}\end{array}$ & $\begin{array}{c}\mathrm{MnO} \\
-\mathrm{t}\end{array}$ & $\begin{array}{c}\mathrm{MoO} \\
-\mathrm{t}\end{array}$ & $\begin{array}{c}\mathrm{MoO} 3 \\
-\mathrm{t}\end{array}$ & $\begin{array}{c}\mathrm{Nb} 2 \mathrm{O} 5 \\
-\mathrm{t}\end{array}$ & $\begin{array}{c}\mathrm{Nd} 2 \mathrm{O} 3 \\
-\mathrm{t}\end{array}$ & $\begin{array}{c}\mathrm{PbO} \\
-\mathrm{t}\end{array}$ & $\begin{array}{c}\mathrm{PdO} 2 \\
-\mathrm{t}\end{array}$ \\
\hline CVS2-27 & & & 0.0000 & 0.0002 & 0.0003 & 0.0003 & & 0.0005 & & & & & 0.0011 & 0.0002 & & & 0.0005 & & 0.0022 & 0.0000 & \\
\hline CVS2-28 & & & 0.0000 & 0.0022 & 0.0027 & 0.0027 & & 0.0054 & & & & & 0.0112 & 0.0022 & & & 0.0054 & & 0.0221 & 0.0000 & \\
\hline CVS2-29 & & & 0.0000 & 0.0022 & 0.0027 & 0.0027 & & 0.0054 & & & & & 0.0112 & 0.0022 & & & 0.0054 & & 0.0221 & 0.0000 & \\
\hline CVS2-30 & & & 0.0000 & 0.0002 & 0.0003 & 0.0003 & & 0.0005 & & & & & 0.0011 & 0.0002 & & & 0.0005 & & 0.0022 & 0.0000 & \\
\hline CVS2-31 & & & 0.0000 & 0.0002 & 0.0003 & 0.0003 & & 0.0005 & & & & & 0.0011 & 0.0002 & & & 0.0005 & & 0.0022 & 0.0000 & \\
\hline CVS2-32 & & & 0.0000 & 0.0002 & 0.0003 & 0.0003 & & 0.0005 & & & & & 0.0011 & 0.0002 & & & 0.0005 & & 0.0022 & 0.0000 & \\
\hline CVS2-33 & & & 0.0000 & 0.0022 & 0.0027 & 0.0027 & & 0.0054 & & & & & 0.0112 & 0.0022 & & & 0.0054 & & 0.0221 & 0.0000 & \\
\hline CVS2-34 & & & 0.0000 & 0.0002 & 0.0003 & 0.0003 & & 0.0005 & & & & & 0.0011 & 0.0002 & & & 0.0005 & & 0.0022 & 0.0000 & \\
\hline CVS2-35 & & & 0.0000 & 0.0002 & 0.0003 & 0.0003 & & 0.0005 & & & & & 0.0011 & 0.0002 & & & 0.0005 & & 0.0022 & 0.0000 & \\
\hline CVS2-36 & & & 0.0000 & 0.0002 & 0.0003 & 0.0003 & & 0.0005 & & & & & 0.0011 & 0.0002 & & & 0.0005 & & 0.0022 & 0.0000 & \\
\hline CVS2-37 & & & 0.0000 & 0.0002 & 0.0003 & 0.0003 & & 0.0005 & & & & & 0.0011 & 0.0002 & & & 0.0005 & & 0.0022 & 0.0000 & \\
\hline CVS2-38 & & & 0.0000 & 0.0002 & 0.0003 & 0.0003 & & 0.0005 & & & & & 0.0011 & 0.0002 & & & 0.0005 & & 0.0022 & 0.0000 & \\
\hline CVS2-39 & & & 0.0000 & 0.0002 & 0.0003 & 0.0003 & & 0.0005 & & & & & 0.0011 & 0.0002 & & & 0.0005 & & 0.0022 & 0.0000 & \\
\hline CVS2-40 & & & 0.0000 & 0.0007 & 0.0008 & 0.0008 & & 0.0016 & & & & & 0.0034 & 0.0007 & & & 0.0016 & & 0.0066 & 0.0000 & \\
\hline CVS2-41 & & & 0.0000 & 0.0007 & 0.0008 & 0.0008 & & 0.0016 & & & & & 0.0034 & 0.0007 & & & 0.0016 & & 0.0066 & 0.0000 & \\
\hline CVS2-42 & & & 0.0000 & 0.0007 & 0.0008 & 0.0008 & & 0.0016 & & & & & 0.0034 & 0.0007 & & & 0.0016 & & 0.0066 & 0.0000 & \\
\hline CVS2-43 & & & 0.0000 & 0.0007 & 0.0008 & 0.0008 & & 0.0016 & & & & & 0.0034 & 0.0007 & & & 0.0016 & & 0.0066 & 0.0000 & \\
\hline CVS2-44 & & & 0.0000 & 0.0007 & 0.0008 & 0.0008 & & 0.0016 & & & & & 0.0034 & 0.0007 & & & 0.0016 & & 0.0066 & 0.0000 & \\
\hline CVS2-45 & & & 0.0000 & 0.0016 & 0.0019 & 0.0019 & & 0.0038 & & & & & 0.0078 & 0.0015 & & & 0.0038 & & 0.0155 & 0.0000 & \\
\hline CVS2-46 & & & 0.0000 & 0.0015 & 0.0018 & 0.0018 & & 0.0035 & & & & & 0.0073 & 0.0014 & & & 0.0035 & & 0.0145 & 0.0000 & \\
\hline CVS2-47 & & & 0.0000 & 0.0016 & 0.0019 & 0.0019 & & 0.0038 & & & & & 0.0078 & 0.0015 & & & 0.0038 & & 0.0155 & 0.0000 & \\
\hline CVS2-48 & & & 0.0000 & 0.0012 & 0.0015 & 0.0015 & & 0.0029 & & & & & 0.0061 & 0.0012 & & & 0.0029 & & 0.0121 & 0.0000 & \\
\hline CVS2-49 & & & 0.0000 & 0.0007 & 0.0008 & 0.0008 & & 0.0016 & & & & & 0.0034 & 0.0007 & & & 0.0016 & & 0.0066 & 0.0000 & \\
\hline CVS2-50 & & & 0.0000 & 0.0009 & 0.0011 & 0.0011 & & 0.0022 & & & & & 0.0046 & 0.0009 & & & 0.0022 & & 0.0090 & 0.0000 & \\
\hline CVS2-51 & & & 0.0000 & 0.0013 & 0.0015 & 0.0016 & & 0.0019 & & & & & 0.0155 & 0.0013 & & & 0.0031 & & 0.0057 & 0.0000 & \\
\hline CVS2-52 & & & 0.0000 & 0.0013 & 0.0016 & 0.0016 & & 0.0032 & & & & & 0.0066 & 0.0013 & & & 0.0032 & & 0.0130 & 0.0000 & \\
\hline CVS2-53 & & & 0.0000 & 0.0018 & 0.0022 & 0.0022 & & 0.0043 & & & & & 0.0090 & 0.0018 & & & 0.0043 & & 0.0000 & 0.0000 & \\
\hline CVS2-54 & & & 0.0000 & 0.0013 & 0.0015 & 0.0016 & & 0.0019 & & & & & 0.0155 & 0.0013 & & & 0.0031 & & 0.0000 & 0.0000 & \\
\hline CVS2-55 & & & 0.0000 & 0.0089 & 0.0000 & 0.0000 & & 0.0030 & & & & & 0.0000 & 0.0280 & & & 0.0000 & & 0.0167 & 0.0000 & \\
\hline CVS2-56 & & & 0.0000 & 0.0072 & 0.0000 & 0.0000 & & 0.0030 & & & & & 0.0000 & 0.0465 & & & 0.0000 & & 0.0000 & 0.0000 & \\
\hline CVS2-57 & & & 0.0000 & 0.0054 & 0.0000 & 0.0000 & & 0.0030 & & & & & 0.0000 & 0.0000 & & & 0.0000 & & 0.0513 & 0.0000 & \\
\hline CVS2-58 & & & 0.0000 & 0.0002 & 0.0003 & 0.0003 & & 0.0005 & & & & & 0.0011 & 0.0002 & & & 0.0005 & & 0.0022 & 0.0000 & \\
\hline CVS2-59 & & & 0.0000 & 0.0002 & 0.0003 & 0.0003 & & 0.0005 & & & & & 0.0011 & 0.0002 & & & 0.0005 & & 0.0023 & 0.0000 & \\
\hline CVS2-60 & & & 0.0000 & 0.0002 & 0.0003 & 0.0003 & & 0.0006 & & & & & 0.0012 & 0.0002 & & & 0.0006 & & 0.0024 & 0.0000 & \\
\hline CVS2-61 & & & 0.0000 & 0.0003 & 0.0003 & 0.0003 & & 0.0007 & & & & & 0.0014 & 0.0003 & & & 0.0007 & & 0.0028 & 0.0000 & \\
\hline CVS2-62 & & & 0.0000 & 0.0002 & 0.0003 & 0.0003 & & 0.0005 & & & & & 0.0011 & 0.0002 & & & 0.0005 & & 0.0021 & 0.0000 & \\
\hline CVS2-63 & & & 0.0000 & 0.0002 & 0.0003 & 0.0003 & & 0.0005 & & & & & 0.0011 & 0.0002 & & & 0.0005 & & 0.0022 & 0.0000 & \\
\hline CVS2-64 & & & 0.0000 & 0.0011 & 0.0026 & 0.0000 & & 0.0003 & & & & & 0.0028 & 0.0000 & & & 0.0024 & & 0.0146 & 0.0028 & \\
\hline CVS2-65 & & & 0.0000 & 0.0048 & 0.0000 & 0.0000 & & 0.0000 & & & & & 0.0004 & 0.0000 & & & 0.0000 & & 0.0062 & 0.0000 & \\
\hline
\end{tabular}


Hanford CVS 2 (Hrma et al. 1994)

\begin{tabular}{|c|c|c|c|c|c|c|c|c|c|c|c|c|c|c|c|c|c|c|c|c|c|}
\hline Glass ID & $\begin{array}{c}\mathrm{PdO} \\
-\mathrm{t}\end{array}$ & $\begin{array}{c}\mathrm{Pr} 2 \mathrm{O} 3 \\
-\mathrm{t}\end{array}$ & $\begin{array}{c}\text { Pr6O11 } \\
-t\end{array}$ & $\begin{array}{c}\mathrm{Rb} 2 \mathrm{O} \\
-\mathrm{t}\end{array}$ & $\begin{array}{c}\mathrm{ReO} \\
-\mathrm{t}\end{array}$ & $\begin{array}{c}\mathrm{ReO} 2 \\
-\mathrm{t}\end{array}$ & $\begin{array}{c}\mathrm{Rh} 2 \mathrm{O} 3 \\
-\mathrm{t}\end{array}$ & $\begin{array}{c}\mathrm{RhO} 2 \\
-\mathrm{t}\end{array}$ & $\begin{array}{c}\mathrm{RuO} 2 \\
-\mathrm{t} \\
\end{array}$ & $\begin{array}{c}\mathrm{Sb} 2 \mathrm{O} 3 \\
-\mathrm{t}\end{array}$ & $\begin{array}{c}\mathrm{Sb} 2 \mathrm{O} 5 \\
-\mathrm{t}\end{array}$ & $\begin{array}{c}\mathrm{SeO} 2 \\
-\mathrm{t}\end{array}$ & $\begin{array}{c}\mathrm{Sm} 2 \mathrm{O} 3 \\
-\mathrm{t}\end{array}$ & $\begin{array}{c}\mathrm{SnO} \\
-\mathrm{t}\end{array}$ & $\begin{array}{c}\mathrm{SnO} 2 \\
-\mathrm{t}\end{array}$ & $\begin{array}{c}\mathrm{SO} 3 \\
-\mathrm{t}\end{array}$ & $\begin{array}{c}\mathrm{SrO} \\
-\mathrm{t}\end{array}$ & $\begin{array}{c}\mathrm{Tc} 2 \mathrm{O} 7 \\
-\mathrm{t}\end{array}$ & $\begin{array}{c}\mathrm{TeO} 2 \\
-\mathrm{t}\end{array}$ & $\begin{array}{c}\mathrm{ThO} 2 \\
-\mathrm{t}\end{array}$ & $\begin{array}{c}\mathrm{TiO} 2 \\
-\mathrm{t}\end{array}$ \\
\hline CVS2-27 & 0.0001 & & 0.0002 & 0.0001 & & & 0.0001 & & 0.0003 & & & & 0.0001 & & & 0.0005 & 0.0002 & & 0.0000 & & 0.0000 \\
\hline CVS2-28 & 0.0009 & & 0.0018 & 0.0009 & & & 0.0009 & & 0.0027 & & & & 0.0009 & & & 0.0049 & 0.0018 & & 0.0000 & & 0.0000 \\
\hline CVS2-29 & 0.0009 & & 0.0018 & 0.0009 & & & 0.0009 & & 0.0027 & & & & 0.0009 & & & 0.0049 & 0.0018 & & 0.0000 & & 0.0000 \\
\hline CVS2-30 & 0.0001 & & 0.0002 & 0.0001 & & & 0.0001 & & 0.0003 & & & & 0.0001 & & & 0.0005 & 0.0002 & & 0.0000 & & 0.0000 \\
\hline CVS2-31 & 0.0001 & & 0.0002 & 0.0001 & & & 0.0001 & & 0.0003 & & & & 0.0001 & & & 0.0005 & 0.0002 & & 0.0000 & & 0.0000 \\
\hline CVS2-32 & 0.0001 & & 0.0002 & 0.0001 & & & 0.0001 & & 0.0003 & & & & 0.0001 & & & 0.0005 & 0.0002 & & 0.0000 & & 0.0000 \\
\hline CVS2-33 & 0.0009 & & 0.0018 & 0.0009 & & & 0.0009 & & 0.0027 & & & & 0.0009 & & & 0.0049 & 0.0018 & & 0.0000 & & 0.0000 \\
\hline CVS2-34 & 0.0001 & & 0.0002 & 0.0001 & & & 0.0001 & & 0.0003 & & & & 0.0001 & & & 0.0005 & 0.0002 & & 0.0000 & & 0.0000 \\
\hline CVS2-35 & 0.0001 & & 0.0002 & 0.0001 & & & 0.0001 & & 0.0003 & & & & 0.0001 & & & 0.0005 & 0.0002 & & 0.0000 & & 0.0000 \\
\hline CVS2-36 & 0.0001 & & 0.0002 & 0.0001 & & & 0.0001 & & 0.0003 & & & & 0.0001 & & & 0.0005 & 0.0002 & & 0.0000 & & 0.0000 \\
\hline CVS2-37 & 0.0001 & & 0.0002 & 0.0001 & & & 0.0001 & & 0.0003 & & & & 0.0001 & & & 0.0005 & 0.0002 & & 0.0000 & & 0.0000 \\
\hline CVS2-38 & 0.0001 & & 0.0002 & 0.0001 & & & 0.0001 & & 0.0003 & & & & 0.0001 & & & 0.0005 & 0.0002 & & 0.0000 & & 0.0000 \\
\hline CVS2-39 & 0.0001 & & 0.0002 & 0.0001 & & & 0.0001 & & 0.0003 & & & & 0.0001 & & & 0.0005 & 0.0002 & & 0.0000 & & 0.0000 \\
\hline CVS2-40 & 0.0003 & & 0.0005 & 0.0003 & & & 0.0003 & & 0.0008 & & & & 0.0003 & & & 0.0015 & 0.0005 & & 0.0000 & & 0.0000 \\
\hline CVS2-41 & 0.0003 & & 0.0005 & 0.0003 & & & 0.0003 & & 0.0008 & & & & 0.0003 & & & 0.0015 & 0.0005 & & 0.0000 & & 0.0000 \\
\hline CVS2-42 & 0.0003 & & 0.0005 & 0.0003 & & & 0.0003 & & 0.0008 & & & & 0.0003 & & & 0.0015 & 0.0005 & & 0.0000 & & 0.0000 \\
\hline CVS2-43 & 0.0003 & & 0.0005 & 0.0003 & & & 0.0003 & & 0.0008 & & & & 0.0003 & & & 0.0015 & 0.0005 & & 0.0000 & & 0.0000 \\
\hline CVS2-44 & 0.0003 & & 0.0005 & 0.0003 & & & 0.0003 & & 0.0008 & & & & 0.0003 & & & 0.0015 & 0.0005 & & 0.0000 & & 0.0000 \\
\hline CVS2-45 & 0.0006 & & 0.0013 & 0.0006 & & & 0.0006 & & 0.0019 & & & & 0.0006 & & & 0.0035 & 0.0013 & & 0.0000 & & 0.0000 \\
\hline CVS2-46 & 0.0006 & & 0.0012 & 0.0006 & & & 0.0006 & & 0.0018 & & & & 0.0006 & & & 0.0032 & 0.0012 & & 0.0000 & & 0.0000 \\
\hline CVS2-47 & 0.0006 & & 0.0013 & 0.0006 & & & 0.0006 & & 0.0019 & & & & 0.0006 & & & 0.0035 & 0.0013 & & 0.0000 & & 0.0000 \\
\hline CVS2-48 & 0.0005 & & 0.0010 & 0.0005 & & & 0.0005 & & 0.0015 & & & & 0.0005 & & & 0.0027 & 0.0010 & & 0.0000 & & 0.0000 \\
\hline CVS2-49 & 0.0003 & & 0.0005 & 0.0003 & & & 0.0003 & & 0.0008 & & & & 0.0003 & & & 0.0015 & 0.0005 & & 0.0000 & & 0.0000 \\
\hline CVS2-50 & 0.0004 & & 0.0007 & 0.0004 & & & 0.0004 & & 0.0011 & & & & 0.0004 & & & 0.0020 & 0.0007 & & 0.0000 & & 0.0000 \\
\hline CVS2-51 & 0.0000 & & 0.0019 & 0.0000 & & & 0.0000 & & 0.0015 & & & & 0.0000 & & & 0.0044 & 0.0000 & & 0.0000 & & 0.0000 \\
\hline CVS2-52 & 0.0005 & & 0.0011 & 0.0005 & & & 0.0005 & & 0.0016 & & & & 0.0005 & & & 0.0029 & 0.0011 & & 0.0000 & & 0.0000 \\
\hline CVS2-53 & 0.0007 & & 0.0014 & 0.0007 & & & 0.0007 & & 0.0022 & & & & 0.0007 & & & 0.0039 & 0.0014 & & 0.0000 & & 0.0000 \\
\hline CVS2-54 & 0.0000 & & 0.0019 & 0.0000 & & & 0.0000 & & 0.0015 & & & & 0.0000 & & & 0.0044 & 0.0000 & & 0.0000 & & 0.0000 \\
\hline CVS2-55 & 0.0000 & & 0.0000 & 0.0000 & & & 0.0000 & & 0.0000 & 0.0000 & & 0.0000 & 0.0000 & & & 0.0030 & 0.0000 & & 0.0000 & & 0.0000 \\
\hline CVS2-56 & 0.0000 & & 0.0000 & 0.0000 & & & 0.0000 & & 0.0000 & 0.0000 & & 0.0000 & 0.0000 & & & 0.0030 & 0.0000 & & 0.0000 & & 0.0000 \\
\hline CVS2-57 & 0.0000 & & 0.0000 & 0.0000 & & & 0.0000 & & 0.0000 & & & & 0.0000 & & & 0.0000 & 0.0000 & & 0.0000 & & 0.0000 \\
\hline CVS2-58 & 0.0001 & & 0.0002 & 0.0001 & & & 0.0001 & & 0.0003 & & & & 0.0001 & & & 0.0005 & 0.0002 & & 0.0000 & & 0.0000 \\
\hline CVS2-59 & 0.0001 & & 0.0002 & 0.0001 & & & 0.0001 & & 0.0003 & & & & 0.0001 & & & 0.0005 & 0.0002 & & 0.0000 & & 0.0000 \\
\hline CVS2-60 & 0.0001 & & 0.0002 & 0.0001 & & & 0.0001 & & 0.0003 & & & & 0.0001 & & & 0.0005 & 0.0002 & & 0.0000 & & 0.0000 \\
\hline CVS2-61 & 0.0001 & & 0.0002 & 0.0001 & & & 0.0001 & & 0.0003 & & & & 0.0001 & & & 0.0006 & 0.0002 & & 0.0000 & & 0.0000 \\
\hline CVS2-62 & 0.0001 & & 0.0002 & 0.0001 & & & 0.0001 & & 0.0003 & & & & 0.0001 & & & 0.0005 & 0.0002 & & 0.0000 & & 0.0000 \\
\hline CVS2-63 & 0.0001 & & 0.0002 & 0.0001 & & & 0.0001 & & 0.0003 & & & & 0.0001 & & & 0.0005 & 0.0002 & & 0.0000 & & 0.0000 \\
\hline CVS2-64 & 0.0005 & & 0.0000 & 0.0000 & & & 0.0005 & & 0.0018 & & & & 0.0000 & & & 0.0028 & 0.0000 & & 0.0000 & & 0.0028 \\
\hline CVS2-65 & 0.0000 & & 0.0000 & 0.0000 & & & 0.0001 & & 0.0000 & & & & 0.0000 & & & 0.0000 & 0.0000 & & 0.0000 & & 0.0000 \\
\hline
\end{tabular}


Hanford CVS 2 (Hrma et al. 1994)

\begin{tabular}{|c|c|c|c|c|c|c|c|c|c|c|c|c|c|c|c|c|c|c|c|c|c|}
\hline Glass ID & $\begin{array}{c}\mathrm{T} 12 \mathrm{O} 3 \\
-\mathrm{t}\end{array}$ & $\begin{array}{c}\mathrm{U} 3 \mathrm{O} 8 \\
-\mathrm{t}\end{array}$ & $\begin{array}{c}\mathrm{UO} 2 \\
-\mathrm{t}\end{array}$ & $\begin{array}{c}\text { UO3 } \\
-t\end{array}$ & $\begin{array}{c}\mathrm{V} 2 \mathrm{O} 5 \\
-\mathrm{t}\end{array}$ & $\begin{array}{c}\text { WO3 } \\
-t\end{array}$ & $\begin{array}{c}\mathrm{Y} 2 \mathrm{O} 3 \\
-\mathrm{t}\end{array}$ & $\begin{array}{c}\mathrm{ZnO} \\
-\mathrm{t}\end{array}$ & $\begin{array}{c}\text { Others } \\
-t\end{array}$ & $\begin{array}{c}\text { Sum } \\
-t\end{array}$ & $\begin{array}{c}\mathrm{A} 12 \mathrm{O} 3 \\
-\mathrm{a}\end{array}$ & $\begin{array}{c}\mathrm{B} 2 \mathrm{O} 3 \\
-\mathrm{a}\end{array}$ & $\begin{array}{c}\mathrm{CaO} \\
-\mathrm{a}\end{array}$ & $\begin{array}{c}\mathrm{Fe} 2 \mathrm{O} 3 \\
-\mathrm{a}\end{array}$ & $\begin{array}{c}\mathrm{FeO} \\
-\mathrm{a}\end{array}$ & $\begin{array}{c}\mathrm{K} 2 \mathrm{O} \\
-\mathrm{a}\end{array}$ & $\begin{array}{c}\mathrm{Li} 2 \mathrm{O} \\
-\mathrm{a}\end{array}$ & $\begin{array}{c}\mathrm{MgO} \\
-\mathrm{a}\end{array}$ & $\begin{array}{c}\mathrm{Na} 2 \mathrm{O} \\
-\mathrm{a}\end{array}$ & $\begin{array}{c}\mathrm{NiO} \\
-\mathrm{a}\end{array}$ & $\begin{array}{c}\mathrm{P} 2 \mathrm{O} 5 \\
-\mathrm{a}\end{array}$ \\
\hline CVS2-27 & & 0.0000 & & & & 0.0000 & 0.0001 & 0.0000 & & 0.9999 & 0.0764 & 0.0492 & 0.0005 & 0.0190 & & 0.0000 & 0.0168 & 0.0715 & 0.2335 & 0.0009 & 0.0002 \\
\hline CVS2-28 & & 0.0000 & & & & 0.0000 & 0.0009 & 0.0000 & & 0.9999 & 0.0486 & 0.0443 & 0.0002 & 0.0175 & & 0.0000 & 0.0103 & 0.0001 & 0.2030 & 0.0093 & 0.0020 \\
\hline CVS2-29 & & 0.0000 & & & & 0.0000 & 0.0009 & 0.0000 & & 0.9999 & 0.0005 & 0.2018 & 0.0758 & 0.0188 & & 0.0000 & 0.0082 & 0.0007 & 0.1080 & 0.0093 & 0.0020 \\
\hline CVS2-30 & & 0.0000 & & & & 0.0000 & 0.0001 & 0.0000 & & 0.9999 & 0.0435 & 0.1960 & 0.0001 & 0.1218 & & 0.0000 & 0.0084 & 0.0001 & 0.1250 & 0.0009 & 0.0002 \\
\hline CVS2-31 & & 0.0000 & & & & 0.0000 & 0.0001 & 0.0000 & & 1.0000 & 0.0514 & 0.1895 & 0.0757 & 0.0193 & & 0.0000 & 0.0295 & 0.0007 & 0.0901 & 0.0010 & 0.0004 \\
\hline CVS2-32 & & 0.0000 & & & & 0.0000 & 0.0001 & 0.0000 & & 1.0000 & 0.0027 & 0.0491 & 0.0005 & 0.0194 & & 0.0000 & 0.0412 & 0.0004 & 0.2445 & 0.0009 & 0.0009 \\
\hline CVS2-33 & & 0.0000 & & & & 0.0000 & 0.0009 & 0.0000 & & 0.9999 & 0.0875 & 0.0540 & 0.0005 & 0.0190 & & 0.0000 & 0.0350 & 0.0710 & 0.2200 & 0.0092 & 0.0010 \\
\hline CVS2-34 & & 0.0000 & & & & 0.0000 & 0.0001 & 0.0000 & & 0.9999 & 0.1350 & 0.1700 & 0.0005 & 0.0195 & & 0.0000 & 0.0350 & 0.0002 & 0.2100 & 0.0009 & 0.0001 \\
\hline CVS2-35 & & 0.0000 & & & & 0.0000 & 0.0001 & 0.0000 & & 1.0000 & 0.1305 & 0.0493 & 0.0776 & 0.0597 & & 0.0000 & 0.0420 & 0.0006 & 0.2450 & 0.0009 & 0.0002 \\
\hline CVS2-36 & & 0.0000 & & & & 0.0000 & 0.0001 & 0.0000 & & 1.0000 & 0.0088 & 0.0497 & 0.0780 & 0.1443 & & 0.0000 & 0.0692 & 0.0005 & 0.1395 & 0.0009 & 0.0002 \\
\hline CVS2-37 & & 0.0000 & & & & 0.0000 & 0.0001 & 0.0000 & & 1.0000 & 0.1358 & 0.0830 & 0.0002 & 0.0190 & & 0.0000 & 0.0642 & 0.0000 & 0.1405 & 0.0009 & 0.0004 \\
\hline CVS2-38 & & 0.0000 & & & & 0.0000 & 0.0001 & 0.0000 & & 1.0000 & 0.0273 & 0.1100 & 0.0002 & 0.1338 & & 0.0000 & 0.0085 & 0.0706 & 0.1435 & 0.0010 & 0.0005 \\
\hline CVS2-39 & & 0.0000 & & & & 0.0000 & 0.0001 & 0.0000 & & 1.0000 & 0.0247 & 0.0483 & 0.0005 & 0.0675 & & 0.0000 & 0.0643 & 0.0690 & 0.1725 & 0.0009 & 0.0005 \\
\hline CVS2-40 & & 0.0000 & & & & 0.0000 & 0.0003 & 0.0000 & & 0.9999 & 0.0970 & 0.0630 & 0.0190 & 0.0190 & & 0.0000 & 0.0400 & 0.0440 & 0.1660 & 0.0028 & 0.0005 \\
\hline CVS2-41 & & 0.0000 & & & & 0.0000 & 0.0003 & 0.0000 & & 0.9999 & 0.0970 & 0.0680 & 0.0480 & 0.0285 & & 0.0000 & 0.0660 & 0.0180 & 0.1200 & 0.0030 & 0.0006 \\
\hline CVS2-42 & & 0.0000 & & & & 0.0000 & 0.0003 & 0.0000 & & 0.9999 & 0.0290 & 0.0490 & 0.0310 & 0.0930 & & 0.0000 & 0.0660 & 0.0335 & 0.0660 & 0.0029 & 0.0006 \\
\hline CVS2-43 & & 0.0000 & & & & 0.0000 & 0.0003 & 0.0000 & & 0.9999 & 0.0477 & 0.1418 & 0.0209 & 0.0283 & & 0.0000 & 0.0593 & 0.0266 & 0.0809 & 0.0030 & 0.0008 \\
\hline CVS2-44 & & 0.0000 & & & & 0.0000 & 0.0003 & 0.0000 & & 0.9999 & 0.0610 & 0.1050 & 0.0470 & 0.0190 & & 0.0000 & 0.0650 & 0.0175 & 0.0530 & 0.0029 & 0.0005 \\
\hline CVS2-45 & & 0.0000 & & & & 0.0000 & 0.0006 & 0.0000 & & 0.9999 & 0.0575 & 0.1100 & 0.0190 & 0.0290 & & 0.0000 & 0.0560 & 0.0445 & 0.0520 & 0.0065 & 0.0010 \\
\hline CVS2-46 & & 0.0000 & & & & 0.0000 & 0.0006 & 0.0000 & & 0.9999 & 0.0721 & 0.1225 & 0.0196 & 0.0194 & & 0.0000 & 0.0632 & 0.0181 & 0.0927 & 0.0065 & 0.0010 \\
\hline CVS2-47 & & 0.0000 & & & & 0.0000 & 0.0006 & 0.0000 & & 0.9999 & 0.0390 & 0.0495 & 0.0490 & 0.0190 & & 0.0000 & 0.0400 & 0.0175 & 0.1300 & 0.0065 & 0.0015 \\
\hline CVS2-48 & & 0.0000 & & & & 0.0000 & 0.0005 & 0.0000 & & 0.9998 & 0.0835 & 0.1300 & 0.0490 & 0.0190 & & 0.0000 & 0.0380 & 0.0175 & 0.1100 & 0.0051 & 0.0010 \\
\hline CVS2-49 & & 0.0000 & & & & 0.0000 & 0.0003 & 0.0000 & & 0.9999 & 0.0763 & 0.1325 & 0.0191 & 0.0494 & & 0.0000 & 0.0368 & 0.0176 & 0.1280 & 0.0031 & 0.0006 \\
\hline CVS2-50 & & 0.0000 & & & & 0.0000 & 0.0004 & 0.0000 & & 0.9999 & 0.0625 & 0.1150 & 0.0270 & 0.0535 & & 0.0000 & 0.0370 & 0.0317 & 0.1100 & 0.0037 & 0.0007 \\
\hline CVS2-51 & & 0.0000 & & & & 0.0000 & 0.0000 & 0.0049 & & 0.9999 & 0.0285 & 0.0975 & 0.0099 & 0.0570 & & 0.0000 & 0.0320 & 0.0072 & 0.1200 & 0.0059 & 0.0015 \\
\hline CVS2-52 & & 0.0000 & & & & 0.0000 & 0.0005 & 0.0000 & & 1.0000 & 0.0260 & 0.0875 & 0.0012 & 0.0755 & & 0.0000 & 0.0830 & 0.0001 & 0.0680 & 0.0000 & 0.0020 \\
\hline CVS2-53 & & & 0.0177 & & & 0.0000 & 0.0007 & 0.0000 & & 0.9996 & 0.0840 & 0.0910 & 0.0018 & 0.0360 & & 0.0000 & 0.0630 & 0.0440 & 0.0650 & 0.0077 & 0.0020 \\
\hline CVS2-54 & & & 0.0057 & & & 0.0000 & 0.0000 & 0.0049 & & 0.9998 & 0.0330 & 0.1100 & 0.0094 & 0.0660 & & 0.0000 & 0.0390 & 0.0075 & 0.1000 & 0.0056 & 0.0010 \\
\hline CVS2-55 & & & 0.0000 & & & 0.0000 & 0.0000 & 0.0000 & & 1.0000 & 0.0270 & 0.1150 & 0.0069 & 0.0765 & & 0.0000 & 0.0405 & 0.0071 & 0.1100 & 0.0000 & 0.0004 \\
\hline CVS2-56 & & & 0.0000 & & & 0.0000 & 0.0000 & 0.0000 & & 1.0000 & 0.0260 & 0.1100 & 0.0066 & 0.0760 & & 0.0000 & 0.0405 & 0.0069 & 0.0940 & 0.0000 & 0.0004 \\
\hline CVS2-57 & & 0.0000 & & & & 0.0000 & 0.0000 & 0.0000 & & 1.0000 & 0.0250 & 0.1100 & 0.0067 & 0.0755 & & 0.0000 & 0.0385 & 0.0068 & 0.1000 & 0.0000 & 0.0006 \\
\hline CVS2-58 & & 0.0000 & & & & 0.0000 & 0.0001 & 0.0000 & & 0.9999 & 0.1500 & 0.2050 & 0.0190 & 0.0210 & & 0.0000 & 0.0715 & 0.0755 & 0.0670 & 0.0007 & 0.0004 \\
\hline CVS2-59 & & 0.0000 & & & & 0.0000 & 0.0001 & 0.0000 & & 1.0000 & 0.1055 & 0.1620 & 0.0331 & 0.0173 & & 0.0000 & 0.0658 & 0.0008 & 0.1140 & 0.0029 & 0.0002 \\
\hline CVS2-60 & & 0.0000 & & & & 0.0000 & 0.0001 & 0.0000 & & 0.9999 & & & & & & & & & & & \\
\hline CVS2-61 & & 0.0000 & & & & 0.0000 & 0.0001 & 0.0000 & & 1.0000 & & & & & & & & & & & \\
\hline CVS2-62 & & 0.0000 & & & & 0.0000 & 0.0001 & 0.0000 & & 1.0000 & & & & & & & & & & & \\
\hline CVS2-63 & & 0.0000 & & & & 0.0000 & 0.0001 & 0.0000 & & 0.9999 & & & & & & & & & & & \\
\hline CVS2-64 & & 0.0000 & & & & 0.0000 & 0.0000 & 0.0015 & & 1.0000 & & & & & & & & & & & \\
\hline CVS2-65 & & 0.0000 & & & & 0.0000 & 0.0000 & 0.0000 & & 1.0000 & & & & & & & & & & & \\
\hline
\end{tabular}


Appendix A. Database - mass fraction

Hanford CVS 2 (Hrma et al. 1994)

\begin{tabular}{|c|c|c|c|c|c|c|c|c|c|c|c|c|c|c|c|c|c|c|c|c|c|}
\hline Glass ID & $\begin{array}{c}\mathrm{SiO} 2 \\
-\mathrm{a}\end{array}$ & $\begin{array}{c}\mathrm{ZrO} 2 \\
-\mathrm{a}\end{array}$ & $\begin{array}{c}\mathrm{Ag} 2 \mathrm{O} \\
-\mathrm{a}\end{array}$ & $\begin{array}{c}\mathrm{As} 2 \mathrm{O} 3 \\
-\mathrm{a}\end{array}$ & $\begin{array}{c}\mathrm{BaO} \\
-\mathrm{a}\end{array}$ & $\begin{array}{c}\mathrm{Bi} 2 \mathrm{O} 3 \\
-\mathrm{a}\end{array}$ & $\begin{array}{l}\mathrm{Br} \\
-\mathrm{a}\end{array}$ & $\begin{array}{c}\mathrm{CdO} \\
-\mathrm{a}\end{array}$ & $\begin{array}{c}\mathrm{Ce} 2 \mathrm{O} 3 \\
-\mathrm{a}\end{array}$ & $\begin{array}{c}\mathrm{CeO} 2 \\
-\mathrm{a}\end{array}$ & $\begin{array}{l}\mathrm{Cl} \\
-\mathrm{a}\end{array}$ & $\begin{array}{c}\mathrm{CoO} \\
-\mathrm{a}\end{array}$ & $\begin{array}{c}\mathrm{Co} 2 \mathrm{O} 3 \\
-\mathrm{a}\end{array}$ & $\begin{array}{c}\mathrm{Cr} 2 \mathrm{O} 3 \\
-\mathrm{a}\end{array}$ & $\begin{array}{c}\mathrm{Cs} 2 \mathrm{O} \\
-\mathrm{a}\end{array}$ & $\begin{array}{c}\mathrm{CuO} \\
-\mathrm{a}\end{array}$ & $\begin{array}{c}\mathrm{Eu} 2 \mathrm{O} 3 \\
-\mathrm{a}\end{array}$ & $\begin{array}{c}\mathrm{F} \\
-\mathrm{a}\end{array}$ & $\begin{array}{c}\mathrm{Ga} 2 \mathrm{O} 3 \\
-\mathrm{a}\end{array}$ & $\begin{array}{c}\mathrm{Gd} 2 \mathrm{O} 3 \\
-\mathrm{a}\end{array}$ & $\begin{array}{c}\mathrm{HgO} \\
-\mathrm{a}\end{array}$ \\
\hline CVS2-27 & 0.5333 & 0.0000 & & & 0.0002 & & & 0.0012 & & 0.0002 & & & & 0.0003 & 0.0003 & 0.0002 & & 0.0005 & & & \\
\hline CVS2-28 & 0.4915 & 0.0000 & & & 0.0015 & & & 0.0109 & & 0.0016 & & & & 0.0012 & 0.0025 & 0.0022 & & 0.0054 & & & \\
\hline CVS2-29 & 0.4170 & 0.0736 & & & 0.0017 & & & 0.0131 & & 0.0065 & & & & 0.0021 & 0.0028 & 0.0025 & & 0.0054 & & & \\
\hline CVS2-30 & 0.4915 & 0.0000 & & & 0.0002 & & & 0.0012 & & 0.0002 & & & & 0.0003 & 0.0003 & 0.0003 & & 0.0005 & & & \\
\hline CVS2-31 & 0.5415 & 0.0000 & & & 0.0002 & & & 0.0013 & & 0.0002 & & & & 0.0001 & 0.0005 & 0.0003 & & 0.0005 & & & \\
\hline CVS2-32 & 0.5383 & 0.1225 & & & 0.0002 & & & 0.0012 & & 0.0055 & & & & 0.0002 & 0.0003 & 0.0002 & & 0.0005 & & & \\
\hline CVS2-33 & 0.4100 & 0.0000 & & & 0.0017 & & & 0.0125 & & 0.0029 & & & & 0.0021 & 0.0028 & 0.0025 & & 0.0054 & & & \\
\hline CVS2-34 & 0.4000 & 0.0009 & & & 0.0002 & & & 0.0012 & & 0.0002 & & & & 0.0002 & 0.0006 & 0.0003 & & 0.0005 & & & \\
\hline CVS2-35 & 0.3978 & 0.0000 & & & 0.0002 & & & 0.0012 & & 0.0002 & & & & 0.0002 & 0.0002 & 0.0002 & & 0.0005 & & & \\
\hline CVS2-36 & 0.5338 & 0.0000 & & & 0.0002 & & & 0.0012 & & 0.0002 & & & & 0.0002 & 0.0002 & 0.0002 & & 0.0005 & & & \\
\hline CVS2-37 & 0.5508 & 0.0000 & & & 0.0002 & & & 0.0012 & & 0.0001 & & & & 0.0003 & 0.0003 & 0.0003 & & 0.0005 & & & \\
\hline CVS2-38 & 0.5005 & 0.0000 & & & 0.0002 & & & 0.0013 & & 0.0002 & & & & 0.0003 & 0.0003 & 0.0003 & & 0.0005 & & & \\
\hline CVS2-39 & 0.4551 & 0.0601 & & & 0.0002 & & & 0.0012 & & 0.0032 & & & & 0.0002 & 0.0004 & 0.0002 & & 0.0005 & & & \\
\hline CVS2-40 & 0.4900 & 0.0160 & & & 0.0005 & & & 0.0038 & & 0.0019 & & & & 0.0007 & 0.0010 & 0.0008 & & 0.0016 & & & \\
\hline CVS2-41 & 0.5100 & 0.0170 & & & 0.0052 & & & 0.0039 & & 0.0019 & & & & 0.0006 & 0.0011 & 0.0008 & & 0.0016 & & & \\
\hline CVS2-42 & 0.5500 & 0.0170 & & & 0.0050 & & & 0.0037 & & 0.0014 & & & & 0.0006 & 0.0009 & 0.0007 & & 0.0016 & & & \\
\hline CVS2-43 & 0.4870 & 0.0610 & & & 0.0005 & & & 0.0037 & & 0.0039 & & & & 0.0007 & 0.0012 & 0.0008 & & 0.0016 & & & \\
\hline CVS2-44 & 0.4900 & 0.0160 & & & 0.0005 & & & 0.0004 & & 0.0018 & & & & 0.0006 & 0.0010 & 0.0008 & & 0.0016 & & & \\
\hline CVS2-45 & 0.4700 & 0.0170 & & & 0.0012 & & & 0.0088 & & 0.0030 & & & & 0.0014 & 0.0022 & 0.0018 & & 0.0038 & & & \\
\hline CVS2-46 & 0.5060 & 0.0150 & & & 0.0011 & & & 0.0081 & & 0.0023 & & & & 0.0014 & 0.0024 & 0.0017 & & 0.0035 & & & \\
\hline CVS2-47 & 0.5200 & 0.0440 & & & 0.0015 & & & 0.0088 & & 0.0041 & & & & 0.0017 & 0.0020 & 0.0018 & & 0.0038 & & & \\
\hline CVS2-48 & 0.4600 & 0.0170 & & & 0.0009 & & & 0.0069 & & 0.0016 & & & & 0.0011 & 0.0017 & 0.0014 & & 0.0029 & & & \\
\hline CVS2-49 & 0.4800 & 0.0167 & & & 0.0005 & & & 0.0038 & & 0.0018 & & & & 0.0001 & 0.0113 & 0.0008 & & 0.0016 & & & \\
\hline CVS2-50 & 0.4400 & 0.0370 & & & 0.0006 & & & 0.0051 & & 0.0030 & & & & 0.0008 & 0.0012 & 0.0001 & & 0.0219 & & & \\
\hline CVS2-51 & 0.4600 & 0.0340 & & & 0.0022 & & & 0.0059 & & 0.0021 & & & & 0.0010 & 0.0016 & 0.0019 & & 0.0019 & & & \\
\hline CVS2-52 & 0.5800 & 0.0390 & & & 0.0011 & & & 0.0075 & & 0.0016 & & & & 0.0013 & 0.0016 & 0.0015 & & 0.0032 & & & \\
\hline CVS2-53 & 0.5000 & 0.0091 & & & 0.0013 & & & 0.0100 & & 0.0020 & & & & 0.0017 & 0.0022 & 0.0020 & & 0.0043 & & & \\
\hline CVS2-54 & 0.5100 & 0.0350 & & & 0.0009 & & & 0.0062 & & 0.0002 & & & & 0.0013 & 0.0015 & 0.0015 & & 0.0019 & & & \\
\hline CVS2-55 & 0.5200 & 0.0390 & & & 0.0000 & & & 0.0000 & & 0.0000 & & & & 0.0082 & 0.0000 & 0.0000 & & 0.0030 & & & \\
\hline CVS2-56 & 0.5000 & 0.0370 & & & 0.0000 & & & 0.0000 & & 0.0002 & & & & 0.0066 & 0.0000 & 0.0066 & & 0.0030 & & & \\
\hline CVS2-57 & 0.4900 & 0.0380 & & & 0.0000 & & & 0.0000 & & 0.0000 & & & & 0.0049 & 0.0000 & 0.0000 & & 0.0030 & & & \\
\hline CVS2-58 & 0.3600 & 0.0086 & & & 0.0002 & & & 0.0012 & & 0.0002 & & & & 0.0002 & 0.0003 & 0.0003 & & 0.0005 & & & \\
\hline CVS2-59 & 0.3980 & 0.0064 & & 0.0000 & 0.0002 & & & 0.0018 & & 0.0003 & & & 0.0002 & 0.0003 & 0.0003 & 0.0003 & 0.0000 & 0.0006 & & 0.0000 & \\
\hline CVS2-60 & & & & & & & & & & & & & & & & & & & & & \\
\hline CVS2-61 & & & & & & & & & & & & & & & & & & & & & \\
\hline CVS2-62 & & & & & & & & & & & & & & & & & & & & & \\
\hline CVS2-63 & & & & & & & & & & & & & & & & & & & & & \\
\hline CVS2-64 & & & & & & & & & & & & & & & & & & & & & \\
\hline CVS2-65 & & & & & & & & & & & & & & & & & & & & & \\
\hline
\end{tabular}


Hanford CVS 2 (Hrma et al. 1994)

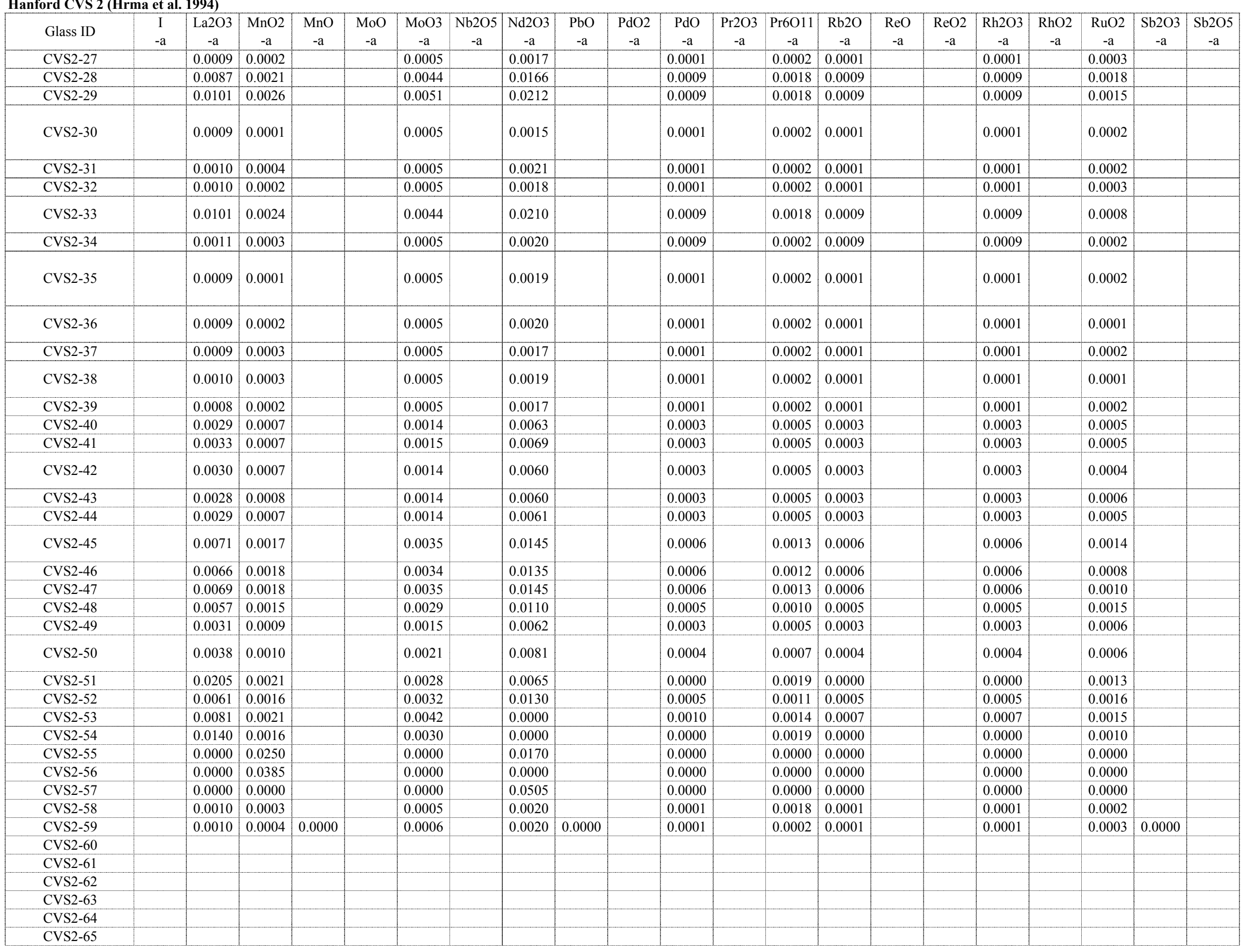


Appendix A. Database - mass fraction

Hanford CVS 2 (Hrma et al. 1994)

\begin{tabular}{|c|c|c|c|c|c|c|c|c|c|c|c|c|c|c|c|c|c|c|c|c|}
\hline Glass ID & $\begin{array}{c}\mathrm{SeO} 2 \\
-\mathrm{a}\end{array}$ & $\begin{array}{c}\mathrm{Sm} 2 \mathrm{O} 3 \\
-\mathrm{a} \\
\end{array}$ & $\begin{array}{c}\mathrm{SnO} \\
-\mathrm{a} \\
\end{array}$ & $\begin{array}{c}\mathrm{SnO} 2 \\
-\mathrm{a} \\
\end{array}$ & $\begin{array}{c}\mathrm{SO} 3 \\
-\mathrm{a} \\
\end{array}$ & $\begin{array}{c}\mathrm{SrO} \\
-\mathrm{a} \\
\end{array}$ & $\begin{array}{c}\mathrm{Tc} 2 \mathrm{O} 7 \\
-\mathrm{a}\end{array}$ & $\begin{array}{c}\mathrm{TeO} 2 \\
-\mathrm{a}\end{array}$ & $\begin{array}{c}\mathrm{ThO} 2 \\
-\mathrm{a}\end{array}$ & $\begin{array}{c}\mathrm{TiO} 2 \\
-\mathrm{a} \\
\end{array}$ & $\begin{array}{c}\mathrm{T} 12 \mathrm{O} 3 \\
-\mathrm{a}\end{array}$ & $\begin{array}{c}\mathrm{U} 3 \mathrm{O} 8 \\
-\mathrm{a}\end{array}$ & $\begin{array}{c}\mathrm{UO} 2 \\
-\mathrm{a}\end{array}$ & $\begin{array}{c}\mathrm{UO} 3 \\
-\mathrm{a}\end{array}$ & $\begin{array}{c}\mathrm{V} 2 \mathrm{O} 5 \\
-\mathrm{a}\end{array}$ & $\begin{array}{c}\text { WO3 } \\
-\mathrm{a}\end{array}$ & $\begin{array}{c}\mathrm{Y} 2 \mathrm{O} 3 \\
-\mathrm{a} \\
\end{array}$ & $\begin{array}{c}\mathrm{ZnO} \\
-\mathrm{a}\end{array}$ & $\begin{array}{c}\text { Others } \\
-\mathrm{a}\end{array}$ & $\begin{array}{c}\text { Sum } \\
-\mathrm{a}\end{array}$ \\
\hline CVS2-27 & & 0.0001 & & & 0.0005 & 0.0002 & & & & 0.0007 & & & & & & & 0.0001 & & & 1.0097 \\
\hline CVS2-28 & & 0.0009 & & & 0.0049 & 0.0014 & & & & 0.0001 & & & & & & & 0.0007 & & & 0.8978 \\
\hline CVS2-29 & & 0.0009 & & & 0.0049 & 0.0017 & & & & 0.0003 & & & & & & & 0.0009 & & & 1.0032 \\
\hline CVS2-30 & & 0.0001 & & & 0.0005 & 0.0002 & & & & 0.0001 & & & & & & & 0.0001 & & & 0.9949 \\
\hline CVS2-31 & & 0.0001 & & & 0.0005 & 0.0002 & & & & 0.0002 & & & & & & & 0.0001 & & & 1.0078 \\
\hline CVS2-32 & & 0.0001 & & & 0.0005 & 0.0002 & & & & 0.0003 & & & & & & & 0.0001 & & & 1.0336 \\
\hline CVS2-33 & & 0.0009 & & & 0.0049 & 0.0017 & & & & 0.0001 & & & & & & & 0.0008 & & & 0.9887 \\
\hline CVS2-34 & & 0.0009 & & & 0.0005 & 0.0002 & & & & 0.0001 & & & & & & & 0.0000 & & & 0.9839 \\
\hline CVS2-35 & & 0.0001 & & & 0.0005 & 0.0002 & & & & 0.0001 & & & & & & & 0.0001 & & & 1.0112 \\
\hline CVS2-36 & & 0.0001 & & & 0.0005 & 0.0002 & & & & 0.0002 & & & & & & & 0.0001 & & & 1.0327 \\
\hline CVS2-37 & & 0.0001 & & & 0.0005 & 0.0002 & & & & 0.0001 & & & & & & & 0.0001 & & & 1.0025 \\
\hline CVS2-38 & & 0.0001 & & & 0.0005 & 0.0002 & & & & 0.0002 & & & & & & & 0.0001 & & & 1.0041 \\
\hline CVS2-39 & & 0.0001 & & & 0.0005 & 0.0002 & & & & 0.0002 & & & & & & & 0.0001 & & & 0.9741 \\
\hline CVS2-40 & & 0.0003 & & & 0.0015 & 0.0001 & & & & 0.0001 & & & & & & & 0.0001 & & & 0.9827 \\
\hline CVS2-41 & & 0.0003 & & & 0.0015 & 0.0006 & & & & 0.0001 & & & & & & & 0.0002 & & & 1.0080 \\
\hline CVS2-42 & & 0.0003 & & & 0.0015 & 0.0005 & & & & 0.0001 & & & & & & & 0.0002 & & & 0.9673 \\
\hline CVS2-43 & & 0.0003 & & & 0.0015 & 0.0005 & & & & 0.0003 & & & & & & & 0.0003 & & & 0.9852 \\
\hline CVS2-44 & & 0.0003 & & & 0.0015 & 0.0005 & & & & 0.0002 & & & & & & & 0.0002 & & & 0.8991 \\
\hline CVS2-45 & & 0.0006 & & & 0.0035 & 0.0012 & & & & 0.0001 & & & & & & & 0.0006 & & & 0.9217 \\
\hline CVS2-46 & & 0.0006 & & & 0.0032 & 0.0011 & & & & 0.0002 & & & & & & & 0.0006 & & & 0.9912 \\
\hline CVS2-47 & & 0.0006 & & & 0.0035 & 0.0012 & & & & 0.0002 & & & & & & & 0.0006 & & & 0.9764 \\
\hline CVS2-48 & & 0.0005 & & & 0.0027 & 0.0009 & & & & 0.0001 & & & & & & & 0.0003 & & & 0.9760 \\
\hline CVS2-49 & & 0.0003 & & & 0.0015 & 0.0005 & & & & 0.0001 & & & & & & & 0.0003 & & & 0.9961 \\
\hline CVS2-50 & & 0.0004 & & & 0.0020 & 0.0007 & & & & 0.0002 & & & & & & & 0.0004 & & & 0.9716 \\
\hline CVS2-51 & & 0.0000 & & & 0.0044 & 0.0000 & & & & 0.0011 & & & & & & & 0.0000 & 0.0043 & & 0.9147 \\
\hline CVS2-52 & & 0.0005 & & & 0.0029 & 0.0011 & & & & 0.0002 & & & & & & & 0.0005 & 0.0000 & & 1.0130 \\
\hline CVS2-53 & & 0.0007 & & & 0.0039 & 0.0014 & & & & 0.0001 & & & & & & & 0.0007 & 0.0000 & & 0.9537 \\
\hline CVS2-54 & & 0.0000 & & & 0.0040 & 0.0000 & & & & 0.0002 & & & & & & & 0.0000 & 0.0045 & & 0.9602 \\
\hline CVS2-55 & & 0.0000 & & & 0.0030 & 0.0000 & & & & 0.0002 & & & & & & & 0.0000 & 0.0000 & & 0.9988 \\
\hline CVS2-56 & & 0.0000 & & & 0.0030 & 0.0000 & & & & 0.0002 & & & & & & & 0.0000 & 0.0000 & & 0.9554 \\
\hline CVS2-57 & & 0.0000 & & & 0.0000 & 0.0000 & & & & 0.0002 & & & & & & & 0.0000 & 0.0000 & & 0.9496 \\
\hline CVS2-58 & & 0.0000 & & & 0.0005 & 0.0000 & & & & 0.0001 & & & & & & & 0.0001 & 0.0000 & & 0.9882 \\
\hline CVS2-59 & 0.0000 & 0.0001 & & & 0.0005 & 0.0002 & & 0.0000 & 0.0000 & 0.0002 & & & 0.0000 & & 0.0000 & & 0.0001 & 0.0000 & & 0.9154 \\
\hline CVS2-60 & & & & & & & & & & & & & & & & & & & & \\
\hline CVS2-61 & & & & & & & & & & & & & & & & & & & & \\
\hline CVS2-62 & & & & & & & & & & & & & & & & & & & & \\
\hline CVS2-63 & & & & & & & & & & & & & & & & & & & & \\
\hline CVS2-64 & & & & & & & & & & & & & & & & & & & & \\
\hline CVS2-65 & & & & & & & & & & & & & & & & & & & & \\
\hline
\end{tabular}


Appendix A. Database - mass fraction

Hanford CVS 2 (Hrma et al. 1994)

\begin{tabular}{|c|c|c|c|c|c|c|c|c|}
\hline Glass ID & $\begin{array}{l}\mathrm{TM} \\
\left({ }^{\circ} \mathrm{C}\right) \\
\end{array}$ & $\begin{array}{l}\text { Gradient } \\
\text { TL }\left({ }^{\circ} \mathrm{C}\right) \\
\end{array}$ & $\begin{array}{l}\text { Uniform } \\
\text { TL }\left({ }^{\circ} \mathrm{C}\right) \\
\end{array}$ & Primary Phase & $\begin{array}{l}\text { Quenched } \\
\text { Visual/OM }\end{array}$ & $\begin{array}{c}\text { Quenched } \\
\text { SEM/EDS or TEM }\end{array}$ & $\begin{array}{l}\text { Quenched } \\
\text { XRD }\end{array}$ & $\begin{array}{c}\text { Quenched } \\
\text { Homogeneous? }\end{array}$ \\
\hline \multicolumn{9}{|l|}{ CVS2-27 } \\
\hline \multicolumn{9}{|l|}{ CVS2-28 } \\
\hline CVS2-29 & & 1108 & & zircon & & & & \\
\hline CVS2-30 & & 1090 & & spinel & & & & \\
\hline CVS2-31 & & 945 & & $\mathrm{SiO} 2$ & & & & \\
\hline CVS2-32 & & 1089 & & $\mathrm{Na}-\mathrm{Zr}$ silicate & & & & \\
\hline CVS2-33 & & $>1038$ & & spinel & & & & \\
\hline CVS2-34 & & 736 & & nepheline & & & & \\
\hline CVS2-35 & & 961 & & nepheline & & & & \\
\hline CVS2-36 & & 887 & & orthopyroxene & & & & \\
\hline CVS2-37 & & 803 & & nepheline & & & & \\
\hline CVS2-38 & & $>1115$ & & spinel & & & & \\
\hline CVS2-39 & & 1029 & & zircon & & & & \\
\hline CVS2-40 & & 862 & & clinopyroxene & & & & \\
\hline CVS2-41 & & 887 & & clinopyroxene & & & & \\
\hline CVS2-42 & & 1093 & & spinel & & & & \\
\hline CVS2-43 & & 1090 & & zircon & & & & \\
\hline CVS2-44 & & 884 & & clinopyroxene & & & & \\
\hline CVS2-45 & & $>956$ & & spinel & & & & \\
\hline CVS2-46 & & 1004 & & spinel & & & & \\
\hline CVS2-47 & & 935 & & clinopyroxene & & & & \\
\hline CVS2-48 & & 868 & & clinopyroxene & & & & \\
\hline CVS2-49 & & 957 & & spinel & & & & \\
\hline CVS2-50 & & 1025 & & spinel & & & & \\
\hline CVS2-51 & & 899 & & spinel & & & & \\
\hline CVS2-52 & & $>1038$ & & spinel & & & & \\
\hline \multicolumn{9}{|l|}{ CVS2-53 } \\
\hline \multicolumn{9}{|l|}{ CVS2-54 } \\
\hline CVS2-55 & & $>1118$ & & spinel & & & & \\
\hline CVS2-56 & & 1117 & & spinel & & & & \\
\hline CVS2-57 & & 1036 & & $\mathrm{Cr} 2 \mathrm{O} 3$ & & & & \\
\hline CVS2-58 & & 921 & & olivine & & & & \\
\hline CVS2-59 & & 715 & & $\mathrm{SiO} 2$ & & & & \\
\hline CVS2-60 & & 788 & & $\mathrm{SiO} 2$ & & & & \\
\hline CVS2-61 & & 792 & & $\mathrm{SiO} 2$ & & & & \\
\hline \multicolumn{9}{|l|}{ CVS2-62 } \\
\hline CVS2-63 & & 927 & & nepheline & & & & \\
\hline CVS2-64 & & 983 & & spinel & & & & \\
\hline CVS2-65 & & 1168 & & zircon & & & & \\
\hline
\end{tabular}


Appendix A. Database - mass fraction

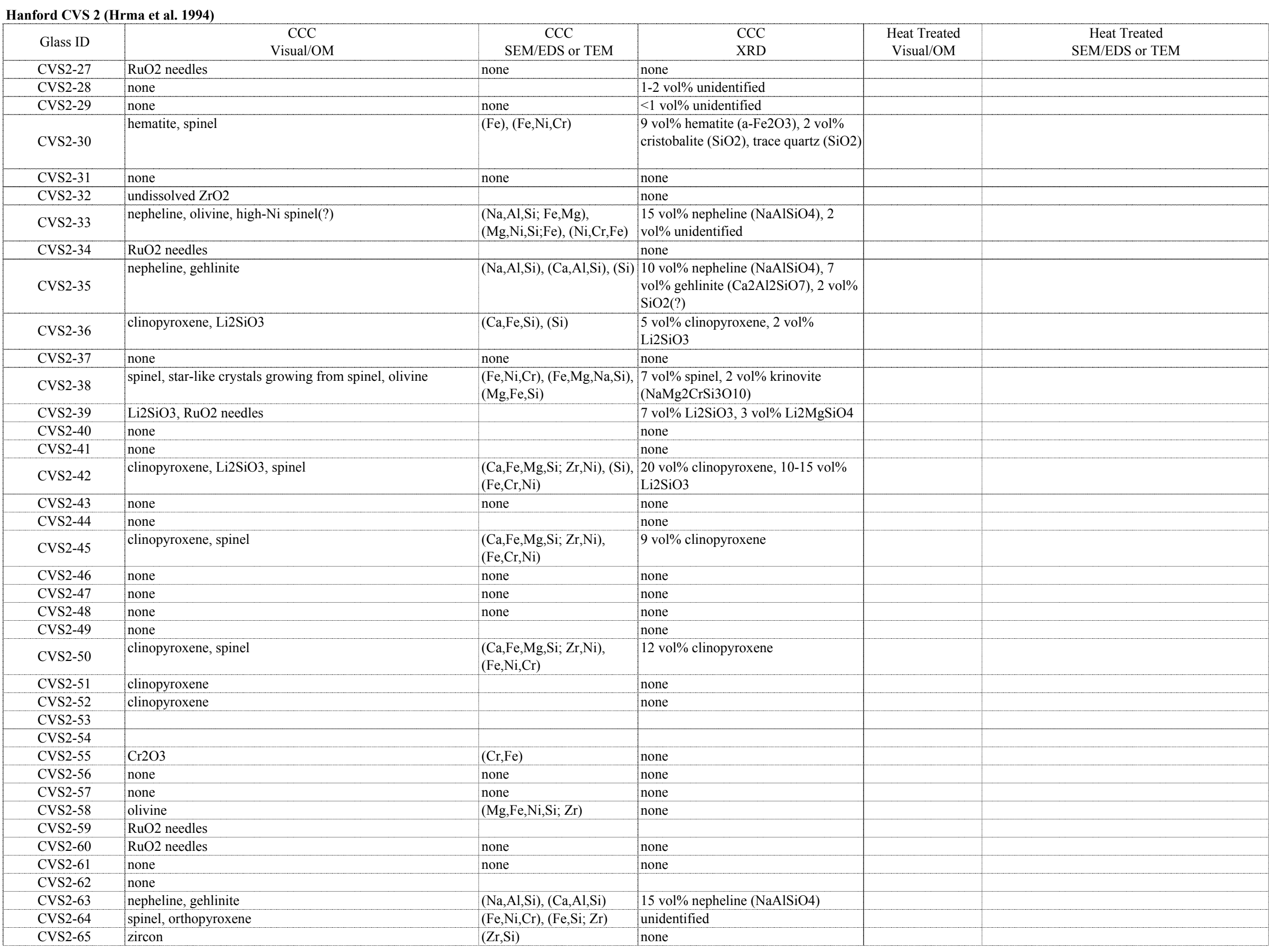


Hanford CVS 2 (Hrma et al. 1994)

\begin{tabular}{|c|c|c|c|c|c|c|c|c|c|c|c|c|c|c|c|}
\hline Glass ID & $\begin{array}{c}\text { Heat Treated } \\
\text { XRD }\end{array}$ & $\begin{array}{l}\text { Density } \\
\left(\mathrm{g} / \mathrm{cm}^{3}\right)\end{array}$ & $\begin{array}{c}\text { Fulc Visc } \\
\text { A }\end{array}$ & $\begin{array}{c}\text { Fulc Visc } \\
\text { B }\end{array}$ & \begin{tabular}{|c|} 
Fulc Visc \\
To
\end{tabular} & $\begin{array}{c}\mathrm{FV} 1150^{\circ} \mathrm{C} \\
(\mathrm{Pa} \cdot \mathrm{s})\end{array}$ & $\begin{array}{c}\text { Arrh Visc } \\
\text { A }\end{array}$ & $\begin{array}{c}\text { Arrh Visc } \\
\text { B }\end{array}$ & $1150^{\circ} \mathrm{C}$ & $\begin{array}{c}\mathrm{T}\left({ }^{\circ} \mathrm{C}\right) \text { at } \\
2 \mathrm{~Pa} \cdot \mathrm{s}\end{array}$ & \begin{tabular}{|c|}
$\mathrm{T}\left({ }^{\circ} \mathrm{C}\right)$ at \\
$5 \mathrm{~Pa} \cdot \mathrm{s}$
\end{tabular} & $\begin{array}{l}\mathrm{T}\left({ }^{\circ} \mathrm{C}\right) \text { at } \\
10 \mathrm{~Pa} \cdot \mathrm{s}\end{array}$ & $\begin{array}{c}\mathrm{T} 1 \\
\left({ }^{\circ} \mathrm{C}\right)\end{array}$ & $\begin{array}{c}\mathrm{V} 1 \\
(\mathrm{~Pa} \cdot \mathrm{s})\end{array}$ & $\begin{array}{c}\mathrm{T} 2 \\
\left({ }^{\circ} \mathrm{C}\right)\end{array}$ \\
\hline CVS2-27 & & 2.5386 & -6.07 & 8126.56 & 220.07 & 14.41 & -11.577 & 20286.0 & 14.57 & & & & 1241 & 6.61 & 1194 \\
\hline CVS2-28 & & 2.6441 & -4.57 & 5824.32 & 337.01 & 13.44 & -11.083 & 19493.3 & 13.68 & & & & 1246 & 6.28 & 1197 \\
\hline CVS2-29 & & 2.7597 & -5.91 & 5180.47 & 466.54 & 5.32 & -16.232 & 25527.3 & 5.51 & & & & 1248 & 2.06 & 1199 \\
\hline CVS2-30 & & 2.4689 & -3.39 & 4967.63 & 408.69 & 27.42 & -10.318 & 19428.3 & 28.08 & & & & 1247 & 12.56 & 1197 \\
\hline CVS2-31 & & 2.4946 & -3.94 & 4316.83 & 462.00 & 10.30 & -12.436 & 21051.0 & 10.56 & & & & 1245 & 4.84 & 1195 \\
\hline CVS2-32 & & 2.7214 & -7.67 & 9122.28 & 214.78 & 8.07 & -13.694 & 22476.2 & 8.17 & & & & 1243 & 3.35 & 1193 \\
\hline CVS2-33 & & 2.6983 & -6.66 & 7113.98 & 191.83 & 2.15 & -10.862 & 16559.9 & 2.17 & & & & 1247 & 1.08 & 1198 \\
\hline CVS2-34 & & 2.5194 & -1.87 & 1173.64 & 671.69 & 1.79 & -9.532 & 14492.6 & 1.92 & & & & 1244 & 1.07 & 1195 \\
\hline CVS2-35 & & 2.6384 & -5.49 & 5647.30 & 283.96 & 2.82 & -10.535 & 16480.3 & 2.85 & & & & 1244 & 1.51 & 1193 \\
\hline CVS2-36 & & 2.7335 & -5.93 & 5863.67 & 258.71 & 1.91 & -10.605 & 16025.4 & 1.93 & & & & 1246 & 1.01 & 1196 \\
\hline CVS2-37 & & 2.4814 & -4.80 & 6610.03 & 246.37 & 12.34 & -9.785 & 17516.1 & 12.49 & & & & 1244 & 6.2 & 1195 \\
\hline CVS2-38 & & 2.6349 & -5.26 & 6086.86 & 364.62 & 12.02 & -12.266 & 21022.0 & 12.27 & & & & 1247 & 5.14 & 1197 \\
\hline CVS2-39 & & 2.7182 & -7.06 & 6961.55 & 250.19 & 1.98 & -12.658 & 18972.9 & 1.96 & & & & 1196 & 1.33 & 1147 \\
\hline CVS2-40 & & 2.601 & -5.75 & 6742.38 & 271.62 & 6.88 & -11.406 & 18994.7 & 6.98 & & & & 1247 & 3.24 & 1197 \\
\hline CVS2-41 & & 2.6035 & -5.40 & 6099.51 & 305.66 & 6.20 & -11.340 & 18754.6 & 6.30 & & & & 1246 & 2.97 & 1197 \\
\hline CVS2-42 & & 2.6886 & -5.74 & 6416.85 & 288.43 & 5.51 & -11.526 & 18851.2 & 5.59 & & & & 1247 & 2.58 & 1197 \\
\hline CVS2-43 & & 2.6258 & -4.86 & 4564.37 & 430.53 & 4.43 & -12.595 & 20073.0 & 4.53 & & & & 1247 & 2.08 & 1197 \\
\hline CVS2-44 & & 2.5685 & -5.65 & 6415.57 & 290.01 & 6.08 & -11.482 & 18930.0 & 6.18 & & & & 1246 & 2.88 & 1197 \\
\hline CVS2-45 & & 2.6326 & -5.46 & 5948.95 & 330.69 & 6.03 & -11.907 & 19526.7 & 6.14 & & & & 1248 & 2.75 & 1198 \\
\hline CVS2-46 & & 2.5955 & -4.81 & 4922.26 & 375.69 & 4.70 & -11.304 & 18317.5 & 4.80 & & & & 1248 & 2.25 & 1199 \\
\hline CVS2-47 & & 2.7207 & -5.90 & 6497.30 & 316.28 & 6.64 & -12.505 & 20515.3 & 6.77 & & & & 1248 & 2.9 & 1198 \\
\hline CVS2-48 & & 2.6336 & -5.06 & 4906.65 & 387.16 & 3.94 & -11.875 & 18879.0 & 4.03 & & & & 1248 & 1.9 & 1198 \\
\hline CVS2-49 & & 2.5893 & -4.99 & 5515.20 & 345.32 & 6.46 & -11.360 & 18847.3 & 6.58 & & & & 1247 & 3.09 & 1198 \\
\hline CVS2-50 & & 2.6811 & -6.13 & 6769.05 & 289.66 & 5.71 & -12.261 & 19948.8 & 5.80 & & & & 1247 & 2.57 & 1197 \\
\hline CVS2-51 & & 2.7014 & -4.93 & 5280.77 & 383.49 & 7.07 & -12.148 & 20101.8 & 7.23 & & & & 1248 & 3.26 & 1198 \\
\hline CVS2-52 & & 2.639 & -4.43 & 5054.41 & 390.32 & 9.22 & -11.534 & 19605.5 & 9.43 & & & & 1249 & 4.26 & 1198 \\
\hline \multicolumn{16}{|l|}{ CVS2-53 } \\
\hline CVS2-54 & & & & & & & & & & & & & & & \\
\hline CVS2-55 & & 2.6795 & -5.48 & 6007.61 & 329.16 & 6.26 & -11.972 & 19671.7 & 6.37 & & & & 1247 & 2.89 & 1197 \\
\hline CVS2-56 & & 2.6725 & -5.22 & 5672.65 & 343.32 & 6.12 & -11.741 & 19312.1 & 6.23 & & & & 1247 & 2.89 & 1197 \\
\hline CVS2-57 & & 2.7002 & -5.51 & 6014.17 & 338.91 & 6.74 & -12.298 & 20241.1 & 6.86 & & & & 1247 & 3.04 & 1197 \\
\hline CVS2-58 & & 2.5394 & -6.53 & 6403.64 & 253.59 & 1.85 & -11.522 & 17290.4 & 1.88 & & & & 1245 & 0.94 & 1195 \\
\hline CVS2-59 & & 2.535 & -5.35 & 4588.72 & 313.48 & 1.15 & -9.974 & 14406.5 & 1.16 & & & & 1246 & 0.62 & 1196 \\
\hline CVS2-60 & & 2.523 & -5.68 & 6413.79 & 184.20 & 2.61 & -9.354 & 14688.7 & 2.63 & & & & 1246 & 1.45 & 1196 \\
\hline CVS2-61 & & 2.4912 & -5.39 & 7522.86 & 203.81 & 12.90 & -10.084 & 18004.5 & 13.05 & & & & 1246 & 6.21 & 1197 \\
\hline CVS2-62 & & 2.4931 & -4.29 & 4595.09 & 452.47 & 9.95 & -12.949 & 21732.8 & 10.21 & & & & 1245 & 4.54 & 1195 \\
\hline CVS2-63 & & 2.5763 & -5.02 & 4410.76 & 400.45 & 2.38 & -11.542 & 17687.4 & 2.43 & & & & 1246 & 1.25 & 1197 \\
\hline CVS2-64 & & 2.7003 & -7.14 & 9409.83 & 140.36 & 8.89 & -11.541 & 19547.1 & 8.99 & & & & 1249 & 3.87 & 1199 \\
\hline CVS2-65 & & 2.6721 & -7.47 & 8445.48 & 268.33 & 8.20 & -14.489 & 23637.6 & 8.35 & & & & 1246 & 3.21 & 1196 \\
\hline
\end{tabular}


Appendix A. Database - mass fraction

Hanford CVS 2 (Hrma et al. 1994)

\begin{tabular}{|c|c|c|c|c|c|c|c|c|c|c|c|c|c|c|c|c|c|c|c|c|c|}
\hline \multicolumn{22}{|c|}{ Hanford CVS } \\
\hline Glass ID & $\begin{array}{c}\mathrm{V} 2 \\
(\mathrm{~Pa} \cdot \mathrm{s})\end{array}$ & $\begin{array}{c}\mathrm{T} 3 \\
\left({ }^{\circ} \mathrm{C}\right)\end{array}$ & $\begin{array}{c}\mathrm{V} 3 \\
(\mathrm{~Pa} \cdot \mathrm{s})\end{array}$ & $\begin{array}{c}\mathrm{T} 4 \\
\left({ }^{\circ} \mathrm{C}\right)\end{array}$ & $\begin{array}{c}\mathrm{V} 4 \\
(\mathrm{~Pa} \cdot \mathrm{s})\end{array}$ & $\begin{array}{c}\mathrm{T} 5 \\
\left({ }^{\circ} \mathrm{C}\right) \\
\end{array}$ & $\begin{array}{c}\text { V5 } \\
(\mathrm{Pa} \cdot \mathrm{s})\end{array}$ & $\begin{array}{c}\mathrm{T} 6 \\
\left({ }^{\circ} \mathrm{C}\right)\end{array}$ & $\begin{array}{c}\mathrm{V6} \\
(\mathrm{Pa} \cdot \mathrm{s})\end{array}$ & $\begin{array}{c}\mathrm{T} 7 \\
\left({ }^{\circ} \mathrm{C}\right) \\
\end{array}$ & $\begin{array}{c}\mathrm{V} 7 \\
(\mathrm{~Pa} \cdot \mathrm{s})\end{array}$ & $\begin{array}{c}\mathrm{T} 8 \\
\left({ }^{\circ} \mathrm{C}\right)\end{array}$ & $\begin{array}{c}\mathrm{V} 8 \\
(\mathrm{~Pa} \cdot \mathrm{s})\end{array}$ & $\begin{array}{c}\mathrm{T} 9 \\
\left({ }^{\circ} \mathrm{C}\right)\end{array}$ & $\begin{array}{c}\mathrm{V} 9 \\
(\mathrm{~Pa} \cdot \mathrm{s})\end{array}$ & $\begin{array}{l}\mathrm{T} 10 \\
\left({ }^{\circ} \mathrm{C}\right)\end{array}$ & $\begin{array}{c}\mathrm{V} 10 \\
(\mathrm{~Pa} \cdot \mathrm{s})\end{array}$ & $\begin{array}{l}\mathrm{T} 11 \\
\left({ }^{\circ} \mathrm{C}\right)\end{array}$ & $\begin{array}{c}\text { V11 } \\
(\mathrm{Pa} \cdot \mathrm{s})\end{array}$ & $\begin{array}{l}\mathrm{T} 12 \\
\left({ }^{\circ} \mathrm{C}\right)\end{array}$ & $\begin{array}{c}\mathrm{V} 12 \\
(\mathrm{~Pa} \cdot \mathrm{s})\end{array}$ \\
\hline CVS2-27 & 9.77 & 1144 & 15.06 & 1144 & 15.11 & 1143 & 15.85 & 1094 & 24.94 & 1094 & 25.1 & 1045 & 42.92 & 993 & 88.73 & 943 & 173.11 & & & & \\
\hline CVS2-28 & 9.21 & 1147 & 13.32 & 1148 & 13.73 & 1146 & 14.54 & 1097 & 21.65 & 1097 & 22.01 & 1046 & 37.5 & 996 & 75.7 & 946 & 145.16 & & & & \\
\hline CVS2-29 & 3.23 & 1149 & 5.22 & 1149 & 5.43 & 1149 & 5.45 & 1099 & 9.75 & 1098 & 9.89 & 1048 & 20.38 & 998 & 46.62 & 949 & 124.81 & & & & \\
\hline CVS2-30 & 18.61 & 1147 & 26.59 & 1148 & 28.22 & 1147 & 28.92 & 1098 & 46.67 & 1096 & 46.78 & 1047 & 78.55 & 998 & 155.75 & & & & & & \\
\hline CVS2-31 & 6.82 & 1146 & 10.5 & 1146 & 10.8 & 1146 & 11.08 & 1096 & 17.58 & 1096 & 17.8 & 1047 & 29.96 & 996 & 64.17 & 947 & 141.91 & & & & \\
\hline CVS2-32 & 5.27 & 1142 & 8.59 & 1143 & 8.66 & 1143 & 8.78 & 1093 & 15.09 & 1092 & 15.71 & 1043 & 28.16 & 994 & 57.52 & 943 & 128.61 & & & & \\
\hline CVS2-33 & 1.52 & 1148 & 2.14 & 1149 & 2.17 & 1148 & 2.24 & 1099 & 3.22 & 1098 & 3.26 & 1048 & 5.2 & 999 & 8.74 & 948 & 15.52 & & & & \\
\hline CVS2-34 & 1.44 & 1146 & 1.96 & 1145 & 1.97 & 1145 & 1.98 & 1097 & 2.77 & 1095 & 2.8 & 1046 & 4.14 & 996 & 6.57 & 945 & 11.29 & & & & \\
\hline CVS2-35 & 2.04 & 1144 & 2.87 & 1144 & 2.95 & 1143 & 3.01 & 1094 & 4.42 & 1093 & 4.45 & 1043 & 7.12 & 993 & 12.06 & 943 & 21.65 & & & & \\
\hline CVS2-36 & 1.38 & 1145 & 1.95 & 1146 & 1.96 & 1145 & 1.98 & 1096 & 2.93 & 1095 & 2.94 & 1045 & 4.67 & 995 & 7.5 & 945 & 13.65 & & & & \\
\hline CVS2-37 & 8.7 & 1146 & 12.43 & 1146 & 12.74 & 1146 & 13.05 & 1097 & 19.48 & 1095 & 20.05 & 1046 & 31.75 & 997 & 54.67 & 947 & 102.97 & & & & \\
\hline CVS2-38 & 7.75 & 1147 & 12.21 & 1147 & 12.41 & 1147 & 12.44 & 1096 & 21.19 & 1097 & 21.27 & 1047 & 38.8 & 998 & 77.06 & 1097 & 21.27 & & & & \\
\hline CVS2-39 & 2.02 & 1143 & 2.08 & 1145 & 2.14 & 1097 & 3.15 & 1095 & 3.34 & 1047 & 5.45 & 994 & 9.61 & 944 & 20 & & & & & & \\
\hline CVS2-40 & 4.72 & 1148 & 6.78 & 1147 & 6.81 & 1147 & 7.16 & 1098 & 11.19 & 1097 & 11.42 & 1048 & 18.83 & 998 & 35.11 & 947 & 68.1 & & & & \\
\hline CVS2-41 & 4.16 & 1147 & 6.3 & 1147 & 6.4 & 1147 & 6.46 & 1098 & 10.01 & 1097 & 10.23 & 1047 & 16.8 & 998 & 29.58 & 947 & 61.73 & & & & \\
\hline CVS2-42 & 3.68 & 1147 & 5.66 & 1148 & 5.71 & 1147 & 5.75 & 1098 & 8.94 & 1096 & 9.05 & 1047 & 14.95 & 998 & 26.7 & 948 & 54.54 & & & & \\
\hline CVS2-43 & 2.94 & 1147 & 4.55 & 1148 & 4.59 & 1147 & 4.7 & 1097 & 7.23 & 1097 & 7.27 & 1047 & 12.43 & 997 & 25.21 & 947 & 53.29 & & & & \\
\hline CVS2-44 & 4.12 & 1147 & 6.19 & 1148 & 6.19 & 1147 & 6.3 & 1098 & 9.8 & 1097 & 10.01 & 1047 & 16.63 & 998 & 30.39 & 948 & 59.96 & & & & \\
\hline CVS2-45 & 4.09 & 1148 & 6.05 & 1149 & 6.08 & 1149 & 6.29 & 1100 & 9.55 & 1098 & 9.73 & 1049 & 16.44 & 998 & 32.61 & 948 & 64.17 & & & & \\
\hline CVS2-46 & 3.27 & 1148 & 4.79 & 1150 & 4.82 & 1148 & 4.9 & 1100 & 7.16 & 1097 & 7.31 & 1048 & 12.26 & 998 & 22.41 & 948 & 44.39 & & & & \\
\hline CVS2-47 & 4.32 & 1148 & 6.6 & 1148 & 6.81 & 1149 & 7.07 & 1098 & 10.99 & 1100 & 11.03 & 1048 & 19.29 & 999 & 37.34 & 948 & 80.59 & & & & \\
\hline CVS2-48 & 2.69 & 1148 & 3.94 & 1149 & 4 & 1148 & 4.06 & 1099 & 6.23 & 1098 & 6.32 & 1048 & 10.51 & 999 & 19.64 & 948 & 39.72 & & & & \\
\hline CVS2-49 & 4.35 & 1147 & 6.63 & 1147 & 6.65 & 1147 & 6.72 & 1098 & 10.31 & 1098 & 10.47 & 1048 & 17.56 & 998 & 31.31 & 948 & 64.85 & & & & \\
\hline CVS2-50 & 3.78 & 1147 & 5.85 & 1147 & 5.87 & 1147 & 5.96 & 1098 & 9.46 & 1096 & 9.57 & 1047 & 16.49 & 998 & 31.28 & 948 & 63.53 & & & & \\
\hline CVS2-51 & 4.75 & 1149 & 6.97 & 1149 & 7.03 & 1148 & 7.28 & 1099 & 11.55 & 1099 & 11.73 & 1048 & 19.96 & 999 & 39.58 & 948 & 82.16 & & & & \\
\hline CVS2-52 & 6.23 & 1148 & 9.2 & 1149 & 9.43 & 1149 & 9.56 & 1099 & 14.71 & 1098 & 14.82 & 1048 & 25.92 & 999 & 48.56 & 949 & 100.65 & & & & \\
\hline CVS2-53 & & & & & & & & & & & & & & & & & & & & & \\
\hline CVS2-54 & & & & & & & & & & & & & & & & & & & & & \\
\hline CVS2-55 & 4.18 & 1147 & 6.35 & 1147 & 6.49 & 1147 & 6.53 & 1097 & 10.36 & 1096 & 10.55 & 1046 & 17.76 & 997 & 33.88 & 946 & 70.3 & & & & \\
\hline CVS2-56 & 4.12 & 1146 & 6.22 & 1147 & 6.32 & 1147 & 6.44 & 1097 & 10.05 & 1096 & 10.07 & 1047 & 17.05 & 996 & 32.52 & 946 & 65.92 & & & & \\
\hline CVS2-57 & 4.49 & 1147 & 6.9 & 1147 & 6.97 & 1147 & 7 & 1097 & 11.29 & 1096 & 11.35 & 1046 & 19.9 & 996 & 38.62 & 945 & 82.58 & & & & \\
\hline CVS2-58 & 1.31 & 1146 & 1.88 & 1146 & 1.92 & 1146 & 1.93 & 1097 & 2.89 & 1096 & 2.96 & 1046 & 4.75 & 997 & 8.05 & 946 & 15.25 & & & & \\
\hline CVS2-59 & 0.89 & 1147 & 1.19 & 1147 & 1.19 & 1147 & 1.23 & 1098 & 1.59 & 1097 & 1.67 & 1048 & 2.41 & 998 & 3.77 & 947 & 6.82 & & & & \\
\hline CVS2-60 & 1.92 & 1147 & 2.62 & 1147 & 2.63 & 1147 & 2.69 & 1098 & 3.78 & 1098 & 3.88 & 1048 & 5.74 & 998 & 9.12 & 947 & 15.18 & & & & \\
\hline CVS2-61 & 8.78 & 1148 & 13.1 & 1147 & 13.14 & 1147 & 13.6 & 1098 & 20.36 & 1097 & 20.57 & 1047 & 33.64 & 998 & 60.13 & 948 & 111.03 & & & & \\
\hline CVS2-62 & 6.7 & 1147 & 9.82 & 1147 & 10.36 & 1146 & 10.58 & 1097 & 17.03 & 1097 & 17.09 & 1047 & 30.72 & 997 & 65.34 & 946 & 149.73 & & & & \\
\hline CVS2-63 & 1.65 & 1147 & 2.4 & 1147 & 2.43 & 1147 & 2.45 & 1098 & 3.68 & 1097 & 3.77 & 1047 & 6.06 & 998 & 10.92 & 947 & 20.93 & & & & \\
\hline CVS2-64 & 5.79 & 1149 & 8.85 & 1149 & 8.88 & 1149 & 9.03 & 1100 & 14.55 & 1099 & 14.82 & 1049 & 24.81 & 999 & 45.69 & 948 & 91.58 & & & & \\
\hline CVS2-65 & 5.06 & 1146 & 8.53 & 1146 & 8.6 & 1146 & 8.73 & 1096 & 15.14 & 1096 & 15.26 & 1046 & 29.64 & 996 & 62.62 & 945 & 149.06 & & & & \\
\hline
\end{tabular}


Appendix A. Database - mass fraction

Hanford CVS 2 (Hrma et al. 1994)

\begin{tabular}{|c|c|c|c|c|c|c|c|c|c|c|c|c|c|c|c|c|}
\hline Glass ID & $\begin{array}{l}\mathrm{T} 13 \\
\left({ }^{\circ} \mathrm{C}\right)\end{array}$ & $\begin{array}{c}\mathrm{V} 13 \\
(\mathrm{~Pa} \cdot \mathrm{s})\end{array}$ & $\begin{array}{l}\mathrm{T} 14 \\
\left({ }^{\circ} \mathrm{C}\right)\end{array}$ & $\begin{array}{c}\mathrm{V} 14 \\
(\mathrm{~Pa} \cdot \mathrm{s})\end{array}$ & $\begin{array}{c}\text { Q PCT } \\
\text { B }\left(\mathrm{g} / \mathrm{m}^{2}\right)\end{array}$ & $\begin{array}{c}\text { Q PCT } \\
\mathrm{Li}\left(\mathrm{g} / \mathrm{m}^{2}\right)\end{array}$ & $\begin{array}{c}\text { Q PCT } \\
\mathrm{Na}\left(\mathrm{g} / \mathrm{m}^{2}\right)\end{array}$ & $\begin{array}{c}\text { Q PCT } \\
\mathrm{Si}\left(\mathrm{g} / \mathrm{m}^{2}\right)\end{array}$ & $\begin{array}{c}\text { Q PCT } \\
\text { pH }\end{array}$ & $\begin{array}{c}\text { CCC PCT } \\
\mathrm{B}\left(\mathrm{g} / \mathrm{m}^{2}\right)\end{array}$ & $\begin{array}{l}\text { CCC PCT } \\
\mathrm{Li}\left(\mathrm{g} / \mathrm{m}^{2}\right)\end{array}$ & $\begin{array}{l}\text { CCC PCT } \\
\mathrm{Na}\left(\mathrm{g} / \mathrm{m}^{2}\right)\end{array}$ & $\begin{array}{c}\text { CCC PCT } \\
\mathrm{Si}\left(\mathrm{g} / \mathrm{m}^{2}\right)\end{array}$ & $\begin{array}{c}\text { CCC PCT } \\
\mathrm{pH}\end{array}$ & $\begin{array}{l}\text { Q PCT B at } \\
20^{\circ} \mathrm{C}\left(\mathrm{g} / \mathrm{m}^{2}\right)\end{array}$ & $\begin{array}{l}\text { Q PCT Li at } \\
20^{\circ} \mathrm{C}\left(\mathrm{g} / \mathrm{m}^{2}\right)\end{array}$ \\
\hline CVS2-27 & & & & & 3.270 & 1.835 & 2.342 & 0.89 & 11.87 & 2.572 & 1.734 & 2.096 & 0.86 & 11.84 & & \\
\hline CVS2-28 & & & & & 5.144 & 3.682 & 3.538 & 1.654 & 11.54 & 3.601 & 3.158 & 3.032 & 1.654 & 11.54 & & \\
\hline CVS2-29 & & & & & 1.286 & 1.355 & 1.273 & 0.192 & 9.11 & 0.949 & 1.075 & 1.108 & 0.243 & 9.22 & & \\
\hline CVS2-30 & & & & & 6.512 & 4.194 & 2.552 & 0.174 & 8.74 & 18.006 & 14.462 & 8.682 & 0.3005 & 8.76 & & \\
\hline CVS2-31 & & & & & 0.411 & 0.460 & 0.318 & 0.121 & 9.54 & 0.584 & 0.682 & 0.539 & 0.172 & 9.60 & & \\
\hline CVS2-32 & & & & & 9.646 & 5.527 & 5.896 & 2.747 & 12.31 & 7.588 & 6.231 & 5.660 & 2.924 & 12.16 & & \\
\hline CVS2-33 & & & & & 1.723 & 1.438 & 1.608 & 0.516 & 11.94 & 11.526 & 5.908 & 7.412 & 1.526 & 12.50 & & \\
\hline CVS2-34 & & & & & 4.340 & 3.468 & 2.700 & 0.265 & 11.55 & 4.520 & 3.497 & 2.594 & 0.28 & 11.42 & & \\
\hline CVS2-35 & & & & & 0.320 & 0.580 & 0.910 & 0.204 & 11.90 & 25.048 & 9.283 & 11.742 & 2.16 & 12.60 & & \\
\hline CVS2-36 & & & & & 0.480 & 0.630 & 0.654 & 0.276 & 11.34 & 0.354 & 0.568 & 0.431 & 0.223 & 11.40 & & \\
\hline CVS2-37 & & & & & 0.246 & 0.300 & 0.177 & 0.206 & 10.45 & 0.230 & 0.320 & 0.177 & 0.236 & 10.35 & & \\
\hline CVS2-38 & & & & & 1.119 & 0.726 & 0.807 & 0.28 & 9.56 & 0.725 & 0.602 & 0.574 & 0.27 & 9.84 & & \\
\hline CVS2-39 & & & & & 12.701 & 6.528 & 7.668 & 1.612 & 12.50 & 15.386 & 5.046 & 8.312 & 1.634 & 12.36 & & \\
\hline CVS2-40 & & & & & 0.337 & 0.328 & 0.399 & 0.172 & 11.13 & 0.317 & 0.347 & 0.364 & 0.171 & 11.12 & & \\
\hline CVS2-41 & & & & & 0.177 & 0.260 & 0.188 & 0.104 & 10.89 & 0.199 & 0.307 & 0.199 & 0.124 & 10.91 & & \\
\hline CVS2-42 & & & & & 1.694 & 1.220 & 1.337 & 0.574 & 11.19 & 6.109 & 4.301 & 3.763 & 2.259 & 11.45 & & \\
\hline CVS2-43 & & & & & 0.767 & 0.697 & 0.451 & 0.196 & 9.97 & 0.697 & 0.593 & 0.445 & 0.198 & 9.88 & & \\
\hline CVS2-44 & & & & & 0.255 & 0.294 & 0.194 & 0.128 & 10.31 & 0.388 & 0.323 & 0.310 & 0.174 & 10.40 & & \\
\hline CVS2-45 & & & & & 0.500 & 0.478 & 0.374 & 0.186 & 10.05 & 0.397 & 0.439 & 0.240 & 0.169 & 9.97 & & \\
\hline CVS2-46 & & & & & 0.317 & 0.346 & 0.188 & 0.148 & 10.19 & 0.294 & 0.307 & 0.199 & 0.146 & 10.09 & & \\
\hline CVS2-47 & & & & & 1.159 & 1.124 & 1.055 & 0.434 & 11.39 & 1.286 & 0.902 & 0.945 & 0.478 & 11.40 & & \\
\hline CVS2-48 & & & & & 0.307 & 0.318 & 0.284 & 0.102 & 10.09 & 0.229 & 0.309 & 0.258 & 0.114 & 10.09 & & \\
\hline CVS2-49 & & & & & 0.303 & 0.334 & 0.217 & 0.136 & 9.97 & 0.206 & 0.279 & 0.204 & 0.141 & 9.91 & & \\
\hline CVS2-50 & & & & & 0.442 & 0.426 & 0.368 & 0.15 & 10.23 & 0.563 & 0.486 & 0.376 & 0.158 & 10.27 & & \\
\hline CVS2-51 & & & & & 1.764 & 1.496 & 1.283 & 0.411 & 10.30 & 1.442 & 1.067 & 0.997 & 0.359 & 10.23 & & \\
\hline CVS2-52 & & & & & 0.557 & 0.554 & 0.260 & 0.294 & 10.16 & 0.551 & 0.478 & 0.270 & 0.285 & 10.08 & & \\
\hline CVS2-53 & & & & & 0.304 & 0.305 & 0.202 & 0.176 & 10.02 & & & & & & & \\
\hline CVS2-54 & & & & & 2.761 & 1.715 & 1.612 & 0.571 & 10.23 & & & & & & & \\
\hline CVS2-55 & & & & & 1.342 & 1.127 & 0.880 & 0.352 & 10.19 & 0.930 & 0.894 & 0.728 & 0.373 & 10.08 & & \\
\hline CVS2-56 & & & & & 1.419 & 1.218 & 0.946 & 0.368 & 10.20 & 1.212 & 1.193 & 1.027 & 0.461 & 10.21 & & \\
\hline CVS2-57 & & & & & 1.164 & 1.081 & 0.842 & 0.346 & 10.26 & 0.828 & 0.778 & 0.657 & 0.281 & 9.91 & & \\
\hline CVS2-58 & & & & & 0.778 & 0.636 & 0.620 & 0.138 & 9.87 & 0.820 & 0.829 & 0.768 & 0.156 & 9.81 & & \\
\hline CVS2-59 & & & & & 1.591 & 1.287 & 1.222 & 0.268 & 11.47 & 1.591 & 1.390 & 1.116 & 0.268 & 11.52 & & \\
\hline CVS2-60 & & & & & 1.624 & 1.158 & 1.523 & 0.415 & 11.96 & 1.542 & 1.621 & 1.457 & 0.577 & 11.99 & & \\
\hline CVS2-61 & & & & & 0.222 & 0.280 & 0.230 & 0.195 & 10.89 & 0.232 & 0.354 & 0.225 & 0.223 & 10.83 & & \\
\hline CVS2-62 & & & & & 1.002 & 0.862 & 0.928 & 0.268 & 9.56 & 1.093 & 1.034 & 0.958 & 0.287 & 9.54 & & \\
\hline CVS2-63 & & & & & 0.332 & 0.222 & 0.390 & 0.079 & 10.59 & 3.652 & 3.584 & 2.163 & 0.102 & 10.29 & & \\
\hline CVS2-64 & & & & & 0.379 & 0.376 & 0.342 & 0.234 & 10.62 & 0.354 & 0.419 & 0.297 & 0.242 & 10.79 & & \\
\hline CVS2-65 & & & & & 0.335 & 0.355 & 0.200 & 0.148 & 10.62 & 0.356 & 0.417 & 0.204 & 0.156 & 10.72 & & \\
\hline
\end{tabular}


Appendix A. Database - mass fraction

Hanford CVS 2 (Hrma et al. 1994)

\begin{tabular}{|c|c|c|c|c|c|c|c|c|c|c|c|c|}
\hline Glass ID & $\begin{array}{l}\text { Q PCT Na at } \\
20^{\circ} \mathrm{C}\left(\mathrm{g} / \mathrm{m}^{2}\right)\end{array}$ & $\begin{array}{l}\text { Q PCT Si at } \\
20^{\circ} \mathrm{C}\left(\mathrm{g} / \mathrm{m}^{2}\right)\end{array}$ & $\begin{array}{c}\mathrm{QpH} \\
\text { at } 20^{\circ} \mathrm{C}\end{array}$ & $\begin{array}{c}\text { TCLP Ag } \\
\text { (ppm) }\end{array}$ & $\begin{array}{c}\text { TCLP As } \\
\text { (ppm) }\end{array}$ & $\begin{array}{l}\text { TCLP Ba } \\
\text { (ppm) }\end{array}$ & $\begin{array}{c}\text { TCLP Cd } \\
\text { (ppm) }\end{array}$ & $\begin{array}{c}\text { TCLP Cr } \\
\text { (ppm) }\end{array}$ & $\begin{array}{c}\text { TCLP Ni } \\
(\mathrm{ppm})\end{array}$ & $\begin{array}{c}\text { TCLP Pb } \\
\text { (ppm) }\end{array}$ & $\begin{array}{c}\text { TCLP Se } \\
\text { (ppm) }\end{array}$ & $\begin{array}{c}\text { TCLP Zn } \\
(\mathrm{ppm})\end{array}$ \\
\hline CVS2-27 & & & & & & & & & & & & \\
\hline CVS2-28 & & & & & & & & & & & & \\
\hline CVS2-29 & & & & & & & & & & & & \\
\hline CVS2-30 & & & & & & & & & & & & \\
\hline CVS2-31 & & & & & & & & & & & & \\
\hline CVS2-32 & & & & & & & & & & & & \\
\hline CVS2-33 & & & & & & & & & & & & \\
\hline CVS2-34 & & & & & & & & & & & & \\
\hline CVS2-35 & & & & & & & & & & & & \\
\hline CVS2-36 & & & & & & & & & & & & \\
\hline CVS2-37 & & & & & & & & & & & & \\
\hline CVS2-38 & & & & & & & & & & & & \\
\hline CVS2-39 & & & & & & & & & & & & \\
\hline CVS2-40 & & & & & & & & & & & & \\
\hline CVS2-41 & & & & & & & & & & & & \\
\hline CVS2-42 & & & & & & & & & & & & \\
\hline CVS2-43 & & & & & & & & & & & & \\
\hline CVS2-44 & & & & & & & & & & & & \\
\hline CVS2-45 & & & & & & & & & & & & \\
\hline CVS2-46 & & & & & & & & & & & & \\
\hline CVS2-47 & & & & & & & & & & & & \\
\hline CVS2-48 & & & & & & & & & & & & \\
\hline CVS2-49 & & & & & & & & & & & & \\
\hline CVS2-50 & & & & & & & & & & & & \\
\hline CVS2-51 & & & & & & & & & & & & \\
\hline CVS2-52 & & & & & & & & & & & & \\
\hline CVS2-53 & & & & & & & & & & & & \\
\hline CVS2-54 & & & & & & & & & & & & \\
\hline CVS2-55 & & & & & & & & & & & & \\
\hline CVS2-56 & & & & & & & & & & & & \\
\hline CVS2-57 & & & & & & & & & & & & \\
\hline CVS2-58 & & & & & & & & & & & & \\
\hline CVS2-59 & & & & & & & & & & & & \\
\hline CVS2-60 & & & & & & & & & & & & \\
\hline CVS2-61 & & & & & & & & & & & & \\
\hline CVS2-62 & & & & & & & & & & & & \\
\hline CVS2-63 & & & & & & & & & & & & \\
\hline CVS2-64 & & & & & & & & & & & & \\
\hline CVS2-65 & & & & & & & & & & & & \\
\hline
\end{tabular}


Hanford CVS 2 (Hrma et al. 1994)

\begin{tabular}{|c|c|c|c|c|c|c|c|c|c|c|c|c|c|c|c|c|c|c|c|c|c|}
\hline Glass ID & $\begin{array}{c}\mathrm{A} 12 \mathrm{O} 3 \\
-\mathrm{t}\end{array}$ & $\begin{array}{c}\mathrm{B} 2 \mathrm{O} 3 \\
-\mathrm{t}\end{array}$ & $\begin{array}{c}\mathrm{CaO} \\
-\mathrm{t} \\
\end{array}$ & $\begin{array}{c}\mathrm{Fe} 2 \mathrm{O} 3 \\
-\mathrm{t}\end{array}$ & $\begin{array}{c}\mathrm{FeO} \\
-\mathrm{t}\end{array}$ & $\begin{array}{c}\mathrm{K} 2 \mathrm{O} \\
-\mathrm{t}\end{array}$ & $\begin{array}{c}\mathrm{Li} 2 \mathrm{O} \\
-\mathrm{t}\end{array}$ & $\begin{array}{c}\mathrm{MgO} \\
-\mathrm{t}\end{array}$ & $\begin{array}{c}\mathrm{Na} 2 \mathrm{O} \\
-\mathrm{t}\end{array}$ & $\begin{array}{c}\mathrm{NiO} \\
-\mathrm{t}\end{array}$ & $\begin{array}{c}\mathrm{P} 2 \mathrm{O} 5 \\
-\mathrm{t}\end{array}$ & $\begin{array}{c}\mathrm{SiO} 2 \\
-\mathrm{t}\end{array}$ & $\begin{array}{c}\mathrm{ZrO} 2 \\
-\mathrm{t}\end{array}$ & $\begin{array}{c}\mathrm{Ag} 2 \mathrm{O} \\
-\mathrm{t}\end{array}$ & $\begin{array}{c}\mathrm{As} 2 \mathrm{O} 3 \\
-\mathrm{t}\end{array}$ & $\begin{array}{c}\mathrm{BaO} \\
-\mathrm{t}\end{array}$ & $\begin{array}{c}\mathrm{Bi} 2 \mathrm{O} 3 \\
-\mathrm{t}\end{array}$ & $\begin{array}{l}\mathrm{Br} \\
-\mathrm{t}\end{array}$ & $\begin{array}{c}\mathrm{CdO} \\
-\mathrm{t}\end{array}$ & $\begin{array}{c}\mathrm{Ce} 2 \mathrm{O} 3 \\
-\mathrm{t} \\
\end{array}$ & $\begin{array}{c}\mathrm{CeO} 2 \\
-\mathrm{t}\end{array}$ \\
\hline CVS2-66 & 0.1183 & 0.0919 & 0.0097 & 0.0389 & & 0.0000 & 0.0524 & 0.0061 & 0.1214 & 0.0052 & 0.0000 & 0.5189 & 0.0026 & 0.0000 & & 0.0005 & 0.0000 & & 0.0000 & & 0.0000 \\
\hline CVS2-67 & 0.2043 & 0.1587 & 0.0024 & 0.0004 & & 0.0000 & 0.0583 & 0.0001 & 0.1086 & 0.0000 & 0.0049 & 0.4596 & 0.0000 & 0.0000 & & 0.0000 & 0.0000 & & 0.0000 & & 0.0000 \\
\hline CVS2-68 & 0.1641 & 0.1356 & 0.0007 & 0.0046 & & 0.0016 & 0.0696 & 0.0002 & 0.0797 & 0.0000 & 0.0000 & 0.5042 & 0.0001 & 0.0000 & & 0.0000 & 0.0000 & & 0.0000 & & 0.0000 \\
\hline CVS2-69 & 0.0818 & 0.0783 & 0.0079 & 0.0334 & & 0.0000 & 0.0715 & 0.0032 & 0.0666 & 0.0000 & 0.0326 & 0.5676 & 0.0005 & 0.0000 & & 0.0000 & 0.0000 & & 0.0011 & & 0.0000 \\
\hline CVS2-70 & 0.1820 & 0.1419 & 0.0008 & 0.0080 & & 0.0000 & 0.0691 & 0.0008 & 0.0813 & 0.0008 & 0.0007 & 0.4857 & 0.0005 & 0.0000 & 0.0011 & 0.0000 & 0.0000 & & 0.0011 & & 0.0013 \\
\hline CVS2-71 & 0.0288 & 0.0509 & 0.0025 & 0.0812 & & 0.0000 & 0.0642 & 0.0008 & 0.0925 & 0.0068 & 0.0012 & 0.5699 & 0.0431 & 0.0000 & & 0.0012 & 0.0000 & & 0.0089 & & 0.0018 \\
\hline CVS2-72 & 0.1180 & 0.0917 & 0.0097 & 0.0388 & & 0.0000 & 0.0523 & 0.0061 & 0.1211 & 0.0043 & 0.0008 & 0.5176 & 0.0026 & 0.0000 & & 0.0008 & 0.0000 & & 0.0057 & & 0.0011 \\
\hline CVS2-73 & 0.1640 & 0.1355 & 0.0007 & 0.0046 & & 0.0000 & 0.0696 & 0.0002 & 0.0797 & 0.0043 & 0.0007 & 0.5040 & 0.0001 & 0.0000 & & 0.0007 & 0.0000 & & 0.0056 & & 0.0011 \\
\hline CVS2-74 & 0.0816 & 0.0781 & 0.0079 & 0.0334 & & 0.0000 & 0.0713 & 0.0032 & 0.0664 & 0.0094 & 0.0016 & 0.5662 & 0.0005 & 0.0000 & & 0.0016 & 0.0000 & & 0.0123 & & 0.0025 \\
\hline CVS2-75 & 0.1819 & 0.1418 & 0.0008 & 0.0080 & & 0.0000 & 0.0691 & 0.0008 & 0.0812 & 0.0031 & 0.0005 & 0.4855 & 0.0005 & 0.0000 & & 0.0005 & 0.0000 & & 0.0041 & & 0.0008 \\
\hline CVS2-76 & 0.0200 & 0.0600 & 0.0400 & 0.1050 & & 0.0000 & 0.0632 & 0.0050 & 0.1800 & 0.0021 & 0.0004 & 0.5018 & 0.0050 & 0.0000 & & 0.0004 & 0.0000 & & 0.0027 & & 0.0005 \\
\hline CVS2-77 & 0.0200 & 0.0600 & 0.0050 & 0.0050 & & 0.0000 & 0.0700 & 0.0050 & 0.1801 & 0.0093 & 0.0016 & 0.4552 & 0.1100 & 0.0000 & & 0.0016 & 0.0000 & & 0.0121 & & 0.0024 \\
\hline CVS2-78 & 0.0200 & 0.1600 & 0.0050 & 0.0699 & & 0.0000 & 0.0254 & 0.0400 & 0.0500 & 0.0021 & 0.0004 & 0.5600 & 0.0497 & 0.0000 & & 0.0004 & 0.0000 & & 0.0027 & & 0.0005 \\
\hline CVS2-79 & 0.0200 & 0.1601 & 0.0050 & 0.1050 & & 0.0000 & 0.0121 & 0.0050 & 0.0500 & 0.0093 & 0.0016 & 0.5481 & 0.0050 & 0.0000 & & 0.0016 & 0.0000 & & 0.0121 & & 0.0024 \\
\hline CVS2-80 & 0.0200 & 0.1600 & 0.0050 & 0.1050 & & 0.0000 & 0.0176 & 0.0400 & 0.0500 & 0.0021 & 0.0004 & 0.5074 & 0.0750 & 0.0000 & & 0.0004 & 0.0000 & & 0.0027 & & 0.0005 \\
\hline CVS2-81 & 0.0200 & 0.0600 & 0.0050 & 0.1050 & & 0.0000 & 0.0700 & 0.0400 & 0.1735 & 0.0084 & 0.0015 & 0.4402 & 0.0050 & 0.0000 & & 0.0015 & 0.0000 & & 0.0110 & & 0.0022 \\
\hline CVS2-82 & 0.0200 & 0.0950 & 0.0050 & 0.0050 & & 0.0000 & 0.0700 & 0.0400 & 0.1800 & 0.0021 & 0.0004 & 0.5600 & 0.0050 & 0.0000 & & 0.0004 & 0.0000 & & 0.0027 & & 0.0005 \\
\hline CVS2-83 & 0.0200 & 0.0951 & 0.0400 & 0.0050 & & 0.0000 & 0.0699 & 0.0050 & 0.1801 & 0.0093 & 0.0016 & 0.4902 & 0.0050 & 0.0000 & & 0.0016 & 0.0000 & & 0.0121 & & 0.0024 \\
\hline CVS2-84 & 0.0200 & 0.0600 & 0.0050 & 0.1050 & & 0.0000 & 0.0700 & 0.0050 & 0.1800 & 0.0021 & 0.0004 & 0.4550 & 0.0800 & 0.0000 & & 0.0004 & 0.0000 & & 0.0027 & & 0.0005 \\
\hline CVS2-85 & 0.1700 & 0.0600 & 0.0050 & 0.0050 & & 0.0000 & 0.0700 & 0.0200 & 0.1800 & 0.0046 & 0.0008 & 0.4401 & 0.0050 & 0.0000 & & 0.0008 & 0.0000 & & 0.0060 & & 0.0012 \\
\hline CVS2-86 & 0.1700 & 0.0600 & 0.0400 & 0.0050 & & 0.0000 & 0.0136 & 0.0050 & 0.1800 & 0.0046 & 0.0008 & 0.4765 & 0.0050 & 0.0000 & & 0.0008 & 0.0000 & & 0.0060 & & 0.0012 \\
\hline CVS2-87 & 0.0987 & 0.0800 & 0.0137 & 0.0250 & & 0.0000 & 0.0180 & 0.0050 & 0.1800 & 0.0021 & 0.0004 & 0.4983 & 0.0613 & 0.0000 & & 0.0004 & 0.0000 & & 0.0027 & & 0.0005 \\
\hline CVS2-88 & 0.1050 & 0.0600 & 0.0400 & 0.0250 & & 0.0000 & 0.0700 & 0.0050 & 0.1403 & 0.0021 & 0.0004 & 0.4597 & 0.0750 & 0.0000 & & 0.0004 & 0.0000 & & 0.0027 & & 0.0005 \\
\hline CVS2-89 & 0.0200 & 0.1171 & 0.0400 & 0.1050 & & 0.0000 & 0.0100 & 0.0050 & 0.1800 & 0.0021 & 0.0004 & 0.4400 & 0.0629 & 0.0000 & & 0.0004 & 0.0000 & & 0.0027 & & 0.0005 \\
\hline CVS2-90 & 0.0200 & 0.1600 & 0.0050 & 0.1008 & & 0.0000 & 0.0700 & 0.0050 & 0.0542 & 0.0021 & 0.0004 & 0.5600 & 0.0050 & 0.0000 & & 0.0004 & 0.0000 & & 0.0027 & & 0.0005 \\
\hline CVS2-91 & 0.0200 & 0.1601 & 0.0050 & 0.0050 & & 0.0000 & 0.0100 & 0.0400 & 0.1050 & 0.0093 & 0.0016 & 0.5602 & 0.0050 & 0.0000 & & 0.0016 & 0.0000 & & 0.0121 & & 0.0024 \\
\hline CVS2-92 & 0.0200 & 0.1601 & 0.0050 & 0.0050 & & 0.0000 & 0.0700 & 0.0400 & 0.1000 & 0.0093 & 0.0016 & 0.4402 & 0.0700 & 0.0000 & & 0.0016 & 0.0000 & & 0.0121 & & 0.0024 \\
\hline CVS2-93 & 0.0200 & 0.1338 & 0.0098 & 0.0986 & & 0.0000 & 0.0700 & 0.0050 & 0.1279 & 0.0093 & 0.0016 & 0.4402 & 0.0050 & 0.0000 & & 0.0016 & 0.0000 & & 0.0121 & & 0.0024 \\
\hline CVS2-94 & 0.0703 & 0.1600 & 0.0400 & 0.0271 & & 0.0000 & 0.0526 & 0.0050 & 0.1800 & 0.0021 & 0.0004 & 0.4400 & 0.0050 & 0.0000 & & 0.0004 & 0.0000 & & 0.0027 & & 0.0005 \\
\hline CVS2-95 & 0.0367 & 0.1112 & 0.0113 & 0.0897 & & 0.0004 & 0.0428 & 0.0166 & 0.1671 & 0.0061 & 0.0000 & 0.4895 & 0.0041 & 0.0000 & & 0.0000 & 0.0000 & & 0.0000 & & 0.0000 \\
\hline CVS2-96 & 0.0636 & 0.1142 & 0.0275 & 0.0568 & & 0.0000 & 0.0376 & 0.0363 & 0.1003 & 0.0042 & 0.0007 & 0.4802 & 0.0429 & 0.0000 & & 0.0007 & 0.0000 & & 0.0055 & & 0.0011 \\
\hline CVS2-97 & 0.0235 & 0.1048 & 0.0082 & 0.0733 & & 0.0000 & 0.0373 & 0.0084 & 0.1129 & 0.0060 & 0.0010 & 0.5330 & 0.0392 & 0.0000 & & 0.0010 & 0.0000 & & 0.0065 & & 0.0002 \\
\hline CVS2-98 & 0.1388 & 0.1743 & 0.0000 & 0.0200 & & 0.0000 & 0.0369 & 0.0000 & 0.2000 & 0.0010 & 0.0002 & 0.4200 & 0.0000 & 0.0000 & & 0.0002 & 0.0000 & & 0.0013 & & 0.0003 \\
\hline CVS2-99 & 0.0523 & 0.0970 & 0.0097 & 0.1019 & & 0.0212 & 0.0356 & 0.0077 & 0.0981 & 0.0029 & 0.0055 & 0.5208 & 0.0199 & 0.0010 & & 0.0005 & 0.0000 & & 0.0030 & & 0.0014 \\
\hline CVS2-100 & 0.0286 & 0.0740 & 0.0035 & 0.1229 & & 0.0164 & 0.0596 & 0.0012 & 0.0626 & 0.0069 & 0.0088 & 0.5331 & 0.0443 & 0.0003 & & 0.0007 & 0.0000 & & 0.0080 & & 0.0000 \\
\hline CVS2-101 & 0.0367 & 0.1112 & 0.0113 & 0.0897 & & 0.0004 & 0.0428 & 0.0166 & 0.1671 & 0.0055 & 0.0000 & 0.4895 & 0.0041 & 0.0000 & 0.0000 & 0.0000 & 0.0000 & & 0.0000 & & 0.0000 \\
\hline CVS2-102 & 0.0231 & 0.1053 & 0.0083 & 0.0719 & & 0.0000 & 0.0375 & 0.0084 & 0.1125 & 0.0061 & 0.0011 & 0.5353 & 0.0385 & 0.0000 & 0.0000 & 0.0011 & 0.0000 & & 0.0080 & & 0.0016 \\
\hline
\end{tabular}


Hanford CVS 2 (Hrma et al. 1994)

\begin{tabular}{|c|c|c|c|c|c|c|c|c|c|c|c|c|c|c|c|c|c|c|c|c|c|}
\hline Glass ID & $\begin{array}{l}\mathrm{Cl} \\
-\mathrm{t}\end{array}$ & $\begin{array}{c}\mathrm{CoO} \\
-\mathrm{t}\end{array}$ & $\begin{array}{c}\mathrm{Co} 2 \mathrm{O} 3 \\
-\mathrm{t}\end{array}$ & $\begin{array}{c}\mathrm{Cr} 2 \mathrm{O} 3 \\
-\mathrm{t} \\
\end{array}$ & $\begin{array}{c}\mathrm{Cs} 2 \mathrm{O} \\
-\mathrm{t}\end{array}$ & $\begin{array}{c}\mathrm{CuO} \\
-\mathrm{t}\end{array}$ & $\begin{array}{c}\mathrm{Eu} 2 \mathrm{O} 3 \\
-\mathrm{t}\end{array}$ & $\begin{array}{l}F \\
-t\end{array}$ & $\begin{array}{c}\mathrm{Ga} 2 \mathrm{O} 3 \\
-\mathrm{t}\end{array}$ & $\begin{array}{c}\mathrm{Gd} 2 \mathrm{O} 3 \\
-\mathrm{t}\end{array}$ & $\begin{array}{c}\mathrm{HgO} \\
-\mathrm{t}\end{array}$ & $\begin{array}{l}I \\
-t\end{array}$ & $\begin{array}{c}\mathrm{La} 2 \mathrm{O} 3 \\
-\mathrm{t}\end{array}$ & $\begin{array}{c}\mathrm{MnO} 2 \\
-\mathrm{t}\end{array}$ & $\begin{array}{c}\mathrm{MnO} \\
-\mathrm{t}\end{array}$ & $\begin{array}{c}\mathrm{MoO} \\
-\mathrm{t}\end{array}$ & $\begin{array}{c}\mathrm{MoO} 3 \\
-\mathrm{t}\end{array}$ & $\begin{array}{c}\mathrm{Nb} 2 \mathrm{O} 5 \\
-\mathrm{t}\end{array}$ & $\begin{array}{c}\mathrm{Nd} 2 \mathrm{O} 3 \\
-\mathrm{t}\end{array}$ & $\begin{array}{c}\mathrm{PbO} \\
-\mathrm{t}\end{array}$ & $\begin{array}{c}\mathrm{PdO} 2 \\
-\mathrm{t}\end{array}$ \\
\hline CVS2-66 & & & 0.0000 & 0.0108 & 0.0000 & 0.0000 & & 0.0006 & & & & & 0.0043 & 0.0119 & & & 0.0063 & & 0.0000 & 0.0000 & \\
\hline CVS2-67 & & & 0.0000 & 0.0014 & 0.0000 & 0.0000 & & 0.0013 & & & & & 0.0000 & 0.0000 & & & 0.0000 & & 0.0000 & 0.0000 & \\
\hline CVS2-68 & & & 0.0000 & 0.0297 & 0.0000 & 0.0000 & & 0.0057 & & & & & 0.0000 & 0.0022 & & & 0.0007 & & 0.0000 & 0.0000 & \\
\hline CVS2-69 & & & 0.0000 & 0.0238 & 0.0000 & 0.0018 & & 0.0011 & & & & & 0.0000 & 0.0122 & & & 0.0000 & & 0.0017 & 0.0015 & \\
\hline CVS2-70 & & & 0.0000 & 0.0117 & 0.0000 & 0.0016 & & 0.0000 & & & & & 0.0005 & 0.0029 & & & 0.0000 & & 0.0005 & 0.0000 & \\
\hline CVS2-71 & & & 0.0000 & 0.0015 & 0.0018 & 0.0018 & & 0.0036 & & & & & 0.0074 & 0.0015 & & & 0.0036 & & 0.0147 & 0.0000 & \\
\hline CVS2-72 & & & 0.0000 & 0.0009 & 0.0011 & 0.0011 & & 0.0023 & & & & & 0.0047 & 0.0009 & & & 0.0023 & & 0.0093 & 0.0000 & \\
\hline CVS2-73 & & & 0.0000 & 0.0009 & 0.0011 & 0.0011 & & 0.0022 & & & & & 0.0047 & 0.0009 & & & 0.0022 & & 0.0092 & 0.0000 & \\
\hline CVS2-74 & & & 0.0000 & 0.0021 & 0.0025 & 0.0025 & & 0.0049 & & & & & 0.0103 & 0.0020 & & & 0.0049 & & 0.0202 & 0.0000 & \\
\hline CVS2-75 & & & 0.0000 & 0.0007 & 0.0008 & 0.0008 & & 0.0016 & & & & & 0.0034 & 0.0007 & & & 0.0016 & & 0.0067 & 0.0000 & \\
\hline CVS2-76 & & & 0.0000 & 0.0004 & 0.0005 & 0.0005 & & 0.0011 & & & & & 0.0022 & 0.0004 & & & 0.0011 & & 0.0044 & 0.0000 & \\
\hline CVS2-77 & & & 0.0000 & 0.0020 & 0.0024 & 0.0024 & & 0.0048 & & & & & 0.0101 & 0.0020 & & & 0.0048 & & 0.0199 & 0.0000 & \\
\hline CVS2-78 & & & 0.0000 & 0.0004 & 0.0005 & 0.0005 & & 0.0011 & & & & & 0.0022 & 0.0004 & & & 0.0011 & & 0.0044 & 0.0000 & \\
\hline CVS2-79 & & & 0.0000 & 0.0020 & 0.0024 & 0.0024 & & 0.0048 & & & & & 0.0101 & 0.0020 & & & 0.0048 & & 0.0199 & 0.0000 & \\
\hline CVS2-80 & & & 0.0000 & 0.0004 & 0.0005 & 0.0005 & & 0.0011 & & & & & 0.0022 & 0.0004 & & & 0.0011 & & 0.0044 & 0.0000 & \\
\hline CVS2-81 & & & 0.0000 & 0.0018 & 0.0022 & 0.0022 & & 0.0044 & & & & & 0.0091 & 0.0018 & & & 0.0044 & & 0.0180 & 0.0000 & \\
\hline CVS2-82 & & & 0.0000 & 0.0004 & 0.0005 & 0.0005 & & 0.0011 & & & & & 0.0022 & 0.0004 & & & 0.0011 & & 0.0044 & 0.0000 & \\
\hline CVS2-83 & & & 0.0000 & 0.0020 & 0.0024 & 0.0024 & & 0.0048 & & & & & 0.0101 & 0.0020 & & & 0.0048 & & 0.0199 & 0.0000 & \\
\hline CVS2-84 & & & 0.0000 & 0.0004 & 0.0005 & 0.0005 & & 0.0011 & & & & & 0.0022 & 0.0004 & & & 0.0011 & & 0.0044 & 0.0000 & \\
\hline CVS2-85 & & & 0.0000 & 0.0010 & 0.0012 & 0.0012 & & 0.0024 & & & & & 0.0050 & 0.0010 & & & 0.0024 & & 0.0099 & 0.0000 & \\
\hline CVS2-86 & & & 0.0000 & 0.0010 & 0.0012 & 0.0012 & & 0.0024 & & & & & 0.0050 & 0.0010 & & & 0.0024 & & 0.0099 & 0.0000 & \\
\hline CVS2-87 & & & 0.0000 & 0.0004 & 0.0005 & 0.0005 & & 0.0011 & & & & & 0.0022 & 0.0004 & & & 0.0011 & & 0.0044 & 0.0000 & \\
\hline CVS2-88 & & & 0.0000 & 0.0004 & 0.0005 & 0.0005 & & 0.0011 & & & & & 0.0022 & 0.0004 & & & 0.0011 & & 0.0044 & 0.0000 & \\
\hline CVS2-89 & & & 0.0000 & 0.0004 & 0.0005 & 0.0005 & & 0.0011 & & & & & 0.0022 & 0.0004 & & & 0.0011 & & 0.0044 & 0.0000 & \\
\hline CVS2-90 & & & 0.0000 & 0.0004 & 0.0005 & 0.0005 & & 0.0011 & & & & & 0.0022 & 0.0004 & & & 0.0011 & & 0.0044 & 0.0000 & \\
\hline CVS2-91 & & & 0.0000 & 0.0020 & 0.0024 & 0.0024 & & 0.0048 & & & & & 0.0101 & 0.0020 & & & 0.0048 & & 0.0199 & 0.0000 & \\
\hline CVS2-92 & & & 0.0000 & 0.0020 & 0.0024 & 0.0024 & & 0.0048 & & & & & 0.0101 & 0.0020 & & & 0.0048 & & 0.0199 & 0.0000 & \\
\hline CVS2-93 & & & 0.0000 & 0.0020 & 0.0024 & 0.0024 & & 0.0048 & & & & & 0.0101 & 0.0020 & & & 0.0048 & & 0.0199 & 0.0000 & \\
\hline CVS2-94 & & & 0.0000 & 0.0004 & 0.0005 & 0.0005 & & 0.0011 & & & & & 0.0022 & 0.0004 & & & 0.0011 & & 0.0044 & 0.0000 & \\
\hline CVS2-95 & & & 0.0000 & 0.0000 & 0.0000 & 0.0000 & & 0.0000 & & & & & 0.0041 & 0.0134 & & & 0.0000 & & 0.0000 & 0.0000 & \\
\hline CVS2-96 & & & 0.0000 & 0.0009 & 0.0011 & 0.0011 & & 0.0022 & & & & & 0.0046 & 0.0009 & & & 0.0022 & & 0.0090 & 0.0000 & \\
\hline CVS2-97 & & & 0.0000 & 0.0013 & 0.0015 & 0.0016 & & 0.0019 & & & & & 0.0155 & 0.0013 & & & 0.0031 & & 0.0057 & 0.0000 & \\
\hline CVS2-98 & & & 0.0000 & 0.0002 & 0.0003 & 0.0003 & & 0.0005 & & & & & 0.0011 & 0.0002 & & & 0.0005 & & 0.0022 & 0.0000 & \\
\hline CVS2-99 & & & 0.0010 & 0.0020 & 0.0000 & 0.0002 & & 0.0000 & & & & & 0.0014 & 0.0039 & & & 0.0001 & & 0.0046 & 0.0020 & \\
\hline CVS2-100 & & & 0.0000 & 0.0006 & 0.0000 & 0.0003 & & 0.0000 & & & & & 0.0046 & 0.0017 & & & 0.0000 & & 0.0145 & 0.0011 & \\
\hline CVS2-101 & & & 0.0000 & 0.0000 & 0.0000 & 0.0000 & & 0.0000 & & & & & 0.0037 & 0.0149 & & & 0.0000 & & 0.0000 & 0.0000 & \\
\hline CVS2-102 & & & 0.0000 & 0.0013 & 0.0016 & 0.0016 & & 0.0032 & & & & & 0.0066 & 0.0016 & & & 0.0032 & & 0.0131 & 0.0000 & \\
\hline
\end{tabular}


Hanford CVS 2 (Hrma et al. 1994)

\begin{tabular}{|c|c|c|c|c|c|c|c|c|c|c|c|c|c|c|c|c|c|c|c|c|c|}
\hline Glass ID & $\begin{array}{c}\mathrm{PdO} \\
-\mathrm{t}\end{array}$ & $\begin{array}{c}\mathrm{Pr} 2 \mathrm{O} 3 \\
-\mathrm{t}\end{array}$ & Pr6O11 & $\begin{array}{c}\mathrm{Rb} 2 \mathrm{O} \\
-\mathrm{t}\end{array}$ & $\begin{array}{c}\mathrm{ReO} \\
-\mathrm{t}\end{array}$ & $\begin{array}{c}\mathrm{ReO} 2 \\
-\mathrm{t}\end{array}$ & $\begin{array}{c}\mathrm{Rh} 2 \mathrm{O} 3 \\
-\mathrm{t}\end{array}$ & $\begin{array}{c}\mathrm{RhO} 2 \\
-\mathrm{t}\end{array}$ & $\begin{array}{c}\mathrm{RuO} 2 \\
-\mathrm{t}\end{array}$ & $\begin{array}{c}\mathrm{Sb} 2 \mathrm{O} 3 \\
-\mathrm{t}\end{array}$ & $\begin{array}{c}\mathrm{Sb} 2 \mathrm{O} 5 \\
-\mathrm{t}\end{array}$ & $\begin{array}{c}\mathrm{SeO} 2 \\
-\mathrm{t}\end{array}$ & $\begin{array}{c}\mathrm{Sm} 2 \mathrm{O} 3 \\
-\mathrm{t}\end{array}$ & $\begin{array}{c}\mathrm{SnO} \\
-\mathrm{t}\end{array}$ & $\begin{array}{c}\mathrm{SnO} 2 \\
-\mathrm{t}\end{array}$ & $\begin{array}{c}\mathrm{SO} 3 \\
-\mathrm{t}\end{array}$ & $\begin{array}{c}\mathrm{SrO} \\
-\mathrm{t}\end{array}$ & $\begin{array}{c}\mathrm{Tc} 2 \mathrm{O} 7 \\
-\mathrm{t}\end{array}$ & $\begin{array}{c}\mathrm{TeO} 2 \\
-\mathrm{t}\end{array}$ & $\begin{array}{c}\mathrm{ThO} 2 \\
-\mathrm{t}\end{array}$ & $\begin{array}{c}\mathrm{TiO} 2 \\
-\mathrm{t}\end{array}$ \\
\hline CVS2-66 & 0.0000 & & 0.0000 & 0.0000 & & & 0.0000 & & 0.0000 & & & & 0.0000 & & & 0.0000 & 0.0000 & & 0.0000 & & 0.0000 \\
\hline CVS2-67 & 0.0000 & & 0.0000 & 0.0000 & & & 0.0000 & & 0.0000 & & & & 0.0000 & & & 0.0000 & 0.0000 & & 0.0000 & & 0.0000 \\
\hline CVS2-68 & 0.0000 & & 0.0000 & 0.0000 & & & 0.0000 & & 0.0000 & & & & 0.0000 & & & 0.0001 & 0.0000 & & 0.0000 & & 0.0000 \\
\hline CVS2-69 & 0.0014 & & 0.0000 & 0.0000 & & & 0.0006 & & 0.0006 & 0.0000 & & 0.0036 & 0.0000 & & & 0.0069 & 0.0000 & & 0.0000 & & 0.0000 \\
\hline CVS2-70 & 0.0014 & & 0.0000 & 0.0000 & & & 0.0007 & & 0.0007 & 0.0005 & & 0.0035 & 0.0000 & & & 0.0003 & 0.0000 & & 0.0005 & & 0.0000 \\
\hline CVS2-71 & 0.0006 & & 0.0012 & 0.0006 & & & 0.0006 & & 0.0018 & & & & 0.0006 & & & 0.0033 & 0.0012 & & 0.0000 & & 0.0000 \\
\hline CVS2-72 & 0.0004 & & 0.0008 & 0.0004 & & & 0.0004 & & 0.0011 & & & & 0.0004 & & & 0.0021 & 0.0008 & & 0.0000 & & 0.0000 \\
\hline CVS2-73 & 0.0004 & & 0.0007 & 0.0004 & & & 0.0004 & & 0.0011 & & & & 0.0004 & & & 0.0021 & 0.0007 & & 0.0000 & & 0.0000 \\
\hline CVS2-74 & 0.0008 & & 0.0016 & 0.0008 & & & 0.0008 & & 0.0025 & & & & 0.0008 & & & 0.0045 & 0.0016 & & 0.0000 & & 0.0000 \\
\hline CVS2-75 & 0.0003 & & 0.0005 & 0.0003 & & & 0.0003 & & 0.0008 & & & & 0.0003 & & & 0.0015 & 0.0005 & & 0.0000 & & 0.0000 \\
\hline CVS2-76 & 0.0002 & & 0.0004 & 0.0002 & & & 0.0002 & & 0.0005 & & & & 0.0002 & & & 0.0010 & 0.0004 & & 0.0000 & & 0.0000 \\
\hline CVS2-77 & 0.0008 & & 0.0016 & 0.0008 & & & 0.0008 & & 0.0024 & & & & 0.0008 & & & 0.0044 & 0.0016 & & 0.0000 & & 0.0000 \\
\hline CVS2-78 & 0.0002 & & 0.0004 & 0.0002 & & & 0.0002 & & 0.0005 & & & & 0.0002 & & & 0.0010 & 0.0004 & & 0.0000 & & 0.0000 \\
\hline CVS2-79 & 0.0008 & & 0.0016 & 0.0008 & & & 0.0008 & & 0.0024 & & & & 0.0008 & & & 0.0044 & 0.0016 & & 0.0000 & & 0.0000 \\
\hline CVS2-80 & 0.0002 & & 0.0004 & 0.0002 & & & 0.0002 & & 0.0005 & & & & 0.0002 & & & 0.0010 & 0.0004 & & 0.0000 & & 0.0000 \\
\hline CVS2-81 & 0.0007 & & 0.0015 & 0.0007 & & & 0.0007 & & 0.0022 & & & & 0.0007 & & & 0.0040 & 0.0015 & & 0.0000 & & 0.0000 \\
\hline CVS2-82 & 0.0002 & & 0.0004 & 0.0002 & & & 0.0002 & & 0.0005 & & & & 0.0002 & & & 0.0010 & 0.0004 & & 0.0000 & & 0.0000 \\
\hline CVS2-83 & 0.0008 & & 0.0016 & 0.0008 & & & 0.0008 & & 0.0024 & & & & 0.0008 & & & 0.0044 & 0.0016 & & 0.0000 & & 0.0000 \\
\hline CVS2-84 & 0.0002 & & 0.0004 & 0.0002 & & & 0.0002 & & 0.0005 & & & & 0.0002 & & & 0.0010 & 0.0004 & & 0.0000 & & 0.0000 \\
\hline CVS2-85 & 0.0004 & & 0.0008 & 0.0004 & & & 0.0004 & & 0.0012 & & & & 0.0004 & & & 0.0022 & 0.0008 & & 0.0000 & & 0.0000 \\
\hline CVS2-86 & 0.0004 & & 0.0008 & 0.0004 & & & 0.0004 & & 0.0012 & & & & 0.0004 & & & 0.0022 & 0.0008 & & 0.0000 & & 0.0000 \\
\hline CVS2-87 & 0.0002 & & 0.0004 & 0.0002 & & & 0.0002 & & 0.0005 & & & & 0.0002 & & & 0.0010 & 0.0004 & & 0.0000 & & 0.0000 \\
\hline CVS2-88 & 0.0002 & & 0.0004 & 0.0002 & & & 0.0002 & & 0.0005 & & & & 0.0002 & & & 0.0010 & 0.0004 & & 0.0000 & & 0.0000 \\
\hline CVS2-89 & 0.0002 & & 0.0004 & 0.0002 & & & 0.0002 & & 0.0005 & & & & 0.0002 & & & 0.0010 & 0.0004 & & 0.0000 & & 0.0000 \\
\hline CVS2-90 & 0.0002 & & 0.0004 & 0.0002 & & & 0.0002 & & 0.0005 & & & & 0.0002 & & & 0.0010 & 0.0004 & & 0.0000 & & 0.0000 \\
\hline CVS2-91 & 0.0008 & & 0.0016 & 0.0008 & & & 0.0008 & & 0.0024 & & & & 0.0008 & & & 0.0044 & 0.0016 & & 0.0000 & & 0.0000 \\
\hline CVS2-92 & 0.0008 & & 0.0016 & 0.0008 & & & 0.0008 & & 0.0024 & & & & 0.0008 & & & 0.0044 & 0.0016 & & 0.0000 & & 0.0000 \\
\hline CVS2-93 & 0.0008 & & 0.0016 & 0.0008 & & & 0.0008 & & 0.0024 & & & & 0.0008 & & & 0.0044 & 0.0016 & & 0.0000 & & 0.0000 \\
\hline CVS2-94 & 0.0002 & & 0.0004 & 0.0002 & & & 0.0002 & & 0.0005 & & & & 0.0002 & & & 0.0010 & 0.0004 & & 0.0000 & & 0.0000 \\
\hline CVS2-95 & 0.0000 & & 0.0000 & 0.0000 & & & 0.0000 & & 0.0000 & & & & 0.0000 & & & 0.0000 & 0.0000 & & 0.0000 & & 0.0071 \\
\hline CVS2-96 & 0.0004 & & 0.0007 & 0.0004 & & & 0.0004 & & 0.0011 & & & & 0.0004 & & & 0.0020 & 0.0007 & & 0.0000 & & 0.0000 \\
\hline CVS2-97 & 0.0000 & & 0.0019 & 0.0000 & & & 0.0000 & & 0.0015 & & & & 0.0000 & & & 0.0044 & 0.0000 & & 0.0000 & & 0.0000 \\
\hline CVS2-98 & 0.0001 & & 0.0002 & 0.0001 & & & 0.0001 & & 0.0003 & & & & 0.0001 & & & 0.0005 & 0.0002 & & 0.0000 & & 0.0000 \\
\hline CVS2-99 & 0.0000 & & 0.0000 & 0.0000 & & & 0.0000 & & 0.0000 & & & & 0.0000 & & & 0.0040 & 0.0004 & & 0.0000 & & 0.0012 \\
\hline CVS2-100 & 0.0000 & & 0.0000 & 0.0000 & & & 0.0000 & & 0.0009 & & & & 0.0000 & & & 0.0032 & 0.0005 & & 0.0005 & & 0.0008 \\
\hline CVS2-101 & 0.0000 & & 0.0000 & 0.0000 & & & 0.0000 & & 0.0000 & 0.0000 & & 0.0000 & 0.0000 & & & 0.0000 & 0.0000 & & 0.0000 & & 0.0065 \\
\hline CVS2-102 & 0.0005 & & 0.0011 & 0.0005 & & & 0.0005 & & 0.0016 & 0.0000 & & 0.0000 & 0.0005 & & & 0.0029 & 0.0011 & & 0.0000 & & 0.0000 \\
\hline
\end{tabular}




\begin{tabular}{|c|c|c|c|c|c|c|c|c|c|c|c|c|c|c|c|c|c|c|c|c|c|}
\hline \multicolumn{22}{|c|}{ Hanford CVS 2 (Hrma et al. 1994) } \\
\hline Glass ID & $\begin{array}{c}\mathrm{T} 12 \mathrm{O} 3 \\
-\mathrm{t}\end{array}$ & $\begin{array}{c}\text { U3O8 } \\
-t\end{array}$ & $\begin{array}{c}\mathrm{UO} 2 \\
-\mathrm{t}\end{array}$ & $\begin{array}{c}\text { UO3 } \\
-t\end{array}$ & $\begin{array}{c}\mathrm{V} 2 \mathrm{O} 5 \\
-\mathrm{t}\end{array}$ & $\begin{array}{c}\text { WO3 } \\
-t\end{array}$ & $\begin{array}{c}\mathrm{Y} 2 \mathrm{O} 3 \\
-\mathrm{t}\end{array}$ & $\begin{array}{c}\mathrm{ZnO} \\
-\mathrm{t}\end{array}$ & $\begin{array}{c}\text { Others } \\
-t\end{array}$ & $\begin{array}{c}\text { Sum } \\
-t\end{array}$ & $\begin{array}{c}\mathrm{A} 12 \mathrm{O} 3 \\
-\mathrm{a}\end{array}$ & $\begin{array}{c}\mathrm{B} 2 \mathrm{O} 3 \\
-\mathrm{a}\end{array}$ & $\begin{array}{c}\mathrm{CaO} \\
-\mathrm{a}\end{array}$ & $\begin{array}{c}\mathrm{Fe} 2 \mathrm{O} 3 \\
-\mathrm{a}\end{array}$ & $\begin{array}{c}\mathrm{FeO} \\
-\mathrm{a}\end{array}$ & $\begin{array}{c}\mathrm{K} 2 \mathrm{O} \\
-\mathrm{a}\end{array}$ & $\begin{array}{c}\mathrm{Li} 2 \mathrm{O} \\
-\mathrm{a}\end{array}$ & $\begin{array}{c}\mathrm{MgO} \\
-\mathrm{a}\end{array}$ & $\begin{array}{c}\mathrm{Na} 2 \mathrm{O} \\
-\mathrm{a}\end{array}$ & $\begin{array}{c}\mathrm{NiO} \\
-\mathrm{a}\end{array}$ & $\begin{array}{c}\mathrm{P} 2 \mathrm{O} 5 \\
-\mathrm{a}\end{array}$ \\
\hline CVS2-66 & & 0.0000 & & & & 0.0000 & 0.0000 & 0.0000 & & 0.9998 & & & & & & & & & & & \\
\hline CVS2-67 & & 0.0000 & & & & 0.0000 & 0.0000 & 0.0000 & & 1.0000 & & & & & & & & & & & \\
\hline CVS2-68 & & 0.0000 & & & & 0.0000 & 0.0000 & 0.0012 & & 0.9999 & & & & & & & & & & & \\
\hline CVS2-69 & & 0.0000 & & & & 0.0000 & 0.0000 & 0.0000 & & 0.9999 & 0.0774 & 0.0768 & 0.0059 & 0.0306 & & 0.0000 & 0.0665 & 0.0030 & 0.0655 & 0.0003 & 0.0167 \\
\hline CVS2-70 & & 0.0000 & & & & 0.0000 & 0.0000 & 0.0000 & & 0.9999 & 0.1670 & 0.1335 & 0.0008 & 0.0064 & & 0.0000 & 0.0623 & 0.0008 & 0.0746 & 0.0008 & 0.0007 \\
\hline CVS2-71 & & 0.0000 & & & & 0.0000 & 0.0006 & 0.0000 & & 0.9999 & & & & & & & & & & & \\
\hline CVS2-72 & & 0.0000 & & & & 0.0000 & 0.0004 & 0.0000 & & 0.9999 & & & & & & & & & & & \\
\hline CVS2-73 & & 0.0000 & & & & 0.0000 & 0.0004 & 0.0000 & & 0.9999 & & & & & & & & & & & \\
\hline CVS2-74 & & 0.0000 & & & & 0.0000 & 0.0008 & 0.0000 & & 0.9998 & 0.0741 & 0.0751 & 0.0070 & 0.0293 & & 0.0000 & 0.0638 & 0.0027 & 0.0623 & 0.0081 & 0.0020 \\
\hline CVS2-75 & & 0.0000 & & & & 0.0000 & 0.0003 & 0.0000 & & 0.9999 & & & & & & & & & & & \\
\hline CVS2-76 & & 0.0000 & & & & 0.0000 & 0.0002 & 0.0000 & & 0.9999 & & & & & & & & & & & \\
\hline CVS2-77 & & 0.0000 & & & & 0.0000 & 0.0008 & 0.0000 & & 0.9999 & & & & & & & & & & & \\
\hline CVS2-78 & & 0.0000 & & & & 0.0000 & 0.0002 & 0.0000 & & 0.9999 & & & & & & & & & & & \\
\hline CVS2-79 & & 0.0000 & & & & 0.0000 & 0.0008 & 0.0000 & & 0.9999 & 0.0200 & 0.1565 & 0.0046 & 0.0999 & & 0.0000 & 0.0115 & 0.0044 & 0.0475 & 0.0085 & 0.0015 \\
\hline CVS2-80 & & 0.0000 & & & & 0.0000 & 0.0002 & 0.0000 & & 0.9999 & & & & & & & & & & & \\
\hline CVS2-81 & & 0.0000 & & & & 0.0000 & 0.0007 & 0.0000 & & 0.9999 & & & & & & & & & & & \\
\hline CVS2-82 & & 0.0000 & & & & 0.0000 & 0.0002 & 0.0000 & & 0.9999 & & & & & & & & & & & \\
\hline CVS2-83 & & 0.0000 & & & & 0.0000 & 0.0008 & 0.0000 & & 0.9999 & & & & & & & & & & & \\
\hline CVS2-84 & & 0.0000 & & & & 0.0000 & 0.0002 & 0.0000 & & 0.9999 & & & & & & & & & & & \\
\hline CVS2-85 & & 0.0000 & & & & 0.0000 & 0.0004 & 0.0000 & & 0.9999 & & & & & & & & & & & \\
\hline CVS2-86 & & 0.0000 & & & & 0.0000 & 0.0004 & 0.0000 & & 0.9999 & & & & & & & & & & & \\
\hline CVS2-87 & & 0.0000 & & & & 0.0000 & 0.0002 & 0.0000 & & 0.9999 & & & & & & & & & & & \\
\hline CVS2-88 & & 0.0000 & & & & 0.0000 & 0.0002 & 0.0000 & & 0.9999 & & & & & & & & & & & \\
\hline CVS2-89 & & 0.0000 & & & & 0.0000 & 0.0002 & 0.0000 & & 0.9999 & & & & & & & & & & & \\
\hline CVS2-90 & & 0.0000 & & & & 0.0000 & 0.0002 & 0.0000 & & 0.9999 & & & & & & & & & & & \\
\hline CVS2-91 & & 0.0000 & & & & 0.0000 & 0.0008 & 0.0000 & & 0.9999 & 0.0185 & 0.1545 & 0.0044 & 0.0036 & & 0.0000 & 0.0091 & 0.0342 & 0.0999 & 0.0081 & 0.0020 \\
\hline CVS2-92 & & 0.0000 & & & & 0.0000 & 0.0008 & 0.0000 & & 0.9999 & & & & & & & & & & & \\
\hline CVS2-93 & & 0.0000 & & & & 0.0000 & 0.0008 & 0.0000 & & 0.9999 & & & & & & & & & & & \\
\hline CVS2-94 & & 0.0000 & & & & 0.0000 & 0.0002 & 0.0000 & & 0.9999 & & & & & & & & & & & \\
\hline CVS2-95 & & 0.0000 & & & & 0.0000 & 0.0000 & 0.0000 & & 1.0001 & 0.0339 & 0.1055 & 0.0107 & 0.0833 & & 0.0000 & 0.0407 & 0.0140 & 0.1600 & 0.0055 & 0.0006 \\
\hline CVS2-96 & & 0.0000 & & & & 0.0000 & 0.0004 & 0.0000 & & 0.9999 & 0.0597 & 0.1120 & 0.0247 & 0.0527 & & 0.0000 & 0.0347 & 0.0317 & 0.0994 & 0.0036 & 0.0007 \\
\hline CVS2-97 & & 0.0000 & & & & 0.0000 & 0.0000 & 0.0049 & & 0.9999 & 0.0287 & 0.0994 & 0.0098 & 0.0590 & & 0.0000 & 0.0328 & 0.0078 & 0.1140 & 0.0060 & 0.0015 \\
\hline CVS2-98 & & 0.0000 & & & & 0.0000 & 0.0001 & 0.0000 & & 0.9999 & 0.1275 & 0.1645 & 0.0008 & \begin{tabular}{|l|}
0.0172 \\
\end{tabular} & & 0.0000 & 0.0333 & 0.0000 & 0.1840 & 0.0008 & 0.0002 \\
\hline CVS2-99 & & 0.0000 & & & & 0.0000 & 0.0000 & 0.0005 & & 0.9999 & & & & & & & & & & & \\
\hline CVS2-100 & & 0.0000 & & & & 0.0000 & 0.0000 & 0.0002 & & 0.9998 & & & & & & & & & & & \\
\hline CVS2-101 & & & 0.0000 & & & 0.0000 & 0.0000 & 0.0000 & & 1.0000 & 0.0341 & 0.1055 & 0.0100 & 0.0850 & & 0.0000 & 0.0415 & 0.0155 & 0.1650 & 0.0054 & \\
\hline CVS2-102 & & & 0.0000 & & & 0.0000 & 0.0005 & 0.0000 & & 1.0000 & & & & & & & & & & & \\
\hline
\end{tabular}


Appendix A. Database - mass fraction

\begin{tabular}{|c|c|c|c|c|c|c|c|c|c|c|c|c|c|c|c|c|c|c|c|c|c|}
\hline Glass ID & $\begin{array}{c}\mathrm{SiO} 2 \\
-\mathrm{a} \\
\end{array}$ & $\begin{array}{c}\mathrm{ZrO} 2 \\
-\mathrm{a}\end{array}$ & $\begin{array}{c}\mathrm{Ag} 2 \mathrm{O} \\
-\mathrm{a}\end{array}$ & $\begin{array}{c}\text { As203 } \\
-\mathrm{a} \\
\end{array}$ & $\begin{array}{c}\mathrm{BaO} \\
-\mathrm{a}\end{array}$ & $\begin{array}{c}\mathrm{Bi} 2 \mathrm{O} 3 \\
-\mathrm{a}\end{array}$ & $\begin{array}{l}\mathrm{Br} \\
-\mathrm{a}\end{array}$ & $\begin{array}{c}\mathrm{CdO} \\
-\mathrm{a}\end{array}$ & $\begin{array}{c}\mathrm{Ce} 2 \mathrm{O} 3 \\
-\mathrm{a}\end{array}$ & $\begin{array}{c}\mathrm{CeO} 2 \\
-\mathrm{a}\end{array}$ & $\begin{array}{l}\mathrm{Cl} \\
-\mathrm{a}\end{array}$ & $\begin{array}{c}\mathrm{CoO} \\
-\mathrm{a}\end{array}$ & $\begin{array}{c}\mathrm{Co} 2 \mathrm{O} 3 \\
-\mathrm{a}\end{array}$ & $\begin{array}{c}\mathrm{Cr} 2 \mathrm{O} 3 \\
-\mathrm{a} \\
\end{array}$ & $\begin{array}{c}\mathrm{Cs} 2 \mathrm{O} \\
-\mathrm{a}\end{array}$ & $\begin{array}{c}\mathrm{CuO} \\
-\mathrm{a} \\
\end{array}$ & $\begin{array}{c}\mathrm{Eu} 2 \mathrm{O} 3 \\
-\mathrm{a}\end{array}$ & $\begin{array}{c}\mathrm{F} \\
-\mathrm{a} \\
\end{array}$ & $\begin{array}{c}\mathrm{Ga} 2 \mathrm{O} 3 \\
-\mathrm{a} \\
\end{array}$ & $\begin{array}{c}\mathrm{Gd} 2 \mathrm{O} 3 \\
-\mathrm{a}\end{array}$ & $\begin{array}{c}\mathrm{HgO} \\
-\mathrm{a}\end{array}$ \\
\hline CVS2-66 & & & & & & & & & & & & & & & & & & & & & \\
\hline CVS2-67 & & & & & & & & & & & & & & & & & & & & & \\
\hline CVS2-68 & & & & & & & & & & & & & & & & & & & & & \\
\hline CVS2-69 & 0.5240 & 0.0005 & & 0.0000 & 0.0000 & & & 0.0008 & & 0.0000 & & & 0.0000 & 0.0223 & 0.0000 & 0.0017 & 0.0000 & 0.0011 & & 0.0000 & \\
\hline CVS2-70 & 0.4460 & 0.0006 & & 0.0008 & 0.0000 & & & 0.0010 & & 0.0010 & & & 0.0000 & 0.0106 & 0.0000 & 0.0015 & 0.0000 & 0.0000 & & 0.0000 & \\
\hline CVS2-71 & & & & & & & & & & & & & & & & & & & & & \\
\hline CVS2-72 & & & & & & & & & & & & & & & & & & & & & \\
\hline CVS2-73 & & & & & & & & & & & & & & & & & & & & & \\
\hline CVS2-74 & 0.5165 & 0.0008 & & 0.0000 & 0.0015 & & & 0.0104 & & 0.0021 & & & 0.0000 & 0.0019 & 0.0025 & 0.0022 & 0.0000 & 0.0049 & & 0.0000 & \\
\hline CVS2-75 & & & & & & & & & & & & & & & & & & & & & \\
\hline CVS2-76 & & & & & & & & & & & & & & & & & & & & & \\
\hline CVS2-77 & & & & & & & & & & & & & & & & & & & & & \\
\hline CVS2-78 & & & & & & & & & & & & & & & & & & & & & \\
\hline CVS2-79 & 0.5240 & 0.0048 & & 0.0000 & 0.0015 & & & 0.0107 & & 0.0021 & & & 0.0000 & 0.0019 & 0.0024 & 0.0023 & 0.0000 & 0.0048 & & 0.0000 & \\
\hline CVS2-80 & & & & & & & & & & & & & & & & & & & & & \\
\hline CVS2-81 & & & & & & & & & & & & & & & & & & & & & \\
\hline CVS2-82 & & & & & & & & & & & & & & & & & & & & & \\
\hline CVS2-83 & & & & & & & & & & & & & & & & & & & & & \\
\hline CVS2-84 & & & & & & & & & & & & & & & & & & & & & \\
\hline CVS2-85 & & & & & & & & & & & & & & & & & & & & & \\
\hline CVS2-86 & & & & & & & & & & & & & & & & & & & & & \\
\hline CVS2-87 & & & & & & & & & & & & & & & & & & & & & \\
\hline CVS2-88 & & & & & & & & & & & & & & & & & & & & & \\
\hline CVS2-89 & & & & & & & & & & & & & & & & & & & & & \\
\hline CVS2-90 & & & & & & & & & & & & & & & & & & & & & \\
\hline CVS2-91 & 0.4895 & 0.0050 & & 0.0000 & 0.0015 & & & 0.0102 & & 0.0021 & & & 0.0000 & 0.0019 & 0.0024 & 0.0022 & 0.0000 & 0.0048 & & 0.0000 & \\
\hline CVS2-92 & & & & & & & & & & & & & & & & & & & & & \\
\hline CVS2-93 & & & & & & & & & & & & & & & & & & & & & \\
\hline CVS2-94 & & & & & & & & & & & & & & & & & & & & & \\
\hline CVS2-95 & 0.4630 & 0.0036 & & 0.0000 & 0.0006 & & & 0.0042 & & 0.0008 & & & 0.0001 & 0.0007 & 0.0008 & 0.0008 & 0.0000 & 0.0017 & & 0.0000 & \\
\hline CVS2-96 & 0.4460 & 0.0398 & & 0.0000 & 0.0007 & & & 0.0047 & & 0.0010 & & & 0.0000 & 0.0009 & 0.0011 & 0.0010 & 0.0000 & 0.0022 & & 0.0000 & \\
\hline CVS2-97 & 0.4850 & 0.0367 & & 0.0000 & 0.0023 & & & 0.0060 & & 0.0002 & & & 0.0004 & 0.0011 & 0.0015 & 0.0020 & 0.0000 & 0.0019 & & 0.0000 & \\
\hline CVS2-98 & 0.3840 & 0.0001 & & 0.0000 & 0.0002 & & & 0.0011 & & 0.0003 & & & 0.0000 & 0.0003 & 0.0003 & 0.0003 & 0.0000 & 0.0005 & & 0.0000 & \\
\hline CVS2-99 & & & & & & & & & & & & & & & & & & & & & \\
\hline CVS2-100 & & & & & & & & & & & & & & & & & & & & & \\
\hline CVS2-101 & 0.4610 & 0.0043 & & & & & & 0.0001 & & & & & 0.0001 & & & & & & & & \\
\hline CVS2-102 & & & & & & & & & & & & & & & & & & & & & \\
\hline
\end{tabular}


Hanford CVS 2 (Hrma et al. 1994)

\begin{tabular}{|c|c|c|c|c|c|c|c|c|c|c|c|c|c|c|c|c|c|c|c|c|c|}
\hline Glass ID & $\begin{array}{c}\mathrm{I} \\
-\mathrm{a}\end{array}$ & $\begin{array}{c}1994) \\
\mathrm{La} 2 \mathrm{O} 3 \\
-\mathrm{a}\end{array}$ & $\begin{array}{c}\mathrm{MnO} 2 \\
-\mathrm{a}\end{array}$ & $\begin{array}{c}\mathrm{MnO} \\
-\mathrm{a}\end{array}$ & $\begin{array}{c}\mathrm{MoO} \\
-\mathrm{a} \\
\end{array}$ & $\begin{array}{c}\mathrm{MoO} 3 \\
-\mathrm{a} \\
\end{array}$ & $\begin{array}{c}\mathrm{Nb} 2 \mathrm{O} 5 \\
-\mathrm{a}\end{array}$ & $\begin{array}{c}\mathrm{Nd} 2 \mathrm{O} 3 \\
-\mathrm{a}\end{array}$ & $\begin{array}{c}\mathrm{PbO} \\
-\mathrm{a}\end{array}$ & $\begin{array}{c}\mathrm{PdO} 2 \\
-\mathrm{a}\end{array}$ & $\begin{array}{c}\mathrm{PdO} \\
-\mathrm{a}\end{array}$ & $\begin{array}{c}\mathrm{Pr} 2 \mathrm{O} 3 \\
-\mathrm{a} \\
\end{array}$ & $\begin{array}{c}\text { Pr6O11 } \\
-\mathrm{a}\end{array}$ & $\begin{array}{c}\mathrm{Rb} 2 \mathrm{O} \\
-\mathrm{a}\end{array}$ & $\begin{array}{c}\mathrm{ReO} \\
-\mathrm{a}\end{array}$ & $\begin{array}{c}\mathrm{ReO} 2 \\
-\mathrm{a}\end{array}$ & $\begin{array}{c}\mathrm{Rh} 2 \mathrm{O} 3 \\
-\mathrm{a} \\
\end{array}$ & $\begin{array}{c}\mathrm{RhO} 2 \\
-\mathrm{a}\end{array}$ & $\begin{array}{c}\mathrm{RuO} 2 \\
-\mathrm{a} \\
\end{array}$ & $\begin{array}{c}\mathrm{Sb} 2 \mathrm{O} 3 \\
-\mathrm{a} \\
\end{array}$ & $\begin{array}{c}\mathrm{Sb} 2 \mathrm{O} 5 \\
-\mathrm{a}\end{array}$ \\
\hline CVS2-66 & & & & & & & & & & & & & & & & & & & & & \\
\hline CVS2-67 & & & & & & & & & & & & & & & & & & & & & \\
\hline CVS2-68 & & & & & & & & & & & & & & & & & & & & & \\
\hline CVS2-69 & & 0.0000 & 0.0142 & 0.0000 & & 0.0000 & & 0.0013 & 0.0020 & & 0.0014 & & 0.0000 & 0.0000 & & & 0.0006 & & 0.0006 & 0.0000 & \\
\hline CVS2-70 & & 0.0004 & 0.0033 & 0.0000 & & 0.0000 & & 0.0004 & 0.0000 & & 0.0014 & & 0.0000 & 0.0000 & & & 0.0007 & & 0.0006 & 0.0005 & \\
\hline CVS2-71 & & & & & & & & & & & & & & & & & & & & & \\
\hline CVS2-72 & & & & & & & & & & & & & & & & & & & & & \\
\hline CVS2-73 & & & & & & & & & & & & & & & & & & & & & \\
\hline CVS2-74 & & 0.0084 & 0.0022 & 0.0000 & & 0.0044 & & 0.0170 & 0.0000 & & 0.0008 & & 0.0016 & 0.0008 & & & 0.0008 & & 0.0017 & 0.0000 & \\
\hline CVS2-75 & & & & & & & & & & & & & & & & & & & & & \\
\hline CVS2-76 & & & & & & & & & & & & & & & & & & & & & \\
\hline CVS2-77 & & & & & & & & & & & & & & & & & & & & & \\
\hline CVS2-78 & & & & & & & & & & & & & & & & & & & & & \\
\hline CVS2-79 & & 0.0086 & 0.0024 & 0.0000 & & 0.0045 & & 0.0174 & 0.0000 & & 0.0008 & & 0.0016 & 0.0008 & & & 0.0008 & & 0.0017 & 0.0000 & \\
\hline CVS2-80 & & & & & & & & & & & & & & & & & & & & & \\
\hline CVS2-81 & & & & & & & & & & & & & & & & & & & & & \\
\hline CVS2-82 & & & & & & & & & & & & & & & & & & & & & \\
\hline CVS2-83 & & & & & & & & & & & & & & & & & & & & & \\
\hline CVS2-84 & & & & & & & & & & & & & & & & & & & & & \\
\hline CVS2-85 & & & & & & & & & & & & & & & & & & & & & \\
\hline CVS2-86 & & & & & & & & & & & & & & & & & & & & & \\
\hline CVS2-87 & & & & & & & & & & & & & & & & & & & & & \\
\hline CVS2-88 & & & & & & & & & & & & & & & & & & & & & \\
\hline CVS2-89 & & & & & & & & & & & & & & & & & & & & & \\
\hline CVS2-90 & & & & & & & & & & & & & & & & & & & & & \\
\hline CVS2-91 & & 0.0083 & 0.0022 & 0.0000 & & 0.0043 & & 0.0167 & 0.0000 & & 0.0008 & & 0.0016 & 0.0008 & & & 0.0008 & & 0.0018 & 0.0000 & \\
\hline CVS2-92 & & & & & & & & & & & & & & & & & & & & & \\
\hline CVS2-93 & & & & & & & & & & & & & & & & & & & & & \\
\hline CVS2-94 & & & & & & & & & & & & & & & & & & & & & \\
\hline CVS2-95 & & 0.0035 & 0.0125 & 0.0000 & & 0.0017 & & 0.0069 & 0.0000 & & 0.0003 & & 0.0006 & 0.0003 & & & 0.0003 & & 0.0008 & 0.0000 & \\
\hline CVS2-96 & & 0.0039 & 0.0011 & 0.0000 & & 0.0020 & & 0.0077 & 0.0000 & & 0.0004 & & 0.0007 & 0.0004 & & & 0.0004 & & 0.0011 & 0.0000 & \\
\hline CVS2-97 & & 0.0196 & 0.0023 & 0.0000 & & 0.0029 & & 0.0066 & 0.0000 & & 0.0000 & & 0.0019 & 0.0000 & & & 0.0000 & & 0.0010 & 0.0000 & \\
\hline CVS2-98 & & 0.0007 & 0.0003 & 0.0000 & & 0.0005 & & 0.0015 & 0.0000 & & 0.0001 & & 0.0002 & 0.0001 & & & 0.0001 & & 0.0003 & 0.0000 & \\
\hline CVS2-99 & & & & & & & & & & & & & & & & & & & & & \\
\hline CVS2-100 & & & & & & & & & & & & & & & & & & & & & \\
\hline CVS2-101 & & 0.0038 & 0.0153 & 0.0000 & & & & & & & & & & & & & & & & & \\
\hline CVS2-102 & & & & & & & & & & & & & & & & & & & & & \\
\hline
\end{tabular}


Appendix A. Database - mass fraction

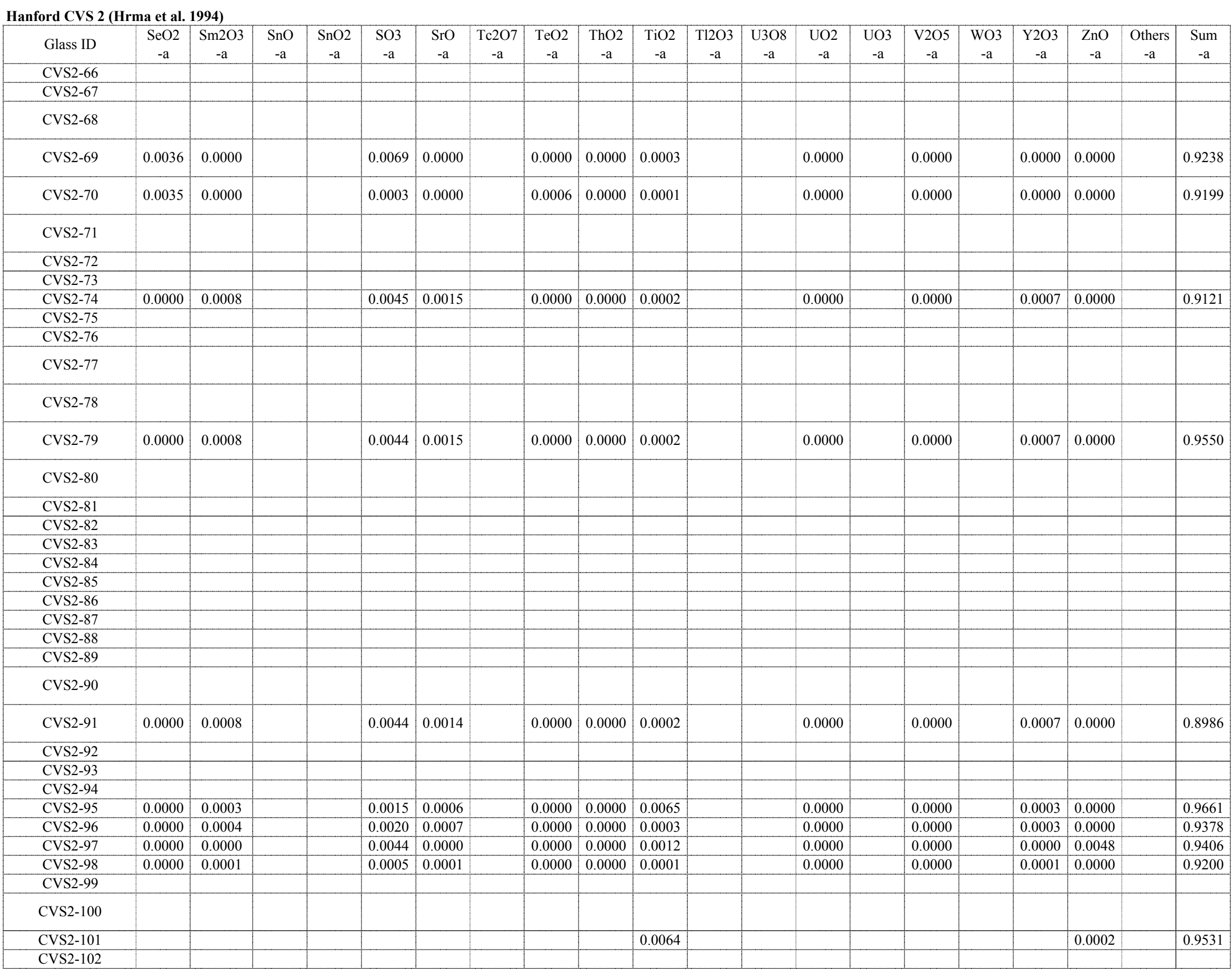


Appendix A. Database - mass fraction

\begin{tabular}{|c|c|c|c|c|c|c|c|c|}
\hline Glass ID & $\begin{array}{l}\mathrm{TM} \\
\left({ }^{\circ} \mathrm{C}\right)\end{array}$ & $\begin{array}{l}\text { Gradient } \\
\text { TL }\left({ }^{\circ} \mathrm{C}\right)\end{array}$ & $\begin{array}{l}\text { Uniform } \\
\text { TL }\left({ }^{\circ} \mathrm{C}\right)\end{array}$ & Primary Phase & $\begin{array}{l}\text { Quenched } \\
\text { Visual/OM }\end{array}$ & $\begin{array}{c}\text { Quenched } \\
\text { SEM/EDS or TEM }\end{array}$ & $\begin{array}{l}\text { Quenched } \\
\text { XRD }\end{array}$ & $\begin{array}{c}\text { Quenched } \\
\text { Homogeneous? }\end{array}$ \\
\hline CVS2-66 & & $>1114$ & & spinel & & & & \\
\hline CVS2-67 & & 1022 & & $\mathrm{Cr} 2 \mathrm{O} 3$ & & & & \\
\hline CVS2-68 & & $>1114$ & & $\mathrm{Cr} 2 \mathrm{O} 3$ & & & & \\
\hline CVS2-69 & & $>1114$ & & spinel & & & & \\
\hline CVS2-70 & & $>1114$ & & $\mathrm{Cr} 2 \mathrm{O} 3$ & & & & \\
\hline CVS2-71 & & 1004 & & spinel & & & & \\
\hline CVS2-72 & & $>868$ & & spinel & & & & \\
\hline CVS2-73 & & 840 & & Li-Al silicate & & & & \\
\hline CVS2-74 & & 1066 & & spinel & & & & \\
\hline CVS2-75 & & 826 & & Li-Al silicate & & & & \\
\hline CVS2-76 & & 761 & & clinopyroxene & & & & \\
\hline CVS2-77 & & 1090 & & Na-Zr silicate & & & & \\
\hline CVS2-78 & & 1129 & & zircon & & & & \\
\hline CVS2-79 & & $>1154$ & & spinel & & & & \\
\hline CVS2-80 & & 1154 & & zircon & & & & \\
\hline CVS2-81 & & $>1154$ & & $\mathrm{Li}_{2} \mathrm{SiO} 3$ & & & & \\
\hline CVS2-82 & & 745 & & $\mathrm{Li} 2 \mathrm{SiO} 3$ & & & & \\
\hline CVS2-83 & & 741 & & $\mathrm{Li} 2 \mathrm{SiO} 3$ & & & & \\
\hline CVS2-84 & & 983 & & Na-Zr silicate & & & & \\
\hline CVS2-85 & & $>1150$ & & $\mathrm{Li} 2 \mathrm{SiO} 3$ & & & & \\
\hline CVS2-86 & & 1015 & & nepheline & & & & \\
\hline CVS2-87 & & & & & & & & \\
\hline CVS2-88 & & 982 & & $\mathrm{ZrO} 2$ & & & & \\
\hline CVS2-89 & & 838 & & clinopyroxene & & & & \\
\hline CVS2-90 & & 897 & & orthopyroxene & & & & \\
\hline CVS2-91 & & $>967$ & & spinel & & & & \\
\hline CVS2-92 & & 897 & & zircon & & & & \\
\hline CVS2-93 & & $>983$ & & spinel & & & & \\
\hline CVS2-94 & & 663 & & $\mathrm{Ca}$ asilicate & & & & \\
\hline CVS2-95 & & 804 & & clinopyroxene & & & & \\
\hline CVS2-96 & & 1010 & & spinel & & & & \\
\hline CVS2-97 & & 887 & & spinel & & & & \\
\hline CVS2-98 & & 740 & & nepheline & & & & \\
\hline CVS2-99 & & 988 & & spinel & & & & \\
\hline CVS2-100 & & 1030 & & spinel & & & & \\
\hline CVS2-101 & & & & & & & & \\
\hline CVS2-102 & & & & & & & & \\
\hline
\end{tabular}


Appendix A. Database - mass fraction

\begin{tabular}{|c|c|c|c|c|c|}
\hline Glass ID & $\begin{array}{c}\mathrm{CCC} \\
\text { Visual/OM }\end{array}$ & $\begin{array}{c}\text { CCC } \\
\text { SEM/EDS or TEM }\end{array}$ & $\begin{array}{l}\mathrm{CCC} \\
\mathrm{XRD}\end{array}$ & $\begin{array}{l}\text { Heat Treated } \\
\text { Visual/OM }\end{array}$ & $\begin{array}{c}\text { Heat Treated } \\
\text { SEM/EDS or TEM }\end{array}$ \\
\hline CVS2-66 & spinel & (Cr; $\mathrm{Mn}, \mathrm{Fe}, \mathrm{Ni})$ & $5 \mathrm{vol} \%$ spinel & & \\
\hline CVS2-67 & none & none & none & & \\
\hline CVS2-68 & $\mathrm{Li}, \mathrm{Al}$ silicate, $\mathrm{Cr} 2 \mathrm{O} 3$ & $(\mathrm{Cr})$ & $\begin{array}{l}8 \text { vol\% Li, Al silicate, } 2 \text { vol\% eskolaite } \\
(\mathrm{Cr} 2 \mathrm{O} 3)\end{array}$ & & \\
\hline CVS2-69 & $\mathrm{Cr} 2 \mathrm{O} 3, \mathrm{Li} 3 \mathrm{PO} 4$ & $(\mathrm{Cr}),(\mathrm{P})$ & $\begin{array}{l}3 \mathrm{vol} \% \text { eskolaite }(\mathrm{Cr} 2 \mathrm{O} 3), 2 \mathrm{vol} \% \\
\mathrm{Li3PO} 4\end{array}$ & & \\
\hline CVS2-70 & $\mathrm{Li}, \mathrm{Al}$ silicate, $\mathrm{Cr} 2 \mathrm{O} 3$ & $(\mathrm{Cr})$ & $\begin{array}{l}10 \mathrm{vol} \% \mathrm{Li}, \mathrm{Al} \text { silicate, }<1 \mathrm{vol} \% \\
\text { eskolaite (Cr2O3) }\end{array}$ & & \\
\hline CVS2-71 & orthopyroxene(?), spinel & $(\mathrm{Fe}, \mathrm{Si}),(\mathrm{Fe}, \mathrm{Ni}, \mathrm{Cr})$ & $\begin{array}{l}<3 \text { vol } \% \text { clinopyroxene(?), }<1 \text { vol } \% \\
\text { spinel }\end{array}$ & & \\
\hline CVS2-72 & none & & & & \\
\hline CVS2-73 & none & & & & \\
\hline CVS2-74 & spinel & $(\mathrm{Cr}, \mathrm{Fe}, \mathrm{Ni})$ & $<5 \mathrm{vol} \%$ spinel & & \\
\hline CVS2-75 & none & & & & \\
\hline CVS2-76 & Li2SiO3, RuO2 needles & $(\mathrm{Si})$ & $5 \mathrm{vol} \% \mathrm{Li} 2 \mathrm{SiO} 3$ & & \\
\hline CVS2-77 & $\mathrm{SiO} 2, \mathrm{Na}-\mathrm{Zr}$ silicate, $\mathrm{RuO} 2$ needles & $(\mathrm{Si}),(\mathrm{Na}, \mathrm{Zr}, \mathrm{Si})$ & $\begin{array}{l}<2 \text { vol } \% \text { cristobalite }(\mathrm{SiO} 2) \text {, } \\
\text { unidentified }\end{array}$ & & \\
\hline CVS2-78 & SiO2, zircon, olivine & $(\mathrm{Zr}, \mathrm{Si}),(\mathrm{Fe}, \mathrm{Mg}, \mathrm{Si} ; \mathrm{Zr}, \mathrm{Ni})$ & $\begin{array}{l}22 \text { vol } \% \text { cristobalite (SiO2), } 3 \text { vol } \% \\
\text { zircon ( } \mathrm{ZrSiO} 4), 3 \text { vol } \% \text { unidentified }\end{array}$ & & \\
\hline CVS2-79 & SiO2, spinel, hematite & $(\mathrm{Si}),(\mathrm{Fe} ; \mathrm{Ni}, \mathrm{Cr}),(\mathrm{Fe})$ & $\begin{array}{l}20 \text { vol } \% \text { cristobalite }(\mathrm{SiO} 2), 5 \text { vol } \% \\
\text { spinel, } 1 \text { vol } \% \text { hematite }(\mathrm{Fe} 2 \mathrm{O} 3)\end{array}$ & & \\
\hline CVS2-80 & SiO2, zircon, olivine & $\begin{array}{l}(\mathrm{Si}),(\mathrm{Zr}, \mathrm{Si}),(\mathrm{Fe}, \mathrm{Mg}, \mathrm{Si} ; \\
\mathrm{Ni})\end{array}$ & $\begin{array}{l}15 \text { vol } \% \text { cristobalite (SiO2), } 9 \text { vol\% } \\
\text { zircon ( } \mathrm{ZrSiO} 4), 6 \text { vol } \% \text { unidentified }\end{array}$ & & \\
\hline CVS2-81 & spinel, Li2SiO3 & $(\mathrm{Fe}, \mathrm{Ni}, \mathrm{Cr}),(\mathrm{Si})$ & 5 vol $\%$ spinel, 4 vol $\%$ Li2 2 SiO3 & & \\
\hline CVS2-82 & $\mathrm{Li} 2 \mathrm{SiO} 3, \mathrm{RuO} 2$ needles & $(\mathrm{Si})$ & 4 vol\% Li2SiO3 & & \\
\hline CVS2-83 & RuO2 needles & none & none & & \\
\hline CVS2-84 & $\mathrm{Li} 2 \mathrm{SiO} 3, \mathrm{RuO} 2$ needles & (Si) & 5 vol\% Li2SiO3 & & \\
\hline CVS2-85 & nepheline, $\mathrm{Li} 2 \mathrm{SiO} 3$ & $(\mathrm{Na}, \mathrm{Al}, \mathrm{Si}),(\mathrm{Si})$ & 33 vol\% nepheline, 30 vol\% Li2SiO3 & & \\
\hline CVS2-86 & nepheline & $(\mathrm{Na}, \mathrm{Al}, \mathrm{Si})$ & 5 vol\% nepheline & & \\
\hline CVS2-87 & none & & & & \\
\hline CVS2-88 & $\mathrm{RuO} 2$ needles & & & & \\
\hline CVS2-89 & $\mathrm{RuO} 2$ needles & & & & \\
\hline CVS2-90 & $\mathrm{SiO} 2$, spinel & $(\mathrm{Si}),(\mathrm{Fe}, \mathrm{Ni})$ & $\begin{array}{l}5 \mathrm{vol} \% \text { cristobalite }(\mathrm{SiO} 2),<1 \mathrm{vol} \% \\
\text { spinel, }<1 \text { vol } \% \text { LiAl silicate(?) }\end{array}$ & & \\
\hline CVS2-91 & $(?)$ & none & $\begin{array}{l}<2 \mathrm{vol} \% \text { unidentified, }<1 \mathrm{vol} \% \\
\text { cristobalite }(\mathrm{SiO} 2)\end{array}$ & & \\
\hline CVS2-92 & none & none & $<2 \mathrm{vol} \%$ unidentified & & \\
\hline CVS2-93 & spinel & $(\mathrm{Fe}, \mathrm{Cr}, \mathrm{Ni})$ & $<4$ vol\% spinel & & \\
\hline CVS2-94 & RuO2 needles & none & none & & \\
\hline CVS2-95 & none & none & none & & \\
\hline CVS2-96 & clinopyroxene, spinel & $(\mathrm{Ca}, \mathrm{Mg}, \mathrm{Fe}, \mathrm{Si})$ & $15 \mathrm{vol} \%$ clinopyroxene & & \\
\hline CVS2-97 & clinopyroxene & $(\mathrm{Ca}, \mathrm{Fe}, \mathrm{Si} ; \mathrm{Mg})$ & none & & \\
\hline CVS2-98 & RuO2 needles & none & none & & \\
\hline CVS2-99 & none & & & & \\
\hline CVS2-100 & orthopyroxene, spinel & $(\mathrm{Fe}, \mathrm{Si}),(\mathrm{Fe}, \mathrm{Ni})$ & $3 \mathrm{vol} \%$ orthopyroxene, 2 vol $\%$ spinel & & \\
\hline \multicolumn{6}{|l|}{ CVS2-101 } \\
\hline CVS2-102 & & & & & \\
\hline
\end{tabular}


Hanford CVS 2 (Hrma et al. 1994)

\begin{tabular}{|c|c|c|c|c|c|c|c|c|c|c|c|c|c|c|c|}
\hline Glass ID & $\begin{array}{c}\text { Heat Treated } \\
\text { XRD }\end{array}$ & $\begin{array}{l}\text { Density } \\
\left(\mathrm{g} / \mathrm{cm}^{3}\right)\end{array}$ & $\begin{array}{c}\text { Fulc Visc } \\
\mathrm{A}\end{array}$ & \begin{tabular}{|c|} 
Fulc Visc \\
$\mathrm{B}$ \\
\end{tabular} & $\begin{array}{c}\text { Fulc Visc } \\
\text { To } \\
\end{array}$ & $\begin{array}{c}\mathrm{FV} 1150^{\circ} \mathrm{C} \\
(\mathrm{Pa} \cdot \mathrm{s})\end{array}$ & \begin{tabular}{|c|} 
Arrh Visc \\
$\mathrm{A}$
\end{tabular} & \begin{tabular}{|c|} 
Arrh Visc \\
B
\end{tabular} & $\begin{array}{c}1150^{\circ} \mathrm{C} \\
\text { (Da.o) }\end{array}$ & \begin{tabular}{|c|}
$\mathrm{T}\left({ }^{\circ} \mathrm{C}\right)$ at \\
$2 \mathrm{~Pa} \cdot \mathrm{s}$ \\
\end{tabular} & $\begin{array}{c}\mathrm{T}\left({ }^{\circ} \mathrm{C}\right) \text { at } \\
5 \mathrm{~Pa} \cdot \mathrm{s}\end{array}$ & \begin{tabular}{|c|}
$\mathrm{T}\left({ }^{\circ} \mathrm{C}\right)$ at \\
$10 \mathrm{~Pa} \cdot \mathrm{s}$ \\
\end{tabular} & $\begin{array}{l}\mathrm{T} 1 \\
\left({ }^{\circ} \mathrm{C}\right) \\
\end{array}$ & $\begin{array}{c}\mathrm{V} 1 \\
(\mathrm{~Pa} \cdot \mathrm{s}) \\
\end{array}$ & $\begin{array}{c}\mathrm{T} 2 \\
\left({ }^{\circ} \mathrm{C}\right) \\
\end{array}$ \\
\hline CVS2-66 & & 2.5259 & -5.34 & 6683.87 & 253.16 & 8.24 & -10.489 & 17948.2 & 8.36 & & & & 1250 & 3.9 & 1200 \\
\hline CVS2-67 & & 2.4243 & -5.23 & 6894.45 & 221.22 & 8.97 & -9.892 & 17214.0 & 9.07 & & & & 1244 & 4.51 & 1195 \\
\hline CVS2-68 & & 2.4447 & -5.99 & 9991.45 & 18.11 & 17.05 & & & & & & & (1250) & (8.54) & (1201) \\
\hline CVS2-69 & & 2.4905 & -6.07 & 8377.90 & 235.63 & 21.97 & & & & & & & (1251) & (8.63) & $(1201)$ \\
\hline CVS2-70 & & 2.4566 & -6.89 & 10305.91 & 37.89 & 10.80 & -9.807 & 17352.4 & 10.89 & & & & 1253 & 4.87 & 1204 \\
\hline CVS2-71 & & 2.6833 & -7.34 & 9636.07 & 133.89 & 8.50 & -11.709 & 19722.3 & 8.59 & & & & 1250 & 3.56 & 1200 \\
\hline CVS2-72 & & 2.5505 & -5.86 & 7570.66 & 193.65 & 7.81 & -10.349 & 17670.1 & 7.91 & & & & 1249 & 3.69 & 1200 \\
\hline CVS2-73 & & 2.4597 & -5.88 & 7844.44 & 174.32 & 8.67 & -10.158 & 17544.2 & 8.77 & & & & 1248 & 4.12 & 1199 \\
\hline CVS2-74 & & 2.5884 & -5.11 & 6427.61 & 277.16 & 9.55 & -10.586 & 18298.2 & 9.71 & & & & 1251 & 4.42 & 1201 \\
\hline CVS2-75 & & 2.46 & -5.34 & 6896.99 & 229.66 & 8.66 & -10.126 & 17499.1 & 8.77 & & & & 1249 & 4.13 & 1200 \\
\hline CVS2-76 & & 2.6688 & -5.33 & 4925.24 & 254.58 & 1.18 & -9.164 & 13296.0 & 1.20 & & & & 1248 & 0.69 & 1198 \\
\hline CVS2-77 & & 2.7897 & -6.28 & 5333.94 & 356.37 & 1.55 & -12.748 & 18792.4 & 1.58 & & & & 1247 & 0.75 & 1199 \\
\hline CVS2-78 & & 2.5264 & -7.05 & 10022.81 & 184.77 & 28.12 & -12.385 & 22393.9 & 28.55 & & & & 1250 & 10.91 & 1200 \\
\hline CVS2-79 & & 2.5481 & -3.37 & 5588.22 & 418.24 & 71.38 & -11.634 & 22619.4 & 70.95 & & & & (1252) & $(14.46)$ & $(1152)$ \\
\hline CVS2-80 & & 2.5691 & -3.97 & 4098.99 & 637.80 & 56.30 & $(-18.56)$ & 32185.898 & $(57.878)$ & & & & (1252) & $(17.32)$ & 1200 \\
\hline CVS2-81 & & 2.7419 & -3.82 & 1666.90 & 667.51 & 0.69 & -13.944 & 19495.3 & 0.78 & & & & 1248 & 0.35 & 1198 \\
\hline CVS2-82 & & 2.5412 & -2.99 & 2066.37 & 550.60 & 1.58 & -10.280 & 15194.6 & 1.49 & & & & (1246) & $(1.02)$ & (1196) \\
\hline CVS2-83 & & 2.6567 & -5.51 & 3926.44 & 395.30 & 0.74 & -11.175 & 15493.5 & 0.75 & & & & 1246 & 0.39 & 1196 \\
\hline CVS2-84 & & 2.755 & -6.13 & 5274.80 & 313.34 & 1.19 & -11.384 & 16472.1 & 1.21 & & & & 1250 & 0.61 & 1201 \\
\hline CVS2-85 & & 2.5555 & -6.99 & 8887.61 & 90.21 & 4.02 & -10.291 & 16635.3 & 4.05 & & & & 1247 & 1.99 & 1198 \\
\hline CVS2-86 & & 2.4915 & -5.33 & 7632.33 & 275.12 & 29.69 & -11.328 & 20971.0 & 30.24 & & & & 1249 & 12.14 & 1199 \\
\hline CVS2-87 & & 2.5798 & -6.86 & 9243.27 & 201.98 & 17.98 & -12.563 & 22009.1 & 18.24 & & & & 1248 & 7.26 & 1198 \\
\hline CVS2-88 & & 2.6556 & 6.28 & 6455.00 & 295.58 & 3.57 & -12.241 & 19255.7 & 3.64 & & & & 1250 & 1.63 & 1200 \\
\hline CVS2-89 & & 2.7538 & -5.75 & 5167.78 & 387.33 & 2.78 & -12.922 & 19876.7 & 2.85 & & & & 1248 & 1.29 & 1199 \\
\hline CVS2-90 & & 2.5718 & -4.95 & 5090.70 & 334.61 & 3.65 & -10.554 & 16883.6 & 3.71 & & & & 1248 & 1.86 & 1199 \\
\hline CVS2-91 & & 2.5271 & -4.20 & 4818.26 & 447.80 & 14.31 & -12.976 & 22294.8 & 14.75 & & & & 1250 & 6.09 & 1200 \\
\hline CVS2-92 & & 2.7211 & -6.34 & 5237.82 & 323.83 & 1.00 & -11.846 & 16872.3 & 1.01 & & & & 1247 & 0.51 & 1197 \\
\hline CVS2-93 & & 2.7521 & -6.17 & 5113.18 & 255.81 & 0.64 & -10.162 & 13835.4 & 0.64 & & & & 1247 & 0.36 & 1198 \\
\hline CVS2-94 & & 2.5884 & -4.78 & 3416.96 & 401.23 & 0.81 & -9.278 & 12906.3 & 0.81 & & & & 1197 & 0.61 & 1148 \\
\hline CVS2-95 & & 2.649 & -4.21 & 3304.95 & 443.20 & 1.60 & -10.066 & 15022.5 & 1.63 & & & & 1251 & 0.89 & 1202 \\
\hline CVS2-96 & & 2.6666 & -5.39 & 5539.08 & 370.69 & 5.55 & -12.531 & 20302.5 & 5.68 & & & & 1250 & 2.51 & 1199 \\
\hline CVS2-97 & & & -5.72 & 6451.33 & 312.06 & 7.25 & -12.122 & 20096.6 & 7.39 & & & & 1250 & 3.16 & 1200 \\
\hline CVS2-98 & & 2.5128 & -4.75 & 4415.06 & 330.91 & 1.90 & -9.549 & 14519.7 & 1.92 & & & & 1246 & 1.08 & 1196 \\
\hline CVS2-99 & & 2.6321 & -5.40 & 6369.71 & 305.31 & 8.53 & -11.593 & 19571.6 & 8.68 & & & & 1247 & 3.93 & 1198 \\
\hline CVS2-100 & & 2.7136 & -5.33 & 5934.93 & 332.06 & 6.85 & -11.813 & 19573.0 & 6.97 & & & & 1247 & 3.19 & 1197 \\
\hline CVS2-101 & & 2.6602 & -5.26 & 4730.46 & 315.57 & 1.51 & -10.009 & 14846.3 & 1.53 & & & & 1251 & 0.81 & 1202 \\
\hline CVS2-102 & & 2.6754 & & & & & & & & & & & & & \\
\hline
\end{tabular}




\section{Hanford CVS 2 (Hrma et al. 1994)}

\begin{tabular}{|c|c|c|c|c|c|c|c|c|c|c|c|c|c|c|c|c|c|c|c|c|c|}
\hline Glass ID & $\begin{array}{c}\mathrm{V} 2 \\
(\mathrm{~Pa} \cdot \mathrm{s})\end{array}$ & $\begin{array}{l}\mathrm{T} 3 \\
\left({ }^{\circ} \mathrm{C}\right)\end{array}$ & $\begin{array}{c}\mathrm{V} 3 \\
(\mathrm{~Pa} \cdot \mathrm{s})\end{array}$ & $\begin{array}{l}\mathrm{T} 4 \\
\left({ }^{\circ} \mathrm{C}\right)\end{array}$ & $\begin{array}{c}\mathrm{V} 4 \\
(\mathrm{~Pa} \cdot \mathrm{s})\end{array}$ & $\begin{array}{l}\text { T5 } \\
\left({ }^{\circ} \mathrm{C}\right)\end{array}$ & $\begin{array}{c}\text { V5 } \\
(\mathrm{Pa} \cdot \mathrm{s})\end{array}$ & $\begin{array}{l}\text { T6 } \\
\left({ }^{\circ} \mathrm{C}\right)\end{array}$ & $\begin{array}{c}\mathrm{V} 6 \\
(\mathrm{~Pa} \cdot \mathrm{s})\end{array}$ & $\begin{array}{l}\text { T7 } \\
\left({ }^{\circ} \mathrm{C}\right)\end{array}$ & $\begin{array}{c}\text { V7 } \\
(\mathrm{Pa} \cdot \mathrm{s})\end{array}$ & $\begin{array}{l}\mathrm{T} 8 \\
\left({ }^{\circ} \mathrm{C}\right)\end{array}$ & $\begin{array}{c}\mathrm{V} 8 \\
(\mathrm{~Pa} \cdot \mathrm{s})\end{array}$ & $\begin{array}{l}\mathrm{T} 9 \\
\left({ }^{\circ} \mathrm{C}\right)\end{array}$ & $\begin{array}{c}\text { V9 } \\
(\mathrm{Pa} \cdot \mathrm{s})\end{array}$ & $\begin{array}{l}\mathrm{T} 10 \\
\left({ }^{\circ} \mathrm{C}\right)\end{array}$ & $\begin{array}{c}\mathrm{V} 10 \\
(\mathrm{~Pa} \cdot \mathrm{s})\end{array}$ & $\begin{array}{l}\text { T11 } \\
\left({ }^{\circ} \mathrm{C}\right)\end{array}$ & $\begin{array}{c}\mathrm{V} 11 \\
(\mathrm{~Pa} \cdot \mathrm{s})\end{array}$ & $\begin{array}{l}\mathrm{T} 12 \\
\left({ }^{\circ} \mathrm{C}\right)\end{array}$ & $\begin{array}{c}\mathrm{V} 12 \\
(\mathrm{~Pa} \cdot \mathrm{s})\end{array}$ \\
\hline CVS2-66 & 5.55 & 1150 & 8.13 & 1150 & 8.28 & 1150 & 8.38 & 1100 & 12.74 & 1100 & 12.86 & 1050 & 20.69 & 1000 & 37.33 & 949 & 70.7 & & & & \\
\hline CVS2-67 & 6.37 & 1146 & 9.17 & 1146 & 9.3 & 1146 & 9.42 & 1096 & 14.2 & 1096 & 14.29 & 1046 & 22.44 & 996 & 39.41 & 946 & 72.63 & & & & \\
\hline CVS2-68 & (11.39) & (1151) & (16.13) & (1150) & (17.68) & (1151) & $(17.83)$ & (1101) & (23.9) & (1101) & (24.29) & (1051) & $(39.77)$ & (1000) & (74.27) & (950) & $(106.71)$ & & & & \\
\hline CVS2-69 & (13.6) & (1151) & (19.77) & (1150) & (21.97) & (1151) & $(22.56)$ & (1101) & $(36.91)$ & (1101) & (36.91) & (1051) & $(66.83)$ & (1000) & $(105.67)$ & & & & & & \\
\hline CVS2-70 & 7.39 & 1154 & 9.98 & 1153 & 10.3 & 1154 & 10.52 & 1105 & 15.97 & 1104 & 16.19 & 1053 & 27.16 & 1003 & 43.48 & 953 & 79.55 & & & & \\
\hline CVS2-71 & 5.49 & 1150 & 8.33 & 1150 & 8.62 & 1150 & 8.84 & 1100 & 13.86 & 1100 & 13.89 & 1050 & 23.4 & 1000 & 43.34 & 950 & 87.96 & & & & \\
\hline CVS2-72 & 5.28 & 1150 & 7.74 & 1150 & 7.84 & 1150 & 8.02 & 1101 & 11.89 & 1100 & 12.16 & 1050 & 19.29 & 1000 & 34.41 & 950 & 63.4 & & & & \\
\hline CVS2-73 & 5.98 & 1149 & 8.72 & 1149 & 8.75 & 1149 & 8.83 & 1100 & 13.37 & 1098 & 13.63 & 1049 & 21.49 & 999 & 38.4 & 949 & 69.65 & & & & \\
\hline CVS2-74 & 6.39 & 1151 & 9.28 & 1151 & 9.54 & 1151 & 9.69 & 1102 & 14.7 & 1101 & 14.73 & 1050 & 24.26 & 1001 & 44.16 & 950 & 84.97 & & & & \\
\hline CVS2-75 & 5.93 & 1150 & 8.53 & 1150 & 8.72 & 1150 & 8.87 & 1100 & 13.19 & 1100 & 13.41 & 1050 & 21.28 & 1000 & 37.21 & 950 & 69.65 & & & & \\
\hline CVS2-76 & 0.88 & 1148 & 1.18 & 1148 & 1.2 & 1148 & 1.23 & 1098 & 1.66 & 1098 & 1.69 & 1048 & 2.47 & 999 & 3.42 & 947 & 6.07 & & & & \\
\hline CVS2-77 & 1.04 & 1150 & 1.52 & 1148 & 1.59 & 1149 & 1.6 & 1099 & 2.44 & 1100 & 2.46 & 1049 & 4.06 & 999 & 7.69 & 948 & 15.29 & & & & \\
\hline CVS2-78 & 16.79 & 1149 & 26.93 & 1149 & 27.31 & 1150 & 28.28 & 1099 & 47.63 & 1100 & 54.86 & 1050 & 97.61 & 999 & 187.57 & & & & & & \\
\hline CVS2-79 & $(32.87)$ & (1203) & (34.75) & 1151 & 69.68 & 1151 & 71.59 & 1101 & 121.28 & 1102 & 124.22 & (1002) & $(199.92)$ & 1051 & 235.79 & & & & & & \\
\hline CVS2-80 & 27.62 & 1150 & 54.77 & 1150 & 57.93 & (1149) & (110.88) & 1101 & 128.15 & 1100 & 136.93 & & & & & & & & & & \\
\hline CVS2-81 & 0.54 & 1150 & 0.72 & 1149 & 0.74 & 1149 & 0.76 & $(1099)$ & $(0.99)$ & $(1099)$ & (1) & $(1050)$ & $(1.4)$ & 1000 & 3.88 & 949 & 7.89 & & & & \\
\hline CVS2-82 & $(1.26)$ & 1146 & 1.52 & 1146 & 1.54 & 1147 & 1.56 & 1098 & 2.24 & 1097 & 2.26 & 1047 & 3.31 & 997 & 5.26 & 946 & 9.17 & & & & \\
\hline CVS2-83 & 0.56 & 1147 & 0.77 & 1148 & 0.77 & 1148 & 0.78 & 1097 & 1.08 & 1098 & 1.08 & 1048 & 1.55 & 999 & 2.75 & 948 & 5 & & & & \\
\hline CVS2-84 & 0.82 & 1152 & 1.16 & 1152 & 1.18 & 1152 & 1.18 & 1101 & 1.75 & 1102 & 1.8 & 1051 & 2.74 & 1002 & 4.6 & 951 & 8.55 & & & & \\
\hline CVS2-85 & 2.79 & 1148 & 4.06 & 1148 & 4.06 & 1148 & 4.16 & 1099 & 6.18 & 1098 & 6.21 & 1049 & 9.66 & 999 & 16.19 & 948 & 29.05 & & & & \\
\hline CVS2-86 & 18.49 & 1150 & 29.75 & 1150 & 30.22 & 1149 & 30.75 & 1100 & 49.92 & 1100 & 50.16 & 1049 & 90.26 & 999 & 185.81 & & & & & & \\
\hline CVS2-87 & 11.13 & 1149 & 17.89 & 1150 & 17.98 & 1150 & 18.13 & 1100 & 30.9 & 1100 & 31.37 & 1050 & 56.56 & 999 & 114.01 & 949 & 247.66 & & & & \\
\hline CVS2-88 & 2.32 & 1152 & 3.5 & 1151 & 3.51 & 1150 & 3.63 & 1102 & 5.58 & 1102 & 5.62 & 1052 & 9.48 & 1001 & 17.69 & 951 & 35.32 & & & & \\
\hline CVS2-89 & 1.85 & 1149 & 2.77 & 1149 & 2.78 & 1149 & 2.83 & 1099 & 4.53 & 1100 & 4.53 & 1049 & 7.86 & 999 & 14.7 & 949 & 31.52 & & & & \\
\hline CVS2-90 & 2.58 & 1149 & 3.64 & 1149 & 3.68 & 1149 & 3.68 & 1099 & 5.52 & 1100 & 5.52 & 1049 & 8.8 & 999 & 15.06 & 948 & 28.52 & & & & \\
\hline CVS2-91 & 9.14 & 1150 & 13.91 & 1150 & 14.29 & 1150 & 14.82 & 1100 & 23.87 & 1100 & 23.93 & 1049 & 44.34 & 1000 & 97.17 & 949 & 219.91 & & & & \\
\hline CVS2-92 & 0.7 & 1149 & 1.00 & 1148 & 1.01 & 1148 & 1.03 & 1099 & 1.52 & 1099 & 1.54 & 1049 & 2.36 & 999 & 4.06 & 949 & 7.72 & & & & \\
\hline CVS2-93 & 0.48 & 1148 & 0.64 & 1148 & 0.64 & 1148 & 0.67 & 1099 & 0.9 & 1099 & 0.9 & 1049 & 1.3 & 999 & 2.04 & 949 & 3.36 & & & & \\
\hline CVS2-94 & 0.81 & 1148 & 0.82 & 1099 & 1.14 & 1098 & 1.17 & 1048 & 1.62 & & & & & & & & & & & & \\
\hline CVS2-95 & 1.18 & 1152 & 1.55 & 1152 & 1.55 & 1152 & 1.59 & 1102 & 2.25 & 1102 & 2.26 & 1051 & 3.43 & 1002 & 5.55 & 951 & 9.95 & & & & \\
\hline CVS2-96 & 3.6 & 1150 & 5.53 & 1149 & 5.56 & 1149 & 5.56 & 1099 & 9.11 & 1100 & 9.17 & 1049 & 15.88 & 999 & 31.16 & 948 & 66.15 & & & & \\
\hline CVS2-97 & 4.72 & 1151 & 7.14 & 1151 & 7.23 & 1150 & 7.49 & 1101 & 11.53 & 1100 & 11.67 & 1051 & 19.97 & 1001 & 39.29 & 950 & 80.52 & & & & \\
\hline CVS2-98 & 1.43 & 1146 & 1.93 & 1147 & 1.95 & 1147 & 1.95 & 1098 & 2.73 & 1096 & 2.75 & 1047 & 4.19 & 997 & 6.52 & 947 & 11.22 & & & & \\
\hline CVS2-99 & 5.67 & 1148 & 8.74 & 1146 & 8.8 & 1146 & 8.81 & 1098 & 14.07 & 1096 & 14.33 & 1045 & 24.52 & 997 & 45.71 & 946 & 93.86 & & & & \\
\hline CVS2-100 & 4.61 & 1147 & 6.92 & 1148 & 6.96 & 1145 & 7.27 & 1098 & 11.22 & 1097 & 11.32 & 1047 & 19.14 & 998 & 36.92 & 947 & 74.36 & & & & \\
\hline CVS2-101 & 1.09 & 1153 & 1.46 & 1152 & 1.5 & 1152 & 1.49 & 1103 & 2.09 & 1103 & 2.11 & 1053 & 3.21 & 1002 & 5.05 & 952 & 8.82 & & & & \\
\hline CVS2-102 & & & & & & & & & & & & & & & & & & & & & \\
\hline
\end{tabular}




\section{Hanford CVS 2 (Hrma et al. 1994)}

\begin{tabular}{|c|c|c|c|c|c|c|c|c|c|c|c|c|c|c|c|c|}
\hline Glass ID & $\begin{array}{l}\mathrm{T} 13 \\
\left({ }^{\circ} \mathrm{C}\right)\end{array}$ & $\begin{array}{c}\mathrm{V} 13 \\
(\mathrm{~Pa} \cdot \mathrm{s})\end{array}$ & $\begin{array}{l}\mathrm{T} 14 \\
\left({ }^{\circ} \mathrm{C}\right)\end{array}$ & $\begin{array}{c}\mathrm{V} 14 \\
(\mathrm{~Pa} \cdot \mathrm{s})\end{array}$ & $\begin{array}{c}\text { Q PCT } \\
\mathrm{B}\left(\mathrm{g} / \mathrm{m}^{2}\right)\end{array}$ & $\begin{array}{c}\text { Q PCT } \\
\mathrm{Li}\left(\mathrm{g} / \mathrm{m}^{2}\right)\end{array}$ & $\begin{array}{c}\text { Q PCT } \\
\mathrm{Na}\left(\mathrm{g} / \mathrm{m}^{2}\right)\end{array}$ & $\begin{array}{c}\text { Q PCT } \\
\mathrm{Si}\left(\mathrm{g} / \mathrm{m}^{2}\right)\end{array}$ & $\begin{array}{c}\text { Q PCT } \\
\text { pH }\end{array}$ & $\begin{array}{c}\text { CCC PCT } \\
\mathrm{B}\left(\mathrm{g} / \mathrm{m}^{2}\right)\end{array}$ & $\begin{array}{l}\text { CCC PCT } \\
\mathrm{Li}\left(\mathrm{g} / \mathrm{m}^{2}\right)\end{array}$ & $\begin{array}{l}\text { CCC PCT } \\
\mathrm{Na}\left(\mathrm{g} / \mathrm{m}^{2}\right)\end{array}$ & $\begin{array}{l}\text { CCC PCT } \\
\mathrm{Si}\left(\mathrm{g} / \mathrm{m}^{2}\right)\end{array}$ & \begin{tabular}{|c|} 
CCC PCT \\
$\mathrm{pH}$
\end{tabular} & $\begin{array}{l}\text { Q PCT B at } \\
20^{\circ} \mathrm{C}\left(\mathrm{g} / \mathrm{m}^{2}\right)\end{array}$ & $\begin{array}{l}\text { Q PCT Li at } \\
20^{\circ} \mathrm{C}\left(\mathrm{g} / \mathrm{m}^{2}\right)\end{array}$ \\
\hline CVS2-66 & & & & & 0.210 & 0.247 & 0.223 & 0.164 & 10.54 & 0.210 & 0.267 & 0.195 & 0.019 & 10.39 & & \\
\hline CVS2-67 & & & & & 0.512 & 0.480 & 0.214 & 0.23 & 9.73 & 0.446 & 0.480 & 0.205 & 0.232 & 9.63 & & \\
\hline CVS2-68 & & & & & 0.308 & 0.340 & 0.127 & 0.254 & 9.84 & 11.320 & 7.153 & 2.934 & 0.6625 & 9.94 & & \\
\hline CVS2-69 & & & & & 0.226 & 0.302 & 0.112 & 0.186 & 10.18 & 0.391 & 0.317 & 0.406 & 0.223 & 10.17 & & \\
\hline CVS2-70 & & & & & 0.312 & 0.381 & 0.128 & 0.286 & 9.89 & 13.152 & 8.138 & 3.643 & 0.5 & 9.86 & & \\
\hline CVS2-71 & & & & & 0.411 & 0.427 & 0.350 & 0.244 & 10.78 & 0.370 & 0.444 & 0.318 & 0.223 & 10.80 & & \\
\hline CVS2-72 & & & & & 0.210 & 0.226 & 0.228 & 0.153 & 10.48 & 0.193 & 0.278 & 0.218 & 0.171 & 10.54 & & \\
\hline CVS2-73 & & & & & 0.244 & 0.301 & 0.074 & 0.179 & 9.96 & 2.658 & 1.869 & 0.680 & 0.2925 & 10.10 & & \\
\hline CVS2-74 & & & & & 0.226 & 0.279 & 0.147 & 0.163 & 10.41 & 0.247 & 0.302 & 0.152 & 0.194 & 10.30 & & \\
\hline CVS2-75 & & & & & 0.278 & 0.334 & 0.100 & 0.22 & 9.88 & 2.426 & 1.619 & 0.704 & 0.274 & 9.96 & & \\
\hline CVS2-76 & & & & & 14.871 & 7.571 & 12.541 & 4.045 & 12.46 & 13.130 & 6.550 & 9.546 & 4.024 & 12.27 & & \\
\hline CVS2-77 & & & & & 9.512 & 6.221 & 6.552 & 2.29 & 12.26 & 8.039 & 41.475 & 4.155 & 1.785 & 12.21 & & \\
\hline CVS2-78 & & & & & 0.934 & 0.698 & 0.593 & 0.191 & 9.21 & 19.092 & 22.013 & 14.825 & 0.248 & 9.29 & & \\
\hline CVS2-79 & & & & & 0.744 & 0.684 & 0.660 & 0.19 & 8.96 & 40.193 & 43.544 & 38.275 & 1.638 & 8.84 & & \\
\hline CVS2-80 & & & & & 0.764 & 0.672 & 0.600 & 0.195 & 9.15 & 25.774 & 29.631 & 21.159 & 0.3485 & 9.28 & & \\
\hline CVS2-81 & & & & & 16.613 & 8.064 & 12.824 & 3.036 & 12.51 & 20.351 & 4.270 & 15.136 & 2.1595 & 12.58 & & \\
\hline CVS2-82 & & & & & 44.000 & 19.969 & 35.377 & 16.884 & 12.18 & 50.093 & 20.123 & 37.436 & 21.558 & 12.09 & & \\
\hline CVS2-83 & & & & & 34.656 & 19.228 & 27.890 & 11.447 & 12.33 & 29.247 & 18.613 & 21.751 & 7.566 & 12.43 & & \\
\hline CVS2-84 & & & & & 12.460 & 6.682 & 8.236 & 0.493 & 12.42 & 20.364 & 8.848 & 12.728 & 3.287 & 12.48 & & \\
\hline CVS2-85 & & & & & 0.456 & 0.422 & 0.749 & 0.291 & 11.68 & 45.552 & 6.605 & 18.344 & 0.364 & 12.36 & & \\
\hline CVS2-86 & & & & & 0.115 & 0.091 & 0.254 & 0.082 & 10.98 & 0.268 & 0.269 & 0.299 & 0.105 & 11.04 & & \\
\hline CVS2-87 & & & & & 0.178 & 0.131 & 0.286 & 0.114 & 10.90 & 0.161 & 0.185 & 0.261 & 0.142 & 10.77 & & \\
\hline CVS2-88 & & & & & 0.308 & 0.376 & 0.473 & 0.162 & 11.52 & 0.340 & 0.484 & 0.463 & 0.173 & 11.66 & & \\
\hline CVS2-89 & & & & & 1.716 & 1.236 & 1.348 & 0.364 & 11.04 & 1.194 & 0.978 & 1.048 & 0.267 & 10.25 & & \\
\hline CVS2-90 & & & & & 5.577 & 3.764 & 3.481 & 0.716 & 9.98 & 6.129 & 4.670 & 3.916 & 0.763 & 9.96 & & \\
\hline CVS2-91 & & & & & 8.642 & 6.774 & 6.738 & 0.468 & 9.38 & 8.240 & 6.667 & 6.418 & 0.515 & 9.73 & & \\
\hline CVS2-92 & & & & & 18.590 & 12.442 & 11.792 & 1.214 & 11.12 & 16.077 & 12.750 & 10.377 & 1.87 & 11.08 & & \\
\hline CVS2-93 & & & & & 13.227 & 7.757 & 10.010 & 1.967 & 11.76 & 14.430 & 9.524 & 12.118 & 1.02 & 11.80 & & \\
\hline CVS2-94 & & & & & 4.070 & 3.066 & 3.350 & 0.935 & 11.84 & 3.195 & 3.700 & 3.594 & 1.02 & 11.89 & & \\
\hline CVS2-95 & & & & & 9.976 & 4.271 & 7.259 & 2.008 & 11.81 & 8.718 & 5.168 & 7.727 & 2.236 & 11.83 & & \\
\hline CVS2-96 & & & & & 0.493 & 0.429 & 0.410 & 0.159 & 10.24 & 0.662 & 0.486 & 0.349 & 0.145 & 10.11 & & \\
\hline CVS2-97 & & & & & 1.434 & 1.052 & 1.134 & 0.391 & 10.25 & 1.105 & 1.182 & 1.158 & 0.401 & 10.29 & & \\
\hline CVS2-98 & & & & & 4.520 & 3.497 & 2.796 & 0.267 & 11.58 & 2.970 & 3.730 & 2.763 & 0.282 & 11.62 & & \\
\hline CVS2-99 & & & & & 0.232 & 0.246 & 0.199 & 0.131 & 10.10 & 0.183 & 0.254 & 0.199 & 0.117 & 9.73 & & \\
\hline CVS2-100 & & & & & 0.326 & 0.343 & 0.226 & 0.17 & 10.34 & 0.326 & 0.386 & 0.269 & 0.206 & 10.41 & & \\
\hline CVS2-101 & & & & & 8.644 & 3.831 & 6.586 & 1.819 & 11.92 & 9.590 & 5.049 & 8.144 & 2.333 & 11.84 & & \\
\hline CVS2-102 & & & & & 2.672 & 1.921 & 1.677 & 0.559 & 10.28 & 2.638 & 1.880 & 1.722 & 0.536 & 10.30 & & \\
\hline
\end{tabular}


Appendix A. Database - mass fraction

Hanford CVS 2 (Hrma et al. 1994)

\begin{tabular}{|c|c|c|c|c|c|c|c|c|c|c|c|c|}
\hline Glass ID & $\begin{array}{l}\text { Q PCT Na at } \\
20^{\circ} \mathrm{C}\left(\mathrm{g} / \mathrm{m}^{2}\right)\end{array}$ & $\begin{array}{l}\text { Q PCT Si at } \\
20^{\circ} \mathrm{C}\left(\mathrm{g} / \mathrm{m}^{2}\right)\end{array}$ & $\begin{array}{c}\mathrm{QpH} \\
\text { at } 20^{\circ} \mathrm{C}\end{array}$ & $\begin{array}{c}\text { TCLP Ag } \\
\text { (ppm) }\end{array}$ & $\begin{array}{c}\text { TCLP As } \\
\text { (ppm) }\end{array}$ & $\begin{array}{c}\text { TCLP Ba } \\
(\mathrm{ppm})\end{array}$ & $\begin{array}{c}\text { TCLP Cd } \\
(\mathrm{ppm})\end{array}$ & $\begin{array}{c}\text { TCLP Cr } \\
(\mathrm{ppm})\end{array}$ & $\begin{array}{c}\text { TCLP Ni } \\
(\mathrm{ppm})\end{array}$ & $\begin{array}{c}\text { TCLP Pb } \\
(\mathrm{ppm})\end{array}$ & $\begin{array}{c}\text { TCLP Se } \\
(\mathrm{ppm})\end{array}$ & $\begin{array}{c}\text { TCLP Zn } \\
(\mathrm{ppm})\end{array}$ \\
\hline CVS2-66 & & & & & & & & & & & & \\
\hline CVS2-67 & & & & & & & & & & & & \\
\hline CVS2-68 & & & & & & & & & & & & \\
\hline CVS2-69 & & & & & & & & & & & & \\
\hline CVS2-70 & & & & & & & & & & & & \\
\hline CVS2-71 & & & & & & & & & & & & \\
\hline CVS2-72 & & & & & & & & & & & & \\
\hline CVS2-73 & & & & & & & & & & & & \\
\hline CVS2-74 & & & & & & & & & & & & \\
\hline CVS2-75 & & & & & & & & & & & & \\
\hline CVS2-76 & & & & & & & & & & & & \\
\hline CVS2-77 & & & & & & & & & & & & \\
\hline CVS2-78 & & & & & & & & & & & & \\
\hline CVS2-79 & & & & & & & & & & & & \\
\hline CVS2-80 & & & & & & & & & & & & \\
\hline CVS2-81 & & & & & & & & & & & & \\
\hline CVS2-82 & & & & & & & & & & & & \\
\hline CVS2-83 & & & & & & & & & & & & \\
\hline CVS2-84 & & & & & & & & & & & & \\
\hline CVS2-85 & & & & & & & & & & & & \\
\hline CVS2-86 & & & & & & & & & & & & \\
\hline CVS2-87 & & & & & & & & & & & & \\
\hline CVS2-88 & & & & & & & & & & & & \\
\hline CVS2-89 & & & & & & & & & & & & \\
\hline CVS2-90 & & & & & & & & & & & & \\
\hline CVS2-91 & & & & & & & & & & & & \\
\hline CVS2-92 & & & & & & & & & & & & \\
\hline CVS2-93 & & & & & & & & & & & & \\
\hline CVS2-94 & & & & & & & & & & & & \\
\hline CVS2-95 & & & & & & & & & & & & \\
\hline CVS2-96 & & & & & & & & & & & & \\
\hline CVS2-97 & & & & & & & & & & & & \\
\hline CVS2-98 & & & & & & & & & & & & \\
\hline CVS2-99 & & & & & & & & & & & & \\
\hline CVS2-100 & & & & & & & & & & & & \\
\hline CVS2-101 & & & & & & & & & & & & \\
\hline CVS2-102 & & & & & & & & & & & & \\
\hline
\end{tabular}


Hanford CVS 2 (Hrma et al. 1994)

\begin{tabular}{|c|c|c|c|c|c|c|c|c|c|c|c|c|c|c|c|c|c|c|c|c|c|}
\hline \multicolumn{22}{|l|}{ Hanford CVS 21} \\
\hline Glass ID & $\begin{array}{c}\mathrm{A} 12 \mathrm{O} 3 \\
-\mathrm{t}\end{array}$ & $\begin{array}{c}\mathrm{B} 2 \mathrm{O} 3 \\
-\mathrm{t}\end{array}$ & $\begin{array}{c}\mathrm{CaO} \\
-\mathrm{t}\end{array}$ & $\begin{array}{c}\mathrm{Fe} 2 \mathrm{O} 3 \\
-\mathrm{t}\end{array}$ & $\begin{array}{c}\mathrm{FeO} \\
-\mathrm{t}\end{array}$ & $\begin{array}{c}\mathrm{K} 2 \mathrm{O} \\
-\mathrm{t}\end{array}$ & $\begin{array}{c}\mathrm{Li} 2 \mathrm{O} \\
-\mathrm{t}\end{array}$ & $\begin{array}{c}\mathrm{MgO} \\
-\mathrm{t}\end{array}$ & $\begin{array}{c}\mathrm{Na} 2 \mathrm{O} \\
-\mathrm{t}\end{array}$ & $\begin{array}{c}\mathrm{NiO} \\
-\mathrm{t}\end{array}$ & $\begin{array}{c}\mathrm{P} 2 \mathrm{O} 5 \\
-\mathrm{t}\end{array}$ & $\begin{array}{c}\mathrm{SiO} 2 \\
-\mathrm{t}\end{array}$ & $\begin{array}{c}\mathrm{ZrO} 2 \\
-\mathrm{t}\end{array}$ & $\begin{array}{c}\mathrm{Ag} 2 \mathrm{O} \\
-\mathrm{t}\end{array}$ & $\begin{array}{c}\mathrm{As} 2 \mathrm{O} 3 \\
-\mathrm{t}\end{array}$ & $\begin{array}{c}\mathrm{BaO} \\
-\mathrm{t}\end{array}$ & $\begin{array}{c}\mathrm{Bi} 2 \mathrm{O} 3 \\
-\mathrm{t}\end{array}$ & $\begin{array}{c}\mathrm{Br} \\
-\mathrm{t}\end{array}$ & $\begin{array}{c}\mathrm{CdO} \\
-\mathrm{t}\end{array}$ & $\begin{array}{c}\mathrm{Ce} 2 \mathrm{O} 3 \\
-\mathrm{t}\end{array}$ & $\begin{array}{c}\mathrm{CeO} 2 \\
-\mathrm{t}\end{array}$ \\
\hline CVS2-103 & 0.0293 & 0.1337 & 0.0105 & 0.0913 & & 0.0000 & 0.0476 & 0.0107 & 0.1428 & 0.0077 & 0.0013 & 0.4100 & 0.0489 & 0.0000 & 0.0000 & 0.0013 & 0.0000 & & 0.0101 & & 0.0020 \\
\hline CVS2-104 & 0.0273 & 0.1246 & 0.0098 & 0.0851 & & 0.0000 & 0.0444 & 0.0099 & 0.1332 & 0.0072 & 0.0013 & 0.4500 & 0.0456 & 0.0000 & 0.0000 & 0.0013 & 0.0000 & & 0.0094 & & 0.0019 \\
\hline CVS2-105 & 0.0254 & 0.1156 & 0.0091 & 0.0789 & & 0.0000 & 0.0412 & 0.0092 & 0.1235 & 0.0067 & 0.0012 & 0.4900 & 0.0423 & 0.0000 & 0.0000 & 0.0012 & 0.0000 & & 0.0087 & & 0.0017 \\
\hline CVS2-106 & 0.0214 & 0.0974 & 0.0077 & 0.0665 & & 0.0000 & 0.0347 & 0.0078 & 0.1041 & 0.0056 & 0.0010 & 0.5700 & 0.0356 & 0.0000 & 0.0000 & 0.0010 & 0.0000 & & 0.0074 & & 0.0015 \\
\hline CVS2-107 & 0.0245 & 0.0500 & 0.0088 & 0.0763 & & 0.0000 & 0.0398 & 0.0089 & 0.1195 & 0.0065 & 0.0011 & 0.5684 & 0.0409 & 0.0000 & 0.0000 & 0.0011 & 0.0000 & & 0.0084 & & 0.0017 \\
\hline CVS2-108 & 0.0220 & 0.1500 & 0.0079 & 0.0683 & & 0.0000 & 0.0356 & 0.0080 & 0.1069 & 0.0058 & 0.0010 & 0.5086 & 0.0366 & 0.0000 & 0.0000 & 0.0010 & 0.0000 & & 0.0076 & & 0.0015 \\
\hline CVS2-109 & 0.0207 & 0.2000 & 0.0074 & 0.0643 & & 0.0000 & 0.0335 & 0.0075 & 0.1006 & 0.0055 & 0.0009 & 0.4786 & 0.0344 & 0.0000 & 0.0000 & 0.0009 & 0.0000 & & 0.0071 & & 0.0014 \\
\hline CVS2-110 & 0.0247 & 0.1127 & 0.0089 & 0.0770 & & 0.0000 & 0.0401 & 0.0090 & 0.0500 & 0.0065 & 0.0011 & 0.5730 & 0.0412 & 0.0000 & 0.0000 & 0.0011 & 0.0000 & & 0.0085 & & 0.0017 \\
\hline CVS2-111 & 0.0221 & 0.1009 & 0.0080 & 0.0689 & & 0.0000 & 0.0359 & 0.0081 & 0.1500 & 0.0058 & 0.0010 & 0.5127 & 0.0369 & 0.0000 & 0.0000 & 0.0010 & 0.0000 & & 0.0076 & & 0.0015 \\
\hline CVS2-112 & 0.0208 & 0.0949 & 0.0075 & 0.0648 & & 0.0000 & 0.0338 & 0.0076 & 0.2000 & 0.0055 & 0.0010 & 0.4825 & 0.0347 & 0.0000 & 0.0000 & 0.0010 & 0.0000 & & 0.0072 & & 0.0014 \\
\hline CVS2-113 & 0.0238 & 0.1083 & 0.0085 & 0.0740 & & 0.0000 & 0.0100 & 0.0086 & 0.1157 & 0.0063 & 0.0011 & 0.5506 & 0.0396 & 0.0000 & 0.0000 & 0.0011 & 0.0000 & & 0.0082 & & 0.0016 \\
\hline CVS2-114 & 0.0226 & 0.1028 & 0.0081 & 0.0702 & & 0.0000 & 0.0600 & 0.0082 & 0.1099 & 0.0060 & 0.0010 & 0.5228 & 0.0376 & 0.0000 & 0.0000 & 0.0010 & 0.0000 & & 0.0078 & & 0.0016 \\
\hline CVS2-115 & 0.0223 & 0.1017 & 0.0080 & 0.0695 & & 0.0000 & 0.0700 & 0.0081 & 0.1087 & 0.0059 & 0.0010 & 0.5172 & 0.0372 & 0.0000 & 0.0000 & 0.0010 & 0.0000 & & 0.0077 & & 0.0015 \\
\hline CVS2-116 & 0.0228 & 0.1041 & 0.0200 & 0.0711 & & 0.0000 & 0.0371 & 0.0083 & 0.1112 & 0.0060 & 0.0010 & 0.5290 & 0.0381 & 0.0000 & 0.0000 & 0.0010 & 0.0000 & & 0.0079 & & 0.0016 \\
\hline CVS2-117 & 0.0233 & 0.1062 & 0.0084 & 0.0725 & & 0.0000 & 0.0378 & 0.0000 & 0.1135 & 0.0061 & 0.0011 & 0.5398 & 0.0388 & 0.0000 & 0.0000 & 0.0011 & 0.0000 & & 0.0080 & & 0.0016 \\
\hline CVS2-118 & 0.0228 & 0.1041 & 0.0082 & 0.0711 & & 0.0000 & 0.0371 & 0.0200 & 0.1112 & 0.0060 & 0.0010 & 0.5290 & 0.0381 & 0.0000 & 0.0000 & 0.0010 & 0.0000 & & 0.0079 & & 0.0016 \\
\hline CVS2-119 & 0.0000 & 0.1078 & 0.0085 & 0.0736 & & 0.0000 & 0.0384 & 0.0086 & 0.1152 & 0.0062 & 0.0011 & 0.5480 & 0.0394 & 0.0000 & 0.0000 & 0.0011 & 0.0000 & & 0.0081 & & 0.0016 \\
\hline CVS2-120 & 0.0500 & 0.1024 & 0.0081 & 0.0699 & & 0.0000 & 0.0365 & 0.0082 & 0.1094 & 0.0059 & 0.0010 & 0.5206 & 0.0374 & 0.0000 & 0.0000 & 0.0010 & 0.0000 & & 0.0077 & & 0.0015 \\
\hline CVS2-121 & 0.1000 & 0.0970 & 0.0077 & 0.0662 & & 0.0000 & 0.0346 & 0.0077 & 0.1036 & 0.0056 & 0.0010 & 0.4932 & 0.0355 & 0.0000 & 0.0000 & 0.0010 & 0.0000 & & 0.0073 & & 0.0015 \\
\hline CVS2-122 & 0.1500 & 0.0916 & 0.0072 & 0.0626 & & 0.0000 & 0.0326 & 0.0073 & 0.0979 & 0.0053 & 0.0009 & 0.4658 & 0.0335 & 0.0000 & 0.0000 & 0.0009 & 0.0000 & & 0.0069 & & 0.0014 \\
\hline CVS2-123 & 0.0235 & 0.1048 & 0.0082 & 0.0733 & & 0.0000 & 0.0373 & 0.0084 & 0.1129 & 0.0060 & 0.0010 & 0.5328 & 0.0392 & 0.0000 & 0.0000 & 0.0010 & 0.0000 & & 0.0065 & & 0.0002 \\
\hline \multicolumn{22}{|c|}{ nna et al. 1996) } \\
\hline CVS3-1 & 0.0487 & 0.0005 & 0.0126 & 0.0680 & & 0.0013 & 0.0286 & 0.0005 & 0.1234 & 0.0140 & 0.0079 & 0.5333 & 0.0438 & 0.0000 & 0.0000 & 0.0000 & 0.0137 & & 0.0006 & & 0.0169 \\
\hline CVS3-2 & 0.0417 & 0.0004 & 0.0108 & 0.0583 & & 0.0011 & 0.0245 & 0.0004 & 0.1057 & 0.0120 & 0.0068 & 0.6000 & 0.0375 & 0.0000 & 0.0000 & 0.0000 & 0.0118 & & 0.0005 & & 0.0145 \\
\hline CVS3-3 & 0.0574 & 0.0005 & 0.0148 & 0.0801 & & 0.0015 & 0.0337 & 0.0006 & 0.1454 & 0.0165 & 0.0094 & 0.4500 & 0.0516 & 0.0000 & 0.0000 & 0.0000 & 0.0162 & & 0.0007 & & 0.0199 \\
\hline CVS3-4 & 0.0626 & 0.0006 & 0.0162 & 0.0874 & & 0.0017 & 0.0367 & 0.0006 & 0.1586 & 0.0180 & 0.0102 & 0.4000 & 0.0563 & 0.0000 & 0.0000 & 0.0000 & 0.0176 & & 0.0007 & & 0.0217 \\
\hline CVS3-5 & 0.0657 & 0.0006 & 0.0170 & 0.0918 & & 0.0018 & 0.0386 & 0.0006 & 0.1665 & 0.0189 & 0.0107 & 0.3700 & 0.0591 & 0.0000 & 0.0000 & 0.0000 & 0.0185 & & 0.0008 & & 0.0228 \\
\hline CVS3-6 & 0.0446 & 0.0837 & 0.0116 & 0.0623 & & 0.0012 & 0.0262 & 0.0004 & 0.1131 & 0.0129 & 0.0073 & 0.4889 & 0.0401 & 0.0000 & 0.0000 & 0.0000 & 0.0126 & & 0.0005 & & 0.0155 \\
\hline CVS3-7 & 0.0468 & 0.0400 & 0.0121 & 0.0653 & & 0.0013 & 0.0274 & 0.0005 & 0.1185 & 0.0135 & 0.0076 & 0.5122 & 0.0420 & 0.0000 & 0.0000 & 0.0000 & 0.0132 & & 0.0005 & & 0.0162 \\
\hline CVS3-8 & 0.0430 & 0.0004 & 0.0111 & 0.0600 & & 0.0012 & 0.0252 & 0.0004 & 0.2258 & 0.0124 & 0.0070 & 0.4710 & 0.0387 & 0.0000 & 0.0000 & 0.0000 & 0.0121 & & 0.0005 & & 0.0149 \\
\hline CVS3-9 & 0.0527 & 0.0005 & 0.0137 & 0.0737 & & 0.0014 & 0.0309 & 0.0005 & 0.0500 & 0.0152 & 0.0086 & 0.5779 & 0.0474 & 0.0000 & 0.0000 & 0.0000 & 0.0149 & & 0.0006 & & 0.0183 \\
\hline CVS3-10 & 0.0465 & 0.0004 & 0.0120 & 0.0650 & & 0.0013 & 0.0716 & 0.0005 & 0.1179 & 0.0134 & 0.0076 & 0.5097 & 0.0418 & 0.0000 & 0.0000 & 0.0000 & 0.0131 & & 0.0005 & & 0.0161 \\
\hline CVS3-11 & 0.0476 & 0.0005 & 0.0123 & 0.0665 & & 0.0013 & 0.0500 & 0.0005 & 0.1206 & 0.0137 & 0.0078 & 0.5215 & 0.0428 & 0.0000 & 0.0000 & 0.0000 & 0.0134 & & 0.0005 & & 0.0165 \\
\hline CVS3-12 & 0.0501 & 0.0005 & 0.0130 & 0.0700 & & 0.0014 & 0.0000 & 0.0005 & 0.1270 & 0.0144 & 0.0082 & 0.5490 & 0.0451 & 0.0000 & 0.0000 & 0.0000 & 0.0141 & & 0.0006 & & 0.0174 \\
\hline CVS3-13 & 0.0444 & 0.0004 & 0.0115 & 0.1500 & & 0.0012 & 0.0260 & 0.0004 & 0.1125 & 0.0128 & 0.0072 & 0.4864 & 0.0399 & 0.0000 & 0.0000 & 0.0000 & 0.0125 & & 0.0005 & & 0.0154 \\
\hline CVS3-14 & 0.0465 & 0.0004 & 0.0120 & 0.1100 & & 0.0013 & 0.0273 & 0.0005 & 0.1178 & 0.0134 & 0.0076 & 0.5093 & 0.0418 & 0.0000 & 0.0000 & 0.0000 & 0.0131 & & 0.0005 & & 0.0161 \\
\hline CVS3-15 & 0.0522 & 0.0005 & 0.0135 & 0.0000 & & 0.0014 & 0.0306 & 0.0005 & 0.1324 & 0.0151 & 0.0085 & 0.5722 & 0.0470 & 0.0000 & 0.0000 & 0.0000 & 0.0147 & & 0.0006 & & 0.0181 \\
\hline CVS3-16 & 0.2500 & 0.0004 & 0.0099 & 0.0536 & & 0.0010 & 0.0225 & 0.0004 & 0.0972 & 0.0111 & 0.0063 & 0.4204 & 0.0345 & 0.0000 & 0.0000 & 0.0000 & 0.0108 & & 0.0004 & & 0.0133 \\
\hline CVS3-17 & 0.1800 & 0.0004 & 0.0109 & 0.0586 & & 0.0011 & 0.0246 & 0.0004 & 0.1063 & 0.0121 & 0.0068 & 0.4597 & 0.0377 & 0.0000 & 0.0000 & 0.0000 & 0.0118 & & 0.0005 & & 0.0146 \\
\hline CVS3-18 & 0.1300 & 0.0004 & 0.0115 & 0.0622 & & 0.0012 & 0.0261 & 0.0004 & 0.1128 & 0.0128 & 0.0073 & 0.4877 & 0.0400 & 0.0000 & 0.0000 & 0.0000 & 0.0126 & & 0.0005 & & 0.0154 \\
\hline CVS3-19 & 0.0000 & 0.0005 & 0.0132 & 0.0715 & & 0.0014 & 0.0300 & 0.0005 & 0.1297 & 0.0148 & 0.0084 & 0.5606 & 0.0460 & 0.0000 & 0.0000 & 0.0000 & 0.0144 & & 0.0006 & & 0.0178 \\
\hline CVS3-20 & 0.0428 & 0.0004 & 0.0111 & 0.0597 & & 0.0012 & 0.0251 & 0.0004 & 0.1084 & 0.0123 & 0.0070 & 0.4685 & 0.1600 & 0.0000 & 0.0000 & 0.0000 & 0.0121 & & 0.0005 & & 0.0148 \\
\hline
\end{tabular}


Hanford CVS 2 (Hrma et al. 1994)

\begin{tabular}{|c|c|c|c|c|c|c|c|c|c|c|c|c|c|c|c|c|c|c|c|c|c|}
\hline Glass ID & $\begin{array}{c}\mathrm{Cl} \\
-\mathrm{t}\end{array}$ & $\begin{array}{c}\mathrm{CoO} \\
-\mathrm{t}\end{array}$ & $\begin{array}{c}\mathrm{Co} 2 \mathrm{O} 3 \\
-\mathrm{t}\end{array}$ & $\begin{array}{c}\mathrm{Cr} 2 \mathrm{O} 3 \\
-\mathrm{t}\end{array}$ & $\begin{array}{c}\mathrm{Cs} 2 \mathrm{O} \\
-\mathrm{t}\end{array}$ & $\begin{array}{c}\mathrm{CuO} \\
-\mathrm{t}\end{array}$ & $\begin{array}{c}\mathrm{Eu} 2 \mathrm{O} 3 \\
-\mathrm{t}\end{array}$ & $\begin{array}{l}F \\
-t\end{array}$ & $\begin{array}{c}\mathrm{Ga} 2 \mathrm{O} 3 \\
-\mathrm{t}\end{array}$ & $\begin{array}{c}\mathrm{Gd} 2 \mathrm{O} 3 \\
-\mathrm{t}\end{array}$ & $\begin{array}{c}\mathrm{HgO} \\
-\mathrm{t}\end{array}$ & $\begin{array}{l}I \\
-t\end{array}$ & $\begin{array}{c}\mathrm{La} 2 \mathrm{O} 3 \\
-\mathrm{t}\end{array}$ & $\begin{array}{c}\mathrm{MnO} 2 \\
-\mathrm{t}\end{array}$ & $\begin{array}{c}\mathrm{MnO} \\
-\mathrm{t}\end{array}$ & $\begin{array}{c}\mathrm{MoO} \\
-\mathrm{t}\end{array}$ & $\begin{array}{c}\mathrm{MoO} 3 \\
-\mathrm{t}\end{array}$ & $\begin{array}{c}\mathrm{Nb} 2 \mathrm{O} 5 \\
-\mathrm{t}\end{array}$ & $\begin{array}{c}\mathrm{Nd} 2 \mathrm{O} 3 \\
-\mathrm{t}\end{array}$ & $\begin{array}{c}\mathrm{PbO} \\
-\mathrm{t}\end{array}$ & $\begin{array}{c}\mathrm{PdO} 2 \\
-\mathrm{t}\end{array}$ \\
\hline CVS2-103 & & & 0.0000 & 0.0017 & 0.0020 & 0.0020 & & 0.0040 & & & & & 0.0084 & 0.0020 & & & 0.0040 & & 0.0166 & 0.0000 & \\
\hline CVS2-104 & & & 0.0000 & 0.0016 & 0.0019 & 0.0019 & & 0.0038 & & & & & 0.0078 & 0.0019 & & & 0.0038 & & 0.0155 & 0.0000 & \\
\hline CVS2-105 & & & 0.0000 & 0.0015 & 0.0017 & 0.0017 & & 0.0035 & & & & & 0.0073 & 0.0017 & & & 0.0035 & & 0.0144 & 0.0000 & \\
\hline CVS2-106 & & & 0.0000 & 0.0012 & 0.0015 & 0.0015 & & 0.0029 & & & & & 0.0061 & 0.0015 & & & 0.0029 & & 0.0121 & 0.0000 & \\
\hline CVS2-107 & & & 0.0000 & 0.0014 & 0.0017 & 0.0017 & & 0.0034 & & & & & 0.0070 & 0.0017 & & & 0.0034 & & 0.0139 & 0.0000 & \\
\hline CVS2-108 & & & 0.0000 & 0.0013 & 0.0015 & 0.0015 & & 0.0030 & & & & & 0.0063 & 0.0015 & & & 0.0030 & & 0.0124 & 0.0000 & \\
\hline CVS2-109 & & & 0.0000 & 0.0012 & 0.0014 & 0.0014 & & 0.0028 & & & & & 0.0059 & 0.0014 & & & 0.0028 & & 0.0117 & 0.0000 & \\
\hline CVS2-110 & & & 0.0000 & 0.0014 & 0.0017 & 0.0017 & & 0.0034 & & & & & 0.0071 & 0.0017 & & & 0.0034 & & 0.0140 & 0.0000 & \\
\hline CVS2-111 & & & 0.0000 & 0.0013 & 0.0015 & 0.0015 & & 0.0030 & & & & & 0.0063 & 0.0015 & & & 0.0030 & & 0.0125 & 0.0000 & \\
\hline CVS2-112 & & & 0.0000 & 0.0012 & 0.0014 & 0.0014 & & 0.0029 & & & & & 0.0060 & 0.0014 & & & 0.0029 & & 0.0118 & 0.0000 & \\
\hline CVS2-113 & & & 0.0000 & 0.0014 & 0.0016 & 0.0016 & & 0.0033 & & & & & 0.0068 & 0.0016 & & & 0.0033 & & 0.0135 & 0.0000 & \\
\hline CVS2-114 & & & 0.0000 & 0.0013 & 0.0016 & 0.0016 & & 0.0031 & & & & & 0.0065 & 0.0016 & & & 0.0031 & & 0.0128 & 0.0000 & \\
\hline CVS2-115 & & & 0.0000 & 0.0013 & 0.0015 & 0.0015 & & 0.0031 & & & & & 0.0064 & 0.0015 & & & 0.0031 & & 0.0126 & 0.0000 & \\
\hline CVS2-116 & & & 0.0000 & 0.0013 & 0.0016 & 0.0016 & & 0.0031 & & & & & 0.0065 & 0.0016 & & & 0.0031 & & 0.0129 & 0.0000 & \\
\hline CVS2-117 & & & 0.0000 & 0.0013 & 0.0016 & 0.0016 & & 0.0032 & & & & & 0.0067 & 0.0016 & & & 0.0032 & & 0.0132 & 0.0000 & \\
\hline CVS2-118 & & & 0.0000 & 0.0013 & 0.0016 & 0.0016 & & 0.0031 & & & & & 0.0065 & 0.0016 & & & 0.0031 & & 0.0129 & 0.0000 & \\
\hline CVS2-119 & & & 0.0000 & 0.0014 & 0.0016 & 0.0016 & & 0.0033 & & & & & 0.0068 & 0.0016 & & & 0.0033 & & 0.0134 & 0.0000 & \\
\hline CVS2-120 & & & 0.0000 & 0.0013 & 0.0015 & 0.0015 & & 0.0031 & & & & & 0.0064 & 0.0015 & & & 0.0031 & & 0.0127 & 0.0000 & \\
\hline CVS2-121 & & & 0.0000 & 0.0012 & 0.0015 & 0.0015 & & 0.0029 & & & & & 0.0061 & 0.0015 & & & 0.0029 & & 0.0120 & 0.0000 & \\
\hline CVS2-122 & & & 0.0000 & 0.0012 & 0.0014 & 0.0014 & & 0.0028 & & & & & 0.0058 & 0.0014 & & & 0.0028 & & 0.0114 & 0.0000 & \\
\hline CVS2-123 & & & 0.0000 & 0.0013 & 0.0015 & 0.0016 & & 0.0019 & & & & & 0.0155 & 0.0016 & & & 0.0031 & & 0.0057 & 0.0000 & \\
\hline \multicolumn{22}{|l|}{ Hanford CVS 3 ( } \\
\hline CVS3-1 & & & 0.0000 & 0.0053 & 0.0000 & 0.0000 & & 0.0034 & & & & & 0.0026 & 0.0112 & & & 0.0005 & & 0.0602 & 0.0000 & \\
\hline CVS3-2 & & & 0.0000 & 0.0045 & 0.0000 & 0.0000 & & 0.0029 & & & & & 0.0023 & 0.0096 & & & 0.0004 & & 0.0516 & 0.0000 & \\
\hline CVS3-3 & & & 0.0000 & 0.0062 & 0.0000 & 0.0000 & & 0.0040 & & & & & 0.0031 & 0.0132 & & & 0.0005 & & 0.0710 & 0.0000 & \\
\hline CVS3-4 & & & 0.0000 & 0.0068 & 0.0000 & 0.0000 & & 0.0044 & & & & & 0.0034 & 0.0144 & & & 0.0006 & & 0.0774 & 0.0000 & \\
\hline CVS3-5 & & & 0.0000 & 0.0071 & 0.0000 & 0.0000 & & 0.0046 & & & & & 0.0036 & 0.0152 & & & 0.0006 & & 0.0813 & 0.0000 & \\
\hline CVS3-6 & & & 0.0000 & 0.0049 & 0.0000 & 0.0000 & & 0.0031 & & & & & 0.0024 & 0.0103 & & & 0.0004 & & 0.0552 & 0.0000 & \\
\hline CVS3-7 & & & 0.0000 & 0.0051 & 0.0000 & 0.0000 & & 0.0033 & & & & & 0.0025 & 0.0108 & & & 0.0004 & & 0.0578 & 0.0000 & \\
\hline CVS3-8 & & & 0.0000 & 0.0047 & 0.0000 & 0.0000 & & 0.0030 & & & & & 0.0023 & 0.0099 & & & 0.0004 & & 0.0532 & 0.0000 & \\
\hline CVS3-9 & & & 0.0000 & 0.0057 & 0.0000 & 0.0000 & & 0.0037 & & & & & 0.0029 & 0.0122 & & & 0.0005 & & 0.0652 & 0.0000 & \\
\hline CVS3-10 & & & 0.0000 & 0.0051 & 0.0000 & 0.0000 & & 0.0032 & & & & & 0.0025 & 0.0107 & & & 0.0004 & & 0.0575 & 0.0000 & \\
\hline CVS3-11 & & & 0.0000 & 0.0052 & 0.0000 & 0.0000 & & 0.0033 & & & & & 0.0026 & 0.0110 & & & 0.0004 & & 0.0589 & 0.0000 & \\
\hline CVS3-12 & & & 0.0000 & 0.0054 & 0.0000 & 0.0000 & & 0.0035 & & & & & 0.0027 & 0.0116 & & & 0.0005 & & 0.0620 & 0.0000 & \\
\hline CVS3-13 & & & 0.0000 & 0.0048 & 0.0000 & 0.0000 & & 0.0031 & & & & & 0.0024 & 0.0102 & & & 0.0004 & & 0.0549 & 0.0000 & \\
\hline CVS3-14 & & & 0.0000 & 0.0051 & 0.0000 & 0.0000 & & 0.0032 & & & & & 0.0025 & 0.0107 & & & 0.0004 & & 0.0575 & 0.0000 & \\
\hline CVS3-15 & & & 0.0000 & 0.0057 & 0.0000 & 0.0000 & & 0.0036 & & & & & 0.0028 & 0.0120 & & & 0.0005 & & 0.0646 & 0.0000 & \\
\hline CVS3-16 & & & 0.0000 & 0.0042 & 0.0000 & 0.0000 & & 0.0027 & & & & & 0.0021 & 0.0089 & & & 0.0004 & & 0.0475 & 0.0000 & \\
\hline CVS3-17 & & & 0.0000 & 0.0046 & 0.0000 & 0.0000 & & 0.0029 & & & & & 0.0023 & 0.0097 & & & 0.0004 & & 0.0519 & 0.0000 & \\
\hline CVS3-18 & & & 0.0000 & 0.0048 & 0.0000 & 0.0000 & & 0.0031 & & & & & 0.0024 & 0.0103 & & & 0.0004 & & 0.0551 & 0.0000 & \\
\hline CVS3-19 & & & 0.0000 & 0.0056 & 0.0000 & 0.0000 & & 0.0036 & & & & & 0.0028 & 0.0118 & & & 0.0005 & & 0.0633 & 0.0000 & \\
\hline CVS3-20 & & & 0.0000 & 0.0047 & 0.0000 & 0.0000 & & 0.0030 & & & & & 0.0023 & 0.0099 & & & 0.0004 & & 0.0529 & 0.0000 & \\
\hline
\end{tabular}


Hanford CVS 2 (Hrma et al. 1994)

\begin{tabular}{|c|c|c|c|c|c|c|c|c|c|c|c|c|c|c|c|c|c|c|c|c|c|}
\hline Glass ID & $\begin{array}{c}\mathrm{PdO} \\
-\mathrm{t}\end{array}$ & $\begin{array}{c}\mathrm{Pr} 2 \mathrm{O} 3 \\
-\mathrm{t}\end{array}$ & Pr6O11 & $\begin{array}{c}\mathrm{Rb} 2 \mathrm{O} \\
-\mathrm{t}\end{array}$ & $\begin{array}{c}\mathrm{ReO} \\
-\mathrm{t}\end{array}$ & $\begin{array}{c}\mathrm{ReO} 2 \\
-\mathrm{t}\end{array}$ & $\begin{array}{c}\mathrm{Rh} 2 \mathrm{O} 3 \\
-\mathrm{t}\end{array}$ & $\begin{array}{c}\mathrm{RhO}^{2} \\
-\mathrm{t}\end{array}$ & $\begin{array}{c}\mathrm{RuO} 2 \\
-\mathrm{t}\end{array}$ & $\begin{array}{c}\mathrm{Sb} 2 \mathrm{O} 3 \\
-\mathrm{t}\end{array}$ & $\begin{array}{c}\mathrm{Sb} 2 \mathrm{O} 5 \\
-\mathrm{t}\end{array}$ & $\begin{array}{c}\mathrm{SeO} 2 \\
-\mathrm{t}\end{array}$ & $\begin{array}{c}\mathrm{Sm} 2 \mathrm{O} 3 \\
-\mathrm{t}\end{array}$ & $\begin{array}{c}\mathrm{SnO} \\
-\mathrm{t}\end{array}$ & $\begin{array}{c}\mathrm{SnO} 2 \\
-\mathrm{t}\end{array}$ & $\begin{array}{c}\mathrm{SO} 3 \\
-\mathrm{t}\end{array}$ & $\begin{array}{c}\mathrm{SrO} \\
-\mathrm{t}\end{array}$ & $\begin{array}{c}\mathrm{Tc} 2 \mathrm{O} 7 \\
-\mathrm{t}\end{array}$ & $\begin{array}{c}\mathrm{TeO} 2 \\
-\mathrm{t}\end{array}$ & $\begin{array}{c}\mathrm{ThO} 2 \\
-\mathrm{t}\end{array}$ & $\begin{array}{c}\mathrm{TiO} 2 \\
-\mathrm{t}\end{array}$ \\
\hline CVS2-103 & 0.0007 & & 0.0013 & 0.0007 & & & 0.0007 & & 0.0020 & 0.0000 & & 0.0000 & 0.0007 & & & 0.0037 & 0.0013 & & 0.0000 & & 0.0000 \\
\hline CVS2-104 & 0.0006 & & 0.0013 & 0.0006 & & & 0.0006 & & 0.0019 & 0.0000 & & 0.0000 & 0.0006 & & & 0.0035 & 0.0013 & & 0.0000 & & 0.0000 \\
\hline CVS2-105 & 0.0006 & & 0.0012 & 0.0006 & & & 0.0006 & & 0.0017 & 0.0000 & & 0.0000 & 0.0006 & & & 0.0032 & 0.0012 & & 0.0000 & & 0.0000 \\
\hline CVS2-106 & 0.0005 & & 0.0010 & 0.0005 & & & 0.0005 & & 0.0015 & 0.0000 & & 0.0000 & 0.0005 & & & 0.0027 & 0.0010 & & 0.0000 & & 0.0000 \\
\hline CVS2-107 & 0.0006 & & 0.0011 & 0.0006 & & & 0.0006 & & 0.0017 & 0.0000 & & 0.0000 & 0.0006 & & & 0.0031 & 0.0011 & & 0.0000 & & 0.0000 \\
\hline CVS2-108 & 0.0005 & & 0.0010 & 0.0005 & & & 0.0005 & & 0.0015 & 0.0000 & & 0.0000 & 0.0005 & & & 0.0028 & 0.0010 & & 0.0000 & & 0.0000 \\
\hline CVS2-109 & 0.0005 & & 0.0009 & 0.0005 & & & 0.0005 & & 0.0014 & 0.0000 & & 0.0000 & 0.0005 & & & 0.0026 & 0.0009 & & 0.0000 & & 0.0000 \\
\hline CVS2-110 & 0.0006 & & 0.0011 & 0.0006 & & & 0.0006 & & 0.0017 & 0.0000 & & 0.0000 & 0.0006 & & & 0.0031 & 0.0011 & & 0.0000 & & 0.0000 \\
\hline CVS2-111 & 0.0005 & & 0.0010 & 0.0005 & & & 0.0005 & & 0.0015 & 0.0000 & & 0.0000 & 0.0005 & & & 0.0028 & 0.0010 & & 0.0000 & & 0.0000 \\
\hline CVS2-112 & 0.0005 & & 0.0010 & 0.0005 & & & 0.0005 & & 0.0014 & 0.0000 & & 0.0000 & 0.0005 & & & 0.0026 & 0.0010 & & 0.0000 & & 0.0000 \\
\hline CVS2-113 & 0.0005 & & 0.0011 & 0.0005 & & & 0.0005 & & 0.0016 & 0.0000 & & 0.0000 & 0.0005 & & & 0.0030 & 0.0011 & & 0.0000 & & 0.0000 \\
\hline CVS2-114 & 0.0005 & & 0.0010 & 0.0005 & & & 0.0005 & & 0.0016 & 0.0000 & & 0.0000 & 0.0005 & & & 0.0029 & 0.0010 & & 0.0000 & & 0.0000 \\
\hline CVS2-115 & 0.0005 & & 0.0010 & 0.0005 & & & 0.0005 & & 0.0015 & 0.0000 & & 0.0000 & 0.0005 & & & 0.0028 & 0.0010 & & 0.0000 & & 0.0000 \\
\hline CVS2-116 & 0.0005 & & 0.0010 & 0.0005 & & & 0.0005 & & 0.0016 & 0.0000 & & 0.0000 & 0.0005 & & & 0.0029 & 0.0010 & & 0.0000 & & 0.0000 \\
\hline CVS2-117 & 0.0005 & & 0.0011 & 0.0005 & & & 0.0005 & & 0.0016 & 0.0000 & & 0.0000 & 0.0005 & & & 0.0029 & 0.0011 & & 0.0000 & & 0.0000 \\
\hline CVS2-118 & 0.0005 & & 0.0010 & 0.0005 & & & 0.0005 & & 0.0016 & 0.0000 & & 0.0000 & 0.0005 & & & 0.0029 & 0.0010 & & 0.0000 & & 0.0000 \\
\hline CVS2-119 & 0.0005 & & 0.0011 & 0.0005 & & & 0.0005 & & 0.0016 & 0.0000 & & 0.0000 & 0.0005 & & & 0.0030 & 0.0011 & & 0.0000 & & 0.0000 \\
\hline CVS2-120 & 0.0005 & & 0.0010 & 0.0005 & & & 0.0005 & & 0.0015 & 0.0000 & & 0.0000 & 0.0005 & & & 0.0028 & 0.0010 & & 0.0000 & & 0.0000 \\
\hline CVS2-121 & 0.0005 & & 0.0010 & 0.0005 & & & 0.0005 & & 0.0015 & 0.0000 & & 0.0000 & 0.0005 & & & 0.0027 & 0.0010 & & 0.0000 & & 0.0000 \\
\hline CVS2-122 & 0.0005 & & 0.0009 & 0.0005 & & & 0.0005 & & 0.0014 & 0.0000 & & 0.0000 & 0.0005 & & & 0.0025 & 0.0009 & & 0.0000 & & 0.0000 \\
\hline CVS2-123 & 0.0000 & & 0.0019 & 0.0000 & & & 0.0000 & & 0.0015 & 0.0000 & & 0.0000 & 0.0000 & & & 0.0044 & 0.0000 & & 0.0000 & & 0.0000 \\
\hline \multicolumn{22}{|c|}{ Hanford CVS 3 (Vienna et al. 1996) } \\
\hline CVS3-1 & 0.0000 & & 0.0000 & 0.0000 & & & 0.0000 & & 0.0000 & 0.0000 & & 0.0000 & 0.0000 & & & 0.0020 & 0.0000 & & 0.0000 & & 0.0000 \\
\hline CVS3-2 & 0.0000 & & 0.0000 & 0.0000 & & & 0.0000 & & 0.0000 & 0.0000 & & 0.0000 & 0.0000 & & & 0.0017 & 0.0000 & & 0.0000 & & 0.0000 \\
\hline CVS3-3 & 0.0000 & & 0.0000 & 0.0000 & & & 0.0000 & & 0.0000 & 0.0000 & & 0.0000 & 0.0000 & & & 0.0024 & 0.0000 & & 0.0000 & & 0.0000 \\
\hline CVS3-4 & 0.0000 & & 0.0000 & 0.0000 & & & 0.0000 & & 0.0000 & 0.0000 & & 0.0000 & 0.0000 & & & 0.0026 & 0.0000 & & 0.0000 & & 0.0000 \\
\hline CVS3-5 & 0.0000 & & 0.0000 & 0.0000 & & & 0.0000 & & 0.0000 & 0.0000 & & 0.0000 & 0.0000 & & & 0.0028 & 0.0000 & & 0.0000 & & 0.0000 \\
\hline CVS3-6 & 0.0000 & & 0.0000 & 0.0000 & & & 0.0000 & & 0.0000 & 0.0000 & & 0.0000 & 0.0000 & & & 0.0019 & 0.0000 & & 0.0000 & & 0.0000 \\
\hline CVS3-7 & 0.0000 & & 0.0000 & 0.0000 & & & 0.0000 & & 0.0000 & 0.0000 & & 0.0000 & 0.0000 & & & 0.0020 & 0.0000 & & 0.0000 & & 0.0000 \\
\hline CVS3-8 & 0.0000 & & 0.0000 & 0.0000 & & & 0.0000 & & 0.0000 & 0.0000 & & 0.0000 & 0.0000 & & & 0.0018 & 0.0000 & & 0.0000 & & 0.0000 \\
\hline CVS3-9 & 0.0000 & & 0.0000 & 0.0000 & & & 0.0000 & & 0.0000 & 0.0000 & & 0.0000 & 0.0000 & & & 0.0022 & 0.0000 & & 0.0000 & & 0.0000 \\
\hline CVS3-10 & 0.0000 & & 0.0000 & 0.0000 & & & 0.0000 & & 0.0000 & 0.0000 & & 0.0000 & 0.0000 & & & 0.0020 & 0.0000 & & 0.0000 & & 0.0000 \\
\hline CVS3-11 & 0.0000 & & 0.0000 & 0.0000 & & & 0.0000 & & 0.0000 & 0.0000 & & 0.0000 & 0.0000 & & & 0.0020 & 0.0000 & & 0.0000 & & 0.0000 \\
\hline CVS3-12 & 0.0000 & & 0.0000 & 0.0000 & & & 0.0000 & & 0.0000 & 0.0000 & & 0.0000 & 0.0000 & & & 0.0021 & 0.0000 & & 0.0000 & & 0.0000 \\
\hline CVS3-13 & 0.0000 & & 0.0000 & 0.0000 & & & 0.0000 & & 0.0000 & 0.0000 & & 0.0000 & 0.0000 & & & 0.0019 & 0.0000 & & 0.0000 & & 0.0000 \\
\hline CVS3-14 & 0.0000 & & 0.0000 & 0.0000 & & & 0.0000 & & 0.0000 & 0.0000 & & 0.0000 & 0.0000 & & & 0.0019 & 0.0000 & & 0.0000 & & 0.0000 \\
\hline CVS3-15 & 0.0000 & & 0.0000 & 0.0000 & & & 0.0000 & & 0.0000 & 0.0000 & & 0.0000 & 0.0000 & & & 0.0022 & 0.0000 & & 0.0000 & & 0.0000 \\
\hline CVS3-16 & 0.0000 & & 0.0000 & 0.0000 & & & 0.0000 & & 0.0000 & 0.0000 & & 0.0000 & 0.0000 & & & 0.0016 & 0.0000 & & 0.0000 & & 0.0000 \\
\hline CVS3-17 & 0.0000 & & 0.0000 & 0.0000 & & & 0.0000 & & 0.0000 & 0.0000 & & 0.0000 & 0.0000 & & & 0.0018 & 0.0000 & & 0.0000 & & 0.0000 \\
\hline CVS3-18 & 0.0000 & & 0.0000 & 0.0000 & & & 0.0000 & & 0.0000 & 0.0000 & & 0.0000 & 0.0000 & & & 0.0019 & 0.0000 & & 0.0000 & & 0.0000 \\
\hline CVS3-19 & 0.0000 & & 0.0000 & 0.0000 & & & 0.0000 & & 0.0000 & 0.0000 & & 0.0000 & 0.0000 & & & 0.0021 & 0.0000 & & 0.0000 & & 0.0000 \\
\hline CVS3-20 & 0.0000 & & 0.0000 & 0.0000 & & & 0.0000 & & 0.0000 & 0.0000 & & 0.0000 & 0.0000 & & & 0.0018 & 0.0000 & & 0.0000 & & 0.0000 \\
\hline
\end{tabular}




\section{Hanford CVS 2 (Hrma et al. 1994)}

\begin{tabular}{|c|c|c|c|c|c|c|c|c|c|c|c|c|c|c|c|c|c|c|c|c|c|}
\hline Glass ID & $\begin{array}{c}\mathrm{T} 12 \mathrm{O} 3 \\
-\mathrm{t}\end{array}$ & $\begin{array}{c}\mathrm{U} 3 \mathrm{O} 8 \\
-\mathrm{t}\end{array}$ & $\begin{array}{c}\mathrm{UO} 2 \\
-\mathrm{t}\end{array}$ & $\begin{array}{c}\mathrm{UO} 3 \\
-\mathrm{t}\end{array}$ & $\begin{array}{c}\mathrm{V} 2 \mathrm{O} 5 \\
-\mathrm{t}\end{array}$ & $\begin{array}{c}\text { WO3 } \\
-t\end{array}$ & $\begin{array}{c}\mathrm{Y} 2 \mathrm{O} 3 \\
-\mathrm{t}\end{array}$ & $\begin{array}{c}\mathrm{ZnO} \\
-\mathrm{t}\end{array}$ & $\begin{array}{c}\text { Others } \\
-t\end{array}$ & $\begin{array}{c}\text { Sum } \\
-t\end{array}$ & $\begin{array}{c}\mathrm{A} 12 \mathrm{O} 3 \\
-\mathrm{a}\end{array}$ & $\begin{array}{c}\mathrm{B} 2 \mathrm{O} 3 \\
-\mathrm{a}\end{array}$ & $\begin{array}{c}\mathrm{CaO} \\
-\mathrm{a} \\
\end{array}$ & $\begin{array}{c}\mathrm{Fe} 2 \mathrm{O} 3 \\
-\mathrm{a}\end{array}$ & $\begin{array}{c}\mathrm{FeO} \\
-\mathrm{a}\end{array}$ & $\begin{array}{c}\mathrm{K} 2 \mathrm{O} \\
-\mathrm{a}\end{array}$ & $\begin{array}{c}\mathrm{Li} 2 \mathrm{O} \\
-\mathrm{a}\end{array}$ & $\begin{array}{c}\mathrm{MgO} \\
-\mathrm{a}\end{array}$ & $\begin{array}{c}\mathrm{Na} 2 \mathrm{O} \\
-\mathrm{a}\end{array}$ & $\begin{array}{c}\mathrm{NiO} \\
-\mathrm{a}\end{array}$ & $\begin{array}{c}\mathrm{P} 2 \mathrm{O} 5 \\
-\mathrm{a}\end{array}$ \\
\hline CVS2-103 & & & 0.0000 & & & 0.0000 & 0.0007 & 0.0000 & & 1.0000 & & & & & & & & & & & \\
\hline CVS2-104 & & & 0.0000 & & & 0.0000 & 0.0006 & 0.0000 & & 1.0000 & & & & & & & & & & & \\
\hline CVS2-105 & & & 0.0000 & & & 0.0000 & 0.0006 & 0.0000 & & 1.0000 & & & & & & & & & & & \\
\hline CVS2-106 & & & 0.0000 & & & 0.0000 & 0.0005 & 0.0000 & & 1.0000 & & & & & & & & & & & \\
\hline CVS2-107 & & & 0.0000 & & & 0.0000 & 0.0006 & 0.0000 & & 1.0000 & & & & & & & & & & & \\
\hline CVS2-108 & & & 0.0000 & & & 0.0000 & 0.0005 & 0.0000 & & 1.0000 & & & & & & & & & & & \\
\hline CVS2-109 & & & 0.0000 & & & 0.0000 & 0.0005 & 0.0000 & & 1.0000 & & & & & & & & & & & \\
\hline CVS2-110 & & & 0.0000 & & & 0.0000 & 0.0006 & 0.0000 & & 1.0000 & & & & & & & & & & & \\
\hline CVS2-111 & & & 0.0000 & & & 0.0000 & 0.0005 & 0.0000 & & 1.0000 & & & & & & & & & & & \\
\hline CVS2-112 & & & 0.0000 & & & 0.0000 & 0.0005 & 0.0000 & & 1.0000 & & & & & & & & & & & \\
\hline CVS2-113 & & & 0.0000 & & & 0.0000 & 0.0005 & 0.0000 & & 1.0000 & & & & & & & & & & & \\
\hline CVS2-114 & & & 0.0000 & & & 0.0000 & 0.0005 & 0.0000 & & 1.0000 & & & & & & & & & & & \\
\hline CVS2-115 & & & 0.0000 & & & 0.0000 & 0.0005 & 0.0000 & & 1.0000 & & & & & & & & & & & \\
\hline CVS2-116 & & & 0.0000 & & & 0.0000 & 0.0005 & 0.0000 & & 1.0000 & & & & & & & & & & & \\
\hline CVS2-117 & & & 0.0000 & & & 0.0000 & 0.0005 & 0.0000 & & 1.0000 & & & & & & & & & & & \\
\hline CVS2-118 & & & 0.0000 & & & 0.0000 & 0.0005 & 0.0000 & & 1.0000 & & & & & & & & & & & \\
\hline CVS2-119 & & & 0.0000 & & & 0.0000 & 0.0005 & 0.0000 & & 1.0000 & & & & & & & & & & & \\
\hline CVS2-120 & & & 0.0000 & & & 0.0000 & 0.0005 & 0.0000 & & 1.0000 & & & & & & & & & & & \\
\hline CVS2-121 & & & 0.0000 & & & 0.0000 & 0.0005 & 0.0000 & & 1.0000 & & & & & & & & & & & \\
\hline CVS2-122 & & & 0.0000 & & & 0.0000 & 0.0005 & 0.0000 & & 1.0000 & & & & & & & & & & & \\
\hline CVS2-123 & & & 0.0000 & & & 0.0000 & 0.0000 & 0.0049 & & 1.0000 & & & & & & & & & & & \\
\hline
\end{tabular}

\section{Hanford CVS 3 (Vienna et al. 1996)}

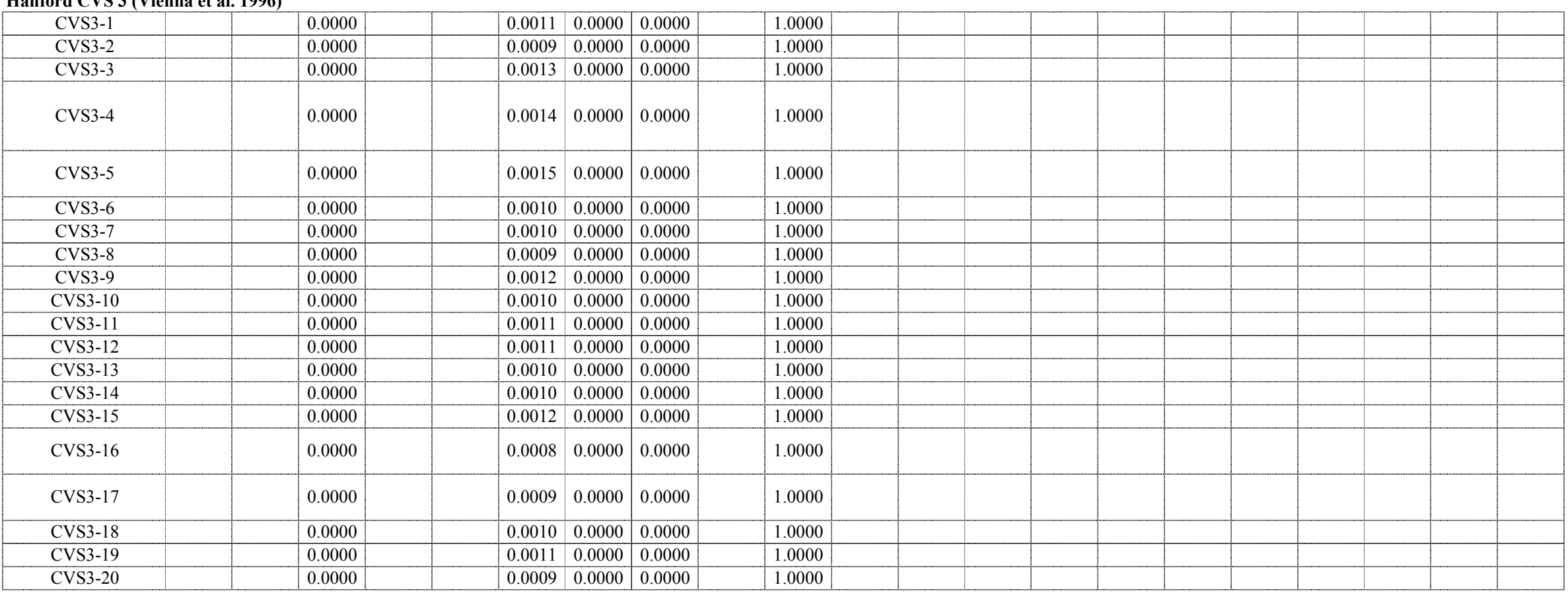


Appendix A. Database - mass fraction

\section{Hanford CVS 2 (Hrma et al. 1994)}

\begin{tabular}{|c|c|c|c|c|c|c|c|c|c|c|c|c|c|c|c|c|c|c|c|c|c|}
\hline Glass ID & $\begin{array}{c}\mathrm{SiO} 2 \\
-\mathrm{a}\end{array}$ & $\begin{array}{c}\mathrm{ZrO} 2 \\
-\mathrm{a}\end{array}$ & $\begin{array}{c}\mathrm{Ag} 2 \mathrm{O} \\
-\mathrm{a}\end{array}$ & $\begin{array}{c}\mathrm{As} 2 \mathrm{O} 3 \\
-\mathrm{a}\end{array}$ & $\begin{array}{c}\mathrm{BaO} \\
-\mathrm{a}\end{array}$ & $\begin{array}{c}\mathrm{Bi} 2 \mathrm{O} 3 \\
-\mathrm{a}\end{array}$ & $\begin{array}{l}\mathrm{Br} \\
-\mathrm{a}\end{array}$ & $\begin{array}{c}\mathrm{CdO} \\
-\mathrm{a}\end{array}$ & $\begin{array}{c}\mathrm{Ce} 2 \mathrm{O} 3 \\
-\mathrm{a}\end{array}$ & $\begin{array}{c}\mathrm{CeO} 2 \\
-\mathrm{a}\end{array}$ & $\begin{array}{l}\mathrm{Cl} \\
-\mathrm{a}\end{array}$ & $\begin{array}{c}\mathrm{CoO} \\
-\mathrm{a}\end{array}$ & $\begin{array}{c}\mathrm{Co} 2 \mathrm{O} 3 \\
-\mathrm{a} \\
\end{array}$ & $\begin{array}{c}\mathrm{Cr} 2 \mathrm{O} 3 \\
-\mathrm{a}\end{array}$ & $\begin{array}{c}\mathrm{Cs} 2 \mathrm{O} \\
-\mathrm{a}\end{array}$ & $\begin{array}{c}\mathrm{CuO} \\
-\mathrm{a}\end{array}$ & $\begin{array}{c}\mathrm{Eu} 2 \mathrm{O} 3 \\
-\mathrm{a}\end{array}$ & $\begin{array}{l}\mathrm{F} \\
-\mathrm{a}\end{array}$ & $\begin{array}{c}\mathrm{Ga} 2 \mathrm{O} 3 \\
-\mathrm{a}\end{array}$ & $\begin{array}{c}\mathrm{Gd} 2 \mathrm{O} 3 \\
-\mathrm{a}\end{array}$ & $\begin{array}{c}\mathrm{HgO} \\
-\mathrm{a}\end{array}$ \\
\hline CVS2-103 & & & & & & & & & & & & & & & & & & & & & \\
\hline CVS2-104 & & & & & & & & & & & & & & & & & & & & & \\
\hline CVS2-105 & & & & & & & & & & & & & & & & & & & & & \\
\hline CVS2-106 & & & & & & & & & & & & & & & & & & & & & \\
\hline CVS2-107 & & & & & & & & & & & & & & & & & & & & & \\
\hline CVS2-108 & & & & & & & & & & & & & & & & & & & & & \\
\hline CVS2-109 & & & & & & & & & & & & & & & & & & & & & \\
\hline CVS2-110 & & & & & & & & & & & & & & & & & & & & & \\
\hline CVS2-111 & & & & & & & & & & & & & & & & & & & & & \\
\hline CVS2-112 & & & & & & & & & & & & & & & & & & & & & \\
\hline CVS2-113 & & & & & & & & & & & & & & & & & & & & & \\
\hline CVS2-114 & & & & & & & & & & & & & & & & & & & & & \\
\hline CVS2-115 & & & & & & & & & & & & & & & & & & & & & \\
\hline CVS2-116 & & & & & & & & & & & & & & & & & & & & & \\
\hline CVS2-117 & & & & & & & & & & & & & & & & & & & & & \\
\hline CVS2-118 & & & & & & & & & & & & & & & & & & & & & \\
\hline CVS2-119 & & & & & & & & & & & & & & & & & & & & & \\
\hline CVS2-120 & & & & & & & & & & & & & & & & & & & & & \\
\hline CVS2-121 & & & & & & & & & & & & & & & & & & & & & \\
\hline CVS2-122 & & & & & & & & & & & & & & & & & & & & & \\
\hline CVS2-123 & & & & & & & & & & & & & & & & & & & & & \\
\hline
\end{tabular}

CVS2-123

\section{Hanford CVS 3 (Vienna et al. 1996)}

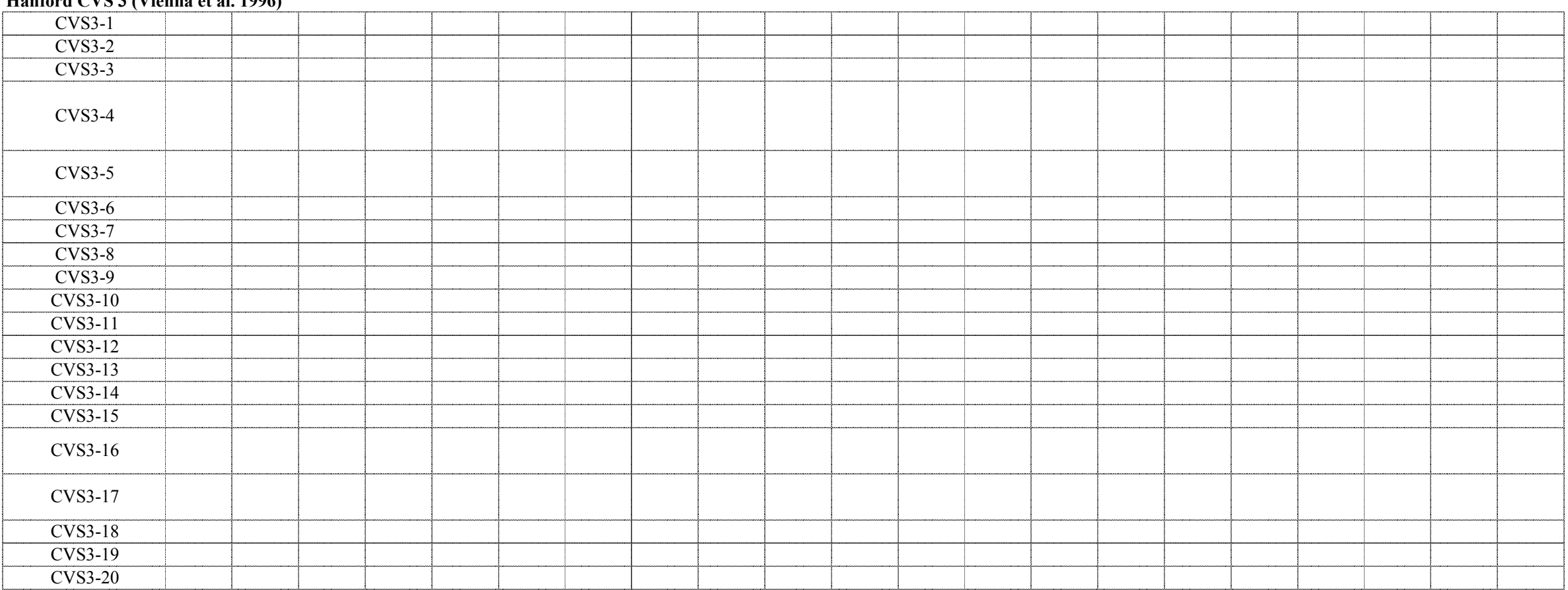


Hanford CVS 2 (Hrma et al. 1994)

\begin{tabular}{|c|c|c|c|c|c|c|c|c|c|c|c|c|c|c|c|c|c|c|c|c|c|}
\hline Glass ID & $\begin{array}{c}\mathrm{I} \\
-\mathrm{a}\end{array}$ & $\begin{array}{c}\mathrm{La} 2 \mathrm{O} 3 \\
-\mathrm{a}\end{array}$ & $\begin{array}{c}\mathrm{MnO} 2 \\
-\mathrm{a}\end{array}$ & $\begin{array}{c}\mathrm{MnO} \\
-\mathrm{a}\end{array}$ & $\begin{array}{c}\mathrm{MoO} \\
-\mathrm{a}\end{array}$ & $\begin{array}{c}\mathrm{MoO} 3 \\
-\mathrm{a}\end{array}$ & $\begin{array}{c}\mathrm{Nb} 2 \mathrm{O} 5 \\
-\mathrm{a}\end{array}$ & $\begin{array}{c}\mathrm{Nd} 2 \mathrm{O} 3 \\
-\mathrm{a}\end{array}$ & $\begin{array}{c}\mathrm{PbO} \\
-\mathrm{a}\end{array}$ & $\begin{array}{c}\mathrm{PdO} 2 \\
-\mathrm{a}\end{array}$ & $\begin{array}{c}\mathrm{PdO} \\
-\mathrm{a}\end{array}$ & $\begin{array}{c}\mathrm{Pr} 2 \mathrm{O} 3 \\
-\mathrm{a}\end{array}$ & $\begin{array}{c}\text { Pr6O11 } \\
-\mathrm{a}\end{array}$ & $\begin{array}{c}\mathrm{Rb} 2 \mathrm{O} \\
-\mathrm{a}\end{array}$ & $\begin{array}{c}\mathrm{ReO} \\
-\mathrm{a}\end{array}$ & $\begin{array}{c}\mathrm{ReO} 2 \\
-\mathrm{a}\end{array}$ & $\begin{array}{c}\mathrm{Rh} 2 \mathrm{O} 3 \\
-\mathrm{a}\end{array}$ & $\begin{array}{c}\mathrm{RhO}_{2} \\
-\mathrm{a}\end{array}$ & $\begin{array}{c}\mathrm{RuO} 2 \\
-\mathrm{a}\end{array}$ & $\begin{array}{c}\mathrm{Sb} 2 \mathrm{O} 3 \\
-\mathrm{a} \\
\end{array}$ & $\begin{array}{c}\mathrm{Sb} 2 \mathrm{O} 5 \\
-\mathrm{a}\end{array}$ \\
\hline \multicolumn{22}{|l|}{ CVS2-103 } \\
\hline \multicolumn{22}{|l|}{ CVS2-104 } \\
\hline \multicolumn{22}{|l|}{ CVS2-105 } \\
\hline \multicolumn{22}{|l|}{ CVS2-106 } \\
\hline \multicolumn{22}{|l|}{ CVS2-107 } \\
\hline \multicolumn{22}{|l|}{ CVS2-108 } \\
\hline \multicolumn{22}{|l|}{ CVS2-109 } \\
\hline \multicolumn{22}{|l|}{ CVS2-110 } \\
\hline \multicolumn{22}{|l|}{ CVS2-111 } \\
\hline \multicolumn{22}{|l|}{ CVS2-112 } \\
\hline \multicolumn{22}{|l|}{ CVS2-113 } \\
\hline \multicolumn{22}{|l|}{ CVS2-114 } \\
\hline \multicolumn{22}{|l|}{ CVS2-115 } \\
\hline \multicolumn{22}{|l|}{ CVS2-116 } \\
\hline \multicolumn{22}{|l|}{ CVS2-117 } \\
\hline \multicolumn{22}{|l|}{ CVS2-118 } \\
\hline \multicolumn{22}{|l|}{ CVS2-119 } \\
\hline \multicolumn{22}{|l|}{ CVS2-120 } \\
\hline \multicolumn{22}{|l|}{ CVS2-121 } \\
\hline \multicolumn{22}{|l|}{ CVS2-122 } \\
\hline CVS2-123 & & & & & & & & & & & & & & & & & & & & & \\
\hline
\end{tabular}

\section{Hanford CVS 3 (Vienna et al. 1996)}

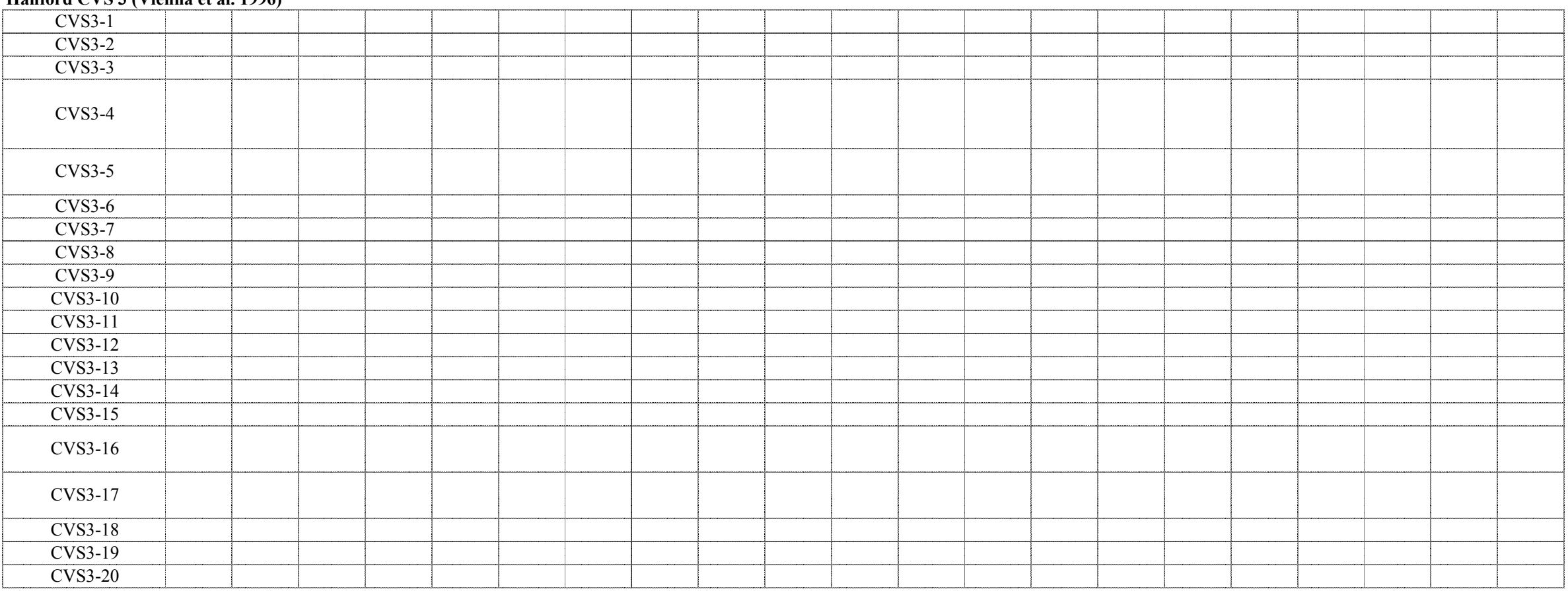


Appendix A. Database - mass fraction

Hanford CVS 2 (Hrma et al. 1994)

\begin{tabular}{|c|c|c|c|c|c|c|c|c|c|c|c|c|c|c|c|c|c|c|c|c|}
\hline Glass ID & $\begin{array}{c}\mathrm{SeO} 2 \\
-\mathrm{a}\end{array}$ & $\begin{array}{c}\mathrm{Sm} 2 \mathrm{O} 3 \\
-\mathrm{a}\end{array}$ & $\begin{array}{c}\mathrm{SnO} \\
-\mathrm{a}\end{array}$ & $\begin{array}{c}\mathrm{SnO} 2 \\
-\mathrm{a}\end{array}$ & $\begin{array}{c}\mathrm{SO} 3 \\
-\mathrm{a}\end{array}$ & $\begin{array}{c}\mathrm{SrO} \\
-\mathrm{a}\end{array}$ & $\begin{array}{c}\mathrm{Tc} 2 \mathrm{O} 7 \\
-\mathrm{a}\end{array}$ & $\begin{array}{c}\mathrm{TeO} 2 \\
-\mathrm{a}\end{array}$ & $\begin{array}{c}\mathrm{ThO} 2 \\
-\mathrm{a}\end{array}$ & $\begin{array}{c}\mathrm{TiO} 2 \\
-\mathrm{a}\end{array}$ & $\begin{array}{c}\mathrm{T} 12 \mathrm{O} 3 \\
-\mathrm{a}\end{array}$ & $\begin{array}{c}\text { U3O8 } \\
-\mathrm{a}\end{array}$ & $\begin{array}{c}\mathrm{UO} 2 \\
-\mathrm{a}\end{array}$ & $\begin{array}{c}\text { UO3 } \\
-\mathrm{a}\end{array}$ & $\begin{array}{c}\mathrm{V} 2 \mathrm{O} 5 \\
-\mathrm{a}\end{array}$ & $\begin{array}{c}\mathrm{WO} 3 \\
-\mathrm{a}\end{array}$ & $\begin{array}{c}\mathrm{Y} 2 \mathrm{O} 3 \\
-\mathrm{a}\end{array}$ & $\begin{array}{c}\mathrm{ZnO} \\
-\mathrm{a}\end{array}$ & $\begin{array}{c}\text { Others } \\
-\mathrm{a}\end{array}$ & $\begin{array}{c}\text { Sum } \\
-\mathrm{a}\end{array}$ \\
\hline CVS2-103 & & & & & & & & & & & & & & & & & & & & \\
\hline CVS2-104 & & & & & & & & & & & & & & & & & & & & \\
\hline CVS2-105 & & & & & & & & & & & & & & & & & & & & \\
\hline CVS2-106 & & & & & & & & & & & & & & & & & & & & \\
\hline CVS2-107 & & & & & & & & & & & & & & & & & & & & \\
\hline CVS2-108 & & & & & & & & & & & & & & & & & & & & \\
\hline CVS2-109 & & & & & & & & & & & & & & & & & & & & \\
\hline CVS2-110 & & & & & & & & & & & & & & & & & & & & \\
\hline CVS2-111 & & & & & & & & & & & & & & & & & & & & \\
\hline CVS2-112 & & & & & & & & & & & & & & & & & & & & \\
\hline CVS2-113 & & & & & & & & & & & & & & & & & & & & \\
\hline CVS2-114 & & & & & & & & & & & & & & & & & & & & \\
\hline CVS2-115 & & & & & & & & & & & & & & & & & & & & \\
\hline CVS2-116 & & & & & & & & & & & & & & & & & & & & \\
\hline CVS2-117 & & & & & & & & & & & & & & & & & & & & \\
\hline CVS2-118 & & & & & & & & & & & & & & & & & & & & \\
\hline CVS2-119 & & & & & & & & & & & & & & & & & & & & \\
\hline CVS2-120 & & & & & & & & & & & & & & & & & & & & \\
\hline CVS2-121 & & & & & & & & & & & & & & & & & & & & \\
\hline CVS2-122 & & & & & & & & & & & & & & & & & & & & \\
\hline CVS2-123 & & & & & & & & & & & & & & & & & & & & \\
\hline
\end{tabular}

\section{Hanford CVS 3 (Vienna et al. 1996)}

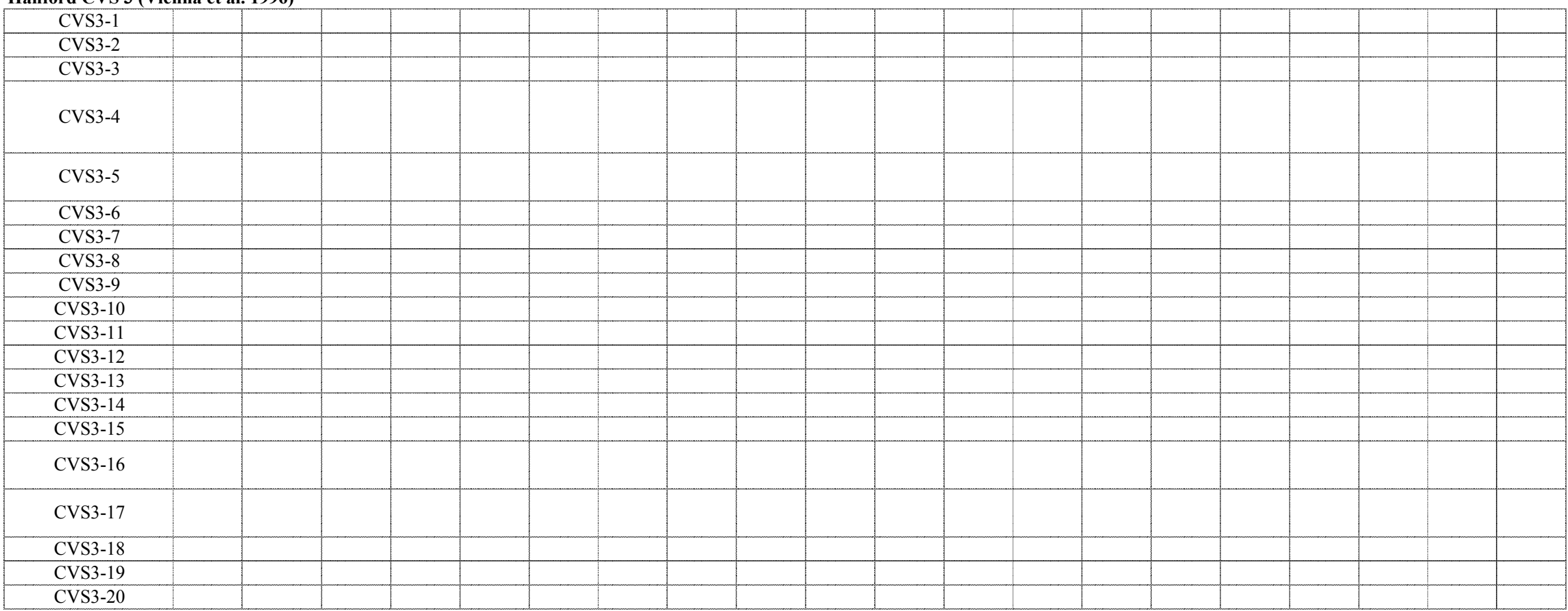


Appendix A. Database - mass fraction

\section{Hanford CVS 2 (Hrma et al. 1994)}

\begin{tabular}{|c|c|c|c|c|c|c|c|c|}
\hline Glass ID & $\begin{array}{l}\mathrm{TM} \\
\left({ }^{\circ} \mathrm{C}\right)\end{array}$ & $\begin{array}{l}\text { Gradient } \\
\text { TL }\left({ }^{\circ} \mathrm{C}\right)\end{array}$ & $\begin{array}{l}\text { Uniform } \\
\text { TL }\left({ }^{\circ} \mathrm{C}\right)\end{array}$ & Primary Phase & $\begin{array}{l}\text { Quenched } \\
\text { Visual/OM }\end{array}$ & $\begin{array}{c}\text { Quenched } \\
\text { SEM/EDS or TEM }\end{array}$ & $\begin{array}{l}\text { Quenched } \\
\text { XRD }\end{array}$ & $\begin{array}{c}\text { Quenched } \\
\text { Homogeneous? }\end{array}$ \\
\hline \multicolumn{9}{|l|}{ CVS2-103 } \\
\hline \multicolumn{9}{|l|}{ CVS2-104 } \\
\hline \multicolumn{9}{|l|}{ CVS2-105 } \\
\hline \multicolumn{9}{|l|}{ CVS2-106 } \\
\hline \multicolumn{9}{|l|}{ CVS2-107 } \\
\hline \multicolumn{9}{|l|}{ CVS2-108 } \\
\hline \multicolumn{9}{|l|}{ CVS2-109 } \\
\hline \multicolumn{9}{|l|}{ CVS2-110 } \\
\hline \multicolumn{9}{|l|}{ CVS2-111 } \\
\hline \multicolumn{9}{|l|}{ CVS2-112 } \\
\hline \multicolumn{9}{|l|}{ CVS2-113 } \\
\hline \multicolumn{9}{|l|}{ CVS2-114 } \\
\hline \multicolumn{9}{|l|}{ CVS2-115 } \\
\hline \multicolumn{9}{|l|}{ CVS2-116 } \\
\hline \multicolumn{9}{|l|}{ CVS2-117 } \\
\hline \multicolumn{9}{|l|}{ CVS2-118 } \\
\hline \multicolumn{9}{|l|}{ CVS2-119 } \\
\hline \multicolumn{9}{|l|}{ CVS2-120 } \\
\hline \multicolumn{9}{|l|}{ CVS2-121 } \\
\hline \multicolumn{9}{|l|}{ CVS2-122 } \\
\hline CVS2-123 & & & & & & & & \\
\hline
\end{tabular}

\section{Hanford CVS 3 (Vienna et al. 1996)}

\begin{tabular}{|c|c|c|c|c|c|}
\hline CVS3-1 & 1153 & spinel & No XL & & \\
\hline CVS3-2 & 1181 & spinel & No XL & & \\
\hline CVS3-3 & 1130 & spinel & $<<.1$ undisolved cluster $<<.1$ spinel & & \\
\hline CVS3-4 & 1186 & $\mathrm{Ce}, \mathrm{Zr}, \mathrm{Oy}$ & $2.6 \%$ spinel, possibly und $\mathrm{Zr}$ & spinel, (Si, Zr, Ce, Nd, [Na?]) & $\begin{array}{l}5 \mathrm{vol} \% \mathrm{XL}, 90 \mathrm{w} \% \mathrm{sp}, \\
10 \mathrm{w} \% \mathrm{ZrCeO}\end{array}$ \\
\hline CVS3-5 & 1232 & $\mathrm{Nd}, \mathrm{Ce}, \mathrm{Zr}, \mathrm{Oy}$ & $3.6 \%$ spinel, possibly und. $\mathrm{Zr}$ & spinel & $\begin{array}{l}3 \mathrm{vol} \% \text { XL, } 75 \text { w } \% \\
\mathrm{NiO}, 25 \mathrm{w} \% \mathrm{sp}\end{array}$ \\
\hline CVS3-6 & 1066 & $?$ & No XL & & \\
\hline CVS3-7 & 1132 & spinel & No XL & & \\
\hline CVS3-8 & 1066 & $\mathrm{Na}, \mathrm{Zr}, \mathrm{Si}$ & No XL & & \\
\hline CVS3-9 & 1374 & spinel & very small particles & & \\
\hline CVS3-10 & 1021 & spinel & No XL & & \\
\hline CVS3-11 & 1075 & spinel & No XL & & \\
\hline CVS3-12 & 1278 & spinel & very small particles & hi Cr spinel & \\
\hline CVS3-13 & 1295 & spinel & $<.1 \%$ spinel & spinel & \\
\hline CVS3-14 & 1227 & spinel & No XL & & \\
\hline CVS3-15 & 1111 & $\mathrm{Cr}$ & No XL & & \\
\hline CVS3-16 & & & $3.8 \% \mathrm{XL}$, inhomo & spinel & $\begin{array}{l}10 \mathrm{vol} \% \text { XL, } 75 \mathrm{w} \% \mathrm{sp}, \\
25 \mathrm{w} \% \mathrm{NdZrO}\end{array}$ \\
\hline CVS3-17 & & & $2.2 \% \mathrm{XL}$, inhomo & & \\
\hline CVS3-18 & 1293 & spinel & very small particles similar to $3-17$ & & \\
\hline CVS3-19 & 1070 & spinel & No XL & & \\
\hline CVS3-20 & & & $3.1 \% \mathrm{XL}$ inhomo, und $\mathrm{Zr}$ and other & $\mathrm{ZrO} 2$ & $10 \% \mathrm{ZrO} 2, \mathrm{YZrO}$ type \\
\hline
\end{tabular}


Appendix A. Database - mass fraction

Hanford CVS 2 (Hrma et al. 1994)

\begin{tabular}{|c|c|c|c|c|c|}
\hline Glass ID & $\begin{array}{c}\mathrm{CCC} \\
\text { Visual/OM }\end{array}$ & $\begin{array}{c}\text { CCC } \\
\text { SEM/EDS or TEM }\end{array}$ & $\begin{array}{l}\text { CCC } \\
\text { XRD }\end{array}$ & $\begin{array}{c}\text { Heat Treated } \\
\text { Visual/OM }\end{array}$ & $\begin{array}{c}\text { Heat Treated } \\
\text { SEM/EDS or TEM }\end{array}$ \\
\hline \multicolumn{6}{|l|}{ CVS2-103 } \\
\hline \multicolumn{6}{|l|}{ CVS2-104 } \\
\hline \multicolumn{6}{|l|}{ CVS2-105 } \\
\hline \multicolumn{6}{|l|}{ CVS2-106 } \\
\hline \multicolumn{6}{|l|}{ CVS2-107 } \\
\hline \multicolumn{6}{|l|}{ CVS2-108 } \\
\hline \multicolumn{6}{|l|}{ CVS2-109 } \\
\hline \multicolumn{6}{|l|}{ CVS2-110 } \\
\hline \multicolumn{6}{|l|}{ CVS2-111 } \\
\hline \multicolumn{6}{|l|}{ CVS2-112 } \\
\hline \multicolumn{6}{|l|}{ CVS2-113 } \\
\hline \multicolumn{6}{|l|}{ CVS2-114 } \\
\hline \multicolumn{6}{|l|}{ CVS2-115 } \\
\hline \multicolumn{6}{|l|}{ CVS2-116 } \\
\hline \multicolumn{6}{|l|}{ CVS2-117 } \\
\hline \multicolumn{6}{|l|}{ CVS2-118 } \\
\hline \multicolumn{6}{|l|}{ CVS2-119 } \\
\hline \multicolumn{6}{|l|}{ CVS2-120 } \\
\hline \multicolumn{6}{|l|}{ CVS2-121 } \\
\hline \multicolumn{6}{|l|}{ CVS2-122 } \\
\hline CVS2-123 & & & & & \\
\hline
\end{tabular}

\section{Hanford CVS 3 (Vienna et al. 1996)}

CVS3-1 $3.7 \%$ XL inhomo

\begin{tabular}{l|l} 
CVS3-3 & $2.2 \%$ XL spinel + ?
\end{tabular}

CVS3-4

$13.2 \%$ XL 2 types

spinel

spinel only

spinel, (Si,Ca,Nd, [Na?]), 35vol\% XL, 45w\%CaNdPO4SiO4,

( $\mathrm{Si}, \mathrm{Zr}, \mathrm{Ce}, \mathrm{Nd}$, [Na?]), $35 \mathrm{w} \%$ spinel, $20 \mathrm{w} \% \mathrm{ZrCeO}$

hiCr sp

CVS3-5 $33 \%$ XL 2 types (same as 3-4)

spinel, (Si,Ca,Nd, [Na?]), 40vol\% XL, 30w\%CaNdPO4SiO4,

( $\mathrm{Si}, \mathrm{Zr}, \mathrm{Ce}, \mathrm{Nd},[\mathrm{Na} ?]) \quad 30 \mathrm{w} \%$ spinel, $40 \mathrm{w} \% \mathrm{ZrCeO}$

\begin{tabular}{l|l} 
CVS3-6 & No XL \\
\hline CVS3-7 & No XL
\end{tabular}

CVS3-7 No XL

\begin{tabular}{l|l} 
CVS3-8 & No XL
\end{tabular}

$\begin{array}{lll}\text { CVS3-9 } & 4.4 \% \text { XL spinel+? }\end{array}$

CVS3-10 <.1\% stars (spinel?)

CVS3-11 No XL

CVS3-12 Very high XL many phases, inhomo

$\begin{array}{ll}\text { CVS3-13 } & 5.9 \% \text { tiny spinel at bottom, } 4.5 \% \text { larger spinel at top }\end{array}$

CVS3-14 sp 2.3\%

CVS3-15 no XL

\begin{tabular}{l|l} 
CVS3-15 & no XL \\
\hline CVS3-16 & Fully XL? two distinct phases maybe more
\end{tabular}

\begin{tabular}{l|l} 
CVS3-17 & $2.9 \% \mathrm{XL}$ seem to be all spinel
\end{tabular}

$\begin{array}{ll}\text { CVS3-18 } & 1.9 \% \text { XL same as 3-17 only smaller }\end{array}$

CVS3-19 No XL

$\begin{array}{lll}\text { CVS3-20 } & 6.7 \% \mathrm{XL}\end{array}$

\begin{tabular}{|l|l|}
\hline & \\
\hline & \\
\hline spinel, hiCr sp & \\
\hline spinel, hiCr sp, hiNi sp & \\
\hline spinel, hiCr sp & 10 vol\% Spinel \\
\hline spinel & \\
\hline & \\
\hline $\begin{array}{l}\text { (Si, Al), (Si, Al, Zr), (Si, } \\
\text { Al, Zr, Nd, Fe), spinel }\end{array}$ & fully XL, 45w\% LiAlSi, 40w\% np, \\
\hline $\begin{array}{l}\text { spinel, (Si, Al, Nd [dend]), } \\
\text { (Fe, Al, Ni [sp?]) }\end{array}$ & 25 vol\% XL, 50w\% NaNdSi, 35w\% sp \\
\hline & \\
\hline Zr, (Si, Fe, Zr, Ni) & $15 \%$ ZrO2, Sp \\
\hline
\end{tabular}


Hanford CVS 2 (Hrma et al. 1994)

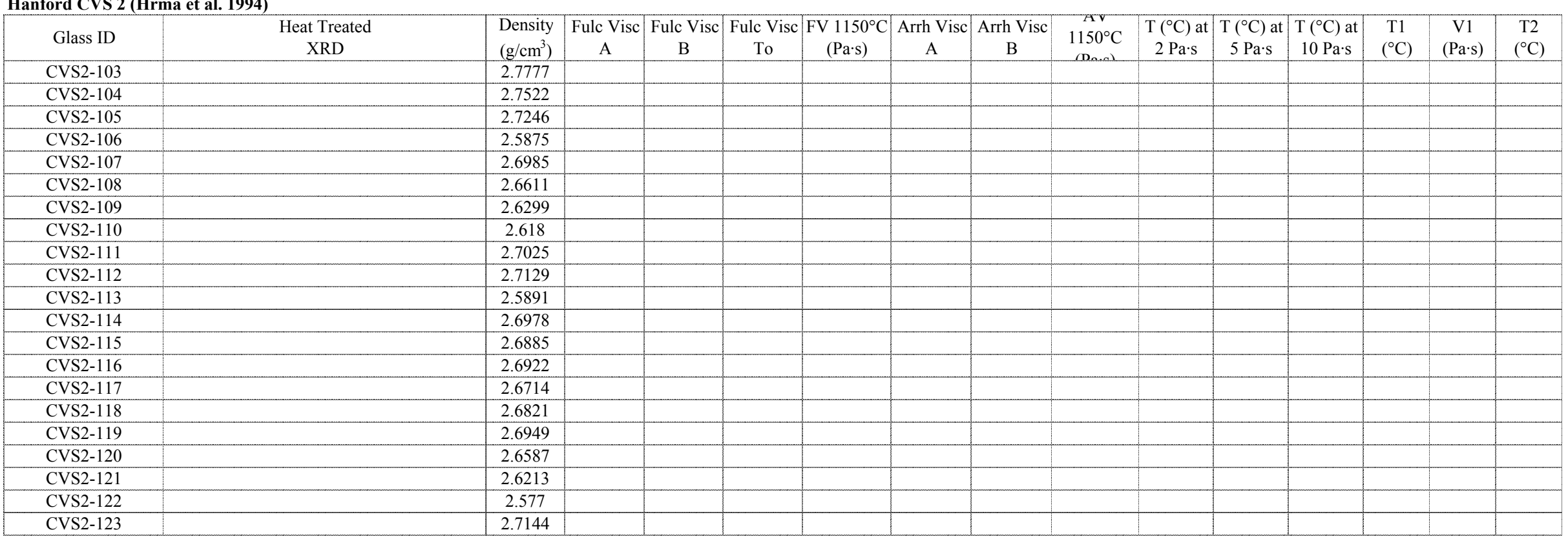

\section{Hanford CVS 3 (Vienna et al. 1996)}

\begin{tabular}{|c|c|c|c|c|c|c|c|c|c|}
\hline CVS3-1 & -7.17 & 10073 & 492.1 & -12.040 & 22335.1 & 38.70 & 1095 & 76.079 & 1145 \\
\hline CVS3-2 & -2.61 & 3388.7 & 979.1 & -11.320 & 22774.9 & 108.29 & 1194 & 74.061 & 1245 \\
\hline CVS3-3 & -8.15 & 9715.8 & 490.4 & -13.610 & 22648.3 & 10.03 & 1000 & 71.0640 & 1100 \\
\hline CVS3-4 & & & & -13.640 & 21802.3 & 5.37 & 1044 & 16.676 & 1094 \\
\hline CVS3-5 & & & & $(-39.99)$ & (59812.9) & (7.7134) & 1051 & 188.3760 & 1126 \\
\hline CVS3-6 & -7.88 & 10857 & 366.6 & -11.240 & 19441.9 & 11.28 & 1043 & 35.037 & 1094 \\
\hline CVS3-7 & -6.1 & 7680.2 & 577.2 & -11.310 & 20355.7 & 19.98 & 1093 & 37.886 & 1144 \\
\hline CVS3-8 & -8.61 & 11477 & 332.8 & -11.950 & 19755.5 & 6.91 & 992 & 40.279 & 1043 \\
\hline CVS3-9 & -3.38 & 4309.2 & 942.4 & -11.530 & 23596.9 & 156.41 & 1244 & 60.786 & 1294 \\
\hline CVS3-10 & -6.51 & 6836.5 & 584 & -12.910 & 20788.1 & 5.47 & 949 & 65.5650 & 1000 \\
\hline CVS3-11 & -6.85 & 8702.9 & 501.4 & -12.050 & 20895.7 & 13.93 & 992 & 93.903 & 1042 \\
\hline CVS3-12 & -3.33 & 4247.6 & 977.9 & -12.580 & 25798.1 & 257.08 & 1245 & 92.087 & 1294 \\
\hline CVS3-13 & & & & $(-12.45)$ & $(22264.6)$ & $(24.44)$ & 1093 & 42.993 & 1144 \\
\hline CVS3-14 & -8.24 & 11874 & 401.9 & -12.190 & 22179.8 & 29.86 & 1093 & 59.388 & 1144 \\
\hline CVS3-15 & -6.9 & 10081 & 508.2 & -11.620 & 22350.5 & 59.54 & 1143 & 66.794 & 1194 \\
\hline CVS3-16 & & & & $(-15.756)$ & $(31073.01)$ & $(437.26)$ & 1244 & 120.44 & 1294 \\
\hline CVS3-17 & -3.82 & 4518.2 & 948.9 & -12.570 & 25176.8 & 167.80 & 1245 & 60.506 & 1295 \\
\hline CVS3-18 & -4.17 & 5022.3 & 863.4 & -12.200 & 23840.3 & 94.97 & 1194 & 61.98 & 1245 \\
\hline CVS3-19 & -6.22 & 7808.3 & 584.3 & -11.660 & 20994.0 & 22.05 & 1095 & 42.114 & 1145 \\
\hline CVS3-20 & & & & $(-16.606)$ & 31488.601 & $(250.22)$ & 1194 & 94.237 & 1245 \\
\hline
\end{tabular}




\section{Hanford CVS 2 (Hrma et al. 1994)}

\begin{tabular}{|c|c|c|c|c|c|c|c|c|c|c|c|c|c|c|c|c|c|c|c|c|c|}
\hline Glass ID & $\begin{array}{c}\mathrm{V} 2 \\
(\mathrm{~Pa} \cdot \mathrm{s})\end{array}$ & $\begin{array}{c}\mathrm{T} 3 \\
\left({ }^{\circ} \mathrm{C}\right)\end{array}$ & $\begin{array}{c}\mathrm{V} 3 \\
(\mathrm{~Pa} \cdot \mathrm{s})\end{array}$ & $\begin{array}{c}\mathrm{T} 4 \\
\left({ }^{\circ} \mathrm{C}\right)\end{array}$ & $\begin{array}{c}\mathrm{V} 4 \\
(\mathrm{~Pa} \cdot \mathrm{s})\end{array}$ & $\begin{array}{c}\mathrm{T} 5 \\
\left({ }^{\circ} \mathrm{C}\right)\end{array}$ & $\begin{array}{c}\mathrm{V} 5 \\
(\mathrm{~Pa} \cdot \mathrm{s})\end{array}$ & $\begin{array}{c}\mathrm{T} 6 \\
\left({ }^{\circ} \mathrm{C}\right)\end{array}$ & $\begin{array}{c}\mathrm{V} 6 \\
(\mathrm{~Pa} \cdot \mathrm{s})\end{array}$ & $\begin{array}{c}\mathrm{T} 7 \\
\left({ }^{\circ} \mathrm{C}\right)\end{array}$ & $\begin{array}{c}\mathrm{V} 7 \\
(\mathrm{~Pa} \cdot \mathrm{s})\end{array}$ & $\begin{array}{c}\mathrm{T} 8 \\
\left({ }^{\circ} \mathrm{C}\right)\end{array}$ & $\begin{array}{c}\mathrm{V} 8 \\
(\mathrm{~Pa} \cdot \mathrm{s})\end{array}$ & $\begin{array}{l}\mathrm{T} 9 \\
\left({ }^{\circ} \mathrm{C}\right)\end{array}$ & $\begin{array}{c}\mathrm{V} 9 \\
(\mathrm{~Pa} \cdot \mathrm{s})\end{array}$ & $\begin{array}{l}\mathrm{T} 10 \\
\left({ }^{\circ} \mathrm{C}\right)\end{array}$ & $\begin{array}{c}\mathrm{V} 10 \\
(\mathrm{~Pa} \cdot \mathrm{s})\end{array}$ & $\begin{array}{l}\mathrm{T} 11 \\
\left({ }^{\circ} \mathrm{C}\right)\end{array}$ & $\begin{array}{c}\mathrm{V} 11 \\
(\mathrm{~Pa} \cdot \mathrm{s})\end{array}$ & $\begin{array}{l}\mathrm{T} 12 \\
\left({ }^{\circ} \mathrm{C}\right)\end{array}$ & $\begin{array}{c}\mathrm{V} 12 \\
(\mathrm{~Pa} \cdot \mathrm{s})\end{array}$ \\
\hline CVS2-103 & & & & & & & & & & & & & & & & & & & & & \\
\hline CVS2-104 & & & & & & & & & & & & & & & & & & & & & \\
\hline CVS2-105 & & & & & & & & & & & & & & & & & & & & & \\
\hline CVS2-106 & & & & & & & & & & & & & & & & & & & & & \\
\hline CVS2-107 & & & & & & & & & & & & & & & & & & & & & \\
\hline CVS2-108 & & & & & & & & & & & & & & & & & & & & & \\
\hline CVS2-109 & & & & & & & & & & & & & & & & & & & & & \\
\hline CVS2-110 & & & & & & & & & & & & & & & & & & & & & \\
\hline CVS2-111 & & & & & & & & & & & & & & & & & & & & & \\
\hline CVS2-112 & & & & & & & & & & & & & & & & & & & & & \\
\hline CVS2-113 & & & & & & & & & & & & & & & & & & & & & \\
\hline CVS2-114 & & & & & & & & & & & & & & & & & & & & & \\
\hline CVS2-115 & & & & & & & & & & & & & & & & & & & & & \\
\hline CVS2-116 & & & & & & & & & & & & & & & & & & & & & \\
\hline CVS2-117 & & & & & & & & & & & & & & & & & & & & & \\
\hline CVS2-118 & & & & & & & & & & & & & & & & & & & & & \\
\hline CVS2-119 & & & & & & & & & & & & & & & & & & & & & \\
\hline CVS2-120 & & & & & & & & & & & & & & & & & & & & & \\
\hline CVS2-121 & & & & & & & & & & & & & & & & & & & & & \\
\hline CVS2-122 & & & & & & & & & & & & & & & & & & & & & \\
\hline CVS2-123 & & & & & & & & & & & & & & & & & & & & & \\
\hline
\end{tabular}

\section{Hanford CVS 3 (Vienna et al. 1996)}

\begin{tabular}{|c|c|c|c|c|c|c|c|c|c|c|c|c|c|c|c|c|c|c|c|c|}
\hline CVS3-1 & 40.684 & 1194 & 23.198 & 1244 & 14.135 & 1245 & 14.357 & 1294 & 8.8702 & 1294 & 9.0916 & 1295 & 9.1074 & 1344 & 5.9372 & 1395 & 4.0161 & & & \\
\hline CVS3-2 & 41.304 & 1294 & 23.435 & 1344 & 14.786 & 1344 & 15.009 & 1394 & 8.8410 & 1395 & 10.266 & 1395 & 10.464 & 1445 & 7.2353 & 1497 & 5.4573 & & & \\
\hline CVS3-3 & 16.723 & 1150 & 9.9264 & 1199 & 5.6753 & 1200 & 5.8163 & 1235 & 4.0115 & 1235 & 4.0538 & 1235 & 3.9480 & 1300 & 2.2222 & 1350 & 1.5454 & & & \\
\hline CVS3-4 & 10.903 & 1143 & 6.1885 & 1144 & 5.8498 & 1193 & 3.3329 & 1194 & 3.5869 & 1194 & 3.1712 & 1244 & 1.9735 & & & & & & & \\
\hline CVS3-5 & 21.037 & 1126 & 21.263 & 1126 & 7.8678 & 1200 & 1.9655 & & & & & & & & & & & & & \\
\hline CVS3-6 & 19.427 & 1144 & 11.768 & 1195 & 7.2584 & 1244 & 4.7183 & 1245 & 4.6799 & 1294 & 3.1712 & 1294 & 3.2097 & 1295 & 3.2328 & 1344 & 2.2353 & & & \\
\hline CVS3-7 & 21.2990 & 1194 & 12.678 & 1244 & 7.8553 & 1244 & 7.9159 & 1294 & 5.1494 & 1294 & 5.3187 & 1295 & 5.3803 & 1344 & 3.6331 & 1395 & 2.5708 & & & \\
\hline CVS3-8 & 21.123 & 1094 & 12.177 & 1144 & 7.2199 & 1194 & 4.4797 & 1194 & 4.4566 & 1243 & 2.8910 & 1244 & 2.9342 & 1245 & 2.9619 & 1294 & 2.0043 & & & \\
\hline CVS3-9 & 34.41 & 1344 & 20.304 & 1394 & 12.935 & 1394 & 13.421 & 1444 & 8.4467 & 1444 & 8.8713 & 1445 & 8.9926 & 1495 & 6.1577 & 1547 & 4.7568 & & & \\
\hline CVS3-10 & 30.851 & 1100 & 8.7279 & 1149 & 5.1042 & 1199 & 3.1373 & 1199 & 3.243 & 1249 & 2.1347 & 1249 & 2.1770 & 1249 & 2.2334 & 1299 & 1.5143 & & & \\
\hline CVS3-11 & 46.147 & 1094 & 24.927 & 1143 & 14.3 & 1193 & 8.5528 & 1193 & 8.6135 & 1243 & 5.5342 & 1243 & 5.6574 & 1244 & 5.7113 & 1294 & 3.7254 & 1344 & 2.5585 & \\
\hline CVS3-12 & 49.166 & 1344 & 27.706 & 1394 & 16.589 & 1394 & 17.232 & 1444 & 10.661 & 1445 & 11.222 & 1446 & 11.116 & 1496 & 7.9159 & 1547 & 5.5112 & & & \\
\hline CVS3-13 & 22.966 & 1194 & 18.479 & 1244 & 10.377 & 1244 & 10.693 & 1294 & 5.8297 & 1294 & 5.9205 & 1295 & 5.1114 & 1344 & 3.5689 & 1396 & 2.2744 & & & \\
\hline CVS3-14 & 30.65 & 1195 & 18.637 & 1244 & 11.358 & 1245 & 10.979 & 1294 & 7.0121 & 1295 & 6.7658 & 1295 & 7.0583 & 1345 & 4.5182 & 1395 & 3.1635 & & & \\
\hline CVS3-15 & 36.803 & 1244 & 21.621 & 1294 & 13.7240 & 1294 & 13.891 & 1343 & 8.6742 & 1344 & 8.9320 & 1345 & 8.9471 & 1394 & 6.1038 & 1445 & 4.1334 & & & \\
\hline CVS3-16 & 45.916 & 1345 & 38.751 & 1395 & 18.508 & 1395 & 20.473 & 1445 & 9.5335 & 1445 & 10.1660 & 1446 & 8.7654 & 1496 & 5.6558 & 1547 & 4.1209 & & & \\
\hline CVS3-17 & 32.075 & 1345 & 19.924 & 1395 & 12.056 & 1395 & 12.374 & 1444 & 7.5355 & 1445 & 7.4913 & 1446 & 7.4307 & 1496 & 5.0570 & 1547 & 4.2873 & & & \\
\hline CVS3-18 & 33.993 & 1294 & 19.3 & 1344 & 12.034 & 1344 & 12.29 & 1394 & 7.6358 & 1395 & 7.8165 & 1396 & 8.0123 & 1446 & 5.2551 & 1496 & 4.0831 & & & \\
\hline CVS3-19 & 23.382 & 1194 & 13.605 & 1244 & 8.5366 & 1244 & 8.6131 & 1294 & 5.5339 & 1294 & 5.6508 & 1295 & 5.6451 & 1345 & 3.8140 & 1395 & 2.6561 & & & \\
\hline CVS3-20 & 35.827 & 1295 & 80.0100 & 1345 & 24.316 & 1345 & 23.201 & 1395 & 11.687 & 1395 & 10.347 & 1396 & 5.7466 & 1446 & 5.1643 & 1496 & 2.3818 & & & \\
\hline
\end{tabular}


Appendix A. Database - mass fraction

Hanford CVS 2 (Hrma et al. 1994)

\begin{tabular}{|c|c|c|c|c|c|c|c|c|c|c|c|c|c|c|c|c|}
\hline Glass ID & $\begin{array}{l}\mathrm{T} 13 \\
\left({ }^{\circ} \mathrm{C}\right)\end{array}$ & $\begin{array}{l}\mathrm{V} 13 \\
(\mathrm{~Pa} \cdot \mathrm{s})\end{array}$ & $\begin{array}{l}\mathrm{T} 14 \\
\left({ }^{\circ} \mathrm{C}\right)\end{array}$ & $\begin{array}{c}\mathrm{V} 14 \\
(\mathrm{~Pa} \cdot \mathrm{s})\end{array}$ & $\begin{array}{c}\text { Q PCT } \\
\mathrm{B}\left(\mathrm{g} / \mathrm{m}^{2}\right)\end{array}$ & $\begin{array}{c}\text { Q PCT } \\
\mathrm{Li}\left(\mathrm{g} / \mathrm{m}^{2}\right)\end{array}$ & $\begin{array}{c}\text { Q PCT } \\
\mathrm{Na}\left(\mathrm{g} / \mathrm{m}^{2}\right)\end{array}$ & $\begin{array}{c}\text { Q PCT } \\
\mathrm{Si}\left(\mathrm{g} / \mathrm{m}^{2}\right)\end{array}$ & $\begin{array}{l}\text { Q PCT } \\
\text { pH }\end{array}$ & $\begin{array}{c}\text { CCC PCT } \\
\text { B }\left(\mathrm{g} / \mathrm{m}^{2}\right)\end{array}$ & \begin{tabular}{|c|} 
CCC PCT \\
$\mathrm{Li}\left(\mathrm{g} / \mathrm{m}^{2}\right)$
\end{tabular} & $\begin{array}{l}\text { CCC PCT } \\
\mathrm{Na}\left(\mathrm{g} / \mathrm{m}^{2}\right)\end{array}$ & $\begin{array}{c}\text { CCC PCT } \\
\mathrm{Si}\left(\mathrm{g} / \mathrm{m}^{2}\right)\end{array}$ & $\begin{array}{c}\text { CCC PCT } \\
\mathrm{pH}\end{array}$ & $\begin{array}{l}\text { Q PCT B at } \\
20^{\circ} \mathrm{C}\left(\mathrm{g} / \mathrm{m}^{2}\right)\end{array}$ & $\begin{array}{l}\text { Q PCT Li at } \\
20^{\circ} \mathrm{C}\left(\mathrm{g} / \mathrm{m}^{2}\right)\end{array}$ \\
\hline CVS2-103 & & & & & 6.073 & 4.066 & 4.082 & 0.691 & 11.61 & 5.901 & 4.397 & 4.250 & 0.780 & 11.49 & & \\
\hline CVS2-104 & & & & & 5.548 & 3.633 & 3.668 & 0.712 & 11.29 & 6.435 & 4.523 & 4.141 & 0.805 & 11.20 & & \\
\hline CVS2-105 & & & & & 4.590 & 3.132 & 2.974 & 0.687 & 10.82 & 3.539 & 3.020 & 2.743 & 0.656 & 10.67 & & \\
\hline CVS2-106 & & & & & 1.651 & 1.348 & 1.165 & 0.447 & 10.18 & 1.330 & 1.102 & 0.936 & 0.393 & 10.03 & & \\
\hline CVS2-107 & & & & & 0.788 & 0.662 & 0.620 & 0.348 & 10.68 & 0.660 & 0.484 & 0.449 & 0.260 & 10.50 & & \\
\hline CVS2-108 & & & & & 2.144 & 1.646 & 1.387 & 0.357 & 9.79 & 2.190 & 1.783 & 1.485 & 0.370 & 9.80 & & \\
\hline CVS2-109 & & & & & 5.707 & 4.494 & 3.584 & 0.313 & 9.54 & 4.331 & 3.868 & 3.032 & 0.331 & 9.40 & & \\
\hline CVS2-110 & & & & & 0.314 & 0.402 & 0.118 & 0.160 & 9.61 & 2.635 & 2.239 & 1.557 & 0.236 & 9.80 & & \\
\hline CVS2-111 & & & & & 6.135 & 3.894 & 4.043 & 1.199 & 11.35 & 4.638 & 4.570 & 4.648 & 1.329 & 11.20 & & \\
\hline CVS2-112 & & & & & 14.400 & 7.794 & 9.603 & 0.454 & 12.04 & 15.325 & 7.945 & 9.908 & 0.454 & 12.05 & & \\
\hline CVS2-113 & & & & & 0.612 & 0.630 & 0.510 & 0.216 & 9.63 & 0.558 & 0.515 & 0.437 & 0.202 & 9.63 & & \\
\hline CVS2-114 & & & & & 7.116 & 4.928 & 4.599 & 1.860 & 11.41 & 7.038 & 4.498 & 4.170 & 1.758 & 11.35 & & \\
\hline CVS2-115 & & & & & 9.406 & 5.837 & 5.765 & 1.942 & 11.55 & 9.472 & 5.704 & 5.611 & 1.827 & 11.55 & & \\
\hline CVS2-116 & & & & & 3.012 & 2.203 & 2.030 & 0.606 & 10.43 & 2.754 & 2.019 & 1.922 & 0.570 & 10.40 & & \\
\hline CVS2-117 & & & & & 1.590 & 1.337 & 1.099 & 0.445 & 10.19 & 1.600 & 1.366 & 1.141 & 0.436 & 10.20 & & \\
\hline CVS2-118 & & & & & 3.630 & 2.609 & 2.394 & 0.606 & 10.40 & 2.808 & 2.106 & 1.968 & 0.555 & 10.30 & & \\
\hline CVS2-119 & & & & & 3.803 & 2.688 & 2.574 & 0.829 & 10.32 & 3.593 & 3.014 & 2.815 & 0.827 & 10.34 & & \\
\hline CVS2-120 & & & & & 0.291 & 0.324 & 0.231 & 0.154 & 10.10 & 0.291 & 0.306 & 0.216 & 0.138 & 10.09 & & \\
\hline CVS2-121 & & & & & 0.199 & 0.311 & 0.137 & 0.129 & 10.06 & 0.175 & 0.270 & 0.135 & 0.119 & 10.01 & & \\
\hline CVS2-122 & & & & & 0.193 & 0.363 & 0.089 & 0.128 & 9.91 & 0.213 & 0.309 & 0.089 & 0.116 & 9.92 & & \\
\hline CVS2-123 & & & & & 1.473 & 1.125 & 1.075 & 0.391 & 10.22 & 1.352 & 1.054 & 1.006 & 0.356 & 10.20 & & \\
\hline
\end{tabular}

\section{Hanford CVS 3 (Vienna et al. 1996)}

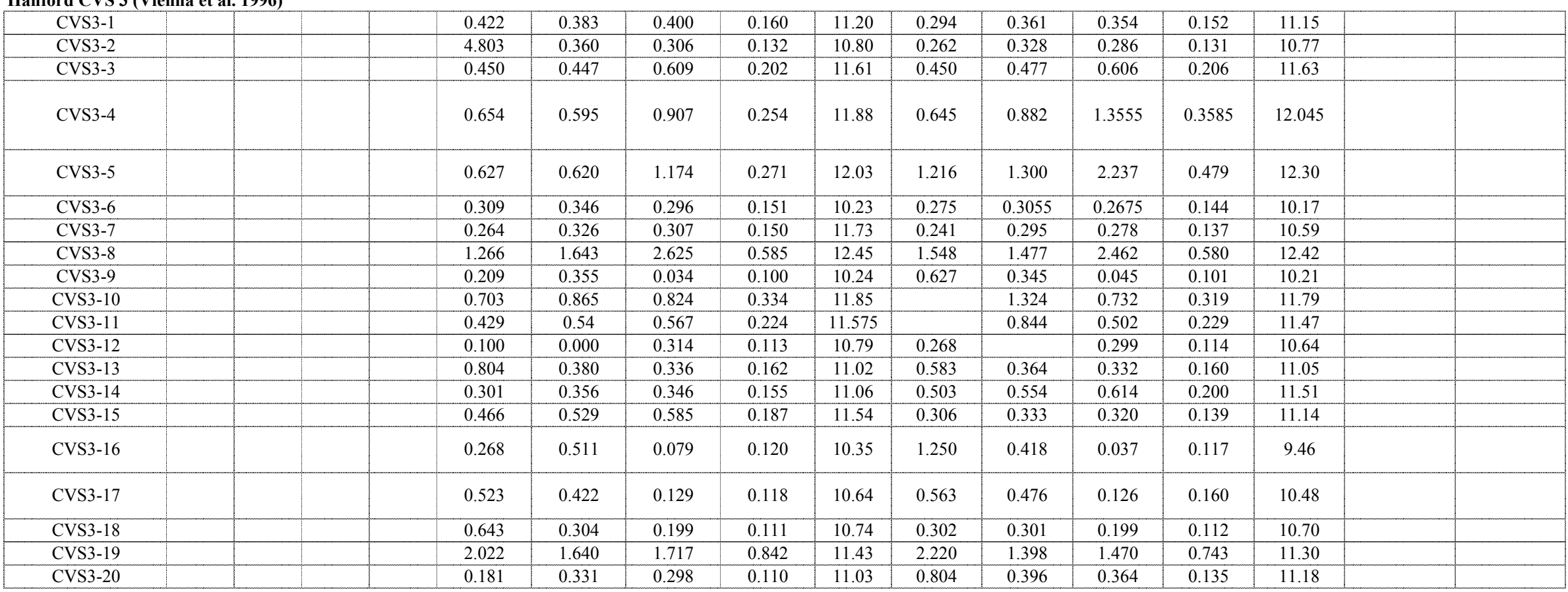


Appendix A. Database - mass fraction

\section{Hanford CVS 2 (Hrma et al. 1994)}

\begin{tabular}{|c|c|c|c|c|c|c|c|c|c|c|c|c|}
\hline Glass ID & $\begin{array}{l}\text { Q PCT Na at } \\
20^{\circ} \mathrm{C}\left(\mathrm{g} / \mathrm{m}^{2}\right)\end{array}$ & $\begin{array}{l}\text { Q PCT Si at } \\
20^{\circ} \mathrm{C}\left(\mathrm{g} / \mathrm{m}^{2}\right)\end{array}$ & $\begin{array}{c}\mathrm{QpH} \\
\text { at } 20^{\circ} \mathrm{C}\end{array}$ & $\begin{array}{c}\text { TCLP Ag } \\
(\mathrm{ppm})\end{array}$ & $\begin{array}{c}\text { TCLP As } \\
(\mathrm{ppm})\end{array}$ & $\begin{array}{c}\text { TCLP Ba } \\
\text { (ppm) }\end{array}$ & $\begin{array}{c}\text { TCLP Cd } \\
(\mathrm{ppm})\end{array}$ & $\begin{array}{c}\text { TCLP Cr } \\
(\mathrm{ppm})\end{array}$ & $\begin{array}{c}\text { TCLP Ni } \\
(\mathrm{ppm})\end{array}$ & $\begin{array}{c}\text { TCLP Pb } \\
(\mathrm{ppm})\end{array}$ & $\begin{array}{c}\text { TCLP Se } \\
(\mathrm{ppm})\end{array}$ & $\begin{array}{c}\text { TCLP Zn } \\
(\mathrm{ppm})\end{array}$ \\
\hline \multicolumn{13}{|c|}{ 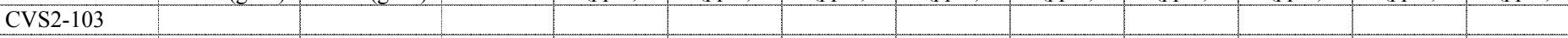 } \\
\hline \multicolumn{13}{|l|}{ CVS2-104 } \\
\hline \multicolumn{13}{|l|}{ CVS2-105 } \\
\hline \multicolumn{13}{|l|}{ CVS2-106 } \\
\hline \multicolumn{13}{|l|}{ CVS2-107 } \\
\hline \multicolumn{13}{|l|}{ CVS2-108 } \\
\hline \multicolumn{13}{|l|}{ CVS2-109 } \\
\hline \multicolumn{13}{|l|}{ CVS2-110 } \\
\hline \multicolumn{13}{|l|}{ CVS2-111 } \\
\hline \multicolumn{13}{|l|}{ CVS2-112 } \\
\hline \multicolumn{13}{|l|}{ CVS2-113 } \\
\hline \multicolumn{13}{|l|}{ CVS2-114 } \\
\hline \multicolumn{13}{|l|}{ CVS2-115 } \\
\hline \multicolumn{13}{|l|}{ CVS2-116 } \\
\hline \multicolumn{13}{|l|}{ CVS2-117 } \\
\hline \multicolumn{13}{|l|}{ CVS2-118 } \\
\hline \multicolumn{13}{|l|}{ CVS2-119 } \\
\hline \multicolumn{13}{|l|}{ CVS2-120 } \\
\hline \multicolumn{13}{|l|}{ CVS2-121 } \\
\hline \multicolumn{13}{|l|}{ CVS2-122 } \\
\hline CVS2-123 & & & & & & & & & & & & \\
\hline
\end{tabular}

\section{Hanford CVS 3 (Vienna et al. 1996)}

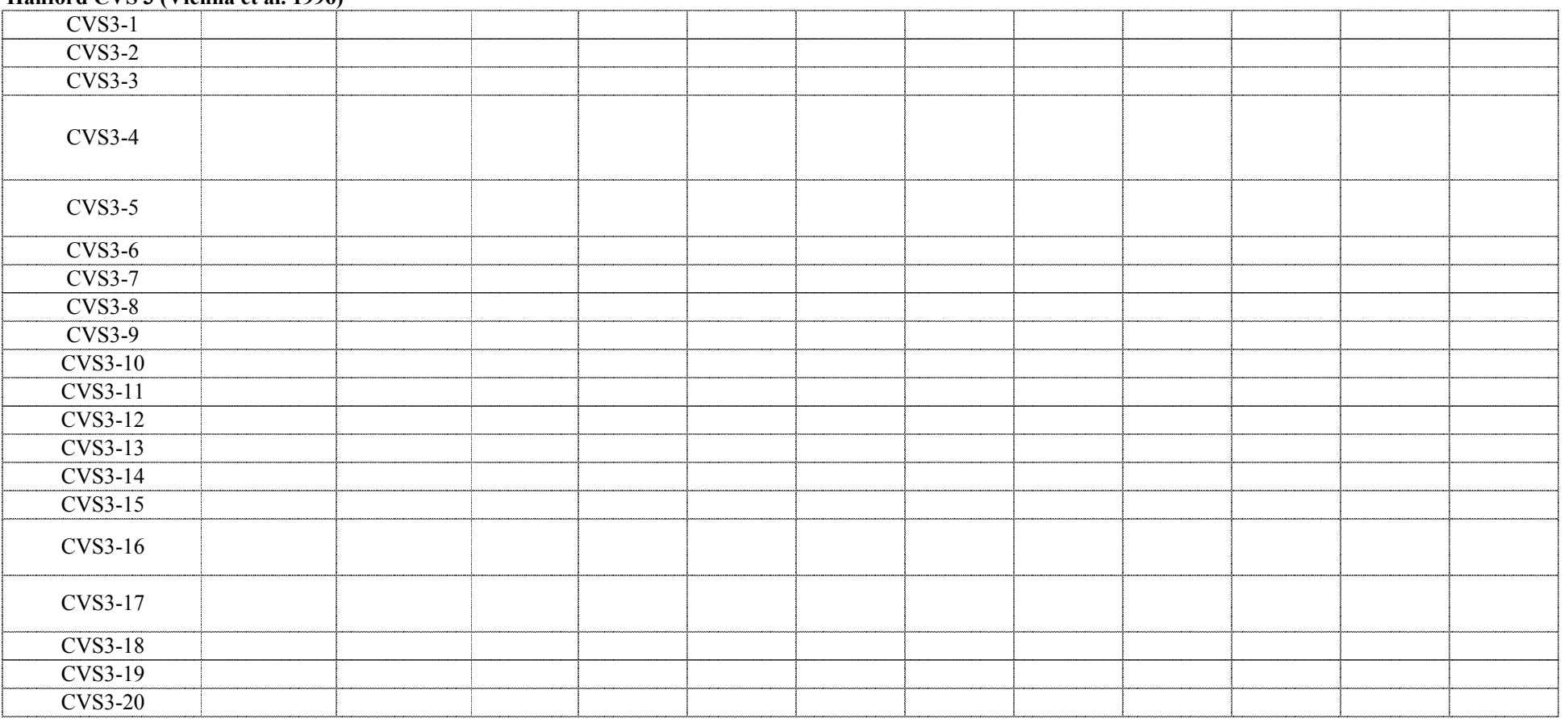




\section{Hanford CVS 3 (Vienna et al. 1996)}

\begin{tabular}{|c|c|c|c|c|c|c|c|c|c|c|c|c|c|c|c|c|c|c|c|c|c|}
\hline Glass ID & $\begin{array}{c}\mathrm{A} 12 \mathrm{O} 3 \\
-\mathrm{t}\end{array}$ & $\begin{array}{c}\mathrm{B} 2 \mathrm{O} 3 \\
-\mathrm{t}\end{array}$ & $\begin{array}{c}\mathrm{CaO} \\
-\mathrm{t}\end{array}$ & $\begin{array}{c}\mathrm{Fe} 2 \mathrm{O} 3 \\
-\mathrm{t}\end{array}$ & $\begin{array}{c}\mathrm{FeO} \\
-\mathrm{t}\end{array}$ & $\begin{array}{c}\mathrm{K} 2 \mathrm{O} \\
-\mathrm{t}\end{array}$ & $\begin{array}{c}\mathrm{Li} 2 \mathrm{O} \\
-\mathrm{t}\end{array}$ & $\begin{array}{c}\mathrm{MgO} \\
-\mathrm{t}\end{array}$ & $\mathrm{Na} 2 \mathrm{O}$ & $\begin{array}{c}\mathrm{NiO} \\
-\mathrm{t}\end{array}$ & $\begin{array}{c}\mathrm{P} 2 \mathrm{O} 5 \\
-\mathrm{t}\end{array}$ & $\begin{array}{c}\mathrm{SiO} 2 \\
-\mathrm{t}\end{array}$ & $\begin{array}{c}\mathrm{ZrO} 2 \\
-\mathrm{t}\end{array}$ & $\begin{array}{c}\mathrm{Ag} 2 \mathrm{O} \\
-\mathrm{t}\end{array}$ & $\begin{array}{c}\mathrm{As} 2 \mathrm{O} 3 \\
-\mathrm{t}\end{array}$ & $\begin{array}{c}\mathrm{BaO} \\
-\mathrm{t}\end{array}$ & $\begin{array}{c}\mathrm{Bi} 2 \mathrm{O} 3 \\
-\mathrm{t}\end{array}$ & $\begin{array}{l}\mathrm{Br} \\
-\mathrm{t}\end{array}$ & $\begin{array}{c}\mathrm{CdO} \\
-\mathrm{t}\end{array}$ & $\begin{array}{c}\mathrm{Ce} 2 \mathrm{O} 3 \\
-\mathrm{t}\end{array}$ & $\begin{array}{c}\mathrm{CeO} 2 \\
-\mathrm{t}\end{array}$ \\
\hline CVS3-21 & 0.0448 & 0.0004 & 0.0116 & 0.0626 & & 0.0012 & 0.0263 & 0.0004 & 0.1135 & 0.0129 & 0.0073 & 0.4908 & 0.1200 & 0.0000 & 0.0000 & 0.0000 & 0.0126 & & 0.0005 & & 0.0155 \\
\hline CVS3-22 & 0.0468 & 0.0004 & 0.0121 & 0.0654 & & 0.0013 & 0.0275 & 0.0005 & 0.1187 & 0.0135 & 0.0076 & 0.5131 & 0.0800 & 0.0000 & 0.0000 & 0.0000 & 0.0132 & & 0.0005 & & 0.0163 \\
\hline CVS3-23 & 0.0509 & 0.0005 & 0.0132 & 0.0711 & & 0.0014 & 0.0299 & 0.0005 & 0.1290 & 0.0147 & 0.0083 & 0.5577 & 0.0000 & 0.0000 & 0.0000 & 0.0000 & 0.0144 & & 0.0006 & & 0.0177 \\
\hline CVS3-24 & 0.0453 & 0.0004 & 0.0117 & 0.0633 & & 0.0019 & 0.0266 & 0.0004 & 0.1149 & 0.0199 & 0.0113 & 0.4966 & 0.0408 & 0.0000 & 0.0000 & 0.0000 & 0.0195 & & 0.0008 & & 0.0240 \\
\hline CVS3-25 & 0.0524 & 0.0005 & 0.0136 & 0.0732 & & 0.0007 & 0.0307 & 0.0005 & 0.1328 & 0.0075 & 0.0042 & 0.5742 & 0.0471 & 0.0000 & 0.0000 & 0.0000 & 0.0073 & & 0.0003 & & 0.0090 \\
\hline CVS3-26 & 0.0235 & 0.1048 & 0.0082 & 0.0733 & & 0.0000 & 0.0373 & 0.0084 & 0.1129 & 0.0060 & 0.0010 & 0.5328 & 0.0392 & 0.0000 & 0.0000 & 0.0010 & 0.0000 & & 0.0065 & & 0.0002 \\
\hline CVS3-27 & 0.0444 & 0.0004 & 0.0115 & 0.1500 & & 0.0012 & 0.0260 & 0.0004 & 0.1125 & 0.0128 & 0.0072 & 0.4864 & 0.0399 & 0.0000 & 0.0000 & 0.0000 & 0.0125 & & 0.0005 & & 0.0154 \\
\hline CVS3-28 & 0.0472 & 0.0005 & 0.0122 & 0.0659 & & 0.0013 & 0.0277 & 0.0005 & 0.1196 & 0.0136 & 0.0077 & 0.5170 & 0.0424 & 0.0000 & 0.0000 & 0.0000 & 0.0437 & & 0.0005 & & 0.0164 \\
\hline CVS3-29 & 0.0462 & 0.0005 & 0.0120 & 0.0645 & & 0.0012 & 0.0271 & 0.0005 & 0.1171 & 0.0133 & 0.0075 & 0.5062 & 0.0416 & 0.0000 & 0.0000 & 0.0000 & 0.0637 & & 0.0005 & & 0.0160 \\
\hline CVS3-30 & 0.0437 & 0.0005 & 0.0113 & 0.0611 & & 0.0012 & 0.0257 & 0.0004 & 0.1109 & 0.0126 & 0.0071 & 0.4792 & 0.0393 & 0.0000 & 0.0000 & 0.0000 & 0.1137 & & 0.0005 & & 0.0152 \\
\hline CVS3-31 & 0.0413 & 0.0004 & 0.0107 & 0.0576 & & 0.0011 & 0.0242 & 0.0004 & 0.1046 & 0.0119 & 0.0067 & 0.4522 & 0.0371 & 0.0000 & 0.0000 & 0.0000 & 0.1637 & & 0.0005 & & 0.0143 \\
\hline CVS3-32 & 0.0452 & 0.0004 & 0.0117 & 0.0631 & & 0.0012 & 0.0287 & 0.0004 & 0.1146 & 0.0130 & 0.0074 & 0.5306 & 0.0407 & 0.0000 & 0.0000 & 0.0000 & 0.0127 & & 0.0005 & & 0.0156 \\
\hline CVS3-33 & 0.0481 & 0.0005 & 0.0124 & 0.0672 & & 0.0013 & 0.0305 & 0.0005 & 0.1219 & 0.0138 & 0.0078 & 0.5644 & 0.0432 & 0.0000 & 0.0000 & 0.0000 & 0.0135 & & 0.0006 & & 0.0166 \\
\hline CVS3-34 & 0.0467 & 0.0004 & 0.0121 & 0.0652 & & 0.0013 & 0.0296 & 0.0005 & 0.1182 & 0.0134 & 0.0076 & 0.5475 & 0.0420 & 0.0000 & 0.0000 & 0.0000 & 0.0131 & & 0.0005 & & 0.0161 \\
\hline CVS3-35 & 0.0438 & 0.0004 & 0.0113 & 0.0611 & & 0.0012 & 0.0277 & 0.0004 & 0.1109 & 0.0126 & 0.0071 & 0.5136 & 0.0394 & 0.0000 & 0.0000 & 0.0000 & 0.0123 & & 0.0005 & & 0.0152 \\
\hline CVS3-36 & 0.0423 & 0.0004 & 0.0110 & 0.0591 & & 0.0011 & 0.0268 & 0.0004 & 0.1072 & 0.0122 & 0.0069 & 0.4966 & 0.0381 & 0.0000 & 0.0000 & 0.0000 & 0.0119 & & 0.0005 & & 0.0147 \\
\hline CVS3-37 & 0.0476 & 0.0005 & 0.0123 & 0.0665 & & 0.0013 & 0.0279 & 0.0005 & 0.1206 & 0.0137 & 0.0300 & 0.5214 & 0.0428 & 0.0000 & 0.0000 & 0.0000 & 0.0134 & & 0.0005 & & 0.0165 \\
\hline CVS3-38 & 0.0466 & 0.0005 & 0.0121 & 0.0651 & & 0.0013 & 0.0274 & 0.0005 & 0.1181 & 0.0134 & 0.0500 & 0.5107 & 0.0419 & 0.0000 & 0.0000 & 0.0000 & 0.0131 & & 0.0005 & & 0.0162 \\
\hline CVS3-39 & 0.0456 & 0.0005 & 0.0118 & 0.0637 & & 0.0012 & 0.0268 & 0.0004 & 0.1156 & 0.0132 & 0.0700 & 0.4999 & 0.0410 & 0.0000 & 0.0000 & 0.0000 & 0.0129 & & 0.0005 & & 0.0158 \\
\hline CVS3-40 & 0.0447 & 0.0005 & 0.0116 & 0.0624 & & 0.0012 & 0.0262 & 0.0004 & 0.1132 & 0.0129 & 0.0900 & 0.4892 & 0.0402 & 0.0000 & 0.0000 & 0.0000 & 0.0126 & & 0.0005 & & 0.0155 \\
\hline
\end{tabular}

TRU Study (Crum et al. 1997)

\begin{tabular}{|c|c|c|c|c|c|c|c|c|c|c|c|c|c|c|c|c|}
\hline TRU-BL-1 & 0.0230 & 0.0815 & 0.0098 & 0.0435 & 0.0027 & 0.0458 & 0.0026 & 0.1139 & 0.0007 & 0.0087 & 0.4676 & 0.1070 & 0.0002 & 0.0004 & 0.0542 & 0.0000 \\
\hline TRU-AZ-1 & 0.0300 & 0.0803 & 0.0096 & 0.0429 & 0.0027 & 0.0452 & 0.0026 & 0.1122 & 0.0007 & 0.0086 & 0.4606 & 0.1130 & 0.0002 & 0.0004 & 0.0533 & 0.0000 \\
\hline TRU-NL-1 & 0.0235 & 0.0834 & 0.0100 & 0.0446 & 0.0028 & 0.0100 & 0.0027 & 0.1300 & 0.0007 & 0.0089 & 0.4786 & 0.1095 & 0.0002 & 0.0005 & 0.0554 & 0.0000 \\
\hline TRU-AB-1 & 0.0400 & 0.1200 & 0.0092 & 0.0408 & 0.0025 & 0.0430 & 0.0025 & 0.1069 & 0.0006 & 0.0082 & 0.4386 & 0.1003 & 0.0002 & 0.0004 & 0.0508 & 0.0000 \\
\hline TRU-NB-1 & 0.0234 & 0.1000 & 0.0099 & 0.0444 & 0.0028 & 0.0467 & 0.0027 & 0.0800 & 0.0007 & 0.0089 & 0.4766 & 0.1090 & 0.0002 & 0.0004 & 0.0552 & 0.0000 \\
\hline TRU-ZN-1 & 0.0221 & 0.0785 & 0.0094 & 0.0419 & 0.0026 & 0.0441 & 0.0025 & 0.1300 & 0.0007 & 0.0084 & 0.4501 & 0.1200 & 0.0002 & 0.0004 & 0.0521 & 0.0000 \\
\hline TRU-ZB-1 & 0.0215 & 0.1200 & 0.0091 & 0.0408 & 0.0025 & 0.0429 & 0.0025 & 0.1067 & 0.0006 & 0.0082 & 0.4379 & 0.1200 & 0.0002 & 0.0004 & 0.0507 & 0.0000 \\
\hline TRU-ANZ-1 & 0.0400 & 0.0744 & 0.0089 & 0.0397 & 0.0025 & 0.0418 & 0.0024 & 0.1500 & 0.0006 & 0.0080 & 0.4267 & 0.1200 & 0.0002 & 0.0004 & 0.0494 & 0.0000 \\
\hline TRU-ABZ-1 & 0.0400 & 0.1000 & 0.0092 & 0.0409 & 0.0025 & 0.0430 & 0.0025 & 0.1069 & 0.0006 & 0.0082 & 0.4388 & 0.1200 & 0.0002 & 0.0004 & 0.0508 & 0.0000 \\
\hline TRU-NBZ-1 & 0.0227 & 0.0600 & 0.0097 & 0.0431 & 0.0027 & 0.0453 & 0.0026 & 0.1300 & 0.0007 & 0.0086 & 0.4625 & 0.1200 & 0.0002 & 0.0004 & 0.0536 & 0.0000 \\
\hline TRU-Al-1 & 0.0600 & 0.0784 & 0.0094 & 0.0419 & 0.0026 & 0.0441 & 0.0025 & 0.1096 & 0.0007 & 0.0084 & 0.4499 & 0.1029 & 0.0002 & 0.0004 & 0.0521 & 0.0000 \\
\hline TRU-Al-6 & 0.0400 & 0.0801 & 0.0096 & 0.0428 & 0.0027 & 0.0450 & 0.0026 & 0.1119 & 0.0007 & 0.0086 & 0.4595 & 0.1051 & 0.0002 & 0.0004 & 0.0532 & 0.0000 \\
\hline
\end{tabular}




\section{Hanford CVS 3 (Vienna et al. 1996)}

\begin{tabular}{|c|c|c|c|c|c|c|c|c|c|c|c|c|c|c|c|c|c|c|c|c|c|}
\hline Glass ID & $\begin{array}{l}\mathrm{Cl} \\
-\mathrm{t}\end{array}$ & $\begin{array}{c}\mathrm{CoO} \\
-\mathrm{t}\end{array}$ & $\mathrm{Co} 2 \mathrm{O} 3$ & $\begin{array}{c}\mathrm{Cr} 2 \mathrm{O} 3 \\
-\mathrm{t}\end{array}$ & $\begin{array}{c}\mathrm{Cs} 2 \mathrm{O} \\
-\mathrm{t}\end{array}$ & $\begin{array}{c}\mathrm{CuO} \\
-\mathrm{t}\end{array}$ & $\begin{array}{c}\mathrm{Eu} 2 \mathrm{O} 3 \\
-\mathrm{t}\end{array}$ & $\begin{array}{l}F \\
-t\end{array}$ & $\begin{array}{c}\mathrm{Ga} 2 \mathrm{O} 3 \\
-\mathrm{t}\end{array}$ & {$\left[\begin{array}{c}\mathrm{Gd} 2 \mathrm{O} 3 \\
-\mathrm{t}\end{array}\right.$} & $\begin{array}{c}\mathrm{HgO} \\
-\mathrm{t}\end{array}$ & $\begin{array}{l}I \\
-t\end{array}$ & $\begin{array}{c}\mathrm{La} 2 \mathrm{O} 3 \\
-\mathrm{t}\end{array}$ & $\begin{array}{c}\mathrm{MnO} 2 \\
-\mathrm{t}\end{array}$ & $\begin{array}{c}\mathrm{MnO} \\
-\mathrm{t}\end{array}$ & $\begin{array}{c}\mathrm{MoO} \\
-\mathrm{t}\end{array}$ & $\begin{array}{c}\mathrm{MoO} 3 \\
-\mathrm{t}\end{array}$ & $\begin{array}{c}\mathrm{Nb} 2 \mathrm{O} 5 \\
-\mathrm{t}\end{array}$ & $\begin{array}{c}\mathrm{Nd} 2 \mathrm{O} 3 \\
-\mathrm{t}\end{array}$ & $\begin{array}{c}\mathrm{PbO} \\
-\mathrm{t}\end{array}$ & $\begin{array}{c}\mathrm{PdO} 2 \\
-\mathrm{t}\end{array}$ \\
\hline CVS3-21 & & & 0.0000 & 0.0049 & 0.0000 & 0.0000 & & 0.0031 & & & & & 0.0024 & 0.0103 & & & 0.0004 & & 0.0554 & 0.0000 & \\
\hline CVS3-22 & & & 0.0000 & 0.0051 & 0.0000 & 0.0000 & & 0.0033 & & & & & 0.0025 & 0.0108 & & & 0.0004 & & 0.0579 & 0.0000 & \\
\hline CVS3-23 & & & 0.0000 & 0.0055 & 0.0000 & 0.0000 & & 0.0036 & & & & & 0.0028 & 0.0117 & & & 0.0005 & & 0.0630 & 0.0000 & \\
\hline CVS3-24 & & & 0.0000 & 0.0075 & 0.0000 & 0.0000 & & 0.0048 & & & & & 0.0038 & 0.0159 & & & 0.0006 & & 0.0855 & 0.0000 & \\
\hline CVS3-25 & & & 0.0000 & 0.0028 & 0.0000 & 0.0000 & & 0.0018 & & & & & 0.0014 & 0.0060 & & & 0.0002 & & 0.0321 & 0.0000 & \\
\hline CVS3-26 & & & 0.0000 & 0.0013 & 0.0015 & 0.0016 & & 0.0019 & & & & & 0.0155 & 0.0016 & & & 0.0031 & & 0.0057 & 0.0000 & \\
\hline CVS3-27 & & & 0.0000 & 0.0048 & 0.0000 & 0.0000 & & 0.0031 & & & & & 0.0024 & 0.0102 & & & 0.0004 & & 0.0549 & 0.0000 & \\
\hline CVS3-28 & & & 0.0000 & 0.0051 & 0.0000 & 0.0000 & & 0.0033 & & & & & 0.0026 & 0.0109 & & & 0.0004 & & 0.0584 & 0.0000 & \\
\hline CVS3-29 & & & 0.0000 & 0.0050 & 0.0000 & 0.0000 & & 0.0032 & & & & & 0.0025 & 0.0107 & & & 0.0004 & & 0.0572 & 0.0000 & \\
\hline CVS3-30 & & & 0.0000 & 0.0048 & 0.0000 & 0.0000 & & 0.0031 & & & & & 0.0024 & 0.0101 & & & 0.0004 & & 0.0541 & 0.0000 & \\
\hline CVS3-31 & & & 0.0000 & 0.0045 & 0.0000 & 0.0000 & & 0.0029 & & & & & 0.0022 & 0.0095 & & & 0.0004 & & 0.0511 & 0.0000 & \\
\hline CVS3-32 & & & 0.0000 & 0.0049 & 0.0000 & 0.0000 & & 0.0031 & & & & & 0.0024 & 0.0104 & & & 0.0004 & & 0.0000 & 0.0000 & \\
\hline CVS3-33 & & & 0.0000 & 0.0052 & 0.0000 & 0.0000 & & 0.0033 & & & & & 0.0026 & 0.0111 & & & 0.0004 & & 0.0000 & 0.0000 & \\
\hline CVS3-34 & & & 0.0000 & 0.0051 & 0.0000 & 0.0000 & & 0.0032 & & & & & 0.0025 & 0.0107 & & & 0.0004 & & 0.0000 & 0.0000 & \\
\hline CVS3-35 & & & 0.0000 & 0.0048 & 0.0000 & 0.0000 & & 0.0030 & & & & & 0.0024 & 0.0101 & & & 0.0004 & & 0.0000 & 0.0000 & \\
\hline CVS3-36 & & & 0.0000 & 0.0046 & 0.0000 & 0.0000 & & 0.0030 & & & & & 0.0023 & 0.0098 & & & 0.0004 & & 0.0000 & 0.0000 & \\
\hline CVS3-37 & & & 0.0000 & 0.0052 & 0.0000 & 0.0000 & & 0.0033 & & & & & 0.0026 & 0.0110 & & & 0.0004 & & 0.0589 & 0.0000 & \\
\hline CVS3-38 & & & 0.0000 & 0.0051 & 0.0000 & 0.0000 & & 0.0033 & & & & & 0.0025 & 0.0107 & & & 0.0004 & & 0.0577 & 0.0000 & \\
\hline CVS3-39 & & & 0.0000 & 0.0050 & 0.0000 & 0.0000 & & 0.0032 & & & & & 0.0025 & 0.0105 & & & 0.0004 & & 0.0564 & 0.0000 & \\
\hline CVS3-40 & & & 0.0000 & 0.0049 & 0.0000 & 0.0000 & & 0.0031 & & & & & 0.0024 & 0.0103 & & & 0.0004 & & 0.0552 & 0.0000 & \\
\hline
\end{tabular}

\begin{tabular}{|c|c|c|c|c|c|c|c|}
\hline TRU-BL-1 & 0.0000 & 0.0036 & 0.0002 & 0.0042 & 0.0108 & 0.0173 & 0.0012 \\
\hline TRU-AZ-1 & 0.0000 & 0.0035 & 0.0002 & 0.0041 & 0.0106 & 0.0170 & 0.0011 \\
\hline TRU-NL-1 & 0.0000 & 0.0037 & 0.0002 & 0.0043 & 0.0111 & 0.0177 & 0.0012 \\
\hline TRU-AB-1 & 0.0000 & 0.0034 & 0.0002 & 0.0039 & 0.0101 & 0.0162 & 0.0011 \\
\hline TRU-NB-1 & 0.0000 & 0.0037 & 0.0002 & 0.0043 & 0.0110 & 0.0176 & 0.0012 \\
\hline TRU-ZN-1 & 0.0000 & 0.0035 & 0.0002 & 0.0040 & 0.0104 & 0.0167 & 0.0011 \\
\hline TRU-ZB-1 & 0.0000 & 0.0034 & 0.0001 & 0.0039 & 0.0101 & 0.0162 & 0.0011 \\
\hline TRU-ANZ-1 & 0.0000 & 0.0033 & 0.0001 & 0.0038 & 0.0099 & 0.0158 & 0.0010 \\
\hline TRU-ABZ-1 & 0.0000 & 0.0034 & 0.0002 & 0.0039 & 0.0101 & 0.0162 & 0.0011 \\
\hline TRU-NBZ-1 & 0.0000 & 0.0036 & 0.0002 & 0.0042 & 0.0107 & 0.0171 & 0.0011 \\
\hline TRU-Al-1 & 0.0000 & 0.0035 & 0.0002 & 0.0040 & 0.0104 & 0.0166 & 0.0011 \\
\hline TRU-Al-6 & 0.0000 & 0.0035 & 0.0002 & 0.0041 & 0.0106 & 0.0170 & 0.0011 \\
\hline
\end{tabular}


Appendix A. Database - mass fraction

Hanford CVS 3 (Vienna et al. 1996)

\begin{tabular}{|c|c|c|c|c|c|c|c|c|c|c|c|c|c|c|c|c|c|c|c|c|c|}
\hline Glass ID & $\begin{array}{c}\mathrm{PdO} \\
-\mathrm{t}\end{array}$ & $\begin{array}{c}\mathrm{Pr} 2 \mathrm{O} 3 \\
-\mathrm{t}\end{array}$ & $\begin{array}{c}\text { Pr6O11 } \\
-\mathrm{t}\end{array}$ & $\begin{array}{c}\mathrm{Rb} 2 \mathrm{O} \\
-\mathrm{t}\end{array}$ & $\begin{array}{c}\mathrm{ReO} \\
-\mathrm{t}\end{array}$ & $\begin{array}{c}\mathrm{ReO} 2 \\
-\mathrm{t}\end{array}$ & $\begin{array}{c}\mathrm{Rh} 2 \mathrm{O} 3 \\
-\mathrm{t}\end{array}$ & $\begin{array}{c}\mathrm{RhO} 2 \\
-\mathrm{t}\end{array}$ & $\begin{array}{c}\mathrm{RuO} 2 \\
-\mathrm{t}\end{array}$ & $\begin{array}{c}\mathrm{Sb} 2 \mathrm{O} 3 \\
-\mathrm{t}\end{array}$ & $\begin{array}{c}\mathrm{Sb} 2 \mathrm{O} 5 \\
-\mathrm{t}\end{array}$ & $\begin{array}{c}\mathrm{SeO} 2 \\
-\mathrm{t}\end{array}$ & $\begin{array}{c}\mathrm{Sm} 2 \mathrm{O} 3 \\
-\mathrm{t}\end{array}$ & $\begin{array}{c}\mathrm{SnO} \\
-\mathrm{t}\end{array}$ & $\begin{array}{c}\mathrm{SnO} 2 \\
-\mathrm{t}\end{array}$ & $\begin{array}{c}\mathrm{SO} 3 \\
-\mathrm{t}\end{array}$ & $\begin{array}{c}\mathrm{SrO} \\
-\mathrm{t}\end{array}$ & $\begin{array}{c}\mathrm{Tc} 2 \mathrm{O} 7 \\
-\mathrm{t}\end{array}$ & $\begin{array}{c}\mathrm{TeO} 2 \\
-\mathrm{t}\end{array}$ & $\begin{array}{c}\text { ThO2 } \\
-t\end{array}$ & $\begin{array}{c}\mathrm{TiO} 2 \\
-\mathrm{t}\end{array}$ \\
\hline CVS3-21 & 0.0000 & & 0.0000 & 0.0000 & & & 0.0000 & & 0.0000 & 0.0000 & & 0.0000 & 0.0000 & & & 0.0019 & 0.0000 & & 0.0000 & & 0.0000 \\
\hline CVS3-22 & 0.0000 & & 0.0000 & 0.0000 & & & 0.0000 & & 0.0000 & 0.0000 & & 0.0000 & 0.0000 & & & 0.0020 & 0.0000 & & 0.0000 & & 0.0000 \\
\hline CVS3-23 & 0.0000 & & 0.0000 & 0.0000 & & & 0.0000 & & 0.0000 & 0.0000 & & 0.0000 & 0.0000 & & & 0.0021 & 0.0000 & & 0.0000 & & 0.0000 \\
\hline CVS3-24 & 0.0000 & & 0.0000 & 0.0000 & & & 0.0000 & & 0.0000 & 0.0000 & & 0.0000 & 0.0000 & & & 0.0029 & 0.0000 & & 0.0000 & & 0.0000 \\
\hline CVS3-25 & 0.0000 & & 0.0000 & 0.0000 & & & 0.0000 & & 0.0000 & 0.0000 & & 0.0000 & 0.0000 & & & 0.0011 & 0.0000 & & 0.0000 & & 0.0000 \\
\hline CVS3-26 & 0.0000 & & 0.0019 & 0.0000 & & & 0.0000 & & 0.0015 & 0.0000 & & 0.0000 & 0.0000 & & & 0.0044 & 0.0000 & & 0.0000 & & 0.0000 \\
\hline CVS3-27 & 0.0000 & & 0.0000 & 0.0000 & & & 0.0000 & & 0.0000 & 0.0000 & & 0.0000 & 0.0000 & & & 0.0019 & 0.0000 & & 0.0000 & & 0.0000 \\
\hline CVS3-28 & 0.0000 & & 0.0000 & 0.0000 & & & 0.0000 & & 0.0000 & 0.0000 & & 0.0000 & 0.0000 & & & 0.0020 & 0.0000 & & 0.0000 & & 0.0000 \\
\hline CVS3-29 & 0.0000 & & 0.0000 & 0.0000 & & & 0.0000 & & 0.0000 & 0.0000 & & 0.0000 & 0.0000 & & & 0.0019 & 0.0000 & & 0.0000 & & 0.0000 \\
\hline CVS3-30 & 0.0000 & & 0.0000 & 0.0000 & & & 0.0000 & & 0.0000 & 0.0000 & & 0.0000 & 0.0000 & & & 0.0018 & 0.0000 & & 0.0000 & & 0.0000 \\
\hline CVS3-31 & 0.0000 & & 0.0000 & 0.0000 & & & 0.0000 & & 0.0000 & 0.0000 & & 0.0000 & 0.0000 & & & 0.0017 & 0.0000 & & 0.0000 & & 0.0000 \\
\hline CVS3-32 & 0.0000 & & 0.0000 & 0.0000 & & & 0.0000 & & 0.0000 & 0.0000 & & 0.0000 & 0.0000 & & & 0.0019 & 0.0000 & & 0.0000 & & 0.0000 \\
\hline CVS3-33 & 0.0000 & & 0.0000 & 0.0000 & & & 0.0000 & & 0.0000 & 0.0000 & & 0.0000 & 0.0000 & & & 0.0020 & 0.0000 & & 0.0000 & & 0.0000 \\
\hline CVS3-34 & 0.0000 & & 0.0000 & 0.0000 & & & 0.0000 & & 0.0000 & 0.0000 & & 0.0000 & 0.0000 & & & 0.0019 & 0.0000 & & 0.0000 & & 0.0000 \\
\hline CVS3-35 & 0.0000 & & 0.0000 & 0.0000 & & & 0.0000 & & 0.0000 & 0.0000 & & 0.0000 & 0.0000 & & & 0.0018 & 0.0000 & & 0.0000 & & 0.0000 \\
\hline CVS3-36 & 0.0000 & & 0.0000 & 0.0000 & & & 0.0000 & & 0.0000 & 0.0000 & & 0.0000 & 0.0000 & & & 0.0018 & 0.0000 & & 0.0000 & & 0.0000 \\
\hline CVS3-37 & 0.0000 & & 0.0000 & 0.0000 & & & 0.0000 & & 0.0000 & 0.0000 & & 0.0000 & 0.0000 & & & 0.0020 & 0.0000 & & 0.0000 & & 0.0000 \\
\hline CVS3-38 & 0.0000 & & 0.0000 & 0.0000 & & & 0.0000 & & 0.0000 & 0.0000 & & 0.0000 & 0.0000 & & & 0.0020 & 0.0000 & & 0.0000 & & 0.0000 \\
\hline CVS3-39 & 0.0000 & & 0.0000 & 0.0000 & & & 0.0000 & & 0.0000 & 0.0000 & & 0.0000 & 0.0000 & & & 0.0019 & 0.0000 & & 0.0000 & & 0.0000 \\
\hline CVS3-40 & 0.0000 & & 0.0000 & 0.0000 & & & 0.0000 & & 0.0000 & 0.0000 & & 0.0000 & 0.0000 & & & 0.0019 & 0.0000 & & 0.0000 & & 0.0000 \\
\hline
\end{tabular}

TRU Study (Crum et al. 1997)

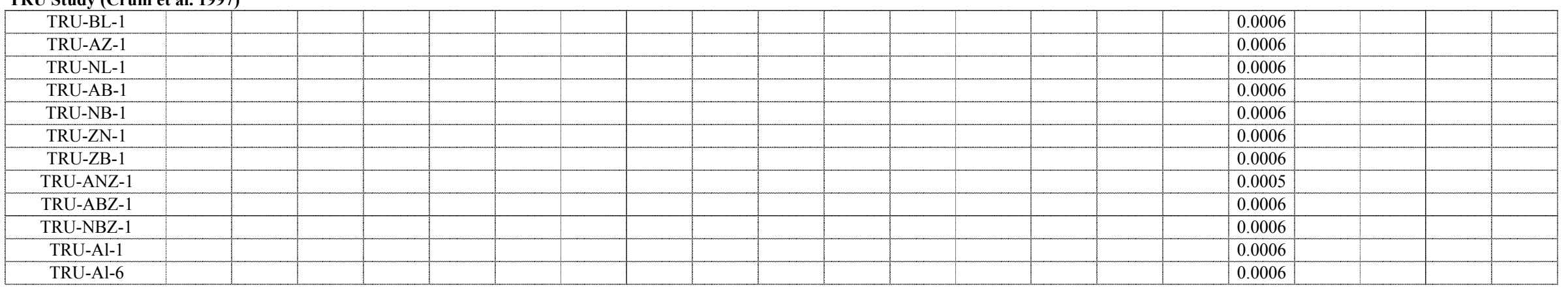


Appendix A. Database - mass fraction

\begin{tabular}{|c|c|c|c|c|c|c|c|c|c|c|c|c|c|c|c|c|c|c|c|c|c|}
\hline CVS3-21 & & & 0.0000 & & & 0.0010 & 0.0000 & 0.0000 & & 1.0000 & & & & & & & & & & & \\
\hline CVS3-22 & & & 0.0000 & & & 0.0010 & 0.0000 & 0.0000 & & 1.0000 & & & & & & & & & & & \\
\hline CVS3-23 & & & 0.0000 & & & 0.0011 & 0.0000 & 0.0000 & & 1.0000 & & & & & & & & & & & \\
\hline CVS3-24 & & & 0.0000 & & & 0.0015 & 0.0000 & 0.0000 & & 1.0000 & & & & & & & & & & & \\
\hline CVS3-25 & & & 0.0000 & & & 0.0006 & 0.0000 & 0.0000 & & 1.0000 & & & & & & & & & & & \\
\hline CVS3-26 & & & 0.0000 & & & 0.0000 & 0.0000 & 0.0049 & & 1.0000 & & & & & & & & & & & \\
\hline CVS3-27 & & & 0.0000 & & & 0.0010 & 0.0000 & 0.0000 & & 1.0000 & & & & & & & & & & & \\
\hline CVS3-28 & & & 0.0000 & & & 0.0010 & 0.0000 & 0.0000 & & 1.0000 & & & & & & & & & & & \\
\hline CVS3-29 & & & 0.0000 & & & 0.0010 & 0.0000 & 0.0000 & & 1.0000 & & & & & & & & & & & \\
\hline CVS3-30 & & & 0.0000 & & & 0.0010 & 0.0000 & 0.0000 & & 1.0000 & & & & & & & & & & & \\
\hline CVS3-31 & & & 0.0000 & & & 0.0009 & 0.0000 & 0.0000 & & 1.0000 & & & & & & & & & & & \\
\hline CVS3-32 & & & 0.0900 & & & 0.0010 & 0.0000 & 0.0000 & & 1.0000 & & & & & & & & & & & \\
\hline CVS3-33 & & & 0.0320 & & & 0.0011 & 0.0000 & 0.0000 & & 1.0000 & & & & & & & & & & & \\
\hline CVS3-34 & & & 0.0610 & & & 0.0010 & 0.0000 & 0.0000 & & 1.0000 & & & & & & & & & & & \\
\hline CVS3-35 & & & 0.1190 & & & 0.0010 & 0.0000 & 0.0000 & & 1.0000 & & & & & & & & & & & \\
\hline CVS3-36 & & & 0.1480 & & & 0.0009 & 0.0000 & 0.0000 & & 1.0000 & & & & & & & & & & & \\
\hline CVS3-37 & & & 0.0000 & & & 0.0011 & 0.0000 & 0.0000 & & 1.0000 & & & & & & & & & & & \\
\hline CVS3-38 & & & 0.0000 & & & 0.0010 & 0.0000 & 0.0000 & & 1.0000 & & & & & & & & & & & \\
\hline CVS3-39 & & & 0.0000 & & & 0.0010 & 0.0000 & 0.0000 & & 1.0000 & & & & & & & & & & & \\
\hline CVS3-40 & & & 0.0000 & & & 0.0010 & 0.0000 & 0.0000 & & 1.0000 & & & & & & & & & & & \\
\hline
\end{tabular}

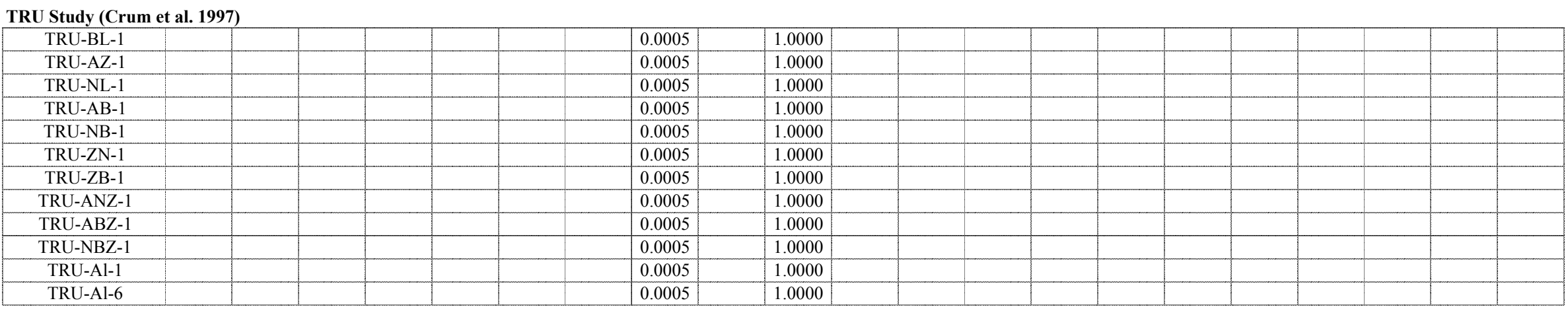


Appendix A. Database - mass fraction

\begin{tabular}{|c|c|c|c|c|c|c|c|c|c|c|c|c|c|c|c|c|c|c|c|c|c|}
\hline \multicolumn{22}{|c|}{ Hanford CVS 3 (Vienna et al. 1996) } \\
\hline Glass ID & $\begin{array}{c}\mathrm{SiO} 2 \\
-\mathrm{a} \\
\end{array}$ & $\begin{array}{c}\mathrm{ZrO} 2 \\
-\mathrm{a}\end{array}$ & $\begin{array}{c}\mathrm{Ag} 2 \mathrm{O} \\
-\mathrm{a}\end{array}$ & $\begin{array}{c}\mathrm{As} 2 \mathrm{O} 3 \\
-\mathrm{a} \\
\end{array}$ & $\begin{array}{c}\mathrm{BaO} \\
-\mathrm{a}\end{array}$ & $\begin{array}{c}\mathrm{Bi} 2 \mathrm{O} 3 \\
-\mathrm{a}\end{array}$ & $\begin{array}{l}\mathrm{Br} \\
-\mathrm{a}\end{array}$ & $\begin{array}{c}\mathrm{CdO} \\
-\mathrm{a}\end{array}$ & $\begin{array}{c}\mathrm{Ce} 2 \mathrm{O} 3 \\
-\mathrm{a}\end{array}$ & $\begin{array}{c}\mathrm{CeO} 2 \\
-\mathrm{a}\end{array}$ & $\begin{array}{l}\mathrm{Cl} \\
-\mathrm{a}\end{array}$ & $\begin{array}{c}\mathrm{CoO} \\
-\mathrm{a}\end{array}$ & $\begin{array}{c}\mathrm{Co} 2 \mathrm{O} 3 \\
-\mathrm{a}\end{array}$ & $\begin{array}{c}\mathrm{Cr} 2 \mathrm{O} 3 \\
-\mathrm{a}\end{array}$ & $\begin{array}{c}\mathrm{Cs} 2 \mathrm{O} \\
-\mathrm{a}\end{array}$ & $\begin{array}{c}\mathrm{CuO} \\
-\mathrm{a}\end{array}$ & $\begin{array}{c}\mathrm{Eu} 2 \mathrm{O} 3 \\
-\mathrm{a}\end{array}$ & $\begin{array}{l}\mathrm{F} \\
-\mathrm{a}\end{array}$ & $\begin{array}{c}\mathrm{Ga} 2 \mathrm{O} 3 \\
-\mathrm{a}\end{array}$ & $\begin{array}{c}\mathrm{Gd} 2 \mathrm{O} 3 \\
-\mathrm{a}\end{array}$ & $\begin{array}{c}\mathrm{HgO} \\
-\mathrm{a}\end{array}$ \\
\hline \multicolumn{22}{|l|}{ CVS3-21 } \\
\hline \multicolumn{22}{|l|}{ CVS3-22 } \\
\hline \multicolumn{22}{|l|}{ CVS3-23 } \\
\hline \multicolumn{22}{|l|}{ CVS3-24 } \\
\hline \multicolumn{22}{|l|}{ CVS3-25 } \\
\hline \multicolumn{22}{|l|}{ CVS3-26 } \\
\hline \multicolumn{22}{|l|}{ CVS3-27 } \\
\hline \multicolumn{22}{|l|}{ CVS3-28 } \\
\hline \multicolumn{22}{|l|}{ CVS3-29 } \\
\hline \multicolumn{22}{|l|}{ CVS3-30 } \\
\hline \multicolumn{22}{|l|}{ CVS3-31 } \\
\hline \multicolumn{22}{|l|}{ CVS3-32 } \\
\hline \multicolumn{22}{|l|}{ CVS3-33 } \\
\hline \multicolumn{22}{|l|}{ CVS3-34 } \\
\hline \multicolumn{22}{|l|}{ CVS3-35 } \\
\hline \multicolumn{22}{|l|}{ CVS3-36 } \\
\hline \multicolumn{22}{|l|}{ CVS3-37 } \\
\hline \multicolumn{22}{|l|}{ CVS3-38 } \\
\hline \multicolumn{22}{|l|}{ CVS3-39 } \\
\hline CVS3-40 & & & & & & & & & & & & & & & & & & & & & \\
\hline
\end{tabular}

TRU Study (Crum et al. 1997)

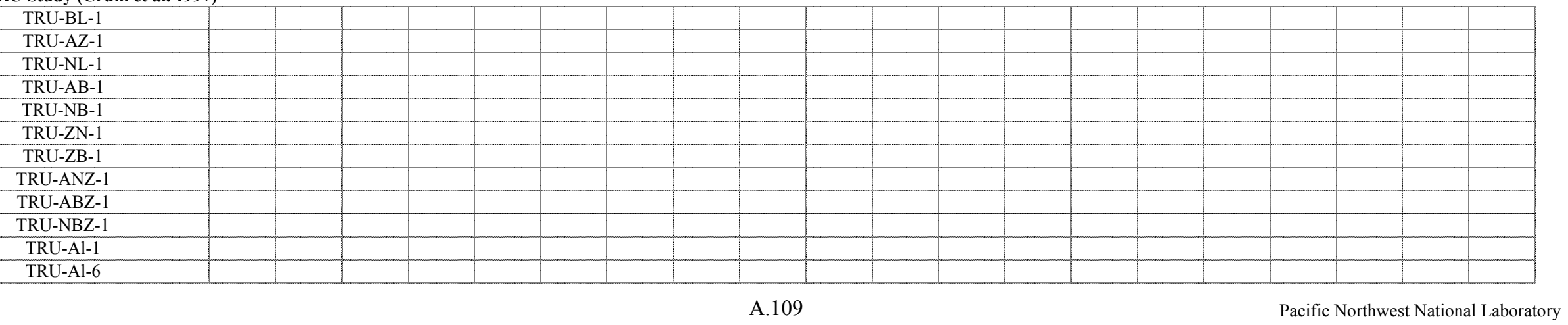


Appendix A. Database - mass fraction

Hanford CVS 3 (Vienna et al. 1996)

\begin{tabular}{|c|c|c|c|c|c|c|c|c|c|c|c|c|c|c|c|c|c|c|c|c|c|}
\hline Glass ID & $\begin{array}{l}\mathrm{I} \\
-\mathrm{a} \\
\end{array}$ & $\begin{array}{c}\mathrm{La} 2 \mathrm{O} 3 \\
-\mathrm{a}\end{array}$ & $\begin{array}{c}\mathrm{MnO} 2 \\
-\mathrm{a}\end{array}$ & $\begin{array}{c}\mathrm{MnO} \\
-\mathrm{a}\end{array}$ & $\begin{array}{c}\mathrm{MoO} \\
-\mathrm{a}\end{array}$ & $\begin{array}{c}\mathrm{MoO} 3 \\
-\mathrm{a}\end{array}$ & $\begin{array}{c}\mathrm{Nb} 2 \mathrm{O} 5 \\
-\mathrm{a}\end{array}$ & $\begin{array}{c}\mathrm{Nd} 2 \mathrm{O} 3 \\
-\mathrm{a}\end{array}$ & $\begin{array}{c}\mathrm{PbO} \\
-\mathrm{a}\end{array}$ & $\begin{array}{c}\mathrm{PdO} 2 \\
-\mathrm{a}\end{array}$ & $\begin{array}{c}\mathrm{PdO} \\
-\mathrm{a}\end{array}$ & $\begin{array}{c}\mathrm{Pr} 2 \mathrm{O} 3 \\
-\mathrm{a}\end{array}$ & $\begin{array}{c}\text { Pr6O11 } \\
-\mathrm{a}\end{array}$ & $\begin{array}{c}\mathrm{Rb} 2 \mathrm{O} \\
-\mathrm{a}\end{array}$ & $\begin{array}{c}\mathrm{ReO} \\
-\mathrm{a}\end{array}$ & $\begin{array}{c}\mathrm{ReO} 2 \\
-\mathrm{a}\end{array}$ & $\begin{array}{c}\mathrm{Rh} 2 \mathrm{O} 3 \\
-\mathrm{a}\end{array}$ & $\begin{array}{c}\mathrm{RhO} 2 \\
-\mathrm{a}\end{array}$ & $\begin{array}{c}\mathrm{RuO} 2 \\
-\mathrm{a}\end{array}$ & $\begin{array}{c}\mathrm{Sb} 2 \mathrm{O} 3 \\
-\mathrm{a}\end{array}$ & $\begin{array}{c}\mathrm{Sb} 2 \mathrm{O} 5 \\
-\mathrm{a}\end{array}$ \\
\hline CVS3-21 & & & & & & & & & & & & & & & & & & & & & \\
\hline CVS3-22 & & & & & & & & & & & & & & & & & & & & & \\
\hline CVS3-23 & & & & & & & & & & & & & & & & & & & & & \\
\hline CVS3-24 & & & & & & & & & & & & & & & & & & & & & \\
\hline CVS3-25 & & & & & & & & & & & & & & & & & & & & & \\
\hline CVS3-26 & & & & & & & & & & & & & & & & & & & & & \\
\hline CVS3-27 & & & & & & & & & & & & & & & & & & & & & \\
\hline CVS3-28 & & & & & & & & & & & & & & & & & & & & & \\
\hline CVS3-29 & & & & & & & & & & & & & & & & & & & & & \\
\hline CVS3-30 & & & & & & & & & & & & & & & & & & & & & \\
\hline CVS3-31 & & & & & & & & & & & & & & & & & & & & & \\
\hline CVS3-32 & & & & & & & & & & & & & & & & & & & & & \\
\hline CVS3-33 & & & & & & & & & & & & & & & & & & & & & \\
\hline CVS3-34 & & & & & & & & & & & & & & & & & & & & & \\
\hline CVS3-35 & & & & & & & & & & & & & & & & & & & & & \\
\hline CVS3-36 & & & & & & & & & & & & & & & & & & & & & \\
\hline CVS3-37 & & & & & & & & & & & & & & & & & & & & & \\
\hline CVS3-38 & & & & & & & & & & & & & & & & & & & & & \\
\hline CVS3-39 & & & & & & & & & & & & & & & & & & & & & \\
\hline CVS3-40 & & & & & & & & & & & & & & & & & & & & & \\
\hline
\end{tabular}

TRU Study (Crum et al. 1997)

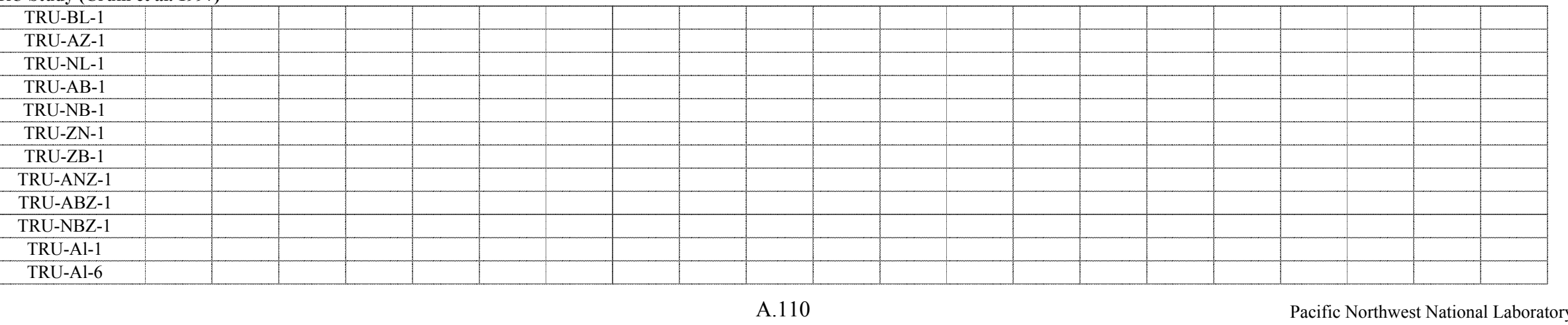


Appendix A. Database - mass fraction

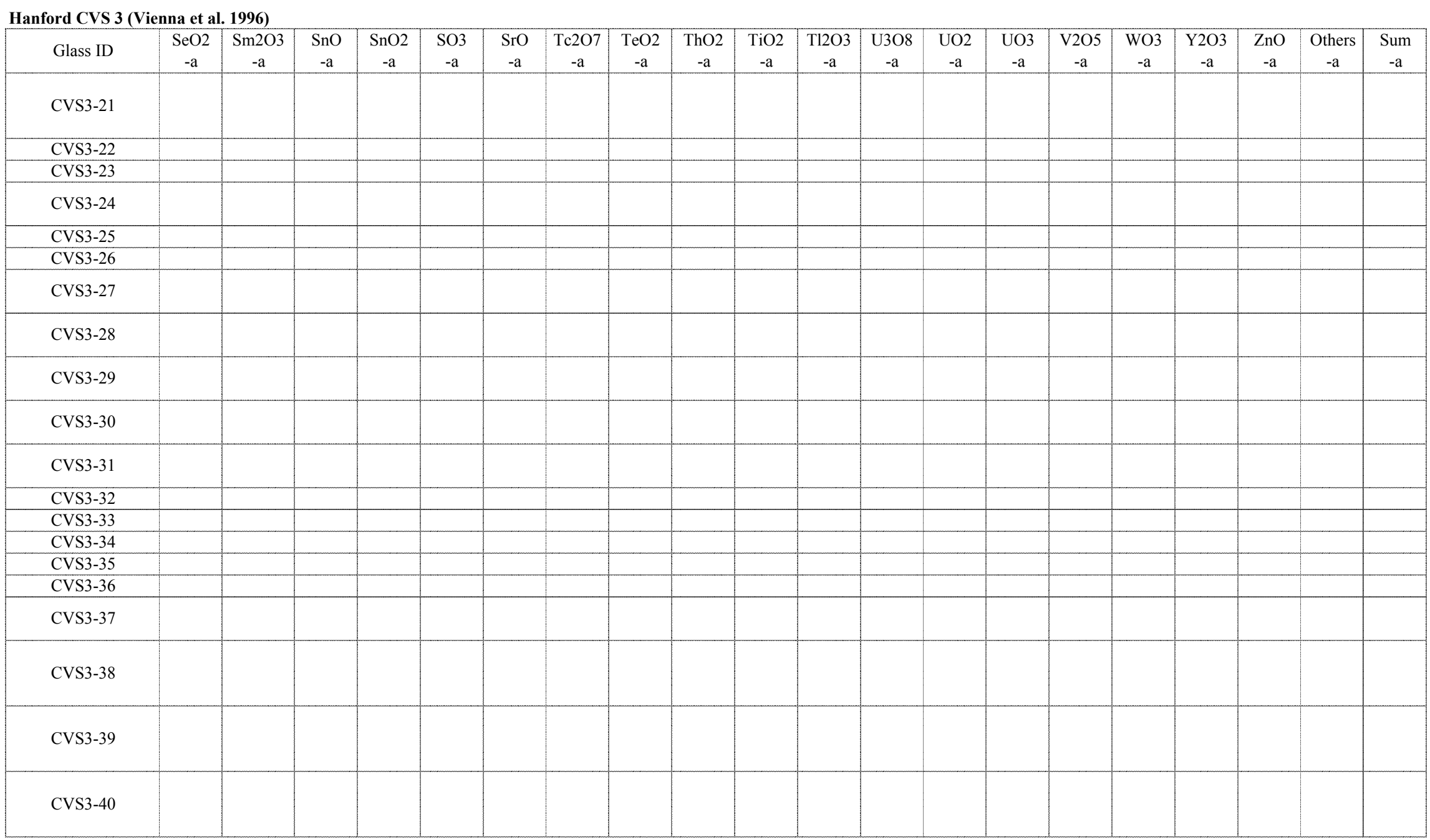

TRU Study (Crum et al. 1997)

TRU-BL-1
TRU-AZ-1


Appendix A. Database - mass fraction

\section{Hanford CVS 3 (Vienna et al. 1996)}

\begin{tabular}{|c|c|c|c|c|c|c|c|c|}
\hline Glass ID & $\begin{array}{l}\mathrm{TM} \\
\left({ }^{\circ} \mathrm{C}\right) \\
\end{array}$ & $\begin{array}{l}\text { Gradient } \\
\text { TL }\left({ }^{\circ} \mathrm{C}\right) \\
\end{array}$ & $\begin{array}{l}\text { Uniform } \\
\text { TL }\left({ }^{\circ} \mathrm{C}\right) \\
\end{array}$ & Primary Phase & $\begin{array}{l}\text { Quenched } \\
\text { Visual/OM }\end{array}$ & $\begin{array}{c}\text { Quenched } \\
\text { SEM/EDS or TEM }\end{array}$ & $\begin{array}{l}\text { Quenched } \\
\text { XRD } \\
\end{array}$ & $\begin{array}{c}\text { Quenched } \\
\text { Homogeneous? }\end{array}$ \\
\hline CVS3-21 & & & 1400 & zircon & $.32 \%$ same as $3-20$ & & & \\
\hline CVS3-22 & & & 1177 & spinel & No XL & & & \\
\hline CVS3-23 & & & 1122 & spinel & No XL & & & \\
\hline CVS3-24 & & & 1127 & spinel & very small particles inhomo & spinel & amorphous & \\
\hline CVS3-25 & & & 1150 & spinel & No XL & & & \\
\hline CVS3-26 & & & 1047 & spinel & & & & \\
\hline CVS3-27 & & & 1285 & spinel & scattered spinel & & & \\
\hline CVS3-28 & & & 1141 & spinel & No XL & & & \\
\hline CVS3-29 & & & 1136 & spinel & No XL & & & \\
\hline CVS3-30 & & & 1121 & spinel & No XL & & & \\
\hline CVS3-31 & & & 1109 & spinel & No XL & & & \\
\hline CVS3-32 & & & & & & & amorphous & \\
\hline CVS3-33 & & & & & & & amorphous & \\
\hline CVS3-34 & & & & & & & amorphous & \\
\hline CVS3-35 & & & & & & & amorphous & \\
\hline CVS3-36 & & & & & & & amorphous & \\
\hline CVS3-37 & & & 1175 & spinel & No XL & & amorphous & \\
\hline CVS3-38 & & & 1171 & spinel & No XL & & 1 vol $\%$ XL, NaNdPO4 & \\
\hline CVS3-39 & & & 1168 & spinel & No XL & & 3 vol $\%$ XL, NaNdPO4 & \\
\hline CVS3-40 & & & 1198 & Zr Re Oy & No XL & phase separated & 7 vol\% XL, NaNdPO4 & \\
\hline
\end{tabular}

TRU Study (Crum et al. 1997)

\begin{tabular}{|c|c|c|c|c|c|}
\hline TRU-BL-1 & & 1012 & Zircon & & \\
\hline TRU-AZ-1 & & 1065 & Zircon & & \\
\hline TRU-NL-1 & & 1095 & Zircon & & \\
\hline TRU-AB-1 & & 1056 & Zircon & & \\
\hline TRU-NB-1 & & 1161 & Zircon & & \\
\hline TRU-ZN-1 & & 988 & Zircon & & \\
\hline TRU-ZB-1 & & 1094 & Zircon & \\
\hline TRU-ANZ-1 & & 1032 & Zircon & \\
\hline TRU-ABZ-1 & & 1151 & Zircon & & \\
\hline TRU-NBZ-1 & & 1010 & Zircon & \\
\hline TRU-Al-1 & & 1196 & ZrO2 & \\
\hline TRU-Al-6 & & 1069 & Zircon & \\
\hline
\end{tabular}


Appendix A. Database - mass fraction

\begin{tabular}{|c|c|c|c|c|c|}
\hline \multicolumn{6}{|c|}{ Hanford CVS 3 (Vienna et al. 1996) } \\
\hline Glass ID & $\begin{array}{c}\mathrm{CCC} \\
\text { Visual/OM }\end{array}$ & $\begin{array}{c}\text { CCC } \\
\text { SEM/EDS or TEM }\end{array}$ & $\begin{array}{l}\text { CCC } \\
\text { XRD }\end{array}$ & $\begin{array}{l}\text { Heat Treated } \\
\text { Visual/OM }\end{array}$ & $\begin{array}{c}\text { Heat Treated } \\
\text { SEM/EDS or TEM }\end{array}$ \\
\hline CVS3-21 & $3.6 \% \mathrm{XL}$ more than one phase & $\begin{array}{l}\text { Zr, spinel, hiCr spinel } \\
\text { (spinel in } \mathrm{Zr} \text { interstecies) } \\
\text { streaks of } \mathrm{Cr} \text { rich area }\end{array}$ & 10 vol\% XL, 60w\% ZrO2, 40w\% sp & & \\
\hline CVS3-22 & $1.6 \% \mathrm{XL}$ same as $3-21$ & & & & \\
\hline CVS3-23 & few tiny $\mathrm{XL}$ & & & & \\
\hline CVS3-24 & $3.4 \% \mathrm{XL}$ many phase inhomo & $\begin{array}{l}(\mathrm{Si}, \mathrm{Ca}, \mathrm{Nd}, \mathrm{Ce}), \mathrm{hiCr} \mathrm{sp} \\
\text { spinel }\end{array}$ & $5 \% \mathrm{Sp}$ & & \\
\hline CVS3-25 & No XL & & & & \\
\hline CVS3-26 & & & & & \\
\hline CVS3-27 & Lots of colorful flowers and Botom agglom of XL & $\begin{array}{l}\text { hiCr sp, spinel, (Nd, Si, Ca, } \\
\mathrm{Ce} \text { ), }\end{array}$ & $\begin{array}{l}\text { 10vol\% XL, 70wt \% Spinel, 30wt\% } \\
\text { Ca5((Si,P,S)O4)3F }\end{array}$ & & \\
\hline CVS3-28 & $<0.01 \mathrm{vol} \% \mathrm{sp}$ & $\begin{array}{l}\text { star shaped } \mathrm{sp}(\mathrm{Cr}, \mathrm{Ni}, \mathrm{Fe}, \\
\mathrm{Mn})\end{array}$ & Amorphous & & \\
\hline CVS3-29 & $<0.01$ vol\% sp & $\begin{array}{l}\text { star shaped } \mathrm{sp}(\mathrm{Cr}, \mathrm{Ni}, \mathrm{Fe}, \\
\mathrm{Mn})\end{array}$ & Amorphous & & \\
\hline CVS3-30 & $<0.01$ vol\% sp & $\begin{array}{l}\text { star shaped } \mathrm{sp}(\mathrm{Cr}, \mathrm{Ni}, \mathrm{Fe}, \\
\mathrm{Mn})\end{array}$ & Amorphous & & \\
\hline CVS3-31 & few tiny $\mathrm{XL}$ & $\begin{array}{l}\text { cube shaped } \mathrm{sp}(\mathrm{Cr}, \mathrm{Ni} \\
\text { mostly) }\end{array}$ & Amorphous & & \\
\hline CVS3-32 & & & Amorphous & & \\
\hline CVS3-33 & & & Amorphous & & \\
\hline CVS3-34 & & & small amt of $\mathrm{Al} 2 \mathrm{O} 3, \mathrm{cp}$ & & \\
\hline CVS3-35 & & & small amt of $\mathrm{Ce} 2 \mathrm{O} 3$ and $\mathrm{cp}$ & & \\
\hline CVS3-36 & & & $\mathrm{cp}, \mathrm{CeO} 2$ type crystals & & \\
\hline CVS3-37 & $<0.01 \mathrm{vol} \%$ & $\mathrm{sp}(\mathrm{Cr}, \mathrm{Ni}, \mathrm{Fe}, \mathrm{Mn})$ & $\begin{array}{l}2 \text { vol\% XL, Na3Nd(PO4)2, Li3PO4, } \\
\text { NdPO4 }\end{array}$ & & \\
\hline CVS3-38 & $0.21 \mathrm{vol} \%$ & $\begin{array}{l}\text { Phase separated region of } \\
\text { NaCaNdCeFe, sp, possible } \\
\text { Li3PO4 }\end{array}$ & $\begin{array}{l}8 \text { vol\% XL, Na3Nd(PO4)2, Li3PO4, } \\
\text { NdPO4, sp }\end{array}$ & & \\
\hline CVS3-39 & $1.58 \mathrm{vol} \%$ & $\begin{array}{l}\text { Phase separated region of } \\
\text { NaCaNdLaCeFePNi, sp, } \\
\text { possible Li3PO4 }\end{array}$ & $\begin{array}{l}17 \text { vol\% XL, Na3Nd(PO4)2, Li3PO4, } \\
\text { NdPO4, sp }\end{array}$ & & \\
\hline CVS3-40 & $3.19 \mathrm{vol} \%$ & $\begin{array}{l}\text { Phase separated region of } \\
\text { NaNdCaPFe, sp, possible } \\
\text { Li3PO4 }\end{array}$ & $\begin{array}{l}22 \text { vol\% XL, Na3Nd(PO4)2, Li3PO4, } \\
\text { NdPO4, sp }\end{array}$ & & \\
\hline
\end{tabular}

TRU Study (Crum et al. 1997)

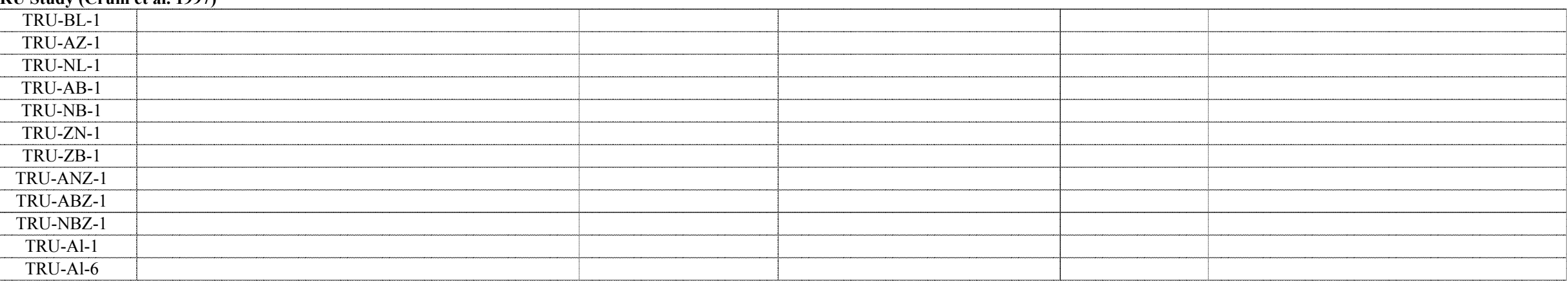


Appendix A. Database - mass fraction

Hanford CVS 3 (Vienna et al. 1996)

\begin{tabular}{|c|c|c|c|c|c|c|c|c|c|c|c|c|c|c|c|}
\hline Glass ID & $\begin{array}{c}\text { Heat Treated } \\
\text { XRD } \\
\end{array}$ & $\begin{array}{l}\text { Density } \\
\left(\mathrm{g} / \mathrm{cm}^{3}\right)\end{array}$ & $\begin{array}{c}\text { Fulc Visc } \\
\mathrm{A}\end{array}$ & \begin{tabular}{|c|} 
Fulc Visc \\
$\mathrm{B}$ \\
\end{tabular} & \begin{tabular}{|c|} 
Fulc Visc \\
To \\
\end{tabular} & $\begin{array}{c}\mathrm{FV} 1150^{\circ} \mathrm{C} \\
(\mathrm{Pa} \cdot \mathrm{s})\end{array}$ & \begin{tabular}{|c|} 
Arrh Visc \\
A
\end{tabular} & \begin{tabular}{|c|} 
Arrh Visc \\
$\mathrm{B}$ \\
\end{tabular} & $\begin{array}{c}1150^{\circ} \mathrm{C} \\
\left(\mathrm{D}_{0.0}\right)\end{array}$ & $\begin{array}{c}\mathrm{T}\left({ }^{\circ} \mathrm{C}\right) \text { at } \\
2 \mathrm{~Pa} \cdot \mathrm{s}\end{array}$ & $\begin{array}{c}\mathrm{T}\left({ }^{\circ} \mathrm{C}\right) \text { at } \\
5 \mathrm{~Pa} \cdot \mathrm{s} \\
\end{array}$ & \begin{tabular}{|c|}
$\mathrm{T}\left({ }^{\circ} \mathrm{C}\right)$ at \\
$10 \mathrm{~Pa} \cdot \mathrm{s}$ \\
\end{tabular} & $\begin{array}{l}\mathrm{T} 1 \\
\left({ }^{\circ} \mathrm{C}\right) \\
\end{array}$ & $\begin{array}{c}\mathrm{V} 1 \\
(\mathrm{~Pa} \cdot \mathrm{s}) \\
\end{array}$ & $\begin{array}{c}\mathrm{T} 2 \\
\left({ }^{\circ} \mathrm{C}\right) \\
\end{array}$ \\
\hline CVS3-21 & & & & & & & -14.540 & 26635.5 & 65.23 & & & & 1144 & 73.108 & 1195 \\
\hline CVS3-22 & & & -6.41 & 7887.1 & 655.4 & & -13.330 & 24483.5 & 48.21 & & & & 1094 & 106.9 & 1144 \\
\hline CVS3-23 & & & -6.13 & 8624.7 & 513 & & -10.230 & 19285.4 & 27.73 & & & & 1144 & 30.252 & 1194 \\
\hline CVS3-24 & & & -10.59 & 17178 & 188.2 & & -12.460 & 22463.8 & 27.83 & & & & 1093 & 54.8630 & 1144 \\
\hline CVS3-25 & & & -6.4 & 9108.6 & 547 & & -10.890 & 21058.0 & 49.81 & & & & 1193 & 33.2710 & 1244 \\
\hline CVS3-26 & & & & & & & & & & & & & & & \\
\hline CVS3-27 & & & & & & & -12.300 & 22012.2 & 23.78 & & & & 1094 & 43.412 & 1144 \\
\hline CVS3-28 & & & -5.28 & 6550.7 & 671.7 & & -11.730 & 21601.3 & 31.50 & & & & 1093 & 63.952 & 1144 \\
\hline CVS3-29 & & & -5.73 & 7242.1 & 623.2 & & -11.620 & 21281.6 & 28.09 & & & & 1093 & 55.661 & 1144 \\
\hline CVS3-30 & & & -5.15 & 6062.3 & 677 & & -12.090 & 21496.7 & 20.42 & & & & 1043 & 76.066 & 1093 \\
\hline CVS3-31 & & & -6.05 & 7152.5 & 601.3 & & -12.850 & 22130.1 & 14.91 & & & & 993 & 110.8590 & 1043 \\
\hline CVS3-32 & & & -4.86 & 5810.8 & 762.6 & & -12.060 & 22652.7 & 47.42 & & & & 1160 & 45.21 & 1241 \\
\hline CVS3-33 & & & -4.05 & 4904.8 & 816.2 & & -11.700 & 22277.1 & 52.20 & & & & 1162 & 48.4700 & 1234 \\
\hline CVS3-34 & & & -2.26 & 2504.3 & 1035.1 & & -12.320 & 23248.5 & 55.57 & & & & 1160 & 56.8020 & 1243 \\
\hline CVS3-35 & & & -3.44 & 3717.8 & 917.3 & & -12.530 & 23315.2 & 47.21 & & & & 1136.5 & 60.795 & 1242 \\
\hline CVS3-36 & & & -1.58 & 1563.1 & 1154.7 & & -10.750 & 20346.7 & 34.76 & & & & $(1136.5)$ & 94.1288 & 1243 \\
\hline CVS3-37 & & & -6.25 & 8214.7 & 606.9 & & -11.860 & 22236.4 & 43.23 & & & & 1144 & 48.668 & 1194 \\
\hline CVS3-38 & & & -6.4 & 8599.9 & 590 & & -11.900 & 22463.8 & 48.73 & & & & 1144 & 54.859 & 1194 \\
\hline CVS3-39 & & & -4.46 & 5383.3 & 795.1 & & -12.000 & 22833.0 & 57.15 & & & & 1144 & 66.2050 & 1194 \\
\hline CVS3-40 & & & -5.8 & 7434.1 & 687.1 & & -12.190 & 23294.0 & 65.34 & & & & 1194 & 42.0180 & 1245 \\
\hline
\end{tabular}

TRU Study (Crum et al. 1997)

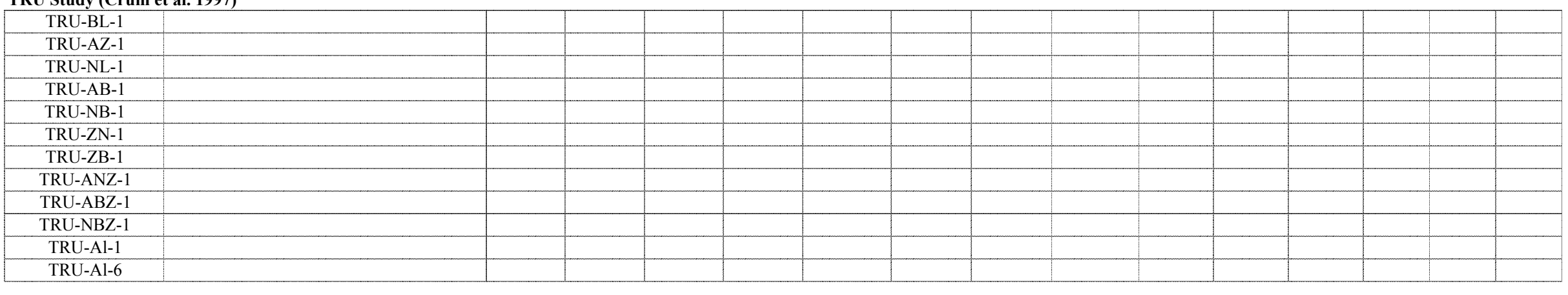




\section{Hanford CVS 3 (Vienna et al. 1996)}

\begin{tabular}{|c|c|c|c|c|c|c|c|c|c|c|c|c|c|c|c|c|c|c|c|c|c|}
\hline Glass ID & $\begin{array}{c}\mathrm{V} 2 \\
(\mathrm{~Pa} \cdot \mathrm{s})\end{array}$ & $\begin{array}{c}\mathrm{T} 3 \\
\left({ }^{\circ} \mathrm{C}\right) \\
\end{array}$ & $\begin{array}{c}\mathrm{V} 3 \\
(\mathrm{~Pa} \cdot \mathrm{s})\end{array}$ & $\begin{array}{c}\mathrm{T} 4 \\
\left({ }^{\circ} \mathrm{C}\right) \\
\end{array}$ & $\begin{array}{c}\mathrm{V} 4 \\
(\mathrm{~Pa} \cdot \mathrm{s})\end{array}$ & $\begin{array}{c}\mathrm{T} 5 \\
\left({ }^{\circ} \mathrm{C}\right) \\
\end{array}$ & $\begin{array}{c}\text { V5 } \\
(\mathrm{Pa} \cdot \mathrm{s})\end{array}$ & $\begin{array}{c}\mathrm{T} 6 \\
\left({ }^{\circ} \mathrm{C}\right) \\
\end{array}$ & $\begin{array}{c}\mathrm{V} 6 \\
(\mathrm{~Pa} \cdot \mathrm{s})\end{array}$ & $\begin{array}{c}\mathrm{T} 7 \\
\left({ }^{\circ} \mathrm{C}\right) \\
\end{array}$ & $\begin{array}{c}\mathrm{V} 7 \\
(\mathrm{~Pa} \cdot \mathrm{s})\end{array}$ & $\begin{array}{c}\mathrm{T} 8 \\
\left({ }^{\circ} \mathrm{C}\right) \\
\end{array}$ & $\begin{array}{c}\mathrm{V} 8 \\
(\mathrm{~Pa} \cdot \mathrm{s})\end{array}$ & $\begin{array}{c}\mathrm{T} 9 \\
\left({ }^{\circ} \mathrm{C}\right) \\
\end{array}$ & $\begin{array}{c}\mathrm{V} 9 \\
\text { (Pa.s) }\end{array}$ & $\begin{array}{l}\mathrm{T} 10 \\
\left({ }^{\circ} \mathrm{C}\right)\end{array}$ & $\begin{array}{c}\mathrm{V} 10 \\
\mathrm{~Pa} \cdot \mathrm{s})\end{array}$ & $\begin{array}{l}\mathrm{T} 11 \\
\left({ }^{\circ} \mathrm{C}\right)\end{array}$ & $\begin{array}{c}\mathrm{V} 11 \\
(\mathrm{~Pa} \cdot \mathrm{s})\end{array}$ & $\begin{array}{l}\mathrm{T} 12 \\
\left({ }^{\circ} \mathrm{C}\right)\end{array}$ & $\begin{array}{c}\mathrm{V} 12 \\
(\mathrm{~Pa} \cdot \mathrm{s})\end{array}$ \\
\hline CVS3-21 & 32.159 & 1245 & 21.558 & 1294 & 12.44 & 1295 & 11.883 & 1344 & 6.7220 & 1345 & 7.1756 & 1346 & 6.1095 & 1395 & 4.1889 & 1446 & 2.5708 & & & & \\
\hline CVS3-22 & 51.787 & 1195 & 27.589 & 1244 & 15.097 & 1294 & 9.2201 & 1295 & 9.4172 & 1344 & 5.9037 & 1345 & 6.0961 & 1345 & 6.1269 & 1395 & 3.9640 & 1446 & 2.7156 & & \\
\hline CVS3-23 & 18.578 & 1244 & 11.646 & 1294 & 7.5975 & 1294 & 7.7946 & 1344 & 5.2110 & 1345 & 5.4496 & 1345 & 5.5727 & 1396 & 3.8101 & 1446 & 2.7525 & & & & \\
\hline CVS3-24 & 29.055 & 1194 & 17.379 & 1244 & 10.373 & 1244 & 10.615 & 1294 & 6.3655 & 1295 & 6.5503 & 1295 & 6.3732 & 1345 & 4.1257 & 1396 & 2.7741 & & & & \\
\hline CVS3-25 & 19.924 & 1293 & 12.738 & 1343 & 8.1889 & 1344 & 8.2799 & 1393 & 5.6497 & 1394 & 5.7267 & 1394 & 5.6728 & 1444 & 3.9025 & 1495 & 2.9126 & & & & \\
\hline CVS3-26 & & & & & & & & & & & & & & & & & & & & & \\
\hline CVS3-27 & 23.025 & 1195 & 16.413 & 1244 & 9.7660 & 1245 & 9.4476 & 1295 & 5.5189 & 1295 & 5.7190 & 1296 & 5.2648 & 1345 & 3.5946 & 1396 & 2.3676 & & & & \\
\hline CVS3-28 & 33.534 & 1194 & 19.153 & 1244 & 11.928 & 1244 & 12.034 & 1293 & 7.6358 & 1294 & 7.6509 & 1295 & 7.6057 & 1344 & 5.2702 & 1395 & 3.6597 & & & & \\
\hline CVS3-29 & 29.98 & 1194 & 17.1000 & 1244 & 10.633 & 1244 & 10.799 & 1294 & 6.9034 & 1294 & 6.9942 & 1295 & 6.9488 & 1345 & 4.8543 & 1395 & 3.2665 & & & & \\
\hline CVS3-30 & 38.35 & 1143 & 20.972 & 1193 & 12.6510 & 1193 & 12.787 & 1243 & 7.8165 & 1243 & 7.9069 & 1245 & 7.8617 & 1294 & 5.1190 & 1344 & 3.7201 & & & & \\
\hline CVS3-31 & 52.279 & 1093 & 27.425 & 1142 & 15.37 & 1143 & 15.135 & 1192 & 9.2624 & 1193 & 9.1569 & 1194 & 9.2774 & 1243 & 5.8902 & 1294 & 3.8638 & & & & \\
\hline CVS3-32 & \begin{tabular}{|l|}
17.379 \\
\end{tabular} & 1289 & 11 & 1289 & 11.034 & 1337 & 7.3502 & 1338 & 7.4016 & 1338 & 7.4701 & 1424 & 3.8293 & & & & & & & & \\
\hline CVS3-33 & 21.132 & 1281 & 13.021 & 1281 & 13.484 & 1329 & 8.7722 & 1329 & 9.1663 & 1330 & 9.2862 & 1401 & 5.2342 & & & & & & & & \\
\hline CVS3-34 & 18.943 & 1289 & 11.993 & 1290 & 11.925 & 1336 & 7.6843 & 1337 & 7.9670 & 1338 & 9.2520 & 1425 & 4.5061 & & & & & & & & \\
\hline CVS3-35 & 16.187 & 1289 & 10.3200 & 1289 & 10.357 & 1336 & 6.8619 & 1337 & 6.8048 & 1338 & 6.9190 & 1424 & 3.8087 & & & & & & & & \\
\hline CVS3-36 & 15.129 & 1289 & 9.7488 & 1290 & 9.6632 & 1336 & 6.4078 & 1338 & 6.3479 & 1339 & 6.3564 & 1424 & 3.6494 & & & & & & & & \\
\hline CVS3-37 & 27.483 & 1244 & 15.575 & 1294 & 10.061 & 1295 & 9.8497 & 1377 & 6.3137 & 1345 & 6.6237 & 1346 & 6.6615 & 1396 & 4.4158 & 1446 & 3.0774 & & & & \\
\hline CVS3-38 & 30.554 & 1244 & 17.3640 & 1294 & 11.13 & 1295 & 10.9040 & 1344 & 7.0395 & 1345 & 7.3117 & 1346 & 7.3269 & 1396 & 4.8619 & 1446 & 3.3421 & & & & \\
\hline CVS3-39 & 35.4260 & 1244 & 20.0920 & 1294 & 12.4400 & 1294 & 12.6360 & 1344 & 7.9220 & 1344 & 8.0880 & 1345 & 8.0270 & 1396 & 5.4590 & 1446 & 4.0000 & & & & \\
\hline CVS3-40 & 23.0540 & 1295 & 14.0670 & 1295 & 14.1720 & 1344 & 8.7200 & 1345 & 8.9160 & 1346 & 8.9910 & 1396 & 5.9810 & 1447 & 4.0230 & & & & & & \\
\hline
\end{tabular}

\section{TRU Study (Crum et al. 1997)}

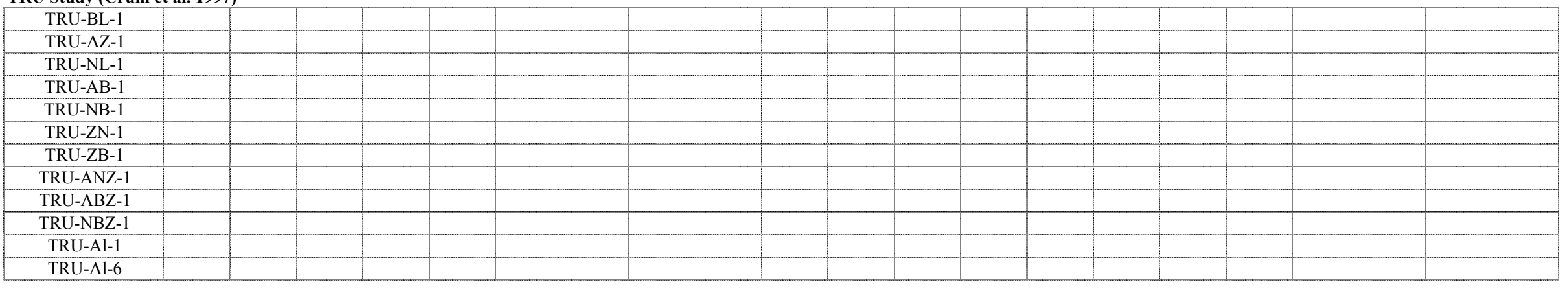


Appendix A. Database - mass fraction

\begin{tabular}{|c|c|c|c|c|c|c|c|c|c|c|c|c|c|c|c|c|}
\hline \multicolumn{17}{|c|}{ Hanford CVS 3 (Vienna et al. 1996) } \\
\hline Glass ID & $\begin{array}{l}\mathrm{T} 13 \\
\left({ }^{\circ} \mathrm{C}\right)\end{array}$ & $\begin{array}{c}\mathrm{V} 13 \\
(\mathrm{~Pa} \cdot \mathrm{s})\end{array}$ & $\begin{array}{l}\mathrm{T} 14 \\
\left({ }^{\circ} \mathrm{C}\right)\end{array}$ & $\begin{array}{c}\text { V14 } \\
(\mathrm{Pa} \cdot \mathrm{s})\end{array}$ & $\begin{array}{c}\text { Q PCT } \\
\text { B }\left(\mathrm{g} / \mathrm{m}^{2}\right)\end{array}$ & $\begin{array}{c}\text { Q PCT } \\
\mathrm{Li}\left(\mathrm{g} / \mathrm{m}^{2}\right)\end{array}$ & $\begin{array}{c}\text { Q PCT } \\
\mathrm{Na}\left(\mathrm{g} / \mathrm{m}^{2}\right)\end{array}$ & $\begin{array}{c}\text { Q PCT } \\
\mathrm{Si}\left(\mathrm{g} / \mathrm{m}^{2}\right)\end{array}$ & $\begin{array}{c}\text { Q PCT } \\
\text { pH }\end{array}$ & $\begin{array}{c}\text { CCC PCT } \\
\text { B }\left(\mathrm{g} / \mathrm{m}^{2}\right)\end{array}$ & $\begin{array}{l}\text { CCC PCT } \\
\mathrm{Li}\left(\mathrm{g} / \mathrm{m}^{2}\right)\end{array}$ & $\begin{array}{l}\text { CCC PCT } \\
\mathrm{Na}\left(\mathrm{g} / \mathrm{m}^{2}\right)\end{array}$ & \begin{tabular}{|c|}
$\mathrm{CCC}$ PCT \\
$\mathrm{Si}\left(\mathrm{g} / \mathrm{m}^{2}\right)$
\end{tabular} & $\begin{array}{c}\text { CCC PCT } \\
\text { pH }\end{array}$ & $\begin{array}{l}\text { Q PCT B at } \\
20^{\circ} \mathrm{C}\left(\mathrm{g} / \mathrm{m}^{2}\right)\end{array}$ & $\begin{array}{l}\text { Q PCT Li at } \\
20^{\circ} \mathrm{C}\left(\mathrm{g} / \mathrm{m}^{2}\right)\end{array}$ \\
\hline CVS3-21 & & & & & 0.196 & 0.301 & 0.254 & 0.097 & 11.03 & 0.530 & 0.359 & 0.322 & 0.124 & 11.14 & & \\
\hline CVS3-22 & & & & & 0.324 & 0.310 & 0.303 & 0.119 & 11.07 & 0.432 & 0.330 & 0.303 & 0.118 & 10.97 & & \\
\hline CVS3-23 & & & & & 0.402 & 0.502 & 0.562 & 0.232 & 11.28 & 0.515 & 0.481 & 0.528 & 0.228 & 11.17 & & \\
\hline CVS3-24 & & & & & 0.261 & 0.355 & 0.380 & 0.151 & 11.07 & 0.362 & 0.356 & 0.342 & 0.155 & 10.97 & & \\
\hline CVS3-25 & & & & & 0.209 & 0.384 & 0.420 & 0.161 & 11.26 & 0.338 & 0.368 & 0.382 & 0.156 & 11.18 & & \\
\hline CVS3-26 & & & & & & & & & & & & & & & & \\
\hline CVS3-27 & & & & & 0.342 & 0.358 & 0.329 & 0.157 & 10.98 & 0.704 & 0.395 & 0.391 & 0.159 & 11.15 & & \\
\hline CVS3-28 & & & & & 0.305 & 0.382 & 0.400 & 0.160 & 11.21 & 1.094 & 0.365 & 0.366 & 0.157 & 11.09 & & \\
\hline CVS3-29 & & & & & 0.241 & 0.411 & 0.432 & 0.189 & 11.22 & 0.531 & 0.376 & 0.381 & 0.155 & 11.16 & & \\
\hline CVS3-30 & & & & & 0.338 & 0.397 & 0.420 & 0.183 & 11.23 & 0.463 & 0.385 & 0.379 & 0.159 & 11.03 & & \\
\hline CVS3-31 & & & & & 0.261 & 0.451 & 0.465 & 0.202 & 11.25 & 2.311 & 0.382 & 0.376 & 0.156 & 11.05 & & \\
\hline CVS3-32 & & & & & 0.487 & 0.370 & 0.326 & 0.159 & 10.88 & 0.412 & 0.367 & 0.289 & 0.155 & 10.84 & & \\
\hline CVS3-33 & & & & & 0.403 & 0.338 & 0.335 & 0.149 & 10.95 & 0.368 & 0.334 & 0.299 & 0.144 & 10.87 & & \\
\hline CVS3-34 & & & & & 0.411 & 0.357 & 0.331 & 0.154 & 10.89 & 0.394 & 0.369 & 0.297 & 0.151 & 10.88 & & \\
\hline CVS3-35 & & & & & 0.422 & 0.395 & 0.325 & 0.163 & 10.78 & 0.441 & 0.468 & 0.343 & 1.740 & 10.94 & & \\
\hline CVS3-36 & & & & & 0.543 & 0.393 & 0.304 & 0.165 & 10.80 & 0.523 & 0.458 & 0.313 & 0.174 & 10.88 & & \\
\hline CVS3-37 & & & & & 0.547 & 0.188 & 0.370 & 0.151 & 10.52 & 0.294 & 0.354 & 0.361 & 0.152 & 10.96 & & \\
\hline CVS3-38 & & & & & 0.418 & 0.292 & 0.327 & 0.147 & 11.03 & 0.370 & 0.326 & 0.283 & 0.120 & 10.90 & & \\
\hline CVS3-39 & & & & & 0.209 & 0.234 & 0.252 & 0.121 & 10.81 & 0.242 & 0.681 & 0.286 & 0.130 & 10.89 & & \\
\hline CVS3-40 & & & & & 0.225 & 0.205 & 0.248 & 0.141 & 10.59 & 0.290 & 1.055 & 0.359 & 0.168 & 10.72 & & \\
\hline
\end{tabular}

TRU Study (Crum et al. 1997)

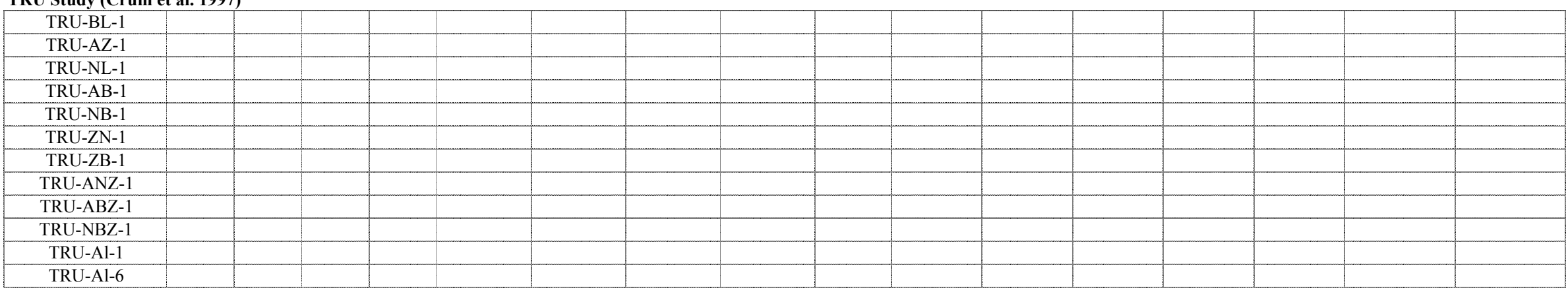


Appendix A. Database - mass fraction

\begin{tabular}{|c|c|c|c|c|c|c|c|c|c|c|c|c|}
\hline Glass ID & $\begin{array}{l}\text { Q PCT Na at } \\
20^{\circ} \mathrm{C}\left(\mathrm{g} / \mathrm{m}^{2}\right)\end{array}$ & $\begin{array}{l}\text { Q PCT Si at } \\
20^{\circ} \mathrm{C}\left(\mathrm{g} / \mathrm{m}^{2}\right)\end{array}$ & $\begin{array}{c}\mathrm{QpH} \\
\text { at } 20^{\circ} \mathrm{C}\end{array}$ & $\begin{array}{c}\text { TCLP Ag } \\
(\mathrm{ppm})\end{array}$ & $\begin{array}{c}\text { TCLP As } \\
\text { (ppm) }\end{array}$ & $\begin{array}{c}\text { TCLP Ba } \\
\text { (ppm) }\end{array}$ & $\begin{array}{c}\text { TCLP Cd } \\
(\mathrm{ppm})\end{array}$ & $\begin{array}{c}\text { TCLP Cr } \\
(\mathrm{ppm})\end{array}$ & $\begin{array}{c}\text { TCLP Ni } \\
(\mathrm{ppm})\end{array}$ & $\begin{array}{c}\text { TCLP Pb } \\
\text { (ppm) }\end{array}$ & $\begin{array}{c}\text { TCLP Se } \\
(\mathrm{ppm})\end{array}$ & $\begin{array}{c}\text { TCLP Zn } \\
(\mathrm{ppm})\end{array}$ \\
\hline \multicolumn{13}{|l|}{ CVS3-21 } \\
\hline \multicolumn{13}{|l|}{ CVS3-22 } \\
\hline \multicolumn{13}{|l|}{ CVS3-23 } \\
\hline \multicolumn{13}{|l|}{ CVS3-24 } \\
\hline \multicolumn{13}{|l|}{ CVS3-25 } \\
\hline \multicolumn{13}{|l|}{ CVS3-26 } \\
\hline \multicolumn{13}{|l|}{ CVS3-27 } \\
\hline \multicolumn{13}{|l|}{ CVS3-28 } \\
\hline \multicolumn{13}{|l|}{ CVS3-29 } \\
\hline \multicolumn{13}{|l|}{ CVS3-30 } \\
\hline \multicolumn{13}{|l|}{ CVS3-31 } \\
\hline \multicolumn{13}{|l|}{ CVS3-32 } \\
\hline \multicolumn{13}{|l|}{ CVS3-33 } \\
\hline \multicolumn{13}{|l|}{ CVS3-34 } \\
\hline \multicolumn{13}{|l|}{ CVS3-35 } \\
\hline \multicolumn{13}{|l|}{ CVS3-36 } \\
\hline \multicolumn{13}{|l|}{ CVS3-37 } \\
\hline \multicolumn{13}{|l|}{ CVS3-38 } \\
\hline \multicolumn{13}{|l|}{ CVS3-39 } \\
\hline CVS3-40 & & & & & & & & & & & & \\
\hline
\end{tabular}

TRU Study (Crum et al. 1997)

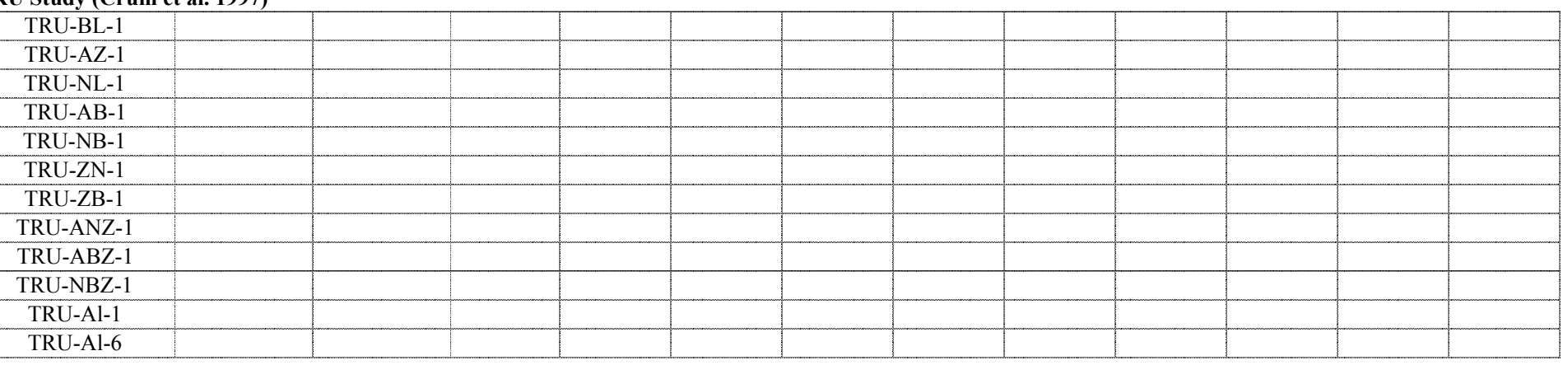


TRU Study (Crum et al. 1997)

\begin{tabular}{|c|c|c|c|c|c|c|c|c|c|c|c|c|c|c|c|c|c|c|c|c|c|}
\hline Glass ID & $\begin{array}{c}\mathrm{A} 12 \mathrm{O} 3 \\
-\mathrm{t}\end{array}$ & $\begin{array}{c}\mathrm{B} 2 \mathrm{O} 3 \\
-\mathrm{t}\end{array}$ & $\begin{array}{c}\mathrm{CaO} \\
-\mathrm{t}\end{array}$ & $\begin{array}{c}\mathrm{Fe} 2 \mathrm{O} 3 \\
-\mathrm{t}\end{array}$ & $\begin{array}{c}\mathrm{FeO} \\
-\mathrm{t}\end{array}$ & $\begin{array}{c}\mathrm{K} 2 \mathrm{O} \\
-\mathrm{t}\end{array}$ & $\begin{array}{c}\mathrm{Li} 2 \mathrm{O} \\
-\mathrm{t}\end{array}$ & $\begin{array}{c}\mathrm{MgO} \\
-\mathrm{t}\end{array}$ & $\begin{array}{c}\mathrm{Na} 2 \mathrm{O} \\
-\mathrm{t}\end{array}$ & $\begin{array}{c}\mathrm{NiO} \\
-\mathrm{t}\end{array}$ & $\begin{array}{c}\mathrm{P} 2 \mathrm{O} 5 \\
-\mathrm{t}\end{array}$ & $\begin{array}{c}\mathrm{SiO} 2 \\
-\mathrm{t}\end{array}$ & $\begin{array}{c}\mathrm{ZrO} 2 \\
-\mathrm{t}\end{array}$ & $\begin{array}{c}\mathrm{Ag} 2 \mathrm{O} \\
-\mathrm{t}\end{array}$ & $\begin{array}{c}\mathrm{As} 2 \mathrm{O} 3 \\
-\mathrm{t}\end{array}$ & $\begin{array}{c}\mathrm{BaO} \\
-\mathrm{t}\end{array}$ & $\begin{array}{c}\mathrm{Bi} 2 \mathrm{O} 3 \\
-\mathrm{t}\end{array}$ & $\begin{array}{c}\mathrm{Br} \\
-\mathrm{t}\end{array}$ & $\begin{array}{c}\mathrm{CdO} \\
-\mathrm{t}\end{array}$ & $\begin{array}{c}\mathrm{Ce} 2 \mathrm{O} 3 \\
-\mathrm{t}\end{array}$ & $\begin{array}{c}\mathrm{CeO} 2 \\
-\mathrm{t}\end{array}$ \\
\hline TRU-B-1 & 0.0250 & 0.0000 & 0.0106 & 0.0474 & & 0.0029 & 0.0499 & 0.0029 & 0.1240 & 0.0007 & 0.0095 & 0.5091 & 0.1165 & 0.0002 & & 0.0005 & 0.0590 & & & & 0.0000 \\
\hline TRU-B-2 & 0.0245 & 0.0200 & 0.0104 & 0.0465 & & 0.0029 & 0.0489 & 0.0028 & 0.1216 & 0.0007 & 0.0093 & 0.4989 & 0.1141 & 0.0002 & & 0.0005 & 0.0578 & & & & 0.0000 \\
\hline TRU-B-3 & 0.0238 & 0.0500 & 0.0101 & 0.0450 & & 0.0028 & 0.0474 & 0.0027 & 0.1178 & 0.0007 & 0.0090 & 0.4836 & 0.1106 & 0.0002 & & 0.0005 & 0.0560 & & & & 0.0000 \\
\hline TRU-B-4 & 0.0220 & 0.1200 & 0.0094 & 0.0417 & & 0.0026 & 0.0439 & 0.0025 & 0.1091 & 0.0007 & 0.0083 & 0.4480 & 0.1025 & 0.0002 & & 0.0004 & 0.0519 & & & & 0.0000 \\
\hline TRU-B-5 & 0.0248 & 0.0100 & 0.0105 & 0.0469 & & 0.0029 & 0.0494 & 0.0028 & 0.1228 & 0.0007 & 0.0094 & 0.5040 & 0.1153 & 0.0002 & & 0.0005 & 0.0584 & & & & 0.0000 \\
\hline TRU-Bi-1 & 0.0243 & 0.0862 & 0.0103 & 0.0460 & & 0.0029 & 0.0485 & 0.0028 & 0.1204 & 0.0007 & 0.0092 & 0.4944 & 0.1131 & 0.0002 & & 0.0005 & 0.0000 & & & & 0.0000 \\
\hline TRU-Bi-2 & 0.0237 & 0.0840 & 0.0101 & 0.0449 & & 0.0028 & 0.0473 & 0.0027 & 0.1174 & 0.0007 & 0.0090 & 0.4820 & 0.1103 & 0.0002 & & 0.0005 & 0.0250 & & & & 0.0000 \\
\hline TRU-Bi-3 & 0.0225 & 0.0797 & 0.0096 & 0.0426 & & 0.0026 & 0.0448 & 0.0026 & 0.1114 & 0.0007 & 0.0085 & 0.4573 & 0.1046 & 0.0002 & & 0.0004 & 0.0750 & & & & 0.0000 \\
\hline TRU-Bi-4 & 0.0219 & 0.0776 & 0.0093 & 0.0414 & & 0.0026 & 0.0436 & 0.0025 & 0.1084 & 0.0006 & 0.0083 & 0.4449 & 0.1018 & 0.0002 & & 0.0004 & 0.1000 & & & & 0.0000 \\
\hline TRU-Ce-1 & 0.0226 & 0.0803 & 0.0096 & 0.0429 & & 0.0027 & 0.0452 & 0.0026 & 0.1122 & 0.0007 & 0.0086 & 0.4606 & 0.1054 & 0.0002 & & 0.0004 & 0.0533 & & & & 0.0150 \\
\hline TRU-Ce-2 & 0.0223 & 0.0791 & 0.0095 & 0.0422 & & 0.0026 & 0.0445 & 0.0026 & 0.1105 & 0.0007 & 0.0085 & 0.4536 & 0.1038 & 0.0002 & & 0.0004 & 0.0525 & & & & 0.0300 \\
\hline TRU-Ce-3 & 0.0219 & 0.0778 & 0.0093 & 0.0416 & & 0.0026 & 0.0438 & 0.0025 & 0.1088 & 0.0006 & 0.0083 & 0.4466 & 0.1022 & 0.0002 & & 0.0004 & 0.0517 & & & & 0.0450 \\
\hline TRU-Li-1 & 0.0241 & 0.0854 & 0.0102 & 0.0456 & & 0.0028 & 0.0000 & 0.0028 & 0.1194 & 0.0007 & 0.0091 & 0.4901 & 0.1121 & 0.0002 & & 0.0005 & 0.0568 & & & & 0.0000 \\
\hline TRU-Li-2 & 0.0237 & 0.0841 & 0.0101 & 0.0450 & & 0.0028 & 0.0150 & 0.0027 & 0.1176 & 0.0007 & 0.0090 & 0.4827 & 0.1104 & 0.0002 & & 0.0005 & 0.0559 & & & & 0.0000 \\
\hline TRU-Li-3 & 0.0234 & 0.0829 & 0.0099 & 0.0443 & & 0.0027 & 0.0300 & 0.0027 & 0.1158 & 0.0007 & 0.0089 & 0.4754 & 0.1087 & 0.0002 & & 0.0004 & 0.0551 & & & & 0.0000 \\
\hline TRU-Li-4 & 0.0226 & 0.0803 & 0.0096 & 0.0429 & & 0.0027 & 0.0600 & 0.0026 & 0.1122 & 0.0007 & 0.0086 & 0.4607 & 0.1054 & 0.0002 & & 0.0004 & 0.0534 & & & & 0.0000 \\
\hline TRU-Na-1 & 0.0248 & 0.0878 & 0.0105 & 0.0469 & & 0.0029 & 0.0494 & 0.0028 & 0.0450 & 0.0007 & 0.0094 & 0.5040 & 0.1153 & 0.0002 & & 0.0005 & 0.0584 & & & & 0.0000 \\
\hline TRU-Na-2 & 0.0241 & 0.0855 & 0.0102 & 0.0457 & & 0.0028 & 0.0481 & 0.0028 & 0.0700 & 0.0007 & 0.0091 & 0.4908 & 0.1123 & 0.0002 & & 0.0005 & 0.0568 & & & & 0.0000 \\
\hline TRU-Na-3 & 0.0235 & 0.0832 & 0.0100 & 0.0445 & & 0.0028 & 0.0468 & 0.0027 & 0.0950 & 0.0007 & 0.0089 & 0.4776 & 0.1093 & 0.0002 & & 0.0004 & 0.0553 & & & & 0.0000 \\
\hline TRU-Na-4 & 0.0226 & 0.0800 & 0.0096 & 0.0428 & & 0.0027 & 0.0450 & 0.0026 & 0.1300 & 0.0007 & 0.0086 & 0.4591 & 0.1050 & 0.0002 & & 0.0004 & 0.0532 & & & & 0.0000 \\
\hline TRU-P-1 & 0.0227 & 0.0806 & 0.0097 & 0.0430 & & 0.0027 & 0.0453 & 0.0026 & 0.1126 & 0.0007 & 0.0200 & 0.4623 & 0.1058 & 0.0002 & & 0.0004 & 0.0535 & & & & 0.0000 \\
\hline TRU-P-2 & 0.0225 & 0.0798 & 0.0096 & 0.0426 & & 0.0026 & 0.0449 & 0.0026 & 0.1115 & 0.0007 & 0.0300 & 0.4576 & 0.1047 & 0.0002 & & 0.0004 & 0.0530 & & & & 0.0000 \\
\hline TRU-P-3 & 0.0222 & 0.0789 & 0.0095 & 0.0422 & & 0.0026 & 0.0444 & 0.0026 & 0.1103 & 0.0007 & 0.0400 & 0.4528 & 0.1036 & 0.0002 & & 0.0004 & 0.0524 & & & & 0.0000 \\
\hline TRU-P-4 & 0.0220 & 0.0781 & 0.0094 & 0.0417 & & 0.0026 & 0.0439 & 0.0025 & 0.1092 & 0.0007 & 0.0500 & 0.4481 & 0.1025 & 0.0002 & & 0.0004 & 0.0519 & & & & 0.0000 \\
\hline TRU-Si-1 & 0.0255 & 0.0903 & 0.0108 & 0.0483 & & 0.0030 & 0.0508 & 0.0029 & 0.1263 & 0.0008 & 0.0097 & 0.4100 & 0.1185 & 0.0002 & & 0.0005 & 0.0600 & & & & 0.0000 \\
\hline TRU-Si-2 & 0.0207 & 0.0735 & 0.0088 & 0.0393 & & 0.0024 & 0.0413 & 0.0024 & 0.1027 & 0.0006 & 0.0079 & 0.5200 & 0.0964 & 0.0002 & & 0.0004 & 0.0488 & & & & 0.0000 \\
\hline TRU-Si-3 & 0.0272 & 0.0964 & 0.0115 & 0.0515 & & 0.0032 & 0.0543 & 0.0031 & 0.1348 & 0.0008 & 0.0103 & 0.3700 & 0.1266 & 0.0002 & & 0.0005 & 0.0641 & & & & 0.0000 \\
\hline TRU-Si-4 & 0.0186 & 0.0658 & 0.0079 & 0.0352 & & 0.0022 & 0.0370 & 0.0021 & 0.0920 & 0.0005 & 0.0070 & 0.5700 & 0.0864 & 0.0002 & & 0.0004 & 0.0437 & & & & 0.0000 \\
\hline TRU-Zr-1 & 0.0237 & 0.0840 & 0.0101 & 0.0449 & & 0.0028 & 0.0472 & 0.0027 & 0.1174 & 0.0007 & 0.0090 & 0.4817 & 0.0800 & 0.0002 & & 0.0005 & 0.0558 & & & & 0.0000 \\
\hline TRU-Zr-2 & 0.0226 & 0.0803 & 0.0096 & 0.0429 & & 0.0027 & 0.0452 & 0.0026 & 0.1123 & 0.0007 & 0.0086 & 0.4608 & 0.1200 & 0.0002 & & 0.0004 & 0.0534 & & & & 0.0000 \\
\hline TRU-Zr-3 & 0.0221 & 0.0785 & 0.0094 & 0.0419 & & 0.0026 & 0.0442 & 0.0025 & 0.1097 & 0.0007 & 0.0084 & 0.4503 & 0.1400 & 0.0002 & & 0.0004 & 0.0522 & & & & 0.0000 \\
\hline TRU-Zr-4 & 0.0216 & 0.0767 & 0.0092 & 0.0410 & & 0.0025 & 0.0431 & 0.0025 & 0.1072 & 0.0006 & 0.0082 & 0.4398 & 0.1600 & 0.0002 & & 0.0004 & 0.0509 & & & & 0.0000 \\
\hline \multicolumn{22}{|c|}{ Zr Study (Vienna et al. 1999) } \\
\hline $\mathrm{Zr}-1$ & 0.0450 & 0.1000 & 0.0009 & & & 0.0042 & 0.0750 & & 0.1100 & & 0.0021 & 0.5114 & 0.1500 & & & & & & & 0.0002 & \\
\hline $\mathrm{Zr}-2$ & 0.0450 & 0.1000 & 0.0008 & & & 0.0038 & 0.0750 & & 0.1116 & & 0.0019 & 0.5256 & 0.1350 & & & & & & & 0.0002 & \\
\hline Zr-3 & 0.0450 & 0.1000 & 0.0009 & & & 0.0047 & 0.0750 & & 0.1084 & & 0.0023 & 0.4971 & 0.1650 & & & & & & & 0.0002 & \\
\hline $\mathrm{Zr}-4$ & 0.0300 & 0.1000 & 0.0009 & & & 0.0042 & 0.0750 & & 0.1083 & & 0.0021 & 0.5281 & 0.1500 & & & & & & & 0.0002 & \\
\hline Zr-5 & 0.0600 & 0.1000 & 0.0009 & & & 0.0042 & 0.0750 & & 0.1118 & & 0.0021 & 0.4946 & 0.1500 & & & & & & & 0.0002 & \\
\hline $\mathrm{Zr}-6$ & 0.0450 & 0.1000 & 0.0009 & & & 0.0042 & 0.0750 & & 0.1100 & & 0.0021 & 0.5114 & 0.1500 & & & & & & & 0.0002 & \\
\hline $\mathrm{Zr}-7$ & 0.0450 & 0.0500 & 0.0009 & & & 0.0042 & 0.0750 & & 0.1480 & & 0.0021 & 0.5234 & 0.1500 & & & & & & & 0.0002 & \\
\hline $\mathrm{Zr}-8$ & 0.0450 & 0.1500 & 0.0009 & & & 0.0042 & 0.0750 & & 0.0721 & & 0.0021 & 0.4993 & 0.1500 & & & & & & & 0.0002 & \\
\hline Zr-9 & 0.0450 & 0.1000 & 0.0009 & & & 0.0042 & 0.0600 & & 0.1425 & & 0.0021 & 0.4939 & 0.1500 & & & & & & & 0.0002 & \\
\hline $\mathrm{Zr}-10$ & 0.0450 & 0.1000 & 0.0009 & & & 0.0042 & 0.0750 & & 0.1100 & & 0.0021 & 0.5114 & 0.1500 & & & & & & & 0.0002 & \\
\hline $\mathrm{Zr}-11$ & 0.0450 & 0.1000 & 0.0009 & & & 0.0042 & 0.0900 & & 0.0776 & & 0.0021 & 0.5288 & 0.1500 & & & & & & & 0.0002 & \\
\hline $\mathrm{Zr}-12$ & 0.0600 & 0.1500 & 0.0009 & & & 0.0047 & 0.0900 & & 0.0398 & & 0.0023 & 0.4858 & 0.1650 & & & & & & & 0.0002 & \\
\hline $\mathrm{Zr}-13$ & 0.0600 & 0.1500 & 0.0009 & & & 0.0047 & 0.0600 & & 0.1046 & & 0.0023 & 0.4509 & 0.1650 & & & & & & & 0.0002 & \\
\hline $\mathrm{Zr}-14$ & 0.0600 & 0.0500 & 0.0009 & & & 0.0047 & 0.0900 & & 0.1157 & & 0.0023 & 0.5098 & 0.1650 & & & & & & & 0.0002 & \\
\hline Zr-15 & 0.0600 & 0.0500 & 0.0009 & & & 0.0047 & 0.0600 & & 0.1805 & & 0.0023 & 0.4750 & 0.1650 & & & & & & & 0.0002 & \\
\hline
\end{tabular}


TRU Study (Crum et al. 1997)

\begin{tabular}{|c|c|c|c|c|c|c|c|c|c|c|c|c|c|c|c|c|c|c|c|c|c|}
\hline Glass ID & $\begin{array}{l}\mathrm{Cl} \\
-\mathrm{t}\end{array}$ & $\begin{array}{c}\mathrm{CoO} \\
-\mathrm{t}\end{array}$ & $\begin{array}{c}\mathrm{Co} 2 \mathrm{O} 3 \\
-\mathrm{t}\end{array}$ & $\begin{array}{c}\mathrm{Cr} 2 \mathrm{O} 3 \\
-\mathrm{t}\end{array}$ & $\begin{array}{c}\mathrm{Cs} 2 \mathrm{O} \\
-\mathrm{t}\end{array}$ & $\begin{array}{c}\mathrm{CuO} \\
-\mathrm{t}\end{array}$ & $\begin{array}{c}\mathrm{Eu} 2 \mathrm{O} 3 \\
-\mathrm{t}\end{array}$ & $\begin{array}{l}F \\
-t\end{array}$ & $\begin{array}{c}\mathrm{Ga} 2 \mathrm{O} 3 \\
-\mathrm{t}\end{array}$ & $\begin{array}{c}\mathrm{Gd} 2 \mathrm{O} 3 \\
-\mathrm{t}\end{array}$ & $\begin{array}{c}\mathrm{HgO} \\
-\mathrm{t}\end{array}$ & $\begin{array}{l}I \\
-t\end{array}$ & $\begin{array}{c}\mathrm{La} 2 \mathrm{O} 3 \\
-\mathrm{t}\end{array}$ & $\begin{array}{c}\mathrm{MnO} 2 \\
-\mathrm{t}\end{array}$ & $\begin{array}{c}\mathrm{MnO} \\
-\mathrm{t}\end{array}$ & $\begin{array}{c}\mathrm{MoO} \\
-\mathrm{t}\end{array}$ & $\begin{array}{c}\mathrm{MoO} 3 \\
-\mathrm{t}\end{array}$ & $\begin{array}{c}\mathrm{Nb} 2 \mathrm{O} 5 \\
-\mathrm{t}\end{array}$ & $\begin{array}{c}\mathrm{Nd} 2 \mathrm{O} 3 \\
-\mathrm{t}\end{array}$ & $\begin{array}{c}\mathrm{PbO} \\
-\mathrm{t}\end{array}$ & $\begin{array}{c}\mathrm{PdO} 2 \\
-\mathrm{t}\end{array}$ \\
\hline TRU-B-1 & & & 0.0000 & 0.0039 & & 0.0002 & & 0.0046 & & & & & 0.0118 & & 0.0188 & & & & & 0.0013 & \\
\hline TRU-B-2 & & & 0.0000 & 0.0038 & & 0.0002 & & 0.0045 & & & & & 0.0115 & & 0.0185 & & & & & 0.0012 & \\
\hline TRU-B-3 & & & 0.0000 & 0.0037 & & 0.0002 & & 0.0043 & & & & & 0.0112 & & 0.0179 & & & & & 0.0012 & \\
\hline TRU-B-4 & & & 0.0000 & 0.0034 & & 0.0002 & & 0.0040 & & & & & 0.0103 & & 0.0166 & & & & & 0.0011 & \\
\hline TRU-B-5 & & & 0.0000 & 0.0039 & & 0.0002 & & 0.0045 & & & & & 0.0116 & & 0.0186 & & & & & 0.0012 & \\
\hline TRU-Bi-1 & & & 0.0000 & 0.0038 & & 0.0002 & & 0.0044 & & & & & 0.0114 & & 0.0183 & & & & & 0.0012 & \\
\hline TRU-Bi-2 & & & 0.0000 & 0.0037 & & 0.0002 & & 0.0043 & & & & & 0.0111 & & 0.0178 & & & & & 0.0012 & \\
\hline TRU-Bi-3 & & & 0.0000 & 0.0035 & & 0.0002 & & 0.0041 & & & & & 0.0106 & & 0.0169 & & & & & 0.0011 & \\
\hline TRU-Bi-4 & & & 0.0000 & 0.0034 & & 0.0002 & & 0.0040 & & & & & 0.0103 & & 0.0165 & & & & & 0.0011 & \\
\hline TRU-Ce-1 & & & 0.0000 & 0.0035 & & 0.0002 & & 0.0041 & & & & & 0.0106 & & 0.0170 & & & & & 0.0011 & \\
\hline TRU-Ce-2 & & & 0.0000 & 0.0035 & & 0.0002 & & 0.0041 & & & & & 0.0105 & & 0.0168 & & & & & 0.0011 & \\
\hline TRU-Ce-3 & & & 0.0000 & 0.0034 & & 0.0002 & & 0.0040 & & & & & 0.0103 & & 0.0165 & & & & & 0.0011 & \\
\hline TRU-Li-1 & & & 0.0000 & 0.0038 & & 0.0002 & & 0.0044 & & & & & 0.0113 & & 0.0181 & & & & & 0.0012 & \\
\hline TRU-Li-2 & & & 0.0000 & 0.0037 & & 0.0002 & & 0.0043 & & & & & 0.0111 & & 0.0179 & & & & & 0.0012 & \\
\hline TRU-Li-3 & & & 0.0000 & 0.0037 & & 0.0002 & & 0.0043 & & & & & 0.0110 & & 0.0176 & & & & & 0.0012 & \\
\hline TRU-Li-4 & & & 0.0000 & 0.0035 & & 0.0002 & & 0.0041 & & & & & 0.0106 & & 0.0170 & & & & & 0.0011 & \\
\hline TRU-Na-1 & & & 0.0000 & 0.0039 & & 0.0002 & & 0.0045 & & & & & 0.0116 & & 0.0186 & & & & & 0.0012 & \\
\hline TRU-Na-2 & & & 0.0000 & 0.0038 & & 0.0002 & & 0.0044 & & & & & 0.0113 & & 0.0182 & & & & & 0.0012 & \\
\hline TRU-Na-3 & & & 0.0000 & 0.0037 & & 0.0002 & & 0.0043 & & & & & 0.0110 & & 0.0177 & & & & & 0.0012 & \\
\hline TRU-Na-4 & & & 0.0000 & 0.0035 & & 0.0002 & & 0.0041 & & & & & 0.0106 & & 0.0170 & & & & & 0.0011 & \\
\hline TRU-P-1 & & & 0.0000 & 0.0036 & & 0.0002 & & 0.0042 & & & & & 0.0107 & & 0.0171 & & & & & 0.0011 & \\
\hline TRU-P-2 & & & 0.0000 & 0.0035 & & 0.0002 & & 0.0041 & & & & & 0.0106 & & 0.0169 & & & & & 0.0011 & \\
\hline TRU-P-3 & & & 0.0000 & 0.0035 & & 0.0002 & & 0.0041 & & & & & 0.0105 & & 0.0168 & & & & & 0.0011 & \\
\hline TRU-P-4 & & & 0.0000 & 0.0035 & & 0.0002 & & 0.0040 & & & & & 0.0104 & & 0.0166 & & & & & 0.0011 & \\
\hline TRU-Si-1 & & & 0.0000 & 0.0040 & & 0.0002 & & 0.0047 & & & & & 0.0120 & & 0.0192 & & & & & 0.0013 & \\
\hline TRU-Si-2 & & & 0.0000 & 0.0032 & & 0.0001 & & 0.0038 & & & & & 0.0097 & & 0.0156 & & & & & 0.0010 & \\
\hline TRU-Si-3 & & & 0.0000 & 0.0043 & & 0.0002 & & 0.0050 & & & & & 0.0128 & & 0.0205 & & & & & 0.0014 & \\
\hline TRU-Si-4 & & & 0.0000 & 0.0029 & & 0.0001 & & 0.0034 & & & & & 0.0087 & & 0.0140 & & & & & 0.0009 & \\
\hline TRU-Zr-1 & & & 0.0000 & 0.0037 & & 0.0002 & & 0.0043 & & & & & 0.0111 & & 0.0178 & & & & & 0.0012 & \\
\hline TRU-Zr-2 & & & 0.0000 & 0.0035 & & 0.0002 & & 0.0041 & & & & & 0.0106 & & 0.0170 & & & & & 0.0011 & \\
\hline TRU-Zr-3 & & & 0.0000 & 0.0035 & & 0.0002 & & 0.0040 & & & & & 0.0104 & & 0.0167 & & & & & 0.0011 & \\
\hline TRU-Zr-4 & & & 0.0000 & 0.0034 & & 0.0002 & & 0.0040 & & & & & 0.0102 & & 0.0163 & & & & & 0.0011 & \\
\hline
\end{tabular}

\section{Zr Study (Vienna et al. 1999)}

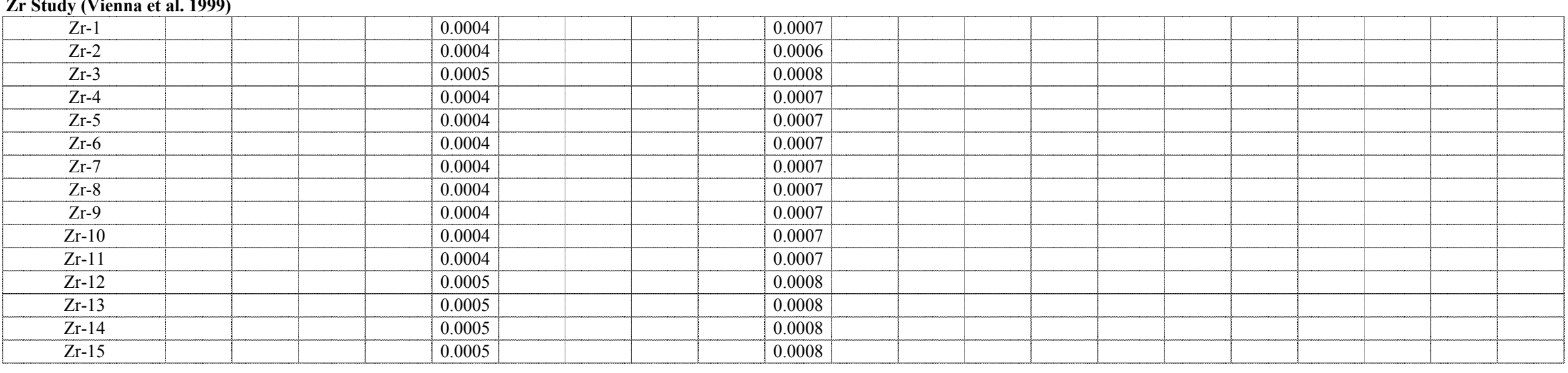


TRU Study (Crum et al. 1997)

\begin{tabular}{|c|c|c|c|c|c|c|c|c|c|c|c|c|c|c|c|c|c|c|c|c|c|}
\hline Glass ID & $\begin{array}{c}\mathrm{PdO} \\
-\mathrm{t}\end{array}$ & $\begin{array}{c}\mathrm{Pr} 2 \mathrm{O} 3 \\
-\mathrm{t}\end{array}$ & $\begin{array}{c}\text { Pr6O11 } \\
-t\end{array}$ & $\begin{array}{c}\mathrm{Rb} 2 \mathrm{O} \\
-\mathrm{t}\end{array}$ & $\begin{array}{c}\mathrm{ReO} \\
-\mathrm{t}\end{array}$ & $\begin{array}{c}\mathrm{ReO} 2 \\
-\mathrm{t}\end{array}$ & $\begin{array}{c}\mathrm{Rh} 2 \mathrm{O} 3 \\
-\mathrm{t}\end{array}$ & $\begin{array}{c}\mathrm{RhO}^{2} \\
-\mathrm{t}\end{array}$ & $\begin{array}{c}\mathrm{RuO} 2 \\
-\mathrm{t}\end{array}$ & $\begin{array}{c}\mathrm{Sb} 2 \mathrm{O} 3 \\
-\mathrm{t}\end{array}$ & $\begin{array}{c}\mathrm{Sb} 2 \mathrm{O} 5 \\
-\mathrm{t}\end{array}$ & $\begin{array}{c}\mathrm{SeO} 2 \\
-\mathrm{t}\end{array}$ & $\begin{array}{c}\mathrm{Sm} 2 \mathrm{O} 3 \\
-\mathrm{t}\end{array}$ & $\begin{array}{c}\mathrm{SnO} \\
-\mathrm{t}\end{array}$ & $\begin{array}{c}\mathrm{SnO} 2 \\
-\mathrm{t}\end{array}$ & $\begin{array}{c}\mathrm{SO} 3 \\
-\mathrm{t}\end{array}$ & $\begin{array}{c}\mathrm{SrO} \\
-\mathrm{t}\end{array}$ & $\begin{array}{c}\mathrm{Tc} 2 \mathrm{O} 7 \\
-\mathrm{t}\end{array}$ & $\begin{array}{c}\mathrm{TeO} 2 \\
-\mathrm{t}\end{array}$ & $\begin{array}{c}\text { ThO2 } \\
-t\end{array}$ & $\begin{array}{c}\mathrm{TiO} 2 \\
-\mathrm{t}\end{array}$ \\
\hline TRU-B-1 & & & & & & & & & & & & & & & & & 0.0007 & & & & \\
\hline TRU-B-2 & & & & & & & & & & & & & & & & & 0.0006 & & & & \\
\hline TRU-B-3 & & & & & & & & & & & & & & & & & 0.0006 & & & & \\
\hline TRU-B-4 & & & & & & & & & & & & & & & & & 0.0006 & & & & \\
\hline TRU-B-5 & & & & & & & & & & & & & & & & & 0.0006 & & & & \\
\hline TRU-Bi-1 & & & & & & & & & & & & & & & & & 0.0006 & & & & \\
\hline TRU-Bi-2 & & & & & & & & & & & & & & & & & 0.0006 & & & & \\
\hline TRU-Bi-3 & & & & & & & & & & & & & & & & & 0.0006 & & & & \\
\hline TRU-Bi-4 & & & & & & & & & & & & & & & & & 0.0006 & & & & \\
\hline TRU-Ce-1 & & & & & & & & & & & & & & & & & 0.0006 & & & & \\
\hline TRU-Ce-2 & & & & & & & & & & & & & & & & & 0.0006 & & & & \\
\hline TRU-Ce-3 & & & & & & & & & & & & & & & & & 0.0006 & & & & \\
\hline TRU-Li-1 & & & & & & & & & & & & & & & & & 0.0006 & & & & \\
\hline TRU-Li-2 & & & & & & & & & & & & & & & & & 0.0006 & & & & \\
\hline TRU-Li-3 & & & & & & & & & & & & & & & & & 0.0006 & & & & \\
\hline TRU-Li-4 & & & & & & & & & & & & & & & & & 0.0006 & & & & \\
\hline TRU-Na-1 & & & & & & & & & & & & & & & & & 0.0006 & & & & \\
\hline TRU-Na-2 & & & & & & & & & & & & & & & & & 0.0006 & & & & \\
\hline TRU-Na-3 & & & & & & & & & & & & & & & & & 0.0006 & & & & \\
\hline TRU-Na-4 & & & & & & & & & & & & & & & & & 0.0006 & & & & \\
\hline TRU-P-1 & & & & & & & & & & & & & & & & & 0.0006 & & & & \\
\hline TRU-P-2 & & & & & & & & & & & & & & & & & 0.0006 & & & & \\
\hline TRU-P-3 & & & & & & & & & & & & & & & & & 0.0006 & & & & \\
\hline TRU-P-4 & & & & & & & & & & & & & & & & & 0.0006 & & & & \\
\hline TRU-Si-1 & & & & & & & & & & & & & & & & & 0.0007 & & & & \\
\hline TRU-Si-2 & & & & & & & & & & & & & & & & & 0.0005 & & & & \\
\hline TRU-Si-3 & & & & & & & & & & & & & & & & & 0.0007 & & & & \\
\hline TRU-Si-4 & & & & & & & & & & & & & & & & & 0.0005 & & & & \\
\hline TRU-Zr-1 & & & & & & & & & & & & & & & & & 0.0006 & & & & \\
\hline TRU-Zr-2 & & & & & & & & & & & & & & & & & 0.0006 & & & & \\
\hline TRU-Zr-3 & & & & & & & & & & & & & & & & & 0.0006 & & & & \\
\hline TRU-Zr-4 & & & & & & & & & & & & & & & & & 0.0006 & & & & \\
\hline
\end{tabular}

\section{Zr Study (Vienna et al. 1999)}

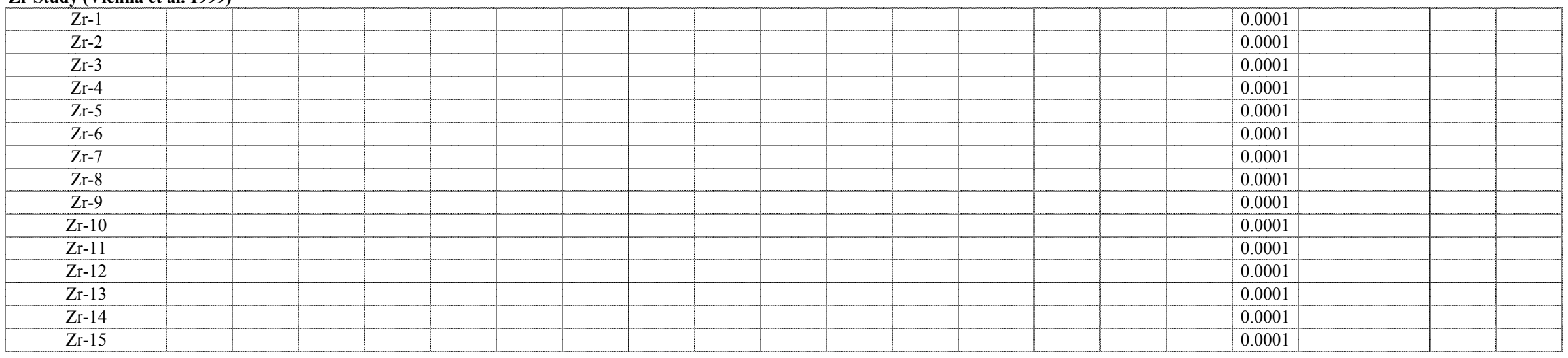


Appendix A. Database - mass fraction

TRU Study (Crum et al. 1997)

\begin{tabular}{|c|c|c|c|c|c|c|c|c|c|c|c|c|c|c|c|c|c|c|c|c|c|}
\hline Glass ID & $\begin{array}{c}\mathrm{T} 12 \mathrm{O} 3 \\
-\mathrm{t}\end{array}$ & $\begin{array}{c}\text { U3O8 } \\
-t\end{array}$ & $\begin{array}{c}\mathrm{UO} 2 \\
-\mathrm{t}\end{array}$ & $\begin{array}{c}\text { UO3 } \\
-t\end{array}$ & $\begin{array}{c}\mathrm{V} 2 \mathrm{O} 5 \\
-\mathrm{t}\end{array}$ & $\begin{array}{c}\text { WO3 } \\
-t\end{array}$ & $\begin{array}{c}\mathrm{Y} 2 \mathrm{O} 3 \\
-\mathrm{t}\end{array}$ & $\begin{array}{c}\mathrm{ZnO} \\
-\mathrm{t}\end{array}$ & $\begin{array}{c}\text { Others } \\
-t\end{array}$ & $\begin{array}{c}\text { Sum } \\
-t\end{array}$ & $\begin{array}{c}\mathrm{A} 12 \mathrm{O} 3 \\
-\mathrm{a}\end{array}$ & $\begin{array}{c}\mathrm{B} 2 \mathrm{O} 3 \\
-\mathrm{a}\end{array}$ & $\begin{array}{c}\mathrm{CaO} \\
-\mathrm{a}\end{array}$ & $\begin{array}{c}\mathrm{Fe} 2 \mathrm{O} 3 \\
-\mathrm{a} \\
\end{array}$ & $\begin{array}{c}\mathrm{FeO} \\
-\mathrm{a} \\
\end{array}$ & $\begin{array}{c}\mathrm{K} 2 \mathrm{O} \\
-\mathrm{a}\end{array}$ & $\begin{array}{c}\mathrm{Li} 2 \mathrm{O} \\
-\mathrm{a}\end{array}$ & $\begin{array}{c}\mathrm{MgO} \\
-\mathrm{a}\end{array}$ & $\begin{array}{c}\mathrm{Na} 2 \mathrm{O} \\
-\mathrm{a}\end{array}$ & $\begin{array}{c}\mathrm{NiO} \\
-\mathrm{a}\end{array}$ & $\begin{array}{c}\mathrm{P} 2 \mathrm{O} 5 \\
-\mathrm{a} \\
\end{array}$ \\
\hline TRU-B-1 & & & & & & & & 0.0006 & & 1.0000 & & & & & & & & & & & \\
\hline TRU-B-2 & & & & & & & & 0.0006 & & 1.0000 & & & & & & & & & & & \\
\hline TRU-B-3 & & & & & & & & 0.0005 & & 1.0000 & & & & & & & & & & & \\
\hline TRU-B-4 & & & & & & & & 0.0005 & & 1.0000 & & & & & & & & & & & \\
\hline TRU-B-5 & & & & & & & & 0.0006 & & 1.0000 & & & & & & & & & & & \\
\hline TRU-Bi-1 & & & & & & & & 0.0005 & & 1.0000 & & & & & & & & & & & \\
\hline TRU-Bi-2 & & & & & & & & 0.0005 & & 1.0000 & & & & & & & & & & & \\
\hline TRU-Bi-3 & & & & & & & & 0.0005 & & 1.0000 & & & & & & & & & & & \\
\hline TRU-Bi-4 & & & & & & & & 0.0005 & & 1.0000 & & & & & & & & & & & \\
\hline TRU-Ce-1 & & & & & & & & 0.0005 & & 1.0000 & & & & & & & & & & & \\
\hline TRU-Ce-2 & & & & & & & & 0.0005 & & 1.0000 & & & & & & & & & & & \\
\hline TRU-Ce-3 & & & & & & & & 0.0005 & & 1.0000 & & & & & & & & & & & \\
\hline TRU-Li-1 & & & & & & & & 0.0005 & & 1.0000 & & & & & & & & & & & \\
\hline TRU-Li-2 & & & & & & & & 0.0005 & & 1.0000 & & & & & & & & & & & \\
\hline TRU-Li-3 & & & & & & & & 0.0005 & & 1.0000 & & & & & & & & & & & \\
\hline TRU-Li-4 & & & & & & & & 0.0005 & & 1.0000 & & & & & & & & & & & \\
\hline TRU-Na-1 & & & & & & & & 0.0006 & & 1.0000 & & & & & & & & & & & \\
\hline TRU-Na-2 & & & & & & & & 0.0005 & & 1.0000 & & & & & & & & & & & \\
\hline TRU-Na-3 & & & & & & & & 0.0005 & & 1.0000 & & & & & & & & & & & \\
\hline TRU-Na-4 & & & & & & & & 0.0005 & & 1.0000 & & & & & & & & & & & \\
\hline TRU-P-1 & & & & & & & & 0.0005 & & 1.0000 & & & & & & & & & & & \\
\hline TRU-P-2 & & & & & & & & 0.0005 & & 1.0000 & & & & & & & & & & & \\
\hline TRU-P-3 & & & & & & & & 0.0005 & & 1.0000 & & & & & & & & & & & \\
\hline TRU-P-4 & & & & & & & & 0.0005 & & 1.0000 & & & & & & & & & & & \\
\hline TRU-Si-1 & & & & & & & & 0.0006 & & 1.0000 & & & & & & & & & & & \\
\hline TRU-Si-2 & & & & & & & & 0.0005 & & 1.0000 & & & & & & & & & & & \\
\hline TRU-Si-3 & & & & & & & & 0.0006 & & 1.0000 & & & & & & & & & & & \\
\hline TRU-Si-4 & & & & & & & & 0.0004 & & 1.0000 & & & & & & & & & & & \\
\hline TRU-Zr-1 & & & & & & & & 0.0005 & & 1.0000 & & & & & & & & & & & \\
\hline TRU-Zr-2 & & & & & & & & 0.0005 & & 1.0000 & & & & & & & & & & & \\
\hline TRU-Zr-3 & & & & & & & & 0.0005 & & 1.0000 & & & & & & & & & & & \\
\hline TRU-Zr-4 & & & & & & & & 0.0005 & & 1.0000 & & & & & & & & & & & \\
\hline
\end{tabular}

Zr Study (Vienna et al. 1999)

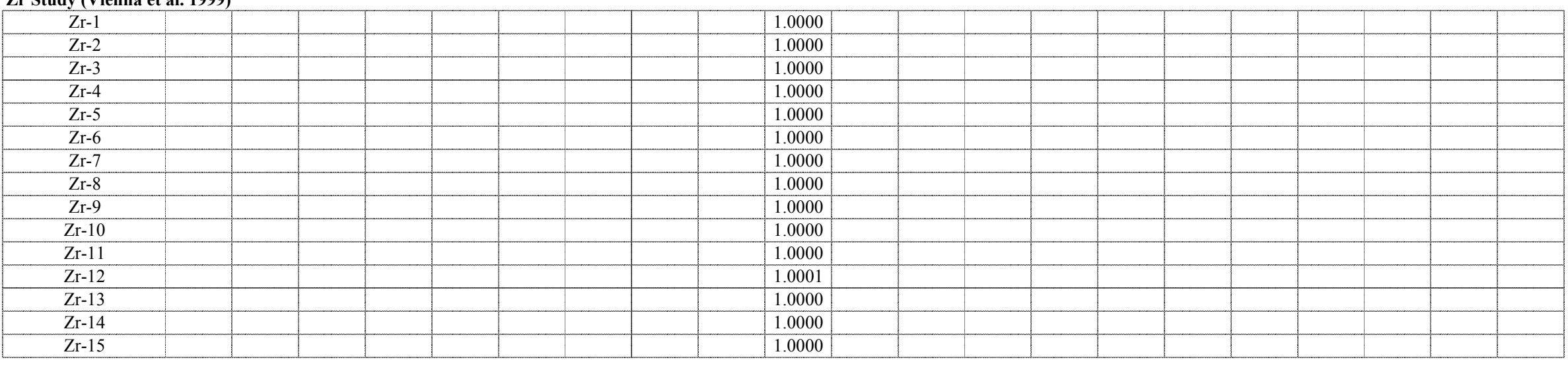


Appendix A. Database - mass fraction

TRU Study (Crum et al. 1997)

\begin{tabular}{|c|c|c|c|c|c|c|c|c|c|c|c|c|c|c|c|c|c|c|c|c|c|}
\hline Glass ID & $\begin{array}{c}\mathrm{SiO} 2 \\
-\mathrm{a}\end{array}$ & $\begin{array}{c}\mathrm{ZrO} 2 \\
-\mathrm{a}\end{array}$ & $\begin{array}{c}\mathrm{Ag} 2 \mathrm{O} \\
-\mathrm{a}\end{array}$ & $\begin{array}{c}\mathrm{As} 2 \mathrm{O} 3 \\
-\mathrm{a}\end{array}$ & $\begin{array}{c}\mathrm{BaO} \\
-\mathrm{a}\end{array}$ & $\begin{array}{c}\mathrm{Bi} 2 \mathrm{O} 3 \\
-\mathrm{a}\end{array}$ & $\begin{array}{l}\mathrm{Br} \\
-\mathrm{a}\end{array}$ & $\begin{array}{c}\mathrm{CdO} \\
-\mathrm{a}\end{array}$ & $\begin{array}{c}\mathrm{Ce} 2 \mathrm{O} 3 \\
-\mathrm{a}\end{array}$ & $\begin{array}{c}\mathrm{CeO} 2 \\
-\mathrm{a}\end{array}$ & $\begin{array}{l}\mathrm{Cl} \\
-\mathrm{a}\end{array}$ & $\begin{array}{c}\mathrm{CoO} \\
-\mathrm{a}\end{array}$ & $\begin{array}{c}\mathrm{Co} 2 \mathrm{O} 3 \\
-\mathrm{a}\end{array}$ & $\begin{array}{c}\mathrm{Cr} 2 \mathrm{O} 3 \\
-\mathrm{a}\end{array}$ & $\begin{array}{c}\text { Cs2O } \\
-\mathrm{a}\end{array}$ & $\begin{array}{c}\mathrm{CuO} \\
-\mathrm{a}\end{array}$ & $\begin{array}{c}\mathrm{Eu} 2 \mathrm{O} 3 \\
-\mathrm{a}\end{array}$ & $\begin{array}{l}\mathrm{F} \\
-\mathrm{a}\end{array}$ & $\begin{array}{c}\mathrm{Ga} 2 \mathrm{O} 3 \\
-\mathrm{a}\end{array}$ & $\begin{array}{c}\mathrm{Gd} 2 \mathrm{O} 3 \\
-\mathrm{a}\end{array}$ & $\begin{array}{c}\mathrm{HgO} \\
-\mathrm{a}\end{array}$ \\
\hline \multicolumn{22}{|l|}{ TRU-B-1 } \\
\hline \multicolumn{22}{|l|}{ TRU-B-2 } \\
\hline \multicolumn{22}{|l|}{ TRU-B-3 } \\
\hline \multicolumn{22}{|l|}{ TRU-B-4 } \\
\hline \multicolumn{22}{|l|}{ TRU-B-5 } \\
\hline \multicolumn{22}{|l|}{ TRU-Bi-1 } \\
\hline \multicolumn{22}{|l|}{ TRU-Bi-2 } \\
\hline \multicolumn{22}{|l|}{ TRU-Bi-3 } \\
\hline \multicolumn{22}{|l|}{ TRU-Bi-4 } \\
\hline \multicolumn{22}{|l|}{ TRU-Ce-1 } \\
\hline \multicolumn{22}{|l|}{ TRU-Ce-2 } \\
\hline \multicolumn{22}{|l|}{ TRU-Ce-3 } \\
\hline \multicolumn{22}{|l|}{ TRU-Li-1 } \\
\hline \multicolumn{22}{|l|}{ TRU-Li-2 } \\
\hline \multicolumn{22}{|l|}{ TRU-Li-3 } \\
\hline \multicolumn{22}{|l|}{ TRU-Li-4 } \\
\hline \multicolumn{22}{|l|}{ TRU-Na-1 } \\
\hline \multicolumn{22}{|l|}{ TRU-Na-2 } \\
\hline \multicolumn{22}{|l|}{ TRU-Na-3 } \\
\hline \multicolumn{22}{|l|}{ TRU-Na-4 } \\
\hline \multicolumn{22}{|l|}{ TRU-P-1 } \\
\hline TRU-P-2 & & & & & & & & & & & & & & & & & & & & & \\
\hline TRU-P-3 & & & & & & & & & & & & & & & & & & & & & \\
\hline TRU-P-4 & & & & & & & & & & & & & & & & & & & & & \\
\hline TRU-Si-1 & & & & & & & & & & & & & & & & & & & & & \\
\hline TRU-Si-2 & & & & & & & & & & & & & & & & & & & & & \\
\hline TRU-Si-3 & & & & & & & & & & & & & & & & & & & & & \\
\hline TRU-Si-4 & & & & & & & & & & & & & & & & & & & & & \\
\hline TRU-Zr-1 & & & & & & & & & & & & & & & & & & & & & \\
\hline TRU-Zr-2 & & & & & & & & & & & & & & & & & & & & & \\
\hline TRU-Zr-3 & & & & & & & & & & & & & & & & & & & & & \\
\hline TRU-Zr-4 & & & & & & & & & & & & & & & & & & & & & \\
\hline
\end{tabular}

Zr Study (Vienna et al. 1999)

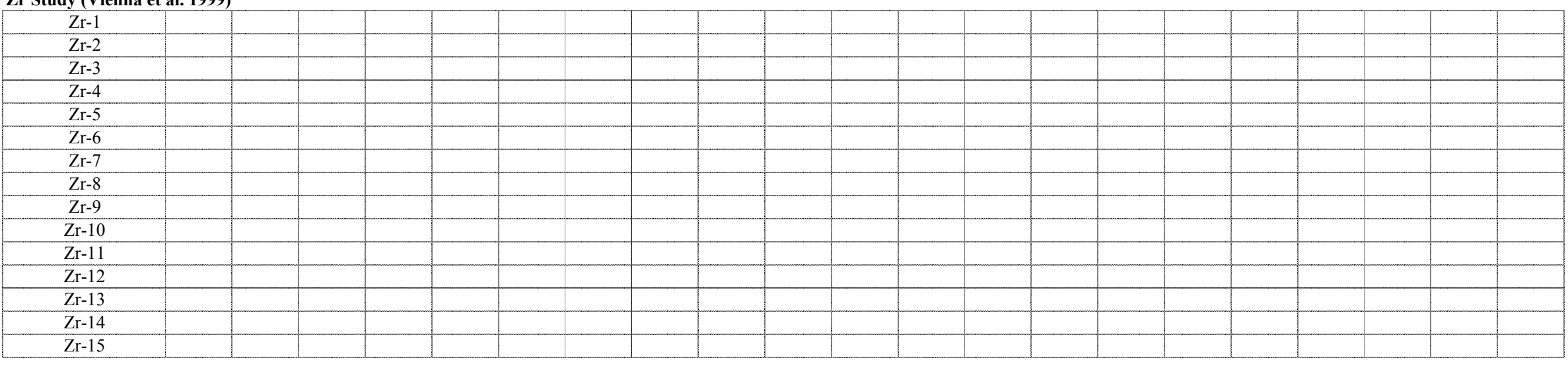


Appendix A. Database - mass fraction

TRU Study (Crum et al. 1997)

\begin{tabular}{|c|c|c|c|c|c|c|c|c|c|c|c|c|c|c|c|c|c|c|c|c|c|}
\hline Glass ID & $\begin{array}{c}\mathrm{I} \\
-\mathrm{a}\end{array}$ & $\begin{array}{c}\mathrm{La} 2 \mathrm{O} 3 \\
-\mathrm{a}\end{array}$ & $\begin{array}{c}\mathrm{MnO} 2 \\
-\mathrm{a}\end{array}$ & $\begin{array}{c}\mathrm{MnO} \\
-\mathrm{a}\end{array}$ & $\begin{array}{c}\mathrm{MoO} \\
-\mathrm{a}\end{array}$ & $\begin{array}{c}\mathrm{MoO} 3 \\
-\mathrm{a}\end{array}$ & $\begin{array}{c}\mathrm{Nb} 2 \mathrm{O} 5 \\
-\mathrm{a}\end{array}$ & $\begin{array}{c}\mathrm{Nd} 2 \mathrm{O} 3 \\
-\mathrm{a}\end{array}$ & $\begin{array}{c}\mathrm{PbO} \\
-\mathrm{a}\end{array}$ & $\begin{array}{c}\mathrm{PdO} 2 \\
-\mathrm{a}\end{array}$ & $\begin{array}{c}\mathrm{PdO} \\
-\mathrm{a}\end{array}$ & $\begin{array}{c}\mathrm{Pr} 2 \mathrm{O} 3 \\
-\mathrm{a}\end{array}$ & $\begin{array}{c}\text { Pr6O11 } \\
-\mathrm{a}\end{array}$ & $\begin{array}{c}\mathrm{Rb} 2 \mathrm{O} \\
-\mathrm{a}\end{array}$ & $\begin{array}{c}\mathrm{ReO} \\
-\mathrm{a}\end{array}$ & $\begin{array}{c}\mathrm{ReO} 2 \\
-\mathrm{a}\end{array}$ & $\begin{array}{c}\mathrm{Rh} 2 \mathrm{O} 3 \\
-\mathrm{a}\end{array}$ & $\begin{array}{c}\mathrm{RhO}^{2} \\
-\mathrm{a}\end{array}$ & $\begin{array}{c}\mathrm{RuO} 2 \\
-\mathrm{a}\end{array}$ & $\begin{array}{c}\mathrm{Sb} 2 \mathrm{O} 3 \\
-\mathrm{a}\end{array}$ & $\begin{array}{c}\mathrm{Sb} 2 \mathrm{O} 5 \\
-\mathrm{a}\end{array}$ \\
\hline TRU-B-1 & & & & & & & & & & & & & & & & & & & & & \\
\hline TRU-B-2 & & & & & & & & & & & & & & & & & & & & & \\
\hline TRU-B-3 & & & & & & & & & & & & & & & & & & & & & \\
\hline TRU-B-4 & & & & & & & & & & & & & & & & & & & & & \\
\hline TRU-B-5 & & & & & & & & & & & & & & & & & & & & & \\
\hline TRU-Bi-1 & & & & & & & & & & & & & & & & & & & & & \\
\hline TRU-Bi-2 & & & & & & & & & & & & & & & & & & & & & \\
\hline TRU-Bi-3 & & & & & & & & & & & & & & & & & & & & & \\
\hline TRU-Bi-4 & & & & & & & & & & & & & & & & & & & & & \\
\hline TRU-Ce-1 & & & & & & & & & & & & & & & & & & & & & \\
\hline TRU-Ce-2 & & & & & & & & & & & & & & & & & & & & & \\
\hline TRU-Ce-3 & & & & & & & & & & & & & & & & & & & & & \\
\hline TRU-Li-1 & & & & & & & & & & & & & & & & & & & & & \\
\hline TRU-Li-2 & & & & & & & & & & & & & & & & & & & & & \\
\hline TRU-Li-3 & & & & & & & & & & & & & & & & & & & & & \\
\hline TRU-Li-4 & & & & & & & & & & & & & & & & & & & & & \\
\hline TRU-Na-1 & & & & & & & & & & & & & & & & & & & & & \\
\hline TRU-Na-2 & & & & & & & & & & & & & & & & & & & & & \\
\hline TRU-Na-3 & & & & & & & & & & & & & & & & & & & & & \\
\hline TRU-Na-4 & & & & & & & & & & & & & & & & & & & & & \\
\hline TRU-P-1 & & & & & & & & & & & & & & & & & & & & & \\
\hline TRU-P-2 & & & & & & & & & & & & & & & & & & & & & \\
\hline TRU-P-3 & & & & & & & & & & & & & & & & & & & & & \\
\hline TRU-P-4 & & & & & & & & & & & & & & & & & & & & & \\
\hline TRU-Si-1 & & & & & & & & & & & & & & & & & & & & & \\
\hline TRU-Si-2 & & & & & & & & & & & & & & & & & & & & & \\
\hline TRU-Si-3 & & & & & & & & & & & & & & & & & & & & & \\
\hline TRU-Si-4 & & & & & & & & & & & & & & & & & & & & & \\
\hline TRU-Zr-1 & & & & & & & & & & & & & & & & & & & & & \\
\hline TRU-Zr-2 & & & & & & & & & & & & & & & & & & & & & \\
\hline TRU-Zr-3 & & & & & & & & & & & & & & & & & & & & & \\
\hline TRU-Zr-4 & & & & & & & & & & & & & & & & & & & & & \\
\hline
\end{tabular}

\section{Zr Study (Vienna et al. 1999)}

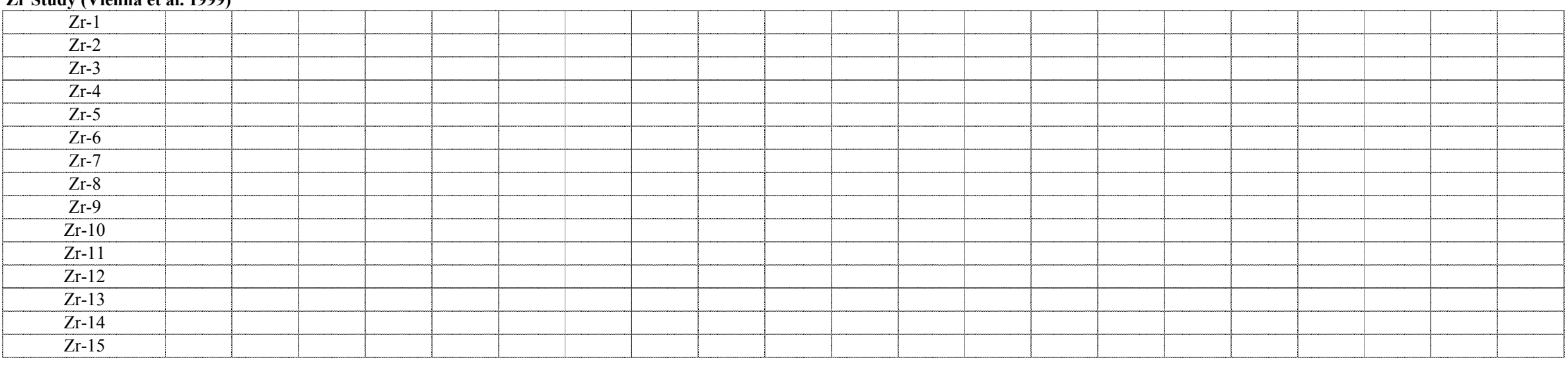


Appendix A. Database - mass fraction

TRU Study (Crum et al. 1997)

\begin{tabular}{|c|c|c|c|c|c|c|c|c|c|c|c|c|c|c|c|c|c|c|c|c|}
\hline Glass ID & $\begin{array}{c}\mathrm{SeO} 2 \\
-\mathrm{a}\end{array}$ & $\begin{array}{c}\mathrm{Sm} 2 \mathrm{O} 3 \\
-\mathrm{a}\end{array}$ & $\begin{array}{c}\mathrm{SnO} \\
-\mathrm{a}\end{array}$ & $\begin{array}{c}\mathrm{SnO} 2 \\
-\mathrm{a}\end{array}$ & $\begin{array}{c}\mathrm{SO} 3 \\
-\mathrm{a}\end{array}$ & $\begin{array}{c}\mathrm{SrO} \\
-\mathrm{a}\end{array}$ & $\begin{array}{c}\mathrm{Tc} 2 \mathrm{O} 7 \\
-\mathrm{a}\end{array}$ & $\begin{array}{c}\mathrm{TeO} 2 \\
-\mathrm{a}\end{array}$ & $\begin{array}{c}\mathrm{ThO} 2 \\
-\mathrm{a}\end{array}$ & $\begin{array}{c}\mathrm{TiO} 2 \\
-\mathrm{a}\end{array}$ & $\begin{array}{c}\mathrm{T} 12 \mathrm{O} 3 \\
-\mathrm{a}\end{array}$ & $\begin{array}{c}\text { U3O8 } \\
-\mathrm{a}\end{array}$ & $\begin{array}{c}\mathrm{UO} 2 \\
-\mathrm{a}\end{array}$ & $\begin{array}{c}\mathrm{UO3} \\
-\mathrm{a}\end{array}$ & $\begin{array}{c}\mathrm{V} 2 \mathrm{O} 5 \\
-\mathrm{a}\end{array}$ & $\begin{array}{c}\text { WO3 } \\
-\mathrm{a}\end{array}$ & $\begin{array}{c}\mathrm{Y} 2 \mathrm{O} 3 \\
-\mathrm{a}\end{array}$ & $\begin{array}{c}\mathrm{ZnO} \\
-\mathrm{a}\end{array}$ & $\begin{array}{c}\text { Others } \\
-\mathrm{a}\end{array}$ & $\begin{array}{c}\text { Sum } \\
-\mathrm{a}\end{array}$ \\
\hline TRU-B-1 & & & & & & & & & & & & & & & & & & & & \\
\hline TRU-B-2 & & & & & & & & & & & & & & & & & & & & \\
\hline TRU-B-3 & & & & & & & & & & & & & & & & & & & & \\
\hline TRU-B-4 & & & & & & & & & & & & & & & & & & & & \\
\hline TRU-B-5 & & & & & & & & & & & & & & & & & & & & \\
\hline TRU-Bi-1 & & & & & & & & & & & & & & & & & & & & \\
\hline TRU-Bi-2 & & & & & & & & & & & & & & & & & & & & \\
\hline TRU-Bi-3 & & & & & & & & & & & & & & & & & & & & \\
\hline TRU-Bi-4 & & & & & & & & & & & & & & & & & & & & \\
\hline TRU-Ce-1 & & & & & & & & & & & & & & & & & & & & \\
\hline TRU-Ce-2 & & & & & & & & & & & & & & & & & & & & \\
\hline TRU-Ce-3 & & & & & & & & & & & & & & & & & & & & \\
\hline TRU-Li-1 & & & & & & & & & & & & & & & & & & & & \\
\hline TRU-Li-2 & & & & & & & & & & & & & & & & & & & & \\
\hline TRU-Li-3 & & & & & & & & & & & & & & & & & & & & \\
\hline TRU-Li-4 & & & & & & & & & & & & & & & & & & & & \\
\hline TRU-Na-1 & & & & & & & & & & & & & & & & & & & & \\
\hline TRU-Na-2 & & & & & & & & & & & & & & & & & & & & \\
\hline TRU-Na-3 & & & & & & & & & & & & & & & & & & & & \\
\hline TRU-Na-4 & & & & & & & & & & & & & & & & & & & & \\
\hline TRU-P-1 & & & & & & & & & & & & & & & & & & & & \\
\hline TRU-P-2 & & & & & & & & & & & & & & & & & & & & \\
\hline TRU-P-3 & & & & & & & & & & & & & & & & & & & & \\
\hline TRU-P-4 & & & & & & & & & & & & & & & & & & & & \\
\hline TRU-Si-1 & & & & & & & & & & & & & & & & & & & & \\
\hline TRU-Si-2 & & & & & & & & & & & & & & & & & & & & \\
\hline TRU-Si-3 & & & & & & & & & & & & & & & & & & & & \\
\hline TRU-Si-4 & & & & & & & & & & & & & & & & & & & & \\
\hline TRU-Zr-1 & & & & & & & & & & & & & & & & & & & & \\
\hline TRU-Zr-2 & & & & & & & & & & & & & & & & & & & & \\
\hline TRU-Zr-3 & & & & & & & & & & & & & & & & & & & & \\
\hline TRU-Zr-4 & & & & & & & & & & & & & & & & & & & & \\
\hline
\end{tabular}

\section{Zr Study (Vienna et al. 1999)}

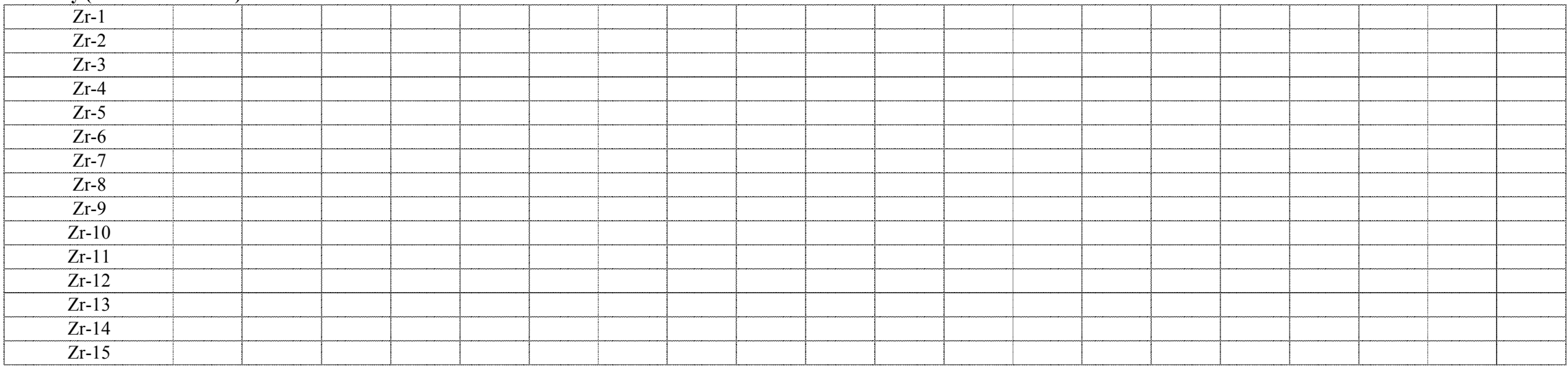


Appendix A. Database - mass fraction

TRU Study (Crum et al. 1997)

\begin{tabular}{|c|c|c|c|c|c|c|c|c|}
\hline Glass ID & $\begin{array}{l}\mathrm{TM} \\
\left({ }^{\circ} \mathrm{C}\right)\end{array}$ & $\begin{array}{l}\text { Gradient } \\
\text { TL }\left({ }^{\circ} \mathrm{C}\right)\end{array}$ & $\begin{array}{l}\text { Uniform } \\
\text { TL }\left({ }^{\circ} \mathrm{C}\right)\end{array}$ & Primary Phase & $\begin{array}{l}\text { Quenched } \\
\text { Visual/OM }\end{array}$ & $\begin{array}{c}\text { Quenched } \\
\text { SEM/EDS or TEM }\end{array}$ & $\begin{array}{l}\text { Quenched } \\
\text { XRD }\end{array}$ & $\begin{array}{c}\text { Quenched } \\
\text { Homogeneous? }\end{array}$ \\
\hline TRU-B-1 & & & 1155 & $\mathrm{ZrO} 2$ & & & & \\
\hline TRU-B-2 & & & 1031 & Zircon & & & & \\
\hline TRU-B-3 & & & 1030 & Zircon & & & & \\
\hline TRU-B-4 & & & 992 & Zircon & & & & \\
\hline TRU-B-5 & & & 1116 & $\mathrm{ZrO} 2$ & & & & \\
\hline TRU-Bi-1 & & & 1015 & Zircon & & & & \\
\hline TRU-Bi-2 & & & 1017 & Zircon & & & & \\
\hline TRU-Bi-3 & & & 1009 & Zircon & & & & \\
\hline TRU-Bi-4 & & & 1023 & Zircon & & & & \\
\hline TRU-Ce-1 & & & 1019 & Zircon & & & & \\
\hline TRU-Ce-2 & & & 1062 & Zircon & & & & \\
\hline TRU-Ce-3 & & & 1120 & $\mathrm{CeO} 2$ & & & & \\
\hline TRU-Li-1 & & & 1271 & $\mathrm{ZrO} 2$ & & & & \\
\hline TRU-Li-2 & & & 1155 & Zircon & & & & \\
\hline TRU-Li-3 & & & 1074 & Zircon & & & & \\
\hline TRU-Li-4 & & & 982 & Zircon & & & & \\
\hline TRU-Na-1 & & & 1350 & Zircon & & & & \\
\hline TRU-Na-2 & & & 1223 & Zircon & & & & \\
\hline TRU-Na-3 & & & 1110 & Zircon & & & & \\
\hline TRU-Na-4 & & & 930 & Zircon & & & & \\
\hline TRU-P-1 & & & 1042 & Zircon & & & & \\
\hline TRU-P-2 & & & 1052 & Zircon & & & & \\
\hline TRU-P-3 & & & 1057 & Zircon & & & & \\
\hline TRU-P-4 & & & 1070 & Zircon & & & & \\
\hline TRU-Si-1 & & & 1012 & Zircon & & & & \\
\hline TRU-Si-2 & & & 1022 & Zircon & & & & \\
\hline TRU-Si-3 & & & & Zircon & & & & \\
\hline TRU-Si-4 & & & 1022 & Zircon & & & & \\
\hline TRU-Zr-1 & & & 937 & Zircon & & & & \\
\hline TRU-Zr-2 & & & 1074 & Zircon & & & & \\
\hline TRU-Zr-3 & & & 1182 & $\mathrm{ZrO} 2$ & & & & \\
\hline TRU-Zr-4 & & & & $\mathrm{ZrO} 2$ & & & & \\
\hline
\end{tabular}

Zr Study (Vienna et al. 1999)

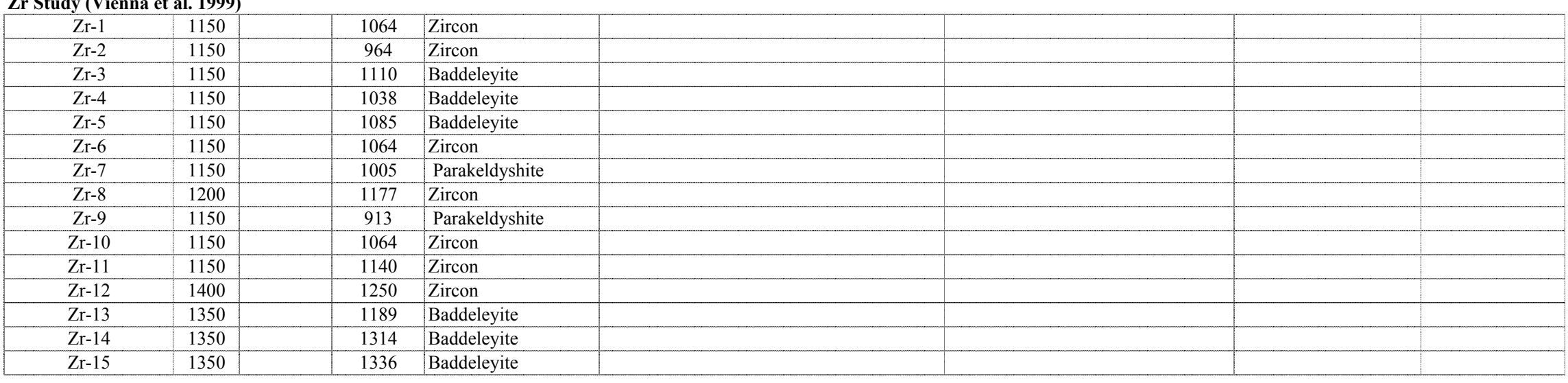


Appendix A. Database - mass fraction

\section{TRU Study (Crum et al. 1997)}

\begin{tabular}{|c|c|c|c|c|c|}
\hline Glass ID & $\begin{array}{c}\mathrm{CCC} \\
\text { Visual/OM }\end{array}$ & $\begin{array}{c}\text { CCC } \\
\text { SEM/EDS or TEM }\end{array}$ & $\begin{array}{l}\text { CCC } \\
\text { XRD }\end{array}$ & $\begin{array}{l}\text { Heat Treated } \\
\text { Visual/OM }\end{array}$ & $\begin{array}{c}\text { Heat Treated } \\
\text { SEM/EDS or TEM }\end{array}$ \\
\hline \multicolumn{6}{|l|}{ TRU-B-1 } \\
\hline \multirow{2}{*}{\multicolumn{6}{|c|}{$\begin{array}{l}\text { TRU-B-2 } \\
\text { TRU-B-3 }\end{array}$}} \\
\hline & & & & & \\
\hline \multicolumn{6}{|l|}{ TRU-B-4 } \\
\hline \multicolumn{6}{|l|}{ TRU-B-5 } \\
\hline \multicolumn{6}{|l|}{ TRU-Bi-1 } \\
\hline \multicolumn{6}{|l|}{ TRU-Bi-2 } \\
\hline \multicolumn{6}{|l|}{ TRU-Bi-3 } \\
\hline \multicolumn{6}{|l|}{ TRU-Bi-4 } \\
\hline \multicolumn{6}{|l|}{ TRU-Ce-1 } \\
\hline \multicolumn{6}{|l|}{ TRU-Ce-2 } \\
\hline \multicolumn{6}{|l|}{ TRU-Ce-3 } \\
\hline \multicolumn{6}{|l|}{ TRU-Li-1 } \\
\hline \multicolumn{6}{|l|}{ TRU-Li-2 } \\
\hline \multicolumn{6}{|l|}{ TRU-Li-3 } \\
\hline \multicolumn{6}{|l|}{ TRU-Li-4 } \\
\hline \multicolumn{6}{|l|}{ TRU-Na-1 } \\
\hline \multicolumn{6}{|l|}{ TRU-Na-2 } \\
\hline \multicolumn{6}{|l|}{ TRU-Na-3 } \\
\hline \multicolumn{6}{|l|}{ TRU-Na-4 } \\
\hline \multicolumn{6}{|l|}{ TRU-P-1 } \\
\hline \multicolumn{6}{|l|}{ TRU-P-2 } \\
\hline \multicolumn{6}{|l|}{ TRU-P-3 } \\
\hline \multicolumn{6}{|l|}{ TRU-P-4 } \\
\hline \multicolumn{6}{|l|}{ TRU-Si-1 } \\
\hline \multicolumn{6}{|l|}{ TRU-Si-2 } \\
\hline \multicolumn{6}{|l|}{ TRU-Si-3 } \\
\hline \multicolumn{6}{|l|}{ TRU-Si-4 } \\
\hline \multicolumn{6}{|l|}{ TRU-Zr-1 } \\
\hline \multicolumn{6}{|l|}{ TRU-Zr-2 } \\
\hline \multicolumn{6}{|l|}{ TRU-Zr-3 } \\
\hline TRU-Zr-4 & & & & & \\
\hline
\end{tabular}

\section{Zr Study (Vienna et al. 1999)}

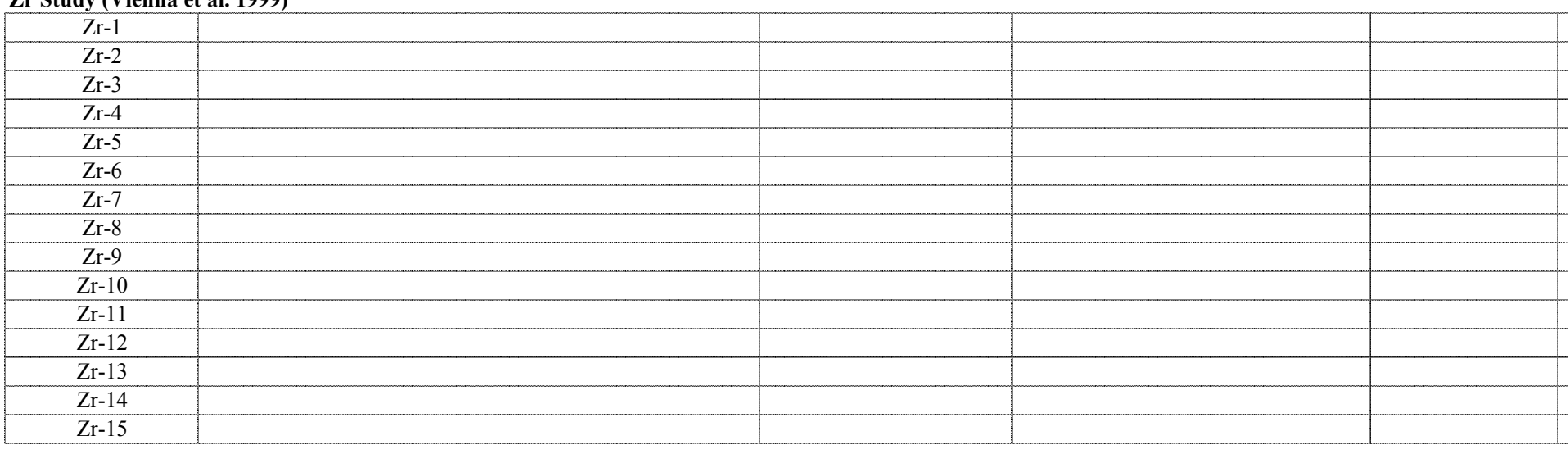


Appendix A. Database - mass fraction

\section{TRU Study (Crum et al. 1997)}

Glass ID

TRU-B-1

TRU-B-2

TRU-B-3

TRU-B-4

TRU-B-5

TRU-Bi-1

TRU-Bi-2

TRU-Bi-3

TRU-Bi-4

TRU-Ce-1

TRU-Ce-2

TRU-Ce-3

TRU-Li-1

TRU-Li-2

TRU-Li-3

TRU-Li-4

TRU-Na-1

TRU-Na-2

TRU-Na-3

TRU-Na-4

TRU-P-1

TRU-P-2

TRU-P-3

TRU-P-4

TRU-Si-1

TRU-Si-2

TRU-Si-3

TRU-Si-4

TRU-Zr-1

TRU-Zr-2

TRU-Zr-3

\section{Zr Study (Vienna et al. 1999)}

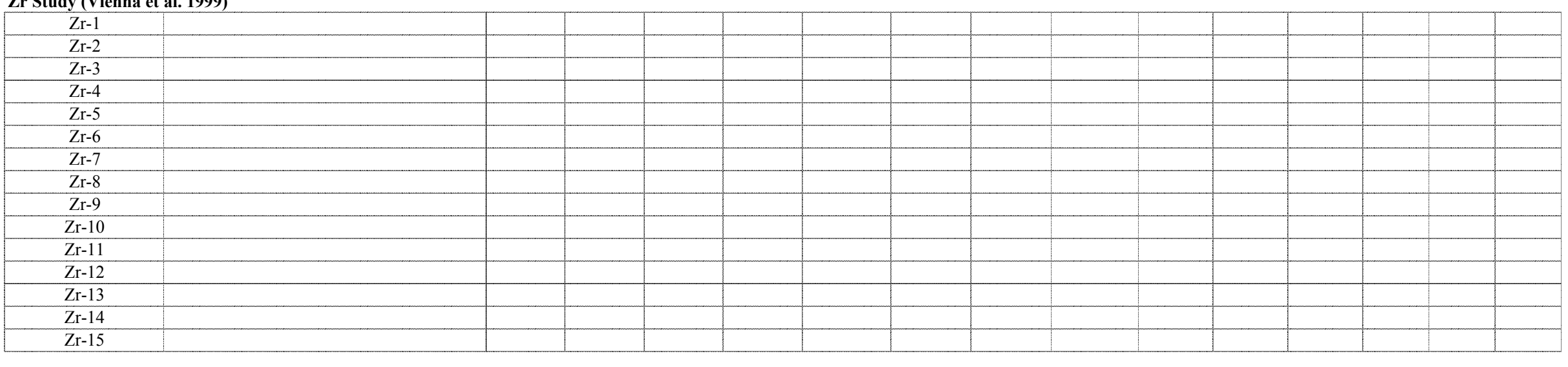

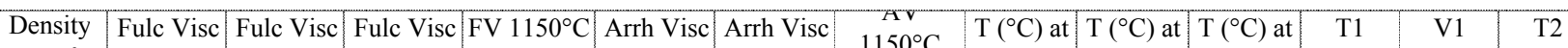

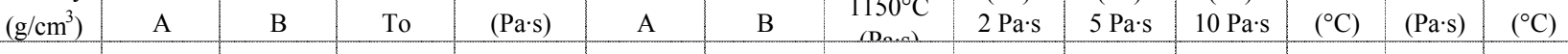

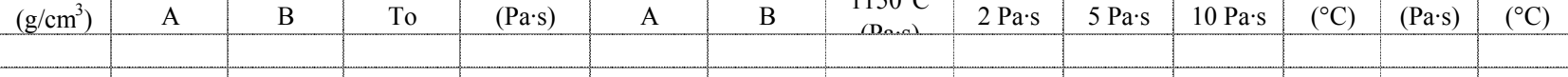

Heat Treated

XRD

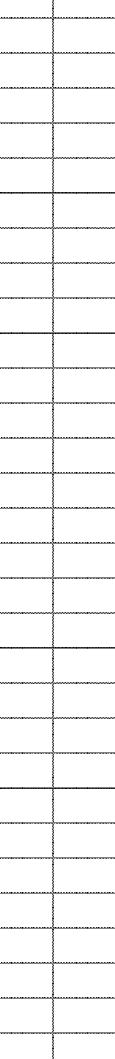

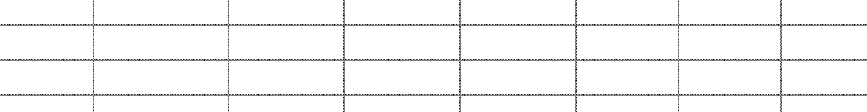

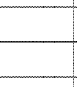

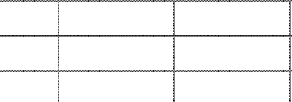

$\left[\begin{array}{l|l}-1 \\ -\end{array}\right.$

\begin{tabular}{|l|l|l|l|l|l|l|}
\hline & & & & & & \\
\hline & & & & & & \\
\hline & & & & & \\
\hline & & & & & \\
\hline & & & & & \\
\hline & & & & & \\
\hline & & & & & \\
\hline & & & & & \\
\hline & & & & & \\
\hline & & & & & \\
\hline
\end{tabular}


Appendix A. Database - mass fraction

TRU Study (Crum et al. 1997)

\begin{tabular}{|c|c|c|c|c|c|c|c|c|c|c|c|c|c|c|c|c|c|c|c|c|c|}
\hline Glass ID & $\begin{array}{c}\mathrm{V} 2 \\
(\mathrm{~Pa} \cdot \mathrm{s})\end{array}$ & $\begin{array}{c}\mathrm{T} 3 \\
\left({ }^{\circ} \mathrm{C}\right)\end{array}$ & $\begin{array}{c}\mathrm{V} 3 \\
(\mathrm{~Pa} \cdot \mathrm{s})\end{array}$ & $\begin{array}{c}\mathrm{T} 4 \\
\left({ }^{\circ} \mathrm{C}\right)\end{array}$ & $\begin{array}{c}\mathrm{V} 4 \\
(\mathrm{~Pa} \cdot \mathrm{s})\end{array}$ & $\begin{array}{c}\mathrm{T} 5 \\
\left({ }^{\circ} \mathrm{C}\right)\end{array}$ & $\begin{array}{c}\mathrm{V} 5 \\
(\mathrm{~Pa} \cdot \mathrm{s})\end{array}$ & $\begin{array}{c}\mathrm{T} 6 \\
\left({ }^{\circ} \mathrm{C}\right)\end{array}$ & $\begin{array}{c}\mathrm{V} 6 \\
(\mathrm{~Pa} \cdot \mathrm{s})\end{array}$ & $\begin{array}{c}\mathrm{T} 7 \\
\left({ }^{\circ} \mathrm{C}\right)\end{array}$ & $\begin{array}{c}\text { V7 } \\
(\mathrm{Pa} \cdot \mathrm{s})\end{array}$ & $\begin{array}{c}\text { T8 } \\
\left({ }^{\circ} \mathrm{C}\right)\end{array}$ & $\begin{array}{c}\mathrm{V} 8 \\
(\mathrm{~Pa} \cdot \mathrm{s})\end{array}$ & $\begin{array}{c}\mathrm{T} 9 \\
\left({ }^{\circ} \mathrm{C}\right)\end{array}$ & $\begin{array}{c}\text { V9 } \\
(\mathrm{Pa} \cdot \mathrm{s})\end{array}$ & $\begin{array}{l}\mathrm{T} 10 \\
\left({ }^{\circ} \mathrm{C}\right)\end{array}$ & $\begin{array}{c}\mathrm{V} 10 \\
(\mathrm{~Pa} \cdot \mathrm{s})\end{array}$ & $\begin{array}{l}\mathrm{T} 11 \\
\left({ }^{\circ} \mathrm{C}\right)\end{array}$ & $\begin{array}{c}\mathrm{V} 11 \\
(\mathrm{~Pa} \cdot \mathrm{s})\end{array}$ & $\begin{array}{l}\mathrm{T} 12 \\
\left({ }^{\circ} \mathrm{C}\right)\end{array}$ & $\begin{array}{c}\mathrm{V} 12 \\
(\mathrm{~Pa} \cdot \mathrm{s})\end{array}$ \\
\hline TRU-B-1 & & & & & & & & & & & & & & & & & & & & & \\
\hline TRU-B-2 & & & & & & & & & & & & & & & & & & & & & \\
\hline TRU-B-3 & & & & & & & & & & & & & & & & & & & & & \\
\hline TRU-B-4 & & & & & & & & & & & & & & & & & & & & & \\
\hline TRU-B-5 & & & & & & & & & & & & & & & & & & & & & \\
\hline TRU-Bi-1 & & & & & & & & & & & & & & & & & & & & & \\
\hline TRU-Bi-2 & & & & & & & & & & & & & & & & & & & & & \\
\hline TRU-Bi-3 & & & & & & & & & & & & & & & & & & & & & \\
\hline TRU-Bi-4 & & & & & & & & & & & & & & & & & & & & & \\
\hline TRU-Ce-1 & & & & & & & & & & & & & & & & & & & & & \\
\hline TRU-Ce-2 & & & & & & & & & & & & & & & & & & & & & \\
\hline TRU-Ce-3 & & & & & & & & & & & & & & & & & & & & & \\
\hline TRU-Li-1 & & & & & & & & & & & & & & & & & & & & & \\
\hline TRU-Li-2 & & & & & & & & & & & & & & & & & & & & & \\
\hline TRU-Li-3 & & & & & & & & & & & & & & & & & & & & & \\
\hline TRU-Li-4 & & & & & & & & & & & & & & & & & & & & & \\
\hline TRU-Na-1 & & & & & & & & & & & & & & & & & & & & & \\
\hline TRU-Na-2 & & & & & & & & & & & & & & & & & & & & & \\
\hline TRU-Na-3 & & & & & & & & & & & & & & & & & & & & & \\
\hline TRU-Na-4 & & & & & & & & & & & & & & & & & & & & & \\
\hline TRU-P-1 & & & & & & & & & & & & & & & & & & & & & \\
\hline TRU-P-2 & & & & & & & & & & & & & & & & & & & & & \\
\hline TRU-P-3 & & & & & & & & & & & & & & & & & & & & & \\
\hline TRU-P-4 & & & & & & & & & & & & & & & & & & & & & \\
\hline TRU-Si-1 & & & & & & & & & & & & & & & & & & & & & \\
\hline TRU-Si-2 & & & & & & & & & & & & & & & & & & & & & \\
\hline TRU-Si-3 & & & & & & & & & & & & & & & & & & & & & \\
\hline TRU-Si-4 & & & & & & & & & & & & & & & & & & & & & \\
\hline TRU-Zr-1 & & & & & & & & & & & & & & & & & & & & & \\
\hline TRU-Zr-2 & & & & & & & & & & & & & & & & & & & & & \\
\hline TRU-Zr-3 & & & & & & & & & & & & & & & & & & & & & \\
\hline TRU-Zr-4 & & & & & & & & & & & & & & & & & & & & & \\
\hline
\end{tabular}

\section{Zr Study (Vienna et al. 1999)}

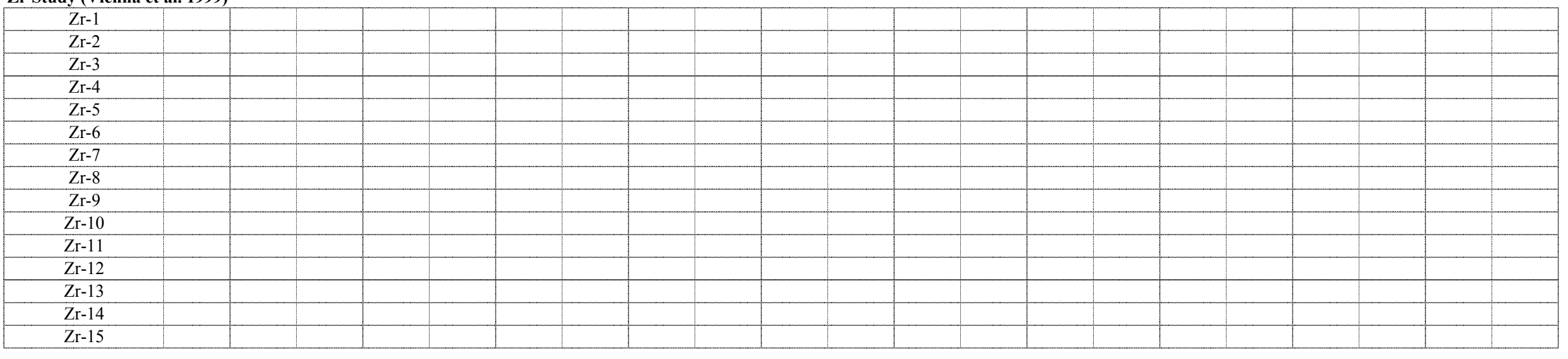


Appendix A. Database - mass fraction

TRU Study (Crum et al. 1997)

\begin{tabular}{|c|c|c|c|c|c|c|c|c|c|c|c|c|c|c|c|c|}
\hline Glass ID & $\begin{array}{l}\mathrm{T} 13 \\
\left({ }^{\circ} \mathrm{C}\right)\end{array}$ & $\begin{array}{c}\mathrm{V} 13 \\
(\mathrm{~Pa} \cdot \mathrm{s})\end{array}$ & $\begin{array}{l}\mathrm{T} 14 \\
\left({ }^{\circ} \mathrm{C}\right)\end{array}$ & $\begin{array}{c}\mathrm{V} 14 \\
(\mathrm{~Pa} \cdot \mathrm{s})\end{array}$ & $\begin{array}{c}\text { Q PCT } \\
\mathrm{B}\left(\mathrm{g} / \mathrm{m}^{2}\right)\end{array}$ & $\begin{array}{c}\text { Q PCT } \\
\mathrm{Li}\left(\mathrm{g} / \mathrm{m}^{2}\right)\end{array}$ & $\begin{array}{c}\text { Q PCT } \\
\mathrm{Na}\left(\mathrm{g} / \mathrm{m}^{2}\right)\end{array}$ & $\begin{array}{c}\text { Q PCT } \\
\mathrm{Si}\left(\mathrm{g} / \mathrm{m}^{2}\right)\end{array}$ & $\begin{array}{c}\text { Q PCT } \\
\text { pH }\end{array}$ & $\begin{array}{c}\text { CCC PCT } \\
\mathrm{B}\left(\mathrm{g} / \mathrm{m}^{2}\right)\end{array}$ & $\begin{array}{l}\text { CCC PCT } \\
\mathrm{Li}\left(\mathrm{g} / \mathrm{m}^{2}\right)\end{array}$ & $\begin{array}{l}\text { CCC PCT } \\
\mathrm{Na}\left(\mathrm{g} / \mathrm{m}^{2}\right)\end{array}$ & $\begin{array}{l}\text { CCC PCT } \\
\mathrm{Si}\left(\mathrm{g} / \mathrm{m}^{2}\right)\end{array}$ & $\begin{array}{c}\text { CCC PCT } \\
\text { pH }\end{array}$ & $\begin{array}{l}\text { Q PCT B at } \\
20^{\circ} \mathrm{C}\left(\mathrm{g} / \mathrm{m}^{2}\right)\end{array}$ & $\begin{array}{l}\text { Q PCT Li at } \\
20^{\circ} \mathrm{C}\left(\mathrm{g} / \mathrm{m}^{2}\right)\end{array}$ \\
\hline \multicolumn{17}{|l|}{ TRU-B-1 } \\
\hline \multirow{2}{*}{\multicolumn{17}{|c|}{$\begin{array}{l}\text { TRU-B-2 } \\
\text { TRU-B-3 }\end{array}$}} \\
\hline & & & & & & & & & & & & & & & & \\
\hline \multicolumn{17}{|l|}{ TRU-B-4 } \\
\hline \multicolumn{17}{|l|}{ TRU-B-5 } \\
\hline \multicolumn{17}{|l|}{ TRU-Bi-1 } \\
\hline \multicolumn{17}{|l|}{ TRU-Bi-2 } \\
\hline \multicolumn{17}{|l|}{ TRU-Bi-3 } \\
\hline \multicolumn{17}{|l|}{ TRU-Bi-4 } \\
\hline \multicolumn{17}{|l|}{ TRU-Ce-1 } \\
\hline \multicolumn{17}{|l|}{ TRU-Ce-2 } \\
\hline \multicolumn{17}{|l|}{ TRU-Ce-3 } \\
\hline \multicolumn{17}{|l|}{ TRU-Li-1 } \\
\hline \multicolumn{17}{|l|}{ TRU-Li-2 } \\
\hline \multirow{2}{*}{\multicolumn{17}{|c|}{$\begin{array}{l}\text { TRU-Li-3 } \\
\text { TRU-Li-4 }\end{array}$}} \\
\hline \multirow{2}{*}{\multicolumn{17}{|c|}{$\frac{\text { TRU-Li-4 }}{\text { TRU-Na-1 }}$}} \\
\hline & & & & & & & & & & & & & & & & \\
\hline \multicolumn{17}{|l|}{ TRU-Na-2 } \\
\hline \multicolumn{17}{|l|}{ TRU-Na-3 } \\
\hline \multicolumn{17}{|l|}{ TRU-Na-4 } \\
\hline \multicolumn{17}{|l|}{ TRU-P-1 } \\
\hline \multicolumn{17}{|l|}{ TRU-P-2 } \\
\hline \multicolumn{17}{|l|}{ TRU-P-3 } \\
\hline \multicolumn{17}{|l|}{ TRU-P-4 } \\
\hline \multicolumn{17}{|l|}{ TRU-Si-1 } \\
\hline \multicolumn{17}{|l|}{ TRU-Si-2 } \\
\hline TRU-Si-3 & & & & & & & & & & & & & & & & \\
\hline TRU-Si-4 & & & & & & & & & & & & & & & & \\
\hline TRU-Zr-1 & & & & & & & & & & & & & & & & \\
\hline TRU-Zr-2 & & & & & & & & & & & & & & & & \\
\hline TRU-Zr-3 & & & & & & & & & & & & & & & & \\
\hline TRU-Zr-4 & & & & & & & & & & & & & & & & \\
\hline
\end{tabular}

Zr Study (Vienna et al. 1999)

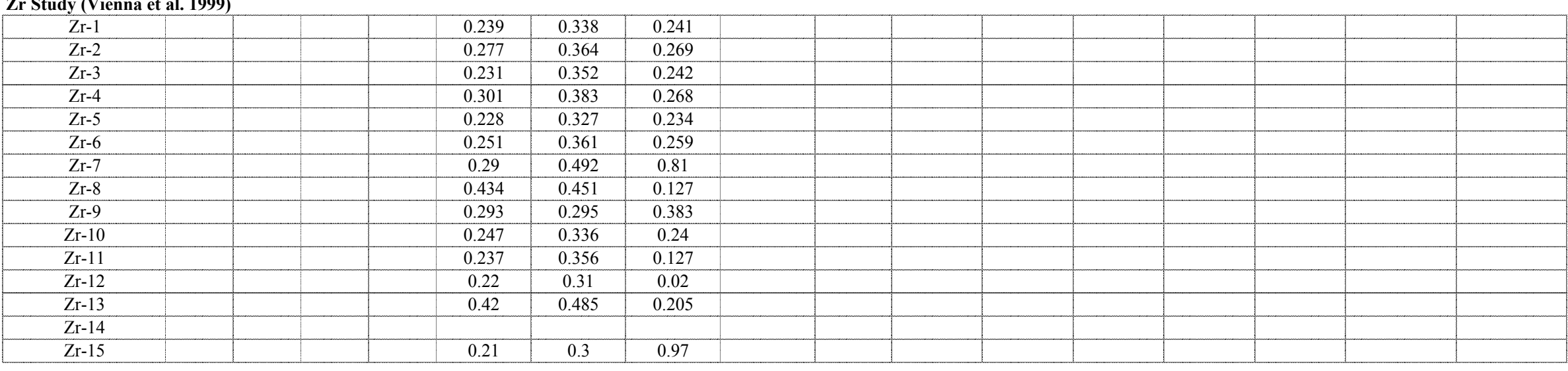


Appendix A. Database - mass fraction

\section{TRU Study (Crum et al. 1997)}

\begin{tabular}{|c|c|c|c|c|c|c|c|c|c|c|c|c|}
\hline Glass ID & $\begin{array}{l}\text { Q PCT Na at } \\
20^{\circ} \mathrm{C}\left(\mathrm{g} / \mathrm{m}^{2}\right)\end{array}$ & $\begin{array}{l}\text { Q PCT Si at } \\
20^{\circ} \mathrm{C}\left(\mathrm{g} / \mathrm{m}^{2}\right)\end{array}$ & $\begin{array}{c}\mathrm{QpH} \\
\text { at } 20^{\circ} \mathrm{C}\end{array}$ & $\begin{array}{c}\text { TCLP Ag } \\
(\mathrm{ppm})\end{array}$ & $\begin{array}{c}\text { TCLP As } \\
(\mathrm{ppm})\end{array}$ & $\begin{array}{c}\text { TCLP Ba } \\
(\mathrm{ppm})\end{array}$ & $\begin{array}{c}\text { TCLP Cd } \\
(\mathrm{ppm})\end{array}$ & $\begin{array}{c}\text { TCLP Cr } \\
(\mathrm{ppm})\end{array}$ & $\begin{array}{c}\text { TCLP Ni } \\
(\mathrm{ppm})\end{array}$ & $\begin{array}{c}\text { TCLP Pb } \\
(\mathrm{ppm})\end{array}$ & $\begin{array}{l}\text { TCLP Se } \\
(\mathrm{ppm})\end{array}$ & $\begin{array}{c}\text { TCLP Zn } \\
(\mathrm{ppm})\end{array}$ \\
\hline \multicolumn{13}{|c|}{ 19 } \\
\hline \multicolumn{13}{|l|}{ TRU-B-2 } \\
\hline \multicolumn{13}{|l|}{ TRU-B-3 } \\
\hline \multicolumn{13}{|l|}{ TRU-B-4 } \\
\hline \multicolumn{13}{|l|}{ TRU-B-5 } \\
\hline \multicolumn{13}{|l|}{ TRU-Bi-1 } \\
\hline \multicolumn{13}{|l|}{ TRU-Bi-2 } \\
\hline \multicolumn{13}{|l|}{ TRU-Bi-3 } \\
\hline \multicolumn{13}{|l|}{ TRU-Bi-4 } \\
\hline \multicolumn{13}{|l|}{ TRU-Ce-1 } \\
\hline \multicolumn{13}{|l|}{ TRU-Ce-2 } \\
\hline \multicolumn{13}{|l|}{ TRU-Ce-3 } \\
\hline \multicolumn{13}{|l|}{ TRU-Li-1 } \\
\hline \multicolumn{13}{|l|}{ TRU-Li-2 } \\
\hline \multicolumn{13}{|l|}{ TRU-Li-3 } \\
\hline \multicolumn{13}{|l|}{ TRU-Li-4 } \\
\hline \multicolumn{13}{|l|}{ TRU-Na-1 } \\
\hline \multicolumn{13}{|l|}{ TRU-Na-2 } \\
\hline \multicolumn{13}{|l|}{ TRU-Na-3 } \\
\hline \multicolumn{13}{|l|}{ TRU-Na-4 } \\
\hline \multicolumn{13}{|l|}{ TRU-P-1 } \\
\hline \multicolumn{13}{|l|}{ TRU-P-2 } \\
\hline \multicolumn{13}{|l|}{ TRU-P-3 } \\
\hline \multicolumn{13}{|l|}{ TRU-P-4 } \\
\hline \multicolumn{13}{|l|}{ TRU-Si-1 } \\
\hline \multicolumn{13}{|l|}{ TRU-Si-2 } \\
\hline TRU-Si-3 & & & & & & & & & & & & \\
\hline TRU-Si-4 & & & & & & & & & & & & \\
\hline TRU-Zr-1 & & & & & & & & & & & & \\
\hline TRU-Zr-2 & & & & & & & & & & & & \\
\hline TRU-Zr-3 & & & & & & & & & & & & \\
\hline TRU-Zr-4 & & & & & & & & & & & & \\
\hline
\end{tabular}

\section{Zr Study (Vienna et al. 1999)}

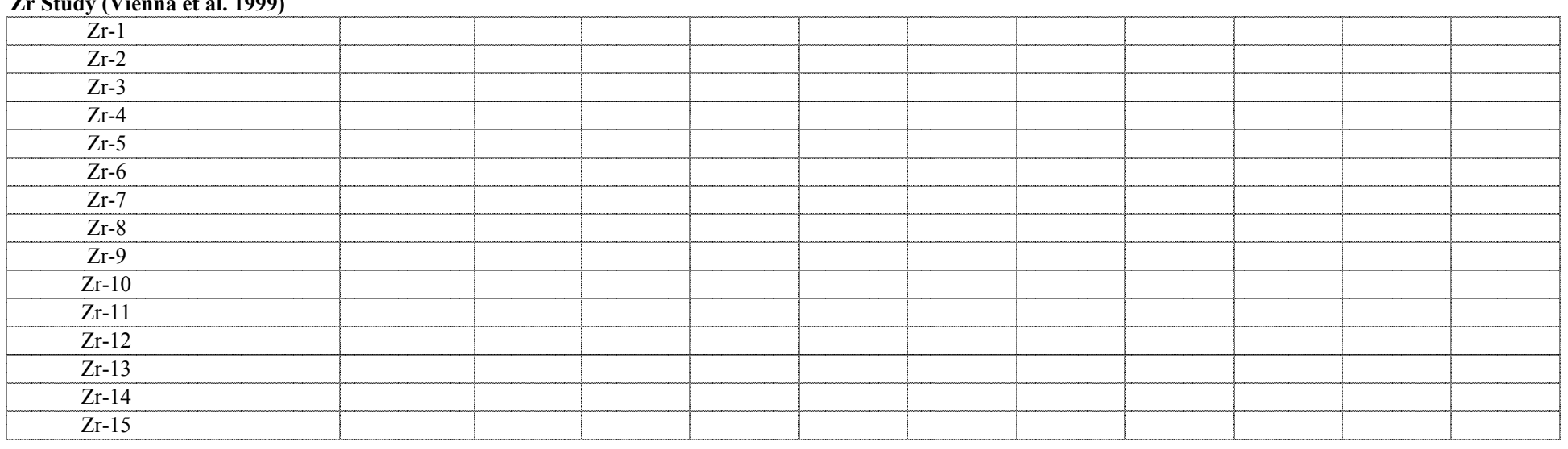


Zr Study (Vienna et al. 1999)

\begin{tabular}{|c|c|c|c|c|c|c|c|c|c|c|c|c|c|c|c|c|c|c|c|c|c|}
\hline Glass ID & $\begin{array}{c}\mathrm{A} 12 \mathrm{O} 3 \\
-\mathrm{t}\end{array}$ & $\begin{array}{c}\mathrm{B} 2 \mathrm{O} 3 \\
-\mathrm{t}\end{array}$ & $\begin{array}{c}\mathrm{CaO} \\
-\mathrm{t}\end{array}$ & $\begin{array}{c}\mathrm{Fe} 2 \mathrm{O} 3 \\
-\mathrm{t}\end{array}$ & $\begin{array}{c}\mathrm{FeO} \\
-\mathrm{t}\end{array}$ & $\begin{array}{c}\mathrm{K} 2 \mathrm{O} \\
-\mathrm{t}\end{array}$ & $\begin{array}{c}\mathrm{Li} 2 \mathrm{O} \\
-\mathrm{t}\end{array}$ & $\begin{array}{c}\mathrm{MgO} \\
-\mathrm{t}\end{array}$ & $\begin{array}{c}\mathrm{Na} 2 \mathrm{O} \\
-\mathrm{t}\end{array}$ & $\begin{array}{c}\mathrm{NiO} \\
-\mathrm{t}\end{array}$ & $\begin{array}{c}\mathrm{P} 2 \mathrm{O} 5 \\
-\mathrm{t}\end{array}$ & $\begin{array}{c}\mathrm{SiO} 2 \\
-\mathrm{t}\end{array}$ & $\begin{array}{c}\mathrm{ZrO} 2 \\
-\mathrm{t}\end{array}$ & $\begin{array}{c}\mathrm{Ag} 2 \mathrm{O} \\
-\mathrm{t}\end{array}$ & $\begin{array}{c}\mathrm{As} 2 \mathrm{O} 3 \\
-\mathrm{t}\end{array}$ & $\begin{array}{c}\mathrm{BaO} \\
-\mathrm{t}\end{array}$ & $\begin{array}{c}\mathrm{Bi} 2 \mathrm{O} 3 \\
-\mathrm{t}\end{array}$ & $\begin{array}{c}\mathrm{Br} \\
-\mathrm{t}\end{array}$ & $\begin{array}{c}\mathrm{CdO} \\
-\mathrm{t}\end{array}$ & $\begin{array}{c}\mathrm{Ce} 2 \mathrm{O} 3 \\
-\mathrm{t}\end{array}$ & $\begin{array}{c}\mathrm{CeO} 2 \\
-\mathrm{t}\end{array}$ \\
\hline $\mathrm{Zr}-16$ & 0.0450 & 0.1000 & 0.0009 & & & 0.0042 & 0.0750 & & 0.1100 & & 0.0021 & 0.5114 & 0.1500 & & & & & & & 0.0002 & \\
\hline $\mathrm{Zr}-17$ & 0.0300 & 0.1500 & 0.0009 & & & 0.0047 & 0.0900 & & 0.0362 & & 0.0023 & 0.5193 & 0.1650 & & & & & & & 0.0002 & \\
\hline $\mathrm{Zr}-18$ & 0.0300 & 0.1500 & 0.0009 & & & 0.0047 & 0.0600 & & 0.1011 & & 0.0023 & 0.4844 & 0.1650 & & & & & & & 0.0002 & \\
\hline $\mathrm{Zr}-19$ & 0.0300 & 0.0500 & 0.0009 & & & 0.0047 & 0.0900 & & 0.1122 & & 0.0023 & 0.5434 & 0.1650 & & & & & & & 0.0002 & \\
\hline $\mathrm{Zr}-20$ & 0.0300 & 0.0500 & 0.0009 & & & 0.0047 & 0.0600 & & 0.1770 & & 0.0023 & 0.5085 & 0.1650 & & & & & & & 0.0002 & \\
\hline $\mathrm{Zr}-21$ & 0.0600 & 0.1500 & 0.0008 & & & 0.0038 & 0.0900 & & 0.0430 & & 0.0019 & 0.5142 & 0.1350 & & & & & & & 0.0002 & \\
\hline $\mathrm{Zr}-22$ & 0.0600 & 0.1500 & 0.0008 & & & 0.0038 & 0.0600 & & 0.1079 & & 0.0019 & 0.4794 & 0.1350 & & & & & & & 0.0002 & \\
\hline $\mathrm{Zr}-23$ & 0.0600 & 0.0500 & 0.0008 & & & 0.0038 & 0.0900 & & 0.1189 & & 0.0019 & 0.5383 & 0.1350 & & & & & & & 0.0002 & \\
\hline $\mathrm{Zr}-24$ & 0.0600 & 0.0500 & 0.0008 & & & 0.0038 & 0.0600 & & 0.1838 & & 0.0019 & 0.5035 & 0.1350 & & & & & & & 0.0002 & \\
\hline $\mathrm{Zr}-25$ & 0.0300 & 0.1500 & 0.0008 & & & 0.0038 & 0.0900 & & 0.0395 & & 0.0019 & 0.5477 & 0.1350 & & & & & & & 0.0002 & \\
\hline $\mathrm{Zr}-26$ & 0.0300 & 0.1500 & 0.0008 & & & 0.0038 & 0.0600 & & 0.1044 & & 0.0019 & 0.5129 & 0.1350 & & & & & & & 0.0002 & \\
\hline $\mathrm{Zr}-27$ & 0.0300 & 0.0500 & 0.0008 & & & 0.0038 & 0.0900 & & 0.1154 & & 0.0019 & 0.5718 & 0.1350 & & & & & & & 0.0002 & \\
\hline $\mathrm{Zr}-28$ & 0.0450 & 0.1000 & 0.0009 & & & 0.0042 & 0.0750 & & 0.1100 & & 0.0021 & 0.5114 & 0.1500 & & & & & & & 0.0002 & \\
\hline $\mathrm{Zr}-29$ & 0.0300 & 0.0500 & 0.0008 & & & 0.0038 & 0.0600 & & 0.1803 & & 0.0019 & 0.5370 & 0.1350 & & & & & & & 0.0002 & \\
\hline
\end{tabular}

\section{SP (Mika et al. 1997)}

\begin{tabular}{l|l|l|l|l|l|} 
SP-1a & 0.0800 & 0.0700 & 0.0100 & 0.1250 \\
\hline
\end{tabular}

\begin{tabular}{l|l|l|l|l|}
\hline SP-1b & 0.0800 & 0.0700 & 0.0100 & 0.1250
\end{tabular}

\begin{tabular}{|l|l|l|l|l|}
\hline SP-1c & 0.0800 & 0.0700 & 0.0100 & 0.1250 \\
\hline
\end{tabular}

\begin{tabular}{|l|l|l|l|l|}
\hline SP-1d & 0.0800 & 0.0700 & 0.0100 & 0.1250 \\
\hline
\end{tabular}

\begin{tabular}{l|l|l|l|l|} 
SP-1e & 0.0800 & 0.0700 & 0.0100 & 0.1250
\end{tabular}

\begin{tabular}{l|l|l|l|l|l|}
\hline SP-1f & 0.0800 & 0.0700 & 0.0100 & 0.1250 \\
\hline
\end{tabular}

\begin{tabular}{l|l|l|l|l|}
\hline SP-1g & 0.0800 & 0.0700 & 0.0100 & 0.1250 \\
\hline
\end{tabular}

\begin{tabular}{|l|l|l|l|l|}
\hline SP-1h & 0.0800 & 0.0700 & 0.0100 & 0.1250 \\
\hline
\end{tabular}

\begin{tabular}{l|l|l|l|l|l} 
SP-1i & 0.0800 & 0.0700 & 0.0100 & 0.1250
\end{tabular}

\begin{tabular}{|l|l|l|l|l|}
\hline SP-Al-1 & 0.0400 & 0.0730 & 0.0104 & 0.1304 \\
\hline
\end{tabular}

\begin{tabular}{l|l|l|l|l|l} 
SP-Al-2 & 0.1200 & 0.0670 & 0.0096 & 0.1196
\end{tabular}

\begin{tabular}{l|l|l|l|l|l|}
\hline SP-Al-3 & 0.1600 & 0.0639 & 0.0091 & 0.1141
\end{tabular}

\begin{tabular}{l|l|l|l|l|l|} 
SP-B-1 & 0.0860 & 0.0000 & 0.0108 & 0.1344 \\
\hline
\end{tabular}

\begin{tabular}{|l|l|l|l|l|}
\hline SP-B-2 & 0.0852 & 0.0100 & 0.0106 & 0.1331 \\
\hline
\end{tabular}

\begin{tabular}{|l|l|l|l|l|}
\hline SP-B-3 & 0.0826 & 0.0400 & 0.0103 & 0.1290 \\
\hline
\end{tabular}

\begin{tabular}{|l|l|l|l|l|}
\hline SP-B-4 & 0.0757 & 0.1200 & 0.0095 & 0.1183 \\
\hline
\end{tabular}

\begin{tabular}{l|l|l|l|l|}
\hline SP-Cr-1 & 0.0802 & 0.0702 & 0.0100 & 0.1253 \\
\hline
\end{tabular}

\begin{tabular}{|l|l|l|l|l|l|}
\hline SP-Cr-2 & 0.0798 & 0.0698 & 0.0100 & 0.1246 \\
\hline
\end{tabular}

\begin{tabular}{|l|l|l|l|l|l|}
\hline SP-Cr-3 & 0.0795 & 0.0696 & 0.0099 & 0.1243 \\
\hline
\end{tabular}

\begin{tabular}{l|l|l|l|l|l|}
\hline SP-Cr-4 & 0.0792 & 0.0693 & 0.0099 & 0.1238 \\
\hline
\end{tabular}

\begin{tabular}{l|l|l|l|l|}
\hline SP-Fe-1 & 0.0859 & 0.0752 & 0.0107 & 0.0600
\end{tabular}

\begin{tabular}{|l|l|l|l|l|}
\hline SP-Fe-2 & 0.0832 & 0.0728 & 0.0104 & 0.0900 \\
\hline
\end{tabular}

\begin{tabular}{l|l|l|l|l|} 
SP-Fe-3 & 0.0777 & 0.0680 & 0.0097 & 0.1500
\end{tabular}

\begin{tabular}{|l|l|l|l|l|l|}
\hline SP-Li-1 & 0.0825 & 0.0722 & 0.0103 & 0.1289 \\
\hline
\end{tabular}

\begin{tabular}{l|l|l|l|l|l|} 
SP-Li-2 & 0.0816 & 0.0714 & 0.0102 & 0.1276
\end{tabular}

\begin{tabular}{l|l|l|l|l|}
\hline SP-Li-3 & 0.0800 & 0.0700 & 0.0100 & 0.1250 \\
\hline
\end{tabular}

\begin{tabular}{l|l|l|l|l|l|}
\hline SP-Mg-1 & 0.0797 & 0.0697 & 0.0100 & 0.1245 \\
\hline
\end{tabular}

\begin{tabular}{|l|l|l|l|l|l|}
\hline SP-Mg-2 & 0.0789 & 0.0690 & 0.0099 & 0.1232 \\
\hline
\end{tabular}

\begin{tabular}{l|l|l|l|l|}
\hline SP-Mg-3 & 0.0757 & 0.0662 & 0.0095 & 0.1182 \\
\hline
\end{tabular}

\begin{tabular}{l|l|l|l|l|l}
\hline SP-Mn-1 & 0.0803 & 0.0703 & 0.0100 & 0.1255 \\
\hline
\end{tabular}

\begin{tabular}{l|l|l|l|l|l|l|l|l}
\hline SP-Mn-2 & 0.0795 & 0.0696 & 0.0099 & 0.1242 \\
\hline
\end{tabular}

\begin{tabular}{l|l|l|l|l|l|}
\hline SP-Mn-3 & 0.0771 & 0.0674 & 0.0096 & 0.1204 \\
\hline
\end{tabular}

\begin{tabular}{l|l|l|l|l|} 
SP-Na-1 & 0.0873 & 0.0764 & 0.0109 & 0.1365
\end{tabular}

\begin{tabular}{|l|l|l|l|l|l|l|l|l|l|l|l|}
0.0028 & 0.0300 & 0.0060 & 0.1573 & 0.0052 & 0.0046 & 0.4600 & 0.0185 & 0.0007 & 0.0000 & 0.0030 & 0.0000 \\
\hline
\end{tabular}

\begin{tabular}{|l|l|l|l|l|l|l|l|l|l|l|l|l|l|l|}
\hline 0.0028 & 0.0300 & 0.0060 & 0.1573 & 0.0052 & 0.0046 & 0.4600 & 0.0185 & 0.0007 & 0.0000 & 0.0030 & 0.0000 \\
\hline
\end{tabular}

\begin{tabular}{|l|l|l|l|l|l|l|l|l|l|l|l|}
0.0028 & 0.0300 & 0.0060 & 0.1573 & 0.0052 & 0.0046 & 0.4600 & 0.0185 & 0.0007 & 0.0000 & 0.0030 & 0.0000 \\
\hline
\end{tabular}

\begin{tabular}{|l|l|l|l|l|l|l|l|l|l|l|l|l|l|l|l|}
0.0028 & 0.0300 & 0.0060 & 0.1573 & 0.0052 & 0.0046 & 0.4600 & 0.0185 & 0.0007 & 0.0000 & 0.0030 & 0.0000 \\
\hline
\end{tabular}

\begin{tabular}{|l|l|l|l|l|l|l|l|l|l|l|l|l|l|}
\hline & 0.028 & 0.0300 & 0.0060 & 0.1573 & 0.0052 & 0.0046 & 0.4600 & 0.0185 & 0.0007 & 0.0000 & 0.0030 & 0.0000 \\
\hline
\end{tabular}

\begin{tabular}{|l|l|l|l|l|l|l|l|l|l|l|l|l|}
0.0028 & 0.0300 & 0.0060 & 0.1573 & 0.0052 & 0.0046 & 0.4600 & 0.0185 & 0.0007 & 0.0000 & 0.0030 & 0.0000 \\
\hline
\end{tabular}

\begin{tabular}{|l|l|l|l|l|l|l|l|l|l|l|l|}
0.0028 & 0.0300 & 0.0060 & 0.1573 & 0.0052 & 0.0046 & 0.4600 & 0.0185 & 0.0007 & 0.0000 & 0.0030 & 0.0000 \\
\hline
\end{tabular}

\begin{tabular}{|l|l|l|l|l|l|l|l|l|l|l|l|l|l|}
0.0028 & 0.0300 & 0.0060 & 0.1573 & 0.0052 & 0.0046 & 0.4600 & 0.0185 & 0.0007 & 0.0000 & 0.0030 & 0.0000 \\
\hline
\end{tabular}

\begin{tabular}{|l|l|l|l|l|l|l|l|l|l|l|l|l|l|}
\hline 0.0028 & 0.0300 & 0.0060 & 0.1573 & 0.0052 & 0.0046 & 0.4600 & 0.0185 & 0.0007 & 0.0000 & 0.0030 & 0.0000 \\
\hline
\end{tabular}

\begin{tabular}{|l|l|l|l|l|l|l|l|l|l|l|l|l|}
\hline 0.0029 & 0.0313 & 0.0063 & 0.1641 & 0.0054 & 0.0048 & 0.4800 & 0.0193 & 0.0007 & 0.0000 & 0.0032 & 0.0000 \\
\hline
\end{tabular}

\begin{tabular}{|l|l|l|l|l|l|l|l|l|l|l|l|l|}
0.0027 & 0.0287 & 0.0057 & 0.1505 & 0.0050 & 0.0044 & 0.4400 & 0.0177 & 0.0007 & 0.0000 & 0.0029 & 0.0000 \\
\hline
\end{tabular}

\begin{tabular}{|l|l|l|l|l|l|l|l|l|l|l|l|l|l|l|}
0.0026 & 0.0274 & 0.0055 & 0.1436 & 0.0047 & 0.0042 & 0.4200 & 0.0169 & 0.0006 & 0.0000 & 0.0028 & 0.0000 \\
\hline
\end{tabular}

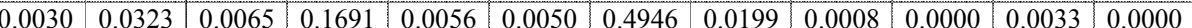

\begin{tabular}{|l|l|l|l|l|l|l|l|l|l|l|l|}
\hline 0.0030 & 0.0319 & 0.0064 & 0.1674 & 0.0055 & 0.0049 & 0.4897 & 0.0197 & 0.0008 & 0.0000 & 0.0032 & 0.0000 \\
\hline
\end{tabular}

\begin{tabular}{|l|l|l|l|l|l|l|l|l|l|l|l|l|}
0.0029 & 0.0310 & 0.0062 & 0.1624 & 0.0054 & 0.0048 & 0.4748 & 0.0191 & 0.0007 & 0.0000 & 0.0031 & 0.0000 \\
\hline
\end{tabular}

\begin{tabular}{|l|l|l|l|l|l|l|l|l|l|l|l|l|l|}
0.0026 & 0.0284 & 0.0057 & 0.1488 & 0.0049 & 0.0044 & 0.4353 & 0.0175 & 0.0007 & 0.0000 & 0.0029 & 0.0000 \\
\hline
\end{tabular}

\begin{tabular}{|l|l|l|l|l|l|l|l|l|l|l|l|l|}
\hline 0.0028 & 0.0301 & 0.0060 & 0.1576 & 0.0052 & 0.0047 & 0.4610 & 0.0185 & 0.0007 & 0.0000 & 0.0030 & 0.0000 \\
\hline
\end{tabular}

\begin{tabular}{|l|l|l|l|l|l|l|l|l|l|l|l|l|}
\hline 0.0028 & 0.0299 & 0.0060 & 0.1569 & 0.0052 & 0.0046 & 0.4587 & 0.0184 & 0.0007 & 0.0000 & 0.0030 & 0.0000 \\
\hline
\end{tabular}

\begin{tabular}{|l|l|l|l|l|l|l|l|l|l|l|l|l|}
0.0028 & 0.0298 & 0.0060 & 0.1564 & 0.0052 & 0.0046 & 0.4573 & 0.0184 & 0.0007 & 0.0000 & 0.0030 & 0.0000 \\
\hline
\end{tabular}

\begin{tabular}{|l|l|l|l|l|l|l|l|l|l|l|l|l|l|l|}
0.0028 & 0.0297 & 0.0059 & 0.1558 & 0.0051 & 0.0046 & 0.4555 & 0.0183 & 0.0007 & 0.0000 & 0.0030 & 0.0000 \\
\hline
\end{tabular}

\begin{tabular}{|l|l|l|l|l|l|l|l|l|l|l|l|l|l|}
\hline 0.0030 & 0.0322 & 0.0064 & 0.1690 & 0.0056 & 0.0050 & 0.4942 & 0.0198 & 0.0008 & 0.0000 & 0.0033 & 0.0000 \\
\hline
\end{tabular}

\begin{tabular}{|l|l|l|l|l|l|l|l|l|l|l|l|l|}
0.0029 & 0.0312 & 0.0062 & 0.1636 & 0.0054 & 0.0048 & 0.4784 & 0.0192 & 0.0007 & 0.0000 & 0.0031 & 0.0000 \\
\hline
\end{tabular}

\begin{tabular}{|l|l|l|l|l|l|l|l|l|l|l|l|}
0.0027 & 0.0291 & 0.0058 & 0.1528 & 0.0051 & 0.0045 & 0.4469 & 0.0179 & 0.0007 & 0.0000 & 0.0029 & 0.0000 \\
\hline
\end{tabular}

\begin{tabular}{|l|l|l|l|l|l|l|l|l|l|l|l|l|l|l|}
\hline 0.0029 & 0.0000 & 0.0062 & 0.1622 & 0.0054 & 0.0048 & 0.4742 & 0.0190 & 0.0007 & 0.0000 & 0.0031 & 0.0000 \\
\hline
\end{tabular}

\begin{tabular}{|l|l|l|l|l|l|l|l|l|l|l|l|l|l|l|}
\hline 0.0029 & 0.0100 & 0.0061 & 0.1605 & 0.0053 & 0.0047 & 0.4695 & 0.0188 & 0.0007 & 0.0000 & 0.0031 & 0.0000 \\
\hline
\end{tabular}

\begin{tabular}{|l|l|l|l|l|l|l|l|l|l|l|l|}
\hline 0.0028 & 0.0300 & 0.0060 & 0.1573 & 0.0052 & 0.0046 & 0.4600 & 0.0185 & 0.0007 & 0.0000 & 0.0030 & 0.0000 \\
\hline
\end{tabular}

\begin{tabular}{|l|l|l|l|l|l|l|l|l|l|l|l|}
0.0028 & 0.0299 & 0.0100 & 0.1567 & 0.0052 & 0.0046 & 0.4581 & 0.0184 & 0.0007 & 0.0000 & 0.0030 & 0.0000 \\
\hline
\end{tabular}

\begin{tabular}{|l|l|l|l|l|l|l|l|l|l|l|l|l|l|}
0.0028 & 0.0296 & 0.0200 & 0.1551 & 0.0051 & 0.0046 & 0.4535 & 0.0182 & 0.0007 & 0.0000 & 0.0030 & 0.0000 \\
\hline
\end{tabular}

\begin{tabular}{|l|l|l|l|l|l|l|l|l|l|l|l|l|}
\hline 0.0026 & 0.0284 & 0.0600 & 0.1488 & 0.0049 & 0.0044 & 0.4350 & 0.0175 & 0.0007 & 0.0000 & 0.0029 & 0.0000 \\
\hline
\end{tabular}

\begin{tabular}{|l|l|l|l|l|l|l|l|l|l|l|l|}
0.0028 & 0.0301 & 0.0060 & 0.1579 & 0.0052 & 0.0047 & 0.4617 & 0.0185 & 0.0007 & 0.0000 & 0.0030 & 0.0000 \\
\hline
\end{tabular}

\begin{tabular}{|l|l|l|l|l|l|l|l|l|l|l|l|l|l|}
\hline 0.0028 & 0.0298 & 0.0060 & 0.1563 & 0.0052 & 0.0046 & 0.4570 & 0.0183 & 0.0007 & 0.0000 & 0.0030 & 0.0000 \\
\hline
\end{tabular}

\begin{tabular}{|l|l|l|l|l|l|l|l|l|l|l|l|l|l|}
0.0027 & 0.0289 & 0.0058 & 0.1516 & 0.0050 & 0.0045 & 0.4432 & 0.0178 & 0.0007 & 0.0000 & 0.0029 & 0.0000 \\
\hline
\end{tabular}

\begin{tabular}{|l|l|l|l|l|l|l|l|l|l|l|l|}
0.0031 & 0.0328 & 0.0066 & 0.0800 & 0.0057 & 0.0051 & 0.5022 & 0.0202 & 0.0008 & 0.0000 & 0.0033 & 0.0000 \\
\hline
\end{tabular}

\begin{tabular}{|c|c|}
\hline 0.0070 & 0.0007 \\
\hline 0.0070 & 0.0007 \\
\hline 0.0070 & 0.0007 \\
\hline 0.0070 & 0.0007 \\
\hline 0.0070 & 0.0007 \\
\hline 0.0070 & 0.0007 \\
\hline 0.0070 & 0.0007 \\
\hline 0.0070 & 0.0007 \\
\hline 0.0070 & 0.0007 \\
\hline 0.0073 & 0.0007 \\
\hline 0.0067 & 0.0007 \\
\hline 0.0064 & 0.0006 \\
\hline 0.0075 & 0.0008 \\
\hline 0.0074 & 0.0008 \\
\hline 0.0072 & 0.0007 \\
\hline 0.0066 & 0.0007 \\
\hline 0.0070 & 0.0007 \\
\hline 0.0069 & 0.0007 \\
\hline 0.0069 & 0.0007 \\
\hline 0.0069 & 0.0007 \\
\hline 0.0075 & 0.0008 \\
\hline 0.0072 & 0.0007 \\
\hline 0.0068 & 0.0007 \\
\hline 0.0072 & 0.0007 \\
\hline 0.0071 & 0.0007 \\
\hline 0.0070 & 0.0007 \\
\hline 0.0069 & 0.0007 \\
\hline 0.0069 & 0.0007 \\
\hline 0.0066 & 0.0007 \\
\hline 0.0070 & 0.0007 \\
\hline 0.0069 & 0.0007 \\
\hline 0.0067 & 0.0007 \\
\hline 0.0076 & 0.0008 \\
\hline
\end{tabular}


Zr Study (Vienna et al. 1999)

\begin{tabular}{|c|c|c|c|c|c|c|c|c|c|c|c|c|c|c|c|c|c|c|c|c|c|}
\hline Glass ID & $\begin{array}{l}\mathrm{Cl} \\
-\mathrm{t}\end{array}$ & $\begin{array}{c}\mathrm{CoO} \\
-\mathrm{t}\end{array}$ & $\begin{array}{c}\mathrm{Co} 2 \mathrm{O} 3 \\
-\mathrm{t}\end{array}$ & $\begin{array}{c}\mathrm{Cr} 2 \mathrm{O} 3 \\
-\mathrm{t}\end{array}$ & $\mid \begin{array}{c}\mathrm{Cs} 2 \mathrm{O} \\
-\mathrm{t}\end{array}$ & $\begin{array}{c}\mathrm{CuO} \\
-\mathrm{t}\end{array}$ & $\begin{array}{c}\mathrm{Eu} 2 \mathrm{O} 3 \\
-\mathrm{t}\end{array}$ & $\begin{array}{l}F \\
-t\end{array}$ & $\begin{array}{c}\mathrm{Ga} 2 \mathrm{O} 3 \\
-\mathrm{t}\end{array}$ & {$\left[\begin{array}{c}\mathrm{Gd} 2 \mathrm{O} 3 \\
-\mathrm{t}\end{array}\right.$} & $\begin{array}{c}\mathrm{HgO} \\
-\mathrm{t}\end{array}$ & $\begin{array}{l}I \\
-t\end{array}$ & $\begin{array}{c}\mathrm{La} 2 \mathrm{O} 3 \\
-\mathrm{t}\end{array}$ & $\begin{array}{c}\mathrm{MnO} 2 \\
-\mathrm{t}\end{array}$ & $\begin{array}{c}\mathrm{MnO} \\
-\mathrm{t}\end{array}$ & $\begin{array}{c}\mathrm{MoO} \\
-\mathrm{t}\end{array}$ & $\begin{array}{c}\mathrm{MoO} 3 \\
-\mathrm{t}\end{array}$ & $\begin{array}{c}\mathrm{Nb} 2 \mathrm{O} 5 \\
-\mathrm{t}\end{array}$ & $\begin{array}{c}\mathrm{Nd} 2 \mathrm{O} 3 \\
-\mathrm{t}\end{array}$ & $\begin{array}{c}\mathrm{PbO} \\
-\mathrm{t}\end{array}$ & $\begin{array}{c}\mathrm{PdO} 2 \\
-\mathrm{t}\end{array}$ \\
\hline $\mathrm{Zr}-16$ & & & & & 0.0004 & & & & & 0.0007 & & & & & & & & & & & \\
\hline $\mathrm{Zr}-17$ & & & & & 0.0005 & & & & & 0.0008 & & & & & & & & & & & \\
\hline $\mathrm{Zr}-18$ & & & & & 0.0005 & & & & & 0.0008 & & & & & & & & & & & \\
\hline $\mathrm{Zr}-19$ & & & & & 0.0005 & & & & & 0.0008 & & & & & & & & & & & \\
\hline $\mathrm{Zr}-20$ & & & & & 0.0005 & & & & & 0.0008 & & & & & & & & & & & \\
\hline $\mathrm{Zr}-21$ & & & & & 0.0005 & & & & & 0.0006 & & & & & & & & & & & \\
\hline $\mathrm{Zr}-22$ & & & & & 0.0004 & & & & & 0.0006 & & & & & & & & & & & \\
\hline $\mathrm{Zr}-23$ & & & & & 0.0004 & & & & & 0.0006 & & & & & & & & & & & \\
\hline $\mathrm{Zr}-24$ & & & & & 0.0004 & & & & & 0.0006 & & & & & & & & & & & \\
\hline $\mathrm{Zr}-25$ & & & & & 0.0004 & & & & & 0.0006 & & & & & & & & & & & \\
\hline $\mathrm{Zr}-26$ & & & & & 0.0004 & & & & & 0.0006 & & & & & & & & & & & \\
\hline $\mathrm{Zr}-27$ & & & & & 0.0004 & & & & & 0.0006 & & & & & & & & & & & \\
\hline $\mathrm{Zr}-28$ & & & & & 0.0004 & & & & & 0.0007 & & & & & & & & & & & \\
\hline $\mathrm{Zr}-29$ & & & & & \begin{tabular}{|l|}
0.0004 \\
\end{tabular} & & & & & 0.0006 & & & & & & & & & & & \\
\hline
\end{tabular}

\section{SP (Mika et al. 1997)}

\begin{tabular}{|c|c|c|c|c|c|c|c|c|c|c|c|}
\hline SP-1a & 0.0001 & 0.0009 & 0.0022 & 0.0000 & 0.0003 & 0.0006 & 0.0027 & 0.0036 & 0.0001 & 0.0018 & 0.0017 \\
\hline SP-1b & 0.0001 & 0.0009 & 0.0022 & 0.0000 & 0.0003 & 0.0006 & 0.0027 & 0.0036 & 0.0001 & 0.0018 & 0.0017 \\
\hline SP-1c & 0.0001 & 0.0009 & 0.0022 & 0.0000 & 0.0003 & 0.0006 & 0.0027 & 0.0036 & 0.0001 & 0.0018 & 0.0017 \\
\hline SP-1d & 0.0001 & 0.0009 & 0.0022 & 0.0000 & 0.0003 & 0.0006 & 0.0027 & 0.0036 & 0.0001 & 0.0018 & 0.0017 \\
\hline SP-1e & 0.0001 & 0.0009 & 0.0022 & 0.0000 & 0.0003 & 0.0006 & 0.0027 & 0.0036 & 0.0001 & 0.0018 & 0.0017 \\
\hline SP-1f & 0.0001 & 0.0009 & 0.0022 & 0.0000 & 0.0003 & 0.0006 & 0.0027 & 0.0036 & 0.0001 & 0.0018 & 0.0017 \\
\hline SP-1g & 0.0001 & 0.0009 & 0.0022 & 0.0000 & 0.0003 & 0.0006 & 0.0027 & 0.0036 & 0.0001 & 0.0018 & 0.0017 \\
\hline SP-1h & 0.0001 & 0.0009 & 0.0022 & 0.0000 & 0.0003 & 0.0006 & 0.0027 & 0.0036 & 0.0001 & 0.0018 & 0.0017 \\
\hline SP-1i & 0.0001 & 0.0009 & 0.0022 & 0.0000 & 0.0003 & 0.0006 & 0.0027 & 0.0036 & 0.0001 & 0.0018 & 0.0017 \\
\hline SP-Al-1 & 0.0001 & 0.0009 & 0.0023 & 0.0000 & 0.0003 & 0.0006 & 0.0028 & 0.0038 & 0.0001 & 0.0019 & 0.0018 \\
\hline SP-Al-2 & 0.0001 & 0.0009 & 0.0021 & 0.0000 & 0.0003 & 0.0006 & 0.0026 & 0.0034 & 0.0001 & 0.0017 & 0.0016 \\
\hline SP-Al-3 & 0.0001 & 0.0008 & 0.0020 & 0.0000 & 0.0003 & 0.0006 & 0.0025 & 0.0033 & 0.0001 & 0.0017 & 0.0016 \\
\hline SP-B-1 & 0.0001 & 0.0010 & 0.0024 & 0.0000 & 0.0003 & 0.0007 & 0.0029 & 0.0039 & 0.0001 & 0.0020 & 0.0018 \\
\hline SP-B-2 & 0.0001 & 0.0010 & 0.0023 & 0.0000 & 0.0003 & 0.0006 & 0.0029 & 0.0038 & 0.0001 & 0.0019 & 0.0018 \\
\hline SP-B-3 & 0.0001 & 0.0009 & 0.0023 & 0.0000 & 0.0003 & 0.0006 & 0.0028 & 0.0037 & 0.0001 & 0.0019 & 0.0018 \\
\hline SP-B-4 & 0.0001 & 0.0009 & 0.0021 & 0.0000 & 0.0003 & 0.0006 & 0.0026 & 0.0034 & 0.0001 & 0.0017 & 0.0016 \\
\hline SP-Cr-1 & 0.0001 & 0.0009 & 0.0000 & 0.0000 & 0.0003 & 0.0006 & 0.0027 & 0.0036 & 0.0001 & 0.0018 & 0.0017 \\
\hline SP-Cr-2 & 0.0001 & 0.0009 & 0.0050 & 0.0000 & 0.0003 & 0.0006 & 0.0027 & 0.0036 & 0.0001 & 0.0018 & 0.0017 \\
\hline SP-Cr-3 & 0.0001 & 0.0009 & 0.0080 & 0.0000 & 0.0003 & 0.0006 & 0.0027 & 0.0036 & 0.0001 & 0.0018 & 0.0017 \\
\hline SP-Cr-4 & 0.0001 & 0.0009 & 0.0120 & 0.0000 & 0.0003 & 0.0006 & 0.0027 & 0.0036 & 0.0001 & 0.0018 & 0.0017 \\
\hline SP-Fe-1 & 0.0001 & 0.0010 & 0.0024 & 0.0000 & 0.0003 & 0.0007 & 0.0029 & 0.0039 & 0.0001 & 0.0020 & 0.0018 \\
\hline SP-Fe-2 & 0.0001 & 0.0009 & 0.0023 & 0.0000 & 0.0003 & 0.0006 & 0.0028 & 0.0037 & 0.0001 & 0.0019 & 0.0018 \\
\hline SP-Fe-3 & 0.0001 & 0.0009 & 0.0021 & 0.0000 & 0.0003 & 0.0006 & 0.0026 & 0.0035 & 0.0001 & 0.0018 & 0.0017 \\
\hline SP-Li-1 & 0.0001 & 0.0009 & 0.0023 & 0.0000 & 0.0003 & 0.0006 & 0.0028 & 0.0037 & 0.0001 & 0.0019 & 0.0018 \\
\hline SP-Li-2 & 0.0001 & 0.0009 & 0.0022 & 0.0000 & 0.0003 & 0.0006 & 0.0028 & 0.0037 & 0.0001 & 0.0019 & 0.0018 \\
\hline SP-Li-3 & 0.0001 & 0.0009 & 0.0022 & 0.0000 & 0.0003 & 0.0006 & 0.0027 & 0.0036 & 0.0001 & 0.0018 & 0.0017 \\
\hline SP-Mg-1 & 0.0001 & 0.0009 & 0.0022 & 0.0000 & 0.0003 & 0.0006 & 0.0027 & 0.0036 & 0.0001 & 0.0018 & 0.0017 \\
\hline SP-Mg-2 & 0.0001 & 0.0009 & 0.0022 & 0.0000 & 0.0003 & 0.0006 & 0.0027 & 0.0035 & 0.0001 & 0.0018 & 0.0017 \\
\hline SP-Mg-3 & 0.0001 & 0.0009 & 0.0021 & 0.0000 & 0.0003 & 0.0006 & 0.0026 & 0.0034 & 0.0001 & 0.0017 & 0.0016 \\
\hline SP-Mn-1 & 0.0001 & 0.0009 & 0.0022 & 0.0000 & 0.0003 & 0.0006 & 0.0027 & 0.0000 & 0.0001 & 0.0018 & 0.0017 \\
\hline SP-Mn-2 & 0.0001 & 0.0009 & 0.0022 & 0.0000 & 0.0003 & 0.0006 & 0.0027 & 0.0100 & 0.0001 & 0.0018 & 0.0017 \\
\hline SP-Mn-3 & 0.0001 & 0.0009 & 0.0021 & 0.0000 & 0.0003 & 0.0006 & 0.0026 & 0.0400 & 0.0001 & 0.0018 & 0.0017 \\
\hline SP-Na-1 & 0.0001 & 0.0010 & 0.0024 & 0.0000 & 0.0003 & 0.0007 & 0.0030 & 0.0039 & 0.0001 & 0.0020 & 0.0019 \\
\hline
\end{tabular}


Zr Study (Vienna et al. 1999)

\begin{tabular}{|c|c|c|c|c|c|c|c|c|c|c|c|c|c|c|c|c|c|c|c|c|c|}
\hline Glass ID & $\begin{array}{c}\mathrm{PdO} \\
-\mathrm{t}\end{array}$ & $\begin{array}{c}\mathrm{Pr} 2 \mathrm{O} 3 \\
-\mathrm{t}\end{array}$ & $\begin{array}{c}\text { Pr6O11 } \\
-t\end{array}$ & $\begin{array}{c}\mathrm{Rb} 2 \mathrm{O} \\
-\mathrm{t}\end{array}$ & $\begin{array}{c}\mathrm{ReO} \\
-\mathrm{t}\end{array}$ & $\begin{array}{c}\mathrm{ReO} 2 \\
-\mathrm{t}\end{array}$ & $\begin{array}{c}\mathrm{Rh} 2 \mathrm{O} 3 \\
-\mathrm{t}\end{array}$ & $\begin{array}{c}\mathrm{RhO} 2 \\
-\mathrm{t}\end{array}$ & $\begin{array}{c}\mathrm{RuO} 2 \\
-\mathrm{t}\end{array}$ & $\begin{array}{c}\mathrm{Sb} 2 \mathrm{O} 3 \\
-\mathrm{t}\end{array}$ & $\begin{array}{c}\mathrm{Sb} 2 \mathrm{O} 5 \\
-\mathrm{t}\end{array}$ & $\begin{array}{c}\mathrm{SeO} 2 \\
-\mathrm{t}\end{array}$ & $\begin{array}{c}\mathrm{Sm} 2 \mathrm{O} 3 \\
-\mathrm{t}\end{array}$ & $\begin{array}{c}\mathrm{SnO} \\
-\mathrm{t}\end{array}$ & $\begin{array}{c}\mathrm{SnO} 2 \\
-\mathrm{t}\end{array}$ & $\begin{array}{c}\mathrm{SO} 3 \\
-\mathrm{t}\end{array}$ & $\begin{array}{c}\mathrm{SrO} \\
-\mathrm{t}\end{array}$ & $\begin{array}{c}\mathrm{Tc} 2 \mathrm{O} 7 \\
-\mathrm{t}\end{array}$ & $\begin{array}{c}\mathrm{TeO} 2 \\
-\mathrm{t}\end{array}$ & $\begin{array}{c}\mathrm{ThO} 2 \\
-\mathrm{t}\end{array}$ & $\begin{array}{c}\mathrm{TiO} 2 \\
-\mathrm{t}\end{array}$ \\
\hline $\mathrm{Zr}-16$ & & & & & & & & & & & & & & & & & 0.0001 & & & & \\
\hline $\mathrm{Zr}-17$ & & & & & & & & & & & & & & & & & 0.0001 & & & & \\
\hline $\mathrm{Zr}-18$ & & & & & & & & & & & & & & & & & 0.0001 & & & & \\
\hline $\mathrm{Zr}-19$ & & & & & & & & & & & & & & & & & 0.0001 & & & & \\
\hline $\mathrm{Zr}-20$ & & & & & & & & & & & & & & & & & 0.0001 & & & & \\
\hline $\mathrm{Zr}-21$ & & & & & & & & & & & & & & & & & 0.0001 & & & & \\
\hline $\mathrm{Zr}-22$ & & & & & & & & & & & & & & & & & 0.0001 & & & & \\
\hline $\mathrm{Zr}-23$ & & & & & & & & & & & & & & & & & 0.0001 & & & & \\
\hline $\mathrm{Zr}-24$ & & & & & & & & & & & & & & & & & 0.0001 & & & & \\
\hline $\mathrm{Zr}-25$ & & & & & & & & & & & & & & & & & 0.0001 & & & & \\
\hline $\mathrm{Zr}-26$ & & & & & & & & & & & & & & & & & 0.0001 & & & & \\
\hline $\mathrm{Zr}-27$ & & & & & & & & & & & & & & & & & 0.0001 & & & & \\
\hline $\mathrm{Zr}-28$ & & & & & & & & & & & & & & & & & 0.0001 & & & & \\
\hline Zr-29 & & & & & & & & & & & & & & & & & 0.0001 & & & & \\
\hline
\end{tabular}

\section{SP (Mika et al. 1997)}

\begin{tabular}{|c|c|c|c|c|c|c|c|c|c|c|c|c|}
\hline SP-1a & \begin{tabular}{|l|}
0.0000 \\
\end{tabular} & 0.0000 & 0.0000 & 0.0003 & 0.0003 & 0.0007 & 0.0009 & 0.0000 & 0.0019 & \begin{tabular}{|l|}
0.0003 \\
\end{tabular} & 0.0000 & 0.0003 \\
\hline SP-1b & 0.0000 & 0.0000 & 0.0000 & 0.0003 & 0.0003 & 0.0007 & 0.0009 & 0.0000 & 0.0019 & \begin{tabular}{|l|}
0.0003 \\
\end{tabular} & 0.0000 & 0.0003 \\
\hline SP-1c & 0.0000 & 0.0000 & 0.0000 & 0.0003 & 0.0003 & 0.0007 & 0.0009 & 0.0000 & 0.0019 & 0.0003 & 0.0000 & 0.0003 \\
\hline SP-1d & 0.0000 & 0.0000 & 0.0000 & 0.0003 & 0.0003 & 0.0007 & 0.0009 & 0.0000 & 0.0019 & 0.0003 & 0.0000 & 0.0003 \\
\hline SP-1e & 0.0000 & 0.0000 & 0.0000 & 0.0003 & 0.0003 & 0.0007 & 0.0009 & 0.0000 & 0.0019 & 0.0003 & 0.0000 & 0.0003 \\
\hline SP-1f & 0.0000 & 0.0000 & 0.0000 & 0.0003 & 0.0003 & 0.0007 & 0.0009 & 0.0000 & 0.0019 & 0.0003 & 0.0000 & 0.0003 \\
\hline SP-1g & 0.0000 & 0.0000 & 0.0000 & 0.0003 & 0.0003 & 0.0007 & 0.0009 & 0.0000 & 0.0019 & 0.0003 & 0.0000 & 0.0003 \\
\hline SP-1h & 0.0000 & 0.0000 & 0.0000 & 0.0003 & 0.0003 & 0.0007 & 0.0009 & 0.0000 & 0.0019 & 0.0003 & 0.0000 & 0.0003 \\
\hline SP-1i & 0.0000 & 0.0000 & 0.0000 & 0.0003 & 0.0003 & 0.0007 & 0.0009 & 0.0000 & 0.0019 & 0.0003 & 0.0000 & 0.0003 \\
\hline SP-Al-1 & 0.0000 & 0.0000 & 0.0000 & 0.0003 & 0.0003 & 0.0007 & 0.0009 & 0.0000 & 0.0020 & 0.0003 & 0.0000 & 0.0003 \\
\hline SP-Al-2 & 0.0000 & 0.0000 & 0.0000 & 0.0003 & 0.0003 & 0.0007 & 0.0009 & 0.0000 & 0.0018 & \begin{tabular}{|l|}
0.0003 \\
\end{tabular} & 0.0000 & 0.0003 \\
\hline SP-Al-3 & 0.0000 & 0.0000 & 0.0000 & 0.0003 & 0.0003 & 0.0006 & 0.0008 & 0.0000 & 0.0017 & 0.0003 & 0.0000 & 0.0003 \\
\hline SP-B-1 & 0.0000 & 0.0000 & 0.0000 & 0.0003 & 0.0003 & 0.0008 & 0.0010 & 0.0000 & 0.0021 & 0.0003 & 0.0000 & 0.0003 \\
\hline SP-B-2 & 0.0000 & 0.0000 & 0.0000 & 0.0003 & 0.0003 & 0.0008 & 0.0010 & 0.0000 & 0.0020 & \begin{tabular}{|l|}
0.0003 \\
\end{tabular} & 0.0000 & 0.0003 \\
\hline SP-B-3 & 0.0000 & 0.0000 & 0.0000 & 0.0003 & 0.0003 & 0.0007 & 0.0009 & 0.0000 & 0.0020 & \begin{tabular}{|l|}
0.0003 \\
\end{tabular} & 0.0000 & 0.0003 \\
\hline SP-B-4 & 0.0000 & 0.0000 & 0.0000 & 0.0003 & 0.0003 & 0.0007 & 0.0009 & 0.0000 & 0.0018 & 0.0003 & 0.0000 & 0.0003 \\
\hline SP-Cr-1 & 0.0000 & 0.0000 & 0.0000 & 0.0003 & 0.0003 & 0.0007 & 0.0009 & 0.0000 & 0.0019 & \begin{tabular}{|l|}
0.0003 \\
\end{tabular} & 0.0000 & 0.0003 \\
\hline SP-Cr-2 & 0.0000 & 0.0000 & 0.0000 & 0.0003 & 0.0003 & 0.0007 & 0.0009 & 0.0000 & 0.0019 & 0.0003 & 0.0000 & 0.0003 \\
\hline SP-Cr-3 & 0.0000 & 0.0000 & 0.0000 & 0.0003 & 0.0003 & 0.0007 & 0.0009 & 0.0000 & 0.0019 & 0.0003 & 0.0000 & 0.0003 \\
\hline SP-Cr-4 & 0.0000 & 0.0000 & 0.0000 & 0.0003 & 0.0003 & 0.0007 & 0.0009 & 0.0000 & 0.0019 & 0.0003 & 0.0000 & 0.0003 \\
\hline SP-Fe-1 & 0.0000 & 0.0000 & 0.0000 & 0.0003 & 0.0003 & 0.0008 & 0.0010 & 0.0000 & 0.0021 & 0.0003 & 0.0000 & 0.0003 \\
\hline SP-Fe-2 & 0.0000 & 0.0000 & 0.0000 & 0.0003 & 0.0003 & 0.0007 & 0.0009 & 0.0000 & 0.0020 & 0.0003 & 0.0000 & 0.0003 \\
\hline SP-Fe-3 & \begin{tabular}{|l|}
0.0000 \\
\end{tabular} & 0.0000 & 0.0000 & 0.0003 & 0.0003 & 0.0007 & 0.0009 & 0.0000 & 0.0019 & \begin{tabular}{|l|}
0.0003 \\
\end{tabular} & 0.0000 & 0.0003 \\
\hline SP-Li-1 & 0.0000 & 0.0000 & 0.0000 & 0.0003 & 0.0003 & 0.0007 & 0.0009 & 0.0000 & 0.0020 & 0.0003 & 0.0000 & 0.0003 \\
\hline SP-Li-2 & 0.0000 & 0.0000 & 0.0000 & 0.0003 & 0.0003 & 0.0007 & 0.0009 & 0.0000 & 0.0020 & 0.0003 & 0.0000 & 0.0003 \\
\hline SP-Li-3 & 0.0000 & 0.0000 & 0.0000 & 0.0003 & 0.0003 & 0.0007 & 0.0009 & 0.0000 & 0.0019 & 0.0003 & 0.0000 & 0.0003 \\
\hline SP-Mg-1 & 0.0000 & 0.0000 & 0.0000 & 0.0003 & 0.0003 & 0.0007 & 0.0009 & 0.0000 & 0.0019 & \begin{tabular}{|l|}
0.0003 \\
\end{tabular} & 0.0000 & 0.0003 \\
\hline SP-Mg-2 & 0.0000 & 0.0000 & 0.0000 & 0.0003 & 0.0003 & 0.0007 & 0.0009 & 0.0000 & 0.0019 & 0.0003 & 0.0000 & 0.0003 \\
\hline SP-Mg-3 & 0.0000 & 0.0000 & 0.0000 & 0.0003 & 0.0003 & 0.0007 & 0.0009 & 0.0000 & 0.0018 & 0.0003 & 0.0000 & 0.0003 \\
\hline SP-Mn-1 & 0.0000 & 0.0000 & 0.0000 & 0.0003 & 0.0003 & 0.0007 & 0.0009 & 0.0000 & 0.0019 & 0.0003 & 0.0000 & 0.0003 \\
\hline SP-Mn-2 & 0.0000 & 0.0000 & 0.0000 & 0.0003 & 0.0003 & 0.0007 & 0.0009 & 0.0000 & 0.0019 & 0.0003 & 0.0000 & 0.0003 \\
\hline SP-Mn-3 & 0.0000 & 0.0000 & 0.0000 & 0.0003 & 0.0003 & 0.0007 & 0.0009 & 0.0000 & 0.0018 & 0.0003 & 0.0000 & 0.0003 \\
\hline SP-Na-1 & 0.0000 & 0.0000 & 0.0000 & 0.0003 & 0.0003 & 0.0008 & 0.0010 & 0.0000 & 0.0021 & \begin{tabular}{|l|}
0.0003 \\
\end{tabular} & 0.0000 & 0.0003 \\
\hline
\end{tabular}


Zr Study (Vienna et al. 1999)

\begin{tabular}{|c|c|c|c|c|c|c|c|c|c|c|c|c|c|c|c|c|c|c|c|c|c|}
\hline Glass ID & $\begin{array}{c}\mathrm{T} 12 \mathrm{O} 3 \\
-\mathrm{t}\end{array}$ & $\begin{array}{c}\mathrm{U} 3 \mathrm{O} 8 \\
-\mathrm{t}\end{array}$ & $\begin{array}{c}\mathrm{UO} 2 \\
-\mathrm{t}\end{array}$ & $\begin{array}{c}\mathrm{UO} 3 \\
-\mathrm{t}\end{array}$ & $\begin{array}{c}\mathrm{V} 2 \mathrm{O} 5 \\
-\mathrm{t}\end{array}$ & $\begin{array}{c}\text { WO3 } \\
-t\end{array}$ & $\begin{array}{c}\mathrm{Y} 2 \mathrm{O} 3 \\
-\mathrm{t}\end{array}$ & $\begin{array}{c}\mathrm{ZnO} \\
-\mathrm{t}\end{array}$ & $\begin{array}{c}\text { Others } \\
-t\end{array}$ & $\begin{array}{c}\text { Sum } \\
-t\end{array}$ & $\begin{array}{c}\mathrm{A} 12 \mathrm{O} 3 \\
-\mathrm{a}\end{array}$ & $\begin{array}{c}\mathrm{B} 2 \mathrm{O} 3 \\
-\mathrm{a}\end{array}$ & $\begin{array}{c}\mathrm{CaO} \\
-\mathrm{a}\end{array}$ & $\begin{array}{c}\mathrm{Fe} 2 \mathrm{O} 3 \\
-\mathrm{a}\end{array}$ & $\begin{array}{c}\mathrm{FeO} \\
-\mathrm{a}\end{array}$ & $\begin{array}{c}\mathrm{K} 2 \mathrm{O} \\
-\mathrm{a}\end{array}$ & $\begin{array}{c}\mathrm{Li} 2 \mathrm{O} \\
-\mathrm{a}\end{array}$ & $\begin{array}{c}\mathrm{MgO} \\
-\mathrm{a}\end{array}$ & $\begin{array}{c}\mathrm{Na} 2 \mathrm{O} \\
-\mathrm{a}\end{array}$ & $\begin{array}{c}\mathrm{NiO} \\
-\mathrm{a}\end{array}$ & $\begin{array}{c}\mathrm{P} 2 \mathrm{O} 5 \\
-\mathrm{a}\end{array}$ \\
\hline $\mathrm{Zr}-16$ & & & & & & & & & & 1.0000 & & & & & & & & & & & \\
\hline $\mathrm{Zr}-17$ & & & & & & & & & & 1.0000 & & & & & & & & & & & \\
\hline $\mathrm{Zr}-18$ & & & & & & & & & & 1.0000 & & & & & & & & & & & \\
\hline Zr-19 & & & & & & & & & & 1.0001 & & & & & & & & & & & \\
\hline $\mathrm{Zr}-20$ & & & & & & & & & & 1.0000 & & & & & & & & & & & \\
\hline $\mathrm{Zr}-21$ & & & & & & & & & & 1.0001 & & & & & & & & & & & \\
\hline $\mathrm{Zr}-22$ & & & & & & & & & & 1.0001 & & & & & & & & & & & \\
\hline $\mathrm{Zr}-23$ & & & & & & & & & & 1.0000 & & & & & & & & & & & \\
\hline $\mathrm{Zr}-24$ & & & & & & & & & & 1.0001 & & & & & & & & & & & \\
\hline $\mathrm{Zr}-25$ & & & & & & & & & & 1.0000 & & & & & & & & & & & \\
\hline $\mathrm{Zr}-26$ & & & & & & & & & & 1.0001 & & & & & & & & & & & \\
\hline $\mathrm{Zr}-27$ & & & & & & & & & & 1.0000 & & & & & & & & & & & \\
\hline $\mathrm{Zr}-28$ & & & & & & & & & & 1.0000 & & & & & & & & & & & \\
\hline $\mathrm{Zr}-29$ & & & & & & & & & & 1.0001 & & & & & & & & & & & \\
\hline
\end{tabular}

\section{SP (Mika et al. 1997)}

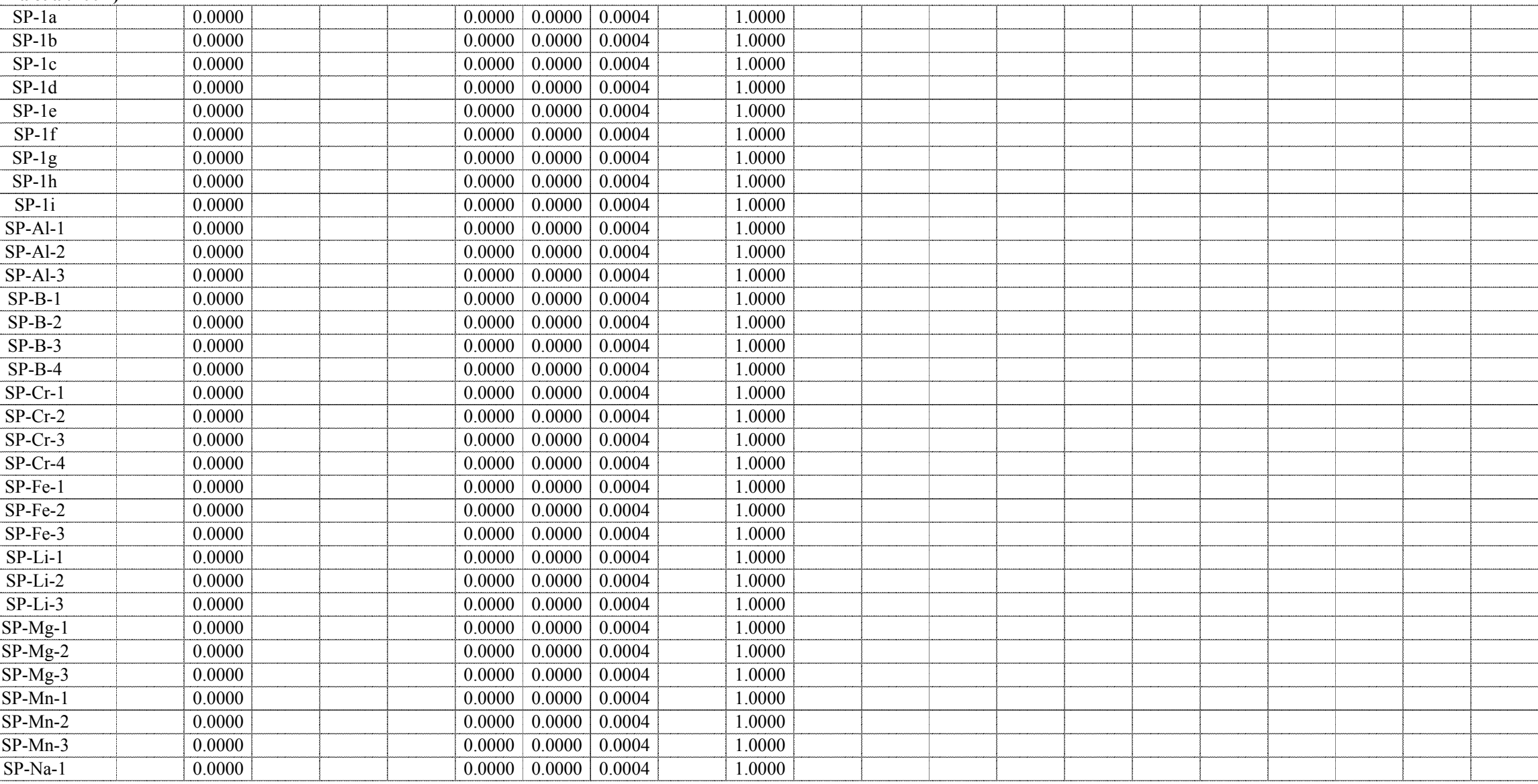


Appendix A. Database - mass fraction

Zr Study (Vienna et al. 1999)

\begin{tabular}{|c|c|c|c|c|c|c|c|c|c|c|c|c|c|c|c|c|c|c|c|c|c|}
\hline Glass ID & $\begin{array}{c}\mathrm{SiO} 2 \\
-\mathrm{a}\end{array}$ & $\begin{array}{c}\mathrm{ZrO} 2 \\
-\mathrm{a}\end{array}$ & $\begin{array}{c}\mathrm{Ag} 2 \mathrm{O} \\
-\mathrm{a}\end{array}$ & $\begin{array}{c}\mathrm{As} 2 \mathrm{O} 3 \\
-\mathrm{a}\end{array}$ & $\begin{array}{c}\mathrm{BaO} \\
-\mathrm{a}\end{array}$ & $\begin{array}{c}\mathrm{Bi} 2 \mathrm{O} 3 \\
-\mathrm{a}\end{array}$ & $\begin{array}{l}\mathrm{Br} \\
-\mathrm{a}\end{array}$ & $\begin{array}{c}\mathrm{CdO} \\
-\mathrm{a}\end{array}$ & $\begin{array}{c}\mathrm{Ce} 2 \mathrm{O} 3 \\
-\mathrm{a}\end{array}$ & $\begin{array}{c}\mathrm{CeO} 2 \\
-\mathrm{a}\end{array}$ & $\begin{array}{l}\mathrm{Cl} \\
-\mathrm{a}\end{array}$ & $\begin{array}{c}\mathrm{CoO} \\
-\mathrm{a}\end{array}$ & $\begin{array}{c}\mathrm{Co} 2 \mathrm{O} 3 \\
-\mathrm{a}\end{array}$ & $\begin{array}{c}\mathrm{Cr} 2 \mathrm{O} 3 \\
-\mathrm{a}\end{array}$ & $\begin{array}{c}\mathrm{Cs} 2 \mathrm{O} \\
-\mathrm{a}\end{array}$ & $\begin{array}{c}\mathrm{CuO} \\
-\mathrm{a}\end{array}$ & $\begin{array}{c}\mathrm{Eu} 2 \mathrm{O} 3 \\
-\mathrm{a}\end{array}$ & $\begin{array}{c}F \\
-a\end{array}$ & $\begin{array}{c}\mathrm{Ga} 2 \mathrm{O} 3 \\
-\mathrm{a}\end{array}$ & $\begin{array}{c}\mathrm{Gd} 2 \mathrm{O} 3 \\
-\mathrm{a}\end{array}$ & $\begin{array}{c}\mathrm{HgO} \\
-\mathrm{a}\end{array}$ \\
\hline $\mathrm{Zr}-16$ & & & & & & & & & & & & & & & & & & & & & \\
\hline $\mathrm{Zr}-17$ & & & & & & & & & & & & & & & & & & & & & \\
\hline $\mathrm{Zr}-18$ & & & & & & & & & & & & & & & & & & & & & \\
\hline $\mathrm{Zr}-19$ & & & & & & & & & & & & & & & & & & & & & \\
\hline $\mathrm{Zr}-20$ & & & & & & & & & & & & & & & & & & & & & \\
\hline $\mathrm{Zr}-21$ & & & & & & & & & & & & & & & & & & & & & \\
\hline $\mathrm{Zr}-22$ & & & & & & & & & & & & & & & & & & & & & \\
\hline $\mathrm{Zr}-23$ & & & & & & & & & & & & & & & & & & & & & \\
\hline $\mathrm{Zr}-24$ & & & & & & & & & & & & & & & & & & & & & \\
\hline $\mathrm{Zr}-25$ & & & & & & & & & & & & & & & & & & & & & \\
\hline $\mathrm{Zr}-26$ & & & & & & & & & & & & & & & & & & & & & \\
\hline $\mathrm{Zr}-27$ & & & & & & & & & & & & & & & & & & & & & \\
\hline $\mathrm{Zr}-28$ & & & & & & & & & & & & & & & & & & & & & \\
\hline $\mathrm{Zr}-29$ & & & & & & & & & & & & & & & & & & & & & \\
\hline
\end{tabular}

\section{SP (Mika et al. 1997)}

SP-1a

SP-1b

SP-1c

SP-1d

SP-1e

SP-1f

SP-1g

SP-1h

SP-1i

SP-Al-1

SP-Al-2

SP-Al-3

SP-B-1

SP-B-2

SP-B-3

SP-B-4

SP-Cr-1

SP-Cr-2

SP-Cr-3

SP-Cr-4

SP-Fe-1

$\mathrm{SP}-\mathrm{Fe}-2$

$\mathrm{SP}-\mathrm{Fe}-3$

SP-Li-1

SP-Li-2

SP-Li-3

SP-Mg-1

SP-Mg-2

SP-Mg-3

SP-Mn-1

SP-Mn-2

SP-Mn-3

SP-Na-1

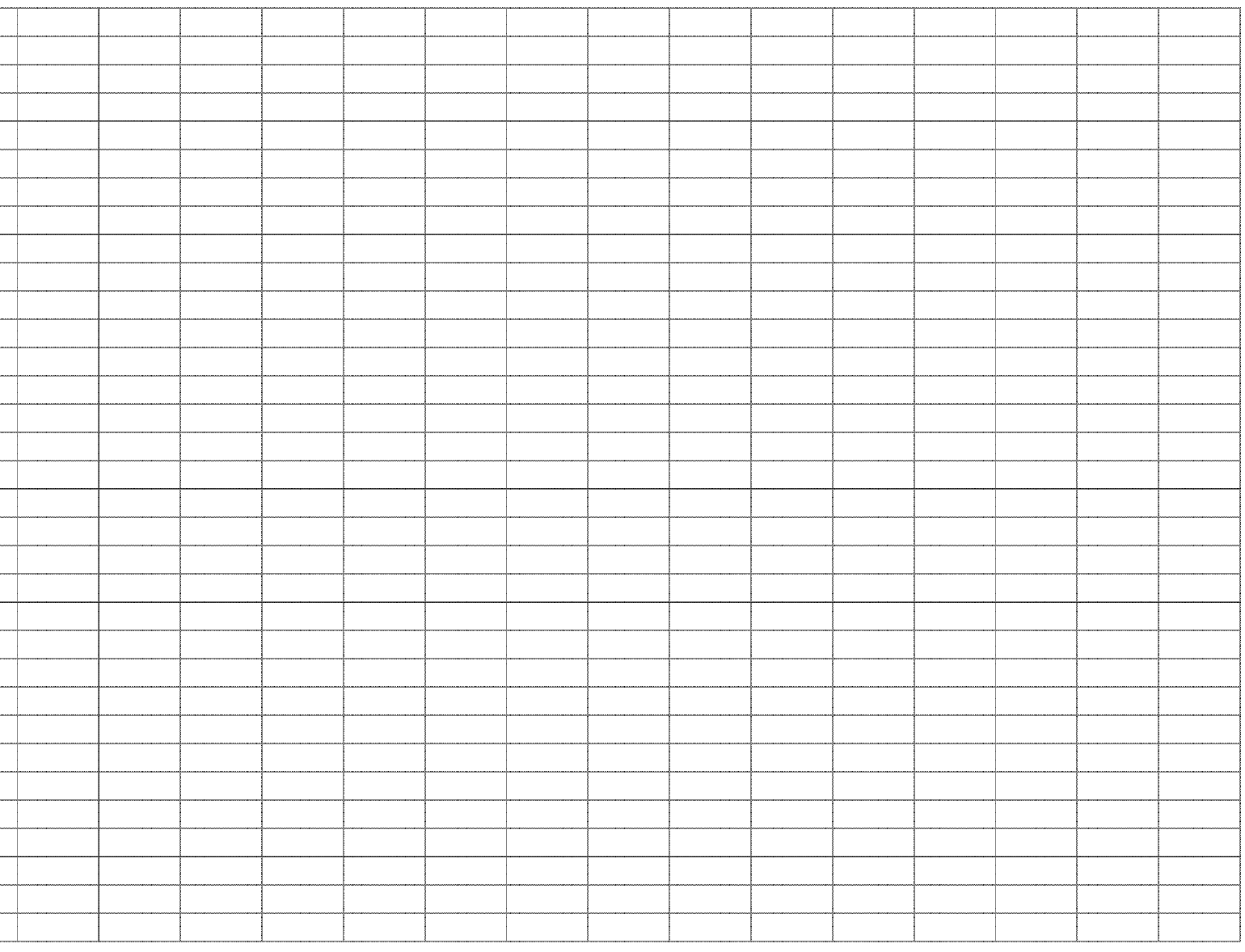


Appendix A. Database - mass fraction

Zr Study (Vienna et al. 1999)

\begin{tabular}{|c|c|c|c|c|c|c|c|c|c|c|c|c|c|c|c|c|c|c|c|c|c|}
\hline Glass ID & $\begin{array}{c}\mathrm{I} \\
-\mathrm{a}\end{array}$ & $\begin{array}{c}\mathrm{La} 2 \mathrm{O} 3 \\
-\mathrm{a}\end{array}$ & $\begin{array}{c}\mathrm{MnO} 2 \\
-\mathrm{a}\end{array}$ & $\begin{array}{c}\mathrm{MnO} \\
-\mathrm{a}\end{array}$ & $\begin{array}{c}\mathrm{MoO} \\
-\mathrm{a}\end{array}$ & $\begin{array}{c}\mathrm{MoO} 3 \\
-\mathrm{a}\end{array}$ & $\begin{array}{c}\mathrm{Nb} 2 \mathrm{O} 5 \\
-\mathrm{a}\end{array}$ & $\begin{array}{c}\mathrm{Nd} 2 \mathrm{O} 3 \\
-\mathrm{a}\end{array}$ & $\begin{array}{c}\mathrm{PbO} \\
-\mathrm{a}\end{array}$ & $\begin{array}{c}\mathrm{PdO} 2 \\
-\mathrm{a}\end{array}$ & $\begin{array}{c}\mathrm{PdO} \\
-\mathrm{a}\end{array}$ & $\begin{array}{c}\mathrm{Pr} 2 \mathrm{O} 3 \\
-\mathrm{a}\end{array}$ & $\begin{array}{c}\text { Pr6O11 } \\
-\mathrm{a}\end{array}$ & $\begin{array}{c}\mathrm{Rb} 2 \mathrm{O} \\
-\mathrm{a}\end{array}$ & $\begin{array}{c}\mathrm{ReO} \\
-\mathrm{a}\end{array}$ & $\begin{array}{c}\mathrm{ReO} 2 \\
-\mathrm{a}\end{array}$ & $\begin{array}{c}\mathrm{Rh} 2 \mathrm{O} 3 \\
-\mathrm{a}\end{array}$ & $\begin{array}{c}\mathrm{RhO} 2 \\
-\mathrm{a}\end{array}$ & $\begin{array}{c}\mathrm{RuO}_{2} \\
-\mathrm{a}\end{array}$ & $\begin{array}{c}\mathrm{Sb} 2 \mathrm{O} 3 \\
-\mathrm{a}\end{array}$ & $\begin{array}{c}\mathrm{Sb} 2 \mathrm{O} 5 \\
-\mathrm{a}\end{array}$ \\
\hline \multicolumn{22}{|c|}{ a } \\
\hline \multicolumn{22}{|l|}{$\mathrm{Zr}-17$} \\
\hline \multicolumn{22}{|l|}{$\mathrm{Zr}-18$} \\
\hline \multicolumn{22}{|l|}{$\mathrm{Zr}-19$} \\
\hline \multicolumn{22}{|l|}{$\mathrm{Zr}-20$} \\
\hline \multicolumn{22}{|l|}{$\mathrm{Zr}-21$} \\
\hline \multicolumn{22}{|l|}{$\mathrm{Zr}-22$} \\
\hline \multicolumn{22}{|l|}{$\mathrm{Zr}-23$} \\
\hline \multicolumn{22}{|l|}{$\mathrm{Zr}-24$} \\
\hline \multicolumn{22}{|l|}{$\begin{array}{l}\mathrm{Zr}-25 \\
\mathrm{Zr}-26\end{array}$} \\
\hline \multicolumn{22}{|l|}{$\mathrm{Zr}-26$} \\
\hline \multicolumn{22}{|l|}{$\mathrm{Zr}-27$} \\
\hline \multirow{2}{*}{\multicolumn{22}{|c|}{$\mathrm{Zr}-28$}} \\
\hline Zr-29 & & & & & & & & & & & & & & & & & & & & & \\
\hline
\end{tabular}

\section{SP (Mika et al. 1997)}

SP-1a

SP-1b

SP-1c

SP-1d

SP-1e

SP-1f

SP-1g

SP-1h

SP-1i

SP-Al-1

SP-Al-2

SP-Al-3

SP-B-1

SP-B-2

SP-B-3

SP-B-4

SP-Cr-1

SP-Cr-2

$\mathrm{SP}-\mathrm{Cr}-3$

SP-Cr-4

SP-Fe-1

$\mathrm{SP}-\mathrm{Fe}-2$

$\mathrm{SP}-\mathrm{Fe}-3$

SP-Li-1

SP-Li-2

SP-Li-3

SP-Mg-1

SP-Mg-2

SP-Mg-3

SP-Mn-1

SP-Mn-2

SP-Mn-3

SP-Na-1

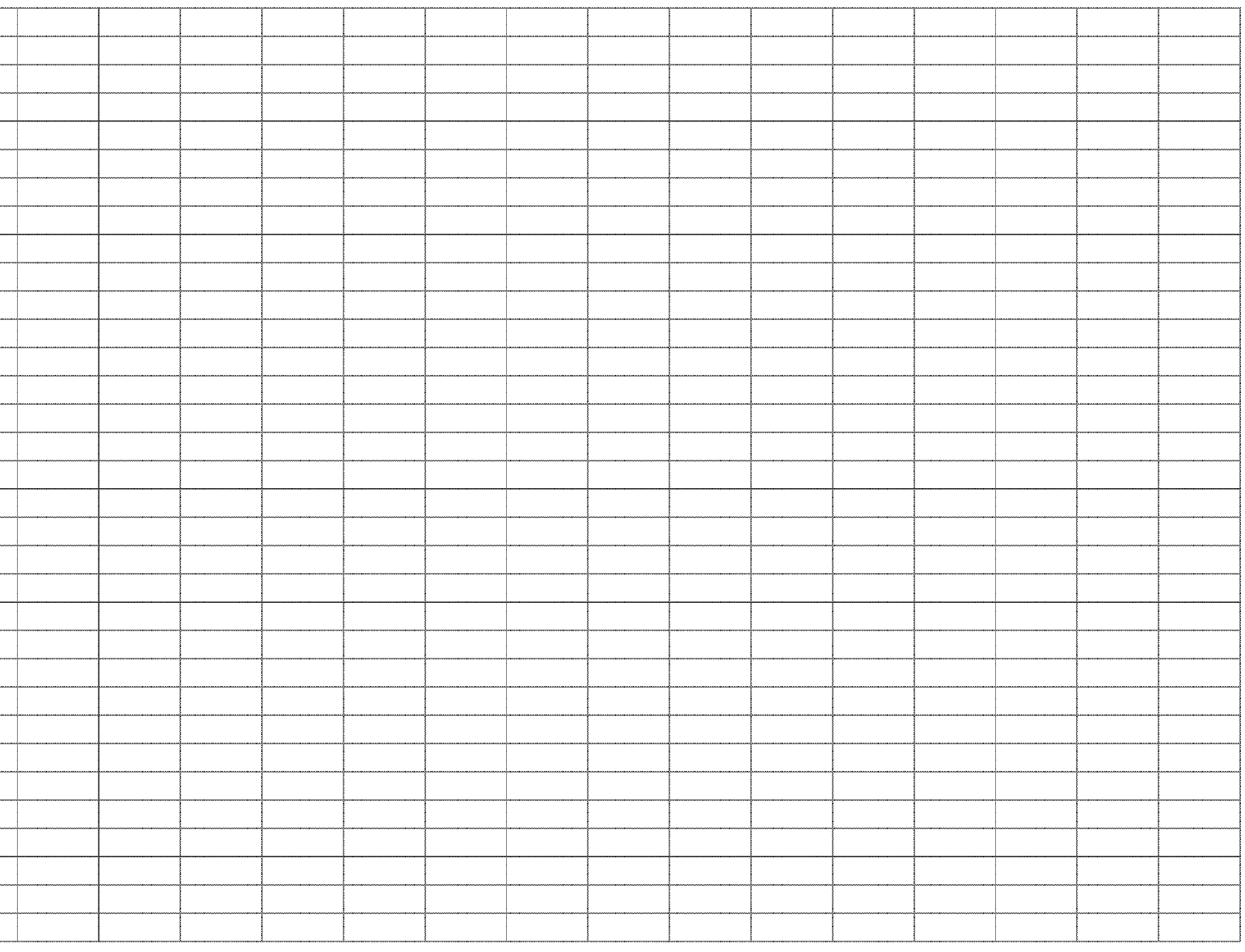


Appendix A. Database - mass fraction

Zr Study (Vienna et al. 1999)

\begin{tabular}{|c|c|c|c|c|c|c|c|c|c|c|c|c|c|c|c|c|c|c|c|c|}
\hline Glass ID & $\begin{array}{c}\mathrm{SeO} 2 \\
-\mathrm{a}\end{array}$ & $\begin{array}{c}\mathrm{Sm} 2 \mathrm{O} 3 \\
-\mathrm{a}\end{array}$ & $\begin{array}{c}\mathrm{SnO} \\
-\mathrm{a}\end{array}$ & $\begin{array}{c}\mathrm{SnO} 2 \\
-\mathrm{a}\end{array}$ & $\begin{array}{c}\mathrm{SO} 3 \\
-\mathrm{a} \\
\end{array}$ & $\begin{array}{c}\mathrm{SrO} \\
-\mathrm{a} \\
\end{array}$ & $\begin{array}{c}\mathrm{Tc} 2 \mathrm{O} 7 \\
-\mathrm{a}\end{array}$ & $\begin{array}{c}\mathrm{TeO} 2 \\
-\mathrm{a}\end{array}$ & $\begin{array}{c}\mathrm{ThO} 2 \\
-\mathrm{a}\end{array}$ & $\begin{array}{c}\mathrm{TiO} 2 \\
-\mathrm{a}\end{array}$ & $\begin{array}{c}\mathrm{T} 12 \mathrm{O} 3 \\
-\mathrm{a}\end{array}$ & $\begin{array}{c}\mathrm{U} 3 \mathrm{O} 8 \\
-\mathrm{a}\end{array}$ & $\begin{array}{c}\mathrm{UO} 2 \\
-\mathrm{a}\end{array}$ & $\begin{array}{c}\mathrm{UO} 3 \\
-\mathrm{a}\end{array}$ & $\begin{array}{c}\mathrm{V} 2 \mathrm{O} 5 \\
-\mathrm{a}\end{array}$ & $\begin{array}{c}\text { WO3 } \\
-\mathrm{a}\end{array}$ & $\begin{array}{c}\mathrm{Y} 2 \mathrm{O} 3 \\
-\mathrm{a}\end{array}$ & $\begin{array}{c}\mathrm{ZnO} \\
-\mathrm{a}\end{array}$ & $\begin{array}{c}\text { Others } \\
-\mathrm{a}\end{array}$ & $\begin{array}{l}\text { Sum } \\
-\mathrm{a}\end{array}$ \\
\hline $\mathrm{Zr}-16$ & & & & & & & & & & & & & & & & & & & & \\
\hline $\mathrm{Zr}-17$ & & & & & & & & & & & & & & & & & & & & \\
\hline $\mathrm{Zr}-18$ & & & & & & & & & & & & & & & & & & & & \\
\hline $\mathrm{Zr}-19$ & & & & & & & & & & & & & & & & & & & & \\
\hline $\mathrm{Zr}-20$ & & & & & & & & & & & & & & & & & & & & \\
\hline $\mathrm{Zr}-21$ & & & & & & & & & & & & & & & & & & & & \\
\hline $\mathrm{Zr}-22$ & & & & & & & & & & & & & & & & & & & & \\
\hline $\mathrm{Zr}-23$ & & & & & & & & & & & & & & & & & & & & \\
\hline $\mathrm{Zr}-24$ & & & & & & & & & & & & & & & & & & & & \\
\hline $\mathrm{Zr}-25$ & & & & & & & & & & & & & & & & & & & & \\
\hline $\mathrm{Zr}-26$ & & & & & & & & & & & & & & & & & & & & \\
\hline $\mathrm{Zr}-27$ & & & & & & & & & & & & & & & & & & & & \\
\hline $\mathrm{Zr}-28$ & & & & & & & & & & & & & & & & & & & & \\
\hline Zr-29 & & & & & & & & & & & & & & & & & & & & \\
\hline
\end{tabular}

\section{SP (Mika et al. 1997)}

SP-1a

SP-1b

SP-1c

SP-1d

SP-1e

SP-1f

SP-1g

SP-1h

SP-1i

SP-Al-1

SP-Al-2

SP-Al-3

SP-B-1

SP-B-2

SP-B-3

SP-B-4

SP-Cr-1

SP-Cr-2

$\mathrm{SP}-\mathrm{Cr}-3$

SP-Cr-4

SP-Fe-1

$\mathrm{SP}-\mathrm{Fe}-2$

$\mathrm{SP}-\mathrm{Fe}-3$

SP-Li-1

SP-Li-2

SP-Li-3

SP-Mg-1

SP-Mg-2

SP-Mg-3

SP-Mn-1

SP-Mn-2

SP-Mn-3

SP-Na-1

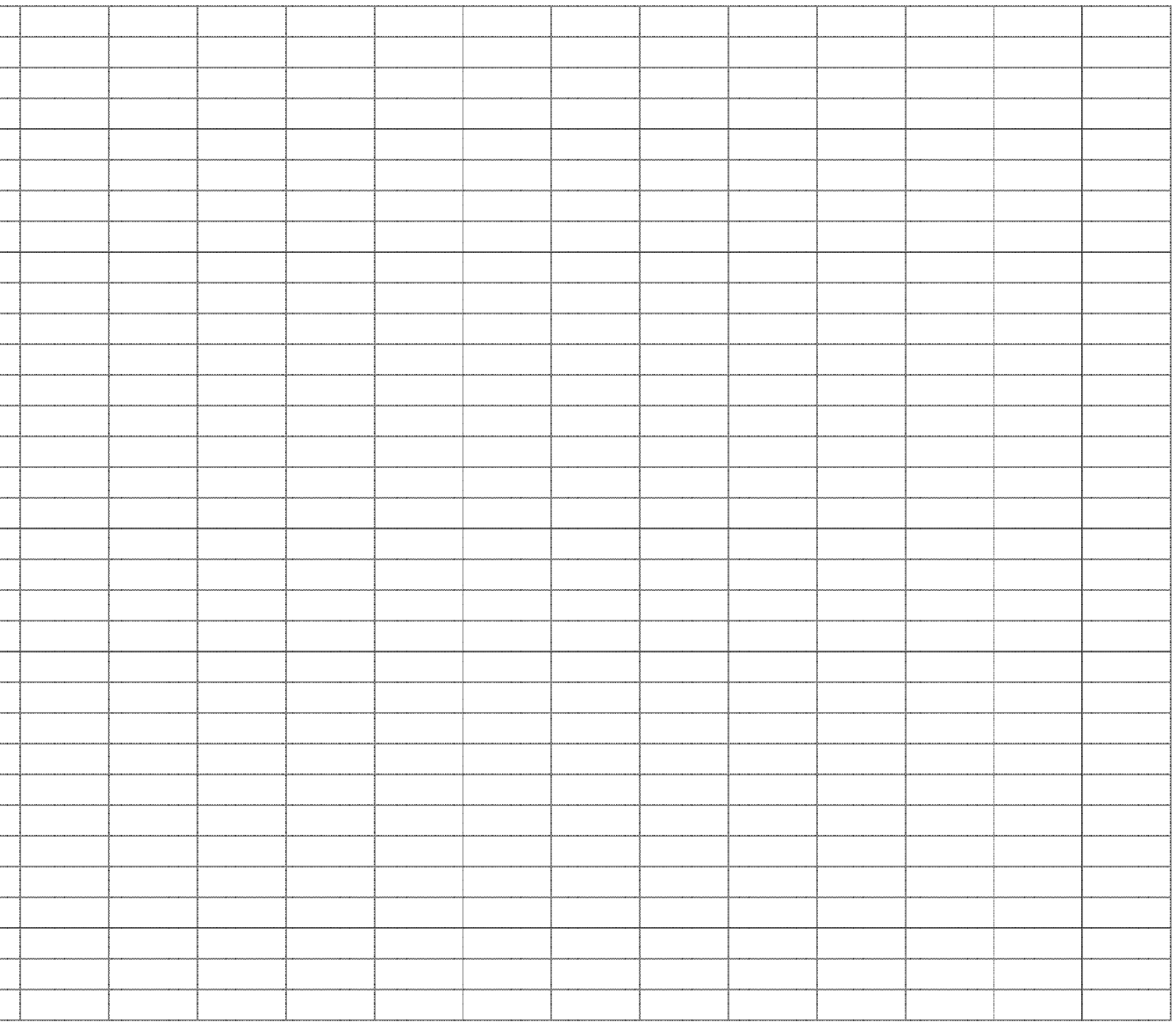


Appendix A. Database - mass fraction

Zr Study (Vienna et al. 1999)

\begin{tabular}{|c|c|c|c|c|c|c|c|c|}
\hline Glass ID & $\begin{array}{l}\mathrm{TM} \\
\left({ }^{\circ} \mathrm{C}\right)\end{array}$ & $\begin{array}{l}\text { Gradient } \\
\text { TL }\left({ }^{\circ} \mathrm{C}\right)\end{array}$ & $\begin{array}{l}\text { Uniform } \\
\text { TL }\left({ }^{\circ} \mathrm{C}\right)\end{array}$ & Primary Phase & $\begin{array}{l}\text { Quenched } \\
\text { Visual/OM }\end{array}$ & $\begin{array}{c}\text { Quenched } \\
\text { SEM/EDS or TEM }\end{array}$ & $\begin{array}{l}\text { Quenched } \\
\text { XRD }\end{array}$ & $\begin{array}{c}\text { Quenched } \\
\text { Homogeneous? }\end{array}$ \\
\hline $\mathrm{Zr}-16$ & 1150 & & 1067 & Zircon & & & & \\
\hline $\mathrm{Zr}-17$ & 1450 & & 1228 & Baddeleyite & & & & \\
\hline $\mathrm{Zr}-18$ & 1350 & & 1125 & Zircon & & & & \\
\hline $\mathrm{Zr}-19$ & 1250 & & 1034 & Zircon & & & & \\
\hline $\mathrm{Zr}-20$ & 1150 & & 1113 & Parakeldyshite & & & & \\
\hline $\mathrm{Zr}-21$ & 1350 & & 1180 & Zircon & & & & \\
\hline $\mathrm{Zr}-22$ & 1150 & & 1087 & Parakeldyshite & & & & \\
\hline $\mathrm{Zr}-23$ & 1150 & & 1003 & Zircon & & & & \\
\hline $\mathrm{Zr}-24$ & 1150 & & 1105 & Parakeldyshite & & & & \\
\hline $\mathrm{Zr}-25$ & 1150 & & 1137 & Zircon & & & & \\
\hline $\mathrm{Zr}-26$ & 1150 & & 1070 & Zircon & & & & \\
\hline Zr-27 & 1150 & & 1081 & Zircon & & & & \\
\hline $\mathrm{Zr}-28$ & 1150 & & 1064 & Zircon & & & & \\
\hline Zr-29 & 1150 & & 1059 & Parakeldyshite & & & & \\
\hline
\end{tabular}

\section{SP (Mika et al. 1997)}

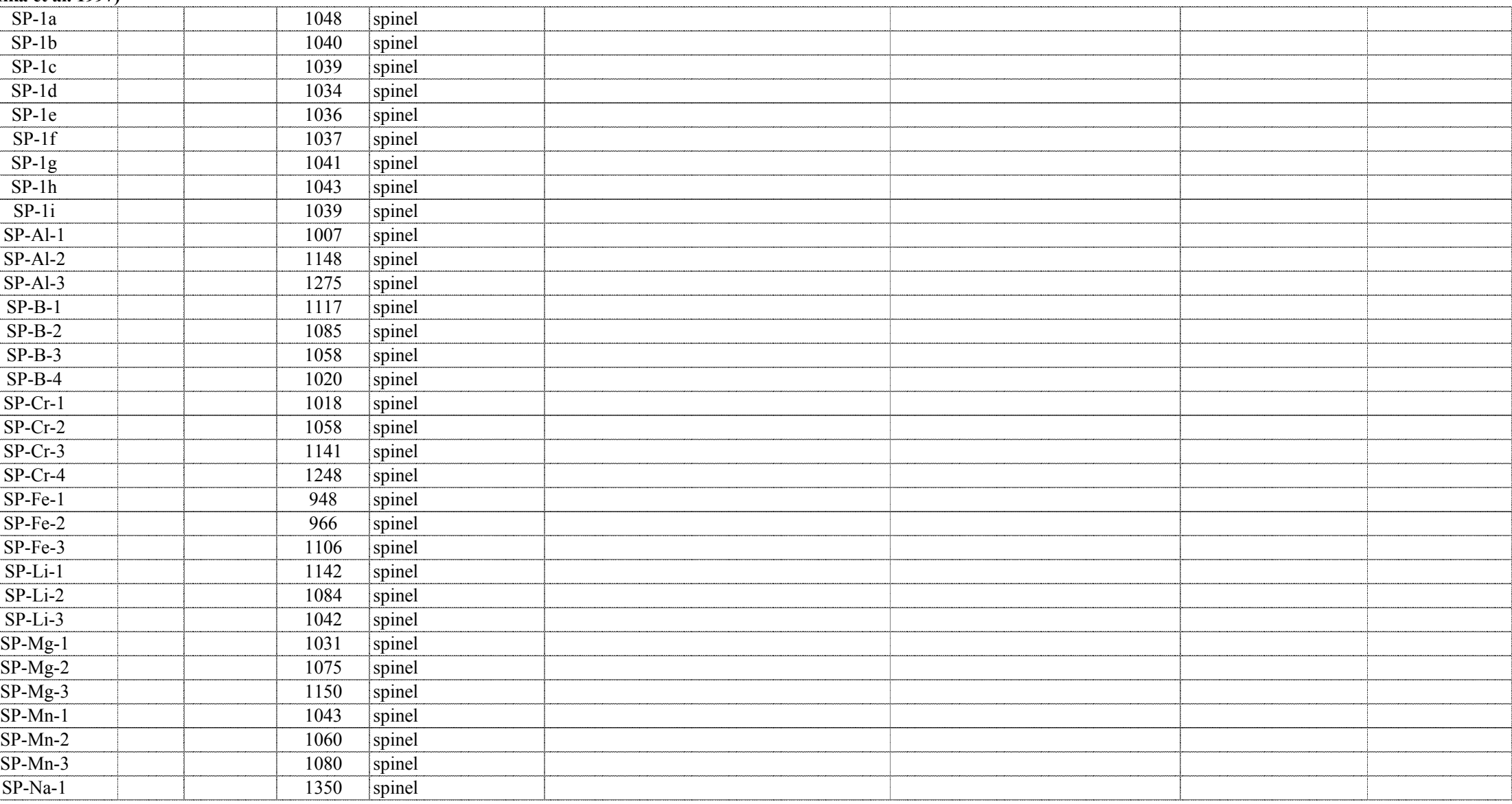


Appendix A. Database - mass fraction

\section{Zr Study (Vienna et al. 1999)}

\begin{tabular}{|c|c|c|c|c|c|}
\hline Glass ID & $\begin{array}{c}\mathrm{CCC} \\
\text { Visual/OM }\end{array}$ & $\begin{array}{c}\text { CCC } \\
\text { SEM/EDS or TEM }\end{array}$ & $\begin{array}{l}\text { CCC } \\
\text { XRD }\end{array}$ & $\begin{array}{c}\text { Heat Treated } \\
\text { Visual/OM }\end{array}$ & $\begin{array}{c}\text { Heat Treated } \\
\text { SEM/EDS or TEM }\end{array}$ \\
\hline \multicolumn{6}{|l|}{$\mathrm{Zr}-16$} \\
\hline \multirow{2}{*}{\multicolumn{6}{|c|}{$\begin{array}{l}\mathrm{Zr}-17 \\
\mathrm{Zr}-18\end{array}$}} \\
\hline & & & & & \\
\hline \multicolumn{6}{|l|}{$\mathrm{Zr}-19$} \\
\hline \multicolumn{6}{|l|}{$\mathrm{Zr}-20$} \\
\hline \multicolumn{6}{|l|}{$\mathrm{Zr}-21$} \\
\hline \multicolumn{6}{|l|}{$\mathrm{Zr}-22$} \\
\hline \multicolumn{6}{|l|}{$\mathrm{Zr}-23$} \\
\hline \multicolumn{6}{|l|}{$\mathrm{Zr}-24$} \\
\hline \multicolumn{6}{|l|}{$\mathrm{Zr}-25$} \\
\hline \multicolumn{6}{|l|}{$\mathrm{Zr}-26$} \\
\hline \multicolumn{6}{|l|}{$\mathrm{Zr}-27$} \\
\hline $\mathrm{Zr}-28$ & & & & & \\
\hline $\mathrm{Zr}-29$ & & & & & \\
\hline
\end{tabular}

\section{SP (Mika et al. 1997)}

SP-1a

SP-1b

SP-1c

SP-10

SP-1e

SP-1f

SP-1g

SP-1h

SP-1i

SP-Al-1

SP-Al-2

SP-Al-3

SP-B-1

SP-B-2

SP-B-3

SP-B-4

SP-Cr-1

$\mathrm{SP}-\mathrm{Cr}-2$

$\mathrm{SP}-\mathrm{Cr}-3$

SP-Cr-4

SP-Fe-1

$\mathrm{SP}-\mathrm{Fe}-2$

SP-Fe-3

SP-Li-1

SP-Li-2

SP-Li-3

SP-Mg-1

SP-Mg-2

SP-Mg-3

SP-Mn-1

SP-Mn-2

SP-Mn-3

SP-Na-1

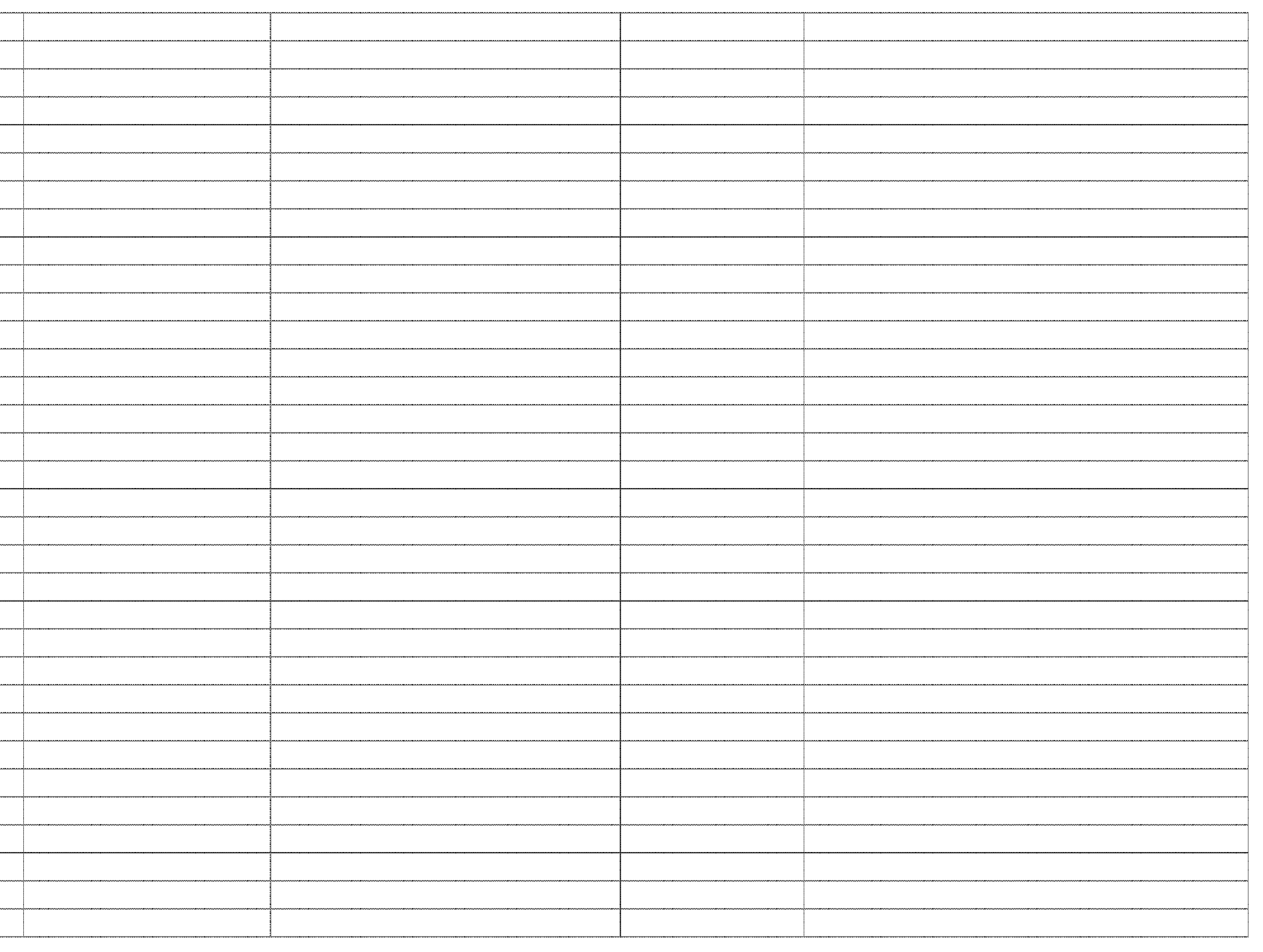


Zr Study (Vienna et al. 1999)

\begin{tabular}{|c|c|c|c|c|c|c|c|c|c|c|c|c|c|c|c|c|c|c|c|c|c|}
\hline Glass ID & $\begin{array}{c}\mathrm{V} 2 \\
(\mathrm{~Pa} \cdot \mathrm{s})\end{array}$ & $\begin{array}{c}\mathrm{T} 3 \\
\left({ }^{\circ} \mathrm{C}\right) \\
\end{array}$ & $\begin{array}{c}\mathrm{V} 3 \\
(\mathrm{~Pa} \cdot \mathrm{s})\end{array}$ & $\begin{array}{c}\mathrm{T} 4 \\
\left({ }^{\circ} \mathrm{C}\right)\end{array}$ & $\begin{array}{c}\mathrm{V} 4 \\
(\mathrm{~Pa} \cdot \mathrm{s})\end{array}$ & $\begin{array}{c}\mathrm{T} 5 \\
\left({ }^{\circ} \mathrm{C}\right)\end{array}$ & $\begin{array}{c}\mathrm{V} 5 \\
(\mathrm{~Pa} \cdot \mathrm{s})\end{array}$ & $\begin{array}{c}\text { T6 } \\
\left({ }^{\circ} \mathrm{C}\right) \\
\end{array}$ & $\begin{array}{c}\mathrm{V} 6 \\
(\mathrm{~Pa} \cdot \mathrm{s})\end{array}$ & $\begin{array}{c}\mathrm{T} 7 \\
\left({ }^{\circ} \mathrm{C}\right)\end{array}$ & $\begin{array}{c}\mathrm{V} 7 \\
(\mathrm{~Pa} \cdot \mathrm{s})\end{array}$ & $\begin{array}{c}\mathrm{T} 8 \\
\left({ }^{\circ} \mathrm{C}\right)\end{array}$ & $\begin{array}{c}\mathrm{V} 8 \\
(\mathrm{~Pa} \cdot \mathrm{s})\end{array}$ & $\begin{array}{c}\mathrm{T} 9 \\
\left({ }^{\circ} \mathrm{C}\right) \\
\end{array}$ & $\begin{array}{c}\mathrm{V} 9 \\
(\mathrm{~Pa} \cdot \mathrm{s})\end{array}$ & $\begin{array}{l}\mathrm{T} 10 \\
\left({ }^{\circ} \mathrm{C}\right)\end{array}$ & $\begin{array}{c}\mathrm{V} 10 \\
(\mathrm{~Pa} \cdot \mathrm{s})\end{array}$ & $\begin{array}{l}\mathrm{T} 11 \\
\left({ }^{\circ} \mathrm{C}\right)\end{array}$ & $\begin{array}{c}\mathrm{V} 11 \\
(\mathrm{~Pa} \cdot \mathrm{s})\end{array}$ & $\begin{array}{l}\mathrm{T} 12 \\
\left({ }^{\circ} \mathrm{C}\right)\end{array}$ & $\begin{array}{c}\mathrm{V} 12 \\
(\mathrm{~Pa} \cdot \mathrm{s})\end{array}$ \\
\hline $\mathrm{Zr}-16$ & & & & & & & & & & & & & & & & & & & & & \\
\hline $\mathrm{Zr}-17$ & & & & & & & & & & & & & & & & & & & & & \\
\hline $\mathrm{Zr}-18$ & & & & & & & & & & & & & & & & & & & & & \\
\hline $\mathrm{Zr}-19$ & & & & & & & & & & & & & & & & & & & & & \\
\hline $\mathrm{Zr}-20$ & & & & & & & & & & & & & & & & & & & & & \\
\hline $\mathrm{Zr}-21$ & & & & & & & & & & & & & & & & & & & & & \\
\hline $\mathrm{Zr}-22$ & & & & & & & & & & & & & & & & & & & & & \\
\hline $\mathrm{Zr}-23$ & & & & & & & & & & & & & & & & & & & & & \\
\hline $\mathrm{Zr}-24$ & & & & & & & & & & & & & & & & & & & & & \\
\hline $\mathrm{Zr}-25$ & & & & & & & & & & & & & & & & & & & & & \\
\hline $\mathrm{Zr}-26$ & & & & & & & & & & & & & & & & & & & & & \\
\hline $\mathrm{Zr}-27$ & & & & & & & & & & & & & & & & & & & & & \\
\hline $\mathrm{Zr}-28$ & & & & & & & & & & & & & & & & & & & & & \\
\hline $\mathrm{Zr}-29$ & & & & & & & & & & & & & & & & & & & & & \\
\hline
\end{tabular}

\section{SP (Mika et al. 1997)}

SP-1a

SP-1b

SP-1c

SP-1d

SP-1e

SP-1f

SP-1g

SP-1h

SP-1i

SP-Al-1

SP-Al-2

SP-Al-3

SP-B-1

SP-B-2

SP-B-3

SP-B-4

SP-Cr-1

SP-Cr-2

SP-Cr-3

SP-Cr-4

SP-Fe-1

$\mathrm{SP}-\mathrm{Fe}-2$

$\mathrm{SP}-\mathrm{Fe}-3$

SP-Li-1

SP-Li-2

SP-Li-3

SP-Mg-1

SP-Mg-2

SP-Mg-3

SP-Mn-1

SP-Mn-2

SP-Mn-3

SP-Na-1

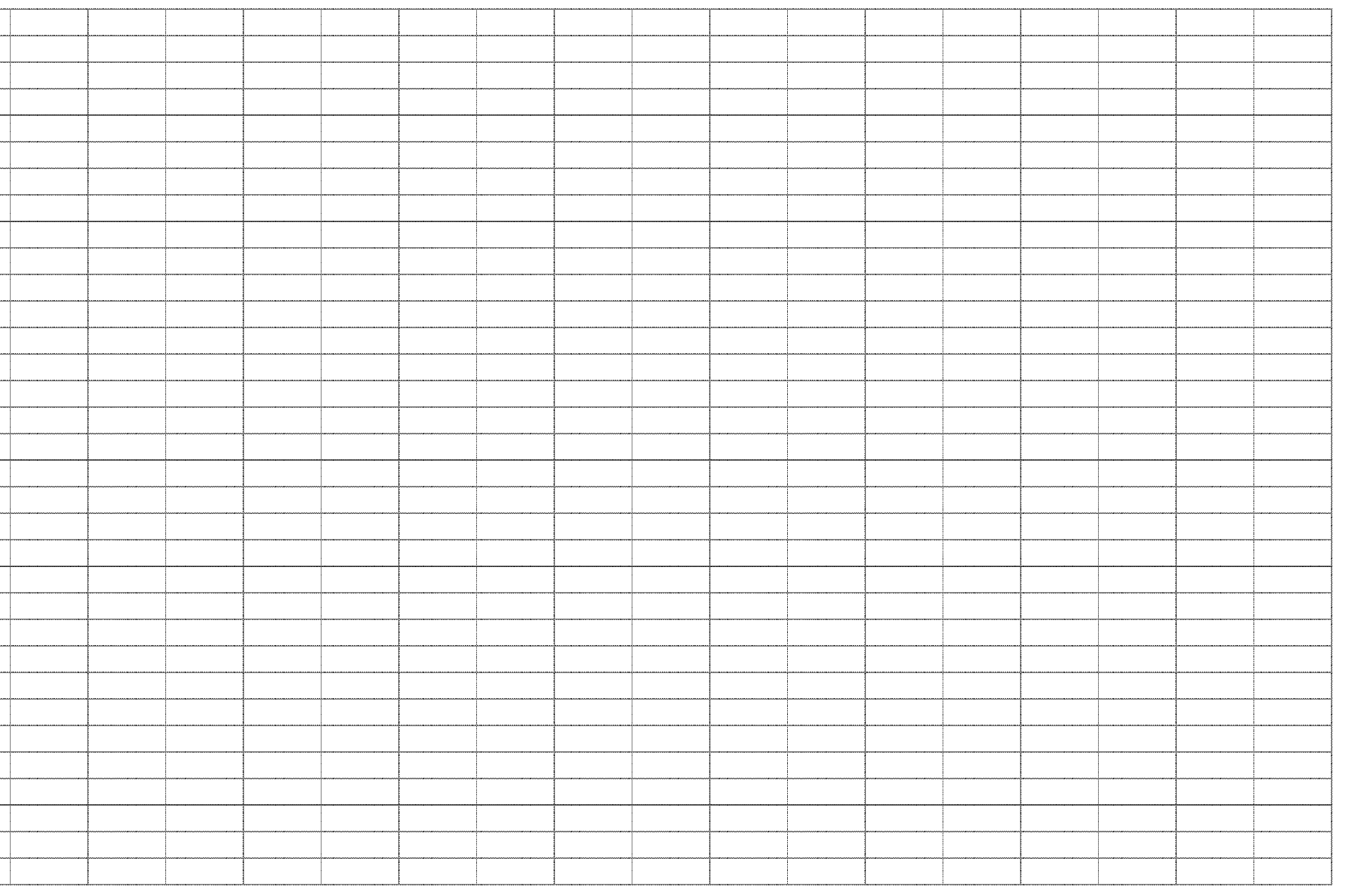


Appendix A. Database - mass fraction

\section{Zr Study (Vienna et al. 1999)}

\begin{tabular}{|c|c|c|c|c|c|c|c|c|c|c|c|c|c|c|c|c|}
\hline Glass ID & $\begin{array}{l}\mathrm{T} 13 \\
\left({ }^{\circ} \mathrm{C}\right) \\
\end{array}$ & $\begin{array}{c}\mathrm{V} 13 \\
(\mathrm{~Pa} \cdot \mathrm{s}) \\
\end{array}$ & $\begin{array}{l}\mathrm{T} 14 \\
\left({ }^{\circ} \mathrm{C}\right) \\
\end{array}$ & $\begin{array}{c}\mathrm{V} 14 \\
(\mathrm{~Pa} \cdot \mathrm{s})\end{array}$ & $\begin{array}{c}\text { Q PCT } \\
\text { B }\left(\mathrm{g} / \mathrm{m}^{2}\right)\end{array}$ & $\begin{array}{c}\text { Q PCT } \\
\mathrm{Li}\left(\mathrm{g} / \mathrm{m}^{2}\right)\end{array}$ & $\begin{array}{c}\text { Q PCT } \\
\mathrm{Na}\left(\mathrm{g} / \mathrm{m}^{2}\right) \\
\end{array}$ & $\begin{array}{c}\text { Q PCT } \\
\mathrm{Si}\left(\mathrm{g} / \mathrm{m}^{2}\right) \\
\end{array}$ & $\begin{array}{c}\text { Q PCT } \\
\mathrm{pH}\end{array}$ & $\begin{array}{c}\text { CCC PCT } \\
\mathrm{B}\left(\mathrm{g} / \mathrm{m}^{2}\right) \\
\end{array}$ & $\begin{array}{l}\text { CCC PCT } \\
\mathrm{Li}\left(\mathrm{g} / \mathrm{m}^{2}\right)\end{array}$ & $\begin{array}{l}\mathrm{CCC} \text { PCT } \\
\mathrm{Na}\left(\mathrm{g} / \mathrm{m}^{2}\right) \\
\end{array}$ & $\begin{array}{l}\text { CCC PCT } \\
\mathrm{Si}\left(\mathrm{g} / \mathrm{m}^{2}\right) \\
\end{array}$ & $\begin{array}{c}\text { CCC PCT } \\
\mathrm{pH}\end{array}$ & $\begin{array}{l}\text { Q PCT B at } \\
20^{\circ} \mathrm{C}\left(\mathrm{g} / \mathrm{m}^{2}\right)\end{array}$ & $\begin{array}{l}\text { Q PCT Li at } \\
20^{\circ} \mathrm{C}\left(\mathrm{g} / \mathrm{m}^{2}\right)\end{array}$ \\
\hline $\mathrm{Zr}-16$ & & & & & 0.185 & 0.34 & 0.23 & & & & & & & & & \\
\hline $\mathrm{Zr}-17$ & & & & & 0.265 & 0.33 & 0.02 & & & & & & & & & \\
\hline $\mathrm{Zr}-18$ & & & & & 0.66 & 0.75 & 0.335 & & & & & & & & & \\
\hline $\mathrm{Zr}-19$ & & & & & 0.275 & 0.54 & 0.495 & & & & & & & & & \\
\hline $\mathrm{Zr}-20$ & & & & & 0.49 & 0.505 & 1.175 & & & & & & & & & \\
\hline $\mathrm{Zr}-21$ & & & & & 0.3 & 0.36 & 0.035 & & & & & & & & & \\
\hline $\mathrm{Zr}-22$ & & & & & 1.03 & 0.965 & 0.525 & & & & & & & & & \\
\hline $\mathrm{Zr}-23$ & & & & & 0.255 & 0.515 & 0.545 & & & & & & & & & \\
\hline $\mathrm{Zr}-24$ & & & & & 0.295 & 0.41 & 1.16 & & & & & & & & & \\
\hline $\mathrm{Zr}-25$ & & & & & 0.405 & 0.45 & 0.065 & & & & & & & & & \\
\hline $\mathrm{Zr}-26$ & & & & & 0.61 & 0.62 & 0.315 & & & & & & & & & \\
\hline $\mathrm{Zr}-27$ & & & & & 0.595 & 0.735 & 0.71 & & & & & & & & & \\
\hline $\mathrm{Zr}-28$ & & & & & 0.24 & 0.34 & 0.26 & & & & & & & & & \\
\hline $\mathrm{Zr}-29$ & & & & & 3.89 & 2.715 & 3.085 & & & & & & & & & \\
\hline
\end{tabular}

\section{SP (Mika et al. 1997)}

SP-1a

SP-1b

SP-1c

SP-1d

SP-1e

SP-1f

SP-1g

SP-1h

SP-1i

SP-Al-1

SP-Al-2

SP-Al-3

SP-B-1

SP-B-2

SP-B-3

SP-B-4

SP-Cr-1

$\mathrm{SP}-\mathrm{Cr}-2$

SP-Cr-3

$\mathrm{SP}-\mathrm{Cr}-4$

SP-Fe-1

$\mathrm{SP}-\mathrm{Fe}-2$

$\mathrm{SP}-\mathrm{Fe}-3$

SP-Li-1

SP-Li-2

SP-Li-3

SP-Mg-1

SP-Mg-2

SP-Mg-3

SP-Mn-1

SP-Mn-2

SP-Mn-3

SP-Na-1

\begin{tabular}{|l|l|l|l|l|l|l|l|}
\hline & & & & & & & \\
\hline & & & & & & & \\
\hline & & & & & & & \\
\hline & & & & & & \\
\hline & & & & & & \\
\hline & & & & & & & \\
\hline & & & & & & & \\
\hline & & & & & & & \\
\hline & & & & & & & \\
\hline & & & & & & & \\
\hline
\end{tabular}


Appendix A. Database - mass fraction

Zr Study (Vienna et al. 1999)

\begin{tabular}{|c|c|c|c|c|c|c|c|c|c|c|c|c|}
\hline Glass ID & $\begin{array}{l}\text { Q PCT Na at } \\
20^{\circ} \mathrm{C}\left(\mathrm{g} / \mathrm{m}^{2}\right)\end{array}$ & $\begin{array}{l}\text { Q PCT Si at } \\
20^{\circ} \mathrm{C}\left(\mathrm{g} / \mathrm{m}^{2}\right)\end{array}$ & $\begin{array}{c}\mathrm{QpH} \\
\text { at } 20^{\circ} \mathrm{C}\end{array}$ & $\begin{array}{c}\text { TCLP Ag } \\
(\mathrm{ppm})\end{array}$ & $\begin{array}{c}\text { TCLP As } \\
(\mathrm{ppm})\end{array}$ & $\begin{array}{c}\text { TCLP Ba } \\
(\mathrm{ppm})\end{array}$ & $\begin{array}{c}\text { TCLP Cd } \\
(\mathrm{ppm})\end{array}$ & $\begin{array}{c}\text { TCLP Cr } \\
(\mathrm{ppm})\end{array}$ & $\begin{array}{c}\text { TCLP Ni } \\
(\mathrm{ppm})\end{array}$ & $\begin{array}{c}\text { TCLP Pb } \\
(\mathrm{ppm})\end{array}$ & $\begin{array}{l}\text { TCLP Se } \\
(\mathrm{ppm})\end{array}$ & $\begin{array}{c}\text { TCLP Zn } \\
(\mathrm{ppm})\end{array}$ \\
\hline \multicolumn{13}{|c|}{ 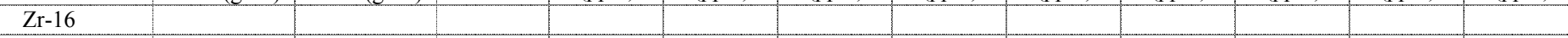 } \\
\hline \multicolumn{13}{|l|}{$\mathrm{Zr}-17$} \\
\hline \multicolumn{13}{|l|}{$\mathrm{Zr}-18$} \\
\hline \multicolumn{13}{|l|}{ Zr-19 } \\
\hline \multicolumn{13}{|l|}{$\mathrm{Zr}-20$} \\
\hline \multicolumn{13}{|l|}{$\mathrm{Zr}-21$} \\
\hline \multicolumn{13}{|l|}{$\mathrm{Zr}-22$} \\
\hline \multicolumn{13}{|l|}{$\mathrm{Zr}-23$} \\
\hline \multicolumn{13}{|l|}{$\mathrm{Zr}-24$} \\
\hline \multicolumn{13}{|l|}{$\mathrm{Zr}-25$} \\
\hline \multicolumn{13}{|l|}{$\mathrm{Zr}-26$} \\
\hline \multicolumn{13}{|l|}{$\mathrm{Zr}-27$} \\
\hline \multicolumn{13}{|l|}{ Zr-28 } \\
\hline $\mathrm{Zr}-29$ & & & & & & & & & & & & \\
\hline
\end{tabular}

SP (Mika et al. 1997)

SP-1a

SP-1b

SP-1d

SP-1e

SP-1f

SP-1g

SP-1h

SP-1i

SP-Al-1

SP-Al-2

SP-Al-3

SP-B-1

SP-B-2

SP-B-3

SP-B-4

SP-Cr-1

SP-Cr-2

SP-Cr-3

SP-Cr-4

SP-Fe-1

$\mathrm{SP}-\mathrm{Fe}-2$

$\mathrm{SP}-\mathrm{Fe}-3$

SP-Li-1

SP-Li-2

SP-Li-3

SP-Mg-1

SP-Mg-2

SP-Mg-3

SP-Mn-1

SP-Mn-2

SP-Mn-3

SP-Na-1

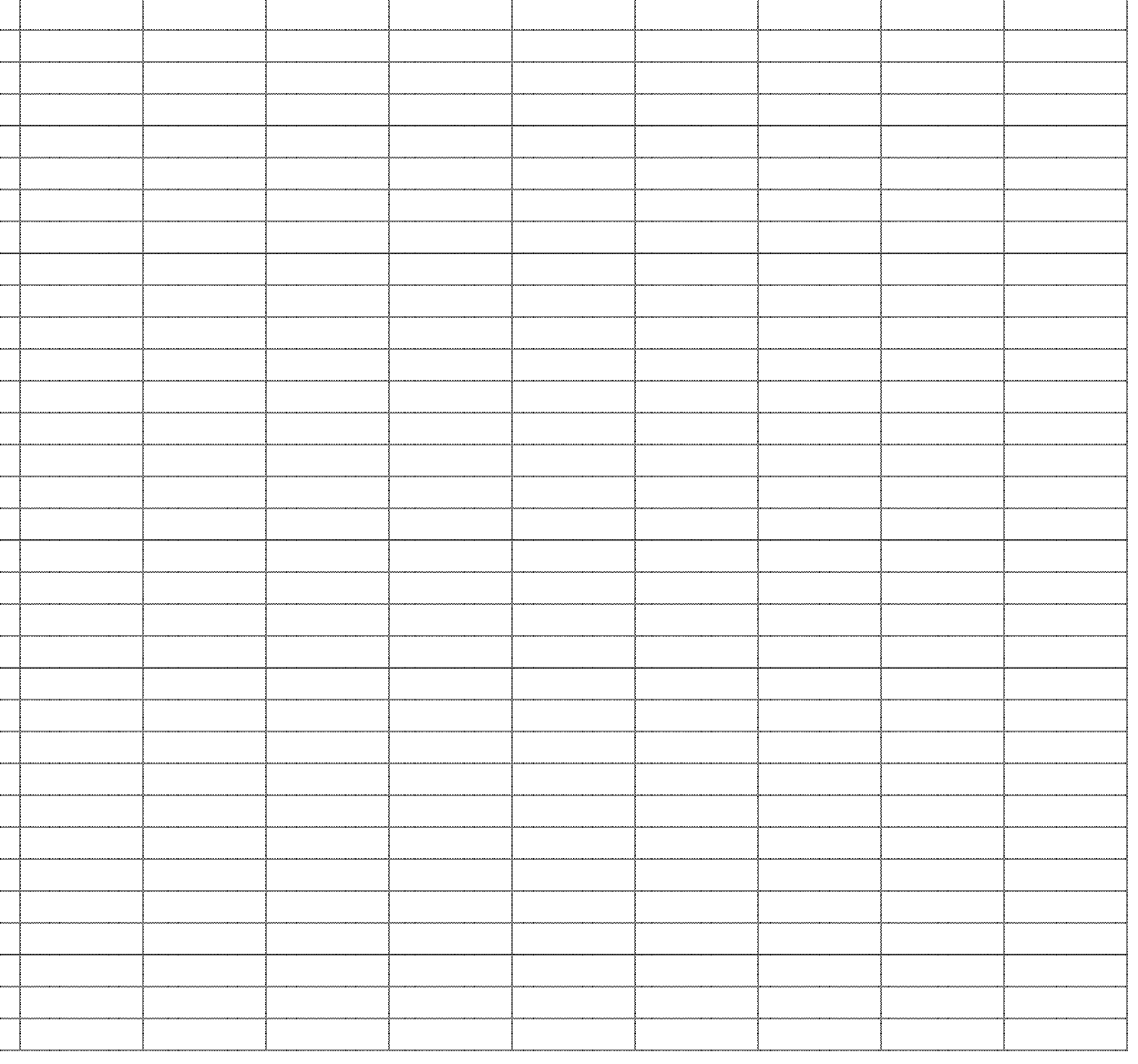


SP (Mika et al. 1997)

\begin{tabular}{l|l|l|l|l|l|l|l|l|l|l|l|l|l|l|l|l|l|l|l|l|l|l|l|l|l|l} 
Glass ID & $\mathrm{Al} 2 \mathrm{O} 3$ & $\mathrm{~B} 2 \mathrm{O} 3$ & $\mathrm{CaO}$ & $\mathrm{Fe} 2 \mathrm{O} 3$ & $\mathrm{FeO}$ & $\mathrm{K} 2 \mathrm{O}$ & $\mathrm{Li} 2 \mathrm{O}$ & $\mathrm{MgO}$ & $\mathrm{Na} 2 \mathrm{O}$ & $\mathrm{NiO}$ & $\mathrm{P} 2 \mathrm{O} 5$ & $\mathrm{SiO} 2$ & $\mathrm{ZrO} 2$ & $\mathrm{Ag} 2 \mathrm{O}$ & $\mathrm{As} 2 \mathrm{O} 3$ & $\mathrm{BaO}$ & $\mathrm{Bi} 2 \mathrm{O} 3$ & $\mathrm{Br}$ & $\mathrm{CdO}$ & $\mathrm{Ce} 2 \mathrm{O} 3$ & $\mathrm{CeO} 2$ \\
\hline
\end{tabular}

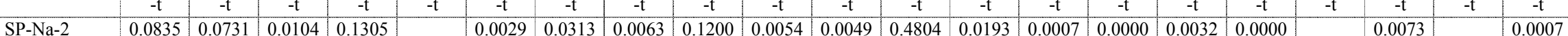

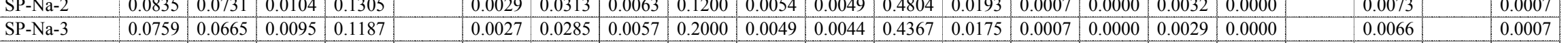

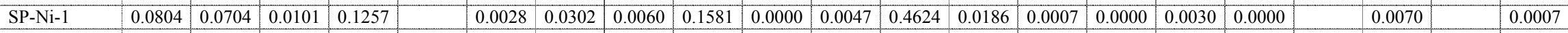

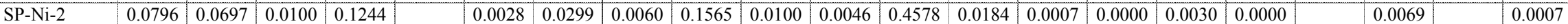

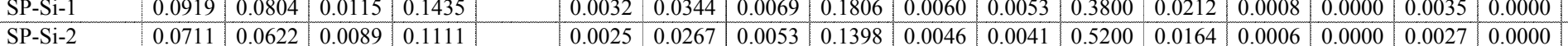

\begin{tabular}{|l|l|l|l|l}
0.0069 & 0.0007 \\
\hline 0.0068 & 0.0007 \\
\hline
\end{tabular}

\begin{tabular}{|l|l|l|l|l|l|l|l|l|l|l|l|l|}
0.0021 & 0.0222 & 0.0044 & 0.1165 & 0.0039 & 0.0034 & 0.6000 & 0.0137 & 0.0005 & 0.0000 & 0.0022 & 0.0000 \\
\hline
\end{tabular}

\begin{tabular}{l|l}
0.0062 & 0.0006 \\
\hline 0.0052 & 0.0005
\end{tabular}

\section{MS (Hrma 1999)}

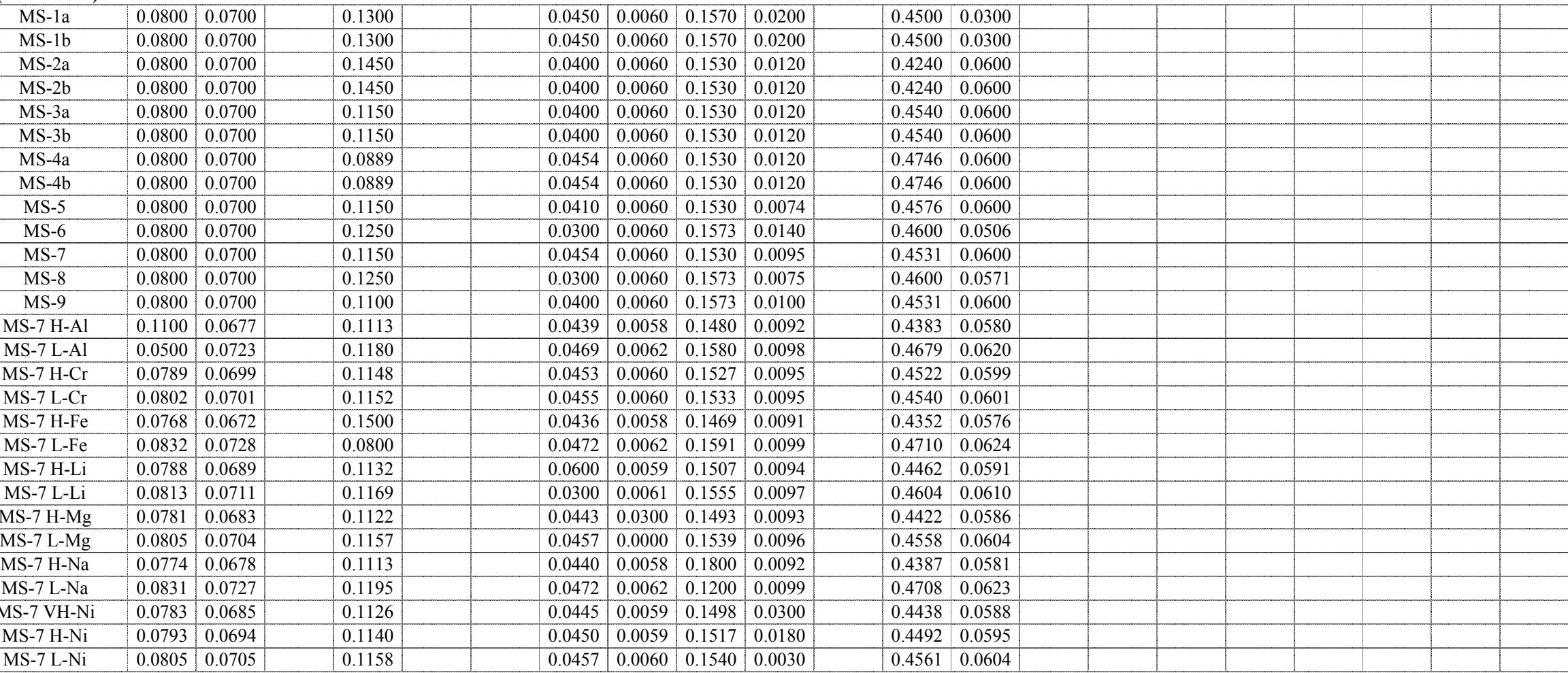

\section{SG (Hrma et al. 1999)}

\begin{tabular}{|c|c|c|c|c|c|c|c|c|c|c|c|c|c|c|c|c|c|c|}
\hline SG01 & 0.0250 & 0.0999 & 0.0200 & 0.1499 & 0.0380 & 0.0599 & 0.0050 & 0.0599 & 0.0200 & 0.0000 & 0.4496 & 0.0000 & 0.0000 & 0.0000 & 0.0000 & 0.0000 & 0.0000 & 0.0000 \\
\hline SG02 & 0.0250 & 0.0500 & 0.0200 & 0.0599 & 0.0380 & 0.0599 & 0.0250 & 0.1099 & 0.0005 & 0.0000 & 0.5785 & 0.0000 & 0.0000 & 0.0000 & 0.0000 & 0.0000 & 0.0000 & 0.0000 \\
\hline SG03 & 0.0390 & 0.0876 & 0.0158 & 0.1202 & 0.0208 & 0.0375 & 0.0200 & 0.0976 & 0.0151 & 0.0000 & 0.4741 & 0.0000 & 0.0000 & 0.0000 & 0.0000 & 0.0000 & 0.0000 & 0.0000 \\
\hline SG04 & 0.0799 & 0.0500 & 0.0030 & 0.1499 & 0.0150 & 0.0599 & 0.0250 & 0.0599 & 0.0200 & 0.0000 & 0.5240 & 0.0000 & 0.0000 & 0.0000 & 0.0000 & 0.0000 & 0.0000 & 0.0000 \\
\hline SG05 & 0.0530 & 0.0752 & 0.0115 & 0.1052 & 0.0266 & 0.0450 & 0.0150 & 0.0852 & 0.0102 & 0.0000 & 0.5186 & 0.0000 & 0.0000 & 0.0000 & 0.0000 & 0.0000 & 0.0000 & 0.0000 \\
\hline SG06 & 0.0799 & 0.0500 & 0.0200 & 0.1499 & 0.0380 & 0.0300 & 0.0050 & 0.1099 & 0.0005 & 0.0000 & 0.4991 & 0.0000 & 0.0000 & 0.0000 & 0.0000 & 0.0000 & 0.0000 & 0.0000 \\
\hline SG07 & 0.0799 & 0.0999 & 0.0030 & 0.0599 & 0.0380 & 0.0599 & 0.0250 & 0.0599 & 0.0005 & 0.0000 & 0.5385 & 0.0000 & 0.0000 & 0.0000 & 0.0000 & 0.0000 & 0.0000 & 0.0000 \\
\hline SG08 & 0.0390 & 0.0626 & 0.0158 & 0.1275 & 0.0323 & 0.0375 & 0.0200 & 0.0726 & 0.0054 & 0.0000 & 0.5397 & 0.0000 & 0.0000 & 0.0000 & 0.0000 & 0.0000 & 0.0000 & 0.0000 \\
\hline
\end{tabular}




\section{SP (Mika et al. 1997)}

\begin{tabular}{|c|c|c|c|c|c|c|c|c|c|c|c|c|c|c|c|c|c|c|c|c|c|}
\hline Glass ID & $\begin{array}{l}\mathrm{Cl} \\
-\mathrm{t}\end{array}$ & $\begin{array}{c}\mathrm{CoO} \\
-\mathrm{t}\end{array}$ & $\begin{array}{c}\mathrm{Co} 2 \mathrm{O} 3 \\
-\mathrm{t} \\
\end{array}$ & $\begin{array}{c}\mathrm{Cr} 2 \mathrm{O} 3 \\
-\mathrm{t} \\
\end{array}$ & $\begin{array}{c}\mathrm{Cs} 2 \mathrm{O} \\
-\mathrm{t}\end{array}$ & $\begin{array}{c}\mathrm{CuO} \\
-\mathrm{t} \\
\end{array}$ & $\begin{array}{c}\mathrm{Eu} 2 \mathrm{O} 3 \\
-\mathrm{t} \\
\end{array}$ & $\begin{array}{l}F \\
-t\end{array}$ & $\begin{array}{c}\mathrm{Ga} 2 \mathrm{O} 3 \\
-\mathrm{t}\end{array}$ & $\begin{array}{c}\mathrm{Gd} 2 \mathrm{O} 3 \\
-\mathrm{t}\end{array}$ & $\begin{array}{c}\mathrm{HgO} \\
-\mathrm{t} \\
\end{array}$ & $\begin{array}{l}I \\
-t\end{array}$ & $\begin{array}{c}\mathrm{La} 2 \mathrm{O} 3 \\
-\mathrm{t} \\
\end{array}$ & $\begin{array}{c}\mathrm{MnO} 2 \\
-\mathrm{t} \\
\end{array}$ & $\begin{array}{c}\mathrm{MnO} \\
-\mathrm{t}\end{array}$ & $\begin{array}{c}\mathrm{MoO} \\
-\mathrm{t}\end{array}$ & $\begin{array}{c}\mathrm{MoO} 3 \\
-\mathrm{t} \\
\end{array}$ & $\begin{array}{c}\mathrm{Nb} 2 \mathrm{O} 5 \\
-\mathrm{t}\end{array}$ & $\begin{array}{c}\mathrm{Nd} 2 \mathrm{O} 3 \\
-\mathrm{t} \\
\end{array}$ & $\begin{array}{c}\mathrm{PbO} \\
-\mathrm{t}\end{array}$ & $\begin{array}{c}\mathrm{PdO} 2 \\
-\mathrm{t} \\
\end{array}$ \\
\hline SP-Na-2 & 0.0001 & & 0.0009 & 0.0023 & 0.0000 & 0.0003 & & 0.0006 & & & & & 0.0028 & & 0.0038 & & 0.0001 & & 0.0019 & 0.0018 & \\
\hline SP-Na-3 & 0.0001 & & 0.0009 & 0.0021 & 0.0000 & 0.0003 & & 0.0006 & & & & & 0.0026 & & 0.0034 & & 0.0001 & & 0.0017 & 0.0016 & \\
\hline SP-Ni-1 & 0.0001 & & 0.0009 & 0.0022 & 0.0000 & 0.0003 & & 0.0006 & & & & & 0.0027 & & 0.0036 & & 0.0001 & & 0.0018 & 0.0017 & \\
\hline SP-Ni-2 & 0.0001 & & 0.0009 & 0.0022 & 0.0000 & 0.0003 & & 0.0006 & & & & & 0.0027 & & 0.0036 & & 0.0001 & & 0.0018 & 0.0017 & \\
\hline SP-Ni-3 & 0.0001 & & 0.0009 & 0.0021 & 0.0000 & 0.0003 & & 0.0006 & & & & & 0.0027 & & 0.0035 & & 0.0001 & & 0.0018 & 0.0017 & \\
\hline SP-Si-1 & 0.0001 & & 0.0010 & 0.0025 & 0.0000 & 0.0003 & & 0.0007 & & & & & 0.0031 & & 0.0041 & & 0.0001 & & 0.0021 & 0.0020 & \\
\hline SP-Si-2 & 0.0001 & & 0.0008 & 0.0020 & 0.0000 & 0.0003 & & 0.0005 & & & & & 0.0024 & & 0.0032 & & 0.0001 & & 0.0016 & 0.0015 & \\
\hline SP-Si-3 & 0.0001 & & 0.0007 & 0.0016 & 0.0000 & 0.0002 & & 0.0004 & & & & & 0.0020 & & 0.0027 & & 0.0001 & & 0.0013 & 0.0013 & \\
\hline
\end{tabular}

\section{MS (Hrma 1999)}

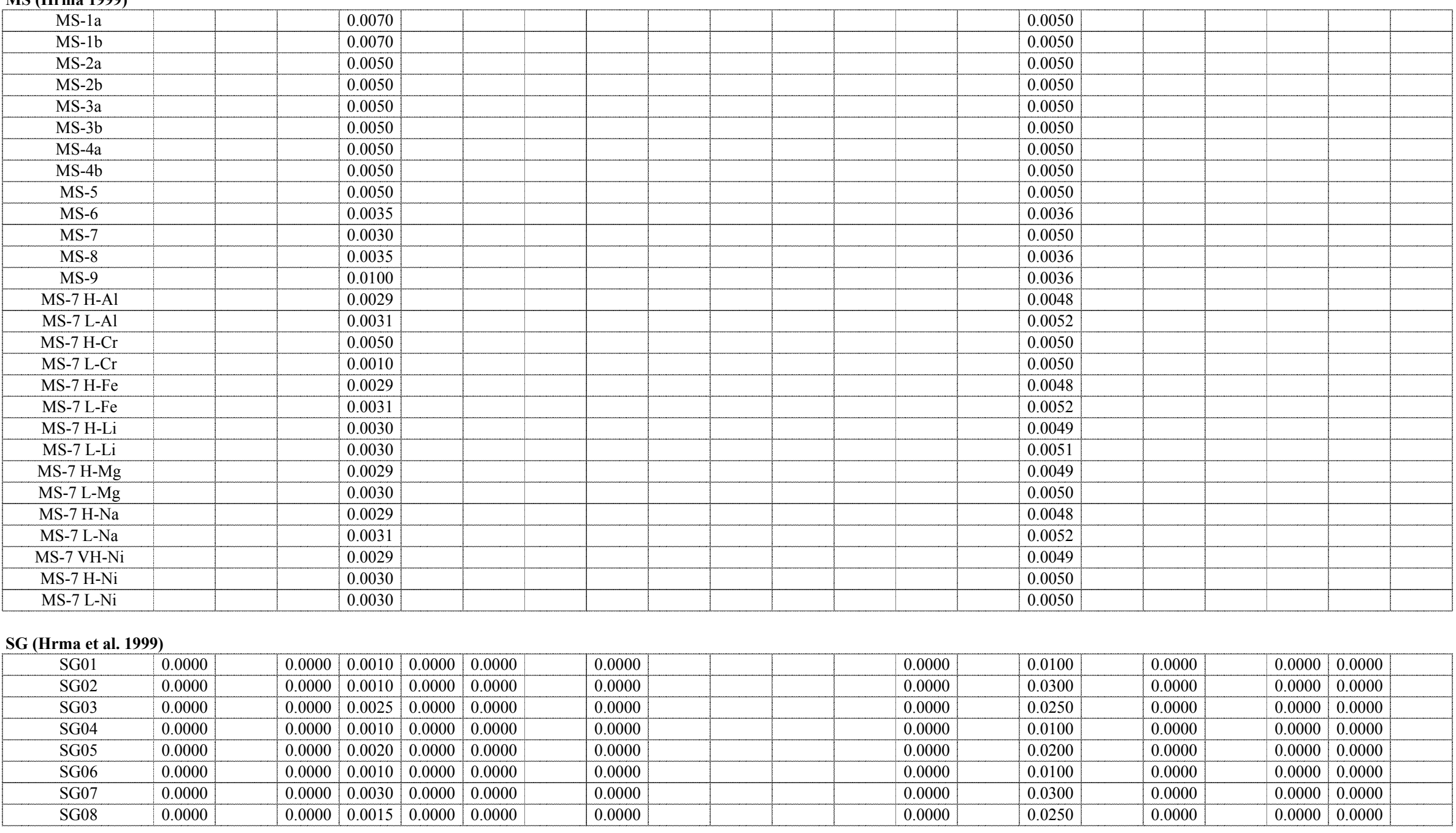


SP (Mika et al. 1997)

\begin{tabular}{|c|c|c|c|c|c|c|c|c|c|c|c|c|c|c|c|c|c|c|c|c|c|}
\hline Glass ID & $\begin{array}{c}\mathrm{PdO} \\
-\mathrm{t}\end{array}$ & $\begin{array}{c}\mathrm{Pr} 2 \mathrm{O} 3 \\
-\mathrm{t} \\
\end{array}$ & {$\left[\begin{array}{c}\text { Pr6O11 } \\
-t\end{array}\right.$} & $\begin{array}{c}\mathrm{Rb} 2 \mathrm{O} \\
-\mathrm{t} \\
\end{array}$ & $\begin{array}{c}\mathrm{ReO} \\
-\mathrm{t} \\
\end{array}$ & $\begin{array}{c}\mathrm{ReO} 2 \\
-\mathrm{t} \\
\end{array}$ & $\begin{array}{c}\mathrm{Rh} 2 \mathrm{O} 3 \\
-\mathrm{t} \\
\end{array}$ & $\begin{array}{c}\mathrm{RhO}^{2} \\
-\mathrm{t}\end{array}$ & $\begin{array}{c}\mathrm{RuO} 2 \\
-\mathrm{t}\end{array}$ & $\begin{array}{c}\mathrm{Sb} 2 \mathrm{O} 3 \\
-\mathrm{t}\end{array}$ & $\begin{array}{c}\mathrm{Sb} 2 \mathrm{O} 5 \\
-\mathrm{t} \\
\end{array}$ & $\begin{array}{c}\mathrm{SeO} 2 \\
-\mathrm{t}\end{array}$ & $\begin{array}{c}\mathrm{Sm} 2 \mathrm{O} 3 \\
-\mathrm{t}\end{array}$ & $\begin{array}{c}\mathrm{SnO} \\
-\mathrm{t} \\
\end{array}$ & $\begin{array}{c}\mathrm{SnO} 2 \\
-\mathrm{t}\end{array}$ & $\begin{array}{c}\mathrm{SO} 3 \\
-\mathrm{t}\end{array}$ & $\begin{array}{c}\mathrm{SrO} \\
-\mathrm{t}\end{array}$ & $\begin{array}{c}\mathrm{Tc} 2 \mathrm{O} 7 \\
-\mathrm{t} \\
\end{array}$ & $\begin{array}{c}\mathrm{TeO} 2 \\
-\mathrm{t}\end{array}$ & $\begin{array}{c}\mathrm{ThO} 2 \\
-\mathrm{t} \\
\end{array}$ & $\begin{array}{c}\mathrm{TiO} 2 \\
-\mathrm{t} \\
\end{array}$ \\
\hline SP-Na-2 & 0.0000 & & 0.0000 & 0.0000 & & & 0.0003 & & 0.0003 & 0.0007 & & 0.0009 & 0.0000 & & & 0.0020 & 0.0003 & & 0.0000 & & 0.0003 \\
\hline SP-Na-3 & 0.0000 & & 0.0000 & 0.0000 & & & 0.0003 & & 0.0003 & 0.0007 & & 0.0009 & 0.0000 & & & 0.0018 & 0.0003 & & 0.0000 & & 0.0003 \\
\hline SP-Ni-1 & 0.0000 & & 0.0000 & 0.0000 & & & 0.0003 & & 0.0003 & 0.0007 & & 0.0009 & 0.0000 & & & 0.0019 & 0.0003 & & 0.0000 & & 0.0003 \\
\hline SP-Ni-2 & 0.0000 & & 0.0000 & 0.0000 & & & 0.0003 & & 0.0003 & 0.0007 & & 0.0009 & 0.0000 & & & 0.0019 & 0.0003 & & 0.0000 & & 0.0003 \\
\hline SP-Ni-3 & 0.0000 & & 0.0000 & 0.0000 & & & 0.0003 & & 0.0003 & 0.0007 & & 0.0009 & 0.0000 & & & 0.0019 & 0.0003 & & 0.0000 & & 0.0003 \\
\hline SP-Si-1 & 0.0000 & & 0.0000 & 0.0000 & & & 0.0003 & & 0.0003 & 0.0008 & & 0.0010 & 0.0000 & & & 0.0022 & 0.0003 & & 0.0000 & & 0.0003 \\
\hline SP-Si-2 & 0.0000 & & 0.0000 & 0.0000 & & & 0.0003 & & 0.0003 & 0.0006 & & 0.0008 & 0.0000 & & & 0.0017 & 0.0003 & & 0.0000 & & 0.0003 \\
\hline SP-Si-3 & 0.0000 & & 0.0000 & 0.0000 & & & 0.0002 & & 0.0002 & 0.0005 & & 0.0007 & 0.0000 & & & 0.0014 & 0.0002 & & 0.0000 & & 0.0002 \\
\hline
\end{tabular}

\section{MS (Hrma 1999)}

MS-1a

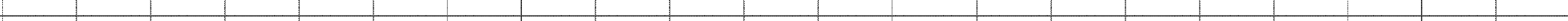

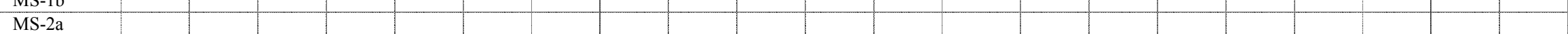

MS-4a

MS-4b

MS-5

MS-6

MS-7

MS-8

MS-9

MS-7 H-Al

MS-7 L-Al

MS-7 H-Cr

MS-7 L-Cr

$\mathrm{MS}-7 \mathrm{H}-\mathrm{Fe}$

MS-7 L-Fe

MS-7 H-Li

MS-7 L-Li

MS-7 H-Mg

MS-7 L-Mg

MS-7 H-Na

MS-7 L-Na

MS-7 VH-Ni

MS-7 H-Ni

MS-7 L-Ni

\section{SG (Hrma et al. 1999)}

\begin{tabular}{|c|c|c|c|c|c|c|c|c|c|c|c|c|}
\hline SG01 & 0.0000 & 0.0000 & 0.0000 & 0.0000 & 0.0009 & 0.0000 & 0.0000 & 0.0000 & 0.0000 & 0.0000 & 0.0000 & 0.0060 \\
\hline SG02 & 0.0000 & 0.0000 & 0.0000 & 0.0000 & 0.0009 & 0.0000 & 0.0000 & 0.0000 & 0.0000 & 0.0000 & 0.0000 & 0.0015 \\
\hline SG03 & 0.0000 & 0.0000 & 0.0000 & 0.0000 & 0.0009 & 0.0000 & 0.0000 & 0.0000 & 0.0000 & 0.0000 & 0.0000 & 0.0026 \\
\hline SG04 & 0.0000 & 0.0000 & 0.0000 & 0.0000 & 0.0009 & 0.0000 & 0.0000 & 0.0000 & 0.0000 & 0.0000 & 0.0000 & 0.0015 \\
\hline SG05 & 0.0000 & 0.0000 & 0.0000 & 0.0000 & 0.0009 & 0.0000 & 0.0000 & 0.0000 & 0.0000 & 0.0000 & 0.0000 & 0.0037 \\
\hline SG06 & 0.0000 & 0.0000 & 0.0000 & 0.0000 & 0.0009 & 0.0000 & 0.0000 & 0.0000 & 0.0000 & 0.0000 & 0.0000 & 0.0060 \\
\hline SG07 & 0.0000 & 0.0000 & 0.0000 & 0.0000 & 0.0009 & 0.0000 & 0.0000 & 0.0000 & 0.0000 & 0.0000 & 0.0000 & 0.0015 \\
\hline SG08 & 0.0000 & 0.0000 & 0.0000 & 0.0000 & 0.0009 & 0.0000 & 0.0000 & 0.0000 & 0.0000 & 0.0000 & 0.0000 & 0.0026 \\
\hline
\end{tabular}


SP (Mika et al. 1997)

\begin{tabular}{|c|c|c|c|c|c|c|c|c|c|c|c|c|c|c|c|c|c|c|c|c|c|}
\hline Glass ID & $\begin{array}{c}\mathrm{T} 12 \mathrm{O} 3 \\
-\mathrm{t}\end{array}$ & $\begin{array}{c}\mathrm{U} 3 \mathrm{O} 8 \\
-\mathrm{t}\end{array}$ & $\begin{array}{c}\mathrm{UO} 2 \\
-\mathrm{t}\end{array}$ & $\begin{array}{c}\text { UO3 } \\
-t\end{array}$ & $\begin{array}{c}\mathrm{V} 2 \mathrm{O} 5 \\
-\mathrm{t}\end{array}$ & $\begin{array}{c}\text { WO3 } \\
-t\end{array}$ & $\begin{array}{c}\mathrm{Y} 2 \mathrm{O} 3 \\
-\mathrm{t}\end{array}$ & $\begin{array}{c}\mathrm{ZnO} \\
-\mathrm{t}\end{array}$ & $\begin{array}{c}\text { Others } \\
-t\end{array}$ & $\begin{array}{c}\text { Sum } \\
-t\end{array}$ & $\begin{array}{c}\mathrm{A} 12 \mathrm{O} 3 \\
-\mathrm{a}\end{array}$ & $\begin{array}{c}\mathrm{B} 2 \mathrm{O} 3 \\
-\mathrm{a}\end{array}$ & $\begin{array}{c}\mathrm{CaO} \\
-\mathrm{a}\end{array}$ & $\begin{array}{c}\mathrm{Fe} 2 \mathrm{O} 3 \\
-\mathrm{a}\end{array}$ & $\begin{array}{c}\mathrm{FeO} \\
-\mathrm{a}\end{array}$ & $\begin{array}{c}\mathrm{K} 2 \mathrm{O} \\
-\mathrm{a}\end{array}$ & $\begin{array}{c}\mathrm{Li} 2 \mathrm{O} \\
-\mathrm{a}\end{array}$ & $\begin{array}{c}\mathrm{MgO} \\
-\mathrm{a}\end{array}$ & $\begin{array}{c}\mathrm{Na} 2 \mathrm{O} \\
-\mathrm{a}\end{array}$ & $\begin{array}{c}\mathrm{NiO} \\
-\mathrm{a}\end{array}$ & $\begin{array}{c}\mathrm{P} 2 \mathrm{O} 5 \\
-\mathrm{a}\end{array}$ \\
\hline SP-Na-2 & & 0.0000 & & & & 0.0000 & 0.0000 & 0.0004 & & 1.0000 & & & & & & & & & & & \\
\hline SP-Na-3 & & 0.0000 & & & & 0.0000 & 0.0000 & 0.0004 & & 1.0000 & & & & & & & & & & & \\
\hline SP-Ni-1 & & 0.0000 & & & & 0.0000 & 0.0000 & 0.0004 & & 1.0000 & & & & & & & & & & & \\
\hline SP-Ni-2 & & 0.0000 & & & & 0.0000 & 0.0000 & 0.0004 & & 1.0000 & & & & & & & & & & & \\
\hline SP-Ni-3 & & 0.0000 & & & & 0.0000 & 0.0000 & 0.0004 & & 1.0000 & & & & & & & & & & & \\
\hline SP-Si-1 & & 0.0000 & & & & 0.0000 & 0.0000 & 0.0005 & & 1.0000 & & & & & & & & & & & \\
\hline SP-Si-2 & & 0.0000 & & & & 0.0000 & 0.0000 & 0.0004 & & 1.0000 & & & & & & & & & & & \\
\hline SP-Si-3 & & 0.0000 & & & & 0.0000 & 0.0000 & 0.0003 & & 1.0000 & & & & & & & & & & & \\
\hline
\end{tabular}

\section{MS (Hrma 1999)}

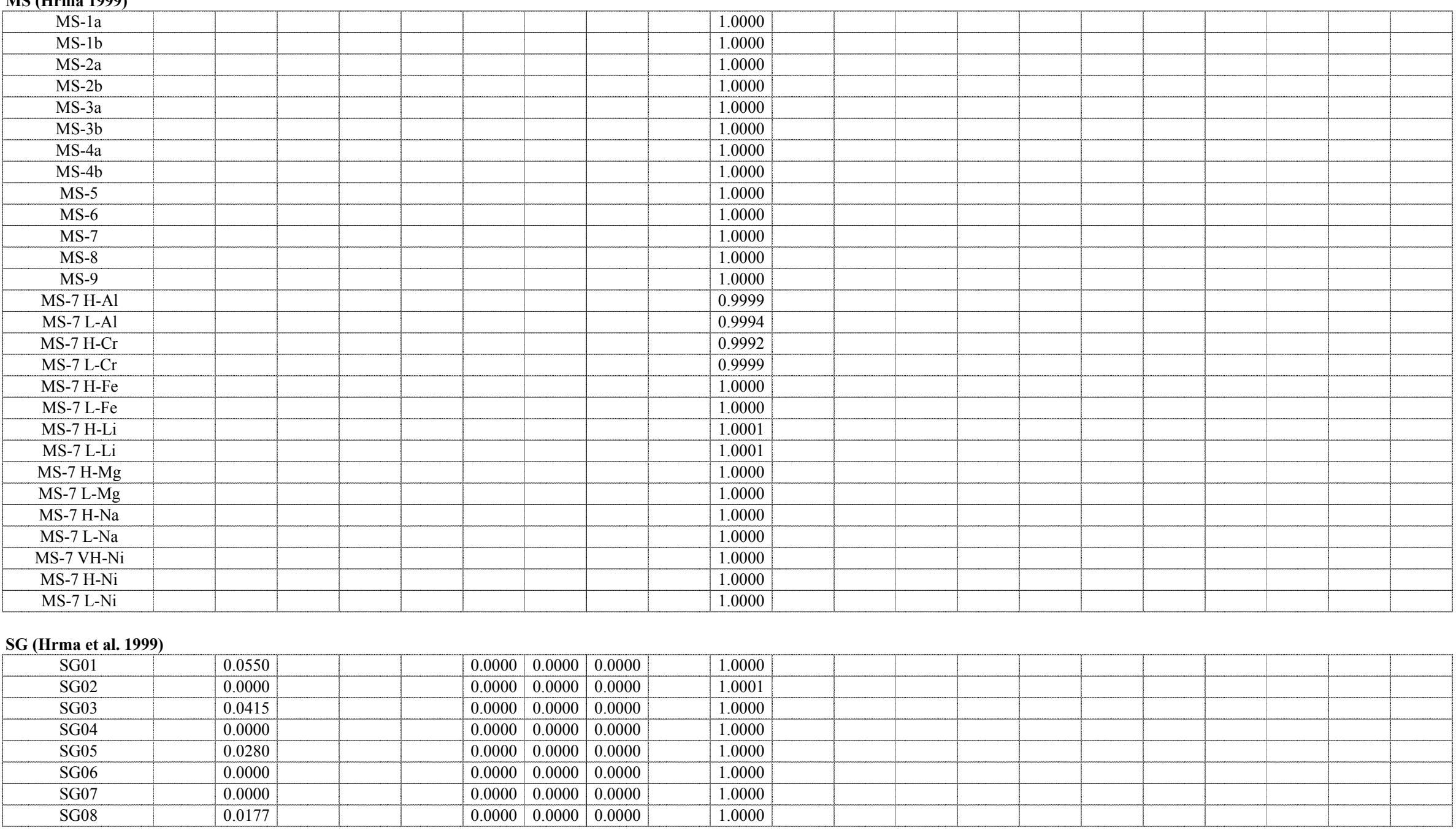


Appendix A. Database - mass fraction

SP (Mika et al. 1997)

\begin{tabular}{|c|c|c|c|c|c|c|c|c|c|c|c|c|c|c|c|c|c|c|c|c|c|}
\hline Glass ID & $\begin{array}{c}\mathrm{SiO} 2 \\
-\mathrm{a}\end{array}$ & $\begin{array}{c}\mathrm{ZrO} 2 \\
-\mathrm{a}\end{array}$ & $\begin{array}{c}\mathrm{Ag} 2 \mathrm{O} \\
-\mathrm{a}\end{array}$ & $\begin{array}{c}\mathrm{As} 2 \mathrm{O} 3 \\
-\mathrm{a}\end{array}$ & $\begin{array}{c}\mathrm{BaO} \\
-\mathrm{a}\end{array}$ & $\begin{array}{c}\mathrm{Bi} 2 \mathrm{O} 3 \\
-\mathrm{a}\end{array}$ & $\begin{array}{l}\mathrm{Br} \\
-\mathrm{a}\end{array}$ & $\begin{array}{c}\mathrm{CdO} \\
-\mathrm{a}\end{array}$ & $\begin{array}{c}\mathrm{Ce} 2 \mathrm{O} 3 \\
-\mathrm{a}\end{array}$ & $\begin{array}{c}\mathrm{CeO} 2 \\
-\mathrm{a}\end{array}$ & $\begin{array}{l}\mathrm{Cl} \\
-\mathrm{a}\end{array}$ & $\begin{array}{c}\mathrm{CoO} \\
-\mathrm{a}\end{array}$ & $\begin{array}{c}\mathrm{Co} 2 \mathrm{O} 3 \\
-\mathrm{a}\end{array}$ & $\begin{array}{c}\mathrm{Cr} 2 \mathrm{O} 3 \\
-\mathrm{a}\end{array}$ & $\begin{array}{c}\mathrm{Cs} 2 \mathrm{O} \\
-\mathrm{a}\end{array}$ & $\begin{array}{c}\mathrm{CuO} \\
-\mathrm{a}\end{array}$ & $\begin{array}{c}\text { Eu2O3 } \\
-a\end{array}$ & $\begin{array}{c}F \\
-a\end{array}$ & $\begin{array}{c}\mathrm{Ga} 2 \mathrm{O} 3 \\
-\mathrm{a}\end{array}$ & $\begin{array}{c}\mathrm{Gd} 2 \mathrm{O} 3 \\
-\mathrm{a}\end{array}$ & $\begin{array}{c}\mathrm{HgO} \\
-\mathrm{a}\end{array}$ \\
\hline SP-Na-2 & & & & & & & & & & & & & & & & & & & & & \\
\hline SP-Na-3 & & & & & & & & & & & & & & & & & & & & & \\
\hline SP-Ni-1 & & & & & & & & & & & & & & & & & & & & & \\
\hline SP-Ni-2 & & & & & & & & & & & & & & & & & & & & & \\
\hline SP-Ni-3 & & & & & & & & & & & & & & & & & & & & & \\
\hline SP-Si-1 & & & & & & & & & & & & & & & & & & & & & \\
\hline SP-Si-2 & & & & & & & & & & & & & & & & & & & & & \\
\hline SP-Si-3 & & & & & & & & & & & & & & & & & & & & & \\
\hline
\end{tabular}

\section{MS (Hrma 1999)}

MS-1a

MS-1b

MS-2a

MS- $2 \mathrm{~b}$

MS-3a

MS-3b

MS-4a

MS-4b

MS-5

MS-6

MS-7

MS-8

MS-9

MS-7 H-Al

MS-7 H-Cr

MS-7 L-Cr

MS-7 H-Fe

MS-7 L-Fe

MS-7 H-Li

MS-7 L-Li

MS-7 H-Mg

MS-7 L-Mg

MS-7 H-Na

MS-7 L-Na

MS-7 VH-Ni

MS-7 H-Ni

MS-7 L-Ni

\section{SG (Hrma et al. 1999)}

SG01

SG02

SG03

SG04

SG05

SG06

SG07

SG08

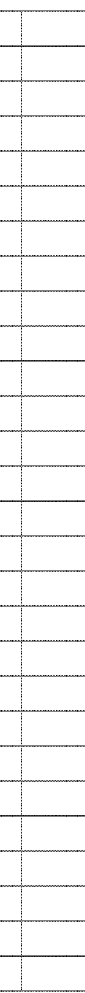

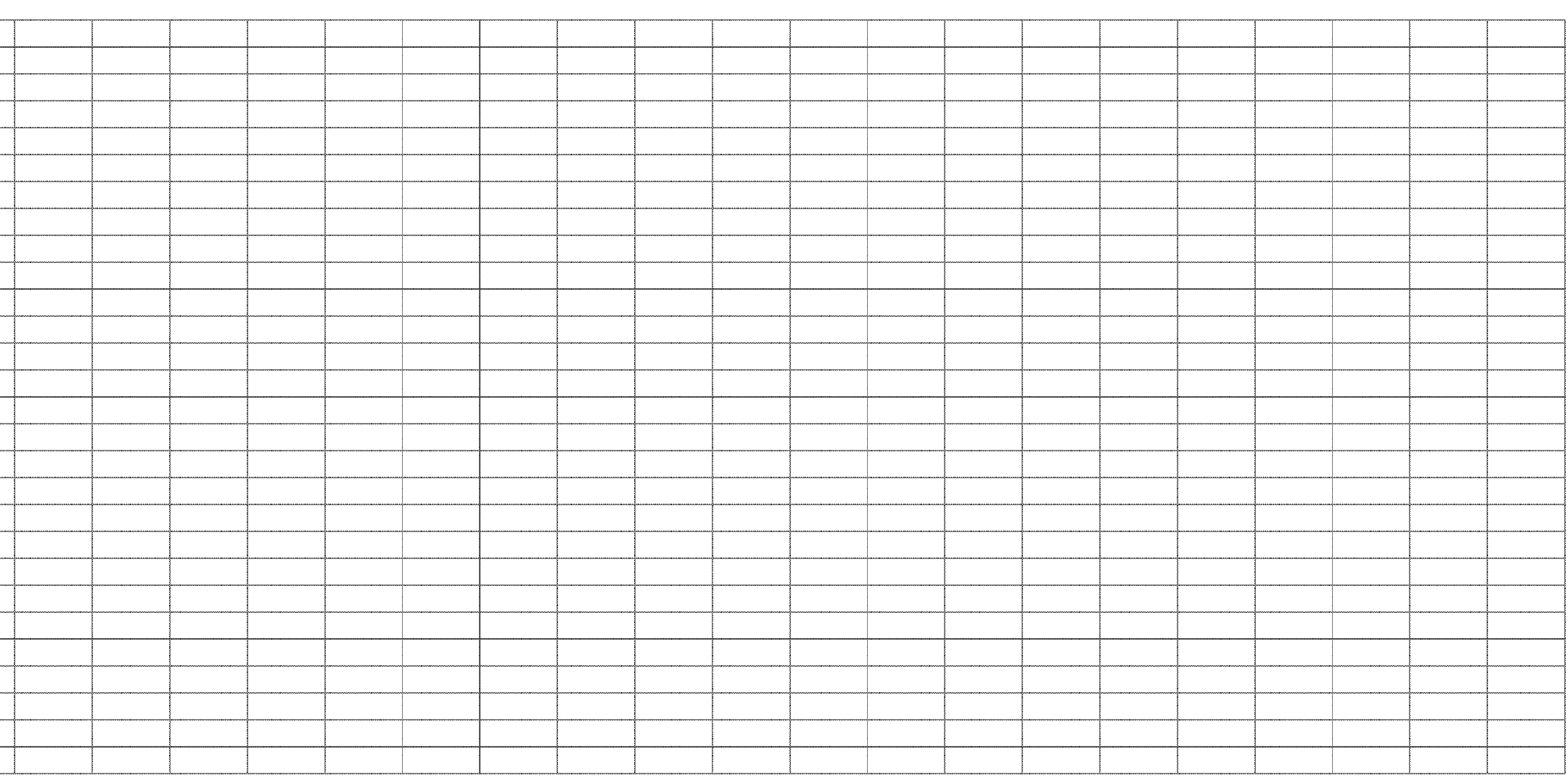

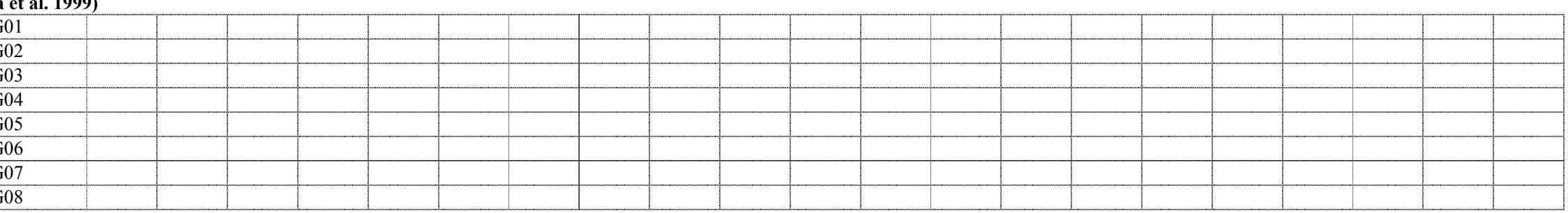




\section{SP (Mika et al. 1997)}

\begin{tabular}{|c|c|c|c|c|c|c|c|c|c|c|c|c|c|c|c|c|c|c|c|c|c|}
\hline Glass ID & $\begin{array}{c}\mathrm{I} \\
-\mathrm{a}\end{array}$ & $\begin{array}{c}\mathrm{La} 2 \mathrm{O} 3 \\
-\mathrm{a}\end{array}$ & $\begin{array}{c}\mathrm{MnO} 2 \\
-\mathrm{a} \\
\end{array}$ & $\begin{array}{c}\mathrm{MnO} \\
-\mathrm{a} \\
\end{array}$ & $\begin{array}{c}\mathrm{MoO} \\
-\mathrm{a}\end{array}$ & $\begin{array}{c}\mathrm{MoO} 3 \\
-\mathrm{a}\end{array}$ & $\begin{array}{c}\mathrm{Nb} 2 \mathrm{O} 5 \\
-\mathrm{a}\end{array}$ & $\begin{array}{c}\mathrm{Nd} 2 \mathrm{O} 3 \\
-\mathrm{a}\end{array}$ & $\begin{array}{c}\mathrm{PbO} \\
-\mathrm{a}\end{array}$ & $\begin{array}{c}\mathrm{PdO} 2 \\
-\mathrm{a} \\
\end{array}$ & $\begin{array}{c}\mathrm{PdO} \\
-\mathrm{a} \\
\end{array}$ & $\begin{array}{c}\mathrm{Pr} 2 \mathrm{O} 3 \\
-\mathrm{a}\end{array}$ & $\begin{array}{c}\text { Pr6O11 } \\
-\mathrm{a}\end{array}$ & $\begin{array}{c}\mathrm{Rb} 2 \mathrm{O} \\
-\mathrm{a}\end{array}$ & $\begin{array}{c}\mathrm{ReO} \\
-\mathrm{a} \\
\end{array}$ & $\begin{array}{c}\mathrm{ReO} 2 \\
-\mathrm{a}\end{array}$ & $\begin{array}{c}\mathrm{Rh} 2 \mathrm{O} 3 \\
-\mathrm{a}\end{array}$ & $\begin{array}{c}\mathrm{RhO} 2 \\
-\mathrm{a}\end{array}$ & $\begin{array}{c}\mathrm{RuO} 2 \\
-\mathrm{a}\end{array}$ & $\begin{array}{c}\mathrm{Sb} 2 \mathrm{O} 3 \\
-\mathrm{a}\end{array}$ & $\begin{array}{c}\mathrm{Sb} 2 \mathrm{O} 5 \\
-\mathrm{a}\end{array}$ \\
\hline SP-Na-2 & & & & & & & & & & & & & & & & & & & & & \\
\hline SP-Na-3 & & & & & & & & & & & & & & & & & & & & & \\
\hline SP-Ni-1 & & & & & & & & & & & & & & & & & & & & & \\
\hline SP-Ni-2 & & & & & & & & & & & & & & & & & & & & & \\
\hline SP-Ni-3 & & & & & & & & & & & & & & & & & & & & & \\
\hline SP-Si-1 & & & & & & & & & & & & & & & & & & & & & \\
\hline SP-Si-2 & & & & & & & & & & & & & & & & & & & & & \\
\hline SP-Si-3 & & & & & & & & & & & & & & & & & & & & & \\
\hline
\end{tabular}

\section{MS (Hrma 1999)}

MS-1a

MS-1b

MS-2a

MS-2b

MS-3a

MS-3b

MS-4a

MS- $4 \mathrm{~b}$

MS-5

MS-6

MS-7

MS-8

MS-9

MS-7 H-Al

MS-7 H-Cr

MS-7 L-Cr

MS-7 H-Fe

MS-7 L-Fe

MS-7 H-Li

MS-7 L-Li

MS-7 H-Mg

MS-7 L-Mg

MS-7 H-Na

MS-7 L-Na

MS-7 VH-N

MS-7 H-Ni

MS-7 L-Ni

\section{SG (Hrma et al. 1999)}

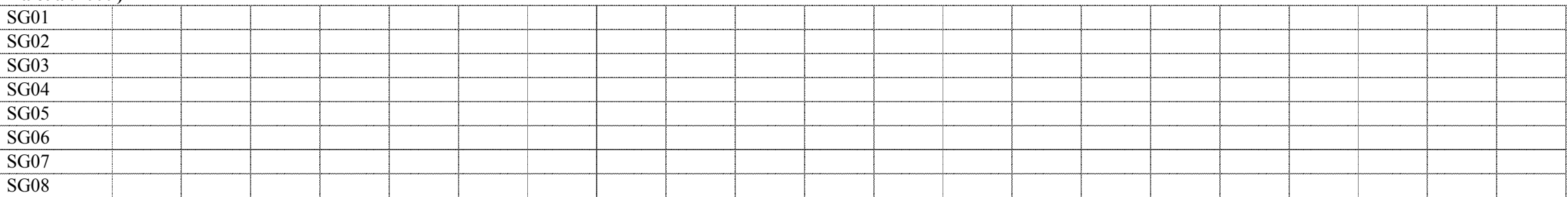


Appendix A. Database - mass fraction

SP (Mika et al. 1997)

\begin{tabular}{|c|c|c|c|c|c|c|c|c|c|c|c|c|c|c|c|c|c|c|c|c|}
\hline Glass ID & $\begin{array}{c}\mathrm{SeO} 2 \\
-\mathrm{a} \\
\end{array}$ & $\begin{array}{c}\mathrm{Sm} 2 \mathrm{O} 3 \\
-\mathrm{a} \\
\end{array}$ & $\begin{array}{c}\mathrm{SnO} \\
-\mathrm{a} \\
\end{array}$ & $\begin{array}{c}\mathrm{SnO} 2 \\
-\mathrm{a} \\
\end{array}$ & $\begin{array}{c}\mathrm{SO} 3 \\
-\mathrm{a} \\
\end{array}$ & $\begin{array}{c}\mathrm{SrO} \\
-\mathrm{a} \\
\end{array}$ & $\begin{array}{c}\mathrm{Tc} 2 \mathrm{O} 7 \\
-\mathrm{a}\end{array}$ & $\begin{array}{c}\mathrm{TeO} 2 \\
-\mathrm{a} \\
\end{array}$ & $\begin{array}{c}\mathrm{ThO} 2 \\
-\mathrm{a}\end{array}$ & $\begin{array}{c}\mathrm{TiO} 2 \\
-\mathrm{a} \\
\end{array}$ & $\begin{array}{c}\mathrm{T} 12 \mathrm{O} 3 \\
-\mathrm{a} \\
\end{array}$ & $\begin{array}{c}\mathrm{U} 3 \mathrm{O} 8 \\
-\mathrm{a}\end{array}$ & $\begin{array}{c}\mathrm{UO} 2 \\
-\mathrm{a}\end{array}$ & $\begin{array}{c}\text { UO3 } \\
-\mathrm{a} \\
\end{array}$ & $\begin{array}{c}\mathrm{V} 2 \mathrm{O} 5 \\
-\mathrm{a} \\
\end{array}$ & $\begin{array}{c}\text { WO3 } \\
-\mathrm{a} \\
\end{array}$ & $\begin{array}{c}\mathrm{Y} 2 \mathrm{O} 3 \\
-\mathrm{a}\end{array}$ & $\begin{array}{c}\mathrm{ZnO} \\
-\mathrm{a} \\
\end{array}$ & $\begin{array}{c}\text { Others } \\
-\mathrm{a}\end{array}$ & $\begin{array}{c}\text { Sum } \\
-\mathrm{a}\end{array}$ \\
\hline SP-Na-2 & & & & & & & & & & & & & & & & & & & & \\
\hline SP-Na-3 & & & & & & & & & & & & & & & & & & & & \\
\hline SP-Ni-1 & & & & & & & & & & & & & & & & & & & & \\
\hline SP-Ni-2 & & & & & & & & & & & & & & & & & & & & \\
\hline SP-Ni-3 & & & & & & & & & & & & & & & & & & & & \\
\hline SP-Si-1 & & & & & & & & & & & & & & & & & & & & \\
\hline SP-Si-2 & & & & & & & & & & & & & & & & & & & & \\
\hline SP-Si-3 & & & & & & & & & & & & & & & & & & & & \\
\hline
\end{tabular}

\section{MS (Hrma 1999)}

MS-1a

MS-1b

MS-2a

MS-2b

MS-3a

MS-3b

MS-4a

MS-4b

MS-5

MS-6

MS-7

MS-8

MS-9

MS-7 H-Al

MS-7 L-Al

MS-7 H-Cr

MS-7 L-Cr

MS-7 H-Fe

MS-7 L-Fe

MS-7 H-Li

MS-7 L-Li

MS-7 H-Mg

MS-7 L-Mg

MS-7 H-Na

MS-7 L-Na

MS-7 VH-N

MS-7 H-Ni

MS-7 L-Ni

\section{SG (Hrma et al. 1999)}

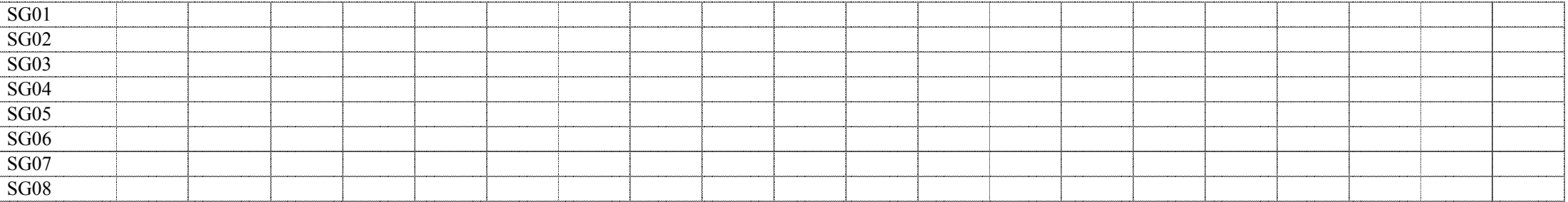


Appendix A. Database - mass fraction

SP (Mika et al. 1997)

\begin{tabular}{|c|c|c|c|c|c|c|c|c|}
\hline Glass ID & $\begin{array}{l}\mathrm{TM} \\
\left({ }^{\circ} \mathrm{C}\right) \\
\end{array}$ & $\begin{array}{l}\text { Gradient } \\
\text { TL }\left({ }^{\circ} \mathrm{C}\right) \\
\end{array}$ & $\begin{array}{l}\text { Uniform } \\
\text { TL }\left({ }^{\circ} \mathrm{C}\right) \\
\end{array}$ & Primary Phase & $\begin{array}{l}\text { Quenched } \\
\text { Visual/OM }\end{array}$ & $\begin{array}{c}\text { Quenched } \\
\text { SEM/EDS or TEM }\end{array}$ & $\begin{array}{l}\text { Quenched } \\
\text { XRD }\end{array}$ & $\begin{array}{c}\text { Quenched } \\
\text { Homogeneous? }\end{array}$ \\
\hline SP-Na-2 & & & 1147 & spinel & & & & \\
\hline SP-Na-3 & & & 941 & spinel & & & & \\
\hline SP-Ni-1 & & & 970 & spinel & & & & \\
\hline SP-Ni-2 & & & 1078 & spinel & & & & \\
\hline SP-Ni-3 & & & 1222 & spinel & & & & \\
\hline SP-Si-1 & & & 1107 & spinel & & & & \\
\hline SP-Si-2 & & & 1007 & spinel & & & & \\
\hline SP-Si-3 & & & 1017 & spinel & & & & \\
\hline
\end{tabular}

\section{MS (Hrma 1999)}

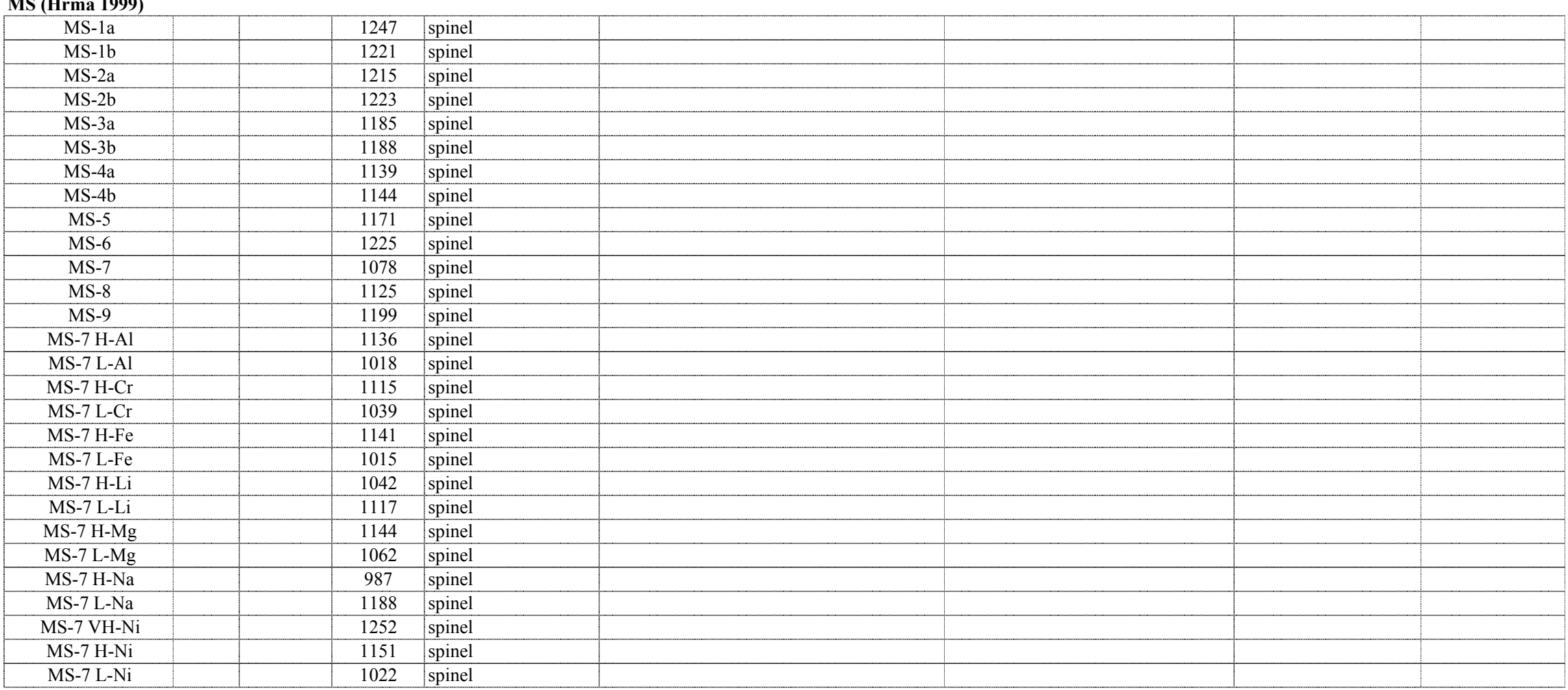

\section{SG (Hrma et al. 1999)}

\begin{tabular}{|l|l|l|l|l|l|l|}
\hline SG01 & 1107 & 1124 & spinel & & \\
\hline SG02 & 1150 & 775 & RuO2, clinopyroxene & & \\
\hline SG03 & 1200 & 1164 & spinel & & \\
\hline SG04 & 1250 & 1261 & spinel & & \\
\hline SG05 & 1250 & 1084 & spinel & & \\
\hline SG06 & 1322 & 911 & RuO2, spinel & & \\
\hline SG07 & 1294 & 950 & spinel & & \\
\hline SG08 & 1330 & 1114 & RuO2, spinel & & \\
\hline
\end{tabular}


Appendix A. Database - mass fraction

\section{SP (Mika et al. 1997)}

\begin{tabular}{|c|c|c|c|c|c|}
\hline Glass ID & $\begin{array}{c}\text { CCC } \\
\text { Visual/OM }\end{array}$ & $\begin{array}{c}\text { CCC } \\
\text { SEM/EDS or TEM }\end{array}$ & $\begin{array}{l}\text { CCC } \\
\text { XRD }\end{array}$ & $\begin{array}{c}\text { Heat Treated } \\
\text { Visual/OM }\end{array}$ & $\begin{array}{c}\text { Heat Treated } \\
\text { SEM/EDS or TEM }\end{array}$ \\
\hline \multicolumn{6}{|l|}{ SP-Na-2 } \\
\hline \multicolumn{6}{|l|}{ SP-Na-3 } \\
\hline \multicolumn{6}{|l|}{ SP-Ni-1 } \\
\hline \multicolumn{6}{|l|}{ SP-Ni-2 } \\
\hline \multicolumn{6}{|l|}{ SP-Ni-3 } \\
\hline \multicolumn{6}{|l|}{ SP-Si-1 } \\
\hline SP-Si-2 & & & & & \\
\hline SP-Si-3 & & & & & \\
\hline
\end{tabular}

MS (Hrma 1999)

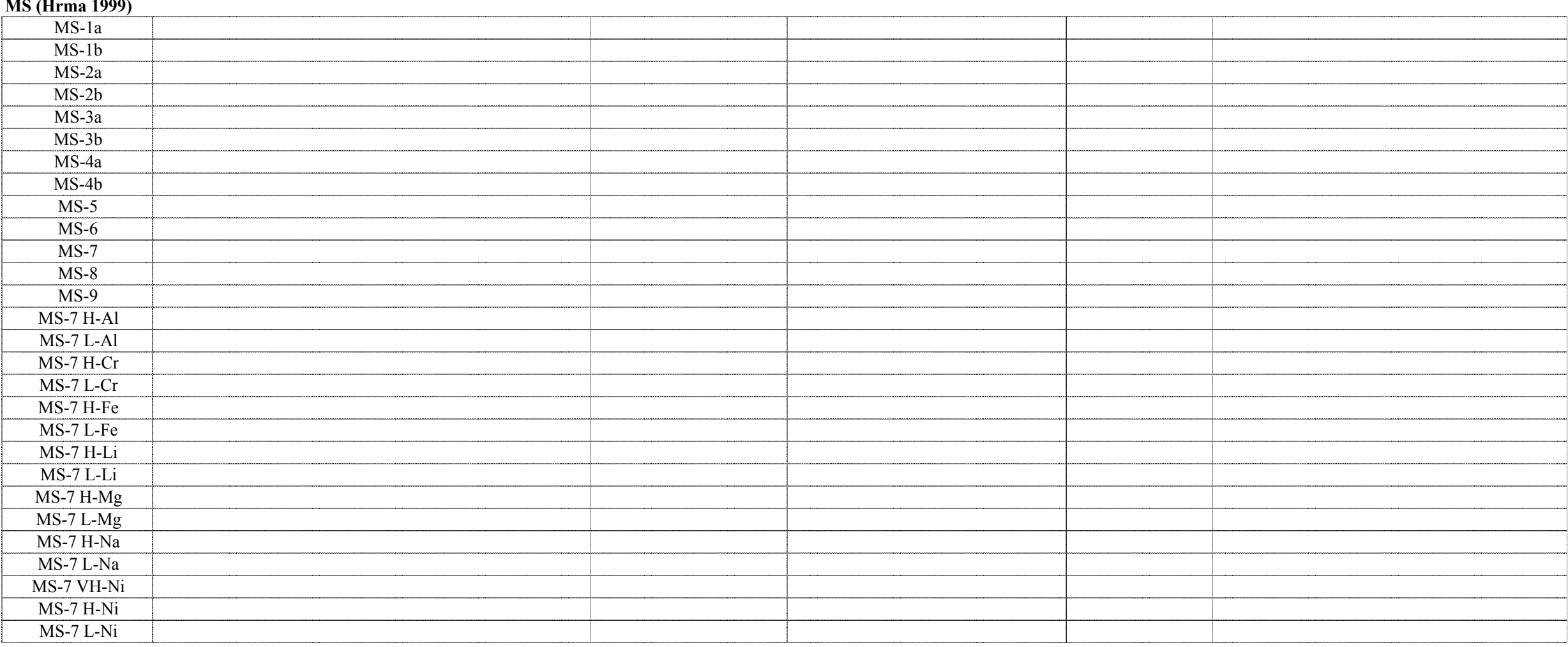

SG (Hrma et al. 1999)

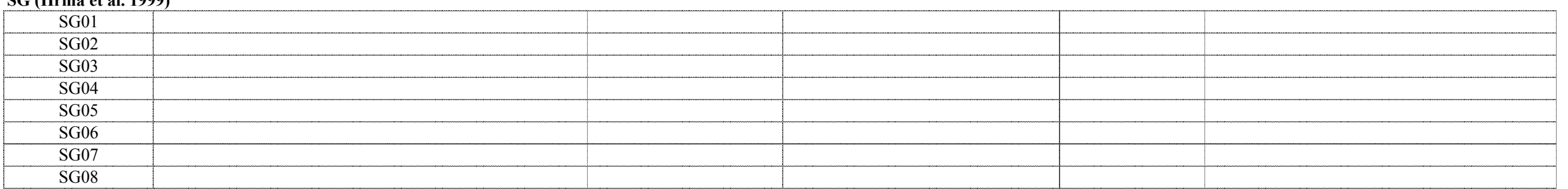


Appendix A. Database - mass fraction

\section{SP (Mika et al. 1997)}

\begin{tabular}{|c|c|c|c|c|c|c|c|c|c|c|c|c|c|c|c|}
\hline Glass ID & $\begin{array}{c}\text { Heat Treated } \\
\text { XRD }\end{array}$ & $\begin{array}{l}\text { Density } \\
\left(\mathrm{g} / \mathrm{cm}^{3}\right)\end{array}$ & $\begin{array}{c}\text { Fulc Visc } \\
\text { A }\end{array}$ & \begin{tabular}{|c|} 
Fulc Visc \\
B
\end{tabular} & \begin{tabular}{|c|} 
Fulc Visc \\
To \\
\end{tabular} & $\begin{array}{c}\mathrm{FV} 1150^{\circ} \mathrm{C} \\
(\mathrm{Pa} \cdot \mathrm{s})\end{array}$ & $\begin{array}{c}\text { Arrh Visc } \\
\text { A }\end{array}$ & $\begin{array}{c}\text { Arrh Visc } \\
\text { B } \\
\end{array}$ & $1150^{\circ} \mathrm{C}$ & $\begin{array}{c}\mathrm{T}\left({ }^{\circ} \mathrm{C}\right) \text { at } \\
2 \mathrm{~Pa} \cdot \mathrm{s} \\
\end{array}$ & $\begin{array}{c}\mathrm{T}\left({ }^{\circ} \mathrm{C}\right) \text { at } \\
5 \mathrm{~Pa} \cdot \mathrm{s}\end{array}$ & $\begin{array}{l}\mathrm{T}\left({ }^{\circ} \mathrm{C}\right) \text { at } \\
10 \mathrm{~Pa} \cdot \mathrm{s}\end{array}$ & $\begin{array}{l}\mathrm{T} 1 \\
\left({ }^{\circ} \mathrm{C}\right)\end{array}$ & $\begin{array}{c}\mathrm{V} 1 \\
(\mathrm{~Pa} \cdot \mathrm{s})\end{array}$ & $\begin{array}{l}\mathrm{T} 2 \\
\left({ }^{\circ} \mathrm{C}\right)\end{array}$ \\
\hline \multicolumn{16}{|l|}{ SP-Na-2 } \\
\hline \multicolumn{16}{|l|}{ SP-Na-3 } \\
\hline \multicolumn{16}{|l|}{ SP-Ni-1 } \\
\hline \multicolumn{16}{|l|}{ SP-Ni-2 } \\
\hline \multicolumn{16}{|l|}{ SP-Ni-3 } \\
\hline \multicolumn{16}{|l|}{ SP-Si-1 } \\
\hline SP-Si-2 & & & & & & & & & & & & & & & \\
\hline SP-Si-3 & & & & & & & & & & & & & & & \\
\hline
\end{tabular}

\section{MS (Hrma 1999)}

MS-1a

MS-1b

MS-2b

MS-3a

MS-3b

MS-4a

MS- $4 \mathrm{~b}$

MS-5

MS-6

MS-7

MS-8

MS-9

MS-7 H-Al

MS-7 H-Cr

MS-7 L-Cr

MS-7 H-Fe

MS-7 L-Fe

MS-7 H-Li

MS-7 L-Li

MS-7 H-Mg

MS-7 L-Mg

MS-7 H-Na

MS-7 L-Na

MS-7 VH-Ni

MS-7 H-Ni

MS-7 L-Ni

\section{SG (Hrma et al. 1999)}

$$
\mathrm{SG} 01
$$

$\mathrm{SG} 02$

$\mathrm{SG} 03$

SG04

SG05

SG06

SG07

SG08

Heat Treated

$\left[\begin{array}{ll}0 \\ 5\end{array}\right.$

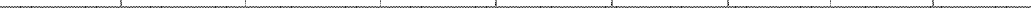


Appendix A. Database - mass fraction

\begin{tabular}{|c|c|c|c|c|c|c|c|c|c|c|c|c|c|c|c|c|c|c|c|c|c|}
\hline Glass ID & $\begin{array}{c}\mathrm{V} 2 \\
(\mathrm{~Pa} \cdot \mathrm{s})\end{array}$ & $\begin{array}{c}\mathrm{T} 3 \\
\left({ }^{\circ} \mathrm{C}\right)\end{array}$ & $\begin{array}{c}\text { V3 } \\
(\mathrm{Pa} \cdot \mathrm{s})\end{array}$ & $\begin{array}{l}\mathrm{T} 4 \\
\left({ }^{\circ} \mathrm{C}\right)\end{array}$ & $\begin{array}{c}\mathrm{V} 4 \\
(\mathrm{~Pa} \cdot \mathrm{s})\end{array}$ & $\begin{array}{l}\text { T5 } \\
\left({ }^{\circ} \mathrm{C}\right)\end{array}$ & $\begin{array}{c}\text { V5 } \\
(\mathrm{Pa} \cdot \mathrm{s})\end{array}$ & $\begin{array}{l}\text { T6 } \\
\left({ }^{\circ} \mathrm{C}\right)\end{array}$ & $\begin{array}{c}\mathrm{V} 6 \\
(\mathrm{~Pa} \cdot \mathrm{s})\end{array}$ & $\begin{array}{l}\mathrm{T} 7 \\
\left({ }^{\circ} \mathrm{C}\right)\end{array}$ & $\begin{array}{c}\mathrm{V} 7 \\
(\mathrm{~Pa} \cdot \mathrm{s})\end{array}$ & $\begin{array}{l}\mathrm{T} 8 \\
\left({ }^{\circ} \mathrm{C}\right)\end{array}$ & $\begin{array}{c}\mathrm{V} 8 \\
(\mathrm{~Pa} \cdot \mathrm{s})\end{array}$ & $\begin{array}{c}\mathrm{T} 9 \\
\left({ }^{\circ} \mathrm{C}\right)\end{array}$ & $\begin{array}{c}\text { V9 } \\
(\mathrm{Pa} \cdot \mathrm{s})\end{array}$ & $\begin{array}{l}\text { T10 } \\
\left({ }^{\circ} \mathrm{C}\right)\end{array}$ & $\begin{array}{c}\mathrm{V} 10 \\
(\mathrm{~Pa} \cdot \mathrm{s})\end{array}$ & $\begin{array}{l}\text { T11 } \\
\left({ }^{\circ} \mathrm{C}\right)\end{array}$ & $\begin{array}{c}\text { V11 } \\
(\mathrm{Pa} \cdot \mathrm{s})\end{array}$ & $\begin{array}{l}\mathrm{T} 12 \\
\left({ }^{\circ} \mathrm{C}\right)\end{array}$ & $\begin{array}{c}\mathrm{V} 12 \\
(\mathrm{~Pa} \cdot \mathrm{s})\end{array}$ \\
\hline SP-Na-2 & & & & & & & & & & & & & & & & & & & & & \\
\hline SP-Na-3 & & & & & & & & & & & & & & & & & & & & & \\
\hline SP-Ni-1 & & & & & & & & & & & & & & & & & & & & & \\
\hline SP-Ni-2 & & & & & & & & & & & & & & & & & & & & & \\
\hline SP-Ni-3 & & & & & & & & & & & & & & & & & & & & & \\
\hline SP-Si-1 & & & & & & & & & & & & & & & & & & & & & \\
\hline SP-Si-2 & & & & & & & & & & & & & & & & & & & & & \\
\hline SP-Si-3 & & & & & & & & & & & & & & & & & & & & & \\
\hline
\end{tabular}

\section{MS (Hrma 1999)}

MS-1a

MS-2a

MS-2b

MS-3a

MS-3b

MS-4a

MS-4b

MS-5

MS-6

MS-7

MS-8

MS-9

MS-7 H-Al

MS-7 H-Cr

MS-7 L-Cr

MS-7 H-Fe

MS-7 L-Fe

MS-7 H-Li

MS-7 L-Li

MS-7 H-Mg

MS-7 L-Mg

MS-7 H-Na

MS-7 L-Na

MS-7 VH-N

MS-7 H-Ni

MS-7 L-Ni

\section{SG (Hrma et al. 1999)}

$$
\text { SG01 }
$$

$\mathrm{SG} 02$

SG03

SG04

SG05

SG06

SG07

SG08

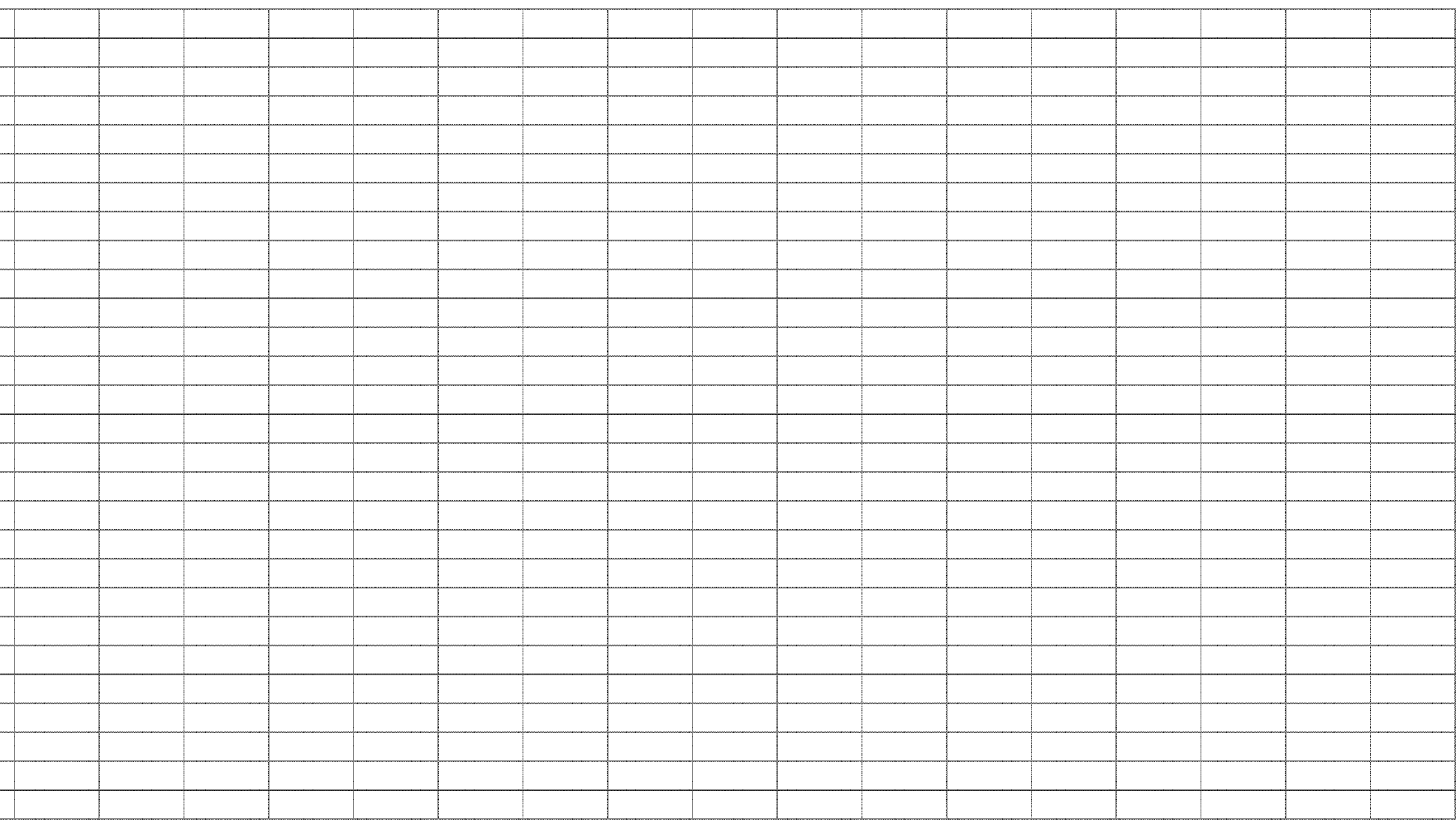


Appendix A. Database - mass fraction

\section{SP (Mika et al. 1997)}

\begin{tabular}{|c|c|c|c|c|c|c|c|c|c|c|c|c|c|c|c|c|}
\hline Glass ID & $\begin{array}{l}\mathrm{T} 13 \\
\left({ }^{\circ} \mathrm{C}\right)\end{array}$ & $\begin{array}{l}\text { V13 } \\
(\mathrm{Pa} \cdot \mathrm{s})\end{array}$ & $\begin{array}{l}\mathrm{T} 14 \\
\left({ }^{\circ} \mathrm{C}\right) \\
\end{array}$ & $\begin{array}{l}\text { V14 } \\
(\mathrm{Pa} \cdot \mathrm{s})\end{array}$ & $\begin{array}{c}\text { Q PCT } \\
\text { B }\left(\mathrm{g} / \mathrm{m}^{2}\right)\end{array}$ & $\begin{array}{c}\text { Q PCT } \\
\mathrm{Li}\left(\mathrm{g} / \mathrm{m}^{2}\right)\end{array}$ & $\begin{array}{c}\text { Q PCT } \\
\mathrm{Na}\left(\mathrm{g} / \mathrm{m}^{2}\right)\end{array}$ & $\begin{array}{c}\text { Q PCT } \\
\mathrm{Si}\left(\mathrm{g} / \mathrm{m}^{2}\right)\end{array}$ & $\begin{array}{c}\text { Q PCT } \\
\text { pH }\end{array}$ & $\begin{array}{c}\text { CCC PCT } \\
\mathrm{B}\left(\mathrm{g} / \mathrm{m}^{2}\right) \\
\end{array}$ & $\begin{array}{l}\text { CCC PCT } \\
\mathrm{Li}\left(\mathrm{g} / \mathrm{m}^{2}\right) \\
\end{array}$ & $\begin{array}{l}\text { CCC PCT } \\
\mathrm{Na}\left(\mathrm{g} / \mathrm{m}^{2}\right) \\
\end{array}$ & $\begin{array}{l}\text { CCC PCT } \\
\mathrm{Si}\left(\mathrm{g} / \mathrm{m}^{2}\right)\end{array}$ & $\begin{array}{c}\text { CCC PCT } \\
\mathrm{pH}\end{array}$ & $\begin{array}{l}\text { Q PCT B at } \\
20^{\circ} \mathrm{C}\left(\mathrm{g} / \mathrm{m}^{2}\right)\end{array}$ & $\begin{array}{l}\text { Q PCT Li at } \\
20^{\circ} \mathrm{C}\left(\mathrm{g} / \mathrm{m}^{2}\right)\end{array}$ \\
\hline \multicolumn{17}{|c|}{ 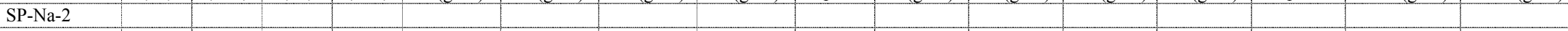 } \\
\hline \multicolumn{17}{|l|}{ SP-Na-3 } \\
\hline \multicolumn{17}{|l|}{ SP-Ni-1 } \\
\hline \multicolumn{17}{|l|}{ SP-Ni-2 } \\
\hline \multicolumn{17}{|l|}{ SP-Ni-3 } \\
\hline \multicolumn{17}{|l|}{ SP-Si-1 } \\
\hline SP-Si-2 & & & & & & & & & & & & & & & & \\
\hline SP-Si-3 & & & & & & & & & & & & & & & & \\
\hline
\end{tabular}

\section{MS (Hrma 1999)}

MS-1a

MS-1b

MS-2a

MS-2b

MS-3a

MS-3b

MS-4a

MS- $4 \mathrm{~b}$

MS-5

MS-6

MS-7

MS-8

MS-9

MS-7 H-Al

MS-7 H-Cr

MS-7 L-Cr

MS-7 H-Fe

MS-7 L-Fe

MS-7 H-Li

MS-7 L-Li

MS-7 H-Mg

MS-7 L-Mg

MS-7 H-Na

MS-7 L-Na

MS-7 VH-Ni

MS-7 H-Ni

MS-7 L-Ni

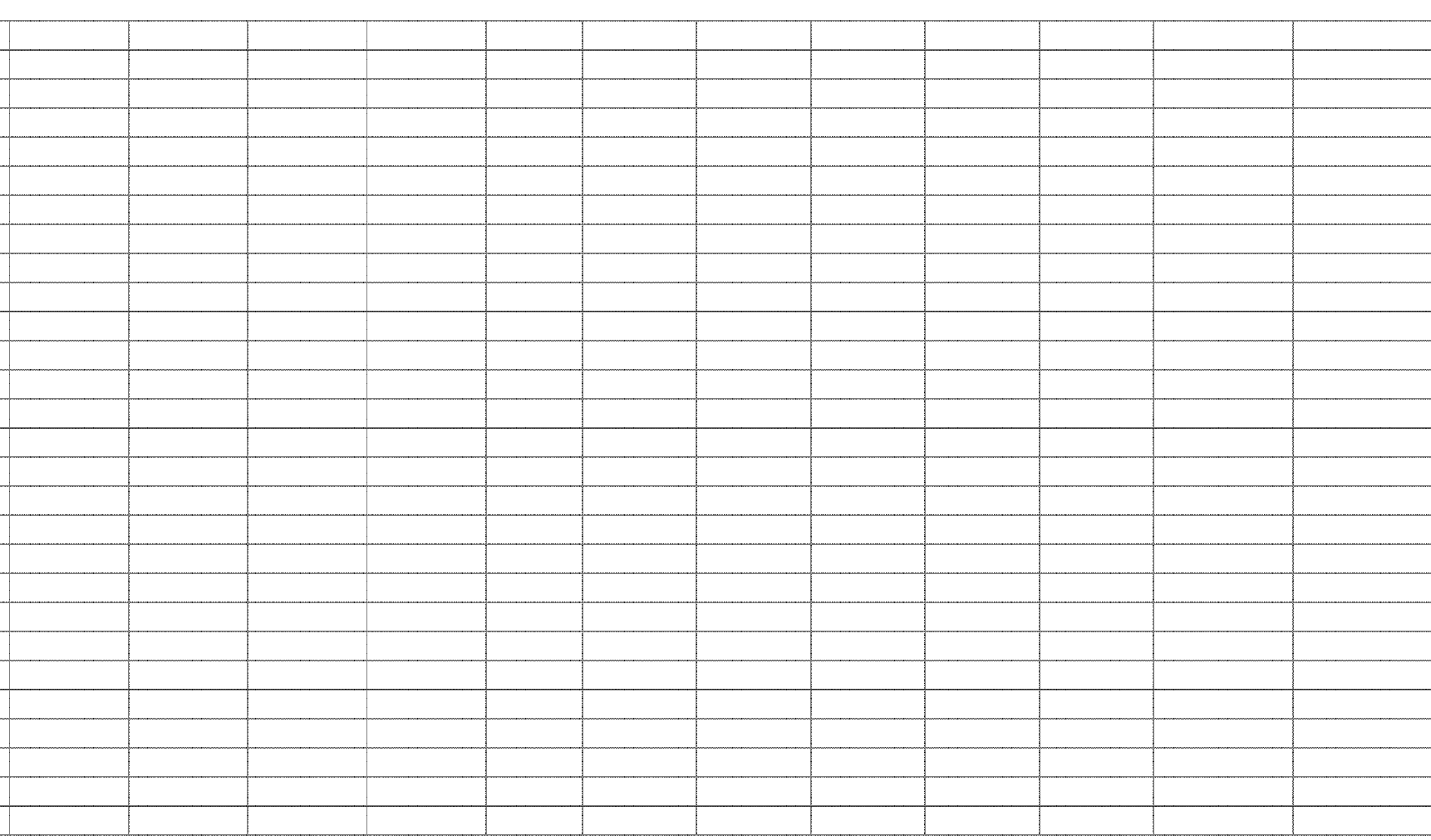

\section{SG (Hrma et al. 1999)}

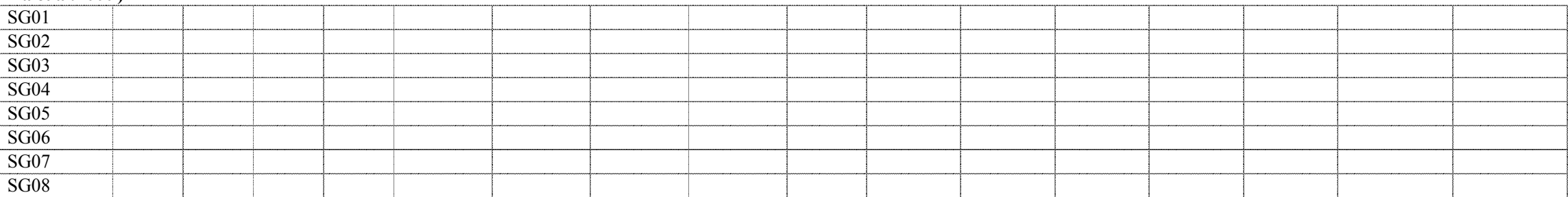


Appendix A. Database - mass fraction

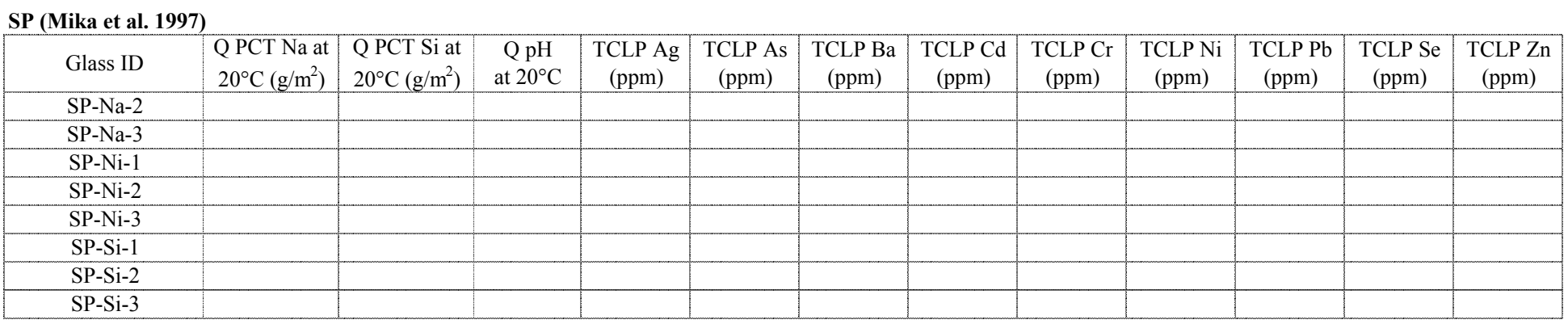

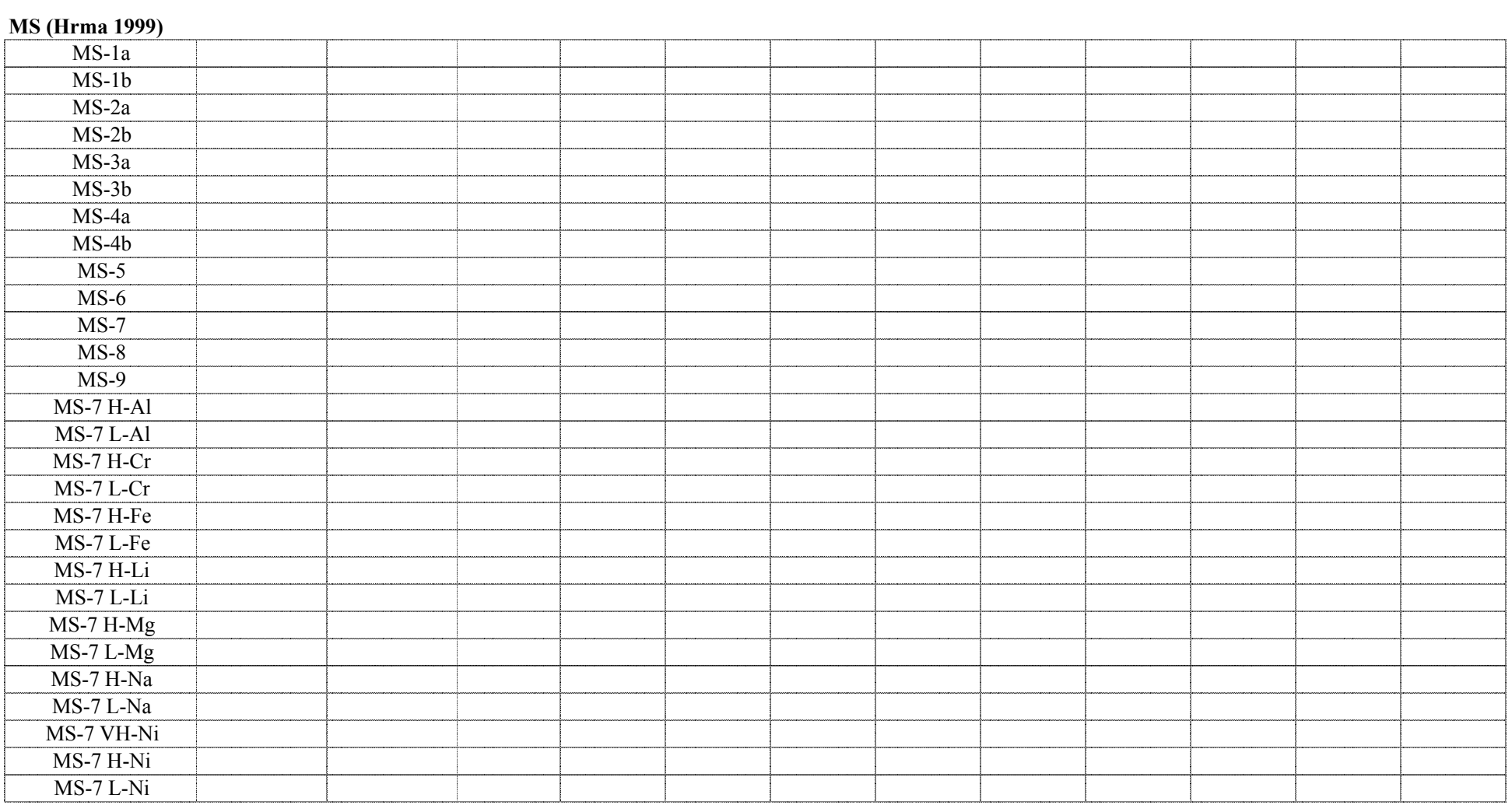

\section{SG (Hrma et al. 1999)}

\begin{tabular}{|c|c|c|c|c|c|c|c|c|}
\hline SG01 & & & & & & & & \\
\hline SG02 & & & & & & \\
\hline SG03 & & & & & & \\
\hline SG04 & & & & & & & \\
\hline SG05 & & & & & & \\
\hline SG06 & & & & & \\
\hline SG07 & & & & & & \\
\hline SG08 & & & & & \\
\hline
\end{tabular}




\begin{tabular}{|c|c|c|c|c|c|c|c|c|c|c|c|c|c|c|c|c|c|c|c|c|c|}
\hline Glass ID & $\begin{array}{c}\mathrm{Al} 2 \mathrm{O} 3 \\
-\mathrm{t}\end{array}$ & $\begin{array}{c}\mathrm{B} 2 \mathrm{O} 3 \\
-\mathrm{t}\end{array}$ & $\begin{array}{c}\mathrm{CaO} \\
-\mathrm{t}\end{array}$ & $\begin{array}{c}\mathrm{Fe} 2 \mathrm{O} 3 \\
-\mathrm{t}\end{array}$ & $\begin{array}{c}\mathrm{FeO} \\
-\mathrm{t}\end{array}$ & $\begin{array}{c}\mathrm{K} 2 \mathrm{O} \\
-\mathrm{t}\end{array}$ & $\begin{array}{c}\mathrm{Li} 2 \mathrm{O} \\
-\mathrm{t}\end{array}$ & $\begin{array}{c}\mathrm{MgO} \\
-\mathrm{t}\end{array}$ & $\begin{array}{c}\mathrm{Na} 2 \mathrm{O} \\
-\mathrm{t}\end{array}$ & $\begin{array}{c}\mathrm{NiO} \\
-\mathrm{t}\end{array}$ & $\begin{array}{c}\mathrm{P} 2 \mathrm{O} 5 \\
-\mathrm{t}\end{array}$ & $\begin{array}{c}\mathrm{SiO} 2 \\
-\mathrm{t}\end{array}$ & $\begin{array}{c}\mathrm{ZrO} 2 \\
-\mathrm{t}\end{array}$ & $\begin{array}{c}\mathrm{Ag} 2 \mathrm{O} \\
-\mathrm{t}\end{array}$ & $\begin{array}{c}\mathrm{As} 2 \mathrm{O} 3 \\
-\mathrm{t}\end{array}$ & $\begin{array}{c}\mathrm{BaO} \\
-\mathrm{t}\end{array}$ & $\begin{array}{c}\mathrm{Bi} 2 \mathrm{O} 3 \\
-\mathrm{t}\end{array}$ & $\begin{array}{c}\mathrm{Br} \\
-\mathrm{t}\end{array}$ & $\begin{array}{c}\mathrm{CdO} \\
-\mathrm{t}\end{array}$ & $\begin{array}{c}\mathrm{Ce} 2 \mathrm{O} 3 \\
-\mathrm{t}\end{array}$ & $\begin{array}{c}\mathrm{CeO} 2 \\
-\mathrm{t}\end{array}$ \\
\hline SG09 & 0.0799 & 0.0999 & 0.0200 & 0.1499 & & 0.0150 & 0.0599 & 0.0050 & 0.0599 & 0.0005 & 0.0000 & 0.4396 & 0.0000 & 0.0000 & 0.0000 & 0.0000 & 0.0000 & & 0.0000 & & 0.0000 \\
\hline SG10 & 0.0390 & 0.0626 & 0.0073 & 0.0825 & & 0.0323 & 0.0525 & 0.0200 & 0.0726 & 0.0151 & 0.0000 & 0.5437 & 0.0000 & 0.0000 & 0.0000 & 0.0000 & 0.0000 & & 0.0000 & & 0.0000 \\
\hline SG11 & 0.0390 & 0.0876 & 0.0073 & 0.0825 & & 0.0208 & 0.0525 & 0.0200 & 0.0976 & 0.0054 & 0.0000 & 0.5497 & 0.0000 & 0.0000 & 0.0000 & 0.0000 & 0.0000 & & 0.0000 & & 0.0000 \\
\hline SG12 & 0.0250 & 0.0500 & 0.0030 & 0.1499 & & 0.0150 & 0.0300 & 0.0250 & 0.1099 & 0.0005 & 0.0000 & 0.5765 & 0.0000 & 0.0000 & 0.0000 & 0.0000 & 0.0000 & & 0.0000 & & 0.0000 \\
\hline SG13 & 0.0250 & 0.0999 & 0.0030 & 0.0874 & & 0.0150 & 0.0599 & 0.0050 & 0.0599 & 0.0200 & 0.0000 & 0.5895 & 0.0000 & 0.0000 & 0.0000 & 0.0000 & 0.0000 & & 0.0000 & & 0.0000 \\
\hline SG14 & 0.0250 & 0.0999 & 0.0030 & 0.1499 & & 0.0380 & 0.0300 & 0.0250 & 0.1099 & 0.0005 & 0.0000 & 0.4306 & 0.0000 & 0.0000 & 0.0000 & 0.0000 & 0.0000 & & 0.0000 & & 0.0000 \\
\hline SG15 & 0.0250 & 0.0999 & 0.0200 & 0.0599 & & 0.0150 & 0.0599 & 0.0250 & 0.0599 & 0.0200 & 0.0000 & 0.5895 & 0.0000 & 0.0000 & 0.0000 & 0.0000 & 0.0000 & & 0.0000 & & 0.0000 \\
\hline SG16 & 0.0664 & 0.0626 & 0.0158 & 0.0825 & & 0.0208 & 0.0525 & 0.0200 & 0.0976 & 0.0054 & 0.0000 & 0.5026 & 0.0000 & 0.0000 & 0.0000 & 0.0000 & 0.0000 & & 0.0000 & & 0.0000 \\
\hline SG17 & 0.0390 & 0.0725 & 0.0158 & 0.1275 & & 0.0323 & 0.0525 & 0.0100 & 0.0976 & 0.0151 & 0.0000 & 0.4741 & 0.0000 & 0.0000 & 0.0000 & 0.0000 & 0.0000 & & 0.0000 & & 0.0000 \\
\hline SG18a & 0.0250 & 0.0999 & 0.0030 & 0.1499 & & 0.0150 & 0.0599 & 0.0050 & 0.1099 & 0.0005 & 0.0000 & 0.4921 & 0.0000 & 0.0000 & 0.0000 & 0.0000 & 0.0000 & & 0.0000 & & 0.0000 \\
\hline SG18b & 0.0250 & 0.0999 & 0.0030 & 0.1499 & & 0.0150 & 0.0599 & 0.0050 & 0.1099 & 0.0005 & 0.0000 & 0.4921 & 0.0000 & 0.0000 & 0.0000 & 0.0000 & 0.0000 & & 0.0000 & & 0.0000 \\
\hline SG18c & 0.0250 & 0.0999 & 0.0030 & 0.1499 & & 0.0150 & 0.0599 & 0.0050 & 0.1099 & 0.0005 & 0.0000 & 0.4921 & 0.0000 & 0.0000 & 0.0000 & 0.0000 & 0.0000 & & 0.0000 & & 0.0000 \\
\hline SG18d & 0.0250 & 0.0999 & 0.0030 & 0.1499 & & 0.0150 & 0.0599 & 0.0050 & 0.1099 & 0.0005 & 0.0000 & 0.4921 & 0.0000 & 0.0000 & 0.0000 & 0.0000 & 0.0000 & & 0.0000 & & 0.0000 \\
\hline SG18e & 0.0250 & 0.0999 & 0.0030 & 0.1499 & & 0.0150 & 0.0599 & 0.0050 & 0.1099 & 0.0005 & 0.0000 & 0.4921 & 0.0000 & 0.0000 & 0.0000 & 0.0000 & 0.0000 & & 0.0000 & & 0.0000 \\
\hline SG18f & 0.0250 & 0.0999 & 0.0030 & 0.1499 & & 0.0150 & 0.0599 & 0.0050 & 0.1099 & 0.0005 & 0.0000 & 0.4921 & 0.0000 & 0.0000 & 0.0000 & 0.0000 & 0.0000 & & 0.0000 & & 0.0000 \\
\hline SG18g & 0.0250 & 0.0999 & 0.0030 & 0.1499 & & 0.0150 & 0.0599 & 0.0050 & 0.1099 & 0.0005 & 0.0000 & 0.4921 & 0.0000 & 0.0000 & 0.0000 & 0.0000 & 0.0000 & & 0.0000 & & 0.0000 \\
\hline SG19 & 0.0799 & 0.0999 & 0.0030 & 0.0599 & & 0.0380 & 0.0599 & 0.0050 & 0.1099 & 0.0200 & 0.0000 & 0.4541 & 0.0000 & 0.0000 & 0.0000 & 0.0000 & 0.0000 & & 0.0000 & & 0.0000 \\
\hline SG20 & 0.0799 & 0.0500 & 0.0200 & 0.0599 & & 0.0150 & 0.0599 & 0.0250 & 0.1099 & 0.0005 & 0.0000 & 0.5070 & 0.0000 & 0.0000 & 0.0000 & 0.0000 & 0.0000 & & 0.0000 & & 0.0000 \\
\hline SG21 & 0390 & 0.0876 & 0.0158 & 0.0825 & & 0.0208 & 0.0525 & 0.0100 & 0.0726 & 0.0151 & 0.0000 & 0.5540 & 0.0000 & 0.0000 & 0.0000 & 0.0000 & 0.0000 & & 0.0000 & & 0.0000 \\
\hline SG22 & 0.0664 & 0.0626 & 0.0158 & 0.1275 & & 0.0208 & 0.0525 & 0.0100 & 0.0976 & 0.0151 & 0.0000 & 0.4931 & 0.0000 & 0.0000 & 0.0000 & 0.0000 & 0.0000 & & 0.0000 & & 0.0000 \\
\hline SG23 & 0.0417 & 0.0626 & 0.0158 & 0.0825 & & 0.0323 & 0.0375 & 0.0200 & 0.0976 & 0.0151 & 0.0000 & 0.5540 & 0.0000 & 0.0000 & 0.0000 & 0.0000 & 0.0000 & & 0.0000 & & 0.0000 \\
\hline SG24 & 0.0250 & 0.0500 & 0.0030 & 0.1194 & & 0.0150 & 0.0599 & 0.0050 & 0.0599 & 0.0005 & 0.0000 & 0.5895 & 0.0000 & 0.0000 & 0.0000 & 0.0000 & 0.0000 & & 0.0000 & & 0.0000 \\
\hline SG25 & 0.0799 & 0.0999 & 0.0030 & 0.1499 & & 0.0380 & 0.0300 & 0.0250 & 0.0599 & 0.0200 & 0.0000 & 0.4811 & 0.0000 & 0.0000 & 0.0000 & 0.0000 & 0.0000 & & 0.0000 & & 0.0000 \\
\hline SG26 & 0.0390 & 0.0626 & 0.0073 & 0.1275 & & 0.0208 & 0.0375 & 0.0100 & 0.0976 & 0.0054 & 0.0000 & 0.5276 & 0.0000 & 0.0000 & 0.0000 & 0.0000 & 0.0000 & & 0.0000 & & 0.0000 \\
\hline SG27 & 0.0664 & 0.0876 & 0.0158 & 0.1109 & & 0.0323 & 0.0525 & 0.0200 & 0.0726 & 0.0054 & 0.0000 & 0.4741 & 0.0000 & 0.0000 & 0.0000 & 0.0000 & 0.0000 & & 0.0000 & & 0.0000 \\
\hline SG28 & 0.0250 & 0.0999 & 0.0200 & 0.1499 & & 0.0150 & 0.0599 & 0.0050 & 0.1099 & 0.0005 & 0.0000 & 0.5015 & 0.0000 & 0.0000 & 0.0000 & 0.0000 & 0.0000 & & 0.0000 & & 0.0000 \\
\hline SG29 & 0.0799 & 0.0500 & 0.0030 & 0.0599 & & 0.0150 & 0.0599 & 0.0050 & 0.1099 & 0.0005 & 0.0000 & 0.5240 & 0.0000 & 0.0000 & 0.0000 & 0.0000 & 0.0000 & & 0.0000 & & 0.0000 \\
\hline SG30 & 0.0799 & 0.0500 & 0.0200 & 0.0599 & & 0.0380 & 0.0599 & 0.0250 & 0.1099 & 0.0200 & 0.0000 & 0.4491 & 0.0000 & 0.0000 & 0.0000 & 0.0000 & 0.0000 & & 0.0000 & & 0.0000 \\
\hline SG31 & 0.0799 & 0.0999 & 0.0200 & 0.1494 & & 0.0380 & 0.0599 & 0.0250 & 0.0599 & 0.0005 & 0.0000 & 0.4296 & 0.0000 & 0.0000 & 0.0000 & 0.0000 & 0.0000 & & 0.0000 & & 0.0000 \\
\hline SG32 & 0.0799 & 0.0999 & 0.0030 & 0.1499 & & 0.0150 & 0.0599 & 0.0050 & 0.1099 & 0.0200 & 0.0000 & 0.4396 & 0.0000 & 0.0000 & 0.0000 & 0.0000 & 0.0000 & & 0.0000 & & 0.0000 \\
\hline SG33 & 0.0799 & 0.0999 & 0.0200 & 0.0599 & & 0.0380 & 0.0599 & 0.0050 & 0.1099 & 0.0200 & 0.0000 & 0.4676 & 0.0000 & 0.0000 & 0.0000 & 0.0000 & 0.0000 & & 0.0000 & & 0.0000 \\
\hline SG34 & 0.0799 & 0.0999 & 0.0200 & 0.1454 & & 0.0150 & 0.0300 & 0.0250 & 0.0599 & 0.0005 & 0.0000 & 0.4296 & 0.0000 & 0.0000 & 0.0000 & 0.0000 & 0.0000 & & 0.0000 & & 0.0000 \\
\hline SG35 & 0.0799 & 0.0500 & 0.0030 & 0.1449 & & 0.0380 & 0.0599 & 0.0250 & 0.1099 & 0.0200 & 0.0000 & 0.4296 & 0.0000 & 0.0000 & 0.0000 & 0.0000 & 0.0000 & & 0.0000 & & 0.0000 \\
\hline SG36 & 0.0250 & 0.0999 & 0.0200 & 0.0599 & & 0.0380 & 0.0300 & 0.0050 & 0.1099 & 0.0005 & 0.0000 & 0.5415 & 0.0000 & 0.0000 & 0.0000 & 0.0000 & 0.0000 & & 0.0000 & & 0.0000 \\
\hline SG37 & 0.0250 & 0.0999 & 0.0200 & 0.0599 & & 0.0380 & 0.0599 & 0.0250 & 0.0599 & 0.0030 & 0.0000 & 0.5895 & 0.0000 & 0.0000 & 0.0000 & 0.0000 & 0.0000 & & 0.0000 & & 0.0000 \\
\hline SG38 & 0.0250 & 0.0999 & 0.0030 & 0.1464 & & 0.0380 & 0.0300 & 0.0250 & 0.1099 & 0.0005 & 0.0000 & 0.4296 & 0.0000 & 0.0000 & 0.0000 & 0.0000 & 0.0000 & & 0.0000 & & 0.0000 \\
\hline SG39 & 0.0250 & 0.0500 & 0.0200 & 0.1499 & & 0.0150 & 0.0300 & 0.0050 & 0.1099 & 0.0200 & 0.0000 & 0.5355 & 0.0000 & 0.0000 & 0.0000 & 0.0000 & 0.0000 & & 0.0000 & & 0.0000 \\
\hline SG40 & 0.0799 & 0.0999 & 0.0030 & 0.0599 & & 0.0150 & 0.0300 & 0.0250 & 0.1099 & 0.0200 & 0.0000 & 0.4826 & 0.0000 & 0.0000 & 0.0000 & 0.0000 & 0.0000 & & 0.0000 & & 0.0000 \\
\hline SG41 & 0.0799 & 0.0999 & 0.0200 & 0.1499 & & 0.0150 & 0.0300 & 0.0050 & 0.0599 & 0.0200 & 0.0000 & 0.4321 & 0.0000 & 0.0000 & 0.0000 & 0.0000 & 0.0000 & & 0.0000 & & 0.0000 \\
\hline SG42 & 0.0449 & 0.0876 & 0.0073 & 0.1275 & & 0.0323 & 0.0525 & 0.0200 & 0.0976 & 0.0054 & 0.0000 & 0.4741 & 0.0000 & 0.0000 & 0.0000 & 0.0000 & 0.0000 & & 0.0000 & & 0.0000 \\
\hline SG43 & 0.0664 & 0.0876 & 0.0073 & 0.0825 & & 0.0323 & 0.0375 & 0.0100 & 0.0976 & 0.0054 & 0.0000 & 0.5257 & 0.0000 & 0.0000 & 0.0000 & 0.0000 & 0.0000 & & 0.0000 & & 0.0000 \\
\hline SG44 & 0.0664 & 0.0876 & 0.0073 & 0.1275 & & 0.0208 & 0.0375 & 0.0200 & 0.0726 & 0.0151 & 0.0000 & 0.5052 & 0.0000 & 0.0000 & 0.0000 & 0.0000 & 0.0000 & & 0.0000 & & 0.0000 \\
\hline SG45 & 0.0250 & 0.0999 & 0.0200 & 0.0599 & & 0.0150 & 0.0300 & 0.0250 & 0.1099 & 0.0200 & 0.0000 & 0.5620 & 0.0000 & 0.0000 & 0.0000 & 0.0000 & 0.0000 & & 0.0000 & & 0.0000 \\
\hline
\end{tabular}




\section{SG (Hrma et al. 1999)}

\begin{tabular}{|c|c|c|c|c|c|c|c|c|c|c|c|c|c|c|c|c|c|c|c|c|c|}
\hline Glass ID & $\begin{array}{l}\mathrm{Cl} \\
-\mathrm{t}\end{array}$ & $\begin{array}{c}\mathrm{CoO} \\
-\mathrm{t}\end{array}$ & $\begin{array}{c}\mathrm{Co} 2 \mathrm{O} 3 \\
-\mathrm{t}\end{array}$ & $\begin{array}{c}\mathrm{Cr} 2 \mathrm{O} 3 \\
-\mathrm{t}\end{array}$ & $\begin{array}{c}\mathrm{Cs} 2 \mathrm{O} \\
-\mathrm{t}\end{array}$ & $\begin{array}{c}\mathrm{CuO} \\
-\mathrm{t}\end{array}$ & $\begin{array}{c}\mathrm{Eu} 2 \mathrm{O} 3 \\
-\mathrm{t}\end{array}$ & $\begin{array}{l}F \\
-t\end{array}$ & $\begin{array}{c}\mathrm{Ga} 2 \mathrm{O} 3 \\
-\mathrm{t}\end{array}$ & {$\left[\begin{array}{c}\mathrm{Gd} 2 \mathrm{O} 3 \\
-\mathrm{t}\end{array}\right.$} & $\begin{array}{c}\mathrm{HgO} \\
-\mathrm{t}\end{array}$ & $\begin{array}{l}I \\
-t\end{array}$ & $\begin{array}{c}\mathrm{La} 2 \mathrm{O} 3 \\
-\mathrm{t}\end{array}$ & $\begin{array}{c}\mathrm{MnO} 2 \\
-\mathrm{t}\end{array}$ & $\begin{array}{c}\mathrm{MnO} \\
-\mathrm{t}\end{array}$ & $\begin{array}{c}\mathrm{MoO} \\
-\mathrm{t}\end{array}$ & $\begin{array}{c}\mathrm{MoO} 3 \\
-\mathrm{t}\end{array}$ & $\begin{array}{c}\mathrm{Nb} 2 \mathrm{O} 5 \\
-\mathrm{t}\end{array}$ & $\begin{array}{c}\mathrm{Nd} 2 \mathrm{O} 3 \\
-\mathrm{t}\end{array}$ & $\begin{array}{c}\mathrm{PbO} \\
-\mathrm{t}\end{array}$ & $\begin{array}{c}\mathrm{PdO} 2 \\
-\mathrm{t}\end{array}$ \\
\hline SG09 & 0.0000 & & 0.0000 & 0.0030 & 0.0000 & 0.0000 & & 0.0000 & & & & & 0.0000 & & 0.0100 & & 0.0000 & & 0.0000 & 0.0000 & \\
\hline SG10 & 0.0000 & & 0.0000 & 0.0025 & 0.0000 & 0.0000 & & 0.0000 & & & & & 0.0000 & & 0.0250 & & 0.0000 & & 0.0000 & 0.0000 & \\
\hline SG11 & 0.0000 & & 0.0000 & 0.0015 & 0.0000 & 0.0000 & & 0.0000 & & & & & 0.0000 & & 0.0150 & & 0.0000 & & 0.0000 & 0.0000 & \\
\hline SG12 & 0.0000 & & 0.0000 & 0.0030 & 0.0000 & 0.0000 & & 0.0000 & & & & & 0.0000 & & 0.0100 & & 0.0000 & & 0.0000 & 0.0000 & \\
\hline SG13 & 0.0000 & & 0.0000 & 0.0030 & 0.0000 & 0.0000 & & 0.0000 & & & & & 0.0000 & & 0.0300 & & 0.0000 & & 0.0000 & 0.0000 & \\
\hline SG14 & 0.0000 & & 0.0000 & 0.0010 & 0.0000 & 0.0000 & & 0.0000 & & & & & 0.0000 & & 0.0300 & & 0.0000 & & 0.0000 & 0.0000 & \\
\hline SG15 & 0.0000 & & 0.0000 & 0.0010 & 0.0000 & 0.0000 & & 0.0000 & & & & & 0.0000 & & 0.0100 & & 0.0000 & & 0.0000 & 0.0000 & \\
\hline SG16 & 0.0000 & & 0.0000 & 0.0015 & 0.0000 & 0.0000 & & 0.0000 & & & & & 0.0000 & & 0.0250 & & 0.0000 & & 0.0000 & 0.0000 & \\
\hline SG17 & 0.0000 & & 0.0000 & 0.0015 & 0.0000 & 0.0000 & & 0.0000 & & & & & 0.0000 & & 0.0150 & & 0.0000 & & 0.0000 & 0.0000 & \\
\hline SG18a & 0.0000 & & 0.0000 & 0.0030 & 0.0000 & 0.0000 & & 0.0000 & & & & & 0.0000 & & 0.0300 & & 0.0000 & & 0.0000 & 0.0000 & \\
\hline SG18b & 0.0000 & & 0.0000 & 0.0030 & 0.0000 & 0.0000 & & 0.0000 & & & & & 0.0000 & & 0.0300 & & 0.0000 & & 0.0000 & 0.0000 & \\
\hline SG18c & 0.0000 & & 0.0000 & 0.0030 & 0.0000 & 0.0000 & & 0.0000 & & & & & 0.0000 & & 0.0300 & & 0.0000 & & 0.0000 & 0.0000 & \\
\hline SG18d & 0.0000 & & 0.0000 & 0.0030 & 0.0000 & 0.0000 & & 0.0000 & & & & & 0.0000 & & 0.0300 & & 0.0000 & & 0.0000 & 0.0000 & \\
\hline SG18e & 0.0000 & & 0.0000 & 0.0030 & 0.0000 & 0.0000 & & 0.0000 & & & & & 0.0000 & & 0.0300 & & 0.0000 & & 0.0000 & 0.0000 & \\
\hline SG18f & 0.0000 & & 0.0000 & 0.0030 & 0.0000 & 0.0000 & & 0.0000 & & & & & 0.0000 & & 0.0300 & & 0.0000 & & 0.0000 & 0.0000 & \\
\hline SG18g & 0.0000 & & 0.0000 & 0.0030 & 0.0000 & 0.0000 & & 0.0000 & & & & & 0.0000 & & 0.0300 & & 0.0000 & & 0.0000 & 0.0000 & \\
\hline SG19 & 0.0000 & & 0.0000 & 0.0030 & 0.0000 & 0.0000 & & 0.0000 & & & & & 0.0000 & & 0.0100 & & 0.0000 & & 0.0000 & 0.0000 & \\
\hline $\mathrm{SG} 20$ & 0.0000 & & 0.0000 & 0.0010 & 0.0000 & 0.0000 & & 0.0000 & & & & & 0.0000 & & 0.0100 & & 0.0000 & & 0.0000 & 0.0000 & \\
\hline SG21 & 0.0000 & & 0.0000 & 0.0025 & 0.0000 & 0.0000 & & 0.0000 & & & & & 0.0000 & & 0.0242 & & 0.0000 & & 0.0000 & 0.0000 & \\
\hline SG22 & 0.0000 & & 0.0000 & 0.0025 & 0.0000 & 0.0000 & & 0.0000 & & & & & 0.0000 & & 0.0150 & & 0.0000 & & 0.0000 & 0.0000 & \\
\hline $\mathrm{SG} 23$ & 0.0000 & & 0.0000 & 0.0025 & 0.0000 & 0.0000 & & 0.0000 & & & & & 0.0000 & & 0.0150 & & 0.0000 & & 0.0000 & 0.0000 & \\
\hline SG24 & 0.0000 & & 0.0000 & 0.0010 & 0.0000 & 0.0000 & & 0.0000 & & & & & 0.0000 & & 0.0100 & & 0.0000 & & 0.0000 & 0.0000 & \\
\hline SG25 & 0.0000 & & 0.0000 & 0.0010 & 0.0000 & 0.0000 & & 0.0000 & & & & & 0.0000 & & 0.0100 & & 0.0000 & & 0.0000 & 0.0000 & \\
\hline SG26 & 0.0000 & & 0.0000 & 0.0025 & 0.0000 & 0.0000 & & 0.0000 & & & & & 0.0000 & & 0.0150 & & 0.0000 & & 0.0000 & 0.0000 & \\
\hline SG27 & 0.0000 & & 0.0000 & 0.0025 & 0.0000 & 0.0000 & & 0.0000 & & & & & 0.0000 & & 0.0150 & & 0.0000 & & 0.0000 & 0.0000 & \\
\hline SG28 & 0.0000 & & 0.0000 & 0.0010 & 0.0000 & 0.0000 & & 0.0000 & & & & & 0.0000 & & 0.0100 & & 0.0000 & & 0.0000 & 0.0000 & \\
\hline SG29 & 0.0000 & & 0.0000 & 0.0010 & 0.0000 & 0.0000 & & 0.0000 & & & & & 0.0000 & & 0.0300 & & 0.0000 & & 0.0000 & 0.0000 & \\
\hline SG30 & 0.0000 & & 0.0000 & 0.0010 & 0.0000 & 0.0000 & & 0.0000 & & & & & 0.0000 & & 0.0300 & & 0.0000 & & 0.0000 & 0.0000 & \\
\hline SG31 & 0.0000 & & 0.0000 & 0.0010 & 0.0000 & 0.0000 & & 0.0000 & & & & & 0.0000 & & 0.0300 & & 0.0000 & & 0.0000 & 0.0000 & \\
\hline SG32 & 0.0000 & & 0.0000 & 0.0010 & 0.0000 & 0.0000 & & 0.0000 & & & & & 0.0000 & & 0.0100 & & 0.0000 & & 0.0000 & 0.0000 & \\
\hline SG33 & 0.0000 & & 0.0000 & 0.0030 & 0.0000 & 0.0000 & & 0.0000 & & & & & 0.0000 & & 0.0300 & & 0.0000 & & 0.0000 & 0.0000 & \\
\hline SG34 & 0.0000 & & 0.0000 & 0.0030 & 0.0000 & 0.0000 & & 0.0000 & & & & & 0.0000 & & 0.0300 & & 0.0000 & & 0.0000 & 0.0000 & \\
\hline SG35 & 0.0000 & & 0.0000 & 0.0030 & 0.0000 & 0.0000 & & 0.0000 & & & & & 0.0000 & & 0.0300 & & 0.0000 & & 0.0000 & 0.0000 & \\
\hline SG36 & 0.0000 & & 0.0000 & 0.0030 & 0.0000 & 0.0000 & & 0.0000 & & & & & 0.0000 & & 0.0100 & & 0.0000 & & 0.0000 & 0.0000 & \\
\hline SG37 & 0.0000 & & 0.0000 & 0.0030 & 0.0000 & 0.0000 & & 0.0000 & & & & & 0.0000 & & 0.0100 & & 0.0000 & & 0.0000 & 0.0000 & \\
\hline SG38 & 0.0000 & & 0.0000 & 0.0010 & 0.0000 & 0.0000 & & 0.0000 & & & & & 0.0000 & & 0.0300 & & 0.0000 & & 0.0000 & 0.0000 & \\
\hline SG39 & 0.0000 & & 0.0000 & 0.0030 & 0.0000 & 0.0000 & & 0.0000 & & & & & 0.0000 & & 0.0300 & & 0.0000 & & 0.0000 & 0.0000 & \\
\hline SG40 & 0.0000 & & 0.0000 & 0.0030 & 0.0000 & 0.0000 & & 0.0000 & & & & & 0.0000 & & 0.0100 & & 0.0000 & & 0.0000 & 0.0000 & \\
\hline SG41 & 0.0000 & & 0.0000 & 0.0010 & 0.0000 & 0.0000 & & 0.0000 & & & & & 0.0000 & & 0.0300 & & 0.0000 & & 0.0000 & 0.0000 & \\
\hline SG42 & 0.0000 & & 0.0000 & 0.0025 & 0.0000 & 0.0000 & & 0.0000 & & & & & 0.0000 & & 0.0250 & & 0.0000 & & 0.0000 & 0.0000 & \\
\hline SG43 & 0.0000 & & 0.0000 & 0.0015 & 0.0000 & 0.0000 & & 0.0000 & & & & & 0.0000 & & 0.0250 & & 0.0000 & & 0.0000 & 0.0000 & \\
\hline SG44 & 0.0000 & & 0.0000 & 0.0015 & 0.0000 & 0.0000 & & 0.0000 & & & & & 0.0000 & & 0.0150 & & 0.0000 & & 0.0000 & 0.0000 & \\
\hline SG45 & 0.0000 & & 0.0000 & 0.0010 & 0.0000 & 0.0000 & & 0.0000 & & & & & 0.0000 & & 0.0300 & & 0.0000 & & 0.0000 & 0.0000 & \\
\hline
\end{tabular}


SG (Hrma et al. 1999)

\begin{tabular}{|c|c|c|c|c|c|c|c|c|c|c|c|c|c|c|c|c|c|c|c|c|c|}
\hline Glass ID & $\begin{array}{c}\mathrm{PdO} \\
-\mathrm{t}\end{array}$ & $\begin{array}{c}\mathrm{Pr} 2 \mathrm{O} 3 \\
-\mathrm{t}\end{array}$ & $\begin{array}{c}\text { Pr6O11 } \\
-\mathrm{t}\end{array}$ & $\begin{array}{c}\mathrm{Rb} 2 \mathrm{O} \\
-\mathrm{t}\end{array}$ & $\begin{array}{c}\mathrm{ReO} \\
-\mathrm{t}\end{array}$ & $\begin{array}{c}\mathrm{ReO} 2 \\
-\mathrm{t}\end{array}$ & $\begin{array}{c}\mathrm{Rh} 2 \mathrm{O} 3 \\
-\mathrm{t}\end{array}$ & $\begin{array}{c}\mathrm{RhO}^{2} \\
-\mathrm{t}\end{array}$ & $\begin{array}{c}\mathrm{RuO} 2 \\
-\mathrm{t}\end{array}$ & $\begin{array}{c}\mathrm{Sb} 2 \mathrm{O} 3 \\
-\mathrm{t}\end{array}$ & $\begin{array}{c}\mathrm{Sb} 2 \mathrm{O} 5 \\
-\mathrm{t}\end{array}$ & $\begin{array}{c}\mathrm{SeO} 2 \\
-\mathrm{t}\end{array}$ & $\begin{array}{c}\mathrm{Sm} 2 \mathrm{O} 3 \\
-\mathrm{t}\end{array}$ & $\begin{array}{c}\mathrm{SnO} \\
-\mathrm{t}\end{array}$ & $\begin{array}{c}\mathrm{SnO} 2 \\
-\mathrm{t}\end{array}$ & $\begin{array}{c}\mathrm{SO} 3 \\
-\mathrm{t}\end{array}$ & $\begin{array}{c}\mathrm{SrO} \\
-\mathrm{t}\end{array}$ & $\begin{array}{c}\mathrm{Tc} 2 \mathrm{O} 7 \\
-\mathrm{t}\end{array}$ & $\begin{array}{c}\mathrm{TeO} 2 \\
-\mathrm{t}\end{array}$ & $\begin{array}{c}\mathrm{ThO} 2 \\
-\mathrm{t}\end{array}$ & $\begin{array}{c}\mathrm{TiO} 2 \\
-\mathrm{t}\end{array}$ \\
\hline SG09 & 0.0000 & & 0.0000 & 0.0000 & & & 0.0000 & & 0.0009 & 0.0000 & & 0.0000 & 0.0000 & & & 0.0000 & 0.0000 & & 0.0000 & & 0.0015 \\
\hline SG10 & 0.0000 & & 0.0000 & 0.0000 & & & 0.0000 & & 0.0009 & 0.0000 & & 0.0000 & 0.0000 & & & 0.0000 & 0.0000 & & 0.0000 & & 0.0026 \\
\hline SG11 & 0.0000 & & 0.0000 & 0.0000 & & & 0.0000 & & 0.0009 & 0.0000 & & 0.0000 & 0.0000 & & & 0.0000 & 0.0000 & & 0.0000 & & 0.0026 \\
\hline SG12 & 0.0000 & & 0.0000 & 0.0000 & & & 0.0000 & & 0.0009 & 0.0000 & & 0.0000 & 0.0000 & & & 0.0000 & 0.0000 & & 0.0000 & & 0.0015 \\
\hline SG13 & 0.0000 & & 0.0000 & 0.0000 & & & 0.0000 & & 0.0009 & 0.0000 & & 0.0000 & 0.0000 & & & 0.0000 & 0.0000 & & 0.0000 & & 0.0015 \\
\hline SG14 & 0.0000 & & 0.0000 & 0.0000 & & & 0.0000 & & 0.0009 & 0.0000 & & 0.0000 & 0.0000 & & & 0.0000 & 0.0000 & & 0.0000 & & 0.0015 \\
\hline SG15 & 0.0000 & & 0.0000 & 0.0000 & & & 0.0000 & & 0.0009 & 0.0000 & & 0.0000 & 0.0000 & & & 0.0000 & 0.0000 & & 0.0000 & & 0.0060 \\
\hline SG16 & 0.0000 & & 0.0000 & 0.0000 & & & 0.0000 & & 0.0009 & 0.0000 & & 0.0000 & 0.0000 & & & 0.0000 & 0.0000 & & 0.0000 & & 0.0049 \\
\hline SG17 & 0.0000 & & 0.0000 & 0.0000 & & & 0.0000 & & 0.0009 & 0.0000 & & 0.0000 & 0.0000 & & & 0.0000 & 0.0000 & & 0.0000 & & 0.0049 \\
\hline SG18a & 0.0000 & & 0.0000 & 0.0000 & & & 0.0000 & & 0.0009 & 0.0000 & & 0.0000 & 0.0000 & & & 0.0000 & 0.0000 & & 0.0000 & & 0.0060 \\
\hline SG18b & 0.0000 & & 0.0000 & 0.0000 & & & 0.0000 & & 0.0009 & 0.0000 & & 0.0000 & 0.0000 & & & 0.0000 & 0.0000 & & 0.0000 & & 0.0060 \\
\hline SG18c & 0.0000 & & 0.0000 & 0.0000 & & & 0.0000 & & 0.0009 & 0.0000 & & 0.0000 & 0.0000 & & & 0.0000 & 0.0000 & & 0.0000 & & 0.0060 \\
\hline SG18d & 0.0000 & & 0.0000 & 0.0000 & & & 0.0000 & & 0.0009 & 0.0000 & & 0.0000 & 0.0000 & & & 0.0000 & 0.0000 & & 0.0000 & & 0.0060 \\
\hline SG18e & 0.0000 & & 0.0000 & 0.0000 & & & 0.0000 & & 0.0009 & 0.0000 & & 0.0000 & 0.0000 & & & 0.0000 & 0.0000 & & 0.0000 & & 0.0060 \\
\hline SG18f & 0.0000 & & 0.0000 & 0.0000 & & & 0.0000 & & 0.0009 & 0.0000 & & 0.0000 & 0.0000 & & & 0.0000 & 0.0000 & & 0.0000 & & 0.0060 \\
\hline SG18g & 0.0000 & & 0.0000 & 0.0000 & & & 0.0000 & & 0.0009 & 0.0000 & & 0.0000 & 0.0000 & & & 0.0000 & 0.0000 & & 0.0000 & & 0.0060 \\
\hline SG19 & 0.0000 & & 0.0000 & 0.0000 & & & 0.0000 & & 0.0009 & 0.0000 & & 0.0000 & 0.0000 & & & 0.0000 & 0.0000 & & 0.0000 & & 0.0015 \\
\hline SG20 & 0.0000 & & 0.0000 & 0.0000 & & & 0.0000 & & 0.0009 & 0.0000 & & 0.0000 & 0.0000 & & & 0.0000 & 0.0000 & & 0.0000 & & 0.0060 \\
\hline $\mathrm{SG} 21$ & 0.0000 & & 0.0000 & 0.0000 & & & 0.0000 & & 0.0009 & 0.0000 & & 0.0000 & 0.0000 & & & 0.0000 & 0.0000 & & 0.0000 & & 0.0049 \\
\hline SG22 & 0.0000 & & 0.0000 & 0.0000 & & & 0.0000 & & 0.0009 & 0.0000 & & 0.0000 & 0.0000 & & & 0.0000 & 0.0000 & & 0.0000 & & 0.0026 \\
\hline SG23 & 0.0000 & & 0.0000 & 0.0000 & & & 0.0000 & & 0.0009 & 0.0000 & & 0.0000 & 0.0000 & & & 0.0000 & 0.0000 & & 0.0000 & & 0.0049 \\
\hline SG24 & 0.0000 & & 0.0000 & 0.0000 & & & 0.0000 & & 0.0009 & 0.0000 & & 0.0000 & 0.0000 & & & 0.0000 & 0.0000 & & 0.0000 & & 0.0060 \\
\hline SG25 & 0.0000 & & 0.0000 & 0.0000 & & & 0.0000 & & 0.0009 & 0.0000 & & 0.0000 & 0.0000 & & & 0.0000 & 0.0000 & & 0.0000 & & 0.0015 \\
\hline SG26 & 0.0000 & & 0.0000 & 0.0000 & & & 0.0000 & & 0.0009 & 0.0000 & & 0.0000 & 0.0000 & & & 0.0000 & 0.0000 & & 0.0000 & & 0.0049 \\
\hline SG27 & 0.0000 & & 0.0000 & 0.0000 & & & 0.0000 & & 0.0009 & 0.0000 & & 0.0000 & 0.0000 & & & 0.0000 & 0.0000 & & 0.0000 & & 0.0026 \\
\hline SG28 & 0.0000 & & 0.0000 & 0.0000 & & & 0.0000 & & 0.0009 & 0.0000 & & 0.0000 & 0.0000 & & & 0.0000 & 0.0000 & & 0.0000 & & 0.0015 \\
\hline SG29 & 0.0000 & & 0.0000 & 0.0000 & & & 0.0000 & & 0.0009 & 0.0000 & & 0.0000 & 0.0000 & & & 0.0000 & 0.0000 & & 0.0000 & & 0.0060 \\
\hline SG30 & 0.0000 & & 0.0000 & 0.0000 & & & 0.0000 & & 0.0009 & 0.0000 & & 0.0000 & 0.0000 & & & 0.0000 & 0.0000 & & 0.0000 & & 0.0015 \\
\hline SG31 & 0.0000 & & 0.0000 & 0.0000 & & & 0.0000 & & 0.0009 & 0.0000 & & 0.0000 & 0.0000 & & & 0.0000 & 0.0000 & & 0.0000 & & 0.0060 \\
\hline SG32 & 0.0000 & & 0.0000 & 0.0000 & & & 0.0000 & & 0.0009 & 0.0000 & & 0.0000 & 0.0000 & & & 0.0000 & 0.0000 & & 0.0000 & & 0.0060 \\
\hline SG33 & 0.0000 & & 0.0000 & 0.0000 & & & 0.0000 & & 0.0009 & 0.0000 & & 0.0000 & 0.0000 & & & 0.0000 & 0.0000 & & 0.0000 & & 0.0060 \\
\hline SG34 & 0.0000 & & 0.0000 & 0.0000 & & & 0.0000 & & 0.0009 & 0.0000 & & 0.0000 & 0.0000 & & & 0.0000 & 0.0000 & & 0.0000 & & 0.0060 \\
\hline SG35 & 0.0000 & & 0.0000 & 0.0000 & & & 0.0000 & & 0.0009 & 0.0000 & & 0.0000 & 0.0000 & & & 0.0000 & 0.0000 & & 0.0000 & & 0.0060 \\
\hline SG36 & 0.0000 & & 0.0000 & 0.0000 & & & 0.0000 & & 0.0009 & 0.0000 & & 0.0000 & 0.0000 & & & 0.0000 & 0.0000 & & 0.0000 & & 0.0015 \\
\hline SG37 & 0.0000 & & 0.0000 & 0.0000 & & & 0.0000 & & 0.0009 & 0.0000 & & 0.0000 & 0.0000 & & & 0.0000 & 0.0000 & & 0.0000 & & 0.0060 \\
\hline SG38 & 0.0000 & & 0.0000 & 0.0000 & & & 0.0000 & & 0.0009 & 0.0000 & & 0.0000 & 0.0000 & & & 0.0000 & 0.0000 & & 0.0000 & & 0.0060 \\
\hline SG39 & 0.0000 & & 0.0000 & 0.0000 & & & 0.0000 & & 0.0009 & 0.0000 & & 0.0000 & 0.0000 & & & 0.0000 & 0.0000 & & 0.0000 & & 0.0060 \\
\hline SG40 & 0.0000 & & 0.0000 & 0.0000 & & & 0.0000 & & 0.0009 & 0.0000 & & 0.0000 & 0.0000 & & & 0.0000 & 0.0000 & & 0.0000 & & 0.0060 \\
\hline SG41 & 0.0000 & & 0.0000 & 0.0000 & & & 0.0000 & & 0.0009 & 0.0000 & & 0.0000 & 0.0000 & & & 0.0000 & 0.0000 & & 0.0000 & & 0.0015 \\
\hline SG42 & 0.0000 & & 0.0000 & 0.0000 & & & 0.0000 & & 0.0009 & 0.0000 & & 0.0000 & 0.0000 & & & 0.0000 & 0.0000 & & 0.0000 & & 0.0049 \\
\hline SG43 & 0.0000 & & 0.0000 & 0.0000 & & & 0.0000 & & 0.0009 & 0.0000 & & 0.0000 & 0.0000 & & & 0.0000 & 0.0000 & & 0.0000 & & 0.0026 \\
\hline SG44 & 0.0000 & & 0.0000 & 0.0000 & & & 0.0000 & & 0.0009 & 0.0000 & & 0.0000 & 0.0000 & & & 0.0000 & 0.0000 & & 0.0000 & & 0.0049 \\
\hline SG45 & 0.0000 & & 0.0000 & 0.0000 & & & 0.0000 & & 0.0009 & 0.0000 & & 0.0000 & 0.0000 & & & 0.0000 & 0.0000 & & 0.0000 & & 0.0015 \\
\hline
\end{tabular}




\section{SG (Hrma et al. 1999)}

\begin{tabular}{|c|c|c|c|c|c|c|c|c|c|c|c|c|c|c|c|c|c|c|c|c|c|}
\hline Glass ID & $\begin{array}{c}\mathrm{T} 12 \mathrm{O} 3 \\
-\mathrm{t}\end{array}$ & $\begin{array}{c}\mathrm{U} 3 \mathrm{O} 8 \\
-\mathrm{t}\end{array}$ & $\begin{array}{c}\mathrm{UO} 2 \\
-\mathrm{t} \\
\end{array}$ & $\begin{array}{c}\mathrm{UO} 3 \\
-\mathrm{t}\end{array}$ & $\begin{array}{c}\mathrm{V} 2 \mathrm{O} 5 \\
-\mathrm{t}\end{array}$ & $\begin{array}{c}\text { WO3 } \\
-t\end{array}$ & $\begin{array}{c}\mathrm{Y} 2 \mathrm{O} 3 \\
-\mathrm{t}\end{array}$ & $\begin{array}{c}\mathrm{ZnO} \\
-\mathrm{t}\end{array}$ & $\begin{array}{c}\text { Others } \\
-t\end{array}$ & $\begin{array}{c}\text { Sum } \\
-t\end{array}$ & $\begin{array}{c}\mathrm{A} 12 \mathrm{O} 3 \\
-\mathrm{a}\end{array}$ & $\begin{array}{c}\mathrm{B} 2 \mathrm{O} 3 \\
-\mathrm{a}\end{array}$ & $\begin{array}{c}\mathrm{CaO} \\
-\mathrm{a} \\
\end{array}$ & $\begin{array}{c}\mathrm{Fe} 2 \mathrm{O} 3 \\
-\mathrm{a} \\
\end{array}$ & $\begin{array}{c}\mathrm{FeO} \\
-\mathrm{a} \\
\end{array}$ & $\begin{array}{c}\mathrm{K} 2 \mathrm{O} \\
-\mathrm{a} \\
\end{array}$ & $\begin{array}{c}\mathrm{Li} 2 \mathrm{O} \\
-\mathrm{a} \\
\end{array}$ & $\begin{array}{c}\mathrm{MgO} \\
-\mathrm{a}\end{array}$ & $\begin{array}{c}\mathrm{Na} 2 \mathrm{O} \\
-\mathrm{a}\end{array}$ & $\begin{array}{c}\mathrm{NiO} \\
-\mathrm{a} \\
\end{array}$ & $\begin{array}{c}\mathrm{P} 2 \mathrm{O} 5 \\
-\mathrm{a}\end{array}$ \\
\hline SG09 & & 0.0550 & & & & 0.0000 & 0.0000 & 0.0000 & & 1.0000 & & & & & & & & & & & \\
\hline SG10 & & 0.0415 & & & & 0.0000 & 0.0000 & 0.0000 & & 1.0000 & & & & & & & & & & & \\
\hline SG11 & & 0.0177 & & & & 0.0000 & 0.0000 & 0.0000 & & 1.0000 & & & & & & & & & & & \\
\hline SG12 & & 0.0000 & & & & 0.0000 & 0.0000 & 0.0000 & & 1.0000 & & & & & & & & & & & \\
\hline SG13 & & 0.0000 & & & & 0.0000 & 0.0000 & 0.0000 & & 1.0000 & & & & & & & & & & & \\
\hline SG14 & & 0.0550 & & & & 0.0000 & 0.0000 & 0.0000 & & 1.0000 & & & & & & & & & & & \\
\hline SG15 & & 0.0080 & & & & 0.0000 & 0.0000 & 0.0000 & & 1.0000 & & & & & & & & & & & \\
\hline SG16 & & 0.0415 & & & & 0.0000 & 0.0000 & 0.0000 & & 1.0000 & & & & & & & & & & & \\
\hline SG17 & & 0.0415 & & & & 0.0000 & 0.0000 & 0.0000 & & 1.0000 & & & & & & & & & & & \\
\hline SG18a & & 0.0000 & & & & 0.0000 & 0.0000 & 0.0000 & & 1.0000 & & & & & & & & & & & \\
\hline SG18b & & 0.0000 & & & & 0.0000 & 0.0000 & 0.0000 & & 1.0000 & & & & & & & & & & & \\
\hline SG18c & & 0.0000 & & & & 0.0000 & 0.0000 & 0.0000 & & 1.0000 & & & & & & & & & & & \\
\hline SG18d & & 0.0000 & & & & 0.0000 & 0.0000 & 0.0000 & & 1.0000 & & & & & & & & & & & \\
\hline SG18e & & 0.0000 & & & & 0.0000 & 0.0000 & 0.0000 & & 1.0000 & & & & & & & & & & & \\
\hline SG18f & & 0.0000 & & & & 0.0000 & 0.0000 & 0.0000 & & 1.0000 & & & & & & & & & & & \\
\hline SG18g & & 0.0000 & & & & 0.0000 & 0.0000 & 0.0000 & & 1.0000 & & & & & & & & & & & \\
\hline SG19 & & 0.0550 & & & & 0.0000 & 0.0000 & 0.0000 & & 1.0000 & & & & & & & & & & & \\
\hline $\mathrm{SG} 20$ & & 0.0550 & & & & 0.0000 & 0.0000 & 0.0000 & & 1.0000 & & & & & & & & & & & \\
\hline SG21 & & 0.0177 & & & & 0.0000 & 0.0000 & 0.0000 & & 1.0000 & & & & & & & & & & & \\
\hline SG22 & & 0.0177 & & & & 0.0000 & 0.0000 & 0.0000 & & 1.0000 & & & & & & & & & & & \\
\hline SG23 & & 0.0177 & & & & 0.0000 & 0.0000 & 0.0000 & & 1.0000 & & & & & & & & & & & \\
\hline SG24 & & 0.0550 & & & & 0.0000 & 0.0000 & 0.0000 & & 1.0001 & & & & & & & & & & & \\
\hline SG25 & & 0.0000 & & & & 0.0000 & 0.0000 & 0.0000 & & 1.0000 & & & & & & & & & & & \\
\hline SG26 & & 0.0415 & & & & 0.0000 & 0.0000 & 0.0000 & & 1.0000 & & & & & & & & & & & \\
\hline SG27 & & 0.0415 & & & & 0.0000 & 0.0000 & 0.0000 & & 1.0000 & & & & & & & & & & & \\
\hline SG28 & & 0.0000 & & & & 0.0000 & 0.0000 & 0.0000 & & 1.0000 & & & & & & & & & & & \\
\hline SG29 & & 0.0550 & & & & 0.0000 & 0.0000 & 0.0000 & & 1.0000 & & & & & & & & & & & \\
\hline SG30 & & 0.0550 & & & & 0.0000 & 0.0000 & 0.0000 & & 1.0000 & & & & & & & & & & & \\
\hline SG31 & & 0.0000 & & & & 0.0000 & 0.0000 & 0.0000 & & 1.0000 & & & & & & & & & & & \\
\hline SG32 & & 0.0000 & & & & 0.0000 & 0.0000 & 0.0000 & & 1.0000 & & & & & & & & & & & \\
\hline SG33 & & 0.0000 & & & & 0.0000 & 0.0000 & 0.0000 & & 1.0000 & & & & & & & & & & & \\
\hline SG34 & & 0.0550 & & & & 0.0000 & 0.0000 & 0.0000 & & 1.0000 & & & & & & & & & & & \\
\hline SG35 & & 0.0000 & & & & 0.0000 & 0.0000 & 0.0000 & & 1.0000 & & & & & & & & & & & \\
\hline SG36 & & 0.0550 & & & & 0.0000 & 0.0000 & 0.0000 & & 1.0001 & & & & & & & & & & & \\
\hline SG37 & & 0.0000 & & & & 0.0000 & 0.0000 & 0.0000 & & 1.0000 & & & & & & & & & & & \\
\hline SG38 & & 0.0550 & & & & 0.0000 & 0.0000 & 0.0000 & & 1.0000 & & & & & & & & & & & \\
\hline SG39 & & 0.0000 & & & & 0.0000 & 0.0000 & 0.0000 & & 1.0000 & & & & & & & & & & & \\
\hline SG40 & & 0.0550 & & & & 0.0000 & 0.0000 & 0.0000 & & 1.0000 & & & & & & & & & & & \\
\hline SG41 & & 0.0550 & & & & 0.0000 & 0.0000 & 0.0000 & & 1.0000 & & & & & & & & & & & \\
\hline SG42 & & 0.0177 & & & & 0.0000 & 0.0000 & 0.0000 & & 1.0000 & & & & & & & & & & & \\
\hline SG43 & & 0.0177 & & & & 0.0000 & 0.0000 & 0.0000 & & 1.0000 & & & & & & & & & & & \\
\hline SG44 & & 0.0177 & & & & 0.0000 & 0.0000 & 0.0000 & & 1.0000 & & & & & & & & & & & \\
\hline SG45 & & 0.0000 & & & & 0.0000 & 0.0000 & 0.0000 & & 1.0000 & & & & & & & & & & & \\
\hline
\end{tabular}


Appendix A. Database - mass fraction

SG (Hrma et al. 1999)

\begin{tabular}{|c|c|c|c|c|c|c|c|c|c|c|c|c|c|c|c|c|c|c|c|c|c|}
\hline Glass ID & $\begin{array}{c}\mathrm{SiO} 2 \\
-\mathrm{a}\end{array}$ & $\begin{array}{c}\mathrm{ZrO} 2 \\
-\mathrm{a}\end{array}$ & $\begin{array}{c}\mathrm{Ag} 2 \mathrm{O} \\
-\mathrm{a}\end{array}$ & $\begin{array}{c}\text { As2O3 } \\
-\mathrm{a}\end{array}$ & $\begin{array}{c}\mathrm{BaO} \\
-\mathrm{a}\end{array}$ & $\begin{array}{c}\mathrm{Bi} 2 \mathrm{O} 3 \\
-\mathrm{a}\end{array}$ & $\begin{array}{l}\mathrm{Br} \\
-\mathrm{a}\end{array}$ & $\begin{array}{c}\mathrm{CdO} \\
-\mathrm{a}\end{array}$ & $\begin{array}{c}\mathrm{Ce} 2 \mathrm{O} 3 \\
-\mathrm{a}\end{array}$ & $\begin{array}{c}\mathrm{CeO} 2 \\
-\mathrm{a}\end{array}$ & $\begin{array}{l}\mathrm{Cl} \\
-\mathrm{a}\end{array}$ & $\begin{array}{c}\mathrm{CoO} \\
-\mathrm{a}\end{array}$ & $\begin{array}{c}\mathrm{Co} 2 \mathrm{O} 3 \\
-\mathrm{a}\end{array}$ & $\begin{array}{c}\mathrm{Cr} 2 \mathrm{O} 3 \\
-\mathrm{a}\end{array}$ & $\begin{array}{c}\mathrm{Cs} 2 \mathrm{O} \\
-\mathrm{a}\end{array}$ & $\begin{array}{c}\mathrm{CuO} \\
-\mathrm{a}\end{array}$ & $\begin{array}{c}\mathrm{Eu} 2 \mathrm{O} 3 \\
-\mathrm{a}\end{array}$ & $\begin{array}{l}\mathrm{F} \\
-\mathrm{a}\end{array}$ & $\begin{array}{c}\mathrm{Ga} 2 \mathrm{O} 3 \\
-\mathrm{a}\end{array}$ & $\begin{array}{c}\mathrm{Gd} 2 \mathrm{O} 3 \\
-\mathrm{a}\end{array}$ & $\begin{array}{c}\mathrm{HgO} \\
-\mathrm{a}\end{array}$ \\
\hline SG09 & & & & & & & & & & & & & & & & & & & & & \\
\hline SG10 & & & & & & & & & & & & & & & & & & & & & \\
\hline SG11 & & & & & & & & & & & & & & & & & & & & & \\
\hline SG12 & & & & & & & & & & & & & & & & & & & & & \\
\hline SG13 & & & & & & & & & & & & & & & & & & & & & \\
\hline SG14 & & & & & & & & & & & & & & & & & & & & & \\
\hline SG15 & & & & & & & & & & & & & & & & & & & & & \\
\hline SG16 & & & & & & & & & & & & & & & & & & & & & \\
\hline SG17 & & & & & & & & & & & & & & & & & & & & & \\
\hline SG18a & & & & & & & & & & & & & & & & & & & & & \\
\hline SG18b & & & & & & & & & & & & & & & & & & & & & \\
\hline SG18c & & & & & & & & & & & & & & & & & & & & & \\
\hline SG18d & & & & & & & & & & & & & & & & & & & & & \\
\hline SG18e & & & & & & & & & & & & & & & & & & & & & \\
\hline SG18f & & & & & & & & & & & & & & & & & & & & & \\
\hline SG18g & & & & & & & & & & & & & & & & & & & & & \\
\hline SG19 & & & & & & & & & & & & & & & & & & & & & \\
\hline SG20 & & & & & & & & & & & & & & & & & & & & & \\
\hline SG21 & & & & & & & & & & & & & & & & & & & & & \\
\hline SG22 & & & & & & & & & & & & & & & & & & & & & \\
\hline SG23 & & & & & & & & & & & & & & & & & & & & & \\
\hline SG24 & & & & & & & & & & & & & & & & & & & & & \\
\hline SG25 & & & & & & & & & & & & & & & & & & & & & \\
\hline SG26 & & & & & & & & & & & & & & & & & & & & & \\
\hline SG27 & & & & & & & & & & & & & & & & & & & & & \\
\hline SG28 & & & & & & & & & & & & & & & & & & & & & \\
\hline SG29 & & & & & & & & & & & & & & & & & & & & & \\
\hline SG30 & & & & & & & & & & & & & & & & & & & & & \\
\hline SG31 & & & & & & & & & & & & & & & & & & & & & \\
\hline SG32 & & & & & & & & & & & & & & & & & & & & & \\
\hline SG33 & & & & & & & & & & & & & & & & & & & & & \\
\hline SG34 & & & & & & & & & & & & & & & & & & & & & \\
\hline SG35 & & & & & & & & & & & & & & & & & & & & & \\
\hline SG36 & & & & & & & & & & & & & & & & & & & & & \\
\hline SG37 & & & & & & & & & & & & & & & & & & & & & \\
\hline SG38 & & & & & & & & & & & & & & & & & & & & & \\
\hline SG39 & & & & & & & & & & & & & & & & & & & & & \\
\hline SG40 & & & & & & & & & & & & & & & & & & & & & \\
\hline SG41 & & & & & & & & & & & & & & & & & & & & & \\
\hline SG42 & & & & & & & & & & & & & & & & & & & & & \\
\hline SG43 & & & & & & & & & & & & & & & & & & & & & \\
\hline SG44 & & & & & & & & & & & & & & & & & & & & & \\
\hline SG45 & & & & & & & & & & & & & & & & & & & & & \\
\hline
\end{tabular}


Appendix A. Database - mass fraction

SG (Hrma et al. 1999)

\begin{tabular}{|c|c|c|c|c|c|c|c|c|c|c|c|c|c|c|c|c|c|c|c|c|c|}
\hline Glass ID & $\begin{array}{c}\mathrm{I} \\
-\mathrm{a} \\
\end{array}$ & \begin{tabular}{|c|}
$\mathrm{La} 2 \mathrm{O} 3$ \\
$-\mathrm{a}$ \\
\end{tabular} & $\begin{array}{c}\mathrm{MnO} 2 \\
-\mathrm{a} \\
\end{array}$ & $\begin{array}{c}\mathrm{MnO} \\
-\mathrm{a} \\
\end{array}$ & $\begin{array}{c}\mathrm{MoO} \\
-\mathrm{a}\end{array}$ & $\begin{array}{c}\mathrm{MoO} 3 \\
-\mathrm{a} \\
\end{array}$ & $\begin{array}{c}\mathrm{Nb} 2 \mathrm{O} 5 \\
-\mathrm{a}\end{array}$ & $\begin{array}{c}\mathrm{Nd} 2 \mathrm{O} 3 \\
-\mathrm{a} \\
\end{array}$ & $\begin{array}{c}\mathrm{PbO} \\
-\mathrm{a} \\
\end{array}$ & $\begin{array}{c}\mathrm{PdO} 2 \\
-\mathrm{a}\end{array}$ & $\begin{array}{c}\mathrm{PdO} \\
-\mathrm{a} \\
\end{array}$ & $\begin{array}{c}\mathrm{Pr} 2 \mathrm{O} 3 \\
-\mathrm{a} \\
\end{array}$ & $\begin{array}{c}\operatorname{Pr} 6011 \\
-\mathrm{a}\end{array}$ & $\begin{array}{c}\mathrm{Rb} 2 \mathrm{O} \\
-\mathrm{a} \\
\end{array}$ & $\begin{array}{c}\mathrm{ReO} \\
-\mathrm{a} \\
\end{array}$ & $\begin{array}{c}\mathrm{ReO} 2 \\
-\mathrm{a}\end{array}$ & $\begin{array}{c}\mathrm{Rh} 2 \mathrm{O} 3 \\
-\mathrm{a}\end{array}$ & $\begin{array}{c}\mathrm{RhO} 2 \\
-\mathrm{a}\end{array}$ & $\begin{array}{c}\mathrm{RuO} 2 \\
-\mathrm{a}\end{array}$ & $\begin{array}{c}\mathrm{Sb} 2 \mathrm{O} 3 \\
-\mathrm{a} \\
\end{array}$ & $\begin{array}{c}\mathrm{Sb} 2 \mathrm{O} 5 \\
-\mathrm{a}\end{array}$ \\
\hline SG09 & & & & & & & & & & & & & & & & & & & & & \\
\hline SG10 & & & & & & & & & & & & & & & & & & & & & \\
\hline SG11 & & & & & & & & & & & & & & & & & & & & & \\
\hline SG12 & & & & & & & & & & & & & & & & & & & & & \\
\hline SG13 & & & & & & & & & & & & & & & & & & & & & \\
\hline SG14 & & & & & & & & & & & & & & & & & & & & & \\
\hline SG15 & & & & & & & & & & & & & & & & & & & & & \\
\hline SG16 & & & & & & & & & & & & & & & & & & & & & \\
\hline SG17 & & & & & & & & & & & & & & & & & & & & & \\
\hline SG18a & & & & & & & & & & & & & & & & & & & & & \\
\hline SG18b & & & & & & & & & & & & & & & & & & & & & \\
\hline SG18c & & & & & & & & & & & & & & & & & & & & & \\
\hline SG18d & & & & & & & & & & & & & & & & & & & & & \\
\hline SG18e & & & & & & & & & & & & & & & & & & & & & \\
\hline SG18f & & & & & & & & & & & & & & & & & & & & & \\
\hline SG18g & & & & & & & & & & & & & & & & & & & & & \\
\hline SG19 & & & & & & & & & & & & & & & & & & & & & \\
\hline SG20 & & & & & & & & & & & & & & & & & & & & & \\
\hline SG21 & & & & & & & & & & & & & & & & & & & & & \\
\hline SG22 & & & & & & & & & & & & & & & & & & & & & \\
\hline SG23 & & & & & & & & & & & & & & & & & & & & & \\
\hline SG24 & & & & & & & & & & & & & & & & & & & & & \\
\hline SG25 & & & & & & & & & & & & & & & & & & & & & \\
\hline SG26 & & & & & & & & & & & & & & & & & & & & & \\
\hline SG27 & & & & & & & & & & & & & & & & & & & & & \\
\hline SG28 & & & & & & & & & & & & & & & & & & & & & \\
\hline SG29 & & & & & & & & & & & & & & & & & & & & & \\
\hline SG30 & & & & & & & & & & & & & & & & & & & & & \\
\hline SG31 & & & & & & & & & & & & & & & & & & & & & \\
\hline SG32 & & & & & & & & & & & & & & & & & & & & & \\
\hline SG33 & & & & & & & & & & & & & & & & & & & & & \\
\hline SG34 & & & & & & & & & & & & & & & & & & & & & \\
\hline SG35 & & & & & & & & & & & & & & & & & & & & & \\
\hline SG36 & & & & & & & & & & & & & & & & & & & & & \\
\hline SG37 & & & & & & & & & & & & & & & & & & & & & \\
\hline SG38 & & & & & & & & & & & & & & & & & & & & & \\
\hline SG39 & & & & & & & & & & & & & & & & & & & & & \\
\hline SG40 & & & & & & & & & & & & & & & & & & & & & \\
\hline SG41 & & & & & & & & & & & & & & & & & & & & & \\
\hline SG42 & & & & & & & & & & & & & & & & & & & & & \\
\hline SG43 & & & & & & & & & & & & & & & & & & & & & \\
\hline SG44 & & & & & & & & & & & & & & & & & & & & & \\
\hline SG45 & & & & & & & & & & & & & & & & & & & & & \\
\hline
\end{tabular}


Appendix A. Database - mass fraction

SG (Hrma et al. 1999)

\begin{tabular}{|c|c|c|c|c|c|c|c|c|c|c|c|c|c|c|c|c|c|c|c|c|}
\hline Glass ID & $\begin{array}{c}\mathrm{SeO} 2 \\
-\mathrm{a}\end{array}$ & $\begin{array}{c}\mathrm{Sm} 2 \mathrm{O} 3 \\
-\mathrm{a}\end{array}$ & $\begin{array}{c}\mathrm{SnO} \\
-\mathrm{a}\end{array}$ & $\begin{array}{c}\mathrm{SnO} 2 \\
-\mathrm{a}\end{array}$ & $\begin{array}{c}\mathrm{SO} 3 \\
-\mathrm{a}\end{array}$ & $\begin{array}{c}\mathrm{SrO} \\
-\mathrm{a}\end{array}$ & $\begin{array}{c}\mathrm{Tc} 2 \mathrm{O} 7 \\
-\mathrm{a}\end{array}$ & $\begin{array}{c}\mathrm{TeO} 2 \\
-\mathrm{a}\end{array}$ & $\begin{array}{c}\mathrm{ThO} 2 \\
-\mathrm{a}\end{array}$ & $\begin{array}{c}\mathrm{TiO} 2 \\
-\mathrm{a}\end{array}$ & $\begin{array}{c}\mathrm{T} 12 \mathrm{O} 3 \\
-\mathrm{a}\end{array}$ & $\begin{array}{c}\mathrm{U} 3 \mathrm{O} 8 \\
-\mathrm{a}\end{array}$ & $\begin{array}{c}\mathrm{UO} 2 \\
-\mathrm{a}\end{array}$ & $\begin{array}{c}\text { UO3 } \\
-\mathrm{a}\end{array}$ & $\begin{array}{c}\mathrm{V} 2 \mathrm{O} 5 \\
-\mathrm{a}\end{array}$ & $\begin{array}{c}\text { WO3 } \\
-\mathrm{a}\end{array}$ & $\begin{array}{c}\mathrm{Y} 2 \mathrm{O} 3 \\
-\mathrm{a}\end{array}$ & $\begin{array}{c}\mathrm{ZnO} \\
-\mathrm{a}\end{array}$ & $\begin{array}{c}\text { Others } \\
-\mathrm{a}\end{array}$ & $\begin{array}{c}\text { Sum } \\
-a\end{array}$ \\
\hline SG09 & & & & & & & & & & & & & & & & & & & & \\
\hline SG10 & & & & & & & & & & & & & & & & & & & & \\
\hline SG11 & & & & & & & & & & & & & & & & & & & & \\
\hline SG12 & & & & & & & & & & & & & & & & & & & & \\
\hline SG13 & & & & & & & & & & & & & & & & & & & & \\
\hline SG14 & & & & & & & & & & & & & & & & & & & & \\
\hline SG15 & & & & & & & & & & & & & & & & & & & & \\
\hline SG16 & & & & & & & & & & & & & & & & & & & & \\
\hline SG17 & & & & & & & & & & & & & & & & & & & & \\
\hline SG18a & & & & & & & & & & & & & & & & & & & & \\
\hline SG18b & & & & & & & & & & & & & & & & & & & & \\
\hline SG18c & & & & & & & & & & & & & & & & & & & & \\
\hline SG18d & & & & & & & & & & & & & & & & & & & & \\
\hline SG18e & & & & & & & & & & & & & & & & & & & & \\
\hline SG18f & & & & & & & & & & & & & & & & & & & & \\
\hline SG18g & & & & & & & & & & & & & & & & & & & & \\
\hline SG19 & & & & & & & & & & & & & & & & & & & & \\
\hline SG20 & & & & & & & & & & & & & & & & & & & & \\
\hline SG21 & & & & & & & & & & & & & & & & & & & & \\
\hline SG22 & & & & & & & & & & & & & & & & & & & & \\
\hline SG23 & & & & & & & & & & & & & & & & & & & & \\
\hline SG24 & & & & & & & & & & & & & & & & & & & & \\
\hline SG25 & & & & & & & & & & & & & & & & & & & & \\
\hline SG26 & & & & & & & & & & & & & & & & & & & & \\
\hline SG27 & & & & & & & & & & & & & & & & & & & & \\
\hline SG28 & & & & & & & & & & & & & & & & & & & & \\
\hline SG29 & & & & & & & & & & & & & & & & & & & & \\
\hline SG30 & & & & & & & & & & & & & & & & & & & & \\
\hline SG31 & & & & & & & & & & & & & & & & & & & & \\
\hline SG32 & & & & & & & & & & & & & & & & & & & & \\
\hline SG33 & & & & & & & & & & & & & & & & & & & & \\
\hline SG34 & & & & & & & & & & & & & & & & & & & & \\
\hline SG35 & & & & & & & & & & & & & & & & & & & & \\
\hline SG36 & & & & & & & & & & & & & & & & & & & & \\
\hline SG37 & & & & & & & & & & & & & & & & & & & & \\
\hline SG38 & & & & & & & & & & & & & & & & & & & & \\
\hline SG39 & & & & & & & & & & & & & & & & & & & & \\
\hline SG40 & & & & & & & & & & & & & & & & & & & & \\
\hline SG41 & & & & & & & & & & & & & & & & & & & & \\
\hline SG42 & & & & & & & & & & & & & & & & & & & & \\
\hline SG43 & & & & & & & & & & & & & & & & & & & & \\
\hline SG44 & & & & & & & & & & & & & & & & & & & & \\
\hline SG45 & & & & & & & & & & & & & & & & & & & & \\
\hline
\end{tabular}


Appendix A. Database - mass fraction

SG (Hrma et al. 1999)

\begin{tabular}{|c|c|c|c|c|c|c|c|c|}
\hline Glass ID & $\begin{array}{l}\mathrm{TM} \\
\left({ }^{\circ} \mathrm{C}\right)\end{array}$ & $\begin{array}{l}\text { Gradient } \\
\text { TL }\left({ }^{\circ} \mathrm{C}\right)\end{array}$ & $\begin{array}{l}\text { Uniform } \\
\text { TL }\left({ }^{\circ} \mathrm{C}\right)\end{array}$ & Primary Phase & $\begin{array}{l}\text { Quenched } \\
\text { Visual/OM }\end{array}$ & $\begin{array}{c}\text { Quenched } \\
\text { SEM/EDS or TEM }\end{array}$ & $\begin{array}{l}\text { Quenched } \\
\text { XRD }\end{array}$ & $\begin{array}{c}\text { Quenched } \\
\text { Homogeneous? }\end{array}$ \\
\hline SG09 & 1200 & & 1173 & spinel & & & & \\
\hline SG10 & 1275 & & 1098 & $\mathrm{RuO} 2$, spinel & & & & \\
\hline SG11 & 1264 & & 895 & $\mathrm{RuO} 2$, spinel & & & & \\
\hline SG12 & 1384 & & 1030 & $\mathrm{RuO} 2$, spinel & & & & \\
\hline SG13 & 1319 & & 1063 & $\mathrm{RuO} 2$, spinel & & & & \\
\hline SG14 & 1160 & & 951 & spinel & & & & \\
\hline SG15 & 1285 & & 935 & RuO2, clinopyroxene & & & & \\
\hline SG16 & 1250 & & 995 & RuO2, spinel & & & & \\
\hline SG17 & 1160 & & 1075 & spinel & & & & \\
\hline SG18a & 1142 & & 879 & spinel & & & & \\
\hline SG18b & 1142 & & 887 & spinel & & & & \\
\hline SG18c & 1142 & & 859 & spinel & & & & \\
\hline SG18d & 1142 & & 883 & spinel & & & & \\
\hline SG18e & 1142 & & 883 & spinel & & & & \\
\hline SG18f & 1142 & & 891 & spinel & & & & \\
\hline SG18g & 1142 & & 882 & spinel & & & & \\
\hline SG19 & 1140 & & 929 & spinel & & & & \\
\hline SG20 & 1240 & & 799 & $\begin{array}{l}\text { RuO2, spinel, } \\
\text { clinopyroxene }\end{array}$ & & & & \\
\hline SG21 & 1284 & & 987 & $\mathrm{RuO} 2$, spinel & & & & \\
\hline SG22 & 1246 & & 1145 & spinel & & & & \\
\hline SG23 & 1304 & & 1069 & $\mathrm{RuO} 2$, spinel & & & & \\
\hline SG24 & 1345 & & 995 & RuO2, clinopyroxene & & & & \\
\hline SG25 & 1333 & & 1310 & spinel & & & & \\
\hline SG26 & 1304 & & 1071 & spinel & & & & \\
\hline SG27 & 1211 & & 1086 & spinel & & & & \\
\hline SG28 & 1150 & & 833 & RuO2, clinopyroxene & & & & \\
\hline SG29 & 1280 & & 811 & spinel & & & & \\
\hline SG30 & 1157 & & 1031 & spinel & & & & \\
\hline SG31 & 1149 & & 1081 & spinel & & & & \\
\hline SG32 & 1250 & & 1132 & spinel & & & & \\
\hline SG33 & 1145 & & 943 & spinel & & & & \\
\hline SG34 & 1285 & & 1282 & spinel & & & & \\
\hline SG35 & 1134 & & 1231 & spinel & & & & \\
\hline SG36 & 1265 & & 813 & 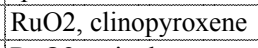 & & & & \\
\hline SG37 & 1268 & & 944 & $\mathrm{RuO} 2$, spinel & & & & \\
\hline SG38 & 1151 & & 897 & spinel & & & & \\
\hline SG39 & 1322 & & 1164 & spinel & & & & \\
\hline SG40 & 1300 & & 1173 & spinel & & & & \\
\hline SG41 & 1300 & & 1304 & spinel & & & & \\
\hline SG42 & 1160 & & 990 & spinel & & & & \\
\hline SG43 & 1317 & & 924 & spinel & & & & \\
\hline SG44 & 1330 & & 1244 & $\mathrm{RuO} 2$, spinel & & & & \\
\hline SG45 & 1317 & & 936 & $\begin{array}{l}\mathrm{RuO} 2 \text {, spinel, } \\
\text { clinopyroxene }\end{array}$ & & & & \\
\hline
\end{tabular}


Appendix A. Database - mass fraction

\section{SG (Hrma et al. 1999)}

\begin{tabular}{|c|c|c|c|c|c|}
\hline Glass ID & $\begin{array}{c}\mathrm{CCC} \\
\text { Visual/OM }\end{array}$ & $\begin{array}{c}\text { CCC } \\
\text { SEM/EDS or TEM }\end{array}$ & $\begin{array}{l}\text { CCC } \\
\text { XRD }\end{array}$ & $\begin{array}{l}\text { Heat Treated } \\
\text { Visual/OM }\end{array}$ & $\begin{array}{c}\text { Heat Treated } \\
\text { SEM/EDS or TEM }\end{array}$ \\
\hline \multicolumn{6}{|l|}{$\begin{array}{l}\text { SG09 } \\
\text { SG10 }\end{array}$} \\
\hline SG10 & & & & & \\
\hline \multicolumn{6}{|l|}{ SG11 } \\
\hline \multicolumn{6}{|l|}{ SG12 } \\
\hline \multicolumn{6}{|l|}{ SG13 } \\
\hline \multicolumn{6}{|l|}{ SG14 } \\
\hline \multicolumn{6}{|l|}{ SG15 } \\
\hline \multicolumn{6}{|l|}{ SG16 } \\
\hline \multicolumn{6}{|l|}{ SG17 } \\
\hline \multicolumn{6}{|l|}{ SG18a } \\
\hline \multicolumn{6}{|l|}{ SG18b } \\
\hline \multicolumn{6}{|l|}{ SG18c } \\
\hline \multicolumn{6}{|l|}{ SG18d } \\
\hline \multicolumn{6}{|l|}{ SG18e } \\
\hline \multicolumn{6}{|l|}{ SG18f } \\
\hline \multicolumn{6}{|l|}{ SG18g } \\
\hline \multicolumn{6}{|l|}{ SG19 } \\
\hline \multicolumn{6}{|l|}{ SG20 } \\
\hline \multicolumn{6}{|l|}{ SG21 } \\
\hline \multicolumn{6}{|l|}{ SG22 } \\
\hline \multicolumn{6}{|l|}{$\mathrm{SG} 23$} \\
\hline \multicolumn{6}{|l|}{ SG24 } \\
\hline \multicolumn{6}{|l|}{ SG25 } \\
\hline \multicolumn{6}{|l|}{ SG26 } \\
\hline \multicolumn{6}{|l|}{ SG27 } \\
\hline SG28 & & & & & \\
\hline SG29 & & & & & \\
\hline SG30 & & & & & \\
\hline SG31 & & & & & \\
\hline SG32 & & & & & \\
\hline SG33 & & & & & \\
\hline SG34 & & & & & \\
\hline SG35 & & & & & \\
\hline SG36 & & & & & \\
\hline SG37 & & & & & \\
\hline SG38 & & & & & \\
\hline SG39 & & & & & \\
\hline SG40 & & & & & \\
\hline SG41 & & & & & \\
\hline SG42 & & & & & \\
\hline SG43 & & & & & \\
\hline SG44 & & & & & \\
\hline SG45 & & & & & \\
\hline
\end{tabular}


Appendix A. Database - mass fraction

\section{SG (Hrma et al. 1999)}

\begin{tabular}{|c|c|c|c|c|c|c|c|c|c|c|c|c|c|c|c|}
\hline Glass ID & $\begin{array}{c}\text { Heat Treated } \\
\text { XRD }\end{array}$ & $\begin{array}{l}\text { Density } \\
\left(\mathrm{g} / \mathrm{cm}^{3}\right)\end{array}$ & $\begin{array}{c}\text { Fulc Visc } \\
\text { A }\end{array}$ & \begin{tabular}{|c|} 
Fulc Visc \\
B
\end{tabular} & \begin{tabular}{|c|} 
Fulc Visc \\
To
\end{tabular} & $\begin{array}{c}\mathrm{FV} 1150^{\circ} \mathrm{C} \\
(\mathrm{Pa} \cdot \mathrm{s})\end{array}$ & $\begin{array}{c}\text { Arrh Visc } \\
\text { A }\end{array}$ & $\begin{array}{c}\text { Arrh Visc } \\
\text { B }\end{array}$ & $1150^{\circ} \mathrm{C}$ & $\begin{array}{c}\mathrm{T}\left({ }^{\circ} \mathrm{C}\right) \text { at } \\
2 \mathrm{~Pa} \cdot \mathrm{s}\end{array}$ & \begin{tabular}{|c|}
$\mathrm{T}\left({ }^{\circ} \mathrm{C}\right)$ at \\
$5 \mathrm{~Pa} \cdot \mathrm{s}$
\end{tabular} & $\begin{array}{l}\mathrm{T}\left({ }^{\circ} \mathrm{C}\right) \text { at } \\
10 \mathrm{~Pa} \cdot \mathrm{s}\end{array}$ & $\begin{array}{l}\mathrm{T} 1 \\
\left({ }^{\circ} \mathrm{C}\right)\end{array}$ & $\begin{array}{c}\mathrm{V} 1 \\
(\mathrm{~Pa} \cdot \mathrm{s})\end{array}$ & $\begin{array}{c}\mathrm{T} 2 \\
\left({ }^{\circ} \mathrm{C}\right) \\
\end{array}$ \\
\hline \multicolumn{16}{|l|}{ SG09 } \\
\hline \multicolumn{16}{|l|}{ SG10 } \\
\hline \multicolumn{16}{|l|}{ SG11 } \\
\hline \multicolumn{16}{|l|}{ SG12 } \\
\hline \multicolumn{16}{|l|}{ SG13 } \\
\hline \multicolumn{16}{|l|}{ SG14 } \\
\hline \multicolumn{16}{|l|}{ SG15 } \\
\hline \multicolumn{16}{|l|}{ SG16 } \\
\hline \multicolumn{16}{|l|}{ SG17 } \\
\hline \multicolumn{16}{|l|}{ SG18a } \\
\hline \multicolumn{16}{|l|}{ SG18b } \\
\hline \multicolumn{16}{|l|}{ SG18c } \\
\hline \multicolumn{16}{|l|}{ SG18d } \\
\hline \multicolumn{16}{|l|}{ SG18e } \\
\hline \multicolumn{16}{|l|}{ SG18f } \\
\hline \multicolumn{16}{|l|}{ SG18g } \\
\hline \multicolumn{16}{|l|}{ SG19 } \\
\hline \multicolumn{16}{|l|}{ SG20 } \\
\hline \multicolumn{16}{|l|}{ SG21 } \\
\hline SG22 & & & & & & & & & & & & & & & \\
\hline SG23 & & & & & & & & & & & & & & & \\
\hline SG24 & & & & & & & & & & & & & & & \\
\hline SG25 & & & & & & & & & & & & & & & \\
\hline SG26 & & & & & & & & & & & & & & & \\
\hline SG27 & & & & & & & & & & & & & & & \\
\hline SG28 & & & & & & & & & & & & & & & \\
\hline SG29 & & & & & & & & & & & & & & & \\
\hline SG30 & & & & & & & & & & & & & & & \\
\hline SG31 & & & & & & & & & & & & & & & \\
\hline SG32 & & & & & & & & & & & & & & & \\
\hline SG33 & & & & & & & & & & & & & & & \\
\hline SG34 & & & & & & & & & & & & & & & \\
\hline SG35 & & & & & & & & & & & & & & & \\
\hline SG36 & & & & & & & & & & & & & & & \\
\hline SG37 & & & & & & & & & & & & & & & \\
\hline SG38 & & & & & & & & & & & & & & & \\
\hline SG39 & & & & & & & & & & & & & & & \\
\hline SG40 & & & & & & & & & & & & & & & \\
\hline SG41 & & & & & & & & & & & & & & & \\
\hline SG42 & & & & & & & & & & & & & & & \\
\hline SG43 & & & & & & & & & & & & & & & \\
\hline SG44 & & & & & & & & & & & & & & & \\
\hline SG45 & & & & & & & & & & & & & & & \\
\hline
\end{tabular}


Appendix A. Database - mass fraction

\begin{tabular}{|c|c|c|c|c|c|c|c|c|c|c|c|c|c|c|c|c|c|c|c|c|c|}
\hline Glass ID & $\begin{array}{c}\text { V2 } \\
(\mathrm{Pa} \cdot \mathrm{s}) \\
\end{array}$ & $\begin{array}{c}\mathrm{T} 3 \\
\left({ }^{\circ} \mathrm{C}\right) \\
\end{array}$ & $\begin{array}{c}\mathrm{V} 3 \\
(\mathrm{~Pa} \cdot \mathrm{s}) \\
\end{array}$ & $\begin{array}{c}\mathrm{T} 4 \\
\left({ }^{\circ} \mathrm{C}\right) \\
\end{array}$ & $\begin{array}{c}\mathrm{V} 4 \\
(\mathrm{~Pa} \cdot \mathrm{s}) \\
\end{array}$ & $\begin{array}{c}\mathrm{T} 5 \\
\left({ }^{\circ} \mathrm{C}\right) \\
\end{array}$ & $\begin{array}{c}\text { V5 } \\
(\mathrm{Pa} \cdot \mathrm{s}) \\
\end{array}$ & $\begin{array}{c}\mathrm{T} 6 \\
\left({ }^{\circ} \mathrm{C}\right) \\
\end{array}$ & $\begin{array}{c}\mathrm{V} 6 \\
(\mathrm{~Pa} \cdot \mathrm{s}) \\
\end{array}$ & $\begin{array}{c}\mathrm{T} 7 \\
\left({ }^{\circ} \mathrm{C}\right) \\
\end{array}$ & $\begin{array}{c}\text { V7 } \\
\text { (Pa.s) } \\
\end{array}$ & $\begin{array}{c}\mathrm{T} 8 \\
\left({ }^{\circ} \mathrm{C}\right) \\
\end{array}$ & $\begin{array}{c}\mathrm{V} 8 \\
\text { (Pa.s) }\end{array}$ & $\begin{array}{c}\mathrm{T} 9 \\
\left({ }^{\circ} \mathrm{C}\right)\end{array}$ & $\begin{array}{c}\mathrm{V} 9 \\
(\mathrm{~Pa} \cdot \mathrm{s})\end{array}$ & $\begin{array}{l}\mathrm{T} 10 \\
\left({ }^{\circ} \mathrm{C}\right)\end{array}$ & $\begin{array}{c}\mathrm{V} 10 \\
(\mathrm{~Pa} \cdot \mathrm{s})\end{array}$ & $\begin{array}{l}\mathrm{T} 11 \\
\left({ }^{\circ} \mathrm{C}\right)\end{array}$ & $\begin{array}{c}\mathrm{V} 11 \\
(\mathrm{~Pa} \cdot \mathrm{s})\end{array}$ & $\begin{array}{l}\mathrm{T} 12 \\
\left({ }^{\circ} \mathrm{C}\right)\end{array}$ & $\begin{array}{c}\mathrm{V} 12 \\
(\mathrm{~Pa} \cdot \mathrm{s})\end{array}$ \\
\hline SG09 & & & & & & & & & & & & & & & & & & & & & \\
\hline SG10 & & & & & & & & & & & & & & & & & & & & & \\
\hline SG11 & & & & & & & & & & & & & & & & & & & & & \\
\hline SG12 & & & & & & & & & & & & & & & & & & & & & \\
\hline SG13 & & & & & & & & & & & & & & & & & & & & & \\
\hline SG14 & & & & & & & & & & & & & & & & & & & & & \\
\hline SG15 & & & & & & & & & & & & & & & & & & & & & \\
\hline SG16 & & & & & & & & & & & & & & & & & & & & & \\
\hline SG17 & & & & & & & & & & & & & & & & & & & & & \\
\hline SG18a & & & & & & & & & & & & & & & & & & & & & \\
\hline SG18b & & & & & & & & & & & & & & & & & & & & & \\
\hline SG18c & & & & & & & & & & & & & & & & & & & & & \\
\hline SG18d & & & & & & & & & & & & & & & & & & & & & \\
\hline SG18e & & & & & & & & & & & & & & & & & & & & & \\
\hline SG18f & & & & & & & & & & & & & & & & & & & & & \\
\hline SG18g & & & & & & & & & & & & & & & & & & & & & \\
\hline SG19 & & & & & & & & & & & & & & & & & & & & & \\
\hline SG20 & & & & & & & & & & & & & & & & & & & & & \\
\hline $\mathrm{SG} 21$ & & & & & & & & & & & & & & & & & & & & & \\
\hline SG22 & & & & & & & & & & & & & & & & & & & & & \\
\hline SG23 & & & & & & & & & & & & & & & & & & & & & \\
\hline SG24 & & & & & & & & & & & & & & & & & & & & & \\
\hline SG25 & & & & & & & & & & & & & & & & & & & & & \\
\hline SG26 & & & & & & & & & & & & & & & & & & & & & \\
\hline SG27 & & & & & & & & & & & & & & & & & & & & & \\
\hline SG28 & & & & & & & & & & & & & & & & & & & & & \\
\hline SG29 & & & & & & & & & & & & & & & & & & & & & \\
\hline SG30 & & & & & & & & & & & & & & & & & & & & & \\
\hline SG31 & & & & & & & & & & & & & & & & & & & & & \\
\hline SG32 & & & & & & & & & & & & & & & & & & & & & \\
\hline SG33 & & & & & & & & & & & & & & & & & & & & & \\
\hline SG34 & & & & & & & & & & & & & & & & & & & & & \\
\hline SG35 & & & & & & & & & & & & & & & & & & & & & \\
\hline SG36 & & & & & & & & & & & & & & & & & & & & & \\
\hline SG37 & & & & & & & & & & & & & & & & & & & & & \\
\hline SG38 & & & & & & & & & & & & & & & & & & & & & \\
\hline SG39 & & & & & & & & & & & & & & & & & & & & & \\
\hline SG40 & & & & & & & & & & & & & & & & & & & & & \\
\hline SG41 & & & & & & & & & & & & & & & & & & & & & \\
\hline SG42 & & & & & & & & & & & & & & & & & & & & & \\
\hline SG43 & & & & & & & & & & & & & & & & & & & & & \\
\hline SG44 & & & & & & & & & & & & & & & & & & & & & \\
\hline SG45 & & & & & & & & & & & & & & & & & & & & & \\
\hline
\end{tabular}


Appendix A. Database - mass fraction

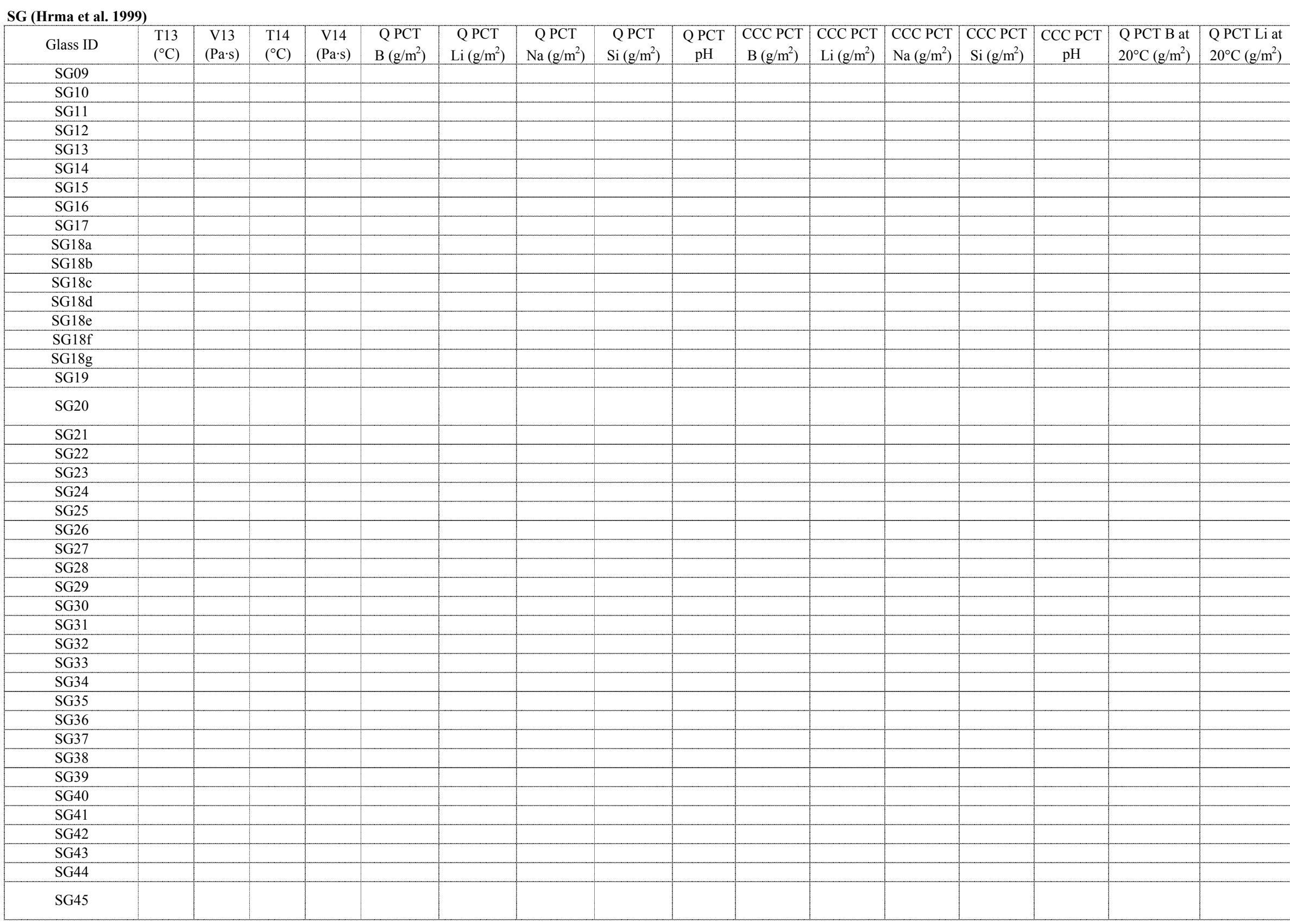


Appendix A. Database - mass fraction

SG (Hrma et al. 1999)

\begin{tabular}{|c|c|c|c|c|c|c|c|c|c|c|c|c|}
\hline Glass ID & $\begin{array}{l}\text { Q PCT Na at } \\
20^{\circ} \mathrm{C}\left(\mathrm{g} / \mathrm{m}^{2}\right)\end{array}$ & $\begin{array}{l}\text { Q PCT Si at } \\
20^{\circ} \mathrm{C}\left(\mathrm{g} / \mathrm{m}^{2}\right)\end{array}$ & $\begin{array}{c}\mathrm{QpH} \\
\text { at } 20^{\circ} \mathrm{C}\end{array}$ & $\begin{array}{c}\text { TCLP Ag } \\
\text { (ppm) }\end{array}$ & $\begin{array}{c}\text { TCLP As } \\
\text { (ppm) }\end{array}$ & $\begin{array}{c}\text { TCLP Ba } \\
\text { (ppm) }\end{array}$ & $\begin{array}{l}\text { TCLP Cd } \\
\text { (ppm) }\end{array}$ & $\begin{array}{c}\text { TCLP Cr } \\
\text { (ppm) }\end{array}$ & $\begin{array}{l}\text { TCLP Ni } \\
(\mathrm{ppm})\end{array}$ & $\begin{array}{c}\text { TCLP Pb } \\
\text { (ppm) }\end{array}$ & $\begin{array}{l}\text { TCLP Se } \\
(\mathrm{ppm})\end{array}$ & $\begin{array}{c}\text { TCLP Zn } \\
\text { (ppm) }\end{array}$ \\
\hline \multicolumn{13}{|l|}{ SG09 } \\
\hline \multicolumn{13}{|l|}{ SG10 } \\
\hline \multicolumn{13}{|l|}{ SG11 } \\
\hline \multicolumn{13}{|l|}{ SG12 } \\
\hline \multicolumn{13}{|l|}{ SG13 } \\
\hline \multicolumn{13}{|l|}{ SG14 } \\
\hline \multicolumn{13}{|l|}{ SG15 } \\
\hline \multicolumn{13}{|l|}{ SG16 } \\
\hline \multicolumn{13}{|l|}{ SG17 } \\
\hline \multicolumn{13}{|l|}{ SG18a } \\
\hline \multicolumn{13}{|l|}{ SG18b } \\
\hline \multicolumn{13}{|l|}{ SG18c } \\
\hline \multicolumn{13}{|l|}{ SG18d } \\
\hline \multicolumn{13}{|l|}{ SG18e } \\
\hline \multicolumn{13}{|l|}{ SG18f } \\
\hline \multicolumn{13}{|l|}{ SG18g } \\
\hline \multicolumn{13}{|l|}{ SG19 } \\
\hline \multicolumn{13}{|l|}{ SG20 } \\
\hline \multicolumn{13}{|l|}{ SG21 } \\
\hline \multicolumn{13}{|l|}{ SG22 } \\
\hline \multicolumn{13}{|l|}{ SG23 } \\
\hline SG24 & & & & & & & & & & & & \\
\hline SG25 & & & & & & & & & & & & \\
\hline SG26 & & & & & & & & & & & & \\
\hline SG27 & & & & & & & & & & & & \\
\hline SG28 & & & & & & & & & & & & \\
\hline SG29 & & & & & & & & & & & & \\
\hline SG30 & & & & & & & & & & & & \\
\hline SG31 & & & & & & & & & & & & \\
\hline SG32 & & & & & & & & & & & & \\
\hline SG33 & & & & & & & & & & & & \\
\hline SG34 & & & & & & & & & & & & \\
\hline SG35 & & & & & & & & & & & & \\
\hline SG36 & & & & & & & & & & & & \\
\hline SG37 & & & & & & & & & & & & \\
\hline SG38 & & & & & & & & & & & & \\
\hline SG39 & & & & & & & & & & & & \\
\hline SG40 & & & & & & & & & & & & \\
\hline SG41 & & & & & & & & & & & & \\
\hline SG42 & & & & & & & & & & & & \\
\hline SG43 & & & & & & & & & & & & \\
\hline SG44 & & & & & & & & & & & & \\
\hline SG45 & & & & & & & & & & & & \\
\hline
\end{tabular}




\begin{tabular}{|c|c|c|c|c|c|c|c|c|c|c|c|c|c|c|c|c|c|c|c|c|c|}
\hline Glass ID & $\begin{array}{c}\mathrm{Al} 2 \mathrm{O} 3 \\
-\mathrm{t}\end{array}$ & $\begin{array}{c}\mathrm{B} 2 \mathrm{O} 3 \\
-\mathrm{t}\end{array}$ & $\begin{array}{c}\mathrm{CaO} \\
-\mathrm{t}\end{array}$ & $\begin{array}{c}\mathrm{Fe} 2 \mathrm{O} 3 \\
-\mathrm{t}\end{array}$ & $\begin{array}{c}\mathrm{FeO} \\
-\mathrm{t}\end{array}$ & $\begin{array}{c}\mathrm{K} 2 \mathrm{O} \\
-\mathrm{t}\end{array}$ & $\begin{array}{c}\mathrm{Li} 2 \mathrm{O} \\
-\mathrm{t}\end{array}$ & $\begin{array}{c}\mathrm{MgO} \\
-\mathrm{t}\end{array}$ & $\begin{array}{c}\mathrm{Na} 2 \mathrm{O} \\
-\mathrm{t}\end{array}$ & $\begin{array}{c}\mathrm{NiO} \\
-\mathrm{t}\end{array}$ & $\begin{array}{c}\mathrm{P} 2 \mathrm{O} 5 \\
-\mathrm{t}\end{array}$ & $\begin{array}{c}\mathrm{SiO} 2 \\
-\mathrm{t}\end{array}$ & $\begin{array}{c}\mathrm{ZrO} 2 \\
-\mathrm{t}\end{array}$ & $\begin{array}{c}\mathrm{Ag} 2 \mathrm{O} \\
-\mathrm{t}\end{array}$ & $\begin{array}{c}\text { As2 } 203 \\
-t\end{array}$ & $\begin{array}{c}\mathrm{BaO} \\
-\mathrm{t}\end{array}$ & $\begin{array}{c}\mathrm{Bi} 2 \mathrm{O} 3 \\
-\mathrm{t}\end{array}$ & $\begin{array}{l}\mathrm{Br} \\
-\mathrm{t}\end{array}$ & $\begin{array}{c}\mathrm{CdO} \\
-\mathrm{t}\end{array}$ & $\begin{array}{c}\mathrm{Ce} 2 \mathrm{O} 3 \\
-\mathrm{t}\end{array}$ & $\begin{array}{c}\mathrm{CeO} 2 \\
-t\end{array}$ \\
\hline SG46 & 0.0250 & 0.0500 & 0.0030 & 0.1499 & & 0.0380 & 0.0599 & 0.0250 & 0.0599 & 0.0200 & 0.0000 & 0.4946 & 0.0000 & 0.0000 & 0.0000 & 0.0000 & 0.0000 & & 0.0000 & & 0.0000 \\
\hline SG47 & .0250 & 0.0500 & 0.0200 & 0.1499 & & 0.0150 & 0.0599 & 0.0250 & 0.1099 & 0.0200 & 0.0000 & 0.4551 & 0.0000 & 0.0000 & 0.0000 & 0.0000 & 0.0000 & & 0.0000 & & 0.0000 \\
\hline SG48 & 0270 & 0.0999 & 0.0030 & 0.0599 & & 0.0380 & 0.0300 & 0.0050 & 0.1099 & 0.0200 & 0.0000 & 0.5895 & 0.0000 & 0.0000 & 0.0000 & 0.0000 & 0.0000 & & 0.0000 & & 0.0000 \\
\hline SG49 & 0.0250 & 0.0500 & 0.0030 & 0.0809 & & 0.0380 & 0.0599 & 0.0050 & 0.0599 & 0.0005 & 0.0000 & 0.5895 & 0.0000 & 0.0000 & 0.0000 & 0.0000 & 0.0000 & & 0.0000 & & 0.0000 \\
\hline SG50 & 0.0250 & 0.0500 & 0.0200 & 0.1499 & & 0.0380 & 0.0300 & 0.0050 & 0.0599 & 0.0200 & 0.0000 & 0.5075 & 0.0000 & 0.0000 & 0.0000 & 0.0000 & 0.0000 & & 0.0000 & & 0.0000 \\
\hline SG51 & 0.0799 & 0.0500 & 0.0200 & 0.1499 & & 0.0380 & 0.0300 & 0.0050 & 0.1099 & 0.0005 & 0.0000 & 0.5015 & 0.0000 & 0.0000 & 0.0000 & 0.0000 & 0.0000 & & 0.0000 & & 0.0000 \\
\hline SG52a & 0.0250 & 0.0999 & 0.0030 & 0.1499 & & 0.0150 & 0.0599 & 0.0050 & 0.1099 & 0.0005 & 0.0000 & 0.4921 & 0.0000 & 0.0000 & 0.0000 & 0.0000 & 0.0000 & & 0.0000 & & 0.0000 \\
\hline SG52b & 0.0250 & 0.0999 & 0.0030 & 0.1499 & & 0.0150 & 0.0599 & 0.0050 & 0.1099 & 0.0005 & 0.0000 & 0.4921 & 0.0000 & 0.0000 & 0.0000 & 0.0000 & 0.0000 & & 0.0000 & & 0.0000 \\
\hline SG52c & 0.0250 & 0.0999 & 0.0030 & 0.1499 & & 0.0150 & 0.0599 & 0.0050 & 0.1099 & 0.0005 & 0.0000 & 0.4921 & 0.0000 & 0.0000 & 0.0000 & 0.0000 & 0.0000 & & 0.0000 & & 0.0000 \\
\hline SG52d & 0.0250 & 0.0999 & 0.0030 & 0.1499 & & 0.0150 & 0.0599 & 0.0050 & 0.1099 & 0.0005 & 0.0000 & 0.4921 & 0.0000 & 0.0000 & 0.0000 & 0.0000 & 0.0000 & & 0.0000 & & 0.0000 \\
\hline SG52e & 0.0250 & 0.0999 & 0.0030 & 0.1499 & & 0.0150 & 0.0599 & 0.0050 & 0.1099 & 0.0005 & 0.0000 & 0.4921 & 0.0000 & 0.0000 & 0.0000 & 0.0000 & 0.0000 & & 0.0000 & & 0.0000 \\
\hline SG53 & 0.0530 & 0.0752 & 0.0115 & 0.1052 & & 0.0266 & 0.0450 & 0.0150 & 0.0852 & 0.0102 & 0.0000 & 0.5186 & 0.0000 & 0.0000 & 0.0000 & 0.0000 & 0.0000 & & 0.0000 & & 0.0000 \\
\hline
\end{tabular}

SP3, SPx4, Misc. (Vienna et al. 2001)

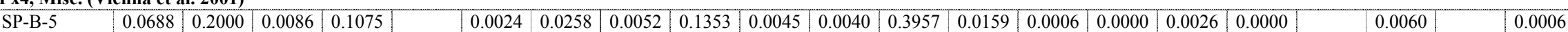

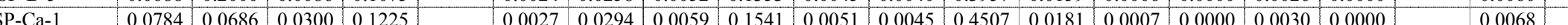

\begin{tabular}{l|l|l|l|l|l|l|l|l} 
& 0.0768 & 0.0672 & 0.0500 & 0.1199
\end{tabular}

\begin{tabular}{|l|l|l|l|l|l|}
\hline SP-Fe-4 & 0.0704 & 0.0616 & 0.0088 & 0.2300 \\
\hline
\end{tabular}

\begin{tabular}{|l|l|l|l|l|l|}
\hline SP-K-1 & 0.0786 & 0.0688 & 0.0098 & 0.1228 \\
\hline
\end{tabular}

\begin{tabular}{l|l|l|l|l|l|l|} 
SP-K-2 & 0.0770 & 0.0674 & 0.0096 & 0.1203
\end{tabular}

\begin{tabular}{l|l|l|l|l|l|l}
\hline SP-Li-5 & 0.0788 & 0.0689 & 0.0098 & 0.1231
\end{tabular}

\begin{tabular}{|l|l|l|l|l|}
\hline SP-Li-6 & 0.0763 & 0.0668 & 0.0095 & 0.1192 \\
\hline
\end{tabular}

\begin{tabular}{|l|l|l|l|l|}
\hline SP-Si-4 & 0.1037 & 0.0907 & 0.0130 & 0.1620 \\
\hline
\end{tabular}

\begin{tabular}{l|l|l|l|l|l|l} 
SP-Ti-1 & 0.0780 & 0.0683 & 0.0098 & 0.1219
\end{tabular}

\begin{tabular}{l|l|l|l|l|l|} 
SP-Ti-2 & 0.0760 & 0.0665 & 0.0095 & 0.1188 \\
\hline
\end{tabular}

\begin{tabular}{|l|l|l|l|l|}
\hline SP-Zr-1 & 0.0782 & 0.0685 & 0.0098 & 0.1223 \\
\hline
\end{tabular}

\begin{tabular}{l|l|l|l|l|l|}
\hline SP-Ru-1 & 0.0800 & 0.0700 & 0.0000 & 0.1249 \\
\hline
\end{tabular}

\begin{tabular}{l|l|l|l|l|l|l|l|l|l|l|l|l}
\hline SP-Ru-2 & 0.0799 & 0.0699 & 0.0000 & 0.1249 \\
\hline
\end{tabular}

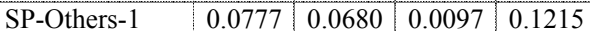

\begin{tabular}{|l|l|l|l|l|l|}
\hline SP3-1(env.D) & 0.1014 & 0.0699 & 0.0404 & 0.2297 \\
\hline
\end{tabular}

\begin{tabular}{l|l|l|l|l|l} 
SP-MC-1 & 0.1600 & 0.1500 & 0.0000 & 0.0500
\end{tabular} \begin{tabular}{|l|l|l|l|l|l|l|l|l|l|l|l|l|l|l|l|l|}
0.0027 & 0.0288 & 0.0058 & 0.1509 & 0.0050 & 0.0044 & 0.4414 & 0.0178 & 0.0007 & 0.0000 & 0.0029 & 0.0000 & & 0.0067 & 0.0007 \\
\hline
\end{tabular} \begin{tabular}{|l|l|l|l|l|l|l|l|l|l|l|l|l|l|l|l|}
\hline 0.0025 & 0.0264 & 0.0053 & 0.1384 & 0.0046 & 0.0041 & 0.4048 & 0.0163 & 0.0006 & 0.0000 & 0.0027 & 0.0000 & & 0.0061 & 0.0006 \\
\hline
\end{tabular} \begin{tabular}{|l|l|l|l|l|l|l|l|l|l|l|l|l|l|l|l|l|}
0.0200 & 0.0295 & 0.0059 & 0.1546 & 0.0051 & 0.0045 & 0.4521 & 0.0182 & 0.0007 & 0.0000 & 0.0030 & 0.0000 & & 0.0068 & & 0.0007 \\
\hline
\end{tabular} \begin{tabular}{|l|l|l|l|l|l|l|l|l|l|l|l|l|l|l|l|l|l|l|}
0.0400 & 0.0289 & 0.0058 & 0.1514 & 0.0050 & 0.0045 & 0.4428 & 0.0178 & 0.0007 & 0.0000 & 0.0029 & 0.0000 & & 0.0067 & 0.0007 \\
\hline
\end{tabular} \begin{tabular}{|l|l|l|l|l|l|l|l|l|l|l|l|l|l|l|l|l|}
0.0028 & 0.0450 & 0.0059 & 0.1549 & 0.0051 & 0.0046 & 0.4529 & 0.0182 & 0.0007 & 0.0000 & 0.0030 & 0.0000 & & 0.0068 & 0.0007 \\
\hline
\end{tabular} \begin{tabular}{|l|l|l|l|l|l|l|l|l|l|l|l|l|l|l|l|l|l|l|}
0.0027 & 0.0750 & 0.0057 & 0.1500 & 0.0050 & 0.0044 & 0.4387 & 0.0176 & 0.0007 & 0.0000 & 0.0029 & 0.0000 & & 0.0066 & 0.0007 \\
\hline
\end{tabular} \begin{tabular}{|l|l|l|l|l|l|l|l|l|l|l|l|l|l|l|l|l|}
0.0036 & 0.0389 & 0.0078 & 0.2039 & 0.0067 & 0.0060 & 0.3000 & 0.0240 & 0.0009 & 0.0000 & 0.0039 & 0.0000 & & 0.0090 & & 0.0009 \\
\hline
\end{tabular} \begin{tabular}{|l|l|l|l|l|l|l|l|l|l|l|l|l|l|l|l|l|l|}
0.0027 & 0.0293 & 0.0059 & 0.1534 & 0.0051 & 0.0045 & 0.4486 & 0.0180 & 0.0007 & 0.0000 & 0.0029 & 0.0000 & & 0.0068 & 0.0007 \\
\hline
\end{tabular} \begin{tabular}{|l|l|l|l|l|l|l|l|l|l|l|l|l|l|l|l|l|l|}
0.0027 & 0.0285 & 0.0057 & 0.1495 & 0.0049 & 0.0044 & 0.4371 & 0.0176 & 0.0007 & 0.0000 & 0.0029 & 0.0000 & & 0.0066 & 0.0007 \\
\hline
\end{tabular} \begin{tabular}{|l|l|l|l|l|l|l|l|l|l|l|l|l|l|l|l|l|l|l|l|l|l|l|l|}
0.0027 & 0.0293 & 0.0059 & 0.1539 & 0.0051 & 0.0045 & 0.4499 & 0.0400 & 0.0007 & 0.0000 & 0.0030 & 0.0000 & & 0.0068 & 0.0007 \\
\hline
\end{tabular} \begin{tabular}{|l|l|l|l|l|l|l|l|l|l|l|l|l|l|l|l|l|l|}
0.0000 & 0.0300 & 0.0060 & 0.1572 & 0.0052 & 0.0059 & 0.4597 & 0.0234 & 0.0009 & 0.0000 & 0.0038 & 0.0000 & & 0.0088 & & 0.0009 \\
\hline
\end{tabular} \begin{tabular}{|l|l|l|l|l|l|l|l|l|l|l|l|l|l|l|l|l|l|}
0.0000 & 0.0300 & 0.0060 & 0.1572 & 0.0052 & 0.0059 & 0.4596 & 0.0234 & 0.0009 & 0.0000 & 0.0038 & 0.0000 & & 0.0088 & 0.0009 \\
\hline
\end{tabular}

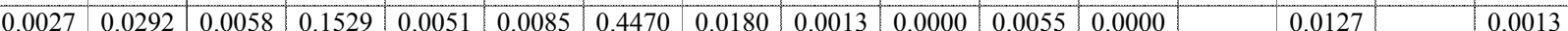
\begin{tabular}{|l|l|l|l|l|l|l|l|l|l|l|l|l|l|l|l|l|}
\hline 0.0086 & 0.0300 & 0.0011 & 0.1575 & 0.0010 & 0.0079 & 0.3021 & 0.0000 & 0.0012 & 0.0000 & 0.0052 & 0.0000 & & 0.0119 & 0.0012 \\
\hline
\end{tabular} \begin{tabular}{|l|l|l|l|l|l|l|l|l|l|l|l|l|l|l|l|l|l|}
0.0400 & 0.0600 & 0.0000 & 0.1000 & 0.0300 & 0.0000 & 0.3600 & 0.0000 & 0.0000 & 0.0000 & 0.0000 & 0.0000 & & 0.0000 & & 0.0000 \\
\hline
\end{tabular}

\begin{tabular}{l|l|l|l|l|l} 
SP-MC-2 & 0.0000 & 0.1500 & 0.0000 & 0.0500
\end{tabular}

\begin{tabular}{l|l|l|l|l|l|l}
\hline SP-MC-8 & 0.0057 & 0.0000 & 0.0500 & 0.0500
\end{tabular}

\begin{tabular}{l|l|l|l|l|l}
\hline SP-MC-9 & 0.0573 & 0.1500 & 0.0500 & 0.0500
\end{tabular} \begin{tabular}{l|l|l|l|l|l|l|}
\hline Sp-LHLL & 0.0500 & 0.0706 & 0.0000 & 0.1260 \\
\hline
\end{tabular} \begin{tabular}{|l|l|l|l|l|l|l|l|l|l|l|l|l|l|l|l|}
\hline 0.0400 & 0.0000 & 0.0300 & 0.1700 & 0.0000 & 0.0085 & 0.3600 & 0.0800 & 0.0013 & 0.0000 & 0.0055 & 0.0000 \\
\hline
\end{tabular} \begin{tabular}{|l|l|l|l|l|l|l|l|l|l|l|l|}
\hline 0.0000 & 0.0600 & 0.0300 & 0.1355 & 0.0300 & 0.0085 & 0.5000 & 0.0288 & 0.0013 & 0.0000 & 0.0055 & 0.0000 \\
\hline
\end{tabular} \begin{tabular}{|l|l|l|l|l|l|l|l|l|l|l|l|l|l|}
\hline 0.0000 & 0.0527 & 0.0300 & 0.0500 & 0.0000 & 0.0000 & 0.5000 & 0.0000 & 0.0000 & 0.0000 & 0.0000 & 0.0000 \\
\hline
\end{tabular} \begin{tabular}{|l|l|l|l|l|l|l|l|l|l|l|l|l|l|}
0.0000 & 0.0302 & 0.0061 & 0.1873 & 0.0010 & 0.0059 & 0.4636 & 0.0236 & 0.0009 & 0.0000 & 0.0039 & 0.0000 \\
\hline
\end{tabular} \begin{tabular}{l|l|l|l|l|l|l} 
Sp-LHLH & 0.0500 & 0.0688 & 0.0000 & 0.1228 \\
\hline
\end{tabular} \begin{tabular}{l|l|l|l|l|l}
\hline Sp-LHHL & 0.0500 & 0.0700 & 0.0000 & 0.1251
\end{tabular} \begin{tabular}{l|l|l|l|l|l|}
\hline Sp-LHHH & 0.0500 & 0.0683 & 0.0000 & 0.1219 \\
\hline
\end{tabular} \begin{tabular}{l|l|l|l|l|}
\hline Sp-LHMM & 0.0500 & 0.0700 & 0.0000 & 0.1250
\end{tabular} \begin{tabular}{|l|l|l|l|l|l|l|l|l|l|l|l|l|l|l|}
\hline 0.0000 & 0.0295 & 0.0059 & 0.1873 & 0.0200 & 0.0058 & 0.4520 & 0.0231 & 0.0009 & 0.0000 & 0.0038 & 0.0000 \\
\hline
\end{tabular} \begin{tabular}{|l|l|l|l|l|l|l|l|l|l|l|l|l|l|}
\hline 0.0000 & 0.0300 & 0.0060 & 0.1873 & 0.0010 & 0.0059 & 0.4602 & 0.0234 & 0.0009 & 0.0000 & 0.0038 & 0.0000 \\
\hline
\end{tabular} \begin{tabular}{|l|l|l|l|l|l|l|l|l|l|l|l|}
0.0000 & 0.0293 & 0.0059 & 0.1873 & 0.0200 & 0.0058 & 0.4487 & 0.0229 & 0.0009 & 0.0000 & 0.0037 & 0.0000 \\
\hline
\end{tabular} \begin{tabular}{|l|l|l|l|l|l|l|l|l|l|l|l|}
0.0000 & 0.0300 & 0.0060 & 0.1873 & 0.0052 & 0.0059 & 0.4600 & 0.0234 & 0.0009 & 0.0000 & 0.0038 & 0.0000 \\
\hline
\end{tabular} \begin{tabular}{l|l|l|l|l|l|l} 
Sp-MMLL & 0.0800 & 0.0706 & 0.0000 & 0.1260
\end{tabular} \begin{tabular}{l|l|l|l|l|l|l|} 
Sp-MMLH & 0.0800 & 0.0688 & 0.0000 & 0.1228 \\
\hline
\end{tabular} \begin{tabular}{l|l|l|l|l|l|}
\hline Sp-MMHL & 0.0800 & 0.0700 & 0.0000 & 0.1251 \\
\hline
\end{tabular} \begin{tabular}{l|l|l|l|l|l|}
\hline Sp-MMHH & 0.0800 & 0.0683 & 0.0000 & 0.1219 \\
\hline
\end{tabular} \begin{tabular}{|l|l|l|l|l|l|l|l|l|l|l|l|l|l|l|}
\hline 0.0000 & 0.0302 & 0.0061 & 0.1573 & 0.0010 & 0.0059 & 0.4636 & 0.0236 & 0.0009 & 0.0000 & 0.0039 & 0.0000 \\
\hline
\end{tabular} \begin{tabular}{|l|l|l|l|l|l|l|l|l|l|l|l|l|l|}
\hline 0.0000 & 0.0295 & 0.0059 & 0.1573 & 0.0200 & 0.0058 & 0.4520 & 0.0231 & 0.0009 & 0.0000 & 0.0038 & 0.0000 \\
\hline
\end{tabular} \begin{tabular}{|l|l|l|l|l|l|l|l|l|l|l|l|}
0.0000 & 0.0300 & 0.0060 & 0.1573 & 0.0010 & 0.0059 & 0.4602 & 0.0234 & 0.0009 & 0.0000 & 0.0038 & 0.0000 \\
\hline
\end{tabular} \begin{tabular}{|l|l|l|l|l|l|l|l|l|l|l|l|}
0.0000 & 0.0293 & 0.0059 & 0.1573 & 0.0200 & 0.0058 & 0.4487 & 0.0229 & 0.0009 & 0.0000 & 0.0037 & 0.0000 \\
\hline
\end{tabular} \begin{tabular}{l|l|l|l|l|l|l} 
Sp-MMMM & 0.0800 & 0.0700 & 0.0000 & 0.1250
\end{tabular} \begin{tabular}{l|l|l|l|l|l|l} 
Sp-HLLL & 0.1100 & 0.0706 & 0.0000 & 0.1260
\end{tabular} \begin{tabular}{l|l|l|l|l|l}
\hline Sp-HLLH & 0.1100 & 0.0688 & 0.0000 & 0.1228 \\
\hline
\end{tabular} \begin{tabular}{l|l|l|l|l|l|l} 
Sp-HLHL & 0.1100 & 0.0700 & 0.0000 & 0.1251
\end{tabular}

\begin{tabular}{l|l|l|l|l|l|l} 
Sp-HLHH & 0.1100 & 0.0683 & 0.0000 & 0.1219
\end{tabular} \begin{tabular}{|l|l|l|l|l|l|l|l|l|l|l|l|l|l|l|}
\hline 0.0000 & 0.0300 & 0.0060 & 0.1573 & 0.0052 & 0.0059 & 0.4600 & 0.0234 & 0.0009 & 0.0000 & 0.0038 & 0.0000 \\
\hline
\end{tabular} \begin{tabular}{|l|l|l|l|l|l|l|l|l|l|l|l|l|}
0.0000 & 0.0302 & 0.0061 & 0.1273 & 0.0010 & 0.0059 & 0.4636 & 0.0236 & 0.0009 & 0.0000 & 0.0039 & 0.0000 \\
\hline
\end{tabular} \begin{tabular}{|l|l|l|l|l|l|l|l|l|l|l|l|l|}
\hline 0.0000 & 0.0295 & 0.0059 & 0.1273 & 0.0200 & 0.0058 & 0.4520 & 0.0231 & 0.0009 & 0.0000 & 0.0038 & 0.0000 \\
\hline
\end{tabular}

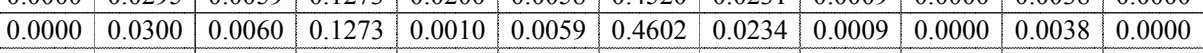
\begin{tabular}{|l|l|l|l|l|l|l|l|l|l|l|l|l|l|l|}
0.0000 & 0.0293 & 0.0059 & 0.1273 & 0.0200 & 0.0058 & 0.4487 & 0.0229 & 0.0009 & 0.0000 & 0.0037 & 0.0000 \\
\hline
\end{tabular} \begin{tabular}{l|l|l|l|l|l}
\hline Sp-HLMM & 0.1100 & 0.0700 & 0.0000 & 0.1250
\end{tabular} 
SG (Hrma et al. 1999)

\begin{tabular}{|c|c|c|c|c|c|c|c|c|c|c|c|c|c|c|c|c|c|c|c|c|c|}
\hline Glass ID & $\begin{array}{l}\mathrm{Cl} \\
-\mathrm{t}\end{array}$ & $\begin{array}{c}\mathrm{CoO} \\
-\mathrm{t}\end{array}$ & $\begin{array}{c}\mathrm{Co} 2 \mathrm{O} 3 \\
-\mathrm{t}\end{array}$ & $\begin{array}{c}\mathrm{Cr} 2 \mathrm{O} 3 \\
-\mathrm{t}\end{array}$ & $\begin{array}{c}\mathrm{Cs} 2 \mathrm{O} \\
-\mathrm{t}\end{array}$ & $\begin{array}{c}\mathrm{CuO} \\
-\mathrm{t}\end{array}$ & $\begin{array}{c}\mathrm{Eu} 2 \mathrm{O} 3 \\
-\mathrm{t}\end{array}$ & $\begin{array}{l}F \\
-t\end{array}$ & $\begin{array}{c}\mathrm{Ga} 2 \mathrm{O} 3 \\
-\mathrm{t}\end{array}$ & $\begin{array}{c}\mathrm{Gd} 2 \mathrm{O} 3 \\
-\mathrm{t}\end{array}$ & $\begin{array}{c}\mathrm{HgO} \\
-\mathrm{t}\end{array}$ & $\begin{array}{l}I \\
-t\end{array}$ & $\begin{array}{c}\mathrm{La} 2 \mathrm{O} 3 \\
-\mathrm{t}\end{array}$ & $\begin{array}{c}\mathrm{MnO} 2 \\
-\mathrm{t}\end{array}$ & $\begin{array}{c}\mathrm{MnO} \\
-\mathrm{t}\end{array}$ & $\begin{array}{c}\mathrm{MoO} \\
-\mathrm{t}\end{array}$ & $\begin{array}{c}\mathrm{MoO} 3 \\
-\mathrm{t}\end{array}$ & $\begin{array}{c}\mathrm{Nb} 2 \mathrm{O} 5 \\
-\mathrm{t}\end{array}$ & $\begin{array}{c}\mathrm{Nd} 2 \mathrm{O} 3 \\
-\mathrm{t}\end{array}$ & $\begin{array}{c}\mathrm{PbO} \\
-\mathrm{t}\end{array}$ & $\begin{array}{c}\mathrm{PdO} 2 \\
-\mathrm{t}\end{array}$ \\
\hline SG46 & 0.0000 & & 0.0000 & 0.0030 & 0.0000 & 0.0000 & & 0.0000 & & & & & 0.0000 & & 0.0100 & & 0.0000 & & 0.0000 & 0.0000 & \\
\hline SG47 & 0.0000 & & 0.0000 & 0.0030 & 0.0000 & 0.0000 & & 0.0000 & & & & & 0.0000 & & 0.0100 & & 0.0000 & & 0.0000 & 0.0000 & \\
\hline SG48 & 0.0000 & & 0.0000 & 0.0010 & 0.0000 & 0.0000 & & 0.0000 & & & & & 0.0000 & & 0.0100 & & 0.0000 & & 0.0000 & 0.0000 & \\
\hline SG49 & 0.0000 & & 0.0000 & 0.0010 & 0.0000 & 0.0000 & & 0.0000 & & & & & 0.0000 & & 0.0300 & & 0.0000 & & 0.0000 & 0.0000 & \\
\hline SG50 & 0.0000 & & 0.0000 & 0.0030 & 0.0000 & 0.0000 & & 0.0000 & & & & & 0.0000 & & 0.0300 & & 0.0000 & & 0.0000 & 0.0000 & \\
\hline SG51 & 0.0000 & & 0.0000 & 0.0030 & 0.0000 & 0.0000 & & 0.0000 & & & & & 0.0000 & & 0.0100 & & 0.0000 & & 0.0000 & 0.0000 & \\
\hline SG52a & 0.0000 & & 0.0000 & 0.0030 & 0.0000 & 0.0000 & & 0.0000 & & & & & 0.0000 & & 0.0300 & & 0.0000 & & 0.0000 & 0.0000 & \\
\hline SG52b & 0.0000 & & 0.0000 & 0.0030 & 0.0000 & 0.0000 & & 0.0000 & & & & & 0.0000 & & 0.0300 & & 0.0000 & & 0.0000 & 0.0000 & \\
\hline SG52c & 0.0000 & & 0.0000 & 0.0030 & 0.0000 & 0.0000 & & 0.0000 & & & & & 0.0000 & & 0.0300 & & 0.0000 & & 0.0000 & 0.0000 & \\
\hline SG52d & 0.0000 & & 0.0000 & 0.0030 & 0.0000 & 0.0000 & & 0.0000 & & & & & 0.0000 & & 0.0300 & & 0.0000 & & 0.0000 & 0.0000 & \\
\hline SG52e & 0.0000 & & 0.0000 & 0.0030 & 0.0000 & 0.0000 & & 0.0000 & & & & & 0.0000 & & 0.0300 & & 0.0000 & & 0.0000 & 0.0000 & \\
\hline SG53 & 0.0000 & & 0.0000 & 0.0020 & 0.0000 & 0.0000 & & 0.0000 & & & & & 0.0000 & & 0.0200 & & 0.0000 & & 0.0000 & 0.0000 & \\
\hline \multicolumn{22}{|c|}{ SP3, SPx4, Misc. (Vienna et al. 2001) } \\
\hline SP-B-5 & 0.0001 & & 0.0008 & 0.0019 & 0.0000 & 0.0003 & & 0.0005 & & & & & 0.0023 & & 0.0031 & & 0.0001 & & 0.0016 & 0.0015 & \\
\hline SP-Ca-1 & 0.0001 & & 0.0009 & 0.0022 & 0.0000 & 0.0003 & & 0.0006 & & & & & 0.0027 & & 0.0036 & & 0.0001 & & 0.0018 & 0.0017 & \\
\hline SP-Ca-2 & 0.0001 & & 0.0009 & 0.0021 & 0.0000 & 0.0003 & & 0.0006 & & & & & 0.0026 & & 0.0035 & & 0.0001 & & 0.0017 & 0.0016 & \\
\hline SP-Fe-4 & 0.0001 & & 0.0008 & 0.0019 & 0.0000 & 0.0003 & & 0.0005 & & & & & 0.0024 & & 0.0032 & & 0.0001 & & 0.0016 & 0.0015 & \\
\hline SP-K-1 & 0.0001 & & 0.0009 & 0.0022 & 0.0000 & 0.0003 & & 0.0006 & & & & & 0.0027 & & 0.0036 & & 0.0001 & & 0.0018 & 0.0017 & \\
\hline SP-K-2 & 0.0001 & & 0.0009 & 0.0021 & 0.0000 & 0.0003 & & 0.0006 & & & & & 0.0026 & & 0.0035 & & 0.0001 & & 0.0017 & 0.0016 & \\
\hline SP-Li-5 & 0.0001 & & 0.0009 & 0.0022 & 0.0000 & 0.0003 & & 0.0006 & & & & & 0.0027 & & 0.0036 & & 0.0001 & & 0.0018 & 0.0017 & \\
\hline SP-Li-6 & 0.0001 & & 0.0009 & 0.0021 & 0.0000 & 0.0003 & & 0.0006 & & & & & 0.0026 & & 0.0035 & & 0.0001 & & 0.0017 & 0.0016 & \\
\hline SP-Si-4 & 0.0001 & & 0.0012 & 0.0029 & 0.0000 & 0.0004 & & 0.0008 & & & & & 0.0035 & & 0.0047 & & 0.0001 & & 0.0023 & 0.0022 & \\
\hline SP-Ti-1 & 0.0001 & & 0.0009 & 0.0021 & 0.0000 & 0.0003 & & 0.0006 & & & & & 0.0026 & & 0.0035 & & 0.0001 & & 0.0018 & 0.0017 & \\
\hline SP-Ti-2 & 0.0001 & & 0.0009 & 0.0021 & 0.0000 & 0.0003 & & 0.0006 & & & & & 0.0026 & & 0.0034 & & 0.0001 & & 0.0017 & 0.0016 & \\
\hline SP-Zr-1 & 0.0001 & & 0.0009 & 0.0022 & 0.0000 & 0.0003 & & 0.0006 & & & & & 0.0027 & & 0.0036 & & 0.0001 & & 0.0018 & 0.0017 & \\
\hline SP-Ru-1 & 0.0001 & & 0.0011 & 0.0022 & 0.0000 & 0.0004 & & 0.0008 & & & & & 0.0035 & & 0.0036 & & 0.0001 & & 0.0023 & 0.0022 & \\
\hline SP-Ru-2 & 0.0001 & & 0.0011 & 0.0022 & 0.0000 & 0.0004 & & 0.0008 & & & & & 0.0034 & & 0.0036 & & 0.0001 & & 0.0023 & 0.0022 & \\
\hline SP-Others-1 & 0.0002 & & 0.0017 & 0.0021 & 0.0000 & 0.0006 & & 0.0011 & & & & & 0.0050 & & 0.0067 & & 0.0002 & & 0.0033 & 0.0031 & \\
\hline SP3-1(env.D) & 0.0002 & & 0.0016 & 0.0004 & 0.0000 & 0.0005 & & 0.0010 & & & & & 0.0047 & & 0.0062 & & 0.0002 & & 0.0031 & 0.0029 & \\
\hline SP-MC-1 & 0.0000 & & 0.0000 & 0.0000 & 0.0000 & 0.0000 & & 0.0000 & & & & & 0.0000 & & 0.0000 & & 0.0000 & & 0.0000 & 0.0000 & \\
\hline SP-MC-2 & 0.0002 & & 0.0017 & 0.0100 & 0.0000 & 0.0006 & & 0.0011 & & & & & 0.0050 & & 0.0067 & & 0.0002 & & 0.0033 & 0.0031 & \\
\hline SP-MC-8 & 0.0002 & & 0.0017 & 0.0000 & 0.0000 & 0.0006 & & 0.0011 & & & & & 0.0050 & & 0.0067 & & 0.0002 & & 0.0033 & 0.0031 & \\
\hline SP-MC-9 & 0.0000 & & 0.0000 & 0.0100 & 0.0000 & 0.0000 & & 0.0000 & & & & & 0.0000 & & 0.0000 & & 0.0000 & & 0.0000 & 0.0000 & \\
\hline Sp-LHLL & 0.0001 & & 0.0012 & 0.0005 & 0.0000 & 0.0004 & & 0.0008 & & & & & 0.0035 & & 0.0036 & & 0.0001 & & 0.0023 & 0.0022 & \\
\hline Sp-LHLH & 0.0001 & & 0.0011 & 0.0005 & 0.0000 & 0.0004 & & 0.0008 & & & & & 0.0034 & & 0.0035 & & 0.0001 & & 0.0023 & 0.0021 & \\
\hline Sp-LHHL & 0.0001 & & 0.0011 & 0.0060 & 0.0000 & 0.0004 & & 0.0008 & & & & & 0.0035 & & 0.0036 & & 0.0001 & & 0.0023 & 0.0022 & \\
\hline Sp-LHHH & 0.0001 & & 0.0011 & 0.0060 & 0.0000 & 0.0004 & & 0.0007 & & & & & 0.0034 & & 0.0035 & & 0.0001 & & 0.0022 & 0.0021 & \\
\hline Sp-LHMM & 0.0001 & & 0.0011 & 0.0022 & 0.0000 & 0.0004 & & 0.0008 & & & & & 0.0035 & & 0.0036 & & 0.0001 & & 0.0023 & 0.0022 & \\
\hline Sp-MMLL & 0.0001 & & 0.0012 & 0.0005 & 0.0000 & 0.0004 & & 0.0008 & & & & & 0.0035 & & 0.0036 & & 0.0001 & & 0.0023 & 0.0022 & \\
\hline Sp-MMLH & 0.0001 & & 0.0011 & 0.0005 & 0.0000 & 0.0004 & & 0.0008 & & & & & 0.0034 & & 0.0035 & & 0.0001 & & 0.0023 & 0.0021 & \\
\hline Sp-MMHL & 0.0001 & & 0.0011 & 0.0060 & 0.0000 & 0.0004 & & 0.0008 & & & & & 0.0035 & & 0.0036 & & 0.0001 & & 0.0023 & 0.0022 & \\
\hline Sp-MMHH & 0.0001 & & 0.0011 & 0.0060 & 0.0000 & 0.0004 & & 0.0007 & & & & & 0.0034 & & 0.0035 & & 0.0001 & & 0.0022 & 0.0021 & \\
\hline Sp-MMMM & 0.0001 & & 0.0011 & 0.0022 & 0.0000 & 0.0004 & & 0.0008 & & & & & 0.0035 & & 0.0036 & & 0.0001 & & 0.0023 & 0.0022 & \\
\hline Sp-HLLL & 0.0001 & & 0.0012 & 0.0005 & 0.0000 & 0.0004 & & 0.0008 & & & & & 0.0035 & & 0.0036 & & 0.0001 & & 0.0023 & 0.0022 & \\
\hline Sp-HLLH & 0.0001 & & 0.0011 & 0.0005 & 0.0000 & 0.0004 & & 0.0008 & & & & & 0.0034 & & 0.0035 & & 0.0001 & & 0.0023 & 0.0021 & \\
\hline Sp-HLHL & 0.0001 & & 0.0011 & 0.0060 & 0.0000 & 0.0004 & & 0.0008 & & & & & 0.0035 & & 0.0036 & & 0.0001 & & 0.0023 & 0.0022 & \\
\hline Sp-HLHH & 0.0001 & & 0.0011 & 0.0060 & 0.0000 & 0.0004 & & 0.0007 & & & & & 0.0034 & & 0.0035 & & 0.0001 & & 0.0022 & 0.0021 & \\
\hline Sp-HLMM & 0.0001 & & 0.0011 & 0.0022 & 0.0000 & 0.0004 & & 0.0008 & & & & & 0.0035 & & 0.0036 & & 0.0001 & & 0.0023 & 0.0022 & \\
\hline
\end{tabular}


SG (Hrma et al. 1999)

\begin{tabular}{|c|c|c|c|c|c|c|c|c|c|c|c|c|c|c|c|c|c|c|c|c|c|}
\hline Glass ID & $\begin{array}{c}\mathrm{PdO} \\
-\mathrm{t} \\
\end{array}$ & $\begin{array}{c}\mathrm{Pr} 2 \mathrm{O} 3 \\
-\mathrm{t}\end{array}$ & $\begin{array}{c}\text { Pr6O11 } \\
-t\end{array}$ & $\begin{array}{c}\mathrm{Rb} 2 \mathrm{O} \\
-\mathrm{t}\end{array}$ & $\begin{array}{c}\mathrm{ReO} \\
-\mathrm{t}\end{array}$ & $\begin{array}{c}\mathrm{ReO} 2 \\
-\mathrm{t}\end{array}$ & $\begin{array}{c}\mathrm{Rh} 2 \mathrm{O} 3 \\
-\mathrm{t}\end{array}$ & $\begin{array}{c}\mathrm{RhO} 2 \\
-\mathrm{t}\end{array}$ & $\begin{array}{c}\mathrm{RuO} 2 \\
-\mathrm{t}\end{array}$ & $\begin{array}{c}\mathrm{Sb} 2 \mathrm{O} 3 \\
-\mathrm{t}\end{array}$ & $\begin{array}{c}\mathrm{Sb} 2 \mathrm{O} 5 \\
-\mathrm{t}\end{array}$ & $\begin{array}{c}\mathrm{SeO} 2 \\
-\mathrm{t}\end{array}$ & $\begin{array}{c}\mathrm{Sm} 2 \mathrm{O} 3 \\
-\mathrm{t}\end{array}$ & $\begin{array}{c}\mathrm{SnO} \\
-\mathrm{t}\end{array}$ & $\begin{array}{c}\mathrm{SnO} 2 \\
-\mathrm{t}\end{array}$ & $\begin{array}{c}\mathrm{SO} 3 \\
-\mathrm{t}\end{array}$ & $\begin{array}{c}\mathrm{SrO} \\
-\mathrm{t}\end{array}$ & $\begin{array}{c}\mathrm{Tc} 2 \mathrm{O} 7 \\
-\mathrm{t}\end{array}$ & $\begin{array}{c}\mathrm{TeO} 2 \\
-\mathrm{t}\end{array}$ & $\begin{array}{c}\mathrm{ThO} 2 \\
-\mathrm{t}\end{array}$ & $\begin{array}{c}\mathrm{TiO} 2 \\
-\mathrm{t}\end{array}$ \\
\hline SG46 & 0.0000 & & 0.0000 & 0.0000 & & & 0.0000 & & 0.0009 & 0.0000 & & 0.0000 & 0.0000 & & & 0.0000 & 0.0000 & & 0.0000 & & 0.0060 \\
\hline SG47 & 0.0000 & & 0.0000 & 0.0000 & & & 0.0000 & & 0.0009 & 0.0000 & & 0.0000 & 0.0000 & & & 0.0000 & 0.0000 & & 0.0000 & & 0.0015 \\
\hline SG48 & 0.0000 & & 0.0000 & 0.0000 & & & 0.0000 & & 0.0009 & 0.0000 & & 0.0000 & 0.0000 & & & 0.0000 & 0.0000 & & 0.0000 & & 0.0060 \\
\hline SG49 & 0.0000 & & 0.0000 & 0.0000 & & & 0.0000 & & 0.0009 & 0.0000 & & 0.0000 & 0.0000 & & & 0.0000 & 0.0000 & & 0.0000 & & 0.0015 \\
\hline SG50 & 0.0000 & & 0.0000 & 0.0000 & & & 0.0000 & & 0.0009 & 0.0000 & & 0.0000 & 0.0000 & & & 0.0000 & 0.0000 & & 0.0000 & & 0.0060 \\
\hline SG51 & 0.0000 & & 0.0000 & 0.0000 & & & 0.0000 & & 0.0009 & 0.0000 & & 0.0000 & 0.0000 & & & 0.0000 & 0.0000 & & 0.0000 & & 0.0015 \\
\hline SG52a & 0.0000 & & 0.0000 & 0.0000 & & & 0.0000 & & 0.0009 & 0.0000 & & 0.0000 & 0.0000 & & & 0.0000 & 0.0000 & & 0.0000 & & 0.0060 \\
\hline SG52b & 0.0000 & & 0.0000 & 0.0000 & & & 0.0000 & & 0.0009 & 0.0000 & & 0.0000 & 0.0000 & & & 0.0000 & 0.0000 & & 0.0000 & & 0.0060 \\
\hline SG52c & 0.0000 & & 0.0000 & 0.0000 & & & 0.0000 & & 0.0009 & 0.0000 & & 0.0000 & 0.0000 & & & 0.0000 & 0.0000 & & 0.0000 & & 0.0060 \\
\hline SG52d & 0.0000 & & 0.0000 & 0.0000 & & & 0.0000 & & 0.0009 & 0.0000 & & 0.0000 & 0.0000 & & & 0.0000 & 0.0000 & & 0.0000 & & 0.0060 \\
\hline SG52e & 0.0000 & & 0.0000 & 0.0000 & & & 0.0000 & & 0.0009 & 0.0000 & & 0.0000 & 0.0000 & & & 0.0000 & 0.0000 & & 0.0000 & & 0.0060 \\
\hline SG53 & 0.0000 & & 0.0000 & 0.0000 & & & 0.0000 & & 0.0009 & 0.0000 & & 0.0000 & 0.0000 & & & 0.0000 & 0.0000 & & 0.0000 & & 0.0037 \\
\hline
\end{tabular}

SP3, SPx4, Misc. (Vienna et al. 2001)

\begin{tabular}{|c|c|c|c|c|c|c|c|c|c|c|c|c|}
\hline SP-B-5 & 0.0000 & 0.0000 & 0.0000 & 0.0003 & 0.0003 & 0.0006 & 0.0008 & 0.0000 & 0.0016 & 0.0003 & 0.0000 & 0.0003 \\
\hline SP-Ca-1 & 0.0000 & 0.0000 & 0.0000 & 0.0003 & 0.0003 & 0.0007 & 0.0009 & 0.0000 & 0.0019 & 0.0003 & 0.0000 & 0.0003 \\
\hline SP-Ca-2 & 0.0000 & 0.0000 & 0.0000 & 0.0003 & 0.0003 & 0.0007 & 0.0009 & 0.0000 & 0.0018 & 0.0003 & 0.0000 & 0.0003 \\
\hline SP-Fe-4 & 0.0000 & 0.0000 & 0.0000 & 0.0003 & 0.0003 & 0.0006 & 0.0008 & 0.0000 & 0.0017 & 0.0003 & 0.0000 & 0.0003 \\
\hline SP-K-1 & 0.0000 & 0.0000 & 0.0000 & 0.0003 & 0.0003 & 0.0007 & 0.0009 & 0.0000 & 0.0019 & 0.0003 & 0.0000 & 0.0003 \\
\hline SP-K-2 & 0.0000 & 0.0000 & 0.0000 & 0.0003 & 0.0003 & 0.0007 & 0.0009 & 0.0000 & 0.0018 & 0.0003 & 0.0000 & 0.0003 \\
\hline SP-Li-5 & 0.0000 & 0.0000 & 0.0000 & 0.0003 & 0.0003 & 0.0007 & 0.0009 & 0.0000 & 0.0019 & 0.0003 & 0.0000 & 0.0003 \\
\hline SP-Li-6 & 0.0000 & 0.0000 & 0.0000 & 0.0003 & 0.0003 & 0.0007 & 0.0009 & 0.0000 & 0.0018 & 0.0003 & 0.0000 & 0.0003 \\
\hline SP-Si-4 & 0.0000 & 0.0000 & 0.0000 & 0.0004 & 0.0004 & 0.0009 & 0.0012 & 0.0000 & 0.0025 & 0.0004 & 0.0000 & 0.0004 \\
\hline SP-Ti-1 & 0.0000 & 0.0000 & 0.0000 & 0.0003 & 0.0003 & 0.0007 & 0.0009 & 0.0000 & 0.0019 & 0.0003 & 0.0000 & 0.0250 \\
\hline SP-Ti-2 & 0.0000 & 0.0000 & 0.0000 & 0.0003 & 0.0003 & 0.0007 & 0.0009 & 0.0000 & 0.0018 & 0.0003 & 0.0000 & 0.0500 \\
\hline SP-Zr-1 & 0.0000 & 0.0000 & 0.0000 & 0.0003 & 0.0003 & 0.0007 & 0.0009 & 0.0000 & 0.0019 & 0.0003 & 0.0000 & 0.0003 \\
\hline SP-Ru-1 & 0.0000 & 0.0000 & 0.0000 & 0.0004 & 0.0009 & 0.0009 & 0.0011 & 0.0000 & 0.0024 & 0.0004 & 0.0000 & 0.0003 \\
\hline SP-Ru-2 & 0.0000 & 0.0000 & 0.0000 & 0.0004 & 0.0012 & 0.0009 & 0.0011 & 0.0000 & 0.0024 & 0.0004 & 0.0000 & 0.0003 \\
\hline SP-Others-1 & 0.0000 & 0.0000 & 0.0000 & 0.0006 & 0.0005 & 0.0013 & 0.0017 & 0.0000 & 0.0035 & 0.0006 & 0.0000 & 0.0003 \\
\hline SP3-1(env.D) & 0.0000 & 0.0000 & 0.0000 & 0.0005 & 0.0005 & 0.0012 & 0.0016 & 0.0000 & 0.0033 & 0.0005 & 0.0000 & 0.0019 \\
\hline SP-MC-1 & 0.0000 & 0.0000 & 0.0000 & 0.0000 & 0.0000 & 0.0000 & 0.0000 & 0.0000 & 0.0000 & 0.0000 & 0.0000 & 0.0500 \\
\hline SP-MC-2 & 0.0000 & 0.0000 & 0.0000 & 0.0006 & 0.0005 & 0.0013 & 0.0017 & 0.0000 & 0.0035 & 0.0006 & 0.0000 & 0.0500 \\
\hline SP-MC-8 & 0.0000 & 0.0000 & 0.0000 & 0.0006 & 0.0005 & 0.0013 & 0.0017 & 0.0000 & 0.0035 & 0.0006 & 0.0000 & 0.0500 \\
\hline SP-MC-9 & 0.0000 & 0.0000 & 0.0000 & 0.0000 & 0.0000 & 0.0000 & 0.0000 & 0.0000 & 0.0000 & 0.0000 & 0.0000 & 0.0500 \\
\hline Sp-LHLL & 0.0000 & 0.0000 & 0.0000 & 0.0004 & 0.0003 & 0.0009 & 0.0012 & 0.0000 & 0.0025 & 0.0004 & 0.0000 & 0.0003 \\
\hline Sp-LHLH & 0.0000 & 0.0000 & 0.0000 & 0.0004 & 0.0003 & 0.0009 & 0.0011 & 0.0000 & 0.0024 & 0.0004 & 0.0000 & 0.0003 \\
\hline Sp-LHHL & 0.0000 & 0.0000 & 0.0000 & 0.0004 & 0.0003 & 0.0009 & 0.0011 & 0.0000 & 0.0024 & 0.0004 & 0.0000 & 0.0003 \\
\hline Sp-LHHH & 0.0000 & 0.0000 & 0.0000 & 0.0004 & 0.0003 & 0.0009 & 0.0011 & 0.0000 & 0.0024 & 0.0004 & 0.0000 & 0.0003 \\
\hline Sp-LHMM & 0.0000 & 0.0000 & 0.0000 & 0.0004 & 0.0003 & 0.0009 & 0.0011 & 0.0000 & 0.0024 & 0.0004 & 0.0000 & 0.0003 \\
\hline Sp-MMLL & 0.0000 & 0.0000 & 0.0000 & 0.0004 & 0.0003 & 0.0009 & 0.0012 & 0.0000 & 0.0025 & 0.0004 & 0.0000 & 0.0003 \\
\hline Sp-MMLH & 0.0000 & 0.0000 & 0.0000 & 0.0004 & 0.0003 & 0.0009 & 0.0011 & 0.0000 & 0.0024 & 0.0004 & 0.0000 & 0.0003 \\
\hline Sp-MMHL & 0.0000 & 0.0000 & 0.0000 & 0.0004 & 0.0003 & 0.0009 & 0.0011 & 0.0000 & 0.0024 & 0.0004 & 0.0000 & 0.0003 \\
\hline Sp-MMHH & 0.0000 & 0.0000 & 0.0000 & 0.0004 & 0.0003 & 0.0009 & 0.0011 & 0.0000 & 0.0024 & 0.0004 & 0.0000 & 0.0003 \\
\hline Sp-MMMM & 0.0000 & 0.0000 & 0.0000 & 0.0004 & 0.0003 & 0.0009 & 0.0011 & 0.0000 & 0.0024 & 0.0004 & 0.0000 & 0.0003 \\
\hline Sp-HLLL & 0.0000 & 0.0000 & 0.0000 & 0.0004 & 0.0003 & 0.0009 & 0.0012 & 0.0000 & 0.0025 & 0.0004 & 0.0000 & 0.0003 \\
\hline Sp-HLLH & 0.0000 & 0.0000 & 0.0000 & 0.0004 & 0.0003 & 0.0009 & 0.0011 & 0.0000 & 0.0024 & 0.0004 & 0.0000 & 0.0003 \\
\hline Sp-HLHL & 0.0000 & 0.0000 & 0.0000 & 0.0004 & 0.0003 & 0.0009 & 0.0011 & 0.0000 & 0.0024 & 0.0004 & 0.0000 & 0.0003 \\
\hline Sp-HLHH & 0.0000 & 0.0000 & 0.0000 & 0.0004 & 0.0003 & 0.0009 & 0.0011 & 0.0000 & 0.0024 & 0.0004 & 0.0000 & 0.0003 \\
\hline Sp-HLMM & 0.0000 & 0.0000 & 0.0000 & 0.0004 & 0.0003 & 0.0009 & 0.0011 & 0.0000 & 0.0024 & 0.0004 & 0.0000 & 0.0003 \\
\hline
\end{tabular}




\section{SG (Hrma et al. 1999)}

\begin{tabular}{|c|c|c|c|c|c|c|c|c|c|c|c|c|c|c|c|c|c|c|c|c|c|}
\hline Glass ID & $\begin{array}{c}\mathrm{T} 12 \mathrm{O} 3 \\
-\mathrm{t}\end{array}$ & $\begin{array}{c}\mathrm{U} 3 \mathrm{O} 8 \\
-\mathrm{t}\end{array}$ & $\begin{array}{c}\mathrm{UO} 2 \\
-\mathrm{t}\end{array}$ & $\begin{array}{c}\text { UO3 } \\
-t\end{array}$ & $\begin{array}{c}\mathrm{V} 2 \mathrm{O} 5 \\
-\mathrm{t}\end{array}$ & $\begin{array}{c}\text { WO3 } \\
-t\end{array}$ & $\begin{array}{c}\mathrm{Y} 2 \mathrm{O} 3 \\
-\mathrm{t}\end{array}$ & $\begin{array}{c}\mathrm{ZnO} \\
-\mathrm{t}\end{array}$ & $\begin{array}{c}\text { Others } \\
-t\end{array}$ & $\begin{array}{c}\text { Sum } \\
-t\end{array}$ & $\begin{array}{c}\mathrm{A} 12 \mathrm{O} 3 \\
-\mathrm{a}\end{array}$ & $\begin{array}{c}\mathrm{B} 2 \mathrm{O} 3 \\
-\mathrm{a}\end{array}$ & $\begin{array}{c}\mathrm{CaO} \\
-\mathrm{a}\end{array}$ & $\begin{array}{c}\mathrm{Fe} 2 \mathrm{O} 3 \\
-\mathrm{a}\end{array}$ & $\begin{array}{c}\mathrm{FeO} \\
-\mathrm{a}\end{array}$ & $\begin{array}{c}\mathrm{K} 2 \mathrm{O} \\
-\mathrm{a}\end{array}$ & $\begin{array}{c}\mathrm{Li} 2 \mathrm{O} \\
-\mathrm{a}\end{array}$ & $\begin{array}{c}\mathrm{MgO} \\
-\mathrm{a}\end{array}$ & $\begin{array}{c}\mathrm{Na} 2 \mathrm{O} \\
-\mathrm{a}\end{array}$ & $\begin{array}{c}\mathrm{NiO} \\
-\mathrm{a}\end{array}$ & $\begin{array}{c}\mathrm{P} 2 \mathrm{O} 5 \\
-\mathrm{a}\end{array}$ \\
\hline SG46 & & 0.0550 & & & & 0.0000 & 0.0000 & 0.0000 & & 1.0000 & & & & & & & & & & & \\
\hline SG47 & & 0.0550 & & & & 0.0000 & 0.0000 & 0.0000 & & 1.0000 & & & & & & & & & & & \\
\hline SG48 & & 0.0000 & & & & 0.0000 & 0.0000 & 0.0000 & & 1.0001 & & & & & & & & & & & \\
\hline SG49 & & 0.0550 & & & & 0.0000 & 0.0000 & 0.0000 & & 1.0001 & & & & & & & & & & & \\
\hline SG50 & & 0.0550 & & & & 0.0000 & 0.0000 & 0.0000 & & 1.0000 & & & & & & & & & & & \\
\hline SG51 & & 0.0000 & & & & 0.0000 & 0.0000 & 0.0000 & & 1.0000 & & & & & & & & & & & \\
\hline SG52a & & 0.0000 & & & & 0.0000 & 0.0000 & 0.0000 & & 1.0000 & & & & & & & & & & & \\
\hline SG52b & & 0.0000 & & & & 0.0000 & 0.0000 & 0.0000 & & 1.0000 & & & & & & & & & & & \\
\hline SG52c & & 0.0000 & & & & 0.0000 & 0.0000 & 0.0000 & & 1.0000 & & & & & & & & & & & \\
\hline SG52d & & 0.0000 & & & & 0.0000 & 0.0000 & 0.0000 & & 1.0000 & & & & & & & & & & & \\
\hline SG52e & & 0.0000 & & & & 0.0000 & 0.0000 & 0.0000 & & 1.0000 & & & & & & & & & & & \\
\hline SG53 & & 0.0280 & & & & 0.0000 & 0.0000 & 0.0000 & & 1.0000 & & & & & & & & & & & \\
\hline
\end{tabular}

SP3, SPx4, Misc. (Vienna et al. 2001)

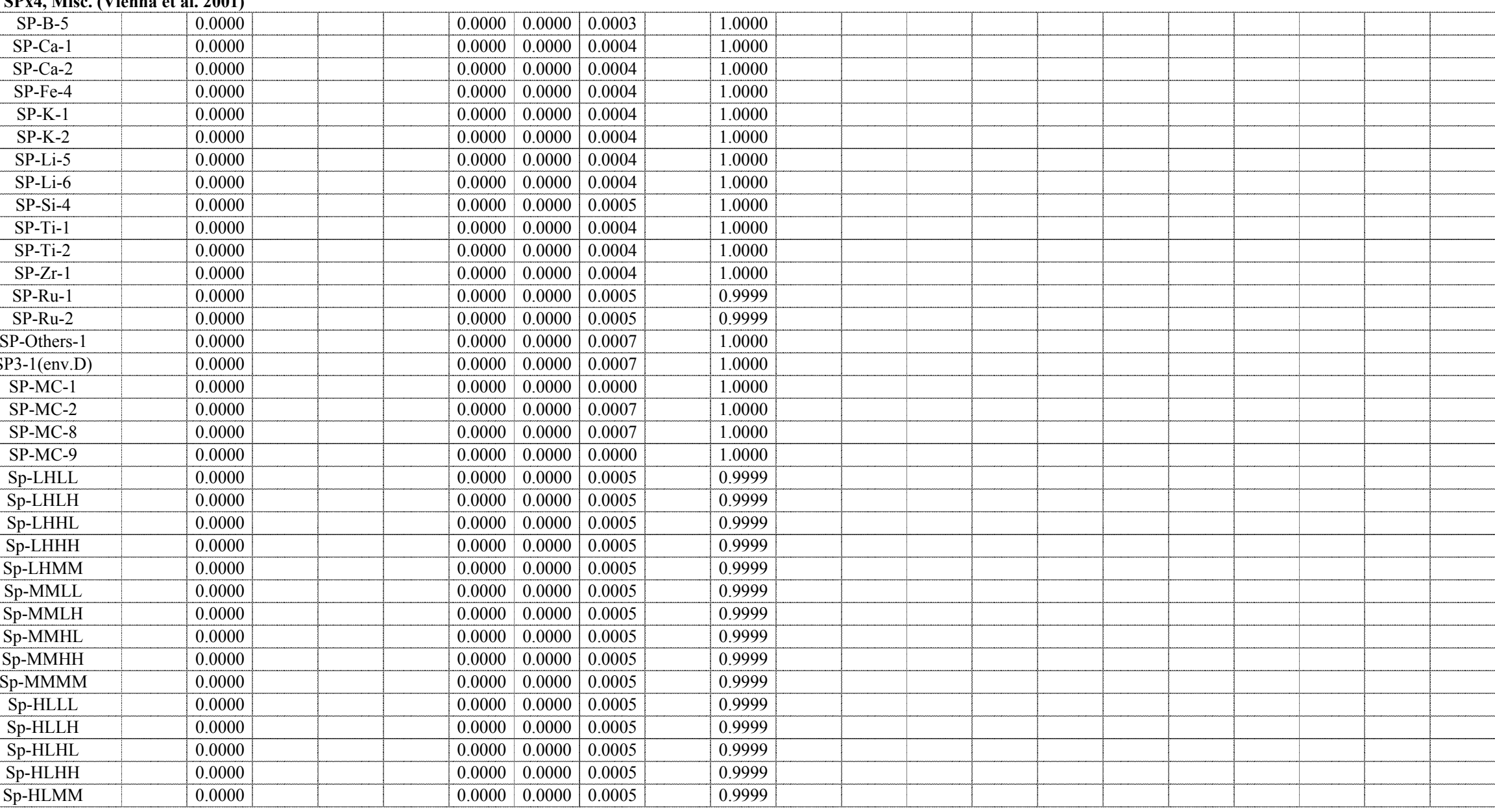


Appendix A. Database - mass fraction

SG (Hrma et al. 1999)

\begin{tabular}{|c|c|c|c|c|c|c|c|c|c|c|c|c|c|c|c|c|c|c|c|c|c|}
\hline Glass ID & $\begin{array}{c}\mathrm{SiO} 2 \\
-\mathrm{a}\end{array}$ & $\begin{array}{c}\mathrm{ZrO} 2 \\
-\mathrm{a} \\
\end{array}$ & $\begin{array}{c}\mathrm{Ag} 2 \mathrm{O} \\
-\mathrm{a}\end{array}$ & $\begin{array}{c}\mathrm{As} 2 \mathrm{O} 3 \\
-\mathrm{a}\end{array}$ & $\begin{array}{c}\mathrm{BaO} \\
-\mathrm{a}\end{array}$ & $\begin{array}{c}\mathrm{Bi} 2 \mathrm{O} 3 \\
-\mathrm{a}\end{array}$ & $\begin{array}{l}\mathrm{Br} \\
-\mathrm{a}\end{array}$ & $\begin{array}{c}\mathrm{CdO} \\
-\mathrm{a}\end{array}$ & $\begin{array}{c}\mathrm{Ce} 2 \mathrm{O} 3 \\
-\mathrm{a}\end{array}$ & $\begin{array}{c}\mathrm{CeO} 2 \\
-\mathrm{a} \\
\end{array}$ & $\begin{array}{l}\mathrm{Cl} \\
-\mathrm{a}\end{array}$ & $\begin{array}{c}\mathrm{CoO} \\
-\mathrm{a} \\
\end{array}$ & $\begin{array}{c}\mathrm{Co} 2 \mathrm{O} 3 \\
-\mathrm{a} \\
\end{array}$ & $\begin{array}{c}\mathrm{Cr} 2 \mathrm{O} 3 \\
-\mathrm{a}\end{array}$ & $\begin{array}{c}\mathrm{Cs} 2 \mathrm{O} \\
-\mathrm{a} \\
\end{array}$ & $\begin{array}{c}\mathrm{CuO} \\
-\mathrm{a}\end{array}$ & $\begin{array}{c}\mathrm{Eu} 2 \mathrm{O} 3 \\
-\mathrm{a}\end{array}$ & $\begin{array}{l}\mathrm{F} \\
-\mathrm{a}\end{array}$ & $\begin{array}{c}\mathrm{Ga} 2 \mathrm{O} 3 \\
-\mathrm{a}\end{array}$ & $\begin{array}{c}\mathrm{Gd} 2 \mathrm{O} 3 \\
-\mathrm{a}\end{array}$ & $\begin{array}{c}\mathrm{HgO} \\
-\mathrm{a}\end{array}$ \\
\hline SG46 & & & & & & & & & & & & & & & & & & & & & \\
\hline SG47 & & & & & & & & & & & & & & & & & & & & & \\
\hline $\mathrm{SG} 48$ & & & & & & & & & & & & & & & & & & & & & \\
\hline SG49 & & & & & & & & & & & & & & & & & & & & & \\
\hline SG50 & & & & & & & & & & & & & & & & & & & & & \\
\hline SG51 & & & & & & & & & & & & & & & & & & & & & \\
\hline SG52a & & & & & & & & & & & & & & & & & & & & & \\
\hline SG52b & & & & & & & & & & & & & & & & & & & & & \\
\hline SG52c & & & & & & & & & & & & & & & & & & & & & \\
\hline SG52d & & & & & & & & & & & & & & & & & & & & & \\
\hline SG52e & & & & & & & & & & & & & & & & & & & & & \\
\hline SG53 & & & & & & & & & & & & & & & & & & & & & \\
\hline
\end{tabular}

SP3, SPx4, Misc. (Vienna et al. 2001)

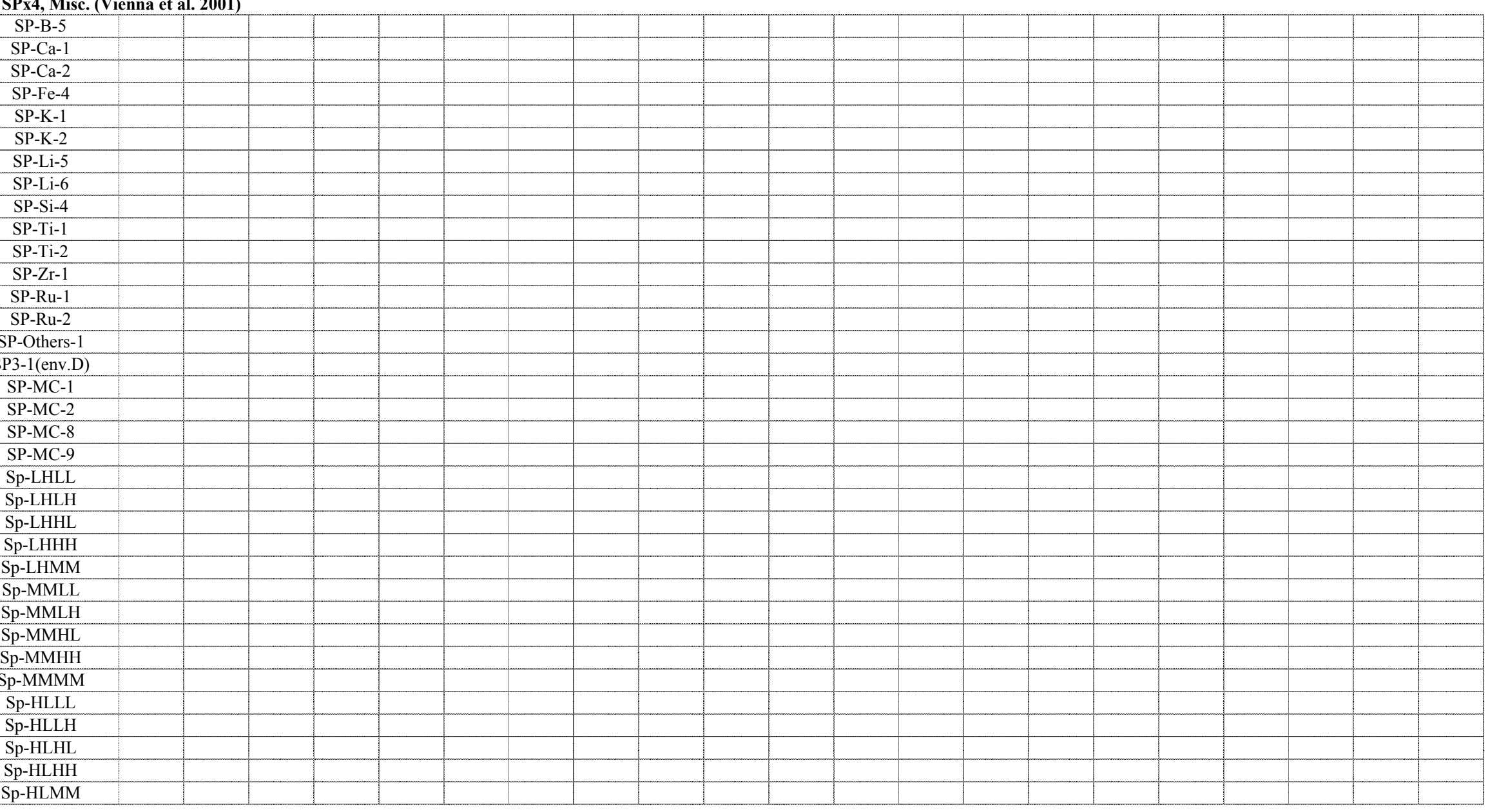


Appendix A. Database - mass fraction

SG (Hrma et al. 1999)

\begin{tabular}{|c|c|c|c|c|c|c|c|c|c|c|c|c|c|c|c|c|c|c|c|c|c|}
\hline Glass ID & $\begin{array}{c}\mathrm{I} \\
-\mathrm{a}\end{array}$ & $\begin{array}{c}\mathrm{La} 2 \mathrm{O} 3 \\
-\mathrm{a}\end{array}$ & $\begin{array}{c}\mathrm{MnO} 2 \\
-\mathrm{a}\end{array}$ & $\begin{array}{c}\mathrm{MnO} \\
-\mathrm{a}\end{array}$ & $\begin{array}{c}\mathrm{MoO} \\
-\mathrm{a}\end{array}$ & $\begin{array}{c}\mathrm{MoO} 3 \\
-\mathrm{a}\end{array}$ & $\begin{array}{c}\mathrm{Nb} 2 \mathrm{O} 5 \\
-\mathrm{a}\end{array}$ & $\begin{array}{c}\mathrm{Nd} 2 \mathrm{O} 3 \\
-\mathrm{a}\end{array}$ & $\begin{array}{c}\mathrm{PbO} \\
-\mathrm{a}\end{array}$ & $\begin{array}{c}\mathrm{PdO} 2 \\
-\mathrm{a}\end{array}$ & $\begin{array}{c}\mathrm{PdO} \\
-\mathrm{a}\end{array}$ & $\begin{array}{c}\mathrm{Pr} 2 \mathrm{O} 3 \\
-\mathrm{a}\end{array}$ & $\begin{array}{c}\text { Pr6O11 } \\
-\mathrm{a}\end{array}$ & $\begin{array}{c}\mathrm{Rb} 2 \mathrm{O} \\
-\mathrm{a}\end{array}$ & $\begin{array}{c}\mathrm{ReO} \\
-\mathrm{a}\end{array}$ & $\begin{array}{c}\mathrm{ReO} 2 \\
-\mathrm{a}\end{array}$ & $\begin{array}{c}\mathrm{Rh} 2 \mathrm{O} 3 \\
-\mathrm{a}\end{array}$ & $\begin{array}{c}\mathrm{RhO} 2 \\
-\mathrm{a}\end{array}$ & $\begin{array}{c}\mathrm{RuO} 2 \\
-\mathrm{a}\end{array}$ & $\begin{array}{c}\mathrm{Sb} 2 \mathrm{O} 3 \\
-\mathrm{a}\end{array}$ & $\begin{array}{c}\mathrm{Sb} 2 \mathrm{O} 5 \\
-\mathrm{a}\end{array}$ \\
\hline \multicolumn{22}{|l|}{ SG46 } \\
\hline \multicolumn{22}{|l|}{ SG47 } \\
\hline \multirow{2}{*}{\multicolumn{22}{|c|}{$\begin{array}{l}\text { SG48 } \\
\text { SG49 }\end{array}$}} \\
\hline & & & & & & & & & & & & & & & & & & & & & \\
\hline \multicolumn{22}{|l|}{ SG50 } \\
\hline \multicolumn{22}{|l|}{ SG51 } \\
\hline \multicolumn{22}{|l|}{ SG52a } \\
\hline \multicolumn{22}{|l|}{ SG52b } \\
\hline \multicolumn{22}{|l|}{ SG52c } \\
\hline \multicolumn{22}{|l|}{ SG52d } \\
\hline SG52e & & & & & & & & & & & & & & & & & & & & & \\
\hline SG53 & & & & & & & & & & & & & & & & & & & & & \\
\hline
\end{tabular}

\section{SP3, SPx4, Misc. (Vienna et al. 2001)}

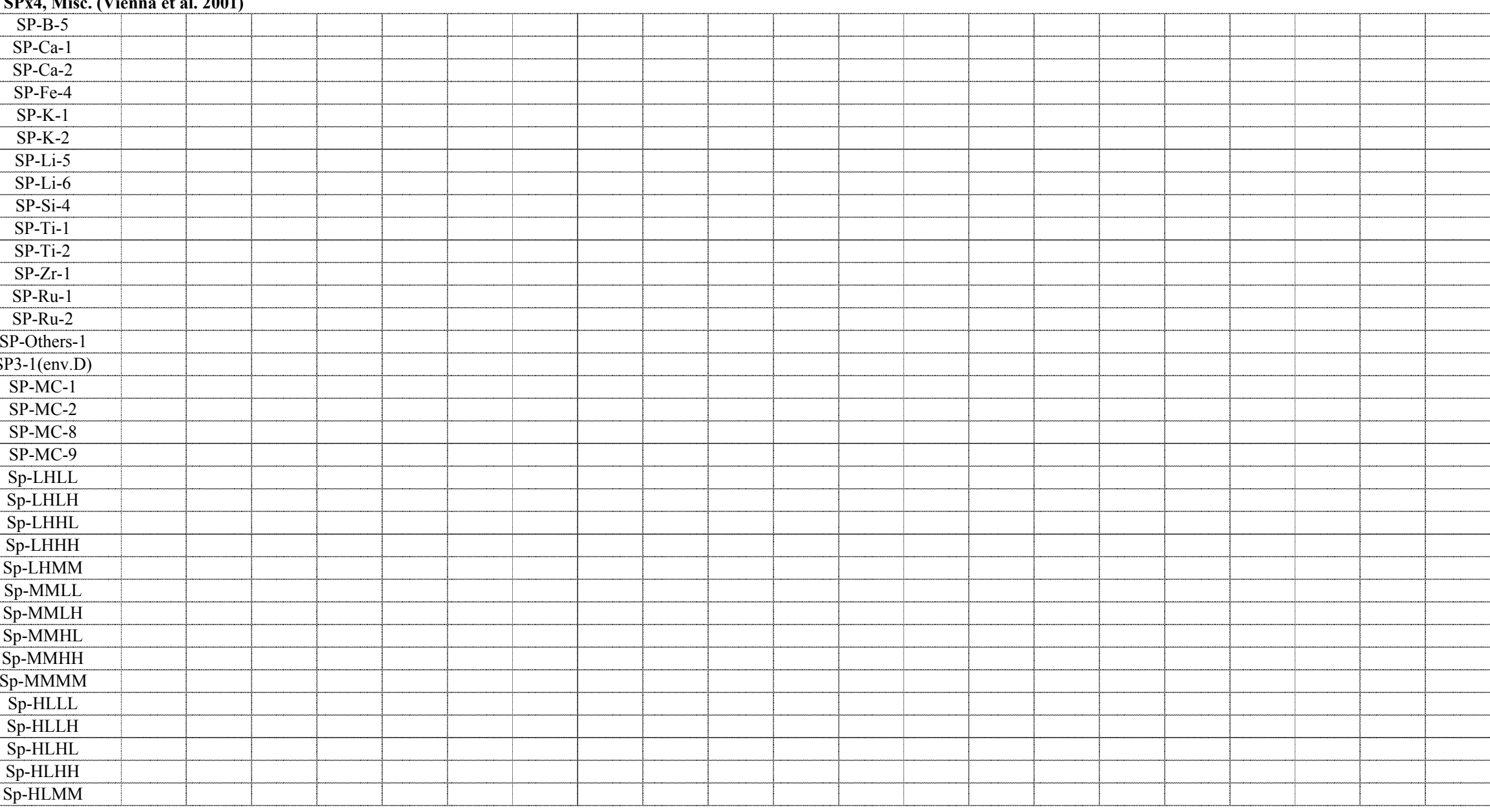


Appendix A. Database - mass fraction

\section{SG (Hrma et al. 1999)}

\begin{tabular}{|c|c|c|c|c|c|c|c|c|c|c|c|c|c|c|c|c|c|c|c|c|}
\hline Glass ID & $\begin{array}{c}\mathrm{SeO} 2 \\
-\mathrm{a}\end{array}$ & $\begin{array}{c}\mathrm{Sm} 2 \mathrm{O} 3 \\
-\mathrm{a}\end{array}$ & $\begin{array}{c}\mathrm{SnO} \\
-\mathrm{a}\end{array}$ & $\begin{array}{c}\mathrm{SnO} 2 \\
-\mathrm{a}\end{array}$ & $\begin{array}{c}\mathrm{SO} 3 \\
-\mathrm{a}\end{array}$ & $\begin{array}{c}\mathrm{SrO} \\
-\mathrm{a}\end{array}$ & $\begin{array}{c}\mathrm{Tc} 2 \mathrm{O} 7 \\
-\mathrm{a}\end{array}$ & $\begin{array}{c}\mathrm{TeO} 2 \\
-\mathrm{a}\end{array}$ & $\begin{array}{c}\mathrm{ThO} 2 \\
-\mathrm{a}\end{array}$ & $\begin{array}{c}\mathrm{TiO} 2 \\
-\mathrm{a}\end{array}$ & $\begin{array}{c}\mathrm{T} 12 \mathrm{O} 3 \\
-\mathrm{a}\end{array}$ & $\begin{array}{c}\mathrm{U} 3 \mathrm{O} 8 \\
-\mathrm{a}\end{array}$ & $\begin{array}{c}\mathrm{UO} 2 \\
-\mathrm{a}\end{array}$ & $\begin{array}{c}\text { UO3 } \\
-\mathrm{a}\end{array}$ & $\begin{array}{c}\mathrm{V} 2 \mathrm{O} 5 \\
-\mathrm{a}\end{array}$ & $\begin{array}{c}\text { WO3 } \\
-\mathrm{a}\end{array}$ & $\begin{array}{c}\mathrm{Y} 2 \mathrm{O} 3 \\
-\mathrm{a}\end{array}$ & $\begin{array}{c}\mathrm{ZnO} \\
-\mathrm{a}\end{array}$ & $\begin{array}{c}\text { Others } \\
-\mathrm{a}\end{array}$ & $\begin{array}{c}\text { Sum } \\
-\mathrm{a}\end{array}$ \\
\hline SG46 & & & & & & & & & & & & & & & & & & & & \\
\hline SG47 & & & & & & & & & & & & & & & & & & & & \\
\hline SG48 & & & & & & & & & & & & & & & & & & & & \\
\hline SG49 & & & & & & & & & & & & & & & & & & & & \\
\hline SG50 & & & & & & & & & & & & & & & & & & & & \\
\hline SG51 & & & & & & & & & & & & & & & & & & & & \\
\hline SG52a & & & & & & & & & & & & & & & & & & & & \\
\hline SG52b & & & & & & & & & & & & & & & & & & & & \\
\hline SG52c & & & & & & & & & & & & & & & & & & & & \\
\hline SG52d & & & & & & & & & & & & & & & & & & & & \\
\hline SG52e & & & & & & & & & & & & & & & & & & & & \\
\hline SG53 & & & & & & & & & & & & & & & & & & & & \\
\hline
\end{tabular}

\section{SP3, SPx4, Misc. (Vienna et al. 2001)}

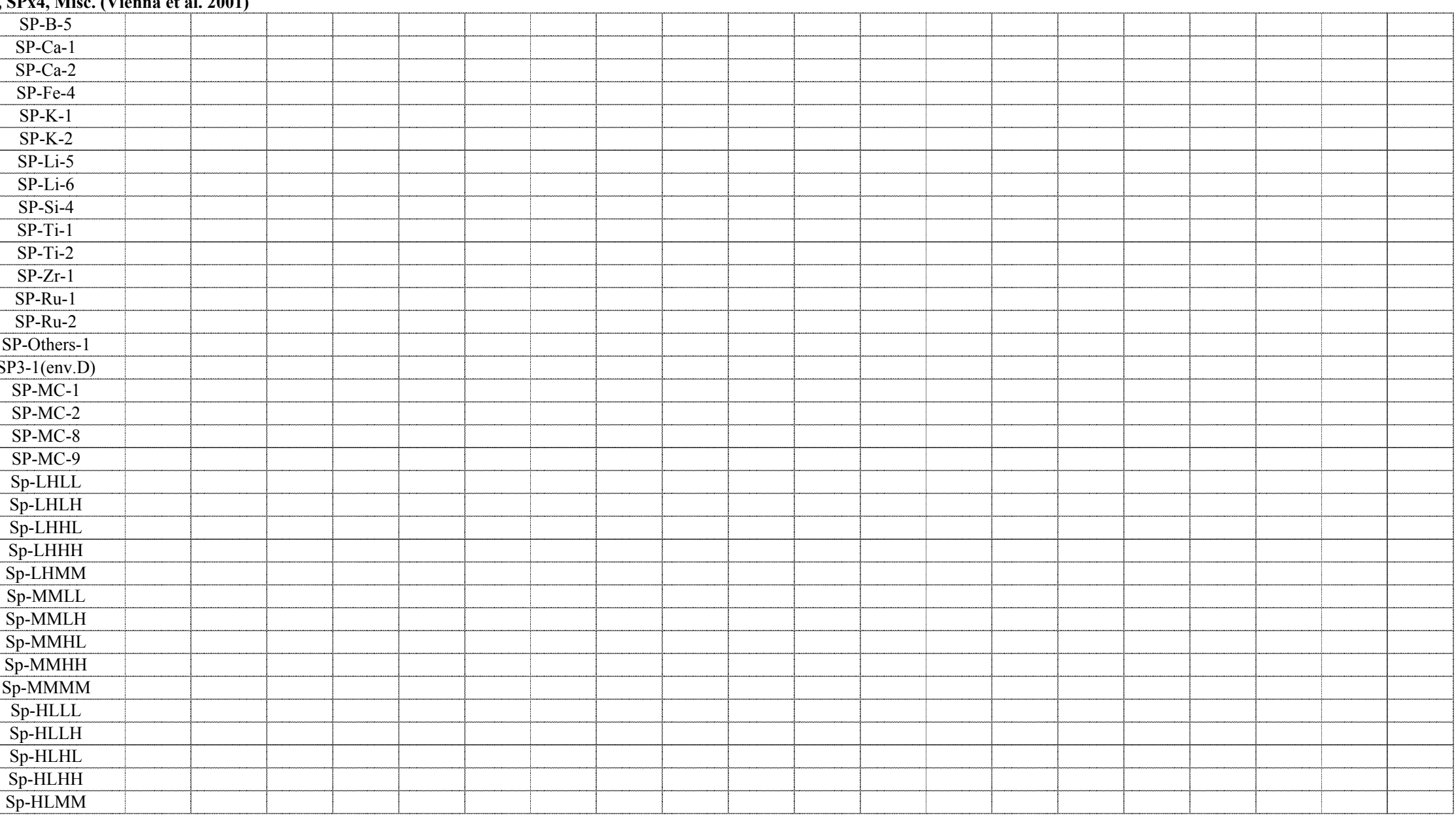


Appendix A. Database - mass fraction

SG (Hrma et al. 1999)

\begin{tabular}{|c|c|c|c|c|c|c|c|c|}
\hline Glass ID & $\begin{array}{l}\mathrm{TM} \\
\left({ }^{\circ} \mathrm{C}\right) \\
\end{array}$ & $\begin{array}{l}\text { Gradient } \\
\text { TL }\left({ }^{\circ} \mathrm{C}\right)\end{array}$ & $\begin{array}{l}\text { Uniform } \\
\text { TL }\left({ }^{\circ} \mathrm{C}\right)\end{array}$ & Primary Phase & $\begin{array}{l}\text { Quenched } \\
\text { Visual/OM }\end{array}$ & $\begin{array}{c}\text { Quenched } \\
\text { SEM/EDS or TEM }\end{array}$ & $\begin{array}{l}\text { Quenched } \\
\text { XRD }\end{array}$ & $\begin{array}{c}\text { Quenched } \\
\text { Homogeneous? }\end{array}$ \\
\hline SG46 & 1257 & & 1247 & spinel & & & & \\
\hline SG47 & 1193 & & 1144 & spinel & & & & \\
\hline SG48 & 1356 & & 862 & clinopyroxene & & & & \\
\hline SG49 & 1315 & & 877 & clinopyroxene & & & & \\
\hline SG50 & 1320 & & 1285 & spinel & & & & \\
\hline SG51 & 1326 & & 1033 & $\mathrm{RuO} 2$, spinel & & & & \\
\hline SG52a & 1142 & & 869 & $\mathrm{RuO} 2$, spinel & & & & \\
\hline SG52b & 1142 & & 883 & $\mathrm{RuO} 2$, spinel & & & & \\
\hline SG52c & 1142 & & 882 & $\mathrm{RuO} 2$, spinel & & & & \\
\hline SG52d & 1142 & & 883 & $\mathrm{RuO} 2$, spinel & & & & \\
\hline SG52e & 1142 & & 891 & $\mathrm{RuO} 2$, spinel & & & & \\
\hline SG53 & 1284 & & 1082 & spinel & & & & \\
\hline
\end{tabular}

SP3, SPx4, Misc. (Vienna et al. 2001)

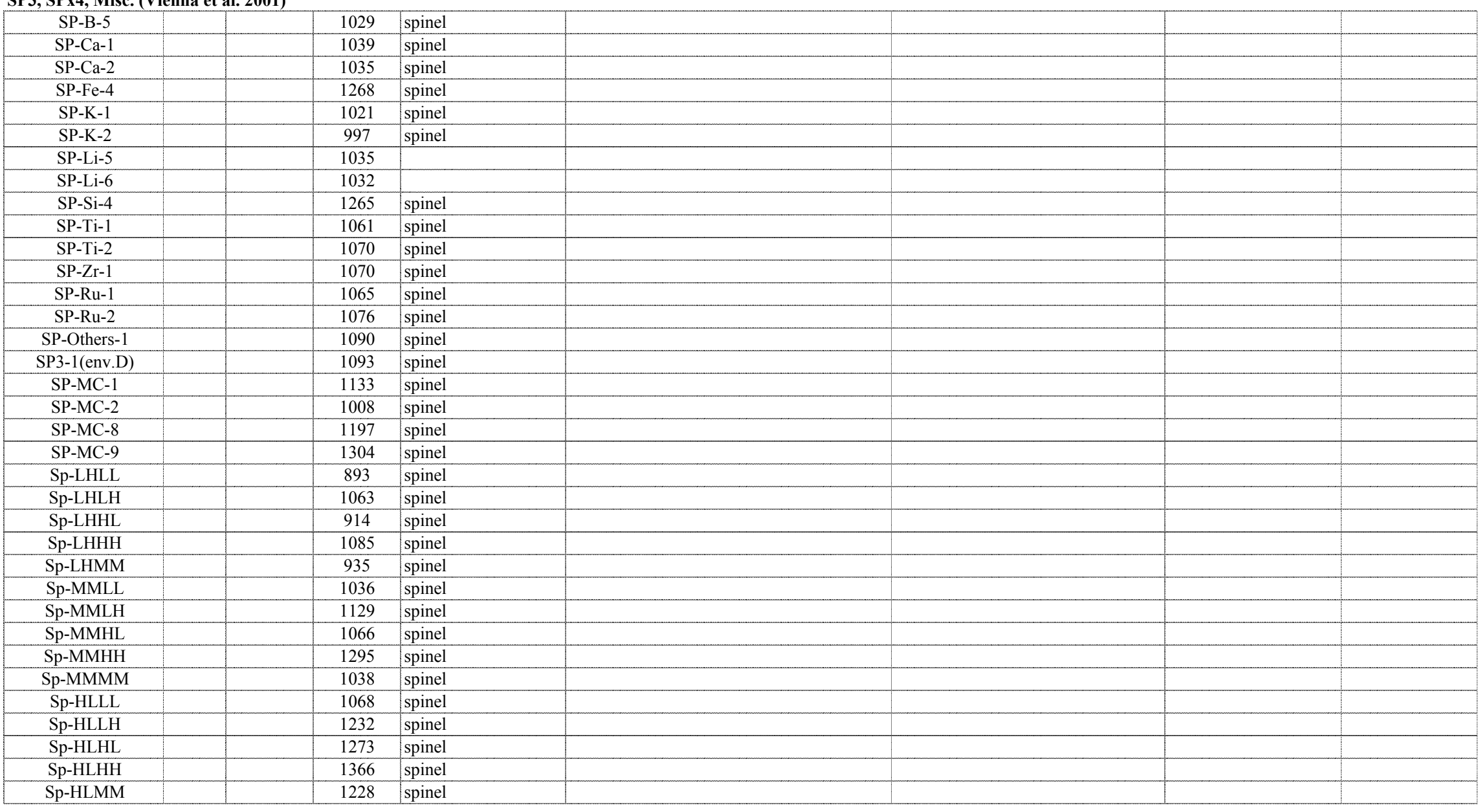


Appendix A. Database - mass fraction

\section{SG (Hrma et al. 1999)}

\begin{tabular}{|c|c|c|c|c|c|}
\hline Glass ID & $\begin{array}{c}\text { CCC } \\
\text { Visual/OM }\end{array}$ & $\begin{array}{c}\text { CCC } \\
\text { SEM/EDS or TEM }\end{array}$ & $\begin{array}{l}\text { CCC } \\
\text { XRD }\end{array}$ & $\begin{array}{c}\text { Heat Treated } \\
\text { Visual/OM }\end{array}$ & $\begin{array}{c}\text { Heat Treated } \\
\text { SEM/EDS or TEM }\end{array}$ \\
\hline \multicolumn{6}{|l|}{ SG46 } \\
\hline \multirow{2}{*}{\multicolumn{6}{|c|}{$\begin{array}{l}\text { SG47 } \\
\text { SG48 }\end{array}$}} \\
\hline & & & & & \\
\hline \multicolumn{6}{|l|}{ SG49 } \\
\hline \multicolumn{6}{|l|}{ SG50 } \\
\hline \multicolumn{6}{|l|}{ SG51 } \\
\hline \multicolumn{6}{|l|}{ SG52a } \\
\hline \multicolumn{6}{|l|}{ SG52b } \\
\hline \multicolumn{6}{|l|}{ SG52c } \\
\hline \multicolumn{6}{|l|}{ SG52d } \\
\hline \multicolumn{6}{|l|}{ SG52e } \\
\hline SG53 & & & & & \\
\hline
\end{tabular}

\section{SP3, SPx4, Misc. (Vienna et al. 2001)} SP-B-5

SP-Ca-1

SP-Ca-2

SP-Fe-4

SP-K-1

SP-K-2

SP-Li-5

SP-Li-6

SP-Si-4

SP-Ti-1

SP-Ti-2

SP-Zr-1

SP-Ru-1

SP-Ru-2

SP-Others-1

SP3-1(env.D)

SP-MC-1

SP-MC-2

SP-MC-8

SP-MC-9

Sp-LHLL

Sp-LHLH

Sp-LHHL

Sp-LHHH

Sp-LHMM

Sp-MMLL

Sp-MMLH

Sp-MMHL

Sp-MMHH

Sp-MMMM

Sp-HLLL

Sp-HLLH

Sp-HLHL

Sp-HLHH

Sp-HLMM

\begin{tabular}{|c|c|c|}
\hline & & \\
\hline & & \\
\hline & & \\
\hline & & \\
\hline & & \\
\hline & & \\
\hline & & \\
\hline & & \\
\hline & & \\
\hline & & \\
\hline & & \\
\hline & 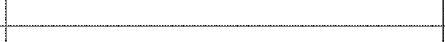 & . \\
\hline & (N) & \\
\hline & & \\
\hline & & \\
\hline & $x_{a}$ & \\
\hline & & \\
\hline & & \\
\hline & & \\
\hline & & \\
\hline & & \\
\hline & & \\
\hline & & \\
\hline & & \\
\hline & & \\
\hline & & \\
\hline & & \\
\hline & & \\
\hline $5 x^{2}=x^{2}$ & 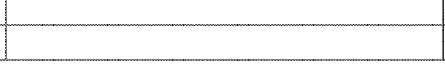 & \\
\hline
\end{tabular}


Appendix A. Database - mass fraction

\section{SG (Hrma et al. 1999)}

\begin{tabular}{|c|c|c|c|c|c|c|c|c|c|c|c|c|c|c|c|}
\hline Glass ID & $\begin{array}{c}\text { Heat Treated } \\
\text { XRD }\end{array}$ & $\begin{array}{l}\text { Density } \\
\left(\mathrm{g} / \mathrm{cm}^{3}\right)\end{array}$ & $\begin{array}{c}\text { Fulc Visc } \\
\text { A }\end{array}$ & $\begin{array}{c}\text { Fulc Visc } \\
\text { B }\end{array}$ & $\begin{array}{c}\text { Fulc Visc } \\
\text { To }\end{array}$ & $\begin{array}{c}\mathrm{FV} 1150^{\circ} \mathrm{C} \\
(\mathrm{Pa} \cdot \mathrm{s})\end{array}$ & $\begin{array}{c}\text { Arrh Visc } \\
\text { A }\end{array}$ & $\begin{array}{c}\text { Arrh Visc } \\
\text { B }\end{array}$ & $1150^{\circ} \mathrm{C}$ & $\begin{array}{c}\mathrm{T}\left({ }^{\circ} \mathrm{C}\right) \text { at } \\
2 \mathrm{~Pa} \cdot \mathrm{s}\end{array}$ & $\begin{array}{c}\mathrm{T}\left({ }^{\circ} \mathrm{C}\right) \text { at } \\
5 \mathrm{~Pa} \cdot \mathrm{s}\end{array}$ & $\begin{array}{c}\mathrm{T}\left({ }^{\circ} \mathrm{C}\right) \text { at } \\
10 \mathrm{~Pa} \cdot \mathrm{s}\end{array}$ & $\begin{array}{c}\mathrm{T} 1 \\
\left({ }^{\circ} \mathrm{C}\right)\end{array}$ & $\begin{array}{c}\mathrm{V} 1 \\
(\mathrm{~Pa} \cdot \mathrm{s})\end{array}$ & $\begin{array}{l}\mathrm{T} 2 \\
\left({ }^{\circ} \mathrm{C}\right)\end{array}$ \\
\hline \multicolumn{16}{|c|}{ 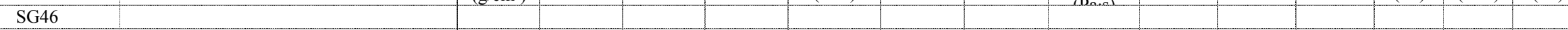 } \\
\hline \multicolumn{16}{|l|}{ SG47 } \\
\hline \multicolumn{16}{|l|}{ SG48 } \\
\hline \multicolumn{16}{|l|}{ SG49 } \\
\hline \multicolumn{16}{|l|}{ SG50 } \\
\hline \multicolumn{16}{|l|}{ SG51 } \\
\hline \multicolumn{16}{|l|}{ SG52a } \\
\hline \multicolumn{16}{|l|}{ SG52b } \\
\hline \multicolumn{16}{|l|}{ SG52c } \\
\hline \multicolumn{16}{|l|}{ SG52d } \\
\hline \multicolumn{16}{|l|}{ SG52e } \\
\hline SG53 & & & & & & & & & & & & & & & \\
\hline
\end{tabular}

\section{SP3, SPx4, Misc. (Vienna et al. 2001)}

SP-Ca-1

SP-Ca-2

SP-Fe-4

SP-K-1

SP-K-2

SP-Li-5

SP-Li-6

SP-Si-4

SP-Ti-1

SP-Ti-2

SP-Zr-1

SP-Ru-1

SP-Ru-2

SP-Others-1

SP3-1(env.D)

SP-MC-1

SP-MC-2

SP-MC-8

SP-MC-9

Sp-LHLL

Sp-LHLH

Sp-LHHL

Sp-LHHH

Sp-LHMM

Sp-MMLL

Sp-MMLH

Sp-MMHL

Sp-MMHH

Sp-MMMM

Sp-HLLL

Sp-HLLH

Sp-HLHL

Sp-HLHH

Sp-HLMM \begin{tabular}{|l|l|l|l|l|l|l|l|l|l|l|l|l|l|l|l|l|l|l|l}
\hline Density & Fulc Visc & Fulc Visc & Fulc Visc & FV $1150^{\circ} \mathrm{C}$ & Arrh Visc & Arrh Visc & $1150^{\circ} \mathrm{C}$ & $\mathrm{T}\left({ }^{\circ} \mathrm{C}\right)$ at & $\mathrm{T}\left({ }^{\circ} \mathrm{C}\right)$ at & $\mathrm{T}\left({ }^{\circ} \mathrm{C}\right)$ at & $\mathrm{T} 1$ & $\mathrm{~V} 1$ & $\mathrm{~T} 2$ \\
\hline
\end{tabular} 
Appendix A. Database - mass fraction

\begin{tabular}{|c|c|c|c|c|c|c|c|c|c|c|c|c|c|c|c|c|c|c|c|c|c|}
\hline \multicolumn{22}{|c|}{ SG (Hrma et al. 1999) } \\
\hline Glass ID & $\begin{array}{c}\mathrm{V} 2 \\
(\mathrm{~Pa} \cdot \mathrm{s})\end{array}$ & $\begin{array}{c}\mathrm{T} 3 \\
\left({ }^{\circ} \mathrm{C}\right)\end{array}$ & $\begin{array}{c}\mathrm{V} 3 \\
(\mathrm{~Pa} \cdot \mathrm{s})\end{array}$ & $\begin{array}{c}\mathrm{T} 4 \\
\left({ }^{\circ} \mathrm{C}\right)\end{array}$ & $\begin{array}{c}\mathrm{V} 4 \\
(\mathrm{~Pa} \cdot \mathrm{s})\end{array}$ & $\begin{array}{c}\mathrm{T} 5 \\
\left({ }^{\circ} \mathrm{C}\right)\end{array}$ & $\begin{array}{c}\mathrm{V} 5 \\
(\mathrm{~Pa} \cdot \mathrm{s})\end{array}$ & $\begin{array}{c}\mathrm{T} 6 \\
\left({ }^{\circ} \mathrm{C}\right)\end{array}$ & $\begin{array}{c}\mathrm{V} 6 \\
(\mathrm{~Pa} \cdot \mathrm{s})\end{array}$ & $\begin{array}{c}\mathrm{T} 7 \\
\left({ }^{\circ} \mathrm{C}\right)\end{array}$ & $\begin{array}{c}\text { V7 } \\
(\mathrm{Pa} \cdot \mathrm{s})\end{array}$ & $\begin{array}{c}\mathrm{T} 8 \\
\left({ }^{\circ} \mathrm{C}\right)\end{array}$ & $\begin{array}{c}\text { V8 } \\
(\mathrm{Pa} \cdot \mathrm{s})\end{array}$ & $\begin{array}{c}\text { T9 } \\
\left({ }^{\circ} \mathrm{C}\right)\end{array}$ & $\begin{array}{c}\mathrm{V} 9 \\
(\mathrm{~Pa} \cdot \mathrm{s})\end{array}$ & $\begin{array}{l}\mathrm{T} 10 \\
\left({ }^{\circ} \mathrm{C}\right)\end{array}$ & $\begin{array}{c}\mathrm{V} 10 \\
(\mathrm{~Pa} \cdot \mathrm{s})\end{array}$ & $\begin{array}{l}\mathrm{T} 11 \\
\left({ }^{\circ} \mathrm{C}\right)\end{array}$ & $\begin{array}{c}\mathrm{V} 11 \\
(\mathrm{~Pa} \cdot \mathrm{s})\end{array}$ & $\begin{array}{l}\mathrm{T} 12 \\
\left({ }^{\circ} \mathrm{C}\right)\end{array}$ & $\begin{array}{c}\mathrm{V} 12 \\
(\mathrm{~Pa} \cdot \mathrm{s})\end{array}$ \\
\hline SG46 & & & & & & & & & & & & & & & & & & & & & \\
\hline SG47 & & & & & & & & & & & & & & & & & & & & & \\
\hline SG48 & & & & & & & & & & & & & & & & & & & & & \\
\hline SG49 & & & & & & & & & & & & & & & & & & & & & \\
\hline SG50 & & & & & & & & & & & & & & & & & & & & & \\
\hline SG51 & & & & & & & & & & & & & & & & & & & & & \\
\hline SG52a & & & & & & & & & & & & & & & & & & & & & \\
\hline SG52b & & & & & & & & & & & & & & & & & & & & & \\
\hline SG52c & & & & & & & & & & & & & & & & & & & & & \\
\hline SG52d & & & & & & & & & & & & & & & & & & & & & \\
\hline SG52e & & & & & & & & & & & & & & & & & & & & & \\
\hline SG53 & & & & & & & & & & & & & & & & & & & & & \\
\hline
\end{tabular}

\section{SP3, SPx4, Misc. (Vienna et al. 2001)}

SP-B-5

SP-Ca-1

SP-Ca-2

SP-Fe-4

SP-K-1

SP-K-2

SP-Li-5

SP-Li-6

SP-Si-4

SP-Ti-1

SP-Ti-2

SP-Zr-1

SP-Ru-1

SP-Ru-2

SP-Others-1

SP3-1(env.D)

SP-MC-1

SP-MC-2

SP-MC-8

SP-MC-9

Sp-LHLL

Sp-LHLH

Sp-LHHL

Sp-LHHH

Sp-LHMM

Sp-MMLL

Sp-MMLH

Sp-MMHL

Sp-MMHH

Sp-MMMM

Sp-HLLL

Sp-HLLH

Sp-HLHL

Sp-HLHH

Sp-HLMM

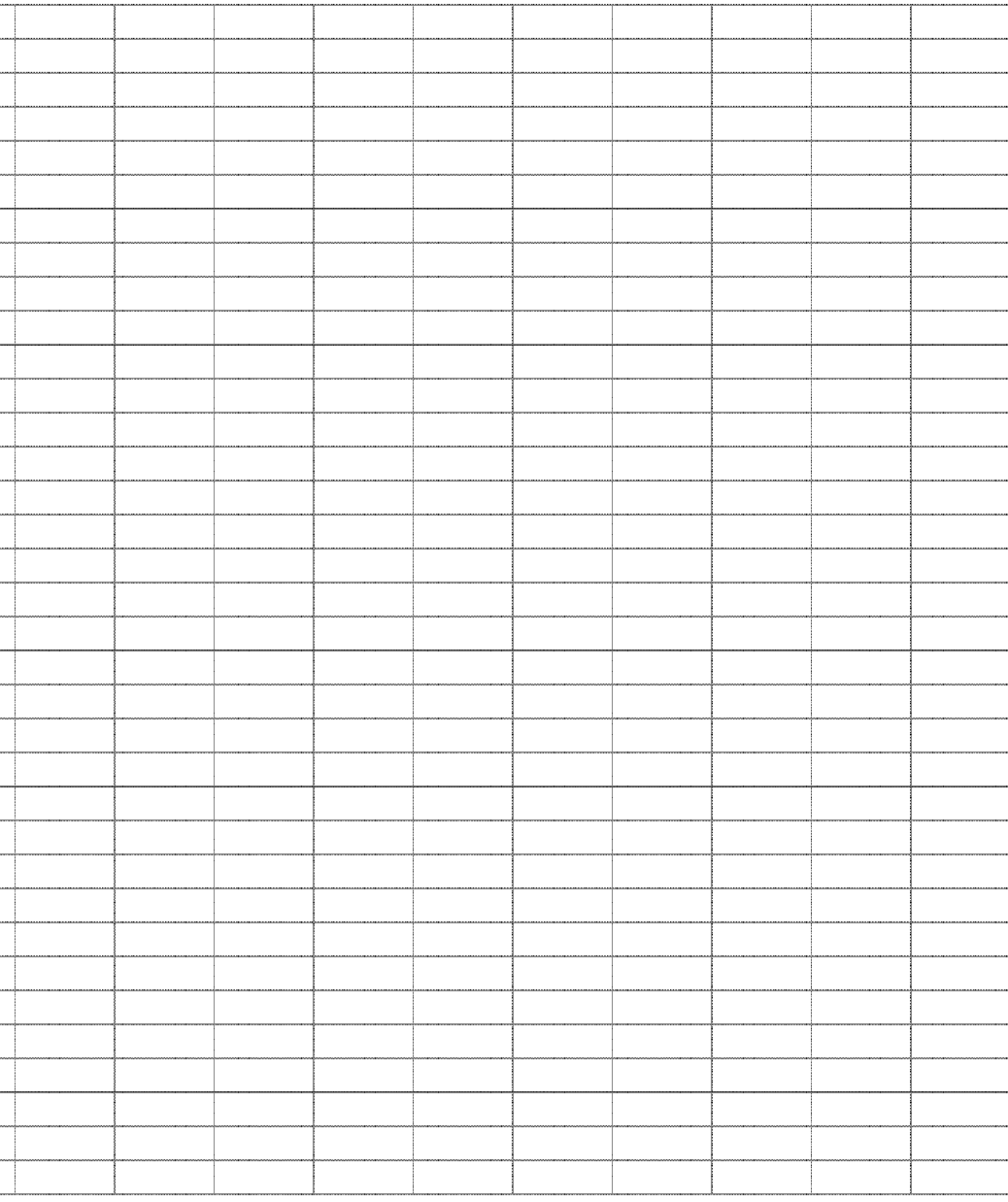


Appendix A. Database - mass fraction

SG (Hrma et al. 1999)

\begin{tabular}{|c|c|c|c|c|c|c|c|c|c|c|c|c|c|c|c|c|}
\hline Glass ID & $\begin{array}{l}\mathrm{T} 13 \\
\left({ }^{\circ} \mathrm{C}\right) \\
\end{array}$ & $\begin{array}{c}\mathrm{V} 13 \\
(\mathrm{~Pa} \cdot \mathrm{s})\end{array}$ & $\begin{array}{l}\mathrm{T} 14 \\
\left({ }^{\circ} \mathrm{C}\right) \\
\end{array}$ & $\begin{array}{c}\mathrm{V} 14 \\
(\mathrm{~Pa} \cdot \mathrm{s}) \\
\end{array}$ & $\begin{array}{l}\text { Q PCT } \\
\text { B }\left(\mathrm{g} / \mathrm{m}^{2}\right)\end{array}$ & $\begin{array}{c}\text { Q PCT } \\
\mathrm{Li}\left(\mathrm{g} / \mathrm{m}^{2}\right)\end{array}$ & $\begin{array}{c}\text { Q PCT } \\
\mathrm{Na}\left(\mathrm{g} / \mathrm{m}^{2}\right)\end{array}$ & $\begin{array}{c}\text { Q PCT } \\
\mathrm{Si}\left(\mathrm{g} / \mathrm{m}^{2}\right)\end{array}$ & $\begin{array}{c}\text { Q PCT } \\
\mathrm{pH}\end{array}$ & $\begin{array}{c}\text { CCC PCT } \\
\mathrm{B}\left(\mathrm{g} / \mathrm{m}^{2}\right) \\
\end{array}$ & $\begin{array}{l}\text { CCC PCT } \\
\mathrm{Li}\left(\mathrm{g} / \mathrm{m}^{2}\right)\end{array}$ & $\begin{array}{l}\mathrm{CCC} \text { PCT } \\
\mathrm{Na}\left(\mathrm{g} / \mathrm{m}^{2}\right) \\
\end{array}$ & $\begin{array}{c}\text { CCC PCT } \\
\mathrm{Si}\left(\mathrm{g} / \mathrm{m}^{2}\right)\end{array}$ & $\begin{array}{c}\text { CCC PCT } \\
\mathrm{pH}\end{array}$ & $\begin{array}{l}\text { Q PCT B at } \\
20^{\circ} \mathrm{C}\left(\mathrm{g} / \mathrm{m}^{2}\right)\end{array}$ & $\begin{array}{l}\text { Q PCT Li at } \\
20^{\circ} \mathrm{C}\left(\mathrm{g} / \mathrm{m}^{2}\right)\end{array}$ \\
\hline \multicolumn{17}{|l|}{ SG46 } \\
\hline \multicolumn{17}{|l|}{ SG47 } \\
\hline \multirow{2}{*}{\multicolumn{17}{|c|}{$\begin{array}{l}\text { SG48 } \\
\text { SG49 }\end{array}$}} \\
\hline \multicolumn{9}{|l|}{ SG49 } & & & & & & & & \\
\hline \multicolumn{17}{|l|}{$\begin{array}{l}\text { SG50 } \\
\text { SG51 }\end{array}$} \\
\hline \multicolumn{17}{|l|}{ SG51 } \\
\hline \multirow{2}{*}{\multicolumn{17}{|c|}{$\begin{array}{l}\text { SG52a } \\
\text { SG52b }\end{array}$}} \\
\hline SG52b & & & & & & & & & & & & & & & & \\
\hline \multicolumn{17}{|l|}{ SG52c } \\
\hline \multicolumn{17}{|l|}{ SG52d } \\
\hline \multicolumn{17}{|l|}{ SG52e } \\
\hline SG53 & & & & & & & & & & & & & & & & \\
\hline
\end{tabular}

SP3, SPx4, Misc. (Vienna et al. 2001)

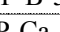

SP-Ca-2

SP-Fe-4

SP-K-1

SP-K-2

SP-Li-5

SP-Li-6

SP-Si-4

SP-Ti-1

SP-Ti-2

SP-Zr-1

SP-Ru-1

SP-Ru-2

SP-Others-1

SP3-1(env.D)

SP-MC-1

SP-MC-2

SP-MC-8

SP-MC-9

Sp-LHLL

Sp-LHLH

Sp-LHHL

Sp-LHHH

Sp-LHMM

Sp-MMLL

Sp-MMLH

Sp-MMHL

Sp-MMHH

Sp-MMMM

Sp-HLLL

Sp-HLLH

Sp-HLHL

Sp-HLHH

Sp-HLMM

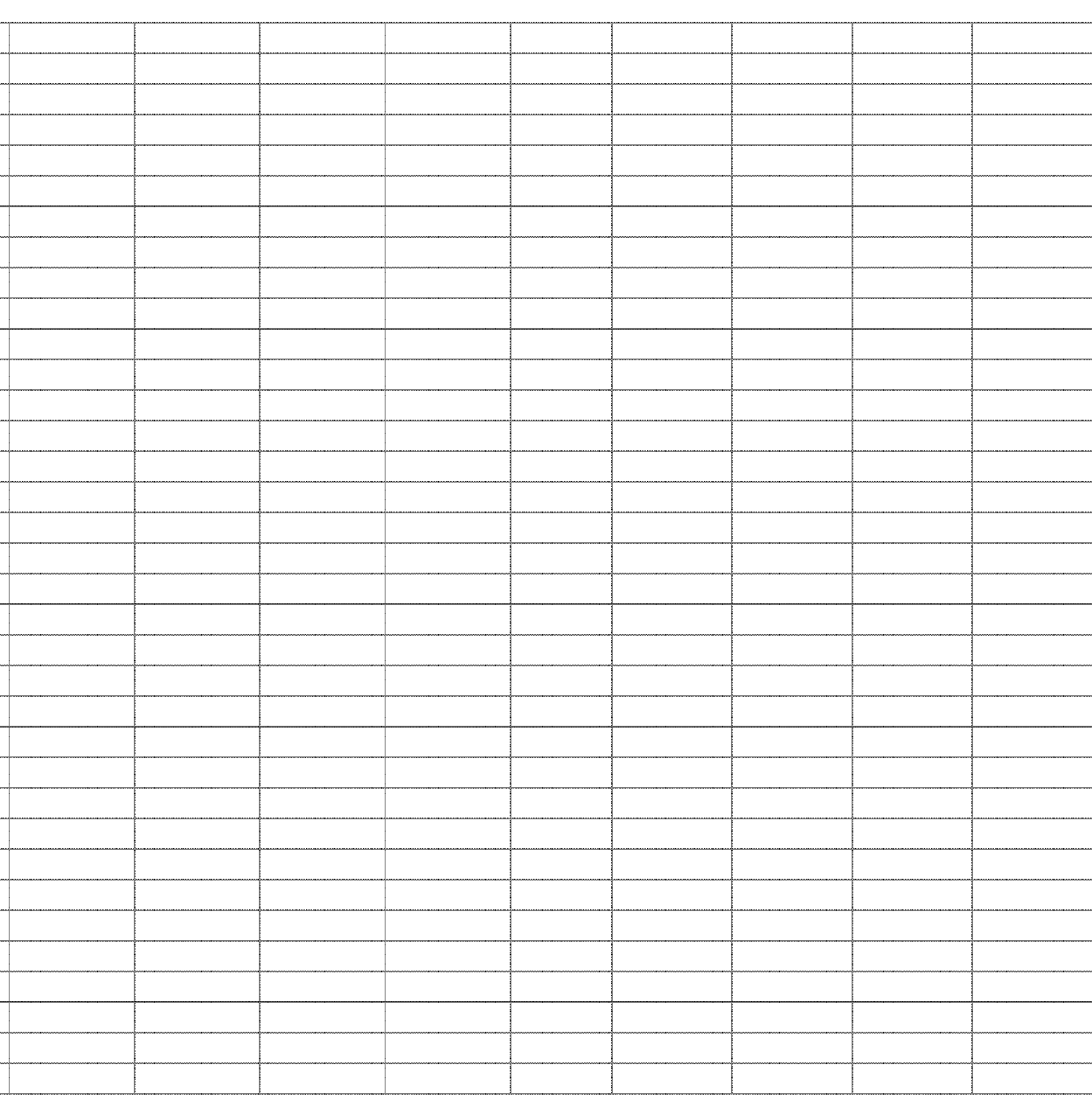


Appendix A. Database - mass fraction

\section{SG (Hrma et al. 1999)}

\begin{tabular}{|c|c|c|c|c|c|c|c|c|c|c|c|c|}
\hline Glass ID & $\begin{array}{l}\text { Q PCT Na at } \\
20^{\circ} \mathrm{C}\left(\mathrm{g} / \mathrm{m}^{2}\right)\end{array}$ & $\begin{array}{l}\text { Q PCT Si at } \\
20^{\circ} \mathrm{C}\left(\mathrm{g} / \mathrm{m}^{2}\right)\end{array}$ & $\begin{array}{c}\mathrm{Q} \mathrm{pH} \\
\text { at } 20^{\circ} \mathrm{C}\end{array}$ & $\begin{array}{c}\text { TCLP Ag } \\
(\mathrm{ppm})\end{array}$ & $\begin{array}{l}\text { TCLP As } \\
(\mathrm{ppm})\end{array}$ & $\begin{array}{c}\text { TCLP Ba } \\
\text { (ppm) }\end{array}$ & $\begin{array}{c}\text { TCLP Cd } \\
\text { (ppm) }\end{array}$ & $\begin{array}{c}\text { TCLP Cr } \\
(\mathrm{ppm})\end{array}$ & $\begin{array}{l}\text { TCLP Ni } \\
(\mathrm{ppm})\end{array}$ & $\begin{array}{c}\text { TCLP Pb } \\
(\mathrm{ppm})\end{array}$ & $\begin{array}{c}\text { TCLP Se } \\
(\mathrm{ppm})\end{array}$ & $\begin{array}{c}\text { TCLP Zn } \\
(\mathrm{ppm})\end{array}$ \\
\hline \multicolumn{13}{|l|}{ SG46 } \\
\hline \multirow{2}{*}{\multicolumn{13}{|c|}{$\begin{array}{l}\text { SG47 } \\
\text { SG48 }\end{array}$}} \\
\hline \multirow{2}{*}{\multicolumn{13}{|c|}{$\begin{array}{l}\text { SG48 } \\
\text { SG49 }\end{array}$}} \\
\hline & & & & & & & & & & & & \\
\hline \multicolumn{13}{|l|}{ SG50 } \\
\hline \multicolumn{13}{|l|}{ SG51 } \\
\hline \multicolumn{13}{|l|}{ SG52a } \\
\hline \multicolumn{13}{|l|}{ SG52b } \\
\hline \multicolumn{13}{|l|}{ SG52c } \\
\hline \multicolumn{13}{|l|}{ SG52d } \\
\hline SG52e & & & & & & & & & & & & \\
\hline SG53 & & & & & & & & & & & & \\
\hline
\end{tabular}

\section{SP3, SPx4, Misc. (Vienna et al. 2001)}

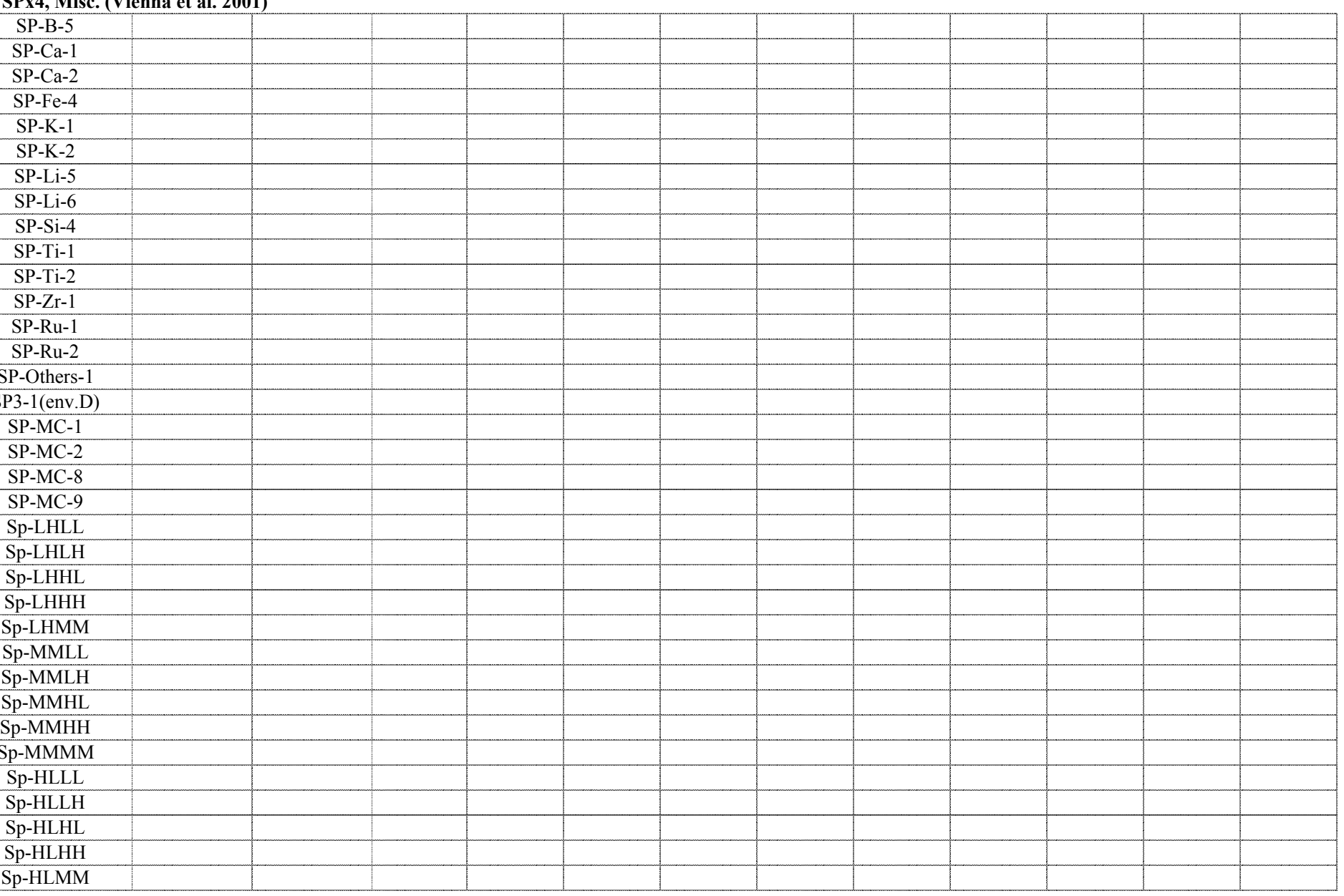


SP3, SPx4, Misc. (Vienna et al. 2001)

\begin{tabular}{|c|c|c|c|c|c|c|c|c|c|c|c|c|c|c|c|c|c|c|c|c|c|}
\hline Glass ID & $\begin{array}{c}\mathrm{A} 12 \mathrm{O} 3 \\
-\mathrm{t}\end{array}$ & $\begin{array}{c}\mathrm{B} 2 \mathrm{O} 3 \\
-\mathrm{t}\end{array}$ & $\begin{array}{c}\mathrm{CaO} \\
-\mathrm{t}\end{array}$ & $\begin{array}{c}\mathrm{Fe} 2 \mathrm{O} 3 \\
-\mathrm{t}\end{array}$ & $\begin{array}{c}\mathrm{FeO} \\
-\mathrm{t}\end{array}$ & $\begin{array}{c}\mathrm{K} 2 \mathrm{O} \\
-\mathrm{t}\end{array}$ & $\begin{array}{c}\mathrm{Li} 2 \mathrm{O} \\
-\mathrm{t}\end{array}$ & $\begin{array}{c}\mathrm{MgO} \\
-\mathrm{t}\end{array}$ & $\begin{array}{c}\mathrm{Na} 2 \mathrm{O} \\
-\mathrm{t}\end{array}$ & $\begin{array}{c}\mathrm{NiO} \\
-\mathrm{t}\end{array}$ & $\begin{array}{c}\mathrm{P} 2 \mathrm{O} 5 \\
-\mathrm{t}\end{array}$ & $\begin{array}{c}\mathrm{SiO} 2 \\
-\mathrm{t} \\
\end{array}$ & $\begin{array}{c}\mathrm{ZrO} 2 \\
-t\end{array}$ & $\begin{array}{c}\mathrm{Ag} 2 \mathrm{O} \\
-\mathrm{t} \\
\end{array}$ & $\begin{array}{c}\mathrm{As} 2 \mathrm{O} 3 \\
-\mathrm{t}\end{array}$ & $\begin{array}{c}\mathrm{BaO} \\
-\mathrm{t} \\
\end{array}$ & $\begin{array}{c}\mathrm{Bi} 2 \mathrm{O} 3 \\
-\mathrm{t} \\
\end{array}$ & $\begin{array}{l}\mathrm{Br} \\
-\mathrm{t}\end{array}$ & $\begin{array}{c}\mathrm{CdO} \\
-\mathrm{t}\end{array}$ & $\begin{array}{c}\mathrm{Ce} 2 \mathrm{O} 3 \\
-\mathrm{t}\end{array}$ & $\begin{array}{c}\mathrm{CeO} 2 \\
-\mathrm{t}\end{array}$ \\
\hline ILH(b) & 00 & 0688 & 0000 & 0.1228 & & 0000 & 0295 & 59 & 0.1873 & 0 & 58 & 20 & .0231 & 0.0009 & 0.0 & .0 & 0.0000 & & .0 & & 0.0 \\
\hline $\mathrm{S}$ & 0 & 706 & 000 & 1260 & & 000 & 0302 & 1 & 573 & 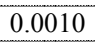 & 0059 & 36 & 36 & 0009 & .0000 & .0039 & .0000 & & .0089 & & .0009 \\
\hline Sp-M & 300 & 683 & 0000 & . & & 0000 & 0293 & 59 & 573 & 200 & 0.0058 & 4487 & .0229 & .0009 & .0000 & .0037 & 0.0000 & & .0086 & & .0009 \\
\hline Sp-HLLL(b) & 100 & 0706 & 0000 & 1260 & & 0000 & .0302 & .0061 & 273 & 10 & 0.0059 & 4636 & 0.0236 & 0.0009 & 0.0000 & 0.0039 & 0.0000 & & 0.0089 & & 0.0009 \\
\hline nom-2 & 681 & 0982 & 0086 & 1076 & 0000 & .0024 & 0.0600 & .0051 & .0913 & 0.0045 & 0.0039 & .5011 & 0.0155 & 0.0006 & 0.0003 & 0.0025 & 0.0000 & & 0.0057 & 0.0000 & 0.0005 \\
\hline-3 & 808 & 1093 & 0102 & 1278 & 0.0000 & 0.0029 & 0.0100 & 0.0060 & .1733 & 0.0053 & 0.0046 & 0.4113 & 0.0184 & 0.0007 & 0.0004 & 0.0030 & 0.0000 & & 0.0068 & 0.0000 & 0.0007 \\
\hline c-1 & 4493 & 0500 & 0119 & 1500 & 0.0000 & 0.0034 & 0.0100 & .0071 & 1883 & 0.0062 & 0.0019 & .4535 & 0.0216 & 0.0008 & 0.0005 & 0.0035 & 0.0000 & & 0.0080 & 0.0000 & 0.0008 \\
\hline $\mathrm{cc}-2$ & 0412 & 0588 & 0100 & 1254 & 0.0000 & 0.0028 & 0.0521 & 0.0059 & 1177 & 0.0052 & 0.0015 & 0.5221 & 0.0181 & 0.0007 & 0.0004 & 0.0029 & 0.0000 & & 0.0067 & 0.0000 & 0.0006 \\
\hline $6 a-2$ & 295 & 0500 & 0278 & 0.1248 & 0.0000 & 0.0027 & 0.0000 & 0.0182 & 2209 & 0.0020 & 0.0095 & 0.3849 & 0.0046 & 0.0010 & 0.0000 & 0.0091 & 0.0000 & & 0.0007 & 0.0000 & 0.0000 \\
\hline $\mathrm{cl}$ & 1214 & .0500 & 0261 & 0.1170 & 0.0000 & 0.0025 & 0.0252 & 0.0171 & 1886 & 0.0019 & 0.0089 & 0.4135 & 0.0044 & 0.0009 & 0.0000 & 0.0086 & 0.0000 & & 0.0006 & 0.0000 & 0.0000 \\
\hline c106a-4 & 9940 & .0831 & 0.0202 & 0.0906 & 0.0000 & 0.0019 & 0.0402 & 0.0132 & .1461 & 0.0015 & 0.0069 & 0.4806 & 0.0034 & 0.0007 & 0.0000 & 0.0066 & 0.0000 & & 0.0005 & 0.0000 & 0.0000 \\
\hline c106b-1 & 1604 & 0.1853 & 0.0020 & 0.0871 & 0.0000 & 0.0000 & 0.0351 & 0.0005 & 1316 & 0000 & 0.0105 & 0.3700 & 0.0006 & 0.0017 & 0.0004 & 0.0003 & 0.0000 & & 0.0000 & 0.0000 & 0.0000 \\
\hline c106b-2 & 1440 & .1094 & 0018 & 0.0781 & 0.0000 & 0.0000 & 0.0599 & 0.0005 & 1311 & 0.0000 & 0.0094 & 0.4501 & 0.0005 & 0.0015 & 0.0003 & 0.0003 & 0.0000 & & 0.0000 & 0.0000 & 0.0000 \\
\hline az-3 & 0664 & 0500 & .0031 & 0.1397 & .0000 & .0032 & 0.0100 & .0011 & 2114 & 0.0079 & 0.0027 & .4296 & 0.0264 & 0.0005 & 0.0006 & 0.0006 & 0.0000 & & 0.0102 & 0.0000 & 0.0010 \\
\hline az-5 & 0.0554 & 0.0974 & 0.0026 & 0.1164 & 0.0000 & 0.0027 & 0.0149 & 0.0009 & 0.1796 & 0.0060 & 0.0023 & 0.4588 & 0.0220 & 0.0004 & 0.0005 & 0.0005 & 0.0000 & & 0.0085 & 0.0000 & 0.0008 \\
\hline
\end{tabular}

\section{DWPF PCT Model (Jantzen et al. 1995)}

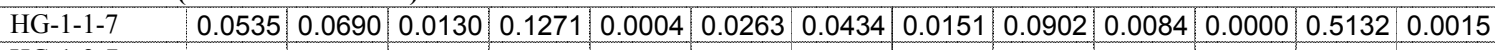
\begin{tabular}{lll|l|l|l|l|l|l|l|l|l|l|l|l|l|l|l}
\hline HG-1-2-7 & 0.0499 & 0.0672 & 0.0104 & 0.1160 & 0.0004 & 0.0297 & 0.0448 & 0.0147 & 0.0849 & 0.0076 & 0.0004 & 0.5349 & 0.0009
\end{tabular}

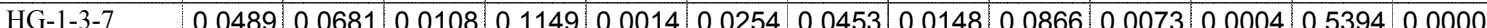

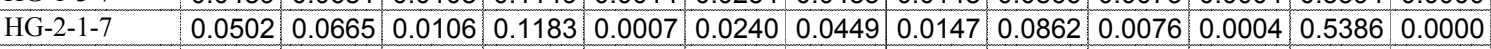
\begin{tabular}{l|l|l|l|l|l|l|l|l|l|l|l|l|l|l|l|l|l}
\hline HG-2-2-7 & 0.0535 & 0.0594 & 0.0108 & 0.1254 & 0.0007 & 0.0177 & 0.0432 & 0.0135 & 0.1015 & 0.0075 & 0.0007 & 0.5255 & 0.0021 \\
\hline
\end{tabular} \begin{tabular}{l|l|l|l|l|l|l|l|l|l|l|l|l|l|l|l|l|l}
\hline HG-2-3-7 & 0.0527 & 0.0592 & 0.0108 & 0.1235 & 0.0007 & 0.0177 & 0.0436 & 0.0135 & 0.1021 & 0.0075 & 0.0000 & 0.5308 & 0.0008
\end{tabular} \begin{tabular}{ll|l|l|l|l|l|l|l|l|l|l|l|l|l|l|l|l|l|l|l} 
HG-3-1-7 & 0.0549 & 0.0602 & 0.0112 & 0.1226 & 0.0051 & 0.0197 & 0.0426 & 0.0134 & 0.0988 & 0.0078 & 0.0004 & 0.5221 & 0.0017
\end{tabular}

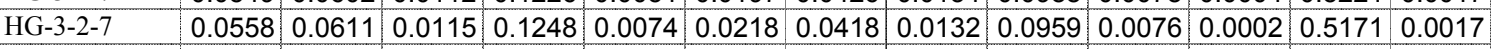

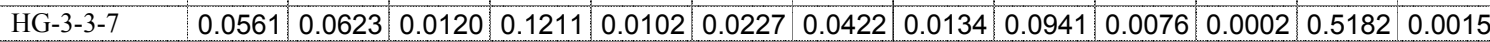

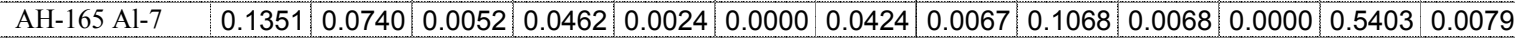
\begin{tabular}{l|l|l|l|l|l|l|l|l|l|l|l|l|l|l|l|l|l|l} 
AH-165 AV-7 & 0.0519 & 0.0660 & 0.0105 & 0.1111 & 0.0047 & 0.0000 & 0.0504 & 0.0066 & 0.1000 & 0.0102 & 0.0000 & 0.5551 & 0.0077
\end{tabular} \begin{tabular}{l|l|l|l|l|l|l|l|l|l|l|l|l|l|l|l|l|l}
\hline AH-165 FE-7 & 0.0144 & 0.0738 & 0.0142 & 0.0920 & 0.0725 & 0.0000 & 0.0411 & 0.0066 & 0.1085 & 0.0301 & 0.0000 & 0.5275 & 0.0085 \\
\hline
\end{tabular}

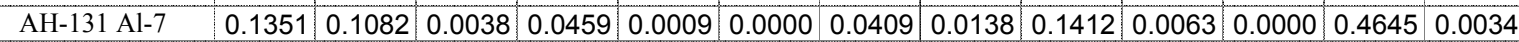

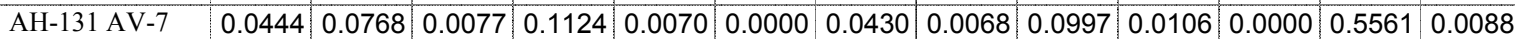
\begin{tabular}{|l|l|l|l|l|l|l|l|l|l|l|l|l|l|l|l|l}
\hline AH-131 FE-7 & 0.0229 & 0.0745 & 0.0103 & 0.0774 & 0.0895 & 0.0000 & 0.0412 & 0.0067 & 0.1108 & 0.0261 & 0.0000 & 0.5224 & 0.0088 \\
\hline
\end{tabular}

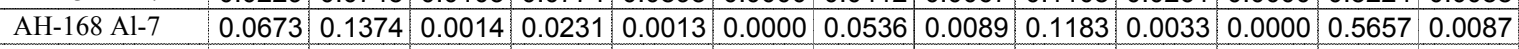

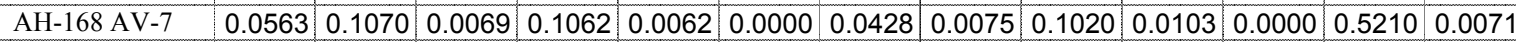
\begin{tabular}{l|l|l|l|l|l|l|l|l|l|l|l|l|l|l|l|l} 
AH-168 FE-7 & 0.0248 & 0.1149 & 0.0136 & 0.0947 & 0.0627 & 0.0000 & 0.0415 & 0.0072 & 0.1089 & 0.0284 & 0.0000 & 0.4867 & 0.0067
\end{tabular} \begin{tabular}{|c|c|c|c|c|c|c|c|c|c|c|c|c|c|c|c|}
\hline AH-200 Al-7 & 0.1347 & 0.1026 & 0.0055 & 0.0441 & 0.0007 & 0.0313 & 0.0266 & 0.0125 & 0.1066 & 0.0061 & 0.0000 & 0.4867 & 0.0004
\end{tabular}

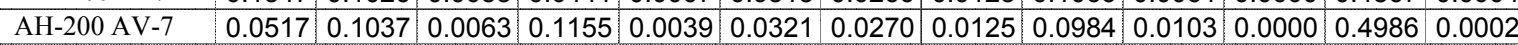
\begin{tabular}{l|l|l|l|l|l|l|l|l|l|l|l|l|l|l|l|l|l} 
AH-200 FE-7 & 0.0209 & 0.1019 & 0.0093 & 0.0988 & 0.0600 & 0.0318 & 0.0262 & 0.0122 & 0.1070 & 0.0259 & 0.0000 & 0.4784 & 0.0002 \\
\hline
\end{tabular} \begin{tabular}{l|l|l|l|l|l|l|l|l|l|l|l|l|l|l} 
AH-202 Al-7 & 0.1397 & 0.0746 & 0.0041 & 0.0433 & 0.0009 & 0.0334 & 0.0420 & 0.0129 & 0.0737 & 0.0062 & 0.0000 & 0.5265 & 0.0002
\end{tabular} \begin{tabular}{ll|l|l|l|l|l|l|l|l|l|l|l|l|l|l|l|l} 
AH-202-AV-7 & 0.0498 & 0.0748 & 0.0072 & 0.1161 & 0.0031 & 0.0334 & 0.0429 & 0.0131 & 0.0658 & 0.0101 & 0.0000 & 0.5437 & 0.0002
\end{tabular} \begin{tabular}{|l|l|l|l|l|l|l|l|l|l|l|l|l|l|l|l|l|l|l|l|l|l|}
\hline AH-202 FE-7 & 0.0137 & 0.0710 & 0.0096 & 0.0966 & 0.0630 & 0.0328 & 0.0428 & 0.0127 & 0.0764 & 0.0274 & 0.0000 & 0.5270 & 0.0002 \\
\hline
\end{tabular} \begin{tabular}{l|l|l|l|l|l|l|l|l|l|l|l|l|l|l|l} 
AH-1-7 & 0.0697 & 0.1029 & 0.0096 & 0.1200 & 0.0000 & 0.0331 & 0.0447 & 0.0066 & 0.1189 & 0.0094 & 0.0000 & 0.4406 & 0.0067 \\
\hline
\end{tabular} \begin{tabular}{l|l|l|l|l|l|l|l|l|l|l|l|l|l|l|l|l} 
AH-2-7 & 0.0657 & 0.1332 & 0.0065 & 0.1143 & 0.0000 & 0.0311 & 0.0377 & 0.0058 & 0.1031 & 0.0093 & 0.0000 & 0.4463 & 0.0063
\end{tabular} \begin{tabular}{l|l|l|l|l|l|l|l|l|l|l|l|l|l|l|l|l|l|l|l} 
AH-4-7 & 0.0471 & 0.0709 & 0.0100 & 0.1103 & 0.0000 & 0.0317 & 0.0411 & 0.0073 & 0.0993 & 0.0098 & 0.0000 & 0.5368 & 0.0000 \\
\hline
\end{tabular}

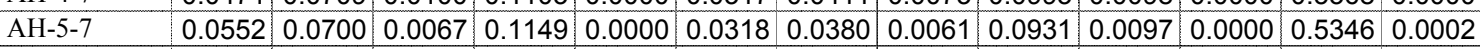
\begin{tabular}{l|l|l|l|l|l|l|l|l|l|l|l|l|l|l|l|l|l|l|l|l}
\hline AH-6-7 & 0.0560 & 0.0940 & 0.0068 & 0.1188 & 0.0000 & 0.0316 & 0.0462 & 0.0061 & 0.1058 & 0.0094 & 0.0000 & 0.4763 & 0.0067
\end{tabular}

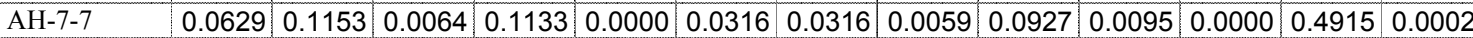
$\mathrm{AH}-8-7$ \begin{tabular}{|l|l|l|l|l|l|l|l|l|l|l|l|l|l|l|l|l}
0.0590 & 0.1015 & 0.0069 & 0.1165 & 0.0000 & 0.0309 & 0.0319 & 0.0121 & 0.0981 & 0.0098 & 0.0000 & 0.4922 & 0.0000
\end{tabular}

\begin{tabular}{|c|c|c|}
\hline & 0.0012 & 0.0000 \\
\hline & 0.0007 & 0.0000 \\
\hline & 0.0007 & 0.0000 \\
\hline & 0.0007 & 0.0000 \\
\hline & 0.0009 & 0.0000 \\
\hline & 0.0007 & 0.0000 \\
\hline & 0.0007 & 0.0000 \\
\hline & 0.0009 & 0.0000 \\
\hline & 0.0009 & 0.0000 \\
\hline & 0.0000 & 0.0000 \\
\hline & 0.0000 & 0.0000 \\
\hline & 0.0000 & 0.0000 \\
\hline & 0.0000 & 0.0000 \\
\hline & 0.0000 & 0.0000 \\
\hline & 0.0000 & 0.0000 \\
\hline & 0.0000 & 0.0000 \\
\hline & 0.0000 & 0.0000 \\
\hline & 0.0000 & 0.0000 \\
\hline & 0.0000 & 0.0000 \\
\hline & 0.0000 & 0.0000 \\
\hline & 0.0000 & 0.0000 \\
\hline & 0.0000 & 0.0000 \\
\hline & 0.0000 & 0.0000 \\
\hline & 0.0000 & 0.0000 \\
\hline & 0.0000 & 0.0000 \\
\hline & 0.0000 & 0.0000 \\
\hline & 0.0000 & 0.0000 \\
\hline & 0.0000 & 0.0000 \\
\hline & 0.0000 & 0.0000 \\
\hline & 0.0000 & 0.0000 \\
\hline & 0.0000 & 0.0000 \\
\hline
\end{tabular}


SP3, SPx4, Misc. (Vienna et al. 2001)

\begin{tabular}{|c|c|c|c|c|c|c|c|c|c|c|c|c|c|c|c|c|c|c|c|c|c|}
\hline Glass ID & $\begin{array}{c}\mathrm{Cl} \\
-\mathrm{t}\end{array}$ & $\begin{array}{c}\mathrm{CoO} \\
-\mathrm{t} \\
\end{array}$ & $\begin{array}{c}\mathrm{Co} 2 \mathrm{O} 3 \\
-\mathrm{t}\end{array}$ & $\begin{array}{c}\mathrm{Cr} 2 \mathrm{O} 3 \\
-\mathrm{t}\end{array}$ & $\begin{array}{c}\mathrm{Cs} 2 \mathrm{O} \\
-\mathrm{t} \\
\end{array}$ & $\begin{array}{c}\mathrm{CuO} \\
-\mathrm{t}\end{array}$ & $\begin{array}{c}\mathrm{Eu} 2 \mathrm{O} 3 \\
-\mathrm{t}\end{array}$ & $\begin{array}{l}F \\
-t\end{array}$ & $\begin{array}{c}\mathrm{Ga} 2 \mathrm{O} 3 \\
-\mathrm{t} \\
\end{array}$ & $\begin{array}{c}\mathrm{Gd} 2 \mathrm{O} 3 \\
-\mathrm{t} \\
\end{array}$ & $\begin{array}{c}\mathrm{HgO} \\
-\mathrm{t} \\
\end{array}$ & $\begin{array}{l}I \\
-t \\
\end{array}$ & $\begin{array}{c}\mathrm{La} 2 \mathrm{O} 3 \\
-\mathrm{t}\end{array}$ & $\begin{array}{c}\mathrm{MnO} 2 \\
-\mathrm{t} \\
\end{array}$ & $\begin{array}{c}\mathrm{MnO} \\
-\mathrm{t}\end{array}$ & $\begin{array}{c}\mathrm{MoO} \\
-\mathrm{t}\end{array}$ & $\begin{array}{c}\mathrm{MoO} 3 \\
-\mathrm{t}\end{array}$ & $\begin{array}{c}\mathrm{Nb} 2 \mathrm{O} 5 \\
-\mathrm{t}\end{array}$ & $\begin{array}{c}\mathrm{Nd} 2 \mathrm{O} 3 \\
-\mathrm{t}\end{array}$ & $\begin{array}{c}\mathrm{PbO} \\
-\mathrm{t}\end{array}$ & $\begin{array}{c}\mathrm{PdO} 2 \\
-\mathrm{t}\end{array}$ \\
\hline Sp-LHLH(b) & 0.0001 & & 0.0011 & 0.0005 & 0.0000 & 0.0004 & & 0.0008 & & & & & 0.0034 & & 0.0035 & & 0.0001 & & 0.0023 & 0.0021 & \\
\hline Sp-MMLL(b) & 0.0001 & & 0.0012 & 0.0005 & 0.0000 & 0.0004 & & 0.0008 & & & & & 0.0035 & & 0.0036 & & 0.0001 & & 0.0023 & 0.0022 & \\
\hline Sp-MMHH(b) & 0.0001 & & 0.0011 & 0.0060 & 0.0000 & 0.0004 & & 0.0007 & & & & & 0.0034 & & 0.0035 & & 0.0001 & & 0.0022 & 0.0021 & \\
\hline Sp-HLLL(b) & 0.0001 & & 0.0012 & 0.0005 & 0.0000 & 0.0004 & & 0.0008 & & & & & 0.0035 & & 0.0036 & & 0.0001 & & 0.0023 & 0.0022 & \\
\hline nom-2 & 0.0000 & & 0.0008 & 0.0018 & 0.0000 & 0.0003 & & 0.0005 & & & & & 0.0023 & & 0.0028 & & 0.0001 & & 0.0015 & 0.0000 & \\
\hline nom-3 & 0.0000 & & 0.0009 & 0.0022 & 0.0000 & 0.0003 & & 0.0006 & & & & & 0.0027 & & 0.0033 & & 0.0001 & & 0.0018 & 0.0000 & \\
\hline nomc-1 & 0.0000 & & 0.0011 & 0.0023 & 0.0000 & 0.0004 & & 0.0007 & & & & & 0.0032 & & 0.0039 & & 0.0001 & & 0.0021 & 0.0000 & \\
\hline nomc-2 & 0.0000 & & 0.0009 & 0.0019 & 0.0000 & 0.0003 & & 0.0005 & & & & & 0.0027 & & 0.0033 & & 0.0001 & & 0.0018 & 0.0000 & \\
\hline c106a-2 & 0.0000 & & 0.0000 & 0.0024 & 0.0000 & 0.0003 & & 0.0000 & & & & & 0.0000 & & 0.0040 & & 0.0000 & & 0.0000 & 0.0000 & \\
\hline c106a-3 & 0.0000 & & 0.0000 & 0.0023 & 0.0000 & 0.0003 & & 0.0000 & & & & & 0.0000 & & 0.0037 & & 0.0000 & & 0.0000 & 0.0000 & \\
\hline c106a-4 & 0.0000 & & 0.0000 & 0.0017 & 0.0000 & 0.0002 & & 0.0000 & & & & & 0.0000 & & 0.0029 & & 0.0000 & & 0.0000 & 0.0000 & \\
\hline c106b-1 & 0.0000 & & 0.0000 & 0.0019 & 0.0000 & 0.0003 & & 0.0000 & & & & & 0.0000 & & 0.0043 & & 0.0000 & & 0.0000 & 0.0000 & \\
\hline c106b-2 & 0.0000 & & 0.0000 & 0.0017 & 0.0000 & 0.0002 & & 0.0000 & & & & & 0.0000 & & 0.0039 & & 0.0000 & & 0.0000 & 0.0000 & \\
\hline$a z-3$ & 0.0000 & & 0.0014 & 0.0022 & 0.0000 & 0.0004 & & 0.0007 & & & & & 0.0041 & & 0.0031 & & 0.0001 & & 0.0027 & 0.0008 & \\
\hline az-5 & 0.0000 & & 0.0012 & 0.0018 & 0.0000 & 0.0003 & & 0.0006 & & & & & 0.0034 & & 0.0026 & & 0.0001 & & 0.0023 & 0.0006 & \\
\hline
\end{tabular}

\section{DWPF PCT Model (Jantzen et al. 1995)}

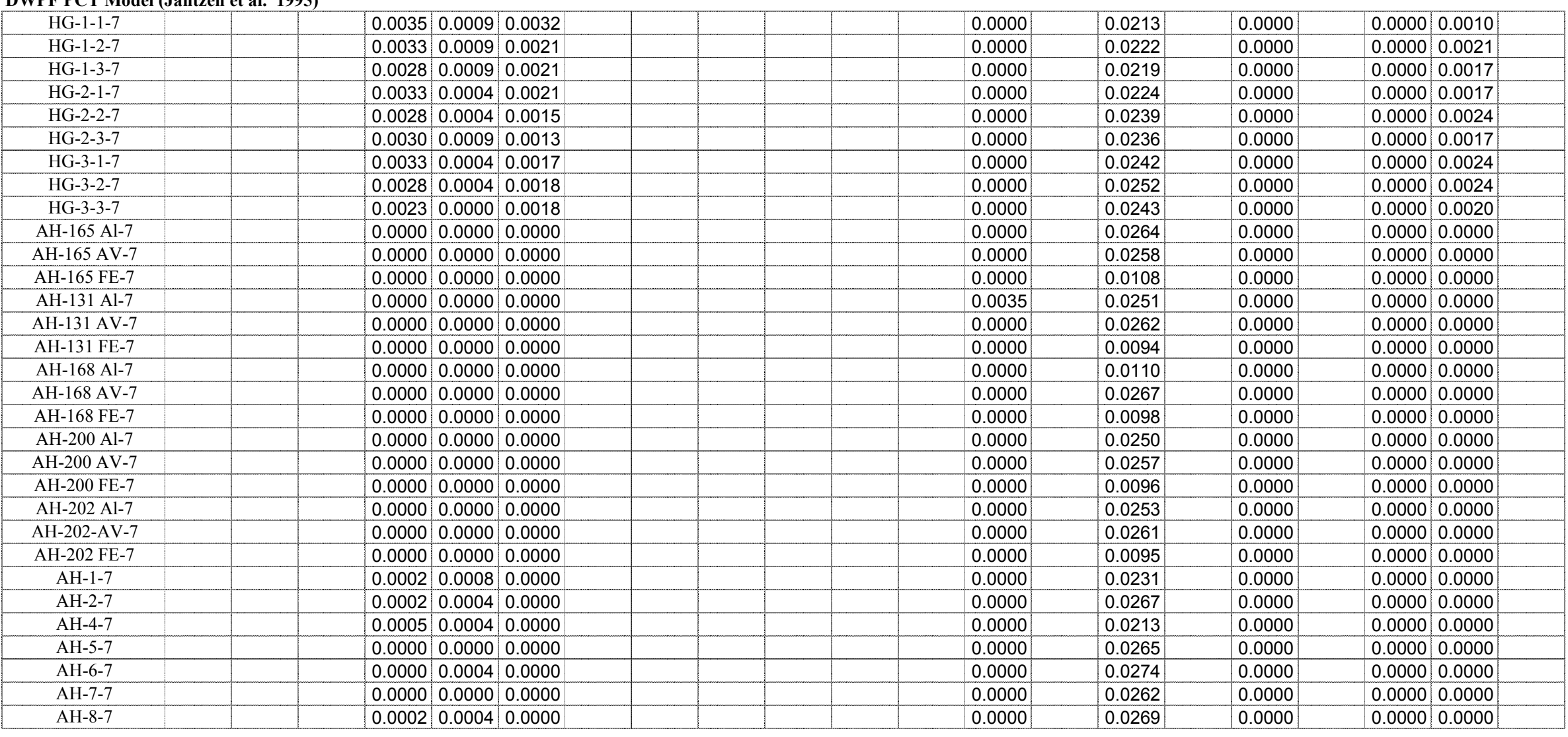


SP3, SPx4, Misc. (Vienna et al. 2001)

\begin{tabular}{|c|c|c|c|c|c|c|c|c|c|c|c|c|c|c|c|c|c|c|c|c|c|}
\hline Glass ID & $\begin{array}{c}\mathrm{PdO} \\
-\mathrm{t}\end{array}$ & $\begin{array}{c}\mathrm{Pr} 2 \mathrm{O} 3 \\
-\mathrm{t}\end{array}$ & $\begin{array}{c}\operatorname{Pr} 6011 \\
-t\end{array}$ & $\begin{array}{c}\mathrm{Rb} 2 \mathrm{O} \\
-\mathrm{t}\end{array}$ & $\begin{array}{c}\mathrm{ReO} \\
-\mathrm{t}\end{array}$ & $\begin{array}{c}\mathrm{ReO} 2 \\
-\mathrm{t}\end{array}$ & $\begin{array}{c}\mathrm{Rh} 2 \mathrm{O} 3 \\
-\mathrm{t}\end{array}$ & $\begin{array}{c}\mathrm{RhO} 2 \\
-\mathrm{t}\end{array}$ & $\begin{array}{c}\mathrm{RuO} 2 \\
-\mathrm{t}\end{array}$ & $\begin{array}{c}\mathrm{Sb} 2 \mathrm{O} 3 \\
-\mathrm{t}\end{array}$ & $\begin{array}{c}\mathrm{Sb} 2 \mathrm{O} 5 \\
-\mathrm{t}\end{array}$ & $\begin{array}{c}\mathrm{SeO} 2 \\
-\mathrm{t}\end{array}$ & $\begin{array}{c}\mathrm{Sm} 2 \mathrm{O} 3 \\
-\mathrm{t} \\
\end{array}$ & $\begin{array}{c}\mathrm{SnO} \\
-\mathrm{t}\end{array}$ & $\begin{array}{c}\mathrm{SnO} 2 \\
-\mathrm{t}\end{array}$ & $\begin{array}{c}\mathrm{SO} 3 \\
-\mathrm{t}\end{array}$ & $\begin{array}{c}\mathrm{SrO} \\
-\mathrm{t} \\
\end{array}$ & $\begin{array}{c}\mathrm{Tc} 2 \mathrm{O} 7 \\
-\mathrm{t}\end{array}$ & $\begin{array}{c}\mathrm{TeO} 2 \\
-\mathrm{t}\end{array}$ & $\begin{array}{c}\mathrm{ThO} 2 \\
-\mathrm{t}\end{array}$ & $\begin{array}{c}\mathrm{TiO} 2 \\
-\mathrm{t}\end{array}$ \\
\hline Sp-LHLH(b) & 0.0000 & & 0.0000 & 0.0000 & & & 0.0004 & & 0.0003 & 0.0009 & & 0.0011 & 0.0000 & & & 0.0024 & 0.0004 & & 0.0000 & & 0.0003 \\
\hline Sp-MMLL(b) & 0.0000 & & 0.0000 & 0.0000 & & & 0.0004 & & 0.0003 & 0.0009 & & 0.0012 & 0.0000 & & & 0.0025 & 0.0004 & & 0.0000 & & 0.0003 \\
\hline Sp-MMHH(b) & 0.0000 & & 0.0000 & 0.0000 & & & 0.0004 & & 0.0003 & 0.0009 & & 0.0011 & 0.0000 & & & 0.0024 & 0.0004 & & 0.0000 & & 0.0003 \\
\hline Sp-HLLL(b) & 0.0000 & & 0.0000 & 0.0000 & & & 0.0004 & & 0.0003 & 0.0009 & & 0.0012 & 0.0000 & & & 0.0025 & 0.0004 & & 0.0000 & & 0.0003 \\
\hline nom-2 & 0.0000 & & 0.0000 & 0.0000 & & & 0.0002 & & 0.0003 & 0.0006 & & 0.0008 & 0.0000 & & & 0.0016 & 0.0003 & & 0.0006 & & 0.0002 \\
\hline nom-3 & 0.0000 & & 0.0000 & 0.0000 & & & 0.0003 & & 0.0003 & 0.0007 & & 0.0009 & 0.0000 & & & 0.0019 & 0.0003 & & 0.0007 & & 0.0003 \\
\hline nomc-1 & 0.0000 & & 0.0000 & 0.0000 & & & 0.0003 & & 0.0004 & 0.0008 & & 0.0011 & 0.0000 & & & 0.0022 & 0.0004 & & 0.0008 & & 0.0003 \\
\hline nomc-2 & 0.0000 & & 0.0000 & 0.0000 & & & 0.0003 & & 0.0003 & 0.0007 & & 0.0009 & 0.0000 & & & 0.0019 & 0.0003 & & 0.0007 & & 0.0003 \\
\hline c106a-2 & 0.0000 & & 0.0000 & 0.0000 & & & 0.0000 & & 0.0000 & 0.0000 & & 0.0000 & 0.0000 & & & 0.0000 & 0.0002 & & 0.0000 & & 0.0000 \\
\hline c106a-3 & 0.0000 & & 0.0000 & 0.0000 & & & 0.0000 & & 0.0000 & 0.0000 & & 0.0000 & 0.0000 & & & 0.0000 & 0.0002 & & 0.0000 & & 0.0000 \\
\hline c106a-4 & 0.0000 & & 0.0000 & 0.0000 & & & 0.0000 & & 0.0000 & 0.0000 & & 0.0000 & 0.0000 & & & 0.0000 & 0.0001 & & 0.0000 & & 0.0000 \\
\hline c106b-1 & 0.0000 & & 0.0000 & 0.0000 & & & 0.0000 & & 0.0000 & 0.0026 & & 0.0013 & 0.0000 & & & 0.0000 & 0.0001 & & 0.0000 & & 0.0004 \\
\hline c106b-2 & 0.0000 & & 0.0000 & 0.0000 & & & 0.0000 & & 0.0000 & 0.0023 & & 0.0012 & 0.0000 & & & 0.0000 & 0.0001 & & 0.0000 & & 0.0004 \\
\hline$a z-3$ & 0.0000 & & 0.0000 & 0.0000 & & & 0.0004 & & 0.0005 & 0.0000 & & 0.0000 & 0.0000 & & & 0.0029 & 0.0004 & & 0.0010 & & 0.0004 \\
\hline$a z-5$ & 0.0000 & & 0.0000 & 0.0000 & & & 0.0004 & & 0.0004 & 0.0000 & & 0.0000 & 0.0000 & & & 0.0025 & 0.0003 & & 0.0008 & & 0.0004 \\
\hline
\end{tabular}

\section{DWPF PCT Model (Jantzen et al. 1995)}

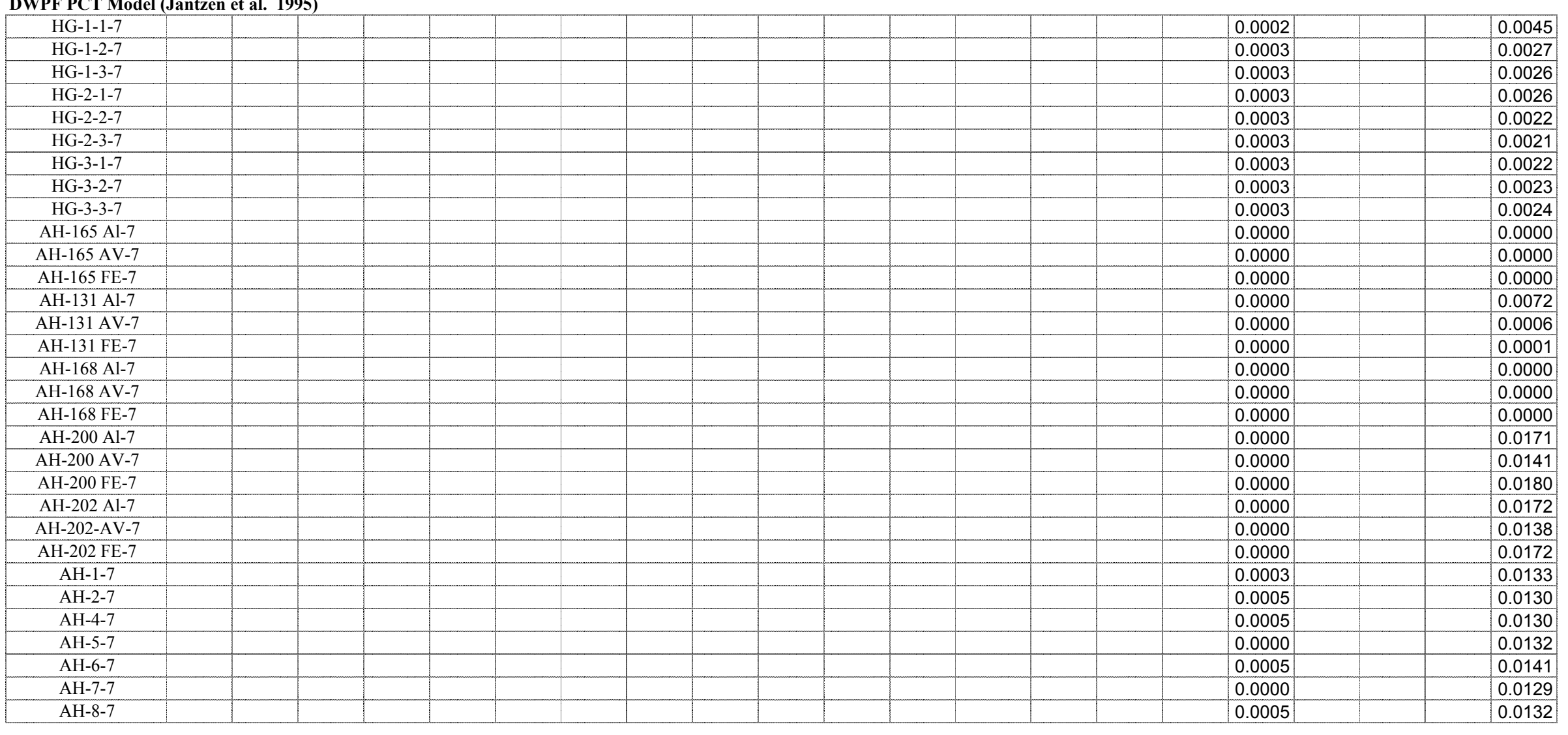


SP3, SPx4, Misc. (Vienna et al. 2001)

\begin{tabular}{|c|c|c|c|c|c|c|c|c|c|c|c|c|c|c|c|c|c|c|c|c|c|}
\hline Glass ID & $\begin{array}{c}\mathrm{T} 12 \mathrm{O} 3 \\
-\mathrm{t}\end{array}$ & $\begin{array}{c}\text { U3O8 } \\
-t\end{array}$ & $\begin{array}{c}\mathrm{UO} 2 \\
-\mathrm{t}\end{array}$ & $\begin{array}{c}\text { UO3 } \\
-t\end{array}$ & $\begin{array}{c}\mathrm{V} 2 \mathrm{O} 5 \\
-\mathrm{t}\end{array}$ & $\begin{array}{c}\text { WO3 } \\
-t\end{array}$ & $\begin{array}{c}\mathrm{Y} 2 \mathrm{O} 3 \\
-\mathrm{t}\end{array}$ & $\begin{array}{c}\mathrm{ZnO} \\
-\mathrm{t}\end{array}$ & $\begin{array}{c}\text { Others } \\
-t\end{array}$ & $\begin{array}{c}\text { Sum } \\
-t\end{array}$ & $\begin{array}{c}\mathrm{A} 12 \mathrm{O} 3 \\
-\mathrm{a}\end{array}$ & $\begin{array}{c}\mathrm{B} 2 \mathrm{O} 3 \\
-\mathrm{a}\end{array}$ & $\begin{array}{c}\mathrm{CaO} \\
-\mathrm{a} \\
\end{array}$ & $\begin{array}{c}\mathrm{Fe} 2 \mathrm{O} 3 \\
-\mathrm{a} \\
\end{array}$ & $\begin{array}{c}\mathrm{FeO} \\
-\mathrm{a} \\
\end{array}$ & $\begin{array}{c}\mathrm{K} 2 \mathrm{O} \\
-\mathrm{a}\end{array}$ & $\begin{array}{c}\mathrm{Li} 2 \mathrm{O} \\
-\mathrm{a}\end{array}$ & $\begin{array}{c}\mathrm{MgO} \\
-\mathrm{a}\end{array}$ & $\begin{array}{c}\mathrm{Na} 2 \mathrm{O} \\
-\mathrm{a}\end{array}$ & $\begin{array}{c}\mathrm{NiO} \\
-\mathrm{a} \\
\end{array}$ & $\begin{array}{c}\mathrm{P} 2 \mathrm{O} 5 \\
-\mathrm{a} \\
\end{array}$ \\
\hline Sp-LHLH(b) & & 0.0000 & & & & 0.0000 & 0.0000 & 0.0005 & & 0.9999 & & & & & & & & & & & \\
\hline Sp-MMLL(b) & & 0.0000 & & & & 0.0000 & 0.0000 & 0.0005 & & 0.9999 & & & & & & & & & & & \\
\hline Sp-MMHH(b) & & 0.0000 & & & & 0.0000 & 0.0000 & 0.0005 & & 0.9999 & & & & & & & & & & & \\
\hline Sp-HLLL(b) & & 0.0000 & & & & 0.0000 & 0.0000 & 0.0005 & & 0.9999 & & & & & & & & & & & \\
\hline nom-2 & & 0.0000 & & & & 0.0000 & 0.0000 & 0.0002 & & 0.9908 & & & & & & & & & & & \\
\hline nom-3 & & 0.0000 & & & & 0.0000 & 0.0000 & 0.0002 & & 0.9891 & & & & & & & & & & & \\
\hline nomc-1 & & 0.0000 & & & & 0.0000 & 0.0000 & 0.0002 & & 0.9871 & & & & & & & & & & & \\
\hline nomc-2 & & 0.0000 & & & & 0.0000 & 0.0000 & 0.0002 & & 0.9892 & & & & & & & & & & & \\
\hline c106a-2 & & 0.0000 & & & & 0.0000 & 0.0000 & 0.0001 & & 0.9926 & & & & & & & & & & & \\
\hline c106a-3 & & 0.0000 & & & & 0.0000 & 0.0000 & 0.0001 & & 0.9931 & & & & & & & & & & & \\
\hline c106a-4 & & 0.0000 & & & & 0.0000 & 0.0000 & 0.0001 & & 0.9946 & & & & & & & & & & & \\
\hline c106b-1 & & 0.0000 & & & & 0.0000 & 0.0000 & 0.0001 & & 0.9964 & & & & & & & & & & & \\
\hline c106b-2 & & 0.0000 & & & & 0.0000 & 0.0000 & 0.0001 & & 0.9967 & & & & & & & & & & & \\
\hline az-3 & & 0.0000 & & & & 0.0000 & 0.0000 & 0.0003 & & 0.9858 & & & & & & & & & & & \\
\hline $\mathrm{az}-5$ & & 0.0000 & & & & 0.0000 & 0.0000 & 0.0002 & & 0.9876 & & & & & & & & & & & \\
\hline
\end{tabular}

\section{DWPF PCT Model (Jantzen et al. 1995)}

HG-1-1-7

HG-1-2-7

HG-1-3-7

HG-2-1-7

HG-2-2-7

HG-2-3-7

HG-3-1-7

HG-3-2-7

HG-3-3-7

AH-165 Al-7

AH-165 AV-7

AH-165 FE-7

AH-131 Al-7

AH-131 AV-7

AH-131 FE-7

AH-168 Al-7

AH-168 AV-7

AH-168 FE-7

AH-200 Al-7

AH-200 AV-7

AH-200 FE-7

$\mathrm{AH}-202 \mathrm{Al}-7$

AH-202-AV-7

AH-202 FE-7

$\mathrm{AH}-1-7$

AH-2-7

AH-4-7

AH-5-7

AH-6-7

AH-8-7
$0.0000 \quad 0.0031$ $0.0000 \quad 0.0038$ $0.0000 \quad 0.0037$ $0.0000 \quad 0.0038$ $0.0000 \quad 0.0040$ $0.0000 \quad 0.0038$ $0.0000 \quad 0.0044$ \begin{tabular}{lll}
0.0000 & 0.0038 \\
\hline 0.0000 & 0.0042
\end{tabular} $0.0000 \quad 0.0042$ $0.0000 \quad 0.0000$ $0.0000 \quad 0.0000$ $0.0000 \quad 0.0000$ $0.0000 \quad 0.0000$ $0.0000 \quad 0.0000$ $0.0000 \quad 0.0000$ 0.00000 .0000 $0.0000 \quad 0.0000$ $0.0000 \quad 0.0000$ $0.0000 \quad 0.0000$ $0.0000 \quad 0.0000$ $0.0000 \quad 0.0000$ $0.0000 \quad 0.0000$ $0.0000 \quad 0.0000$ $0.0000 \quad 0.0000$ $0.0000 \quad 0.0000$ $0.0000 \quad 0.0000$ $0.0000 \quad 0.0000$ $0.0000 \quad 0.0000$ $0.0000 \quad 0.0000$ $0.0000 \quad 0.0000$ $0.0000 \quad 0.0000$

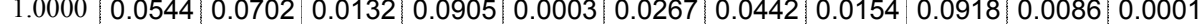
\begin{tabular}{ll|l|l|l|l|l|l|l|l|l|l|l|l|l}
1.0000 & 0.0516 & 0.0695 & 0.0108 & 0.1200 & 0.0005 & 0.0307 & 0.0463 & 0.0152 & 0.0878 & 0.0079 & 0.0005 \\
\hline
\end{tabular} \begin{tabular}{ll|l|l|l|l|l|l|l|l|l|l|l|l|l|l|l|l|l|l|l}
1.0000 & 0.0501 & 0.0697 & 0.0111 & 0.1175 & 0.0015 & 0.0260 & 0.0464 & 0.0151 & 0.0886 & 0.0075 & 0.0005 \\
\hline
\end{tabular} \begin{tabular}{ll|l|l|l|l|l|l|l|l|l|l|l|l}
1.0000 & 0.0515 & 0.0683 & 0.0109 & 0.1215 & 0.0007 & 0.0246 & 0.0462 & 0.0151 & 0.0885 & 0.0078 & 0.0004 \\
\hline
\end{tabular} \begin{tabular}{ll|l|l|l|l|l|l|l|l|l|l|l|l|l|l}
1.0000 & 0.0559 & 0.0619 & 0.0113 & 0.1308 & 0.0007 & 0.0185 & 0.0451 & 0.0140 & 0.1059 & 0.0079 & 0.0006 \\
\hline
\end{tabular} \begin{tabular}{|l|l|l|l|l|l|l|l|l|l|l|l|l|}
1.0000 & 0.0542 & 0.0610 & 0.0111 & 0.1273 & 0.0007 & 0.0182 & 0.0449 & 0.0139 & 0.1052 & 0.0077 \\
\hline
\end{tabular}

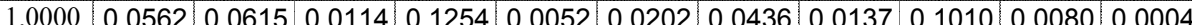
\begin{tabular}{l|l|l|l|l|l|l|l|l|l|l|l|l}
1.0000 & 0.0573 & 0.0628 & 0.0118 & 0.1285 & 0.0077 & 0.0224 & 0.0430 & 0.0136 & 0.0986 & 0.0079 & 0.0002 \\
\hline
\end{tabular} \begin{tabular}{ll|l|l|l|l|l|l|l|l|l|l|l|l|l|l}
1.0000 & 0.0579 & 0.0643 & 0.0124 & 0.1248 & 0.0105 & 0.0235 & 0.0435 & 0.0138 & 0.0970 & 0.0079 & 0.0002 \\
\hline
\end{tabular} \begin{tabular}{ll|l|l|l|l|l|l|l|l}
1.0000 & 0.1340 & 0.0734 & 0.0051 & 0.0457 & 0.0024
\end{tabular}

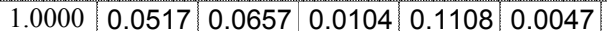
\begin{tabular}{l|l|l|l|l|l|l|l|}
1.0000 & 0.0142 & 0.0728 & 0.0140 & 0.0907 & 0.0714 \\
\hline
\end{tabular} \begin{tabular}{|l|l|l|l|l|l|l|l|l|l}
1.0000 & 0.1350 & 0.1080 & 0.0038 & 0.0457 & 0.0009 \\
\hline
\end{tabular}

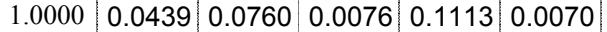

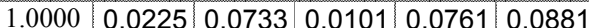
\begin{tabular}{|l|l|l|l|l|l|l|}
\hline 1.0000 & 0.0672 & 0.1370 & 0.0014 & 0.0231 & 0.0014 \\
\hline
\end{tabular}

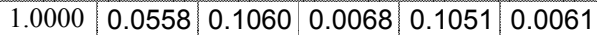
\begin{tabular}{ll|l|l|l|l|l|l|l|l|l|l|l|l}
1.0000 & 0.0247 & 0.1140 & 0.0135 & 0.0939 & 0.0622 \\
\hline
\end{tabular} \begin{tabular}{ll|l|l|l|l|l|l|l}
0.0420 & 0.0066 & 0.1060 & 0.0067
\end{tabular} $\begin{array}{lllllll}0.0502 & 0.0066 & 0.0996 & 0.0101\end{array}$

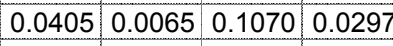
\begin{tabular}{ll|l|l|l|l|l|l}
0.0409 & 0.0138 & 0.1410 & 0.0063
\end{tabular} \begin{tabular}{ll|l|l|l|l|l|l|l}
0.0425 & 0.0067 & 0.0986 & 0.0104 \\
\hline
\end{tabular} \begin{tabular}{ll|l|l|l|l|l|l}
0.0406 & 0.0066 & 0.1090 & 0.0256
\end{tabular}

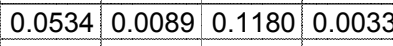
\begin{tabular}{ll|l|l|l|l|l|l|l}
0.0424 & 0.0074 & 0.1010 & 0.0102
\end{tabular}

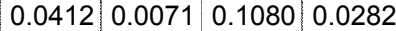
\begin{tabular}{ll|l|l|l|l|l|l|l|l|l|l|l|l}
1.0000 & 0.1340 & 0.1020 & 0.0054 & 0.0439 & 0.0007 & 0.0312 & 0.0265 & 0.0125 & 0.1060 & 0.0061
\end{tabular} \begin{tabular}{|l|l|l|l|l|l|l|l|l|l|l|l|l|}
1.0000 & 0.0514 & 0.1030 & 0.0063 & 0.1147 & 0.0039 & 0.0318 & 0.0268 & 0.0124 & 0.0977 & 0.0102 \\
\hline
\end{tabular} \begin{tabular}{ll|l|l|l|l|l|l|l|l|l|l|l|}
1.0000 & 0.0207 & 0.1010 & 0.0092 & 0.0980 & 0.0594 & 0.0315 & 0.0259 & 0.0121 & 0.1060 & 0.0257 \\
\hline
\end{tabular} \begin{tabular}{|l|l|l|l|l|l|l|l|l|l|l|l|l|}
1.0000 & 0.1390 & 0.0742 & 0.0041 & 0.0431 & 0.0008 & 0.0332 & 0.0418 & 0.0128 & 0.0734 & 0.0062 \\
\hline
\end{tabular} \begin{tabular}{ll|l|l|l|l|l|l|l|l|l|l|l|l|}
1.0000 & 0.0496 & 0.0744 & 0.0072 & 0.1156 & 0.0031 & 0.0333 & 0.0427 & 0.0130 & 0.0655 & 0.0100
\end{tabular} \begin{tabular}{|l|l|l|l|l|l|l|l|l|l|l|l|}
1.0000 & 0.0136 & 0.0708 & 0.0096 & 0.0962 & 0.0628 & 0.0328 & 0.0427 & 0.0126 & 0.0762 & 0.0273 \\
\hline
\end{tabular}

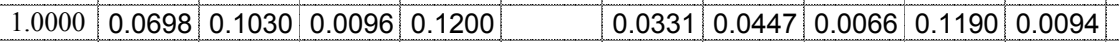

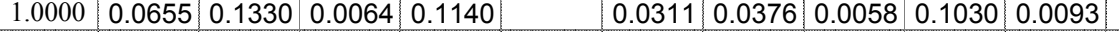

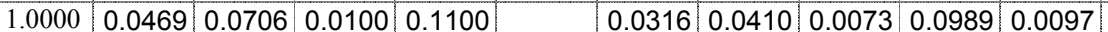

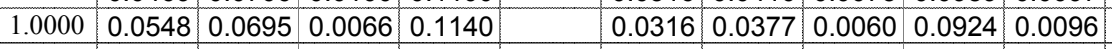
\begin{tabular}{ll|l|l|l|l|l|l|l|l|l|l|l|l|l}
1.0000 & 0.0556 & 0.0933 & 0.0068 & 0.1180 & & 0.0314 & 0.0459 & 0.0060 & 0.1050 & 0.0094
\end{tabular}

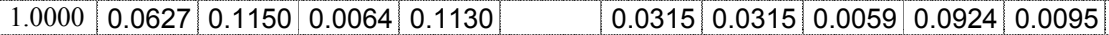
\begin{tabular}{ll|l|l|l|l|l|l|l}
1.0000 & 0.0588 & 0.1010 & 0.0069 & 0.1160
\end{tabular} \begin{tabular}{|l|l|l|l|l|l|l|l|l|l}
0.0308 & 0.0317 & 0.0120 & 0.0976 & 0.0097 \\
\hline
\end{tabular} 
SP3, SPx4, Misc. (Vienna et al. 2001)

\begin{tabular}{|c|c|c|c|c|c|c|c|c|c|c|c|c|c|c|c|c|c|c|c|c|c|}
\hline Glass ID & $\begin{array}{c}\mathrm{SiO} 2 \\
-\mathrm{a}\end{array}$ & $\begin{array}{c}\mathrm{ZrO} 2 \\
-\mathrm{a}\end{array}$ & $\begin{array}{c}\mathrm{Ag} 2 \mathrm{O} \\
-\mathrm{a}\end{array}$ & $\begin{array}{c}\mathrm{As} 2 \mathrm{O} 3 \\
-\mathrm{a} \\
\end{array}$ & $\begin{array}{c}\mathrm{BaO} \\
-\mathrm{a}\end{array}$ & $\begin{array}{c}\mathrm{Bi} 2 \mathrm{O} 3 \\
-\mathrm{a}\end{array}$ & $\begin{array}{l}\mathrm{Br} \\
-\mathrm{a}\end{array}$ & $\begin{array}{c}\mathrm{CdO} \\
-\mathrm{a}\end{array}$ & $\begin{array}{c}\mathrm{Ce} 2 \mathrm{O} 3 \\
-\mathrm{a}\end{array}$ & $\begin{array}{c}\mathrm{CeO} 2 \\
-\mathrm{a}\end{array}$ & $\begin{array}{l}\mathrm{Cl} \\
-\mathrm{a}\end{array}$ & $\begin{array}{c}\mathrm{CoO} \\
-\mathrm{a}\end{array}$ & $\begin{array}{c}\mathrm{Co} 2 \mathrm{O} 3 \\
-\mathrm{a}\end{array}$ & $\begin{array}{c}\mathrm{Cr} 2 \mathrm{O} 3 \\
-\mathrm{a}\end{array}$ & $\begin{array}{c}\mathrm{Cs} 2 \mathrm{O} \\
-\mathrm{a}\end{array}$ & $\begin{array}{c}\mathrm{CuO} \\
-\mathrm{a}\end{array}$ & $\begin{array}{c}\mathrm{Eu} 2 \mathrm{O} 3 \\
-\mathrm{a}\end{array}$ & $\begin{array}{c}\mathrm{F} \\
-\mathrm{a} \\
\end{array}$ & $\begin{array}{c}\mathrm{Ga} 2 \mathrm{O} 3 \\
-\mathrm{a}\end{array}$ & $\begin{array}{c}\mathrm{Gd} 2 \mathrm{O} 3 \\
-\mathrm{a}\end{array}$ & $\begin{array}{c}\mathrm{HgO} \\
-\mathrm{a}\end{array}$ \\
\hline Sp-LHLH(b) & & & & & & & & & & & & & & & & & & & & & \\
\hline Sp-MMLL(b) & & & & & & & & & & & & & & & & & & & & & \\
\hline Sp-MMHH(b) & & & & & & & & & & & & & & & & & & & & & \\
\hline Sp-HLLL(b) & & & & & & & & & & & & & & & & & & & & & \\
\hline nom-2 & & & & & & & & & & & & & & & & & & & & & \\
\hline nom-3 & & & & & & & & & & & & & & & & & & & & & \\
\hline nomc-1 & & & & & & & & & & & & & & & & & & & & & \\
\hline nomc-2 & & & & & & & & & & & & & & & & & & & & & \\
\hline c106a-2 & & & & & & & & & & & & & & & & & & & & & \\
\hline c106a-3 & & & & & & & & & & & & & & & & & & & & & \\
\hline c106a-4 & & & & & & & & & & & & & & & & & & & & & \\
\hline c106b-1 & & & & & & & & & & & & & & & & & & & & & \\
\hline c106b-2 & & & & & & & & & & & & & & & & & & & & & \\
\hline $\mathrm{az}-3$ & & & & & & & & & & & & & & & & & & & & & \\
\hline $\mathrm{az}-5$ & & & & & & & & & & & & & & & & & & & & & \\
\hline
\end{tabular}

\section{DWPF PCT Model (Jantzen et al. 1995)}

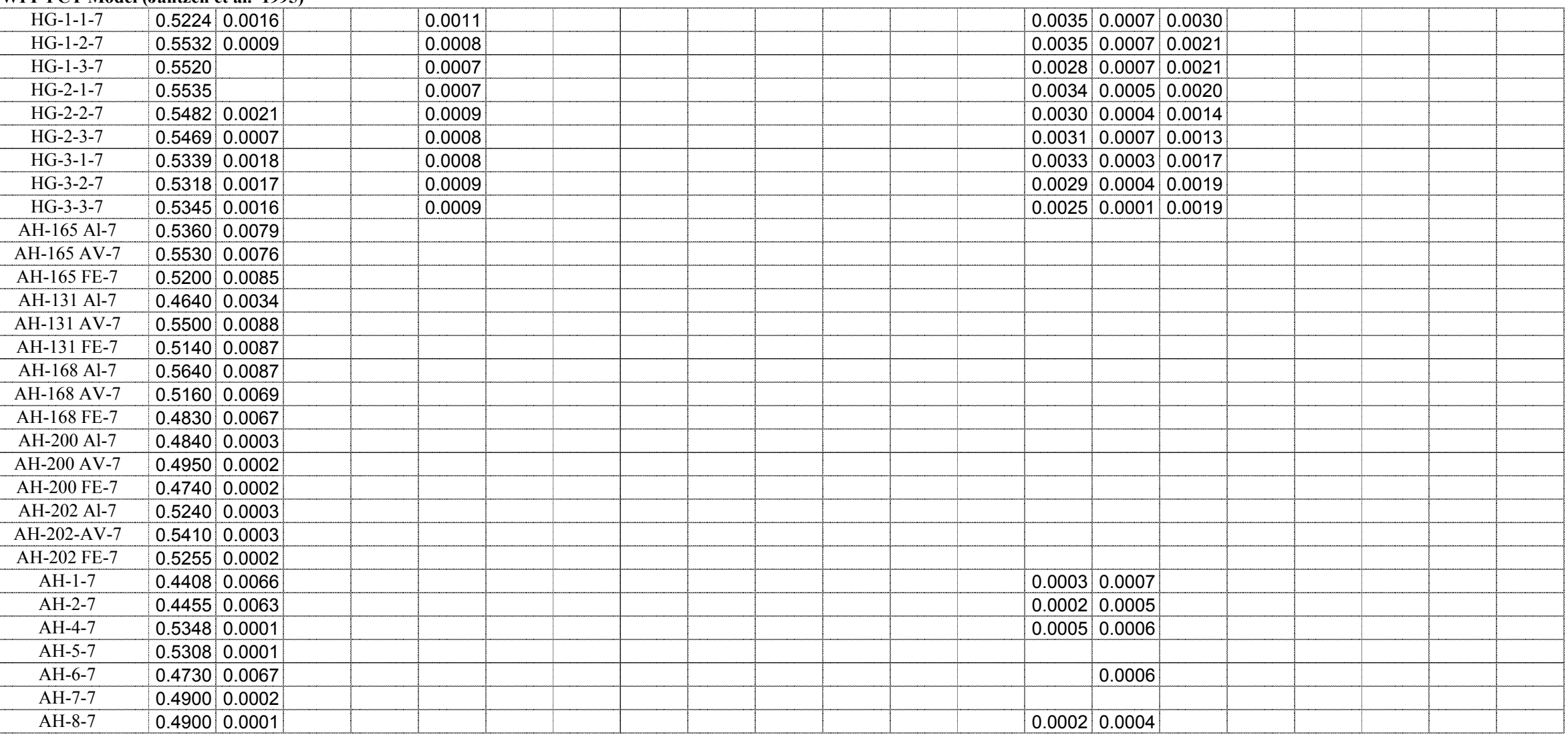


SP3, SPx4, Misc. (Vienna et al. 2001)

\begin{tabular}{|c|c|c|c|c|c|c|c|c|c|c|c|c|c|c|c|c|c|c|c|c|c|}
\hline Glass ID & $\begin{array}{c} \\
-\mathrm{a} \\
\end{array}$ & $\begin{array}{c}\mathrm{La} 2 \mathrm{O} 3 \\
-\mathrm{a}\end{array}$ & $\begin{array}{c}\mathrm{MnO} 2 \\
-\mathrm{a}\end{array}$ & $\begin{array}{c}\mathrm{MnO} \\
-\mathrm{a}\end{array}$ & $\begin{array}{c}\mathrm{MoO} \\
-\mathrm{a}\end{array}$ & $\begin{array}{c}\mathrm{MoO} 3 \\
-\mathrm{a}\end{array}$ & $\begin{array}{c}\mathrm{Nb} 2 \mathrm{O} 5 \\
-\mathrm{a}\end{array}$ & $\begin{array}{c}\mathrm{Nd} 2 \mathrm{O} 3 \\
-\mathrm{a}\end{array}$ & $\begin{array}{c}\mathrm{PbO} \\
-\mathrm{a}\end{array}$ & $\begin{array}{c}\mathrm{PdO} 2 \\
-\mathrm{a}\end{array}$ & $\begin{array}{c}\mathrm{PdO} \\
-\mathrm{a}\end{array}$ & $\begin{array}{c}\mathrm{Pr} 2 \mathrm{O} 3 \\
-\mathrm{a}\end{array}$ & $\begin{array}{c}\text { Pr6O11 } \\
-\mathrm{a}\end{array}$ & $\begin{array}{c}\mathrm{Rb} 2 \mathrm{O} \\
-\mathrm{a}\end{array}$ & $\begin{array}{c}\mathrm{ReO} \\
-\mathrm{a}\end{array}$ & $\begin{array}{c}\mathrm{ReO} 2 \\
-\mathrm{a}\end{array}$ & $\begin{array}{c}\mathrm{Rh} 2 \mathrm{O} 3 \\
-\mathrm{a}\end{array}$ & $\begin{array}{c}\mathrm{RhO} 2 \\
-\mathrm{a}\end{array}$ & $\begin{array}{c}\mathrm{RuO} 2 \\
-\mathrm{a}\end{array}$ & \begin{tabular}{|c}
$\mathrm{Sb} 2 \mathrm{O} 3$ \\
$-\mathrm{a}$
\end{tabular} & $\begin{array}{c}\mathrm{Sb} 2 \mathrm{O} 5 \\
-\mathrm{a}\end{array}$ \\
\hline \multicolumn{22}{|c|}{ Sp-LHLH(b) } \\
\hline \multicolumn{22}{|c|}{ Sp-MMLL(b) } \\
\hline \multicolumn{22}{|c|}{ Sp-MMHH(b) } \\
\hline \multirow{2}{*}{\multicolumn{22}{|c|}{ Sp-HLLL(b) }} \\
\hline \multirow{2}{*}{\multicolumn{22}{|c|}{$\frac{\text { nom-2 }}{\text { nom-3 }}$}} \\
\hline & & & & & & & & & & & & & & & & & & & & & \\
\hline \multicolumn{22}{|l|}{ nomc-1 } \\
\hline \multicolumn{22}{|l|}{ nomc-2 } \\
\hline \multicolumn{22}{|l|}{ c106a-2 } \\
\hline \multicolumn{22}{|l|}{ c106a-3 } \\
\hline \multicolumn{22}{|l|}{ c106a-4 } \\
\hline \multirow{2}{*}{\multicolumn{22}{|c|}{$\begin{array}{l}\text { c106b-1 } \\
\text { c106b-2 }\end{array}$}} \\
\hline \multirow{2}{*}{\multicolumn{22}{|c|}{$\frac{c 106 b-2}{a z-3}$}} \\
\hline & & & & & & & & & & & & & & & & & & & & & \\
\hline az-5 & & & & & & & & & & & & & & & & & & & & & \\
\hline
\end{tabular}

\section{DWPF PCT Model (Jantzen et al. 1995)}

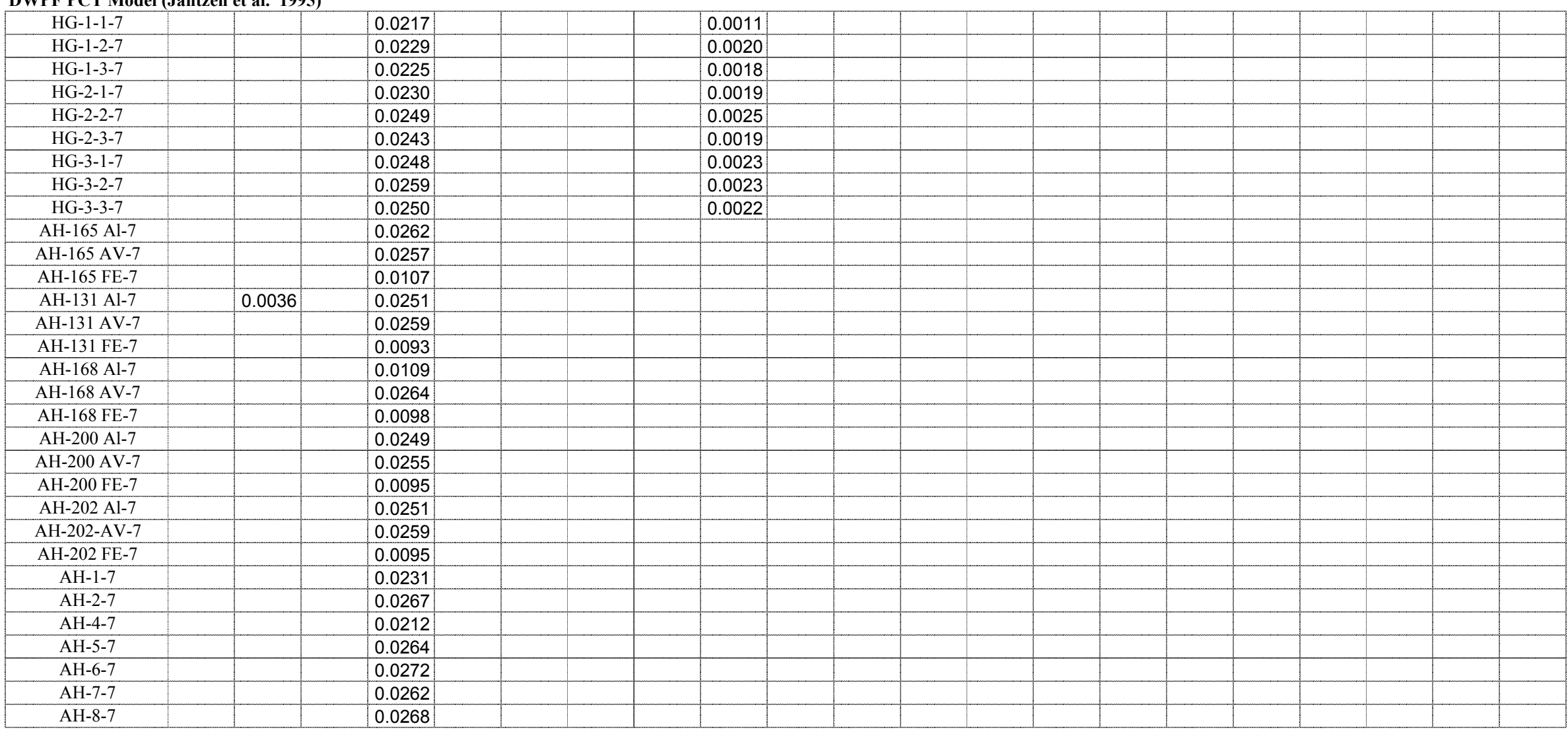


Appendix A. Database - mass fraction

SP3, SPx4, Misc. (Vienna et al. 2001)

\begin{tabular}{|c|c|c|c|c|c|c|c|c|c|c|c|c|c|c|c|c|c|c|c|c|}
\hline Glass ID & $\begin{array}{c}\mathrm{SeO} 2 \\
-\mathrm{a}\end{array}$ & $\begin{array}{c}\mathrm{Sm} 2 \mathrm{O} 3 \\
-\mathrm{a}\end{array}$ & $\begin{array}{c}\mathrm{SnO} \\
-\mathrm{a}\end{array}$ & $\begin{array}{c}\mathrm{SnO} 2 \\
-\mathrm{a}\end{array}$ & $\begin{array}{c}\mathrm{SO} 3 \\
-\mathrm{a}\end{array}$ & $\begin{array}{c}\mathrm{SrO} \\
-\mathrm{a}\end{array}$ & $\begin{array}{c}\mathrm{Tc} 2 \mathrm{O} 7 \\
-\mathrm{a}\end{array}$ & $\begin{array}{c}\mathrm{TeO} 2 \\
-\mathrm{a}\end{array}$ & $\begin{array}{c}\text { ThO2 } \\
-\mathrm{a}\end{array}$ & $\begin{array}{c}\mathrm{TiO} 2 \\
-\mathrm{a}\end{array}$ & $\begin{array}{c}\mathrm{T} 12 \mathrm{O} 3 \\
-\mathrm{a} \\
\end{array}$ & $\begin{array}{c}\mathrm{U} 3 \mathrm{O} 8 \\
-\mathrm{a}\end{array}$ & $\begin{array}{c}\mathrm{UO} 2 \\
-\mathrm{a} \\
\end{array}$ & $\begin{array}{c}\mathrm{UO} 3 \\
-\mathrm{a} \\
\end{array}$ & $\begin{array}{c}\mathrm{V} 2 \mathrm{O} 5 \\
-\mathrm{a}\end{array}$ & $\begin{array}{c}\text { WO3 } \\
-\mathrm{a} \\
\end{array}$ & $\begin{array}{c}\mathrm{Y} 2 \mathrm{O} 3 \\
-\mathrm{a}\end{array}$ & $\begin{array}{c}\mathrm{ZnO} \\
-\mathrm{a} \\
\end{array}$ & $\begin{array}{c}\text { Others } \\
-\mathrm{a}\end{array}$ & $\begin{array}{c}\text { Sum } \\
-\mathrm{a}\end{array}$ \\
\hline \multicolumn{21}{|c|}{ Sp-LHLH(b) } \\
\hline \multicolumn{21}{|c|}{ Sp-MMLL(b) } \\
\hline \multicolumn{21}{|c|}{ Sp-MMHH(b) } \\
\hline \multicolumn{21}{|c|}{ Sp-HLLL(b) } \\
\hline \multicolumn{21}{|c|}{ nom-2 } \\
\hline \multicolumn{21}{|l|}{ nom-3 } \\
\hline \multicolumn{21}{|l|}{ nomc-1 } \\
\hline \multicolumn{21}{|l|}{ nomc-2 } \\
\hline \multicolumn{21}{|l|}{ c106a-2 } \\
\hline \multicolumn{21}{|l|}{ c106a-3 } \\
\hline \multicolumn{21}{|l|}{ c106a-4 } \\
\hline \multicolumn{21}{|l|}{ c106b-1 } \\
\hline \multicolumn{21}{|l|}{ c106b-2 } \\
\hline $\mathrm{az}-3$ & & & & & & & & & & & & & & & & & & & & \\
\hline az-5 & & & & & & & & & & & & & & & & & & & & \\
\hline
\end{tabular}

\section{DWPF PCT Model (Jantzen et al. 1995)}

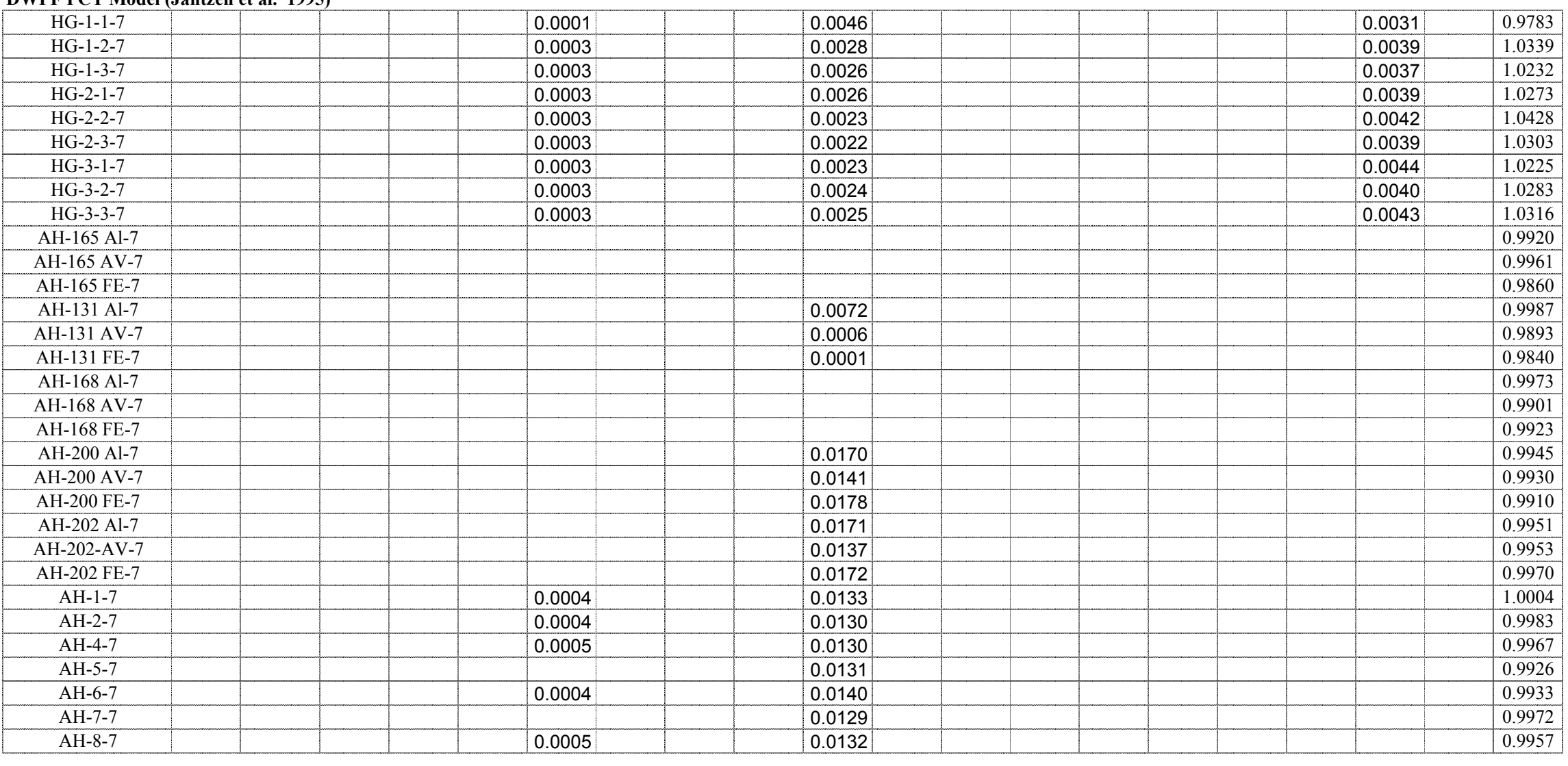


Appendix A. Database - mass fraction

SP3, SPx4, Misc. (Vienna et al. 2001)

\begin{tabular}{|c|c|c|c|c|c|c|c|c|}
\hline Glass ID & $\begin{array}{l}\mathrm{TM} \\
\left({ }^{\circ} \mathrm{C}\right) \\
\end{array}$ & $\begin{array}{l}\text { Gradient } \\
\text { TL }\left({ }^{\circ} \mathrm{C}\right)\end{array}$ & $\begin{array}{l}\text { Uniform } \\
\text { TL }\left({ }^{\circ} \mathrm{C}\right)\end{array}$ & Primary Phase & $\begin{array}{l}\text { Quenched } \\
\text { Visual/OM }\end{array}$ & $\begin{array}{c}\text { Quenched } \\
\text { SEM/EDS or TEM }\end{array}$ & $\begin{array}{l}\text { Quenched } \\
\text { XRD }\end{array}$ & $\begin{array}{c}\text { Quenched } \\
\text { Homogeneous? }\end{array}$ \\
\hline Sp-LHLH(b) & & & 1102 & triangle shaped spinels & & & & \\
\hline Sp-MMLL(b) & & & 1033 & spinel & & & & \\
\hline Sp-MMHH(b) & & & 1288 & spinel & & & & \\
\hline Sp-HLLL(b) & & & 1089 & spinel & & & & \\
\hline nom-2 & & & 1009 & spinel & & & & \\
\hline nom-3 & & & 1047 & spinel & & & & \\
\hline nomc-1 & & & 1005 & spinel & & & & \\
\hline nomc-2 & & & 998 & spinel & & & & \\
\hline c106a-2 & & & 1004 & spinel & & & & \\
\hline c106a-3 & & & 1029 & spinel & & & & \\
\hline c106a-4 & & & 885 & spinel & & & & \\
\hline c106b-1 & & & 986 & spinel & & & & \\
\hline c106b-2 & & & 894 & spinel & & & & \\
\hline$a z-3$ & & & 1048 & spinel & & & & \\
\hline az-5 & & & 953 & spinel & & & & \\
\hline
\end{tabular}

\section{DWPF PCT Model (Jantzen et al. 1995)}

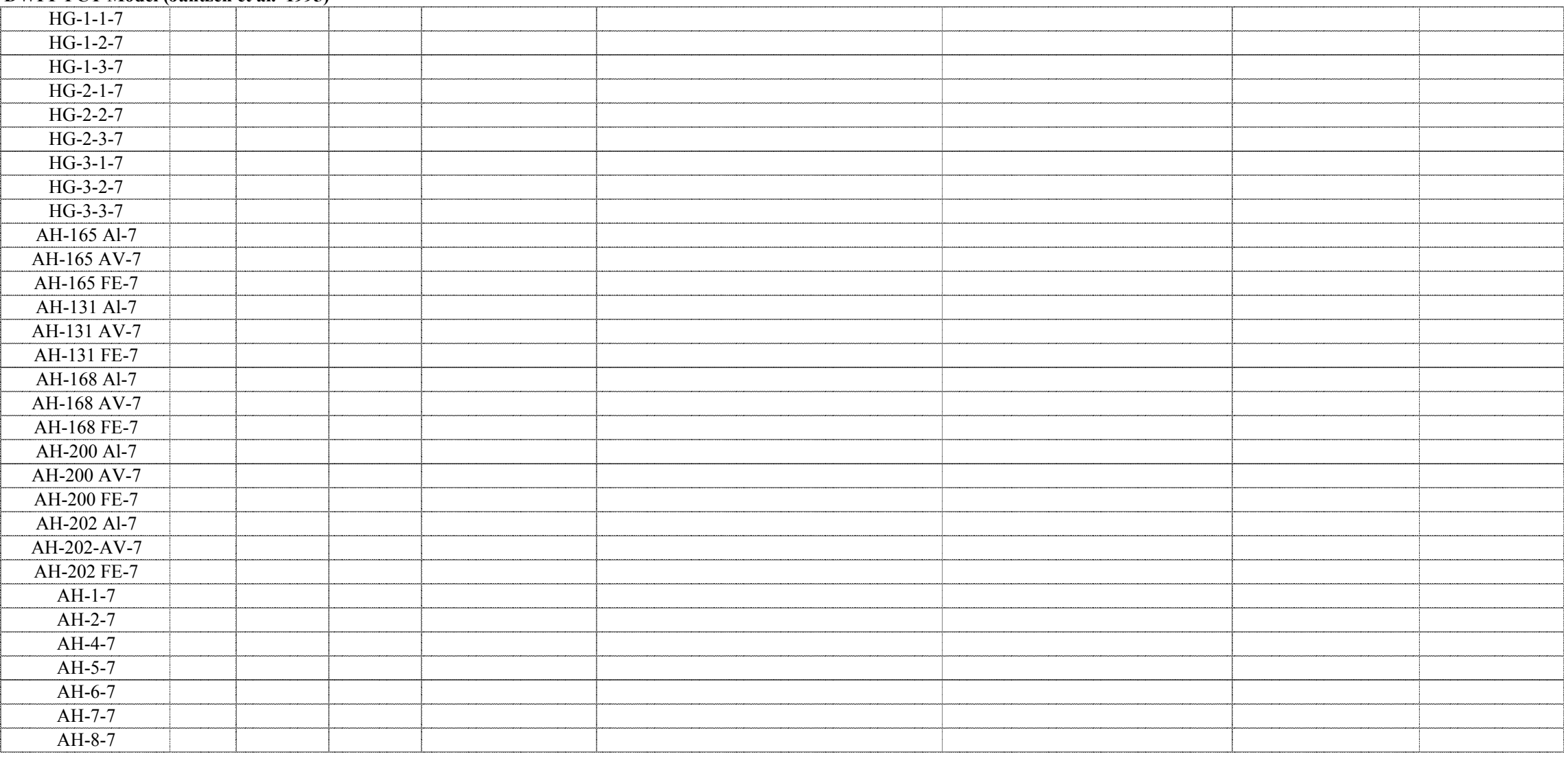


Appendix A. Database - mass fraction

SP3, SPx4, Misc. (Vienna et al. 2001)

\begin{tabular}{|c|c|c|c|c|c|}
\hline Glass ID & $\begin{array}{c}\mathrm{CCC} \\
\text { Visual/OM }\end{array}$ & $\begin{array}{c}\text { CCC } \\
\text { SEM/EDS or TEM }\end{array}$ & $\begin{array}{l}\mathrm{CCC} \\
\mathrm{XRD} \\
\end{array}$ & $\begin{array}{c}\text { Heat Treated } \\
\text { Visual/OM }\end{array}$ & $\begin{array}{c}\text { Heat Treated } \\
\text { SEM/EDS or TEM }\end{array}$ \\
\hline \multicolumn{6}{|c|}{ Sp-LHLH(b) } \\
\hline \multicolumn{6}{|c|}{ Sp-MMLL(b) } \\
\hline \multicolumn{6}{|c|}{ Sp-MMHH(b) } \\
\hline \multicolumn{6}{|c|}{ Sp-HLLL(b) } \\
\hline \multicolumn{6}{|l|}{ nom-2 } \\
\hline \multicolumn{6}{|l|}{ nom-3 } \\
\hline \multicolumn{6}{|l|}{ nomc-1 } \\
\hline \multicolumn{6}{|l|}{ nomc-2 } \\
\hline \multicolumn{6}{|l|}{ c106a-2 } \\
\hline \multicolumn{6}{|l|}{ c106a-3 } \\
\hline \multicolumn{6}{|l|}{ c106a-4 } \\
\hline \multicolumn{6}{|l|}{ c106b-1 } \\
\hline \multicolumn{6}{|l|}{ c106b-2 } \\
\hline$a z-3$ & & & & & \\
\hline az-5 & & & & & \\
\hline
\end{tabular}

\section{DWPF PCT Model (Jantzen et al. 1995)}

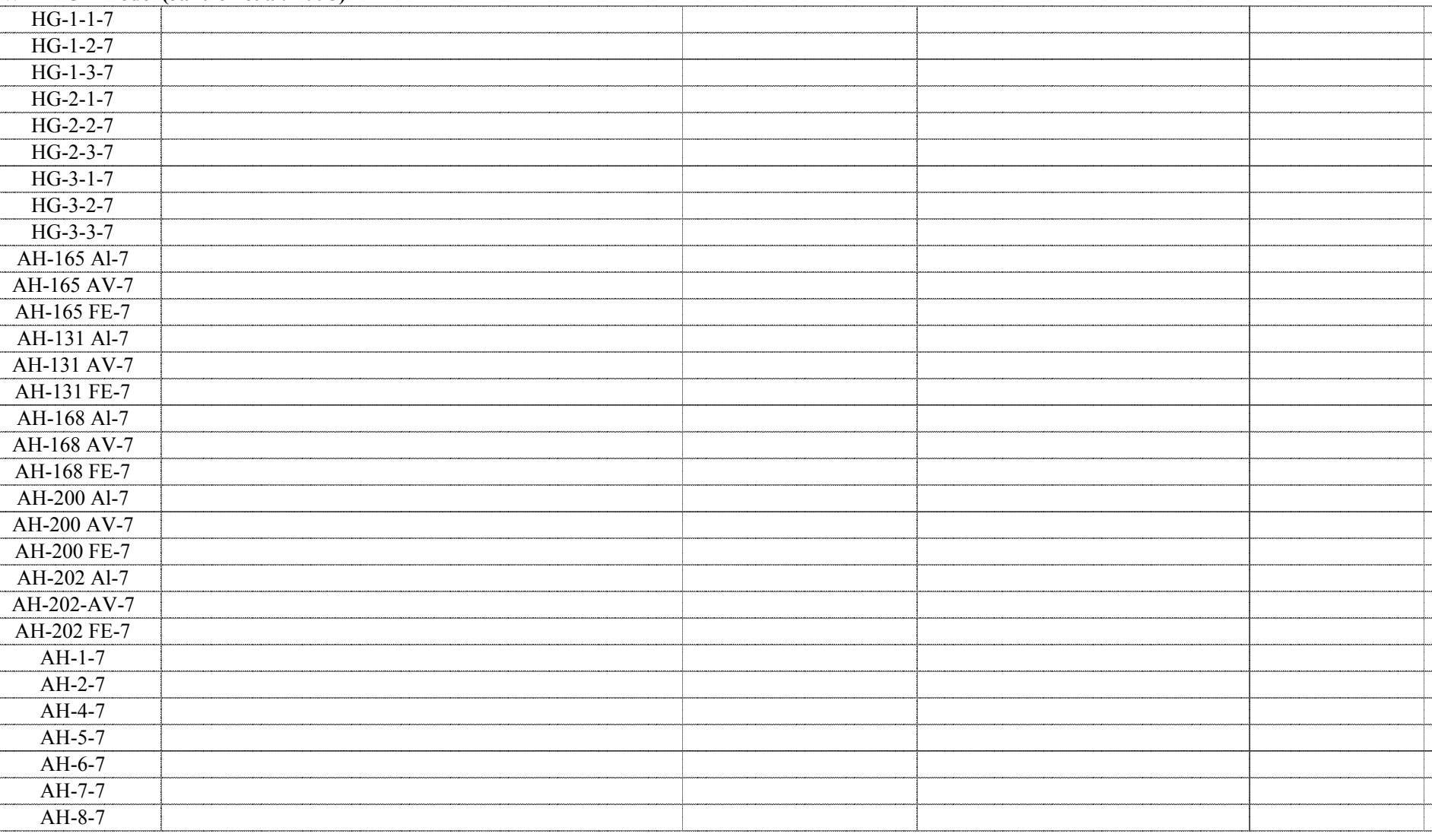


Appendix A. Database - mass fraction

SP3, SPx4, Misc. (Vienna et al. 2001)

\begin{tabular}{|c|c|c|c|c|c|c|c|c|c|c|c|c|c|c|c|}
\hline Glass ID & $\begin{array}{c}\text { Heat Treated } \\
\text { XRD } \\
\end{array}$ & $\begin{array}{l}\text { Density } \\
\left(\mathrm{g} / \mathrm{cm}^{3}\right)\end{array}$ & $\begin{array}{c}\text { Fulc Visc } \\
\text { A }\end{array}$ & \begin{tabular}{|c|}
$\begin{array}{c}\text { Fulc Visc } \\
\mathrm{B}\end{array}$ \\
\end{tabular} & \begin{tabular}{|c|} 
Fulc Visc \\
To \\
\end{tabular} & $\begin{array}{c}\mathrm{FV} 1150^{\circ} \mathrm{C} \\
(\mathrm{Pa} \cdot \mathrm{s})\end{array}$ & \begin{tabular}{|c|} 
Arrh Visc \\
A
\end{tabular} & $\begin{array}{c}\text { Arrh Visc } \\
\text { B } \\
\end{array}$ & $\begin{array}{c}\mathrm{Ar} \\
1150^{\circ} \mathrm{C} \\
\left(\mathrm{D}_{0.0+}\right)\end{array}$ & \begin{tabular}{|c|}
$\mathrm{T}\left({ }^{\circ} \mathrm{C}\right)$ at \\
$2 \mathrm{~Pa} \cdot \mathrm{s}$ \\
\end{tabular} & \begin{tabular}{|c|}
$\mathrm{T}\left({ }^{\circ} \mathrm{C}\right)$ at \\
$5 \mathrm{~Pa} \cdot \mathrm{s}$ \\
\end{tabular} & $\begin{array}{c}\mathrm{T}\left({ }^{\circ} \mathrm{C}\right) \text { at } \\
10 \mathrm{~Pa} \cdot \mathrm{s}\end{array}$ & $\begin{array}{l}\mathrm{T} 1 \\
\left({ }^{\circ} \mathrm{C}\right) \\
\end{array}$ & $\begin{array}{c}\mathrm{V} 1 \\
(\mathrm{~Pa} \cdot \mathrm{s}) \\
\end{array}$ & $\begin{array}{l}\mathrm{T} 2 \\
\left({ }^{\circ} \mathrm{C}\right) \\
\end{array}$ \\
\hline \multicolumn{16}{|c|}{ Sp-LHLH(b) } \\
\hline \multicolumn{16}{|c|}{ Sp-MMLL(b) } \\
\hline \multicolumn{16}{|c|}{ Sp-MMHH(b) } \\
\hline \multicolumn{16}{|c|}{ Sp-HLLL(b) } \\
\hline \multicolumn{16}{|l|}{ nom-2 } \\
\hline \multicolumn{16}{|l|}{ nom-3 } \\
\hline \multicolumn{16}{|l|}{ nomc-1 } \\
\hline \multicolumn{16}{|l|}{ nomc-2 } \\
\hline \multicolumn{16}{|l|}{ c106a-2 } \\
\hline \multicolumn{16}{|l|}{ c106a-3 } \\
\hline \multicolumn{16}{|l|}{ c106a-4 } \\
\hline \multicolumn{16}{|l|}{ c106b-1 } \\
\hline \multicolumn{16}{|l|}{ c106b-2 } \\
\hline$a z-3$ & & & & & & & & & & & & & & & \\
\hline $\mathrm{az}-5$ & & & & & & & & & & & & & & & \\
\hline
\end{tabular}

\section{DWPF PCT Model (Jantzen et al. 1995)}

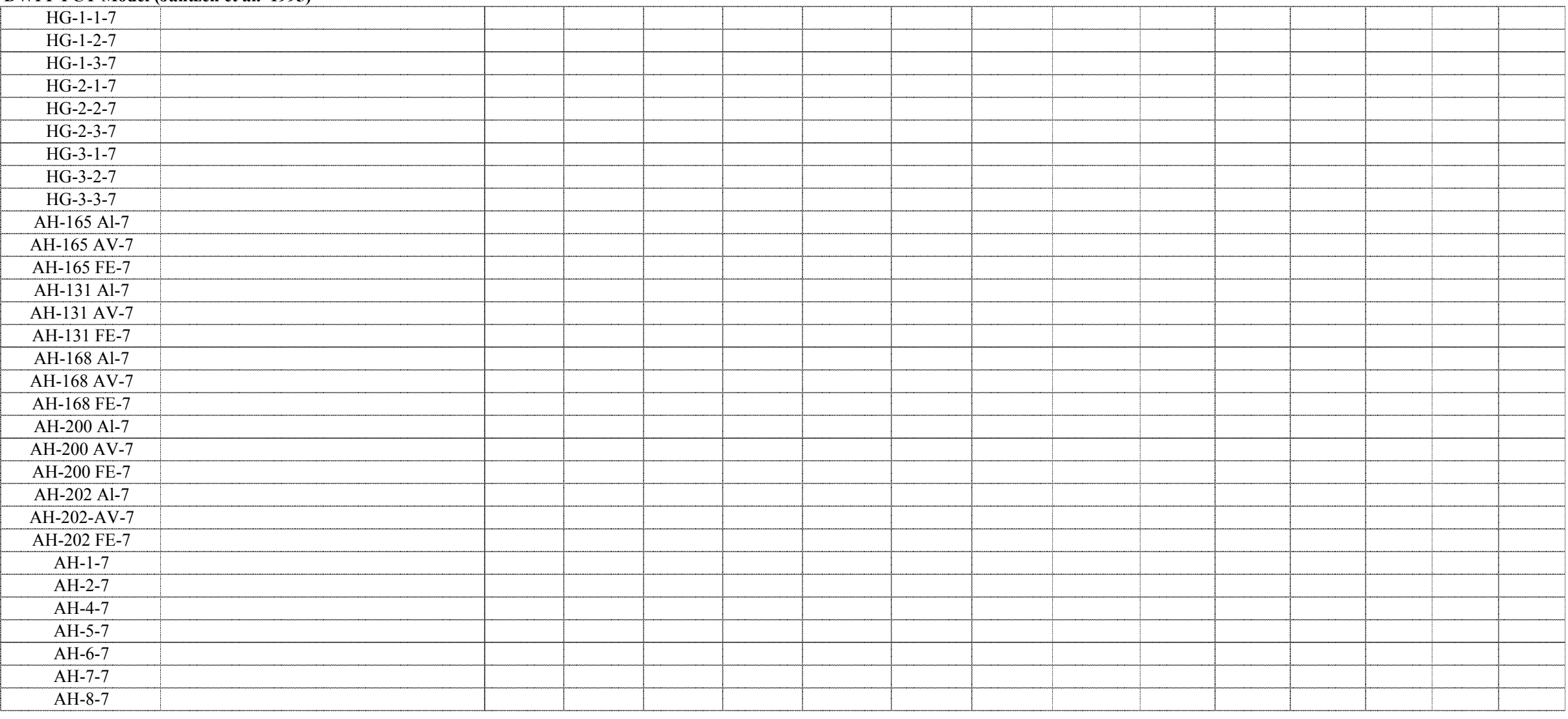


Appendix A. Database - mass fraction

SP3, SPx4, Misc. (Vienna et al. 2001)

\begin{tabular}{|c|c|c|c|c|c|c|c|c|c|c|c|c|c|c|c|c|c|c|c|c|c|}
\hline Glass ID & $\begin{array}{c}\mathrm{V} 2 \\
(\mathrm{~Pa} \cdot \mathrm{s})\end{array}$ & $\begin{array}{c}\mathrm{T} 3 \\
\left({ }^{\circ} \mathrm{C}\right) \\
\end{array}$ & $\begin{array}{c}\mathrm{V} 3 \\
(\mathrm{~Pa} \cdot \mathrm{s})\end{array}$ & $\begin{array}{c}\mathrm{T} 4 \\
\left({ }^{\circ} \mathrm{C}\right) \\
\end{array}$ & $\begin{array}{c}\mathrm{V} 4 \\
(\mathrm{~Pa} \cdot \mathrm{s})\end{array}$ & $\begin{array}{c}\mathrm{T} 5 \\
\left({ }^{\circ} \mathrm{C}\right) \\
\end{array}$ & $\begin{array}{c}\text { V5 } \\
(\mathrm{Pa} \cdot \mathrm{s})\end{array}$ & $\begin{array}{c}\mathrm{T} 6 \\
\left({ }^{\circ} \mathrm{C}\right) \\
\end{array}$ & $\begin{array}{c}\mathrm{V} 6 \\
(\mathrm{~Pa} \cdot \mathrm{s})\end{array}$ & $\begin{array}{c}\mathrm{T} 7 \\
\left({ }^{\circ} \mathrm{C}\right) \\
\end{array}$ & $\begin{array}{c}\mathrm{V} 7 \\
(\mathrm{~Pa} \cdot \mathrm{s})\end{array}$ & $\begin{array}{c}\mathrm{T} 8 \\
\left({ }^{\circ} \mathrm{C}\right) \\
\end{array}$ & $\begin{array}{c}\mathrm{V} 8 \\
(\mathrm{~Pa} \cdot \mathrm{s})\end{array}$ & $\begin{array}{c}\mathrm{T} 9 \\
\left({ }^{\circ} \mathrm{C}\right) \\
\end{array}$ & $\begin{array}{c}\mathrm{V} 9 \\
(\mathrm{~Pa} \cdot \mathrm{s})\end{array}$ & $\begin{array}{l}\mathrm{T} 10 \\
\left({ }^{\circ} \mathrm{C}\right)\end{array}$ & $\begin{array}{c}\mathrm{V} 10 \\
(\mathrm{~Pa} \cdot \mathrm{s})\end{array}$ & $\begin{array}{l}\mathrm{T} 11 \\
\left({ }^{\circ} \mathrm{C}\right)\end{array}$ & $\begin{array}{c}\mathrm{V} 11 \\
(\mathrm{~Pa} \cdot \mathrm{s})\end{array}$ & $\begin{array}{l}\mathrm{T} 12 \\
\left({ }^{\circ} \mathrm{C}\right)\end{array}$ & $\begin{array}{c}\mathrm{V} 12 \\
(\mathrm{~Pa} \cdot \mathrm{s})\end{array}$ \\
\hline \multicolumn{22}{|c|}{ Sp-LHLH(b) } \\
\hline \multicolumn{22}{|c|}{ Sp-MMLL(b) } \\
\hline \multicolumn{22}{|c|}{ Sp-MMHH(b) } \\
\hline \multicolumn{22}{|c|}{ Sp-HLLL(b) } \\
\hline \multicolumn{22}{|l|}{ nom-2 } \\
\hline \multirow{2}{*}{\multicolumn{22}{|c|}{ nom-3 }} \\
\hline \multirow{2}{*}{\multicolumn{22}{|c|}{$\begin{array}{l}\text { nomc-1 } \\
\text { nomc-2 }\end{array}$}} \\
\hline \multirow{2}{*}{\multicolumn{22}{|c|}{ nomc-2 }} \\
\hline & & & & & & & & & & & & & & & & & & & & & \\
\hline \multicolumn{22}{|l|}{ c106a-3 } \\
\hline \multicolumn{22}{|l|}{ c106a-4 } \\
\hline \multicolumn{22}{|l|}{ c106b-1 } \\
\hline \multicolumn{22}{|l|}{ c106b-2 } \\
\hline $\mathrm{az}-3$ & & & & & & & & & & & & & & & & & & & & & \\
\hline $\mathrm{az}-5$ & & & & & & & & & & & & & & & & & & & & & \\
\hline
\end{tabular}

\section{DWPF PCT Model (Jantzen et al. 1995)}

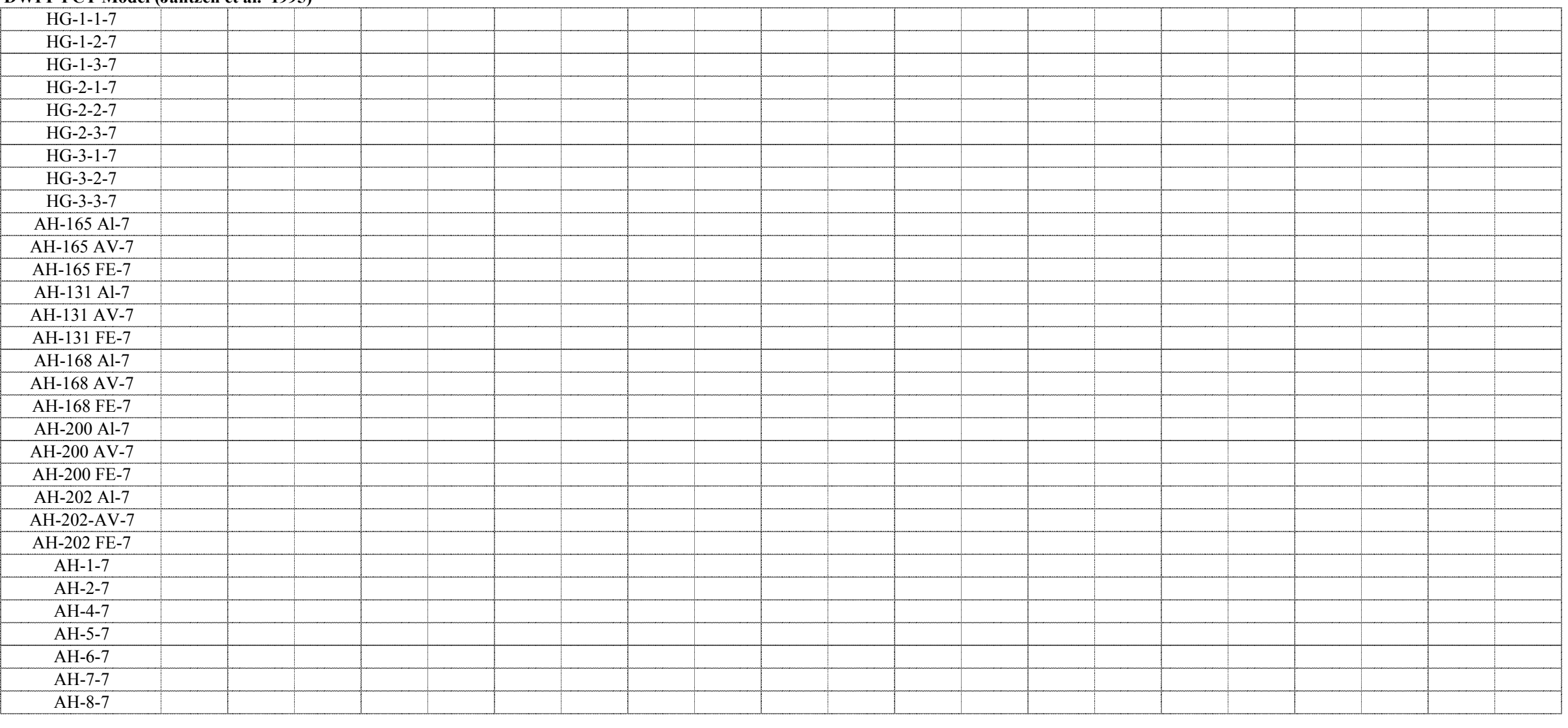




\section{SP3, SPx4, Misc. (Vienna et al. 2001)}

\begin{tabular}{|c|c|c|c|c|c|c|c|c|c|c|c|c|c|c|c|c|}
\hline Glass ID & $\begin{array}{l}\mathrm{T} 13 \\
\left({ }^{\circ} \mathrm{C}\right) \\
\end{array}$ & $\begin{array}{l}\mathrm{V} 13 \\
(\mathrm{~Pa} \cdot \mathrm{s})\end{array}$ & $\begin{array}{l}\mathrm{T} 14 \\
\left({ }^{\circ} \mathrm{C}\right) \\
\end{array}$ & $\begin{array}{c}\mathrm{V} 14 \\
(\mathrm{~Pa} \cdot \mathrm{s}) \\
\end{array}$ & $\begin{array}{c}\text { Q PCT } \\
\text { B }\left(\mathrm{g} / \mathrm{m}^{2}\right)\end{array}$ & $\begin{array}{c}\text { Q PCT } \\
\mathrm{Li}\left(\mathrm{g} / \mathrm{m}^{2}\right)\end{array}$ & $\begin{array}{c}\text { Q PCT } \\
\mathrm{Na}\left(\mathrm{g} / \mathrm{m}^{2}\right)\end{array}$ & $\begin{array}{c}\text { Q PCT } \\
\mathrm{Si}\left(\mathrm{g} / \mathrm{m}^{2}\right) \\
\end{array}$ & $\begin{array}{c}\text { Q PCT } \\
\mathrm{pH}\end{array}$ & $\begin{array}{c}\text { CCC PCT } \\
\text { B }\left(\mathrm{g} / \mathrm{m}^{2}\right)\end{array}$ & $\begin{array}{l}\text { CCC PCT } \\
\mathrm{Li}\left(\mathrm{g} / \mathrm{m}^{2}\right)\end{array}$ & $\begin{array}{l}\text { CCC PCT } \\
\mathrm{Na}\left(\mathrm{g} / \mathrm{m}^{2}\right)\end{array}$ & $\begin{array}{l}\text { CCC PCT } \\
\mathrm{Si}\left(\mathrm{g} / \mathrm{m}^{2}\right)\end{array}$ & $\begin{array}{c}\begin{array}{c}\text { CCC PCT } \\
\mathrm{pH}\end{array} \\
\end{array}$ & $\begin{array}{l}\text { Q PCT B at } \\
20^{\circ} \mathrm{C}\left(\mathrm{g} / \mathrm{m}^{2}\right)\end{array}$ & $\begin{array}{l}\text { Q PCT Li at } \\
20^{\circ} \mathrm{C}\left(\mathrm{g} / \mathrm{m}^{2}\right)\end{array}$ \\
\hline \multicolumn{17}{|c|}{ Sp-LHLH(b) } \\
\hline \multicolumn{17}{|c|}{ Sp-MMLL(b) } \\
\hline \multicolumn{17}{|c|}{ Sp-MMHH(b) } \\
\hline \multicolumn{17}{|c|}{ Sp-HLLL(b) } \\
\hline \multirow{2}{*}{\multicolumn{17}{|c|}{$\begin{array}{l}\text { nom-2 } \\
\text { nom-3 }\end{array}$}} \\
\hline & & & & & & & & & & & & & & & & \\
\hline \multicolumn{17}{|l|}{ nomc-1 } \\
\hline \multicolumn{17}{|l|}{ nomc-2 } \\
\hline \multicolumn{17}{|l|}{ c106a-2 } \\
\hline \multicolumn{17}{|l|}{ c106a-3 } \\
\hline \multicolumn{17}{|l|}{ c106a-4 } \\
\hline \multicolumn{17}{|l|}{ c106b-1 } \\
\hline \multicolumn{17}{|l|}{ c106b-2 } \\
\hline$a z-3$ & & & & & & & & & & & & & & & & \\
\hline az-5 & & & & & & & & & & & & & & & & \\
\hline
\end{tabular}

\section{DWPF PCT Model (Jantzen et al. 1995)}

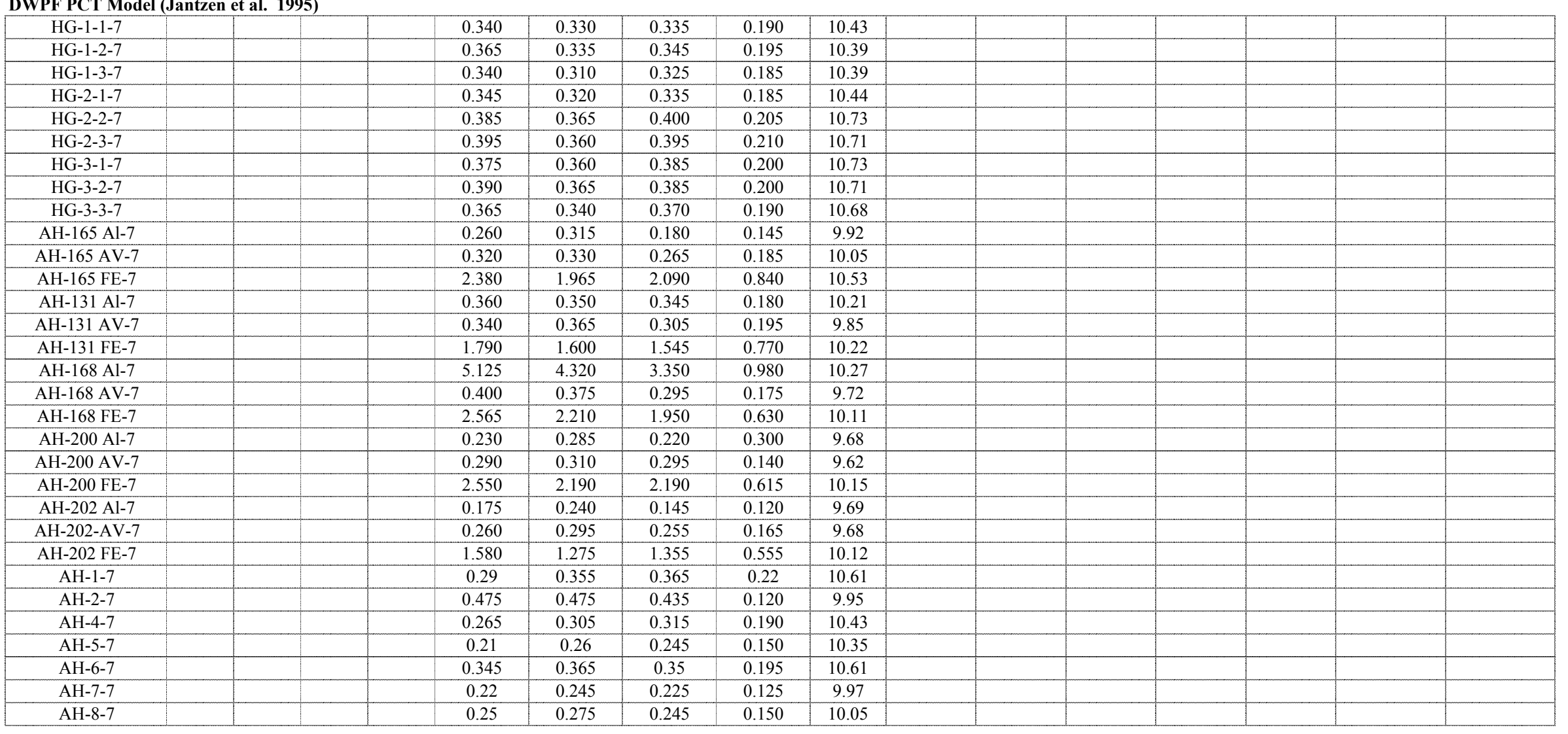


Appendix A. Database - mass fraction

SP3, SPx4, Misc. (Vienna et al. 2001)

\begin{tabular}{|c|c|c|c|c|c|c|c|c|c|c|c|c|}
\hline Glass ID & $\begin{array}{l}\text { Q PCT Na at } \\
20^{\circ} \mathrm{C}\left(\mathrm{g} / \mathrm{m}^{2}\right)\end{array}$ & $\begin{array}{l}\text { Q PCT Si at } \\
20^{\circ} \mathrm{C}\left(\mathrm{g} / \mathrm{m}^{2}\right)\end{array}$ & $\begin{array}{c}\mathrm{Q} \mathrm{pH} \\
\text { at } 20^{\circ} \mathrm{C}\end{array}$ & $\begin{array}{c}\text { TCLP Ag } \\
(\mathrm{ppm})\end{array}$ & $\begin{array}{l}\text { TCLP As } \\
\text { (ppm) }\end{array}$ & $\begin{array}{c}\text { TCLP Ba } \\
\text { (ppm) }\end{array}$ & $\begin{array}{c}\text { TCLP Cd } \\
\text { (ppm) }\end{array}$ & $\begin{array}{c}\text { TCLP Cr } \\
(\mathrm{ppm})\end{array}$ & $\begin{array}{l}\text { TCLP Ni } \\
(\mathrm{ppm})\end{array}$ & $\begin{array}{c}\text { TCLP Pb } \\
(\mathrm{ppm})\end{array}$ & $\begin{array}{c}\text { TCLP Se } \\
(\mathrm{ppm})\end{array}$ & $\begin{array}{c}\text { TCLP Zn } \\
(\mathrm{ppm})\end{array}$ \\
\hline \multicolumn{13}{|c|}{ Sp-LHLH(b) } \\
\hline \multicolumn{13}{|c|}{ Sp-MMLL(b) } \\
\hline \multicolumn{13}{|c|}{ Sp-MMHH(b) } \\
\hline \multicolumn{13}{|c|}{ Sp-HLLL(b) } \\
\hline \multirow{2}{*}{\multicolumn{13}{|c|}{ nom-2 }} \\
\hline \multirow{2}{*}{\multicolumn{13}{|c|}{ nom-3 }} \\
\hline \multirow{2}{*}{\multicolumn{13}{|c|}{$\begin{array}{l}\text { nomc-1 } \\
\text { nomc-2 }\end{array}$}} \\
\hline & & & & & & & & & & & & \\
\hline \multicolumn{13}{|l|}{ c106a-2 } \\
\hline \multicolumn{13}{|l|}{ c106a-3 } \\
\hline \multicolumn{13}{|l|}{ c106a-4 } \\
\hline \multicolumn{13}{|l|}{ c106b-1 } \\
\hline \multicolumn{13}{|l|}{ c106b-2 } \\
\hline az-3 & & & & & & & & & & & & \\
\hline az-5 & & & & & & & & & & & & \\
\hline
\end{tabular}

\section{DWPF PCT Model (Jantzen et al. 1995)}

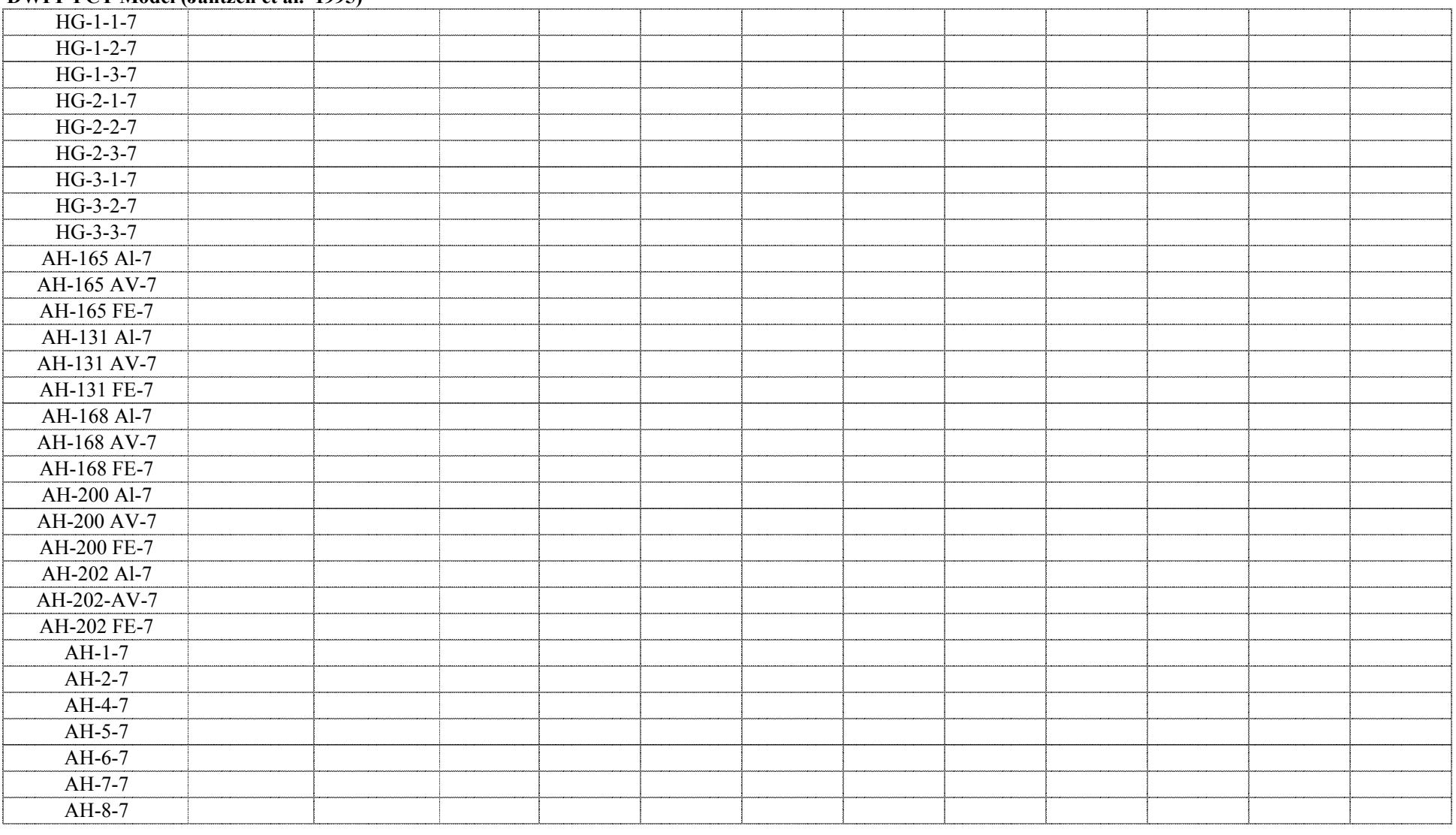


DWPF PCT Model (Jantzen et al. 1995)

\begin{tabular}{|c|c|c|c|c|c|c|c|c|c|c|c|c|c|c|c|c|c|c|c|c|c|}
\hline Glass ID & $\begin{array}{c}\mathrm{A} 12 \mathrm{O} 3 \\
-\mathrm{t}\end{array}$ & $\begin{array}{c}\mathrm{B} 2 \mathrm{O} 3 \\
-\mathrm{t}\end{array}$ & $\begin{array}{c}\mathrm{CaO} \\
-\mathrm{t}\end{array}$ & $\begin{array}{c}\mathrm{Fe} 2 \mathrm{O} 3 \\
-\mathrm{t}\end{array}$ & $\begin{array}{c}\mathrm{FeO} \\
-\mathrm{t}\end{array}$ & $\begin{array}{c}\mathrm{K} 2 \mathrm{O} \\
-\mathrm{t}\end{array}$ & $\begin{array}{c}\mathrm{Li} 2 \mathrm{O} \\
-\mathrm{t}\end{array}$ & $\begin{array}{c}\mathrm{MgO} \\
-\mathrm{t}\end{array}$ & $\begin{array}{c}\mathrm{Na} 2 \mathrm{O} \\
-\mathrm{t}\end{array}$ & $\begin{array}{c}\mathrm{NiO} \\
-\mathrm{t}\end{array}$ & $\begin{array}{c}\mathrm{P} 2 \mathrm{O} 5 \\
-\mathrm{t}\end{array}$ & $\begin{array}{c}\mathrm{SiO} 2 \\
-\mathrm{t}\end{array}$ & $\begin{array}{c}\mathrm{ZrO} 2 \\
-\mathrm{t}\end{array}$ & $\begin{array}{c}\mathrm{Ag} 2 \mathrm{O} \\
-\mathrm{t}\end{array}$ & $\begin{array}{c}\mathrm{As} 2 \mathrm{O} 3 \\
-\mathrm{t}\end{array}$ & $\begin{array}{c}\mathrm{BaO} \\
-\mathrm{t}\end{array}$ & $\begin{array}{c}\mathrm{Bi} 2 \mathrm{O} 3 \\
-\mathrm{t}\end{array}$ & $\begin{array}{c}\mathrm{Br} \\
-\mathrm{t}\end{array}$ & $\begin{array}{c}\mathrm{CdO} \\
-\mathrm{t}\end{array}$ & $\begin{array}{c}\mathrm{Ce} 2 \mathrm{O} 3 \\
-\mathrm{t}\end{array}$ & $\begin{array}{c}\mathrm{CeO} 2 \\
-\mathrm{t}\end{array}$ \\
\hline AH-9-7 & 0.0608 & 0.0881 & 0.0069 & 0.1166 & 0.0000 & 0.0315 & 0.0349 & 0.0058 & 0.0926 & 0.0098 & 0.0000 & 0.5120 & 0.0000 & & & 0.0000 & & & & 0.0000 & \\
\hline AH-10-7 & 0.0517 & 0.0764 & 0.0068 & 0.1136 & 0.0000 & 0.0311 & 0.0447 & 0.0112 & 0.0687 & 0.0097 & 0.0000 & 0.5453 & 0.0000 & & & 0.0000 & & & & 0.0000 & \\
\hline AH-11-7 & 0.0576 & 0.1214 & 0.0065 & 0.1113 & 0.0000 & 0.0303 & 0.0366 & 0.0112 & 0.0649 & 0.0094 & 0.0000 & 0.5099 & 0.0000 & & & 0.0000 & & & & 0.0000 & \\
\hline AH-12-7 & 0.0608 & 0.0881 & 0.0069 & 0.1166 & 0.0000 & 0.0315 & 0.0349 & 0.0058 & 0.0926 & 0.0098 & 0.0000 & 0.5121 & 0.0000 & & & 0.0000 & & & & 0.0000 & \\
\hline AH-13-7 & 0.0660 & 0.0653 & 0.0127 & 0.1386 & 0.0000 & 0.0311 & 0.0338 & 0.0050 & 0.0896 & 0.0116 & 0.0000 & 0.4989 & 0.0002 & & & 0.0000 & & & & 0.0000 & \\
\hline AH-14-7 & 0.0715 & 0.0832 & 0.0126 & 0.1402 & 0.0000 & 0.0311 & 0.0398 & 0.0054 & 0.0985 & 0.0114 & 0.0000 & 0.4521 & 0.0058 & & & 0.0000 & & & & 0.0000 & \\
\hline AH-15-7 & 0.0696 & 0.0932 & 0.0128 & 0.1378 & 0.0000 & 0.0314 & 0.0284 & 0.0104 & 0.0953 & 0.0114 & 0.0000 & 0.4619 & 0.0000 & & & 0.0000 & & & & 0.0000 & \\
\hline AH-16-7 & 0.0643 & 0.0728 & 0.0127 & 0.1354 & 0.0000 & 0.0310 & 0.0411 & 0.0101 & 0.0661 & 0.0111 & 0.0000 & 0.5077 & 0.0000 & & & 0.0000 & & & & 0.0000 & \\
\hline AH-17-7 & 0.0578 & 0.0820 & 0.0066 & 0.1151 & 0.0000 & 0.0309 & 0.0473 & 0.0058 & 0.0667 & 0.0092 & 0.0000 & 0.5298 & 0.0066 & & & 0.0000 & & & & 0.0000 & \\
\hline SFRIT1 & 0.0462 & 0.0855 & 0.0148 & 0.1428 & 0.0000 & 0.0271 & 0.0326 & 0.0085 & 0.1158 & 0.0111 & 0.0000 & 0.4813 & 0.0011 & & & 0.0009 & & & & 0.0000 & \\
\hline SFRIT2 & 0.0462 & 0.0855 & 0.0148 & 0.1428 & 0.0000 & 0.0271 & 0.0326 & 0.0085 & 0.1158 & 0.0111 & 0.0000 & 0.4813 & 0.0011 & & & 0.0009 & & & & 0.0000 & \\
\hline SFRIT3 & 0.0462 & 0.0855 & 0.0148 & 0.1428 & 0.0000 & 0.0271 & 0.0326 & 0.0085 & 0.1158 & 0.0111 & 0.0000 & 0.4813 & 0.0011 & & & 0.0009 & & & & 0.0000 & \\
\hline $202 \mathrm{P}$ w/o Mn-7 & 0.0429 & 0.0858 & 0.0121 & 0.1068 & 0.0000 & 0.0571 & 0.0341 & 0.0291 & 0.0917 & 0.0070 & 0.0000 & 0.5106 & 0.0000 & & & 0.0019 & & & & 0.0000 & \\
\hline $202 \mathrm{G}$ w/o Mn-7 & 0.0461 & 0.0603 & 0.0130 & 0.1209 & 0.0000 & 0.0274 & 0.0377 & 0.0318 & 0.0824 & 0.0067 & 0.0000 & 0.5602 & 0.0000 & & & 0.0017 & & & & 0.0000 & \\
\hline $200 \mathrm{R}-7$ & 0.0478 & 0.0979 & 0.0152 & 0.1272 & 0.0000 & 0.0362 & 0.0311 & 0.0129 & 0.1375 & 0.0055 & 0.0000 & 0.4666 & 0.0000 & & & 0.0000 & & & & 0.0000 & \\
\hline NBS SRM 623-7 & 0.0630 & 0.1071 & 0.0070 & 0.0000 & 0.0000 & 0.0061 & 0.0000 & 0.0000 & 0.0641 & 0.0000 & 0.0000 & 0.7306 & 0.0000 & & & 0.0221 & & & & 0.0000 & \\
\hline 165 CGW STD-7 & 0.0420 & 0.0673 & 0.0154 & 0.1296 & 0.0000 & 0.0000 & 0.0502 & 0.0103 & 0.1047 & 0.0097 & 0.0000 & 0.5401 & 0.0050 & & & 0.0000 & & & & 0.0000 & \\
\hline ARM-1-7 (4/88) & 0.0558 & 0.1128 & 0.0223 & 0.0000 & 0.0000 & 0.0000 & 0.0507 & 0.0000 & 0.0966 & 0.0000 & 0.0065 & 0.4645 & 0.0179 & & & 0.0066 & & & & 0.0273 & \\
\hline ARM-1-7 (5/89) & 0.0558 & 0.1128 & 0.0223 & 0.0000 & 0.0000 & 0.0000 & 0.0507 & 0.0000 & 0.0966 & 0.0000 & 0.0065 & 0.4645 & 0.0179 & & & 0.0066 & & & & 0.0273 & \\
\hline ARM-1-7 (7/90) & 0.0558 & 0.1128 & 0.0223 & 0.0000 & 0.0000 & 0.0000 & 0.0507 & 0.0000 & 0.0966 & 0.0000 & 0.0065 & 0.4645 & 0.0179 & & & 0.0066 & & & & 0.0273 & \\
\hline ARM-1-7 (12/90) & 0.0558 & 0.1128 & 0.0223 & 0.0000 & 0.0000 & 0.0000 & 0.0507 & 0.0000 & 0.0966 & 0.0000 & 0.0065 & 0.4645 & 0.0179 & & & 0.0066 & & & & 0.0273 & \\
\hline ARM-1-7 (5/91) & 0.0558 & 0.1128 & 0.0223 & 0.0000 & 0.0000 & 0.0000 & 0.0507 & 0.0000 & 0.0966 & 0.0000 & 0.0065 & 0.4645 & 0.0179 & & & 0.0066 & & & & 0.0273 & \\
\hline ARM-1-7 (10/91) & 0.0558 & 0.1128 & 0.0223 & 0.0000 & 0.0000 & 0.0000 & 0.0507 & 0.0000 & 0.0966 & 0.0000 & 0.0065 & 0.4645 & 0.0179 & & & 0.0066 & & & & 0.0273 & \\
\hline ARM-1-7 (10/92) & 0.0558 & 0.1128 & 0.0223 & 0.0000 & 0.0000 & 0.0000 & 0.0507 & 0.0000 & 0.0966 & 0.0000 & 0.0065 & 0.4645 & 0.0179 & & & 0.0066 & & & & 0.0273 & \\
\hline ARM-1-7 (4/93) & 0.0558 & 0.1128 & 0.0223 & 0.0000 & 0.0000 & 0.0000 & 0.0507 & 0.0000 & 0.0966 & 0.0000 & 0.0065 & 0.4645 & 0.0179 & & & 0.0066 & & & & 0.0273 & \\
\hline ARM-1-7 (6/93) & 0.0558 & 0.1128 & 0.0223 & 0.0000 & 0.0000 & 0.0000 & 0.0507 & 0.0000 & 0.0966 & 0.0000 & 0.0065 & 0.4645 & 0.0179 & & & 0.0066 & & & & 0.0273 & \\
\hline ARM-1-7 (8/93) & 0.0558 & 0.1128 & 0.0223 & 0.0000 & 0.0000 & 0.0000 & 0.0507 & 0.0000 & 0.0966 & 0.0000 & 0.0065 & 0.4645 & 0.0179 & & & 0.0066 & & & & 0.0273 & \\
\hline ARM-1-7 & 0.0558 & 0.1128 & 0.0223 & 0.0000 & 0.0000 & 0.0000 & 0.0507 & 0.0000 & 0.0966 & 0.0000 & 0.0065 & 0.4645 & 0.0179 & & & 0.0066 & & & & 0.0273 & \\
\hline T-ARM-1 & 0.0558 & 0.1128 & 0.0223 & 0.0000 & 0.0000 & 0.0000 & 0.0507 & 0.0000 & 0.0966 & 0.0000 & 0.0065 & 0.4645 & 0.0179 & & & 0.0066 & & & & 0.0273 & \\
\hline SS-ARM-1 & 0.0558 & 0.1128 & 0.0223 & 0.0000 & 0.0000 & 0.0000 & 0.0507 & 0.0000 & 0.0966 & 0.0000 & 0.0065 & 0.4645 & 0.0179 & & & 0.0066 & & & & 0.0273 & \\
\hline SS-ARM-1 & 0.0558 & 0.1128 & 0.0223 & 0.0000 & 0.0000 & 0.0000 & 0.0507 & 0.0000 & 0.0966 & 0.0000 & 0.0065 & 0.4645 & 0.0179 & & & 0.0066 & & & & 0.0273 & \\
\hline EA-1-7 & 0.0370 & 0.1130 & 0.0112 & 0.0738 & 0.0145 & 0.0005 & 0.0426 & 0.0172 & 0.1681 & 0.0057 & 0.0000 & 0.4873 & 0.0045 & & & 0.0000 & & & & 0.0000 & \\
\hline EA-1-7 & 0.0370 & 0.1130 & 0.0112 & 0.0738 & 0.0145 & 0.0005 & 0.0426 & 0.0172 & 0.1681 & 0.0057 & 0.0000 & 0.4873 & 0.0045 & & & 0.0000 & & & & 0.0000 & \\
\hline EA-2-7 & 0.0370 & 0.1130 & 0.0112 & 0.0738 & 0.0145 & 0.0005 & 0.0426 & 0.0172 & 0.1681 & 0.0057 & 0.0000 & 0.4873 & 0.0045 & & & 0.0000 & & & & 0.0000 & \\
\hline EA-7 & 0.0370 & 0.1130 & 0.0112 & 0.0738 & 0.0145 & 0.0005 & 0.0426 & 0.0172 & 0.1681 & 0.0057 & 0.0000 & 0.4873 & 0.0045 & & & 0.0000 & & & & 0.0000 & \\
\hline T-EA & 0.0370 & 0.1130 & 0.0112 & 0.0738 & 0.0145 & 0.0005 & 0.0426 & 0.0172 & 0.1681 & 0.0057 & 0.0000 & 0.4873 & 0.0045 & & & 0.0000 & & & & 0.0000 & \\
\hline SS-EA-19 & 0.0370 & 0.1130 & 0.0112 & 0.0738 & 0.0145 & 0.0005 & 0.0426 & 0.0172 & 0.1681 & 0.0057 & 0.0000 & 0.4873 & 0.0045 & & & 0.0000 & & & & 0.0000 & \\
\hline SS-EA-15 & 0.0370 & 0.1130 & 0.0112 & 0.0738 & 0.0145 & 0.0005 & 0.0426 & 0.0172 & 0.1681 & 0.0057 & 0.0000 & 0.4873 & 0.0045 & & & 0.0000 & & & & 0.0000 & \\
\hline SS-EA-1-7 & 0.0370 & 0.1130 & 0.0112 & 0.0738 & 0.0145 & 0.0005 & 0.0426 & 0.0172 & 0.1681 & 0.0057 & 0.0000 & 0.4873 & 0.0045 & & & 0.0000 & & & & 0.0000 & \\
\hline SS-EA-2-7 & 0.0370 & 0.1130 & 0.0112 & 0.0738 & 0.0145 & 0.0005 & 0.0426 & 0.0172 & 0.1681 & 0.0057 & 0.0000 & 0.4873 & 0.0045 & & & 0.0000 & & & & 0.0000 & \\
\hline SRS-SEA-A-7 & 0.0370 & 0.1130 & 0.0112 & 0.0738 & 0.0145 & 0.0005 & 0.0426 & 0.0172 & 0.1681 & 0.0057 & 0.0000 & 0.4873 & 0.0045 & & & 0.0000 & & & & 0.0000 & \\
\hline SRS-SEA-B-7 & 0.0370 & 0.1130 & 0.0112 & 0.0738 & 0.0145 & 0.0005 & 0.0426 & 0.0172 & 0.1681 & 0.0057 & 0.0000 & 0.4873 & 0.0045 & & & 0.0000 & & & & 0.0000 & \\
\hline CUASEA-A-7 & 0.0370 & 0.1130 & 0.0112 & 0.0738 & 0.0145 & 0.0005 & 0.0426 & 0.0172 & 0.1681 & 0.0057 & 0.0000 & 0.4873 & 0.0045 & & & 0.0000 & & & & 0.0000 & \\
\hline CUASEA-B-7 & 0.0370 & 0.1130 & 0.0112 & 0.0738 & 0.0145 & 0.0005 & 0.0426 & 0.0172 & 0.1681 & 0.0057 & 0.0000 & 0.4873 & 0.0045 & & & 0.0000 & & & & 0.0000 & \\
\hline 131-TDS-EA-7 & 0.0571 & 0.1259 & 0.0091 & 0.1418 & 0.0000 & 0.0004 & 0.0479 & 0.0152 & 0.1447 & 0.0143 & 0.0000 & 0.3947 & 0.0043 & & & 0.0000 & & & & 0.0000 & \\
\hline 131-TDS-3A-SOPER & 0.0548 & 0.1073 & 0.0091 & 0.1133 & 0.0000 & 0.0004 & 0.0418 & 0.0140 & 0.1361 & 0.0153 & 0.0000 & 0.4590 & 0.0034 & & & 0.0000 & & & & 0.0000 & \\
\hline BLEND 1-7 & 0.0418 & 0.0809 & 0.0104 & 0.1095 & 0.0000 & 0.0370 & 0.0446 & 0.0142 & 0.0917 & 0.0090 & 0.0000 & 0.5215 & 0.0013 & & & 0.0019 & & & & 0.0000 & \\
\hline BLEND 1-7 (2) & 0.0418 & 0.0809 & 0.0104 & 0.1095 & 0.0000 & 0.0370 & 0.0446 & 0.0142 & 0.0917 & 0.0090 & 0.0000 & 0.5215 & 0.0013 & & & 0.0019 & & & & 0.0000 & \\
\hline BLEND 1.6-7 & 0.0418 & 0.0809 & 0.0104 & 0.1095 & 0.0000 & 0.0370 & 0.0446 & 0.0142 & 0.0917 & 0.0090 & 0.0000 & 0.5215 & 0.0013 & & & 0.0019 & & & & 0.0000 & \\
\hline
\end{tabular}


DWPF PCT Model (Jantzen et al. 1995)

\begin{tabular}{|c|c|c|c|c|c|c|c|c|c|c|c|c|c|c|c|c|c|c|c|c|c|}
\hline Glass ID & $\begin{array}{l}\mathrm{Cl} \\
-\mathrm{t}\end{array}$ & $\begin{array}{c}\mathrm{CoO} \\
-\mathrm{t}\end{array}$ & $\begin{array}{c}\mathrm{Co} 2 \mathrm{O} 3 \\
-\mathrm{t}\end{array}$ & $\begin{array}{c}\mathrm{Cr} 2 \mathrm{O} 3 \\
-\mathrm{t} \\
\end{array}$ & $\begin{array}{c}\mathrm{Cs} 2 \mathrm{O} \\
-\mathrm{t} \\
\end{array}$ & $\begin{array}{c}\mathrm{CuO} \\
-\mathrm{t} \\
\end{array}$ & $\begin{array}{c}\mathrm{Eu} 2 \mathrm{O} 3 \\
-\mathrm{t}\end{array}$ & $\begin{array}{l}F \\
-t\end{array}$ & $\begin{array}{c}\mathrm{Ga} 2 \mathrm{O} 3 \\
-\mathrm{t}\end{array}$ & $\begin{array}{c}\mathrm{Gd} 2 \mathrm{O} 3 \\
-\mathrm{t}\end{array}$ & $\begin{array}{c}\mathrm{HgO} \\
-\mathrm{t}\end{array}$ & $\begin{array}{l}I \\
-t\end{array}$ & $\begin{array}{c}\mathrm{La} 2 \mathrm{O} 3 \\
-\mathrm{t}\end{array}$ & $\begin{array}{c}\mathrm{MnO} 2 \\
-\mathrm{t} \\
\end{array}$ & $\begin{array}{c}\mathrm{MnO} \\
-\mathrm{t}\end{array}$ & $\begin{array}{c}\mathrm{MoO} \\
-\mathrm{t}\end{array}$ & $\begin{array}{c}\mathrm{MoO} 3 \\
-\mathrm{t}\end{array}$ & $\begin{array}{c}\mathrm{Nb} 2 \mathrm{O} 5 \\
-\mathrm{t}\end{array}$ & $\begin{array}{c}\mathrm{Nd} 2 \mathrm{O} 3 \\
-\mathrm{t}\end{array}$ & $\begin{array}{c}\mathrm{PbO} \\
-\mathrm{t}\end{array}$ & $\begin{array}{c}\mathrm{PdO} 2 \\
-\mathrm{t}\end{array}$ \\
\hline AH-9-7 & & & & 0.0002 & 0.0004 & 0.0000 & & & & & & & 0.0000 & & 0.0266 & & 0.0000 & & 0.0000 & 0.0000 & \\
\hline AH-10-7 & & & & 0.0000 & 0.0004 & 0.0000 & & & & & & & 0.0000 & & 0.0269 & & 0.0000 & & 0.0000 & 0.0000 & \\
\hline AH-11-7 & & & & 0.0002 & 0.0004 & 0.0000 & & & & & & & 0.0000 & & 0.0266 & & 0.0000 & & 0.0000 & 0.0000 & \\
\hline AH-12-7 & & & & 0.0000 & 0.0004 & 0.0000 & & & & & & & 0.0000 & & 0.0266 & & 0.0000 & & 0.0000 & 0.0000 & \\
\hline AH-13-7 & & & & 0.0000 & 0.0004 & 0.0000 & & & & & & & 0.0000 & & 0.0331 & & 0.0000 & & 0.0000 & 0.0000 & \\
\hline AH-14-7 & & & & 0.0000 & 0.0008 & 0.0000 & & & & & & & 0.0000 & & 0.0337 & & 0.0000 & & 0.0000 & 0.0000 & \\
\hline AH-15-7 & & & & 0.0002 & 0.0004 & 0.0000 & & & & & & & 0.0000 & & 0.0330 & & 0.0000 & & 0.0000 & 0.0000 & \\
\hline AH-16-7 & & & & 0.0007 & 0.0004 & 0.0000 & & & & & & & 0.0000 & & 0.0326 & & 0.0000 & & 0.0000 & 0.0000 & \\
\hline AH-17-7 & & & & 0.0009 & 0.0004 & 0.0000 & & & & & & & 0.0000 & & 0.0273 & & 0.0000 & & 0.0000 & 0.0000 & \\
\hline SFRIT1 & & & & 0.0009 & 0.0000 & 0.0000 & & & & & & & 0.0000 & & 0.0195 & & 0.0000 & & 0.0000 & 0.0000 & \\
\hline SFRIT2 & & & & 0.0009 & 0.0000 & 0.0000 & & & & & & & 0.0000 & & 0.0195 & & 0.0000 & & 0.0000 & 0.0000 & \\
\hline SFRIT3 & & & & 0.0009 & 0.0000 & 0.0000 & & & & & & & 0.0000 & & 0.0195 & & 0.0000 & & 0.0000 & 0.0000 & \\
\hline $202 \mathrm{P}$ w/o Mn-7 & & & & 0.0007 & 0.0013 & 0.0066 & & & & & & & 0.0000 & & 0.0000 & & 0.0000 & & 0.0000 & 0.0000 & \\
\hline 202G w/o Mn-7 & & & & 0.0007 & 0.0004 & 0.0040 & & & & & & & 0.0000 & & 0.0000 & & 0.0000 & & 0.0000 & 0.0000 & \\
\hline $200 \mathrm{R}-7$ & & & & 0.0000 & 0.0000 & 0.0000 & & & & & & & 0.0000 & & 0.0220 & & 0.0000 & & 0.0000 & 0.0000 & \\
\hline NBS SRM 623-7 & & & & 0.0000 & 0.0000 & 0.0000 & & & & & & & 0.0000 & & 0.0000 & & 0.0000 & & 0.0000 & 0.0000 & \\
\hline 165 CGW STD-7 & & & & 0.0000 & 0.0000 & 0.0000 & & & & & & & 0.0000 & & 0.0257 & & 0.0000 & & 0.0000 & 0.0000 & \\
\hline ARM-1-7 (4/88) & & & & 0.0000 & 0.0117 & 0.0000 & & & & & & & 0.0000 & & 0.0000 & & 0.0167 & & 0.0594 & 0.0000 & \\
\hline ARM-1-7 (5/89) & & & & 0.0000 & 0.0117 & 0.0000 & & & & & & & 0.0000 & & 0.0000 & & 0.0167 & & 0.0594 & 0.0000 & \\
\hline ARM-1-7 (7/90) & & & & 0.0000 & 0.0117 & 0.0000 & & & & & & & 0.0000 & & 0.0000 & & 0.0167 & & 0.0594 & 0.0000 & \\
\hline ARM-1-7 (12/90) & & & & 0.0000 & 0.0117 & 0.0000 & & & & & & & 0.0000 & & 0.0000 & & 0.0167 & & 0.0594 & 0.0000 & \\
\hline ARM-1-7 (5/91) & & & & 0.0000 & 0.0117 & 0.0000 & & & & & & & 0.0000 & & 0.0000 & & 0.0167 & & 0.0594 & 0.0000 & \\
\hline ARM-1-7 (10/91) & & & & 0.0000 & 0.0117 & 0.0000 & & & & & & & 0.0000 & & 0.0000 & & 0.0167 & & 0.0594 & 0.0000 & \\
\hline ARM-1-7 (10/92) & & & & 0.0000 & 0.0117 & 0.0000 & & & & & & & 0.0000 & & 0.0000 & & 0.0167 & & 0.0594 & 0.0000 & \\
\hline ARM-1-7 (4/93) & & & & 0.0000 & 0.0117 & 0.0000 & & & & & & & 0.0000 & & 0.0000 & & 0.0167 & & 0.0594 & 0.0000 & \\
\hline ARM-1-7 (6/93) & & & & 0.0000 & 0.0117 & 0.0000 & & & & & & & 0.0000 & & 0.0000 & & 0.0167 & & 0.0594 & 0.0000 & \\
\hline ARM-1-7 (8/93) & & & & 0.0000 & 0.0117 & 0.0000 & & & & & & & 0.0000 & & 0.0000 & & 0.0167 & & 0.0594 & 0.0000 & \\
\hline ARM-1-7 & & & & 0.0000 & 0.0117 & 0.0000 & & & & & & & 0.0000 & & 0.0000 & & 0.0167 & & 0.0594 & 0.0000 & \\
\hline T-ARM-1 & & & & 0.0000 & 0.0117 & 0.0000 & & & & & & & 0.0000 & & 0.0000 & & 0.0167 & & 0.0594 & 0.0000 & \\
\hline SS-ARM-1 & & & & 0.0000 & 0.0117 & 0.0000 & & & & & & & 0.0000 & & 0.0000 & & 0.0167 & & 0.0594 & 0.0000 & \\
\hline SS-ARM-1 & & & & 0.0000 & 0.0117 & 0.0000 & & & & & & & 0.0000 & & 0.0000 & & 0.0167 & & 0.0594 & 0.0000 & \\
\hline EA-1-7 & & & & 0.0000 & 0.0000 & 0.0000 & & & & & & & 0.0042 & & 0.0134 & & 0.0000 & & 0.0000 & 0.0000 & \\
\hline EA-1-7 & & & & 0.0000 & 0.0000 & 0.0000 & & & & & & & 0.0042 & & 0.0134 & & 0.0000 & & 0.0000 & 0.0000 & \\
\hline EA-2-7 & & & & 0.0000 & 0.0000 & 0.0000 & & & & & & & 0.0042 & & 0.0134 & & 0.0000 & & 0.0000 & 0.0000 & \\
\hline EA-7 & & & & 0.0000 & 0.0000 & 0.0000 & & & & & & & 0.0042 & & 0.0134 & & 0.0000 & & 0.0000 & 0.0000 & \\
\hline T-EA & & & & 0.0000 & 0.0000 & 0.0000 & & & & & & & 0.0042 & & 0.0134 & & 0.0000 & & 0.0000 & 0.0000 & \\
\hline SS-EA-19 & & & & 0.0000 & 0.0000 & 0.0000 & & & & & & & 0.0042 & & 0.0134 & & 0.0000 & & 0.0000 & 0.0000 & \\
\hline SS-EA-15 & & & & 0.0000 & 0.0000 & 0.0000 & & & & & & & 0.0042 & & 0.0134 & & 0.0000 & & 0.0000 & 0.0000 & \\
\hline SS-EA-1-7 & & & & 0.0000 & 0.0000 & 0.0000 & & & & & & & 0.0042 & & 0.0134 & & 0.0000 & & 0.0000 & 0.0000 & \\
\hline SS-EA-2-7 & & & & 0.0000 & 0.0000 & 0.0000 & & & & & & & 0.0042 & & 0.0134 & & 0.0000 & & 0.0000 & 0.0000 & \\
\hline SRS-SEA-A-7 & & & & 0.0000 & 0.0000 & 0.0000 & & & & & & & 0.0042 & & 0.0134 & & 0.0000 & & 0.0000 & 0.0000 & \\
\hline SRS-SEA-B-7 & & & & 0.0000 & 0.0000 & 0.0000 & & & & & & & 0.0042 & & 0.0134 & & 0.0000 & & 0.0000 & 0.0000 & \\
\hline CUASEA-A-7 & & & & 0.0000 & 0.0000 & 0.0000 & & & & & & & 0.0042 & & 0.0134 & & 0.0000 & & 0.0000 & 0.0000 & \\
\hline CUASEA-B-7 & & & & 0.0000 & 0.0000 & 0.0000 & & & & & & & 0.0042 & & 0.0134 & & 0.0000 & & 0.0000 & 0.0000 & \\
\hline 131-TDS-EA-7 & & & & 0.0005 & 0.0000 & 0.0000 & & & & & & & 0.0034 & & 0.0314 & & 0.0000 & & 0.0000 & 0.0000 & \\
\hline 131-TDS-3A-SOPER- & & & & 0.0012 & 0.0000 & 0.0000 & & & & & & & 0.0035 & & 0.0334 & & 0.0000 & & 0.0000 & 0.0000 & \\
\hline BLEND 1-7 & & & & 0.0014 & 0.0009 & 0.0044 & & & & & & & 0.0000 & & 0.0168 & & 0.0016 & & 0.0021 & 0.0000 & \\
\hline BLEND 1-7 (2) & & & & 0.0014 & 0.0009 & 0.0044 & & & & & & & 0.0000 & & 0.0168 & & 0.0016 & & 0.0021 & 0.0000 & \\
\hline BLEND 1.6-7 & & & & 0.0014 & 0.0009 & 0.0044 & & & & & & & 0.0000 & & 0.0168 & & 0.0016 & & 0.0021 & 0.0000 & \\
\hline
\end{tabular}


DWPF PCT Model (Jantzen et al. 1995)

\begin{tabular}{|c|c|c|c|c|c|c|c|c|c|c|c|c|c|c|c|c|c|c|c|c|c|}
\hline Glass ID & $\begin{array}{c}\mathrm{PdO} \\
-\mathrm{t}\end{array}$ & $\begin{array}{c}\mathrm{Pr} 2 \mathrm{O} 3 \\
-\mathrm{t}\end{array}$ & {$\left[\begin{array}{c}\operatorname{Pr} 6 \mathrm{O} 11 \\
-\mathrm{t}\end{array}\right.$} & $\begin{array}{c}\mathrm{Rb} 2 \mathrm{O} \\
-\mathrm{t}\end{array}$ & $\begin{array}{c}\mathrm{ReO} \\
-\mathrm{t}\end{array}$ & $\begin{array}{c}\mathrm{ReO} 2 \\
-\mathrm{t}\end{array}$ & $\begin{array}{c}\mathrm{Rh} 2 \mathrm{O} 3 \\
-\mathrm{t}\end{array}$ & $\begin{array}{c}\mathrm{RhO} 2 \\
-\mathrm{t}\end{array}$ & $\begin{array}{c}\mathrm{RuO}_{2} \\
-\mathrm{t}\end{array}$ & $\begin{array}{c}\mathrm{Sb} 2 \mathrm{O} 3 \\
-\mathrm{t}\end{array}$ & $\begin{array}{c}\mathrm{Sb} 2 \mathrm{O} 5 \\
-\mathrm{t}\end{array}$ & $\begin{array}{c}\mathrm{SeO} 2 \\
-\mathrm{t}\end{array}$ & $\begin{array}{c}\mathrm{Sm} 2 \mathrm{O} 3 \\
-\mathrm{t}\end{array}$ & $\begin{array}{c}\mathrm{SnO} \\
-\mathrm{t}\end{array}$ & $\begin{array}{c}\mathrm{SnO} 2 \\
-\mathrm{t}\end{array}$ & $\begin{array}{c}\mathrm{SO} 3 \\
-\mathrm{t}\end{array}$ & $\begin{array}{c}\mathrm{SrO} \\
-\mathrm{t}\end{array}$ & $\begin{array}{c}\mathrm{Tc} 2 \mathrm{O} 7 \\
-\mathrm{t}\end{array}$ & $\begin{array}{c}\mathrm{TeO} 2 \\
-\mathrm{t}\end{array}$ & $\begin{array}{c}\mathrm{ThO} 2 \\
-\mathrm{t}\end{array}$ & $\begin{array}{c}\mathrm{TiO} 2 \\
-\mathrm{t}\end{array}$ \\
\hline AH-9-7 & & & & & & & & & & & & & & & & & 0.0005 & & & & 0.0134 \\
\hline AH-10-7 & & & & & & & & & & & & & & & & & 0.0005 & & & & 0.0130 \\
\hline AH-11-7 & & & & & & & & & & & & & & & & & 0.0005 & & & & 0.0131 \\
\hline AH-12-7 & & & & & & & & & & & & & & & & & 0.0005 & & & & 0.0134 \\
\hline AH-13-7 & & & & & & & & & & & & & & & & & 0.0006 & & & & 0.0131 \\
\hline AH-14-7 & & & & & & & & & & & & & & & & & 0.0006 & & & & 0.0133 \\
\hline AH-15-7 & & & & & & & & & & & & & & & & & 0.0006 & & & & 0.0136 \\
\hline AH-16-7 & & & & & & & & & & & & & & & & & 0.0008 & & & & 0.0132 \\
\hline AH-17-7 & & & & & & & & & & & & & & & & & 0.0003 & & & & 0.0132 \\
\hline SFRIT1 & & & & & & & & & & & & & & & & & 0.0000 & & & & 0.0119 \\
\hline SFRIT2 & & & & & & & & & & & & & & & & & 0.0000 & & & & 0.0119 \\
\hline SFRIT3 & & & & & & & & & & & & & & & & & 0.0000 & & & & 0.0119 \\
\hline 202P w/o Mn-7 & & & & & & & & & & & & & & & & & 0.0000 & & & & 0.0124 \\
\hline 202G w/o Mn-7 & & & & & & & & & & & & & & & & & 0.0000 & & & & 0.0068 \\
\hline 200R-7 & & & & & & & & & & & & & & & & & 0.0000 & & & & 0.0000 \\
\hline NBS SRM 623-7 & & & & & & & & & & & & & & & & & 0.0000 & & & & 0.0000 \\
\hline 165 CGW STD-7 & & & & & & & & & & & & & & & & & 0.0000 & & & & 0.0000 \\
\hline ARM-1-7 (4/88) & & & & & & & & & & & & & & & & & 0.0045 & & & & 0.0321 \\
\hline ARM-1-7 (5/89) & & & & & & & & & & & & & & & & & 0.0045 & & & & 0.0321 \\
\hline ARM-1-7 (7/90) & & & & & & & & & & & & & & & & & 0.0045 & & & & 0.0321 \\
\hline ARM-1-7 (12/90) & & & & & & & & & & & & & & & & & 0.0045 & & & & 0.0321 \\
\hline ARM-1-7 (5/91) & & & & & & & & & & & & & & & & & 0.0045 & & & & 0.0321 \\
\hline ARM-1-7 (10/91) & & & & & & & & & & & & & & & & & 0.0045 & & & & 0.0321 \\
\hline ARM-1-7 (10/92) & & & & & & & & & & & & & & & & & 0.0045 & & & & 0.0321 \\
\hline ARM-1-7 (4/93) & & & & & & & & & & & & & & & & & 0.0045 & & & & 0.0321 \\
\hline ARM-1-7 (6/93) & & & & & & & & & & & & & & & & & 0.0045 & & & & 0.0321 \\
\hline ARM-1-7 (8/93) & & & & & & & & & & & & & & & & & 0.0045 & & & & 0.0321 \\
\hline ARM-1-7 & & & & & & & & & & & & & & & & & 0.0045 & & & & 0.0321 \\
\hline T-ARM-1 & & & & & & & & & & & & & & & & & 0.0045 & & & & 0.0321 \\
\hline SS-ARM-1 & & & & & & & & & & & & & & & & & 0.0045 & & & & 0.0321 \\
\hline SS-ARM-1 & & & & & & & & & & & & & & & & & 0.0045 & & & & 0.0321 \\
\hline EA-1-7 & & & & & & & & & & & & & & & & & 0.0000 & & & & 0.0070 \\
\hline EA-1-7 & & & & & & & & & & & & & & & & & 0.0000 & & & & 0.0070 \\
\hline EA-2-7 & & & & & & & & & & & & & & & & & 0.0000 & & & & 0.0070 \\
\hline EA-7 & & & & & & & & & & & & & & & & & 0.0000 & & & & 0.0070 \\
\hline T-EA & & & & & & & & & & & & & & & & & 0.0000 & & & & 0.0070 \\
\hline SS-EA-19 & & & & & & & & & & & & & & & & & 0.0000 & & & & 0.0070 \\
\hline SS-EA-15 & & & & & & & & & & & & & & & & & 0.0000 & & & & 0.0070 \\
\hline SS-EA-1-7 & & & & & & & & & & & & & & & & & 0.0000 & & & & 0.0070 \\
\hline SS-EA-2-7 & & & & & & & & & & & & & & & & & 0.0000 & & & & 0.0070 \\
\hline SRS-SEA-A-7 & & & & & & & & & & & & & & & & & 0.0000 & & & & 0.0070 \\
\hline SRS-SEA-B-7 & & & & & & & & & & & & & & & & & 0.0000 & & & & 0.0070 \\
\hline CUASEA-A-7 & & & & & & & & & & & & & & & & & 0.0000 & & & & 0.0070 \\
\hline CUASEA-B-7 & & & & & & & & & & & & & & & & & 0.0000 & & & & 0.0070 \\
\hline 131-TDS-EA-7 & & & & & & & & & & & & & & & & & 0.0000 & & & & 0.0092 \\
\hline 131-TDS-3A-SOPER- & & & & & & & & & & & & & & & & & 0.0000 & & & & 0.0074 \\
\hline BLEND 1-7 & & & & & & & & & & & & & & & & & 0.0000 & & & & 0.0090 \\
\hline BLEND 1-7 (2) & & & & & & & & & & & & & & & & & 0.0000 & & & & 0.0090 \\
\hline BLEND 1.6-7 & & & & & & & & & & & & & & & & & 0.0000 & & & & 0.0090 \\
\hline
\end{tabular}


DWPF PCT Model (Jantzen et al. 1995)

\begin{tabular}{|c|c|c|c|c|c|c|c|c|c|c|c|c|c|c|c|c|c|c|c|c|c|}
\hline Glass ID & $\begin{array}{c}\mathrm{T} 12 \mathrm{O} 3 \\
-\mathrm{t}\end{array}$ & $\begin{array}{c}\mathrm{U} 3 \mathrm{O} 8 \\
-\mathrm{t}\end{array}$ & $\begin{array}{c}\mathrm{UO} 2 \\
-\mathrm{t}\end{array}$ & $\begin{array}{c}\mathrm{UO} 3 \\
-\mathrm{t}\end{array}$ & $\begin{array}{c}\mathrm{V} 2 \mathrm{O} 5 \\
-\mathrm{t}\end{array}$ & $\begin{array}{c}\text { WO3 } \\
-t\end{array}$ & $\begin{array}{c}\mathrm{Y} 2 \mathrm{O} 3 \\
-\mathrm{t}\end{array}$ & $\begin{array}{c}\mathrm{ZnO} \\
-\mathrm{t}\end{array}$ & $\begin{array}{c}\text { Others } \\
-t\end{array}$ & $\begin{array}{c}\text { Sum } \\
-t\end{array}$ & $\begin{array}{c}\mathrm{A} 12 \mathrm{O} 3 \\
-\mathrm{a}\end{array}$ & $\begin{array}{c}\mathrm{B} 2 \mathrm{O} 3 \\
-\mathrm{a}\end{array}$ & $\begin{array}{c}\mathrm{CaO} \\
-\mathrm{a}\end{array}$ & $\begin{array}{c}\mathrm{Fe} 2 \mathrm{O} 3 \\
-\mathrm{a}\end{array}$ & $\begin{array}{c}\mathrm{FeO} \\
-\mathrm{a}\end{array}$ & $\begin{array}{c}\mathrm{K} 2 \mathrm{O} \\
-\mathrm{a}\end{array}$ & $\begin{array}{c}\mathrm{Li} 2 \mathrm{O} \\
-\mathrm{a}\end{array}$ & $\begin{array}{c}\mathrm{MgO} \\
-\mathrm{a}\end{array}$ & $\begin{array}{c}\mathrm{Na} 2 \mathrm{O} \\
-\mathrm{a}\end{array}$ & $\begin{array}{c}\mathrm{NiO} \\
-\mathrm{a}\end{array}$ & $\begin{array}{c}\mathrm{P} 2 \mathrm{O} 5 \\
-\mathrm{a}\end{array}$ \\
\hline $\mathrm{AH}-9-7$ & & & & & & & 0.0000 & 0.0000 & & 1.0000 & 0.0604 & 0.0875 & 0.0069 & 0.1160 & & 0.0313 & 0.0347 & 0.0058 & 0.0920 & 0.0097 & \\
\hline AH-10-7 & & & & & & & 0.0000 & 0.0000 & & 1.0000 & 0.0514 & 0.0759 & 0.0068 & 0.1130 & & 0.0309 & 0.0444 & 0.0111 & 0.0683 & 0.0096 & \\
\hline AH-11-7 & & & & & & & 0.0000 & 0.0000 & & 1.0000 & 0.0570 & 0.1200 & 0.0065 & 0.1100 & & 0.0299 & 0.0362 & 0.0111 & 0.0642 & 0.0093 & \\
\hline AH-12-7 & & & & & & & 0.0000 & 0.0000 & & 1.0000 & 0.0604 & 0.0875 & 0.0069 & 0.1160 & & 0.0313 & 0.0347 & 0.0058 & 0.0920 & 0.0097 & \\
\hline AH-13-7 & & & & & & & 0.0000 & 0.0000 & & 1.0000 & 0.0648 & 0.0641 & 0.0125 & 0.1360 & & 0.0306 & 0.0332 & 0.0049 & 0.0880 & 0.0114 & \\
\hline AH-14-7 & & & & & & & 0.0000 & 0.0000 & & 1.0000 & 0.0708 & 0.0825 & 0.0125 & 0.1390 & & 0.0308 & 0.0394 & 0.0053 & 0.0976 & 0.0113 & \\
\hline AH-15-7 & & & & & & & 0.0000 & 0.0000 & & 1.0000 & 0.0687 & 0.0920 & 0.0127 & 0.1360 & & 0.0310 & 0.0280 & 0.0102 & 0.0941 & 0.0113 & \\
\hline AH-16-7 & & & & & & & 0.0000 & 0.0000 & & 1.0000 & 0.0636 & 0.0720 & 0.0126 & 0.1340 & & 0.0306 & 0.0406 & 0.0100 & 0.0654 & 0.0110 & \\
\hline AH-17-7 & & & & & & & 0.0000 & 0.0000 & & 1.0000 & 0.0572 & 0.0812 & 0.0065 & 0.1140 & & 0.0306 & 0.0469 & 0.0058 & 0.0661 & 0.0092 & \\
\hline SFRIT1 & & & & & & & 0.0000 & 0.0000 & & 1.0000 & 0.0460 & 0.0851 & 0.0147 & 0.1420 & & 0.0270 & 0.0325 & 0.0084 & 0.1153 & 0.0111 & \\
\hline SFRIT2 & & & & & & & 0.0000 & 0.0000 & & 1.0000 & & & & & & & & & & & \\
\hline SFRIT3 & & & & & & & 0.0000 & 0.0000 & & 1.0000 & & & & & & & & & & & \\
\hline $202 \mathrm{P}$ w/o Mn-7 & & & & & & & 0.0000 & 0.0000 & & 1.0000 & 0.0430 & 0.0860 & 0.0122 & 0.1070 & & 0.0573 & 0.0342 & 0.0292 & 0.0920 & 0.0070 & \\
\hline $202 \mathrm{G}$ w/o Mn-7 & & & & & & & 0.0000 & 0.0000 & & 1.0000 & 0.0469 & 0.0613 & 0.0132 & 0.1230 & & 0.0278 & 0.0383 & 0.0324 & 0.0838 & 0.0068 & \\
\hline $200 \mathrm{R}-7$ & & & & & & & 0.0000 & 0.0000 & & 1.0000 & 0.0462 & 0.0946 & 0.0147 & 0.1230 & & 0.0349 & 0.0301 & 0.0125 & 0.1329 & 0.0053 & \\
\hline NBS SRM 623-7 & & & & & & & 0.0000 & 0.0000 & & 1.0000 & 0.0630 & 0.1070 & 0.0070 & & & 0.0060 & & & 0.0640 & & \\
\hline 165 CGW STD-7 & & & & & & & 0.0000 & 0.0000 & & 1.0000 & 0.0419 & 0.0671 & 0.0154 & 0.1292 & & & 0.0500 & 0.0102 & 0.1044 & 0.0097 & \\
\hline ARM-1-7 (4/88) & & & & & & & 0.0000 & 0.0146 & & 1.0000 & 0.0559 & 0.1130 & 0.0223 & & & & 0.0508 & & 0.0967 & & 0.0065 \\
\hline ARM-1-7 (5/89) & & & & & & & 0.0000 & 0.0146 & & 1.0000 & & & & & & & & & & & \\
\hline ARM-1-7 (7/90) & & & & & & & 0.0000 & 0.0146 & & 1.0000 & & & & & & & & & & & \\
\hline ARM-1-7 (12/90) & & & & & & & 0.0000 & 0.0146 & & 1.0000 & & & & & & & & & & & \\
\hline ARM-1-7 (5/91) & & & & & & & 0.0000 & 0.0146 & & 1.0000 & & & & & & & & & & & \\
\hline ARM-1-7 (10/91) & & & & & & & 0.0000 & 0.0146 & & 1.0000 & & & & & & & & & & & \\
\hline ARM-1-7 (10/92) & & & & & & & 0.0000 & 0.0146 & & 1.0000 & & & & & & & & & & & \\
\hline ARM-1-7 (4/93) & & & & & & & 0.0000 & 0.0146 & & 1.0000 & & & & & & & & & & & \\
\hline ARM-1-7 (6/93) & & & & & & & 0.0000 & 0.0146 & & 1.0000 & & & & & & & & & & & \\
\hline ARM-1-7 (8/93) & & & & & & & 0.0000 & 0.0146 & & 1.0000 & & & & & & & & & & & \\
\hline ARM-1-7 & & & & & & & 0.0000 & 0.0146 & & 1.0000 & & & & & & & & & & & \\
\hline T-ARM-1 & & & & & & & 0.0000 & 0.0146 & & 1.0000 & & & & & & & & & & & \\
\hline SS-ARM-1 & & & & & & & 0.0000 & 0.0146 & & 1.0000 & & & & & & & & & & & \\
\hline SS-ARM-1 & & & & & & & 0.0000 & 0.0146 & & 1.0000 & & & & & & & & & & & \\
\hline EA-1-7 & & & & & & & 0.0000 & 0.0000 & & 1.0000 & 0.0370 & 0.1130 & 0.0112 & 0.0738 & 0.0145 & 0.0004 & 0.0426 & 0.0172 & 0.1680 & 0.0057 & \\
\hline EA-1-7 & & & & & & & 0.0000 & 0.0000 & & 1.0000 & & & & & & & & & & & \\
\hline EA-2-7 & & & & & & & 0.0000 & 0.0000 & & 1.0000 & & & & & & & & & & & \\
\hline EA-7 & & & & & & & 0.0000 & 0.0000 & & 1.0000 & & & & & & & & & & & \\
\hline T-EA & & & & & & & 0.0000 & 0.0000 & & 1.0000 & & & & & & & & & & & \\
\hline SS-EA-19 & & & & & & & 0.0000 & 0.0000 & & 1.0000 & & & & & & & & & & & \\
\hline SS-EA-15 & & & & & & & 0.0000 & 0.0000 & & 1.0000 & & & & & & & & & & & \\
\hline SS-EA-1-7 & & & & & & & 0.0000 & 0.0000 & & 1.0000 & & & & & & & & & & & \\
\hline SS-EA-2-7 & & & & & & & 0.0000 & 0.0000 & & 1.0000 & & & & & & & & & & & \\
\hline SRS-SEA-A-7 & & & & & & & 0.0000 & 0.0000 & & 1.0000 & & & & & & & & & & & \\
\hline SRS-SEA-B-7 & & & & & & & 0.0000 & 0.0000 & & 1.0000 & & & & & & & & & & & \\
\hline CUASEA-A-7 & & & & & & & 0.0000 & 0.0000 & & 1.0000 & & & & & & & & & & & \\
\hline CUASEA-B-7 & & & & & & & 0.0000 & 0.0000 & & 1.0000 & & & & & & & & & & & \\
\hline 131-TDS-EA-7 & & & & & & & 0.0000 & 0.0000 & & 1.0000 & 0.0577 & 0.1270 & 0.0092 & 0.1430 & & 0.0004 & 0.0483 & 0.0153 & 0.1460 & 0.0144 & \\
\hline 131-TDS-3A-SOPER- & & & & & & & 0.0000 & 0.0000 & & 1.0000 & 0.0551 & 0.1080 & 0.0092 & 0.1140 & & 0.0004 & 0.0421 & 0.0141 & 0.1370 & 0.0153 & \\
\hline BLEND 1-7 & & & & & & & 0.0000 & 0.0000 & & 1.0000 & 0.0416 & 0.0805 & 0.0103 & 0.1091 & & 0.0368 & 0.0444 & 0.0141 & 0.0913 & 0.0089 & \\
\hline BLEND 1-7 (2) & & & & & & & 0.0000 & 0.0000 & & 1.0000 & & & & & & & & & & & \\
\hline BLEND 1.6-7 & & & & & & & 0.0000 & 0.0000 & & 1.0000 & & & & & & & & & & & \\
\hline
\end{tabular}


DWPF PCT Model (Jantzen et al. 1995)

\begin{tabular}{|c|c|c|c|c|c|c|c|c|c|c|c|c|c|c|c|c|c|c|c|c|c|}
\hline Glass ID & $\begin{array}{c}\mathrm{SiO} 2 \\
-\mathrm{a}\end{array}$ & $\begin{array}{c}\mathrm{ZrO} 2 \\
-\mathrm{a}\end{array}$ & $\begin{array}{c}\mathrm{Ag} 2 \mathrm{O} \\
-\mathrm{a}\end{array}$ & $\begin{array}{c}\text { As2O3 } \\
-\mathrm{a}\end{array}$ & $\begin{array}{c}\mathrm{BaO} \\
-\mathrm{a}\end{array}$ & $\begin{array}{c}\mathrm{Bi} 2 \mathrm{O} 3 \\
-\mathrm{a}\end{array}$ & $\begin{array}{l}\mathrm{Br} \\
-\mathrm{a}\end{array}$ & $\begin{array}{c}\mathrm{CdO} \\
-\mathrm{a}\end{array}$ & $\begin{array}{c}\mathrm{Ce} 2 \mathrm{O} 3 \\
-\mathrm{a}\end{array}$ & $\begin{array}{c}\mathrm{CeO} 2 \\
-\mathrm{a}\end{array}$ & $\begin{array}{l}\mathrm{Cl} \\
-\mathrm{a}\end{array}$ & $\begin{array}{c}\mathrm{CoO} \\
-\mathrm{a}\end{array}$ & $\begin{array}{c}\mathrm{Co} 2 \mathrm{O} 3 \\
-\mathrm{a}\end{array}$ & $\begin{array}{c}\mathrm{Cr} 2 \mathrm{O} 3 \\
-\mathrm{a}\end{array}$ & $\begin{array}{c}\mathrm{Cs} 2 \mathrm{O} \\
-\mathrm{a}\end{array}$ & $\begin{array}{c}\mathrm{CuO} \\
-\mathrm{a}\end{array}$ & $\begin{array}{c}\mathrm{Eu} 2 \mathrm{O} 3 \\
-\mathrm{a} \\
\end{array}$ & $\begin{array}{l}F \\
-a\end{array}$ & $\begin{array}{c}\mathrm{Ga} 2 \mathrm{O} 3 \\
-\mathrm{a}\end{array}$ & $\begin{array}{c}\mathrm{Gd} 2 \mathrm{O} 3 \\
-\mathrm{a}\end{array}$ & $\begin{array}{c}\mathrm{HgO} \\
-\mathrm{a}\end{array}$ \\
\hline AH-9-7 & 0.5088 & 0.0001 & & & & & & & & & & & & 0.0001 & 0.0006 & & & & & & \\
\hline AH-10-7 & 0.5420 & 0.0001 & & & & & & & & & & & & 0.0001 & 0.0006 & & & & & & \\
\hline $\mathrm{AH}-11-7$ & 0.5040 & 0.0001 & & & & & & & & & & & & 0.0001 & 0.0006 & & & & & & \\
\hline AH-12-7 & 0.5090 & 0.0001 & & & & & & & & & & & & & 0.0006 & & & & & & \\
\hline AH-13-7 & 0.4900 & 0.0003 & & & & & & & & & & & & 0.0001 & 0.0005 & & & & & & \\
\hline AH-14-7 & 0.4480 & 0.0058 & & & & & & & & & & & & 0.0001 & 0.0006 & & & & & & \\
\hline AH-15-7 & 0.4560 & 0.0001 & & & & & & & & & & & & 0.0001 & 0.0005 & & & & & & \\
\hline AH-16-7 & 0.5020 & 0.0001 & & & & & & & & & & & & 0.0008 & 0.0003 & & & & & & \\
\hline AH-17-7 & 0.5250 & 0.0066 & & & & & & & & & & & & 0.0009 & 0.0006 & & & & & & \\
\hline SFRIT1 & 0.4790 & 0.0011 & & & & & & & & & & & & 0.0009 & & & & & & & \\
\hline \multicolumn{22}{|l|}{ SFRIT2 } \\
\hline \multicolumn{22}{|l|}{ SFRIT3 } \\
\hline $202 \mathrm{P}$ w/o Mn-7 & 0.5120 & & & & & & & & & & & & & 0.0007 & 0.0015 & 0.0063 & & & & & \\
\hline $202 \mathrm{G}$ w/o Mn-7 & 0.5700 & & & & & & & & & & & & & 0.0008 & 0.0005 & 0.0038 & & & & & \\
\hline $200 \mathrm{R}-7$ & 0.4510 & & & & & & & & & & & & & & & & & & & & \\
\hline NBS SRM 623-7 & 0.7300 & & & & 0.0220 & & & & & & & & & & & & & & & & \\
\hline 165 CGW STD-7 & 0.5384 & 0.0050 & & & & & & & & & & & & & & & & & & & \\
\hline ARM-1-7 (4/88) & 0.4650 & 0.0180 & & & 0.0066 & & & & 0.0144 & & & & & & 0.0116 & & & & & & \\
\hline ARM-1-7 (5/89) & & & & & & & & & & & & & & & & & & & & & \\
\hline \multicolumn{22}{|l|}{ ARM-1-7 (7/90) } \\
\hline \multicolumn{22}{|l|}{ ARM-1-7 (12/90) } \\
\hline \multicolumn{22}{|l|}{ ARM-1-7 (5/91) } \\
\hline \multicolumn{22}{|l|}{ ARM-1-7 (10/91) } \\
\hline \multicolumn{22}{|l|}{ ARM-1-7 (10/92) } \\
\hline \multicolumn{22}{|l|}{ ARM-1-7 (4/93) } \\
\hline \multicolumn{22}{|l|}{ ARM-1-7 (6/93) } \\
\hline \multicolumn{22}{|l|}{ ARM-1-7 (8/93) } \\
\hline \multicolumn{22}{|l|}{ ARM-1-7 } \\
\hline \multicolumn{22}{|l|}{ T-ARM-1 } \\
\hline \multicolumn{22}{|l|}{ SS-ARM-1 } \\
\hline SS-ARM-1 & & & & & & & & & & & & & & & & & & & & & \\
\hline EA-1-7 & 0.4873 & 0.0046 & & & & & & & & & & & & & & & & & & & \\
\hline EA-1-7 & & & & & & & & & & & & & & & & & & & & & \\
\hline EA-2-7 & & & & & & & & & & & & & & & & & & & & & \\
\hline EA-7 & & & & & & & & & & & & & & & & & & & & & \\
\hline T-EA & & & & & & & & & & & & & & & & & & & & & \\
\hline SS-EA-19 & & & & & & & & & & & & & & & & & & & & & \\
\hline SS-EA-15 & & & & & & & & & & & & & & & & & & & & & \\
\hline SS-EA-1-7 & & & & & & & & & & & & & & & & & & & & & \\
\hline SS-EA-2-7 & & & & & & & & & & & & & & & & & & & & & \\
\hline SRS-SEA-A-7 & & & & & & & & & & & & & & & & & & & & & \\
\hline SRS-SEA-B-7 & & & & & & & & & & & & & & & & & & & & & \\
\hline CUASEA-A-7 & & & & & & & & & & & & & & & & & & & & & \\
\hline CUASEA-B-7 & & & & & & & & & & & & & & & & & & & & & \\
\hline 131-TDS-EA-7 & 0.3980 & 0.0043 & & & & & & & & & & & & 0.0004 & & & & & & & \\
\hline 131-TDS-3A-SOPER- & 0.4620 & 0.0034 & & & & & & & & & & & & 0.0012 & & & & & & & \\
\hline BLEND 1-7 & 0.5190 & 0.0014 & & & 0.0018 & & & & & & & & & 0.0013 & 0.0008 & 0.0042 & & & & & \\
\hline BLEND 1-7 (2) & & & & & & & & & & & & & & & & & & & & & \\
\hline BLEND 1.6-7 & & & & & & & & & & & & & & & & & & & & & \\
\hline
\end{tabular}


Appendix A. Database - mass fraction

DWPF PCT Model (Jantzen et al. 1995)

\begin{tabular}{|c|c|c|c|c|c|c|c|c|c|c|c|c|c|c|c|c|c|c|c|c|c|}
\hline Glass ID & $\begin{array}{c}I \\
-a\end{array}$ & $\begin{array}{c}\mathrm{La} 2 \mathrm{O} 3 \\
-\mathrm{a}\end{array}$ & $\begin{array}{c}\mathrm{MnO} 2 \\
-\mathrm{a}\end{array}$ & $\begin{array}{c}\mathrm{MnO} \\
-\mathrm{a}\end{array}$ & $\begin{array}{c}\mathrm{MoO} \\
-\mathrm{a}\end{array}$ & $\begin{array}{c}\mathrm{MoO} 3 \\
-\mathrm{a}\end{array}$ & $\begin{array}{c}\mathrm{Nb} 2 \mathrm{O} 5 \\
-\mathrm{a}\end{array}$ & $\begin{array}{c}\mathrm{Nd} 2 \mathrm{O} 3 \\
-\mathrm{a}\end{array}$ & $\begin{array}{c}\mathrm{PbO} \\
-\mathrm{a}\end{array}$ & $\begin{array}{c}\mathrm{PdO} 2 \\
-\mathrm{a}\end{array}$ & $\begin{array}{c}\mathrm{PdO} \\
-\mathrm{a}\end{array}$ & $\begin{array}{c}\mathrm{Pr} 2 \mathrm{O} 3 \\
-\mathrm{a}\end{array}$ & $\begin{array}{c}\operatorname{Pr} 6 \mathrm{O} 11 \\
-\mathrm{a}\end{array}$ & $\begin{array}{c}\mathrm{Rb} 2 \mathrm{O} \\
-\mathrm{a}\end{array}$ & $\begin{array}{c}\mathrm{ReO} \\
-\mathrm{a}\end{array}$ & $\begin{array}{c}\mathrm{ReO} 2 \\
-\mathrm{a}\end{array}$ & $\begin{array}{c}\mathrm{Rh} 2 \mathrm{O} 3 \\
-\mathrm{a}\end{array}$ & $\begin{array}{c}\mathrm{RhO} 2 \\
-\mathrm{a}\end{array}$ & $\begin{array}{c}\mathrm{RuO} 2 \\
-\mathrm{a}\end{array}$ & $\begin{array}{c}\mathrm{Sb} 2 \mathrm{O} 3 \\
-\mathrm{a}\end{array}$ & $\begin{array}{c}\mathrm{Sb} 2 \mathrm{O} 5 \\
-\mathrm{a}\end{array}$ \\
\hline AH-9-7 & & & & 0.0264 & & & & & & & & & & & & & & & & & \\
\hline AH-10-7 & & & & 0.0267 & & & & & & & & & & & & & & & & & \\
\hline AH-11-7 & & & & 0.0263 & & & & & & & & & & & & & & & & & \\
\hline AH-12-7 & & & & 0.0264 & & & & & & & & & & & & & & & & & \\
\hline AH-13-7 & & & & 0.0325 & & & & & & & & & & & & & & & & & \\
\hline AH-14-7 & & & & 0.0334 & & & & & & & & & & & & & & & & & \\
\hline AH-15-7 & & & & 0.0326 & & & & & & & & & & & & & & & & & \\
\hline AH-16-7 & & & & 0.0322 & & & & & & & & & & & & & & & & & \\
\hline AH-17-7 & & & & 0.0271 & & & & & & & & & & & & & & & & & \\
\hline SFRIT1 & & & & 0.0193 & & & & & 0.0010 & & & & & & & & & & & & \\
\hline SFRIT2 & & & & & & & & & & & & & & & & & & & & & \\
\hline SFRIT3 & & & & & & & & & & & & & & & & & & & & & \\
\hline $202 \mathrm{P}$ w/o Mn-7 & & & & & & & & & 0.0020 & & & & & & & & & & & & \\
\hline $202 \mathrm{G} \mathrm{w} / \mathrm{o} \mathrm{Mn}-7$ & & & & & & & & & 0.0016 & & & & & & & & & & & & \\
\hline $200 \mathrm{R}-7$ & & & & 0.0213 & & & & & & & & & & & & & & & & & \\
\hline NBS SRM 623-7 & & & & & & & & & & & & & & & & & & & & & \\
\hline 165 CGW STD-7 & & & & 0.0256 & & & & & & & & & & & & & & & & & \\
\hline ARM-1-7 (4/88) & & & & & & 0.0167 & & 0.0596 & & & & & & & & & & & & & \\
\hline ARM-1-7 (5/89) & & & & & & & & & & & & & & & & & & & & & \\
\hline ARM-1-7 (7/90) & & & & & & & & & & & & & & & & & & & & & \\
\hline ARM-1-7 (12/90) & & & & & & & & & & & & & & & & & & & & & \\
\hline ARM-1-7 (5/91) & & & & & & & & & & & & & & & & & & & & & \\
\hline ARM-1-7 (10/91) & & & & & & & & & & & & & & & & & & & & & \\
\hline ARM-1-7 (10/92) & & & & & & & & & & & & & & & & & & & & & \\
\hline ARM-1-7 (4/93) & & & & & & & & & & & & & & & & & & & & & \\
\hline ARM-1-7 (6/93) & & & & & & & & & & & & & & & & & & & & & \\
\hline ARM-1-7 (8/93) & & & & & & & & & & & & & & & & & & & & & \\
\hline ARM-1-7 & & & & & & & & & & & & & & & & & & & & & \\
\hline T-ARM-1 & & & & & & & & & & & & & & & & & & & & & \\
\hline SS-ARM-1 & & & & & & & & & & & & & & & & & & & & & \\
\hline SS-ARM-1 & & & & & & & & & & & & & & & & & & & & & \\
\hline EA-1-7 & & 0.0042 & & 0.0134 & & & & & & & & & & & & & & & & & \\
\hline EA-1-7 & & & & & & & & & & & & & & & & & & & & & \\
\hline EA-2-7 & & & & & & & & & & & & & & & & & & & & & \\
\hline EA-7 & & & & & & & & & & & & & & & & & & & & & \\
\hline T-EA & & & & & & & & & & & & & & & & & & & & & \\
\hline SS-EA-19 & & & & & & & & & & & & & & & & & & & & & \\
\hline SS-EA-15 & & & & & & & & & & & & & & & & & & & & & \\
\hline SS-EA-1-7 & & & & & & & & & & & & & & & & & & & & & \\
\hline SS-EA-2-7 & & & & & & & & & & & & & & & & & & & & & \\
\hline SRS-SEA-A-7 & & & & & & & & & & & & & & & & & & & & & \\
\hline SRS-SEA-B-7 & & & & & & & & & & & & & & & & & & & & & \\
\hline CUASEA-A-7 & & & & & & & & & & & & & & & & & & & & & \\
\hline CUASEA-B-7 & & & & & & & & & & & & & & & & & & & & & \\
\hline 131-TDS-EA-7 & & 0.0037 & & 0.0317 & & & & & & & & & & & & & & & & & \\
\hline 131-TDS-3A-SOPER-7 & & 0.0035 & & 0.0336 & & & & & & & & & & & & & & & & & \\
\hline BLEND 1-7 & & & & 0.0167 & & 0.0015 & & 0.0022 & & & & & & & & & & & & & \\
\hline BLEND 1-7 (2) & & & & & & & & & & & & & & & & & & & & & \\
\hline BLEND 1.6-7 & & & & & & & & & & & & & & & & & & & & & \\
\hline
\end{tabular}


Appendix A. Database - mass fraction

DWPF PCT Model (Jantzen et al. 1995)

\begin{tabular}{|c|c|c|c|c|c|c|c|c|c|c|c|c|c|c|c|c|c|c|c|c|}
\hline Glass ID & $\begin{array}{c}\mathrm{SeO} 2 \\
-\mathrm{a}\end{array}$ & $\begin{array}{c}\mathrm{Sm} 2 \mathrm{O} 3 \\
-\mathrm{a}\end{array}$ & $\begin{array}{c}\mathrm{SnO} \\
-\mathrm{a}\end{array}$ & $\begin{array}{c}\mathrm{SnO} 2 \\
-\mathrm{a}\end{array}$ & $\begin{array}{c}\mathrm{SO} 3 \\
-\mathrm{a}\end{array}$ & $\begin{array}{c}\mathrm{SrO} \\
-\mathrm{a}\end{array}$ & $\begin{array}{c}\mathrm{Tc} 2 \mathrm{O} 7 \\
-\mathrm{a}\end{array}$ & $\begin{array}{c}\mathrm{TeO} 2 \\
-\mathrm{a}\end{array}$ & $\begin{array}{c}\mathrm{ThO} 2 \\
-\mathrm{a}\end{array}$ & $\begin{array}{c}\mathrm{TiO} 2 \\
-\mathrm{a}\end{array}$ & $\begin{array}{c}\mathrm{T} 12 \mathrm{O} 3 \\
-\mathrm{a}\end{array}$ & $\begin{array}{c}\mathrm{U} 3 \mathrm{O} 8 \\
-\mathrm{a}\end{array}$ & $\begin{array}{c}\mathrm{UO} 2 \\
-\mathrm{a}\end{array}$ & $\begin{array}{c}\text { UO3 } \\
-\mathrm{a}\end{array}$ & $\begin{array}{c}\mathrm{V} 2 \mathrm{O} 5 \\
-\mathrm{a}\end{array}$ & $\begin{array}{c}\text { WO3 } \\
-\mathrm{a}\end{array}$ & $\begin{array}{c}\mathrm{Y} 2 \mathrm{O} 3 \\
-\mathrm{a}\end{array}$ & $\begin{array}{c}\mathrm{ZnO} \\
-\mathrm{a}\end{array}$ & $\begin{array}{c}\text { Others } \\
-\mathrm{a}\end{array}$ & $\begin{array}{c}\text { Sum } \\
-\mathrm{a}\end{array}$ \\
\hline AH-9-7 & & & & & & 0.0005 & & & & 0.0133 & & & & & & & & & & 0.9941 \\
\hline $\mathrm{AH}-10-7$ & & & & & & 0.0005 & & & & 0.0130 & & & & & & & & & & 0.9944 \\
\hline AH-11-7 & & & & & & 0.0004 & & & & 0.0129 & & & & & & & & & & 0.9886 \\
\hline AH-12-7 & & & & & & 0.0005 & & & & 0.0133 & & & & & & & & & & 0.9942 \\
\hline AH-13-7 & & & & & & 0.0005 & & & & 0.0129 & & & & & & & & & & 0.9823 \\
\hline AH-14-7 & & & & & & 0.0007 & & & & 0.0132 & & & & & & & & & & 0.9910 \\
\hline AH-15-7 & & & & & & 0.0006 & & & & 0.0134 & & & & & & & & & & 0.9873 \\
\hline AH-16-7 & & & & & & 0.0007 & & & & 0.0130 & & & & & & & & & & 0.9889 \\
\hline AH-17-7 & & & & & & 0.0003 & & & & 0.0130 & & & & & & & & & & 0.9910 \\
\hline SFRIT1 & & & & & & & & & & 0.0118 & & & & & & & & & & 0.9952 \\
\hline SFRIT2 & & & & & & & & & & & & & & & & & & & & \\
\hline SFRIT3 & & & & & & & & & & & & & & & & & & & & \\
\hline $202 \mathrm{P} \mathrm{w} / \mathrm{o} \mathrm{Mn}-7$ & & & & & & & & & & 0.0124 & & & & & & & & & & 1.0028 \\
\hline $202 \mathrm{G}$ w/o Mn-7 & & & & & & & & & & 0.0070 & & & & & & & & & & 1.0172 \\
\hline $200 \mathrm{R}-7$ & & & & & & & & & & & & 0.0004 & & & & & & & & 0.9669 \\
\hline NBS SRM 623-7 & & & & & & & & & & & & & & & & & & & & 0.9990 \\
\hline 165 CGW STD-7 & & & & & & & & & & & & & & & & & & & & 0.9969 \\
\hline ARM-1-7 (4/88) & & & & & & 0.0045 & & & & 0.0321 & & & & & & & & 0.0146 & & 0.9883 \\
\hline ARM-1-7 (5/89) & & & & & & & & & & & & & & & & & & & & \\
\hline ARM-1-7 (7/90) & & & & & & & & & & & & & & & & & & & & \\
\hline ARM-1-7 (12/90) & & & & & & & & & & & & & & & & & & & & \\
\hline ARM-1-7 (5/91) & & & & & & & & & & & & & & & & & & & & \\
\hline ARM-1-7 (10/91) & & & & & & & & & & & & & & & & & & & & \\
\hline ARM-1-7 (10/92) & & & & & & & & & & & & & & & & & & & & \\
\hline ARM-1-7 (4/93) & & & & & & & & & & & & & & & & & & & & \\
\hline ARM-1-7 (6/93) & & & & & & & & & & & & & & & & & & & & \\
\hline ARM-1-7 (8/93) & & & & & & & & & & & & & & & & & & & & \\
\hline ARM-1-7 & & & & & & & & & & & & & & & & & & & & \\
\hline T-ARM-1 & & & & & & & & & & & & & & & & & & & & \\
\hline SS-ARM-1 & & & & & & & & & & & & & & & & & & & & \\
\hline SS-ARM-1 & & & & & & & & & & & & & & & & & & & & \\
\hline EA-1-7 & & & & & & & & & & 0.0070 & & & & & & & & & & 0.9999 \\
\hline EA-1-7 & & & & & & & & & & & & & & & & & & & & \\
\hline EA-2-7 & & & & & & & & & & & & & & & & & & & & \\
\hline EA-7 & & & & & & & & & & & & & & & & & & & & \\
\hline T-EA & & & & & & & & & & & & & & & & & & & & \\
\hline SS-EA-19 & & & & & & & & & & & & & & & & & & & & \\
\hline SS-EA-15 & & & & & & & & & & & & & & & & & & & & \\
\hline SS-EA-1-7 & & & & & & & & & & & & & & & & & & & & \\
\hline SS-EA-2-7 & & & & & & & & & & & & & & & & & & & & \\
\hline SRS-SEA-A-7 & & & & & & & & & & & & & & & & & & & & \\
\hline SRS-SEA-B-7 & & & & & & & & & & & & & & & & & & & & \\
\hline CUASEA-A-7 & & & & & & & & & & & & & & & & & & & & \\
\hline CUASEA-B-7 & & & & & & & & & & & & & & & & & & & & \\
\hline 131-TDS-EA-7 & & & & & & 0.0001 & & & & 0.0093 & & & & & & & & & & 1.0088 \\
\hline 131-TDS-3A-SOPER- & & & & & & & & & & 0.0074 & & & & & & & & & & 1.0063 \\
\hline BLEND 1-7 & & & & & & & & & & 0.0089 & & & & & & & & & & 0.9948 \\
\hline BLEND 1-7 (2) & & & & & & & & & & & & & & & & & & & & \\
\hline BLEND 1.6-7 & & & & & & & & & & & & & & & & & & & & \\
\hline
\end{tabular}


Appendix A. Database - mass fraction

DWPF PCT Model (Jantzen et al. 1995)

\begin{tabular}{|c|c|c|c|c|c|c|c|c|}
\hline Glass ID & $\begin{array}{l}\mathrm{TM} \\
\left({ }^{\circ} \mathrm{C}\right)\end{array}$ & $\begin{array}{l}\text { Gradient } \\
\text { TL }\left({ }^{\circ} \mathrm{C}\right)\end{array}$ & $\begin{array}{l}\text { Uniform } \\
\text { TL }\left({ }^{\circ} \mathrm{C}\right)\end{array}$ & Primary Phase & $\begin{array}{l}\text { Quenched } \\
\text { Visual/OM }\end{array}$ & $\begin{array}{c}\text { Quenched } \\
\text { SEM/EDS or TEM }\end{array}$ & $\begin{array}{l}\text { Quenched } \\
\text { XRD }\end{array}$ & $\begin{array}{c}\text { Quenched } \\
\text { Homogeneous? }\end{array}$ \\
\hline \multicolumn{9}{|l|}{ AH-9-7 } \\
\hline \multicolumn{9}{|l|}{$\begin{array}{l}\mathrm{AH}-10-7 \\
\mathrm{AH}-11-7\end{array}$} \\
\hline \multicolumn{9}{|l|}{$\mathrm{AH}-11-7$} \\
\hline \multirow{2}{*}{\multicolumn{9}{|c|}{$\begin{array}{l}\mathrm{AH}-12-7 \\
\mathrm{AH}-13-7\end{array}$}} \\
\hline & & & & & & & & \\
\hline \multicolumn{9}{|l|}{$\mathrm{AH}-14-7$} \\
\hline \multicolumn{9}{|l|}{$\mathrm{AH}-15-7$} \\
\hline \multicolumn{9}{|l|}{$\mathrm{AH}-16-7$} \\
\hline \multicolumn{9}{|l|}{$\mathrm{AH}-17-7$} \\
\hline \multirow{2}{*}{\multicolumn{9}{|c|}{$\begin{array}{l}\text { SFRIT1 } \\
\text { SFRIT2 }\end{array}$}} \\
\hline SFRIT2 & & & & & & & & \\
\hline \multicolumn{9}{|l|}{ SFRIT3 } \\
\hline \multicolumn{9}{|l|}{$202 \mathrm{P}$ w/o Mn-7 } \\
\hline $202 \mathrm{G}$ w/o Mn-7 & & & & & & & & \\
\hline \multicolumn{9}{|l|}{$200 \mathrm{R}-7$} \\
\hline \multirow{2}{*}{\multicolumn{9}{|c|}{$\begin{array}{l}\text { NBS SRM 623-7 } \\
165 \text { CGW STD-7 }\end{array}$}} \\
\hline \multicolumn{8}{|l|}{$\begin{array}{l}165 \text { CGW STD-7 } \\
\text { ARM-1-7 }(4 / 88)\end{array}$} & \\
\hline \multicolumn{9}{|l|}{ ARM-1-7 (4/88) } \\
\hline \multicolumn{9}{|l|}{ ARM-1-7 (5/89) } \\
\hline \multicolumn{9}{|l|}{ ARM-1-7 (7/90) } \\
\hline \multirow{2}{*}{\multicolumn{9}{|c|}{$\begin{array}{cc}\text { ARM-1-7 } & (12 / 90) \\
\text { ARM-1-7 } & (5 / 91)\end{array}$}} \\
\hline ARM-1-7 (5/91) & & & & & & & & \\
\hline \multicolumn{9}{|l|}{ ARM-1-7 (10/91) } \\
\hline ARM-1-7 (10/92) & & & & & & & & \\
\hline ARM-1-7 (4/93) & & & & & & & & \\
\hline ARM-1-7 (6/93) & & & & & & & & \\
\hline ARM-1-7 (8/93) & & & & & & & & \\
\hline ARM-1-7 & & & & & & & & \\
\hline T-ARM-1 & & & & & & & & \\
\hline SS-ARM-1 & & & & & & & & \\
\hline SS-ARM-1 & & & & & & & & \\
\hline EA-1-7 & & & & & & & & \\
\hline EA-1-7 & & & & & & & & \\
\hline EA-2-7 & & & & & & & & \\
\hline EA-7 & & & & & & & & \\
\hline T-EA & & & & & & & & \\
\hline SS-EA-19 & & & & & & & & \\
\hline SS-EA-15 & & & & & & & & \\
\hline SS-EA-1-7 & & & & & & & & \\
\hline SS-EA-2-7 & & & & & & & & \\
\hline SRS-SEA-A-7 & & & & & & & & \\
\hline SRS-SEA-B-7 & & & & & & & & \\
\hline CUASEA-A-7 & & & & & & & & \\
\hline CUASEA-B-7 & & & & & & & & \\
\hline 131-TDS-EA-7 & & & & & & & & \\
\hline 131-TDS-3A-SOPEI & & & & & & & & \\
\hline BLEND 1-7 & & & & & & & & \\
\hline BLEND 1-7 (2) & & & & & & & & \\
\hline BLEND 1.6-7 & & & & & & & & \\
\hline
\end{tabular}


Appendix A. Database - mass fraction

\section{DWPF PCT Model (Jantzen et al. 1995)}

\begin{tabular}{|c|c|c|c|c|c|}
\hline Glass ID & $\begin{array}{c}\mathrm{CCC} \\
\text { Visual } / \mathrm{OM} \\
\end{array}$ & $\begin{array}{c}\text { CCC } \\
\text { SEM/EDS or TEM }\end{array}$ & $\begin{array}{l}\text { CCC } \\
\text { XRD }\end{array}$ & $\begin{array}{c}\text { Heat Treated } \\
\text { Visual/OM }\end{array}$ & $\begin{array}{c}\text { Heat Treated } \\
\text { SEM/EDS or TEM }\end{array}$ \\
\hline \multicolumn{6}{|l|}{ AH-9-7 } \\
\hline \multicolumn{6}{|l|}{ AH-10-7 } \\
\hline \multicolumn{6}{|l|}{ AH-11-7 } \\
\hline \multicolumn{6}{|l|}{ AH-12-7 } \\
\hline \multicolumn{6}{|l|}{ AH-13-7 } \\
\hline \multicolumn{6}{|l|}{ AH-14-7 } \\
\hline \multicolumn{6}{|l|}{ AH-15-7 } \\
\hline \multicolumn{6}{|l|}{ AH-16-7 } \\
\hline \multicolumn{6}{|l|}{ AH-17-7 } \\
\hline \multicolumn{6}{|l|}{ SFRIT1 } \\
\hline \multicolumn{6}{|l|}{ SFRIT2 } \\
\hline \multicolumn{6}{|l|}{ SFRIT3 } \\
\hline \multicolumn{6}{|l|}{$202 \mathrm{P}$ w/o Mn-7 } \\
\hline \multicolumn{6}{|l|}{$202 \mathrm{G}$ w/o Mn-7 } \\
\hline \multicolumn{6}{|l|}{ 200R-7 } \\
\hline \multicolumn{6}{|l|}{ NBS SRM 623-7 } \\
\hline \multicolumn{6}{|l|}{165 CGW STD-7 } \\
\hline \multicolumn{6}{|l|}{ ARM-1-7 (4/88) } \\
\hline \multicolumn{6}{|l|}{ ARM-1-7 (5/89) } \\
\hline \multicolumn{6}{|l|}{ ARM-1-7 (7/90) } \\
\hline \multicolumn{6}{|l|}{ ARM-1-7 (12/90) } \\
\hline ARM-1-7 (5/91) & & & & & \\
\hline ARM-1-7 (10/91) & & & & & \\
\hline ARM-1-7 (10/92) & & & & & \\
\hline ARM-1-7 (4/93) & & & & & \\
\hline ARM-1-7 (6/93) & & & & & \\
\hline ARM-1-7 (8/93) & & & & & \\
\hline ARM-1-7 & & & & & \\
\hline T-ARM-1 & & & & & \\
\hline SS-ARM-1 & & & & & \\
\hline SS-ARM-1 & & & & & \\
\hline EA-1-7 & & & & & \\
\hline EA-1-7 & & & & & \\
\hline EA-2-7 & & & & & \\
\hline EA-7 & & & & & \\
\hline T-EA & & & & & \\
\hline SS-EA-19 & & & & & \\
\hline SS-EA-15 & & & & & \\
\hline SS-EA-1-7 & & & & & \\
\hline SS-EA-2-7 & & & & & \\
\hline SRS-SEA-A-7 & & & & & \\
\hline SRS-SEA-B-7 & & & & & \\
\hline CUASEA-A-7 & & & & & \\
\hline CUASEA-B-7 & & & & & \\
\hline 131-TDS-EA-7 & & & & & \\
\hline 131-TDS-3A-SOPE & & & & & \\
\hline BLEND 1-7 & & & & & \\
\hline BLEND 1-7 (2) & & & & & \\
\hline BLEND 1.6-7 & & & & & \\
\hline
\end{tabular}


Appendix A. Database - mass fraction

\section{DWPF PCT Model (Jantzen et al. 1995)}

\begin{tabular}{|c|c|c|c|c|c|c|c|c|c|c|c|c|c|c|c|}
\hline Glass ID & $\begin{array}{c}\text { Heat Treated } \\
\text { XRD }\end{array}$ & $\begin{array}{l}\text { Density } \\
\left(\mathrm{g} / \mathrm{cm}^{3}\right)\end{array}$ & $\begin{array}{c}\text { Fulc Visc } \\
\text { A }\end{array}$ & $\begin{array}{c}\text { Fulc Visc } \\
\text { B }\end{array}$ & $\begin{array}{c}\text { Fulc Visc } \\
\text { To }\end{array}$ & $\begin{array}{c}\mathrm{FV} 1150^{\circ} \mathrm{C} \\
(\mathrm{Pa} \cdot \mathrm{s})\end{array}$ & $\begin{array}{c}\text { Arrh Visc } \\
\text { A }\end{array}$ & $\begin{array}{c}\text { Arrh Visc } \\
\text { B }\end{array}$ & $1150^{\circ} \mathrm{C}$ & $\begin{array}{l}\mathrm{T}\left({ }^{\circ} \mathrm{C}\right) \text { at } \\
2 \mathrm{~Pa} \cdot \mathrm{s}\end{array}$ & $\begin{array}{c}\mathrm{T}\left({ }^{\circ} \mathrm{C}\right) \text { at } \\
5 \mathrm{~Pa} \cdot \mathrm{s}\end{array}$ & $\begin{array}{l}\mathrm{T}\left({ }^{\circ} \mathrm{C}\right) \text { at } \\
10 \mathrm{~Pa} \cdot \mathrm{s}\end{array}$ & $\begin{array}{l}\mathrm{T} 1 \\
\left({ }^{\circ} \mathrm{C}\right)\end{array}$ & $\begin{array}{c}\mathrm{V} 1 \\
(\mathrm{~Pa} \cdot \mathrm{s}) \\
\end{array}$ & $\begin{array}{l}\mathrm{T} 2 \\
\left({ }^{\circ} \mathrm{C}\right)\end{array}$ \\
\hline \multicolumn{16}{|c|}{ (2) Wach } \\
\hline \multicolumn{16}{|l|}{$\mathrm{AH}-10-7$} \\
\hline \multicolumn{16}{|l|}{$\mathrm{AH}-11-7$} \\
\hline \multirow{2}{*}{\multicolumn{16}{|c|}{$\begin{array}{l}\mathrm{AH}-12-7 \\
\mathrm{AH}-13-7\end{array}$}} \\
\hline & & & & & & & & & & & & & & & \\
\hline \multicolumn{16}{|l|}{ AH-14-7 } \\
\hline \multicolumn{16}{|l|}{$\mathrm{AH}-15-7$} \\
\hline \multicolumn{16}{|l|}{$\mathrm{AH}-16-7$} \\
\hline \multirow{2}{*}{\multicolumn{16}{|c|}{$\begin{array}{l}\text { AH-17-7 } \\
\text { SERIT1 }\end{array}$}} \\
\hline \multicolumn{13}{|l|}{$\begin{array}{l}\text { SFRIT1 } \\
\text { SFRIT2 }\end{array}$} & & & \\
\hline \multirow{2}{*}{\multicolumn{16}{|c|}{$\begin{array}{l}\text { SFRIT2 } \\
\text { SFRIT3 }\end{array}$}} \\
\hline & & & & & & & & & & & & & & & \\
\hline \multicolumn{16}{|l|}{ 202P w/o Mn-7 } \\
\hline \multicolumn{16}{|l|}{$202 \mathrm{G} \mathrm{w} / \mathrm{o} \mathrm{Mn-7}$} \\
\hline \multirow{2}{*}{\multicolumn{16}{|c|}{$\begin{array}{c}200 R-7 \\
\text { NBS SRM 623-7 }\end{array}$}} \\
\hline \multirow{2}{*}{\multicolumn{16}{|c|}{ NBS SRM 623-7 }} \\
\hline \multicolumn{15}{|l|}{165 CGW STD-7 } & \\
\hline \multicolumn{16}{|l|}{ ARM-1-7 (4/88) } \\
\hline ARM-1-7 (5/89) & & & & & & & & & & & & & & & \\
\hline ARM-1-7 (7/90) & & & & & & & & & & & & & & & \\
\hline ARM-1-7 (12/90) & & & & & & & & & & & & & & & \\
\hline ARM-1-7 (5/91) & & & & & & & & & & & & & & & \\
\hline ARM-1-7 (10/91) & & & & & & & & & & & & & & & \\
\hline ARM-1-7 (10/92) & & & & & & & & & & & & & & & \\
\hline ARM-1-7 (4/93) & & & & & & & & & & & & & & & \\
\hline ARM-1-7 (6/93) & & & & & & & & & & & & & & & \\
\hline ARM-1-7 (8/93) & & & & & & & & & & & & & & & \\
\hline ARM-1-7 & & & & & & & & & & & & & & & \\
\hline T-ARM-1 & & & & & & & & & & & & & & & \\
\hline SS-ARM-1 & & & & & & & & & & & & & & & \\
\hline SS-ARM-1 & & & & & & & & & & & & & & & \\
\hline EA-1-7 & & & & & & & & & & & & & & & \\
\hline EA-1-7 & & & & & & & & & & & & & & & \\
\hline EA-2-7 & & & & & & & & & & & & & & & \\
\hline EA-7 & & & & & & & & & & & & & & & \\
\hline T-EA & & & & & & & & & & & & & & & \\
\hline SS-EA-19 & & & & & & & & & & & & & & & \\
\hline SS-EA-15 & & & & & & & & & & & & & & & \\
\hline SS-EA-1-7 & & & & & & & & & & & & & & & \\
\hline SS-EA-2-7 & & & & & & & & & & & & & & & \\
\hline SRS-SEA-A-7 & & & & & & & & & & & & & & & \\
\hline SRS-SEA-B-7 & & & & & & & & & & & & & & & \\
\hline CUASEA-A-7 & & & & & & & & & & & & & & & \\
\hline CUASEA-B-7 & & & & & & & & & & & & & & & \\
\hline 131-TDS-EA-7 & & & & & & & & & & & & & & & \\
\hline 131-TDS-3A-SOPER & & & & & & & & & & & & & & & \\
\hline BLEND 1-7 & & & & & & & & & & & & & & & \\
\hline BLEND 1-7 (2) & & & & & & & & & & & & & & & \\
\hline BLEND 1.6-7 & & & & & & & & & & & & & & & \\
\hline
\end{tabular}


Appendix A. Database - mass fraction

DWPF PCT Model (Jantzen et al. 1995)

\begin{tabular}{|c|c|c|c|c|c|c|c|c|c|c|c|c|c|c|c|c|c|c|c|c|c|}
\hline Glass ID & $\begin{array}{c}\mathrm{V} 2 \\
(\mathrm{~Pa} \cdot \mathrm{s})\end{array}$ & $\begin{array}{c}\mathrm{T} 3 \\
\left({ }^{\circ} \mathrm{C}\right)\end{array}$ & $\begin{array}{c}\mathrm{V} 3 \\
(\mathrm{~Pa} \cdot \mathrm{s})\end{array}$ & $\begin{array}{c}\mathrm{T} 4 \\
\left({ }^{\circ} \mathrm{C}\right)\end{array}$ & $\begin{array}{c}\mathrm{V} 4 \\
(\mathrm{~Pa} \cdot \mathrm{s})\end{array}$ & $\begin{array}{c}\mathrm{T} 5 \\
\left({ }^{\circ} \mathrm{C}\right)\end{array}$ & $\begin{array}{c}\text { V5 } \\
(\mathrm{Pa} \cdot \mathrm{s})\end{array}$ & $\begin{array}{l}\mathrm{T} 6 \\
\left({ }^{\circ} \mathrm{C}\right)\end{array}$ & $\begin{array}{c}\mathrm{V} 6 \\
(\mathrm{~Pa} \cdot \mathrm{s})\end{array}$ & $\begin{array}{l}\mathrm{T} 7 \\
\left({ }^{\circ} \mathrm{C}\right)\end{array}$ & $\begin{array}{c}\mathrm{V} 7 \\
(\mathrm{~Pa} \cdot \mathrm{s})\end{array}$ & $\begin{array}{l}\mathrm{T} 8 \\
\left({ }^{\circ} \mathrm{C}\right)\end{array}$ & $\begin{array}{c}\mathrm{V} 8 \\
(\mathrm{~Pa} \cdot \mathrm{s})\end{array}$ & $\begin{array}{l}\mathrm{T} 9 \\
\left({ }^{\circ} \mathrm{C}\right)\end{array}$ & $\begin{array}{c}\mathrm{V} 9 \\
(\mathrm{~Pa} \cdot \mathrm{s})\end{array}$ & $\begin{array}{l}\mathrm{T} 10 \\
\left({ }^{\circ} \mathrm{C}\right)\end{array}$ & $\begin{array}{c}\mathrm{V} 10 \\
(\mathrm{~Pa} \cdot \mathrm{s})\end{array}$ & $\begin{array}{l}\mathrm{T} 11 \\
\left({ }^{\circ} \mathrm{C}\right)\end{array}$ & $\begin{array}{c}\text { V11 } \\
(\mathrm{Pa} \cdot \mathrm{s})\end{array}$ & $\begin{array}{l}\mathrm{T} 12 \\
\left({ }^{\circ} \mathrm{C}\right)\end{array}$ & $\begin{array}{c}\mathrm{V} 12 \\
(\mathrm{~Pa} \cdot \mathrm{s})\end{array}$ \\
\hline AH-9-7 & & & & & & & & & & & & & & & & & & & & & \\
\hline AH-10-7 & & & & & & & & & & & & & & & & & & & & & \\
\hline AH-11-7 & & & & & & & & & & & & & & & & & & & & & \\
\hline AH-12-7 & & & & & & & & & & & & & & & & & & & & & \\
\hline AH-13-7 & & & & & & & & & & & & & & & & & & & & & \\
\hline AH-14-7 & & & & & & & & & & & & & & & & & & & & & \\
\hline AH-15-7 & & & & & & & & & & & & & & & & & & & & & \\
\hline AH-16-7 & & & & & & & & & & & & & & & & & & & & & \\
\hline AH-17-7 & & & & & & & & & & & & & & & & & & & & & \\
\hline SFRIT1 & & & & & & & & & & & & & & & & & & & & & \\
\hline SFRIT2 & & & & & & & & & & & & & & & & & & & & & \\
\hline SFRIT3 & & & & & & & & & & & & & & & & & & & & & \\
\hline $202 \mathrm{P}$ w/o Mn-7 & & & & & & & & & & & & & & & & & & & & & \\
\hline $202 \mathrm{G}$ w/o Mn-7 & & & & & & & & & & & & & & & & & & & & & \\
\hline $200 \mathrm{R}-7$ & & & & & & & & & & & & & & & & & & & & & \\
\hline NBS SRM 623-7 & & & & & & & & & & & & & & & & & & & & & \\
\hline 165 CGW STD-7 & & & & & & & & & & & & & & & & & & & & & \\
\hline ARM-1-7 (4/88) & & & & & & & & & & & & & & & & & & & & & \\
\hline ARM-1-7 (5/89) & & & & & & & & & & & & & & & & & & & & & \\
\hline ARM-1-7 (7/90) & & & & & & & & & & & & & & & & & & & & & \\
\hline ARM-1-7 (12/90) & & & & & & & & & & & & & & & & & & & & & \\
\hline ARM-1-7 (5/91) & & & & & & & & & & & & & & & & & & & & & \\
\hline ARM-1-7 (10/91) & & & & & & & & & & & & & & & & & & & & & \\
\hline ARM-1-7 (10/92) & & & & & & & & & & & & & & & & & & & & & \\
\hline ARM-1-7 (4/93) & & & & & & & & & & & & & & & & & & & & & \\
\hline ARM-1-7 (6/93) & & & & & & & & & & & & & & & & & & & & & \\
\hline ARM-1-7 (8/93) & & & & & & & & & & & & & & & & & & & & & \\
\hline ARM-1-7 & & & & & & & & & & & & & & & & & & & & & \\
\hline T-ARM-1 & & & & & & & & & & & & & & & & & & & & & \\
\hline SS-ARM-1 & & & & & & & & & & & & & & & & & & & & & \\
\hline SS-ARM-1 & & & & & & & & & & & & & & & & & & & & & \\
\hline EA-1-7 & & & & & & & & & & & & & & & & & & & & & \\
\hline EA-1-7 & & & & & & & & & & & & & & & & & & & & & \\
\hline EA-2-7 & & & & & & & & & & & & & & & & & & & & & \\
\hline EA-7 & & & & & & & & & & & & & & & & & & & & & \\
\hline T-EA & & & & & & & & & & & & & & & & & & & & & \\
\hline SS-EA-19 & & & & & & & & & & & & & & & & & & & & & \\
\hline SS-EA-15 & & & & & & & & & & & & & & & & & & & & & \\
\hline SS-EA-1-7 & & & & & & & & & & & & & & & & & & & & & \\
\hline SS-EA-2-7 & & & & & & & & & & & & & & & & & & & & & \\
\hline SRS-SEA-A-7 & & & & & & & & & & & & & & & & & & & & & \\
\hline SRS-SEA-B-7 & & & & & & & & & & & & & & & & & & & & & \\
\hline CUASEA-A-7 & & & & & & & & & & & & & & & & & & & & & \\
\hline CUASEA-B-7 & & & & & & & & & & & & & & & & & & & & & \\
\hline 131-TDS-EA-7 & & & & & & & & & & & & & & & & & & & & & \\
\hline 131-TDS-3A-SOPE & & & & & & & & & & & & & & & & & & & & & \\
\hline BLEND 1-7 & & & & & & & & & & & & & & & & & & & & & \\
\hline BLEND 1-7 (2) & & & & & & & & & & & & & & & & & & & & & \\
\hline BLEND 1.6-7 & & & & & & & & & & & & & & & & & & & & & \\
\hline
\end{tabular}


Appendix A. Database - mass fraction

DWPF PCT Model (Jantzen et al. 1995)

\begin{tabular}{|c|c|c|c|c|c|c|c|c|c|c|c|c|c|c|c|c|}
\hline Glass ID & $\begin{array}{l}\mathrm{T} 13 \\
\left({ }^{\circ} \mathrm{C}\right) \\
\end{array}$ & $\begin{array}{c}\mathrm{V} 13 \\
(\mathrm{~Pa} \cdot \mathrm{s})\end{array}$ & $\begin{array}{l}\mathrm{T} 14 \\
\left({ }^{\circ} \mathrm{C}\right) \\
\end{array}$ & $\begin{array}{l}\mathrm{V} 14 \\
(\mathrm{~Pa} \cdot \mathrm{s}) \\
\end{array}$ & $\begin{array}{c}\text { Q PCT } \\
\mathrm{B}\left(\mathrm{g} / \mathrm{m}^{2}\right)\end{array}$ & $\begin{array}{c}\text { Q PCT } \\
\mathrm{Li}\left(\mathrm{g} / \mathrm{m}^{2}\right)\end{array}$ & $\begin{array}{c}\text { Q PCT } \\
\mathrm{Na}\left(\mathrm{g} / \mathrm{m}^{2}\right)\end{array}$ & $\begin{array}{c}\text { Q PCT } \\
\mathrm{Si}\left(\mathrm{g} / \mathrm{m}^{2}\right) \\
\end{array}$ & $\begin{array}{c}\text { Q PCT } \\
\mathrm{pH}\end{array}$ & $\begin{array}{c}\text { CCC PCT } \\
\mathrm{B}\left(\mathrm{g} / \mathrm{m}^{2}\right) \\
\end{array}$ & $\begin{array}{l}\text { CCC PCT } \\
\mathrm{Li}\left(\mathrm{g} / \mathrm{m}^{2}\right) \\
\end{array}$ & $\begin{array}{l}\text { CCC PCT } \\
\mathrm{Na}\left(\mathrm{g} / \mathrm{m}^{2}\right)\end{array}$ & $\begin{array}{c}\text { CCC PCT } \\
\mathrm{Si}\left(\mathrm{g} / \mathrm{m}^{2}\right)\end{array}$ & $\begin{array}{c}\text { CCC PCT } \\
\mathrm{pH} \\
\end{array}$ & $\begin{array}{l}\text { Q PCT B at } \\
20^{\circ} \mathrm{C}\left(\mathrm{g} / \mathrm{m}^{2}\right)\end{array}$ & $\begin{array}{l}\text { Q PCT Li at } \\
20^{\circ} \mathrm{C}\left(\mathrm{g} / \mathrm{m}^{2}\right)\end{array}$ \\
\hline AH-9-7 & & & & & 0.22 & 0.255 & 0.23 & 0.140 & 10.19 & & & & & & & \\
\hline AH-10-7 & & & & & 0.215 & 0.26 & 0.215 & 0.155 & 10.06 & & & & & & & \\
\hline AH-11-7 & & & & & 0.235 & 0.285 & 0.225 & 0.135 & 9.8 & & & & & & & \\
\hline AH-12-7 & & & & & 0.425 & 0.305 & 0.365 & 0.095 & 10.12 & & & & & & & \\
\hline AH-13-7 & & & & & 0.215 & 0.26 & 0.235 & 0.145 & 10.33 & & & & & & & \\
\hline AH-14-7 & & & & & 0.26 & 0.29 & 0.305 & 0.155 & 10.66 & & & & & & & \\
\hline AH-15-7 & & & & & 0.215 & 0.24 & 0.225 & 0.120 & 10.18 & & & & & & & \\
\hline AH-16-7 & & & & & 0.21 & 0.24 & 0.22 & 0.140 & 10.12 & & & & & & & \\
\hline AH-17-7 & & & & & 0.18 & 0.225 & 0.185 & 0.145 & 10.09 & & & & & & & \\
\hline SFRIT1 & & & & & 0.375 & 0.345 & 0.355 & 0.095 & 9.74 & & & & & & & \\
\hline SFRIT2 & & & & & 0.365 & 0.350 & 0.350 & 0.175 & 10.09 & & & & & & & \\
\hline SFRIT3 & & & & & 0.380 & 0.410 & 0.435 & 0.215 & 10.21 & & & & & & & \\
\hline 202P w/o Mn-7 & & & & & 0.320 & 0.255 & 0.370 & 0.195 & 9.95 & & & & & & & \\
\hline $202 \mathrm{G}$ w/o Mn-7 & & & & & 0.195 & 0.195 & 0.260 & 0.175 & 9.79 & & & & & & & \\
\hline $200 \mathrm{R}-7$ & & & & & 0.575 & 0.485 & 0.505 & 0.230 & 10.54 & & & & & & & \\
\hline NBS SRM 623-7 & & & & & 0.055 & 0.005 & 0.075 & 0.045 & 8.48 & & & & & & & \\
\hline 165 CGW STD-7 & & & & & 0.420 & 0.365 & 0.410 & 0.265 & 10.32 & & & & & & & \\
\hline ARM-1-7 (4/88) & & & & & 0.290 & 0.375 & 0.345 & 0.175 & 10.13 & & & & & & & \\
\hline ARM-1-7 (5/89) & & & & & 0.295 & 0.280 & 0.235 & 0.160 & 9.64 & & & & & & & \\
\hline ARM-1-7 (7/90) & & & & & 0.240 & 0.285 & 0.255 & 0.140 & 10.22 & & & & & & & \\
\hline ARM-1-7 (12/90) & & & & & 0.250 & 0.295 & 0.260 & 0.140 & 10.52 & & & & & & & \\
\hline ARM-1-7 (5/91) & & & & & 0.270 & 0.315 & 0.270 & 0.145 & 10.42 & & & & & & & \\
\hline ARM-1-7 (10/91) & & & & & 0.255 & 0.300 & 0.270 & 0.140 & 10.33 & & & & & & & \\
\hline ARM-1-7 (10/92) & & & & & 0.220 & 0.250 & 0.225 & 0.130 & 10.16 & & & & & & & \\
\hline ARM-1-7 (4/93) & & & & & 0.240 & 0.290 & 0.250 & 0.135 & 9.9 & & & & & & & \\
\hline ARM-1-7 (6/93) & & & & & 0.235 & 0.260 & 0.240 & 0.130 & 9.89 & & & & & & & \\
\hline ARM-1-7 (8/93) & & & & & 0.205 & 0.235 & 0.215 & 0.120 & 10.24 & & & & & & & \\
\hline ARM-1-7 & & & & & 0.255 & 0.300 & 0.255 & 0.135 & 9.38 & & & & & & & \\
\hline T-ARM-1 & & & & & 0.245 & 0.300 & 0.265 & 0.135 & 10.32 & & & & & & & \\
\hline SS-ARM-1 & & & & & 0.265 & 0.315 & 0.275 & 0.145 & 10.32 & & & & & & & \\
\hline SS-ARM-1 & & & & & 0.270 & 0.320 & 0.280 & 0.145 & 10.36 & & & & & & & \\
\hline EA-1-7 & & & & & 7.000 & 3.925 & 5.760 & 2.330 & 11.8 & & & & & & & \\
\hline EA-1-7 & & & & & 8.395 & 5.345 & 7.245 & 2.330 & 11.8 & & & & & & & \\
\hline EA-2-7 & & & & & 7.605 & 4.715 & 6.39 & 2.005 & 11.87 & & & & & & & \\
\hline EA-7 & & & & & 8.86 & 4.945 & 6.945 & 2.040 & 12.01 & & & & & & & \\
\hline T-EA & & & & & 7.375 & 4.26 & 5.91 & 1.710 & 11.89 & & & & & & & \\
\hline SS-EA-19 & & & & & 8.205 & 4.56 & 6.545 & 1.750 & 11.92 & & & & & & & \\
\hline SS-EA-15 & & & & & 8.395 & 4.87 & 6.8 & 1.855 & 11.95 & & & & & & & \\
\hline SS-EA-1-7 & & & & & 9.25 & 5.14 & 7.165 & 2.090 & 11.78 & & & & & & & \\
\hline SS-EA-2-7 & & & & & 8.880 & 4.885 & 6.86 & 2.005 & 11.75 & & & & & & & \\
\hline SRS-SEA-A-7 & & & & & 8.225 & 4.67 & 6.425 & 1.895 & 11.78 & & & & & & & \\
\hline SRS-SEA-B-7 & & & & & 8.495 & 4.56 & 6.755 & 1.960 & 11.78 & & & & & & & \\
\hline CUASEA-A-7 & & & & & 8.425 & 4.835 & 6.475 & 1.935 & 11.77 & & & & & & & \\
\hline CUASEA-B-7 & & & & & 8.23 & 4.605 & 6.71 & 1.965 & 11.77 & & & & & & & \\
\hline 131-TDS-EA-7 & & & & & 2.215 & 1.925 & 2.14 & 1.190 & 10.59 & & & & & & & \\
\hline 131-TDS-3A-SOPER & & & & & 1.405 & 1.225 & 1.15 & 0.445 & 10.57 & & & & & & & \\
\hline BLEND 1-7 & & & & & 0.36 & 0.405 & 0.39 & 0.205 & 10.22 & & & & & & & \\
\hline BLEND 1-7 (2) & & & & & 0.39 & 0.4 & 0.395 & 0.210 & 10.45 & & & & & & & \\
\hline BLEND 1.6-7 & & & & & 0.36 & 0.38 & 0.375 & 0.210 & 10.17 & & & & & & & \\
\hline
\end{tabular}


Appendix A. Database - mass fraction

DWPF PCT Model (Jantzen et al. 1995)

\begin{tabular}{|c|c|c|c|c|c|c|c|c|c|c|c|c|}
\hline Glass ID & $\begin{array}{l}\text { Q PCT Na at } \\
20^{\circ} \mathrm{C}\left(\mathrm{g} / \mathrm{m}^{2}\right)\end{array}$ & $\begin{array}{l}\text { Q PCT Si at } \\
20^{\circ} \mathrm{C}\left(\mathrm{g} / \mathrm{m}^{2}\right)\end{array}$ & $\begin{array}{c}\mathrm{QpH} \\
\text { at } 20^{\circ} \mathrm{C}\end{array}$ & $\begin{array}{c}\text { TCLP Ag } \\
\text { (ppm) }\end{array}$ & $\begin{array}{c}\text { TCLP As } \\
(\mathrm{ppm})\end{array}$ & $\begin{array}{c}\text { TCLP Ba } \\
(\mathrm{ppm})\end{array}$ & $\begin{array}{c}\text { TCLP Cd } \\
(\mathrm{ppm})\end{array}$ & $\begin{array}{c}\text { TCLP Cr } \\
(\mathrm{ppm})\end{array}$ & $\begin{array}{c}\text { TCLP Ni } \\
(\mathrm{ppm})\end{array}$ & $\begin{array}{c}\text { TCLP Pb } \\
\text { (ppm) }\end{array}$ & $\begin{array}{c}\text { TCLP Se } \\
(\mathrm{ppm})\end{array}$ & $\begin{array}{c}\text { TCLP Zn } \\
\text { (ppm) }\end{array}$ \\
\hline \multicolumn{13}{|c|}{ 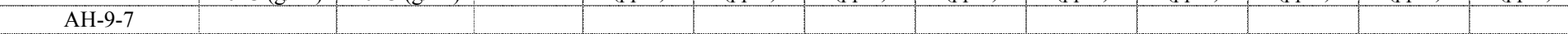 } \\
\hline \multicolumn{13}{|l|}{$\mathrm{AH}-10-7$} \\
\hline \multicolumn{13}{|l|}{$\begin{array}{l}\mathrm{AH}-11-7 \\
\mathrm{AH}-12-7\end{array}$} \\
\hline \multicolumn{13}{|l|}{$\mathrm{AH}-12-7$} \\
\hline \multicolumn{13}{|l|}{$\mathrm{AH}-13-7$} \\
\hline \multicolumn{13}{|l|}{$\mathrm{AH}-14-7$} \\
\hline \multicolumn{13}{|l|}{$\mathrm{AH}-15-7$} \\
\hline \multicolumn{13}{|l|}{$\mathrm{AH}-16-7$} \\
\hline \multicolumn{13}{|l|}{$\begin{array}{l}\text { AH-17-7 } \\
\text { SERIT1 }\end{array}$} \\
\hline \multicolumn{13}{|l|}{$\begin{array}{l}\text { SFRIT1 } \\
\text { SFRIT2 }\end{array}$} \\
\hline \multirow{2}{*}{\multicolumn{13}{|c|}{$\begin{array}{l}\text { SFRIT2 } \\
\text { SFRIT3 }\end{array}$}} \\
\hline & & & & & & & & & & & & \\
\hline \multicolumn{13}{|l|}{$202 \mathrm{P}$ w/o Mn-7 } \\
\hline \multicolumn{13}{|l|}{$202 \mathrm{G} \mathrm{w} / \mathrm{O} \mathrm{Mn-7}$} \\
\hline \multicolumn{13}{|l|}{$200 \mathrm{R}-7$} \\
\hline \multicolumn{13}{|l|}{ NBS SRM 623-7 } \\
\hline \multicolumn{13}{|l|}{165 CGW STD-7 } \\
\hline \multicolumn{13}{|l|}{ ARM-1-7 (4/88) } \\
\hline \multicolumn{13}{|l|}{ ARM-1-7 (5/89) } \\
\hline ARM-1-7 (7/90) & & & & & & & & & & & & \\
\hline ARM-1-7 (12/90) & & & & & & & & & & & & \\
\hline ARM-1-7 (5/91) & & & & & & & & & & & & \\
\hline ARM-1-7 (10/91) & & & & & & & & & & & & \\
\hline ARM-1-7 (10/92) & & & & & & & & & & & & \\
\hline ARM-1-7 (4/93) & & & & & & & & & & & & \\
\hline ARM-1-7 (6/93) & & & & & & & & & & & & \\
\hline ARM-1-7 (8/93) & & & & & & & & & & & & \\
\hline ARM-1-7 & & & & & & & & & & & & \\
\hline T-ARM-1 & & & & & & & & & & & & \\
\hline SS-ARM-1 & & & & & & & & & & & & \\
\hline SS-ARM-1 & & & & & & & & & & & & \\
\hline EA-1-7 & & & & & & & & & & & & \\
\hline EA-1-7 & & & & & & & & & & & & \\
\hline EA-2-7 & & & & & & & & & & & & \\
\hline EA-7 & & & & & & & & & & & & \\
\hline T-EA & & & & & & & & & & & & \\
\hline SS-EA-19 & & & & & & & & & & & & \\
\hline SS-EA-15 & & & & & & & & & & & & \\
\hline SS-EA-1-7 & & & & & & & & & & & & \\
\hline SS-EA-2-7 & & & & & & & & & & & & \\
\hline SRS-SEA-A-7 & & & & & & & & & & & & \\
\hline SRS-SEA-B-7 & & & & & & & & & & & & \\
\hline CUASEA-A-7 & & & & & & & & & & & & \\
\hline CUASEA-B-7 & & & & & & & & & & & & \\
\hline 131-TDS-EA-7 & & & & & & & & & & & & \\
\hline 131-TDS-3A-SOPER & & & & & & & & & & & & \\
\hline BLEND 1-7 & & & & & & & & & & & & \\
\hline BLEND 1-7 (2) & & & & & & & & & & & & \\
\hline BLEND 1.6-7 & & & & & & & & & & & & \\
\hline
\end{tabular}


DWPF PCT Model (Jantzen et al. 1995)

\begin{tabular}{l|l|l|l|l|l|l|l|l|l|l|l|l|l|l|l|l|l|l|l|l|l|l|l} 
Glass ID & $\mathrm{A} 2 \mathrm{O} 3$ & $\mathrm{~B} 2 \mathrm{O} 3$ & $\mathrm{CaO}$ & $\mathrm{Fe} 2 \mathrm{O} 3$ & $\mathrm{FeO}$ & $\mathrm{K} 2 \mathrm{O}$ & $\mathrm{Li} 2 \mathrm{O}$ & $\mathrm{MgO}$ & $\mathrm{Na} 2 \mathrm{O}$ & $\mathrm{NiO}$ & $\mathrm{P} 2 \mathrm{O} 5$ & $\mathrm{SiO} 2$ & $\mathrm{ZrO} 2$ & $\mathrm{Ag} 2 \mathrm{O}$ & $\mathrm{As} 2 \mathrm{O} 3$ & $\mathrm{BaO}$ & $\mathrm{Bi} 2 \mathrm{O} 3$ & $\mathrm{Br}$ & $\mathrm{CdO}$ & $\mathrm{Ce} 2 \mathrm{O} 3$ & $\mathrm{CeO} 2$ \\
\hline
\end{tabular}

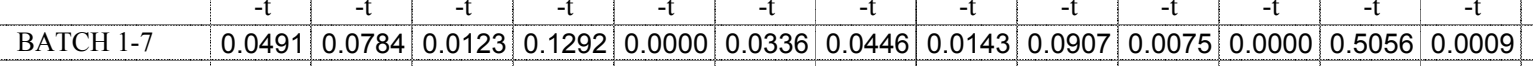

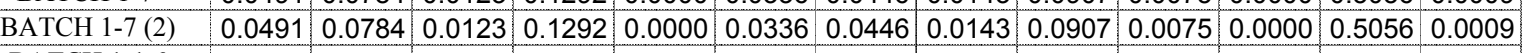

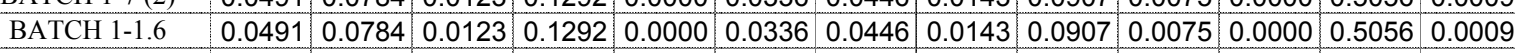

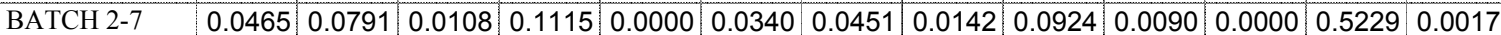

\begin{tabular}{|c|c|c|c|c|c|c|c|c|c|c|c|c|c|c|}
\hline BATCH 2-7 (2) & 0.0465 & 0.0791 & 0.0108 & 0.1115 & 0.0000 & 0.0340 & 0.0451 & 0.0142 & 0.0924 & 0.0090 & 0.0000 & 0.5229 & 0.0017
\end{tabular}

\begin{tabular}{|c|c|c|c|c|c|c|c|c|c|c|c|c|c|c|}
\hline BATCH 2-1.6 & 0.0465 & 0.0791 & 0.0108 & 0.1115 & 0.0000 & 0.0340 & 0.0451 & 0.0142 & 0.0924 & 0.0090 & 0.0000 & 0.5229 & 0.0017 \\
\hline
\end{tabular}

\begin{tabular}{|l|l|l|l|l|l|l|l|l|l|l|l|l|l|l|l|}
\hline BATCH 3-7 & 0.0346 & 0.0775 & 0.0100 & 0.1181 & 0.0000 & 0.0343 & 0.0455 & 0.0143 & 0.0908 & 0.0106 & 0.0000 & 0.5303 & 0.0011
\end{tabular}

\begin{tabular}{ll|l|l|l|l|l|l|l|l|l|l|l|l|l|l|l|l}
\hline BATCH 3-7 (2) & 0.0346 & 0.0775 & 0.0100 & 0.1181 & 0.0000 & 0.0343 & 0.0455 & 0.0143 & 0.0908 & 0.0106 & 0.0000 & 0.5303 & 0.0011
\end{tabular}

\begin{tabular}{ll|l|l|l|l|l|l|l|l|l|l|l|l|l} 
BATCH 3-7 (3) & 0.0346 & 0.0775 & 0.0100 & 0.1181 & 0.0000 & 0.0343 & 0.0455 & 0.0143 & 0.0908 & 0.0106 & 0.0000 & 0.5303 & 0.0011
\end{tabular}

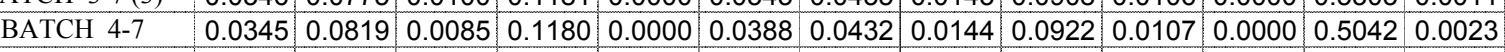

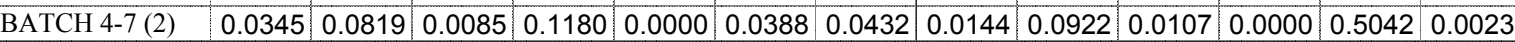

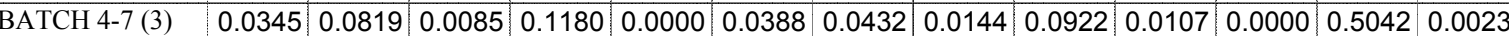

$\mathrm{HM}-1-7$

\begin{tabular}{ll|l|l|l|l|l|l|l|l|l|l|l|l|l|l|}
0.0715 & 0.0703 & 0.0101 & 0.0779 & 0.0000 & 0.0222 & 0.0462 & 0.0149 & 0.0856 & 0.0041 & 0.0000 & 0.5582 & 0.0033
\end{tabular}

\begin{tabular}{ll|l|l|l|l|l|l|l|l|l|l|l|l|l|l|l|l|l} 
HM-1-7 (2) & 0.0715 & 0.0703 & 0.0101 & 0.0779 & 0.0000 & 0.0222 & 0.0462 & 0.0149 & 0.0856 & 0.0041 & 0.0000 & 0.5582 & 0.0033 \\
\hline
\end{tabular}

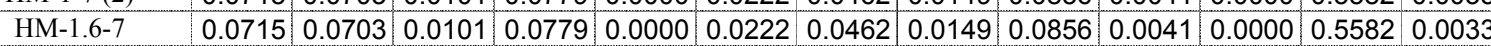

\begin{tabular}{ll|l|l|l|l|l|l|l|l|l|l|l|l|l} 
PUREX 1-7 & 0.0300 & 0.1039 & 0.0110 & 0.1333 & 0.0000 & 0.0343 & 0.0324 & 0.0142 & 0.1270 & 0.0120 & 0.0000 & 0.4681 & 0.0004
\end{tabular}

\begin{tabular}{|l|l|l|l|l|l|l|l|l|l|l|l|l|l|l|}
\hline PUREX 1-7 (2) & 0.0300 & 0.1039 & 0.0110 & 0.1333 & 0.0000 & 0.0343 & 0.0324 & 0.0142 & 0.1270 & 0.0120 & 0.0000 & 0.4681 & 0.0004
\end{tabular}

\begin{tabular}{|c|c|c|c|c|c|c|c|c|c|c|c|c|c|c|c|}
\hline PUREX 1.6-7 & 0.0300 & 0.1039 & 0.0110 & 0.1333 & 0.0000 & 0.0343 & 0.0324 & 0.0142 & 0.1270 & 0.0120 & 0.0000 & 0.4681 & 0.0004 \\
\hline
\end{tabular}

\begin{tabular}{|l|l|l|l|l|l|l|l|l|l|l|l|l|l|l|} 
PUREX SRSS 1.6 & 0.0300 & 0.1039 & 0.0110 & 0.1333 & 0.0000 & 0.0343 & 0.0324 & 0.0142 & 0.1270 & 0.0120 & 0.0000 & 0.4681 & 0.0004
\end{tabular}

\begin{tabular}{ll|l|l|l|l|l|l|l|l|l|l|l|l|l|l}
\hline PUREX SRST-4.0 & 0.0300 & 0.1039 & 0.0110 & 0.1333 & 0.0000 & 0.0343 & 0.0324 & 0.0142 & 0.1270 & 0.0120 & 0.0000 & 0.4681 & 0.0004
\end{tabular}

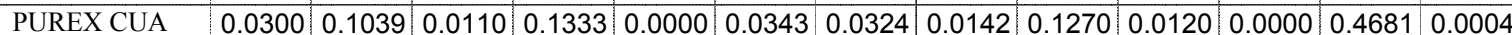

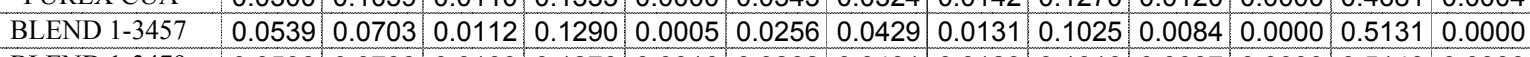

\begin{tabular}{|l|l|l|l|l|l|l|l|l|l|l|l|l|l|l|l|l|l|l|l|l|}
\hline BLEND 1-3479 & 0.0538 & 0.0708 & 0.0109 & 0.1270 & 0.0016 & 0.0262 & 0.0431 & 0.0129 & 0.1016 & 0.0087 & 0.0000 & 0.5140 & 0.0000 \\
\hline
\end{tabular}

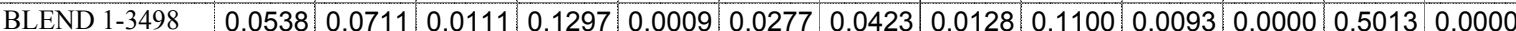

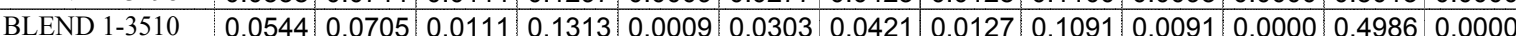

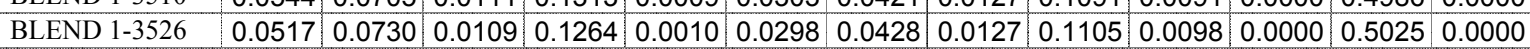

\begin{tabular}{l|l|l|l|l|l|l|l|l|l|l|l|l|l|l|l|l|l|l|l|l}
\hline BLEND 2-3611 & 0.0523 & 0.0684 & 0.0099 & 0.1232 & 0.0000 & 0.0242 & 0.0433 & 0.0134 & 0.1039 & 0.0098 & 0.0000 & 0.5230 & 0.0000
\end{tabular}

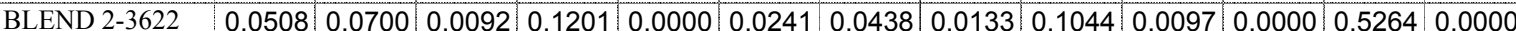

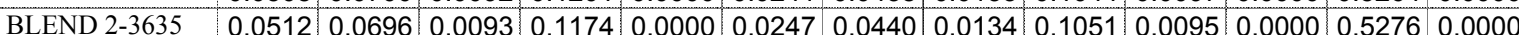

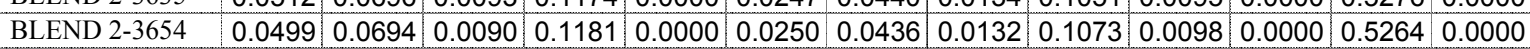

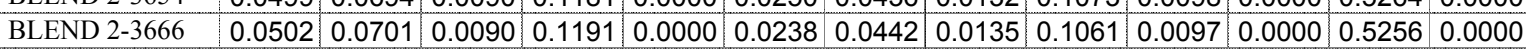

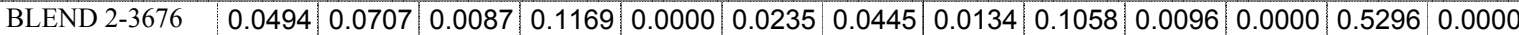

\begin{tabular}{|l|l|l|l|l|l|l|l|l|l|l|l|l|l|l|l|l|l|l|l|l|}
\hline BLEND 3-3768 & 0.0509 & 0.0710 & 0.0092 & 0.1176 & 0.0011 & 0.0234 & 0.0450 & 0.0141 & 0.1069 & 0.0098 & 0.0000 & 0.5198 & 0.0006 \\
\hline
\end{tabular}

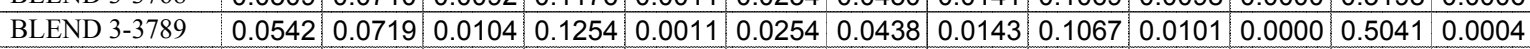

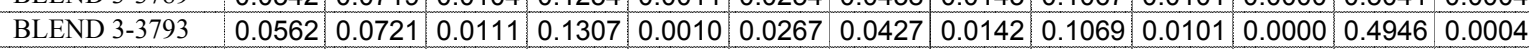

\begin{tabular}{l|l|l|l|l|l|l|l|l|l|l|l|l|l|l}
\hline BLEND 3-3802B & 0.0561 & 0.0717 & 0.0108 & 0.1304 & 0.0025 & 0.0277 & 0.0428 & 0.0143 & 0.1032 & 0.0099 & 0.0000 & 0.4971 & 0.0004
\end{tabular}

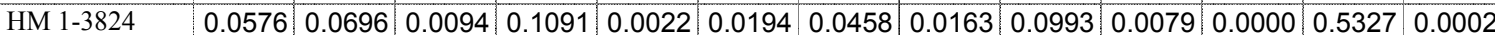

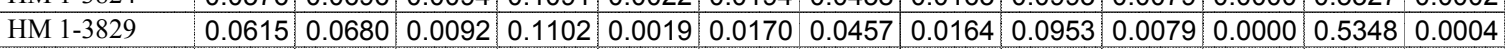

\begin{tabular}{l|l|l|l|l|l|l|l|l|l|l|l|l|l|l|l}
\hline HM 1-3851 & 0.0624 & 0.0669 & 0.0094 & 0.1073 & 0.0018 & 0.0145 & 0.0456 & 0.0166 & 0.1012 & 0.0078 & 0.0000 & 0.5342 & 0.0004
\end{tabular}

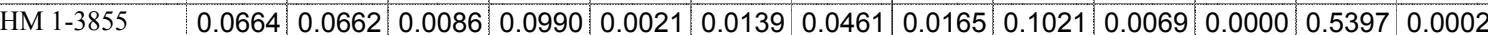

\begin{tabular}{ll|l|l|l|l|l|l|l|l|l|l|l|l|l} 
HM-2-1 & $(3979 \mathrm{C})$ & 0.0750 & 0.0664 & 0.0075 & 0.0992 & 0.0000 & 0.0159 & 0.0439 & 0.0154 & 0.1017 & 0.0068 & 0.0000 & 0.5289 & 0.0008
\end{tabular}

\begin{tabular}{ll|l|l|l|l|l|l|l|l|l|l|l|l|l|l|l|l|l|l|l}
\hline HM-2-2 & $(4099 \mathrm{~A})$ & 0.0769 & 0.0666 & 0.0072 & 0.0989 & 0.0000 & 0.0182 & 0.0435 & 0.0151 & 0.1037 & 0.0066 & 0.0000 & 0.5235 & 0.0006 \\
\hline
\end{tabular}

\begin{tabular}{|l|l|l|l|l|l|l|l|l|l|l|l|l|l|l|l|l|l}
\hline HM-2-3 & $(4120 \mathrm{~B})$ & 0.0748 & 0.0674 & 0.0070 & 0.0938 & 0.0000 & 0.0194 & 0.0463 & 0.0153 & 0.1052 & 0.0060 & 0.0000 & 0.5270 & 0.0008 \\
\hline
\end{tabular}

\begin{tabular}{|l|l|l|l|l|l|l|l|l|l|l|l|l|l|l|l|}
\hline HM-3-1 & $(4176)$ & 0.0774 & 0.0646 & 0.0068 & 0.0951 & 0.0000 & 0.0217 & 0.0416 & 0.0148 & 0.1082 & 0.0058 & 0.0000 & 0.5281 & 0.0006
\end{tabular}

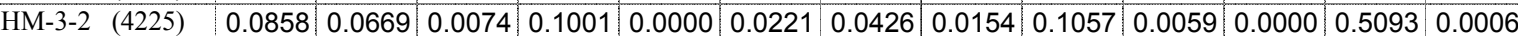

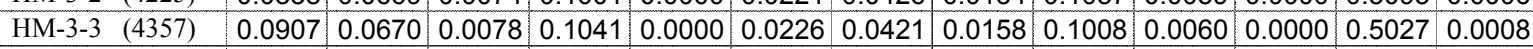

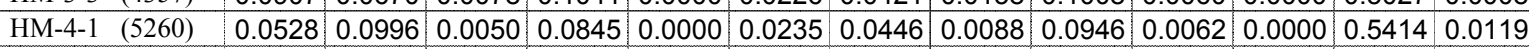

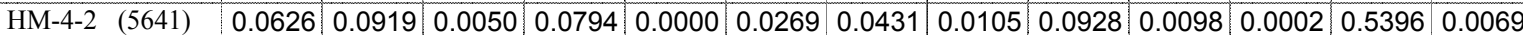

\begin{tabular}{|l|l|l|l|l|l|l|l|l|l|l|l|l|l|l}
\hline HM-4-3 & $(5748)$ & 0.0566 & 0.0880 & 0.0049 & 0.0727 & 0.0000 & 0.0279 & 0.0444 & 0.0117 & 0.0934 & 0.0052 & 0.0000 & 0.5631 & 0.0045 \\
\hline
\end{tabular}

\begin{tabular}{|c|c|c|c|c|c|c|c|}
\hline $\begin{array}{l}\mathrm{Ag}_{2} \mathrm{O} \\
-\mathrm{t}\end{array}$ & $\begin{array}{c}\text { As } 203 \\
-t\end{array}$ & $\begin{array}{c}\mathrm{BaO} \\
-\mathrm{t}\end{array}$ & $\begin{array}{c}\text { B1203 } \\
-t\end{array}$ & $\begin{array}{l}\mathrm{Br} \\
-\mathrm{t}\end{array}$ & $-t$ & $\begin{array}{c}c e 203 \\
-t\end{array}$ & $-t$ \\
\hline & & 0.0014 & & & & 0.0000 & \\
\hline & & 0.0014 & & & & 0.0000 & \\
\hline & & 0.0014 & & & & 0.0000 & \\
\hline & & 0.0017 & & & & 0.0000 & \\
\hline & & 0.0017 & & & & 0.0000 & \\
\hline & & 0.0017 & & & & 0.0000 & \\
\hline & & 0.0019 & & & & 0.0000 & \\
\hline & & 0.0019 & & & & 0.0000 & \\
\hline & & 0.0019 & & & & 0.0000 & \\
\hline & & 0.0026 & & & & 0.0000 & \\
\hline & & 0.0026 & & & & 0.0000 & \\
\hline & & 0.0026 & & & & 0.0000 & \\
\hline & & 0.0012 & & & & 0.0000 & \\
\hline & & 0.0012 & & & & 0.0000 & \\
\hline & & 0.0012 & & & & 0.0000 & \\
\hline & & 0.0021 & & & & 0.0000 & \\
\hline & & 0.0021 & & & & 0.0000 & \\
\hline & & 0.0021 & & & & 0.0000 & \\
\hline & & 0.0021 & & & & 0.0000 & \\
\hline & & 0.0021 & & & & 0.0000 & \\
\hline & & 0.0021 & & & & 0.0000 & \\
\hline & & 0.0009 & & & & 0.0000 & \\
\hline & & 0.0009 & & & & 0.0000 & \\
\hline & & 0.0009 & & & & 0.0000 & \\
\hline & & 0.0009 & & & & 0.0000 & \\
\hline & & 0.0009 & & & & 0.0000 & \\
\hline & & 0.0009 & & & & 0.0000 & \\
\hline & & 0.0009 & & & & 0.0000 & \\
\hline & & 0.0009 & & & & 0.0000 & \\
\hline & & 0.0009 & & & & 0.0000 & \\
\hline & & 0.0009 & & & & 0.0000 & \\
\hline & & 0.0009 & & & & 0.0000 & \\
\hline & & 0.0012 & & & & 0.0000 & \\
\hline & & 0.0014 & & & & 0.0000 & \\
\hline & & 0.0014 & & & & 0.0000 & \\
\hline & & 0.0014 & & & & 0.0000 & \\
\hline & & 0.0010 & & & & 0.0000 & \\
\hline & & 0.0010 & & & & 0.0000 & \\
\hline & & 0.0010 & & & & 0.0000 & \\
\hline & & 0.0010 & & & & 0.0000 & \\
\hline & & 0.0009 & & & & 0.0000 & \\
\hline & & 0.0009 & & & & 0.0000 & \\
\hline & & 0.0010 & & & & 0.0000 & \\
\hline & & 0.0007 & & & & 0.0000 & \\
\hline & & 0.0009 & & & & 0.0000 & \\
\hline & & 0.0009 & & & & 0.0000 & \\
\hline & & 0.0005 & & & & 0.0000 & \\
\hline & & 0.0005 & & & & 0.0000 & \\
\hline & & 0.0005 & & & & 0.0000 & \\
\hline
\end{tabular}


DWPF PCT Model (Jantzen et al. 1995)

\begin{tabular}{|c|c|c|c|c|c|c|c|c|c|c|c|c|c|c|c|c|c|c|c|c|c|}
\hline Glass ID & $\begin{array}{l}\mathrm{Cl} \\
-\mathrm{t}\end{array}$ & $\begin{array}{c}\mathrm{CoO} \\
-\mathrm{t}\end{array}$ & $\begin{array}{c}\mathrm{Co} 2 \mathrm{O} 3 \\
-\mathrm{t}\end{array}$ & $\begin{array}{c}\mathrm{Cr} 2 \mathrm{O} 3 \\
-\mathrm{t}\end{array}$ & $\begin{array}{c}\mathrm{Cs} 2 \mathrm{O} \\
-\mathrm{t} \\
\end{array}$ & $\begin{array}{c}\mathrm{CuO} \\
-\mathrm{t}\end{array}$ & $\begin{array}{c}\mathrm{Eu} 2 \mathrm{O} 3 \\
-\mathrm{t}\end{array}$ & $\begin{array}{l}F \\
-t\end{array}$ & $\begin{array}{c}\mathrm{Ga} 2 \mathrm{O} 3 \\
-\mathrm{t}\end{array}$ & $\begin{array}{c}\mathrm{Gd} 2 \mathrm{O} 3 \\
-\mathrm{t}\end{array}$ & $\begin{array}{c}\mathrm{HgO} \\
-\mathrm{t}\end{array}$ & $\begin{array}{l}I \\
-t\end{array}$ & $\begin{array}{c}\mathrm{La} 2 \mathrm{O} 3 \\
-\mathrm{t}\end{array}$ & $\begin{array}{c}\mathrm{MnO} 2 \\
-\mathrm{t} \\
\end{array}$ & $\begin{array}{c}\mathrm{MnO} \\
-\mathrm{t}\end{array}$ & $\begin{array}{c}\mathrm{MoO} \\
-\mathrm{t}\end{array}$ & $\begin{array}{c}\mathrm{MoO} 3 \\
-\mathrm{t}\end{array}$ & $\begin{array}{c}\mathrm{Nb} 2 \mathrm{O} 5 \\
-\mathrm{t}\end{array}$ & {$\left[\begin{array}{c}\mathrm{Nd} 2 \mathrm{O} 3 \\
-\mathrm{t}\end{array}\right.$} & $\begin{array}{c}\mathrm{PbO} \\
-\mathrm{t}\end{array}$ & $\begin{array}{c}\mathrm{PdO} 2 \\
-\mathrm{t}\end{array}$ \\
\hline BATCH 1-7 & & & & 0.0012 & 0.0004 & 0.0040 & & & & & & & 0.0000 & & 0.0173 & & 0.0011 & & 0.0015 & 0.0000 & \\
\hline BATCH 1-7 (2) & & & & 0.0012 & 0.0004 & 0.0040 & & & & & & & 0.0000 & & 0.0173 & & 0.0011 & & 0.0015 & 0.0000 & \\
\hline BATCH 1-1.6 & & & & 0.0012 & 0.0004 & 0.0040 & & & & & & & 0.0000 & & 0.0173 & & 0.0011 & & 0.0015 & 0.0000 & \\
\hline BATCH 2-7 & & & & 0.0014 & 0.0000 & 0.0042 & & & & & & & 0.0000 & & 0.0141 & & 0.0018 & & 0.0026 & 0.0000 & \\
\hline BATCH 2-7 (2) & & & & 0.0014 & 0.0000 & 0.0042 & & & & & & & 0.0000 & & 0.0141 & & 0.0018 & & 0.0026 & 0.0000 & \\
\hline BATCH 2-1.6 & & & & 0.0014 & 0.0000 & 0.0042 & & & & & & & 0.0000 & & 0.0141 & & 0.0018 & & 0.0026 & 0.0000 & \\
\hline BATCH 3-7 & & & & 0.0014 & 0.0004 & 0.0041 & & & & & & & 0.0000 & & 0.0153 & & 0.0011 & & 0.0016 & 0.0000 & \\
\hline BATCH 3-7 (2) & & & & 0.0014 & 0.0004 & 0.0041 & & & & & & & 0.0000 & & 0.0153 & & 0.0011 & & 0.0016 & 0.0000 & \\
\hline BATCH 3-7 (3) & & & & 0.0014 & 0.0004 & 0.0041 & & & & & & & 0.0000 & & 0.0153 & & 0.0011 & & 0.0016 & 0.0000 & \\
\hline BATCH $4-7$ & & & & 0.0014 & 0.0009 & 0.0045 & & & & & & & 0.0000 & & 0.0255 & & 0.0020 & & 0.0041 & 0.0000 & \\
\hline BATCH 4-7 (2) & & & & 0.0014 & 0.0009 & 0.0045 & & & & & & & 0.0000 & & 0.0255 & & 0.0020 & & 0.0041 & 0.0000 & \\
\hline BATCH 4-7 (3) & & & & 0.0014 & 0.0009 & 0.0045 & & & & & & & 0.0000 & & 0.0255 & & 0.0020 & & 0.0041 & 0.0000 & \\
\hline HM-1-7 & & & & 0.0010 & 0.0004 & 0.0025 & & & & & & & 0.0000 & & 0.0176 & & 0.0023 & & 0.0053 & 0.0000 & \\
\hline HM-1-7 (2) & & & & 0.0010 & 0.0004 & 0.0025 & & & & & & & 0.0000 & & 0.0176 & & 0.0023 & & 0.0053 & 0.0000 & \\
\hline HM-1.6-7 & & & & 0.0010 & 0.0004 & 0.0025 & & & & & & & 0.0000 & & 0.0176 & & 0.0023 & & 0.0053 & 0.0000 & \\
\hline PUREX 1-7 & & & & 0.0016 & 0.0004 & 0.0042 & & & & & & & 0.0000 & & 0.0170 & & 0.0009 & & 0.0005 & 0.0000 & \\
\hline PUREX 1-7 (2) & & & & 0.0016 & 0.0004 & 0.0042 & & & & & & & 0.0000 & & 0.0170 & & 0.0009 & & 0.0005 & 0.0000 & \\
\hline PUREX 1.6-7 & & & & 0.0016 & 0.0004 & 0.0042 & & & & & & & 0.0000 & & 0.0170 & & 0.0009 & & 0.0005 & 0.0000 & \\
\hline PUREX SRSS 1.6 & & & & 0.0016 & 0.0004 & 0.0042 & & & & & & & 0.0000 & & 0.0170 & & 0.0009 & & 0.0005 & 0.0000 & \\
\hline PUREX SRST-4.0 & & & & 0.0016 & 0.0004 & 0.0042 & & & & & & & 0.0000 & & 0.0170 & & 0.0009 & & 0.0005 & 0.0000 & \\
\hline PUREX CUA & & & & 0.0016 & 0.0004 & 0.0042 & & & & & & & 0.0000 & & 0.0170 & & 0.0009 & & 0.0005 & 0.0000 & \\
\hline BLEND 1-3457 & & & & 0.0023 & 0.0000 & 0.0022 & & & & & & & 0.0000 & & 0.0216 & & 0.0000 & & 0.0000 & 0.0000 & \\
\hline BLEND 1-3479 & & & & 0.0023 & 0.0000 & 0.0018 & & & & & & & 0.0000 & & 0.0220 & & 0.0000 & & 0.0000 & 0.0000 & \\
\hline BLEND 1-3498 & & & & 0.0023 & 0.0000 & 0.0019 & & & & & & & 0.0000 & & 0.0228 & & 0.0000 & & 0.0000 & 0.0000 & \\
\hline BLEND 1-3510 & & & & 0.0023 & 0.0000 & 0.0019 & & & & & & & 0.0000 & & 0.0229 & & 0.0000 & & 0.0000 & 0.0000 & \\
\hline BLEND 1-3526 & & & & 0.0021 & 0.0000 & 0.0018 & & & & & & & 0.0000 & & 0.0223 & & 0.0000 & & 0.0000 & 0.0000 & \\
\hline BLEND 2-3611 & & & & 0.0026 & 0.0000 & 0.0017 & & & & & & & 0.0000 & & 0.0218 & & 0.0000 & & 0.0000 & 0.0000 & \\
\hline BLEND 2-3622 & & & & 0.0023 & 0.0000 & 0.0017 & & & & & & & 0.0000 & & 0.0218 & & 0.0000 & & 0.0000 & 0.0000 & \\
\hline BLEND 2-3635 & & & & 0.0023 & 0.0000 & 0.0017 & & & & & & & 0.0000 & & 0.0216 & & 0.0000 & & 0.0000 & 0.0000 & \\
\hline BLEND 2-3654 & & & & 0.0023 & 0.0000 & 0.0017 & & & & & & & 0.0000 & & 0.0218 & & 0.0000 & & 0.0000 & 0.0000 & \\
\hline BLEND 2-3666 & & & & 0.0026 & 0.0000 & 0.0017 & & & & & & & 0.0000 & & 0.0220 & & 0.0000 & & 0.0000 & 0.0000 & \\
\hline BLEND 2-3676 & & & & 0.0024 & 0.0000 & 0.0017 & & & & & & & 0.0000 & & 0.0215 & & 0.0000 & & 0.0000 & 0.0000 & \\
\hline BLEND 3-3768 & & & & 0.0031 & 0.0000 & 0.0020 & & & & & & & 0.0000 & & 0.0230 & & 0.0000 & & 0.0000 & 0.0000 & \\
\hline BLEND 3-3789 & & & & 0.0026 & 0.0000 & 0.0021 & & & & & & & 0.0000 & & 0.0250 & & 0.0000 & & 0.0000 & 0.0000 & \\
\hline BLEND 3-3793 & & & & 0.0025 & 0.0000 & 0.0023 & & & & & & & 0.0000 & & 0.0258 & & 0.0000 & & 0.0000 & 0.0000 & \\
\hline BLEND 3-3802B & & & & 0.0025 & 0.0000 & 0.0022 & & & & & & & 0.0000 & & 0.0258 & & 0.0000 & & 0.0000 & 0.0000 & \\
\hline HM 1-3824 & & & & 0.0024 & 0.0000 & 0.0015 & & & & & & & 0.0000 & & 0.0247 & & 0.0000 & & 0.0000 & 0.0000 & \\
\hline HM 1-3829 & & & & 0.0024 & 0.0000 & 0.0015 & & & & & & & 0.0000 & & 0.0260 & & 0.0000 & & 0.0000 & 0.0000 & \\
\hline HM 1-3851 & & & & 0.0024 & 0.0000 & 0.0015 & & & & & & & 0.0000 & & 0.0261 & & 0.0000 & & 0.0000 & 0.0000 & \\
\hline HM 1-3855 & & & & 0.0021 & 0.0000 & 0.0014 & & & & & & & 0.0000 & & 0.0269 & & 0.0000 & & 0.0000 & 0.0000 & \\
\hline HM-2-1 (3979C) & & & & 0.0049 & 0.0000 & 0.0014 & & & & & & & 0.0000 & & 0.0273 & & 0.0000 & & 0.0000 & 0.0007 & \\
\hline HM-2-2 (4099A) & & & & 0.0047 & 0.0000 & 0.0015 & & & & & & & 0.0000 & & 0.0275 & & 0.0000 & & 0.0000 & 0.0007 & \\
\hline HM-2-3 (4120B) & & & & 0.0040 & 0.0000 & 0.0014 & & & & & & & 0.0000 & & 0.0266 & & 0.0000 & & 0.0000 & 0.0007 & \\
\hline HM-3-1 (4176) & & & & 0.0040 & 0.0000 & 0.0015 & & & & & & & 0.0000 & & 0.0256 & & 0.0000 & & 0.0000 & 0.0003 & \\
\hline HM-3-2 (4225) & & & & 0.0040 & 0.0000 & 0.0016 & & & & & & & 0.0000 & & 0.0280 & & 0.0000 & & 0.0000 & 0.0003 & \\
\hline HM-3-3 (4357) & & & & 0.0042 & 0.0000 & 0.0016 & & & & & & & 0.0000 & & 0.0293 & & 0.0000 & & 0.0000 & 0.0003 & \\
\hline HM-4-1 (5260) & & & & 0.0045 & 0.0000 & 0.0010 & & & & & & & 0.0000 & & 0.0150 & & 0.0007 & & 0.0000 & 0.0010 & \\
\hline HM-4-2 (5641) & & & & 0.0066 & 0.0000 & 0.0020 & & & & & & & 0.0000 & & 0.0165 & & 0.0004 & & 0.0000 & 0.0007 & \\
\hline HM-4-3 (5748) & & & & 0.0038 & 0.0000 & 0.0019 & & & & & & & 0.0000 & & 0.0165 & & 0.0002 & & 0.0000 & 0.0004 & \\
\hline
\end{tabular}


DWPF PCT Model (Jantzen et al. 1995)

\begin{tabular}{|c|c|c|c|c|c|c|c|c|c|c|c|c|c|c|c|c|c|c|c|c|c|}
\hline Glass ID & $\begin{array}{c}\mathrm{PdO} \\
-\mathrm{t}\end{array}$ & $\begin{array}{c}\mathrm{Pr} 2 \mathrm{O} 3 \\
-\mathrm{t}\end{array}$ & Pr6O11 & $\begin{array}{c}\mathrm{Rb} 2 \mathrm{O} \\
-\mathrm{t}\end{array}$ & $\begin{array}{c}\mathrm{ReO} \\
-\mathrm{t}\end{array}$ & $\begin{array}{c}\mathrm{ReO} 2 \\
-\mathrm{t}\end{array}$ & $\begin{array}{c}\mathrm{Rh} 2 \mathrm{O} 3 \\
-\mathrm{t}\end{array}$ & $\begin{array}{c}\mathrm{RhO}_{2} \\
-\mathrm{t}\end{array}$ & $\begin{array}{c}\mathrm{RuO} 2 \\
-\mathrm{t}\end{array}$ & $\begin{array}{c}\mathrm{Sb} 2 \mathrm{O} 3 \\
-\mathrm{t}\end{array}$ & $\begin{array}{c}\mathrm{Sb} 2 \mathrm{O} 5 \\
-\mathrm{t}\end{array}$ & $\begin{array}{c}\mathrm{SeO} 2 \\
-\mathrm{t}\end{array}$ & $\begin{array}{c}\mathrm{Sm} 2 \mathrm{O} 3 \\
-\mathrm{t}\end{array}$ & $\begin{array}{c}\mathrm{SnO} \\
-\mathrm{t}\end{array}$ & $\begin{array}{c}\mathrm{SnO} 2 \\
-\mathrm{t}\end{array}$ & $\begin{array}{c}\mathrm{SO} 3 \\
-\mathrm{t}\end{array}$ & $\begin{array}{c}\mathrm{SrO} \\
-\mathrm{t}\end{array}$ & $\begin{array}{c}\mathrm{Tc} 2 \mathrm{O} 7 \\
-\mathrm{t}\end{array}$ & $\begin{array}{c}\mathrm{TeO} 2 \\
-\mathrm{t}\end{array}$ & $\begin{array}{c}\mathrm{ThO} 2 \\
-\mathrm{t}\end{array}$ & $\begin{array}{c}\mathrm{TiO} 2 \\
-\mathrm{t}\end{array}$ \\
\hline BATCH 1-7 & & & & & & & & & & & & & & & & & 0.0000 & & & & 0.0068 \\
\hline BATCH 1-7 (2) & & & & & & & & & & & & & & & & & 0.0000 & & & & 0.0068 \\
\hline BATCH 1-1.6 & & & & & & & & & & & & & & & & & 0.0000 & & & & 0.0068 \\
\hline BATCH 2-7 & & & & & & & & & & & & & & & & & 0.0000 & & & & 0.0069 \\
\hline BATCH 2-7 (2) & & & & & & & & & & & & & & & & & 0.0000 & & & & 0.0069 \\
\hline BATCH 2-1.6 & & & & & & & & & & & & & & & & & 0.0000 & & & & 0.0069 \\
\hline BATCH 3-7 & & & & & & & & & & & & & & & & & 0.0000 & & & & 0.0069 \\
\hline BATCH 3-7 (2) & & & & & & & & & & & & & & & & & 0.0000 & & & & 0.0069 \\
\hline BATCH 3-7 (3) & & & & & & & & & & & & & & & & & 0.0000 & & & & 0.0069 \\
\hline BATCH 4-7 & & & & & & & & & & & & & & & & & 0.0000 & & & & 0.0104 \\
\hline BATCH 4-7 (2) & & & & & & & & & & & & & & & & & 0.0000 & & & & 0.0104 \\
\hline BATCH 4-7 (3) & & & & & & & & & & & & & & & & & 0.0000 & & & & 0.0104 \\
\hline HM-1-7 & & & & & & & & & & & & & & & & & 0.0000 & & & & 0.0056 \\
\hline HM-1-7 (2) & & & & & & & & & & & & & & & & & 0.0000 & & & & 0.0056 \\
\hline HM-1.6-7 & & & & & & & & & & & & & & & & & 0.0000 & & & & 0.0056 \\
\hline PUREX 1-7 & & & & & & & & & & & & & & & & & 0.0000 & & & & 0.0068 \\
\hline PUREX 1-7 (2) & & & & & & & & & & & & & & & & & 0.0000 & & & & 0.0068 \\
\hline PUREX 1.6-7 & & & & & & & & & & & & & & & & & 0.0000 & & & & 0.0068 \\
\hline PUREX SRSS 1.6 & & & & & & & & & & & & & & & & & 0.0000 & & & & 0.0068 \\
\hline PUREX SRST-4.0 & & & & & & & & & & & & & & & & & 0.0000 & & & & 0.0068 \\
\hline PUREX CUA & & & & & & & & & & & & & & & & & 0.0000 & & & & 0.0068 \\
\hline BLEND 1-3457 & & & & & & & & & & & & & & & & & 0.0000 & & & & 0.0024 \\
\hline BLEND 1-3479 & & & & & & & & & & & & & & & & & 0.0000 & & & & 0.0023 \\
\hline BLEND 1-3498 & & & & & & & & & & & & & & & & & 0.0000 & & & & 0.0021 \\
\hline BLEND 1-3510 & & & & & & & & & & & & & & & & & 0.0000 & & & & 0.0019 \\
\hline BLEND 1-3526 & & & & & & & & & & & & & & & & & 0.0000 & & & & 0.0018 \\
\hline BLEND 2-3611 & & & & & & & & & & & & & & & & & 0.0000 & & & & 0.0017 \\
\hline BLEND 2-3622 & & & & & & & & & & & & & & & & & 0.0000 & & & & 0.0016 \\
\hline BLEND 2-3635 & & & & & & & & & & & & & & & & & 0.0000 & & & & 0.0017 \\
\hline BLEND 2-3654 & & & & & & & & & & & & & & & & & 0.0000 & & & & 0.0015 \\
\hline BLEND 2-3666 & & & & & & & & & & & & & & & & & 0.0000 & & & & 0.0015 \\
\hline BLEND 2-3676 & & & & & & & & & & & & & & & & & 0.0000 & & & & 0.0015 \\
\hline BLEND 3-3768 & & & & & & & & & & & & & & & & & 0.0000 & & & & 0.0014 \\
\hline BLEND 3-3789 & & & & & & & & & & & & & & & & & 0.0000 & & & & 0.0013 \\
\hline BLEND 3-3793 & & & & & & & & & & & & & & & & & 0.0000 & & & & 0.0012 \\
\hline BLEND 3-3802B & & & & & & & & & & & & & & & & & 0.0000 & & & & 0.0012 \\
\hline HM 1-3824 & & & & & & & & & & & & & & & & & 0.0000 & & & & 0.0009 \\
\hline HM 1-3829 & & & & & & & & & & & & & & & & & 0.0000 & & & & 0.0010 \\
\hline HM 1-3851 & & & & & & & & & & & & & & & & & 0.0000 & & & & 0.0009 \\
\hline HM 1-3855 & & & & & & & & & & & & & & & & & 0.0000 & & & & 0.0009 \\
\hline HM-2-1 (3979C) & & & & & & & & & & & & & & & & & 0.0014 & & & & 0.0009 \\
\hline HM-2-2 (4099A) & & & & & & & & & & & & & & & & & 0.0016 & & & & 0.0012 \\
\hline HM-2-3 (4120B) & & & & & & & & & & & & & & & & & 0.0015 & & & & 0.0010 \\
\hline HM-3-1 (4176) & & & & & & & & & & & & & & & & & 0.0014 & & & & 0.0010 \\
\hline HM-3-2 (4225) & & & & & & & & & & & & & & & & & 0.0014 & & & & 0.0010 \\
\hline HM-3-3 (4357) & & & & & & & & & & & & & & & & & 0.0016 & & & & 0.0010 \\
\hline HM-4-1 (5260) & & & & & & & & & & & & & & & & & 0.0008 & & & & 0.0025 \\
\hline HM-4-2 (5641) & & & & & & & & & & & & & & & & & 0.0008 & & & & 0.0026 \\
\hline HM-4-3 (5748) & & & & & & & & & & & & & & & & & 0.0008 & & & & 0.0025 \\
\hline
\end{tabular}


DWPF PCT Model (Jantzen et al. 1995)

\begin{tabular}{|c|c|c|c|c|c|c|c|c|c|c|c|c|c|c|c|c|c|c|c|c|c|}
\hline Glass ID & $\begin{array}{c}\mathrm{T} 12 \mathrm{O} 3 \\
-\mathrm{t}\end{array}$ & $\begin{array}{c}\text { U3O8 } \\
-t\end{array}$ & $\begin{array}{c}\mathrm{UO} 2 \\
-\mathrm{t}\end{array}$ & $\begin{array}{c}\text { UO3 } \\
-t\end{array}$ & $\begin{array}{c}\mathrm{V} 2 \mathrm{O} 5 \\
-\mathrm{t}\end{array}$ & $\begin{array}{c}\text { WO3 } \\
-\mathrm{t}\end{array}$ & $\begin{array}{c}\mathrm{Y} 2 \mathrm{O} 3 \\
-\mathrm{t}\end{array}$ & $\begin{array}{c}\mathrm{ZnO} \\
-\mathrm{t}\end{array}$ & $\begin{array}{c}\text { Others } \\
-t\end{array}$ & $\begin{array}{c}\text { Sum } \\
-t\end{array}$ & $\begin{array}{c}\mathrm{A} 12 \mathrm{O} 3 \\
-\mathrm{a}\end{array}$ & $\begin{array}{c}\mathrm{B} 2 \mathrm{O} 3 \\
-\mathrm{a}\end{array}$ & $\begin{array}{c}\mathrm{CaO} \\
-\mathrm{a} \\
\end{array}$ & $\begin{array}{c}\mathrm{Fe} 2 \mathrm{O} 3 \\
-\mathrm{a} \\
\end{array}$ & $\begin{array}{c}\mathrm{FeO} \\
-\mathrm{a} \\
\end{array}$ & $\begin{array}{c}\mathrm{K} 2 \mathrm{O} \\
-\mathrm{a}\end{array}$ & $\begin{array}{c}\mathrm{Li} 2 \mathrm{O} \\
-\mathrm{a}\end{array}$ & $\begin{array}{c}\mathrm{MgO} \\
-\mathrm{a}\end{array}$ & $\begin{array}{c}\mathrm{Na} 2 \mathrm{O} \\
-\mathrm{a}\end{array}$ & $\begin{array}{c}\mathrm{NiO} \\
-\mathrm{a} \\
\end{array}$ & $\begin{array}{c}\mathrm{P} 2 \mathrm{O} 5 \\
-\mathrm{a}\end{array}$ \\
\hline BATCH 1-7 & & & & & & & 0.0000 & 0.0000 & & 1.0000 & 0.0488 & 0.0778 & 0.0122 & 0.1284 & & 0.0333 & 0.0443 & 0.0142 & 0.0900 & 0.0075 & \\
\hline BATCH 1-7 (2) & & & & & & & 0.0000 & 0.0000 & & 1.0000 & & & & & & & & & & & \\
\hline BATCH 1-1.6 & & & & & & & 0.0000 & 0.0000 & & 1.0000 & & & & & & & & & & & \\
\hline BATCH 2-7 & & & & & & & 0.0000 & 0.0000 & & 1.0000 & 0.0463 & 0.0788 & 0.0108 & 0.1112 & & 0.0338 & 0.0450 & 0.0142 & 0.0921 & 0.0090 & \\
\hline BATCH 2-7 (2) & & & & & & & 0.0000 & 0.0000 & & 1.0000 & & & & & & & & & & & \\
\hline BATCH 2-1.6 & & & & & & & 0.0000 & 0.0000 & & 1.0000 & & & & & & & & & & & \\
\hline BATCH 3-7 & & & & & & & 0.0000 & 0.0000 & & 1.0000 & 0.0344 & 0.0769 & 0.0099 & 0.1171 & & 0.0340 & 0.0451 & 0.0142 & 0.0901 & 0.0105 & \\
\hline BATCH 3-7 (2) & & & & & & & 0.0000 & 0.0000 & & 1.0000 & & & & & & & & & & & \\
\hline BATCH 3-7 (3) & & & & & & & 0.0000 & 0.0000 & & 1.0000 & & & & & & & & & & & \\
\hline BATCH 4-7 & & & & & & & 0.0000 & 0.0000 & & 1.0000 & 0.0343 & 0.0814 & 0.0084 & 0.1171 & & 0.0386 & 0.0429 & 0.0143 & 0.0916 & 0.0106 & \\
\hline BATCH 4-7 (2) & & & & & & & 0.0000 & 0.0000 & & 1.0000 & & & & & & & & & & & \\
\hline BATCH 4-7 (3) & & & & & & & 0.0000 & 0.0000 & & 1.0000 & & & & & & & & & & & \\
\hline HM-1-7 & & & & & & & 0.0000 & 0.0000 & & 1.0000 & 0.0715 & 0.0703 & 0.0101 & 0.0778 & & 0.0221 & 0.0462 & 0.0149 & 0.0856 & 0.0041 & \\
\hline HM-1-7 (2) & & & & & & & 0.0000 & 0.0000 & & 1.0000 & & & & & & & & & & & \\
\hline HM-1.6-7 & & & & & & & 0.0000 & 0.0000 & & 1.0000 & & & & & & & & & & & \\
\hline PUREX 1-7 & & & & & & & 0.0000 & 0.0000 & & 1.0000 & 0.0299 & 0.1033 & 0.0109 & 0.1325 & & 0.0341 & 0.0322 & 0.0141 & 0.1262 & 0.0119 & \\
\hline PUREX 1-7 (2) & & & & & & & 0.0000 & 0.0000 & & 1.0000 & & & & & & & & & & & \\
\hline PUREX 1.6-7 & & & & & & & 0.0000 & 0.0000 & & 1.0000 & & & & & & & & & & & \\
\hline PUREX SRSS 1.6 & & & & & & & 0.0000 & 0.0000 & & 1.0000 & & & & & & & & & & & \\
\hline PUREX SRST-4.0 & & & & & & & 0.0000 & 0.0000 & & 1.0000 & & & & & & & & & & & \\
\hline PUREX CUA & & & & & & & 0.0000 & 0.0000 & & 1.0000 & & & & & & & & & & & \\
\hline BLEND 1-3457 & & & & & & & 0.0000 & 0.0000 & & 1.0000 & 0.0518 & 0.0676 & 0.0108 & 0.1241 & 0.0006 & 0.0247 & 0.0412 & 0.0126 & 0.0987 & 0.0081 & \\
\hline BLEND 1-3479 & & & & & & & 0.0000 & 0.0000 & & 1.0000 & 0.0515 & 0.0678 & 0.0104 & 0.1216 & 0.0016 & 0.0251 & 0.0413 & 0.0124 & 0.0974 & 0.0083 & \\
\hline BLEND 1-3498 & & & & & & & 0.0000 & 0.0000 & & 1.0000 & 0.0516 & 0.0682 & 0.0107 & 0.1243 & 0.0008 & 0.0265 & 0.0406 & 0.0122 & 0.1054 & 0.0089 & \\
\hline BLEND 1-3510 & & & & & & & 0.0000 & 0.0000 & & 1.0000 & 0.0521 & 0.0674 & 0.0106 & 0.1256 & 0.0008 & 0.0291 & 0.0403 & 0.0122 & 0.1044 & 0.0087 & \\
\hline BLEND 1-3526 & & & & & & & 0.0000 & 0.0000 & & 1.0000 & 0.0489 & 0.0689 & 0.0103 & 0.1194 & 0.0010 & 0.0281 & 0.0404 & 0.0121 & 0.1043 & 0.0093 & \\
\hline BLEND 2-3611 & & & & & & & 0.0000 & 0.0000 & & 1.0000 & 0.0502 & 0.0656 & 0.0095 & 0.1182 & & 0.0232 & 0.0415 & 0.0128 & 0.0997 & 0.0094 & \\
\hline BLEND 2-3622 & & & & & & & 0.0000 & 0.0000 & & 1.0000 & 0.0489 & 0.0675 & 0.0089 & 0.1158 & & 0.0232 & 0.0422 & 0.0128 & 0.1007 & 0.0093 & \\
\hline BLEND 2-3635 & & & & & & & 0.0000 & 0.0000 & & 1.0000 & 0.0489 & 0.0666 & 0.0089 & 0.1123 & & 0.0236 & 0.0421 & 0.0128 & 0.1005 & 0.0091 & \\
\hline BLEND 2-3654 & & & & & & & 0.0000 & 0.0000 & & 1.0000 & 0.0472 & 0.0656 & 0.0085 & 0.1115 & & 0.0237 & 0.0412 & 0.0125 & 0.1005 & 0.0093 & \\
\hline BLEND 2-3666 & & & & & & & 0.0000 & 0.0000 & & 1.0000 & 0.0480 & 0.0670 & 0.0086 & 0.1137 & & 0.0228 & 0.0422 & 0.0129 & 0.1013 & 0.0092 & \\
\hline BLEND 2-3676 & & & & & & & 0.0000 & 0.0000 & & 1.0000 & 0.0473 & 0.0676 & 0.0083 & 0.1119 & & 0.0225 & 0.0426 & 0.0128 & 0.1012 & 0.0092 & \\
\hline BLEND 3-3768 & & & & & & & 0.0000 & 0.0000 & & 1.0000 & 0.0489 & 0.0683 & 0.0088 & 0.1131 & 0.0011 & 0.0225 & 0.0433 & 0.0136 & 0.1028 & 0.0094 & \\
\hline BLEND 3-3789 & & & & & & & 0.0000 & 0.0000 & & 1.0000 & 0.0528 & 0.0702 & 0.0101 & 0.1223 & 0.0011 & 0.0248 & 0.0427 & 0.0139 & 0.1041 & 0.0098 & \\
\hline BLEND 3-3793 & & & & & & & 0.0000 & 0.0000 & & 1.0000 & 0.0546 & 0.0701 & 0.0108 & 0.1269 & 0.0010 & 0.0260 & 0.0415 & 0.0138 & 0.1039 & 0.0098 & \\
\hline BLEND 3-3802B & & & & & & & 0.0000 & 0.0000 & & 1.0000 & 0.0539 & 0.0688 & 0.0104 & 0.1253 & 0.0024 & 0.0266 & 0.0411 & 0.0137 & 0.0992 & 0.0095 & \\
\hline HM 1-3824 & & & & & & & 0.0000 & 0.0000 & & 1.0000 & 0.0558 & 0.0674 & 0.0091 & 0.1057 & 0.0021 & 0.0188 & 0.0444 & 0.0158 & 0.0962 & 0.0077 & \\
\hline HM 1-3829 & & & & & & & 0.0000 & 0.0000 & & 1.0000 & 0.0600 & 0.0662 & 0.0090 & 0.1073 & 0.0018 & 0.0166 & 0.0445 & 0.0160 & 0.0929 & 0.0077 & \\
\hline HM 1-3851 & & & & & & & 0.0000 & 0.0000 & & 1.0000 & 0.0608 & 0.0651 & 0.0091 & 0.1045 & 0.0017 & 0.0142 & 0.0444 & 0.0162 & 0.0985 & 0.0076 & \\
\hline HM 1-3855 & & & & & & & 0.0000 & 0.0000 & & 1.0000 & 0.0650 & 0.0649 & 0.0084 & 0.0971 & 0.0021 & 0.0136 & 0.0452 & 0.0162 & 0.1001 & 0.0068 & \\
\hline HM-2-1 (3979C) & & & & & & & 0.0000 & 0.0010 & & 1.0000 & 0.0766 & 0.0677 & 0.0076 & 0.1012 & & 0.0162 & 0.0448 & 0.0157 & 0.1038 & 0.0070 & \\
\hline HM-2-2 (4099A) & & & & & & & 0.0000 & 0.0010 & & 1.0000 & 0.0782 & 0.0678 & 0.0073 & 0.1008 & & 0.0185 & 0.0443 & 0.0154 & 0.1056 & 0.0067 & \\
\hline HM-2-3 (4120B) & & & & & & & 0.0000 & 0.0009 & & 1.0000 & 0.0754 & 0.0680 & 0.0071 & 0.0946 & & 0.0195 & 0.0467 & 0.0155 & 0.1060 & 0.0061 & \\
\hline HM-3-1 (4176) & & & & & & & 0.0000 & 0.0009 & & 1.0000 & 0.0749 & 0.0625 & 0.0066 & 0.0920 & & 0.0211 & 0.0402 & 0.0144 & 0.1048 & 0.0056 & \\
\hline HM-3-2 (4225) & & & & & & & 0.0000 & 0.0009 & & 1.0000 & 0.0838 & 0.0653 & 0.0072 & 0.0976 & & 0.0217 & 0.0416 & 0.0151 & 0.1032 & 0.0057 & \\
\hline HM-3-3 (4357) & & & & & & & 0.0000 & 0.0009 & & 1.0000 & 0.0896 & 0.0661 & 0.0077 & 0.1027 & & 0.0223 & 0.0415 & 0.0155 & 0.0995 & 0.0059 & \\
\hline HM-4-1 (5260) & & & & & & & 0.0000 & 0.0011 & & 1.0000 & 0.0533 & 0.1005 & 0.0050 & 0.0854 & & 0.0237 & 0.0450 & 0.0090 & 0.0955 & 0.0062 & \\
\hline HM-4-2 (5641) & & & & & & & 0.0000 & 0.0011 & & 1.0000 & 0.0648 & 0.0952 & 0.0051 & 0.0823 & & 0.0280 & 0.0447 & 0.0109 & 0.0962 & 0.0101 & 0.0002 \\
\hline HM-4-3 (5748) & & & & & & & 0.0000 & 0.0010 & & 1.0000 & 0.0587 & 0.0912 & 0.0051 & 0.0753 & & 0.0289 & 0.0459 & 0.0121 & 0.0968 & 0.0054 & \\
\hline
\end{tabular}




\section{DWPF PCT Model (Jantzen et al. 1995)}

\begin{tabular}{|c|c|c|c|c|c|c|c|c|c|c|c|c|c|c|c|c|c|c|c|c|c|}
\hline Glass ID & $\begin{array}{c}\mathrm{SiO} 2 \\
-\mathrm{a}\end{array}$ & $\begin{array}{c}\mathrm{ZrO} 2 \\
-\mathrm{a}\end{array}$ & $\begin{array}{c}\mathrm{Ag} 2 \mathrm{O} \\
-\mathrm{a}\end{array}$ & $\begin{array}{c}\mathrm{As} 2 \mathrm{O} 3 \\
-\mathrm{a}\end{array}$ & $\begin{array}{c}\mathrm{BaO} \\
-\mathrm{a}\end{array}$ & $\begin{array}{c}\mathrm{Bi} 2 \mathrm{O} 3 \\
-\mathrm{a}\end{array}$ & $\begin{array}{l}\mathrm{Br} \\
-\mathrm{a}\end{array}$ & $\begin{array}{c}\mathrm{CdO} \\
-\mathrm{a}\end{array}$ & $\begin{array}{c}\mathrm{Ce} 2 \mathrm{O} 3 \\
-\mathrm{a}\end{array}$ & $\begin{array}{c}\mathrm{CeO} 2 \\
-\mathrm{a}\end{array}$ & $\begin{array}{l}\mathrm{Cl} \\
-\mathrm{a}\end{array}$ & $\begin{array}{c}\mathrm{CoO} \\
-\mathrm{a}\end{array}$ & $\begin{array}{c}\mathrm{Co} 2 \mathrm{O} 3 \\
-\mathrm{a}\end{array}$ & $\begin{array}{c}\mathrm{Cr} 2 \mathrm{O} 3 \\
-\mathrm{a}\end{array}$ & $\begin{array}{c}\mathrm{Cs} 2 \mathrm{O} \\
-\mathrm{a}\end{array}$ & $\begin{array}{c}\mathrm{CuO} \\
-\mathrm{a}\end{array}$ & $\begin{array}{c}\mathrm{Eu} 2 \mathrm{O} 3 \\
-\mathrm{a}\end{array}$ & $\begin{array}{c}\mathrm{F} \\
-\mathrm{a}\end{array}$ & $\begin{array}{c}\mathrm{Ga} 2 \mathrm{O} 3 \\
-\mathrm{a}\end{array}$ & $\begin{array}{c}\mathrm{Gd} 2 \mathrm{O} 3 \\
-\mathrm{a}\end{array}$ & $\begin{array}{c}\mathrm{HgO} \\
-\mathrm{a}\end{array}$ \\
\hline BATCH 1-7 & 0.5020 & 0.0010 & & & 0.0015 & & & & & & & & & 0.0011 & 0.0006 & 0.0038 & & & & & \\
\hline BATCH 1-7 (2) & & & & & & & & & & & & & & & & & & & & & \\
\hline BATCH 1-1.6 & & & & & & & & & & & & & & & & & & & & & \\
\hline BATCH 2-7 & 0.5210 & 0.0017 & & & 0.0016 & & & & & & & & & 0.0013 & 0.0002 & 0.0040 & & & & & \\
\hline BATCH 2-7 (2) & & & & & & & & & & & & & & & & & & & & & \\
\hline BATCH 2-1.6 & & & & & & & & & & & & & & & & & & & & & \\
\hline BATCH 3-7 & 0.5260 & 0.0012 & & & 0.0018 & & & & & & & & & 0.0014 & 0.0006 & 0.0038 & & & & & \\
\hline BATCH 3-7 (2) & & & & & & & & & & & & & & & & & & & & & \\
\hline BATCH 3-7 (3) & & & & & & & & & & & & & & & & & & & & & \\
\hline BATCH 4-7 & 0.5010 & 0.0022 & & & 0.0025 & & & & & & & & & 0.0014 & 0.0009 & 0.0043 & & & & & \\
\hline BATCH 4-7 (2) & & & & & & & & & & & & & & & & & & & & & \\
\hline BATCH 4-7 (3) & & & & & & & & & & & & & & & & & & & & & \\
\hline HM-1-7 & 0.5580 & 0.0033 & & & 0.0011 & & & & & & & & & 0.0009 & 0.0006 & 0.0024 & & & & & \\
\hline HM-1-7 (2) & & & & & & & & & & & & & & & & & & & & & \\
\hline HM-1.6-7 & & & & & & & & & & & & & & & & & & & & & \\
\hline PUREX 1-7 & 0.4650 & 0.0005 & & & 0.0020 & & & & & & & & & 0.0015 & 0.0006 & 0.0040 & & & & & \\
\hline PUREX 1-7 (2) & & & & & & & & & & & & & & & & & & & & & \\
\hline PUREX 1.6-7 & & & & & & & & & & & & & & & & & & & & & \\
\hline PUREX SRSS 1.6 & & & & & & & & & & & & & & & & & & & & & \\
\hline PUREX SRST-4.0 & & & & & & & & & & & & & & & & & & & & & \\
\hline PUREX CUA & & & & & & & & & & & & & & & & & & & & & \\
\hline BLEND 1-3457 & 0.4935 & & & & 0.0009 & & & & & & & & & 0.0023 & & 0.0020 & & & & & \\
\hline BLEND 1-3479 & 0.4925 & & & & 0.0010 & & & & & & & & & 0.0022 & & 0.0017 & & & & & \\
\hline BLEND 1-3498 & 0.4806 & & & & 0.0010 & & & & & & & & & 0.0023 & & 0.0019 & & & & & \\
\hline BLEND 1-3510 & 0.4772 & & & & 0.0010 & & & & & & & & & 0.0022 & & 0.0019 & & & & & \\
\hline BLEND 1-3526 & 0.4745 & & & & 0.0009 & & & & & & & & & 0.0020 & & 0.0017 & & & & & \\
\hline BLEND 2-3611 & 0.5019 & & & & 0.0010 & & & & & & & & & 0.0026 & & 0.0017 & & & & & \\
\hline BLEND 2-3622 & 0.5078 & & & & 0.0010 & & & & & & & & & 0.0023 & & 0.0015 & & & & & \\
\hline BLEND 2-3635 & 0.5045 & & & & 0.0010 & & & & & & & & & 0.0023 & & 0.0015 & & & & & \\
\hline BLEND 2-3654 & 0.4977 & & & & 0.0010 & & & & & & & & & 0.0023 & & 0.0015 & & & & & \\
\hline BLEND 2-3666 & 0.5021 & & & & 0.0010 & & & & & & & & & 0.0025 & & 0.0015 & & & & & \\
\hline BLEND 2-3676 & 0.5066 & & & & 0.0010 & & & & & & & & & 0.0022 & & 0.0015 & & & & & \\
\hline BLEND 3-3768 & 0.5000 & 0.0005 & & & 0.0012 & & & & & & & & & 0.0028 & & 0.0019 & & & & & \\
\hline BLEND 3-3789 & 0.4918 & 0.0004 & & & 0.0013 & & & & & & & & & 0.0025 & & 0.0019 & & & & & \\
\hline BLEND 3-3793 & 0.4804 & 0.0003 & & & 0.0014 & & & & & & & & & 0.0025 & & 0.0021 & & & & & \\
\hline BLEND 3-3802B & 0.4775 & 0.0004 & & & 0.0013 & & & & & & & & & 0.0025 & & 0.0020 & & & & & \\
\hline HM 1-3824 & 0.5160 & 0.0002 & & & 0.0010 & & & & & & & & & 0.0022 & & 0.0015 & & & & & \\
\hline HM 1-3829 & 0.5213 & 0.0004 & & & 0.0010 & & & & & & & & & 0.0023 & & 0.0013 & & & & & \\
\hline HM 1-3851 & 0.5200 & 0.0003 & & & 0.0010 & & & & & & & & & 0.0023 & & 0.0013 & & & & & \\
\hline HM 1-3855 & 0.5290 & 0.0002 & & & 0.0010 & & & & & & & & & 0.0022 & & 0.0013 & & & & & \\
\hline HM-2-1 (3979C) & 0.5396 & 0.0008 & & & 0.0009 & & & & & & & & & 0.0051 & & 0.0013 & & & & & \\
\hline HM-2-2 (4099A) & 0.5328 & 0.0006 & & & 0.0009 & & & & & & & & & 0.0047 & & 0.0015 & & & & & \\
\hline HM-2-3 (4120B) & 0.5311 & 0.0007 & & & 0.0009 & & & & & & & & & 0.0039 & & 0.0013 & & & & & \\
\hline HM-3-1 (4176) & 0.5111 & 0.0005 & & & 0.0008 & & & & & & & & & 0.0038 & & 0.0013 & & & & & \\
\hline HM-3-2 (4225) & 0.4973 & 0.0005 & & & 0.0008 & & & & & & & & & 0.0038 & & 0.0015 & & & & & \\
\hline HM-3-3 (4357) & 0.4960 & 0.0007 & & & 0.0009 & & & & & & & & & 0.0041 & & 0.0015 & & & & & \\
\hline HM-4-1 (5260) & 0.5462 & 0.0120 & & & 0.0006 & & & & & & & & & 0.0045 & & 0.0011 & & & & & \\
\hline HM-4-2 (5641) & 0.5595 & 0.0071 & & & 0.0005 & & & & & & & & & 0.0069 & & 0.0020 & & & & & \\
\hline HM-4-3 (5748) & 0.5836 & 0.0046 & & & 0.0005 & & & & & & & & & 0.0039 & & 0.0016 & & & & & \\
\hline
\end{tabular}




\section{DWPF PCT Model (Jantzen et al. 1995)}

\begin{tabular}{|c|c|c|c|c|c|c|c|c|c|c|c|c|c|c|c|c|c|c|c|c|c|}
\hline Glass ID & $\begin{array}{c}\mathrm{I} \\
-\mathrm{a}\end{array}$ & $\begin{array}{c}\mathrm{La} 2 \mathrm{O} 3 \\
-\mathrm{a}\end{array}$ & $\begin{array}{c}\mathrm{MnO} 2 \\
-\mathrm{a}\end{array}$ & $\begin{array}{c}\mathrm{MnO} \\
-\mathrm{a}\end{array}$ & $\begin{array}{c}\mathrm{MoO} \\
-\mathrm{a}\end{array}$ & $\begin{array}{c}\mathrm{MoO} 3 \\
-\mathrm{a}\end{array}$ & $\begin{array}{c}\mathrm{Nb} 2 \mathrm{O} 5 \\
-\mathrm{a}\end{array}$ & $\begin{array}{c}\mathrm{Nd} 2 \mathrm{O} 3 \\
-\mathrm{a}\end{array}$ & $\begin{array}{c}\mathrm{PbO} \\
-\mathrm{a}\end{array}$ & $\begin{array}{c}\mathrm{PdO} 2 \\
-\mathrm{a}\end{array}$ & $\begin{array}{c}\mathrm{PdO} \\
-\mathrm{a}\end{array}$ & $\begin{array}{c}\mathrm{Pr} 2 \mathrm{O} 3 \\
-\mathrm{a}\end{array}$ & $\begin{array}{c}\text { Pr6O11 } \\
-\mathrm{a}\end{array}$ & $\begin{array}{c}\mathrm{Rb} 2 \mathrm{O} \\
-\mathrm{a}\end{array}$ & $\begin{array}{c}\mathrm{ReO} \\
-\mathrm{a}\end{array}$ & $\begin{array}{c}\mathrm{ReO} 2 \\
-\mathrm{a}\end{array}$ & $\begin{array}{c}\mathrm{Rh} 2 \mathrm{O} 3 \\
-\mathrm{a}\end{array}$ & $\begin{array}{c}\mathrm{RhO} 2 \\
-\mathrm{a}\end{array}$ & $\begin{array}{c}\mathrm{RuO} 2 \\
-\mathrm{a}\end{array}$ & $\begin{array}{c}\mathrm{Sb} 2 \mathrm{O} 3 \\
-\mathrm{a}\end{array}$ & $\begin{array}{c}\mathrm{Sb} 2 \mathrm{O} 5 \\
-\mathrm{a}\end{array}$ \\
\hline BATCH 1-7 & & & & 0.0172 & & 0.0011 & & 0.0015 & & & & & & & & & & & & & \\
\hline BATCH 1-7 (2) & & & & & & & & & & & & & & & & & & & & & \\
\hline BATCH 1-1.6 & & & & & & & & & & & & & & & & & & & & & \\
\hline BATCH 2-7 & & & & 0.0141 & & 0.0017 & & 0.0026 & & & & & & & & & & & & & \\
\hline BATCH 2-7 (2) & & & & & & & & & & & & & & & & & & & & & \\
\hline BATCH 2-1.6 & & & & & & & & & & & & & & & & & & & & & \\
\hline BATCH 3-7 & & & & 0.0153 & & 0.0012 & & 0.0017 & & & & & & & & & & & & & \\
\hline BATCH 3-7 (2) & & & & & & & & & & & & & & & & & & & & & \\
\hline BATCH 3-7 (3) & & & & & & & & & & & & & & & & & & & & & \\
\hline BATCH 4-7 & & & & 0.0254 & & 0.0020 & & 0.0039 & & & & & & & & & & & & & \\
\hline BATCH 4-7 (2) & & & & & & & & & & & & & & & & & & & & & \\
\hline BATCH 4-7 (3) & & & & & & & & & & & & & & & & & & & & & \\
\hline HM-1-7 & & & & 0.0175 & & 0.0022 & & 0.0055 & & & & & & & & & & & & & \\
\hline HM-1-7 (2) & & & & & & & & & & & & & & & & & & & & & \\
\hline HM-1.6-7 & & & & & & & & & & & & & & & & & & & & & \\
\hline PUREX 1-7 & & & & 0.0169 & & 0.0008 & & 0.0006 & & & & & & & & & & & & & \\
\hline PUREX 1-7 (2) & & & & & & & & & & & & & & & & & & & & & \\
\hline PUREX 1.6-7 & & & & & & & & & & & & & & & & & & & & & \\
\hline PUREX SRSS 1.6 & & & & & & & & & & & & & & & & & & & & & \\
\hline PUREX SRST-4.0 & & & & & & & & & & & & & & & & & & & & & \\
\hline PUREX CUA & & & & & & & & & & & & & & & & & & & & & \\
\hline BLEND 1-3457 & & & & 0.0207 & & & & & & & & & & & & & & & & & \\
\hline BLEND 1-3479 & & & & 0.0211 & & & & & & & & & & & & & & & & & \\
\hline BLEND 1-3498 & & & & 0.0219 & & & & & & & & & & & & & & & & & \\
\hline BLEND 1-3510 & & & & 0.0219 & & & & & & & & & & & & & & & & & \\
\hline BLEND 1-3526 & & & & 0.0211 & & & & & & & & & & & & & & & & & \\
\hline BLEND 2-3611 & & & & 0.0209 & & & & & & & & & & & & & & & & & \\
\hline BLEND 2-3622 & & & & 0.0210 & & & & & & & & & & & & & & & & & \\
\hline BLEND 2-3635 & & & & 0.0206 & & & & & & & & & & & & & & & & & \\
\hline BLEND 2-3654 & & & & 0.0206 & & & & & & & & & & & & & & & & & \\
\hline BLEND 2-3666 & & & & 0.0210 & & & & & & & & & & & & & & & & & \\
\hline BLEND 2-3676 & & & & 0.0206 & & & & & & & & & & & & & & & & & \\
\hline BLEND 3-3768 & & & & 0.0221 & & & & & & & & & & & & & & & & & \\
\hline BLEND 3-3789 & & & & 0.0244 & & & & & & & & & & & & & & & & & \\
\hline BLEND 3-3793 & & & & 0.0251 & & & & & & & & & & & & & & & & & \\
\hline BLEND 3-3802B & & & & 0.0248 & & & & & & & & & & & & & & & & & \\
\hline HM 1-3824 & & & & 0.0240 & & & & & & & & & & & & & & & & & \\
\hline HM 1-3829 & & & & 0.0254 & & & & & & & & & & & & & & & & & \\
\hline HM 1-3851 & & & & 0.0254 & & & & & & & & & & & & & & & & & \\
\hline HM 1-3855 & & & & 0.0263 & & & & & & & & & & & & & & & & & \\
\hline HM-2-1 (3979C) & & & & 0.0279 & & & & & 0.0005 & & & & & & & & & & & & \\
\hline HM-2-2 (4099A) & & & & 0.0280 & & & & & 0.0006 & & & & & & & & & & & & \\
\hline HM-2-3 (4120B) & & & & 0.0268 & & & & & 0.0005 & & & & & & & & & & & & \\
\hline HM-3-1 (4176) & & & & 0.0248 & & & & & 0.0004 & & & & & & & & & & & & \\
\hline HM-3-2 (4225) & & & & 0.0273 & & & & & 0.0005 & & & & & & & & & & & & \\
\hline HM-3-3 (4357) & & & & 0.0290 & & & & & 0.0004 & & & & & & & & & & & & \\
\hline HM-4-1 (5260) & & & & 0.0151 & & 0.0007 & & & 0.0010 & & & & & & & & & & & & \\
\hline HM-4-2 (5641) & & 0.0002 & & 0.0172 & & 0.0004 & & & 0.0007 & & & & & & & & & & & & \\
\hline HM-4-3 (5748) & & & & 0.0172 & & 0.0002 & & & 0.0003 & & & & & & & & & & & & \\
\hline
\end{tabular}


Appendix A. Database - mass fraction

DWPF PCT Model (Jantzen et al. 1995)

\begin{tabular}{|c|c|c|c|c|c|c|c|c|c|c|c|c|c|c|c|c|c|c|c|c|}
\hline Glass ID & $\begin{array}{c}\mathrm{SeO} 2 \\
-\mathrm{a}\end{array}$ & $\begin{array}{c}\mathrm{Sm} 2 \mathrm{O} 3 \\
-\mathrm{a}\end{array}$ & $\begin{array}{c}\mathrm{SnO} \\
-\mathrm{a}\end{array}$ & $\begin{array}{c}\mathrm{SnO} 2 \\
-\mathrm{a}\end{array}$ & $\begin{array}{c}\mathrm{SO} 3 \\
-\mathrm{a}\end{array}$ & $\begin{array}{c}\mathrm{SrO} \\
-\mathrm{a}\end{array}$ & $\begin{array}{c}\mathrm{Tc} 2 \mathrm{O} 7 \\
-\mathrm{a}\end{array}$ & $\begin{array}{c}\mathrm{TeO} 2 \\
-\mathrm{a}\end{array}$ & $\begin{array}{c}\mathrm{ThO} 2 \\
-\mathrm{a}\end{array}$ & $\begin{array}{c}\mathrm{TiO} 2 \\
-\mathrm{a}\end{array}$ & $\begin{array}{c}\mathrm{T} 12 \mathrm{O} 3 \\
-\mathrm{a}\end{array}$ & $\begin{array}{c}\mathrm{U} 3 \mathrm{O} 8 \\
-\mathrm{a}\end{array}$ & $\begin{array}{c}\mathrm{UO} 2 \\
-\mathrm{a}\end{array}$ & $\begin{array}{c}\mathrm{UO} 3 \\
-\mathrm{a}\end{array}$ & $\begin{array}{c}\mathrm{V} 2 \mathrm{O} 5 \\
-\mathrm{a}\end{array}$ & $\begin{array}{c}\text { WO3 } \\
-\mathrm{a}\end{array}$ & $\begin{array}{c}\mathrm{Y} 2 \mathrm{O} 3 \\
-\mathrm{a}\end{array}$ & $\begin{array}{c}\mathrm{ZnO} \\
-\mathrm{a}\end{array}$ & $\begin{array}{c}\text { Others } \\
-\mathrm{a}\end{array}$ & $\begin{array}{c}\text { Sum } \\
-\mathrm{a}\end{array}$ \\
\hline BATCH 1-7 & & & & & & & & & & 0.0068 & & & & & & & & & & 0.9931 \\
\hline BATCH 1-7 (2) & & & & & & & & & & & & & & & & & & & & \\
\hline BATCH 1-1.6 & & & & & & & & & & & & & & & & & & & & \\
\hline BATCH 2-7 & & & & & & & & & & 0.0069 & & & & & & & & & & 0.9963 \\
\hline BATCH 2-7 (2) & & & & & & & & & & & & & & & & & & & & \\
\hline BATCH 2-1.6 & & & & & & & & & & & & & & & & & & & & \\
\hline BATCH 3-7 & & & & & & & & & & 0.0068 & & & & & & & & & & 0.9920 \\
\hline BATCH 3-7 (2) & & & & & & & & & & & & & & & & & & & & \\
\hline BATCH 3-7 (3) & & & & & & & & & & & & & & & & & & & & \\
\hline BATCH $4-7$ & & & & & & & & & & 0.0103 & & & & & & & & & & 0.9931 \\
\hline BATCH 4-7 (2) & & & & & & & & & & & & & & & & & & & & \\
\hline BATCH 4-7 (3) & & & & & & & & & & & & & & & & & & & & \\
\hline HM-1-7 & & & & & & & & & & 0.0056 & & & & & & & & & & 0.9997 \\
\hline HM-1-7 (2) & & & & & & & & & & & & & & & & & & & & \\
\hline HM-1.6-7 & & & & & & & & & & & & & & & & & & & & \\
\hline PUREX 1-7 & & & & & & & & & & 0.0068 & & & & & & & & & & 0.9938 \\
\hline PUREX 1-7 (2) & & & & & & & & & & & & & & & & & & & & \\
\hline PUREX 1.6-7 & & & & & & & & & & & & & & & & & & & & \\
\hline PUREX SRSS 1.6 & & & & & & & & & & & & & & & & & & & & \\
\hline PUREX SRST-4.0 & & & & & & & & & & & & & & & & & & & & \\
\hline PUREX CUA & & & & & & & & & & & & & & & & & & & & \\
\hline BLEND 1-3457 & & & & & & & & & & 0.0024 & & & & & & & & & & 0.9620 \\
\hline BLEND 1-3479 & & & & & & & & & & 0.0022 & & & & & & & & & & 0.9581 \\
\hline BLEND 1-3498 & & & & & & & & & & 0.0019 & & & & & & & & & & 0.9588 \\
\hline BLEND 1-3510 & & & & & & & & & & 0.0019 & & & & & & & & & & 0.9573 \\
\hline BLEND 1-3526 & & & & & & & & & & 0.0017 & & & & & & & & & & 0.9446 \\
\hline BLEND 2-3611 & & & & & & & & & & 0.0017 & & & & & & & & & & 0.9599 \\
\hline BLEND 2-3622 & & & & & & & & & & 0.0015 & & & & & & & & & & 0.9644 \\
\hline BLEND 2-3635 & & & & & & & & & & 0.0017 & & & & & & & & & & 0.9564 \\
\hline BLEND 2-3654 & & & & & & & & & & 0.0014 & & & & & & & & & & 0.9445 \\
\hline BLEND 2-3666 & & & & & & & & & & 0.0014 & & & & & & & & & & 0.9552 \\
\hline BLEND 2-3676 & & & & & & & & & & 0.0014 & & & & & & & & & & 0.9567 \\
\hline BLEND 3-3768 & & & & & & & & & & 0.0013 & & & & & & & & & & 0.9616 \\
\hline BLEND 3-3789 & & & & & & & & & & 0.0013 & & & & & & & & & & 0.9754 \\
\hline BLEND 3-3793 & & & & & & & & & & 0.0012 & & & & & & & & & & 0.9714 \\
\hline BLEND 3-3802B & & & & & & & & & & 0.0012 & & & & & & & & & & 0.9606 \\
\hline HM 1-3824 & & & & & & & & & & 0.0009 & & & & & & & & & & 0.9688 \\
\hline HM 1-3829 & & & & & & & & & & 0.0010 & & & & & & & & & & 0.9747 \\
\hline HM 1-3851 & & & & & & & & & & 0.0009 & & & & & & & & & & 0.9733 \\
\hline HM 1-3855 & & & & & & & & & & 0.0009 & & & & & & & & & & 0.9803 \\
\hline HM-2-1 (3979C) & & & & & & 0.0015 & & & & 0.0009 & & & & & & & & 0.0010 & & 1.0201 \\
\hline HM-2-2 (4099A) & & & & & & 0.0016 & & & & 0.0012 & & & & & & & & 0.0010 & & 1.0175 \\
\hline HM-2-3 (4120B) & & & & & & 0.0015 & & & & 0.0011 & & & & & & & & 0.0009 & & 1.0076 \\
\hline HM-3-1 (4176) & & & & & & 0.0014 & & & & 0.0010 & & & & & & & & 0.0008 & & 0.9680 \\
\hline HM-3-2 (4225) & & & & & & 0.0015 & & & & 0.0010 & & & & & & & & 0.0009 & & 0.9763 \\
\hline HM-3-3 (4357) & & & & & & 0.0015 & & & & 0.0010 & & & & & & & & 0.0008 & & 0.9867 \\
\hline HM-4-1 (5260) & & & & & & 0.0008 & & & & 0.0026 & & & & & & & & 0.0012 & & 1.0094 \\
\hline HM-4-2 (5641) & & & & & & 0.0009 & & & & 0.0027 & & & & & & & & 0.0011 & & 1.0367 \\
\hline HM-4-3 (5748) & & & & & & 0.0009 & & & & 0.0027 & & & & & & & & 0.0011 & & 1.0360 \\
\hline
\end{tabular}

A. 215

Pacific Northwest National Laboratory 
Appendix A. Database - mass fraction

\section{DWPF PCT Model (Jantzen et al. 1995)}

\begin{tabular}{|c|c|c|c|c|c|c|c|c|}
\hline Glass ID & $\begin{array}{l}\mathrm{TM} \\
\left({ }^{\circ} \mathrm{C}\right) \\
\end{array}$ & $\begin{array}{l}\text { Gradient } \\
\text { TL }\left({ }^{\circ} \mathrm{C}\right) \\
\end{array}$ & $\begin{array}{l}\text { Uniform } \\
\text { TL }\left({ }^{\circ} \mathrm{C}\right)\end{array}$ & Primary Phase & $\begin{array}{l}\text { Quenched } \\
\text { Visual/OM }\end{array}$ & $\begin{array}{c}\text { Quenched } \\
\text { SEM/EDS or TEM }\end{array}$ & $\begin{array}{l}\text { Quenched } \\
\text { XRD } \\
\end{array}$ & $\begin{array}{c}\text { Quenched } \\
\text { Homogeneous? }\end{array}$ \\
\hline \multicolumn{9}{|l|}{ BATCH $1-7$} \\
\hline \multicolumn{9}{|l|}{ BATCH 1-7 (2) } \\
\hline \multicolumn{9}{|l|}{ BATCH 1-1.6 } \\
\hline \multicolumn{9}{|l|}{ BATCH 2-7 } \\
\hline \multicolumn{9}{|l|}{ BATCH 2-7 (2) } \\
\hline \multicolumn{9}{|l|}{ BATCH 2-1.6 } \\
\hline \multicolumn{9}{|l|}{ BATCH 3-7 } \\
\hline \multicolumn{9}{|l|}{ BATCH 3-7 (2) } \\
\hline \multicolumn{9}{|l|}{ BATCH 3-7 (3) } \\
\hline \multicolumn{9}{|l|}{ BATCH 4-7 } \\
\hline \multicolumn{9}{|l|}{ BATCH 4-7 (2) } \\
\hline \multicolumn{9}{|l|}{ BATCH 4-7 (3) } \\
\hline \multicolumn{9}{|l|}{ HM-1-7 } \\
\hline \multicolumn{9}{|l|}{ HM-1-7 (2) } \\
\hline \multicolumn{9}{|l|}{ HM-1.6-7 } \\
\hline \multicolumn{9}{|l|}{ PUREX 1-7 } \\
\hline \multicolumn{9}{|l|}{ PUREX 1-7 (2) } \\
\hline \multicolumn{9}{|l|}{ PUREX 1.6-7 } \\
\hline \multicolumn{9}{|l|}{ PUREX SRSS 1.6} \\
\hline \multicolumn{9}{|l|}{ PUREX SRST-4.0 } \\
\hline PUREX CUA & & & & & & & & \\
\hline BLEND 1-3457 & & & & & & & & \\
\hline BLEND 1-3479 & & & & & & & & \\
\hline BLEND 1-3498 & & & & & & & & \\
\hline BLEND 1-3510 & & & & & & & & \\
\hline BLEND 1-3526 & & & & & & & & \\
\hline BLEND 2-3611 & & & & & & & & \\
\hline BLEND 2-3622 & & & & & & & & \\
\hline BLEND 2-3635 & & & & & & & & \\
\hline BLEND 2-3654 & & & & & & & & \\
\hline BLEND 2-3666 & & & & & & & & \\
\hline BLEND 2-3676 & & & & & & & & \\
\hline BLEND 3-3768 & & & & & & & & \\
\hline BLEND 3-3789 & & & & & & & & \\
\hline BLEND 3-3793 & & & & & & & & \\
\hline BLEND 3-3802B & & & & & & & & \\
\hline HM 1-3824 & & & & & & & & \\
\hline HM 1-3829 & & & & & & & & \\
\hline HM 1-3851 & & & & & & & & \\
\hline HM 1-3855 & & & & & & & & \\
\hline HM-2-1 (3979C) & & & & & & & & \\
\hline HM-2-2 (4099A) & & & & & & & & \\
\hline HM-2-3 (4120B) & & & & & & & & \\
\hline HM-3-1 (4176) & & & & & & & & \\
\hline HM-3-2 (4225) & & & & & & & & \\
\hline HM-3-3 (4357) & & & & & & & & \\
\hline HM-4-1 (5260) & & & & & & & & \\
\hline HM-4-2 (5641) & & & & & & & & \\
\hline HM-4-3 (5748) & & & & & & & & \\
\hline
\end{tabular}


Appendix A. Database - mass fraction

\section{DWPF PCT Model (Jantzen et al. 1995)}

\begin{tabular}{|c|c|c|c|c|c|}
\hline Glass ID & $\begin{array}{c}\mathrm{CCC} \\
\text { Visual/OM }\end{array}$ & $\begin{array}{c}\text { CCC } \\
\text { SEM/EDS or TEM }\end{array}$ & $\begin{array}{l}\text { CCC } \\
\text { XRD }\end{array}$ & $\begin{array}{l}\text { Heat Treated } \\
\text { Visual/OM }\end{array}$ & $\begin{array}{c}\text { Heat Treated } \\
\text { SEM/EDS or TEM }\end{array}$ \\
\hline \multicolumn{6}{|l|}{ BATCH 1-7 } \\
\hline \multicolumn{6}{|l|}{ BATCH 1-7 (2) } \\
\hline \multicolumn{6}{|l|}{ BATCH 1-1.6 } \\
\hline \multicolumn{6}{|l|}{ BATCH 2-7 } \\
\hline \multicolumn{6}{|l|}{ BATCH 2-7 (2) } \\
\hline \multicolumn{6}{|l|}{ BATCH 2-1.6 } \\
\hline \multicolumn{6}{|l|}{ BATCH 3-7 } \\
\hline \multicolumn{6}{|l|}{ BATCH 3-7 (2) } \\
\hline \multicolumn{6}{|l|}{ BATCH 3-7 (3) } \\
\hline \multicolumn{6}{|l|}{ BATCH 4-7 } \\
\hline \multicolumn{6}{|l|}{ BATCH 4-7 (2) } \\
\hline \multicolumn{6}{|l|}{ BATCH 4-7 (3) } \\
\hline \multicolumn{6}{|l|}{ HM-1-7 } \\
\hline \multicolumn{6}{|l|}{ HM-1-7 (2) } \\
\hline \multicolumn{6}{|l|}{ HM-1.6-7 } \\
\hline \multicolumn{6}{|l|}{ PUREX 1-7 } \\
\hline \multicolumn{6}{|l|}{ PUREX 1-7 (2) } \\
\hline \multicolumn{6}{|l|}{ PUREX 1.6-7 } \\
\hline \multicolumn{6}{|l|}{ PUREX SRSS 1.6} \\
\hline \multicolumn{6}{|l|}{ PUREX SRST-4.0 } \\
\hline \multicolumn{6}{|l|}{ PUREX CUA } \\
\hline BLEND 1-3457 & & & & & \\
\hline BLEND 1-3479 & & & & & \\
\hline BLEND 1-3498 & & & & & \\
\hline BLEND 1-3510 & & & & & \\
\hline BLEND 1-3526 & & & & & \\
\hline BLEND 2-3611 & & & & & \\
\hline BLEND 2-3622 & & & & & \\
\hline BLEND 2-3635 & & & & & \\
\hline BLEND 2-3654 & & & & & \\
\hline BLEND 2-3666 & & & & & \\
\hline BLEND 2-3676 & & & & & \\
\hline BLEND 3-3768 & & & & & \\
\hline BLEND 3-3789 & & & & & \\
\hline BLEND 3-3793 & & & & & \\
\hline BLEND 3-3802B & & & & & \\
\hline HM 1-3824 & & & & & \\
\hline HM 1-3829 & & & & & \\
\hline HM 1-3851 & & & & & \\
\hline HM 1-3855 & & & & & \\
\hline HM-2-1 (3979C) & & & & & \\
\hline HM-2-2 (4099A) & & & & & \\
\hline HM-2-3 (4120B) & & & & & \\
\hline HM-3-1 (4176) & & & & & \\
\hline HM-3-2 (4225) & & & & & \\
\hline HM-3-3 (4357) & & & & & \\
\hline HM-4-1 (5260) & & & & & \\
\hline HM-4-2 (5641) & & & & & \\
\hline HM-4-3 (5748) & & & & & \\
\hline
\end{tabular}


Appendix A. Database - mass fraction

\section{DWPF PCT Model (Jantzen et al. 1995)}

\begin{tabular}{|c|c|c|c|c|c|c|c|c|c|c|c|c|c|c|c|}
\hline Glass ID & $\begin{array}{c}\text { Heat Treated } \\
\text { XRD } \\
\end{array}$ & $\begin{array}{l}\text { Density } \\
\left(\mathrm{g} / \mathrm{cm}^{3}\right)\end{array}$ & \begin{tabular}{|c|} 
Fulc Visc \\
$\mathrm{A}$
\end{tabular} & \begin{tabular}{|c|} 
Fulc Visc \\
$\mathrm{B}$
\end{tabular} & \begin{tabular}{|c|} 
Fulc Visc \\
To
\end{tabular} & $\begin{array}{c}\mathrm{FV} 1150^{\circ} \mathrm{C} \\
(\mathrm{Pa} \cdot \mathrm{s})\end{array}$ & $\begin{array}{c}\text { Arrh Visc } \\
\text { A }\end{array}$ & \begin{tabular}{|c|} 
Arrh Visc \\
B
\end{tabular} & $1150^{\circ} \mathrm{C}$ & $\begin{array}{c}\mathrm{T}\left({ }^{\circ} \mathrm{C}\right) \text { at } \\
2 \mathrm{~Pa} \cdot \mathrm{s}\end{array}$ & \begin{tabular}{|c|}
$\mathrm{T}\left({ }^{\circ} \mathrm{C}\right)$ at \\
$5 \mathrm{~Pa} \cdot \mathrm{s}$ \\
\end{tabular} & $\begin{array}{l}\mathrm{T}\left({ }^{\circ} \mathrm{C}\right) \text { at } \\
10 \mathrm{~Pa} \cdot \mathrm{s}\end{array}$ & $\begin{array}{l}\mathrm{T} 1 \\
\left({ }^{\circ} \mathrm{C}\right)\end{array}$ & $\begin{array}{c}\mathrm{V} 1 \\
(\mathrm{~Pa} \cdot \mathrm{s})\end{array}$ & $\begin{array}{l}\mathrm{T} 2 \\
\left({ }^{\circ} \mathrm{C}\right) \\
\end{array}$ \\
\hline \multicolumn{16}{|c|}{ 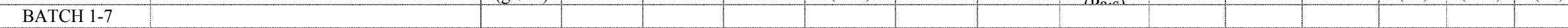 } \\
\hline \multicolumn{16}{|l|}{ BATCH 1-7 (2) } \\
\hline \multicolumn{16}{|l|}{ BATCH 1-1.6 } \\
\hline \multicolumn{16}{|l|}{ BATCH 2-7 } \\
\hline \multirow{2}{*}{\multicolumn{16}{|c|}{ BATCH 2-7 (2) }} \\
\hline \multirow{2}{*}{\multicolumn{15}{|c|}{$\begin{array}{c}\text { BATCH 2-1.6 } \\
\text { BATCH 3-7 }\end{array}$}} & \\
\hline & & & & & & & & & & & & & & & \\
\hline \multicolumn{16}{|l|}{ BATCH 3-7 (2) } \\
\hline \multicolumn{16}{|l|}{ BATCH 3-7 (3) } \\
\hline \multirow{2}{*}{\multicolumn{16}{|c|}{$\begin{array}{l}\text { BATCH 4-7 } \\
\text { BATCH 4-7 (2) }\end{array}$}} \\
\hline \multicolumn{15}{|l|}{ BATCH 4-7 (2) } & \\
\hline BATCH 4-7 (3) & & & & & & & & & & & & & & & \\
\hline HM-1-7 & & & & & & & & & & & & & & & \\
\hline \multicolumn{16}{|l|}{ HM-1-7 (2) } \\
\hline \multicolumn{16}{|l|}{ HM-1.6-7 } \\
\hline \multicolumn{16}{|l|}{ PUREX 1-7 } \\
\hline \multirow{2}{*}{\multicolumn{16}{|c|}{$\begin{array}{c}\text { PUREX 1-7 (2) } \\
\text { PUREX 1.6-7 }\end{array}$}} \\
\hline PUREX 1.6-7 & & & & & & & & & & & & & & & \\
\hline PUREX SRSS 1.6 & & & & & & & & & & & & & & & \\
\hline PUREX SRST-4.0 & & & & & & & & & & & & & & & \\
\hline PUREX CUA & & & & & & & & & & & & & & & \\
\hline BLEND 1-3457 & & & & & & & & & & & & & & & \\
\hline BLEND 1-3479 & & & & & & & & & & & & & & & \\
\hline BLEND 1-3498 & & & & & & & & & & & & & & & \\
\hline BLEND 1-3510 & & & & & & & & & & & & & & & \\
\hline BLEND 1-3526 & & & & & & & & & & & & & & & \\
\hline BLEND 2-3611 & & & & & & & & & & & & & & & \\
\hline BLEND 2-3622 & & & & & & & & & & & & & & & \\
\hline BLEND 2-3635 & & & & & & & & & & & & & & & \\
\hline BLEND 2-3654 & & & & & & & & & & & & & & & \\
\hline BLEND 2-3666 & & & & & & & & & & & & & & & \\
\hline BLEND 2-3676 & & & & & & & & & & & & & & & \\
\hline BLEND 3-3768 & & & & & & & & & & & & & & & \\
\hline BLEND 3-3789 & & & & & & & & & & & & & & & \\
\hline BLEND 3-3793 & & & & & & & & & & & & & & & \\
\hline BLEND 3-3802B & & & & & & & & & & & & & & & \\
\hline HM 1-3824 & & & & & & & & & & & & & & & \\
\hline HM 1-3829 & & & & & & & & & & & & & & & \\
\hline HM 1-3851 & & & & & & & & & & & & & & & \\
\hline HM 1-3855 & & & & & & & & & & & & & & & \\
\hline HM-2-1 (3979C) & & & & & & & & & & & & & & & \\
\hline HM-2-2 (4099A) & & & & & & & & & & & & & & & \\
\hline HM-2-3 (4120B) & & & & & & & & & & & & & & & \\
\hline HM-3-1 (4176) & & & & & & & & & & & & & & & \\
\hline HM-3-2 (4225) & & & & & & & & & & & & & & & \\
\hline HM-3-3 (4357) & & & & & & & & & & & & & & & \\
\hline HM-4-1 (5260) & & & & & & & & & & & & & & & \\
\hline HM-4-2 (5641) & & & & & & & & & & & & & & & \\
\hline HM-4-3 (5748) & & & & & & & & & & & & & & & \\
\hline
\end{tabular}


Appendix A. Database - mass fraction

DWPF PCT Model (Jantzen et al. 1995)

\begin{tabular}{|c|c|c|c|c|c|c|c|c|c|c|c|c|c|c|c|c|c|c|c|c|c|}
\hline Glass ID & $\begin{array}{c}\mathrm{V} 2 \\
(\mathrm{~Pa} \cdot \mathrm{s})\end{array}$ & $\begin{array}{c}\mathrm{T} 3 \\
\left({ }^{\circ} \mathrm{C}\right)\end{array}$ & $\begin{array}{c}\mathrm{V} 3 \\
(\mathrm{~Pa} \cdot \mathrm{s})\end{array}$ & $\begin{array}{l}\mathrm{T} 4 \\
\left({ }^{\circ} \mathrm{C}\right)\end{array}$ & $\begin{array}{c}\mathrm{V} 4 \\
(\mathrm{~Pa} \cdot \mathrm{s})\end{array}$ & $\begin{array}{l}\text { T5 } \\
\left({ }^{\circ} \mathrm{C}\right)\end{array}$ & $\begin{array}{c}\text { V5 } \\
(\mathrm{Pa} \cdot \mathrm{s})\end{array}$ & $\begin{array}{c}\mathrm{T} 6 \\
\left({ }^{\circ} \mathrm{C}\right)\end{array}$ & $\begin{array}{c}\mathrm{V} 6 \\
(\mathrm{~Pa} \cdot \mathrm{s})\end{array}$ & $\begin{array}{c}\mathrm{T} 7 \\
\left({ }^{\circ} \mathrm{C}\right)\end{array}$ & $\begin{array}{c}\text { V7 } \\
(\mathrm{Pa} \cdot \mathrm{s})\end{array}$ & $\begin{array}{c}\mathrm{T} 8 \\
\left({ }^{\circ} \mathrm{C}\right)\end{array}$ & $\begin{array}{c}\mathrm{V} 8 \\
(\mathrm{~Pa} \cdot \mathrm{s})\end{array}$ & $\begin{array}{c}\mathrm{T} 9 \\
\left({ }^{\circ} \mathrm{C}\right)\end{array}$ & $\begin{array}{c}\mathrm{V} 9 \\
(\mathrm{~Pa} \cdot \mathrm{s})\end{array}$ & $\begin{array}{l}\mathrm{T} 10 \\
\left({ }^{\circ} \mathrm{C}\right)\end{array}$ & $\begin{array}{c}\mathrm{V} 10 \\
(\mathrm{~Pa} \cdot \mathrm{s})\end{array}$ & $\begin{array}{l}\mathrm{T} 11 \\
\left({ }^{\circ} \mathrm{C}\right)\end{array}$ & $\begin{array}{c}\mathrm{V} 11 \\
(\mathrm{~Pa} \cdot \mathrm{s})\end{array}$ & $\begin{array}{l}\mathrm{T} 12 \\
\left({ }^{\circ} \mathrm{C}\right)\end{array}$ & $\begin{array}{c}\mathrm{V} 12 \\
(\mathrm{~Pa} \cdot \mathrm{s})\end{array}$ \\
\hline BATCH 1-7 & & & & & & & & & & & & & & & & & & & & & \\
\hline BATCH 1-7 (2) & & & & & & & & & & & & & & & & & & & & & \\
\hline BATCH 1-1.6 & & & & & & & & & & & & & & & & & & & & & \\
\hline BATCH 2-7 & & & & & & & & & & & & & & & & & & & & & \\
\hline BATCH 2-7 (2) & & & & & & & & & & & & & & & & & & & & & \\
\hline BATCH 2-1.6 & & & & & & & & & & & & & & & & & & & & & \\
\hline BATCH 3-7 & & & & & & & & & & & & & & & & & & & & & \\
\hline BATCH 3-7 (2) & & & & & & & & & & & & & & & & & & & & & \\
\hline BATCH 3-7 (3) & & & & & & & & & & & & & & & & & & & & & \\
\hline BATCH 4-7 & & & & & & & & & & & & & & & & & & & & & \\
\hline BATCH 4-7 (2) & & & & & & & & & & & & & & & & & & & & & \\
\hline BATCH 4-7 (3) & & & & & & & & & & & & & & & & & & & & & \\
\hline HM-1-7 & & & & & & & & & & & & & & & & & & & & & \\
\hline HM-1-7 (2) & & & & & & & & & & & & & & & & & & & & & \\
\hline HM-1.6-7 & & & & & & & & & & & & & & & & & & & & & \\
\hline PUREX 1-7 & & & & & & & & & & & & & & & & & & & & & \\
\hline PUREX 1-7 (2) & & & & & & & & & & & & & & & & & & & & & \\
\hline PUREX 1.6-7 & & & & & & & & & & & & & & & & & & & & & \\
\hline PUREX SRSS 1.6 & & & & & & & & & & & & & & & & & & & & & \\
\hline PUREX SRST-4.0 & & & & & & & & & & & & & & & & & & & & & \\
\hline PUREX CUA & & & & & & & & & & & & & & & & & & & & & \\
\hline BLEND 1-3457 & & & & & & & & & & & & & & & & & & & & & \\
\hline BLEND 1-3479 & & & & & & & & & & & & & & & & & & & & & \\
\hline BLEND 1-3498 & & & & & & & & & & & & & & & & & & & & & \\
\hline BLEND 1-3510 & & & & & & & & & & & & & & & & & & & & & \\
\hline BLEND 1-3526 & & & & & & & & & & & & & & & & & & & & & \\
\hline BLEND 2-3611 & & & & & & & & & & & & & & & & & & & & & \\
\hline BLEND 2-3622 & & & & & & & & & & & & & & & & & & & & & \\
\hline BLEND 2-3635 & & & & & & & & & & & & & & & & & & & & & \\
\hline BLEND 2-3654 & & & & & & & & & & & & & & & & & & & & & \\
\hline BLEND 2-3666 & & & & & & & & & & & & & & & & & & & & & \\
\hline BLEND 2-3676 & & & & & & & & & & & & & & & & & & & & & \\
\hline BLEND 3-3768 & & & & & & & & & & & & & & & & & & & & & \\
\hline BLEND 3-3789 & & & & & & & & & & & & & & & & & & & & & \\
\hline BLEND 3-3793 & & & & & & & & & & & & & & & & & & & & & \\
\hline BLEND 3-3802B & & & & & & & & & & & & & & & & & & & & & \\
\hline HM 1-3824 & & & & & & & & & & & & & & & & & & & & & \\
\hline HM 1-3829 & & & & & & & & & & & & & & & & & & & & & \\
\hline HM 1-3851 & & & & & & & & & & & & & & & & & & & & & \\
\hline HM 1-3855 & & & & & & & & & & & & & & & & & & & & & \\
\hline HM-2-1 (3979C) & & & & & & & & & & & & & & & & & & & & & \\
\hline HM-2-2 (4099A) & & & & & & & & & & & & & & & & & & & & & \\
\hline HM-2-3 (4120B) & & & & & & & & & & & & & & & & & & & & & \\
\hline HM-3-1 (4176) & & & & & & & & & & & & & & & & & & & & & \\
\hline HM-3-2 (4225) & & & & & & & & & & & & & & & & & & & & & \\
\hline HM-3-3 (4357) & & & & & & & & & & & & & & & & & & & & & \\
\hline HM-4-1 (5260) & & & & & & & & & & & & & & & & & & & & & \\
\hline HM-4-2 (5641) & & & & & & & & & & & & & & & & & & & & & \\
\hline HM-4-3 (5748) & & & & & & & & & & & & & & & & & & & & & \\
\hline
\end{tabular}




\section{DWPF PCT Model (Jantzen et al. 1995)}

\begin{tabular}{|c|c|c|c|c|c|c|c|c|c|c|c|c|c|c|c|c|}
\hline Glass ID & $\begin{array}{l}\mathrm{T} 13 \\
\left({ }^{\circ} \mathrm{C}\right) \\
\end{array}$ & $\begin{array}{c}\mathrm{V} 13 \\
(\mathrm{~Pa} \cdot \mathrm{s}) \\
\end{array}$ & $\begin{array}{l}\mathrm{T} 14 \\
\left({ }^{\circ} \mathrm{C}\right) \\
\end{array}$ & $\begin{array}{c}\mathrm{V} 14 \\
(\mathrm{~Pa} \cdot \mathrm{s}) \\
\end{array}$ & $\begin{array}{c}\text { Q PCT } \\
\mathrm{B}\left(\mathrm{g} / \mathrm{m}^{2}\right)\end{array}$ & $\begin{array}{c}\text { Q PCT } \\
\mathrm{Li}\left(\mathrm{g} / \mathrm{m}^{2}\right)\end{array}$ & $\begin{array}{c}\text { Q PCT } \\
\mathrm{Na}\left(\mathrm{g} / \mathrm{m}^{2}\right)\end{array}$ & $\begin{array}{c}\text { Q PCT } \\
\mathrm{Si}\left(\mathrm{g} / \mathrm{m}^{2}\right)\end{array}$ & $\begin{array}{c}\text { Q PCT } \\
\text { pH }\end{array}$ & $\begin{array}{c}\text { CCC PCT } \\
\mathrm{B}\left(\mathrm{g} / \mathrm{m}^{2}\right) \\
\end{array}$ & $\begin{array}{l}\text { CCC PCT } \\
\mathrm{Li}\left(\mathrm{g} / \mathrm{m}^{2}\right) \\
\end{array}$ & $\begin{array}{l}\text { CCC PCT } \\
\mathrm{Na}\left(\mathrm{g} / \mathrm{m}^{2}\right)\end{array}$ & $\begin{array}{c}\text { CCC PCT } \\
\mathrm{Si}\left(\mathrm{g} / \mathrm{m}^{2}\right)\end{array}$ & $\begin{array}{c}\text { CCC PCT } \\
\mathrm{pH} \\
\end{array}$ & $\begin{array}{l}\text { Q PCT B at } \\
20^{\circ} \mathrm{C}\left(\mathrm{g} / \mathrm{m}^{2}\right)\end{array}$ & $\begin{array}{l}\text { Q PCT Li at } \\
20^{\circ} \mathrm{C}\left(\mathrm{g} / \mathrm{m}^{2}\right)\end{array}$ \\
\hline BATCH 1-7 & & & & & 0.365 & 0.405 & 0.4 & 0.210 & 10.31 & & & & & & & \\
\hline BATCH 1-7 (2) & & & & & 0.365 & 0.38 & 0.375 & 0.205 & 10.47 & & & & & & & \\
\hline BATCH 1-1.6 & & & & & 0.355 & 0.38 & 0.37 & 0.215 & 10.19 & & & & & & & \\
\hline BATCH 2-7 & & & & & 0.33 & 0.385 & 0.365 & 0.200 & 10.18 & & & & & & & \\
\hline BATCH 2-7 (2) & & & & & 0.335 & 0.36 & 0.345 & 0.200 & 10.42 & & & & & & & \\
\hline BATCH 2-1.6 & & & & & 0.32 & 0.355 & 0.335 & 0.200 & 10.09 & & & & & & & \\
\hline BATCH 3-7 & & & & & 0.43 & 0.45 & 0.445 & 0.230 & 10.31 & & & & & & & \\
\hline BATCH 3-7 (2) & & & & & 0.445 & 0.425 & 0.425 & 0.235 & 10.37 & & & & & & & \\
\hline BATCH 3-7 (3) & & & & & 0.425 & 0.425 & 0.415 & 0.240 & 10.11 & & & & & & & \\
\hline BATCH 4-7 & & & & & 0.485 & 0.5 & 0.5 & 0.250 & 10.2 & & & & & & & \\
\hline BATCH 4-7 (2) & & & & & 0.45 & 0.445 & 0.435 & 0.230 & 10.43 & & & & & & & \\
\hline BATCH 4-7 (3) & & & & & 0.47 & 0.47 & 0.47 & 0.250 & 10.24 & & & & & & & \\
\hline HM-1-7 & & & & & 0.23 & 0.31 & 0.245 & 0.150 & 10.09 & & & & & & & \\
\hline HM-1-7 (2) & & & & & 0.23 & 0.285 & 0.23 & 0.150 & 10.28 & & & & & & & \\
\hline HM-1.6-7 & & & & & 0.215 & 0.28 & 0.22 & 0.155 & 9.87 & & & & & & & \\
\hline PUREX 1-7 & & & & & 1.095 & 0.93 & 1.045 & 0.405 & 10.6 & & & & & & & \\
\hline PUREX 1-7 (2) & & & & & 0.985 & 0.825 & 0.88 & 0.360 & 10.64 & & & & & & & \\
\hline PUREX 1.6-7 & & & & & 2.23 & 1.625 & 2.13 & 0.585 & 10.88 & & & & & & & \\
\hline PUREX SRSS 1.6 & & & & & 1.19 & 1.02 & 1.04 & 0.430 & 10.63 & & & & & & & \\
\hline PUREX SRST-4.0 & & & & & 1.13 & 0.98 & 0.995 & 0.425 & 10.62 & & & & & & & \\
\hline PUREX CUA & & & & & 1.425 & 1.185 & 1.21 & 0.480 & 10.75 & & & & & & & \\
\hline BLEND 1-3457 & & & & & 0.3 & 0.335 & 0.325 & 0.190 & 10.22 & & & & & & & \\
\hline BLEND 1-3479 & & & & & 0.29 & 0.32 & 0.315 & 0.185 & 10.26 & & & & & & & \\
\hline BLEND 1-3498 & & & & & 0.315 & 0.35 & 0.335 & 0.195 & 10.31 & & & & & & & \\
\hline BLEND 1-3510 & & & & & 0.34 & 0.37 & 0.365 & 0.205 & 10.37 & & & & & & & \\
\hline BLEND 1-3526 & & & & & 0.335 & 0.37 & 0.36 & 0.210 & 10.28 & & & & & & & \\
\hline BLEND 2-3611 & & & & & 0.305 & 0.335 & 0.335 & 0.195 & 10.21 & & & & & & & \\
\hline BLEND 2-3622 & & & & & 0.305 & 0.33 & 0.33 & 0.195 & 10.24 & & & & & & & \\
\hline BLEND 2-3635 & & & & & 0.305 & 0.325 & 0.33 & 0.195 & 10.14 & & & & & & & \\
\hline BLEND 2-3654 & & & & & 0.3 & 0.325 & 0.32 & 0.195 & 10.23 & & & & & & & \\
\hline BLEND 2-3666 & & & & & 0.3 & 0.325 & 0.32 & 0.195 & 10.1 & & & & & & & \\
\hline BLEND 2-3676 & & & & & 0.295 & 0.32 & 0.315 & 0.195 & 10.07 & & & & & & & \\
\hline BLEND 3-3768 & & & & & 0.3 & 0.32 & 0.33 & 0.185 & 9.99 & & & & & & & \\
\hline BLEND 3-3789 & & & & & 0.315 & 0.33 & 0.35 & 0.185 & 10.14 & & & & & & & \\
\hline BLEND 3-3793 & & & & & 0.315 & 0.33 & 0.355 & 0.190 & 10.13 & & & & & & & \\
\hline BLEND 3-3802B & & & & & 0.35 & 0.355 & 0.385 & 0.195 & 10.18 & & & & & & & \\
\hline HM 1-3824 & & & & & 0.295 & 0.315 & 0.31 & 0.180 & 9.98 & & & & & & & \\
\hline HM 1-3829 & & & & & 0.285 & 0.315 & 0.305 & 0.175 & 9.96 & & & & & & & \\
\hline HM 1-3851 & & & & & 0.26 & 0.3 & 0.275 & 0.180 & 9.98 & & & & & & & \\
\hline HM 1-3855 & & & & & 0.265 & 0.3 & 0.275 & 0.170 & 9.99 & & & & & & & \\
\hline HM-2-1 (3979C) & & & & & 0.265 & 0.305 & 0.27 & 0.185 & 9.56 & & & & & & & \\
\hline HM-2-2 (4099A) & & & & & 0.275 & 0.315 & 0.28 & 0.180 & 9.6 & & & & & & & \\
\hline HM-2-3 (4120B) & & & & & 0.265 & 0.31 & 0.27 & 0.180 & 9.22 & & & & & & & \\
\hline HM-3-1 (4176) & & & & & 0.28 & 0.325 & 0.265 & 0.180 & 9.22 & & & & & & & \\
\hline HM-3-2 (4225) & & & & & 0.275 & 0.32 & 0.26 & 0.180 & 9.22 & & & & & & & \\
\hline HM-3-3 (4357) & & & & & 0.255 & 0.295 & 0.265 & 0.175 & 9.2 & & & & & & & \\
\hline HM-4-1 (5260) & & & & & 0.375 & 0.38 & 0.32 & 0.175 & 9.29 & & & & & & & \\
\hline HM-4-2 (5641) & & & & & 0.265 & 0.285 & 0.26 & 0.150 & 9.32 & & & & & & & \\
\hline HM-4-3 (5748) & & & & & 0.35 & 0.355 & 0.325 & 0.170 & 9.7 & & & & & & & \\
\hline
\end{tabular}


Appendix A. Database - mass fraction

DWPF PCT Model (Jantzen et al. 1995)

\begin{tabular}{|c|c|c|c|c|c|c|c|c|c|c|c|c|}
\hline Glass ID & $\begin{array}{l}\text { Q PCT Na at } \\
20^{\circ} \mathrm{C}\left(\mathrm{g} / \mathrm{m}^{2}\right)\end{array}$ & $\begin{array}{l}\text { Q PCT Si at } \\
20^{\circ} \mathrm{C}\left(\mathrm{g} / \mathrm{m}^{2}\right)\end{array}$ & $\begin{array}{c}\mathrm{QpH} \\
\text { at } 20^{\circ} \mathrm{C}\end{array}$ & $\begin{array}{c}\text { TCLP Ag } \\
\text { (ppm) }\end{array}$ & $\begin{array}{c}\text { TCLP As } \\
(\mathrm{ppm})\end{array}$ & $\begin{array}{c}\text { TCLP Ba } \\
(\mathrm{ppm})\end{array}$ & $\begin{array}{c}\text { TCLP Cd } \\
(\mathrm{ppm})\end{array}$ & $\begin{array}{c}\text { TCLP Cr } \\
(\mathrm{ppm})\end{array}$ & $\begin{array}{c}\text { TCLP Ni } \\
(\mathrm{ppm})\end{array}$ & $\begin{array}{c}\text { TCLP Pb } \\
(\mathrm{ppm})\end{array}$ & $\begin{array}{c}\text { TCLP Se } \\
(\mathrm{ppm})\end{array}$ & $\begin{array}{c}\text { TCLP Zn } \\
\text { (ppm) }\end{array}$ \\
\hline \multicolumn{13}{|l|}{ BATCH 1-7 } \\
\hline \multicolumn{13}{|l|}{ BATCH 1-7 (2) } \\
\hline \multicolumn{13}{|l|}{ BATCH 1-1.6 } \\
\hline \multicolumn{13}{|l|}{ BATCH 2-7 } \\
\hline \multicolumn{13}{|l|}{ BATCH 2-7 (2) } \\
\hline \multicolumn{13}{|l|}{ BATCH 2-1.6 } \\
\hline \multicolumn{13}{|l|}{ BATCH 3-7 } \\
\hline \multicolumn{13}{|l|}{ BATCH 3-7 (2) } \\
\hline \multicolumn{13}{|l|}{ BATCH 3-7 (3) } \\
\hline \multicolumn{13}{|l|}{ BATCH 4-7 } \\
\hline \multicolumn{13}{|l|}{ BATCH 4-7 (2) } \\
\hline \multicolumn{13}{|l|}{ BATCH 4-7 (3) } \\
\hline \multicolumn{13}{|l|}{ HM-1-7 } \\
\hline \multicolumn{13}{|l|}{ HM-1-7 (2) } \\
\hline \multicolumn{13}{|l|}{ HM-1.6-7 } \\
\hline \multicolumn{13}{|l|}{ PUREX 1-7 } \\
\hline \multicolumn{13}{|l|}{ PUREX 1-7 (2) } \\
\hline \multicolumn{13}{|l|}{ PUREX 1.6-7 } \\
\hline PUREX SRSS 1.6 & & & & & & & & & & & & \\
\hline PUREX SRST-4.0 & & & & & & & & & & & & \\
\hline PUREX CUA & & & & & & & & & & & & \\
\hline BLEND 1-3457 & & & & & & & & & & & & \\
\hline BLEND 1-3479 & & & & & & & & & & & & \\
\hline BLEND 1-3498 & & & & & & & & & & & & \\
\hline BLEND 1-3510 & & & & & & & & & & & & \\
\hline BLEND 1-3526 & & & & & & & & & & & & \\
\hline BLEND 2-3611 & & & & & & & & & & & & \\
\hline BLEND 2-3622 & & & & & & & & & & & & \\
\hline BLEND 2-3635 & & & & & & & & & & & & \\
\hline BLEND 2-3654 & & & & & & & & & & & & \\
\hline BLEND 2-3666 & & & & & & & & & & & & \\
\hline BLEND 2-3676 & & & & & & & & & & & & \\
\hline BLEND 3-3768 & & & & & & & & & & & & \\
\hline BLEND 3-3789 & & & & & & & & & & & & \\
\hline BLEND 3-3793 & & & & & & & & & & & & \\
\hline BLEND 3-3802B & & & & & & & & & & & & \\
\hline HM 1-3824 & & & & & & & & & & & & \\
\hline HM 1-3829 & & & & & & & & & & & & \\
\hline HM 1-3851 & & & & & & & & & & & & \\
\hline HM 1-3855 & & & & & & & & & & & & \\
\hline HM-2-1 (3979C) & & & & & & & & & & & & \\
\hline HM-2-2 (4099A) & & & & & & & & & & & & \\
\hline HM-2-3 (4120B) & & & & & & & & & & & & \\
\hline HM-3-1 (4176) & & & & & & & & & & & & \\
\hline HM-3-2 (4225) & & & & & & & & & & & & \\
\hline HM-3-3 (4357) & & & & & & & & & & & & \\
\hline HM-4-1 (5260) & & & & & & & & & & & & \\
\hline HM-4-2 (5641) & & & & & & & & & & & & \\
\hline HM-4-3 (5748) & & & & & & & & & & & & \\
\hline
\end{tabular}


DWPF PCT Model (Jantzen et al. 1995)

\begin{tabular}{|c|c|c|c|c|c|c|c|c|c|c|c|c|c|c|c|c|c|c|c|c|c|}
\hline Glass ID & $\begin{array}{c}\mathrm{A} 12 \mathrm{O} 3 \\
-\mathrm{t}\end{array}$ & $\begin{array}{c}\mathrm{B} 2 \mathrm{O} 3 \\
-\mathrm{t}\end{array}$ & $\begin{array}{c}\mathrm{CaO} \\
-\mathrm{t}\end{array}$ & $\begin{array}{c}\mathrm{Fe} 2 \mathrm{O} 3 \\
-\mathrm{t}\end{array}$ & $\begin{array}{c}\mathrm{FeO} \\
-\mathrm{t}\end{array}$ & $\begin{array}{c}\mathrm{K} 2 \mathrm{O} \\
-\mathrm{t} \\
\end{array}$ & $\begin{array}{c}\mathrm{Li} 2 \mathrm{O} \\
-\mathrm{t}\end{array}$ & $\begin{array}{c}\mathrm{MgO} \\
-\mathrm{t}\end{array}$ & $\begin{array}{c}\mathrm{Na} 2 \mathrm{O} \\
-\mathrm{t}\end{array}$ & $\begin{array}{c}\mathrm{NiO} \\
-\mathrm{t}\end{array}$ & $\begin{array}{c}\mathrm{P} 2 \mathrm{O} 5 \\
-\mathrm{t}\end{array}$ & $\begin{array}{c}\mathrm{SiO} 2 \\
-\mathrm{t} \\
\end{array}$ & $\begin{array}{c}\mathrm{ZrO} 2 \\
-\mathrm{t} \\
\end{array}$ & $\begin{array}{c}\mathrm{Ag} 2 \mathrm{O} \\
-\mathrm{t} \\
\end{array}$ & $\begin{array}{c}\text { As2O3 } \\
-\mathrm{t} \\
\end{array}$ & $\begin{array}{c}\mathrm{BaO} \\
-\mathrm{t}\end{array}$ & $\begin{array}{c}\mathrm{Bi} 2 \mathrm{O} 3 \\
-\mathrm{t}\end{array}$ & $\begin{array}{c}\mathrm{Br} \\
-\mathrm{t}\end{array}$ & $\begin{array}{c}\mathrm{CdO} \\
-\mathrm{t} \\
\end{array}$ & $\begin{array}{c}\mathrm{Ce} 2 \mathrm{O} 3 \\
-\mathrm{t}\end{array}$ & $\begin{array}{c}\mathrm{CeO} 2 \\
-\mathrm{t}\end{array}$ \\
\hline PX 1-1 (4643) & 0.0533 & 0.0742 & 0.0148 & 0.1071 & 0.0000 & 0.0293 & 0.0436 & 0.0148 & 0.0991 & 0.0162 & 0.0000 & 0.5003 & 0.0008 & & & 0.0019 & & & & 0.0000 & \\
\hline PX 1-2 (4726) & 0.0490 & 0.0726 & 0.0148 & 0.1103 & 0.0000 & 0.0299 & 0.0428 & 0.0142 & 0.0989 & 0.0169 & 0.0002 & 0.4995 & 0.0008 & & & 0.0019 & & & & 0.0000 & \\
\hline PX 1-3 (4776) & 0.0435 & 0.0727 & 0.0133 & 0.0999 & 0.0000 & 0.0277 & 0.0450 & 0.0147 & 0.0958 & 0.0157 & 0.0000 & 0.5256 & 0.0008 & & & 0.0017 & & & & 0.0000 & \\
\hline PX 2-1 (4455) & 0.0846 & 0.0682 & 0.0079 & 0.0946 & 0.0000 & 0.0214 & 0.0431 & 0.0158 & 0.1038 & 0.0063 & 0.0000 & 0.5153 & 0.0006 & & & 0.0009 & & & & 0.0000 & \\
\hline PX 2-2 (4509) & 0.0696 & 0.0719 & 0.0114 & 0.1100 & 0.0000 & 0.0248 & 0.0432 & 0.0155 & 0.0955 & 0.0118 & 0.0000 & 0.5020 & 0.0006 & & & 0.0014 & & & & 0.0000 & \\
\hline PX 2-3 (4566) & 0.0601 & 0.0744 & 0.0146 & 0.1095 & 0.0000 & 0.0303 & 0.0431 & 0.0151 & 0.0989 & 0.0152 & 0.0000 & 0.4907 & 0.0008 & & & 0.0019 & & & & 0.0000 & \\
\hline PX 3-1 (5780) & 0.0409 & 0.0793 & 0.0066 & 0.0875 & 0.0000 & 0.0234 & 0.0463 & 0.0138 & 0.0851 & 0.0071 & 0.0002 & 0.5762 & 0.0070 & & & 0.0005 & & & & 0.0000 & \\
\hline PX 3-2 (5818) & 0.0374 & 0.0770 & 0.0070 & 0.0953 & 0.0000 & 0.0221 & 0.0464 & 0.0142 & 0.0817 & 0.0076 & 0.0002 & 0.5765 & 0.0070 & & & 0.0007 & & & & 0.0000 & \\
\hline PX 3-3 (5880) & 0.0310 & 0.0729 & 0.0072 & 0.0989 & 0.0000 & 0.0229 & 0.0474 & 0.0149 & 0.0886 & 0.0085 & 0.0000 & 0.5723 & 0.0089 & & & 0.0007 & & & & 0.0000 & \\
\hline PX 4-1 (6390) & 0.0291 & 0.0819 & 0.0085 & 0.1063 & 0.0000 & 0.0268 & 0.0501 & 0.0169 & 0.0898 & 0.0096 & 0.0007 & 0.5423 & 0.0085 & & & 0.0007 & & & & 0.0000 & \\
\hline PX 4-2 (6434) & 0.0264 & 0.0819 & 0.0092 & 0.1079 & 0.0000 & 0.0279 & 0.0489 & 0.0170 & 0.0886 & 0.0100 & 0.0004 & 0.5433 & 0.0083 & & & 0.0007 & & & & 0.0000 & \\
\hline PX 4-3 (6458) & 0.0262 & 0.0824 & 0.0091 & 0.1082 & 0.0003 & 0.0272 & 0.0494 & 0.0173 & 0.0850 & 0.0101 & 0.0007 & 0.5454 & 0.0077 & & & 0.0010 & & & & 0.0000 & \\
\hline PX 5-1 (6787) & 0.0338 & 0.0823 & 0.0083 & 0.0970 & 0.0000 & 0.0230 & 0.0498 & 0.0172 & 0.0854 & 0.0114 & 0.0000 & 0.5548 & 0.0066 & & & 0.0010 & & & & 0.0000 & \\
\hline PX 5-2 (6795) & 0.0358 & 0.0798 & 0.0092 & 0.1124 & 0.0000 & 0.0286 & 0.0447 & 0.0155 & 0.1164 & 0.0135 & 0.0000 & 0.4998 & 0.0095 & & & 0.0009 & & & & 0.0000 & \\
\hline PX 5-3 (6812) & 0.0342 & 0.0801 & 0.0096 & 0.1197 & 0.0000 & 0.0333 & 0.0432 & 0.0149 & 0.1323 & 0.0131 & 0.0000 & 0.4761 & 0.0075 & & & 0.0009 & & & & 0.0000 & \\
\hline PX 5-4 (6820) & 0.0322 & 0.0795 & 0.0094 & 0.1138 & 0.0000 & 0.0329 & 0.0446 & 0.0151 & 0.1330 & 0.0129 & 0.0000 & 0.4838 & 0.0072 & & & 0.0009 & & & & 0.0000 & \\
\hline PX 5-5 (6839) & 0.0304 & 0.0775 & 0.0093 & 0.1092 & 0.0000 & 0.0330 & 0.0437 & 0.0152 & 0.1373 & 0.0123 & 0.0000 & 0.4881 & 0.0093 & & & 0.0009 & & & & 0.0000 & \\
\hline PX 5-6 (6862) & 0.0283 & 0.0806 & 0.0083 & 0.0955 & 0.0000 & 0.0287 & 0.0463 & 0.0157 & 0.1291 & 0.0116 & 0.0000 & 0.5166 & 0.0081 & & & 0.0007 & & & & 0.0000 & \\
\hline PX 5-7 (6871) & 0.0265 & 0.0839 & 0.0080 & 0.0914 & 0.0000 & 0.0278 & 0.0470 & 0.0159 & 0.1316 & 0.0104 & 0.0000 & 0.5201 & 0.0078 & & & 0.0007 & & & & 0.0000 & \\
\hline PX 5-8 (6884) & 0.0279 & 0.0948 & 0.0082 & 0.0948 & 0.0000 & 0.0303 & 0.0443 & 0.0152 & 0.1365 & 0.0120 & 0.0000 & 0.4971 & 0.0079 & & & 0.0007 & & & & 0.0000 & \\
\hline PX 5-9 (6960) & 0.0268 & 0.1087 & 0.0076 & 0.0916 & 0.0000 & 0.0294 & 0.0438 & 0.0145 & 0.1364 & 0.0112 & 0.0000 & 0.4928 & 0.0075 & & & 0.0007 & & & & 0.0000 & \\
\hline PX 5-10 (6972) & 0.0266 & 0.1095 & 0.0075 & 0.0872 & 0.0000 & 0.0279 & 0.0439 & 0.0145 & 0.1354 & 0.0111 & 0.0000 & 0.5000 & 0.0072 & & & 0.0007 & & & & 0.0000 & \\
\hline PX 6-1 (7340) & 0.0207 & 0.0863 & 0.0084 & 0.0991 & 0.0000 & 0.0281 & 0.0487 & 0.0159 & 0.1090 & 0.0114 & 0.0000 & 0.5336 & 0.0101 & & & 0.0007 & & & & 0.0000 & \\
\hline ATCH 1 STUDY-6- & 0.0256 & 0.1144 & 0.0053 & 0.0260 & 0.0163 & 0.0771 & 0.0467 & 0.0137 & 0.0912 & 0.0028 & 0.0002 & 0.5432 & 0.0016 & & & 0.0020 & & & & 0.0000 & \\
\hline ATCH 1 STUDY-1 & 0.0270 & 0.0990 & 0.0016 & 0.0278 & 0.0156 & 0.0421 & 0.0526 & 0.0160 & 0.0823 & 0.0026 & 0.0000 & 0.6038 & 0.0020 & & & 0.0015 & & & & 0.0000 & \\
\hline ATCH 1 STUDY-1 & 0.0256 & 0.0810 & 0.0056 & 0.0320 & 0.0112 & 0.0225 & 0.0563 & 0.0170 & 0.0723 & 0.0027 & 0.0000 & 0.6528 & 0.0019 & & & 0.0010 & & & & 0.0000 & \\
\hline H-GLAS-0112 & 0.0407 & 0.0826 & 0.0133 & 0.0984 & 0.0000 & 0.0159 & 0.0462 & 0.0136 & 0.0938 & 0.0145 & 0.0007 & 0.5295 & 0.0062 & & & 0.0017 & & & & 0.0000 & \\
\hline H-GLAS-0130 & 0.0386 & 0.0945 & 0.0126 & 0.0945 & 0.0006 & 0.0162 & 0.0471 & 0.0116 & 0.0887 & 0.0131 & 0.0009 & 0.5374 & 0.0062 & & & 0.0014 & & & & 0.0000 & \\
\hline H-GLAS-0162 & 0.0324 & 0.1161 & 0.0078 & 0.0859 & 0.0002 & 0.0082 & 0.0479 & 0.0069 & 0.0799 & 0.0100 & 0.0009 & 0.5458 & 0.0261 & & & 0.0010 & & & & 0.0000 & \\
\hline H-GLAS-0244 & 0.0435 & 0.0940 & 0.0100 & 0.1102 & 0.0004 & 0.0144 & 0.0453 & 0.0100 & 0.0928 & 0.0091 & 0.0007 & 0.5236 & 0.0133 & & & 0.0009 & & & & 0.0000 & \\
\hline H-GLAS-0254 & 0.0319 & 0.1216 & 0.0068 & 0.0827 & 0.0000 & 0.0066 & 0.0486 & 0.0059 & 0.0769 & 0.0092 & 0.0011 & 0.5529 & 0.0283 & & & 0.0010 & & & & 0.0000 & \\
\hline H-GLAS-0278 & 0.0322 & 0.1187 & 0.0057 & 0.0889 & 0.0000 & 0.0060 & 0.0472 & 0.0054 & 0.0763 & 0.0085 & 0.0009 & 0.5543 & 0.0293 & & & 0.0007 & & & & 0.0000 & \\
\hline H-GLAS-0293 & 0.0292 & 0.1229 & 0.0056 & 0.0938 & 0.0006 & 0.0063 & 0.0480 & 0.0050 & 0.0813 & 0.0085 & 0.0011 & 0.5559 & 0.0158 & & & 0.0007 & & & & 0.0000 & \\
\hline H-GLAS-0308 & 0.0310 & 0.1264 & 0.0058 & 0.0841 & 0.0002 & 0.0051 & 0.0503 & 0.0046 & 0.0791 & 0.0101 & 0.0011 & 0.5417 & 0.0323 & & & 0.0007 & & & & 0.0000 & \\
\hline H-GLAS-0334 & 0.0306 & 0.1296 & 0.0052 & 0.0968 & 0.0000 & 0.0047 & 0.0492 & 0.0043 & 0.0805 & 0.0093 & 0.0009 & 0.5457 & 0.0175 & & & 0.0007 & & & & 0.0000 & \\
\hline H-GLAS-0352 & 0.0290 & 0.1318 & 0.0047 & 0.0931 & 0.0000 & 0.0041 & 0.0494 & 0.0034 & 0.0791 & 0.0095 & 0.0009 & 0.5356 & 0.0353 & & & 0.0007 & & & & 0.0000 & \\
\hline H-GLAS-0387 & 0.0283 & 0.1360 & 0.0040 & 0.0805 & 0.0000 & 0.0032 & 0.0499 & 0.0029 & 0.0764 & 0.0080 & 0.0009 & 0.5496 & 0.0371 & & & 0.0007 & & & & 0.0000 & \\
\hline H-GLAS-0421 & 0.0303 & 0.1376 & 0.0043 & 0.0891 & 0.0000 & 0.0030 & 0.0508 & 0.0028 & 0.0797 & 0.0085 & 0.0009 & 0.5510 & 0.0191 & & & 0.0007 & & & & 0.0000 & \\
\hline H-GLAS-0466 & 0.0285 & 0.1373 & 0.0039 & 0.0921 & 0.0000 & 0.0032 & 0.0502 & 0.0024 & 0.0789 & 0.0075 & 0.0009 & 0.5345 & 0.0379 & & & 0.0007 & & & & 0.0000 & \\
\hline FRIT-202-CLEAR & 0.0039 & 0.0776 & 0.0010 & 0.0000 & 0.0000 & 0.0012 & 0.0656 & 0.0189 & 0.0576 & 0.0000 & 0.0000 & 0.7654 & 0.0000 & & & 0.0000 & & & & 0.0000 & \\
\hline FRIT-202-INT & 0.0039 & 0.0776 & 0.0010 & 0.0000 & 0.0000 & 0.0012 & 0.0656 & 0.0189 & 0.0576 & 0.0000 & 0.0000 & 0.7654 & 0.0000 & & & 0.0000 & & & & 0.0000 & \\
\hline FRIT-202-WHITE & 0.0039 & 0.0776 & 0.0010 & 0.0000 & 0.0000 & 0.0012 & 0.0656 & 0.0189 & 0.0576 & 0.0000 & 0.0000 & 0.7654 & 0.0000 & & & 0.0000 & & & & 0.0000 & \\
\hline FRIT-165-7 & 0.0000 & 0.0939 & 0.0000 & 0.0000 & 0.0000 & 0.0000 & 0.0701 & 0.0100 & 0.1224 & 0.0000 & 0.0000 & 0.6937 & 0.0099 & & & 0.0000 & & & & 0.0000 & \\
\hline FRIT-131-7 & 0.0036 & 0.1511 & 0.0009 & 0.0005 & 0.0000 & 0.0019 & 0.0539 & 0.0178 & 0.1977 & 0.0010 & 0.0000 & 0.5473 & 0.0048 & & & 0.0000 & & & & 0.0000 & \\
\hline MG 9-7 & 0.0020 & 0.0563 & 0.0925 & 0.0000 & 0.0000 & 0.0000 & 0.0000 & 0.0000 & 0.2597 & 0.0000 & 0.0000 & 0.5894 & 0.0000 & & & 0.0000 & & & & 0.0000 & \\
\hline MG $18-7$ & 0.0094 & 0.1610 & 0.0933 & 0.0000 & 0.0000 & 0.0000 & 0.0000 & 0.0000 & 0.2585 & 0.0000 & 0.0000 & 0.4779 & 0.0000 & & & 0.0000 & & & & 0.0000 & \\
\hline MG 25-7 & 0.1296 & 0.1371 & 0.0301 & 0.0823 & 0.0116 & 0.0000 & 0.0000 & 0.0000 & 0.1547 & 0.0000 & 0.0000 & 0.4545 & 0.0000 & & & 0.0000 & & & & 0.0000 & \\
\hline MG 28-7 & 0.1463 & 0.0923 & 0.0000 & 0.1119 & 0.0215 & 0.0000 & 0.0000 & 0.0000 & 0.1685 & 0.0000 & 0.0000 & 0.4594 & 0.0000 & & & 0.0000 & & & & 0.0000 & \\
\hline
\end{tabular}


DWPF PCT Model (Jantzen et al. 1995)

\begin{tabular}{|c|c|c|c|c|c|c|c|c|c|c|c|c|c|c|c|c|c|c|c|c|c|}
\hline Glass ID & $\begin{array}{c}\mathrm{Cl} \\
-\mathrm{t} \\
\end{array}$ & $\begin{array}{c}\mathrm{CoO} \\
-\mathrm{t}\end{array}$ & $\begin{array}{c}\mathrm{Co} 2 \mathrm{O} 3 \\
-\mathrm{t}\end{array}$ & $\begin{array}{c}\mathrm{Cr} 2 \mathrm{O} 3 \\
-\mathrm{t}\end{array}$ & $\begin{array}{c}\mathrm{Cs} 2 \mathrm{O} \\
-\mathrm{t}\end{array}$ & $\begin{array}{c}\mathrm{CuO} \\
-\mathrm{t} \\
\end{array}$ & $\begin{array}{c}\mathrm{Eu} 2 \mathrm{O} 3 \\
-\mathrm{t} \\
\end{array}$ & $\begin{array}{l}F \\
-t\end{array}$ & $\begin{array}{c}\mathrm{Ga} 2 \mathrm{O} 3 \\
-\mathrm{t}\end{array}$ & $\begin{array}{c}\mathrm{Gd} 2 \mathrm{O} 3 \\
-\mathrm{t}\end{array}$ & $\begin{array}{c}\mathrm{HgO} \\
-\mathrm{t}\end{array}$ & $\begin{array}{l}I \\
-t \\
\end{array}$ & $\begin{array}{c}\mathrm{La} 2 \mathrm{O} 3 \\
-\mathrm{t}\end{array}$ & $\begin{array}{c}\mathrm{MnO} 2 \\
-\mathrm{t} \\
\end{array}$ & $\begin{array}{c}\mathrm{MnO} \\
-\mathrm{t} \\
\end{array}$ & $\begin{array}{c}\mathrm{MoO} \\
-\mathrm{t}\end{array}$ & $\begin{array}{c}\mathrm{MoO} 3 \\
-\mathrm{t}\end{array}$ & $\begin{array}{c}\mathrm{Nb} 2 \mathrm{O} 5 \\
-\mathrm{t}\end{array}$ & $\begin{array}{c}\mathrm{Nd} 2 \mathrm{O} 3 \\
-\mathrm{t}\end{array}$ & $\begin{array}{c}\mathrm{PbO} \\
-\mathrm{t}\end{array}$ & $\begin{array}{c}\mathrm{PdO} 2 \\
-\mathrm{t}\end{array}$ \\
\hline PX 1-1 (4643) & & & & 0.0047 & 0.0000 & 0.0031 & & & & & & & 0.0000 & & 0.0315 & & 0.0000 & & 0.0000 & 0.0017 & \\
\hline PX 1-2 (4726) & & & & 0.0054 & 0.0000 & 0.0032 & & & & & & & 0.0000 & & 0.0322 & & 0.0000 & & 0.0000 & 0.0017 & \\
\hline PX 1-3 (4776) & & & & 0.0052 & 0.0000 & 0.0028 & & & & & & & 0.0000 & & 0.0284 & & 0.0000 & & 0.0000 & 0.0017 & \\
\hline PX 2-1 (4455) & & & & 0.0040 & 0.0000 & 0.0016 & & & & & & & 0.0000 & & 0.0281 & & 0.0000 & & 0.0000 & 0.0003 & \\
\hline PX 2-2 (4509) & & & & 0.0047 & 0.0000 & 0.0023 & & & & & & & 0.0000 & & 0.0304 & & 0.0000 & & 0.0000 & 0.0010 & \\
\hline PX 2-3 (4566) & & & & 0.0047 & 0.0000 & 0.0028 & & & & & & & 0.0000 & & 0.0326 & & 0.0000 & & 0.0000 & 0.0014 & \\
\hline PX 3-1 (5780) & & & & 0.0034 & 0.0000 & 0.0019 & & & & & & & 0.0000 & & 0.0167 & & 0.0000 & & 0.0000 & 0.0004 & \\
\hline PX 3-2 (5818) & & & & 0.0034 & 0.0000 & 0.0019 & & & & & & & 0.0000 & & 0.0168 & & 0.0002 & & 0.0000 & 0.0007 & \\
\hline PX 3-3 (5880) & & & & 0.0031 & 0.0000 & 0.0019 & & & & & & & 0.0000 & & 0.0164 & & 0.0000 & & 0.0000 & 0.0007 & \\
\hline PX 4-1 (6390) & & & & 0.0036 & 0.0000 & 0.0029 & & & & & & & 0.0000 & & 0.0173 & & 0.0000 & & 0.0000 & 0.0011 & \\
\hline PX 4-2 (6434) & & & & 0.0036 & 0.0000 & 0.0034 & & & & & & & 0.0000 & & 0.0172 & & 0.0000 & & 0.0000 & 0.0010 & \\
\hline PX 4-3 (6458) & & & & 0.0038 & 0.0000 & 0.0035 & & & & & & & 0.0000 & & 0.0172 & & 0.0002 & & 0.0000 & 0.0011 & \\
\hline PX 5-1 (6787) & & & & 0.0041 & 0.0004 & 0.0030 & & & & & & & 0.0000 & & 0.0159 & & 0.0000 & & 0.0005 & 0.0014 & \\
\hline PX 5-2 (6795) & & & & 0.0037 & 0.0004 & 0.0039 & & & & & & & 0.0000 & & 0.0180 & & 0.0000 & & 0.0016 & 0.0017 & \\
\hline PX 5-3 (6812) & & & & 0.0035 & 0.0009 & 0.0044 & & & & & & & 0.0000 & & 0.0194 & & 0.0000 & & 0.0010 & 0.0014 & \\
\hline PX 5-4 (6820) & & & & 0.0042 & 0.0009 & 0.0043 & & & & & & & 0.0000 & & 0.0185 & & 0.0000 & & 0.0010 & 0.0014 & \\
\hline PX 5-5 (6839) & & & & 0.0038 & 0.0004 & 0.0042 & & & & & & & 0.0000 & & 0.0185 & & 0.0000 & & 0.0010 & 0.0014 & \\
\hline PX 5-6 (6862) & & & & 0.0043 & 0.0004 & 0.0036 & & & & & & & 0.0000 & & 0.0163 & & 0.0000 & & 0.0011 & 0.0011 & \\
\hline PX 5-7 (6871) & & & & 0.0034 & 0.0004 & 0.0036 & & & & & & & 0.0000 & & 0.0158 & & 0.0000 & & 0.0011 & 0.0011 & \\
\hline PX 5-8 (6884) & & & & 0.0050 & 0.0004 & 0.0037 & & & & & & & 0.0000 & & 0.0161 & & 0.0000 & & 0.0005 & 0.0010 & \\
\hline PX 5-9 (6960) & & & & 0.0040 & 0.0004 & 0.0036 & & & & & & & 0.0000 & & 0.0154 & & 0.0000 & & 0.0011 & 0.0010 & \\
\hline PX 5-10 (6972) & & & & 0.0045 & 0.0004 & 0.0035 & & & & & & & 0.0000 & & 0.0149 & & 0.0000 & & 0.0011 & 0.0011 & \\
\hline PX 6-1 (7340) & & & & 0.0038 & 0.0000 & 0.0028 & & & & & & & 0.0000 & & 0.0172 & & 0.0002 & & 0.0000 & 0.0014 & \\
\hline BATCH 1 STUDY-6- & & & & 0.0005 & 0.0018 & 0.0078 & & & & & & & 0.0000 & & 0.0076 & & 0.0000 & & 0.0000 & 0.0004 & \\
\hline BATCH 1 STUDY-10 & & & & 0.0005 & 0.0014 & 0.0035 & & & & & & & 0.0000 & & 0.0074 & & 0.0000 & & 0.0022 & 0.0000 & \\
\hline BATCH 1 STUDY-15 & & & & 0.0003 & 0.0005 & 0.0021 & & & & & & & 0.0000 & & 0.0072 & & 0.0000 & & 0.0022 & 0.0000 & \\
\hline H-GLAS-0112 & & & & 0.0045 & 0.0000 & 0.0026 & & & & & & & 0.0005 & & 0.0255 & & 0.0002 & & 0.0000 & 0.0021 & \\
\hline H-GLAS-0130 & & & & 0.0043 & 0.0000 & 0.0022 & & & & & & & 0.0000 & & 0.0214 & & 0.0007 & & 0.0000 & 0.0021 & \\
\hline H-GLAS-0162 & & & & 0.0043 & 0.0000 & 0.0014 & & & & & & & 0.0025 & & 0.0129 & & 0.0011 & & 0.0000 & 0.0017 & \\
\hline H-GLAS-0244 & & & & 0.0037 & 0.0000 & 0.0017 & & & & & & & 0.0010 & & 0.0179 & & 0.0007 & & 0.0000 & 0.0017 & \\
\hline H-GLAS-0254 & & & & 0.0038 & 0.0000 & 0.0011 & & & & & & & 0.0025 & & 0.0113 & & 0.0011 & & 0.0000 & 0.0017 & \\
\hline H-GLAS-0278 & & & & 0.0038 & 0.0000 & 0.0012 & & & & & & & 0.0025 & & 0.0099 & & 0.0013 & & 0.0000 & 0.0017 & \\
\hline H-GLAS-0293 & & & & 0.0038 & 0.0000 & 0.0010 & & & & & & & 0.0025 & & 0.0100 & & 0.0013 & & 0.0000 & 0.0017 & \\
\hline H-GLAS-0308 & & & & 0.0052 & 0.0000 & 0.0010 & & & & & & & 0.0025 & & 0.0097 & & 0.0016 & & 0.0000 & 0.0017 & \\
\hline H-GLAS-0334 & & & & 0.0045 & 0.0000 & 0.0007 & & & & & & & 0.0025 & & 0.0091 & & 0.0016 & & 0.0000 & 0.0017 & \\
\hline H-GLAS-0352 & & & & 0.0040 & 0.0000 & 0.0007 & & & & & & & 0.0025 & & 0.0079 & & 0.0016 & & 0.0000 & 0.0017 & \\
\hline H-GLAS-0387 & & & & 0.0040 & 0.0000 & 0.0006 & & & & & & & 0.0020 & & 0.0070 & & 0.0016 & & 0.0000 & 0.0017 & \\
\hline H-GLAS-0421 & & & & 0.0045 & 0.0000 & 0.0006 & & & & & & & 0.0020 & & 0.0070 & & 0.0016 & & 0.0000 & 0.0017 & \\
\hline H-GLAS-0466 & & & & 0.0038 & 0.0000 & 0.0007 & & & & & & & 0.0025 & & 0.0066 & & 0.0018 & & 0.0000 & 0.0021 & \\
\hline FRIT-202-CLEAR & & & & 0.0000 & 0.0089 & 0.0000 & & & & & & & 0.0000 & & 0.0000 & & 0.0000 & & 0.0000 & 0.0000 & \\
\hline FRIT-202-INT & & & & 0.0000 & 0.0089 & 0.0000 & & & & & & & 0.0000 & & 0.0000 & & 0.0000 & & 0.0000 & 0.0000 & \\
\hline FRIT-202-WHITE & & & & 0.0000 & 0.0089 & 0.0000 & & & & & & & 0.0000 & & 0.0000 & & 0.0000 & & 0.0000 & 0.0000 & \\
\hline FRIT-165-7 & & & & 0.0000 & 0.0000 & 0.0000 & & & & & & & 0.0000 & & 0.0000 & & 0.0000 & & 0.0000 & 0.0000 & \\
\hline FRIT-131-7 & & & & 0.0000 & 0.0000 & 0.0000 & & & & & & & 0.0000 & & 0.0015 & & 0.0000 & & 0.0052 & 0.0000 & \\
\hline MG 9-7 & & & & 0.0000 & 0.0000 & 0.0000 & & & & & & & 0.0000 & & 0.0000 & & 0.0000 & & 0.0000 & 0.0000 & \\
\hline MG $18-7$ & & & & 0.0000 & 0.0000 & 0.0000 & & & & & & & 0.0000 & & 0.0000 & & 0.0000 & & 0.0000 & 0.0000 & \\
\hline MG 25-7 & & & & 0.0000 & 0.0000 & 0.0000 & & & & & & & 0.0000 & & 0.0000 & & 0.0000 & & 0.0000 & 0.0000 & \\
\hline MG 28-7 & & & & 0.0000 & 0.0000 & 0.0000 & & & & & & & 0.0000 & & 0.0000 & & 0.0000 & & 0.0000 & 0.0000 & \\
\hline
\end{tabular}


DWPF PCT Model (Jantzen et al. 1995)

\begin{tabular}{|c|c|c|c|c|c|c|c|c|c|c|c|c|c|c|c|c|c|c|c|c|c|}
\hline Glass ID & $\begin{array}{c}\mathrm{PdO} \\
-\mathrm{t}\end{array}$ & $\begin{array}{c}\mathrm{Pr} 2 \mathrm{O} 3 \\
-\mathrm{t}\end{array}$ & \begin{tabular}{|c|} 
Pr6O11 \\
$-t$
\end{tabular} & $\begin{array}{c}\mathrm{Rb} 2 \mathrm{O} \\
-\mathrm{t}\end{array}$ & $\begin{array}{c}\mathrm{ReO} \\
-\mathrm{t}\end{array}$ & $\begin{array}{c}\mathrm{ReO} 2 \\
-\mathrm{t}\end{array}$ & $\begin{array}{c}\mathrm{Rh} 2 \mathrm{O} 3 \\
-\mathrm{t}\end{array}$ & $\begin{array}{c}\mathrm{RhO}_{2} \\
-\mathrm{t}\end{array}$ & $\begin{array}{c}\mathrm{RuO}_{2} \\
-\mathrm{t}\end{array}$ & $\begin{array}{c}\mathrm{Sb} 2 \mathrm{O} 3 \\
-\mathrm{t}\end{array}$ & $\begin{array}{c}\mathrm{Sb} 2 \mathrm{O} 5 \\
-\mathrm{t}\end{array}$ & $\begin{array}{c}\mathrm{SeO} 2 \\
-\mathrm{t}\end{array}$ & $\begin{array}{c}\mathrm{Sm} 2 \mathrm{O} 3 \\
-\mathrm{t}\end{array}$ & $\begin{array}{c}\mathrm{SnO} \\
-\mathrm{t}\end{array}$ & $\begin{array}{c}\mathrm{SnO} 2 \\
-\mathrm{t}\end{array}$ & $\begin{array}{c}\mathrm{SO} 3 \\
-\mathrm{t}\end{array}$ & $\begin{array}{c}\mathrm{SrO} \\
-\mathrm{t}\end{array}$ & $\begin{array}{c}\mathrm{Tc} 2 \mathrm{O} 7 \\
-\mathrm{t}\end{array}$ & $\begin{array}{c}\mathrm{TeO} 2 \\
-\mathrm{t}\end{array}$ & $\begin{array}{c}\mathrm{ThO} 2 \\
-\mathrm{t}\end{array}$ & $\begin{array}{c}\mathrm{TiO} 2 \\
-\mathrm{t}\end{array}$ \\
\hline PX 1-1 (4643) & & & & & & & & & & & & & & & & & 0.0003 & & & & 0.0017 \\
\hline PX 1-2 (4726) & & & & & & & & & & & & & & & & & 0.0003 & & & & 0.0037 \\
\hline PX 1-3 (4776) & & & & & & & & & & & & & & & & & 0.0002 & & & & 0.0036 \\
\hline PX 2-1 (4455) & & & & & & & & & & & & & & & & & 0.0014 & & & & 0.0010 \\
\hline PX 2-2 (4509) & & & & & & & & & & & & & & & & & 0.0008 & & & & 0.0017 \\
\hline PX 2-3 (4566) & & & & & & & & & & & & & & & & & 0.0005 & & & & 0.0022 \\
\hline PX 3-1 (5780) & & & & & & & & & & & & & & & & & 0.0005 & & & & 0.0023 \\
\hline PX 3-2 (5818) & & & & & & & & & & & & & & & & & 0.0005 & & & & 0.0023 \\
\hline PX 3-3 (5880) & & & & & & & & & & & & & & & & & 0.0003 & & & & 0.0020 \\
\hline PX 4-1 (6390) & & & & & & & & & & & & & & & & & 0.0002 & & & & 0.0026 \\
\hline PX 4-2 (6434) & & & & & & & & & & & & & & & & & 0.0002 & & & & 0.0028 \\
\hline PX 4-3 (6458) & & & & & & & & & & & & & & & & & 0.0002 & & & & 0.0029 \\
\hline PX 5-1 (6787) & & & & & & & & & & & & & & & & & 0.0002 & & & & 0.0029 \\
\hline PX 5-2 (6795) & & & & & & & & & & & & & & & & & 0.0003 & & & & 0.0030 \\
\hline PX 5-3 (6812) & & & & & & & & & & & & & & & & & 0.0003 & & & & 0.0031 \\
\hline PX 5-4 (6820) & & & & & & & & & & & & & & & & & 0.0003 & & & & 0.0028 \\
\hline PX 5-5 (6839) & & & & & & & & & & & & & & & & & 0.0003 & & & & 0.0028 \\
\hline PX 5-6 (6862) & & & & & & & & & & & & & & & & & 0.0002 & & & & 0.0025 \\
\hline PX 5-7 (6871) & & & & & & & & & & & & & & & & & 0.0002 & & & & 0.0024 \\
\hline PX 5-8 (6884) & & & & & & & & & & & & & & & & & 0.0002 & & & & 0.0024 \\
\hline PX 5-9 (6960) & & & & & & & & & & & & & & & & & 0.0002 & & & & 0.0022 \\
\hline PX 5-10 (6972) & & & & & & & & & & & & & & & & & 0.0000 & & & & 0.0021 \\
\hline PX 6-1 (7340) & & & & & & & & & & & & & & & & & 0.0000 & & & & 0.0015 \\
\hline BATCH 1 STUDY-6- & & & & & & & & & & & & & & & & & 0.0002 & & & & 0.0153 \\
\hline BATCH 1 STUDY-10 & & & & & & & & & & & & & & & & & 0.0000 & & & & 0.0104 \\
\hline BATCH 1 STUDY-15 & & & & & & & & & & & & & & & & & 0.0000 & & & & 0.0052 \\
\hline H-GLAS-0112 & & & & & & & & & & & & & & & & & 0.0003 & & & & 0.0039 \\
\hline H-GLAS-0130 & & & & & & & & & & & & & & & & & 0.0003 & & & & 0.0028 \\
\hline H-GLAS-0162 & & & & & & & & & & & & & & & & & 0.0003 & & & & 0.0021 \\
\hline H-GLAS-0244 & & & & & & & & & & & & & & & & & 0.0003 & & & & 0.0020 \\
\hline H-GLAS-0254 & & & & & & & & & & & & & & & & & 0.0003 & & & & 0.0019 \\
\hline H-GLAS-0278 & & & & & & & & & & & & & & & & & 0.0003 & & & & 0.0017 \\
\hline H-GLAS-0293 & & & & & & & & & & & & & & & & & 0.0003 & & & & 0.0017 \\
\hline H-GLAS-0308 & & & & & & & & & & & & & & & & & 0.0003 & & & & 0.0017 \\
\hline H-GLAS-0334 & & & & & & & & & & & & & & & & & 0.0003 & & & & 0.0016 \\
\hline H-GLAS-0352 & & & & & & & & & & & & & & & & & 0.0003 & & & & 0.0016 \\
\hline H-GLAS-0387 & & & & & & & & & & & & & & & & & 0.0003 & & & & 0.0015 \\
\hline H-GLAS-0421 & & & & & & & & & & & & & & & & & 0.0003 & & & & 0.0015 \\
\hline H-GLAS-0466 & & & & & & & & & & & & & & & & & 0.0003 & & & & 0.0016 \\
\hline FRIT-202-CLEAR & & & & & & & & & & & & & & & & & 0.0000 & & & & 0.0000 \\
\hline FRIT-202-INT & & & & & & & & & & & & & & & & & 0.0000 & & & & 0.0000 \\
\hline FRIT-202-WHITE & & & & & & & & & & & & & & & & & 0.0000 & & & & 0.0000 \\
\hline FRIT-165-7 & & & & & & & & & & & & & & & & & 0.0000 & & & & 0.0000 \\
\hline FRIT-131-7 & & & & & & & & & & & & & & & & & 0.0000 & & & & 0.0099 \\
\hline MG 9-7 & & & & & & & & & & & & & & & & & 0.0000 & & & & 0.0000 \\
\hline MG 18-7 & & & & & & & & & & & & & & & & & 0.0000 & & & & 0.0000 \\
\hline MG 25-7 & & & & & & & & & & & & & & & & & 0.0000 & & & & 0.0000 \\
\hline MG 28-7 & & & & & & & & & & & & & & & & & 0.0000 & & & & 0.0000 \\
\hline
\end{tabular}


DWPF PCT Model (Jantzen et al. 1995)

\begin{tabular}{|c|c|c|c|c|c|c|c|c|c|c|c|c|c|c|c|c|c|c|c|c|c|}
\hline Glass ID & $\begin{array}{c}\mathrm{T} 2 \mathrm{O} 3 \\
-\mathrm{t}\end{array}$ & $\begin{array}{c}\mathrm{U} 3 \mathrm{O} 8 \\
-\mathrm{t}\end{array}$ & $\begin{array}{c}\mathrm{UO} 2 \\
-\mathrm{t}\end{array}$ & $\begin{array}{c}\text { UO3 } \\
-t\end{array}$ & $\begin{array}{c}\mathrm{V} 2 \mathrm{O} 5 \\
-\mathrm{t}\end{array}$ & $\begin{array}{c}\text { WO3 } \\
-t\end{array}$ & $\begin{array}{c}\mathrm{Y} 2 \mathrm{O} 3 \\
-\mathrm{t}\end{array}$ & $\begin{array}{c}\mathrm{ZnO} \\
-\mathrm{t}\end{array}$ & $\begin{array}{c}\text { Others } \\
-t\end{array}$ & $\begin{array}{c}\text { Sum } \\
-t\end{array}$ & $\begin{array}{c}\mathrm{A} 2 \mathrm{O} 3 \\
-\mathrm{a}\end{array}$ & $\begin{array}{c}\mathrm{B} 2 \mathrm{O} 3 \\
-\mathrm{a}\end{array}$ & $\begin{array}{c}\mathrm{CaO} \\
-\mathrm{a}\end{array}$ & \begin{tabular}{|c|}
$\mathrm{Fe} 2 \mathrm{O} 3$ \\
$-\mathrm{a}$
\end{tabular} & $\begin{array}{c}\mathrm{FeO} \\
-\mathrm{a} \\
\end{array}$ & $\begin{array}{c}\mathrm{K} 2 \mathrm{O} \\
-\mathrm{a} \\
\end{array}$ & $\begin{array}{c}\mathrm{Li} 2 \mathrm{O} \\
-\mathrm{a}\end{array}$ & $\begin{array}{c}\mathrm{MgO} \\
-\mathrm{a} \\
\end{array}$ & $\begin{array}{c}\mathrm{Na} 2 \mathrm{O} \\
-\mathrm{a} \\
\end{array}$ & $\begin{array}{c}\mathrm{NiO} \\
-\mathrm{a}\end{array}$ & $\begin{array}{c}\mathrm{P} 2 \mathrm{O} 5 \\
-\mathrm{a} \\
\end{array}$ \\
\hline PX 1-1 (4643) & & & & & & & 0.0000 & 0.0016 & & 1.0000 & 0.0523 & 0.0729 & 0.0146 & 0.1051 & & 0.0288 & 0.0428 & 0.0146 & 0.0973 & 0.0159 & \\
\hline PX 1-2 (4726) & & & & & & & 0.0000 & 0.0017 & & 1.0000 & 0.0496 & 0.0736 & 0.0150 & 0.1117 & & 0.0303 & 0.0434 & 0.0144 & 0.1002 & 0.0171 & \\
\hline PX 1-3 (4776) & & & & & & & 0.0000 & 0.0016 & & 1.0000 & 0.0434 & 0.0725 & 0.0133 & 0.0996 & & 0.0277 & 0.0449 & 0.0147 & 0.0955 & 0.0157 & \\
\hline PX 2-1 (4455) & & & & & & & 0.0000 & 0.0010 & & 1.0000 & 0.0852 & 0.0687 & 0.0079 & 0.0952 & & 0.0215 & 0.0433 & 0.0159 & 0.1045 & 0.0064 & \\
\hline PX 2-2 (4509) & & & & & & & 0.0000 & 0.0014 & & 1.0000 & 0.0697 & 0.0719 & 0.0114 & 0.1099 & & 0.0248 & 0.0432 & 0.0156 & 0.0955 & 0.0118 & \\
\hline PX 2-3 (4566) & & & & & & & 0.0000 & 0.0015 & & 1.0000 & 0.0599 & 0.0740 & 0.0145 & 0.1090 & & 0.0302 & 0.0429 & 0.0151 & 0.0984 & 0.0151 & \\
\hline PX 3-1 (5780) & & & & & & & 0.0000 & 0.0010 & & 1.0000 & 0.0423 & 0.0821 & 0.0068 & 0.0908 & & 0.0241 & 0.0480 & 0.0143 & 0.0881 & 0.0074 & \\
\hline PX 3-2 (5818) & & & & & & & 0.0000 & 0.0012 & & 1.0000 & 0.0392 & 0.0806 & 0.0074 & 0.0999 & & 0.0232 & 0.0486 & 0.0149 & 0.0856 & 0.0081 & 0.0003 \\
\hline PX 3-3 (5880) & & & & & & & 0.0000 & 0.0013 & & 1.0000 & 0.0294 & 0.0690 & 0.0069 & 0.0938 & & 0.0216 & 0.0449 & 0.0141 & 0.0840 & 0.0080 & \\
\hline PX 4-1 (6390) & & & & & & & 0.0000 & 0.0013 & & 1.0000 & 0.0281 & 0.0789 & 0.0081 & 0.1026 & & 0.0259 & 0.0483 & 0.0163 & 0.0866 & 0.0092 & 0.0007 \\
\hline PX 4-2 (6434) & & & & & & & 0.0000 & 0.0013 & & 1.0000 & 0.0257 & 0.0801 & 0.0090 & 0.1055 & & 0.0274 & 0.0478 & 0.0166 & 0.0867 & 0.0097 & 0.0005 \\
\hline PX 4-3 (6458) & & & & & & & 0.0000 & 0.0013 & & 1.0000 & 0.0256 & 0.0807 & 0.0088 & 0.1057 & 0.0003 & 0.0266 & 0.0483 & 0.0169 & 0.0831 & 0.0099 & 0.0007 \\
\hline PX 5-1 (6787) & & & & & & & 0.0000 & 0.0009 & & 1.0000 & 0.0331 & 0.0807 & 0.0082 & 0.0951 & & 0.0226 & 0.0488 & 0.0169 & 0.0838 & 0.0112 & \\
\hline PX 5-2 (6795) & & & & & & & 0.0000 & 0.0011 & & 1.0000 & 0.0347 & 0.0774 & 0.0090 & 0.1090 & & 0.0278 & 0.0434 & 0.0150 & 0.1129 & 0.0131 & \\
\hline PX 5-3 (6812) & & & & & & & 0.0000 & 0.0011 & & 1.0000 & 0.0326 & 0.0764 & 0.0092 & 0.1263 & & 0.0318 & 0.0412 & 0.0143 & 0.1143 & 0.0125 & \\
\hline PX 5-4 (6820) & & & & & & & 0.0000 & 0.0011 & & 1.0000 & 0.0305 & 0.0755 & 0.0089 & 0.1078 & & 0.0313 & 0.0423 & 0.0143 & 0.1262 & 0.0121 & \\
\hline PX 5-5 (6839) & & & & & & & 0.0000 & 0.0011 & & 1.0000 & 0.0299 & 0.0762 & 0.0091 & 0.1073 & & 0.0325 & 0.0430 & 0.0150 & 0.1351 & 0.0121 & \\
\hline PX 5-6 (6862) & & & & & & & 0.0000 & 0.0010 & & 1.0000 & 0.0277 & 0.0789 & 0.0081 & 0.0935 & & 0.0280 & 0.0453 & 0.0154 & 0.1263 & 0.0114 & \\
\hline PX 5-7 (6871) & & & & & & & 0.0000 & 0.0009 & & 1.0000 & 0.0258 & 0.0815 & 0.0078 & 0.0887 & & 0.0270 & 0.0457 & 0.0155 & 0.1278 & 0.0101 & \\
\hline PX 5-8 (6884) & & & & & & & 0.0000 & 0.0009 & & 1.0000 & 0.0274 & 0.0935 & 0.0081 & 0.0934 & & 0.0299 & 0.0437 & 0.0149 & 0.1346 & 0.0119 & \\
\hline PX 5-9 (6960) & & & & & & & 0.0000 & 0.0009 & & 1.0000 & 0.0262 & 0.1064 & 0.0075 & 0.0896 & & 0.0288 & 0.0429 & 0.0142 & 0.1335 & 0.0110 & \\
\hline PX 5-10 (6972) & & & & & & & 0.0000 & 0.0009 & & 1.0000 & 0.0262 & 0.1080 & 0.0074 & 0.0859 & & 0.0275 & 0.0433 & 0.0143 & 0.1335 & 0.0110 & \\
\hline PX 6-1 (7340) & & & & & & & 0.0000 & 0.0010 & & 1.0000 & 0.0207 & 0.0861 & 0.0084 & 0.0988 & & 0.0279 & 0.0486 & 0.0158 & 0.1086 & 0.0114 & \\
\hline BATCH 1 STUDY-6- & & & & & & & 0.0000 & 0.0003 & & 1.0000 & 0.0613 & 0.0796 & 0.0152 & 0.1012 & 0.0372 & 0.0481 & 0.0366 & 0.0122 & 0.0952 & 0.0084 & 0.0003 \\
\hline BATCH 1 STUDY-10 & $\mathrm{B}-7$ & & & & & & 0.0000 & 0.0005 & & 1.0000 & 0.0265 & 0.0970 & 0.0055 & 0.0120 & 0.0247 & 0.0413 & 0.0515 & 0.0157 & 0.0805 & 0.0025 & 0.0001 \\
\hline BATCH 1 STUDY-15 & & & & & & & 0.0000 & 0.0005 & & 1.0000 & 0.0254 & 0.0803 & 0.0056 & 0.0269 & 0.0257 & 0.0223 & 0.0558 & 0.0168 & 0.0717 & 0.0027 & 0.0002 \\
\hline H-GLAS-0112 & & & & & & & 0.0000 & 0.0033 & & 1.0000 & 0.0392 & 0.0793 & 0.0128 & 0.0946 & & 0.0153 & 0.0444 & 0.0131 & 0.0902 & 0.0139 & 0.0006 \\
\hline H-GLAS-0130 & & & & & & & 0.0000 & 0.0028 & & 1.0000 & 0.0372 & 0.0909 & 0.0120 & 0.0908 & 0.0006 & 0.0156 & 0.0453 & 0.0111 & 0.0852 & 0.0126 & 0.0008 \\
\hline H-GLAS-0162 & & & & & & & 0.0000 & 0.0044 & & 1.0000 & 0.0308 & 0.1104 & 0.0074 & 0.0818 & 0.0003 & 0.0078 & 0.0456 & 0.0065 & 0.0761 & 0.0095 & 0.0009 \\
\hline H-GLAS-0244 & & & & & & & 0.0000 & 0.0029 & & 1.0000 & 0.0417 & 0.0907 & 0.0096 & 0.1057 & 0.0004 & 0.0138 & 0.0436 & 0.0096 & 0.0890 & 0.0088 & 0.0007 \\
\hline H-GLAS-0254 & & & & & & & 0.0000 & 0.0027 & & 1.0000 & 0.0311 & 0.1182 & 0.0067 & 0.0803 & & 0.0064 & 0.0473 & 0.0058 & 0.0746 & 0.0090 & 0.0011 \\
\hline H-GLAS-0278 & & & & & & & 0.0000 & 0.0033 & & 1.0000 & 0.0306 & 0.1132 & 0.0054 & 0.0845 & & 0.0057 & 0.0450 & 0.0052 & 0.0726 & 0.0081 & 0.0008 \\
\hline H-GLAS-0293 & & & & & & & 0.0000 & 0.0028 & & 1.0000 & 0.0271 & 0.1143 & 0.0052 & 0.0874 & 0.0005 & 0.0059 & 0.0446 & 0.0047 & 0.0756 & 0.0079 & 0.0011 \\
\hline H-GLAS-0308 & & & & & & & 0.0000 & 0.0034 & & 1.0000 & 0.0299 & 0.1218 & 0.0055 & 0.0811 & 0.0002 & 0.0050 & 0.0485 & 0.0045 & 0.0762 & 0.0097 & 0.0010 \\
\hline H-GLAS-0334 & & & & & & & 0.0000 & 0.0030 & & 1.0000 & 0.0293 & 0.1241 & 0.0049 & 0.0925 & & 0.0045 & 0.0471 & 0.0041 & 0.0771 & 0.0089 & 0.0009 \\
\hline H-GLAS-0352 & & & & & & & 0.0000 & 0.0030 & & 1.0000 & 0.0281 & 0.1265 & 0.0048 & 0.0854 & & 0.0039 & 0.0473 & 0.0036 & 0.0761 & 0.0087 & 0.0010 \\
\hline H-GLAS-0387 & & & & & & & 0.0000 & 0.0034 & & 1.0000 & 0.0270 & 0.1304 & 0.0036 & 0.0762 & & 0.0029 & 0.0477 & 0.0028 & 0.0724 & 0.0075 & 0.0007 \\
\hline H-GLAS-0421 & & & & & & & 0.0000 & 0.0028 & & 1.0000 & 0.0282 & 0.1314 & 0.0041 & 0.0794 & & 0.0027 & 0.0483 & 0.0027 & 0.0777 & 0.0081 & 0.0006 \\
\hline H-GLAS-0466 & & & & & & & 0.0000 & 0.0026 & & 1.0000 & 0.0275 & 0.1328 & 0.0037 & 0.0891 & & 0.0031 & 0.0486 & 0.0023 & 0.0763 & 0.0073 & 0.0008 \\
\hline FRIT-202-CLEAR & & & & & & & 0.0000 & 0.0000 & & 1.0000 & 0.0040 & 0.0781 & 0.0010 & & & 0.0030 & 0.0660 & 0.0190 & 0.0580 & & \\
\hline FRIT-202-INT & & & & & & & 0.0000 & 0.0000 & & 1.0000 & & & & & & & & & & & \\
\hline FRIT-202-WHITE & & & & & & & 0.0000 & 0.0000 & & 1.0000 & & & & & & & & & & & \\
\hline FRIT-165-7 & & & & & & & 0.0000 & 0.0000 & & 1.0000 & & 0.0932 & & & & & 0.0696 & 0.0099 & 0.1216 & & \\
\hline FRIT-131-7 & & & & & & & 0.0029 & 0.0000 & & 1.0000 & 0.0035 & 0.1437 & 0.0008 & 0.0006 & & 0.0018 & 0.0512 & 0.0169 & 0.1880 & 0.0010 & \\
\hline MG 9-7 & & & & & & & 0.0000 & 0.0000 & & 1.0000 & & & & & & & & & & & \\
\hline MG $18-7$ & & & & & & & 0.0000 & 0.0000 & & 1.0000 & & & & & & & & & & & \\
\hline MG 25-7 & & & & & & & 0.0000 & 0.0000 & & 1.0000 & & & & & & & & & & & \\
\hline MG 28-7 & & & & & & & 0.0000 & 0.0000 & & 1.0000 & & & & & & & & & & & \\
\hline
\end{tabular}


Appendix A. Database - mass fraction

DWPF PCT Model (Jantzen et al. 1995)

\begin{tabular}{|c|c|c|c|c|c|c|c|c|c|c|c|c|c|c|c|c|c|c|c|c|c|}
\hline Glass ID & $\begin{array}{c}\mathrm{SiO} 2 \\
-\mathrm{a}\end{array}$ & $\begin{array}{c}\mathrm{ZrO} 2 \\
-\mathrm{a}\end{array}$ & $\begin{array}{c}\mathrm{Ag} 2 \mathrm{O} \\
-\mathrm{a}\end{array}$ & $\begin{array}{c}\text { As2O3 } \\
-\mathrm{a}\end{array}$ & $\begin{array}{c}\mathrm{BaO} \\
-\mathrm{a}\end{array}$ & $\begin{array}{c}\mathrm{Bi} 2 \mathrm{O} 3 \\
-\mathrm{a}\end{array}$ & $\begin{array}{l}\mathrm{Br} \\
-\mathrm{a}\end{array}$ & $\begin{array}{c}\mathrm{CdO} \\
-\mathrm{a}\end{array}$ & $\begin{array}{c}\mathrm{Ce} 2 \mathrm{O} 3 \\
-\mathrm{a}\end{array}$ & $\begin{array}{c}\mathrm{CeO} 2 \\
-\mathrm{a}\end{array}$ & $\begin{array}{l}\mathrm{Cl} \\
-\mathrm{a}\end{array}$ & $\begin{array}{c}\mathrm{CoO} \\
-\mathrm{a}\end{array}$ & $\begin{array}{c}\mathrm{Co} 2 \mathrm{O} 3 \\
-\mathrm{a}\end{array}$ & $\begin{array}{c}\mathrm{Cr} 2 \mathrm{O} 3 \\
-\mathrm{a}\end{array}$ & $\begin{array}{c}\mathrm{Cs} 2 \mathrm{O} \\
-\mathrm{a}\end{array}$ & $\begin{array}{c}\mathrm{CuO} \\
-\mathrm{a}\end{array}$ & $\begin{array}{c}\mathrm{Eu} 2 \mathrm{O} 3 \\
-\mathrm{a}\end{array}$ & $\begin{array}{c}F \\
-a\end{array}$ & $\begin{array}{c}\mathrm{Ga} 2 \mathrm{O} 3 \\
-\mathrm{a}\end{array}$ & $\begin{array}{c}\mathrm{Gd} 2 \mathrm{O} 3 \\
-\mathrm{a}\end{array}$ & $\begin{array}{c}\mathrm{HgO} \\
-\mathrm{a}\end{array}$ \\
\hline PX 1-1 (4643) & 0.4914 & 0.0008 & & & 0.0019 & & & & & & & & & 0.0046 & & 0.0016 & & & & & \\
\hline PX 1-2 (4726) & 0.5058 & 0.0007 & & & 0.0020 & & & & & & & & & 0.0055 & & 0.0032 & & & & & \\
\hline PX 1-3 (4776) & 0.5242 & 0.0008 & & & 0.0018 & & & & & & & & & 0.0052 & & 0.0028 & & & & & \\
\hline PX 2-1 (4455) & 0.5186 & 0.0006 & & & 0.0009 & & & & & & & & & 0.0039 & & 0.0015 & & & & & \\
\hline PX 2-2 (4509) & 0.5020 & 0.0006 & & & 0.0014 & & & & & & & & & 0.0045 & & 0.0022 & & & & & \\
\hline PX 2-3 (4566) & 0.4886 & 0.0007 & & & 0.0018 & & & & & & & & & 0.0046 & & 0.0027 & & & & & \\
\hline PX 3-1 (5780) & 0.5969 & 0.0073 & & & 0.0006 & & & & & & & & & 0.0035 & & 0.0016 & & & & & \\
\hline PX 3-2 (5818) & 0.6040 & 0.0072 & & & 0.0007 & & & & & & & & & 0.0036 & & 0.0020 & & & & & \\
\hline PX 3-3 (5880) & 0.5425 & 0.0085 & & & 0.0006 & & & & & & & & & 0.0029 & & 0.0018 & & & & & \\
\hline PX 4-1 (6390) & 0.5229 & 0.0082 & & & 0.0008 & & & & & & & & & 0.0035 & & 0.0028 & & & & & \\
\hline PX 4-2 (6434) & 0.5314 & 0.0081 & & & 0.0008 & & & & & & & & & 0.0035 & & 0.0032 & & & & & \\
\hline PX 4-3 (6458) & 0.5336 & 0.0077 & & & 0.0008 & & & & & & & & & 0.0038 & & 0.0034 & & & & & \\
\hline PX 5-1 (6787) & 0.5439 & 0.0066 & & & 0.0009 & & & & & & & & & 0.0040 & 0.0005 & 0.0028 & & & & & \\
\hline PX 5-2 (6795) & 0.4847 & 0.0092 & & & 0.0009 & & & & & & & & & 0.0036 & 0.0005 & 0.0036 & & & & & \\
\hline PX 5-3 (6812) & 0.4542 & 0.0071 & & & 0.0009 & & & & & & & & & 0.0033 & 0.0007 & 0.0039 & & & & & \\
\hline PX 5-4 (6820) & 0.4585 & 0.0069 & & & 0.0009 & & & & & & & & & 0.0039 & 0.0006 & 0.0039 & & & & & \\
\hline PX 5-5 (6839) & 0.4797 & 0.0092 & & & 0.0008 & & & & & & & & & 0.0038 & 0.0006 & 0.0039 & & & & & \\
\hline PX 5-6 (6862) & 0.5051 & 0.0079 & & & 0.0007 & & & & & & & & & 0.0042 & 0.0006 & 0.0034 & & & & & \\
\hline PX 5-7 (6871) & 0.5051 & 0.0076 & & & 0.0007 & & & & & & & & & 0.0033 & 0.0006 & 0.0034 & & & & & \\
\hline PX 5-8 (6884) & 0.4900 & 0.0079 & & & 0.0007 & & & & & & & & & 0.0050 & 0.0006 & 0.0036 & & & & & \\
\hline PX 5-9 (6960) & 0.4820 & 0.0073 & & & 0.0007 & & & & & & & & & 0.0041 & 0.0006 & 0.0034 & & & & & \\
\hline PX 5-10 (6972) & 0.4927 & 0.0071 & & & 0.0006 & & & & & & & & & 0.0044 & 0.0006 & 0.0032 & & & & & \\
\hline PX 6-1 (7340) & 0.5319 & 0.0101 & & & 0.0008 & & & & & & & & & 0.0040 & & 0.0026 & & & & & \\
\hline BATCH 1 STUDY-6- & 0.4353 & 0.0048 & & & 0.0020 & & & & & & & & & 0.0004 & 0.0012 & 0.0049 & & & & & \\
\hline BATCH 1 STUDY-10 & 0.5910 & 0.0020 & & & 0.0015 & & & & & & & & & 0.0004 & 0.0012 & 0.0032 & & & & & \\
\hline BATCH 1 STUDY-1 & 0.6468 & 0.0018 & & & 0.0009 & & & & & & & & & 0.0004 & 0.0005 & 0.0021 & & & & & \\
\hline H-GLAS-0112 & 0.5091 & 0.0060 & & & 0.0016 & & & & & & & & & 0.0043 & & 0.0026 & & & & & \\
\hline H-GLAS-0130 & 0.5165 & 0.0060 & & & 0.0014 & & & & & & & & & 0.0041 & & 0.0022 & & & & & \\
\hline H-GLAS-0162 & 0.5195 & 0.0248 & & & 0.0009 & & & & & & & & & 0.0041 & & 0.0013 & & & & & \\
\hline H-GLAS-0244 & 0.5038 & 0.0129 & & & 0.0010 & & & & & & & & & 0.0036 & & 0.0008 & & & & & \\
\hline H-GLAS-0254 & 0.5377 & 0.0275 & & & 0.0008 & & & & & & & & & 0.0038 & & 0.0007 & & & & & \\
\hline H-GLAS-0278 & 0.5273 & 0.0279 & & & 0.0008 & & & & & & & & & 0.0035 & & 0.0010 & & & & & \\
\hline H-GLAS-0293 & 0.5168 & 0.0148 & & & 0.0007 & & & & & & & & & 0.0036 & & 0.0009 & & & & & \\
\hline H-GLAS-0308 & 0.5217 & 0.0312 & & & 0.0007 & & & & & & & & & 0.0049 & & 0.0009 & & & & & \\
\hline H-GLAS-0334 & 0.5223 & 0.0167 & & & 0.0007 & & & & & & & & & 0.0043 & & 0.0008 & & & & & \\
\hline H-GLAS-0352 & 0.5173 & 0.0338 & & & 0.0006 & & & & & & & & & 0.0038 & & 0.0007 & & & & & \\
\hline H-GLAS-0387 & 0.5220 & 0.0352 & & & 0.0006 & & & & & & & & & 0.0039 & & 0.0007 & & & & & \\
\hline H-GLAS-0421 & 0.5239 & 0.0366 & & & 0.0006 & & & & & & & & & 0.0045 & & 0.0007 & & & & & \\
\hline H-GLAS-0466 & 0.5171 & 0.0367 & & & 0.0006 & & & & & & & & & 0.0036 & & 0.0007 & & & & & \\
\hline FRIT-202-CLEAR & 0.7704 & & & & & & & & & & & & & & & & & & & & \\
\hline \multicolumn{22}{|l|}{ FRIT-202-INT } \\
\hline \multicolumn{22}{|l|}{ FRIT-202-WHITE } \\
\hline FRIT-165-7 & 0.6889 & 0.0099 & & & & & & & & & & & & & & & & & & & \\
\hline FRIT-131-7 & 0.5206 & 0.0046 & & & & & & & & & & & & & & & & & & & \\
\hline \multicolumn{22}{|l|}{ MG 9-7 } \\
\hline \multicolumn{22}{|l|}{ MG 18-7 } \\
\hline MG 25-7 & & & & & & & & & & & & & & & & & & & & & \\
\hline MG 28-7 & & & & & & & & & & & & & & & & & & & & & \\
\hline
\end{tabular}


Appendix A. Database - mass fraction

DWPF PCT Model (Jantzen et al. 1995)

\begin{tabular}{|c|c|c|c|c|c|c|c|c|c|c|c|c|c|c|c|c|c|c|c|c|c|}
\hline Glass ID & $\begin{array}{c}\mathrm{I} \\
-\mathrm{a}\end{array}$ & $\begin{array}{c}\mathrm{La} 2 \mathrm{O} 3 \\
-\mathrm{a}\end{array}$ & $\begin{array}{c}\mathrm{MnO} 2 \\
-\mathrm{a}\end{array}$ & $\begin{array}{c}\mathrm{MnO} \\
-\mathrm{a}\end{array}$ & $\begin{array}{c}\mathrm{MoO} \\
-\mathrm{a}\end{array}$ & $\begin{array}{c}\mathrm{MoO} 3 \\
-\mathrm{a}\end{array}$ & $\begin{array}{c}\mathrm{Nb} 2 \mathrm{O} 5 \\
-\mathrm{a}\end{array}$ & $\begin{array}{c}\mathrm{Nd} 2 \mathrm{O} 3 \\
-\mathrm{a}\end{array}$ & $\begin{array}{c}\mathrm{PbO} \\
-\mathrm{a}\end{array}$ & $\begin{array}{c}\mathrm{PdO} 2 \\
-\mathrm{a}\end{array}$ & $\begin{array}{c}\mathrm{PdO} \\
-\mathrm{a}\end{array}$ & $\begin{array}{c}\mathrm{Pr} 2 \mathrm{O} 3 \\
-\mathrm{a}\end{array}$ & $\begin{array}{c}\text { Pr6O11 } \\
-\mathrm{a}\end{array}$ & $\begin{array}{c}\mathrm{Rb} 2 \mathrm{O} \\
-\mathrm{a}\end{array}$ & $\begin{array}{c}\mathrm{ReO} \\
-\mathrm{a}\end{array}$ & $\begin{array}{c}\mathrm{ReO} 2 \\
-\mathrm{a}\end{array}$ & $\begin{array}{c}\mathrm{Rh} 2 \mathrm{O} 3 \\
-\mathrm{a}\end{array}$ & $\begin{array}{c}\mathrm{RhO} 2 \\
-\mathrm{a}\end{array}$ & $\begin{array}{c}\mathrm{RuO} 2 \\
-\mathrm{a}\end{array}$ & $\begin{array}{c}\mathrm{Sb} 2 \mathrm{O} 3 \\
-\mathrm{a}\end{array}$ & $\begin{array}{c}\mathrm{Sb} 2 \mathrm{O} 5 \\
-\mathrm{a}\end{array}$ \\
\hline PX 1-1 (4643) & & & & 0.0310 & & & & & 0.0017 & & & & & & & & & & & & \\
\hline PX 1-2 (4726) & & & & 0.0326 & & & & & 0.0019 & & & & & & & & & & & & \\
\hline PX 1-3 (4776) & & & & 0.0284 & & & & & 0.0016 & & & & & & & & & & & & \\
\hline PX 2-1 (4455) & & & & 0.0284 & & & & & 0.0003 & & & & & & & & & & & & \\
\hline PX 2-2 (4509) & & & & 0.0304 & & & & & 0.0011 & & & & & & & & & & & & \\
\hline PX 2-3 (4566) & & & & 0.0324 & & & & & 0.0015 & & & & & & & & & & & & \\
\hline PX 3-1 (5780) & & & & 0.0173 & & 0.0001 & & & 0.0005 & & & & & & & & & & & & \\
\hline PX 3-2 (5818) & & 0.0001 & & 0.0175 & & 0.0001 & & & 0.0007 & & & & & & & & & & & & \\
\hline PX 3-3 (5880) & & & & 0.0156 & & 0.0001 & & & 0.0007 & & & & & & & & & & & & \\
\hline PX 4-1 (6390) & & & & 0.0166 & & 0.0001 & & & 0.0010 & & & & & & & & & & & & \\
\hline PX 4-2 (6434) & & & & 0.0168 & & 0.0001 & & & 0.0011 & & & & & & & & & & & & \\
\hline PX 4-3 (6458) & & & & 0.0169 & & 0.0001 & & & 0.0011 & & & & & & & & & & & & \\
\hline PX 5-1 (6787) & & & & 0.0156 & & & & 0.0010 & 0.0013 & & & & & & & & & & & & \\
\hline PX 5-2 (6795) & & & & 0.0175 & & & & 0.0013 & 0.0015 & & & & & & & & & & & & \\
\hline PX 5-3 (6812) & & & & 0.0185 & & & & 0.0010 & 0.0014 & & & & & & & & & & & & \\
\hline PX 5-4 (6820) & & & & 0.0175 & & & & 0.0009 & 0.0014 & & & & & & & & & & & & \\
\hline PX 5-5 (6839) & & & & 0.0182 & & & & 0.0006 & 0.0014 & & & & & & & & & & & & \\
\hline PX 5-6 (6862) & & & & 0.0158 & & & & 0.0007 & 0.0012 & & & & & & & & & & & & \\
\hline PX 5-7 (6871) & & & & 0.0153 & & & & 0.0009 & 0.0012 & & & & & & & & & & & & \\
\hline PX 5-8 (6884) & & & & 0.0159 & & & & 0.0007 & 0.0012 & & & & & & & & & & & & \\
\hline PX 5-9 (6960) & & & & 0.0151 & & & & 0.0005 & 0.0011 & & & & & & & & & & & & \\
\hline PX 5-10 (6972) & & & & 0.0147 & & & & 0.0005 & 0.0011 & & & & & & & & & & & & \\
\hline PX 6-1 (7340) & & 0.0001 & & 0.0171 & & 0.0002 & & & 0.0014 & & & & & & & & & & & & \\
\hline \multicolumn{2}{|c|}{ BATCH 1 STUDY-6-7 } & & & 0.0236 & & & & 0.0067 & 0.0013 & & & & & & & & & & & & \\
\hline \multicolumn{2}{|c|}{ BATCH 1 STUDY-10B-7 } & & & 0.0072 & & & & 0.0022 & 0.0004 & & & & & & & & & & & & \\
\hline \multicolumn{2}{|c|}{ BATCH 1 STUDY-15-7 } & & & 0.0072 & & & & 0.0022 & 0.0004 & & & & & & & & & & & & \\
\hline H-GLAS-0112 & & 0.0004 & & 0.0244 & & 0.0003 & & & 0.0020 & & & & & & & & & & & & \\
\hline H-GLAS-0130 & & & & 0.0206 & & 0.0006 & & & 0.0021 & & & & & & & & & & & & \\
\hline H-GLAS-0162 & & 0.0022 & & 0.0124 & & 0.0011 & & & 0.0018 & & & & & & & & & & & & \\
\hline H-GLAS-0244 & & 0.0011 & & 0.0171 & & 0.0006 & & & 0.0016 & & & & & & & & & & & & \\
\hline H-GLAS-0254 & & 0.0025 & & 0.0110 & & 0.0012 & & & 0.0017 & & & & & & & & & & & & \\
\hline H-GLAS-0278 & & 0.0024 & & 0.0095 & & 0.0012 & & & 0.0017 & & & & & & & & & & & & \\
\hline H-GLAS-0293 & & 0.0025 & & 0.0093 & & 0.0012 & & & 0.0016 & & & & & & & & & & & & \\
\hline H-GLAS-0308 & & 0.0025 & & 0.0094 & & 0.0014 & & & 0.0017 & & & & & & & & & & & & \\
\hline H-GLAS-0334 & & 0.0024 & & 0.0087 & & 0.0015 & & & 0.0016 & & & & & & & & & & & & \\
\hline H-GLAS-0352 & & 0.0023 & & 0.0078 & & 0.0015 & & & 0.0017 & & & & & & & & & & & & \\
\hline H-GLAS-0387 & & 0.0021 & & 0.0066 & & 0.0015 & & & 0.0015 & & & & & & & & & & & & \\
\hline H-GLAS-0421 & & 0.0021 & & 0.0066 & & 0.0016 & & & 0.0017 & & & & & & & & & & & & \\
\hline H-GLAS-0466 & & 0.0022 & & 0.0063 & & 0.0016 & & & 0.0020 & & & & & & & & & & & & \\
\hline \multicolumn{22}{|l|}{ FRIT-202-CLEAR } \\
\hline \multicolumn{22}{|l|}{ FRIT-202-INT } \\
\hline \multicolumn{22}{|l|}{ FRIT-202-WHITE } \\
\hline \multicolumn{22}{|l|}{ FRIT-165-7 } \\
\hline FRIT-131-7 & & & & 0.0013 & & 0.0027 & & 0.0050 & & & & & & & & & & & & & \\
\hline \multicolumn{22}{|l|}{ MG 9-7 } \\
\hline \multicolumn{22}{|l|}{ MG 18-7 } \\
\hline \multicolumn{22}{|l|}{ MG 25-7 } \\
\hline MG 28-7 & & & & & & & & & & & & & & & & & & & & & \\
\hline
\end{tabular}


Appendix A. Database - mass fraction

DWPF PCT Model (Jantzen et al. 1995)

\begin{tabular}{|c|c|c|c|c|c|c|c|c|c|c|c|c|c|c|c|c|c|c|c|c|}
\hline Glass ID & $\begin{array}{c}\mathrm{SeO} 2 \\
-\mathrm{a}\end{array}$ & $\begin{array}{c}\mathrm{Sm} 2 \mathrm{O} 3 \\
-\mathrm{a} \\
\end{array}$ & $\begin{array}{c}\mathrm{SnO} \\
-\mathrm{a} \\
\end{array}$ & $\begin{array}{c}\mathrm{SnO} 2 \\
-\mathrm{a}\end{array}$ & $\begin{array}{c}\mathrm{SO} 3 \\
-\mathrm{a} \\
\end{array}$ & $\begin{array}{c}\text { SrO } \\
-\mathrm{a} \\
\end{array}$ & $\begin{array}{c}\mathrm{Tc} 2 \mathrm{O} 7 \\
-\mathrm{a}\end{array}$ & $\begin{array}{c}\mathrm{TeO} 2 \\
-\mathrm{a}\end{array}$ & $\begin{array}{c}\mathrm{ThO} 2 \\
-\mathrm{a}\end{array}$ & $\begin{array}{c}\mathrm{TiO} 2 \\
-\mathrm{a} \\
\end{array}$ & $\begin{array}{c}\mathrm{T} 12 \mathrm{O} 3 \\
-\mathrm{a} \\
\end{array}$ & $\begin{array}{c}\mathrm{U} 3 \mathrm{O} 8 \\
-\mathrm{a}\end{array}$ & $\begin{array}{c}\mathrm{UO} 2 \\
-\mathrm{a}\end{array}$ & $\begin{array}{c}\mathrm{UO} 3 \\
-\mathrm{a}\end{array}$ & $\begin{array}{c}\mathrm{V} 2 \mathrm{O} 5 \\
-\mathrm{a} \\
\end{array}$ & $\begin{array}{c}\text { WO3 } \\
-\mathrm{a}\end{array}$ & $\begin{array}{c}\mathrm{Y} 2 \mathrm{O} 3 \\
-\mathrm{a}\end{array}$ & $\begin{array}{c}\mathrm{ZnO} \\
-\mathrm{a} \\
\end{array}$ & $\begin{array}{c}\text { Others } \\
-\mathrm{a}\end{array}$ & $\begin{array}{c}\text { Sum } \\
-\mathrm{a}\end{array}$ \\
\hline PX 1-1 (4643) & & & & & & 0.0003 & & & & 0.0017 & & & & & & & & 0.0016 & & 0.9809 \\
\hline PX 1-2 (4726) & & & & & & 0.0003 & & & & 0.0037 & & & & & & & & 0.0017 & & 1.0127 \\
\hline PX 1-3 (4776) & & & & & & 0.0002 & & & & 0.0035 & & & & & & & & 0.0017 & & 0.9975 \\
\hline PX 2-1 (4455) & & & & & & 0.0014 & & & & 0.0010 & & & & & & & & 0.0010 & & 1.0062 \\
\hline PX 2-2 (4509) & & & & & & 0.0008 & & & & 0.0017 & & & & & & & & 0.0013 & & 0.9998 \\
\hline PX 2-3 (4566) & & & & & & 0.0005 & & & & 0.0022 & & & & & & & & 0.0015 & & 0.9956 \\
\hline PX 3-1 (5780) & & & & & & 0.0005 & & & & 0.0024 & & & & & & & & 0.0011 & & 1.0357 \\
\hline PX 3-2 (5818) & & & & & & 0.0004 & & & & 0.0024 & & & & & & & & 0.0012 & & 1.0477 \\
\hline PX 3-3 (5880) & & & & & & 0.0002 & & & & 0.0019 & & & & & & & & 0.0013 & & 0.9478 \\
\hline PX 4-1 (6390) & & & & & & 0.0002 & & & & 0.0025 & & & & & & & & 0.0012 & & 0.9645 \\
\hline PX 4-2 (6434) & & & & & & 0.0002 & & & & 0.0027 & & & & & & & & 0.0012 & & 0.9781 \\
\hline PX 4-3 (6458) & & & & & & 0.0002 & & & & 0.0028 & & & & & & & & 0.0012 & & 0.9782 \\
\hline PX 5-1 (6787) & & & & & & 0.0002 & & & & 0.0028 & & & & & & & & 0.0009 & & 0.9809 \\
\hline PX 5-2 (6795) & & & & & & 0.0002 & & & & 0.0029 & & & & & & & & 0.0011 & & 0.9693 \\
\hline PX 5-3 (6812) & & & & & & 0.0002 & & & & 0.0029 & & & & & & & & 0.0011 & & 0.9538 \\
\hline PX 5-4 (6820) & & & & & & 0.0002 & & & & 0.0027 & & & & & & & & 0.0011 & & 0.9474 \\
\hline PX 5-5 (6839) & & & & & & 0.0002 & & & & 0.0027 & & & & & & & & 0.0011 & & 0.9824 \\
\hline PX 5-6 (6862) & & & & & & 0.0001 & & & & 0.0025 & & & & & & & & 0.0010 & & 0.9778 \\
\hline PX 5-7 (6871) & & & & & & 0.0001 & & & & 0.0023 & & & & & & & & 0.0009 & & 0.9713 \\
\hline PX 5-8 (6884) & & & & & & 0.0001 & & & & 0.0024 & & & & & & & & 0.0009 & & 0.9864 \\
\hline PX 5-9 (6960) & & & & & & 0.0001 & & & & 0.0022 & & & & & & & & 0.0009 & & 0.9781 \\
\hline PX 5-10 (6972) & & & & & & 0.0001 & & & & 0.0021 & & & & & & & & 0.0009 & & 0.9851 \\
\hline PX 6-1 (7340) & & & & & & & & & & 0.0016 & & & & & & & & 0.0010 & & 0.9971 \\
\hline \multicolumn{2}{|l|}{ BATCH 1 STUDY-6-7 } & & & & & 0.0003 & & & & 0.0099 & & & & & & & 0.0001 & 0.0003 & & 0.9861 \\
\hline \multicolumn{2}{|c|}{ BATCH 1 STUDY-10B-7 } & & & & & 0.0001 & & & & 0.0101 & & & & & & & & 0.0003 & & 0.9769 \\
\hline \multicolumn{2}{|c|}{ BATCH 1 STUDY-15-7 } & & & & & 0.0001 & & & & 0.0051 & & & & & & & & 0.0005 & & 1.0014 \\
\hline H-GLAS-0112 & & & & & & 0.0003 & & & & 0.0037 & & & & & & & & 0.0032 & & 0.9613 \\
\hline H-GLAS-0130 & & & & & & 0.0004 & & & & 0.0026 & & & & & & & & 0.0027 & & 0.9613 \\
\hline H-GLAS-0162 & & & & & & 0.0003 & & & & 0.0020 & & & & & & & & 0.0042 & & 0.9517 \\
\hline H-GLAS-0244 & & & & & & 0.0004 & & & & 0.0019 & & & & & & & & 0.0028 & & 0.9612 \\
\hline H-GLAS-0254 & & & & & & 0.0003 & & & & 0.0018 & & & & & & & & 0.0026 & & 0.9721 \\
\hline H-GLAS-0278 & & & & & & 0.0003 & & & & 0.0017 & & & & & & & & 0.0032 & & 0.9516 \\
\hline H-GLAS-0293 & & & & & & 0.0003 & & & & 0.0016 & & & & & & & & 0.0026 & & 0.9302 \\
\hline H-GLAS-0308 & & & & & & 0.0003 & & & & 0.0016 & & & & & & & & 0.0033 & & 0.9630 \\
\hline H-GLAS-0334 & & & & & & 0.0003 & & & & 0.0016 & & & & & & & & 0.0027 & & 0.9570 \\
\hline H-GLAS-0352 & & & & & & 0.0003 & & & & 0.0016 & & & & & & & & 0.0029 & & 0.9597 \\
\hline H-GLAS-0387 & & & & & & 0.0003 & & & & 0.0014 & & & & & & & & 0.0033 & & 0.9503 \\
\hline H-GLAS-0421 & & & & & & 0.0003 & & & & 0.0014 & & & & & & & & 0.0029 & & 0.9661 \\
\hline H-GLAS-0466 & & & & & & 0.0004 & & & & 0.0015 & & & & & & & & 0.0026 & & 0.9668 \\
\hline FRIT-202-CLEAR & & & & & & & & & & 0.0010 & & & & & & & & & & 1.0005 \\
\hline \multicolumn{21}{|l|}{ FRIT-202-INT } \\
\hline \multicolumn{21}{|l|}{ FRIT-202-WHITE } \\
\hline FRIT-165-7 & & & & & & & & & & & & & & & & & & & & 0.9931 \\
\hline FRIT-131-7 & & & & & & & & & & 0.0095 & & & & & & & & & & 0.9512 \\
\hline \multicolumn{21}{|l|}{ MG 9-7 } \\
\hline \multicolumn{21}{|l|}{ MG 18-7 } \\
\hline \multicolumn{21}{|l|}{ MG 25-7 } \\
\hline MG 28-7 & & & & & & & & & & & & & & & & & & & & \\
\hline
\end{tabular}


Appendix A. Database - mass fraction

DWPF PCT Model (Jantzen et al. 1995)

\begin{tabular}{|c|c|c|c|c|c|c|c|c|}
\hline Glass ID & $\begin{array}{l}\mathrm{TM} \\
\left({ }^{\circ} \mathrm{C}\right)\end{array}$ & $\begin{array}{l}\text { Gradient } \\
\text { TL }\left({ }^{\circ} \mathrm{C}\right)\end{array}$ & $\begin{array}{l}\text { Uniform } \\
\text { TL }\left({ }^{\circ} \mathrm{C}\right)\end{array}$ & Primary Phase & $\begin{array}{l}\text { Quenched } \\
\text { Visual/OM }\end{array}$ & $\begin{array}{c}\text { Quenched } \\
\text { SEM/EDS or TEM }\end{array}$ & $\begin{array}{l}\text { Quenched } \\
\text { XRD }\end{array}$ & $\begin{array}{c}\text { Quenched } \\
\text { Homogeneous? }\end{array}$ \\
\hline \multicolumn{9}{|l|}{ PX 1-1 (4643) } \\
\hline \multicolumn{9}{|l|}{ PX 1-2 (4726) } \\
\hline \multicolumn{9}{|l|}{ PX 1-3 (4776) } \\
\hline \multicolumn{9}{|l|}{ PX 2-1 (4455) } \\
\hline \multicolumn{9}{|l|}{ PX 2-2 (4509) } \\
\hline \multicolumn{9}{|l|}{ PX 2-3 (4566) } \\
\hline \multicolumn{9}{|l|}{ PX 3-1 (5780) } \\
\hline \multicolumn{9}{|l|}{ PX 3-2 (5818) } \\
\hline \multicolumn{9}{|l|}{ PX 3-3 (5880) } \\
\hline \multicolumn{9}{|l|}{ PX 4-1 (6390) } \\
\hline \multicolumn{9}{|l|}{ PX 4-2 (6434) } \\
\hline \multicolumn{9}{|l|}{ PX 4-3 (6458) } \\
\hline \multicolumn{9}{|l|}{ PX 5-1 (6787) } \\
\hline \multicolumn{9}{|l|}{ PX 5-2 (6795) } \\
\hline \multicolumn{9}{|l|}{ PX 5-3 (6812) } \\
\hline \multicolumn{9}{|l|}{ PX 5-4 (6820) } \\
\hline \multicolumn{9}{|l|}{ PX 5-5 (6839) } \\
\hline \multicolumn{9}{|l|}{ PX 5-6 (6862) } \\
\hline \multicolumn{9}{|l|}{ PX 5-7 (6871) } \\
\hline \multicolumn{9}{|l|}{ PX 5-8 (6884) } \\
\hline PX 5-9 (6960) & & & & & & & & \\
\hline PX 5-10 (6972) & & & & & & & & \\
\hline PX 6-1 (7340) & & & & & & & & \\
\hline BATCH 1 STUDY-6 & & & & & & & & \\
\hline BATCH 1 STUDY-1 & & & & & & & & \\
\hline BATCH 1 STUDY-1 & & & & & & & & \\
\hline H-GLAS-0112 & & & & & & & & \\
\hline H-GLAS-0130 & & & & & & & & \\
\hline H-GLAS-0162 & & & & & & & & \\
\hline H-GLAS-0244 & & & & & & & & \\
\hline H-GLAS-0254 & & & & & & & & \\
\hline H-GLAS-0278 & & & & & & & & \\
\hline H-GLAS-0293 & & & & & & & & \\
\hline H-GLAS-0308 & & & & & & & & \\
\hline H-GLAS-0334 & & & & & & & & \\
\hline H-GLAS-0352 & & & & & & & & \\
\hline H-GLAS-0387 & & & & & & & & \\
\hline H-GLAS-0421 & & & & & & & & \\
\hline H-GLAS-0466 & & & & & & & & \\
\hline FRIT-202-CLEAR & & & & & & & & \\
\hline FRIT-202-INT & & & & & & & & \\
\hline FRIT-202-WHITE & & & & & & & & \\
\hline FRIT-165-7 & & & & & & & & \\
\hline FRIT-131-7 & & & & & & & & \\
\hline MG 9-7 & & & & & & & & \\
\hline MG 18-7 & & & & & & & & \\
\hline MG 25-7 & & & & & & & & \\
\hline MG 28-7 & & & & & & & & \\
\hline
\end{tabular}


Appendix A. Database - mass fraction

\section{DWPF PCT Model (Jantzen et al. 1995)}

\begin{tabular}{|c|c|c|c|c|c|}
\hline Glass ID & $\begin{array}{c}\mathrm{CCC} \\
\text { Visual/OM }\end{array}$ & $\begin{array}{c}\text { CCC } \\
\text { SEM/EDS or TEM } \\
\end{array}$ & $\begin{array}{l}\mathrm{CCC} \\
\mathrm{XRD}\end{array}$ & $\begin{array}{l}\text { Heat Treated } \\
\text { Visual/OM }\end{array}$ & $\begin{array}{c}\text { Heat Treated } \\
\text { SEM/EDS or TEM }\end{array}$ \\
\hline \multicolumn{6}{|l|}{ PX 1-1 (4643) } \\
\hline PX 1-2 (4726) & & & & & \\
\hline \multicolumn{6}{|l|}{ PX 1-3 (4776) } \\
\hline \multicolumn{6}{|l|}{ PX 2-1 (4455) } \\
\hline \multicolumn{6}{|l|}{ PX 2-2 (4509) } \\
\hline \multicolumn{6}{|l|}{ PX 2-3 (4566) } \\
\hline \multicolumn{6}{|l|}{ PX 3-1 (5780) } \\
\hline \multicolumn{6}{|l|}{ PX 3-2 (5818) } \\
\hline \multicolumn{6}{|l|}{ PX 3-3 (5880) } \\
\hline \multicolumn{6}{|l|}{ PX 4-1 (6390) } \\
\hline \multicolumn{6}{|l|}{ PX 4-2 (6434) } \\
\hline \multicolumn{6}{|l|}{ PX 4-3 (6458) } \\
\hline \multicolumn{6}{|l|}{ PX 5-1 (6787) } \\
\hline \multicolumn{6}{|l|}{ PX 5-2 (6795) } \\
\hline \multicolumn{6}{|l|}{ PX 5-3 (6812) } \\
\hline \multicolumn{6}{|l|}{ PX 5-4 (6820) } \\
\hline \multicolumn{6}{|l|}{ PX 5-5 (6839) } \\
\hline \multicolumn{6}{|l|}{ PX 5-6 (6862) } \\
\hline \multicolumn{6}{|l|}{ PX 5-7 (6871) } \\
\hline \multicolumn{6}{|l|}{ PX 5-8 (6884) } \\
\hline \multicolumn{6}{|l|}{ PX 5-9 (6960) } \\
\hline \multicolumn{6}{|l|}{ PX 5-10 (6972) } \\
\hline \multicolumn{6}{|l|}{ PX 6-1 (7340) } \\
\hline BATCH 1 STUDY- & & & & & \\
\hline BATCH 1 STUDY- & $0 \mathrm{~B}-7$ & & & & \\
\hline BATCH 1 STUDY- & & & & & \\
\hline H-GLAS-0112 & & & & & \\
\hline H-GLAS-0130 & & & & & \\
\hline H-GLAS-0162 & & & & & \\
\hline H-GLAS-0244 & & & & & \\
\hline H-GLAS-0254 & & & & & \\
\hline H-GLAS-0278 & & & & & \\
\hline H-GLAS-0293 & & & & & \\
\hline H-GLAS-0308 & & & & & \\
\hline H-GLAS-0334 & & & & & \\
\hline H-GLAS-0352 & & & & & \\
\hline H-GLAS-0387 & & & & & \\
\hline H-GLAS-0421 & & & & & \\
\hline H-GLAS-0466 & & & & & \\
\hline FRIT-202-CLEAR & & & & & \\
\hline FRIT-202-INT & & & & & \\
\hline FRIT-202-WHITE & & & & & \\
\hline FRIT-165-7 & & & & & \\
\hline FRIT-131-7 & & & & & \\
\hline MG 9-7 & & & & & \\
\hline MG 18-7 & & & & & \\
\hline MG 25-7 & & & & & \\
\hline MG 28-7 & & & & & \\
\hline
\end{tabular}


Appendix A. Database - mass fraction

\section{DWPF PCT Model (Jantzen et al. 1995)}

\section{Glass ID}

PX 1-1 (4643)

PX 1-2 (4726)

PX 1-3 (4776)

PX 2-1 (4455)

PX 2-2 (4509)

PX 2-3 (4566)

PX 3-1 (5780)

PX 3-2 (5818)

PX 3-3 (5880)

PX 4-1 (6390)

PX 4-2 (6434)

PX 4-3 (6458)

PX 5-1 (6787)

PX 5-2 (6795)

PX 5-3 (6812)

PX 5-4 (6820)

PX 5-5 (6839)

PX 5-6 (6862)

PX 5-7 (6871)

PX 5-8 (6884)

PX 5-9 (6960)

PX 5-10 (6972)

PX 6-1 (7340)

BATCH 1 STUDY-6-7

BATCH 1 STUDY-10B-7

BATCH 1 STUDY-15-7

H-GLAS-0112

H-GLAS-0130

H-GLAS-0162

H-GLAS-0244

H-GLAS-0254

H-GLAS-0278

H-GLAS-0293

H-GLAS-0308

H-GLAS-0334

H-GLAS-0352

H-GLAS-0387

H-GLAS-0421

H-GLAS-0466

FRIT-202-CLEAR

FRIT-202-INT

FRIT-202-WHITE

FRIT-165-7

FRIT-131-7

MG 9-7

MG 18-7

MG 25-7

MG 28-7

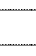

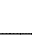

Heat Treated

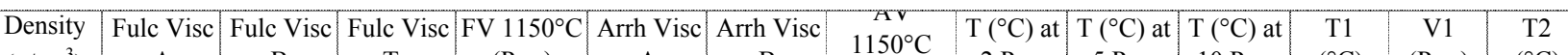

\begin{tabular}{l|l|l|l|l|l|l|l|l|l|l|l|l|l}
$\left(\mathrm{g} / \mathrm{cm}^{3}\right)$ & $\mathrm{A}$ & $\mathrm{B}$ & To & $(\mathrm{Pa} \cdot \mathrm{s})$ & $\mathrm{A}$ & $\mathrm{B}$ & $1150^{\circ} \mathrm{C}$ & $2 \mathrm{~Pa} \cdot \mathrm{s}$ & $5 \mathrm{~Pa} \cdot \mathrm{s}$ & $10 \mathrm{~Pa} \cdot \mathrm{s}$ & $\left({ }^{\circ} \mathrm{C}\right)$ & $(\mathrm{Pa} \cdot \mathrm{s})$ & $\left({ }^{\circ} \mathrm{C}\right)$ \\
\hline
\end{tabular}

2

\begin{tabular}{c|c|c}
$n-1$ \\
\hdashline
\end{tabular}

\begin{tabular}{|l|l|l|l|l|l|l|}
\hline & & & & & \\
\hline
\end{tabular}

$+$

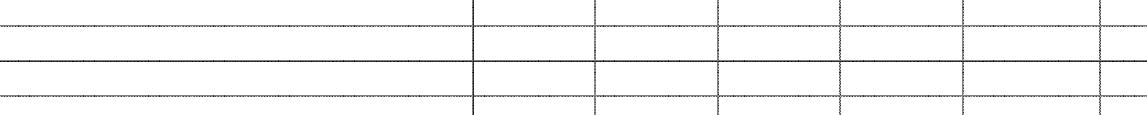

\begin{tabular}{l|l|l|l|l|l|l|l|l|l}
0 & & & \\
\hline
\end{tabular}

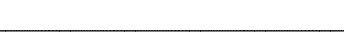

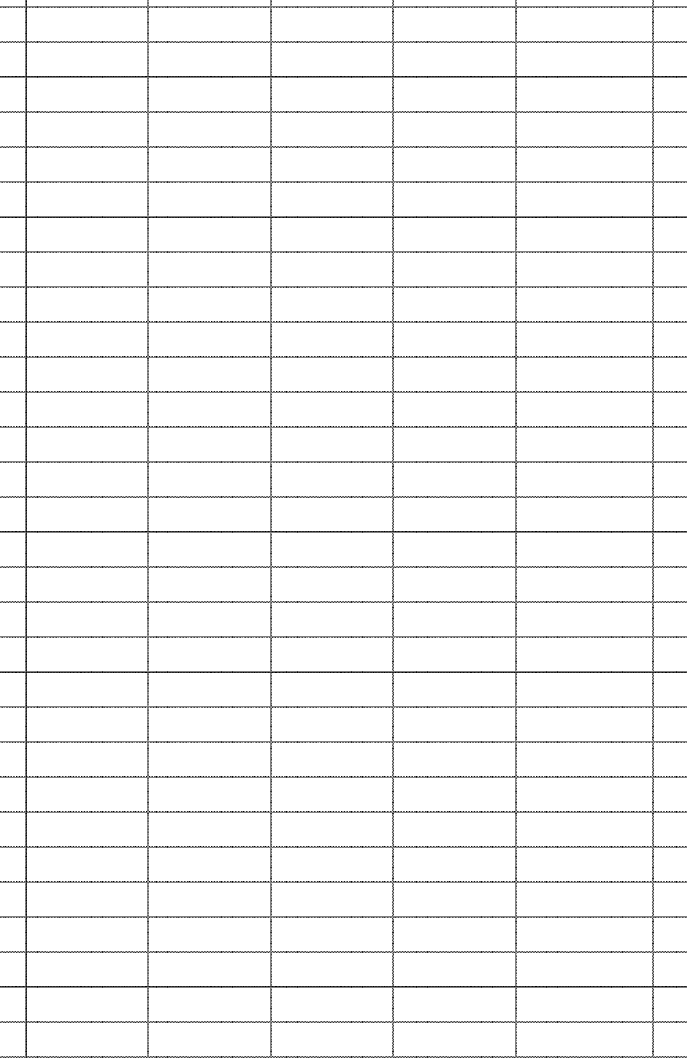


Appendix A. Database - mass fraction

DWPF PCT Model (Jantzen et al. 1995)

\begin{tabular}{|c|c|c|c|c|c|c|c|c|c|c|c|c|c|c|c|c|c|c|c|c|c|}
\hline Glass ID & $\begin{array}{c}\mathrm{V} 2 \\
(\mathrm{~Pa} \cdot \mathrm{s})\end{array}$ & $\begin{array}{c}\mathrm{T} 3 \\
\left({ }^{\circ} \mathrm{C}\right) \\
\end{array}$ & $\begin{array}{c}\mathrm{V} 3 \\
(\mathrm{~Pa} \cdot \mathrm{s})\end{array}$ & $\begin{array}{c}\mathrm{T} 4 \\
\left({ }^{\circ} \mathrm{C}\right)\end{array}$ & $\begin{array}{c}\mathrm{V} 4 \\
(\mathrm{~Pa} \cdot \mathrm{s})\end{array}$ & $\begin{array}{c}\mathrm{T} 5 \\
\left({ }^{\circ} \mathrm{C}\right)\end{array}$ & $\begin{array}{c}\mathrm{V} 5 \\
(\mathrm{~Pa} \cdot \mathrm{s})\end{array}$ & $\begin{array}{c}\mathrm{T} 6 \\
\left({ }^{\circ} \mathrm{C}\right) \\
\end{array}$ & $\begin{array}{c}\mathrm{V} 6 \\
(\mathrm{~Pa} \cdot \mathrm{s})\end{array}$ & $\begin{array}{c}\mathrm{T} 7 \\
\left({ }^{\circ} \mathrm{C}\right) \\
\end{array}$ & $\begin{array}{c}\mathrm{V} 7 \\
(\mathrm{~Pa} \cdot \mathrm{s})\end{array}$ & $\begin{array}{c}\mathrm{T} 8 \\
\left({ }^{\circ} \mathrm{C}\right) \\
\end{array}$ & $\begin{array}{c}\mathrm{V} 8 \\
(\mathrm{~Pa} \cdot \mathrm{s})\end{array}$ & $\begin{array}{c}\mathrm{T} 9 \\
\left({ }^{\circ} \mathrm{C}\right) \\
\end{array}$ & $\begin{array}{c}\mathrm{V} 9 \\
(\mathrm{~Pa} \cdot \mathrm{s})\end{array}$ & $\begin{array}{l}\mathrm{T} 10 \\
\left({ }^{\circ} \mathrm{C}\right) \\
\end{array}$ & $\begin{array}{c}\mathrm{V} 10 \\
(\mathrm{~Pa} \cdot \mathrm{s})\end{array}$ & $\begin{array}{l}\mathrm{T} 11 \\
\left({ }^{\circ} \mathrm{C}\right) \\
\end{array}$ & $\begin{array}{c}\mathrm{V} 11 \\
(\mathrm{~Pa} \cdot \mathrm{s})\end{array}$ & $\begin{array}{l}\mathrm{T} 12 \\
\left({ }^{\circ} \mathrm{C}\right)\end{array}$ & $\begin{array}{c}\mathrm{V} 12 \\
(\mathrm{~Pa} \cdot \mathrm{s})\end{array}$ \\
\hline PX 1-1 (4643) & & & & & & & & & & & & & & & & & & & & & \\
\hline PX 1-2 (4726) & & & & & & & & & & & & & & & & & & & & & \\
\hline PX 1-3 (4776) & & & & & & & & & & & & & & & & & & & & & \\
\hline PX 2-1 (4455) & & & & & & & & & & & & & & & & & & & & & \\
\hline PX 2-2 (4509) & & & & & & & & & & & & & & & & & & & & & \\
\hline PX 2-3 (4566) & & & & & & & & & & & & & & & & & & & & & \\
\hline PX 3-1 (5780) & & & & & & & & & & & & & & & & & & & & & \\
\hline PX 3-2 (5818) & & & & & & & & & & & & & & & & & & & & & \\
\hline PX 3-3 (5880) & & & & & & & & & & & & & & & & & & & & & \\
\hline PX 4-1 (6390) & & & & & & & & & & & & & & & & & & & & & \\
\hline PX 4-2 (6434) & & & & & & & & & & & & & & & & & & & & & \\
\hline PX 4-3 (6458) & & & & & & & & & & & & & & & & & & & & & \\
\hline PX 5-1 (6787) & & & & & & & & & & & & & & & & & & & & & \\
\hline PX 5-2 (6795) & & & & & & & & & & & & & & & & & & & & & \\
\hline PX 5-3 (6812) & & & & & & & & & & & & & & & & & & & & & \\
\hline PX 5-4 (6820) & & & & & & & & & & & & & & & & & & & & & \\
\hline PX 5-5 (6839) & & & & & & & & & & & & & & & & & & & & & \\
\hline PX 5-6 (6862) & & & & & & & & & & & & & & & & & & & & & \\
\hline PX 5-7 (6871) & & & & & & & & & & & & & & & & & & & & & \\
\hline PX 5-8 (6884) & & & & & & & & & & & & & & & & & & & & & \\
\hline PX 5-9 (6960) & & & & & & & & & & & & & & & & & & & & & \\
\hline PX 5-10 (6972) & & & & & & & & & & & & & & & & & & & & & \\
\hline PX 6-1 (7340) & & & & & & & & & & & & & & & & & & & & & \\
\hline BATCH 1 STUDY-6 & & & & & & & & & & & & & & & & & & & & & \\
\hline BATCH 1 STUDY-1 & B-7 & & & & & & & & & & & & & & & & & & & & \\
\hline BATCH 1 STUDY-1 & & & & & & & & & & & & & & & & & & & & & \\
\hline H-GLAS-0112 & & & & & & & & & & & & & & & & & & & & & \\
\hline H-GLAS-0130 & & & & & & & & & & & & & & & & & & & & & \\
\hline H-GLAS-0162 & & & & & & & & & & & & & & & & & & & & & \\
\hline H-GLAS-0244 & & & & & & & & & & & & & & & & & & & & & \\
\hline H-GLAS-0254 & & & & & & & & & & & & & & & & & & & & & \\
\hline H-GLAS-0278 & & & & & & & & & & & & & & & & & & & & & \\
\hline H-GLAS-0293 & & & & & & & & & & & & & & & & & & & & & \\
\hline H-GLAS-0308 & & & & & & & & & & & & & & & & & & & & & \\
\hline H-GLAS-0334 & & & & & & & & & & & & & & & & & & & & & \\
\hline H-GLAS-0352 & & & & & & & & & & & & & & & & & & & & & \\
\hline H-GLAS-0387 & & & & & & & & & & & & & & & & & & & & & \\
\hline H-GLAS-0421 & & & & & & & & & & & & & & & & & & & & & \\
\hline H-GLAS-0466 & & & & & & & & & & & & & & & & & & & & & \\
\hline FRIT-202-CLEAR & & & & & & & & & & & & & & & & & & & & & \\
\hline FRIT-202-INT & & & & & & & & & & & & & & & & & & & & & \\
\hline FRIT-202-WHITE & & & & & & & & & & & & & & & & & & & & & \\
\hline FRIT-165-7 & & & & & & & & & & & & & & & & & & & & & \\
\hline FRIT-131-7 & & & & & & & & & & & & & & & & & & & & & \\
\hline MG 9-7 & & & & & & & & & & & & & & & & & & & & & \\
\hline MG 18-7 & & & & & & & & & & & & & & & & & & & & & \\
\hline MG 25-7 & & & & & & & & & & & & & & & & & & & & & \\
\hline MG 28-7 & & & & & & & & & & & & & & & & & & & & & \\
\hline
\end{tabular}


Appendix A. Database - mass fraction

DWPF PCT Model (Jantzen et al. 1995)

\begin{tabular}{|c|c|c|c|c|c|c|c|c|c|c|c|c|c|c|c|c|}
\hline Glass ID & $\begin{array}{l}\mathrm{T} 13 \\
\left({ }^{\circ} \mathrm{C}\right)\end{array}$ & $\begin{array}{l}\text { V13 } \\
(\mathrm{Pa} \cdot \mathrm{s})\end{array}$ & $\begin{array}{l}\mathrm{T} 14 \\
\left({ }^{\circ} \mathrm{C}\right)\end{array}$ & $\begin{array}{l}\text { V14 } \\
(\mathrm{Pa} \cdot \mathrm{s})\end{array}$ & $\begin{array}{c}\text { Q PCT } \\
\mathrm{B}\left(\mathrm{g} / \mathrm{m}^{2}\right)\end{array}$ & $\begin{array}{c}\text { Q PCT } \\
\mathrm{Li}\left(\mathrm{g} / \mathrm{m}^{2}\right)\end{array}$ & $\begin{array}{c}\text { Q PCT } \\
\mathrm{Na}\left(\mathrm{g} / \mathrm{m}^{2}\right)\end{array}$ & $\begin{array}{c}\text { Q PCT } \\
\mathrm{Si}\left(\mathrm{g} / \mathrm{m}^{2}\right)\end{array}$ & $\begin{array}{c}\text { Q PCT } \\
\text { pH }\end{array}$ & $\begin{array}{c}\text { CCC PCT } \\
\mathrm{B}\left(\mathrm{g} / \mathrm{m}^{2}\right)\end{array}$ & $\begin{array}{l}\text { CCC PCT } \\
\operatorname{Li}\left(\mathrm{g} / \mathrm{m}^{2}\right)\end{array}$ & $\begin{array}{l}\text { CCC PCT } \\
\mathrm{Na}\left(\mathrm{g} / \mathrm{m}^{2}\right)\end{array}$ & $\begin{array}{l}\text { CCC PCT } \\
\mathrm{Si}\left(\mathrm{g} / \mathrm{m}^{2}\right)\end{array}$ & $\begin{array}{c}\text { CCC PCT } \\
\mathrm{pH}\end{array}$ & $\begin{array}{l}\text { Q PCT B at } \\
20^{\circ} \mathrm{C}\left(\mathrm{g} / \mathrm{m}^{2}\right)\end{array}$ & $\begin{array}{l}\text { Q PCT Li at } \\
20^{\circ} \mathrm{C}\left(\mathrm{g} / \mathrm{m}^{2}\right)\end{array}$ \\
\hline PX 1-1 (4643) & & & & & 0.36 & 0.39 & 0.365 & 0.210 & 9.46 & & & & & & & \\
\hline PX 1-2 (4726) & & & & & 0.355 & 0.38 & 0.36 & 0.215 & 9.36 & & & & & & & \\
\hline PX 1-3 (4776) & & & & & 0.395 & 0.415 & 0.385 & 0.230 & 9.38 & & & & & & & \\
\hline PX 2-1 (4455) & & & & & 0.24 & 0.285 & 0.24 & 0.165 & 9.32 & & & & & & & \\
\hline PX 2-2 (4509) & & & & & 0.29 & 0.325 & 0.305 & 0.185 & 9.35 & & & & & & & \\
\hline PX 2-3 (4566) & & & & & 0.365 & 0.39 & 0.365 & 0.205 & 9.49 & & & & & & & \\
\hline PX 3-1 (5780) & & & & & 0.42 & 0.41 & 0.335 & 0.210 & 9.68 & & & & & & & \\
\hline PX 3-2 (5818) & & & & & 0.54 & 0.505 & 0.425 & 0.245 & 9.7 & & & & & & & \\
\hline PX 3-3 (5880) & & & & & 0.675 & 0.605 & 0.52 & 0.315 & 9.62 & & & & & & & \\
\hline PX 4-1 (6390) & & & & & 1.135 & 0.985 & 0.92 & 0.465 & 10.05 & & & & & & & \\
\hline PX 4-2 (6434) & & & & & 2.27 & 1.815 & 1.8 & 0.780 & 10.52 & & & & & & & \\
\hline PX 4-3 (6458) & & & & & 2.185 & 1.745 & 1.825 & 0.765 & 10.42 & & & & & & & \\
\hline PX 5-1 (6787) & & & & & 0.535 & 0.525 & 0.53 & 0.250 & 10.36 & & & & & & & \\
\hline PX 5-2 (6795) & & & & & 4.58 & 3.095 & 4.085 & 0.995 & 11.61 & & & & & & & \\
\hline PX 5-3 (6812) & & & & & 9.6 & 5.305 & 8.235 & 1.965 & 12.04 & & & & & & & \\
\hline PX 5-4 (6820) & & & & & 10.5 & 5.645 & 8.94 & 2.225 & 12.01 & & & & & & & \\
\hline PX 5-5 (6839) & & & & & 10.515 & 5.7 & 8.485 & 2.330 & 11.99 & & & & & & & \\
\hline PX 5-6 (6862) & & & & & 9.485 & 5.405 & 7.56 & 2.285 & 11.84 & & & & & & & \\
\hline PX 5-7 (6871) & & & & & 9.29 & 5.21 & 7.215 & 2.315 & 11.84 & & & & & & & \\
\hline PX 5-8 (6884) & & & & & 10.79 & 5.9 & 8.575 & 2.350 & 11.87 & & & & & & & \\
\hline PX 5-9 (6960) & & & & & 8.57 & 4.79 & 6.85 & 2.195 & 11.78 & & & & & & & \\
\hline PX 5-10 (6972) & & & & & 10.95 & 4.745 & 8.685 & 1.615 & 11.73 & & & & & & & \\
\hline PX 6-1 (7340) & & & & & 7.56 & 4.805 & 8.415 & 1.950 & 11.65 & & & & & & & \\
\hline BATCH 1 STUDY-6- & & & & & 8.595 & 6.81 & 7.725 & 2.270 & 11.34 & & & & & & & \\
\hline BATCH 1 STUDY-10 & & & & & 5.265 & 4.495 & 4.765 & 1.565 & 10.9 & & & & & & & \\
\hline BATCH 1 STUDY-15 & & & & & 0.83 & 0.76 & 0.71 & 0.360 & 10.35 & & & & & & & \\
\hline H-GLAS-0112 & & & & & 0.44 & 0.415 & 0.375 & 0.220 & 9.83 & & & & & & & \\
\hline H-GLAS-0130 & & & & & 0.58 & 0.515 & 0.46 & 0.245 & 9.63 & & & & & & & \\
\hline H-GLAS-0162 & & & & & 1.005 & 0.835 & 0.69 & 0.320 & 9.69 & & & & & & & \\
\hline H-GLAS-0244 & & & & & 1.475 & 1.085 & 0.69 & 0.365 & 9.89 & & & & & & & \\
\hline H-GLAS-0254 & & & & & 1.05 & 0.91 & 0.715 & 0.320 & 9.79 & & & & & & & \\
\hline H-GLAS-0278 & & & & & 1.25 & 1.06 & 0.77 & 0.350 & 9.82 & & & & & & & \\
\hline H-GLAS-0293 & & & & & 1.54 & 1.29 & 0.925 & 0.380 & 9.84 & & & & & & & \\
\hline H-GLAS-0308 & & & & & 1.01 & 0.865 & 0.66 & 0.300 & 9.73 & & & & & & & \\
\hline H-GLAS-0334 & & & & & 1.205 & 1.075 & 0.775 & 0.325 & 9.76 & & & & & & & \\
\hline H-GLAS-0352 & & & & & 1.33 & 1.18 & 0.835 & 0.340 & 9.62 & & & & & & & \\
\hline H-GLAS-0387 & & & & & 1.29 & 1.155 & 0.785 & 0.330 & 9.67 & & & & & & & \\
\hline H-GLAS-0421 & & & & & 1.14 & 1.04 & 0.685 & 0.305 & 9.62 & & & & & & & \\
\hline H-GLAS-0466 & & & & & 1.395 & 1.205 & 0.83 & 0.330 & 9.69 & & & & & & & \\
\hline FRIT-202-CLEAR & & & & & 15.98 & 14.445 & 15.19 & 5.755 & & & & & & & & \\
\hline FRIT-202-INT & & & & & 21.95 & 18.635 & 19.54 & 10.485 & & & & & & & & \\
\hline FRIT-202-WHITE & & & & & 31.535 & 29.13 & 28.72 & 1.055 & & & & & & & & \\
\hline FRIT-165-7 & & & & & 40.16 & 42.155 & 37.08 & 30.515 & & & & & & & & \\
\hline FRIT-131-7 & & & & & 25.15 & 24.3 & 21.565 & 14.830 & & & & & & & & \\
\hline MG 9-7 & & & & & 35.12 & & 28.21 & 17.005 & 12.63 & & & & & & & \\
\hline MG 18-7 & & & & & 28.91 & & 23.885 & 8.340 & 12.01 & & & & & & & \\
\hline MG 25-7 & & & & & 0.415 & & 0.395 & 0.120 & 10.05 & & & & & & & \\
\hline MG 28-7 & & & & & 0.315 & & 0.36 & 0.160 & 10.65 & & & & & & & \\
\hline
\end{tabular}


Appendix A. Database - mass fraction

DWPF PCT Model (Jantzen et al. 1995)

\begin{tabular}{|c|c|c|c|c|c|c|c|c|c|c|c|c|}
\hline Glass ID & $\begin{array}{l}\text { Q PCT Na at } \\
20^{\circ} \mathrm{C}\left(\mathrm{g} / \mathrm{m}^{2}\right)\end{array}$ & $\begin{array}{l}\text { Q PCT Si at } \\
20^{\circ} \mathrm{C}\left(\mathrm{g} / \mathrm{m}^{2}\right)\end{array}$ & $\begin{array}{c}\mathrm{QpH} \\
\text { at } 20^{\circ} \mathrm{C}\end{array}$ & $\begin{array}{c}\text { TCLP Ag } \\
(\mathrm{ppm})\end{array}$ & $\begin{array}{c}\text { TCLP As } \\
\text { (ppm) }\end{array}$ & $\begin{array}{c}\text { TCLP Ba } \\
\text { (ppm) }\end{array}$ & $\begin{array}{c}\text { TCLP Cd } \\
(\mathrm{ppm})\end{array}$ & $\begin{array}{c}\text { TCLP Cr } \\
(\mathrm{ppm})\end{array}$ & $\begin{array}{c}\text { TCLP Ni } \\
(\mathrm{ppm})\end{array}$ & $\begin{array}{c}\text { TCLP Pb } \\
(\mathrm{ppm})\end{array}$ & $\begin{array}{l}\text { TCLP Se } \\
(\mathrm{ppm})\end{array}$ & $\begin{array}{c}\text { TCLP Zn } \\
(\mathrm{ppm})\end{array}$ \\
\hline PX 1-1 (4643) & & & & & & & & & & & & \\
\hline PX 1-2 (4726) & & & & & & & & & & & & \\
\hline PX 1-3 (4776) & & & & & & & & & & & & \\
\hline PX 2-1 (4455) & & & & & & & & & & & & \\
\hline PX 2-2 (4509) & & & & & & & & & & & & \\
\hline PX 2-3 (4566) & & & & & & & & & & & & \\
\hline PX 3-1 (5780) & & & & & & & & & & & & \\
\hline PX 3-2 (5818) & & & & & & & & & & & & \\
\hline PX 3-3 (5880) & & & & & & & & & & & & \\
\hline PX 4-1 (6390) & & & & & & & & & & & & \\
\hline PX 4-2 (6434) & & & & & & & & & & & & \\
\hline PX 4-3 (6458) & & & & & & & & & & & & \\
\hline PX 5-1 (6787) & & & & & & & & & & & & \\
\hline PX 5-2 (6795) & & & & & & & & & & & & \\
\hline PX 5-3 (6812) & & & & & & & & & & & & \\
\hline PX 5-4 (6820) & & & & & & & & & & & & \\
\hline PX 5-5 (6839) & & & & & & & & & & & & \\
\hline PX 5-6 (6862) & & & & & & & & & & & & \\
\hline PX 5-7 (6871) & & & & & & & & & & & & \\
\hline PX 5-8 (6884) & & & & & & & & & & & & \\
\hline PX 5-9 (6960) & & & & & & & & & & & & \\
\hline PX 5-10 (6972) & & & & & & & & & & & & \\
\hline PX 6-1 (7340) & & & & & & & & & & & & \\
\hline BATCH 1 STUDY-6 & & & & & & & & & & & & \\
\hline BATCH 1 STUDY-1 & B-7 & & & & & & & & & & & \\
\hline BATCH 1 STUDY-1 & & & & & & & & & & & & \\
\hline H-GLAS-0112 & & & & & & & & & & & & \\
\hline H-GLAS-0130 & & & & & & & & & & & & \\
\hline H-GLAS-0162 & & & & & & & & & & & & \\
\hline H-GLAS-0244 & & & & & & & & & & & & \\
\hline H-GLAS-0254 & & & & & & & & & & & & \\
\hline H-GLAS-0278 & & & & & & & & & & & & \\
\hline H-GLAS-0293 & & & & & & & & & & & & \\
\hline H-GLAS-0308 & & & & & & & & & & & & \\
\hline H-GLAS-0334 & & & & & & & & & & & & \\
\hline H-GLAS-0352 & & & & & & & & & & & & \\
\hline H-GLAS-0387 & & & & & & & & & & & & \\
\hline H-GLAS-0421 & & & & & & & & & & & & \\
\hline H-GLAS-0466 & & & & & & & & & & & & \\
\hline FRIT-202-CLEAR & & & & & & & & & & & & \\
\hline FRIT-202-INT & & & & & & & & & & & & \\
\hline FRIT-202-WHITE & & & & & & & & & & & & \\
\hline FRIT-165-7 & & & & & & & & & & & & \\
\hline FRIT-131-7 & & & & & & & & & & & & \\
\hline MG 9-7 & & & & & & & & & & & & \\
\hline MG 18-7 & & & & & & & & & & & & \\
\hline MG 25-7 & & & & & & & & & & & & \\
\hline MG 28-7 & & & & & & & & & & & & \\
\hline
\end{tabular}


Plutonium Vitrification (Bulkley and Vienna 1997)

\begin{tabular}{|c|c|c|c|c|c|c|c|c|c|c|c|c|c|c|c|c|c|c|c|c|c|}
\hline Glass ID & $\begin{array}{c}\mathrm{Al} 2 \mathrm{O} 3 \\
-\mathrm{t}\end{array}$ & $\begin{array}{c}\mathrm{B} 2 \mathrm{O} 3 \\
-\mathrm{t}\end{array}$ & $\begin{array}{c}\mathrm{CaO} \\
-\mathrm{t}\end{array}$ & $\begin{array}{c}\mathrm{Fe} 2 \mathrm{O} 3 \\
-\mathrm{t}\end{array}$ & $\begin{array}{c}\mathrm{FeO} \\
-\mathrm{t}\end{array}$ & $\begin{array}{c}\mathrm{K} 2 \mathrm{O} \\
-\mathrm{t}\end{array}$ & $\begin{array}{c}\mathrm{Li} 2 \mathrm{O} \\
-\mathrm{t}\end{array}$ & $\begin{array}{c}\mathrm{MgO} \\
-\mathrm{t}\end{array}$ & $\begin{array}{c}\mathrm{Na} 2 \mathrm{O} \\
-\mathrm{t}\end{array}$ & $\begin{array}{c}\mathrm{NiO} \\
-\mathrm{t}\end{array}$ & $\begin{array}{c}\mathrm{P} 2 \mathrm{O} 5 \\
-\mathrm{t}\end{array}$ & $\begin{array}{c}\mathrm{SiO} 2 \\
-\mathrm{t}\end{array}$ & $\begin{array}{c}\mathrm{ZrO} 2 \\
-\mathrm{t}\end{array}$ & $\begin{array}{c}\mathrm{Ag} 2 \mathrm{O} \\
-\mathrm{t}\end{array}$ & $\begin{array}{c}\mathrm{As} 2 \mathrm{O} 3 \\
-\mathrm{t}\end{array}$ & $\begin{array}{c}\mathrm{BaO} \\
-\mathrm{t}\end{array}$ & $\begin{array}{c}\mathrm{Bi} 2 \mathrm{O} 3 \\
-\mathrm{t}\end{array}$ & $\begin{array}{c}\mathrm{Br} \\
-\mathrm{t}\end{array}$ & $\begin{array}{c}\mathrm{CdO} \\
-\mathrm{t}\end{array}$ & $\begin{array}{c}\mathrm{Ce} 2 \mathrm{O} 3 \\
-\mathrm{t}\end{array}$ & $\begin{array}{c}\mathrm{CeO} 2 \\
-\mathrm{t}\end{array}$ \\
\hline $\mathrm{PBG} 3 / \mathrm{Ce}$ & 0.0500 & 0.0600 & 0.0490 & 0.0160 & & 0.0300 & 0.0400 & 0.0200 & 0.1000 & 0.0050 & 0.0030 & 0.4500 & 0.0300 & & & 0.0022 & & & & & 0.0450 \\
\hline $\mathrm{PBG} 3 / 0 \mathrm{x}$ & 0.0500 & 0.0600 & 0.0490 & 0.0160 & & 0.0300 & 0.0400 & 0.0200 & 0.1000 & 0.0050 & 0.0030 & 0.4500 & 0.0300 & & & 0.0022 & & & & & 0.0450 \\
\hline PBG3-.5C & 0.0500 & 0.0600 & 0.0490 & 0.0160 & & 0.0300 & 0.0400 & 0.0200 & 0.1000 & 0.0050 & 0.0030 & 0.4500 & 0.0300 & & & 0.0022 & & & & & 0.0450 \\
\hline SRC-Al-1 & 0.0000 & 0.0632 & 0.0516 & 0.0168 & & 0.0316 & 0.0421 & 0.0211 & 0.1053 & 0.0053 & 0.0032 & 0.4737 & 0.0316 & & & 0.0023 & & & & & 0.0474 \\
\hline SRC-Al-2 & 0.1000 & 0.0568 & 0.0464 & 0.0152 & & 0.0284 & 0.0379 & 0.0189 & 0.0947 & 0.0047 & 0.0028 & 0.4263 & 0.0284 & & & 0.0021 & & & & & 0.0426 \\
\hline SRC-B-1 & 0.0532 & 0.0000 & 0.0521 & 0.0170 & & 0.0319 & 0.0426 & 0.0213 & 0.1064 & 0.0053 & 0.0032 & 0.4787 & 0.0319 & & & 0.0023 & & & & & 0.0479 \\
\hline SRC-B-2 & 0.0511 & 0.0400 & 0.0500 & 0.0163 & & 0.0306 & 0.0409 & 0.0204 & 0.1021 & 0.0051 & 0.0031 & 0.4596 & 0.0306 & & & 0.0022 & & & & & 0.0460 \\
\hline SRC-B-3 & 0.0468 & 0.1200 & 0.0459 & 0.0150 & & 0.0281 & 0.0374 & 0.0187 & 0.0936 & 0.0047 & 0.0028 & 0.4213 & 0.0281 & & & 0.0021 & & & & & 0.0421 \\
\hline SRC-Ca-1 & 0.0526 & 0.0631 & 0.0000 & 0.0168 & & 0.0315 & 0.0421 & 0.0210 & 0.1052 & 0.0053 & 0.0032 & 0.4732 & 0.0315 & & & 0.0023 & & & & & 0.0473 \\
\hline SRC-Ca-2 & 0.0515 & 0.0618 & 0.0200 & 0.0165 & & 0.0309 & 0.0412 & 0.0206 & 0.1030 & 0.0052 & 0.0031 & 0.4637 & 0.0309 & & & 0.0023 & & & & & 0.0464 \\
\hline SRC-Ca-3 & 0.0484 & 0.0580 & 0.0800 & 0.0155 & & 0.0290 & 0.0387 & 0.0193 & 0.0967 & 0.0048 & 0.0029 & 0.4353 & 0.0290 & & & 0.0021 & & & & & 0.0435 \\
\hline SRC-Ce-1 & 0.0524 & 0.0628 & 0.0513 & 0.0168 & & 0.0314 & 0.0419 & 0.0209 & 0.1047 & 0.0052 & 0.0031 & 0.4712 & 0.0314 & & & 0.0023 & & & & & 0.0000 \\
\hline SRC-Ce-2 & 0.0510 & 0.0613 & 0.0500 & 0.0163 & & 0.0306 & 0.0408 & 0.0204 & 0.1021 & 0.0051 & 0.0031 & 0.4594 & 0.0306 & & & 0.0022 & & & & & 0.0250 \\
\hline SRC-Ce-3 & 0.0490 & 0.0587 & 0.0480 & 0.0157 & & 0.0294 & 0.0392 & 0.0196 & 0.0979 & 0.0049 & 0.0029 & 0.4406 & 0.0294 & & & 0.0022 & & & & & 0.0650 \\
\hline SRC-Ce-4 & 0.0482 & 0.0578 & 0.0472 & 0.0154 & & 0.0289 & 0.0385 & 0.0193 & 0.0963 & 0.0048 & 0.0029 & 0.4335 & 0.0289 & & & 0.0021 & & & & & 0.0800 \\
\hline SRC-Eu-1 & 0.0501 & 0.0602 & 0.0491 & 0.0160 & & 0.0301 & 0.0401 & 0.0201 & 0.1003 & 0.0050 & 0.0030 & 0.4512 & 0.0301 & & & 0.0022 & & & & & 0.0424 \\
\hline SRC-Gd-1 & 0.0524 & 0.0628 & 0.0513 & 0.0168 & & 0.0314 & 0.0419 & 0.0209 & 0.1047 & 0.0052 & 0.0031 & 0.4712 & 0.0314 & & & 0.0023 & & & & & 0.0471 \\
\hline SRC-Gd-2 & 0.0510 & 0.0613 & 0.0500 & 0.0163 & & 0.0306 & 0.0408 & 0.0204 & 0.1021 & 0.0051 & 0.0031 & 0.4594 & 0.0306 & & & 0.0022 & & & & & 0.0459 \\
\hline SRC-Gd-3 & 0.0490 & 0.0587 & 0.0480 & 0.0157 & & 0.0294 & 0.0392 & 0.0196 & 0.0979 & 0.0049 & 0.0029 & 0.4406 & 0.0294 & & & 0.0022 & & & & & 0.0441 \\
\hline SRC-Gd-4 & 0.0482 & 0.0578 & 0.0472 & 0.0154 & & 0.0289 & 0.0385 & 0.0193 & 0.0963 & 0.0048 & 0.0029 & 0.4335 & 0.0289 & & & 0.0021 & & & & & 0.0434 \\
\hline SRC-K-1 & 0.0515 & 0.0619 & 0.0505 & 0.0165 & & 0.0000 & 0.0412 & 0.0206 & 0.1031 & 0.0052 & 0.0031 & 0.4639 & 0.0309 & & & 0.0023 & & & & & 0.0464 \\
\hline SRC-K-2 & 0.0485 & 0.0581 & 0.0475 & 0.0155 & & 0.0600 & 0.0388 & 0.0194 & 0.0969 & 0.0048 & 0.0029 & 0.4361 & 0.0291 & & & 0.0021 & & & & & 0.0436 \\
\hline SRC-K-3 & 0.0469 & 0.0563 & 0.0460 & 0.0150 & & 0.0900 & 0.0375 & 0.0188 & 0.0938 & 0.0047 & 0.0028 & 0.4222 & 0.0281 & & & 0.0021 & & & & & 0.0422 \\
\hline SRC-K-3i & 0.0469 & 0.0563 & 0.0460 & 0.0150 & & 0.0900 & 0.0375 & 0.0188 & 0.0938 & 0.0047 & 0.0028 & 0.4222 & 0.0281 & & & 0.0021 & & & & & 0.0422 \\
\hline SRC-Li-1 & 0.0521 & 0.0625 & 0.0510 & 0.0167 & & 0.0313 & 0.0000 & 0.0208 & 0.1042 & 0.0052 & 0.0031 & 0.4688 & 0.0313 & & & 0.0023 & & & & & 0.0469 \\
\hline SRC-Li-2 & 0.0513 & 0.0616 & 0.0503 & 0.0164 & & 0.0308 & 0.0150 & 0.0205 & 0.1026 & 0.0051 & 0.0031 & 0.4617 & 0.0308 & & & 0.0023 & & & & & 0.0462 \\
\hline SRC-Li-3 & 0.0497 & 0.0597 & 0.0487 & 0.0159 & & 0.0298 & 0.0450 & 0.0199 & 0.0995 & 0.0050 & 0.0030 & 0.4477 & 0.0298 & & & 0.0022 & & & & & 0.0448 \\
\hline SRC-Mg-1 & 0.0485 & 0.0582 & 0.0475 & 0.0155 & & 0.0291 & 0.0388 & 0.0500 & 0.0969 & 0.0048 & 0.0029 & 0.4362 & 0.0291 & & & 0.0021 & & & & & 0.0436 \\
\hline SRC-Mg-2 & 0.0469 & 0.0563 & 0.0460 & 0.0150 & & 0.0282 & 0.0376 & 0.0800 & 0.0939 & 0.0047 & 0.0028 & 0.4224 & 0.0282 & & & 0.0021 & & & & & 0.0422 \\
\hline SRC-Mg-3 & 0.0449 & 0.0539 & 0.0440 & 0.0144 & & 0.0269 & 0.0359 & 0.1200 & 0.0898 & 0.0045 & 0.0027 & 0.4041 & 0.0269 & & & 0.0020 & & & & & 0.0404 \\
\hline SRC-Na-1 & 0.0551 & 0.0661 & 0.0540 & 0.0176 & & 0.0331 & 0.0441 & 0.0220 & 0.0081 & 0.0055 & 0.0033 & 0.4960 & 0.0331 & & & 0.0024 & & & & & 0.0496 \\
\hline SRC-Na-2 & 0.0511 & 0.0613 & 0.0501 & 0.0164 & & 0.0307 & 0.0409 & 0.0204 & 0.0800 & 0.0051 & 0.0031 & 0.4600 & 0.0307 & & & 0.0022 & & & & & 0.0460 \\
\hline SRC-Pb-1 & 0.0505 & 0.0606 & 0.0495 & 0.0162 & & 0.0303 & 0.0404 & 0.0202 & 0.1010 & 0.0051 & 0.0030 & 0.4545 & 0.0303 & & & 0.0022 & & & & & 0.0455 \\
\hline SRC-Pb-2 & 0.0480 & 0.0576 & 0.0470 & 0.0154 & & 0.0288 & 0.0384 & 0.0192 & 0.0960 & 0.0048 & 0.0029 & 0.4318 & 0.0288 & & & 0.0021 & & & & & 0.0432 \\
\hline SRC-Pb-3 & 0.0454 & 0.0545 & 0.0445 & 0.0145 & & 0.0273 & 0.0363 & 0.0182 & 0.0909 & 0.0045 & 0.0027 & 0.4091 & 0.0273 & & & 0.0020 & & & & & 0.0409 \\
\hline SRC-Si-1 & 0.0545 & 0.0655 & 0.0535 & 0.0175 & & 0.0327 & 0.0436 & 0.0218 & 0.1091 & 0.0055 & 0.0033 & 0.4000 & 0.0327 & & & 0.0024 & & & & & 0.0491 \\
\hline SRC-Si-2 & 0.0409 & 0.0491 & 0.0401 & 0.0131 & & 0.0245 & 0.0327 & 0.0164 & 0.0818 & 0.0041 & 0.0025 & 0.5500 & 0.0245 & & & 0.0018 & & & & & 0.0368 \\
\hline SRC-Sm-1 & 0.0501 & 0.0602 & 0.0491 & 0.0160 & & 0.0301 & 0.0401 & 0.0201 & 0.1003 & 0.0050 & 0.0030 & 0.4512 & 0.0301 & & & 0.0022 & & & & & 0.0424 \\
\hline SRC-Sn-1 & 0.0490 & 0.0588 & 0.0480 & 0.0157 & & 0.0294 & 0.0392 & 0.0196 & 0.0980 & 0.0049 & 0.0029 & 0.4410 & 0.0294 & & & 0.0022 & & & & & 0.0441 \\
\hline SRC-Sn-2 & 0.0480 & 0.0576 & 0.0470 & 0.0154 & & 0.0288 & 0.0384 & 0.0192 & 0.0960 & 0.0048 & 0.0029 & 0.4320 & 0.0288 & & & 0.0021 & & & & & 0.0432 \\
\hline SRC-Sn-3 & 0.0470 & 0.0564 & 0.0461 & 0.0150 & & 0.0282 & 0.0376 & 0.0188 & 0.0940 & 0.0047 & 0.0028 & 0.4230 & 0.0282 & & & 0.0021 & & & & & 0.0423 \\
\hline SRC-Ti-1 & 0.0505 & 0.0606 & 0.0495 & 0.0162 & & 0.0303 & 0.0404 & 0.0202 & 0.1010 & 0.0051 & 0.0030 & 0.4545 & 0.0303 & & & 0.0022 & & & & & 0.0455 \\
\hline SRC-Ti-2 & 0.0480 & 0.0576 & 0.0470 & 0.0154 & & 0.0288 & 0.0384 & 0.0192 & 0.0960 & 0.0048 & 0.0029 & 0.4318 & 0.0288 & & & 0.0021 & & & & & 0.0432 \\
\hline SRC-Zr-1 & 0.0515 & 0.0619 & 0.0505 & 0.0165 & & 0.0309 & 0.0412 & 0.0206 & 0.1031 & 0.0052 & 0.0031 & 0.4639 & 0.0000 & & & 0.0023 & & & & & 0.0464 \\
\hline SRC-Zr-2 & 0.0490 & 0.0588 & 0.0480 & 0.0157 & & 0.0294 & 0.0392 & 0.0196 & 0.0979 & 0.0049 & 0.0029 & 0.4407 & 0.0500 & & & 0.0022 & & & & & 0.0441 \\
\hline
\end{tabular}


Plutonium Vitrification (Bulkley and Vienna 1997)

\begin{tabular}{|c|c|c|c|c|c|c|c|c|c|c|c|c|c|c|c|c|c|c|c|c|c|}
\hline Glass ID & $\begin{array}{l}\mathrm{Cl} \\
-\mathrm{t}\end{array}$ & $\begin{array}{c}\mathrm{CoO} \\
-\mathrm{t}\end{array}$ & $\begin{array}{c}\mathrm{Co} 2 \mathrm{O} 3 \\
-\mathrm{t}\end{array}$ & $\begin{array}{c}\mathrm{Cr} 2 \mathrm{O} 3 \\
-\mathrm{t}\end{array}$ & $\begin{array}{c}\mathrm{Cs} 2 \mathrm{O} \\
-\mathrm{t}\end{array}$ & $\begin{array}{c}\mathrm{CuO} \\
-\mathrm{t}\end{array}$ & $\begin{array}{c}\mathrm{Eu} 2 \mathrm{O} 3 \\
-\mathrm{t}\end{array}$ & $\begin{array}{l}F \\
-t\end{array}$ & $\begin{array}{c}\mathrm{Ga} 2 \mathrm{O} 3 \\
-\mathrm{t}\end{array}$ & $\begin{array}{c}\mathrm{Gd} 2 \mathrm{O} 3 \\
-\mathrm{t}\end{array}$ & $\begin{array}{c}\mathrm{HgO} \\
-\mathrm{t}\end{array}$ & $\begin{array}{l}I \\
-t\end{array}$ & $\begin{array}{c}\mathrm{La} 2 \mathrm{O} 3 \\
-\mathrm{t}\end{array}$ & $\begin{array}{c}\mathrm{MnO} 2 \\
-\mathrm{t}\end{array}$ & $\begin{array}{c}\mathrm{MnO} \\
-\mathrm{t}\end{array}$ & $\begin{array}{c}\mathrm{MoO} \\
-\mathrm{t}\end{array}$ & $\begin{array}{c}\mathrm{MoO} 3 \\
-\mathrm{t}\end{array}$ & $\begin{array}{c}\mathrm{Nb} 2 \mathrm{O} 5 \\
-\mathrm{t}\end{array}$ & $\begin{array}{c}\mathrm{Nd} 2 \mathrm{O} 3 \\
-\mathrm{t}\end{array}$ & $\begin{array}{c}\mathrm{PbO} \\
-\mathrm{t}\end{array}$ & $\begin{array}{c}\mathrm{PdO} 2 \\
-\mathrm{t}\end{array}$ \\
\hline $\mathrm{PBG} 3 / \mathrm{Ce}$ & 0.0050 & & & 0.0040 & 0.0100 & 0.0004 & & 0.0010 & & 0.0450 & & & & & & & 0.0064 & & 0.0080 & 0.0100 & \\
\hline $\mathrm{PBG} 3 / 0 \mathrm{x}$ & 0.0050 & & & 0.0040 & 0.0100 & 0.0004 & & 0.0010 & & 0.0450 & & & & & & & 0.0064 & & 0.0080 & 0.0100 & \\
\hline PBG3-.5C & 0.0050 & & & 0.0040 & 0.0100 & 0.0004 & & 0.0010 & & 0.0450 & & & & & & & 0.0064 & & 0.0080 & 0.0100 & \\
\hline SRC-Al-1 & 0.0053 & & & 0.0042 & 0.0105 & 0.0004 & & 0.0011 & & 0.0474 & & & & & & & 0.0067 & & 0.0084 & 0.0105 & \\
\hline SRC-Al-2 & 0.0047 & & & 0.0038 & 0.0095 & 0.0004 & & 0.0009 & & 0.0426 & & & & & & & 0.0061 & & 0.0076 & 0.0095 & \\
\hline SRC-B-1 & 0.0053 & & & 0.0043 & 0.0106 & 0.0004 & & 0.0011 & & \begin{tabular}{|l|}
0.0479 \\
\end{tabular} & & & & & & & 0.0068 & & 0.0085 & 0.0106 & \\
\hline SRC-B-2 & 0.0051 & & & 0.0041 & 0.0102 & 0.0004 & & 0.0010 & & 0.0460 & & & & & & & 0.0065 & & 0.0082 & 0.0102 & \\
\hline SRC-B-3 & 0.0047 & & & 0.0037 & 0.0094 & 0.0004 & & 0.0009 & & 0.0421 & & & & & & & 0.0060 & & 0.0075 & 0.0094 & \\
\hline SRC-Ca-1 & 0.0053 & & & 0.0042 & 0.0105 & 0.0004 & & 0.0011 & & 0.0473 & & & & & & & 0.0067 & & 0.0084 & 0.0105 & \\
\hline SRC-Ca-2 & 0.0052 & & & 0.0041 & 0.0103 & 0.0004 & & 0.0010 & & 0.0464 & & & & & & & 0.0066 & & 0.0082 & 0.0103 & \\
\hline SRC-Ca-3 & 0.0048 & & & 0.0039 & 0.0097 & 0.0004 & & 0.0010 & & 0.0435 & & & & & & & 0.0062 & & 0.0077 & 0.0097 & \\
\hline SRC-Ce-1 & 0.0052 & & & 0.0042 & 0.0105 & 0.0004 & & 0.0010 & & 0.0471 & & & & & & & 0.0067 & & 0.0084 & 0.0105 & \\
\hline SRC-Ce-2 & 0.0051 & & & 0.0041 & 0.0102 & 0.0004 & & 0.0010 & & 0.0459 & & & & & & & 0.0065 & & 0.0082 & 0.0102 & \\
\hline SRC-Ce-3 & 0.0049 & & & 0.0039 & 0.0098 & 0.0004 & & 0.0010 & & 0.0441 & & & & & & & 0.0063 & & 0.0078 & 0.0098 & \\
\hline SRC-Ce-4 & 0.0048 & & & 0.0039 & 0.0096 & 0.0004 & & 0.0010 & & 0.0434 & & & & & & & 0.0062 & & 0.0077 & 0.0096 & \\
\hline SRC-Eu-1 & 0.0050 & & & 0.0040 & 0.0100 & 0.0004 & 0.0451 & 0.0010 & & 0.0000 & & & & & & & 0.0064 & & 0.0080 & 0.0100 & \\
\hline SRC-Gd-1 & 0.0052 & & & 0.0042 & 0.0105 & 0.0004 & & 0.0010 & & 0.0000 & & & & & & & 0.0067 & & 0.0084 & 0.0105 & \\
\hline SRC-Gd-2 & 0.0051 & & & 0.0041 & 0.0102 & 0.0004 & & 0.0010 & & 0.0250 & & & & & & & 0.0065 & & 0.0082 & 0.0102 & \\
\hline SRC-Gd-3 & 0.0049 & & & 0.0039 & 0.0098 & 0.0004 & & 0.0010 & & 0.0650 & & & & & & & 0.0063 & & 0.0078 & 0.0098 & \\
\hline SRC-Gd-4 & 0.0048 & & & 0.0039 & 0.0096 & 0.0004 & & 0.0010 & & 0.0800 & & & & & & & 0.0062 & & 0.0077 & 0.0096 & \\
\hline SRC-K-1 & 0.0052 & & & 0.0041 & 0.0103 & 0.0004 & & 0.0010 & & 0.0464 & & & & & & & 0.0066 & & 0.0082 & 0.0103 & \\
\hline SRC-K-2 & 0.0048 & & & 0.0039 & 0.0097 & 0.0004 & & 0.0010 & & 0.0436 & & & & & & & 0.0062 & & 0.0078 & 0.0097 & \\
\hline SRC-K-3 & 0.0047 & & & 0.0038 & 0.0094 & 0.0004 & & 0.0009 & & 0.0422 & & & & & & & 0.0060 & & 0.0075 & 0.0094 & \\
\hline SRC-K-3i & 0.0047 & & & 0.0038 & 0.0094 & 0.0004 & & 0.0009 & & 0.0422 & & & & & & & 0.0060 & & 0.0075 & 0.0094 & \\
\hline SRC-Li-1 & 0.0052 & & & 0.0042 & 0.0104 & 0.0004 & & 0.0010 & & 0.0469 & & & & & & & 0.0067 & & 0.0083 & 0.0104 & \\
\hline SRC-Li-2 & 0.0051 & & & 0.0041 & 0.0103 & 0.0004 & & 0.0010 & & 0.0462 & & & & & & & 0.0066 & & 0.0082 & 0.0103 & \\
\hline SRC-Li-3 & 0.0050 & & & 0.0040 & 0.0099 & 0.0004 & & 0.0010 & & 0.0448 & & & & & & & 0.0064 & & 0.0080 & 0.0099 & \\
\hline SRC-Mg-1 & 0.0048 & & & 0.0039 & 0.0097 & 0.0004 & & 0.0010 & & 0.0436 & & & & & & & 0.0062 & & 0.0078 & 0.0097 & \\
\hline SRC-Mg-2 & 0.0047 & & & 0.0038 & 0.0094 & 0.0004 & & 0.0009 & & 0.0422 & & & & & & & 0.0060 & & 0.0075 & 0.0094 & \\
\hline SRC-Mg-3 & 0.0045 & & & 0.0036 & 0.0090 & 0.0004 & & 0.0009 & & 0.0404 & & & & & & & 0.0057 & & 0.0072 & 0.0090 & \\
\hline SRC-Na-1 & 0.0055 & & & 0.0044 & 0.0110 & 0.0004 & & 0.0011 & & 0.0496 & & & & & & & 0.0071 & & 0.0088 & 0.0110 & \\
\hline SRC-Na-2 & 0.0051 & & & 0.0041 & 0.0102 & 0.0004 & & 0.0010 & & 0.0460 & & & & & & & 0.0065 & & 0.0082 & 0.0102 & \\
\hline SRC-Pb-1 & 0.0051 & & & 0.0040 & 0.0101 & 0.0004 & & 0.0010 & & 0.0455 & & & & & & & 0.0065 & & 0.0081 & 0.0000 & \\
\hline SRC-Pb-2 & 0.0048 & & & 0.0038 & 0.0096 & 0.0004 & & 0.0010 & & 0.0432 & & & & & & & 0.0061 & & 0.0077 & 0.0500 & \\
\hline SRC-Pb-3 & 0.0045 & & & 0.0036 & 0.0091 & 0.0004 & & 0.0009 & & 0.0409 & & & & & & & 0.0058 & & 0.0073 & 0.1000 & \\
\hline SRC-Si-1 & 0.0055 & & & 0.0044 & 0.0109 & 0.0004 & & 0.0011 & & 0.0491 & & & & & & & 0.0070 & & 0.0087 & 0.0109 & \\
\hline SRC-Si-2 & 0.0041 & & & 0.0033 & 0.0082 & 0.0003 & & 0.0008 & & 0.0368 & & & & & & & 0.0052 & & 0.0065 & 0.0082 & \\
\hline SRC-Sm-1 & 0.0050 & & & 0.0040 & 0.0100 & 0.0004 & & 0.0010 & & 0.0000 & & & & & & & 0.0064 & & 0.0080 & 0.0100 & \\
\hline SRC-Sn-1 & 0.0049 & & & 0.0039 & 0.0098 & 0.0004 & & 0.0010 & & 0.0441 & & & & & & & 0.0063 & & 0.0078 & 0.0098 & \\
\hline SRC-Sn-2 & 0.0048 & & & 0.0038 & 0.0096 & 0.0004 & & 0.0010 & & 0.0432 & & & & & & & 0.0061 & & 0.0077 & 0.0096 & \\
\hline SRC-Sn-3 & 0.0047 & & & 0.0038 & 0.0094 & 0.0004 & & 0.0009 & & 0.0423 & & & & & & & 0.0060 & & 0.0075 & 0.0094 & \\
\hline SRC-Ti-1 & 0.0051 & & & 0.0040 & 0.0101 & 0.0004 & & 0.0010 & & 0.0455 & & & & & & & 0.0065 & & 0.0081 & 0.0101 & \\
\hline SRC-Ti-2 & 0.0048 & & & 0.0038 & 0.0096 & 0.0004 & & 0.0010 & & 0.0432 & & & & & & & 0.0061 & & 0.0077 & 0.0096 & \\
\hline SRC-Zr-1 & 0.0052 & & & 0.0041 & 0.0103 & 0.0004 & & 0.0010 & & 0.0464 & & & & & & & 0.0066 & & 0.0082 & 0.0103 & \\
\hline SRC-Zr-2 & 0.0049 & & & 0.0039 & 0.0098 & 0.0004 & & 0.0010 & & 0.0441 & & & & & & & 0.0063 & & 0.0078 & 0.0098 & \\
\hline
\end{tabular}


Appendix A. Database - mass fraction

Plutonium Vitrification (Bulkley and Vienna 1997)

\begin{tabular}{|c|c|c|c|c|c|c|c|c|c|c|c|c|c|c|c|c|c|c|c|c|c|}
\hline Glass ID & $\begin{array}{c}\mathrm{PdO} \\
-\mathrm{t}\end{array}$ & $\begin{array}{c}\mathrm{Pr} 2 \mathrm{O} 3 \\
-\mathrm{t} \\
\end{array}$ & $\begin{array}{c}\operatorname{Pr} 6011 \\
-t\end{array}$ & $\begin{array}{c}\mathrm{Rb} 2 \mathrm{O} \\
-\mathrm{t}\end{array}$ & $\begin{array}{c}\mathrm{ReO} \\
-\mathrm{t}\end{array}$ & $\begin{array}{c}\mathrm{ReO} 2 \\
-\mathrm{t}\end{array}$ & $\begin{array}{c}\mathrm{Rh} 2 \mathrm{O} 3 \\
-\mathrm{t}\end{array}$ & $\begin{array}{c}\mathrm{RhO}^{2} \\
-\mathrm{t}\end{array}$ & $\begin{array}{c}\mathrm{RuO}_{2} \\
-\mathrm{t}\end{array}$ & $\begin{array}{c}\mathrm{Sb} 2 \mathrm{O} 3 \\
-\mathrm{t}\end{array}$ & $\begin{array}{c}\mathrm{Sb} 2 \mathrm{O} 5 \\
-\mathrm{t}\end{array}$ & $\begin{array}{c}\mathrm{SeO} 2 \\
-\mathrm{t}\end{array}$ & $\begin{array}{c}\mathrm{Sm} 2 \mathrm{O} 3 \\
-\mathrm{t}\end{array}$ & $\begin{array}{c}\mathrm{SnO} \\
-\mathrm{t}\end{array}$ & $\begin{array}{c}\mathrm{SnO} 2 \\
-\mathrm{t} \\
\end{array}$ & $\begin{array}{c}\mathrm{SO} 3 \\
-\mathrm{t}\end{array}$ & $\begin{array}{c}\mathrm{SrO} \\
-\mathrm{t}\end{array}$ & $\begin{array}{c}\mathrm{Tc} 2 \mathrm{O} 7 \\
-\mathrm{t}\end{array}$ & $\begin{array}{c}\mathrm{TeO} 2 \\
-\mathrm{t}\end{array}$ & $\begin{array}{c}\mathrm{ThO}_{2} \\
-\mathrm{t}\end{array}$ & $\begin{array}{c}\mathrm{TiO} 2 \\
-\mathrm{t}\end{array}$ \\
\hline $\mathrm{PBG} 3 / \mathrm{Ce}$ & & & & & & & & & & & & & & & & & & & & & 0.0100 \\
\hline $\mathrm{PBG} 3 / 0 \mathrm{x}$ & & & & & & & & & & & & & & & & & & & & & 0.0100 \\
\hline PBG3-.5C & & & & & & & & & & & & & & & & & & & & & 0.0100 \\
\hline SRC-Al-1 & & & & & & & & & & & & & & & & & & & & & 0.0105 \\
\hline SRC-Al-2 & & & & & & & & & & & & & & & & & & & & & 0.0095 \\
\hline SRC-B-1 & & & & & & & & & & & & & & & & & & & & & 0.0106 \\
\hline SRC-B-2 & & & & & & & & & & & & & & & & & & & & & 0.0102 \\
\hline SRC-B-3 & & & & & & & & & & & & & & & & & & & & & 0.0094 \\
\hline SRC-Ca-1 & & & & & & & & & & & & & & & & & & & & & 0.0105 \\
\hline SRC-Ca-2 & & & & & & & & & & & & & & & & & & & & & 0.0103 \\
\hline SRC-Ca-3 & & & & & & & & & & & & & & & & & & & & & 0.0097 \\
\hline SRC-Ce-1 & & & & & & & & & & & & & & & & & & & & & 0.0105 \\
\hline SRC-Ce-2 & & & & & & & & & & & & & & & & & & & & & 0.0102 \\
\hline SRC-Ce-3 & & & & & & & & & & & & & & & & & & & & & 0.0098 \\
\hline SRC-Ce-4 & & & & & & & & & & & & & & & & & & & & & 0.0096 \\
\hline SRC-Eu-1 & & & & & & & & & & & & & & & & & & & & & 0.0100 \\
\hline SRC-Gd-1 & & & & & & & & & & & & & & & & & & & & & 0.0105 \\
\hline SRC-Gd-2 & & & & & & & & & & & & & & & & & & & & & 0.0102 \\
\hline SRC-Gd-3 & & & & & & & & & & & & & & & & & & & & & 0.0098 \\
\hline SRC-Gd-4 & & & & & & & & & & & & & & & & & & & & & 0.0096 \\
\hline SRC-K-1 & & & & & & & & & & & & & & & & & & & & & 0.0103 \\
\hline SRC-K-2 & & & & & & & & & & & & & & & & & & & & & 0.0097 \\
\hline SRC-K-3 & & & & & & & & & & & & & & & & & & & & & 0.0094 \\
\hline SRC-K-3i & & & & & & & & & & & & & & & & & & & & & 0.0094 \\
\hline SRC-Li-1 & & & & & & & & & & & & & & & & & & & & & 0.0104 \\
\hline SRC-Li-2 & & & & & & & & & & & & & & & & & & & & & 0.0103 \\
\hline SRC-Li-3 & & & & & & & & & & & & & & & & & & & & & 0.0099 \\
\hline SRC-Mg-1 & & & & & & & & & & & & & & & & & & & & & 0.0097 \\
\hline SRC-Mg-2 & & & & & & & & & & & & & & & & & & & & & 0.0094 \\
\hline SRC-Mg-3 & & & & & & & & & & & & & & & & & & & & & 0.0090 \\
\hline SRC-Na-1 & & & & & & & & & & & & & & & & & & & & & 0.0110 \\
\hline SRC-Na-2 & & & & & & & & & & & & & & & & & & & & & 0.0102 \\
\hline SRC-Pb-1 & & & & & & & & & & & & & & & & & & & & & 0.0101 \\
\hline SRC-Pb-2 & & & & & & & & & & & & & & & & & & & & & 0.0096 \\
\hline SRC-Pb-3 & & & & & & & & & & & & & & & & & & & & & 0.0091 \\
\hline SRC-Si-1 & & & & & & & & & & & & & & & & & & & & & 0.0109 \\
\hline SRC-Si-2 & & & & & & & & & & & & & & & & & & & & & 0.0082 \\
\hline SRC-Sm-1 & & & & & & & & & & & & & 0.0451 & & & & & & & & 0.0100 \\
\hline SRC-Sn-1 & & & & & & & & & & & & & & & 0.0200 & & & & & & 0.0098 \\
\hline SRC-Sn-2 & & & & & & & & & & & & & & & 0.0400 & & & & & & 0.0096 \\
\hline SRC-Sn-3 & & & & & & & & & & & & & & & 0.0600 & & & & & & 0.0094 \\
\hline SRC-Ti-1 & & & & & & & & & & & & & & & & & & & & & 0.0000 \\
\hline SRC-Ti-2 & & & & & & & & & & & & & & & & & & & & & 0.0500 \\
\hline SRC-Zr-1 & & & & & & & & & & & & & & & & & & & & & 0.0103 \\
\hline SRC-Zr-2 & & & & & & & & & & & & & & & & & & & & & 0.0098 \\
\hline
\end{tabular}


Appendix A. Database - mass fraction

Plutonium Vitrification (Bulkley and Vienna 1997)

\begin{tabular}{|c|c|c|c|c|c|c|c|c|c|c|c|c|c|c|c|c|c|c|c|c|c|}
\hline Glass ID & $\begin{array}{c}\mathrm{T} 12 \mathrm{O} 3 \\
-\mathrm{t}\end{array}$ & $\begin{array}{c}\mathrm{U} 3 \mathrm{O} 8 \\
-\mathrm{t}\end{array}$ & $\begin{array}{c}\mathrm{UO} 2 \\
-\mathrm{t}\end{array}$ & $\begin{array}{c}\mathrm{UO} 3 \\
-\mathrm{t}\end{array}$ & $\begin{array}{c}\mathrm{V} 2 \mathrm{O} 5 \\
-\mathrm{t}\end{array}$ & $\begin{array}{c}\text { WO3 } \\
-t\end{array}$ & $\begin{array}{c}\mathrm{Y} 2 \mathrm{O} 3 \\
-\mathrm{t}\end{array}$ & $\begin{array}{c}\mathrm{ZnO} \\
-\mathrm{t}\end{array}$ & $\begin{array}{c}\text { Others } \\
-t\end{array}$ & $\begin{array}{c}\text { Sum } \\
-t\end{array}$ & $\begin{array}{c}\mathrm{A} 12 \mathrm{O} 3 \\
-\mathrm{a}\end{array}$ & $\begin{array}{c}\mathrm{B} 2 \mathrm{O} 3 \\
-\mathrm{a}\end{array}$ & $\begin{array}{c}\mathrm{CaO} \\
-\mathrm{a}\end{array}$ & $\begin{array}{c}\mathrm{Fe} 2 \mathrm{O} 3 \\
-\mathrm{a}\end{array}$ & $\begin{array}{c}\mathrm{FeO} \\
-\mathrm{a}\end{array}$ & $\begin{array}{c}\mathrm{K} 2 \mathrm{O} \\
-\mathrm{a}\end{array}$ & $\begin{array}{c}\mathrm{Li} 2 \mathrm{O} \\
-\mathrm{a}\end{array}$ & $\begin{array}{c}\mathrm{MgO} \\
-\mathrm{a}\end{array}$ & $\begin{array}{c}\mathrm{Na} 2 \mathrm{O} \\
-\mathrm{a}\end{array}$ & $\begin{array}{c}\mathrm{NiO} \\
-\mathrm{a}\end{array}$ & $\begin{array}{c}\mathrm{P} 2 \mathrm{O} 5 \\
-\mathrm{a}\end{array}$ \\
\hline $\mathrm{PBG} 3 / \mathrm{Ce}$ & & & & & & & & & & 1.0000 & & & & & & & & & & & \\
\hline $\mathrm{PBG} 3 / 0 \mathrm{x}$ & & & & & & & & & & 1.0000 & & & & & & & & & & & \\
\hline PBG3-.5C & & & & & & & & & & 1.0000 & & & & & & & & & & & \\
\hline SRC-Al-1 & & & & & & & & & & 1.0002 & & & & & & & & & & & \\
\hline SRC-Al-2 & & & & & & & & & & 0.9998 & & & & & & & & & & & \\
\hline SRC-B-1 & & & & & & & & & & 0.9999 & & & & & & & & & & & \\
\hline SRC-B-2 & & & & & & & & & & 0.9999 & & & & & & & & & & & \\
\hline SRC-B-3 & & & & & & & & & & 1.0001 & & & & & & & & & & & \\
\hline SRC-Ca-1 & & & & & & & & & & 1.0000 & & & & & & & & & & & \\
\hline SRC-Ca-2 & & & & & & & & & & 0.9999 & & & & & & & & & & & \\
\hline SRC-Ca-3 & & & & & & & & & & 0.9998 & & & & & & & & & & & \\
\hline SRC-Ce-1 & & & & & & & & & & 0.9999 & & & & & & & & & & & \\
\hline SRC-Ce-2 & & & & & & & & & & 0.9997 & & & & & & & & & & & \\
\hline SRC-Ce-3 & & & & & & & & & & 1.0003 & & & & & & & & & & & \\
\hline SRC-Ce-4 & & & & & & & & & & 1.0000 & & & & & & & & & & & \\
\hline SRC-Eu-1 & & & & & & & & & & 0.9998 & & & & & & & & & & & \\
\hline SRC-Gd-1 & & & & & & & & & & 0.9999 & & & & & & & & & & & \\
\hline SRC-Gd-2 & & & & & & & & & & 0.9997 & & & & & & & & & & & \\
\hline SRC-Gd-3 & & & & & & & & & & 1.0003 & & & & & & & & & & & \\
\hline SRC-Gd-4 & & & & & & & & & & 1.0000 & & & & & & & & & & & \\
\hline SRC-K-1 & & & & & & & & & & 0.9999 & & & & & & & & & & & \\
\hline SRC-K-2 & & & & & & & & & & 1.0001 & & & & & & & & & & & \\
\hline SRC-K-3 & & & & & & & & & & 1.0001 & & & & & & & & & & & \\
\hline SRC-K-3i & & & & & & & & & & 1.0001 & & & & & & & & & & & \\
\hline SRC-Li-1 & & & & & & & & & & 1.0001 & & & & & & & & & & & \\
\hline SRC-Li-2 & & & & & & & & & & 1.0002 & & & & & & & & & & & \\
\hline SRC-Li-3 & & & & & & & & & & 1.0000 & & & & & & & & & & & \\
\hline SRC-Mg-1 & & & & & & & & & & 1.0000 & & & & & & & & & & & \\
\hline SRC-Mg-2 & & & & & & & & & & 1.0000 & & & & & & & & & & & \\
\hline SRC-Mg-3 & & & & & & & & & & 1.0001 & & & & & & & & & & & \\
\hline SRC-Na-1 & & & & & & & & & & 0.9999 & & & & & & & & & & & \\
\hline SRC-Na-2 & & & & & & & & & & 0.9999 & & & & & & & & & & & \\
\hline SRC-Pb-1 & & & & & & & & & & 1.0001 & & & & & & & & & & & \\
\hline SRC-Pb-2 & & & & & & & & & & 1.0002 & & & & & & & & & & & \\
\hline SRC-Pb-3 & & & & & & & & & & 0.9997 & & & & & & & & & & & \\
\hline SRC-Si-1 & & & & & & & & & & 1.0001 & & & & & & & & & & & \\
\hline SRC-Si-2 & & & & & & & & & & 0.9999 & & & & & & & & & & & \\
\hline SRC-Sm-1 & & & & & & & & & & 0.9998 & & & & & & & & & & & \\
\hline SRC-Sn-1 & & & & & & & & & & 1.0000 & & & & & & & & & & & \\
\hline SRC-Sn-2 & & & & & & & & & & 1.0000 & & & & & & & & & & & \\
\hline SRC-Sn-3 & & & & & & & & & & 1.0000 & & & & & & & & & & & \\
\hline SRC-Ti-1 & & & & & & & & & & 1.0001 & & & & & & & & & & & \\
\hline SRC-Ti-2 & & & & & & & & & & 1.0002 & & & & & & & & & & & \\
\hline SRC-Zr-1 & & & & & & & & & & 0.9999 & & & & & & & & & & & \\
\hline SRC-Zr-2 & & & & & & & & & & 1.0002 & & & & & & & & & & & \\
\hline
\end{tabular}


Appendix A. Database - mass fraction

Plutonium Vitrification (Bulkley and Vienna 1997)

\begin{tabular}{|c|c|c|c|c|c|c|c|c|c|c|c|c|c|c|c|c|c|c|c|c|c|}
\hline Glass ID & $\begin{array}{c}\mathrm{SiO} 2 \\
-\mathrm{a}\end{array}$ & $\begin{array}{c}\mathrm{ZrO} 2 \\
-\mathrm{a}\end{array}$ & $\begin{array}{c}\mathrm{Ag} 2 \mathrm{O} \\
-\mathrm{a}\end{array}$ & $\begin{array}{c}\text { As2O3 } \\
-\mathrm{a}\end{array}$ & $\begin{array}{c}\mathrm{BaO} \\
-\mathrm{a}\end{array}$ & $\begin{array}{c}\mathrm{Bi} 2 \mathrm{O} 3 \\
-\mathrm{a}\end{array}$ & $\begin{array}{l}\mathrm{Br} \\
-\mathrm{a}\end{array}$ & $\begin{array}{c}\mathrm{CdO} \\
-\mathrm{a}\end{array}$ & $\begin{array}{c}\mathrm{Ce} 2 \mathrm{O} 3 \\
-\mathrm{a}\end{array}$ & $\begin{array}{c}\mathrm{CeO} 2 \\
-\mathrm{a}\end{array}$ & $\begin{array}{l}\mathrm{Cl} \\
-\mathrm{a}\end{array}$ & $\begin{array}{c}\mathrm{CoO} \\
-\mathrm{a}\end{array}$ & $\begin{array}{c}\mathrm{Co} 2 \mathrm{O} 3 \\
-\mathrm{a}\end{array}$ & $\begin{array}{c}\mathrm{Cr} 2 \mathrm{O} 3 \\
-\mathrm{a}\end{array}$ & $\begin{array}{c}\mathrm{Cs} 2 \mathrm{O} \\
-\mathrm{a}\end{array}$ & $\begin{array}{c}\mathrm{CuO} \\
-\mathrm{a}\end{array}$ & $\begin{array}{c}\mathrm{Eu} 2 \mathrm{O} 3 \\
-\mathrm{a}\end{array}$ & $\begin{array}{l}\mathrm{F} \\
-\mathrm{a}\end{array}$ & $\begin{array}{c}\mathrm{Ga} 2 \mathrm{O} 3 \\
-\mathrm{a}\end{array}$ & $\begin{array}{c}\mathrm{Gd} 2 \mathrm{O} 3 \\
-\mathrm{a}\end{array}$ & $\begin{array}{c}\mathrm{HgO} \\
-\mathrm{a}\end{array}$ \\
\hline $\mathrm{PBG} 3 / \mathrm{Ce}$ & & & & & & & & & & & & & & & & & & & & & \\
\hline PBG3/0x & & & & & & & & & & & & & & & & & & & & & \\
\hline PBG3-.5C & & & & & & & & & & & & & & & & & & & & & \\
\hline SRC-Al-1 & & & & & & & & & & & & & & & & & & & & & \\
\hline SRC-Al-2 & & & & & & & & & & & & & & & & & & & & & \\
\hline SRC-B-1 & & & & & & & & & & & & & & & & & & & & & \\
\hline SRC-B-2 & & & & & & & & & & & & & & & & & & & & & \\
\hline SRC-B-3 & & & & & & & & & & & & & & & & & & & & & \\
\hline SRC-Ca-1 & & & & & & & & & & & & & & & & & & & & & \\
\hline SRC-Ca-2 & & & & & & & & & & & & & & & & & & & & & \\
\hline SRC-Ca-3 & & & & & & & & & & & & & & & & & & & & & \\
\hline SRC-Ce-1 & & & & & & & & & & & & & & & & & & & & & \\
\hline SRC-Ce-2 & & & & & & & & & & & & & & & & & & & & & \\
\hline SRC-Ce-3 & & & & & & & & & & & & & & & & & & & & & \\
\hline SRC-Ce-4 & & & & & & & & & & & & & & & & & & & & & \\
\hline SRC-Eu-1 & & & & & & & & & & & & & & & & & & & & & \\
\hline SRC-Gd-1 & & & & & & & & & & & & & & & & & & & & & \\
\hline SRC-Gd-2 & & & & & & & & & & & & & & & & & & & & & \\
\hline SRC-Gd-3 & & & & & & & & & & & & & & & & & & & & & \\
\hline SRC-Gd-4 & & & & & & & & & & & & & & & & & & & & & \\
\hline SRC-K-1 & & & & & & & & & & & & & & & & & & & & & \\
\hline SRC-K-2 & & & & & & & & & & & & & & & & & & & & & \\
\hline SRC-K-3 & & & & & & & & & & & & & & & & & & & & & \\
\hline SRC-K-3i & & & & & & & & & & & & & & & & & & & & & \\
\hline SRC-Li-1 & & & & & & & & & & & & & & & & & & & & & \\
\hline SRC-Li-2 & & & & & & & & & & & & & & & & & & & & & \\
\hline SRC-Li-3 & & & & & & & & & & & & & & & & & & & & & \\
\hline SRC-Mg-1 & & & & & & & & & & & & & & & & & & & & & \\
\hline SRC-Mg-2 & & & & & & & & & & & & & & & & & & & & & \\
\hline SRC-Mg-3 & & & & & & & & & & & & & & & & & & & & & \\
\hline SRC-Na-1 & & & & & & & & & & & & & & & & & & & & & \\
\hline SRC-Na-2 & & & & & & & & & & & & & & & & & & & & & \\
\hline SRC-Pb-1 & & & & & & & & & & & & & & & & & & & & & \\
\hline SRC-Pb-2 & & & & & & & & & & & & & & & & & & & & & \\
\hline SRC-Pb-3 & & & & & & & & & & & & & & & & & & & & & \\
\hline SRC-Si-1 & & & & & & & & & & & & & & & & & & & & & \\
\hline SRC-Si-2 & & & & & & & & & & & & & & & & & & & & & \\
\hline SRC-Sm-1 & & & & & & & & & & & & & & & & & & & & & \\
\hline SRC-Sn-1 & & & & & & & & & & & & & & & & & & & & & \\
\hline SRC-Sn-2 & & & & & & & & & & & & & & & & & & & & & \\
\hline SRC-Sn-3 & & & & & & & & & & & & & & & & & & & & & \\
\hline SRC-Ti-1 & & & & & & & & & & & & & & & & & & & & & \\
\hline SRC-Ti-2 & & & & & & & & & & & & & & & & & & & & & \\
\hline SRC-Zr-1 & & & & & & & & & & & & & & & & & & & & & \\
\hline SRC-Zr-2 & & & & & & & & & & & & & & & & & & & & & \\
\hline
\end{tabular}


Appendix A. Database - mass fraction

Plutonium Vitrification (Bulkley and Vienna 1997)

\begin{tabular}{|c|c|c|c|c|c|c|c|c|c|c|c|c|c|c|c|c|c|c|c|c|c|}
\hline Glass ID & $\begin{array}{c}\mathrm{I} \\
-\mathrm{a}\end{array}$ & $\begin{array}{c}\mathrm{La} 2 \mathrm{O} 3 \\
-\mathrm{a}\end{array}$ & $\begin{array}{c}\mathrm{MnO} 2 \\
-\mathrm{a}\end{array}$ & $\begin{array}{c}\mathrm{MnO} \\
-\mathrm{a}\end{array}$ & $\begin{array}{c}\mathrm{MoO} \\
-\mathrm{a}\end{array}$ & $\begin{array}{c}\mathrm{MoO} 3 \\
-\mathrm{a}\end{array}$ & $\begin{array}{c}\mathrm{Nb} 2 \mathrm{O} 5 \\
-\mathrm{a}\end{array}$ & $\begin{array}{c}\mathrm{Nd} 2 \mathrm{O} 3 \\
-\mathrm{a}\end{array}$ & $\begin{array}{c}\mathrm{PbO} \\
-\mathrm{a}\end{array}$ & $\begin{array}{c}\mathrm{PdO} 2 \\
-\mathrm{a}\end{array}$ & $\begin{array}{c}\mathrm{PdO} \\
-\mathrm{a}\end{array}$ & $\begin{array}{c}\mathrm{Pr} 2 \mathrm{O} 3 \\
-\mathrm{a}\end{array}$ & $\begin{array}{c}\text { Pr6O11 } \\
-\mathrm{a}\end{array}$ & $\begin{array}{c}\mathrm{Rb} 2 \mathrm{O} \\
-\mathrm{a}\end{array}$ & $\begin{array}{c}\mathrm{ReO} \\
-\mathrm{a}\end{array}$ & $\begin{array}{c}\mathrm{ReO} 2 \\
-\mathrm{a}\end{array}$ & $\begin{array}{c}\mathrm{Rh} 2 \mathrm{O} 3 \\
-\mathrm{a}\end{array}$ & $\begin{array}{c}\mathrm{RhO} 2 \\
-\mathrm{a}\end{array}$ & $\begin{array}{c}\mathrm{RuO} 2 \\
-\mathrm{a}\end{array}$ & $\begin{array}{c}\mathrm{Sb} 2 \mathrm{O} 3 \\
-\mathrm{a}\end{array}$ & $\begin{array}{c}\mathrm{Sb} 2 \mathrm{O} 5 \\
-\mathrm{a}\end{array}$ \\
\hline $\mathrm{PBG} 3 / \mathrm{Ce}$ & & & & & & & & & & & & & & & & & & & & & \\
\hline $\mathrm{PBG} 3 / 0 \mathrm{x}$ & & & & & & & & & & & & & & & & & & & & & \\
\hline PBG3-.5C & & & & & & & & & & & & & & & & & & & & & \\
\hline SRC-Al-1 & & & & & & & & & & & & & & & & & & & & & \\
\hline SRC-Al-2 & & & & & & & & & & & & & & & & & & & & & \\
\hline SRC-B-1 & & & & & & & & & & & & & & & & & & & & & \\
\hline SRC-B-2 & & & & & & & & & & & & & & & & & & & & & \\
\hline SRC-B-3 & & & & & & & & & & & & & & & & & & & & & \\
\hline SRC-Ca-1 & & & & & & & & & & & & & & & & & & & & & \\
\hline SRC-Ca-2 & & & & & & & & & & & & & & & & & & & & & \\
\hline SRC-Ca-3 & & & & & & & & & & & & & & & & & & & & & \\
\hline SRC-Ce-1 & & & & & & & & & & & & & & & & & & & & & \\
\hline SRC-Ce-2 & & & & & & & & & & & & & & & & & & & & & \\
\hline SRC-Ce-3 & & & & & & & & & & & & & & & & & & & & & \\
\hline SRC-Ce-4 & & & & & & & & & & & & & & & & & & & & & \\
\hline SRC-Eu-1 & & & & & & & & & & & & & & & & & & & & & \\
\hline SRC-Gd-1 & & & & & & & & & & & & & & & & & & & & & \\
\hline SRC-Gd-2 & & & & & & & & & & & & & & & & & & & & & \\
\hline SRC-Gd-3 & & & & & & & & & & & & & & & & & & & & & \\
\hline SRC-Gd-4 & & & & & & & & & & & & & & & & & & & & & \\
\hline SRC-K-1 & & & & & & & & & & & & & & & & & & & & & \\
\hline SRC-K-2 & & & & & & & & & & & & & & & & & & & & & \\
\hline SRC-K-3 & & & & & & & & & & & & & & & & & & & & & \\
\hline SRC-K-3i & & & & & & & & & & & & & & & & & & & & & \\
\hline SRC-Li-1 & & & & & & & & & & & & & & & & & & & & & \\
\hline SRC-Li-2 & & & & & & & & & & & & & & & & & & & & & \\
\hline SRC-Li-3 & & & & & & & & & & & & & & & & & & & & & \\
\hline SRC-Mg-1 & & & & & & & & & & & & & & & & & & & & & \\
\hline SRC-Mg-2 & & & & & & & & & & & & & & & & & & & & & \\
\hline SRC-Mg-3 & & & & & & & & & & & & & & & & & & & & & \\
\hline SRC-Na-1 & & & & & & & & & & & & & & & & & & & & & \\
\hline SRC-Na-2 & & & & & & & & & & & & & & & & & & & & & \\
\hline SRC-Pb-1 & & & & & & & & & & & & & & & & & & & & & \\
\hline SRC-Pb-2 & & & & & & & & & & & & & & & & & & & & & \\
\hline SRC-Pb-3 & & & & & & & & & & & & & & & & & & & & & \\
\hline SRC-Si-1 & & & & & & & & & & & & & & & & & & & & & \\
\hline SRC-Si-2 & & & & & & & & & & & & & & & & & & & & & \\
\hline SRC-Sm-1 & & & & & & & & & & & & & & & & & & & & & \\
\hline SRC-Sn-1 & & & & & & & & & & & & & & & & & & & & & \\
\hline SRC-Sn-2 & & & & & & & & & & & & & & & & & & & & & \\
\hline SRC-Sn-3 & & & & & & & & & & & & & & & & & & & & & \\
\hline SRC-Ti-1 & & & & & & & & & & & & & & & & & & & & & \\
\hline SRC-Ti-2 & & & & & & & & & & & & & & & & & & & & & \\
\hline SRC-Zr-1 & & & & & & & & & & & & & & & & & & & & & \\
\hline SRC-Zr-2 & & & & & & & & & & & & & & & & & & & & & \\
\hline
\end{tabular}


Appendix A. Database - mass fraction

Plutonium Vitrification (Bulkley and Vienna 1997)

\begin{tabular}{|c|c|c|c|c|c|c|c|c|c|c|c|c|c|c|c|c|c|c|c|c|}
\hline Glass ID & $\begin{array}{c}\mathrm{SeO} 2 \\
-\mathrm{a}\end{array}$ & $\begin{array}{c}\mathrm{Sm} 2 \mathrm{O} 3 \\
-\mathrm{a}\end{array}$ & $\begin{array}{c}\mathrm{SnO} \\
-\mathrm{a}\end{array}$ & $\begin{array}{c}\mathrm{SnO} 2 \\
-\mathrm{a}\end{array}$ & $\begin{array}{c}\mathrm{SO} 3 \\
-\mathrm{a}\end{array}$ & $\begin{array}{c}\mathrm{SrO} \\
-\mathrm{a}\end{array}$ & $\begin{array}{c}\mathrm{Tc} 2 \mathrm{O} 7 \\
-\mathrm{a}\end{array}$ & $\begin{array}{c}\mathrm{TeO} 2 \\
-\mathrm{a}\end{array}$ & $\begin{array}{c}\mathrm{ThO} 2 \\
-\mathrm{a}\end{array}$ & $\begin{array}{c}\mathrm{TiO} 2 \\
-\mathrm{a}\end{array}$ & $\begin{array}{c}\mathrm{T} 12 \mathrm{O} 3 \\
-\mathrm{a}\end{array}$ & $\begin{array}{c}\mathrm{U} 3 \mathrm{O} 8 \\
-\mathrm{a}\end{array}$ & $\begin{array}{c}\mathrm{UO} 2 \\
-\mathrm{a}\end{array}$ & $\begin{array}{c}\text { UO3 } \\
-\mathrm{a}\end{array}$ & $\begin{array}{c}\mathrm{V} 2 \mathrm{O} 5 \\
-\mathrm{a}\end{array}$ & $\begin{array}{c}\text { WO3 } \\
-\mathrm{a}\end{array}$ & $\begin{array}{c}\mathrm{Y} 2 \mathrm{O} 3 \\
-\mathrm{a}\end{array}$ & $\begin{array}{c}\mathrm{ZnO} \\
-\mathrm{a}\end{array}$ & $\begin{array}{c}\text { Others } \\
-\mathrm{a}\end{array}$ & $\begin{array}{l}\text { Sum } \\
-\mathrm{a}\end{array}$ \\
\hline $\mathrm{PBG} 3 / \mathrm{Ce}$ & & & & & & & & & & & & & & & & & & & & \\
\hline PBG3/0x & & & & & & & & & & & & & & & & & & & & \\
\hline PBG3-.5C & & & & & & & & & & & & & & & & & & & & \\
\hline SRC-Al-1 & & & & & & & & & & & & & & & & & & & & \\
\hline SRC-Al-2 & & & & & & & & & & & & & & & & & & & & \\
\hline SRC-B-1 & & & & & & & & & & & & & & & & & & & & \\
\hline SRC-B-2 & & & & & & & & & & & & & & & & & & & & \\
\hline SRC-B-3 & & & & & & & & & & & & & & & & & & & & \\
\hline SRC-Ca-1 & & & & & & & & & & & & & & & & & & & & \\
\hline SRC-Ca-2 & & & & & & & & & & & & & & & & & & & & \\
\hline SRC-Ca-3 & & & & & & & & & & & & & & & & & & & & \\
\hline SRC-Ce-1 & & & & & & & & & & & & & & & & & & & & \\
\hline SRC-Ce-2 & & & & & & & & & & & & & & & & & & & & \\
\hline SRC-Ce-3 & & & & & & & & & & & & & & & & & & & & \\
\hline SRC-Ce-4 & & & & & & & & & & & & & & & & & & & & \\
\hline SRC-Eu-1 & & & & & & & & & & & & & & & & & & & & \\
\hline SRC-Gd-1 & & & & & & & & & & & & & & & & & & & & \\
\hline SRC-Gd-2 & & & & & & & & & & & & & & & & & & & & \\
\hline SRC-Gd-3 & & & & & & & & & & & & & & & & & & & & \\
\hline SRC-Gd-4 & & & & & & & & & & & & & & & & & & & & \\
\hline SRC-K-1 & & & & & & & & & & & & & & & & & & & & \\
\hline SRC-K-2 & & & & & & & & & & & & & & & & & & & & \\
\hline SRC-K-3 & & & & & & & & & & & & & & & & & & & & \\
\hline SRC-K-3i & & & & & & & & & & & & & & & & & & & & \\
\hline SRC-Li-1 & & & & & & & & & & & & & & & & & & & & \\
\hline SRC-Li-2 & & & & & & & & & & & & & & & & & & & & \\
\hline SRC-Li-3 & & & & & & & & & & & & & & & & & & & & \\
\hline SRC-Mg-1 & & & & & & & & & & & & & & & & & & & & \\
\hline SRC-Mg-2 & & & & & & & & & & & & & & & & & & & & \\
\hline SRC-Mg-3 & & & & & & & & & & & & & & & & & & & & \\
\hline SRC-Na-1 & & & & & & & & & & & & & & & & & & & & \\
\hline SRC-Na-2 & & & & & & & & & & & & & & & & & & & & \\
\hline SRC-Pb-1 & & & & & & & & & & & & & & & & & & & & \\
\hline SRC-Pb-2 & & & & & & & & & & & & & & & & & & & & \\
\hline SRC-Pb-3 & & & & & & & & & & & & & & & & & & & & \\
\hline SRC-Si-1 & & & & & & & & & & & & & & & & & & & & \\
\hline SRC-Si-2 & & & & & & & & & & & & & & & & & & & & \\
\hline SRC-Sm-1 & & & & & & & & & & & & & & & & & & & & \\
\hline SRC-Sn-1 & & & & & & & & & & & & & & & & & & & & \\
\hline SRC-Sn-2 & & & & & & & & & & & & & & & & & & & & \\
\hline SRC-Sn-3 & & & & & & & & & & & & & & & & & & & & \\
\hline SRC-Ti-1 & & & & & & & & & & & & & & & & & & & & \\
\hline SRC-Ti-2 & & & & & & & & & & & & & & & & & & & & \\
\hline SRC-Zr-1 & & & & & & & & & & & & & & & & & & & & \\
\hline SRC-Zr-2 & & & & & & & & & & & & & & & & & & & & \\
\hline
\end{tabular}


Appendix A. Database - mass fraction

Plutonium Vitrification (Bulkley and Vienna 1997)

\begin{tabular}{|c|c|c|c|c|c|c|c|c|}
\hline Glass ID & $\begin{array}{l}\mathrm{TM} \\
\left({ }^{\circ} \mathrm{C}\right) \\
\end{array}$ & $\begin{array}{l}\text { Gradient } \\
\text { TL }\left({ }^{\circ} \mathrm{C}\right)\end{array}$ & $\begin{array}{l}\text { Uniform } \\
\text { TL }\left({ }^{\circ} \mathrm{C}\right) \\
\end{array}$ & Primary Phase & $\begin{array}{l}\text { Quenched } \\
\text { Visual/OM }\end{array}$ & $\begin{array}{c}\text { Quenched } \\
\text { SEM/EDS or TEM }\end{array}$ & $\begin{array}{l}\text { Quenched } \\
\text { XRD } \\
\end{array}$ & $\begin{array}{c}\text { Quenched } \\
\text { Homogeneous? }\end{array}$ \\
\hline $\mathrm{PBG} 3 / \mathrm{Ce}$ & 1240 & & & & & & & \\
\hline $\mathrm{PBG} 3 / 0 \mathrm{x}$ & 1240 & & & & & & & \\
\hline PBG3-.5C & 1240 & & & & & & & \\
\hline SRC-Al-1 & 1180 & & & & & & & \\
\hline SRC-Al-2 & 1242 & & & & & & & \\
\hline SRC-B-1 & 1268 & & & & & & & \\
\hline SRC-B-2 & 1242 & & & & & & & \\
\hline SRC-B-3 & 1095 & & & & & & & \\
\hline SRC-Ca-1 & 1258 & & & & & & & \\
\hline SRC-Ca-2 & 1250 & & & & & & & \\
\hline SRC-Ca-3 & 1143 & & & & & & & \\
\hline \multicolumn{9}{|l|}{ SRC-Ce-1 } \\
\hline SRC-Ce-2 & 1200 & & & & & & & \\
\hline SRC-Ce-3 & 1243 & & & & & & & \\
\hline \multicolumn{9}{|l|}{ SRC-Ce-4 } \\
\hline SRC-Eu-1 & 1248 & & & & & & & \\
\hline \multicolumn{9}{|l|}{ SRC-Gd-1 } \\
\hline SRC-Gd-2 & 1192 & & & & & & & \\
\hline SRC-Gd-3 & 1243 & & & & & & & \\
\hline \multicolumn{9}{|l|}{ SRC-Gd-4 } \\
\hline SRC-K-1 & 1264 & & & & & & & \\
\hline SRC-K-2 & 1169 & & & & & & & \\
\hline SRC-K-3 & 1136 & & & & & & & \\
\hline SRC-K-3i & 1146 & & & & & & & \\
\hline SRC-Li-1 & 1390 & & & & & & & \\
\hline SRC-Li-2 & 1293 & & & & & & & \\
\hline SRC-Li-3 & 1152 & & & & & & & \\
\hline SRC-Mg-1 & 1189 & & & & & & & \\
\hline SRC-Mg-2 & 1158 & & & & & & & \\
\hline SRC-Mg-3 & 1138 & & & & & & & \\
\hline SRC-Na-1 & 1368 & & & & & & & \\
\hline SRC-Na-2 & 1215 & & & & & & & \\
\hline SRC-Pb-1 & 1246 & & & & & & & \\
\hline SRC-Pb-2 & 1126 & & & & & & & \\
\hline SRC-Pb-3 & 1083 & & & & & & & \\
\hline SRC-Si-1 & 1250 & & & & & & & \\
\hline SRC-Si-2 & 1365 & & & & & & & \\
\hline SRC-Sm-1 & 1250 & & & & & & & \\
\hline SRC-Sn-1 & 1180 & & & & & & & \\
\hline SRC-Sn-2 & 1203 & & & & & & & \\
\hline SRC-Sn-3 & 1306 & & & & & & & \\
\hline SRC-Ti-1 & 1184 & & & & & & & \\
\hline SRC-Ti-2 & 1208 & & & & & & & \\
\hline SRC-Zr-1 & 1169 & & & & & & & \\
\hline SRC-Zr-2 & 1170 & & & & & & & \\
\hline
\end{tabular}


Appendix A. Database - mass fraction

Plutonium Vitrification (Bulkley and Vienna 1997)

\begin{tabular}{|c|c|c|c|c|c|}
\hline Glass ID & $\begin{array}{c}\text { CCC } \\
\text { Visual/OM }\end{array}$ & $\begin{array}{c}\text { CCC } \\
\text { SEM/EDS or TEM }\end{array}$ & $\begin{array}{l}\text { CCC } \\
\text { XRD }\end{array}$ & $\begin{array}{c}\text { Heat Treated } \\
\text { Visual/OM }\end{array}$ & $\begin{array}{c}\text { Heat Treated } \\
\text { SEM/EDS or TEM }\end{array}$ \\
\hline \multicolumn{6}{|l|}{$\mathrm{PBG} 3 / \mathrm{Ce}$} \\
\hline \multicolumn{6}{|l|}{ PBG3/0x } \\
\hline \multicolumn{6}{|l|}{ PBG3-.5C } \\
\hline \multicolumn{6}{|l|}{ SRC-Al-1 } \\
\hline \multicolumn{6}{|l|}{ SRC-Al-2 } \\
\hline \multicolumn{6}{|l|}{ SRC-B-1 } \\
\hline \multicolumn{6}{|l|}{ SRC-B-2 } \\
\hline \multicolumn{6}{|l|}{ SRC-B-3 } \\
\hline \multicolumn{6}{|l|}{ SRC-Ca-1 } \\
\hline \multicolumn{6}{|l|}{ SRC-Ca-2 } \\
\hline \multicolumn{6}{|l|}{ SRC-Ca-3 } \\
\hline \multicolumn{6}{|l|}{ SRC-Ce-1 } \\
\hline \multicolumn{6}{|l|}{ SRC-Ce-2 } \\
\hline \multicolumn{6}{|l|}{ SRC-Ce-3 } \\
\hline \multicolumn{6}{|l|}{ SRC-Ce-4 } \\
\hline \multicolumn{6}{|l|}{ SRC-Eu-1 } \\
\hline \multicolumn{6}{|l|}{ SRC-Gd-1 } \\
\hline \multicolumn{6}{|l|}{ SRC-Gd-2 } \\
\hline \multicolumn{6}{|l|}{ SRC-Gd-3 } \\
\hline \multicolumn{6}{|l|}{ SRC-Gd-4 } \\
\hline \multicolumn{6}{|l|}{ SRC-K-1 } \\
\hline \multicolumn{6}{|l|}{ SRC-K-2 } \\
\hline \multicolumn{6}{|l|}{ SRC-K-3 } \\
\hline SRC-K-3i & & & & & \\
\hline SRC-Li-1 & & & & & \\
\hline SRC-Li-2 & & & & & \\
\hline SRC-Li-3 & & & & & \\
\hline SRC-Mg-1 & & & & & \\
\hline SRC-Mg-2 & & & & & \\
\hline SRC-Mg-3 & & & & & \\
\hline SRC-Na-1 & & & & & \\
\hline SRC-Na-2 & & & & & \\
\hline SRC-Pb-1 & & & & & \\
\hline SRC-Pb-2 & & & & & \\
\hline SRC-Pb-3 & & & & & \\
\hline SRC-Si-1 & & & & & \\
\hline SRC-Si-2 & & & & & \\
\hline SRC-Sm-1 & & & & & \\
\hline SRC-Sn-1 & & & & & \\
\hline SRC-Sn-2 & & & & & \\
\hline SRC-Sn-3 & & & & & \\
\hline SRC-Ti-1 & & & & & \\
\hline SRC-Ti-2 & & & & & \\
\hline SRC-Zr-1 & & & & & \\
\hline SRC-Zr-2 & & & & & \\
\hline
\end{tabular}


Plutonium Vitrification (Bulkley and Vienna 1997)

\begin{tabular}{|c|c|c|c|c|c|c|c|c|c|c|c|c|c|c|c|}
\hline Glass ID & $\begin{array}{l}\text { Heat Treated } \\
\text { XRD }\end{array}$ & $\begin{array}{l}\text { Density } \\
\left(\mathrm{g} / \mathrm{cm}^{3}\right)\end{array}$ & $\begin{array}{c}\text { Fulc Visc } \\
\mathrm{A}\end{array}$ & $\begin{array}{c}\text { Fulc Visc } \\
\text { B }\end{array}$ & \begin{tabular}{|c|} 
Fulc Visc \\
To
\end{tabular} & $\begin{array}{c}\mathrm{FV} 1150^{\circ} \mathrm{C} \\
(\mathrm{Pa} \cdot \mathrm{s})\end{array}$ & \begin{tabular}{|c|} 
Arrh Visc \\
A \\
\end{tabular} & \begin{tabular}{|c|} 
Arrh Visc \\
B
\end{tabular} & $1150^{\circ} \mathrm{C}$ & \begin{tabular}{|c|}
$\mathrm{T}\left({ }^{\circ} \mathrm{C}\right)$ at \\
$2 \mathrm{~Pa} \cdot \mathrm{s}$ \\
\end{tabular} & $\begin{array}{c}\mathrm{T}\left({ }^{\circ} \mathrm{C}\right) \text { at } \\
5 \mathrm{~Pa} \cdot \mathrm{s}\end{array}$ & \begin{tabular}{|c|}
$\mathrm{T}\left({ }^{\circ} \mathrm{C}\right)$ at \\
$10 \mathrm{~Pa} \cdot \mathrm{s}$
\end{tabular} & $\begin{array}{c}\mathrm{T} 1 \\
\left({ }^{\circ} \mathrm{C}\right) \\
\end{array}$ & $\begin{array}{c}\mathrm{V} 1 \\
(\mathrm{~Pa} \cdot \mathrm{s})\end{array}$ & $\begin{array}{c}\mathrm{T} 2 \\
\left({ }^{\circ} \mathrm{C}\right) \\
\end{array}$ \\
\hline $\mathrm{PBG} 3 / \mathrm{Ce}$ & & & & & & & -13.500 & 20914.0 & $\begin{array}{c}\text { Donat } \\
3.31\end{array}$ & & 1114 & 1053 & 1096 & 6.094 & 1046 \\
\hline \multicolumn{16}{|l|}{ PBG3/0x } \\
\hline PBG3-.5C & & & & & & & -12.700 & 19967.0 & 3.79 & & 1120 & 1056 & 1195 & 2.355 & 996 \\
\hline SRC-Al-1 & & & & & & & -13.200 & 19930.0 & 2.24 & & 1075 & 1014 & 1190 & 1.587 & 1141 \\
\hline SRC-Al-2 & & & & & & & -12.500 & 20656.0 & 7.51 & & 1195 & 1126 & 1148 & 9.887 & 1247 \\
\hline SRC-B-1 & & & & & & & -12.700 & 21231.0 & 9.21 & & 1209 & 1141 & 1288 & 2.371 & 1239 \\
\hline SRC-B-2 & & & & & & & -11.600 & 18852.0 & 5.20 & & 1154 & 1083 & 1239 & 2.358 & 1190 \\
\hline SRC-B-3 & & & & & & & -12.700 & 19085.0 & 2.04 & & 1065 & 1003 & 1189 & 1.536 & 1140 \\
\hline SRC-Ca-1 & & & & & & & -11.000 & 18423.0 & 7.00 & & 1188 & 1112 & 1290 & 2.131 & 1241 \\
\hline SRC-Ca-2 & & & & & & & -11.000 & 18084.0 & 5.52 & & 1158 & 1084 & 1239 & 2.51 & 1190 \\
\hline SRC-Ca-3 & & & & & & & -13.400 & 20707.0 & 3.16 & & 1103 & 1042 & 1097 & 5.624 & 1147 \\
\hline SRC-Ce-1 & & & & & & & -12.700 & 19944.0 & 3.73 & & 1124 & 1060 & 1096 & 6.692 & 1046 \\
\hline SRC-Ce-2 & & & & & & & -11.900 & 18913.0 & 4.02 & & 1129 & 1061 & 1191 & 2.881 & 1142 \\
\hline SRC-Ce-3 & & & & & & & -12.800 & 20042.0 & 3.61 & & 1120 & 1056 & 1239 & 1.592 & 1190 \\
\hline SRC-Ce-4 & & & & & & & -14.000 & 21866.0 & 3.92 & & 1130 & 1070 & 1240 & 1.638 & 1191 \\
\hline SRC-Eu-1 & & & & & & & -12.100 & 19223.0 & 4.09 & & 1134 & 1066 & 1241 & 1.881 & 1191 \\
\hline SRC-Gd-1 & & & & & & & -11.900 & 18941.0 & 4.10 & & 1124 & 1056 & 1239 & 1.74 & 1191 \\
\hline SRC-Gd-2 & & & & & & & -11.500 & 18418.0 & 4.23 & & 1132 & 1061 & 1239 & 1.964 & 1190 \\
\hline SRC-Gd-3 & & & & & & & -12.100 & 19150.0 & 3.89 & & 1124 & 1057 & 1239 & 1.763 & 1190 \\
\hline SRC-Gd-4 & & & & & & & -13.700 & 21362.0 & 3.71 & & 1127 & 1066 & 1097 & 7.035 & 1047 \\
\hline SRC-K-1 & & & & & & & -11.200 & 18126.0 & 4.65 & & 1138 & 1066 & 1289 & 1.46 & 1239 \\
\hline SRC-K-2 & & & & & & & -12.600 & 19605.0 & 3.25 & & 1107 & 1043 & 1098 & 5.601 & 1148 \\
\hline \multicolumn{16}{|l|}{ SRC-K-3 } \\
\hline \multicolumn{16}{|l|}{ SRC-K-3i } \\
\hline SRC-Li-1 & & & & & & & -12.100 & 22194.0 & 33.00 & & 1343 & 1266 & 1389 & 3.399 & 1339 \\
\hline SRC-Li-2 & & & & & & & -11.600 & 20126.0 & 12.72 & & 1248 & 1173 & 1338 & 2.378 & 1288 \\
\hline SRC-Li-3 & & & & & & & -12.700 & 19675.0 & 3.08 & & 1106 & 1043 & 1097 & 5.871 & 1147 \\
\hline SRC-Mg-1 & & & & & & & -14.000 & 21647.0 & 3.36 & & 1111 & 1052 & 1190 & 2.1 & 1141 \\
\hline SRC-Mg-2 & & & & & & & -12.900 & 19912.0 & 2.98 & & 1103 & 1040 & 1239 & 1.408 & 1189 \\
\hline \multicolumn{16}{|l|}{ SRC-Mg-3 } \\
\hline SRC-Na-1 & & & & & & & -12.000 & 21104.0 & 16.96 & & 1282 & 1206 & 1388 & 2.059 & 1339 \\
\hline SRC-Na-2 & & & & & & & -12.300 & 19914.0 & 5.44 & & 1159 & 1091 & 1238 & 2.393 & 1188 \\
\hline SRC-Pb-1 & & & & & & & -11.800 & 18771.0 & 4.02 & & 1128 & 1059 & 1239 & 1.901 & 1190 \\
\hline SRC-Pb-2 & & & & & & & -12.700 & 19628.0 & 2.98 & & 1103 & 1039 & 1196 & 2.033 & 1146 \\
\hline SRC-Pb-3 & & & & & & & -11.300 & 17621.0 & 2.95 & & 1096 & 1026 & 1239 & 1.566 & 1190 \\
\hline SRC-Si-1 & & & & & & & -11.000 & 16823.0 & 2.28 & & 1059 & 990 & 1239 & 1.184 & 1190 \\
\hline SRC-Si-2 & & & & & & & -11.100 & 20166.0 & 21.57 & & 1314 & 1232 & 1386 & 2.737 & 1337 \\
\hline SRC-Sm-1 & & & & & & & -11.800 & 18947.0 & 4.55 & & 1136 & 1067 & 1240 & 1.947 & 1190 \\
\hline SRC-Sn-1 & & & & & & & -12.900 & 20472.0 & 4.42 & & 1135 & 1071 & 1239 & 1.839 & 1189 \\
\hline SRC-Sn-2 & & & & & & & -14.200 & 22304.0 & 4.37 & & 1139 & 1080 & 1239 & 1.769 & 1189 \\
\hline \multicolumn{16}{|l|}{ SRC-Sn-3 } \\
\hline SRC-Ti-1 & & & & & & & -11.700 & 18672.0 & 4.14 & & 1130 & 1060 & 1239 & 1.898 & 1190 \\
\hline SRC-Ti-2 & & & & & & & -12.800 & 19802.0 & 3.05 & & 1098 & 1035 & 1239 & 1.329 & 1189 \\
\hline SRC-Zr-1 & & & & & & & -10.700 & 17113.0 & 3.77 & & 1116 & 1042 & 1239 & 1.822 & 1190 \\
\hline SRC-Zr-2 & & & & & & & -13.300 & 20889.0 & 3.97 & & 1131 & 1068 & 1239 & 1.765 & 1189 \\
\hline
\end{tabular}


Plutonium Vitrification (Bulkley and Vienna 1997)

\begin{tabular}{|c|c|c|c|c|c|c|c|c|c|c|c|c|c|c|c|c|c|c|c|c|c|}
\hline Glass ID & $\begin{array}{c}\mathrm{V} 2 \\
(\mathrm{~Pa} \cdot \mathrm{s})\end{array}$ & $\begin{array}{c}\mathrm{T} 3 \\
\left({ }^{\circ} \mathrm{C}\right)\end{array}$ & $\begin{array}{c}\mathrm{V} 3 \\
(\mathrm{~Pa} \cdot \mathrm{s})\end{array}$ & $\begin{array}{c}\mathrm{T} 4 \\
\left({ }^{\circ} \mathrm{C}\right)\end{array}$ & $\begin{array}{c}\mathrm{V} 4 \\
(\mathrm{~Pa} \cdot \mathrm{s})\end{array}$ & $\begin{array}{c}\mathrm{T} 5 \\
\left({ }^{\circ} \mathrm{C}\right)\end{array}$ & $\begin{array}{c}\mathrm{V} 5 \\
(\mathrm{~Pa} \cdot \mathrm{s})\end{array}$ & $\begin{array}{c}\mathrm{T} 6 \\
\left({ }^{\circ} \mathrm{C}\right)\end{array}$ & $\begin{array}{c}\mathrm{V} 6 \\
(\mathrm{~Pa} \cdot \mathrm{s})\end{array}$ & $\begin{array}{c}\mathrm{T} 7 \\
\left({ }^{\circ} \mathrm{C}\right)\end{array}$ & $\begin{array}{c}\mathrm{V} 7 \\
(\mathrm{~Pa} \cdot \mathrm{s})\end{array}$ & $\begin{array}{c}\mathrm{T} 8 \\
\left({ }^{\circ} \mathrm{C}\right)\end{array}$ & $\begin{array}{c}\mathrm{V} 8 \\
(\mathrm{~Pa} \cdot \mathrm{s})\end{array}$ & $\begin{array}{c}\mathrm{T} 9 \\
\left({ }^{\circ} \mathrm{C}\right)\end{array}$ & $\begin{array}{c}\mathrm{V} 9 \\
(\mathrm{~Pa} \cdot \mathrm{s})\end{array}$ & $\begin{array}{l}\mathrm{T} 10 \\
\left({ }^{\circ} \mathrm{C}\right)\end{array}$ & $\begin{array}{c}\mathrm{V} 10 \\
(\mathrm{~Pa} \cdot \mathrm{s})\end{array}$ & $\begin{array}{l}\mathrm{T} 11 \\
\left({ }^{\circ} \mathrm{C}\right)\end{array}$ & $\begin{array}{c}\mathrm{V} 11 \\
(\mathrm{~Pa} \cdot \mathrm{s})\end{array}$ & $\begin{array}{l}\mathrm{T} 12 \\
\left({ }^{\circ} \mathrm{C}\right)\end{array}$ & $\begin{array}{c}\mathrm{V} 12 \\
(\mathrm{~Pa} \cdot \mathrm{s})\end{array}$ \\
\hline PBG3/Ce & 10.507 & 996 & 19.44 & 1045 & 10.634 & 1096 & 6.134 & 1146 & 3.609 & 1196 & 2.253 & 1096 & 6.23 & 945 & 39.523 & 895 & 91.36 & & & & \\
\hline \multicolumn{22}{|l|}{$\mathrm{PBG} 3 / 0 \mathrm{x}$} \\
\hline PBG3-.5C & 20.376 & 1195 & 2.415 & 1145 & 3.864 & 1096 & 6.429 & 1046 & 11.032 & 996 & 20.255 & 1046 & 11.286 & 1195 & 2.435 & 1146 & 3.92 & 1096 & 6.493 & & \\
\hline SRC-Al-1 & 2.466 & 1092 & 4.135 & 1042 & 7.042 & 993 & 12.971 & 1190 & 1.558 & 1141 & 2.457 & 1092 & 4.182 & 1042 & 7.194 & 993 & 13.178 & & & & \\
\hline SRC-Al-2 & 2.929 & 1297 & 1.922 & 1198 & 4.533 & 1148 & 7.201 & 1098 & 12.622 & 1047 & 23.304 & 1147 & 8.218 & 1197 & 5.146 & 1248 & 3.145 & & & & \\
\hline SRC-B-1 & 3.848 & 1189 & 6.079 & 1141 & 10.062 & 1090 & 17.025 & 1288 & 2.371 & 1239 & 3.824 & 1189 & 6.134 & 1140 & 10.158 & 1090 & 17.388 & & & & \\
\hline SRC-B-2 & 3.584 & 1141 & 5.756 & 1092 & 8.796 & 1042 & 15.272 & 1240 & 2.345 & 1190 & 3.624 & 1141 & 5.772 & 1092 & 8.946 & 1042 & 15.67 & & & & \\
\hline SRC-B-3 & 2.317 & 1091 & 3.736 & 1041 & 6.325 & 993 & 11.445 & 1189 & 1.52 & 1140 & 2.326 & 1091 & 3.784 & 1041 & 6.453 & 993 & 11.604 & & & & \\
\hline SRC-Ca-1 & 3.213 & 1191 & 4.874 & 1142 & 7.64 & 1093 & 11.524 & 1290 & 2.181 & 1241 & 3.266 & 1192 & 4.93 & 1141 & 7.801 & 1093 & 11.973 & & & & \\
\hline SRC-Ca-2 & 3.816 & 1141 & 5.949 & 1092 & 9.021 & 1042 & 15.158 & 1240 & 2.5 & 1190 & 3.8 & 1141 & 5.997 & 1092 & 9.156 & 1042 & 15.3 & & & & \\
\hline SRC-Ca-3 & 3.242 & 1196 & 1.871 & 1147 & 3.083 & 1097 & 5.266 & 1047 & 9.331 & 997 & 17.146 & 1096 & 5.369 & 1146 & 3.131 & 1196 & 1.899 & & & & \\
\hline SRC-Ce-1 & 11.27 & 996 & 19.984 & 1045 & 11.207 & 1096 & 6.652 & 1146 & 4.063 & 1196 & 2.642 & 1096 & 6.803 & 945 & 40.152 & 895 & 89.305 & & & & \\
\hline SRC-Ce-2 & 4.498 & 1092 & 7.295 & 1043 & 11.778 & 994 & 21.187 & 1191 & 2.75 & 1141 & 4.409 & 1093 & 7.231 & 1043 & 11.733 & 995 & 21.13 & & & & \\
\hline SRC-Ce-3 & 2.447 & 1142 & 3.896 & 1092 & 6.59 & 1042 & 11.149 & 1240 & 1.628 & 1190 & 2.529 & 1141 & 4.073 & 1092 & 7.007 & 1042 & 12.168 & & & & \\
\hline SRC-Ce-4 & 2.819 & 1142 & 5.323 & 1093 & 9.246 & 1043 & 16.978 & 1239 & 1.523 & 1190 & 2.391 & 1141 & 4.033 & 1092 & 6.494 & 1042 & 11.074 & & & & \\
\hline SRC-Eu-1 & 3.016 & 1142 & 4.698 & 1092 & 7.889 & 1043 & 12.842 & 1240 & 1.832 & 1191 & 2.983 & 1142 & 4.746 & 1093 & 7.253 & 1043 & 12.468 & & & & \\
\hline SRC-Gd-1 & 2.69 & 1142 & 4.273 & 1092 & 6.951 & 1042 & 11.209 & 1239 & 1.737 & 1190 & 2.736 & 1141 & 4.346 & 1092 & 7.159 & 1042 & 11.329 & & & & \\
\hline SRC-Gd-2 & 2.94 & 1141 & 4.562 & 1092 & 7.456 & 1042 & 11.778 & 1240 & 1.95 & 1190 & 2.957 & 1141 & 4.602 & 1092 & 7.544 & 1042 & 12.258 & & & & \\
\hline SRC-Gd-3 & 2.684 & 1140 & 4.249 & 1091 & 7.095 & 1042 & 11.614 & 1239 & 1.763 & 1190 & 2.704 & 1141 & 4.281 & 1092 & 7.119 & 1041 & 11.748 & & & & \\
\hline SRC-Gd-4 & 13.543 & 997 & 27.138 & 1047 & 13.893 & 1097 & 7.266 & 1147 & 3.975 & 1197 & 2.212 & 1097 & 6.238 & 947 & 41.354 & 897 & 95.606 & & & & \\
\hline SRC-K-1 & 2.049 & 1190 & 3.108 & 1141 & 4.906 & 1092 & 7.568 & 1289 & 1.464 & 1240 & 2.079 & 1190 & 3.151 & 1141 & 4.995 & 1092 & 7.717 & & & & \\
\hline SRC-K-2 & 3.37 & 1198 & 2.049 & 1148 & 3.258 & 1098 & 5.489 & 1048 & 9.379 & 998 & 16.663 & 1097 & 5.601 & 1147 & 3.354 & 1197 & 2.081 & & & & \\
\hline \multicolumn{22}{|l|}{ SRC-K-3 } \\
\hline \multicolumn{22}{|l|}{ SRC-K-3i } \\
\hline SRC-Li-1 & 5.243 & 1290 & 7.657 & 1240 & 12.573 & 1189 & 21.13 & 1388 & 3.471 & 1339 & 5.371 & 1289 & 7.942 & 1240 & 12.977 & 1189 & 21.813 & & & & \\
\hline SRC-Li-2 & 3.568 & 1239 & 5.484 & 1190 & 8.092 & 1140 & 13.696 & 1338 & 2.348 & 1289 & 3.608 & 1239 & 5.588 & 1190 & 8.302 & 1140 & 14.086 & & & & \\
\hline SRC-Li-3 & 3.418 & 1196 & 2.036 & 1147 & 3.211 & 1097 & 5.441 & 1047 & 9.426 & 997 & 16.693 & 1096 & 5.648 & 1146 & 3.338 & 1197 & 2.084 & & & & \\
\hline SRC-Mg-1 & 3.728 & 1092 & 6.294 & 1042 & 11.429 & 994 & 21.433 & 1190 & 2.094 & 1141 & 3.665 & 1092 & 6.182 & 1042 & 11.111 & 994 & 20.769 & & & & \\
\hline SRC-Mg-2 & 2.071 & 1140 & 3.314 & 1091 & 5.608 & 1042 & 9.903 & 1239 & 1.386 & 1190 & 2.068 & 1140 & 3.33 & 1091 & 5.672 & 1042 & 9.935 & & & & \\
\hline \multicolumn{22}{|l|}{ SRC-Mg-3 } \\
\hline SRC-Na-1 & 3.042 & 1289 & 4.73 & 1240 & 7.408 & 1190 & 11.269 & 1388 & 2.059 & 1339 & 3.125 & 1289 & 4.77 & 1240 & 7.504 & 1190 & 11.464 & & & & \\
\hline SRC-Na-2 & 3.776 & 1139 & 6.015 & 1090 & 10.046 & 1040 & 17.267 & 1238 & 2.419 & 1188 & 3.864 & 1139 & 6.166 & 1090 & 10.285 & 1040 & 17.871 & & & & \\
\hline SRC-Pb-1 & 2.848 & 1141 & 4.441 & 1091 & 7.263 & 1041 & 11.898 & 1240 & 1.822 & 1190 & 2.802 & 1141 & 4.441 & 1091 & 7.311 & 1042 & 12.048 & & & & \\
\hline SRC-Pb-2 & 3.179 & 1096 & 5.274 & 1046 & 9.029 & 996 & 16.422 & 1196 & 2.014 & 1146 & 3.29 & 1096 & 5.433 & 1046 & 9.379 & 996 & 16.844 & & & & \\
\hline SRC-Pb-3 & 2.089 & 1141 & 3.271 & 1091 & 5.307 & 1041 & 8.452 & 1239 & 1.477 & 1190 & 2.144 & 1140 & 3.327 & 1091 & 5.411 & 1041 & 8.646 & & & & \\
\hline SRC-Si-1 & 1.473 & 1141 & 2.325 & 1092 & 3.96 & 1240 & 1.164 & 1190 & 1.539 & 1142 & 2.25 & 1092 & 3.752 & & & & & & & & \\
\hline SRC-Si-2 & 4.151 & 1288 & 6.158 & 1239 & 9.315 & 1189 & 14.248 & 1388 & 2.842 & 1338 & 4.254 & 1289 & 6.294 & 1240 & 9.633 & 1190 & 14.58 & & & & \\
\hline SRC-Sm-1 & 2.977 & 1141 & 4.746 & 1092 & 7.841 & 1042 & 12.767 & 1240 & 2.019 & 1190 & 3.085 & 1141 & 4.85 & 1091 & 7.977 & 1042 & 13.067 & & & & \\
\hline SRC-Sn-1 & 2.878 & 1141 & 4.626 & 1050 & 13.217 & 1240 & 1.845 & 1190 & 2.901 & 1142 & 4.682 & 1092 & 7.969 & 1042 & 13.502 & & & & & & \\
\hline SRC-Sn-2 & 2.842 & 1140 & 4.884 & 1091 & 8.536 & 1042 & 15.667 & 1239 & 1.797 & 1190 & 2.9 & 1140 & 4.971 & 1091 & 8.886 & 1042 & 16.663 & & & & \\
\hline \multicolumn{22}{|l|}{ SRC-Sn-3 } \\
\hline SRC-Ti-1 & 2.888 & 1141 & 4.361 & 1091 & 7.336 & 1042 & 11.943 & 1240 & 1.894 & 1190 & 2.898 & 1141 & 4.465 & 1091 & 7.464 & 1042 & 12.108 & & & & \\
\hline SRC-Ti-2 & 2.02 & 1140 & 3.179 & 1092 & 5.282 & 1042 & 9.188 & 1189 & 2.008 & 1140 & 3.226 & 1092 & 5.354 & 1042 & 9.379 & & & & & & \\
\hline SRC-Zr-1 & 2.661 & 1141 & 3.976 & 1092 & 6.309 & 1042 & 9.695 & 1240 & 1.806 & 1190 & 2.677 & 1140 & 4.033 & 1092 & 6.382 & 1042 & 9.905 & & & & \\
\hline SRC-Zr-2 & 2.734 & 1141 & 4.445 & 1091 & 7.64 & 1042 & 13.718 & 1239 & 1.708 & 1189 & 2.702 & 1141 & 4.525 & 1091 & 7.68 & 1041 & 13.798 & & & & \\
\hline
\end{tabular}


Appendix A. Database - mass fraction

Plutonium Vitrification (Bulkley and Vienna 1997)

\begin{tabular}{|c|c|c|c|c|c|c|c|c|c|c|c|c|c|c|c|c|}
\hline Glass ID & $\begin{array}{l}\mathrm{T} 13 \\
\left({ }^{\circ} \mathrm{C}\right)\end{array}$ & $\begin{array}{l}\text { V13 } \\
(\mathrm{Pa} \cdot \mathrm{s})\end{array}$ & $\begin{array}{l}\mathrm{T} 14 \\
\left({ }^{\circ} \mathrm{C}\right)\end{array}$ & $\begin{array}{c}\mathrm{V} 14 \\
(\mathrm{~Pa} \cdot \mathrm{s})\end{array}$ & $\begin{array}{c}\text { Q PCT } \\
\text { B }\left(\mathrm{g} / \mathrm{m}^{2}\right)\end{array}$ & $\begin{array}{c}\text { Q PCT } \\
\mathrm{Li}\left(\mathrm{g} / \mathrm{m}^{2}\right)\end{array}$ & $\begin{array}{c}\text { Q PCT } \\
\mathrm{Na}\left(\mathrm{g} / \mathrm{m}^{2}\right)\end{array}$ & $\begin{array}{c}\text { Q PCT } \\
\mathrm{Si}\left(\mathrm{g} / \mathrm{m}^{2}\right)\end{array}$ & $\begin{array}{c}\text { Q PCT } \\
\text { pH }\end{array}$ & $\begin{array}{c}\text { CCC PCT } \\
\text { B }\left(\mathrm{g} / \mathrm{m}^{2}\right)\end{array}$ & \begin{tabular}{|c|} 
CCC PCT \\
$\mathrm{Li}\left(\mathrm{g} / \mathrm{m}^{2}\right)$
\end{tabular} & $\begin{array}{l}\text { CCC PCT } \\
\mathrm{Na}\left(\mathrm{g} / \mathrm{m}^{2}\right)\end{array}$ & $\begin{array}{l}\text { CCC PCT } \\
\mathrm{Si}\left(\mathrm{g} / \mathrm{m}^{2}\right)\end{array}$ & $\begin{array}{c}\text { CCC PCT } \\
\text { pH }\end{array}$ & $\begin{array}{l}\text { Q PCT B at } \\
20^{\circ} \mathrm{C}\left(\mathrm{g} / \mathrm{m}^{2}\right)\end{array}$ & $\begin{array}{l}\text { Q PCT Li at } \\
20^{\circ} \mathrm{C}\left(\mathrm{g} / \mathrm{m}^{2}\right)\end{array}$ \\
\hline \multicolumn{17}{|c|}{ 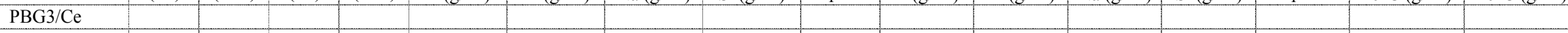 } \\
\hline \multicolumn{17}{|l|}{$\mathrm{PBG} 3 / 0 \mathrm{x}$} \\
\hline PBG3-.5C & & & & & 0.483 & 0.554 & 0.554 & 0.197 & & & & & & & & \\
\hline SRC-Al-1 & & & & & 6.096 & 4.909 & 4.961 & 1.536 & 11.88 & & & & & & & \\
\hline SRC-Al-2 & & & & & 0.226 & 0.306 & 0.291 & 0.104 & 10.95 & & & & & & & \\
\hline SRC-B-1 & & & & & & 0.627 & 0.603 & 0.197 & 11.53 & & & & & & & \\
\hline SRC-B-2 & & & & & 0.347 & 0.306 & 0.292 & 0.101 & 10.97 & & & & & & & \\
\hline SRC-B-3 & & & & & 1.787 & 1.697 & 1.541 & 0.349 & 10.93 & & & & & & & \\
\hline SRC-Ca-1 & & & & & 0.334 & 0.411 & 0.394 & 0.173 & 10.90 & & & & & & & \\
\hline SRC-Ca-2 & & & & & 0.411 & 0.503 & 0.480 & 0.191 & 11.09 & & & & & & & \\
\hline SRC-Ca-3 & & & & & 0.630 & 0.696 & 0.670 & 0.216 & 11.33 & & & & & & & \\
\hline SRC-Ce-1 & & & & & 0.626 & 0.669 & 0.635 & 0.231 & 11.18 & & & & & & & \\
\hline SRC-Ce-2 & & & & & 0.605 & 0.663 & 0.618 & 0.228 & 11.23 & & & & & & & \\
\hline SRC-Ce-3 & & & & & 0.593 & 0.645 & 0.604 & 0.219 & 11.29 & & & & & & & \\
\hline SRC-Ce-4 & & & & & 0.626 & 0.669 & 0.631 & 0.227 & 11.19 & & & & & & & \\
\hline SRC-Eu-1 & & & & & 0.718 & 0.773 & 0.731 & 0.269 & 11.26 & & & & & & & \\
\hline SRC-Gd-1 & & & & & 0.342 & 0.431 & 0.416 & 0.160 & 11.03 & & & & & & & \\
\hline SRC-Gd-2 & & & & & 0.471 & 0.554 & 0.541 & 0.201 & 11.18 & & & & & & & \\
\hline SRC-Gd-3 & & & & & 0.558 & 0.592 & 0.579 & 0.216 & 11.19 & & & & & & & \\
\hline SRC-Gd-4 & & & & & 0.580 & 0.620 & 0.588 & 0.202 & 11.18 & & & & & & & \\
\hline SRC-K-1 & & & & & 0.472 & 0.504 & 0.481 & 0.182 & 11.10 & & & & & & & \\
\hline SRC-K-2 & & & & & 0.602 & 0.675 & 0.639 & 0.222 & 11.31 & & & & & & & \\
\hline \multicolumn{17}{|l|}{ SRC-K-3 } \\
\hline \multicolumn{17}{|l|}{ SRC-K-3i } \\
\hline SRC-Li-1 & & & & & 0.253 & & 0.314 & 0.108 & 10.26 & & & & & & & \\
\hline SRC-Li-2 & & & & & 0.315 & 0.361 & 0.363 & 0.130 & 10.61 & & & & & & & \\
\hline SRC-Li-3 & & & & & 0.617 & 0.668 & 0.639 & 0.243 & 11.30 & & & & & & & \\
\hline SRC-Mg-1 & & & & & 1.270 & 1.187 & 1.116 & 0.320 & 11.55 & & & & & & & \\
\hline SRC-Mg-2 & & & & & 4.042 & 3.419 & 3.306 & 0.651 & 11.98 & & & & & & & \\
\hline \multicolumn{17}{|l|}{ SRC-Mg-3 } \\
\hline SRC-Na-1 & & & & & 0.140 & 0.239 & 0.165 & 0.088 & 10.16 & & & & & & & \\
\hline SRC-Na-2 & & & & & 0.422 & 0.484 & 0.454 & 0.170 & 10.92 & & & & & & & \\
\hline SRC-Pb-1 & & & & & 0.542 & 0.618 & 0.571 & 0.200 & 11.24 & & & & & & & \\
\hline SRC-Pb-2 & & & & & 0.498 & 0.573 & 0.534 & 0.193 & 11.21 & & & & & & & \\
\hline SRC-Pb-3 & & & & & 0.465 & 0.568 & 0.537 & 0.191 & 11.20 & & & & & & & \\
\hline SRC-Si-1 & & & & & 0.935 & 0.990 & 0.940 & 0.285 & 11.55 & & & & & & & \\
\hline SRC-Si-2 & & & & & 0.275 & 0.359 & 0.332 & 0.151 & 10.45 & & & & & & & \\
\hline SRC-Sm-1 & & & & & 0.792 & 0.826 & 0.795 & 0.290 & 11.38 & & & & & & & \\
\hline SRC-Sn-1 & & & & & 0.541 & 0.615 & 0.568 & 0.202 & 11.17 & & & & & & & \\
\hline SRC-Sn-2 & & & & & 0.440 & 0.513 & 0.474 & 0.180 & 11.09 & & & & & & & \\
\hline \multicolumn{17}{|l|}{ SRC-Sn-3 } \\
\hline SRC-Ti-1 & & & & & 0.550 & 0.616 & 0.576 & 0.199 & 11.27 & & & & & & & \\
\hline SRC-Ti-2 & & & & & 0.483 & 0.538 & 0.493 & 0.188 & 11.11 & & & & & & & \\
\hline SRC-Zr-1 & & & & & 0.582 & 0.670 & 0.631 & 0.235 & 11.24 & & & & & & & \\
\hline SRC-Zr-2 & & & & & 0.462 & 0.538 & 0.498 & 0.168 & 11.23 & & & & & & & \\
\hline
\end{tabular}


Appendix A. Database - mass fraction

Plutonium Vitrification (Bulkley and Vienna 1997)

\begin{tabular}{|c|c|c|c|c|c|c|c|c|c|c|c|c|}
\hline Glass ID & $\begin{array}{l}\text { Q PCT Na at } \\
20^{\circ} \mathrm{C}\left(\mathrm{g} / \mathrm{m}^{2}\right)\end{array}$ & $\begin{array}{l}\text { Q PCT Si at } \\
20^{\circ} \mathrm{C}\left(\mathrm{g} / \mathrm{m}^{2}\right)\end{array}$ & $\begin{array}{c}\mathrm{QpH} \\
\text { at } 20^{\circ} \mathrm{C}\end{array}$ & $\begin{array}{c}\text { TCLP Ag } \\
(\mathrm{ppm})\end{array}$ & $\begin{array}{c}\text { TCLP As } \\
\text { (ppm) }\end{array}$ & $\begin{array}{l}\text { TCLP Ba } \\
\text { (ppm) }\end{array}$ & $\begin{array}{c}\text { TCLP Cd } \\
\text { (ppm) }\end{array}$ & $\begin{array}{l}\text { TCLP Cr } \\
\text { (ppm) }\end{array}$ & $\begin{array}{l}\text { TCLP Ni } \\
(\mathrm{ppm})\end{array}$ & $\begin{array}{c}\text { TCLP Pb } \\
\text { (ppm) }\end{array}$ & $\begin{array}{l}\text { TCLP Se } \\
(\mathrm{ppm})\end{array}$ & $\begin{array}{c}\text { TCLP Zn } \\
(\mathrm{ppm})\end{array}$ \\
\hline \multicolumn{13}{|l|}{$\mathrm{PBG} 3 / \mathrm{Ce}$} \\
\hline \multicolumn{13}{|l|}{$\mathrm{PBG} 3 / 0 \mathrm{x}$} \\
\hline \multicolumn{13}{|l|}{ PBG3-.5C } \\
\hline \multicolumn{13}{|l|}{ SRC-Al-1 } \\
\hline \multicolumn{13}{|l|}{ SRC-Al-2 } \\
\hline \multicolumn{13}{|l|}{ SRC-B-1 } \\
\hline \multicolumn{13}{|l|}{ SRC-B-2 } \\
\hline \multicolumn{13}{|l|}{ SRC-B-3 } \\
\hline \multicolumn{13}{|l|}{ SRC-Ca-1 } \\
\hline \multicolumn{13}{|l|}{ SRC-Ca-2 } \\
\hline \multicolumn{13}{|l|}{ SRC-Ca-3 } \\
\hline \multicolumn{13}{|l|}{ SRC-Ce-1 } \\
\hline \multicolumn{13}{|l|}{ SRC-Ce-2 } \\
\hline \multicolumn{13}{|l|}{ SRC-Ce-3 } \\
\hline \multicolumn{13}{|l|}{ SRC-Ce-4 } \\
\hline \multicolumn{13}{|l|}{ SRC-Eu-1 } \\
\hline \multicolumn{13}{|l|}{ SRC-Gd-1 } \\
\hline \multicolumn{13}{|l|}{ SRC-Gd-2 } \\
\hline \multicolumn{13}{|l|}{ SRC-Gd-3 } \\
\hline \multicolumn{13}{|l|}{ SRC-Gd-4 } \\
\hline SRC-K-1 & & & & & & & & & & & & \\
\hline SRC-K-2 & & & & & & & & & & & & \\
\hline SRC-K-3 & & & & & & & & & & & & \\
\hline SRC-K-3i & & & & & & & & & & & & \\
\hline SRC-Li-1 & & & & & & & & & & & & \\
\hline SRC-Li-2 & & & & & & & & & & & & \\
\hline SRC-Li-3 & & & & & & & & & & & & \\
\hline SRC-Mg-1 & & & & & & & & & & & & \\
\hline SRC-Mg-2 & & & & & & & & & & & & \\
\hline SRC-Mg-3 & & & & & & & & & & & & \\
\hline SRC-Na-1 & & & & & & & & & & & & \\
\hline SRC-Na-2 & & & & & & & & & & & & \\
\hline SRC-Pb-1 & & & & & & & & & & & & \\
\hline SRC-Pb-2 & & & & & & & & & & & & \\
\hline SRC-Pb-3 & & & & & & & & & & & & \\
\hline SRC-Si-1 & & & & & & & & & & & & \\
\hline SRC-Si-2 & & & & & & & & & & & & \\
\hline SRC-Sm-1 & & & & & & & & & & & & \\
\hline SRC-Sn-1 & & & & & & & & & & & & \\
\hline SRC-Sn-2 & & & & & & & & & & & & \\
\hline SRC-Sn-3 & & & & & & & & & & & & \\
\hline SRC-Ti-1 & & & & & & & & & & & & \\
\hline SRC-Ti-2 & & & & & & & & & & & & \\
\hline SRC-Zr-1 & & & & & & & & & & & & \\
\hline SRC-Zr-2 & & & & & & & & & & & & \\
\hline
\end{tabular}


Appendix A. Database - mass fraction

Comp. Vs Properties Study (Chick et al. 1981)

\begin{tabular}{|c|c|c|c|c|c|c|c|c|c|c|c|c|c|c|c|c|c|c|c|c|c|}
\hline Glass ID & $\begin{array}{c}\mathrm{A} 12 \mathrm{O} 3 \\
-\mathrm{t}\end{array}$ & $\begin{array}{c}\mathrm{B} 2 \mathrm{O} 3 \\
-\mathrm{t}\end{array}$ & $\begin{array}{c}\mathrm{CaO} \\
-\mathrm{t}\end{array}$ & $\begin{array}{c}\mathrm{Fe} 2 \mathrm{O} 3 \\
-\mathrm{t}\end{array}$ & $\begin{array}{c}\mathrm{FeO} \\
-\mathrm{t}\end{array}$ & $\begin{array}{c}\mathrm{K} 2 \mathrm{O} \\
-\mathrm{t}\end{array}$ & $\begin{array}{c}\mathrm{Li} 2 \mathrm{O} \\
-\mathrm{t}\end{array}$ & $\begin{array}{c}\mathrm{MgO} \\
-\mathrm{t}\end{array}$ & $\begin{array}{c}\mathrm{Na} 2 \mathrm{O} \\
-\mathrm{t}\end{array}$ & $\begin{array}{c}\mathrm{NiO} \\
-\mathrm{t}\end{array}$ & $\begin{array}{c}\mathrm{P} 2 \mathrm{O} 5 \\
-\mathrm{t}\end{array}$ & $\begin{array}{c}\mathrm{SiO} 2 \\
-\mathrm{t}\end{array}$ & $\begin{array}{c}\mathrm{ZrO} 2 \\
-\mathrm{t}\end{array}$ & $\begin{array}{c}\mathrm{Ag} 2 \mathrm{O} \\
-\mathrm{t}\end{array}$ & $\begin{array}{c}\mathrm{As} 2 \mathrm{O} 3 \\
-\mathrm{t}\end{array}$ & $\begin{array}{c}\mathrm{BaO} \\
-\mathrm{t}\end{array}$ & $\begin{array}{c}\mathrm{Bi} 2 \mathrm{O} 3 \\
-\mathrm{t}\end{array}$ & $\begin{array}{l}\mathrm{Br} \\
-\mathrm{t}\end{array}$ & $\begin{array}{c}\mathrm{CdO} \\
-\mathrm{t}\end{array}$ & $\mathrm{Ce} 2 \mathrm{O} 3$ & $\begin{array}{c}\mathrm{CeO} 2 \\
-\mathrm{t}\end{array}$ \\
\hline 1 & & & & & & & & & & & & & & & & & & & & & \\
\hline 2 & & & & & & & & & & & & & & & & & & & & & \\
\hline 3 & & & & & & & & & & & & & & & & & & & & & \\
\hline 4 & & & & & & & & & & & & & & & & & & & & & \\
\hline 5 & & & & & & & & & & & & & & & & & & & & & \\
\hline 6 & & & & & & & & & & & & & & & & & & & & & \\
\hline 7 & & & & & & & & & & & & & & & & & & & & & \\
\hline 8 & & & & & & & & & & & & & & & & & & & & & \\
\hline 9 & & & & & & & & & & & & & & & & & & & & & \\
\hline 10 & & & & & & & & & & & & & & & & & & & & & \\
\hline 11 & & & & & & & & & & & & & & & & & & & & & \\
\hline 12 & & & & & & & & & & & & & & & & & & & & & \\
\hline 13 & & & & & & & & & & & & & & & & & & & & & \\
\hline 14 & & & & & & & & & & & & & & & & & & & & & \\
\hline 15 & & & & & & & & & & & & & & & & & & & & & \\
\hline 16 & & & & & & & & & & & & & & & & & & & & & \\
\hline 17 & & & & & & & & & & & & & & & & & & & & & \\
\hline 18 & & & & & & & & & & & & & & & & & & & & & \\
\hline 19 & & & & & & & & & & & & & & & & & & & & & \\
\hline 20 & & & & & & & & & & & & & & & & & & & & & \\
\hline 21 & & & & & & & & & & & & & & & & & & & & & \\
\hline 22 & & & & & & & & & & & & & & & & & & & & & \\
\hline 23 & & & & & & & & & & & & & & & & & & & & & \\
\hline 24 & & & & & & & & & & & & & & & & & & & & & \\
\hline 25 & & & & & & & & & & & & & & & & & & & & & \\
\hline 26 & & & & & & & & & & & & & & & & & & & & & \\
\hline 27 & & & & & & & & & & & & & & & & & & & & & \\
\hline 28 & & & & & & & & & & & & & & & & & & & & & \\
\hline 29 & & & & & & & & & & & & & & & & & & & & & \\
\hline 30 & & & & & & & & & & & & & & & & & & & & & \\
\hline 31 & & & & & & & & & & & & & & & & & & & & & \\
\hline 32 & & & & & & & & & & & & & & & & & & & & & \\
\hline 33 & & & & & & & & & & & & & & & & & & & & & \\
\hline 34 & & & & & & & & & & & & & & & & & & & & & \\
\hline 35 & & & & & & & & & & & & & & & & & & & & & \\
\hline 36 & & & & & & & & & & & & & & & & & & & & & \\
\hline 37 & & & & & & & & & & & & & & & & & & & & & \\
\hline 38 & & & & & & & & & & & & & & & & & & & & & \\
\hline 39 & & & & & & & & & & & & & & & & & & & & & \\
\hline 40 & & & & & & & & & & & & & & & & & & & & & \\
\hline 41 & & & & & & & & & & & & & & & & & & & & & \\
\hline
\end{tabular}


Comp. Vs Properties Study (Chick et al. 1981)

\begin{tabular}{|c|c|c|c|c|c|c|c|c|c|c|c|c|c|c|c|c|c|c|c|c|c|}
\hline Glass ID & $\begin{array}{l}\mathrm{Cl} \\
-\mathrm{t}\end{array}$ & $\begin{array}{c}\mathrm{CoO} \\
-\mathrm{t}\end{array}$ & $\begin{array}{c}\mathrm{Co} 2 \mathrm{O} 3 \\
-\mathrm{t}\end{array}$ & $\begin{array}{c}\mathrm{Cr} 2 \mathrm{O} 3 \\
-\mathrm{t}\end{array}$ & $\begin{array}{c}\mathrm{Cs} 2 \mathrm{O} \\
-\mathrm{t}\end{array}$ & $\begin{array}{c}\mathrm{CuO} \\
-\mathrm{t}\end{array}$ & $\begin{array}{c}\mathrm{Eu} 2 \mathrm{O} 3 \\
-\mathrm{t}\end{array}$ & $\begin{array}{l}F \\
-t\end{array}$ & $\begin{array}{c}\mathrm{Ga} 2 \mathrm{O} 3 \\
-\mathrm{t}\end{array}$ & $\begin{array}{c}\mathrm{Gd} 2 \mathrm{O} 3 \\
-\mathrm{t}\end{array}$ & $\begin{array}{c}\mathrm{HgO} \\
-\mathrm{t}\end{array}$ & $\begin{array}{l}I \\
-t\end{array}$ & $\begin{array}{c}\mathrm{La} 2 \mathrm{O} 3 \\
-\mathrm{t}\end{array}$ & $\begin{array}{c}\mathrm{MnO} 2 \\
-\mathrm{t}\end{array}$ & $\begin{array}{c}\mathrm{MnO} \\
-\mathrm{t}\end{array}$ & $\begin{array}{c}\mathrm{MoO} \\
-\mathrm{t}\end{array}$ & $\begin{array}{c}\mathrm{MoO} 3 \\
-\mathrm{t}\end{array}$ & $\begin{array}{c}\mathrm{Nb} 2 \mathrm{O} 5 \\
-\mathrm{t}\end{array}$ & $\begin{array}{c}\mathrm{Nd} 2 \mathrm{O} 3 \\
-\mathrm{t}\end{array}$ & $\begin{array}{c}\mathrm{PbO} \\
-\mathrm{t}\end{array}$ & $\begin{array}{c}\mathrm{PdO} 2 \\
-\mathrm{t}\end{array}$ \\
\hline 1 & & & & & & & & & & & & & & & & & & & & & \\
\hline 2 & & & & & & & & & & & & & & & & & & & & & \\
\hline 3 & & & & & & & & & & & & & & & & & & & & & \\
\hline 4 & & & & & & & & & & & & & & & & & & & & & \\
\hline 5 & & & & & & & & & & & & & & & & & & & & & \\
\hline 6 & & & & & & & & & & & & & & & & & & & & & \\
\hline 7 & & & & & & & & & & & & & & & & & & & & & \\
\hline 8 & & & & & & & & & & & & & & & & & & & & & \\
\hline 9 & & & & & & & & & & & & & & & & & & & & & \\
\hline 10 & & & & & & & & & & & & & & & & & & & & & \\
\hline 11 & & & & & & & & & & & & & & & & & & & & & \\
\hline 12 & & & & & & & & & & & & & & & & & & & & & \\
\hline 13 & & & & & & & & & & & & & & & & & & & & & \\
\hline 14 & & & & & & & & & & & & & & & & & & & & & \\
\hline 15 & & & & & & & & & & & & & & & & & & & & & \\
\hline 16 & & & & & & & & & & & & & & & & & & & & & \\
\hline 17 & & & & & & & & & & & & & & & & & & & & & \\
\hline 18 & & & & & & & & & & & & & & & & & & & & & \\
\hline 19 & & & & & & & & & & & & & & & & & & & & & \\
\hline 20 & & & & & & & & & & & & & & & & & & & & & \\
\hline 21 & & & & & & & & & & & & & & & & & & & & & \\
\hline 22 & & & & & & & & & & & & & & & & & & & & & \\
\hline 23 & & & & & & & & & & & & & & & & & & & & & \\
\hline 24 & & & & & & & & & & & & & & & & & & & & & \\
\hline 25 & & & & & & & & & & & & & & & & & & & & & \\
\hline 26 & & & & & & & & & & & & & & & & & & & & & \\
\hline 27 & & & & & & & & & & & & & & & & & & & & & \\
\hline 28 & & & & & & & & & & & & & & & & & & & & & \\
\hline 29 & & & & & & & & & & & & & & & & & & & & & \\
\hline 30 & & & & & & & & & & & & & & & & & & & & & \\
\hline 31 & & & & & & & & & & & & & & & & & & & & & \\
\hline 32 & & & & & & & & & & & & & & & & & & & & & \\
\hline 33 & & & & & & & & & & & & & & & & & & & & & \\
\hline 34 & & & & & & & & & & & & & & & & & & & & & \\
\hline 35 & & & & & & & & & & & & & & & & & & & & & \\
\hline 36 & & & & & & & & & & & & & & & & & & & & & \\
\hline 37 & & & & & & & & & & & & & & & & & & & & & \\
\hline 38 & & & & & & & & & & & & & & & & & & & & & \\
\hline 39 & & & & & & & & & & & & & & & & & & & & & \\
\hline 40 & & & & & & & & & & & & & & & & & & & & & \\
\hline 41 & & & & & & & & & & & & & & & & & & & & & \\
\hline
\end{tabular}


Comp. Vs Properties Study (Chick et al. 1981)

\begin{tabular}{|c|c|c|c|c|c|c|c|c|c|c|c|c|c|c|c|c|c|c|c|c|c|}
\hline Glass ID & $\begin{array}{c}\mathrm{PdO} \\
-\mathrm{t}\end{array}$ & $\begin{array}{c}\mathrm{Pr} 2 \mathrm{O} 3 \\
-\mathrm{t}\end{array}$ & $\begin{array}{c}\text { Pr6O11 } \\
-t\end{array}$ & $\begin{array}{c}\mathrm{Rb} 2 \mathrm{O} \\
-\mathrm{t}\end{array}$ & $\begin{array}{c}\mathrm{ReO} \\
-\mathrm{t}\end{array}$ & $\begin{array}{c}\mathrm{ReO} 2 \\
-\mathrm{t}\end{array}$ & $\begin{array}{c}\mathrm{Rh} 2 \mathrm{O} 3 \\
-\mathrm{t}\end{array}$ & $\begin{array}{c}\mathrm{RhO} 2 \\
-\mathrm{t}\end{array}$ & $\begin{array}{c}\mathrm{RuO}^{2} \\
-\mathrm{t}\end{array}$ & $\begin{array}{c}\mathrm{Sb} 2 \mathrm{O} 3 \\
-\mathrm{t}\end{array}$ & $\begin{array}{c}\mathrm{Sb} 2 \mathrm{O} 5 \\
-\mathrm{t}\end{array}$ & $\begin{array}{c}\mathrm{SeO} 2 \\
-\mathrm{t}\end{array}$ & $\begin{array}{c}\mathrm{Sm} 2 \mathrm{O} 3 \\
-\mathrm{t}\end{array}$ & $\begin{array}{c}\mathrm{SnO} \\
-\mathrm{t}\end{array}$ & $\begin{array}{c}\mathrm{SnO} 2 \\
-\mathrm{t}\end{array}$ & $\begin{array}{c}\mathrm{SO} 3 \\
-\mathrm{t}\end{array}$ & $\begin{array}{c}\mathrm{SrO} \\
-\mathrm{t}\end{array}$ & $\begin{array}{c}\mathrm{Tc} 2 \mathrm{O} 7 \\
-\mathrm{t}\end{array}$ & $\begin{array}{c}\mathrm{TeO} 2 \\
-\mathrm{t}\end{array}$ & $\begin{array}{c}\mathrm{ThO} 2 \\
-\mathrm{t}\end{array}$ & $\begin{array}{c}\mathrm{TiO} 2 \\
-\mathrm{t}\end{array}$ \\
\hline 1 & & & & & & & & & & & & & & & & & & & & & \\
\hline 2 & & & & & & & & & & & & & & & & & & & & & \\
\hline 3 & & & & & & & & & & & & & & & & & & & & & \\
\hline 4 & & & & & & & & & & & & & & & & & & & & & \\
\hline 5 & & & & & & & & & & & & & & & & & & & & & \\
\hline 6 & & & & & & & & & & & & & & & & & & & & & \\
\hline 7 & & & & & & & & & & & & & & & & & & & & & \\
\hline 8 & & & & & & & & & & & & & & & & & & & & & \\
\hline 9 & & & & & & & & & & & & & & & & & & & & & \\
\hline 10 & & & & & & & & & & & & & & & & & & & & & \\
\hline 11 & & & & & & & & & & & & & & & & & & & & & \\
\hline 12 & & & & & & & & & & & & & & & & & & & & & \\
\hline 13 & & & & & & & & & & & & & & & & & & & & & \\
\hline 14 & & & & & & & & & & & & & & & & & & & & & \\
\hline 15 & & & & & & & & & & & & & & & & & & & & & \\
\hline 16 & & & & & & & & & & & & & & & & & & & & & \\
\hline 17 & & & & & & & & & & & & & & & & & & & & & \\
\hline 18 & & & & & & & & & & & & & & & & & & & & & \\
\hline 19 & & & & & & & & & & & & & & & & & & & & & \\
\hline 20 & & & & & & & & & & & & & & & & & & & & & \\
\hline 21 & & & & & & & & & & & & & & & & & & & & & \\
\hline 22 & & & & & & & & & & & & & & & & & & & & & \\
\hline 23 & & & & & & & & & & & & & & & & & & & & & \\
\hline 24 & & & & & & & & & & & & & & & & & & & & & \\
\hline 25 & & & & & & & & & & & & & & & & & & & & & \\
\hline 26 & & & & & & & & & & & & & & & & & & & & & \\
\hline 27 & & & & & & & & & & & & & & & & & & & & & \\
\hline 28 & & & & & & & & & & & & & & & & & & & & & \\
\hline 29 & & & & & & & & & & & & & & & & & & & & & \\
\hline 30 & & & & & & & & & & & & & & & & & & & & & \\
\hline 31 & & & & & & & & & & & & & & & & & & & & & \\
\hline 32 & & & & & & & & & & & & & & & & & & & & & \\
\hline 33 & & & & & & & & & & & & & & & & & & & & & \\
\hline 34 & & & & & & & & & & & & & & & & & & & & & \\
\hline 35 & & & & & & & & & & & & & & & & & & & & & \\
\hline 36 & & & & & & & & & & & & & & & & & & & & & \\
\hline 37 & & & & & & & & & & & & & & & & & & & & & \\
\hline 38 & & & & & & & & & & & & & & & & & & & & & \\
\hline 39 & & & & & & & & & & & & & & & & & & & & & \\
\hline 40 & & & & & & & & & & & & & & & & & & & & & \\
\hline 41 & & & & & & & & & & & & & & & & & & & & & \\
\hline
\end{tabular}


Comp. Vs Properties Study (Chick et al. 1981)

\begin{tabular}{|c|c|c|c|c|c|c|c|c|c|c|c|c|c|c|c|c|c|c|c|c|c|}
\hline Glass ID & $\begin{array}{c}\mathrm{T} 12 \mathrm{O} 3 \\
-\mathrm{t}\end{array}$ & $\begin{array}{c}\mathrm{U} 3 \mathrm{O} 8 \\
-\mathrm{t}\end{array}$ & $\begin{array}{c}\mathrm{UO} 2 \\
-\mathrm{t}\end{array}$ & $\begin{array}{c}\text { UO3 } \\
-t\end{array}$ & $\begin{array}{c}\mathrm{V} 2 \mathrm{O} 5 \\
-\mathrm{t}\end{array}$ & $\begin{array}{c}\text { WO3 } \\
-t\end{array}$ & $\begin{array}{c}\mathrm{Y} 2 \mathrm{O} 3 \\
-\mathrm{t}\end{array}$ & $\begin{array}{c}\mathrm{ZnO} \\
-\mathrm{t}\end{array}$ & $\begin{array}{c}\text { Others } \\
-t\end{array}$ & $\begin{array}{c}\text { Sum } \\
-t\end{array}$ & $\begin{array}{c}\mathrm{A} 12 \mathrm{O} 3 \\
-\mathrm{a}\end{array}$ & $\begin{array}{c}\mathrm{B} 2 \mathrm{O} 3 \\
-\mathrm{a}\end{array}$ & $\begin{array}{c}\mathrm{CaO} \\
-\mathrm{a} \\
\end{array}$ & $\begin{array}{c}\mathrm{Fe} 2 \mathrm{O} 3 \\
-\mathrm{a}\end{array}$ & $\begin{array}{c}\mathrm{FeO} \\
-\mathrm{a}\end{array}$ & $\begin{array}{c}\mathrm{K} 2 \mathrm{O} \\
-\mathrm{a}\end{array}$ & $\begin{array}{c}\mathrm{Li} 2 \mathrm{O} \\
-\mathrm{a}\end{array}$ & $\begin{array}{c}\mathrm{MgO} \\
-\mathrm{a}\end{array}$ & $\begin{array}{c}\mathrm{Na} 2 \mathrm{O} \\
-\mathrm{a}\end{array}$ & $\begin{array}{c}\mathrm{NiO} \\
-\mathrm{a}\end{array}$ & $\begin{array}{c}\mathrm{P} 2 \mathrm{O} 5 \\
-\mathrm{a}\end{array}$ \\
\hline 1 & & & & & & & & & & & 0.0177 & 0.1257 & 0.0267 & 0.0692 & & & & 0.0000 & 0.0931 & 0.0302 & \\
\hline 2 & & & & & & & & & & & 0.0000 & 0.1554 & 0.0000 & 0.0000 & & & & 0.0483 & 0.1303 & 0.0218 & \\
\hline 3 & & & & & & & & & & & 0.0000 & 0.0620 & 0.1144 & 0.0748 & & & & 0.0244 & 0.1414 & 0.0000 & \\
\hline 4 & & & & & & & & & & & 0.0000 & 0.0956 & 0.0858 & 0.0000 & & & & 0.0491 & 0.1393 & 0.0000 & \\
\hline 5 & & & & & & & & & & & 0.0000 & 0.0912 & 0.0830 & 0.0000 & & & & 0.0491 & 0.1385 & 0.0000 & \\
\hline 6 & & & & & & & & & & & 0.0000 & 0.0894 & 0.0825 & 0.0000 & & & & 0.0480 & 0.1439 & 0.0000 & \\
\hline 7 & & & & & & & & & & & 0.2094 & 0.0610 & 0.0314 & 0.0000 & & & & 0.0440 & 0.0944 & 0.0290 & \\
\hline 8 & & & & & & & & & & & 0.2072 & 0.1171 & 0.0000 & 0.0448 & & & & 0.0000 & 0.1155 & 0.0283 & \\
\hline 9 & & & & & & & & & & & 0.1930 & 0.1255 & 0.0000 & 0.0000 & & & & 0.0067 & 0.1424 & 0.0000 & \\
\hline 10 & & & & & & & & & & & 0.0000 & 0.0734 & 0.1227 & 0.0000 & & & & 0.0131 & 0.1035 & 0.0327 & \\
\hline 11 & & & & & & & & & & & 0.0000 & 0.1161 & 0.1154 & 0.0743 & & & & 0.0478 & 0.1015 & 0.0000 & \\
\hline 12 & & & & & & & & & & & 0.2130 & 0.0920 & 0.1020 & 0.0703 & & & & 0.0000 & 0.1383 & 0.0276 & \\
\hline 13 & & & & & & & & & & & 0.0774 & 0.1409 & 0.0000 & 0.0741 & & & & 0.0181 & 0.1429 & 0.0000 & \\
\hline 14 & & & & & & & & & & & 0.2184 & 0.0690 & 0.0000 & 0.0000 & & & & 0.0000 & 0.0984 & 0.0000 & \\
\hline 15 & & & & & & & & & & & 0.0213 & 0.0697 & 0.0000 & 0.0716 & & & & 0.0458 & 0.1296 & 0.0000 & \\
\hline 16 & & & & & & & & & & & 0.0710 & 0.1277 & 0.1076 & 0.0704 & & & & 0.0000 & 0.1128 & 0.0000 & \\
\hline 17 & & & & & & & & & & & 0.2143 & 0.0579 & 0.0254 & 0.0000 & & & & 0.0000 & 0.1330 & 0.0000 & \\
\hline 18 & & & & & & & & & & & 0.1071 & 0.0968 & 0.0547 & 0.0354 & & & & 0.0221 & 0.1146 & 0.0155 & \\
\hline 19 & & & & & & & & & & & 0.1085 & 0.0978 & 0.0531 & 0.0354 & & & & 0.0226 & 0.1155 & 0.0144 & \\
\hline 20 & & & & & & & & & & & 0.1088 & 0.0898 & 0.0532 & 0.0331 & & & & 0.0227 & 0.1185 & 0.0144 & \\
\hline 21 & & & & & & & & & & & 0.2062 & 0.1219 & 0.0000 & 0.0682 & & & & 0.0419 & 0.0944 & 0.0000 & \\
\hline 22 & & & & & & & & & & & 0.0000 & 0.0664 & 0.1121 & 0.0726 & & & & 0.0000 & 0.1005 & 0.0000 & \\
\hline 23 & & & & & & & & & & & 0.2042 & 0.1170 & 0.0754 & 0.0000 & & & & 0.0000 & 0.0955 & 0.0000 & \\
\hline 24 & & & & & & & & & & & 0.0000 & 0.1425 & 0.0924 & 0.0000 & & & & 0.0503 & 0.1630 & 0.0348 & \\
\hline 25 & & & & & & & & & & & 0.2017 & 0.0596 & 0.0000 & 0.0650 & & & & 0.0407 & 0.0991 & 0.0252 & \\
\hline 26 & & & & & & & & & & & 0.1988 & 0.0602 & 0.0000 & 0.0636 & & & & 0.0000 & 0.1404 & 0.0256 & \\
\hline 27 & & & & & & & & & & & 0.0000 & 0.0679 & 0.1198 & 0.0000 & & & & 0.0000 & 0.1478 & 0.0000 & \\
\hline 28 & & & & & & & & & & & 0.0000 & 0.1246 & 0.0000 & 0.0000 & & & & 0.0000 & 0.1414 & 0.0000 & \\
\hline 29 & & & & & & & & & & & 0.0187 & 0.0650 & 0.0000 & 0.0685 & & & & 0.0488 & 0.1025 & 0.0000 & \\
\hline 30 & & & & & & & & & & & 0.0000 & 0.1406 & 0.0881 & 0.0230 & & & & 0.0510 & 0.1570 & 0.0335 & \\
\hline 31 & & & & & & & & & & & 0.0000 & 0.0657 & 0.1157 & 0.0729 & & & & 0.0000 & 0.1452 & 0.0319 & \\
\hline 32 & & & & & & & & & & & 0.2190 & 0.0619 & 0.1154 & 0.0000 & & & & 0.0078 & 0.1395 & 0.0000 & \\
\hline 33 & & & & & & & & & & & 0.2152 & 0.1265 & 0.1134 & 0.0000 & & & & 0.0000 & 0.0954 & 0.0000 & \\
\hline 34 & & & & & & & & & & & 0.0000 & 0.1291 & 0.0858 & 0.0642 & & & & 0.0498 & 0.0967 & 0.0000 & \\
\hline 35 & & & & & & & & & & & 0.2124 & 0.1117 & 0.0095 & 0.0562 & & & & 0.0437 & 0.1308 & 0.0000 & \\
\hline 36 & & & & & & & & & & & 0.2065 & 0.1177 & 0.0078 & 0.0647 & & & & 0.0439 & 0.1394 & 0.0000 & \\
\hline 37 & & & & & & & & & & & 0.2240 & 0.0628 & 0.0000 & 0.0000 & & & & 0.0461 & 0.1035 & 0.0303 & \\
\hline 38 & & & & & & & & & & & 0.2063 & 0.0552 & 0.0974 & 0.0546 & & & & 0.0000 & 0.0924 & 0.0000 & \\
\hline 39 & & & & & & & & & & & 0.0000 & 0.1331 & 0.1186 & 0.0626 & & & & 0.0000 & 0.1068 & 0.0328 & \\
\hline 40 & & & & & & & & & & & 0.0000 & 0.1335 & 0.1172 & 0.0697 & & & & 0.0000 & 0.1082 & 0.0326 & \\
\hline 41 & & & & & & & & & & & 0.0000 & 0.0727 & 0.1189 & 0.0000 & & & & 0.0510 & 0.1029 & 0.0331 & \\
\hline
\end{tabular}


Appendix A. Database - mass fraction

Comp. Vs Properties Study (Chick et al. 1981)

\begin{tabular}{|c|c|c|c|c|c|c|c|c|c|c|c|c|c|c|c|c|c|c|c|c|c|}
\hline Glass ID & $\begin{array}{c}\mathrm{SiO} 2 \\
-\mathrm{a}\end{array}$ & $\begin{array}{c}\mathrm{ZrO} 2 \\
-\mathrm{a}\end{array}$ & $\begin{array}{c}\mathrm{Ag} 2 \mathrm{O} \\
-\mathrm{a}\end{array}$ & $\begin{array}{c}\mathrm{As} 2 \mathrm{O} 3 \\
-\mathrm{a}\end{array}$ & $\begin{array}{c}\mathrm{BaO} \\
-\mathrm{a}\end{array}$ & $\begin{array}{c}\mathrm{Bi} 2 \mathrm{O} 3 \\
-\mathrm{a}\end{array}$ & $\begin{array}{l}\mathrm{Br} \\
-\mathrm{a}\end{array}$ & $\begin{array}{c}\mathrm{CdO} \\
-\mathrm{a}\end{array}$ & $\begin{array}{c}\mathrm{Ce} 2 \mathrm{O} 3 \\
-\mathrm{a}\end{array}$ & $\begin{array}{c}\mathrm{CeO} 2 \\
-\mathrm{a}\end{array}$ & $\begin{array}{l}\mathrm{Cl} \\
-\mathrm{a}\end{array}$ & $\begin{array}{c}\mathrm{CoO} \\
-\mathrm{a}\end{array}$ & $\begin{array}{c}\mathrm{Co} 2 \mathrm{O} 3 \\
-\mathrm{a}\end{array}$ & $\begin{array}{c}\mathrm{Cr} 2 \mathrm{O} 3 \\
-\mathrm{a} \\
\end{array}$ & $\begin{array}{c}\mathrm{Cs} 2 \mathrm{O} \\
-\mathrm{a}\end{array}$ & $\begin{array}{c}\mathrm{CuO} \\
-\mathrm{a}\end{array}$ & $\begin{array}{c}\mathrm{Eu} 2 \mathrm{O} 3 \\
-\mathrm{a}\end{array}$ & $\begin{array}{c}F \\
-a\end{array}$ & $\begin{array}{c}\mathrm{Ga} 2 \mathrm{O} 3 \\
-\mathrm{a}\end{array}$ & $\begin{array}{c}\mathrm{Gd} 2 \mathrm{O} 3 \\
-\mathrm{a}\end{array}$ & $\begin{array}{c}\mathrm{HgO} \\
-\mathrm{a}\end{array}$ \\
\hline 1 & 0.5018 & & & & & & & & & & & & & 0.0637 & & & & & & & \\
\hline 2 & 0.5550 & & & & & & & & & & & & & 0.0000 & & & & & & & \\
\hline 3 & 0.4738 & & & & & & & & & & & & & 0.0712 & & & & & & & \\
\hline 4 & 0.4097 & & & & & & & & & & & & & 0.0546 & & & & & & & \\
\hline 5 & 0.3982 & & & & & & & & & & & & & 0.0750 & & & & & & & \\
\hline 6 & 0.4045 & & & & & & & & & & & & & 0.0682 & & & & & & & \\
\hline 7 & 0.3952 & & & & & & & & & & & & & 0.0655 & & & & & & & \\
\hline 8 & 0.4076 & & & & & & & & & & & & & 0.0000 & & & & & & & \\
\hline 9 & 0.5325 & & & & & & & & & & & & & 0.0000 & & & & & & & \\
\hline 10 & 0.5728 & & & & & & & & & & & & & 0.0000 & & & & & & & \\
\hline 11 & 0.3952 & & & & & & & & & & & & & 0.0753 & & & & & & & \\
\hline 12 & 0.3569 & & & & & & & & & & & & & 0.0000 & & & & & & & \\
\hline 13 & 0.3995 & & & & & & & & & & & & & 0.0705 & & & & & & & \\
\hline 14 & 0.5245 & & & & & & & & & & & & & 0.0000 & & & & & & & \\
\hline 15 & 0.5161 & & & & & & & & & & & & & 0.0613 & & & & & & & \\
\hline 16 & 0.3707 & & & & & & & & & & & & & 0.0670 & & & & & & & \\
\hline 17 & 0.4726 & & & & & & & & & & & & & 0.0000 & & & & & & & \\
\hline 18 & 0.4426 & & & & & & & & & & & & & 0.0337 & & & & & & & \\
\hline 19 & 0.4415 & & & & & & & & & & & & & \begin{tabular}{|l|}
0.0337 \\
\end{tabular} & & & & & & & \\
\hline 20 & 0.4443 & & & & & & & & & & & & & 0.0315 & & & & & & & \\
\hline 21 & 0.3978 & & & & & & & & & & & & & 0.0000 & & & & & & & \\
\hline 22 & 0.4897 & & & & & & & & & & & & & 0.0000 & & & & & & & \\
\hline 23 & 0.3593 & & & & & & & & & & & & & 0.0681 & & & & & & & \\
\hline 24 & 0.5170 & & & & & & & & & & & & & 0.0000 & & & & & & & \\
\hline 25 & 0.4468 & & & & & & & & & & & & & 0.0618 & & & & & & & \\
\hline 26 & 0.3696 & & & & & & & & & & & & & 0.0000 & & & & & & & \\
\hline 27 & 0.5061 & & & & & & & & & & & & & 0.0706 & & & & & & & \\
\hline 28 & 0.5063 & & & & & & & & & & & & & 0.0703 & & & & & & & \\
\hline 29 & 0.5347 & & & & & & & & & & & & & 0.0000 & & & & & & & \\
\hline 30 & 0.4170 & & & & & & & & & & & & & 0.0000 & & & & & & & \\
\hline 31 & 0.4303 & & & & & & & & & & & & & 0.0627 & & & & & & & \\
\hline 32 & 0.3817 & & & & & & & & & & & & & 0.0000 & & & & & & & \\
\hline 33 & 0.3778 & & & & & & & & & & & & & 0.0000 & & & & & & & \\
\hline 34 & 0.4113 & & & & & & & & & & & & & 0.0000 & & & & & & & \\
\hline 35 & 0.3780 & & & & & & & & & & & & & 0.0578 & & & & & & & \\
\hline 36 & 0.3584 & & & & & & & & & & & & & 0.0616 & & & & & & & \\
\hline 37 & 0.4972 & & & & & & & & & & & & & 0.0000 & & & & & & & \\
\hline 38 & 0.3565 & & & & & & & & & & & & & 0.0623 & & & & & & & \\
\hline 39 & 0.5461 & & & & & & & & & & & & & 0.0000 & & & & & & & \\
\hline 40 & 0.5387 & & & & & & & & & & & & & 0.0000 & & & & & & & \\
\hline 41 & 0.4733 & & & & & & & & & & & & & 0.0721 & & & & & & & \\
\hline
\end{tabular}


Comp. Vs Properties Study (Chick et al. 1981)

\begin{tabular}{|c|c|c|c|c|c|c|c|c|c|c|c|c|c|c|c|c|c|c|c|c|c|}
\hline Glass ID & $\begin{array}{c}\mathrm{I} \\
-\mathrm{a}\end{array}$ & $\begin{array}{c}\mathrm{La} 2 \mathrm{O} 3 \\
-\mathrm{a}\end{array}$ & $\begin{array}{c}\mathrm{MnO} 2 \\
-\mathrm{a}\end{array}$ & $\begin{array}{c}\mathrm{MnO} \\
-\mathrm{a}\end{array}$ & $\begin{array}{c}\mathrm{MoO} \\
-\mathrm{a}\end{array}$ & $\begin{array}{c}\mathrm{MoO} 3 \\
-\mathrm{a}\end{array}$ & $\begin{array}{c}\mathrm{Nb} 2 \mathrm{O} 5 \\
-\mathrm{a}\end{array}$ & $\begin{array}{c}\mathrm{Nd} 2 \mathrm{O} 3 \\
-\mathrm{a}\end{array}$ & $\begin{array}{c}\mathrm{PbO} \\
-\mathrm{a}\end{array}$ & $\begin{array}{c}\mathrm{PdO} 2 \\
-\mathrm{a}\end{array}$ & $\begin{array}{c}\mathrm{PdO} \\
-\mathrm{a}\end{array}$ & $\begin{array}{c}\mathrm{Pr} 2 \mathrm{O} 3 \\
-\mathrm{a}\end{array}$ & $\begin{array}{c}\text { Pr6O11 } \\
-\mathrm{a}\end{array}$ & $\begin{array}{c}\mathrm{Rb} 2 \mathrm{O} \\
-\mathrm{a}\end{array}$ & $\begin{array}{c}\mathrm{ReO} \\
-\mathrm{a}\end{array}$ & $\begin{array}{c}\mathrm{ReO} 2 \\
-\mathrm{a}\end{array}$ & $\begin{array}{c}\mathrm{Rh} 2 \mathrm{O} 3 \\
-\mathrm{a}\end{array}$ & $\begin{array}{c}\mathrm{RhO} 2 \\
-\mathrm{a}\end{array}$ & $\begin{array}{c}\mathrm{RuO} 2 \\
-\mathrm{a}\end{array}$ & $\begin{array}{c}\mathrm{Sb} 2 \mathrm{O} 3 \\
-\mathrm{a}\end{array}$ & $\begin{array}{c}\mathrm{Sb} 2 \mathrm{O} 5 \\
-\mathrm{a}\end{array}$ \\
\hline 1 & & & & & & & & & & & & & & & & & & & & & \\
\hline 2 & & & & & & & & & & & & & & & & & & & & & \\
\hline 3 & & & & & & & & & & & & & & & & & & & & & \\
\hline 4 & & & & & & & & & & & & & & & & & & & & & \\
\hline 5 & & & & & & & & & & & & & & & & & & & & & \\
\hline 6 & & & & & & & & & & & & & & & & & & & & & \\
\hline 7 & & & & & & & & & & & & & & & & & & & & & \\
\hline 8 & & & & & & & & & & & & & & & & & & & & & \\
\hline 9 & & & & & & & & & & & & & & & & & & & & & \\
\hline 10 & & & & & & & & & & & & & & & & & & & & & \\
\hline 11 & & & & & & & & & & & & & & & & & & & & & \\
\hline 12 & & & & & & & & & & & & & & & & & & & & & \\
\hline 13 & & & & & & & & & & & & & & & & & & & & & \\
\hline 14 & & & & & & & & & & & & & & & & & & & & & \\
\hline 15 & & & & & & & & & & & & & & & & & & & & & \\
\hline 16 & & & & & & & & & & & & & & & & & & & & & \\
\hline 17 & & & & & & & & & & & & & & & & & & & & & \\
\hline 18 & & & & & & & & & & & & & & & & & & & & & \\
\hline 19 & & & & & & & & & & & & & & & & & & & & & \\
\hline 20 & & & & & & & & & & & & & & & & & & & & & \\
\hline 21 & & & & & & & & & & & & & & & & & & & & & \\
\hline 22 & & & & & & & & & & & & & & & & & & & & & \\
\hline 23 & & & & & & & & & & & & & & & & & & & & & \\
\hline 24 & & & & & & & & & & & & & & & & & & & & & \\
\hline 25 & & & & & & & & & & & & & & & & & & & & & \\
\hline 26 & & & & & & & & & & & & & & & & & & & & & \\
\hline 27 & & & & & & & & & & & & & & & & & & & & & \\
\hline 28 & & & & & & & & & & & & & & & & & & & & & \\
\hline 29 & & & & & & & & & & & & & & & & & & & & & \\
\hline 30 & & & & & & & & & & & & & & & & & & & & & \\
\hline 31 & & & & & & & & & & & & & & & & & & & & & \\
\hline 32 & & & & & & & & & & & & & & & & & & & & & \\
\hline 33 & & & & & & & & & & & & & & & & & & & & & \\
\hline 34 & & & & & & & & & & & & & & & & & & & & & \\
\hline 35 & & & & & & & & & & & & & & & & & & & & & \\
\hline 36 & & & & & & & & & & & & & & & & & & & & & \\
\hline 37 & & & & & & & & & & & & & & & & & & & & & \\
\hline 38 & & & & & & & & & & & & & & & & & & & & & \\
\hline 39 & & & & & & & & & & & & & & & & & & & & & \\
\hline 40 & & & & & & & & & & & & & & & & & & & & & \\
\hline 41 & & & & & & & & & & & & & & & & & & & & & \\
\hline
\end{tabular}


Appendix A. Database - mass fraction

Comp. Vs Properties Study (Chick et al. 1981)

\begin{tabular}{|c|c|c|c|c|c|c|c|c|c|c|c|c|c|c|c|c|c|c|c|c|}
\hline Glass ID & $\begin{array}{c}\mathrm{SeO} 2 \\
-\mathrm{a}\end{array}$ & $\begin{array}{c}\mathrm{Sm} 2 \mathrm{O} 3 \\
-\mathrm{a}\end{array}$ & $\begin{array}{c}\mathrm{SnO} \\
-\mathrm{a}\end{array}$ & $\begin{array}{c}\mathrm{SnO} 2 \\
-\mathrm{a}\end{array}$ & $\begin{array}{c}\mathrm{SO} 3 \\
-\mathrm{a}\end{array}$ & $\begin{array}{c}\mathrm{SrO} \\
-\mathrm{a}\end{array}$ & $\begin{array}{c}\mathrm{Tc} 2 \mathrm{O} 7 \\
-\mathrm{a}\end{array}$ & $\begin{array}{c}\mathrm{TeO} 2 \\
-\mathrm{a}\end{array}$ & $\begin{array}{c}\mathrm{ThO} 2 \\
-\mathrm{a}\end{array}$ & $\begin{array}{c}\mathrm{TiO} 2 \\
-\mathrm{a}\end{array}$ & $\begin{array}{c}\mathrm{T} 12 \mathrm{O} 3 \\
-\mathrm{a}\end{array}$ & $\begin{array}{c}\mathrm{U} 3 \mathrm{O} 8 \\
-\mathrm{a}\end{array}$ & $\begin{array}{c}\mathrm{UO} 2 \\
-\mathrm{a}\end{array}$ & $\begin{array}{c}\mathrm{UO3} \\
-\mathrm{a}\end{array}$ & $\begin{array}{c}\mathrm{V} 2 \mathrm{O} 5 \\
-\mathrm{a}\end{array}$ & $\begin{array}{c}\text { WO3 } \\
-\mathrm{a}\end{array}$ & $\begin{array}{c}\mathrm{Y} 2 \mathrm{O} 3 \\
-\mathrm{a}\end{array}$ & $\begin{array}{c}\mathrm{ZnO} \\
-\mathrm{a}\end{array}$ & $\begin{array}{c}\text { Others } \\
-\mathrm{a}\end{array}$ & $\begin{array}{c}\text { Sum } \\
-\mathrm{a}\end{array}$ \\
\hline 1 & & & & & & & & & & 0.0000 & & & & & & & & 0.0717 & & 1.0000 \\
\hline 2 & & & & & & & & & & 0.0892 & & & & & & & & 0.0000 & & 1.0000 \\
\hline 3 & & & & & & & & & & 0.0000 & & & & & & & & 0.0381 & & 1.0000 \\
\hline 4 & & & & & & & & & & 0.0885 & & & & & & & & 0.0775 & & 1.0000 \\
\hline 5 & & & & & & & & & & 0.0874 & & & & & & & & 0.0778 & & 1.0000 \\
\hline 6 & & & & & & & & & & 0.0866 & & & & & & & & 0.0768 & & 1.0000 \\
\hline 7 & & & & & & & & & & 0.0000 & & & & & & & & 0.0701 & & 1.0000 \\
\hline 8 & & & & & & & & & & 0.0795 & & & & & & & & 0.0000 & & 1.0000 \\
\hline 9 & & & & & & & & & & 0.0000 & & & & & & & & 0.0000 & & 1.0000 \\
\hline 10 & & & & & & & & & & 0.0000 & & & & & & & & 0.0818 & & 1.0000 \\
\hline 11 & & & & & & & & & & 0.0744 & & & & & & & & 0.0000 & & 1.0000 \\
\hline 12 & & & & & & & & & & 0.0000 & & & & & & & & 0.0000 & & 1.0000 \\
\hline 13 & & & & & & & & & & 0.0000 & & & & & & & & 0.0766 & & 1.0000 \\
\hline 14 & & & & & & & & & & 0.0897 & & & & & & & & 0.0000 & & 1.0000 \\
\hline 15 & & & & & & & & & & 0.0847 & & & & & & & & 0.0000 & & 1.0000 \\
\hline 16 & & & & & & & & & & 0.0000 & & & & & & & & 0.0729 & & 1.0000 \\
\hline 17 & & & & & & & & & & 0.0245 & & & & & & & & 0.0724 & & 1.0000 \\
\hline 18 & & & & & & & & & & 0.0414 & & & & & & & & 0.0361 & & 1.0000 \\
\hline 19 & & & & & & & & & & 0.0413 & & & & & & & & 0.0361 & & 1.0000 \\
\hline 20 & & & & & & & & & & 0.0403 & & & & & & & & 0.0434 & & 1.0000 \\
\hline 21 & & & & & & & & & & 0.0000 & & & & & & & & 0.0695 & & 1.0000 \\
\hline 22 & & & & & & & & & & 0.0835 & & & & & & & & 0.0752 & & 1.0000 \\
\hline 23 & & & & & & & & & & 0.0806 & & & & & & & & 0.0000 & & 1.0000 \\
\hline 24 & & & & & & & & & & 0.0000 & & & & & & & & 0.0000 & & 1.0000 \\
\hline 25 & & & & & & & & & & 0.0000 & & & & & & & & 0.0000 & & 1.0000 \\
\hline 26 & & & & & & & & & & 0.0757 & & & & & & & & 0.0659 & & 1.0000 \\
\hline 27 & & & & & & & & & & 0.0878 & & & & & & & & 0.0000 & & 1.0000 \\
\hline 28 & & & & & & & & & & 0.0834 & & & & & & & & 0.0740 & & 1.0000 \\
\hline 29 & & & & & & & & & & 0.0857 & & & & & & & & 0.0760 & & 1.0000 \\
\hline 30 & & & & & & & & & & 0.0897 & & & & & & & & 0.0000 & & 1.0000 \\
\hline 31 & & & & & & & & & & 0.0000 & & & & & & & & 0.0755 & & 1.0000 \\
\hline 32 & & & & & & & & & & 0.0000 & & & & & & & & 0.0747 & & 1.0000 \\
\hline 33 & & & & & & & & & & 0.0000 & & & & & & & & 0.0716 & & 1.0000 \\
\hline 34 & & & & & & & & & & 0.0864 & & & & & & & & 0.0767 & & 1.0000 \\
\hline 35 & & & & & & & & & & 0.0000 & & & & & & & & 0.0000 & & 1.0000 \\
\hline 36 & & & & & & & & & & 0.0000 & & & & & & & & 0.0000 & & 1.0000 \\
\hline 37 & & & & & & & & & & 0.0361 & & & & & & & & 0.0000 & & 1.0000 \\
\hline 38 & & & & & & & & & & 0.0754 & & & & & & & & 0.0000 & & 1.0000 \\
\hline 39 & & & & & & & & & & 0.0000 & & & & & & & & 0.0000 & & 1.0000 \\
\hline 40 & & & & & & & & & & 0.0000 & & & & & & & & 0.0000 & & 1.0000 \\
\hline 41 & & & & & & & & & & 0.0000 & & & & & & & & 0.0760 & & 1.0000 \\
\hline
\end{tabular}


Appendix A. Database - mass fraction

Comp. Vs Properties Study (Chick et al. 1981)

\begin{tabular}{|c|c|c|c|c|c|c|c|c|}
\hline Glass ID & $\begin{array}{l}\mathrm{TM} \\
\left({ }^{\circ} \mathrm{C}\right)\end{array}$ & $\begin{array}{l}\text { Gradient } \\
\text { TL }\left({ }^{\circ} \mathrm{C}\right)\end{array}$ & $\begin{array}{l}\text { Uniform } \\
\text { TL }\left({ }^{\circ} \mathrm{C}\right)\end{array}$ & Primary Phase & $\begin{array}{l}\text { Quenched } \\
\text { Visual/OM }\end{array}$ & $\begin{array}{c}\text { Quenched } \\
\text { SEM/EDS or TEM }\end{array}$ & $\begin{array}{l}\text { Quenched } \\
\text { XRD }\end{array}$ & $\begin{array}{c}\text { Quenched } \\
\text { Homogeneous? }\end{array}$ \\
\hline 1 & 1250 & & & & & & & \\
\hline 2 & 1250 & & & & & & & \\
\hline 3 & 1250 & & & & & & & \\
\hline 4 & 1250 & & & & & & & \\
\hline 5 & 1250 & & & & & & & \\
\hline 6 & 1250 & & & & & & & \\
\hline 7 & 1350 & & & & & & & \\
\hline 8 & 1350 & & & & & & & \\
\hline 9 & 1350 & & & & & & & \\
\hline 10 & 1250 & & & & & & & \\
\hline 11 & 1350 & & & & & & & \\
\hline 12 & 1250 & & & & & & & \\
\hline 13 & 1160 & & & & & & & \\
\hline 14 & 1450 & & & & & & & \\
\hline 15 & 1250 & & & & & & & \\
\hline 16 & 1250 & & & & & & & \\
\hline 17 & 1350 & & & & & & & \\
\hline 18 & 1160 & & & & & & & \\
\hline 19 & 1160 & & & & & & & \\
\hline 20 & 1160 & & & & & & & \\
\hline 21 & 1450 & & & & & & & \\
\hline 22 & 1150 & & & & & & & \\
\hline 23 & 1350 & & & & & & & \\
\hline 24 & 1150 & & & & & & & \\
\hline 25 & 1350 & & & & & & & \\
\hline 26 & 1350 & & & & & & $\begin{array}{l}24.5 \mathrm{wt} \% \\
\mathrm{NiFe} 2 \mathrm{O} 4 / \mathrm{Zn} 2 \mathrm{Ti} 3 \mathrm{O} 8\end{array}$ & \\
\hline 27 & 1250 & & & & & & $\begin{array}{l}3.5 \mathrm{wt} \% \\
\mathrm{Cr} 2 \mathrm{O} / \mathrm{CaTiSiO} 5\end{array}$ & \\
\hline 28 & 1250 & & & & & & $15 \mathrm{wt} \% \mathrm{ZnCr} 2 \mathrm{O} 4$ & \\
\hline 29 & 1250 & & & & & & amorphous & \\
\hline 30 & 1150 & & & & & & amorphous & \\
\hline 31 & 1150 & & & & & & $\begin{array}{l}10.5 \mathrm{wt} \%(\mathrm{Ni}, \\
\mathrm{Zn})(\mathrm{Fe}, \mathrm{Cr}) 2 \mathrm{O} 4\end{array}$ & \\
\hline 32 & 1400 & & & & & & amorphous & \\
\hline 33 & 1380 & & & & & & amorphous & \\
\hline 34 & 1150 & & & & & & amorphous & \\
\hline 35 & 1320 & & & & & & $\begin{array}{l}15.5 \mathrm{wt} \% \\
\mathrm{Mg}(\mathrm{Cr}, \mathrm{Fe}, \mathrm{Al}) 2 \mathrm{O} 4\end{array}$ & \\
\hline 36 & 1320 & & & & & & $\begin{array}{l}15.5 \mathrm{wt} \% \\
\mathrm{Mg}(\mathrm{Cr}, \mathrm{Fe}, \mathrm{Al}) 2 \mathrm{O} 4\end{array}$ & \\
\hline 37 & 1400 & & & & & & $4 \mathrm{wt} \%$ AlNi2Ti & \\
\hline 38 & 1450 & & & & & & $6.5 \mathrm{wt} \%(\mathrm{Fe}, \mathrm{Ti}) \mathrm{Cr} 2 \mathrm{O} 4$ & \\
\hline 39 & 1150 & & & & & & amorphous & \\
\hline 40 & 1150 & & & & & & amorphous & \\
\hline 41 & 1250 & & & & & & $\begin{array}{l}13.5 \mathrm{wt} \% \\
(\mathrm{Zn}, \mathrm{Ni}) \mathrm{Cr} 2 \mathrm{O} 4 / \mathrm{Cr} 2 \mathrm{O} 3\end{array}$ & \\
\hline
\end{tabular}


Appendix A. Database - mass fraction

Comp. Vs Properties Study (Chick et al. 1981)

\begin{tabular}{|c|c|c|c|c|c|}
\hline Glass ID & $\begin{array}{c}\mathrm{CCC} \\
\text { Visual/OM }\end{array}$ & $\begin{array}{c}\text { CCC } \\
\text { SEM/EDS or TEM }\end{array}$ & $\begin{array}{l}\text { CCC } \\
\text { XRD }\end{array}$ & $\begin{array}{c}\text { Heat Treated } \\
\text { Visual/OM }\end{array}$ & $\begin{array}{c}\text { Heat Treated } \\
\text { SEM/EDS or TEM }\end{array}$ \\
\hline 1 & & & & & \\
\hline 2 & & & & & \\
\hline 3 & & & & & \\
\hline 4 & & & & & \\
\hline 5 & & & & & \\
\hline 6 & & & & & \\
\hline 7 & & & & & \\
\hline 8 & & & & & \\
\hline 9 & & & & & \\
\hline 10 & & & & & \\
\hline 11 & & & & & \\
\hline 12 & & & & & \\
\hline 13 & & & & & \\
\hline 14 & & & & & \\
\hline 15 & & & & & \\
\hline 16 & & & & & \\
\hline 17 & & & & & \\
\hline 18 & & & & & \\
\hline 19 & & & & & \\
\hline 20 & & & & & \\
\hline 21 & & & & & \\
\hline 22 & & & & & \\
\hline 23 & & & & & \\
\hline 24 & & & & & \\
\hline 25 & & & & & \\
\hline 26 & & & $65 \mathrm{wt} \% \mathrm{NaAlSiO} 4 / \mathrm{NiFe} 2 \mathrm{O} 4$ & & \\
\hline 27 & & & $18 \mathrm{wt} \% \mathrm{CaTiSiO} 5 / \mathrm{Cr} 2 \mathrm{O} 3$ & & \\
\hline 28 & & & $21 \mathrm{wt} \% \mathrm{ZnCr} 2 \mathrm{O} 4 / \mathrm{TiO} 2$ & & \\
\hline 29 & & & $12.5 \mathrm{wt} \% \mathrm{MgTi} 2 \mathrm{O} 5$ & & \\
\hline 30 & & & $27 \mathrm{wt} \% \mathrm{CaTiSiO} / \mathrm{NiFe} 2 \mathrm{O} 4$ & & \\
\hline 31 & & & $18 \mathrm{wt} \%(\mathrm{Ni}, \mathrm{Zn})(\mathrm{Fe}, \mathrm{Cr}) \mathrm{CrO} 4$ & & \\
\hline 32 & & & $93.5 \mathrm{wt} \% \mathrm{NaAlSiO} 4 / \mathrm{ZnAl} 2 \mathrm{O} 4$ & & \\
\hline 33 & & & $11.5 \mathrm{wt} \% \mathrm{ZnAl} 2 \mathrm{O} 4$ & & \\
\hline 34 & & & $11.5 \mathrm{wt} \% \mathrm{MgTi} 2 \mathrm{O} 5$ & & \\
\hline 35 & & & $19 \mathrm{wt} \% \mathrm{Mg}(\mathrm{Cr}, \mathrm{Fe}, \mathrm{Al}) 2 \mathrm{O} 4$ & & \\
\hline 36 & & & $22 \mathrm{wt} \% \mathrm{Mg}(\mathrm{Cr}, \mathrm{Fe}, \mathrm{Al}) 2 \mathrm{O} 4$ & & \\
\hline 37 & & & $16.5 \mathrm{wt} \% \mathrm{MgAl} 2 \mathrm{O} 4 / \mathrm{MgTi} 2 \mathrm{O} 5$ & & \\
\hline 38 & & & $49.5 \mathrm{wt} \% \mathrm{NaAlSiO} 4 /(\mathrm{Fe}, \mathrm{Cr}) \mathrm{Cr} 2 \mathrm{O} 3$ & & \\
\hline 39 & & & $3 \mathrm{wt} \%$ NiFe $2 \mathrm{O} 4 /(\mathrm{Ca}, \mathrm{Na}) \mathrm{FeSi} 2 \mathrm{O} 6$ & & \\
\hline 40 & & & $4.5 \mathrm{wt} \% \mathrm{NiFe} 2 \mathrm{O} 4 /(\mathrm{Ca}, \mathrm{Na}) \mathrm{FeSi} 2 \mathrm{O} 6$ & & \\
\hline 41 & & & $\begin{array}{l}42 \mathrm{wt} \% \\
(\mathrm{Ca}, \mathrm{Na}) \mathrm{FeSi} 2 \mathrm{O} 6 /(\mathrm{Zn}, \mathrm{Ni}) \mathrm{Cr} 2 \mathrm{O} 4\end{array}$ & & \\
\hline
\end{tabular}


Appendix A. Database - mass fraction

\section{Comp. Vs Properties Study (Chick et al. 1981)}

\begin{tabular}{|c|c|c|c|c|c|c|c|c|c|c|c|c|c|c|c|}
\hline Glass ID & $\begin{array}{c}\text { Heat Treated } \\
\text { XRD }\end{array}$ & $\begin{array}{l}\text { Density } \\
\left(\mathrm{g} / \mathrm{cm}^{3}\right)\end{array}$ & $\begin{array}{c}\text { Fulc Visc } \\
\text { A }\end{array}$ & \begin{tabular}{|c|} 
Fulc Visc \\
B
\end{tabular} & \begin{tabular}{|c|} 
Fulc Visc \\
To \\
\end{tabular} & $\begin{array}{c}\mathrm{FV} 1150^{\circ} \mathrm{C} \\
(\mathrm{Pa} \cdot \mathrm{s})\end{array}$ & $\begin{array}{c}\text { Arrh Visc } \\
\text { A }\end{array}$ & $\begin{array}{c}\text { Arrh Visc } \\
\text { B }\end{array}$ & $1150^{\circ} \mathrm{C}$ & $\begin{array}{c}\mathrm{T}\left({ }^{\circ} \mathrm{C}\right) \text { at } \\
2 \mathrm{~Pa} \cdot \mathrm{s} \\
\end{array}$ & $\begin{array}{c}\mathrm{T}\left({ }^{\circ} \mathrm{C}\right) \text { at } \\
5 \mathrm{~Pa} \cdot \mathrm{s}\end{array}$ & $\begin{array}{l}\mathrm{T}\left({ }^{\circ} \mathrm{C}\right) \text { at } \\
10 \mathrm{~Pa} \cdot \mathrm{s}\end{array}$ & $\begin{array}{l}\mathrm{T} 1 \\
\left({ }^{\circ} \mathrm{C}\right)\end{array}$ & $\begin{array}{c}\mathrm{V} 1 \\
(\mathrm{~Pa} \cdot \mathrm{s})\end{array}$ & $\begin{array}{l}\mathrm{T} 2 \\
\left({ }^{\circ} \mathrm{C}\right)\end{array}$ \\
\hline 1 & & & & & & & & & & & & & 1250 & $>580$ & \\
\hline 2 & & & & & & & & & & & & & 1250 & 2.2 & \\
\hline 3 & & & & & & & & & & & & & 1250 & 2.4 & \\
\hline 4 & & & & & & & & & & & & & 1250 & 1.3 & \\
\hline 5 & & & & & & & & & & & & & 1250 & 1.8 & \\
\hline 6 & & & & & & & & & & & & & 1250 & 1.2 & \\
\hline 7 & & & & & & & & & & & & & 1250 & $>580$ & \\
\hline 8 & & & & & & & & & & & & & 1250 & 74 & \\
\hline 9 & & & & & & & & & & & & & 1250 & 97 & \\
\hline 10 & & & & & & & & & & & & & 1250 & 1.8 & \\
\hline 11 & & & & & & & & & & & & & 1250 & 6 & \\
\hline 12 & & & & & & & & & & & & & 1250 & 10.5 & \\
\hline 13 & & & & & & & & & & & & & 1250 & 35 & \\
\hline 14 & & & & & & & & & & & & & 1250 & $>580$ & \\
\hline 15 & & & & & & & & & & & & & 1250 & 47 & \\
\hline 16 & & & & & & & & & & & & & 1250 & 2.5 & \\
\hline 17 & & & & & & & & & & & & & 1250 & 82 & \\
\hline 18 & & & & & & & & & & & & & 1250 & 33 & \\
\hline 19 & & & & & & & & & & & & & 1250 & 30 & \\
\hline 20 & & & & & & & & & & & & & 1250 & 28 & \\
\hline 21 & & & & & & & & & & & & & 1250 & 96 & \\
\hline 22 & & & & & & & & & & & & & 1250 & 1.2 & \\
\hline 23 & & & & & & & & & & & & & 1250 & 64 & \\
\hline 24 & & & & & & & & & & & & & 1250 & 0.55 & \\
\hline 25 & & & & & & & & & & & & & 1250 & $>580$ & \\
\hline 26 & & & & & & & & & & & & & 1250 & $>580$ & \\
\hline 27 & & & & & & & & & & & & & 1250 & 4.4 & \\
\hline 28 & & & & & & & & & & & & & 1250 & 8.3 & \\
\hline 29 & & & & & & & & & & & & & 1250 & 7.7 & \\
\hline 30 & & & & & & & & & & & & & 1250 & 0.13 & \\
\hline 31 & & & & & & & & & & & & & 1250 & 6.6 & \\
\hline 32 & & & & & & & & & & & & & 1250 & 8.2 & \\
\hline 33 & & & & & & & & & & & & & 1250 & 8.4 & \\
\hline 34 & & & & & & & & & & & & & 1250 & 0.62 & \\
\hline 35 & & & & & & & & & & & & & 1250 & $>580$ & \\
\hline 36 & & & & & & & & & & & & & 1250 & $>580$ & \\
\hline 37 & & & & & & & & & & & & & 1250 & 140 & \\
\hline 38 & & & & & & & & & & & & & 1250 & 49.5 & \\
\hline 39 & & & & & & & & & & & & & 1250 & 3.4 & \\
\hline 40 & & & & & & & & & & & & & 1250 & 3.3 & \\
\hline 41 & & & & & & & & & & & & & 1250 & 6.8 & \\
\hline
\end{tabular}


Appendix A. Database - mass fraction

\begin{tabular}{|c|c|c|c|c|c|c|c|c|c|c|c|c|c|c|c|c|c|c|c|c|c|}
\hline Glass ID & $\begin{array}{c}\mathrm{V} 2 \\
(\mathrm{~Pa} \cdot \mathrm{s})\end{array}$ & $\begin{array}{c}\mathrm{T} 3 \\
\left({ }^{\circ} \mathrm{C}\right) \\
\end{array}$ & $\begin{array}{c}\mathrm{V} 3 \\
(\mathrm{~Pa} \cdot \mathrm{s})\end{array}$ & $\begin{array}{c}\mathrm{T} 4 \\
\left({ }^{\circ} \mathrm{C}\right) \\
\end{array}$ & $\begin{array}{c}\mathrm{V} 4 \\
(\mathrm{~Pa} \cdot \mathrm{s})\end{array}$ & $\begin{array}{c}\mathrm{T} 5 \\
\left({ }^{\circ} \mathrm{C}\right) \\
\end{array}$ & $\begin{array}{c}\mathrm{V} 5 \\
(\mathrm{~Pa} \cdot \mathrm{s}) \\
\end{array}$ & $\begin{array}{l}\mathrm{T} 6 \\
\left({ }^{\circ} \mathrm{C}\right) \\
\end{array}$ & $\begin{array}{c}\mathrm{V} 6 \\
(\mathrm{~Pa} \cdot \mathrm{s})\end{array}$ & $\begin{array}{c}\mathrm{T} 7 \\
\left({ }^{\circ} \mathrm{C}\right) \\
\end{array}$ & $\begin{array}{c}\mathrm{V} 7 \\
(\mathrm{~Pa} \cdot \mathrm{s}) \\
\end{array}$ & $\begin{array}{c}\mathrm{T} 8 \\
\left({ }^{\circ} \mathrm{C}\right) \\
\end{array}$ & $\begin{array}{c}\mathrm{V} 8 \\
(\mathrm{~Pa} \cdot \mathrm{s})\end{array}$ & $\begin{array}{c}\mathrm{T} 9 \\
\left({ }^{\circ} \mathrm{C}\right) \\
\end{array}$ & $\begin{array}{c}\mathrm{V} 9 \\
(\mathrm{~Pa} \cdot \mathrm{s})\end{array}$ & $\begin{array}{l}\mathrm{T} 10 \\
\left({ }^{\circ} \mathrm{C}\right) \\
\end{array}$ & $\begin{array}{c}\mathrm{V} 10 \\
(\mathrm{~Pa} \cdot \mathrm{s})\end{array}$ & $\begin{array}{l}\mathrm{T} 11 \\
\left({ }^{\circ} \mathrm{C}\right) \\
\end{array}$ & $\begin{array}{c}\mathrm{V} 11 \\
(\mathrm{~Pa} \cdot \mathrm{s})\end{array}$ & $\begin{array}{l}\mathrm{T} 12 \\
\left({ }^{\circ} \mathrm{C}\right) \\
\end{array}$ & $\begin{array}{c}\mathrm{V} 12 \\
(\mathrm{~Pa} \cdot \mathrm{s})\end{array}$ \\
\hline 1 & & & & & & & & & & & & & & & & & & & & & \\
\hline 2 & & & & & & & & & & & & & & & & & & & & & \\
\hline 3 & & & & & & & & & & & & & & & & & & & & & \\
\hline 4 & & & & & & & & & & & & & & & & & & & & & \\
\hline 5 & & & & & & & & & & & & & & & & & & & & & \\
\hline 6 & & & & & & & & & & & & & & & & & & & & & \\
\hline 7 & & & & & & & & & & & & & & & & & & & & & \\
\hline 8 & & & & & & & & & & & & & & & & & & & & & \\
\hline 9 & & & & & & & & & & & & & & & & & & & & & \\
\hline 10 & & & & & & & & & & & & & & & & & & & & & \\
\hline 11 & & & & & & & & & & & & & & & & & & & & & \\
\hline 12 & & & & & & & & & & & & & & & & & & & & & \\
\hline 13 & & & & & & & & & & & & & & & & & & & & & \\
\hline 14 & & & & & & & & & & & & & & & & & & & & & \\
\hline 15 & & & & & & & & & & & & & & & & & & & & & \\
\hline 16 & & & & & & & & & & & & & & & & & & & & & \\
\hline 17 & & & & & & & & & & & & & & & & & & & & & \\
\hline 18 & & & & & & & & & & & & & & & & & & & & & \\
\hline 19 & & & & & & & & & & & & & & & & & & & & & \\
\hline 20 & & & & & & & & & & & & & & & & & & & & & \\
\hline 21 & & & & & & & & & & & & & & & & & & & & & \\
\hline 22 & & & & & & & & & & & & & & & & & & & & & \\
\hline 23 & & & & & & & & & & & & & & & & & & & & & \\
\hline 24 & & & & & & & & & & & & & & & & & & & & & \\
\hline 25 & & & & & & & & & & & & & & & & & & & & & \\
\hline 26 & & & & & & & & & & & & & & & & & & & & & \\
\hline 27 & & & & & & & & & & & & & & & & & & & & & \\
\hline 28 & & & & & & & & & & & & & & & & & & & & & \\
\hline 29 & & & & & & & & & & & & & & & & & & & & & \\
\hline 30 & & & & & & & & & & & & & & & & & & & & & \\
\hline 31 & & & & & & & & & & & & & & & & & & & & & \\
\hline 32 & & & & & & & & & & & & & & & & & & & & & \\
\hline 33 & & & & & & & & & & & & & & & & & & & & & \\
\hline 34 & & & & & & & & & & & & & & & & & & & & & \\
\hline 35 & & & & & & & & & & & & & & & & & & & & & \\
\hline 36 & & & & & & & & & & & & & & & & & & & & & \\
\hline 37 & & & & & & & & & & & & & & & & & & & & & \\
\hline 38 & & & & & & & & & & & & & & & & & & & & & \\
\hline 39 & & & & & & & & & & & & & & & & & & & & & \\
\hline 40 & & & & & & & & & & & & & & & & & & & & & \\
\hline 41 & & & & & & & & & & & & & & & & & & & & & \\
\hline
\end{tabular}


Appendix A. Database - mass fraction

Comp. Vs Properties Study (Chick et al. 1981)

\begin{tabular}{|c|c|c|c|c|c|c|c|c|c|c|c|c|c|c|c|c|}
\hline Glass ID & $\begin{array}{l}\mathrm{T} 13 \\
\left({ }^{\circ} \mathrm{C}\right) \\
\end{array}$ & $\begin{array}{c}\mathrm{V} 13 \\
(\mathrm{~Pa} \cdot \mathrm{s})\end{array}$ & $\begin{array}{l}\mathrm{T} 14 \\
\left({ }^{\circ} \mathrm{C}\right) \\
\end{array}$ & $\begin{array}{c}\mathrm{V} 14 \\
(\mathrm{~Pa} \cdot \mathrm{s})\end{array}$ & $\begin{array}{c}\text { Q PCT } \\
\mathrm{B}\left(\mathrm{g} / \mathrm{m}^{2}\right)\end{array}$ & $\begin{array}{c}\text { Q PCT } \\
\mathrm{Li}\left(\mathrm{g} / \mathrm{m}^{2}\right)\end{array}$ & $\begin{array}{c}\text { Q PCT } \\
\mathrm{Na}\left(\mathrm{g} / \mathrm{m}^{2}\right) \\
\end{array}$ & $\begin{array}{c}\text { Q PCT } \\
\mathrm{Si}\left(\mathrm{g} / \mathrm{m}^{2}\right)\end{array}$ & $\begin{array}{c}\text { Q PCT } \\
\text { pH }\end{array}$ & $\begin{array}{c}\text { CCC PCT } \\
\text { B }\left(\mathrm{g} / \mathrm{m}^{2}\right)\end{array}$ & $\begin{array}{c}\text { CCC PCT } \\
\mathrm{Li}\left(\mathrm{g} / \mathrm{m}^{2}\right) \\
\end{array}$ & $\begin{array}{l}\text { CCC PCT } \\
\mathrm{Na}\left(\mathrm{g} / \mathrm{m}^{2}\right)\end{array}$ & $\begin{array}{c}\text { CCC PCT } \\
\mathrm{Si}\left(\mathrm{g} / \mathrm{m}^{2}\right) \\
\end{array}$ & $\begin{array}{c}\text { CCC PCT } \\
\text { pH }\end{array}$ & $\begin{array}{l}\text { Q PCT B at } \\
20^{\circ} \mathrm{C}\left(\mathrm{g} / \mathrm{m}^{2}\right)\end{array}$ & $\begin{array}{l}\text { Q PCT Li at } \\
20^{\circ} \mathrm{C}\left(\mathrm{g} / \mathrm{m}^{2}\right)\end{array}$ \\
\hline 1 & & & & & & & & & & & & & & & & \\
\hline 2 & & & & & & & & & & & & & & & & \\
\hline 3 & & & & & & & & & & & & & & & & \\
\hline 4 & & & & & & & & & & & & & & & & \\
\hline 5 & & & & & & & & & & & & & & & & \\
\hline 6 & & & & & & & & & & & & & & & & \\
\hline 7 & & & & & & & & & & & & & & & & \\
\hline 8 & & & & & & & & & & & & & & & & \\
\hline 9 & & & & & & & & & & & & & & & & \\
\hline 10 & & & & & & & & & & & & & & & & \\
\hline 11 & & & & & & & & & & & & & & & & \\
\hline 12 & & & & & & & & & & & & & & & & \\
\hline 13 & & & & & & & & & & & & & & & & \\
\hline 14 & & & & & & & & & & & & & & & & \\
\hline 15 & & & & & & & & & & & & & & & & \\
\hline 16 & & & & & & & & & & & & & & & & \\
\hline 17 & & & & & & & & & & & & & & & & \\
\hline 18 & & & & & & & & & & & & & & & & \\
\hline 19 & & & & & & & & & & & & & & & & \\
\hline 20 & & & & & & & & & & & & & & & & \\
\hline 21 & & & & & & & & & & & & & & & & \\
\hline 22 & & & & & & & & & & & & & & & & \\
\hline 23 & & & & & & & & & & & & & & & & \\
\hline 24 & & & & & & & & & & & & & & & & \\
\hline 25 & & & & & & & & & & & & & & & & \\
\hline 26 & & & & & & & & & & & & & & & & \\
\hline 27 & & & & & & & & & & & & & & & & \\
\hline 28 & & & & & & & & & & & & & & & & \\
\hline 29 & & & & & & & & & & & & & & & & \\
\hline 30 & & & & & & & & & & & & & & & & \\
\hline 31 & & & & & & & & & & & & & & & & \\
\hline 32 & & & & & & & & & & & & & & & & \\
\hline 33 & & & & & & & & & & & & & & & & \\
\hline 34 & & & & & & & & & & & & & & & & \\
\hline 35 & & & & & & & & & & & & & & & & \\
\hline 36 & & & & & & & & & & & & & & & & \\
\hline 37 & & & & & & & & & & & & & & & & \\
\hline 38 & & & & & & & & & & & & & & & & \\
\hline 39 & & & & & & & & & & & & & & & & \\
\hline 40 & & & & & & & & & & & & & & & & \\
\hline 41 & & & & & & & & & & & & & & & & \\
\hline
\end{tabular}


Appendix A. Database - mass fraction

\begin{tabular}{|c|c|c|c|c|c|c|c|c|c|c|c|c|}
\hline Glass ID & $\begin{array}{l}\text { Q PCT Na at } \\
20^{\circ} \mathrm{C}\left(\mathrm{g} / \mathrm{m}^{2}\right)\end{array}$ & $\begin{array}{l}\text { Q PCT Si at } \\
20^{\circ} \mathrm{C}\left(\mathrm{g} / \mathrm{m}^{2}\right)\end{array}$ & $\begin{array}{c}\mathrm{QpH} \\
\text { at } 20^{\circ} \mathrm{C}\end{array}$ & $\begin{array}{c}\text { TCLP Ag } \\
(\mathrm{ppm})\end{array}$ & $\begin{array}{c}\text { TCLP As } \\
\text { (ppm) }\end{array}$ & $\begin{array}{c}\text { TCLP Ba } \\
\text { (ppm) }\end{array}$ & $\begin{array}{c}\text { TCLP Cd } \\
\text { (ppm) }\end{array}$ & $\begin{array}{c}\text { TCLP Cr } \\
\text { (ppm) }\end{array}$ & $\begin{array}{l}\text { TCLP Ni } \\
(\mathrm{ppm})\end{array}$ & $\begin{array}{c}\text { TCLP Pb } \\
\text { (ppm) }\end{array}$ & $\begin{array}{l}\text { TCLP Se } \\
(\mathrm{ppm})\end{array}$ & $\begin{array}{c}\text { TCLP Zn } \\
(\mathrm{ppm})\end{array}$ \\
\hline \multicolumn{13}{|l|}{1} \\
\hline \multicolumn{13}{|l|}{2} \\
\hline \multirow{2}{*}{\multicolumn{13}{|c|}{$\frac{3}{4}$}} \\
\hline 4 & & & & & & & & & & & & \\
\hline \multicolumn{13}{|l|}{5} \\
\hline \multicolumn{13}{|l|}{6} \\
\hline \multicolumn{13}{|l|}{7} \\
\hline \multicolumn{13}{|l|}{8} \\
\hline \multicolumn{13}{|l|}{9} \\
\hline \multicolumn{13}{|l|}{10} \\
\hline \multicolumn{13}{|l|}{11} \\
\hline \multicolumn{13}{|l|}{12} \\
\hline \multicolumn{13}{|l|}{13} \\
\hline \multicolumn{13}{|l|}{14} \\
\hline \multicolumn{13}{|l|}{15} \\
\hline \multicolumn{13}{|l|}{16} \\
\hline \multicolumn{13}{|l|}{17} \\
\hline \multicolumn{13}{|l|}{18} \\
\hline \multicolumn{13}{|l|}{19} \\
\hline \multicolumn{13}{|l|}{20} \\
\hline \multicolumn{13}{|l|}{21} \\
\hline \multicolumn{13}{|l|}{22} \\
\hline \multicolumn{13}{|l|}{23} \\
\hline 24 & & & & & & & & & & & & \\
\hline 25 & & & & & & & & & & & & \\
\hline 26 & & & & & & & & & & & & \\
\hline 27 & & & & & & & & & & & & \\
\hline 28 & & & & & & & & & & & & \\
\hline 29 & & & & & & & & & & & & \\
\hline 30 & & & & & & & & & & & & \\
\hline 31 & & & & & & & & & & & & \\
\hline 32 & & & & & & & & & & & & \\
\hline 33 & & & & & & & & & & & & \\
\hline 34 & & & & & & & & & & & & \\
\hline 35 & & & & & & & & & & & & \\
\hline 36 & & & & & & & & & & & & \\
\hline 37 & & & & & & & & & & & & \\
\hline 38 & & & & & & & & & & & & \\
\hline 39 & & & & & & & & & & & & \\
\hline 40 & & & & & & & & & & & & \\
\hline 41 & & & & & & & & & & & & \\
\hline
\end{tabular}


Appendix A. Database - mass fraction

Comp. Vs Properties Study (Chick et al. 1981)

\begin{tabular}{|c|c|c|c|c|c|c|c|c|c|c|c|c|c|c|c|c|c|c|c|c|c|}
\hline Glass ID & $\begin{array}{c}\mathrm{A} 12 \mathrm{O} 3 \\
-\mathrm{t}\end{array}$ & $\begin{array}{c}\mathrm{B} 2 \mathrm{O} 3 \\
-\mathrm{t}\end{array}$ & $\begin{array}{c}\mathrm{CaO} \\
-\mathrm{t}\end{array}$ & $\begin{array}{c}\mathrm{Fe} 2 \mathrm{O} 3 \\
-\mathrm{t}\end{array}$ & $\begin{array}{c}\mathrm{FeO} \\
-\mathrm{t} \\
\end{array}$ & $\begin{array}{c}\mathrm{K} 2 \mathrm{O} \\
-\mathrm{t} \\
\end{array}$ & $\begin{array}{c}\mathrm{Li} 2 \mathrm{O} \\
-\mathrm{t}\end{array}$ & $\begin{array}{c}\mathrm{MgO} \\
-\mathrm{t}\end{array}$ & $\begin{array}{c}\mathrm{Na} 2 \mathrm{O} \\
-\mathrm{t} \\
\end{array}$ & $\begin{array}{c}\mathrm{NiO} \\
-\mathrm{t} \\
\end{array}$ & $\begin{array}{c}\mathrm{P} 2 \mathrm{O} 5 \\
-\mathrm{t} \\
\end{array}$ & $\begin{array}{c}\mathrm{SiO} 2 \\
-\mathrm{t}\end{array}$ & $\begin{array}{c}\mathrm{ZrO} 2 \\
-\mathrm{t}\end{array}$ & $\begin{array}{c}\mathrm{Ag} 2 \mathrm{O} \\
-\mathrm{t}\end{array}$ & $\begin{array}{c}\mathrm{As} 2 \mathrm{O} 3 \\
-\mathrm{t}\end{array}$ & $\begin{array}{c}\mathrm{BaO} \\
-\mathrm{t} \\
\end{array}$ & $\begin{array}{c}\mathrm{Bi} 2 \mathrm{O} 3 \\
-\mathrm{t} \\
\end{array}$ & $\begin{array}{c}\mathrm{Br} \\
-\mathrm{t}\end{array}$ & $\begin{array}{c}\mathrm{CdO} \\
-\mathrm{t} \\
\end{array}$ & $\begin{array}{c}\mathrm{Ce} 2 \mathrm{O} 3 \\
-\mathrm{t} \\
\end{array}$ & $\begin{array}{c}\mathrm{CeO} 2 \\
-\mathrm{t}\end{array}$ \\
\hline \multicolumn{22}{|l|}{42} \\
\hline \multicolumn{22}{|l|}{43} \\
\hline \multicolumn{22}{|l|}{44} \\
\hline \multicolumn{22}{|l|}{45} \\
\hline \multicolumn{22}{|l|}{46} \\
\hline \multicolumn{22}{|l|}{47} \\
\hline \multicolumn{22}{|l|}{48} \\
\hline \multicolumn{22}{|l|}{49} \\
\hline \multicolumn{22}{|l|}{50} \\
\hline \multicolumn{22}{|l|}{51} \\
\hline \multicolumn{22}{|l|}{52} \\
\hline \multicolumn{22}{|l|}{53} \\
\hline \multicolumn{22}{|l|}{54} \\
\hline \multicolumn{22}{|l|}{55} \\
\hline \multicolumn{22}{|l|}{56} \\
\hline \multicolumn{22}{|l|}{57} \\
\hline \multicolumn{22}{|l|}{58} \\
\hline \multicolumn{22}{|l|}{59} \\
\hline \multirow{2}{*}{\multicolumn{22}{|c|}{$\frac{60}{61}$}} \\
\hline & & & & & & & & & & & & & & & & & & & & & \\
\hline \multicolumn{22}{|l|}{62} \\
\hline \multicolumn{22}{|l|}{63} \\
\hline \multicolumn{22}{|l|}{64} \\
\hline \multicolumn{22}{|l|}{65} \\
\hline 66 & & & & & & & & & & & & & & & & & & & & & \\
\hline 67 & & & & & & & & & & & & & & & & & & & & & \\
\hline 68 & & & & & & & & & & & & & & & & & & & & & \\
\hline 69 & & & & & & & & & & & & & & & & & & & & & \\
\hline 70 & & & & & & & & & & & & & & & & & & & & & \\
\hline
\end{tabular}


Comp. Vs Properties Study (Chick et al. 1981)

\begin{tabular}{|c|c|c|c|c|c|c|c|c|c|c|c|c|c|c|c|c|c|c|c|c|c|}
\hline Glass ID & $\begin{array}{l}\mathrm{Cl} \\
-\mathrm{t}\end{array}$ & $\begin{array}{c}\mathrm{CoO} \\
-\mathrm{t}\end{array}$ & $\begin{array}{c}\mathrm{Co} 2 \mathrm{O} 3 \\
-\mathrm{t}\end{array}$ & $\begin{array}{c}\mathrm{Cr} 2 \mathrm{O} 3 \\
-\mathrm{t}\end{array}$ & $\begin{array}{c}\mathrm{Cs} 2 \mathrm{O} \\
-\mathrm{t}\end{array}$ & $\begin{array}{c}\mathrm{CuO} \\
-\mathrm{t}\end{array}$ & $\begin{array}{c}\mathrm{Eu} 2 \mathrm{O} 3 \\
-\mathrm{t}\end{array}$ & $\begin{array}{l}F \\
-t\end{array}$ & $\begin{array}{c}\mathrm{Ga} 2 \mathrm{O} 3 \\
-\mathrm{t}\end{array}$ & $\begin{array}{c}\mathrm{Gd} 2 \mathrm{O} 3 \\
-\mathrm{t}\end{array}$ & $\begin{array}{c}\mathrm{HgO} \\
-\mathrm{t}\end{array}$ & $\begin{array}{l}I \\
-t\end{array}$ & {$\left[\begin{array}{c}\mathrm{La} 2 \mathrm{O} 3 \\
-\mathrm{t}\end{array}\right.$} & $\begin{array}{c}\mathrm{MnO} 2 \\
-\mathrm{t}\end{array}$ & $\begin{array}{c}\mathrm{MnO} \\
-\mathrm{t}\end{array}$ & $\begin{array}{c}\mathrm{MoO} \\
-\mathrm{t}\end{array}$ & $\begin{array}{c}\mathrm{MoO} 3 \\
-\mathrm{t}\end{array}$ & $\begin{array}{c}\mathrm{Nb} 2 \mathrm{O} 5 \\
-\mathrm{t}\end{array}$ & $\begin{array}{c}\mathrm{Nd} 2 \mathrm{O} 3 \\
-\mathrm{t}\end{array}$ & $\begin{array}{c}\mathrm{PbO} \\
-\mathrm{t}\end{array}$ & $\begin{array}{c}\mathrm{PdO} 2 \\
-\mathrm{t}\end{array}$ \\
\hline \multicolumn{22}{|l|}{42} \\
\hline \multicolumn{22}{|l|}{43} \\
\hline \multicolumn{22}{|l|}{44} \\
\hline \multicolumn{22}{|l|}{45} \\
\hline \multicolumn{22}{|l|}{46} \\
\hline \multicolumn{22}{|l|}{47} \\
\hline \multicolumn{22}{|l|}{48} \\
\hline \multicolumn{22}{|l|}{49} \\
\hline \multicolumn{22}{|l|}{50} \\
\hline \multicolumn{22}{|l|}{51} \\
\hline \multicolumn{22}{|l|}{52} \\
\hline \multicolumn{22}{|l|}{53} \\
\hline \multicolumn{22}{|l|}{54} \\
\hline \multicolumn{22}{|l|}{55} \\
\hline \multicolumn{22}{|l|}{56} \\
\hline \multicolumn{22}{|l|}{57} \\
\hline \multicolumn{22}{|l|}{58} \\
\hline \multirow{2}{*}{\multicolumn{22}{|c|}{59}} \\
\hline \multirow{2}{*}{\multicolumn{22}{|c|}{$\frac{60}{61}$}} \\
\hline & & & & & & & & & & & & & & & & & & & & & \\
\hline \multicolumn{22}{|l|}{62} \\
\hline \multicolumn{22}{|l|}{63} \\
\hline \multicolumn{22}{|l|}{64} \\
\hline \multirow{2}{*}{\multicolumn{22}{|c|}{$\frac{65}{66}$}} \\
\hline & & & & & & & & & & & & & & & & & & & & & \\
\hline 67 & & & & & & & & & & & & & & & & & & & & & \\
\hline 68 & & & & & & & & & & & & & & & & & & & & & \\
\hline 69 & & & & & & & & & & & & & & & & & & & & & \\
\hline 70 & & & & & & & & & & & & & & & & & & & & & \\
\hline
\end{tabular}


Comp. Vs Properties Study (Chick et al. 1981)

\begin{tabular}{|c|c|c|c|c|c|c|c|c|c|c|c|c|c|c|c|c|c|c|c|c|c|}
\hline Glass ID & $\begin{array}{c}\mathrm{PdO} \\
-\mathrm{t}\end{array}$ & $\begin{array}{c}\mathrm{Pr} 2 \mathrm{O} 3 \\
-\mathrm{t}\end{array}$ & $\begin{array}{c}\text { Pr6O11 } \\
-t\end{array}$ & $\begin{array}{c}\mathrm{Rb} 2 \mathrm{O} \\
-\mathrm{t}\end{array}$ & $\begin{array}{c}\mathrm{ReO} \\
-\mathrm{t}\end{array}$ & $\begin{array}{c}\mathrm{ReO} 2 \\
-\mathrm{t}\end{array}$ & $\begin{array}{c}\mathrm{Rh} 2 \mathrm{O} 3 \\
-\mathrm{t}\end{array}$ & $\begin{array}{c}\mathrm{RhO} 2 \\
-\mathrm{t}\end{array}$ & $\begin{array}{c}\mathrm{RuO} 2 \\
-\mathrm{t}\end{array}$ & $\begin{array}{c}\mathrm{Sb} 2 \mathrm{O} 3 \\
-\mathrm{t}\end{array}$ & $\begin{array}{c}\mathrm{Sb} 2 \mathrm{O} 5 \\
-\mathrm{t}\end{array}$ & $\begin{array}{c}\mathrm{SeO} 2 \\
-\mathrm{t} \\
\end{array}$ & $\begin{array}{c}\mathrm{Sm} 2 \mathrm{O} 3 \\
-\mathrm{t}\end{array}$ & $\begin{array}{c}\mathrm{SnO} \\
-\mathrm{t}\end{array}$ & $\begin{array}{c}\mathrm{SnO} 2 \\
-\mathrm{t}\end{array}$ & $\begin{array}{c}\mathrm{SO} 3 \\
-\mathrm{t} \\
\end{array}$ & $\begin{array}{c}\mathrm{SrO} \\
-\mathrm{t} \\
\end{array}$ & $\begin{array}{c}\mathrm{Tc} 2 \mathrm{O} 7 \\
-\mathrm{t} \\
\end{array}$ & $\begin{array}{c}\mathrm{TeO} 2 \\
-\mathrm{t}\end{array}$ & $\begin{array}{c}\mathrm{ThO}_{2} \\
-\mathrm{t}\end{array}$ & $\begin{array}{c}\mathrm{TiO} 2 \\
-\mathrm{t}\end{array}$ \\
\hline 42 & & & & & & & & & & & & & & & & & & & & & \\
\hline 43 & & & & & & & & & & & & & & & & & & & & & \\
\hline 44 & & & & & & & & & & & & & & & & & & & & & \\
\hline 45 & & & & & & & & & & & & & & & & & & & & & \\
\hline 46 & & & & & & & & & & & & & & & & & & & & & \\
\hline 47 & & & & & & & & & & & & & & & & & & & & & \\
\hline 48 & & & & & & & & & & & & & & & & & & & & & \\
\hline 49 & & & & & & & & & & & & & & & & & & & & & \\
\hline 50 & & & & & & & & & & & & & & & & & & & & & \\
\hline 51 & & & & & & & & & & & & & & & & & & & & & \\
\hline 52 & & & & & & & & & & & & & & & & & & & & & \\
\hline 53 & & & & & & & & & & & & & & & & & & & & & \\
\hline 54 & & & & & & & & & & & & & & & & & & & & & \\
\hline 55 & & & & & & & & & & & & & & & & & & & & & \\
\hline 56 & & & & & & & & & & & & & & & & & & & & & \\
\hline 57 & & & & & & & & & & & & & & & & & & & & & \\
\hline 58 & & & & & & & & & & & & & & & & & & & & & \\
\hline 59 & & & & & & & & & & & & & & & & & & & & & \\
\hline 60 & & & & & & & & & & & & & & & & & & & & & \\
\hline 61 & & & & & & & & & & & & & & & & & & & & & \\
\hline 62 & & & & & & & & & & & & & & & & & & & & & \\
\hline 63 & & & & & & & & & & & & & & & & & & & & & \\
\hline 64 & & & & & & & & & & & & & & & & & & & & & \\
\hline 65 & & & & & & & & & & & & & & & & & & & & & \\
\hline 66 & & & & & & & & & & & & & & & & & & & & & \\
\hline 67 & & & & & & & & & & & & & & & & & & & & & \\
\hline 68 & & & & & & & & & & & & & & & & & & & & & \\
\hline 69 & & & & & & & & & & & & & & & & & & & & & \\
\hline 70 & & & & & & & & & & & & & & & & & & & & & \\
\hline
\end{tabular}


Comp. Vs Properties Study (Chick et al. 1981)

\begin{tabular}{|c|c|c|c|c|c|c|c|c|c|c|c|c|c|c|c|c|c|c|c|c|c|}
\hline Glass ID & $\begin{array}{c}\mathrm{T} 12 \mathrm{O} 3 \\
-\mathrm{t}\end{array}$ & $\begin{array}{c}\text { U3O8 } \\
-t\end{array}$ & $\begin{array}{c}\mathrm{UO} 2 \\
-\mathrm{t}\end{array}$ & $\begin{array}{c}\text { UO3 } \\
-t\end{array}$ & $\begin{array}{c}\mathrm{V} 2 \mathrm{O} 5 \\
-\mathrm{t}\end{array}$ & $\begin{array}{c}\text { WO3 } \\
-t\end{array}$ & $\begin{array}{c}\mathrm{Y} 2 \mathrm{O} 3 \\
-\mathrm{t}\end{array}$ & $\begin{array}{c}\mathrm{ZnO} \\
-\mathrm{t}\end{array}$ & $\begin{array}{c}\text { Others } \\
-t\end{array}$ & $\begin{array}{c}\text { Sum } \\
-t\end{array}$ & $\begin{array}{c}\mathrm{A} 12 \mathrm{O} 3 \\
-\mathrm{a}\end{array}$ & $\begin{array}{c}\mathrm{B} 2 \mathrm{O} 3 \\
-\mathrm{a}\end{array}$ & $\begin{array}{c}\mathrm{CaO} \\
-\mathrm{a} \\
\end{array}$ & $\begin{array}{c}\mathrm{Fe} 2 \mathrm{O} 3 \\
-\mathrm{a}\end{array}$ & $\begin{array}{c}\mathrm{FeO} \\
-\mathrm{a}\end{array}$ & $\begin{array}{c}\mathrm{K} 2 \mathrm{O} \\
-\mathrm{a}\end{array}$ & $\begin{array}{c}\mathrm{Li} 2 \mathrm{O} \\
-\mathrm{a}\end{array}$ & $\begin{array}{c}\mathrm{MgO} \\
-\mathrm{a}\end{array}$ & $\begin{array}{c}\mathrm{Na} 2 \mathrm{O} \\
-\mathrm{a}\end{array}$ & $\begin{array}{c}\mathrm{NiO} \\
-\mathrm{a} \\
\end{array}$ & $\begin{array}{c}\mathrm{P} 2 \mathrm{O} 5 \\
-\mathrm{a}\end{array}$ \\
\hline 42 & & & & & & & & & & & 0.2042 & 0.1135 & 0.0000 & 0.0000 & & & & 0.0173 & 0.0873 & 0.0279 & \\
\hline 43 & & & & & & & & & & & 0.1086 & 0.0947 & 0.0556 & 0.0354 & & & & 0.0226 & 0.1146 & 0.0155 & \\
\hline 44 & & & & & & & & & & & 0.1088 & 0.0939 & 0.0549 & 0.0355 & & & & 0.0233 & 0.1185 & 0.0155 & \\
\hline 45 & & & & & & & & & & & 0.1088 & 0.0939 & 0.0557 & 0.0355 & & & & 0.0233 & 0.1167 & 0.0155 & \\
\hline 46 & & & & & & & & & & & 0.0000 & 0.1337 & 0.0086 & 0.0000 & & & & 0.0223 & 0.1553 & 0.0000 & \\
\hline 47 & & & & & & & & & & & 0.1122 & 0.0714 & 0.0989 & 0.0650 & & & & 0.0511 & 0.1009 & 0.0270 & \\
\hline 48 & & & & & & & & & & & 0.2142 & 0.0604 & 0.0357 & 0.0476 & & & & 0.0000 & 0.1336 & 0.0192 & \\
\hline 49 & & & & & & & & & & & 0.0000 & 0.1493 & 0.0000 & 0.0267 & & & & 0.0000 & 0.1471 & 0.0000 & \\
\hline 50 & & & & & & & & & & & 0.0361 & 0.1359 & 0.0000 & 0.0000 & & & & 0.0533 & 0.0896 & 0.0000 & \\
\hline 51 & & & & & & & & & & & 0.0375 & 0.1344 & 0.0000 & 0.0000 & & & & 0.0488 & 0.0883 & 0.0000 & \\
\hline 52 & & & & & & & & & & & 0.0361 & 0.1371 & 0.0000 & 0.0000 & & & & 0.0508 & 0.0867 & 0.0000 & \\
\hline 53 & & & & & & & & & & & 0.0000 & 0.0739 & 0.0000 & 0.0773 & & & & 0.0554 & 0.1413 & 0.0315 & \\
\hline 54 & & & & & & & & & & & 0.2153 & 0.0615 & 0.0557 & 0.0573 & & & & 0.0000 & 0.0966 & 0.0000 & \\
\hline 55 & & & & & & & & & & & 0.2230 & 0.1272 & 0.0081 & 0.0000 & & & & 0.0464 & 0.1445 & 0.0226 & \\
\hline 56 & & & & & & & & & & & 0.0485 & 0.1422 & 0.1119 & 0.0000 & & & & 0.0000 & 0.1370 & 0.0000 & \\
\hline 57 & & & & & & & & & & & 0.1981 & 0.0638 & 0.0000 & 0.0000 & & & & 0.0000 & 0.1229 & 0.0225 & \\
\hline 58 & & & & & & & & & & & 0.2093 & 0.1346 & 0.0000 & 0.0000 & & & & 0.0498 & 0.1429 & 0.0000 & \\
\hline 59 & & & & & & & & & & & 0.0000 & 0.0701 & 0.0976 & 0.0000 & & & & 0.0000 & 0.1022 & 0.0308 & \\
\hline 60 & & & & & & & & & & & 0.0491 & 0.0725 & 0.1116 & 0.0621 & & & & 0.0000 & 0.1590 & 0.0000 & \\
\hline 61 & & & & & & & & & & & 0.2236 & 0.0720 & 0.1091 & 0.0000 & & & & 0.0517 & 0.1130 & 0.0000 & \\
\hline 62 & & & & & & & & & & & 0.1984 & 0.1278 & 0.0292 & 0.0656 & & & & 0.0000 & 0.0790 & 0.0000 & \\
\hline 63 & & & & & & & & & & & 0.0190 & 0.1446 & 0.1122 & 0.0743 & & & & 0.0519 & 0.0923 & 0.0290 & \\
\hline 64 & & & & & & & & & & & 0.1919 & 0.0701 & 0.1088 & 0.0000 & & & & 0.0000 & 0.1405 & 0.0288 & \\
\hline 65 & & & & & & & & & & & 0.2072 & 0.0687 & 0.1082 & 0.0000 & & & & 0.0000 & 0.1332 & 0.0286 & \\
\hline 66 & & & & & & & & & & & 0.2087 & 0.0687 & 0.1090 & 0.0000 & & & & 0.0000 & 0.1360 & 0.0231 & \\
\hline 67 & & & & & & & & & & & 0.2219 & 0.1274 & 0.0089 & 0.0000 & & & & 0.0465 & 0.1474 & 0.0248 & \\
\hline 68 & & & & & & & & & & & 0.2089 & 0.1317 & 0.1013 & 0.0000 & & & & 0.0000 & 0.0979 & 0.0255 & \\
\hline 69 & & & & & & & & & & & 0.0000 & 0.0713 & 0.0093 & 0.0000 & & & & 0.0000 & 0.1270 & 0.0281 & \\
\hline 70 & & & & & & & & & & & 0.1220 & 0.0975 & 0.0646 & 0.0419 & & & & 0.0265 & 0.1338 & 0.0174 & \\
\hline
\end{tabular}


Appendix A. Database - mass fraction

Comp. Vs Properties Study (Chick et al. 1981)

\begin{tabular}{|c|c|c|c|c|c|c|c|c|c|c|c|c|c|c|c|c|c|c|c|c|c|}
\hline Glass ID & $\begin{array}{c}\mathrm{SiO} 2 \\
-\mathrm{a}\end{array}$ & $\begin{array}{c}\mathrm{ZrO} 2 \\
-\mathrm{a}\end{array}$ & $\begin{array}{c}\mathrm{Ag} 2 \mathrm{O} \\
-\mathrm{a}\end{array}$ & $\begin{array}{c}\mathrm{As} 2 \mathrm{O} 3 \\
-\mathrm{a} \\
\end{array}$ & $\begin{array}{c}\mathrm{BaO} \\
-\mathrm{a}\end{array}$ & $\begin{array}{c}\mathrm{Bi} 2 \mathrm{O} 3 \\
-\mathrm{a}\end{array}$ & $\begin{array}{l}\mathrm{Br} \\
-\mathrm{a} \\
\end{array}$ & $\begin{array}{c}\mathrm{CdO} \\
-\mathrm{a}\end{array}$ & $\begin{array}{c}\mathrm{Ce} 2 \mathrm{O} 3 \\
-\mathrm{a}\end{array}$ & $\begin{array}{c}\mathrm{CeO} 2 \\
-\mathrm{a}\end{array}$ & $\begin{array}{l}\mathrm{Cl} \\
-\mathrm{a} \\
\end{array}$ & $\begin{array}{c}\mathrm{CoO} \\
-\mathrm{a}\end{array}$ & $\begin{array}{c}\mathrm{Co} 2 \mathrm{O} 3 \\
-\mathrm{a}\end{array}$ & $\begin{array}{c}\mathrm{Cr} 2 \mathrm{O} 3 \\
-\mathrm{a}\end{array}$ & $\begin{array}{c}\mathrm{Cs} 2 \mathrm{O} \\
-\mathrm{a}\end{array}$ & $\begin{array}{c}\mathrm{CuO} \\
-\mathrm{a}\end{array}$ & $\begin{array}{c}\mathrm{Eu} 2 \mathrm{O} 3 \\
-\mathrm{a}\end{array}$ & $\begin{array}{c}\mathrm{F} \\
-\mathrm{a}\end{array}$ & $\begin{array}{c}\mathrm{Ga} 2 \mathrm{O} 3 \\
-\mathrm{a}\end{array}$ & $\begin{array}{c}\mathrm{Gd} 2 \mathrm{O} 3 \\
-\mathrm{a}\end{array}$ & $\begin{array}{c}\mathrm{HgO} \\
-\mathrm{a}\end{array}$ \\
\hline 42 & 0.3610 & & & & & & & & & & & & & 0.0651 & & & & & & & \\
\hline 43 & 0.4407 & & & & & & & & & & & & & 0.0337 & & & & & & & \\
\hline 44 & 0.4427 & & & & & & & & & & & & & 0.0315 & & & & & & & \\
\hline 45 & 0.4426 & & & & & & & & & & & & & 0.0315 & & & & & & & \\
\hline 46 & 0.5217 & & & & & & & & & & & & & 0.0724 & & & & & & & \\
\hline 47 & 0.3878 & & & & & & & & & & & & & 0.0000 & & & & & & & \\
\hline 48 & 0.3526 & & & & & & & & & & & & & 0.0577 & & & & & & & \\
\hline 49 & 0.5028 & & & & & & & & & & & & & 0.0000 & & & & & & & \\
\hline 50 & 0.5330 & & & & & & & & & & & & & 0.0771 & & & & & & & \\
\hline 51 & 0.5395 & & & & & & & & & & & & & 0.0792 & & & & & & & \\
\hline 52 & 0.5406 & & & & & & & & & & & & & 0.0748 & & & & & & & \\
\hline 53 & 0.5432 & & & & & & & & & & & & & 0.0000 & & & & & & & \\
\hline 54 & 0.4486 & & & & & & & & & & & & & 0.0650 & & & & & & & \\
\hline 55 & 0.3605 & & & & & & & & & & & & & 0.0678 & & & & & & & \\
\hline 56 & 0.3958 & & & & & & & & & & & & & 0.0000 & & & & & & & \\
\hline 57 & 0.3732 & & & & & & & & & & & & & 0.0686 & & & & & & & \\
\hline 58 & 0.3754 & & & & & & & & & & & & & 0.0000 & & & & & & & \\
\hline 59 & 0.5265 & & & & & & & & & & & & & 0.0789 & & & & & & & \\
\hline 60 & 0.5456 & & & & & & & & & & & & & 0.0000 & & & & & & & \\
\hline 61 & 0.4306 & & & & & & & & & & & & & 0.0000 & & & & & & & \\
\hline 62 & 0.3565 & & & & & & & & & & & & & 0.0000 & & & & & & & \\
\hline 63 & 0.4024 & & & & & & & & & & & & & 0.0000 & & & & & & & \\
\hline 64 & 0.3863 & & & & & & & & & & & & & 0.0000 & & & & & & & \\
\hline 65 & 0.3822 & & & & & & & & & & & & & 0.0000 & & & & & & & \\
\hline 66 & 0.3814 & & & & & & & & & & & & & 0.0000 & & & & & & & \\
\hline 67 & 0.3576 & & & & & & & & & & & & & 0.0657 & & & & & & & \\
\hline 68 & 0.3676 & & & & & & & & & & & & & 0.0670 & & & & & & & \\
\hline 69 & 0.5394 & & & & & & & & & & & & & 0.0549 & & & & & & & \\
\hline 70 & 0.3690 & & & & & & & & & & & & & 0.0333 & & & & & & & \\
\hline
\end{tabular}


Comp. Vs Properties Study (Chick et al. 1981)

\begin{tabular}{|c|c|c|c|c|c|c|c|c|c|c|c|c|c|c|c|c|c|c|c|c|c|}
\hline Glass ID & $\begin{array}{c}\mathrm{I} \\
-\mathrm{a} \\
\end{array}$ & $\begin{array}{c}\mathrm{La} 2 \mathrm{O} 3 \\
-\mathrm{a} \\
\end{array}$ & $\begin{array}{c}\mathrm{MnO} 2 \\
-\mathrm{a} \\
\end{array}$ & $\begin{array}{c}\mathrm{MnO} \\
-\mathrm{a}\end{array}$ & $\begin{array}{c}\mathrm{MoO} \\
-\mathrm{a}\end{array}$ & $\begin{array}{c}\mathrm{MoO} 3 \\
-\mathrm{a} \\
\end{array}$ & $\begin{array}{c}\mathrm{Nb} 2 \mathrm{O} 5 \\
-\mathrm{a}\end{array}$ & $\begin{array}{c}\mathrm{Nd} 2 \mathrm{O} 3 \\
-\mathrm{a}\end{array}$ & $\begin{array}{c}\mathrm{PbO} \\
-\mathrm{a} \\
\end{array}$ & $\begin{array}{c}\mathrm{PdO} 2 \\
-\mathrm{a}\end{array}$ & $\begin{array}{c}\mathrm{PdO} \\
-\mathrm{a} \\
\end{array}$ & $\begin{array}{c}\mathrm{Pr} 2 \mathrm{O} 3 \\
-\mathrm{a} \\
\end{array}$ & $\begin{array}{c}\text { Pr6O11 } \\
-\mathrm{a}\end{array}$ & $\begin{array}{c}\mathrm{Rb} 2 \mathrm{O} \\
-\mathrm{a}\end{array}$ & $\begin{array}{c}\mathrm{ReO} \\
-\mathrm{a} \\
\end{array}$ & $\begin{array}{c}\mathrm{ReO} 2 \\
-\mathrm{a} \\
\end{array}$ & $\begin{array}{c}\mathrm{Rh} 2 \mathrm{O} 3 \\
-\mathrm{a} \\
\end{array}$ & $\begin{array}{c}\mathrm{RhO} 2 \\
-\mathrm{a}\end{array}$ & $\begin{array}{c}\mathrm{RuO} 2 \\
-\mathrm{a} \\
\end{array}$ & $\begin{array}{c}\mathrm{Sb} 2 \mathrm{O} 3 \\
-\mathrm{a}\end{array}$ & $\begin{array}{c}\mathrm{Sb} 2 \mathrm{O} 5 \\
-\mathrm{a}\end{array}$ \\
\hline \multicolumn{22}{|l|}{42} \\
\hline \multicolumn{22}{|l|}{43} \\
\hline \multicolumn{22}{|l|}{44} \\
\hline \multicolumn{22}{|l|}{45} \\
\hline \multicolumn{22}{|l|}{46} \\
\hline \multicolumn{22}{|l|}{47} \\
\hline \multicolumn{22}{|l|}{48} \\
\hline \multicolumn{22}{|l|}{49} \\
\hline \multicolumn{22}{|l|}{50} \\
\hline \multicolumn{22}{|l|}{51} \\
\hline \multicolumn{22}{|l|}{52} \\
\hline \multicolumn{22}{|l|}{53} \\
\hline \multicolumn{22}{|l|}{54} \\
\hline \multicolumn{22}{|l|}{55} \\
\hline \multicolumn{22}{|l|}{56} \\
\hline \multicolumn{22}{|l|}{57} \\
\hline \multicolumn{22}{|l|}{58} \\
\hline \multirow{2}{*}{\multicolumn{22}{|c|}{$\begin{array}{l}59 \\
60\end{array}$}} \\
\hline \multirow{2}{*}{\multicolumn{14}{|c|}{$\begin{array}{l}60 \\
61\end{array}$}} & & & & & & & & \\
\hline & & & & & & & & & & & & & & & & & & & & & \\
\hline \multicolumn{22}{|l|}{62} \\
\hline \multicolumn{22}{|l|}{63} \\
\hline \multicolumn{22}{|l|}{64} \\
\hline \multirow{2}{*}{\multicolumn{22}{|c|}{65}} \\
\hline 66 & & & & & & & & & & & & & & & & & & & & & \\
\hline 67 & & & & & & & & & & & & & & & & & & & & & \\
\hline 68 & & & & & & & & & & & & & & & & & & & & & \\
\hline 69 & & & & & & & & & & & & & & & & & & & & & \\
\hline 70 & & & & & & & & & & & & & & & & & & & & & \\
\hline
\end{tabular}


Appendix A. Database - mass fraction

Comp. Vs Properties Study (Chick et al. 1981)

\begin{tabular}{|c|c|c|c|c|c|c|c|c|c|c|c|c|c|c|c|c|c|c|c|c|}
\hline Glass ID & $\begin{array}{c}\mathrm{SeO} 2 \\
-\mathrm{a}\end{array}$ & $\begin{array}{c}\mathrm{Sm} 2 \mathrm{O} 3 \\
-\mathrm{a}\end{array}$ & $\begin{array}{c}\mathrm{SnO} \\
-\mathrm{a} \\
\end{array}$ & $\begin{array}{c}\mathrm{SnO} 2 \\
-\mathrm{a}\end{array}$ & $\begin{array}{c}\mathrm{SO} 3 \\
-\mathrm{a} \\
\end{array}$ & $\begin{array}{c}\mathrm{SrO} \\
-\mathrm{a} \\
\end{array}$ & $\begin{array}{c}\mathrm{Tc} 2 \mathrm{O} 7 \\
-\mathrm{a}\end{array}$ & $\begin{array}{c}\mathrm{TeO} 2 \\
-\mathrm{a} \\
\end{array}$ & $\begin{array}{c}\mathrm{ThO} 2 \\
-\mathrm{a}\end{array}$ & $\begin{array}{c}\mathrm{TiO} 2 \\
-\mathrm{a} \\
\end{array}$ & $\begin{array}{c}\mathrm{T} 12 \mathrm{O} 3 \\
-\mathrm{a}\end{array}$ & $\begin{array}{c}\mathrm{U} 3 \mathrm{O} 8 \\
-\mathrm{a}\end{array}$ & $\begin{array}{c}\mathrm{UO} 2 \\
-\mathrm{a}\end{array}$ & $\begin{array}{c}\mathrm{UO} 3 \\
-\mathrm{a}\end{array}$ & $\begin{array}{c}\mathrm{V} 2 \mathrm{O} 5 \\
-\mathrm{a} \\
\end{array}$ & $\begin{array}{c}\text { WO3 } \\
-\mathrm{a}\end{array}$ & $\begin{array}{c}\mathrm{Y} 2 \mathrm{O} 3 \\
-\mathrm{a}\end{array}$ & $\begin{array}{c}\mathrm{ZnO} \\
-\mathrm{a} \\
\end{array}$ & $\begin{array}{c}\text { Others } \\
-\mathrm{a}\end{array}$ & $\begin{array}{c}\text { Sum } \\
-\mathrm{a}\end{array}$ \\
\hline 42 & & & & & & & & & & 0.0563 & & & & & & & & 0.0674 & & 1.0000 \\
\hline 43 & & & & & & & & & & 0.0414 & & & & & & & & 0.0373 & & 1.0000 \\
\hline 44 & & & & & & & & & & 0.0403 & & & & & & & & 0.0350 & & 1.0000 \\
\hline 45 & & & & & & & & & & 0.0403 & & & & & & & & 0.0362 & & 1.0000 \\
\hline 46 & & & & & & & & & & 0.0860 & & & & & & & & 0.0000 & & 1.0000 \\
\hline 47 & & & & & & & & & & 0.0855 & & & & & & & & 0.0000 & & 1.0000 \\
\hline 48 & & & & & & & & & & 0.0790 & & & & & & & & 0.0000 & & 1.0000 \\
\hline 49 & & & & & & & & & & 0.0960 & & & & & & & & 0.0780 & & 1.0000 \\
\hline 50 & & & & & & & & & & 0.0000 & & & & & & & & 0.0751 & & 1.0000 \\
\hline 51 & & & & & & & & & & 0.0000 & & & & & & & & 0.0723 & & 1.0000 \\
\hline 52 & & & & & & & & & & 0.0000 & & & & & & & & 0.0738 & & 1.0000 \\
\hline 53 & & & & & & & & & & 0.0000 & & & & & & & & 0.0775 & & 1.0000 \\
\hline 54 & & & & & & & & & & 0.0000 & & & & & & & & 0.0000 & & 1.0000 \\
\hline 55 & & & & & & & & & & 0.0000 & & & & & & & & 0.0000 & & 1.0000 \\
\hline 56 & & & & & & & & & & 0.0908 & & & & & & & & 0.0737 & & 1.0000 \\
\hline 57 & & & & & & & & & & 0.0842 & & & & & & & & 0.0668 & & 1.0000 \\
\hline 58 & & & & & & & & & & 0.0880 & & & & & & & & 0.0000 & & 1.0000 \\
\hline 59 & & & & & & & & & & 0.0939 & & & & & & & & 0.0000 & & 1.0000 \\
\hline 60 & & & & & & & & & & 0.0000 & & & & & & & & 0.0000 & & 1.0000 \\
\hline 61 & & & & & & & & & & 0.0000 & & & & & & & & 0.0000 & & 1.0000 \\
\hline 62 & & & & & & & & & & 0.0788 & & & & & & & & 0.0647 & & 1.0000 \\
\hline 63 & & & & & & & & & & 0.0000 & & & & & & & & 0.0744 & & 1.0000 \\
\hline 64 & & & & & & & & & & 0.0000 & & & & & & & & 0.0735 & & 1.0000 \\
\hline 65 & & & & & & & & & & 0.0000 & & & & & & & & 0.0719 & & 1.0000 \\
\hline 66 & & & & & & & & & & 0.0000 & & & & & & & & 0.0731 & & 1.0000 \\
\hline 67 & & & & & & & & & & 0.0000 & & & & & & & & 0.0000 & & 1.0000 \\
\hline 68 & & & & & & & & & & 0.0000 & & & & & & & & 0.0000 & & 1.0000 \\
\hline 69 & & & & & & & & & & 0.0927 & & & & & & & & 0.0772 & & 1.0000 \\
\hline 70 & & & & & & & & & & 0.0536 & & & & & & & & 0.0404 & & 1.0000 \\
\hline
\end{tabular}


Appendix A. Database - mass fraction

Comp. Vs Properties Study (Chick et al. 1981)

\begin{tabular}{|c|c|c|c|c|c|c|c|c|}
\hline Glass ID & $\begin{array}{l}\mathrm{TM} \\
\left({ }^{\circ} \mathrm{C}\right) \\
\end{array}$ & $\begin{array}{l}\text { Gradient } \\
\text { TL }\left({ }^{\circ} \mathrm{C}\right) \\
\end{array}$ & $\begin{array}{l}\text { Uniform } \\
\text { TL }\left({ }^{\circ} \mathrm{C}\right) \\
\end{array}$ & Primary Phase & $\begin{array}{l}\text { Quenched } \\
\text { Visual/OM }\end{array}$ & $\begin{array}{c}\text { Quenched } \\
\text { SEM/EDS or TEM }\end{array}$ & $\begin{array}{l}\text { Quenched } \\
\text { XRD } \\
\end{array}$ & $\begin{array}{c}\text { Quenched } \\
\text { Homogeneous? }\end{array}$ \\
\hline 42 & 1550 & & & & & & $\begin{array}{l}30 \mathrm{wt} \% \\
(\mathrm{Mg}, \mathrm{Ni}, \mathrm{Zn})(\mathrm{Al}, \mathrm{Cr}) 2 \mathrm{O} 4 / \\
\mathrm{TiO} 2\end{array}$ & \\
\hline 43 & 1250 & & & & & & $\begin{array}{l}9 \mathrm{wt} \% \\
(\mathrm{Mg}, \mathrm{Ni}, \mathrm{Zn})(\mathrm{Cr}, \mathrm{Fe}) 2 \mathrm{O} 4\end{array}$ & \\
\hline 44 & 1250 & & & & & & $\begin{array}{l}9 \mathrm{wt} \% \\
(\mathrm{Mg}, \mathrm{Ni}, \mathrm{Zn})(\mathrm{Cr}, \mathrm{Fe}) 2 \mathrm{O} 4\end{array}$ & \\
\hline 45 & 1250 & & & & & & $\begin{array}{l}9 \mathrm{wt} \% \\
(\mathrm{Mg}, \mathrm{Ni}, \mathrm{Zn})(\mathrm{Cr}, \mathrm{Fe}) 2 \mathrm{O} 4\end{array}$ & \\
\hline 46 & 1250 & & & & & & $\begin{array}{l}7 \mathrm{wt} \% \\
\mathrm{MgCr} 2 \mathrm{O} 4 / \mathrm{Cr} 2 \mathrm{O} 3\end{array}$ & \\
\hline 47 & 1250 & & & & & & $4.5 \mathrm{wt} \% \mathrm{Ni}(\mathrm{Fe}, \mathrm{Ti}) 2 \mathrm{O} 4$ & \\
\hline 48 & 1300 & & & & & & $\begin{array}{l}13.5 \mathrm{wt} \% \\
\mathrm{Cr} 2 \mathrm{O} 3 / \mathrm{Ni}(\mathrm{Cr}, \mathrm{Ti}, \mathrm{Fe}) 2 \mathrm{O} \\
4\end{array}$ & \\
\hline 49 & 1200 & & & & & & amorphous & \\
\hline 50 & 1200 & & & & & & $\begin{array}{l}18.5 \mathrm{wt} \% \\
(\mathrm{Mg}, \mathrm{Zn}) \mathrm{Cr} 2 \mathrm{O} 4\end{array}$ & \\
\hline 51 & 1200 & & & & & & $\begin{array}{l}20.5 \mathrm{wt} \% \\
(\mathrm{Mg}, \mathrm{Zn}) \mathrm{Cr} 2 \mathrm{O} 4\end{array}$ & \\
\hline 52 & 1200 & & & & & & $20 \mathrm{wt} \%(\mathrm{Mg}, \mathrm{Zn}) \mathrm{Cr} 2 \mathrm{O} 4$ & \\
\hline 53 & 1200 & & & & & & amorphous & \\
\hline 54 & 1250 & & & & & & $9.5 \mathrm{wt} \%(\mathrm{Cr}, \mathrm{Fe}) \mathrm{CrO} 3$ & \\
\hline 55 & 1250 & & & & & & $\begin{array}{l}16.5 \mathrm{wt} \% \\
(\mathrm{Ni}, \mathrm{Mg})(\mathrm{Cr}, \mathrm{Al}) \mathrm{CrO} 4\end{array}$ & \\
\hline 56 & 1050 & & & & & & amorphous & \\
\hline 57 & 1150 & & & & & & $\begin{array}{l}27.5 \mathrm{wt} \% \\
(\mathrm{Ni}, \mathrm{Zn})(\mathrm{Cr}, \mathrm{Ti}, \mathrm{Al}) \mathrm{CrO} 4\end{array}$ & \\
\hline 58 & 1150 & & & & & & amorphous & \\
\hline 59 & 1300 & & & & & & $11.5 \mathrm{wt} \% \mathrm{Cr} 2 \mathrm{O} 3 / \mathrm{TiO} 2$ & \\
\hline 60 & 1050 & & & & & & amorphous & \\
\hline 61 & 1250 & & & & & & amorphous & \\
\hline 62 & 1300 & & & & & & $16.5 \mathrm{wt} \%$ unidentifiable & \\
\hline 63 & 1150 & & & & & & amorphous & \\
\hline 64 & 1200 & & & & & & amorphous & \\
\hline 65 & 1200 & & & & & & amorphous & \\
\hline 66 & 1200 & & & & & & amorphous & \\
\hline 67 & 1150 & & & & & & $\begin{array}{l}18.5 \mathrm{wt} \% \\
(\mathrm{Mg}, \mathrm{Ni})(\mathrm{Al}, \mathrm{Cr}) \mathrm{CrO} 4\end{array}$ & \\
\hline 68 & 1400 & & & & & & $\begin{array}{l}12.5 \mathrm{wt} \% \\
\mathrm{Cr} 2 \mathrm{O} 3 / \mathrm{NiCr} 2 \mathrm{O} 4\end{array}$ & \\
\hline 69 & 1400 & & & & & & $14 \mathrm{wt} \%(\mathrm{Ni}, \mathrm{Zn}) \mathrm{Cr} 2 \mathrm{O} 4$ & \\
\hline 70 & 1280 & & & & & & $14 \mathrm{wt} \%$ spinel & \\
\hline
\end{tabular}


Appendix A. Database - mass fraction

Comp. Vs Properties Study (Chick et al. 1981)

\begin{tabular}{|c|c|c|c|c|c|}
\hline Glass ID & $\begin{array}{c}\mathrm{CCC} \\
\text { Visual/OM } \\
\end{array}$ & $\begin{array}{c}\text { CCC } \\
\text { SEM/EDS or TEM }\end{array}$ & $\begin{array}{l}\mathrm{CCC} \\
\mathrm{XRD}\end{array}$ & $\begin{array}{l}\text { Heat Treated } \\
\text { Visual/OM }\end{array}$ & $\begin{array}{c}\text { Heat Treated } \\
\text { SEM/EDS or TEM }\end{array}$ \\
\hline 42 & & & $\begin{array}{l}35.5 \mathrm{wt} \% \\
(\mathrm{Ca}, \mathrm{Na}) \mathrm{FeSi} 2 \mathrm{O} 6 /(\mathrm{Zn}, \mathrm{Ni}) \mathrm{Cr} 2 \mathrm{O} 4\end{array}$ & & \\
\hline 43 & & & $\begin{array}{l}18.5 \mathrm{wt} \% \\
(\mathrm{Mg}, \mathrm{Ni}, \mathrm{Zn})(\mathrm{Cr}, \mathrm{Fe}) 2 \mathrm{O} 4 /(\mathrm{Mg}, \mathrm{Fe}) \mathrm{Al} 2 \mathrm{O} 4\end{array}$ & & \\
\hline 44 & & & $\begin{array}{l}22.5 \mathrm{wt} \% \\
(\mathrm{Mg}, \mathrm{Ni}, \mathrm{Zn})(\mathrm{Cr}, \mathrm{Fe}) 2 \mathrm{O} 4 /(\mathrm{Mg}, \mathrm{Fe}) \mathrm{Al} 2 \mathrm{O} 4\end{array}$ & & \\
\hline 45 & & & $\begin{array}{l}20.5 \mathrm{wt} \% \\
(\mathrm{Mg}, \mathrm{Ni}, \mathrm{Zn})(\mathrm{Cr}, \mathrm{Fe}) 2 \mathrm{O} 4 /(\mathrm{Mg}, \mathrm{Fe}) \mathrm{Al} 2 \mathrm{O} 4\end{array}$ & & \\
\hline 46 & & & $14 \mathrm{wt} \% \mathrm{Cr} 2 \mathrm{O} 3 / \mathrm{TiO} 2$ & & \\
\hline 47 & & & $32 \mathrm{wt} \%(\mathrm{Ca}, \mathrm{Fe}, \mathrm{Mg}) \mathrm{SiO} 3 / \mathrm{CaTiSiO} 5$ & & \\
\hline 48 & & & $55 \mathrm{wt} \% \mathrm{NaAlSiO} 4 / \mathrm{TiO} 2$ & & \\
\hline 49 & & & $4 \mathrm{wt} \% \mathrm{TiO} 2$ & & \\
\hline 50 & & & $18.5 \mathrm{wt} \%(\mathrm{Mg}, \mathrm{Zn}) \mathrm{Cr} 2 \mathrm{O} 4$ & & \\
\hline 51 & & & $19 \mathrm{wt} \%(\mathrm{Mg}, \mathrm{Zn}) \mathrm{Cr} 2 \mathrm{O} 4$ & & \\
\hline 52 & & & $18.5 \mathrm{wt} \%(\mathrm{Mg}, \mathrm{Zn}) \mathrm{Cr} 2 \mathrm{O} 4$ & & \\
\hline 53 & & & $6.5 \mathrm{wt} \% \mathrm{NiSiO} 4$ & & \\
\hline 54 & & & $\begin{array}{l}2.4 \mathrm{wt} \% \\
(\mathrm{Cr}, \mathrm{Fe}) \mathrm{CrO} 3 /(\mathrm{Na}, \mathrm{Ca}) \mathrm{Al} 3 \mathrm{Si} 5 \mathrm{O} 16\end{array}$ & & \\
\hline 55 & & & $\begin{array}{l}17 \mathrm{wt} \% \\
(\mathrm{Ni}, \mathrm{Mg})(\mathrm{Cr}, \mathrm{Al}) \mathrm{CrO} 4 / \mathrm{NiAl} 2 \mathrm{O} 4\end{array}$ & & \\
\hline 56 & & & $4 \mathrm{wt} \%$ Ca4Ti3O 10 & & \\
\hline 57 & & & $\begin{array}{l}40.5 \mathrm{wt} \% \\
(\mathrm{Ni}, \mathrm{Zn})(\mathrm{Cr}, \mathrm{Ti}, \mathrm{Al}) \mathrm{CrO} 4 / \mathrm{NaAlSiO} 4\end{array}$ & & \\
\hline 58 & & & $15.5 \mathrm{wt} \% \mathrm{NaA} 1 \mathrm{SiO} 4 / \mathrm{MgTi} 2 \mathrm{O} 5$ & & \\
\hline 59 & & & $19.5 \mathrm{wt} \% \mathrm{Cr} 2 \mathrm{O} 3 / \mathrm{CaTiSiO} 5$ & & \\
\hline 60 & & & amorphous & & \\
\hline 61 & & & $21 \mathrm{wt} \%$ NaAlSiO4/CaAl2SiO6 & & \\
\hline 62 & & & $16 \mathrm{wt} \% \mathrm{Fe} 2 \mathrm{TiO} / \mathrm{ZnAl} 2 \mathrm{O} 4$ & & \\
\hline 63 & & & $30 \mathrm{wt} \%(\mathrm{Ca}, \mathrm{Fe}, \mathrm{Mg}) \mathrm{SiO} 3 / \mathrm{NiFe} 2 \mathrm{O} 4$ & & \\
\hline 64 & & & $76.5 \mathrm{wt} \% \mathrm{NaAlSiO} 4 / \mathrm{Ca} 2 \mathrm{Al} 2 \mathrm{SiO} 7$ & & \\
\hline 65 & & & $76 \mathrm{wt} \% \mathrm{NaA} 1 \mathrm{SiO} 4 / \mathrm{Ca} 2 \mathrm{~A} 12 \mathrm{SiO} 7$ & & \\
\hline 66 & & & $78 \mathrm{wt} \% \mathrm{NaA} 1 \mathrm{SiO} 4 / \mathrm{Ca} 2 \mathrm{Al} 2 \mathrm{SiO} 7$ & & \\
\hline 67 & & & $18 \mathrm{wt} \%(\mathrm{Mg}, \mathrm{Ni})(\mathrm{Al}, \mathrm{Cr}) \mathrm{CrO} 4$ & & \\
\hline 68 & & & $16.5 \mathrm{wt} \% \mathrm{NaAlSiO} 4 / \mathrm{Cr} 2 \mathrm{O} 3$ & & \\
\hline 69 & & & $26 \mathrm{wt} \%(\mathrm{Ni}, \mathrm{Zn}) \mathrm{Cr} 2 \mathrm{O} 4 / \mathrm{TiO} 2$ & & \\
\hline 70 & & & & & \\
\hline
\end{tabular}


Appendix A. Database - mass fraction

Comp. Vs Properties Study (Chick et al. 1981)

\begin{tabular}{|c|c|c|c|c|c|c|c|c|c|c|c|c|c|c|c|}
\hline Glass ID & $\begin{array}{c}\text { Heat Treated } \\
\text { XRD } \\
\end{array}$ & $\begin{array}{l}\text { Density } \\
\left(\mathrm{g} / \mathrm{cm}^{3}\right) \\
\end{array}$ & \begin{tabular}{|c|} 
Fulc Visc \\
$\mathrm{A}$
\end{tabular} & \begin{tabular}{|c|}
$\begin{array}{c}\text { Fulc Visc } \\
\mathrm{B}\end{array}$ \\
\end{tabular} & \begin{tabular}{|c|} 
Fulc Visc \\
To \\
\end{tabular} & $\begin{array}{c}\mathrm{FV} 1150^{\circ} \mathrm{C} \\
(\mathrm{Pa} \cdot \mathrm{s})\end{array}$ & $\begin{array}{c}\text { Arrh Visc } \\
\text { A }\end{array}$ & $\begin{array}{c}\text { Arrh Visc } \\
\text { B } \\
\end{array}$ & $\begin{array}{c}\mathrm{Av} \\
1150^{\circ} \mathrm{C} \\
(\mathrm{Dawa})\end{array}$ & $\begin{array}{c}\mathrm{T}\left({ }^{\circ} \mathrm{C}\right) \text { at } \\
2 \mathrm{~Pa} \cdot \mathrm{s} \\
\end{array}$ & \begin{tabular}{|c|}
$\mathrm{T}\left({ }^{\circ} \mathrm{C}\right)$ at \\
$5 \mathrm{~Pa} \cdot \mathrm{s}$ \\
\end{tabular} & \begin{tabular}{|c|}
$\mathrm{T}\left({ }^{\circ} \mathrm{C}\right)$ at \\
$10 \mathrm{~Pa} \cdot \mathrm{s}$ \\
\end{tabular} & $\begin{array}{c}\mathrm{T} 1 \\
\left({ }^{\circ} \mathrm{C}\right) \\
\end{array}$ & $\begin{array}{c}\mathrm{V} 1 \\
(\mathrm{~Pa} \cdot \mathrm{s}) \\
\end{array}$ & $\begin{array}{c}\mathrm{T} 2 \\
\left({ }^{\circ} \mathrm{C}\right) \\
\end{array}$ \\
\hline 42 & & & & & & & & & & & & & 1250 & $>580$ & \\
\hline 43 & & & & & & & & & & & & & 1250 & 22 & \\
\hline 44 & & & & & & & & & & & & & 1250 & 17.5 & \\
\hline 45 & & & & & & & & & & & & & 1250 & 24.3 & \\
\hline 46 & & & & & & & & & & & & & 1250 & 15 & \\
\hline 47 & & & & & & & & & & & & & 1250 & 1.8 & \\
\hline 48 & & & & & & & & & & & & & 1250 & 80 & \\
\hline 49 & & & & & & & & & & & & & 1250 & 2.3 & \\
\hline 50 & & & & & & & & & & & & & 1250 & 52.3 & \\
\hline 51 & & & & & & & & & & & & & 1250 & 60 & \\
\hline 52 & & & & & & & & & & & & & 1250 & 35 & \\
\hline 53 & & & & & & & & & & & & & 1250 & 1.3 & \\
\hline 54 & & & & & & & & & & & & & 1250 & $>580$ & \\
\hline 55 & & & & & & & & & & & & & 1250 & 58.5 & \\
\hline 56 & & & & & & & & & & & & & 1250 & 0.39 & \\
\hline 57 & & & & & & & & & & & & & 1250 & $>580$ & \\
\hline 58 & & & & & & & & & & & & & 1250 & 7.4 & \\
\hline 59 & & & & & & & & & & & & & 1250 & 29.3 & \\
\hline 60 & & & & & & & & & & & & & 1250 & 3.7 & \\
\hline 61 & & & & & & & & & & & & & 1250 & 13 & \\
\hline 62 & & & & & & & & & & & & & 1250 & 9 & \\
\hline 63 & & & & & & & & & & & & & 1250 & 0.9 & \\
\hline 64 & & & & & & & & & & & & & 1250 & 5.1 & \\
\hline 65 & & & & & & & & & & & & & 1250 & 6.8 & \\
\hline 66 & & & & & & & & & & & & & 1250 & 5.7 & \\
\hline 67 & & & & & & & & & & & & & 1250 & 68 & \\
\hline 68 & & & & & & & & & & & & & 1250 & 52 & \\
\hline 69 & & & & & & & & & & & & & 1250 & 15 & \\
\hline 70 & & & & & & & & & & & & & 1250 & 11.5 & \\
\hline
\end{tabular}


Appendix A. Database - mass fraction

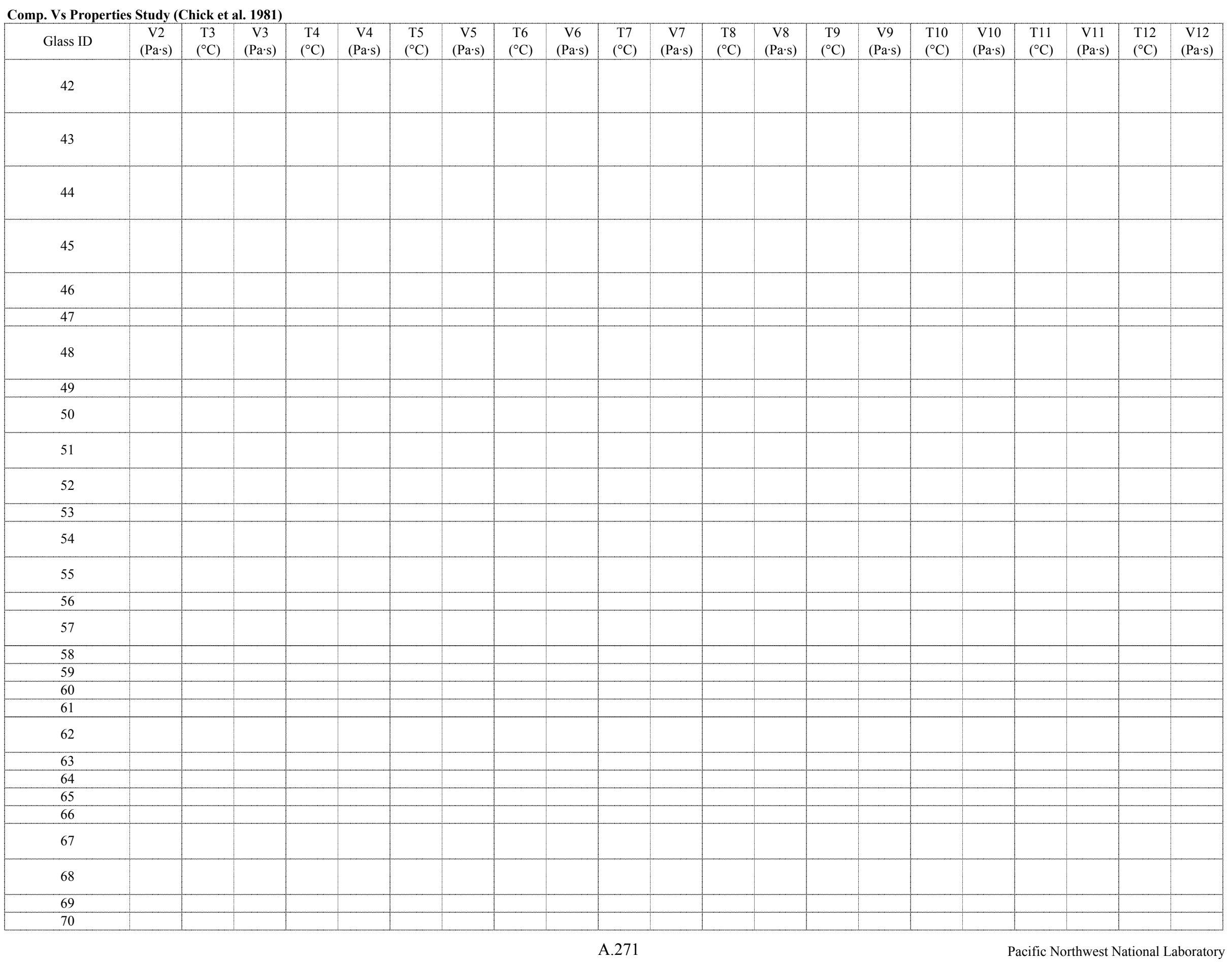


Appendix A. Database - mass fraction

Comp. Vs Properties Study (Chick et al. 1981)

\begin{tabular}{|c|c|c|c|c|c|c|c|c|c|c|c|c|c|c|c|c|}
\hline \multicolumn{17}{|c|}{ Comp. Vs Properties Study (Chick et al. 1981) } \\
\hline Glass ID & $\begin{array}{l}\mathrm{T} 13 \\
\left({ }^{\circ} \mathrm{C}\right) \\
\end{array}$ & $\begin{array}{c}\mathrm{V} 13 \\
(\mathrm{~Pa} \cdot \mathrm{s}) \\
\end{array}$ & $\begin{array}{l}\mathrm{T} 14 \\
\left({ }^{\circ} \mathrm{C}\right) \\
\end{array}$ & $\begin{array}{c}\mathrm{V} 14 \\
(\mathrm{~Pa} \cdot \mathrm{s})\end{array}$ & $\begin{array}{c}\text { Q PCT } \\
\mathrm{B}\left(\mathrm{g} / \mathrm{m}^{2}\right)\end{array}$ & $\begin{array}{c}\text { Q PCT } \\
\mathrm{Li}\left(\mathrm{g} / \mathrm{m}^{2}\right)\end{array}$ & $\begin{array}{c}\text { Q PCT } \\
\mathrm{Na}\left(\mathrm{g} / \mathrm{m}^{2}\right)\end{array}$ & $\begin{array}{c}\text { Q PCT } \\
\mathrm{Si}\left(\mathrm{g} / \mathrm{m}^{2}\right)\end{array}$ & $\begin{array}{c}\text { Q PCT } \\
\mathrm{pH}\end{array}$ & \begin{tabular}{|c|} 
CCC PCT \\
$\mathrm{B}\left(\mathrm{g} / \mathrm{m}^{2}\right)$ \\
\end{tabular} & $\begin{array}{l}\text { CCC PCT } \\
\mathrm{Li}\left(\mathrm{g} / \mathrm{m}^{2}\right) \\
\end{array}$ & $\begin{array}{l}\text { CCC PCT } \\
\mathrm{Na}\left(\mathrm{g} / \mathrm{m}^{2}\right) \\
\end{array}$ & \begin{tabular}{|c|} 
CCC PCT \\
$\mathrm{Si}\left(\mathrm{g} / \mathrm{m}^{2}\right)$ \\
\end{tabular} & \begin{tabular}{|c|}
$\begin{array}{c}\text { CCC PCT } \\
\mathrm{pH}\end{array}$ \\
\end{tabular} & $\begin{array}{l}\text { Q PCT B at } \\
20^{\circ} \mathrm{C}\left(\mathrm{g} / \mathrm{m}^{2}\right)\end{array}$ & $\begin{array}{l}\text { Q PCT Li at } \\
20^{\circ} \mathrm{C}\left(\mathrm{g} / \mathrm{m}^{2}\right)\end{array}$ \\
\hline \multicolumn{17}{|l|}{42} \\
\hline \multicolumn{17}{|l|}{43} \\
\hline \multicolumn{17}{|l|}{44} \\
\hline \multicolumn{17}{|l|}{45} \\
\hline \multicolumn{17}{|l|}{46} \\
\hline \multicolumn{17}{|l|}{47} \\
\hline \multicolumn{17}{|l|}{48} \\
\hline \multicolumn{17}{|l|}{49} \\
\hline \multicolumn{17}{|l|}{50} \\
\hline \multicolumn{17}{|l|}{51} \\
\hline \multicolumn{17}{|l|}{52} \\
\hline \multicolumn{17}{|l|}{53} \\
\hline \multicolumn{17}{|l|}{54} \\
\hline \multicolumn{17}{|l|}{55} \\
\hline \multicolumn{17}{|l|}{56} \\
\hline \multicolumn{17}{|l|}{57} \\
\hline \multicolumn{17}{|l|}{58} \\
\hline \multicolumn{17}{|l|}{59} \\
\hline \multicolumn{17}{|l|}{60} \\
\hline \multicolumn{17}{|l|}{61} \\
\hline \multicolumn{17}{|l|}{62} \\
\hline \multicolumn{17}{|l|}{63} \\
\hline \multicolumn{17}{|l|}{64} \\
\hline \multirow{2}{*}{\multicolumn{17}{|c|}{$\frac{65}{66}$}} \\
\hline 66 & & & & & & & & & & & & & & & & \\
\hline 67 & & & & & & & & & & & & & & & & \\
\hline 68 & & & & & & & & & & & & & & & & \\
\hline 69 & & & & & & & & & & & & & & & & \\
\hline 70 & & & & & & & & & & & & & & & & \\
\hline
\end{tabular}


Appendix A. Database - mass fraction

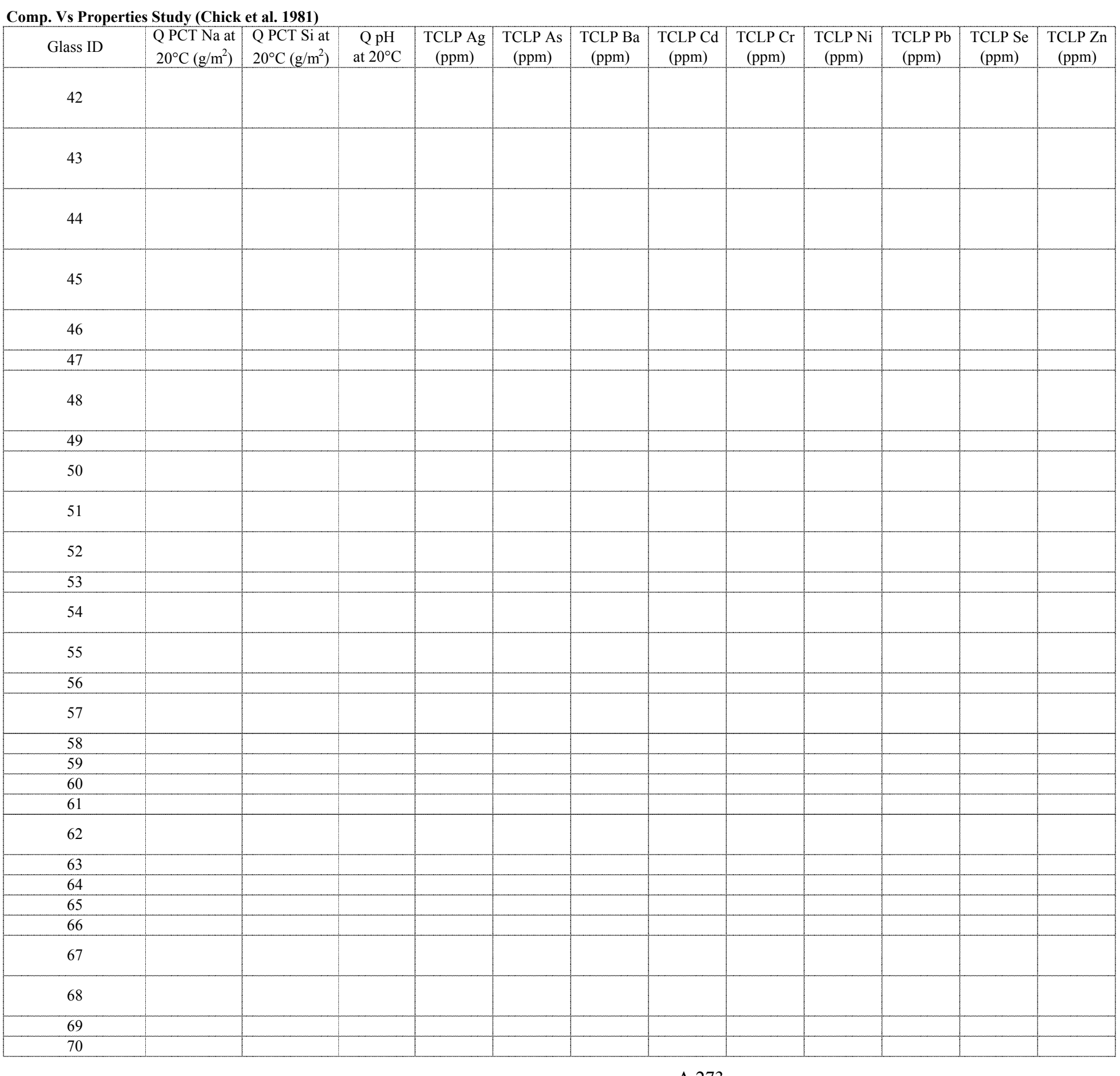


Comp. Vs Properties Study (Chick et al. 1981)

\begin{tabular}{|c|c|c|c|c|c|c|c|c|c|c|c|c|c|c|c|c|c|c|c|c|c|}
\hline Glass ID & $\begin{array}{c}\mathrm{A} 12 \mathrm{O} 3 \\
-\mathrm{t}\end{array}$ & $\begin{array}{c}\mathrm{B} 2 \mathrm{O} 3 \\
-\mathrm{t}\end{array}$ & $\begin{array}{c}\mathrm{CaO} \\
-\mathrm{t}\end{array}$ & $\begin{array}{c}\mathrm{Fe} 2 \mathrm{O} 3 \\
-\mathrm{t}\end{array}$ & $\begin{array}{c}\mathrm{FeO} \\
-\mathrm{t}\end{array}$ & $\begin{array}{c}\mathrm{K} 2 \mathrm{O} \\
-\mathrm{t}\end{array}$ & $\begin{array}{c}\mathrm{Li} 2 \mathrm{O} \\
-\mathrm{t}\end{array}$ & $\begin{array}{c}\mathrm{MgO} \\
-\mathrm{t}\end{array}$ & $\begin{array}{c}\mathrm{Na} 2 \mathrm{O} \\
-\mathrm{t}\end{array}$ & $\begin{array}{c}\mathrm{NiO} \\
-\mathrm{t}\end{array}$ & $\begin{array}{c}\mathrm{P} 2 \mathrm{O} 5 \\
-\mathrm{t}\end{array}$ & $\begin{array}{c}\mathrm{SiO} 2 \\
-\mathrm{t}\end{array}$ & $\begin{array}{c}\mathrm{ZrO} 2 \\
-\mathrm{t}\end{array}$ & $\begin{array}{c}\mathrm{Ag} 2 \mathrm{O} \\
-\mathrm{t}\end{array}$ & $\begin{array}{c}\mathrm{As} 2 \mathrm{O} 3 \\
-\mathrm{t}\end{array}$ & $\begin{array}{c}\mathrm{BaO} \\
-\mathrm{t}\end{array}$ & $\begin{array}{c}\mathrm{Bi} 2 \mathrm{O} 3 \\
-\mathrm{t}\end{array}$ & $\begin{array}{c}\mathrm{Br} \\
-\mathrm{t}\end{array}$ & $\begin{array}{c}\mathrm{CdO} \\
-\mathrm{t}\end{array}$ & $\begin{array}{c}\mathrm{Ce} 2 \mathrm{O} 3 \\
-\mathrm{t}\end{array}$ & $\begin{array}{c}\mathrm{CeO} 2 \\
-\mathrm{t}\end{array}$ \\
\hline 71 & & & & & & & & & & & & & & & & & & & & & \\
\hline 72 & & & & & & & & & & & & & & & & & & & & & \\
\hline 73 & & & & & & & & & & & & & & & & & & & & & \\
\hline 74 & & & & & & & & & & & & & & & & & & & & & \\
\hline 75 & & & & & & & & & & & & & & & & & & & & & \\
\hline 76 & & & & & & & & & & & & & & & & & & & & & \\
\hline 77 & & & & & & & & & & & & & & & & & & & & & \\
\hline 78 & & & & & & & & & & & & & & & & & & & & & \\
\hline 79 & & & & & & & & & & & & & & & & & & & & & \\
\hline 80 & & & & & & & & & & & & & & & & & & & & & \\
\hline 81 & & & & & & & & & & & & & & & & & & & & & \\
\hline 82 & & & & & & & & & & & & & & & & & & & & & \\
\hline 83 & & & & & & & & & & & & & & & & & & & & & \\
\hline 84 & & & & & & & & & & & & & & & & & & & & & \\
\hline 85 & & & & & & & & & & & & & & & & & & & & & \\
\hline 86 & & & & & & & & & & & & & & & & & & & & & \\
\hline 87 & & & & & & & & & & & & & & & & & & & & & \\
\hline 88 & & & & & & & & & & & & & & & & & & & & & \\
\hline 89 & & & & & & & & & & & & & & & & & & & & & \\
\hline 90 & & & & & & & & & & & & & & & & & & & & & \\
\hline 91 & & & & & & & & & & & & & & & & & & & & & \\
\hline 92 & & & & & & & & & & & & & & & & & & & & & \\
\hline 93 & & & & & & & & & & & & & & & & & & & & & \\
\hline 94 & & & & & & & & & & & & & & & & & & & & & \\
\hline 95 & & & & & & & & & & & & & & & & & & & & & \\
\hline 96 & & & & & & & & & & & & & & & & & & & & & \\
\hline 97 & & & & & & & & & & & & & & & & & & & & & \\
\hline 98 & & & & & & & & & & & & & & & & & & & & & \\
\hline 99 & & & & & & & & & & & & & & & & & & & & & \\
\hline 100 & & & & & & & & & & & & & & & & & & & & & \\
\hline 101 & & & & & & & & & & & & & & & & & & & & & \\
\hline 102 & & & & & & & & & & & & & & & & & & & & & \\
\hline 31-01 & 0.0800 & 0.0000 & 0.1500 & 0.0000 & & & & & 0.1700 & & & 0.6000 & & & & & & & & & \\
\hline 73-01 & 0.0800 & 0.0000 & 0.1500 & 0.0000 & & & & & 0.1700 & & & 0.6000 & & & & & & & & & \\
\hline $27-02$ & 0.0800 & 0.0200 & 0.0000 & 0.0000 & & & & & 0.3000 & & & 0.6000 & & & & & & & & & \\
\hline $72-02$ & 0.0800 & 0.0200 & 0.0000 & 0.0000 & & & & & 0.3000 & & & 0.6000 & & & & & & & & & \\
\hline 09-03 & 0.0800 & 0.0000 & 0.0000 & 0.2000 & & & & & 0.1200 & & & 0.6000 & & & & & & & & & \\
\hline $47-04$ & 0.0000 & 0.0600 & 0.0000 & 0.0000 & & 0.0009 & & & 0.1200 & 0.0077 & 0.0598 & 0.6000 & 0.0044 & & & & & & & & 0.0026 \\
\hline $50-05$ & 0.0000 & 0.0000 & 0.0000 & 0.0000 & & 0.0009 & & & 0.1800 & 0.0077 & 0.0598 & 0.6000 & 0.0044 & & & & & & & & 0.0026 \\
\hline 33-06 & 0.0800 & 0.2000 & 0.0000 & 0.0000 & & & & & 0.1200 & & & 0.6000 & & & & & & & & & \\
\hline $59-06$ & 0.0800 & 0.2000 & 0.0000 & 0.0000 & & & & & 0.1200 & & & 0.6000 & & & & & & & & & \\
\hline $57-07$ & 0.0000 & 0.0000 & 0.0400 & 0.2400 & & & & & 0.1200 & & & 0.6000 & & & & & & & & & \\
\hline 83-08 & 0.0000 & 0.2000 & 0.0000 & 0.0000 & & & & & 0.2000 & & & 0.6000 & & & & & & & & & \\
\hline 01-09 & 0.0000 & 0.0000 & 0.0000 & 0.2400 & & & & & 0.1600 & & & 0.6000 & & & & & & & & & \\
\hline $26-10$ & 0.0000 & 0.2000 & 0.0000 & 0.0800 & & & & & 0.1200 & & & 0.6000 & & & & & & & & & \\
\hline $43-11$ & 0.0600 & 0.0000 & 0.0000 & 0.0000 & & 0.0009 & & & 0.1200 & 0.0077 & 0.0598 & 0.6000 & 0.0044 & & & & & & & & 0.0026 \\
\hline 74-12 & 0.0000 & 0.0000 & 0.1500 & 0.0000 & & & & & 0.3000 & & & 0.5500 & & & & & & & & & \\
\hline $49-13$ & 0.0000 & 0.2000 & 0.1500 & 0.0000 & & & & & 0.1200 & & & 0.5300 & & & & & & & & & \\
\hline $30-14$ & 0.0800 & 0.0000 & 0.1500 & 0.0000 & & 0.0009 & & & 0.1200 & 0.0077 & 0.0598 & 0.4300 & 0.0044 & & & & & & & & 0.0026 \\
\hline
\end{tabular}


Comp. Vs Properties Study (Chick et al. 1981)

\begin{tabular}{|c|c|c|c|c|c|c|c|c|c|c|c|c|c|c|c|c|c|c|c|c|c|}
\hline Glass ID & $\begin{array}{l}\mathrm{Cl} \\
-\mathrm{t}\end{array}$ & $\begin{array}{c}\mathrm{CoO} \\
-\mathrm{t}\end{array}$ & $\begin{array}{c}\mathrm{Co} 2 \mathrm{O} 3 \\
-\mathrm{t}\end{array}$ & $\begin{array}{c}\mathrm{Cr} 2 \mathrm{O} 3 \\
-\mathrm{t}\end{array}$ & $\begin{array}{c}\mathrm{Cs} 2 \mathrm{O} \\
-\mathrm{t}\end{array}$ & $\begin{array}{c}\mathrm{CuO} \\
-\mathrm{t}\end{array}$ & $\begin{array}{c}\mathrm{Eu} 2 \mathrm{O} 3 \\
-\mathrm{t}\end{array}$ & $\begin{array}{l}F \\
-t\end{array}$ & $\begin{array}{c}\mathrm{Ga} 2 \mathrm{O} 3 \\
-\mathrm{t}\end{array}$ & $\begin{array}{c}\mathrm{Gd} 2 \mathrm{O} 3 \\
-\mathrm{t}\end{array}$ & $\begin{array}{c}\mathrm{HgO} \\
-\mathrm{t}\end{array}$ & $\begin{array}{l}I \\
-t\end{array}$ & $\begin{array}{c}\mathrm{La} 2 \mathrm{O} 3 \\
-\mathrm{t}\end{array}$ & $\begin{array}{c}\mathrm{MnO} 2 \\
-\mathrm{t}\end{array}$ & $\begin{array}{c}\mathrm{MnO} \\
-\mathrm{t}\end{array}$ & $\begin{array}{c}\mathrm{MoO} \\
-\mathrm{t}\end{array}$ & $\begin{array}{c}\mathrm{MoO} 3 \\
-\mathrm{t}\end{array}$ & $\begin{array}{c}\mathrm{Nb} 2 \mathrm{O} 5 \\
-\mathrm{t}\end{array}$ & $\begin{array}{c}\mathrm{Nd} 2 \mathrm{O} 3 \\
-\mathrm{t}\end{array}$ & $\begin{array}{c}\mathrm{PbO} \\
-\mathrm{t}\end{array}$ & $\begin{array}{c}\mathrm{PdO} 2 \\
-\mathrm{t}\end{array}$ \\
\hline 71 & & & & & & & & & & & & & & & & & & & & & \\
\hline 72 & & & & & & & & & & & & & & & & & & & & & \\
\hline 73 & & & & & & & & & & & & & & & & & & & & & \\
\hline 74 & & & & & & & & & & & & & & & & & & & & & \\
\hline 75 & & & & & & & & & & & & & & & & & & & & & \\
\hline 76 & & & & & & & & & & & & & & & & & & & & & \\
\hline 77 & & & & & & & & & & & & & & & & & & & & & \\
\hline 78 & & & & & & & & & & & & & & & & & & & & & \\
\hline 79 & & & & & & & & & & & & & & & & & & & & & \\
\hline 80 & & & & & & & & & & & & & & & & & & & & & \\
\hline 81 & & & & & & & & & & & & & & & & & & & & & \\
\hline 82 & & & & & & & & & & & & & & & & & & & & & \\
\hline 83 & & & & & & & & & & & & & & & & & & & & & \\
\hline 84 & & & & & & & & & & & & & & & & & & & & & \\
\hline 85 & & & & & & & & & & & & & & & & & & & & & \\
\hline 86 & & & & & & & & & & & & & & & & & & & & & \\
\hline 87 & & & & & & & & & & & & & & & & & & & & & \\
\hline 88 & & & & & & & & & & & & & & & & & & & & & \\
\hline 89 & & & & & & & & & & & & & & & & & & & & & \\
\hline 90 & & & & & & & & & & & & & & & & & & & & & \\
\hline 91 & & & & & & & & & & & & & & & & & & & & & \\
\hline 92 & & & & & & & & & & & & & & & & & & & & & \\
\hline 93 & & & & & & & & & & & & & & & & & & & & & \\
\hline 94 & & & & & & & & & & & & & & & & & & & & & \\
\hline 95 & & & & & & & & & & & & & & & & & & & & & \\
\hline 96 & & & & & & & & & & & & & & & & & & & & & \\
\hline 97 & & & & & & & & & & & & & & & & & & & & & \\
\hline 98 & & & & & & & & & & & & & & & & & & & & & \\
\hline 99 & & & & & & & & & & & & & & & & & & & & & \\
\hline 100 & & & & & & & & & & & & & & & & & & & & & \\
\hline 101 & & & & & & & & & & & & & & & & & & & & & \\
\hline 102 & & & & & & & & & & & & & & & & & & & & & \\
\hline 31-01 & & & & & & & & & & & & & & & & & & & & & \\
\hline 73-01 & & & & & & & & & & & & & & & & & & & & & \\
\hline $27-02$ & & & & & & & & & & & & & & & & & & & & & \\
\hline $72-02$ & & & & & & & & & & & & & & & & & & & & & \\
\hline $09-03$ & & & & & & & & & & & & & & & & & & & & & \\
\hline $47-04$ & & & & 0.0163 & 0.0033 & & & & & & & & & 0.0101 & & & 0.0042 & & 0.0040 & & \\
\hline $50-05$ & & & & 0.0163 & 0.0033 & & & & & & & & & 0.0101 & & & 0.0042 & & 0.0040 & & \\
\hline 33-06 & & & & & & & & & & & & & & & & & & & & & \\
\hline $59-06$ & & & & & & & & & & & & & & & & & & & & & \\
\hline $57-07$ & & & & & & & & & & & & & & & & & & & & & \\
\hline $83-08$ & & & & & & & & & & & & & & & & & & & & & \\
\hline 01-09 & & & & & & & & & & & & & & & & & & & & & \\
\hline $26-10$ & & & & & & & & & & & & & & & & & & & & & \\
\hline $43-11$ & & & & 0.0163 & 0.0033 & & & & & & & & & 0.0101 & & & 0.0042 & & 0.0040 & & \\
\hline $74-12$ & & & & & & & & & & & & & & & & & & & & & \\
\hline $49-13$ & & & & & & & & & & & & & & & & & & & & & \\
\hline $30-14$ & & & & 0.0163 & 0.0033 & & & & & & & & & 0.0101 & & & 0.0042 & & 0.0040 & & \\
\hline
\end{tabular}


Comp. Vs Properties Study (Chick et al. 1981)

\begin{tabular}{|c|c|c|c|c|c|c|c|c|c|c|c|c|c|c|c|c|c|c|c|c|c|}
\hline Glass ID & $\begin{array}{c}\mathrm{PdO} \\
-\mathrm{t}\end{array}$ & $\begin{array}{c}\mathrm{Pr} 2 \mathrm{O} 3 \\
-\mathrm{t} \\
\end{array}$ & $\begin{array}{c}\text { Pr6O11 } \\
-\mathrm{t}\end{array}$ & $\begin{array}{c}\mathrm{Rb} 2 \mathrm{O} \\
-\mathrm{t}\end{array}$ & $\begin{array}{c}\mathrm{ReO} \\
-\mathrm{t}\end{array}$ & $\begin{array}{c}\mathrm{ReO} 2 \\
-\mathrm{t}\end{array}$ & $\begin{array}{c}\mathrm{Rh} 2 \mathrm{O} 3 \\
-\mathrm{t}\end{array}$ & $\begin{array}{c}\mathrm{RhO}^{2} \\
-\mathrm{t}\end{array}$ & $\begin{array}{c}\mathrm{RuO}_{2} \\
-\mathrm{t}\end{array}$ & {$\left[\begin{array}{c}\mathrm{Sb} 2 \mathrm{O} 3 \\
-\mathrm{t}\end{array}\right.$} & $\begin{array}{c}\mathrm{Sb} 2 \mathrm{O} 5 \\
-\mathrm{t}\end{array}$ & $\begin{array}{c}\mathrm{SeO} 2 \\
-\mathrm{t}\end{array}$ & $\begin{array}{c}\mathrm{Sm} 2 \mathrm{O} 3 \\
-\mathrm{t}\end{array}$ & $\begin{array}{c}\mathrm{SnO} \\
-\mathrm{t}\end{array}$ & $\begin{array}{c}\mathrm{SnO} 2 \\
-t\end{array}$ & $\begin{array}{c}\mathrm{SO} 3 \\
-\mathrm{t}\end{array}$ & $\begin{array}{c}\mathrm{SrO} \\
-\mathrm{t}\end{array}$ & $\begin{array}{c}\mathrm{Tc} 2 \mathrm{O} 7 \\
-\mathrm{t}\end{array}$ & $\begin{array}{c}\mathrm{TeO} 2 \\
-\mathrm{t}\end{array}$ & $\begin{array}{c}\mathrm{ThO} 2 \\
-\mathrm{t}\end{array}$ & $\begin{array}{c}\mathrm{TiO} 2 \\
-\mathrm{t} \\
\end{array}$ \\
\hline \multicolumn{22}{|l|}{71} \\
\hline \multirow{2}{*}{\multicolumn{22}{|c|}{$\frac{72}{73}$}} \\
\hline \multirow{2}{*}{\multicolumn{22}{|c|}{$\frac{13}{74}$}} \\
\hline & & & & & & & & & & & & & & & & & & & & & \\
\hline \multicolumn{22}{|l|}{75} \\
\hline \multicolumn{22}{|l|}{76} \\
\hline \multicolumn{22}{|l|}{77} \\
\hline \multicolumn{22}{|l|}{78} \\
\hline \multicolumn{22}{|l|}{79} \\
\hline \multicolumn{22}{|l|}{80} \\
\hline \multicolumn{22}{|l|}{81} \\
\hline \multicolumn{22}{|l|}{82} \\
\hline \multicolumn{22}{|l|}{83} \\
\hline \multicolumn{22}{|l|}{84} \\
\hline 85 & & & & & & & & & & & & & & & & & & & & & \\
\hline 86 & & & & & & & & & & & & & & & & & & & & & \\
\hline 87 & & & & & & & & & & & & & & & & & & & & & \\
\hline 88 & & & & & & & & & & & & & & & & & & & & & \\
\hline 89 & & & & & & & & & & & & & & & & & & & & & \\
\hline 90 & & & & & & & & & & & & & & & & & & & & & \\
\hline 91 & & & & & & & & & & & & & & & & & & & & & \\
\hline 92 & & & & & & & & & & & & & & & & & & & & & \\
\hline 93 & & & & & & & & & & & & & & & & & & & & & \\
\hline 94 & & & & & & & & & & & & & & & & & & & & & \\
\hline 95 & & & & & & & & & & & & & & & & & & & & & \\
\hline 96 & & & & & & & & & & & & & & & & & & & & & \\
\hline 97 & & & & & & & & & & & & & & & & & & & & & \\
\hline 98 & & & & & & & & & & & & & & & & & & & & & \\
\hline 99 & & & & & & & & & & & & & & & & & & & & & \\
\hline 100 & & & & & & & & & & & & & & & & & & & & & \\
\hline 101 & & & & & & & & & & & & & & & & & & & & & \\
\hline 102 & & & & & & & & & & & & & & & & & & & & & \\
\hline $31-01$ & & & & & & & & & & & & & & & & & & & & & \\
\hline 73-01 & & & & & & & & & & & & & & & & & & & & & \\
\hline $27-02$ & & & & & & & & & & & & & & & & & & & & & \\
\hline $72-02$ & & & & & & & & & & & & & & & & & & & & & \\
\hline 09-03 & & & & & & & & & & & & & & & & & & & & & \\
\hline $47-04$ & & & & & & & & & & & & & & & & & 0.0033 & & & 0.0781 & \\
\hline $50-05$ & & & & & & & & & & & & & & & & & 0.0033 & & & 0.0781 & \\
\hline $33-06$ & & & & & & & & & & & & & & & & & & & & & \\
\hline 59-06 & & & & & & & & & & & & & & & & & & & & & \\
\hline $57-07$ & & & & & & & & & & & & & & & & & & & & & \\
\hline $83-08$ & & & & & & & & & & & & & & & & & & & & & \\
\hline 01-09 & & & & & & & & & & & & & & & & & & & & & \\
\hline $26-10$ & & & & & & & & & & & & & & & & & & & & & \\
\hline $43-11$ & & & & & & & & & & & & & & & & & 0.0033 & & & 0.0781 & \\
\hline $74-12$ & & & & & & & & & & & & & & & & & & & & & \\
\hline $49-13$ & & & & & & & & & & & & & & & & & & & & & \\
\hline $30-14$ & & & & & & & & & & & & & & & & & 0.0033 & & & 0.0781 & \\
\hline
\end{tabular}


Comp. Vs Properties Study (Chick et al. 1981)

\begin{tabular}{|c|c|c|c|c|c|c|c|c|c|c|c|c|c|c|c|c|c|c|c|c|c|}
\hline Glass ID & $\begin{array}{c}\mathrm{Tl} 2 \mathrm{O} 3 \\
-\mathrm{t}\end{array}$ & $\begin{array}{c}\mathrm{U} 3 \mathrm{O} 8 \\
-\mathrm{t}\end{array}$ & $\begin{array}{c}\mathrm{UO} 2 \\
-\mathrm{t}\end{array}$ & $\begin{array}{c}\mathrm{UO} 3 \\
-\mathrm{t}\end{array}$ & $\begin{array}{c}\mathrm{V} 2 \mathrm{O} 5 \\
-\mathrm{t}\end{array}$ & $\begin{array}{c}\text { WO3 } \\
-\mathrm{t}\end{array}$ & $\begin{array}{c}\mathrm{Y} 2 \mathrm{O} 3 \\
-\mathrm{t}\end{array}$ & $\begin{array}{c}\mathrm{ZnO} \\
-\mathrm{t}\end{array}$ & $\begin{array}{c}\text { Others } \\
-t\end{array}$ & $\begin{array}{c}\text { Sum } \\
-t\end{array}$ & $\begin{array}{c}\mathrm{A} 12 \mathrm{O} 3 \\
-\mathrm{a}\end{array}$ & $\begin{array}{c}\mathrm{B} 2 \mathrm{O} 3 \\
-\mathrm{a}\end{array}$ & $\begin{array}{c}\mathrm{CaO} \\
-\mathrm{a}\end{array}$ & $\begin{array}{c}\mathrm{Fe} 2 \mathrm{O} 3 \\
-\mathrm{a}\end{array}$ & $\begin{array}{c}\mathrm{FeO} \\
-\mathrm{a}\end{array}$ & $\begin{array}{c}\mathrm{K} 2 \mathrm{O} \\
-\mathrm{a}\end{array}$ & $\begin{array}{c}\mathrm{Li} 2 \mathrm{O} \\
-\mathrm{a}\end{array}$ & $\begin{array}{c}\mathrm{MgO} \\
-\mathrm{a}\end{array}$ & $\begin{array}{c}\mathrm{Na} 2 \mathrm{O} \\
-\mathrm{a}\end{array}$ & $\begin{array}{c}\mathrm{NiO} \\
-\mathrm{a}\end{array}$ & $\begin{array}{c}\mathrm{P} 2 \mathrm{O} 5 \\
-\mathrm{a}\end{array}$ \\
\hline 71 & & & & & & & & & & & 0.0777 & 0.0764 & 0.0393 & 0.0268 & & & & 0.0178 & 0.1124 & 0.0125 & \\
\hline 72 & & & & & & & & & & & 0.1099 & 0.0555 & 0.0580 & 0.0401 & & & & 0.0244 & 0.1290 & 0.0176 & \\
\hline 73 & & & & & & & & & & & 0.0882 & 0.1484 & 0.0468 & 0.0309 & & & & 0.0204 & 0.1238 & 0.0145 & \\
\hline 74 & & & & & & & & & & & 0.0000 & 0.1017 & 0.0664 & 0.0442 & & & & 0.0298 & 0.1392 & 0.0195 & \\
\hline 75 & & & & & & & & & & & 0.2204 & 0.0768 & 0.0401 & 0.0252 & & & & 0.0167 & 0.1091 & 0.0107 & \\
\hline 76 & & & & & & & & & & & 0.1168 & 0.0919 & 0.0000 & 0.0417 & & & & 0.0269 & 0.1303 & 0.0173 & \\
\hline 77 & & & & & & & & & & & 0.0836 & 0.0867 & 0.1141 & 0.0291 & & & & 0.0196 & 0.1233 & 0.0147 & \\
\hline 78 & & & & & & & & & & & 0.1095 & 0.0889 & 0.0578 & 0.0394 & & & & 0.0000 & 0.1287 & 0.0174 & \\
\hline 79 & & & & & & & & & & & 0.0917 & 0.0891 & 0.0504 & 0.0341 & & & & 0.0541 & 0.1256 & 0.0148 & \\
\hline 80 & & & & & & & & & & & 0.1096 & 0.0902 & 0.0586 & 0.0376 & & & & 0.0243 & 0.0849 & 0.0165 & \\
\hline 81 & & & & & & & & & & & 0.0930 & 0.0875 & 0.0503 & 0.0334 & & & & 0.0205 & 0.1557 & 0.0145 & \\
\hline 82 & & & & & & & & & & & 0.1077 & 0.0942 & 0.0567 & 0.0380 & & & & 0.0246 & 0.1291 & 0.0200 & \\
\hline 83 & & & & & & & & & & & 0.0948 & 0.0873 & 0.0397 & 0.0330 & & & & 0.0220 & 0.1235 & 0.0154 & \\
\hline 84 & & & & & & & & & & & 0.1093 & 0.0933 & 0.0584 & 0.0404 & & & & 0.0264 & 0.1319 & 0.0189 & \\
\hline 85 & & & & & & & & & & & 0.0918 & 0.0843 & 0.0488 & 0.0330 & & & & 0.0214 & 0.1207 & 0.0143 & \\
\hline 86 & & & & & & & & & & & 0.1077 & 0.0946 & 0.0576 & 0.0386 & & & & 0.0255 & 0.1310 & 0.0169 & \\
\hline 87 & & & & & & & & & & & 0.0962 & 0.0778 & 0.0505 & 0.0324 & & & & 0.0211 & 0.1151 & 0.0152 & \\
\hline 88 & & & & & & & & & & & 0.1080 & 0.0927 & 0.0569 & 0.0000 & & & & 0.0244 & 0.1285 & 0.0170 & \\
\hline 89 & & & & & & & & & & & 0.0960 & 0.0867 & 0.0487 & 0.0786 & & & & 0.0228 & 0.1274 & 0.0151 & \\
\hline 90 & & & & & & & & & & & 0.1045 & 0.0931 & 0.0550 & 0.0356 & & & & 0.0233 & 0.1280 & 0.0000 & \\
\hline 91 & & & & & & & & & & & 0.0968 & 0.0867 & 0.0507 & 0.0355 & & & & 0.0227 & 0.1241 & 0.0377 & \\
\hline 92 & & & & & & & & & & & 0.2040 & 0.0504 & 0.0318 & 0.0793 & & & & 0.0000 & 0.0871 & 0.0360 & \\
\hline 93 & & & & & & & & & & & 0.2143 & 0.0532 & 0.0330 & 0.0000 & & & & 0.0000 & 0.0929 & 0.0000 & \\
\hline 94 & & & & & & & & & & & 0.2073 & 0.0287 & 0.0327 & 0.0000 & & & & 0.0000 & 0.0873 & 0.0000 & \\
\hline 95 & & & & & & & & & & & 0.2411 & 0.0552 & 0.0000 & 0.0000 & & & & 0.0000 & 0.1466 & 0.0329 & \\
\hline 96 & & & & & & & & & & & 0.0000 & 0.1204 & 0.0899 & 0.0764 & & & & 0.0000 & 0.0889 & 0.0347 & \\
\hline 97 & & & & & & & & & & & 0.0000 & 0.1538 & 0.0189 & 0.0955 & & & & 0.0593 & 0.1654 & 0.0000 & \\
\hline 98 & & & & & & & & & & & 0.0000 & 0.1384 & 0.1176 & 0.0000 & & & & 0.0557 & 0.0972 & 0.0394 & \\
\hline 99 & & & & & & & & & & & 0.0000 & 0.1194 & 0.1115 & 0.0805 & & & & 0.0000 & 0.1527 & 0.0161 & \\
\hline 100 & & & & & & & & & & & 0.1519 & 0.1183 & 0.0363 & 0.0000 & & & & 0.0000 & 0.1231 & 0.0000 & \\
\hline 101 & & & & & & & & & & & 0.0000 & 0.1320 & 0.0391 & 0.0824 & & & & 0.0000 & 0.1326 & 0.0000 & \\
\hline 102 & & & & & & & & & & & 0.0411 & 0.0982 & 0.1087 & 0.0520 & & & & 0.0388 & 0.1336 & 0.0000 & \\
\hline $31-01$ & & & & & & & & & & 1.0000 & 0.0815 & 0.0000 & 0.1496 & 0.0000 & & & & & 0.1665 & & \\
\hline 73-01 & & & & & & & & & & 1.0000 & 0.0830 & 0.0000 & 0.1530 & 0.0000 & & & & & 0.1740 & & \\
\hline $27-02$ & & & & & & & & & & 1.0000 & 0.0838 & 0.0000 & 0.0021 & 0.0000 & & & & & 0.2996 & & \\
\hline $72-02$ & & & & & & & & & & 1.0000 & 0.0836 & 0.0000 & 0.0033 & 0.0000 & & & & & 0.3084 & & \\
\hline $09-03$ & & & & & & & & & & 1.0000 & 0.0814 & 0.0000 & 0.0000 & 0.1719 & & & & & 0.1200 & & \\
\hline $47-04$ & & & 0.0253 & & & & & & & 1.0000 & 0.0041 & 0.0601 & 0.0015 & 0.0000 & & 0.0008 & & & 0.1328 & 0.0069 & 0.0533 \\
\hline $50-05$ & & & 0.0253 & & & & & & & 1.0000 & 0.0104 & 0.0000 & 0.0013 & 0.0000 & & 0.0008 & & & 0.1816 & 0.0070 & 0.0547 \\
\hline $33-06$ & & & & & & & & & & 1.0000 & 0.0832 & 0.1922 & 0.0014 & 0.0000 & & & & & 0.1189 & & \\
\hline $59-06$ & & & & & & & & & & 1.0000 & 0.0832 & 0.1945 & 0.0016 & 0.0000 & & & & & 0.1193 & & \\
\hline $57-07$ & & & & & & & & & & 1.0000 & 0.0111 & 0.0000 & 0.0423 & 0.2002 & & & & & 0.1227 & & \\
\hline $83-08$ & & & & & & & & & & 1.0000 & 0.0044 & 0.1947 & 0.0025 & 0.0000 & & & & & 0.2103 & & \\
\hline 01-09 & & & & & & & & & & 1.0000 & 0.0102 & 0.0000 & 0.0039 & 0.2043 & & & & & 0.1636 & & \\
\hline $26-10$ & & & & & & & & & & 1.0000 & 0.0064 & 0.1931 & 0.0020 & 0.0680 & & & & & 0.1186 & & \\
\hline $43-11$ & & & 0.0253 & & & & & & & 1.0000 & 0.0642 & 0.0000 & 0.0031 & 0.0000 & & 0.0008 & & & 0.1204 & 0.0074 & 0.0571 \\
\hline $74-12$ & & & & & & & & & & 1.0000 & 0.0038 & 0.0000 & 0.1492 & 0.0000 & & & & & 0.3034 & & \\
\hline $49-13$ & & & & & & & & & & 1.0000 & 0.0029 & 0.2005 & 0.1511 & 0.0000 & & & & & 0.1280 & & \\
\hline $30-14$ & & & 0.0253 & & & & & & & 1.0000 & 0.0880 & 0.0000 & 0.1548 & 0.0000 & & 0.0009 & & & 0.1272 & 0.0075 & 0.0580 \\
\hline
\end{tabular}


Appendix A. Database - mass fraction

Comp. Vs Properties Study (Chick et al. 1981)

\begin{tabular}{|c|c|c|c|c|c|c|c|c|c|c|c|c|c|c|c|c|c|c|c|c|c|}
\hline Glass ID & $\begin{array}{c}\mathrm{SiO} 2 \\
-\mathrm{a}\end{array}$ & $\begin{array}{c}\mathrm{ZrO} 2 \\
-\mathrm{a}\end{array}$ & $\begin{array}{c}\mathrm{Ag} 2 \mathrm{O} \\
-\mathrm{a}\end{array}$ & $\begin{array}{c}\mathrm{As} 2 \mathrm{O} 3 \\
-\mathrm{a}\end{array}$ & $\begin{array}{c}\mathrm{BaO} \\
-\mathrm{a}\end{array}$ & $\begin{array}{c}\mathrm{Bi} 2 \mathrm{O} 3 \\
-\mathrm{a}\end{array}$ & $\begin{array}{l}\mathrm{Br} \\
-\mathrm{a}\end{array}$ & $\begin{array}{c}\mathrm{CdO} \\
-\mathrm{a}\end{array}$ & $\begin{array}{c}\mathrm{Ce} 2 \mathrm{O} 3 \\
-\mathrm{a}\end{array}$ & $\begin{array}{c}\mathrm{CeO} 2 \\
-\mathrm{a}\end{array}$ & $\begin{array}{l}\mathrm{Cl} \\
-\mathrm{a}\end{array}$ & $\begin{array}{c}\mathrm{CoO} \\
-\mathrm{a}\end{array}$ & $\begin{array}{c}\mathrm{Co} 2 \mathrm{O} 3 \\
-\mathrm{a}\end{array}$ & $\begin{array}{c}\mathrm{Cr} 2 \mathrm{O} 3 \\
-\mathrm{a}\end{array}$ & $\begin{array}{c}\mathrm{Cs} 2 \mathrm{O} \\
-\mathrm{a}\end{array}$ & $\begin{array}{c}\mathrm{CuO} \\
-\mathrm{a}\end{array}$ & $\begin{array}{c}\mathrm{Eu} 2 \mathrm{O} 3 \\
-\mathrm{a}\end{array}$ & $\begin{array}{l}\mathrm{F} \\
-\mathrm{a}\end{array}$ & $\begin{array}{c}\mathrm{Ga} 2 \mathrm{O} 3 \\
-\mathrm{a}\end{array}$ & $\begin{array}{c}\mathrm{Gd} 2 \mathrm{O} 3 \\
-\mathrm{a}\end{array}$ & $\begin{array}{c}\mathrm{HgO} \\
-\mathrm{a}\end{array}$ \\
\hline 71 & 0.5514 & & & & & & & & & & & & & 0.0255 & & & & & & & \\
\hline 72 & 0.4427 & & & & & & & & & & & & & 0.0359 & & & & & & & \\
\hline 73 & 0.4290 & & & & & & & & & & & & & 0.0295 & & & & & & & \\
\hline 74 & 0.4676 & & & & & & & & & & & & & 0.0351 & & & & & & & \\
\hline 75 & 0.4181 & & & & & & & & & & & & & 0.0218 & & & & & & & \\
\hline 76 & 0.4409 & & & & & & & & & & & & & 0.0397 & & & & & & & \\
\hline 77 & 0.4315 & & & & & & & & & & & & & 0.0277 & & & & & & & \\
\hline 78 & 0.4388 & & & & & & & & & & & & & 0.0353 & & & & & & & \\
\hline 79 & 0.4377 & & & & & & & & & & & & & 0.0301 & & & & & & & \\
\hline 80 & 0.4512 & & & & & & & & & & & & & 0.0358 & & & & & & & \\
\hline 81 & 0.4421 & & & & & & & & & & & & & 0.0295 & & & & & & & \\
\hline 82 & 0.4460 & & & & & & & & & & & & & 0.0362 & & & & & & & \\
\hline 83 & 0.4371 & & & & & & & & & & & & & 0.0314 & & & & & & & \\
\hline 84 & 0.4464 & & & & & & & & & & & & & 0.0362 & & & & & & & \\
\hline 85 & 0.4274 & & & & & & & & & & & & & 0.0314 & & & & & & & \\
\hline 86 & 0.4443 & & & & & & & & & & & & & 0.0000 & & & & & & & \\
\hline 87 & 0.4385 & & & & & & & & & & & & & 0.0750 & & & & & & & \\
\hline 88 & 0.4538 & & & & & & & & & & & & & 0.0345 & & & & & & & \\
\hline 89 & 0.4193 & & & & & & & & & & & & & 0.0308 & & & & & & & \\
\hline 90 & 0.4453 & & & & & & & & & & & & & 0.0339 & & & & & & & \\
\hline 91 & 0.4366 & & & & & & & & & & & & & 0.0316 & & & & & & & \\
\hline 92 & 0.5115 & & & & & & & & & & & & & 0.0000 & & & & & & & \\
\hline 93 & 0.5210 & & & & & & & & & & & & & 0.0000 & & & & & & & \\
\hline 94 & 0.5263 & & & & & & & & & & & & & 0.0756 & & & & & & & \\
\hline 95 & 0.5242 & & & & & & & & & & & & & 0.0000 & & & & & & & \\
\hline 96 & 0.3507 & & & & & & & & & & & & & 0.0770 & & & & & & & \\
\hline 97 & 0.4184 & & & & & & & & & & & & & 0.0000 & & & & & & & \\
\hline 98 & 0.3798 & & & & & & & & & & & & & 0.0850 & & & & & & & \\
\hline 99 & 0.3645 & & & & & & & & & & & & & 0.0789 & & & & & & & \\
\hline 100 & 0.5080 & & & & & & & & & & & & & 0.0000 & & & & & & & \\
\hline 101 & 0.5460 & & & & & & & & & & & & & 0.0000 & & & & & & & \\
\hline 102 & 0.4193 & & & & & & & & & & & & & 0.0024 & & & & & & & \\
\hline $31-01$ & 0.6025 & & & & & & & & & & & & & & & & & & & & \\
\hline $73-01$ & 0.5900 & & & & & & & & & & & & & & & & & & & & \\
\hline $27-02$ & 0.6145 & & & & & & & & & & & & & & & & & & & & \\
\hline $72-02$ & 0.6047 & & & & & & & & & & & & & & & & & & & & \\
\hline 09-03 & 0.6267 & & & & & & & & & & & & & & & & & & & & \\
\hline $47-04$ & 0.6055 & 0.0039 & & & & & & & & 0.0024 & & & & 0.0145 & 0.0029 & & & & & & \\
\hline $50-05$ & 0.6053 & 0.0040 & & & & & & & & 0.0024 & & & & 0.0149 & 0.0030 & & & & & & \\
\hline $33-06$ & 0.6043 & & & & & & & & & & & & & & & & & & & & \\
\hline $59-06$ & 0.6014 & & & & & & & & & & & & & & & & & & & & \\
\hline $57-07$ & 0.6237 & & & & & & & & & & & & & & & & & & & & \\
\hline 83-08 & 0.5881 & & & & & & & & & & & & & & & & & & & & \\
\hline 01-09 & 0.6179 & & & & & & & & & & & & & & & & & & & & \\
\hline $26-10$ & 0.6120 & & & & & & & & & & & & & & & & & & & & \\
\hline $43-11$ & 0.6022 & 0.0042 & & & & & & & & 0.0025 & & & & 0.0156 & 0.0032 & & & & & & \\
\hline $74-12$ & 0.5436 & & & & & & & & & & & & & & & & & & & & \\
\hline $49-13$ & 0.5174 & & & & & & & & & & & & & & & & & & & & \\
\hline $30-14$ & 0.4167 & 0.0043 & & & & & & & & 0.0026 & & & & 0.0158 & 0.0032 & & & & & & \\
\hline
\end{tabular}


Comp. Vs Properties Study (Chick et al. 1981)

\begin{tabular}{|c|c|c|c|c|c|c|c|c|c|c|c|c|c|c|c|c|c|c|c|c|c|}
\hline Glass ID & $\begin{array}{c}\mathrm{I} \\
-\mathrm{a}\end{array}$ & $\begin{array}{c}\mathrm{La} 2 \mathrm{O} 3 \\
-\mathrm{a}\end{array}$ & $\begin{array}{c}\mathrm{MnO} 2 \\
-\mathrm{a}\end{array}$ & $\begin{array}{c}\mathrm{MnO} \\
-\mathrm{a}\end{array}$ & $\begin{array}{c}\mathrm{MoO} \\
-\mathrm{a}\end{array}$ & $\begin{array}{c}\mathrm{MoO} 3 \\
-\mathrm{a}\end{array}$ & $\begin{array}{c}\mathrm{Nb} 2 \mathrm{O} 5 \\
-\mathrm{a}\end{array}$ & $\begin{array}{c}\mathrm{Nd} 2 \mathrm{O} 3 \\
-\mathrm{a}\end{array}$ & $\begin{array}{c}\mathrm{PbO} \\
-\mathrm{a}\end{array}$ & $\begin{array}{c}\mathrm{PdO} 2 \\
-\mathrm{a}\end{array}$ & $\begin{array}{c}\mathrm{PdO} \\
-\mathrm{a}\end{array}$ & $\begin{array}{c}\mathrm{Pr} 2 \mathrm{O} 3 \\
-\mathrm{a}\end{array}$ & $\begin{array}{c}\text { Pr6O11 } \\
-\mathrm{a}\end{array}$ & $\begin{array}{c}\mathrm{Rb} 2 \mathrm{O} \\
-\mathrm{a}\end{array}$ & $\begin{array}{c}\mathrm{ReO} \\
-\mathrm{a}\end{array}$ & $\begin{array}{c}\mathrm{ReO} 2 \\
-\mathrm{a}\end{array}$ & $\begin{array}{c}\mathrm{Rh} 2 \mathrm{O} 3 \\
-\mathrm{a}\end{array}$ & $\begin{array}{c}\mathrm{RhO} 2 \\
-\mathrm{a}\end{array}$ & $\begin{array}{c}\mathrm{RuO} 2 \\
-\mathrm{a}\end{array}$ & $\begin{array}{c}\mathrm{Sb} 2 \mathrm{O} 3 \\
-\mathrm{a}\end{array}$ & $\begin{array}{c}\mathrm{Sb} 2 \mathrm{O} 5 \\
-\mathrm{a}\end{array}$ \\
\hline 71 & & & & & & & & & & & & & & & & & & & & & \\
\hline 72 & & & & & & & & & & & & & & & & & & & & & \\
\hline 73 & & & & & & & & & & & & & & & & & & & & & \\
\hline 74 & & & & & & & & & & & & & & & & & & & & & \\
\hline 75 & & & & & & & & & & & & & & & & & & & & & \\
\hline 76 & & & & & & & & & & & & & & & & & & & & & \\
\hline 77 & & & & & & & & & & & & & & & & & & & & & \\
\hline 78 & & & & & & & & & & & & & & & & & & & & & \\
\hline 79 & & & & & & & & & & & & & & & & & & & & & \\
\hline 80 & & & & & & & & & & & & & & & & & & & & & \\
\hline 81 & & & & & & & & & & & & & & & & & & & & & \\
\hline 82 & & & & & & & & & & & & & & & & & & & & & \\
\hline 83 & & & & & & & & & & & & & & & & & & & & & \\
\hline 84 & & & & & & & & & & & & & & & & & & & & & \\
\hline 85 & & & & & & & & & & & & & & & & & & & & & \\
\hline 86 & & & & & & & & & & & & & & & & & & & & & \\
\hline 87 & & & & & & & & & & & & & & & & & & & & & \\
\hline 88 & & & & & & & & & & & & & & & & & & & & & \\
\hline 89 & & & & & & & & & & & & & & & & & & & & & \\
\hline 90 & & & & & & & & & & & & & & & & & & & & & \\
\hline 91 & & & & & & & & & & & & & & & & & & & & & \\
\hline 92 & & & & & & & & & & & & & & & & & & & & & \\
\hline 93 & & & & & & & & & & & & & & & & & & & & & \\
\hline 94 & & & & & & & & & & & & & & & & & & & & & \\
\hline 95 & & & & & & & & & & & & & & & & & & & & & \\
\hline 96 & & & & & & & & & & & & & & & & & & & & & \\
\hline 97 & & & & & & & & & & & & & & & & & & & & & \\
\hline 98 & & & & & & & & & & & & & & & & & & & & & \\
\hline 99 & & & & & & & & & & & & & & & & & & & & & \\
\hline 100 & & & & & & & & & & & & & & & & & & & & & \\
\hline 101 & & & & & & & & & & & & & & & & & & & & & \\
\hline 102 & & & & & & & & & & & & & & & & & & & & & \\
\hline 31-01 & & & & & & & & & & & & & & & & & & & & & \\
\hline 73-01 & & & & & & & & & & & & & & & & & & & & & \\
\hline $27-02$ & & & & & & & & & & & & & & & & & & & & & \\
\hline $72-02$ & & & & & & & & & & & & & & & & & & & & & \\
\hline $09-03$ & & & & & & & & & & & & & & & & & & & & & \\
\hline $47-04$ & & & 0.0090 & & & 0.0037 & & 0.0035 & & & & & & & & & & & & & \\
\hline $50-05$ & & & 0.0092 & & & 0.0038 & & 0.0036 & & & & & & & & & & & & & \\
\hline $33-06$ & & & & & & & & & & & & & & & & & & & & & \\
\hline $59-06$ & & & & & & & & & & & & & & & & & & & & & \\
\hline $57-07$ & & & & & & & & & & & & & & & & & & & & & \\
\hline 83-08 & & & & & & & & & & & & & & & & & & & & & \\
\hline 01-09 & & & & & & & & & & & & & & & & & & & & & \\
\hline $26-10$ & & & & & & & & & & & & & & & & & & & & & \\
\hline $43-11$ & & & 0.0096 & & & 0.0040 & & 0.0038 & & & & & & & & & & & & & \\
\hline $74-12$ & & & & & & & & & & & & & & & & & & & & & \\
\hline $49-13$ & & & & & & & & & & & & & & & & & & & & & \\
\hline $30-14$ & & & 0.0098 & & & 0.0041 & & 0.0038 & & & & & & & & & & & & & \\
\hline
\end{tabular}


Appendix A. Database - mass fraction

Comp. Vs Properties Study (Chick et al. 1981)

\begin{tabular}{|c|c|c|c|c|c|c|c|c|c|c|c|c|c|c|c|c|c|c|c|c|}
\hline Glass ID & $\begin{array}{c}\mathrm{SeO} 2 \\
-\mathrm{a}\end{array}$ & $\begin{array}{c}\mathrm{Sm} 2 \mathrm{O} 3 \\
-\mathrm{a} \\
\end{array}$ & $\begin{array}{c}\mathrm{SnO} \\
-\mathrm{a} \\
\end{array}$ & $\begin{array}{c}\mathrm{SnO} 2 \\
-\mathrm{a}\end{array}$ & $\begin{array}{c}\mathrm{SO} 3 \\
-\mathrm{a} \\
\end{array}$ & $\begin{array}{c}\mathrm{SrO} \\
-\mathrm{a} \\
\end{array}$ & $\begin{array}{c}\mathrm{Tc} 2 \mathrm{O} 7 \\
-\mathrm{a} \\
\end{array}$ & $\begin{array}{c}\mathrm{TeO} 2 \\
-\mathrm{a}\end{array}$ & $\begin{array}{c}\mathrm{ThO} 2 \\
-\mathrm{a} \\
\end{array}$ & $\begin{array}{c}\mathrm{TiO} 2 \\
-\mathrm{a} \\
\end{array}$ & $\begin{array}{c}\mathrm{T} 12 \mathrm{O} 3 \\
-\mathrm{a} \\
\end{array}$ & $\begin{array}{c}\mathrm{U} 3 \mathrm{O} 8 \\
-\mathrm{a}\end{array}$ & $\begin{array}{c}\mathrm{UO} 2 \\
-\mathrm{a}\end{array}$ & $\begin{array}{c}\mathrm{UO} 3 \\
-\mathrm{a} \\
\end{array}$ & $\begin{array}{c}\mathrm{V} 2 \mathrm{O} 5 \\
-\mathrm{a}\end{array}$ & $\begin{array}{c}\mathrm{WO} 3 \\
-\mathrm{a} \\
\end{array}$ & $\begin{array}{c}\mathrm{Y} 2 \mathrm{O} 3 \\
-\mathrm{a}\end{array}$ & $\begin{array}{c}\mathrm{ZnO} \\
-\mathrm{a}\end{array}$ & $\begin{array}{c}\text { Others } \\
-\mathrm{a}\end{array}$ & $\begin{array}{c}\text { Sum } \\
-\mathrm{a}\end{array}$ \\
\hline 71 & & & & & & & & & & 0.0341 & & & & & & & & 0.0261 & & 1.0000 \\
\hline 72 & & & & & & & & & & 0.0484 & & & & & & & & 0.0385 & & 1.0000 \\
\hline 73 & & & & & & & & & & 0.0381 & & & & & & & & 0.0303 & & 1.0000 \\
\hline 74 & & & & & & & & & & 0.0541 & & & & & & & & 0.0426 & & 1.0000 \\
\hline 75 & & & & & & & & & & 0.0355 & & & & & & & & 0.0256 & & 1.0000 \\
\hline 76 & & & & & & & & & & 0.0533 & & & & & & & & 0.0413 & & 1.0000 \\
\hline 77 & & & & & & & & & & 0.0412 & & & & & & & & 0.0284 & & 1.0000 \\
\hline 78 & & & & & & & & & & 0.0476 & & & & & & & & 0.0366 & & 1.0000 \\
\hline 79 & & & & & & & & & & 0.0402 & & & & & & & & 0.0322 & & 1.0000 \\
\hline 80 & & & & & & & & & & 0.0529 & & & & & & & & 0.0383 & & 1.0000 \\
\hline 81 & & & & & & & & & & 0.0418 & & & & & & & & 0.0316 & & 1.0000 \\
\hline 82 & & & & & & & & & & 0.0475 & & & & & & & & 0.0000 & & 1.0000 \\
\hline 83 & & & & & & & & & & 0.0413 & & & & & & & & 0.0745 & & 1.0000 \\
\hline 84 & & & & & & & & & & 0.0000 & & & & & & & & 0.0388 & & 1.0000 \\
\hline 85 & & & & & & & & & & 0.0943 & & & & & & & & 0.0324 & & 1.0000 \\
\hline 86 & & & & & & & & & & 0.0470 & & & & & & & & 0.0368 & & 1.0000 \\
\hline 87 & & & & & & & & & & 0.0452 & & & & & & & & 0.0331 & & 1.0000 \\
\hline 88 & & & & & & & & & & 0.0472 & & & & & & & & 0.0370 & & 1.0000 \\
\hline 89 & & & & & & & & & & 0.0416 & & & & & & & & 0.0330 & & 1.0000 \\
\hline 90 & & & & & & & & & & 0.0463 & & & & & & & & 0.0351 & & 1.0000 \\
\hline 91 & & & & & & & & & & 0.0438 & & & & & & & & 0.0338 & & 1.0000 \\
\hline 92 & & & & & & & & & & 0.0000 & & & & & & & & 0.0000 & & 1.0000 \\
\hline 93 & & & & & & & & & & 0.0857 & & & & & & & & 0.0000 & & 1.0000 \\
\hline 94 & & & & & & & & & & 0.0420 & & & & & & & & 0.0000 & & 1.0000 \\
\hline 95 & & & & & & & & & & 0.0000 & & & & & & & & 0.0000 & & 1.0000 \\
\hline 96 & & & & & & & & & & 0.0888 & & & & & & & & 0.0733 & & 1.0000 \\
\hline 97 & & & & & & & & & & 0.0000 & & & & & & & & 0.0886 & & 1.0000 \\
\hline 98 & & & & & & & & & & 0.0869 & & & & & & & & 0.0000 & & 1.0000 \\
\hline 99 & & & & & & & & & & 0.0000 & & & & & & & & 0.0762 & & 1.0000 \\
\hline 100 & & & & & & & & & & 0.0000 & & & & & & & & 0.0624 & & 1.0000 \\
\hline 101 & & & & & & & & & & 0.0000 & & & & & & & & 0.0679 & & 1.0000 \\
\hline 102 & & & & & & & & & & 0.0719 & & & & & & & & 0.0341 & & 1.0000 \\
\hline $31-01$ & & & & & & & & & & & & & & & & & & & & 1.0001 \\
\hline 73-01 & & & & & & & & & & & & & & & & & & & & 1.0000 \\
\hline $27-02$ & & & & & & & & & & & & & & & & & & & & 1.0000 \\
\hline 72-02 & & & & & & & & & & & & & & & & & & & & 1.0000 \\
\hline 09-03 & & & & & & & & & & & & & & & & & & & & 1.0000 \\
\hline $47-04$ & & & & & & 0.0029 & & & 0.0696 & & & & 0.0225 & & & & & & & 1.0000 \\
\hline 50-05 & & & & & & 0.0030 & & & 0.0715 & & & & 0.0232 & & & & & & & 1.0000 \\
\hline $33-06$ & & & & & & & & & & & & & & & & & & & & 1.0000 \\
\hline $59-06$ & & & & & & & & & & & & & & & & & & & & 1.0000 \\
\hline $57-07$ & & & & & & & & & & & & & & & & & & & & 1.0000 \\
\hline 83-08 & & & & & & & & & & & & & & & & & & & & 1.0000 \\
\hline 01-09 & & & & & & & & & & & & & & & & & & & & 0.9999 \\
\hline $26-10$ & & & & & & & & & & & & & & & & & & & & 1.0001 \\
\hline $43-11$ & & & & & & 0.0032 & & & 0.0746 & & & & 0.0242 & & & & & & & 0.9999 \\
\hline $74-12$ & & & & & & & & & & & & & & & & & & & & 1.0000 \\
\hline $49-13$ & & & & & & & & & & & & & & & & & & & & 0.9999 \\
\hline $30-14$ & & & & & & 0.0032 & & & 0.0757 & & & & 0.0245 & & & & & & & 0.9999 \\
\hline
\end{tabular}


Appendix A. Database - mass fraction

Comp. Vs Properties Study (Chick et al. 1981)

\begin{tabular}{|c|c|c|c|c|c|c|c|c|}
\hline Glass ID & $\begin{array}{l}\mathrm{TM} \\
\left({ }^{\circ} \mathrm{C}\right)\end{array}$ & $\begin{array}{l}\text { Gradient } \\
\text { TL }\left({ }^{\circ} \mathrm{C}\right) \\
\end{array}$ & $\begin{array}{l}\text { Uniform } \\
\text { TL }\left({ }^{\circ} \mathrm{C}\right)\end{array}$ & Primary Phase & $\begin{array}{l}\text { Quenched } \\
\text { Visual/OM }\end{array}$ & $\begin{array}{c}\text { Quenched } \\
\text { SEM/EDS or TEM }\end{array}$ & $\begin{array}{l}\text { Quenched } \\
\text { XRD }\end{array}$ & $\begin{array}{c}\text { Quenched } \\
\text { Homogeneous? }\end{array}$ \\
\hline 71 & 1570 & & & & & & $5.9 \mathrm{wt} \%$ spinel & \\
\hline 72 & 1350 & & & & & & $13 \mathrm{wt} \%$ spinel & \\
\hline 73 & 1250 & & & & & & $9.4 \mathrm{wt} \%$ spinel & \\
\hline 74 & 1150 & & & & & & $9.9 \mathrm{wt} \%$ spinel & \\
\hline 75 & 1600 & & & & & & $13.9 \mathrm{wt} \%$ spinel & \\
\hline 76 & 1450 & & & & & & $19.5 \mathrm{wt} \%$ spinel & \\
\hline 77 & 1150 & & & & & & $7.2 \mathrm{wt} \%$ spinel & \\
\hline 78 & 1350 & & & & & & $12.6 \mathrm{wt} \%$ spinel & \\
\hline 79 & 1250 & & & & & & $11.3 \mathrm{wt} \%$ spinel & \\
\hline 80 & 1450 & & & & & & $13.3 \mathrm{wt} \%$ spinel & \\
\hline 81 & 1210 & & & & & & $7 \mathrm{wt} \%$ spinel & \\
\hline 82 & 1250 & & & & & & $9.4 \mathrm{wt} \%$ spinel & \\
\hline 83 & 1250 & & & & & & $12.9 \mathrm{wt} \%$ spinel & \\
\hline 84 & 1250 & & & & & & $12.7 \mathrm{wt} \%$ spinel & \\
\hline 85 & 1350 & & & & & & $10.2 \mathrm{wt} \%$ spinel & \\
\hline 86 & 1250 & & & & & & amorphous & \\
\hline 87 & 1550 & & & & & & $18.8 \mathrm{wt} \%$ spinel & \\
\hline 88 & 1350 & & & & & & $10.7 \mathrm{wt} \%$ spinel & \\
\hline 89 & 1300 & & & & & & $12.8 \mathrm{wt} \%$ spinel & \\
\hline 90 & 1410 & & & & & & $10.1 \mathrm{wt} \%$ spinel & \\
\hline 91 & 1250 & & & & & & $15.5 \mathrm{wt} \%$ spinel & \\
\hline 92 & 1610 & & & & & & $4.3 \mathrm{wt} \%$ unknown & \\
\hline 93 & 1600 & & & & & & amorphous & \\
\hline 94 & 1720 & & & & & & $10.7 \mathrm{wt} \% \mathrm{FeNiBO} 4$ & \\
\hline 95 & 1600 & & & & & & $2 \mathrm{wt} \%$ unknown & \\
\hline 96 & 1450 & & & & & & $18.4 \mathrm{wt} \%$ spinel & \\
\hline 97 & 1050 & & & & & & amorphous & \\
\hline 98 & 1350 & & & & & & $10.9 \mathrm{wt} \% \mathrm{Cr} 2 \mathrm{O} 3$ & \\
\hline 99 & 1450 & & & & & & $13.4 \mathrm{wt} \%$ spinel & \\
\hline 100 & 1400 & & & & & & amorphous & \\
\hline 101 & 1250 & & & & & & amorphous & \\
\hline 102 & 1200 & & & & & & amorphous & \\
\hline $31-01$ & & & & & amorphous & & & \\
\hline 73-01 & & & & & amorphous & & & \\
\hline $27-02$ & & & & & amorphous & & & \\
\hline $72-02$ & & & & & amorphous & & & \\
\hline $09-03$ & & & & & amorphous & & & \\
\hline $47-04$ & & & & & 0.05 crystallinity & & & \\
\hline $50-05$ & & & & & amorphous & & & \\
\hline $33-06$ & & & & & amorphous & & & \\
\hline $59-06$ & & & & & amorphous & & & \\
\hline $57-07$ & & & & & amorphous & & & \\
\hline $83-08$ & & & & & amorphous & & & \\
\hline $01-09$ & & & & & amorphous & & & \\
\hline $26-10$ & & & & & amorphous & & & \\
\hline $43-11$ & & & & & 0.16 crystallinity & & & \\
\hline $74-12$ & & & & & amorphous & & & \\
\hline $49-13$ & & & & & amorphous & & & \\
\hline $30-14$ & & & & & amorphous & & & \\
\hline
\end{tabular}


Appendix A. Database - mass fraction

Comp. Vs Properties Study (Chick et al. 1981)

\begin{tabular}{|c|c|c|c|c|c|}
\hline Glass ID & $\begin{array}{c}\mathrm{CCC} \\
\text { Visual/OM } \\
\end{array}$ & $\begin{array}{c}\text { CCC } \\
\text { SEM/EDS or TEM }\end{array}$ & $\begin{array}{l}\mathrm{CCC} \\
\mathrm{XRD}\end{array}$ & $\begin{array}{l}\text { Heat Treated } \\
\text { Visual/OM }\end{array}$ & $\begin{array}{c}\text { Heat Treated } \\
\text { SEM/EDS or TEM }\end{array}$ \\
\hline \multicolumn{6}{|l|}{71} \\
\hline \multirow{2}{*}{\multicolumn{6}{|c|}{$\begin{array}{l}72 \\
73\end{array}$}} \\
\hline & & & & & \\
\hline \multicolumn{6}{|l|}{74} \\
\hline \multicolumn{6}{|l|}{75} \\
\hline \multicolumn{6}{|l|}{76} \\
\hline \multicolumn{6}{|l|}{77} \\
\hline \multicolumn{6}{|l|}{78} \\
\hline \multicolumn{6}{|l|}{79} \\
\hline \multicolumn{6}{|l|}{80} \\
\hline \multicolumn{6}{|l|}{81} \\
\hline \multicolumn{6}{|l|}{82} \\
\hline \multicolumn{6}{|l|}{83} \\
\hline \multicolumn{6}{|l|}{84} \\
\hline \multicolumn{6}{|l|}{85} \\
\hline \multicolumn{6}{|l|}{86} \\
\hline \multicolumn{6}{|l|}{87} \\
\hline \multicolumn{6}{|l|}{88} \\
\hline \multicolumn{6}{|l|}{89} \\
\hline \multicolumn{6}{|l|}{90} \\
\hline \multicolumn{6}{|l|}{91} \\
\hline \multicolumn{6}{|l|}{92} \\
\hline 93 & & & & & \\
\hline 94 & & & & & \\
\hline 95 & & & & & \\
\hline 96 & & & & & \\
\hline 97 & & & & & \\
\hline 98 & & & & & \\
\hline 99 & & & & & \\
\hline 100 & & & & & \\
\hline 101 & & & & & \\
\hline 102 & & & & & \\
\hline $31-01$ & 0.17 crystallinity & & & 0.05 crystallinity & \\
\hline 73-01 & 0.19 crystallinity & & & 0.06 crystallinity & \\
\hline $27-02$ & amorphous & & & amorphous & \\
\hline $72-02$ & amorphous & & & amorphous & \\
\hline $09-03$ & 0.06 crystallinity & & & amorphous & \\
\hline $47-04$ & amorphous & & & 0.04 crystallinity & \\
\hline $50-05$ & 0.11 crystallinity & & & amorphous & \\
\hline $33-06$ & amorphous & & & amorphous & \\
\hline $59-06$ & amorphous & & & amorphous & \\
\hline $57-07$ & amorphous & & & amorphous & \\
\hline $83-08$ & amorphous & & & amorphous & \\
\hline $01-09$ & amorphous & & & amorphous & \\
\hline $26-10$ & amorphous & & & amorphous & \\
\hline $43-11$ & 0.31 crystallinity & & & 0.05 crystallinity & \\
\hline $74-12$ & 0.84 crystallinity & & & 0.76 crystallinity & \\
\hline $49-13$ & amorphous & & & amorphous & \\
\hline $30-14$ & 0.64 crystallinity & & & 0.41 crystallinity & \\
\hline
\end{tabular}


Appendix A. Database - mass fraction

Comp. Vs Properties Study (Chick et al. 1981)

\begin{tabular}{|c|c|c|c|c|c|c|c|c|c|c|c|c|c|c|c|}
\hline Glass ID & $\begin{array}{l}\text { Heat Treated } \\
\text { XRD }\end{array}$ & $\begin{array}{l}\text { Density } \\
\left(\mathrm{g} / \mathrm{cm}^{3}\right)\end{array}$ & \begin{tabular}{|c|} 
Fulc Visc \\
A
\end{tabular} & \begin{tabular}{|c|} 
Fulc Visc \\
$\mathrm{B}$
\end{tabular} & \begin{tabular}{|c|} 
Fulc Visc \\
To
\end{tabular} & $\begin{array}{c}\mathrm{FV} 1150^{\circ} \mathrm{C} \\
(\mathrm{Pa} \cdot \mathrm{s})\end{array}$ & $\begin{array}{c}\text { Arrh Visc } \\
\text { A }\end{array}$ & \begin{tabular}{|c|} 
Arrh Visc \\
B
\end{tabular} & $1150^{\circ} \mathrm{C}$ & $\begin{array}{c}\mathrm{T}\left({ }^{\circ} \mathrm{C}\right) \text { at } \\
2 \mathrm{~Pa} \cdot \mathrm{s}\end{array}$ & \begin{tabular}{|c|}
$\mathrm{T}\left({ }^{\circ} \mathrm{C}\right)$ at \\
$5 \mathrm{~Pa} \cdot \mathrm{s}$
\end{tabular} & \begin{tabular}{|c|}
$\mathrm{T}\left({ }^{\circ} \mathrm{C}\right)$ at \\
$10 \mathrm{~Pa} \cdot \mathrm{s}$ \\
\end{tabular} & $\begin{array}{l}\mathrm{T} 1 \\
\left({ }^{\circ} \mathrm{C}\right)\end{array}$ & $\begin{array}{c}\mathrm{V} 1 \\
(\mathrm{~Pa} \cdot \mathrm{s})\end{array}$ & $\begin{array}{c}\mathrm{T} 2 \\
\left({ }^{\circ} \mathrm{C}\right) \\
\end{array}$ \\
\hline 71 & & & & & & & & & & & & & 1250 & 23 & \\
\hline 72 & & & & & & & & & & & & & 1250 & 25 & \\
\hline 73 & & & & & & & & & & & & & 1250 & 6.1 & \\
\hline 74 & & & & & & & & & & & & & 1250 & 4.6 & \\
\hline 75 & & & & & & & & & & & & & 1250 & 59 & \\
\hline 76 & & & & & & & & & & & & & 1250 & 72 & \\
\hline 77 & & & & & & & & & & & & & 1250 & 2.8 & \\
\hline 78 & & & & & & & & & & & & & 1250 & 18 & \\
\hline 79 & & & & & & & & & & & & & 1250 & 13 & \\
\hline 80 & & & & & & & & & & & & & 1250 & 35 & \\
\hline 81 & & & & & & & & & & & & & 1250 & 7 & \\
\hline 82 & & & & & & & & & & & & & 1250 & 10 & \\
\hline 83 & & & & & & & & & & & & & 1250 & 14 & \\
\hline 84 & & & & & & & & & & & & & 1250 & 15 & \\
\hline 85 & & & & & & & & & & & & & 1250 & 8.2 & \\
\hline 86 & & & & & & & & & & & & & 1250 & 2.7 & \\
\hline 87 & & & & & & & & & & & & & 1250 & 43 & \\
\hline 88 & & & & & & & & & & & & & 1250 & 8.6 & \\
\hline 89 & & & & & & & & & & & & & 1250 & 21 & \\
\hline 90 & & & & & & & & & & & & & 1250 & & \\
\hline 91 & & & & & & & & & & & & & 1250 & 6.1 & \\
\hline 92 & & & & & & & & & & & & & 1250 & 64 & \\
\hline 93 & & & & & & & & & & & & & 1250 & 180 & \\
\hline 94 & & & & & & & & & & & & & 1250 & & \\
\hline 95 & & & & & & & & & & & & & 1250 & 230 & \\
\hline 96 & & & & & & & & & & & & & 1250 & 19 & \\
\hline 97 & & & & & & & & & & & & & 1250 & 0.13 & \\
\hline 98 & & & & & & & & & & & & & 1250 & 4 & \\
\hline 99 & & & & & & & & & & & & & 1250 & 11 & \\
\hline 100 & & & & & & & & & & & & & 1250 & 16 & \\
\hline 101 & & & & & & & & & & & & & 1250 & 1.6 & \\
\hline 102 & & & & & & & & & & & & & 1250 & 0.45 & \\
\hline 31-01 & & & & & & & & & & & & 1294 & 1454 & 3 & \\
\hline 73-01 & & & & & & & & & & & & 1307 & 1460 & 3 & \\
\hline $27-02$ & & & & & & & & & & & & 1240 & 1431 & 3 & \\
\hline 72-02 & & & & & & & & & & & & 1168 & 1332 & 3 & \\
\hline 09-03 & & & & & & & & & & & & 1586 & 1747 & 3 & \\
\hline $47-04$ & & & & & & & & & & & & 1425 & 1600 & 3 & \\
\hline 50-05 & & & & & & & & & & & & 1364 & 1522 & 3 & \\
\hline $33-06$ & & & & & & & & & & & & 1327 & 1503 & 3 & \\
\hline 59-06 & & & & & & & & & & & & 1402 & 1589 & 3 & \\
\hline $57-07$ & & & & & & & & & & & & 1270 & 1445 & 3 & \\
\hline 83-08 & & & & & & & & & & & & 1050 & 1176 & 3 & \\
\hline 01-09 & & & & & & & & & & & & 1345 & 1503 & 3 & \\
\hline $26-10$ & & & & & & & & & & & & 1198 & 1364 & 3 & \\
\hline $43-11$ & & & & & & & & & & & & 1625 & 1806 & 3 & \\
\hline $74-12$ & & & & & & & & & & & & & 1176 & 3 & \\
\hline $49-13$ & & & & & & & & & & & & 1032 & 1152 & 3 & \\
\hline $30-14$ & & & & & & & & & & & & 1220 & 1383 & 3 & \\
\hline
\end{tabular}


Comp. Vs Properties Study (Chick et al. 1981)

\begin{tabular}{|c|c|c|c|c|c|c|c|c|c|c|c|c|c|c|c|c|c|c|c|c|c|}
\hline Glass ID & $\begin{array}{c}\mathrm{V} 2 \\
(\mathrm{~Pa} \cdot \mathrm{s})\end{array}$ & $\begin{array}{c}\mathrm{T} 3 \\
\left({ }^{\circ} \mathrm{C}\right) \\
\end{array}$ & $\begin{array}{c}\mathrm{V} 3 \\
(\mathrm{~Pa} \cdot \mathrm{s})\end{array}$ & $\begin{array}{c}\mathrm{T} 4 \\
\left({ }^{\circ} \mathrm{C}\right)\end{array}$ & $\begin{array}{c}\mathrm{V} 4 \\
(\mathrm{~Pa} \cdot \mathrm{s})\end{array}$ & $\begin{array}{c}\mathrm{T} 5 \\
\left({ }^{\circ} \mathrm{C}\right) \\
\end{array}$ & $\begin{array}{c}\mathrm{V} 5 \\
(\mathrm{~Pa} \cdot \mathrm{s})\end{array}$ & $\begin{array}{c}\mathrm{T} 6 \\
\left({ }^{\circ} \mathrm{C}\right) \\
\end{array}$ & $\begin{array}{c}\mathrm{V} 6 \\
(\mathrm{~Pa} \cdot \mathrm{s})\end{array}$ & $\begin{array}{c}\mathrm{T} 7 \\
\left({ }^{\circ} \mathrm{C}\right) \\
\end{array}$ & $\begin{array}{c}\mathrm{V} 7 \\
(\mathrm{~Pa} \cdot \mathrm{s})\end{array}$ & $\begin{array}{c}\mathrm{T} 8 \\
\left({ }^{\circ} \mathrm{C}\right)\end{array}$ & $\begin{array}{c}\mathrm{V} 8 \\
(\mathrm{~Pa} \cdot \mathrm{s})\end{array}$ & $\begin{array}{c}\mathrm{T} 9 \\
\left({ }^{\circ} \mathrm{C}\right) \\
\end{array}$ & $\begin{array}{c}\mathrm{V} 9 \\
(\mathrm{~Pa} \cdot \mathrm{s})\end{array}$ & $\begin{array}{l}\mathrm{T} 10 \\
\left({ }^{\circ} \mathrm{C}\right)\end{array}$ & $\begin{array}{c}\mathrm{V} 10 \\
(\mathrm{~Pa} \cdot \mathrm{s})\end{array}$ & $\begin{array}{l}\mathrm{T} 11 \\
\left({ }^{\circ} \mathrm{C}\right)\end{array}$ & $\begin{array}{c}\text { V11 } \\
(\mathrm{Pa} \cdot \mathrm{s})\end{array}$ & $\begin{array}{l}\mathrm{T} 12 \\
\left({ }^{\circ} \mathrm{C}\right)\end{array}$ & $\begin{array}{c}\mathrm{V} 12 \\
(\mathrm{~Pa} \cdot \mathrm{s})\end{array}$ \\
\hline \multirow{2}{*}{\multicolumn{17}{|c|}{$\frac{71}{72}$}} & & & & & \\
\hline & & & & & & & & & & & & & & & & & & & & & \\
\hline \multicolumn{22}{|l|}{73} \\
\hline \multicolumn{22}{|l|}{74} \\
\hline \multicolumn{22}{|l|}{75} \\
\hline \multicolumn{22}{|l|}{76} \\
\hline \multicolumn{22}{|l|}{77} \\
\hline \multicolumn{22}{|l|}{78} \\
\hline \multicolumn{22}{|l|}{79} \\
\hline \multicolumn{22}{|l|}{80} \\
\hline \multicolumn{22}{|l|}{81} \\
\hline \multicolumn{22}{|l|}{82} \\
\hline \multicolumn{22}{|l|}{83} \\
\hline \multicolumn{22}{|l|}{84} \\
\hline 85 & & & & & & & & & & & & & & & & & & & & & \\
\hline 86 & & & & & & & & & & & & & & & & & & & & & \\
\hline 87 & & & & & & & & & & & & & & & & & & & & & \\
\hline 88 & & & & & & & & & & & & & & & & & & & & & \\
\hline 89 & & & & & & & & & & & & & & & & & & & & & \\
\hline 90 & & & & & & & & & & & & & & & & & & & & & \\
\hline 91 & & & & & & & & & & & & & & & & & & & & & \\
\hline 92 & & & & & & & & & & & & & & & & & & & & & \\
\hline 93 & & & & & & & & & & & & & & & & & & & & & \\
\hline 94 & & & & & & & & & & & & & & & & & & & & & \\
\hline 95 & & & & & & & & & & & & & & & & & & & & & \\
\hline 96 & & & & & & & & & & & & & & & & & & & & & \\
\hline 97 & & & & & & & & & & & & & & & & & & & & & \\
\hline 98 & & & & & & & & & & & & & & & & & & & & & \\
\hline 99 & & & & & & & & & & & & & & & & & & & & & \\
\hline 100 & & & & & & & & & & & & & & & & & & & & & \\
\hline 101 & & & & & & & & & & & & & & & & & & & & & \\
\hline 102 & & & & & & & & & & & & & & & & & & & & & \\
\hline 31-01 & & & & & & & & & & & & & & & & & & & & & \\
\hline 73-01 & & & & & & & & & & & & & & & & & & & & & \\
\hline $27-02$ & & & & & & & & & & & & & & & & & & & & & \\
\hline $72-02$ & & & & & & & & & & & & & & & & & & & & & \\
\hline 09-03 & & & & & & & & & & & & & & & & & & & & & \\
\hline $47-04$ & & & & & & & & & & & & & & & & & & & & & \\
\hline $50-05$ & & & & & & & & & & & & & & & & & & & & & \\
\hline $33-06$ & & & & & & & & & & & & & & & & & & & & & \\
\hline $59-06$ & & & & & & & & & & & & & & & & & & & & & \\
\hline $57-07$ & & & & & & & & & & & & & & & & & & & & & \\
\hline 83-08 & & & & & & & & & & & & & & & & & & & & & \\
\hline 01-09 & & & & & & & & & & & & & & & & & & & & & \\
\hline $26-10$ & & & & & & & & & & & & & & & & & & & & & \\
\hline $43-11$ & & & & & & & & & & & & & & & & & & & & & \\
\hline $74-12$ & & & & & & & & & & & & & & & & & & & & & \\
\hline $49-13$ & & & & & & & & & & & & & & & & & & & & & \\
\hline $30-14$ & & & & & & & & & & & & & & & & & & & & & \\
\hline
\end{tabular}


Appendix A. Database - mass fraction

Comp. Vs Properties Study (Chick et al. 1981)

\begin{tabular}{|c|c|c|c|c|c|c|c|c|c|c|c|c|c|c|c|c|}
\hline Glass ID & $\begin{array}{l}\mathrm{T} 13 \\
\left({ }^{\circ} \mathrm{C}\right) \\
\end{array}$ & $\begin{array}{l}\text { V13 } \\
(\mathrm{Pa} \cdot \mathrm{s})\end{array}$ & $\begin{array}{l}\mathrm{T} 14 \\
\left({ }^{\circ} \mathrm{C}\right) \\
\end{array}$ & $\begin{array}{l}\mathrm{V} 14 \\
(\mathrm{~Pa} \cdot \mathrm{s})\end{array}$ & $\begin{array}{c}\text { Q PCT } \\
\text { B }\left(\mathrm{g} / \mathrm{m}^{2}\right)\end{array}$ & $\begin{array}{c}\text { Q PCT } \\
\mathrm{Li}\left(\mathrm{g} / \mathrm{m}^{2}\right)\end{array}$ & $\begin{array}{c}\text { Q PCT } \\
\mathrm{Na}\left(\mathrm{g} / \mathrm{m}^{2}\right)\end{array}$ & $\begin{array}{c}\text { Q PCT } \\
\mathrm{Si}\left(\mathrm{g} / \mathrm{m}^{2}\right)\end{array}$ & $\begin{array}{c}\text { Q PCT } \\
\mathrm{pH}\end{array}$ & $\begin{array}{l}\text { CCC PCT } \\
\mathrm{B}\left(\mathrm{g} / \mathrm{m}^{2}\right)\end{array}$ & $\begin{array}{l}\text { CCC PCT } \\
\mathrm{Li}\left(\mathrm{g} / \mathrm{m}^{2}\right)\end{array}$ & $\begin{array}{l}\text { CCC PCT } \\
\mathrm{Na}\left(\mathrm{g} / \mathrm{m}^{2}\right)\end{array}$ & $\begin{array}{c}\text { CCC PCT } \\
\mathrm{Si}\left(\mathrm{g} / \mathrm{m}^{2}\right)\end{array}$ & $\begin{array}{c}\begin{array}{c}\text { CCC PCT } \\
\mathrm{pH}\end{array} \\
\end{array}$ & $\begin{array}{l}\text { Q PCT B at } \\
20^{\circ} \mathrm{C}\left(\mathrm{g} / \mathrm{m}^{2}\right)\end{array}$ & $\begin{array}{l}\text { Q PCT Li at } \\
20^{\circ} \mathrm{C}\left(\mathrm{g} / \mathrm{m}^{2}\right)\end{array}$ \\
\hline \multicolumn{17}{|l|}{71} \\
\hline \multirow{2}{*}{\multicolumn{17}{|c|}{$\begin{array}{l}72 \\
73\end{array}$}} \\
\hline & & & & & & & & & & & & & & & & \\
\hline \multicolumn{17}{|l|}{74} \\
\hline \multicolumn{17}{|l|}{75} \\
\hline \multicolumn{17}{|l|}{76} \\
\hline \multicolumn{17}{|l|}{77} \\
\hline \multicolumn{17}{|l|}{78} \\
\hline \multicolumn{17}{|l|}{79} \\
\hline \multicolumn{17}{|l|}{80} \\
\hline \multicolumn{17}{|l|}{81} \\
\hline \multicolumn{17}{|l|}{82} \\
\hline \multicolumn{17}{|l|}{83} \\
\hline \multicolumn{17}{|l|}{84} \\
\hline \multicolumn{17}{|l|}{85} \\
\hline \multicolumn{17}{|l|}{86} \\
\hline \multicolumn{17}{|l|}{87} \\
\hline 88 & & & & & & & & & & & & & & & & \\
\hline 89 & & & & & & & & & & & & & & & & \\
\hline 90 & & & & & & & & & & & & & & & & \\
\hline 91 & & & & & & & & & & & & & & & & \\
\hline 92 & & & & & & & & & & & & & & & & \\
\hline 93 & & & & & & & & & & & & & & & & \\
\hline 94 & & & & & & & & & & & & & & & & \\
\hline 95 & & & & & & & & & & & & & & & & \\
\hline 96 & & & & & & & & & & & & & & & & \\
\hline 97 & & & & & & & & & & & & & & & & \\
\hline 98 & & & & & & & & & & & & & & & & \\
\hline 99 & & & & & & & & & & & & & & & & \\
\hline 100 & & & & & & & & & & & & & & & & \\
\hline 101 & & & & & & & & & & & & & & & & \\
\hline 102 & & & & & & & & & & & & & & & & \\
\hline $31-01$ & & & & & & & & & & & & & & & & \\
\hline 73-01 & & & & & & & & & & & & & & & & \\
\hline $27-02$ & & & & & & & & & & & & & & & & \\
\hline $72-02$ & & & & & & & & & & & & & & & & \\
\hline 09-03 & & & & & & & & & & & & & & & & \\
\hline $47-04$ & & & & & & & & & & & & & & & & \\
\hline $50-05$ & & & & & & & & & & & & & & & & \\
\hline $33-06$ & & & & & & & & & & & & & & & & \\
\hline 59-06 & & & & & & & & & & & & & & & & \\
\hline $57-07$ & & & & & & & & & & & & & & & & \\
\hline $83-08$ & & & & & & & & & & & & & & & & \\
\hline 01-09 & & & & & & & & & & & & & & & & \\
\hline $26-10$ & & & & & & & & & & & & & & & & \\
\hline $43-11$ & & & & & & & & & & & & & & & & \\
\hline $74-12$ & & & & & & & & & & & & & & & & \\
\hline $49-13$ & & & & & & & & & & & & & & & & \\
\hline $30-14$ & & & & & & & & & & & & & & & & \\
\hline
\end{tabular}


Appendix A. Database - mass fraction

Comp. Vs Properties Study (Chick et al. 1981)

\begin{tabular}{|c|c|c|c|c|c|c|c|c|c|c|c|c|}
\hline Glass ID & $\begin{array}{l}\text { Q PCT Na at } \\
20^{\circ} \mathrm{C}\left(\mathrm{g} / \mathrm{m}^{2}\right)\end{array}$ & $\begin{array}{l}\text { Q PCT Si at } \\
20^{\circ} \mathrm{C}\left(\mathrm{g} / \mathrm{m}^{2}\right)\end{array}$ & $\begin{array}{c}\mathrm{QpH} \\
\text { at } 20^{\circ} \mathrm{C}\end{array}$ & $\begin{array}{l}\text { TCLP Ag } \\
(\mathrm{ppm})\end{array}$ & $\begin{array}{c}\text { TCLP As } \\
(\mathrm{ppm})\end{array}$ & $\begin{array}{c}\text { TCLP Ba } \\
(\mathrm{ppm})\end{array}$ & $\begin{array}{c}\text { TCLP Cd } \\
(\mathrm{ppm})\end{array}$ & $\begin{array}{c}\text { TCLP Cr } \\
(\mathrm{ppm})\end{array}$ & $\begin{array}{l}\text { TCLP Ni } \\
(\mathrm{ppm})\end{array}$ & $\begin{array}{c}\text { TCLP Pb } \\
\text { (ppm) }\end{array}$ & $\begin{array}{c}\text { TCLP Se } \\
(\mathrm{ppm})\end{array}$ & $\begin{array}{c}\text { TCLP Zn } \\
(\mathrm{ppm})\end{array}$ \\
\hline \multicolumn{13}{|l|}{71} \\
\hline \multirow{2}{*}{\multicolumn{13}{|c|}{$\begin{array}{l}72 \\
73\end{array}$}} \\
\hline \multirow{2}{*}{\multicolumn{13}{|c|}{$\begin{array}{l}73 \\
74\end{array}$}} \\
\hline & & & & & & & & & & & & \\
\hline \multicolumn{13}{|l|}{75} \\
\hline \multicolumn{13}{|l|}{76} \\
\hline \multicolumn{13}{|l|}{77} \\
\hline \multicolumn{13}{|l|}{78} \\
\hline \multicolumn{13}{|l|}{79} \\
\hline \multicolumn{13}{|l|}{80} \\
\hline \multicolumn{13}{|l|}{81} \\
\hline \multicolumn{13}{|l|}{82} \\
\hline \multirow{2}{*}{\multicolumn{13}{|c|}{$\begin{array}{l}83 \\
84\end{array}$}} \\
\hline & & & & & & & & & & & & \\
\hline \multicolumn{13}{|l|}{85} \\
\hline \multicolumn{13}{|l|}{86} \\
\hline \multicolumn{13}{|l|}{87} \\
\hline \multicolumn{13}{|l|}{88} \\
\hline \multirow{2}{*}{\multicolumn{13}{|c|}{$\begin{array}{l}89 \\
90\end{array}$}} \\
\hline \multirow{2}{*}{\multicolumn{13}{|c|}{$\frac{90}{91}$}} \\
\hline & & & & & & & & & & & & \\
\hline 92 & & & & & & & & & & & & \\
\hline 93 & & & & & & & & & & & & \\
\hline 94 & & & & & & & & & & & & \\
\hline 95 & & & & & & & & & & & & \\
\hline 96 & & & & & & & & & & & & \\
\hline 97 & & & & & & & & & & & & \\
\hline 98 & & & & & & & & & & & & \\
\hline 99 & & & & & & & & & & & & \\
\hline 100 & & & & & & & & & & & & \\
\hline 101 & & & & & & & & & & & & \\
\hline 102 & & & & & & & & & & & & \\
\hline $31-01$ & & & & & & & & & & & & \\
\hline 73-01 & & & & & & & & & & & & \\
\hline $27-02$ & & & & & & & & & & & & \\
\hline $72-02$ & & & & & & & & & & & & \\
\hline 09-03 & & & & & & & & & & & & \\
\hline $47-04$ & & & & & & & & & & & & \\
\hline $50-05$ & & & & & & & & & & & & \\
\hline $33-06$ & & & & & & & & & & & & \\
\hline 59-06 & & & & & & & & & & & & \\
\hline $57-07$ & & & & & & & & & & & & \\
\hline $83-08$ & & & & & & & & & & & & \\
\hline 01-09 & & & & & & & & & & & & \\
\hline $26-10$ & & & & & & & & & & & & \\
\hline $43-11$ & & & & & & & & & & & & \\
\hline $74-12$ & & & & & & & & & & & & \\
\hline $49-13$ & & & & & & & & & & & & \\
\hline $30-14$ & & & & & & & & & & & & \\
\hline
\end{tabular}


Comp. Vs Properties Study (Chick et al. 1981)

\begin{tabular}{|c|c|c|c|c|c|c|c|c|c|c|c|c|c|c|c|c|c|c|c|c|c|}
\hline Glass ID & $\begin{array}{c}\mathrm{A} 12 \mathrm{O} 3 \\
-\mathrm{t} \\
\end{array}$ & $\begin{array}{c}\mathrm{B} 2 \mathrm{O} 3 \\
-\mathrm{t} \\
\end{array}$ & $\begin{array}{c}\mathrm{CaO} \\
-\mathrm{t}\end{array}$ & $\begin{array}{c}\mathrm{Fe} 2 \mathrm{O} 3 \\
-\mathrm{t} \\
\end{array}$ & $\begin{array}{c}\mathrm{FeO} \\
-\mathrm{t} \\
\end{array}$ & $\begin{array}{c}\mathrm{K} 2 \mathrm{O} \\
-\mathrm{t}\end{array}$ & $\begin{array}{c}\mathrm{Li} 2 \mathrm{O} \\
-\mathrm{t}\end{array}$ & $\begin{array}{c}\mathrm{MgO} \\
-\mathrm{t}\end{array}$ & $\begin{array}{c}\mathrm{Na} 2 \mathrm{O} \\
-\mathrm{t}\end{array}$ & $\begin{array}{c}\mathrm{NiO} \\
-\mathrm{t}\end{array}$ & $\begin{array}{c}\mathrm{P} 2 \mathrm{O} 5 \\
-\mathrm{t} \\
\end{array}$ & $\begin{array}{c}\mathrm{SiO} 2 \\
-\mathrm{t}\end{array}$ & $\begin{array}{c}\mathrm{ZrO} 2 \\
-\mathrm{t} \\
\end{array}$ & $\begin{array}{c}\mathrm{Ag} 2 \mathrm{O} \\
-\mathrm{t}\end{array}$ & $\begin{array}{c}\mathrm{As} 2 \mathrm{O} 3 \\
-\mathrm{t}\end{array}$ & $\begin{array}{c}\mathrm{BaO} \\
-\mathrm{t} \\
\end{array}$ & $\begin{array}{c}\mathrm{Bi} 2 \mathrm{O} 3 \\
-\mathrm{t} \\
\end{array}$ & $\begin{array}{l}\mathrm{Br} \\
-\mathrm{t} \\
\end{array}$ & $\begin{array}{c}\mathrm{CdO} \\
-\mathrm{t}\end{array}$ & {$\left[\begin{array}{c}\mathrm{Ce} 2 \mathrm{O} 3 \\
-\mathrm{t}\end{array}\right.$} & $\begin{array}{c}\mathrm{CeO} 2 \\
-\mathrm{t}\end{array}$ \\
\hline $03-15$ & 0.0000 & 0.0000 & 0.0000 & 0.2400 & & 0.0009 & & & 0.1200 & 0.0077 & 0.0598 & 0.4200 & 0.0044 & & & & & & & & 0.0026 \\
\hline $69-16$ & 0.0800 & 0.0000 & 0.1500 & 0.2400 & & & & & 0.1200 & & & 0.4100 & & & & & & & & & \\
\hline $85-16$ & 0.0800 & 0.0000 & 0.1500 & 0.2400 & & & & & 0.1200 & & & 0.4100 & & & & & & & & & \\
\hline $45-17$ & 0.0800 & 0.0000 & 0.0000 & 0.0000 & & 0.0009 & & & 0.3000 & 0.0077 & 0.0598 & 0.4000 & 0.0044 & & & & & & & & 0.0026 \\
\hline $34-18$ & 0.0800 & 0.2000 & 0.0000 & 0.0000 & & 0.0009 & & & 0.1200 & 0.0077 & 0.0598 & 0.3800 & 0.0044 & & & & & & & & 0.0026 \\
\hline $36-19$ & 0.0800 & 0.0000 & 0.0000 & 0.2400 & & & & & 0.3000 & & & 0.3800 & & & & & & & & & \\
\hline $60-20$ & 0.0800 & 0.2000 & 0.0000 & 0.2400 & & & & & 0.1200 & & & 0.3600 & & & & & & & & & \\
\hline $17-21$ & 0.0000 & 0.1100 & 0.0000 & 0.2400 & & & & & 0.3000 & & & 0.3500 & & & & & & & & & \\
\hline $20-22$ & 0.0000 & 0.0000 & 0.0000 & 0.2400 & & 0.0004 & & & 0.3000 & 0.0039 & 0.0299 & 0.3500 & 0.0022 & & & & & & & & 0.0013 \\
\hline $44-23$ & 0.0000 & 0.0000 & 0.0000 & 0.1300 & & 0.0009 & & & 0.3000 & 0.0077 & 0.0598 & 0.3500 & 0.0044 & & & & & & & & 0.0026 \\
\hline $82-24$ & 0.0000 & 0.2000 & 0.0000 & 0.0000 & & 0.0006 & & & 0.3000 & 0.0053 & 0.0408 & 0.3500 & 0.0030 & & & & & & & & 0.0018 \\
\hline $77-25$ & 0.0800 & 0.2000 & 0.1500 & 0.1000 & & & & & 0.1200 & & & 0.3500 & & & & & & & & & \\
\hline $75-26$ & 0.0800 & 0.0800 & 0.1500 & 0.0000 & & 0.0009 & & & 0.1200 & 0.0077 & 0.0598 & 0.3500 & 0.0044 & & & & & & & & 0.0026 \\
\hline $22-27$ & 0.0800 & 0.2000 & 0.0700 & 0.0000 & & & & & 0.3000 & & & 0.3500 & & & & & & & & & \\
\hline $55-27$ & 0.0800 & 0.2000 & 0.0700 & 0.0000 & & & & & 0.3000 & & & 0.3500 & & & & & & & & & \\
\hline $64-28$ & 0.0000 & 0.2000 & 0.0900 & 0.2400 & & & & & 0.1200 & & & 0.3500 & & & & & & & & & \\
\hline $76-29$ & 0.0000 & 0.2000 & 0.1100 & 0.0000 & & 0.0009 & & & 0.1200 & 0.0077 & 0.0598 & 0.3500 & 0.0044 & & & & & & & & 0.0026 \\
\hline $10-30$ & 0.0000 & 0.0000 & 0.1500 & 0.2000 & & & & & 0.3000 & & & 0.3500 & & & & & & & & & \\
\hline $37-31$ & 0.0000 & 0.0000 & 0.1500 & 0.0000 & & 0.0009 & & & 0.2800 & 0.0077 & 0.0598 & 0.3500 & 0.0044 & & & & & & & & 0.0026 \\
\hline $48-32$ & 0.0800 & 0.0000 & 0.0000 & 0.2300 & & 0.0009 & & & 0.1200 & 0.0077 & 0.0598 & 0.3500 & 0.0044 & & & & & & & & 0.0026 \\
\hline $66-33$ & 0.0000 & 0.2000 & 0.1500 & 0.0000 & & & & & 0.3000 & & & 0.3500 & & & & & & & & & \\
\hline $71-33$ & 0.0000 & 0.2000 & 0.1500 & 0.0000 & & & & & 0.3000 & & & 0.3500 & & & & & & & & & \\
\hline $07-34$ & 0.0374 & 0.0809 & 0.0630 & 0.0926 & & 0.0003 & & & 0.1939 & 0.0031 & 0.0237 & 0.4450 & 0.0017 & & & & & & & & 0.0010 \\
\hline $42-34$ & 0.0374 & 0.0809 & 0.0630 & 0.0926 & & 0.0003 & & & 0.1939 & 0.0031 & 0.0237 & 0.4450 & 0.0017 & & & & & & & & 0.0010 \\
\hline $63-34$ & 0.0374 & 0.0809 & 0.0630 & 0.0926 & & 0.0003 & & & 0.1939 & 0.0031 & 0.0237 & 0.4450 & 0.0017 & & & & & & & & 0.0010 \\
\hline $81-34$ & 0.0374 & 0.0809 & 0.0630 & 0.0926 & & 0.0003 & & & 0.1939 & 0.0031 & 0.0237 & 0.4450 & 0.0017 & & & & & & & & 0.0010 \\
\hline $35-35$ & 0.0456 & 0.0986 & 0.0768 & 0.1128 & & 0.0004 & & & 0.2100 & 0.0037 & 0.0289 & 0.3500 & 0.0021 & & & & & & & & 0.0013 \\
\hline $21-36$ & 0.0241 & 0.0521 & 0.0406 & 0.0596 & & 0.0002 & & & 0.1676 & 0.0020 & 0.0152 & 0.5999 & 0.0011 & & & & & & & & 0.0007 \\
\hline $29-37$ & 0.0441 & 0.0000 & 0.0743 & 0.1093 & & 0.0004 & & & 0.2072 & 0.0036 & 0.0280 & 0.4621 & 0.0021 & & & & & & & & 0.0012 \\
\hline $02-38$ & 0.0275 & 0.2000 & 0.0463 & 0.0680 & & 0.0003 & & & 0.1743 & 0.0022 & 0.0174 & 0.4198 & 0.0013 & & & & & & & & 0.0008 \\
\hline $58-39$ & 0.0435 & 0.0940 & 0.0732 & 0.1076 & & 0.0004 & & & 0.1200 & 0.0035 & 0.0275 & 0.4604 & 0.0020 & & & & & & & & 0.0012 \\
\hline $05-40$ & 0.0287 & 0.0621 & 0.0483 & 0.0711 & & 0.0003 & & & 0.3000 & 0.0023 & 0.0182 & 0.4229 & 0.0013 & & & & & & & & 0.0008 \\
\hline $24-41$ & 0.0424 & 0.0918 & 0.0000 & 0.1051 & & 0.0004 & & & 0.2039 & 0.0035 & 0.0269 & 0.4578 & 0.0020 & & & & & & & & 0.0012 \\
\hline $52-42$ & 0.0304 & 0.0658 & 0.1500 & 0.0753 & & 0.0003 & & & 0.1801 & 0.0025 & 0.0193 & 0.4273 & 0.0014 & & & & & & & & 0.0009 \\
\hline $79-43$ & 0.0000 & 0.0870 & 0.0678 & 0.0996 & & 0.0004 & & & 0.1995 & 0.0033 & 0.0255 & 0.4522 & 0.0019 & & & & & & & & 0.0011 \\
\hline $04-44$ & 0.0800 & 0.0739 & 0.0576 & 0.0846 & & 0.0003 & & & 0.1875 & 0.0028 & 0.0217 & 0.4368 & 0.0016 & & & & & & & & 0.0010 \\
\hline $28-45$ & 0.0453 & 0.0980 & 0.0763 & 0.0000 & & 0.0004 & & & 0.2095 & 0.0037 & 0.0287 & 0.4651 & 0.0021 & & & & & & & & 0.0013 \\
\hline $80-46$ & 0.0248 & 0.0536 & 0.0418 & 0.2400 & & 0.0002 & & & 0.1690 & 0.0020 & 0.0157 & 0.4130 & 0.0012 & & & & & & & & 0.0007 \\
\hline $40-47$ & 0.0448 & 0.0968 & 0.0754 & 0.1108 & & & & & 0.2085 & & & 0.4637 & & & & & & & & & \\
\hline $78-48$ & 0.0262 & 0.0566 & 0.0441 & 0.0648 & & 0.0009 & & & 0.1717 & 0.0077 & 0.0598 & 0.4165 & 0.0044 & & & & & & & & 0.0026 \\
\hline $65-49$ & 0.0000 & 0.0000 & 0.1500 & 0.2000 & & 0.0009 & & & 0.1200 & 0.0063 & 0.0489 & 0.3500 & 0.0036 & & & & & & & & 0.0022 \\
\hline $68-50$ & 0.0800 & 0.0000 & 0.1500 & 0.0600 & & 0.0009 & & & 0.3000 & 0.0021 & 0.0163 & 0.3500 & 0.0012 & & & & & & & & 0.0007 \\
\hline 84-51 & 0.0000 & 0.2000 & 0.0000 & 0.1750 & & 0.0009 & & & 0.1200 & 0.0054 & 0.0421 & 0.3500 & 0.0031 & & & & & & & & 0.0019 \\
\hline $53-52$ & 0.0000 & 0.0000 & 0.1500 & 0.0650 & & 0.0009 & & & 0.1200 & 0.0023 & 0.0177 & 0.6000 & 0.0013 & & & & & & & & 0.0008 \\
\hline $38-53$ & 0.0000 & 0.0000 & 0.0000 & 0.0500 & & 0.0009 & & & 0.3000 & 0.0018 & 0.0136 & 0.6000 & 0.0010 & & & & & & & & 0.0006 \\
\hline $62-54$ & 0.0400 & 0.2000 & 0.1500 & 0.0000 & & 0.0009 & & & 0.1200 & 0.0049 & 0.0381 & 0.3500 & 0.0028 & & & & & & & & 0.0017 \\
\hline $70-55$ & 0.0400 & 0.2000 & 0.0000 & 0.1100 & & & & & 0.3000 & & & 0.3500 & & & & & & & & & \\
\hline $46-56$ & 0.0800 & 0.0000 & 0.0750 & 0.2400 & & 0.0009 & & & 0.1200 & 0.0047 & 0.0367 & 0.3500 & 0.0027 & & & & & & & & 0.0016 \\
\hline $11-57$ & 0.0400 & 0.0900 & 0.0000 & 0.0000 & & 0.0009 & & & 0.3000 & 0.0077 & 0.0598 & 0.3500 & 0.0044 & & & & & & & & 0.0026 \\
\hline
\end{tabular}


Comp. Vs Properties Study (Chick et al. 1981)

\begin{tabular}{|c|c|c|c|c|c|c|c|c|c|c|c|c|c|c|c|c|c|c|c|c|c|}
\hline Glass ID & $\begin{array}{l}\mathrm{Cl} \\
-\mathrm{t}\end{array}$ & $\begin{array}{c}\mathrm{CoO} \\
-\mathrm{t}\end{array}$ & $\begin{array}{c}\mathrm{Co} 2 \mathrm{O} 3 \\
-\mathrm{t}\end{array}$ & $\begin{array}{c}\mathrm{Cr} 2 \mathrm{O} 3 \\
-\mathrm{t}\end{array}$ & $\begin{array}{c}\mathrm{Cs} 2 \mathrm{O} \\
-\mathrm{t}\end{array}$ & $\begin{array}{c}\mathrm{CuO} \\
-\mathrm{t}\end{array}$ & $\begin{array}{c}\mathrm{Eu} 2 \mathrm{O} 3 \\
-\mathrm{t}\end{array}$ & $\begin{array}{l}F \\
-t\end{array}$ & $\begin{array}{c}\mathrm{Ga} 2 \mathrm{O} 3 \\
-\mathrm{t}\end{array}$ & $\begin{array}{c}\mathrm{Gd} 2 \mathrm{O} 3 \\
-\mathrm{t}\end{array}$ & $\begin{array}{c}\mathrm{HgO} \\
-\mathrm{t}\end{array}$ & $\begin{array}{l}I \\
-t\end{array}$ & $\begin{array}{c}\mathrm{La} 2 \mathrm{O} 3 \\
-\mathrm{t}\end{array}$ & $\begin{array}{c}\mathrm{MnO} 2 \\
-\mathrm{t}\end{array}$ & $\begin{array}{c}\mathrm{MnO} \\
-\mathrm{t}\end{array}$ & $\begin{array}{c}\mathrm{MoO} \\
-\mathrm{t}\end{array}$ & $\begin{array}{c}\mathrm{MoO} 3 \\
-\mathrm{t}\end{array}$ & $\begin{array}{c}\mathrm{Nb} 2 \mathrm{O} 5 \\
-\mathrm{t}\end{array}$ & $\begin{array}{c}\mathrm{Nd} 2 \mathrm{O} 3 \\
-\mathrm{t}\end{array}$ & $\begin{array}{c}\mathrm{PbO} \\
-\mathrm{t}\end{array}$ & $\begin{array}{c}\mathrm{PdO} 2 \\
-\mathrm{t}\end{array}$ \\
\hline $03-15$ & & & & 0.0163 & 0.0033 & & & & & & & & & 0.0101 & & & 0.0042 & & 0.0040 & & \\
\hline \multicolumn{22}{|l|}{$69-16$} \\
\hline \multicolumn{22}{|l|}{$85-16$} \\
\hline $45-17$ & & & & 0.0163 & 0.0033 & & & & & & & & & 0.0101 & & & 0.0042 & & 0.0040 & & \\
\hline $34-18$ & & & & 0.0163 & 0.0033 & & & & & & & & & 0.0101 & & & 0.0042 & & 0.0040 & & \\
\hline \multicolumn{22}{|l|}{$36-19$} \\
\hline \multicolumn{22}{|l|}{$60-20$} \\
\hline \multicolumn{22}{|l|}{$17-21$} \\
\hline $20-22$ & & & & 0.0081 & 0.0017 & & & & & & & & & 0.0051 & & & 0.0021 & & 0.0020 & & \\
\hline $44-23$ & & & & 0.0163 & 0.0033 & & & & & & & & & 0.0101 & & & 0.0042 & & 0.0040 & & \\
\hline $82-24$ & & & & 0.0111 & 0.0023 & & & & & & & & & 0.0069 & & & 0.0029 & & 0.0027 & & \\
\hline \multicolumn{22}{|l|}{$77-25$} \\
\hline $75-26$ & & & & 0.0163 & 0.0033 & & & & & & & & & 0.0101 & & & 0.0042 & & 0.0040 & & \\
\hline \multicolumn{22}{|l|}{$22-27$} \\
\hline \multicolumn{22}{|l|}{ 55-27 } \\
\hline \multicolumn{22}{|l|}{$64-28$} \\
\hline $76-29$ & & & & 0.0163 & 0.0033 & & & & & & & & & 0.0101 & & & 0.0042 & & 0.0040 & & \\
\hline \multicolumn{22}{|l|}{$10-30$} \\
\hline $37-31$ & & & & 0.0163 & 0.0033 & & & & & & & & & 0.0101 & & & 0.0042 & & 0.0040 & & \\
\hline $48-32$ & & & & 0.0163 & 0.0033 & & & & & & & & & 0.0101 & & & 0.0042 & & 0.0040 & & \\
\hline \multicolumn{22}{|l|}{ 66-33 } \\
\hline \multicolumn{22}{|l|}{$71-33$} \\
\hline $07-34$ & & & & 0.0065 & 0.0013 & & & & & & & & & 0.0040 & & & 0.0017 & & 0.0016 & & \\
\hline $42-34$ & & & & 0.0065 & 0.0013 & & & & & & & & & 0.0040 & & & 0.0017 & & 0.0016 & & \\
\hline 63-34 & & & & 0.0065 & 0.0013 & & & & & & & & & 0.0040 & & & 0.0017 & & 0.0016 & & \\
\hline $81-34$ & & & & 0.0065 & 0.0013 & & & & & & & & & 0.0040 & & & 0.0017 & & 0.0016 & & \\
\hline $35-35$ & & & & 0.0079 & 0.0016 & & & & & & & & & 0.0049 & & & 0.0020 & & 0.0019 & & \\
\hline $21-36$ & & & & 0.0042 & 0.0008 & & & & & & & & & 0.0026 & & & 0.0011 & & 0.0010 & & \\
\hline $29-37$ & & & & 0.0076 & 0.0015 & & & & & & & & & 0.0047 & & & 0.0020 & & 0.0019 & & \\
\hline $02-38$ & & & & 0.0047 & 0.0010 & & & & & & & & & 0.0029 & & & 0.0012 & & 0.0012 & & \\
\hline $58-39$ & & & & 0.0075 & 0.0015 & & & & & & & & & 0.0047 & & & 0.0019 & & 0.0018 & & \\
\hline $05-40$ & & & & 0.0050 & 0.0010 & & & & & & & & & 0.0031 & & & 0.0013 & & 0.0012 & & \\
\hline $24-41$ & & & & 0.0073 & 0.0015 & & & & & & & & & 0.0045 & & & 0.0019 & & 0.0018 & & \\
\hline $52-42$ & & & & 0.0053 & 0.0011 & & & & & & & & & 0.0033 & & & 0.0013 & & 0.0013 & & \\
\hline $79-43$ & & & & 0.0069 & 0.0014 & & & & & & & & & 0.0043 & & & 0.0018 & & 0.0017 & & \\
\hline $04-44$ & & & & 0.0059 & 0.0012 & & & & & & & & & 0.0037 & & & 0.0015 & & 0.0014 & & \\
\hline $28-45$ & & & & 0.0078 & 0.0016 & & & & & & & & & 0.0049 & & & 0.0020 & & 0.0019 & & \\
\hline $80-46$ & & & & 0.0043 & 0.0009 & & & & & & & & & 0.0027 & & & 0.0011 & & 0.0010 & & \\
\hline \multicolumn{22}{|l|}{$40-47$} \\
\hline $78-48$ & & & & 0.0163 & 0.0033 & & & & & & & & & 0.0101 & & & 0.0042 & & 0.0040 & & \\
\hline $65-49$ & & & & 0.0133 & 0.0027 & & & & & & & & & 0.0083 & & & 0.0034 & & 0.0032 & & \\
\hline $68-50$ & & & & 0.0044 & 0.0009 & & & & & & & & & 0.0028 & & & 0.0011 & & 0.0011 & & \\
\hline $84-51$ & & & & 0.0115 & 0.0023 & & & & & & & & & 0.0071 & & & 0.0029 & & 0.0028 & & \\
\hline $53-52$ & & & & 0.0048 & 0.0010 & & & & & & & & & 0.0030 & & & 0.0012 & & 0.0012 & & \\
\hline $38-53$ & & & & 0.0037 & 0.0008 & & & & & & & & & 0.0023 & & & 0.0010 & & 0.0009 & & \\
\hline $62-54$ & & & & 0.0104 & 0.0021 & & & & & & & & & 0.0064 & & & 0.0027 & & 0.0025 & & \\
\hline $70-55$ & & & & & & & & & & & & & & & & & & & & & \\
\hline $46-56$ & & & & 0.0100 & 0.0020 & & & & & & & & & 0.0062 & & & 0.0026 & & 0.0024 & & \\
\hline $11-57$ & & & & 0.0163 & 0.0033 & & & & & & & & & 0.0101 & & & 0.0042 & & 0.0040 & & \\
\hline
\end{tabular}


Comp. Vs Properties Study (Chick et al. 1981)

\begin{tabular}{|c|c|c|c|c|c|c|c|c|c|c|c|c|c|c|c|c|c|c|c|c|c|}
\hline Glass ID & $\begin{array}{c}\mathrm{PdO} \\
-\mathrm{t}\end{array}$ & $\begin{array}{c}\mathrm{Pr} 2 \mathrm{O} 3 \\
-\mathrm{t}\end{array}$ & $\begin{array}{c}\operatorname{Pr} 6011 \\
-t\end{array}$ & $\begin{array}{c}\mathrm{Rb} 2 \mathrm{O} \\
-\mathrm{t}\end{array}$ & $\begin{array}{c}\mathrm{ReO} \\
-\mathrm{t}\end{array}$ & $\begin{array}{c}\mathrm{ReO} 2 \\
-\mathrm{t}\end{array}$ & $\begin{array}{c}\mathrm{Rh} 2 \mathrm{O} 3 \\
-\mathrm{t}\end{array}$ & $\begin{array}{c}\mathrm{RhO} 2 \\
-\mathrm{t}\end{array}$ & $\begin{array}{c}\mathrm{RuO} 2 \\
-\mathrm{t}\end{array}$ & $\begin{array}{c}\mathrm{Sb} 2 \mathrm{O} 3 \\
-\mathrm{t}\end{array}$ & $\begin{array}{c}\mathrm{Sb} 2 \mathrm{O} 5 \\
-\mathrm{t}\end{array}$ & $\begin{array}{c}\mathrm{SeO} 2 \\
-t\end{array}$ & $\begin{array}{c}\mathrm{Sm} 2 \mathrm{O} 3 \\
-\mathrm{t}\end{array}$ & $\begin{array}{c}\mathrm{SnO} \\
-\mathrm{t}\end{array}$ & $\begin{array}{c}\mathrm{SnO} 2 \\
-\mathrm{t}\end{array}$ & $\begin{array}{c}\mathrm{SO} 3 \\
-\mathrm{t}\end{array}$ & $\begin{array}{c}\mathrm{SrO} \\
-\mathrm{t}\end{array}$ & $\begin{array}{c}\mathrm{Tc} 2 \mathrm{O} 7 \\
-\mathrm{t}\end{array}$ & $\begin{array}{c}\mathrm{TeO} 2 \\
-\mathrm{t}\end{array}$ & $\begin{array}{c}\mathrm{ThO} 2 \\
-\mathrm{t}\end{array}$ & $\begin{array}{c}\mathrm{TiO} 2 \\
-\mathrm{t}\end{array}$ \\
\hline $03-15$ & & & & & & & & & & & & & & & & & 0.0033 & & & 0.0781 & \\
\hline $69-16$ & & & & & & & & & & & & & & & & & & & & & \\
\hline $85-16$ & & & & & & & & & & & & & & & & & & & & & \\
\hline $45-17$ & & & & & & & & & & & & & & & & & 0.0033 & & & 0.0781 & \\
\hline $34-18$ & & & & & & & & & & & & & & & & & 0.0033 & & & 0.0781 & \\
\hline $36-19$ & & & & & & & & & & & & & & & & & & & & & \\
\hline $60-20$ & & & & & & & & & & & & & & & & & & & & & \\
\hline $17-21$ & & & & & & & & & & & & & & & & & & & & & \\
\hline $20-22$ & & & & & & & & & & & & & & & & & 0.0017 & & & 0.0391 & \\
\hline $44-23$ & & & & & & & & & & & & & & & & & 0.0033 & & & 0.0781 & \\
\hline $82-24$ & & & & & & & & & & & & & & & & & 0.0023 & & & 0.0533 & \\
\hline $77-25$ & & & & & & & & & & & & & & & & & & & & & \\
\hline $75-26$ & & & & & & & & & & & & & & & & & 0.0033 & & & 0.0781 & \\
\hline $22-27$ & & & & & & & & & & & & & & & & & & & & & \\
\hline $55-27$ & & & & & & & & & & & & & & & & & & & & & \\
\hline $64-28$ & & & & & & & & & & & & & & & & & & & & & \\
\hline $76-29$ & & & & & & & & & & & & & & & & & 0.0033 & & & 0.0781 & \\
\hline $10-30$ & & & & & & & & & & & & & & & & & & & & & \\
\hline $37-31$ & & & & & & & & & & & & & & & & & 0.0033 & & & 0.0781 & \\
\hline $48-32$ & & & & & & & & & & & & & & & & & 0.0033 & & & 0.0781 & \\
\hline $66-33$ & & & & & & & & & & & & & & & & & & & & & \\
\hline $71-33$ & & & & & & & & & & & & & & & & & & & & & \\
\hline $07-34$ & & & & & & & & & & & & & & & & & 0.0013 & & & 0.0310 & \\
\hline $42-34$ & & & & & & & & & & & & & & & & & 0.0013 & & & 0.0310 & \\
\hline $63-34$ & & & & & & & & & & & & & & & & & 0.0013 & & & 0.0310 & \\
\hline $81-34$ & & & & & & & & & & & & & & & & & 0.0013 & & & 0.0310 & \\
\hline $35-35$ & & & & & & & & & & & & & & & & & 0.0016 & & & 0.0377 & \\
\hline $21-36$ & & & & & & & & & & & & & & & & & 0.0008 & & & 0.0199 & \\
\hline $29-37$ & & & & & & & & & & & & & & & & & 0.0015 & & & 0.0365 & \\
\hline $02-38$ & & & & & & & & & & & & & & & & & 0.0010 & & & 0.0228 & \\
\hline $58-39$ & & & & & & & & & & & & & & & & & 0.0015 & & & 0.0360 & \\
\hline $05-40$ & & & & & & & & & & & & & & & & & 0.0010 & & & 0.0237 & \\
\hline $24-41$ & & & & & & & & & & & & & & & & & 0.0015 & & & 0.0351 & \\
\hline $52-42$ & & & & & & & & & & & & & & & & & 0.0011 & & & 0.0252 & \\
\hline $79-43$ & & & & & & & & & & & & & & & & & 0.0014 & & & 0.0333 & \\
\hline 04-44 & & & & & & & & & & & & & & & & & 0.0012 & & & 0.0283 & \\
\hline $28-45$ & & & & & & & & & & & & & & & & & 0.0016 & & & 0.0375 & \\
\hline $80-46$ & & & & & & & & & & & & & & & & & 0.0009 & & & 0.0205 & \\
\hline $40-47$ & & & & & & & & & & & & & & & & & & & & & \\
\hline $78-48$ & & & & & & & & & & & & & & & & & 0.0033 & & & 0.0781 & \\
\hline $65-49$ & & & & & & & & & & & & & & & & & 0.0027 & & & 0.0639 & \\
\hline $68-50$ & & & & & & & & & & & & & & & & & 0.0009 & & & 0.0213 & \\
\hline $84-51$ & & & & & & & & & & & & & & & & & 0.0023 & & & 0.0550 & \\
\hline $53-52$ & & & & & & & & & & & & & & & & & 0.0010 & & & 0.0231 & \\
\hline $38-53$ & & & & & & & & & & & & & & & & & 0.0008 & & & 0.0178 & \\
\hline $62-54$ & & & & & & & & & & & & & & & & & 0.0021 & & & 0.0497 & \\
\hline $70-55$ & & & & & & & & & & & & & & & & & & & & & \\
\hline $46-56$ & & & & & & & & & & & & & & & & & 0.0020 & & & 0.0479 & \\
\hline $11-57$ & & & & & & & & & & & & & & & & & 0.0033 & & & 0.0781 & \\
\hline
\end{tabular}


Comp. Vs Properties Study (Chick et al. 1981)

\begin{tabular}{|c|c|c|c|c|c|c|c|c|c|c|c|c|c|c|c|c|c|c|c|c|c|}
\hline Glass ID & $\begin{array}{c}\mathrm{T} 12 \mathrm{O} 3 \\
-\mathrm{t}\end{array}$ & $\begin{array}{c}\mathrm{U} 3 \mathrm{O} 8 \\
-\mathrm{t}\end{array}$ & $\begin{array}{c}\mathrm{UO} 2 \\
-\mathrm{t}\end{array}$ & $\begin{array}{c}\text { UO3 } \\
-t\end{array}$ & $\begin{array}{c}\mathrm{V} 2 \mathrm{O} 5 \\
-\mathrm{t}\end{array}$ & $\begin{array}{c}\text { WO3 } \\
-t\end{array}$ & $\begin{array}{c}\mathrm{Y} 2 \mathrm{O} 3 \\
-\mathrm{t}\end{array}$ & $\begin{array}{c}\mathrm{ZnO} \\
-\mathrm{t}\end{array}$ & $\begin{array}{c}\text { Others } \\
-t\end{array}$ & $\begin{array}{c}\text { Sum } \\
-t\end{array}$ & $\begin{array}{c}\mathrm{A} 12 \mathrm{O} 3 \\
-\mathrm{a}\end{array}$ & $\begin{array}{c}\mathrm{B} 2 \mathrm{O} 3 \\
-\mathrm{a}\end{array}$ & $\begin{array}{c}\mathrm{CaO} \\
-\mathrm{a} \\
\end{array}$ & $\begin{array}{c}\mathrm{Fe} 2 \mathrm{O} 3 \\
-\mathrm{a}\end{array}$ & $\begin{array}{c}\mathrm{FeO} \\
-\mathrm{a}\end{array}$ & $\begin{array}{c}\mathrm{K} 2 \mathrm{O} \\
-\mathrm{a}\end{array}$ & $\begin{array}{c}\mathrm{Li} 2 \mathrm{O} \\
-\mathrm{a}\end{array}$ & $\begin{array}{c}\mathrm{MgO} \\
-\mathrm{a}\end{array}$ & $\begin{array}{c}\mathrm{Na} 2 \mathrm{O} \\
-\mathrm{a}\end{array}$ & $\begin{array}{c}\mathrm{NiO} \\
-\mathrm{a}\end{array}$ & $\begin{array}{c}\mathrm{P} 2 \mathrm{O} 5 \\
-\mathrm{a}\end{array}$ \\
\hline $03-15$ & & & 0.0253 & & & & & & & 1.0000 & 0.0134 & 0.0000 & 0.0031 & 0.2086 & & 0.0008 & & & 0.1239 & 0.0074 & 0.0574 \\
\hline $69-16$ & & & & & & & & & & 1.0000 & 0.0896 & 0.0000 & 0.1568 & 0.2037 & & & & & 0.1273 & & \\
\hline $85-16$ & & & & & & & & & & 1.0000 & 0.0883 & 0.0000 & 0.1584 & 0.2000 & & & & & 0.1259 & & \\
\hline $45-17$ & & & 0.0253 & & & & & & & 1.0000 & 0.0825 & 0.0000 & 0.0007 & 0.0000 & & 0.0008 & & & 0.2984 & 0.0074 & 0.0576 \\
\hline $34-18$ & & & 0.0253 & & & & & & & 1.0000 & 0.0847 & 0.1992 & 0.0020 & 0.0000 & & 0.0008 & & & 0.1215 & 0.0071 & 0.0553 \\
\hline $36-19$ & & & & & & & & & & 1.0000 & 0.0898 & 0.0000 & 0.0026 & 0.2031 & & & & & 0.2984 & & \\
\hline $60-20$ & & & & & & & & & & 1.0000 & 0.0848 & 0.2356 & 0.0024 & 0.1943 & & & & & 0.1172 & & \\
\hline $17-21$ & & & & & & & & & & 1.0000 & 0.0081 & 0.1126 & 0.0022 & 0.2050 & & & & & 0.2979 & & \\
\hline $20-22$ & & & 0.0127 & & & & & & & 1.0000 & 0.0104 & 0.0000 & 0.0039 & 0.2085 & & 0.0004 & & & 0.2956 & 0.0038 & 0.0292 \\
\hline $44-23$ & & & 0.0253 & & & & & & & 1.0000 & 0.0090 & 0.0000 & 0.0016 & 0.1151 & & 0.0008 & & & 0.3013 & 0.0073 & 0.0570 \\
\hline $82-24$ & & & 0.0173 & & & & & & & 1.0003 & 0.0034 & 0.1909 & 0.0000 & 0.0000 & & 0.0007 & & & 0.2933 & 0.0062 & 0.0474 \\
\hline $77-25$ & & & & & & & & & & 1.0000 & 0.0845 & 0.1976 & 0.1548 & 0.0845 & & & & & 0.1253 & & \\
\hline $75-26$ & & & 0.0253 & & & & & & & 1.0000 & 0.0854 & 0.0830 & 0.1502 & 0.0000 & & 0.0008 & & & 0.1229 & 0.0072 & 0.0558 \\
\hline $22-27$ & & & & & & & & & & 1.0000 & 0.0827 & 0.1982 & 0.0746 & 0.0000 & & & & & 0.2891 & & \\
\hline $55-27$ & & & & & & & & & & 1.0000 & 0.0817 & 0.1962 & 0.0707 & 0.0000 & & & & & 0.3038 & & \\
\hline $64-28$ & & & & & & & & & & 1.0000 & 0.0085 & 0.1997 & 0.0918 & 0.2017 & & & & & 0.1251 & & \\
\hline $76-29$ & & & 0.0253 & & & & & & & 1.0000 & 0.0046 & 0.1925 & 0.1112 & 0.0000 & & 0.0009 & & & 0.1221 & 0.0075 & 0.0581 \\
\hline $10-30$ & & & & & & & & & & 1.0000 & 0.0069 & 0.0000 & 0.1527 & 0.1701 & & & & & 0.3003 & & \\
\hline $37-31$ & & & 0.0253 & & & & & & & 1.0000 & 0.0040 & 0.0000 & 0.1531 & 0.0000 & & 0.0009 & & & 0.2813 & 0.0075 & 0.0581 \\
\hline $48-32$ & & & 0.0253 & & & & & & & 1.0000 & 0.0818 & 0.0000 & 0.0040 & 0.1749 & & 0.0008 & & & 0.1097 & 0.0069 & 0.0532 \\
\hline $66-33$ & & & & & & & & & & 1.0000 & 0.0025 & 0.1918 & 0.1535 & 0.0000 & & & & & 0.3069 & & \\
\hline $71-33$ & & & & & & & & & & 1.0000 & 0.0428 & 0.0911 & 0.0666 & 0.0000 & & & & & 0.2745 & & \\
\hline $07-34$ & & & 0.0100 & & & & & & & 1.0000 & 0.0451 & 0.0841 & 0.0625 & 0.0810 & & 0.0003 & & & 0.1917 & 0.0029 & 0.0224 \\
\hline $42-34$ & & & 0.0100 & & & & & & & 1.0000 & 0.0446 & 0.0810 & 0.0633 & 0.0800 & & 0.0003 & & & 0.1973 & 0.0027 & 0.0209 \\
\hline $63-34$ & & & 0.0100 & & & & & & & 1.0000 & 0.0442 & 0.0802 & 0.0627 & 0.0792 & & 0.0003 & & & 0.1984 & 0.0029 & 0.0225 \\
\hline $81-34$ & & & 0.0100 & & & & & & & 1.0000 & 0.0442 & 0.0802 & 0.0637 & 0.0791 & & 0.0003 & & & 0.1963 & 0.0030 & 0.0229 \\
\hline $35-35$ & & & 0.0122 & & & & & & & 1.0000 & 0.0551 & 0.1044 & 0.0805 & 0.0580 & & 0.0004 & & & 0.2193 & 0.0033 & 0.0254 \\
\hline $21-36$ & & & 0.0065 & & & & & & & 1.0000 & 0.0292 & 0.0514 & 0.0423 & 0.0524 & & 0.0002 & & & 0.1633 & 0.0021 & 0.0161 \\
\hline $29-37$ & & & 0.0118 & & & & & & & 0.9999 & 0.0524 & 0.0000 & 0.0768 & 0.0956 & & 0.0004 & & & 0.2095 & 0.0032 & 0.0251 \\
\hline $02-38$ & & & 0.0074 & & & & & & & 1.0000 & 0.0329 & 0.2044 & 0.0462 & 0.0596 & & 0.0002 & & & 0.1736 & 0.0022 & 0.0169 \\
\hline $58-39$ & & & 0.0116 & & & & & & & 1.0000 & 0.0524 & 0.0929 & 0.0709 & 0.0919 & & 0.0004 & & & 0.1237 & 0.0031 & 0.0244 \\
\hline $05-40$ & & & 0.0077 & & & & & & & 1.0000 & 0.0346 & 0.0631 & 0.0508 & 0.0620 & & 0.0003 & & & 0.2873 & 0.0028 & 0.0218 \\
\hline $24-41$ & & & 0.0114 & & & & & & & 1.0000 & 0.0498 & 0.0916 & 0.0060 & 0.0906 & & 0.0004 & & & 0.2010 & 0.0034 & 0.0267 \\
\hline $52-42$ & & & 0.0082 & & & & & & & 0.9999 & 0.0365 & 0.0657 & 0.1511 & 0.0646 & & 0.0002 & & & 0.1814 & 0.0021 & 0.0166 \\
\hline $79-43$ & & & 0.0108 & & & & & & & 0.9999 & 0.0077 & 0.0870 & 0.0686 & 0.0849 & & 0.0004 & & & 0.2077 & 0.0031 & 0.0244 \\
\hline $04-44$ & & & 0.0092 & & & & & & & 1.0001 & 0.0875 & 0.0763 & 0.0590 & 0.0743 & & 0.0003 & & & 0.1872 & 0.0026 & 0.0201 \\
\hline $28-45$ & & & 0.0122 & & & & & & & 0.9999 & 0.0504 & 0.0977 & 0.0793 & 0.0000 & & 0.0004 & & & 0.2082 & 0.0032 & 0.0251 \\
\hline $80-46$ & & & 0.0066 & & & & & & & 1.0000 & 0.0359 & 0.0553 & 0.0430 & 0.1998 & & 0.0002 & & & 0.1742 & 0.0021 & 0.0167 \\
\hline $40-47$ & & & & & & & & & & 1.0000 & 0.0507 & 0.0954 & 0.0775 & 0.0974 & & & & & 0.2078 & & \\
\hline $78-48$ & & & 0.0253 & & & & & & & 0.9999 & 0.0350 & 0.0577 & 0.0433 & 0.0566 & & 0.0008 & & & 0.1792 & 0.0072 & 0.0557 \\
\hline $65-49$ & & & 0.0207 & & & & & & & 1.0001 & 0.0095 & 0.0000 & 0.1545 & 0.1713 & & 0.0008 & & & 0.1251 & 0.0056 & 0.0438 \\
\hline $68-50$ & & & 0.0069 & & & & & & & 1.0006 & 0.0842 & 0.0000 & 0.1500 & 0.0503 & & 0.0009 & & & 0.3010 & 0.0021 & 0.0166 \\
\hline $84-51$ & & & 0.0178 & & & & & & & 1.0002 & 0.0093 & 0.1974 & 0.0020 & 0.1490 & & 0.0009 & & & 0.1234 & 0.0054 & 0.0416 \\
\hline $53-52$ & & & 0.0075 & & & & & & & 1.0006 & 0.0064 & 0.0000 & 0.1489 & 0.0557 & & 0.0009 & & & 0.1205 & 0.0023 & 0.0176 \\
\hline $38-53$ & & & 0.0058 & & & & & & & 1.0007 & 0.0064 & 0.0000 & 0.0017 & 0.0427 & & 0.0009 & & & 0.2944 & 0.0018 & 0.0139 \\
\hline $62-54$ & & & 0.0161 & & & & & & & 1.0003 & 0.0438 & 0.2004 & 0.1545 & 0.0000 & & 0.0008 & & & 0.1232 & 0.0043 & 0.0335 \\
\hline $70-55$ & & & & & & & & & & 1.0000 & 0.0452 & 0.1977 & 0.0025 & 0.0933 & & & & & 0.3101 & & \\
\hline $46-56$ & & & 0.0155 & & & & & & & 1.0003 & 0.0913 & 0.0000 & 0.0810 & 0.2020 & & 0.0009 & & & 0.1231 & 0.0047 & 0.0362 \\
\hline $11-57$ & & & 0.0253 & & & & & & & 1.0000 & 0.0440 & 0.0911 & 0.0021 & 0.0000 & & 0.0008 & & & 0.2977 & 0.0072 & 0.0558 \\
\hline
\end{tabular}


Appendix A. Database - mass fraction

Comp. Vs Properties Study (Chick et al. 1981)

\begin{tabular}{|c|c|c|c|c|c|c|c|c|c|c|c|c|c|c|c|c|c|c|c|c|c|}
\hline Glass ID & $\begin{array}{c}\mathrm{SiO} 2 \\
-\mathrm{a}\end{array}$ & $\begin{array}{c}\mathrm{ZrO} 2 \\
-\mathrm{a}\end{array}$ & $\begin{array}{c}\mathrm{Ag} 2 \mathrm{O} \\
-\mathrm{a}\end{array}$ & $\begin{array}{c}\mathrm{As} 2 \mathrm{O} 3 \\
-\mathrm{a}\end{array}$ & $\begin{array}{c}\mathrm{BaO} \\
-\mathrm{a}\end{array}$ & $\begin{array}{c}\mathrm{Bi} 2 \mathrm{O} 3 \\
-\mathrm{a}\end{array}$ & $\begin{array}{l}\mathrm{Br} \\
-\mathrm{a}\end{array}$ & $\begin{array}{c}\mathrm{CdO} \\
-\mathrm{a}\end{array}$ & $\begin{array}{c}\mathrm{Ce} 2 \mathrm{O} 3 \\
-\mathrm{a}\end{array}$ & $\begin{array}{c}\mathrm{CeO} 2 \\
-\mathrm{a}\end{array}$ & $\begin{array}{l}\mathrm{Cl} \\
-\mathrm{a}\end{array}$ & $\begin{array}{c}\mathrm{CoO} \\
-\mathrm{a}\end{array}$ & $\begin{array}{c}\mathrm{Co} 2 \mathrm{O} 3 \\
-\mathrm{a}\end{array}$ & $\begin{array}{c}\mathrm{Cr} 2 \mathrm{O} 3 \\
-\mathrm{a}\end{array}$ & $\begin{array}{c}\mathrm{Cs} 2 \mathrm{O} \\
-\mathrm{a}\end{array}$ & $\begin{array}{c}\mathrm{CuO} \\
-\mathrm{a}\end{array}$ & $\begin{array}{c}\mathrm{Eu} 2 \mathrm{O} 3 \\
-\mathrm{a}\end{array}$ & $\begin{array}{c}F \\
-a\end{array}$ & $\begin{array}{c}\mathrm{Ga} 2 \mathrm{O} 3 \\
-\mathrm{a}\end{array}$ & $\begin{array}{c}\mathrm{Gd} 2 \mathrm{O} 3 \\
-\mathrm{a}\end{array}$ & $\begin{array}{c}\mathrm{HgO} \\
-\mathrm{a}\end{array}$ \\
\hline $03-15$ & 0.4398 & 0.0042 & & & & & & & & 0.0025 & & & & 0.0156 & 0.0032 & & & & & & \\
\hline $69-16$ & 0.4226 & & & & & & & & & & & & & & & & & & & & \\
\hline $85-16$ & 0.4274 & & & & & & & & & & & & & & & & & & & & \\
\hline $45-17$ & 0.4063 & 0.0042 & & & & & & & & 0.0025 & & & & 0.0157 & 0.0032 & & & & & & \\
\hline $34-18$ & 0.3892 & 0.0041 & & & & & & & & 0.0024 & & & & 0.0151 & 0.0031 & & & & & & \\
\hline $36-19$ & 0.4062 & & & & & & & & & & & & & & & & & & & & \\
\hline $60-20$ & 0.3657 & & & & & & & & & & & & & & & & & & & & \\
\hline $17-21$ & 0.3742 & & & & & & & & & & & & & & & & & & & & \\
\hline $20-22$ & 0.3740 & 0.0022 & & & & & & & & 0.0013 & & & & 0.0080 & 0.0016 & & & & & & \\
\hline $44-23$ & 0.3634 & 0.0042 & & & & & & & & 0.0025 & & & & 0.0155 & 0.0031 & & & & & & \\
\hline $82-24$ & 0.3381 & 0.0035 & & & & & & & & 0.0021 & & & & 0.0129 & 0.0027 & & & & & & \\
\hline $77-25$ & 0.3534 & & & & & & & & & & & & & & & & & & & & \\
\hline $75-26$ & 0.3533 & 0.0041 & & & & & & & & 0.0025 & & & & 0.0152 & 0.0031 & & & & & & \\
\hline $22-27$ & 0.3555 & & & & & & & & & & & & & & & & & & & & \\
\hline $55-27$ & 0.3476 & & & & & & & & & & & & & & & & & & & & \\
\hline $64-28$ & 0.3732 & & & & & & & & & & & & & & & & & & & & \\
\hline $76-29$ & 0.3559 & 0.0043 & & & & & & & & 0.0026 & & & & 0.0158 & 0.0032 & & & & & & \\
\hline $10-30$ & 0.3700 & & & & & & & & & & & & & & & & & & & & \\
\hline $37-31$ & 0.3478 & 0.0043 & & & & & & & & 0.0026 & & & & 0.0158 & 0.0032 & & & & & & \\
\hline $48-32$ & 0.4336 & 0.0039 & & & & & & & & 0.0024 & & & & 0.0145 & 0.0029 & & & & & & \\
\hline $66-33$ & 0.3453 & & & & & & & & & & & & & & & & & & & & \\
\hline $71-33$ & 0.3426 & & & & & & & & & & & & & & & & & & & & \\
\hline $07-34$ & 0.4532 & 0.0016 & & & & & & & & 0.0009 & & & & 0.0061 & 0.0012 & & & & & & \\
\hline $42-34$ & 0.4569 & 0.0015 & & & & & & & & 0.0009 & & & & 0.0057 & 0.0011 & & & & & & \\
\hline $63-34$ & 0.4524 & 0.0016 & & & & & & & & 0.0010 & & & & 0.0062 & 0.0012 & & & & & & \\
\hline $81-34$ & 0.4523 & 0.0016 & & & & & & & & 0.0010 & & & & 0.0063 & 0.0013 & & & & & & \\
\hline $35-35$ & 0.3894 & 0.0018 & & & & & & & & 0.0011 & & & & 0.0069 & 0.0014 & & & & & & \\
\hline $21-36$ & 0.6020 & 0.0012 & & & & & & & & 0.0007 & & & & 0.0044 & 0.0009 & & & & & & \\
\hline $29-37$ & 0.4735 & 0.0018 & & & & & & & & 0.0011 & & & & 0.0068 & 0.0014 & & & & & & \\
\hline $02-38$ & 0.4212 & 0.0012 & & & & & & & & 0.0007 & & & & 0.0046 & 0.0009 & & & & & & \\
\hline $58-39$ & 0.4785 & 0.0018 & & & & & & & & 0.0011 & & & & 0.0066 & 0.0013 & & & & & & \\
\hline $05-40$ & 0.4220 & 0.0016 & & & & & & & & 0.0010 & & & & 0.0059 & 0.0012 & & & & & & \\
\hline $24-41$ & 0.4628 & 0.0020 & & & & & & & & 0.0012 & & & & 0.0073 & 0.0015 & & & & & & \\
\hline $52-42$ & 0.4399 & 0.0012 & & & & & & & & 0.0007 & & & & 0.0045 & 0.0009 & & & & & & \\
\hline $79-43$ & 0.4544 & 0.0018 & & & & & & & & 0.0011 & & & & 0.0067 & 0.0013 & & & & & & \\
\hline $04-44$ & 0.4416 & 0.0015 & & & & & & & & 0.0009 & & & & 0.0055 & 0.0011 & & & & & & \\
\hline $28-45$ & 0.4721 & 0.0018 & & & & & & & & 0.0011 & & & & 0.0068 & 0.0014 & & & & & & \\
\hline $80-46$ & 0.4303 & 0.0012 & & & & & & & & 0.0007 & & & & 0.0045 & 0.0009 & & & & & & \\
\hline $40-47$ & 0.4712 & & & & & & & & & & & & & & & & & & & & \\
\hline $78-48$ & 0.4233 & 0.0041 & & & & & & & & 0.0025 & & & & 0.0152 & 0.0031 & & & & & & \\
\hline $65-49$ & 0.3784 & 0.0032 & & & & & & & & 0.0019 & & & & 0.0119 & 0.0024 & & & & & & \\
\hline $68-50$ & 0.3534 & 0.0012 & & & & & & & & 0.0007 & & & & 0.0045 & 0.0009 & & & & & & \\
\hline $84-51$ & 0.3659 & 0.0031 & & & & & & & & 0.0018 & & & & 0.0113 & 0.0023 & & & & & & \\
\hline $53-52$ & 0.6036 & 0.0013 & & & & & & & & 0.0008 & & & & 0.0048 & 0.0010 & & & & & & \\
\hline $38-53$ & 0.6038 & 0.0010 & & & & & & & & 0.0006 & & & & 0.0038 & 0.0008 & & & & & & \\
\hline $62-54$ & 0.3549 & 0.0025 & & & & & & & & 0.0015 & & & & 0.0091 & 0.0018 & & & & & & \\
\hline $70-55$ & 0.3512 & & & & & & & & & & & & & & & & & & & & \\
\hline $46-56$ & 0.3692 & 0.0027 & & & & & & & & 0.0016 & & & & 0.0099 & 0.0020 & & & & & & \\
\hline $11-57$ & 0.3598 & 0.0041 & & & & & & & & 0.0025 & & & & 0.0152 & 0.0031 & & & & & & \\
\hline
\end{tabular}


Appendix A. Database - mass fraction

Comp. Vs Properties Study (Chick et al. 1981)

\begin{tabular}{|c|c|c|c|c|c|c|c|c|c|c|c|c|c|c|c|c|c|c|c|c|c|}
\hline Glass ID & $\begin{array}{c}\mathrm{I} \\
-\mathrm{a}\end{array}$ & $\begin{array}{c}\mathrm{La} 2 \mathrm{O} 3 \\
-\mathrm{a}\end{array}$ & $\begin{array}{c}\mathrm{MnO} 2 \\
-\mathrm{a}\end{array}$ & $\begin{array}{c}\mathrm{MnO} \\
-\mathrm{a}\end{array}$ & $\begin{array}{c}\mathrm{MoO} \\
-\mathrm{a}\end{array}$ & $\begin{array}{c}\mathrm{MoO} 3 \\
-\mathrm{a}\end{array}$ & $\begin{array}{c}\mathrm{Nb} 2 \mathrm{O} 5 \\
-\mathrm{a}\end{array}$ & $\begin{array}{c}\mathrm{Nd} 2 \mathrm{O} 3 \\
-\mathrm{a}\end{array}$ & $\begin{array}{c}\mathrm{PbO} \\
-\mathrm{a}\end{array}$ & $\begin{array}{c}\mathrm{PdO} 2 \\
-\mathrm{a}\end{array}$ & $\begin{array}{c}\mathrm{PdO} \\
-\mathrm{a}\end{array}$ & $\begin{array}{c}\mathrm{Pr} 2 \mathrm{O} 3 \\
-\mathrm{a}\end{array}$ & $\begin{array}{c}\mathrm{Pr} 6 \mathrm{O} 11 \\
-\mathrm{a}\end{array}$ & $\begin{array}{c}\mathrm{Rb} 2 \mathrm{O} \\
-\mathrm{a}\end{array}$ & $\begin{array}{c}\mathrm{ReO} \\
-\mathrm{a}\end{array}$ & $\begin{array}{c}\mathrm{ReO} 2 \\
-\mathrm{a}\end{array}$ & $\begin{array}{c}\mathrm{Rh} 2 \mathrm{O} 3 \\
-\mathrm{a}\end{array}$ & $\begin{array}{c}\mathrm{RhO} 2 \\
-\mathrm{a}\end{array}$ & $\begin{array}{c}\mathrm{RuO} 2 \\
-\mathrm{a}\end{array}$ & $\begin{array}{c}\mathrm{Sb} 2 \mathrm{O} 3 \\
-\mathrm{a}\end{array}$ & $\begin{array}{c}\mathrm{Sb} 2 \mathrm{O} 5 \\
-\mathrm{a}\end{array}$ \\
\hline 03-15 & & & 0.0097 & & & 0.0040 & & 0.0038 & & & & & & & & & & & & & \\
\hline $69-16$ & & & & & & & & & & & & & & & & & & & & & \\
\hline $85-16$ & & & & & & & & & & & & & & & & & & & & & \\
\hline $45-17$ & & & 0.0097 & & & 0.0040 & & 0.0038 & & & & & & & & & & & & & \\
\hline $34-18$ & & & 0.0093 & & & 0.0039 & & 0.0037 & & & & & & & & & & & & & \\
\hline $36-19$ & & & & & & & & & & & & & & & & & & & & & \\
\hline $60-20$ & & & & & & & & & & & & & & & & & & & & & \\
\hline $17-21$ & & & & & & & & & & & & & & & & & & & & & \\
\hline $20-22$ & & & 0.0050 & & & 0.0021 & & 0.0019 & & & & & & & & & & & & & \\
\hline $44-23$ & & & 0.0096 & & & 0.0040 & & 0.0038 & & & & & & & & & & & & & \\
\hline $82-24$ & & & 0.0080 & & & 0.0034 & & 0.0031 & & & & & & & & & & & & & \\
\hline $77-25$ & & & & & & & & & & & & & & & & & & & & & \\
\hline $75-26$ & & & 0.0094 & & & 0.0039 & & 0.0037 & & & & & & & & & & & & & \\
\hline $22-27$ & & & & & & & & & & & & & & & & & & & & & \\
\hline $55-27$ & & & & & & & & & & & & & & & & & & & & & \\
\hline $64-28$ & & & & & & & & & & & & & & & & & & & & & \\
\hline $76-29$ & & & 0.0098 & & & 0.0041 & & 0.0038 & & & & & & & & & & & & & \\
\hline $10-30$ & & & & & & & & & & & & & & & & & & & & & \\
\hline $37-31$ & & & 0.0098 & & & 0.0041 & & 0.0039 & & & & & & & & & & & & & \\
\hline $48-32$ & & & 0.0090 & & & 0.0037 & & 0.0035 & & & & & & & & & & & & & \\
\hline $66-33$ & & & & & & & & & & & & & & & & & & & & & \\
\hline $71-33$ & & & & & & & & & & & & & & & & & & & & & \\
\hline $07-34$ & & & 0.0038 & & & 0.0016 & & 0.0015 & & & & & & & & & & & & & \\
\hline $42-34$ & & & 0.0035 & & & 0.0015 & & 0.0014 & & & & & & & & & & & & & \\
\hline $63-34$ & & & 0.0038 & & & 0.0016 & & 0.0015 & & & & & & & & & & & & & \\
\hline $81-34$ & & & 0.0039 & & & 0.0016 & & 0.0015 & & & & & & & & & & & & & \\
\hline $35-35$ & & & 0.0043 & & & 0.0018 & & 0.0017 & & & & & & & & & & & & & \\
\hline $21-36$ & & & 0.0027 & & & 0.0011 & & 0.0011 & & & & & & & & & & & & & \\
\hline $29-37$ & & & 0.0042 & & & 0.0018 & & 0.0017 & & & & & & & & & & & & & \\
\hline $02-38$ & & & 0.0028 & & & 0.0012 & & 0.0011 & & & & & & & & & & & & & \\
\hline 58-39 & & & 0.0041 & & & 0.0017 & & 0.0016 & & & & & & & & & & & & & \\
\hline $05-40$ & & & 0.0037 & & & 0.0015 & & 0.0014 & & & & & & & & & & & & & \\
\hline $24-41$ & & & 0.0045 & & & 0.0019 & & 0.0018 & & & & & & & & & & & & & \\
\hline $52-42$ & & & 0.0028 & & & 0.0012 & & 0.0011 & & & & & & & & & & & & & \\
\hline $79-43$ & & & 0.0041 & & & 0.0017 & & 0.0016 & & & & & & & & & & & & & \\
\hline $04-44$ & & & 0.0034 & & & 0.0014 & & 0.0013 & & & & & & & & & & & & & \\
\hline $28-45$ & & & 0.0042 & & & 0.0018 & & 0.0017 & & & & & & & & & & & & & \\
\hline $80-46$ & & & 0.0028 & & & 0.0012 & & 0.0011 & & & & & & & & & & & & & \\
\hline $40-47$ & & & & & & & & & & & & & & & & & & & & & \\
\hline $78-48$ & & & 0.0094 & & & 0.0039 & & 0.0037 & & & & & & & & & & & & & \\
\hline $65-49$ & & & 0.0074 & & & 0.0031 & & 0.0029 & & & & & & & & & & & & & \\
\hline $68-50$ & & & 0.0028 & & & 0.0012 & & 0.0011 & & & & & & & & & & & & & \\
\hline $84-51$ & & & 0.0070 & & & 0.0029 & & 0.0028 & & & & & & & & & & & & & \\
\hline $53-52$ & & & 0.0030 & & & 0.0012 & & 0.0012 & & & & & & & & & & & & & \\
\hline $38-53$ & & & 0.0023 & & & 0.0010 & & 0.0009 & & & & & & & & & & & & & \\
\hline $62-54$ & & & 0.0057 & & & 0.0023 & & 0.0022 & & & & & & & & & & & & & \\
\hline $70-55$ & & & & & & & & & & & & & & & & & & & & & \\
\hline $46-56$ & & & 0.0061 & & & 0.0025 & & 0.0024 & & & & & & & & & & & & & \\
\hline $11-57$ & & & 0.0094 & & & 0.0039 & & 0.0037 & & & & & & & & & & & & & \\
\hline
\end{tabular}


Appendix A. Database - mass fraction

Comp. Vs Properties Study (Chick et al. 1981)

\begin{tabular}{|c|c|c|c|c|c|c|c|c|c|c|c|c|c|c|c|c|c|c|c|c|}
\hline Glass ID & $\begin{array}{c}\mathrm{SeO} 2 \\
-\mathrm{a}\end{array}$ & $\begin{array}{c}\mathrm{Sm} 2 \mathrm{O} 3 \\
-\mathrm{a}\end{array}$ & $\begin{array}{c}\mathrm{SnO} \\
-\mathrm{a}\end{array}$ & $\begin{array}{c}\mathrm{SnO} 2 \\
-\mathrm{a}\end{array}$ & $\begin{array}{c}\mathrm{SO} 3 \\
-\mathrm{a}\end{array}$ & $\begin{array}{c}\mathrm{SrO} \\
-\mathrm{a}\end{array}$ & $\begin{array}{c}\mathrm{Tc} 2 \mathrm{O} 7 \\
-\mathrm{a}\end{array}$ & $\begin{array}{c}\mathrm{TeO} 2 \\
-\mathrm{a}\end{array}$ & $\begin{array}{c}\mathrm{ThO} 2 \\
-\mathrm{a}\end{array}$ & $\begin{array}{c}\mathrm{TiO} 2 \\
-\mathrm{a}\end{array}$ & $\begin{array}{c}\mathrm{T} 12 \mathrm{O} 3 \\
-\mathrm{a}\end{array}$ & $\begin{array}{c}\mathrm{U} 3 \mathrm{O} 8 \\
-\mathrm{a}\end{array}$ & $\begin{array}{c}\mathrm{UO} 2 \\
-\mathrm{a}\end{array}$ & $\begin{array}{c}\text { UO3 } \\
-\mathrm{a}\end{array}$ & $\begin{array}{c}\mathrm{V} 2 \mathrm{O} 5 \\
-\mathrm{a}\end{array}$ & $\begin{array}{c}\text { WO3 } \\
-\mathrm{a}\end{array}$ & $\begin{array}{c}\mathrm{Y} 2 \mathrm{O} 3 \\
-\mathrm{a}\end{array}$ & $\begin{array}{c}\mathrm{ZnO} \\
-\mathrm{a}\end{array}$ & $\begin{array}{c}\text { Others } \\
-\mathrm{a}\end{array}$ & $\begin{array}{c}\text { Sum } \\
-\mathrm{a}\end{array}$ \\
\hline 03-15 & & & & & & 0.0032 & & & 0.0750 & & & & 0.0243 & & & & & & & 1.0000 \\
\hline $69-16$ & & & & & & & & & & & & & & & & & & & & 1.0000 \\
\hline $85-16$ & & & & & & & & & & & & & & & & & & & & 1.0000 \\
\hline $45-17$ & & & & & & 0.0032 & & & 0.0753 & & & & 0.0244 & & & & & & & 0.9999 \\
\hline 34-18 & & & & & & 0.0031 & & & 0.0722 & & & & 0.0234 & & & & & & & 1.0000 \\
\hline $36-19$ & & & & & & & & & & & & & & & & & & & & 1.0001 \\
\hline $60-20$ & & & & & & & & & & & & & & & & & & & & 1.0000 \\
\hline $17-21$ & & & & & & & & & & & & & & & & & & & & 1.0000 \\
\hline $20-22$ & & & & & & 0.0016 & & & 0.0382 & & & & 0.0124 & & & & & & & 0.9999 \\
\hline $44-23$ & & & & & & 0.0031 & & & 0.0744 & & & & 0.0241 & & & & & & & 1.0000 \\
\hline $82-24$ & & & & & & 0.0027 & & & 0.0620 & & & & 0.0201 & & & & & & & 1.0004 \\
\hline $77-25$ & & & & & & & & & & & & & & & & & & & & 1.0001 \\
\hline $75-26$ & & & & & & 0.0031 & & & 0.0728 & & & & 0.0236 & & & & & & & 1.0000 \\
\hline $22-27$ & & & & & & & & & & & & & & & & & & & & 1.0001 \\
\hline $55-27$ & & & & & & & & & & & & & & & & & & & & 1.0000 \\
\hline $64-28$ & & & & & & & & & & & & & & & & & & & & 1.0000 \\
\hline $76-29$ & & & & & & 0.0032 & & & 0.0758 & & & & 0.0246 & & & & & & & 0.9999 \\
\hline $10-30$ & & & & & & & & & & & & & & & & & & & & 1.0000 \\
\hline $37-31$ & & & & & & 0.0032 & & & 0.0759 & & & & 0.0246 & & & & & & & 1.0001 \\
\hline $48-32$ & & & & & & 0.0029 & & & 0.0695 & & & & 0.0225 & & & & & & & 0.9999 \\
\hline $66-33$ & & & & & & & & & & & & & & & & & & & & 1.0000 \\
\hline $71-33$ & & & & & & & & & & & & & & & & & & & & 0.8176 \\
\hline $07-34$ & & & & & & 0.0012 & & & 0.0293 & & & & 0.0094 & & & & & & & 0.9999 \\
\hline $42-34$ & & & & & & 0.0011 & & & 0.0273 & & & & 0.0088 & & & & & & & 1.0000 \\
\hline 63-34 & & & & & & 0.0012 & & & 0.0295 & & & & 0.0095 & & & & & & & 1.0000 \\
\hline $81-34$ & & & & & & 0.0013 & & & 0.0299 & & & & 0.0097 & & & & & & & 1.0000 \\
\hline $35-35$ & & & & & & 0.0014 & & & 0.0331 & & & & 0.0107 & & & & & & & 1.0000 \\
\hline $21-36$ & & & & & & 0.0009 & & & 0.0210 & & & & 0.0068 & & & & & & & 0.9998 \\
\hline $29-37$ & & & & & & 0.0014 & & & 0.0327 & & & & 0.0106 & & & & & & & 1.0000 \\
\hline $02-38$ & & & & & & 0.0009 & & & 0.0220 & & & & 0.0071 & & & & & & & 0.9999 \\
\hline $58-39$ & & & & & & 0.0013 & & & 0.0318 & & & & 0.0103 & & & & & & & 1.0000 \\
\hline $05-40$ & & & & & & 0.0012 & & & 0.0284 & & & & 0.0092 & & & & & & & 0.9999 \\
\hline $24-41$ & & & & & & 0.0015 & & & 0.0349 & & & & 0.0113 & & & & & & & 1.0001 \\
\hline $52-42$ & & & & & & 0.0009 & & & 0.0216 & & & & 0.0070 & & & & & & & 1.0001 \\
\hline $79-43$ & & & & & & 0.0013 & & & 0.0319 & & & & 0.0103 & & & & & & & 1.0001 \\
\hline 04-44 & & & & & & 0.0011 & & & 0.0263 & & & & 0.0085 & & & & & & & 1.0000 \\
\hline $28-45$ & & & & & & 0.0014 & & & 0.0328 & & & & 0.0106 & & & & & & & 1.0000 \\
\hline $80-46$ & & & & & & 0.0009 & & & 0.0218 & & & & 0.0071 & & & & & & & 0.9999 \\
\hline $40-47$ & & & & & & & & & & & & & & & & & & & & 1.0000 \\
\hline $78-48$ & & & & & & 0.0031 & & & 0.0727 & & & & 0.0236 & & & & & & & 1.0000 \\
\hline $65-49$ & & & & & & 0.0024 & & & 0.0572 & & & & 0.0185 & & & & & & & 1.0001 \\
\hline $68-50$ & & & & & & 0.0009 & & & 0.0217 & & & & 0.0070 & & & & & & & 1.0005 \\
\hline 84-51 & & & & & & 0.0023 & & & 0.0544 & & & & 0.0176 & & & & & & & 1.0003 \\
\hline $53-52$ & & & & & & 0.0010 & & & 0.0230 & & & & 0.0075 & & & & & & & 1.0006 \\
\hline $38-53$ & & & & & & 0.0008 & & & 0.0181 & & & & 0.0059 & & & & & & & 1.0007 \\
\hline $62-54$ & & & & & & 0.0018 & & & 0.0437 & & & & 0.0142 & & & & & & & 1.0003 \\
\hline $70-55$ & & & & & & & & & & & & & & & & & & & & 1.0000 \\
\hline $46-56$ & & & & & & 0.0020 & & & 0.0473 & & & & 0.0153 & & & & & & & 1.0002 \\
\hline $11-57$ & & & & & & 0.0031 & & & 0.0729 & & & & 0.0236 & & & & & & & 1.0000 \\
\hline
\end{tabular}


Appendix A. Database - mass fraction

Comp. Vs Properties Study (Chick et al. 1981)

\begin{tabular}{|c|c|c|c|c|c|c|c|c|}
\hline Glass ID & $\begin{array}{l}\mathrm{TM} \\
\left({ }^{\circ} \mathrm{C}\right) \\
\end{array}$ & $\begin{array}{l}\text { Gradient } \\
\text { TL }\left({ }^{\circ} \mathrm{C}\right) \\
\end{array}$ & $\begin{array}{l}\text { Uniform } \\
\text { TL }\left({ }^{\circ} \mathrm{C}\right) \\
\end{array}$ & Primary Phase & $\begin{array}{l}\text { Quenched } \\
\text { Visual/OM }\end{array}$ & $\begin{array}{c}\text { Quenched } \\
\text { SEM/EDS or TEM }\end{array}$ & $\begin{array}{l}\text { Quenched } \\
\text { XRD }\end{array}$ & $\begin{array}{c}\text { Quenched } \\
\text { Homogeneous? }\end{array}$ \\
\hline $03-15$ & & & & & amorphous & & & \\
\hline $69-16$ & & & & & amorphous & & & \\
\hline $85-16$ & & & & & amorphous & & & \\
\hline $45-17$ & & & & & 0.14 crystallinity & & & \\
\hline $34-18$ & & & & & amorphous & & & \\
\hline $36-19$ & & & & & amorphous & & & \\
\hline $60-20$ & & & & & 0.12 crystallinity & & & \\
\hline $17-21$ & & & & & amorphous & & & \\
\hline $20-22$ & & & & & 0.71 crystallinity & & & \\
\hline $44-23$ & & & & & 0.42 crystallinity & & & \\
\hline $82-24$ & & & & & amorphous & & & \\
\hline $77-25$ & & & & & amorphous & & & \\
\hline $75-26$ & & & & & amorphous & & & \\
\hline $22-27$ & & & & & amorphous & & & \\
\hline $55-27$ & & & & & amorphous & & & \\
\hline $64-28$ & & & & & amorphous & & & \\
\hline $76-29$ & & & & & amorphous & & & \\
\hline $10-30$ & & & & & 0.83 crystallinity & & & \\
\hline $37-31$ & & & & & 1.88 crystallinity & & & \\
\hline $48-32$ & & & & & 0.52 crystallinity & & & \\
\hline $66-33$ & & & & & amorphous & & & \\
\hline $71-33$ & & & & & amorphous & & & \\
\hline $07-34$ & & & & & amorphous & & & \\
\hline $42-34$ & & & & & amorphous & & & \\
\hline $63-34$ & & & & & amorphous & & & \\
\hline $81-34$ & & & & & amorphous & & & \\
\hline $35-35$ & & & & & 0.09 crystallinity & & & \\
\hline $21-36$ & & & & & amorphous & & & \\
\hline $29-37$ & & & & & amorphous & & & \\
\hline $02-38$ & & & & & amorphous & & & \\
\hline $58-39$ & & & & & amorphous & & & \\
\hline $05-40$ & & & & & amorphous & & & \\
\hline $24-41$ & & & & & amorphous & & & \\
\hline $52-42$ & & & & & amorphous & & & \\
\hline $79-43$ & & & & & 0.09 crystallinity & & & \\
\hline $04-44$ & & & & & amorphous & & & \\
\hline $28-45$ & & & & & amorphous & & & \\
\hline $80-46$ & & & & & amorphous & & & \\
\hline $40-47$ & & & & & amorphous & & & \\
\hline $78-48$ & & & & & amorphous & & & \\
\hline $65-49$ & & & & & amorphous & & & \\
\hline $68-50$ & & & & & 1.61 crystallinity & & & \\
\hline $84-51$ & & & & & amorphous & & & \\
\hline $53-52$ & & & & & amorphous & & & \\
\hline $38-53$ & & & & & amorphous & & & \\
\hline $62-54$ & & & & & amorphous & & & \\
\hline $70-55$ & & & & & amorphous & & & \\
\hline $46-56$ & & & & & 0.42 crystallinity & & & \\
\hline $11-57$ & & & & & amorphous & & & \\
\hline
\end{tabular}


Appendix A. Database - mass fraction

Comp. Vs Properties Study (Chick et al. 1981)

\begin{tabular}{|c|c|c|c|c|c|}
\hline Glass ID & $\begin{array}{c}\mathrm{CCC} \\
\text { Visual/OM }\end{array}$ & $\begin{array}{c}\text { CCC } \\
\text { SEM/EDS or TEM }\end{array}$ & $\begin{array}{l}\text { CCC } \\
\text { XRD }\end{array}$ & $\begin{array}{l}\text { Heat Treated } \\
\text { Visual/OM }\end{array}$ & $\begin{array}{c}\text { Heat Treated } \\
\text { SEM/EDS or TEM }\end{array}$ \\
\hline $03-15$ & 0.45 crystallinity & & & 0.53 crystallinity & \\
\hline $69-16$ & 1.66 crystallinity & & & 0.25 crystallinity & \\
\hline $85-16$ & 1.60 crystallinity & & & 0.43 crystallinity & \\
\hline $45-17$ & 0.38 crystallinity & & & 0.31 crystallinity & \\
\hline $34-18$ & 0.88 crystallinity & & & 0.38 crystallinity & \\
\hline $36-19$ & 0.04 crystallinity & & & amorphous & \\
\hline $60-20$ & 0.34 crystallinity & & & 0.18 crystallinity & \\
\hline $17-21$ & amorphous & & & amorphous & \\
\hline $20-22$ & 0.02 crystallinity & & & amorphous & \\
\hline $44-23$ & 0.40 crystallinity & & & 0.27 crystallinity & \\
\hline $82-24$ & amorphous & & & 0.02 crystallinity & \\
\hline $77-25$ & amorphous & & & amorphous & \\
\hline $75-26$ & 0.52 crystallinity & & & 0.25 crystallinity & \\
\hline $22-27$ & 0.14 crystallinity & & & 0.14 crystallinity & \\
\hline $55-27$ & 0.06 crystallinity & & & 0.14 crystallinity & \\
\hline $64-28$ & amorphous & & & amorphous & \\
\hline $76-29$ & 0.06 crystallinity & & & 0.07 crystallinity & \\
\hline $10-30$ & 1.26 crystallinity & & & 1.18 crystallinity & \\
\hline $37-31$ & 2.20 crystallinity & & & 1.83 crystallinity & \\
\hline $48-32$ & 1.41 crystallinity & & & 1.03 crystallinity & \\
\hline $66-33$ & 0.91 crystallinity & & & 0.67 crystallinity & \\
\hline $71-33$ & 0.87 crystallinity & & & 0.58 crystallinity & \\
\hline $07-34$ & amorphous & & & amorphous & \\
\hline $42-34$ & amorphous & & & amorphous & \\
\hline $63-34$ & amorphous & & & amorphous & \\
\hline $81-34$ & amorphous & & & amorphous & \\
\hline $35-35$ & amorphous & & & amorphous & \\
\hline $21-36$ & amorphous & & & amorphous & \\
\hline $29-37$ & amorphous & & & amorphous & \\
\hline $02-38$ & amorphous & & & amorphous & \\
\hline $58-39$ & amorphous & & & amorphous & \\
\hline $05-40$ & amorphous & & & 0.22 crystallinity & \\
\hline $24-41$ & amorphous & & & amorphous & \\
\hline $52-42$ & amorphous & & & 0.07 crystallinity & \\
\hline $79-43$ & amorphous & & & amorphous & \\
\hline $04-44$ & amorphous & & & 0.59 crystallinity & \\
\hline $28-45$ & amorphous & & & amorphous & \\
\hline $80-46$ & amorphous & & & amorphous & \\
\hline $40-47$ & amorphous & & & amorphous & \\
\hline $78-48$ & 0.09 crystallinity & & & 0.05 crystallinity & \\
\hline $65-49$ & 0.59 crystallinity & & & 0.29 crystallinity & \\
\hline $68-50$ & 0.99 crystallinity & & & 0.85 crystallinity & \\
\hline $84-51$ & 0.38 crystallinity & & & 0.25 crystallinity & \\
\hline $53-52$ & amorphous & & & amorphous & \\
\hline $38-53$ & amorphous & & & amorphous & \\
\hline $62-54$ & 0.07 crystallinity & & & 0.05 crystallinity & \\
\hline $70-55$ & amorphous & & & amorphous & \\
\hline $46-56$ & 0.98 crystallinity & & & 0.55 crystallinity & \\
\hline $11-57$ & 0.07 crystallinity & & & 0.07 crystallinity & \\
\hline
\end{tabular}


Appendix A. Database - mass fraction

Comp. Vs Properties Study (Chick et al. 1981)

\begin{tabular}{|c|c|c|c|c|c|c|c|c|c|c|c|c|c|c|c|}
\hline Glass ID & $\begin{array}{c}\text { Heat Treated } \\
\text { XRD }\end{array}$ & $\begin{array}{l}\text { Density } \\
\left(\mathrm{g} / \mathrm{cm}^{3}\right)\end{array}$ & $\begin{array}{c}\text { Fulc Visc } \\
\mathrm{A}\end{array}$ & $\begin{array}{c}\text { Fulc Visc } \\
\mathrm{B}\end{array}$ & \begin{tabular}{|c|} 
Fulc Visc \\
To
\end{tabular} & $\begin{array}{c}\mathrm{FV} 1150^{\circ} \mathrm{C} \\
(\mathrm{Pa} \cdot \mathrm{s})\end{array}$ & $\begin{array}{c}\text { Arrh Visc } \\
\text { A }\end{array}$ & \begin{tabular}{|c|} 
Arrh Visc \\
B
\end{tabular} & $1150^{\circ} \mathrm{C}$ & $\begin{array}{c}\mathrm{T}\left({ }^{\circ} \mathrm{C}\right) \text { at } \\
2 \mathrm{~Pa} \cdot \mathrm{s}\end{array}$ & $\begin{array}{c}\mathrm{T}\left({ }^{\circ} \mathrm{C}\right) \text { at } \\
5 \mathrm{~Pa} \cdot \mathrm{s}\end{array}$ & \begin{tabular}{|c|}
$\mathrm{T}\left({ }^{\circ} \mathrm{C}\right)$ at \\
$10 \mathrm{~Pa} \cdot \mathrm{s}$
\end{tabular} & $\begin{array}{c}\mathrm{T} 1 \\
\left({ }^{\circ} \mathrm{C}\right)\end{array}$ & $\begin{array}{c}\mathrm{V} 1 \\
(\mathrm{~Pa} \cdot \mathrm{s})\end{array}$ & $\begin{array}{l}\mathrm{T} 2 \\
\left({ }^{\circ} \mathrm{C}\right)\end{array}$ \\
\hline $03-15$ & & & & & & & & & & & & 1327 & 1399 & 3 & \\
\hline $69-16$ & & & & & & & & & & & & 1187 & 1317 & 3 & \\
\hline $85-16$ & & & & & & & & & & & & 1166 & 1307 & 3 & \\
\hline $45-17$ & & & & & & & & & & & & 1191 & 1330 & 3 & \\
\hline $34-18$ & & & & & & & & & & & & 1189 & 1343 & 3 & \\
\hline $36-19$ & & & & & & & & & & & & 1112 & 1249 & 3 & \\
\hline $60-20$ & & & & & & & & & & & & & 1258 & 3 & \\
\hline $17-21$ & & & & & & & & & & & & 758 & 870 & 3 & \\
\hline $20-22$ & & & & & & & & & & & & 951 & 1073 & 3 & \\
\hline $44-23$ & & & & & & & & & & & & & & & \\
\hline $82-24$ & & & & & & & & & & & & 820 & 886 & 3 & \\
\hline $77-25$ & & & & & & & & & & & & & 1043 & 3 & \\
\hline $75-26$ & & & & & & & & & & & & & 1195 & 3 & \\
\hline $22-27$ & & & & & & & & & & & & 810 & 897 & 3 & \\
\hline $55-27$ & & & & & & & & & & & & 807 & 910 & 3 & \\
\hline $64-28$ & & & & & & & & & & & & 951 & 1055 & 3 & \\
\hline $76-29$ & & & & & & & & & & & & & & & \\
\hline $10-30$ & & & & & & & & & & & & 1034 & 1064 & 3 & \\
\hline $37-31$ & & & & & & & & & & & & & & & \\
\hline $48-32$ & & & & & & & & & & & & 1324 & 1513 & 3 & \\
\hline $66-33$ & & & & & & & & & & & & & & & \\
\hline $71-33$ & & & & & & & & & & & & & & & \\
\hline $07-34$ & & & & & & & & & & & & 1052 & 1172 & 3 & \\
\hline $42-34$ & & & & & & & & & & & & 1048 & 1183 & 3 & \\
\hline $63-34$ & & & & & & & & & & & & 1078 & 1222 & 3 & \\
\hline $81-34$ & & & & & & & & & & & & 1043 & 1195 & 3 & \\
\hline $35-35$ & & & & & & & & & & & & 941 & 1039 & 3 & \\
\hline $21-36$ & & & & & & & & & & & & 1265 & 1436 & 3 & \\
\hline $29-37$ & & & & & & & & & & & & 1124 & 1297 & 3 & \\
\hline $02-38$ & & & & & & & & & & & & 977 & 1062 & 3 & \\
\hline 58-39 & & & & & & & & & & & & 1180 & 1312 & 3 & \\
\hline $05-40$ & & & & & & & & & & & & 919 & 1026 & 3 & \\
\hline $24-41$ & & & & & & & & & & & & 1089 & 1240 & 3 & \\
\hline $52-42$ & & & & & & & & & & & & 1006 & 1124 & 3 & \\
\hline $79-43$ & & & & & & & & & & & & 1001 & 1126 & 3 & \\
\hline 04-44 & & & & & & & & & & & & 1122 & 1254 & 3 & \\
\hline $28-45$ & & & & & & & & & & & & 1026 & 1168 & 3 & \\
\hline $80-46$ & & & & & & & & & & & & 1067 & 1215 & 3 & \\
\hline $40-47$ & & & & & & & & & & & & 1021 & 1147 & 3 & \\
\hline $78-48$ & & & & & & & & & & & & 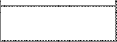 & 1231 & 3 & \\
\hline $65-49$ & & & & & & & & & & & & & & & \\
\hline $68-50$ & & & & & & & & & & & & 1007 & 1114 & 3 & \\
\hline $84-51$ & & & & & & & & & & & & 1060 & 1162 & 3 & \\
\hline $53-52$ & & & & & & & & & & & & 1268 & 1416 & 3 & \\
\hline $38-53$ & & & & & & & & & & & & 1191 & 1374 & 3 & \\
\hline $62-54$ & & & & & & & & & & & & & & & \\
\hline $70-55$ & & & & & & & & & & & & & 886 & 3 & \\
\hline $46-56$ & & & & & & & & & & & & 1202 & 1317 & 3 & \\
\hline $11-57$ & & & & & & & & & & & & & 998 & 3 & \\
\hline
\end{tabular}


Appendix A. Database - mass fraction

Comp. Vs Properties Study (Chick et al. 1981)

\begin{tabular}{|c|c|c|c|c|c|c|c|c|c|c|c|c|c|c|c|c|c|c|c|c|c|}
\hline Glass ID & $\begin{array}{c}\mathrm{V} 2 \\
(\mathrm{~Pa} \cdot \mathrm{s})\end{array}$ & $\begin{array}{c}\mathrm{T} 3 \\
\left({ }^{\circ} \mathrm{C}\right)\end{array}$ & $\begin{array}{c}\mathrm{V} 3 \\
(\mathrm{~Pa} \cdot \mathrm{s})\end{array}$ & $\begin{array}{c}\mathrm{T} 4 \\
\left({ }^{\circ} \mathrm{C}\right)\end{array}$ & $\begin{array}{c}\mathrm{V} 4 \\
(\mathrm{~Pa} \cdot \mathrm{s})\end{array}$ & $\begin{array}{c}\mathrm{T} 5 \\
\left({ }^{\circ} \mathrm{C}\right)\end{array}$ & $\begin{array}{c}\text { V5 } \\
(\mathrm{Pa} \cdot \mathrm{s})\end{array}$ & $\begin{array}{c}\mathrm{T} 6 \\
\left({ }^{\circ} \mathrm{C}\right)\end{array}$ & $\begin{array}{c}\mathrm{V} 6 \\
(\mathrm{~Pa} \cdot \mathrm{s})\end{array}$ & $\begin{array}{c}\mathrm{T} 7 \\
\left({ }^{\circ} \mathrm{C}\right)\end{array}$ & $\begin{array}{c}\mathrm{V} 7 \\
(\mathrm{~Pa} \cdot \mathrm{s})\end{array}$ & $\begin{array}{c}\mathrm{T} 8 \\
\left({ }^{\circ} \mathrm{C}\right)\end{array}$ & $\begin{array}{c}\mathrm{V} 8 \\
(\mathrm{~Pa} \cdot \mathrm{s})\end{array}$ & $\begin{array}{c}\mathrm{T} 9 \\
\left({ }^{\circ} \mathrm{C}\right)\end{array}$ & $\begin{array}{c}\mathrm{V} 9 \\
(\mathrm{~Pa} \cdot \mathrm{s})\end{array}$ & $\begin{array}{l}\mathrm{T} 10 \\
\left({ }^{\circ} \mathrm{C}\right)\end{array}$ & $\begin{array}{c}\mathrm{V} 10 \\
(\mathrm{~Pa} \cdot \mathrm{s})\end{array}$ & $\begin{array}{l}\mathrm{T} 11 \\
\left({ }^{\circ} \mathrm{C}\right)\end{array}$ & $\begin{array}{c}\text { V11 } \\
(\mathrm{Pa} \cdot \mathrm{s})\end{array}$ & $\begin{array}{l}\mathrm{T} 12 \\
\left({ }^{\circ} \mathrm{C}\right)\end{array}$ & $\begin{array}{c}\mathrm{V} 12 \\
(\mathrm{~Pa} \cdot \mathrm{s})\end{array}$ \\
\hline \multicolumn{22}{|l|}{$03-15$} \\
\hline \multicolumn{22}{|l|}{$69-16$} \\
\hline \multicolumn{22}{|l|}{$85-16$} \\
\hline \multicolumn{22}{|l|}{$45-17$} \\
\hline \multicolumn{22}{|l|}{$34-18$} \\
\hline \multicolumn{22}{|l|}{$36-19$} \\
\hline \multicolumn{22}{|l|}{$60-20$} \\
\hline \multicolumn{22}{|l|}{$17-21$} \\
\hline \multicolumn{22}{|l|}{$20-22$} \\
\hline \multicolumn{22}{|l|}{$44-23$} \\
\hline \multicolumn{22}{|l|}{$82-24$} \\
\hline \multicolumn{22}{|l|}{$77-25$} \\
\hline \multicolumn{22}{|l|}{$75-26$} \\
\hline $22-27$ & & & & & & & & & & & & & & & & & & & & & \\
\hline $55-27$ & & & & & & & & & & & & & & & & & & & & & \\
\hline $64-28$ & & & & & & & & & & & & & & & & & & & & & \\
\hline $76-29$ & & & & & & & & & & & & & & & & & & & & & \\
\hline $10-30$ & & & & & & & & & & & & & & & & & & & & & \\
\hline $37-31$ & & & & & & & & & & & & & & & & & & & & & \\
\hline $48-32$ & & & & & & & & & & & & & & & & & & & & & \\
\hline $66-33$ & & & & & & & & & & & & & & & & & & & & & \\
\hline $71-33$ & & & & & & & & & & & & & & & & & & & & & \\
\hline $07-34$ & & & & & & & & & & & & & & & & & & & & & \\
\hline $42-34$ & & & & & & & & & & & & & & & & & & & & & \\
\hline $63-34$ & & & & & & & & & & & & & & & & & & & & & \\
\hline $81-34$ & & & & & & & & & & & & & & & & & & & & & \\
\hline $35-35$ & & & & & & & & & & & & & & & & & & & & & \\
\hline $21-36$ & & & & & & & & & & & & & & & & & & & & & \\
\hline $29-37$ & & & & & & & & & & & & & & & & & & & & & \\
\hline $02-38$ & & & & & & & & & & & & & & & & & & & & & \\
\hline $58-39$ & & & & & & & & & & & & & & & & & & & & & \\
\hline $05-40$ & & & & & & & & & & & & & & & & & & & & & \\
\hline $24-41$ & & & & & & & & & & & & & & & & & & & & & \\
\hline $52-42$ & & & & & & & & & & & & & & & & & & & & & \\
\hline $79-43$ & & & & & & & & & & & & & & & & & & & & & \\
\hline $04-44$ & & & & & & & & & & & & & & & & & & & & & \\
\hline $28-45$ & & & & & & & & & & & & & & & & & & & & & \\
\hline $80-46$ & & & & & & & & & & & & & & & & & & & & & \\
\hline $40-47$ & & & & & & & & & & & & & & & & & & & & & \\
\hline $78-48$ & & & & & & & & & & & & & & & & & & & & & \\
\hline $65-49$ & & & & & & & & & & & & & & & & & & & & & \\
\hline $68-50$ & & & & & & & & & & & & & & & & & & & & & \\
\hline $84-51$ & & & & & & & & & & & & & & & & & & & & & \\
\hline $53-52$ & & & & & & & & & & & & & & & & & & & & & \\
\hline $38-53$ & & & & & & & & & & & & & & & & & & & & & \\
\hline $62-54$ & & & & & & & & & & & & & & & & & & & & & \\
\hline $70-55$ & & & & & & & & & & & & & & & & & & & & & \\
\hline $46-56$ & & & & & & & & & & & & & & & & & & & & & \\
\hline $11-57$ & & & & & & & & & & & & & & & & & & & & & \\
\hline
\end{tabular}


Appendix A. Database - mass fraction

Comp. Vs Properties Study (Chick et al. 1981)

\begin{tabular}{|c|c|c|c|c|c|c|c|c|c|c|c|c|c|c|c|c|}
\hline Glass ID & $\begin{array}{l}\mathrm{T} 13 \\
\left({ }^{\circ} \mathrm{C}\right) \\
\end{array}$ & $\begin{array}{c}\mathrm{V} 13 \\
(\mathrm{~Pa} \cdot \mathrm{s})\end{array}$ & $\begin{array}{l}\mathrm{T} 14 \\
\left({ }^{\circ} \mathrm{C}\right) \\
\end{array}$ & $\begin{array}{c}\mathrm{V} 14 \\
(\mathrm{~Pa} \cdot \mathrm{s})\end{array}$ & $\begin{array}{c}\text { Q PCT } \\
B\left(\mathrm{~g} / \mathrm{m}^{2}\right)\end{array}$ & $\begin{array}{c}\text { Q PCT } \\
\mathrm{Li}\left(\mathrm{g} / \mathrm{m}^{2}\right)\end{array}$ & $\begin{array}{c}\text { Q PCT } \\
\mathrm{Na}\left(\mathrm{g} / \mathrm{m}^{2}\right)\end{array}$ & $\begin{array}{c}\text { Q PCT } \\
\mathrm{Si}\left(\mathrm{g} / \mathrm{m}^{2}\right)\end{array}$ & $\begin{array}{c}\text { Q PCT } \\
\text { pH }\end{array}$ & $\begin{array}{c}\text { CCC PCT } \\
\mathrm{B}\left(\mathrm{g} / \mathrm{m}^{2}\right) \\
\end{array}$ & $\begin{array}{l}\text { CCC PCT } \\
\mathrm{Li}\left(\mathrm{g} / \mathrm{m}^{2}\right)\end{array}$ & $\begin{array}{l}\text { CCC PCT } \\
\mathrm{Na}\left(\mathrm{g} / \mathrm{m}^{2}\right)\end{array}$ & $\begin{array}{l}\text { CCC PCT } \\
\mathrm{Si}\left(\mathrm{g} / \mathrm{m}^{2}\right)\end{array}$ & $\begin{array}{c}\text { CCC PCT } \\
\mathrm{pH}\end{array}$ & $\begin{array}{l}\text { Q PCT B at } \\
20^{\circ} \mathrm{C}\left(\mathrm{g} / \mathrm{m}^{2}\right)\end{array}$ & $\begin{array}{l}\text { Q PCT Li at } \\
20^{\circ} \mathrm{C}\left(\mathrm{g} / \mathrm{m}^{2}\right)\end{array}$ \\
\hline \multicolumn{17}{|l|}{$03-15$} \\
\hline \multirow{2}{*}{\multicolumn{17}{|c|}{$\begin{array}{l}69-16 \\
85-16\end{array}$}} \\
\hline $85-16$ & & & & & & & & & & & & & & & & \\
\hline \multicolumn{17}{|l|}{$45-17$} \\
\hline \multicolumn{17}{|l|}{$34-18$} \\
\hline \multicolumn{17}{|l|}{$36-19$} \\
\hline \multicolumn{17}{|l|}{$60-20$} \\
\hline \multicolumn{17}{|l|}{$17-21$} \\
\hline \multirow{2}{*}{\multicolumn{17}{|c|}{$\frac{20-22}{44-23}$}} \\
\hline $44-23$ & & & & & & & & & & & & & & & & \\
\hline \multicolumn{17}{|l|}{$82-24$} \\
\hline \multicolumn{17}{|l|}{$77-25$} \\
\hline \multicolumn{17}{|l|}{$75-26$} \\
\hline \multicolumn{17}{|l|}{$22-27$} \\
\hline \multirow{2}{*}{\multicolumn{17}{|c|}{$\begin{array}{l}55-27 \\
64-28\end{array}$}} \\
\hline \multicolumn{10}{|l|}{$\frac{64-28}{76-29}$} & & & & & & & \\
\hline $76-29$ & & & & & & & & & & & & & & & & \\
\hline \multicolumn{17}{|l|}{$10-30$} \\
\hline \multicolumn{17}{|l|}{$37-31$} \\
\hline $48-32$ & & & & & & & & & & & & & & & & \\
\hline $66-33$ & & & & & & & & & & & & & & & & \\
\hline $71-33$ & & & & & & & & & & & & & & & & \\
\hline $07-34$ & & & & & & & & & & & & & & & & \\
\hline $42-34$ & & & & & & & & & & & & & & & & \\
\hline $63-34$ & & & & & & & & & & & & & & & & \\
\hline $81-34$ & & & & & & & & & & & & & & & & \\
\hline $35-35$ & & & & & & & & & & & & & & & & \\
\hline $21-36$ & & & & & & & & & & & & & & & & \\
\hline $29-37$ & & & & & & & & & & & & & & & & \\
\hline $02-38$ & & & & & & & & & & & & & & & & \\
\hline $58-39$ & & & & & & & & & & & & & & & & \\
\hline $05-40$ & & & & & & & & & & & & & & & & \\
\hline $24-41$ & & & & & & & & & & & & & & & & \\
\hline $52-42$ & & & & & & & & & & & & & & & & \\
\hline $79-43$ & & & & & & & & & & & & & & & & \\
\hline $04-44$ & & & & & & & & & & & & & & & & \\
\hline $28-45$ & & & & & & & & & & & & & & & & \\
\hline $80-46$ & & & & & & & & & & & & & & & & \\
\hline $40-47$ & & & & & & & & & & & & & & & & \\
\hline $78-48$ & & & & & & & & & & & & & & & & \\
\hline $65-49$ & & & & & & & & & & & & & & & & \\
\hline $68-50$ & & & & & & & & & & & & & & & & \\
\hline $84-51$ & & & & & & & & & & & & & & & & \\
\hline $53-52$ & & & & & & & & & & & & & & & & \\
\hline $38-53$ & & & & & & & & & & & & & & & & \\
\hline $62-54$ & & & & & & & & & & & & & & & & \\
\hline $70-55$ & & & & & & & & & & & & & & & & \\
\hline $46-56$ & & & & & & & & & & & & & & & & \\
\hline $11-57$ & & & & & & & & & & & & & & & & \\
\hline
\end{tabular}


Appendix A. Database - mass fraction

Comp. Vs Properties Study (Chick et al. 1981)

\begin{tabular}{|c|c|c|c|c|c|c|c|c|c|c|c|c|}
\hline Glass ID & $\begin{array}{l}\text { Q PCT Na at } \\
20^{\circ} \mathrm{C}\left(\mathrm{g} / \mathrm{m}^{2}\right)\end{array}$ & $\begin{array}{l}\text { Q PCT Si at } \\
20^{\circ} \mathrm{C}\left(\mathrm{g} / \mathrm{m}^{2}\right)\end{array}$ & $\begin{array}{c}\mathrm{QpH} \\
\text { at } 20^{\circ} \mathrm{C}\end{array}$ & $\begin{array}{l}\text { TCLP Ag } \\
(\mathrm{ppm})\end{array}$ & $\begin{array}{c}\text { TCLP As } \\
\text { (ppm) }\end{array}$ & $\begin{array}{c}\text { TCLP Ba } \\
(\mathrm{ppm})\end{array}$ & $\begin{array}{c}\text { TCLP Cd } \\
(\mathrm{ppm})\end{array}$ & $\begin{array}{c}\text { TCLP Cr } \\
(\mathrm{ppm})\end{array}$ & $\begin{array}{l}\text { TCLP Ni } \\
(\mathrm{ppm})\end{array}$ & $\begin{array}{c}\text { TCLP Pb } \\
(\mathrm{ppm})\end{array}$ & $\begin{array}{c}\text { TCLP Se } \\
(\mathrm{ppm})\end{array}$ & $\begin{array}{c}\text { TCLP Zn } \\
(\mathrm{ppm})\end{array}$ \\
\hline \multicolumn{13}{|l|}{$03-15$} \\
\hline \multicolumn{13}{|l|}{$69-16$} \\
\hline \multicolumn{13}{|l|}{$85-16$} \\
\hline \multicolumn{13}{|l|}{$45-17$} \\
\hline \multicolumn{13}{|l|}{$34-18$} \\
\hline \multicolumn{13}{|l|}{$36-19$} \\
\hline \multicolumn{13}{|l|}{$60-20$} \\
\hline \multicolumn{13}{|l|}{$17-21$} \\
\hline \multicolumn{13}{|l|}{$20-22$} \\
\hline \multicolumn{13}{|l|}{$44-23$} \\
\hline \multicolumn{13}{|l|}{$82-24$} \\
\hline \multicolumn{13}{|l|}{$77-25$} \\
\hline \multicolumn{13}{|l|}{$75-26$} \\
\hline \multicolumn{13}{|l|}{$22-27$} \\
\hline \multicolumn{13}{|l|}{$55-27$} \\
\hline \multicolumn{13}{|l|}{$64-28$} \\
\hline \multicolumn{13}{|l|}{$76-29$} \\
\hline \multicolumn{13}{|l|}{$10-30$} \\
\hline $37-31$ & & & & & & & & & & & & \\
\hline $48-32$ & & & & & & & & & & & & \\
\hline $66-33$ & & & & & & & & & & & & \\
\hline $71-33$ & & & & & & & & & & & & \\
\hline $07-34$ & & & & & & & & & & & & \\
\hline $42-34$ & & & & & & & & & & & & \\
\hline 63-34 & & & & & & & & & & & & \\
\hline $81-34$ & & & & & & & & & & & & \\
\hline $35-35$ & & & & & & & & & & & & \\
\hline $21-36$ & & & & & & & & & & & & \\
\hline $29-37$ & & & & & & & & & & & & \\
\hline $02-38$ & & & & & & & & & & & & \\
\hline 58-39 & & & & & & & & & & & & \\
\hline $05-40$ & & & & & & & & & & & & \\
\hline $24-41$ & & & & & & & & & & & & \\
\hline $52-42$ & & & & & & & & & & & & \\
\hline $79-43$ & & & & & & & & & & & & \\
\hline $04-44$ & & & & & & & & & & & & \\
\hline $28-45$ & & & & & & & & & & & & \\
\hline $80-46$ & & & & & & & & & & & & \\
\hline $40-47$ & & & & & & & & & & & & \\
\hline $78-48$ & & & & & & & & & & & & \\
\hline $65-49$ & & & & & & & & & & & & \\
\hline $68-50$ & & & & & & & & & & & & \\
\hline $84-51$ & & & & & & & & & & & & \\
\hline $53-52$ & & & & & & & & & & & & \\
\hline $38-53$ & & & & & & & & & & & & \\
\hline $62-54$ & & & & & & & & & & & & \\
\hline $70-55$ & & & & & & & & & & & & \\
\hline $46-56$ & & & & & & & & & & & & \\
\hline $11-57$ & & & & & & & & & & & & \\
\hline
\end{tabular}


Comp. Vs Properties Study (Chick et al. 1981)

\begin{tabular}{|c|c|c|c|c|c|c|c|c|c|c|c|c|c|c|c|c|c|c|c|c|c|}
\hline Glass ID & $\begin{array}{c}\mathrm{A} 12 \mathrm{O} 3 \\
-\mathrm{t}\end{array}$ & $\begin{array}{c}\mathrm{B} 2 \mathrm{O} 3 \\
-\mathrm{t}\end{array}$ & $\begin{array}{c}\mathrm{CaO} \\
-\mathrm{t}\end{array}$ & $\begin{array}{c}\mathrm{Fe} 2 \mathrm{O} 3 \\
-\mathrm{t} \\
\end{array}$ & $\begin{array}{c}\mathrm{FeO} \\
-\mathrm{t}\end{array}$ & $\begin{array}{c}\mathrm{K} 2 \mathrm{O} \\
-\mathrm{t}\end{array}$ & $\begin{array}{c}\mathrm{Li} 2 \mathrm{O} \\
-\mathrm{t}\end{array}$ & $\begin{array}{c}\mathrm{MgO} \\
-\mathrm{t}\end{array}$ & $\begin{array}{c}\mathrm{Na} 2 \mathrm{O} \\
-\mathrm{t}\end{array}$ & $\begin{array}{c}\mathrm{NiO} \\
-\mathrm{t}\end{array}$ & $\begin{array}{c}\mathrm{P} 2 \mathrm{O} 5 \\
-\mathrm{t}\end{array}$ & $\begin{array}{c}\mathrm{SiO} 2 \\
-\mathrm{t}\end{array}$ & $\begin{array}{c}\mathrm{ZrO} 2 \\
-\mathrm{t}\end{array}$ & $\begin{array}{c}\mathrm{Ag} 2 \mathrm{O} \\
-\mathrm{t}\end{array}$ & $\begin{array}{c}\mathrm{As} 2 \mathrm{O} 3 \\
-\mathrm{t}\end{array}$ & $\begin{array}{c}\mathrm{BaO} \\
-\mathrm{t}\end{array}$ & $\begin{array}{c}\mathrm{Bi} 2 \mathrm{O} 3 \\
-\mathrm{t}\end{array}$ & $\begin{array}{l}\mathrm{Br} \\
-\mathrm{t}\end{array}$ & $\begin{array}{c}\mathrm{CdO} \\
-\mathrm{t} \\
\end{array}$ & $\begin{array}{c}\mathrm{Ce} 2 \mathrm{O} 3 \\
-\mathrm{t}\end{array}$ & $\begin{array}{c}\mathrm{CeO} 2 \\
-\mathrm{t}\end{array}$ \\
\hline 16-58 & 0.0000 & 0.0700 & 0.1500 & 0.2400 & & & & & 0.1900 & & & 0.3500 & & & & & & & & & \\
\hline $39-59$ & 0.1200 & 0.0000 & 0.1300 & 0.0600 & & 0.0009 & & & 0.0700 & 0.0021 & 0.0163 & 0.5600 & 0.0012 & & & & & & & & 0.0007 \\
\hline $13-60$ & 0.0000 & 0.0900 & 0.0200 & 0.1550 & & 0.0005 & & & 0.1600 & 0.0040 & 0.0313 & 0.4600 & 0.0023 & & & & & & & & 0.0014 \\
\hline $61-60$ & 0.0000 & 0.0900 & 0.0200 & 0.1550 & & 0.0005 & & & 0.1600 & 0.0040 & 0.0313 & 0.4600 & 0.0023 & & & & & & & & 0.0014 \\
\hline $23-61$ & 0.0000 & 0.0650 & 0.0100 & 0.1550 & & 0.0005 & & & 0.1350 & 0.0040 & 0.0313 & 0.5200 & 0.0023 & & & & & & & & 0.0014 \\
\hline $08-62$ & 0.0550 & 0.1050 & 0.0100 & 0.1350 & & & & & 0.1650 & & & 0.5300 & & & & & & & & & \\
\hline $41-63$ & 0.1200 & 0.1050 & 0.0100 & 0.0700 & & & & & 0.1650 & & & 0.5300 & & & & & & & & & \\
\hline $25-64$ & 0.1200 & 0.1200 & 0.0000 & 0.0000 & & & & & 0.1600 & & & 0.6000 & & & & & & & & & \\
\hline $12-65$ & 0.0000 & 0.1200 & 0.0000 & 0.1200 & & & & & 0.1600 & & & 0.6000 & & & & & & & & & \\
\hline $51-66$ & 0.0600 & 0.1200 & 0.0000 & 0.0600 & & & & & 0.1600 & & & 0.6000 & & & & & & & & & \\
\hline $14-67$ & 0.0000 & 0.0900 & 0.0000 & 0.2400 & & & & & 0.0700 & & & 0.6000 & & & & & & & & & \\
\hline $56-68$ & 0.0000 & 0.0000 & 0.1100 & 0.0000 & & 0.0009 & & & 0.0700 & 0.0077 & 0.0598 & 0.6000 & 0.0044 & & & & & & & & 0.0026 \\
\hline $18-69$ & 0.1200 & 0.0000 & 0.0000 & 0.0000 & & 0.0009 & & & 0.3000 & 0.0077 & 0.0598 & 0.3600 & 0.0044 & & & & & & & & 0.0026 \\
\hline $32-70$ & 0.1200 & 0.2000 & 0.0000 & 0.0000 & & 0.0009 & & & 0.1100 & 0.0077 & 0.0598 & 0.3500 & 0.0044 & & & & & & & & 0.0026 \\
\hline $06-71$ & 0.1200 & 0.0000 & 0.1500 & 0.0800 & & & & & 0.3000 & & & 0.3500 & & & & & & & & & \\
\hline $54-72$ & 0.1200 & 0.2000 & 0.1500 & 0.0000 & & & & & 0.1800 & & & 0.3500 & & & & & & & & & \\
\hline $19-73$ & 0.1200 & 0.0900 & 0.1500 & 0.0000 & & 0.0009 & & & 0.0700 & 0.0077 & 0.0598 & 0.3500 & 0.0044 & & & & & & & & 0.0026 \\
\hline $15-74$ & 0.1200 & 0.2000 & 0.0000 & 0.2400 & & & & & 0.0900 & & & 0.3500 & & & & & & & & & \\
\hline $67-75$ & 0.1200 & 0.0000 & 0.0000 & 0.2350 & & & & & 0.2950 & & & 0.3500 & & & & & & & & & \\
\hline $96-76$ & 0.0000 & 0.0000 & 0.1330 & 0.0000 & & & & & 0.2670 & & & 0.6000 & & & & & & & & & \\
\hline $89-77$ & 0.0710 & 0.1770 & 0.0000 & 0.2120 & & & & & 0.1200 & & & 0.4200 & & & & & & & & & \\
\hline $99-78$ & 0.0000 & 0.1000 & 0.0000 & 0.2180 & & & & & 0.2720 & & & 0.4100 & & & & & & & & & \\
\hline $98-79$ & 0.0000 & 0.0000 & 0.0000 & 0.1130 & & 0.0009 & & & 0.2610 & 0.0067 & 0.0519 & 0.4350 & 0.0038 & & & & & & & & 0.0023 \\
\hline $101-80$ & 0.0690 & 0.1740 & 0.1300 & 0.0870 & & & & & 0.1200 & & & 0.4200 & & & & & & & & & \\
\hline $93-81$ & 0.0000 & 0.1530 & 0.0840 & 0.0000 & & 0.0009 & & & 0.1200 & 0.0059 & 0.0457 & 0.4750 & 0.0034 & & & & & & & & 0.0020 \\
\hline $97-82$ & 0.0000 & 0.0000 & 0.1150 & 0.0000 & & 0.0009 & & & 0.2160 & 0.0059 & 0.0459 & 0.5000 & 0.0034 & & & & & & & & 0.0020 \\
\hline $103-83$ & 0.0000 & 0.1450 & 0.1080 & 0.0000 & & & & & 0.2170 & & & 0.5300 & & & & & & & & & \\
\hline $92-84$ & 0.0313 & 0.0677 & 0.0527 & 0.0775 & & 0.0009 & & & 0.1817 & 0.0056 & 0.0435 & 0.4293 & 0.0032 & & & & & & & & 0.0019 \\
\hline $90-85$ & 0.0000 & 0.0000 & 0.1200 & 0.1600 & & 0.0009 & & & 0.1200 & 0.0050 & 0.0391 & 0.4560 & 0.0029 & & & & & & & & 0.0017 \\
\hline $87-86$ & 0.0730 & 0.0000 & 0.1360 & 0.0540 & & 0.0009 & & & 0.2720 & 0.0019 & 0.0147 & 0.4110 & 0.0011 & & & & & & & & 0.0006 \\
\hline $102-87$ & 0.1090 & 0.1820 & 0.0000 & 0.0000 & & 0.0009 & & & 0.1000 & 0.0070 & 0.0544 & 0.4090 & 0.0040 & & & & & & & & 0.0024 \\
\hline $86-88$ & 0.1200 & 0.0000 & 0.0000 & 0.0000 & & & & & 0.2800 & & & 0.6000 & & & & & & & & & \\
\hline $91-89$ & 0.1200 & 0.1000 & 0.0000 & 0.1200 & & 0.0004 & & & 0.1500 & 0.0038 & 0.0294 & 0.4020 & 0.0022 & & & & & & & & 0.0013 \\
\hline $95-90$ & 0.1200 & 0.0000 & 0.1000 & 0.1200 & & 0.0004 & & & 0.1500 & 0.0038 & 0.0294 & 0.4020 & 0.0022 & & & & & & & & 0.0013 \\
\hline $88-91$ & 0.0000 & 0.0600 & 0.1500 & 0.0300 & & 0.0009 & & & 0.1800 & 0.0021 & 0.0163 & 0.5200 & 0.0012 & & & & & & & & 0.0007 \\
\hline 94-92 & 0.0300 & 0.0600 & 0.0000 & 0.0000 & & 0.0009 & & & 0.1800 & 0.0074 & 0.0571 & 0.5200 & 0.0042 & & & & & & & & 0.0025 \\
\hline $100-93$ & 0.0400 & 0.0000 & 0.0800 & 0.1280 & & 0.0004 & & & 0.1000 & 0.0039 & 0.0299 & 0.5420 & 0.0022 & & & & & & & & 0.0013 \\
\hline
\end{tabular}

HWVP 85 (Bates 1985)

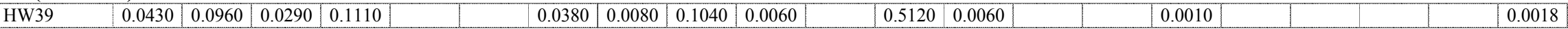

\section{WV Glasses by CUA and PNL (Johnston et al. 1990)}

DWRG

\begin{tabular}{l|l|l|l|l|l|l|l|l|l|l|l|l|l|l|l|l}
\hline SF10 & & & & \\
\hline TC27 & & & \\
\hline
\end{tabular}

WV205

WVCM42

WVCM44

WVCM45 
Comp. Vs Properties Study (Chick et al. 1981)

\begin{tabular}{|c|c|c|c|c|c|c|c|c|c|c|c|c|c|c|c|c|c|c|c|c|c|}
\hline Glass ID & $\begin{array}{l}\mathrm{Cl} \\
-\mathrm{t}\end{array}$ & $\begin{array}{c}\mathrm{CoO} \\
-\mathrm{t}\end{array}$ & $\begin{array}{c}\mathrm{Co} 2 \mathrm{O} 3 \\
-\mathrm{t}\end{array}$ & $\begin{array}{c}\mathrm{Cr} 2 \mathrm{O} 3 \\
-\mathrm{t} \\
\end{array}$ & $\begin{array}{c}\mathrm{Cs} 2 \mathrm{O} \\
-\mathrm{t} \\
\end{array}$ & $\begin{array}{c}\mathrm{CuO} \\
-\mathrm{t}\end{array}$ & $\begin{array}{c}\mathrm{Eu} 2 \mathrm{O} 3 \\
-\mathrm{t}\end{array}$ & $\begin{array}{l}F \\
-t\end{array}$ & $\begin{array}{c}\mathrm{Ga} 2 \mathrm{O} 3 \\
-\mathrm{t}\end{array}$ & $\begin{array}{c}\mathrm{Gd} 2 \mathrm{O} 3 \\
-\mathrm{t}\end{array}$ & $\begin{array}{c}\mathrm{HgO} \\
-\mathrm{t}\end{array}$ & $\begin{array}{l}I \\
-t\end{array}$ & $\begin{array}{c}\mathrm{La} 2 \mathrm{O} 3 \\
-\mathrm{t} \\
\end{array}$ & $\begin{array}{c}\mathrm{MnO} 2 \\
-\mathrm{t}\end{array}$ & $\begin{array}{c}\mathrm{MnO} \\
-\mathrm{t}\end{array}$ & $\begin{array}{c}\mathrm{MoO} \\
-\mathrm{t}\end{array}$ & $\begin{array}{c}\mathrm{MoO} 3 \\
-\mathrm{t}\end{array}$ & $\begin{array}{c}\mathrm{Nb} 2 \mathrm{O} 5 \\
-\mathrm{t}\end{array}$ & $\begin{array}{c}\mathrm{Nd} 2 \mathrm{O} 3 \\
-\mathrm{t}\end{array}$ & $\begin{array}{c}\mathrm{PbO} \\
-\mathrm{t}\end{array}$ & $\begin{array}{c}\mathrm{PdO} 2 \\
-\mathrm{t}\end{array}$ \\
\hline \multicolumn{22}{|l|}{$16-58$} \\
\hline $39-59$ & & & & 0.0044 & 0.0009 & & & & & & & & & 0.0028 & & & 0.0011 & & 0.0011 & & \\
\hline $13-60$ & & & & 0.0085 & 0.0017 & & & & & & & & & 0.0053 & & & 0.0022 & & 0.0021 & & \\
\hline $61-60$ & & & & 0.0085 & 0.0017 & & & & & & & & & 0.0053 & & & 0.0022 & & 0.0021 & & \\
\hline $23-61$ & & & & 0.0085 & 0.0017 & & & & & & & & & 0.0053 & & & 0.0022 & & 0.0021 & & \\
\hline \multicolumn{22}{|l|}{$08-62$} \\
\hline \multicolumn{22}{|l|}{$41-63$} \\
\hline \multicolumn{22}{|l|}{ 25-64 } \\
\hline \multicolumn{22}{|l|}{$12-65$} \\
\hline \multirow{2}{*}{\multicolumn{22}{|c|}{$\frac{51-66}{14-67}$}} \\
\hline & & & & & & & & & & & & & & & & & & & & & \\
\hline $56-68$ & & & & 0.0163 & 0.0033 & & & & & & & & & 0.0101 & & & 0.0042 & & 0.0040 & & \\
\hline $18-69$ & & & & 0.0163 & 0.0033 & & & & & & & & & 0.0101 & & & 0.0042 & & 0.0040 & & \\
\hline $32-70$ & & & & 0.0163 & 0.0033 & & & & & & & & & 0.0101 & & & 0.0042 & & 0.0040 & & \\
\hline \multicolumn{22}{|l|}{$06-71$} \\
\hline \multicolumn{22}{|l|}{$54-72$} \\
\hline $19-73$ & & & & 0.0163 & 0.0033 & & & & & & & & & 0.0101 & & & 0.0042 & & 0.0040 & & \\
\hline \multicolumn{22}{|l|}{$15-74$} \\
\hline \multicolumn{22}{|l|}{$67-75$} \\
\hline \multicolumn{22}{|l|}{$96-76$} \\
\hline \multicolumn{22}{|l|}{$89-77$} \\
\hline \multicolumn{22}{|l|}{$99-78$} \\
\hline $98-79$ & & & & 0.0142 & 0.0029 & & & & & & & & & 0.0088 & & & 0.0036 & & 0.0034 & & \\
\hline \multicolumn{22}{|l|}{$101-80$} \\
\hline $93-81$ & & & & 0.0124 & 0.0025 & & & & & & & & & 0.0077 & & & 0.0032 & & 0.0030 & & \\
\hline $97-82$ & & & & 0.0125 & 0.0025 & & & & & & & & & 0.0078 & & & 0.0032 & & 0.0030 & & \\
\hline \multicolumn{22}{|l|}{$103-83$} \\
\hline $92-84$ & & & & 0.0118 & 0.0024 & & & & & & & & & 0.0073 & & & 0.0030 & & 0.0029 & & \\
\hline $90-85$ & & & & 0.0107 & 0.0022 & & & & & & & & & 0.0066 & & & 0.0027 & & 0.0026 & & \\
\hline $87-86$ & & & & 0.0040 & 0.0008 & & & & & & & & & 0.0025 & & & 0.0010 & & 0.0010 & & \\
\hline $102-87$ & & & & 0.0148 & 0.0030 & & & & & & & & & 0.0092 & & & 0.0038 & & 0.0036 & & \\
\hline \multicolumn{22}{|l|}{$86-88$} \\
\hline $91-89$ & & & & 0.0080 & 0.0016 & & & & & & & & & 0.0050 & & & 0.0021 & & 0.0019 & & \\
\hline $95-90$ & & & & 0.0080 & 0.0016 & & & & & & & & & 0.0050 & & & 0.0021 & & 0.0019 & & \\
\hline $88-91$ & & & & 0.0044 & 0.0009 & & & & & & & & & 0.0028 & & & 0.0011 & & 0.0011 & & \\
\hline $94-92$ & & & & 0.0156 & 0.0032 & & & & & & & & & 0.0096 & & & 0.0040 & & 0.0038 & & \\
\hline $100-93$ & & & & 0.0081 & 0.0017 & & & & & & & & & 0.0051 & & & 0.0021 & & 0.0020 & & \\
\hline
\end{tabular}

\section{HWVP 85 (Bates 1985)}

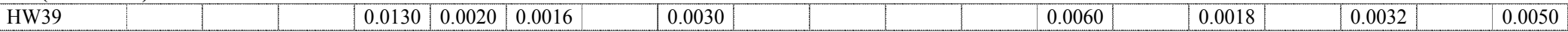

\section{WV Glasses by CUA and PNL (Johnston et al. 1990)}

DWRG

SF10

TC27

WV205

WVCM42

WVCM44

WVCM45 
Comp. Vs Properties Study (Chick et al. 1981)

\begin{tabular}{|c|c|c|c|c|c|c|c|c|c|c|c|c|c|c|c|c|c|c|c|c|c|}
\hline Glass ID & $\begin{array}{c}\mathrm{PdO} \\
-\mathrm{t}\end{array}$ & $\begin{array}{c}\mathrm{Pr} 2 \mathrm{O} 3 \\
-\mathrm{t}\end{array}$ & $\begin{array}{c}\operatorname{Pr6O11} \\
-\mathrm{t}\end{array}$ & $\begin{array}{c}\mathrm{Rb} 2 \mathrm{O} \\
-\mathrm{t}\end{array}$ & $\begin{array}{c}\mathrm{ReO} \\
-\mathrm{t}\end{array}$ & $\begin{array}{c}\mathrm{ReO} 2 \\
-\mathrm{t}\end{array}$ & $\begin{array}{c}\mathrm{Rh} 2 \mathrm{O} 3 \\
-\mathrm{t}\end{array}$ & $\begin{array}{c}\mathrm{RhO} 2 \\
-\mathrm{t}\end{array}$ & $\begin{array}{c}\mathrm{RuO} 2 \\
-\mathrm{t}\end{array}$ & $\begin{array}{c}\mathrm{Sb} 2 \mathrm{O} 3 \\
-\mathrm{t}\end{array}$ & $\begin{array}{c}\mathrm{Sb} 2 \mathrm{O} 5 \\
-\mathrm{t}\end{array}$ & $\begin{array}{c}\mathrm{SeO} 2 \\
-t\end{array}$ & $\begin{array}{c}\mathrm{Sm} 2 \mathrm{O} 3 \\
-\mathrm{t}\end{array}$ & $\begin{array}{c}\mathrm{SnO} \\
-\mathrm{t}\end{array}$ & $\begin{array}{c}\mathrm{SnO} 2 \\
-\mathrm{t}\end{array}$ & $\begin{array}{c}\mathrm{SO} 3 \\
-\mathrm{t}\end{array}$ & $\begin{array}{c}\mathrm{SrO} \\
-\mathrm{t}\end{array}$ & $\begin{array}{c}\mathrm{Tc} 2 \mathrm{O} 7 \\
-\mathrm{t}\end{array}$ & $\begin{array}{c}\mathrm{TeO} 2 \\
-\mathrm{t}\end{array}$ & $\begin{array}{c}\text { ThO2 } \\
-t\end{array}$ & $\begin{array}{c}\mathrm{TiO} 2 \\
-\mathrm{t}\end{array}$ \\
\hline \multicolumn{22}{|l|}{ 16-58 } \\
\hline $39-59$ & & & & & & & & & & & & & & & & & 0.0009 & & & 0.0213 & \\
\hline $13-60$ & & & & & & & & & & & & & & & & & 0.0017 & & & 0.0408 & \\
\hline $61-60$ & & & & & & & & & & & & & & & & & 0.0017 & & & 0.0408 & \\
\hline $23-61$ & & & & & & & & & & & & & & & & & 0.0017 & & & 0.0408 & \\
\hline \multicolumn{22}{|l|}{$08-62$} \\
\hline \multicolumn{22}{|l|}{$41-63$} \\
\hline \multicolumn{22}{|l|}{$25-64$} \\
\hline \multicolumn{22}{|l|}{$12-65$} \\
\hline \multicolumn{22}{|l|}{$51-66$} \\
\hline \multicolumn{22}{|l|}{$14-67$} \\
\hline $56-68$ & & & & & & & & & & & & & & & & & 0.0033 & & & 0.0781 & \\
\hline $18-69$ & & & & & & & & & & & & & & & & & 0.0033 & & & 0.0781 & \\
\hline $32-70$ & & & & & & & & & & & & & & & & & 0.0033 & & & 0.0781 & \\
\hline \multicolumn{22}{|l|}{ 06-71 } \\
\hline \multicolumn{22}{|l|}{$54-72$} \\
\hline $19-73$ & & & & & & & & & & & & & & & & & 0.0033 & & & 0.0781 & \\
\hline \multicolumn{22}{|l|}{$15-74$} \\
\hline \multicolumn{22}{|l|}{$67-75$} \\
\hline \multicolumn{22}{|l|}{$96-76$} \\
\hline \multicolumn{22}{|l|}{ 89-77 } \\
\hline \multicolumn{22}{|l|}{$99-78$} \\
\hline 98-79 & & & & & & & & & & & & & & & & & 0.0029 & & & 0.0678 & \\
\hline \multicolumn{22}{|l|}{$101-80$} \\
\hline 93-81 & & & & & & & & & & & & & & & & & 0.0025 & & & 0.0596 & \\
\hline $97-82$ & & & & & & & & & & & & & & & & & 0.0025 & & & 0.0600 & \\
\hline \multicolumn{22}{|l|}{$103-83$} \\
\hline $92-84$ & & & & & & & & & & & & & & & & & 0.0024 & & & 0.0568 & \\
\hline $90-85$ & & & & & & & & & & & & & & & & & 0.0022 & & & 0.0511 & \\
\hline $87-86$ & & & & & & & & & & & & & & & & & 0.0008 & & & 0.0192 & \\
\hline $102-87$ & & & & & & & & & & & & & & & & & 0.0030 & & & 0.0710 & \\
\hline \multicolumn{22}{|l|}{$86-88$} \\
\hline $91-89$ & & & & & & & & & & & & & & & & & 0.0016 & & & 0.0383 & \\
\hline $95-90$ & & & & & & & & & & & & & & & & & 0.0016 & & & 0.0383 & \\
\hline $88-91$ & & & & & & & & & & & & & & & & & 0.0009 & & & 0.0213 & \\
\hline $94-92$ & & & & & & & & & & & & & & & & & 0.0032 & & & 0.0746 & \\
\hline $100-93$ & & & & & & & & & & & & & & & & & 0.0017 & & & 0.0391 & \\
\hline
\end{tabular}

\section{HWVP 85 (Bates 1985)}

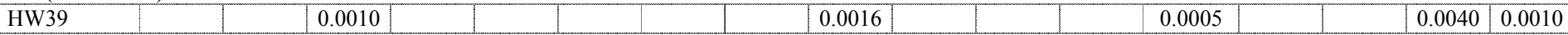

\section{WV Glasses by CUA and PNL (Johnston et al. 1990)}

DWRG

SF10

WVCM42

WVCM44

WVCM45

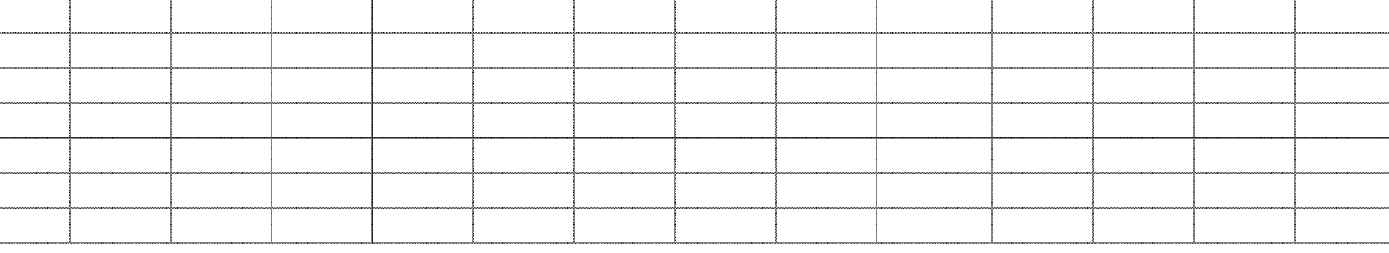


Comp. Vs Properties Study (Chick et al. 1981)

\begin{tabular}{|c|c|c|c|c|c|c|c|c|c|c|c|c|c|c|c|c|c|c|c|c|c|}
\hline Glass ID & $\begin{array}{c}\mathrm{T} 12 \mathrm{O} 3 \\
-\mathrm{t}\end{array}$ & $\begin{array}{c}\text { U3O8 } \\
-t\end{array}$ & $\begin{array}{c}\mathrm{UO} 2 \\
-\mathrm{t}\end{array}$ & $\begin{array}{c}\text { UO3 } \\
-t\end{array}$ & $\begin{array}{c}\mathrm{V} 2 \mathrm{O} 5 \\
-\mathrm{t}\end{array}$ & $\begin{array}{c}\text { WO3 } \\
-t\end{array}$ & $\begin{array}{c}\mathrm{Y} 2 \mathrm{O} 3 \\
-\mathrm{t}\end{array}$ & $\begin{array}{c}\mathrm{ZnO} \\
-\mathrm{t}\end{array}$ & $\begin{array}{c}\text { Others } \\
-t\end{array}$ & $\begin{array}{c}\text { Sum } \\
-t\end{array}$ & $\begin{array}{c}\mathrm{A} 12 \mathrm{O} 3 \\
-\mathrm{a}\end{array}$ & $\begin{array}{c}\mathrm{B} 2 \mathrm{O} 3 \\
-\mathrm{a}\end{array}$ & $\begin{array}{c}\mathrm{CaO} \\
-\mathrm{a}\end{array}$ & $\begin{array}{c}\mathrm{Fe} 2 \mathrm{O} 3 \\
-\mathrm{a}\end{array}$ & $\begin{array}{c}\mathrm{FeO} \\
-\mathrm{a}\end{array}$ & $\begin{array}{c}\mathrm{K} 2 \mathrm{O} \\
-\mathrm{a}\end{array}$ & $\begin{array}{c}\mathrm{Li} 2 \mathrm{O} \\
-\mathrm{a}\end{array}$ & $\begin{array}{c}\mathrm{MgO} \\
-\mathrm{a}\end{array}$ & $\begin{array}{c}\mathrm{Na} 2 \mathrm{O} \\
-\mathrm{a}\end{array}$ & $\begin{array}{c}\mathrm{NiO} \\
-\mathrm{a}\end{array}$ & $\begin{array}{c}\mathrm{P} 2 \mathrm{O} 5 \\
-\mathrm{a}\end{array}$ \\
\hline $16-58$ & & & & & & & & & & 1.0000 & 0.0101 & 0.0717 & 0.1515 & 0.2121 & & & & & 0.1818 & & \\
\hline $39-59$ & & & 0.0069 & & & & & & & 1.0006 & 0.1240 & 0.0000 & 0.1370 & 0.0430 & & 0.0008 & & & 0.0700 & 0.0020 & 0.0152 \\
\hline $13-60$ & & & 0.0132 & & & & & & & 1.0000 & 0.0092 & 0.0898 & 0.0225 & 0.1348 & & 0.0005 & & & 0.1613 & 0.0037 & 0.0290 \\
\hline $61-60$ & & & 0.0132 & & & & & & & 1.0000 & 0.0101 & 0.0891 & 0.0246 & 0.1281 & & 0.0005 & & & 0.1629 & 0.0040 & 0.0309 \\
\hline $23-61$ & & & 0.0132 & & & & & & & 1.0000 & 0.0091 & 0.0637 & 0.0131 & 0.1344 & & 0.0005 & & & 0.1344 & 0.0038 & 0.0299 \\
\hline $08-62$ & & & & & & & & & & 1.0000 & 0.0564 & 0.1101 & 0.0123 & 0.1163 & & & & & 0.1639 & & \\
\hline $41-63$ & & & & & & & & & & 1.0000 & 0.1254 & 0.0973 & 0.0120 & 0.0602 & & & & & 0.1625 & & \\
\hline $25-64$ & & & & & & & & & & 1.0000 & 0.1249 & 0.1159 & 0.0042 & 0.0000 & & & & & 0.1563 & & \\
\hline $12-65$ & & & & & & & & & & 1.0000 & 0.0079 & 0.1186 & 0.0000 & 0.1090 & & & & & 0.1561 & & \\
\hline $51-66$ & & & & & & & & & & 1.0000 & 0.0563 & 0.1031 & 0.0053 & 0.0473 & & & & & 0.1454 & & \\
\hline $14-67$ & & & & & & & & & & 1.0000 & 0.0098 & 0.0859 & 0.0040 & 0.2063 & & & & & 0.0689 & & \\
\hline $56-68$ & & & 0.0253 & & & & & & & 1.0000 & 0.0083 & 0.0000 & 0.1094 & 0.0000 & & 0.0009 & & & 0.0733 & 0.0077 & 0.0597 \\
\hline $18-69$ & & & 0.0253 & & & & & & & 1.0000 & 0.1283 & 0.0000 & 0.0030 & 0.0000 & & 0.0008 & & & 0.2921 & 0.0074 & 0.0573 \\
\hline $32-70$ & & & 0.0253 & & & & & & & 1.0000 & 0.1255 & 0.1983 & 0.0021 & 0.0000 & & 0.0008 & & & 0.1123 & 0.0071 & 0.0555 \\
\hline $06-71$ & & & & & & & & & & 1.0000 & 0.1230 & 0.0000 & 0.1516 & 0.0697 & & & & & 0.3002 & & \\
\hline $54-72$ & & & & & & & & & & 1.0000 & 0.1208 & 0.1974 & 0.1511 & 0.0000 & & & & & 0.1833 & & \\
\hline $19-73$ & & & 0.0253 & & & & & & & 1.0000 & 0.1244 & 0.1455 & 0.0943 & 0.0000 & & 0.0008 & & & 0.0752 & 0.0073 & 0.0563 \\
\hline $15-74$ & & & & & & & & & & 1.0000 & 0.1221 & 0.2096 & 0.0000 & 0.1974 & & & & & 0.0926 & & \\
\hline $67-75$ & & & & & & & & & & 1.0000 & 0.1282 & 0.0000 & 0.0019 & 0.1968 & & & & & 0.3048 & & \\
\hline $96-76$ & & & & & & & & & & 1.0000 & 0.0032 & 0.0000 & 0.1297 & 0.0000 & & & & & 0.2654 & & \\
\hline $89-77$ & & & & & & & & & & 1.0000 & 0.0800 & 0.1666 & 0.0016 & 0.1798 & & & & & 0.1219 & & \\
\hline $99-78$ & & & & & & & & & & 1.0000 & 0.0092 & 0.0000 & 0.1217 & 0.1454 & & & & & 0.1279 & & \\
\hline $98-79$ & & & 0.0220 & & & & & & & 1.0001 & 0.0061 & 0.0000 & 0.0044 & 0.0964 & & 0.0009 & & & 0.2658 & 0.0065 & 0.0503 \\
\hline $101-80$ & & & & & & & & & & 1.0000 & 0.0727 & 0.1707 & 0.1309 & 0.0747 & & & & & 0.1196 & & \\
\hline $93-81$ & & & 0.0193 & & & & & & & 1.0002 & 0.0031 & 0.1749 & 0.0838 & 0.0000 & & 0.0007 & & & 0.1288 & 0.0047 & 0.0365 \\
\hline $97-82$ & & & 0.0194 & & & & & & & 1.0002 & 0.0027 & 0.0000 & 0.1176 & 0.0000 & & 0.0009 & & & 0.2270 & 0.0059 & 0.0460 \\
\hline $103-83$ & & & & & & & & & & 1.0000 & 0.0033 & 0.1456 & 0.1084 & 0.0000 & & & & & 0.2210 & & \\
\hline $92-84$ & & & 0.0184 & & & & & & & 1.0003 & 0.0364 & 0.0673 & 0.0512 & 0.0664 & & 0.0008 & & & 0.1871 & 0.0052 & 0.0407 \\
\hline $90-85$ & & & 0.0166 & & & & & & & 1.0003 & 0.0070 & 0.0000 & 0.1228 & 0.1264 & & 0.0011 & & & 0.1218 & 0.0060 & 0.0468 \\
\hline $87-86$ & & & 0.0062 & & & & & & & 1.0007 & 0.0791 & 0.0000 & 0.1384 & 0.0462 & & 0.0010 & & & 0.2811 & 0.0021 & 0.0161 \\
\hline $102-87$ & & & 0.0230 & & & & & & & 1.0000 & 0.1165 & 0.1689 & 0.0019 & 0.0000 & & 0.0008 & & & 0.0997 & 0.0065 & 0.0504 \\
\hline $86-88$ & & & & & & & & & & 1.0000 & 0.1222 & 0.0000 & 0.0010 & 0.0000 & & & & & 0.2668 & & \\
\hline $91-89$ & & & 0.0124 & & & & & & & 1.0000 & 0.1246 & 0.0961 & 0.0019 & 0.1014 & & 0.0004 & & & 0.1513 & 0.0038 & 0.0293 \\
\hline $95-90$ & & & 0.0124 & & & & & & & 1.0000 & 0.1281 & 0.0000 & 0.0997 & 0.1027 & & 0.0004 & & & 0.1525 & 0.0038 & 0.0297 \\
\hline $88-91$ & & & 0.0069 & & & & & & & 1.0006 & 0.0050 & 0.0592 & 0.1517 & 0.0256 & & 0.0009 & & & 0.1819 & 0.0021 & 0.0166 \\
\hline 94-92 & & & 0.0242 & & & & & & & 1.0000 & 0.0344 & 0.0587 & 0.0025 & 0.0000 & & 0.0009 & & & 0.1875 & 0.0072 & 0.0557 \\
\hline $100-93$ & & & 0.0127 & & & & & & & 1.0000 & 0.0461 & 0.0000 & 0.0781 & 0.1086 & & 0.0004 & & & 0.1026 & 0.0039 & 0.0301 \\
\hline
\end{tabular}

\section{HWVP 85 (Bates 1985)}

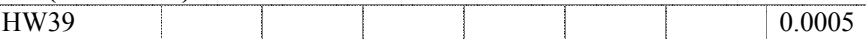

\section{WV Glasses by CUA and PNL (Johnston et al. 1990)}

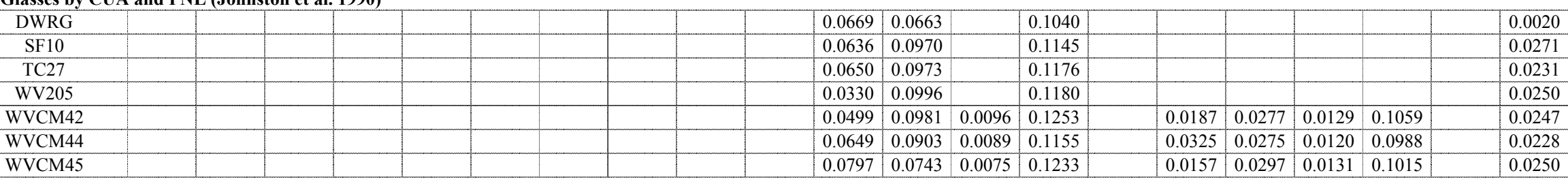


Comp. Vs Properties Study (Chick et al. 1981)

\begin{tabular}{|c|c|c|c|c|c|c|c|c|c|c|c|c|c|c|c|c|c|c|c|c|c|}
\hline Glass ID & $\begin{array}{c}\mathrm{SiO} 2 \\
-\mathrm{a}\end{array}$ & $\begin{array}{c}\mathrm{ZrO} 2 \\
-\mathrm{a}\end{array}$ & $\begin{array}{c}\mathrm{Ag} 2 \mathrm{O} \\
-\mathrm{a}\end{array}$ & $\begin{array}{c}\mathrm{As} 2 \mathrm{O} 3 \\
-\mathrm{a}\end{array}$ & $\begin{array}{c}\mathrm{BaO} \\
-\mathrm{a}\end{array}$ & $\begin{array}{c}\mathrm{Bi} 2 \mathrm{O} 3 \\
-\mathrm{a}\end{array}$ & $\begin{array}{l}\mathrm{Br} \\
-\mathrm{a}\end{array}$ & $\begin{array}{c}\mathrm{CdO} \\
-\mathrm{a}\end{array}$ & $\begin{array}{c}\mathrm{Ce} 2 \mathrm{O} 3 \\
-\mathrm{a}\end{array}$ & $\begin{array}{c}\mathrm{CeO} 2 \\
-\mathrm{a}\end{array}$ & $\begin{array}{l}\mathrm{Cl} \\
-\mathrm{a}\end{array}$ & $\begin{array}{c}\mathrm{CoO} \\
-\mathrm{a}\end{array}$ & $\begin{array}{c}\mathrm{Co} 2 \mathrm{O} 3 \\
-\mathrm{a}\end{array}$ & $\begin{array}{c}\mathrm{Cr} 2 \mathrm{O} 3 \\
-\mathrm{a}\end{array}$ & $\begin{array}{c}\mathrm{Cs} 2 \mathrm{O} \\
-\mathrm{a}\end{array}$ & $\begin{array}{c}\mathrm{CuO} \\
-\mathrm{a}\end{array}$ & $\begin{array}{c}\mathrm{Eu} 2 \mathrm{O} 3 \\
-\mathrm{a}\end{array}$ & $\begin{array}{l}\mathrm{F} \\
-\mathrm{a}\end{array}$ & $\begin{array}{c}\mathrm{Ga} 2 \mathrm{O} 3 \\
-\mathrm{a}\end{array}$ & {$\left[\begin{array}{c}\mathrm{Gd} 2 \mathrm{O} 3 \\
-\mathrm{a}\end{array}\right.$} & $\begin{array}{c}\mathrm{HgO} \\
-\mathrm{a}\end{array}$ \\
\hline $16-58$ & 0.3727 & & & & & & & & & & & & & & & & & & & & \\
\hline $39-59$ & 0.5699 & 0.0011 & & & & & & & & 0.0007 & & & & 0.0042 & 0.0008 & & & & & & \\
\hline $13-60$ & 0.4757 & 0.0021 & & & & & & & & 0.0013 & & & & 0.0079 & 0.0016 & & & & & & \\
\hline $61-60$ & 0.4714 & 0.0023 & & & & & & & & 0.0014 & & & & 0.0084 & 0.0017 & & & & & & \\
\hline $23-61$ & 0.5355 & 0.0022 & & & & & & & & 0.0013 & & & & 0.0081 & 0.0016 & & & & & & \\
\hline $08-62$ & 0.5410 & & & & & & & & & & & & & & & & & & & & \\
\hline $41-63$ & 0.5426 & & & & & & & & & & & & & & & & & & & & \\
\hline $25-64$ & 0.5988 & & & & & & & & & & & & & & & & & & & & \\
\hline $12-65$ & 0.6083 & & & & & & & & & & & & & & & & & & & & \\
\hline $51-66$ & 0.6057 & & & & & & & & & & & & & & & & & & & & \\
\hline 14-67 & 0.6250 & & & & & & & & & & & & & & & & & & & & \\
\hline $56-68$ & 0.5894 & 0.0044 & & & & & & & & 0.0026 & & & & 0.0163 & 0.0033 & & & & & & \\
\hline $18-69$ & 0.3658 & 0.0042 & & & & & & & & 0.0025 & & & & 0.0156 & 0.0032 & & & & & & \\
\hline $32-70$ & 0.3577 & 0.0041 & & & & & & & & 0.0024 & & & & 0.0151 & 0.0031 & & & & & & \\
\hline $06-71$ & 0.3555 & & & & & & & & & & & & & & & & & & & & \\
\hline $54-72$ & 0.3474 & & & & & & & & & & & & & & & & & & & & \\
\hline 19-73 & 0.3533 & 0.0041 & & & & & & & & 0.0025 & & & & 0.0154 & 0.0031 & & & & & & \\
\hline $15-74$ & 0.3784 & & & & & & & & & & & & & & & & & & & & \\
\hline $67-75$ & 0.3684 & & & & & & & & & & & & & & & & & & & & \\
\hline $96-76$ & 0.6017 & & & & & & & & & & & & & & & & & & & & \\
\hline 89-77 & 0.4501 & & & & & & & & & & & & & & & & & & & & \\
\hline $99-78$ & 0.4889 & & & & & & & & & & & & & & & & & & & & \\
\hline $98-79$ & 0.4424 & 0.0037 & & & & & & & & 0.0022 & & & & 0.0137 & 0.0028 & & & & & & \\
\hline $101-80$ & 0.4314 & & & & & & & & & & & & & & & & & & & & \\
\hline $93-81$ & 0.4754 & 0.0027 & & & & & & & & 0.0016 & & & & 0.0099 & 0.0020 & & & & & & \\
\hline $97-82$ & 0.4834 & 0.0034 & & & & & & & & 0.0020 & & & & 0.0125 & 0.0025 & & & & & & \\
\hline $103-83$ & 0.5216 & & & & & & & & & & & & & & & & & & & & \\
\hline $92-84$ & 0.4417 & 0.0030 & & & & & & & & 0.0018 & & & & 0.0111 & 0.0022 & & & & & & \\
\hline $90-85$ & 0.4497 & 0.0034 & & & & & & & & 0.0021 & & & & 0.0128 & 0.0026 & & & & & & \\
\hline $87-86$ & 0.3959 & 0.0012 & & & & & & & & 0.0007 & & & & 0.0044 & 0.0009 & & & & & & \\
\hline $102-87$ & 0.4276 & 0.0037 & & & & & & & & 0.0022 & & & & 0.0137 & 0.0028 & & & & & & \\
\hline $86-88$ & 0.6100 & & & & & & & & & & & & & & & & & & & & \\
\hline $91-89$ & 0.4170 & 0.0022 & & & & & & & & 0.0013 & & & & 0.0080 & 0.0016 & & & & & & \\
\hline $95-90$ & 0.4077 & 0.0022 & & & & & & & & 0.0013 & & & & 0.0081 & 0.0016 & & & & & & \\
\hline $88-91$ & 0.5154 & 0.0012 & & & & & & & & 0.0007 & & & & 0.0045 & 0.0009 & & & & & & \\
\hline 94-92 & 0.5117 & 0.0041 & & & & & & & & 0.0025 & & & & 0.0152 & 0.0031 & & & & & & \\
\hline $100-93$ & 0.5541 & 0.0022 & & & & & & & & 0.0013 & & & & 0.0082 & 0.0017 & & & & & & \\
\hline
\end{tabular}

\section{HWVP 85 (Bates 1985)}

HW39

\section{WV Glasses by CUA and PNL (Johnston et al. 1990)}

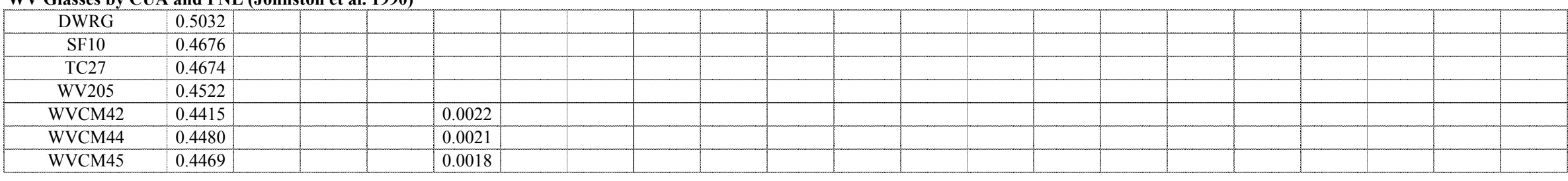


Appendix A. Database - mass fraction

Comp. Vs Properties Study (Chick et al. 1981)

\begin{tabular}{|c|c|c|c|c|c|c|c|c|c|c|c|c|c|c|c|c|c|c|c|c|c|}
\hline Glass ID & $\begin{array}{c}\mathrm{I} \\
-\mathrm{a}\end{array}$ & $\begin{array}{c}\mathrm{La} 2 \mathrm{O} 3 \\
-\mathrm{a}\end{array}$ & $\begin{array}{c}\mathrm{MnO} 2 \\
-\mathrm{a}\end{array}$ & $\begin{array}{c}\mathrm{MnO} \\
-\mathrm{a}\end{array}$ & $\begin{array}{c}\mathrm{MoO} \\
-\mathrm{a}\end{array}$ & $\begin{array}{c}\mathrm{MoO} 3 \\
-\mathrm{a}\end{array}$ & $\begin{array}{c}\mathrm{Nb} 2 \mathrm{O} 5 \\
-\mathrm{a}\end{array}$ & $\begin{array}{c}\mathrm{Nd} 2 \mathrm{O} 3 \\
-\mathrm{a}\end{array}$ & $\begin{array}{c}\mathrm{PbO} \\
-\mathrm{a}\end{array}$ & $\begin{array}{c}\mathrm{PdO} 2 \\
-\mathrm{a}\end{array}$ & $\begin{array}{c}\mathrm{PdO} \\
-\mathrm{a}\end{array}$ & $\begin{array}{c}\mathrm{Pr} 2 \mathrm{O} 3 \\
-\mathrm{a}\end{array}$ & $\begin{array}{c}\operatorname{Pr} 6011 \\
-\mathrm{a}\end{array}$ & $\begin{array}{c}\mathrm{Rb} 2 \mathrm{O} \\
-\mathrm{a}\end{array}$ & $\begin{array}{c}\mathrm{ReO} \\
-\mathrm{a}\end{array}$ & $\begin{array}{c}\mathrm{ReO} 2 \\
-\mathrm{a}\end{array}$ & $\begin{array}{c}\mathrm{Rh} 2 \mathrm{O} 3 \\
-\mathrm{a}\end{array}$ & $\begin{array}{c}\mathrm{RhO} 2 \\
-\mathrm{a}\end{array}$ & $\begin{array}{c}\mathrm{RuO} 2 \\
-\mathrm{a}\end{array}$ & $\begin{array}{c}\mathrm{Sb} 2 \mathrm{O} 3 \\
-\mathrm{a}\end{array}$ & $\begin{array}{c}\mathrm{Sb} 2 \mathrm{O} 5 \\
-\mathrm{a}\end{array}$ \\
\hline \multicolumn{22}{|l|}{$16-58$} \\
\hline $39-59$ & & & 0.0026 & & & 0.0011 & & 0.0010 & & & & & & & & & & & & & \\
\hline $13-60$ & & & 0.0049 & & & 0.0020 & & 0.0019 & & & & & & & & & & & & & \\
\hline $61-60$ & & & 0.0052 & & & 0.0022 & & 0.0021 & & & & & & & & & & & & & \\
\hline $23-61$ & & & 0.0051 & & & 0.0021 & & 0.0020 & & & & & & & & & & & & & \\
\hline \multicolumn{22}{|l|}{$08-62$} \\
\hline \multicolumn{22}{|l|}{$41-63$} \\
\hline \multicolumn{22}{|l|}{$25-64$} \\
\hline \multicolumn{22}{|l|}{$12-65$} \\
\hline \multicolumn{22}{|l|}{$51-66$} \\
\hline \multicolumn{22}{|l|}{$14-67$} \\
\hline $56-68$ & & & 0.0101 & & & 0.0042 & & 0.0040 & & & & & & & & & & & & & \\
\hline $18-69$ & & & 0.0097 & & & 0.0040 & & 0.0038 & & & & & & & & & & & & & \\
\hline $32-70$ & & & 0.0094 & & & 0.0039 & & 0.0037 & & & & & & & & & & & & & \\
\hline \multicolumn{22}{|l|}{$06-71$} \\
\hline \multicolumn{22}{|l|}{$54-72$} \\
\hline $19-73$ & & & 0.0095 & & & 0.0039 & & 0.0037 & & & & & & & & & & & & & \\
\hline \multicolumn{22}{|l|}{$15-74$} \\
\hline \multicolumn{22}{|l|}{$67-75$} \\
\hline \multicolumn{22}{|l|}{$96-76$} \\
\hline \multicolumn{22}{|l|}{$89-77$} \\
\hline \multicolumn{22}{|l|}{$99-78$} \\
\hline $98-79$ & & & 0.0085 & & & 0.0035 & & 0.0033 & & & & & & & & & & & & & \\
\hline \multicolumn{22}{|l|}{$101-80$} \\
\hline $93-81$ & & & 0.0062 & & & 0.0025 & & 0.0024 & & & & & & & & & & & & & \\
\hline $97-82$ & & & 0.0078 & & & 0.0032 & & 0.0030 & & & & & & & & & & & & & \\
\hline \multicolumn{22}{|l|}{$103-83$} \\
\hline $92-84$ & & & 0.0069 & & & 0.0028 & & 0.0027 & & & & & & & & & & & & & \\
\hline $90-85$ & & & 0.0079 & & & 0.0033 & & 0.0031 & & & & & & & & & & & & & \\
\hline $87-86$ & & & 0.0027 & & & 0.0011 & & 0.0011 & & & & & & & & & & & & & \\
\hline $102-87$ & & & 0.0085 & & & 0.0035 & & 0.0033 & & & & & & & & & & & & & \\
\hline \multicolumn{22}{|l|}{$86-88$} \\
\hline $91-89$ & & & 0.0050 & & & 0.0021 & & 0.0019 & & & & & & & & & & & & & \\
\hline $95-90$ & & & 0.0051 & & & 0.0021 & & 0.0019 & & & & & & & & & & & & & \\
\hline $88-91$ & & & 0.0028 & & & 0.0012 & & 0.0011 & & & & & & & & & & & & & \\
\hline $94-92$ & & & 0.0094 & & & 0.0039 & & 0.0037 & & & & & & & & & & & & & \\
\hline $100-93$ & & & 0.0051 & & & 0.0021 & & 0.0020 & & & & & & & & & & & & & \\
\hline
\end{tabular}

\section{HWVP 85 (Bates 1985)}

HW39

\section{WV Glasses by CUA and PNL (Johnston et al. 1990)}

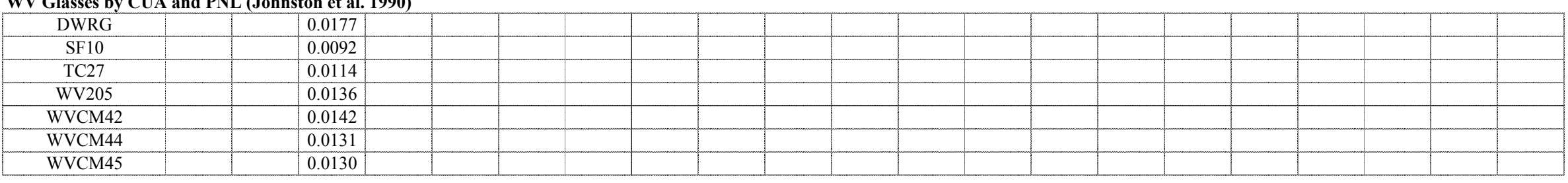


Appendix A. Database - mass fraction

Comp. Vs Properties Study (Chick et al. 1981)

\begin{tabular}{|c|c|c|c|c|c|c|c|c|c|c|c|c|c|c|c|c|c|c|c|c|}
\hline Glass ID & $\begin{array}{c}\mathrm{SeO} 2 \\
-\mathrm{a}\end{array}$ & $\begin{array}{c}\mathrm{Sm} 2 \mathrm{O} 3 \\
-\mathrm{a}\end{array}$ & $\begin{array}{c}\mathrm{SnO} \\
-\mathrm{a}\end{array}$ & $\begin{array}{c}\mathrm{SnO} 2 \\
-\mathrm{a}\end{array}$ & $\begin{array}{c}\mathrm{SO} 3 \\
-\mathrm{a}\end{array}$ & $\begin{array}{c}\mathrm{SrO} \\
-\mathrm{a}\end{array}$ & $\begin{array}{c}\mathrm{Tc} 2 \mathrm{O} 7 \\
-\mathrm{a}\end{array}$ & $\begin{array}{c}\mathrm{TeO} 2 \\
-\mathrm{a}\end{array}$ & $\begin{array}{c}\mathrm{ThO} 2 \\
-\mathrm{a}\end{array}$ & $\begin{array}{c}\mathrm{TiO} 2 \\
-\mathrm{a}\end{array}$ & $\begin{array}{c}\mathrm{T} 12 \mathrm{O} 3 \\
-\mathrm{a}\end{array}$ & $\begin{array}{c}\mathrm{U} 3 \mathrm{O} 8 \\
-\mathrm{a}\end{array}$ & $\begin{array}{c}\mathrm{UO} 2 \\
-\mathrm{a}\end{array}$ & $\begin{array}{c}\mathrm{UO} 3 \\
-\mathrm{a}\end{array}$ & $\begin{array}{c}\mathrm{V} 2 \mathrm{O} 5 \\
-\mathrm{a}\end{array}$ & $\begin{array}{c}\text { WO3 } \\
-\mathrm{a}\end{array}$ & $\begin{array}{c}\mathrm{Y} 2 \mathrm{O} 3 \\
-\mathrm{a}\end{array}$ & $\begin{array}{c}\mathrm{ZnO} \\
-\mathrm{a}\end{array}$ & $\begin{array}{c}\text { Others } \\
-\mathrm{a}\end{array}$ & $\begin{array}{c}\text { Sum } \\
-\mathrm{a}\end{array}$ \\
\hline $16-58$ & & & & & & & & & & & & & & & & & & & & 0.9999 \\
\hline $39-59$ & & & & & & 0.0008 & & & 0.0199 & & & & 0.0065 & & & & & & & 1.0006 \\
\hline $13-60$ & & & & & & 0.0016 & & & 0.0379 & & & & 0.0122 & & & & & & & 1.0000 \\
\hline $61-60$ & & & & & & 0.0017 & & & 0.0403 & & & & 0.0131 & & & & & & & 0.9999 \\
\hline $23-61$ & & & & & & 0.0016 & & & 0.0390 & & & & 0.0126 & & & & & & & 1.0001 \\
\hline $08-62$ & & & & & & & & & & & & & & & & & & & & 1.0000 \\
\hline $41-63$ & & & & & & & & & & & & & & & & & & & & 1.0000 \\
\hline $25-64$ & & & & & & & & & & & & & & & & & & & & 1.0001 \\
\hline $12-65$ & & & & & & & & & & & & & & & & & & & & 0.9999 \\
\hline $51-66$ & & & & & & & & & & & & & & & & & & & & 0.9631 \\
\hline 14-67 & & & & & & & & & & & & & & & & & & & & 0.9999 \\
\hline $56-68$ & & & & & & 0.0033 & & & 0.0780 & & & & 0.0253 & & & & & & & 1.0000 \\
\hline 18-69 & & & & & & 0.0032 & & & 0.0748 & & & & 0.0242 & & & & & & & 0.9999 \\
\hline $32-70$ & & & & & & 0.0031 & & & 0.0724 & & & & 0.0235 & & & & & & & 0.9999 \\
\hline $06-71$ & & & & & & & & & & & & & & & & & & & & 1.0000 \\
\hline $54-72$ & & & & & & & & & & & & & & & & & & & & 1.0000 \\
\hline 19-73 & & & & & & 0.0031 & & & 0.0736 & & & & 0.0238 & & & & & & & 1.0000 \\
\hline $15-74$ & & & & & & & & & & & & & & & & & & & & 1.0001 \\
\hline $67-75$ & & & & & & & & & & & & & & & & & & & & 1.0001 \\
\hline $96-76$ & & & & & & & & & & & & & & & & & & & & 1.0000 \\
\hline $89-77$ & & & & & & & & & & & & & & & & & & & & 1.0000 \\
\hline $99-78$ & & & & & & & & & & & & & & & & & & & & 0.8931 \\
\hline $98-79$ & & & & & & 0.0028 & & & 0.0656 & & & & 0.0213 & & & & & & & 1.0001 \\
\hline $101-80$ & & & & & & & & & & & & & & & & & & & & 1.0000 \\
\hline $93-81$ & & & & & & 0.0020 & & & 0.0476 & & & & 0.0154 & & & & & & & 1.0002 \\
\hline $97-82$ & & & & & & 0.0025 & & & 0.0601 & & & & 0.0195 & & & & & & & 1.0001 \\
\hline 103-83 & & & & & & & & & & & & & & & & & & & & 0.9999 \\
\hline $92-84$ & & & & & & 0.0022 & & & 0.0532 & & & & 0.0172 & & & & & & & 1.0002 \\
\hline $90-85$ & & & & & & 0.0026 & & & 0.0612 & & & & 0.0198 & & & & & & & 1.0003 \\
\hline $87-86$ & & & & & & 0.0009 & & & 0.0211 & & & & 0.0068 & & & & & & & 1.0007 \\
\hline $102-87$ & & & & & & 0.0028 & & & 0.0658 & & & & 0.0213 & & & & & & & 0.9999 \\
\hline $86-88$ & & & & & & & & & & & & & & & & & & & & 1.0000 \\
\hline 91-89 & & & & & & 0.0016 & & & 0.0382 & & & & 0.0124 & & & & & & & 1.0001 \\
\hline $95-90$ & & & & & & 0.0016 & & & 0.0388 & & & & 0.0125 & & & & & & & 1.0000 \\
\hline $88-91$ & & & & & & 0.0009 & & & 0.0217 & & & & 0.0070 & & & & & & & 1.0006 \\
\hline 94-92 & & & & & & 0.0031 & & & 0.0728 & & & & 0.0236 & & & & & & & 0.9999 \\
\hline $100-93$ & & & & & & 0.0017 & & & 0.0393 & & & & 0.0127 & & & & & & & 1.0001 \\
\hline
\end{tabular}

HWVP 85 (Bates 1985)

HW39

WV Glasses by CUA and PNL (Johnston et al. 1990)

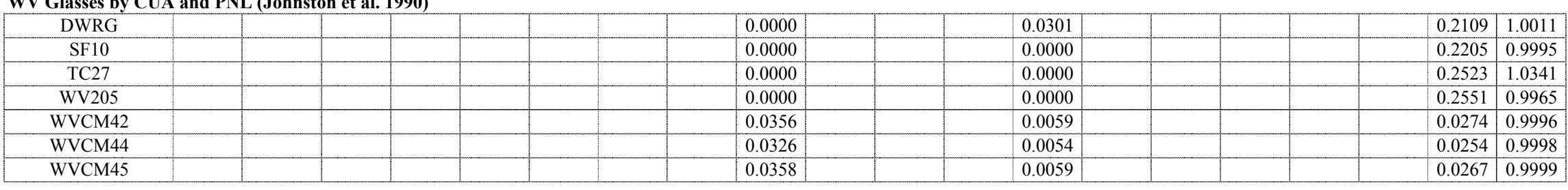


Appendix A. Database - mass fraction

Comp. Vs Properties Study (Chick et al. 1981)

\begin{tabular}{|c|c|c|c|c|c|c|c|c|}
\hline Glass ID & $\begin{array}{l}\mathrm{TM} \\
\left({ }^{\circ} \mathrm{C}\right) \\
\end{array}$ & $\begin{array}{l}\text { Gradient } \\
\text { TL }\left({ }^{\circ} \mathrm{C}\right)\end{array}$ & $\begin{array}{l}\text { Uniform } \\
\text { TL }\left({ }^{\circ} \mathrm{C}\right)\end{array}$ & Primary Phase & $\begin{array}{l}\text { Quenched } \\
\text { Visual/OM }\end{array}$ & $\begin{array}{c}\text { Quenched } \\
\text { SEM/EDS or TEM }\end{array}$ & $\begin{array}{l}\text { Quenched } \\
\text { XRD }\end{array}$ & $\begin{array}{c}\text { Quenched } \\
\text { Homogeneous? }\end{array}$ \\
\hline $16-58$ & & & & & 0.04 crystallinity & & & \\
\hline $39-59$ & & & & & amorphous & & & \\
\hline $13-60$ & & & & & amorphous & & & \\
\hline $61-60$ & & & & & amorphous & & & \\
\hline $23-61$ & & & & & amorphous & & & \\
\hline $08-62$ & & & & & amorphous & & & \\
\hline $41-63$ & & & & & amorphous & & & \\
\hline $25-64$ & & & & & amorphous & & & \\
\hline $12-65$ & & & & & amorphous & & & \\
\hline $51-66$ & & & & & amorphous & & & \\
\hline $14-67$ & & & & & amorphous & & & \\
\hline $56-68$ & & & & & 0.10 crystallinity & & & \\
\hline $18-69$ & & & & & 0.47 crystallinity & & & \\
\hline $32-70$ & & & & & amorphous & & & \\
\hline $06-71$ & & & & & 0.06 crystallinity & & & \\
\hline $54-72$ & & & & & amorphous & & & \\
\hline $19-73$ & & & & & 0.07 crystallinity & & & \\
\hline $15-74$ & & & & & 0.39 crystallinity & & & \\
\hline $67-75$ & & & & & amorphous & & & \\
\hline $96-76$ & & & & & amorphous & & & \\
\hline $89-77$ & & & & & amorphous & & & \\
\hline $99-78$ & & & & & amorphous & & & \\
\hline $98-79$ & & & & & amorphous & & & \\
\hline $101-80$ & & & & & amorphous & & & \\
\hline $93-81$ & & & & & 0.03 crystallinity & & & \\
\hline $97-82$ & & & & & amorphous & & & \\
\hline $103-83$ & & & & & amorphous & & & \\
\hline $92-84$ & & & & & amorphous & & & \\
\hline $90-85$ & & & & & amorphous & & & \\
\hline $87-86$ & & & & & 0.01 crystallinity & & & \\
\hline $102-87$ & & & & & amorphous & & & \\
\hline $86-88$ & & & & & amorphous & & & \\
\hline $91-89$ & & & & & amorphous & & & \\
\hline $95-90$ & & & & & amorphous & & & \\
\hline $88-91$ & & & & & amorphous & & & \\
\hline $94-92$ & & & & & amorphous & & & \\
\hline $100-93$ & & & & & amorphous & & & \\
\hline
\end{tabular}

\section{HWVP 85 (Bates 1985)}

HW39

\section{WV Glasses by CUA and PNL (Johnston et al. 1990)}

DWRG

\begin{tabular}{|c|c|c|c|c|c|c|}
\hline DWRG & & & & & \\
\hline SF10 & & & & & & \\
\hline TC27 & & & & & & \\
\hline WV205 & & & & & & \\
\hline WVCM42 & & & & & \\
\hline WVCM44 & & & & & \\
\hline WVCM45 & & & & & & \\
\hline
\end{tabular}


Appendix A. Database - mass fraction

Comp. Vs Properties Study (Chick et al. 1981)

\begin{tabular}{|c|c|c|c|c|c|}
\hline Glass ID & $\begin{array}{c}\mathrm{CCC} \\
\text { Visual/OM } \\
\end{array}$ & $\begin{array}{c}\text { CCC } \\
\text { SEM/EDS or TEM }\end{array}$ & $\begin{array}{l}\text { CCC } \\
\text { XRD }\end{array}$ & $\begin{array}{l}\text { Heat Treated } \\
\text { Visual/OM }\end{array}$ & $\begin{array}{c}\text { Heat Treated } \\
\text { SEM/EDS or TEM }\end{array}$ \\
\hline $16-58$ & 1.20 crystallinity & & & 0.65 crystallinity & \\
\hline $39-59$ & amorphous & & & amorphous & \\
\hline $13-60$ & amorphous & & & amorphous & \\
\hline $61-60$ & amorphous & & & amorphous & \\
\hline $23-61$ & amorphous & & & amorphous & \\
\hline $08-62$ & amorphous & & & amorphous & \\
\hline $41-63$ & amorphous & & & amorphous & \\
\hline $25-64$ & amorphous & & & amorphous & \\
\hline $12-65$ & amorphous & & & amorphous & \\
\hline $51-66$ & 0.05 crystallinity & & & amorphous & \\
\hline $14-67$ & 0.31 crystallinity & & & 0.06 crystallinity & \\
\hline $56-68$ & 0.47 crystallinity & & & 0.24 crystallinity & \\
\hline $18-69$ & 0.80 crystallinity & & & 0.65 crystallinity & \\
\hline $32-70$ & 0.88 crystallinity & & & 0.58 crystallinity & \\
\hline $06-71$ & 1.07 crystallinity & & & 0.73 crystallinity & \\
\hline $54-72$ & amorphous & & & amorphous & \\
\hline $19-73$ & 0.59 crystallinity & & & amorphous & \\
\hline $15-74$ & 0.47 crystallinity & & & 0.21 crystallinity & \\
\hline $67-75$ & 1.01 crystallinity & & & amorphous & \\
\hline $96-76$ & 0.66 crystallinity & & & 0.47 crystallinity & \\
\hline $89-77$ & 0.23 crystallinity & & & 0.11 crystallinity & \\
\hline $99-78$ & amorphous & & & 0.04 crystallinity & \\
\hline $98-79$ & 0.07 crystallinity & & & 0.03 crystallinity & \\
\hline $101-80$ & amorphous & & & amorphous & \\
\hline $93-81$ & amorphous & & & 0.04 crystallinity & \\
\hline $97-82$ & amorphous & & & 0.06 crystallinity & \\
\hline 103-83 & amorphous & & & amorphous & \\
\hline $92-84$ & 0.05 crystallinity & & & amorphous & \\
\hline $90-85$ & 0.06 crystallinity & & & .013 crystallinity & \\
\hline $87-86$ & 1.51 crystallinity & & & 1.64 crystallinity & \\
\hline $102-87$ & 0.75 crystallinity & & & 0.53 crystallinity & \\
\hline $86-88$ & amorphous & & & amorphous & \\
\hline $91-89$ & 0.05 crystallinity & & & 0.16 crystallinity & \\
\hline $95-90$ & 0.26 crystallinity & & & 0.24 crystallinity & \\
\hline $88-91$ & amorphous & & & 0.05 crystallinity & \\
\hline $94-92$ & 0.14 crystallinity & & & 0.03 crystallinity & \\
\hline $100-93$ & 0.04 crystallinity & & & 0.01 crystallinity & \\
\hline
\end{tabular}

\section{HWVP 85 (Bates 1985)}

HW39

\section{WV Glasses by CUA and PNL (Johnston et al. 1990)}

DWRG

SF10

TC27

WV205

WVCM42

WVCM44

WVCM45 
Appendix A. Database - mass fraction

Comp. Vs Properties Study (Chick et al. 1981)

\begin{tabular}{|c|c|c|c|c|c|c|c|c|c|c|c|c|c|c|c|}
\hline Glass ID & $\begin{array}{c}\text { Heat Treated } \\
\text { XRD }\end{array}$ & $\begin{array}{l}\text { Density } \\
\left(\mathrm{g} / \mathrm{cm}^{3}\right)\end{array}$ & $\begin{array}{c}\text { Fulc Visc } \\
\text { A }\end{array}$ & \begin{tabular}{|c|} 
Fulc Visc \\
B
\end{tabular} & \begin{tabular}{|c|} 
Fulc Visc \\
To
\end{tabular} & $\begin{array}{c}\mathrm{FV} 1150^{\circ} \mathrm{C} \\
(\mathrm{Pa} \cdot \mathrm{s})\end{array}$ & $\begin{array}{c}\text { Arrh Visc } \\
\text { A }\end{array}$ & \begin{tabular}{|c} 
Arrh Visc \\
B
\end{tabular} & $1150^{\circ} \mathrm{C}$ & $\begin{array}{c}\mathrm{T}\left({ }^{\circ} \mathrm{C}\right) \text { at } \\
2 \mathrm{~Pa} \cdot \mathrm{s} \\
\end{array}$ & $\begin{array}{c}\mathrm{T}\left({ }^{\circ} \mathrm{C}\right) \text { at } \\
5 \mathrm{~Pa} \cdot \mathrm{s}\end{array}$ & $\begin{array}{l}\mathrm{T}\left({ }^{\circ} \mathrm{C}\right) \text { at } \\
10 \mathrm{~Pa} \cdot \mathrm{s}\end{array}$ & $\begin{array}{l}\mathrm{T} 1 \\
\left({ }^{\circ} \mathrm{C}\right)\end{array}$ & $\begin{array}{c}\mathrm{V} 1 \\
(\mathrm{~Pa} \cdot \mathrm{s})\end{array}$ & $\begin{array}{l}\mathrm{T} 2 \\
\left({ }^{\circ} \mathrm{C}\right)\end{array}$ \\
\hline $16-58$ & & & & & & & & & & & & 854 & 960 & 3 & \\
\hline $39-59$ & & & & & & & & & & & & 1481 & 1632 & 3 & \\
\hline $13-60$ & & & & & & & & & & & & 1078 & 1224 & 3 & \\
\hline $61-60$ & & & & & & & & & & & & 1110 & 1222 & 3 & \\
\hline $23-61$ & & & & & & & & & & & & 1238 & 1419 & 3 & \\
\hline $08-62$ & & & & & & & & & & & & 1215 & 1391 & 3 & \\
\hline $41-63$ & & & & & & & & & & & & 1366 & 1555 & 3 & \\
\hline $25-64$ & & & & & & & & & & & & 1380 & 1582 & 3 & \\
\hline $12-65$ & & & & & & & & & & & & 1180 & 1335 & 3 & \\
\hline $51-66$ & & & & & & & & & & & & 1335 & 1542 & 3 & \\
\hline $14-67$ & & & & & & & & & & & & 1497 & 1699 & 3 & \\
\hline $56-68$ & & & & & & & & & & & & 1436 & 1582 & 3 & \\
\hline 18-69 & & & & & & & & & & & & 1231 & 1337 & 3 & \\
\hline $32-70$ & & & & & & & & & & & & 1233 & 1457 & 3 & \\
\hline $06-71$ & & & & & & & & & & & & 1031 & 1147 & 3 & \\
\hline $54-72$ & & & & & & & & & & & & 962 & 1057 & 3 & \\
\hline $19-73$ & & & & & & & & & & & & 1217 & 1343 & 3 & \\
\hline $15-74$ & & & & & & & & & & & & 1285 & 1350 & 3 & \\
\hline $67-75$ & & & & & & & & & & & & 1191 & 1335 & 3 & \\
\hline $96-76$ & & & & & & & & & & & & 1152 & 1324 & 3 & \\
\hline $89-77$ & & & & & & & & & & & & 1231 & 1422 & 3 & \\
\hline $99-78$ & & & & & & & & & & & & 874 & 1006 & 3 & \\
\hline $98-79$ & & & & & & & & & & & & 1110 & 1261 & 3 & \\
\hline $101-80$ & & & & & & & & & & & & 1060 & 1183 & 3 & \\
\hline $93-81$ & & & & & & & & & & & & & 1224 & 3 & \\
\hline $97-82$ & & & & & & & & & & & & 1131 & 1290 & 3 & \\
\hline $103-83$ & & & & & & & & & & & & 965 & 1086 & 3 & \\
\hline $92-84$ & & & & & & & & & & & & 1106 & 1282 & 3 & \\
\hline $90-85$ & & & & & & & & & & & & 1152 & 1287 & 3 & \\
\hline $87-86$ & & & & & & & & & & & & 1078 & 1200 & 3 & \\
\hline $102-87$ & & & & & & & & & & & & 1388 & 1562 & 3 & \\
\hline 86-88 & & & & & & & & & & & & 1348 & 1542 & 3 & \\
\hline $91-89$ & & & & & & & & & & & & 1327 & 1469 & 3 & \\
\hline $95-90$ & & & & & & & & & & & & 1275 & 1428 & 3 & \\
\hline $88-91$ & & & & & & & & & & & & 1060 & 1204 & 3 & \\
\hline 94-92 & & & & & & & & & & & & 1277 & 1451 & 3 & \\
\hline $100-93$ & & & & & & & & & & & & 1383 & 1545 & 3 & \\
\hline
\end{tabular}

\section{HWVP 85 (Bates 1985)}

HW39

\section{WV Glasses by CUA and PNL (Johnston et al. 1990)}

DWRG

$\mathrm{SF} 10$

WV205

WVCM44

WVCM45

\begin{tabular}{|l|l|l|l|l|l|l|l|}
\hline & & & & & & & \\
\hline & & & & & & & \\
\hline & & & & & & & \\
\hline & & & & & & & \\
\hline
\end{tabular}


Appendix A. Database - mass fraction

Comp. Vs Properties Study (Chick et al. 1981)

\begin{tabular}{|c|c|c|c|c|c|c|c|c|c|c|c|c|c|c|c|c|c|c|c|c|c|}
\hline Glass ID & $\begin{array}{c}\mathrm{V} 2 \\
(\mathrm{~Pa} \cdot \mathrm{s})\end{array}$ & $\begin{array}{c}\mathrm{T} 3 \\
\left({ }^{\circ} \mathrm{C}\right)\end{array}$ & $\begin{array}{c}\mathrm{V3} \\
(\mathrm{Pa} \cdot \mathrm{s})\end{array}$ & $\begin{array}{c}\mathrm{T} 4 \\
\left({ }^{\circ} \mathrm{C}\right)\end{array}$ & $\begin{array}{c}\mathrm{V} 4 \\
(\mathrm{~Pa} \cdot \mathrm{s})\end{array}$ & $\begin{array}{c}\mathrm{T} 5 \\
\left({ }^{\circ} \mathrm{C}\right)\end{array}$ & $\begin{array}{c}\text { V5 } \\
(\mathrm{Pa} \cdot \mathrm{s})\end{array}$ & $\begin{array}{c}\text { T6 } \\
\left({ }^{\circ} \mathrm{C}\right)\end{array}$ & $\begin{array}{c}\mathrm{V} 6 \\
(\mathrm{~Pa} \cdot \mathrm{s})\end{array}$ & $\begin{array}{c}\mathrm{T} 7 \\
\left({ }^{\circ} \mathrm{C}\right)\end{array}$ & $\begin{array}{c}\text { V7 } \\
(\mathrm{Pa} \cdot \mathrm{s})\end{array}$ & $\begin{array}{c}\mathrm{T} 8 \\
\left({ }^{\circ} \mathrm{C}\right)\end{array}$ & $\begin{array}{c}\mathrm{V} 8 \\
(\mathrm{~Pa} \cdot \mathrm{s})\end{array}$ & $\begin{array}{c}\text { T9 } \\
\left({ }^{\circ} \mathrm{C}\right)\end{array}$ & $\begin{array}{c}\text { V9 } \\
(\mathrm{Pa} \cdot \mathrm{s})\end{array}$ & $\begin{array}{l}\mathrm{T} 10 \\
\left({ }^{\circ} \mathrm{C}\right)\end{array}$ & $\begin{array}{c}\text { V10 } \\
(\mathrm{Pa} \cdot \mathrm{s})\end{array}$ & $\begin{array}{l}\text { T11 } \\
\left({ }^{\circ} \mathrm{C}\right)\end{array}$ & $\begin{array}{c}\text { V11 } \\
(\mathrm{Pa} \cdot \mathrm{s})\end{array}$ & $\begin{array}{l}\mathrm{T} 12 \\
\left({ }^{\circ} \mathrm{C}\right)\end{array}$ & $\begin{array}{c}\mathrm{V} 12 \\
(\mathrm{~Pa} \cdot \mathrm{s})\end{array}$ \\
\hline $16-58$ & & & & & & & & & & & & & & & & & & & & & \\
\hline $39-59$ & & & & & & & & & & & & & & & & & & & & & \\
\hline $13-60$ & & & & & & & & & & & & & & & & & & & & & \\
\hline $61-60$ & & & & & & & & & & & & & & & & & & & & & \\
\hline $23-61$ & & & & & & & & & & & & & & & & & & & & & \\
\hline $08-62$ & & & & & & & & & & & & & & & & & & & & & \\
\hline $41-63$ & & & & & & & & & & & & & & & & & & & & & \\
\hline $25-64$ & & & & & & & & & & & & & & & & & & & & & \\
\hline $12-65$ & & & & & & & & & & & & & & & & & & & & & \\
\hline $51-66$ & & & & & & & & & & & & & & & & & & & & & \\
\hline $14-67$ & & & & & & & & & & & & & & & & & & & & & \\
\hline $56-68$ & & & & & & & & & & & & & & & & & & & & & \\
\hline $18-69$ & & & & & & & & & & & & & & & & & & & & & \\
\hline $32-70$ & & & & & & & & & & & & & & & & & & & & & \\
\hline $06-71$ & & & & & & & & & & & & & & & & & & & & & \\
\hline $54-72$ & & & & & & & & & & & & & & & & & & & & & \\
\hline $19-73$ & & & & & & & & & & & & & & & & & & & & & \\
\hline $15-74$ & & & & & & & & & & & & & & & & & & & & & \\
\hline $67-75$ & & & & & & & & & & & & & & & & & & & & & \\
\hline $96-76$ & & & & & & & & & & & & & & & & & & & & & \\
\hline $89-77$ & & & & & & & & & & & & & & & & & & & & & \\
\hline $99-78$ & & & & & & & & & & & & & & & & & & & & & \\
\hline 98-79 & & & & & & & & & & & & & & & & & & & & & \\
\hline $101-80$ & & & & & & & & & & & & & & & & & & & & & \\
\hline $93-81$ & & & & & & & & & & & & & & & & & & & & & \\
\hline $97-82$ & & & & & & & & & & & & & & & & & & & & & \\
\hline 103-83 & & & & & & & & & & & & & & & & & & & & & \\
\hline $92-84$ & & & & & & & & & & & & & & & & & & & & & \\
\hline $90-85$ & & & & & & & & & & & & & & & & & & & & & \\
\hline $87-86$ & & & & & & & & & & & & & & & & & & & & & \\
\hline $102-87$ & & & & & & & & & & & & & & & & & & & & & \\
\hline $86-88$ & & & & & & & & & & & & & & & & & & & & & \\
\hline $91-89$ & & & & & & & & & & & & & & & & & & & & & \\
\hline 95-90 & & & & & & & & & & & & & & & & & & & & & \\
\hline $88-91$ & & & & & & & & & & & & & & & & & & & & & \\
\hline $94-92$ & & & & & & & & & & & & & & & & & & & & & \\
\hline $100-93$ & & & & & & & & & & & & & & & & & & & & & \\
\hline
\end{tabular}

\section{HWVP 85 (Bates 1985)}

\begin{tabular}{|l|l|l|l|l|l}
\hline HW39 & 18.00 & 1125 & 9.20 & 1200 & 5.10 \\
\hline
\end{tabular}

\section{WV Glasses by CUA and PNL (Johnston et al. 1990)}

DWRG

SF10

TC27

WV205

WVCM42

WVCM44

WVCM45

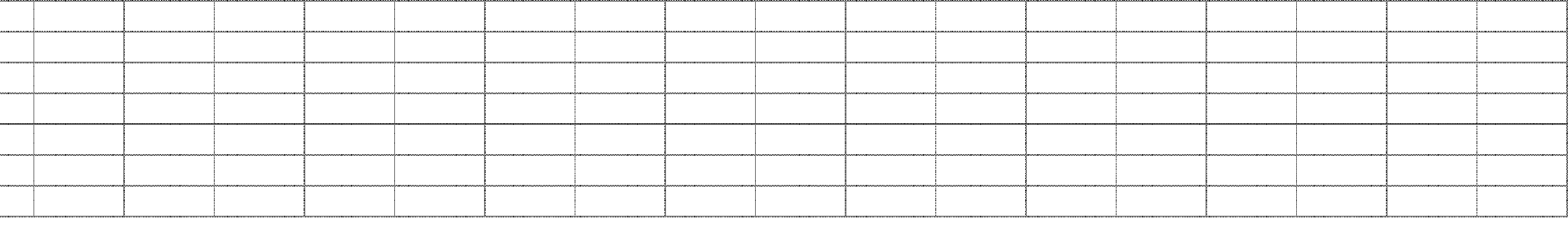


Appendix A. Database - mass fraction

Comp. Vs Properties Study (Chick et al. 1981)

\begin{tabular}{|c|c|c|c|c|c|c|c|c|c|c|c|c|c|c|c|c|}
\hline Glass ID & $\begin{array}{l}\mathrm{T} 13 \\
\left({ }^{\circ} \mathrm{C}\right)\end{array}$ & $\begin{array}{l}\mathrm{V} 13 \\
(\mathrm{~Pa} \cdot \mathrm{s})\end{array}$ & $\begin{array}{l}\mathrm{T} 14 \\
\left({ }^{\circ} \mathrm{C}\right)\end{array}$ & $\begin{array}{c}\text { V14 } \\
(\mathrm{Pa} \cdot \mathrm{s})\end{array}$ & $\begin{array}{c}\text { Q PCT } \\
\mathrm{B}\left(\mathrm{g} / \mathrm{m}^{2}\right)\end{array}$ & $\begin{array}{c}\text { Q PCT } \\
\mathrm{Li}\left(\mathrm{g} / \mathrm{m}^{2}\right)\end{array}$ & $\begin{array}{c}\text { Q PCT } \\
\mathrm{Na}\left(\mathrm{g} / \mathrm{m}^{2}\right)\end{array}$ & $\begin{array}{c}\text { Q PCT } \\
\mathrm{Si}\left(\mathrm{g} / \mathrm{m}^{2}\right)\end{array}$ & $\begin{array}{c}\text { Q PCT } \\
\mathrm{pH}\end{array}$ & $\begin{array}{l}\text { CCC PCT } \\
\text { B }\left(\mathrm{g} / \mathrm{m}^{2}\right)\end{array}$ & $\begin{array}{l}\text { CCC PCT } \\
\operatorname{Li}\left(\mathrm{g} / \mathrm{m}^{2}\right)\end{array}$ & $\begin{array}{l}\text { CCC PCT } \\
\mathrm{Na}\left(\mathrm{g} / \mathrm{m}^{2}\right)\end{array}$ & $\begin{array}{l}\text { CCC PCT } \\
\mathrm{Si}\left(\mathrm{g} / \mathrm{m}^{2}\right)\end{array}$ & $\begin{array}{c}\text { CCC PCT } \\
\mathrm{pH}\end{array}$ & $\begin{array}{l}\text { Q PCT B at } \\
20^{\circ} \mathrm{C}\left(\mathrm{g} / \mathrm{m}^{2}\right)\end{array}$ & $\begin{array}{l}\text { Q PCT Li at } \\
20^{\circ} \mathrm{C}\left(\mathrm{g} / \mathrm{m}^{2}\right)\end{array}$ \\
\hline \multicolumn{17}{|c|}{ P } \\
\hline \multicolumn{17}{|l|}{$39-59$} \\
\hline \multicolumn{17}{|l|}{$13-60$} \\
\hline \multicolumn{17}{|l|}{$61-60$} \\
\hline \multicolumn{17}{|l|}{$23-61$} \\
\hline \multicolumn{17}{|l|}{$08-62$} \\
\hline \multicolumn{17}{|l|}{$41-63$} \\
\hline \multicolumn{17}{|l|}{$25-64$} \\
\hline \multicolumn{17}{|l|}{$12-65$} \\
\hline \multicolumn{17}{|l|}{$51-66$} \\
\hline \multicolumn{17}{|l|}{$14-67$} \\
\hline \multicolumn{17}{|l|}{$56-68$} \\
\hline \multicolumn{17}{|l|}{$18-69$} \\
\hline \multicolumn{17}{|l|}{$32-70$} \\
\hline \multicolumn{17}{|l|}{$06-71$} \\
\hline \multicolumn{17}{|l|}{$54-72$} \\
\hline \multicolumn{17}{|l|}{$19-73$} \\
\hline \multicolumn{17}{|l|}{$15-74$} \\
\hline \multicolumn{17}{|l|}{$67-75$} \\
\hline \multicolumn{17}{|l|}{$96-76$} \\
\hline \multicolumn{17}{|l|}{$89-77$} \\
\hline \multicolumn{17}{|l|}{$99-78$} \\
\hline $98-79$ & & & & & & & & & & & & & & & & \\
\hline $101-80$ & & & & & & & & & & & & & & & & \\
\hline $93-81$ & & & & & & & & & & & & & & & & \\
\hline $97-82$ & & & & & & & & & & & & & & & & \\
\hline $103-83$ & & & & & & & & & & & & & & & & \\
\hline $92-84$ & & & & & & & & & & & & & & & & \\
\hline $90-85$ & & & & & & & & & & & & & & & & \\
\hline $87-86$ & & & & & & & & & & & & & & & & \\
\hline $102-87$ & & & & & & & & & & & & & & & & \\
\hline 86-88 & & & & & & & & & & & & & & & & \\
\hline $91-89$ & & & & & & & & & & & & & & & & \\
\hline $95-90$ & & & & & & & & & & & & & & & & \\
\hline $88-91$ & & & & & & & & & & & & & & & & \\
\hline $94-92$ & & & & & & & & & & & & & & & & \\
\hline $100-93$ & & & & & & & & & & & & & & & & \\
\hline
\end{tabular}

\section{HWVP 85 (Bates 1985)}

HW39

\section{WV Glasses by CUA and PNL (Johnston et al. 1990)}

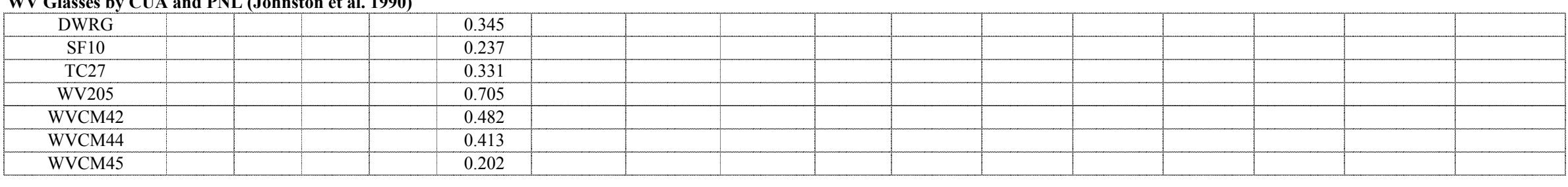


Appendix A. Database - mass fraction

Comp. Vs Properties Study (Chick et al. 1981)

\begin{tabular}{|c|c|c|c|c|c|c|c|c|c|c|c|c|}
\hline Glass ID & $\begin{array}{l}\text { Q PCT Na at } \\
20^{\circ} \mathrm{C}\left(\mathrm{g} / \mathrm{m}^{2}\right)\end{array}$ & $\begin{array}{l}\text { Q PCT Si at } \\
20^{\circ} \mathrm{C}\left(\mathrm{g} / \mathrm{m}^{2}\right)\end{array}$ & $\begin{array}{c}\mathrm{QpH} \\
\text { at } 20^{\circ} \mathrm{C}\end{array}$ & $\begin{array}{c}\text { TCLP Ag } \\
(\mathrm{ppm})\end{array}$ & $\begin{array}{c}\text { TCLP As } \\
(\mathrm{ppm})\end{array}$ & $\begin{array}{c}\text { TCLP Ba } \\
(\mathrm{ppm})\end{array}$ & $\begin{array}{c}\text { TCLP Cd } \\
\text { (ppm) }\end{array}$ & $\begin{array}{c}\text { TCLP Cr } \\
(\mathrm{ppm})\end{array}$ & $\begin{array}{c}\text { TCLP Ni } \\
(\mathrm{ppm})\end{array}$ & $\begin{array}{c}\text { TCLP Pb } \\
(\mathrm{ppm})\end{array}$ & $\begin{array}{l}\text { TCLP Se } \\
(\mathrm{ppm})\end{array}$ & $\begin{array}{c}\text { TCLP Zn } \\
(\mathrm{ppm})\end{array}$ \\
\hline \multicolumn{13}{|l|}{$16-58$} \\
\hline \multicolumn{13}{|l|}{$39-59$} \\
\hline \multicolumn{13}{|l|}{$13-60$} \\
\hline \multicolumn{13}{|l|}{ 61-60 } \\
\hline \multicolumn{13}{|l|}{$23-61$} \\
\hline \multicolumn{13}{|l|}{ 08-62 } \\
\hline \multicolumn{13}{|l|}{$41-63$} \\
\hline \multicolumn{13}{|l|}{$25-64$} \\
\hline \multicolumn{13}{|l|}{$12-65$} \\
\hline \multicolumn{13}{|l|}{$51-66$} \\
\hline \multicolumn{13}{|l|}{ 14-67 } \\
\hline \multicolumn{13}{|l|}{$56-68$} \\
\hline \multicolumn{13}{|l|}{$18-69$} \\
\hline \multicolumn{13}{|l|}{$32-70$} \\
\hline \multicolumn{13}{|l|}{$06-71$} \\
\hline \multicolumn{13}{|l|}{$54-72$} \\
\hline \multicolumn{13}{|l|}{ 19-73 } \\
\hline \multicolumn{13}{|l|}{$15-74$} \\
\hline \multicolumn{13}{|l|}{$67-75$} \\
\hline \multicolumn{13}{|l|}{$96-76$} \\
\hline \multicolumn{13}{|l|}{ 89-77 } \\
\hline \multicolumn{13}{|l|}{$99-78$} \\
\hline \multicolumn{13}{|l|}{$98-79$} \\
\hline \multicolumn{13}{|l|}{$101-80$} \\
\hline $93-81$ & & & & & & & & & & & & \\
\hline $97-82$ & & & & & & & & & & & & \\
\hline $103-83$ & & & & & & & & & & & & \\
\hline $92-84$ & & & & & & & & & & & & \\
\hline $90-85$ & & & & & & & & & & & & \\
\hline $87-86$ & & & & & & & & & & & & \\
\hline $102-87$ & & & & & & & & & & & & \\
\hline $86-88$ & & & & & & & & & & & & \\
\hline $91-89$ & & & & & & & & & & & & \\
\hline $95-90$ & & & & & & & & & & & & \\
\hline $88-91$ & & & & & & & & & & & & \\
\hline 94-92 & & & & & & & & & & & & \\
\hline $100-93$ & & & & & & & & & & & & \\
\hline
\end{tabular}

\section{HWVP 85 (Bates 1985)}

HW39

\section{WV Glasses by CUA and PNL (Johnston et al. 1990)}

DWRG

\begin{tabular}{|c|l|l|l|l|l|l|l|l|l|}
\hline DWRG & & & & & & & \\
\hline SF10 & & & & & & & & \\
\hline TC27 & & & & & & & \\
\hline WV205 & & & & & & & \\
\hline WVCM42 & & & & & & & \\
\hline WVCM44 & & & & & & \\
\hline
\end{tabular}


Appendix A. Database - mass fraction

\section{WV Glasses by CUA and PNL (Johnston et al. 1990)}

\begin{tabular}{|c|c|c|c|c|c|c|c|c|c|c|c|c|c|c|c|c|c|c|c|c|c|}
\hline Glass ID & $\begin{array}{c}\mathrm{Al} 2 \mathrm{O} 3 \\
-\mathrm{t}\end{array}$ & $\begin{array}{c}\mathrm{B} 2 \mathrm{O} 3 \\
-\mathrm{t}\end{array}$ & $\begin{array}{c}\mathrm{CaO} \\
-\mathrm{t}\end{array}$ & $\begin{array}{c}\mathrm{Fe} 2 \mathrm{O} 3 \\
-\mathrm{t}\end{array}$ & $\begin{array}{c}\mathrm{FeO} \\
-\mathrm{t}\end{array}$ & $\begin{array}{c}\mathrm{K} 2 \mathrm{O} \\
-\mathrm{t}\end{array}$ & $\begin{array}{c}\mathrm{Li} 2 \mathrm{O} \\
-\mathrm{t}\end{array}$ & $\begin{array}{c}\mathrm{MgO} \\
-\mathrm{t}\end{array}$ & $\begin{array}{c}\mathrm{Na} 2 \mathrm{O} \\
-\mathrm{t}\end{array}$ & $\begin{array}{c}\mathrm{NiO} \\
-\mathrm{t}\end{array}$ & $\begin{array}{c}\mathrm{P} 2 \mathrm{O} 5 \\
-\mathrm{t}\end{array}$ & $\begin{array}{c}\mathrm{SiO} 2 \\
-\mathrm{t}\end{array}$ & $\begin{array}{c}\mathrm{ZrO} 2 \\
-\mathrm{t}\end{array}$ & $\begin{array}{c}\mathrm{Ag} 2 \mathrm{O} \\
-\mathrm{t}\end{array}$ & $\begin{array}{c}\mathrm{As} 2 \mathrm{O} 3 \\
-\mathrm{t}\end{array}$ & $\begin{array}{c}\mathrm{BaO} \\
-\mathrm{t}\end{array}$ & $\begin{array}{c}\mathrm{Bi} 2 \mathrm{O} 3 \\
-\mathrm{t}\end{array}$ & $\begin{array}{l}\mathrm{Br} \\
-\mathrm{t}\end{array}$ & $\begin{array}{c}\mathrm{CdO} \\
-\mathrm{t}\end{array}$ & $\begin{array}{c}\mathrm{Ce} 2 \mathrm{O} 3 \\
-\mathrm{t}\end{array}$ & $\begin{array}{c}\mathrm{CeO} 2 \\
-\mathrm{t}\end{array}$ \\
\hline \multicolumn{22}{|l|}{ WVCM46 } \\
\hline \multicolumn{22}{|l|}{ WVCM47 } \\
\hline \multicolumn{22}{|l|}{ WVCM48 } \\
\hline \multicolumn{22}{|l|}{ WVCM50 } \\
\hline \multicolumn{22}{|l|}{ WVCM53 } \\
\hline \multicolumn{22}{|l|}{ WVCM55 } \\
\hline \multicolumn{22}{|l|}{ WVCM56 } \\
\hline \multicolumn{22}{|l|}{ WVCM57 } \\
\hline \multicolumn{22}{|l|}{ WVCM59 } \\
\hline \multicolumn{22}{|l|}{ WVUTH7 } \\
\hline \multicolumn{22}{|l|}{ WVUTH8 } \\
\hline \multicolumn{22}{|l|}{ WVUTH12 } \\
\hline WVUTH13 & & & & & & & & & & & & & & & & & & & & & \\
\hline WVUTH14 & & & & & & & & & & & & & & & & & & & & & \\
\hline WVUTH15 & & & & & & & & & & & & & & & & & & & & & \\
\hline WVUTH17 & & & & & & & & & & & & & & & & & & & & & \\
\hline WVUTH18 & & & & & & & & & & & & & & & & & & & & & \\
\hline WVUTH19 & & & & & & & & & & & & & & & & & & & & & \\
\hline WVUTH21 & & & & & & & & & & & & & & & & & & & & & \\
\hline WVUTH22 & & & & & & & & & & & & & & & & & & & & & \\
\hline WVUTH23 & & & & & & & & & & & & & & & & & & & & & \\
\hline WVUTH28 & & & & & & & & & & & & & & & & & & & & & \\
\hline WVUTH30 & & & & & & & & & & & & & & & & & & & & & \\
\hline WVUTH31 & & & & & & & & & & & & & & & & & & & & & \\
\hline WVUTH32 & & & & & & & & & & & & & & & & & & & & & \\
\hline WVUTH34 & & & & & & & & & & & & & & & & & & & & & \\
\hline WVUTH35 & & & & & & & & & & & & & & & & & & & & & \\
\hline WVUTH36 & & & & & & & & & & & & & & & & & & & & & \\
\hline WVUTH37 & & & & & & & & & & & & & & & & & & & & & \\
\hline WVUTH38 & & & & & & & & & & & & & & & & & & & & & \\
\hline WVUTH39 & & & & & & & & & & & & & & & & & & & & & \\
\hline WVUTH40 & & & & & & & & & & & & & & & & & & & & & \\
\hline WVUTH41 & & & & & & & & & & & & & & & & & & & & & \\
\hline WVUTH42 & & & & & & & & & & & & & & & & & & & & & \\
\hline WVUTH48 & & & & & & & & & & & & & & & & & & & & & \\
\hline WVUTH49 & & & & & & & & & & & & & & & & & & & & & \\
\hline WVUTH51 & & & & & & & & & & & & & & & & & & & & & \\
\hline WVUTH52 & & & & & & & & & & & & & & & & & & & & & \\
\hline WVUTH53 & & & & & & & & & & & & & & & & & & & & & \\
\hline WVUTH54 & & & & & & & & & & & & & & & & & & & & & \\
\hline WVUTH55 & & & & & & & & & & & & & & & & & & & & & \\
\hline WVUTH56 & & & & & & & & & & & & & & & & & & & & & \\
\hline WVUTH57 & & & & & & & & & & & & & & & & & & & & & \\
\hline WVUTH58 & & & & & & & & & & & & & & & & & & & & & \\
\hline WVUTH59 & & & & & & & & & & & & & & & & & & & & & \\
\hline WVUTH61 & & & & & & & & & & & & & & & & & & & & & \\
\hline WVUTH62 & & & & & & & & & & & & & & & & & & & & & \\
\hline WVUTH63 & & & & & & & & & & & & & & & & & & & & & \\
\hline WVUTH64 & & & & & & & & & & & & & & & & & & & & & \\
\hline
\end{tabular}


Appendix A. Database - mass fraction

WV Glasses by CUA and PNL (Johnston et al. 1990)

\begin{tabular}{|c|c|c|c|c|c|c|c|c|c|c|c|c|c|c|c|c|c|c|c|c|c|}
\hline Glass ID & $\begin{array}{l}\mathrm{Cl} \\
-\mathrm{t}\end{array}$ & $\begin{array}{c}\mathrm{CoO} \\
-\mathrm{t}\end{array}$ & $\begin{array}{c}\mathrm{Co} 2 \mathrm{O} 3 \\
-\mathrm{t}\end{array}$ & $\begin{array}{c}\mathrm{Cr} 2 \mathrm{O} 3 \\
-\mathrm{t}\end{array}$ & $\begin{array}{c}\mathrm{Cs} 2 \mathrm{O} \\
-\mathrm{t}\end{array}$ & $\begin{array}{c}\mathrm{CuO} \\
-\mathrm{t}\end{array}$ & $\begin{array}{c}\mathrm{Eu} 2 \mathrm{O} 3 \\
-\mathrm{t}\end{array}$ & $\begin{array}{l}F \\
-t\end{array}$ & $\begin{array}{c}\mathrm{Ga} 2 \mathrm{O} 3 \\
-\mathrm{t}\end{array}$ & $\begin{array}{c}\mathrm{Gd} 2 \mathrm{O} 3 \\
-\mathrm{t}\end{array}$ & $\begin{array}{c}\mathrm{HgO} \\
-\mathrm{t}\end{array}$ & $\begin{array}{l}I \\
-t\end{array}$ & $\begin{array}{c}\mathrm{La} 2 \mathrm{O} 3 \\
-\mathrm{t}\end{array}$ & $\begin{array}{c}\mathrm{MnO} 2 \\
-\mathrm{t}\end{array}$ & $\begin{array}{c}\mathrm{MnO} \\
-\mathrm{t}\end{array}$ & $\begin{array}{c}\mathrm{MoO} \\
-\mathrm{t}\end{array}$ & $\begin{array}{c}\mathrm{MoO} 3 \\
-\mathrm{t}\end{array}$ & $\begin{array}{c}\mathrm{Nb} 2 \mathrm{O} 5 \\
-\mathrm{t}\end{array}$ & $\begin{array}{c}\mathrm{Nd} 2 \mathrm{O} 3 \\
-\mathrm{t}\end{array}$ & $\begin{array}{c}\mathrm{PbO} \\
-\mathrm{t}\end{array}$ & $\begin{array}{c}\mathrm{PdO} 2 \\
-\mathrm{t}\end{array}$ \\
\hline WVCM46 & & & & & & & & & & & & & & & & & & & & & \\
\hline WVCM47 & & & & & & & & & & & & & & & & & & & & & \\
\hline WVCM48 & & & & & & & & & & & & & & & & & & & & & \\
\hline WVCM50 & & & & & & & & & & & & & & & & & & & & & \\
\hline WVCM53 & & & & & & & & & & & & & & & & & & & & & \\
\hline WVCM55 & & & & & & & & & & & & & & & & & & & & & \\
\hline WVCM56 & & & & & & & & & & & & & & & & & & & & & \\
\hline WVCM57 & & & & & & & & & & & & & & & & & & & & & \\
\hline WVCM59 & & & & & & & & & & & & & & & & & & & & & \\
\hline WVUTH7 & & & & & & & & & & & & & & & & & & & & & \\
\hline WVUTH8 & & & & & & & & & & & & & & & & & & & & & \\
\hline WVUTH12 & & & & & & & & & & & & & & & & & & & & & \\
\hline WVUTH13 & & & & & & & & & & & & & & & & & & & & & \\
\hline WVUTH14 & & & & & & & & & & & & & & & & & & & & & \\
\hline WVUTH15 & & & & & & & & & & & & & & & & & & & & & \\
\hline WVUTH17 & & & & & & & & & & & & & & & & & & & & & \\
\hline WVUTH18 & & & & & & & & & & & & & & & & & & & & & \\
\hline WVUTH19 & & & & & & & & & & & & & & & & & & & & & \\
\hline WVUTH21 & & & & & & & & & & & & & & & & & & & & & \\
\hline WVUTH22 & & & & & & & & & & & & & & & & & & & & & \\
\hline WVUTH23 & & & & & & & & & & & & & & & & & & & & & \\
\hline WVUTH28 & & & & & & & & & & & & & & & & & & & & & \\
\hline WVUTH30 & & & & & & & & & & & & & & & & & & & & & \\
\hline WVUTH31 & & & & & & & & & & & & & & & & & & & & & \\
\hline WVUTH32 & & & & & & & & & & & & & & & & & & & & & \\
\hline WVUTH34 & & & & & & & & & & & & & & & & & & & & & \\
\hline WVUTH35 & & & & & & & & & & & & & & & & & & & & & \\
\hline WVUTH36 & & & & & & & & & & & & & & & & & & & & & \\
\hline WVUTH37 & & & & & & & & & & & & & & & & & & & & & \\
\hline WVUTH38 & & & & & & & & & & & & & & & & & & & & & \\
\hline WVUTH39 & & & & & & & & & & & & & & & & & & & & & \\
\hline WVUTH40 & & & & & & & & & & & & & & & & & & & & & \\
\hline WVUTH41 & & & & & & & & & & & & & & & & & & & & & \\
\hline WVUTH42 & & & & & & & & & & & & & & & & & & & & & \\
\hline WVUTH48 & & & & & & & & & & & & & & & & & & & & & \\
\hline WVUTH49 & & & & & & & & & & & & & & & & & & & & & \\
\hline WVUTH51 & & & & & & & & & & & & & & & & & & & & & \\
\hline WVUTH52 & & & & & & & & & & & & & & & & & & & & & \\
\hline WVUTH53 & & & & & & & & & & & & & & & & & & & & & \\
\hline WVUTH54 & & & & & & & & & & & & & & & & & & & & & \\
\hline WVUTH55 & & & & & & & & & & & & & & & & & & & & & \\
\hline WVUTH56 & & & & & & & & & & & & & & & & & & & & & \\
\hline WVUTH57 & & & & & & & & & & & & & & & & & & & & & \\
\hline WVUTH58 & & & & & & & & & & & & & & & & & & & & & \\
\hline WVUTH59 & & & & & & & & & & & & & & & & & & & & & \\
\hline WVUTH61 & & & & & & & & & & & & & & & & & & & & & \\
\hline WVUTH62 & & & & & & & & & & & & & & & & & & & & & \\
\hline WVUTH63 & & & & & & & & & & & & & & & & & & & & & \\
\hline WVUTH64 & & & & & & & & & & & & & & & & & & & & & \\
\hline
\end{tabular}


Appendix A. Database - mass fraction

WV Glasses by CUA and PNL (Johnston et al. 1990)

\begin{tabular}{|c|c|c|c|c|c|c|c|c|c|c|c|c|c|c|c|c|c|c|c|c|c|}
\hline Glass ID & $\begin{array}{c}\mathrm{PdO} \\
-\mathrm{t}\end{array}$ & $\begin{array}{c}\mathrm{Pr} 2 \mathrm{O} 3 \\
-\mathrm{t}\end{array}$ & $\begin{array}{c}\text { Pr6O11 } \\
-\mathrm{t}\end{array}$ & $\begin{array}{c}\mathrm{Rb} 2 \mathrm{O} \\
-\mathrm{t}\end{array}$ & $\begin{array}{c}\mathrm{ReO} \\
-\mathrm{t}\end{array}$ & $\begin{array}{c}\mathrm{ReO} 2 \\
-\mathrm{t}\end{array}$ & $\begin{array}{c}\mathrm{Rh} 2 \mathrm{O} 3 \\
-\mathrm{t}\end{array}$ & $\begin{array}{c}\mathrm{RhO} 2 \\
-\mathrm{t}\end{array}$ & $\begin{array}{c}\mathrm{RuO} 2 \\
-\mathrm{t}\end{array}$ & $\begin{array}{c}\mathrm{Sb} 2 \mathrm{O} 3 \\
-\mathrm{t}\end{array}$ & $\begin{array}{c}\mathrm{Sb} 2 \mathrm{O} 5 \\
-\mathrm{t}\end{array}$ & $\begin{array}{c}\mathrm{SeO} 2 \\
-\mathrm{t}\end{array}$ & $\begin{array}{c}\mathrm{Sm} 2 \mathrm{O} 3 \\
-\mathrm{t}\end{array}$ & $\begin{array}{c}\mathrm{SnO} \\
-\mathrm{t}\end{array}$ & $\begin{array}{c}\mathrm{SnO} 2 \\
-\mathrm{t}\end{array}$ & $\begin{array}{c}\mathrm{SO} 3 \\
-\mathrm{t}\end{array}$ & $\begin{array}{c}\mathrm{SrO} \\
-\mathrm{t}\end{array}$ & $\begin{array}{c}\mathrm{Tc} 2 \mathrm{O} 7 \\
-\mathrm{t}\end{array}$ & $\begin{array}{c}\mathrm{TeO} 2 \\
-\mathrm{t}\end{array}$ & $\begin{array}{c}\mathrm{ThO} 2 \\
-\mathrm{t}\end{array}$ & $\begin{array}{c}\mathrm{TiO} 2 \\
-\mathrm{t}\end{array}$ \\
\hline WVCM46 & & & & & & & & & & & & & & & & & & & & & \\
\hline WVCM47 & & & & & & & & & & & & & & & & & & & & & \\
\hline WVCM48 & & & & & & & & & & & & & & & & & & & & & \\
\hline WVCM50 & & & & & & & & & & & & & & & & & & & & & \\
\hline WVCM53 & & & & & & & & & & & & & & & & & & & & & \\
\hline WVCM55 & & & & & & & & & & & & & & & & & & & & & \\
\hline WVCM56 & & & & & & & & & & & & & & & & & & & & & \\
\hline WVCM57 & & & & & & & & & & & & & & & & & & & & & \\
\hline WVCM59 & & & & & & & & & & & & & & & & & & & & & \\
\hline WVUTH7 & & & & & & & & & & & & & & & & & & & & & \\
\hline WVUTH8 & & & & & & & & & & & & & & & & & & & & & \\
\hline WVUTH12 & & & & & & & & & & & & & & & & & & & & & \\
\hline WVUTH13 & & & & & & & & & & & & & & & & & & & & & \\
\hline WVUTH14 & & & & & & & & & & & & & & & & & & & & & \\
\hline WVUTH15 & & & & & & & & & & & & & & & & & & & & & \\
\hline WVUTH17 & & & & & & & & & & & & & & & & & & & & & \\
\hline WVUTH18 & & & & & & & & & & & & & & & & & & & & & \\
\hline WVUTH19 & & & & & & & & & & & & & & & & & & & & & \\
\hline WVUTH21 & & & & & & & & & & & & & & & & & & & & & \\
\hline WVUTH22 & & & & & & & & & & & & & & & & & & & & & \\
\hline WVUTH 23 & & & & & & & & & & & & & & & & & & & & & \\
\hline WVUTH28 & & & & & & & & & & & & & & & & & & & & & \\
\hline WVUTH30 & & & & & & & & & & & & & & & & & & & & & \\
\hline WVUTH31 & & & & & & & & & & & & & & & & & & & & & \\
\hline WVUTH32 & & & & & & & & & & & & & & & & & & & & & \\
\hline WVUTH34 & & & & & & & & & & & & & & & & & & & & & \\
\hline WVUTH35 & & & & & & & & & & & & & & & & & & & & & \\
\hline WVUTH36 & & & & & & & & & & & & & & & & & & & & & \\
\hline WVUTH37 & & & & & & & & & & & & & & & & & & & & & \\
\hline WVUTH38 & & & & & & & & & & & & & & & & & & & & & \\
\hline WVUTH39 & & & & & & & & & & & & & & & & & & & & & \\
\hline WVUTH40 & & & & & & & & & & & & & & & & & & & & & \\
\hline WVUTH41 & & & & & & & & & & & & & & & & & & & & & \\
\hline WVUTH42 & & & & & & & & & & & & & & & & & & & & & \\
\hline WVUTH48 & & & & & & & & & & & & & & & & & & & & & \\
\hline WVUTH49 & & & & & & & & & & & & & & & & & & & & & \\
\hline WVUTH51 & & & & & & & & & & & & & & & & & & & & & \\
\hline WVUTH52 & & & & & & & & & & & & & & & & & & & & & \\
\hline WVUTH53 & & & & & & & & & & & & & & & & & & & & & \\
\hline WVUTH54 & & & & & & & & & & & & & & & & & & & & & \\
\hline WVUTH55 & & & & & & & & & & & & & & & & & & & & & \\
\hline WVUTH56 & & & & & & & & & & & & & & & & & & & & & \\
\hline WVUTH57 & & & & & & & & & & & & & & & & & & & & & \\
\hline WVUTH58 & & & & & & & & & & & & & & & & & & & & & \\
\hline WVUTH59 & & & & & & & & & & & & & & & & & & & & & \\
\hline WVUTH61 & & & & & & & & & & & & & & & & & & & & & \\
\hline WVUTH62 & & & & & & & & & & & & & & & & & & & & & \\
\hline WVUTH63 & & & & & & & & & & & & & & & & & & & & & \\
\hline WVUTH64 & & & & & & & & & & & & & & & & & & & & & \\
\hline
\end{tabular}


WV Glasses by CUA and PNL (Johnston et al. 1990)

\begin{tabular}{|c|c|c|c|c|c|c|c|c|c|c|c|c|c|c|c|c|c|c|c|c|c|}
\hline Glass ID & $\begin{array}{c}\mathrm{T} 12 \mathrm{O} 3 \\
-\mathrm{t}\end{array}$ & $\begin{array}{c}\mathrm{U} 3 \mathrm{O} 8 \\
-\mathrm{t}\end{array}$ & $\begin{array}{c}\mathrm{UO} 2 \\
-\mathrm{t}\end{array}$ & $\begin{array}{c}\text { UO3 } \\
-t\end{array}$ & $\begin{array}{c}\mathrm{V} 2 \mathrm{O} 5 \\
-\mathrm{t}\end{array}$ & $\begin{array}{c}\text { WO3 } \\
-t\end{array}$ & $\begin{array}{c}\mathrm{Y} 2 \mathrm{O} 3 \\
-\mathrm{t}\end{array}$ & $\begin{array}{c}\mathrm{ZnO} \\
-\mathrm{t}\end{array}$ & $\begin{array}{c}\text { Others } \\
-t\end{array}$ & $\begin{array}{c}\text { Sum } \\
-t\end{array}$ & $\begin{array}{c}\mathrm{A} 12 \mathrm{O} 3 \\
-\mathrm{a}\end{array}$ & $\begin{array}{c}\mathrm{B} 2 \mathrm{O} 3 \\
-\mathrm{a}\end{array}$ & $\begin{array}{c}\mathrm{CaO} \\
-\mathrm{a}\end{array}$ & $\begin{array}{c}\mathrm{Fe} 2 \mathrm{O} 3 \\
-\mathrm{a}\end{array}$ & $\begin{array}{c}\mathrm{FeO} \\
-\mathrm{a}\end{array}$ & $\begin{array}{c}\mathrm{K} 2 \mathrm{O} \\
-\mathrm{a}\end{array}$ & $\begin{array}{c}\mathrm{Li} 2 \mathrm{O} \\
-\mathrm{a}\end{array}$ & $\begin{array}{c}\mathrm{MgO} \\
-\mathrm{a}\end{array}$ & $\begin{array}{c}\mathrm{Na} 2 \mathrm{O} \\
-\mathrm{a}\end{array}$ & $\begin{array}{c}\mathrm{NiO} \\
-\mathrm{a}\end{array}$ & $\begin{array}{c}\mathrm{P} 2 \mathrm{O} 5 \\
-\mathrm{a}\end{array}$ \\
\hline WVCM46 & & & & & & & & & & & 0.0796 & 0.0992 & 0.0068 & 0.1214 & & 0.0157 & 0.0202 & 0.0131 & 0.0890 & & 0.0250 \\
\hline WVCM47 & & & & & & & & & & & 0.0798 & 0.1243 & 0.0060 & 0.1212 & & 0.0128 & 0.0202 & 0.0132 & 0.0921 & & 0.0250 \\
\hline WVCM48 & & & & & & & & & & & 0.0861 & 0.0960 & 0.0096 & 0.1311 & & 0.0245 & 0.0293 & 0.0093 & 0.1071 & & 0.0064 \\
\hline WVCM50 & & & & & & & & & & & 0.0986 & 0.1227 & 0.0082 & 0.1193 & & 0.0160 & 0.0222 & 0.0079 & 0.0979 & & 0.0247 \\
\hline WVCM53 & & & & & & & & & & & 0.1021 & 0.1262 & 0.0024 & 0.1223 & & 0.0095 & 0.0204 & & 0.1087 & & 0.0261 \\
\hline WVCM55 & & & & & & & & & & & 0.1044 & 0.1336 & 0.0069 & 0.1264 & & 0.0135 & 0.0459 & 0.0067 & 0.0857 & & 0.0264 \\
\hline WVCM56 & & & & & & & & & & & 0.0883 & 0.1403 & 0.0081 & 0.1086 & & 0.0158 & 0.0240 & 0.0088 & 0.0887 & & 0.0055 \\
\hline WVCM57 & & & & & & & & & & & 0.0480 & 0.0944 & & 0.1287 & & & & & & & 0.0252 \\
\hline WVCM59 & & & & & & & & & & & 0.0647 & 0.1029 & 0.0068 & 0.1204 & & 0.0362 & 0.0316 & 0.0089 & 0.1117 & & 0.0238 \\
\hline WVUTH7 & & & & & & & & & & & 0.0815 & 0.0931 & 0.0092 & 0.1163 & & 0.0334 & 0.0282 & 0.0122 & 0.1018 & & 0.0234 \\
\hline WVUTH8 & & & & & & & & & & & 0.0285 & 0.0934 & 0.0092 & 0.1166 & & 0.0335 & 0.0283 & 0.0122 & 0.1021 & & 0.0235 \\
\hline WVUTH12 & & & & & & & & & & & 0.0995 & 0.1234 & 0.0087 & 0.1197 & & 0.0249 & 0.0268 & 0.0124 & 0.0978 & & 0.0058 \\
\hline WVUTH13 & & & & & & & & & & & 0.1194 & 0.1234 & 0.0087 & 0.1196 & & 0.0249 & 0.0268 & 0.0125 & 0.0978 & & 0.0058 \\
\hline WVUTH14 & & & & & & & & & & & 0.0995 & 0.1236 & 0.0088 & 0.1198 & & 0.0249 & 0.0268 & 0.0125 & 0.0979 & & 0.0244 \\
\hline WVUTH15 & & & & & & & & & & & 0.0997 & 0.1036 & 0.0087 & 0.1198 & & 0.0249 & 0.0268 & 0.0125 & 0.0979 & & 0.0246 \\
\hline WVUTH17 & & & & & & & & & & & 0.0986 & 0.1227 & 0.0082 & 0.1193 & & 0.0160 & 0.0222 & 0.0079 & 0.0979 & & 0.0247 \\
\hline WVUTH18 & & & & & & & & & & & 0.0969 & 0.1206 & 0.0080 & 0.1172 & & 0.0157 & 0.0218 & 0.0078 & 0.0962 & & 0.0243 \\
\hline WVUTH19 & & & & & & & & & & & 0.0967 & 0.1203 & 0.0080 & 0.1169 & & 0.0157 & 0.0217 & 0.0078 & 0.1156 & & 0.0242 \\
\hline WVUTH21 & & & & & & & & & & & 0.1010 & 0.1249 & 0.0083 & 0.1217 & & 0.0162 & 0.0229 & 0.0080 & 0.0998 & & 0.0254 \\
\hline WVUTH22 & & & & & & & & & & & 0.0966 & 0.1408 & 0.0080 & 0.1168 & & 0.0156 & 0.0217 & 0.0078 & 0.0958 & & 0.0242 \\
\hline WVUTH23 & & & & & & & & & & & 0.0948 & 0.1180 & 0.0079 & 0.1531 & & 0.0154 & 0.0213 & 0.0076 & 0.0941 & & 0.0238 \\
\hline WVUTH 28 & & & & & & & & & & & 0.0986 & 0.1027 & 0.0071 & 0.1194 & & 0.0191 & 0.0195 & 0.0126 & 0.1175 & & 0.0247 \\
\hline WVUTH30 & & & & & & & & & & & 0.0981 & 0.1220 & 0.0081 & 0.1186 & & 0.0159 & 0.0220 & 0.0079 & 0.0973 & & 0.0246 \\
\hline WVUTH31 & & & & & & & & & & & 0.0983 & 0.1223 & 0.0071 & 0.1190 & & 0.0190 & 0.0211 & 0.0125 & 0.0891 & & 0.0346 \\
\hline WVUTH32 & & & & & & & & & & & 0.1011 & 0.1250 & 0.0083 & 0.1217 & & 0.0162 & 0.0229 & 0.0080 & 0.0998 & & 0.0254 \\
\hline WVUTH34 & & & & & & & & & & & 0.1004 & 0.1241 & 0.0084 & 0.1202 & & 0.0093 & 0.0207 & 0.0081 & 0.1069 & & 0.0257 \\
\hline WVUTH35 & & & & & & & & & & & 0.1004 & 0.1241 & 0.0084 & 0.1203 & & 0.0093 & 0.0207 & & 0.1069 & & 0.0257 \\
\hline WVUTH36 & & & & & & & & & & & 0.0987 & 0.1264 & 0.0081 & 0.1196 & & 0.0128 & 0.0434 & 0.0088 & 0.0811 & & 0.0249 \\
\hline WVUTH37 & & & & & & & & & & & 0.1005 & 0.1287 & 0.0083 & 0.1217 & & 0.0130 & 0.0442 & 0.0089 & 0.0825 & & 0.0254 \\
\hline WVUTH38 & & & & & & & & & & & 0.0987 & 0.1264 & 0.0081 & 0.1196 & & 0.0128 & 0.0434 & 0.0088 & 0.0810 & & 0.0249 \\
\hline WVUTH39 & & & & & & & & & & & 0.1006 & 0.1288 & 0.0083 & 0.1218 & & 0.0130 & 0.0442 & 0.0089 & 0.0826 & & 0.0254 \\
\hline WVUTH40 & & & & & & & & & & & 0.1384 & 0.1222 & 0.0080 & 0.1192 & & 0.0157 & 0.0227 & 0.0078 & 0.0977 & & 0.0251 \\
\hline WVUTH41 & & & & & & & & & & & \begin{tabular}{|l|}
0.0992 \\
\end{tabular} & 0.1221 & 0.0080 & 0.1591 & & 0.0157 & 0.0227 & 0.0078 & 0.0976 & & 0.0250 \\
\hline WVUTH42 & & & & & & & & & & & 0.0988 & 0.1221 & 0.0083 & 0.1184 & & 0.0092 & 0.0204 & 0.0159 & 0.1052 & & 0.0253 \\
\hline WVUTH48 & & & & & & & & & & & 0.0993 & 0.1221 & 0.0080 & 0.1192 & & 0.0157 & 0.0227 & 0.0078 & 0.0977 & & 0.0251 \\
\hline WVUTH49 & & & & & & & & & & & 0.0992 & 0.1221 & 0.0080 & 0.1191 & & 0.0157 & 0.0227 & 0.0078 & 0.0976 & & 0.0250 \\
\hline WVUTH51 & & & & & & & & & & & 0.0996 & 0.1231 & 0.0084 & 0.1193 & & 0.0092 & 0.0205 & 0.0081 & 0.1060 & & 0.0255 \\
\hline WVUTH52 & & & & & & & & & & & 0.0996 & 0.1231 & 0.0024 & 0.1193 & & 0.0092 & 0.0205 & 0.0141 & 0.1060 & & 0.0255 \\
\hline WVUTH53 & & & & & & & & & & & 0.0994 & 0.1272 & 0.0082 & 0.1204 & & 0.0129 & 0.0437 & 0.0088 & 0.0816 & & 0.0251 \\
\hline WVUTH54 & & & & & & & & & & & 0.1004 & 0.1286 & 0.0083 & 0.1214 & & 0.0142 & 0.0216 & 0.0079 & 0.0850 & & 0.0252 \\
\hline WVUTH55 & & & & & & & & & & & 0.0807 & 0.1283 & 0.0083 & 0.1212 & & 0.0162 & 0.0225 & 0.0080 & 0.1021 & & 0.0251 \\
\hline WVUTH56 & & & & & & & & & & & 0.0993 & 0.1273 & 0.0082 & 0.1203 & & 0.0161 & 0.0223 & 0.0080 & 0.1000 & & 0.0148 \\
\hline WVUTH57 & & & & & & & & & & & 0.0983 & 0.1252 & 0.0088 & 0.1190 & & 0.0336 & 0.0289 & 0.0096 & 0.1047 & & 0.0247 \\
\hline WVUTH58 & & & & & & & & & & & 0.0984 & 0.1224 & 0.0081 & 0.1190 & & 0.0159 & 0.0221 & 0.0079 & 0.0977 & & 0.0247 \\
\hline WVUTH59 & & & & & & & & & & & 0.0967 & 0.1202 & 0.0080 & 0.1369 & & 0.0157 & 0.0217 & 0.0078 & 0.0959 & & 0.0242 \\
\hline WVUTH61 & & & & & & & & & & & 0.0963 & 0.1198 & 0.0080 & 0.1165 & & 0.0156 & 0.0216 & 0.0077 & 0.0956 & & 0.0241 \\
\hline WVUTH62 & & & & & & & & & & & 0.0450 & 0.1137 & 0.0082 & 0.1205 & & 0.0405 & 0.0348 & 0.0090 & 0.1261 & & 0.0236 \\
\hline WVUTH63 & & & & & & & & & & & 0.0450 & 0.0957 & 0.0082 & 0.1205 & & 0.0341 & 0.0292 & 0.0090 & 0.1061 & & 0.0236 \\
\hline WVUTH64 & & & & & & & & & & & 0.0650 & 0.1065 & 0.0082 & 0.1205 & & 0.0379 & 0.0326 & 0.0090 & 0.1181 & & 0.0236 \\
\hline
\end{tabular}


Appendix A. Database - mass fraction

\section{WV Glasses by CUA and PNL (Johnston et al. 1990)}

\begin{tabular}{|c|c|c|c|c|c|c|c|c|c|c|c|c|c|c|c|c|c|c|c|c|c|}
\hline Glass ID & $\begin{array}{c}\mathrm{SiO} 2 \\
-\mathrm{a}\end{array}$ & $\begin{array}{c}\mathrm{ZrO} 2 \\
-\mathrm{a}\end{array}$ & $\begin{array}{c}\mathrm{Ag} 2 \mathrm{O} \\
-\mathrm{a}\end{array}$ & $\begin{array}{c}\mathrm{As} 2 \mathrm{O} 3 \\
-\mathrm{a}\end{array}$ & $\begin{array}{c}\mathrm{BaO} \\
-\mathrm{a}\end{array}$ & $\begin{array}{c}\mathrm{Bi} 2 \mathrm{O} 3 \\
-\mathrm{a}\end{array}$ & $\begin{array}{l}\mathrm{Br} \\
-\mathrm{a}\end{array}$ & $\begin{array}{c}\mathrm{CdO} \\
-\mathrm{a}\end{array}$ & $\begin{array}{c}\mathrm{Ce} 2 \mathrm{O} 3 \\
-\mathrm{a}\end{array}$ & $\begin{array}{c}\mathrm{CeO} 2 \\
-\mathrm{a}\end{array}$ & $\begin{array}{l}\mathrm{Cl} \\
-\mathrm{a}\end{array}$ & $\begin{array}{c}\mathrm{CoO} \\
-\mathrm{a}\end{array}$ & $\begin{array}{c}\mathrm{Co} 2 \mathrm{O} 3 \\
-\mathrm{a}\end{array}$ & $\begin{array}{c}\mathrm{Cr} 2 \mathrm{O} 3 \\
-\mathrm{a}\end{array}$ & $\begin{array}{c}\mathrm{Cs} 2 \mathrm{O} \\
-\mathrm{a}\end{array}$ & $\begin{array}{c}\mathrm{CuO} \\
-\mathrm{a}\end{array}$ & \begin{tabular}{|c|}
$\mathrm{Eu} 2 \mathrm{O} 3$ \\
$-\mathrm{a}$
\end{tabular} & $\begin{array}{c}F \\
-a\end{array}$ & $\begin{array}{c}\mathrm{Ga} 2 \mathrm{O} 3 \\
-\mathrm{a}\end{array}$ & $\begin{array}{c}\mathrm{Gd} 2 \mathrm{O} 3 \\
-\mathrm{a}\end{array}$ & $\begin{array}{c}\mathrm{HgO} \\
-\mathrm{a}\end{array}$ \\
\hline WVCM46 & 0.4473 & & & & 0.0016 & & & & & & & & & & & & & & & & \\
\hline WVCM47 & 0.4230 & & & & 0.0013 & & & & & & & & & & & & & & & & \\
\hline WVCM48 & 0.4101 & & & & 0.0022 & & & & & & & & & & & & & & & & \\
\hline WVCM50 & 0.3960 & & & & 0.0019 & & & & & & & & & & & & & & & & \\
\hline WVCM53 & 0.4112 & & & & & & & & & & & & & & & & & & & & \\
\hline WVCM55 & 0.4199 & & & & 0.0016 & & & & & & & & & & & & & & & & \\
\hline WVCM56 & 0.4202 & & & & 0.0019 & & & & & & & & & & & & & & & & \\
\hline WVCM57 & 0.4260 & & & & & & & & & & & & & & & & & & & & \\
\hline WVCM59 & 0.4093 & & & & 0.0016 & & & & & & & & & & & & & & & & \\
\hline WVUTH7 & 0.4191 & & & & 0.0021 & & & & & & & & & & & & & & & & \\
\hline WVUTH8 & 0.4204 & & & & 0.0022 & & & & & & & & & & & & & & & & \\
\hline WVUTH12 & 0.3984 & & & & 0.0020 & & & & & & & & & & & & & & & & \\
\hline WVUTH13 & 0.3784 & & & & 0.0020 & & & & & & & & & & & & & & & & \\
\hline WVUTH14 & 0.3790 & & & & 0.0020 & & & & & & & & & & & & & & & & \\
\hline WVUTH15 & 0.3987 & & & & 0.0020 & & & & & & & & & & & & & & & & \\
\hline WVUTH17 & 0.3960 & & & & 0.0019 & & & & & & & & & & & & & & & & \\
\hline WVUTH18 & 0.3891 & & & & 0.0019 & & & & & & & & & & & & & & & & \\
\hline WVUTH19 & 0.3882 & & & & 0.0019 & & & & & & & & & & & & & & & & \\
\hline WVUTH21 & 0.3848 & & & & 0.0019 & & & & & & & & & & & & & & & & \\
\hline WVUTH22 & 0.3878 & & & & 0.0019 & & & & & & & & & & & & & & & & \\
\hline WVUTH23 & 0.3807 & & & & 0.0180 & & & & & & & & & & & & & & & & \\
\hline WVUTH 28 & 0.3960 & & & & 0.0017 & & & & & & & & & & & & & & & & \\
\hline WVUTH30 & 0.3937 & & & & 0.0019 & & & & & & & & & & & & & & & & \\
\hline WVUTH31 & 0.3946 & & & & 0.0017 & & & & & & & & & & & & & & & & \\
\hline WVUTH32 & 0.3849 & & & & 0.0019 & & & & & & & & & & & & & & & & \\
\hline WVUTH34 & 0.4043 & & & & & & & & & & & & & & & & & & & & \\
\hline WVUTH35 & 0.4043 & & & & & & & & & & & & & & & & & & & & \\
\hline WVUTH36 & 0.3972 & & & & 0.0015 & & & & & & & & & & & & & & & & \\
\hline WVUTH37 & 0.4043 & & & & 0.0016 & & & & & & & & & & & & & & & & \\
\hline WVUTH38 & 0.3971 & & & & 0.0015 & & & & & & & & & & & & & & & & \\
\hline WVUTH39 & 0.4046 & & & & 0.0016 & & & & & & & & & & & & & & & & \\
\hline WVUTH40 & 0.3592 & & & & 0.0019 & & & & & & & & & & & & & & & & \\
\hline WVUTH41 & 0.3588 & & & & 0.0019 & & & & & & & & & & & & & & & & \\
\hline WVUTH42 & 0.3979 & & & & & & & & & & & & & & & & & & & & \\
\hline WVUTH48 & 0.3984 & & & & 0.0019 & & & & & & & & & & & & & & & & \\
\hline WVUTH49 & 0.3588 & & & & 0.0019 & & & & & & & & & & & & & & & & \\
\hline WVUTH51 & 0.4010 & & & & 0.0000 & & & & & & & & & & & & & & & & \\
\hline WVUTH52 & 0.4010 & & & & 0.0000 & & & & & & & & & & & & & & & & \\
\hline WVUTH53 & 0.3997 & & & & 0.0015 & & & & & & & & & & & & & & & & \\
\hline WVUTH54 & 0.4033 & & & & 0.0017 & & & & & & & & & & & & & & & & \\
\hline WVUTH55 & 0.4023 & & & & 0.0017 & & & & & & & & & & & & & & & & \\
\hline WVUTH56 & 0.3991 & & & & 0.0017 & & & & & & & & & & & & & & & & \\
\hline WVUTH57 & 0.3751 & & & & 0.0021 & & & & & & & & & & & & & & & & \\
\hline WVUTH58 & 0.3952 & & & & 0.0019 & & & & & & & & & & & & & & & & \\
\hline WVUTH59 & 0.3880 & & & & 0.0019 & & & & & & & & & & & & & & & & \\
\hline WVUTH61 & 0.4101 & & & & 0.0019 & & & & & & & & & & & & & & & & \\
\hline WVUTH62 & 0.3991 & & & & 0.0019 & & & & & & & & & & & & & & & & \\
\hline WVUTH63 & 0.4491 & & & & 0.0019 & & & & & & & & & & & & & & & & \\
\hline WVUTH64 & 0.3991 & & & & 0.0019 & & & & & & & & & & & & & & & & \\
\hline
\end{tabular}


Appendix A. Database - mass fraction

WV Glasses by CUA and PNL (Johnston et al. 1990)

\begin{tabular}{|c|c|c|c|c|c|c|c|c|c|c|c|c|c|c|c|c|c|c|c|c|c|}
\hline Glass ID & $\begin{array}{c}\mathrm{I} \\
-\mathrm{a}\end{array}$ & $\begin{array}{c}\mathrm{La} 2 \mathrm{O} 3 \\
-\mathrm{a}\end{array}$ & $\begin{array}{c}\mathrm{MnO} 2 \\
-\mathrm{a}\end{array}$ & $\begin{array}{c}\mathrm{MnO} \\
-\mathrm{a}\end{array}$ & $\begin{array}{c}\mathrm{MoO} \\
-\mathrm{a}\end{array}$ & $\begin{array}{c}\mathrm{MoO} 3 \\
-\mathrm{a}\end{array}$ & $\begin{array}{c}\mathrm{Nb} 2 \mathrm{O} 5 \\
-\mathrm{a}\end{array}$ & $\begin{array}{c}\mathrm{Nd} 2 \mathrm{O} 3 \\
-\mathrm{a}\end{array}$ & $\begin{array}{c}\mathrm{PbO} \\
-\mathrm{a}\end{array}$ & $\begin{array}{c}\mathrm{PdO} 2 \\
-\mathrm{a}\end{array}$ & $\begin{array}{c}\mathrm{PdO} \\
-\mathrm{a}\end{array}$ & $\begin{array}{c}\mathrm{Pr} 2 \mathrm{O} 3 \\
-\mathrm{a}\end{array}$ & $\begin{array}{c}\operatorname{Pr} 6011 \\
-\mathrm{a}\end{array}$ & $\begin{array}{c}\mathrm{Rb} 2 \mathrm{O} \\
-\mathrm{a}\end{array}$ & $\begin{array}{c}\mathrm{ReO} \\
-\mathrm{a}\end{array}$ & $\begin{array}{c}\mathrm{ReO} 2 \\
-\mathrm{a}\end{array}$ & $\begin{array}{c}\mathrm{Rh} 2 \mathrm{O} 3 \\
-\mathrm{a}\end{array}$ & $\begin{array}{c}\mathrm{RhO} 2 \\
-\mathrm{a}\end{array}$ & $\begin{array}{c}\mathrm{RuO} 2 \\
-\mathrm{a}\end{array}$ & $\begin{array}{c}\mathrm{Sb} 2 \mathrm{O} 3 \\
-\mathrm{a}\end{array}$ & $\begin{array}{c}\mathrm{Sb} 2 \mathrm{O} 5 \\
-\mathrm{a}\end{array}$ \\
\hline WVCM46 & & & 0.0130 & & & & & & & & & & & & & & & & & & \\
\hline WVCM47 & & & 0.0131 & & & & & & & & & & & & & & & & & & \\
\hline WVCM48 & & & 0.0142 & & & & & & & & & & & & & & & & & & \\
\hline WVCM50 & & & 0.0121 & & & & & & & & & & & & & & & & & & \\
\hline WVCM53 & & & 0.0100 & & & & & & & & & & & & & & & & & & \\
\hline WVCM55 & & & 0.0103 & & & & & & & & & & & & & & & & & & \\
\hline WVCM56 & & & 0.0120 & & & & & & & & & & & & & & & & & & \\
\hline WVCM57 & & & 0.0130 & & & & & & & & & & & & & & & & & & \\
\hline WVCM59 & & & 0.0101 & & & & & & & & & & & & & & & & & & \\
\hline WVUTH7 & & & 0.0136 & & & & & & & & & & & & & & & & & & \\
\hline WVUTH8 & & & 0.0136 & & & & & & & & & & & & & & & & & & \\
\hline WVUTH12 & & & 0.0129 & & & & & & & & & & & & & & & & & & \\
\hline WVUTH13 & & & 0.0129 & & & & & & & & & & & & & & & & & & \\
\hline WVUTH14 & & & 0.0130 & & & & & & & & & & & & & & & & & & \\
\hline WVUTH15 & & & 0.0130 & & & & & & & & & & & & & & & & & & \\
\hline WVUTH17 & & & 0.0121 & & & & & & & & & & & & & & & & & & \\
\hline WVUTH18 & & & 0.0119 & & & & & & & & & & & & & & & & & & \\
\hline WVUTH19 & & & 0.0119 & & & & & & & & & & & & & & & & & & \\
\hline WVUTH21 & & & 0.0122 & & & & & & & & & & & & & & & & & & \\
\hline WVUTH22 & & & 0.0118 & & & & & & & & & & & & & & & & & & \\
\hline WVUTH 23 & & & 0.0116 & & & & & & & & & & & & & & & & & & \\
\hline WVUTH28 & & & 0.0128 & & & & & & & & & & & & & & & & & & \\
\hline WVUTH30 & & & 0.0120 & & & & & & & & & & & & & & & & & & \\
\hline WVUTH31 & & & 0.0128 & & & & & & & & & & & & & & & & & & \\
\hline WVUTH32 & & & 0.0122 & & & & & & & & & & & & & & & & & & \\
\hline WVUTH34 & & & 0.0099 & & & & & & & & & & & & & & & & & & \\
\hline WVUTH35 & & & 0.0099 & & & & & & & & & & & & & & & & & & \\
\hline WVUTH36 & & & 0.0121 & & & & & & & & & & & & & & & & & & \\
\hline WVUTH37 & & & 0.0123 & & & & & & & & & & & & & & & & & & \\
\hline WVUTH38 & & & 0.0121 & & & & & & & & & & & & & & & & & & \\
\hline WVUTH39 & & & 0.0123 & & & & & & & & & & & & & & & & & & \\
\hline WVUTH40 & & & 0.0119 & & & & & & & & & & & & & & & & & & \\
\hline WVUTH41 & & & 0.0119 & & & & & & & & & & & & & & & & & & \\
\hline WVUTH42 & & & 0.0097 & & & & & & & & & & & & & & & & & & \\
\hline WVUTH48 & & & 0.0119 & & & & & & & & & & & & & & & & & & \\
\hline WVUTH49 & & & 0.0119 & & & & & & & & & & & & & & & & & & \\
\hline WVUTH51 & & & 0.0098 & & & & & & & & & & & & & & & & & & \\
\hline WVUTH52 & & & 0.0098 & & & & & & & & & & & & & & & & & & \\
\hline WVUTH53 & & & 0.0098 & & & & & & & & & & & & & & & & & & \\
\hline WVUTH54 & & & 0.0123 & & & & & & & & & & & & & & & & & & \\
\hline WVUTH55 & & & 0.0123 & & & & & & & & & & & & & & & & & & \\
\hline WVUTH56 & & & 0.0122 & & & & & & & & & & & & & & & & & & \\
\hline WVUTH57 & & & 0.0121 & & & & & & & & & & & & & & & & & & \\
\hline WVUTH58 & & & 0.0121 & & & & & & & & & & & & & & & & & & \\
\hline WVUTH59 & & & 0.0119 & & & & & & & & & & & & & & & & & & \\
\hline WVUTH61 & & & 0.0118 & & & & & & & & & & & & & & & & & & \\
\hline WVUTH62 & & & 0.0122 & & & & & & & & & & & & & & & & & & \\
\hline WVUTH63 & & & 0.0122 & & & & & & & & & & & & & & & & & & \\
\hline WVUTH64 & & & 0.0122 & & & & & & & & & & & & & & & & & & \\
\hline
\end{tabular}




\section{WV Glasses by CUA and PNL (Johnston et al. 1990)}

\begin{tabular}{|c|c|c|c|c|c|c|c|c|c|c|c|c|c|c|c|c|c|c|c|c|}
\hline Glass ID & $\begin{array}{c}\mathrm{SeO} 2 \\
-\mathrm{a}\end{array}$ & $\begin{array}{c}\mathrm{Sm} 2 \mathrm{O} 3 \\
-\mathrm{a}\end{array}$ & $\begin{array}{c}\mathrm{SnO} \\
-\mathrm{a}\end{array}$ & $\begin{array}{c}\mathrm{SnO} 2 \\
-\mathrm{a}\end{array}$ & $\begin{array}{c}\mathrm{SO} 3 \\
-\mathrm{a}\end{array}$ & $\begin{array}{c}\mathrm{SrO} \\
-\mathrm{a}\end{array}$ & $\begin{array}{c}\mathrm{Tc} 2 \mathrm{O} 7 \\
-\mathrm{a}\end{array}$ & $\begin{array}{c}\mathrm{TeO} 2 \\
-\mathrm{a}\end{array}$ & $\begin{array}{c}\mathrm{ThO} 2 \\
-\mathrm{a}\end{array}$ & $\begin{array}{c}\mathrm{TiO} 2 \\
-\mathrm{a}\end{array}$ & $\begin{array}{c}\mathrm{T} 12 \mathrm{O} 3 \\
-\mathrm{a}\end{array}$ & $\begin{array}{c}\mathrm{U} 3 \mathrm{O} 8 \\
-\mathrm{a}\end{array}$ & $\begin{array}{c}\mathrm{UO} 2 \\
-\mathrm{a}\end{array}$ & $\begin{array}{c}\mathrm{UO} 3 \\
-\mathrm{a}\end{array}$ & $\begin{array}{c}\mathrm{V} 2 \mathrm{O} 5 \\
-\mathrm{a}\end{array}$ & $\begin{array}{c}\text { WO3 } \\
-\mathrm{a}\end{array}$ & $\begin{array}{c}\mathrm{Y} 2 \mathrm{O} 3 \\
-\mathrm{a}\end{array}$ & $\begin{array}{c}\mathrm{ZnO} \\
-\mathrm{a}\end{array}$ & $\begin{array}{c}\text { Others } \\
-\mathrm{a}\end{array}$ & $\begin{array}{c}\text { Sum } \\
-\mathrm{a}\end{array}$ \\
\hline WVCM46 & & & & & & & & & 0.0357 & & & & 0.0059 & & & & & & 0.0261 & 0.9996 \\
\hline WVCM47 & & & & & & & & & 0.0358 & & & & 0.0060 & & & & & & 0.0261 & 0.9999 \\
\hline WVCM48 & & & & & & & & & 0.0387 & & & & 0.0064 & & & & & & 0.0286 & 0.9996 \\
\hline WVCM50 & & & & & & & & & 0.0352 & & & & 0.0061 & & & & & & 0.0308 & 0.9996 \\
\hline WVCM53 & & & & & & & & & 0.0361 & & & & 0.0059 & & & & & & 0.0190 & 0.9999 \\
\hline WVCM55 & & & & & & & & & 0.0000 & & & & 0.0000 & & & & & & 0.0186 & 0.9999 \\
\hline WVCM56 & & & & & & & & & 0.0391 & & & & 0.0066 & & & & & & 0.0319 & 0.9998 \\
\hline WVCM57 & & & & & & & & & 0.0380 & & & & 0.0062 & & & & & & 0.2221 & 1.0016 \\
\hline WVCM59 & & & & & & & & & 0.0360 & & & & 0.0058 & & & & & & 0.0297 & 0.9995 \\
\hline WVUTH7 & & & & & & & & & 0.0338 & & & & 0.0056 & & & & & & 0.0264 & 0.9997 \\
\hline WVUTH8 & & & & & & & & & 0.0339 & & & & 0.0056 & & & & & & 0.0767 & 0.9997 \\
\hline WVUTH12 & & & & & & & & & 0.0353 & & & & 0.0059 & & & & & & 0.0262 & 0.9997 \\
\hline WVUTH13 & & & & & & & & & 0.0353 & & & & 0.0059 & & & & & & 0.0262 & 0.9996 \\
\hline WVUTH14 & & & & & & & & & 0.0354 & & & & 0.0059 & & & & & & 0.0263 & 0.9998 \\
\hline WVUTH15 & & & & & & & & & 0.0354 & & & & 0.0059 & & & & & & 0.0262 & 0.9997 \\
\hline WVUTH17 & & & & & & & & & 0.0352 & & & & 0.0061 & & & & & & 0.0308 & 0.9996 \\
\hline WVUTH18 & & & & & & & & & 0.0519 & & & & 0.0060 & & & & & & 0.0303 & 0.9996 \\
\hline WVUTH19 & & & & & & & & & 0.0345 & & & & 0.0060 & & & & & & 0.0303 & 0.9997 \\
\hline WVUTH21 & & & & & & & & & 0.0359 & & & & 0.0061 & & & & & & 0.0303 & 0.9994 \\
\hline WVUTH22 & & & & & & & & & 0.0345 & & & & 0.0060 & & & & & & 0.0303 & 0.9996 \\
\hline WVUTH23 & & & & & & & & & 0.0339 & & & & 0.0059 & & & & & & 0.0298 & 1.0159 \\
\hline WVUTH28 & & & & & & & & & 0.0352 & & & & 0.0058 & & & & & & 0.0274 & 1.0001 \\
\hline WVUTH30 & & & & & & & & & 0.0350 & & & & 0.0119 & & & & & & 0.0307 & 0.9997 \\
\hline WVUTH31 & & & & & & & & & 0.0351 & & & & 0.0058 & & & & & & 0.0271 & 1.0001 \\
\hline WVUTH32 & & & & & & & & & 0.0359 & & & & 0.0061 & & & & & & 0.0301 & 0.9995 \\
\hline WVUTH34 & & & & & & & & & 0.0355 & & & & 0.0058 & & & & & & 0.0207 & 1.0000 \\
\hline WVUTH35 & & & & & & & & & 0.0355 & & & & 0.0058 & & & & & & 0.0288 & 1.0001 \\
\hline WVUTH36 & & & & & & & & & 0.0349 & & & & 0.0000 & & & & & & 0.0300 & 0.9995 \\
\hline WVUTH37 & & & & & & & & & 0.0178 & & & & 0.0000 & & & & & & 0.0306 & 0.9998 \\
\hline WVUTH38 & & & & & & & & & 0.0000 & & & & 0.0000 & & & & & & 0.0652 & 0.9996 \\
\hline WVUTH39 & & & & & & & & & 0.0000 & & & & 0.0000 & & & & & & 0.0475 & 0.9996 \\
\hline WVUTH40 & & & & & & & & & 0.0352 & & & & 0.0058 & & & & & & 0.0290 & 0.9998 \\
\hline WVUTH41 & & & & & & & & & 0.0352 & & & & 0.0058 & & & & & & 0.0289 & 0.9997 \\
\hline WVUTH42 & & & & & & & & & 0.0349 & & & & 0.0057 & & & & & & 0.0283 & 1.0001 \\
\hline WVUTH48 & & & & & & & & & 0.0352 & & & & 0.0058 & & & & & & 0.0290 & 0.9998 \\
\hline WVUTH49 & & & & & & & & & 0.0352 & & & & 0.0058 & & & & & & 0.0689 & 0.9997 \\
\hline WVUTH51 & & & & & & & & & 0.0352 & & & & 0.0058 & & & & & & 0.0285 & 1.0000 \\
\hline WVUTH52 & & & & & & & & & 0.0352 & & & & 0.0058 & & & & & & 0.0285 & 1.0000 \\
\hline WVUTH53 & & & & & & & & & 0.0351 & & & & 0.0060 & & & & & & 0.0205 & 0.9999 \\
\hline WVUTH54 & & & & & & & & & 0.0352 & & & & 0.0060 & & & & & & 0.0288 & 0.9999 \\
\hline WVUTH55 & & & & & & & & & 0.0358 & & & & 0.0061 & & & & & & 0.0292 & 0.9998 \\
\hline WVUTH56 & & & & & & & & & 0.0355 & & & & 0.0060 & & & & & & 0.0290 & 0.9998 \\
\hline WVUTH57 & & & & & & & & & 0.0349 & & & & 0.0059 & & & & & & 0.0285 & 1.0114 \\
\hline WVUTH58 & & & & & & & & & 0.0351 & & & & 0.0061 & & & & & & 0.0327 & 0.9993 \\
\hline WVUTH59 & & & & & & & & & 0.0345 & & & & 0.0060 & & & & & & 0.0303 & 0.9997 \\
\hline WVUTH61 & & & & & & & & & 0.0344 & & & & 0.0060 & & & & & & 0.0302 & 0.9996 \\
\hline WVUTH62 & & & & & & & & & 0.0356 & & & & 0.0058 & & & & & & 0.0304 & 1.0064 \\
\hline WVUTH63 & & & & & & & & & 0.0356 & & & & 0.0058 & & & & & & 0.0304 & 1.0064 \\
\hline WVUTH64 & & & & & & & & & 0.0356 & & & & 0.0058 & & & & & & 0.0304 & 1.0064 \\
\hline
\end{tabular}


Appendix A. Database - mass fraction

\begin{tabular}{|c|c|c|c|c|c|c|c|c|}
\hline Glass ID & $\begin{array}{l}\mathrm{TM} \\
\left({ }^{\circ} \mathrm{C}\right) \\
\end{array}$ & $\begin{array}{l}\text { Gradient } \\
\text { TL }\left({ }^{\circ} \mathrm{C}\right) \\
\end{array}$ & $\begin{array}{l}\text { Uniform } \\
\text { TL }\left({ }^{\circ} \mathrm{C}\right) \\
\end{array}$ & Primary Phase & $\begin{array}{l}\text { Quenched } \\
\text { Visual/OM }\end{array}$ & $\begin{array}{c}\text { Quenched } \\
\text { SEM/EDS or TEM }\end{array}$ & $\begin{array}{l}\text { Quenched } \\
\text { XRD }\end{array}$ & $\begin{array}{c}\text { Quenched } \\
\text { Homogeneous? }\end{array}$ \\
\hline WVCM46 & & & & & & & & \\
\hline WVCM47 & & & & & & & & \\
\hline WVCM48 & & & & & & & & \\
\hline WVCM50 & & & & & & & & \\
\hline WVCM53 & & & & & & & & \\
\hline WVCM55 & & & & & & & & \\
\hline WVCM56 & & & & & & & & \\
\hline WVCM57 & & & & & & & & \\
\hline WVCM59 & & & & & & & & \\
\hline WVUTH7 & & & & & & & & \\
\hline WVUTH8 & & & & & & & & \\
\hline WVUTH12 & & & & & & & & \\
\hline WVUTH13 & & & & & & & & \\
\hline WVUTH14 & & & & & & & & \\
\hline WVUTH15 & & & & & & & & \\
\hline WVUTH17 & & & & & & & & \\
\hline WVUTH18 & & & & & & & & \\
\hline WVUTH19 & & & & & & & & \\
\hline WVUTH21 & & & & & & & & \\
\hline WVUTH22 & & & & & & & & \\
\hline WVUTH23 & & & & & & & & \\
\hline WVUTH28 & & & & & & & & \\
\hline WVUTH30 & & & & & & & & \\
\hline WVUTH31 & & & & & & & & \\
\hline WVUTH32 & & & & & & & & \\
\hline WVUTH34 & & & & & & & & \\
\hline WVUTH35 & & & & & & & & \\
\hline WVUTH36 & & & & & & & & \\
\hline WVUTH37 & & & & & & & & \\
\hline WVUTH38 & & & & & & & & \\
\hline WVUTH39 & & & & & & & & \\
\hline WVUTH40 & & & & & & & & \\
\hline WVUTH41 & & & & & & & & \\
\hline WVUTH42 & & & & & & & & \\
\hline WVUTH48 & & & & & & & & \\
\hline WVUTH49 & & & & & & & & \\
\hline WVUTH51 & & & & & & & & \\
\hline WVUTH52 & & & & & & & & \\
\hline WVUTH53 & & & & & & & & \\
\hline WVUTH54 & & & & & & & & \\
\hline WVUTH55 & & & & & & & & \\
\hline WVUTH56 & & & & & & & & \\
\hline WVUTH57 & & & & & & & & \\
\hline WVUTH58 & & & & & & & & \\
\hline WVUTH59 & & & & & & & & \\
\hline WVUTH61 & & & & & & & & \\
\hline WVUTH62 & & & & & & & & \\
\hline WVUTH63 & & & & & & & & \\
\hline WVUTH64 & & & & & & & & \\
\hline
\end{tabular}


Appendix A. Database - mass fraction

\section{WV Glasses by CUA and PNL (Johnston et al. 1990)}

\begin{tabular}{|c|c|c|c|c|c|}
\hline Glass ID & $\begin{array}{c}\mathrm{CCC} \\
\text { Visual/OM }\end{array}$ & $\begin{array}{c}\text { CCC } \\
\text { SEM/EDS or TEM }\end{array}$ & $\begin{array}{l}\text { CCC } \\
\text { XRD }\end{array}$ & $\begin{array}{l}\text { Heat Treated } \\
\text { Visual/OM }\end{array}$ & $\begin{array}{c}\text { Heat Treated } \\
\text { SEM/EDS or TEM }\end{array}$ \\
\hline \multicolumn{6}{|l|}{ WVCM46 } \\
\hline \multicolumn{6}{|l|}{ WVCM47 } \\
\hline \multicolumn{6}{|l|}{ WVCM48 } \\
\hline \multicolumn{6}{|l|}{ WVCM50 } \\
\hline \multicolumn{6}{|l|}{ WVCM53 } \\
\hline \multicolumn{6}{|l|}{ WVCM55 } \\
\hline \multicolumn{6}{|l|}{ WVCM56 } \\
\hline \multicolumn{6}{|l|}{ WVCM57 } \\
\hline \multicolumn{6}{|l|}{ WVCM59 } \\
\hline \multicolumn{6}{|l|}{ WVUTH7 } \\
\hline \multicolumn{6}{|l|}{ WVUTH8 } \\
\hline \multicolumn{6}{|l|}{ WVUTH12 } \\
\hline \multicolumn{6}{|l|}{ WVUTH13 } \\
\hline \multicolumn{6}{|l|}{ WVUTH14 } \\
\hline \multicolumn{6}{|l|}{ WVUTH15 } \\
\hline \multicolumn{6}{|l|}{ WVUTH17 } \\
\hline \multicolumn{6}{|l|}{ WVUTH18 } \\
\hline \multicolumn{6}{|l|}{ WVUTH19 } \\
\hline \multicolumn{6}{|l|}{ WVUTH21 } \\
\hline \multicolumn{6}{|l|}{ WVUTH22 } \\
\hline \multicolumn{6}{|l|}{ WVUTH 23} \\
\hline WVUTH28 & & & & & \\
\hline WVUTH30 & & & & & \\
\hline WVUTH31 & & & & & \\
\hline WVUTH32 & & & & & \\
\hline WVUTH34 & & & & & \\
\hline WVUTH35 & & & & & \\
\hline WVUTH36 & & & & & \\
\hline WVUTH37 & & & & & \\
\hline WVUTH38 & & & & & \\
\hline WVUTH39 & & & & & \\
\hline WVUTH40 & & & & & \\
\hline WVUTH41 & & & & & \\
\hline WVUTH42 & & & & & \\
\hline WVUTH48 & & & & & \\
\hline WVUTH49 & & & & & \\
\hline WVUTH51 & & & & & \\
\hline WVUTH52 & & & & & \\
\hline WVUTH53 & & & & & \\
\hline WVUTH54 & & & & & \\
\hline WVUTH55 & & & & & \\
\hline WVUTH56 & & & & & \\
\hline WVUTH57 & & & & & \\
\hline WVUTH58 & & & & & \\
\hline WVUTH59 & & & & & \\
\hline WVUTH61 & & & & & \\
\hline WVUTH62 & & & & & \\
\hline WVUTH63 & & & & & \\
\hline WVUTH64 & & & & & \\
\hline
\end{tabular}


Appendix A. Database - mass fraction

WV Glasses by CUA and PNL (Johnston et al. 1990)

\begin{tabular}{|c|c|c|c|c|c|c|c|c|c|c|c|c|c|c|c|}
\hline Glass ID & $\begin{array}{c}\text { Heat Treated } \\
\text { XRD }\end{array}$ & $\begin{array}{l}\text { Density } \\
\left(\mathrm{g} / \mathrm{cm}^{3}\right)\end{array}$ & $\begin{array}{c}\text { Fulc Visc } \\
\mathrm{A}\end{array}$ & $\begin{array}{c}\text { Fulc Visc } \\
\text { B } \\
\end{array}$ & $\begin{array}{c}\text { Fulc Visc } \\
\text { To } \\
\end{array}$ & $\begin{array}{c}\mathrm{FV} 1150^{\circ} \mathrm{C} \\
(\mathrm{Pa} \cdot \mathrm{s})\end{array}$ & $\begin{array}{c}\text { Arrh Visc } \\
\text { A }\end{array}$ & $\begin{array}{c}\text { Arrh Visc } \\
\text { B }\end{array}$ & $\begin{array}{c}\mathrm{Av} \\
1150^{\circ} \mathrm{C} \\
\left(\mathrm{D}_{0.0} \mathrm{C}\right)\end{array}$ & $\begin{array}{c}\mathrm{T}\left({ }^{\circ} \mathrm{C}\right) \text { at } \\
2 \mathrm{~Pa} \cdot \mathrm{s} \\
\end{array}$ & $\begin{array}{c}\mathrm{T}\left({ }^{\circ} \mathrm{C}\right) \text { at } \\
5 \mathrm{~Pa} \cdot \mathrm{s}\end{array}$ & $\begin{array}{c}\mathrm{T}\left({ }^{\circ} \mathrm{C}\right) \text { at } \\
10 \mathrm{~Pa} \cdot \mathrm{s} \\
\end{array}$ & $\begin{array}{c}\mathrm{T} 1 \\
\left({ }^{\circ} \mathrm{C}\right) \\
\end{array}$ & $\begin{array}{c}\mathrm{V} 1 \\
(\mathrm{~Pa} \cdot \mathrm{s}) \\
\end{array}$ & $\begin{array}{c}\mathrm{T} 2 \\
\left({ }^{\circ} \mathrm{C}\right) \\
\end{array}$ \\
\hline WVCM46 & & & & & & & & & & & & & & & \\
\hline WVCM47 & & & & & & & & & & & & & & & \\
\hline WVCM48 & & & & & & & & & & & & & & & \\
\hline WVCM50 & & & & & & & & & & & & & & & \\
\hline WVCM53 & & & & & & & & & & & & & & & \\
\hline WVCM55 & & & & & & & & & & & & & & & \\
\hline WVCM56 & & & & & & & & & & & & & & & \\
\hline WVCM57 & & & & & & & & & & & & & & & \\
\hline WVCM59 & & & & & & & & & & & & & & & \\
\hline WVUTH7 & & & & & & & & & & & & & & & \\
\hline WVUTH8 & & & & & & & & & & & & & & & \\
\hline WVUTH12 & & & & & & & & & & & & & & & \\
\hline WVUTH13 & & & & & & & & & & & & & & & \\
\hline WVUTH14 & & & & & & & & & & & & & & & \\
\hline WVUTH15 & & & & & & & & & & & & & & & \\
\hline WVUTH17 & & & & & & & & & & & & & & & \\
\hline WVUTH18 & & & & & & & & & & & & & & & \\
\hline WVUTH19 & & & & & & & & & & & & & & & \\
\hline WVUTH21 & & & & & & & & & & & & & & & \\
\hline WVUTH22 & & & & & & & & & & & & & & & \\
\hline WVUTH23 & & & & & & & & & & & & & & & \\
\hline WVUTH28 & & & & & & & & & & & & & & & \\
\hline WVUTH30 & & & & & & & & & & & & & & & \\
\hline WVUTH31 & & & & & & & & & & & & & & & \\
\hline WVUTH32 & & & & & & & & & & & & & & & \\
\hline WVUTH34 & & & & & & & & & & & & & & & \\
\hline WVUTH35 & & & & & & & & & & & & & & & \\
\hline WVUTH36 & & & & & & & & & & & & & & & \\
\hline WVUTH37 & & & & & & & & & & & & & & & \\
\hline WVUTH38 & & & & & & & & & & & & & & & \\
\hline WVUTH39 & & & & & & & & & & & & & & & \\
\hline WVUTH40 & & & & & & & & & & & & & & & \\
\hline WVUTH41 & & & & & & & & & & & & & & & \\
\hline WVUTH42 & & & & & & & & & & & & & & & \\
\hline WVUTH48 & & & & & & & & & & & & & & & \\
\hline WVUTH49 & & & & & & & & & & & & & & & \\
\hline WVUTH51 & & & & & & & & & & & & & & & \\
\hline WVUTH52 & & & & & & & & & & & & & & & \\
\hline WVUTH53 & & & & & & & & & & & & & & & \\
\hline WVUTH54 & & & & & & & & & & & & & & & \\
\hline WVUTH55 & & & & & & & & & & & & & & & \\
\hline WVUTH56 & & & & & & & & & & & & & & & \\
\hline WVUTH57 & & & & & & & & & & & & & & & \\
\hline WVUTH58 & & & & & & & & & & & & & & & \\
\hline WVUTH59 & & & & & & & & & & & & & & & \\
\hline WVUTH61 & & & & & & & & & & & & & & & \\
\hline WVUTH62 & & & & & & & & & & & & & & & \\
\hline WVUTH63 & & & & & & & & & & & & & & & \\
\hline WVUTH64 & & & & & & & & & & & & & & & \\
\hline
\end{tabular}


Appendix A. Database - mass fraction

WV Glasses by CUA and PNL (Johnston et al. 1990)

\begin{tabular}{|c|c|c|c|c|c|c|c|c|c|c|c|c|c|c|c|c|c|c|c|c|c|}
\hline Glass ID & $\begin{array}{c}\mathrm{V} 2 \\
(\mathrm{~Pa} \cdot \mathrm{s})\end{array}$ & $\begin{array}{c}\mathrm{T} 3 \\
\left({ }^{\circ} \mathrm{C}\right)\end{array}$ & $\begin{array}{c}\mathrm{V} 3 \\
(\mathrm{~Pa} \cdot \mathrm{s})\end{array}$ & $\begin{array}{c}\mathrm{T} 4 \\
\left({ }^{\circ} \mathrm{C}\right)\end{array}$ & $\begin{array}{c}\mathrm{V} 4 \\
(\mathrm{~Pa} \cdot \mathrm{s})\end{array}$ & $\begin{array}{c}\mathrm{T} 5 \\
\left({ }^{\circ} \mathrm{C}\right)\end{array}$ & $\begin{array}{c}\mathrm{V} 5 \\
(\mathrm{~Pa} \cdot \mathrm{s})\end{array}$ & $\begin{array}{c}\text { T6 } \\
\left({ }^{\circ} \mathrm{C}\right)\end{array}$ & $\begin{array}{c}\mathrm{V} 6 \\
(\mathrm{~Pa} \cdot \mathrm{s})\end{array}$ & $\begin{array}{c}\mathrm{T} 7 \\
\left({ }^{\circ} \mathrm{C}\right)\end{array}$ & $\begin{array}{c}\mathrm{V7} \\
(\mathrm{Pa} \cdot \mathrm{s})\end{array}$ & $\begin{array}{c}\mathrm{T} 8 \\
\left({ }^{\circ} \mathrm{C}\right)\end{array}$ & $\begin{array}{c}\mathrm{V} 8 \\
(\mathrm{~Pa} \cdot \mathrm{s})\end{array}$ & $\begin{array}{c}\mathrm{T} 9 \\
\left({ }^{\circ} \mathrm{C}\right)\end{array}$ & $\begin{array}{c}\mathrm{V} 9 \\
(\mathrm{~Pa} \cdot \mathrm{s})\end{array}$ & $\begin{array}{l}\mathrm{T} 10 \\
\left({ }^{\circ} \mathrm{C}\right)\end{array}$ & $\begin{array}{c}\mathrm{V} 10 \\
(\mathrm{~Pa} \cdot \mathrm{s})\end{array}$ & $\begin{array}{l}\mathrm{T} 11 \\
\left({ }^{\circ} \mathrm{C}\right)\end{array}$ & $\begin{array}{c}\mathrm{V} 11 \\
(\mathrm{~Pa} \cdot \mathrm{s})\end{array}$ & $\begin{array}{l}\mathrm{T} 12 \\
\left({ }^{\circ} \mathrm{C}\right)\end{array}$ & $\begin{array}{c}\mathrm{V} 12 \\
(\mathrm{~Pa} \cdot \mathrm{s})\end{array}$ \\
\hline \multicolumn{22}{|l|}{ WVCM46 } \\
\hline \multicolumn{22}{|l|}{ WVCM47 } \\
\hline \multicolumn{22}{|l|}{ WVCM48 } \\
\hline \multicolumn{22}{|l|}{ WVCM50 } \\
\hline WVCM53 & & & & & & & & & & & & & & & & & & & & & \\
\hline \multicolumn{22}{|l|}{ WVCM55 } \\
\hline \multicolumn{22}{|l|}{ WVCM56 } \\
\hline \multicolumn{22}{|l|}{ WVCM57 } \\
\hline \multicolumn{22}{|l|}{ WVCM59 } \\
\hline \multicolumn{22}{|l|}{ WVUTH7 } \\
\hline \multirow{2}{*}{\multicolumn{22}{|c|}{$\begin{array}{l}\text { WVUTH8 } \\
\text { WVUTH12 }\end{array}$}} \\
\hline & & & & & & & & & & & & & & & & & & & & & \\
\hline \multicolumn{22}{|l|}{ WVUTH13 } \\
\hline \multicolumn{22}{|l|}{ WVUTH14 } \\
\hline \multicolumn{22}{|l|}{ WVUTH15 } \\
\hline WVUTH17 & & & & & & & & & & & & & & & & & & & & & \\
\hline WVUTH18 & & & & & & & & & & & & & & & & & & & & & \\
\hline WVUTH19 & & & & & & & & & & & & & & & & & & & & & \\
\hline WVUTH21 & & & & & & & & & & & & & & & & & & & & & \\
\hline WVUTH22 & & & & & & & & & & & & & & & & & & & & & \\
\hline WVUTH23 & & & & & & & & & & & & & & & & & & & & & \\
\hline WVUTH 28 & & & & & & & & & & & & & & & & & & & & & \\
\hline WVUTH30 & & & & & & & & & & & & & & & & & & & & & \\
\hline WVUTH31 & & & & & & & & & & & & & & & & & & & & & \\
\hline WVUTH32 & & & & & & & & & & & & & & & & & & & & & \\
\hline WVUTH34 & & & & & & & & & & & & & & & & & & & & & \\
\hline WVUTH35 & & & & & & & & & & & & & & & & & & & & & \\
\hline WVUTH36 & & & & & & & & & & & & & & & & & & & & & \\
\hline WVUTH37 & & & & & & & & & & & & & & & & & & & & & \\
\hline WVUTH38 & & & & & & & & & & & & & & & & & & & & & \\
\hline WVUTH39 & & & & & & & & & & & & & & & & & & & & & \\
\hline WVUTH40 & & & & & & & & & & & & & & & & & & & & & \\
\hline WVUTH41 & & & & & & & & & & & & & & & & & & & & & \\
\hline WVUTH42 & & & & & & & & & & & & & & & & & & & & & \\
\hline WVUTH48 & & & & & & & & & & & & & & & & & & & & & \\
\hline WVUTH49 & & & & & & & & & & & & & & & & & & & & & \\
\hline WVUTH51 & & & & & & & & & & & & & & & & & & & & & \\
\hline WVUTH52 & & & & & & & & & & & & & & & & & & & & & \\
\hline WVUTH53 & & & & & & & & & & & & & & & & & & & & & \\
\hline WVUTH54 & & & & & & & & & & & & & & & & & & & & & \\
\hline WVUTH55 & & & & & & & & & & & & & & & & & & & & & \\
\hline WVUTH56 & & & & & & & & & & & & & & & & & & & & & \\
\hline WVUTH57 & & & & & & & & & & & & & & & & & & & & & \\
\hline WVUTH58 & & & & & & & & & & & & & & & & & & & & & \\
\hline WVUTH59 & & & & & & & & & & & & & & & & & & & & & \\
\hline WVUTH61 & & & & & & & & & & & & & & & & & & & & & \\
\hline WVUTH62 & & & & & & & & & & & & & & & & & & & & & \\
\hline WVUTH63 & & & & & & & & & & & & & & & & & & & & & \\
\hline WVUTH64 & & & & & & & & & & & & & & & & & & & & & \\
\hline
\end{tabular}


Appendix A. Database - mass fraction

WV Glasses by CUA and PNL (Johnston et al. 1990)

\begin{tabular}{|c|c|c|c|c|c|c|c|c|c|c|c|c|c|c|c|c|}
\hline Glass ID & $\begin{array}{l}\mathrm{T} 13 \\
\left({ }^{\circ} \mathrm{C}\right)\end{array}$ & $\begin{array}{c}\mathrm{V} 13 \\
(\mathrm{~Pa} \cdot \mathrm{s})\end{array}$ & $\begin{array}{l}\mathrm{T} 14 \\
\left({ }^{\circ} \mathrm{C}\right)\end{array}$ & $\begin{array}{c}\mathrm{V} 14 \\
(\mathrm{~Pa} \cdot \mathrm{s})\end{array}$ & $\begin{array}{c}\text { Q PCT } \\
\mathrm{B}\left(\mathrm{g} / \mathrm{m}^{2}\right)\end{array}$ & $\begin{array}{c}\text { Q PCT } \\
\mathrm{Li}\left(\mathrm{g} / \mathrm{m}^{2}\right)\end{array}$ & $\begin{array}{c}\text { Q PCT } \\
\mathrm{Na}\left(\mathrm{g} / \mathrm{m}^{2}\right)\end{array}$ & $\begin{array}{c}\text { Q PCT } \\
\mathrm{Si}\left(\mathrm{g} / \mathrm{m}^{2}\right)\end{array}$ & $\begin{array}{l}\text { Q PCT } \\
\text { pH }\end{array}$ & $\begin{array}{c}\text { CCC PCT } \\
\text { B }\left(\mathrm{g} / \mathrm{m}^{2}\right)\end{array}$ & $\begin{array}{l}\text { CCC PCT } \\
\mathrm{Li}\left(\mathrm{g} / \mathrm{m}^{2}\right)\end{array}$ & $\begin{array}{l}\text { CCC PCT } \\
\mathrm{Na}\left(\mathrm{g} / \mathrm{m}^{2}\right)\end{array}$ & \begin{tabular}{|c|} 
CCC PCT \\
$\mathrm{Si}\left(\mathrm{g} / \mathrm{m}^{2}\right)$
\end{tabular} & $\begin{array}{c}\mathrm{CCC} \text { PCT } \\
\mathrm{pH}\end{array}$ & $\begin{array}{l}\text { Q PCT B at } \\
20^{\circ} \mathrm{C}\left(\mathrm{g} / \mathrm{m}^{2}\right)\end{array}$ & $\begin{array}{l}\text { Q PCT Li at } \\
20^{\circ} \mathrm{C}\left(\mathrm{g} / \mathrm{m}^{2}\right)\end{array}$ \\
\hline WVCM46 & & & & & 0.146 & & & & & & & & & & & \\
\hline WVCM47 & & & & & 0.163 & & & & & & & & & & & \\
\hline WVCM48 & & & & & 0.282 & & & & & & & & & & & \\
\hline WVCM50 & & & & & 0.206 & & & & & & & & & & & \\
\hline WVCM53 & & & & & 0.268 & & & & & & & & & & & \\
\hline WVCM55 & & & & & 0.286 & & & & & & & & & & & \\
\hline WVCM56 & & & & & 0.189 & & & & & & & & & & & \\
\hline WVCM57 & & & & & 0.334 & & & & & & & & & & & \\
\hline WVCM59 & & & & & 0.622 & & & & & & & & & & & \\
\hline WVUTH7 & & & & & 0.249 & & & & & & & & & & & \\
\hline WVUTH8 & & & & & 0.349 & & & & & & & & & & & \\
\hline WVUTH12 & & & & & 0.215 & & & & & & & & & & & \\
\hline WVUTH13 & & & & & 0.238 & & & & & & & & & & & \\
\hline WVUTH14 & & & & & 0.25 & & & & & & & & & & & \\
\hline WVUTH15 & & & & & 0.201 & & & & & & & & & & & \\
\hline WVUTH17 & & & & & 0.174 & & & & & & & & & & & \\
\hline WVUTH18 & & & & & 0.194 & & & & & & & & & & & \\
\hline WVUTH19 & & & & & 0.268 & & & & & & & & & & & \\
\hline WVUTH21 & & & & & 0.236 & & & & & & & & & & & \\
\hline WVUTH22 & & & & & 0.243 & & & & & & & & & & & \\
\hline WVUTH23 & & & & & 0.206 & & & & & & & & & & & \\
\hline WVUTH 28 & & & & & 0.213 & & & & & & & & & & & \\
\hline WVUTH30 & & & & & 0.216 & & & & & & & & & & & \\
\hline WVUTH31 & & & & & 0.234 & & & & & & & & & & & \\
\hline WVUTH32 & & & & & 0.416 & & & & & & & & & & & \\
\hline WVUTH34 & & & & & 0.251 & & & & & & & & & & & \\
\hline WVUTH35 & & & & & 0.221 & & & & & & & & & & & \\
\hline WVUTH36 & & & & & 0.223 & & & & & & & & & & & \\
\hline WVUTH37 & & & & & 0.298 & & & & & & & & & & & \\
\hline WVUTH38 & & & & & 0.231 & & & & & & & & & & & \\
\hline WVUTH39 & & & & & 0.211 & & & & & & & & & & & \\
\hline WVUTH40 & & & & & 0.227 & & & & & & & & & & & \\
\hline WVUTH41 & & & & & 0.222 & & & & & & & & & & & \\
\hline WVUTH42 & & & & & 0.228 & & & & & & & & & & & \\
\hline WVUTH48 & & & & & 0.208 & & & & & & & & & & & \\
\hline WVUTH49 & & & & & 0.177 & & & & & & & & & & & \\
\hline WVUTH51 & & & & & 0.23 & & & & & & & & & & & \\
\hline WVUTH52 & & & & & 0.244 & & & & & & & & & & & \\
\hline WVUTH53 & & & & & 0.244 & & & & & & & & & & & \\
\hline WVUTH54 & & & & & 0.168 & & & & & & & & & & & \\
\hline WVUTH55 & & & & & 0.285 & & & & & & & & & & & \\
\hline WVUTH56 & & & & & 0.197 & & & & & & & & & & & \\
\hline WVUTH57 & & & & & 0.25 & & & & & & & & & & & \\
\hline WVUTH58 & & & & & 0.201 & & & & & & & & & & & \\
\hline WVUTH59 & & & & & 0.208 & & & & & & & & & & & \\
\hline WVUTH61 & & & & & 0.201 & & & & & & & & & & & \\
\hline WVUTH62 & & & & & 0.926 & & & & & & & & & & & \\
\hline WVUTH63 & & & & & 0.408 & & & & & & & & & & & \\
\hline WVUTH64 & & & & & 0.469 & & & & & & & & & & & \\
\hline
\end{tabular}


Appendix A. Database - mass fraction

WV Glasses by CUA and PNL (Johnston et al. 1990)

\begin{tabular}{|c|c|c|c|c|c|c|c|c|c|c|c|c|}
\hline Glass ID & $\begin{array}{l}\text { Q PCT Na at } \\
20^{\circ} \mathrm{C}\left(\mathrm{g} / \mathrm{m}^{2}\right)\end{array}$ & $\begin{array}{l}\text { Q PCT Si at } \\
20^{\circ} \mathrm{C}\left(\mathrm{g} / \mathrm{m}^{2}\right)\end{array}$ & $\begin{array}{c}\mathrm{QpH} \\
\text { at } 20^{\circ} \mathrm{C}\end{array}$ & $\begin{array}{l}\text { TCLP Ag } \\
(\mathrm{ppm})\end{array}$ & $\begin{array}{c}\text { TCLP As } \\
(\mathrm{ppm})\end{array}$ & $\begin{array}{c}\text { TCLP Ba } \\
(\mathrm{ppm})\end{array}$ & $\begin{array}{c}\text { TCLP Cd } \\
(\mathrm{ppm})\end{array}$ & $\begin{array}{c}\text { TCLP Cr } \\
(\mathrm{ppm})\end{array}$ & $\begin{array}{l}\text { TCLP Ni } \\
(\mathrm{ppm})\end{array}$ & $\begin{array}{c}\text { TCLP Pb } \\
(\mathrm{ppm})\end{array}$ & $\begin{array}{c}\text { TCLP Se } \\
(\mathrm{ppm})\end{array}$ & $\begin{array}{c}\text { TCLP Zn } \\
(\mathrm{ppm})\end{array}$ \\
\hline WVCM46 & & & & & & & & & & & & \\
\hline WVCM47 & & & & & & & & & & & & \\
\hline WVCM48 & & & & & & & & & & & & \\
\hline WVCM50 & & & & & & & & & & & & \\
\hline WVCM53 & & & & & & & & & & & & \\
\hline WVCM55 & & & & & & & & & & & & \\
\hline WVCM56 & & & & & & & & & & & & \\
\hline WVCM57 & & & & & & & & & & & & \\
\hline WVCM59 & & & & & & & & & & & & \\
\hline WVUTH7 & & & & & & & & & & & & \\
\hline WVUTH8 & & & & & & & & & & & & \\
\hline WVUTH12 & & & & & & & & & & & & \\
\hline WVUTH13 & & & & & & & & & & & & \\
\hline WVUTH14 & & & & & & & & & & & & \\
\hline WVUTH15 & & & & & & & & & & & & \\
\hline WVUTH17 & & & & & & & & & & & & \\
\hline WVUTH18 & & & & & & & & & & & & \\
\hline WVUTH19 & & & & & & & & & & & & \\
\hline WVUTH21 & & & & & & & & & & & & \\
\hline WVUTH22 & & & & & & & & & & & & \\
\hline WVUTH 23 & & & & & & & & & & & & \\
\hline WVUTH28 & & & & & & & & & & & & \\
\hline WVUTH30 & & & & & & & & & & & & \\
\hline WVUTH31 & & & & & & & & & & & & \\
\hline WVUTH32 & & & & & & & & & & & & \\
\hline WVUTH34 & & & & & & & & & & & & \\
\hline WVUTH35 & & & & & & & & & & & & \\
\hline WVUTH36 & & & & & & & & & & & & \\
\hline WVUTH37 & & & & & & & & & & & & \\
\hline WVUTH38 & & & & & & & & & & & & \\
\hline WVUTH39 & & & & & & & & & & & & \\
\hline WVUTH40 & & & & & & & & & & & & \\
\hline WVUTH41 & & & & & & & & & & & & \\
\hline WVUTH42 & & & & & & & & & & & & \\
\hline WVUTH48 & & & & & & & & & & & & \\
\hline WVUTH49 & & & & & & & & & & & & \\
\hline WVUTH51 & & & & & & & & & & & & \\
\hline WVUTH52 & & & & & & & & & & & & \\
\hline WVUTH53 & & & & & & & & & & & & \\
\hline WVUTH54 & & & & & & & & & & & & \\
\hline WVUTH55 & & & & & & & & & & & & \\
\hline WVUTH56 & & & & & & & & & & & & \\
\hline WVUTH57 & & & & & & & & & & & & \\
\hline WVUTH58 & & & & & & & & & & & & \\
\hline WVUTH59 & & & & & & & & & & & & \\
\hline WVUTH61 & & & & & & & & & & & & \\
\hline WVUTH62 & & & & & & & & & & & & \\
\hline WVUTH63 & & & & & & & & & & & & \\
\hline WVUTH64 & & & & & & & & & & & & \\
\hline
\end{tabular}


Appendix A. Database - mass fraction

WV Glasses by CUA and PNL (Johnston et al. 1990)

\begin{tabular}{|c|c|c|c|c|c|c|c|c|c|c|c|c|c|c|c|c|c|c|c|c|c|}
\hline Glass ID & $\begin{array}{c}\mathrm{A} 12 \mathrm{O} 3 \\
-\mathrm{t}\end{array}$ & $\begin{array}{c}\mathrm{B} 2 \mathrm{O} 3 \\
-\mathrm{t}\end{array}$ & $\begin{array}{c}\mathrm{CaO} \\
-\mathrm{t}\end{array}$ & $\begin{array}{c}\mathrm{Fe} 2 \mathrm{O} 3 \\
-\mathrm{t}\end{array}$ & $\begin{array}{c}\mathrm{FeO} \\
-\mathrm{t}\end{array}$ & $\begin{array}{c}\mathrm{K} 2 \mathrm{O} \\
-\mathrm{t}\end{array}$ & $\begin{array}{c}\mathrm{Li} 2 \mathrm{O} \\
-\mathrm{t}\end{array}$ & $\begin{array}{c}\mathrm{MgO} \\
-\mathrm{t}\end{array}$ & $\begin{array}{c}\mathrm{Na} 2 \mathrm{O} \\
-\mathrm{t}\end{array}$ & $\begin{array}{c}\mathrm{NiO} \\
-\mathrm{t}\end{array}$ & $\begin{array}{c}\mathrm{P} 2 \mathrm{O} 5 \\
-\mathrm{t}\end{array}$ & $\begin{array}{c}\mathrm{SiO} 2 \\
-\mathrm{t}\end{array}$ & $\begin{array}{c}\mathrm{ZrO} 2 \\
-\mathrm{t}\end{array}$ & $\begin{array}{c}\mathrm{Ag} 2 \mathrm{O} \\
-\mathrm{t}\end{array}$ & $\begin{array}{c}\mathrm{As} 2 \mathrm{O} 3 \\
-\mathrm{t}\end{array}$ & $\begin{array}{c}\mathrm{BaO} \\
-\mathrm{t}\end{array}$ & $\begin{array}{c}\mathrm{Bi} 2 \mathrm{O} 3 \\
-\mathrm{t}\end{array}$ & $\begin{array}{l}\mathrm{Br} \\
-\mathrm{t}\end{array}$ & $\begin{array}{c}\mathrm{CdO} \\
-\mathrm{t}\end{array}$ & $\begin{array}{c}\mathrm{Ce} 2 \mathrm{O} 3 \\
-\mathrm{t}\end{array}$ & $\begin{array}{c}\mathrm{CeO} 2 \\
-\mathrm{t}\end{array}$ \\
\hline WVUTH6 & & & & & & & & & & & & & & & & & & & & & \\
\hline WVUTH6 & & & & & & & & & & & & & & & & & & & & & \\
\hline WVUTH6 & & & & & & & & & & & & & & & & & & & & & \\
\hline WVUTH6 & & & & & & & & & & & & & & & & & & & & & \\
\hline WVUTH6 & & & & & & & & & & & & & & & & & & & & & \\
\hline WVUTH7 & & & & & & & & & & & & & & & & & & & & & \\
\hline WVUTH7 & & & & & & & & & & & & & & & & & & & & & \\
\hline WVUTH7 & & & & & & & & & & & & & & & & & & & & & \\
\hline WVUTH7 & & & & & & & & & & & & & & & & & & & & & \\
\hline WVUTH7 & & & & & & & & & & & & & & & & & & & & & \\
\hline WVUTH7 & & & & & & & & & & & & & & & & & & & & & \\
\hline WVUTH7 & & & & & & & & & & & & & & & & & & & & & \\
\hline WVUTH7 & & & & & & & & & & & & & & & & & & & & & \\
\hline WVUTH7 & & & & & & & & & & & & & & & & & & & & & \\
\hline WVUTH8 & & & & & & & & & & & & & & & & & & & & & \\
\hline WVUTH8 & & & & & & & & & & & & & & & & & & & & & \\
\hline WVUTH8 & & & & & & & & & & & & & & & & & & & & & \\
\hline WVUTH8 & & & & & & & & & & & & & & & & & & & & & \\
\hline WVUTH8 & & & & & & & & & & & & & & & & & & & & & \\
\hline WVUTH8 & & & & & & & & & & & & & & & & & & & & & \\
\hline WVUTH9 & & & & & & & & & & & & & & & & & & & & & \\
\hline WVUTH9 & & & & & & & & & & & & & & & & & & & & & \\
\hline WVUTH9 & & & & & & & & & & & & & & & & & & & & & \\
\hline WVUTH9 & & & & & & & & & & & & & & & & & & & & & \\
\hline WVUTH9 & & & & & & & & & & & & & & & & & & & & & \\
\hline WVUTH9 & & & & & & & & & & & & & & & & & & & & & \\
\hline WVUTH9 & & & & & & & & & & & & & & & & & & & & & \\
\hline WVUTH9 & & & & & & & & & & & & & & & & & & & & & \\
\hline WVUTH10 & & & & & & & & & & & & & & & & & & & & & \\
\hline WVUTH10 & & & & & & & & & & & & & & & & & & & & & \\
\hline WVUTH10 & & & & & & & & & & & & & & & & & & & & & \\
\hline CUOD & & & & & & & & & & & & & & & & & & & & & \\
\hline CUOI & & & & & & & & & & & & & & & & & & & & & \\
\hline CUOJ & & & & & & & & & & & & & & & & & & & & & \\
\hline CUOK & & & & & & & & & & & & & & & & & & & & & \\
\hline CUOL & & & & & & & & & & & & & & & & & & & & & \\
\hline CUOB & 0.0670 & 0.0960 & 0.0060 & 0.1140 & & 0.0340 & 0.0300 & 0.0130 & 0.1070 & 0.0070 & 0.0240 & 0.4380 & 0.0300 & & & 0.0060 & & & & & 0.0020 \\
\hline CUOC & 0.0310 & 0.0930 & 0.0060 & 0.1110 & & 0.0330 & 0.0290 & 0.0120 & 0.1030 & 0.0070 & 0.0230 & 0.4240 & 0.0950 & & & 0.0060 & & & & & 0.0020 \\
\hline CUOE & 0.0320 & 0.0970 & 0.0060 & 0.1150 & & 0.0340 & 0.0300 & 0.0130 & 0.1070 & 0.0070 & 0.0240 & 0.4700 & 0.0300 & & & 0.0060 & & & & & 0.0020 \\
\hline CUOF & 0.0310 & 0.0940 & 0.0060 & 0.1120 & & 0.0330 & 0.0290 & 0.0120 & 0.1040 & 0.0070 & 0.0240 & 0.4850 & 0.0290 & & & 0.0060 & & & & & 0.0020 \\
\hline CUOG & 0.0320 & 0.0980 & 0.0060 & 0.1160 & & 0.0340 & 0.0300 & 0.0130 & 0.1080 & 0.0070 & 0.0250 & 0.4450 & 0.0500 & & & 0.0060 & & & & & 0.0020 \\
\hline $\mathrm{CUOH}$ & 0.0320 & 0.0970 & 0.0060 & 0.1400 & & 0.0340 & 0.0300 & 0.0130 & 0.1080 & 0.0070 & 0.0240 & 0.4430 & 0.0300 & & & 0.0060 & & & & & 0.0020 \\
\hline CUOO & 0.0330 & 0.1000 & 0.0060 & 0.1180 & & 0.0350 & 0.0310 & 0.0130 & 0.1100 & 0.0070 & 0.0250 & 0.4520 & 0.0310 & & & 0.0060 & & & & & 0.0020 \\
\hline CUOP & 0.0310 & 0.0940 & 0.0060 & 0.1120 & & 0.0330 & 0.0290 & 0.0120 & 0.1040 & 0.0070 & 0.0240 & 0.4850 & 0.0290 & & & 0.0060 & & & & & 0.0020 \\
\hline CUOR & 0.0320 & 0.0980 & 0.0060 & 0.1160 & & 0.0340 & 0.0300 & 0.0130 & 0.1080 & 0.0070 & 0.0250 & 0.4640 & 0.0300 & & & 0.0060 & & & & & 0.0020 \\
\hline CUOU & 0.0330 & 0.0990 & 0.0060 & 0.1170 & & 0.0350 & 0.0310 & 0.0130 & 0.1090 & 0.0070 & 0.0250 & 0.4490 & 0.0410 & & & 0.0060 & & & & & 0.0020 \\
\hline CUOV & 0.0320 & 0.0970 & 0.0060 & 0.1150 & & 0.0340 & 0.0300 & 0.0130 & 0.1070 & 0.0070 & 0.0240 & 0.4400 & 0.0590 & & & 0.0060 & & & & & 0.0020 \\
\hline CU30 & 0.0520 & 0.0980 & 0.0060 & 0.1160 & & 0.0340 & 0.0300 & 0.0130 & 0.1080 & 0.0070 & 0.0250 & 0.4450 & 0.0300 & & & 0.0060 & & & & & 0.0020 \\
\hline CU31 & 0.0610 & 0.0970 & 0.0060 & 0.1150 & & 0.0340 & 0.0300 & 0.0130 & 0.1070 & 0.0070 & 0.0240 & 0.4400 & 0.0300 & & & 0.0060 & & & & & 0.0020 \\
\hline
\end{tabular}


WV Glasses by CUA and PNL (Johnston et al. 1990)

\begin{tabular}{|c|c|c|c|c|c|c|c|c|c|c|c|c|c|c|c|c|c|c|c|c|c|}
\hline Glass ID & $\begin{array}{l}\mathrm{Cl} \\
-\mathrm{t}\end{array}$ & $\begin{array}{c}\mathrm{CoO} \\
-\mathrm{t}\end{array}$ & $\begin{array}{c}\mathrm{Co} 2 \mathrm{O} 3 \\
-\mathrm{t}\end{array}$ & $\begin{array}{c}\mathrm{Cr} 2 \mathrm{O} 3 \\
-\mathrm{t}\end{array}$ & $\begin{array}{c}\mathrm{Cs} 2 \mathrm{O} \\
-\mathrm{t}\end{array}$ & $\begin{array}{c}\mathrm{CuO} \\
-\mathrm{t}\end{array}$ & $\begin{array}{c}\mathrm{Eu} 2 \mathrm{O} 3 \\
-\mathrm{t}\end{array}$ & $\begin{array}{l}F \\
-t\end{array}$ & {$\left[\begin{array}{c}\mathrm{Ga} 2 \mathrm{O} 3 \\
-\mathrm{t}\end{array}\right.$} & $\begin{array}{c}\mathrm{Gd} 2 \mathrm{O} 3 \\
-\mathrm{t}\end{array}$ & $\begin{array}{c}\mathrm{HgO} \\
-\mathrm{t}\end{array}$ & $\begin{array}{l}I \\
-t\end{array}$ & $\begin{array}{c}\mathrm{La} 2 \mathrm{O} 3 \\
-\mathrm{t}\end{array}$ & $\begin{array}{c}\mathrm{MnO} 2 \\
-\mathrm{t}\end{array}$ & $\begin{array}{c}\mathrm{MnO} \\
-\mathrm{t}\end{array}$ & $\begin{array}{c}\mathrm{MoO} \\
-\mathrm{t}\end{array}$ & $\begin{array}{c}\mathrm{MoO} 3 \\
-\mathrm{t}\end{array}$ & $\begin{array}{c}\mathrm{Nb} 2 \mathrm{O} 5 \\
-\mathrm{t}\end{array}$ & $\begin{array}{c}\mathrm{Nd} 2 \mathrm{O} 3 \\
-\mathrm{t}\end{array}$ & $\begin{array}{c}\mathrm{PbO} \\
-\mathrm{t}\end{array}$ & $\begin{array}{c}\mathrm{PdO} 2 \\
-\mathrm{t}\end{array}$ \\
\hline WVUTH65 & & & & & & & & & & & & & & & & & & & & & \\
\hline WVUTH66 & & & & & & & & & & & & & & & & & & & & & \\
\hline WVUTH67 & & & & & & & & & & & & & & & & & & & & & \\
\hline WVUTH68 & & & & & & & & & & & & & & & & & & & & & \\
\hline WVUTH69 & & & & & & & & & & & & & & & & & & & & & \\
\hline WVUTH70 & & & & & & & & & & & & & & & & & & & & & \\
\hline WVUTH71 & & & & & & & & & & & & & & & & & & & & & \\
\hline WVUTH72 & & & & & & & & & & & & & & & & & & & & & \\
\hline WVUTH73 & & & & & & & & & & & & & & & & & & & & & \\
\hline WVUTH74 & & & & & & & & & & & & & & & & & & & & & \\
\hline WVUTH75 & & & & & & & & & & & & & & & & & & & & & \\
\hline WVUTH77 & & & & & & & & & & & & & & & & & & & & & \\
\hline WVUTH78 & & & & & & & & & & & & & & & & & & & & & \\
\hline WVUTH79 & & & & & & & & & & & & & & & & & & & & & \\
\hline WVUTH80 & & & & & & & & & & & & & & & & & & & & & \\
\hline WVUTH81 & & & & & & & & & & & & & & & & & & & & & \\
\hline WVUTH82 & & & & & & & & & & & & & & & & & & & & & \\
\hline WVUTH83 & & & & & & & & & & & & & & & & & & & & & \\
\hline WVUTH84 & & & & & & & & & & & & & & & & & & & & & \\
\hline WVUTH89 & & & & & & & & & & & & & & & & & & & & & \\
\hline WVUTH90 & & & & & & & & & & & & & & & & & & & & & \\
\hline WVUTH91 & & & & & & & & & & & & & & & & & & & & & \\
\hline WVUTH92 & & & & & & & & & & & & & & & & & & & & & \\
\hline WVUTH93 & & & & & & & & & & & & & & & & & & & & & \\
\hline WVUTH94 & & & & & & & & & & & & & & & & & & & & & \\
\hline WVUTH96 & & & & & & & & & & & & & & & & & & & & & \\
\hline WVUTH98 & & & & & & & & & & & & & & & & & & & & & \\
\hline WVUTH99 & & & & & & & & & & & & & & & & & & & & & \\
\hline WVUTH100 & & & & & & & & & & & & & & & & & & & & & \\
\hline WVUTH102 & & & & & & & & & & & & & & & & & & & & & \\
\hline WVUTH103 & & & & & & & & & & & & & & & & & & & & & \\
\hline CUOD & & & & & & & & & & & & & & & & & & & & & \\
\hline CUOI & & & & & & & & & & & & & & & & & & & & & \\
\hline CUOJ & & & & & & & & & & & & & & & & & & & & & \\
\hline CUOK & & & & & & & & & & & & & & & & & & & & & \\
\hline CUOL & & & & & & & & & & & & & & & & & & & & & \\
\hline CUOB & & & & 0.0020 & 0.0010 & 0.0000 & & & & & & & 0.0000 & 0.0130 & & & & & 0.0000 & & \\
\hline CUOC & & & & 0.0020 & 0.0010 & 0.0000 & & & & & & & 0.0000 & 0.0130 & & & & & 0.0000 & & \\
\hline CUOE & & & & 0.0020 & 0.0010 & 0.0000 & & & & & & & 0.0000 & 0.0140 & & & & & 0.0000 & & \\
\hline CUOF & & & & 0.0020 & 0.0010 & 0.0000 & & & & & & & 0.0000 & 0.0130 & & & & & 0.0000 & & \\
\hline CUOG & & & & 0.0020 & 0.0010 & 0.0000 & & & & & & & 0.0000 & 0.0140 & & & & & 0.0000 & & \\
\hline $\mathrm{CUOH}$ & & & & 0.0020 & 0.0010 & 0.0000 & & & & & & & 0.0000 & 0.0140 & & & & & 0.0000 & & \\
\hline CUOO & & & & 0.0020 & 0.0010 & 0.0000 & & & & & & & 0.0000 & 0.0140 & & & & & 0.0000 & & \\
\hline CUOP & & & & 0.0020 & 0.0010 & 0.0000 & & & & & & & 0.0000 & 0.0130 & & & & & 0.0000 & & \\
\hline CUOR & & & & 0.0020 & 0.0010 & 0.0000 & & & & & & & 0.0000 & 0.0140 & & & & & 0.0000 & & \\
\hline CUOU & & & & 0.0020 & 0.0010 & 0.0000 & & & & & & & 0.0000 & 0.0140 & & & & & 0.0000 & & \\
\hline CUOV & & & & 0.0020 & 0.0010 & 0.0000 & & & & & & & 0.0000 & 0.0140 & & & & & 0.0000 & & \\
\hline CU30 & & & & 0.0020 & 0.0010 & 0.0000 & & & & & & & 0.0000 & 0.0140 & & & & & 0.0000 & & \\
\hline CU31 & & & & 0.0020 & 0.0010 & 0.0000 & & & & & & & 0.0000 & 0.0140 & & & & & 0.0000 & & \\
\hline
\end{tabular}


Appendix A. Database - mass fraction

WV Glasses by CUA and PNL (Johnston et al. 1990)

\begin{tabular}{|c|c|c|c|c|c|c|c|c|c|c|c|c|c|c|c|c|c|c|c|c|c|}
\hline Glass ID & $\begin{array}{c}\mathrm{PdO} \\
-\mathrm{t}\end{array}$ & $\begin{array}{c}\mathrm{Pr} 2 \mathrm{O} 3 \\
-\mathrm{t}\end{array}$ & $\begin{array}{c}\text { Pr6O11 } \\
-t\end{array}$ & $\begin{array}{c}\mathrm{Rb} 2 \mathrm{O} \\
-\mathrm{t}\end{array}$ & $\begin{array}{c}\mathrm{ReO} \\
-\mathrm{t}\end{array}$ & $\begin{array}{c}\mathrm{ReO} 2 \\
-\mathrm{t}\end{array}$ & $\begin{array}{c}\mathrm{Rh} 2 \mathrm{O} 3 \\
-\mathrm{t}\end{array}$ & $\begin{array}{c}\mathrm{RhO} 2 \\
-\mathrm{t}\end{array}$ & $\begin{array}{c}\mathrm{RuO}^{2} \\
-\mathrm{t}\end{array}$ & $\begin{array}{c}\mathrm{Sb} 2 \mathrm{O} 3 \\
-\mathrm{t}\end{array}$ & $\begin{array}{c}\mathrm{Sb} 2 \mathrm{O} 5 \\
-\mathrm{t}\end{array}$ & $\begin{array}{c}\mathrm{SeO} 2 \\
-\mathrm{t}\end{array}$ & $\begin{array}{c}\mathrm{Sm} 2 \mathrm{O} 3 \\
-\mathrm{t}\end{array}$ & $\begin{array}{c}\mathrm{SnO} \\
-\mathrm{t}\end{array}$ & $\begin{array}{c}\mathrm{SnO} 2 \\
-t\end{array}$ & $\begin{array}{c}\mathrm{SO} 3 \\
-\mathrm{t}\end{array}$ & $\begin{array}{c}\mathrm{SrO} \\
-\mathrm{t}\end{array}$ & $\begin{array}{c}\mathrm{Tc} 2 \mathrm{O} 7 \\
-\mathrm{t}\end{array}$ & $\begin{array}{c}\mathrm{TeO} 2 \\
-\mathrm{t}\end{array}$ & $\begin{array}{c}\mathrm{ThO} 2 \\
-\mathrm{t}\end{array}$ & $\begin{array}{c}\mathrm{TiO} 2 \\
-\mathrm{t}\end{array}$ \\
\hline WVUTH65 & & & & & & & & & & & & & & & & & & & & & \\
\hline WVUTH66 & & & & & & & & & & & & & & & & & & & & & \\
\hline WVUTH67 & & & & & & & & & & & & & & & & & & & & & \\
\hline WVUTH68 & & & & & & & & & & & & & & & & & & & & & \\
\hline WVUTH69 & & & & & & & & & & & & & & & & & & & & & \\
\hline WVUTH70 & & & & & & & & & & & & & & & & & & & & & \\
\hline WVUTH71 & & & & & & & & & & & & & & & & & & & & & \\
\hline WVUTH72 & & & & & & & & & & & & & & & & & & & & & \\
\hline WVUTH73 & & & & & & & & & & & & & & & & & & & & & \\
\hline WVUTH74 & & & & & & & & & & & & & & & & & & & & & \\
\hline WVUTH75 & & & & & & & & & & & & & & & & & & & & & \\
\hline WVUTH77 & & & & & & & & & & & & & & & & & & & & & \\
\hline WVUTH78 & & & & & & & & & & & & & & & & & & & & & \\
\hline WVUTH79 & & & & & & & & & & & & & & & & & & & & & \\
\hline WVUTH80 & & & & & & & & & & & & & & & & & & & & & \\
\hline WVUTH81 & & & & & & & & & & & & & & & & & & & & & \\
\hline WVUTH82 & & & & & & & & & & & & & & & & & & & & & \\
\hline WVUTH83 & & & & & & & & & & & & & & & & & & & & & \\
\hline WVUTH84 & & & & & & & & & & & & & & & & & & & & & \\
\hline WVUTH89 & & & & & & & & & & & & & & & & & & & & & \\
\hline WVUTH90 & & & & & & & & & & & & & & & & & & & & & \\
\hline WVUTH91 & & & & & & & & & & & & & & & & & & & & & \\
\hline WVUTH92 & & & & & & & & & & & & & & & & & & & & & \\
\hline WVUTH93 & & & & & & & & & & & & & & & & & & & & & \\
\hline WVUTH94 & & & & & & & & & & & & & & & & & & & & & \\
\hline WVUTH96 & & & & & & & & & & & & & & & & & & & & & \\
\hline WVUTH98 & & & & & & & & & & & & & & & & & & & & & \\
\hline WVUTH99 & & & & & & & & & & & & & & & & & & & & & \\
\hline WVUTH10 & & & & & & & & & & & & & & & & & & & & & \\
\hline WVUTH10 & & & & & & & & & & & & & & & & & & & & & \\
\hline WVUTH10 & & & & & & & & & & & & & & & & & & & & & \\
\hline CUOD & & & & & & & & & & & & & & & & & & & & & \\
\hline CUOI & & & & & & & & & & & & & & & & & & & & & \\
\hline CUOJ & & & & & & & & & & & & & & & & & & & & & \\
\hline CUOK & & & & & & & & & & & & & & & & & & & & & \\
\hline CUOL & & & & & & & & & & & & & & & & & & & & & \\
\hline CUOB & & & & & & & & & & & & & & & & 0.0010 & & & & 0.0000 & 0.0100 \\
\hline CUOC & & & & & & & & & & & & & & & & 0.0010 & & & & 0.0000 & 0.0090 \\
\hline CUOE & & & & & & & & & & & & & & & & 0.0010 & & & & 0.0000 & 0.0100 \\
\hline CUOF & & & & & & & & & & & & & & & & 0.0010 & & & & 0.0000 & 0.0090 \\
\hline CUOG & & & & & & & & & & & & & & & & 0.0010 & & & & 0.0000 & 0.0100 \\
\hline $\mathrm{CUOH}$ & & & & & & & & & & & & & & & & 0.0010 & & & & 0.0000 & 0.0100 \\
\hline CUOO & & & & & & & & & & & & & & & & 0.0010 & & & & 0.0000 & 0.0100 \\
\hline CUOP & & & & & & & & & & & & & & & & 0.0010 & & & & 0.0000 & 0.0090 \\
\hline CUOR & & & & & & & & & & & & & & & & 0.0010 & & & & 0.0000 & 0.0100 \\
\hline CUOU & & & & & & & & & & & & & & & & 0.0010 & & & & 0.0000 & 0.0100 \\
\hline CUOV & & & & & & & & & & & & & & & & 0.0010 & & & & 0.0000 & 0.0100 \\
\hline CU30 & & & & & & & & & & & & & & & & 0.0010 & & & & 0.0000 & 0.0100 \\
\hline CU31 & & & & & & & & & & & & & & & & 0.0010 & & & & 0.0000 & 0.0100 \\
\hline
\end{tabular}


WV Glasses by CUA and PNL (Johnston et al. 1990)

\begin{tabular}{|c|c|c|c|c|c|c|c|c|c|c|c|c|c|c|c|c|c|c|c|c|c|}
\hline Glass ID & $\begin{array}{c}\mathrm{T} 12 \mathrm{O} 3 \\
-\mathrm{t}\end{array}$ & $\begin{array}{c}\mathrm{U} 3 \mathrm{O} 8 \\
-\mathrm{t}\end{array}$ & $\begin{array}{c}\mathrm{UO} 2 \\
-\mathrm{t}\end{array}$ & $\begin{array}{c}\mathrm{UO} 3 \\
-\mathrm{t}\end{array}$ & $\begin{array}{c}\mathrm{V} 2 \mathrm{O} 5 \\
-\mathrm{t}\end{array}$ & $\begin{array}{c}\text { WO3 } \\
-t\end{array}$ & $\begin{array}{c}\mathrm{Y} 2 \mathrm{O} 3 \\
-\mathrm{t}\end{array}$ & $\begin{array}{c}\mathrm{ZnO} \\
-\mathrm{t}\end{array}$ & $\begin{array}{c}\text { Others } \\
-t\end{array}$ & $\begin{array}{c}\text { Sum } \\
-t\end{array}$ & $\begin{array}{c}\mathrm{A} 12 \mathrm{O} 3 \\
-\mathrm{a}\end{array}$ & $\begin{array}{c}\mathrm{B} 2 \mathrm{O} 3 \\
-\mathrm{a}\end{array}$ & $\begin{array}{c}\mathrm{CaO} \\
-\mathrm{a}\end{array}$ & $\begin{array}{c}\mathrm{Fe} 2 \mathrm{O} 3 \\
-\mathrm{a} \\
\end{array}$ & $\begin{array}{c}\mathrm{FeO} \\
-\mathrm{a}\end{array}$ & $\begin{array}{c}\mathrm{K} 2 \mathrm{O} \\
-\mathrm{a}\end{array}$ & $\begin{array}{c}\mathrm{Li} 2 \mathrm{O} \\
-\mathrm{a}\end{array}$ & $\begin{array}{c}\mathrm{MgO} \\
-\mathrm{a}\end{array}$ & $\begin{array}{c}\mathrm{Na} 2 \mathrm{O} \\
-\mathrm{a}\end{array}$ & $\begin{array}{c}\mathrm{NiO} \\
-\mathrm{a}\end{array}$ & $\begin{array}{c}\mathrm{P} 2 \mathrm{O} 5 \\
-\mathrm{a} \\
\end{array}$ \\
\hline WVUTH65 & & & & & & & & & & & 0.0644 & 0.0879 & 0.0082 & 0.1197 & & 0.0313 & 0.0269 & 0.0089 & 0.0974 & & 0.0235 \\
\hline WVUTH66 & & & & & & & & & & & 0.0445 & 0.1031 & 0.0081 & 0.1194 & & 0.0359 & 0.0306 & 0.0089 & 0.1107 & & 0.0234 \\
\hline WVUTH67 & & & & & & & & & & & 0.0741 & 0.1055 & 0.0081 & 0.1193 & & 0.0364 & 0.0311 & 0.0089 & 0.1126 & & 0.0234 \\
\hline WVUTH68 & & & & & & & & & & & 0.0545 & 0.0923 & 0.0082 & 0.1196 & & 0.0326 & 0.0279 & 0.0089 & 0.1012 & & 0.0235 \\
\hline WVUTH69 & & & & & & & & & & & 0.0840 & 0.1012 & 0.0082 & 0.1194 & & 0.0352 & 0.0301 & 0.0089 & 0.1088 & & 0.0234 \\
\hline WVUTH70 & & & & & & & & & & & 0.0842 & 0.0923 & 0.0082 & 0.1196 & & 0.0326 & 0.0279 & 0.0089 & 0.1012 & & 0.0235 \\
\hline WVUTH71 & & & & & & & & & & & 0.0989 & 0.0945 & 0.0082 & 0.1196 & & 0.0332 & 0.0285 & 0.0089 & 0.1032 & & 0.0235 \\
\hline WVUTH72 & & & & & & & & & & & 0.0447 & 0.0878 & 0.0082 & 0.1197 & & 0.0313 & 0.0269 & 0.0089 & 0.0974 & & 0.0235 \\
\hline WVUTH73 & & & & & & & & & & & 0.0445 & 0.1099 & 0.0081 & 0.1192 & & 0.0378 & 0.0322 & 0.0089 & 0.1163 & & 0.0234 \\
\hline WVUTH74 & & & & & & & & & & & 0.0444 & 0.1186 & 0.0081 & 0.1190 & & 0.0403 & 0.0343 & 0.0089 & 0.1238 & & 0.0233 \\
\hline WVUTH75 & & & & & & & & & & & 0.0637 & 0.1481 & 0.0081 & 0.1182 & & 0.0309 & 0.0265 & 0.0088 & 0.0962 & & 0.0232 \\
\hline WVUTH77 & & & & & & & & & & & 0.0637 & 0.1482 & 0.0081 & 0.1182 & & 0.0309 & 0.0265 & 0.0088 & 0.0962 & & 0.0232 \\
\hline WVUTH78 & & & & & & & & & & & 0.0645 & 0.0878 & 0.0082 & 0.1694 & & 0.0313 & 0.0269 & 0.0089 & 0.0974 & & 0.0235 \\
\hline WVUTH79 & & & & & & & & & & & 0.0632 & 0.0862 & 0.0080 & 0.1175 & & 0.0307 & 0.0264 & 0.0088 & 0.0956 & & 0.0230 \\
\hline WVUTH80 & & & & & & & & & & & 0.0447 & 0.0879 & 0.0082 & 0.1198 & & 0.0313 & 0.0269 & 0.0089 & 0.0975 & & 0.0235 \\
\hline WVUTH81 & & & & & & & & & & & 0.0447 & 0.0879 & 0.0082 & 0.1197 & & 0.0313 & 0.0269 & 0.0089 & 0.0974 & & 0.0235 \\
\hline WVUTH82 & & & & & & & & & & & 0.0447 & 0.0879 & 0.0082 & 0.1395 & & 0.0313 & 0.0269 & 0.0089 & 0.0975 & & 0.0235 \\
\hline WVUTH83 & & & & & & & & & & & 0.0650 & 0.0885 & 0.0082 & 0.1206 & & 0.0420 & 0.0283 & 0.0090 & 0.1286 & & 0.0236 \\
\hline WVUTH84 & & & & & & & & & & & 0.0450 & 0.1201 & 0.0082 & 0.1206 & & 0.0409 & 0.0348 & 0.0090 & 0.1259 & & 0.0236 \\
\hline WVUTH89 & & & & & & & & & & & 0.0550 & 0.1045 & 0.0082 & 0.1206 & & 0.0361 & 0.0310 & 0.0090 & 0.1120 & & 0.0236 \\
\hline WVUTH90 & & & & & & & & & & & 0.0650 & 0.0976 & 0.0082 & 0.1506 & & 0.0342 & 0.0293 & 0.0090 & 0.1060 & & 0.0236 \\
\hline WVUTH91 & & & & & & & & & & & 0.0450 & 0.1203 & 0.0082 & 0.1206 & & 0.0700 & 0.0348 & 0.0090 & 0.0981 & & 0.0236 \\
\hline WVUTH92 & & & & & & & & & & & 0.0649 & 0.1018 & 0.0069 & 0.1205 & & 0.0371 & 0.0313 & 0.0089 & 0.1134 & & 0.0238 \\
\hline WVUTH93 & & & & & & & & & & & 0.0648 & 0.1241 & 0.0068 & 0.0905 & & 0.0442 & 0.0382 & 0.0089 & 0.1366 & & 0.0238 \\
\hline WVUTH94 & & & & & & & & & & & 0.0580 & 0.1058 & 0.0076 & 0.1106 & & 0.0382 & 0.0323 & 0.0083 & 0.1234 & & 0.0217 \\
\hline WVUTH96 & & & & & & & & & & & 0.0510 & 0.1476 & 0.0081 & 0.1191 & & 0.0330 & 0.0281 & 0.0089 & 0.1015 & & 0.0233 \\
\hline WVUTH98 & & & & & & & & & & & 0.0648 & 0.1175 & 0.0068 & 0.1106 & & 0.0416 & 0.0360 & 0.0089 & 0.1286 & & 0.0238 \\
\hline WVUTH99 & & & & & & & & & & & 0.0510 & 0.1162 & 0.0081 & 0.1191 & & 0.0395 & 0.0335 & 0.0089 & 0.1211 & & 0.0233 \\
\hline WVUTH100 & & & & & & & & & & & 0.0649 & 0.1019 & 0.0068 & 0.1205 & & 0.0371 & 0.0313 & 0.0089 & 0.1135 & & 0.0239 \\
\hline WVUTH102 & & & & & & & & & & & 0.0648 & 0.1017 & 0.0080 & 0.1202 & & 0.0370 & 0.0312 & 0.0087 & 0.1131 & & 0.0229 \\
\hline WVUTH103 & & & & & & & & & & & 0.0509 & 0.0872 & 0.0081 & 0.1189 & & 0.0455 & 0.0381 & 0.0089 & 0.1396 & & 0.0233 \\
\hline CUOD & & & & & & & & & & & 0.0681 & 0.0947 & & 0.1122 & & & & & & & 0.0238 \\
\hline CUOI & & & & & & & & & & & 0.0602 & 0.0951 & & 0.1127 & & & & & & & 0.0239 \\
\hline CUOJ & & & & & & & & & & & 0.0321 & 0.0970 & & 0.1149 & & & & & & & 0.0244 \\
\hline CUOK & & & & & & & & & & & 0.0648 & 0.0950 & & 0.1125 & & & & & & & 0.0238 \\
\hline CUOL & & & & & & & & & & & 0.0312 & 0.0942 & & \begin{tabular}{|l|}
0.1117 \\
\end{tabular} & & & & & & & 0.0237 \\
\hline CUOB & & & 0.0000 & & & & & 0.0000 & & 1.0010 & 0.0668 & 0.0964 & & 0.1142 & & & & & & & 0.0242 \\
\hline CUOC & & & 0.0000 & & & & & 0.0000 & & 1.0010 & 0.0309 & 0.0933 & & 0.1105 & & & & & & & 0.0234 \\
\hline CUOE & & & 0.0000 & & & & & 0.0000 & & 1.0010 & 0.0321 & 0.0970 & & 0.1149 & & & & & & & 0.0244 \\
\hline CUOF & & & 0.0000 & & & & & 0.0000 & & 1.0000 & 0.0312 & 0.0942 & & 0.1117 & & & & & & & 0.0237 \\
\hline CUOG & & & 0.0000 & & & & & 0.0000 & & 1.0000 & 0.0325 & 0.0980 & & 0.1160 & & & & & & & 0.0246 \\
\hline $\mathrm{CUOH}$ & & & 0.0000 & & & & & 0.0000 & & 1.0000 & 0.0323 & 0.0975 & & 0.1399 & & & & & & & 0.0245 \\
\hline CUOO & & & 0.0000 & & & & & 0.0000 & & 0.9970 & & & & & & & & & & & \\
\hline CUOP & & & 0.0000 & & & & & 0.0000 & & 1.0000 & 0.0312 & 0.0942 & & 0.1117 & & & & & & & 0.0237 \\
\hline CUOR & & & 0.0000 & & & & & 0.0000 & & 0.9990 & 0.0325 & 0.0980 & & 0.1160 & & & & & & & 0.0246 \\
\hline CUOU & & & 0.0000 & & & & & 0.0000 & & 1.0010 & & & & & & & & & & & \\
\hline CUOV & & & 0.0000 & & & & & 0.0000 & & 1.0000 & & & & & & & & & & & \\
\hline CU30 & & & 0.0000 & & & & & 0.0000 & & 1.0000 & & & & & & & & & & & \\
\hline CU31 & & & 0.0000 & & & & & 0.0000 & & 1.0000 & & & & & & & & & & & \\
\hline
\end{tabular}


Appendix A. Database - mass fraction

\section{WV Glasses by CUA and PNL (Johnston et al. 1990)}

\begin{tabular}{|c|c|c|c|c|c|c|c|c|c|c|c|c|c|c|c|c|c|c|c|c|c|}
\hline Glass ID & $\begin{array}{c}\mathrm{SiO}^{2} \\
-\mathrm{a}\end{array}$ & $\begin{array}{c}\mathrm{ZrO} 2 \\
-\mathrm{a}\end{array}$ & $\begin{array}{c}\mathrm{Ag} 2 \mathrm{O} \\
-\mathrm{a}\end{array}$ & $\begin{array}{c}\mathrm{As} 2 \mathrm{O} 3 \\
-\mathrm{a}\end{array}$ & $\begin{array}{c}\mathrm{BaO} \\
-\mathrm{a}\end{array}$ & $\begin{array}{c}\mathrm{Bi} 2 \mathrm{O} 3 \\
-\mathrm{a}\end{array}$ & $\begin{array}{l}\mathrm{Br} \\
-\mathrm{a}\end{array}$ & $\begin{array}{c}\mathrm{CdO} \\
-\mathrm{a}\end{array}$ & $\begin{array}{c}\mathrm{Ce} 2 \mathrm{O} 3 \\
-\mathrm{a}\end{array}$ & $\begin{array}{c}\mathrm{CeO} 2 \\
-\mathrm{a}\end{array}$ & $\begin{array}{l}\mathrm{Cl} \\
-\mathrm{a}\end{array}$ & $\begin{array}{c}\mathrm{CoO} \\
-\mathrm{a}\end{array}$ & $\begin{array}{c}\mathrm{Co} 2 \mathrm{O} 3 \\
-\mathrm{a}\end{array}$ & $\begin{array}{c}\mathrm{Cr} 2 \mathrm{O} 3 \\
-\mathrm{a}\end{array}$ & $\begin{array}{c}\mathrm{Cs} 2 \mathrm{O} \\
-\mathrm{a}\end{array}$ & $\begin{array}{c}\mathrm{CuO} \\
-\mathrm{a}\end{array}$ & \begin{tabular}{|c|}
$\mathrm{Eu} 2 \mathrm{O} 3$ \\
$-\mathrm{a}$
\end{tabular} & $\begin{array}{c}F \\
-a\end{array}$ & $\begin{array}{c}\mathrm{Ga} 2 \mathrm{O} 3 \\
-\mathrm{a}\end{array}$ & $\begin{array}{c}\mathrm{Gd} 2 \mathrm{O} 3 \\
-\mathrm{a}\end{array}$ & $\begin{array}{c}\mathrm{HgO} \\
-\mathrm{a}\end{array}$ \\
\hline WVUTH65 & 0.4461 & & & & 0.0019 & & & & & & & & & & & & & & & & \\
\hline WVUTH66 & 0.4299 & & & & 0.0019 & & & & & & & & & & & & & & & & \\
\hline WVUTH67 & 0.3951 & & & & 0.0019 & & & & & & & & & & & & & & & & \\
\hline WVUTH68 & 0.4457 & & & & 0.0019 & & & & & & & & & & & & & & & & \\
\hline WVUTH69 & 0.3954 & & & & 0.0019 & & & & & & & & & & & & & & & & \\
\hline WVUTH70 & 0.4160 & & & & 0.0019 & & & & & & & & & & & & & & & & \\
\hline WVUTH71 & 0.3960 & & & & 0.0019 & & & & & & & & & & & & & & & & \\
\hline WVUTH72 & 0.4660 & & & & 0.0019 & & & & & & & & & & & & & & & & \\
\hline WVUTH73 & 0.4145 & & & & 0.0019 & & & & & & & & & & & & & & & & \\
\hline WVUTH74 & 0.3940 & & & & 0.0019 & & & & & & & & & & & & & & & & \\
\hline WVUTH75 & 0.3916 & & & & 0.0019 & & & & & & & & & & & & & & & & \\
\hline WVUTH77 & 0.3915 & & & & 0.0019 & & & & & & & & & & & & & & & & \\
\hline WVUTH78 & 0.3964 & & & & 0.0019 & & & & & & & & & & & & & & & & \\
\hline WVUTH79 & 0.4375 & & & & 0.0019 & & & & & & & & & & & & & & & & \\
\hline WVUTH 80 & 0.4462 & & & & 0.0019 & & & & & & & & & & & & & & & & \\
\hline WVUTH81 & 0.4461 & & & & 0.0019 & & & & & & & & & & & & & & & & \\
\hline WVUTH82 & 0.4460 & & & & 0.0019 & & & & & & & & & & & & & & & & \\
\hline WVUTH83 & 0.3992 & & & & 0.0020 & & & & & & & & & & & & & & & & \\
\hline WVUTH84 & 0.3992 & & & & 0.0020 & & & & & & & & & & & & & & & & \\
\hline WVUTH89 & 0.4242 & & & & 0.0020 & & & & & & & & & & & & & & & & \\
\hline WVUTH90 & 0.3992 & & & & 0.0020 & & & & & & & & & & & & & & & & \\
\hline WVUTH91 & 0.3992 & & & & 0.0020 & & & & & & & & & & & & & & & & \\
\hline WVUTH92 & 0.4096 & & & & 0.0016 & & & & & & & & & & & & & & & & \\
\hline WVUTH93 & 0.3801 & & & & 0.0016 & & & & & & & & & & & & & & & & \\
\hline WVUTH94 & 0.4151 & & & & 0.0018 & & & & & & & & & & & & & & & & \\
\hline WVUTH96 & 0.3941 & & & & 0.0019 & & & & & & & & & & & & & & & & \\
\hline WVUTH98 & 0.3801 & & & & 0.0016 & & & & & & & & & & & & & & & & \\
\hline WVUTH99 & 0.3941 & & & & 0.0019 & & & & & & & & & & & & & & & & \\
\hline WVUTH100 & 0.4097 & & & & 0.0016 & & & & & & & & & & & & & & & & \\
\hline WVUTH102 & 0.4087 & & & & 0.0019 & & & & & & & & & & & & & & & & \\
\hline WVUTH103 & 0.3937 & & & & 0.0019 & & & & & & & & & & & & & & & & \\
\hline CUOD & 0.4298 & & & & & & & & & & & & & & & & & & & & \\
\hline CUOI & 0.4320 & & & & & & & & & & & & & & & & & & & & \\
\hline CUOJ & 0.4501 & & & & & & & & & & & & & & & & & & & & \\
\hline CUOK & 0.4473 & & & & & & & & & & & & & & & & & & & & \\
\hline CUOL & 0.4468 & & & & & & & & & & & & & & & & & & & & \\
\hline CUOB & 0.4378 & & & & & & & & & & & & & & & & & & & & \\
\hline CUOC & 0.4235 & & & & & & & & & & & & & & & & & & & & \\
\hline CUOE & 0.4696 & & & & & & & & & & & & & & & & & & & & \\
\hline CUOF & 0.4846 & & & & & & & & & & & & & & & & & & & & \\
\hline CUOG & 0.4447 & & & & & & & & & & & & & & & & & & & & \\
\hline $\mathrm{CUOH}$ & 0.4425 & & & & & & & & & & & & & & & & & & & & \\
\hline \multicolumn{22}{|l|}{ CUOO } \\
\hline CUOP & 0.4846 & & & & & & & & & & & & & & & & & & & & \\
\hline CUOR & 0.4644 & & & & & & & & & & & & & & & & & & & & \\
\hline \multicolumn{22}{|l|}{ CUOU } \\
\hline \multicolumn{22}{|l|}{ CUOV } \\
\hline \multicolumn{22}{|l|}{ CU30 } \\
\hline CU31 & & & & & & & & & & & & & & & & & & & & & \\
\hline
\end{tabular}


Appendix A. Database - mass fraction

WV Glasses by CUA and PNL (Johnston et al. 1990)

\begin{tabular}{|c|c|c|c|c|c|c|c|c|c|c|c|c|c|c|c|c|c|c|c|c|c|}
\hline Glass ID & $\begin{array}{c}\mathrm{I} \\
-\mathrm{a}\end{array}$ & $\begin{array}{c}\mathrm{La} 2 \mathrm{O} 3 \\
-\mathrm{a}\end{array}$ & $\begin{array}{c}\mathrm{MnO} 2 \\
-\mathrm{a}\end{array}$ & $\begin{array}{c}\mathrm{MnO} \\
-\mathrm{a}\end{array}$ & $\begin{array}{c}\mathrm{MoO} \\
-\mathrm{a}\end{array}$ & $\begin{array}{c}\mathrm{MoO} 3 \\
-\mathrm{a}\end{array}$ & $\begin{array}{c}\mathrm{Nb} 2 \mathrm{O} 5 \\
-\mathrm{a}\end{array}$ & $\begin{array}{c}\mathrm{Nd} 2 \mathrm{O} 3 \\
-\mathrm{a}\end{array}$ & $\begin{array}{c}\mathrm{PbO} \\
-\mathrm{a}\end{array}$ & $\begin{array}{c}\mathrm{PdO} 2 \\
-\mathrm{a}\end{array}$ & $\begin{array}{c}\mathrm{PdO} \\
-\mathrm{a}\end{array}$ & $\begin{array}{c}\mathrm{Pr} 2 \mathrm{O} 3 \\
-\mathrm{a}\end{array}$ & $\begin{array}{c}\operatorname{Pr} 6011 \\
-\mathrm{a}\end{array}$ & $\begin{array}{c}\mathrm{Rb} 2 \mathrm{O} \\
-\mathrm{a}\end{array}$ & $\begin{array}{c}\mathrm{ReO} \\
-\mathrm{a}\end{array}$ & $\begin{array}{c}\mathrm{ReO} 2 \\
-\mathrm{a}\end{array}$ & $\begin{array}{c}\mathrm{Rh} 2 \mathrm{O} 3 \\
-\mathrm{a}\end{array}$ & $\begin{array}{c}\mathrm{RhO} 2 \\
-\mathrm{a}\end{array}$ & $\begin{array}{c}\mathrm{RuO}_{2} \\
-\mathrm{a}\end{array}$ & $\begin{array}{c}\mathrm{Sb} 2 \mathrm{O} 3 \\
-\mathrm{a}\end{array}$ & $\begin{array}{c}\mathrm{Sb} 2 \mathrm{O} 5 \\
-\mathrm{a}\end{array}$ \\
\hline WVUTH65 & & & 0.0121 & & & & & & & & & & & & & & & & & & \\
\hline WVUTH66 & & & 0.0121 & & & & & & & & & & & & & & & & & & \\
\hline WVUTH67 & & & 0.0121 & & & & & & & & & & & & & & & & & & \\
\hline WVUTH68 & & & 0.0121 & & & & & & & & & & & & & & & & & & \\
\hline WVUTH69 & & & 0.0121 & & & & & & & & & & & & & & & & & & \\
\hline WVUTH70 & & & 0.0121 & & & & & & & & & & & & & & & & & & \\
\hline WVUTH71 & & & 0.0121 & & & & & & & & & & & & & & & & & & \\
\hline WVUTH72 & & & 0.0121 & & & & & & & & & & & & & & & & & & \\
\hline WVUTH73 & & & 0.0121 & & & & & & & & & & & & & & & & & & \\
\hline WVUTH74 & & & 0.0120 & & & & & & & & & & & & & & & & & & \\
\hline WVUTH75 & & & 0.0120 & & & & & & & & & & & & & & & & & & \\
\hline WVUTH77 & & & 0.0120 & & & & & & & & & & & & & & & & & & \\
\hline WVUTH78 & & & 0.0121 & & & & & & & & & & & & & & & & & & \\
\hline WVUTH79 & & & 0.0119 & & & & & & & & & & & & & & & & & & \\
\hline WVUTH80 & & & 0.0121 & & & & & & & & & & & & & & & & & & \\
\hline WVUTH81 & & & 0.0121 & & & & & & & & & & & & & & & & & & \\
\hline WVUTH82 & & & 0.0121 & & & & & & & & & & & & & & & & & & \\
\hline WVUTH83 & & & 0.0122 & & & & & & & & & & & & & & & & & & \\
\hline WVUTH84 & & & 0.0122 & & & & & & & & & & & & & & & & & & \\
\hline WVUTH89 & & & 0.0122 & & & & & & & & & & & & & & & & & & \\
\hline WVUTH90 & & & 0.0122 & & & & & & & & & & & & & & & & & & \\
\hline WVUTH91 & & & 0.0122 & & & & & & & & & & & & & & & & & & \\
\hline WVUTH92 & & & 0.0102 & & & & & & & & & & & & & & & & & & \\
\hline WVUTH93 & & & 0.0102 & & & & & & & & & & & & & & & & & & \\
\hline WVUTH94 & & & 0.0112 & & & & & & & & & & & & & & & & & & \\
\hline WVUTH96 & & & 0.0120 & & & & & & & & & & & & & & & & & & \\
\hline WVUTH98 & & & 0.0102 & & & & & & & & & & & & & & & & & & \\
\hline WVUTH99 & & & 0.0120 & & & & & & & & & & & & & & & & & & \\
\hline WVUTH100 & & & 0.0102 & & & & & & & & & & & & & & & & & & \\
\hline WVUTH102 & & & 0.0118 & & & & & & & & & & & & & & & & & & \\
\hline WVUTH103 & & & 0.0120 & & & & & & & & & & & & & & & & & & \\
\hline CUOD & & & 0.0132 & & & & & & & & & & & & & & & & & & \\
\hline CUOI & & & 0.0133 & & & & & & & & & & & & & & & & & & \\
\hline CUOJ & & & 0.0135 & & & & & & & & & & & & & & & & & & \\
\hline CUOK & & & 0.0132 & & & & & & & & & & & & & & & & & & \\
\hline CUOL & & & 0.0131 & & & & & & & & & & & & & & & & & & \\
\hline CUOB & & & 0.0134 & & & & & & & & & & & & & & & & & & \\
\hline CUOC & & & 0.0130 & & & & & & & & & & & & & & & & & & \\
\hline CUOE & & & 0.0135 & & & & & & & & & & & & & & & & & & \\
\hline CUOF & & & 0.0131 & & & & & & & & & & & & & & & & & & \\
\hline CUOG & & & 0.0136 & & & & & & & & & & & & & & & & & & \\
\hline $\mathrm{CUOH}$ & & & 0.0136 & & & & & & & & & & & & & & & & & & \\
\hline \multicolumn{22}{|l|}{ CUOO } \\
\hline CUOP & & & 0.0131 & & & & & & & & & & & & & & & & & & \\
\hline CUOR & & & 0.0136 & & & & & & & & & & & & & & & & & & \\
\hline \multicolumn{22}{|l|}{ CUOU } \\
\hline \multicolumn{22}{|l|}{ CUOV } \\
\hline CU30 & & & & & & & & & & & & & & & & & & & & & \\
\hline CU31 & & & & & & & & & & & & & & & & & & & & & \\
\hline
\end{tabular}


Appendix A. Database - mass fraction

WV Glasses by CUA and PNL (Johnston et al. 1990)

\begin{tabular}{|c|c|c|c|c|c|c|c|c|c|c|c|c|c|c|c|c|c|c|c|c|}
\hline Glass ID & $\begin{array}{c}\mathrm{SeO} 2 \\
-\mathrm{a}\end{array}$ & $\begin{array}{c}\mathrm{Sm} 2 \mathrm{O} 3 \\
-\mathrm{a}\end{array}$ & $\begin{array}{c}\mathrm{SnO} \\
-\mathrm{a}\end{array}$ & $\begin{array}{c}\mathrm{SnO} 2 \\
-\mathrm{a}\end{array}$ & $\begin{array}{c}\mathrm{SO} 3 \\
-\mathrm{a}\end{array}$ & $\begin{array}{c}\mathrm{SrO} \\
-\mathrm{a}\end{array}$ & $\begin{array}{c}\mathrm{Tc} 2 \mathrm{O} 7 \\
-\mathrm{a}\end{array}$ & $\begin{array}{c}\mathrm{TeO} 2 \\
-\mathrm{a}\end{array}$ & $\begin{array}{c}\mathrm{ThO} 2 \\
-\mathrm{a}\end{array}$ & $\begin{array}{c}\mathrm{TiO} 2 \\
-\mathrm{a}\end{array}$ & $\begin{array}{c}\mathrm{T} 12 \mathrm{O} 3 \\
-\mathrm{a}\end{array}$ & $\begin{array}{c}\mathrm{U} 3 \mathrm{O} 8 \\
-\mathrm{a}\end{array}$ & $\begin{array}{c}\mathrm{UO} 2 \\
-\mathrm{a}\end{array}$ & $\begin{array}{c}\mathrm{UO} 3 \\
-\mathrm{a}\end{array}$ & $\begin{array}{c}\mathrm{V} 2 \mathrm{O} 5 \\
-\mathrm{a}\end{array}$ & $\begin{array}{c}\text { WO3 } \\
-\mathrm{a}\end{array}$ & $\begin{array}{c}\mathrm{Y} 2 \mathrm{O} 3 \\
-\mathrm{a}\end{array}$ & $\begin{array}{c}\mathrm{ZnO} \\
-\mathrm{a}\end{array}$ & $\begin{array}{c}\text { Others } \\
\text {-a }\end{array}$ & $\begin{array}{c}\text { Sum } \\
-\mathrm{a}\end{array}$ \\
\hline WVUTH65 & & & & & & & & & 0.0353 & & & & 0.0058 & & & & & & 0.0302 & 0.9996 \\
\hline WVUTH66 & & & & & & & & & 0.0352 & & & & 0.0058 & & & & & & 0.0301 & 0.9996 \\
\hline WVUTH67 & & & & & & & & & 0.0352 & & & & 0.0058 & & & & & & 0.0301 & 0.9996 \\
\hline WVUTH68 & & & & & & & & & 0.0353 & & & & 0.0058 & & & & & & 0.0302 & 0.9997 \\
\hline WVUTH69 & & & & & & & & & 0.0352 & & & & 0.0058 & & & & & & 0.0301 & 0.9997 \\
\hline WVUTH70 & & & & & & & & & 0.0353 & & & & 0.0058 & & & & & & 0.0302 & 0.9997 \\
\hline WVUTH71 & & & & & & & & & 0.0353 & & & & 0.0058 & & & & & & 0.0302 & 0.9998 \\
\hline WVUTH72 & & & & & & & & & 0.0353 & & & & 0.0058 & & & & & & 0.0302 & 0.9997 \\
\hline WVUTH73 & & & & & & & & & 0.0352 & & & & 0.0058 & & & & & & 0.0301 & 0.9999 \\
\hline WVUTH74 & & & & & & & & & 0.0351 & & & & 0.0058 & & & & & & 0.0300 & 0.9995 \\
\hline WVUTH75 & & & & & & & & & 0.0349 & & & & 0.0057 & & & & & & 0.0297 & 0.9995 \\
\hline WVUTH77 & & & & & & & & & 0.0349 & & & & 0.0057 & & & & & & 0.0297 & 0.9995 \\
\hline WVUTH78 & & & & & & & & & 0.0353 & & & & 0.0058 & & & & & & 0.0302 & 0.9996 \\
\hline WVUTH79 & & & & & & & & & 0.0538 & & & & 0.0057 & & & & & & 0.0297 & 0.9999 \\
\hline WVUTH80 & & & & & & & & & 0.0549 & & & & 0.0058 & & & & & & 0.0302 & 0.9998 \\
\hline WVUTH81 & & & & & & & & & 0.0353 & & & & 0.0058 & & & & & & 0.0500 & 0.9997 \\
\hline WVUTH82 & & & & & & & & & 0.0353 & & & & 0.0058 & & & & & & 0.0302 & 0.9997 \\
\hline WVUTH83 & & & & & & & & & 0.0356 & & & & 0.0058 & & & & & & 0.0306 & 0.9992 \\
\hline WVUTH84 & & & & & & & & & 0.0356 & & & & 0.0058 & & & & & & 0.0306 & 1.0135 \\
\hline WVUTH89 & & & & & & & & & 0.0356 & & & & 0.0058 & & & & & & 0.0306 & 1.0104 \\
\hline WVUTH90 & & & & & & & & & 0.0356 & & & & 0.0058 & & & & & & 0.0306 & 1.0089 \\
\hline WVUTH91 & & & & & & & & & 0.0356 & & & & 0.0058 & & & & & & 0.0306 & 1.0150 \\
\hline WVUTH92 & & & & & & & & & 0.0351 & & & & 0.0058 & & & & & & 0.0290 & 0.9999 \\
\hline WVUTH93 & & & & & & & & & 0.0351 & & & & 0.0058 & & & & & & 0.0289 & 0.9996 \\
\hline WVUTH94 & & & & & & & & & 0.0327 & & & & 0.0053 & & & & & & 0.0277 & 0.9997 \\
\hline WVUTH96 & & & & & & & & & 0.0352 & & & & 0.0057 & & & & & & 0.0301 & 0.9996 \\
\hline WVUTH98 & & & & & & & & & 0.0351 & & & & 0.0058 & & & & & & 0.0290 & 1.0004 \\
\hline WVUTH99 & & & & & & & & & 0.0352 & & & & 0.0052 & & & & & & 0.0301 & 0.9992 \\
\hline WVUTH100 & & & & & & & & & 0.0351 & & & & 0.0058 & & & & & & 0.0289 & 1.0001 \\
\hline WVUTH102 & & & & & & & & & 0.0346 & & & & 0.0056 & & & & & & 0.0297 & 0.9999 \\
\hline WVUTH103 & & & & & & & & & 0.0351 & & & & 0.0057 & & & & & & 0.0313 & 1.0002 \\
\hline CUOD & & & & & & & & & 0.0000 & & & & 0.0000 & & & & & & 0.2586 & 1.0004 \\
\hline CUOI & & & & & & & & & 0.0000 & & & & 0.0000 & & & & & & 0.2628 & 1.0000 \\
\hline CUOJ & & & & & & & & & 0.0000 & & & & 0.0000 & & & & & & 0.2680 & 1.0000 \\
\hline CUOK & & & & & & & & & 0.0000 & & & & 0.0000 & & & & & & 0.2434 & 1.0000 \\
\hline CUOL & & & & & & & & & 0.0000 & & & & 0.0000 & & & & & & 0.2794 & 1.0001 \\
\hline CUOB & & & & & & & & & 0.0000 & & & & 0.0000 & & & & & & 0.2472 & 1.0000 \\
\hline CUOC & & & & & & & & & 0.0000 & & & & 0.0000 & & & & & & 0.3054 & 1.0000 \\
\hline CUOE & & & & & & & & & 0.0000 & & & & 0.0000 & & & & & & 0.2485 & 1.0000 \\
\hline CUOF & & & & & & & & & 0.0000 & & & & 0.0000 & & & & & & 0.2415 & 1.0000 \\
\hline CUOG & & & & & & & & & 0.0000 & & & & 0.0000 & & & & & & 0.2708 & 1.0002 \\
\hline $\mathrm{CUOH}$ & & & & & & & & & 0.0000 & & & & 0.0000 & & & & & & 0.2500 & 1.0003 \\
\hline \multicolumn{21}{|l|}{ CUOO } \\
\hline CUOP & & & & & & & & & 0.0000 & & & & 0.0000 & & & & & & 0.2415 & 1.0000 \\
\hline CUOR & & & & & & & & & 0.0000 & & & & 0.0000 & & & & & & 0.2511 & 1.0002 \\
\hline \multicolumn{21}{|l|}{ CUOU } \\
\hline \multicolumn{21}{|l|}{ CUOV } \\
\hline CU30 & & & & & & & & & & & & & & & & & & & & \\
\hline CU31 & & & & & & & & & & & & & & & & & & & & \\
\hline
\end{tabular}


Appendix A. Database - mass fraction

\begin{tabular}{|c|c|c|c|c|c|c|c|c|}
\hline Glass ID & $\begin{array}{l}\mathrm{TM} \\
\left({ }^{\circ} \mathrm{C}\right) \\
\end{array}$ & $\begin{array}{l}\text { Gradient } \\
\text { TL }\left({ }^{\circ} \mathrm{C}\right) \\
\end{array}$ & $\begin{array}{l}\text { Uniform } \\
\text { TL }\left({ }^{\circ} \mathrm{C}\right)\end{array}$ & Primary Phase & $\begin{array}{l}\text { Quenched } \\
\text { Visual/OM }\end{array}$ & $\begin{array}{c}\text { Quenched } \\
\text { SEM/EDS or TEM }\end{array}$ & $\begin{array}{l}\text { Quenched } \\
\text { XRD }\end{array}$ & $\begin{array}{c}\text { Quenched } \\
\text { Homogeneous? }\end{array}$ \\
\hline \multicolumn{9}{|c|}{ WVUTH65 } \\
\hline \multicolumn{9}{|c|}{ WVUTH66 } \\
\hline \multicolumn{9}{|c|}{ WVUTH67 } \\
\hline \multicolumn{9}{|c|}{ WVUTH68 } \\
\hline \multicolumn{9}{|c|}{ WVUTH69 } \\
\hline \multicolumn{9}{|c|}{ WVUTH70 } \\
\hline \multicolumn{9}{|c|}{ WVUTH71 } \\
\hline \multicolumn{9}{|c|}{ WVUTH72 } \\
\hline \multicolumn{9}{|c|}{ WVUTH73 } \\
\hline \multicolumn{9}{|c|}{ WVUTH74 } \\
\hline \multicolumn{9}{|c|}{ WVUTH75 } \\
\hline \multicolumn{9}{|c|}{ WVUTH77 } \\
\hline \multicolumn{9}{|c|}{ WVUTH78 } \\
\hline \multicolumn{9}{|c|}{ WVUTH79 } \\
\hline \multicolumn{9}{|c|}{ WVUTH80 } \\
\hline \multicolumn{9}{|c|}{ WVUTH81 } \\
\hline \multicolumn{9}{|c|}{ WVUTH82 } \\
\hline \multicolumn{9}{|c|}{ WVUTH83 } \\
\hline \multicolumn{9}{|c|}{ WVUTH84 } \\
\hline \multicolumn{9}{|c|}{ WVUTH89 } \\
\hline WVUTHS & & & & & & & & \\
\hline WVUTHS & & & & & & & & \\
\hline WVUTHS & & & & & & & & \\
\hline WVUTHS & & & & & & & & \\
\hline WVUTHS & & & & & & & & \\
\hline WVUTHS & & & & & & & & \\
\hline WVUTHS & & & & & & & & \\
\hline WVUTHS & & & & & & & & \\
\hline WVUTH1 & & & & & & & & \\
\hline WVUTH1 & & & & & & & & \\
\hline WVUTH1 & & & & & & & & \\
\hline CUOD & & & & & & & & \\
\hline CUOI & & & & & & & & \\
\hline CUOJ & & & & & & & & \\
\hline CUOK & & & & & & & & \\
\hline CUOL & & & & & & & & \\
\hline CUOB & & & & & & & & \\
\hline CUOC & & & & & & & & \\
\hline CUOE & & & & & & & & \\
\hline CUOF & & & & & & & & \\
\hline CUOG & & & & & & & & \\
\hline $\mathrm{CUOH}$ & & & & & & & & \\
\hline CUOO & & & & & & & & \\
\hline CUOP & & & & & & & & \\
\hline CUOR & & & & & & & & \\
\hline CUOU & & & & & & & & \\
\hline CUOV & & & & & & & & \\
\hline CU30 & & & & & & & & \\
\hline CU31 & & & & & & & & \\
\hline
\end{tabular}


Appendix A. Database - mass fraction

\section{WV Glasses by CUA and PNL (Johnston et al. 1990)}

\begin{tabular}{|c|c|c|c|c|c|}
\hline Glass ID & $\begin{array}{c}\mathrm{CCC} \\
\text { Visual/OM }\end{array}$ & $\begin{array}{c}\text { CCC } \\
\text { SEM/EDS or TEM }\end{array}$ & $\begin{array}{l}\text { CCC } \\
\text { XRD }\end{array}$ & $\begin{array}{l}\text { Heat Treated } \\
\text { Visual/OM }\end{array}$ & $\begin{array}{c}\text { Heat Treated } \\
\text { SEM/EDS or TEM }\end{array}$ \\
\hline \multicolumn{6}{|l|}{ WVUTH65 } \\
\hline \multicolumn{6}{|l|}{ WVUTH66 } \\
\hline \multicolumn{6}{|c|}{ WVUTH67 } \\
\hline \multicolumn{6}{|c|}{ WVUTH68 } \\
\hline \multicolumn{6}{|c|}{ WVUTH69 } \\
\hline \multicolumn{6}{|c|}{ WVUTH70 } \\
\hline \multicolumn{6}{|c|}{ WVUTH71 } \\
\hline \multicolumn{6}{|c|}{ WVUTH72 } \\
\hline \multicolumn{6}{|c|}{ WVUTH73 } \\
\hline \multicolumn{6}{|c|}{ WVUTH74 } \\
\hline \multicolumn{6}{|c|}{ WVUTH75 } \\
\hline \multicolumn{6}{|c|}{ WVUTH77 } \\
\hline \multicolumn{6}{|l|}{ WVUTH78 } \\
\hline \multicolumn{6}{|c|}{ WVUTH79 } \\
\hline \multicolumn{6}{|c|}{ WVUTH80 } \\
\hline \multicolumn{6}{|c|}{ WVUTH81 } \\
\hline \multicolumn{6}{|c|}{ WVUTH82 } \\
\hline \multicolumn{6}{|c|}{ WVUTH83 } \\
\hline \multicolumn{6}{|c|}{ WVUTH84 } \\
\hline \multicolumn{6}{|c|}{ WVUTH89 } \\
\hline \multicolumn{6}{|c|}{ WVUTH90 } \\
\hline WVUTH91 & & & & & \\
\hline WVUTH92 & & & & & \\
\hline WVUTH93 & & & & & \\
\hline WVUTH94 & & & & & \\
\hline WVUTH96 & & & & & \\
\hline WVUTH98 & & & & & \\
\hline WVUTH99 & & & & & \\
\hline WVUTH10 & & & & & \\
\hline WVUTH10 & & & & & \\
\hline WVUTH10 & & & & & \\
\hline CUOD & & & & & \\
\hline CUOI & & & & & \\
\hline CUOJ & & & & & \\
\hline CUOK & & & & & \\
\hline CUOL & & & & & \\
\hline CUOB & & & & & \\
\hline CUOC & & & & & \\
\hline CUOE & & & & & \\
\hline CUOF & & & & & \\
\hline CUOG & & & & & \\
\hline $\mathrm{CUOH}$ & & & & & \\
\hline CUOO & & & & & \\
\hline CUOP & & & & & \\
\hline CUOR & & & & & \\
\hline CUOU & & & & & \\
\hline CUOV & & & & & \\
\hline CU30 & & & & & \\
\hline CU31 & & & & & \\
\hline
\end{tabular}


Appendix A. Database - mass fraction

WV Glasses by CUA and PNL (Johnston et al. 1990)

\begin{tabular}{|c|c|c|c|c|c|c|c|c|c|c|c|c|c|c|c|}
\hline Glass ID & $\begin{array}{c}\text { Heat Treated } \\
\text { XRD }\end{array}$ & $\begin{array}{l}\text { Density } \\
\left(\mathrm{g} / \mathrm{cm}^{3}\right)\end{array}$ & $\begin{array}{c}\text { Fulc Visc } \\
\mathrm{A}\end{array}$ & $\begin{array}{c}\text { Fulc Visc } \\
\text { B }\end{array}$ & $\begin{array}{c}\text { Fulc Visc } \\
\text { To } \\
\end{array}$ & $\begin{array}{c}\mathrm{FV} 1150^{\circ} \mathrm{C} \\
(\mathrm{Pa} \cdot \mathrm{s})\end{array}$ & \begin{tabular}{|c|} 
Arrh Visc \\
$\mathrm{A}$
\end{tabular} & \begin{tabular}{|c|} 
Arrh Visc \\
B
\end{tabular} & $\begin{array}{c}1150^{\circ} \mathrm{C} \\
\left(\mathrm{D}_{\mathrm{a}, 0}\right)\end{array}$ & $\begin{array}{c}\mathrm{T}\left({ }^{\circ} \mathrm{C}\right) \text { at } \\
2 \mathrm{~Pa} \cdot \mathrm{s}\end{array}$ & \begin{tabular}{|c|}
$\mathrm{T}\left({ }^{\circ} \mathrm{C}\right)$ at \\
$5 \mathrm{~Pa} \cdot \mathrm{s}$ \\
\end{tabular} & $\begin{array}{l}\mathrm{T}\left({ }^{\circ} \mathrm{C}\right) \text { at } \\
10 \mathrm{~Pa} \cdot \mathrm{s}\end{array}$ & $\begin{array}{l}\mathrm{T} 1 \\
\left({ }^{\circ} \mathrm{C}\right) \\
\end{array}$ & \begin{tabular}{c|}
$\mathrm{V} 1$ \\
$(\mathrm{~Pa} \cdot \mathrm{s})$ \\
\end{tabular} & $\begin{array}{l}\mathrm{T} 2 \\
\left({ }^{\circ} \mathrm{C}\right) \\
\end{array}$ \\
\hline WVUTH6 & & & & & & & & & & & & & & & \\
\hline WVUTH6 & & & & & & & & & & & & & & & \\
\hline WVUTH6 & & & & & & & & & & & & & & & \\
\hline WVUTH6 & & & & & & & & & & & & & & & \\
\hline WVUTH6 & & & & & & & & & & & & & & & \\
\hline WVUTH7 & & & & & & & & & & & & & & & \\
\hline WVUTH7 & & & & & & & & & & & & & & & \\
\hline WVUTH7 & & & & & & & & & & & & & & & \\
\hline WVUTH7 & & & & & & & & & & & & & & & \\
\hline WVUTH7 & & & & & & & & & & & & & & & \\
\hline WVUTH7 & & & & & & & & & & & & & & & \\
\hline WVUTH7 & & & & & & & & & & & & & & & \\
\hline WVUTH7 & & & & & & & & & & & & & & & \\
\hline WVUTH7 & & & & & & & & & & & & & & & \\
\hline WVUTH8 & & & & & & & & & & & & & & & \\
\hline WVUTH8 & & & & & & & & & & & & & & & \\
\hline WVUTH8 & & & & & & & & & & & & & & & \\
\hline WVUTH8 & & & & & & & & & & & & & & & \\
\hline WVUTH8 & & & & & & & & & & & & & & & \\
\hline WVUTH8 & & & & & & & & & & & & & & & \\
\hline WVUTH9 & & & & & & & & & & & & & & & \\
\hline WVUTH9 & & & & & & & & & & & & & & & \\
\hline WVUTH9 & & & & & & & & & & & & & & & \\
\hline WVUTH9 & & & & & & & & & & & & & & & \\
\hline WVUTH9 & & & & & & & & & & & & & & & \\
\hline WVUTH9 & & & & & & & & & & & & & & & \\
\hline WVUTH9 & & & & & & & & & & & & & & & \\
\hline WVUTH9 & & & & & & & & & & & & & & & \\
\hline WVUTH1C & & & & & & & & & & & & & & & \\
\hline WVUTH10 & & & & & & & & & & & & & & & \\
\hline WVUTH10 & & & & & & & & & & & & & & & \\
\hline CUOD & & & & & & & & & & & & & & & \\
\hline CUOI & & & & & & & & & & & & & & & \\
\hline CUOJ & & & & & & & & & & & & & & & \\
\hline CUOK & & & & & & & & & & & & & & & \\
\hline CUOL & & & & & & & & & & & & & & & \\
\hline CUOB & & & & & & & & & & & & & & & \\
\hline CUOC & & & & & & & & & & & & & & & \\
\hline CUOE & & & & & & & & & & & & & & & \\
\hline CUOF & & & & & & & & & & & & & & & \\
\hline CUOG & & & & & & & & & & & & & & & \\
\hline $\mathrm{CUOH}$ & & & & & & & & & & & & & & & \\
\hline CUOO & & & & & & & & & & & & & & & \\
\hline CUOP & & & & & & & & & & & & & & & \\
\hline CUOR & & & & & & & & & & & & & & & \\
\hline CUOU & & & & & & & & & & & & & & & \\
\hline CUOV & & & & & & & & & & & & & & & \\
\hline CU30 & & & & & & & & & & & & & & & \\
\hline CU31 & & & & & & & & & & & & & & & \\
\hline
\end{tabular}


Appendix A. Database - mass fraction

\begin{tabular}{|c|c|c|c|c|c|c|c|c|c|c|c|c|c|c|c|c|c|c|c|c|c|}
\hline Glass ID & $\begin{array}{c}\mathrm{V} 2 \\
(\mathrm{~Pa} \cdot \mathrm{s})\end{array}$ & $\begin{array}{c}\mathrm{T} 3 \\
\left({ }^{\circ} \mathrm{C}\right) \\
\end{array}$ & $\begin{array}{c}\mathrm{V} 3 \\
(\mathrm{~Pa} \cdot \mathrm{s})\end{array}$ & $\begin{array}{c}\mathrm{T} 4 \\
\left({ }^{\circ} \mathrm{C}\right) \\
\end{array}$ & $\begin{array}{c}\mathrm{V} 4 \\
(\mathrm{~Pa} \cdot \mathrm{s})\end{array}$ & $\begin{array}{c}\mathrm{T} 5 \\
\left({ }^{\circ} \mathrm{C}\right) \\
\end{array}$ & $\begin{array}{c}\mathrm{V} 5 \\
(\mathrm{~Pa} \cdot \mathrm{s})\end{array}$ & $\begin{array}{c}\mathrm{T} 6 \\
\left({ }^{\circ} \mathrm{C}\right) \\
\end{array}$ & $\begin{array}{c}\mathrm{V} 6 \\
(\mathrm{~Pa} \cdot \mathrm{s})\end{array}$ & $\begin{array}{c}\mathrm{T} 7 \\
\left({ }^{\circ} \mathrm{C}\right) \\
\end{array}$ & $\begin{array}{c}\mathrm{V} 7 \\
(\mathrm{~Pa} \cdot \mathrm{s})\end{array}$ & $\begin{array}{c}\mathrm{T} 8 \\
\left({ }^{\circ} \mathrm{C}\right) \\
\end{array}$ & $\begin{array}{c}\mathrm{V} 8 \\
(\mathrm{~Pa} \cdot \mathrm{s})\end{array}$ & $\begin{array}{c}\mathrm{T} 9 \\
\left({ }^{\circ} \mathrm{C}\right) \\
\end{array}$ & $\begin{array}{c}\mathrm{V} 9 \\
(\mathrm{~Pa} \cdot \mathrm{s})\end{array}$ & $\begin{array}{l}\mathrm{T} 10 \\
\left({ }^{\circ} \mathrm{C}\right) \\
\end{array}$ & $\begin{array}{c}\mathrm{V} 10 \\
(\mathrm{~Pa} \cdot \mathrm{s})\end{array}$ & $\begin{array}{l}\mathrm{T} 11 \\
\left({ }^{\circ} \mathrm{C}\right) \\
\end{array}$ & $\begin{array}{c}\mathrm{V} 11 \\
(\mathrm{~Pa} \cdot \mathrm{s})\end{array}$ & $\begin{array}{l}\mathrm{T} 12 \\
\left({ }^{\circ} \mathrm{C}\right)\end{array}$ & $\begin{array}{c}\mathrm{V} 12 \\
(\mathrm{~Pa} \cdot \mathrm{s})\end{array}$ \\
\hline \multicolumn{22}{|c|}{ WVUTH65 } \\
\hline \multicolumn{22}{|l|}{ WVUTH66 } \\
\hline \multicolumn{22}{|l|}{ WVUTH67 } \\
\hline \multicolumn{22}{|l|}{ WVUTH68 } \\
\hline \multicolumn{22}{|l|}{ WVUTH69 } \\
\hline \multicolumn{22}{|c|}{ WVUTH70 } \\
\hline \multicolumn{22}{|l|}{ WVUTH71 } \\
\hline \multicolumn{22}{|l|}{ WVUTH72 } \\
\hline \multicolumn{22}{|l|}{ WVUTH73 } \\
\hline \multicolumn{22}{|l|}{ WVUTH74 } \\
\hline \multicolumn{22}{|l|}{ WVUTH75 } \\
\hline \multicolumn{22}{|l|}{ WVUTH77 } \\
\hline \multicolumn{22}{|l|}{ WVUTH78 } \\
\hline WVUTH7 & & & & & & & & & & & & & & & & & & & & & \\
\hline WVUTH8C & & & & & & & & & & & & & & & & & & & & & \\
\hline WVUTH8 & & & & & & & & & & & & & & & & & & & & & \\
\hline WVUTH8 & & & & & & & & & & & & & & & & & & & & & \\
\hline WVUTH 8 & & & & & & & & & & & & & & & & & & & & & \\
\hline WVUTH8 & & & & & & & & & & & & & & & & & & & & & \\
\hline WVUTH8 & & & & & & & & & & & & & & & & & & & & & \\
\hline WVUTH9 & & & & & & & & & & & & & & & & & & & & & \\
\hline WVUTH9 & & & & & & & & & & & & & & & & & & & & & \\
\hline WVUTH92 & & & & & & & & & & & & & & & & & & & & & \\
\hline WVUTH9 & & & & & & & & & & & & & & & & & & & & & \\
\hline WVUTH9 & & & & & & & & & & & & & & & & & & & & & \\
\hline WVUTH9 & & & & & & & & & & & & & & & & & & & & & \\
\hline WVUTH98 & & & & & & & & & & & & & & & & & & & & & \\
\hline WVUTH9S & & & & & & & & & & & & & & & & & & & & & \\
\hline WVUTH10 & & & & & & & & & & & & & & & & & & & & & \\
\hline WVUTH10 & & & & & & & & & & & & & & & & & & & & & \\
\hline WVUTH10 & & & & & & & & & & & & & & & & & & & & & \\
\hline CUOD & & & & & & & & & & & & & & & & & & & & & \\
\hline CUOI & & & & & & & & & & & & & & & & & & & & & \\
\hline CUOJ & & & & & & & & & & & & & & & & & & & & & \\
\hline CUOK & & & & & & & & & & & & & & & & & & & & & \\
\hline CUOL & & & & & & & & & & & & & & & & & & & & & \\
\hline CUOB & & & & & & & & & & & & & & & & & & & & & \\
\hline CUOC & & & & & & & & & & & & & & & & & & & & & \\
\hline CUOE & & & & & & & & & & & & & & & & & & & & & \\
\hline CUOF & & & & & & & & & & & & & & & & & & & & & \\
\hline CUOG & & & & & & & & & & & & & & & & & & & & & \\
\hline $\mathrm{CUOH}$ & & & & & & & & & & & & & & & & & & & & & \\
\hline CUOO & & & & & & & & & & & & & & & & & & & & & \\
\hline CUOP & & & & & & & & & & & & & & & & & & & & & \\
\hline CUOR & & & & & & & & & & & & & & & & & & & & & \\
\hline CUOU & & & & & & & & & & & & & & & & & & & & & \\
\hline CUOV & & & & & & & & & & & & & & & & & & & & & \\
\hline CU30 & & & & & & & & & & & & & & & & & & & & & \\
\hline CU31 & & & & & & & & & & & & & & & & & & & & & \\
\hline
\end{tabular}


Appendix A. Database - mass fraction

WV Glasses by CUA and PNL (Johnston et al. 1990)

\begin{tabular}{|c|c|c|c|c|c|c|c|c|c|c|c|c|c|c|c|c|}
\hline Glass ID & $\begin{array}{l}\mathrm{T} 13 \\
\left({ }^{\circ} \mathrm{C}\right)\end{array}$ & $\begin{array}{c}\mathrm{V} 13 \\
(\mathrm{~Pa} \cdot \mathrm{s})\end{array}$ & $\begin{array}{l}\mathrm{T} 14 \\
\left({ }^{\circ} \mathrm{C}\right)\end{array}$ & $\begin{array}{c}\mathrm{V} 14 \\
(\mathrm{~Pa} \cdot \mathrm{s})\end{array}$ & $\begin{array}{c}\text { Q PCT } \\
\mathrm{B}\left(\mathrm{g} / \mathrm{m}^{2}\right)\end{array}$ & $\begin{array}{c}\text { Q PCT } \\
\mathrm{Li}\left(\mathrm{g} / \mathrm{m}^{2}\right)\end{array}$ & $\begin{array}{c}\text { Q PCT } \\
\mathrm{Na}\left(\mathrm{g} / \mathrm{m}^{2}\right)\end{array}$ & $\begin{array}{c}\text { Q PCT } \\
\mathrm{Si}\left(\mathrm{g} / \mathrm{m}^{2}\right)\end{array}$ & $\begin{array}{l}\text { Q PCT } \\
\text { pH }\end{array}$ & $\begin{array}{c}\text { CCC PCT } \\
\text { B }\left(\mathrm{g} / \mathrm{m}^{2}\right)\end{array}$ & $\begin{array}{l}\text { CCC PCT } \\
\mathrm{Li}\left(\mathrm{g} / \mathrm{m}^{2}\right)\end{array}$ & $\begin{array}{l}\text { CCC PCT } \\
\mathrm{Na}\left(\mathrm{g} / \mathrm{m}^{2}\right)\end{array}$ & \begin{tabular}{|c|} 
CCC PCT \\
$\mathrm{Si}\left(\mathrm{g} / \mathrm{m}^{2}\right)$
\end{tabular} & $\begin{array}{c}\mathrm{CCC} \text { PCT } \\
\mathrm{pH}\end{array}$ & $\begin{array}{l}\text { Q PCT B at } \\
20^{\circ} \mathrm{C}\left(\mathrm{g} / \mathrm{m}^{2}\right)\end{array}$ & $\begin{array}{l}\text { Q PCT Li at } \\
20^{\circ} \mathrm{C}\left(\mathrm{g} / \mathrm{m}^{2}\right)\end{array}$ \\
\hline WVUTH65 & & & & & 0.246 & & & & & & & & & & & \\
\hline WVUTH66 & & & & & 0.408 & & & & & & & & & & & \\
\hline WVUTH67 & & & & & 0.314 & & & & & & & & & & & \\
\hline WVUTH68 & & & & & 0.298 & & & & & & & & & & & \\
\hline WVUTH69 & & & & & 0.251 & & & & & & & & & & & \\
\hline WVUTH70 & & & & & 0.245 & & & & & & & & & & & \\
\hline WVUTH71 & & & & & 0.231 & & & & & & & & & & & \\
\hline WVUTH72 & & & & & 0.253 & & & & & & & & & & & \\
\hline WVUTH73 & & & & & 0.548 & & & & & & & & & & & \\
\hline WVUTH74 & & & & & 0.807 & & & & & & & & & & & \\
\hline WVUTH75 & & & & & 0.281 & & & & & & & & & & & \\
\hline WVUTH77 & & & & & 0.358 & & & & & & & & & & & \\
\hline WVUTH78 & & & & & 0.325 & & & & & & & & & & & \\
\hline WVUTH79 & & & & & 0.275 & & & & & & & & & & & \\
\hline WVUTH80 & & & & & 0.363 & & & & & & & & & & & \\
\hline WVUTH81 & & & & & 0.315 & & & & & & & & & & & \\
\hline WVUTH82 & & & & & 0.385 & & & & & & & & & & & \\
\hline WVUTH83 & & & & & 0.478 & & & & & & & & & & & \\
\hline WVUTH84 & & & & & 1.113 & & & & & & & & & & & \\
\hline WVUTH89 & & & & & 0.452 & & & & & & & & & & & \\
\hline WVUTH90 & & & & & 0.349 & & & & & & & & & & & \\
\hline WVUTH91 & & & & & 0.864 & & & & & & & & & & & \\
\hline WVUTH92 & & & & & 0.304 & & & & & & & & & & & \\
\hline WVUTH93 & & & & & 1.294 & & & & & & & & & & & \\
\hline WVUTH94 & & & & & 0.421 & & & & & & & & & & & \\
\hline WVUTH96 & & & & & 0.393 & & & & & & & & & & & \\
\hline WVUTH98 & & & & & 0.684 & & & & & & & & & & & \\
\hline WVUTH99 & & & & & 0.629 & & & & & & & & & & & \\
\hline WVUTH100 & & & & & 0.346 & & & & & & & & & & & \\
\hline WVUTH102 & & & & & 0.327 & & & & & & & & & & & \\
\hline WVUTH103 & & & & & 0.878 & & & & & & & & & & & \\
\hline CUOD & & & & & 0.220 & & & & & & & & & & & \\
\hline CUOI & & & & & 0.211 & & & & & & & & & & & \\
\hline CUOJ & & & & & 0.374 & & & & & & & & & & & \\
\hline CUOK & & & & & 0.202 & & & & & & & & & & & \\
\hline CUOL & & & & & 0.271 & & & & & & & & & & & \\
\hline CUOB & & & & & 0.213 & 0.226 & 0.202 & 0.107 & 9.6 & & & & & & & \\
\hline CUOC & & & & & 0.218 & 0.241 & 0.204 & 0.092 & 9.8 & & & & & & & \\
\hline CUOE & & & & & 0.412 & 0.395 & 0.346 & 0.135 & 9.8 & & & & & & & \\
\hline CUOF & & & & & 0.351 & 0.419 & 0.313 & 0.135 & 9.8 & & & & & & & \\
\hline CUOG & & & & & 0.414 & 0.391 & 0.343 & 0.123 & 9.9 & & & & & & & \\
\hline $\mathrm{CUOH}$ & & & & & 0.672 & 0.581 & 0.502 & 0.167 & 10.1 & & & & & & & \\
\hline CUOO & & & & & 0.617 & 0.521 & 0.338 & 0.159 & 10.0 & & & & & & & \\
\hline CUOP & & & & & 0.334 & 0.386 & 0.299 & 0.132 & 9.8 & & & & & & & \\
\hline CUOR & & & & & 0.522 & 0.552 & 0.453 & 0.163 & 10.0 & & & & & & & \\
\hline CUOU & & & & & 0.364 & 0.371 & 0.344 & 0.130 & 9.8 & & & & & & & \\
\hline CUOV & & & & & 0.249 & 0.305 & 0.270 & 0.103 & 9.8 & & & & & & & \\
\hline CU30 & & & & & 0.276 & 0.330 & 0.276 & 0.111 & 9.8 & & & & & & & \\
\hline CU31 & & & & & 0.266 & 0.301 & 0.288 & 0.121 & 9.8 & & & & & & & \\
\hline
\end{tabular}


Appendix A. Database - mass fraction

WV Glasses by CUA and PNL (Johnston et al. 1990)

\begin{tabular}{|c|c|c|c|c|c|c|c|c|c|c|c|c|}
\hline Glass ID & $\begin{array}{l}\text { Q PCT Na at } \\
20^{\circ} \mathrm{C}\left(\mathrm{g} / \mathrm{m}^{2}\right)\end{array}$ & $\begin{array}{l}\text { Q PCT Si at } \\
20^{\circ} \mathrm{C}\left(\mathrm{g} / \mathrm{m}^{2}\right)\end{array}$ & $\begin{array}{c}\mathrm{QpH} \\
\text { at } 20^{\circ} \mathrm{C}\end{array}$ & $\begin{array}{l}\text { TCLP Ag } \\
(\mathrm{ppm})\end{array}$ & $\begin{array}{c}\text { TCLP As } \\
(\mathrm{ppm})\end{array}$ & $\begin{array}{c}\text { TCLP Ba } \\
(\mathrm{ppm})\end{array}$ & $\begin{array}{c}\text { TCLP Cd } \\
(\mathrm{ppm})\end{array}$ & $\begin{array}{c}\text { TCLP Cr } \\
(\mathrm{ppm})\end{array}$ & $\begin{array}{l}\text { TCLP Ni } \\
(\mathrm{ppm})\end{array}$ & $\begin{array}{c}\text { TCLP Pb } \\
(\mathrm{ppm})\end{array}$ & $\begin{array}{c}\text { TCLP Se } \\
(\mathrm{ppm})\end{array}$ & $\begin{array}{c}\text { TCLP Zn } \\
(\mathrm{ppm})\end{array}$ \\
\hline \multicolumn{13}{|c|}{ WVUTH65 } \\
\hline \multicolumn{13}{|c|}{ WVUTH66 } \\
\hline \multicolumn{13}{|c|}{ WVUTH67 } \\
\hline \multicolumn{13}{|c|}{ WVUTH68 } \\
\hline \multicolumn{13}{|c|}{ WVUTH69 } \\
\hline \multicolumn{13}{|c|}{ WVUTH70 } \\
\hline \multicolumn{13}{|c|}{ WVUTH71 } \\
\hline \multicolumn{13}{|c|}{ WVUTH72 } \\
\hline \multicolumn{13}{|c|}{ WVUTH73 } \\
\hline \multicolumn{13}{|c|}{ WVUTH74 } \\
\hline \multicolumn{13}{|c|}{ WVUTH75 } \\
\hline \multicolumn{13}{|c|}{ WVUTH77 } \\
\hline \multicolumn{13}{|c|}{ WVUTH78 } \\
\hline \multicolumn{13}{|c|}{ WVUTH79 } \\
\hline \multicolumn{13}{|c|}{ WVUTH80 } \\
\hline \multicolumn{13}{|c|}{ WVUTH81 } \\
\hline \multicolumn{13}{|c|}{ WVUTH82 } \\
\hline \multicolumn{13}{|c|}{ WVUTH83 } \\
\hline WVUTH8 & & & & & & & & & & & & \\
\hline WVUTH8 & & & & & & & & & & & & \\
\hline WVUTH9 & & & & & & & & & & & & \\
\hline WVUTH9 & & & & & & & & & & & & \\
\hline WVUTH9 & & & & & & & & & & & & \\
\hline WVUTH9 & & & & & & & & & & & & \\
\hline WVUTH9 & & & & & & & & & & & & \\
\hline WVUTH9 & & & & & & & & & & & & \\
\hline WVUTH9 & & & & & & & & & & & & \\
\hline WVUTH9 & & & & & & & & & & & & \\
\hline WVUTH1 & & & & & & & & & & & & \\
\hline WVUTH1 & & & & & & & & & & & & \\
\hline WVUTH1 & & & & & & & & & & & & \\
\hline CUOD & & & & & & & & & & & & \\
\hline CUOI & & & & & & & & & & & & \\
\hline CUOJ & & & & & & & & & & & & \\
\hline CUOK & & & & & & & & & & & & \\
\hline CUOL & & & & & & & & & & & & \\
\hline CUOB & & & & & & & & & & & & \\
\hline CUOC & & & & & & & & & & & & \\
\hline CUOE & & & & & & & & & & & & \\
\hline CUOF & & & & & & & & & & & & \\
\hline CUOG & & & & & & & & & & & & \\
\hline $\mathrm{CUOH}$ & & & & & & & & & & & & \\
\hline CUOO & & & & & & & & & & & & \\
\hline CUOP & & & & & & & & & & & & \\
\hline CUOR & & & & & & & & & & & & \\
\hline CUOU & & & & & & & & & & & & \\
\hline CUOV & & & & & & & & & & & & \\
\hline CU30 & & & & & & & & & & & & \\
\hline CU31 & & & & & & & & & & & & \\
\hline
\end{tabular}




\section{WV Glasses by CUA and PNL (Johnston et al. 1990)}

\begin{tabular}{|c|c|c|c|c|c|c|c|c|c|c|c|c|c|c|c|c|c|c|c|c|c|}
\hline Glass ID & $\begin{array}{c}\mathrm{A} 12 \mathrm{O} 3 \\
-\mathrm{t}\end{array}$ & $\begin{array}{c}\mathrm{B} 2 \mathrm{O} 3 \\
-\mathrm{t}\end{array}$ & $\begin{array}{c}\mathrm{CaO} \\
-\mathrm{t}\end{array}$ & $\begin{array}{c}\mathrm{Fe} 2 \mathrm{O} 3 \\
-\mathrm{t}\end{array}$ & $\begin{array}{c}\mathrm{FeO} \\
-\mathrm{t}\end{array}$ & $\begin{array}{c}\mathrm{K} 2 \mathrm{O} \\
-\mathrm{t}\end{array}$ & $\begin{array}{c}\mathrm{Li} 2 \mathrm{O} \\
-\mathrm{t}\end{array}$ & $\begin{array}{c}\mathrm{MgO} \\
-\mathrm{t}\end{array}$ & $\begin{array}{c}\mathrm{Na} 2 \mathrm{O} \\
-\mathrm{t}\end{array}$ & $\begin{array}{c}\mathrm{NiO} \\
-\mathrm{t}\end{array}$ & $\begin{array}{c}\mathrm{P} 2 \mathrm{O} 5 \\
-\mathrm{t}\end{array}$ & $\begin{array}{c}\mathrm{SiO} 2 \\
-\mathrm{t}\end{array}$ & $\begin{array}{c}\mathrm{ZrO} 2 \\
-\mathrm{t}\end{array}$ & $\begin{array}{c}\mathrm{Ag} 2 \mathrm{O} \\
-\mathrm{t}\end{array}$ & $\begin{array}{c}\mathrm{As} 2 \mathrm{O} 3 \\
-\mathrm{t}\end{array}$ & $\begin{array}{c}\mathrm{BaO} \\
-\mathrm{t}\end{array}$ & $\begin{array}{c}\mathrm{Bi} 2 \mathrm{O} 3 \\
-\mathrm{t}\end{array}$ & $\begin{array}{c}\mathrm{Br} \\
-\mathrm{t}\end{array}$ & $\begin{array}{c}\mathrm{CdO} \\
-\mathrm{t}\end{array}$ & $\begin{array}{c}\mathrm{Ce} 2 \mathrm{O} 3 \\
-\mathrm{t}\end{array}$ & $\begin{array}{c}\mathrm{CeO} 2 \\
-\mathrm{t}\end{array}$ \\
\hline CU33 & 0.0790 & 0.0950 & 0.0060 & 0.1130 & & 0.0330 & 0.0300 & 0.0120 & 0.1050 & 0.0070 & 0.0240 & 0.4320 & 0.0300 & & & 0.0060 & & & & & 0.0020 \\
\hline CU34 & 0.0880 & 0.0940 & 0.0060 & 0.1120 & & 0.0330 & 0.0290 & 0.0120 & 0.1040 & 0.0070 & 0.0240 & 0.4280 & 0.0290 & & & 0.0060 & & & & & 0.0020 \\
\hline CU35 & 0.0320 & 0.0980 & 0.0060 & 0.1160 & & 0.0340 & 0.0300 & 0.0130 & 0.1080 & 0.0070 & 0.0250 & 0.4450 & 0.0300 & & & 0.0060 & & & & & 0.0020 \\
\hline CU36 & 0.0320 & 0.0960 & 0.0060 & 0.1140 & & 0.0340 & 0.0300 & 0.0130 & 0.1060 & 0.0070 & 0.0240 & 0.4360 & 0.0300 & & & 0.0060 & & & & & 0.0020 \\
\hline CU37 & 0.0310 & 0.0940 & 0.0060 & 0.1120 & & 0.0330 & 0.0290 & 0.0120 & 0.1040 & 0.0070 & 0.0240 & 0.4280 & 0.0290 & & & 0.0060 & & & & & 0.0020 \\
\hline CU38 & 0.0320 & 0.0980 & 0.0060 & 0.1160 & & 0.0340 & 0.0300 & 0.0130 & 0.1080 & 0.0070 & 0.0250 & 0.4450 & 0.0300 & & & 0.0060 & & & & & 0.0020 \\
\hline CU39 & 0.0320 & 0.0960 & 0.0060 & 0.1140 & & 0.0340 & 0.0300 & 0.0130 & 0.1060 & 0.0070 & 0.0240 & 0.4360 & 0.0300 & & & 0.0060 & & & & & 0.0020 \\
\hline CU40 & 0.0310 & 0.0940 & 0.0060 & 0.1120 & & 0.0330 & 0.0290 & 0.0120 & 0.1040 & 0.0070 & 0.0240 & 0.4280 & 0.0290 & & & 0.0060 & & & & & 0.0020 \\
\hline CU41 & 0.0320 & 0.0980 & 0.0060 & 0.1160 & & 0.0340 & 0.0300 & 0.0130 & 0.1080 & 0.0070 & 0.0250 & 0.4450 & 0.0300 & & & 0.0060 & & & & & 0.0020 \\
\hline CU42 & 0.0320 & 0.0960 & 0.0060 & 0.1140 & & 0.0340 & 0.0300 & 0.0130 & 0.1060 & 0.0070 & 0.0240 & 0.4360 & 0.0300 & & & 0.0060 & & & & & 0.0020 \\
\hline CU43 & 0.0310 & 0.0940 & 0.0060 & 0.1120 & & 0.0330 & 0.0290 & 0.0120 & 0.1040 & 0.0070 & 0.0240 & 0.4280 & 0.0290 & & & 0.0060 & & & & & 0.0020 \\
\hline CU44 & 0.0320 & 0.0980 & 0.0060 & 0.1160 & & 0.0340 & 0.0300 & 0.0130 & 0.1080 & 0.0070 & 0.0250 & 0.4450 & 0.0300 & & & 0.0060 & & & & & 0.0020 \\
\hline CU46 & 0.0310 & 0.0940 & 0.0060 & 0.1120 & & 0.0330 & 0.0290 & 0.0120 & 0.1040 & 0.0070 & 0.0240 & 0.4280 & 0.0290 & & & 0.0060 & & & & & 0.0020 \\
\hline CU47 & 0.0320 & 0.0980 & 0.0060 & 0.1160 & & 0.0340 & 0.0300 & 0.0130 & 0.1080 & 0.0270 & 0.0250 & 0.4450 & 0.0300 & & & 0.0060 & & & & & 0.0020 \\
\hline CU49 & 0.0310 & 0.0940 & 0.0060 & 0.1120 & & 0.0330 & 0.0290 & 0.0120 & 0.1040 & 0.0630 & 0.0240 & 0.4280 & 0.0290 & & & 0.0060 & & & & & 0.0020 \\
\hline CU50 & 0.0320 & 0.0980 & 0.0060 & 0.1160 & & 0.0340 & 0.0300 & 0.0130 & 0.1080 & 0.0070 & 0.0250 & 0.4450 & 0.0300 & & & 0.0060 & & & & & 0.0020 \\
\hline CU52 & 0.0310 & 0.0940 & 0.0060 & 0.1120 & & 0.0330 & 0.0290 & 0.0120 & 0.1040 & 0.0070 & 0.0240 & 0.4280 & 0.0290 & & & 0.0060 & & & & & 0.0020 \\
\hline \multicolumn{22}{|l|}{ CU53 } \\
\hline \multicolumn{22}{|l|}{ CU54 } \\
\hline \multicolumn{22}{|l|}{ CU55 } \\
\hline \multicolumn{22}{|l|}{ CU56 } \\
\hline \multicolumn{22}{|l|}{ CU57 } \\
\hline CU58 & & & & & & & & & & & & & & & & & & & & & \\
\hline
\end{tabular}

WVDP Support (Olson 1993 and 1994)

\begin{tabular}{|c|c|c|c|c|c|c|c|c|c|c|c|c|c|c|}
\hline PNL 1 & 0.0771 & 0.1656 & 0.0048 & 0.1545 & 0.0313 & 0.0232 & 0.0089 & 0.0572 & 0.0025 & 0.0120 & 0.3949 & 0.0132 & 0.0026 & 0.0031 \\
\hline PNL 2 & 0.0429 & 0.1656 & 0.0048 & 0.0859 & 0.0688 & 0.0232 & 0.0089 & 0.1028 & 0.0025 & 0.0120 & 0.4146 & 0.0132 & 0.0026 & 0.0031 \\
\hline PNL 3 & 0.0771 & 0.1656 & 0.0048 & 0.0859 & 0.0688 & 0.0510 & 0.0089 & 0.1028 & 0.0025 & 0.0120 & 0.3526 & 0.0132 & 0.0026 & 0.0031 \\
\hline PNL 4 & 0.0429 & 0.0922 & 0.0048 & 0.1545 & 0.0688 & 0.0232 & 0.0089 & 0.0572 & 0.0025 & 0.0120 & 0.4650 & 0.0132 & 0.0026 & 0.0031 \\
\hline PNL 5 & 0.0429 & 0.1656 & 0.0048 & 0.0859 & 0.0313 & 0.0510 & 0.0089 & 0.0572 & 0.0025 & 0.0120 & 0.4699 & 0.0132 & 0.0026 & 0.0031 \\
\hline PNL 6 & 0.0771 & 0.0922 & 0.0048 & 0.0859 & 0.0313 & 0.0432 & 0.0089 & 0.1028 & 0.0025 & 0.0120 & 0.4713 & 0.0132 & 0.0026 & 0.0031 \\
\hline PNL 7 & 0.0771 & 0.0922 & 0.0048 & 0.0859 & 0.0688 & 0.0232 & 0.0089 & 0.0572 & 0.0025 & 0.0120 & 0.4638 & 0.0132 & 0.0026 & 0.0031 \\
\hline PNL 8 & 0.0429 & 0.1652 & 0.0048 & 0.1545 & 0.0313 & 0.0232 & 0.0089 & 0.1028 & 0.0025 & 0.0120 & 0.3483 & 0.0132 & 0.0026 & 0.0031 \\
\hline PNL 9 & 0.0771 & 0.0922 & 0.0048 & 0.1545 & 0.0688 & 0.0245 & 0.0089 & 0.1028 & 0.0025 & 0.0120 & 0.3483 & 0.0132 & 0.0026 & 0.0031 \\
\hline PNL 10 & 0.0429 & 0.0922 & 0.0048 & 0.0859 & 0.0313 & 0.0510 & 0.0089 & 0.1028 & 0.0025 & 0.0120 & 0.4621 & 0.0132 & 0.0026 & 0.0031 \\
\hline Alkali1 & 0.0657 & 0.1050 & 0.0048 & 0.1250 & 0.0470 & 0.0360 & 0.0089 & 0.0740 & 0.0025 & 0.0120 & 0.4276 & 0.0145 & 0.0026 & 0.0031 \\
\hline Alkali2 & 0.0590 & 0.1460 & 0.0048 & 0.1242 & 0.0460 & 0.0410 & 0.0089 & 0.0760 & 0.0025 & 0.0120 & 0.3880 & 0.0144 & 0.0026 & 0.0031 \\
\hline Alkali3 & 0.0596 & 0.1330 & 0.0048 & 0.1300 & 0.0480 & 0.0340 & 0.0089 & 0.0820 & 0.0025 & 0.0120 & 0.4000 & 0.0152 & 0.0026 & 0.0031 \\
\hline Alkali4 & 0.0635 & 0.1110 & 0.0048 & 0.1150 & 0.0450 & 0.0350 & 0.0089 & 0.0780 & 0.0025 & 0.0120 & 0.4350 & 0.0153 & 0.0026 & 0.0031 \\
\hline Alkali5 & 0.0620 & 0.1450 & 0.0048 & 0.1100 & 0.0460 & 0.0365 & 0.0089 & 0.0800 & 0.0025 & 0.0120 & 0.4043 & 0.0125 & 0.0026 & 0.0031 \\
\hline Alkali6 & 0.0644 & 0.1180 & 0.0048 & 0.1350 & 0.0510 & 0.0380 & 0.0089 & 0.0775 & 0.0025 & 0.0120 & 0.4025 & 0.0139 & 0.0026 & 0.0031 \\
\hline Alkali7 & 0.0580 & 0.1390 & 0.0048 & 0.1150 & 0.0540 & 0.0400 & 0.0089 & 0.0815 & 0.0025 & 0.0120 & 0.3900 & 0.0160 & 0.0026 & 0.0031 \\
\hline Alkali8 & 0.0560 & 0.1250 & 0.0048 & 0.1150 & 0.0550 & 0.0330 & 0.0089 & 0.0900 & 0.0025 & 0.0120 & 0.4070 & 0.0144 & 0.0026 & 0.0031 \\
\hline Alkali9 & 0.0667 & 0.1250 & 0.0048 & 0.1065 & 0.0510 & 0.0340 & 0.0089 & 0.0720 & 0.0025 & 0.0120 & 0.4215 & 0.0162 & 0.0026 & 0.0031 \\
\hline Ref6Qtr2 & 0.0600 & 0.1289 & 0.0048 & 0.1202 & 0.0500 & 0.0371 & 0.0089 & 0.0800 & 0.0025 & 0.0120 & 0.4098 & 0.0132 & 0.0026 & 0.0031 \\
\hline
\end{tabular}




\section{WV Glasses by CUA and PNL (Johnston et al. 1990)}

\begin{tabular}{|c|c|c|c|c|c|c|c|c|c|c|c|c|c|c|c|c|c|c|c|c|c|}
\hline Glass ID & $\begin{array}{l}\mathrm{Cl} \\
-\mathrm{t}\end{array}$ & $\begin{array}{c}\mathrm{CoO} \\
-\mathrm{t}\end{array}$ & $\begin{array}{c}\mathrm{Co} 2 \mathrm{O} 3 \\
-\mathrm{t}\end{array}$ & $\begin{array}{c}\mathrm{Cr} 2 \mathrm{O} 3 \\
-\mathrm{t}\end{array}$ & $\begin{array}{c}\mathrm{Cs} 2 \mathrm{O} \\
-\mathrm{t}\end{array}$ & $\begin{array}{c}\mathrm{CuO} \\
-\mathrm{t}\end{array}$ & $\begin{array}{c}\mathrm{Eu} 2 \mathrm{O} 3 \\
-\mathrm{t}\end{array}$ & $\begin{array}{l}F \\
-t\end{array}$ & $\begin{array}{c}\mathrm{Ga} 2 \mathrm{O} 3 \\
-\mathrm{t}\end{array}$ & $\begin{array}{c}\mathrm{Gd} 2 \mathrm{O} 3 \\
-\mathrm{t}\end{array}$ & $\begin{array}{c}\mathrm{HgO} \\
-\mathrm{t}\end{array}$ & $\begin{array}{l}I \\
-t\end{array}$ & $\begin{array}{c}\mathrm{La} 2 \mathrm{O} 3 \\
-\mathrm{t}\end{array}$ & $\begin{array}{c}\mathrm{MnO} 2 \\
-\mathrm{t}\end{array}$ & $\begin{array}{c}\mathrm{MnO} \\
-\mathrm{t}\end{array}$ & $\begin{array}{c}\mathrm{MoO} \\
-\mathrm{t}\end{array}$ & $\begin{array}{c}\mathrm{MoO} 3 \\
-\mathrm{t}\end{array}$ & $\begin{array}{c}\mathrm{Nb} 2 \mathrm{O} 5 \\
-\mathrm{t}\end{array}$ & $\begin{array}{c}\mathrm{Nd} 2 \mathrm{O} 3 \\
-\mathrm{t}\end{array}$ & $\begin{array}{c}\mathrm{PbO} \\
-\mathrm{t}\end{array}$ & $\begin{array}{c}\mathrm{PdO} 2 \\
-\mathrm{t}\end{array}$ \\
\hline CU33 & & & & 0.0020 & 0.0010 & 0.0000 & & & & & & & 0.0000 & 0.0130 & & & & & 0.0000 & & \\
\hline CU34 & & & & 0.0020 & 0.0010 & 0.0000 & & & & & & & 0.0000 & 0.0130 & & & & & 0.0000 & & \\
\hline CU35 & & & & 0.0020 & 0.0010 & 0.0000 & & & & & & & 0.0000 & 0.0140 & & & & & 0.0000 & & \\
\hline CU36 & & & & 0.0020 & 0.0010 & 0.0000 & & & & & & & 0.0000 & 0.0130 & & & & & 0.0000 & & \\
\hline CU37 & & & & 0.0020 & 0.0010 & 0.0000 & & & & & & & 0.0000 & 0.0130 & & & & & 0.0000 & & \\
\hline CU38 & & & & 0.0220 & 0.0010 & 0.0000 & & & & & & & 0.0000 & 0.0140 & & & & & 0.0000 & & \\
\hline CU39 & & & & 0.0410 & 0.0010 & 0.0000 & & & & & & & 0.0000 & 0.0130 & & & & & 0.0000 & & \\
\hline CU40 & & & & 0.0590 & 0.0010 & 0.0000 & & & & & & & 0.0000 & 0.0130 & & & & & 0.0000 & & \\
\hline CU41 & & & & 0.0020 & 0.0010 & 0.0000 & & & & & & & 0.0000 & 0.0140 & & & & & 0.0000 & & \\
\hline CU42 & & & & 0.0020 & 0.0010 & 0.0000 & & & & & & & 0.0000 & 0.0130 & & & & & 0.0000 & & \\
\hline CU43 & & & & 0.0020 & 0.0010 & 0.0000 & & & & & & & 0.0000 & 0.0130 & & & & & 0.0000 & & \\
\hline CU44 & & & & 0.0020 & 0.0010 & 0.0200 & & & & & & & 0.0000 & 0.0140 & & & & & 0.0000 & & \\
\hline CU46 & & & & 0.0020 & 0.0010 & 0.0570 & & & & & & & 0.0000 & 0.0130 & & & & & 0.0000 & & \\
\hline CU47 & & & & 0.0020 & 0.0010 & 0.0000 & & & & & & & 0.0000 & 0.0140 & & & & & 0.0000 & & \\
\hline CU49 & & & & 0.0020 & 0.0010 & 0.0000 & & & & & & & 0.0000 & 0.0130 & & & & & 0.0000 & & \\
\hline CU50 & & & & 0.0020 & 0.0010 & 0.0000 & & & & & & & 0.0200 & 0.0140 & & & & & 0.0000 & & \\
\hline CU52 & & & & 0.0020 & 0.0010 & 0.0000 & & & & & & & 0.0570 & 0.0130 & & & & & 0.0000 & & \\
\hline CU53 & & & & & & & & & & & & & & & & & & & & & \\
\hline CU54 & & & & & & & & & & & & & & & & & & & & & \\
\hline CU55 & & & & & & & & & & & & & & & & & & & & & \\
\hline CU56 & & & & & & & & & & & & & & & & & & & & & \\
\hline CU57 & & & & & & & & & & & & & & & & & & & & & \\
\hline CU58 & & & & & & & & & & & & & & & & & & & & & \\
\hline
\end{tabular}

WVDP Support (Olson 1993 and 1994)

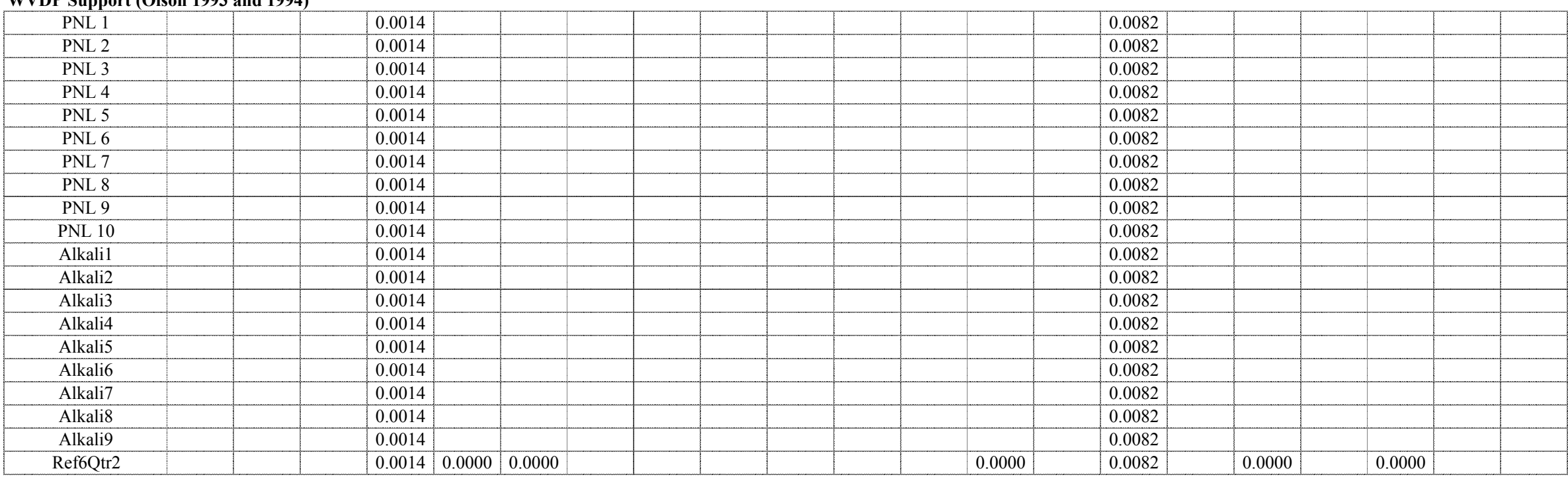




\section{WV Glasses by CUA and PNL (Johnston et al. 1990)}

\begin{tabular}{|c|c|c|c|c|c|c|c|c|c|c|c|c|c|c|c|c|c|c|c|c|c|}
\hline Glass ID & $\begin{array}{c}\mathrm{PdO} \\
-\mathrm{t} \\
\end{array}$ & $\begin{array}{c}\mathrm{P} 2 \mathrm{O} 3 \\
-\mathrm{t}\end{array}$ & {$\left[\begin{array}{c}\text { Pr6O11 } \\
-t\end{array}\right.$} & $\begin{array}{c}\mathrm{Rb} 2 \mathrm{O} \\
-\mathrm{t}\end{array}$ & $\begin{array}{c}\mathrm{ReO} \\
-\mathrm{t} \\
\end{array}$ & $\begin{array}{c}\mathrm{ReO} 2 \\
-\mathrm{t}\end{array}$ & $\begin{array}{c}\mathrm{Rh} 2 \mathrm{O} 3 \\
-\mathrm{t}\end{array}$ & $\begin{array}{c}\mathrm{RhO}^{2} \\
-\mathrm{t}\end{array}$ & $\begin{array}{c}\mathrm{RuO} 2 \\
-\mathrm{t}\end{array}$ & $\begin{array}{c}\mathrm{Sb} 2 \mathrm{O} 3 \\
-\mathrm{t}\end{array}$ & $\begin{array}{c}\mathrm{Sb} 2 \mathrm{O} 5 \\
-\mathrm{t} \\
\end{array}$ & $\begin{array}{c}\mathrm{SeO} 2 \\
-\mathrm{t}\end{array}$ & $\begin{array}{c}\mathrm{Sm} 2 \mathrm{O} 3 \\
-\mathrm{t}\end{array}$ & $\begin{array}{c}\mathrm{SnO} \\
-\mathrm{t} \\
\end{array}$ & $\begin{array}{c}\mathrm{SnO} 2 \\
-\mathrm{t}\end{array}$ & $\begin{array}{c}\text { SO3 } \\
-t \\
\end{array}$ & $\begin{array}{c}\mathrm{SrO} \\
-\mathrm{t} \\
\end{array}$ & $\begin{array}{c}\mathrm{Tc} 2 \mathrm{O} 7 \\
-\mathrm{t} \\
\end{array}$ & $\begin{array}{c}\mathrm{TeO} 2 \\
-\mathrm{t}\end{array}$ & $\begin{array}{c}\mathrm{ThO} 2 \\
-\mathrm{t} \\
\end{array}$ & $\begin{array}{c}\mathrm{TiO} 2 \\
-\mathrm{t}\end{array}$ \\
\hline CU33 & & & & & & & & & & & & & & & & 0.0010 & & & & 0.0000 & 0.0100 \\
\hline CU34 & & & & & & & & & & & & & & & & 0.0010 & & & & 0.0000 & 0.0090 \\
\hline CU35 & & & & & & & & & & & & & & & & 0.0010 & & & & 0.0000 & 0.0300 \\
\hline CU36 & & & & & & & & & & & & & & & & 0.0010 & & & & 0.0000 & 0.0480 \\
\hline CU37 & & & & & & & & & & & & & & & & 0.0010 & & & & 0.0000 & 0.0660 \\
\hline CU38 & & & & & & & & & & & & & & & & 0.0010 & & & & 0.0000 & 0.0100 \\
\hline CU39 & & & & & & & & & & & & & & & & 0.0010 & & & & 0.0000 & 0.0100 \\
\hline CU40 & & & & & & & & & & & & & & & & 0.0010 & & & & 0.0000 & 0.0090 \\
\hline CU41 & & & & & & & & & & & & & & & & 0.0010 & & & & 0.0000 & 0.0100 \\
\hline CU42 & & & & & & & & & & & & & & & & 0.0010 & & & & 0.0000 & 0.0100 \\
\hline CU43 & & & & & & & & & & & & & & & & 0.0010 & & & & 0.0000 & 0.0090 \\
\hline CU44 & & & & & & & & & & & & & & & & 0.0010 & & & & 0.0000 & 0.0100 \\
\hline CU46 & & & & & & & & & & & & & & & & 0.0010 & & & & 0.0000 & 0.0090 \\
\hline CU47 & & & & & & & & & & & & & & & & 0.0010 & & & & 0.0000 & 0.0100 \\
\hline CU49 & & & & & & & & & & & & & & & & 0.0010 & & & & 0.0000 & 0.0090 \\
\hline CU50 & & & & & & & & & & & & & & & & 0.0010 & & & & 0.0000 & 0.0100 \\
\hline CU52 & & & & & & & & & & & & & & & & 0.0010 & & & & 0.0000 & 0.0090 \\
\hline \multicolumn{22}{|l|}{ CU53 } \\
\hline \multicolumn{22}{|l|}{ CU54 } \\
\hline \multicolumn{22}{|l|}{ CU55 } \\
\hline \multicolumn{22}{|l|}{ CU56 } \\
\hline \multicolumn{22}{|l|}{ CU57 } \\
\hline CU58 & & & & & & & & & & & & & & & & & & & & & \\
\hline
\end{tabular}

WVDP Support (Olson 1993 and 1994)

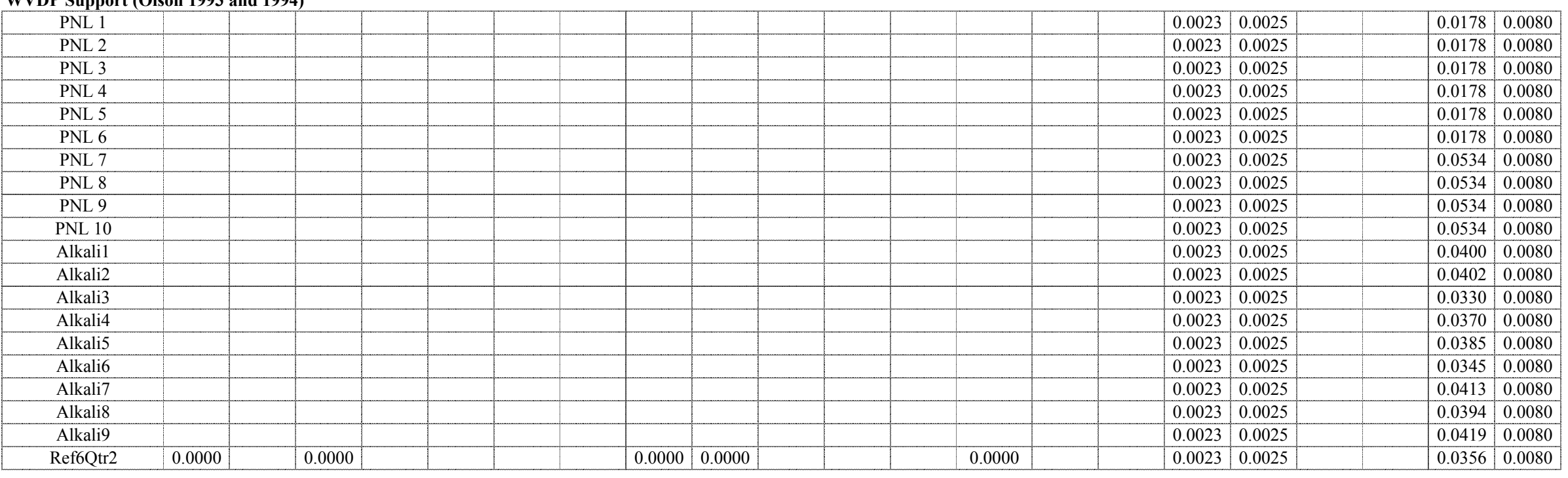


Appendix A. Database - mass fraction

WV Glasses by CUA and PNL (Johnston et al. 1990)

\begin{tabular}{|c|c|c|c|c|c|c|c|c|c|c|c|c|c|c|c|c|c|c|c|c|c|}
\hline Glass ID & $\begin{array}{c}\mathrm{T} 12 \mathrm{O} 3 \\
-\mathrm{t}\end{array}$ & $\begin{array}{c}\mathrm{U} 3 \mathrm{O} 8 \\
-\mathrm{t}\end{array}$ & $\begin{array}{c}\mathrm{UO} 2 \\
-\mathrm{t}\end{array}$ & $\begin{array}{c}\mathrm{UO} 3 \\
-\mathrm{t}\end{array}$ & $\begin{array}{c}\mathrm{V} 2 \mathrm{O} 5 \\
-\mathrm{t}\end{array}$ & $\begin{array}{c}\text { WO3 } \\
-t\end{array}$ & $\begin{array}{c}\mathrm{Y} 2 \mathrm{O} 3 \\
-\mathrm{t}\end{array}$ & $\begin{array}{c}\mathrm{ZnO} \\
-\mathrm{t}\end{array}$ & $\begin{array}{c}\text { Others } \\
-t\end{array}$ & $\begin{array}{c}\text { Sum } \\
-t\end{array}$ & $\begin{array}{c}\mathrm{A} 12 \mathrm{O} 3 \\
-\mathrm{a}\end{array}$ & $\begin{array}{c}\mathrm{B} 2 \mathrm{O} 3 \\
-\mathrm{a}\end{array}$ & $\begin{array}{c}\mathrm{CaO} \\
-\mathrm{a}\end{array}$ & $\begin{array}{c}\mathrm{Fe} 2 \mathrm{O} 3 \\
-\mathrm{a}\end{array}$ & $\begin{array}{c}\mathrm{FeO} \\
-\mathrm{a}\end{array}$ & $\begin{array}{c}\mathrm{K} 2 \mathrm{O} \\
-\mathrm{a}\end{array}$ & $\begin{array}{c}\mathrm{Li} 2 \mathrm{O} \\
-\mathrm{a}\end{array}$ & $\begin{array}{c}\mathrm{MgO} \\
-\mathrm{a}\end{array}$ & $\begin{array}{c}\mathrm{Na} 2 \mathrm{O} \\
-\mathrm{a}\end{array}$ & $\begin{array}{c}\mathrm{NiO} \\
-\mathrm{a}\end{array}$ & $\begin{array}{c}\mathrm{P} 2 \mathrm{O} 5 \\
-\mathrm{a}\end{array}$ \\
\hline CU33 & & & 0.0000 & & & & & 0.0000 & & 1.0010 & & & & & & & & & & & \\
\hline CU34 & & & 0.0000 & & & & & 0.0000 & & 1.0000 & & & & & & & & & & & \\
\hline CU35 & & & 0.0000 & & & & & 0.0000 & & 1.0000 & & & & & & & & & & & \\
\hline CU36 & & & 0.0000 & & & & & 0.0000 & & 1.0010 & & & & & & & & & & & \\
\hline CU37 & & & 0.0000 & & & & & 0.0000 & & 1.0000 & & & & & & & & & & & \\
\hline CU38 & & & 0.0000 & & & & & 0.0000 & & 1.0000 & & & & & & & & & & & \\
\hline CU39 & & & 0.0000 & & & & & 0.0000 & & 1.0020 & & & & & & & & & & & \\
\hline CU40 & & & 0.0000 & & & & & 0.0000 & & 1.0000 & & & & & & & & & & & \\
\hline CU41 & & & 0.0000 & & & & & 0.0200 & & 1.0000 & & & & & & & & & & & \\
\hline CU42 & & & 0.0000 & & & & & 0.0390 & & 1.0020 & & & & & & & & & & & \\
\hline CU43 & & & 0.0000 & & & & & 0.0570 & & 1.0000 & & & & & & & & & & & \\
\hline CU44 & & & 0.0000 & & & & & 0.0000 & & 1.0000 & & & & & & & & & & & \\
\hline CU46 & & & 0.0000 & & & & & 0.0000 & & 1.0000 & & & & & & & & & & & \\
\hline CU47 & & & 0.0000 & & & & & 0.0000 & & 1.0000 & & & & & & & & & & & \\
\hline CU49 & & & 0.0000 & & & & & 0.0000 & & 0.9990 & & & & & & & & & & & \\
\hline CU50 & & & 0.0000 & & & & & 0.0000 & & 1.0000 & & & & & & & & & & & \\
\hline CU52 & & & 0.0000 & & & & & 0.0000 & & 1.0000 & & & & & & & & & & & \\
\hline CU53 & & & & & & & & & & 0.0000 & 0.0594 & 0.0949 & & 0.1125 & & & & & & & 0.0238 \\
\hline CU54 & & & & & & & & & & 0.0000 & 0.0586 & 0.0942 & & 0.1115 & & & & & & & 0.0236 \\
\hline CU55 & & & & & & & & & & 0.0000 & 0.0569 & 0.0923 & & 0.1094 & & & & & & & 0.0232 \\
\hline CU56 & & & & & & & & & & 0.0000 & 0.0584 & 0.0939 & & 0.1112 & & & & & & & 0.0236 \\
\hline CU57 & & & & & & & & & & 0.0000 & 0.0599 & 0.0955 & & 0.1131 & & & & & & & 0.0240 \\
\hline CU58 & & & & & & & & & & 0.0000 & 0.0330 & 0.0996 & & 0.1180 & & & & & & & 0.0250 \\
\hline
\end{tabular}

WVDP Support (Olson 1993 and 1994)

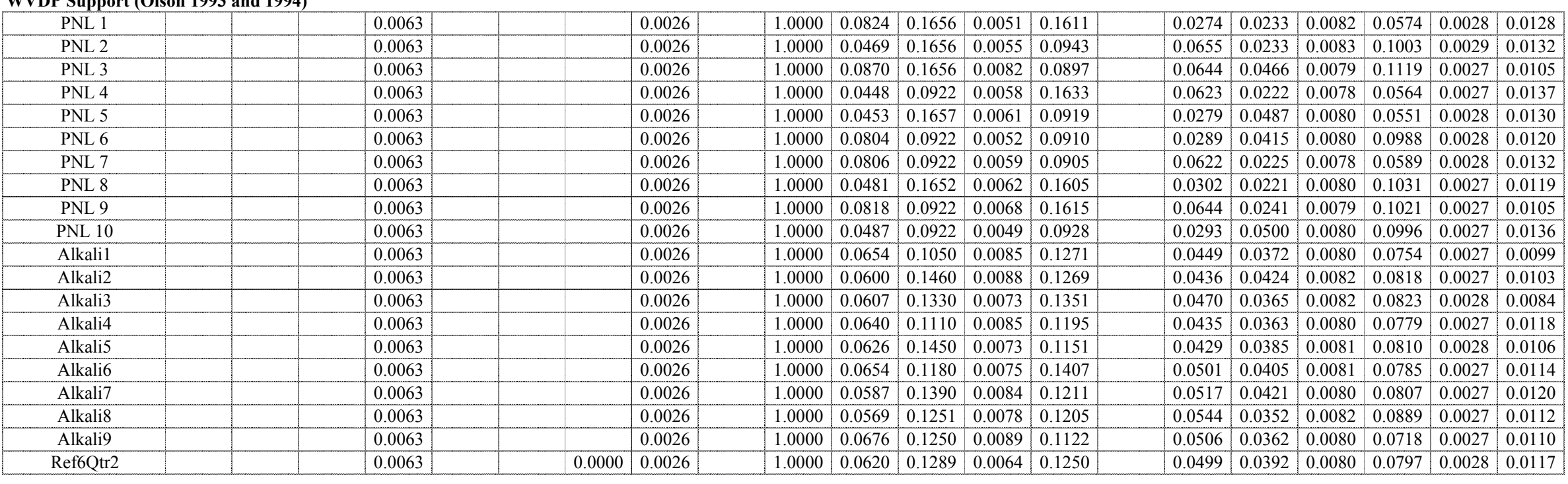


Appendix A. Database - mass fraction

WV Glasses by CUA and PNL (Johnston et al. 1990)

\begin{tabular}{|c|c|c|c|c|c|c|c|c|c|c|c|c|c|c|c|c|c|c|c|c|c|}
\hline Glass ID & $\begin{array}{c}\mathrm{SiO} 2 \\
-\mathrm{a} \\
\end{array}$ & $\begin{array}{c}\mathrm{ZrO} 2 \\
-\mathrm{a}\end{array}$ & $\begin{array}{c}\mathrm{Ag} 2 \mathrm{O} \\
-\mathrm{a}\end{array}$ & $\begin{array}{c}\mathrm{As} 2 \mathrm{O} 3 \\
-\mathrm{a} \\
\end{array}$ & $\begin{array}{c}\mathrm{BaO} \\
-\mathrm{a} \\
\end{array}$ & $\begin{array}{c}\mathrm{Bi} 2 \mathrm{O} 3 \\
-\mathrm{a} \\
\end{array}$ & $\begin{array}{l}\mathrm{Br} \\
-\mathrm{a} \\
\end{array}$ & $\begin{array}{c}\mathrm{CdO} \\
-\mathrm{a} \\
\end{array}$ & $\begin{array}{c}\mathrm{Ce} 2 \mathrm{O} 3 \\
-\mathrm{a}\end{array}$ & $\begin{array}{c}\mathrm{CeO} 2 \\
-\mathrm{a}\end{array}$ & $\begin{array}{l}\mathrm{Cl} \\
-\mathrm{a} \\
\end{array}$ & $\begin{array}{c}\mathrm{CoO} \\
-\mathrm{a}\end{array}$ & $\begin{array}{c}\mathrm{Co} 2 \mathrm{O} 3 \\
-\mathrm{a} \\
\end{array}$ & $\begin{array}{c}\mathrm{Cr} 2 \mathrm{O} 3 \\
-\mathrm{a}\end{array}$ & $\begin{array}{c}\mathrm{Cs} 2 \mathrm{O} \\
-\mathrm{a} \\
\end{array}$ & $\begin{array}{c}\mathrm{CuO} \\
-\mathrm{a}\end{array}$ & \begin{tabular}{|c|}
$\mathrm{Eu} 2 \mathrm{O} 3$ \\
$-\mathrm{a}$ \\
\end{tabular} & $\begin{array}{c}\mathrm{F} \\
-\mathrm{a} \\
\end{array}$ & $\begin{array}{c}\mathrm{Ga} 2 \mathrm{O} 3 \\
-\mathrm{a}\end{array}$ & $\begin{array}{c}\mathrm{Gd} 2 \mathrm{O} 3 \\
-\mathrm{a}\end{array}$ & $\begin{array}{c}\mathrm{HgO} \\
-\mathrm{a} \\
\end{array}$ \\
\hline \multicolumn{22}{|l|}{ CU33 } \\
\hline \multicolumn{22}{|l|}{ CU34 } \\
\hline \multirow{2}{*}{\multicolumn{22}{|c|}{$\frac{\text { CU35 }}{\text { CU36 }}$}} \\
\hline CU36 & & & & & & & & & & & & & & & & & & & & & \\
\hline \multicolumn{22}{|l|}{ CU37 } \\
\hline \multicolumn{22}{|l|}{ CU38 } \\
\hline \multirow{2}{*}{\multicolumn{22}{|c|}{$\begin{array}{l}\text { CU39 } \\
\text { CU40 }\end{array}$}} \\
\hline CU40 & & & & & & & & & & & & & & & & & & & & & \\
\hline \multicolumn{22}{|l|}{ CU41 } \\
\hline \multicolumn{22}{|l|}{ CU42 } \\
\hline \multicolumn{22}{|l|}{ CU43 } \\
\hline CU44 & & & & & & & & & & & & & & & & & & & & & \\
\hline CU46 & & & & & & & & & & & & & & & & & & & & & \\
\hline \multicolumn{22}{|l|}{ CU47 } \\
\hline \multicolumn{22}{|l|}{ CU49 } \\
\hline CU50 & & & & & & & & & & & & & & & & & & & & & \\
\hline CU52 & & & & & & & & & & & & & & & & & & & & & \\
\hline CU53 & 0.4310 & & & & & & & & & & & & & & & & & & & & \\
\hline CU54 & 0.4275 & & & & & & & & & & & & & & & & & & & & \\
\hline CU55 & 0.4191 & & & & & & & & & & & & & & & & & & & & \\
\hline CU56 & 0.4261 & & & & & & & & & & & & & & & & & & & & \\
\hline CU57 & 0.4334 & & & & & & & & & & & & & & & & & & & & \\
\hline CU58 & 0.4522 & & & & & & & & & & & & & & & & & & & & \\
\hline
\end{tabular}

WVDP Support (Olson 1993 and 1994)

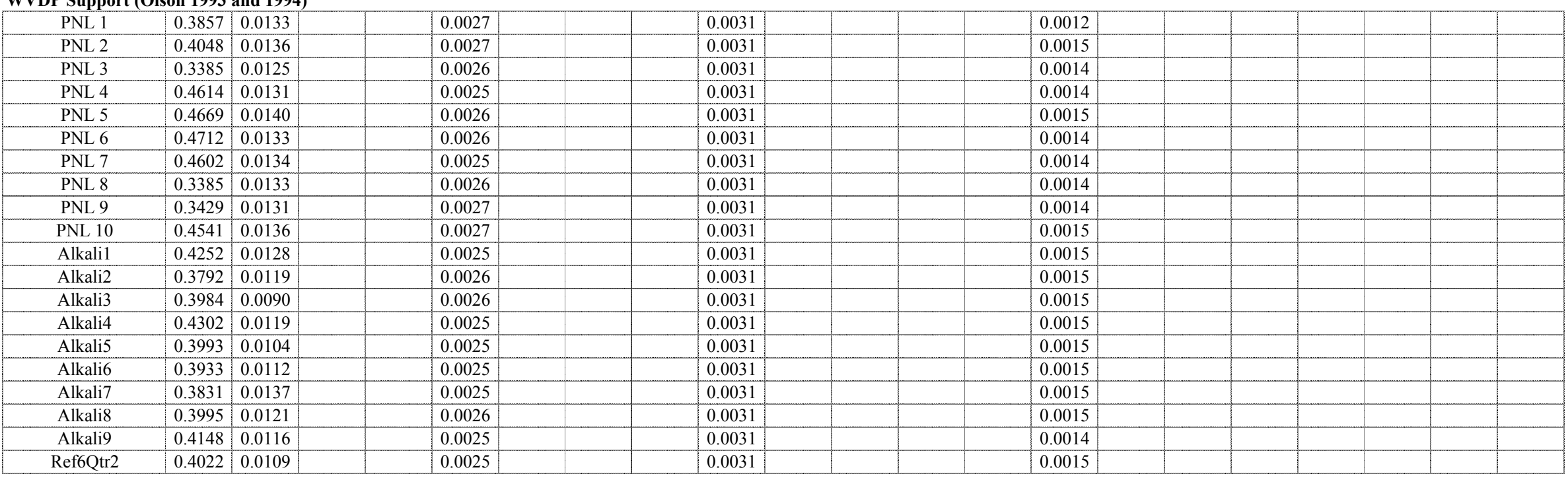


WV Glasses by CUA and PNL (Johnston et al. 1990)

\begin{tabular}{|c|c|c|c|c|c|c|c|c|c|c|c|c|c|c|c|c|c|c|c|c|c|}
\hline Glass ID & $\begin{array}{c}\mathrm{I} \\
-\mathrm{a} \\
\end{array}$ & $\begin{array}{c}\mathrm{La} 2 \mathrm{O} 3 \\
-\mathrm{a}\end{array}$ & $\begin{array}{c}\mathrm{MnO} 2 \\
-\mathrm{a}\end{array}$ & $\begin{array}{c}\mathrm{MnO} \\
-\mathrm{a}\end{array}$ & $\begin{array}{c}\mathrm{MoO} \\
-\mathrm{a}\end{array}$ & $\begin{array}{c}\mathrm{MoO} 3 \\
-\mathrm{a}\end{array}$ & $\begin{array}{c}\mathrm{Nb} 2 \mathrm{O} 5 \\
-\mathrm{a}\end{array}$ & $\begin{array}{c}\mathrm{Nd} 2 \mathrm{O} 3 \\
-\mathrm{a}\end{array}$ & $\begin{array}{c}\mathrm{PbO} \\
-\mathrm{a}\end{array}$ & $\begin{array}{c}\mathrm{PdO} 2 \\
-\mathrm{a}\end{array}$ & $\begin{array}{c}\mathrm{PdO} \\
-\mathrm{a} \\
\end{array}$ & $\begin{array}{c}\mathrm{Pr} 2 \mathrm{O} 3 \\
-\mathrm{a}\end{array}$ & $\begin{array}{c}\text { Pr6O11 } \\
-\mathrm{a}\end{array}$ & $\begin{array}{c}\mathrm{Rb} 2 \mathrm{O} \\
-\mathrm{a}\end{array}$ & $\begin{array}{c}\mathrm{ReO} \\
-\mathrm{a}\end{array}$ & $\begin{array}{c}\mathrm{ReO} 2 \\
-\mathrm{a}\end{array}$ & $\begin{array}{c}\mathrm{Rh} 2 \mathrm{O} 3 \\
-\mathrm{a}\end{array}$ & $\begin{array}{c}\mathrm{RhO} 2 \\
-\mathrm{a}\end{array}$ & $\begin{array}{c}\mathrm{RuO} 2 \\
-\mathrm{a}\end{array}$ & $\begin{array}{c}\mathrm{Sb} 2 \mathrm{O} 3 \\
-\mathrm{a}\end{array}$ & $\begin{array}{c}\mathrm{Sb} 2 \mathrm{O} 5 \\
-\mathrm{a}\end{array}$ \\
\hline CU33 & & & & & & & & & & & & & & & & & & & & & \\
\hline CU34 & & & & & & & & & & & & & & & & & & & & & \\
\hline CU35 & & & & & & & & & & & & & & & & & & & & & \\
\hline CU36 & & & & & & & & & & & & & & & & & & & & & \\
\hline CU37 & & & & & & & & & & & & & & & & & & & & & \\
\hline CU38 & & & & & & & & & & & & & & & & & & & & & \\
\hline CU39 & & & & & & & & & & & & & & & & & & & & & \\
\hline CU40 & & & & & & & & & & & & & & & & & & & & & \\
\hline CU41 & & & & & & & & & & & & & & & & & & & & & \\
\hline CU42 & & & & & & & & & & & & & & & & & & & & & \\
\hline CU43 & & & & & & & & & & & & & & & & & & & & & \\
\hline CU44 & & & & & & & & & & & & & & & & & & & & & \\
\hline CU46 & & & & & & & & & & & & & & & & & & & & & \\
\hline CU47 & & & & & & & & & & & & & & & & & & & & & \\
\hline CU49 & & & & & & & & & & & & & & & & & & & & & \\
\hline CU50 & & & & & & & & & & & & & & & & & & & & & \\
\hline CU52 & & & & & & & & & & & & & & & & & & & & & \\
\hline CU53 & & & 0.0132 & & & & & & & & & & & & & & & & & & \\
\hline CU54 & & & 0.0131 & & & & & & & & & & & & & & & & & & \\
\hline CU55 & & & 0.0129 & & & & & & & & & & & & & & & & & & \\
\hline CU56 & & & 0.0131 & & & & & & & & & & & & & & & & & & \\
\hline CU57 & & & 0.0133 & & & & & & & & & & & & & & & & & & \\
\hline CU58 & & & 0.0136 & & & & & & & & & & & & & & & & & & \\
\hline
\end{tabular}

WVDP Support (Olson 1993 and 1994)

\begin{tabular}{|l|l|l}
\hline PNL 1 & & 0.0087 \\
\hline PNL 2 & & 0.0089 \\
\hline PNL 3 & & 0.0083 \\
\hline PNL 4 & & 0.0085 \\
\hline PNL 5 & & 0.0084 \\
\hline PNL 6 & & 0.0085 \\
\hline PNL 7 & & 0.0083 \\
\hline PNL 8 & & 0.0084 \\
\hline PNL 9 & & 0.0083 \\
\hline PNL 10 & & 0.0085 \\
\hline Alkali1 & & 0.0094 \\
\hline Alkali2 & & 0.0093 \\
\hline Alkali3 & & 0.0095 \\
\hline Alkali4 & & 0.0093 \\
\hline Alkali5 & & 0.0093 \\
\hline Alkali6 & & 0.0095 \\
\hline Alkali7 & & 0.0093 \\
\hline Alkali8 & & 0.0093 \\
\hline Alkali9 & & 0.0092 \\
\hline Ref6Qtr2 & & 0.0091 \\
\hline
\end{tabular}


Appendix A. Database - mass fraction

WV Glasses by CUA and PNL (Johnston et al. 1990)

\begin{tabular}{|c|c|c|c|c|c|c|c|c|c|c|c|c|c|c|c|c|c|c|c|c|}
\hline Glass ID & $\begin{array}{c}\mathrm{SeO} 2 \\
-\mathrm{a}\end{array}$ & $\begin{array}{c}\mathrm{Sm} 2 \mathrm{O} 3 \\
-\mathrm{a}\end{array}$ & $\begin{array}{c}\mathrm{SnO} \\
-\mathrm{a}\end{array}$ & $\begin{array}{c}\mathrm{SnO}^{2} \\
-\mathrm{a}\end{array}$ & $\begin{array}{c}\mathrm{SO} 3 \\
-\mathrm{a}\end{array}$ & $\begin{array}{c}\mathrm{SrO} \\
-\mathrm{a}\end{array}$ & $\begin{array}{c}\mathrm{Tc} 2 \mathrm{O} 7 \\
-\mathrm{a}\end{array}$ & $\begin{array}{c}\mathrm{TeO} 2 \\
-\mathrm{a}\end{array}$ & $\begin{array}{c}\mathrm{ThO} 2 \\
-\mathrm{a}\end{array}$ & $\begin{array}{c}\mathrm{TiO} 2 \\
-\mathrm{a}\end{array}$ & $\begin{array}{c}\mathrm{T} 12 \mathrm{O} 3 \\
-\mathrm{a}\end{array}$ & $\begin{array}{c}\mathrm{U} 3 \mathrm{O} 8 \\
-\mathrm{a}\end{array}$ & $\begin{array}{c}\mathrm{UO} 2 \\
-\mathrm{a}\end{array}$ & $\begin{array}{c}\mathrm{UO3} \\
-\mathrm{a}\end{array}$ & $\begin{array}{c}\mathrm{V} 2 \mathrm{O} 5 \\
-\mathrm{a}\end{array}$ & $\begin{array}{c}\text { WO3 } \\
-\mathrm{a}\end{array}$ & $\begin{array}{c}\mathrm{Y} 2 \mathrm{O} 3 \\
-\mathrm{a}\end{array}$ & $\begin{array}{c}\mathrm{ZnO} \\
-\mathrm{a}\end{array}$ & $\begin{array}{c}\text { Others } \\
-\mathrm{a}\end{array}$ & $\begin{array}{c}\text { Sum } \\
-\mathrm{a}\end{array}$ \\
\hline \multicolumn{21}{|l|}{ CU33 } \\
\hline \multicolumn{21}{|l|}{ CU34 } \\
\hline \multicolumn{21}{|l|}{ CU35 } \\
\hline \multicolumn{21}{|l|}{ CU36 } \\
\hline \multicolumn{21}{|l|}{ CU37 } \\
\hline \multicolumn{21}{|l|}{ CU38 } \\
\hline \multicolumn{21}{|l|}{ CU39 } \\
\hline \multicolumn{21}{|l|}{ CU40 } \\
\hline \multicolumn{21}{|l|}{ CU41 } \\
\hline \multicolumn{21}{|l|}{ CU42 } \\
\hline \multicolumn{21}{|l|}{ CU43 } \\
\hline \multicolumn{21}{|l|}{ CU44 } \\
\hline \multicolumn{21}{|l|}{ CU46 } \\
\hline \multicolumn{21}{|l|}{ CU47 } \\
\hline \multicolumn{21}{|l|}{ CU49 } \\
\hline \multicolumn{21}{|l|}{ CU50 } \\
\hline \multicolumn{21}{|l|}{ CU52 } \\
\hline CU53 & & & & & & & & & 0.0000 & & & & 0.0000 & & & & & & 0.2652 & 1.0000 \\
\hline CU54 & & & & & & & & & 0.0000 & & & & 0.0000 & & & & & & 0.2716 & 1.0001 \\
\hline CU55 & & & & & & & & & 0.0000 & & & & 0.0000 & & & & & & 0.2864 & 1.0002 \\
\hline CU56 & & & & & & & & & 0.0000 & & & & 0.0000 & & & & & & 0.2739 & 1.0002 \\
\hline CU57 & & & & & & & & & 0.0000 & & & & 0.0000 & & & & & & 0.2611 & 1.0003 \\
\hline CU58 & & & & & & & & & 0.0000 & & & & 0.0000 & & & & & & 0.2551 & 0.9965 \\
\hline
\end{tabular}

WVDP Support (Olson 1993 and 1994)

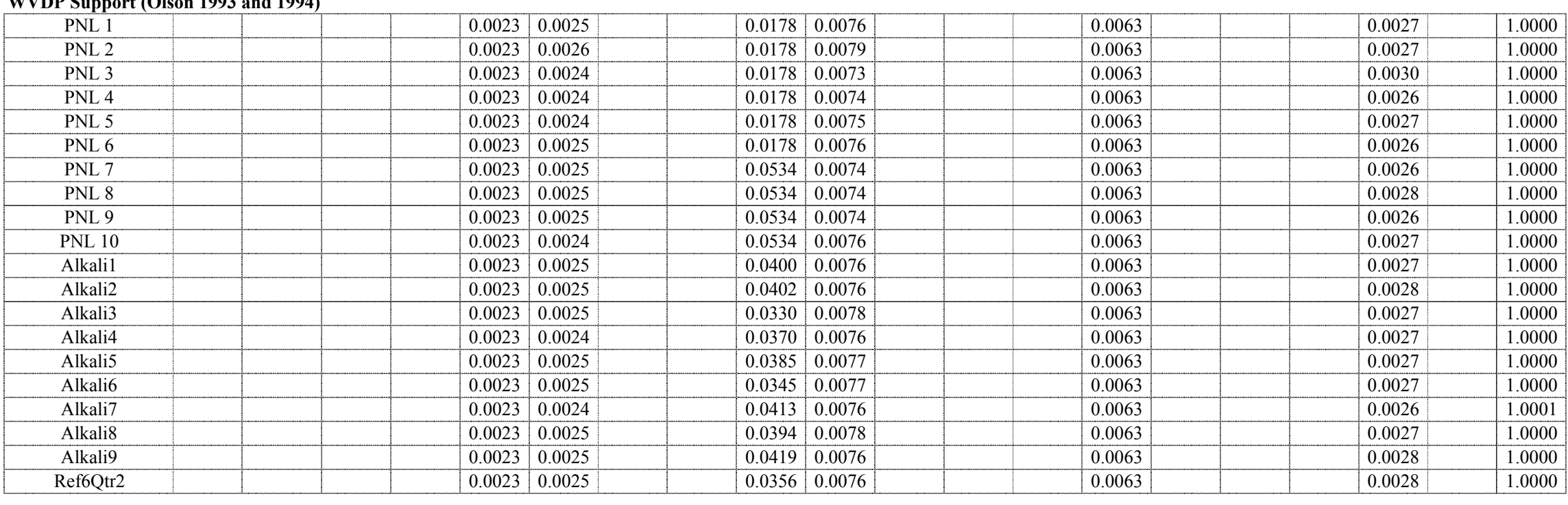


Appendix A. Database - mass fraction

\section{WV Glasses by CUA and PNL (Johnston et al. 1990)}

\begin{tabular}{|c|c|c|c|c|c|c|c|c|}
\hline Glass ID & $\begin{array}{l}\mathrm{TM} \\
\left({ }^{\circ} \mathrm{C}\right) \\
\end{array}$ & $\begin{array}{l}\text { Gradient } \\
\text { TL }\left({ }^{\circ} \mathrm{C}\right)\end{array}$ & $\begin{array}{l}\text { Uniform } \\
\text { TL }\left({ }^{\circ} \mathrm{C}\right)\end{array}$ & Primary Phase & $\begin{array}{l}\text { Quenched } \\
\text { Visual/OM }\end{array}$ & $\begin{array}{c}\text { Quenched } \\
\text { SEM/EDS or TEM }\end{array}$ & $\begin{array}{l}\text { Quenched } \\
\text { XRD }\end{array}$ & $\begin{array}{c}\text { Quenched } \\
\text { Homogeneous? }\end{array}$ \\
\hline \multicolumn{9}{|l|}{ CU33 } \\
\hline \multicolumn{9}{|l|}{ CU34 } \\
\hline \multicolumn{9}{|l|}{ CU35 } \\
\hline \multicolumn{9}{|l|}{ CU36 } \\
\hline \multicolumn{9}{|l|}{ CU37 } \\
\hline \multicolumn{9}{|l|}{ CU38 } \\
\hline \multicolumn{9}{|l|}{ CU39 } \\
\hline \multicolumn{9}{|l|}{ CU40 } \\
\hline \multicolumn{9}{|l|}{ CU41 } \\
\hline \multicolumn{9}{|l|}{ CU42 } \\
\hline \multicolumn{9}{|l|}{ CU43 } \\
\hline \multicolumn{9}{|l|}{ CU44 } \\
\hline \multicolumn{9}{|l|}{ CU46 } \\
\hline \multicolumn{9}{|l|}{ CU47 } \\
\hline \multicolumn{9}{|l|}{ CU49 } \\
\hline \multicolumn{9}{|l|}{ CU50 } \\
\hline \multicolumn{9}{|l|}{ CU52 } \\
\hline \multicolumn{9}{|l|}{ CU53 } \\
\hline \multicolumn{9}{|l|}{ CU54 } \\
\hline \multicolumn{9}{|l|}{ CU55 } \\
\hline \multicolumn{9}{|l|}{ CU56 } \\
\hline CU57 & & & & & & & & \\
\hline CU58 & & & & & & & & \\
\hline
\end{tabular}

WVDP Support (Olson 1993 and 1994)

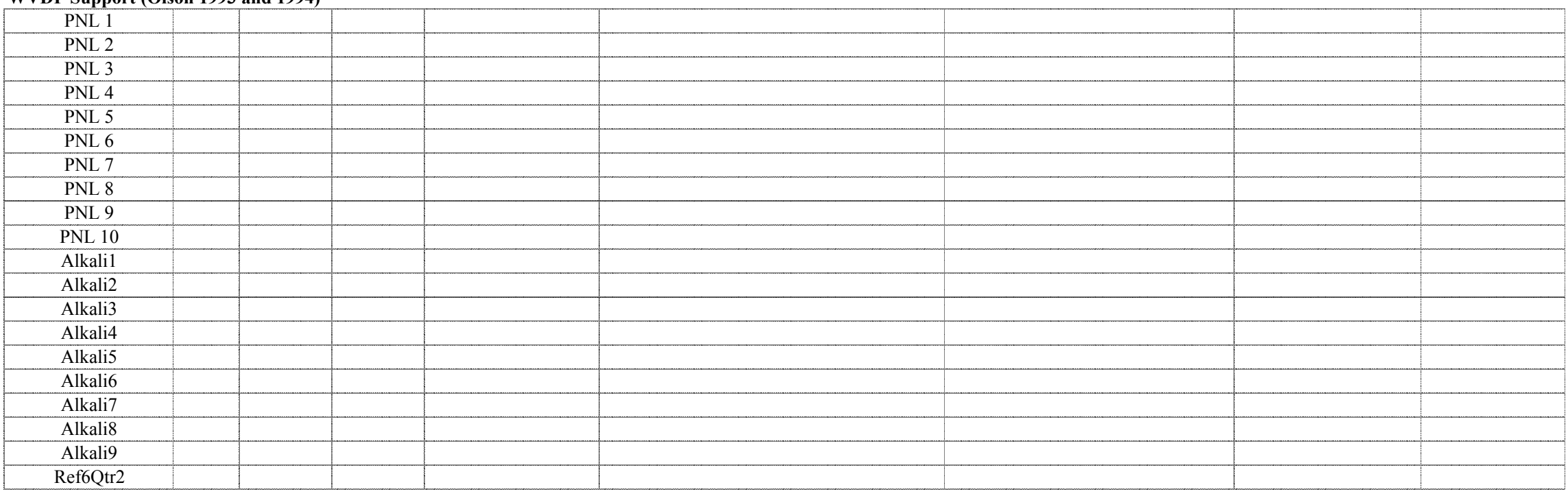


Appendix A. Database - mass fraction

WV Glasses by CUA and PNL (Johnston et al. 1990)

\begin{tabular}{|c|c|c|c|c|c|}
\hline Glass ID & $\begin{array}{c}\text { CCC } \\
\text { Visual/OM }\end{array}$ & $\begin{array}{c}\text { CCC } \\
\text { SEM/EDS or TEM }\end{array}$ & $\begin{array}{l}\text { CCC } \\
\text { XRD }\end{array}$ & $\begin{array}{c}\text { Heat Treated } \\
\text { Visual/OM }\end{array}$ & $\begin{array}{c}\text { Heat Treated } \\
\text { SEM/EDS or TEM }\end{array}$ \\
\hline \multicolumn{6}{|l|}{ CU33 } \\
\hline \multicolumn{6}{|l|}{ CU34 } \\
\hline \multicolumn{6}{|l|}{ CU35 } \\
\hline \multicolumn{6}{|l|}{ CU36 } \\
\hline \multicolumn{6}{|l|}{ CU37 } \\
\hline \multicolumn{6}{|l|}{ CU38 } \\
\hline \multicolumn{6}{|l|}{ CU39 } \\
\hline \multicolumn{6}{|l|}{ CU40 } \\
\hline \multicolumn{6}{|l|}{ CU41 } \\
\hline \multicolumn{6}{|l|}{ CU42 } \\
\hline \multicolumn{6}{|l|}{ CU43 } \\
\hline \multicolumn{6}{|l|}{ CU44 } \\
\hline \multicolumn{6}{|l|}{ CU46 } \\
\hline \multicolumn{6}{|l|}{ CU47 } \\
\hline \multicolumn{6}{|l|}{ CU49 } \\
\hline \multicolumn{6}{|l|}{ CU50 } \\
\hline \multicolumn{6}{|l|}{ CU52 } \\
\hline \multicolumn{6}{|l|}{ CU53 } \\
\hline \multicolumn{6}{|l|}{ CU54 } \\
\hline \multicolumn{6}{|l|}{ CU55 } \\
\hline \multicolumn{6}{|l|}{ CU56 } \\
\hline \multicolumn{6}{|l|}{ CU57 } \\
\hline CU58 & & & & & \\
\hline
\end{tabular}

WVDP Support (Olson 1993 and 1994)

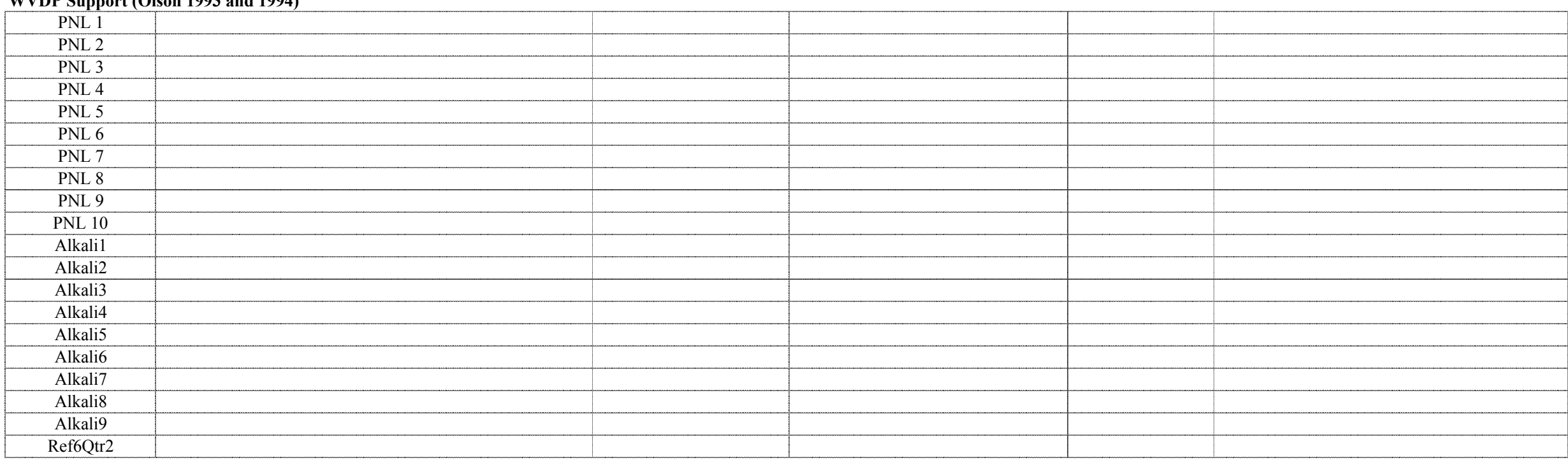


Appendix A. Database - mass fraction

WV Glasses by CUA and PNL (Johnston et al. 1990)

\begin{tabular}{|c|c|c|c|c|c|c|c|c|c|c|c|c|c|c|c|}
\hline Glass ID & $\begin{array}{l}\text { Heat Treated } \\
\text { XRD }\end{array}$ & $\begin{array}{l}\text { Density } \\
\left(\mathrm{g} / \mathrm{cm}^{3}\right)\end{array}$ & $\begin{array}{c}\text { Fulc Visc } \\
\mathrm{A}\end{array}$ & $\begin{array}{c}\text { Fulc Visc } \\
\text { B }\end{array}$ & $\begin{array}{c}\text { Fulc Visc } \\
\text { To } \\
\end{array}$ & $\begin{array}{c}\mathrm{FV} 1150^{\circ} \mathrm{C} \\
(\mathrm{Pa} \cdot \mathrm{s})\end{array}$ & $\begin{array}{c}\text { Arrh Visc } \\
\text { A }\end{array}$ & $\begin{array}{c}\text { Arrh Visc } \\
\text { B }\end{array}$ & $1150^{\circ} \mathrm{C}$ & $\begin{array}{c}\mathrm{T}\left({ }^{\circ} \mathrm{C}\right) \text { at } \\
2 \mathrm{~Pa} \cdot \mathrm{s}\end{array}$ & $\begin{array}{c}\mathrm{T}\left({ }^{\circ} \mathrm{C}\right) \text { at } \\
5 \mathrm{~Pa} \cdot \mathrm{s}\end{array}$ & $\begin{array}{l}\mathrm{T}\left({ }^{\circ} \mathrm{C}\right) \text { at } \\
10 \mathrm{~Pa} \cdot \mathrm{s}\end{array}$ & $\begin{array}{l}\mathrm{T} 1 \\
\left({ }^{\circ} \mathrm{C}\right)\end{array}$ & $\begin{array}{c}\mathrm{V} 1 \\
(\mathrm{~Pa} \cdot \mathrm{s}) \\
\end{array}$ & $\begin{array}{c}\mathrm{T} 2 \\
\left({ }^{\circ} \mathrm{C}\right) \\
\end{array}$ \\
\hline \multicolumn{16}{|c|}{ 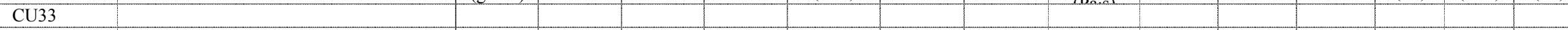 } \\
\hline \multicolumn{16}{|l|}{ CU34 } \\
\hline \multicolumn{16}{|l|}{ CU35 } \\
\hline \multicolumn{16}{|l|}{ CU36 } \\
\hline \multicolumn{16}{|l|}{ CU37 } \\
\hline \multicolumn{16}{|l|}{ CU38 } \\
\hline \multicolumn{16}{|l|}{ CU39 } \\
\hline \multicolumn{16}{|l|}{ CU40 } \\
\hline \multicolumn{16}{|l|}{ CU41 } \\
\hline \multicolumn{16}{|l|}{ CU42 } \\
\hline \multicolumn{16}{|l|}{ CU43 } \\
\hline \multicolumn{16}{|l|}{ CU44 } \\
\hline \multicolumn{16}{|l|}{ CU46 } \\
\hline \multicolumn{16}{|l|}{ CU47 } \\
\hline \multicolumn{16}{|l|}{ CU49 } \\
\hline \multicolumn{16}{|l|}{ CU50 } \\
\hline \multicolumn{16}{|l|}{ CU52 } \\
\hline \multicolumn{16}{|l|}{ CU53 } \\
\hline \multicolumn{16}{|l|}{ CU54 } \\
\hline \multicolumn{16}{|l|}{ CU55 } \\
\hline \multicolumn{16}{|l|}{ CU56 } \\
\hline \multicolumn{16}{|l|}{ CU57 } \\
\hline CU58 & & & & & & & & & & & & & & & \\
\hline
\end{tabular}

WVDP Support (Olson 1993 and 1994)

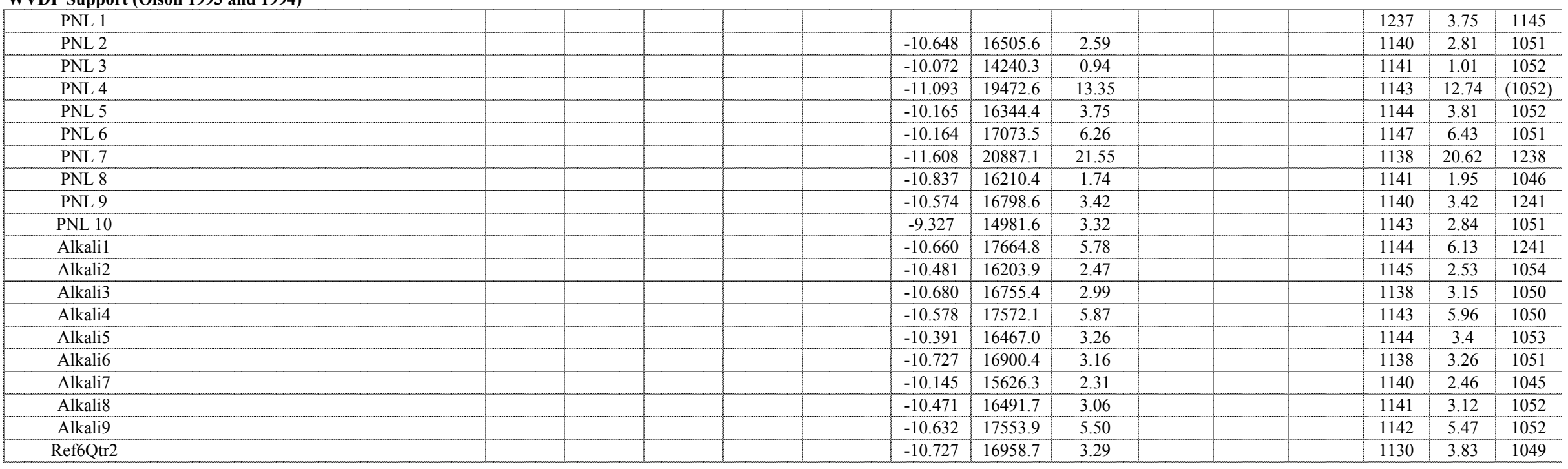




\section{WV Glasses by CUA and PNL (Johnston et al. 1990)}

\begin{tabular}{|c|c|c|c|c|c|c|c|c|c|c|c|c|c|c|c|c|c|c|c|c|c|}
\hline Glass ID & $\begin{array}{c}\mathrm{V} 2 \\
(\mathrm{~Pa} \cdot \mathrm{s})\end{array}$ & $\begin{array}{c}\mathrm{T} 3 \\
\left({ }^{\circ} \mathrm{C}\right) \\
\end{array}$ & $\begin{array}{c}\mathrm{V} 3 \\
(\mathrm{~Pa} \cdot \mathrm{s})\end{array}$ & $\begin{array}{c}\mathrm{T} 4 \\
\left({ }^{\circ} \mathrm{C}\right) \\
\end{array}$ & $\begin{array}{c}\mathrm{V} 4 \\
(\mathrm{~Pa} \cdot \mathrm{s})\end{array}$ & $\begin{array}{c}\mathrm{T} 5 \\
\left({ }^{\circ} \mathrm{C}\right) \\
\end{array}$ & $\begin{array}{c}\mathrm{V} 5 \\
(\mathrm{~Pa} \cdot \mathrm{s}) \\
\end{array}$ & $\begin{array}{c}\mathrm{T} 6 \\
\left({ }^{\circ} \mathrm{C}\right) \\
\end{array}$ & $\begin{array}{c}\mathrm{V} 6 \\
(\mathrm{~Pa} \cdot \mathrm{s})\end{array}$ & $\begin{array}{c}\mathrm{T} 7 \\
\left({ }^{\circ} \mathrm{C}\right) \\
\end{array}$ & $\begin{array}{c}\mathrm{V} 7 \\
(\mathrm{~Pa} \cdot \mathrm{s}) \\
\end{array}$ & $\begin{array}{c}\mathrm{T} 8 \\
\left({ }^{\circ} \mathrm{C}\right) \\
\end{array}$ & $\begin{array}{c}\mathrm{V} 8 \\
(\mathrm{~Pa} \cdot \mathrm{s})\end{array}$ & $\begin{array}{c}\mathrm{T} 9 \\
\left({ }^{\circ} \mathrm{C}\right) \\
\end{array}$ & $\begin{array}{c}\mathrm{V} 9 \\
(\mathrm{~Pa} \cdot \mathrm{s})\end{array}$ & $\begin{array}{l}\mathrm{T} 10 \\
\left({ }^{\circ} \mathrm{C}\right)\end{array}$ & $\begin{array}{c}\mathrm{V} 10 \\
(\mathrm{~Pa} \cdot \mathrm{s})\end{array}$ & $\begin{array}{l}\mathrm{T} 11 \\
\left({ }^{\circ} \mathrm{C}\right) \\
\end{array}$ & $\begin{array}{c}\mathrm{V} 11 \\
(\mathrm{~Pa} \cdot \mathrm{s})\end{array}$ & $\begin{array}{l}\mathrm{T} 12 \\
\left({ }^{\circ} \mathrm{C}\right)\end{array}$ & $\begin{array}{c}\mathrm{V} 12 \\
(\mathrm{~Pa} \cdot \mathrm{s})\end{array}$ \\
\hline \multicolumn{22}{|l|}{ CU33 } \\
\hline \multirow{2}{*}{\multicolumn{22}{|c|}{$\begin{array}{l}\text { CU34 } \\
\text { CU35 }\end{array}$}} \\
\hline CU35 & & & & & & & & & & & & & & & & & & & & & \\
\hline \multicolumn{22}{|l|}{ CU36 } \\
\hline \multicolumn{22}{|l|}{ CU37 } \\
\hline \multicolumn{22}{|l|}{ CU38 } \\
\hline \multicolumn{22}{|l|}{ CU39 } \\
\hline \multicolumn{22}{|l|}{ CU40 } \\
\hline \multicolumn{22}{|l|}{ CU41 } \\
\hline \multicolumn{22}{|l|}{ CU42 } \\
\hline \multicolumn{22}{|l|}{ CU43 } \\
\hline \multicolumn{22}{|l|}{ CU44 } \\
\hline \multicolumn{22}{|l|}{ CU46 } \\
\hline \multicolumn{22}{|l|}{ CU47 } \\
\hline \multicolumn{22}{|l|}{ CU49 } \\
\hline \multicolumn{22}{|l|}{ CU50 } \\
\hline \multicolumn{22}{|l|}{ CU52 } \\
\hline \multicolumn{22}{|l|}{ CU53 } \\
\hline \multicolumn{22}{|l|}{ CU54 } \\
\hline \multicolumn{22}{|l|}{ CU55 } \\
\hline \multicolumn{22}{|l|}{ CU56 } \\
\hline CU57 & & & & & & & & & & & & & & & & & & & & & \\
\hline CU58 & & & & & & & & & & & & & & & & & & & & & \\
\hline
\end{tabular}

WVDP Support (Olson 1993 and 1994)

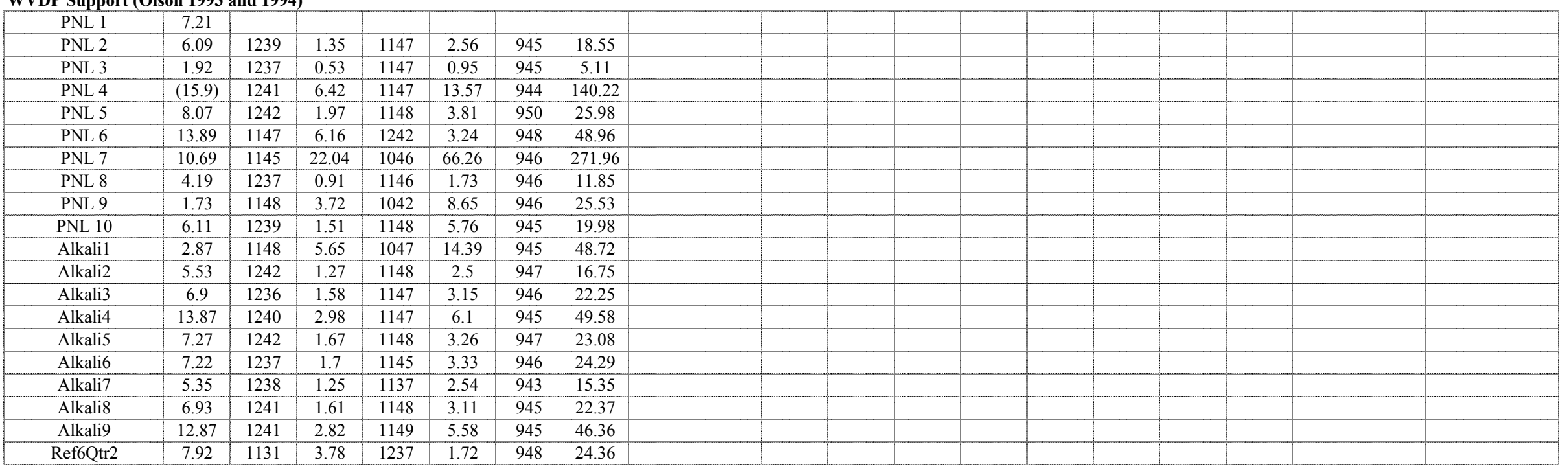


Appendix A. Database - mass fraction

WV Glasses by CUA and PNL (Johnston et al. 1990)

\begin{tabular}{|c|c|c|c|c|c|c|c|c|c|c|c|c|c|c|c|c|}
\hline Glass ID & $\begin{array}{l}\mathrm{T} 13 \\
\left({ }^{\circ} \mathrm{C}\right) \\
\end{array}$ & $\begin{array}{c}\mathrm{V} 13 \\
(\mathrm{~Pa} \cdot \mathrm{s}) \\
\end{array}$ & $\begin{array}{l}\mathrm{T} 14 \\
\left({ }^{\circ} \mathrm{C}\right) \\
\end{array}$ & $\begin{array}{c}\mathrm{V} 14 \\
(\mathrm{~Pa} \cdot \mathrm{s}) \\
\end{array}$ & $\begin{array}{l}\text { Q PCT } \\
\text { B }\left(\mathrm{g} / \mathrm{m}^{2}\right)\end{array}$ & $\begin{array}{c}\text { Q PCT } \\
\mathrm{Li}\left(\mathrm{g} / \mathrm{m}^{2}\right)\end{array}$ & $\begin{array}{c}\text { Q PCT } \\
\mathrm{Na}\left(\mathrm{g} / \mathrm{m}^{2}\right) \\
\end{array}$ & $\begin{array}{c}\text { Q PCT } \\
\mathrm{Si}\left(\mathrm{g} / \mathrm{m}^{2}\right)\end{array}$ & $\begin{array}{c}\text { Q PCT } \\
\mathrm{pH}\end{array}$ & $\begin{array}{c}\text { CCC PCT } \\
\mathrm{B}\left(\mathrm{g} / \mathrm{m}^{2}\right) \\
\end{array}$ & $\begin{array}{l}\text { CCC PCT } \\
\mathrm{Li}\left(\mathrm{g} / \mathrm{m}^{2}\right)\end{array}$ & $\begin{array}{l}\text { CCC PCT } \\
\mathrm{Na}\left(\mathrm{g} / \mathrm{m}^{2}\right) \\
\end{array}$ & $\begin{array}{c}\text { CCC PCT } \\
\mathrm{Si}\left(\mathrm{g} / \mathrm{m}^{2}\right)\end{array}$ & $\begin{array}{c}\text { CCC PCT } \\
\mathrm{pH}\end{array}$ & $\begin{array}{l}\text { Q PCT B at } \\
20^{\circ} \mathrm{C}\left(\mathrm{g} / \mathrm{m}^{2}\right)\end{array}$ & $\begin{array}{l}\text { Q PCT Li at } \\
20^{\circ} \mathrm{C}\left(\mathrm{g} / \mathrm{m}^{2}\right)\end{array}$ \\
\hline CU33 & & & & & 0.230 & 0.251 & 0.234 & 0.116 & 9.9 & & & & & & & \\
\hline CU34 & & & & & 0.207 & 0.256 & 0.224 & 0.104 & 9.9 & & & & & & & \\
\hline CU35 & & & & & 0.973 & 0.825 & 0.808 & 0.229 & 10.2 & & & & & & & \\
\hline CU36 & & & & & 0.444 & 0.491 & 0.413 & 0.158 & 10.1 & & & & & & & \\
\hline CU37 & & & & & 0.379 & 0.382 & 0.367 & 0.159 & 10.0 & & & & & & & \\
\hline CU38 & & & & & 0.578 & 0.538 & 0.501 & 0.191 & 9.9 & & & & & & & \\
\hline CU39 & & & & & 0.481 & 0.445 & 0.463 & 0.166 & 10.0 & & & & & & & \\
\hline CU40 & & & & & 0.540 & 0.497 & 0.525 & 0.184 & 10.1 & & & & & & & \\
\hline CU41 & & & & & 1.955 & 1.471 & 1.510 & 0.312 & 10.2 & & & & & & & \\
\hline CU42 & & & & & 1.566 & 1.245 & 1.303 & 0.265 & 10.2 & & & & & & & \\
\hline CU43 & & & & & 1.764 & 1.499 & 1.321 & 0.322 & 10.4 & & & & & & & \\
\hline CU44 & & & & & 0.879 & 0.703 & 0.693 & 0.216 & 10.1 & & & & & & & \\
\hline CU46 & & & & & 0.579 & 0.560 & 0.542 & 0.157 & 9.6 & & & & & & & \\
\hline CU47 & & & & & 2.891 & 2.038 & 1.747 & 0.382 & 10.4 & & & & & & & \\
\hline CU49 & & & & & 3.331 & 2.490 & 2.390 & 0.356 & 10.4 & & & & & & & \\
\hline CU50 & & & & & 0.706 & 0.825 & 0.849 & 0.185 & 7.7 & & & & & & & \\
\hline CU52 & & & & & 1.040 & 0.935 & 0.929 & 0.218 & 10.2 & & & & & & & \\
\hline CU53 & & & & & 0.268 & & & & & & & & & & & \\
\hline CU54 & & & & & 0.349 & & & & & & & & & & & \\
\hline CU55 & & & & & 0.361 & & & & & & & & & & & \\
\hline CU56 & & & & & 0.349 & & & & & & & & & & & \\
\hline CU57 & & & & & 0.332 & & & & & & & & & & & \\
\hline CU58 & & & & & 0.823 & & & & & & & & & & & \\
\hline
\end{tabular}

WVDP Support (Olson 1993 and 1994)

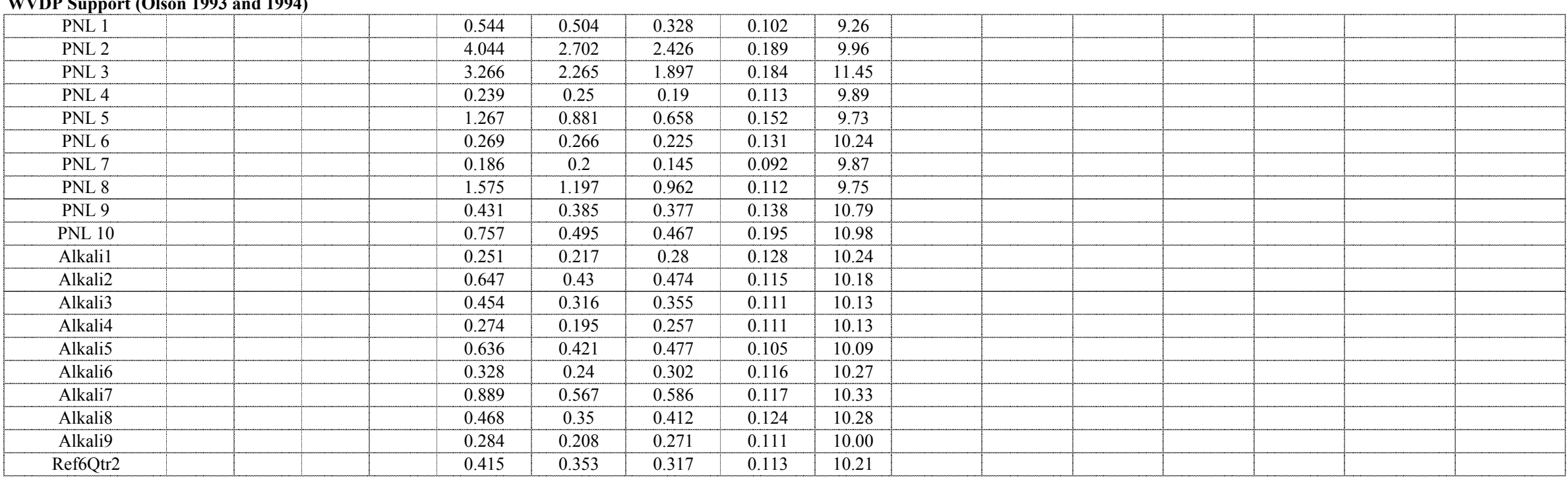


Appendix A. Database - mass fraction

WV Glasses by CUA and PNL (Johnston et al. 1990)

\begin{tabular}{|c|c|c|c|c|c|c|c|c|c|c|c|c|}
\hline Glass ID & $\begin{array}{l}\text { Q PCT Na at } \\
20^{\circ} \mathrm{C}\left(\mathrm{g} / \mathrm{m}^{2}\right)\end{array}$ & $\begin{array}{l}\text { Q PCT Si at } \\
20^{\circ} \mathrm{C}\left(\mathrm{g} / \mathrm{m}^{2}\right)\end{array}$ & $\begin{array}{c}\mathrm{QpH} \\
\text { at } 20^{\circ} \mathrm{C}\end{array}$ & $\begin{array}{c}\text { TCLP Ag } \\
(\mathrm{ppm})\end{array}$ & $\begin{array}{c}\text { TCLP As } \\
(\mathrm{ppm})\end{array}$ & $\begin{array}{c}\text { TCLP Ba } \\
(\mathrm{ppm})\end{array}$ & $\begin{array}{c}\text { TCLP Cd } \\
(\mathrm{ppm})\end{array}$ & $\begin{array}{c}\text { TCLP Cr } \\
(\mathrm{ppm})\end{array}$ & $\begin{array}{c}\text { TCLP Ni } \\
(\mathrm{ppm})\end{array}$ & $\begin{array}{c}\text { TCLP Pb } \\
(\mathrm{ppm})\end{array}$ & $\begin{array}{l}\text { TCLP Se } \\
(\mathrm{ppm})\end{array}$ & $\begin{array}{c}\text { TCLP Zn } \\
(\mathrm{ppm})\end{array}$ \\
\hline \multicolumn{13}{|c|}{ 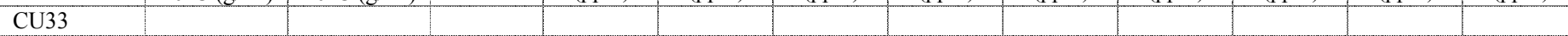 } \\
\hline \multicolumn{13}{|l|}{ CU34 } \\
\hline \multicolumn{13}{|l|}{ CU35 } \\
\hline \multicolumn{13}{|l|}{ CU36 } \\
\hline \multicolumn{13}{|l|}{ CU37 } \\
\hline \multicolumn{13}{|l|}{ CU38 } \\
\hline \multicolumn{13}{|l|}{ CU39 } \\
\hline \multicolumn{13}{|l|}{ CU40 } \\
\hline \multicolumn{13}{|l|}{ CU41 } \\
\hline \multicolumn{13}{|l|}{ CU42 } \\
\hline \multicolumn{13}{|l|}{ CU43 } \\
\hline \multicolumn{13}{|l|}{ CU44 } \\
\hline \multicolumn{13}{|l|}{ CU46 } \\
\hline \multicolumn{13}{|l|}{ CU47 } \\
\hline \multicolumn{13}{|l|}{ CU49 } \\
\hline \multicolumn{13}{|l|}{ CU50 } \\
\hline \multicolumn{13}{|l|}{ CU52 } \\
\hline \multicolumn{13}{|l|}{ CU53 } \\
\hline \multicolumn{13}{|l|}{ CU54 } \\
\hline \multicolumn{13}{|l|}{ CU55 } \\
\hline \multicolumn{13}{|l|}{ CU56 } \\
\hline \multicolumn{13}{|l|}{ CU57 } \\
\hline CU58 & & & & & & & & & & & & \\
\hline
\end{tabular}

WVDP Support (Olson 1993 and 1994)

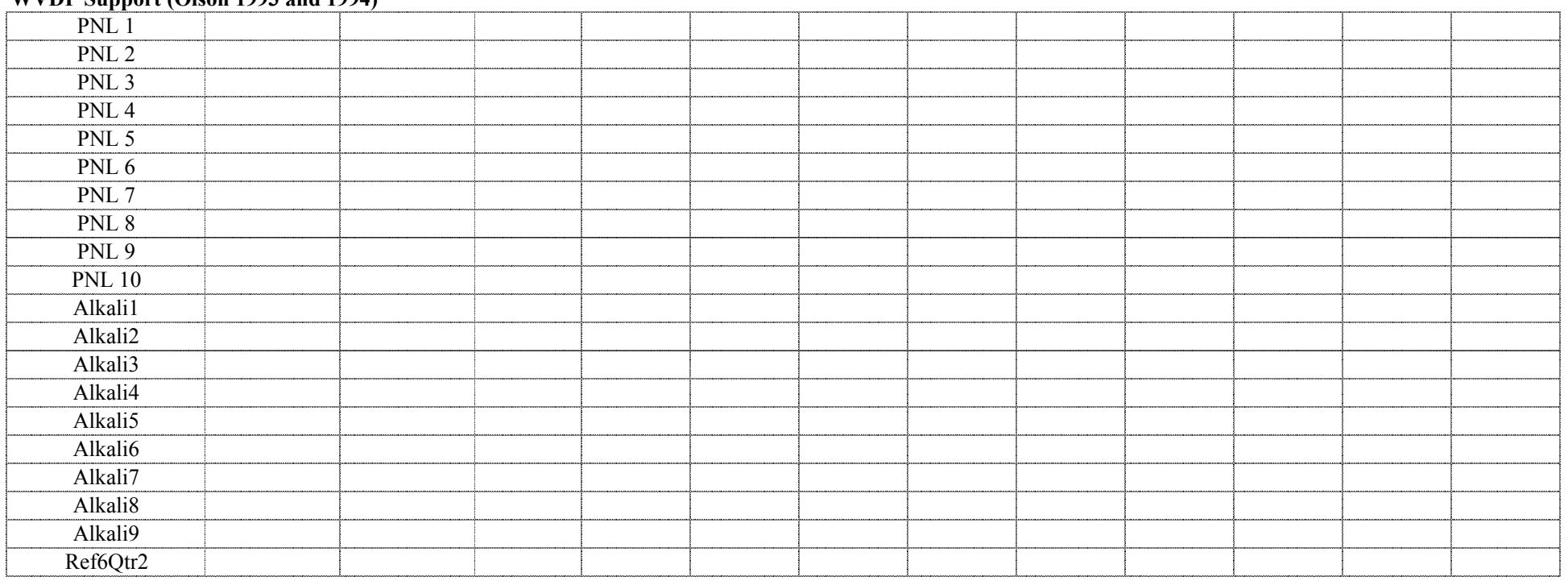


Appendix A. Database - mass fraction

\begin{tabular}{|c|c|c|c|c|c|c|c|c|c|c|c|c|c|c|c|c|c|c|c|c|c|}
\hline \multicolumn{22}{|c|}{ West Valley CVS Glasses PCT (Olson et al. 1994) } \\
\hline Glass ID & $\begin{array}{c}\mathrm{A} 12 \mathrm{O} 3 \\
-\mathrm{t}\end{array}$ & $\begin{array}{c}\mathrm{B} 2 \mathrm{O} 3 \\
-\mathrm{t}\end{array}$ & $\begin{array}{c}\mathrm{CaO} \\
-\mathrm{t}\end{array}$ & $\begin{array}{c}\mathrm{Fe} 2 \mathrm{O} 3 \\
-\mathrm{t}\end{array}$ & $\begin{array}{c}\mathrm{FeO} \\
-\mathrm{t}\end{array}$ & $\begin{array}{c}\mathrm{K} 2 \mathrm{O} \\
-\mathrm{t}\end{array}$ & $\begin{array}{c}\mathrm{Li} 2 \mathrm{O} \\
-\mathrm{t}\end{array}$ & $\begin{array}{c}\mathrm{MgO} \\
-\mathrm{t}\end{array}$ & $\begin{array}{c}\mathrm{Na} 2 \mathrm{O} \\
-\mathrm{t}\end{array}$ & $\begin{array}{c}\mathrm{NiO} \\
-\mathrm{t}\end{array}$ & $\begin{array}{c}\mathrm{P} 2 \mathrm{O} 5 \\
-\mathrm{t}\end{array}$ & $\begin{array}{c}\mathrm{SiO} 2 \\
-\mathrm{t}\end{array}$ & $\mathrm{ZrO} 2$ & $\begin{array}{c}\mathrm{Ag} 2 \mathrm{O} \\
-t\end{array}$ & As2O3 & $\mathrm{BaO}$ & $\mathrm{Bi} 2 \mathrm{O} 3$ & $\mathrm{Br}$ & $\mathrm{CdO}$ & $\mathrm{Ce} 2 \mathrm{O} 3$ & $\begin{array}{c}\mathrm{CeO} 2 \\
-t\end{array}$ \\
\hline \multicolumn{22}{|l|}{ WVDG-1 } \\
\hline \multicolumn{22}{|l|}{ WVDG-2 } \\
\hline \multicolumn{22}{|l|}{ WVDG-3 } \\
\hline \multicolumn{22}{|l|}{ WVDG-4 } \\
\hline \multicolumn{22}{|l|}{ WVDG-5 } \\
\hline \multicolumn{22}{|l|}{ WVDG-6 } \\
\hline \multicolumn{22}{|l|}{ WVDG-7 } \\
\hline \multicolumn{22}{|l|}{ WVDG-8 } \\
\hline \multicolumn{22}{|l|}{ WVDG-11R } \\
\hline \multicolumn{22}{|l|}{ WVDG-12R } \\
\hline \multirow{2}{*}{\multicolumn{22}{|c|}{$\begin{array}{l}\text { WVDG-13R } \\
\text { WVDG-14R }\end{array}$}} \\
\hline \multicolumn{15}{|l|}{ WVDG-14R } & & & & & & & \\
\hline \multirow{2}{*}{\multicolumn{22}{|c|}{$\begin{array}{l}\text { WVDG-15 } \\
\text { WVDG-16 }\end{array}$}} \\
\hline & & & & & & & & & & & & & & & & & & & & & \\
\hline WVDG-17 & & & & & & & & & & & & & & & & & & & & & \\
\hline WVDG-18 & & & & & & & & & & & & & & & & & & & & & \\
\hline WVDG-19 & & & & & & & & & & & & & & & & & & & & & \\
\hline WVDG-20 & & & & & & & & & & & & & & & & & & & & & \\
\hline WVDG-21 & & & & & & & & & & & & & & & & & & & & & \\
\hline WVDG-22 & & & & & & & & & & & & & & & & & & & & & \\
\hline WVDG-23 & & & & & & & & & & & & & & & & & & & & & \\
\hline WVDG-24 & & & & & & & & & & & & & & & & & & & & & \\
\hline WVDG-25 & & & & & & & & & & & & & & & & & & & & & \\
\hline WVDG-26 & & & & & & & & & & & & & & & & & & & & & \\
\hline WVDG-27 & & & & & & & & & & & & & & & & & & & & & \\
\hline WVDG-28 & & & & & & & & & & & & & & & & & & & & & \\
\hline WVDG-29 & & & & & & & & & & & & & & & & & & & & & \\
\hline WVDG-30 & & & & & & & & & & & & & & & & & & & & & \\
\hline WVDG-33 & & & & & & & & & & & & & & & & & & & & & \\
\hline WVDG-34 & & & & & & & & & & & & & & & & & & & & & \\
\hline WVDG-35 & & & & & & & & & & & & & & & & & & & & & \\
\hline WVDG-36 & & & & & & & & & & & & & & & & & & & & & \\
\hline WVDG-37 & & & & & & & & & & & & & & & & & & & & & \\
\hline WVDG-38 & & & & & & & & & & & & & & & & & & & & & \\
\hline WVDG-39 & & & & & & & & & & & & & & & & & & & & & \\
\hline WVDG-40 & & & & & & & & & & & & & & & & & & & & & \\
\hline WVDG-41 & & & & & & & & & & & & & & & & & & & & & \\
\hline WVDG-42 & & & & & & & & & & & & & & & & & & & & & \\
\hline WVDG-43 & & & & & & & & & & & & & & & & & & & & & \\
\hline WVDG-44 & & & & & & & & & & & & & & & & & & & & & \\
\hline WVDG-45 & & & & & & & & & & & & & & & & & & & & & \\
\hline WVDG-46 & & & & & & & & & & & & & & & & & & & & & \\
\hline WVDG-47 & & & & & & & & & & & & & & & & & & & & & \\
\hline WVDG-48 & & & & & & & & & & & & & & & & & & & & & \\
\hline
\end{tabular}


Appendix A. Database - mass fraction

West Valley CVS Glasses PCT (Olson et al. 1994)

\begin{tabular}{|c|c|c|c|c|c|c|c|c|c|c|c|c|c|c|c|c|c|c|c|c|c|}
\hline Glass ID & $\begin{array}{c}\mathrm{Cl} \\
-\mathrm{t}\end{array}$ & $\begin{array}{c}\mathrm{CoO} \\
-\mathrm{t}\end{array}$ & $\begin{array}{c}\mathrm{Co} 2 \mathrm{O} 3 \\
-\mathrm{t}\end{array}$ & $\begin{array}{c}\mathrm{Cr} 2 \mathrm{O} 3 \\
-\mathrm{t}\end{array}$ & $\begin{array}{c}\mathrm{Cs} 2 \mathrm{O} \\
-\mathrm{t}\end{array}$ & $\begin{array}{c}\mathrm{CuO} \\
-\mathrm{t}\end{array}$ & $\begin{array}{c}\mathrm{Eu} 2 \mathrm{O} 3 \\
-\mathrm{t}\end{array}$ & $\begin{array}{l}F \\
-t\end{array}$ & $\begin{array}{c}\mathrm{Ga} 2 \mathrm{O} 3 \\
-\mathrm{t}\end{array}$ & $\begin{array}{c}\mathrm{Gd} 2 \mathrm{O} 3 \\
-\mathrm{t}\end{array}$ & $\begin{array}{c}\mathrm{HgO} \\
-\mathrm{t}\end{array}$ & $\begin{array}{l}I \\
-t\end{array}$ & $\begin{array}{c}\mathrm{La} 2 \mathrm{O} 3 \\
-\mathrm{t}\end{array}$ & $\begin{array}{c}\mathrm{MnO} 2 \\
-\mathrm{t}\end{array}$ & $\begin{array}{c}\mathrm{MnO} \\
-\mathrm{t}\end{array}$ & $\begin{array}{c}\mathrm{MoO} \\
-\mathrm{t}\end{array}$ & $\begin{array}{c}\mathrm{MoO} 3 \\
-\mathrm{t}\end{array}$ & $\begin{array}{c}\mathrm{Nb} 2 \mathrm{O} 5 \\
-\mathrm{t}\end{array}$ & $\begin{array}{c}\mathrm{Nd} 2 \mathrm{O} 3 \\
-\mathrm{t}\end{array}$ & $\begin{array}{c}\mathrm{PbO} \\
-\mathrm{t}\end{array}$ & $\begin{array}{c}\mathrm{PdO} 2 \\
-\mathrm{t}\end{array}$ \\
\hline WVDG-1 & & & & & & & & & & & & & & & & & & & & & \\
\hline WVDG-2 & & & & & & & & & & & & & & & & & & & & & \\
\hline WVDG-3 & & & & & & & & & & & & & & & & & & & & & \\
\hline WVDG-4 & & & & & & & & & & & & & & & & & & & & & \\
\hline WVDG-5 & & & & & & & & & & & & & & & & & & & & & \\
\hline WVDG-6 & & & & & & & & & & & & & & & & & & & & & \\
\hline WVDG-7 & & & & & & & & & & & & & & & & & & & & & \\
\hline WVDG-8 & & & & & & & & & & & & & & & & & & & & & \\
\hline WVDG-11R & & & & & & & & & & & & & & & & & & & & & \\
\hline WVDG-12R & & & & & & & & & & & & & & & & & & & & & \\
\hline WVDG-13R & & & & & & & & & & & & & & & & & & & & & \\
\hline WVDG-14R & & & & & & & & & & & & & & & & & & & & & \\
\hline WVDG-15 & & & & & & & & & & & & & & & & & & & & & \\
\hline WVDG-16 & & & & & & & & & & & & & & & & & & & & & \\
\hline WVDG-17 & & & & & & & & & & & & & & & & & & & & & \\
\hline WVDG-18 & & & & & & & & & & & & & & & & & & & & & \\
\hline WVDG-19 & & & & & & & & & & & & & & & & & & & & & \\
\hline WVDG-20 & & & & & & & & & & & & & & & & & & & & & \\
\hline WVDG-21 & & & & & & & & & & & & & & & & & & & & & \\
\hline WVDG-22 & & & & & & & & & & & & & & & & & & & & & \\
\hline WVDG-23 & & & & & & & & & & & & & & & & & & & & & \\
\hline WVDG-24 & & & & & & & & & & & & & & & & & & & & & \\
\hline WVDG-25 & & & & & & & & & & & & & & & & & & & & & \\
\hline WVDG-26 & & & & & & & & & & & & & & & & & & & & & \\
\hline WVDG-27 & & & & & & & & & & & & & & & & & & & & & \\
\hline WVDG-28 & & & & & & & & & & & & & & & & & & & & & \\
\hline WVDG-29 & & & & & & & & & & & & & & & & & & & & & \\
\hline WVDG-30 & & & & & & & & & & & & & & & & & & & & & \\
\hline WVDG-33 & & & & & & & & & & & & & & & & & & & & & \\
\hline WVDG-34 & & & & & & & & & & & & & & & & & & & & & \\
\hline WVDG-35 & & & & & & & & & & & & & & & & & & & & & \\
\hline WVDG-36 & & & & & & & & & & & & & & & & & & & & & \\
\hline WVDG-37 & & & & & & & & & & & & & & & & & & & & & \\
\hline WVDG-38 & & & & & & & & & & & & & & & & & & & & & \\
\hline WVDG-39 & & & & & & & & & & & & & & & & & & & & & \\
\hline WVDG-40 & & & & & & & & & & & & & & & & & & & & & \\
\hline WVDG-41 & & & & & & & & & & & & & & & & & & & & & \\
\hline WVDG-42 & & & & & & & & & & & & & & & & & & & & & \\
\hline WVDG-43 & & & & & & & & & & & & & & & & & & & & & \\
\hline WVDG-44 & & & & & & & & & & & & & & & & & & & & & \\
\hline WVDG-45 & & & & & & & & & & & & & & & & & & & & & \\
\hline WVDG-46 & & & & & & & & & & & & & & & & & & & & & \\
\hline WVDG-47 & & & & & & & & & & & & & & & & & & & & & \\
\hline WVDG-48 & & & & & & & & & & & & & & & & & & & & & \\
\hline
\end{tabular}


Appendix A. Database - mass fraction

West Valley CVS Glasses PCT (Olson et al. 1994)

\begin{tabular}{|c|c|c|c|c|c|c|c|c|c|c|c|c|c|c|c|c|c|c|c|c|c|}
\hline Glass ID & $\begin{array}{c}\mathrm{PdO} \\
-\mathrm{t}\end{array}$ & $\begin{array}{c}\mathrm{Pr} 2 \mathrm{O} 3 \\
-\mathrm{t}\end{array}$ & $\begin{array}{c}\text { Pr6O11 } \\
-t\end{array}$ & $\begin{array}{c}\mathrm{Rb} 2 \mathrm{O} \\
-\mathrm{t}\end{array}$ & $\begin{array}{c}\mathrm{ReO} \\
-\mathrm{t}\end{array}$ & $\begin{array}{c}\mathrm{ReO} 2 \\
-\mathrm{t}\end{array}$ & $\begin{array}{c}\mathrm{Rh} 2 \mathrm{O} 3 \\
-\mathrm{t}\end{array}$ & $\begin{array}{c}\mathrm{RhO} 2 \\
-\mathrm{t}\end{array}$ & $\begin{array}{c}\mathrm{RuO}^{2} \\
-\mathrm{t}\end{array}$ & $\begin{array}{c}\mathrm{Sb} 2 \mathrm{O} 3 \\
-\mathrm{t}\end{array}$ & $\begin{array}{c}\mathrm{Sb} 2 \mathrm{O} 5 \\
-\mathrm{t}\end{array}$ & $\begin{array}{c}\mathrm{SeO} 2 \\
-\mathrm{t}\end{array}$ & $\begin{array}{c}\mathrm{Sm} 2 \mathrm{O} 3 \\
-\mathrm{t}\end{array}$ & $\begin{array}{c}\mathrm{SnO} \\
-\mathrm{t}\end{array}$ & $\begin{array}{c}\mathrm{SnO} 2 \\
-\mathrm{t}\end{array}$ & $\begin{array}{c}\mathrm{SO} 3 \\
-\mathrm{t}\end{array}$ & $\begin{array}{c}\mathrm{SrO} \\
-\mathrm{t}\end{array}$ & $\begin{array}{c}\mathrm{Tc} 2 \mathrm{O} 7 \\
-\mathrm{t}\end{array}$ & $\begin{array}{c}\mathrm{TeO} 2 \\
-\mathrm{t}\end{array}$ & $\begin{array}{c}\mathrm{ThO} 2 \\
-\mathrm{t}\end{array}$ & $\begin{array}{c}\mathrm{TiO} 2 \\
-\mathrm{t}\end{array}$ \\
\hline WVDG-1 & & & & & & & & & & & & & & & & & & & & & \\
\hline WVDG-2 & & & & & & & & & & & & & & & & & & & & & \\
\hline WVDG-3 & & & & & & & & & & & & & & & & & & & & & \\
\hline WVDG-4 & & & & & & & & & & & & & & & & & & & & & \\
\hline WVDG-5 & & & & & & & & & & & & & & & & & & & & & \\
\hline WVDG-6 & & & & & & & & & & & & & & & & & & & & & \\
\hline WVDG-7 & & & & & & & & & & & & & & & & & & & & & \\
\hline WVDG-8 & & & & & & & & & & & & & & & & & & & & & \\
\hline WVDG-11R & & & & & & & & & & & & & & & & & & & & & \\
\hline WVDG-12R & & & & & & & & & & & & & & & & & & & & & \\
\hline WVDG-13R & & & & & & & & & & & & & & & & & & & & & \\
\hline WVDG-14R & & & & & & & & & & & & & & & & & & & & & \\
\hline WVDG-15 & & & & & & & & & & & & & & & & & & & & & \\
\hline WVDG-16 & & & & & & & & & & & & & & & & & & & & & \\
\hline WVDG-17 & & & & & & & & & & & & & & & & & & & & & \\
\hline WVDG-18 & & & & & & & & & & & & & & & & & & & & & \\
\hline WVDG-19 & & & & & & & & & & & & & & & & & & & & & \\
\hline WVDG-20 & & & & & & & & & & & & & & & & & & & & & \\
\hline WVDG-21 & & & & & & & & & & & & & & & & & & & & & \\
\hline WVDG-22 & & & & & & & & & & & & & & & & & & & & & \\
\hline WVDG-23 & & & & & & & & & & & & & & & & & & & & & \\
\hline WVDG-24 & & & & & & & & & & & & & & & & & & & & & \\
\hline WVDG-25 & & & & & & & & & & & & & & & & & & & & & \\
\hline WVDG-26 & & & & & & & & & & & & & & & & & & & & & \\
\hline WVDG-27 & & & & & & & & & & & & & & & & & & & & & \\
\hline WVDG-28 & & & & & & & & & & & & & & & & & & & & & \\
\hline WVDG-29 & & & & & & & & & & & & & & & & & & & & & \\
\hline WVDG-30 & & & & & & & & & & & & & & & & & & & & & \\
\hline WVDG-33 & & & & & & & & & & & & & & & & & & & & & \\
\hline WVDG-34 & & & & & & & & & & & & & & & & & & & & & \\
\hline WVDG-35 & & & & & & & & & & & & & & & & & & & & & \\
\hline WVDG-36 & & & & & & & & & & & & & & & & & & & & & \\
\hline WVDG-37 & & & & & & & & & & & & & & & & & & & & & \\
\hline WVDG-38 & & & & & & & & & & & & & & & & & & & & & \\
\hline WVDG-39 & & & & & & & & & & & & & & & & & & & & & \\
\hline WVDG-40 & & & & & & & & & & & & & & & & & & & & & \\
\hline WVDG-41 & & & & & & & & & & & & & & & & & & & & & \\
\hline WVDG-42 & & & & & & & & & & & & & & & & & & & & & \\
\hline WVDG-43 & & & & & & & & & & & & & & & & & & & & & \\
\hline WVDG-44 & & & & & & & & & & & & & & & & & & & & & \\
\hline WVDG-45 & & & & & & & & & & & & & & & & & & & & & \\
\hline WVDG-46 & & & & & & & & & & & & & & & & & & & & & \\
\hline WVDG-47 & & & & & & & & & & & & & & & & & & & & & \\
\hline WVDG-48 & & & & & & & & & & & & & & & & & & & & & \\
\hline
\end{tabular}


West Valley CVS Glasses PCT (Olson et al. 1994)

\begin{tabular}{|c|c|c|c|c|c|c|c|c|c|c|c|c|c|c|c|c|c|c|c|c|c|}
\hline Glass ID & $\begin{array}{c}\mathrm{T} 12 \mathrm{O} 3 \\
-\mathrm{t}\end{array}$ & $\begin{array}{c}\mathrm{U} 3 \mathrm{O} 8 \\
-\mathrm{t}\end{array}$ & $\begin{array}{c}\mathrm{UO} 2 \\
-\mathrm{t}\end{array}$ & $\begin{array}{c}\mathrm{UO} 3 \\
-\mathrm{t}\end{array}$ & $\begin{array}{c}\mathrm{V} 2 \mathrm{O} 5 \\
-\mathrm{t}\end{array}$ & $\begin{array}{c}\text { WO3 } \\
-t\end{array}$ & $\begin{array}{c}\mathrm{Y} 2 \mathrm{O} 3 \\
-\mathrm{t}\end{array}$ & $\begin{array}{c}\mathrm{ZnO} \\
-\mathrm{t}\end{array}$ & $\begin{array}{c}\text { Others } \\
-t\end{array}$ & $\begin{array}{c}\text { Sum } \\
-t\end{array}$ & $\begin{array}{c}\mathrm{A} 12 \mathrm{O} 3 \\
-\mathrm{a}\end{array}$ & $\begin{array}{c}\mathrm{B} 2 \mathrm{O} 3 \\
-\mathrm{a}\end{array}$ & $\begin{array}{c}\mathrm{CaO} \\
-\mathrm{a}\end{array}$ & $\begin{array}{c}\mathrm{Fe} 2 \mathrm{O} 3 \\
-\mathrm{a}\end{array}$ & $\begin{array}{c}\mathrm{FeO} \\
-\mathrm{a}\end{array}$ & $\begin{array}{c}\mathrm{K} 2 \mathrm{O} \\
-\mathrm{a}\end{array}$ & $\begin{array}{c}\mathrm{Li} 2 \mathrm{O} \\
-\mathrm{a}\end{array}$ & $\begin{array}{c}\mathrm{MgO} \\
-\mathrm{a}\end{array}$ & $\begin{array}{c}\mathrm{Na} 2 \mathrm{O} \\
-\mathrm{a}\end{array}$ & $\begin{array}{c}\mathrm{NiO} \\
-\mathrm{a}\end{array}$ & $\begin{array}{c}\mathrm{P} 2 \mathrm{O} 5 \\
-\mathrm{a}\end{array}$ \\
\hline WVDG-1 & & & & & & & & & & & 0.0874 & 0.1215 & 0.0068 & 0.1174 & & 0.0116 & 0.0174 & 0.0132 & 0.0913 & 0.0031 & 0.0258 \\
\hline WVDG-2 & & & & & & & & & & & 0.0861 & 0.1217 & 0.0083 & 0.1172 & & 0.0091 & 0.0176 & 0.0130 & 0.0904 & 0.0027 & 0.0260 \\
\hline WVDG-3 & & & & & & & & & & & 0.0716 & 0.0933 & 0.0063 & 0.1081 & & 0.0192 & 0.0234 & 0.0170 & 0.0982 & 0.0036 & 0.0241 \\
\hline WVDG-4 & & & & & & & & & & & 0.0838 & 0.1177 & 0.0068 & 0.1106 & & 0.0171 & 0.0171 & 0.0129 & 0.1147 & 0.0027 & 0.0254 \\
\hline WVDG-5 & & & & & & & & & & & 0.0690 & 0.1241 & 0.0068 & 0.1191 & & 0.0150 & 0.0176 & 0.0135 & 0.0910 & 0.0030 & 0.0263 \\
\hline WVDG-6 & & & & & & & & & & & 0.0855 & 0.1215 & 0.0069 & 0.1155 & & 0.0070 & 0.0171 & 0.0129 & 0.0913 & 0.0028 & 0.0356 \\
\hline WVDG-7 & & & & & & & & & & & 0.0884 & 0.1215 & 0.0065 & 0.1155 & & 0.0127 & 0.0171 & 0.0128 & 0.0868 & 0.0028 & 0.0251 \\
\hline WVDG-8 & & & & & & & & & & & 0.0901 & 0.0751 & 0.0067 & 0.1229 & & 0.0171 & 0.0184 & 0.0139 & 0.0938 & 0.0030 & 0.0268 \\
\hline WVDG-11R & & & & & & & & & & & 0.1046 & 0.1097 & 0.0062 & 0.1190 & & 0.0267 & 0.0263 & 0.0125 & 0.0952 & 0.0031 & 0.0248 \\
\hline WVDG-12R & & & & & & & & & & & 0.1052 & 0.1082 & 0.0063 & 0.1182 & & 0.0285 & 0.0257 & 0.0125 & 0.0958 & 0.0029 & 0.0252 \\
\hline WVDG-13R & & & & & & & & & & & 0.1058 & 0.1078 & 0.0061 & 0.1190 & & 0.0287 & 0.0259 & 0.0126 & 0.0938 & 0.0031 & 0.0250 \\
\hline WVDG-14R & & & & & & & & & & & 0.1058 & 0.1098 & 0.0061 & 0.1199 & & 0.0219 & 0.0261 & 0.0125 & 0.0951 & 0.0029 & 0.0250 \\
\hline WVDG-15 & & & & & & & & & & & 0.0960 & 0.1258 & 0.0136 & 0.0880 & & 0.0164 & 0.0273 & 0.0127 & 0.1017 & 0.0053 & 0.0133 \\
\hline WVDG-16 & & & & & & & & & & & 0.0943 & 0.1261 & 0.0137 & 0.1442 & & 0.0131 & 0.0191 & 0.0122 & 0.0864 & 0.0016 & 0.0323 \\
\hline WVDG-17 & & & & & & & & & & & 0.0943 & 0.0978 & 0.0143 & 0.0887 & & 0.0207 & 0.0234 & 0.0129 & 0.1057 & 0.0058 & 0.0124 \\
\hline WVDG-18 & & & & & & & & & & & 0.0947 & 0.1062 & 0.0054 & 0.1444 & & 0.0175 & 0.0244 & 0.0044 & 0.1114 & 0.0017 & 0.0155 \\
\hline WVDG-19 & & & & & & & & & & & 0.1223 & 0.1008 & 0.0131 & 0.1420 & & 0.0197 & 0.0207 & 0.0128 & 0.0988 & 0.0016 & 0.0135 \\
\hline WVDG-20 & & & & & & & & & & & 0.1224 & 0.1062 & 0.0134 & 0.0875 & & 0.0223 & 0.0204 & 0.0142 & 0.0863 & 0.0058 & 0.0334 \\
\hline WVDG-21 & & & & & & & & & & & 0.1251 & 0.1005 & 0.0050 & 0.1467 & & 0.0123 & 0.0231 & 0.0053 & 0.1046 & 0.0017 & 0.0338 \\
\hline WVDG-22 & & & & & & & & & & & 0.1250 & 0.1157 & 0.0050 & 0.1403 & & 0.0184 & 0.0182 & 0.0048 & 0.0831 & 0.0057 & 0.0143 \\
\hline WVDG-23 & & & & & & & & & & & 0.0942 & 0.1248 & 0.0050 & 0.1424 & & 0.0186 & 0.0180 & 0.0054 & 0.0823 & 0.0018 & 0.0330 \\
\hline WVDG-24 & & & & & & & & & & & 0.1232 & 0.1322 & 0.0048 & 0.0867 & & 0.0252 & 0.0205 & 0.0056 & 0.0956 & 0.0017 & 0.0141 \\
\hline WVDG-25 & & & & & & & & & & & 0.0930 & 0.1090 & 0.0050 & 0.0867 & & 0.0202 & 0.0203 & 0.0053 & 0.0909 & 0.0019 & 0.0313 \\
\hline WVDG-26 & & & & & & & & & & & 0.1227 & 0.1338 & 0.0134 & 0.0867 & & 0.0264 & 0.0200 & 0.0135 & 0.0909 & 0.0019 & 0.0162 \\
\hline WVDG-27 & & & & & & & & & & & 0.1217 & 0.1258 & 0.0049 & 0.0868 & & 0.0245 & 0.0228 & 0.0062 & 0.1014 & 0.0062 & 0.0348 \\
\hline WVDG-28 & & & & & & & & & & & 0.0968 & 0.1066 & 0.0048 & 0.1211 & & 0.0135 & 0.0205 & 0.0062 & 0.0886 & 0.0059 & 0.0153 \\
\hline WVDG-29 & & & & & & & & & & & 0.0928 & 0.1145 & 0.0133 & 0.0874 & & 0.0184 & 0.0257 & 0.0136 & 0.1074 & 0.0019 & 0.0327 \\
\hline WVDG-30 & & & & & & & & & & & 0.1007 & 0.1188 & 0.0084 & 0.1148 & & 0.0181 & 0.0210 & 0.0101 & 0.0963 & 0.0035 & 0.0242 \\
\hline WVDG-33 & & & & & & & & & & & 0.0822 & 0.0903 & 0.0040 & 0.1145 & & 0.0344 & 0.0291 & 0.0098 & 0.1051 & 0.0029 & 0.0247 \\
\hline WVDG-34 & & & & & & & & & & & 0.0517 & 0.1081 & 0.0018 & 0.0977 & & 0.0362 & 0.0307 & 0.0045 & 0.1107 & 0.0082 & 0.0187 \\
\hline WVDG-35 & & & & & & & & & & & 0.1002 & 0.0715 & 0.0070 & 0.1498 & & 0.0371 & 0.0314 & 0.0174 & 0.1134 & 0.0014 & 0.0006 \\
\hline WVDG-36 & & & & & & & & & & & 0.0569 & 0.1080 & 0.0016 & 0.1466 & & 0.0254 & 0.0215 & 0.0040 & 0.0775 & 0.0081 & 0.0059 \\
\hline WVDG-37 & & & & & & & & & & & 0.0998 & 0.0778 & 0.0014 & 0.0977 & & 0.0390 & 0.0330 & 0.0036 & 0.1191 & 0.0080 & 0.0050 \\
\hline WVDG-38 & & & & & & & & & & & 0.1024 & 0.0758 & 0.0016 & 0.0967 & & 0.0326 & 0.0276 & 0.0039 & 0.0995 & 0.0083 & 0.0060 \\
\hline WVDG-39 & & & & & & & & & & & 0.1005 & 0.1122 & 0.0066 & 0.0928 & & 0.0362 & 0.0306 & 0.0164 & 0.1104 & 0.0022 & 0.0381 \\
\hline WVDG-40 & & & & & & & & & & & 0.0609 & 0.0753 & 0.0072 & 0.1535 & & 0.0317 & 0.0269 & 0.0178 & 0.0968 & 0.0018 & 0.0060 \\
\hline WVDG-41 & & & & & & & & & & & 0.0592 & 0.0726 & 0.0020 & 0.0948 & & 0.0375 & 0.0317 & 0.0051 & 0.1144 & 0.0021 & 0.0380 \\
\hline WVDG-42 & & & & & & & & & & & 0.0555 & 0.0675 & 0.0068 & 0.0953 & & 0.0347 & 0.0294 & 0.0017 & 0.1060 & 0.0080 & 0.0368 \\
\hline WVDG-43 & & & & & & & & & & & 0.1004 & 0.1243 & 0.0020 & 0.0953 & & 0.0268 & 0.0227 & 0.0049 & 0.0817 & 0.0018 & 0.0239 \\
\hline WVDG-44 & & & & & & & & & & & 0.0832 & 0.1150 & 0.0019 & 0.1581 & & 0.0353 & 0.0299 & 0.0048 & 0.1079 & 0.0017 & 0.0062 \\
\hline WVDG-45 & & & & & & & & & & & 0.1021 & 0.0713 & 0.0016 & 0.1551 & & 0.0384 & 0.0325 & 0.0040 & 0.1173 & 0.0018 & 0.0390 \\
\hline WVDG-46 & & & & & & & & & & & 0.0700 & 0.1223 & 0.0068 & 0.1039 & & 0.0337 & 0.0286 & 0.0167 & 0.1030 & 0.0016 & 0.0059 \\
\hline WVDG-47 & & & & & & & & & & & 0.0569 & 0.1121 & 0.0016 & 0.1494 & & 0.0267 & 0.0226 & 0.0040 & 0.0818 & 0.0015 & 0.0393 \\
\hline WVDG-48 & & & & & & & & & & & 0.0943 & 0.1056 & 0.0068 & 0.0924 & & 0.0268 & 0.0227 & 0.0167 & 0.0818 & 0.0088 & 0.0428 \\
\hline
\end{tabular}




\section{West Valley CVS Glasses PCT (Olson et al. 1994)}

\begin{tabular}{|c|c|c|c|c|c|c|c|c|c|c|c|c|c|c|c|c|c|c|c|c|c|}
\hline Glass ID & $\begin{array}{c}\mathrm{SiO} 2 \\
-\mathrm{a}\end{array}$ & $\begin{array}{c}\mathrm{ZrO} 2 \\
-\mathrm{a}\end{array}$ & $\begin{array}{c}\mathrm{Ag} 2 \mathrm{O} \\
-\mathrm{a}\end{array}$ & $\begin{array}{c}\mathrm{As} 2 \mathrm{O} 3 \\
-\mathrm{a} \\
\end{array}$ & $\begin{array}{c}\mathrm{BaO} \\
-\mathrm{a}\end{array}$ & $\begin{array}{c}\mathrm{Bi} 2 \mathrm{O} 3 \\
-\mathrm{a}\end{array}$ & $\begin{array}{l}\mathrm{Br} \\
-\mathrm{a}\end{array}$ & $\begin{array}{c}\mathrm{CdO} \\
-\mathrm{a}\end{array}$ & $\begin{array}{c}\mathrm{Ce} 2 \mathrm{O} 3 \\
-\mathrm{a}\end{array}$ & $\begin{array}{c}\mathrm{CeO} 2 \\
-\mathrm{a}\end{array}$ & $\begin{array}{l}\mathrm{Cl} \\
-\mathrm{a}\end{array}$ & $\begin{array}{c}\mathrm{CoO} \\
-\mathrm{a}\end{array}$ & $\begin{array}{c}\mathrm{Co} 2 \mathrm{O} 3 \\
-\mathrm{a}\end{array}$ & \begin{tabular}{|c|}
$\mathrm{Cr} 2 \mathrm{O} 3$ \\
$-\mathrm{a}$ \\
\end{tabular} & $\begin{array}{c}\mathrm{Cs} 2 \mathrm{O} \\
-\mathrm{a} \\
\end{array}$ & $\begin{array}{c}\mathrm{CuO} \\
-\mathrm{a}\end{array}$ & $\begin{array}{c}\mathrm{Eu} 2 \mathrm{O} 3 \\
-\mathrm{a}\end{array}$ & $\begin{array}{c}\mathrm{F} \\
-\mathrm{a}\end{array}$ & $\begin{array}{c}\mathrm{Ga} 2 \mathrm{O} 3 \\
-\mathrm{a}\end{array}$ & $\begin{array}{c}\mathrm{Gd} 2 \mathrm{O} 3 \\
-\mathrm{a}\end{array}$ & $\begin{array}{c}\mathrm{HgO} \\
-\mathrm{a}\end{array}$ \\
\hline WVDG-1 & 0.4325 & 0.0059 & & & 0.0005 & & & & & 0.0007 & & & & 0.0019 & 0.0005 & 0.0000 & & & & & \\
\hline WVDG-2 & 0.4360 & 0.0056 & & & 0.0005 & & & & & 0.0007 & & & & 0.0020 & 0.0005 & 0.0000 & & & & & \\
\hline WVDG-3 & 0.4546 & 0.0064 & & & 0.0007 & & & & & 0.0010 & & & & 0.0029 & 0.0006 & 0.0000 & & & & & \\
\hline WVDG-4 & 0.4194 & 0.0054 & & & 0.0005 & & & & & 0.0007 & & & & 0.0020 & 0.0005 & 0.0000 & & & & & \\
\hline WVDG-5 & 0.4443 & 0.0060 & & & 0.0005 & & & & & 0.0009 & & & & 0.0021 & 0.0005 & 0.0000 & & & & & \\
\hline WVDG-6 & 0.4342 & 0.0056 & & & 0.0005 & & & & & 0.0005 & & & & 0.0019 & 0.0005 & 0.0000 & & & & & \\
\hline WVDG-7 & 0.4303 & 0.0062 & & & 0.0005 & & & & & 0.0007 & & & & 0.0020 & 0.0004 & 0.0000 & & & & & \\
\hline WVDG-8 & 0.4583 & 0.0057 & & & 0.0005 & & & & & 0.0010 & & & & 0.0019 & 0.0014 & 0.0000 & & & & & \\
\hline WVDG-11R & 0.4061 & 0.0046 & & & 0.0005 & & & & & 0.0007 & & & & 0.0017 & 0.0009 & 0.0000 & & & & & \\
\hline WVDG-12R & 0.4016 & 0.0054 & & & 0.0005 & & & & & 0.0007 & & & & 0.0017 & 0.0010 & 0.0000 & & & & & \\
\hline WVDG-13R & 0.4018 & 0.0052 & & & 0.0005 & & & & & 0.0007 & & & & 0.0017 & 0.0012 & 0.0000 & & & & & \\
\hline WVDG-14R & 0.4020 & 0.0073 & & & 0.0005 & & & & & 0.0007 & & & & 0.0017 & 0.0013 & 0.0000 & & & & & \\
\hline WVDG-15 & 0.3979 & 0.0069 & & & 0.0033 & & & & & 0.0124 & & & & 0.0024 & 0.0012 & 0.0002 & & & & & \\
\hline WVDG-16 & 0.3995 & 0.0021 & & & 0.0032 & & & & & 0.0038 & & & & 0.0007 & 0.0004 & 0.0008 & & & & & \\
\hline WVDG-17 & 0.3957 & 0.0075 & & & 0.0032 & & & & & 0.0136 & & & & 0.0026 & 0.0013 & 0.0002 & & & & & \\
\hline WVDG-18 & 0.4320 & 0.0022 & & & 0.0011 & & & & & 0.0039 & & & & 0.0008 & 0.0004 & 0.0002 & & & & & \\
\hline WVDG-19 & 0.3601 & 0.0021 & & & 0.0032 & & & & & 0.0038 & & & & 0.0007 & 0.0004 & 0.0007 & & & & & \\
\hline WVDG-20 & 0.3995 & 0.0075 & & & 0.0031 & & & & & 0.0135 & & & & 0.0026 & 0.0013 & 0.0002 & & & & & \\
\hline WVDG-21 & 0.3754 & 0.0022 & & & 0.0011 & & & & & 0.0039 & & & & 0.0008 & 0.0004 & 0.0007 & & & & & \\
\hline WVDG-22 & 0.3738 & 0.0073 & & & 0.0011 & & & & & 0.0132 & & & & 0.0026 & 0.0013 & 0.0002 & & & & & \\
\hline WVDG-23 & 0.3931 & 0.0024 & & & 0.0011 & & & & & 0.0042 & & & & 0.0008 & 0.0004 & 0.0000 & & & & & \\
\hline WVDG-24 & 0.4210 & 0.0022 & & & 0.0011 & & & & & 0.0040 & & & & 0.0008 & 0.0004 & 0.0002 & & & & & \\
\hline WVDG-25 & 0.4500 & 0.0024 & & & 0.0010 & & & & & 0.0044 & & & & 0.0008 & 0.0004 & 0.0002 & & & & & \\
\hline WVDG-26 & 0.4095 & 0.0025 & & & 0.0030 & & & & & 0.0045 & & & & 0.0009 & 0.0004 & 0.0003 & & & & & \\
\hline WVDG-27 & 0.3672 & 0.0080 & & & 0.0011 & & & & & 0.0144 & & & & 0.0028 & 0.0014 & 0.0008 & & & & & \\
\hline WVDG-28 & 0.4338 & 0.0077 & & & 0.0011 & & & & & 0.0138 & & & & 0.0027 & 0.0013 & 0.0008 & & & & & \\
\hline WVDG-29 & 0.4468 & 0.0024 & & & 0.0032 & & & & & 0.0044 & & & & 0.0008 & 0.0004 & 0.0002 & & & & & \\
\hline WVDG-30 & 0.4006 & 0.0045 & & & 0.0020 & & & & & 0.0080 & & & & 0.0016 & 0.0008 & 0.0004 & & & & & \\
\hline WVDG-33 & 0.4250 & 0.0037 & & & 0.0006 & & & & & 0.0067 & & & & 0.0013 & 0.0007 & 0.0004 & & & & & \\
\hline WVDG-34 & 0.4000 & 0.0106 & & & 0.0003 & & & & & 0.0191 & & & & 0.0037 & 0.0019 & 0.0011 & & & & & \\
\hline WVDG-35 & 0.3800 & 0.0018 & & & 0.0010 & & & & & 0.0032 & & & & 0.0006 & 0.0003 & 0.0002 & & & & & \\
\hline WVDG-36 & 0.4110 & 0.0104 & & & 0.0002 & & & & & 0.0188 & & & & 0.0037 & 0.0018 & 0.0010 & & & & & \\
\hline WVDG-37 & 0.4200 & 0.0103 & & & 0.0002 & & & & & 0.0186 & & & & 0.0036 & 0.0018 & 0.0010 & & & & & \\
\hline WVDG-38 & 0.4090 & 0.0107 & & & 0.0000 & & & & & 0.0193 & & & & 0.0037 & 0.0019 & 0.0011 & & & & & \\
\hline WVDG-39 & 0.3800 & 0.0028 & & & 0.0009 & & & & & 0.0051 & & & & 0.0010 & 0.0005 & 0.0003 & & & & & \\
\hline WVDG-40 & 0.4436 & 0.0023 & & & 0.0010 & & & & & 0.0042 & & & & 0.0008 & 0.0004 & 0.0002 & & & & & \\
\hline WVDG-41 & 0.4500 & 0.0027 & & & 0.0003 & & & & & 0.0048 & & & & 0.0009 & 0.0005 & 0.0003 & & & & & \\
\hline WVDG-42 & 0.4000 & 0.0103 & & & 0.0010 & & & & & 0.0185 & & & & 0.0036 & 0.0018 & 0.0010 & & & & & \\
\hline WVDG-43 & 0.4200 & 0.0023 & & & 0.0003 & & & & & 0.0041 & & & & 0.0008 & 0.0004 & 0.0002 & & & & & \\
\hline WVDG-44 & 0.3800 & 0.0022 & & & 0.0003 & & & & & 0.0040 & & & & 0.0008 & 0.0004 & 0.0002 & & & & & \\
\hline WVDG-45 & 0.3800 & 0.0023 & & & 0.0002 & & & & & 0.0041 & & & & 0.0008 & 0.0004 & 0.0002 & & & & & \\
\hline WVDG-46 & 0.4500 & 0.0021 & & & 0.0010 & & & & & 0.0037 & & & & 0.0007 & 0.0004 & 0.0002 & & & & & \\
\hline WVDG-47 & 0.4500 & 0.0020 & & & 0.0002 & & & & & 0.0036 & & & & 0.0007 & 0.0004 & 0.0002 & & & & & \\
\hline WVDG-48 & 0.3800 & 0.0114 & & & 0.0010 & & & & & 0.0205 & & & & 0.0040 & 0.0020 & 0.0011 & & & & & \\
\hline
\end{tabular}


West Valley CVS Glasses PCT (Olson et al. 1994)

\begin{tabular}{|c|c|c|c|c|c|c|c|c|c|c|c|c|c|c|c|c|c|c|c|c|c|}
\hline Glass ID & $\begin{array}{c}\mathrm{I} \\
-\mathrm{a}\end{array}$ & $\begin{array}{c}\mathrm{La} 2 \mathrm{O} 3 \\
-\mathrm{a}\end{array}$ & $\begin{array}{c}\mathrm{MnO} 2 \\
-\mathrm{a}\end{array}$ & $\begin{array}{c}\mathrm{MnO} \\
-\mathrm{a}\end{array}$ & $\begin{array}{c}\mathrm{MoO} \\
-\mathrm{a}\end{array}$ & $\begin{array}{c}\mathrm{MoO} 3 \\
-\mathrm{a}\end{array}$ & $\begin{array}{c}\mathrm{Nb} 2 \mathrm{O} 5 \\
-\mathrm{a}\end{array}$ & $\begin{array}{c}\mathrm{Nd} 2 \mathrm{O} 3 \\
-\mathrm{a}\end{array}$ & $\begin{array}{c}\mathrm{PbO} \\
-\mathrm{a}\end{array}$ & $\begin{array}{c}\mathrm{PdO} 2 \\
-\mathrm{a}\end{array}$ & $\begin{array}{c}\mathrm{PdO} \\
-\mathrm{a}\end{array}$ & $\begin{array}{c}\mathrm{Pr} 2 \mathrm{O} 3 \\
-\mathrm{a}\end{array}$ & $\begin{array}{c}\text { Pr6O11 } \\
-\mathrm{a}\end{array}$ & $\begin{array}{c}\mathrm{Rb} 2 \mathrm{O} \\
-\mathrm{a}\end{array}$ & $\begin{array}{c}\mathrm{ReO} \\
-\mathrm{a}\end{array}$ & $\begin{array}{c}\mathrm{ReO} 2 \\
-\mathrm{a}\end{array}$ & $\begin{array}{c}\mathrm{Rh} 2 \mathrm{O} 3 \\
-\mathrm{a}\end{array}$ & $\begin{array}{c}\mathrm{RhO}_{2} \\
-\mathrm{a}\end{array}$ & $\begin{array}{c}\mathrm{RuO} 2 \\
-\mathrm{a}\end{array}$ & $\begin{array}{c}\mathrm{Sb} 2 \mathrm{O} 3 \\
-\mathrm{a}\end{array}$ & $\begin{array}{c}\mathrm{Sb} 2 \mathrm{O} 5 \\
-\mathrm{a}\end{array}$ \\
\hline WVDG-1 & & 0.0004 & 0.0130 & & & 0.0001 & & 0.0000 & & 0.0000 & & & 0.0003 & & & & & 0.0000 & 0.0000 & & \\
\hline WVDG-2 & & 0.0004 & 0.0132 & & & 0.0001 & & 0.0000 & & 0.0001 & & & 0.0003 & & & & & 0.0001 & 0.0008 & & \\
\hline WVDG-3 & & 0.0005 & 0.0176 & & & 0.0000 & & 0.0000 & & 0.0001 & & & 0.0000 & & & & & 0.0001 & 0.0007 & & \\
\hline WVDG-4 & & 0.0004 & 0.0130 & & & 0.0001 & & 0.0000 & & 0.0000 & & & 0.0003 & & & & & 0.0000 & 0.0000 & & \\
\hline WVDG-5 & & 0.0004 & 0.0136 & & & 0.0001 & & 0.0000 & & 0.0000 & & & 0.0003 & & & & & 0.0000 & 0.0000 & & \\
\hline WVDG-6 & & 0.0004 & 0.0130 & & & 0.0001 & & 0.0000 & & 0.0000 & & & 0.0003 & & & & & 0.0000 & 0.0000 & & \\
\hline WVDG-7 & & 0.0004 & 0.0129 & & & 0.0001 & & 0.0000 & & 0.0000 & & & 0.0003 & & & & & 0.0000 & 0.0000 & & \\
\hline WVDG-8 & & 0.0004 & 0.0137 & & & 0.0001 & & 0.0000 & & 0.0000 & & & 0.0003 & & & & & 0.0000 & 0.0000 & & \\
\hline WVDG-11R & & 0.0003 & 0.0132 & & & 0.0001 & & 0.0000 & & 0.0001 & & & 0.0003 & & & & & 0.0001 & 0.0008 & & \\
\hline WVDG-12R & & 0.0003 & 0.0131 & & & 0.0001 & & 0.0000 & & 0.0001 & & & 0.0003 & & & & & 0.0001 & 0.0008 & & \\
\hline WVDG-13R & & 0.0003 & 0.0131 & & & 0.0001 & & 0.0000 & & 0.0001 & & & 0.0003 & & & & & 0.0001 & 0.0008 & & \\
\hline WVDG-14R & & 0.0003 & 0.0131 & & & 0.0001 & & 0.0000 & & 0.0001 & & & 0.0003 & & & & & 0.0001 & 0.0008 & & \\
\hline WVDG-15 & & 0.0007 & 0.0251 & & & 0.0007 & & 0.0024 & & & & & 0.0007 & & & & & & & & \\
\hline WVDG-16 & & 0.0002 & 0.0223 & & & 0.0002 & & 0.0007 & & & & & 0.0002 & & & & & & & & \\
\hline WVDG-17 & & 0.0008 & 0.0249 & & & 0.0008 & & 0.0026 & & & & & 0.0008 & & & & & & & & \\
\hline WVDG-18 & & 0.0002 & 0.0054 & & & 0.0002 & & 0.0008 & & & & & 0.0002 & & & & & & & & \\
\hline WVDG-19 & & 0.0002 & 0.0050 & & & 0.0002 & & 0.0007 & & & & & 0.0002 & & & & & & & & \\
\hline WVDG-20 & & 0.0007 & 0.0052 & & & 0.0007 & & 0.0026 & & & & & 0.0007 & & & & & & & & \\
\hline WVDG-21 & & 0.0002 & 0.0250 & & & 0.0002 & & 0.0008 & & & & & 0.0002 & & & & & & & & \\
\hline WVDG-22 & & 0.0007 & 0.0246 & & & 0.0007 & & 0.0026 & & & & & 0.0007 & & & & & & & & \\
\hline WVDG-23 & & 0.0002 & 0.0051 & & & 0.0002 & & 0.0008 & & & & & 0.0002 & & & & & & & & \\
\hline WVDG-24 & & 0.0002 & 0.0244 & & & 0.0002 & & 0.0008 & & & & & 0.0002 & & & & & & & & \\
\hline WVDG-25 & & 0.0002 & 0.0240 & & & 0.0002 & & 0.0008 & & & & & 0.0002 & & & & & & & & \\
\hline WVDG-26 & & 0.0003 & 0.0055 & & & 0.0003 & & 0.0009 & & & & & 0.0003 & & & & & & & & \\
\hline WVDG-27 & & 0.0008 & 0.0051 & & & 0.0008 & & 0.0028 & & & & & 0.0008 & & & & & & & & \\
\hline WVDG-28 & & 0.0008 & 0.0052 & & & 0.0008 & & 0.0027 & & & & & 0.0008 & & & & & & & & \\
\hline WVDG-29 & & 0.0002 & 0.0050 & & & 0.0002 & & 0.0008 & & & & & 0.0002 & & & & & & & & \\
\hline WVDG-30 & & 0.0004 & 0.0120 & & & 0.0004 & & 0.0016 & & & & & 0.0004 & & & & & & & & \\
\hline WVDG-33 & & 0.0004 & 0.0128 & & & 0.0004 & & 0.0013 & & 0.0000 & & & 0.0004 & & & & & 0.0000 & 0.0000 & & \\
\hline WVDG-34 & & 0.0011 & 0.0198 & & & 0.0011 & & 0.0037 & & 0.0000 & & & 0.0011 & & & & & 0.0000 & 0.0000 & & \\
\hline WVDG-35 & & 0.0002 & 0.0195 & & & 0.0002 & & 0.0006 & & 0.0000 & & & 0.0002 & & & & & 0.0000 & 0.0000 & & \\
\hline WVDG-36 & & 0.0010 & 0.0012 & & & 0.0010 & & 0.0037 & & 0.0000 & & & 0.0010 & & & & & 0.0000 & 0.0000 & & \\
\hline WVDG-37 & & 0.0010 & 0.0013 & & & 0.0010 & & 0.0036 & & 0.0000 & & & 0.0010 & & & & & 0.0000 & 0.0000 & & \\
\hline WVDG-38 & & 0.0011 & 0.0161 & & & 0.0011 & & 0.0037 & & 0.0000 & & & 0.0011 & & & & & 0.0000 & 0.0000 & & \\
\hline WVDG-39 & & 0.0003 & 0.0011 & & & 0.0003 & & 0.0010 & & 0.0000 & & & 0.0003 & & & & & 0.0000 & 0.0000 & & \\
\hline WVDG-40 & & 0.0002 & 0.0012 & & & 0.0002 & & 0.0008 & & & & & 0.0002 & & & & & & & & \\
\hline WVDG-41 & & 0.0003 & 0.0012 & & & 0.0003 & & 0.0009 & & 0.0000 & & & 0.0003 & & & & & 0.0000 & 0.0000 & & \\
\hline WVDG-42 & & 0.0010 & 0.0195 & & & 0.0010 & & 0.0036 & & 0.0000 & & & 0.0010 & & & & & 0.0000 & 0.0000 & & \\
\hline WVDG-43 & & 0.0002 & 0.0194 & & & 0.0002 & & 0.0008 & & 0.0000 & & & 0.0002 & & & & & 0.0000 & 0.0000 & & \\
\hline WVDG-44 & & 0.0002 & 0.0013 & & & 0.0002 & & 0.0008 & & 0.0000 & & & 0.0002 & & & & & 0.0000 & 0.0000 & & \\
\hline WVDG-45 & & 0.0002 & 0.0201 & & & 0.0002 & & 0.0008 & & 0.0000 & & & 0.0002 & & & & & 0.0000 & 0.0000 & & \\
\hline WVDG-46 & & 0.0002 & 0.0196 & & & 0.0002 & & 0.0007 & & & & & 0.0002 & & & & & & & & \\
\hline WVDG-47 & & 0.0002 & 0.0194 & & & 0.0002 & & 0.0007 & & 0.0000 & & & 0.0002 & & & & & 0.0000 & 0.0000 & & \\
\hline WVDG-48 & & 0.0011 & 0.0012 & & & 0.0011 & & 0.0040 & & 0.0000 & & & 0.0011 & & & & & 0.0000 & 0.0000 & & \\
\hline
\end{tabular}




\section{West Valley CVS Glasses PCT (Olson et al. 1994)}

\begin{tabular}{|c|c|c|c|c|c|c|c|c|c|c|c|c|c|c|c|c|c|c|c|c|}
\hline Glass ID & $\begin{array}{c}\mathrm{SeO} 2 \\
-\mathrm{a}\end{array}$ & $\begin{array}{c}\mathrm{Sm} 2 \mathrm{O} 3 \\
-\mathrm{a} \\
\end{array}$ & $\begin{array}{c}\mathrm{SnO} \\
-\mathrm{a} \\
\end{array}$ & $\begin{array}{c}\mathrm{SnO} 2 \\
-\mathrm{a}\end{array}$ & $\begin{array}{c}\mathrm{SO} 3 \\
-\mathrm{a} \\
\end{array}$ & $\begin{array}{c}\mathrm{SrO} \\
-\mathrm{a} \\
\end{array}$ & $\begin{array}{c}\mathrm{Tc} 2 \mathrm{O} 7 \\
-\mathrm{a} \\
\end{array}$ & $\begin{array}{c}\mathrm{TeO} 2 \\
-\mathrm{a}\end{array}$ & $\begin{array}{c}\mathrm{ThO} 2 \\
-\mathrm{a}\end{array}$ & $\begin{array}{c}\mathrm{TiO} 2 \\
-\mathrm{a} \\
\end{array}$ & $\begin{array}{c}\mathrm{T} 12 \mathrm{O} 3 \\
-\mathrm{a}\end{array}$ & $\begin{array}{c}\mathrm{U} 3 \mathrm{O} 8 \\
-\mathrm{a}\end{array}$ & $\begin{array}{c}\mathrm{UO} 2 \\
-\mathrm{a}\end{array}$ & $\begin{array}{c}\mathrm{UO} 3 \\
-\mathrm{a}\end{array}$ & $\begin{array}{c}\mathrm{V} 2 \mathrm{O} 5 \\
-\mathrm{a} \\
\end{array}$ & $\begin{array}{c}\text { WO3 } \\
-\mathrm{a}\end{array}$ & $\begin{array}{c}\mathrm{Y} 2 \mathrm{O} 3 \\
-\mathrm{a}\end{array}$ & $\begin{array}{c}\mathrm{ZnO} \\
-\mathrm{a} \\
\end{array}$ & $\begin{array}{c}\text { Others } \\
-\mathrm{a}\end{array}$ & $\begin{array}{c}\text { Sum } \\
-\mathrm{a}\end{array}$ \\
\hline WVDG-1 & & 0.0003 & & & 0.0030 & 0.0003 & & & 0.0286 & 0.0096 & & & 0.0054 & & & & 0.0002 & 0.0000 & & 0.9987 \\
\hline WVDG-2 & & 0.0003 & & & 0.0030 & 0.0003 & & & 0.0280 & 0.0096 & & & 0.0053 & & & & 0.0002 & 0.0000 & & 0.9991 \\
\hline WVDG-3 & & 0.0000 & & & 0.0027 & 0.0004 & & & 0.0273 & 0.0122 & & & 0.0054 & & & & 0.0003 & 0.0000 & & 0.9983 \\
\hline WVDG-4 & & 0.0003 & & & 0.0029 & 0.0003 & & & 0.0294 & 0.0090 & & & 0.0054 & & & & 0.0002 & 0.0000 & & 0.9986 \\
\hline WVDG-5 & & 0.0003 & & & 0.0031 & 0.0003 & & & 0.0311 & 0.0097 & & & 0.0057 & & & & 0.0002 & 0.0000 & & 1.0045 \\
\hline WVDG-6 & & 0.0003 & & & 0.0030 & 0.0003 & & & 0.0275 & 0.0093 & & & 0.0053 & & & & 0.0002 & 0.0000 & & 0.9990 \\
\hline WVDG-7 & & 0.0003 & & & 0.0029 & 0.0003 & & & 0.0383 & 0.0096 & & & 0.0046 & & & & 0.0002 & 0.0000 & & 0.9992 \\
\hline WVDG-8 & & 0.0003 & & & 0.0032 & 0.0003 & & & 0.0275 & 0.0101 & & & 0.0058 & & & & 0.0002 & 0.0005 & & 0.9990 \\
\hline WVDG-11R & & 0.0003 & & & 0.0031 & 0.0003 & & & 0.0220 & 0.0095 & & & 0.0063 & & & & 0.0002 & 0.0002 & & 0.9994 \\
\hline WVDG-12R & & 0.0003 & & & 0.0030 & 0.0003 & & & 0.0253 & 0.0094 & & & 0.0065 & & & & 0.0001 & 0.0006 & & 0.9997 \\
\hline WVDG-13R & & 0.0003 & & & 0.0031 & 0.0003 & & & 0.0248 & 0.0096 & & & 0.0069 & & & & 0.0002 & 0.0000 & & 0.9989 \\
\hline WVDG-14R & & 0.0003 & & & 0.0030 & 0.0003 & & & 0.0259 & 0.0096 & & & 0.0064 & & & & 0.0002 & 0.0000 & & 0.9991 \\
\hline WVDG-15 & & 0.0003 & & & 0.0040 & 0.0003 & & & 0.0166 & 0.0144 & & & 0.0092 & & & & 0.0003 & 0.0002 & & 0.9993 \\
\hline WVDG-16 & & 0.0001 & & & 0.0012 & 0.0001 & & & 0.0160 & 0.0044 & & & 0.0013 & & & & 0.0001 & 0.0002 & & 1.0005 \\
\hline WVDG-17 & & 0.0004 & & & 0.0043 & 0.0004 & & & 0.0469 & 0.0158 & & & 0.0008 & & & & 0.0004 & 0.0006 & & 0.9996 \\
\hline WVDG-18 & & 0.0001 & & & 0.0013 & 0.0001 & & & 0.0196 & 0.0046 & & & 0.0009 & & & & 0.0001 & 0.0000 & & 0.9997 \\
\hline WVDG-19 & & 0.0001 & & & 0.0012 & 0.0001 & & & 0.0524 & 0.0044 & & & 0.0093 & & & & 0.0001 & 0.0000 & & 0.9902 \\
\hline WVDG-20 & & 0.0004 & & & 0.0043 & 0.0004 & & & 0.0187 & 0.0157 & & & 0.0095 & & & & 0.0004 & 0.0002 & & 0.9991 \\
\hline WVDG-21 & & 0.0001 & & & 0.0012 & 0.0001 & & & 0.0159 & 0.0046 & & & 0.0092 & & & & 0.0001 & 0.0006 & & 1.0008 \\
\hline WVDG-22 & & 0.0004 & & & 0.0042 & 0.0004 & & & 0.0175 & 0.0154 & & & 0.0011 & & & & 0.0004 & 0.0002 & & 0.9989 \\
\hline WVDG-23 & & 0.0001 & & & 0.0014 & 0.0001 & & & 0.0495 & 0.0050 & & & 0.0093 & & & & 0.0001 & 0.0002 & & 0.9997 \\
\hline WVDG-24 & & 0.0001 & & & 0.0013 & 0.0001 & & & 0.0188 & 0.0047 & & & 0.0093 & & & & 0.0001 & 0.0002 & & 0.9997 \\
\hline WVDG-25 & & 0.0001 & & & 0.0014 & 0.0001 & & & 0.0436 & 0.0051 & & & 0.0010 & & & & 0.0001 & 0.0000 & & 0.9996 \\
\hline WVDG-26 & & 0.0001 & & & 0.0014 & 0.0001 & & & 0.0380 & 0.0053 & & & 0.0010 & & & & 0.0001 & 0.0002 & & 1.0001 \\
\hline WVDG-27 & & 0.0004 & & & 0.0046 & 0.0004 & & & 0.0346 & 0.0168 & & & 0.0011 & & & & 0.0004 & 0.0006 & & 1.0000 \\
\hline WVDG-28 & & 0.0004 & & & 0.0044 & 0.0004 & & & 0.0172 & 0.0161 & & & 0.0096 & & & & 0.0004 & 0.0006 & & 0.9999 \\
\hline WVDG-29 & & 0.0001 & & & 0.0014 & 0.0001 & & & 0.0193 & 0.0051 & & & 0.0010 & & & & 0.0001 & 0.0002 & & 0.9996 \\
\hline WVDG-30 & & 0.0002 & & & 0.0026 & 0.0002 & & & 0.0326 & 0.0094 & & & 0.0059 & & & & 0.0002 & 0.0003 & & 1.0000 \\
\hline WVDG-33 & & 0.0002 & & & 0.0021 & 0.0002 & & & 0.0337 & 0.0078 & & & 0.0050 & & & & 0.0002 & 0.0003 & & 1.0002 \\
\hline WVDG-34 & & 0.0005 & & & 0.0061 & 0.0005 & & & 0.0192 & 0.0223 & & & 0.0183 & & & & 0.0005 & 0.0008 & & 1.0000 \\
\hline WVDG-35 & & 0.0001 & & & 0.0010 & 0.0001 & & & 0.0278 & 0.0038 & & & 0.0240 & & & & 0.0001 & 0.0001 & & 0.9946 \\
\hline WVDG-36 & & 0.0005 & & & 0.0060 & 0.0005 & & & 0.0581 & 0.0219 & & & 0.0013 & & & & 0.0005 & 0.0008 & & 0.9999 \\
\hline WVDG-37 & & 0.0005 & & & 0.0060 & 0.0005 & & & 0.0209 & 0.0216 & & & 0.0014 & & & & 0.0005 & 0.0008 & & 1.0000 \\
\hline WVDG-38 & & 0.0005 & & & 0.0061 & 0.0005 & & & 0.0413 & 0.0225 & & & 0.0009 & & & & 0.0005 & 0.0008 & & 0.9963 \\
\hline WVDG-39 & & 0.0001 & & & 0.0016 & 0.0001 & & & 0.0511 & 0.0060 & & & 0.0010 & & & & 0.0001 & 0.0002 & & 0.9998 \\
\hline WVDG-40 & & 0.0001 & & & 0.0013 & 0.0001 & & & 0.0419 & 0.0049 & & & 0.0182 & & & & 0.0001 & 0.0002 & & 0.9998 \\
\hline WVDG-41 & & 0.0001 & & & 0.0015 & 0.0001 & & & 0.0527 & 0.0056 & & & 0.0199 & & & & 0.0001 & 0.0002 & & 1.0001 \\
\hline WVDG-42 & & 0.0005 & & & 0.0059 & 0.0005 & & & 0.0500 & 0.0216 & & & 0.0012 & & & & 0.0005 & 0.0008 & & 0.9850 \\
\hline WVDG-43 & & 0.0001 & & & 0.0013 & 0.0001 & & & 0.0414 & 0.0048 & & & 0.0192 & & & & 0.0001 & 0.0002 & & 0.9999 \\
\hline WVDG-44 & & 0.0001 & & & 0.0013 & 0.0001 & & & 0.0371 & 0.0047 & & & 0.0215 & & & & 0.0001 & 0.0002 & & 0.9997 \\
\hline WVDG-45 & & 0.0001 & & & 0.0013 & 0.0001 & & & 0.0179 & 0.0048 & & & 0.0010 & & & & 0.0001 & 0.0002 & & 0.9981 \\
\hline WVDG-46 & & 0.0001 & & & 0.0012 & 0.0001 & & & 0.0216 & 0.0042 & & & 0.0011 & & & & 0.0001 & 0.0002 & & 1.0001 \\
\hline WVDG-47 & & 0.0001 & & & 0.0011 & 0.0001 & & & 0.0195 & 0.0042 & & & 0.0012 & & & & 0.0001 & 0.0002 & & 1.0002 \\
\hline WVDG-48 & & 0.0006 & & & 0.0066 & 0.0006 & & & 0.0206 & 0.0239 & & & 0.0190 & & & & 0.0006 & 0.0009 & & 1.0000 \\
\hline
\end{tabular}


Appendix A. Database - mass fraction

\begin{tabular}{|c|c|c|c|c|c|c|c|c|}
\hline \multicolumn{9}{|c|}{ West Valley CVS Glasses PCT (Olson et al. 1994) } \\
\hline Glass ID & $\begin{array}{l}\mathrm{TM} \\
\left({ }^{\circ} \mathrm{C}\right)\end{array}$ & $\begin{array}{l}\text { Gradient } \\
\text { TL }\left({ }^{\circ} \mathrm{C}\right)\end{array}$ & $\begin{array}{l}\text { Uniform } \\
\text { TL }\left({ }^{\circ} \mathrm{C}\right)\end{array}$ & Primary Phase & $\begin{array}{l}\text { Quenched } \\
\text { Visual/OM }\end{array}$ & $\begin{array}{c}\text { Quenched } \\
\text { SEM/EDS or TEM }\end{array}$ & $\begin{array}{l}\text { Quenched } \\
\text { XRD }\end{array}$ & $\begin{array}{c}\text { Quenched } \\
\text { Homogeneous? }\end{array}$ \\
\hline \multicolumn{9}{|l|}{ WVDG-1 } \\
\hline \multicolumn{9}{|l|}{ WVDG-2 } \\
\hline \multirow{2}{*}{\multicolumn{9}{|c|}{$\frac{\text { WVDG-3 }}{\text { WVDG-4 }}$}} \\
\hline & & & & & & & & \\
\hline \multicolumn{9}{|l|}{ WVDG-5 } \\
\hline \multicolumn{9}{|l|}{ WVDG-6 } \\
\hline \multicolumn{9}{|l|}{ WVDG-7 } \\
\hline \multicolumn{9}{|l|}{ WVDG-8 } \\
\hline \multicolumn{9}{|l|}{ WVDG-11R } \\
\hline \multicolumn{9}{|l|}{ WVDG-12R } \\
\hline \multicolumn{9}{|l|}{ WVDG-13R } \\
\hline \multicolumn{9}{|l|}{ WVDG-14R } \\
\hline \multicolumn{9}{|l|}{ WVDG-15 } \\
\hline \multicolumn{9}{|l|}{ WVDG-16 } \\
\hline \multicolumn{9}{|l|}{ WVDG-17 } \\
\hline \multicolumn{9}{|l|}{ WVDG-18 } \\
\hline \multicolumn{9}{|l|}{ WVDG-19 } \\
\hline \multicolumn{9}{|l|}{ WVDG-20 } \\
\hline \multicolumn{9}{|l|}{ WVDG-21 } \\
\hline \multicolumn{9}{|l|}{ WVDG-22 } \\
\hline \multicolumn{9}{|l|}{ WVDG-23 } \\
\hline \multicolumn{9}{|l|}{ WVDG-24 } \\
\hline WVDG-25 & & & & & & & & \\
\hline WVDG-26 & & & & & & & & \\
\hline WVDG-27 & & & & & & & & \\
\hline WVDG-28 & & & & & & & & \\
\hline WVDG-29 & & & & & & & & \\
\hline WVDG-30 & & & & & & & & \\
\hline WVDG-33 & & & & & & & & \\
\hline WVDG-34 & & & & & & & & \\
\hline WVDG-35 & & & & & & & & \\
\hline WVDG-36 & & & & & & & & \\
\hline WVDG-37 & & & & & & & & \\
\hline WVDG-38 & & & & & & & & \\
\hline WVDG-39 & & & & & & & & \\
\hline WVDG-40 & & & & & & & & \\
\hline WVDG-41 & & & & & & & & \\
\hline WVDG-42 & & & & & & & & \\
\hline WVDG-43 & & & & & & & & \\
\hline WVDG-44 & & & & & & & & \\
\hline WVDG-45 & & & & & & & & \\
\hline WVDG-46 & & & & & & & & \\
\hline WVDG-47 & & & & & & & & \\
\hline WVDG-48 & & & & & & & & \\
\hline
\end{tabular}


Appendix A. Database - mass fraction

\begin{tabular}{|c|c|c|c|c|c|}
\hline \multicolumn{6}{|c|}{ West Valley CVS Glasses PCT (Olson et al. 1994) } \\
\hline Glass ID & $\begin{array}{c}\text { CCC } \\
\text { Visual/OM }\end{array}$ & $\begin{array}{c}\text { CCC } \\
\text { SEM/EDS or TEM }\end{array}$ & $\begin{array}{l}\text { CCC } \\
\text { XRD }\end{array}$ & $\begin{array}{l}\text { Heat Treated } \\
\text { Visual/OM }\end{array}$ & $\begin{array}{c}\text { Heat Treated } \\
\text { SEM/EDS or TEM }\end{array}$ \\
\hline \multicolumn{6}{|l|}{ WVDG-1 } \\
\hline \multicolumn{6}{|l|}{ WVDG-2 } \\
\hline \multicolumn{6}{|l|}{ WVDG-3 } \\
\hline \multicolumn{6}{|l|}{ WVDG-4 } \\
\hline \multicolumn{6}{|l|}{ WVDG-5 } \\
\hline \multicolumn{6}{|l|}{ WVDG-6 } \\
\hline \multicolumn{6}{|l|}{ WVDG-7 } \\
\hline \multicolumn{6}{|l|}{ WVDG-8 } \\
\hline \multicolumn{6}{|l|}{ WVDG-11R } \\
\hline \multicolumn{6}{|l|}{ WVDG-12R } \\
\hline \multicolumn{6}{|l|}{ WVDG-13R } \\
\hline \multicolumn{6}{|l|}{ WVDG-14R } \\
\hline \multicolumn{6}{|l|}{ WVDG-15 } \\
\hline \multicolumn{6}{|l|}{ WVDG-16 } \\
\hline \multicolumn{6}{|l|}{ WVDG-17 } \\
\hline \multicolumn{6}{|l|}{ WVDG-18 } \\
\hline \multicolumn{6}{|l|}{ WVDG-19 } \\
\hline \multicolumn{6}{|l|}{ WVDG-20 } \\
\hline \multicolumn{6}{|l|}{ WVDG-21 } \\
\hline \multicolumn{6}{|l|}{ WVDG-22 } \\
\hline \multicolumn{6}{|l|}{ WVDG-23 } \\
\hline \multicolumn{6}{|l|}{ WVDG-24 } \\
\hline WVDG-25 & & & & & \\
\hline WVDG-26 & & & & & \\
\hline WVDG-27 & & & & & \\
\hline WVDG-28 & & & & & \\
\hline WVDG-29 & & & & & \\
\hline WVDG-30 & & & & & \\
\hline WVDG-33 & & & & & \\
\hline WVDG-34 & & & & & \\
\hline WVDG-35 & & & & & \\
\hline WVDG-36 & & & & & \\
\hline WVDG-37 & & & & & \\
\hline WVDG-38 & & & & & \\
\hline WVDG-39 & & & & & \\
\hline WVDG-40 & & & & & \\
\hline WVDG-41 & & & & & \\
\hline WVDG-42 & & & & & \\
\hline WVDG-43 & & & & & \\
\hline WVDG-44 & & & & & \\
\hline WVDG-45 & & & & & \\
\hline WVDG-46 & & & & & \\
\hline WVDG-47 & & & & & \\
\hline WVDG-48 & & & & & \\
\hline
\end{tabular}


Appendix A. Database - mass fraction

West Valley CVS Glasses PCT (Olson et al. 1994)

\begin{tabular}{|c|c|c|c|c|c|c|c|c|c|c|c|c|c|c|c|}
\hline Glass ID & $\begin{array}{c}\text { Heat Treated } \\
\text { XRD } \\
\end{array}$ & $\begin{array}{l}\text { Density } \\
\left(\mathrm{g} / \mathrm{cm}^{3}\right)\end{array}$ & $\begin{array}{c}\text { Fulc Visc } \\
\mathrm{A}\end{array}$ & $\begin{array}{c}\text { Fulc Visc } \\
\text { B } \\
\end{array}$ & $\begin{array}{c}\text { Fulc Visc } \\
\text { To } \\
\end{array}$ & $\begin{array}{c}\mathrm{FV} 1150^{\circ} \mathrm{C} \\
(\mathrm{Pa} \cdot \mathrm{s})\end{array}$ & \begin{tabular}{|c|} 
Arrh Visc \\
A
\end{tabular} & \begin{tabular}{|c|} 
Arrh Visc \\
$\mathrm{B}$ \\
\end{tabular} & $\begin{array}{c}1150^{\circ} \mathrm{C} \\
\left(\mathrm{D}_{0.0}\right)\end{array}$ & $\begin{array}{c}\mathrm{T}\left({ }^{\circ} \mathrm{C}\right) \text { at } \\
2 \mathrm{~Pa} \cdot \mathrm{s}\end{array}$ & $\begin{array}{c}\mathrm{T}\left({ }^{\circ} \mathrm{C}\right) \text { at } \\
5 \mathrm{~Pa} \cdot \mathrm{s} \\
\end{array}$ & $\begin{array}{c}\mathrm{T}\left({ }^{\circ} \mathrm{C}\right) \text { at } \\
10 \mathrm{~Pa} \cdot \mathrm{s} \\
\end{array}$ & $\begin{array}{l}\mathrm{T} 1 \\
\left({ }^{\circ} \mathrm{C}\right) \\
\end{array}$ & $\begin{array}{c}\mathrm{V} 1 \\
(\mathrm{~Pa} \cdot \mathrm{s})\end{array}$ & $\begin{array}{c}\mathrm{T} 2 \\
\left({ }^{\circ} \mathrm{C}\right) \\
\end{array}$ \\
\hline WVDG-1 & & & & & & & & & & & & & & & \\
\hline WVDG-2 & & & & & & & & & & & & & & & \\
\hline WVDG-3 & & & & & & & & & & & & & & & \\
\hline WVDG-4 & & & & & & & & & & & & & & & \\
\hline WVDG-5 & & & & & & & & & & & & & & & \\
\hline WVDG-6 & & & & & & & & & & & & & & & \\
\hline WVDG-7 & & & & & & & & & & & & & & & \\
\hline WVDG-8 & & & & & & & & & & & & & & & \\
\hline WVDG-11R & & & & & & & & & & & & & & & \\
\hline WVDG-12R & & & & & & & & & & & & & & & \\
\hline WVDG-13R & & & & & & & & & & & & & & & \\
\hline WVDG-14R & & & & & & & & & & & & & & & \\
\hline WVDG-15 & & & & & & & & & & & & & & & \\
\hline WVDG-16 & & & & & & & & & & & & & & & \\
\hline WVDG-17 & & & & & & & & & & & & & & & \\
\hline WVDG-18 & & & & & & & & & & & & & & & \\
\hline WVDG-19 & & & & & & & & & & & & & & & \\
\hline WVDG-20 & & & & & & & & & & & & & & & \\
\hline WVDG-21 & & & & & & & & & & & & & & & \\
\hline WVDG-22 & & & & & & & & & & & & & & & \\
\hline WVDG-23 & & & & & & & & & & & & & & & \\
\hline WVDG-24 & & & & & & & & & & & & & & & \\
\hline WVDG-25 & & & & & & & & & & & & & & & \\
\hline WVDG-26 & & & & & & & & & & & & & & & \\
\hline WVDG-27 & & & & & & & & & & & & & & & \\
\hline WVDG-28 & & & & & & & & & & & & & & & \\
\hline WVDG-29 & & & & & & & & & & & & & & & \\
\hline WVDG-30 & & & & & & & & & & & & & & & \\
\hline WVDG-33 & & & & & & & & & & & & & & & \\
\hline WVDG-34 & & & & & & & & & & & & & & & \\
\hline WVDG-35 & & & & & & & & & & & & & & & \\
\hline WVDG-36 & & & & & & & & & & & & & & & \\
\hline WVDG-37 & & & & & & & & & & & & & & & \\
\hline WVDG-38 & & & & & & & & & & & & & & & \\
\hline WVDG-39 & & & & & & & & & & & & & & & \\
\hline WVDG-40 & & & & & & & & & & & & & & & \\
\hline WVDG-41 & & & & & & & & & & & & & & & \\
\hline WVDG-42 & & & & & & & & & & & & & & & \\
\hline WVDG-43 & & & & & & & & & & & & & & & \\
\hline WVDG-44 & & & & & & & & & & & & & & & \\
\hline WVDG-45 & & & & & & & & & & & & & & & \\
\hline WVDG-46 & & & & & & & & & & & & & & & \\
\hline WVDG-47 & & & & & & & & & & & & & & & \\
\hline WVDG-48 & & & & & & & & & & & & & & & \\
\hline
\end{tabular}


Appendix A. Database - mass fraction

\begin{tabular}{|c|c|c|c|c|c|c|c|c|c|c|c|c|c|c|c|c|c|c|c|c|c|}
\hline \multicolumn{22}{|c|}{ West Valley CVS Glasses PCT (Olson et al. 1994) } \\
\hline Glass ID & $\begin{array}{c}\mathrm{V} 2 \\
(\mathrm{~Pa} \cdot \mathrm{s})\end{array}$ & $\begin{array}{c}\mathrm{T} 3 \\
\left({ }^{\circ} \mathrm{C}\right) \\
\end{array}$ & $\begin{array}{c}\mathrm{V} 3 \\
(\mathrm{~Pa} \cdot \mathrm{s}) \\
\end{array}$ & $\begin{array}{c}\mathrm{T} 4 \\
\left({ }^{\circ} \mathrm{C}\right) \\
\end{array}$ & $\begin{array}{c}\mathrm{V} 4 \\
(\mathrm{~Pa} \cdot \mathrm{s})\end{array}$ & $\begin{array}{c}\mathrm{T} 5 \\
\left({ }^{\circ} \mathrm{C}\right) \\
\end{array}$ & $\begin{array}{c}\mathrm{V} 5 \\
(\mathrm{~Pa} \cdot \mathrm{s}) \\
\end{array}$ & $\begin{array}{c}\mathrm{T} 6 \\
\left({ }^{\circ} \mathrm{C}\right) \\
\end{array}$ & $\begin{array}{c}\mathrm{V} 6 \\
(\mathrm{~Pa} \cdot \mathrm{s})\end{array}$ & $\begin{array}{c}\mathrm{T} 7 \\
\left({ }^{\circ} \mathrm{C}\right) \\
\end{array}$ & $\begin{array}{c}\mathrm{V} 7 \\
(\mathrm{~Pa} \cdot \mathrm{s})\end{array}$ & $\begin{array}{c}\mathrm{T} 8 \\
\left({ }^{\circ} \mathrm{C}\right) \\
\end{array}$ & $\begin{array}{c}\mathrm{V} 8 \\
(\mathrm{~Pa} \cdot \mathrm{s}) \\
\end{array}$ & $\begin{array}{c}\text { T9 } \\
\left({ }^{\circ} \mathrm{C}\right) \\
\end{array}$ & $\begin{array}{c}\mathrm{V} 9 \\
(\mathrm{~Pa} \cdot \mathrm{s})\end{array}$ & $\begin{array}{l}\mathrm{T} 10 \\
\left({ }^{\circ} \mathrm{C}\right) \\
\end{array}$ & $\begin{array}{c}\mathrm{V} 10 \\
(\mathrm{~Pa} \cdot \mathrm{s}) \\
\end{array}$ & $\begin{array}{l}\mathrm{T} 11 \\
\left({ }^{\circ} \mathrm{C}\right) \\
\end{array}$ & $\begin{array}{c}\mathrm{V} 11 \\
(\mathrm{~Pa} \cdot \mathrm{s})\end{array}$ & $\begin{array}{l}\mathrm{T} 12 \\
\left({ }^{\circ} \mathrm{C}\right) \\
\end{array}$ & $\begin{array}{c}\mathrm{V} 12 \\
(\mathrm{~Pa} \cdot \mathrm{s})\end{array}$ \\
\hline \multicolumn{22}{|l|}{ WVDG-1 } \\
\hline \multicolumn{22}{|l|}{ WVDG-2 } \\
\hline \multicolumn{22}{|l|}{ WVDG-3 } \\
\hline \multicolumn{22}{|l|}{ WVDG-4 } \\
\hline \multicolumn{22}{|l|}{ WVDG-5 } \\
\hline \multicolumn{22}{|l|}{ WVDG-6 } \\
\hline \multicolumn{22}{|l|}{ WVDG-7 } \\
\hline \multirow{2}{*}{\multicolumn{22}{|c|}{$\begin{array}{l}\text { WVDG-8 } \\
\text { WVDG-11R }\end{array}$}} \\
\hline \multirow{2}{*}{\multicolumn{22}{|c|}{$\begin{array}{l}\text { WVDG-11R } \\
\text { WVDG-12R }\end{array}$}} \\
\hline & & & & & & & & & & & & & & & & & & & & & \\
\hline \multicolumn{22}{|l|}{ WVDG-13R } \\
\hline \multicolumn{22}{|l|}{ WVDG-14R } \\
\hline \multirow{2}{*}{\multicolumn{22}{|c|}{$\begin{array}{l}\text { WVDG-15 } \\
\text { WVDG-16 }\end{array}$}} \\
\hline \multirow{2}{*}{\multicolumn{22}{|c|}{$\begin{array}{l}\text { WVDG-16 } \\
\text { WVDG-17 }\end{array}$}} \\
\hline & & & & & & & & & & & & & & & & & & & & & \\
\hline \multicolumn{22}{|l|}{ WVDG-18 } \\
\hline WVDG-19 & & & & & & & & & & & & & & & & & & & & & \\
\hline WVDG-20 & & & & & & & & & & & & & & & & & & & & & \\
\hline WVDG-21 & & & & & & & & & & & & & & & & & & & & & \\
\hline WVDG-22 & & & & & & & & & & & & & & & & & & & & & \\
\hline WVDG-23 & & & & & & & & & & & & & & & & & & & & & \\
\hline WVDG-24 & & & & & & & & & & & & & & & & & & & & & \\
\hline WVDG-25 & & & & & & & & & & & & & & & & & & & & & \\
\hline WVDG-26 & & & & & & & & & & & & & & & & & & & & & \\
\hline WVDG-27 & & & & & & & & & & & & & & & & & & & & & \\
\hline WVDG-28 & & & & & & & & & & & & & & & & & & & & & \\
\hline WVDG-29 & & & & & & & & & & & & & & & & & & & & & \\
\hline WVDG-30 & & & & & & & & & & & & & & & & & & & & & \\
\hline WVDG-33 & & & & & & & & & & & & & & & & & & & & & \\
\hline WVDG-34 & & & & & & & & & & & & & & & & & & & & & \\
\hline WVDG-35 & & & & & & & & & & & & & & & & & & & & & \\
\hline WVDG-36 & & & & & & & & & & & & & & & & & & & & & \\
\hline WVDG-37 & & & & & & & & & & & & & & & & & & & & & \\
\hline WVDG-38 & & & & & & & & & & & & & & & & & & & & & \\
\hline WVDG-39 & & & & & & & & & & & & & & & & & & & & & \\
\hline WVDG-40 & & & & & & & & & & & & & & & & & & & & & \\
\hline WVDG-41 & & & & & & & & & & & & & & & & & & & & & \\
\hline WVDG-42 & & & & & & & & & & & & & & & & & & & & & \\
\hline WVDG-43 & & & & & & & & & & & & & & & & & & & & & \\
\hline WVDG-44 & & & & & & & & & & & & & & & & & & & & & \\
\hline WVDG-45 & & & & & & & & & & & & & & & & & & & & & \\
\hline WVDG-46 & & & & & & & & & & & & & & & & & & & & & \\
\hline WVDG-47 & & & & & & & & & & & & & & & & & & & & & \\
\hline WVDG-48 & & & & & & & & & & & & & & & & & & & & & \\
\hline
\end{tabular}


Appendix A. Database - mass fraction

West Valley CVS Glasses PCT (Olson et al. 1994)

\begin{tabular}{|c|c|c|c|c|c|c|c|c|c|c|c|c|c|c|c|c|}
\hline Glass ID & $\begin{array}{l}\mathrm{T} 13 \\
\left({ }^{\circ} \mathrm{C}\right)\end{array}$ & $\begin{array}{l}\text { V13 } \\
(\mathrm{Pa} \cdot \mathrm{s})\end{array}$ & $\begin{array}{l}\mathrm{T} 14 \\
\left({ }^{\circ} \mathrm{C}\right)\end{array}$ & $\begin{array}{l}\mathrm{V} 14 \\
(\mathrm{~Pa} \cdot \mathrm{s})\end{array}$ & $\begin{array}{c}\text { Q PCT } \\
\mathrm{B}\left(\mathrm{g} / \mathrm{m}^{2}\right)\end{array}$ & $\begin{array}{c}\text { Q PCT } \\
\mathrm{Li}\left(\mathrm{g} / \mathrm{m}^{2}\right)\end{array}$ & $\begin{array}{c}\text { Q PCT } \\
\mathrm{Na}\left(\mathrm{g} / \mathrm{m}^{2}\right)\end{array}$ & $\begin{array}{c}\text { Q PCT } \\
\mathrm{Si}\left(\mathrm{g} / \mathrm{m}^{2}\right)\end{array}$ & $\begin{array}{l}\text { Q PCT } \\
\text { pH }\end{array}$ & $\begin{array}{c}\text { CCC PCT } \\
\text { B }\left(\mathrm{g} / \mathrm{m}^{2}\right)\end{array}$ & $\begin{array}{l}\text { CCC PCT } \\
\mathrm{Li}\left(\mathrm{g} / \mathrm{m}^{2}\right)\end{array}$ & $\begin{array}{l}\text { CCC PCT } \\
\mathrm{Na}\left(\mathrm{g} / \mathrm{m}^{2}\right)\end{array}$ & $\begin{array}{l}\text { CCC PCT } \\
\mathrm{Si}\left(\mathrm{g} / \mathrm{m}^{2}\right)\end{array}$ & $\begin{array}{c}\text { CCC PCT } \\
\text { pH }\end{array}$ & $\begin{array}{l}\text { Q PCT B at } \\
20^{\circ} \mathrm{C}\left(\mathrm{g} / \mathrm{m}^{2}\right)\end{array}$ & $\begin{array}{l}\text { Q PCT Li at } \\
20^{\circ} \mathrm{C}\left(\mathrm{g} / \mathrm{m}^{2}\right)\end{array}$ \\
\hline WVDG-1 & & & & & 0.180 & 0.250 & 0.159 & 0.127 & 9.40 & & & & & & & \\
\hline WVDG-2 & & & & & 0.207 & 0.311 & 0.181 & 0.133 & 9.34 & & & & & & & \\
\hline WVDG-3 & & & & & 0.238 & 0.291 & 0.233 & 0.139 & 9.77 & & & & & & & \\
\hline WVDG-4 & & & & & 0.261 & 0.303 & 0.266 & 0.134 & 9.78 & & & & & & & \\
\hline WVDG-5 & & & & & 0.214 & 0.285 & 0.198 & 0.128 & 9.46 & & & & & & & \\
\hline WVDG-6 & & & & & 0.199 & 0.303 & 0.169 & 0.131 & 9.50 & & & & & & & \\
\hline WVDG-7 & & & & & 0.180 & 0.267 & 0.169 & 0.125 & 9.37 & & & & & & & \\
\hline WVDG-8 & & & & & 0.173 & 0.273 & 0.174 & 0.121 & 9.73 & & & & & & & \\
\hline WVDG-11R & & & & & 0.205 & 0.246 & 0.204 & 0.122 & 9.81 & & & & & & & \\
\hline WVDG-12R & & & & & 0.217 & 0.263 & 0.214 & 0.123 & 9.81 & & & & & & & \\
\hline WVDG-13R & & & & & 0.237 & 0.279 & 0.243 & 0.128 & 9.74 & & & & & & & \\
\hline WVDG-14R & & & & & 0.288 & 0.333 & 0.302 & 0.136 & 9.95 & & & & & & & \\
\hline WVDG-15 & & & & & 0.296 & 0.3 & 0.264 & 0.132 & 9.89 & & & & & & & \\
\hline WVDG-16 & & & & & 0.189 & 0.235 & 0.165 & 0.09 & 9.42 & & & & & & & \\
\hline WVDG-17 & & & & & 0.292 & 0.292 & 0.273 & 0.128 & 10.12 & & & & & & & \\
\hline WVDG-18 & & & & & 0.225 & 0.269 & 0.219 & 0.137 & 9.94 & & & & & & & \\
\hline WVDG-19 & & & & & 0.214 & 0.282 & 0.207 & 0.124 & 9.97 & & & & & & & \\
\hline WVDG-20 & & & & & 0.16 & 0.242 & 0.139 & 0.113 & 9.46 & & & & & & & \\
\hline WVDG-21 & & & & & 0.225 & 0.252 & 0.222 & 0.152 & 9.71 & & & & & & & \\
\hline WVDG-22 & & & & & 0.21 & 0.3 & 0.179 & 0.145 & 9.39 & & & & & & & \\
\hline WVDG-23 & & & & & 0.253 & 0.307 & 0.187 & 0.146 & 9.3 & & & & & & & \\
\hline WVDG-24 & & & & & 0.213 & 0.286 & 0.173 & 0.124 & 9.45 & & & & & & & \\
\hline WVDG-25 & & & & & 0.188 & 0.253 & 0.164 & 0.131 & 9.58 & & & & & & & \\
\hline WVDG-26 & & & & & 0.185 & 0.257 & 0.156 & 0.105 & 9.48 & & & & & & & \\
\hline WVDG-27 & & & & & 0.309 & 0.327 & 0.243 & 0.154 & 9.55 & & & & & & & \\
\hline WVDG-28 & & & & & 0.201 & 0.289 & 0.169 & 0.139 & 9.51 & & & & & & & \\
\hline WVDG-29 & & & & & 0.217 & 0.018 & 0.214 & 0.12 & 9.79 & & & & & & & \\
\hline WVDG-30 & & & & & 0.209 & 0.007 & 0.196 & 0.126 & 9.63 & & & & & & & \\
\hline WVDG-33 & & & & & 0.247 & 0.263 & 0.245 & 0.138 & 10.17 & & & & & & & \\
\hline WVDG-34 & & & & & 0.417 & 0.388 & 0.363 & 0.168 & 10.14 & & & & & & & \\
\hline WVDG-35 & & & & & 0.400 & 0.394 & 0.399 & 0.185 & 10.83 & & & & & & & \\
\hline WVDG-36 & & & & & 0.244 & 0.251 & 0.164 & 0.134 & 9.68 & & & & & & & \\
\hline WVDG-37 & & & & & 0.231 & 0.267 & 0.267 & 0.148 & 10.38 & & & & & & & \\
\hline WVDG-38 & & & & & 0.197 & 0.240 & 0.197 & 0.133 & 10.23 & & & & & & & \\
\hline WVDG-39 & & & & & 0.271 & 0.249 & 0.247 & 0.128 & 9.9 & & & & & & & \\
\hline WVDG-40 & & & & & 0.305 & 0.305 & 0.289 & 0.154 & 10.32 & & & & & & & \\
\hline WVDG-41 & & & & & 0.314 & 0.299 & 0.369 & 0.161 & 10.52 & & & & & & & \\
\hline WVDG-42 & & & & & 0.380 & 0.355 & 0.364 & 0.173 & 10.44 & & & & & & & \\
\hline WVDG-43 & & & & & 0.208 & 0.257 & 0.138 & 0.138 & 9.35 & & & & & & & \\
\hline WVDG-44 & & & & & 0.357 & 0.358 & 0.331 & 0.152 & 10.29 & & & & & & & \\
\hline WVDG-45 & & & & & 0.260 & 0.265 & 0.300 & 0.155 & 10.57 & & & & & & & \\
\hline WVDG-46 & & & & & 0.372 & 0.317 & 0.299 & 0.145 & 10.01 & & & & & & & \\
\hline WVDG-47 & & & & & 0.224 & 0.233 & 0.156 & 0.14 & 9.3 & & & & & & & \\
\hline WVDG-48 & & & & & 0.179 & 0.206 & 0.129 & 0.123 & 9.32 & & & & & & & \\
\hline
\end{tabular}


Appendix A. Database - mass fraction

\begin{tabular}{|c|c|c|c|c|c|c|c|c|c|c|c|c|}
\hline \multicolumn{13}{|c|}{ West Valley CVS Glasses PCT (Olson et al. 1994) } \\
\hline Glass ID & $\begin{array}{l}\text { Q PCT Na at } \\
20^{\circ} \mathrm{C}\left(\mathrm{g} / \mathrm{m}^{2}\right)\end{array}$ & $\begin{array}{l}\text { Q PCT Si at } \\
20^{\circ} \mathrm{C}\left(\mathrm{g} / \mathrm{m}^{2}\right)\end{array}$ & $\begin{array}{c}\mathrm{QpH} \\
\text { at } 20^{\circ} \mathrm{C}\end{array}$ & $\begin{array}{l}\text { TCLP Ag } \\
\text { (ppm) }\end{array}$ & $\begin{array}{c}\text { TCLP As } \\
\text { (ppm) }\end{array}$ & $\begin{array}{l}\text { TCLP Ba } \\
(\mathrm{ppm})\end{array}$ & $\begin{array}{c}\text { TCLP Cd } \\
(\mathrm{ppm})\end{array}$ & $\begin{array}{l}\text { TCLP Cr } \\
(\mathrm{ppm})\end{array}$ & $\begin{array}{c}\text { TCLP Ni } \\
\text { (ppm) }\end{array}$ & $\begin{array}{c}\text { TCLP Pb } \\
\text { (ppm) }\end{array}$ & $\begin{array}{c}\text { TCLP Se } \\
\text { (ppm) }\end{array}$ & $\begin{array}{c}\text { TCLP Zn } \\
\text { (ppm) }\end{array}$ \\
\hline \multicolumn{13}{|l|}{ WVDG-1 } \\
\hline \multicolumn{13}{|l|}{ WVDG-2 } \\
\hline \multicolumn{13}{|l|}{ WVDG-3 } \\
\hline \multicolumn{13}{|l|}{ WVDG-4 } \\
\hline \multicolumn{13}{|l|}{ WVDG-5 } \\
\hline \multicolumn{13}{|l|}{ WVDG-6 } \\
\hline \multicolumn{13}{|l|}{ WVDG-7 } \\
\hline \multicolumn{13}{|l|}{ WVDG-8 } \\
\hline \multicolumn{13}{|l|}{ WVDG-11R } \\
\hline \multicolumn{13}{|l|}{ WVDG-12R } \\
\hline \multicolumn{13}{|l|}{ WVDG-13R } \\
\hline \multicolumn{13}{|l|}{ WVDG-14R } \\
\hline \multicolumn{13}{|l|}{ WVDG-15 } \\
\hline \multicolumn{13}{|l|}{ WVDG-16 } \\
\hline \multicolumn{13}{|l|}{ WVDG-17 } \\
\hline \multicolumn{13}{|l|}{ WVDG-18 } \\
\hline \multicolumn{13}{|l|}{ WVDG-19 } \\
\hline \multicolumn{13}{|l|}{ WVDG-20 } \\
\hline \multicolumn{13}{|l|}{ WVDG-21 } \\
\hline WVDG-22 & & & & & & & & & & & & \\
\hline WVDG-23 & & & & & & & & & & & & \\
\hline WVDG-24 & & & & & & & & & & & & \\
\hline WVDG-25 & & & & & & & & & & & & \\
\hline WVDG-26 & & & & & & & & & & & & \\
\hline WVDG-27 & & & & & & & & & & & & \\
\hline WVDG-28 & & & & & & & & & & & & \\
\hline WVDG-29 & & & & & & & & & & & & \\
\hline WVDG-30 & & & & & & & & & & & & \\
\hline WVDG-33 & & & & & & & & & & & & \\
\hline WVDG-34 & & & & & & & & & & & & \\
\hline WVDG-35 & & & & & & & & & & & & \\
\hline WVDG-36 & & & & & & & & & & & & \\
\hline WVDG-37 & & & & & & & & & & & & \\
\hline WVDG-38 & & & & & & & & & & & & \\
\hline WVDG-39 & & & & & & & & & & & & \\
\hline WVDG-40 & & & & & & & & & & & & \\
\hline WVDG-41 & & & & & & & & & & & & \\
\hline WVDG-42 & & & & & & & & & & & & \\
\hline WVDG-43 & & & & & & & & & & & & \\
\hline WVDG-44 & & & & & & & & & & & & \\
\hline WVDG-45 & & & & & & & & & & & & \\
\hline WVDG-46 & & & & & & & & & & & & \\
\hline WVDG-47 & & & & & & & & & & & & \\
\hline WVDG-48 & & & & & & & & & & & & \\
\hline
\end{tabular}


Appendix A. Database - mass fraction

West Valley WQR (West Valley Nuclear Services 1995)

\begin{tabular}{|c|c|c|c|c|c|c|c|c|c|c|c|c|c|c|c|c|c|c|c|c|c|}
\hline Glass ID & $\begin{array}{c}\mathrm{A} 12 \mathrm{O} 3 \\
-\mathrm{t}\end{array}$ & $\begin{array}{c}\mathrm{B} 2 \mathrm{O} 3 \\
-\mathrm{t}\end{array}$ & $\begin{array}{c}\mathrm{CaO} \\
-\mathrm{t}\end{array}$ & $\begin{array}{c}\mathrm{Fe} 2 \mathrm{O} 3 \\
-\mathrm{t} \\
\end{array}$ & $\begin{array}{c}\mathrm{FeO} \\
-\mathrm{t} \\
\end{array}$ & $\begin{array}{c}\mathrm{K} 2 \mathrm{O} \\
-\mathrm{t}\end{array}$ & $\begin{array}{c}\mathrm{Li} 2 \mathrm{O} \\
-\mathrm{t}\end{array}$ & $\begin{array}{c}\mathrm{MgO} \\
-\mathrm{t} \\
\end{array}$ & $\begin{array}{c}\mathrm{Na} 2 \mathrm{O} \\
-\mathrm{t}\end{array}$ & $\begin{array}{c}\mathrm{NiO} \\
-\mathrm{t}\end{array}$ & $\begin{array}{c}\mathrm{P} 2 \mathrm{O} 5 \\
-\mathrm{t}\end{array}$ & $\begin{array}{c}\mathrm{SiO} 2 \\
-\mathrm{t}\end{array}$ & $\begin{array}{c}\mathrm{ZrO} 2 \\
-\mathrm{t}\end{array}$ & $\begin{array}{c}\mathrm{Ag} 2 \mathrm{O} \\
-\mathrm{t}\end{array}$ & $\begin{array}{c}\mathrm{As} 2 \mathrm{O} 3 \\
-\mathrm{t}\end{array}$ & $\begin{array}{c}\mathrm{BaO} \\
-\mathrm{t}\end{array}$ & $\begin{array}{c}\mathrm{Bi} 2 \mathrm{O} 3 \\
-\mathrm{t}\end{array}$ & $\begin{array}{l}\mathrm{Br} \\
-\mathrm{t}\end{array}$ & $\begin{array}{c}\mathrm{CdO} \\
-\mathrm{t} \\
\end{array}$ & $\begin{array}{c}\mathrm{Ce} 2 \mathrm{O} 3 \\
-\mathrm{t}\end{array}$ & $\begin{array}{c}\mathrm{CeO} 2 \\
-\mathrm{t}\end{array}$ \\
\hline \multicolumn{22}{|l|}{ WVDG-40 } \\
\hline \multicolumn{22}{|l|}{ WVDG-46 } \\
\hline \multicolumn{22}{|l|}{ FY92\#5 } \\
\hline \multicolumn{22}{|l|}{ FY92\#6 } \\
\hline \multicolumn{22}{|l|}{ FY92\#7 } \\
\hline \multicolumn{22}{|l|}{ FY92\#9 } \\
\hline \multicolumn{22}{|l|}{ FY92\#10 } \\
\hline \multicolumn{22}{|l|}{ FY92Ref5 } \\
\hline \multicolumn{22}{|l|}{ Ratio2 } \\
\hline \multicolumn{22}{|l|}{ Ratio4 } \\
\hline \multicolumn{22}{|l|}{ Ratio5 } \\
\hline \multicolumn{22}{|l|}{ LoTh2 } \\
\hline \multicolumn{22}{|l|}{ LoTh4 } \\
\hline \multicolumn{22}{|l|}{ LoTh5 } \\
\hline $\mathrm{HiFe} 2$ & & & & & & & & & & & & & & & & & & & & & \\
\hline HiFe3 & & & & & & & & & & & & & & & & & & & & & \\
\hline HiFe4 & & & & & & & & & & & & & & & & & & & & & \\
\hline PNL190 & & & & & & & & & & & & & & & & & & & & & \\
\hline FY93\#1 & & & & & & & & & & & & & & & & & & & & & \\
\hline FY93\#2 & & & & & & & & & & & & & & & & & & & & & \\
\hline FY93\#3 & & & & & & & & & & & & & & & & & & & & & \\
\hline FY93\#4 & & & & & & & & & & & & & & & & & & & & & \\
\hline FY93\#5 & & & & & & & & & & & & & & & & & & & & & \\
\hline FY93\#6 & & & & & & & & & & & & & & & & & & & & & \\
\hline FY93\#7 & & & & & & & & & & & & & & & & & & & & & \\
\hline FY93\#8 & & & & & & & & & & & & & & & & & & & & & \\
\hline FY93\#9 & & & & & & & & & & & & & & & & & & & & & \\
\hline FY93\#10 & & & & & & & & & & & & & & & & & & & & & \\
\hline FY94\#1 & & & & & & & & & & & & & & & & & & & & & \\
\hline FY94\#2 & & & & & & & & & & & & & & & & & & & & & \\
\hline FY94\#3 & & & & & & & & & & & & & & & & & & & & & \\
\hline FY94\#4 & & & & & & & & & & & & & & & & & & & & & \\
\hline FY94\#5 & & & & & & & & & & & & & & & & & & & & & \\
\hline FY94\#6 & & & & & & & & & & & & & & & & & & & & & \\
\hline FY94\#7 & & & & & & & & & & & & & & & & & & & & & \\
\hline FY94\#8 & & & & & & & & & & & & & & & & & & & & & \\
\hline FY94\#9 & & & & & & & & & & & & & & & & & & & & & \\
\hline FY94\#10 & & & & & & & & & & & & & & & & & & & & & \\
\hline Sigma1 & & & & & & & & & & & & & & & & & & & & & \\
\hline Sigma2 & & & & & & & & & & & & & & & & & & & & & \\
\hline Sigma3 & & & & & & & & & & & & & & & & & & & & & \\
\hline Sigma4 & & & & & & & & & & & & & & & & & & & & & \\
\hline Sigma5 & & & & & & & & & & & & & & & & & & & & & \\
\hline Sigma6 & & & & & & & & & & & & & & & & & & & & & \\
\hline Sigma7 & & & & & & & & & & & & & & & & & & & & & \\
\hline Sigma8 & & & & & & & & & & & & & & & & & & & & & \\
\hline Sigma9 & & & & & & & & & & & & & & & & & & & & & \\
\hline Sigma10 & & & & & & & & & & & & & & & & & & & & & \\
\hline
\end{tabular}


West Valley WQR (West Valley Nuclear Services 1995)

\begin{tabular}{|c|c|c|c|c|c|c|c|c|c|c|c|c|c|c|c|c|c|c|c|c|c|}
\hline Glass ID & $\begin{array}{l}\mathrm{Cl} \\
-\mathrm{t}\end{array}$ & $\begin{array}{c}\mathrm{CoO} \\
-\mathrm{t}\end{array}$ & $\begin{array}{c}\mathrm{Co} 2 \mathrm{O} 3 \\
-\mathrm{t}\end{array}$ & $\begin{array}{c}\mathrm{Cr} 2 \mathrm{O} 3 \\
-\mathrm{t}\end{array}$ & $\begin{array}{c}\mathrm{Cs} 2 \mathrm{O} \\
-\mathrm{t}\end{array}$ & $\begin{array}{c}\mathrm{CuO} \\
-\mathrm{t}\end{array}$ & $\begin{array}{c}\mathrm{Eu} 2 \mathrm{O} 3 \\
-\mathrm{t}\end{array}$ & $\begin{array}{l}F \\
-t\end{array}$ & $\begin{array}{c}\mathrm{Ga} 2 \mathrm{O} 3 \\
-\mathrm{t}\end{array}$ & $\begin{array}{c}\mathrm{Gd} 2 \mathrm{O} 3 \\
-\mathrm{t}\end{array}$ & $\begin{array}{c}\mathrm{HgO} \\
-\mathrm{t}\end{array}$ & $\begin{array}{c}I \\
-t\end{array}$ & $\begin{array}{c}\mathrm{La} 2 \mathrm{O} 3 \\
-\mathrm{t}\end{array}$ & $\begin{array}{c}\mathrm{MnO} 2 \\
-\mathrm{t}\end{array}$ & $\begin{array}{c}\mathrm{MnO} \\
-\mathrm{t}\end{array}$ & $\begin{array}{c}\mathrm{MoO} \\
-\mathrm{t}\end{array}$ & $\begin{array}{c}\mathrm{MoO} 3 \\
-\mathrm{t}\end{array}$ & $\begin{array}{c}\mathrm{Nb} 2 \mathrm{O} 5 \\
-\mathrm{t}\end{array}$ & $\begin{array}{c}\mathrm{Nd} 2 \mathrm{O} 3 \\
-\mathrm{t}\end{array}$ & $\begin{array}{c}\mathrm{PbO} \\
-\mathrm{t}\end{array}$ & $\begin{array}{c}\mathrm{PdO} 2 \\
-\mathrm{t}\end{array}$ \\
\hline WVDG-40 & & & & & & & & & & & & & & & & & & & & & \\
\hline WVDG-46 & & & & & & & & & & & & & & & & & & & & & \\
\hline FY92\#5 & & & & & & & & & & & & & & & & & & & & & \\
\hline FY92\#6 & & & & & & & & & & & & & & & & & & & & & \\
\hline FY92\#7 & & & & & & & & & & & & & & & & & & & & & \\
\hline FY92\#9 & & & & & & & & & & & & & & & & & & & & & \\
\hline FY92\#10 & & & & & & & & & & & & & & & & & & & & & \\
\hline FY92Ref5 & & & & & & & & & & & & & & & & & & & & & \\
\hline Ratio2 & & & & & & & & & & & & & & & & & & & & & \\
\hline Ratio4 & & & & & & & & & & & & & & & & & & & & & \\
\hline Ratio5 & & & & & & & & & & & & & & & & & & & & & \\
\hline LoTh2 & & & & & & & & & & & & & & & & & & & & & \\
\hline LoTh4 & & & & & & & & & & & & & & & & & & & & & \\
\hline LoTh5 & & & & & & & & & & & & & & & & & & & & & \\
\hline $\mathrm{HiFe} 2$ & & & & & & & & & & & & & & & & & & & & & \\
\hline $\mathrm{HiFe} 3$ & & & & & & & & & & & & & & & & & & & & & \\
\hline $\mathrm{HiFe} 4$ & & & & & & & & & & & & & & & & & & & & & \\
\hline PNL190 & & & & & & & & & & & & & & & & & & & & & \\
\hline FY93\#1 & & & & & & & & & & & & & & & & & & & & & \\
\hline FY93\#2 & & & & & & & & & & & & & & & & & & & & & \\
\hline FY93\#3 & & & & & & & & & & & & & & & & & & & & & \\
\hline FY93\#4 & & & & & & & & & & & & & & & & & & & & & \\
\hline FY93\#5 & & & & & & & & & & & & & & & & & & & & & \\
\hline FY93\#6 & & & & & & & & & & & & & & & & & & & & & \\
\hline FY93\#7 & & & & & & & & & & & & & & & & & & & & & \\
\hline FY93\#8 & & & & & & & & & & & & & & & & & & & & & \\
\hline FY93\#9 & & & & & & & & & & & & & & & & & & & & & \\
\hline FY93\#10 & & & & & & & & & & & & & & & & & & & & & \\
\hline FY94\#1 & & & & & & & & & & & & & & & & & & & & & \\
\hline FY94\#2 & & & & & & & & & & & & & & & & & & & & & \\
\hline FY94\#3 & & & & & & & & & & & & & & & & & & & & & \\
\hline FY94\#4 & & & & & & & & & & & & & & & & & & & & & \\
\hline FY94\#5 & & & & & & & & & & & & & & & & & & & & & \\
\hline FY94\#6 & & & & & & & & & & & & & & & & & & & & & \\
\hline FY94\#7 & & & & & & & & & & & & & & & & & & & & & \\
\hline FY94\#8 & & & & & & & & & & & & & & & & & & & & & \\
\hline FY94\#9 & & & & & & & & & & & & & & & & & & & & & \\
\hline FY94\#10 & & & & & & & & & & & & & & & & & & & & & \\
\hline Sigma1 & & & & & & & & & & & & & & & & & & & & & \\
\hline Sigma2 & & & & & & & & & & & & & & & & & & & & & \\
\hline Sigma3 & & & & & & & & & & & & & & & & & & & & & \\
\hline Sigma4 & & & & & & & & & & & & & & & & & & & & & \\
\hline Sigma5 & & & & & & & & & & & & & & & & & & & & & \\
\hline Sigma6 & & & & & & & & & & & & & & & & & & & & & \\
\hline Sigma7 & & & & & & & & & & & & & & & & & & & & & \\
\hline Sigma8 & & & & & & & & & & & & & & & & & & & & & \\
\hline Sigma9 & & & & & & & & & & & & & & & & & & & & & \\
\hline Sigma10 & & & & & & & & & & & & & & & & & & & & & \\
\hline
\end{tabular}


West Valley WQR (West Valley Nuclear Services 1995)

\begin{tabular}{|c|c|c|c|c|c|c|c|c|c|c|c|c|c|c|c|c|c|c|c|c|c|}
\hline Glass ID & $\begin{array}{c}\mathrm{PdO} \\
-\mathrm{t}\end{array}$ & $\begin{array}{c}\mathrm{Pr} 2 \mathrm{O} 3 \\
-\mathrm{t}\end{array}$ & $\begin{array}{c}\operatorname{Pr} 6 \mathrm{O} 11 \\
-\mathrm{t}\end{array}$ & $\begin{array}{c}\mathrm{Rb} 2 \mathrm{O} \\
-\mathrm{t}\end{array}$ & $\begin{array}{c}\mathrm{ReO} \\
-\mathrm{t}\end{array}$ & $\begin{array}{c}\mathrm{ReO} 2 \\
-\mathrm{t}\end{array}$ & $\begin{array}{c}\mathrm{Rh} 2 \mathrm{O} 3 \\
-\mathrm{t}\end{array}$ & $\begin{array}{c}\mathrm{RhO} 2 \\
-\mathrm{t}\end{array}$ & $\begin{array}{c}\mathrm{RuO} 2 \\
-\mathrm{t}\end{array}$ & $\begin{array}{c}\mathrm{Sb} 2 \mathrm{O} 3 \\
-\mathrm{t}\end{array}$ & $\begin{array}{c}\mathrm{Sb} 2 \mathrm{O} 5 \\
-\mathrm{t}\end{array}$ & $\begin{array}{c}\mathrm{SeO} 2 \\
-\mathrm{t}\end{array}$ & $\begin{array}{c}\mathrm{Sm} 2 \mathrm{O} 3 \\
-\mathrm{t}\end{array}$ & $\begin{array}{c}\mathrm{SnO} \\
-\mathrm{t}\end{array}$ & $\begin{array}{c}\mathrm{SnO} 2 \\
-\mathrm{t}\end{array}$ & $\begin{array}{c}\mathrm{SO} 3 \\
-\mathrm{t}\end{array}$ & $\begin{array}{c}\mathrm{SrO} \\
-\mathrm{t}\end{array}$ & $\begin{array}{c}\mathrm{Tc} 2 \mathrm{O} 7 \\
-\mathrm{t}\end{array}$ & $\begin{array}{c}\mathrm{TeO} 2 \\
-\mathrm{t}\end{array}$ & $\begin{array}{c}\text { ThO2 } \\
-t\end{array}$ & $\begin{array}{c}\mathrm{TiO} 2 \\
-\mathrm{t}\end{array}$ \\
\hline WVDG-40 & & & & & & & & & & & & & & & & & & & & & \\
\hline WVDG-46 & & & & & & & & & & & & & & & & & & & & & \\
\hline FY92\#5 & & & & & & & & & & & & & & & & & & & & & \\
\hline FY92\#6 & & & & & & & & & & & & & & & & & & & & & \\
\hline FY92\#7 & & & & & & & & & & & & & & & & & & & & & \\
\hline FY92\#9 & & & & & & & & & & & & & & & & & & & & & \\
\hline FY92\#10 & & & & & & & & & & & & & & & & & & & & & \\
\hline FY92Ref5 & & & & & & & & & & & & & & & & & & & & & \\
\hline Ratio2 & & & & & & & & & & & & & & & & & & & & & \\
\hline Ratio4 & & & & & & & & & & & & & & & & & & & & & \\
\hline Ratio5 & & & & & & & & & & & & & & & & & & & & & \\
\hline LoTh2 & & & & & & & & & & & & & & & & & & & & & \\
\hline LoTh4 & & & & & & & & & & & & & & & & & & & & & \\
\hline LoTh5 & & & & & & & & & & & & & & & & & & & & & \\
\hline $\mathrm{HiFe} 2$ & & & & & & & & & & & & & & & & & & & & & \\
\hline HiFe3 & & & & & & & & & & & & & & & & & & & & & \\
\hline HiFe4 & & & & & & & & & & & & & & & & & & & & & \\
\hline PNL190 & & & & & & & & & & & & & & & & & & & & & \\
\hline FY93\#1 & & & & & & & & & & & & & & & & & & & & & \\
\hline FY93\#2 & & & & & & & & & & & & & & & & & & & & & \\
\hline FY93\#3 & & & & & & & & & & & & & & & & & & & & & \\
\hline FY93\#4 & & & & & & & & & & & & & & & & & & & & & \\
\hline FY93\#5 & & & & & & & & & & & & & & & & & & & & & \\
\hline FY93\#6 & & & & & & & & & & & & & & & & & & & & & \\
\hline FY93\#7 & & & & & & & & & & & & & & & & & & & & & \\
\hline FY93\#8 & & & & & & & & & & & & & & & & & & & & & \\
\hline FY93\#9 & & & & & & & & & & & & & & & & & & & & & \\
\hline FY93\#10 & & & & & & & & & & & & & & & & & & & & & \\
\hline FY94\#1 & & & & & & & & & & & & & & & & & & & & & \\
\hline FY94\#2 & & & & & & & & & & & & & & & & & & & & & \\
\hline FY94\#3 & & & & & & & & & & & & & & & & & & & & & \\
\hline FY94\#4 & & & & & & & & & & & & & & & & & & & & & \\
\hline FY94\#5 & & & & & & & & & & & & & & & & & & & & & \\
\hline FY94\#6 & & & & & & & & & & & & & & & & & & & & & \\
\hline FY94\#7 & & & & & & & & & & & & & & & & & & & & & \\
\hline FY94\#8 & & & & & & & & & & & & & & & & & & & & & \\
\hline FY94\#9 & & & & & & & & & & & & & & & & & & & & & \\
\hline FY94\#10 & & & & & & & & & & & & & & & & & & & & & \\
\hline Sigma1 & & & & & & & & & & & & & & & & & & & & & \\
\hline Sigma2 & & & & & & & & & & & & & & & & & & & & & \\
\hline Sigma3 & & & & & & & & & & & & & & & & & & & & & \\
\hline Sigma4 & & & & & & & & & & & & & & & & & & & & & \\
\hline Sigma5 & & & & & & & & & & & & & & & & & & & & & \\
\hline Sigma6 & & & & & & & & & & & & & & & & & & & & & \\
\hline Sigma7 & & & & & & & & & & & & & & & & & & & & & \\
\hline Sigma8 & & & & & & & & & & & & & & & & & & & & & \\
\hline Sigma9 & & & & & & & & & & & & & & & & & & & & & \\
\hline Sigma10 & & & & & & & & & & & & & & & & & & & & & \\
\hline
\end{tabular}


West Valley WQR (West Valley Nuclear Services 1995)

\begin{tabular}{|c|c|c|c|c|c|c|c|c|c|c|c|c|c|c|c|c|c|c|c|c|c|}
\hline Glass ID & $\begin{array}{c}\mathrm{T} 12 \mathrm{O} 3 \\
-\mathrm{t}\end{array}$ & $\begin{array}{c}\mathrm{U} 3 \mathrm{O} 8 \\
-\mathrm{t}\end{array}$ & $\begin{array}{c}\mathrm{UO} 2 \\
-\mathrm{t}\end{array}$ & $\begin{array}{c}\text { UO3 } \\
-t\end{array}$ & $\begin{array}{c}\mathrm{V} 2 \mathrm{O} 5 \\
-\mathrm{t}\end{array}$ & $\begin{array}{c}\text { WO3 } \\
-t\end{array}$ & $\begin{array}{c}\mathrm{Y} 2 \mathrm{O} 3 \\
-\mathrm{t}\end{array}$ & $\begin{array}{c}\mathrm{ZnO} \\
-\mathrm{t}\end{array}$ & $\begin{array}{c}\text { Others } \\
-t\end{array}$ & $\begin{array}{c}\text { Sum } \\
-t\end{array}$ & $\begin{array}{c}\mathrm{A} 12 \mathrm{O} 3 \\
-\mathrm{a}\end{array}$ & $\begin{array}{c}\mathrm{B} 2 \mathrm{O} 3 \\
-\mathrm{a}\end{array}$ & $\begin{array}{c}\mathrm{CaO} \\
-\mathrm{a}\end{array}$ & $\begin{array}{c}\mathrm{Fe} 2 \mathrm{O} 3 \\
-\mathrm{a}\end{array}$ & $\begin{array}{c}\mathrm{FeO} \\
-\mathrm{a}\end{array}$ & $\begin{array}{c}\mathrm{K} 2 \mathrm{O} \\
-\mathrm{a}\end{array}$ & $\begin{array}{c}\mathrm{Li} 2 \mathrm{O} \\
-\mathrm{a}\end{array}$ & $\begin{array}{c}\mathrm{MgO} \\
-\mathrm{a}\end{array}$ & $\begin{array}{c}\mathrm{Na} 2 \mathrm{O} \\
-\mathrm{a}\end{array}$ & $\begin{array}{c}\mathrm{NiO} \\
-\mathrm{a}\end{array}$ & $\begin{array}{c}\mathrm{P} 2 \mathrm{O} 5 \\
-\mathrm{a}\end{array}$ \\
\hline WVDG-40 & & & & & & & & & & & 0.0609 & 0.0753 & 0.0072 & 0.1535 & & 0.0317 & 0.0269 & 0.0178 & 0.0968 & 0.0018 & 0.0060 \\
\hline WVDG-46 & & & & & & & & & & & 0.0700 & 0.1223 & 0.0068 & 0.1039 & & 0.0337 & 0.0286 & 0.0167 & 0.1030 & 0.0016 & 0.0059 \\
\hline FY92\#5 & & & & & & & & & & & 0.0714 & 0.1044 & 0.0048 & 0.1430 & & 0.0688 & 0.0510 & 0.0089 & 0.0875 & 0.0025 & 0.0120 \\
\hline FY92\#6 & & & & & & & & & & & 0.0486 & 0.1044 & 0.0048 & 0.0974 & & 0.0688 & 0.0232 & 0.0089 & 0.1028 & 0.0025 & 0.0120 \\
\hline FY92\#7 & & & & & & & & & & & 0.0486 & 0.1044 & 0.0048 & 0.1430 & & 0.0688 & 0.0232 & 0.0089 & 0.0572 & 0.0025 & 0.0120 \\
\hline FY92\#9 & & & & & & & & & & & 0.0486 & 0.1044 & 0.0048 & 0.1430 & & 0.0313 & 0.0510 & 0.0089 & 0.0572 & 0.0025 & 0.0120 \\
\hline FY92\#10 & & & & & & & & & & & 0.0714 & 0.1534 & 0.0048 & 0.0974 & & 0.0688 & 0.0232 & 0.0089 & 0.0572 & 0.0025 & 0.0120 \\
\hline FY92Ref5 & & & & & & & & & & & 0.0645 & 0.1289 & 0.0068 & 0.1202 & & 0.0318 & 0.0271 & 0.0089 & 0.0982 & 0.0025 & 0.0237 \\
\hline Ratio2 & & & & & & & & & & & 0.0667 & 0.1100 & 0.0048 & 0.1606 & & 0.0550 & 0.0370 & 0.0089 & 0.0880 & 0.0025 & 0.0120 \\
\hline Ratio4 & & & & & & & & & & & 0.0570 & 0.1120 & 0.0048 & 0.1008 & & 0.0560 & 0.0410 & 0.0089 & 0.0720 & 0.0025 & 0.0120 \\
\hline Ratio5 & & & & & & & & & & & 0.0520 & 0.1460 & 0.0048 & 0.1298 & & 0.0500 & 0.0410 & 0.0089 & 0.0900 & 0.0025 & 0.0120 \\
\hline LoTh2 & & & & & & & & & & & 0.0621 & 0.1170 & 0.0048 & 0.1300 & & 0.0450 & 0.0392 & 0.0089 & 0.0890 & 0.0025 & 0.0120 \\
\hline LoTh4 & & & & & & & & & & & 0.0608 & 0.1380 & 0.0048 & 0.1050 & & 0.0480 & 0.0330 & 0.0089 & 0.0890 & 0.0025 & 0.0120 \\
\hline LoTh5 & & & & & & & & & & & 0.0548 & 0.1433 & 0.0048 & 0.1380 & & 0.0437 & 0.0325 & 0.0089 & 0.0700 & 0.0025 & 0.0120 \\
\hline $\mathrm{HiFe} 2$ & & & & & & & & & & & 0.0534 & 0.1100 & 0.0048 & 0.1550 & & 0.0540 & 0.0400 & 0.0089 & 0.0880 & 0.0025 & 0.0120 \\
\hline $\mathrm{HiFe} 3$ & & & & & & & & & & & 0.0555 & 0.1123 & 0.0048 & 0.1470 & & 0.0575 & 0.0424 & 0.0089 & 0.0920 & 0.0025 & 0.0120 \\
\hline HiFe4 & & & & & & & & & & & 0.0545 & 0.1470 & 0.0048 & 0.1360 & & 0.0435 & 0.0415 & 0.0089 & 0.0830 & 0.0025 & 0.0120 \\
\hline PNL190 & & & & & & & & & & & 0.0490 & 0.1040 & 0.0048 & 0.0970 & & 0.0688 & 0.0510 & 0.0089 & 0.1030 & 0.0025 & 0.0120 \\
\hline FY93\#1 & & & & & & & & & & & 0.0771 & 0.1656 & 0.0048 & 0.1545 & & 0.0313 & 0.0232 & 0.0089 & 0.0572 & 0.0025 & 0.0120 \\
\hline FY93\#2 & & & & & & & & & & & 0.0429 & 0.1656 & 0.0048 & 0.0859 & & 0.0688 & 0.0232 & 0.0089 & 0.1028 & 0.0025 & 0.0120 \\
\hline FY93\#3 & & & & & & & & & & & 0.0771 & 0.1656 & 0.0048 & 0.0859 & & 0.0688 & 0.0510 & 0.0089 & 0.1028 & 0.0025 & 0.0120 \\
\hline FY93\#4 & & & & & & & & & & & 0.0429 & 0.0922 & 0.0048 & 0.1545 & & 0.0688 & 0.0232 & 0.0089 & 0.0572 & 0.0025 & 0.0120 \\
\hline FY93\#5 & & & & & & & & & & & 0.0429 & 0.1656 & 0.0048 & 0.0859 & & 0.0313 & 0.0510 & 0.0089 & 0.0572 & 0.0025 & 0.0120 \\
\hline FY93\#6 & & & & & & & & & & & 0.0771 & 0.0922 & 0.0048 & 0.0859 & & 0.0313 & 0.0432 & 0.0089 & 0.1028 & 0.0025 & 0.0120 \\
\hline FY93\#7 & & & & & & & & & & & 0.0771 & 0.0922 & 0.0048 & 0.0859 & & 0.0688 & 0.0232 & 0.0089 & 0.0572 & 0.0025 & 0.0120 \\
\hline FY93\#8 & & & & & & & & & & & 0.0429 & 0.1652 & 0.0048 & 0.1545 & & 0.0313 & 0.0232 & 0.0089 & 0.1028 & 0.0025 & 0.0120 \\
\hline FY93\#9 & & & & & & & & & & & 0.0771 & 0.0922 & 0.0048 & 0.1545 & & 0.0688 & 0.0245 & 0.0089 & 0.1028 & 0.0025 & 0.0120 \\
\hline FY93\#10 & & & & & & & & & & & 0.0429 & 0.0922 & 0.0048 & 0.0859 & & 0.0313 & 0.0510 & 0.0089 & 0.1028 & 0.0025 & 0.0120 \\
\hline FY94\#1 & & & & & & & & & & & 0.0740 & 0.0900 & 0.0048 & 0.0661 & & 0.0600 & 0.0280 & 0.0089 & 0.0840 & 0.0025 & 0.0120 \\
\hline FY94\#2 & & & & & & & & & & & 0.0740 & 0.0709 & 0.0048 & 0.1743 & & 0.0590 & 0.0400 & 0.0089 & 0.0520 & 0.0025 & 0.0120 \\
\hline FY94\#3 & & & & & & & & & & & 0.0566 & 0.1869 & 0.0048 & 0.1100 & & 0.0460 & 0.0280 & 0.0089 & 0.1100 & 0.0025 & 0.0120 \\
\hline FY94\#4 & & & & & & & & & & & 0.0430 & 0.1710 & 0.0048 & 0.1695 & & 0.0530 & 0.0460 & 0.0089 & 0.0910 & 0.0025 & 0.0120 \\
\hline FY94\#5 & & & & & & & & & & & 0.0550 & 0.0709 & 0.0048 & 0.0661 & & 0.0660 & 0.0445 & 0.0089 & 0.1070 & 0.0025 & 0.0120 \\
\hline FY94\#6 & & & & & & & & & & & 0.0771 & 0.0709 & 0.0048 & 0.1743 & & 0.0420 & 0.0470 & 0.0089 & 0.0600 & 0.0025 & 0.0120 \\
\hline FY94\#7 & & & & & & & & & & & 0.0630 & 0.1753 & 0.0048 & 0.0661 & & 0.0400 & 0.0240 & 0.0089 & 0.0660 & 0.0025 & 0.0120 \\
\hline FY94\#8 & & & & & & & & & & & 0.0440 & 0.1800 & 0.0048 & 0.1743 & & 0.0380 & 0.0380 & 0.0089 & 0.0854 & 0.0025 & 0.0120 \\
\hline FY94\#9 & & & & & & & & & & & 0.0460 & 0.1350 & 0.0048 & 0.1400 & & 0.0640 & 0.0340 & 0.0089 & 0.0612 & 0.0025 & 0.0120 \\
\hline FY94\#10 & & & & & & & & & & & 0.0612 & 0.1869 & 0.0048 & 0.0661 & & 0.0313 & 0.0480 & 0.0089 & 0.0780 & 0.0025 & 0.0120 \\
\hline Sigma1 & & & & & & & & & & & 0.0429 & 0.1869 & 0.0048 & 0.1168 & & 0.0688 & 0.0510 & 0.0089 & 0.1100 & 0.0025 & 0.0120 \\
\hline Sigma2 & & & & & & & & & & & 0.0429 & 0.1869 & 0.0048 & 0.0741 & & 0.0688 & 0.0510 & 0.0089 & 0.1100 & 0.0025 & 0.0120 \\
\hline Sigma3 & & & & & & & & & & & 0.0460 & 0.0761 & 0.0048 & 0.1743 & & 0.0670 & 0.0490 & 0.0089 & 0.1090 & 0.0025 & 0.0120 \\
\hline Sigma4 & & & & & & & & & & & 0.0490 & 0.1850 & 0.0048 & 0.1107 & & 0.0640 & 0.0500 & 0.0089 & 0.1030 & 0.0025 & 0.0120 \\
\hline Sigma5 & & & & & & & & & & & 0.0460 & 0.1800 & 0.0048 & 0.1208 & & 0.0670 & 0.0490 & 0.0089 & 0.1050 & 0.0025 & 0.0120 \\
\hline Sigma6 & & & & & & & & & & & 0.0470 & 0.1750 & 0.0048 & 0.1036 & & 0.0650 & 0.0480 & 0.0089 & 0.1010 & 0.0025 & 0.0120 \\
\hline Sigma7 & & & & & & & & & & & 0.0510 & 0.1700 & 0.0048 & 0.1125 & & 0.0660 & 0.0495 & 0.0089 & 0.1070 & 0.0025 & 0.0120 \\
\hline Sigma8 & & & & & & & & & & & 0.0480 & 0.1600 & 0.0048 & 0.1299 & & 0.0630 & 0.0485 & 0.0089 & 0.0970 & 0.0025 & 0.0120 \\
\hline Sigma9 & & & & & & & & & & & 0.0520 & 0.1550 & 0.0048 & 0.0938 & & 0.0610 & 0.0475 & 0.0089 & 0.0990 & 0.0025 & 0.0120 \\
\hline Sigma10 & & & & & & & & & & & 0.0500 & 0.1580 & 0.0048 & 0.0845 & & 0.0620 & 0.0470 & 0.0089 & 0.0950 & 0.0025 & 0.0120 \\
\hline
\end{tabular}


West Valley WQR (West Valley Nuclear Services 1995)

\begin{tabular}{|c|c|c|c|c|c|c|c|c|c|c|c|c|c|c|c|c|c|c|c|c|c|}
\hline Glass ID & $\begin{array}{c}\mathrm{SiO} 2 \\
-\mathrm{a}\end{array}$ & $\begin{array}{c}\mathrm{ZrO} 2 \\
-\mathrm{a}\end{array}$ & $\begin{array}{c}\mathrm{Ag} 2 \mathrm{O} \\
-\mathrm{a}\end{array}$ & $\begin{array}{c}\mathrm{As} 2 \mathrm{O} 3 \\
-\mathrm{a}\end{array}$ & $\begin{array}{c}\mathrm{BaO} \\
-\mathrm{a}\end{array}$ & $\begin{array}{c}\mathrm{Bi} 2 \mathrm{O} 3 \\
-\mathrm{a}\end{array}$ & $\begin{array}{l}\mathrm{Br} \\
-\mathrm{a}\end{array}$ & $\begin{array}{c}\mathrm{CdO} \\
-\mathrm{a}\end{array}$ & $\begin{array}{c}\mathrm{Ce} 2 \mathrm{O} 3 \\
-\mathrm{a}\end{array}$ & $\begin{array}{c}\mathrm{CeO} 2 \\
-\mathrm{a}\end{array}$ & $\begin{array}{l}\mathrm{Cl} \\
-\mathrm{a}\end{array}$ & $\begin{array}{c}\mathrm{CoO} \\
-\mathrm{a}\end{array}$ & $\begin{array}{c}\mathrm{Co} 2 \mathrm{O} 3 \\
-\mathrm{a}\end{array}$ & $\begin{array}{c}\mathrm{Cr} 2 \mathrm{O} 3 \\
-\mathrm{a}\end{array}$ & $\begin{array}{c}\mathrm{Cs} 2 \mathrm{O} \\
-\mathrm{a}\end{array}$ & $\begin{array}{c}\mathrm{CuO} \\
-\mathrm{a}\end{array}$ & $\begin{array}{c}\mathrm{Eu} 2 \mathrm{O} 3 \\
-\mathrm{a}\end{array}$ & $\begin{array}{c}F \\
-a\end{array}$ & $\begin{array}{c}\mathrm{Ga} 2 \mathrm{O} 3 \\
-\mathrm{a}\end{array}$ & $\begin{array}{c}\mathrm{Gd} 2 \mathrm{O} 3 \\
-\mathrm{a}\end{array}$ & $\begin{array}{c}\mathrm{HgO} \\
-\mathrm{a}\end{array}$ \\
\hline WVDG-40 & 0.4436 & 0.0023 & & & 0.0010 & & & & 0.0040 & & & & & 0.0008 & 0.0004 & 0.0002 & & & & & \\
\hline WVDG-46 & 0.4500 & 0.0021 & & & 0.0010 & & & & 0.0035 & & & & & 0.0007 & 0.0004 & 0.0002 & & & & & \\
\hline FY92\#5 & 0.3688 & 0.0132 & & & 0.0026 & & & & 0.0031 & & & & & 0.0014 & 0.0000 & 0.0000 & & & & & \\
\hline FY92\#6 & 0.4497 & 0.0132 & & & 0.0026 & & & & 0.0031 & & & & & 0.0014 & 0.0000 & 0.0000 & & & & & \\
\hline FY92\#7 & 0.4497 & 0.0132 & & & 0.0026 & & & & 0.0031 & & & & & 0.0014 & 0.0000 & 0.0000 & & & & & \\
\hline FY92\#9 & 0.4416 & 0.0132 & & & 0.0026 & & & & 0.0031 & & & & & 0.0014 & 0.0000 & 0.0000 & & & & & \\
\hline FY92\#10 & 0.4057 & 0.0132 & & & 0.0026 & & & & 0.0031 & & & & & 0.0014 & 0.0000 & 0.0000 & & & & & \\
\hline FY92Ref5 & 0.4116 & 0.0032 & & & 0.0016 & & & & 0.0015 & & & & & 0.0014 & 0.0008 & 0.0003 & & & & & \\
\hline Ratio2 & 0.3900 & 0.0120 & & & 0.0026 & & & & 0.0031 & & & & & 0.0014 & 0.0000 & 0.0000 & & & & & \\
\hline Ratio4 & 0.4340 & 0.0190 & & & 0.0026 & & & & 0.0031 & & & & & 0.0014 & 0.0000 & 0.0000 & & & & & \\
\hline Ratio5 & 0.3880 & 0.0100 & & & 0.0026 & & & & 0.0031 & & & & & 0.0014 & 0.0000 & 0.0000 & & & & & \\
\hline LoTh2 & 0.4250 & 0.0135 & & & 0.0026 & & & & 0.0031 & & & & & 0.0014 & 0.0000 & 0.0000 & & & & & \\
\hline LoTh4 & 0.4310 & 0.0120 & & & 0.0026 & & & & 0.0031 & & & & & 0.0014 & 0.0000 & 0.0000 & & & & & \\
\hline LoTh5 & 0.4170 & 0.0115 & & & 0.0026 & & & & 0.0031 & & & & & 0.0014 & 0.0000 & 0.0000 & & & & & \\
\hline $\mathrm{HiFe} 2$ & 0.3926 & 0.0114 & & & 0.0026 & & & & 0.0031 & & & & & 0.0014 & 0.0000 & 0.0000 & & & & & \\
\hline $\mathrm{HiFe} 3$ & 0.3879 & 0.0112 & & & 0.0026 & & & & 0.0031 & & & & & 0.0014 & 0.0000 & 0.0000 & & & & & \\
\hline $\mathrm{HiFe} 4$ & 0.3880 & 0.0093 & & & 0.0026 & & & & 0.0031 & & & & & 0.0014 & 0.0000 & 0.0000 & & & & & \\
\hline PNL190 & 0.4040 & 0.0130 & & & 0.0026 & & & & 0.0031 & & & & & 0.0014 & 0.0000 & 0.0000 & & & & & \\
\hline FY93\#1 & 0.3949 & 0.0132 & & & 0.0026 & & & & 0.0031 & & & & & 0.0014 & & & & & & & \\
\hline FY93\#2 & 0.4146 & 0.0132 & & & 0.0026 & & & & 0.0031 & & & & & 0.0014 & & & & & & & \\
\hline FY93\#3 & 0.3526 & 0.0132 & & & 0.0026 & & & & 0.0031 & & & & & 0.0014 & & & & & & & \\
\hline FY93\#4 & 0.4650 & 0.0132 & & & 0.0026 & & & & 0.0031 & & & & & 0.0014 & & & & & & & \\
\hline FY93\#5 & 0.4699 & 0.0132 & & & 0.0026 & & & & 0.0031 & & & & & 0.0014 & & & & & & & \\
\hline FY93\#6 & 0.4713 & 0.0132 & & & 0.0026 & & & & 0.0031 & & & & & 0.0014 & & & & & & & \\
\hline FY93\#7 & 0.4638 & 0.0132 & & & 0.0026 & & & & 0.0031 & & & & & 0.0014 & & & & & & & \\
\hline FY93\#8 & 0.3483 & 0.0132 & & & 0.0026 & & & & 0.0031 & & & & & 0.0014 & & & & & & & \\
\hline FY93\#9 & 0.3483 & 0.0132 & & & 0.0026 & & & & 0.0031 & & & & & 0.0014 & & & & & & & \\
\hline FY93\#10 & 0.4621 & 0.0132 & & & 0.0026 & & & & 0.0031 & & & & & 0.0014 & & & & & & & \\
\hline FY94\#1 & 0.4774 & 0.0073 & & & 0.0026 & & & & 0.0031 & & & & & 0.0014 & 0.0000 & 0.0000 & & & & & \\
\hline FY94\#2 & 0.4300 & 0.0073 & & & 0.0026 & & & & 0.0031 & & & & & 0.0014 & 0.0000 & 0.0000 & & & & & \\
\hline FY94\#3 & 0.3700 & 0.0073 & & & 0.0026 & & & & 0.0031 & & & & & 0.0014 & 0.0000 & 0.0000 & & & & & \\
\hline FY94\#4 & 0.3450 & 0.0073 & & & 0.0026 & & & & 0.0031 & & & & & 0.0014 & 0.0000 & 0.0000 & & & & & \\
\hline FY94\#5 & 0.4770 & 0.0173 & & & 0.0026 & & & & 0.0031 & & & & & 0.0014 & 0.0000 & 0.0000 & & & & & \\
\hline FY94\#6 & 0.3928 & 0.0191 & & & 0.0026 & & & & 0.0031 & & & & & 0.0014 & 0.0000 & 0.0000 & & & & & \\
\hline FY94\#7 & 0.4350 & 0.0184 & & & 0.0026 & & & & 0.0031 & & & & & 0.0014 & 0.0000 & 0.0000 & & & & & \\
\hline FY94\#8 & 0.3450 & 0.0191 & & & 0.0026 & & & & 0.0031 & & & & & 0.0014 & 0.0000 & 0.0000 & & & & & \\
\hline FY94\#9 & 0.4126 & 0.0180 & & & 0.0026 & & & & 0.0031 & & & & & 0.0014 & 0.0000 & 0.0000 & & & & & \\
\hline FY94\#10 & 0.4200 & 0.0073 & & & 0.0026 & & & & 0.0031 & & & & & 0.0014 & 0.0000 & 0.0000 & & & & & \\
\hline Sigma1 & 0.3422 & 0.0073 & & & 0.0026 & & & & 0.0031 & & & & & 0.0014 & 0.0000 & 0.0000 & & & & & \\
\hline Sigma2 & 0.3422 & 0.0073 & & & 0.0026 & & & & 0.0031 & & & & & 0.0014 & 0.0000 & 0.0000 & & & & & \\
\hline Sigma3 & 0.3672 & 0.0078 & & & 0.0026 & & & & 0.0031 & & & & & 0.0014 & 0.0000 & 0.0000 & & & & & \\
\hline Sigma4 & 0.3550 & 0.0081 & & & 0.0026 & & & & 0.0031 & & & & & 0.0014 & 0.0000 & 0.0000 & & & & & \\
\hline Sigma5 & 0.3500 & 0.0080 & & & 0.0026 & & & & 0.0031 & & & & & 0.0014 & 0.0000 & 0.0000 & & & & & \\
\hline Sigma6 & 0.3650 & 0.0082 & & & 0.0026 & & & & 0.0031 & & & & & 0.0014 & 0.0000 & 0.0000 & & & & & \\
\hline Sigma7 & 0.3600 & 0.0078 & & & 0.0026 & & & & 0.0031 & & & & & 0.0014 & 0.0000 & 0.0000 & & & & & \\
\hline Sigma8 & 0.3700 & 0.0084 & & & 0.0026 & & & & 0.0031 & & & & & 0.0014 & 0.0000 & 0.0000 & & & & & \\
\hline Sigma9 & 0.3750 & 0.0085 & & & 0.0026 & & & & 0.0031 & & & & & 0.0014 & 0.0000 & 0.0000 & & & & & \\
\hline Sigma10 & 0.3800 & 0.0083 & & & 0.0026 & & & & 0.0031 & & & & & 0.0014 & 0.0000 & 0.0000 & & & & & \\
\hline
\end{tabular}


West Valley WQR (West Valley Nuclear Services 1995)

\begin{tabular}{|c|c|c|c|c|c|c|c|c|c|c|c|c|c|c|c|c|c|c|c|c|c|}
\hline Glass ID & $\begin{array}{c}\mathrm{I} \\
-\mathrm{a}\end{array}$ & $\begin{array}{c}\mathrm{La} 2 \mathrm{O} 3 \\
-\mathrm{a}\end{array}$ & $\begin{array}{c}\mathrm{MnO} 2 \\
-\mathrm{a}\end{array}$ & $\begin{array}{c}\mathrm{MnO} \\
-\mathrm{a}\end{array}$ & $\begin{array}{c}\mathrm{MoO} \\
-\mathrm{a}\end{array}$ & $\begin{array}{c}\mathrm{MoO} 3 \\
-\mathrm{a}\end{array}$ & $\begin{array}{c}\mathrm{Nb} 2 \mathrm{O} 5 \\
-\mathrm{a}\end{array}$ & $\begin{array}{c}\mathrm{Nd} 2 \mathrm{O} 3 \\
-\mathrm{a}\end{array}$ & $\begin{array}{c}\mathrm{PbO} \\
-\mathrm{a}\end{array}$ & $\begin{array}{c}\mathrm{PdO} 2 \\
-\mathrm{a}\end{array}$ & $\begin{array}{c}\mathrm{PdO} \\
-\mathrm{a}\end{array}$ & $\begin{array}{c}\mathrm{Pr} 2 \mathrm{O} 3 \\
-\mathrm{a}\end{array}$ & $\begin{array}{c}\operatorname{Pr} 6011 \\
-\mathrm{a}\end{array}$ & $\begin{array}{c}\mathrm{Rb} 2 \mathrm{O} \\
-\mathrm{a}\end{array}$ & $\begin{array}{c}\mathrm{ReO} \\
-\mathrm{a}\end{array}$ & $\begin{array}{c}\mathrm{ReO} 2 \\
-\mathrm{a}\end{array}$ & $\begin{array}{c}\mathrm{Rh} 2 \mathrm{O} 3 \\
-\mathrm{a}\end{array}$ & $\begin{array}{c}\mathrm{RhO}_{2} \\
-\mathrm{a}\end{array}$ & $\begin{array}{c}\mathrm{RuO} 2 \\
-\mathrm{a}\end{array}$ & $\begin{array}{c}\mathrm{Sb} 2 \mathrm{O} 3 \\
-\mathrm{a}\end{array}$ & $\begin{array}{c}\mathrm{Sb} 2 \mathrm{O} 5 \\
-\mathrm{a}\end{array}$ \\
\hline WVDG-40 & & 0.0002 & & 0.0010 & & 0.0002 & & 0.0008 & & & 0.0000 & & 0.0002 & & & & & 0.0000 & 0.0000 & & \\
\hline WVDG-46 & & 0.0002 & & 0.0160 & & 0.0002 & & 0.0007 & & & 0.0000 & & 0.0002 & & & & & 0.0000 & 0.0000 & & \\
\hline FY92\#5 & & 0.0000 & & 0.0082 & & 0.0000 & & 0.0000 & & & 0.0000 & & 0.0000 & & & & & 0.0000 & 0.0000 & & \\
\hline FY92\#6 & & 0.0000 & & 0.0082 & & 0.0000 & & 0.0000 & & & 0.0000 & & 0.0000 & & & & & 0.0000 & 0.0000 & & \\
\hline FY92\#7 & & 0.0000 & & 0.0082 & & 0.0000 & & 0.0000 & & & 0.0000 & & 0.0000 & & & & & 0.0000 & 0.0000 & & \\
\hline FY92\#9 & & 0.0000 & & 0.0082 & & 0.0000 & & 0.0000 & & & 0.0000 & & 0.0000 & & & & & 0.0000 & 0.0000 & & \\
\hline FY92\#10 & & 0.0000 & & 0.0082 & & 0.0000 & & 0.0000 & & & 0.0000 & & 0.0000 & & & & & 0.0000 & 0.0000 & & \\
\hline FY92Ref5 & & 0.0004 & & 0.0082 & & 0.0004 & & 0.0014 & & & 0.0003 & & 0.0004 & & & & & 0.0002 & 0.0008 & & \\
\hline Ratio2 & & 0.0000 & & 0.0082 & & 0.0000 & & 0.0000 & & & 0.0000 & & 0.0000 & & & & & 0.0000 & 0.0000 & & \\
\hline Ratio4 & & 0.0000 & & 0.0082 & & 0.0000 & & 0.0000 & & & 0.0000 & & 0.0000 & & & & & 0.0000 & 0.0000 & & \\
\hline Ratio5 & & 0.0000 & & 0.0082 & & 0.0000 & & 0.0000 & & & 0.0000 & & 0.0000 & & & & & 0.0000 & 0.0000 & & \\
\hline LoTh2 & & 0.0000 & & 0.0082 & & 0.0000 & & 0.0000 & & & 0.0000 & & 0.0000 & & & & & 0.0000 & 0.0000 & & \\
\hline LoTh4 & & 0.0000 & & 0.0082 & & 0.0000 & & 0.0000 & & & 0.0000 & & 0.0000 & & & & & 0.0000 & 0.0000 & & \\
\hline LoTh5 & & 0.0000 & & 0.0082 & & 0.0000 & & 0.0000 & & & 0.0000 & & 0.0000 & & & & & 0.0000 & 0.0000 & & \\
\hline $\mathrm{HiFe} 2$ & & 0.0000 & & 0.0082 & & 0.0000 & & 0.0000 & & & 0.0000 & & 0.0000 & & & & & 0.0000 & 0.0000 & & \\
\hline HiFe3 & & 0.0000 & & 0.0082 & & 0.0000 & & 0.0000 & & & 0.0000 & & 0.0000 & & & & & 0.0000 & 0.0000 & & \\
\hline $\mathrm{HiFe} 4$ & & 0.0000 & & 0.0082 & & 0.0000 & & 0.0000 & & & 0.0000 & & 0.0000 & & & & & 0.0000 & 0.0000 & & \\
\hline PNL190 & & 0.0000 & & 0.0082 & & 0.0000 & & 0.0000 & & & 0.0000 & & 0.0000 & & & & & 0.0000 & 0.0000 & & \\
\hline FY93\#1 & & & & 0.0082 & & & & & & & & & & & & & & & & & \\
\hline FY93\#2 & & & & 0.0082 & & & & & & & & & & & & & & & & & \\
\hline FY93\#3 & & & & 0.0082 & & & & & & & & & & & & & & & & & \\
\hline FY93\#4 & & & & 0.0082 & & & & & & & & & & & & & & & & & \\
\hline FY93\#5 & & & & 0.0082 & & & & & & & & & & & & & & & & & \\
\hline FY93\#6 & & & & 0.0082 & & & & & & & & & & & & & & & & & \\
\hline FY93\#7 & & & & 0.0082 & & & & & & & & & & & & & & & & & \\
\hline FY93\#8 & & & & 0.0082 & & & & & & & & & & & & & & & & & \\
\hline FY93\#9 & & & & 0.0082 & & & & & & & & & & & & & & & & & \\
\hline FY93\#10 & & & & 0.0082 & & & & & & & & & & & & & & & & & \\
\hline FY94\#1 & & 0.0000 & & 0.0082 & & 0.0000 & & & & & 0.0000 & & 0.0000 & & & & & 0.0000 & 0.0000 & & \\
\hline FY94\#2 & & 0.0000 & & 0.0082 & & 0.0000 & & & & & 0.0000 & & 0.0000 & & & & & 0.0000 & 0.0000 & & \\
\hline FY94\#3 & & 0.0000 & & 0.0082 & & 0.0000 & & & & & 0.0000 & & 0.0000 & & & & & 0.0000 & 0.0000 & & \\
\hline FY94\#4 & & 0.0000 & & 0.0082 & & 0.0000 & & & & & 0.0000 & & 0.0000 & & & & & 0.0000 & 0.0000 & & \\
\hline FY94\#5 & & 0.0000 & & 0.0082 & & 0.0000 & & & & & 0.0000 & & 0.0000 & & & & & 0.0000 & 0.0000 & & \\
\hline FY94\#6 & & 0.0000 & & 0.0082 & & 0.0000 & & & & & 0.0000 & & 0.0000 & & & & & 0.0000 & 0.0000 & & \\
\hline FY94\#7 & & 0.0000 & & 0.0082 & & 0.0000 & & & & & 0.0000 & & 0.0000 & & & & & 0.0000 & 0.0000 & & \\
\hline FY94\#8 & & 0.0000 & & 0.0082 & & 0.0000 & & & & & 0.0000 & & 0.0000 & & & & & 0.0000 & 0.0000 & & \\
\hline FY94\#9 & & 0.0000 & & 0.0082 & & 0.0000 & & & & & 0.0000 & & 0.0000 & & & & & 0.0000 & 0.0000 & & \\
\hline FY94\#10 & & 0.0000 & & 0.0082 & & 0.0000 & & & & & 0.0000 & & 0.0000 & & & & & 0.0000 & 0.0000 & & \\
\hline Sigma1 & & 0.0000 & & 0.0082 & & 0.0000 & & & & & 0.0000 & & 0.0000 & & & & & 0.0000 & 0.0000 & & \\
\hline Sigma2 & & 0.0000 & & 0.0082 & & 0.0000 & & & & & 0.0000 & & 0.0000 & & & & & 0.0000 & 0.0000 & & \\
\hline Sigma3 & & 0.0000 & & 0.0082 & & 0.0000 & & & & & 0.0000 & & 0.0000 & & & & & 0.0000 & 0.0000 & & \\
\hline Sigma4 & & 0.0000 & & 0.0082 & & 0.0000 & & & & & 0.0000 & & 0.0000 & & & & & 0.0000 & 0.0000 & & \\
\hline Sigma5 & & 0.0000 & & 0.0082 & & 0.0000 & & & & & 0.0000 & & 0.0000 & & & & & 0.0000 & 0.0000 & & \\
\hline Sigma6 & & 0.0000 & & 0.0082 & & 0.0000 & & & & & 0.0000 & & 0.0000 & & & & & 0.0000 & 0.0000 & & \\
\hline Sigma7 & & 0.0000 & & 0.0082 & & 0.0000 & & & & & 0.0000 & & 0.0000 & & & & & 0.0000 & 0.0000 & & \\
\hline Sigma8 & & 0.0000 & & 0.0082 & & 0.0000 & & & & & 0.0000 & & 0.0000 & & & & & 0.0000 & 0.0000 & & \\
\hline Sigma9 & & 0.0000 & & 0.0082 & & 0.0000 & & & & & 0.0000 & & 0.0000 & & & & & 0.0000 & 0.0000 & & \\
\hline Sigma10 & & 0.0000 & & 0.0082 & & 0.0000 & & & & & 0.0000 & & 0.0000 & & & & & 0.0000 & 0.0000 & & \\
\hline
\end{tabular}


West Valley WQR (West Valley Nuclear Services 1995)

\begin{tabular}{|c|c|c|c|c|c|c|c|c|c|c|c|c|c|c|c|c|c|c|c|c|}
\hline Glass ID & $\begin{array}{c}\mathrm{SeO} 2 \\
-\mathrm{a}\end{array}$ & $\begin{array}{c}\mathrm{Sm} 2 \mathrm{O} 3 \\
-\mathrm{a}\end{array}$ & $\begin{array}{c}\mathrm{SnO} \\
-\mathrm{a} \\
\end{array}$ & $\begin{array}{c}\mathrm{SnO} 2 \\
-\mathrm{a}\end{array}$ & $\begin{array}{c}\mathrm{SO} 3 \\
-\mathrm{a} \\
\end{array}$ & $\begin{array}{c}\mathrm{SrO} \\
-\mathrm{a}\end{array}$ & $\begin{array}{c}\mathrm{Tc} 2 \mathrm{O} 7 \\
-\mathrm{a}\end{array}$ & $\begin{array}{c}\mathrm{TeO} 2 \\
-\mathrm{a}\end{array}$ & $\begin{array}{c}\mathrm{ThO} 2 \\
-\mathrm{a} \\
\end{array}$ & $\begin{array}{c}\mathrm{TiO} 2 \\
-\mathrm{a} \\
\end{array}$ & $\begin{array}{c}\mathrm{T} 12 \mathrm{O} 3 \\
-\mathrm{a} \\
\end{array}$ & $\begin{array}{c}\mathrm{U} 3 \mathrm{O} 8 \\
-\mathrm{a} \\
\end{array}$ & $\begin{array}{c}\mathrm{UO} 2 \\
-\mathrm{a} \\
\end{array}$ & $\begin{array}{c}\text { UO3 } \\
-\mathrm{a} \\
\end{array}$ & $\begin{array}{c}\mathrm{V} 2 \mathrm{O} 5 \\
-\mathrm{a} \\
\end{array}$ & $\begin{array}{c}\text { WO3 } \\
-\mathrm{a}\end{array}$ & $\begin{array}{c}\mathrm{Y} 2 \mathrm{O} 3 \\
-\mathrm{a} \\
\end{array}$ & $\begin{array}{c}\mathrm{ZnO} \\
-\mathrm{a}\end{array}$ & $\begin{array}{c}\text { Others } \\
-\mathrm{a}\end{array}$ & $\begin{array}{c}\text { Sum } \\
-\mathrm{a}\end{array}$ \\
\hline WVDG-40 & & 0.0001 & & & 0.0013 & 0.0001 & & & 0.0419 & 0.0049 & & 0.0189 & & & & & 0.0001 & 0.0002 & & 1.0001 \\
\hline WVDG-46 & & 0.0001 & & & 0.0012 & 0.0001 & & & 0.0216 & 0.0043 & & 0.0011 & & & & & 0.0001 & 0.0002 & & 0.9965 \\
\hline FY92\#5 & & 0.0000 & & & 0.0023 & 0.0025 & & & 0.0267 & 0.0080 & & 0.0062 & & & & & 0.0000 & 0.0026 & & 0.9999 \\
\hline FY92\#6 & & 0.0000 & & & 0.0023 & 0.0025 & & & 0.0267 & 0.0080 & & 0.0062 & & & & & 0.0000 & 0.0026 & & 0.9999 \\
\hline FY92\#7 & & 0.0000 & & & 0.0023 & 0.0025 & & & 0.0267 & 0.0080 & & 0.0062 & & & & & 0.0000 & 0.0026 & & 0.9999 \\
\hline FY92\#9 & & 0.0000 & & & 0.0023 & 0.0025 & & & 0.0445 & 0.0080 & & 0.0062 & & & & & 0.0000 & 0.0026 & & 0.9999 \\
\hline FY92\#10 & & 0.0000 & & & 0.0023 & 0.0025 & & & 0.0445 & 0.0080 & & 0.0062 & & & & & 0.0000 & 0.0026 & & 0.9999 \\
\hline FY92Ref5 & & 0.0003 & & & 0.0023 & 0.0025 & & & 0.0356 & 0.0080 & & 0.0061 & & & & & 0.0002 & 0.0002 & & 1.0004 \\
\hline Ratio2 & & 0.0000 & & & 0.0023 & 0.0025 & & & 0.0130 & 0.0080 & & 0.0062 & & & & & 0.0000 & 0.0026 & & 0.9974 \\
\hline Ratio4 & & 0.0000 & & & 0.0023 & 0.0025 & & & 0.0430 & 0.0080 & & 0.0062 & & & & & 0.0000 & 0.0026 & & 0.9999 \\
\hline Ratio5 & & 0.0000 & & & 0.0023 & 0.0025 & & & 0.0280 & 0.0080 & & 0.0062 & & & & & 0.0000 & 0.0026 & & 0.9999 \\
\hline LoTh2 & & 0.0000 & & & 0.0023 & 0.0025 & & & 0.0140 & 0.0080 & & 0.0062 & & & & & 0.0000 & 0.0026 & & 0.9999 \\
\hline LoTh4 & & 0.0000 & & & 0.0023 & 0.0025 & & & 0.0180 & 0.0080 & & 0.0062 & & & & & 0.0000 & 0.0026 & & 0.9999 \\
\hline LoTh5 & & 0.0000 & & & 0.0023 & 0.0025 & & & 0.0240 & 0.0080 & & 0.0062 & & & & & 0.0000 & 0.0026 & & 0.9999 \\
\hline $\mathrm{HiFe} 2$ & & 0.0000 & & & 0.0023 & 0.0025 & & & 0.0304 & 0.0080 & & 0.0062 & & & & & 0.0000 & 0.0026 & & 0.9999 \\
\hline HiFe3 & & 0.0000 & & & 0.0023 & 0.0025 & & & 0.0290 & 0.0080 & & 0.0062 & & & & & 0.0000 & 0.0026 & & 0.9999 \\
\hline $\mathrm{HiFe} 4$ & & 0.0000 & & & 0.0023 & 0.0025 & & & 0.0320 & 0.0080 & & 0.0062 & & & & & 0.0000 & 0.0026 & & 0.9999 \\
\hline PNL190 & & 0.0000 & & & 0.0023 & 0.0025 & & & 0.0450 & 0.0080 & & 0.0062 & & & & & 0.0000 & 0.0026 & & 0.9999 \\
\hline FY93\#1 & & & & & 0.0023 & 0.0025 & & & 0.0178 & 0.0080 & & 0.0062 & & & & & & 0.0026 & & 0.9999 \\
\hline FY93\#2 & & & & & 0.0023 & 0.0025 & & & 0.0178 & 0.0080 & & 0.0062 & & & & & & 0.0026 & & 0.9999 \\
\hline FY93\#3 & & & & & 0.0023 & 0.0025 & & & 0.0178 & 0.0080 & & 0.0062 & & & & & & 0.0026 & & 0.9999 \\
\hline FY93\#4 & & & & & 0.0023 & 0.0025 & & & 0.0178 & 0.0080 & & 0.0062 & & & & & & 0.0026 & & 0.9999 \\
\hline FY93\#5 & & & & & 0.0023 & 0.0025 & & & 0.0178 & 0.0080 & & 0.0062 & & & & & & 0.0026 & & 0.9999 \\
\hline FY93\#6 & & & & & 0.0023 & 0.0025 & & & 0.0178 & 0.0080 & & 0.0062 & & & & & & 0.0026 & & 0.9999 \\
\hline FY93\#7 & & & & & 0.0023 & 0.0025 & & & 0.0534 & 0.0080 & & 0.0062 & & & & & & 0.0026 & & 0.9999 \\
\hline FY93\#8 & & & & & 0.0023 & 0.0025 & & & 0.0534 & 0.0080 & & 0.0062 & & & & & & 0.0026 & & 0.9999 \\
\hline FY93\#9 & & & & & 0.0023 & 0.0025 & & & 0.0534 & 0.0080 & & 0.0062 & & & & & & 0.0026 & & 0.9999 \\
\hline FY93\#10 & & & & & 0.0023 & 0.0025 & & & 0.0534 & 0.0080 & & 0.0062 & & & & & & 0.0026 & & 0.9999 \\
\hline FY94\#1 & & 0.0000 & & & 0.0023 & 0.0025 & & & 0.0480 & 0.0080 & & 0.0062 & & & & & 0.0000 & 0.0026 & & 0.9999 \\
\hline FY94\#2 & & 0.0000 & & & 0.0023 & 0.0025 & & & 0.0273 & 0.0080 & & 0.0062 & & & & & 0.0000 & 0.0026 & & 0.9999 \\
\hline FY94\#3 & & 0.0000 & & & 0.0023 & 0.0025 & & & 0.0200 & 0.0080 & & 0.0062 & & & & & 0.0000 & 0.0026 & & 0.9999 \\
\hline FY94\#4 & & 0.0000 & & & 0.0023 & 0.0025 & & & 0.0090 & 0.0080 & & 0.0062 & & & & & 0.0000 & 0.0026 & & 0.9999 \\
\hline FY94\#5 & & 0.0000 & & & 0.0023 & 0.0025 & & & 0.0310 & 0.0080 & & 0.0062 & & & & & 0.0000 & 0.0026 & & 0.9999 \\
\hline FY94\#6 & & 0.0000 & & & 0.0023 & 0.0025 & & & 0.0516 & 0.0080 & & 0.0062 & & & & & 0.0000 & 0.0026 & & 0.9999 \\
\hline FY94\#7 & & 0.0000 & & & 0.0023 & 0.0025 & & & 0.0470 & 0.0080 & & 0.0062 & & & & & 0.0000 & 0.0026 & & 0.9999 \\
\hline FY94\#8 & & 0.0000 & & & 0.0023 & 0.0025 & & & 0.0110 & 0.0080 & & 0.0062 & & & & & 0.0000 & 0.0026 & & 0.9999 \\
\hline FY94\#9 & & 0.0000 & & & 0.0023 & 0.0025 & & & 0.0240 & 0.0080 & & 0.0062 & & & & & 0.0000 & 0.0026 & & 0.9999 \\
\hline FY94\#10 & & 0.0000 & & & 0.0023 & 0.0025 & & & 0.0360 & 0.0080 & & 0.0062 & & & & & 0.0000 & 0.0026 & & 0.9999 \\
\hline Sigma1 & & 0.0000 & & & 0.0023 & 0.0025 & & & 0.0089 & 0.0080 & & 0.0062 & & & & & 0.0000 & 0.0026 & & 0.9999 \\
\hline Sigma2 & & 0.0000 & & & 0.0023 & 0.0025 & & & 0.0516 & 0.0080 & & 0.0062 & & & & & 0.0000 & 0.0026 & & 0.9999 \\
\hline Sigma3 & & 0.0000 & & & 0.0023 & 0.0025 & & & 0.0384 & 0.0080 & & 0.0062 & & & & & 0.0000 & 0.0026 & & 0.9999 \\
\hline Sigma4 & & 0.0000 & & & 0.0023 & 0.0025 & & & 0.0100 & 0.0080 & & 0.0062 & & & & & 0.0000 & 0.0026 & & 0.9999 \\
\hline Sigma5 & & 0.0000 & & & 0.0023 & 0.0025 & & & 0.0090 & 0.0080 & & 0.0062 & & & & & 0.0000 & 0.0026 & & 0.9999 \\
\hline Sigma6 & & 0.0000 & & & 0.0023 & 0.0025 & & & 0.0220 & 0.0080 & & 0.0062 & & & & & 0.0000 & 0.0026 & & 0.9999 \\
\hline Sigma7 & & 0.0000 & & & 0.0023 & 0.0025 & & & 0.0110 & 0.0080 & & 0.0062 & & & & & 0.0000 & 0.0026 & & 0.9999 \\
\hline Sigma8 & & 0.0000 & & & 0.0023 & 0.0025 & & & 0.0100 & 0.0080 & & 0.0062 & & & & & 0.0000 & 0.0026 & & 0.9999 \\
\hline Sigma9 & & 0.0000 & & & 0.0023 & 0.0025 & & & 0.0430 & 0.0080 & & 0.0062 & & & & & 0.0000 & 0.0026 & & 0.9999 \\
\hline Sigma10 & & 0.0000 & & & 0.0023 & 0.0025 & & & 0.0500 & 0.0080 & & 0.0062 & & & & & 0.0000 & 0.0026 & & 0.9999 \\
\hline
\end{tabular}


Appendix A. Database - mass fraction

West Valley WQR (West Valley Nuclear Services 1995)

\begin{tabular}{|c|c|c|c|c|c|c|c|c|}
\hline Glass ID & $\begin{array}{l}\mathrm{TM} \\
\left({ }^{\circ} \mathrm{C}\right) \\
\end{array}$ & $\begin{array}{l}\text { Gradient } \\
\text { TL }\left({ }^{\circ} \mathrm{C}\right) \\
\end{array}$ & $\begin{array}{l}\text { Uniform } \\
\text { TL }\left({ }^{\circ} \mathrm{C}\right)\end{array}$ & Primary Phase & $\begin{array}{l}\text { Quenched } \\
\text { Visual/OM }\end{array}$ & $\begin{array}{c}\text { Quenched } \\
\text { SEM/EDS or TEM }\end{array}$ & $\begin{array}{l}\text { Quenched } \\
\text { XRD }\end{array}$ & $\begin{array}{c}\text { Quenched } \\
\text { Homogeneous? }\end{array}$ \\
\hline \multicolumn{9}{|l|}{ WVDG-40 } \\
\hline \multicolumn{9}{|l|}{ WVDG-46 } \\
\hline \multicolumn{9}{|l|}{ FY92\#5 } \\
\hline \multicolumn{9}{|l|}{ FY92\#6 } \\
\hline \multicolumn{9}{|l|}{ FY92\#7 } \\
\hline \multicolumn{9}{|l|}{ FY92\#9 } \\
\hline \multicolumn{9}{|l|}{ FY92\#10 } \\
\hline \multicolumn{9}{|l|}{ FY92Ref5 } \\
\hline \multicolumn{9}{|l|}{ Ratio2 } \\
\hline \multicolumn{9}{|l|}{ Ratio4 } \\
\hline \multicolumn{9}{|l|}{ Ratio5 } \\
\hline \multicolumn{9}{|l|}{ LoTh2 } \\
\hline \multicolumn{9}{|l|}{ LoTh4 } \\
\hline \multicolumn{9}{|l|}{ LoTh5 } \\
\hline \multicolumn{9}{|l|}{$\mathrm{HiFe} 2$} \\
\hline \multicolumn{9}{|l|}{$\mathrm{HiFe} 3$} \\
\hline \multicolumn{9}{|l|}{ HiFe4 } \\
\hline \multicolumn{9}{|l|}{ PNL190 } \\
\hline \multicolumn{9}{|l|}{ FY93\#1 } \\
\hline \multicolumn{9}{|l|}{ FY93\#2 } \\
\hline FY93\#3 & & & & & & & & \\
\hline FY93\#4 & & & & & & & & \\
\hline FY93\#5 & & & & & & & & \\
\hline FY93\#6 & & & & & & & & \\
\hline FY93\#7 & & & & & & & & \\
\hline FY93\#8 & & & & & & & & \\
\hline FY93\#9 & & & & & & & & \\
\hline FY93\#10 & & & & & & & & \\
\hline FY94\#1 & & & & & & & & \\
\hline FY94\#2 & & & & & & & & \\
\hline FY94\#3 & & & & & & & & \\
\hline FY94\#4 & & & & & & & & \\
\hline FY94\#5 & & & & & & & & \\
\hline FY94\#6 & & & & & & & & \\
\hline FY94\#7 & & & & & & & & \\
\hline FY94\#8 & & & & & & & & \\
\hline FY94\#9 & & & & & & & & \\
\hline FY94\#10 & & & & & & & & \\
\hline Sigma1 & & & & & & & & \\
\hline Sigma2 & & & & & & & & \\
\hline Sigma3 & & & & & & & & \\
\hline Sigma4 & & & & & & & & \\
\hline Sigma5 & & & & & & & & \\
\hline Sigma6 & & & & & & & & \\
\hline Sigma7 & & & & & & & & \\
\hline Sigma8 & & & & & & & & \\
\hline Sigma9 & & & & & & & & \\
\hline Sigma10 & & & & & & & & \\
\hline
\end{tabular}


Appendix A. Database - mass fraction

\begin{tabular}{|c|c|c|c|c|c|}
\hline \multicolumn{6}{|c|}{ West Valley WQR (West Valley Nuclear Services 1995) } \\
\hline Glass ID & $\begin{array}{c}\text { CCC } \\
\text { Visual/OM }\end{array}$ & $\begin{array}{c}\text { CCC } \\
\text { SEM/EDS or TEM }\end{array}$ & $\begin{array}{l}\mathrm{CCC} \\
\mathrm{XRD}\end{array}$ & $\begin{array}{c}\text { Heat Treated } \\
\text { Visual/OM }\end{array}$ & $\begin{array}{c}\text { Heat Treated } \\
\text { SEM/EDS or TEM }\end{array}$ \\
\hline \multicolumn{6}{|l|}{ WVDG-40 } \\
\hline \multicolumn{6}{|l|}{ WVDG-46 } \\
\hline \multicolumn{6}{|l|}{ FY92\#5 } \\
\hline \multicolumn{6}{|l|}{ FY92\#6 } \\
\hline \multicolumn{6}{|l|}{ FY92\#7 } \\
\hline \multicolumn{6}{|l|}{ FY92\#9 } \\
\hline \multicolumn{6}{|l|}{ FY92\#10 } \\
\hline \multicolumn{6}{|l|}{ FY92Ref5 } \\
\hline \multicolumn{6}{|l|}{ Ratio2 } \\
\hline \multicolumn{6}{|l|}{ Ratio4 } \\
\hline \multicolumn{6}{|l|}{ Ratio5 } \\
\hline \multicolumn{6}{|l|}{ LoTh2 } \\
\hline \multicolumn{6}{|l|}{ LoTh4 } \\
\hline \multicolumn{6}{|l|}{ LoTh5 } \\
\hline \multicolumn{6}{|l|}{$\mathrm{HiFe} 2$} \\
\hline \multicolumn{6}{|l|}{$\mathrm{HiFe} 3$} \\
\hline \multicolumn{6}{|l|}{$\mathrm{HiFe} 4$} \\
\hline \multicolumn{6}{|l|}{ PNL190 } \\
\hline \multicolumn{6}{|l|}{ FY93\#1 } \\
\hline \multicolumn{6}{|l|}{ FY93\#2 } \\
\hline FY93\#3 & & & & & \\
\hline FY93\#4 & & & & & \\
\hline FY93\#5 & & & & & \\
\hline FY93\#6 & & & & & \\
\hline FY93\#7 & & & & & \\
\hline FY93\#8 & & & & & \\
\hline FY93\#9 & & & & & \\
\hline FY93\#10 & & & & & \\
\hline FY94\#1 & & & & & \\
\hline FY94\#2 & & & & & \\
\hline FY94\#3 & & & & & \\
\hline FY94\#4 & & & & & \\
\hline FY94\#5 & & & & & \\
\hline FY94\#6 & & & & & \\
\hline FY94\#7 & & & & & \\
\hline FY94\#8 & & & & & \\
\hline FY94\#9 & & & & & \\
\hline FY94\#10 & & & & & \\
\hline Sigmal & & & & & \\
\hline Sigma2 & & & & & \\
\hline Sigma3 & & & & & \\
\hline Sigma4 & & & & & \\
\hline Sigma5 & & & & & \\
\hline Sigma6 & & & & & \\
\hline Sigma7 & & & & & \\
\hline Sigma8 & & & & & \\
\hline Sigma9 & & & & & \\
\hline Sigma10 & & & & & \\
\hline
\end{tabular}


Appendix A. Database - mass fraction

West Valley WQR (West Valley Nuclear Services 1995)

\begin{tabular}{|c|c|c|c|c|c|c|c|c|c|c|c|c|c|c|c|}
\hline Glass ID & $\begin{array}{c}\text { Heat Treated } \\
\text { XRD }\end{array}$ & $\begin{array}{l}\text { Density } \\
\left(\mathrm{g} / \mathrm{cm}^{3}\right)\end{array}$ & $\begin{array}{c}\text { Fulc Visc } \\
\mathrm{A}\end{array}$ & $\begin{array}{c}\text { Fulc Visc } \\
\text { B } \\
\end{array}$ & $\begin{array}{c}\text { Fulc Visc } \\
\text { To } \\
\end{array}$ & $\begin{array}{c}\mathrm{FV} 1150^{\circ} \mathrm{C} \\
(\mathrm{Pa} \cdot \mathrm{s})\end{array}$ & \begin{tabular}{|c|} 
Arrh Visc \\
$\mathrm{A}$
\end{tabular} & $\begin{array}{c}\text { Arrh Visc } \\
\text { B } \\
\end{array}$ & $\begin{array}{l}\text { Av } \\
1150^{\circ} \mathrm{C} \\
(\mathrm{D} a, 0)\end{array}$ & $\begin{array}{c}\mathrm{T}\left({ }^{\circ} \mathrm{C}\right) \text { at } \\
2 \mathrm{~Pa} \cdot \mathrm{s}\end{array}$ & $\begin{array}{c}\mathrm{T}\left({ }^{\circ} \mathrm{C}\right) \text { at } \\
5 \mathrm{~Pa} \cdot \mathrm{s}\end{array}$ & \begin{tabular}{|c|}
$\mathrm{T}\left({ }^{\circ} \mathrm{C}\right)$ at \\
$10 \mathrm{~Pa} \cdot \mathrm{s}$
\end{tabular} & $\begin{array}{c}\mathrm{T} 1 \\
\left({ }^{\circ} \mathrm{C}\right) \\
\end{array}$ & $\begin{array}{c}\mathrm{V} 1 \\
(\mathrm{~Pa} \cdot \mathrm{s})\end{array}$ & $\begin{array}{c}\mathrm{T} 2 \\
\left({ }^{\circ} \mathrm{C}\right) \\
\end{array}$ \\
\hline \multicolumn{16}{|l|}{ WVDG-40 } \\
\hline \multicolumn{16}{|l|}{ WVDG-46 } \\
\hline \multicolumn{16}{|l|}{ FY92\#5 } \\
\hline \multicolumn{16}{|l|}{ FY92\#6 } \\
\hline \multicolumn{16}{|l|}{ FY92\#7 } \\
\hline \multicolumn{16}{|l|}{ FY92\#9 } \\
\hline \multicolumn{16}{|l|}{ FY92\#10 } \\
\hline \multicolumn{16}{|l|}{ FY92Ref5 } \\
\hline Ratio2 & & & & & & & -10.164 & 15260.5 & 1.75 & & & & 1146 & 1.79 & 1052 \\
\hline Ratio4 & & & & & & & -10.583 & 17165.6 & 4.39 & & & & 1144 & 4.55 & 1051 \\
\hline Ratio5 & & & & & & & -10.696 & 16675.3 & 2.78 & & & & 1144 & 2.77 & 1100 \\
\hline LoTh2 & & & & & & & -10.372 & 16482.7 & 3.36 & & & & 1144 & 3.44 & 1099 \\
\hline LoTh4 & & & & & & & -10.422 & 16805.5 & 4.01 & & & & 1146 & 3.92 & 1051 \\
\hline LoTh5 & & & & & & & -10.691 & 17189.8 & 4.01 & & & & 1141 & 4.19 & 1049 \\
\hline $\mathrm{HiFe} 2$ & & & & & & & -9.571 & 14750.1 & 2.21 & & & & 1185 & 1.76 & 1241 \\
\hline HiFe3 & & & & & & & -10.350 & 15917.1 & 2.31 & & & & 1145 & 2.31 & 1052 \\
\hline HiFe4 & & & & & & & -10.253 & 15473.3 & 1.86 & & & & 1142 & 1.94 & 1099 \\
\hline PNL190 & & & & & & & -10.013 & 14801.8 & 1.48 & & & & 1144 & 1.49 & 1052 \\
\hline \multicolumn{16}{|l|}{ FY93\#1 } \\
\hline \multicolumn{16}{|l|}{ FY93\#2 } \\
\hline \multicolumn{16}{|l|}{ FY93\#3 } \\
\hline \multicolumn{16}{|l|}{ FY93\#4 } \\
\hline \multicolumn{16}{|l|}{ FY93\#5 } \\
\hline \multicolumn{16}{|l|}{ FY93\#6 } \\
\hline \multicolumn{16}{|l|}{ FY93\#7 } \\
\hline \multicolumn{16}{|l|}{ FY93\#8 } \\
\hline \multicolumn{16}{|l|}{ FY93\#9 } \\
\hline FY93\#10 & & & & & & & & & & & & & & & \\
\hline FY94\#1 & & & & & & & & & & & & & & & \\
\hline FY94\#2 & & & & & & & & & & & & & & & \\
\hline FY94\#3 & & & & & & & & & & & & & & & \\
\hline FY94\#4 & & & & & & & & & & & & & & & \\
\hline FY94\#5 & & & & & & & & & & & & & & & \\
\hline FY94\#6 & & & & & & & & & & & & & & & \\
\hline FY94\#7 & & & & & & & & & & & & & & & \\
\hline FY94\#8 & & & & & & & & & & & & & & & \\
\hline FY94\#9 & & & & & & & & & & & & & & & \\
\hline FY94\#10 & & & & & & & & & & & & & & & \\
\hline Sigma1 & & & & & & & & & & & & & & & \\
\hline Sigma2 & & & & & & & & & & & & & & & \\
\hline Sigma3 & & & & & & & & & & & & & & & \\
\hline Sigma4 & & & & & & & & & & & & & & & \\
\hline Sigma5 & & & & & & & & & & & & & & & \\
\hline Sigma6 & & & & & & & & & & & & & & & \\
\hline Sigma7 & & & & & & & & & & & & & & & \\
\hline Sigma8 & & & & & & & & & & & & & & & \\
\hline Sigma9 & & & & & & & & & & & & & & & \\
\hline Sigma10 & & & & & & & & & & & & & & & \\
\hline
\end{tabular}


Appendix A. Database - mass fraction

\begin{tabular}{|c|c|c|c|c|c|c|c|c|c|c|c|c|c|c|c|c|c|c|c|c|c|}
\hline \multicolumn{22}{|c|}{ West Valley WQR (West Valley Nuclear Services 1995) } \\
\hline Glass ID & $\begin{array}{c}\mathrm{V} 2 \\
(\mathrm{~Pa} \cdot \mathrm{s})\end{array}$ & $\begin{array}{c}\mathrm{T} 3 \\
\left({ }^{\circ} \mathrm{C}\right)\end{array}$ & $\begin{array}{c}\mathrm{V} 3 \\
(\mathrm{~Pa} \cdot \mathrm{s})\end{array}$ & $\begin{array}{c}\mathrm{T} 4 \\
\left({ }^{\circ} \mathrm{C}\right)\end{array}$ & $\begin{array}{c}\mathrm{V} 4 \\
(\mathrm{~Pa} \cdot \mathrm{s})\end{array}$ & $\begin{array}{l}\mathrm{T} 5 \\
\left({ }^{\circ} \mathrm{C}\right)\end{array}$ & $\begin{array}{c}\text { V5 } \\
(\mathrm{Pa} \cdot \mathrm{s})\end{array}$ & $\begin{array}{c}\text { T6 } \\
\left({ }^{\circ} \mathrm{C}\right)\end{array}$ & $\begin{array}{c}\mathrm{V} 6 \\
(\mathrm{~Pa} \cdot \mathrm{s})\end{array}$ & $\begin{array}{c}\mathrm{T} 7 \\
\left({ }^{\circ} \mathrm{C}\right)\end{array}$ & $\begin{array}{c}\text { V7 } \\
(\mathrm{Pa} \cdot \mathrm{s})\end{array}$ & $\begin{array}{c}\mathrm{T} 8 \\
\left({ }^{\circ} \mathrm{C}\right)\end{array}$ & $\begin{array}{c}\mathrm{V} 8 \\
(\mathrm{~Pa} \cdot \mathrm{s})\end{array}$ & $\begin{array}{c}\mathrm{T} 9 \\
\left({ }^{\circ} \mathrm{C}\right)\end{array}$ & $\begin{array}{c}\mathrm{V} 9 \\
(\mathrm{~Pa} \cdot \mathrm{s})\end{array}$ & $\begin{array}{l}\mathrm{T} 10 \\
\left({ }^{\circ} \mathrm{C}\right)\end{array}$ & $\begin{array}{c}\mathrm{V} 10 \\
(\mathrm{~Pa} \cdot \mathrm{s})\end{array}$ & $\begin{array}{l}\mathrm{T} 11 \\
\left({ }^{\circ} \mathrm{C}\right)\end{array}$ & $\begin{array}{c}\mathrm{V} 11 \\
(\mathrm{~Pa} \cdot \mathrm{s})\end{array}$ & $\begin{array}{l}\mathrm{T} 12 \\
\left({ }^{\circ} \mathrm{C}\right)\end{array}$ & $\begin{array}{c}\mathrm{V} 12 \\
(\mathrm{~Pa} \cdot \mathrm{s})\end{array}$ \\
\hline \multicolumn{22}{|l|}{ WVDG-40 } \\
\hline \multicolumn{22}{|l|}{ WVDG-46 } \\
\hline \multicolumn{22}{|l|}{ FY92\#5 } \\
\hline \multicolumn{22}{|l|}{ FY92\#6 } \\
\hline \multicolumn{22}{|l|}{ FY92\#7 } \\
\hline \multicolumn{22}{|l|}{ FY92\#9 } \\
\hline \multicolumn{22}{|l|}{ FY92\#10 } \\
\hline \multicolumn{22}{|l|}{ FY92Ref5 } \\
\hline Ratio2 & 3.74 & 1242 & 0.94 & 1149 & 1.74 & 946 & 10.81 & & & & & & & & & & & & & & \\
\hline Ratio4 & 10.5 & 1239 & 2.23 & 1146 & 4.5 & 943 & 35.12 & & & & & & & & & & & & & & \\
\hline Ratio5 & 4.21 & 1051 & 6.59 & 1146 & 2.8 & 1194 & 1.97 & 1243 & 1.42 & 1148 & 2.86 & 1003 & 10.95 & 952 & 18.74 & & & & & & \\
\hline LoTh2 & 5.01 & 1050 & 7.7 & 1146 & 3.42 & 1195 & 2.35 & 1243 & 1.72 & 1148 & 3.5 & 1003 & 12.94 & 953 & 22.34 & & & & & & \\
\hline LoTh4 & 8.96 & 1243 & 2.06 & 1148 & 4.14 & 946 & 30.62 & & & & & & & & & & & & & & \\
\hline LoTh5 & 9.46 & 1238 & 2.08 & 1146 & 4.17 & 945 & 32.11 & & & & & & & & & & & & & & \\
\hline $\mathrm{HiFe} 2$ & 1.21 & 1148 & 2.19 & 1051 & 4.61 & 944 & 13.16 & & & & & & & & & & & & & & \\
\hline $\mathrm{HiFe} 3$ & 5.14 & 1240 & 1.23 & 1148 & 2.35 & 944 & 15.71 & & & & & & & & & & & & & & \\
\hline $\mathrm{HiFe} 4$ & 2.73 & 1050 & 4.18 & 1145 & 1.93 & 1195 & 1.4 & 1243 & 0.97 & 1147 & 1.84 & 1002 & 6.56 & 951 & 11.22 & & & & & & \\
\hline PNL190 & 3.09 & 1240 & 0.82 & 1148 & 1.51 & 944 & 8.8 & & & & & & & & & & & & & & \\
\hline \multicolumn{22}{|l|}{ FY93\#1 } \\
\hline \multicolumn{22}{|l|}{ FY93\#2 } \\
\hline \multicolumn{22}{|l|}{ FY93\#3 } \\
\hline \multicolumn{22}{|l|}{ FY93\#4 } \\
\hline FY93\#5 & & & & & & & & & & & & & & & & & & & & & \\
\hline FY93\#6 & & & & & & & & & & & & & & & & & & & & & \\
\hline FY93\#7 & & & & & & & & & & & & & & & & & & & & & \\
\hline FY93\#8 & & & & & & & & & & & & & & & & & & & & & \\
\hline FY93\#9 & & & & & & & & & & & & & & & & & & & & & \\
\hline FY93\#10 & & & & & & & & & & & & & & & & & & & & & \\
\hline FY94\#1 & & & & & & & & & & & & & & & & & & & & & \\
\hline FY94\#2 & & & & & & & & & & & & & & & & & & & & & \\
\hline FY94\#3 & & & & & & & & & & & & & & & & & & & & & \\
\hline FY94\#4 & & & & & & & & & & & & & & & & & & & & & \\
\hline FY94\#5 & & & & & & & & & & & & & & & & & & & & & \\
\hline FY94\#6 & & & & & & & & & & & & & & & & & & & & & \\
\hline FY94\#7 & & & & & & & & & & & & & & & & & & & & & \\
\hline FY94\#8 & & & & & & & & & & & & & & & & & & & & & \\
\hline FY94\#9 & & & & & & & & & & & & & & & & & & & & & \\
\hline FY94\#10 & & & & & & & & & & & & & & & & & & & & & \\
\hline Sigma1 & & & & & & & & & & & & & & & & & & & & & \\
\hline Sigma2 & & & & & & & & & & & & & & & & & & & & & \\
\hline Sigma3 & & & & & & & & & & & & & & & & & & & & & \\
\hline Sigma4 & & & & & & & & & & & & & & & & & & & & & \\
\hline Sigma5 & & & & & & & & & & & & & & & & & & & & & \\
\hline Sigma6 & & & & & & & & & & & & & & & & & & & & & \\
\hline Sigma7 & & & & & & & & & & & & & & & & & & & & & \\
\hline Sigma8 & & & & & & & & & & & & & & & & & & & & & \\
\hline Sigma9 & & & & & & & & & & & & & & & & & & & & & \\
\hline Sigma10 & & & & & & & & & & & & & & & & & & & & & \\
\hline
\end{tabular}


Appendix A. Database - mass fraction

West Valley WQR (West Valley Nuclear Services 1995)

\begin{tabular}{|c|c|c|c|c|c|c|c|c|c|c|c|c|c|c|c|c|}
\hline Glass ID & $\begin{array}{l}\mathrm{T} 13 \\
\left({ }^{\circ} \mathrm{C}\right) \\
\end{array}$ & $\begin{array}{c}\mathrm{V} 13 \\
(\mathrm{~Pa} \cdot \mathrm{s})\end{array}$ & $\begin{array}{l}\mathrm{T} 14 \\
\left({ }^{\circ} \mathrm{C}\right) \\
\end{array}$ & $\begin{array}{c}\mathrm{V} 14 \\
(\mathrm{~Pa} \cdot \mathrm{s})\end{array}$ & $\begin{array}{c}\text { Q PCT } \\
\mathrm{B}\left(\mathrm{g} / \mathrm{m}^{2}\right)\end{array}$ & $\begin{array}{c}\text { Q PCT } \\
\mathrm{Li}\left(\mathrm{g} / \mathrm{m}^{2}\right)\end{array}$ & $\begin{array}{c}\text { Q PCT } \\
\mathrm{Na}\left(\mathrm{g} / \mathrm{m}^{2}\right)\end{array}$ & $\begin{array}{c}\text { Q PCT } \\
\mathrm{Si}\left(\mathrm{g} / \mathrm{m}^{2}\right)\end{array}$ & $\begin{array}{c}\text { Q PCT } \\
\text { pH }\end{array}$ & $\begin{array}{c}\text { CCC PCT } \\
\mathrm{B}\left(\mathrm{g} / \mathrm{m}^{2}\right)\end{array}$ & $\begin{array}{l}\text { CCC PCT } \\
\mathrm{Li}\left(\mathrm{g} / \mathrm{m}^{2}\right) \\
\end{array}$ & \begin{tabular}{|l|}
$\mathrm{CCC}$ PCT \\
$\mathrm{Na}\left(\mathrm{g} / \mathrm{m}^{2}\right)$ \\
\end{tabular} & $\begin{array}{l}\text { CCC PCT } \\
\mathrm{Si}\left(\mathrm{g} / \mathrm{m}^{2}\right) \\
\end{array}$ & $\begin{array}{c}\text { CCC PCT } \\
\mathrm{pH}\end{array}$ & $\begin{array}{l}\text { Q PCT B at } \\
20^{\circ} \mathrm{C}\left(\mathrm{g} / \mathrm{m}^{2}\right)\end{array}$ & $\begin{array}{l}\text { Q PCT Li at } \\
20^{\circ} \mathrm{C}\left(\mathrm{g} / \mathrm{m}^{2}\right)\end{array}$ \\
\hline WVDG-40 & & & & & 0.306 & 0.305 & 0.29 & & & & & & & & & \\
\hline WVDG-46 & & & & & 0.373 & 0.318 & 0.3 & & & & & & & & & \\
\hline FY92\#5 & & & & & 0.694 & 0.584 & 0.529 & & & & & & & & & \\
\hline FY92\#6 & & & & & 0.278 & 0.357 & 0.267 & & & & & & & & & \\
\hline FY92\#7 & & & & & 0.211 & 0.264 & 0.208 & & & & & & & & & \\
\hline FY92\#9 & & & & & 0.262 & 0.295 & 0.196 & & & & & & & & & \\
\hline FY92\#10 & & & & & 0.822 & 0.711 & 0.569 & & & & & & & & & \\
\hline FY92Ref5 & & & & & 0.196 & 0.275 & 0.256 & & & & & & & & & \\
\hline Ratio2 & & & & & 1.546 & 1.129 & 0.909 & & & & & & & & & \\
\hline Ratio4 & & & & & 0.261 & 0.29 & 0.255 & & & & & & & & & \\
\hline Ratio5 & & & & & 0.244 & 0.305 & 0.297 & & & & & & & & & \\
\hline LoTh2 & & & & & 0.393 & 0.283 & 0.229 & & & & & & & & & \\
\hline LoTh4 & & & & & 0.644 & 0.577 & 0.487 & & & & & & & & & \\
\hline LoTh5 & & & & & 0.242 & 0.433 & 0.385 & & & & & & & & & \\
\hline $\mathrm{HiFe} 2$ & & & & & 0.588 & 0.555 & 0.498 & & & & & & & & & \\
\hline $\mathrm{HiFe} 3$ & & & & & 0.418 & 0.423 & 0.382 & & & & & & & & & \\
\hline $\mathrm{HiFe} 4$ & & & & & 0.741 & 0.652 & 0.572 & & & & & & & & & \\
\hline PNL190 & & & & & 1.227 & 1.069 & 1.014 & & & & & & & & & \\
\hline FY93\#1 & & & & & 0.544 & 0.328 & 0.504 & & & & & & & & & \\
\hline FY93\#2 & & & & & 4.044 & 2.426 & 2.702 & & & & & & & & & \\
\hline FY93\#3 & & & & & 3.266 & 1.897 & 2.265 & & & & & & & & & \\
\hline FY93\#4 & & & & & 0.239 & 0.19 & 0.25 & & & & & & & & & \\
\hline FY93\#5 & & & & & 1.267 & 0.658 & 0.881 & & & & & & & & & \\
\hline FY93\#6 & & & & & 0.269 & 0.225 & 0.266 & & & & & & & & & \\
\hline FY93\#7 & & & & & 0.186 & 0.145 & 0.2 & & & & & & & & & \\
\hline FY93\#8 & & & & & 1.575 & 0.962 & 1.197 & & & & & & & & & \\
\hline FY93\#9 & & & & & 0.431 & 0.377 & 0.385 & & & & & & & & & \\
\hline FY93\#10 & & & & & 0.757 & 0.467 & 0.495 & & & & & & & & & \\
\hline FY94\#1 & & & & & 0.186 & 0.253 & 0.208 & & & & & & & & & \\
\hline FY94\#2 & & & & & 0.215 & 0.297 & 0.222 & & & & & & & & & \\
\hline FY94\#3 & & & & & 4.057 & 3.559 & 2.553 & & & & & & & & & \\
\hline FY94\#4 & & & & & 3.336 & 2.605 & 2.296 & & & & & & & & & \\
\hline FY94\#5 & & & & & 0.453 & 0.532 & 0.495 & & & & & & & & & \\
\hline FY94\#6 & & & & & 0.288 & 0.364 & 0.266 & & & & & & & & & \\
\hline FY94\#7 & & & & & 1.047 & 0.949 & 0.693 & & & & & & & & & \\
\hline FY94\#8 & & & & & 2.242 & 1.92 & 1.565 & & & & & & & & & \\
\hline FY94\#9 & & & & & 0.435 & 0.447 & 0.368 & & & & & & & & & \\
\hline FY94\#10 & & & & & 3.385 & 2.885 & 2.151 & & & & & & & & & \\
\hline Sigmal & & & & & 6.609 & 5.312 & 4.92 & & & & & & & & & \\
\hline Sigma2 & & & & & 10.278 & 7.535 & 7.965 & & & & & & & & & \\
\hline Sigma3 & & & & & 7.742 & 4.071 & 5.429 & & & & & & & & & \\
\hline Sigma4 & & & & & 4.655 & 3.839 & 3.551 & & & & & & & & & \\
\hline Sigma5 & & & & & 5.268 & 4.262 & 3.93 & & & & & & & & & \\
\hline Sigma6 & & & & & 4.921 & 3.969 & 3.683 & & & & & & & & & \\
\hline Sigma7 & & & & & 4.429 & 3.545 & 3.326 & & & & & & & & & \\
\hline Sigma8 & & & & & 3.407 & 2.73 & 2.559 & & & & & & & & & \\
\hline Sigma9 & & & & & 3.542 & 2.833 & 2.485 & & & & & & & & & \\
\hline Sigma10 & & & & & 4.028 & 3.245 & 2.913 & & & & & & & & & \\
\hline
\end{tabular}


Appendix A. Database - mass fraction

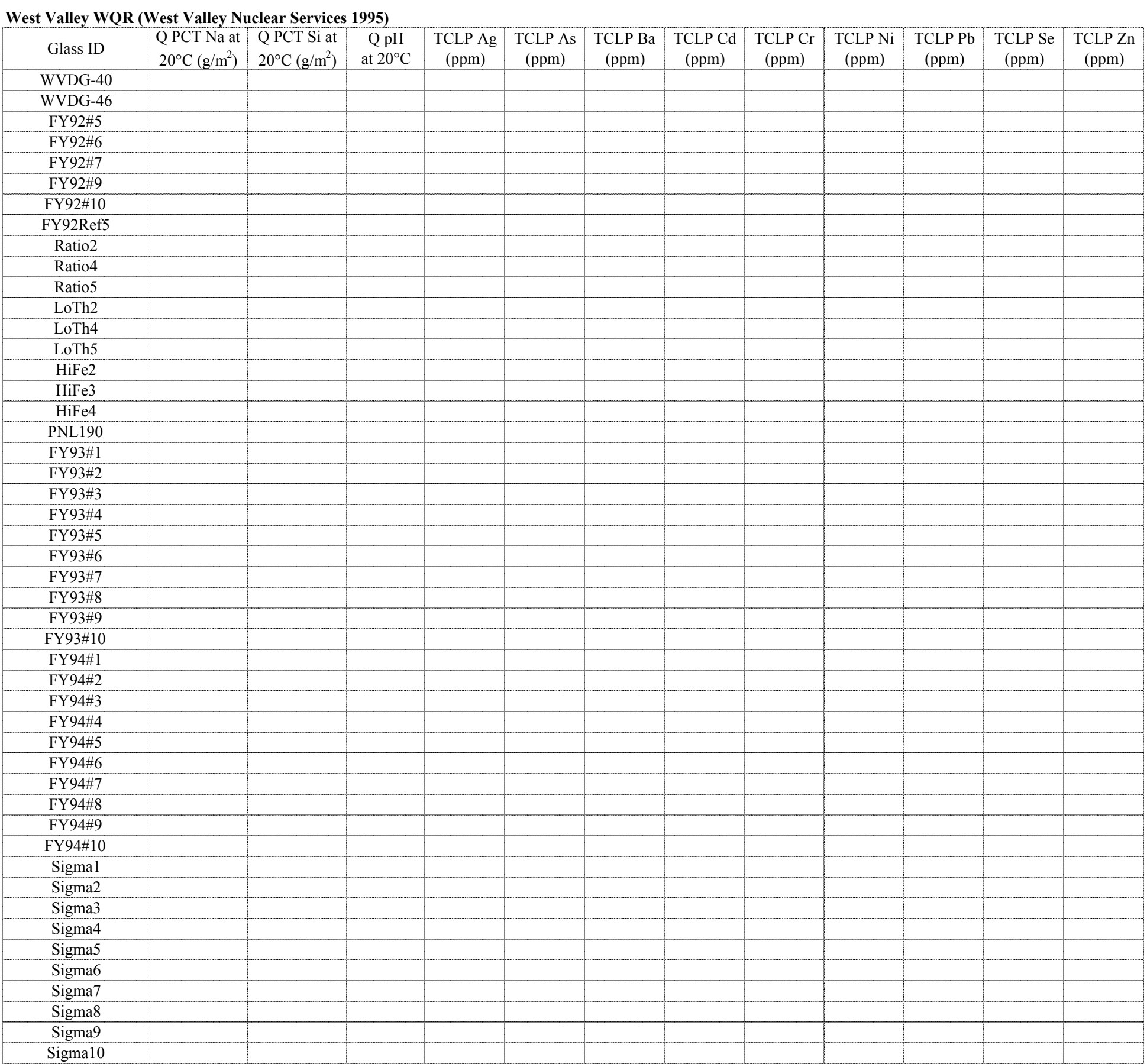


Canonical Correlation (Ramsey) (Oksoy et al. 1994)

\begin{tabular}{|c|c|c|c|c|c|c|c|c|c|c|c|c|c|c|c|c|c|c|c|c|c|}
\hline Glass ID & $\begin{array}{c}\mathrm{A} 12 \mathrm{O} 3 \\
-\mathrm{t}\end{array}$ & $\begin{array}{c}\mathrm{B} 2 \mathrm{O} 3 \\
-\mathrm{t}\end{array}$ & $\begin{array}{c}\mathrm{CaO} \\
-\mathrm{t}\end{array}$ & $\begin{array}{c}\mathrm{Fe} 2 \mathrm{O} 3 \\
-\mathrm{t}\end{array}$ & $\begin{array}{c}\mathrm{FeO} \\
-\mathrm{t}\end{array}$ & $\begin{array}{c}\mathrm{K} 2 \mathrm{O} \\
-\mathrm{t}\end{array}$ & $\begin{array}{c}\mathrm{Li} 2 \mathrm{O} \\
-\mathrm{t}\end{array}$ & $\begin{array}{c}\mathrm{MgO} \\
-\mathrm{t}\end{array}$ & $\begin{array}{c}\mathrm{Na} 2 \mathrm{O} \\
-\mathrm{t}\end{array}$ & $\begin{array}{c}\mathrm{NiO} \\
-\mathrm{t}\end{array}$ & $\begin{array}{c}\mathrm{P} 2 \mathrm{O} 5 \\
-\mathrm{t}\end{array}$ & $\begin{array}{c}\mathrm{SiO} 2 \\
-\mathrm{t}\end{array}$ & $\begin{array}{c}\mathrm{ZrO} 2 \\
-\mathrm{t}\end{array}$ & $\begin{array}{c}\mathrm{Ag} 2 \mathrm{O} \\
-\mathrm{t}\end{array}$ & $\begin{array}{c}\mathrm{As} 2 \mathrm{O} 3 \\
-\mathrm{t}\end{array}$ & $\begin{array}{c}\mathrm{BaO} \\
-\mathrm{t}\end{array}$ & $\begin{array}{c}\mathrm{Bi} 2 \mathrm{O} 3 \\
-\mathrm{t}\end{array}$ & $\begin{array}{c}\mathrm{Br} \\
-\mathrm{t}\end{array}$ & $\begin{array}{c}\mathrm{CdO} \\
-\mathrm{t}\end{array}$ & $\begin{array}{c}\mathrm{Ce} 2 \mathrm{O} 3 \\
-\mathrm{t}\end{array}$ & $\begin{array}{c}\mathrm{CeO} 2 \\
-\mathrm{t}\end{array}$ \\
\hline 1 & 0.1545 & 0.1583 & 0.0000 & 0.0000 & & & & & 0.1409 & & & 0.5463 & & & & & & & & & \\
\hline 2 & 0.1578 & 0.0539 & 0.0868 & 0.0000 & & & & & 0.1438 & & & 0.5578 & & & & & & & & & \\
\hline 4 & 0.1563 & 0.0534 & 0.0000 & 0.0000 & & & & & 0.2376 & & & 0.5527 & & & & & & & & & \\
\hline 5 & 0.0000 & 0.1701 & 0.0913 & 0.0000 & & & & & 0.1514 & & & 0.5872 & & & & & & & & & \\
\hline 6 & 0.0000 & 0.1455 & 0.0000 & 0.2225 & & & & & 0.1296 & & & 0.5024 & & & & & & & & & \\
\hline 7 & 0.0000 & 0.1685 & 0.0000 & 0.0000 & & & & & 0.2500 & & & 0.5816 & & & & & & & & & \\
\hline 8 & 0.0000 & 0.0494 & 0.0797 & 0.2268 & & & & & 0.1320 & & & 0.5120 & & & & & & & & & \\
\hline 9 & 0.0000 & 0.0574 & 0.0925 & 0.0000 & & & & & 0.2555 & & & 0.5946 & & & & & & & & & \\
\hline 10 & 0.0000 & 0.0490 & 0.0000 & 0.2249 & & & & & 0.2182 & & & 0.5078 & & & & & & & & & \\
\hline 11 & 0.1555 & 0.1592 & 0.0855 & 0.0000 & & & & & 0.1418 & & & 0.4581 & & & & & & & & & \\
\hline 13 & 0.1541 & 0.1578 & 0.0000 & 0.0000 & & & & & 0.2341 & & & 0.4540 & & & & & & & & & \\
\hline 15 & 0.1573 & 0.0537 & 0.0865 & 0.0000 & & & & & 0.2390 & & & 0.4635 & & & & & & & & & \\
\hline 16 & 0.1356 & 0.0463 & 0.0000 & 0.2124 & & & & & 0.2061 & & & 0.3996 & & & & & & & & & \\
\hline 17 & 0.0000 & 0.1463 & 0.0786 & 0.2238 & & & & & 0.1303 & & & 0.4210 & & & & & & & & & \\
\hline 18 & 0.0000 & 0.1696 & 0.0911 & 0.0000 & & & & & 0.2516 & & & 0.4878 & & & & & & & & & \\
\hline 19 & 0.0000 & 0.1451 & 0.0000 & 0.2220 & & & & & 0.2154 & & & 0.4175 & & & & & & & & & \\
\hline 20 & 0.0000 & 0.0493 & 0.0794 & 0.2262 & & & & & 0.2195 & & & 0.4256 & & & & & & & & & \\
\hline 21 & 0.0611 & 0.0938 & 0.0336 & 0.0956 & & & & & 0.1763 & & & 0.5397 & & & & & & & & & \\
\hline 22 & 0.0877 & 0.1097 & 0.0482 & 0.1373 & & & & & 0.1865 & & & 0.4305 & & & & & & & & & \\
\hline 23 & 0.1471 & 0.0904 & 0.0324 & 0.0922 & & & & & 0.1699 & & & 0.4681 & & & & & & & & & \\
\hline 24 & 0.0000 & 0.1138 & 0.0500 & 0.1424 & & & & & 0.1935 & & & 0.5002 & & & & & & & & & \\
\hline 25 & 0.0605 & 0.1550 & 0.0333 & 0.0948 & & & & & 0.1748 & & & 0.4816 & & & & & & & & & \\
\hline 26 & 0.0884 & 0.0503 & 0.0486 & 0.1384 & & & & & 0.1881 & & & 0.4862 & & & & & & & & & \\
\hline 27 & 0.0613 & 0.0941 & 0.0843 & 0.0960 & & & & & 0.1769 & & & 0.4875 & & & & & & & & & \\
\hline 28 & 0.0874 & 0.1094 & 0.0000 & 0.1368 & & & & & 0.1859 & & & 0.4805 & & & & & & & & & \\
\hline 29 & 0.0560 & 0.0861 & 0.0308 & 0.2194 & & & & & 0.1618 & & & 0.4458 & & & & & & & & & \\
\hline 30 & 0.0959 & 0.1200 & 0.0527 & 0.0000 & & & & & 0.2040 & & & 0.5274 & & & & & & & & & \\
\hline 31 & 0.0609 & 0.0936 & 0.0335 & 0.0955 & & & & & 0.2316 & & & 0.4849 & & & & & & & & & \\
\hline 32 & 0.0878 & 0.1099 & 0.0483 & 0.1375 & & & & & 0.1334 & & & 0.4830 & & & & & & & & & \\
\hline 33 & 0.0747 & 0.1019 & 0.0411 & 0.1169 & & & & & 0.1815 & & & 0.4839 & & & & & & & & & \\
\hline
\end{tabular}

TWRS LAW Formulation (Muller and Pegg 1998)

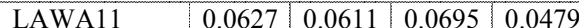

\begin{tabular}{|l|l|l|l|l|}
\hline LAWA12 & 0.0627 & 0.0611 & 0.0389 & 0.0479 \\
\hline
\end{tabular}

\begin{tabular}{l|l|l|l|l|l|}
\hline LAWA13 & 0.0932 & 0.0611 & 0.0695 & 0.0479 \\
\hline
\end{tabular}

\begin{tabular}{l|l|l|l|l|l|l|}
\hline LAWA14 & 0.0627 & 0.0611 & 0.0695 & 0.0479 \\
\hline
\end{tabular}

\begin{tabular}{l|l|l|l|l|l|l|}
\hline LAWA15 & 0.0627 & 0.0611 & 0.0695 & 0.0479 \\
\hline
\end{tabular}

\begin{tabular}{|l|l|l|l|l|l|}
\hline LAWA16 & 0.0627 & 0.0916 & 0.0389 & 0.0479 \\
\hline
\end{tabular}

\begin{tabular}{|l|l|l|l|l|}
\hline LAWA17 & 0.1482 & 0.0611 & 0.0206 & 0.0479 \\
\hline
\end{tabular}

\begin{tabular}{|l|l|l|l|l|l|}
\hline LAWA18 & 0.1237 & 0.0611 & 0.0206 & 0.0479 \\
\hline
\end{tabular}

\begin{tabular}{|l|l|l|l|l|l|} 
LAWA19 & 0.1237 & 0.0611 & 0.0206 & 0.0479 \\
\hline
\end{tabular}

\begin{tabular}{l|l|l|l|l|l} 
LAWA20 & 0.1482 & 0.0458 & 0.0206 & 0.0479
\end{tabular}

\begin{tabular}{|l|l|l|l|l|l|l|l|l|}
0.0548 & 0.0000 & 0.0000 & 0.2004 & 0.0014 & 0.0174 & 0.4093 & 0.0428 \\
\hline
\end{tabular}

\begin{tabular}{|l|l|l|l|l|l|l|l|l|}
\hline 0.0548 & 0.0000 & 0.0305 & 0.2004 & 0.0014 & 0.0174 & 0.4093 & 0.0428 \\
\hline
\end{tabular}

\begin{tabular}{|l|l|l|l|l|l|l|l|l|l|}
\hline 0.0548 & 0.0000 & 0.0000 & 0.2004 & 0.0014 & 0.0174 & 0.3788 & 0.0428 \\
\hline
\end{tabular}

\begin{tabular}{|l|l|l|l|l|l|l|l|l|l|}
\hline 0.0548 & 0.0000 & 0.0000 & 0.2004 & 0.0014 & 0.0174 & 0.3788 & 0.0428 \\
\hline
\end{tabular}

\begin{tabular}{|l|l|l|l|l|l|l|l|l|l|}
0.0548 & 0.0000 & 0.0000 & 0.2004 & 0.0014 & 0.0174 & 0.4093 & 0.0122 \\
\hline
\end{tabular}

\begin{tabular}{|l|l|l|l|l|l|l|l|l|}
0.0548 & 0.0000 & 0.0000 & 0.2004 & 0.0014 & 0.0174 & 0.4093 & 0.0428 \\
\hline
\end{tabular}

\begin{tabular}{|l|l|l|l|l|l|l|l|}
\hline 0.0548 & 0.0000 & 0.0000 & 0.2004 & 0.0014 & 0.0174 & 0.3543 & 0.0305 \\
\hline
\end{tabular}

\begin{tabular}{|l|l|l|l|l|l|l|l|l|}
\hline 0.0548 & 0.0000 & 0.0000 & 0.2004 & 0.0014 & 0.0174 & 0.3543 & 0.0305 \\
\hline
\end{tabular}

\begin{tabular}{ll|l|l|l|l|l|l|l|l|l|}
0.0548 & 0.0000 & 0.0000 & 0.2004 & 0.0014 & 0.0174 & 0.3543 & 0.0305 \\
\hline
\end{tabular}

\begin{tabular}{l|l|l|l|l} 
LAWA21 & 0.0917 & 0.0290 & 0.0970 & 0.0601 \\
\hline
\end{tabular}

\begin{tabular}{|l|l|l|l|l|l|l|l|l|}
0.0548 & 0.0153 & 0.0000 & 0.2004 & 0.0014 & 0.0174 & 0.3543 & 0.0305
\end{tabular}

0.0001

0.0001

0.0001

\begin{tabular}{|l|l|l|l|l|l|l|l|}
\hline 0.0548 & 0.0000 & 0.0000 & 0.2004 & 0.0014 & 0.0174 & 0.3873 & 0.0281 \\
\hline
\end{tabular}

\begin{tabular}{l|l|l|l|l|l|}
\hline LAWA22 & 0.0986 & 0.0292 & 0.0438 & 0.0730 \\
\hline
\end{tabular}

\begin{tabular}{|l|l|l|l|l|l|}
\hline LAWA23 & 0.0986 & 0.0423 & 0.0438 & 0.0730
\end{tabular}

\begin{tabular}{l|l|l|l|l|l|} 
LAWA24 & 0.1237 & 0.0611 & 0.0328 & 0.0601 \\
\hline
\end{tabular}

LAWA25

\begin{tabular}{|l|l|l|l|}
\hline 0.1048 & 0.0178 & 0.0165 & 0.0560 \\
\hline
\end{tabular}

\begin{tabular}{|l|l|l|l|l|l|l|l|l|}
0.0310 & 0.0204 & 0.0204 & 0.2000 & 0.0000 & 0.0008 & 0.4146 & 0.0299 \\
\hline
\end{tabular}

\begin{tabular}{|l|l|l|l|l|l|l|l|}
\hline 0.0310 & 0.0204 & 0.0204 & 0.2000 & 0.0000 & 0.0008 & 0.4015 & 0.0299 \\
\hline
\end{tabular}

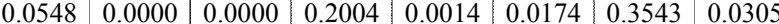

\begin{tabular}{|l|l|l|l|l|l|l|l|}
0.0402 & 0.0000 & 0.0100 & 0.2506 & 0.0017 & 0.0199 & 0.3897 & 0.0278 \\
\hline
\end{tabular} 
Canonical Correlation (Ramsey) (Oksoy et al. 1994)

\begin{tabular}{|c|c|c|c|c|c|c|c|c|c|c|c|c|c|c|c|c|c|c|c|c|c|}
\hline Glass ID & $\begin{array}{l}\mathrm{Cl} \\
-\mathrm{t}\end{array}$ & $\begin{array}{c}\mathrm{CoO} \\
-\mathrm{t}\end{array}$ & $\begin{array}{c}\mathrm{Co} 2 \mathrm{O} 3 \\
-\mathrm{t}\end{array}$ & $\begin{array}{c}\mathrm{Cr} 2 \mathrm{O} 3 \\
-\mathrm{t}\end{array}$ & $\begin{array}{c}\mathrm{Cs} 2 \mathrm{O} \\
-\mathrm{t}\end{array}$ & $\begin{array}{c}\mathrm{CuO} \\
-\mathrm{t}\end{array}$ & $\begin{array}{c}\mathrm{Eu} 2 \mathrm{O} 3 \\
-\mathrm{t}\end{array}$ & $\begin{array}{l}F \\
-t\end{array}$ & $\begin{array}{c}\mathrm{Ga} 2 \mathrm{O} 3 \\
-\mathrm{t}\end{array}$ & $\begin{array}{c}\mathrm{Gd} 2 \mathrm{O} 3 \\
-\mathrm{t}\end{array}$ & $\begin{array}{c}\mathrm{HgO} \\
-\mathrm{t}\end{array}$ & $\begin{array}{l}I \\
-t\end{array}$ & $\begin{array}{c}\mathrm{La} 2 \mathrm{O} 3 \\
-\mathrm{t}\end{array}$ & $\begin{array}{c}\mathrm{MnO} 2 \\
-\mathrm{t}\end{array}$ & $\begin{array}{c}\mathrm{MnO} \\
-\mathrm{t}\end{array}$ & $\begin{array}{c}\mathrm{MoO} \\
-\mathrm{t}\end{array}$ & $\begin{array}{c}\mathrm{MoO} 3 \\
-\mathrm{t}\end{array}$ & $\begin{array}{c}\mathrm{Nb} 2 \mathrm{O} 5 \\
-\mathrm{t}\end{array}$ & $\begin{array}{c}\mathrm{Nd} 2 \mathrm{O} 3 \\
-\mathrm{t}\end{array}$ & $\begin{array}{c}\mathrm{PbO} \\
-\mathrm{t}\end{array}$ & $\begin{array}{c}\mathrm{PdO} 2 \\
-\mathrm{t}\end{array}$ \\
\hline 1 & & & & & & & & & & & & & & & & & & & & & \\
\hline 2 & & & & & & & & & & & & & & & & & & & & & \\
\hline 4 & & & & & & & & & & & & & & & & & & & & & \\
\hline 5 & & & & & & & & & & & & & & & & & & & & & \\
\hline 6 & & & & & & & & & & & & & & & & & & & & & \\
\hline 7 & & & & & & & & & & & & & & & & & & & & & \\
\hline 8 & & & & & & & & & & & & & & & & & & & & & \\
\hline 9 & & & & & & & & & & & & & & & & & & & & & \\
\hline 10 & & & & & & & & & & & & & & & & & & & & & \\
\hline 11 & & & & & & & & & & & & & & & & & & & & & \\
\hline 13 & & & & & & & & & & & & & & & & & & & & & \\
\hline 15 & & & & & & & & & & & & & & & & & & & & & \\
\hline 16 & & & & & & & & & & & & & & & & & & & & & \\
\hline 17 & & & & & & & & & & & & & & & & & & & & & \\
\hline 18 & & & & & & & & & & & & & & & & & & & & & \\
\hline 19 & & & & & & & & & & & & & & & & & & & & & \\
\hline 20 & & & & & & & & & & & & & & & & & & & & & \\
\hline 21 & & & & & & & & & & & & & & & & & & & & & \\
\hline 22 & & & & & & & & & & & & & & & & & & & & & \\
\hline 23 & & & & & & & & & & & & & & & & & & & & & \\
\hline 24 & & & & & & & & & & & & & & & & & & & & & \\
\hline 25 & & & & & & & & & & & & & & & & & & & & & \\
\hline 26 & & & & & & & & & & & & & & & & & & & & & \\
\hline 27 & & & & & & & & & & & & & & & & & & & & & \\
\hline 28 & & & & & & & & & & & & & & & & & & & & & \\
\hline 29 & & & & & & & & & & & & & & & & & & & & & \\
\hline 30 & & & & & & & & & & & & & & & & & & & & & \\
\hline 31 & & & & & & & & & & & & & & & & & & & & & \\
\hline 32 & & & & & & & & & & & & & & & & & & & & & \\
\hline 33 & & & & & & & & & & & & & & & & & & & & & \\
\hline
\end{tabular}

TWRS LAW Formulation (Muller and Pegg 1998)

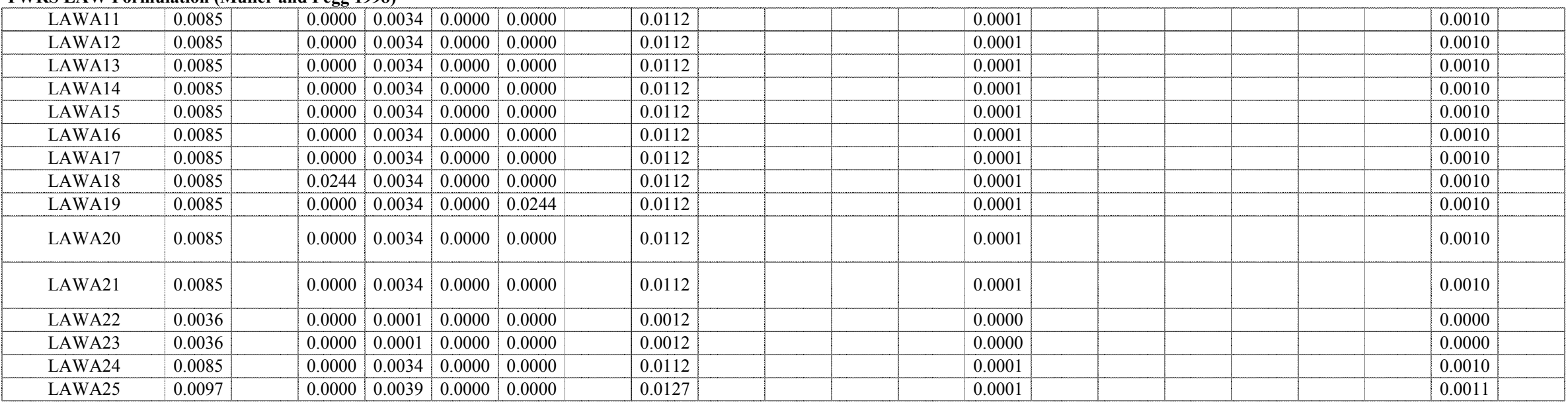


Canonical Correlation (Ramsey) (Oksoy et al. 1994)

\begin{tabular}{|c|c|c|c|c|c|c|c|c|c|c|c|c|c|c|c|c|c|c|c|c|c|}
\hline Glass ID & $\begin{array}{c}\mathrm{PdO} \\
-\mathrm{t}\end{array}$ & $\begin{array}{c}\mathrm{Pr} 2 \mathrm{O} 3 \\
-\mathrm{t}\end{array}$ & $\begin{array}{c}\text { Pr6O11 } \\
-t\end{array}$ & $\begin{array}{c}\mathrm{Rb} 2 \mathrm{O} \\
-\mathrm{t}\end{array}$ & $\begin{array}{c}\mathrm{ReO} \\
-\mathrm{t}\end{array}$ & $\begin{array}{c}\mathrm{ReO} 2 \\
-\mathrm{t}\end{array}$ & $\begin{array}{c}\mathrm{Rh} 2 \mathrm{O} 3 \\
-\mathrm{t}\end{array}$ & $\begin{array}{c}\mathrm{RhO} 2 \\
-\mathrm{t}\end{array}$ & $\begin{array}{c}\mathrm{RuO} 2 \\
-\mathrm{t}\end{array}$ & $\begin{array}{c}\mathrm{Sb} 2 \mathrm{O} 3 \\
-\mathrm{t}\end{array}$ & $\begin{array}{c}\mathrm{Sb} 2 \mathrm{O} 5 \\
-\mathrm{t}\end{array}$ & $\begin{array}{c}\mathrm{SeO} 2 \\
-\mathrm{t}\end{array}$ & $\begin{array}{c}\mathrm{Sm} 2 \mathrm{O} 3 \\
-\mathrm{t}\end{array}$ & $\begin{array}{c}\mathrm{SnO} \\
-\mathrm{t}\end{array}$ & $\begin{array}{c}\mathrm{SnO} 2 \\
-\mathrm{t}\end{array}$ & $\begin{array}{c}\mathrm{SO} 3 \\
-\mathrm{t}\end{array}$ & $\begin{array}{c}\mathrm{SrO} \\
-\mathrm{t}\end{array}$ & $\begin{array}{c}\mathrm{Tc} 2 \mathrm{O} 7 \\
-\mathrm{t}\end{array}$ & $\begin{array}{c}\mathrm{TeO} 2 \\
-\mathrm{t}\end{array}$ & $\begin{array}{c}\text { ThO2 } \\
-t\end{array}$ & $\begin{array}{c}\mathrm{TiO} 2 \\
-\mathrm{t}\end{array}$ \\
\hline 1 & & & & & & & & & & & & & & & & & & & & & \\
\hline 2 & & & & & & & & & & & & & & & & & & & & & \\
\hline 4 & & & & & & & & & & & & & & & & & & & & & \\
\hline 5 & & & & & & & & & & & & & & & & & & & & & \\
\hline 6 & & & & & & & & & & & & & & & & & & & & & \\
\hline 7 & & & & & & & & & & & & & & & & & & & & & \\
\hline 8 & & & & & & & & & & & & & & & & & & & & & \\
\hline 9 & & & & & & & & & & & & & & & & & & & & & \\
\hline 10 & & & & & & & & & & & & & & & & & & & & & \\
\hline 11 & & & & & & & & & & & & & & & & & & & & & \\
\hline 13 & & & & & & & & & & & & & & & & & & & & & \\
\hline 15 & & & & & & & & & & & & & & & & & & & & & \\
\hline 16 & & & & & & & & & & & & & & & & & & & & & \\
\hline 17 & & & & & & & & & & & & & & & & & & & & & \\
\hline 18 & & & & & & & & & & & & & & & & & & & & & \\
\hline 19 & & & & & & & & & & & & & & & & & & & & & \\
\hline 20 & & & & & & & & & & & & & & & & & & & & & \\
\hline 21 & & & & & & & & & & & & & & & & & & & & & \\
\hline 22 & & & & & & & & & & & & & & & & & & & & & \\
\hline 23 & & & & & & & & & & & & & & & & & & & & & \\
\hline 24 & & & & & & & & & & & & & & & & & & & & & \\
\hline 25 & & & & & & & & & & & & & & & & & & & & & \\
\hline 26 & & & & & & & & & & & & & & & & & & & & & \\
\hline 27 & & & & & & & & & & & & & & & & & & & & & \\
\hline 28 & & & & & & & & & & & & & & & & & & & & & \\
\hline 29 & & & & & & & & & & & & & & & & & & & & & \\
\hline 30 & & & & & & & & & & & & & & & & & & & & & \\
\hline 31 & & & & & & & & & & & & & & & & & & & & & \\
\hline 32 & & & & & & & & & & & & & & & & & & & & & \\
\hline 33 & & & & & & & & & & & & & & & & & & & & & \\
\hline
\end{tabular}

TWRS LAW Formulation (Muller and Pegg 1998)

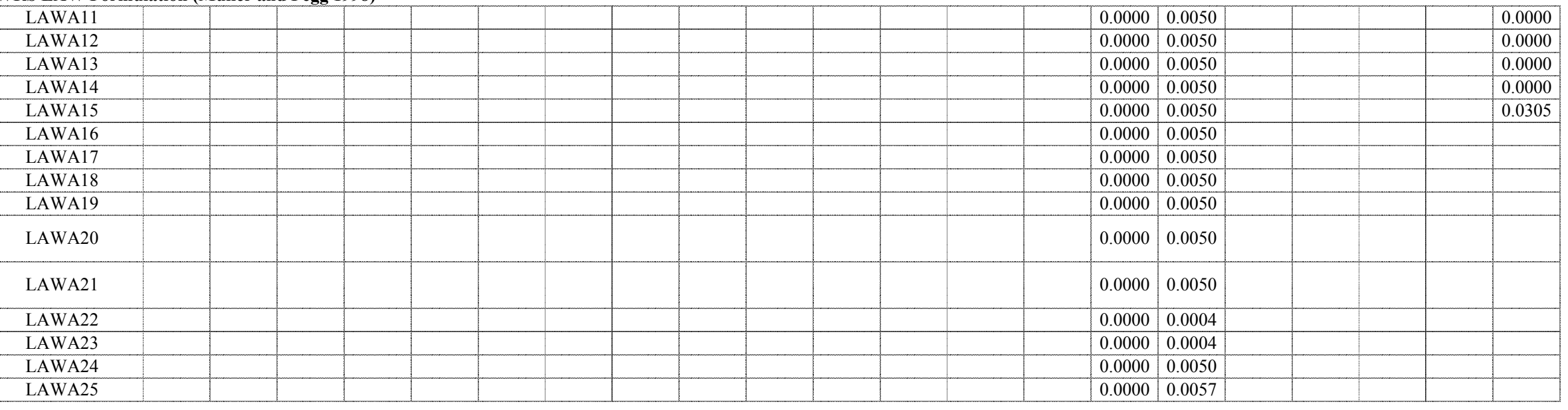


Canonical Correlation (Ramsey) (Oksoy et al. 1994)

\begin{tabular}{|c|c|c|c|c|c|c|c|c|c|c|c|c|c|c|c|c|c|c|c|c|c|}
\hline Glass ID & $\begin{array}{c}\mathrm{T} 12 \mathrm{O} 3 \\
-\mathrm{t}\end{array}$ & $\begin{array}{c}\text { U3O8 } \\
-t\end{array}$ & $\begin{array}{c}\mathrm{UO} 2 \\
-\mathrm{t}\end{array}$ & $\begin{array}{c}\text { UO3 } \\
-t\end{array}$ & $\begin{array}{c}\mathrm{V} 2 \mathrm{O} 5 \\
-\mathrm{t}\end{array}$ & $\begin{array}{c}\text { WO3 } \\
-t\end{array}$ & $\begin{array}{c}\mathrm{Y} 2 \mathrm{O} 3 \\
-\mathrm{t}\end{array}$ & $\begin{array}{c}\mathrm{ZnO} \\
-\mathrm{t}\end{array}$ & $\begin{array}{c}\text { Others } \\
-t\end{array}$ & $\begin{array}{c}\text { Sum } \\
-t\end{array}$ & $\begin{array}{c}\mathrm{A} 12 \mathrm{O} 3 \\
-\mathrm{a}\end{array}$ & $\begin{array}{c}\mathrm{B} 2 \mathrm{O} 3 \\
-\mathrm{a}\end{array}$ & $\begin{array}{c}\mathrm{CaO} \\
-\mathrm{a}\end{array}$ & $\begin{array}{c}\mathrm{Fe} 2 \mathrm{O} 3 \\
-\mathrm{a} \\
\end{array}$ & $\begin{array}{c}\mathrm{FeO} \\
-\mathrm{a}\end{array}$ & $\begin{array}{c}\mathrm{K} 2 \mathrm{O} \\
-\mathrm{a}\end{array}$ & $\begin{array}{c}\mathrm{Li} 2 \mathrm{O} \\
-\mathrm{a}\end{array}$ & $\begin{array}{c}\mathrm{MgO} \\
-\mathrm{a}\end{array}$ & $\begin{array}{c}\mathrm{Na} 2 \mathrm{O} \\
-\mathrm{a}\end{array}$ & $\begin{array}{c}\mathrm{NiO} \\
-\mathrm{a}\end{array}$ & $\begin{array}{c}\mathrm{P} 2 \mathrm{O} 5 \\
-\mathrm{a} \\
\end{array}$ \\
\hline 1 & & & & & & & & & & 1.0000 & 0.1614 & 0.1482 & 0.0000 & 0.0000 & 0.0000 & & & & 0.1406 & & \\
\hline 2 & & & & & & & & & & 1.0000 & 0.1633 & 0.0533 & 0.0895 & 0.0000 & 0.0000 & & & & 0.1463 & & \\
\hline 4 & & & & & & & & & & 1.0000 & 0.1721 & 0.0456 & 0.0000 & 0.0000 & 0.0000 & & & & 0.2319 & & \\
\hline 5 & & & & & & & & & & 1.0000 & 0.0568 & 0.1514 & 0.0859 & 0.0000 & 0.0000 & & & & 0.1420 & & \\
\hline 6 & & & & & & & & & & 1.0000 & 0.0421 & 0.1271 & 0.0000 & 0.1937 & 0.0256 & & & & 0.1214 & & \\
\hline 7 & & & & & & & & & & 1.0000 & 0.0101 & 0.1618 & 0.0000 & 0.0000 & 0.0000 & & & & 0.2546 & & \\
\hline 8 & & & & & & & & & & 1.0000 & 0.0367 & 0.0469 & 0.0748 & 0.1886 & 0.0297 & & & & 0.1260 & & \\
\hline 9 & & & & & & & & & & 1.0000 & 0.0020 & 0.0564 & 0.0926 & 0.0000 & 0.0000 & & & & 0.2597 & & \\
\hline 10 & & & & & & & & & & 1.0000 & 0.0240 & 0.0408 & 0.0000 & 0.1814 & 0.0302 & & & & 0.2270 & & \\
\hline 11 & & & & & & & & & & 1.0000 & 0.1675 & 0.1628 & 0.0884 & 0.0000 & 0.0000 & & & & 0.1425 & & \\
\hline 13 & & & & & & & & & & 1.0000 & 0.1826 & 0.1447 & 0.0000 & 0.0000 & 0.0000 & & & & 0.2235 & & \\
\hline 15 & & & & & & & & & & 1.0000 & 0.1751 & 0.0483 & 0.0843 & 0.0000 & 0.0000 & & & & 0.2289 & & \\
\hline 16 & & & & & & & & & & 1.0000 & 0.1484 & 0.0411 & 0.0000 & 0.1875 & 0.0218 & & & & 0.1941 & & \\
\hline 17 & & & & & & & & & & 1.0000 & 0.1145 & 0.1297 & 0.0696 & 0.1582 & 0.0338 & & & & 0.1154 & & \\
\hline 18 & & & & & & & & & & 1.0000 & 0.0094 & 0.1611 & 0.0931 & 0.0000 & 0.0000 & & & & 0.2586 & & \\
\hline 19 & & & & & & & & & & 1.0000 & 0.0368 & 0.1273 & 0.0000 & 0.1996 & 0.0146 & & & & 0.2347 & & \\
\hline 20 & & & & & & & & & & 1.0000 & 0.0096 & 0.0487 & 0.0786 & 0.2200 & 0.0136 & & & & 0.2155 & & \\
\hline 21 & & & & & & & & & & 1.0000 & 0.1126 & 0.0880 & 0.0316 & 0.0877 & 0.0084 & & & & 0.1699 & & \\
\hline 22 & & & & & & & & & & 1.0000 & 0.1843 & 0.0947 & 0.0416 & 0.1090 & 0.0166 & & & & 0.1601 & & \\
\hline 23 & & & & & & & & & & 1.0000 & 0.2261 & 0.0762 & 0.0279 & 0.0666 & 0.0164 & & & & 0.1580 & & \\
\hline 24 & & & & & & & & & & 1.0000 & 0.0742 & 0.1049 & 0.0457 & 0.1225 & 0.0173 & & & & 0.1721 & & \\
\hline 25 & & & & & & & & & & 1.0000 & 0.1296 & 0.1372 & 0.0300 & 0.0823 & 0.0116 & & & & 0.1547 & & \\
\hline 26 & & & & & & & & & & 1.0000 & 0.1116 & 0.0436 & 0.0458 & 0.1141 & 0.0254 & & & & 0.1761 & & \\
\hline 27 & & & & & & & & & & 1.0000 & 0.1450 & 0.0809 & 0.0768 & 0.0778 & 0.0129 & & & & 0.1570 & & \\
\hline 28 & & & & & & & & & & 1.0000 & 0.1463 & 0.0922 & 0.0000 & 0.1119 & 0.0215 & & & & 0.1687 & & \\
\hline 29 & & & & & & & & & & 1.0000 & 0.1148 & 0.0735 & 0.0266 & 0.1725 & 0.0298 & & & & 0.1400 & & \\
\hline 30 & & & & & & & & & & 1.0000 & 0.1070 & 0.1227 & 0.0552 & 0.0000 & 0.0000 & & & & 0.1957 & & \\
\hline 31 & & & & & & & & & & 1.0000 & 0.1484 & 0.0784 & 0.0299 & 0.0803 & 0.0113 & & & & 0.2070 & & \\
\hline 32 & & & & & & & & & & 1.0000 & 0.1634 & 0.0930 & 0.0430 & 0.1001 & 0.0288 & & & & 0.1231 & & \\
\hline 33 & & & & & & & & & & 1.0000 & 0.1793 & 0.0871 & 0.0343 & 0.0847 & 0.0185 & & & & 0.1580 & & \\
\hline
\end{tabular}

\section{TWRS LAW Formulation (Muller and Pegg 1998)}

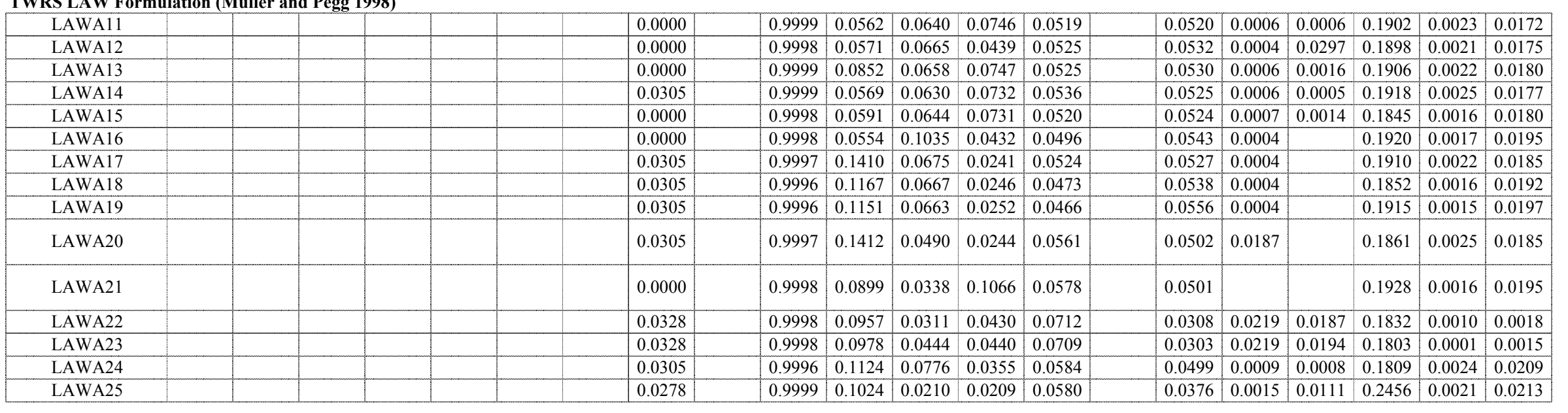




\section{Canonical Correlation (Ramsey) (Oksoy et al. 1994)}

\begin{tabular}{|c|c|c|c|c|c|c|c|c|c|c|c|c|c|c|c|c|c|c|c|c|c|}
\hline Glass ID & $\begin{array}{c}\mathrm{SiO} 2 \\
-\mathrm{a}\end{array}$ & $\begin{array}{c}\mathrm{ZrO} 2 \\
-\mathrm{a}\end{array}$ & $\begin{array}{c}\mathrm{Ag} 2 \mathrm{O} \\
-\mathrm{a}\end{array}$ & $\begin{array}{c}\mathrm{As} 2 \mathrm{O} 3 \\
-\mathrm{a}\end{array}$ & $\begin{array}{c}\mathrm{BaO} \\
-\mathrm{a}\end{array}$ & $\begin{array}{c}\mathrm{Bi} 2 \mathrm{O} 3 \\
-\mathrm{a}\end{array}$ & $\begin{array}{l}\mathrm{Br} \\
-\mathrm{a}\end{array}$ & $\begin{array}{c}\mathrm{CdO} \\
-\mathrm{a}\end{array}$ & $\begin{array}{c}\mathrm{Ce} 2 \mathrm{O} 3 \\
-\mathrm{a}\end{array}$ & $\begin{array}{c}\mathrm{CeO} 2 \\
-\mathrm{a}\end{array}$ & $\begin{array}{l}\mathrm{Cl} \\
-\mathrm{a}\end{array}$ & $\begin{array}{c}\mathrm{CoO} \\
-\mathrm{a}\end{array}$ & $\begin{array}{c}\mathrm{Co} 2 \mathrm{O} 3 \\
-\mathrm{a}\end{array}$ & $\begin{array}{c}\mathrm{Cr} 2 \mathrm{O} 3 \\
-\mathrm{a}\end{array}$ & $\begin{array}{c}\mathrm{Cs} 2 \mathrm{O} \\
-\mathrm{a}\end{array}$ & $\begin{array}{c}\mathrm{CuO} \\
-\mathrm{a}\end{array}$ & $\begin{array}{c}\mathrm{Eu} 2 \mathrm{O} 3 \\
-\mathrm{a}\end{array}$ & $\begin{array}{c}F \\
-a\end{array}$ & $\begin{array}{c}\mathrm{Ga} 2 \mathrm{O} 3 \\
-\mathrm{a}\end{array}$ & $\begin{array}{c}\mathrm{Gd} 2 \mathrm{O} 3 \\
-\mathrm{a}\end{array}$ & $\begin{array}{c}\mathrm{HgO} \\
-\mathrm{a}\end{array}$ \\
\hline 1 & 0.5498 & & & & & & & & & & & & & & & & & & & & \\
\hline 2 & 0.5475 & & & & & & & & & & & & & & & & & & & & \\
\hline 4 & 0.5504 & & & & & & & & & & & & & & & & & & & & \\
\hline 5 & 0.5638 & & & & & & & & & & & & & & & & & & & & \\
\hline 6 & 0.4900 & & & & & & & & & & & & & & & & & & & & \\
\hline 7 & 0.5734 & & & & & & & & & & & & & & & & & & & & \\
\hline 8 & 0.4973 & & & & & & & & & & & & & & & & & & & & \\
\hline 9 & 0.5894 & & & & & & & & & & & & & & & & & & & & \\
\hline 10 & 0.4966 & & & & & & & & & & & & & & & & & & & & \\
\hline 11 & 0.4388 & & & & & & & & & & & & & & & & & & & & \\
\hline 13 & 0.4492 & & & & & & & & & & & & & & & & & & & & \\
\hline 15 & 0.4635 & & & & & & & & & & & & & & & & & & & & \\
\hline 16 & 0.4072 & & & & & & & & & & & & & & & & & & & & \\
\hline 17 & 0.3788 & & & & & & & & & & & & & & & & & & & & \\
\hline 18 & 0.4778 & & & & & & & & & & & & & & & & & & & & \\
\hline 19 & 0.3870 & & & & & & & & & & & & & & & & & & & & \\
\hline 20 & 0.4140 & & & & & & & & & & & & & & & & & & & & \\
\hline 21 & 0.5018 & & & & & & & & & & & & & & & & & & & & \\
\hline 22 & 0.3937 & & & & & & & & & & & & & & & & & & & & \\
\hline 23 & 0.4288 & & & & & & & & & & & & & & & & & & & & \\
\hline 24 & 0.4634 & & & & & & & & & & & & & & & & & & & & \\
\hline 25 & 0.4546 & & & & & & & & & & & & & & & & & & & & \\
\hline 26 & 0.4835 & & & & & & & & & & & & & & & & & & & & \\
\hline 27 & 0.4495 & & & & & & & & & & & & & & & & & & & & \\
\hline 28 & 0.4594 & & & & & & & & & & & & & & & & & & & & \\
\hline 29 & 0.4428 & & & & & & & & & & & & & & & & & & & & \\
\hline 30 & 0.5193 & & & & & & & & & & & & & & & & & & & & \\
\hline 31 & 0.4448 & & & & & & & & & & & & & & & & & & & & \\
\hline 32 & 0.4486 & & & & & & & & & & & & & & & & & & & & \\
\hline 33 & 0.4381 & & & & & & & & & & & & & & & & & & & & \\
\hline
\end{tabular}

TWRS LAW Formulation (Muller and Pegg 1998)

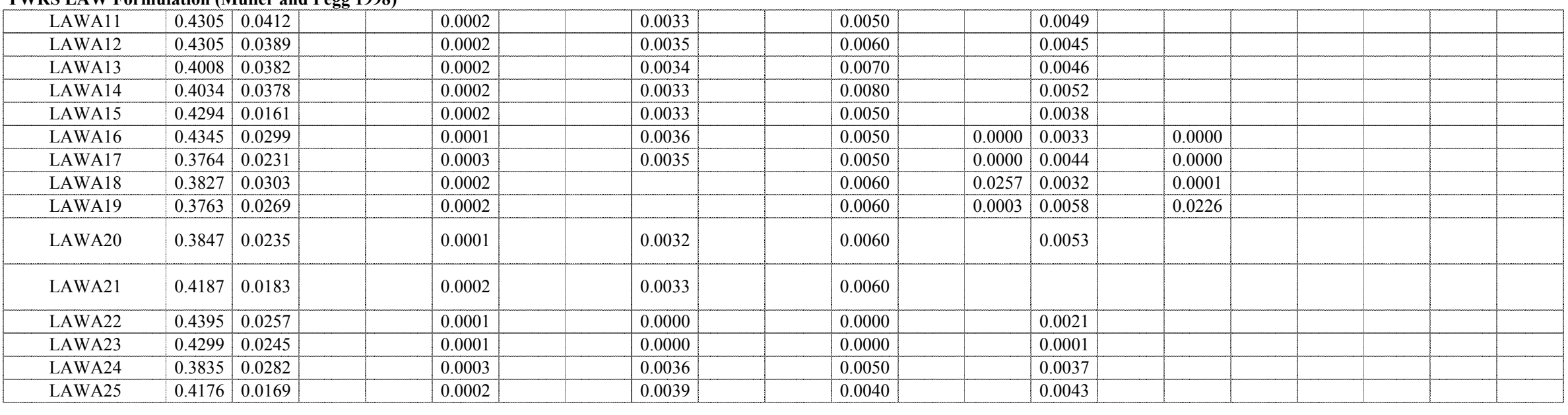


Canonical Correlation (Ramsey) (Oksoy et al. 1994)

\begin{tabular}{|c|c|c|c|c|c|c|c|c|c|c|c|c|c|c|c|c|c|c|c|c|c|}
\hline Glass ID & $\begin{array}{c}\mathrm{I} \\
-\mathrm{a} \\
\end{array}$ & $\begin{array}{c}\mathrm{La} 2 \mathrm{O} 3 \\
-\mathrm{a}\end{array}$ & $\begin{array}{c}\mathrm{MnO} 2 \\
-\mathrm{a}\end{array}$ & $\begin{array}{c}\mathrm{MnO} \\
-\mathrm{a}\end{array}$ & $\begin{array}{c}\mathrm{MoO} \\
-\mathrm{a}\end{array}$ & $\begin{array}{c}\mathrm{MoO} 3 \\
-\mathrm{a}\end{array}$ & $\begin{array}{c}\mathrm{Nb} 2 \mathrm{O} 5 \\
-\mathrm{a}\end{array}$ & $\begin{array}{c}\mathrm{Nd} 2 \mathrm{O} 3 \\
-\mathrm{a}\end{array}$ & $\begin{array}{c}\mathrm{PbO} \\
-\mathrm{a}\end{array}$ & $\begin{array}{c}\mathrm{PdO} 2 \\
-\mathrm{a}\end{array}$ & $\begin{array}{c}\mathrm{PdO} \\
-\mathrm{a}\end{array}$ & $\begin{array}{c}\mathrm{Pr} 2 \mathrm{O} 3 \\
-\mathrm{a}\end{array}$ & $\begin{array}{c}\text { Pr6O11 } \\
-\mathrm{a}\end{array}$ & $\begin{array}{c}\mathrm{Rb} 2 \mathrm{O} \\
-\mathrm{a}\end{array}$ & $\begin{array}{c}\mathrm{ReO} \\
-\mathrm{a}\end{array}$ & $\begin{array}{c}\mathrm{ReO} 2 \\
-\mathrm{a}\end{array}$ & $\begin{array}{c}\mathrm{Rh} 2 \mathrm{O} 3 \\
-\mathrm{a}\end{array}$ & $\begin{array}{c}\mathrm{RhO} 2 \\
-\mathrm{a}\end{array}$ & $\begin{array}{c}\mathrm{RuO} 2 \\
-\mathrm{a}\end{array}$ & $\begin{array}{c}\mathrm{Sb} 2 \mathrm{O} 3 \\
-\mathrm{a}\end{array}$ & $\begin{array}{c}\mathrm{Sb} 2 \mathrm{O} 5 \\
-\mathrm{a}\end{array}$ \\
\hline 1 & & & & & & & & & & & & & & & & & & & & & \\
\hline 2 & & & & & & & & & & & & & & & & & & & & & \\
\hline 4 & & & & & & & & & & & & & & & & & & & & & \\
\hline 5 & & & & & & & & & & & & & & & & & & & & & \\
\hline 6 & & & & & & & & & & & & & & & & & & & & & \\
\hline 7 & & & & & & & & & & & & & & & & & & & & & \\
\hline 8 & & & & & & & & & & & & & & & & & & & & & \\
\hline 9 & & & & & & & & & & & & & & & & & & & & & \\
\hline 10 & & & & & & & & & & & & & & & & & & & & & \\
\hline 11 & & & & & & & & & & & & & & & & & & & & & \\
\hline 13 & & & & & & & & & & & & & & & & & & & & & \\
\hline 15 & & & & & & & & & & & & & & & & & & & & & \\
\hline 16 & & & & & & & & & & & & & & & & & & & & & \\
\hline 17 & & & & & & & & & & & & & & & & & & & & & \\
\hline 18 & & & & & & & & & & & & & & & & & & & & & \\
\hline 19 & & & & & & & & & & & & & & & & & & & & & \\
\hline 20 & & & & & & & & & & & & & & & & & & & & & \\
\hline 21 & & & & & & & & & & & & & & & & & & & & & \\
\hline 22 & & & & & & & & & & & & & & & & & & & & & \\
\hline 23 & & & & & & & & & & & & & & & & & & & & & \\
\hline 24 & & & & & & & & & & & & & & & & & & & & & \\
\hline 25 & & & & & & & & & & & & & & & & & & & & & \\
\hline 26 & & & & & & & & & & & & & & & & & & & & & \\
\hline 27 & & & & & & & & & & & & & & & & & & & & & \\
\hline 28 & & & & & & & & & & & & & & & & & & & & & \\
\hline 29 & & & & & & & & & & & & & & & & & & & & & \\
\hline 30 & & & & & & & & & & & & & & & & & & & & & \\
\hline 31 & & & & & & & & & & & & & & & & & & & & & \\
\hline 32 & & & & & & & & & & & & & & & & & & & & & \\
\hline 33 & & & & & & & & & & & & & & & & & & & & & \\
\hline
\end{tabular}

TWRS LAW Formulation (Muller and Pegg 1998)

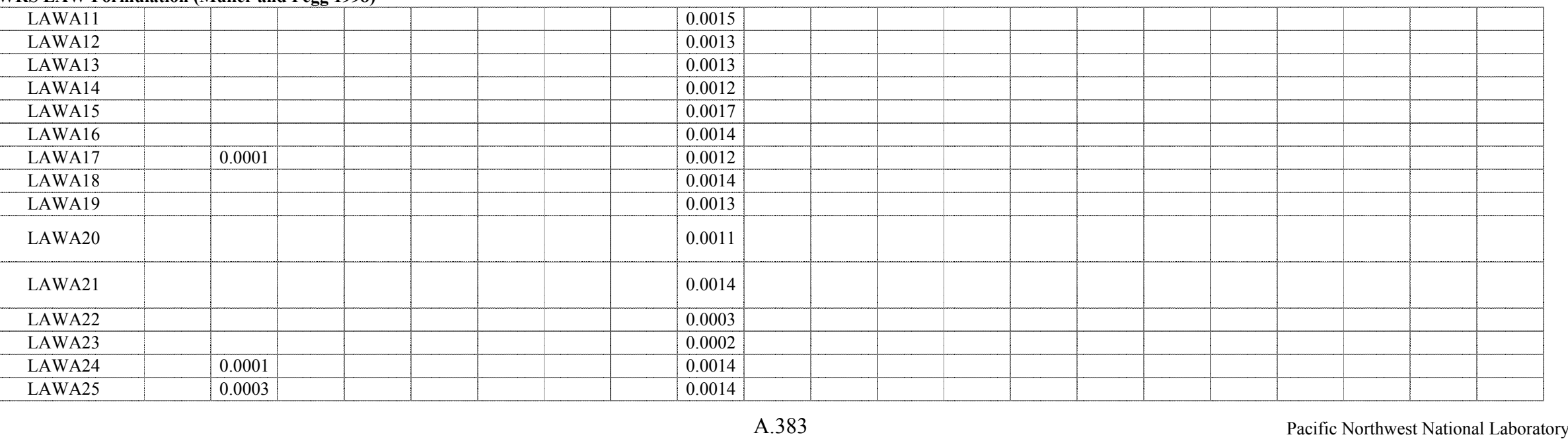


Appendix A. Database - mass fraction

Canonical Correlation (Ramsey) (Oksoy et al. 1994)

\begin{tabular}{|c|c|c|c|c|c|c|c|c|c|c|c|c|c|c|c|c|c|c|c|c|}
\hline Glass ID & $\begin{array}{c}\mathrm{SeO} 2 \\
-\mathrm{a}\end{array}$ & $\begin{array}{c}\mathrm{Sm} 2 \mathrm{O} 3 \\
-\mathrm{a}\end{array}$ & $\begin{array}{c}\mathrm{SnO} \\
-\mathrm{a} \\
\end{array}$ & $\begin{array}{c}\mathrm{SnO} 2 \\
-\mathrm{a}\end{array}$ & $\begin{array}{c}\mathrm{SO} 3 \\
-\mathrm{a}\end{array}$ & $\begin{array}{c}\mathrm{SrO} \\
-\mathrm{a}\end{array}$ & $\begin{array}{c}\mathrm{Tc} 2 \mathrm{O} 7 \\
-\mathrm{a}\end{array}$ & $\begin{array}{c}\mathrm{TeO} 2 \\
-\mathrm{a}\end{array}$ & $\begin{array}{c}\mathrm{ThO} 2 \\
-\mathrm{a}\end{array}$ & $\begin{array}{c}\mathrm{TiO} 2 \\
-\mathrm{a}\end{array}$ & $\begin{array}{c}\mathrm{T} 12 \mathrm{O} 3 \\
-\mathrm{a}\end{array}$ & $\begin{array}{c}\mathrm{U} 3 \mathrm{O} 8 \\
-\mathrm{a}\end{array}$ & $\begin{array}{c}\mathrm{UO} 2 \\
-\mathrm{a}\end{array}$ & $\begin{array}{c}\text { UO3 } \\
-\mathrm{a}\end{array}$ & $\begin{array}{c}\mathrm{V} 2 \mathrm{O} 5 \\
-\mathrm{a}\end{array}$ & $\begin{array}{c}\text { WO3 } \\
-\mathrm{a}\end{array}$ & $\begin{array}{c}\mathrm{Y} 2 \mathrm{O} 3 \\
-\mathrm{a}\end{array}$ & $\begin{array}{c}\mathrm{ZnO} \\
-\mathrm{a}\end{array}$ & $\begin{array}{c}\text { Others } \\
-\mathrm{a}\end{array}$ & $\begin{array}{c}\text { Sum } \\
-\mathrm{a}\end{array}$ \\
\hline 1 & & & & & & & & & & & & & & & & & & & & 1.0000 \\
\hline 2 & & & & & & & & & & & & & & & & & & & & 0.9999 \\
\hline 4 & & & & & & & & & & & & & & & & & & & & 1.0000 \\
\hline 5 & & & & & & & & & & & & & & & & & & & & 0.9999 \\
\hline 6 & & & & & & & & & & & & & & & & & & & & 0.9999 \\
\hline 7 & & & & & & & & & & & & & & & & & & & & 0.9999 \\
\hline 8 & & & & & & & & & & & & & & & & & & & & 1.0000 \\
\hline 9 & & & & & & & & & & & & & & & & & & & & 1.0001 \\
\hline 10 & & & & & & & & & & & & & & & & & & & & 1.0000 \\
\hline 11 & & & & & & & & & & & & & & & & & & & & 1.0000 \\
\hline 13 & & & & & & & & & & & & & & & & & & & & 1.0000 \\
\hline 15 & & & & & & & & & & & & & & & & & & & & 1.0001 \\
\hline 16 & & & & & & & & & & & & & & & & & & & & 1.0001 \\
\hline 17 & & & & & & & & & & & & & & & & & & & & 1.0000 \\
\hline 18 & & & & & & & & & & & & & & & & & & & & 1.0000 \\
\hline 19 & & & & & & & & & & & & & & & & & & & & 1.0000 \\
\hline 20 & & & & & & & & & & & & & & & & & & & & 1.0000 \\
\hline 21 & & & & & & & & & & & & & & & & & & & & 1.0000 \\
\hline 22 & & & & & & & & & & & & & & & & & & & & 1.0000 \\
\hline 23 & & & & & & & & & & & & & & & & & & & & 1.0000 \\
\hline 24 & & & & & & & & & & & & & & & & & & & & 1.0001 \\
\hline 25 & & & & & & & & & & & & & & & & & & & & 1.0000 \\
\hline 26 & & & & & & & & & & & & & & & & & & & & 1.0001 \\
\hline 27 & & & & & & & & & & & & & & & & & & & & 0.9999 \\
\hline 28 & & & & & & & & & & & & & & & & & & & & 1.0000 \\
\hline 29 & & & & & & & & & & & & & & & & & & & & 1.0000 \\
\hline 30 & & & & & & & & & & & & & & & & & & & & 0.9999 \\
\hline 31 & & & & & & & & & & & & & & & & & & & & 1.0001 \\
\hline 32 & & & & & & & & & & & & & & & & & & & & 1.0000 \\
\hline 33 & & & & & & & & & & & & & & & & & & & & 1.0000 \\
\hline
\end{tabular}

TWRS LAW Formulation (Muller and Pegg 1998)

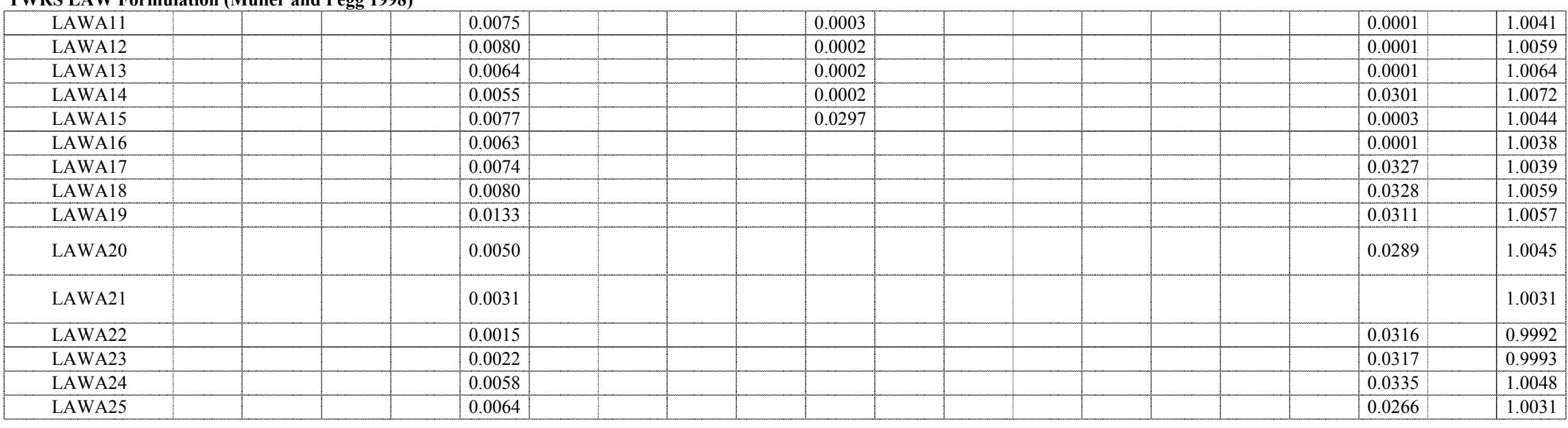


Appendix A. Database - mass fraction

Canonical Correlation (Ramsey) (Oksoy et al. 1994)

\begin{tabular}{|c|c|c|c|c|c|c|c|c|}
\hline Glass ID & $\begin{array}{l}\mathrm{TM} \\
\left({ }^{\circ} \mathrm{C}\right) \\
\end{array}$ & $\begin{array}{l}\text { Gradient } \\
\text { TL }\left({ }^{\circ} \mathrm{C}\right)\end{array}$ & $\begin{array}{l}\text { Uniform } \\
\text { TL }\left({ }^{\circ} \mathrm{C}\right)\end{array}$ & Primary Phase & $\begin{array}{l}\text { Quenched } \\
\text { Visual/OM }\end{array}$ & $\begin{array}{c}\text { Quenched } \\
\text { SEM/EDS or TEM }\end{array}$ & $\begin{array}{l}\text { Quenched } \\
\text { XRD }\end{array}$ & $\begin{array}{c}\text { Quenched } \\
\text { Homogeneous? }\end{array}$ \\
\hline \multicolumn{9}{|l|}{1} \\
\hline \multirow{2}{*}{\multicolumn{9}{|c|}{$\frac{2}{4}$}} \\
\hline & & & & & & & & \\
\hline \multicolumn{9}{|l|}{5} \\
\hline \multicolumn{9}{|l|}{6} \\
\hline \multicolumn{9}{|l|}{7} \\
\hline \multicolumn{9}{|l|}{8} \\
\hline \multicolumn{9}{|l|}{9} \\
\hline \multicolumn{9}{|l|}{10} \\
\hline \multicolumn{9}{|l|}{11} \\
\hline \multicolumn{9}{|l|}{13} \\
\hline \multicolumn{9}{|l|}{15} \\
\hline \multicolumn{9}{|l|}{16} \\
\hline \multicolumn{9}{|l|}{17} \\
\hline \multicolumn{9}{|l|}{18} \\
\hline \multicolumn{9}{|l|}{19} \\
\hline \multicolumn{9}{|l|}{20} \\
\hline \multirow{2}{*}{\multicolumn{9}{|c|}{$\frac{21}{22}$}} \\
\hline \multirow{2}{*}{\multicolumn{9}{|c|}{$\frac{22}{23}$}} \\
\hline \multirow{2}{*}{\multicolumn{9}{|c|}{24}} \\
\hline & & & & & & & & \\
\hline \multicolumn{9}{|l|}{25} \\
\hline \multicolumn{9}{|l|}{26} \\
\hline \multicolumn{9}{|l|}{27} \\
\hline \multicolumn{9}{|l|}{28} \\
\hline \multicolumn{9}{|l|}{29} \\
\hline \multicolumn{9}{|l|}{30} \\
\hline \multicolumn{9}{|l|}{31} \\
\hline \multicolumn{9}{|l|}{32} \\
\hline 33 & & & & & & & & \\
\hline
\end{tabular}

TWRS LAW Formulation (Muller and Pegg 1998)

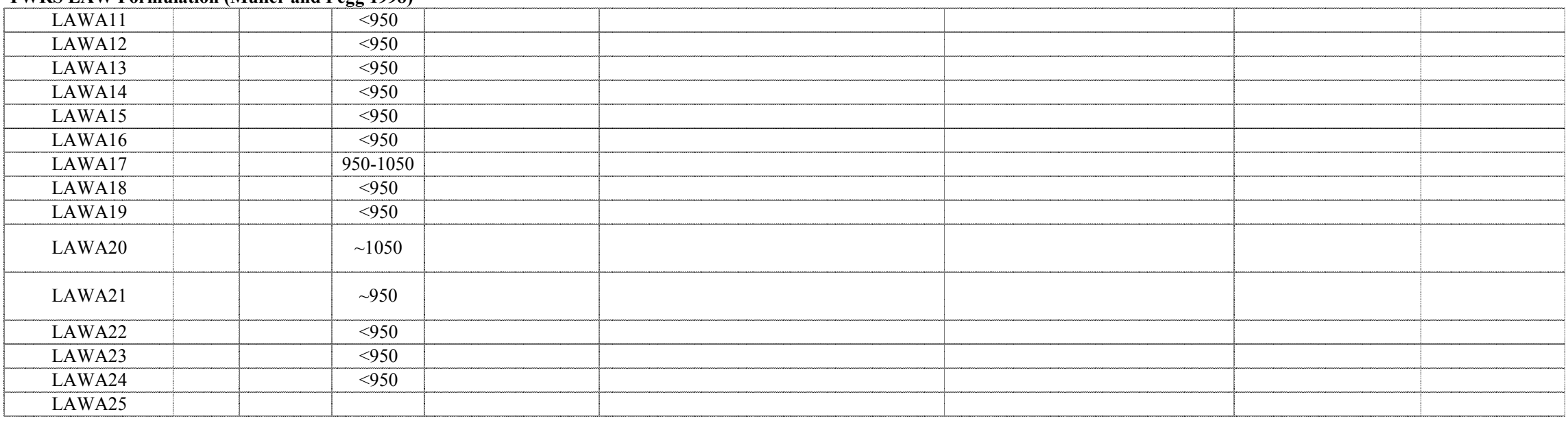


Appendix A. Database - mass fraction

Canonical Correlation (Ramsey) (Oksoy et al. 1994)

\begin{tabular}{|c|c|c|c|c|c|}
\hline Glass ID & $\begin{array}{c}\text { CCC } \\
\text { Visual/OM }\end{array}$ & $\begin{array}{c}\text { CCC } \\
\text { SEM/EDS or TEM }\end{array}$ & $\begin{array}{l}\text { CCC } \\
\text { XRD }\end{array}$ & $\begin{array}{c}\text { Heat Treated } \\
\text { Visual/OM }\end{array}$ & $\begin{array}{c}\text { Heat Treated } \\
\text { SEM/EDS or TEM }\end{array}$ \\
\hline \multicolumn{6}{|l|}{1} \\
\hline \multicolumn{6}{|l|}{2} \\
\hline \multirow{2}{*}{\multicolumn{6}{|c|}{$\frac{4}{5}$}} \\
\hline \multirow{2}{*}{\multicolumn{6}{|c|}{$\frac{5}{6}$}} \\
\hline & & & & & \\
\hline \multicolumn{6}{|l|}{7} \\
\hline \multicolumn{6}{|l|}{8} \\
\hline \multicolumn{6}{|l|}{9} \\
\hline \multicolumn{6}{|l|}{10} \\
\hline \multicolumn{6}{|l|}{11} \\
\hline \multicolumn{6}{|l|}{13} \\
\hline \multicolumn{6}{|l|}{15} \\
\hline \multicolumn{6}{|l|}{16} \\
\hline \multicolumn{6}{|l|}{17} \\
\hline \multicolumn{6}{|l|}{18} \\
\hline \multicolumn{6}{|l|}{19} \\
\hline \multicolumn{6}{|l|}{20} \\
\hline \multicolumn{6}{|l|}{21} \\
\hline \multicolumn{6}{|l|}{22} \\
\hline \multicolumn{6}{|l|}{23} \\
\hline \multicolumn{6}{|l|}{24} \\
\hline \multicolumn{6}{|l|}{25} \\
\hline \multicolumn{6}{|l|}{26} \\
\hline \multicolumn{6}{|l|}{27} \\
\hline \multicolumn{6}{|l|}{28} \\
\hline \multicolumn{6}{|l|}{29} \\
\hline \multicolumn{6}{|l|}{30} \\
\hline \multicolumn{6}{|l|}{31} \\
\hline 32 & & & & & \\
\hline 33 & & & & & \\
\hline
\end{tabular}

TWRS LAW Formulation (Muller and Pegg 1998)

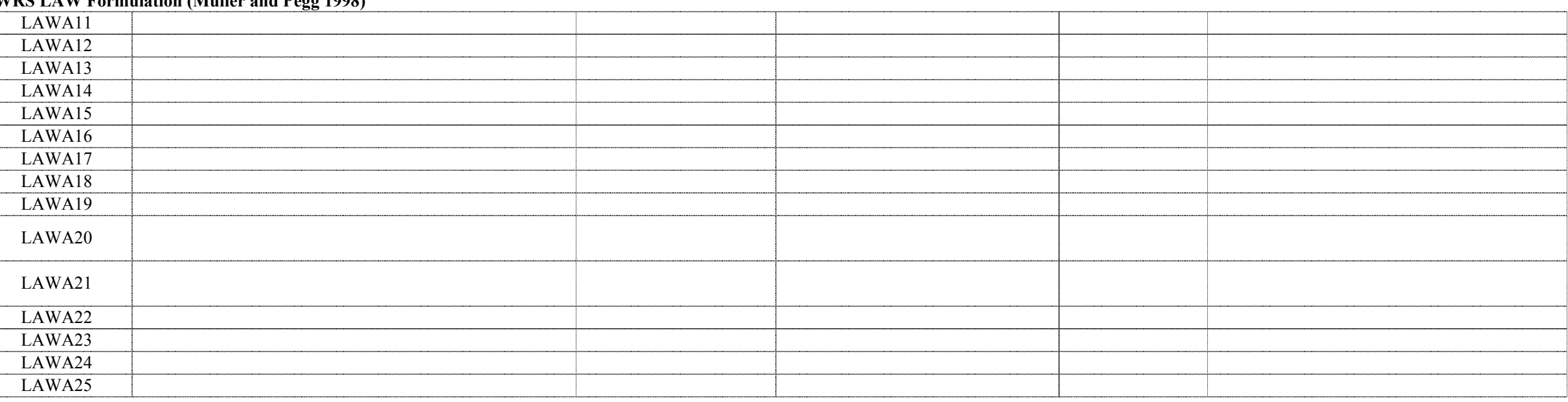


Canonical Correlation (Ramsey) (Oksoy et al. 1994)

\begin{tabular}{|c|c|c|c|c|c|c|c|c|c|c|c|c|c|c|c|}
\hline Glass ID & $\begin{array}{c}\text { Heat Treated } \\
\text { XRD }\end{array}$ & $\begin{array}{l}\text { Density } \\
\left(\mathrm{g} / \mathrm{cm}^{3}\right)\end{array}$ & $\begin{array}{c}\text { Fulc Visc } \\
\mathrm{A}\end{array}$ & $\begin{array}{c}\text { Fulc Visc } \\
\text { B }\end{array}$ & $\begin{array}{c}\text { Fulc Visc } \\
\text { To } \\
\end{array}$ & $\begin{array}{c}\mathrm{FV} 1150^{\circ} \mathrm{C} \\
(\mathrm{Pa} \cdot \mathrm{s})\end{array}$ & \begin{tabular}{|c|} 
Arrh Visc \\
$\mathrm{A}$
\end{tabular} & \begin{tabular}{|c|} 
Arrh Visc \\
B
\end{tabular} & $\begin{array}{l}1150^{\circ} \mathrm{C} \\
\left(\mathrm{D}_{\text {a.col }}\right.\end{array}$ & $\begin{array}{c}\mathrm{T}\left({ }^{\circ} \mathrm{C}\right) \text { at } \\
2 \mathrm{~Pa} \cdot \mathrm{s}\end{array}$ & \begin{tabular}{|c|}
$\mathrm{T}\left({ }^{\circ} \mathrm{C}\right)$ at \\
$5 \mathrm{~Pa} \cdot \mathrm{s}$ \\
\end{tabular} & $\begin{array}{l}\mathrm{T}\left({ }^{\circ} \mathrm{C}\right) \text { at } \\
10 \mathrm{~Pa} \cdot \mathrm{s}\end{array}$ & $\begin{array}{l}\mathrm{T} 1 \\
\left({ }^{\circ} \mathrm{C}\right) \\
\end{array}$ & $\begin{array}{c}\mathrm{V} 1 \\
(\mathrm{~Pa} \cdot \mathrm{s})\end{array}$ & $\begin{array}{l}\mathrm{T} 2 \\
\left({ }^{\circ} \mathrm{C}\right) \\
\end{array}$ \\
\hline \multicolumn{16}{|l|}{1} \\
\hline \multirow{2}{*}{\multicolumn{16}{|c|}{$\frac{2}{4}$}} \\
\hline 4 & & & & & & & & & & & & & & & \\
\hline \multicolumn{16}{|l|}{5} \\
\hline \multicolumn{16}{|l|}{6} \\
\hline \multicolumn{16}{|l|}{7} \\
\hline \multicolumn{16}{|l|}{8} \\
\hline \multicolumn{16}{|l|}{9} \\
\hline \multicolumn{16}{|l|}{10} \\
\hline \multicolumn{16}{|l|}{11} \\
\hline \multicolumn{16}{|l|}{13} \\
\hline \multicolumn{16}{|l|}{15} \\
\hline \multicolumn{16}{|l|}{16} \\
\hline \multicolumn{16}{|l|}{17} \\
\hline \multicolumn{16}{|l|}{18} \\
\hline \multicolumn{16}{|l|}{19} \\
\hline \multicolumn{16}{|l|}{20} \\
\hline \multicolumn{16}{|l|}{21} \\
\hline \multicolumn{16}{|l|}{22} \\
\hline \multicolumn{16}{|l|}{23} \\
\hline \multicolumn{16}{|l|}{24} \\
\hline \multicolumn{16}{|l|}{25} \\
\hline \multicolumn{16}{|l|}{26} \\
\hline \multicolumn{16}{|l|}{27} \\
\hline \multicolumn{16}{|l|}{28} \\
\hline \multicolumn{16}{|l|}{29} \\
\hline \multicolumn{16}{|l|}{30} \\
\hline 31 & & & & & & & & & & & & & & & \\
\hline 32 & & & & & & & & & & & & & & & \\
\hline 33 & & & & & & & & & & & & & & & \\
\hline
\end{tabular}

TWRS LAW Formulation (Muller and Pegg 1998)

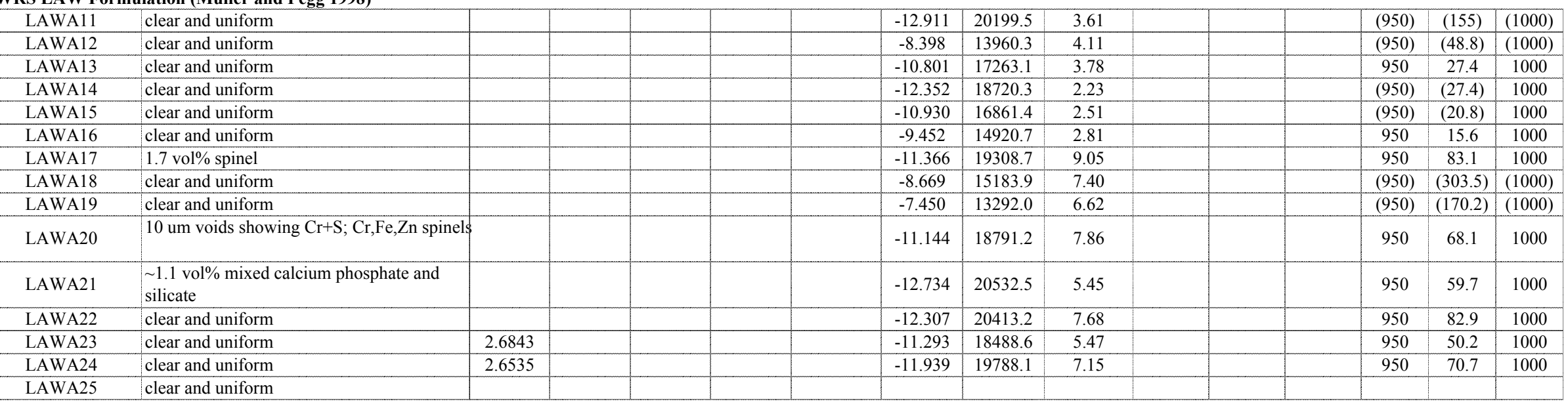


Canonical Correlation (Ramsey) (Oksoy et al. 1994)

\begin{tabular}{|c|c|c|c|c|c|c|c|c|c|c|c|c|c|c|c|c|c|c|c|c|c|}
\hline Glass ID & $\begin{array}{c}\mathrm{V} 2 \\
(\mathrm{~Pa} \cdot \mathrm{s})\end{array}$ & $\begin{array}{c}\mathrm{T} 3 \\
\left({ }^{\circ} \mathrm{C}\right) \\
\end{array}$ & $\begin{array}{c}\mathrm{V} 3 \\
(\mathrm{~Pa} \cdot \mathrm{s})\end{array}$ & $\begin{array}{c}\mathrm{T} 4 \\
\left({ }^{\circ} \mathrm{C}\right)\end{array}$ & $\begin{array}{c}\mathrm{V} 4 \\
(\mathrm{~Pa} \cdot \mathrm{s})\end{array}$ & $\begin{array}{c}\mathrm{T} 5 \\
\left({ }^{\circ} \mathrm{C}\right)\end{array}$ & $\begin{array}{c}\text { V5 } \\
(\mathrm{Pa} \cdot \mathrm{s})\end{array}$ & $\begin{array}{c}\mathrm{T} 6 \\
\left({ }^{\circ} \mathrm{C}\right)\end{array}$ & $\begin{array}{c}\mathrm{V} 6 \\
(\mathrm{~Pa} \cdot \mathrm{s})\end{array}$ & $\begin{array}{c}\mathrm{T} 7 \\
\left({ }^{\circ} \mathrm{C}\right)\end{array}$ & $\begin{array}{c}\mathrm{V} 7 \\
(\mathrm{~Pa} \cdot \mathrm{s})\end{array}$ & $\begin{array}{c}\mathrm{T} 8 \\
\left({ }^{\circ} \mathrm{C}\right)\end{array}$ & $\begin{array}{c}\mathrm{V} 8 \\
(\mathrm{~Pa} \cdot \mathrm{s})\end{array}$ & $\begin{array}{c}\mathrm{T} 9 \\
\left({ }^{\circ} \mathrm{C}\right)\end{array}$ & $\begin{array}{c}\mathrm{V} 9 \\
(\mathrm{~Pa} \cdot \mathrm{s})\end{array}$ & $\begin{array}{l}\mathrm{T} 10 \\
\left({ }^{\circ} \mathrm{C}\right)\end{array}$ & $\begin{array}{c}\mathrm{V} 10 \\
(\mathrm{~Pa} \cdot \mathrm{s})\end{array}$ & $\begin{array}{l}\mathrm{T} 11 \\
\left({ }^{\circ} \mathrm{C}\right)\end{array}$ & $\begin{array}{c}\mathrm{V} 11 \\
(\mathrm{~Pa} \cdot \mathrm{s})\end{array}$ & $\begin{array}{l}\mathrm{T} 12 \\
\left({ }^{\circ} \mathrm{C}\right)\end{array}$ & $\begin{array}{c}\mathrm{V} 12 \\
(\mathrm{~Pa} \cdot \mathrm{s})\end{array}$ \\
\hline \multicolumn{22}{|l|}{1} \\
\hline \multirow{2}{*}{\multicolumn{22}{|c|}{$\frac{2}{4}$}} \\
\hline & & & & & & & & & & & & & & & & & & & & & \\
\hline \multicolumn{22}{|l|}{5} \\
\hline \multicolumn{22}{|l|}{6} \\
\hline \multicolumn{22}{|l|}{7} \\
\hline \multicolumn{22}{|l|}{8} \\
\hline \multicolumn{22}{|l|}{9} \\
\hline \multicolumn{22}{|l|}{10} \\
\hline \multirow{2}{*}{\multicolumn{22}{|c|}{$\frac{11}{13}$}} \\
\hline \multirow{2}{*}{\multicolumn{22}{|c|}{$\begin{array}{l}13 \\
15\end{array}$}} \\
\hline 15 & & & & & & & & & & & & & & & & & & & & & \\
\hline \multicolumn{22}{|l|}{16} \\
\hline \multicolumn{22}{|l|}{17} \\
\hline \multicolumn{22}{|l|}{18} \\
\hline \multicolumn{22}{|l|}{19} \\
\hline \multicolumn{22}{|l|}{20} \\
\hline \multicolumn{22}{|l|}{21} \\
\hline \multicolumn{22}{|l|}{22} \\
\hline \multicolumn{22}{|l|}{23} \\
\hline \multicolumn{22}{|l|}{24} \\
\hline \multicolumn{22}{|l|}{25} \\
\hline \multicolumn{22}{|l|}{26} \\
\hline \multicolumn{22}{|l|}{27} \\
\hline \multicolumn{22}{|l|}{28} \\
\hline 29 & & & & & & & & & & & & & & & & & & & & & \\
\hline 30 & & & & & & & & & & & & & & & & & & & & & \\
\hline 31 & & & & & & & & & & & & & & & & & & & & & \\
\hline 32 & & & & & & & & & & & & & & & & & & & & & \\
\hline 33 & & & & & & & & & & & & & & & & & & & & & \\
\hline
\end{tabular}

TWRS LAW Formulation (Muller and Pegg 1998)

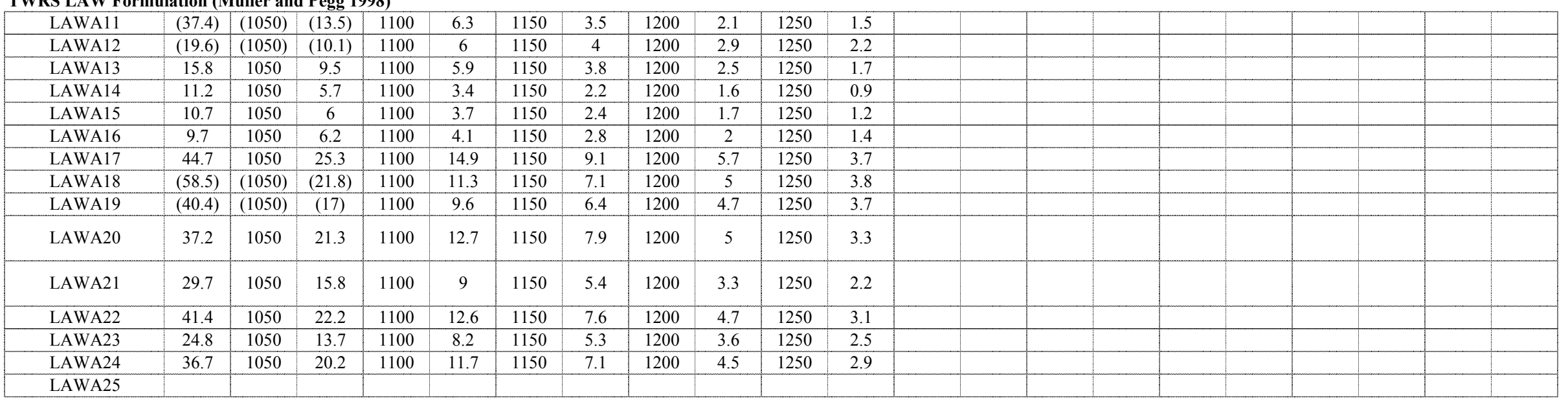


Appendix A. Database - mass fraction

Canonical Correlation (Ramsey) (Oksoy et al. 1994)

\begin{tabular}{|c|c|c|c|c|c|c|c|c|c|c|c|c|c|c|c|c|}
\hline Glass ID & $\begin{array}{l}\mathrm{T} 13 \\
\left({ }^{\circ} \mathrm{C}\right) \\
\end{array}$ & $\begin{array}{c}\mathrm{V} 13 \\
(\mathrm{~Pa} \cdot \mathrm{s})\end{array}$ & $\begin{array}{l}\mathrm{T} 14 \\
\left({ }^{\circ} \mathrm{C}\right) \\
\end{array}$ & $\begin{array}{c}\mathrm{V} 14 \\
(\mathrm{~Pa} \cdot \mathrm{s}) \\
\end{array}$ & $\begin{array}{l}\text { Q PCT } \\
\text { B }\left(\mathrm{g} / \mathrm{m}^{2}\right)\end{array}$ & $\begin{array}{c}\text { Q PCT } \\
\mathrm{Li}\left(\mathrm{g} / \mathrm{m}^{2}\right)\end{array}$ & $\begin{array}{c}\text { Q PCT } \\
\mathrm{Na}\left(\mathrm{g} / \mathrm{m}^{2}\right) \\
\end{array}$ & $\begin{array}{c}\text { Q PCT } \\
\mathrm{Si}\left(\mathrm{g} / \mathrm{m}^{2}\right)\end{array}$ & $\begin{array}{c}\text { Q PCT } \\
\mathrm{pH}\end{array}$ & $\begin{array}{c}\text { CCC PCT } \\
\mathrm{B}\left(\mathrm{g} / \mathrm{m}^{2}\right) \\
\end{array}$ & $\begin{array}{l}\text { CCC PCT } \\
\mathrm{Li}\left(\mathrm{g} / \mathrm{m}^{2}\right)\end{array}$ & $\begin{array}{l}\text { CCC PCT } \\
\mathrm{Na}\left(\mathrm{g} / \mathrm{m}^{2}\right) \\
\end{array}$ & $\begin{array}{c}\text { CCC PCT } \\
\mathrm{Si}\left(\mathrm{g} / \mathrm{m}^{2}\right)\end{array}$ & $\begin{array}{c}\text { CCC PCT } \\
\mathrm{pH}\end{array}$ & $\begin{array}{l}\text { Q PCT B at } \\
20^{\circ} \mathrm{C}\left(\mathrm{g} / \mathrm{m}^{2}\right)\end{array}$ & $\begin{array}{l}\text { Q PCT Li at } \\
20^{\circ} \mathrm{C}\left(\mathrm{g} / \mathrm{m}^{2}\right)\end{array}$ \\
\hline 1 & & & & & 0.555 & & 0.239 & 0.093 & 9.05 & & & & & & & \\
\hline 2 & & & & & 0.075 & & 0.187 & 0.045 & 10.92 & & & & & & & \\
\hline 4 & & & & & 0.368 & & 0.685 & 0.187 & 11.76 & & & & & & & \\
\hline 5 & & & & & 0.361 & & 0.364 & 0.179 & 10 & & & & & & & \\
\hline 6 & & & & & 0.817 & & 0.512 & 0.162 & 9.53 & & & & & & & \\
\hline 7 & & & & & 29.834 & & 25.044 & 15.029 & 11.39 & & & & & & & \\
\hline 8 & & & & & 0.257 & & 0.597 & 0.108 & 11.45 & & & & & & & \\
\hline 9 & & & & & 38.709 & & 27.827 & 16.036 & 12.63 & & & & & & & \\
\hline 10 & & & & & 1.702 & & 1.508 & 0.71 & 11.95 & & & & & & & \\
\hline 11 & & & & & 0.254 & & 0.286 & 0.047 & 10.18 & & & & & & & \\
\hline 13 & & & & & 2.122 & & 1.133 & 0.085 & 10.26 & & & & & & & \\
\hline 15 & & & & & 0.174 & & 0.762 & 0.126 & 12 & & & & & & & \\
\hline 16 & & & & & 0.493 & & 0.62 & 0.218 & 11.69 & & & & & & & \\
\hline 17 & & & & & 0.184 & & 0.481 & 0.078 & 11.18 & & & & & & & \\
\hline 18 & & & & & 31.162 & & 22.973 & 7.624 & 12.01 & & & & & & & \\
\hline 19 & & & & & 5.016 & & 2.621 & 0.367 & 10.97 & & & & & & & \\
\hline 20 & & & & & 0.77 & & 1.748 & 0.361 & 12.32 & & & & & & & \\
\hline 21 & & & & & 0.275 & & 0.378 & 0.126 & 11.06 & & & & & & & \\
\hline 22 & & & & & 0.165 & & 0.24 & 0.084 & 10.75 & & & & & & & \\
\hline 23 & & & & & 0.152 & & 0.205 & 0.086 & 10.47 & & & & & & & \\
\hline 24 & & & & & 0.436 & & 0.531 & 0.128 & 11.16 & & & & & & & \\
\hline 25 & & & & & 0.417 & & 0.353 & 0.103 & 10.05 & & & & & & & \\
\hline 26 & & & & & 0.254 & & 0.472 & 0.123 & 11.46 & & & & & & & \\
\hline 27 & & & & & 0.137 & & 0.277 & 0.058 & 11.04 & & & & & & & \\
\hline 28 & & & & & 0.307 & & 0.312 & 0.134 & 10.65 & & & & & & & \\
\hline 29 & & & & & 0.276 & & 0.382 & 0.12 & 10.95 & & & & & & & \\
\hline 30 & & & & & 0.847 & & 0.817 & 0.138 & 11.47 & & & & & & & \\
\hline 31 & & & & & 0.31 & & 0.469 & 0.147 & 11.49 & & & & & & & \\
\hline 32 & & & & & 0.123 & & 0.202 & 0.05 & 10.42 & & & & & & & \\
\hline 33 & & & & & 0.168 & & 0.237 & 0.088 & 10.72 & & & & & & & \\
\hline
\end{tabular}

TWRS LAW Formulation (Muller and Pegg 1998)

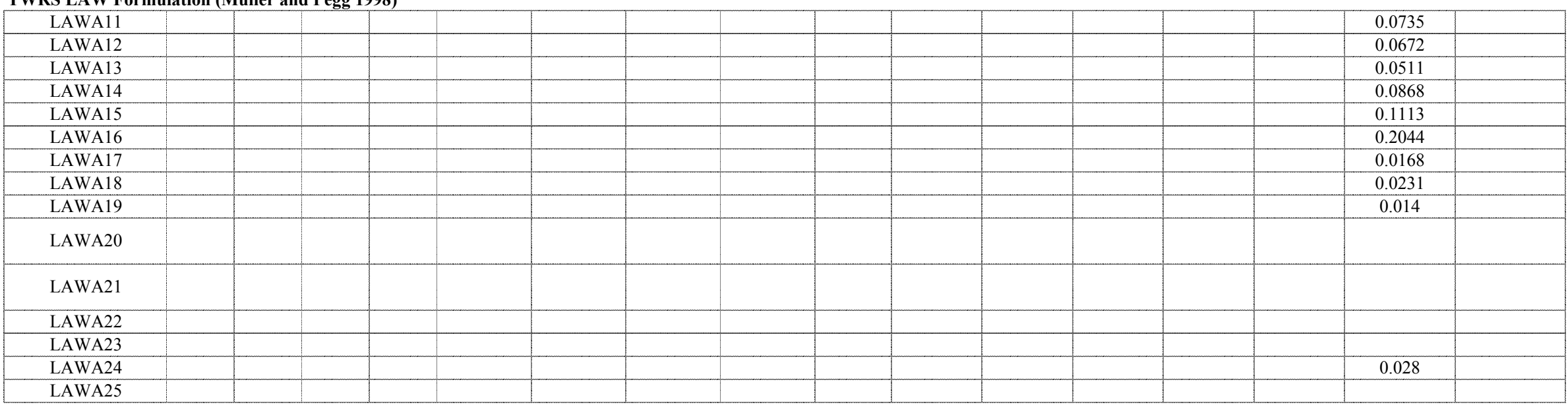


Appendix A. Database - mass fraction

Canonical Correlation (Ramsey) (Oksoy et al. 1994)

\begin{tabular}{|c|c|c|c|c|c|c|c|c|c|c|c|c|}
\hline Glass ID & $\begin{array}{l}\text { Q PCT Na at } \\
20^{\circ} \mathrm{C}\left(\mathrm{g} / \mathrm{m}^{2}\right)\end{array}$ & $\begin{array}{l}\text { Q PCT Si at } \\
20^{\circ} \mathrm{C}\left(\mathrm{g} / \mathrm{m}^{2}\right)\end{array}$ & $\begin{array}{c}\mathrm{QpH} \\
\text { at } 20^{\circ} \mathrm{C}\end{array}$ & $\begin{array}{c}\text { TCLP Ag } \\
(\mathrm{ppm})\end{array}$ & $\begin{array}{c}\text { TCLP As } \\
\text { (ppm) }\end{array}$ & $\begin{array}{c}\text { TCLP Ba } \\
(\mathrm{ppm})\end{array}$ & $\begin{array}{c}\text { TCLP Cd } \\
(\mathrm{ppm})\end{array}$ & $\begin{array}{c}\text { TCLP Cr } \\
(\mathrm{ppm})\end{array}$ & $\begin{array}{c}\text { TCLP Ni } \\
(\mathrm{ppm})\end{array}$ & $\begin{array}{c}\text { TCLP Pb } \\
(\mathrm{ppm})\end{array}$ & $\begin{array}{c}\text { TCLP Se } \\
(\mathrm{ppm})\end{array}$ & $\begin{array}{c}\text { TCLP Zn } \\
(\mathrm{ppm})\end{array}$ \\
\hline \multicolumn{13}{|c|}{ 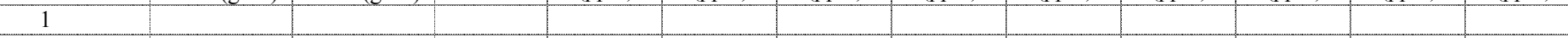 } \\
\hline \multicolumn{13}{|l|}{2} \\
\hline \multicolumn{13}{|l|}{4} \\
\hline \multicolumn{13}{|l|}{5} \\
\hline \multicolumn{13}{|l|}{6} \\
\hline \multicolumn{13}{|l|}{7} \\
\hline \multicolumn{13}{|l|}{8} \\
\hline \multicolumn{13}{|l|}{9} \\
\hline \multicolumn{13}{|l|}{10} \\
\hline \multicolumn{13}{|l|}{11} \\
\hline \multicolumn{13}{|l|}{13} \\
\hline \multicolumn{13}{|l|}{15} \\
\hline \multicolumn{13}{|l|}{16} \\
\hline \multicolumn{13}{|l|}{17} \\
\hline \multicolumn{13}{|l|}{18} \\
\hline \multicolumn{13}{|l|}{19} \\
\hline \multicolumn{13}{|l|}{20} \\
\hline \multicolumn{13}{|l|}{21} \\
\hline \multicolumn{13}{|l|}{22} \\
\hline \multicolumn{13}{|l|}{23} \\
\hline \multicolumn{13}{|l|}{24} \\
\hline \multicolumn{13}{|l|}{25} \\
\hline \multicolumn{13}{|l|}{26} \\
\hline \multicolumn{13}{|l|}{27} \\
\hline \multicolumn{13}{|l|}{28} \\
\hline \multicolumn{13}{|l|}{29} \\
\hline \multicolumn{13}{|l|}{30} \\
\hline 31 & & & & & & & & & & & & \\
\hline 32 & & & & & & & & & & & & \\
\hline 33 & & & & & & & & & & & & \\
\hline
\end{tabular}

TWRS LAW Formulation (Muller and Pegg 1998)

\begin{tabular}{|c|c|c|c|c|c|c|c|c|c|}
\hline LAWA11 & 0.0833 & 0.0252 & 9.35 & & & & & & \\
\hline LAWA12 & 0.0672 & 0.0224 & 9.23 & & & & & & \\
\hline LAWA13 & 0.0658 & 0.014 & 9.36 & & & & & & \\
\hline LAWA14 & 0.0686 & 0.0168 & 9.35 & & & & & & \\
\hline LAWA15 & 0.0882 & 0.0266 & 9.37 & & & & & & \\
\hline LAWA16 & 0.182 & 0.0476 & 9.26 & & & & & & \\
\hline LAWA17 & 0.042 & 0.0091 & 9.3 & & & & & & \\
\hline LAWA18 & 0.056 & 0.0091 & 8.17 & & & & & & \\
\hline LAWA19 & 0.0469 & 0.0084 & 8.86 & & & & & & \\
\hline LAWA20 & 0.0532 & 0.0105 & 8.56 & & & & & & \\
\hline LAWA21 & 1.2061 & 0.1638 & 8.58 & & & & & & \\
\hline LAWA22 & 0.0378 & 0.0084 & 8.11 & & & & & & \\
\hline LAWA23 & 0.0378 & 0.0077 & 8.15 & $<0.98$ & 0.003 & $<0.0055$ & $<0.0079$ & 0.078 & 1.29 \\
\hline LAWA24 & 0.042 & 0.0098 & 8.32 & $<0.92$ & 0.275 & 0.15 & 0.097 & 0.078 & 2.35 \\
\hline LAWA25 & 0.0875 & 0.0105 & 9.13 & & & & & & \\
\hline
\end{tabular}


TWRS LAW Formulation (Muller and Pegg 1998)

\begin{tabular}{|c|c|c|c|c|c|c|c|c|c|c|c|c|c|c|c|c|c|c|c|c|c|}
\hline Glass ID & $\begin{array}{c}\mathrm{A} 12 \mathrm{O} 3 \\
-\mathrm{t}\end{array}$ & $\begin{array}{c}\mathrm{B} 2 \mathrm{O} 3 \\
-\mathrm{t}\end{array}$ & $\begin{array}{c}\mathrm{CaO} \\
-\mathrm{t}\end{array}$ & $\begin{array}{c}\mathrm{Fe} 2 \mathrm{O} 3 \\
-\mathrm{t}\end{array}$ & $\begin{array}{c}\mathrm{FeO} \\
-\mathrm{t}\end{array}$ & $\begin{array}{c}\mathrm{K} 2 \mathrm{O} \\
-\mathrm{t}\end{array}$ & $\begin{array}{c}\mathrm{Li} 2 \mathrm{O} \\
-\mathrm{t}\end{array}$ & $\begin{array}{c}\mathrm{MgO} \\
-\mathrm{t}\end{array}$ & $\begin{array}{c}\mathrm{Na} 2 \mathrm{O} \\
-\mathrm{t}\end{array}$ & $\begin{array}{c}\mathrm{NiO} \\
-\mathrm{t}\end{array}$ & $\begin{array}{c}\mathrm{P} 2 \mathrm{O} 5 \\
-\mathrm{t}\end{array}$ & $\begin{array}{c}\mathrm{SiO} 2 \\
-\mathrm{t}\end{array}$ & $\begin{array}{c}\mathrm{ZrO} 2 \\
-\mathrm{t}\end{array}$ & $\begin{array}{c}\mathrm{Ag} 2 \mathrm{O} \\
-\mathrm{t}\end{array}$ & $\begin{array}{c}\mathrm{As} 2 \mathrm{O} 3 \\
-\mathrm{t}\end{array}$ & $\begin{array}{c}\mathrm{BaO} \\
-\mathrm{t}\end{array}$ & $\begin{array}{c}\mathrm{Bi} 2 \mathrm{O} 3 \\
-\mathrm{t}\end{array}$ & $\begin{array}{c}\mathrm{Br} \\
-\mathrm{t}\end{array}$ & $\begin{array}{c}\mathrm{CdO} \\
-\mathrm{t}\end{array}$ & $\begin{array}{c}\mathrm{Ce} 2 \mathrm{O} 3 \\
-\mathrm{t}\end{array}$ & $\begin{array}{c}\mathrm{CeO} 2 \\
-\mathrm{t}\end{array}$ \\
\hline LAWA25H & 0.1067 & 0.0181 & 0.0168 & 0.0570 & & 0.0409 & 0.0000 & 0.0102 & 0.2551 & 0.0017 & 0.0101 & 0.3967 & 0.0283 & & & 0.0001 & & & \begin{tabular}{|l|}
0.0039 \\
\end{tabular} & & \\
\hline LAWA26 & 0.1048 & 0.0000 & 0.0165 & 0.0393 & & 0.0402 & 0.0111 & 0.0000 & 0.2506 & 0.0017 & 0.0199 & 0.4231 & 0.0278 & & & 0.0001 & & & 0.0038 & & \\
\hline LAWA26H & 0.1067 & 0.0000 & 0.0168 & 0.0400 & & 0.0409 & 0.0113 & 0.0000 & 0.2551 & 0.0017 & 0.0101 & 0.4307 & 0.0283 & & & 0.0001 & & & 0.0039 & & \\
\hline LAWB11 & 0.0642 & 0.0978 & 0.1032 & 0.0834 & & 0.0205 & 0.0000 & 0.0326 & 0.0751 & 0.0005 & 0.0223 & 0.4237 & 0.0407 & & & 0.0000 & & & 0.0012 & & \\
\hline LAWB12 & 0.0642 & 0.0978 & 0.0543 & 0.0834 & & 0.0205 & 0.0000 & 0.0815 & 0.0751 & 0.0005 & 0.0223 & 0.4237 & 0.0407 & & & 0.0000 & & & 0.0012 & & \\
\hline LAWB13 & 0.0642 & 0.0978 & 0.0543 & 0.0834 & & 0.0205 & 0.0000 & 0.0815 & 0.0751 & 0.0005 & 0.0223 & 0.3830 & 0.0407 & & & 0.0000 & & & 0.0012 & & \\
\hline LAWB14 & 0.0642 & 0.0978 & 0.0951 & 0.0834 & & 0.0205 & 0.0000 & 0.0815 & 0.0751 & 0.0005 & 0.0223 & 0.3830 & 0.0407 & & & 0.0000 & & & 0.0012 & & \\
\hline LAWB15 & 0.0642 & 0.1222 & 0.1032 & 0.0834 & & 0.0205 & 0.0163 & 0.0326 & 0.0751 & 0.0005 & 0.0223 & 0.3830 & 0.0407 & & & 0.0000 & & & 0.0012 & & \\
\hline LAWB16 & 0.0642 & 0.0978 & 0.0054 & 0.0834 & & 0.0205 & 0.0000 & 0.0326 & 0.0751 & 0.0005 & 0.0223 & 0.4237 & 0.0407 & & & 0.0000 & & & 0.0012 & & \\
\hline LAWB17 & 0.0642 & 0.0652 & 0.0543 & 0.0834 & & 0.0205 & 0.0407 & 0.0326 & 0.0751 & 0.0005 & 0.0223 & 0.4237 & 0.0407 & & & 0.0000 & & & 0.0012 & & \\
\hline LAWB18 & 0.0642 & 0.0652 & 0.0543 & 0.0834 & & 0.0205 & 0.0407 & 0.0326 & 0.0751 & 0.0005 & 0.0223 & 0.4237 & 0.0407 & & & 0.0000 & & & 0.0012 & & \\
\hline LAWB19 & 0.0988 & 0.0599 & 0.0364 & 0.0834 & & 0.0205 & 0.0204 & 0.0204 & 0.1199 & 0.0005 & 0.0223 & 0.4189 & 0.0302 & & & 0.0000 & & & 0.0012 & & \\
\hline LAWB20 & 0.0887 & 0.0599 & 0.0364 & 0.0834 & & 0.0205 & 0.0302 & 0.0204 & 0.1301 & 0.0005 & 0.0223 & 0.4091 & 0.0302 & & & 0.0000 & & & 0.0012 & & \\
\hline LAWB21 & 0.0887 & 0.0599 & 0.0364 & 0.0631 & & 0.0205 & 0.0302 & 0.0204 & 0.1504 & 0.0005 & 0.0223 & 0.4091 & 0.0302 & & & 0.0000 & & & 0.0012 & & \\
\hline LAWB22 & 0.0887 & 0.0599 & 0.0364 & 0.0631 & & 0.0205 & 0.0000 & 0.0204 & 0.1806 & 0.0005 & 0.0223 & 0.4091 & 0.0302 & & & 0.0000 & & & 0.0012 & & \\
\hline LAWB23 & 0.0901 & 0.0609 & 0.0370 & 0.0641 & & 0.0209 & 0.0306 & 0.0207 & 0.1529 & 0.0006 & 0.0227 & 0.4157 & 0.0306 & & & 0.0000 & & & 0.0013 & & \\
\hline LAWB24 & 0.0896 & 0.0605 & 0.0368 & 0.0637 & & 0.0208 & 0.0305 & 0.0206 & 0.1521 & 0.0005 & 0.0226 & 0.4135 & 0.0305 & & & 0.0000 & & & 0.0013 & & \\
\hline LAWB25 & 0.0891 & 0.0602 & 0.0366 & 0.0634 & & 0.0206 & 0.0303 & 0.0205 & 0.1512 & 0.0005 & 0.0225 & 0.4113 & 0.0303 & & & 0.0000 & & & 0.0013 & & \\
\hline LAWB26 & 0.0797 & 0.0807 & 0.0454 & 0.0635 & & 0.0205 & 0.0407 & 0.0228 & 0.0852 & 0.0005 & 0.0223 & 0.4319 & 0.0302 & & & 0.0000 & & & 0.0012 & & \\
\hline LAWB27 & 0.0797 & 0.0807 & 0.0454 & 0.0635 & & 0.0205 & 0.0302 & 0.0228 & 0.1052 & 0.0005 & 0.0223 & 0.4225 & 0.0302 & & & 0.0000 & & & \begin{tabular}{|l|}
0.0012 \\
\end{tabular} & & \\
\hline LAWB28 & 0.0797 & 0.0807 & 0.0454 & 0.0635 & & 0.0205 & 0.0200 & 0.0228 & 0.1252 & 0.0005 & 0.0223 & 0.4127 & 0.0302 & & & 0.0000 & & & 0.0012 & & \\
\hline LAWB29 & 0.0803 & 0.0807 & 0.0703 & 0.0803 & & 0.0036 & 0.0408 & 0.0300 & 0.1000 & 0.0000 & 0.0001 & 0.4393 & 0.0304 & & & 0.0000 & & & 0.0000 & & \\
\hline LAWC1 & 0.0993 & 0.0610 & 0.0206 & 0.0967 & & 0.0548 & 0.0000 & 0.0000 & 0.2004 & 0.0014 & 0.0174 & 0.3540 & 0.0305 & & & 0.0001 & & & 0.0033 & & \\
\hline LAWC2 & 0.0993 & 0.0610 & 0.0206 & 0.0479 & & 0.0548 & 0.0000 & 0.0000 & 0.2004 & 0.0014 & 0.0174 & 0.3540 & 0.0305 & & & 0.0001 & & & 0.0033 & & \\
\hline LAWC3 & 0.0993 & 0.0610 & 0.0206 & 0.0479 & & 0.0548 & 0.0000 & 0.0000 & 0.2004 & 0.0014 & 0.0174 & 0.3540 & 0.0305 & & & 0.0001 & & & 0.0033 & & \\
\hline LAWC4 & 0.0961 & 0.0300 & 0.1031 & 0.0647 & & 0.0505 & 0.0000 & 0.0000 & 0.1845 & 0.0013 & 0.0080 & 0.3999 & 0.0300 & & & 0.0001 & & & 0.0031 & & \\
\hline LAWC5 & 0.0916 & 0.0290 & 0.0682 & 0.0601 & & 0.0548 & 0.0000 & 0.0000 & 0.2004 & 0.0014 & 0.0174 & 0.3869 & 0.0281 & & & 0.0001 & & & 0.0033 & & \\
\hline LAWPC1 & 0.0987 & 0.0396 & 0.0271 & 0.0632 & & 0.0401 & 0.0000 & 0.0198 & 0.2000 & 0.0011 & 0.0128 & 0.4094 & 0.0297 & & & 0.0001 & & & 0.0024 & & \\
\hline LAWPC2 & 0.0987 & 0.0396 & 0.0271 & 0.0632 & & 0.0401 & 0.0000 & 0.0198 & 0.2000 & 0.0011 & 0.0128 & 0.3797 & 0.0297 & & & 0.0001 & & & 0.0024 & & \\
\hline LAWPC3 & 0.0987 & 0.0396 & 0.0271 & 0.0632 & & 0.0401 & 0.0000 & 0.0198 & 0.2000 & 0.0011 & 0.0128 & 0.3797 & 0.0297 & & & 0.0001 & & & 0.0024 & & \\
\hline LAWPC5 & 0.0987 & 0.0099 & 0.0271 & 0.0632 & & 0.0401 & 0.0000 & 0.0198 & 0.2000 & 0.0011 & 0.0128 & 0.3995 & 0.0297 & & & 0.0001 & & & 0.0024 & & \\
\hline LAWPC6 & 0.0987 & 0.0198 & 0.0271 & 0.0632 & & 0.0401 & 0.0198 & 0.0198 & 0.2000 & 0.0011 & 0.0128 & 0.4094 & 0.0297 & & & 0.0001 & & & 0.0024 & & \\
\hline LAWPC7 & 0.0988 & 0.0300 & 0.0479 & 0.0630 & & 0.0012 & 0.0403 & 0.0300 & 0.2000 & 0.0004 & 0.0005 & 0.4192 & 0.0300 & & & 0.0000 & & & 0.0000 & & \\
\hline LAWPC8 & 0.0988 & 0.0300 & 0.0479 & 0.0630 & & 0.0012 & 0.0403 & 0.0300 & 0.2000 & 0.0004 & 0.0005 & 0.4192 & 0.0300 & & & 0.0000 & & & 0.0000 & & \\
\hline LAWPC9 & 0.0984 & 0.0394 & 0.0467 & 0.0629 & & 0.0401 & 0.0000 & 0.0197 & 0.2000 & 0.0011 & 0.0128 & 0.3873 & 0.0295 & & & 0.0001 & & & 0.0024 & & \\
\hline LAWPC10 & 0.0988 & 0.0300 & 0.0479 & 0.0630 & & 0.0012 & 0.0403 & 0.0300 & 0.2000 & 0.0004 & 0.0005 & 0.4192 & 0.0300 & & & 0.0000 & & & 0.0000 & & \\
\hline
\end{tabular}


TWRS LAW Formulation (Muller and Pegg 1998)

\begin{tabular}{|c|c|c|c|c|c|c|c|c|c|c|c|c|c|c|c|c|c|c|c|c|c|}
\hline Glass ID & $\begin{array}{l}\mathrm{Cl} \\
-\mathrm{t}\end{array}$ & $\begin{array}{c}\mathrm{CoO} \\
-\mathrm{t} \\
\end{array}$ & $\begin{array}{c}\mathrm{Co} 2 \mathrm{O} 3 \\
-\mathrm{t}\end{array}$ & $\begin{array}{c}\mathrm{Cr} 2 \mathrm{O} 3 \\
-\mathrm{t} \\
\end{array}$ & $\begin{array}{c}\mathrm{Cs} 2 \mathrm{O} \\
-\mathrm{t} \\
\end{array}$ & $\begin{array}{c}\mathrm{CuO} \\
-\mathrm{t}\end{array}$ & $\begin{array}{c}\mathrm{Eu} 2 \mathrm{O} 3 \\
-\mathrm{t}\end{array}$ & $\begin{array}{l}F \\
-t\end{array}$ & $\begin{array}{c}\mathrm{Ga} 2 \mathrm{O} 3 \\
-\mathrm{t} \\
\end{array}$ & $\begin{array}{c}\mathrm{Gd} 2 \mathrm{O} 3 \\
-\mathrm{t} \\
\end{array}$ & $\begin{array}{c}\mathrm{HgO} \\
-\mathrm{t} \\
\end{array}$ & $\begin{array}{l}I \\
-t\end{array}$ & $\begin{array}{c}\mathrm{La} 2 \mathrm{O} 3 \\
-\mathrm{t} \\
\end{array}$ & $\begin{array}{c}\mathrm{MnO} 2 \\
-\mathrm{t}\end{array}$ & $\begin{array}{c}\mathrm{MnO} \\
-\mathrm{t}\end{array}$ & $\begin{array}{c}\mathrm{MoO} \\
-\mathrm{t}\end{array}$ & $\begin{array}{c}\mathrm{MoO} 3 \\
-\mathrm{t}\end{array}$ & $\begin{array}{c}\mathrm{Nb} 2 \mathrm{O} 5 \\
-\mathrm{t}\end{array}$ & $\begin{array}{c}\mathrm{Nd} 2 \mathrm{O} 3 \\
-\mathrm{t}\end{array}$ & $\begin{array}{c}\mathrm{PbO} \\
-\mathrm{t}\end{array}$ & $\begin{array}{c}\mathrm{PdO} 2 \\
-\mathrm{t}\end{array}$ \\
\hline LAWA25H & 0.0049 & & 0.0000 & 0.0039 & 0.0000 & 0.0000 & & 0.0130 & & & & & 0.0001 & & & & & & & 0.0011 & \\
\hline LAWA26 & 0.0097 & & 0.0000 & 0.0039 & 0.0000 & 0.0000 & & 0.0127 & & & & & 0.0001 & & & & & & & 0.0011 & \\
\hline LAWA26H & 0.0049 & & 0.0000 & 0.0039 & 0.0000 & 0.0000 & & 0.0130 & & & & & 0.0001 & & & & & & & 0.0011 & \\
\hline LAWB11 & 0.0076 & & 0.0000 & 0.0037 & 0.0000 & 0.0000 & & 0.0092 & & & & & 0.0000 & & & & & & & 0.0004 & \\
\hline LAWB12 & 0.0076 & & 0.0000 & 0.0037 & 0.0000 & 0.0000 & & 0.0092 & & & & & 0.0000 & & & & & & & 0.0004 & \\
\hline LAWB13 & 0.0076 & & 0.0000 & 0.0037 & 0.0000 & 0.0000 & & 0.0092 & & & & & 0.0000 & & & & & & & 0.0004 & \\
\hline LAWB14 & 0.0076 & & 0.0000 & 0.0037 & 0.0000 & 0.0000 & & 0.0092 & & & & & 0.0000 & & & & & & & 0.0004 & \\
\hline LAWB15 & 0.0076 & & 0.0000 & 0.0037 & 0.0000 & 0.0000 & & 0.0092 & & & & & 0.0000 & & & & & & & 0.0004 & \\
\hline LAWB16 & 0.0076 & & 0.0000 & 0.0037 & 0.0000 & 0.0000 & & 0.0092 & & & & & 0.0000 & & & & & & & 0.0004 & \\
\hline LAWB17 & 0.0076 & & 0.0000 & 0.0037 & 0.0000 & 0.0000 & & 0.0092 & & & & & 0.0000 & & & & & & & 0.0004 & \\
\hline LAWB18 & 0.0076 & & 0.0000 & 0.0037 & 0.0000 & 0.0000 & & 0.0092 & & & & & 0.0000 & & & & & & & 0.0004 & \\
\hline LAWB19 & 0.0076 & & 0.0000 & 0.0037 & 0.0000 & 0.0000 & & 0.0092 & & & & & 0.0000 & & & & & & & 0.0004 & \\
\hline LAWB20 & 0.0076 & & 0.0000 & 0.0037 & 0.0000 & 0.0000 & & 0.0092 & & & & & 0.0000 & & & & & & & 0.0004 & \\
\hline LAWB21 & 0.0076 & & 0.0000 & 0.0037 & 0.0000 & 0.0000 & & 0.0092 & & & & & 0.0000 & & & & & & & 0.0004 & \\
\hline LAWB22 & 0.0076 & & 0.0000 & 0.0037 & 0.0000 & 0.0000 & & 0.0092 & & & & & 0.0000 & & & & & & & 0.0004 & \\
\hline LAWB23 & 0.0019 & & 0.0000 & 0.0037 & 0.0000 & 0.0000 & & 0.0094 & & & & & 0.0000 & & & & & & & 0.0004 & \\
\hline LAWB24 & 0.0038 & & 0.0000 & 0.0037 & 0.0000 & 0.0000 & & 0.0093 & & & & & 0.0000 & & & & & & & 0.0004 & \\
\hline LAWB25 & 0.0057 & & 0.0000 & 0.0037 & 0.0000 & 0.0000 & & 0.0093 & & & & & 0.0000 & & & & & & & 0.0004 & \\
\hline LAWB26 & 0.0076 & & 0.0000 & 0.0037 & 0.0000 & 0.0000 & & 0.0092 & & & & & 0.0000 & & & & & & & 0.0004 & \\
\hline LAWB27 & 0.0076 & & 0.0000 & 0.0037 & 0.0000 & 0.0000 & & 0.0092 & & & & & 0.0000 & & & & & & & 0.0004 & \\
\hline LAWB28 & 0.0076 & & 0.0000 & 0.0037 & 0.0000 & 0.0000 & & 0.0092 & & & & & 0.0000 & & & & & & & 0.0004 & \\
\hline LAWB29 & 0.0029 & & 0.0000 & 0.0010 & 0.0000 & 0.0000 & & 0.0000 & & & & & 0.0000 & & & & & & & 0.0000 & \\
\hline LAWC1 & 0.0085 & & 0.0000 & 0.0034 & 0.0000 & 0.0000 & & 0.0112 & & & & & 0.0001 & & & & & & & 0.0010 & \\
\hline LAWC2 & 0.0085 & & 0.0000 & 0.0034 & 0.0000 & 0.0000 & & 0.0112 & & & & & 0.0489 & & & & & & & 0.0010 & \\
\hline LAWC3 & 0.0085 & & 0.0000 & 0.0034 & 0.0000 & 0.0000 & & 0.0112 & & & & & 0.0001 & & & & & & & 0.0010 & \\
\hline LAWC4 & 0.0078 & & 0.0000 & 0.0031 & 0.0000 & 0.0000 & & 0.0103 & & & & & 0.0001 & & & & & & & 0.0009 & \\
\hline LAWC5 & 0.0085 & & 0.0000 & 0.0034 & 0.0000 & 0.0000 & & 0.0112 & & & & & 0.0001 & & & & & & & 0.0010 & \\
\hline LAWPC1 & 0.0062 & & 0.0000 & 0.0025 & 0.0008 & 0.0000 & & 0.0082 & & & & & 0.0000 & & & & & & & 0.0007 & \\
\hline LAWPC2 & 0.0062 & & 0.0000 & 0.0025 & 0.0008 & 0.0297 & & 0.0082 & & & & & 0.0000 & & & & & & & 0.0007 & \\
\hline LAWPC3 & 0.0062 & & 0.0000 & 0.0025 & 0.0008 & 0.0000 & & 0.0082 & & & & & 0.0000 & & & & & & & 0.0007 & \\
\hline LAWPC5 & 0.0062 & & 0.0000 & 0.0025 & 0.0008 & 0.0198 & & 0.0082 & & & & & 0.0000 & & & & & & & 0.0007 & \\
\hline LAWPC6 & 0.0062 & & 0.0000 & 0.0025 & 0.0008 & 0.0000 & & 0.0082 & & & & & 0.0000 & & & & & & & 0.0007 & \\
\hline LAWPC7 & 0.0010 & & 0.0000 & 0.0001 & 0.0000 & 0.0000 & & 0.0001 & & & & & 0.0000 & & & & & & & 0.0002 & \\
\hline LAWPC8 & 0.0010 & & 0.0000 & 0.0001 & 0.0000 & 0.0000 & & 0.0001 & & & & & 0.0000 & & & & & & & 0.0002 & \\
\hline LAWPC9 & 0.0062 & & 0.0000 & 0.0025 & 0.0008 & 0.0000 & & 0.0082 & & & & & 0.0000 & & & & & & & 0.0007 & \\
\hline LAWPC10 & 0.0010 & & 0.0000 & 0.0001 & 0.0000 & 0.0000 & & 0.0001 & & & & & 0.0000 & & & & & & & 0.0002 & \\
\hline
\end{tabular}


TWRS LAW Formulation (Muller and Pegg 1998)

\begin{tabular}{|c|c|c|c|c|c|c|c|c|c|c|c|c|c|c|c|c|c|c|c|c|c|}
\hline Glass ID & $\begin{array}{c}\mathrm{PdO} \\
-\mathrm{t}\end{array}$ & $\begin{array}{c}\mathrm{Pr} 2 \mathrm{O} 3 \\
-\mathrm{t}\end{array}$ & Pr6O11 & $\begin{array}{c}\mathrm{Rb} 2 \mathrm{O} \\
-\mathrm{t}\end{array}$ & $\begin{array}{c}\mathrm{ReO} \\
-\mathrm{t}\end{array}$ & $\begin{array}{c}\mathrm{ReO} 2 \\
-\mathrm{t}\end{array}$ & $\begin{array}{c}\mathrm{Rh} 2 \mathrm{O} 3 \\
-\mathrm{t}\end{array}$ & $\begin{array}{c}\mathrm{RhO}^{2} \\
-\mathrm{t}\end{array}$ & $\begin{array}{c}\mathrm{RuO} 2 \\
-\mathrm{t}\end{array}$ & $\begin{array}{c}\mathrm{Sb} 2 \mathrm{O} 3 \\
-\mathrm{t}\end{array}$ & $\begin{array}{c}\mathrm{Sb} 2 \mathrm{O} 5 \\
-\mathrm{t}\end{array}$ & $\begin{array}{c}\mathrm{SeO} 2 \\
-\mathrm{t}\end{array}$ & $\begin{array}{c}\mathrm{Sm} 2 \mathrm{O} 3 \\
-\mathrm{t}\end{array}$ & $\begin{array}{c}\mathrm{SnO} \\
-\mathrm{t}\end{array}$ & $\begin{array}{c}\mathrm{SnO} 2 \\
-\mathrm{t}\end{array}$ & $\begin{array}{c}\mathrm{SO} 3 \\
-\mathrm{t}\end{array}$ & $\begin{array}{c}\mathrm{SrO} \\
-\mathrm{t}\end{array}$ & $\begin{array}{c}\mathrm{Tc} 2 \mathrm{O} 7 \\
-\mathrm{t}\end{array}$ & $\begin{array}{c}\mathrm{TeO} 2 \\
-\mathrm{t}\end{array}$ & $\begin{array}{c}\mathrm{ThO} 2 \\
-\mathrm{t}\end{array}$ & $\begin{array}{c}\mathrm{TiO} 2 \\
-\mathrm{t}\end{array}$ \\
\hline LAWA25H & & & & & & & & & & & & & & & 0.0000 & 0.0029 & & & & & \\
\hline LAWA26 & & & & & & & & & & & & & & & 0.0000 & 0.0057 & & & & & \\
\hline LAWA26H & & & & & & & & & & & & & & & 0.0000 & 0.0029 & & & & & \\
\hline LAWB11 & & & & & & & & & & & & & & & 0.0000 & 0.0136 & & & & & \\
\hline LAWB12 & & & & & & & & & & & & & & & 0.0000 & 0.0136 & & & & & \\
\hline LAWB13 & & & & & & & & & & & & & & & 0.0000 & 0.0136 & & & & & \\
\hline LAWB14 & & & & & & & & & & & & & & & 0.0000 & 0.0136 & & & & & \\
\hline LAWB15 & & & & & & & & & & & & & & & 0.0000 & 0.0136 & & & & & \\
\hline LAWB16 & & & & & & & & & & & & & & & 0.0000 & 0.0136 & & & & & \\
\hline LAWB17 & & & & & & & & & & & & & & & 0.0000 & 0.0136 & 0.0000 & & 0.0000 & 0.0000 & 0.0000 \\
\hline LAWB18 & & & & & & & & & & & & & & & 0.0000 & 0.0136 & 0.0000 & & 0.0000 & 0.0000 & 0.0000 \\
\hline LAWB19 & & & & & & & & & & & & & & & 0.0000 & 0.0136 & 0.0000 & & 0.0000 & 0.0000 & 0.0000 \\
\hline LAWB20 & & & & & & & & & & & & & & & 0.0000 & 0.0136 & 0.0000 & & 0.0000 & 0.0000 & 0.0000 \\
\hline LAWB21 & & & & & & & & & & & & & & & 0.0000 & 0.0136 & 0.0000 & & 0.0000 & 0.0000 & 0.0000 \\
\hline LAWB22 & & & & & & & & & & & & & & & 0.0000 & 0.0136 & 0.0000 & & 0.0000 & 0.0000 & 0.0000 \\
\hline LAWB23 & & & & & & & & & & & & & & & 0.0000 & 0.0035 & 0.0000 & & 0.0000 & 0.0000 & 0.0000 \\
\hline LAWB24 & & & & & & & & & & & & & & & 0.0000 & 0.0069 & 0.0000 & & 0.0000 & 0.0000 & 0.0000 \\
\hline LAWB25 & & & & & & & & & & & & & & & 0.0000 & 0.0103 & 0.0000 & & 0.0000 & 0.0000 & 0.0000 \\
\hline LAWB26 & & & & & & & & & & & & & & & 0.0000 & 0.0136 & 0.0000 & & 0.0000 & 0.0000 & 0.0000 \\
\hline LAWB27 & & & & & & & & & & & & & & & 0.0000 & 0.0136 & 0.0000 & & 0.0000 & 0.0000 & 0.0000 \\
\hline LAWB28 & & & & & & & & & & & & & & & 0.0000 & 0.0136 & 0.0000 & & 0.0000 & 0.0000 & 0.0000 \\
\hline LAWB29 & & & & & & & & & & & & & & & 0.0000 & 0.0002 & 0.0000 & & 0.0000 & 0.0000 & 0.0000 \\
\hline LAWC1 & & & & & & & & & & & & & & & 0.0000 & 0.0050 & 0.0007 & & & & \\
\hline LAWC2 & & & & & & & & & & & & & & & 0.0000 & 0.0050 & 0.0007 & & & & \\
\hline LAWC3 & & & & & & & & & & & & & & & 0.0488 & 0.0050 & 0.0007 & & & & \\
\hline LAWC4 & & & & & & & & & & & & & & & 0.0000 & 0.0046 & 0.0006 & & & & \\
\hline LAWC5 & & & & & & & & & & & & & & & 0.0000 & 0.0050 & 0.0007 & & & & \\
\hline LAWPC1 & & & & & & & & & & & & & & & 0.0000 & 0.0037 & 0.0008 & & 0.0000 & 0.0000 & 0.0000 \\
\hline LAWPC2 & & & & & & & & & & & & & & & 0.0000 & 0.0037 & 0.0008 & & 0.0000 & 0.0000 & 0.0000 \\
\hline LAWPC3 & & & & & & & & & & & & & & & 0.0000 & 0.0037 & 0.0008 & & 0.0000 & 0.0000 & 0.0000 \\
\hline LAWPC5 & & & & & & & & & & & & & & & 0.0000 & 0.0037 & 0.0008 & & 0.0000 & 0.0000 & 0.0000 \\
\hline LAWPC6 & & & & & & & & & & & & & & & 0.0000 & 0.0037 & 0.0008 & & 0.0000 & 0.0000 & 0.0000 \\
\hline LAWPC7 & & & & & & & & & & & & & & & 0.0000 & 0.0038 & 0.0000 & & 0.0000 & 0.0000 & 0.0000 \\
\hline LAWPC8 & & & & & & & & & & & & & & & 0.0000 & 0.0038 & 0.0000 & & 0.0000 & 0.0000 & 0.0000 \\
\hline LAWPC9 & & & & & & & & & & & & & & & 0.0000 & 0.0076 & 0.0008 & & 0.0000 & 0.0000 & 0.0000 \\
\hline LAWPC10 & & & & & & & & & & & & & & & 0.0000 & 0.0038 & 0.0000 & & 0.0000 & 0.0000 & 0.0000 \\
\hline
\end{tabular}


TWRS LAW Formulation (Muller and Pegg 1998)

\begin{tabular}{|c|c|c|c|c|c|c|c|c|c|c|c|c|c|c|c|c|c|c|c|c|c|}
\hline Glass ID & $\begin{array}{c}\mathrm{T} 12 \mathrm{O} 3 \\
-\mathrm{t}\end{array}$ & $\begin{array}{c}\mathrm{U} 3 \mathrm{O} 8 \\
-\mathrm{t}\end{array}$ & $\begin{array}{c}\mathrm{UO} 2 \\
-\mathrm{t}\end{array}$ & $\begin{array}{c}\text { UO3 } \\
-t\end{array}$ & $\begin{array}{c}\mathrm{V} 2 \mathrm{O} 5 \\
-\mathrm{t}\end{array}$ & $\begin{array}{c}\text { WO3 } \\
-t\end{array}$ & $\begin{array}{c}\mathrm{Y} 2 \mathrm{O} 3 \\
-\mathrm{t}\end{array}$ & $\begin{array}{c}\mathrm{ZnO} \\
-\mathrm{t}\end{array}$ & $\begin{array}{c}\text { Others } \\
-t\end{array}$ & $\begin{array}{c}\text { Sum } \\
-t\end{array}$ & $\begin{array}{c}\mathrm{A} 12 \mathrm{O} 3 \\
-\mathrm{a}\end{array}$ & $\begin{array}{c}\mathrm{B} 2 \mathrm{O} 3 \\
-\mathrm{a}\end{array}$ & $\begin{array}{c}\mathrm{CaO} \\
-\mathrm{a}\end{array}$ & $\begin{array}{c}\mathrm{Fe} 2 \mathrm{O} 3 \\
-\mathrm{a}\end{array}$ & $\begin{array}{c}\mathrm{FeO} \\
-\mathrm{a}\end{array}$ & $\begin{array}{c}\mathrm{K} 2 \mathrm{O} \\
-\mathrm{a}\end{array}$ & $\begin{array}{c}\mathrm{Li} 2 \mathrm{O} \\
-\mathrm{a}\end{array}$ & $\begin{array}{c}\mathrm{MgO} \\
-\mathrm{a}\end{array}$ & $\begin{array}{c}\mathrm{Na} 2 \mathrm{O} \\
-\mathrm{a}\end{array}$ & $\begin{array}{c}\mathrm{NiO} \\
-\mathrm{a}\end{array}$ & $\begin{array}{c}\mathrm{P} 2 \mathrm{O} 5 \\
-\mathrm{a}\end{array}$ \\
\hline LAWA25H & & & & & & & & 0.0283 & & 0.9998 & 0.0983 & 0.0239 & 0.0208 & 0.0632 & & 0.0380 & 0.0015 & 0.0120 & \begin{tabular}{|l|}
0.2369 \\
\end{tabular} & 0.0027 & 0.0124 \\
\hline LAWA26 & & & & & & & & 0.0278 & & 0.9999 & 0.1038 & 0.0012 & 0.0201 & 0.0427 & & 0.0382 & 0.0135 & 0.0003 & 0.2419 & 0.0018 & 0.0218 \\
\hline LAWA26H & & & & & & & & 0.0283 & & 0.9998 & 0.1093 & 0.0016 & 0.0203 & 0.0437 & & 0.0410 & 0.0143 & & 0.2540 & 0.0023 & 0.0111 \\
\hline LAWB11 & & & & & & & & 0.0000 & & 0.9997 & 0.0584 & 0.1038 & 0.1091 & 0.0877 & & 0.0204 & 0.0008 & 0.0314 & 0.0727 & 0.0015 & 0.0227 \\
\hline LAWB12 & & & & & & & & 0.0000 & & 0.9997 & 0.0600 & 0.1036 & 0.0578 & 0.0908 & & 0.0199 & 0.0000 & 0.0005 & 0.0708 & 0.0015 & 0.0220 \\
\hline LAWB13 & & & & & & & & 0.0407 & & 0.9997 & 0.0598 & 0.1076 & 0.0586 & 0.0907 & & 0.0196 & 0.0005 & 0.0777 & \begin{tabular}{|l|}
0.0710 \\
\end{tabular} & 0.0013 & 0.0225 \\
\hline LAWB14 & & & & & & & & 0.0000 & & 0.9998 & 0.0578 & 0.1084 & 0.1037 & 0.0847 & & 0.0228 & 0.0006 & 0.0790 & 0.0731 & 0.0006 & 0.0229 \\
\hline LAWB15 & & & & & & & & 0.0000 & & 0.9997 & 0.0565 & 0.1365 & 0.1093 & 0.0839 & & 0.0234 & 0.0178 & 0.0354 & 0.0701 & 0.0006 & 0.0242 \\
\hline LAWB16 & & & & & & & & 0.0978 & & 0.9997 & 0.0619 & 0.1047 & 0.0067 & 0.0895 & & 0.0218 & 0.0003 & 0.0308 & 0.0723 & 0.0023 & 0.0227 \\
\hline LAWB17 & & 0.0000 & 0.0000 & & & & 0.0000 & 0.0407 & & 0.9996 & 0.0621 & 0.0783 & 0.0555 & 0.0809 & & 0.0225 & 0.0406 & 0.0320 & 0.0700 & 0.0007 & 0.0233 \\
\hline LAWB18 & & 0.0000 & 0.0000 & & & & 0.0000 & 0.0000 & & 0.9589 & 0.0610 & 0.0805 & 0.0555 & 0.0810 & & 0.0229 & 0.0403 & 0.0330 & 0.0683 & 0.0007 & 0.0239 \\
\hline LAWB19 & & 0.0000 & 0.0000 & & & & 0.0000 & 0.0326 & & 0.9999 & 0.0921 & 0.0643 & 0.0389 & 0.0932 & & 0.0211 & 0.0215 & 0.0203 & 0.1084 & 0.0020 & 0.0218 \\
\hline LAWB20 & & 0.0000 & 0.0000 & & & & 0.0000 & 0.0326 & & 1.0000 & 0.0819 & 0.0651 & 0.0383 & 0.0914 & & 0.0213 & 0.0323 & 0.0210 & 0.1154 & 0.0024 & 0.0231 \\
\hline LAWB21 & & 0.0000 & 0.0000 & & & & 0.0000 & 0.0326 & & 1.0000 & 0.0872 & 0.0659 & 0.0418 & 0.0623 & & 0.0215 & 0.0326 & 0.0203 & 0.1379 & 0.0007 & 0.0248 \\
\hline LAWB22 & & 0.0000 & 0.0000 & & & & 0.0000 & 0.0326 & & 1.0000 & 0.0856 & 0.0659 & 0.0414 & 0.0671 & & 0.0212 & 0.0012 & 0.0204 & 0.1644 & 0.0007 & 0.0243 \\
\hline LAWB23 & & 0.0000 & 0.0000 & & & & 0.0000 & 0.0331 & & 1.0001 & & & & & & & & & & & \\
\hline LAWB24 & & 0.0000 & 0.0000 & & & & 0.0000 & 0.0329 & & 1.0000 & & & & & & & & & & & \\
\hline LAWB25 & & 0.0000 & 0.0000 & & & & 0.0000 & 0.0328 & & 1.0000 & & & & & & & & & & & \\
\hline LAWB26 & & 0.0000 & 0.0000 & & & & 0.0000 & 0.0407 & & 0.9998 & 0.0771 & 0.0879 & 0.0515 & 0.0696 & & 0.0210 & 0.0412 & 0.0211 & 0.0766 & 0.0018 & 0.0217 \\
\hline LAWB27 & & 0.0000 & 0.0000 & & & & 0.0000 & 0.0407 & & 0.9999 & 0.0741 & 0.0872 & 0.0520 & 0.0752 & & 0.0228 & 0.0336 & 0.0223 & 0.1012 & 0.0020 & 0.0223 \\
\hline LAWB28 & & 0.0000 & 0.0000 & & & & 0.0000 & 0.0407 & & 0.9999 & 0.0687 & 0.0918 & 0.0508 & 0.0688 & & 0.0233 & 0.0220 & 0.0215 & 0.1175 & 0.0017 & 0.0210 \\
\hline LAWB29 & & 0.0000 & 0.0000 & & & & 0.0000 & 0.0399 & & 0.9998 & 0.0846 & 0.0787 & 0.0726 & 0.0730 & & 0.0050 & 0.0398 & 0.0287 & 0.0993 & 0.0000 & 0.0018 \\
\hline LAWC1 & & & & & & & & 0.0305 & & 0.9999 & 0.0941 & 0.0660 & 0.0243 & 0.0997 & & 0.0545 & & & 0.1912 & 0.0021 & 0.0187 \\
\hline LAWC2 & & & & & & & & 0.0305 & & 0.9999 & 0.0941 & 0.0661 & 0.0237 & 0.0540 & & 0.0557 & & & 0.1934 & 0.0021 & 0.0182 \\
\hline LAWC3 & & & & & & & & 0.0305 & & 0.9999 & 0.0952 & 0.0675 & 0.0250 & 0.0539 & & 0.0533 & & & 0.1906 & 0.0024 & 0.0185 \\
\hline LAWC4 & & & & & & & & 0.0000 & & 0.9987 & 0.0920 & 0.0331 & 0.1121 & 0.0653 & & 0.0469 & & & 0.1731 & 0.0018 & 0.0096 \\
\hline LAWC5 & & & & & & & & 0.0287 & & 0.9999 & 0.0862 & 0.0316 & 0.0759 & 0.0670 & & 0.0511 & 0.0006 & 0.0005 & \begin{tabular}{|l|}
0.1882 \\
\end{tabular} & 0.0024 & 0.0177 \\
\hline LAWPC1 & & 0.0000 & 0.0000 & & & & 0.0000 & 0.0330 & & 0.9999 & 0.0938 & 0.0442 & 0.0301 & 0.0717 & & 0.0381 & 0.0006 & 0.0206 & 0.1854 & 0.0024 & 0.0138 \\
\hline LAWPC2 & & 0.0000 & 0.0000 & & & & 0.0000 & 0.0330 & & 0.9999 & 0.0948 & 0.0439 & 0.0304 & 0.0697 & & 0.0372 & 0.0004 & 0.0206 & 0.1861 & 0.0023 & 0.0143 \\
\hline LAWPC3 & & 0.0000 & 0.0000 & & & & 0.0297 & 0.0330 & & 0.9999 & 0.0941 & 0.0437 & 0.0295 & 0.0716 & & 0.0386 & 0.0003 & 0.0203 & 0.1973 & 0.0021 & 0.0136 \\
\hline LAWPC5 & & 0.0000 & 0.0000 & & & & 0.0198 & 0.0330 & & 0.9999 & 0.0951 & 0.0106 & 0.0300 & 0.0749 & & 0.0386 & 0.0004 & 0.0199 & 0.1935 & 0.0022 & 0.0141 \\
\hline LAWPC6 & & 0.0000 & 0.0000 & & & & 0.0000 & 0.0330 & & 0.9999 & 0.0909 & 0.0205 & 0.0289 & 0.0719 & & 0.0374 & 0.0219 & 0.0196 & 0.1943 & 0.0024 & 0.0138 \\
\hline LAWPC7 & & 0.0000 & 0.0000 & & & & 0.0000 & 0.0332 & & 0.9997 & 0.0806 & 0.0250 & 0.0409 & 0.0567 & & 0.0026 & 0.0340 & 0.0228 & 0.1439 & 0.0015 & 0.0013 \\
\hline LAWPC8 & & 0.0000 & 0.0000 & & & & 0.0000 & 0.0332 & & 0.9997 & 0.0872 & 0.0285 & 0.0442 & 0.0640 & & 0.0022 & 0.0374 & 0.0257 & 0.1601 & 0.0017 & 0.0019 \\
\hline LAWPC9 & & 0.0000 & 0.0000 & & & & 0.0000 & 0.0328 & & 1.0000 & 0.0935 & 0.0446 & 0.0409 & 0.0667 & & 0.0375 & 0.0005 & 0.0204 & 0.2007 & 0.0020 & 0.0130 \\
\hline LAWPC10 & & 0.0000 & 0.0000 & & & & 0.0000 & 0.0332 & & 0.9997 & 0.0910 & 0.0340 & 0.0511 & 0.0704 & & 0.0020 & 0.0447 & 0.0164 & 0.1911 & & 0.0014 \\
\hline
\end{tabular}


Appendix A. Database - mass fraction

TWRS LAW Formulation (Muller and Pegg 1998)

\begin{tabular}{|c|c|c|c|c|c|c|c|c|c|c|c|c|c|c|c|c|c|c|c|c|c|}
\hline Glass ID & $\begin{array}{c}\mathrm{SiO} 2 \\
-\mathrm{a}\end{array}$ & $\begin{array}{c}\mathrm{ZrO} 2 \\
-\mathrm{a}\end{array}$ & $\begin{array}{c}\mathrm{Ag} 2 \mathrm{O} \\
-\mathrm{a}\end{array}$ & $\begin{array}{c}\text { As2O3 } \\
-\mathrm{a}\end{array}$ & $\begin{array}{c}\mathrm{BaO} \\
-\mathrm{a}\end{array}$ & $\begin{array}{c}\mathrm{Bi} 2 \mathrm{O} 3 \\
-\mathrm{a}\end{array}$ & $\begin{array}{l}\mathrm{Br} \\
-\mathrm{a}\end{array}$ & $\begin{array}{c}\mathrm{CdO} \\
-\mathrm{a}\end{array}$ & $\begin{array}{c}\mathrm{Ce} 2 \mathrm{O} 3 \\
-\mathrm{a}\end{array}$ & $\begin{array}{c}\mathrm{CeO} 2 \\
-\mathrm{a}\end{array}$ & $\begin{array}{l}\mathrm{Cl} \\
-\mathrm{a}\end{array}$ & $\begin{array}{c}\mathrm{CoO} \\
-\mathrm{a}\end{array}$ & $\begin{array}{c}\mathrm{Co} 2 \mathrm{O} 3 \\
-\mathrm{a}\end{array}$ & $\begin{array}{c}\mathrm{Cr} 2 \mathrm{O} 3 \\
-\mathrm{a}\end{array}$ & $\begin{array}{c}\mathrm{Cs} 2 \mathrm{O} \\
-\mathrm{a}\end{array}$ & $\begin{array}{c}\mathrm{CuO} \\
-\mathrm{a}\end{array}$ & $\begin{array}{c}\mathrm{Eu} 2 \mathrm{O} 3 \\
-\mathrm{a}\end{array}$ & $\begin{array}{l}\mathrm{F} \\
-\mathrm{a}\end{array}$ & $\begin{array}{c}\mathrm{Ga} 2 \mathrm{O} 3 \\
-\mathrm{a}\end{array}$ & $\begin{array}{c}\mathrm{Gd} 2 \mathrm{O} 3 \\
-\mathrm{a}\end{array}$ & $\begin{array}{c}\mathrm{HgO} \\
-\mathrm{a}\end{array}$ \\
\hline LAWA25H & 0.4255 & 0.0168 & & & 0.0003 & & & 0.0041 & & & & & & 0.0058 & & & & & & & \\
\hline LAWA26 & 0.4460 & 0.0231 & & & 0.0002 & & & 0.0041 & & & 0.0040 & & & 0.0039 & & & & & & & \\
\hline LAWA26H & 0.4439 & 0.0161 & & & 0.0002 & & & 0.0040 & & & & & & 0.0056 & & & & & & & \\
\hline LAWB11 & 0.4395 & 0.0338 & & & 0.0002 & & & 0.0013 & & & 0.0050 & & & 0.0032 & & & & & & & \\
\hline LAWB12 & 0.4504 & 0.0311 & & & 0.0001 & & & 0.0012 & & & 0.0040 & & & 0.0041 & & & & & & & \\
\hline LAWB13 & 0.4081 & 0.0289 & & & 0.0001 & & & 0.0012 & & & 0.0030 & & & 0.0032 & & & & & & & \\
\hline LAWB14 & 0.4113 & 0.0221 & & & 0.0001 & & & 0.0012 & & & 0.0030 & & & 0.0024 & & & & & & & \\
\hline LAWB15 & 0.4013 & 0.0264 & & & 0.0001 & & & 0.0013 & & & 0.0030 & & & 0.0024 & & & & & & & \\
\hline LAWB16 & 0.4551 & 0.0234 & & & 0.0003 & & & 0.0013 & & & 0.0040 & & & 0.0039 & & & & & & & \\
\hline LAWB17 & 0.4417 & 0.0351 & & & 0.0001 & & & & & & 0.0050 & & & 0.0037 & & & & & & & \\
\hline LAWB18 & 0.4489 & 0.0294 & & & 0.0004 & & & 0.0012 & & & 0.0040 & & & 0.0033 & & & & & & & \\
\hline LAWB19 & 0.4377 & 0.0257 & & & 0.0001 & & & 0.0012 & & & 0.0030 & & & 0.0059 & & & & & & & \\
\hline LAWB20 & 0.4273 & 0.0268 & & & 0.0004 & & & 0.0012 & & & 0.0030 & & & 0.0071 & & & & & & & \\
\hline LAWB21 & 0.4310 & 0.0291 & & & 0.0003 & & & 0.0013 & & & 0.0030 & & & 0.0035 & & & & & & & \\
\hline LAWB22 & 0.4349 & 0.0273 & & & 0.0002 & & & 0.0013 & & & 0.0030 & & & 0.0037 & & & & & & & \\
\hline LAWB23 & & & & & & & & & & & & & & & & & & & & & \\
\hline LAWB24 & & & & & & & & & & & & & & & & & & & & & \\
\hline LAWB25 & & & & & & & & & & & & & & & & & & & & & \\
\hline LAWB26 & 0.4514 & 0.0249 & & & 0.0001 & & & 0.0013 & & & 0.0020 & & & 0.0056 & & & & & & & \\
\hline LAWB27 & 0.4174 & 0.0296 & & & 0.0001 & & & 0.0014 & & & 0.0000 & & & 0.0067 & & & & & & & \\
\hline LAWB28 & 0.4343 & 0.0225 & & & 0.0001 & & & 0.0014 & & & 0.0030 & & & 0.0052 & & & & & & & \\
\hline LAWB29 & 0.4511 & 0.0214 & & & 0.0001 & & & 0.0000 & & & 0.0000 & & & 0.0010 & & & & & & & \\
\hline LAWC1 & 0.3757 & 0.0251 & & & 0.0001 & & & 0.0036 & & & 0.0050 & & & 0.0049 & & & & & & & \\
\hline LAWC2 & 0.3769 & 0.0216 & & & 0.0001 & & & 0.0034 & & & 0.0060 & & & 0.0044 & & & & & & & \\
\hline LAWC3 & 0.3875 & 0.0184 & & & 0.0002 & & & 0.0034 & & & 0.0060 & & & 0.0043 & & & & & & & \\
\hline LAWC4 & 0.4290 & 0.0198 & & & 0.0002 & & & 0.0032 & & & 0.0060 & & & 0.0060 & & & & & & & \\
\hline LAWC5 & 0.4120 & 0.0201 & & & 0.0002 & & & 0.0036 & & & 0.0060 & & & 0.0052 & & & & & & & \\
\hline LAWPC1 & 0.4256 & 0.0249 & & & 0.0003 & & & 0.0026 & & & 0.0000 & & & 0.0049 & & & & & & & \\
\hline LAWPC2 & 0.3986 & 0.0215 & & & 0.0003 & & & 0.0026 & & & 0.0000 & & & 0.0052 & & 0.0312 & & & & & \\
\hline LAWPC3 & 0.4128 & 0.0252 & & & 0.0002 & & & 0.0025 & & & 0.0020 & & & 0.0043 & & 0.0001 & & & & & \\
\hline LAWPC5 & 0.4232 & 0.0255 & & & 0.0004 & & & 0.0024 & & & 0.0030 & & & 0.0051 & & 0.0208 & & & & & \\
\hline LAWPC6 & 0.4276 & 0.0246 & & & 0.0002 & & & 0.0024 & & & 0.0090 & & & 0.0057 & & 0.0001 & & & & & \\
\hline LAWPC7 & 0.5335 & 0.0228 & & & 0.0001 & & & 0.0000 & & & 0.0000 & & & 0.0024 & & & & & & & \\
\hline LAWPC8 & 0.4868 & 0.0249 & & & 0.0005 & & & 0.0005 & & & 0.0000 & & & 0.0027 & & & & & & & \\
\hline LAWPC9 & 0.4079 & 0.0235 & & & 0.0003 & & & 0.0028 & & & 0.0030 & & & 0.0040 & & & & & & & \\
\hline LAWPC10 & 0.4337 & 0.0269 & & & 0.0000 & & & 0.0000 & & & 0.0000 & & & 0.0024 & & & & & & & \\
\hline
\end{tabular}


Appendix A. Database - mass fraction

TWRS LAW Formulation (Muller and Pegg 1998)

\begin{tabular}{|c|c|c|c|c|c|c|c|c|c|c|c|c|c|c|c|c|c|c|c|c|c|}
\hline Glass ID & $\begin{array}{l}\mathrm{I} \\
-\mathrm{a}\end{array}$ & $\begin{array}{c}\mathrm{La} 2 \mathrm{O} 3 \\
-\mathrm{a}\end{array}$ & $\begin{array}{c}\mathrm{MnO} 2 \\
\text {-a }\end{array}$ & $\begin{array}{c}\mathrm{MnO} \\
-\mathrm{a}\end{array}$ & $\begin{array}{c}\mathrm{MoO} \\
-\mathrm{a} \\
\end{array}$ & $\begin{array}{c}\mathrm{MoO} 3 \\
-\mathrm{a}\end{array}$ & $\begin{array}{c}\mathrm{Nb} 2 \mathrm{O} 5 \\
-\mathrm{a}\end{array}$ & $\begin{array}{c}\mathrm{Nd} 2 \mathrm{O} 3 \\
-\mathrm{a} \\
\end{array}$ & $\begin{array}{c}\mathrm{PbO} \\
-\mathrm{a} \\
\end{array}$ & $\begin{array}{c}\mathrm{PdO} 2 \\
-\mathrm{a}\end{array}$ & $\begin{array}{c}\mathrm{PdO} \\
-\mathrm{a} \\
\end{array}$ & $\begin{array}{c}\mathrm{Pr} 2 \mathrm{O} 3 \\
-\mathrm{a}\end{array}$ & $\begin{array}{c}\text { Pr6O11 } \\
-\mathrm{a}\end{array}$ & $\begin{array}{c}\mathrm{Rb} 2 \mathrm{O} \\
-\mathrm{a}\end{array}$ & $\begin{array}{c}\mathrm{ReO} \\
-\mathrm{a}\end{array}$ & $\begin{array}{c}\mathrm{ReO} 2 \\
-\mathrm{a}\end{array}$ & $\begin{array}{c}\mathrm{Rh} 2 \mathrm{O} 3 \\
-\mathrm{a}\end{array}$ & $\begin{array}{c}\mathrm{RhO}_{2} \\
-\mathrm{a}\end{array}$ & $\begin{array}{c}\mathrm{RuO} 2 \\
-\mathrm{a}\end{array}$ & $\begin{array}{c}\mathrm{Sb} 2 \mathrm{O} 3 \\
-\mathrm{a}\end{array}$ & $\begin{array}{c}\mathrm{Sb} 2 \mathrm{O} 5 \\
-\mathrm{a}\end{array}$ \\
\hline LAWA25H & & 0.0003 & & & & & & & 0.0014 & & & & & & & & & & & & \\
\hline LAWA26 & & & & & & & & & 0.0013 & & & & & & & & & & & & \\
\hline LAWA26H & & 0.0001 & & & & & & & 0.0013 & & & & & & & & & & & & \\
\hline LAWB11 & & & & & & & & & 0.0008 & & & & & & & & & & & & \\
\hline LAWB12 & & & & & & & & & 0.0007 & & & & & & & & & & & & \\
\hline LAWB13 & & & & & & & & & 0.0007 & & & & & & & & & & & & \\
\hline LAWB14 & & & & & & & & & 0.0007 & & & & & & & & & & & & \\
\hline LAWB15 & & & & & & & & & 0.0008 & & & & & & & & & & & & \\
\hline LAWB16 & & & & & & & & & 0.0006 & & & & & & & & & & & & \\
\hline LAWB17 & & & & & & & & & 0.0007 & & & & & & & & & & & & \\
\hline LAWB18 & & & & & & & & & 0.0007 & & & & & & & & & & & & \\
\hline LAWB19 & & & & & & & & & 0.0006 & & & & & & & & & & & & \\
\hline LAWB20 & & & & & & & & & 0.0006 & & & & & & & & & & & & \\
\hline LAWB21 & & & & & & & & & 0.0006 & & & & & & & & & & & & \\
\hline LAWB22 & & & & & & & & & 0.0006 & & & & & & & & & & & & \\
\hline LAWB23 & & & & & & & & & & & & & & & & & & & & & \\
\hline LAWB24 & & & & & & & & & & & & & & & & & & & & & \\
\hline LAWB25 & & & & & & & & & & & & & & & & & & & & & \\
\hline LAWB26 & & & & & & & & & 0.0007 & & & & & & & & & & & & \\
\hline LAWB27 & & & & & & & & & 0.0007 & & & & & & & & & & & & \\
\hline LAWB28 & & & & & & & & & 0.0006 & & & & & & & & & & & & \\
\hline LAWB29 & & & & & & & & & 0.0000 & & & & & & & & & & & & \\
\hline LAWC1 & & 0.0002 & & & & & & & 0.0014 & & & & & & & & & & & & \\
\hline LAWC2 & & 0.0497 & & & & & & & 0.0011 & & & & & & & & & & & & \\
\hline LAWC3 & & & & & & & & & 0.0012 & & & & & & & & & & & & \\
\hline LAWC4 & & & & & & & & & 0.0012 & & & & & & & & & & & & \\
\hline LAWC5 & & & & & & & & & 0.0012 & & & & & & & & & & & & \\
\hline LAWPC1 & & & & & & & & & 0.0008 & & & & & & & & & & & & \\
\hline LAWPC2 & & & & & & & & & 0.0010 & & & & & & & & & & & & \\
\hline LAWPC3 & & & & & & & & & 0.0011 & & & & & & & & & & & & \\
\hline LAWPC5 & & & & & & & & & 0.0010 & & & & & & & & & & & & \\
\hline LAWPC6 & & & & & & & & & 0.0011 & & & & & & & & & & & & \\
\hline LAWPC7 & & & & & & & & & 0.0003 & & & & & & & & & & & & \\
\hline LAWPC8 & & & & & & & & & 0.0004 & & & & & & & & & & & & \\
\hline LAWPC9 & & & & & & & & & 0.0011 & & & & & & & & & & & & \\
\hline LAWPC10 & & & & & & & & & & & & & & & & & & & & & \\
\hline
\end{tabular}


Appendix A. Database - mass fraction

TWRS LAW Formulation (Muller and Pegg 1998)

\begin{tabular}{|c|c|c|c|c|c|c|c|c|c|c|c|c|c|c|c|c|c|c|c|c|}
\hline Glass ID & $\begin{array}{c}\mathrm{SeO} 2 \\
-\mathrm{a} \\
\end{array}$ & $\begin{array}{c}\mathrm{Sm} 2 \mathrm{O} 3 \\
-\mathrm{a} \\
\end{array}$ & $\begin{array}{c}\mathrm{SnO} \\
-\mathrm{a}\end{array}$ & $\begin{array}{c}\mathrm{SnO} 2 \\
-\mathrm{a}\end{array}$ & $\begin{array}{c}\mathrm{SO} 3 \\
-\mathrm{a} \\
\end{array}$ & $\begin{array}{c}\mathrm{SrO} \\
-\mathrm{a} \\
\end{array}$ & $\begin{array}{c}\mathrm{Tc} 2 \mathrm{O} 7 \\
-\mathrm{a}\end{array}$ & $\begin{array}{c}\mathrm{TeO} 2 \\
-\mathrm{a}\end{array}$ & $\begin{array}{c}\mathrm{ThO} 2 \\
-\mathrm{a}\end{array}$ & $\begin{array}{c}\mathrm{TiO} 2 \\
-\mathrm{a} \\
\end{array}$ & $\begin{array}{c}\mathrm{T} 12 \mathrm{O} 3 \\
-\mathrm{a} \\
\end{array}$ & $\begin{array}{c}\mathrm{U} 3 \mathrm{O} 8 \\
-\mathrm{a}\end{array}$ & $\begin{array}{c}\mathrm{UO} 2 \\
-\mathrm{a} \\
\end{array}$ & $\begin{array}{c}\mathrm{UO} 3 \\
-\mathrm{a} \\
\end{array}$ & $\begin{array}{c}\mathrm{V} 2 \mathrm{O} 5 \\
-\mathrm{a}\end{array}$ & $\begin{array}{c}\mathrm{WO} 3 \\
-\mathrm{a} \\
\end{array}$ & $\begin{array}{c}\mathrm{Y} 2 \mathrm{O} 3 \\
-\mathrm{a} \\
\end{array}$ & $\begin{array}{c}\mathrm{ZnO} \\
-\mathrm{a}\end{array}$ & $\begin{array}{c}\text { Others } \\
-\mathrm{a}\end{array}$ & $\begin{array}{l}\text { Sum } \\
-\mathrm{a}\end{array}$ \\
\hline LAWA25H & & & & & 0.0048 & & & & & & & & & & & & & 0.0301 & & 0.9988 \\
\hline LAWA26 & & & & & 0.0056 & & & & & & & & & & & & & 0.0294 & & 1.0029 \\
\hline LAWA26H & & & & & 0.0019 & & & & & & & & & & & & & 0.0274 & & 0.9981 \\
\hline LAWB11 & & & & & 0.0113 & & & & & & & & & & & & & 0.0003 & & 1.0039 \\
\hline LAWB12 & & & & & 0.0093 & & & & & & & & & & & & & 0.0001 & & 0.9279 \\
\hline LAWB13 & & & & & 0.0067 & & & & & & & & & & & & & 0.0408 & & 1.0020 \\
\hline LAWB14 & & & & & 0.0076 & & & & & & & & & & & & & 0.0000 & & 1.0020 \\
\hline LAWB15 & & & & & 0.0091 & & & & & & & & & & & & & 0.0000 & & 1.0021 \\
\hline LAWB16 & & & & & 0.0054 & & & & & & & & & & & & & 0.0967 & & 1.0037 \\
\hline LAWB17 & & & & & 0.0123 & & & & & & & & & & & & & 0.0407 & & 1.0052 \\
\hline LAWB18 & & & & & 0.0112 & & & & & & & & & & & & & 0.0007 & & 0.9669 \\
\hline LAWB19 & & & & & 0.0120 & & & & & & & & & & & & & 0.0322 & & 1.0020 \\
\hline LAWB20 & & & & & 0.0109 & & & & & & & & & & & & & 0.0328 & & 1.0023 \\
\hline LAWB21 & & & & & 0.0064 & & & & & & & & & & & & & 0.0322 & & 1.0024 \\
\hline LAWB22 & & & & & 0.0047 & & & & & & & & & & & & & 0.0348 & & 1.0027 \\
\hline LAWB23 & & & & & & & & & & & & & & & & & & & & \\
\hline LAWB24 & & & & & & & & & & & & & & & & & & & & \\
\hline LAWB25 & & & & & & & & & & & & & & & & & & & & \\
\hline LAWB26 & & & & & 0.0058 & & & & & & & & & & & & & 0.0404 & & 1.0017 \\
\hline LAWB27 & & & & & 0.0079 & & & & & & & & & & & & & 0.0424 & & 0.9989 \\
\hline LAWB28 & & & & & 0.0121 & & & & & & & & & & & & & 0.0359 & & 1.0022 \\
\hline LAWB29 & & & & & 0.0019 & & & & & & & & & & & & & 0.0400 & & 0.9990 \\
\hline LAWC1 & & & & & 0.0053 & 0.0009 & & & & & & & & & & & & 0.0315 & & 1.0043 \\
\hline LAWC2 & & & & & 0.0034 & 0.0008 & & & & & & & & & & & & 0.0309 & & 1.0056 \\
\hline LAWC3 & & & & 0.0448 & 0.0039 & 0.0008 & & & & & & & & & & & & 0.0276 & & 1.0045 \\
\hline LAWC4 & & & & & 0.0037 & 0.0009 & & & & & & & & & & & & 0.0003 & & 1.0042 \\
\hline LAWC5 & & & & & 0.0059 & 0.0009 & & & & & & & & & & & & 0.0292 & & 1.0055 \\
\hline LAWPC1 & & & & & 0.0057 & 0.0012 & & & & & & & & & & & & 0.0329 & & 0.9996 \\
\hline LAWPC2 & & & & & 0.0046 & 0.0024 & & & & & & & & & & & & 0.0319 & & 0.9990 \\
\hline LAWPC3 & & & & & 0.0061 & 0.0011 & & & & & & & & & & & & 0.0328 & & 0.9993 \\
\hline LAWPC5 & & & & & 0.0051 & 0.0011 & & & & & & & & & & & & 0.0331 & & 1.0000 \\
\hline LAWPC6 & & & & & 0.0034 & 0.0012 & & & & & & & & & & & & 0.0311 & & 1.0080 \\
\hline LAWPC7 & & & & & 0.0045 & 0.0001 & & & & & & & & & & & & 0.0251 & & 0.9981 \\
\hline LAWPC8 & & & & & & 0.0004 & & & & & & & & & & & & 0.0291 & & 0.9982 \\
\hline LAWPC9 & & & & & 0.0071 & 0.0012 & & & & & & & & & & & & 0.0325 & & 1.0032 \\
\hline LAWPC10 & & & & & & & & & & & & & & & & & & 0.0317 & & 0.9968 \\
\hline
\end{tabular}


Appendix A. Database - mass fraction

\begin{tabular}{|c|c|c|c|c|c|c|c|c|}
\hline Glass ID & $\begin{array}{l}\mathrm{TM} \\
\left({ }^{\circ} \mathrm{C}\right) \\
\end{array}$ & $\begin{array}{l}\text { Gradient } \\
\text { TL }\left({ }^{\circ} \mathrm{C}\right) \\
\end{array}$ & $\begin{array}{l}\text { Uniform } \\
\text { TL }\left({ }^{\circ} \mathrm{C}\right) \\
\end{array}$ & Primary Phase & $\begin{array}{l}\text { Quenched } \\
\text { Visual/OM }\end{array}$ & $\begin{array}{c}\text { Quenched } \\
\text { SEM/EDS or TEM }\end{array}$ & $\begin{array}{l}\text { Quenched } \\
\text { XRD }\end{array}$ & $\begin{array}{c}\text { Quenched } \\
\text { Homogeneous? }\end{array}$ \\
\hline LAWA25H & & & $<950$ & & & & & \\
\hline \multicolumn{9}{|l|}{ LAWA26 } \\
\hline LAWA26H & & & $<950$ & & & & & \\
\hline LAWB11 & & & $950-1050$ & & & & & \\
\hline LAWB12 & & & $950-1050$ & & & & & \\
\hline LAWB13 & & & $\sim 1050$ & & & & & \\
\hline LAWB14 & & & $950-1050$ & & & & & \\
\hline LAWB15 & & & $\sim 950$ & & & & & \\
\hline LAWB16 & & & $>1050$ & & & & & \\
\hline LAWB17 & & & $950-1050$ & & & & & \\
\hline LAWB18 & & & $<950$ & & & & & \\
\hline LAWB19 & & & $\sim 1050$ & & & & & \\
\hline LAWB20 & & & $\sim 950$ & & & & & \\
\hline LAWB21 & & & $<950$ & & & & & \\
\hline LAWB22 & & & $\sim 950$ & & & & & \\
\hline \multicolumn{9}{|l|}{ LAWB23 } \\
\hline \multicolumn{9}{|l|}{ LAWB24 } \\
\hline LAWB25 & & & & & & & & \\
\hline LAWB26 & & & $\sim 950$ & & & & & \\
\hline LAWB27 & & & $<950$ & & & & & \\
\hline LAWB28 & & & $<950$ & & & & & \\
\hline LAWB29 & & & $<950$ & & & & & \\
\hline LAWC1 & & & $\sim 950$ & & & & & \\
\hline LAWC2 & & & $\sim 950$ & & & & & \\
\hline LAWC3 & & & $>1050$ & & & & & \\
\hline LAWC4 & & & $\sim 1050$ & & & & & \\
\hline LAWC5 & & & $<950$ & & & & & \\
\hline LAWPC1 & & & $<950$ & & & & & \\
\hline LAWPC2 & & & $<950$ & & & & & \\
\hline LAWPC3 & & & $\sim 950$ & & & & & \\
\hline LAWPC5 & & & $\sim 950$ & & & & & \\
\hline LAWPC6 & & & $<950$ & & & & & \\
\hline LAWPC7 & & & $<950$ & & & & & \\
\hline LAWPC8 & & & $<950$ & & & & & \\
\hline LAWPC9 & & & $<950$ & & & & & \\
\hline LAWPC10 & & & $<950$ & & & & & \\
\hline
\end{tabular}


Appendix A. Database - mass fraction

TWRS LAW Formulation (Muller and Pegg 1998)

\begin{tabular}{|c|c|c|c|c|c|}
\hline Glass ID & $\begin{array}{c}\mathrm{CCC} \\
\text { Visual/OM }\end{array}$ & $\begin{array}{c}\text { CCC } \\
\text { SEM/EDS or TEM }\end{array}$ & $\begin{array}{l}\text { CCC } \\
\text { XRD }\end{array}$ & $\begin{array}{l}\text { Heat Treated } \\
\text { Visual/OM }\end{array}$ & $\begin{array}{c}\text { Heat Treated } \\
\text { SEM/EDS or TEM }\end{array}$ \\
\hline \multicolumn{6}{|l|}{ LAWA25H } \\
\hline \multicolumn{6}{|l|}{ LAWA26 } \\
\hline \multicolumn{6}{|l|}{ LAWA26H } \\
\hline \multicolumn{6}{|l|}{ LAWB11 } \\
\hline \multicolumn{6}{|l|}{ LAWB12 } \\
\hline \multicolumn{6}{|l|}{ LAWB13 } \\
\hline \multicolumn{6}{|l|}{ LAWB14 } \\
\hline \multicolumn{6}{|l|}{ LAWB15 } \\
\hline \multicolumn{6}{|l|}{ LAWB16 } \\
\hline \multicolumn{6}{|l|}{ LAWB17 } \\
\hline \multicolumn{6}{|l|}{ LAWB18 } \\
\hline \multicolumn{6}{|l|}{ LAWB19 } \\
\hline \multicolumn{6}{|l|}{ LAWB20 } \\
\hline \multicolumn{6}{|l|}{ LAWB21 } \\
\hline \multicolumn{6}{|l|}{ LAWB22 } \\
\hline \multicolumn{6}{|l|}{ LAWB23 } \\
\hline \multicolumn{6}{|l|}{ LAWB24 } \\
\hline \multicolumn{6}{|l|}{ LAWB25 } \\
\hline \multicolumn{6}{|l|}{ LAWB26 } \\
\hline \multicolumn{6}{|l|}{ LAWB27 } \\
\hline \multicolumn{6}{|l|}{ LAWB28 } \\
\hline \multicolumn{6}{|l|}{ LAWB29 } \\
\hline \multicolumn{6}{|l|}{ LAWC1 } \\
\hline \multicolumn{6}{|l|}{ LAWC2 } \\
\hline \multicolumn{6}{|l|}{ LAWC3 } \\
\hline \multicolumn{6}{|l|}{ LAWC4 } \\
\hline \multicolumn{6}{|l|}{ LAWC5 } \\
\hline \multicolumn{6}{|l|}{ LAWPC1 } \\
\hline LAWPC2 & & & & & \\
\hline LAWPC3 & & & & & \\
\hline LAWPC5 & & & & & \\
\hline LAWPC6 & & & & & \\
\hline LAWPC7 & & & & & \\
\hline LAWPC8 & & & & & \\
\hline LAWPC9 & & & & & \\
\hline LAWPC10 & & & & & \\
\hline
\end{tabular}


TWRS LAW Formulation (Muller and Pegg 1998)

\begin{tabular}{|c|c|c|c|c|c|c|c|c|c|c|c|c|c|c|c|}
\hline Glass ID & $\begin{array}{c}\text { Heat Treated } \\
\text { XRD } \\
\end{array}$ & $\begin{array}{l}\text { Density } \\
\left(\mathrm{g} / \mathrm{cm}^{3}\right)\end{array}$ & \begin{tabular}{|c|} 
Fulc Visc \\
A
\end{tabular} & \begin{tabular}{|c|} 
Fulc Visc \\
B
\end{tabular} & \begin{tabular}{|c|} 
Fulc Visc \\
To
\end{tabular} & $\begin{array}{c}\mathrm{FV} 1150^{\circ} \mathrm{C} \\
(\mathrm{Pa} \cdot \mathrm{s})\end{array}$ & $\begin{array}{c}\text { Arrh Visc } \\
\text { A }\end{array}$ & $\begin{array}{c}\text { Arrh Visc } \\
\text { B }\end{array}$ & $1150^{\circ} \mathrm{C}$ & $\begin{array}{c}\mathrm{T}\left({ }^{\circ} \mathrm{C}\right) \text { at } \\
2 \mathrm{~Pa} \cdot \mathrm{s}\end{array}$ & $\begin{array}{c}\mathrm{T}\left({ }^{\circ} \mathrm{C}\right) \text { at } \\
5 \mathrm{~Pa} \cdot \mathrm{s}\end{array}$ & $\begin{array}{c}\mathrm{T}\left({ }^{\circ} \mathrm{C}\right) \text { at } \\
10 \mathrm{~Pa} \cdot \mathrm{s} \\
\end{array}$ & $\begin{array}{l}\mathrm{T} 1 \\
\left({ }^{\circ} \mathrm{C}\right)\end{array}$ & \begin{tabular}{c|c}
$\mathrm{V} 1$ \\
$(\mathrm{~Pa} \cdot \mathrm{s})$ \\
\end{tabular} & $\begin{array}{l}\mathrm{T} 2 \\
\left({ }^{\circ} \mathrm{C}\right)\end{array}$ \\
\hline LAWA25H & clear and uniform & & & & & & -12.372 & 20653.8 & 8.52 & & & & 950 & 94.7 & 1000 \\
\hline LAWA26 & shows spatial variations in $\mathrm{SO} 3$ & & & & & & & & & & & & & & \\
\hline LAWA26H & clear and uniform & & & & & & -12.171 & 20540.2 & 9.62 & & & & 950 & 103.7 & 1000 \\
\hline LAWB11 & $\begin{array}{l}\sim 5-8 \text { vol } \% \text { Ca-Mg-Fe silicates, } \sim 2-3 \text { vol } \% \\
\text { Ca3(PO4), } 2 \text { vol } \% \text { spinel, } \sim 2 \text { vol } \% \text { ZrSiO4, } \\
\sim 2 \text { vol } \% \text { ZrO2 }\end{array}$ & & & & & & -13.850 & 23282.5 & 12.32 & & & & 950 & 178.8 & 1000 \\
\hline LAWB12 & $\begin{array}{l}\sim 10 \text { vol\% } \% \text { Ca-Mg-Fe- silicates, } \sim 4 \text { vol } \% \\
\text { Ca3(PO4), } \sim 2.5 \text { vol } \% \text { spinel, } \sim 1.5 \text { vol } \% \\
\text { ZrSiO4 }\end{array}$ & & & & & & -9.085 & 16094.4 & 9.26 & & & & $(950)$ & $(160.8)$ & $(1000)$ \\
\hline LAWB13 & $\sim 2.2 \mathrm{vol} \% \mathrm{Ca} 3(\mathrm{PO} 4), \sim 2.2 \mathrm{vol} \%$ spinel & & & & & & -9.991 & 16728.9 & 5.84 & & & & & & $(1000)$ \\
\hline LAWB14 & $\begin{array}{l}\sim 22 \text { vol\% } \% \text { Ca-Mg-Fe silicates, } \sim 2.6 \text { vol } \% \\
\text { Ca3(PO4), traces of spinel }\end{array}$ & & & & & & -10.744 & 17686.3 & 5.39 & & & & & & $(1000)$ \\
\hline LAWB15 & $\begin{array}{l}\sim 0.5 \mathrm{vol} \% \text { Ca-Mg-Fe silicates, } \sim 3.3 \mathrm{vol} \% \\
\text { Ca3(PO4), traces of spinel }\end{array}$ & & & & & & -12.252 & 19622.7 & 4.65 & & & & & & 1000 \\
\hline LAWB16 & $\begin{array}{l}\sim 1.9 \mathrm{vol} \% \text { spinel, } \sim 1.6 \text { vol } \% \mathrm{ZrSiO} 4,<0.1 \\
\text { vol } \% \mathrm{ZrO} 2\end{array}$ & & & & & & -10.845 & 19712.5 & 20.24 & & & & & & $(1000)$ \\
\hline LAWB17 & $\begin{array}{l}\sim 1 \text { vol\% small spinel, } \sim 1 \text { vol\% phosphate } \\
\text { and } \mathrm{ZrO} 2 \text { at glass-crucible interface }\end{array}$ & & & & & & -11.695 & 18746.8 & 4.39 & & & & & & 1000 \\
\hline LAWB18 & clear and uniform & & & & & & -9.152 & 15150.9 & 4.46 & & & & & & $(1000)$ \\
\hline LAWB19 & $\begin{array}{l}\sim 1.6 \text { vol\% } \mathrm{Ca} 3(\mathrm{PO} 4), \sim 0.9 \text { vol\% spinel } \\
(\mathrm{Cr}, \mathrm{Fe}, \mathrm{Ni} \text {, and } \mathrm{Zn})\end{array}$ & & & & & & -12.175 & 20758.3 & 11.16 & & & & & & $(1000)$ \\
\hline LAWB20 & very tiny spinels on the surface & & & & & & -12.495 & 20536.6 & 6.94 & & & & 950 & 77.2 & 1000 \\
\hline LAWB21 & clear and uniform & & & & & & -11.631 & 18738.0 & 4.65 & & & & 950 & 43.2 & 1000 \\
\hline LAWB22 & $\begin{array}{l}\text { very tiny spinels on the surface and traces of } \\
\text { phosphate }(\sim 0.1 \mathrm{vol} \%)\end{array}$ & & & & & & -13.388 & 22412.5 & 10.61 & & & & 950 & 145.4 & 1000 \\
\hline \multicolumn{16}{|l|}{ LAWB23 } \\
\hline \multicolumn{16}{|l|}{ LAWB24 } \\
\hline LAWB25 & & & & & & & & & & & & & & & \\
\hline LAWB26 & $\begin{array}{l}\sim 1.7 \mathrm{vol} \% \mathrm{Ca} 3(\mathrm{PO} 4), \sim 0.2 \mathrm{vol} \% \text { spinel } \\
(\mathrm{Cr}, \mathrm{Fe}, \mathrm{Ni} \text {, and } \mathrm{Zn})\end{array}$ & & & & & & -12.454 & 20166.6 & 5.57 & & & & 950 & 59.7 & 1000 \\
\hline LAWB27 & clear and uniform & & & & & & -12.087 & 19941.8 & 6.87 & & & & 950 & 70 & 1000 \\
\hline LAWB28 & clear and uniform & & & & & & -12.646 & 20417.1 & 5.48 & & & & 950 & 61.9 & 1000 \\
\hline LAWB29 & clear and uniform & 2.7268 & & & & & -12.840 & 20391.3 & 4.44 & & & & 950 & 48.8 & 1000 \\
\hline LAWC1 & very tiny spinels on the surface & & & & & & -8.475 & 13991.7 & 3.89 & & & & $(950)$ & $(82.4)$ & $(1000)$ \\
\hline LAWC2 & very tiny spinels on the surface & & & & & & -9.568 & 15446.0 & 3.62 & & & & $(950)$ & $(52.8)$ & $(1000)$ \\
\hline LAWC3 & $1.5 \mathrm{vol} \% \mathrm{SnO} 2$ platelets & & & & & & -13.427 & 21571.2 & 5.65 & & & & (950) & $(118.4)$ & $(1000)$ \\
\hline LAWC4 & $\begin{array}{l}\text { voids showing traces of sodium sulfate and } \\
\text { chromate }\end{array}$ & & & & & & -13.433 & 22026.8 & 7.74 & & & & $(950)$ & $(129.1)$ & 1000 \\
\hline LAWC5 & clear and uniform & & & & & & -9.634 & 16148.3 & 5.55 & & & & & & $(1000)$ \\
\hline LAWPC1 & clear and uniform & & & & & & -9.611 & 16914.4 & 9.73 & & & & $(950)$ & $(175.7)$ & $(1000)$ \\
\hline LAWPC2 & clear and uniform & & & & & & -12.779 & 20841.1 & 6.47 & & & & 950 & 75.6 & 1000 \\
\hline LAWPC3 & small spinels & & & & & & -13.165 & 21464.9 & 6.82 & & & & 950 & 82.6 & 1000 \\
\hline LAWPC5 & clear and uniform & & & & & & -13.199 & 22343.5 & 12.22 & & & & & & 1000 \\
\hline LAWPC6 & clear and uniform & & & & & & -12.177 & 19812.5 & 5.73 & & & & 950 & 58.5 & 1000 \\
\hline LAWPC7 & clear and uniform & & & & & & -10.785 & 19409.1 & 17.37 & & & & 950 & 160.9 & 1000 \\
\hline LAWPC8 & clear and uniform & & & & & & -11.965 & 20052.2 & 8.39 & & & & 950 & 88.6 & 1000 \\
\hline LAWPC9 & clear and uniform & & & & & & -12.895 & 21297.9 & 7.94 & & & & 950 & 102.5 & 1000 \\
\hline LAWPC10 & clear and uniform & 2.7072 & & & & & -10.554 & 16685.3 & 3.23 & & & & 950 & 23.7 & 1000 \\
\hline
\end{tabular}


TWRS LAW Formulation (Muller and Pegg 1998)

\begin{tabular}{|c|c|c|c|c|c|c|c|c|c|c|c|c|c|c|c|c|c|c|c|c|c|}
\hline Glass ID & $\begin{array}{c}\mathrm{V} 2 \\
(\mathrm{~Pa} \cdot \mathrm{s})\end{array}$ & $\begin{array}{c}\mathrm{T} 3 \\
\left({ }^{\circ} \mathrm{C}\right) \\
\end{array}$ & $\begin{array}{c}\mathrm{V} 3 \\
(\mathrm{~Pa} \cdot \mathrm{s})\end{array}$ & $\begin{array}{l}\mathrm{T} 4 \\
\left({ }^{\circ} \mathrm{C}\right) \\
\end{array}$ & $\begin{array}{c}\mathrm{V} 4 \\
(\mathrm{~Pa} \cdot \mathrm{s})\end{array}$ & $\begin{array}{l}\mathrm{T} 5 \\
\left({ }^{\circ} \mathrm{C}\right) \\
\end{array}$ & $\begin{array}{c}\mathrm{V} 5 \\
(\mathrm{~Pa} \cdot \mathrm{s}) \\
\end{array}$ & $\begin{array}{l}\mathrm{T} 6 \\
\left({ }^{\circ} \mathrm{C}\right) \\
\end{array}$ & $\begin{array}{c}\mathrm{V} 6 \\
(\mathrm{~Pa} \cdot \mathrm{s}) \\
\end{array}$ & $\begin{array}{l}\mathrm{T} 7 \\
\left({ }^{\circ} \mathrm{C}\right) \\
\end{array}$ & $\begin{array}{c}\mathrm{V} 7 \\
(\mathrm{~Pa} \cdot \mathrm{s}) \\
\end{array}$ & $\begin{array}{l}\mathrm{T} 8 \\
\left({ }^{\circ} \mathrm{C}\right) \\
\end{array}$ & $\begin{array}{c}\mathrm{V} 8 \\
(\mathrm{~Pa} \cdot \mathrm{s})\end{array}$ & $\begin{array}{l}\mathrm{T} 9 \\
\left({ }^{\circ} \mathrm{C}\right)\end{array}$ & $\begin{array}{c}\mathrm{V} 9 \\
(\mathrm{~Pa} \cdot \mathrm{s})\end{array}$ & $\begin{array}{l}\mathrm{T} 10 \\
\left({ }^{\circ} \mathrm{C}\right)\end{array}$ & $\begin{array}{l}\mathrm{V} 10 \\
(\mathrm{~Pa} \cdot \mathrm{s})\end{array}$ & $\begin{array}{l}\mathrm{T} 11 \\
\left({ }^{\circ} \mathrm{C}\right)\end{array}$ & $\begin{array}{l}\mathrm{V} 11 \\
(\mathrm{~Pa} \cdot \mathrm{s})\end{array}$ & $\begin{array}{l}\mathrm{T} 12 \\
\left({ }^{\circ} \mathrm{C}\right)\end{array}$ & $\begin{array}{c}\mathrm{V} 12 \\
(\mathrm{~Pa} \cdot \mathrm{s})\end{array}$ \\
\hline LAWA25H & 46.8 & 1050 & 24.9 & 1100 & 14.1 & 1150 & 8.4 & 1200 & 5.2 & 1250 & 3.4 & & & & & & & & & & \\
\hline \multicolumn{22}{|l|}{ LAWA26 } \\
\hline LAWA26H & 52.6 & 1050 & 28.3 & 1100 & 16.1 & 1150 & 9.5 & 1200 & 5.9 & 1250 & 3.8 & & & & & & & & & & \\
\hline LAWB11 & 84.8 & 1050 & 42.5 & 1100 & 22.4 & 1150 & 12.3 & 1200 & 7.1 & 1250 & 4.2 & & & & & & & & & & \\
\hline LAWB12 & $(55.7)$ & (1050) & (25.7) & 1100 & 14.3 & 1150 & 9 & 1200 & 6.2 & 1250 & 4.5 & & & & & & & & & & \\
\hline LAWB13 & (56) & $(1050)$ & (19) & 1100 & 9.3 & 1150 & 5.6 & 1200 & 3.8 & 1250 & 2.8 & & & & & & & & & & \\
\hline LAWB14 & $(64.4)$ & (1050) & (19.4) & 1100 & 8.9 & 1150 & 5.1 & 1200 & 3.4 & 1250 & 2.5 & & & & & & & & & & \\
\hline LAWB15 & 23.7 & 1050 & 13.2 & 1100 & 7.7 & 1150 & 4.6 & 1200 & 2.9 & 1250 & 1.9 & & & & & & & & & & \\
\hline LAWB16 & (179.3) & (1050) & $(70.5)$ & 1100 & 34.5 & 1150 & 19.6 & 1200 & 12.3 & 1250 & 8.4 & & & & & & & & & & \\
\hline LAWB17 & 21.3 & 1050 & 11.8 & 1100 & 6.9 & 1150 & 4.3 & 1200 & 2.8 & 1250 & 1.9 & & & & & & & & & & \\
\hline LAWB18 & $(29.2)$ & $(1050)$ & (12.5) & 1100 & 6.8 & 1150 & 4.3 & 1200 & 3 & 1250 & 2.3 & & & & & & & & & & \\
\hline LAWB19 & (164.2) & (1050) & (47) & 1100 & 19.9 & 1150 & 10.6 & 1200 & 6.5 & 1250 & 4.5 & & & & & & & & & & \\
\hline LAWB20 & 37.7 & 1050 & 19.9 & 1100 & 11.3 & 1150 & 6.8 & 1200 & 4.3 & 1250 & 2.8 & & & & & & & & & & \\
\hline LAWB21 & 21.7 & 1050 & 11.9 & 1100 & 7.1 & 1150 & 4.5 & 1200 & 3 & 1250 & 2.1 & & & & & & & & & & \\
\hline LAWB22 & 67.4 & 1050 & 33.9 & 1100 & 18.3 & 1150 & 10.4 & 1200 & 6.3 & 1250 & 3.9 & & & & & & & & & & \\
\hline \multicolumn{22}{|l|}{ LAWB23 } \\
\hline \multirow{2}{*}{\multicolumn{22}{|c|}{$\begin{array}{l}\text { LAWB24 } \\
\text { LAWB25 }\end{array}$}} \\
\hline & & & & & & & & & & & & & & & & & & & & & \\
\hline LAWB26 & 29.3 & 1050 & 15.7 & 1100 & 9 & 1150 & 5.4 & 1200 & 3.5 & 1250 & 2.3 & & & & & & & & & & \\
\hline LAWB27 & 35.6 & 1050 & 19.4 & 1100 & 11.2 & 1150 & 6.8 & 1200 & 4.3 & 1250 & 2.8 & & & & & & & & & & \\
\hline LAWB28 & 29.4 & 1050 & 15.4 & 1100 & 8.7 & 1150 & 5.3 & 1200 & 3.4 & 1250 & 2.3 & & & & & & & & & & \\
\hline LAWB29 & 23.8 & 1050 & 12.6 & 1100 & 7.2 & 1150 & 4.3 & 1200 & 2.8 & 1250 & 1.8 & & & & & & & & & & \\
\hline LAWC1 & $(22.8)$ & (1050) & (10) & 1100 & 5.7 & 1150 & 3.8 & 1200 & 2.7 & 1250 & 2.1 & & & & & & & & & & \\
\hline LAWC2 & $(19.7)$ & (1050) & $(9.5)$ & 1100 & 5.5 & 1150 & 3.5 & 1200 & 2.5 & 1250 & 1.8 & & & & & & & & & & \\
\hline LAWC3 & (42.1) & 1050 & 18.4 & (1100) & $(42.1)$ & 1150 & 5.3 & 1200 & 3.3 & 1250 & 2.2 & & & & & & & & & & \\
\hline LAWC4 & 51.8 & 1050 & 24.3 & 1100 & 12.7 & 1150 & 7.3 & 1200 & 4.6 & 1250 & 3 & & & & & & & & & & \\
\hline LAWC5 & $(38.8)$ & $(1050)$ & $(16.2)$ & 1100 & 8.6 & 1150 & 5.4 & 1200 & 3.7 & 1250 & 2.7 & & & & & & & & & & \\
\hline LAWPC1 & $(61.9)$ & $(1050)$ & $(28.3)$ & 1100 & 15.4 & 1150 & 9.5 & 1200 & 6.3 & 1250 & 4.6 & & & & & & & & & & \\
\hline LAWPC2 & 35.9 & 1050 & 18.7 & 1100 & 10.5 & 1150 & 6.3 & 1200 & 4 & 1250 & 2.6 & & & & & & & & & & \\
\hline LAWPC3 & 40.1 & 1050 & 20.9 & 1100 & 11.6 & 1150 & 6.7 & 1200 & 4.1 & 1250 & 2.6 & & & & & & & & & & \\
\hline LAWPC5 & 82.8 & 1050 & 39.1 & 1100 & 20.5 & 1150 & 11.7 & 1200 & 7.2 & 1250 & 4.6 & & & & & & & & & & \\
\hline LAWPC6 & 29.4 & 1050 & 15.9 & 1100 & 9.2 & 1150 & 5.6 & 1200 & 3.6 & 1250 & 2.4 & & & & & & & & & & \\
\hline LAWPC7 & 86.3 & 1050 & 49.5 & 1100 & 28.5 & 1150 & 17.3 & 1200 & 10.9 & 1250 & 7.1 & & & & & & & & & & \\
\hline LAWPC8 & 43.7 & 1050 & 23.4 & 1100 & 13.5 & 1150 & 8.2 & 1200 & 5.2 & 1250 & 3.5 & & & & & & & & & & \\
\hline LAWPC9 & 45.4 & 1050 & 22.8 & 1100 & 12.6 & 1150 & 7.6 & 1200 & 4.8 & 1250 & 3.3 & & & & & & & & & & \\
\hline LAWPC10 & 12.7 & 1050 & 7.4 & 1100 & 4.7 & 1150 & 3.1 & 1200 & 2.2 & 1250 & 1.6 & & & & & & & & & & \\
\hline
\end{tabular}


Appendix A. Database - mass fraction

TWRS LAW Formulation (Muller and Pegg 1998)

\begin{tabular}{|c|c|c|c|c|c|c|c|c|c|c|c|c|c|c|c|c|}
\hline Glass ID & $\begin{array}{l}\mathrm{T} 13 \\
\left({ }^{\circ} \mathrm{C}\right) \\
\end{array}$ & $\begin{array}{l}\mathrm{V} 13 \\
(\mathrm{~Pa} \cdot \mathrm{s}) \\
\end{array}$ & $\begin{array}{l}\mathrm{T} 14 \\
\left({ }^{\circ} \mathrm{C}\right) \\
\end{array}$ & $\begin{array}{c}\mathrm{V} 14 \\
(\mathrm{~Pa} \cdot \mathrm{s}) \\
\end{array}$ & $\begin{array}{c}\text { Q PCT } \\
\mathrm{B}\left(\mathrm{g} / \mathrm{m}^{2}\right)\end{array}$ & $\begin{array}{c}\text { Q PCT } \\
\mathrm{Li}\left(\mathrm{g} / \mathrm{m}^{2}\right)\end{array}$ & $\begin{array}{c}\text { Q PCT } \\
\mathrm{Na}\left(\mathrm{g} / \mathrm{m}^{2}\right) \\
\end{array}$ & $\begin{array}{c}\text { Q PCT } \\
\mathrm{Si}\left(\mathrm{g} / \mathrm{m}^{2}\right)\end{array}$ & $\begin{array}{c}\text { Q PCT } \\
\text { pH }\end{array}$ & $\begin{array}{c}\text { CCC PCT } \\
\text { B }\left(\mathrm{g} / \mathrm{m}^{2}\right)\end{array}$ & $\begin{array}{c}\text { CCC PCT } \\
\mathrm{Li}\left(\mathrm{g} / \mathrm{m}^{2}\right) \\
\end{array}$ & $\begin{array}{l}\text { CCC PCT } \\
\mathrm{Na}\left(\mathrm{g} / \mathrm{m}^{2}\right)\end{array}$ & $\begin{array}{c}\text { CCC PCT } \\
\mathrm{Si}\left(\mathrm{g} / \mathrm{m}^{2}\right)\end{array}$ & $\begin{array}{c}\text { CCC PCT } \\
\mathrm{pH}\end{array}$ & $\begin{array}{l}\text { Q PCT B at } \\
20^{\circ} \mathrm{C}\left(\mathrm{g} / \mathrm{m}^{2}\right)\end{array}$ & $\begin{array}{l}\text { Q PCT Li at } \\
20^{\circ} \mathrm{C}\left(\mathrm{g} / \mathrm{m}^{2}\right)\end{array}$ \\
\hline \multicolumn{17}{|l|}{ LAWA25H } \\
\hline \multirow{2}{*}{\multicolumn{17}{|c|}{$\frac{\text { LAWA26 }}{\text { LAWA26H }}$}} \\
\hline & & & & & & & & & & & & & & & & \\
\hline LAWB11 & & & & & & & & & & & & & & & 0.0847 & \\
\hline LAWB12 & & & & & & & & & & & & & & & 0.0917 & \\
\hline LAWB13 & & & & & & & & & & & & & & & 0.0406 & \\
\hline LAWB14 & & & & & & & & & & & & & & & 0.0567 & \\
\hline LAWB15 & & & & & & & & & & & & & & & 0.0805 & \\
\hline LAWB16 & & & & & & & & & & & & & & & 0.0147 & \\
\hline \multicolumn{17}{|l|}{ LAWB17 } \\
\hline LAWB18 & & & & & & & & & & & & & & & 0.0245 & \\
\hline \multicolumn{17}{|l|}{ LAWB19 } \\
\hline \multicolumn{17}{|l|}{ LAWB20 } \\
\hline LAWB21 & & & & & & & & & & & & & & & 0.0147 & \\
\hline LAWB22 & & & & & & & & & & & & & & & 0.0406 & \\
\hline LAWB23 & & & & & & & & & & & & & & & 0.0175 & \\
\hline \multicolumn{17}{|l|}{ LAWB24 } \\
\hline \multicolumn{17}{|l|}{ LAWB25 } \\
\hline LAWB26 & & & & & & & & & & & & & & & 0.0252 & \\
\hline LAWB27 & & & & & & & & & & & & & & & 0.0105 & \\
\hline LAWB28 & & & & & & & & & & & & & & & 0.0175 & \\
\hline \multicolumn{17}{|l|}{ LAWB29 } \\
\hline LAWC1 & & & & & & & & & & & & & & & 0.0217 & \\
\hline LAWC2 & & & & & & & & & & & & & & & 0.0189 & \\
\hline LAWC3 & & & & & & & & & & & & & & & 0.0196 & \\
\hline \multicolumn{17}{|l|}{ LAWC4 } \\
\hline \multicolumn{17}{|l|}{ LAWC5 } \\
\hline \multicolumn{17}{|l|}{ LAWPC1 } \\
\hline \multicolumn{17}{|l|}{ LAWPC2 } \\
\hline \multicolumn{17}{|l|}{ LAWPC3 } \\
\hline LAWPC5 & & & & & & & & & & & & & & & 0.1106 & \\
\hline LAWPC6 & & & & & & & & & & & & & & & 0.0231 & \\
\hline \multicolumn{17}{|l|}{ LAWPC7 } \\
\hline \multicolumn{17}{|l|}{ LAWPC8 } \\
\hline LAWPC9 & & & & & & & & & & & & & & & & \\
\hline LAWPC10 & & & & & & & & & & & & & & & & \\
\hline
\end{tabular}


TWRS LAW Formulation (Muller and Pegg 1998)

\begin{tabular}{|c|c|c|c|c|c|c|c|c|c|c|c|c|}
\hline Glass ID & $\begin{array}{l}\text { Q PCT Na at } \\
20^{\circ} \mathrm{C}\left(\mathrm{g} / \mathrm{m}^{2}\right)\end{array}$ & $\begin{array}{l}\text { Q PCT Si at } \\
20^{\circ} \mathrm{C}\left(\mathrm{g} / \mathrm{m}^{2}\right)\end{array}$ & $\begin{array}{c}\mathrm{QpH} \\
\text { at } 20^{\circ} \mathrm{C}\end{array}$ & $\begin{array}{c}\text { TCLP Ag } \\
\text { (ppm) }\end{array}$ & $\begin{array}{c}\text { TCLP As } \\
\text { (ppm) }\end{array}$ & $\begin{array}{c}\text { TCLP Ba } \\
\text { (ppm) }\end{array}$ & $\begin{array}{c}\text { TCLP Cd } \\
(\mathrm{ppm})\end{array}$ & $\begin{array}{c}\text { TCLP Cr } \\
(\mathrm{ppm})\end{array}$ & $\begin{array}{c}\text { TCLP Ni } \\
(\mathrm{ppm})\end{array}$ & $\begin{array}{c}\text { TCLP Pb } \\
\text { (ppm) }\end{array}$ & $\begin{array}{c}\text { TCLP Se } \\
(\mathrm{ppm})\end{array}$ & $\begin{array}{c}\text { TCLP Zn } \\
\text { (ppm) }\end{array}$ \\
\hline LAWA25H & 0.1078 & 0.0098 & 8.78 & & & & & & & & & \\
\hline LAWA26 & 0.1274 & 0.0126 & 9.38 & & & & & & & & & \\
\hline LAWA26H & 0.1316 & 0.0126 & 9.32 & & & & & & & & & \\
\hline LAWB11 & 0.0476 & 0.0196 & 8.54 & & & & & & & & & \\
\hline LAWB12 & 0.0595 & 0.0189 & 8.83 & & & & & & & & & \\
\hline LAWB13 & 0.0294 & 0.0147 & 8.76 & & & & & & & & & \\
\hline LAWB14 & 0.0539 & 0.0175 & 8.55 & & & & & & & & & \\
\hline LAWB15 & 0.0476 & 0.0147 & 8.85 & & & & & & & & & \\
\hline LAWB16 & 0.0252 & 0.0063 & 8.1 & & & & & & & & & \\
\hline LAWB17 & 0.0252 & 0.0084 & 7.76 & & & & & & & & & \\
\hline LAWB18 & 0.0252 & 0.0084 & 7.8 & & & & & & & & & \\
\hline LAWB19 & 0.014 & 0.0063 & 7.77 & & & & & & & & & \\
\hline LAWB20 & 0.0217 & 0.0084 & 7.74 & & & & & & & & & \\
\hline LAWB21 & 0.0217 & 0.0084 & 7.85 & & & & & & & & & \\
\hline LAWB22 & 0.0224 & 0.0077 & 7.75 & & & & & & & & & \\
\hline LAWB23 & 0.0224 & 0.0077 & 7.84 & & & & & & & & & \\
\hline LAWB24 & 0.0217 & 0.007 & 7.84 & & & & & & & & & \\
\hline LAWB25 & 0.0224 & 0.0077 & 7.84 & & & & & & & & & \\
\hline LAWB26 & 0.0161 & 0.0077 & 7.71 & & & & & & & & & \\
\hline LAWB27 & 0.0168 & 0.0091 & 7.58 & & & & & & & & & \\
\hline LAWB28 & 0.0287 & 0.0147 & 7.74 & & & & & & & & & \\
\hline LAWB29 & 0.0161 & 0.0084 & 7.72 & & & $<1.0$ & 0.004 & 0.009 & 0.02 & 0.026 & & 1.85 \\
\hline LAWC1 & 0.0532 & 0.0119 & 8.25 & & & & & & & & & \\
\hline LAWC2 & 0.0644 & 0.0112 & 8.87 & & & & & & & & & \\
\hline LAWC3 & 0.0595 & 0.0098 & 8.22 & & & & & & & & & \\
\hline LAWC4 & 0.8974 & 0.1239 & 8.33 & & & & & & & & & \\
\hline LAWC5 & 1.029 & 0.1344 & 8.41 & & & & & & & & & \\
\hline LAWPC1 & 0.0462 & 0.0077 & 8.22 & & & & & & & & & \\
\hline LAWPC2 & 0.0518 & 0.0077 & 8.2 & & & & & & & & & \\
\hline LAWPC3 & 0.056 & 0.0091 & 8.21 & & & & & & & & & \\
\hline LAWPC5 & 0.0567 & 0.0077 & 8.24 & & & & & & & & & \\
\hline LAWPC6 & 0.0679 & 0.0091 & 8.28 & & & & & & & & & \\
\hline LAWPC7 & 0.0336 & 0.0063 & 7.99 & & & & & & & & & \\
\hline LAWPC8 & 0.0301 & 0.0084 & 8.12 & & & & & & & & & \\
\hline LAWPC9 & 0.0504 & 0.0091 & 8.15 & & & & & & & & & \\
\hline LAWPC10 & 0.0651 & 0.0098 & 8.11 & & & $<0.98$ & $<0.0027$ & $<0.0055$ & 0.02 & 0.025 & & 2.45 \\
\hline
\end{tabular}


TWRS LAW Formulation 2 (Ferrara et al. 1998)

\begin{tabular}{|c|c|c|c|c|c|c|c|c|c|c|c|c|c|c|c|c|c|c|c|c|c|}
\hline Glass ID & $\begin{array}{c}\mathrm{A} 12 \mathrm{O} 3 \\
-\mathrm{t}\end{array}$ & $\begin{array}{c}\mathrm{B} 2 \mathrm{O} 3 \\
-\mathrm{t}\end{array}$ & $\begin{array}{c}\mathrm{CaO} \\
-\mathrm{t}\end{array}$ & $\begin{array}{c}\mathrm{Fe} 2 \mathrm{O} 3 \\
-\mathrm{t}\end{array}$ & $\begin{array}{c}\mathrm{FeO} \\
-\mathrm{t}\end{array}$ & $\begin{array}{c}\mathrm{K} 2 \mathrm{O} \\
-\mathrm{t}\end{array}$ & $\begin{array}{c}\mathrm{Li} 2 \mathrm{O} \\
-\mathrm{t}\end{array}$ & $\begin{array}{c}\mathrm{MgO} \\
-\mathrm{t}\end{array}$ & $\begin{array}{c}\mathrm{Na} 2 \mathrm{O} \\
-\mathrm{t}\end{array}$ & $\begin{array}{c}\mathrm{NiO} \\
-\mathrm{t}\end{array}$ & $\begin{array}{c}\mathrm{P} 2 \mathrm{O} 5 \\
-\mathrm{t}\end{array}$ & $\begin{array}{c}\mathrm{SiO} 2 \\
-\mathrm{t}\end{array}$ & $\begin{array}{c}\mathrm{ZrO} 2 \\
-\mathrm{t}\end{array}$ & $\begin{array}{c}\mathrm{Ag} 2 \mathrm{O} \\
-\mathrm{t}\end{array}$ & $\begin{array}{c}\mathrm{As} 2 \mathrm{O} 3 \\
-\mathrm{t}\end{array}$ & $\begin{array}{c}\mathrm{BaO} \\
-\mathrm{t}\end{array}$ & $\begin{array}{c}\mathrm{Bi} 2 \mathrm{O} 3 \\
-\mathrm{t}\end{array}$ & $\begin{array}{c}\mathrm{Br} \\
-\mathrm{t}\end{array}$ & $\begin{array}{c}\mathrm{CdO} \\
-\mathrm{t}\end{array}$ & $\begin{array}{c}\mathrm{Ce} 2 \mathrm{O} 3 \\
-\mathrm{t}\end{array}$ & $\begin{array}{c}\mathrm{CeO} 2 \\
-\mathrm{t}\end{array}$ \\
\hline Envelope A & 0.0970 & 0.0430 & 0.0450 & 0.0740 & & 0.0230 & 0.0210 & 0.0210 & 0.2000 & & & 0.4100 & 0.0310 & & & & & & & & \\
\hline Envelope B & 0.0810 & 0.0810 & 0.0700 & 0.0800 & & 0.0037 & 0.0410 & 0.0300 & 0.1000 & & & 0.4400 & 0.0300 & & & & & & & & \\
\hline Envelope C & 0.0990 & 0.0300 & 0.0480 & 0.0640 & & 0.0037 & 0.0400 & 0.0300 & 0.2000 & & & 0.4200 & 0.0300 & & & & & & & & \\
\hline
\end{tabular}

\section{ISV Glass (Carter et al. 1988)}

ORNL ISV

Hanford ISV

Hanford ISV (CaO adjusted)

Hanford ISV ( $\mathrm{Na} 2 \mathrm{O}$ adjusted)

TWRS Envelop D HLW Glass (Crawford et al. 1998)

Envelope D $: 0.1013$ : $0.0699: 0.0404: 0.2295$

\section{SRS M-Area Mixed Waste Glass (Fu et al. 1997)}

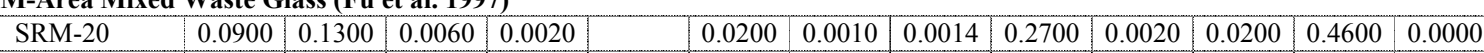

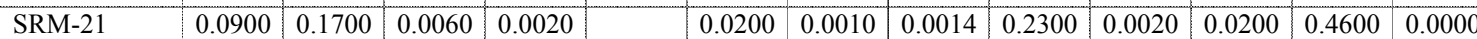

\begin{tabular}{|l|l|l|l|l|l|l|l|l|l|l|l|l|l|l|l|l|l|l|l}
\hline SRM-22 & 0.0900 & 0.2100 & 0.0060 & 0.0020 & & 0.0200 & 0.0010 & 0.0014 & 0.1900 & 0.0020 & 0.0200 & 0.4600 & 0.0000 \\
\hline
\end{tabular}

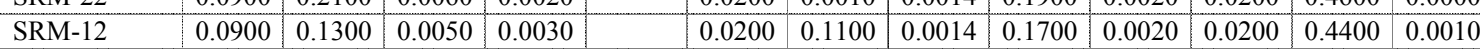

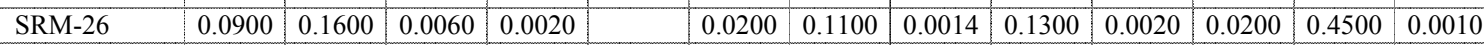

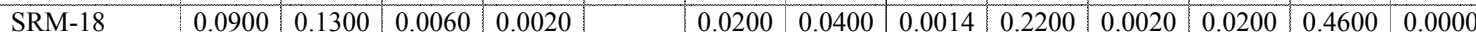

0.0008

0.0002

Kinetics of Spinel Crystallization (Reynolds and Hrma 1997)

\begin{tabular}{l|l|l|l|l|l}
\hline HLW & 0.0833 & 0.0500 & 0.0132 & 0.0705 \\
\hline
\end{tabular}

\begin{tabular}{|l|l|l|l|l|l|l|l|}
\hline 0.0014 & 0.0186 & 0.0005 & 0.1621 & 0.0145 & 0.0302 & 0.3960 & 0.0454 \\
\hline
\end{tabular}

0.0006

0.0175

\section{TWRS HLW Glass Formulation (Fu and Pegg 1998)}

\begin{tabular}{|c|c|c|c|c|c|c|c|c|c|c|c|c|c|c|c|c|c|}
\hline HLWD1-01 & 0.0760 & 0.0842 & 0.0288 & 0.1191 & 0.0047 & 0.0009 & 0.0101 & 0.0788 & 0.0087 & 0.0116 & 0.3629 & 0.0582 & 0.0000 & 0.0146 & 0.0000 & 0.0150 & 0.0029 \\
\hline HLWD1-02 & 0.0753 & 0.0835 & 0.0285 & 0.1181 & 0.0046 & 0.0009 & 0.0100 & 0.1292 & 0.0086 & 0.0115 & 0.3086 & 0.0576 & 0.0000 & 0.0145 & 0.0089 & 0.0148 & 0.0028 \\
\hline HLWD1-03 & 0.0753 & 0.1345 & 0.0285 & 0.1181 & 0.0046 & 0.0009 & 0.0100 & 0.0781 & 0.0086 & 0.0115 & 0.3086 & 0.0576 & 0.0000 & 0.0145 & 0.0089 & 0.0148 & 0.0028 \\
\hline HLWD1-04 & 0.0753 & 0.0835 & 0.0796 & 0.1181 & 0.0046 & 0.0009 & 0.0100 & 0.0781 & 0.0086 & 0.0115 & 0.3086 & 0.0576 & 0.0000 & 0.0145 & 0.0089 & 0.0148 & 0.0028 \\
\hline HLWD1-05 & 0.0753 & 0.0835 & 0.0285 & 0.1181 & 0.0046 & 0.0315 & 0.0100 & 0.0781 & 0.0086 & 0.0115 & 0.3290 & 0.0576 & 0.0000 & 0.0145 & 0.0089 & 0.0148 & 0.0028 \\
\hline HLWD1-07 & 0.0328 & 0.0679 & 0.0000 & 0.1897 & 0.0001 & 0.0291 & 0.0000 & 0.0709 & 0.0009 & 0.0165 & 0.3202 & 0.0000 & 0.0000 & 0.0209 & 0.0128 & 0.0214 & 0.0041 \\
\hline HLWD1-08 & 0.0320 & 0.0969 & 0.0000 & 0.1855 & 0.0001 & 0.0291 & 0.0000 & 0.0418 & 0.0121 & 0.0161 & 0.3199 & 0.0000 & 0.0000 & 0.0204 & 0.0125 & 0.0209 & 0.0040 \\
\hline HLWD1-09 & 0.0320 & 0.0485 & 0.0000 & 0.1855 & 0.0001 & 0.0291 & 0.0000 & 0.0903 & 0.0121 & 0.0161 & 0.3199 & 0.0000 & 0.0000 & 0.0204 & 0.0125 & 0.0209 & 0.0040 \\
\hline
\end{tabular}


TWRS LAW Formulation 2 (Ferrara et al. 1998)

\begin{tabular}{|c|c|c|c|c|c|c|c|c|c|c|c|c|c|c|c|c|c|c|c|c|c|}
\hline Glass ID & $\begin{array}{l}\mathrm{Cl} \\
-\mathrm{t}\end{array}$ & $\begin{array}{c}\mathrm{CoO} \\
-\mathrm{t}\end{array}$ & $\begin{array}{c}\mathrm{Co} 2 \mathrm{O} 3 \\
-\mathrm{t}\end{array}$ & $\begin{array}{c}\mathrm{Cr} 2 \mathrm{O} 3 \\
-\mathrm{t}\end{array}$ & $\begin{array}{c}\mathrm{Cs} 2 \mathrm{O} \\
-\mathrm{t}\end{array}$ & $\begin{array}{c}\mathrm{CuO} \\
-\mathrm{t}\end{array}$ & $\begin{array}{c}\mathrm{Eu} 2 \mathrm{O} 3 \\
-\mathrm{t}\end{array}$ & $\begin{array}{l}F \\
-t\end{array}$ & $\mathrm{Ga} 2 \mathrm{O} 3$ & $\begin{array}{c}\mathrm{Gd} 2 \mathrm{O} 3 \\
-\mathrm{t}\end{array}$ & $\begin{array}{c}\mathrm{HgO} \\
-\mathrm{t}\end{array}$ & $\begin{array}{l}I \\
-t\end{array}$ & $\begin{array}{c}\mathrm{La} 2 \mathrm{O} 3 \\
-\mathrm{t}\end{array}$ & $\begin{array}{c}\mathrm{MnO} 2 \\
-\mathrm{t}\end{array}$ & $\begin{array}{c}\mathrm{MnO} \\
-\mathrm{t}\end{array}$ & $\begin{array}{c}\mathrm{MoO} \\
-\mathrm{t}\end{array}$ & $\begin{array}{c}\mathrm{MoO} 3 \\
-\mathrm{t}\end{array}$ & $\begin{array}{c}\mathrm{Nb} 2 \mathrm{O} 5 \\
-\mathrm{t}\end{array}$ & $\begin{array}{c}\mathrm{Nd} 2 \mathrm{O} 3 \\
-\mathrm{t}\end{array}$ & $\begin{array}{c}\mathrm{PbO} \\
-\mathrm{t}\end{array}$ & $\begin{array}{c}\mathrm{PdO} 2 \\
-\mathrm{t}\end{array}$ \\
\hline Envelope A & & & & 0.0001 & & & & & & & & & & & & & & & & & \\
\hline Envelope B & & & & 0.0010 & & & & & & & & & & & & & & & & & \\
\hline Envelope C & & & & 0.0001 & & & & & & & & & & & & & & & & & \\
\hline
\end{tabular}

ISV Glass (Carter et al. 1988)

ORNL ISV

Hanford ISV

Hanford ISV ( $\mathrm{CaO}$ adjusted)

Hanford ISV (Na2O adjusted)

TWRS Envelop D HLW Glass (Crawford et al. 1998)

0.0041

0.0019

SRS M-Area Mixed Waste Glass (Fu et al. 1997)

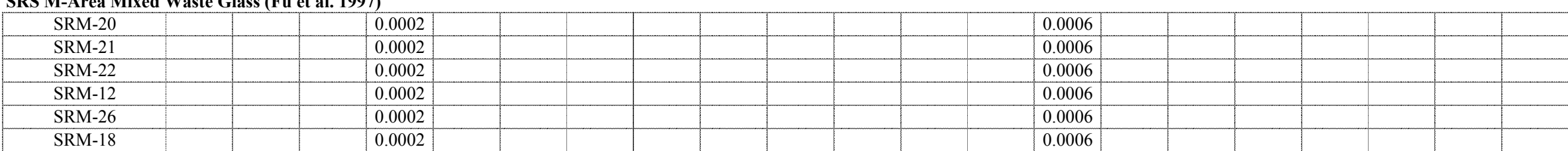

Kinetics of Spinel Crystallization (Reynolds and Hrma 1997)

\begin{tabular}{l|l|l|l|} 
HLW & & 0.0029 \\
\hline
\end{tabular}

0.0036

0.0027

0.0095

0.0005

\begin{tabular}{|l|l|}
\hline 0.0560 & 0.0023 \\
\hline
\end{tabular}

\section{TWRS HLW Glass Formulation (Fu and Pegg 1998)}

\begin{tabular}{|c|c|c|c|c|c|c|c|c|c|c|c|}
\hline HLWD1-01 & 0.0000 & 0.0017 & 0.0029 & 0.0018 & 0.0018 & 0.0081 & 0.0088 & 0.0299 & 0.0022 & 0.0058 & 0.0034 \\
\hline HLWD1-02 & 0.0000 & 0.0017 & 0.0028 & 0.0018 & 0.0017 & 0.0080 & 0.0087 & 0.0296 & 0.0022 & 0.0057 & 0.0034 \\
\hline HLWD1-03 & 0.0000 & 0.0017 & 0.0028 & 0.0018 & 0.0017 & 0.0080 & 0.0087 & 0.0296 & 0.0022 & 0.0057 & 0.0034 \\
\hline HLWD1-04 & 0.0000 & 0.0017 & 0.0028 & 0.0018 & 0.0017 & 0.0080 & 0.0087 & 0.0296 & 0.0022 & 0.0057 & 0.0034 \\
\hline HLWD1-05 & 0.0000 & 0.0017 & 0.0028 & 0.0018 & 0.0017 & 0.0080 & 0.0087 & 0.0296 & 0.0022 & 0.0057 & 0.0034 \\
\hline HLWD1-07 & 0.0000 & 0.0024 & 0.0041 & 0.0026 & 0.0025 & 0.0152 & 0.0125 & 0.0426 & 0.0031 & 0.0083 & 0.0049 \\
\hline HLWD1-08 & 0.0000 & 0.0023 & 0.0040 & 0.0025 & 0.0024 & 0.0152 & 0.0122 & 0.0415 & 0.0030 & 0.0081 & 0.0048 \\
\hline HLWD1-09 & 0.0000 & 0.0023 & 0.0040 & 0.0025 & 0.0024 & 0.0152 & 0.0122 & 0.0415 & 0.0030 & 0.0081 & 0.0048 \\
\hline
\end{tabular}


TWRS LAW Formulation 2 (Ferrara et al. 1998)

\begin{tabular}{|c|c|c|c|c|c|c|c|c|c|c|c|c|c|c|c|c|c|c|c|c|c|}
\hline Glass ID & $\begin{array}{c}\mathrm{PdO} \\
-\mathrm{t}\end{array}$ & $\begin{array}{c}\mathrm{Pr} 2 \mathrm{O} 3 \\
-\mathrm{t}\end{array}$ & $\begin{array}{c}\text { Pr6O11 } \\
-t\end{array}$ & $\begin{array}{c}\mathrm{Rb} 2 \mathrm{O} \\
-\mathrm{t}\end{array}$ & $\begin{array}{c}\mathrm{ReO} \\
-\mathrm{t}\end{array}$ & $\begin{array}{c}\mathrm{ReO} 2 \\
-\mathrm{t}\end{array}$ & $\begin{array}{c}\mathrm{Rh} 2 \mathrm{O} 3 \\
-\mathrm{t}\end{array}$ & $\begin{array}{c}\mathrm{RhO}^{2} \\
-\mathrm{t}\end{array}$ & $\begin{array}{c}\mathrm{RuO}_{2} \\
-\mathrm{t}\end{array}$ & $\begin{array}{c}\mathrm{Sb} 2 \mathrm{O} 3 \\
-\mathrm{t}\end{array}$ & $\begin{array}{c}\mathrm{Sb} 2 \mathrm{O} 5 \\
-\mathrm{t}\end{array}$ & $\begin{array}{c}\mathrm{SeO} 2 \\
-\mathrm{t}\end{array}$ & $\begin{array}{c}\mathrm{Sm} 2 \mathrm{O} 3 \\
-\mathrm{t}\end{array}$ & $\begin{array}{c}\mathrm{SnO} \\
-\mathrm{t}\end{array}$ & $\begin{array}{c}\mathrm{SnO} 2 \\
-\mathrm{t}\end{array}$ & $\begin{array}{c}\mathrm{SO} 3 \\
-\mathrm{t}\end{array}$ & $\begin{array}{c}\mathrm{SrO} \\
-\mathrm{t}\end{array}$ & $\begin{array}{c}\mathrm{Tc} 2 \mathrm{O} 7 \\
-\mathrm{t}\end{array}$ & $\begin{array}{c}\mathrm{TeO} 2 \\
-\mathrm{t}\end{array}$ & $\begin{array}{c}\mathrm{ThO} 2 \\
-\mathrm{t}\end{array}$ & $\begin{array}{c}\mathrm{TiO} 2 \\
-\mathrm{t}\end{array}$ \\
\hline Envelope A & & & & & & & & & & & & & & & & & & & & & \\
\hline Envelope B & & & & & & & & & & & & & & & & & & & & & \\
\hline Envelope C & & & & & & & & & & & & & & & & & & & & & \\
\hline
\end{tabular}

ISV Glass (Carter et al. 1988)

$$
\text { ORNL ISV }
$$

Hanford ISV ( $\mathrm{CaO}$ adjusted)

Hanford ISV ( $\mathrm{Na} 2 \mathrm{O}$ adjusted)

\section{TWRS Envelop D HLW Glass (Crawford et al. 1998)}

Envelope D

0.0416

$0 . 0 0 0 0 \longdiv { 0 . 0 0 1 9 }$

SRS M-Area Mixed Waste Glass (Fu et al. 1997)

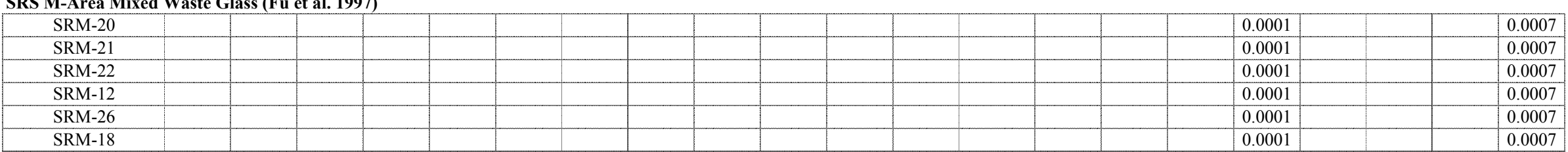

Kinetics of Spinel Crystallization (Reynolds and Hrma 1997)

HLW

\begin{tabular}{|c|c|c|c|c|c|c|c|c|c|c|c|c|c|}
\hline HLWD1-01 & 0.0000 & 0.0000 & 0.0000 & 0.0000 & 0.0000 & 0.0000 & 0.0000 & 0.0000 & 0.0047 & 0.0018 & 0.0000 & 0.0000 & 0.0062 \\
\hline HLWD1-02 & 0.0000 & 0.0000 & 0.0000 & 0.0000 & 0.0000 & 0.0000 & 0.0000 & 0.0000 & 0.0046 & 0.0018 & 0.0000 & 0.0000 & 0.0062 \\
\hline HLWD1-03 & 0.0000 & 0.0000 & 0.0000 & 0.0000 & 0.0000 & 0.0000 & 0.0000 & 0.0000 & 0.0046 & 0.0018 & 0.0000 & 0.0000 & 0.0062 \\
\hline HLWD1-04 & 0.0000 & 0.0000 & 0.0000 & 0.0000 & 0.0000 & 0.0000 & 0.0000 & 0.0000 & 0.0046 & 0.0018 & 0.0000 & 0.0000 & 0.0062 \\
\hline HLWD1-05 & 0.0000 & 0.0000 & 0.0000 & 0.0000 & 0.0000 & 0.0000 & 0.0000 & 0.0000 & 0.0046 & 0.0018 & 0.0000 & 0.0000 & 0.0062 \\
\hline HLWD1-07 & 0.0000 & 0.0000 & 0.0000 & 0.0000 & 0.0000 & 0.0000 & 0.0000 & 0.0000 & 0.0067 & 0.0307 & 0.0000 & 0.0000 & 0.0089 \\
\hline HLWD1-08 & 0.0000 & 0.0000 & 0.0000 & 0.0000 & 0.0000 & 0.0000 & 0.0000 & 0.0000 & 0.0065 & 0.0306 & 0.0000 & 0.0000 & 0.0087 \\
\hline HLWD1-09 & 0.0000 & 0.0000 & 0.0000 & 0.0000 & 0.0000 & 0.0000 & 0.0000 & 0.0000 & 0.0065 & 0.0306 & 0.0000 & 0.0000 & 0.0087 \\
\hline
\end{tabular}

\section{TWRS HLW Glass Formulation (Fu and Pegg 1998)}


TWRS LAW Formulation 2 (Ferrara et al. 1998)

\begin{tabular}{|c|c|c|c|c|c|c|c|c|c|c|c|c|c|c|c|c|c|c|c|c|c|}
\hline Glass ID & $\begin{array}{c}\mathrm{T} 12 \mathrm{O} 3 \\
-\mathrm{t}\end{array}$ & $\begin{array}{c}\text { U3O8 } \\
-t\end{array}$ & $\begin{array}{c}\mathrm{UO} 2 \\
-\mathrm{t}\end{array}$ & $\begin{array}{c}\text { UO3 } \\
-t\end{array}$ & $\begin{array}{c}\mathrm{V} 2 \mathrm{O} 5 \\
-\mathrm{t}\end{array}$ & $\begin{array}{c}\text { WO3 } \\
-t\end{array}$ & $\begin{array}{c}\mathrm{Y} 2 \mathrm{O} 3 \\
-\mathrm{t}\end{array}$ & $\begin{array}{c}\mathrm{ZnO} \\
-\mathrm{t}\end{array}$ & $\begin{array}{c}\text { Others } \\
-t\end{array}$ & $\begin{array}{c}\text { Sum } \\
-t\end{array}$ & $\begin{array}{c}\mathrm{A} 12 \mathrm{O} 3 \\
-\mathrm{a}\end{array}$ & $\begin{array}{c}\mathrm{B} 2 \mathrm{O} 3 \\
-\mathrm{a}\end{array}$ & $\begin{array}{c}\mathrm{CaO} \\
-\mathrm{a}\end{array}$ & $\begin{array}{c}\mathrm{Fe} 2 \mathrm{O} 3 \\
-\mathrm{a}\end{array}$ & $\begin{array}{c}\mathrm{FeO} \\
-\mathrm{a}\end{array}$ & $\begin{array}{c}\mathrm{K} 2 \mathrm{O} \\
-\mathrm{a}\end{array}$ & $\begin{array}{c}\mathrm{Li} 2 \mathrm{O} \\
-\mathrm{a}\end{array}$ & $\begin{array}{c}\mathrm{MgO} \\
-\mathrm{a}\end{array}$ & $\begin{array}{c}\mathrm{Na} 2 \mathrm{O} \\
-\mathrm{a}\end{array}$ & $\begin{array}{c}\mathrm{NiO} \\
-\mathrm{a}\end{array}$ & $\begin{array}{c}\mathrm{P} 2 \mathrm{O} 5 \\
-\mathrm{a}\end{array}$ \\
\hline Envelope A & & & & & & & & 0.0330 & & 0.9981 & 0.0940 & 0.0420 & 0.0440 & 0.0810 & & 0.0220 & 0.0210 & 0.0200 & 0.2000 & 0.0014 & 0.0007 \\
\hline Envelope B & & & & & & & & 0.0400 & & 0.9977 & 0.0770 & 0.0750 & 0.0650 & 0.0870 & & 0.0038 & 0.0400 & 0.0280 & 0.1300 & 0.0016 & 0.0002 \\
\hline Envelope C & & & & & & & & 0.0330 & & 0.9978 & 0.0910 & 0.0300 & 0.0460 & 0.0690 & & 0.0017 & 0.0390 & 0.0280 & 0.2200 & 0.0017 & 0.0006 \\
\hline \multicolumn{22}{|c|}{ V Glass (Carter et al. 1988) } \\
\hline ORNL ISV & & & & & & & & & & & 0.1360 & 0.0016 & 0.1390 & 0.0378 & & 0.0320 & 0.0008 & 0.1010 & 0.0105 & 0.0004 & \\
\hline Hanford ISV & & & & & & & & & & & 0.1220 & 0.0009 & 0.0454 & 0.0657 & & 0.0190 & & 0.0221 & 0.0313 & 0.0005 & \\
\hline \multicolumn{2}{|c|}{ Hanford ISV ( $\mathrm{CaO}$ adjusted) } & & & & & & & & & & 0.1020 & 0.0008 & 0.1810 & 0.0550 & & 0.0150 & 0.0005 & 0.0192 & 0.0301 & 0.0025 & $\mathrm{Ha}$ \\
\hline \multicolumn{2}{|c|}{ Hanford ISV ( $\mathrm{Na} 2 \mathrm{O}$ adjusted) } & & & & & & & & & & 0.1030 & & 0.0393 & 0.0559 & & 0.0090 & 0.0003 & 0.0183 & 0.1780 & 0.0004 & Har \\
\hline
\end{tabular}

TWRS Envelop D HLW Glass (Crawford et al. 1998)

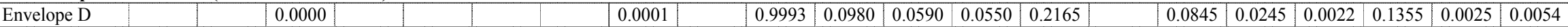

SRS M-Area Mixed Waste Glass (Fu et al. 1997)

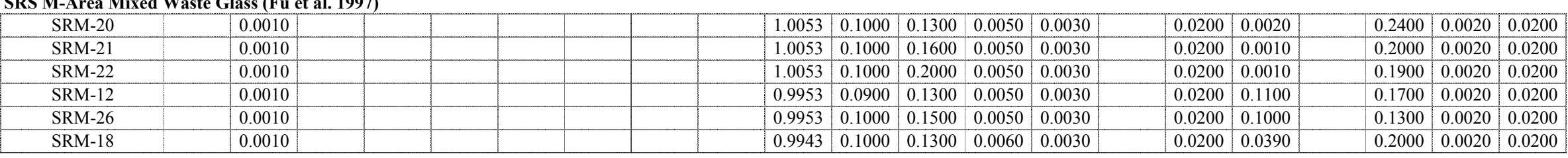

Kinetics of Spinel Crystallization (Reynolds and Hrma 1997)

HLW $\left[\begin{array}{l|l|l|l|l|} & \\ & & & \end{array}\right.$

TWRS HLW Glass Formulation (Fu and Pegg 1998)

\begin{tabular}{|c|c|c|c|c|c|c|c|c|c|c|c|c|c|c|c|c|c|}
\hline HLWD1-01 & 0.0000 & 0.0446 & 0.0000 & 0.0000 & 0.0000 & 0.0000 & 1.0002 & 0.0621 & 0.0803 & 0.0267 & 0.1016 & 0.0052 & 0.0017 & 0.0100 & 0.0637 & 0.0077 & 0.0330 \\
\hline HLWD1-02 & 0.0000 & 0.0442 & 0.0000 & 0.0000 & 0.0000 & 0.0000 & 0.9998 & 0.0677 & 0.0786 & 0.0286 & 0.1048 & 0.0048 & 0.0014 & 0.0102 & 0.1068 & 0.0079 & 0.0097 \\
\hline HLWD1-03 & 0.0000 & 0.0442 & 0.0000 & 0.0000 & 0.0000 & 0.0000 & 0.9997 & 0.0673 & 0.1235 & 0.0280 & 0.1036 & 0.0047 & 0.0015 & 0.0100 & 0.0674 & 0.0042 & 0.0100 \\
\hline HLWD1-04 & 0.0000 & 0.0442 & 0.0000 & 0.0000 & 0.0000 & 0.0000 & 0.9998 & 0.0704 & 0.0823 & 0.0747 & 0.1038 & 0.0047 & 0.0018 & 0.0109 & 0.0699 & 0.0075 & 0.0125 \\
\hline HLWD1-05 & 0.0000 & 0.0442 & 0.0000 & 0.0000 & 0.0000 & 0.0000 & 0.9997 & 0.0628 & 0.0833 & 0.0269 & 0.1030 & 0.0049 & 0.0286 & 0.0104 & 0.0657 & 0.0071 & 0.0136 \\
\hline HLWD1-07 & & 0.0637 & & 0.0000 & 0.0000 & 0.0000 & 0.9955 & 0.0324 & 0.0751 & 0.0052 & 0.1601 & 0.0003 & 0.0293 & 0.0002 & 0.0700 & 0.0009 & 0.0182 \\
\hline HLWD1-08 & & 0.0621 & & 0.0000 & 0.0000 & 0.0000 & 0.9952 & 0.0340 & 0.0958 & 0.0007 & 0.1621 & 0.0005 & 0.0280 & 0.0002 & 0.0417 & 0.0107 & 0.0184 \\
\hline HLWD1-09 & & 0.0621 & & 0.0000 & 0.0000 & 0.0000 & 0.9953 & 0.0336 & 0.0503 & 0.0007 & 0.1672 & 0.0004 & 0.0302 & 0.0002 & 0.0816 & 0.0110 & 0.0185 \\
\hline
\end{tabular}


TWRS LAW Formulation 2 (Ferrara et al. 1998)

\begin{tabular}{|c|c|c|c|c|c|c|c|c|c|c|c|c|c|c|c|c|c|c|c|c|c|}
\hline Glass ID & $\begin{array}{c}\mathrm{SiO} 2 \\
-\mathrm{a}\end{array}$ & $\begin{array}{c}\mathrm{ZrO} 2 \\
-\mathrm{a}\end{array}$ & $\begin{array}{c}\mathrm{Ag} 2 \mathrm{O} \\
-\mathrm{a}\end{array}$ & $\begin{array}{c}\mathrm{As} 2 \mathrm{O} 3 \\
-\mathrm{a}\end{array}$ & $\begin{array}{c}\mathrm{BaO} \\
-\mathrm{a}\end{array}$ & $\begin{array}{c}\mathrm{Bi} 2 \mathrm{O} 3 \\
-\mathrm{a}\end{array}$ & $\begin{array}{l}\mathrm{Br} \\
-\mathrm{a}\end{array}$ & $\begin{array}{c}\mathrm{CdO} \\
-\mathrm{a}\end{array}$ & $\begin{array}{c}\mathrm{Ce} 2 \mathrm{O} 3 \\
-\mathrm{a}\end{array}$ & $\begin{array}{c}\mathrm{CeO} 2 \\
-\mathrm{a}\end{array}$ & $\begin{array}{l}\mathrm{Cl} \\
-\mathrm{a}\end{array}$ & $\begin{array}{c}\mathrm{CoO} \\
-\mathrm{a}\end{array}$ & $\begin{array}{c}\mathrm{Co} 2 \mathrm{O} 3 \\
-\mathrm{a}\end{array}$ & $\begin{array}{c}\mathrm{Cr} 2 \mathrm{O} 3 \\
-\mathrm{a}\end{array}$ & $\begin{array}{c}\mathrm{Cs} 2 \mathrm{O} \\
-\mathrm{a}\end{array}$ & $\begin{array}{c}\mathrm{CuO} \\
-\mathrm{a}\end{array}$ & $\begin{array}{c}\mathrm{Eu} 2 \mathrm{O} 3 \\
-\mathrm{a}\end{array}$ & $\begin{array}{l}\mathrm{F} \\
-\mathrm{a}\end{array}$ & $\begin{array}{c}\mathrm{Ga} 2 \mathrm{O} 3 \\
-\mathrm{a}\end{array}$ & $\begin{array}{c}\mathrm{Gd} 2 \mathrm{O} 3 \\
-\mathrm{a}\end{array}$ & $\begin{array}{c}\mathrm{HgO} \\
-\mathrm{a}\end{array}$ \\
\hline Envelope A & 0.4100 & 0.0310 & 0.0000 & 0.0000 & 0.0000 & 0.0000 & & 0.0000 & & 0.0000 & 0.0007 & & & 0.0022 & 0.0017 & 0.0006 & & 0.0003 & & & \\
\hline Envelope B & 0.4300 & 0.0300 & 0.0000 & 0.0000 & 0.0000 & 0.0000 & & 0.0000 & & 0.0000 & 0.0058 & & & 0.0035 & 0.0016 & 0.0013 & & 0.0000 & & & \\
\hline Envelope C & 0.4100 & 0.0330 & 0.0000 & 0.0000 & 0.0001 & 0.0000 & & 0.0001 & & 0.0000 & 0.0027 & & & 0.0020 & & 0.0008 & & 0.0008 & & & \\
\hline
\end{tabular}

\section{ISV Glass (Carter et al. 1988)}

\begin{tabular}{|l|l|l|l|l|l|l|l|l|}
\hline ORNL ISV & 0.4850 & 0.0003 \\
\hline
\end{tabular} \begin{tabular}{l|l|l|} 
Hanford ISV & 0.6160 & 0.0004 \\
\hline
\end{tabular}

nford ISV $(\mathrm{CaO}$ adjust $0.5110 \quad 0.0002$

iford ISV (Na2O adjus $0.5220 \quad 0.0003$

\begin{tabular}{|l|l|l|l|l|l|l|l|l|l|}
\hline .0010 & & & & & & & & & 0.0196 \\
\hline .0012 & & & & & & & & & 0.0218 \\
\hline .0011 & & & & & & & & 0.0008 & 0.0195
\end{tabular}

TWRS Envelop D HLW Glass (Crawford et al. 1998)

\begin{tabular}{|l|l|l|l|l|l|l|l|l|l|l|}
\hline Envelope D & 0.2570 & 0.0022 & 0.0004 & & & 0.0037
\end{tabular}

\section{SRS M-Area Mixed Waste Glass (Fu et al. 1997)}

\begin{tabular}{|l|l|l|}
\hline SRM-20 & 0.4800 & 0.0000 \\
\hline SRM-21 & 0.4900 & 0.0000 \\
\hline SRM-22 & 0.4700 & 0.0000 \\
\hline SRM-12 & 0.4500 & 0.0100 \\
\hline SRM-26 & 0.4600 & 0.0090 \\
\hline SRM-18 & 0.4800 & 0.0000 \\
\hline
\end{tabular}

Kinetics of Spinel Crystallization (Reynolds and Hrma 1997) HLW

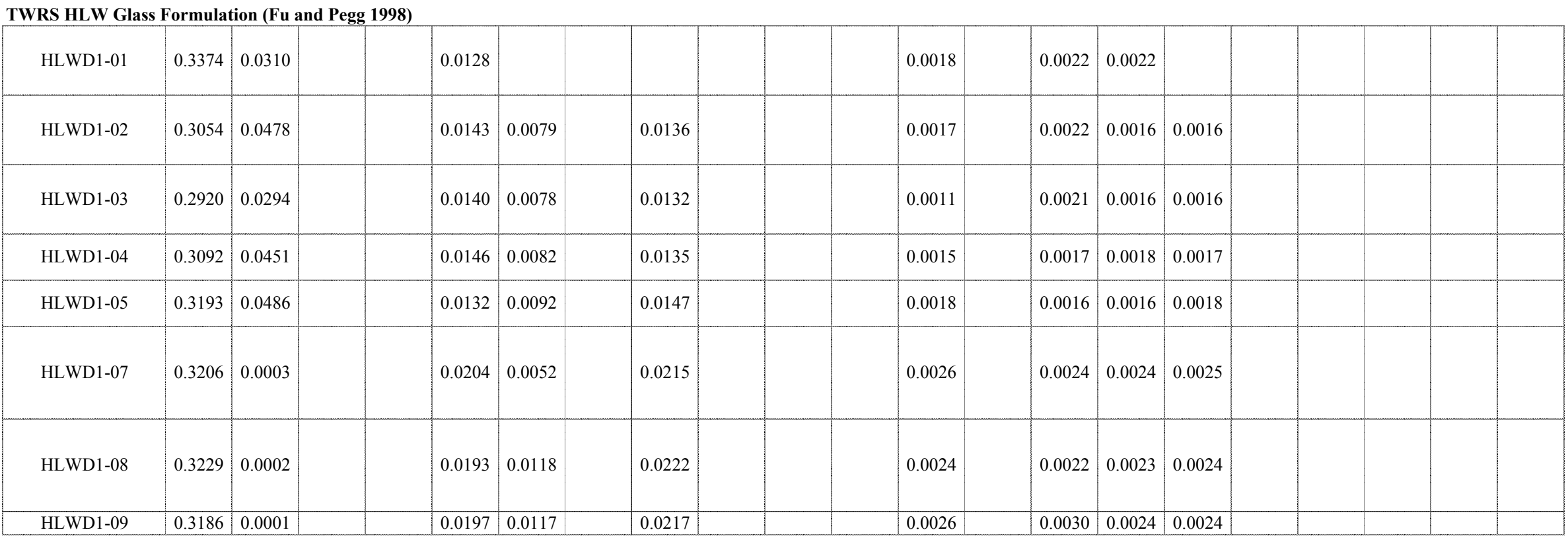


TWRS LAW Formulation 2 (Ferrara et al. 1998)

\begin{tabular}{|c|c|c|c|c|c|c|c|c|c|c|c|c|c|c|c|c|c|c|c|c|c|}
\hline Glass ID & $\begin{array}{c}I \\
-a\end{array}$ & $\begin{array}{c}\mathrm{La} 2 \mathrm{O} 3 \\
-\mathrm{a}\end{array}$ & $\begin{array}{c}\mathrm{MnO} 2 \\
-\mathrm{a}\end{array}$ & $\begin{array}{c}\mathrm{MnO} \\
-\mathrm{a}\end{array}$ & $\begin{array}{c}\mathrm{MoO} \\
-\mathrm{a}\end{array}$ & $\begin{array}{c}\mathrm{MoO} 3 \\
-\mathrm{a}\end{array}$ & $\begin{array}{c}\mathrm{Nb} 2 \mathrm{O} 5 \\
-\mathrm{a}\end{array}$ & $\begin{array}{c}\mathrm{Nd} 2 \mathrm{O} 3 \\
-\mathrm{a}\end{array}$ & $\begin{array}{c}\mathrm{PbO} \\
-\mathrm{a}\end{array}$ & $\begin{array}{c}\mathrm{PdO} 2 \\
-\mathrm{a}\end{array}$ & $\begin{array}{c}\mathrm{PdO} \\
-\mathrm{a}\end{array}$ & $\begin{array}{c}\mathrm{Pr} 2 \mathrm{O} 3 \\
-\mathrm{a}\end{array}$ & $\begin{array}{c}\text { Pr6O11 } \\
-\mathrm{a}\end{array}$ & $\begin{array}{c}\mathrm{Rb} 2 \mathrm{O} \\
-\mathrm{a}\end{array}$ & $\begin{array}{c}\mathrm{ReO} \\
-\mathrm{a}\end{array}$ & $\begin{array}{c}\mathrm{ReO} 2 \\
-\mathrm{a}\end{array}$ & $\begin{array}{c}\mathrm{Rh} 2 \mathrm{O} 3 \\
-\mathrm{a}\end{array}$ & $\begin{array}{c}\mathrm{RhO}_{2} \\
-\mathrm{a}\end{array}$ & $\begin{array}{c}\mathrm{RuO} 2 \\
-\mathrm{a}\end{array}$ & $\begin{array}{c}\mathrm{Sb} 2 \mathrm{O} 3 \\
-\mathrm{a}\end{array}$ & $\begin{array}{c}\mathrm{Sb} 2 \mathrm{O} 5 \\
-\mathrm{a}\end{array}$ \\
\hline Envelope A & & 0.0000 & 0.0002 & & & 0.0002 & & 0.0000 & 0.0001 & & & & & & & & & & & 0.0000 & \\
\hline Envelope B & & 0.0000 & 0.0003 & & & 0.0001 & & 0.0000 & 0.0001 & & & & & & & & & & & 0.0000 & \\
\hline Envelope C & & 0.0000 & 0.0003 & & & 0.0001 & & 0.0000 & 0.0003 & & & & & & & & & & & 0.0000 & \\
\hline
\end{tabular}

ISV Glass (Carter et al. 1988)

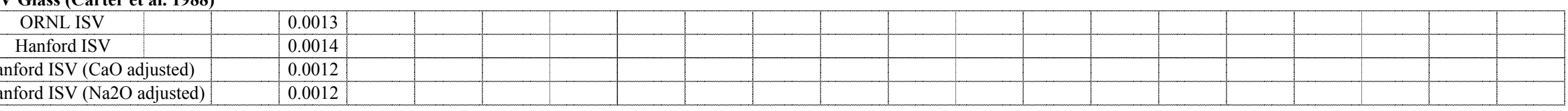

TWRS Envelop D HLW Glass (Crawford et al. 1998)

\begin{tabular}{|l|l|l|l|l|l|l|} 
Envelope D & 0.0003 & 0.0051 & 0.0005 & 0.0037 & & 0.0002
\end{tabular}

SRS M-Area Mixed Waste Glass (Fu et al. 1997)

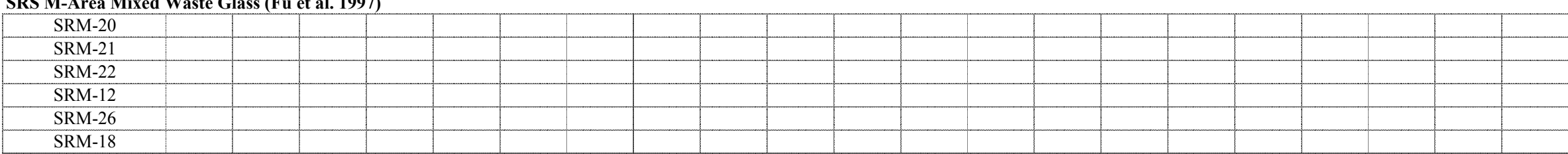

Kinetics of Spinel Crystallization (Reynolds and Hrma 1997)

HLW

TWRS HLW Glass Formulation (Fu and Pegg 1998)

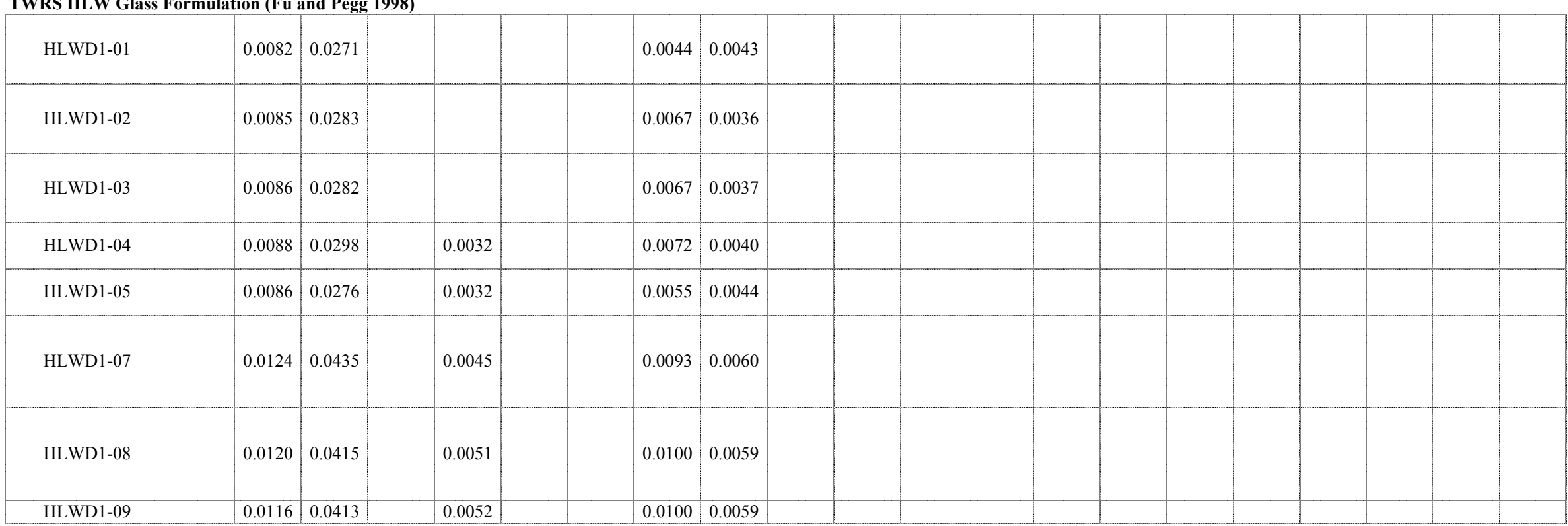


TWRS LAW Formulation 2 (Ferrara et al. 1998)

\begin{tabular}{|c|c|c|c|c|c|c|c|c|c|c|c|c|c|c|c|c|c|c|c|c|}
\hline Glass ID & $\begin{array}{c}\mathrm{SeO} 2 \\
-\mathrm{a}\end{array}$ & $\begin{array}{c}\mathrm{Sm} 2 \mathrm{O} 3 \\
-\mathrm{a}\end{array}$ & $\begin{array}{c}\mathrm{SnO} \\
-\mathrm{a}\end{array}$ & $\begin{array}{c}\mathrm{SnO} 2 \\
-\mathrm{a}\end{array}$ & $\begin{array}{c}\mathrm{SO} 3 \\
-\mathrm{a}\end{array}$ & $\begin{array}{c}\mathrm{SrO} \\
-\mathrm{a}\end{array}$ & $\begin{array}{c}\mathrm{Tc} 2 \mathrm{O} 7 \\
-\mathrm{a}\end{array}$ & $\begin{array}{c}\mathrm{TeO} 2 \\
-\mathrm{a}\end{array}$ & $\begin{array}{c}\mathrm{ThO} 2 \\
-\mathrm{a}\end{array}$ & $\begin{array}{c}\mathrm{TiO} 2 \\
-\mathrm{a}\end{array}$ & $\begin{array}{c}\mathrm{T} 12 \mathrm{O} 3 \\
-\mathrm{a}\end{array}$ & $\begin{array}{c}\mathrm{U} 3 \mathrm{O} 8 \\
-\mathrm{a}\end{array}$ & $\begin{array}{c}\mathrm{UO} 2 \\
-\mathrm{a}\end{array}$ & $\begin{array}{c}\mathrm{UO} 3 \\
-\mathrm{a}\end{array}$ & $\begin{array}{c}\mathrm{V} 2 \mathrm{O} 5 \\
-\mathrm{a}\end{array}$ & $\begin{array}{c}\text { WO3 } \\
-\mathrm{a}\end{array}$ & $\begin{array}{c}\mathrm{Y} 2 \mathrm{O} 3 \\
-\mathrm{a}\end{array}$ & $\begin{array}{c}\mathrm{ZnO} \\
-\mathrm{a}\end{array}$ & $\begin{array}{c}\text { Others } \\
-\mathrm{a}\end{array}$ & $\begin{array}{c}\text { Sum } \\
-\mathrm{a}\end{array}$ \\
\hline Envelope A & 0.0000 & & & & 0.0009 & & & 0.0000 & & 0.0003 & & & 0.0000 & & 0.0008 & & & 0.0320 & & 1.0072 \\
\hline Envelope B & 0.0000 & & & & 0.0002 & & & 0.0000 & & 0.0000 & & & 0.0000 & & 0.0000 & & & 0.0370 & & 1.0175 \\
\hline Envelope C & 0.0000 & & & & 0.0043 & & & 0.0000 & & 0.0002 & & & 0.0000 & & 0.0003 & & & 0.0300 & & 1.0119 \\
\hline \multicolumn{21}{|c|}{ ISV Glass (Carter et al. 1988) } \\
\hline ORNL ISV & & & & & & 0.0416 & & & & 0.0062 & & & & & & & & & & 1.0141 \\
\hline Hanford ISV & & & & & & 0.0372 & & & & 0.0113 & & & & & 0.0003 & & & & & 0.9965 \\
\hline \multicolumn{2}{|c|}{ Hanford ISV ( $\mathrm{CaO}$ adjusted) } & & & & & 0.0364 & & & & 0.0094 & & & & & 0.0002 & & & & & 0.9859 \\
\hline \multicolumn{2}{|c|}{ Hanford ISV ( $\mathrm{Na} 2 \mathrm{O}$ adjusted) } & & & & & 0.0373 & & & & 0.0096 & & & & & 0.0002 & & & & & 0.9974 \\
\hline
\end{tabular}

TWRS Envelop D HLW Glass (Crawford et al. 1998)

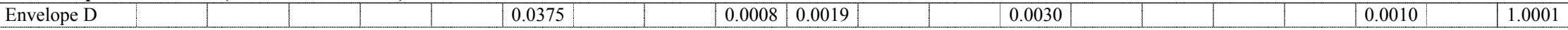

SRS M-Area Mixed Waste Glass (Fu et al. 1997)

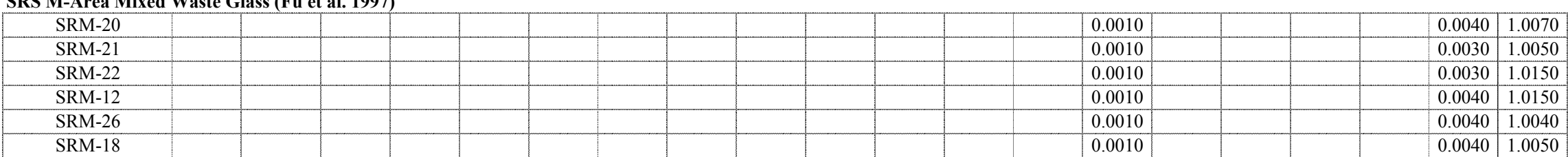

Kinetics of Spinel Crystallization (Reynolds and Hrma 1997)

HLW

TWRS HLW Glass Formulation (Fu and Pegg 1998)

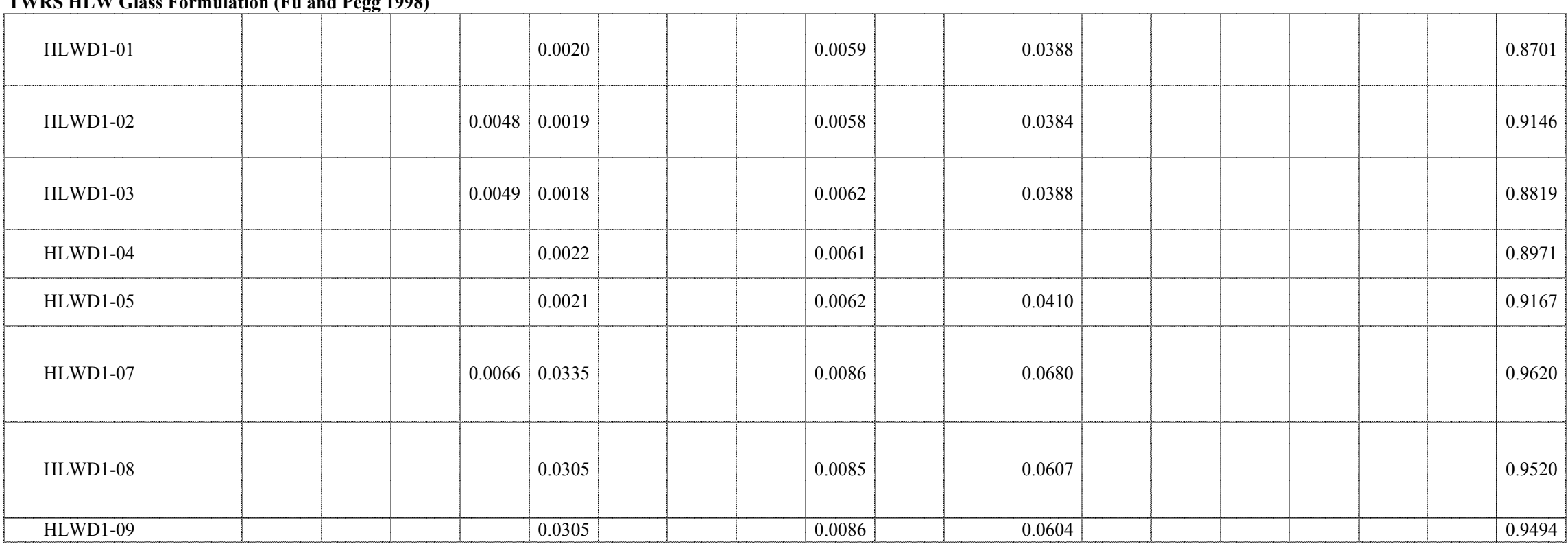


Appendix A. Database - mass fraction

TWRS LAW Formulation 2 (Ferrara et al. 1998)

\begin{tabular}{|c|c|c|c|c|c|c|c|c|}
\hline Glass ID & $\begin{array}{l}\mathrm{TM} \\
\left({ }^{\circ} \mathrm{C}\right)\end{array}$ & $\begin{array}{l}\text { Gradient } \\
\text { TL }\left({ }^{\circ} \mathrm{C}\right)\end{array}$ & $\begin{array}{l}\text { Uniform } \\
\text { TL }\left({ }^{\circ} \mathrm{C}\right)\end{array}$ & Primary Phase & $\begin{array}{l}\text { Quenched } \\
\text { Visual/OM }\end{array}$ & $\begin{array}{c}\text { Quenched } \\
\text { SEM/EDS or TEM }\end{array}$ & $\begin{array}{l}\text { Quenched } \\
\text { XRD }\end{array}$ & $\begin{array}{c}\text { Quenched } \\
\text { Homogeneous? }\end{array}$ \\
\hline Envelope A & & & & & & amorphous & & \\
\hline Envelope B & & & & & & amorphous & & \\
\hline Envelope C & & & & & & amorphous & & \\
\hline
\end{tabular}

ISV Glass (Carter et al. 1988)

ORNL IS

Hanford ISV

Hanford ISV ( $\mathrm{CaO}$ adjusted)

Hanford ISV (Na2O adjusted)

TWRS Envelop D HLW Glass (Crawford et al. 1998)
Envelope D
spinel
$\mathrm{NiFe} 2 \mathrm{O} 4$

SRS M-Area Mixed Waste Glass (Fu et al. 1997)

\begin{tabular}{|c|c|c|c|c|c|c|c|}
\hline SRM-20 & 1150 & & & \\
\hline SRM-21 & 1150 & & & & \\
\hline SRM-22 & 1150 & & & & \\
\hline SRM-12 & 1150 & & & & \\
\hline SRM-26 & 1150 & & & \\
\hline SRM-18 & 1150 & & & \\
\hline
\end{tabular}

Kinetics of Spinel Crystallization (Reynolds and Hrma 1997)

\begin{tabular}{|l|l|l|l|l}
\hline HLW & 1150 & & 1064 & spinel \\
\hline
\end{tabular}

\section{TWRS HLW Glass Formulation (Fu and Pegg 1998)}

\begin{tabular}{|c|c|c|c|c|}
\hline HLWD1-01 & 1150 & $<1050$ & clear dark brown glass & $2.4 \mathrm{vol} \%$ zircon, $0.6 \mathrm{vol} \%$ spinel \\
\hline HLWD1-02 & 1150 & $<1050$ & clear dark brown glass & $0.5 \mathrm{vol} \% \mathrm{ZrO} 2$ and $1 \mathrm{vol} \%$ spinel \\
\hline HLWD1-03 & 1150 & 1050 & clear dark brown glass & $1.5 \mathrm{vol} \%$ zircon and $1 \mathrm{vol} \%$ spinel \\
\hline HLWD1-04 & 1150 & $<1050$ & clear dark brown glass & $\sim 3$ vol\% zircon, spinel, and $\mathrm{ZrO} 2$ \\
\hline HLWD1-05 & 1150 & 950 & clear dark brown glass & $\begin{array}{l}\sim 2 \mathrm{vol} \% \mathrm{ZrO} 2 \text { and spinel with } \mathrm{U} \\
\text { incorporated }\end{array}$ \\
\hline HLWD1-07 & 1150 & $<1050$ & glassy brown & $\begin{array}{l}\text { Clear in bulk, but on bottom of } \\
\text { crucible, about } 10 \text { vol } \% \\
\text { eskolaite/hematite solid solution and } \\
2 \text { vol\% spinel }\end{array}$ \\
\hline HLWD1-08 & 1150 & $<1050$ & glassy brown & \\
\hline HLWD1-09 & 1150 & $<1050$ & glassy brown & \\
\hline
\end{tabular}


Appendix A. Database - mass fraction

TWRS LAW Formulation 2 (Ferrara et al. 1998)

\begin{tabular}{|c|c|c|c|c|c|}
\hline Glass ID & $\begin{array}{c}\text { CCC } \\
\text { Visual/OM }\end{array}$ & $\begin{array}{c}\text { CCC } \\
\text { SEM/EDS or TEM }\end{array}$ & $\begin{array}{l}\text { CCC } \\
\text { XRD }\end{array}$ & $\begin{array}{c}\text { Heat Treated } \\
\text { Visual/OM }\end{array}$ & $\begin{array}{c}\text { Heat Treated } \\
\text { SEM/EDS or TEM }\end{array}$ \\
\hline \multicolumn{6}{|l|}{ Envelope A } \\
\hline Envelope B & & & & & \\
\hline Envelope C & & & & & \\
\hline
\end{tabular}

ISV Glass (Carter et al. 1988)

ORNL ISV

Hanford ISV

Hanford ISV ( $\mathrm{CaO}$ adjusted)

Hanford ISV (Na2O adjusted)

TWRS Envelop D HLW Glass (Crawford et al. 1998)

Envelope D

SRS M-Area Mixed Waste Glass (Fu et al. 1997)

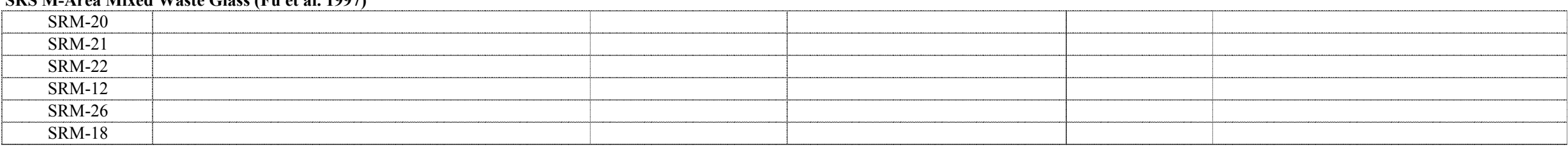

Kinetics of Spinel Crystallization (Reynolds and Hrma 1997)

HLW

\section{TWRS HLW Glass Formulation (Fu and Pegg 1998)}

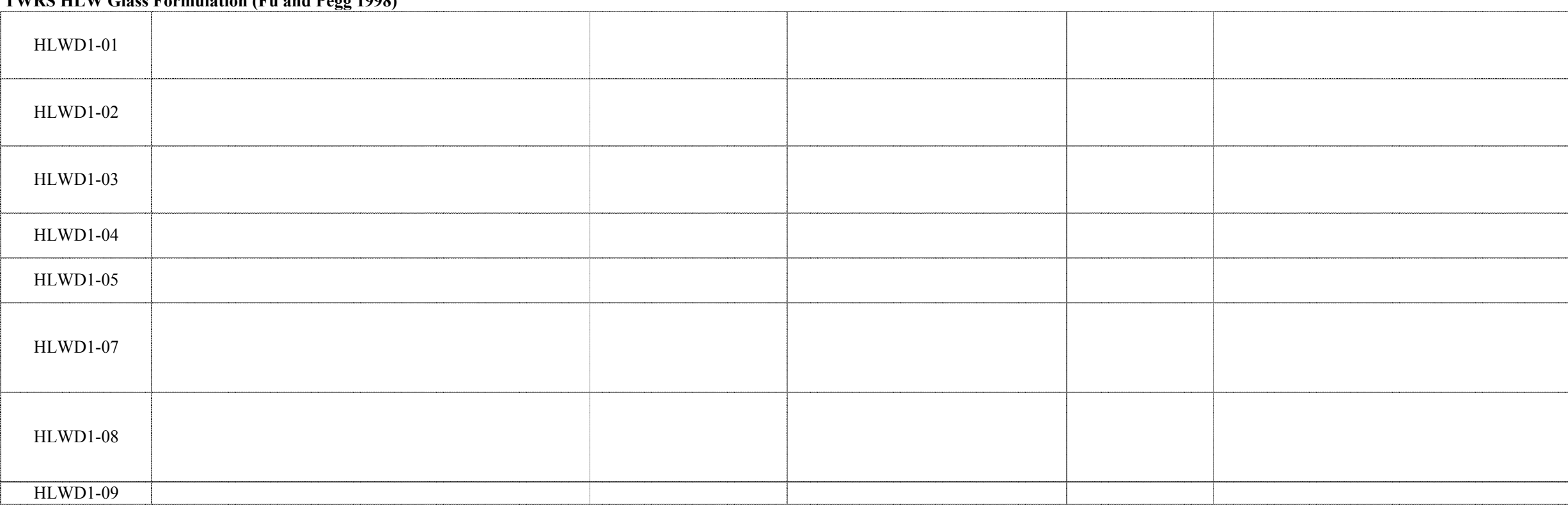


TWRS LAW Formulation 2 (Ferrara et al. 1998)

\begin{tabular}{|c|c|c|c|c|c|c|c|c|c|c|c|c|c|c|c|}
\hline Glass ID & $\begin{array}{c}\text { Heat Treated } \\
\text { XRD }\end{array}$ & $\begin{array}{l}\text { Density } \\
\left(\mathrm{g} / \mathrm{cm}^{3}\right)\end{array}$ & $\begin{array}{c}\text { Fulc Visc } \\
\text { A }\end{array}$ & \begin{tabular}{|c|} 
Fulc Visc \\
B \\
\end{tabular} & \begin{tabular}{|c|} 
Fulc Visc \\
To \\
\end{tabular} & $\begin{array}{c}\mathrm{FV} 1150^{\circ} \mathrm{C} \\
(\mathrm{Pa} \cdot \mathrm{s})\end{array}$ & $\begin{array}{c}\text { Arrh Visc } \\
\text { A }\end{array}$ & $\begin{array}{c}\text { Arrh Visc } \\
\text { B }\end{array}$ & $1150^{\circ} \mathrm{C}$ & \begin{tabular}{|c|}
$\mathrm{T}\left({ }^{\circ} \mathrm{C}\right)$ at \\
$2 \mathrm{~Pa} \cdot \mathrm{s}$
\end{tabular} & \begin{tabular}{|c|}
$\mathrm{T}\left({ }^{\circ} \mathrm{C}\right)$ at \\
$5 \mathrm{~Pa} \cdot \mathrm{s}$
\end{tabular} & \begin{tabular}{|c|}
$\mathrm{T}\left({ }^{\circ} \mathrm{C}\right)$ at \\
$10 \mathrm{~Pa} \cdot \mathrm{s}$ \\
\end{tabular} & $\begin{array}{c}\mathrm{T} 1 \\
\left({ }^{\circ} \mathrm{C}\right)\end{array}$ & $\begin{array}{c}\mathrm{V} 1 \\
(\mathrm{~Pa} \cdot \mathrm{s})\end{array}$ & $\begin{array}{c}\mathrm{T} 2 \\
\left({ }^{\circ} \mathrm{C}\right)\end{array}$ \\
\hline Envelope A & & & & & & & & & & & & & & & \\
\hline Envelope B & & & & & & & & & & & & & & & \\
\hline Envelope C & & & & & & & & & & & & & & & \\
\hline
\end{tabular}

ISV Glass (Carter et al. 1988)

ORNL ISV

Hanford ISV

Hanford ISV ( $\mathrm{CaO}$ adjusted $)$

Hanford ISV (Na2O adjusted)

\section{TWRS Envelop D HLW Glass (Crawford et al. 1998)}

Envelope D

\section{SRS M-Area Mixed Waste Glass (Fu et al. 1997)}

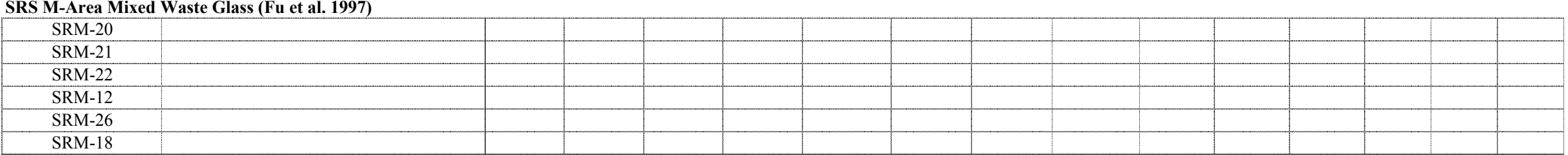

\section{Kinetics of Spinel Crystallization (Reynolds and Hrma 1997)}

$$
\text { HLW }
$$

\section{TWRS HLW Glass Formulation (Fu and Pegg 1998)}

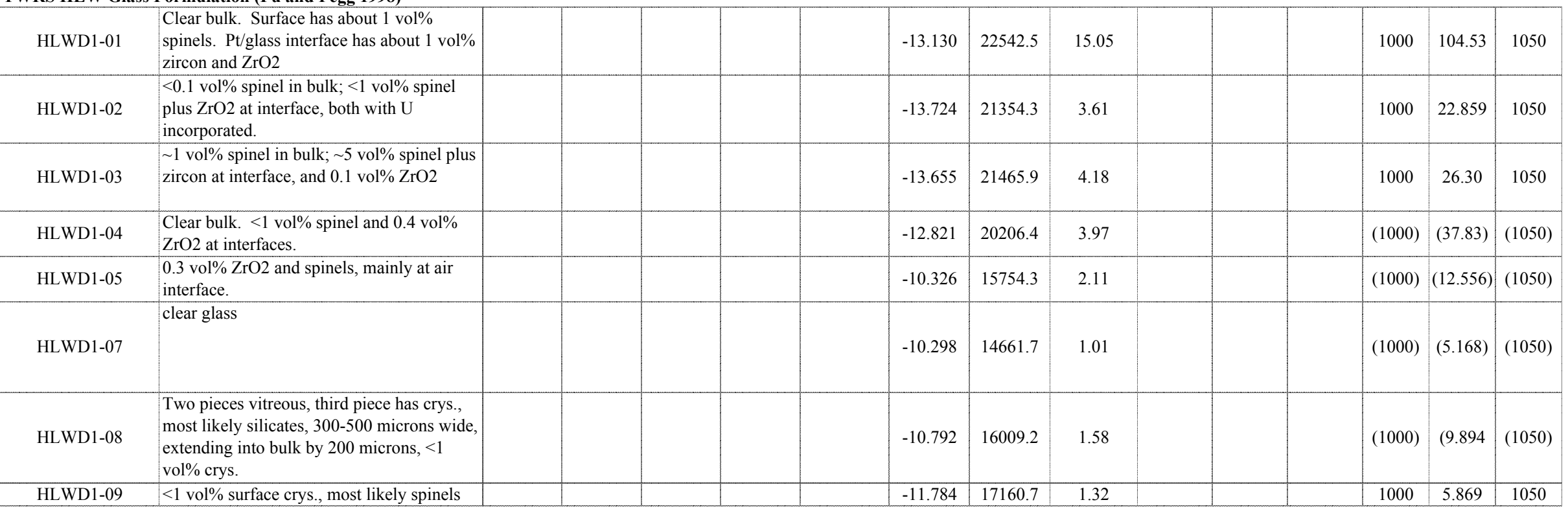


TWRS LAW Formulation 2 (Ferrara et al. 1998)

\begin{tabular}{|c|c|c|c|c|c|c|c|c|c|c|c|c|c|c|c|c|c|c|c|c|c|}
\hline Glass ID & $\begin{array}{c}\mathrm{V} 2 \\
(\mathrm{~Pa} \cdot \mathrm{s})\end{array}$ & $\begin{array}{c}\mathrm{T} 3 \\
\left({ }^{\circ} \mathrm{C}\right)\end{array}$ & $\begin{array}{c}\mathrm{V3} \\
(\mathrm{Pa} \cdot \mathrm{s})\end{array}$ & $\begin{array}{c}\mathrm{T} 4 \\
\left({ }^{\circ} \mathrm{C}\right)\end{array}$ & $\begin{array}{c}\mathrm{V} 4 \\
(\mathrm{~Pa} \cdot \mathrm{s})\end{array}$ & $\begin{array}{c}\mathrm{T} 5 \\
\left({ }^{\circ} \mathrm{C}\right)\end{array}$ & $\begin{array}{c}\text { V5 } \\
(\mathrm{Pa} \cdot \mathrm{s})\end{array}$ & $\begin{array}{c}\mathrm{T} 6 \\
\left({ }^{\circ} \mathrm{C}\right)\end{array}$ & $\begin{array}{c}\mathrm{V} 6 \\
(\mathrm{~Pa} \cdot \mathrm{s})\end{array}$ & $\begin{array}{c}\mathrm{T} 7 \\
\left({ }^{\circ} \mathrm{C}\right)\end{array}$ & $\begin{array}{c}\text { V7 } \\
(\mathrm{Pa} \cdot \mathrm{s})\end{array}$ & $\begin{array}{c}\mathrm{T} 8 \\
\left({ }^{\circ} \mathrm{C}\right)\end{array}$ & $\begin{array}{c}\mathrm{V} 8 \\
(\mathrm{~Pa} \cdot \mathrm{s})\end{array}$ & $\begin{array}{c}\mathrm{T} 9 \\
\left({ }^{\circ} \mathrm{C}\right)\end{array}$ & $\begin{array}{c}\text { V9 } \\
(\mathrm{Pa} \cdot \mathrm{s})\end{array}$ & $\begin{array}{l}\mathrm{T} 10 \\
\left({ }^{\circ} \mathrm{C}\right)\end{array}$ & $\begin{array}{c}\text { V10 } \\
(\mathrm{Pa} \cdot \mathrm{s})\end{array}$ & $\begin{array}{l}\mathrm{T} 11 \\
\left({ }^{\circ} \mathrm{C}\right)\end{array}$ & $\begin{array}{c}\text { V11 } \\
(\mathrm{Pa} \cdot \mathrm{s})\end{array}$ & $\begin{array}{l}\mathrm{T} 12 \\
\left({ }^{\circ} \mathrm{C}\right)\end{array}$ & $\begin{array}{c}\mathrm{V} 12 \\
(\mathrm{~Pa} \cdot \mathrm{s})\end{array}$ \\
\hline Envelope A & & & & & & & & & & & & & & & & & & & & & \\
\hline Envelope B & & & & & & & & & & & & & & & & & & & & & \\
\hline Envelope C & & & & & & & & & & & & & & & & & & & & & \\
\hline
\end{tabular}

ISV Glass (Carter et al. 1988)

$$
\text { ORNL ISV }
$$

Hanford ISV ( $\mathrm{CaO}$ adjusted)

Hanford ISV (Na2O adjusted)

\section{TWRS Envelop D HLW Glass (Crawford et al. 1998)}

Envelope D

\section{SRS M-Area Mixed Waste Glass (Fu et al. 1997)}

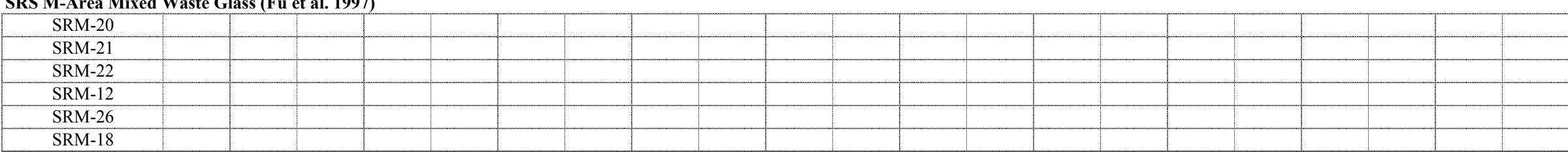

\section{Kinetics of Spinel Crystallization (Reynolds and Hrma 1997)}

$$
\text { HLW }
$$

\section{TWRS HLW Glass Formulation (Fu and Pegg 1998)}

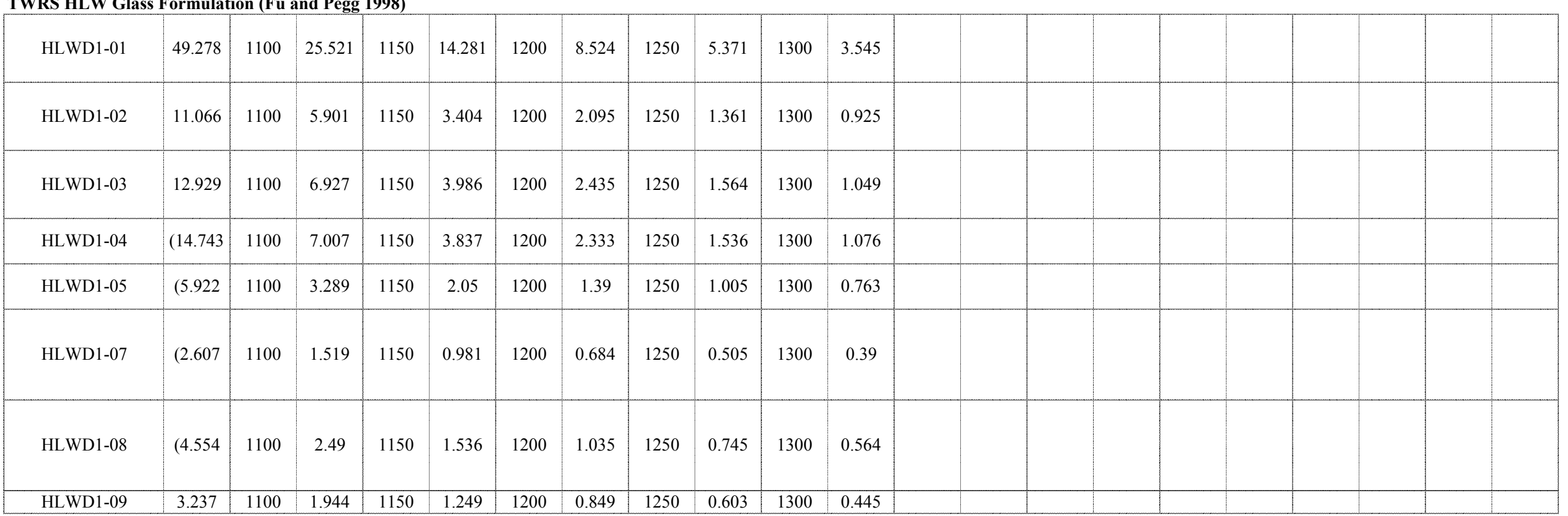


TWRS LAW Formulation 2 (Ferrara et al. 1998)

\begin{tabular}{|c|c|c|c|c|c|c|c|c|c|c|c|c|c|c|c|c|}
\hline Glass ID & $\begin{array}{l}\mathrm{T} 13 \\
\left({ }^{\circ} \mathrm{C}\right) \\
\end{array}$ & $\begin{array}{c}\mathrm{V} 13 \\
(\mathrm{~Pa} \cdot \mathrm{s})\end{array}$ & $\begin{array}{l}\mathrm{T} 14 \\
\left({ }^{\circ} \mathrm{C}\right) \\
\end{array}$ & $\begin{array}{c}\mathrm{V} 14 \\
(\mathrm{~Pa} \cdot \mathrm{s})\end{array}$ & $\begin{array}{c}\text { Q PCT } \\
\text { B }\left(\mathrm{g} / \mathrm{m}^{2}\right)\end{array}$ & $\begin{array}{c}\text { Q PCT } \\
\mathrm{Li}\left(\mathrm{g} / \mathrm{m}^{2}\right)\end{array}$ & $\begin{array}{c}\text { Q PCT } \\
\mathrm{Na}\left(\mathrm{g} / \mathrm{m}^{2}\right)\end{array}$ & $\begin{array}{c}\text { Q PCT } \\
\mathrm{Si}\left(\mathrm{g} / \mathrm{m}^{2}\right) \\
\end{array}$ & $\begin{array}{c}\text { Q PCT } \\
\mathrm{pH}\end{array}$ & $\begin{array}{c}\text { CCC PCT } \\
\text { B }\left(\mathrm{g} / \mathrm{m}^{2}\right)\end{array}$ & \begin{tabular}{|c|} 
CCC PCT \\
$\mathrm{Li}\left(\mathrm{g} / \mathrm{m}^{2}\right)$ \\
\end{tabular} & $\begin{array}{l}\mathrm{CCC} \text { PCT } \\
\mathrm{Na}\left(\mathrm{g} / \mathrm{m}^{2}\right)\end{array}$ & $\begin{array}{c}\text { CCC PCT } \\
\mathrm{Si}\left(\mathrm{g} / \mathrm{m}^{2}\right)\end{array}$ & $\begin{array}{c}\begin{array}{c}\text { CCC PCT } \\
\text { pH }\end{array} \\
\end{array}$ & $\begin{array}{l}\text { Q PCT B at } \\
20^{\circ} \mathrm{C}\left(\mathrm{g} / \mathrm{m}^{2}\right)\end{array}$ & $\begin{array}{l}\text { Q PCT Li at } \\
20^{\circ} \mathrm{C}\left(\mathrm{g} / \mathrm{m}^{2}\right)\end{array}$ \\
\hline Envelope A & & & & & & & & & & & & & & & 0.011 & 0.021 \\
\hline Envelope B & & & & & & & & & & & & & & & 0.0165 & 0.0195 \\
\hline Envelope C & & & & & & & & & & & & & & & 0.013 & 0.0405 \\
\hline
\end{tabular}

ISV Glass (Carter et al. 1988)

ORNL ISV

Hanford ISV

Hanford ISV ( $\mathrm{CaO}$ adjusted $)$

Hanford ISV (Na2O adjusted)

TWRS Envelop D HLW Glass (Crawford et al. 1998)

Envelope D

\begin{tabular}{l|l|l}
0.33 & 0.51 & 0.83
\end{tabular}

SRS M-Area Mixed Waste Glass (Fu et al. 1997)

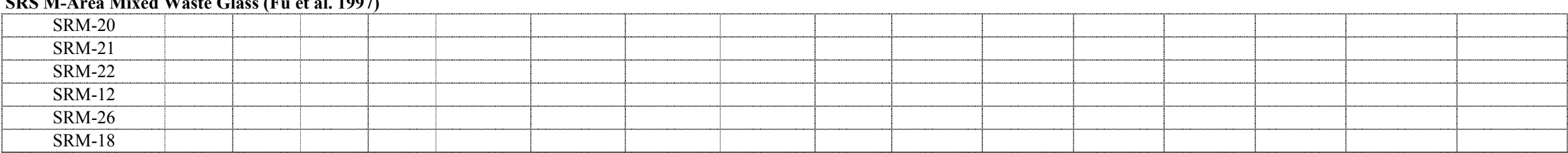

Kinetics of Spinel Crystallization (Reynolds and Hrma 1997)

HLW

TWRS HLW Glass Formulation (Fu and Pegg 1998)

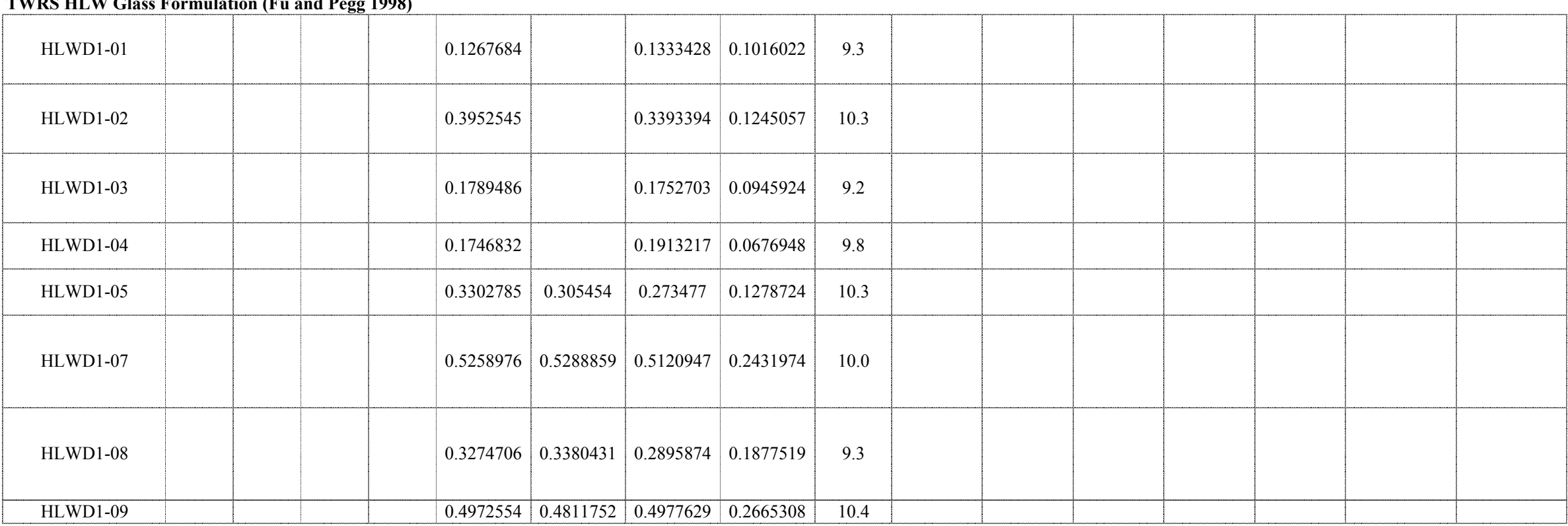


TWRS LAW Formulation 2 (Ferrara et al. 1998)

\begin{tabular}{|c|c|c|c|c|c|c|c|c|c|c|c|c|}
\hline Glass ID & $\begin{array}{l}\text { Q PCT Na at } \\
20^{\circ} \mathrm{C}\left(\mathrm{g} / \mathrm{m}^{2}\right)\end{array}$ & $\begin{array}{l}\text { Q PCT Si at } \\
20^{\circ} \mathrm{C}\left(\mathrm{g} / \mathrm{m}^{2}\right)\end{array}$ & $\begin{array}{c}\mathrm{QpH} \\
\text { at } 20^{\circ} \mathrm{C}\end{array}$ & $\begin{array}{c}\text { TCLP Ag } \\
(\mathrm{ppm})\end{array}$ & $\begin{array}{c}\text { TCLP As } \\
(\mathrm{ppm})\end{array}$ & $\begin{array}{c}\text { TCLP Ba } \\
(\mathrm{ppm})\end{array}$ & $\begin{array}{c}\text { TCLP Cd } \\
(\mathrm{ppm}) \\
\end{array}$ & $\begin{array}{c}\text { TCLP Cr } \\
(\mathrm{ppm})\end{array}$ & $\begin{array}{c}\text { TCLP Ni } \\
(\mathrm{ppm})\end{array}$ & $\begin{array}{c}\text { TCLP Pb } \\
(\mathrm{ppm})\end{array}$ & $\begin{array}{c}\text { TCLP Se } \\
(\mathrm{ppm})\end{array}$ & $\begin{array}{c}\text { TCLP Zn } \\
(\mathrm{ppm})\end{array}$ \\
\hline Envelope A & 0.04 & 0.0095 & 10.1 & & & $<3$ & $<1$ & 4.8 & & $<4$ & & \\
\hline Envelope B & 0.023 & 0.004 & 9.7 & & & $<3$ & $<1$ & $<3$ & & $<4$ & & \\
\hline Envelope C & 0.07 & 0.01 & 10.4 & & & 0.28 & 0.072 & $<4$ & & 0.5 & & \\
\hline
\end{tabular}

ISV Glass (Carter et al. 1988)

$$
\text { ORNL ISV }
$$

Hanford ISV

Hanford ISV ( $\mathrm{CaO}$ adjusted $)$

Hanford ISV (Na2O adjusted)

TWRS Envelop D HLW Glass (Crawford et al. 1998)

Envelope D

$0.34 \quad<0.09$

SRS M-Area Mixed Waste Glass (Fu et al. 1997)

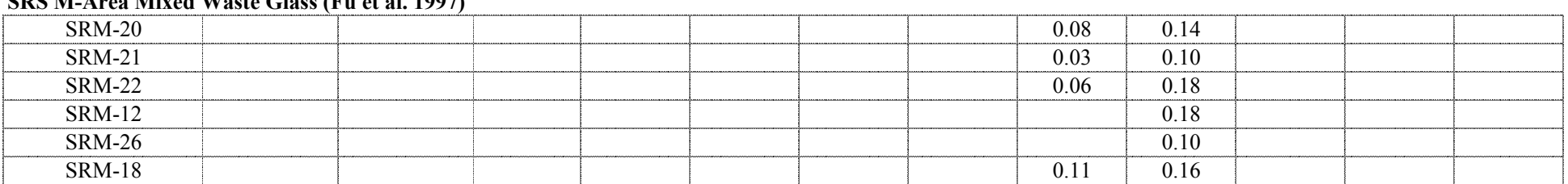

Kinetics of Spinel Crystallization (Reynolds and Hrma 1997)

HLW

TWRS HLW Glass Formulation (Fu and Pegg 1998)

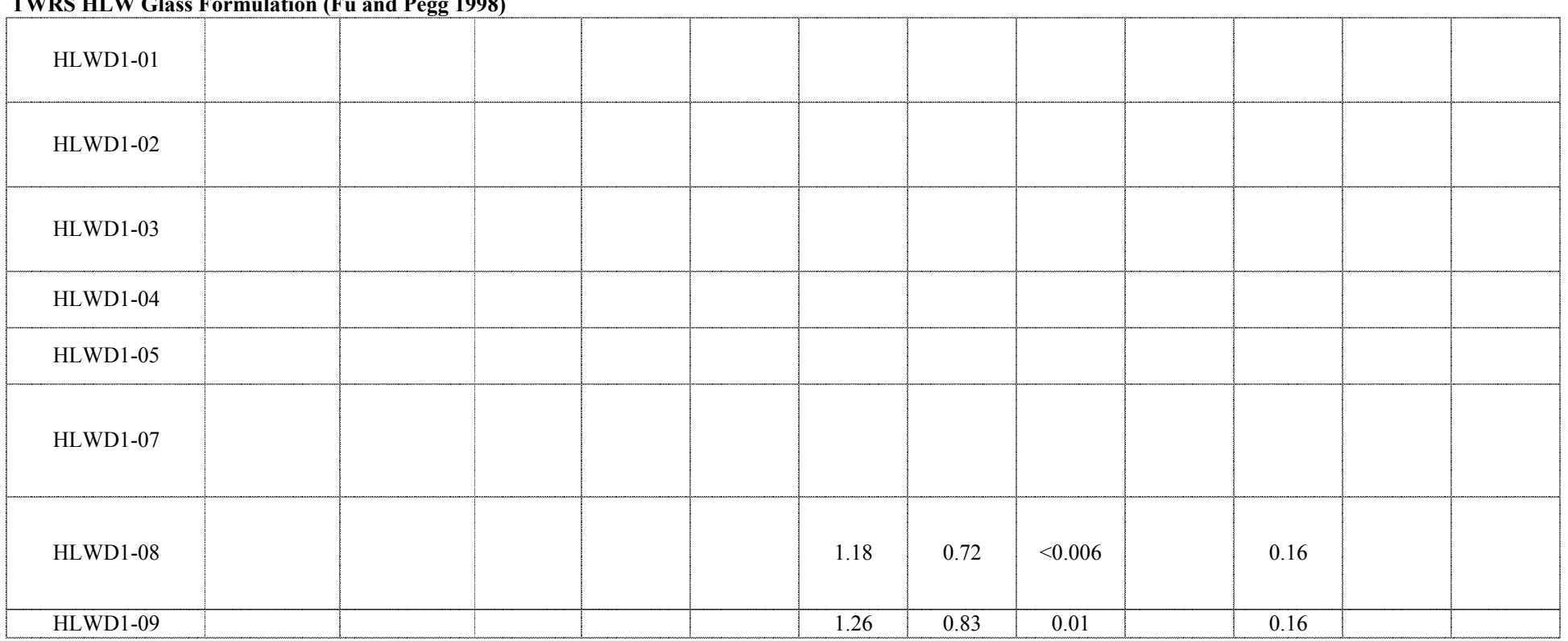


TWRS HLW Glass Formulation (Fu and Pegg 1998)

\begin{tabular}{|c|c|c|c|c|c|c|c|c|c|c|c|c|c|c|c|c|c|c|c|c|c|}
\hline Glass ID & $\begin{array}{c}\mathrm{A} 12 \mathrm{O} 3 \\
-\mathrm{t}\end{array}$ & $\begin{array}{c}\mathrm{B} 2 \mathrm{O} 3 \\
-\mathrm{t}\end{array}$ & $\begin{array}{c}\mathrm{CaO} \\
-\mathrm{t}\end{array}$ & $\begin{array}{c}\mathrm{Fe} 2 \mathrm{O} 3 \\
-\mathrm{t}\end{array}$ & $\begin{array}{c}\mathrm{FeO} \\
-\mathrm{t}\end{array}$ & $\begin{array}{c}\mathrm{K} 2 \mathrm{O} \\
-\mathrm{t}\end{array}$ & $\begin{array}{c}\mathrm{Li} 2 \mathrm{O} \\
-\mathrm{t}\end{array}$ & $\begin{array}{c}\mathrm{MgO} \\
-\mathrm{t}\end{array}$ & $\begin{array}{c}\mathrm{Na} 2 \mathrm{O} \\
-\mathrm{t}\end{array}$ & $\begin{array}{c}\mathrm{NiO} \\
-\mathrm{t}\end{array}$ & $\begin{array}{c}\mathrm{P} 2 \mathrm{O} 5 \\
-\mathrm{t}\end{array}$ & $\begin{array}{c}\mathrm{SiO} 2 \\
-\mathrm{t}\end{array}$ & $\begin{array}{c}\mathrm{ZrO} 2 \\
-\mathrm{t}\end{array}$ & $\begin{array}{c}\mathrm{Ag} 2 \mathrm{O} \\
-\mathrm{t}\end{array}$ & $\begin{array}{c}\mathrm{As} 2 \mathrm{O} 3 \\
-\mathrm{t}\end{array}$ & $\begin{array}{c}\mathrm{BaO} \\
-\mathrm{t}\end{array}$ & $\begin{array}{c}\mathrm{Bi} 2 \mathrm{O} 3 \\
-\mathrm{t}\end{array}$ & $\begin{array}{l}\mathrm{Br} \\
-\mathrm{t}\end{array}$ & $\begin{array}{c}\mathrm{CdO} \\
-\mathrm{t}\end{array}$ & $\begin{array}{c}\mathrm{Ce} 2 \mathrm{O} 3 \\
-\mathrm{t}\end{array}$ & $\begin{array}{c}\mathrm{CeO} 2 \\
-\mathrm{t}\end{array}$ \\
\hline HLWD1-10 & 0.0301 & 0.0921 & 0.0000 & 0.1756 & & 0.0873 & 0.0000 & 0.0000 & 0.0396 & 0.0114 & 0.0152 & 0.3005 & 0.0000 & 0.0000 & & 0.0192 & 0.0118 & & 0.0196 & & 0.0038 \\
\hline HLWD1-11 & 0.1177 & 0.0607 & 0.0163 & 0.2626 & & 0.0112 & 0.0301 & 0.0025 & 0.1463 & 0.0026 & 0.0107 & 0.2845 & 0.0018 & 0.0000 & & 0.0006 & 0.0006 & & 0.0001 & & 0.0003 \\
\hline HLWD1-12 & 0.1332 & 0.0665 & 0.0052 & 0.2185 & & 0.0053 & 0.0402 & 0.0014 & 0.1110 & 0.0079 & 0.0002 & 0.2731 & 0.0662 & 0.0000 & & 0.0012 & 0.0000 & & 0.0090 & & 0.0000 \\
\hline HLWD1-13 & 0.0382 & 0.0668 & 0.0763 & 0.2289 & & 0.0105 & 0.0315 & 0.0229 & 0.1049 & 0.0215 & 0.0000 & 0.2957 & 0.0000 & 0.0000 & & 0.0000 & 0.0000 & & 0.0000 & & 0.0000 \\
\hline HLWD1-14 & 0.0378 & 0.1606 & 0.0000 & 0.0756 & & 0.0000 & 0.0312 & 0.0000 & 0.1417 & 0.0017 & 0.0000 & 0.2740 & 0.1228 & 0.0000 & & 0.0000 & 0.0000 & & 0.0000 & & 0.0059 \\
\hline HLWD1-15 & 0.0661 & 0.1134 & 0.0756 & 0.2362 & & 0.0000 & 0.0312 & 0.0000 & 0.0945 & 0.0017 & 0.0000 & 0.2362 & 0.1228 & 0.0000 & & 0.0000 & 0.0000 & & 0.0000 & & 0.0000 \\
\hline HLWD1-16 & 0.1606 & 0.0661 & 0.0189 & 0.0567 & & 0.0126 & 0.0312 & 0.0000 & 0.0945 & 0.0017 & 0.0000 & 0.2929 & 0.1228 & 0.0047 & & 0.0000 & 0.0000 & & 0.0000 & & 0.0000 \\
\hline HLWD1-17 & 0.0378 & 0.1606 & 0.0000 & 0.2362 & & 0.0000 & 0.0312 & 0.0000 & 0.0945 & 0.0017 & 0.0000 & 0.2835 & 0.0000 & 0.0000 & & 0.0000 & 0.0000 & & 0.0000 & & 0.0059 \\
\hline HLWD1-18 & 0.1604 & 0.1510 & 0.0000 & 0.0472 & & 0.0105 & 0.0311 & 0.0229 & 0.0944 & 0.0214 & 0.0000 & 0.2359 & 0.1227 & 0.0000 & & 0.0000 & 0.0000 & & 0.0000 & & 0.0000 \\
\hline HLWD1-19 & 0.0378 & 0.0661 & 0.0756 & 0.2079 & & 0.0126 & 0.0312 & 0.0000 & 0.1890 & 0.0017 & 0.0000 & 0.2362 & 0.0000 & 0.0047 & & 0.0000 & 0.0000 & & 0.0000 & & 0.0000 \\
\hline HLWD1-20 & 0.0378 & 0.0661 & 0.0756 & 0.0472 & & 0.0126 & 0.0312 & 0.0000 & 0.1890 & 0.0017 & 0.0000 & 0.2740 & 0.1228 & 0.0047 & & 0.0000 & 0.0000 & & 0.0000 & & 0.0000 \\
\hline HLWD1-21 & 0.0997 & 0.1526 & 0.0055 & 0.2212 & & 0.0001 & 0.0410 & 0.0022 & 0.1127 & 0.0021 & 0.0078 & 0.3071 & 0.0015 & 0.0035 & 0.0004 & 0.0005 & 0.0005 & & 0.0001 & & 0.0003 \\
\hline HLWD1-23 & 0.0603 & 0.1549 & 0.0132 & 0.1431 & & 0.0044 & 0.0403 & 0.0044 & 0.1360 & 0.0044 & 0.0088 & 0.3200 & 0.0009 & 0.0021 & 0.0004 & 0.0044 & 0.0004 & & 0.0066 & & 0.0014 \\
\hline HLWD1-24 & 0.0750 & 0.1117 & 0.0164 & 0.1779 & & 0.0055 & 0.0202 & 0.0055 & 0.1691 & 0.0055 & 0.0109 & 0.2654 & 0.0012 & 0.0026 & 0.0005 & 0.0055 & 0.0005 & & 0.0082 & & 0.0017 \\
\hline HLWD1-25 & 0.0692 & 0.1115 & 0.0152 & 0.1642 & & 0.0051 & 0.0200 & 0.0051 & 0.1561 & 0.0051 & 0.0101 & 0.3121 & 0.0000 & 0.0024 & 0.0005 & 0.0051 & 0.0005 & & 0.0076 & & 0.0016 \\
\hline HLWD1-26 & 0.0607 & 0.1321 & 0.0100 & 0.1201 & & 0.0033 & 0.0375 & 0.0033 & 0.1027 & 0.0033 & 0.0066 & 0.4239 & 0.0139 & 0.0016 & 0.0003 & 0.0033 & 0.0003 & & 0.0050 & & 0.0010 \\
\hline HLWD1-27 & 0.0606 & 0.1107 & 0.0634 & 0.1437 & & 0.0044 & 0.0200 & 0.0044 & 0.1366 & 0.0044 & 0.0088 & 0.3324 & 0.0009 & 0.0021 & 0.0004 & 0.0044 & 0.0004 & & 0.0066 & & 0.0014 \\
\hline HLWD1-28 & 0.0948 & 0.1036 & 0.0607 & 0.1557 & & 0.0038 & 0.0308 & 0.0010 & 0.0795 & 0.0056 & 0.0002 & 0.3511 & 0.0471 & 0.0000 & & 0.0008 & 0.0000 & & 0.0064 & & 0.0000 \\
\hline HLWD2-01 & 0.0846 & 0.0669 & 0.0231 & 0.1587 & & 0.0038 & 0.0293 & 0.0081 & 0.0714 & 0.0095 & 0.0093 & 0.3194 & 0.0466 & 0.0000 & & 0.0117 & 0.0072 & & 0.0120 & & 0.0023 \\
\hline HLWD2-02 & 0.0174 & 0.0599 & 0.0000 & 0.2414 & & 0.0001 & 0.0300 & 0.0000 & 0.0704 & 0.0151 & 0.0147 & 0.2996 & 0.0000 & 0.0000 & & 0.0186 & 0.0114 & & 0.0190 & & 0.0037 \\
\hline HLWD2-03 & 0.1606 & 0.0661 & 0.0756 & 0.0472 & & 0.0000 & 0.0312 & 0.0000 & 0.1890 & 0.0017 & 0.0000 & 0.2740 & 0.0000 & 0.0000 & & 0.0000 & 0.0000 & & 0.0000 & & 0.0059 \\
\hline HLWD2-04 & 0.0850 & 0.0661 & 0.0000 & 0.2362 & & 0.0000 & 0.0312 & 0.0000 & 0.1890 & 0.0017 & 0.0305 & 0.2362 & 0.0000 & 0.0000 & & 0.0386 & 0.0237 & & 0.0395 & & 0.0000 \\
\hline
\end{tabular}


Appendix A. Database - mass fraction

TWRS HLW Glass Formulation (Fu and Pegg 1998)

\begin{tabular}{|c|c|c|c|c|c|c|c|c|c|c|c|c|c|c|c|c|c|c|c|c|c|}
\hline Glass ID & $\begin{array}{l}\mathrm{Cl} \\
-\mathrm{t} \\
\end{array}$ & $\begin{array}{c}\mathrm{CoO} \\
-\mathrm{t} \\
\end{array}$ & $\begin{array}{c}\mathrm{Co} 2 \mathrm{O} 3 \\
-\mathrm{t}\end{array}$ & $\begin{array}{c}\mathrm{Cr} 2 \mathrm{O} 3 \\
-\mathrm{t}\end{array}$ & $\begin{array}{c}\mathrm{Cs} 2 \mathrm{O} \\
-\mathrm{t}\end{array}$ & $\begin{array}{c}\mathrm{CuO} \\
-\mathrm{t}\end{array}$ & $\begin{array}{c}\mathrm{Eu} 2 \mathrm{O} 3 \\
-\mathrm{t}\end{array}$ & $\begin{array}{l}F \\
-t\end{array}$ & $\begin{array}{c}\mathrm{Ga} 2 \mathrm{O} 3 \\
-\mathrm{t}\end{array}$ & $\begin{array}{c}\mathrm{Gd} 2 \mathrm{O} 3 \\
-\mathrm{t}\end{array}$ & $\begin{array}{c}\mathrm{HgO} \\
-\mathrm{t}\end{array}$ & $\begin{array}{l}\mathrm{I} \\
-\mathrm{t} \\
\end{array}$ & $\begin{array}{c}\mathrm{La} 2 \mathrm{O} 3 \\
-\mathrm{t}\end{array}$ & $\begin{array}{c}\mathrm{MnO} 2 \\
-\mathrm{t}\end{array}$ & $\begin{array}{c}\mathrm{MnO} \\
-\mathrm{t}\end{array}$ & $\begin{array}{c}\mathrm{MoO} \\
-\mathrm{t} \\
\end{array}$ & $\begin{array}{c}\mathrm{MoO} 3 \\
-\mathrm{t}\end{array}$ & $\begin{array}{c}\mathrm{Nb} 2 \mathrm{O} 5 \\
-\mathrm{t}\end{array}$ & $\begin{array}{c}\mathrm{Nd} 2 \mathrm{O} 3 \\
-\mathrm{t}\end{array}$ & $\begin{array}{c}\mathrm{PbO} \\
-\mathrm{t}\end{array}$ & $\begin{array}{c}\mathrm{PdO} 2 \\
-\mathrm{t}\end{array}$ \\
\hline HLWD1-10 & 0.0000 & 0.0022 & & 0.0038 & 0.0023 & 0.0023 & & 0.0152 & & & & & 0.0115 & 0.0391 & & 0.0029 & & & 0.0076 & 0.0045 & \\
\hline HLWD1-11 & 0.0000 & 0.0000 & & 0.0014 & 0.0000 & 0.0002 & & 0.0035 & & & & & 0.0001 & 0.0063 & & 0.0001 & & & 0.0003 & 0.0039 & \\
\hline HLWD1-12 & 0.0000 & 0.0000 & & 0.0015 & 0.0001 & 0.0008 & & 0.0014 & & & & & 0.0061 & 0.0068 & & 0.0000 & & & 0.0000 & 0.0009 & \\
\hline HLWD1-13 & 0.0000 & 0.0038 & & 0.0065 & 0.0014 & 0.0000 & & 0.0000 & & & & & 0.0000 & 0.0672 & & 0.0000 & & & 0.0000 & 0.0000 & \\
\hline HLWD1-14 & 0.0000 & 0.0000 & & 0.0000 & 0.0014 & 0.0000 & & 0.0000 & & & & & 0.0179 & 0.0000 & & 0.0000 & & & 0.0118 & 0.0000 & \\
\hline HLWD1-15 & 0.0000 & 0.0000 & & 0.0000 & 0.0014 & 0.0000 & & 0.0000 & & & & & 0.0000 & 0.0000 & & 0.0000 & & & 0.0000 & 0.0000 & \\
\hline HLWD1-16 & 0.0025 & 0.0000 & & 0.0000 & 0.0063 & 0.0048 & & 0.0280 & & & & & 0.0000 & 0.0000 & & 0.0059 & & & 0.0000 & 0.0093 & \\
\hline HLWD1-17 & 0.0000 & 0.0000 & & 0.0000 & 0.0014 & 0.0000 & & 0.0000 & & & & & 0.0179 & 0.0000 & & 0.0000 & & & 0.0118 & 0.0000 & \\
\hline HLWD1-18 & 0.0000 & 0.0038 & & 0.0065 & 0.0014 & 0.0000 & & 0.0000 & & & & & 0.0000 & 0.0672 & & 0.0000 & & & 0.0000 & 0.0000 & \\
\hline HLWD1-19 & 0.0025 & 0.0000 & & 0.0065 & 0.0063 & 0.0048 & & 0.0280 & & & & & 0.0000 & 0.0000 & & 0.0059 & & & 0.0000 & 0.0093 & \\
\hline HLWD1-20 & 0.0025 & 0.0000 & & 0.0000 & 0.0063 & 0.0048 & & 0.0280 & & & & & 0.0000 & 0.0000 & & 0.0059 & & & 0.0000 & 0.0093 & \\
\hline HLWD1-21 & 0.0001 & 0.0000 & & 0.0012 & 0.0005 & 0.0002 & & 0.0000 & & & & & 0.0001 & 0.0048 & & 0.0001 & & & 0.0003 & 0.0038 & \\
\hline HLWD1-23 & 0.0011 & 0.0013 & & 0.0031 & 0.0015 & 0.0004 & & 0.0009 & & & & & 0.0018 & 0.0044 & & 0.0000 & & & 0.0000 & 0.0023 & \\
\hline HLWD1-24 & 0.0014 & 0.0016 & & 0.0038 & 0.0018 & 0.0005 & & 0.0011 & & & & & 0.0022 & 0.0055 & & 0.0000 & & & 0.0000 & 0.0029 & \\
\hline HLWD1-25 & 0.0013 & 0.0015 & & 0.0035 & 0.0017 & 0.0005 & & 0.0010 & & & & & 0.0020 & 0.0051 & & 0.0000 & & & 0.0000 & 0.0026 & \\
\hline HLWD1-26 & 0.0009 & 0.0010 & & 0.0023 & 0.0011 & 0.0003 & & 0.0007 & & & & & 0.0013 & 0.0033 & & 0.0000 & & & 0.0000 & 0.0017 & \\
\hline HLWD1-27 & 0.0011 & 0.0013 & & 0.0031 & 0.0015 & 0.0004 & & 0.0009 & & & & & 0.0018 & 0.0044 & & 0.0000 & & & 0.0000 & 0.0023 & \\
\hline HLWD1-28 & 0.0001 & 0.0000 & & 0.0011 & 0.0001 & 0.0006 & & 0.0010 & & & & & 0.0043 & 0.0049 & & 0.0000 & & & 0.0000 & 0.0006 & \\
\hline HLWD2-01 & 0.0000 & 0.0013 & & 0.0046 & 0.0014 & 0.0014 & & 0.0082 & & & & & 0.0070 & 0.0240 & & 0.0018 & & & 0.0046 & 0.0027 & \\
\hline HLWD2-02 & 0.0000 & 0.0021 & & 0.0073 & 0.0023 & 0.0022 & & 0.0131 & & & & & 0.0112 & 0.0379 & & 0.0028 & & & 0.0074 & 0.0044 & \\
\hline HLWD2-03 & 0.0000 & 0.0000 & & 0.0000 & 0.0014 & 0.0000 & & 0.0000 & & & & & 0.0179 & 0.0000 & & 0.0000 & & & 0.0118 & 0.0000 & \\
\hline HLWD2-04 & 0.0000 & 0.0000 & & 0.0000 & 0.0014 & 0.0000 & & 0.0000 & & & & & 0.0000 & 0.0000 & & 0.0000 & & & 0.0000 & 0.0000 & \\
\hline
\end{tabular}


TWRS HLW Glass Formulation (Fu and Pegg 1998)

\begin{tabular}{|c|c|c|c|c|c|c|c|c|c|c|c|c|c|c|c|c|c|c|c|c|c|}
\hline Glass ID & $\begin{array}{c}\mathrm{PdO} \\
-\mathrm{t}\end{array}$ & $\begin{array}{c}\mathrm{Pr} 2 \mathrm{O} 3 \\
-\mathrm{t}\end{array}$ & $\begin{array}{c}\text { Pr6O11 } \\
-t\end{array}$ & $\begin{array}{c}\mathrm{Rb} 2 \mathrm{O} \\
-\mathrm{t}\end{array}$ & $\begin{array}{c}\mathrm{ReO} \\
-\mathrm{t}\end{array}$ & $\begin{array}{c}\mathrm{ReO} 2 \\
-\mathrm{t}\end{array}$ & $\begin{array}{c}\mathrm{Rh} 2 \mathrm{O} 3 \\
-\mathrm{t}\end{array}$ & $\begin{array}{c}\mathrm{RhO} 2 \\
-\mathrm{t}\end{array}$ & $\begin{array}{c}\mathrm{RuO} 2 \\
-\mathrm{t}\end{array}$ & $\begin{array}{c}\mathrm{Sb} 2 \mathrm{O} 3 \\
-\mathrm{t}\end{array}$ & $\begin{array}{c}\mathrm{Sb} 2 \mathrm{O} 5 \\
-\mathrm{t}\end{array}$ & $\begin{array}{c}\mathrm{SeO} 2 \\
-\mathrm{t}\end{array}$ & $\begin{array}{c}\mathrm{Sm} 2 \mathrm{O} 3 \\
-\mathrm{t}\end{array}$ & $\begin{array}{c}\mathrm{SnO} \\
-\mathrm{t}\end{array}$ & $\begin{array}{c}\mathrm{SnO} 2 \\
-\mathrm{t}\end{array}$ & $\begin{array}{c}\mathrm{SO} 3 \\
-\mathrm{t}\end{array}$ & $\begin{array}{c}\mathrm{SrO} \\
-\mathrm{t}\end{array}$ & $\begin{array}{c}\mathrm{Tc} 2 \mathrm{O} 7 \\
-\mathrm{t}\end{array}$ & $\begin{array}{c}\mathrm{TeO} 2 \\
-\mathrm{t}\end{array}$ & $\begin{array}{c}\mathrm{ThO} 2 \\
-\mathrm{t}\end{array}$ & $\begin{array}{c}\mathrm{TiO} 2 \\
-\mathrm{t}\end{array}$ \\
\hline HLWD1-10 & 0.0000 & 0.0000 & & & & 0.0000 & & & 0.0000 & & 0.0000 & 0.0000 & 0.0000 & 0.0000 & & 0.0061 & 0.0305 & & 0.0000 & 0.0000 & 0.0082 \\
\hline HLWD1-11 & 0.0000 & 0.0000 & & & & 0.0000 & & & 0.0000 & & 0.0000 & 0.0000 & 0.0000 & 0.0000 & & 0.0005 & 0.0257 & & 0.0000 & 0.0000 & 0.0027 \\
\hline HLWD1-12 & 0.0000 & 0.0000 & & & & 0.0000 & & & 0.0000 & & 0.0000 & 0.0000 & 0.0000 & 0.0000 & & 0.0000 & 0.0290 & & 0.0000 & 0.0000 & 0.0014 \\
\hline HLWD1-13 & 0.0000 & 0.0000 & & & & 0.0000 & & & 0.0000 & & 0.0000 & 0.0000 & 0.0000 & 0.0003 & & 0.0000 & 0.0182 & & 0.0000 & 0.0000 & 0.0000 \\
\hline HLWD1-14 & 0.0000 & 0.0026 & & & & 0.0000 & & & 0.0000 & & 0.0000 & 0.0000 & 0.0000 & 0.0000 & & 0.0000 & 0.0180 & & 0.0000 & 0.0035 & 0.0000 \\
\hline HLWD1-15 & 0.0000 & 0.0000 & & & & 0.0000 & & & 0.0000 & & 0.0000 & 0.0000 & 0.0000 & 0.0000 & & 0.0000 & 0.0180 & & 0.0000 & 0.0000 & 0.0000 \\
\hline HLWD1-16 & 0.0000 & 0.0000 & & & & 0.0000 & & & 0.0000 & & 0.0088 & 0.0057 & 0.0000 & 0.0000 & & 0.0127 & 0.0228 & & 0.0000 & 0.0000 & 0.0170 \\
\hline HLWD1-17 & 0.0000 & 0.0026 & & & & 0.0000 & & & 0.0000 & & 0.0000 & 0.0000 & 0.0000 & 0.0000 & & 0.0000 & 0.0180 & & 0.0000 & 0.0035 & 0.0000 \\
\hline HLWD1-18 & 0.0000 & 0.0000 & & & & 0.0000 & & & 0.0000 & & 0.0000 & 0.0000 & 0.0000 & 0.0003 & & 0.0000 & 0.0180 & & 0.0000 & 0.0000 & 0.0000 \\
\hline HLWD1-19 & 0.0000 & 0.0000 & & & & 0.0000 & & & 0.0000 & & 0.0088 & 0.0049 & 0.0000 & 0.0000 & & 0.0127 & 0.0264 & & 0.0000 & 0.0000 & 0.0170 \\
\hline HLWD1-20 & 0.0000 & 0.0000 & & & & 0.0000 & & & 0.0000 & & 0.0088 & 0.0049 & 0.0000 & 0.0000 & & 0.0127 & 0.0228 & & 0.0000 & 0.0000 & 0.0170 \\
\hline HLWD1-21 & 0.0000 & 0.0000 & & & & 0.0000 & & & 0.0000 & & 0.0000 & 0.0000 & 0.0001 & 0.0000 & & 0.0004 & 0.0206 & & 0.0000 & 0.0000 & 0.0023 \\
\hline HLWD1-23 & 0.0007 & 0.0000 & & & & 0.0005 & & & 0.0021 & & 0.0013 & 0.0015 & 0.0000 & 0.0000 & & 0.0044 & 0.0275 & & 0.0013 & 0.0011 & 0.0014 \\
\hline HLWD1-24 & 0.0008 & 0.0000 & & & & 0.0006 & & & 0.0022 & & 0.0016 & 0.0016 & 0.0000 & 0.0000 & & 0.0055 & 0.0341 & & 0.0016 & 0.0013 & 0.0017 \\
\hline HLWD1-25 & 0.0008 & 0.0000 & & & & 0.0006 & & & 0.0024 & & 0.0015 & 0.0018 & 0.0000 & 0.0000 & & 0.0051 & 0.0315 & & 0.0000 & 0.0000 & 0.0000 \\
\hline HLWD1-26 & 0.0005 & 0.0000 & & & & 0.0004 & & & 0.0014 & & 0.0010 & 0.0010 & 0.0000 & 0.0000 & & 0.0033 & 0.0207 & & 0.0010 & 0.0008 & 0.0010 \\
\hline HLWD1-27 & 0.0007 & 0.0000 & & & & 0.0005 & & & 0.0018 & & 0.0013 & 0.0013 & 0.0000 & 0.0000 & & 0.0044 & 0.0276 & & 0.0013 & 0.0011 & 0.0014 \\
\hline HLWD1-28 & 0.0000 & 0.0000 & & & & 0.0000 & & & 0.0000 & & 0.0035 & 0.0021 & 0.0000 & 0.0000 & & 0.0000 & 0.0240 & & 0.0019 & 0.0000 & 0.0010 \\
\hline HLWD2-01 & 0.0000 & 0.0000 & & & & 0.0000 & & & 0.0000 & & 0.0000 & 0.0000 & 0.0000 & 0.0000 & & 0.0047 & 0.0292 & & 0.0000 & 0.0000 & 0.0050 \\
\hline HLWD2-02 & 0.0000 & 0.0000 & & & & 0.0000 & & & 0.0000 & & 0.0000 & 0.0000 & 0.0000 & 0.0000 & & 0.0074 & 0.0313 & & 0.0000 & 0.0000 & 0.0079 \\
\hline HLWD2-03 & 0.0000 & 0.0026 & & & & 0.0000 & & & 0.0000 & & 0.0000 & 0.0000 & 0.0000 & 0.0000 & & 0.0000 & 0.0180 & & 0.0000 & 0.0035 & 0.0000 \\
\hline HLWD2-04 & 0.0000 & 0.0000 & & & & 0.0000 & & & 0.0000 & & 0.0000 & 0.0000 & 0.0000 & 0.0000 & & 0.0000 & 0.0180 & & 0.0000 & 0.0000 & 0.0000 \\
\hline
\end{tabular}




\section{TWRS HLW Glass Formulation (Fu and Pegg 1998)}

\begin{tabular}{|c|c|c|c|c|c|c|c|c|c|c|c|c|c|c|c|c|c|c|c|c|c|}
\hline Glass ID & $\begin{array}{c}\mathrm{T} 12 \mathrm{O} 3 \\
-\mathrm{t}\end{array}$ & $\begin{array}{c}\mathrm{U} 3 \mathrm{O} 8 \\
-\mathrm{t}\end{array}$ & $\begin{array}{c}\mathrm{UO} 2 \\
-\mathrm{t}\end{array}$ & $\begin{array}{c}\text { UO3 } \\
-t \\
\end{array}$ & $\begin{array}{c}\mathrm{V} 2 \mathrm{O} 5 \\
-\mathrm{t}\end{array}$ & $\begin{array}{c}\text { WO3 } \\
-t\end{array}$ & $\begin{array}{c}\mathrm{Y} 2 \mathrm{O} 3 \\
-\mathrm{t}\end{array}$ & $\begin{array}{c}\mathrm{ZnO} \\
-\mathrm{t}\end{array}$ & $\begin{array}{c}\text { Others } \\
-t\end{array}$ & $\begin{array}{c}\text { Sum } \\
-t\end{array}$ & $\begin{array}{c}\mathrm{Al} 2 \mathrm{O} 3 \\
-\mathrm{a}\end{array}$ & $\begin{array}{c}\mathrm{B} 2 \mathrm{O} 3 \\
-\mathrm{a}\end{array}$ & $\begin{array}{c}\mathrm{CaO} \\
-\mathrm{a} \\
\end{array}$ & $\begin{array}{c}\mathrm{Fe} 2 \mathrm{O} 3 \\
-\mathrm{a}\end{array}$ & $\begin{array}{c}\mathrm{FeO} \\
-\mathrm{a} \\
\end{array}$ & $\begin{array}{c}\mathrm{K} 2 \mathrm{O} \\
-\mathrm{a} \\
\end{array}$ & $\begin{array}{c}\mathrm{Li} 2 \mathrm{O} \\
-\mathrm{a} \\
\end{array}$ & $\begin{array}{c}\mathrm{MgO} \\
-\mathrm{a}\end{array}$ & $\begin{array}{c}\mathrm{Na} 2 \mathrm{O} \\
-\mathrm{a}\end{array}$ & $\begin{array}{c}\mathrm{NiO} \\
-\mathrm{a} \\
\end{array}$ & $\begin{array}{c}\mathrm{P} 2 \mathrm{O} 5 \\
-\mathrm{a}\end{array}$ \\
\hline HLWD1-10 & & & 0.0584 & & & 0.0000 & 0.0000 & 0.0000 & & 1.0008 & 0.0309 & 0.0908 & 0.0006 & 0.1501 & & 0.0909 & 0.0005 & 0.0001 & 0.0397 & 0.0090 & 0.0166 \\
\hline HLWD1-11 & & & 0.0026 & & & 0.0000 & 0.0000 & 0.0000 & & 0.9959 & 0.1000 & 0.0568 & 0.0149 & 0.2198 & & 0.0109 & 0.0279 & 0.0022 & 0.1236 & 0.0024 & 0.0115 \\
\hline HLWD1-12 & & & 0.0087 & & & 0.0000 & 0.0000 & 0.0000 & & 0.9956 & & & & & & & & & & & \\
\hline HLWD1-13 & & & 0.0000 & & & 0.0020 & 0.0000 & 0.0000 & 0.0006 & 0.9972 & 0.0391 & 0.0615 & 0.0616 & 0.2011 & & 0.0099 & 0.0290 & 0.0201 & 0.0799 & 0.0195 & 0.0007 \\
\hline HLWD1-14 & & & 0.0909 & & & 0.0000 & 0.0000 & 0.0000 & & 0.9974 & 0.0389 & 0.1472 & 0.0015 & 0.0669 & & 0.0006 & 0.0299 & 0.0006 & 0.1061 & 0.0019 & 0.0010 \\
\hline HLWD1-15 & & & 0.0000 & & & 0.0000 & 0.0000 & 0.0000 & & 0.9971 & & & & & & & & & & & \\
\hline HLWD1-16 & & & 0.0000 & & & 0.0000 & 0.0000 & 0.0041 & & 0.9906 & & & & & & & & & & & \\
\hline HLWD1-17 & & & 0.0909 & & & 0.0000 & 0.0000 & 0.0000 & & 0.9975 & 0.0414 & 0.1650 & 0.0006 & 0.1812 & & 0.0004 & 0.0315 & 0.0002 & 0.0819 & 0.0019 & 0.0006 \\
\hline HLWD1-18 & & & 0.0000 & & & 0.0020 & 0.0000 & 0.0000 & 0.0006 & 0.9973 & 0.1393 & 0.1444 & 0.0013 & 0.0522 & & 0.0096 & 0.0296 & 0.0191 & 0.0836 & 0.0197 & 0.0008 \\
\hline HLWD1-19 & & & 0.0000 & & & 0.0000 & 0.0000 & 0.0041 & & 1.0000 & 0.0395 & 0.0607 & 0.0638 & 0.1911 & & 0.0118 & 0.0294 & 0.0008 & 0.1482 & 0.0021 & 0.0011 \\
\hline HLWD1-20 & & & 0.0000 & & & 0.0000 & 0.0000 & 0.0041 & & 0.9898 & 0.0395 & 0.0675 & 0.0675 & 0.0466 & & 0.0126 & 0.0338 & 0.0006 & 0.1583 & 0.0017 & 0.0013 \\
\hline HLWD1-21 & & & 0.0022 & & & 0.0006 & 0.0001 & 0.0004 & & 0.9966 & 0.0938 & 0.1522 & 0.0066 & 0.1840 & & 0.0007 & 0.0388 & 0.0023 & 0.1009 & 0.0019 & 0.0084 \\
\hline HLWD1-23 & 0.0046 & & 0.0264 & & & 0.0000 & 0.0000 & 0.0009 & & 0.9965 & 0.0519 & 0.1531 & 0.0139 & 0.1354 & & 0.0054 & 0.0404 & 0.0048 & 0.1297 & 0.0044 & 0.0100 \\
\hline HLWD1-24 & 0.0055 & & 0.0328 & & & 0.0000 & 0.0000 & 0.0011 & & 0.9945 & 0.0629 & 0.1133 & 0.0171 & 0.1695 & & 0.0063 & 0.0208 & 0.0058 & 0.1574 & 0.0054 & 0.0126 \\
\hline HLWD1-25 & & & 0.0000 & & & 0.0000 & 0.0000 & 0.0000 & & 0.9543 & 0.0561 & 0.1100 & 0.0157 & 0.1479 & & 0.0058 & 0.0208 & 0.0053 & 0.1430 & 0.0050 & 0.0114 \\
\hline HLWD1-26 & 0.0033 & & 0.0199 & & & 0.0000 & 0.0000 & 0.0007 & & 0.9965 & & & & & & & & & & & \\
\hline HLWD1-27 & 0.0044 & & 0.0265 & & & 0.0000 & 0.0000 & 0.0009 & & 0.9956 & 0.0520 & 0.1177 & 0.0612 & 0.1391 & & 0.0050 & 0.0213 & 0.0049 & 0.1260 & 0.0045 & 0.0099 \\
\hline HLWD1-28 & 0.0007 & & 0.0062 & & & 0.0000 & 0.0000 & 0.0005 & & 0.9937 & 0.0815 & 0.1031 & 0.0547 & 0.1421 & & 0.0042 & 0.0298 & 0.0013 & 0.0665 & 0.0052 & 0.0010 \\
\hline HLWD2-01 & 0.0000 & & 0.0357 & & & 0.0000 & 0.0000 & 0.0000 & & 0.9955 & 0.0785 & 0.0622 & 0.0229 & 0.1416 & & 0.0038 & 0.0292 & 0.0082 & 0.0664 & 0.0078 & 0.0111 \\
\hline HLWD2-02 & 0.0000 & & 0.0567 & & & 0.0000 & 0.0000 & 0.0000 & & 0.9953 & 0.0220 & 0.0578 & 0.0014 & 0.2155 & & 0.0007 & 0.0271 & 0.0003 & 0.0605 & 0.0129 & 0.0160 \\
\hline HLWD2-03 & & & 0.0909 & & & 0.0000 & 0.0000 & 0.0000 & & 0.9974 & 0.1565 & 0.0720 & 0.0823 & 0.0515 & & & 0.0340 & & 0.1684 & 0.0019 & \\
\hline HLWD2-04 & & & 0.0000 & & & 0.0000 & 0.0000 & 0.0000 & & 0.9971 & 0.0749 & 0.0624 & 0.0024 & 0.2076 & & 0.0006 & 0.0298 & 0.0002 & 0.1614 & 0.0021 & 0.0303 \\
\hline
\end{tabular}


Appendix A. Database - mass fraction

TWRS HLW Glass Formulation (Fu and Pegg 1998)

\begin{tabular}{|c|c|c|c|c|c|c|c|c|c|c|c|c|c|c|c|c|c|c|c|c|c|}
\hline Glass ID & $\begin{array}{c}\mathrm{SiO} 2 \\
-\mathrm{a}\end{array}$ & $\begin{array}{c}\mathrm{ZrO} 2 \\
-\mathrm{a}\end{array}$ & $\begin{array}{c}\mathrm{Ag} 2 \mathrm{O} \\
-\mathrm{a}\end{array}$ & $\begin{array}{c}\mathrm{As} 2 \mathrm{O} 3 \\
-\mathrm{a} \\
\end{array}$ & $\begin{array}{c}\mathrm{BaO} \\
-\mathrm{a}\end{array}$ & $\begin{array}{c}\mathrm{Bi} 2 \mathrm{O} 3 \\
-\mathrm{a}\end{array}$ & $\begin{array}{l}\mathrm{Br} \\
-\mathrm{a}\end{array}$ & $\begin{array}{c}\mathrm{CdO} \\
-\mathrm{a}\end{array}$ & $\begin{array}{c}\mathrm{Ce} 2 \mathrm{O} 3 \\
-\mathrm{a}\end{array}$ & $\begin{array}{c}\mathrm{CeO} 2 \\
-\mathrm{a}\end{array}$ & $\begin{array}{l}\mathrm{Cl} \\
-\mathrm{a}\end{array}$ & $\begin{array}{c}\mathrm{CoO} \\
-\mathrm{a}\end{array}$ & $\begin{array}{c}\mathrm{Co} 2 \mathrm{O} 3 \\
-\mathrm{a}\end{array}$ & $\begin{array}{c}\mathrm{Cr} 2 \mathrm{O} 3 \\
-\mathrm{a} \\
\end{array}$ & $\begin{array}{c}\mathrm{Cs} 2 \mathrm{O} \\
-\mathrm{a} \\
\end{array}$ & $\begin{array}{c}\mathrm{CuO} \\
-\mathrm{a} \\
\end{array}$ & $\begin{array}{c}\mathrm{Eu} 2 \mathrm{O} 3 \\
-\mathrm{a}\end{array}$ & $\begin{array}{l}\mathrm{F} \\
-\mathrm{a} \\
\end{array}$ & $\begin{array}{c}\mathrm{Ga} 2 \mathrm{O} 3 \\
-\mathrm{a}\end{array}$ & $\begin{array}{c}\mathrm{Gd} 2 \mathrm{O} 3 \\
-\mathrm{a}\end{array}$ & $\begin{array}{c}\mathrm{HgO} \\
-\mathrm{a}\end{array}$ \\
\hline HLWD1-10 & 0.2976 & 0.0001 & & & 0.0183 & 0.0111 & & 0.0188 & & & & 0.0022 & & 0.0029 & 0.0022 & 0.0023 & & & & & \\
\hline HLWD1-11 & 0.2995 & 0.0018 & & & 0.0011 & & & & & & & & & 0.0011 & & & & & & & \\
\hline \multicolumn{22}{|l|}{ HLWD1-12 } \\
\hline HLWD1-13 & 0.3274 & 0.0003 & & & 0.0001 & & & & & & & 0.0034 & & 0.0077 & 0.0013 & & & & & & \\
\hline HLWD1-14 & 0.2968 & 0.1013 & & & 0.0003 & & & & & & & 0.0006 & & 0.0002 & 0.0015 & & & & & & \\
\hline \multicolumn{22}{|l|}{ HLWD1-15 } \\
\hline \multicolumn{22}{|l|}{ HLWD1-16 } \\
\hline HLWD1-17 & 0.2972 & 0.0015 & & & 0.0002 & & & & & & & & & 0.0001 & 0.0016 & & & & & & \\
\hline HLWD1-18 & 0.2509 & 0.1002 & & & 0.0001 & & & & & & & 0.0040 & & 0.0068 & 0.0014 & & & & & & \\
\hline HLWD1-19 & 0.2749 & 0.0004 & & & 0.0001 & & & & & & & 0.0001 & & 0.0014 & & & & & & & \\
\hline HLWD1-20 & 0.2994 & 0.0735 & 0.0026 & & 0.0004 & & & 0.0003 & & & & & & 0.0001 & & 0.0048 & & & & & \\
\hline HLWD1-21 & 0.3204 & 0.0016 & 0.0032 & & 0.0007 & 0.0007 & & 0.0001 & & 0.0012 & & & & 0.0011 & 0.0001 & 0.0004 & & & & & \\
\hline HLWD1-23 & 0.3375 & 0.0015 & 0.0021 & & 0.0051 & 0.0008 & & 0.0066 & & 0.0021 & & 0.0016 & & 0.0029 & 0.0014 & 0.0006 & & & & & \\
\hline HLWD1-24 & 0.2802 & 0.0014 & 0.0025 & & 0.0064 & 0.0008 & & 0.0082 & & 0.0026 & & 0.0020 & & 0.0039 & 0.0017 & 0.0007 & & & & & \\
\hline HLWD1-25 & 0.3171 & & 0.0020 & 0.0000 & 0.0061 & 0.0009 & & 0.0076 & & 0.0024 & & 0.0018 & & 0.0035 & 0.0016 & 0.0006 & & & & & \\
\hline \multicolumn{22}{|l|}{ HLWD1-26 } \\
\hline HLWD1-27 & 0.3504 & 0.0012 & 0.0021 & & 0.0054 & 0.0008 & & 0.0069 & & & & 0.0016 & & 0.0029 & & 0.0007 & & & & & \\
\hline HLWD1-28 & 0.3438 & 0.0383 & 0.0002 & & 0.0011 & 0.0003 & & 0.0062 & & & & & & 0.0008 & 0.0001 & 0.0007 & & & & & \\
\hline HLWD2-01 & 0.3172 & 0.0421 & & & 0.0111 & 0.0075 & & 0.0117 & & & & 0.0015 & & 0.0026 & 0.0014 & 0.0014 & & & & & \\
\hline HLWD2-02 & 0.2954 & 0.0007 & & & 0.0166 & 0.0140 & & 0.0219 & & & & 0.0026 & & 0.0047 & 0.0020 & 0.0025 & & & & & \\
\hline HLWD2-03 & 0.3296 & & & & & & & & & 0.0064 & & & & & 0.0015 & & & & & & \\
\hline HLWD2-04 & 0.2606 & 0.0002 & & & 0.0336 & 0.0220 & & 0.0114 & & & & & & 0.0016 & 0.0013 & & & & & & \\
\hline
\end{tabular}


Appendix A. Database - mass fraction

TWRS HLW Glass Formulation (Fu and Pegg 1998)

\begin{tabular}{|c|c|c|c|c|c|c|c|c|c|c|c|c|c|c|c|c|c|c|c|c|c|}
\hline Glass ID & $\begin{array}{c}\text { I } \\
-\mathrm{a} \\
\end{array}$ & $\begin{array}{c}\mathrm{La} 2 \mathrm{O} 3 \\
-\mathrm{a} \\
\end{array}$ & $\begin{array}{c}\mathrm{MnO} 2 \\
-\mathrm{a}\end{array}$ & $\begin{array}{c}\mathrm{MnO} \\
-\mathrm{a}\end{array}$ & $\begin{array}{c}\mathrm{MoO} \\
-\mathrm{a}\end{array}$ & $\begin{array}{c}\mathrm{MoO} 3 \\
-\mathrm{a}\end{array}$ & $\begin{array}{c}\mathrm{Nb} 2 \mathrm{O} 5 \\
-\mathrm{a}\end{array}$ & $\begin{array}{c}\mathrm{Nd} 2 \mathrm{O} 3 \\
-\mathrm{a} \\
\end{array}$ & $\begin{array}{c}\mathrm{PbO} \\
-\mathrm{a}\end{array}$ & $\begin{array}{c}\mathrm{PdO} 2 \\
-\mathrm{a}\end{array}$ & $\begin{array}{c}\mathrm{PdO} \\
-\mathrm{a}\end{array}$ & $\begin{array}{c}\mathrm{Pr} 2 \mathrm{O} 3 \\
-\mathrm{a}\end{array}$ & $\begin{array}{c}\text { Pr6O11 } \\
-\mathrm{a}\end{array}$ & $\begin{array}{c}\mathrm{Rb} 2 \mathrm{O} \\
-\mathrm{a}\end{array}$ & $\begin{array}{c}\mathrm{ReO} \\
-\mathrm{a}\end{array}$ & $\begin{array}{c}\mathrm{ReO} 2 \\
-\mathrm{a}\end{array}$ & $\begin{array}{c}\mathrm{Rh} 2 \mathrm{O} 3 \\
-\mathrm{a}\end{array}$ & $\begin{array}{c}\mathrm{RhO} 2 \\
-\mathrm{a}\end{array}$ & $\begin{array}{c}\mathrm{RuO} 2 \\
-\mathrm{a}\end{array}$ & $\begin{array}{c}\mathrm{Sb} 2 \mathrm{O} 3 \\
-\mathrm{a} \\
\end{array}$ & $\begin{array}{c}\mathrm{Sb} 2 \mathrm{O} 5 \\
-\mathrm{a}\end{array}$ \\
\hline HLWD1-10 & & 0.0113 & 0.0376 & & 0.0049 & & & 0.0096 & 0.0052 & & & & & & & & & & & & \\
\hline HLWD1-11 & & 0.0005 & 0.0070 & & & & & 0.0007 & & & & & & & & & & & & & \\
\hline \multicolumn{22}{|l|}{ HLWD1-12 } \\
\hline HLWD1-13 & & & 0.0588 & & & & & 0.0002 & & & & 0.0002 & & & & & & & & & \\
\hline HLWD1-14 & & 0.0174 & 0.0011 & & & & & 0.0117 & & & & 0.0010 & & & & & & & & & \\
\hline HLWD1-15 & & & & & & & & & & & & & & & & & & & & & \\
\hline \multicolumn{22}{|l|}{ HLWD1-16 } \\
\hline HLWD1-17 & & & 0.0003 & & & & & & & & & & & & & & & & & & \\
\hline HLWD1-18 & & & 0.0639 & & & & & & & & & & & & & & & & & & \\
\hline HLWD1-19 & & & 0.0002 & & & & & & & & & & & & & & & & & & \\
\hline HLWD1-20 & & & 0.0002 & & 0.0070 & & & & 0.0099 & & & & & & & & & & & & \\
\hline HLWD1-21 & & 0.0001 & 0.0050 & & 0.0002 & & & 0.0004 & & & & & & & & & & & & & \\
\hline HLWD1-23 & & 0.0018 & 0.0051 & & & & & 0.0002 & & & 0.0008 & & & & & 0.0002 & & & 0.0002 & 0.0013 & \\
\hline HLWD1-24 & & 0.0023 & 0.0063 & & & & & 0.0002 & & & 0.0010 & & & & & 0.0003 & & & 0.0001 & 0.0016 & \\
\hline HLWD1-25 & & 0.0022 & 0.0058 & & & & & 0.0002 & & & 0.0004 & & & & & 0.0003 & & & 0.0002 & 0.0015 & \\
\hline \multicolumn{22}{|l|}{ HLWD1-26 } \\
\hline HLWD1-27 & & 0.0023 & 0.0053 & & & & & 0.0005 & & & 0.0007 & & & & & 0.0006 & & & 0.0006 & & \\
\hline HLWD1-28 & & 0.0041 & 0.0051 & & & & & 0.0002 & 0.0014 & & & & & & & & & & & & \\
\hline HLWD2-01 & & 0.0067 & 0.0235 & & 0.0019 & & & 0.0049 & 0.0036 & & & & & & & & & & & & \\
\hline HLWD2-02 & & 0.0110 & 0.0346 & & 0.0029 & & & 0.0069 & 0.0077 & & & & & & & & & & & & \\
\hline HLWD2-03 & & 0.0195 & & & & & & 0.0128 & & & & 0.0028 & & & & & & & & & \\
\hline HLWD2-04 & & & 0.0002 & & & & & & & & & & & & & & & & & & \\
\hline
\end{tabular}


Appendix A. Database - mass fraction

TWRS HLW Glass Formulation (Fu and Pegg 1998)

\begin{tabular}{|c|c|c|c|c|c|c|c|c|c|c|c|c|c|c|c|c|c|c|c|c|}
\hline Glass ID & $\begin{array}{c}\mathrm{SeO} 2 \\
-\mathrm{a}\end{array}$ & $\begin{array}{c}\mathrm{Sm} 2 \mathrm{O} 3 \\
-\mathrm{a}\end{array}$ & $\begin{array}{c}\mathrm{SnO} \\
-\mathrm{a}\end{array}$ & $\begin{array}{c}\mathrm{SnO} 2 \\
-\mathrm{a}\end{array}$ & $\begin{array}{c}\mathrm{SO} 3 \\
-\mathrm{a}\end{array}$ & $\begin{array}{c}\mathrm{SrO} \\
-\mathrm{a} \\
\end{array}$ & $\begin{array}{c}\mathrm{Tc} 2 \mathrm{O} 7 \\
-\mathrm{a}\end{array}$ & $\begin{array}{c}\mathrm{TeO} 2 \\
-\mathrm{a}\end{array}$ & $\begin{array}{c}\mathrm{ThO} 2 \\
-\mathrm{a}\end{array}$ & $\begin{array}{c}\mathrm{TiO} 2 \\
-\mathrm{a} \\
\end{array}$ & $\begin{array}{c}\mathrm{T} 12 \mathrm{O} 3 \\
-\mathrm{a}\end{array}$ & $\begin{array}{c}\mathrm{U} 3 \mathrm{O} 8 \\
-\mathrm{a}\end{array}$ & $\begin{array}{c}\mathrm{UO} 2 \\
-\mathrm{a}\end{array}$ & $\begin{array}{c}\mathrm{UO} 3 \\
-\mathrm{a}\end{array}$ & $\begin{array}{c}\mathrm{V} 2 \mathrm{O} 5 \\
-\mathrm{a}\end{array}$ & $\begin{array}{c}\text { WO3 } \\
-\mathrm{a}\end{array}$ & $\begin{array}{c}\mathrm{Y} 2 \mathrm{O} 3 \\
-\mathrm{a}\end{array}$ & $\begin{array}{c}\mathrm{ZnO} \\
-\mathrm{a} \\
\end{array}$ & $\begin{array}{c}\text { Others } \\
-\mathrm{a}\end{array}$ & $\begin{array}{c}\text { Sum } \\
-\mathrm{a}\end{array}$ \\
\hline HLWD1-10 & & & & & 0.0065 & 0.0298 & & & & 0.0080 & & & 0.0606 & & & & & & & 0.9582 \\
\hline HLWD1-11 & & & & & 0.0020 & 0.0267 & & & & 0.0028 & & & 0.0045 & & & & & & & 0.9177 \\
\hline \multicolumn{21}{|l|}{ HLWD1-12 } \\
\hline HLWD1-13 & & & 0.0006 & & & 0.0193 & & & & 0.0005 & & & 0.0010 & & & & & & & 0.9432 \\
\hline HLWD1-14 & & & 0.0026 & & & 0.0188 & & & 0.0041 & 0.0006 & & & 0.0818 & & & & & & & 0.9344 \\
\hline HLWD1-15 & & & & & & & & & & & & & & & & & & & & \\
\hline \multicolumn{21}{|l|}{ HLWD1-16 } \\
\hline HLWD1-17 & & & & & & 0.0181 & & & & 0.0002 & & & 0.0754 & & & & & & & 0.8993 \\
\hline HLWD1-18 & & & 0.0010 & & & 0.0167 & & & & 0.0004 & & & 0.0008 & & & & & & & 0.9458 \\
\hline HLWD1-19 & & & 0.0002 & & 0.0096 & 0.0210 & & & & 0.0163 & & & 0.0010 & & & & & 0.0041 & & 0.8778 \\
\hline HLWD1-20 & & & & & 0.0130 & 0.0195 & & & & 0.0163 & & & 0.0009 & & & & & 0.0042 & & 0.8815 \\
\hline HLWD1-21 & & & 0.0002 & & 0.0020 & 0.0196 & & & 0.0003 & 0.0023 & & & 0.0031 & & 0.0002 & & & & & 0.9525 \\
\hline HLWD1-23 & & & 0.0010 & & 0.0089 & 0.0250 & & 0.0014 & 0.0007 & 0.0017 & 0.0017 & & 0.0273 & & 0.0002 & & & & & 0.9887 \\
\hline HLWD1-24 & & & 0.0012 & & 0.0092 & 0.0315 & & 0.0018 & 0.0010 & 0.0020 & 0.0017 & & 0.0322 & & 0.0002 & & & & & 0.9741 \\
\hline HLWD1-25 & & & 0.0011 & & 0.0084 & 0.0350 & & & & & & & & & & & & & & 0.9197 \\
\hline \multicolumn{21}{|l|}{ HLWD1-26 } \\
\hline HLWD1-27 & & & & & & 0.0258 & & & 0.0011 & 0.0016 & & & 0.0257 & & & & & & & 0.9778 \\
\hline HLWD1-28 & 0.0012 & & & & & 0.0226 & & 0.0018 & & 0.0013 & 0.0005 & & 0.0067 & & & & & 0.0006 & & 0.9264 \\
\hline HLWD2-01 & & & & & 0.0051 & 0.0311 & & & 0.0014 & 0.0049 & & & 0.0360 & & & & & & & 0.9473 \\
\hline HLWD2-02 & & & & & 0.0065 & 0.0270 & & & & 0.0077 & & & 0.0552 & & & & & & & 0.9341 \\
\hline HLWD2-03 & & & & & & 0.0196 & & & 0.0038 & & & & 0.0375 & & & & & & & 1.0001 \\
\hline HLWD2-04 & & & & & & 0.0176 & & & & 0.0006 & & & 0.0005 & & & & & & & 0.9213 \\
\hline
\end{tabular}


Appendix A. Database - mass fraction

\begin{tabular}{|c|c|c|c|c|c|c|c|c|}
\hline Glass ID & $\begin{array}{l}\mathrm{TM} \\
\left({ }^{\circ} \mathrm{C}\right) \\
\end{array}$ & $\begin{array}{l}\text { Gradient } \\
\text { TL }\left({ }^{\circ} \mathrm{C}\right)\end{array}$ & $\begin{array}{l}\text { Uniform } \\
\text { TL }\left({ }^{\circ} \mathrm{C}\right)\end{array}$ & Primary Phase & $\begin{array}{c}\text { Quenched } \\
\text { Visual/OM }\end{array}$ & $\begin{array}{c}\text { Quenched } \\
\text { SEM/EDS or TEM }\end{array}$ & $\begin{array}{l}\text { Quenched } \\
\text { XRD }\end{array}$ & $\begin{array}{c}\text { Quenched } \\
\text { Homogeneous? }\end{array}$ \\
\hline HLWD1-10 & 1150 & & $<1050$ & & glassy brown & & & \\
\hline HLWD1-11 & 1150 & & $>1050$ & & glassy brown & $\begin{array}{l}2-5 \text { vol } \% \text { spinel } ;<1 \text { vol } \% \text { hematite; } \\
<1 \text { vol } \% \text { silicate \& zircon }\end{array}$ & & \\
\hline HLWD1-12 & 1150 & & $>1050$ & & opaque brown & spinels and hematite & & \\
\hline HLWD1-13 & 1150 & & $>1050$ & & opaque brown & & & \\
\hline HLWD1-14 & 1150 & & $>1050$ & & clear on top and bottom but crystallized bulk & & & \\
\hline HLWD1-15 & 1150 & & $>1050$ & & heavily crystallized & & & \\
\hline HLWD1-16 & 1150 & & $>1050$ & & heavily crystallized & & & \\
\hline HLWD1-17 & 1150 & & $<1050$ & & $\begin{array}{l}\text { clear top and bottom with middle section } \\
\text { crystallized }\end{array}$ & & & \\
\hline HLWD1-18 & 1150 & & $>1050$ & & brown glass & & & \\
\hline HLWD1-19 & 1150 & & $>1050$ & & brown and yellow scum on top of brown glass & & & \\
\hline HLWD1-20 & 1150 & & 1050 & & opaque green w/ white spots on top & & & \\
\hline HLWD1-21 & 1150 & & $<1050$ & & $\begin{array}{l}\text { opaque brown glass } \mathrm{w} / \text { brown spots on } \\
\text { bottom }\end{array}$ & $0.1-0.2 \mathrm{vol} \% \mathrm{Ag}, 0.1 \mathrm{um}$ diameter & & \\
\hline HLWD1-23 & 1150 & & 1050 & & glassy brown but crucible is heterogeneous & $\begin{array}{l}\text { glass on bottom of crucible has } \\
\text { spinels. Pd along sides of crucible. }\end{array}$ & & \\
\hline HLWD1-24 & 1150 & & 1050 & & glassy brown but crucible is heterogeneous & $\begin{array}{l}\text { glass on bottom of crucible has } \\
\text { spinels. Pd along sides of crucible. }\end{array}$ & & \\
\hline HLWD1-25 & 1150 & & $<1050$ & & glassy brown but crucible is heterogeneous & & & \\
\hline HLWD1-26 & 1150 & & $<1050$ & & $\begin{array}{l}\text { brown glass with opaque brown \& yellow } \\
\text { streaks \& heterogeneous crucible }\end{array}$ & $\begin{array}{l}\text { Yellow coloration due to } \mathrm{Ag} \text { particles } \\
\text { Brown band has Ru crystal clusters. }\end{array}$ & & \\
\hline HLWD1-27 & 1150 & & $<1050$ & & glassy brown but crucible is heterogeneous & & & \\
\hline HLWD1-28 & 1150 & & $<1050$ & & glassy brown & & & \\
\hline HLWD2-01 & 1150 & & $<1050$ & & opaque dark brown glass & $\begin{array}{l}0.5 \mathrm{vol} \% \mathrm{ZrO} 2,3.6 \mathrm{vol} \% \text { spinel, both } \\
\text { with U incorp., trace hematite }\end{array}$ & & \\
\hline HLWD2-02 & 1150 & & $<1050$ & & glassy brown & & & \\
\hline HLWD2-03 & 1150 & & $<1050$ & & clear light brown & & & yes \\
\hline HLWD2-04 & 1150 & & $<1050$ & & glassy brown & & & \\
\hline
\end{tabular}


Appendix A. Database - mass fraction

TWRS HLW Glass Formulation (Fu and Pegg 1998)

\begin{tabular}{|c|c|c|c|c|c|}
\hline Glass ID & $\begin{array}{c}\mathrm{CCC} \\
\text { Visual/OM }\end{array}$ & $\begin{array}{c}\text { CCC } \\
\text { SEM/EDS or TEM } \\
\end{array}$ & $\begin{array}{l}\text { CCC } \\
\text { XRD } \\
\end{array}$ & $\begin{array}{c}\text { Heat Treated } \\
\text { Visual/OM }\end{array}$ & $\begin{array}{c}\text { Heat Treated } \\
\text { SEM/EDS or TEM }\end{array}$ \\
\hline \multicolumn{6}{|l|}{ HLWD1-10 } \\
\hline \multicolumn{6}{|l|}{ HLWD1-11 } \\
\hline \multicolumn{6}{|l|}{ HLWD1-12 } \\
\hline \multicolumn{6}{|l|}{ HLWD1-13 } \\
\hline \multicolumn{6}{|l|}{ HLWD1-14 } \\
\hline \multicolumn{6}{|l|}{ HLWD1-15 } \\
\hline \multicolumn{6}{|l|}{ HLWD1-16 } \\
\hline \multicolumn{6}{|l|}{ HLWD1-17 } \\
\hline \multicolumn{6}{|l|}{ HLWD1-18 } \\
\hline \multicolumn{6}{|l|}{ HLWD1-19 } \\
\hline \multicolumn{6}{|l|}{ HLWD1-20 } \\
\hline \multicolumn{6}{|l|}{ HLWD1-21 } \\
\hline \multicolumn{6}{|l|}{ HLWD1-23 } \\
\hline \multicolumn{6}{|l|}{ HLWD1-24 } \\
\hline \multicolumn{6}{|l|}{ HLWD1-25 } \\
\hline \multicolumn{6}{|l|}{ HLWD1-26 } \\
\hline \multicolumn{6}{|l|}{ HLWD1-27 } \\
\hline \multicolumn{6}{|l|}{ HLWD1-28 } \\
\hline \multicolumn{6}{|l|}{ HLWD2-01 } \\
\hline \multicolumn{6}{|l|}{ HLWD2-02 } \\
\hline HLWD2-03 & & & & & \\
\hline HLWD2-04 & & & & & \\
\hline
\end{tabular}


Appendix A. Database - mass fraction

TWRS HLW Glass Formulation (Fu and Pegg 1998)

\begin{tabular}{|c|c|c|c|c|c|c|c|c|c|c|c|c|c|c|c|}
\hline Glass ID & $\begin{array}{c}\text { Heat Treated } \\
\text { XRD } \\
\end{array}$ & $\begin{array}{l}\text { Density } \\
\left(\mathrm{g} / \mathrm{cm}^{3}\right)\end{array}$ & \begin{tabular}{|c|} 
Fulc Visc \\
A
\end{tabular} & \begin{tabular}{|c|} 
Fulc Visc \\
B
\end{tabular} & \begin{tabular}{|c|} 
Fulc Visc \\
To
\end{tabular} & $\begin{array}{c}\mathrm{FV} 1150^{\circ} \mathrm{C} \\
(\mathrm{Pa} \cdot \mathrm{s})\end{array}$ & $\begin{array}{c}\text { Arrh Visc } \\
\mathrm{A}\end{array}$ & $\begin{array}{c}\text { Arrh Visc } \\
\text { B }\end{array}$ & $1150^{\circ} \mathrm{C}$ & \begin{tabular}{|c|}
$\mathrm{T}\left({ }^{\circ} \mathrm{C}\right)$ at \\
$2 \mathrm{~Pa} \cdot \mathrm{s}$ \\
\end{tabular} & $\begin{array}{c}\mathrm{T}\left({ }^{\circ} \mathrm{C}\right) \text { at } \\
5 \mathrm{~Pa} \cdot \mathrm{s}\end{array}$ & $\begin{array}{c}\mathrm{T}\left({ }^{\circ} \mathrm{C}\right) \text { at } \\
10 \mathrm{~Pa} \cdot \mathrm{s} \\
\end{array}$ & $\begin{array}{c}\mathrm{T} 1 \\
\left({ }^{\circ} \mathrm{C}\right)\end{array}$ & \begin{tabular}{c|c}
$\mathrm{V} 1$ \\
$(\mathrm{~Pa} \cdot \mathrm{s})$ \\
\end{tabular} & $\begin{array}{l}\mathrm{T} 2 \\
\left({ }^{\circ} \mathrm{C}\right)\end{array}$ \\
\hline HLWD1-10 & $\begin{array}{l}\text { surface crystallized, }<1 \text { vol } \% \text { bulk crys., } \\
\text { most likely spinels }\end{array}$ & & & & & & -12.419 & 19557.4 & $\begin{array}{l}\text { (D., } \\
3.76\end{array}$ & & & & 1000 & 20.641 & 1050 \\
\hline HLWD1-11 & $\begin{array}{l}1 \mathrm{~mm} \text { wide band of crystals at Pt/glass } \\
\text { interface. } 23 \mathrm{vol} \% \text { crystals in this band. } \\
\text { Mostly } \mathrm{Fe} 3 \mathrm{O} 4 \mathrm{w} / \mathrm{Mg}, \mathrm{Al}, \mathrm{Si}, \mathrm{Cr}, \mathrm{Mn}, \& \mathrm{Ni} \text {. } \\
\text { Rest of glass has }<1 \mathrm{vol} \% \text { crystals. }\end{array}$ & & & & & & -10.616 & 16471.7 & 2.61 & & & & $(1000)$ & $(14.681$ & $(1050)$ \\
\hline HLWD1-12 & About $5 \% \mathrm{ZrO} 2$ and spinel & & & & & & & & & & & & & & \\
\hline HLWD1-13 & $\begin{array}{l}\text { About } 3 \% \text { hematite or spinel and } 5-10 \% \\
\text { silicate }\end{array}$ & & & & & & -13.159 & 18650.4 & 0.95 & & & & 1000 & 4.445 & 1050 \\
\hline HLWD1-14 & About 2 vol $\%$ ZrO2 & & & & & & -12.716 & 18386.0 & 1.23 & & & & 1000 & 5.865 & 1050 \\
\hline HLWD1-15 & About $25 \%$ spinels and silicates & & & & & & & & & & & & & & \\
\hline HLWD1-16 & $\begin{array}{l}6 \mathrm{vol} \% \mathrm{ZrO} 2,<1 \text { vol } \% \text { Zr-Sb-Ca-Ti-Fe } \\
\text { crystals \& one patch of Fe2O3 crystals }\end{array}$ & & & & & & & & & & & & & & \\
\hline HLWD1-17 & $<1 \mathrm{vol} \%$ crystals & & & & & & -10.424 & 14507.4 & 0.80 & & & & 1000 & 2.75 & 1050 \\
\hline HLWD1-18 & $\begin{array}{l}10 \text { vol } \% \text { crys. From top to center and on } \\
\text { bottom. Clear in middle section. Lots of } \\
\text { bubbles in upper area. }\end{array}$ & & & & & & -11.147 & 18683.3 & 7.26 & & & & $(1000)$ & $\sim 100$ & $(1050)$ \\
\hline HLWD1-19 & $<1$ vol $\%$ spinels and $0.5 \mathrm{vol} \% \mathrm{Ag}$ & & & & & & -10.396 & 13242.8 & 0.34 & & & & 1000 & 1.027 & 1050 \\
\hline HLWD1-20 & 1 vol $\%$ crys. & & & & & & & & & & & & & & \\
\hline HLWD1-21 & $\begin{array}{l}<1 \mathrm{vol} \% \text { crystals, mainly spinels on bottom } \\
\text { of crucible and some drops high in Ag (may } \\
\text { occur during cooling) }\end{array}$ & & & & & & -9.917 & 14458.8 & 1.28 & & & & 1000 & 4.367 & 1050 \\
\hline HLWD1-23 & Clear except for $0.1-1$ um $\mathrm{Ag} \sim 1 \mathrm{vol} \% \mathrm{Ag}$ & & & & & & -10.046 & 13685.2 & 0.65 & & & & 1000 & 2.106 & 1050 \\
\hline HLWD1-24 & $\begin{array}{l}\text { Ag particles and } \mathrm{Fe}-\mathrm{Ni} \text { enriched crystals, } 1 \\
\text { vol } \% \text {. }\end{array}$ & & & & & & & & & & & & & & \\
\hline HLWD1-25 & $<1$ vol $\%$ crystals & & & & & & & & & & & & & & \\
\hline HLWD1-26 & $\begin{array}{l}\text { Several Ru clusters varying in size from } \\
50 \times 100 \text { um to } 5 \times 5 \text { um. Much less than } 1 \\
\text { vol } \% \text { crystals. }\end{array}$ & & & & & & & & & & & & & & \\
\hline HLWD1-27 & clear glass & & & & & & -10.788 & 15581.2 & 1.18 & & & & 1000 & 4.539 & 1050 \\
\hline HLWD1-28 & $\begin{array}{l}\text { Fe-Ni spinels with } \mathrm{Cr}, \mathrm{Mn}, \mathrm{Al} \text {, and } \mathrm{Mg} \\
\text { incorporation along the glass } / \text { crucible } \\
\text { interface. About } 0.2 \mathrm{vol} \% \text { total crystals. }\end{array}$ & & & & & & & & & & & & & & \\
\hline HLWD2-01 & $\begin{array}{l}0.02 \text { vol } \% \text { spinel at interfaces. } 0.2 \text { vol } \% \\
\mathrm{ZrO} 2 \text { at interfaces extending down to bulk. }\end{array}$ & & & & & & & & & & & & (1100) & $(11.679$ & $(1050)$ \\
\hline HLWD2-02 & clear except for one $3 \times 5 u m$ Sn particle & & & & & & -14.645 & 21451.7 & 1.54 & & & & 1050 & 4.94 & 1100 \\
\hline HLWD2-03 & clear glass & & & & & & -10.328 & 16674.7 & 4.01 & & & & 1000 & 17.138 & 1050 \\
\hline HLWD2-04 & $\begin{array}{l}\text { Clear for bulk. Crystals (probably spinels) } \\
\text { on bottom and center of meniscus. }<1 \text { vol } \% \\
\text { total crystals }\end{array}$ & & & & & & -9.648 & 13594.6 & 0.91 & & & & 1000 & 2.881 & 1050 \\
\hline
\end{tabular}


Appendix A. Database - mass fraction

\begin{tabular}{|c|c|c|c|c|c|c|c|c|c|c|c|c|c|c|c|c|c|c|c|c|c|}
\hline \multicolumn{22}{|c|}{ CWRS HLW Glass Formulation (Fu and Pegg 1998) } \\
\hline Glass ID & $\begin{array}{c}\mathrm{V} 2 \\
(\mathrm{~Pa} \cdot \mathrm{s})\end{array}$ & $\begin{array}{c}\mathrm{T3} \\
\left({ }^{\circ} \mathrm{C}\right) \\
\end{array}$ & $\begin{array}{c}\mathrm{V} 3 \\
(\mathrm{~Pa} \cdot \mathrm{s})\end{array}$ & $\begin{array}{c}\mathrm{T} 4 \\
\left({ }^{\circ} \mathrm{C}\right) \\
\end{array}$ & $\begin{array}{c}\mathrm{V} 4 \\
(\mathrm{~Pa} \cdot \mathrm{s})\end{array}$ & $\begin{array}{c}\mathrm{T} 5 \\
\left({ }^{\circ} \mathrm{C}\right) \\
\end{array}$ & $\begin{array}{c}\mathrm{V} 5 \\
(\mathrm{~Pa} \cdot \mathrm{s})\end{array}$ & $\begin{array}{c}\mathrm{T} 6 \\
\left({ }^{\circ} \mathrm{C}\right) \\
\end{array}$ & $\begin{array}{c}\mathrm{V} 6 \\
(\mathrm{~Pa} \cdot \mathrm{s})\end{array}$ & $\begin{array}{c}\mathrm{T} 7 \\
\left({ }^{\circ} \mathrm{C}\right) \\
\end{array}$ & $\begin{array}{c}\mathrm{V} 7 \\
(\mathrm{~Pa} \cdot \mathrm{s})\end{array}$ & $\begin{array}{c}\mathrm{T} 8 \\
\left({ }^{\circ} \mathrm{C}\right) \\
\end{array}$ & $\begin{array}{c}\mathrm{V} 8 \\
(\mathrm{~Pa} \cdot \mathrm{s})\end{array}$ & $\begin{array}{c}\mathrm{T} 9 \\
\left({ }^{\circ} \mathrm{C}\right) \\
\end{array}$ & $\begin{array}{c}\mathrm{V} 9 \\
(\mathrm{~Pa} \cdot \mathrm{s})\end{array}$ & $\begin{array}{l}\mathrm{T} 10 \\
\left({ }^{\circ} \mathrm{C}\right) \\
\end{array}$ & $\begin{array}{c}\mathrm{V} 10 \\
(\mathrm{~Pa} \cdot \mathrm{s})\end{array}$ & $\begin{array}{l}\mathrm{T} 11 \\
\left({ }^{\circ} \mathrm{C}\right) \\
\end{array}$ & $\begin{array}{c}\mathrm{V} 11 \\
(\mathrm{~Pa} \cdot \mathrm{s})\end{array}$ & $\begin{array}{l}\mathrm{T} 12 \\
\left({ }^{\circ} \mathrm{C}\right) \\
\end{array}$ & $\begin{array}{c}\mathrm{V} 12 \\
(\mathrm{~Pa} \cdot \mathrm{s})\end{array}$ \\
\hline HLWD1-10 & 10.475 & 1100 & 5.857 & 1150 & 3.538 & 1200 & 2.277 & 1250 & 1.543 & 1300 & 1.092 & & & & & & & & & & \\
\hline HLWD1-11 & (7.294 & 1100 & 4.115 & 1150 & 2.554 & 1200 & 1.706 & 1250 & 1.207 & 1300 & 0.894 & & & & & & & & & & \\
\hline \multicolumn{22}{|l|}{ HLWD1-12 } \\
\hline HLWD1-13 & 2.555 & 1100 & 1.529 & 1150 & 0.949 & 1200 & 0.608 & 1250 & 0.401 & 1300 & 0.272 & & & & & & & & & & \\
\hline HLWD1-14 & 3.236 & 1100 & 1.907 & 1150 & 1.189 & 1200 & 0.776 & 1250 & 0.528 & 1300 & 0.372 & & & & & & & & & & \\
\hline \multicolumn{22}{|l|}{ HLWD1-15 } \\
\hline \multicolumn{22}{|l|}{ HLWD1-16 } \\
\hline HLWD1-17 & 1.708 & 1100 & 1.122 & 1150 & 0.772 & 1200 & 0.553 & 1250 & 0.409 & 1300 & 0.312 & & & & & & & & & & \\
\hline HLWD1-18 & $\sim 40$ & (1100) & $\sim 20$ & $(1150)$ & $\sim 10$ & 1200 & 4.70 & 1250 & 3.00 & 1300 & 2.10 & & & & & & & & & & \\
\hline HLWD1-19 & 0.677 & 1100 & 0.466 & 1150 & 0.331 & 1200 & 0.243 & 1250 & 0.183 & 1300 & 0.141 & & & & & & & & & & \\
\hline \multicolumn{22}{|l|}{ HLWD1-20 } \\
\hline HLWD1-21 & 2.736 & 1100 & 1.805 & 1150 & 1.244 & 1200 & 0.89 & 1250 & 0.658 & 1300 & 0.499 & & & & & & & & & & \\
\hline HLWD1-23 & 1.338 & 1100 & 0.899 & 1150 & 0.632 & 1200 & 0.462 & 1250 & 0.348 & 1300 & 0.27 & & & & & & & & & & \\
\hline \multicolumn{22}{|l|}{ HLWD1-24 } \\
\hline \multicolumn{22}{|l|}{ HLWD1-25 } \\
\hline \multicolumn{22}{|l|}{ HLWD1-26 } \\
\hline HLWD1-27 & 2.66 & 1100 & 1.677 & 1150 & 1.123 & 1200 & 0.789 & 1250 & 0.577 & 1300 & 0.437 & & & & & & & & & & \\
\hline \multicolumn{22}{|l|}{ HLWD1-28 } \\
\hline HLWD2-01 & (4.941 & (1200) & $(3.71$ & $(1250)$ & (3.214 & & & & & & & & & & & & & & & & \\
\hline HLWD2-02 & 2.615 & 1150 & 1.496 & 1200 & 0.913 & 1250 & 0.587 & & & & & & & & & & & & & & \\
\hline HLWD2-03 & 9.612 & 1100 & 5.855 & 1150 & 3.81 & 1200 & 2.617 & 1250 & 1.878 & 1300 & 1.398 & & & & & & & & & & \\
\hline HLWD2-04 & 1.866 & 1100 & 1.266 & 1150 & 0.892 & 1200 & 0.65 & 1250 & 0.488 & 1300 & 0.375 & & & & & & & & & & \\
\hline
\end{tabular}


Appendix A. Database - mass fraction

TWRS HLW Glass Formulation (Fu and Pegg 1998)

\begin{tabular}{|c|c|c|c|c|c|c|c|c|c|c|c|c|c|c|c|c|}
\hline Glass ID & $\begin{array}{l}\mathrm{T} 13 \\
\left({ }^{\circ} \mathrm{C}\right) \\
\end{array}$ & $\begin{array}{c}\mathrm{V} 13 \\
(\mathrm{~Pa} \cdot \mathrm{s}) \\
\end{array}$ & $\begin{array}{l}\mathrm{T} 14 \\
\left({ }^{\circ} \mathrm{C}\right) \\
\end{array}$ & $\begin{array}{c}\mathrm{V} 14 \\
(\mathrm{~Pa} \cdot \mathrm{s}) \\
\end{array}$ & $\begin{array}{c}\text { Q PCT } \\
\text { B }\left(\mathrm{g} / \mathrm{m}^{2}\right)\end{array}$ & $\begin{array}{c}\text { Q PCT } \\
\mathrm{Li}\left(\mathrm{g} / \mathrm{m}^{2}\right)\end{array}$ & $\begin{array}{c}\text { Q PCT } \\
\mathrm{Na}\left(\mathrm{g} / \mathrm{m}^{2}\right)\end{array}$ & $\begin{array}{c}\text { Q PCT } \\
\mathrm{Si}\left(\mathrm{g} / \mathrm{m}^{2}\right)\end{array}$ & $\begin{array}{c}\text { Q PCT } \\
\mathrm{pH}\end{array}$ & $\begin{array}{c}\text { CCC PCT } \\
\text { B }\left(\mathrm{g} / \mathrm{m}^{2}\right)\end{array}$ & $\begin{array}{l}\text { CCC PCT } \\
\mathrm{Li}\left(\mathrm{g} / \mathrm{m}^{2}\right)\end{array}$ & $\begin{array}{l}\text { CCC PCT } \\
\mathrm{Na}\left(\mathrm{g} / \mathrm{m}^{2}\right)\end{array}$ & $\begin{array}{l}\text { CCC PCT } \\
\mathrm{Si}\left(\mathrm{g} / \mathrm{m}^{2}\right)\end{array}$ & $\begin{array}{c}\begin{array}{c}\text { CCC PCT } \\
\mathrm{pH}\end{array} \\
\end{array}$ & $\begin{array}{l}\text { Q PCT B at } \\
20^{\circ} \mathrm{C}\left(\mathrm{g} / \mathrm{m}^{2}\right)\end{array}$ & $\begin{array}{l}\text { Q PCT Li at } \\
20^{\circ} \mathrm{C}\left(\mathrm{g} / \mathrm{m}^{2}\right)\end{array}$ \\
\hline HLWD1-10 & & & & & 0.3677863 & & 0.3710316 & 0.1574067 & 9.3 & & & & & & & \\
\hline HLWD1-11 & & & & & 0.8012563 & 0.67 & 0.6334435 & 0.3083801 & 11.3 & & & & & & & \\
\hline \multicolumn{17}{|l|}{ HLWD1-12 } \\
\hline HLWD1-13 & & & & & 0.1942531 & 0.250445 & 0.2630401 & 0.1599618 & 10.5 & & & & & & & \\
\hline HLWD1-14 & & & & & 1.33 & 0.98 & 0.84 & 0.14 & 10.1 & & & & & & & \\
\hline \multicolumn{17}{|l|}{ HLWD1-15 } \\
\hline \multicolumn{17}{|l|}{ HLWD1-16 } \\
\hline HLWD1-17 & & & & & 1.2209873 & 1.0093427 & 0.7923769 & 0.2182348 & 10.7 & & & & & & & \\
\hline HLWD1-18 & & & & & 0.6478138 & 0.5360557 & 0.4770007 & 0.1760258 & 9.6 & & & & & & & \\
\hline HLWD1-19 & & & & & 1.47 & 1.4 & 1.89 & 0.49 & 11.8 & & & & & & & \\
\hline HLWD1-20 & & & & & 0.6120691 & 0.5646938 & 0.7392427 & 0.2287683 & 11.6 & & & & & & & \\
\hline HLWD1-21 & & & & & 0.6081054 & 0.5126693 & 0.4320195 & 0.1979808 & 10.1 & & & & & & & \\
\hline HLWD1-23 & & & & & 2.4933787 & 2.0059108 & 1.6943758 & 0.379732 & 10.82 & & & & & & & \\
\hline \multicolumn{17}{|l|}{ HLWD1-24 } \\
\hline HLWD1-25 & & & & & 1.9608046 & 1.5578925 & 1.5236959 & 0.3735782 & 10.3 & & & & & & & \\
\hline \multicolumn{17}{|l|}{ HLWD1-26 } \\
\hline HLWD1-27 & & & & & 1.05 & 0.98 & 1.12 & 0.28 & 11.1 & & & & & & & \\
\hline \multicolumn{17}{|l|}{ HLWD1-28 } \\
\hline HLWD2-01 & & & & & 0.23 & 0.2527201 & 0.2131451 & 0.1114544 & 10.0 & & & & & & & \\
\hline HLWD2-02 & & & & & 0.47 & 0.4391158 & 0.4323454 & 0.2861254 & 10.0 & & & & & & & \\
\hline HLWD2-03 & & & & & 0.2265118 & 0.3415069 & 0.5263493 & 0.1849666 & 11.2 & & & & & & & \\
\hline HLWD2-04 & & & & & 3.57 & 2.87 & 3.01 & 0.63 & 11.9 & & & & & & & \\
\hline
\end{tabular}


Appendix A. Database - mass fraction

TWRS HLW Glass Formulation (Fu and Pegg 1998)

\begin{tabular}{|c|c|c|c|c|c|c|c|c|c|c|c|c|}
\hline Glass ID & $\begin{array}{l}\text { Q PCT Na at } \\
20^{\circ} \mathrm{C}\left(\mathrm{g} / \mathrm{m}^{2}\right)\end{array}$ & $\begin{array}{l}\text { Q PCT Si at } \\
20^{\circ} \mathrm{C}\left(\mathrm{g} / \mathrm{m}^{2}\right)\end{array}$ & $\begin{array}{c}\mathrm{QpH} \\
\text { at } 20^{\circ} \mathrm{C}\end{array}$ & $\begin{array}{c}\text { TCLP Ag } \\
\text { (ppm) }\end{array}$ & $\begin{array}{c}\text { TCLP As } \\
\text { (ppm) }\end{array}$ & $\begin{array}{c}\text { TCLP Ba } \\
\text { (ppm) }\end{array}$ & $\begin{array}{c}\text { TCLP Cd } \\
(\mathrm{ppm})\end{array}$ & $\begin{array}{c}\text { TCLP Cr } \\
(\mathrm{ppm})\end{array}$ & $\begin{array}{c}\text { TCLP Ni } \\
(\mathrm{ppm})\end{array}$ & $\begin{array}{c}\text { TCLP Pb } \\
\text { (ppm) }\end{array}$ & $\begin{array}{c}\text { TCLP Se } \\
(\mathrm{ppm})\end{array}$ & $\begin{array}{c}\text { TCLP Zn } \\
(\mathrm{ppm})\end{array}$ \\
\hline HLWD1-10 & & & & & & 1.55 & 1.14 & 0.01 & & 0.21 & & \\
\hline \multicolumn{13}{|l|}{ HLWD1-11 } \\
\hline \multicolumn{13}{|l|}{ HLWD1-12 } \\
\hline \multicolumn{13}{|l|}{ HLWD1-13 } \\
\hline \multicolumn{13}{|l|}{ HLWD1-14 } \\
\hline \multicolumn{13}{|l|}{ HLWD1-15 } \\
\hline \multicolumn{13}{|l|}{ HLWD1-16 } \\
\hline \multicolumn{13}{|l|}{ HLWD1-17 } \\
\hline \multicolumn{13}{|l|}{ HLWD1-18 } \\
\hline \multicolumn{13}{|l|}{ HLWD1-19 } \\
\hline \multicolumn{13}{|l|}{ HLWD1-20 } \\
\hline \multicolumn{13}{|l|}{ HLWD1-21 } \\
\hline \multicolumn{13}{|l|}{ HLWD1-23 } \\
\hline \multicolumn{13}{|l|}{ HLWD1-24 } \\
\hline \multicolumn{13}{|l|}{ HLWD1-25 } \\
\hline \multicolumn{13}{|l|}{ HLWD1-26 } \\
\hline \multicolumn{13}{|l|}{ HLWD1-27 } \\
\hline \multicolumn{13}{|l|}{ HLWD1-28 } \\
\hline \multicolumn{13}{|l|}{ HLWD2-01 } \\
\hline \multicolumn{13}{|l|}{ HLWD2-02 } \\
\hline HLWD2-03 & & & & & & & & & & & & \\
\hline HLWD2-04 & & & & & & & & & & & & \\
\hline
\end{tabular}


TWRS HLW Glass Formulation (Fu and Pegg 1998)

\begin{tabular}{|c|c|c|c|c|c|c|c|c|c|c|c|c|c|c|c|c|c|c|c|c|c|}
\hline Glass ID & $\begin{array}{c}\mathrm{Al} 2 \mathrm{O} 3 \\
-\mathrm{t}\end{array}$ & $\begin{array}{c}\mathrm{B} 2 \mathrm{O} 3 \\
-\mathrm{t}\end{array}$ & $\begin{array}{c}\mathrm{CaO} \\
-\mathrm{t}\end{array}$ & $\begin{array}{c}\mathrm{Fe} 2 \mathrm{O} 3 \\
-\mathrm{t}\end{array}$ & $\begin{array}{c}\mathrm{FeO} \\
-\mathrm{t}\end{array}$ & $\begin{array}{c}\mathrm{K} 2 \mathrm{O} \\
-\mathrm{t}\end{array}$ & $\begin{array}{c}\mathrm{Li} 2 \mathrm{O} \\
-\mathrm{t}\end{array}$ & $\begin{array}{c}\mathrm{MgO} \\
-\mathrm{t}\end{array}$ & $\begin{array}{c}\mathrm{Na} 2 \mathrm{O} \\
-\mathrm{t}\end{array}$ & $\begin{array}{c}\mathrm{NiO} \\
-\mathrm{t}\end{array}$ & $\begin{array}{c}\mathrm{P} 2 \mathrm{O} 5 \\
-\mathrm{t}\end{array}$ & $\begin{array}{c}\mathrm{SiO} 2 \\
-\mathrm{t}\end{array}$ & $\begin{array}{c}\mathrm{ZrO} 2 \\
-\mathrm{t}\end{array}$ & $\begin{array}{c}\mathrm{Ag} 2 \mathrm{O} \\
-\mathrm{t}\end{array}$ & $\begin{array}{c}\mathrm{As} 2 \mathrm{O} 3 \\
-\mathrm{t}\end{array}$ & $\begin{array}{c}\mathrm{BaO} \\
-\mathrm{t}\end{array}$ & $\begin{array}{c}\mathrm{Bi} 2 \mathrm{O} 3 \\
-\mathrm{t}\end{array}$ & $\begin{array}{c}\mathrm{Br} \\
-\mathrm{t}\end{array}$ & $\begin{array}{c}\mathrm{CdO} \\
-\mathrm{t}\end{array}$ & $\begin{array}{c}\mathrm{Ce} 2 \mathrm{O} 3 \\
-\mathrm{t}\end{array}$ & $\begin{array}{c}\mathrm{CeO} 2 \\
-\mathrm{t}\end{array}$ \\
\hline HLWD2-05 & 0.0378 & 0.0661 & 0.0756 & 0.0850 & & 0.0104 & 0.0312 & 0.0227 & 0.1890 & 0.0213 & 0.0000 & 0.2362 & 0.1228 & 0.0000 & & 0.0000 & 0.0000 & & 0.0000 & & 0.0000 \\
\hline HLWD2-06 & 0.0917 & 0.1199 & 0.0564 & 0.1703 & & 0.0022 & 0.0299 & 0.0022 & 0.1136 & 0.0116 & 0.0043 & 0.3380 & 0.0005 & 0.0010 & 0.0002 & 0.0022 & 0.0002 & & 0.0033 & & 0.0007 \\
\hline HLWD2-07 & 0.0446 & 0.1488 & 0.0593 & 0.1059 & & 0.0033 & 0.0297 & 0.0033 & 0.1007 & 0.0376 & 0.0065 & 0.3648 & 0.0007 & 0.0016 & 0.0003 & 0.0033 & 0.0003 & & 0.0049 & & 0.0010 \\
\hline HLWD3-01 & 0.0832 & 0.0707 & 0.0204 & 0.0970 & & 0.0210 & 0.0006 & 0.0070 & 0.1947 & 0.0060 & 0.0188 & 0.3018 & 0.0403 & 0.0000 & & 0.0101 & 0.0000 & & 0.0103 & & 0.0026 \\
\hline HLWD3-02 & 0.0466 & 0.0714 & 0.0004 & 0.1175 & & 0.0153 & 0.0000 & 0.0000 & 0.1584 & 0.0075 & 0.0195 & 0.4004 & 0.0001 & 0.0000 & & 0.0129 & 0.0000 & & 0.0132 & & 0.0031 \\
\hline HLWD3-03 & 0.1622 & 0.0763 & 0.0763 & 0.1049 & & 0.0000 & 0.0315 & 0.0000 & 0.0954 & 0.0017 & 0.0308 & 0.2957 & 0.0000 & 0.0000 & & 0.0390 & 0.0239 & & 0.0399 & & 0.0000 \\
\hline HLWD3-04 & 0.1606 & 0.1606 & 0.0756 & 0.0850 & & 0.0126 & 0.0312 & 0.0000 & 0.0945 & 0.0017 & 0.0000 & 0.2362 & 0.0000 & 0.0047 & & 0.0000 & 0.0000 & & 0.0000 & & 0.0000 \\
\hline HLWD3-05 & 0.1606 & 0.0661 & 0.0000 & 0.0472 & & 0.0000 & 0.0312 & 0.0000 & 0.1890 & 0.0017 & 0.0284 & 0.2362 & 0.1228 & 0.0000 & & 0.0358 & 0.0220 & & 0.0367 & & 0.0000 \\
\hline HLWD3-06 & 0.0378 & 0.1606 & 0.0756 & 0.0472 & & 0.0000 & 0.0312 & 0.0000 & 0.0945 & 0.0017 & 0.0305 & 0.2929 & 0.1039 & 0.0000 & & 0.0386 & 0.0237 & & 0.0395 & & 0.0000 \\
\hline HLWD3-07 & 0.1606 & 0.1134 & 0.0000 & 0.1890 & & 0.0000 & 0.0312 & 0.0000 & 0.1890 & 0.0017 & 0.0000 & 0.2929 & 0.0000 & 0.0000 & & 0.0000 & 0.0000 & & 0.0000 & & 0.0000 \\
\hline HLWD3-08 & 0.1708 & 0.0887 & 0.0147 & 0.1058 & & 0.0094 & 0.0000 & 0.0032 & 0.2203 & 0.0034 & 0.0084 & 0.2863 & 0.0016 & 0.0015 & 0.0003 & 0.0032 & 0.0003 & & 0.0047 & & 0.0009 \\
\hline EnvDSR1 & 0.0900 & 0.0686 & 0.0558 & 0.2255 & & 0.0010 & 0.0294 & 0.0011 & 0.1555 & 0.0010 & 0.0069 & 0.3143 & 0.0000 & 0.0003 & & 0.0002 & 0.0000 & & 0.0000 & & 0.0000 \\
\hline
\end{tabular}

INEEL DZr Process Demonstration (Musick et al. 2000)

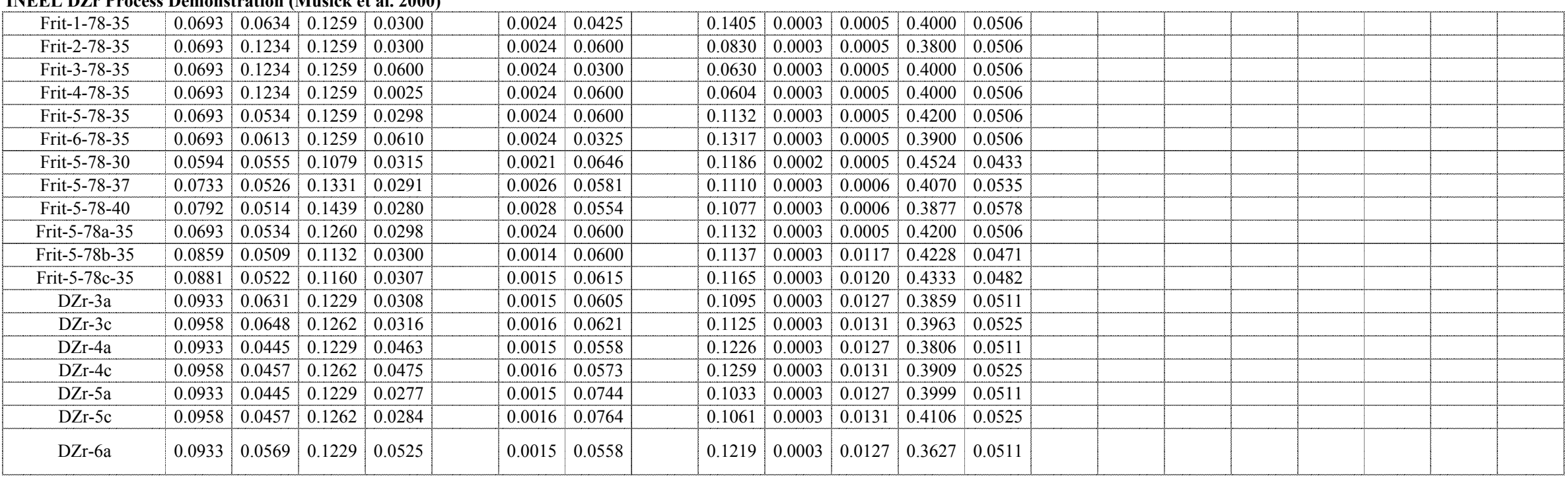


Appendix A. Database - mass fraction

TWRS HLW Glass Formulation (Fu and Pegg 1998)

\begin{tabular}{|c|c|c|c|c|c|c|c|c|c|c|c|c|c|c|c|c|c|c|c|c|c|}
\hline Glass ID & $\begin{array}{l}\mathrm{Cl} \\
-\mathrm{t}\end{array}$ & $\begin{array}{c}\mathrm{CoO} \\
-\mathrm{t} \\
\end{array}$ & $\begin{array}{c}\mathrm{Co} 2 \mathrm{O} 3 \\
-\mathrm{t}\end{array}$ & $\begin{array}{c}\mathrm{Cr} 2 \mathrm{O} 3 \\
-\mathrm{t} \\
\end{array}$ & $\begin{array}{c}\mathrm{Cs} 2 \mathrm{O} \\
-\mathrm{t}\end{array}$ & $\begin{array}{c}\mathrm{CuO} \\
-\mathrm{t} \\
\end{array}$ & $\begin{array}{c}\mathrm{Eu} 2 \mathrm{O} 3 \\
-\mathrm{t}\end{array}$ & $\begin{array}{l}F \\
-t\end{array}$ & $\begin{array}{c}\mathrm{Ga} 2 \mathrm{O} 3 \\
-\mathrm{t}\end{array}$ & $\begin{array}{c}\mathrm{Gd} 2 \mathrm{O} 3 \\
-\mathrm{t}\end{array}$ & $\begin{array}{c}\mathrm{HgO} \\
-\mathrm{t}\end{array}$ & $\begin{array}{l}I \\
-t\end{array}$ & $\begin{array}{c}\mathrm{La} 2 \mathrm{O} 3 \\
-\mathrm{t}\end{array}$ & $\begin{array}{c}\mathrm{MnO} 2 \\
-\mathrm{t} \\
\end{array}$ & $\begin{array}{c}\mathrm{MnO} \\
-\mathrm{t}\end{array}$ & $\begin{array}{c}\mathrm{MoO} \\
-\mathrm{t}\end{array}$ & $\begin{array}{c}\mathrm{MoO} 3 \\
-\mathrm{t} \\
\end{array}$ & $\begin{array}{c}\mathrm{Nb} 2 \mathrm{O} 5 \\
-\mathrm{t}\end{array}$ & $\begin{array}{c}\mathrm{Nd} 2 \mathrm{O} 3 \\
-\mathrm{t}\end{array}$ & $\begin{array}{c}\mathrm{PbO} \\
-\mathrm{t}\end{array}$ & $\begin{array}{c}\mathrm{PdO} 2 \\
-\mathrm{t}\end{array}$ \\
\hline HLWD2-05 & 0.0000 & 0.0037 & & 0.0065 & 0.0014 & 0.0000 & & 0.0000 & & & & & 0.0000 & 0.0666 & & 0.0000 & & & 0.0000 & 0.0000 & \\
\hline HLWD2-06 & 0.0006 & 0.0007 & & 0.0056 & 0.0007 & 0.0002 & & 0.0004 & & & & & 0.0009 & 0.0022 & & 0.0000 & & & 0.0000 & 0.0011 & \\
\hline HLWD2-07 & 0.0008 & 0.0010 & & 0.0128 & 0.0011 & 0.0003 & & 0.0007 & & & & & 0.0013 & 0.0033 & & 0.0000 & & & 0.0000 & 0.0017 & \\
\hline HLWD3-01 & 0.0000 & 0.0013 & & 0.0020 & 0.0013 & 0.0012 & & 0.0071 & & & & & 0.0061 & 0.0210 & & 0.0015 & & & 0.0040 & 0.0024 & \\
\hline HLWD3-02 & 0.0000 & 0.0015 & & 0.0025 & 0.0016 & 0.0015 & & 0.0091 & & & & & 0.0077 & 0.0265 & & 0.0019 & & & 0.0051 & 0.0030 & \\
\hline HLWD3-03 & 0.0000 & 0.0000 & & 0.0000 & 0.0014 & 0.0000 & & 0.0000 & & & & & 0.0000 & 0.0000 & & 0.0000 & & & 0.0000 & 0.0000 & \\
\hline HLWD3-04 & 0.0025 & 0.0000 & & 0.0000 & 0.0063 & 0.0048 & & 0.0280 & & & & & 0.0000 & 0.0000 & & 0.0059 & & & 0.0000 & 0.0093 & \\
\hline HLWD3-05 & 0.0000 & 0.0000 & & 0.0000 & 0.0014 & 0.0000 & & 0.0000 & & & & & 0.0000 & 0.0000 & & 0.0000 & & & 0.0000 & 0.0000 & \\
\hline HLWD3-06 & 0.0000 & 0.0000 & & 0.0000 & 0.0014 & 0.0000 & & 0.0000 & & & & & 0.0000 & 0.0000 & & 0.0000 & & & 0.0000 & 0.0000 & \\
\hline HLWD3-07 & 0.0000 & 0.0000 & & 0.0000 & 0.0014 & 0.0000 & & 0.0000 & & & & & 0.0000 & 0.0000 & & 0.0000 & & & 0.0000 & 0.0000 & \\
\hline HLWD3-08 & 0.0008 & 0.0009 & & 0.0077 & 0.0010 & 0.0003 & & 0.0006 & & & & & 0.0013 & 0.0066 & & 0.0000 & & & 0.0000 & 0.0019 & \\
\hline EnvDSR1 & 0.0000 & 0.0000 & & 0.0004 & 0.0010 & 0.0000 & & 0.0000 & & & & & 0.0000 & 0.0039 & & 0.0000 & & & 0.0000 & 0.0018 & \\
\hline
\end{tabular}

INEEL DZr Process Demonstration (Musick et al. 2000)

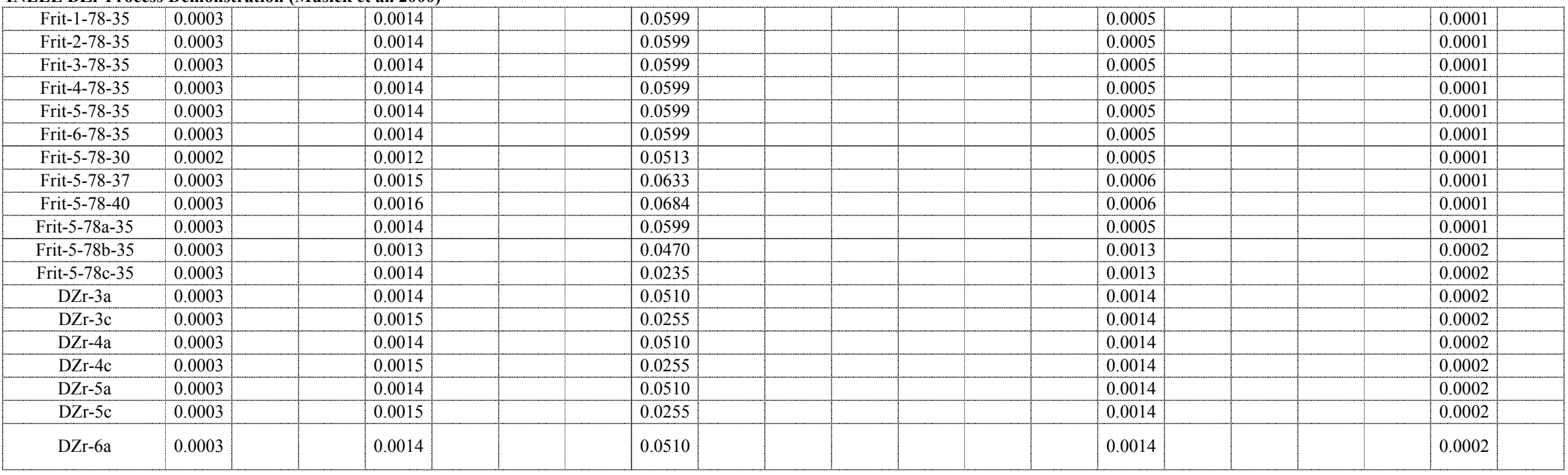


TWRS HLW Glass Formulation (Fu and Pegg 1998)

\begin{tabular}{|c|c|c|c|c|c|c|c|c|c|c|c|c|c|c|c|c|c|c|c|c|c|}
\hline Glass ID & $\begin{array}{c}\mathrm{PdO} \\
-\mathrm{t}\end{array}$ & $\begin{array}{c}\mathrm{Pr} 2 \mathrm{O} 3 \\
-\mathrm{t}\end{array}$ & $\begin{array}{c}\text { Pr6O11 } \\
-t\end{array}$ & $\begin{array}{c}\mathrm{Rb} 2 \mathrm{O} \\
-\mathrm{t}\end{array}$ & $\begin{array}{c}\mathrm{ReO} \\
-\mathrm{t}\end{array}$ & $\begin{array}{c}\mathrm{ReO} 2 \\
-\mathrm{t}\end{array}$ & $\begin{array}{c}\mathrm{Rh} 2 \mathrm{O} 3 \\
-\mathrm{t}\end{array}$ & $\begin{array}{c}\mathrm{RhO} 2 \\
-\mathrm{t}\end{array}$ & $\begin{array}{c}\mathrm{RuO} 2 \\
-\mathrm{t}\end{array}$ & $\begin{array}{c}\mathrm{Sb} 2 \mathrm{O} 3 \\
-\mathrm{t} \\
\end{array}$ & $\begin{array}{c}\mathrm{Sb} 2 \mathrm{O} 5 \\
-\mathrm{t}\end{array}$ & $\begin{array}{c}\mathrm{SeO} 2 \\
-\mathrm{t}\end{array}$ & $\begin{array}{c}\mathrm{Sm} 2 \mathrm{O} 3 \\
-\mathrm{t}\end{array}$ & $\begin{array}{c}\mathrm{SnO} \\
-\mathrm{t}\end{array}$ & $\begin{array}{c}\mathrm{SnO} 2 \\
-\mathrm{t}\end{array}$ & $\begin{array}{c}\mathrm{SO} 3 \\
-\mathrm{t}\end{array}$ & $\begin{array}{c}\mathrm{SrO} \\
-\mathrm{t} \\
\end{array}$ & $\begin{array}{c}\mathrm{Tc} 2 \mathrm{O} 7 \\
-\mathrm{t}\end{array}$ & $\begin{array}{c}\mathrm{TeO} 2 \\
-\mathrm{t}\end{array}$ & $\begin{array}{c}\mathrm{ThO} 2 \\
-\mathrm{t}\end{array}$ & $\begin{array}{c}\mathrm{TiO} 2 \\
-\mathrm{t}\end{array}$ \\
\hline HLWD2-05 & 0.0000 & 0.0000 & & & & 0.0000 & & & 0.0000 & & 0.0000 & 0.0000 & 0.0000 & 0.0003 & & 0.0000 & 0.0180 & & 0.0000 & 0.0000 & 0.0000 \\
\hline HLWD2-06 & 0.0003 & 0.0000 & & & & 0.0003 & & & 0.0009 & & 0.0007 & 0.0008 & 0.0000 & 0.0000 & & 0.0057 & 0.0135 & & 0.0007 & 0.0005 & 0.0007 \\
\hline HLWD2-07 & 0.0005 & 0.0000 & & & & 0.0004 & & & 0.0015 & & 0.0010 & 0.0011 & 0.0000 & 0.0000 & & 0.0094 & 0.0203 & & 0.0010 & 0.0008 & 0.0010 \\
\hline HLWD3-01 & 0.0000 & 0.0000 & & & & 0.0000 & & & 0.0000 & & 0.0000 & 0.0000 & 0.0000 & 0.0000 & & 0.0039 & 0.0249 & & 0.0000 & 0.0000 & 0.0043 \\
\hline HLWD3-02 & 0.0000 & 0.0000 & & & & 0.0000 & & & 0.0000 & & 0.0000 & 0.0000 & 0.0000 & 0.0000 & & 0.0047 & 0.0195 & & 0.0000 & 0.0015 & 0.0055 \\
\hline HLWD3-03 & 0.0000 & 0.0000 & & & & 0.0000 & & & 0.0000 & & 0.0000 & 0.0000 & 0.0000 & 0.0000 & & 0.0000 & 0.0182 & & 0.0000 & 0.0000 & 0.0000 \\
\hline HLWD3-04 & 0.0000 & 0.0000 & & & & 0.0000 & & & 0.0000 & & 0.0088 & 0.0057 & 0.0000 & 0.0000 & & 0.0127 & 0.0228 & & 0.0000 & 0.0000 & 0.0170 \\
\hline HLWD3-05 & 0.0000 & 0.0000 & & & & 0.0000 & & & 0.0000 & & 0.0000 & 0.0000 & 0.0000 & 0.0000 & & 0.0000 & 0.0180 & & 0.0000 & 0.0000 & 0.0000 \\
\hline HLWD3-06 & 0.0000 & 0.0000 & & & & 0.0000 & & & 0.0000 & & 0.0000 & 0.0000 & 0.0000 & 0.0000 & & 0.0000 & 0.0180 & & 0.0000 & 0.0000 & 0.0000 \\
\hline HLWD3-07 & 0.0000 & 0.0000 & & & & 0.0000 & & & 0.0000 & & 0.0000 & 0.0000 & 0.0000 & 0.0000 & & 0.0000 & 0.0180 & & 0.0000 & 0.0000 & 0.0000 \\
\hline HLWD3-08 & 0.0005 & 0.0000 & & & & 0.0003 & & & 0.0013 & & 0.0009 & 0.0009 & 0.0000 & 0.0000 & & 0.0032 & 0.0227 & & 0.0009 & 0.0008 & 0.0010 \\
\hline EnvDSR1 & 0.0000 & 0.0000 & & & & 0.0000 & & & 0.0000 & & 0.0000 & 0.0000 & 0.0000 & 0.0000 & & 0.0000 & 0.0412 & & 0.0000 & 0.0000 & 0.0019 \\
\hline
\end{tabular}

INEEL DZr Process Demonstration (Musick et al. 2000)

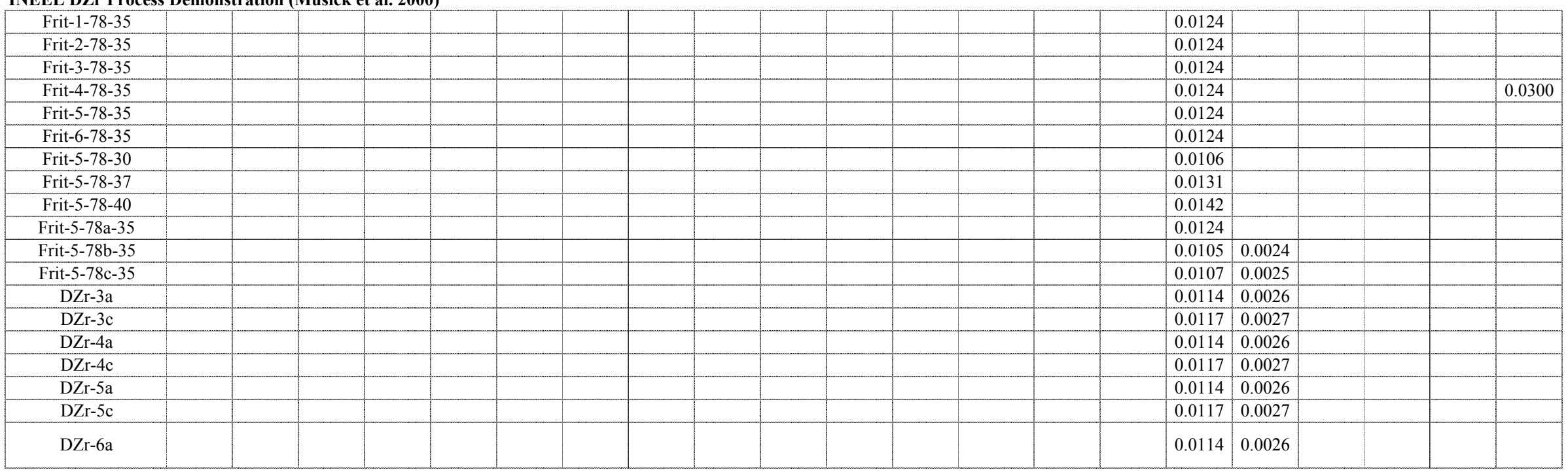


Appendix A. Database - mass fraction

TWRS HLW Glass Formulation (Fu and Pegg 1998)

\begin{tabular}{|c|c|c|c|c|c|c|c|c|c|c|c|c|c|c|c|c|c|c|c|c|c|}
\hline Glass ID & $\begin{array}{c}\mathrm{T} 12 \mathrm{O} 3 \\
-\mathrm{t}\end{array}$ & $\begin{array}{c}\mathrm{U} 3 \mathrm{O} 8 \\
-\mathrm{t}\end{array}$ & $\begin{array}{c}\mathrm{UO} 2 \\
-\mathrm{t}\end{array}$ & $\begin{array}{c}\text { UO3 } \\
-t \\
\end{array}$ & $\begin{array}{c}\mathrm{V} 2 \mathrm{O} 5 \\
-\mathrm{t}\end{array}$ & $\begin{array}{c}\text { WO3 } \\
-t\end{array}$ & $\begin{array}{c}\mathrm{Y} 2 \mathrm{O} 3 \\
-\mathrm{t}\end{array}$ & $\begin{array}{c}\mathrm{ZnO} \\
-\mathrm{t} \\
\end{array}$ & $\begin{array}{c}\text { Others } \\
-t\end{array}$ & $\begin{array}{c}\text { Sum } \\
-t\end{array}$ & $\begin{array}{c}\mathrm{Al} 2 \mathrm{O} 3 \\
-\mathrm{a}\end{array}$ & $\begin{array}{c}\mathrm{B} 2 \mathrm{O} 3 \\
-\mathrm{a}\end{array}$ & $\begin{array}{c}\mathrm{CaO} \\
-\mathrm{a} \\
\end{array}$ & $\begin{array}{c}\mathrm{Fe} 2 \mathrm{O} 3 \\
-\mathrm{a}\end{array}$ & $\begin{array}{c}\mathrm{FeO} \\
-\mathrm{a} \\
\end{array}$ & $\begin{array}{c}\mathrm{K} 2 \mathrm{O} \\
-\mathrm{a}\end{array}$ & $\begin{array}{c}\mathrm{Li} 2 \mathrm{O} \\
-\mathrm{a} \\
\end{array}$ & $\begin{array}{c}\mathrm{MgO} \\
-\mathrm{a}\end{array}$ & $\begin{array}{c}\mathrm{Na} 2 \mathrm{O} \\
-\mathrm{a}\end{array}$ & $\begin{array}{c}\mathrm{NiO} \\
-\mathrm{a}\end{array}$ & $\begin{array}{c}\mathrm{P} 2 \mathrm{O} 5 \\
-\mathrm{a}\end{array}$ \\
\hline HLWD2-05 & & & 0.0000 & & & 0.0020 & 0.0000 & 0.0000 & & 0.9966 & 0.0416 & 0.0631 & 0.0631 & 0.0729 & & 0.0105 & 0.0297 & 0.0194 & 0.1409 & 0.0181 & 0.0005 \\
\hline HLWD2-06 & 0.0022 & & 0.0130 & & & 0.0000 & 0.0000 & 0.0004 & & 1.0003 & 0.0770 & 0.1214 & 0.0496 & 0.1499 & & 0.0024 & 0.0289 & 0.0024 & 0.0937 & 0.0101 & 0.0051 \\
\hline HLWD2-07 & 0.0034 & & 0.0196 & & & 0.0000 & 0.0000 & 0.0007 & & 1.0003 & 0.0384 & 0.1452 & 0.0540 & 0.0903 & & 0.0039 & 0.0295 & 0.0035 & 0.0835 & 0.0283 & 0.0079 \\
\hline HLWD3-01 & & & 0.0308 & & & 0.0000 & 0.0000 & 0.0000 & & 0.9963 & 0.0736 & 0.0672 & 0.0193 & 0.0864 & & 0.0175 & 0.0013 & 0.0074 & 0.1648 & 0.0055 & 0.0202 \\
\hline HLWD3-02 & & & 0.0393 & & & 0.0000 & 0.0000 & 0.0000 & & 0.9972 & 0.0481 & 0.0737 & 0.0004 & 0.1212 & & 0.0158 & & & 0.1349 & 0.0078 & 0.0201 \\
\hline HLWD3-03 & & & 0.0000 & & & 0.0000 & 0.0000 & 0.0000 & & 0.9972 & 0.1407 & 0.0763 & 0.0666 & 0.0917 & & 0.0010 & 0.0325 & 0.0006 & 0.0810 & 0.0016 & 0.0279 \\
\hline HLWD3-04 & 0.0040 & & 0.0000 & & & 0.0000 & 0.0000 & 0.0041 & & 0.9946 & 0.1490 & 0.1661 & 0.0720 & 0.0795 & & 0.0125 & 0.0348 & & 0.0810 & 0.0018 & \\
\hline HLWD3-05 & & & 0.0000 & & & 0.0000 & 0.0000 & 0.0000 & & 0.9971 & & & & & & & & & & & \\
\hline HLWD3-06 & & & 0.0000 & & & 0.0000 & 0.0000 & 0.0000 & & 0.9971 & 0.0326 & 0.1466 & 0.0666 & 0.0510 & & 0.0005 & 0.0291 & 0.0006 & 0.0822 & 0.0029 & 0.0299 \\
\hline HLWD3-07 & & & 0.0000 & & & 0.0000 & 0.0000 & 0.0000 & & 0.9972 & 0.1327 & 0.1152 & 0.0008 & 0.1756 & & 0.0005 & 0.0312 & 0.0002 & 0.1617 & 0.0016 & 0.0005 \\
\hline HLWD3-08 & 0.0032 & & 0.0189 & & & 0.0000 & 0.0000 & 0.0008 & & 1.0000 & 0.1647 & 0.0898 & 0.0155 & 0.0959 & & 0.0090 & 0.0005 & 0.0041 & 0.2010 & 0.0032 & 0.0092 \\
\hline EnvDSR1 & & & 0.0000 & & & 0.0000 & 0.0000 & 0.0001 & & 0.9999 & 0.0757 & 0.0687 & 0.0529 & 0.2046 & & 0.0021 & 0.0298 & 0.0026 & 0.1278 & 0.0028 & 0.0043 \\
\hline
\end{tabular}

INEEL DZr Process Demonstration (Musick et al. 2000)

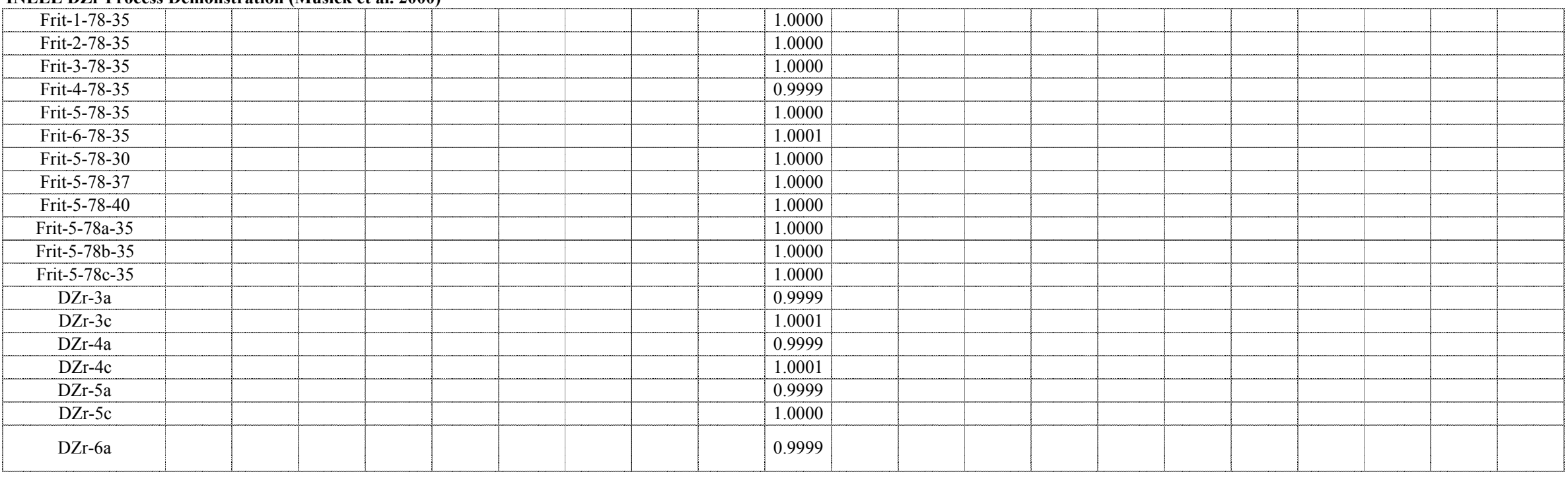


Appendix A. Database - mass fraction

TWRS HLW Glass Formulation (Fu and Pegg 1998)

\begin{tabular}{|c|c|c|c|c|c|c|c|c|c|c|c|c|c|c|c|c|c|c|c|c|c|}
\hline Glass ID & $\begin{array}{c}\mathrm{SiO} 2 \\
-\mathrm{a}\end{array}$ & $\begin{array}{c}\mathrm{ZrO} 2 \\
-\mathrm{a}\end{array}$ & $\begin{array}{c}\mathrm{Ag} 2 \mathrm{O} \\
-\mathrm{a}\end{array}$ & $\begin{array}{c}\mathrm{As} 2 \mathrm{O} 3 \\
-\mathrm{a} \\
\end{array}$ & $\begin{array}{c}\mathrm{BaO} \\
-\mathrm{a}\end{array}$ & $\begin{array}{c}\mathrm{Bi} 2 \mathrm{O} 3 \\
-\mathrm{a}\end{array}$ & $\begin{array}{l}\mathrm{Br} \\
-\mathrm{a}\end{array}$ & $\begin{array}{c}\mathrm{CdO} \\
-\mathrm{a}\end{array}$ & $\begin{array}{c}\mathrm{Ce} 2 \mathrm{O} 3 \\
-\mathrm{a}\end{array}$ & $\begin{array}{c}\mathrm{CeO} 2 \\
-\mathrm{a}\end{array}$ & $\begin{array}{l}\mathrm{Cl} \\
-\mathrm{a}\end{array}$ & $\begin{array}{c}\mathrm{CoO} \\
-\mathrm{a}\end{array}$ & $\begin{array}{c}\mathrm{Co} 2 \mathrm{O} 3 \\
-\mathrm{a}\end{array}$ & $\begin{array}{c}\mathrm{Cr} 2 \mathrm{O} 3 \\
-\mathrm{a}\end{array}$ & $\begin{array}{c}\mathrm{Cs} 2 \mathrm{O} \\
-\mathrm{a} \\
\end{array}$ & $\begin{array}{c}\mathrm{CuO} \\
-\mathrm{a} \\
\end{array}$ & $\begin{array}{c}\mathrm{Eu} 2 \mathrm{O} 3 \\
-\mathrm{a}\end{array}$ & $\begin{array}{c}\mathrm{F} \\
-\mathrm{a}\end{array}$ & $\begin{array}{c}\mathrm{Ga} 2 \mathrm{O} 3 \\
-\mathrm{a}\end{array}$ & $\begin{array}{c}\mathrm{Gd} 2 \mathrm{O} 3 \\
-\mathrm{a}\end{array}$ & $\begin{array}{c}\mathrm{HgO} \\
-\mathrm{a}\end{array}$ \\
\hline HLWD2-05 & 0.2832 & 0.0832 & & & 0.0001 & & & & & & & 0.0038 & & 0.0061 & & & & & & & \\
\hline HLWD2-06 & 0.3300 & 0.0006 & 0.0012 & 0.0031 & 0.0025 & 0.0006 & & 0.0033 & & & & 0.0007 & & 0.0041 & 0.0009 & 0.0004 & & & & & \\
\hline HLWD2-07 & 0.3593 & 0.0010 & 0.0015 & 0.0000 & 0.0039 & 0.0008 & & 0.0047 & & & & 0.0010 & & 0.0049 & 0.0012 & 0.0005 & & & & & \\
\hline HLWD3-01 & 0.3078 & 0.0323 & & & 0.0099 & 0.0007 & & 0.0099 & & & & 0.0015 & & 0.0017 & 0.0011 & 0.0017 & & & & & \\
\hline HLWD3-02 & 0.4130 & 0.0001 & & & 0.0133 & & & 0.0136 & & 0.0032 & & 0.0015 & & 0.0026 & 0.0016 & 0.0016 & & 0.0094 & & & \\
\hline HLWD3-03 & 0.3010 & 0.0006 & & & 0.0348 & 0.0237 & & 0.0372 & & & & 0.0001 & & 0.0001 & 0.0000 & & & & & & \\
\hline HLWD3-04 & 0.2662 & & 0.0035 & & 0.0002 & & & & & & 0.0027 & & & & 0.0066 & 0.0051 & & & & & \\
\hline HLWD3-05 & & & & & & & & & & & & & & & & & & & & & \\
\hline HLWD3-06 & 0.3038 & 0.0868 & & & 0.0341 & 0.0219 & & 0.0378 & & & & & & 0.0024 & 0.0013 & & & & & & \\
\hline HLWD3-07 & 0.3040 & 0.0004 & & & 0.0002 & & & & & & & & & 0.0001 & 0.0014 & & & & & & \\
\hline HLWD3-08 & 0.2894 & 0.0016 & 0.0014 & & 0.0034 & 0.0006 & & 0.0048 & & & & 0.0011 & & 0.0058 & 0.0009 & 0.0004 & & & & & \\
\hline EnvDSR1 & 0.3139 & 0.0048 & & & 0.0008 & & & & & & & & & 0.0027 & & & & & & & \\
\hline
\end{tabular}

INEEL DZr Process Demonstration (Musick et al. 2000)

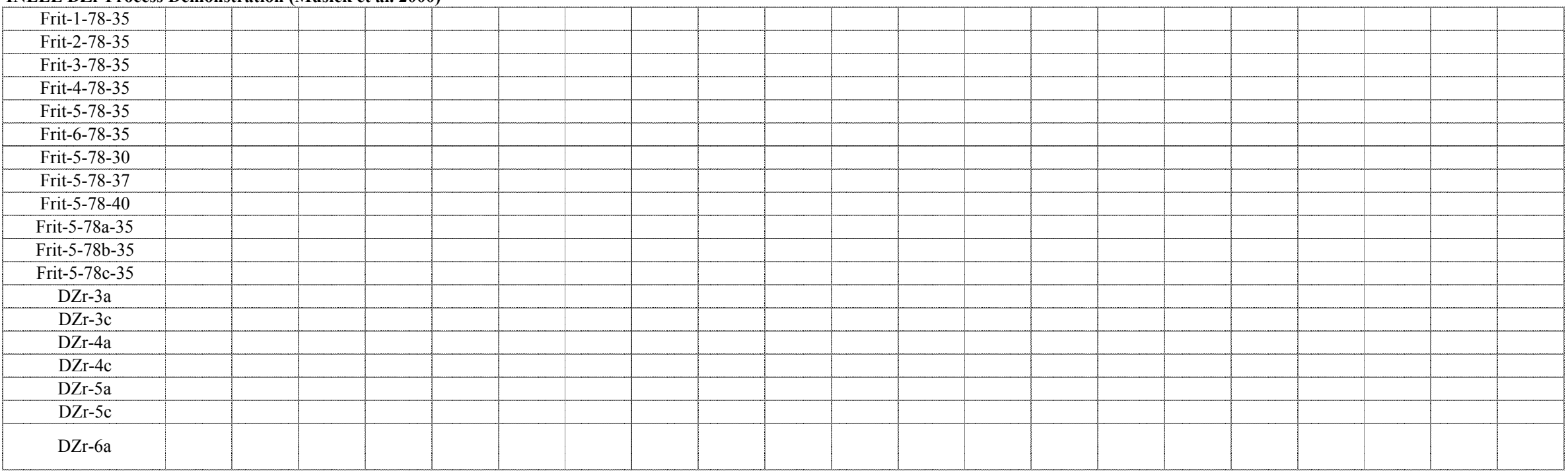


Appendix A. Database - mass fraction

TWRS HLW Glass Formulation (Fu and Pegg 1998)

\begin{tabular}{|c|c|c|c|c|c|c|c|c|c|c|c|c|c|c|c|c|c|c|c|c|c|}
\hline Glass ID & $\begin{array}{c}\mathrm{I} \\
-\mathrm{a}\end{array}$ & $\begin{array}{c}\mathrm{La} 2 \mathrm{O} 3 \\
-\mathrm{a}\end{array}$ & $\begin{array}{c}\mathrm{MnO} 2 \\
-\mathrm{a}\end{array}$ & $\begin{array}{c}\mathrm{MnO} \\
-\mathrm{a}\end{array}$ & $\begin{array}{c}\mathrm{MoO} \\
-\mathrm{a}\end{array}$ & $\begin{array}{c}\mathrm{MoO} 3 \\
-\mathrm{a}\end{array}$ & $\begin{array}{c}\mathrm{Nb} 2 \mathrm{O} 5 \\
-\mathrm{a}\end{array}$ & $\begin{array}{c}\mathrm{Nd} 2 \mathrm{O} 3 \\
-\mathrm{a}\end{array}$ & $\begin{array}{c}\mathrm{PbO} \\
-\mathrm{a}\end{array}$ & $\begin{array}{c}\mathrm{PdO} 2 \\
-\mathrm{a}\end{array}$ & $\begin{array}{c}\mathrm{PdO} \\
-\mathrm{a}\end{array}$ & $\begin{array}{c}\mathrm{Pr} 2 \mathrm{O} 3 \\
-\mathrm{a}\end{array}$ & $\begin{array}{c}\text { Pr6O11 } \\
-\mathrm{a}\end{array}$ & $\begin{array}{c}\mathrm{Rb} 2 \mathrm{O} \\
-\mathrm{a}\end{array}$ & $\begin{array}{c}\mathrm{ReO} \\
-\mathrm{a}\end{array}$ & $\begin{array}{c}\mathrm{ReO} 2 \\
-\mathrm{a}\end{array}$ & $\begin{array}{c}\mathrm{Rh} 2 \mathrm{O} 3 \\
-\mathrm{a}\end{array}$ & $\begin{array}{c}\mathrm{RhO} 2 \\
-\mathrm{a}\end{array}$ & $\begin{array}{c}\mathrm{RuO} 2 \\
-\mathrm{a}\end{array}$ & $\begin{array}{c}\mathrm{Sb} 2 \mathrm{O} 3 \\
-\mathrm{a}\end{array}$ & $\begin{array}{c}\mathrm{Sb} 2 \mathrm{O} 5 \\
-\mathrm{a}\end{array}$ \\
\hline HLWD2-05 & & & & & & & & & 0.0005 & & & & & & & & & & & & \\
\hline HLWD2-06 & & 0.0009 & 0.0024 & & & & & & 0.0019 & & 0.0010 & & & & & 0.0003 & & & 0.0004 & 0.0008 & \\
\hline HLWD2-07 & & 0.0011 & 0.0034 & & & & & 0.0002 & 0.0024 & & & & & & & & & & & & \\
\hline HLWD3-01 & & 0.0060 & 0.0204 & & & & & 0.0041 & 0.0032 & & & & & & & & & & & & \\
\hline HLWD3-02 & & 0.0080 & 0.0273 & & 0.0020 & & & 0.0053 & 0.0031 & & & & & & & & & & & & \\
\hline HLWD3-03 & & & 0.0004 & & & & & & 0.0002 & & & & & & & & & & & & \\
\hline HLWD3-04 & & & & & 0.0059 & & & & 0.0100 & & & & & & & & & & & & 0.0084 \\
\hline HLWD3-05 & & & & & & & & & & & & & & & & & & & & & \\
\hline HLWD3-06 & & 0.0001 & 0.0006 & & & & & & 0.0004 & & & 0.0002 & & & & & & & & & \\
\hline HLWD3-07 & & & 0.0001 & & & & & & & & & & & & & & & & & & \\
\hline HLWD3-08 & & 0.0013 & 0.0064 & & 0.0001 & & & 0.0001 & & & 0.0002 & & & & & 0.0006 & & & 0.0005 & & \\
\hline EnvDSR1 & & & 0.0076 & & & & & & & & & & & & & & & & & & \\
\hline
\end{tabular}

INEEL DZr Process Demonstration (Musick et al. 2000)

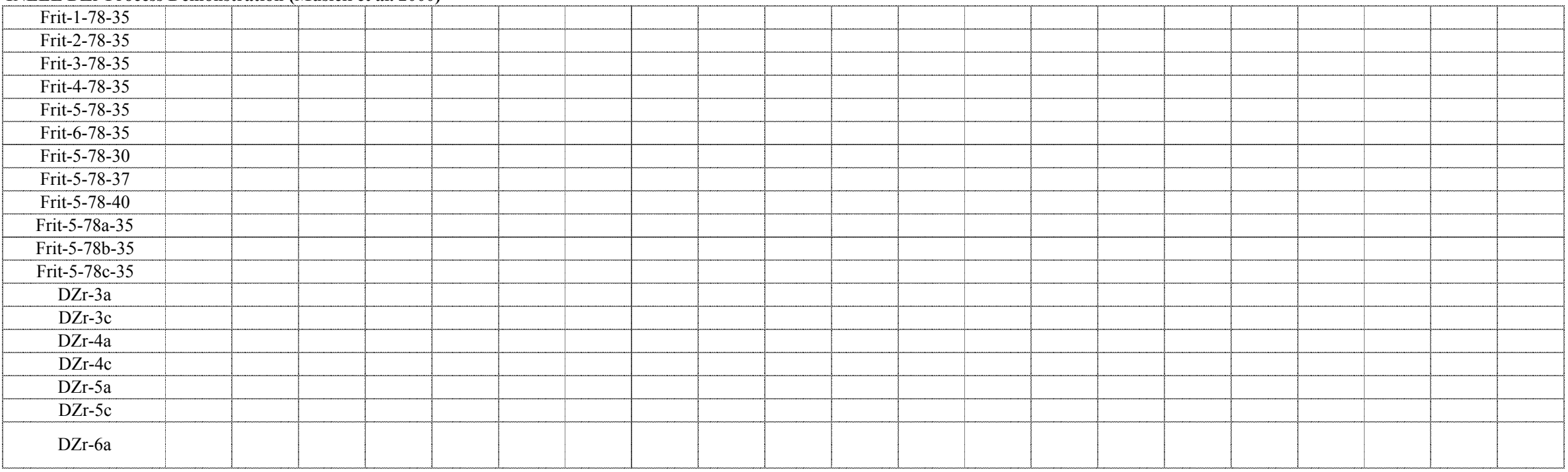


Appendix A. Database - mass fraction

TWRS HLW Glass Formulation (Fu and Pegg 1998)

\begin{tabular}{|c|c|c|c|c|c|c|c|c|c|c|c|c|c|c|c|c|c|c|c|c|}
\hline Glass ID & $\begin{array}{c}\mathrm{SeO} 2 \\
-\mathrm{a}\end{array}$ & $\begin{array}{c}\mathrm{Sm} 2 \mathrm{O} 3 \\
-\mathrm{a}\end{array}$ & $\begin{array}{c}\mathrm{SnO} \\
-\mathrm{a}\end{array}$ & $\begin{array}{c}\mathrm{SnO} 2 \\
-\mathrm{a}\end{array}$ & $\begin{array}{c}\mathrm{SO} 3 \\
-\mathrm{a}\end{array}$ & $\begin{array}{c}\mathrm{SrO} \\
-\mathrm{a}\end{array}$ & $\begin{array}{c}\mathrm{Tc} 2 \mathrm{O} 7 \\
-\mathrm{a}\end{array}$ & $\begin{array}{c}\mathrm{TeO} 2 \\
-\mathrm{a}\end{array}$ & $\begin{array}{c}\mathrm{ThO} 2 \\
-\mathrm{a}\end{array}$ & $\begin{array}{c}\mathrm{TiO} 2 \\
-\mathrm{a}\end{array}$ & $\begin{array}{c}\mathrm{T} 12 \mathrm{O} 3 \\
-\mathrm{a}\end{array}$ & $\begin{array}{c}\mathrm{U} 3 \mathrm{O} 8 \\
-\mathrm{a}\end{array}$ & $\begin{array}{c}\mathrm{UO} 2 \\
-\mathrm{a}\end{array}$ & $\begin{array}{c}\mathrm{UO3} \\
-\mathrm{a}\end{array}$ & $\begin{array}{c}\mathrm{V} 2 \mathrm{O} 5 \\
-\mathrm{a}\end{array}$ & $\begin{array}{c}\text { WO3 } \\
-\mathrm{a}\end{array}$ & $\begin{array}{c}\mathrm{Y} 2 \mathrm{O} 3 \\
-\mathrm{a}\end{array}$ & $\begin{array}{c}\mathrm{ZnO} \\
-\mathrm{a}\end{array}$ & $\begin{array}{c}\text { Others } \\
-\mathrm{a}\end{array}$ & $\begin{array}{c}\text { Sum } \\
-\mathrm{a}\end{array}$ \\
\hline HLWD2-05 & & & & & 0.0005 & 0.0166 & & & & & & & & & & & & 0.0001 & & 0.8539 \\
\hline HLWD2-06 & & & & & 0.0058 & 0.0129 & & & 0.0006 & 0.0008 & 0.0013 & & 0.0124 & & & & & 0.0005 & & 0.9299 \\
\hline HLWD2-07 & 0.0006 & & & & 0.0061 & 0.0196 & & 0.0009 & 0.0008 & 0.0013 & 0.0015 & & 0.0185 & & & & & 0.0007 & & 0.9204 \\
\hline HLWD3-01 & & & & & 0.0035 & 0.0264 & & & & 0.0043 & & & 0.0300 & & & & & & & 0.9277 \\
\hline HLWD3-02 & & & & & 0.0048 & 0.0201 & & & 0.0015 & 0.0057 & & & 0.0405 & & & & & & & 1.0002 \\
\hline HLWD3-03 & & & & & 0.0001 & 0.0181 & & & & 0.0003 & & & 0.0005 & & & & & 0.0001 & & 0.9371 \\
\hline HLWD3-04 & 0.0060 & & & & 0.0097 & 0.0240 & & & & 0.0168 & 0.0008 & & 0.0008 & & & & & 0.0042 & & 0.9676 \\
\hline HLWD3-05 & & & & & & & & & & & & & & & & & & & & \\
\hline HLWD3-06 & & & 0.0005 & & 0.0013 & 0.0168 & & & 0.0003 & 0.0004 & & & 0.0010 & & & & & & & 0.9517 \\
\hline HLWD3-07 & & & 0.0001 & & 0.0018 & 0.0175 & & & & 0.0002 & & & 0.0005 & & & & & & & 0.9463 \\
\hline HLWD3-08 & & & & & 0.0041 & 0.0227 & & & 0.0009 & 0.0011 & & & 0.0187 & & & & & & & 0.9590 \\
\hline EnvDSR1 & & & & & & 0.0342 & & & & 0.0019 & & & & & & & & 0.0004 & & 0.9376 \\
\hline
\end{tabular}

INEEL DZr Process Demonstration (Musick et al. 2000)

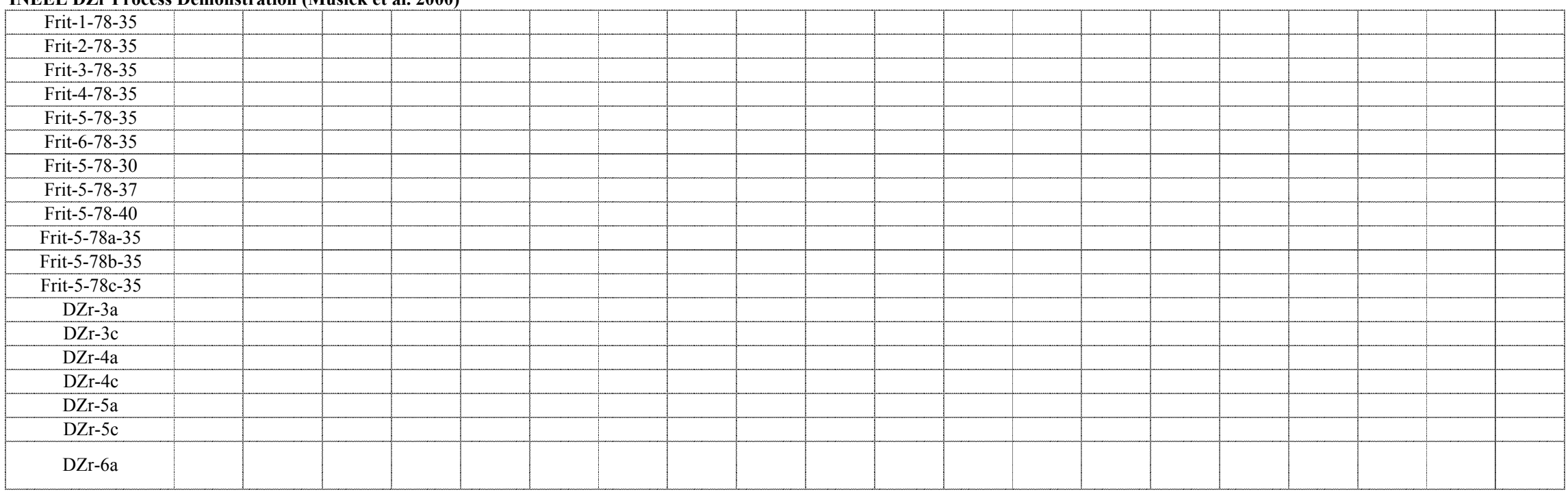


Appendix A. Database - mass fraction

TWRS HLW Glass Formulation (Fu and Pegg 1998)

\begin{tabular}{|c|c|c|c|c|c|c|c|c|}
\hline Glass ID & $\begin{array}{l}\mathrm{TM} \\
\left({ }^{\circ} \mathrm{C}\right)\end{array}$ & $\begin{array}{l}\text { Gradient } \\
\text { TL }\left({ }^{\circ} \mathrm{C}\right)\end{array}$ & $\begin{array}{l}\text { Uniform } \\
\text { TL }\left({ }^{\circ} \mathrm{C}\right)\end{array}$ & Primary Phase & $\begin{array}{r}\text { Quenched } \\
\text { Visual/OM }\end{array}$ & $\begin{array}{c}\text { Quenched } \\
\text { SEM/EDS or TEM }\end{array}$ & $\begin{array}{l}\text { Quenched } \\
\text { XRD }\end{array}$ & $\begin{array}{c}\text { Quenched } \\
\text { Homogeneous? }\end{array}$ \\
\hline HLWD2-05 & 1150 & & $>1050$ & & brown glass w/ blue metallic coating & & & no \\
\hline HLWD2-06 & 1150 & & $>1050$ & & clear brown glass & & & \\
\hline HLWD2-07 & 1150 & & $<1050$ & & glassy brown & & & \\
\hline HLWD3-01 & 1150 & & $<950$ & & glassy brown & & & \\
\hline HLWD3-02 & 1150 & & $<950$ & & glassy brown & & & \\
\hline HLWD3-03 & 1150 & & $<1050$ & & clear brown glass & & & \\
\hline HLWD3-04 & 1150 & & $<1050$ & & opaque brown w/ white spots on top & & & \\
\hline HLWD3-05 & 1150 & & $>1050$ & & opaque yellowish green glass w/ white spots & $\begin{array}{l}\text { white spots are zirconia crystals about } \\
300 \mathrm{X} 500 \text { microns. } \sim 5 \mathrm{vol} \% \mathrm{ZrO} 2\end{array}$ & & \\
\hline HLWD3-06 & 1150 & & $<1050$ & & clear light brown & & & \\
\hline HLWD3-07 & 1150 & & $<1050$ & & glassy brown & & & \\
\hline HLWD3-08 & 1150 & & $<1050$ & & glassy brown & & & \\
\hline EnvDSR1 & 1150 & & $<1050$ & & glassy brown & & & \\
\hline
\end{tabular}

INEEL DZr Process Demonstration (Musick et al. 2000)

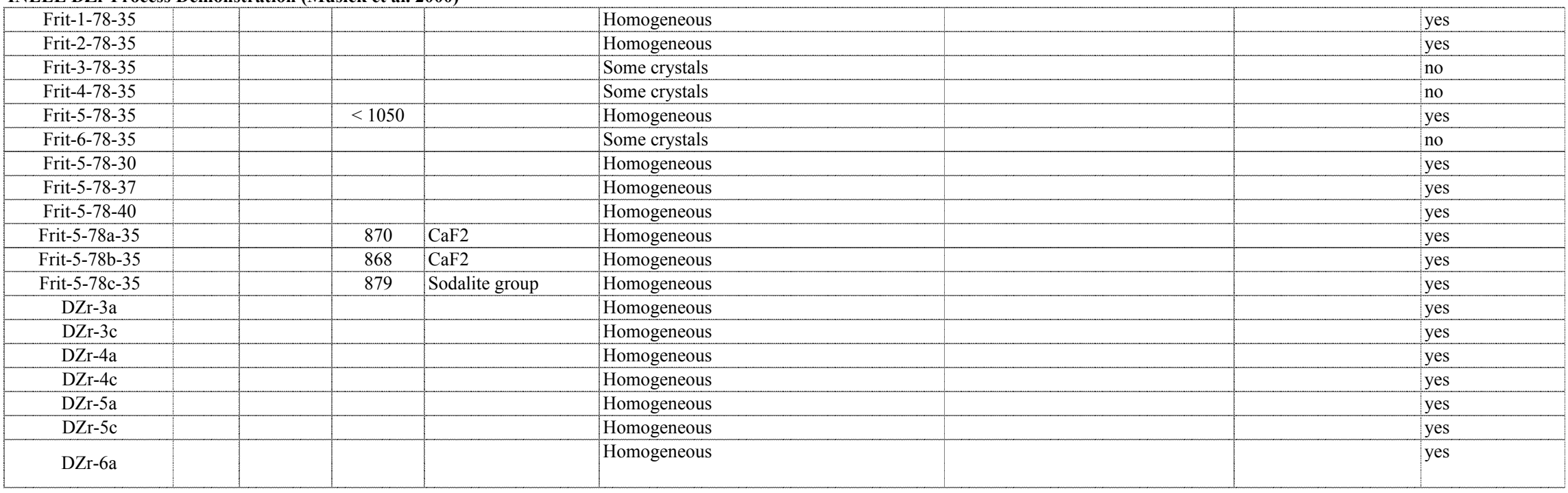


Appendix A. Database - mass fraction

TWRS HLW Glass Formulation (Fu and Pegg 1998)

\begin{tabular}{|c|c|c|c|c|c|}
\hline Glass ID & $\begin{array}{c}\text { CCC } \\
\text { Visual/OM }\end{array}$ & $\begin{array}{c}\text { CCC } \\
\text { SEM/EDS or TEM }\end{array}$ & $\begin{array}{l}\text { CCC } \\
\text { XRD } \\
\end{array}$ & $\begin{array}{c}\text { Heat Treated } \\
\text { Visual/OM }\end{array}$ & $\begin{array}{c}\text { Heat Treated } \\
\text { SEM/EDS or TEM }\end{array}$ \\
\hline \multicolumn{6}{|l|}{ HLWD2-05 } \\
\hline \multicolumn{6}{|l|}{ HLWD2-06 } \\
\hline \multicolumn{6}{|l|}{ HLWD2-07 } \\
\hline \multicolumn{6}{|l|}{ HLWD3-01 } \\
\hline \multicolumn{6}{|l|}{ HLWD3-02 } \\
\hline \multicolumn{6}{|l|}{ HLWD3-03 } \\
\hline \multicolumn{6}{|l|}{ HLWD3-04 } \\
\hline \multicolumn{6}{|l|}{ HLWD3-05 } \\
\hline \multicolumn{6}{|l|}{ HLWD3-06 } \\
\hline \multicolumn{6}{|l|}{ HLWD3-07 } \\
\hline \multicolumn{6}{|l|}{ HLWD3-08 } \\
\hline EnvDSR1 & & & & & \\
\hline
\end{tabular}

INEEL DZr Process Demonstration (Musick et al. 2000)

\begin{tabular}{|c|c|c|}
\hline \multicolumn{3}{|l|}{ Frit-1-78-35 } \\
\hline Frit-2-78-35 & & \\
\hline Frit-3-78-35 & & \\
\hline Frit-4-78-35 & & \\
\hline Frit-5-78-35 & & \\
\hline Frit-6-78-35 & & \\
\hline Frit-5-78-30 & & \\
\hline Frit-5-78-37 & & \\
\hline Frit-5-78-40 & & \\
\hline Frit-5-78a-35 & & \\
\hline Frit-5-78b-35 & & \\
\hline Frit-5-78c-35 & & \\
\hline DZr-3a & $\sim 5 \mathrm{vol} \%$ crystallinity & \\
\hline $\mathrm{DZr}-3 \mathrm{c}$ & $2 \%$ crystallinity & \\
\hline DZr-4a & $10-20 \%$ crystallinity & \\
\hline $\mathrm{DZr}-4 \mathrm{c}$ & $10-20 \%$ crystallinity & \\
\hline DZr-5a & $5-10 \%$ crystallinity & \\
\hline DZr-5c & $5-10 \%$ crystallinity & \\
\hline DZr-6a & $\begin{array}{l}\text { surface fully crystallized, lots of } \\
\text { crystallinity in bulk }\end{array}$ & \\
\hline
\end{tabular}




\section{TWRS HLW Glass Formulation (Fu and Pegg 1998)}

\begin{tabular}{|c|c|c|c|c|c|c|c|c|c|c|c|c|c|c|c|}
\hline Glass ID & $\begin{array}{c}\text { Heat Treated } \\
\text { XRD } \\
\end{array}$ & $\begin{array}{l}\text { Density } \\
\left(\mathrm{g} / \mathrm{cm}^{3}\right)\end{array}$ & $\begin{array}{c}\text { Fulc Visc } \\
\mathrm{A}\end{array}$ & \begin{tabular}{|c|}
$\begin{array}{c}\text { Fulc Visc } \\
\text { B }\end{array}$ \\
\end{tabular} & \begin{tabular}{|c|}
$\begin{array}{c}\text { Fulc Visc } \\
\text { To }\end{array}$ \\
\end{tabular} & $\begin{array}{c}\mathrm{FV} 1150^{\circ} \mathrm{C} \\
(\mathrm{Pa} \cdot \mathrm{s})\end{array}$ & $\begin{array}{c}\text { Arrh Visc } \\
\mathrm{A}\end{array}$ & $\begin{array}{c}\text { Arrh Visc } \\
\mathrm{B} \\
\end{array}$ & $\begin{array}{l}\text { Av } \\
1150^{\circ} \mathrm{C} \\
\left(\mathrm{Danac}^{\circ}\right.\end{array}$ & \begin{tabular}{|c|}
$\mathrm{T}\left({ }^{\circ} \mathrm{C}\right)$ at \\
$2 \mathrm{~Pa} \cdot \mathrm{s}$ \\
\end{tabular} & \begin{tabular}{|c|}
$\mathrm{T}\left({ }^{\circ} \mathrm{C}\right)$ at \\
$5 \mathrm{~Pa} \cdot \mathrm{s}$ \\
\end{tabular} & $\begin{array}{c}\mathrm{T}\left({ }^{\circ} \mathrm{C}\right) \text { at } \\
10 \mathrm{~Pa} \cdot \mathrm{s}\end{array}$ & $\begin{array}{l}\mathrm{T} 1 \\
\left({ }^{\circ} \mathrm{C}\right) \\
\end{array}$ & $\begin{array}{c}\mathrm{V} 1 \\
(\mathrm{~Pa} \cdot \mathrm{s})\end{array}$ & $\begin{array}{l}\mathrm{T} 2 \\
\left({ }^{\circ} \mathrm{C}\right) \\
\end{array}$ \\
\hline HLWD2-05 & $\begin{array}{l}10-20 \text { vol } \% \text { crystals. May be spinels. } \\
\text { Metallic coating on top. }\end{array}$ & & & & & & -11.556 & 17553.7 & 2.18 & & & & (1000) & (13.71 & (1050) \\
\hline HLWD2-06 & $\begin{array}{l}\text { some spinel crystals on bottom. Overall, } 1-5 \\
\text { vol } \% \text { crystals }\end{array}$ & & & & & & -10.732 & 16124.9 & 1.82 & & & & 1000 & 7.178 & 1050 \\
\hline HLWD2-07 & $\begin{array}{l}\mathrm{Cr}-\mathrm{Fe}-\mathrm{Ni} \text { spinels at glass/crucible interface. } \\
\text { Some } \mathrm{Ru}, \mathrm{Al} \text {, and } \mathrm{Mg} \text { incorporation into } \\
\text { these spinels. About } 0.3 \text { vol\% crystals. }\end{array}$ & & & & & & & & 1.00 & & & & & & \\
\hline HLWD3-01 & $\begin{array}{l}\text { clear except for one } 8 \times 10 \text { um phase of } \mathrm{Fe}-\mathrm{Cr}- \\
\mathrm{Ni}-\mathrm{Si}\end{array}$ & & & & & & -11.835 & 18215.9 & 2.63 & & & & 1000 & 12.544 & 1050 \\
\hline HLWD3-02 & clear glass & & & & & & -9.612 & 16069.4 & 5.37 & & & & $(1000)$ & $(28.15$ & (1050) \\
\hline HLWD3-03 & $\begin{array}{l}<1 \text { vol\% crys. total, mainly in the glass- } \\
\text { crucible interface. Most likely some Fe } 2 \mathrm{O} 3 \\
\text { in glass }\end{array}$ & & & & & & -11.354 & 17846.2 & 3.28 & & & & 1000 & 14.80 & 1050 \\
\hline HLWD3-04 & clear glass & & & & & & -10.428 & 14798.5 & 0.97 & & & & 1000 & 3.467 & 1050 \\
\hline HLWD3-05 & $\begin{array}{l}50 \mathrm{vol} \% \text { crys. In bottom layer. } 5 \text { vol } \% \text { in } \\
\text { rest of glass }\end{array}$ & & & & & & & & & & & & & & \\
\hline HLWD3-06 & clear glass & & & & & & -12.427 & 17625.0 & 0.96 & & & & 1000 & 4.319 & 1050 \\
\hline HLWD3-07 & $\begin{array}{l}<1 \text { vol\% crystals in glass, most likely spinels } \\
\text { Most crystals settled to bottom. }\end{array}$ & & & & & & -9.153 & 13968.5 & 1.94 & & & & 1000 & 6.282 & 1050 \\
\hline HLWD3-08 & $\begin{array}{l}\text { Two types of crystals on bottom, most likely } \\
\text { spinel and silicate, }<1 \mathrm{vol} \% \text {. }\end{array}$ & & & & & & -11.596 & 19441.1 & 7.89 & & & & 1000 & 43.264 & 1050 \\
\hline EnvDSR1 & $\begin{array}{l}<1 \text { vol\% spinel-like crystals at the } \\
\text { glass/crucible interface. }\end{array}$ & & & & & & -11.653 & 17137.7 & 1.48 & & & & 1000 & 6.413 & 1050 \\
\hline
\end{tabular}

INEEL DZr Process Demonstration (Musick et al. 2000)

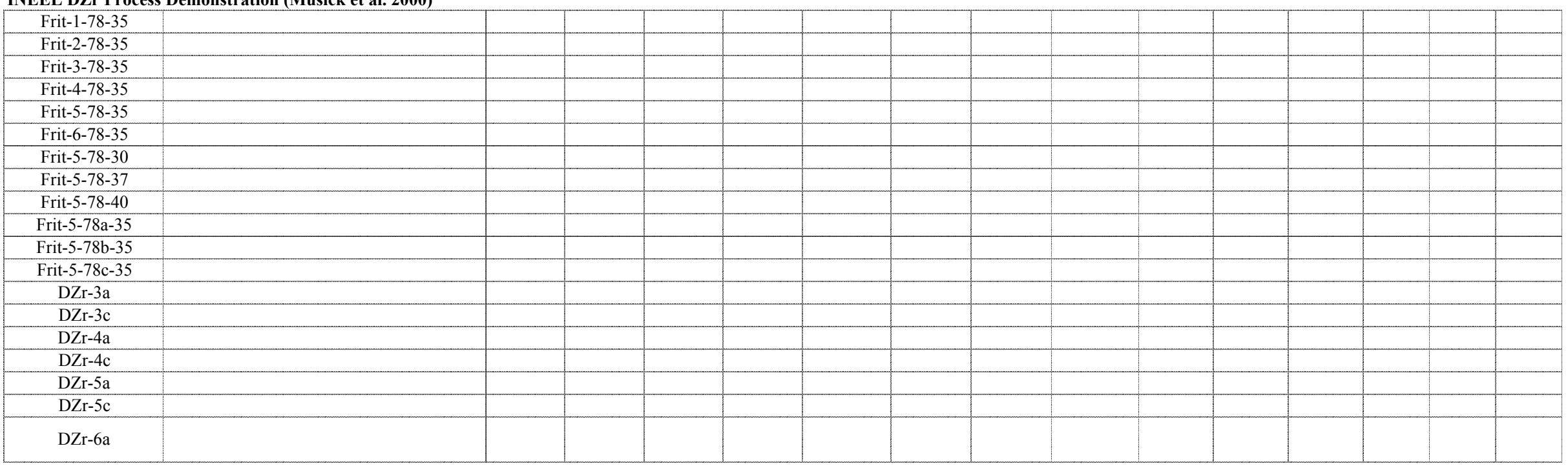


Appendix A. Database - mass fraction

\begin{tabular}{|c|c|c|c|c|c|c|c|c|c|c|c|c|c|c|c|c|c|c|c|c|c|}
\hline \multicolumn{22}{|c|}{ WRS HLW Glass Formulation (Fu and Pegg 1998) } \\
\hline Glass ID & $\begin{array}{c}\mathrm{V} 2 \\
(\mathrm{~Pa} \cdot \mathrm{s}) \\
\end{array}$ & $\begin{array}{c}\mathrm{T} 3 \\
\left({ }^{\circ} \mathrm{C}\right) \\
\end{array}$ & $\begin{array}{c}\mathrm{V} 3 \\
(\mathrm{~Pa} \cdot \mathrm{s})\end{array}$ & $\begin{array}{c}\mathrm{T} 4 \\
\left({ }^{\circ} \mathrm{C}\right) \\
\end{array}$ & $\begin{array}{c}\mathrm{V} 4 \\
(\mathrm{~Pa} \cdot \mathrm{s})\end{array}$ & $\begin{array}{c}\mathrm{T} 5 \\
\left({ }^{\circ} \mathrm{C}\right) \\
\end{array}$ & $\begin{array}{c}\mathrm{V} 5 \\
(\mathrm{~Pa} \cdot \mathrm{s})\end{array}$ & $\begin{array}{c}\mathrm{T} 6 \\
\left({ }^{\circ} \mathrm{C}\right) \\
\end{array}$ & $\begin{array}{c}\mathrm{V} 6 \\
(\mathrm{~Pa} \cdot \mathrm{s})\end{array}$ & $\begin{array}{c}\mathrm{T} 7 \\
\left({ }^{\circ} \mathrm{C}\right) \\
\end{array}$ & $\begin{array}{c}\mathrm{V} 7 \\
(\mathrm{~Pa} \cdot \mathrm{s})\end{array}$ & $\begin{array}{c}\mathrm{T} 8 \\
\left({ }^{\circ} \mathrm{C}\right) \\
\end{array}$ & $\begin{array}{c}\mathrm{V} 8 \\
(\mathrm{~Pa} \cdot \mathrm{s})\end{array}$ & $\begin{array}{c}\mathrm{T} 9 \\
\left({ }^{\circ} \mathrm{C}\right) \\
\end{array}$ & $\begin{array}{c}\mathrm{V} 9 \\
(\mathrm{~Pa} \cdot \mathrm{s})\end{array}$ & $\begin{array}{l}\mathrm{T} 10 \\
\left({ }^{\circ} \mathrm{C}\right) \\
\end{array}$ & $\begin{array}{c}\mathrm{V} 10 \\
(\mathrm{~Pa} \cdot \mathrm{s})\end{array}$ & $\begin{array}{l}\mathrm{T} 11 \\
\left({ }^{\circ} \mathrm{C}\right) \\
\end{array}$ & $\begin{array}{c}\mathrm{V} 11 \\
(\mathrm{~Pa} \cdot \mathrm{s})\end{array}$ & $\begin{array}{l}\mathrm{T} 12 \\
\left({ }^{\circ} \mathrm{C}\right) \\
\end{array}$ & $\begin{array}{c}\mathrm{V} 12 \\
(\mathrm{~Pa} \cdot \mathrm{s})\end{array}$ \\
\hline HLWD2-05 & $(6.513$ & 1100 & 3.541 & 1150 & 2.13 & 1200 & 1.385 & 1250 & 0.958 & 1300 & 0.696 & & & & & & & & & & \\
\hline HLWD2-06 & 4.266 & 1100 & 2.685 & 1150 & 1.773 & 1200 & 1.22 & 1250 & 0.87 & 1300 & 0.639 & & & & & & & & & & \\
\hline \multicolumn{22}{|l|}{ HLWD2-07 } \\
\hline HLWD3-01 & 6.78 & 1100 & 4.001 & 1150 & 2.533 & 1200 & 1.698 & 1250 & 1.193 & & & & & & & & & & & & \\
\hline HLWD3-02 & $(14.472$ & 1100 & 8.352 & 1150 & 5.264 & 1200 & 3.552 & 1250 & 2.531 & 1300 & 1.884 & & & & & & & & & & \\
\hline HLWD3-03 & 8.426 & 1100 & 5.075 & 1150 & 3.208 & 1200 & 2.114 & 1250 & 1.445 & 1300 & 1.019 & & & & & & & & & & \\
\hline HLWD3-04 & 2.117 & 1100 & 1.374 & 1150 & 0.939 & 1200 & 0.669 & 1250 & 0.494 & 1300 & 0.376 & & & & & & & & & & \\
\hline \multicolumn{22}{|l|}{ HLWD3-05 } \\
\hline HLWD3-06 & 2.43 & 1100 & 1.46 & 1150 & 0.927 & 1200 & 0.617 & 1250 & 0.428 & 1300 & 0.307 & & & & & & & & & & \\
\hline HLWD3-07 & 4.065 & 1100 & 2.74 & 1150 & 1.914 & 1200 & 1.379 & 1250 & 1.021 & 1300 & 0.774 & & & & & & & & & & \\
\hline HLWD3-08 & 21.802 & 1100 & 12.179 & 1150 & 7.381 & 1200 & 4.776 & 1250 & 3.26 & 1300 & 2.325 & & & & & & & & & & \\
\hline EnvDSR1 & 3.641 & 1100 & 2.214 & 1150 & 1.424 & 1200 & 0.96 & 1250 & 0.674 & 1300 & 0.49 & & & & & & & & & & \\
\hline
\end{tabular}

\section{INEEL DZr Process Demonstration (Musick et al. 2000)}

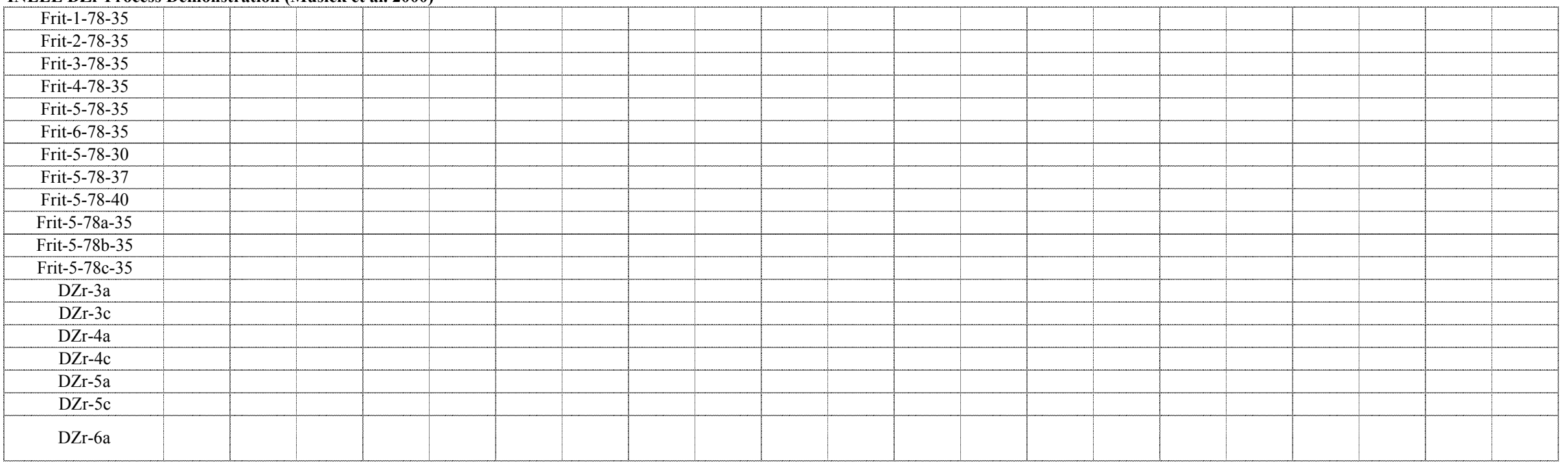


Appendix A. Database - mass fraction

TWRS HLW Glass Formulation (Fu and Pegg 1998)

\begin{tabular}{|c|c|c|c|c|c|c|c|c|c|c|c|c|c|c|c|c|}
\hline Glass ID & $\begin{array}{l}\mathrm{T} 13 \\
\left({ }^{\circ} \mathrm{C}\right) \\
\end{array}$ & $\begin{array}{c}\text { V13 } \\
(\mathrm{Pa} \cdot \mathrm{s})\end{array}$ & $\begin{array}{l}\mathrm{T} 14 \\
\left({ }^{\circ} \mathrm{C}\right)\end{array}$ & $\begin{array}{c}\text { V14 } \\
(\mathrm{Pa} \cdot \mathrm{s}) \\
\end{array}$ & $\begin{array}{c}\text { Q PCT } \\
\mathrm{B}\left(\mathrm{g} / \mathrm{m}^{2}\right)\end{array}$ & $\begin{array}{c}\text { Q PCT } \\
\mathrm{Li}\left(\mathrm{g} / \mathrm{m}^{2}\right)\end{array}$ & $\begin{array}{c}\text { Q PCT } \\
\mathrm{Na}\left(\mathrm{g} / \mathrm{m}^{2}\right)\end{array}$ & $\begin{array}{c}\text { Q PCT } \\
\mathrm{Si}\left(\mathrm{g} / \mathrm{m}^{2}\right)\end{array}$ & $\begin{array}{c}\text { Q PCT } \\
\mathrm{pH}\end{array}$ & $\begin{array}{c}\text { CCC PCT } \\
\mathrm{B}\left(\mathrm{g} / \mathrm{m}^{2}\right) \\
\end{array}$ & $\begin{array}{l}\text { CCC PCT } \\
\mathrm{Li}\left(\mathrm{g} / \mathrm{m}^{2}\right)\end{array}$ & $\begin{array}{l}\text { CCC PCT } \\
\mathrm{Na}\left(\mathrm{g} / \mathrm{m}^{2}\right)\end{array}$ & $\begin{array}{l}\text { CCC PCT } \\
\mathrm{Si}\left(\mathrm{g} / \mathrm{m}^{2}\right)\end{array}$ & $\begin{array}{c}\text { CCC PCT } \\
\mathrm{pH} \\
\end{array}$ & $\begin{array}{l}\text { Q PCT B at } \\
20^{\circ} \mathrm{C}\left(\mathrm{g} / \mathrm{m}^{2}\right)\end{array}$ & $\begin{array}{l}\text { Q PCT Li at } \\
20^{\circ} \mathrm{C}\left(\mathrm{g} / \mathrm{m}^{2}\right)\end{array}$ \\
\hline HLWD2-05 & & & & & 5.81 & 4.20 & 4.69 & 0.56 & 12.2 & & & & & & & \\
\hline HLWD2-06 & & & & & 0.21 & 0.21 & 0.28 & 0.14 & 10.2 & & & & & & & \\
\hline \multicolumn{17}{|l|}{ HLWD2-07 } \\
\hline HLWD3-01 & & & & & 0.77 & 0.70 & 0.91 & 0.21 & 11.3 & & & & & & & \\
\hline HLWD3-02 & & & & & 0.42 & & 0.49 & 0.21 & 10.2 & & & & & & & \\
\hline HLWD3-03 & & & & & 0.1090876 & 0.147602 & 0.1497036 & 0.0663794 & 10.3 & & & & & & & \\
\hline HLWD3-04 & & & & & 0.2792833 & 0.2976974 & 0.2817181 & 0.0714168 & 9.4 & & & & & & & \\
\hline \multicolumn{17}{|l|}{ HLWD3-05 } \\
\hline HLWD3-06 & & & & & 0.561173 & 0.5122604 & 0.5351218 & 0.1444362 & 9.9 & & & & & & & \\
\hline HLWD3-07 & & & & & 0.69 & 0.4650013 & 0.5819798 & 0.2535211 & 10.9 & & & & & & & \\
\hline HLWD3-08 & & & & & 4.66 & & 2.493098 & 0.222863 & 11.5 & & & & & & & \\
\hline EnvDSR1 & & & & & 0.63 & 0.70 & 0.84 & 0.28 & 11.3 & & & & & & & \\
\hline
\end{tabular}

INEEL DZr Process Demonstration (Musick et al. 2000)

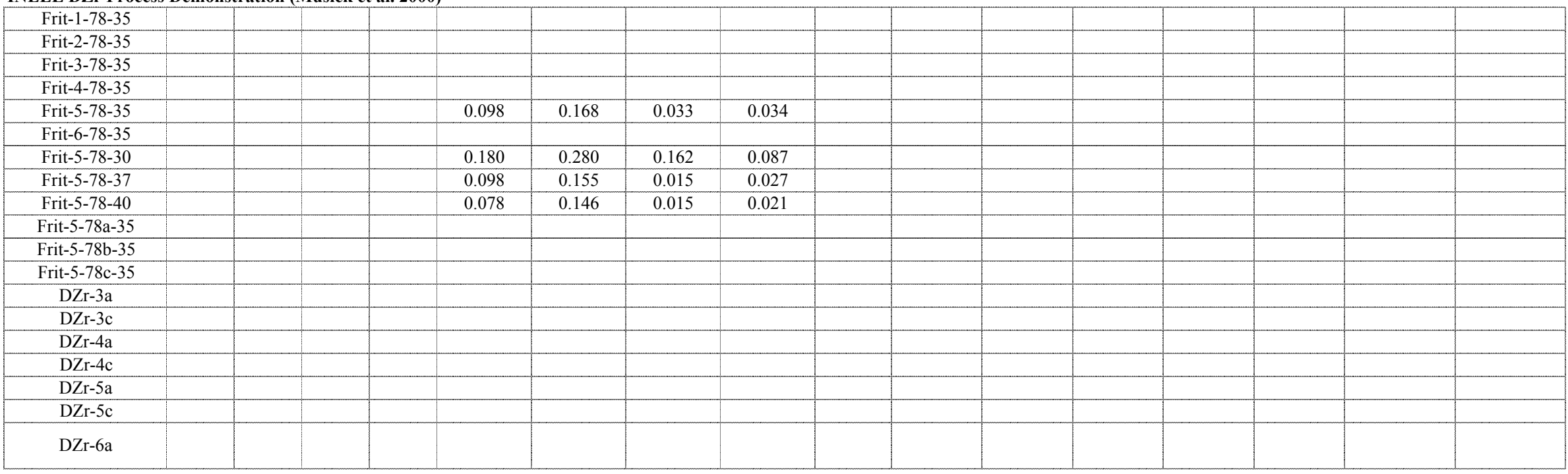


Appendix A. Database - mass fraction

TWRS HLW Glass Formulation (Fu and Pegg 1998)

\begin{tabular}{|c|c|c|c|c|c|c|c|c|c|c|c|c|}
\hline Glass ID & $\begin{array}{l}\text { Q PCT Na at } \\
20^{\circ} \mathrm{C}\left(\mathrm{g} / \mathrm{m}^{2}\right)\end{array}$ & $\begin{array}{l}\text { Q PCT Si at } \\
20^{\circ} \mathrm{C}\left(\mathrm{g} / \mathrm{m}^{2}\right)\end{array}$ & $\begin{array}{c}\mathrm{QpH} \\
\text { at } 20^{\circ} \mathrm{C}\end{array}$ & $\begin{array}{c}\text { TCLP Ag } \\
\text { (ppm) }\end{array}$ & $\begin{array}{c}\text { TCLP As } \\
\text { (ppm) }\end{array}$ & $\begin{array}{c}\text { TCLP Ba } \\
(\mathrm{ppm})\end{array}$ & $\begin{array}{c}\text { TCLP Cd } \\
(\mathrm{ppm})\end{array}$ & $\begin{array}{c}\text { TCLP Cr } \\
(\mathrm{ppm})\end{array}$ & $\begin{array}{c}\text { TCLP Ni } \\
(\mathrm{ppm})\end{array}$ & $\begin{array}{c}\text { TCLP Pb } \\
(\mathrm{ppm})\end{array}$ & $\begin{array}{c}\text { TCLP Se } \\
(\mathrm{ppm})\end{array}$ & $\begin{array}{c}\text { TCLP Zn } \\
(\mathrm{ppm})\end{array}$ \\
\hline \multicolumn{13}{|l|}{ HLWD2-05 } \\
\hline \multicolumn{13}{|l|}{ HLWD2-06 } \\
\hline \multicolumn{13}{|l|}{ HLWD2-07 } \\
\hline \multicolumn{13}{|l|}{ HLWD3-01 } \\
\hline \multicolumn{13}{|l|}{ HLWD3-02 } \\
\hline HLWD3-03 & & & & & & 5.43 & 5.24 & $<0.006$ & & 0.04 & & \\
\hline HLWD3-04 & & & & & & 0.76 & 0.04 & 0.02 & & 0.76 & & \\
\hline \multicolumn{13}{|l|}{ HLWD3-05 } \\
\hline HLWD3-06 & & & & & & 8.45 & 9.22 & $<0.006$ & & 0.07 & & \\
\hline \multicolumn{13}{|l|}{ HLWD3-07 } \\
\hline HLWD3-08 & & & & & & 2.19 & 2.62 & 2.32 & & 1.02 & & \\
\hline EnvDSR1 & & & & & & 0.08 & $<0.003$ & $<0.006$ & & 0.12 & & \\
\hline
\end{tabular}

INEEL DZr Process Demonstration (Musick et al. 2000)

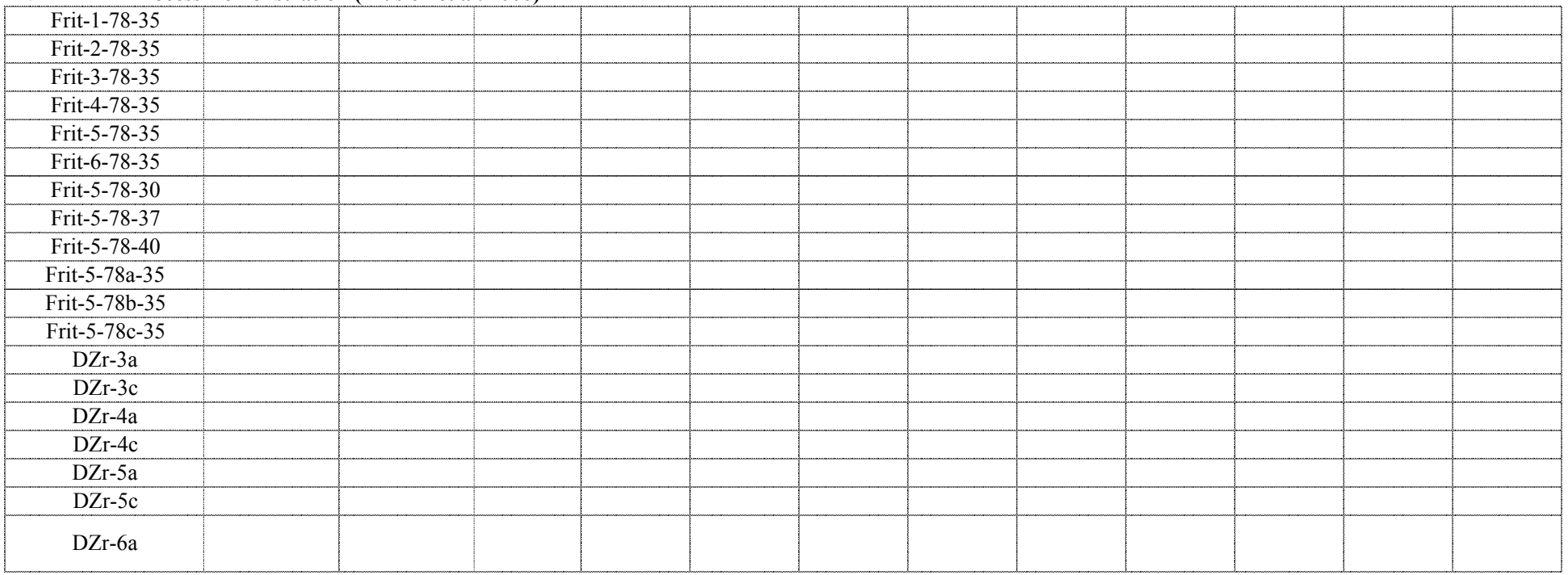


Appendix A. Database - mass fraction

INEEL DZr Process Demonstration (Musick et al. 2000)

\begin{tabular}{|c|c|c|c|c|c|c|c|c|c|c|c|c|c|c|c|c|c|c|c|c|c|}
\hline Glass ID & $\begin{array}{c}\mathrm{Al} 2 \mathrm{O} 3 \\
-\mathrm{t}\end{array}$ & $\begin{array}{c}\mathrm{B} 2 \mathrm{O} 3 \\
-\mathrm{t}\end{array}$ & $\begin{array}{c}\mathrm{CaO} \\
-\mathrm{t}\end{array}$ & $\begin{array}{c}\mathrm{Fe} 2 \mathrm{O} 3 \\
-\mathrm{t}\end{array}$ & $\begin{array}{c}\mathrm{FeO} \\
-\mathrm{t}\end{array}$ & $\begin{array}{c}\mathrm{K} 2 \mathrm{O} \\
-\mathrm{t}\end{array}$ & $\begin{array}{c}\mathrm{Li} 2 \mathrm{O} \\
-\mathrm{t}\end{array}$ & $\begin{array}{c}\mathrm{MgO} \\
-\mathrm{t}\end{array}$ & $\begin{array}{c}\mathrm{Na} 2 \mathrm{O} \\
-\mathrm{t}\end{array}$ & $\begin{array}{c}\mathrm{NiO} \\
-\mathrm{t}\end{array}$ & $\begin{array}{c}\mathrm{P} 2 \mathrm{O} 5 \\
-\mathrm{t}\end{array}$ & $\begin{array}{c}\mathrm{SiO} 2 \\
-\mathrm{t}\end{array}$ & $\begin{array}{c}\mathrm{ZrO} 2 \\
-\mathrm{t}\end{array}$ & $\begin{array}{c}\mathrm{Ag} 2 \mathrm{O} \\
-\mathrm{t}\end{array}$ & $\begin{array}{c}\mathrm{As} 2 \mathrm{O} 3 \\
-\mathrm{t}\end{array}$ & $\begin{array}{c}\mathrm{BaO} \\
-\mathrm{t}\end{array}$ & $\begin{array}{c}\mathrm{Bi} 2 \mathrm{O} 3 \\
-\mathrm{t}\end{array}$ & $\begin{array}{c}\mathrm{Br} \\
-\mathrm{t}\end{array}$ & $\begin{array}{c}\mathrm{CdO} \\
-\mathrm{t}\end{array}$ & $\begin{array}{c}\mathrm{Ce} 2 \mathrm{O} 3 \\
-\mathrm{t}\end{array}$ & $\begin{array}{c}\mathrm{CeO} 2 \\
-\mathrm{t}\end{array}$ \\
\hline DZr-6c & 0.0958 & 0.0584 & 0.1262 & 0.0539 & & 0.0016 & 0.0573 & & 0.1252 & 0.0003 & 0.0131 & 0.3724 & 0.0525 & & & & & & & & \\
\hline DZr-7a & 0.0933 & 0.0631 & 0.1229 & 0.0029 & & 0.0015 & 0.0605 & & 0.1095 & 0.0003 & 0.0127 & 0.3859 & 0.0511 & & & & & & & & \\
\hline $\mathrm{DZr}-7 \mathrm{c}$ & 0.0958 & 0.0648 & 0.1262 & 0.0030 & & 0.0016 & 0.0621 & & 0.1125 & 0.0003 & 0.0131 & 0.3963 & 0.0525 & & & & & & & & \\
\hline DZr-8a & 0.0933 & 0.0569 & 0.1229 & 0.0215 & & 0.0015 & 0.0558 & & 0.1219 & 0.0003 & 0.0127 & 0.3627 & 0.0511 & & & & & & & & \\
\hline DZr-8c & 0.0958 & 0.0584 & 0.1262 & 0.0221 & & 0.0016 & 0.0573 & & 0.1252 & 0.0003 & 0.0131 & 0.3724 & 0.0525 & & & & & & & & \\
\hline DZr-9-78-38 & 0.0933 & 0.0724 & 0.1229 & 0.0029 & & 0.0015 & 0.0620 & & 0.1071 & 0.0003 & 0.0127 & 0.3707 & 0.0610 & & & & & & & & \\
\hline DZr-9-78-40 & 0.0982 & 0.0707 & 0.1294 & 0.0031 & & 0.0016 & 0.0600 & & 0.1050 & 0.0003 & 0.0134 & 0.3590 & 0.0634 & & & & & & & & \\
\hline DZr-10-78-38 & 0.0933 & 0.0724 & 0.1229 & 0.0277 & & 0.0015 & 0.0620 & & 0.1071 & 0.0003 & 0.0127 & 0.3707 & 0.0610 & & & & & & & & \\
\hline DZr-10-78-40 & 0.0982 & 0.0707 & 0.1294 & 0.0271 & & 0.0016 & 0.0600 & & 0.1050 & 0.0003 & 0.0134 & 0.3590 & 0.0634 & & & & & & & & \\
\hline GLA 78-21 & & & & & & & & & & & & & & & & & & & & & \\
\hline GLA 78-22 & & & & & & & & & & & & & & & & & & & & & \\
\hline GLA 78-23 & & & & & & & & & & & & & & & & & & & & & \\
\hline GLA 78-9-11 & & & & & & & & & & & & & & & & & & & & & \\
\hline GLA 78-9-15 & & & & & & & & & & & & & & & & & & & & & \\
\hline GLA 78-9-18 & & & & & & & & & & & & & & & & & & & & & \\
\hline GLA 78-10-14 & & & & & & & & & & & & & & & & & & & & & \\
\hline GLA 78-10-15 & & & & & & & & & & & & & & & & & & & & & \\
\hline GLA $78-10-16$ & & & & & & & & & & & & & & & & & & & & & \\
\hline
\end{tabular}

INEEL CVS 2a (Peeler et al. 1999)

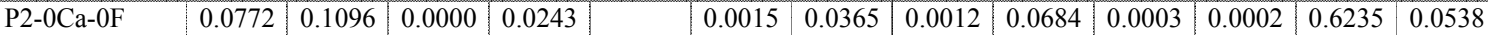

\begin{tabular}{l|l|l|l|l|l|l|l|l|l|l|l|l|l|l} 
P2-0Ca-1F & 0.0694 & 0.0986 & 0.0000 & 0.0219 & & 0.0013 & 0.0329 & 0.0011 & 0.1556 & 0.0003 & 0.0001 & 0.5612 & 0.0485 \\
\hline
\end{tabular}

\begin{tabular}{|l|l|l|l|l|l|l|l|l|l|l|l|l|l|l|l|l|l|l|l|l|}
$\mathrm{P} 2-0 \mathrm{Ca}-2 \mathrm{~F}$ & 0.0691 & 0.0981 & 0.0000 & 0.0218 & & 0.0013 & 0.0327 & 0.0011 & 0.1548 & 0.0003 & 0.0001 & 0.5581 & 0.0481 \\
\hline
\end{tabular}

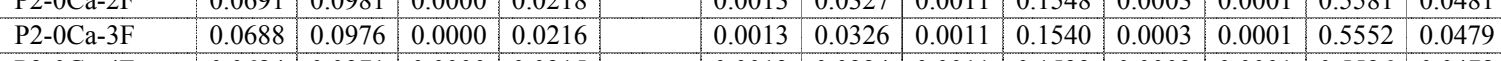

\begin{tabular}{l|l|l|l|l|l|l|l|l|l|l|l|l|l|l|l}
$\mathrm{P} 2-0 \mathrm{Ca}-4 \mathrm{~F}$ & 0.0684 & 0.0971 & 0.0000 & 0.0215 & & 0.0013 & 0.0324 & 0.0011 & 0.1533 & 0.0003 & 0.0001 & 0.5526 & 0.0478 \\
\hline
\end{tabular}

\begin{tabular}{|l|l|l|l|l|l|l|l|l|l|l|l|l|l|}
\hline $\mathrm{P} 2-0 \mathrm{Ca}-5 \mathrm{~F}$ & 0.0679 & 0.0964 & 0.0000 & 0.0214 & & 0.0013 & 0.0322 & 0.0011 & 0.1521 & 0.0003 & 0.0001 & 0.5485 & 0.0473 \\
\hline
\end{tabular}

\begin{tabular}{|l|l|l|l|l|l|l|l|l|l|l|l|l|l|l|l|l}
$\mathrm{P} 2-3 \mathrm{Ca}-\mathrm{OF}$ & 0.0749 & 0.1063 & 0.0300 & 0.0236 & & 0.0014 & 0.0354 & 0.0012 & 0.0664 & 0.0003 & 0.0001 & 0.6048 & 0.0522 \\
\hline
\end{tabular}

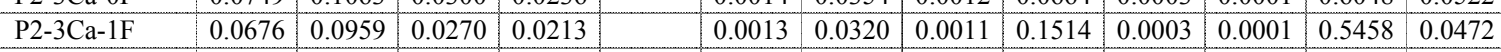

\begin{tabular}{|l|l|l|l|l|l|l|l|l|l|l|l|l|l|l|}
\hline P2-3Ca-2F & 0.0672 & 0.0954 & 0.0269 & 0.0212 & & 0.0013 & 0.0318 & 0.0011 & 0.1505 & 0.0003 & 0.0001 & 0.5428 & 0.0468 \\
\hline
\end{tabular}

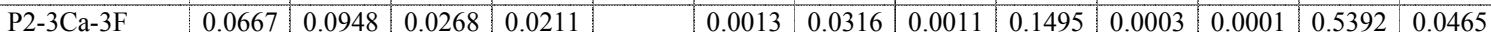

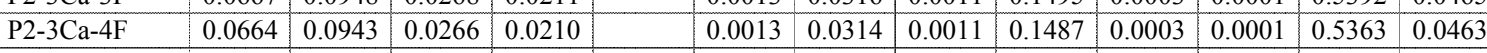

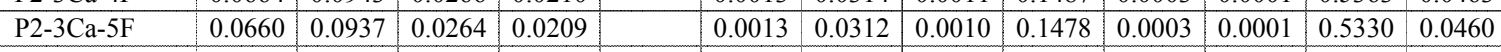

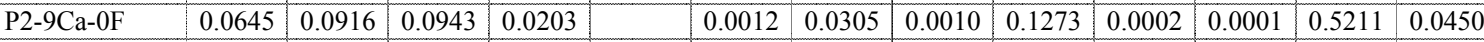

P2-9Ca-1F

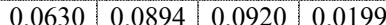

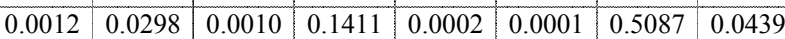


Appendix A. Database - mass fraction

INEEL DZr Process Demonstration (Musick et al. 2000)

\begin{tabular}{|c|c|c|c|c|c|c|c|c|c|c|c|c|c|c|c|c|c|c|c|c|c|}
\hline Glass ID & $\begin{array}{c}\mathrm{Cl} \\
-\mathrm{t}\end{array}$ & $\begin{array}{c}\mathrm{CoO} \\
-\mathrm{t} \\
\end{array}$ & $\begin{array}{c}\mathrm{Co} 2 \mathrm{O} 3 \\
-\mathrm{t}\end{array}$ & $\begin{array}{c}\mathrm{Cr} 2 \mathrm{O} 3 \\
-\mathrm{t} \\
\end{array}$ & $\begin{array}{c}\mathrm{Cs} 2 \mathrm{O} \\
-\mathrm{t}\end{array}$ & $\begin{array}{c}\mathrm{CuO} \\
-\mathrm{t} \\
\end{array}$ & $\begin{array}{c}\begin{array}{c}\mathrm{Eu} 2 \mathrm{O} 3 \\
-\mathrm{t}\end{array} \\
\end{array}$ & $\begin{array}{l}F \\
-t\end{array}$ & $\begin{array}{c}\mathrm{Ga} 2 \mathrm{O} 3 \\
-\mathrm{t} \\
\end{array}$ & $\begin{array}{c}\mathrm{Gd} 2 \mathrm{O} 3 \\
-\mathrm{t} \\
\end{array}$ & $\begin{array}{c}\mathrm{HgO} \\
-\mathrm{t}\end{array}$ & $\begin{array}{l}\mathrm{I} \\
-\mathrm{t}\end{array}$ & $\begin{array}{c}\mathrm{La} 2 \mathrm{O} 3 \\
-\mathrm{t} \\
\end{array}$ & $\begin{array}{c}\mathrm{MnO} 2 \\
-\mathrm{t} \\
\end{array}$ & $\begin{array}{c}\mathrm{MnO} \\
-\mathrm{t} \\
\end{array}$ & $\begin{array}{c}\mathrm{MoO} \\
-\mathrm{t}\end{array}$ & $\begin{array}{c}\mathrm{MoO} 3 \\
-\mathrm{t}\end{array}$ & $\begin{array}{c}\mathrm{Nb} 2 \mathrm{O} 5 \\
-\mathrm{t}\end{array}$ & $\begin{array}{c}\mathrm{Nd} 2 \mathrm{O} 3 \\
-\mathrm{t}\end{array}$ & $\begin{array}{c}\mathrm{PbO} \\
-\mathrm{t}\end{array}$ & $\begin{array}{c}\mathrm{PdO} 2 \\
-\mathrm{t}\end{array}$ \\
\hline DZr-6c & 0.0003 & & & 0.0015 & & & & 0.0255 & & & & & & & 0.0014 & & & & & 0.0002 & \\
\hline DZr-7a & 0.0003 & & & 0.0014 & & & & 0.0510 & & & & & 0.0279 & & 0.0014 & & & & & 0.0002 & \\
\hline DZr-7c & 0.0003 & & & 0.0015 & & & & 0.0255 & & & & & 0.0287 & & 0.0014 & & & & & 0.0002 & \\
\hline $\mathrm{DZr}-8 \mathrm{a}$ & 0.0003 & & & 0.0014 & & & & 0.0510 & & & & & 0.0310 & & 0.0014 & & & & & 0.0002 & \\
\hline DZr-8c & 0.0003 & & & 0.0015 & & & & 0.0255 & & & & & 0.0318 & & 0.0014 & & & & & 0.0002 & \\
\hline DZr-9-78-38 & 0.0003 & & & 0.0014 & & & & 0.0510 & & & & & 0.0248 & & 0.0014 & & & & & 0.0002 & \\
\hline DZr-9-78-40 & 0.0003 & & & 0.0015 & & & & 0.0537 & & & & & 0.0240 & & 0.0014 & & & & & 0.0002 & \\
\hline DZr-10-78-38 & 0.0003 & & & 0.0014 & & & & 0.0510 & & & & & & & 0.0014 & & & & & 0.0002 & \\
\hline DZr-10-78-40 & 0.0003 & & & 0.0015 & & & & 0.0537 & & & & & & & 0.0014 & & & & & 0.0002 & \\
\hline GLA 78-21 & & & & & & & & & & & & & & & & & & & & & \\
\hline GLA $78-22$ & & & & & & & & & & & & & & & & & & & & & \\
\hline GLA $78-23$ & & & & & & & & & & & & & & & & & & & & & \\
\hline GLA 78-9-11 & & & & & & & & & & & & & & & & & & & & & \\
\hline GLA 78-9-15 & & & & & & & & & & & & & & & & & & & & & \\
\hline GLA 78-9-18 & & & & & & & & & & & & & & & & & & & & & \\
\hline GLA 78-10-14 & & & & & & & & & & & & & & & & & & & & & \\
\hline GLA 78-10-15 & & & & & & & & & & & & & & & & & & & & & \\
\hline GLA 78-10-16 & & & & & & & & & & & & & & & & & & & & & \\
\hline
\end{tabular}

INEEL CVS 2a (Peeler et al. 1999)

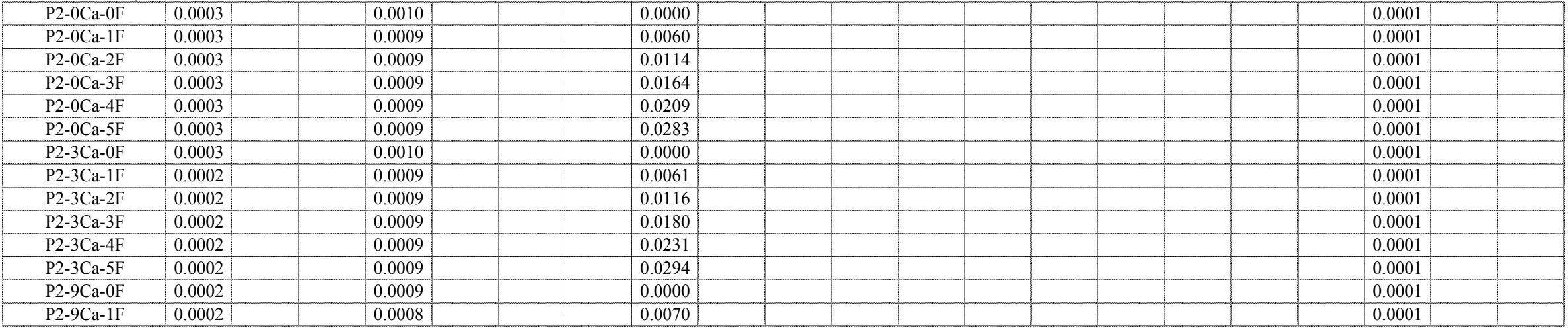


INEEL DZr Process Demonstration (Musick et al. 2000)

\begin{tabular}{|c|c|c|c|c|c|c|c|c|c|c|c|c|c|c|c|c|c|c|c|c|c|}
\hline Glass ID & $\begin{array}{c}\mathrm{PdO} \\
-\mathrm{t}\end{array}$ & $\begin{array}{c}\mathrm{Pr} 2 \mathrm{O} 3 \\
-\mathrm{t}\end{array}$ & $\begin{array}{c}\text { Pr6O11 } \\
-t\end{array}$ & $\begin{array}{c}\mathrm{Rb} 2 \mathrm{O} \\
-\mathrm{t}\end{array}$ & $\begin{array}{c}\mathrm{ReO} \\
-\mathrm{t}\end{array}$ & $\begin{array}{c}\mathrm{ReO} 2 \\
-\mathrm{t}\end{array}$ & $\begin{array}{c}\mathrm{Rh} 2 \mathrm{O} 3 \\
-\mathrm{t}\end{array}$ & $\begin{array}{c}\mathrm{RhO} 2 \\
-\mathrm{t}\end{array}$ & $\begin{array}{c}\mathrm{RuO} 2 \\
-\mathrm{t}\end{array}$ & $\begin{array}{c}\mathrm{Sb} 2 \mathrm{O} 3 \\
-\mathrm{t}\end{array}$ & $\begin{array}{c}\mathrm{Sb} 2 \mathrm{O} 5 \\
-\mathrm{t}\end{array}$ & $\begin{array}{c}\mathrm{SeO} 2 \\
-\mathrm{t}\end{array}$ & $\begin{array}{c}\mathrm{Sm} 2 \mathrm{O} 3 \\
-\mathrm{t}\end{array}$ & $\begin{array}{c}\mathrm{SnO} \\
-\mathrm{t}\end{array}$ & $\begin{array}{c}\mathrm{SnO} 2 \\
-\mathrm{t}\end{array}$ & $\begin{array}{c}\mathrm{SO} 3 \\
-\mathrm{t}\end{array}$ & $\begin{array}{c}\mathrm{SrO} \\
-\mathrm{t}\end{array}$ & $\begin{array}{c}\mathrm{Tc} 2 \mathrm{O} 7 \\
-\mathrm{t}\end{array}$ & $\begin{array}{c}\mathrm{TeO} 2 \\
-\mathrm{t}\end{array}$ & $\begin{array}{c}\text { ThO2 } \\
-t\end{array}$ & $\begin{array}{c}\mathrm{TiO} 2 \\
-\mathrm{t}\end{array}$ \\
\hline DZr-6c & & & & & & & & & & & & & & & & 0.0117 & 0.0027 & & & & \\
\hline DZr-7a & & & & & & & & & & & & & & & & 0.0114 & 0.0026 & & & & \\
\hline DZr-7c & & & & & & & & & & & & & & & & 0.0117 & 0.0027 & & & & \\
\hline DZr-8a & & & & & & & & & & & & & & & & 0.0114 & 0.0026 & & & & \\
\hline DZr-8c & & & & & & & & & & & & & & & & 0.0117 & 0.0027 & & & & \\
\hline DZr-9-78-38 & & & & & & & & & & & & & & & & 0.0114 & 0.0026 & & & & \\
\hline DZr-9-78-40 & & & & & & & & & & & & & & & & 0.0120 & 0.0027 & & & & \\
\hline DZr-10-78-38 & & & & & & & & & & & & & & & & 0.0114 & 0.0026 & & & & \\
\hline DZr-10-78-40 & & & & & & & & & & & & & & & & 0.0120 & 0.0027 & & & & \\
\hline GLA 78-21 & & & & & & & & & & & & & & & & & & & & & \\
\hline GLA 78-22 & & & & & & & & & & & & & & & & & & & & & \\
\hline GLA 78-23 & & & & & & & & & & & & & & & & & & & & & \\
\hline GLA 78-9-11 & & & & & & & & & & & & & & & & & & & & & \\
\hline GLA 78-9-15 & & & & & & & & & & & & & & & & & & & & & \\
\hline GLA 78-9-18 & & & & & & & & & & & & & & & & & & & & & \\
\hline GLA 78-10-14 & & & & & & & & & & & & & & & & & & & & & \\
\hline GLA 78-10-15 & & & & & & & & & & & & & & & & & & & & & \\
\hline GLA 78-10-16 & & & & & & & & & & & & & & & & & & & & & \\
\hline
\end{tabular}

\section{INEEL CVS 2a (Peeler et al. 1999)}

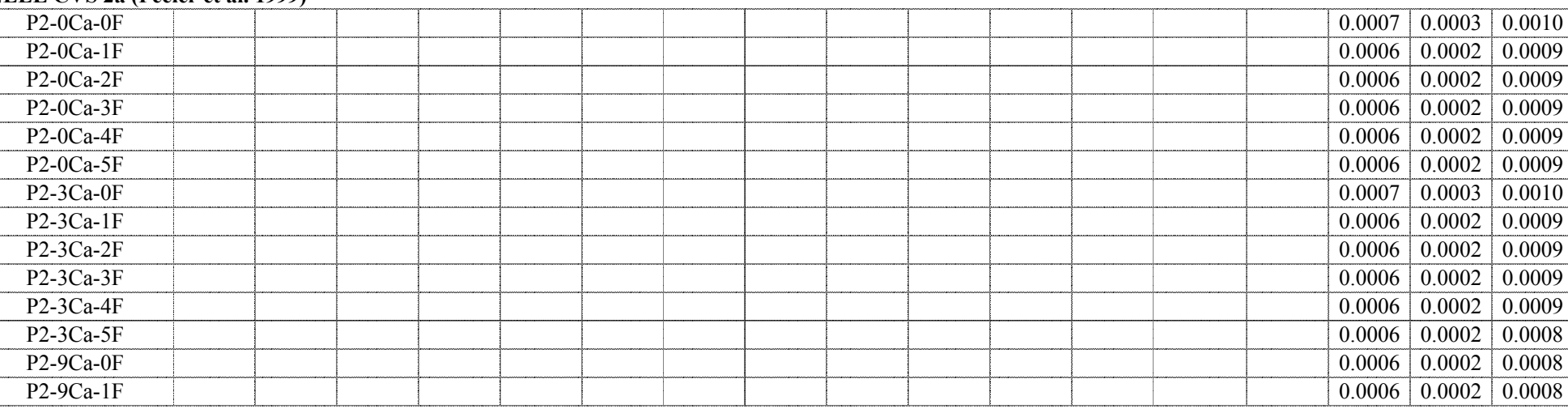


Appendix A. Database - mass fraction

INEEL DZr Process Demonstration (Musick et al. 2000)

\begin{tabular}{|c|c|c|c|c|c|c|c|c|c|c|c|c|c|c|c|c|c|c|c|c|c|}
\hline Glass ID & $\begin{array}{c}\mathrm{T} 12 \mathrm{O} 3 \\
-\mathrm{t}\end{array}$ & $\begin{array}{c}\mathrm{U} 3 \mathrm{O} 8 \\
-\mathrm{t}\end{array}$ & $\begin{array}{c}\mathrm{UO} 2 \\
-\mathrm{t}\end{array}$ & $\begin{array}{c}\mathrm{UO} 3 \\
-\mathrm{t}\end{array}$ & $\begin{array}{c}\mathrm{V} 2 \mathrm{O} 5 \\
-\mathrm{t}\end{array}$ & $\begin{array}{c}\text { WO3 } \\
-t\end{array}$ & $\begin{array}{c}\mathrm{Y} 2 \mathrm{O} 3 \\
-\mathrm{t}\end{array}$ & $\begin{array}{c}\mathrm{ZnO} \\
-\mathrm{t}\end{array}$ & $\begin{array}{c}\text { Others } \\
-t\end{array}$ & $\begin{array}{c}\text { Sum } \\
-t\end{array}$ & $\begin{array}{c}\mathrm{A} 12 \mathrm{O} 3 \\
-\mathrm{a}\end{array}$ & $\begin{array}{c}\mathrm{B} 2 \mathrm{O} 3 \\
-\mathrm{a}\end{array}$ & $\begin{array}{c}\mathrm{CaO} \\
-\mathrm{a}\end{array}$ & $\begin{array}{c}\mathrm{Fe} 2 \mathrm{O} 3 \\
-\mathrm{a}\end{array}$ & $\begin{array}{c}\mathrm{FeO} \\
-\mathrm{a}\end{array}$ & $\begin{array}{c}\mathrm{K} 2 \mathrm{O} \\
-\mathrm{a}\end{array}$ & $\begin{array}{c}\mathrm{Li} 2 \mathrm{O} \\
-\mathrm{a}\end{array}$ & $\begin{array}{c}\mathrm{MgO} \\
-\mathrm{a}\end{array}$ & $\begin{array}{c}\mathrm{Na} 2 \mathrm{O} \\
-\mathrm{a}\end{array}$ & $\begin{array}{c}\mathrm{NiO} \\
-\mathrm{a}\end{array}$ & $\begin{array}{c}\mathrm{P} 2 \mathrm{O} 5 \\
-\mathrm{a}\end{array}$ \\
\hline DZr-6c & & & & & & & & & & 1.0000 & & & & & & & & & & & \\
\hline DZr-7a & & & & & & & & & & 0.9999 & & & & & & & & & & & \\
\hline $\mathrm{DZr}-7 \mathrm{c}$ & & & & & & & & & & 1.0002 & & & & & & & & & & & \\
\hline DZr-8a & & & & & & & & & & 0.9999 & & & & & & & & & & & \\
\hline $\mathrm{DZr}-8 \mathrm{c}$ & & & & & & & & & & 1.0000 & & & & & & & & & & & \\
\hline DZr-9-78-38 & & & & & & & & & & 1.0000 & & & & & & & & & & & \\
\hline DZr-9-78-40 & & & & & & & & & & 1.0000 & & & & & & & & & & & \\
\hline DZr-10-78-38 & & & & & & & & & & 1.0000 & & & & & & & & & & & \\
\hline DZr-10-78-40 & & & & & & & & & & 1.0000 & & & & & & & & & & & \\
\hline GLA 78-21 & & & & & & & & & & & 0.0599 & 0.0539 & 0.1199 & 0.0233 & & 0.0003 & 0.0633 & & 0.1205 & 0.0002 & 0.0295 \\
\hline GLA 78-22 & & & & & & & & & & & 0.0444 & 0.0570 & 0.1236 & 0.0235 & & 0.0003 & 0.0643 & & 0.1267 & 0.0002 & 0.0273 \\
\hline GLA 78-23 & & & & & & & & & & & 0.0440 & 0.0559 & 0.1220 & 0.0237 & & 0.0003 & 0.0627 & & 0.1207 & 0.0001 & 0.0329 \\
\hline GLA 78-9-11 & & & & & & & & & & & 0.0824 & 0.0720 & 0.1149 & 0.0075 & & 0.0011 & 0.0601 & & 0.1122 & 0.0002 & 0.0312 \\
\hline GLA 78-9-15 & & & & & & & & & & & 0.0772 & 0.0991 & 0.1114 & 0.0033 & & 0.0009 & 0.0569 & & 0.1057 & 0.0003 & 0.0290 \\
\hline GLA 78-9-18 & & & & & & & & & & & 0.0661 & 0.0737 & 0.1269 & 0.0032 & & 0.0000 & 0.0588 & & 0.1077 & 0.0003 & 0.0315 \\
\hline GLA 78-10-14 & & & & & & & & & & & 0.1034 & 0.0808 & 0.0695 & 0.0170 & & 0.0032 & 0.0597 & & 0.1176 & 0.0001 & 0.0382 \\
\hline GLA $78-10-15$ & & & & & & & & & & & 0.0931 & 0.0918 & 0.0688 & 0.0175 & & 0.0029 & 0.0550 & & 0.1208 & 0.0002 & 0.0362 \\
\hline GLA $78-10-16$ & & & & & & & & & & & 0.0873 & 0.0849 & 0.0671 & 0.0174 & & 0.0030 & 0.0600 & & 0.1229 & 0.0002 & 0.0394 \\
\hline
\end{tabular}

\section{INEEL CVS 2a (Peeler et al. 1999)}

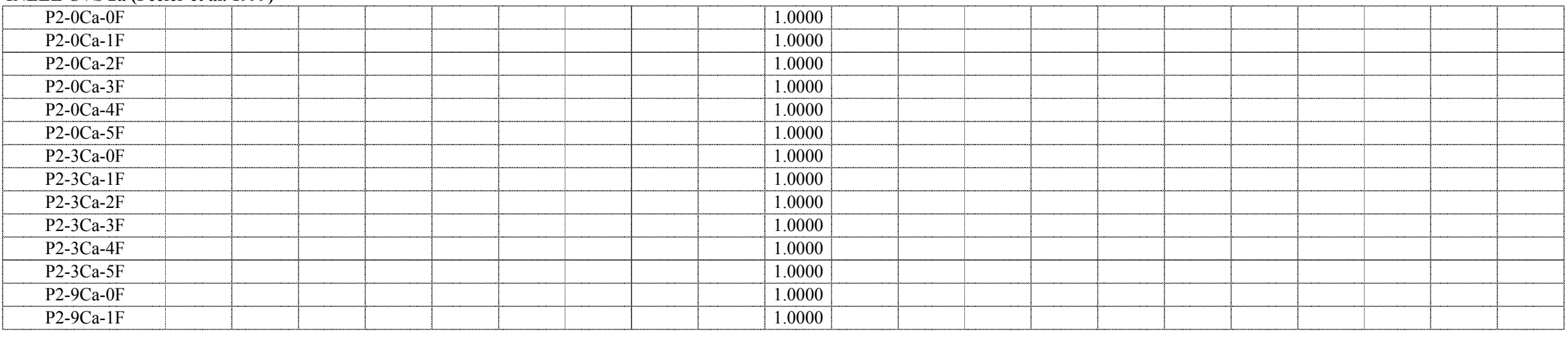


Appendix A. Database - mass fraction

INEEL DZr Process Demonstration (Musick et al. 2000)

\begin{tabular}{|c|c|c|c|c|c|c|c|c|c|c|c|c|c|c|c|c|c|c|c|c|c|}
\hline Glass ID & $\begin{array}{c}\mathrm{SiO} 2 \\
-\mathrm{a}\end{array}$ & $\begin{array}{c}\mathrm{ZrO} 2 \\
-\mathrm{a}\end{array}$ & $\begin{array}{c}\mathrm{Ag} 2 \mathrm{O} \\
-\mathrm{a}\end{array}$ & $\begin{array}{c}\text { As2 } 203 \\
-a\end{array}$ & $\begin{array}{c}\mathrm{BaO} \\
-\mathrm{a}\end{array}$ & $\begin{array}{c}\mathrm{Bi} 2 \mathrm{O} 3 \\
-\mathrm{a}\end{array}$ & $\begin{array}{l}\mathrm{Br} \\
-\mathrm{a}\end{array}$ & $\begin{array}{c}\mathrm{CdO} \\
-\mathrm{a} \\
\end{array}$ & $\begin{array}{c}\mathrm{Ce} 2 \mathrm{O} 3 \\
-\mathrm{a}\end{array}$ & $\begin{array}{c}\mathrm{CeO} 2 \\
-\mathrm{a} \\
\end{array}$ & $\begin{array}{l}\mathrm{Cl} \\
-\mathrm{a}\end{array}$ & $\begin{array}{c}\mathrm{CoO} \\
-\mathrm{a} \\
\end{array}$ & $\begin{array}{c}\mathrm{Co} 2 \mathrm{O} 3 \\
-\mathrm{a}\end{array}$ & $\begin{array}{c}\mathrm{Cr} 2 \mathrm{O} 3 \\
-\mathrm{a}\end{array}$ & $\begin{array}{c}\mathrm{Cs} 2 \mathrm{O} \\
-\mathrm{a} \\
\end{array}$ & $\begin{array}{c}\mathrm{CuO} \\
-\mathrm{a} \\
\end{array}$ & $\begin{array}{c}\mathrm{Eu} 2 \mathrm{O} 3 \\
-\mathrm{a}\end{array}$ & $\begin{array}{c}\mathrm{F} \\
-\mathrm{a} \\
\end{array}$ & $\begin{array}{c}\mathrm{Ga} 2 \mathrm{O} 3 \\
-\mathrm{a} \\
\end{array}$ & $\begin{array}{c}\mathrm{Gd} 2 \mathrm{O} 3 \\
-\mathrm{a}\end{array}$ & $\begin{array}{c}\mathrm{HgO} \\
-\mathrm{a}\end{array}$ \\
\hline \multicolumn{22}{|l|}{ DZr-6c } \\
\hline \multicolumn{22}{|l|}{ DZr-7a } \\
\hline \multirow{2}{*}{\multicolumn{22}{|c|}{$\begin{array}{l}\text { DZr-/c } \\
\text { DZr-8a }\end{array}$}} \\
\hline & & & & & & & & & & & & & & & & & & & & & \\
\hline \multicolumn{22}{|l|}{ DZr-8c } \\
\hline \multicolumn{22}{|l|}{ DZr-9-78-38 } \\
\hline \multirow{2}{*}{\multicolumn{22}{|c|}{$\begin{array}{c}\text { DZr-9-78-40 } \\
\text { DZr-10-78-38 }\end{array}$}} \\
\hline & & & & & & & & & & & & & & & & & & & & & \\
\hline \multicolumn{22}{|l|}{ DZr-10-78-40 } \\
\hline GLA 78-21 & 0.4075 & 0.0587 & & & & & & & & & 0.0003 & & & 0.0012 & & & & 0.0545 & & & \\
\hline GLA $78-22$ & 0.4043 & 0.0632 & & & & & & & & & 0.0003 & & & 0.0015 & & & & 0.0567 & & & \\
\hline GLA 78-23 & 0.4064 & 0.0597 & & & & & & & & & 0.0003 & & & 0.0014 & & & & 0.0630 & & & \\
\hline GLA 78-9-11 & 0.3698 & 0.0565 & & & & & & & & & & & & 0.0010 & & & & 0.0600 & & & \\
\hline GLA 78-9-15 & 0.3627 & 0.0547 & & & & & & & & & & & & 0.0010 & & & & 0.0649 & & & \\
\hline GLA 78-9-18 & 0.3679 & 0.0570 & & & & & & & & & & & & 0.0012 & & & & 0.0724 & & & \\
\hline GLA 78-10-14 & 0.4047 & 0.0677 & & & & & & & & & 0.0003 & & & 0.0013 & & & & 0.0292 & & & \\
\hline GLA $78-10-15$ & 0.4110 & 0.0696 & & & & & & & & & 0.0004 & & & 0.0014 & & & & 0.0248 & & & \\
\hline GLA 78-10-16 & 0.4119 & 0.0708 & & & & & & & & & 0.0003 & & & 0.0014 & & & & 0.0265 & & & \\
\hline
\end{tabular}

\section{INEEL CVS 2a (Peeler et al. 1999)}

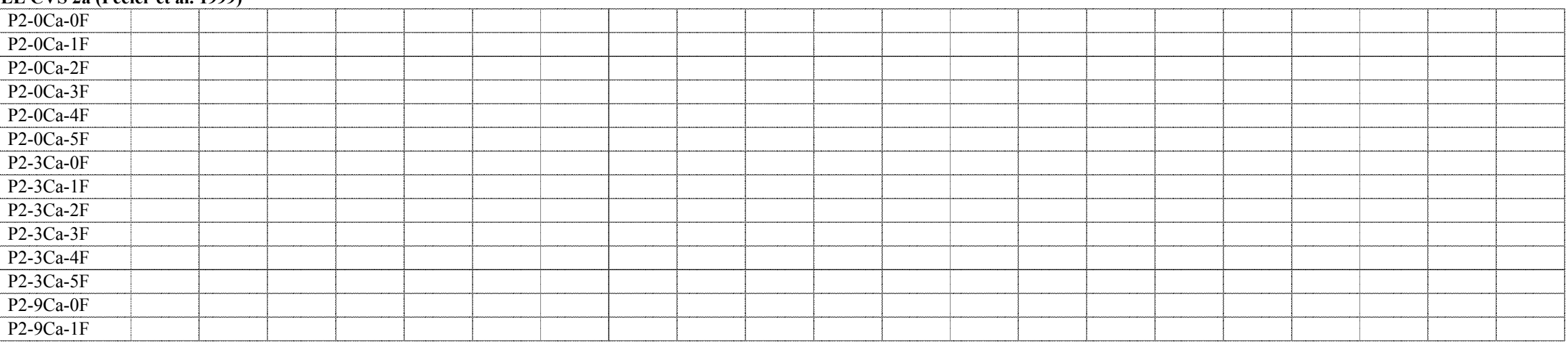


Appendix A. Database - mass fraction

INEEL DZr Process Demonstration (Musick et al. 2000)

\begin{tabular}{|c|c|c|c|c|c|c|c|c|c|c|c|c|c|c|c|c|c|c|c|c|c|}
\hline Glass ID & $\begin{array}{c}\mathrm{I} \\
-\mathrm{a}\end{array}$ & $\begin{array}{c}\mathrm{La} 2 \mathrm{O} 3 \\
-\mathrm{a}\end{array}$ & $\begin{array}{c}\mathrm{MnO} 2 \\
-\mathrm{a}\end{array}$ & $\begin{array}{c}\mathrm{MnO} \\
-\mathrm{a}\end{array}$ & $\begin{array}{c}\mathrm{MoO} \\
-\mathrm{a}\end{array}$ & $\begin{array}{c}\mathrm{MoO} 3 \\
-\mathrm{a}\end{array}$ & $\begin{array}{c}\mathrm{Nb} 2 \mathrm{O} 5 \\
-\mathrm{a}\end{array}$ & $\begin{array}{c}\mathrm{Nd} 2 \mathrm{O} 3 \\
-\mathrm{a}\end{array}$ & $\begin{array}{c}\mathrm{PbO} \\
-\mathrm{a}\end{array}$ & $\begin{array}{c}\mathrm{PdO} 2 \\
-\mathrm{a}\end{array}$ & $\begin{array}{c}\mathrm{PdO} \\
-\mathrm{a}\end{array}$ & $\begin{array}{c}\mathrm{Pr} 2 \mathrm{O} 3 \\
-\mathrm{a} \\
\end{array}$ & $\begin{array}{c}\text { Pr6O11 } \\
-\mathrm{a}\end{array}$ & $\begin{array}{c}\mathrm{Rb} 2 \mathrm{O} \\
-\mathrm{a}\end{array}$ & $\begin{array}{c}\mathrm{ReO} \\
-\mathrm{a}\end{array}$ & $\begin{array}{c}\mathrm{ReO} 2 \\
-\mathrm{a} \\
\end{array}$ & $\begin{array}{c}\mathrm{Rh} 2 \mathrm{O} 3 \\
-\mathrm{a}\end{array}$ & $\begin{array}{c}\mathrm{RhO} 2 \\
-\mathrm{a}\end{array}$ & $\begin{array}{c}\mathrm{RuO} 2 \\
-\mathrm{a}\end{array}$ & $\begin{array}{c}\mathrm{Sb} 2 \mathrm{O} 3 \\
-\mathrm{a} \\
\end{array}$ & $\begin{array}{c}\mathrm{Sb} 2 \mathrm{O} 5 \\
-\mathrm{a}\end{array}$ \\
\hline \multicolumn{22}{|l|}{ DZr-6c } \\
\hline \multicolumn{22}{|l|}{ DZr-7a } \\
\hline \multirow{2}{*}{\multicolumn{22}{|c|}{$\begin{array}{l}\text { DZr-7c } \\
\text { DZr-8a }\end{array}$}} \\
\hline & & & & & & & & & & & & & & & & & & & & & \\
\hline \multicolumn{22}{|l|}{$\mathrm{DZr}-8 \mathrm{c}$} \\
\hline \multicolumn{22}{|l|}{ DZr-9-78-38 } \\
\hline \multicolumn{22}{|l|}{ DZr-9-78-40 } \\
\hline \multicolumn{22}{|l|}{ DZr-10-78-38 } \\
\hline \multicolumn{22}{|l|}{ DZr-10-78-40 } \\
\hline GLA 78-21 & & 0.0002 & & 0.0007 & & & & & 0.0001 & & & & & & & & & & & & \\
\hline GLA $78-22$ & & 0.0001 & & 0.0005 & & & & & 0.0000 & & & & & & & & & & & & \\
\hline GLA 78-23 & & 0.0002 & & 0.0005 & & & & & 0.0001 & & & & & & & & & & & & \\
\hline GLA 78-9-11 & & 0.0246 & & 0.0009 & & & & & 0.0000 & & & & & & & & & & & & \\
\hline GLA 78-9-15 & & 0.0277 & & 0.0007 & & & & & 0.0000 & & & & & & & & & & & & \\
\hline GLA 78-9-18 & & 0.0282 & & 0.0004 & & & & & 0.0001 & & & & & & & & & & & & \\
\hline GLA $78-10-14$ & & 0.0035 & & 0.0010 & & & & & 0.0000 & & & & & & & & & & & & \\
\hline GLA $78-10-15$ & & 0.0032 & & 0.0009 & & & & & 0.0000 & & & & & & & & & & & & \\
\hline GLA $78-10-16$ & & 0.0032 & & 0.0010 & & & & & 0.0000 & & & & & & & & & & & & \\
\hline
\end{tabular}

INEEL CVS 2a (Peeler et al. 1999)

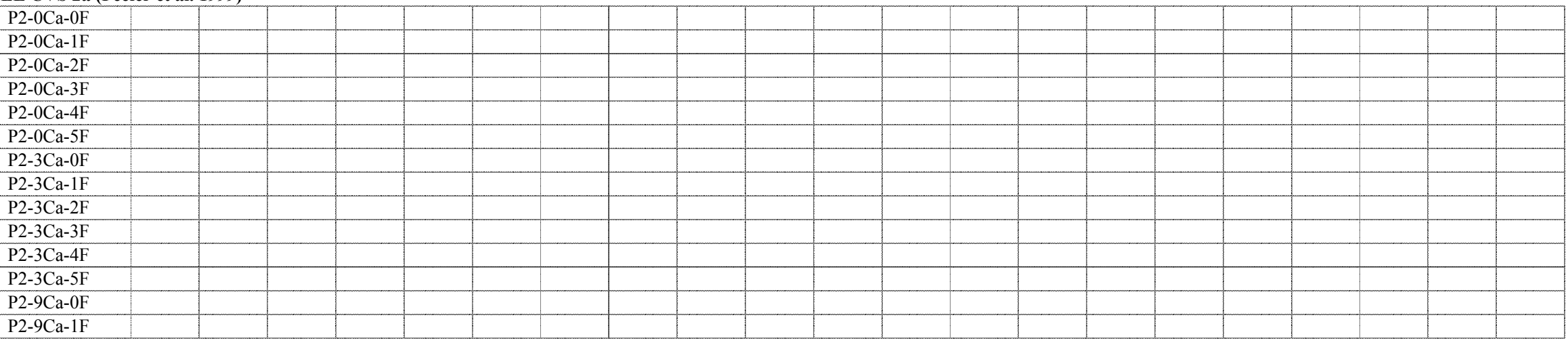


Appendix A. Database - mass fraction

INEEL DZr Process Demonstration (Musick et al. 2000)

\begin{tabular}{|c|c|c|c|c|c|c|c|c|c|c|c|c|c|c|c|c|c|c|c|c|}
\hline Glass ID & $\begin{array}{c}\mathrm{SeO} 2 \\
-\mathrm{a}\end{array}$ & $\begin{array}{c}\mathrm{Sm} 2 \mathrm{O} 3 \\
-\mathrm{a} \\
\end{array}$ & $\begin{array}{c}\mathrm{SnO} \\
-\mathrm{a} \\
\end{array}$ & $\begin{array}{c}\mathrm{SnO} 2 \\
-\mathrm{a} \\
\end{array}$ & $\begin{array}{c}\mathrm{SO} 3 \\
-\mathrm{a}\end{array}$ & $\begin{array}{c}\mathrm{SrO} \\
-\mathrm{a} \\
\end{array}$ & $\begin{array}{c}\mathrm{Tc} 2 \mathrm{O} 7 \\
-\mathrm{a}\end{array}$ & $\begin{array}{c}\mathrm{TeO} 2 \\
-\mathrm{a}\end{array}$ & $\begin{array}{c}\text { ThO2 } \\
-\mathrm{a}\end{array}$ & $\begin{array}{c}\mathrm{TiO} 2 \\
-\mathrm{a} \\
\end{array}$ & $\begin{array}{c}\mathrm{T} 12 \mathrm{O} 3 \\
-\mathrm{a} \\
\end{array}$ & $\begin{array}{c}\mathrm{U} 3 \mathrm{O} 8 \\
-\mathrm{a}\end{array}$ & $\begin{array}{c}\mathrm{UO} 2 \\
-\mathrm{a} \\
\end{array}$ & $\begin{array}{c}\text { UO3 } \\
-\mathrm{a} \\
\end{array}$ & $\begin{array}{c}\mathrm{V} 2 \mathrm{O} 5 \\
-\mathrm{a}\end{array}$ & $\begin{array}{c}\text { WO3 } \\
-\mathrm{a} \\
\end{array}$ & $\begin{array}{c}\mathrm{Y} 2 \mathrm{O} 3 \\
-\mathrm{a}\end{array}$ & $\begin{array}{c}\mathrm{ZnO} \\
-\mathrm{a} \\
\end{array}$ & $\begin{array}{c}\text { Others } \\
-\mathrm{a}\end{array}$ & $\begin{array}{c}\text { Sum } \\
-\mathrm{a}\end{array}$ \\
\hline \multicolumn{21}{|l|}{ DZr-6c } \\
\hline \multicolumn{21}{|l|}{ DZr-7a } \\
\hline \multicolumn{21}{|l|}{ DZr-7c } \\
\hline \multicolumn{21}{|l|}{ DZr-8a } \\
\hline \multicolumn{21}{|l|}{ DZr-8c } \\
\hline \multicolumn{21}{|l|}{ DZr-9-78-38 } \\
\hline \multicolumn{21}{|l|}{ DZr-9-78-40 } \\
\hline \multicolumn{21}{|l|}{ DZr-10-78-38 } \\
\hline \multicolumn{21}{|l|}{ DZr-10-78-40 } \\
\hline GLA 78-21 & & & & & 0.0037 & 0.0022 & & & & & & & & & & & & & & 0.9999 \\
\hline GLA 78-22 & & & & & 0.0025 & 0.0032 & & & & & & & & & & & & & & 0.9996 \\
\hline GLA 78-23 & & & & & 0.0025 & 0.0031 & & & & & & & & & & & & & & 0.9995 \\
\hline GLA 78-9-11 & & & & & 0.0055 & & & & & & & & & & & & & & & 0.9999 \\
\hline GLA 78-9-15 & & & & & 0.0045 & & & & & & & & & & & & & & & 1.0000 \\
\hline GLA 78-9-18 & & & & & 0.0045 & & & & & & & & & & & & & & & 0.9999 \\
\hline GLA $78-10-14$ & & & & & 0.0027 & & & & & & & & & & & & & & & 0.9999 \\
\hline GLA $78-10-15$ & & & & & 0.0022 & & & & & & & & & & & & & & & 0.9998 \\
\hline GLA $78-10-16$ & & & & & 0.0026 & & & & & & & & & & & & & & & 0.9999 \\
\hline
\end{tabular}

INEEL CVS 2a (Peeler et al. 1999)

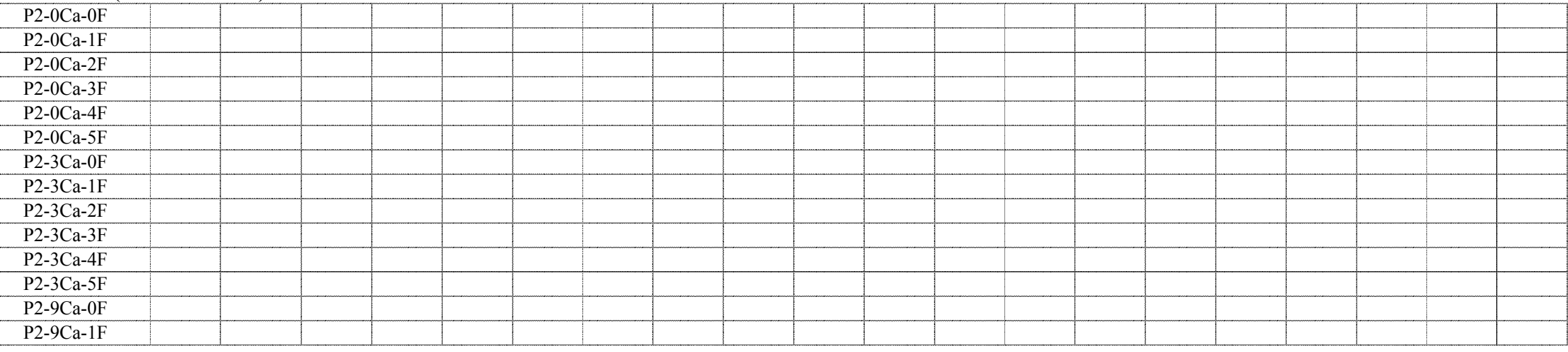


Appendix A. Database - mass fraction

INEEL DZr Process Demonstration (Musick et al. 2000)

\begin{tabular}{|c|c|c|c|c|c|c|c|c|}
\hline Glass ID & $\begin{array}{l}\mathrm{TM} \\
\left({ }^{\circ} \mathrm{C}\right)\end{array}$ & $\begin{array}{l}\text { Gradient } \\
\text { TL }\left({ }^{\circ} \mathrm{C}\right)\end{array}$ & $\begin{array}{l}\text { Uniform } \\
\text { TL }\left({ }^{\circ} \mathrm{C}\right)\end{array}$ & Primary Phase & $\begin{array}{l}\text { Quenched } \\
\text { Visual/OM }\end{array}$ & $\begin{array}{c}\text { Quenched } \\
\text { SEM/EDS or TEM }\end{array}$ & $\begin{array}{l}\text { Quenched } \\
\text { XRD }\end{array}$ & $\begin{array}{c}\text { Quenched } \\
\text { Homogeneous? }\end{array}$ \\
\hline DZr-6c & & & & & Homogeneous & & & yes \\
\hline DZr-7a & & & & & Homogeneous & & & yes \\
\hline DZr-7c & & & & & Homogeneous & & & yes \\
\hline DZr-8a & & & & & Homogeneous & & & yes \\
\hline DZr-8c & & & & & Homogeneous & & & yes \\
\hline DZr-9-78-38 & & & $<950$ & & Homogeneous & & & yes \\
\hline DZr-9-78-40 & & & & & Homogeneous & & & yes \\
\hline DZr-10-78-38 & & & $<950$ & & Homogeneous & & & yes \\
\hline DZr-10-78-40 & & & & & Homogeneous & & & yes \\
\hline GLA 78-21 & & & & & small $(\sim 1 \mathrm{~mm})$ black particles & iron and nickel sulfides & $<0.5 \%$ crystallinity & no \\
\hline GLA 78-22 & & & & & & iron and nickel sulfides & $<0.5 \%$ crystallinity & no \\
\hline GLA $78-23$ & & & & & $\begin{array}{l}\text { small }(\sim 1 \mathrm{~mm}) \text { black particles and larger } \\
\text { cuspidine crystals }\end{array}$ & & $<1$ vol\% cuspidine & no \\
\hline GLA 78-9-11 & & & & & $\begin{array}{l}\text { multiple phases and a transparent green glass } \\
\text { with streaks of white material throughout }\end{array}$ & $\begin{array}{l}\text { a cluster of undissolved batch } \\
\text { material along with dispersed } \\
\text { particles ( } \mathrm{Zr} \text { and } \mathrm{Cr} 2 \mathrm{O} 3 \text { rich) in a } \\
\text { glass matrix }\end{array}$ & $\begin{array}{l}\sim 1.3 \mathrm{vol} \% \text { cuspidine } \\
\text { and } \sim 3.1 \text { vol } \% \mathrm{CaF} 2\end{array}$ & no \\
\hline GLA 78-9-15 & & & & & $\begin{array}{l}\text { multiple phases and a transparent green glass } \\
\text { with streaks of white material throughout }\end{array}$ & $\begin{array}{l}\text { a cluster of undissolved batch } \\
\text { material along with dispersed } \\
\text { particles ( } \mathrm{Zr} \text { and } \mathrm{Cr} 2 \mathrm{O} 3 \text { rich) in a } \\
\text { glass matrix }\end{array}$ & $\begin{array}{l}\sim 8.2 \text { vol } \% \text { cuspidine } \\
\text { and } \sim 1.6 \text { vol } \% \mathrm{CaF} 2\end{array}$ & no \\
\hline GLA 78-9-18 & & & & & $\begin{array}{l}\text { multiple phases and a transparent green glass } \\
\text { with streaks of white material throughout }\end{array}$ & & $\sim 0.5 \mathrm{vol} \%$ crystallinity & no \\
\hline GLA 78-10-14 & & & $\sim 950$ & & & & & \\
\hline GLA 78-10-15 & & & $\sim 950$ & & & & & \\
\hline GLA 78-10-16 & & & $\sim 950$ & & & & & \\
\hline
\end{tabular}

INEEL CVS 2a (Peeler et al. 1999)

\begin{tabular}{|c|c|c|c|c|c|c|}
\hline P2-OCa-OF & & & & & \\
\hline P2-0Ca-1F & & & & & & \\
\hline P2-0Ca-2F & & & & & \\
\hline P2-0Ca-3F & & & & & & \\
\hline P2-0Ca-4F & & & & & & \\
\hline P2-0Ca-5F & & & & & \\
\hline P2-3Ca-0F & & & & \\
\hline P2-3Ca-1F & & & & & \\
\hline P2-3Ca-2F & & & & & \\
\hline P2-3Ca-3F & & & & & \\
\hline P2-3Ca-4F & & & & & \\
\hline P2-3Ca-5F & & & & & \\
\hline P2-9Ca-0F & & & & \\
\hline P2-9Ca-1F & & & & \\
\hline
\end{tabular}


Appendix A. Database - mass fraction

\section{INEEL DZr Process Demonstration (Musick et al. 2000)}

\begin{tabular}{|c|c|c|c|c|c|}
\hline Glass ID & $\begin{array}{c}\mathrm{CCC} \\
\text { Visual/OM } \\
\end{array}$ & $\begin{array}{c}\text { CCC } \\
\text { SEM/EDS or TEM }\end{array}$ & $\begin{array}{l}\mathrm{CCC} \\
\mathrm{XRD}\end{array}$ & $\begin{array}{l}\text { Heat Treated } \\
\text { Visual/OM }\end{array}$ & $\begin{array}{c}\text { Heat Treated } \\
\text { SEM/EDS or TEM }\end{array}$ \\
\hline DZr-6c & & & $\begin{array}{l}\text { homogenous on top, crystallization on } \\
\text { bottom, } \sim 20 \% \text { overall crystallization }\end{array}$ & & \\
\hline DZr-7a & & & $\sim 30 \%$ overall crystallization & & \\
\hline DZr-7c & & & some mottling, likely crystallization & & \\
\hline DZr-8a & & & fully crystallized, like a rock & & \\
\hline DZr-8c & & & $\begin{array}{l}\text { homogenous on top, crystallization on } \\
\text { bottom, } \sim 30 \% \text { overall crystallization }\end{array}$ & & \\
\hline DZr-9-78-38 & & & $\sim 1 \mathrm{vol} \%$ (bulk) crystallinity & & \\
\hline DZr-9-78-40 & & & $20-40$ vol $\%$ crystallinity & & \\
\hline DZr-10-78-38 & & & $<5 \mathrm{vol} \%$ (surface) crystallinity & & \\
\hline DZr-10-78-40 & & & $>50 \mathrm{vol} \%$ crystallinity & & \\
\hline GLA 78-21 & & & $\begin{array}{l}\sim 1-2 \text { vol\% dendritic crystals on all } \\
\text { surfaces and spinel }\end{array}$ & & \\
\hline GLA 78-22 & & & $\begin{array}{l}\sim 5-10 \text { vol } \% \text { dendritic crystals on all } \\
\text { surfaces and spinel }\end{array}$ & & \\
\hline GLA 78-23 & & & $\begin{array}{l}2 \text { vol } \% \text { dendritic crystals on all } \\
\text { surfaces and spinel }\end{array}$ & & \\
\hline GLA 78-9-11 & & & $\begin{array}{l}1-2 \text { vol\% dendritic crystals with } \\
\text { distorted edges }\end{array}$ & & \\
\hline GLA 78-9-15 & & & $\begin{array}{l}\text { 1-2 vol\% dendritic crystals with } \\
\text { distorted edges }\end{array}$ & & \\
\hline GLA 78-9-18 & & & $\begin{array}{l}\sim 25-50 \% \text { dendritic crystals with } \\
\text { distorted edges }\end{array}$ & & \\
\hline GLA $78-10-14$ & & & $\sim 1 \mathrm{vol} \%$ hematite and spinel & & \\
\hline GLA $78-10-15$ & & & $\sim 1 \mathrm{vol} \%$ hematite and spinel & & \\
\hline GLA $78-10-16$ & & & $\sim 1 \mathrm{vol} \%$ hematite and spinel & & \\
\hline
\end{tabular}

\section{INEEL CVS 2a (Peeler et al. 1999)}

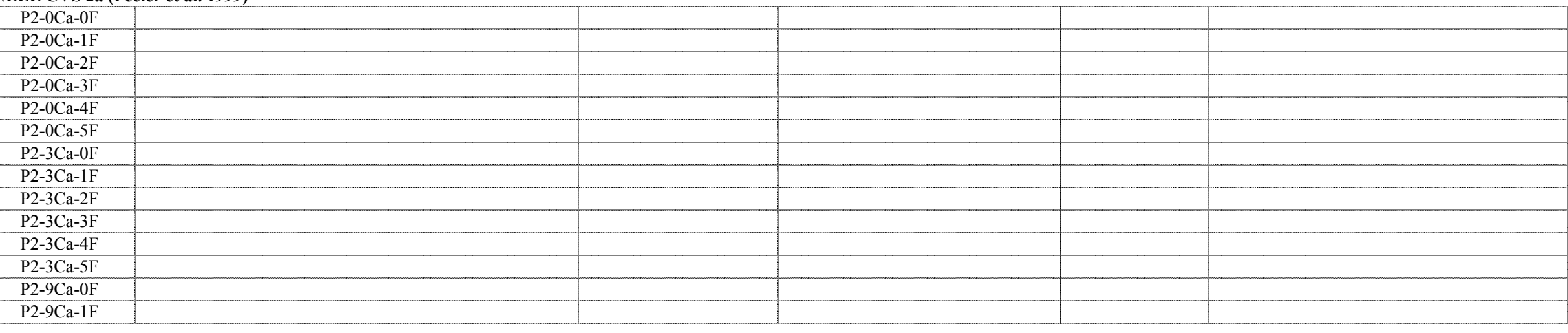


Appendix A. Database - mass fraction

INEEL DZr Process Demonstration (Musick et al. 2000)

\begin{tabular}{|c|c|c|c|c|c|c|c|c|c|c|c|c|c|c|c|}
\hline Glass ID & $\begin{array}{c}\text { Heat Treated } \\
\text { XRD }\end{array}$ & $\begin{array}{l}\text { Density } \\
\left(\mathrm{g} / \mathrm{cm}^{3}\right)\end{array}$ & $\begin{array}{c}\text { Fulc Visc } \\
\mathrm{A}\end{array}$ & \begin{tabular}{|c|} 
Fulc Visc \\
$\mathrm{B}$
\end{tabular} & \begin{tabular}{|c|} 
Fulc Visc \\
To \\
\end{tabular} & $\begin{array}{c}\mathrm{FV} 1150^{\circ} \mathrm{C} \\
(\mathrm{Pa} \cdot \mathrm{s})\end{array}$ & $\begin{array}{c}\text { Arrh Visc } \\
\mathrm{A}\end{array}$ & $\begin{array}{c}\text { Arrh Visc } \\
\text { B }\end{array}$ & $\begin{array}{l}\text { Av } \\
1150^{\circ} \mathrm{C} \\
\left(D_{0} \ldots+c\right)\end{array}$ & $\begin{array}{c}\mathrm{T}\left({ }^{\circ} \mathrm{C}\right) \text { at } \\
2 \mathrm{~Pa} \cdot \mathrm{s} \\
\end{array}$ & $\begin{array}{c}\mathrm{T}\left({ }^{\circ} \mathrm{C}\right) \text { at } \\
5 \mathrm{~Pa} \cdot \mathrm{s}\end{array}$ & $\begin{array}{c}\mathrm{T}\left({ }^{\circ} \mathrm{C}\right) \text { at } \\
10 \mathrm{~Pa} \cdot \mathrm{s}\end{array}$ & $\begin{array}{l}\mathrm{T} 1 \\
\left({ }^{\circ} \mathrm{C}\right) \\
\end{array}$ & $\begin{array}{c}\mathrm{V} 1 \\
(\mathrm{~Pa} \cdot \mathrm{s}) \\
\end{array}$ & $\begin{array}{c}\mathrm{T} 2 \\
\left({ }^{\circ} \mathrm{C}\right)\end{array}$ \\
\hline \multicolumn{16}{|l|}{ DZr-6c } \\
\hline \multicolumn{16}{|l|}{ DZr-7a } \\
\hline \multicolumn{16}{|l|}{ DZr-7c } \\
\hline \multicolumn{16}{|l|}{ DZr-8a } \\
\hline \multicolumn{16}{|l|}{ DZr-8c } \\
\hline \multicolumn{16}{|l|}{ DZr-9-78-38 } \\
\hline \multicolumn{16}{|l|}{ DZr-9-78-40 } \\
\hline \multicolumn{16}{|l|}{ DZr-10-78-38 } \\
\hline \multicolumn{16}{|l|}{ DZr-10-78-40 } \\
\hline \multicolumn{16}{|l|}{ GLA 78-21 } \\
\hline \multicolumn{16}{|l|}{ GLA 78-22 } \\
\hline \multicolumn{16}{|l|}{ GLA 78-23 } \\
\hline \multicolumn{16}{|l|}{ GLA 78-9-11 } \\
\hline \multicolumn{16}{|l|}{ GLA 78-9-15 } \\
\hline \multicolumn{16}{|l|}{ GLA 78-9-18 } \\
\hline \multicolumn{16}{|l|}{ GLA $78-10-14$} \\
\hline \multicolumn{16}{|l|}{ GLA 78-10-15 } \\
\hline GLA $78-10-16$ & & & & & & & & & & & & & & & \\
\hline
\end{tabular}

\section{INEEL CVS 2a (Peeler et al. 1999)}

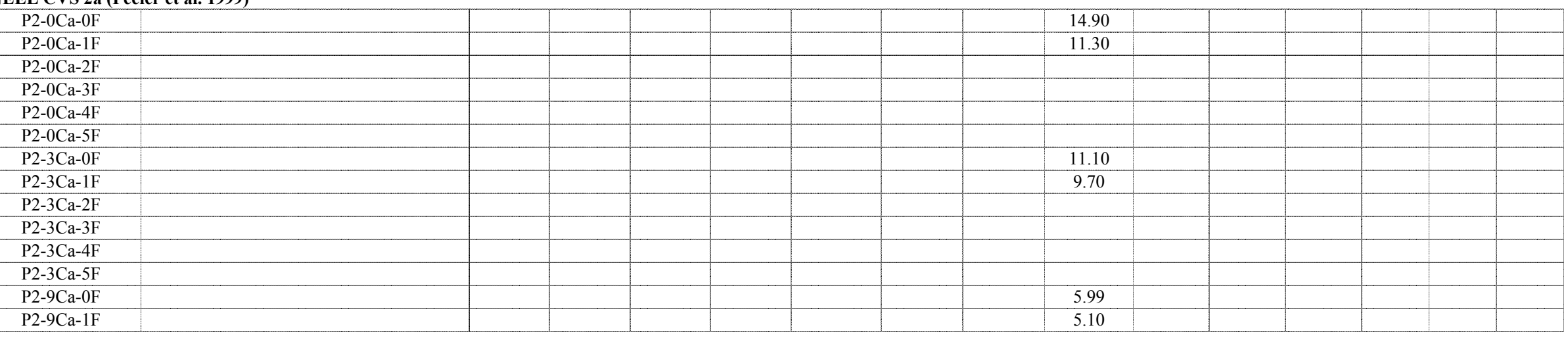


Appendix A. Database - mass fraction

\begin{tabular}{|c|c|c|c|c|c|c|c|c|c|c|c|c|c|c|c|c|c|c|c|c|c|}
\hline Glass ID & $\begin{array}{c}\mathrm{V} 2 \\
(\mathrm{~Pa} \cdot \mathrm{s})\end{array}$ & $\begin{array}{c}\mathrm{T} 3 \\
\left({ }^{\circ} \mathrm{C}\right) \\
\end{array}$ & $\begin{array}{c}\mathrm{V} 3 \\
(\mathrm{~Pa} \cdot \mathrm{s})\end{array}$ & $\begin{array}{c}\mathrm{T} 4 \\
\left({ }^{\circ} \mathrm{C}\right)\end{array}$ & $\begin{array}{c}\mathrm{V} 4 \\
(\mathrm{~Pa} \cdot \mathrm{s})\end{array}$ & $\begin{array}{c}\mathrm{T} 5 \\
\left({ }^{\circ} \mathrm{C}\right) \\
\end{array}$ & $\begin{array}{c}\mathrm{V} 5 \\
(\mathrm{~Pa} \cdot \mathrm{s})\end{array}$ & $\begin{array}{c}\mathrm{T} 6 \\
\left({ }^{\circ} \mathrm{C}\right) \\
\end{array}$ & $\begin{array}{c}\mathrm{V} 6 \\
(\mathrm{~Pa} \cdot \mathrm{s})\end{array}$ & $\begin{array}{c}\mathrm{T} 7 \\
\left({ }^{\circ} \mathrm{C}\right) \\
\end{array}$ & $\begin{array}{c}\mathrm{V} 7 \\
(\mathrm{~Pa} \cdot \mathrm{s})\end{array}$ & $\begin{array}{c}\mathrm{T} 8 \\
\left({ }^{\circ} \mathrm{C}\right) \\
\end{array}$ & $\begin{array}{c}\mathrm{V} 8 \\
(\mathrm{~Pa} \cdot \mathrm{s})\end{array}$ & $\begin{array}{c}\mathrm{T} 9 \\
\left({ }^{\circ} \mathrm{C}\right) \\
\end{array}$ & $\begin{array}{c}\mathrm{V} 9 \\
(\mathrm{~Pa} \cdot \mathrm{s})\end{array}$ & $\begin{array}{l}\mathrm{T} 10 \\
\left({ }^{\circ} \mathrm{C}\right) \\
\end{array}$ & $\begin{array}{c}\mathrm{V} 10 \\
(\mathrm{~Pa} \cdot \mathrm{s})\end{array}$ & $\begin{array}{l}\mathrm{T} 11 \\
\left({ }^{\circ} \mathrm{C}\right) \\
\end{array}$ & $\begin{array}{c}\mathrm{V} 11 \\
(\mathrm{~Pa} \cdot \mathrm{s})\end{array}$ & $\begin{array}{l}\mathrm{T} 12 \\
\left({ }^{\circ} \mathrm{C}\right) \\
\end{array}$ & $\begin{array}{c}\mathrm{V} 12 \\
(\mathrm{~Pa} \cdot \mathrm{s})\end{array}$ \\
\hline \multicolumn{22}{|l|}{ DZr-6c } \\
\hline \multicolumn{22}{|l|}{ DZr-7a } \\
\hline \multicolumn{22}{|l|}{$\mathrm{DZr}-7 \mathrm{c}$} \\
\hline \multicolumn{22}{|l|}{ DZr-8a } \\
\hline \multicolumn{22}{|l|}{ DZr-8c } \\
\hline \multicolumn{22}{|l|}{ DZr-9-78-38 } \\
\hline \multicolumn{22}{|l|}{ DZr-9-78-40 } \\
\hline \multicolumn{22}{|l|}{ DZr-10-78-38 } \\
\hline \multicolumn{22}{|l|}{ DZr-10-78-40 } \\
\hline \multicolumn{22}{|l|}{ GLA 78-21 } \\
\hline \multicolumn{22}{|l|}{ GLA 78-22 } \\
\hline \multicolumn{22}{|l|}{ GLA 78-23 } \\
\hline \multicolumn{22}{|l|}{ GLA 78-9-11 } \\
\hline \multicolumn{22}{|l|}{ GLA 78-9-15 } \\
\hline \multicolumn{22}{|l|}{ GLA 78-9-18 } \\
\hline \multicolumn{22}{|l|}{ GLA 78-10-14 } \\
\hline GLA $78-10-15$ & & & & & & & & & & & & & & & & & & & & & \\
\hline GLA $78-10-16$ & & & & & & & & & & & & & & & & & & & & & \\
\hline
\end{tabular}

INEEL CVS 2a (Peeler et al. 1999)

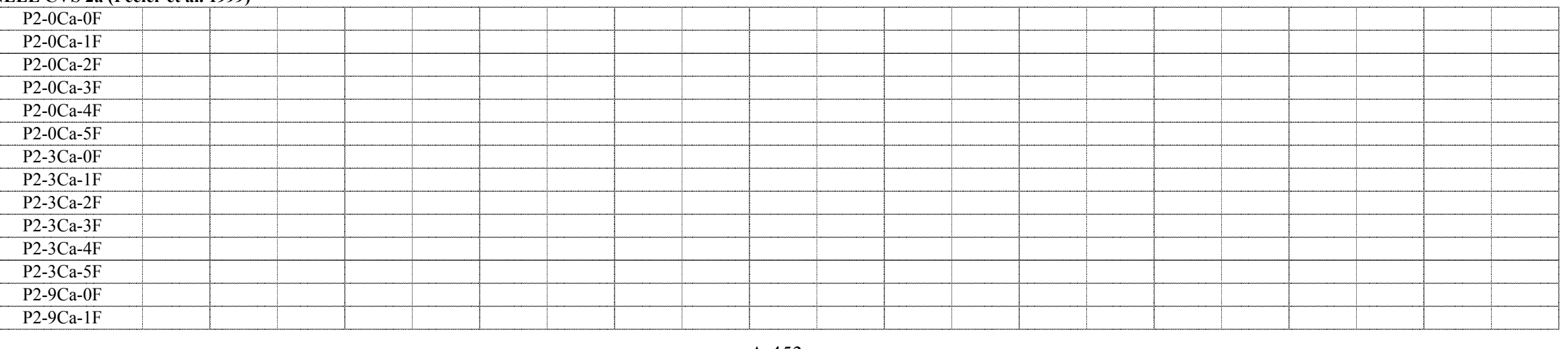


Appendix A. Database - mass fraction

INEEL DZr Process Demonstration (Musick et al. 2000)

\begin{tabular}{|c|c|c|c|c|c|c|c|c|c|c|c|c|c|c|c|c|}
\hline Glass ID & $\begin{array}{l}\mathrm{T} 13 \\
\left({ }^{\circ} \mathrm{C}\right) \\
\end{array}$ & $\begin{array}{c}\mathrm{V} 13 \\
(\mathrm{~Pa} \cdot \mathrm{s}) \\
\end{array}$ & $\begin{array}{l}\mathrm{T} 14 \\
\left({ }^{\circ} \mathrm{C}\right) \\
\end{array}$ & $\begin{array}{c}\text { V14 } \\
(\mathrm{Pa} \cdot \mathrm{s}) \\
\end{array}$ & $\begin{array}{c}\text { Q PCT } \\
\text { B }\left(\mathrm{g} / \mathrm{m}^{2}\right)\end{array}$ & $\begin{array}{c}\text { Q PCT } \\
\mathrm{Li}\left(\mathrm{g} / \mathrm{m}^{2}\right)\end{array}$ & $\begin{array}{c}\text { Q PCT } \\
\mathrm{Na}\left(\mathrm{g} / \mathrm{m}^{2}\right)\end{array}$ & $\begin{array}{c}\text { Q PCT } \\
\mathrm{Si}\left(\mathrm{g} / \mathrm{m}^{2}\right)\end{array}$ & $\begin{array}{c}\text { Q PCT } \\
\mathrm{pH}\end{array}$ & $\begin{array}{c}\text { CCC PCT } \\
\mathrm{B}\left(\mathrm{g} / \mathrm{m}^{2}\right)\end{array}$ & $\begin{array}{l}\text { CCC PCT } \\
\mathrm{Li}\left(\mathrm{g} / \mathrm{m}^{2}\right)\end{array}$ & $\begin{array}{l}\text { CCC PCT } \\
\mathrm{Na}\left(\mathrm{g} / \mathrm{m}^{2}\right)\end{array}$ & $\begin{array}{l}\text { CCC PCT } \\
\mathrm{Si}\left(\mathrm{g} / \mathrm{m}^{2}\right)\end{array}$ & $\begin{array}{c}\text { CCC PCT } \\
\mathrm{pH} \\
\end{array}$ & $\begin{array}{l}\text { Q PCT B at } \\
20^{\circ} \mathrm{C}\left(\mathrm{g} / \mathrm{m}^{2}\right)\end{array}$ & $\begin{array}{l}\text { Q PCT Li at } \\
20^{\circ} \mathrm{C}\left(\mathrm{g} / \mathrm{m}^{2}\right)\end{array}$ \\
\hline \multicolumn{17}{|l|}{ DZr-6c } \\
\hline \multirow{2}{*}{\multicolumn{15}{|c|}{$\frac{D Z r-7 a}{D Z r-7 c}$}} & & \\
\hline & & & & & & & & & & & & & & & & \\
\hline \multicolumn{17}{|l|}{ DZr-8a } \\
\hline \multicolumn{17}{|l|}{ DZr-8c } \\
\hline DZr-9-78-38 & & & & & 0.199 & & 0.418 & 0.065 & & & & & & & & \\
\hline DZr-9-78-40 & & & & & 0.19 & & 0.32 & 0.061 & & & & & & & & \\
\hline DZr-10-78-38 & & & & & 0.208 & & 0.339 & 0.065 & & & & & & & & \\
\hline DZr-10-78-40 & & & & & 0.169 & & 0.295 & 0.058 & & & & & & & & \\
\hline GLA 78-21 & & & & & 0.228 & 0.348 & 0.395 & 0.091 & 11.20 & & & & & & & \\
\hline GLA $78-22$ & & & & & 0.180 & 0.304 & 0.330 & 0.077 & 11.15 & & & & & & & \\
\hline GLA 78-23 & & & & & 0.169 & 0.293 & 0.336 & 0.089 & 11.11 & & & & & & & \\
\hline GLA 78-9-11 & & & & & 0.188 & 0.281 & 0.302 & 0.076 & 10.93 & & & & & & & \\
\hline GLA 78-9-15 & & & & & 0.173 & 0.304 & 0.349 & 0.100 & 10.79 & & & & & & & \\
\hline GLA 78-9-18 & & & & & 0.222 & 0.284 & 0.338 & 0.079 & 10.90 & & & & & & & \\
\hline GLA 78-10-14 & & & & & 0.260 & 0.369 & 0.326 & 0.113 & 10.86 & & & & & & & \\
\hline GLA 78-10-15 & & & & & 0.231 & 0.403 & 0.326 & 0.112 & 10.93 & & & & & & & \\
\hline GLA 78-10-16 & & & & & 0.230 & 0.310 & 0.250 & 0.113 & 10.92 & & & & & & & \\
\hline
\end{tabular}

INEEL CVS 2a (Peeler et al. 1999)

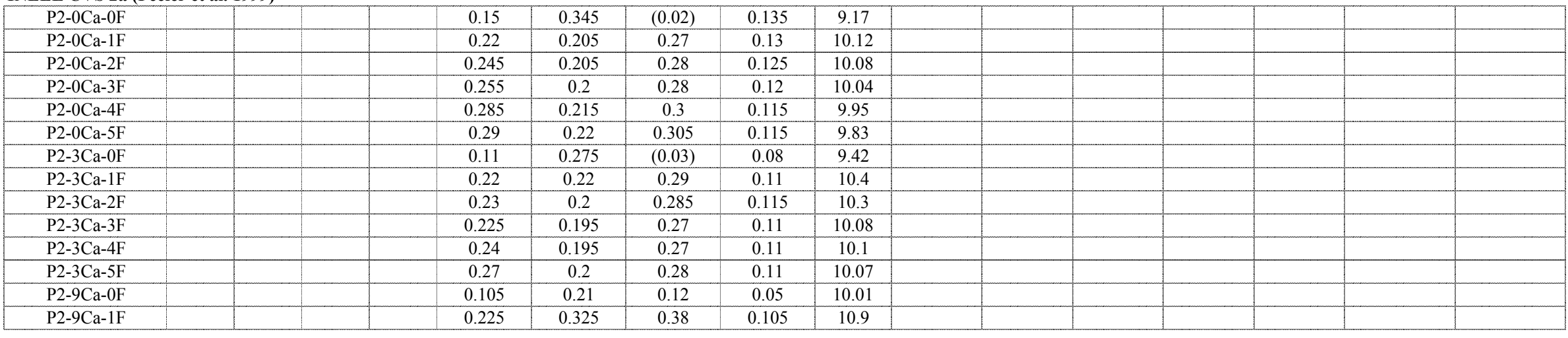


Appendix A. Database - mass fraction

\begin{tabular}{|c|c|c|c|c|c|c|c|c|c|c|c|c|}
\hline \multicolumn{13}{|c|}{ INEEL DZr Process Demonstration (Musick et al. 2000) } \\
\hline Glass ID & $\begin{array}{l}\text { Q PCT Na at } \\
20^{\circ} \mathrm{C}\left(\mathrm{g} / \mathrm{m}^{2}\right)\end{array}$ & $\begin{array}{l}\text { Q PCT Si at } \\
20^{\circ} \mathrm{C}\left(\mathrm{g} / \mathrm{m}^{2}\right)\end{array}$ & $\begin{array}{c}\mathrm{QpH} \\
\text { at } 20^{\circ} \mathrm{C}\end{array}$ & $\begin{array}{c}\text { TCLP Ag } \\
\text { (ppm) }\end{array}$ & $\begin{array}{c}\text { TCLP As } \\
\text { (ppm) }\end{array}$ & $\begin{array}{c}\text { TCLP Ba } \\
\text { (ppm) }\end{array}$ & $\begin{array}{c}\text { TCLP Cd } \\
\text { (ppm) }\end{array}$ & $\begin{array}{c}\text { TCLP Cr } \\
(\mathrm{ppm})\end{array}$ & $\begin{array}{c}\text { TCLP Ni } \\
(\mathrm{ppm})\end{array}$ & $\begin{array}{c}\text { TCLP Pb } \\
(\mathrm{ppm})\end{array}$ & $\begin{array}{c}\text { TCLP Se } \\
(\mathrm{ppm})\end{array}$ & $\begin{array}{c}\text { TCLP Zn } \\
(\mathrm{ppm})\end{array}$ \\
\hline \multicolumn{13}{|l|}{ DZr-6c } \\
\hline \multicolumn{13}{|l|}{ DZr-7a } \\
\hline \multicolumn{13}{|l|}{ DZr-7c } \\
\hline \multicolumn{13}{|l|}{ DZr-8a } \\
\hline \multicolumn{13}{|l|}{$\mathrm{DZr}-8 \mathrm{c}$} \\
\hline \multicolumn{13}{|l|}{ DZr-9-78-38 } \\
\hline \multicolumn{13}{|l|}{ DZr-9-78-40 } \\
\hline \multicolumn{13}{|l|}{ DZr-10-78-38 } \\
\hline \multicolumn{13}{|l|}{ DZr-10-78-40 } \\
\hline \multicolumn{13}{|l|}{ GLA 78-21 } \\
\hline \multicolumn{13}{|l|}{ GLA 78-22 } \\
\hline \multicolumn{13}{|l|}{ GLA 78-23 } \\
\hline \multicolumn{13}{|l|}{ GLA 78-9-11 } \\
\hline \multicolumn{13}{|l|}{ GLA 78-9-15 } \\
\hline \multicolumn{13}{|l|}{ GLA 78-9-18 } \\
\hline \multicolumn{13}{|l|}{ GLA 78-10-14 } \\
\hline \multicolumn{13}{|l|}{ GLA 78-10-15 } \\
\hline GLA 78-10-16 & & & & & & & & & & & & \\
\hline
\end{tabular}

INEEL CVS 2a (Peeler et al. 1999)

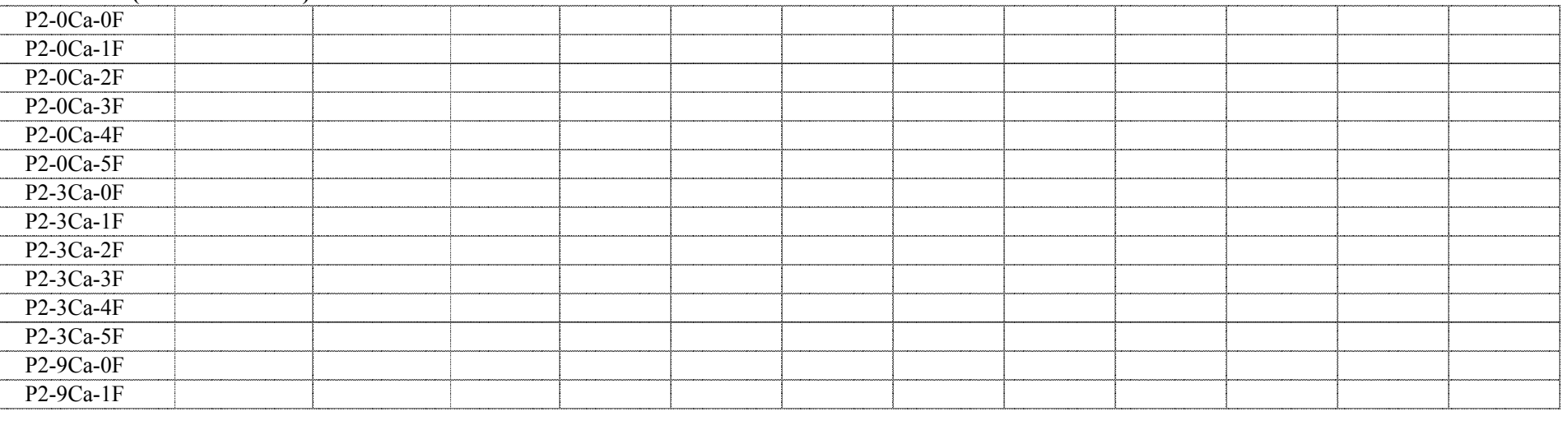


INEEL CVS 2a (Peeler et al. 1999)

\begin{tabular}{|c|c|c|c|c|c|c|c|c|c|c|c|c|c|c|c|c|c|c|c|c|c|}
\hline Glass ID & $\begin{array}{c}\mathrm{A} 12 \mathrm{O} 3 \\
-\mathrm{t}\end{array}$ & $\begin{array}{c}\mathrm{B} 2 \mathrm{O} 3 \\
-\mathrm{t}\end{array}$ & $\begin{array}{c}\mathrm{CaO} \\
-\mathrm{t}\end{array}$ & $\begin{array}{c}\mathrm{Fe} 2 \mathrm{O} 3 \\
-\mathrm{t}\end{array}$ & $\begin{array}{c}\mathrm{FeO} \\
-\mathrm{t}\end{array}$ & $\begin{array}{c}\mathrm{K} 2 \mathrm{O} \\
-\mathrm{t}\end{array}$ & $\begin{array}{c}\mathrm{Li} 2 \mathrm{O} \\
-\mathrm{t}\end{array}$ & $\begin{array}{c}\mathrm{MgO} \\
-\mathrm{t}\end{array}$ & $\begin{array}{c}\mathrm{Na} 2 \mathrm{O} \\
-\mathrm{t}\end{array}$ & $\begin{array}{c}\mathrm{NiO} \\
-\mathrm{t}\end{array}$ & $\begin{array}{c}\mathrm{P} 2 \mathrm{O} 5 \\
-\mathrm{t}\end{array}$ & $\begin{array}{c}\mathrm{SiO} 2 \\
-\mathrm{t}\end{array}$ & $\begin{array}{c}\mathrm{ZrO} 2 \\
-\mathrm{t}\end{array}$ & $\begin{array}{c}\mathrm{Ag} 2 \mathrm{O} \\
-\mathrm{t}\end{array}$ & $\begin{array}{c}\text { As2 } 203 \\
-t\end{array}$ & $\begin{array}{c}\mathrm{BaO} \\
-\mathrm{t}\end{array}$ & $\begin{array}{c}\mathrm{Bi} 2 \mathrm{O} 3 \\
-\mathrm{t}\end{array}$ & $\begin{array}{c}\mathrm{Br} \\
-\mathrm{t}\end{array}$ & $\begin{array}{c}\mathrm{CdO} \\
-\mathrm{t}\end{array}$ & $\begin{array}{c}\mathrm{Ce} 2 \mathrm{O} 3 \\
-\mathrm{t}\end{array}$ & $\begin{array}{c}\mathrm{CeO} 2 \\
-\mathrm{t}\end{array}$ \\
\hline P2-9Ca-2F & 0.0624 & 0.0886 & 0.0913 & 0.0197 & & 0.0012 & 0.0295 & 0.0010 & 0.1398 & 0.0002 & 0.0001 & 0.5043 & 0.0435 & & & & & & & & \\
\hline P2-9Ca-3F & 0.0620 & 0.0880 & 0.0906 & 0.0195 & & 0.0012 & 0.0294 & 0.0010 & 0.1388 & 0.0002 & 0.0001 & 0.5007 & 0.0432 & & & & & & & & \\
\hline P2-9Ca-4F & 0.0616 & 0.0875 & 0.0901 & 0.0195 & & 0.0012 & 0.0292 & 0.0010 & 0.1380 & 0.0002 & 0.0001 & 0.4977 & 0.0430 & & & & & & & & \\
\hline P2-9Ca-5F & 0.0616 & 0.0875 & 0.0900 & 0.0194 & & 0.0012 & 0.0291 & 0.0010 & 0.1380 & 0.0002 & 0.0001 & 0.4975 & 0.0430 & & & & & & & & \\
\hline
\end{tabular}

\section{SRL 165 Glasses (Pye 1985)}

\begin{tabular}{|l|l|l|l|l|l|l|l|l|l|l|l|l|l|l|l|l|l|l|}
\hline SRL 165 HiFe & 0.0177 & 0.0684 & 0.0113 & 0.1454 & & 0.0476 & 0.0074 & 0.1154 & 0.0183 & & 0.4991 & 0.0091 & \\
\hline SRL 165 TDS & 0.0418 & 0.0692 & 0.0153 & 0.1202 & 0.0479 & 0.0081 & 0.1049 & 0.0000 & & 0.5509 & 0.0122 & \\
\hline SRL 165 HiAl & 0.0928 & 0.0739 & 0.0114 & 0.0635 & & 0.0514 & 0.0080 & 0.1176 & 0.0068 & & 0.5429 & 0.0101 & \\
\hline
\end{tabular}

\section{RPP-WTP HLW Formulation (Kot and Pegg 2001)}

\begin{tabular}{|c|c|c|c|c|c|c|c|c|c|c|c|c|c|c|c|c|c|c|}
\hline HLW98-01 & 0.1071 & 0.1062 & 0.0097 & 0.1169 & 0.0043 & 0.0195 & 0.0097 & 0.1305 & 0.0043 & 0.0085 & 0.3213 & 0.0604 & 0.0019 & 0.0005 & 0.0043 & 0.0004 & 0.0063 & 0.0049 \\
\hline HLW98-02 & 0.1106 & 0.1096 & 0.0101 & 0.1207 & 0.0044 & 0.0201 & 0.0101 & 0.1347 & 0.0044 & 0.0087 & 0.3318 & 0.0302 & 0.0020 & 0.0005 & 0.0045 & 0.0004 & 0.0065 & 0.0051 \\
\hline HLW98-03 & 0.1179 & 0.1000 & 0.0142 & 0.0943 & 0.0024 & 0.0300 & 0.0094 & 0.1179 & 0.0024 & 0.0094 & 0.3943 & 0.0377 & 0.0009 & 0.0005 & 0.0024 & 0.0005 & 0.0024 & 0.0024 \\
\hline HLW98-04 & 0.1179 & 0.1300 & 0.0142 & 0.0943 & 0.0024 & 0.0000 & 0.0094 & 0.1179 & 0.0024 & 0.0094 & 0.3943 & 0.0377 & 0.0009 & 0.0005 & 0.0024 & 0.0005 & 0.0024 & 0.0024 \\
\hline HLW98-05 & 0.1146 & 0.1300 & 0.0137 & 0.1217 & 0.0023 & & 0.0092 & 0.1146 & 0.0023 & 0.0092 & 0.4117 & & 0.0009 & 0.0005 & 0.0023 & 0.0005 & 0.0046 & 0.0023 \\
\hline HLW98-06 & 0.0851 & 0.1000 & 0.0043 & 0.1276 & 0.0043 & 0.0300 & 0.0085 & 0.1106 & 0.0043 & 0.0064 & 0.4276 & 0.0213 & 0.0009 & 0.0009 & 0.0043 & 0.0004 & 0.0034 & 0.0013 \\
\hline HLW98-07 & 0.0882 & 0.1000 & 0.0044 & 0.1323 & 0.0044 & 0.0100 & 0.0088 & 0.1146 & 0.0044 & 0.0066 & 0.4323 & 0.0220 & 0.0009 & 0.0009 & 0.0044 & 0.0004 & 0.0035 & 0.0013 \\
\hline HLW98-08 & 0.0743 & 0.1200 & 0.0153 & 0.1226 & 0.0044 & 0.0300 & 0.0087 & 0.0918 & 0.0044 & 0.0066 & 0.4093 & 0.0219 & 0.0009 & 0.0009 & 0.0044 & 0.0004 & 0.0035 & 0.0013 \\
\hline HLW98-09 & 0.0797 & 0.1200 & 0.0040 & 0.1196 & 0.0040 & 0.0300 & 0.0080 & 0.0837 & 0.0040 & 0.0060 & 0.4496 & 0.0199 & 0.0008 & 0.0008 & 0.0040 & 0.0004 & 0.0032 & 0.0012 \\
\hline HLW98-10 & 0.0701 & 0.1200 & 0.0144 & 0.1157 & 0.0041 & 0.0300 & 0.0084 & 0.0867 & 0.0041 & 0.0062 & 0.4332 & 0.0206 & 0.0008 & 0.0008 & 0.0041 & 0.0004 & 0.0033 & 0.0012 \\
\hline HLW98-11 & 0.0729 & 0.1200 & 0.0150 & 0.1202 & 0.0043 & 0.0300 & 0.0086 & 0.0901 & 0.0043 & 0.0064 & 0.4173 & 0.0215 & 0.0009 & 0.0009 & 0.0043 & 0.0004 & 0.0034 & 0.0013 \\
\hline HLW98-12 & 0.0749 & 0.1500 & 0.0027 & 0.1267 & 0.0030 & 0.0700 & 0.0009 & 0.0293 & 0.0046 & 0.0059 & 0.4099 & 0.0388 & 0.0008 & 0.0006 & 0.0006 & & 0.0053 & 0.0012 \\
\hline HLW98-13 & 0.0666 & 0.1500 & 0.0024 & 0.1126 & 0.0026 & 0.0300 & 0.0008 & 0.1060 & 0.0041 & 0.0053 & 0.4088 & 0.0344 & 0.0007 & 0.0005 & 0.0005 & & 0.0047 & 0.0011 \\
\hline HLW98-14 & 0.0870 & 0.1200 & 0.0190 & 0.0876 & 0.0019 & 0.0300 & 0.0114 & 0.1404 & 0.0016 & 0.0065 & 0.4169 & 0.0031 & 0.0011 & & 0.0059 & 0.0006 & 0.0004 & \\
\hline HLW98-15 & 0.0870 & 0.1500 & 0.0190 & 0.0876 & 0.0019 & 0.0000 & 0.0114 & 0.1404 & 0.0016 & 0.0065 & 0.4169 & 0.0031 & 0.0011 & & 0.0059 & 0.0006 & 0.0004 & \\
\hline HLW98-16 & 0.0720 & 0.1200 & 0.0148 & 0.1187 & 0.0043 & 0.0171 & 0.0085 & 0.0890 & 0.0043 & 0.0064 & 0.4356 & 0.0212 & & 0.0009 & 0.0043 & 0.0004 & 0.0034 & 0.0013 \\
\hline HLW98-17 & 0.0703 & 0.1200 & 0.0145 & 0.1154 & 0.0042 & 0.0300 & 0.0083 & 0.0867 & 0.0042 & 0.0062 & 0.4323 & 0.0207 & & 0.0009 & 0.0042 & 0.0004 & 0.0033 & 0.0013 \\
\hline HLW98-18 & 0.0772 & 0.0500 & 0.0028 & 0.1296 & 0.0031 & 0.0700 & 0.0009 & 0.0302 & 0.0048 & 0.0061 & 0.5102 & 0.0400 & & 0.0006 & 0.0006 & & 0.0054 & 0.0012 \\
\hline HLW98-19 & 0.0915 & 0.1200 & 0.0200 & 0.0921 & 0.0020 & 0.0000 & 0.0120 & 0.1477 & 0.0017 & 0.0068 & 0.4256 & 0.0033 & 0.0012 & & 0.0062 & 0.0006 & 0.0004 & \\
\hline
\end{tabular}


INEEL CVS 2a (Peeler et al. 1999)

\begin{tabular}{|c|c|c|c|c|c|c|c|c|c|c|c|c|c|c|c|c|c|c|c|c|c|}
\hline Glass ID & $\begin{array}{l}\mathrm{Cl} \\
-\mathrm{t}\end{array}$ & $\begin{array}{c}\mathrm{CoO} \\
-\mathrm{t}\end{array}$ & $\mathrm{Co} 2 \mathrm{O} 3$ & $\begin{array}{c}\mathrm{Cr} 2 \mathrm{O} 3 \\
-\mathrm{t}\end{array}$ & $\begin{array}{c}\mathrm{Cs} 2 \mathrm{O} \\
-\mathrm{t}\end{array}$ & $\begin{array}{c}\mathrm{CuO} \\
-\mathrm{t}\end{array}$ & $\begin{array}{c}\mathrm{Eu} 2 \mathrm{O} 3 \\
-\mathrm{t}\end{array}$ & $\begin{array}{l}F \\
-t\end{array}$ & $\mathrm{Ga} 2 \mathrm{O} 3$ & $\begin{array}{c}\mathrm{Gd} 2 \mathrm{O} 3 \\
-\mathrm{t}\end{array}$ & $\begin{array}{c}\mathrm{HgO} \\
-\mathrm{t}\end{array}$ & $\begin{array}{l}I \\
-t\end{array}$ & $\begin{array}{c}\mathrm{La} 2 \mathrm{O} 3 \\
-\mathrm{t}\end{array}$ & $\begin{array}{c}\mathrm{MnO} 2 \\
-\mathrm{t}\end{array}$ & $\begin{array}{c}\mathrm{MnO} \\
-\mathrm{t}\end{array}$ & $\begin{array}{c}\mathrm{MoO} \\
-\mathrm{t}\end{array}$ & $\begin{array}{c}\mathrm{MoO} 3 \\
-\mathrm{t}\end{array}$ & $\begin{array}{c}\mathrm{Nb} 2 \mathrm{O} 5 \\
-\mathrm{t}\end{array}$ & $\begin{array}{c}\mathrm{Nd} 2 \mathrm{O} 3 \\
-\mathrm{t}\end{array}$ & $\begin{array}{c}\mathrm{PbO} \\
-\mathrm{t}\end{array}$ & $\begin{array}{c}\mathrm{PdO} 2 \\
-\mathrm{t}\end{array}$ \\
\hline P2-9Ca-2F & 0.0002 & & & 0.0008 & & & & 0.0157 & & & & & & & & & & & 0.0001 & & \\
\hline P2-9Ca-3F & 0.0002 & & & 0.0008 & & & & 0.0225 & & & & & & & & & & & 0.0001 & & \\
\hline P2-9Ca-4F & 0.0002 & & & 0.0008 & & & & 0.0282 & & & & & & & & & & & 0.0001 & & \\
\hline P2-9Ca-5F & 0.0002 & & & 0.0008 & & & & 0.0286 & & & & & & & & & & & 0.0001 & & \\
\hline
\end{tabular}

\section{SRL 165 Glasses (Pye 1985)}

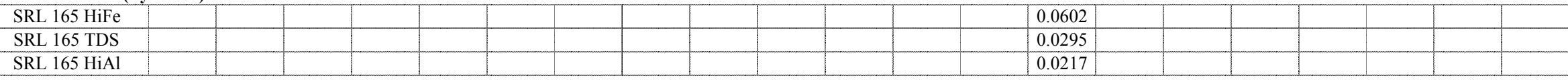

RPP-WTP HLW Formulation (Kot and Pegg 2001)

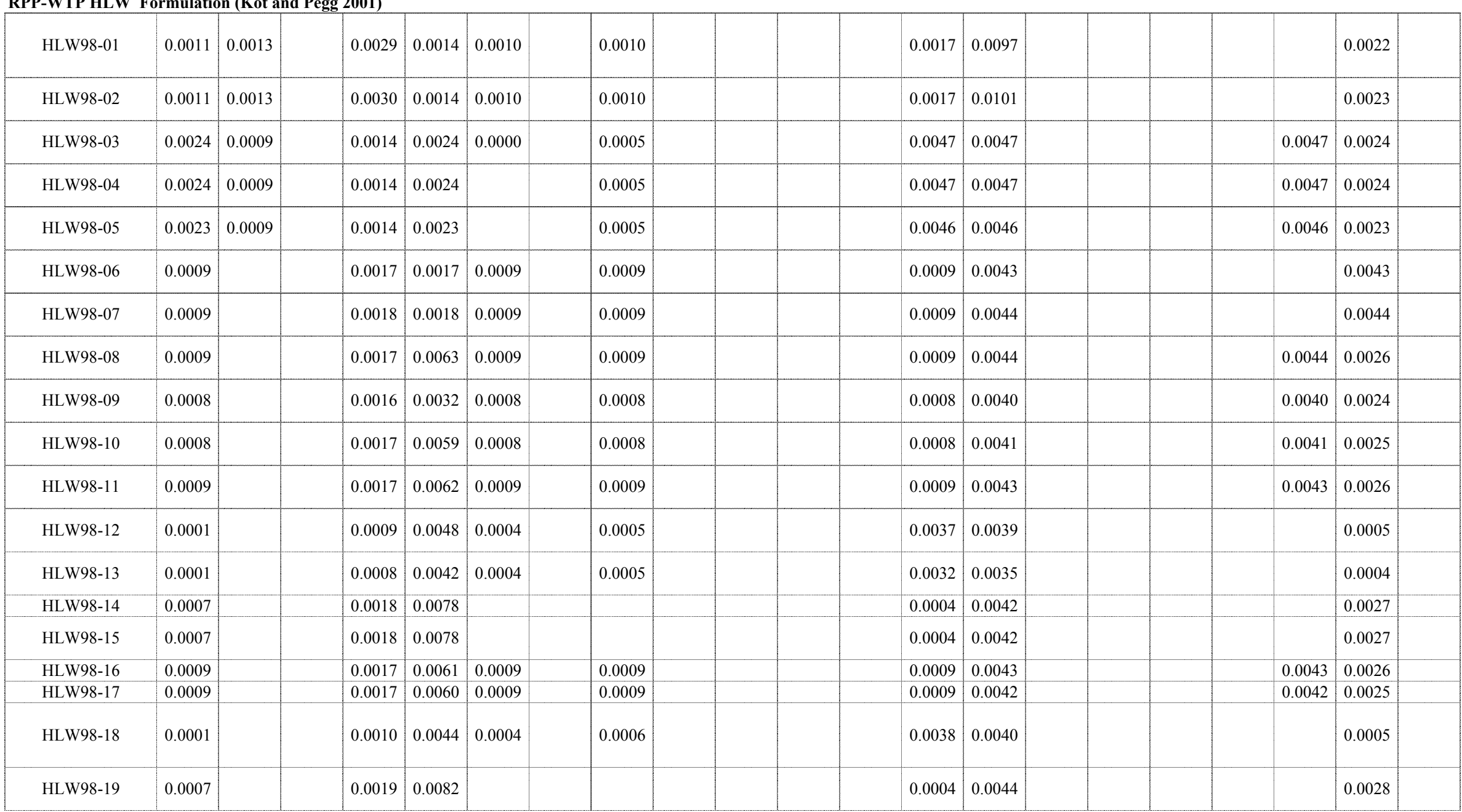


INEEL CVS 2a (Peeler et al. 1999)

\begin{tabular}{|c|c|c|c|c|c|c|c|c|c|c|c|c|c|c|c|c|c|c|c|c|c|}
\hline Glass ID & $\begin{array}{c}\mathrm{PdO} \\
-t\end{array}$ & $\begin{array}{c}\mathrm{Pr} 2 \mathrm{O} 3 \\
-\mathrm{t}\end{array}$ & Pr6O11 & $\begin{array}{c}\mathrm{Rb} 2 \mathrm{O} \\
-\mathrm{t}\end{array}$ & $\begin{array}{c}\mathrm{ReO} \\
-\mathrm{t}\end{array}$ & $\mathrm{ReO}_{-t}$ & $\begin{array}{c}\mathrm{Rh} 2 \mathrm{O} 3 \\
-\mathrm{t}\end{array}$ & $\mathrm{RhO}_{-\mathrm{t}}$ & $\mathrm{RuO}_{-t}$ & $\begin{array}{c}\mathrm{Sb} 2 \mathrm{O} 3 \\
-t\end{array}$ & $\begin{array}{c}\mathrm{Sb} 2 \mathrm{O} 5 \\
-\mathrm{t}\end{array}$ & $\mathrm{SeO} 2$ & $\mathrm{Sm} 2 \mathrm{O} 3$ & $\begin{array}{c}\mathrm{SnO} \\
-t\end{array}$ & $\begin{array}{c}\mathrm{SnO} 2 \\
-t\end{array}$ & $\begin{array}{c}\mathrm{SO} 3 \\
-t\end{array}$ & $\begin{array}{c}\mathrm{SrO} \\
-\mathrm{t}\end{array}$ & $\begin{array}{c}\mathrm{Tc} 2 \mathrm{O} 7 \\
-1\end{array}$ & $\mathrm{TeO}_{-t}$ & $\mathrm{ThO}_{-1}$ & $\mathrm{TiO}_{-t}$ \\
\hline P2-9Ca-2F & & & & & & & & & & & & & & & 0.0006 & 0.0002 & 0.0008 & & & & \\
\hline P2-9Ca-3F & & & & & & & & & & & & & & & 0.0006 & 0.0002 & 0.0008 & & & & \\
\hline P2-9Ca-4F & & & & & & & & & & & & & & & 0.0006 & 0.0002 & 0.0008 & & & & \\
\hline P2-9Ca-5F & & & & & & & & & & & & & & & 0.0006 & 0.0002 & 0.0008 & & & & \\
\hline
\end{tabular}

\section{SRL 165 Glasses (Pye 1985)}

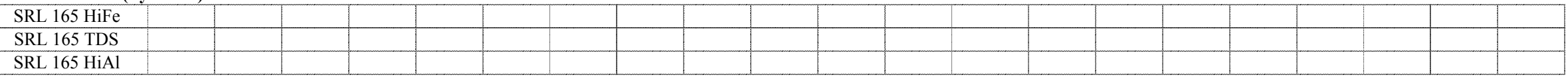

RPP-WTP HLW Formulation (Kot and Pegg 2001)

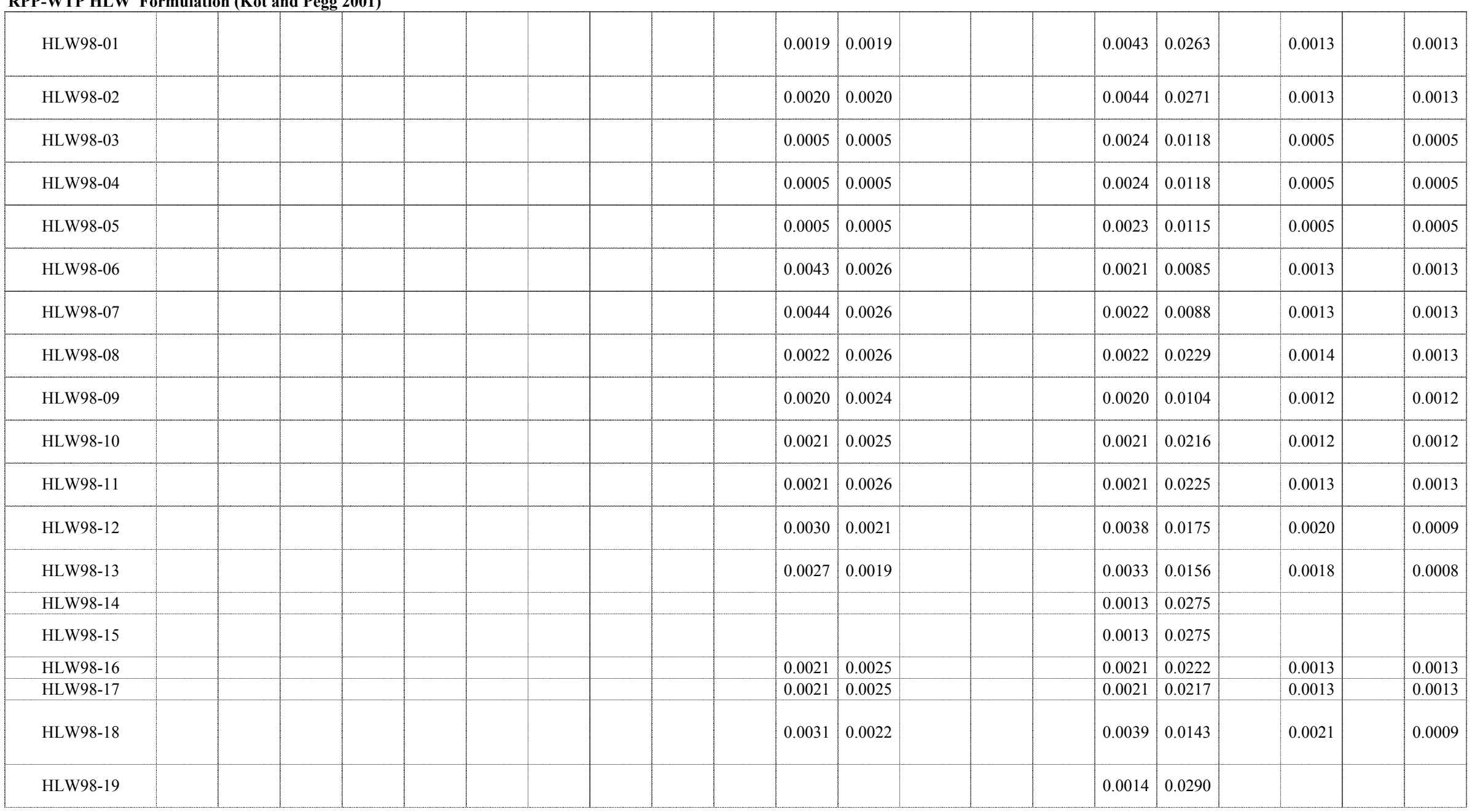


INEEL CVS 2a (Peeler et al. 1999)

\begin{tabular}{|c|c|c|c|c|c|c|c|c|c|c|c|c|c|c|c|c|c|c|c|c|c|}
\hline Glass ID & $\begin{array}{c}\mathrm{T} 12 \mathrm{O} 3 \\
-\mathrm{t}\end{array}$ & $\begin{array}{c}\mathrm{U} 3 \mathrm{O} 8 \\
-\mathrm{t}\end{array}$ & $\begin{array}{c}\mathrm{UO} 2 \\
-\mathrm{t}\end{array}$ & $\begin{array}{c}\text { UO3 } \\
-t\end{array}$ & $\begin{array}{c}\mathrm{V} 2 \mathrm{O} 5 \\
-\mathrm{t}\end{array}$ & $\begin{array}{c}\text { WO3 } \\
-t\end{array}$ & $\begin{array}{c}\mathrm{Y} 2 \mathrm{O} 3 \\
-\mathrm{t}\end{array}$ & $\begin{array}{c}\mathrm{ZnO} \\
-\mathrm{t}\end{array}$ & $\begin{array}{c}\text { Others } \\
-t\end{array}$ & $\begin{array}{c}\text { Sum } \\
-t\end{array}$ & $\begin{array}{c}\mathrm{A} 12 \mathrm{O} 3 \\
-\mathrm{a}\end{array}$ & $\begin{array}{c}\mathrm{B} 2 \mathrm{O} 3 \\
-\mathrm{a}\end{array}$ & $\begin{array}{c}\mathrm{CaO} \\
-\mathrm{a}\end{array}$ & $\begin{array}{c}\mathrm{Fe} 2 \mathrm{O} 3 \\
-\mathrm{a}\end{array}$ & $\begin{array}{c}\mathrm{FeO} \\
-\mathrm{a}\end{array}$ & $\begin{array}{c}\mathrm{K} 2 \mathrm{O} \\
-\mathrm{a}\end{array}$ & $\begin{array}{c}\mathrm{Li} 2 \mathrm{O} \\
-\mathrm{a}\end{array}$ & $\begin{array}{c}\mathrm{MgO} \\
-\mathrm{a}\end{array}$ & $\begin{array}{c}\mathrm{Na} 2 \mathrm{O} \\
-\mathrm{a}\end{array}$ & $\begin{array}{c}\mathrm{NiO} \\
-\mathrm{a}\end{array}$ & $\begin{array}{c}\mathrm{P} 2 \mathrm{O} 5 \\
-\mathrm{a}\end{array}$ \\
\hline P2-9Ca-2F & & & & & & & & & & 1.0000 & & & & & & & & & & & \\
\hline P2-9Ca-3F & & & & & & & & & & 1.0000 & & & & & & & & & & & \\
\hline P2-9Ca-4F & & & & & & & & & & 1.0000 & & & & & & & & & & & \\
\hline P2-9Ca-5F & & & & & & & & & & 1.0000 & & & & & & & & & & & \\
\hline
\end{tabular}

\section{SRL 165 Glasses (Pye 1985)}

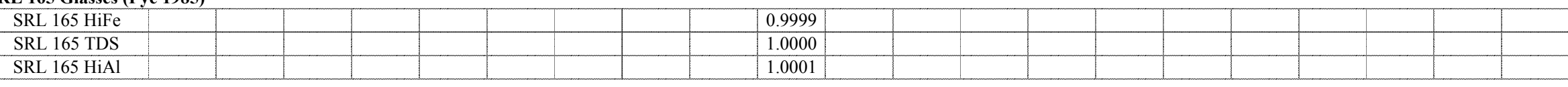

\section{RPP-WTP HLW Formulation (Kot and Pegg 2001)}

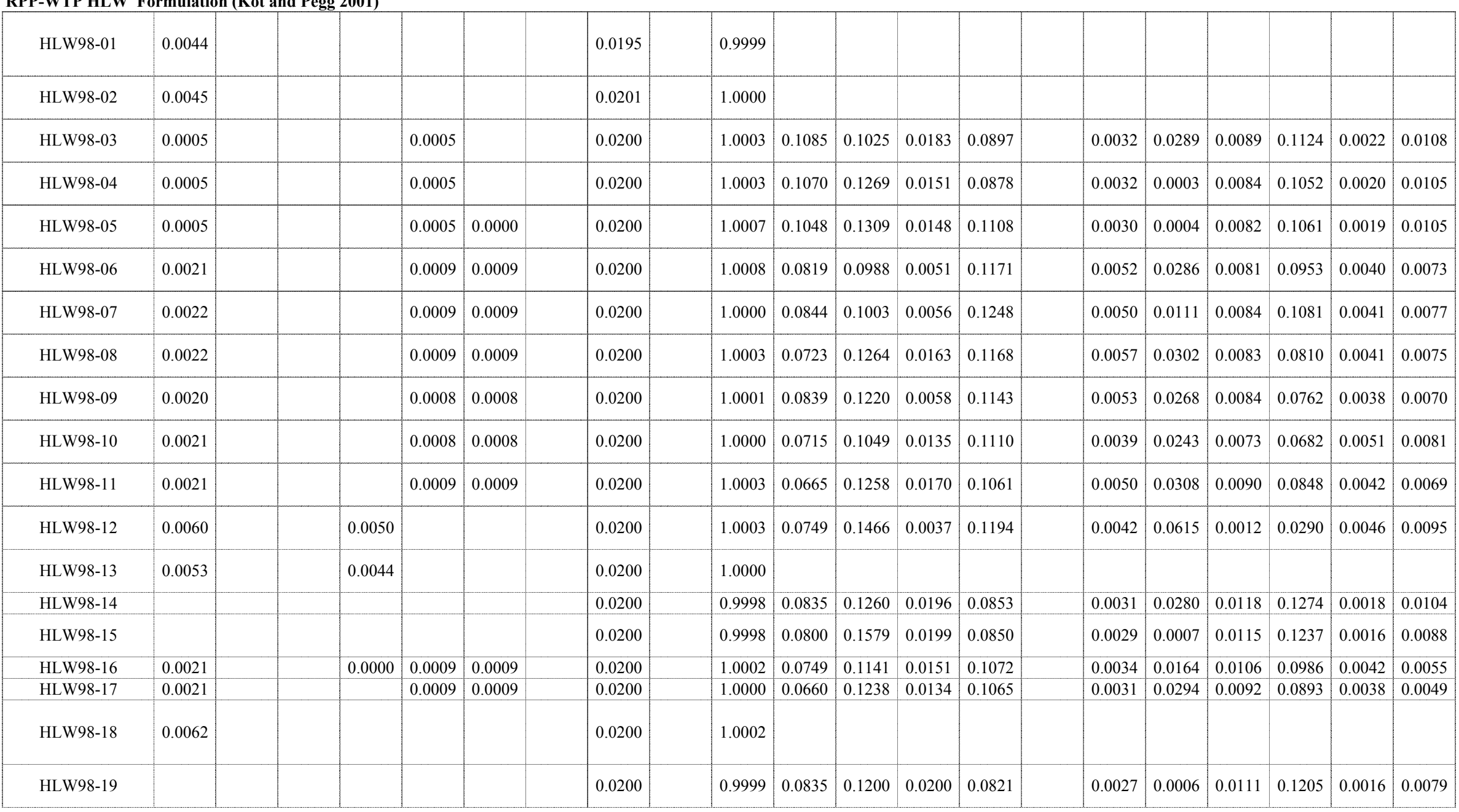


INEEL CVS 2a (Peeler et al. 1999)

\begin{tabular}{|c|c|c|c|c|c|c|c|c|c|c|c|c|c|c|c|c|c|c|c|c|c|}
\hline Glass ID & $\begin{array}{c}\mathrm{SiO} 2 \\
-\mathrm{a}\end{array}$ & $\begin{array}{c}\mathrm{ZrO} 2 \\
-\mathrm{a}\end{array}$ & $\begin{array}{c}\mathrm{Ag} 2 \mathrm{O} \\
-\mathrm{a}\end{array}$ & $\begin{array}{c}\mathrm{As} 2 \mathrm{O} 3 \\
-\mathrm{a}\end{array}$ & $\begin{array}{c}\mathrm{BaO} \\
-\mathrm{a}\end{array}$ & $\begin{array}{c}\mathrm{Bi} 2 \mathrm{O} 3 \\
-\mathrm{a}\end{array}$ & $\begin{array}{l}\mathrm{Br} \\
-\mathrm{a}\end{array}$ & $\begin{array}{c}\mathrm{CdO} \\
-\mathrm{a}\end{array}$ & $\begin{array}{c}\mathrm{Ce} 2 \mathrm{O} 3 \\
-\mathrm{a}\end{array}$ & $\begin{array}{c}\mathrm{CeO} 2 \\
-\mathrm{a}\end{array}$ & $\begin{array}{l}\mathrm{Cl} \\
-\mathrm{a}\end{array}$ & $\begin{array}{c}\mathrm{CoO} \\
-\mathrm{a}\end{array}$ & $\begin{array}{c}\mathrm{Co} 2 \mathrm{O} 3 \\
-\mathrm{a}\end{array}$ & $\begin{array}{c}\mathrm{Cr} 2 \mathrm{O} 3 \\
-\mathrm{a}\end{array}$ & $\begin{array}{c}\mathrm{Cs} 2 \mathrm{O} \\
-\mathrm{a}\end{array}$ & $\begin{array}{c}\mathrm{CuO} \\
-\mathrm{a}\end{array}$ & $\begin{array}{c}\mathrm{Eu} 2 \mathrm{O} 3 \\
-\mathrm{a}\end{array}$ & $\begin{array}{c}F \\
-a\end{array}$ & $\begin{array}{c}\mathrm{Ga} 2 \mathrm{O} 3 \\
-\mathrm{a}\end{array}$ & $\begin{array}{c}\mathrm{Gd} 2 \mathrm{O} 3 \\
-\mathrm{a}\end{array}$ & $\begin{array}{c}\mathrm{HgO} \\
-\mathrm{a}\end{array}$ \\
\hline $\mathrm{P} 2-9 \mathrm{Ca}-2 \mathrm{~F}$ & & & & & & & & & & & & & & & & & & & & & \\
\hline $\mathrm{P} 2-9 \mathrm{Ca}-3 \mathrm{~F}$ & & & & & & & & & & & & & & & & & & & & & \\
\hline P2-9Ca-4F & & & & & & & & & & & & & & & & & & & & & \\
\hline P2-9Ca-5F & & & & & & & & & & & & & & & & & & & & & \\
\hline
\end{tabular}

\section{SRL 165 Glasses (Pye 1985)}

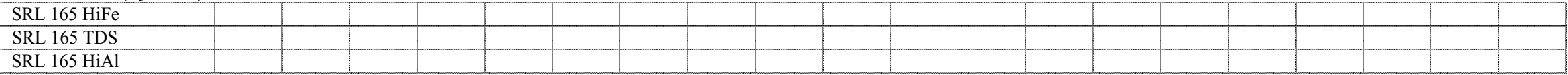

\section{RPP-WTP HLW Formulation (Kot and Pegg 2001)}

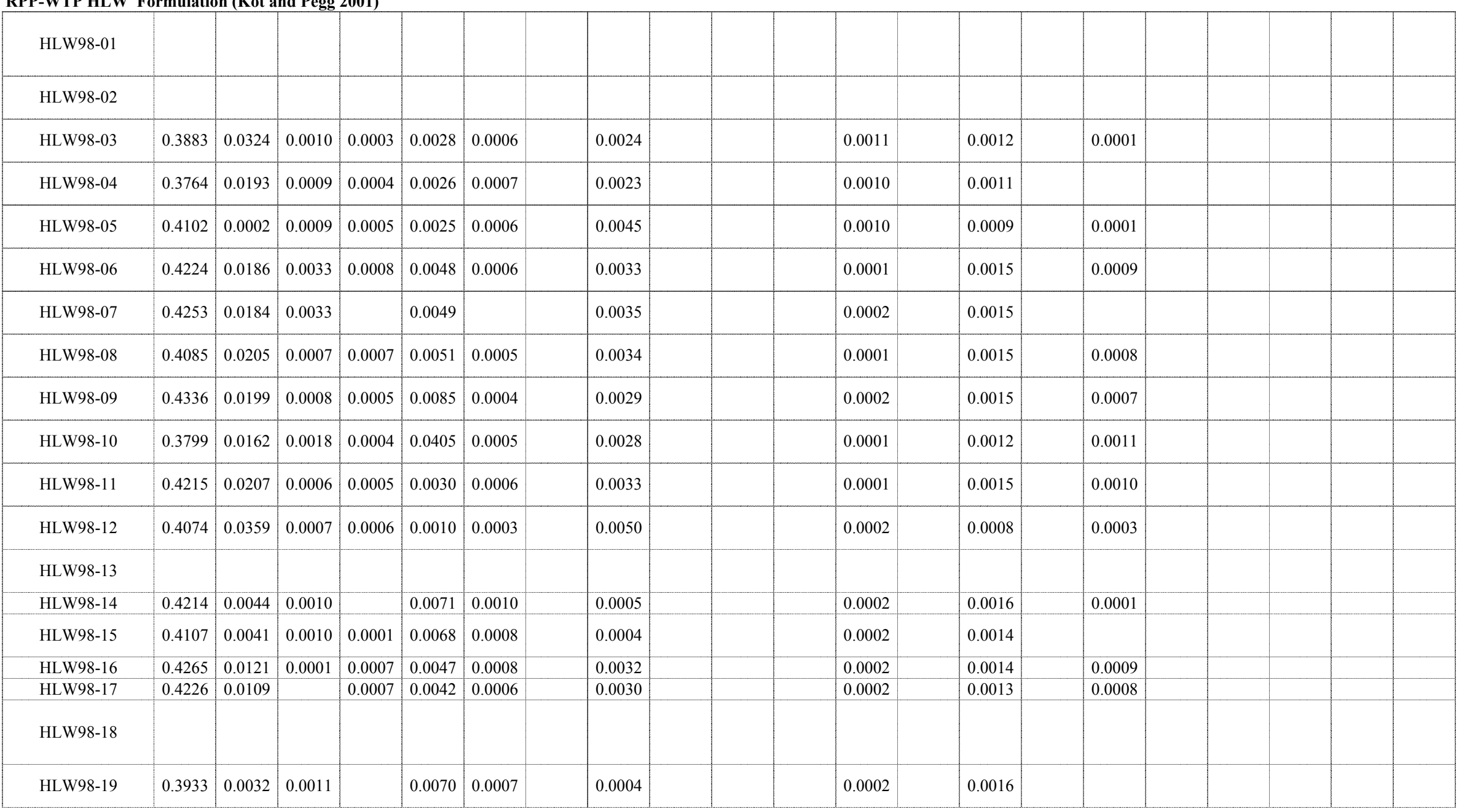


INEEL CVS 2a (Peeler et al. 1999)

\begin{tabular}{|c|c|c|c|c|c|c|c|c|c|c|c|c|c|c|c|c|c|c|c|c|c|}
\hline Glass ID & $\begin{array}{c}\mathrm{I} \\
-\mathrm{a}\end{array}$ & $\begin{array}{c}\mathrm{La} 2 \mathrm{O} 3 \\
-\mathrm{a}\end{array}$ & $\begin{array}{c}\mathrm{MnO} 2 \\
-\mathrm{a}\end{array}$ & $\begin{array}{c}\mathrm{MnO} \\
-\mathrm{a}\end{array}$ & $\begin{array}{c}\mathrm{MoO} \\
-\mathrm{a}\end{array}$ & $\begin{array}{c}\mathrm{MoO} 3 \\
-\mathrm{a}\end{array}$ & $\begin{array}{c}\mathrm{Nb} 2 \mathrm{O} 5 \\
-\mathrm{a}\end{array}$ & $\begin{array}{c}\mathrm{Nd} 2 \mathrm{O} 3 \\
-\mathrm{a}\end{array}$ & $\begin{array}{c}\mathrm{PbO} \\
-\mathrm{a}\end{array}$ & $\begin{array}{c}\mathrm{PdO} 2 \\
-\mathrm{a}\end{array}$ & $\begin{array}{c}\mathrm{PdO} \\
-\mathrm{a}\end{array}$ & $\begin{array}{c}\mathrm{Pr} 2 \mathrm{O} 3 \\
-\mathrm{a}\end{array}$ & $\begin{array}{c}\text { Pr6O11 } \\
-\mathrm{a}\end{array}$ & $\begin{array}{c}\mathrm{Rb} 2 \mathrm{O} \\
-\mathrm{a}\end{array}$ & $\begin{array}{c}\mathrm{ReO} \\
-\mathrm{a}\end{array}$ & $\begin{array}{c}\mathrm{ReO} 2 \\
-\mathrm{a}\end{array}$ & $\begin{array}{c}\mathrm{Rh} 2 \mathrm{O} 3 \\
-\mathrm{a}\end{array}$ & $\begin{array}{c}\mathrm{RhO} 2 \\
-\mathrm{a}\end{array}$ & $\begin{array}{c}\mathrm{RuO} 2 \\
-\mathrm{a}\end{array}$ & $\begin{array}{c}\mathrm{Sb} 2 \mathrm{O} 3 \\
-\mathrm{a}\end{array}$ & $\begin{array}{c}\mathrm{Sb} 2 \mathrm{O} 5 \\
-\mathrm{a}\end{array}$ \\
\hline $\mathrm{P} 2-9 \mathrm{Ca}-2 \mathrm{~F}$ & & & & & & & & & & & & & & & & & & & & & \\
\hline P2-9Ca-3F & & & & & & & & & & & & & & & & & & & & & \\
\hline P2-9Ca-4F & & & & & & & & & & & & & & & & & & & & & \\
\hline P2-9Ca-5F & & & & & & & & & & & & & & & & & & & & & \\
\hline
\end{tabular}

\section{SRL 165 Glasses (Pye 1985)}

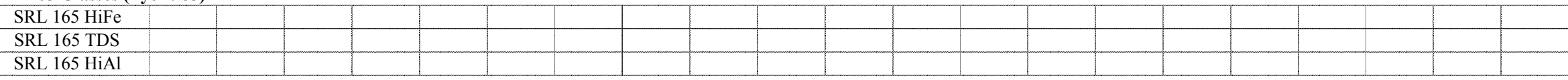

RPP-WTP HLW Formulation (Kot and Pegg 2001)

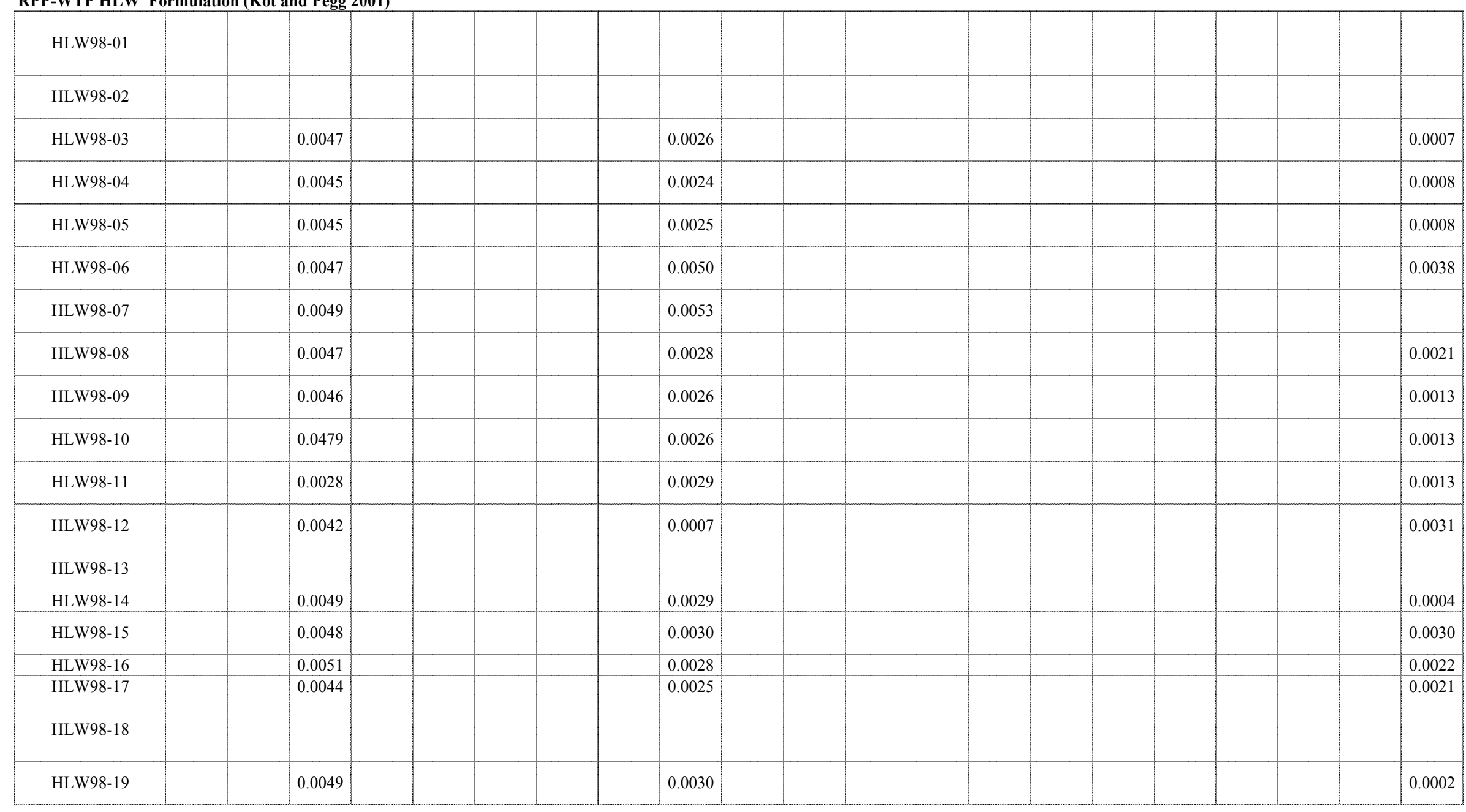


Appendix A. Database - mass fraction

INEEL CVS 2a (Peeler et al. 1999)

\begin{tabular}{|c|c|c|c|c|c|c|c|c|c|c|c|c|c|c|c|c|c|c|c|c|}
\hline Glass ID & $\begin{array}{c}\mathrm{SeO} 2 \\
-\mathrm{a}\end{array}$ & $\begin{array}{c}\mathrm{Sm} 2 \mathrm{O} 3 \\
-\mathrm{a}\end{array}$ & $\begin{array}{c}\mathrm{SnO} \\
-\mathrm{a}\end{array}$ & $\begin{array}{c}\mathrm{SnO} 2 \\
-\mathrm{a}\end{array}$ & $\begin{array}{c}\mathrm{SO} 3 \\
-\mathrm{a}\end{array}$ & $\begin{array}{c}\mathrm{SrO} \\
-\mathrm{a}\end{array}$ & $\begin{array}{c}\mathrm{Tc} 2 \mathrm{O} 7 \\
-\mathrm{a}\end{array}$ & $\begin{array}{c}\mathrm{TeO} 2 \\
-\mathrm{a}\end{array}$ & $\begin{array}{c}\mathrm{ThO} 2 \\
-\mathrm{a}\end{array}$ & $\begin{array}{c}\mathrm{TiO} 2 \\
-\mathrm{a}\end{array}$ & $\begin{array}{c}\mathrm{T} 12 \mathrm{O} 3 \\
-\mathrm{a}\end{array}$ & $\begin{array}{c}\text { U3O8 } \\
-\mathrm{a}\end{array}$ & $\begin{array}{c}\mathrm{UO} 2 \\
-\mathrm{a}\end{array}$ & $\begin{array}{c}\text { UO3 } \\
-\mathrm{a}\end{array}$ & $\begin{array}{c}\mathrm{V} 2 \mathrm{O} 5 \\
-\mathrm{a}\end{array}$ & $\begin{array}{c}\mathrm{WO} 3 \\
-\mathrm{a}\end{array}$ & $\begin{array}{c}\mathrm{Y} 2 \mathrm{O} 3 \\
-\mathrm{a}\end{array}$ & $\begin{array}{c}\mathrm{ZnO} \\
-\mathrm{a}\end{array}$ & $\begin{array}{c}\text { Others } \\
-\mathrm{a}\end{array}$ & $\begin{array}{c}\text { Sum } \\
-\mathrm{a}\end{array}$ \\
\hline P2-9Ca-2F & & & & & & & & & & & & & & & & & & & & \\
\hline P2-9Ca-3F & & & & & & & & & & & & & & & & & & & & \\
\hline P2-9Ca-4F & & & & & & & & & & & & & & & & & & & & \\
\hline P2-9Ca-5F & & & & & & & & & & & & & & & & & & & & \\
\hline
\end{tabular}

\section{SRL 165 Glasses (Pye 1985)}

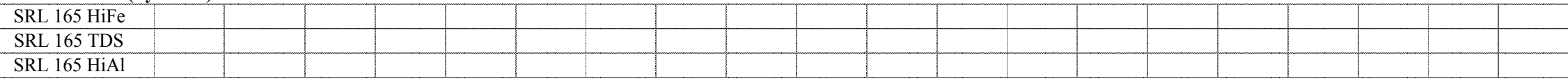

RPP-WTP HLW Formulation (Kot and Pegg 2001)

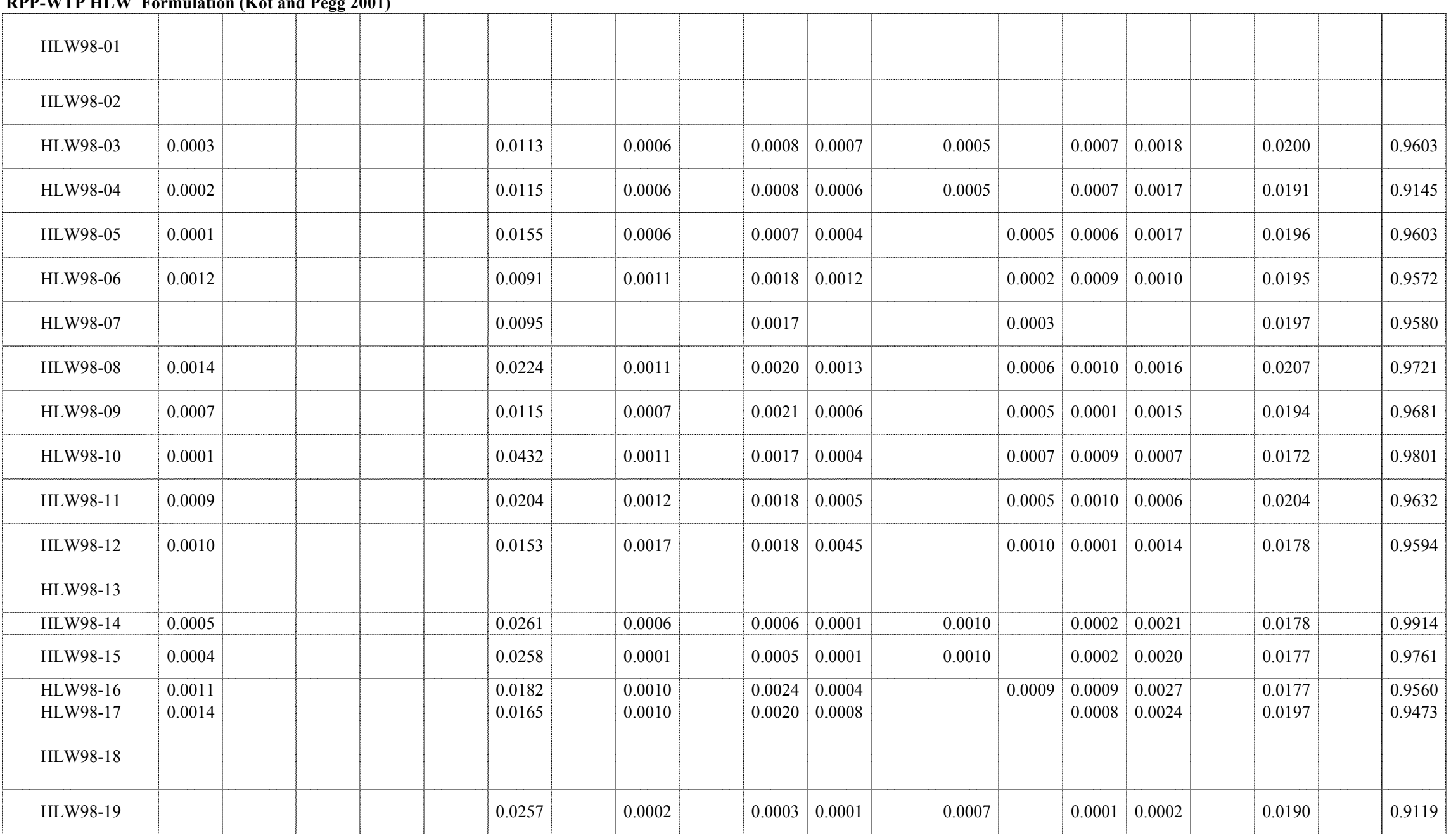


Appendix A. Database - mass fraction

INEEL CVS 2a (Peeler et al. 1999)

\begin{tabular}{|c|c|c|c|c|c|c|}
\hline Glass ID & $\begin{array}{c}\text { TM } \\
\left({ }^{\circ} \mathrm{C}\right)\end{array}$ & $\begin{array}{c}\text { Gradient } \\
\mathrm{TL}\left({ }^{\circ} \mathrm{C}\right)\end{array}$ & $\begin{array}{c}\text { Uniform } \\
\mathrm{TL}\left({ }^{\circ} \mathrm{C}\right)\end{array}$ & Primary Phase & $\begin{array}{c}\text { Quenched } \\
\text { Visual/OM }\end{array}$ & $\begin{array}{c}\text { Quenched } \\
\text { SEM/EDS or TEM }\end{array}$ \\
\hline P2-9Ca-2F & & & & & & \\
\hline P2-9Ca-3F & & & & & & \\
\hline P2-9Ca-4F & & & & & \\
\hline P2-9Ca-5F & & & & & \\
\hline
\end{tabular}

\section{SRL 165 Glasses (Pye 1985)}

\begin{tabular}{|l|l|l|l|l|l|l|}
\hline SRL 165 HiFe & & & & \\
\hline SRL 165 TDS & & & & & \\
\hline SRL 165 HiAl & & & & & \\
\hline
\end{tabular}

RPP-WTP HLW Formulation (Kot and Pegg 2001)

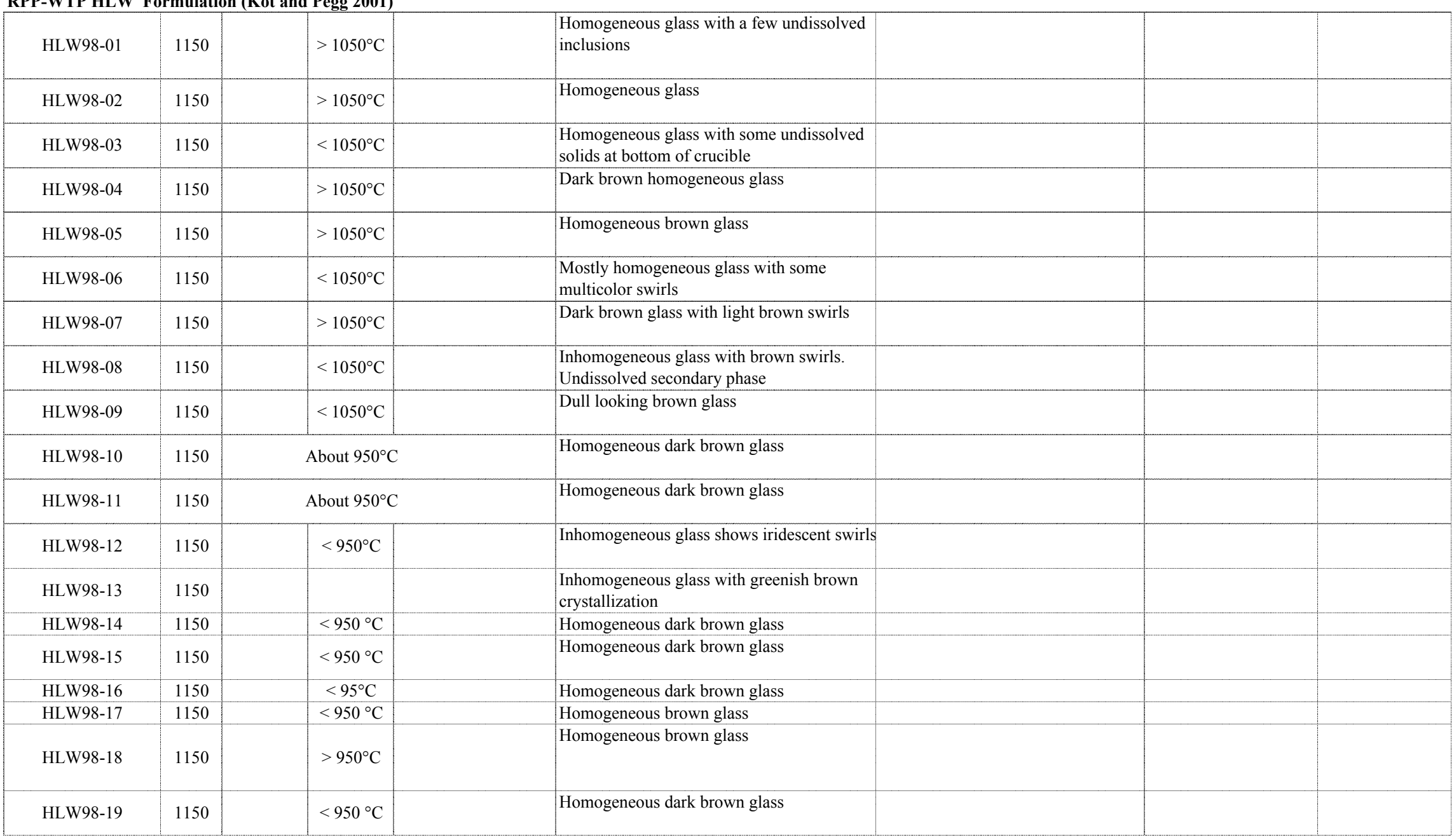


Appendix A. Database - mass fraction

INEEL CVS 2a (Peeler et al. 1999)

\begin{tabular}{|c|c|c|c|c|c|}
\hline Glass ID & $\begin{array}{c}\mathrm{CCC} \\
\text { Visual/OM }\end{array}$ & $\begin{array}{c}\text { CCC } \\
\text { SEM/EDS or TEM }\end{array}$ & $\begin{array}{l}\text { CCC } \\
\text { XRD }\end{array}$ & $\begin{array}{c}\text { Heat Treated } \\
\text { Visual/OM }\end{array}$ & $\begin{array}{c}\text { Heat Treated } \\
\text { SEM/EDS or TEM }\end{array}$ \\
\hline \multicolumn{6}{|l|}{ P2-9Ca-2F } \\
\hline $\mathrm{P} 2-9 \mathrm{Ca}-3 \mathrm{~F}$ & & & & & \\
\hline P2-9Ca-4F & & & & & \\
\hline P2-9Ca-5F & & & & & \\
\hline
\end{tabular}

SRL 165 Glasses (Pye 1985)

SRL $165 \mathrm{HiFe}$

SRL 165 TDS

SRL $165 \mathrm{HiAl}$

RPP-WTP HLW Formulation (Kot and Pegg 2001)

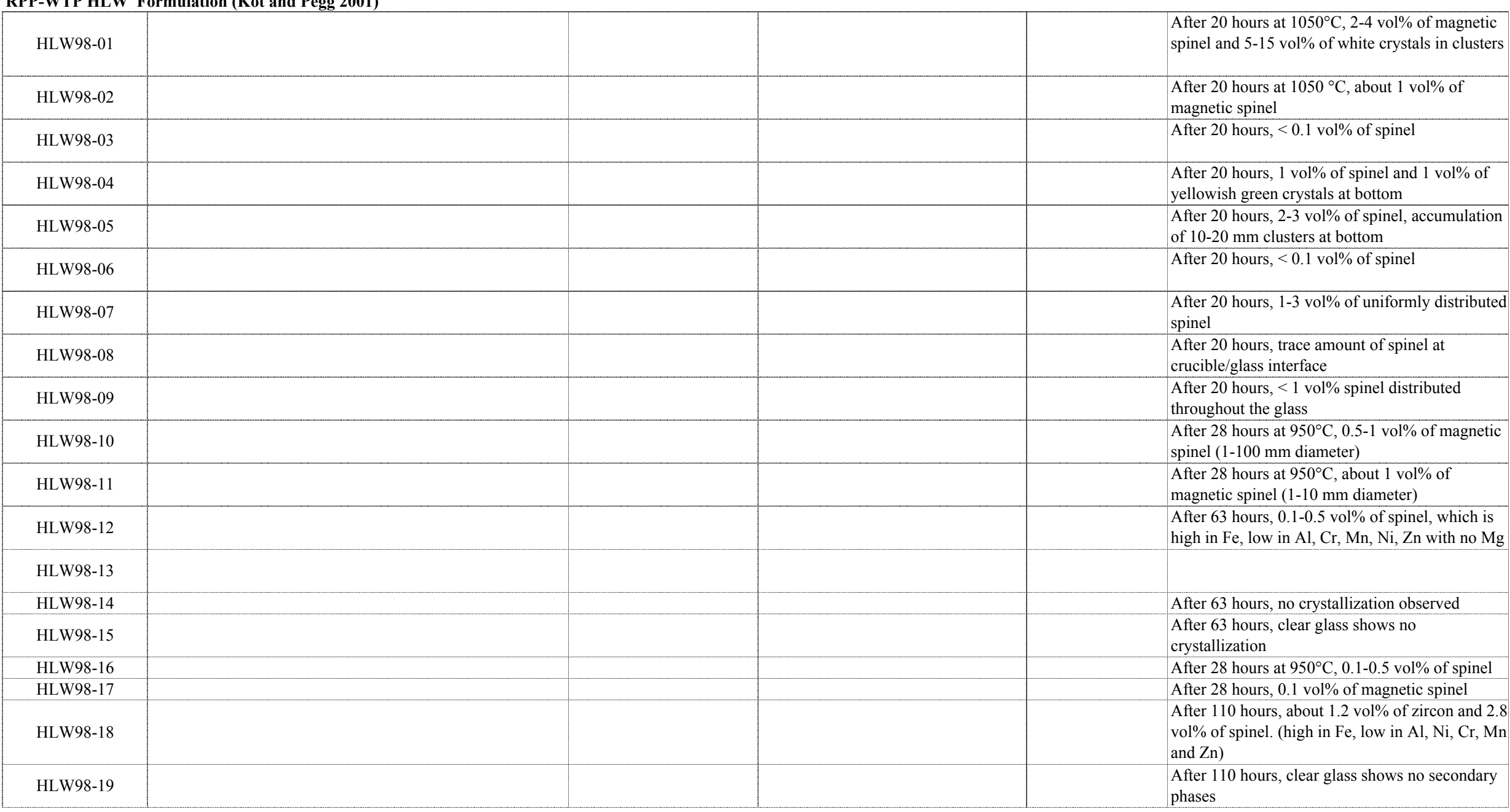


Appendix A. Database - mass fraction

INEEL CVS 2a (Peeler et al. 1999)

\begin{tabular}{|c|c|c|c|c|c|c|c|c|c|c|c|c|c|c|c|}
\hline Glass ID & $\begin{array}{c}\text { Heat Treated } \\
\text { XRD }\end{array}$ & $\begin{array}{l}\text { Density } \\
\left(\mathrm{g} / \mathrm{cm}^{3}\right)\end{array}$ & $\begin{array}{c}\text { Fulc Visc } \\
\text { A }\end{array}$ & $\begin{array}{c}\text { Fulc Visc } \\
\text { B }\end{array}$ & \begin{tabular}{|c|} 
Fulc Visc \\
To
\end{tabular} & $\begin{array}{c}\mathrm{FV} 1150^{\circ} \mathrm{C} \\
(\mathrm{Pa} \cdot \mathrm{s})\end{array}$ & $\begin{array}{c}\text { Arrh Visc } \\
\text { A }\end{array}$ & $\begin{array}{c}\text { Arrh Visc } \\
\text { B }\end{array}$ & $1150^{\circ} \mathrm{C}$ & $\begin{array}{c}\mathrm{T}\left({ }^{\circ} \mathrm{C}\right) \text { at } \\
2 \mathrm{~Pa} \cdot \mathrm{s}\end{array}$ & $\begin{array}{c}\mathrm{T}\left({ }^{\circ} \mathrm{C}\right) \text { at } \\
5 \mathrm{~Pa} \cdot \mathrm{s}\end{array}$ & \begin{tabular}{c|}
$\mathrm{T}\left({ }^{\circ} \mathrm{C}\right)$ at \\
$10 \mathrm{~Pa} \cdot \mathrm{s}$
\end{tabular} & $\begin{array}{l}\mathrm{T} 1 \\
\left({ }^{\circ} \mathrm{C}\right)\end{array}$ & $\begin{array}{c}\mathrm{V} 1 \\
(\mathrm{~Pa} \cdot \mathrm{s})\end{array}$ & $\begin{array}{c}\mathrm{T} 2 \\
\left({ }^{\circ} \mathrm{C}\right)\end{array}$ \\
\hline P2-9Ca-2F & & & & & & & & & & & & & & & \\
\hline P2-9Ca-3F & & & & & & & & & & & & & & & \\
\hline P2-9Ca-4F & & & & & & & & & & & & & & & \\
\hline P2-9Ca-5F & & & & & & & & & & & & & & & \\
\hline
\end{tabular}

SRL 165 Glasses (Pye 1985)

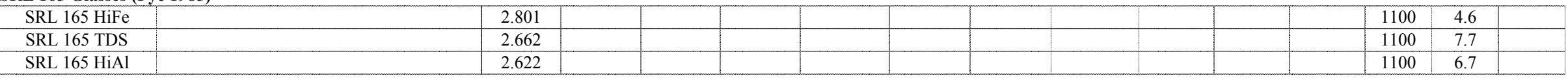

RPP-WTP HLW Formulation (Kot and Pegg 2001)

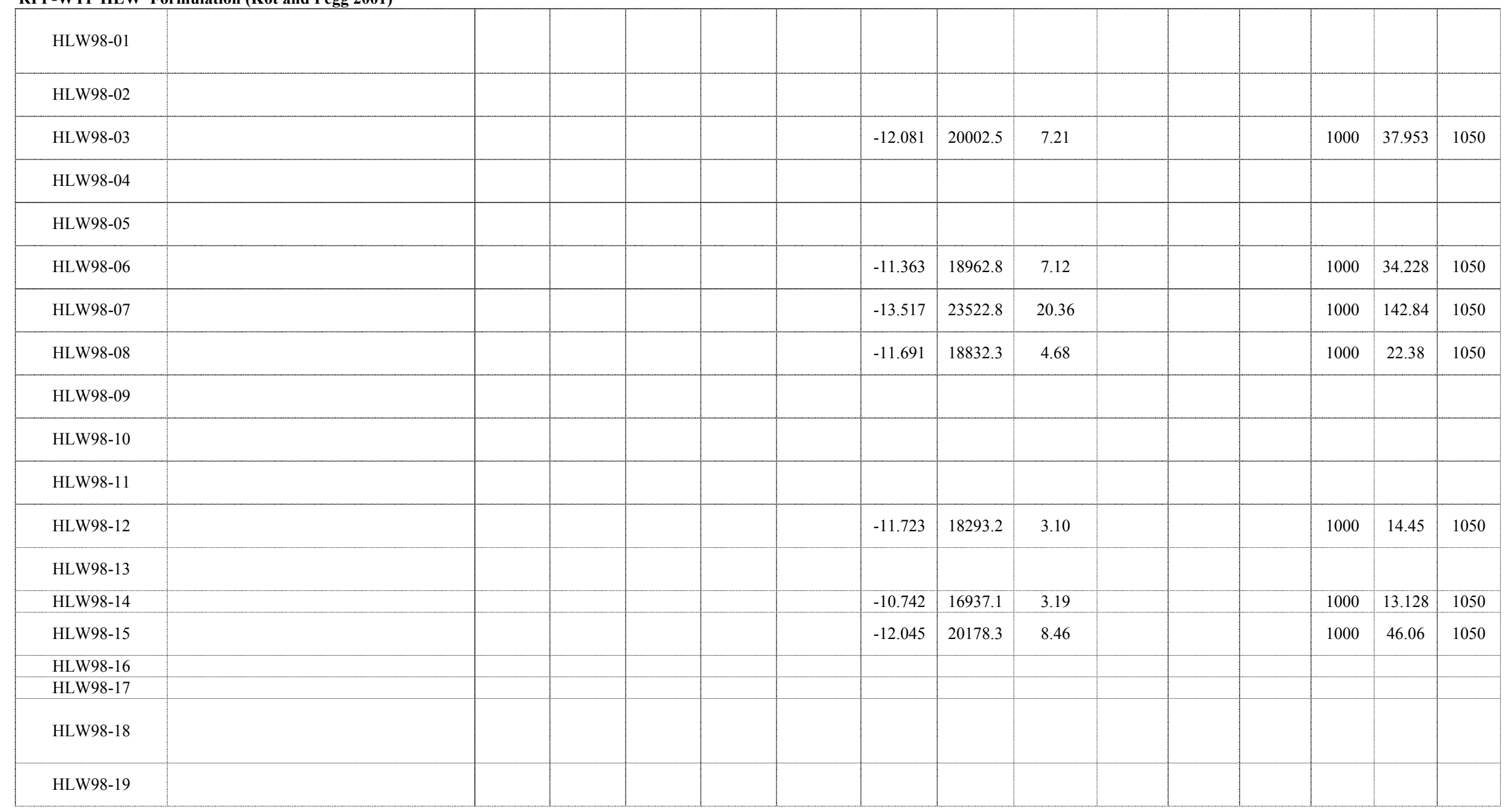


Appendix A. Database - mass fraction

\begin{tabular}{|c|c|c|c|c|c|c|c|c|c|c|c|c|c|c|c|c|c|c|c|c|c|}
\hline \multicolumn{22}{|c|}{ INEEL CVS 2a (Peeler et al. 1999) } \\
\hline Glass ID & $\begin{array}{c}\mathrm{V} 2 \\
(\mathrm{~Pa} \cdot \mathrm{s})\end{array}$ & $\begin{array}{c}\mathrm{T} 3 \\
\left({ }^{\circ} \mathrm{C}\right)\end{array}$ & $\begin{array}{c}\mathrm{V} 3 \\
(\mathrm{~Pa} \cdot \mathrm{s})\end{array}$ & $\begin{array}{c}\mathrm{T} 4 \\
\left({ }^{\circ} \mathrm{C}\right)\end{array}$ & $\begin{array}{c}\mathrm{V} 4 \\
(\mathrm{~Pa} \cdot \mathrm{s})\end{array}$ & $\begin{array}{c}\mathrm{T} 5 \\
\left({ }^{\circ} \mathrm{C}\right)\end{array}$ & $\begin{array}{c}\text { V5 } \\
(\mathrm{Pa} \cdot \mathrm{s})\end{array}$ & $\begin{array}{c}\mathrm{T} 6 \\
\left({ }^{\circ} \mathrm{C}\right)\end{array}$ & $\begin{array}{c}\mathrm{V} 6 \\
(\mathrm{~Pa} \cdot \mathrm{s})\end{array}$ & $\begin{array}{l}\mathrm{T} 7 \\
\left({ }^{\circ} \mathrm{C}\right)\end{array}$ & $\begin{array}{c}\text { V7 } \\
(\mathrm{Pa} \cdot \mathrm{s})\end{array}$ & $\begin{array}{c}\mathrm{T} 8 \\
\left({ }^{\circ} \mathrm{C}\right)\end{array}$ & $\begin{array}{c}\mathrm{V} 8 \\
(\mathrm{~Pa} \cdot \mathrm{s})\end{array}$ & $\begin{array}{l}\text { T9 } \\
\left({ }^{\circ} \mathrm{C}\right)\end{array}$ & $\begin{array}{c}\mathrm{V} 9 \\
(\mathrm{~Pa} \cdot \mathrm{s})\end{array}$ & $\begin{array}{l}\mathrm{T} 10 \\
\left({ }^{\circ} \mathrm{C}\right)\end{array}$ & $\begin{array}{c}\mathrm{V} 10 \\
(\mathrm{~Pa} \cdot \mathrm{s})\end{array}$ & $\begin{array}{l}\mathrm{T} 11 \\
\left({ }^{\circ} \mathrm{C}\right)\end{array}$ & $\begin{array}{c}\mathrm{V} 11 \\
(\mathrm{~Pa} \cdot \mathrm{s})\end{array}$ & $\begin{array}{l}\mathrm{T} 12 \\
\left({ }^{\circ} \mathrm{C}\right)\end{array}$ & $\begin{array}{c}\mathrm{V} 12 \\
(\mathrm{~Pa} \cdot \mathrm{s})\end{array}$ \\
\hline \multicolumn{22}{|l|}{ P2-9Ca-2F } \\
\hline P2-9Ca-3F & & & & & & & & & & & & & & & & & & & & & \\
\hline P2-9Ca-4F & & & & & & & & & & & & & & & & & & & & & \\
\hline P2-9Ca-5F & & & & & & & & & & & & & & & & & & & & & \\
\hline
\end{tabular}

\section{SRL 165 Glasses (Pye 1985)}

SRL $165 \mathrm{HiFe}$

SRL 165 TDS

SRL $165 \mathrm{HiAl}$

\section{RPP-WTP HLW Formulation (Kot and Pegg 2001)}

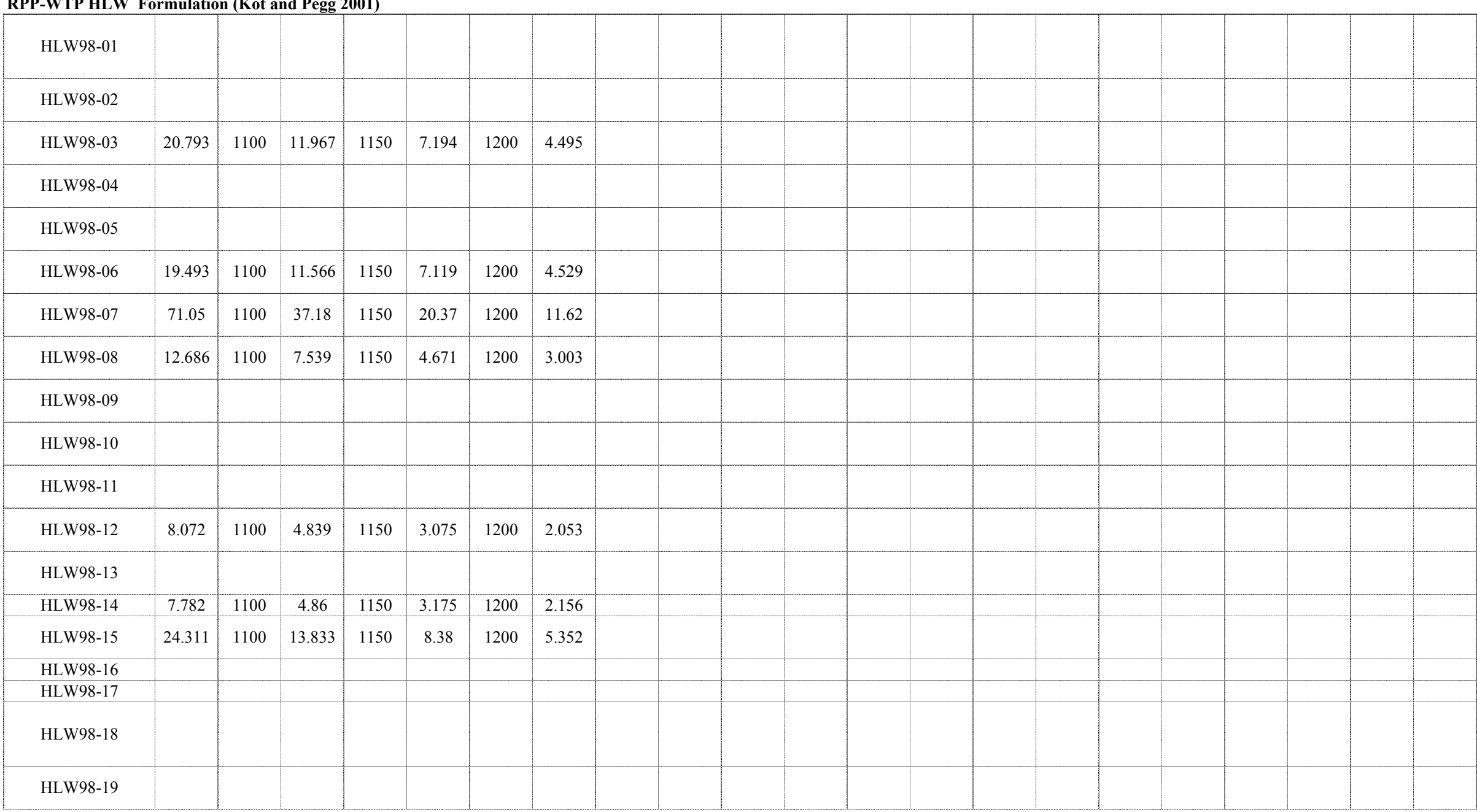


Appendix A. Database - mass fraction

INEEL CVS 2a (Peeler et al. 1999)

\begin{tabular}{|c|c|c|c|c|c|c|c|c|c|c|c|c|c|c|c|c|}
\hline Glass ID & $\begin{array}{l}\mathrm{T} 13 \\
\left({ }^{\circ} \mathrm{C}\right) \\
\end{array}$ & $\begin{array}{c}\mathrm{V} 13 \\
(\mathrm{~Pa} \cdot \mathrm{s})\end{array}$ & $\begin{array}{l}\mathrm{T} 14 \\
\left({ }^{\circ} \mathrm{C}\right) \\
\end{array}$ & $\begin{array}{c}\mathrm{V} 14 \\
(\mathrm{~Pa} \cdot \mathrm{s}) \\
\end{array}$ & $\begin{array}{c}\text { Q PCT } \\
\mathrm{B}\left(\mathrm{g} / \mathrm{m}^{2}\right)\end{array}$ & $\begin{array}{c}\text { Q PCT } \\
\mathrm{Li}\left(\mathrm{g} / \mathrm{m}^{2}\right)\end{array}$ & $\begin{array}{c}\text { Q PCT } \\
\mathrm{Na}\left(\mathrm{g} / \mathrm{m}^{2}\right)\end{array}$ & $\begin{array}{c}\text { Q PCT } \\
\mathrm{Si}\left(\mathrm{g} / \mathrm{m}^{2}\right)\end{array}$ & $\begin{array}{c}\text { Q PCT } \\
\mathrm{pH}\end{array}$ & $\begin{array}{c}\text { CCC PCT } \\
\mathrm{B}\left(\mathrm{g} / \mathrm{m}^{2}\right) \\
\end{array}$ & $\begin{array}{l}\text { CCC PCT } \\
\mathrm{Li}\left(\mathrm{g} / \mathrm{m}^{2}\right)\end{array}$ & $\begin{array}{l}\text { CCC PCT } \\
\mathrm{Na}\left(\mathrm{g} / \mathrm{m}^{2}\right) \\
\end{array}$ & $\begin{array}{c}\text { CCC PCT } \\
\mathrm{Si}\left(\mathrm{g} / \mathrm{m}^{2}\right)\end{array}$ & $\begin{array}{c}\text { CCC PCT } \\
\mathrm{pH}\end{array}$ & $\begin{array}{l}\text { Q PCT B at } \\
20^{\circ} \mathrm{C}\left(\mathrm{g} / \mathrm{m}^{2}\right)\end{array}$ & $\begin{array}{l}\text { Q PCT Li at } \\
20^{\circ} \mathrm{C}\left(\mathrm{g} / \mathrm{m}^{2}\right) \\
\end{array}$ \\
\hline P2-9Ca-2F & & & & & 0.26 & 0.29 & 0.36 & 0.105 & 10.81 & & & & & & & \\
\hline P2-9Ca-3F & & & & & 0.285 & 0.325 & 0.405 & 0.12 & 10.83 & & & & & & & \\
\hline $\mathrm{P} 2-9 \mathrm{Ca}-4 \mathrm{~F}$ & & & & & 0.3 & 0.32 & 0.39 & 0.115 & 10.8 & & & & & & & \\
\hline P2-9Ca-5F & & & & & 0.28 & 0.29 & 0.37 & 0.1 & 10.71 & & & & & & & \\
\hline
\end{tabular}

\section{SRL 165 Glasses (Pye 1985)}

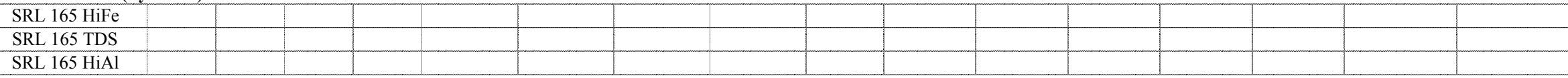

\section{RPP-WTP HLW Formulation (Kot and Pegg 2001)}

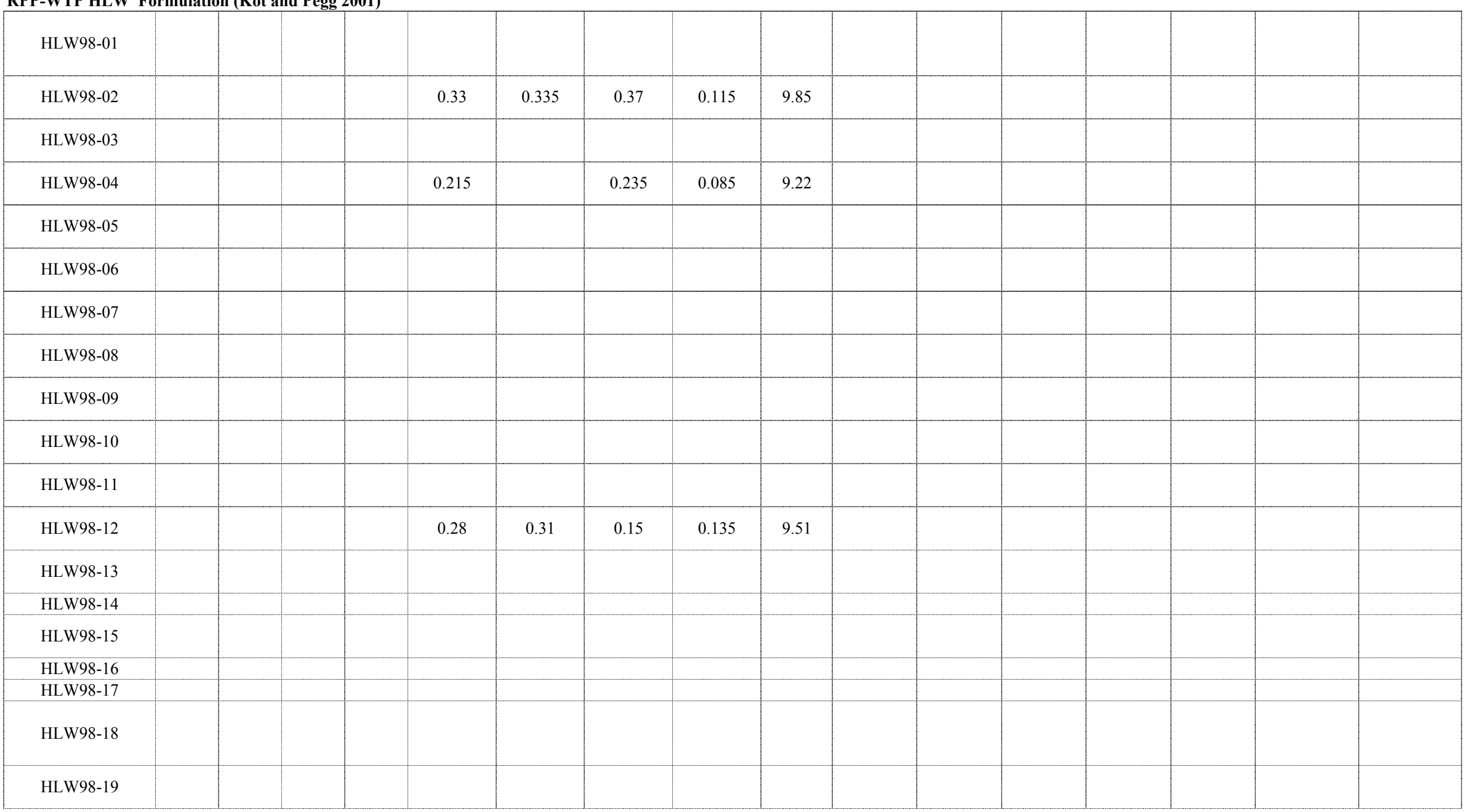


INEEL CVS 2a (Peeler et al. 1999)

\begin{tabular}{|c|c|c|c|c|c|c|c|c|c|c|c|c|}
\hline Glass ID & $\begin{array}{l}\text { Q PCT Na at } \\
20^{\circ} \mathrm{C}\left(\mathrm{g} / \mathrm{m}^{2}\right)\end{array}$ & $\begin{array}{l}\text { Q PCT Si at } \\
20^{\circ} \mathrm{C}\left(\mathrm{g} / \mathrm{m}^{2}\right)\end{array}$ & $\begin{array}{c}\mathrm{QpH} \\
\text { at } 20^{\circ} \mathrm{C}\end{array}$ & $\begin{array}{c}\text { TCLP Ag } \\
\text { (ppm) }\end{array}$ & $\begin{array}{c}\text { TCLP As } \\
\text { (ppm) }\end{array}$ & $\begin{array}{c}\text { TCLP Ba } \\
\text { (ppm) }\end{array}$ & $\begin{array}{c}\text { TCLP Cd } \\
\text { (ppm) }\end{array}$ & $\begin{array}{l}\text { TCLP Cr } \\
\text { (ppm) }\end{array}$ & $\begin{array}{l}\text { TCLP Ni } \\
\text { (ppm) }\end{array}$ & $\begin{array}{c}\text { TCLP Pb } \\
\text { (ppm) }\end{array}$ & $\begin{array}{c}\text { TCLP Se } \\
\text { (ppm) }\end{array}$ & $\begin{array}{c}\text { TCLP Zn } \\
(\mathrm{ppm})\end{array}$ \\
\hline P2-9Ca-2F & & & & & & & & & & & & \\
\hline P2-9Ca-3F & & & & & & & & & & & & \\
\hline P2-9Ca-4F & & & & & & & & & & & & \\
\hline P2-9Ca-5F & & & & & & & & & & & & \\
\hline
\end{tabular}

\section{SRL 165 Glasses (Pye 1985)}

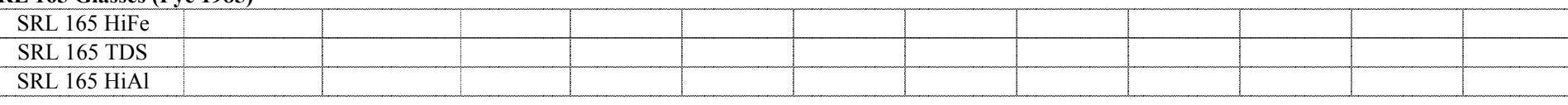

\section{RPP-WTP HLW Formulation (Kot and Pegg 2001)}

\begin{tabular}{|c|c|c|c|c|c|c|c|c|c|}
\hline HLW98-01 & 0.071 & 0.05 & 0.285 & 0.318 & 0.025 & 0.136 & 0.121 & 0.0532 & 0.894 \\
\hline HLW98-02 & 0.098 & 0.07 & 0.317 & 0.365 & 0.023 & 0.17 & 0.137 & 0.0532 & 1.02 \\
\hline HLW98-03 & 0.015 & 0.05 & 0.091 & 0.064 & 0.014 & 0.052 & 0.089 & 0.0532 & 0.488 \\
\hline HLW98-04 & 0.013 & 0.05 & 0.097 & 0.067 & 0.019 & 0.05 & 0.087 & 0.0532 & 0.505 \\
\hline HLW98-05 & 0.015 & 0.05 & 0.082 & 0.116 & 0.012 & 0.04 & 0.09 & 0.0532 & 0.45 \\
\hline HLW98-06 & 0.014 & 0.05 & 0.112 & 0.072 & 0.01 & 0.07 & 0.08 & 0.0532 & 0.44 \\
\hline HLW98-07 & 0.017 & 0.05 & 0.098 & 0.06 & $<0.006$ & 0.04 & 0.08 & 0.0532 & 0.35 \\
\hline HLW98-08 & 0.03 & 0.05 & 0.13 & 0.08 & 0.01 & 0.08 & 0.07 & 0.0532 & 0.46 \\
\hline HLW98-09 & 0.02 & 0.05 & 0.22 & 0.07 & $<0.01$ & 0.06 & 0.06 & 0.0532 & 0.42 \\
\hline \multicolumn{10}{|l|}{ HLW98-10 } \\
\hline \multicolumn{10}{|l|}{ HLW98-11 } \\
\hline HLW98-12 & 0.02 & 0.05 & 0.03 & 0.11 & 0.01 & 0.06 & $<0.03$ & 0.0532 & 0.401 \\
\hline HLW98-13 & 0.033 & 0.012 & 0.053 & 0.179 & 0.01 & 0.111 & 0.261 & 0.038 & 0.77 \\
\hline HLW98-14 & 0.037 & 0.075 & 0.303 & 0.05 & 0.02 & 0.05 & 0.11 & 0.0532 & 0.79 \\
\hline HLW98-15 & 0.029 & 0.08 & 0.224 & 0.03 & 0.01 & 0.03 & 0.1 & 0.0532 & 0.54 \\
\hline \multirow{2}{*}{\multicolumn{10}{|c|}{$\begin{array}{l}\text { HLW98-16 } \\
\text { HLW98-17 }\end{array}$}} \\
\hline & & & & & & & & & \\
\hline HLW98-18 & 0.002 & 0.017 & 0.026 & 0.076 & 0.011 & 0.031 & $<0.03$ & 0.0532 & 0.007 \\
\hline HLW98-19 & 0.023 & 0.05 & 0.228 & 0.01 & 0.01 & 0.015 & 0.08 & 0.0532 & 0.529 \\
\hline
\end{tabular}


RPP-WTP HLW Formulation (Kot and Pegg 2001)

\begin{tabular}{|c|c|c|c|c|c|c|c|c|c|c|c|c|c|c|c|c|c|c|c|c|c|}
\hline Glass ID & $\begin{array}{c}\mathrm{A} 12 \mathrm{O} 3 \\
-\mathrm{t}\end{array}$ & $\begin{array}{c}\mathrm{B} 2 \mathrm{O} 3 \\
-\mathrm{t}\end{array}$ & $\begin{array}{c}\mathrm{CaO} \\
-\mathrm{t}\end{array}$ & $\begin{array}{c}\mathrm{Fe} 2 \mathrm{O} 3 \\
-\mathrm{t}\end{array}$ & $\begin{array}{c}\mathrm{FeO} \\
-\mathrm{t}\end{array}$ & $\begin{array}{c}\mathrm{K} 2 \mathrm{O} \\
-\mathrm{t}\end{array}$ & $\begin{array}{c}\mathrm{Li} 2 \mathrm{O} \\
-\mathrm{t}\end{array}$ & $\begin{array}{c}\mathrm{MgO} \\
-\mathrm{t}\end{array}$ & $\begin{array}{c}\mathrm{Na} 2 \mathrm{O} \\
-\mathrm{t}\end{array}$ & $\begin{array}{c}\mathrm{NiO} \\
-\mathrm{t}\end{array}$ & $\begin{array}{c}\mathrm{P} 2 \mathrm{O} 5 \\
-\mathrm{t}\end{array}$ & $\begin{array}{c}\mathrm{SiO} 2 \\
-\mathrm{t}\end{array}$ & $\begin{array}{c}\mathrm{ZrO} 2 \\
-\mathrm{t}\end{array}$ & $\begin{array}{c}\mathrm{Ag} 2 \mathrm{O} \\
-\mathrm{t}\end{array}$ & $\begin{array}{c}\mathrm{As} 2 \mathrm{O} 3 \\
-\mathrm{t}\end{array}$ & $\begin{array}{c}\mathrm{BaO} \\
-\mathrm{t}\end{array}$ & $\begin{array}{c}\mathrm{Bi} 2 \mathrm{O} 3 \\
-\mathrm{t}\end{array}$ & $\begin{array}{c}\mathrm{Br} \\
-\mathrm{t} \\
\end{array}$ & $\begin{array}{c}\mathrm{CdO} \\
-\mathrm{t}\end{array}$ & $\begin{array}{c}\mathrm{Ce} 2 \mathrm{O} 3 \\
-\mathrm{t}\end{array}$ & $\begin{array}{c}\mathrm{CeO} 2 \\
-\mathrm{t}\end{array}$ \\
\hline HLW98-20 & 0.0891 & 0.1200 & 0.0195 & 0.0978 & & 0.0020 & 0.0150 & 0.0117 & 0.1438 & 0.0016 & 0.0066 & 0.4360 & 0.0032 & 0.0000 & & 0.0061 & 0.0006 & & 0.0004 & & \\
\hline HLW98-21 & 0.0677 & 0.0700 & 0.0024 & 0.1136 & & 0.0027 & 0.0600 & 0.0008 & 0.0664 & 0.0042 & 0.0053 & 0.5089 & 0.0350 & & 0.0005 & 0.0005 & & & 0.0048 & & 0.0011 \\
\hline HLW98-22 & 0.0535 & 0.1200 & 0.0117 & 0.1462 & & 0.0012 & 0.0300 & 0.0070 & 0.0863 & 0.0010 & 0.0040 & 0.4026 & 0.0019 & & & 0.0036 & 0.0004 & & 0.0002 & & \\
\hline HLW98-23 & 0.0485 & 0.1200 & 0.0106 & 0.1324 & & 0.0011 & 0.0300 & 0.0064 & 0.0782 & 0.0009 & 0.0036 & 0.4429 & 0.0017 & & & 0.0033 & 0.0003 & & 0.0002 & & \\
\hline HLW98-24 & 0.0677 & 0.1100 & 0.0024 & 0.1136 & & 0.0027 & 0.0600 & 0.0008 & 0.0664 & 0.0042 & 0.0053 & 0.4689 & 0.0350 & & 0.0005 & 0.0005 & & & 0.0048 & & 0.0011 \\
\hline HLW98-25 & 0.0664 & 0.1000 & 0.0024 & 0.1114 & & 0.0026 & 0.0600 & 0.0008 & 0.0659 & 0.0041 & 0.0052 & 0.4688 & 0.0343 & 0.0007 & 0.0005 & 0.0005 & & & 0.0047 & & 0.0011 \\
\hline HLW98-26 & 0.0781 & 0.0700 & 0.0026 & 0.1305 & & 0.0018 & 0.0650 & 0.0007 & 0.0611 & 0.0057 & 0.0013 & 0.4626 & 0.0279 & & 0.0004 & 0.0004 & & & 0.0039 & & 0.0009 \\
\hline HLW98-27B & 0.0781 & 0.0400 & 0.0026 & 0.1305 & & 0.0018 & 0.0450 & 0.0007 & 0.1435 & 0.0057 & 0.0013 & 0.4302 & 0.0279 & & 0.0004 & 0.0004 & & & 0.0039 & & 0.0009 \\
\hline HLW98-28 & 0.0781 & 0.0200 & 0.0026 & 0.1305 & & 0.0018 & 0.0400 & 0.0007 & 0.1435 & 0.0057 & 0.0013 & 0.4552 & 0.0279 & 0.0000 & 0.0004 & 0.0004 & & & 0.0039 & & 0.0009 \\
\hline HLW98-29 & 0.0781 & 0.1289 & 0.0026 & 0.1305 & & 0.0018 & 0.0400 & 0.0007 & 0.0796 & 0.0057 & 0.0013 & 0.4102 & 0.0279 & 0.0000 & 0.0004 & 0.0004 & & & 0.0039 & & 0.0009 \\
\hline HLW98-30 & 0.0781 & 0.1000 & 0.0026 & 0.1097 & & 0.0018 & 0.0600 & 0.0007 & 0.0661 & 0.0057 & 0.0013 & 0.4534 & 0.0279 & 0.0000 & 0.0004 & 0.0004 & & & 0.0039 & & 0.0009 \\
\hline HLW98-31 & 0.0740 & 0.1000 & 0.0025 & 0.1039 & & 0.0017 & 0.0600 & 0.0006 & 0.0659 & 0.0054 & 0.0013 & 0.4553 & 0.0356 & 0.0000 & 0.0004 & 0.0004 & & & 0.0037 & & 0.0008 \\
\hline HLW98-32A & 0.1368 & 0.0900 & 0.0024 & 0.1110 & & 0.0005 & 0.0700 & 0.0007 & 0.0358 & 0.0067 & 0.0005 & 0.4550 & 0.0146 & 0.0000 & 0.0004 & 0.0003 & & & 0.0078 & & 0.0004 \\
\hline HLW98-33 & 0.1003 & 0.0700 & 0.0067 & 0.1092 & & 0.0003 & 0.0300 & 0.0020 & 0.0879 & 0.0014 & 0.0014 & 0.3773 & 0.0011 & 0.0022 & 0.0000 & 0.0006 & & & 0.0004 & & 0.0003 \\
\hline HLW98-34 & 0.1078 & 0.0700 & 0.0071 & 0.1173 & & 0.0003 & 0.0400 & 0.0022 & 0.0936 & 0.0015 & 0.0015 & 0.4089 & 0.0012 & 0.0024 & 0.0000 & 0.0007 & & & 0.0004 & & 0.0003 \\
\hline HLW98-35 & 0.1034 & 0.0700 & 0.0176 & 0.1206 & & 0.0006 & 0.0500 & 0.0008 & 0.0971 & 0.0073 & 0.0006 & 0.4354 & 0.0158 & 0.0000 & 0.0004 & 0.0003 & & & 0.0085 & & 0.0005 \\
\hline HLW98-36 & 0.0939 & 0.0900 & 0.0281 & 0.0382 & & 0.0008 & 0.0500 & 0.0003 & 0.1263 & 0.0024 & 0.0021 & 0.4350 & 0.0784 & 0.0000 & 0.0000 & 0.0000 & & & 0.0006 & & 0.0000 \\
\hline HLW98-37 & 0.0443 & 0.0505 & 0.0045 & 0.0561 & & 0.0000 & 0.0450 & 0.0005 & 0.1528 & 0.0020 & 0.0007 & 0.4177 & 0.1156 & 0.0000 & 0.0000 & 0.0000 & & & 0.0009 & & 0.0000 \\
\hline HLW98-38 & 0.0336 & 0.0500 & 0.0064 & 0.0663 & & 0.0010 & 0.0450 & 0.0008 & 0.1143 & 0.0033 & 0.0047 & 0.3823 & 0.0684 & 0.0009 & 0.0000 & 0.0003 & & & 0.0009 & & 0.0012 \\
\hline HLW98-39 & 0.0370 & 0.0300 & 0.0070 & 0.0730 & & 0.0011 & 0.0400 & 0.0009 & 0.1223 & 0.0036 & 0.0051 & 0.3696 & 0.0753 & 0.0010 & 0.0000 & 0.0003 & & & 0.0009 & & 0.0013 \\
\hline HLW98-40 & 0.0823 & 0.0600 & 0.0026 & 0.1189 & & 0.0006 & 0.0450 & 0.0008 & 0.1419 & 0.0072 & 0.0006 & 0.4353 & 0.0156 & 0.0004 & 0.0004 & 0.0003 & & & 0.0084 & & 0.0005 \\
\hline
\end{tabular}


Appendix A. Database - mass fraction

RPP-WTP HLW Formulation (Kot and Pegg 2001)

\begin{tabular}{|c|c|c|c|c|c|c|c|c|c|c|c|c|c|c|c|c|c|c|c|c|c|}
\hline Glass ID & $\begin{array}{l}\mathrm{Cl} \\
-\mathrm{t}\end{array}$ & $\begin{array}{c}\mathrm{CoO} \\
-\mathrm{t}\end{array}$ & $\begin{array}{c}\mathrm{Co} 2 \mathrm{O} 3 \\
-\mathrm{t}\end{array}$ & $\begin{array}{c}\mathrm{Cr} 2 \mathrm{O} 3 \\
-\mathrm{t}\end{array}$ & $\begin{array}{c}\mathrm{Cs} 2 \mathrm{O} \\
-\mathrm{t}\end{array}$ & $\begin{array}{c}\mathrm{CuO} \\
-\mathrm{t}\end{array}$ & $\begin{array}{c}\mathrm{Eu} 2 \mathrm{O} 3 \\
-\mathrm{t}\end{array}$ & $\begin{array}{l}F \\
-t\end{array}$ & $\begin{array}{c}\mathrm{Ga} 2 \mathrm{O} 3 \\
-\mathrm{t}\end{array}$ & $\begin{array}{c}\mathrm{Gd} 2 \mathrm{O} 3 \\
-\mathrm{t}\end{array}$ & $\begin{array}{c}\mathrm{HgO} \\
-\mathrm{t}\end{array}$ & $\begin{array}{l} \\
-t\end{array}$ & $\begin{array}{c}\mathrm{La} 2 \mathrm{O} 3 \\
-\mathrm{t}\end{array}$ & $\begin{array}{c}\mathrm{MnO} 2 \\
-\mathrm{t}\end{array}$ & $\begin{array}{c}\mathrm{MnO} \\
-\mathrm{t}\end{array}$ & $\begin{array}{c}\mathrm{MoO} \\
-\mathrm{t}\end{array}$ & $\begin{array}{c}\mathrm{MoO} 3 \\
-\mathrm{t}\end{array}$ & $\begin{array}{c}\mathrm{Nb} 2 \mathrm{O} 5 \\
-\mathrm{t}\end{array}$ & $\begin{array}{c}\mathrm{Nd} 2 \mathrm{O} 3 \\
-\mathrm{t}\end{array}$ & $\begin{array}{c}\mathrm{PbO} \\
-\mathrm{t}\end{array}$ & $\begin{array}{c}\mathrm{PdO} 2 \\
-\mathrm{t}\end{array}$ \\
\hline HLW98-20 & 0.0007 & & & 0.0018 & 0.0038 & & & & & & & & 0.0004 & 0.0043 & & & & & & 0.0027 & \\
\hline HLW98-21 & 0.0001 & & & 0.0009 & 0.0039 & 0.0004 & & 0.0005 & & & & & 0.0033 & 0.0035 & & & & & & 0.0004 & \\
\hline HLW98-22 & 0.0004 & & & 0.0011 & 0.0023 & & & & & & & & 0.0003 & 0.0026 & & & & & & 0.0016 & \\
\hline HLW98-23 & 0.0004 & & & 0.0010 & 0.0021 & & & & & & & & 0.0002 & 0.0023 & & & & & & 0.0015 & \\
\hline HLW98-24 & 0.0001 & & & 0.0009 & 0.0039 & 0.0004 & & 0.0005 & & & & & 0.0033 & 0.0035 & & & & & & 0.0004 & \\
\hline HLW98-25 & 0.0001 & & & 0.0008 & 0.0038 & 0.0004 & & 0.0005 & & & & & 0.0032 & 0.0192 & & & & & & 0.0004 & \\
\hline HLW98-26 & 0.0001 & & & 0.0005 & 0.0008 & 0.0003 & & 0.0004 & & & & & 0.0034 & 0.0271 & & & & & & 0.0016 & \\
\hline HLW98-27B & 0.0001 & & & 0.0005 & 0.0008 & 0.0003 & & 0.0004 & & & & & 0.0034 & 0.0271 & & & & & & 0.0016 & \\
\hline HLW98-28 & 0.0001 & 0.0000 & & 0.0005 & 0.0008 & 0.0003 & & 0.0004 & & & 0.0000 & & 0.0034 & 0.0271 & 0.0000 & & & & 0.0000 & 0.0016 & \\
\hline HLW98-29 & 0.0001 & 0.0000 & & 0.0005 & 0.0008 & 0.0003 & & 0.0004 & & & 0.0000 & & 0.0034 & 0.0271 & 0.0000 & & & & 0.0000 & 0.0016 & \\
\hline HLW98-30 & 0.0001 & 0.0000 & & 0.0005 & 0.0008 & 0.0003 & & 0.0004 & & & 0.0000 & & 0.0034 & 0.0271 & 0.0000 & & & & 0.0000 & 0.0016 & \\
\hline HLW98-31 & 0.0001 & 0.0000 & & 0.0004 & 0.0008 & 0.0003 & & 0.0004 & & & 0.0000 & & 0.0032 & 0.0000 & 0.0303 & & & & 0.0000 & 0.0015 & \\
\hline HLW98-32A & 0.0001 & 0.0000 & & 0.0008 & 0.0009 & 0.0002 & & 0.0001 & & & 0.0000 & & 0.0031 & 0.0141 & 0.0000 & & & & 0.0000 & 0.0007 & \\
\hline HLW98-33 & 0.0003 & 0.0000 & & 0.0015 & 0.0009 & 0.0000 & & 0.0003 & & & 0.0000 & & 0.0010 & 0.0000 & 0.0664 & & & & 0.0000 & 0.0020 & \\
\hline HLW98-34 & 0.0002 & 0.0000 & & 0.0016 & 0.0010 & 0.0000 & & 0.0002 & & & 0.0000 & & 0.0011 & 0.0000 & 0.0444 & & & & 0.0000 & 0.0022 & \\
\hline HLW98-35 & 0.0002 & 0.0000 & & 0.0008 & 0.0010 & 0.0002 & & 0.0002 & & & 0.0000 & & 0.0034 & 0.0153 & 0.0000 & & & & 0.0000 & 0.0008 & \\
\hline HLW98-36 & 0.0001 & 0.0005 & & 0.0014 & 0.0000 & 0.0000 & & 0.0001 & & & 0.0000 & & 0.0000 & 0.0000 & 0.0059 & & & & 0.0000 & 0.0005 & \\
\hline HLW98-37 & 0.0000 & 0.0007 & & 0.0011 & 0.0011 & 0.0000 & & 0.0000 & & & 0.0000 & & 0.0001 & 0.0000 & 0.0244 & & & & 0.0000 & 0.0008 & \\
\hline HLW98-38 & 0.0000 & 0.0000 & & 0.0019 & 0.0000 & 0.0004 & & 0.0000 & & & 0.0000 & & 0.0003 & 0.0000 & 0.0364 & & & & 0.0007 & 0.0022 & \\
\hline HLW98-39 & 0.0000 & 0.0000 & & 0.0021 & 0.0000 & 0.0004 & & 0.0000 & & & 0.0000 & & 0.0003 & 0.0000 & 0.0401 & & & & 0.0009 & 0.0024 & \\
\hline HLW98-40 & 0.0000 & 0.0000 & & 0.0008 & 0.0010 & 0.0002 & & 0.0002 & & & 0.0000 & & 0.0033 & 0.0151 & 0.0000 & & & & 0.0000 & 0.0007 & \\
\hline
\end{tabular}


Appendix A. Database - mass fraction

RPP-WTP HLW Formulation (Kot and Pegg 2001)

\begin{tabular}{|c|c|c|c|c|c|c|c|c|c|c|c|c|c|c|c|c|c|c|c|c|c|}
\hline Glass ID & $\begin{array}{c}\mathrm{PdO} \\
-\mathrm{t}\end{array}$ & $\begin{array}{c}\mathrm{Pr} 2 \mathrm{O} 3 \\
-\mathrm{t}\end{array}$ & $\begin{array}{c}\text { Pr6O11 } \\
-t\end{array}$ & $\begin{array}{c}\mathrm{Rb} 2 \mathrm{O} \\
-\mathrm{t}\end{array}$ & $\begin{array}{c}\mathrm{ReO} \\
-\mathrm{t} \\
\end{array}$ & $\begin{array}{c}\mathrm{ReO} 2 \\
-\mathrm{t}\end{array}$ & $\begin{array}{c}\mathrm{Rh} 2 \mathrm{O} 3 \\
-\mathrm{t}\end{array}$ & $\begin{array}{c}\mathrm{RhO}_{2} \\
-\mathrm{t}\end{array}$ & $\begin{array}{c}\mathrm{RuO}^{2} \\
-\mathrm{t}\end{array}$ & $\begin{array}{c}\mathrm{Sb} 2 \mathrm{O} 3 \\
-\mathrm{t}\end{array}$ & $\begin{array}{c}\mathrm{Sb} 2 \mathrm{O} 5 \\
-\mathrm{t}\end{array}$ & $\begin{array}{c}\mathrm{SeO} 2 \\
-\mathrm{t} \\
\end{array}$ & $\begin{array}{c}\mathrm{Sm} 2 \mathrm{O} 3 \\
-\mathrm{t}\end{array}$ & $\begin{array}{c}\mathrm{SnO} \\
-\mathrm{t}\end{array}$ & $\begin{array}{c}\mathrm{SnO} 2 \\
-\mathrm{t}\end{array}$ & $\begin{array}{c}\mathrm{SO} 3 \\
-\mathrm{t}\end{array}$ & $\begin{array}{c}\mathrm{SrO} \\
-\mathrm{t}\end{array}$ & $\begin{array}{c}\mathrm{Tc} 2 \mathrm{O} 7 \\
-\mathrm{t}\end{array}$ & $\begin{array}{c}\mathrm{TeO} 2 \\
-\mathrm{t}\end{array}$ & $\begin{array}{c}\mathrm{ThO} 2 \\
-\mathrm{t}\end{array}$ & $\begin{array}{c}\mathrm{TiO} 2 \\
-\mathrm{t} \\
\end{array}$ \\
\hline HLW98-20 & & & & & & & & & & & & & & & & 0.0014 & 0.0116 & & & & \\
\hline HLW98-21 & & & & & & & & & & & 0.0027 & 0.0019 & & & & 0.0034 & 0.0125 & & 0.0018 & & 0.0008 \\
\hline HLW98-22 & & & & & & & & & & & & & & & & 0.0008 & 0.1013 & & & & \\
\hline HLW98-23 & & & & & & & & & & & & & & & & 0.0007 & 0.0917 & & & & \\
\hline HLW98-24 & & & & & & & & & & & 0.0027 & 0.0019 & & & & 0.0034 & 0.0125 & & 0.0018 & & 0.0008 \\
\hline HLW98-25 & & & & & & & & & & & 0.0027 & 0.0019 & & & & 0.0033 & 0.0117 & & 0.0018 & & 0.0008 \\
\hline HLW98-26 & & & & & & & & & & & 0.0022 & 0.0015 & & & & 0.0026 & 0.0245 & & 0.0015 & & 0.0007 \\
\hline HLW98-27B & & & & & & & & & & & 0.0022 & 0.0015 & & & & 0.0026 & 0.0245 & & 0.0015 & & 0.0007 \\
\hline HLW98-28 & 0.0000 & & & & & & 0.0000 & & 0.0000 & & 0.0022 & 0.0015 & & & 0.0000 & 0.0026 & 0.0245 & & 0.0015 & 0.0000 & 0.0007 \\
\hline HLW98-29 & 0.0000 & & & & & & 0.0000 & & 0.0000 & & 0.0022 & 0.0015 & & & 0.0000 & 0.0026 & 0.0245 & & 0.0015 & 0.0000 & 0.0007 \\
\hline HLW98-30 & 0.0000 & & & & & & 0.0000 & & 0.0000 & & 0.0022 & 0.0015 & & & 0.0000 & 0.0026 & 0.0245 & & 0.0015 & 0.0000 & 0.0007 \\
\hline HLW98-31 & 0.0000 & & & & & & 0.0000 & & 0.0000 & & 0.0021 & 0.0015 & & & 0.0000 & 0.0025 & 0.0232 & & 0.0014 & 0.0000 & 0.0006 \\
\hline HLW98-32A & 0.0000 & & & & & & 0.0000 & & 0.0000 & & 0.0034 & 0.0006 & & & 0.0000 & 0.0002 & 0.0226 & & 0.0002 & 0.0000 & 0.0000 \\
\hline HLW98-33 & 0.0000 & & & & & & 0.0000 & & 0.0000 & & 0.0000 & 0.0000 & & & 0.0000 & 0.0003 & 0.1156 & & 0.0000 & 0.0000 & 0.0004 \\
\hline HLW98-34 & 0.0000 & & & & & & 0.0000 & & 0.0000 & & 0.0000 & 0.0000 & & & 0.0000 & 0.0002 & 0.0735 & & 0.0000 & 0.0000 & 0.0004 \\
\hline HLW98-35 & 0.0000 & & & & & & 0.0000 & & 0.0000 & & 0.0037 & 0.0007 & & & 0.0000 & 0.0003 & 0.0245 & & 0.0002 & 0.0000 & 0.0000 \\
\hline HLW98-36 & 0.0000 & & & & & & 0.0000 & & 0.0000 & & 0.0000 & 0.0000 & & & 0.0000 & 0.0001 & 0.0000 & & 0.0000 & 0.0000 & 0.0000 \\
\hline HLW98-37 & 0.0000 & & & & & & 0.0000 & & 0.0000 & & 0.0000 & 0.0000 & & & 0.0000 & 0.0000 & 0.0296 & & 0.0000 & 0.0000 & 0.0000 \\
\hline HLW98-38 & 0.0000 & & & & & & 0.0005 & & 0.0002 & & 0.0000 & 0.0000 & & & 0.0010 & 0.0000 & 0.0480 & & 0.0000 & 0.0579 & 0.0002 \\
\hline HLW98-39 & 0.0002 & & & & & & 0.0005 & & 0.0003 & & 0.0000 & 0.0000 & & & 0.0011 & 0.0000 & 0.0529 & & 0.0000 & 0.0637 & 0.0003 \\
\hline HLW98-40 & 0.0000 & & & & & & 0.0000 & & 0.0000 & & 0.0037 & 0.0006 & & & 0.0000 & 0.0003 & 0.0242 & & 0.0002 & 0.0000 & 0.0000 \\
\hline
\end{tabular}


Appendix A. Database - mass fraction

RPP-WTP HLW Formulation (Kot and Pegg 2001)

\begin{tabular}{|c|c|c|c|c|c|c|c|c|c|c|c|c|c|c|c|c|c|c|c|c|c|}
\hline Glass ID & $\begin{array}{c}\mathrm{T} 12 \mathrm{O} 3 \\
-\mathrm{t}\end{array}$ & $\begin{array}{c}\mathrm{U} 3 \mathrm{O} 8 \\
-\mathrm{t}\end{array}$ & $\begin{array}{c}\mathrm{UO} 2 \\
-\mathrm{t}\end{array}$ & $\begin{array}{c}\mathrm{UO3} \\
-\mathrm{t} \\
\end{array}$ & $\begin{array}{c}\mathrm{V} 2 \mathrm{O} 5 \\
-\mathrm{t}\end{array}$ & $\begin{array}{c}\mathrm{WO} 3 \\
-\mathrm{t}\end{array}$ & $\begin{array}{c}\mathrm{Y} 2 \mathrm{O} 3 \\
-\mathrm{t}\end{array}$ & $\begin{array}{c}\mathrm{ZnO} \\
-\mathrm{t}\end{array}$ & $\begin{array}{c}\text { Others } \\
-t\end{array}$ & $\begin{array}{c}\text { Sum } \\
-t\end{array}$ & $\begin{array}{c}\mathrm{A} 12 \mathrm{O} 3 \\
-\mathrm{a}\end{array}$ & $\begin{array}{c}\mathrm{B} 2 \mathrm{O} 3 \\
-\mathrm{a}\end{array}$ & $\begin{array}{c}\mathrm{CaO} \\
-\mathrm{a} \\
\end{array}$ & $\begin{array}{c}\mathrm{Fe} 2 \mathrm{O} 3 \\
-\mathrm{a}\end{array}$ & $\begin{array}{c}\mathrm{FeO} \\
-\mathrm{a} \\
\end{array}$ & $\begin{array}{c}\mathrm{K} 2 \mathrm{O} \\
-\mathrm{a}\end{array}$ & $\begin{array}{c}\mathrm{Li} 2 \mathrm{O} \\
-\mathrm{a} \\
\end{array}$ & $\begin{array}{c}\mathrm{MgO} \\
-\mathrm{a}\end{array}$ & $\begin{array}{c}\mathrm{Na} 2 \mathrm{O} \\
-\mathrm{a}\end{array}$ & $\begin{array}{c}\mathrm{NiO} \\
-\mathrm{a}\end{array}$ & $\begin{array}{c}\mathrm{P} 2 \mathrm{O} 5 \\
-\mathrm{a}\end{array}$ \\
\hline HLW98-20 & & & & & & & & 0.0200 & & 1.0001 & 0.0844 & 0.1245 & 0.0203 & 0.0922 & & 0.0027 & 0.0167 & 0.0112 & 0.1188 & 0.0022 & 0.0077 \\
\hline HLW98-21 & & & & & & & & 0.0200 & & 1.0000 & 0.0623 & 0.0728 & 0.0033 & 0.1058 & & 0.0032 & 0.0609 & 0.0010 & 0.0622 & 0.0047 & 0.0073 \\
\hline HLW98-22 & & & & & & & & 0.0200 & & 1.0000 & 0.0559 & 0.1176 & 0.0129 & 0.1274 & & 0.0019 & 0.0301 & 0.0067 & 0.0775 & 0.0010 & 0.0048 \\
\hline HLW98-23 & & & & & & & & 0.0200 & & 1.0000 & 0.0481 & 0.1158 & 0.0117 & 0.1189 & & 0.0016 & 0.0289 & 0.0059 & 0.0699 & 0.0008 & 0.0041 \\
\hline HLW98-24 & & & & & & & & 0.0200 & & 1.0000 & 0.0668 & 0.1133 & 0.0032 & 0.1056 & & 0.0036 & 0.0552 & 0.0010 & 0.0642 & 0.0041 & 0.0061 \\
\hline HLW98-25 & & & & & & & & 0.0200 & & 1.0000 & & & & & & & & & & & \\
\hline HLW98-26 & & & & & & & & 0.0200 & & 1.0001 & & & & & & & & & & & \\
\hline HLW98-27B & & & & & & & & 0.0200 & & 1.0001 & 0.0784 & 0.0388 & 0.0040 & 0.1238 & & 0.0035 & 0.0403 & 0.0011 & 0.1267 & 0.0054 & 0.0019 \\
\hline HLW98-28 & & & 0.0000 & & & & & 0.0200 & & 1.0001 & & & & & & & & & & & \\
\hline HLW98-29 & & & 0.0000 & & & & & 0.0200 & & 1.0001 & & & & & & & & & & & \\
\hline HLW98-30 & & & 0.0000 & & & & & 0.0200 & & 1.0001 & & & & & & & & & & & \\
\hline HLW98-31 & & & 0.0000 & & & & & 0.0200 & & 0.9998 & 0.0740 & 0.0950 & 0.0029 & 0.0964 & & 0.0024 & 0.0520 & 0.0009 & 0.0568 & 0.0048 & 0.0023 \\
\hline HLW98-32A & & & 0.0000 & & & & & 0.0200 & & 0.9999 & 0.1170 & 0.0900 & 0.0029 & 0.1014 & & 0.0011 & 0.0634 & 0.0010 & 0.0342 & 0.0063 & 0.0022 \\
\hline HLW98-33 & & & 0.0000 & & & & & 0.0200 & & 1.0000 & & & & & & & & & & & \\
\hline HLW98-34 & & & 0.0000 & & & & & 0.0200 & & 1.0000 & 0.1040 & 0.0678 & 0.0086 & 0.1101 & & 0.0018 & 0.0362 & 0.0029 & 0.0880 & 0.0014 & 0.0023 \\
\hline HLW98-35 & & & 0.0000 & & & & & 0.0200 & & 1.0002 & 0.0947 & 0.0671 & 0.0163 & 0.1095 & & 0.0023 & 0.0425 & 0.0016 & 0.0852 & 0.0067 & 0.0017 \\
\hline HLW98-36 & & & 0.0249 & & & & & 0.0200 & & 1.0000 & 0.0844 & 0.0868 & 0.0279 & 0.0376 & & 0.0018 & 0.0462 & 0.0005 & 0.1217 & 0.0026 & 0.0036 \\
\hline HLW98-37 & & & 0.0316 & & & & & 0.0200 & & 1.0000 & 0.0453 & 0.0519 & 0.0053 & 0.0537 & & 0.0010 & 0.0404 & 0.0007 & 0.1373 & 0.0023 & 0.0016 \\
\hline HLW98-38 & & & 0.0511 & & & & & 0.0200 & & 1.0002 & 0.0340 & 0.0529 & 0.0075 & 0.0656 & & 0.0017 & 0.0410 & 0.0010 & 0.1033 & 0.0036 & 0.0063 \\
\hline HLW98-39 & & & 0.0562 & & & & & 0.0100 & & 0.9998 & 0.0401 & 0.0323 & 0.0087 & 0.0711 & & 0.0019 & 0.0368 & 0.0013 & 0.1116 & 0.0039 & 0.0065 \\
\hline HLW98-40 & & & 0.0090 & & & & & 0.0200 & & 1.0001 & 0.0767 & 0.0603 & 0.0035 & 0.1123 & & 0.0014 & 0.0397 & 0.0010 & 0.1274 & 0.0070 & 0.0020 \\
\hline
\end{tabular}


Appendix A. Database - mass fraction

RPP-WTP HLW Formulation (Kot and Pegg 2001)

\begin{tabular}{|c|c|c|c|c|c|c|c|c|c|c|c|c|c|c|c|c|c|c|c|c|c|}
\hline Glass ID & $\begin{array}{c}\mathrm{SiO} 2 \\
-\mathrm{a}\end{array}$ & $\begin{array}{c}\mathrm{ZrO} 2 \\
-\mathrm{a}\end{array}$ & $\begin{array}{c}\mathrm{Ag} 2 \mathrm{O} \\
-\mathrm{a}\end{array}$ & $\begin{array}{c}\mathrm{As} 2 \mathrm{O} 3 \\
-\mathrm{a} \\
\end{array}$ & $\begin{array}{c}\mathrm{BaO} \\
-\mathrm{a}\end{array}$ & $\begin{array}{c}\mathrm{Bi} 2 \mathrm{O} 3 \\
-\mathrm{a}\end{array}$ & $\begin{array}{l}\mathrm{Br} \\
-\mathrm{a}\end{array}$ & $\begin{array}{c}\mathrm{CdO} \\
-\mathrm{a}\end{array}$ & $\begin{array}{c}\mathrm{Ce} 2 \mathrm{O} 3 \\
-\mathrm{a}\end{array}$ & $\begin{array}{c}\mathrm{CeO} 2 \\
-\mathrm{a}\end{array}$ & $\begin{array}{l}\mathrm{Cl} \\
-\mathrm{a}\end{array}$ & $\begin{array}{c}\mathrm{CoO} \\
-\mathrm{a}\end{array}$ & $\begin{array}{c}\mathrm{Co} 2 \mathrm{O} 3 \\
-\mathrm{a}\end{array}$ & $\begin{array}{c}\mathrm{Cr} 2 \mathrm{O} 3 \\
-\mathrm{a}\end{array}$ & $\begin{array}{c}\mathrm{Cs} 2 \mathrm{O} \\
-\mathrm{a}\end{array}$ & $\begin{array}{c}\mathrm{CuO} \\
-\mathrm{a} \\
\end{array}$ & $\begin{array}{c}\mathrm{Eu} 2 \mathrm{O} 3 \\
-\mathrm{a}\end{array}$ & $\begin{array}{c}\mathrm{F} \\
-\mathrm{a}\end{array}$ & $\begin{array}{c}\mathrm{Ga} 2 \mathrm{O} 3 \\
-\mathrm{a}\end{array}$ & $\begin{array}{c}\mathrm{Gd} 2 \mathrm{O} 3 \\
-\mathrm{a}\end{array}$ & $\begin{array}{c}\mathrm{HgO} \\
-\mathrm{a}\end{array}$ \\
\hline HLW98-20 & 0.4113 & 0.0032 & 0.0000 & & 0.0067 & 0.0008 & & 0.0004 & & & & 0.0003 & & 0.0028 & & 0.0001 & & & & & \\
\hline HLW98-21 & 0.4804 & 0.0307 & 0.0001 & 0.0006 & 0.0009 & 0.0003 & & 0.0047 & & & & 0.0003 & & 0.0017 & & 0.0004 & & & & & \\
\hline HLW98-22 & 0.3734 & 0.0018 & & 0.0002 & 0.0055 & 0.0005 & & 0.0003 & & & & 0.0002 & & 0.0010 & & & & & & & \\
\hline HLW98-23 & 0.4169 & 0.0017 & & & 0.0049 & 0.0006 & & 0.0002 & & & & 0.0001 & & 0.0009 & & 0.0001 & & & & & \\
\hline HLW98-24 & 0.4516 & 0.0307 & 0.0001 & 0.0007 & 0.0009 & 0.0002 & & 0.0045 & & & & 0.0001 & & 0.0007 & & 0.0004 & & & & & \\
\hline HLW98-25 & & & & & & & & & & & & & & & & & & & & & \\
\hline HLW98-26 & & & & & & & & & & & & & & & & & & & & & \\
\hline HLW98-27B & 0.4232 & 0.0241 & 0.0001 & 0.0003 & 0.0015 & 0.0003 & & 0.0041 & & & & 0.0002 & & 0.0004 & & 0.0006 & & & & & \\
\hline HLW98-28 & & & & & & & & & & & & & & & & & & & & & \\
\hline HLW98-29 & & & & & & & & & & & & & & & & & & & & & \\
\hline HLW98-30 & & & & & & & & & & & & & & & & & & & & & \\
\hline HLW98-31 & 0.4434 & 0.0312 & 0.0001 & 0.0007 & 0.0008 & 0.0001 & & 0.0034 & & & & 0.0002 & & 0.0003 & & 0.0004 & & & & & \\
\hline HLW98-32A & 0.4353 & 0.0139 & 0.0001 & 0.0005 & 0.0008 & 0.0000 & & 0.0075 & & & & 0.0002 & & 0.0007 & & 0.0004 & & & & & \\
\hline HLW98-33 & & & & & & & & & & & & & & & & & & & & & \\
\hline HLW98-34 & 0.4019 & 0.0019 & 0.0023 & 0.0002 & 0.0020 & 0.0001 & & 0.0004 & & & & 0.0002 & & 0.0014 & & 0.0002 & & & & & \\
\hline HLW98-35 & 0.4119 & 0.0132 & 0.0001 & 0.0003 & 0.0010 & 0.0001 & & 0.0078 & & & & 0.0002 & & 0.0008 & & 0.0005 & & & & & \\
\hline HLW98-36 & 0.4324 & 0.0710 & 0.0000 & 0.0000 & 0.0002 & 0.0000 & & 0.0007 & & & & 0.0013 & & 0.0016 & & 0.0000 & & & & & \\
\hline HLW98-37 & 0.4299 & 0.1058 & 0.0003 & 0.0000 & 0.0006 & 0.0000 & & 0.0009 & & & & 0.0013 & & 0.0011 & & 0.0000 & & & & & \\
\hline HLW98-38 & 0.3700 & 0.0681 & 0.0011 & 0.0000 & 0.0012 & 0.0000 & & 0.0009 & & & & 0.0006 & & 0.0019 & & 0.0005 & & & & & \\
\hline HLW98-39 & 0.3695 & 0.0692 & 0.0012 & 0.0000 & 0.0014 & 0.0002 & & 0.0010 & & & & 0.0007 & & 0.0020 & & 0.0006 & & & & & \\
\hline HLW98-40 & 0.4235 & 0.0142 & 0.0005 & 0.0000 & 0.0009 & 0.0000 & & 0.0073 & & & & 0.0001 & & 0.0009 & & 0.0000 & & & & & \\
\hline
\end{tabular}


Appendix A. Database - mass fraction

RPP-WTP HLW Formulation (Kot and Pegg 2001)

\begin{tabular}{|c|c|c|c|c|c|c|c|c|c|c|c|c|c|c|c|c|c|c|c|c|c|}
\hline Glass ID & $\begin{array}{c} \\
-\mathrm{a} \\
\end{array}$ & $\begin{array}{c}\mathrm{La} 2 \mathrm{O} 3 \\
-\mathrm{a}\end{array}$ & $\begin{array}{c}\mathrm{MnO} 2 \\
-\mathrm{a}\end{array}$ & $\begin{array}{c}\mathrm{MnO} \\
-\mathrm{a}\end{array}$ & $\begin{array}{c}\mathrm{MoO} \\
-\mathrm{a}\end{array}$ & $\begin{array}{c}\mathrm{MoO} 3 \\
-\mathrm{a}\end{array}$ & $\begin{array}{c}\mathrm{Nb} 2 \mathrm{O} 5 \\
-\mathrm{a}\end{array}$ & $\begin{array}{c}\mathrm{Nd} 2 \mathrm{O} 3 \\
-\mathrm{a}\end{array}$ & $\begin{array}{c}\mathrm{PbO} \\
-\mathrm{a} \\
\end{array}$ & $\begin{array}{c}\mathrm{PdO} 2 \\
-\mathrm{a}\end{array}$ & $\begin{array}{c}\mathrm{PdO} \\
-\mathrm{a} \\
\end{array}$ & $\begin{array}{c}\mathrm{Pr} 2 \mathrm{O} 3 \\
-\mathrm{a} \\
\end{array}$ & \begin{tabular}{|c|} 
Pr6O11 \\
$-\mathrm{a}$
\end{tabular} & $\begin{array}{c}\mathrm{Rb} 2 \mathrm{O} \\
-\mathrm{a} \\
\end{array}$ & $\begin{array}{c}\mathrm{ReO} \\
-\mathrm{a}\end{array}$ & $\begin{array}{c}\mathrm{ReO} 2 \\
-\mathrm{a} \\
\end{array}$ & $\begin{array}{c}\mathrm{Rh} 2 \mathrm{O} 3 \\
-\mathrm{a}\end{array}$ & $\begin{array}{c}\mathrm{RhO}_{2} \\
-\mathrm{a}\end{array}$ & $\begin{array}{c}\mathrm{RuO} 2 \\
-\mathrm{a} \\
\end{array}$ & $\begin{array}{c}\mathrm{Sb} 2 \mathrm{O} 3 \\
-\mathrm{a} \\
\end{array}$ & $\begin{array}{c}\mathrm{Sb} 2 \mathrm{O} 5 \\
-\mathrm{a}\end{array}$ \\
\hline HLW98-20 & & & 0.0049 & & & & & & 0.0030 & & & & & & & & & & & & 0.0003 \\
\hline HLW98-21 & & & 0.0043 & & & & & & 0.0007 & & & & & & & & & & & & 0.0030 \\
\hline HLW98-22 & & & 0.0029 & & & & & & 0.0020 & & & & & & & & & & & 0.0003 & \\
\hline HLW98-23 & & & 0.0027 & & & & & & 0.0019 & & & & & & & & & & & 0.0004 & \\
\hline HLW98-24 & & & 0.0038 & & & & & & 0.0007 & & & & & & & & & & & & 0.0029 \\
\hline HLW98-25 & & & & & & & & & & & & & & & & & & & & & \\
\hline HLW98-26 & & & & & & & & & & & & & & & & & & & & & \\
\hline HLW98-27B & & & 0.0263 & & & & & & 0.0018 & & & & & & & & & & & & 0.0023 \\
\hline HLW98-28 & & & & & & & & & & & & & & & & & & & & & \\
\hline HLW98-29 & & & & & & & & & & & & & & & & & & & & & \\
\hline HLW98-30 & & & & & & & & & & & & & & & & & & & & & \\
\hline HLW98-31 & & & 0.0000 & 0.0363 & & & & & 0.0019 & & 0.0000 & & & & & & & & & & 0.0026 \\
\hline HLW98-32A & & & 0.0144 & 0.0000 & & & & & 0.0009 & & 0.0000 & & & & & & & & & & 0.0036 \\
\hline HLW98-33 & & & & & & & & & & & & & & & & & & & & & \\
\hline HLW98-34 & & & 0.0000 & 0.0415 & & & & & 0.0028 & & 0.0000 & & & & & & & & & & 0.0004 \\
\hline HLW98-35 & & & 0.0146 & 0.0000 & & & & & 0.0012 & & 0.0000 & & & & & & & & & & 0.0041 \\
\hline HLW98-36 & & & 0.0000 & 0.0060 & & & & & 0.0011 & & 0.0000 & & & & & & & & & & \\
\hline HLW98-37 & & & 0.0000 & 0.0246 & & & & & 0.0013 & & 0.0002 & & & & & & 0.0001 & & & & 0.0007 \\
\hline HLW98-38 & & & 0.0000 & 0.0353 & & & & & 0.0030 & & 0.0004 & & & & & & 0.0003 & & 0.0002 & & 0.0008 \\
\hline HLW98-39 & & & 0.0000 & 0.0367 & & & & & 0.0036 & & 0.0005 & & & & & & 0.0003 & & 0.0002 & & 0.0008 \\
\hline HLW98-40 & & & 0.0160 & 0.0000 & & & & & 0.0013 & & 0.0000 & & & & & & & & 0.0001 & & 0.0039 \\
\hline
\end{tabular}


Appendix A. Database - mass fraction

RPP-WTP HLW Formulation (Kot and Pegg 2001)

\begin{tabular}{|c|c|c|c|c|c|c|c|c|c|c|c|c|c|c|c|c|c|c|c|c|}
\hline Glass ID & $\begin{array}{c}\mathrm{SeO} 2 \\
-\mathrm{a}\end{array}$ & $\begin{array}{c}\mathrm{Sm} 2 \mathrm{O} 3 \\
-\mathrm{a}\end{array}$ & $\begin{array}{c}\mathrm{SnO} \\
-\mathrm{a}\end{array}$ & $\begin{array}{c}\mathrm{SnO}_{2} \\
-\mathrm{a}\end{array}$ & $\begin{array}{c}\mathrm{SO} 3 \\
-\mathrm{a}\end{array}$ & $\begin{array}{c}\mathrm{SrO} \\
-\mathrm{a} \\
\end{array}$ & $\begin{array}{c}\mathrm{Tc} 2 \mathrm{O} 7 \\
-\mathrm{a}\end{array}$ & $\begin{array}{c}\mathrm{TeO} 2 \\
-\mathrm{a}\end{array}$ & $\begin{array}{c}\mathrm{ThO} 2 \\
-\mathrm{a}\end{array}$ & $\begin{array}{c}\mathrm{TiO} 2 \\
-\mathrm{a}\end{array}$ & $\begin{array}{c}\mathrm{T} 12 \mathrm{O} 3 \\
-\mathrm{a}\end{array}$ & $\begin{array}{c}\mathrm{U} 3 \mathrm{O} 8 \\
-\mathrm{a}\end{array}$ & $\begin{array}{c}\mathrm{UO} 2 \\
-\mathrm{a}\end{array}$ & $\begin{array}{c}\mathrm{UO3} \\
-\mathrm{a}\end{array}$ & $\begin{array}{c}\mathrm{V} 2 \mathrm{O} 5 \\
-\mathrm{a}\end{array}$ & $\begin{array}{c}\text { WO3 } \\
-\mathrm{a}\end{array}$ & $\begin{array}{c}\mathrm{Y} 2 \mathrm{O} 3 \\
-\mathrm{a}\end{array}$ & $\begin{array}{c}\mathrm{ZnO} \\
-\mathrm{a}\end{array}$ & $\begin{array}{c}\text { Others } \\
-\mathrm{a}\end{array}$ & $\begin{array}{l}\text { Sum } \\
-\mathrm{a}\end{array}$ \\
\hline HLW98-20 & & & & & & 0.0118 & & & & 0.0002 & 0.0001 & & 0.0006 & & 0.0001 & 0.0006 & & 0.0193 & & 0.9472 \\
\hline HLW98-21 & 0.0006 & & & & & 0.0121 & & 0.0019 & & 0.0012 & 0.0003 & & & 0.0005 & 0.0001 & 0.0008 & & 0.0193 & & 0.9484 \\
\hline HLW98-22 & & & & & & 0.0817 & & 0.0001 & & 0.0006 & 0.0002 & & 0.0014 & & 0.0001 & 0.0003 & & 0.0191 & & 0.9274 \\
\hline HLW98-23 & & & & & & 0.0740 & & & & 0.0004 & 0.0001 & & 0.0012 & & 0.0001 & 0.0003 & & 0.0193 & & 0.9315 \\
\hline HLW98-24 & 0.0007 & & & & & 0.0116 & & 0.0017 & & 0.0014 & 0.0004 & & & 0.0004 & 0.0005 & 0.0002 & & 0.0197 & & 0.9570 \\
\hline HLW98-25 & & & & & & & & & & & & & & & & & & & & \\
\hline HLW98-26 & & & & & & & & & & & & & & & & & & & & \\
\hline HLW98-27B & 0.0005 & & & & & 0.0212 & & 0.0013 & & 0.0014 & 0.0003 & & & 0.0004 & 0.0001 & 0.0002 & & 0.0195 & & 0.9540 \\
\hline HLW98-28 & & & & & & & & & & & & & & & & & & & & \\
\hline HLW98-29 & & & & & & & & & & & & & & & & & & & & \\
\hline HLW98-30 & & & & & & & & & & & & & & & & & & & & \\
\hline HLW98-31 & 0.0006 & & & & & 0.0219 & & 0.0015 & & 0.0013 & 0.0005 & & 0.0006 & & 0.0001 & 0.0004 & & 0.0208 & & 0.9566 \\
\hline HLW98-32A & 0.0000 & & & & & 0.0226 & & 0.0004 & & 0.0003 & 0.0002 & & 0.0005 & & 0.0001 & 0.0002 & & 0.0194 & & 0.9415 \\
\hline HLW98-33 & & & & & & & & & & & & & & & & & & & & \\
\hline HLW98-34 & & & & & & 0.0669 & & 0.0001 & & 0.0007 & 0.0004 & & 0.0009 & & 0.0001 & & & 0.0207 & & 0.9682 \\
\hline HLW98-35 & 0.0005 & & & & & 0.0209 & & 0.0004 & & 0.0012 & 0.0002 & & 0.0008 & & 0.0001 & & & 0.0204 & & 0.9279 \\
\hline HLW98-36 & & & & & & 0.0001 & & & & 0.0005 & 0.0005 & & 0.0219 & & & & & 0.0185 & & 0.9689 \\
\hline HLW98-37 & & & & & & 0.0277 & & 0.0001 & & 0.0005 & & & 0.0255 & & & & & 0.0201 & & 0.9802 \\
\hline HLW98-38 & & & & 0.0018 & & 0.0422 & & 0.0004 & & 0.0006 & 0.0010 & & 0.0473 & & 0.0003 & 0.0006 & & 0.0192 & & 0.9146 \\
\hline HLW98-39 & & & & 0.0019 & & 0.0462 & & 0.0006 & & 0.0007 & 0.0010 & & 0.0508 & & 0.0003 & & & 0.0103 & & 0.9139 \\
\hline HLW98-40 & 0.0000 & & & & & 0.0222 & & 0.0004 & & 0.0003 & & & 0.0095 & & & & & 0.0191 & & 0.9515 \\
\hline
\end{tabular}


Appendix A. Database - mass fraction

\begin{tabular}{|c|c|c|c|c|c|c|c|c|}
\hline Glass ID & $\begin{array}{l}\mathrm{TM} \\
\left({ }^{\circ} \mathrm{C}\right) \\
\end{array}$ & $\begin{array}{l}\text { Gradient } \\
\text { TL }\left({ }^{\circ} \mathrm{C}\right)\end{array}$ & $\begin{array}{l}\text { Uniform } \\
\text { TL }\left({ }^{\circ} \mathrm{C}\right)\end{array}$ & Primary Phase & $\begin{array}{c}\text { Quenched } \\
\text { Visual/OM }\end{array}$ & $\begin{array}{c}\text { Quenched } \\
\text { SEM/EDS or TEM }\end{array}$ & $\begin{array}{l}\text { Quenched } \\
\text { XRD }\end{array}$ & $\begin{array}{c}\text { Quenched } \\
\text { Homogeneous? }\end{array}$ \\
\hline HLW98-20 & 1150 & & $<950{ }^{\circ} \mathrm{C}$ & & Homogeneous dark brown glass & & & \\
\hline HLW98-21 & 1150 & & $<950^{\circ} \mathrm{C}$ & & $\begin{array}{l}\text { Homogeneous glass with some undissolved } \\
\text { solids }\end{array}$ & & & \\
\hline HLW98-22 & 1150 & & $<950{ }^{\circ} \mathrm{C}$ & & Homogeneous dark brown glass & & & \\
\hline HLW98-23 & 1150 & & $<950^{\circ} \mathrm{C}$ & & Homogeneous dark brown glass & & & \\
\hline HLW98-24 & 1150 & & $<950^{\circ} \mathrm{C}$ & & Dark brown homogeneous glass & & & \\
\hline HLW98-25 & 1150 & & $<950{ }^{\circ} \mathrm{C}$ & & Inhomogeneous glass shows multicolor swirls & & & \\
\hline HLW98-26 & 1150 & & $>950^{\circ} \mathrm{C}$ & & Homogeneous dark brown glass & & & \\
\hline HLW98-27B & 1150 & & $>950^{\circ} \mathrm{C}$ & & Homogeneous dark brown glas & & & \\
\hline HLW98-28 & 1150 & & $>950^{\circ} \mathrm{C}$ & & Homogeneous glass & & & \\
\hline HLW98-29 & 1150 & & $>950^{\circ} \mathrm{C}$ & & Homogeneous dark brown glass & & & \\
\hline HLW98-30 & 1150 & & $<950{ }^{\circ} \mathrm{C}$ & & Homogeneous amber tinted glass & & & \\
\hline HLW98-31 & 1150 & & $<950{ }^{\circ} \mathrm{C}$ & & Homogeneous dark brown glass & & & \\
\hline HLW98-32A & 1150 & & $>950^{\circ} \mathrm{C}$ & & Homogeneous dark brown glass & & & \\
\hline HLW98-33 & 1150 & & $>950^{\circ} \mathrm{C}$ & & $\begin{array}{l}\text { Mostly homogeneous with small amount of } \\
\text { undissolved solids }\end{array}$ & & & \\
\hline HLW98-34 & 1150 & & $<950{ }^{\circ} \mathrm{C}$ & & Homogeneous dark brown glass & & & \\
\hline HLW98-35 & 1150 & & ${ }^{3} 950^{\circ} \mathrm{C}$ & & Homogeneous dark brown glass & & & \\
\hline HLW98-36 & 1150 & & $<950{ }^{\circ} \mathrm{C}$ & & Homogeneous dark brown glass & & & \\
\hline HLW98-37 & 1150 & & $>950^{\circ} \mathrm{C}$ & & Homogeneous dark brown glass & & & \\
\hline HLW98-38 & 1150 & & $>950^{\circ} \mathrm{C}$ & & $\begin{array}{l}\text { Homogeneous dark brown glass with metallii } \\
\text { appearance }\end{array}$ & & & \\
\hline HLW98-39 & 1150 & & $>950^{\circ} \mathrm{C}$ & & $\begin{array}{l}\text { Homogeneous dark brown glass with bluish } \\
\text { metallic appearance }\end{array}$ & & & \\
\hline HLW98-40 & 1150 & & & & Inhomogeneous glass with multicolor swirls & & & \\
\hline
\end{tabular}


Appendix A. Database - mass fraction

RPP-WTP HLW Formulation (Kot and Pegg 2001)

\begin{tabular}{|c|c|c|c|c|c|}
\hline Glass ID & $\begin{array}{c}\mathrm{CCC} \\
\text { Visual/OM }\end{array}$ & $\begin{array}{c}\text { CCC } \\
\text { SEM/EDS or TEM }\end{array}$ & $\begin{array}{l}\mathrm{CCC} \\
\mathrm{XRD}\end{array}$ & $\begin{array}{l}\text { Heat Treated } \\
\text { Visual/OM }\end{array}$ & $\begin{array}{c}\text { Heat Treated } \\
\text { SEM/EDS or TEM }\end{array}$ \\
\hline HLW98-20 & & & & & $\begin{array}{l}\text { After } 110 \text { hours, clear glass shows no secondary } \\
\text { phases }\end{array}$ \\
\hline HLW98-21 & & & & & $\begin{array}{l}\text { After } 74 \text { hours, } 0.05-0.2 \text { vol\% of highly magnetic } \\
\text { spinel (high in } \mathrm{Fe} \text {, low in } \mathrm{Al}, \mathrm{Ni}, \mathrm{Cr}, \mathrm{Mn} \text { and } \mathrm{Zn} \text {, } \\
\text { no } \mathrm{Mg} \text { ) }\end{array}$ \\
\hline HLW98-22 & & & & & $\begin{array}{l}\text { After } 65 \text { hours, }<0.01 \text { vol } \% \text { of spinel found at the } \\
\text { bottom }\end{array}$ \\
\hline HLW98-23 & & & & & $\begin{array}{l}\text { After } 65 \text { hours, clear glass shows no } \\
\text { crystallization }\end{array}$ \\
\hline HLW98-24 & & & & & $\begin{array}{l}\text { After } 110 \text { hours, } 0.1 \text { vol\% of spinel, (high in Fe, } \\
\text { low in } \mathrm{Al}, \mathrm{Cr}, \mathrm{Mn}, \mathrm{Ni} \text { and } \mathrm{Zn} \text {; no } \mathrm{Mg} \text { ). Spinel } \\
\text { cluster along air/glass/crucible interface }\end{array}$ \\
\hline HLW98-25 & & & & & $\begin{array}{l}\text { After } 72 \text { hours, }<0.1 \text { vol\% of magnetic spinel } \\
\text { (high in } \mathrm{Fe} \text {, low in } \mathrm{Al}, \mathrm{Cr}, \mathrm{Mg}, \mathrm{Ni} \text { and } \mathrm{Zn} \text { ) }\end{array}$ \\
\hline HLW98-26 & & & & & $\begin{array}{l}\text { After } 66 \text { hours, about } 2.0 \text { vol\% of spinel. (high in } \\
\mathrm{Fe} \text {, low in } \mathrm{Al}, \mathrm{Cr}, \mathrm{Mn}, \mathrm{Ni} \text { and } \mathrm{Zn} \text {, no } \mathrm{Mg} \text { ) }\end{array}$ \\
\hline HLW98-27B & & & & & $\begin{array}{l}\text { After } 60 \text { hours, } 1.0-2.5 \text { vol } \% \text { of spinel (high in } \mathrm{Fe} \\
\text { low in } \mathrm{Al}, \mathrm{Cr}, \mathrm{Mn} \mathrm{Ni} \text { and } \mathrm{Sb} \text {, no } \mathrm{Mg} \text { ) }\end{array}$ \\
\hline HLW98-28 & & & & & After 16 hours, about $1.0 \mathrm{vol} \%$ of spinel \\
\hline HLW98-29 & & & & & $\begin{array}{l}\text { After } 94 \text { hours, about } 2 \text { vol\% of magnetic spinel, } \\
\text { mostly at the bottom, sides, and air-glass interface }\end{array}$ \\
\hline HLW98-30 & & & & & $\begin{array}{l}\text { After } 21 \text { hours, } 0.14 \text { vol\% of spinel. (high in Fe, } \\
\text { low in } \mathrm{Al}, \mathrm{Cr}, \mathrm{Mg}, \mathrm{Mn}, \mathrm{Ni} \text { and } \mathrm{Zn} \text { ), mostly at the } \\
\text { bottom sides and air-glass surface }\end{array}$ \\
\hline HLW98-31 & & & & & $\begin{array}{l}\text { After } 70 \text { hours, } 0.2-0.5 \text { vol } \% \text { of magnetic spinel, } \\
\text { mostly at the sides and bottom }\end{array}$ \\
\hline HLW98-32A & & & & & $\begin{array}{l}\text { After } 68 \text { hours, } 3-7 \text { vol\% of magnetic spinel (high } \\
\text { in } \mathrm{Fe} \text {, low in } \mathrm{Al}, \mathrm{Cr}, \mathrm{Mn}, \mathrm{Ni} \text { and } \mathrm{Zn} \text { ) }\end{array}$ \\
\hline HLW98-33 & & & & & $\begin{array}{l}\text { After } 48 \text { hours, } 2-5 \mathrm{vol} \% \text { of magnetic spinel (high } \\
\text { in } \mathrm{Fe}, \mathrm{Mn} \text { and low in } \mathrm{Al}, \mathrm{Ni} \text { and } \mathrm{Zn} \text { ) }\end{array}$ \\
\hline HLW98-34 & & & & & $\begin{array}{l}\text { After } 48 \text { hours, } 0.75 \text { vol\% of magnetic spinel } \\
\text { (high in Fe, medium in } \mathrm{Mn} \text {, and low in } \mathrm{Al}, \mathrm{Cr}, \mathrm{Ni} \\
\text { and } \mathrm{Zn} \text { ) }\end{array}$ \\
\hline HLW98-35 & & & & & After 72 hours, about 1 vol $\%$ of magnetic spinel \\
\hline HLW98-36 & & & & & $\begin{array}{l}\text { After } 72 \text { hours, no secondary phases were } \\
\text { observed }\end{array}$ \\
\hline HLW98-37 & & & & & $\begin{array}{l}\text { After } 72 \text { hours, } 3-7 \mathrm{vol} \% \text { of white crystals plus } \\
\text { traces of dark magnetic spinel }\end{array}$ \\
\hline HLW98-38 & & & & & $\begin{array}{l}\text { After } 72 \text { hours, } 0.1-0.2 \text { vo\% of spinel }(\mathrm{Cr}, \mathrm{Fe}, \mathrm{Mn} \\
\mathrm{Ni}, \mathrm{Zn}) \text { and } 1-2 \text { vol } \% \text { of white ThO2 crystals }\end{array}$ \\
\hline HLW98-39 & & & & & $\begin{array}{l}\text { After } 72 \text { hours, } 1-2 \text { vol\% of magnetic spinel }(\mathrm{Cr} \text {, } \\
\mathrm{Mn}, \mathrm{Fe}, \mathrm{Ni}, \mathrm{Zn}) \text { and } 25-50 \mathrm{vol} \% \text { of white crystal } \\
\text { high in } \mathrm{Th} \text { and } \mathrm{Zr} \text { with some } \mathrm{Si}\end{array}$ \\
\hline HLW98-40 & & & & & \\
\hline
\end{tabular}


Appendix A. Database - mass fraction

RPP-WTP HLW Formulation (Kot and Pegg 2001)

\begin{tabular}{|c|c|c|c|c|c|c|c|c|c|c|c|c|c|c|c|}
\hline Glass ID & $\begin{array}{c}\text { Heat Treated } \\
\text { XRD } \\
\end{array}$ & $\begin{array}{l}\text { Density } \\
\left(\mathrm{g} / \mathrm{cm}^{3}\right)\end{array}$ & $\begin{array}{c}\text { Fulc Visc } \\
\mathrm{A}\end{array}$ & $\begin{array}{c}\text { Fulc Visc } \\
\text { B } \\
\end{array}$ & $\begin{array}{c}\text { Fulc Visc } \\
\text { To } \\
\end{array}$ & $\begin{array}{c}\mathrm{FV} 1150^{\circ} \mathrm{C} \\
(\mathrm{Pa} \cdot \mathrm{s})\end{array}$ & \begin{tabular}{|c|} 
Arrh Visc \\
A
\end{tabular} & \begin{tabular}{|c|} 
Arrh Visc \\
B \\
\end{tabular} & $\begin{array}{c}\text { Av } \\
1150^{\circ} \mathrm{C} \\
\text { (Da.o) }\end{array}$ & $\begin{array}{c}\mathrm{T}\left({ }^{\circ} \mathrm{C}\right) \text { at } \\
2 \mathrm{~Pa} \cdot \mathrm{s}\end{array}$ & $\begin{array}{c}\mathrm{T}\left({ }^{\circ} \mathrm{C}\right) \text { at } \\
5 \mathrm{~Pa} \cdot \mathrm{s}\end{array}$ & $\begin{array}{l}\mathrm{T}\left({ }^{\circ} \mathrm{C}\right) \text { at } \\
10 \mathrm{~Pa} \cdot \mathrm{s}\end{array}$ & $\begin{array}{c}\mathrm{T} 1 \\
\left({ }^{\circ} \mathrm{C}\right) \\
\end{array}$ & $\begin{array}{c}\mathrm{V} 1 \\
(\mathrm{~Pa} \cdot \mathrm{s})\end{array}$ & $\begin{array}{c}\mathrm{T} 2 \\
\left({ }^{\circ} \mathrm{C}\right) \\
\end{array}$ \\
\hline \multicolumn{16}{|l|}{ HLW98-20 } \\
\hline HLW98-21 & & & & & & & -11.278 & 19350.5 & 10.18 & & & & 1000 & 51.088 & 1050 \\
\hline \multicolumn{16}{|l|}{ HLW98-22 } \\
\hline HLW98-23 & & & & & & & -10.746 & 17369.6 & 4.31 & & & & 1000 & 18.481 & 1050 \\
\hline HLW98-24 & & & & & & & -10.833 & 17819.0 & 5.41 & & & & 1000 & 24.045 & 1050 \\
\hline \multicolumn{16}{|l|}{ HLW98-25 } \\
\hline \multicolumn{16}{|l|}{ HLW98-26 } \\
\hline \multicolumn{16}{|l|}{ HLW98-27B } \\
\hline \multicolumn{16}{|l|}{ HLW98-28 } \\
\hline \multicolumn{16}{|l|}{ HLW98-29 } \\
\hline \multicolumn{16}{|l|}{ HLW98-30 } \\
\hline HLW98-31 & & 2.75 & & & & & -11.094 & 17871.5 & 4.33 & & & & 1000 & 19.199 & 1050 \\
\hline \multicolumn{16}{|l|}{ HLW98-32A } \\
\hline \multicolumn{16}{|l|}{ HLW98-33 } \\
\hline HLW98-34 & & 2.83 & & & & & -11.803 & 18764.9 & 3.99 & & & & 1000 & 19.235 & 1050 \\
\hline \multicolumn{16}{|l|}{ HLW98-35 } \\
\hline \multicolumn{16}{|l|}{ HLW98-36 } \\
\hline \multicolumn{16}{|l|}{ HLW98-37 } \\
\hline \multicolumn{16}{|l|}{ HLW98-38 } \\
\hline \multicolumn{16}{|l|}{ HLW98-39 } \\
\hline HLW98-40 & & & & & & & & & & & & & & & \\
\hline
\end{tabular}


Appendix A. Database - mass fraction

\begin{tabular}{|c|c|c|c|c|c|c|c|c|c|c|c|c|c|c|c|c|c|c|c|c|c|}
\hline Glass ID & $\begin{array}{c}\mathrm{V} 2 \\
(\mathrm{~Pa} \cdot \mathrm{s})\end{array}$ & $\begin{array}{c}\mathrm{T} 3 \\
\left({ }^{\circ} \mathrm{C}\right) \\
\end{array}$ & $\begin{array}{c}\mathrm{V} 3 \\
(\mathrm{~Pa} \cdot \mathrm{s}) \\
\end{array}$ & $\begin{array}{c}\mathrm{T} 4 \\
\left({ }^{\circ} \mathrm{C}\right) \\
\end{array}$ & $\begin{array}{c}\mathrm{V} 4 \\
(\mathrm{~Pa} \cdot \mathrm{s}) \\
\end{array}$ & $\begin{array}{l}\text { T5 } \\
\left({ }^{\circ} \mathrm{C}\right) \\
\end{array}$ & $\begin{array}{c}\text { V5 } \\
(\mathrm{Pa} \cdot \mathrm{s}) \\
\end{array}$ & $\begin{array}{c}\mathrm{T} 6 \\
\left({ }^{\circ} \mathrm{C}\right) \\
\end{array}$ & $\begin{array}{c}\mathrm{V} 6 \\
(\mathrm{~Pa} \cdot \mathrm{s})\end{array}$ & $\begin{array}{c}\mathrm{T} 7 \\
\left({ }^{\circ} \mathrm{C}\right) \\
\end{array}$ & $\begin{array}{c}\text { V7 } \\
(\mathrm{Pa} \cdot \mathrm{s}) \\
\end{array}$ & $\begin{array}{c}\mathrm{T} 8 \\
\left({ }^{\circ} \mathrm{C}\right) \\
\end{array}$ & $\begin{array}{c}\mathrm{V} 8 \\
(\mathrm{~Pa} \cdot \mathrm{s})\end{array}$ & $\begin{array}{c}\mathrm{T} 9 \\
\left({ }^{\circ} \mathrm{C}\right) \\
\end{array}$ & $\begin{array}{c}\mathrm{V} 9 \\
(\mathrm{~Pa} \cdot \mathrm{s}) \\
\end{array}$ & $\begin{array}{l}\mathrm{T} 10 \\
\left({ }^{\circ} \mathrm{C}\right) \\
\end{array}$ & $\begin{array}{c}\mathrm{V} 10 \\
(\mathrm{~Pa} \cdot \mathrm{s})\end{array}$ & $\begin{array}{l}\mathrm{T} 11 \\
\left({ }^{\circ} \mathrm{C}\right) \\
\end{array}$ & $\begin{array}{c}\text { V11 } \\
(\mathrm{Pa} \cdot \mathrm{s})\end{array}$ & $\begin{array}{l}\mathrm{T} 12 \\
\left({ }^{\circ} \mathrm{C}\right) \\
\end{array}$ & $\begin{array}{c}\mathrm{V} 12 \\
(\mathrm{~Pa} \cdot \mathrm{s})\end{array}$ \\
\hline \multicolumn{22}{|l|}{ HLW98-20 } \\
\hline HLW98-21 & 28.278 & 1100 & 16.537 & 1150 & 10.144 & 1200 & 6.486 & & & & & & & & & & & & & & \\
\hline \multicolumn{22}{|l|}{ HLW98-22 } \\
\hline HLW98-23 & 10.717 & 1100 & 6.602 & 1150 & 4.28 & 1200 & 2.898 & & & & & & & & & & & & & & \\
\hline HLW98-24 & 13.813 & 1100 & 8.412 & 1150 & 5.383 & 1200 & 3.594 & & & & & & & & & & & & & & \\
\hline \multicolumn{22}{|l|}{ HLW98-25 } \\
\hline \multicolumn{22}{|l|}{ HLW98-26 } \\
\hline \multicolumn{22}{|l|}{ HLW98-27B } \\
\hline \multicolumn{22}{|l|}{ HLW98-28 } \\
\hline \multicolumn{22}{|l|}{ HLW98-29 } \\
\hline \multicolumn{22}{|l|}{ HLW98-30 } \\
\hline HLW98-31 & 11.114 & 1100 & 6.771 & 1150 & 4.312 & 1200 & 2.854 & & & & & & & & & & & & & & \\
\hline \multicolumn{22}{|l|}{ HLW98-32A } \\
\hline \multicolumn{22}{|l|}{ HLW98-33 } \\
\hline HLW98-34 & 10.676 & 1100 & 6.326 & 1150 & 3.961 & 1200 & 2.599 & & & & & & & & & & & & & & \\
\hline \multicolumn{22}{|l|}{ HLW98-35 } \\
\hline \multicolumn{22}{|l|}{ HLW98-36 } \\
\hline \multicolumn{22}{|l|}{ HLW98-37 } \\
\hline \multicolumn{22}{|l|}{ HLW98-38 } \\
\hline \multicolumn{22}{|l|}{ HLW98-39 } \\
\hline HLW98-40 & & & & & & & & & & & & & & & & & & & & & \\
\hline
\end{tabular}


Appendix A. Database - mass fraction

RPP-WTP HLW Formulation (Kot and Pegg 2001)

\begin{tabular}{|c|c|c|c|c|c|c|c|c|c|c|c|c|c|c|c|c|}
\hline Glass ID & $\begin{array}{l}\mathrm{T} 13 \\
\left({ }^{\circ} \mathrm{C}\right) \\
\end{array}$ & $\begin{array}{c}\mathrm{V} 13 \\
(\mathrm{~Pa} \cdot \mathrm{s}) \\
\end{array}$ & $\begin{array}{l}\mathrm{T} 14 \\
\left({ }^{\circ} \mathrm{C}\right) \\
\end{array}$ & $\begin{array}{c}\mathrm{V} 14 \\
(\mathrm{~Pa} \cdot \mathrm{s})\end{array}$ & $\begin{array}{c}\text { Q PCT } \\
\text { B }\left(\mathrm{g} / \mathrm{m}^{2}\right)\end{array}$ & $\begin{array}{c}\text { Q PCT } \\
\mathrm{Li}\left(\mathrm{g} / \mathrm{m}^{2}\right)\end{array}$ & $\begin{array}{c}\text { Q PCT } \\
\mathrm{Na}\left(\mathrm{g} / \mathrm{m}^{2}\right) \\
\end{array}$ & $\begin{array}{c}\text { Q PCT } \\
\mathrm{Si}\left(\mathrm{g} / \mathrm{m}^{2}\right)\end{array}$ & $\begin{array}{c}\text { Q PCT } \\
\text { pH }\end{array}$ & $\begin{array}{c}\text { CCC PCT } \\
\mathrm{B}\left(\mathrm{g} / \mathrm{m}^{2}\right) \\
\end{array}$ & $\begin{array}{c}\text { CCC PCT } \\
\mathrm{Li}\left(\mathrm{g} / \mathrm{m}^{2}\right) \\
\end{array}$ & $\begin{array}{l}\text { CCC PCT } \\
\mathrm{Na}\left(\mathrm{g} / \mathrm{m}^{2}\right) \\
\end{array}$ & $\begin{array}{c}\text { CCC PCT } \\
\mathrm{Si}\left(\mathrm{g} / \mathrm{m}^{2}\right) \\
\end{array}$ & $\begin{array}{c}\text { CCC PCT } \\
\mathrm{pH}\end{array}$ & $\begin{array}{l}\text { Q PCT B at } \\
20^{\circ} \mathrm{C}\left(\mathrm{g} / \mathrm{m}^{2}\right)\end{array}$ & $\begin{array}{l}\text { Q PCT Li at } \\
20^{\circ} \mathrm{C}\left(\mathrm{g} / \mathrm{m}^{2}\right)\end{array}$ \\
\hline HLW98-20 & & & & & 0.295 & 0.225 & 0.25 & 0.145 & 9.52 & & & & & & & \\
\hline HLW98-21 & & & & & 0.28 & 0.29 & 0.135 & 0.165 & 9.81 & & & & & & & \\
\hline HLW98-22 & & & & & 0.215 & 0.23 & 0.215 & 0.1 & 9.67 & & & & & & & \\
\hline HLW98-23 & & & & & 0.22 & 0.235 & 0.205 & 0.11 & 9.51 & & & & & & & \\
\hline \multicolumn{17}{|l|}{ HLW98-24 } \\
\hline \multicolumn{17}{|l|}{ HLW98-25 } \\
\hline \multicolumn{17}{|l|}{ HLW98-26 } \\
\hline HLW98-27B & & & & & 0.355 & 0.34 & 0.42 & 0.215 & 10.85 & & & & & & & \\
\hline \multicolumn{17}{|l|}{ HLW98-28 } \\
\hline \multicolumn{17}{|l|}{ HLW98-29 } \\
\hline \multicolumn{17}{|l|}{ HLW98-30 } \\
\hline HLW98-31 & & & & & 0.365 & 0.26 & 0.19 & 0.16 & 9.84 & & & & & & & \\
\hline HLW98-32A & & & & & 0.235 & 0.215 & 0.075 & 0.155 & 9.54 & & & & & & & \\
\hline \multicolumn{17}{|l|}{ HLW98-33 } \\
\hline HLW98-34 & & & & & 0.235 & 0.235 & 0.205 & 0.115 & 9.75 & & & & & & & \\
\hline \multicolumn{17}{|l|}{ HLW98-35 } \\
\hline \multicolumn{17}{|l|}{ HLW98-36 } \\
\hline \multicolumn{17}{|l|}{ HLW98-37 } \\
\hline \multicolumn{17}{|l|}{ HLW98-38 } \\
\hline \multicolumn{17}{|l|}{ HLW98-39 } \\
\hline HLW98-40 & & & & & & & & & & & & & & & & \\
\hline
\end{tabular}


Appendix A. Database - mass fraction

RPP-WTP HLW Formulation (Kot and Pegg 2001)

\begin{tabular}{|c|c|c|c|c|c|c|c|c|c|c|c|c|}
\hline Glass ID & $\begin{array}{l}\text { Q PCT Na at } \\
20^{\circ} \mathrm{C}\left(\mathrm{g} / \mathrm{m}^{2}\right)\end{array}$ & $\begin{array}{l}\text { Q PCT Si at } \\
20^{\circ} \mathrm{C}\left(\mathrm{g} / \mathrm{m}^{2}\right)\end{array}$ & $\begin{array}{c}\mathrm{QpH} \\
\text { at } 20^{\circ} \mathrm{C}\end{array}$ & $\begin{array}{c}\text { TCLP Ag } \\
\text { (ppm) }\end{array}$ & $\begin{array}{c}\text { TCLP As } \\
\text { (ppm) }\end{array}$ & $\begin{array}{c}\text { TCLP Ba } \\
\text { (ppm) }\end{array}$ & $\begin{array}{c}\text { TCLP Cd } \\
(\mathrm{ppm})\end{array}$ & $\begin{array}{c}\text { TCLP Cr } \\
(\mathrm{ppm})\end{array}$ & $\begin{array}{c}\text { TCLP Ni } \\
(\mathrm{ppm})\end{array}$ & $\begin{array}{c}\text { TCLP Pb } \\
\text { (ppm) }\end{array}$ & $\begin{array}{l}\text { TCLP Se } \\
\text { (ppm) }\end{array}$ & $\begin{array}{c}\text { TCLP Zn } \\
(\mathrm{ppm})\end{array}$ \\
\hline HLW98-20 & & & & $<0.004$ & 0.05 & 0.208 & 0.009 & 0.008 & 0.032 & 0.09 & 0.0532 & 0.531 \\
\hline HLW98-21 & & & & $<0.003$ & 0.05 & 0.022 & 0.058 & $<0.006$ & 0.04 & 0.036 & 0.0532 & 0.246 \\
\hline HLW98-22 & & & & $<0.003$ & 0.05 & 0.2 & 0.008 & 0.01 & 0.04 & 0.065 & 0.0532 & 0.59 \\
\hline HLW98-23 & & & & $<0.003$ & 0.05 & 0.16 & 0.004 & $<0.006$ & 0.01 & 0.057 & 0.0532 & 0.51 \\
\hline HLW98-24 & & & & $<0.003$ & 0.05 & 0.027 & 0.071 & $<0.006$ & 0.046 & $<0.03$ & 0.0532 & 0.29 \\
\hline HLW98-25 & & & & 0.014 & 0.05 & 0.039 & 0.091 & 0.009 & 0.06 & $<0.03$ & 0.0532 & 0.343 \\
\hline HLW98-26 & & & & 0.01 & 0.05 & 0.02 & 0.06 & $<0.006$ & 0.06 & 0.04 & 0.0532 & 0.34 \\
\hline HLW98-27B & & & & $<.0031$ & 0.05 & 0.04 & 0.09 & 0.03 & 0.11 & 0.06 & 0.0532 & 0.45 \\
\hline HLW98-28 & & & & $<.0031$ & 0.05 & 0.03 & 0.08 & 0.02 & 0.09 & 0.07 & 0.0532 & 0.37 \\
\hline HLW98-29 & & & & $<.0031$ & 0.05 & 0.03 & 0.08 & 0.01 & 0.1 & 0.04 & 0.0532 & 0.41 \\
\hline HLW98-30 & & & & 0.01 & 0.06 & 0.16 & 0.15 & 0.01 & 0.15 & 0.03 & 0.0532 & 0.41 \\
\hline HLW98-31 & & & & $<0.0031$ & 0.05 & 0.03 & 0.07 & 0.02 & 0.09 & 0.03 & 0.09 & 0.33 \\
\hline HLW98-32A & & & & $<0.0031$ & 0.061 & 0.097 & 0.172 & $<0.0055$ & 0.088 & 0.026 & 0.0532 & 0.337 \\
\hline HLW98-33 & & & & 0.07 & 0.05 & 0.118 & 0.014 & 0.007 & 0.034 & 0.203 & 0.0532 & 0.607 \\
\hline HLW98-34 & & & & 0.064 & 0.05 & 0.193 & 0.079 & 0.012 & 0.107 & 0.081 & 0.0532 & 0.547 \\
\hline \multicolumn{13}{|l|}{ HLW98-35 } \\
\hline \multicolumn{13}{|l|}{ HLW98-36 } \\
\hline \multicolumn{13}{|l|}{ HLW98-37 } \\
\hline \multicolumn{13}{|l|}{ HLW98-38 } \\
\hline \multicolumn{13}{|l|}{ HLW98-39 } \\
\hline HLW98-40 & & & & & & & & & & & & \\
\hline
\end{tabular}


RPP-WTP HLW Formulation (Kot and Pegg 2001)

\begin{tabular}{|c|c|c|c|c|c|c|c|c|c|c|c|c|c|c|c|c|c|c|c|c|c|}
\hline Glass ID & $\begin{array}{c}\mathrm{A} 12 \mathrm{O} 3 \\
-\mathrm{t}\end{array}$ & $\begin{array}{c}\mathrm{B} 2 \mathrm{O} 3 \\
-\mathrm{t}\end{array}$ & $\begin{array}{c}\mathrm{CaO} \\
-\mathrm{t}\end{array}$ & $\begin{array}{c}\mathrm{Fe} 2 \mathrm{O} 3 \\
-\mathrm{t} \\
\end{array}$ & $\begin{array}{c}\mathrm{FeO} \\
-\mathrm{t}\end{array}$ & $\begin{array}{c}\mathrm{K} 2 \mathrm{O} \\
-\mathrm{t}\end{array}$ & $\begin{array}{c}\mathrm{Li} 2 \mathrm{O} \\
-\mathrm{t}\end{array}$ & $\begin{array}{c}\mathrm{MgO} \\
-\mathrm{t}\end{array}$ & $\begin{array}{c}\mathrm{Na} 2 \mathrm{O} \\
-\mathrm{t}\end{array}$ & $\begin{array}{c}\mathrm{NiO} \\
-\mathrm{t}\end{array}$ & $\begin{array}{c}\mathrm{P} 2 \mathrm{O} 5 \\
-\mathrm{t}\end{array}$ & $\begin{array}{c}\mathrm{SiO} 2 \\
-\mathrm{t}\end{array}$ & $\begin{array}{c}\mathrm{ZrO} 2 \\
-\mathrm{t}\end{array}$ & $\begin{array}{c}\mathrm{Ag} 2 \mathrm{O} \\
-\mathrm{t}\end{array}$ & $\begin{array}{c}\mathrm{As} 2 \mathrm{O} 3 \\
-\mathrm{t}\end{array}$ & $\begin{array}{c}\mathrm{BaO} \\
-\mathrm{t}\end{array}$ & $\begin{array}{c}\mathrm{Bi} 2 \mathrm{O} 3 \\
-\mathrm{t}\end{array}$ & $\begin{array}{l}\mathrm{Br} \\
-\mathrm{t}\end{array}$ & $\begin{array}{c}\mathrm{CdO} \\
-\mathrm{t}\end{array}$ & $\begin{array}{c}\mathrm{Ce} 2 \mathrm{O} 3 \\
-\mathrm{t}\end{array}$ & $\begin{array}{c}\mathrm{CeO} 2 \\
-\mathrm{t}\end{array}$ \\
\hline HLW98-41 & 0.0343 & 0.0300 & 0.0065 & 0.0677 & & 0.0010 & 0.0500 & 0.0008 & 0.1404 & 0.0033 & 0.0048 & 0.3628 & 0.0698 & 0.0009 & 0.0000 & 0.0003 & & & 0.0009 & & 0.0012 \\
\hline HLW98-42 & 0.0797 & 0.0850 & 0.0025 & 0.1152 & & 0.0006 & 0.0700 & 0.0007 & 0.0889 & 0.0069 & 0.0006 & 0.4477 & 0.0151 & 0.0004 & 0.0004 & 0.0003 & & & 0.0081 & & 0.0005 \\
\hline HLW98-43 & 0.0325 & 0.0500 & 0.0062 & 0.0641 & & 0.0010 & 0.0600 & 0.0008 & 0.1275 & 0.0032 & 0.0045 & 0.3666 & 0.0661 & 0.0009 & 0.0000 & 0.0003 & & & 0.0008 & & 0.0011 \\
\hline HLW98-44 & 0.0325 & 0.0700 & 0.0062 & 0.0641 & & 0.0010 & 0.0600 & 0.0008 & 0.1025 & 0.0032 & 0.0045 & 0.3716 & 0.0661 & 0.0009 & 0.0000 & 0.0003 & 0.0000 & & 0.0008 & & 0.0011 \\
\hline HLW98-45 & 0.0314 & 0.0700 & 0.0060 & 0.0620 & & 0.0010 & 0.0600 & 0.0007 & 0.1033 & 0.0031 & 0.0044 & 0.3834 & 0.0639 & 0.0009 & 0.0000 & 0.0003 & 0.0000 & & 0.0008 & & 0.0011 \\
\hline HLW98-46 & 0.0300 & 0.0750 & 0.0057 & 0.0591 & & 0.0009 & 0.0700 & 0.0007 & 0.0984 & 0.0029 & 0.0042 & 0.3899 & 0.0610 & 0.0008 & 0.0000 & 0.0002 & 0.0000 & & 0.0008 & & 0.0011 \\
\hline HLW98-47 & 0.0287 & 0.0750 & 0.0055 & 0.0567 & & 0.0009 & 0.0700 & 0.0007 & 0.1014 & 0.0028 & 0.0040 & 0.4016 & 0.0584 & 0.0008 & 0.0000 & 0.0002 & 0.0000 & & 0.0007 & & 0.0010 \\
\hline HLW98-48 & 0.0449 & 0.0900 & 0.0047 & 0.0492 & & 0.0008 & 0.0500 & 0.0006 & 0.1103 & 0.0024 & 0.0035 & 0.4216 & 0.0507 & 0.0007 & 0.0000 & 0.0002 & 0.0000 & & 0.0006 & & 0.0009 \\
\hline HLW98-49 & 0.0588 & 0.0700 & 0.0045 & 0.0470 & & 0.0007 & 0.0400 & 0.0006 & 0.1385 & 0.0023 & 0.0033 & 0.4208 & 0.0485 & 0.0007 & 0.0000 & 0.0002 & 0.0000 & & 0.0006 & & 0.0008 \\
\hline HLW98-50 & 0.0250 & 0.1100 & 0.0043 & 0.0458 & & 0.0002 & 0.0400 & 0.0006 & 0.1359 & 0.0026 & 0.0035 & 0.4544 & 0.0546 & 0.0007 & 0.0000 & 0.0002 & 0.0000 & & 0.0007 & & 0.0008 \\
\hline HLW98-51R & 0.0238 & 0.0900 & 0.0045 & 0.0470 & & 0.0007 & 0.0500 & 0.0006 & 0.0860 & 0.0023 & 0.0033 & 0.4783 & 0.0485 & 0.0007 & 0.0000 & 0.0002 & 0.0000 & & 0.0006 & & 0.0008 \\
\hline HLW98-52 & 0.0797 & 0.0700 & 0.0025 & 0.1152 & & 0.0006 & 0.0600 & 0.0007 & 0.0989 & 0.0069 & 0.0006 & 0.4626 & 0.0151 & 0.0004 & 0.0004 & 0.0003 & & & 0.0081 & & 0.0005 \\
\hline HLW98-53A & 0.0811 & 0.0725 & 0.0025 & 0.1172 & & 0.0006 & 0.0600 & 0.0007 & 0.0992 & 0.0071 & 0.0006 & 0.4638 & 0.0154 & 0.0004 & 0.0004 & 0.0003 & & & 0.0083 & & 0.0005 \\
\hline HLW98-54 & 0.0797 & 0.1000 & 0.0025 & 0.1152 & & 0.0006 & 0.0600 & 0.0007 & 0.0564 & 0.0069 & 0.0006 & 0.4752 & 0.0151 & 0.0004 & 0.0004 & 0.0003 & & & 0.0081 & & 0.0005 \\
\hline HLW98-55 & 0.0797 & 0.0700 & 0.0025 & 0.1152 & & 0.0006 & 0.0700 & 0.0007 & 0.0914 & 0.0069 & 0.0006 & 0.4886 & 0.0151 & 0.0004 & 0.0004 & 0.0003 & & & 0.0081 & & 0.0005 \\
\hline HLW98-56 & 0.0756 & 0.0700 & 0.0058 & 0.1179 & & 0.0000 & 0.0700 & 0.0012 & 0.0954 & 0.0077 & 0.0024 & 0.4972 & 0.0165 & 0.0002 & 0.0000 & 0.0004 & 0.0000 & & 0.0137 & & 0.0007 \\
\hline HLW98-57 & 0.0744 & 0.0400 & 0.0062 & 0.1195 & & 0.0003 & 0.0500 & 0.0012 & 0.1302 & 0.0075 & 0.0025 & 0.4713 & 0.0162 & 0.0002 & 0.0000 & 0.0004 & 0.0000 & & 0.0133 & & 0.0008 \\
\hline HLW98-58 & 0.0763 & 0.0400 & 0.0061 & 0.1208 & & 0.0003 & 0.0500 & 0.0012 & 0.1376 & 0.0078 & 0.0025 & 0.4787 & 0.0166 & 0.0002 & 0.0000 & 0.0004 & 0.0000 & & 0.0137 & & 0.0008 \\
\hline HLW98-59 & 0.0800 & 0.0400 & 0.0025 & 0.1156 & & 0.0006 & 0.0500 & 0.0007 & 0.1315 & 0.0070 & 0.0006 & 0.4775 & 0.0152 & 0.0004 & 0.0004 & 0.0003 & & & 0.0082 & & 0.0005 \\
\hline HLW98-60 & 0.0760 & 0.0400 & 0.0059 & 0.1194 & & 0.0003 & 0.0500 & 0.0012 & 0.1325 & 0.0077 & 0.0024 & 0.4765 & 0.0166 & 0.0002 & 0.0000 & 0.0004 & 0.0000 & & 0.0137 & & 0.0007 \\
\hline HLW98-61 & 0.0770 & 0.0400 & 0.0049 & 0.1224 & & 0.0003 & 0.0500 & 0.0012 & 0.1391 & 0.0076 & 0.0046 & 0.4708 & 0.0147 & 0.0002 & 0.0000 & 0.0004 & 0.0000 & & 0.0139 & & 0.0006 \\
\hline HLW98-62 & 0.0967 & 0.1200 & 0.0231 & 0.0393 & & 0.0008 & 0.0500 & 0.0003 & 0.0964 & 0.0025 & 0.0022 & 0.4255 & 0.0808 & 0.0000 & 0.0000 & 0.0000 & & & 0.0006 & & 0.0000 \\
\hline HLW98-63 & 0.1185 & 0.0627 & 0.0079 & 0.1290 & & 0.0003 & 0.0358 & 0.0024 & 0.1010 & 0.0017 & 0.0017 & 0.3783 & 0.0013 & 0.0026 & 0.0000 & 0.0007 & & & 0.0005 & & 0.0003 \\
\hline HLW98-64 & 0.0814 & 0.0956 & 0.0027 & 0.1143 & & 0.0019 & 0.0574 & 0.0007 & 0.0639 & 0.0059 & 0.0014 & 0.4354 & 0.0392 & 0.0000 & 0.0004 & 0.0005 & & & 0.0041 & & 0.0009 \\
\hline
\end{tabular}


Appendix A. Database - mass fraction

RPP-WTP HLW Formulation (Kot and Pegg 2001)

\begin{tabular}{|c|c|c|c|c|c|c|c|c|c|c|c|c|c|c|c|c|c|c|c|c|c|}
\hline Glass ID & $\begin{array}{l}\mathrm{Cl} \\
-\mathrm{t} \\
\end{array}$ & $\begin{array}{c}\mathrm{CoO} \\
-\mathrm{t}\end{array}$ & $\begin{array}{c}\mathrm{Co} 2 \mathrm{O} 3 \\
-\mathrm{t}\end{array}$ & $\begin{array}{c}\mathrm{Cr} 2 \mathrm{O} 3 \\
-\mathrm{t}\end{array}$ & $\begin{array}{c}\mathrm{Cs} 2 \mathrm{O} \\
-\mathrm{t}\end{array}$ & $\begin{array}{c}\mathrm{CuO} \\
-\mathrm{t}\end{array}$ & $\begin{array}{c}\mathrm{Eu} 2 \mathrm{O} 3 \\
-\mathrm{t}\end{array}$ & $\begin{array}{l}F \\
-t\end{array}$ & $\begin{array}{c}\mathrm{Ga} 2 \mathrm{O} 3 \\
-\mathrm{t}\end{array}$ & $\begin{array}{c}\mathrm{Gd} 2 \mathrm{O} 3 \\
-\mathrm{t}\end{array}$ & $\begin{array}{c}\mathrm{HgO} \\
-\mathrm{t}\end{array}$ & $\begin{array}{l}I \\
-t\end{array}$ & $\begin{array}{c}\mathrm{La} 2 \mathrm{O} 3 \\
-\mathrm{t}\end{array}$ & $\begin{array}{c}\mathrm{MnO} 2 \\
-\mathrm{t}\end{array}$ & $\begin{array}{c}\mathrm{MnO} \\
-\mathrm{t}\end{array}$ & $\begin{array}{c}\mathrm{MoO} \\
-\mathrm{t}\end{array}$ & $\begin{array}{c}\mathrm{MoO} 3 \\
-\mathrm{t}\end{array}$ & $\begin{array}{c}\mathrm{Nb} 2 \mathrm{O} 5 \\
-\mathrm{t}\end{array}$ & $\begin{array}{c}\mathrm{Nd} 2 \mathrm{O} 3 \\
-\mathrm{t}\end{array}$ & $\begin{array}{c}\mathrm{PbO} \\
-\mathrm{t}\end{array}$ & $\begin{array}{c}\mathrm{PdO} 2 \\
-\mathrm{t}\end{array}$ \\
\hline HLW98-41 & 0.0000 & 0.0000 & & 0.0020 & 0.0000 & 0.0004 & & 0.0000 & & & 0.0000 & & 0.0003 & 0.0000 & 0.0372 & & & & 0.0008 & 0.0022 & \\
\hline HLW98-42 & 0.0000 & 0.0000 & & 0.0008 & 0.0009 & 0.0002 & & 0.0002 & & & 0.0000 & & 0.0032 & 0.0146 & 0.0000 & & & & 0.0000 & 0.0007 & \\
\hline HLW98-43 & 0.0000 & 0.0000 & & 0.0019 & 0.0000 & 0.0004 & & 0.0000 & & & 0.0000 & & 0.0003 & 0.0000 & 0.0352 & & & & 0.0008 & 0.0021 & \\
\hline HLW98-44 & 0.0000 & 0.0000 & & 0.0019 & 0.0000 & 0.0004 & & 0.0000 & & & & & 0.0003 & & 0.0352 & 0.0000 & & & 0.0008 & 0.0021 & \\
\hline HLW98-45 & 0.0000 & 0.0000 & & 0.0018 & 0.0000 & 0.0003 & & 0.0000 & & & & & 0.0003 & & 0.0340 & 0.0000 & & & 0.0008 & 0.0020 & \\
\hline HLW98-46 & 0.0000 & 0.0000 & & 0.0017 & 0.0000 & 0.0003 & & 0.0000 & & & & & 0.0002 & & 0.0325 & 0.0000 & & & 0.0007 & 0.0019 & \\
\hline HLW98-47 & 0.0000 & 0.0000 & & 0.0017 & 0.0000 & 0.0003 & & 0.0000 & & & & & 0.0002 & & 0.0311 & 0.0000 & & & 0.0007 & 0.0019 & \\
\hline HLW98-48 & 0.0000 & 0.0000 & & 0.0014 & 0.0000 & 0.0003 & & 0.0000 & & & & & 0.0002 & & 0.0270 & 0.0000 & & & 0.0006 & 0.0016 & \\
\hline HLW98-49 & 0.0000 & 0.0000 & & 0.0014 & 0.0000 & 0.0003 & & 0.0000 & & & & & 0.0002 & & 0.0258 & 0.0000 & & & 0.0006 & 0.0016 & \\
\hline HLW98-50 & 0.0000 & 0.0000 & & 0.0010 & 0.0000 & 0.0002 & & 0.0000 & & & & & 0.0001 & & 0.0091 & 0.0000 & & & 0.0004 & 0.0011 & \\
\hline HLW98-51R & 0.0000 & 0.0000 & & 0.0014 & 0.0000 & 0.0003 & & 0.0000 & & & & & 0.0002 & & 0.0258 & 0.0000 & & & 0.0006 & 0.0016 & \\
\hline HLW98-52 & 0.0000 & 0.0000 & & 0.0008 & 0.0009 & 0.0002 & & 0.0002 & & & 0.0000 & & 0.0032 & 0.0000 & 0.0146 & & & & 0.0000 & 0.0007 & \\
\hline HLW98-53A & 0.0000 & 0.0000 & & 0.0008 & 0.0010 & 0.0002 & & 0.0002 & & & 0.0000 & & 0.0033 & 0.0000 & 0.0149 & & & & 0.0000 & 0.0007 & \\
\hline HLW98-54 & 0.0000 & 0.0000 & & 0.0008 & 0.0009 & 0.0002 & & 0.0002 & & & 0.0000 & & 0.0032 & 0.0000 & 0.0146 & & & & 0.0000 & 0.0007 & \\
\hline HLW98-55 & 0.0000 & 0.0000 & & 0.0008 & 0.0009 & 0.0002 & & 0.0002 & & & 0.0000 & & 0.0032 & 0.0000 & 0.0146 & & & & 0.0000 & 0.0007 & \\
\hline HLW98-56 & 0.0005 & 0.0000 & & 0.0009 & 0.0000 & 0.0000 & & 0.0001 & & & & & 0.0031 & & 0.0026 & 0.0000 & & & 0.0001 & 0.0000 & \\
\hline HLW98-57 & 0.0005 & 0.0001 & & 0.0012 & 0.0000 & 0.0000 & & 0.0001 & & & & & 0.0031 & & 0.0162 & 0.0000 & & & 0.0003 & 0.0005 & \\
\hline HLW98-58 & 0.0005 & 0.0001 & & 0.0011 & 0.0000 & 0.0000 & & 0.0001 & & & & & 0.0031 & & 0.0097 & 0.0000 & & & 0.0002 & 0.0003 & \\
\hline HLW98-59 & 0.0002 & 0.0000 & & 0.0008 & 0.0009 & 0.0002 & & 0.0002 & & & 0.0000 & & 0.0033 & 0.0000 & 0.0147 & & & & 0.0000 & 0.0007 & \\
\hline HLW98-60 & 0.0005 & 0.0001 & & 0.0010 & 0.0000 & 0.0000 & & 0.0001 & & & & & 0.0031 & & 0.0061 & 0.0000 & & & 0.0002 & 0.0001 & \\
\hline HLW98-61 & 0.0003 & 0.0001 & & 0.0011 & 0.0000 & 0.0003 & & 0.0001 & & & & & 0.0030 & & 0.0103 & 0.0000 & & & 0.0022 & 0.0011 & \\
\hline HLW98-62 & 0.0002 & 0.0005 & & 0.0014 & 0.0007 & 0.0000 & & 0.0002 & & & 0.0001 & & 0.0000 & 0.0000 & 0.0171 & & & & 0.0000 & 0.0005 & \\
\hline HLW98-63 & 0.0003 & 0.0000 & & 0.0018 & 0.0011 & 0.0000 & & 0.0003 & & & 0.0000 & & 0.0012 & 0.0000 & 0.0488 & & & & 0.0000 & 0.0024 & \\
\hline HLW98-64 & 0.0001 & 0.0000 & & 0.0005 & 0.0009 & 0.0003 & & 0.0004 & & & 0.0000 & & 0.0036 & 0.0333 & 0.0000 & & & & 0.0000 & 0.0017 & \\
\hline
\end{tabular}


Appendix A. Database - mass fraction

RPP-WTP HLW Formulation (Kot and Pegg 2001)

\begin{tabular}{|c|c|c|c|c|c|c|c|c|c|c|c|c|c|c|c|c|c|c|c|c|c|}
\hline Glass ID & $\begin{array}{c}\mathrm{PdO} \\
-\mathrm{t} \\
\end{array}$ & $\begin{array}{c}\mathrm{Pr} 2 \mathrm{O} 3 \\
-\mathrm{t}\end{array}$ & $\begin{array}{c}\text { Pr6O11 } \\
-t\end{array}$ & $\begin{array}{c}\mathrm{Rb} 2 \mathrm{O} \\
-\mathrm{t}\end{array}$ & $\begin{array}{c}\mathrm{ReO} \\
-\mathrm{t}\end{array}$ & $\begin{array}{c}\mathrm{ReO} 2 \\
-\mathrm{t}\end{array}$ & $\begin{array}{c}\mathrm{Rh} 2 \mathrm{O} 3 \\
-\mathrm{t}\end{array}$ & $\begin{array}{c}\mathrm{RhO} 2 \\
-\mathrm{t}\end{array}$ & $\begin{array}{c}\mathrm{RuO} 2 \\
-\mathrm{t}\end{array}$ & $\begin{array}{c}\mathrm{Sb} 2 \mathrm{O} 3 \\
-\mathrm{t}\end{array}$ & $\begin{array}{c}\mathrm{Sb} 2 \mathrm{O} 5 \\
-\mathrm{t}\end{array}$ & $\begin{array}{c}\mathrm{SeO} 2 \\
-\mathrm{t}\end{array}$ & $\begin{array}{c}\mathrm{Sm} 2 \mathrm{O} 3 \\
-\mathrm{t}\end{array}$ & $\begin{array}{c}\mathrm{SnO} \\
-\mathrm{t} \\
\end{array}$ & $\begin{array}{c}\mathrm{SnO} 2 \\
-\mathrm{t} \\
\end{array}$ & $\begin{array}{c}\mathrm{SO} 3 \\
-\mathrm{t}\end{array}$ & $\begin{array}{c}\mathrm{SrO} \\
-\mathrm{t}\end{array}$ & $\begin{array}{c}\mathrm{Tc} 2 \mathrm{O} 7 \\
-\mathrm{t}\end{array}$ & $\begin{array}{c}\mathrm{TeO} 2 \\
-\mathrm{t}\end{array}$ & $\begin{array}{c}\mathrm{ThO} 2 \\
-\mathrm{t}\end{array}$ & $\begin{array}{c}\mathrm{TiO}_{2} \\
-\mathrm{t}\end{array}$ \\
\hline HLW98-41 & 0.0002 & & & & & & 0.0005 & & 0.0002 & & 0.0000 & 0.0000 & & & 0.0010 & 0.0000 & 0.0490 & & 0.0000 & 0.0591 & 0.0002 \\
\hline HLW98-42 & 0.0000 & & & & & & 0.0000 & & 0.0000 & & 0.0036 & 0.0006 & & & 0.0000 & 0.0003 & 0.0234 & & 0.0002 & 0.0000 & 0.0000 \\
\hline HLW98-43 & 0.0002 & & & & & & 0.0004 & & 0.0002 & & 0.0000 & 0.0000 & & & 0.0009 & 0.0000 & 0.0464 & & 0.0000 & 0.0560 & 0.0002 \\
\hline HLW98-44 & 0.0002 & & & & 0.0000 & & 0.0004 & & 0.0002 & & 0.0000 & 0.0000 & & & 0.0009 & 0.0000 & 0.0464 & 0.0000 & 0.0000 & 0.0560 & 0.0002 \\
\hline HLW98-45 & 0.0002 & & & & 0.0000 & & 0.0004 & & 0.0002 & & 0.0000 & 0.0000 & & & 0.0009 & 0.0000 & 0.0449 & 0.0000 & 0.0000 & 0.0541 & 0.0002 \\
\hline HLW98-46 & 0.0001 & & & & 0.0000 & & 0.0004 & & 0.0002 & & 0.0000 & 0.0000 & & & 0.0009 & 0.0000 & 0.0428 & 0.0000 & 0.0000 & 0.0516 & 0.0002 \\
\hline HLW98-47 & 0.0001 & & & & 0.0000 & & 0.0004 & & 0.0002 & & 0.0000 & 0.0000 & & & 0.0008 & 0.0000 & 0.0410 & 0.0000 & 0.0000 & 0.0494 & 0.0002 \\
\hline HLW98-48 & 0.0001 & & & & 0.0000 & & 0.0003 & & 0.0002 & & 0.0000 & 0.0000 & & & 0.0007 & 0.0000 & 0.0356 & 0.0000 & 0.0000 & 0.0429 & 0.0002 \\
\hline HLW98-49 & 0.0001 & & & & 0.0000 & & 0.0003 & & 0.0002 & & 0.0000 & 0.0000 & & & 0.0007 & 0.0000 & 0.0340 & 0.0000 & 0.0000 & 0.0410 & 0.0002 \\
\hline HLW98-50 & 0.0000 & & & & 0.0000 & & 0.0004 & & 0.0002 & & 0.0000 & 0.0000 & & & 0.0008 & 0.0000 & 0.0001 & 0.0000 & 0.0000 & 0.0463 & 0.0002 \\
\hline HLW98-51R & 0.0001 & & & & 0.0000 & & 0.0003 & & 0.0002 & & 0.0000 & 0.0000 & & & 0.0007 & 0.0000 & 0.0340 & 0.0000 & 0.0000 & 0.0410 & 0.0002 \\
\hline HLW98-52 & 0.0000 & & & & & & 0.0000 & & 0.0000 & & 0.0036 & 0.0006 & & & 0.0000 & 0.0003 & 0.0234 & & 0.0002 & 0.0000 & 0.0000 \\
\hline HLW98-53A & 0.0000 & & & & & & 0.0000 & & 0.0000 & & 0.0036 & 0.0006 & & & 0.0000 & 0.0003 & 0.0238 & & 0.0002 & 0.0000 & 0.0000 \\
\hline HLW98-54 & 0.0000 & & & & & & 0.0000 & & 0.0000 & & 0.0036 & 0.0006 & & & 0.0000 & 0.0003 & 0.0234 & & 0.0002 & 0.0000 & 0.0000 \\
\hline HLW98-55 & 0.0000 & & & & & & 0.0000 & & 0.0000 & & 0.0036 & 0.0006 & & & 0.0000 & 0.0003 & 0.0234 & & 0.0002 & 0.0000 & 0.0000 \\
\hline HLW98-56 & 0.0000 & & & & 0.0000 & & 0.0000 & & 0.0000 & & 0.0000 & 0.0000 & & & 0.0000 & 0.0002 & 0.0000 & 0.0000 & 0.0000 & 0.0000 & 0.0001 \\
\hline HLW98-57 & 0.0001 & & & & 0.0000 & & 0.0000 & & 0.0000 & & 0.0000 & 0.0000 & & & 0.0000 & 0.0003 & 0.0262 & 0.0000 & 0.0000 & 0.0001 & 0.0001 \\
\hline HLW98-58 & 0.0000 & & & & 0.0000 & & 0.0000 & & 0.0000 & & 0.0000 & 0.0000 & & & 0.0000 & 0.0003 & 0.0136 & 0.0000 & 0.0000 & 0.0001 & 0.0001 \\
\hline HLW98-59 & 0.0000 & & & & & & 0.0000 & & 0.0000 & & 0.0036 & 0.0006 & & & 0.0000 & 0.0003 & 0.0235 & & 0.0002 & 0.0000 & 0.0000 \\
\hline HLW98-60 & 0.0000 & & & & 0.0000 & & 0.0000 & & 0.0000 & & 0.0000 & 0.0000 & & & 0.0000 & 0.0002 & 0.0068 & 0.0000 & 0.0000 & 0.0000 & 0.0001 \\
\hline HLW98-61 & 0.0001 & & & & 0.0000 & & 0.0000 & & 0.0000 & & 0.0000 & 0.0000 & & & 0.0016 & 0.0002 & 0.0150 & 0.0000 & 0.0000 & 0.0001 & 0.0001 \\
\hline HLW98-62 & 0.0000 & & & & & & 0.0000 & & 0.0000 & & 0.0000 & 0.0000 & & & 0.0000 & 0.0002 & 0.0208 & & 0.0000 & 0.0000 & 0.0000 \\
\hline HLW98-63 & 0.0000 & & & & & & 0.0000 & & 0.0000 & & 0.0000 & 0.0000 & & & 0.0000 & 0.0003 & 0.0808 & & 0.0000 & 0.0000 & 0.0004 \\
\hline HLW98-64 & 0.0000 & & & & & & 0.0000 & & 0.0000 & & 0.0023 & 0.0016 & & & 0.0000 & 0.0027 & 0.0255 & & 0.0016 & 0.0000 & 0.0007 \\
\hline
\end{tabular}


Appendix A. Database - mass fraction

RPP-WTP HLW Formulation (Kot and Pegg 2001)

\begin{tabular}{|c|c|c|c|c|c|c|c|c|c|c|c|c|c|c|c|c|c|c|c|c|c|}
\hline Glass ID & $\begin{array}{c}\mathrm{T} 12 \mathrm{O} 3 \\
-\mathrm{t}\end{array}$ & $\begin{array}{c}\text { U3O8 } \\
-t\end{array}$ & $\begin{array}{c}\mathrm{UO} 2 \\
-\mathrm{t}\end{array}$ & $\begin{array}{c}\text { UO3 } \\
-t\end{array}$ & $\begin{array}{c}\mathrm{V} 2 \mathrm{O} 5 \\
-\mathrm{t}\end{array}$ & $\begin{array}{c}\text { WO3 } \\
-t\end{array}$ & $\begin{array}{c}\mathrm{Y} 2 \mathrm{O} 3 \\
-\mathrm{t}\end{array}$ & $\begin{array}{c}\mathrm{ZnO} \\
-\mathrm{t}\end{array}$ & $\begin{array}{c}\text { Others } \\
-t\end{array}$ & $\begin{array}{c}\text { Sum } \\
-t\end{array}$ & $\begin{array}{c}\mathrm{A} 12 \mathrm{O} 3 \\
-\mathrm{a}\end{array}$ & $\begin{array}{c}\mathrm{B} 2 \mathrm{O} 3 \\
-\mathrm{a}\end{array}$ & $\begin{array}{c}\mathrm{CaO} \\
-\mathrm{a}\end{array}$ & $\begin{array}{c}\mathrm{Fe} 2 \mathrm{O} 3 \\
-\mathrm{a} \\
\end{array}$ & $\begin{array}{c}\mathrm{FeO} \\
-\mathrm{a}\end{array}$ & $\begin{array}{c}\mathrm{K} 2 \mathrm{O} \\
-\mathrm{a}\end{array}$ & $\begin{array}{c}\mathrm{Li} 2 \mathrm{O} \\
-\mathrm{a}\end{array}$ & $\begin{array}{c}\mathrm{MgO} \\
-\mathrm{a}\end{array}$ & $\begin{array}{c}\mathrm{Na} 2 \mathrm{O} \\
-\mathrm{a}\end{array}$ & $\begin{array}{c}\mathrm{NiO} \\
-\mathrm{a}\end{array}$ & $\begin{array}{c}\mathrm{P} 2 \mathrm{O} 5 \\
-\mathrm{a}\end{array}$ \\
\hline HLW98-41 & & & 0.0521 & & & & & 0.0200 & & 0.9999 & 0.0485 & 0.0359 & 0.0120 & 0.0672 & & 0.0034 & 0.0428 & 0.0027 & 0.1249 & 0.0039 & 0.0072 \\
\hline HLW98-42 & & & 0.0087 & & & & & 0.0200 & & 1.0000 & & & & & & & & & & & \\
\hline HLW98-43 & & & 0.0494 & & & & & 0.0200 & & 1.0000 & 0.0322 & 0.0598 & 0.0066 & 0.0610 & & 0.0013 & 0.0539 & 0.0010 & 0.1035 & 0.0033 & 0.0055 \\
\hline HLW98-44 & 0.0000 & & 0.0494 & & 0.0000 & 0.0000 & 0.0000 & 0.0200 & & 1.0000 & 0.0303 & 0.0705 & 0.0071 & 0.0599 & & 0.0014 & 0.0538 & 0.0010 & 0.0878 & 0.0033 & 0.0058 \\
\hline HLW98-45 & 0.0000 & & 0.0477 & & 0.0000 & 0.0000 & 0.0000 & 0.0200 & & 1.0001 & 0.0313 & 0.0716 & 0.0070 & 0.0600 & & 0.0015 & 0.0556 & 0.0010 & 0.0926 & 0.0032 & 0.0059 \\
\hline HLW98-46 & 0.0000 & & 0.0455 & & 0.0000 & 0.0000 & 0.0000 & 0.0200 & & 0.9997 & 0.0315 & 0.0730 & 0.0067 & 0.0568 & & 0.0015 & 0.0646 & 0.0010 & 0.0898 & 0.0031 & 0.0057 \\
\hline HLW98-47 & 0.0000 & & 0.0436 & & 0.0000 & 0.0000 & 0.0000 & 0.0200 & & 1.0000 & 0.0280 & 0.0749 & 0.0070 & 0.0532 & & 0.0015 & 0.0619 & 0.0010 & 0.0893 & 0.0031 & 0.0052 \\
\hline HLW98-48 & 0.0000 & & 0.0379 & & 0.0000 & 0.0000 & 0.0000 & 0.0200 & & 1.0001 & 0.0423 & 0.0903 & 0.0056 & 0.0470 & & 0.0013 & 0.0440 & 0.0007 & 0.0864 & 0.0027 & 0.0050 \\
\hline HLW98-49 & 0.0000 & & 0.0362 & & 0.0000 & 0.0000 & 0.0000 & 0.0200 & & 0.9999 & 0.0562 & 0.0717 & 0.0052 & 0.0457 & & 0.0014 & 0.0359 & 0.0007 & 0.1213 & 0.0025 & 0.0049 \\
\hline HLW98-50 & 0.0000 & & 0.0408 & & 0.0000 & 0.0000 & 0.0000 & 0.0200 & & 1.0000 & 0.0275 & 0.1117 & 0.0051 & 0.0444 & & 0.0008 & 0.0370 & 0.0009 & 0.1255 & 0.0028 & 0.0055 \\
\hline HLW98-51R & 0.0000 & & 0.0362 & & 0.0000 & 0.0000 & 0.0000 & 0.0200 & & 0.9999 & 0.0282 & 0.0952 & 0.0055 & 0.0473 & & 0.0013 & 0.0479 & 0.0008 & 0.0732 & 0.0026 & 0.0048 \\
\hline HLW98-52 & & & 0.0087 & & & & & 0.0200 & & 0.9999 & & & & & & & & & & & \\
\hline HLW98-53A & & & 0.0000 & & & & & 0.0200 & & 1.0002 & 0.0756 & 0.0716 & 0.0036 & 0.1173 & & 0.0010 & 0.0558 & 0.0010 & 0.0857 & 0.0088 & 0.0012 \\
\hline HLW98-54 & & & 0.0087 & & & & & 0.0200 & & 1.0000 & 0.0577 & 0.1014 & 0.0030 & 0.1161 & & 0.0010 & 0.0534 & 0.0008 & 0.0522 & 0.0068 & 0.0017 \\
\hline HLW98-55 & & & 0.0000 & & & & & 0.0002 & & 0.9999 & & & & & & & & & & & \\
\hline HLW98-56 & 0.0000 & & 0.0172 & & 0.0000 & 0.0000 & 0.0001 & 0.0004 & & 1.0000 & 0.0723 & 0.0745 & 0.0069 & 0.1128 & & 0.0003 & 0.0672 & 0.0015 & 0.0811 & 0.0072 & 0.0014 \\
\hline HLW98-57 & 0.0000 & & 0.0168 & & 0.0000 & 0.0000 & 0.0001 & 0.0005 & & 1.0002 & & & & & & & & & & & \\
\hline HLW98-58 & 0.0000 & & 0.0173 & & 0.0000 & 0.0000 & 0.0001 & 0.0005 & & 1.0001 & 0.0697 & 0.0397 & 0.0075 & 0.1086 & & 0.0009 & 0.0436 & 0.0017 & 0.1179 & 0.0076 & 0.0040 \\
\hline HLW98-59 & & & 0.0000 & & & & & 0.0200 & & 1.0002 & 0.0766 & 0.0427 & 0.0033 & 0.1055 & & 0.0009 & 0.0459 & 0.0010 & 0.1159 & 0.0067 & 0.0022 \\
\hline HLW98-60 & 0.0000 & & 0.0173 & & 0.0000 & 0.0000 & 0.0001 & 0.0204 & & 0.9996 & 0.0694 & 0.0402 & 0.0075 & 0.1088 & & 0.0008 & 0.0433 & 0.0015 & 0.1143 & 0.0081 & 0.0037 \\
\hline HLW98-61 & 0.0000 & & 0.0162 & & 0.0000 & 0.0000 & 0.0001 & 0.0004 & & 1.0000 & 0.0665 & 0.0396 & 0.0084 & 0.0904 & & 0.0019 & 0.0383 & 0.0018 & 0.1015 & 0.0061 & 0.0067 \\
\hline HLW98-62 & & & 0.0000 & & & & & 0.0201 & & 1.0000 & 0.0903 & 0.1180 & 0.0231 & 0.0436 & & 0.0012 & 0.0483 & 0.0007 & 0.0905 & 0.0034 & 0.0047 \\
\hline HLW98-63 & & & 0.0000 & & & & & 0.0179 & & 1.0000 & 0.1122 & 0.0651 & 0.0095 & 0.1129 & & 0.0010 & 0.0330 & 0.0028 & 0.0937 & 0.0015 & 0.0030 \\
\hline HLW98-64 & & & 0.0000 & & & & & 0.0191 & & 1.0000 & & & & & & & & & & & \\
\hline
\end{tabular}


Appendix A. Database - mass fraction

RPP-WTP HLW Formulation (Kot and Pegg 2001)

\begin{tabular}{|c|c|c|c|c|c|c|c|c|c|c|c|c|c|c|c|c|c|c|c|c|c|}
\hline Glass ID & $\begin{array}{c}\mathrm{SiO} 2 \\
-\mathrm{a}\end{array}$ & $\begin{array}{c}\mathrm{ZrO} 2 \\
-\mathrm{a}\end{array}$ & $\begin{array}{c}\mathrm{Ag} 2 \mathrm{O} \\
-\mathrm{a}\end{array}$ & $\begin{array}{c}\mathrm{As} 2 \mathrm{O} 3 \\
-\mathrm{a}\end{array}$ & $\begin{array}{c}\mathrm{BaO} \\
-\mathrm{a} \\
\end{array}$ & $\begin{array}{c}\mathrm{Bi} 2 \mathrm{O} 3 \\
-\mathrm{a}\end{array}$ & $\begin{array}{l}\mathrm{Br} \\
-\mathrm{a}\end{array}$ & $\begin{array}{c}\mathrm{CdO} \\
-\mathrm{a} \\
\end{array}$ & $\begin{array}{c}\mathrm{Ce} 2 \mathrm{O} 3 \\
-\mathrm{a}\end{array}$ & $\begin{array}{c}\mathrm{CeO} 2 \\
-\mathrm{a}\end{array}$ & $\begin{array}{l}\mathrm{Cl} \\
-\mathrm{a} \\
\end{array}$ & $\begin{array}{c}\mathrm{CoO} \\
-\mathrm{a} \\
\end{array}$ & $\begin{array}{c}\mathrm{Co} 2 \mathrm{O} 3 \\
-\mathrm{a}\end{array}$ & $\begin{array}{c}\mathrm{Cr} 2 \mathrm{O} 3 \\
-\mathrm{a}\end{array}$ & $\begin{array}{c}\mathrm{Cs} 2 \mathrm{O} \\
-\mathrm{a}\end{array}$ & $\begin{array}{c}\mathrm{CuO} \\
-\mathrm{a} \\
\end{array}$ & $\begin{array}{c}\mathrm{Eu} 2 \mathrm{O} 3 \\
-\mathrm{a}\end{array}$ & $\begin{array}{l}\mathrm{F} \\
-\mathrm{a}\end{array}$ & $\begin{array}{c}\mathrm{Ga} 2 \mathrm{O} 3 \\
-\mathrm{a}\end{array}$ & $\begin{array}{c}\mathrm{Gd} 2 \mathrm{O} 3 \\
-\mathrm{a}\end{array}$ & $\begin{array}{c}\mathrm{HgO} \\
-\mathrm{a}\end{array}$ \\
\hline HLW98-41 & 0.3526 & 0.0565 & 0.0002 & 0.0000 & 0.0015 & 0.0005 & & 0.0011 & & & & 0.0007 & & 0.0022 & & 0.0010 & & & & & \\
\hline \multicolumn{22}{|l|}{ HLW98-42 } \\
\hline HLW98-43 & 0.3383 & 0.0560 & 0.0011 & 0.0003 & 0.0008 & 0.0003 & & 0.0014 & & & & 0.0004 & & 0.0017 & & 0.0005 & & & & & \\
\hline HLW98-44 & 0.3534 & 0.0575 & 0.0012 & 0.0006 & 0.0011 & 0.0004 & & 0.0010 & & & & 0.0005 & & 0.0017 & & 0.0005 & & & & & \\
\hline HLW98-45 & 0.3786 & 0.0593 & 0.0011 & 0.0000 & 0.0012 & 0.0002 & & 0.0008 & & & & 0.0005 & & 0.0017 & & 0.0004 & & & & & \\
\hline HLW98-46 & 0.3759 & 0.0566 & 0.0010 & 0.0000 & 0.0011 & 0.0000 & & 0.0007 & & & & 0.0005 & & 0.0016 & & 0.0004 & & & & & \\
\hline HLW98-47 & 0.3905 & 0.0526 & 0.0100 & & 0.0013 & & & 0.0007 & & & & 0.0006 & & 0.0018 & & & & & & & \\
\hline HLW98-48 & 0.3755 & 0.0428 & 0.0010 & & 0.0056 & & & 0.0006 & & & & 0.0006 & & 0.0014 & & & & & & & \\
\hline HLW98-49 & 0.4234 & 0.0456 & 0.0009 & & 0.0006 & & & 0.0006 & & & & 0.0006 & & 0.0015 & & & & & & & \\
\hline HLW98-50 & 0.4480 & 0.0515 & 0.0008 & 0.0000 & 0.0003 & 0.0005 & & 0.0008 & & & & 0.0005 & & 0.0010 & & 0.0003 & & & & & \\
\hline HLW98-51R & 0.4484 & 0.0478 & 0.0009 & & 0.0006 & & & 0.0006 & & & & 0.0005 & & 0.0013 & & & & & & & \\
\hline \multicolumn{22}{|l|}{ HLW98-52 } \\
\hline HLW98-53A & 0.4367 & 0.0146 & 0.0006 & 0.0003 & 0.0009 & 0.0001 & & 0.0079 & & & & 0.0002 & & 0.0038 & & 0.0003 & & & & & \\
\hline HLW98-54 & 0.4739 & 0.0139 & 0.0005 & 0.0000 & 0.0009 & 0.0000 & & 0.0073 & & & & 0.0003 & & 0.0009 & & 0.0000 & & & & & \\
\hline \multicolumn{22}{|l|}{ HLW98-55 } \\
\hline HLW98-56 & 0.4889 & 0.0168 & 0.0004 & & 0.0006 & & & 0.0131 & & & & 0.0003 & & 0.0008 & & & & & & & \\
\hline \multicolumn{22}{|l|}{ HLW98-57 } \\
\hline HLW98-58 & 0.4574 & 0.0161 & 0.0002 & & 0.0009 & & & 0.0124 & & & & 0.0004 & & 0.0011 & & & & & & & \\
\hline HLW98-59 & 0.4564 & 0.0149 & 0.0004 & 0.0000 & 0.0009 & 0.0000 & & 0.0076 & & & & 0.0002 & & 0.0007 & & 0.0002 & & & & & \\
\hline HLW98-60 & 0.4603 & 0.0160 & 0.0003 & & 0.0008 & & & 0.0125 & & & & 0.0003 & & 0.0010 & & & & & & & \\
\hline HLW98-61 & 0.4961 & 0.0132 & 0.0003 & 0.0002 & 0.0008 & 0.0006 & & 0.0110 & & & & 0.0004 & & 0.0010 & & 0.0007 & & & & & \\
\hline HLW98-62 & 0.4224 & 0.0876 & 0.0001 & 0.0002 & 0.0005 & 0.0006 & & 0.0006 & & & & 0.0008 & & 0.0027 & & 0.0001 & & & & & \\
\hline HLW98-63 & 0.3702 & 0.0018 & 0.0028 & 0.0000 & 0.0024 & 0.0000 & & 0.0005 & & & & 0.0002 & & 0.0016 & & 0.0001 & & & & & \\
\hline HLW98-64 & & & & & & & & & & & & & & & & & & & & & \\
\hline
\end{tabular}


Appendix A. Database - mass fraction

RPP-WTP HLW Formulation (Kot and Pegg 2001)

\begin{tabular}{|c|c|c|c|c|c|c|c|c|c|c|c|c|c|c|c|c|c|c|c|c|c|}
\hline Glass ID & $\begin{array}{c}\mathrm{I} \\
-\mathrm{a} \\
\end{array}$ & $\begin{array}{c}\mathrm{La} 2 \mathrm{O} 3 \\
-\mathrm{a}\end{array}$ & $\begin{array}{c}\mathrm{MnO} 2 \\
-\mathrm{a} \\
\end{array}$ & $\begin{array}{c}\mathrm{MnO} \\
-\mathrm{a} \\
\end{array}$ & $\begin{array}{c}\mathrm{MoO} \\
-\mathrm{a} \\
\end{array}$ & $\begin{array}{c}\mathrm{MoO} 3 \\
-\mathrm{a}\end{array}$ & $\begin{array}{c}\mathrm{Nb} 2 \mathrm{O} 5 \\
-\mathrm{a} \\
\end{array}$ & $\begin{array}{c}\mathrm{Nd} 2 \mathrm{O} 3 \\
-\mathrm{a} \\
\end{array}$ & $\begin{array}{c}\mathrm{PbO} \\
-\mathrm{a}\end{array}$ & $\begin{array}{c}\mathrm{PdO} 2 \\
-\mathrm{a}\end{array}$ & $\begin{array}{c}\mathrm{PdO} \\
-\mathrm{a}\end{array}$ & $\begin{array}{c}\mathrm{Pr} 2 \mathrm{O} 3 \\
-\mathrm{a}\end{array}$ & $\begin{array}{c}\text { Pr6O11 } \\
-\mathrm{a}\end{array}$ & $\begin{array}{c}\mathrm{Rb} 2 \mathrm{O} \\
-\mathrm{a}\end{array}$ & $\begin{array}{c}\mathrm{ReO} \\
-\mathrm{a}\end{array}$ & $\begin{array}{c}\mathrm{ReO} 2 \\
-\mathrm{a}\end{array}$ & $\begin{array}{c}\mathrm{Rh} 2 \mathrm{O} 3 \\
-\mathrm{a}\end{array}$ & $\begin{array}{c}\mathrm{RhO}_{2} \\
-\mathrm{a}\end{array}$ & $\begin{array}{c}\mathrm{RuO} 2 \\
-\mathrm{a}\end{array}$ & $\begin{array}{c}\mathrm{Sb} 2 \mathrm{O} 3 \\
-\mathrm{a}\end{array}$ & $\begin{array}{c}\mathrm{Sb} 2 \mathrm{O} 5 \\
-\mathrm{a}\end{array}$ \\
\hline HLW98-41 & & & 0.0000 & 0.0319 & & & & & 0.0045 & & 0.0003 & & & & & & 0.0003 & & 0.0001 & & 0.0016 \\
\hline \multicolumn{22}{|l|}{ HLW98-42 } \\
\hline HLW98-43 & & & 0.0000 & 0.0326 & & & & & 0.0028 & & 0.0002 & & & & & & 0.0003 & & 0.0001 & & 0.0005 \\
\hline HLW98-44 & & & & 0.0413 & & & & & 0.0029 & & 0.0002 & & & & & & 0.0003 & & 0.0001 & & 0.0005 \\
\hline HLW98-45 & & & & 0.0334 & & & & & 0.0030 & & 0.0004 & & & & & & 0.0004 & & 0.0001 & & 0.0008 \\
\hline HLW98-46 & & & & 0.0317 & & & & & 0.0028 & & 0.0004 & & & & & & 0.0004 & & 0.0001 & & 0.0009 \\
\hline HLW98-47 & & & & 0.0290 & & & & & 0.0027 & & 0.0003 & & & & & & 0.0002 & & 0.0002 & & 0.0004 \\
\hline HLW98-48 & & & & 0.0268 & & & & & 0.0020 & & 0.0002 & & & & & & 0.0002 & & 0.0001 & & 0.0005 \\
\hline HLW98-49 & & & & 0.0261 & & & & & 0.0019 & & 0.0002 & & & & & & 0.0001 & & 0.0002 & & 0.0006 \\
\hline HLW98-50 & & & & 0.0100 & & & & & 0.0017 & & 0.0003 & & & & & & 0.0004 & & 0.0002 & & 0.0008 \\
\hline HLW98-51R & & & & 0.0271 & & & & & 0.0020 & & 0.0041 & & & & & & 0.0002 & & 0.0002 & & \\
\hline \multicolumn{22}{|l|}{ HLW98-52 } \\
\hline HLW98-53A & & & 0.0000 & 0.0159 & & & & & 0.0011 & & 0.0001 & & & & & & & & 0.0001 & & 0.0045 \\
\hline HLW98-54 & & & 0.0000 & 0.0157 & & & & & 0.0010 & & 0.0000 & & & & & & 0.0001 & & 0.0001 & & 0.0041 \\
\hline \multicolumn{22}{|l|}{ HLW98-55 } \\
\hline HLW98-56 & & & & 0.0028 & & & & & 0.0004 & & & & & & & & & & & & \\
\hline \multicolumn{22}{|l|}{ HLW98-57 } \\
\hline HLW98-58 & & & & 0.0106 & & & & & 0.0005 & & 0.0001 & & & & & & 0.0001 & & 0.0001 & & 0.0006 \\
\hline HLW98-59 & & & 0.0000 & 0.0161 & & & & & 0.0010 & & 0.0000 & & & & & & & & & & 0.0038 \\
\hline HLW98-60 & & & & 0.0072 & & & & & 0.0005 & & 0.0000 & & & & & & 0.0000 & & 0.0001 & & \\
\hline HLW98-61 & & & & 0.0112 & & & & & 0.0019 & & 0.0000 & & & & & & 0.0000 & & 0.0001 & & 0.0007 \\
\hline HLW98-62 & & & 0.0000 & 0.0210 & & & & & 0.0009 & & 0.0001 & & & & & & 0.0001 & & & & 0.0011 \\
\hline HLW98-63 & & & 0.0000 & 0.0562 & & & & & 0.0030 & & 0.0000 & & & & & & & & 0.0001 & & 0.0005 \\
\hline HLW98-64 & & & & & & & & & & & & & & & & & & & & & \\
\hline
\end{tabular}


Appendix A. Database - mass fraction

RPP-WTP HLW Formulation (Kot and Pegg 2001)

\begin{tabular}{|c|c|c|c|c|c|c|c|c|c|c|c|c|c|c|c|c|c|c|c|c|}
\hline Glass ID & $\begin{array}{c}\mathrm{SeO} 2 \\
-\mathrm{a}\end{array}$ & $\begin{array}{c}\mathrm{Sm} 2 \mathrm{O} 3 \\
-\mathrm{a}\end{array}$ & $\begin{array}{c}\mathrm{SnO} \\
-\mathrm{a}\end{array}$ & $\begin{array}{c}\mathrm{SnO} 2 \\
-\mathrm{a}\end{array}$ & $\begin{array}{c}\mathrm{SO} 3 \\
-\mathrm{a}\end{array}$ & $\begin{array}{c}\mathrm{SrO} \\
-\mathrm{a}\end{array}$ & $\begin{array}{c}\mathrm{Tc} 2 \mathrm{O} 7 \\
-\mathrm{a}\end{array}$ & $\begin{array}{c}\mathrm{TeO} 2 \\
-\mathrm{a}\end{array}$ & $\begin{array}{c}\mathrm{ThO} 2 \\
-\mathrm{a}\end{array}$ & $\begin{array}{c}\mathrm{TiO} 2 \\
-\mathrm{a}\end{array}$ & $\begin{array}{c}\mathrm{T} 12 \mathrm{O} 3 \\
-\mathrm{a}\end{array}$ & $\begin{array}{c}\mathrm{U} 3 \mathrm{O} 8 \\
-\mathrm{a}\end{array}$ & $\begin{array}{c}\mathrm{UO} 2 \\
-\mathrm{a}\end{array}$ & $\begin{array}{c}\text { UO3 } \\
-\mathrm{a}\end{array}$ & $\begin{array}{c}\mathrm{V} 2 \mathrm{O} 5 \\
-\mathrm{a}\end{array}$ & $\begin{array}{c}\text { WO3 } \\
-\mathrm{a}\end{array}$ & $\begin{array}{c}\mathrm{Y} 2 \mathrm{O} 3 \\
-\mathrm{a}\end{array}$ & $\begin{array}{c}\mathrm{ZnO} \\
-\mathrm{a}\end{array}$ & $\begin{array}{c}\text { Others } \\
-\mathrm{a}\end{array}$ & $\begin{array}{c}\text { Sum } \\
-\mathrm{a}\end{array}$ \\
\hline HLW98-41 & & & & 0.0017 & & 0.0382 & & 0.0006 & & 0.0010 & 0.0010 & & 0.0497 & & 0.0005 & 0.0006 & & 0.0183 & & 0.9151 \\
\hline \multicolumn{21}{|l|}{ HLW98-42 } \\
\hline HLW98-43 & & & & & & 0.0337 & & 0.0007 & & 0.0005 & 0.0008 & & 0.0396 & & & 0.0006 & & 0.0185 & & 0.8598 \\
\hline HLW98-44 & 0.0000 & & & & & 0.0367 & & 0.0007 & & 0.0005 & 0.0009 & & 0.0424 & & & 0.0006 & & 0.0195 & & 0.8854 \\
\hline HLW98-45 & 0.0000 & & & 0.0016 & & 0.0416 & & 0.0003 & & 0.0006 & 0.0011 & & 0.0414 & & 0.0002 & 0.0005 & & 0.0194 & & 0.9183 \\
\hline HLW98-46 & 0.0000 & & & 0.0016 & & 0.0395 & & 0.0004 & & 0.0006 & & & 0.0412 & & & & & 0.0192 & & 0.9103 \\
\hline HLW98-47 & 0.0000 & & & & & 0.0366 & & & & 0.0006 & & & 0.0391 & & & & & 0.0190 & & 0.9107 \\
\hline HLW98-48 & 0.0001 & & & & & 0.0316 & & 0.0003 & & 0.0005 & & & 0.0309 & & & & & 0.0188 & & 0.8648 \\
\hline HLW98-49 & 0.0000 & & & & & 0.0314 & & 0.0004 & & 0.0006 & & & 0.0289 & & & & & 0.0195 & & 0.9286 \\
\hline HLW98-50 & 0.0002 & & & 0.0012 & & 0.0007 & & 0.0005 & & 0.0006 & 0.0007 & & 0.0341 & & 0.0002 & 0.0004 & & 0.0191 & & 0.9360 \\
\hline HLW98-51R & & & & & & 0.0317 & & & & 0.0006 & & & 0.0305 & & & & & 0.0204 & & 0.9237 \\
\hline \multicolumn{21}{|l|}{ HLW98-52 } \\
\hline HLW98-53A & 0.0001 & & & & & 0.0219 & & 0.0005 & & 0.0002 & 0.0003 & & 0.0006 & & 0.0001 & 0.0006 & & 0.0196 & & 0.9526 \\
\hline HLW98-54 & & & & & & 0.0218 & & 0.0007 & & 0.0003 & & & 0.0097 & & & & & 0.0196 & & 0.9649 \\
\hline \multicolumn{21}{|l|}{ HLW98-55 } \\
\hline HLW98-56 & & & & & & 0.0010 & & & & 0.0003 & & & 0.0154 & & & & & 0.0011 & & 0.9671 \\
\hline \multicolumn{21}{|l|}{ HLW98-57 } \\
\hline HLW98-58 & 0.0001 & & & & & 0.0120 & & & & 0.0003 & & & 0.0146 & & & & & 0.0009 & & 0.9296 \\
\hline HLW98-59 & 0.0004 & & & & & 0.0247 & & 0.0008 & & & & & 0.0008 & & & & & 0.0190 & & 0.9486 \\
\hline HLW98-60 & & & & & & 0.0085 & & & & 0.0004 & & & 0.0147 & & & & & 0.0195 & & 0.9397 \\
\hline HLW98-61 & 0.0000 & & & 0.0018 & & 0.0109 & & 0.0009 & & 0.0006 & 0.0003 & & 0.0158 & & 0.0005 & & & 0.0013 & & 0.9315 \\
\hline HLW98-62 & & & & 0.0007 & & 0.0196 & & & & 0.0003 & 0.0011 & & 0.0015 & & 0.0001 & 0.0005 & & 0.0196 & & 1.0060 \\
\hline HLW98-63 & 0.0002 & & & 0.0004 & & 0.0731 & & 0.0002 & & 0.0007 & 0.0005 & & 0.0018 & & 0.0001 & 0.0003 & & 0.0171 & & 0.9685 \\
\hline HLW98-64 & & & & & & & & & & & & & & & & & & & & \\
\hline
\end{tabular}


Appendix A. Database - mass fraction

RPP-WTP HLW Formulation (Kot and Pegg 2001)

\begin{tabular}{|c|c|c|c|c|c|c|c|c|}
\hline Glass ID & $\begin{array}{l}\mathrm{TM} \\
\left({ }^{\circ} \mathrm{C}\right) \\
\end{array}$ & $\begin{array}{l}\text { Gradient } \\
\text { TL }\left({ }^{\circ} \mathrm{C}\right)\end{array}$ & \begin{tabular}{l|} 
Uniform \\
TL $\left({ }^{\circ} \mathrm{C}\right)$
\end{tabular} & Primary Phase & $\begin{array}{c}\text { Quenched } \\
\text { Visual/OM } \\
\end{array}$ & $\begin{array}{c}\text { Quenched } \\
\text { SEM/EDS or TEM }\end{array}$ & $\begin{array}{l}\text { Quenched } \\
\text { XRD }\end{array}$ & $\begin{array}{c}\text { Quenched } \\
\text { Homogeneous? }\end{array}$ \\
\hline HLW98-41 & 1150 & & $>950^{\circ} \mathrm{C}$ & & $\begin{array}{l}\text { Metallic looking glass with surface } \\
\text { crystallization }\end{array}$ & & & \\
\hline HLW98-42 & 1150 & & $<950^{\circ} \mathrm{C}$ & & Homogeneous dark brown glass & & & \\
\hline HLW98-43 & 1150 & & $>950^{\circ} \mathrm{C}$ & & Homogeneous dark brown glass & & & \\
\hline HLW98-44 & 1150 & & $>950^{\circ} \mathrm{C}$ & & Homogeneous dark brown glass & & & \\
\hline HLW98-45 & 1150 & & $>950^{\circ} \mathrm{C}$ & & Homogeneous dark brown glass & & & \\
\hline HLW98-46 & 1150 & & $<950^{\circ} \mathrm{C}$ & & Homogeneous dark brown glass & & & \\
\hline HLW98-47 & 1150 & & $<950{ }^{\circ} \mathrm{C}$ & & $\begin{array}{l}\text { Dark brown glass with very small amount of } \\
\text { undissolved solids }\end{array}$ & & & \\
\hline HLW98-48 & 1150 & & $<950^{\circ} \mathrm{C}$ & & Homogeneous dark brown glass & & & \\
\hline HLW98-49 & 1150 & & $<950{ }^{\circ} \mathrm{C}$ & & Homogeneous dark brown glass & & & \\
\hline HLW98-50 & 1150 & & $<950^{\circ} \mathrm{C}$ & & Clear, homogeneous dark brown glass & & & \\
\hline HLW98-51R & 1150 & & $<950^{\circ} \mathrm{C}$ & & Homogeneous dark brown glass & & & \\
\hline HLW98-52 & 1150 & & $<950^{\circ} \mathrm{C}$ & & $\begin{array}{l}\text { Inhomogeneous brown opaque glass with } \\
\text { light color swirls }\end{array}$ & & & \\
\hline HLW98-53A & 1150 & & $<950^{\circ} \mathrm{C}$ & & $\begin{array}{l}\text { Inhomogeneous brown glass with apparently } \\
\text { undissolved fractions }\end{array}$ & & & \\
\hline HLW98-54 & 1150 & & $<950{ }^{\circ} \mathrm{C}$ & & $\begin{array}{l}\text { Inhomogeneous brown glass with multicolor } \\
\text { swirls }\end{array}$ & & & \\
\hline HLW98-55 & 1150 & & & & $\begin{array}{l}\text { Inhomogeneous glass with many brown and } \\
\text { green streaks }\end{array}$ & & & \\
\hline HLW98-56 & 1150 & & $<950{ }^{\circ} \mathrm{C}$ & & Homogeneous brown glass & & & \\
\hline HLW98-57 & 1150 & & $<950^{\circ} \mathrm{C}$ & & Homogeneous dark brown glass & & & \\
\hline HLW98-58 & 1150 & & $<950{ }^{\circ} \mathrm{C}$ & & Homogeneous dark brown glass & & & \\
\hline HLW98-59 & 1150 & & $<950{ }^{\circ} \mathrm{C}$ & & $\begin{array}{l}\text { Inhomogeneous brown glass with swirling } \\
\text { patterns throughout }\end{array}$ & & & \\
\hline HLW98-60 & 1150 & & » $950{ }^{\circ} \mathrm{C}$ & & $\begin{array}{l}\text { Homogeneous dark brown glass with some } \\
\text { unmelted particles }\end{array}$ & & & \\
\hline HLW98-61 & 1150 & & $<950{ }^{\circ} \mathrm{C}$ & & Homogeneous dark brown glass & & & \\
\hline HLW98-62 & 1150 & & $<950{ }^{\circ} \mathrm{C}$ & & Homogeneous dark brown glass & & & \\
\hline HLW98-63 & 1150 & & $>1050^{\circ} \mathrm{C}$ & & Homogeneous dark brown glass & & & \\
\hline HLW98-64 & 1150 & \multicolumn{3}{|c|}{ About $1050^{\circ} \mathrm{C}$} & Homogeneous brown glass & & & \\
\hline
\end{tabular}


Appendix A. Database - mass fraction

RPP-WTP HLW Formulation (Kot and Pegg 2001)

\begin{tabular}{|c|c|c|c|c|c|}
\hline Glass ID & $\begin{array}{c}\mathrm{CCC} \\
\text { Visual/OM }\end{array}$ & $\begin{array}{c}\text { CCC } \\
\text { SEM/EDS or TEM }\end{array}$ & $\begin{array}{l}\mathrm{CCC} \\
\mathrm{XRD}\end{array}$ & $\begin{array}{l}\text { Heat Treated } \\
\text { Visual/OM }\end{array}$ & $\begin{array}{c}\text { Heat Treated } \\
\text { SEM/EDS or TEM }\end{array}$ \\
\hline HLW98-41 & & & & & $\begin{array}{l}\text { After } 72 \text { hours, } 5-7 \mathrm{vol} \% \text { of white crystals and } 0.2 \\
0.5 \mathrm{vol} \% \text { of spinel }\end{array}$ \\
\hline HLW98-42 & & & & & After 72 hours, $0.2-0.5 \mathrm{vol} \%$ of magnetic spinel \\
\hline HLW98-43 & & & & & $\begin{array}{l}\text { After } 72 \text { hours, } 1-2 \mathrm{vol} \% \text { of white/clear octahedra } \\
\text { crystals and } 0.1-0.2 \mathrm{vol} \% \text { of magnetic spinel }\end{array}$ \\
\hline HLW98-44 & & & & & $\begin{array}{l}\text { After } 72 \text { hours, } 1-2 \mathrm{vol} \% \text { of white/clear crystals } \\
\text { and } 0.1-0.2 \mathrm{vol} \% \text { of magnetic spinel }\end{array}$ \\
\hline HLW98-45 & & & & & $\begin{array}{l}\text { After } 72 \text { hours, about } 1 \mathrm{vol} \% \text { of white crystals } \\
\text { (ThO2 with small amounts of } \mathrm{Ce} \text { and } \mathrm{Zr} \text { ) and }< \\
0.1 \mathrm{vol} \% \text { of spinel }(\mathrm{Cr}, \mathrm{Mn}, \mathrm{Fe}, \mathrm{Ni}, \mathrm{Zn})\end{array}$ \\
\hline HLW98-46 & & & & & $\begin{array}{l}\text { After } 68 \text { hours, } 0.1 \text { vol } \% \text { of clear crystals and }< \\
0.1 \mathrm{vol} \% \text { of small dark crystals, probably spinel }\end{array}$ \\
\hline HLW98-47 & & & & & $\begin{array}{l}\text { After } 68 \text { hours, much less than } 1 \text { vol\% of clear } \\
\text { crystals }\end{array}$ \\
\hline HLW98-48 & & & & & $\begin{array}{l}\text { Clusters of dark globu-lar crystals }(0.1 \mathrm{vol} \%, 20- \\
200 \mathrm{~mm}) \text {. A few larger white crystals }(0.1 \mathrm{vol} \%)\end{array}$ \\
\hline HLW98-49 & & & & & $\begin{array}{l}\text { After } 72 \text { hours, }<0.1 \text { vol } \% \text { of dark, globular } \\
\text { clusters of acicular crystals }\end{array}$ \\
\hline HLW98-50 & & & & & After 72 hours, $<0.1$ vol $\%$ of dark crystalss \\
\hline HLW98-51R & & & & & $\begin{array}{l}\text { After } 68 \text { hours, }<0.1 \text { vol } \% \text { of dark crystals. No } \\
\text { evidence of magnetic crystals }\end{array}$ \\
\hline HLW98-52 & & & & & $\begin{array}{l}\text { After } 72 \text { hours, } 0.2-0.4 \text { vol } \% \text { of strongly magnetic } \\
\text { spinel }\end{array}$ \\
\hline HLW98-53A & & & & & After 72 hours, $0.2-0.5 \mathrm{vol} \%$ of magnetic spinel \\
\hline HLW98-54 & & & & & After 72 hours, $0.2-0.5 \mathrm{vol} \%$ of magnetic spinel \\
\hline \multicolumn{6}{|l|}{ HLW98-55 } \\
\hline HLW98-56 & & & & & After 68 hours, $0.01-0.05$ vol $\%$ of magnetic spine \\
\hline HLW98-57 & & & & & After 68 hours, $0.1 \mathrm{vol} \%$ of magnetic spinel \\
\hline HLW98-58 & & & & & $\begin{array}{l}\text { After } 70 \text { hours, } 0.1-0.2 \mathrm{vol} \% \text { of magnetic spinel } \\
\text { (large crystals) and trace amount of a white } \\
\text { granular phase }\end{array}$ \\
\hline HLW98-59 & & & & & After 70 hours, $0.1-0.2 \mathrm{vol} \%$ of magnetic spinel \\
\hline HLW98-60 & & & & & After 70 hours, $0.5-1.0 \mathrm{vol} \%$ of magnetic spinel \\
\hline HLW98-61 & & & & & After 70 hours, about $0.1 \mathrm{vol} \%$ of magnetic spinel \\
\hline HLW98-62 & & & & & $\begin{array}{l}\text { After } 24 \text { hours, reddish brown glass showed }<0.1 \\
\text { vol } \% \text { of magnetic spinel }\end{array}$ \\
\hline HLW98-63 & & & & & $\begin{array}{l}\text { Dark reddish-brown glass with 5-7 vol } \% \text { of } \\
\text { magnetic spinel }(30-50 \mathrm{~mm})\end{array}$ \\
\hline HLW98-64 & & & & & $\begin{array}{l}\text { After } 70 \text { hours, about } 1 \mathrm{vol} \% \text { of magnetic spinel } \\
(30-50 \mathrm{~mm})\end{array}$ \\
\hline
\end{tabular}


Appendix A. Database - mass fraction

RPP-WTP HLW Formulation (Kot and Pegg 2001)

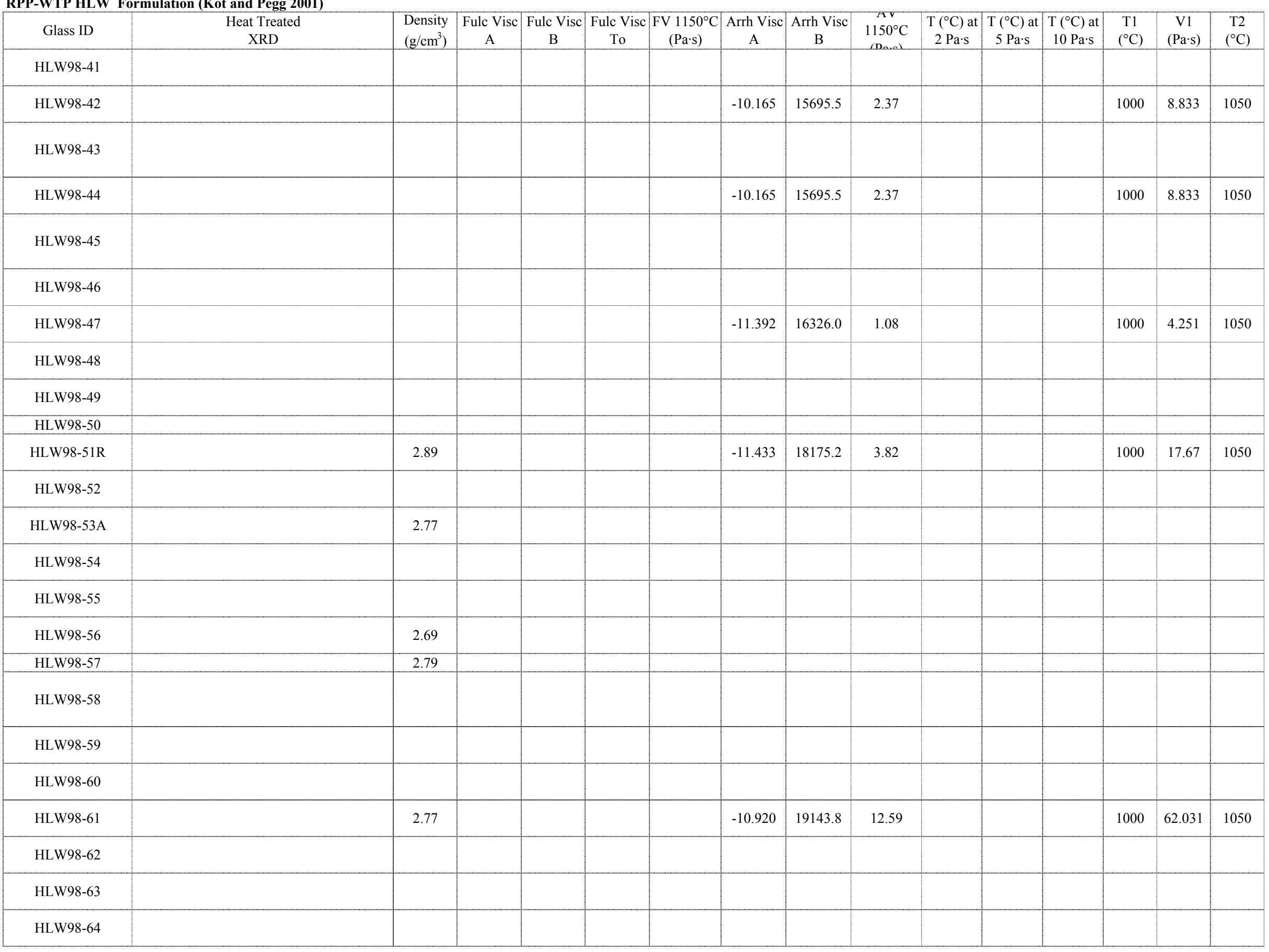


Appendix A. Database - mass fraction

RPP-WTP HLW Formulation (Kot and Pegg 2001)

\begin{tabular}{|c|c|c|c|c|c|c|c|c|c|c|c|c|c|c|c|c|c|c|c|c|c|}
\hline Glass ID & $\begin{array}{c}\mathrm{V} 2 \\
(\mathrm{~Pa} \cdot \mathrm{s}) \\
\end{array}$ & $\begin{array}{c}\mathrm{T} 3 \\
\left({ }^{\circ} \mathrm{C}\right) \\
\end{array}$ & $\begin{array}{c}\mathrm{V} 3 \\
(\mathrm{~Pa} \cdot \mathrm{s})\end{array}$ & $\begin{array}{c}\mathrm{T} 4 \\
\left({ }^{\circ} \mathrm{C}\right) \\
\end{array}$ & $\begin{array}{c}\mathrm{V} 4 \\
(\mathrm{~Pa} \cdot \mathrm{s}) \\
\end{array}$ & $\begin{array}{c}\mathrm{T} 5 \\
\left({ }^{\circ} \mathrm{C}\right) \\
\end{array}$ & $\begin{array}{c}\mathrm{V5} \\
(\mathrm{Pa} \cdot \mathrm{s}) \\
\end{array}$ & $\begin{array}{c}\mathrm{T} 6 \\
\left({ }^{\circ} \mathrm{C}\right) \\
\end{array}$ & $\begin{array}{c}\mathrm{V} 6 \\
(\mathrm{~Pa} \cdot \mathrm{s}) \\
\end{array}$ & $\begin{array}{c}\mathrm{T} 7 \\
\left({ }^{\circ} \mathrm{C}\right) \\
\end{array}$ & $\begin{array}{c}\text { V7 } \\
(\mathrm{Pa} \cdot \mathrm{s}) \\
\end{array}$ & $\begin{array}{c}\mathrm{T} 8 \\
\left({ }^{\circ} \mathrm{C}\right) \\
\end{array}$ & $\begin{array}{c}\mathrm{V} 8 \\
(\mathrm{~Pa} \cdot \mathrm{s})\end{array}$ & $\begin{array}{c}\mathrm{T} 9 \\
\left({ }^{\circ} \mathrm{C}\right) \\
\end{array}$ & $\begin{array}{c}\mathrm{V} 9 \\
(\mathrm{~Pa} \cdot \mathrm{s})\end{array}$ & $\begin{array}{l}\mathrm{T} 10 \\
\left({ }^{\circ} \mathrm{C}\right) \\
\end{array}$ & $\begin{array}{c}\mathrm{V} 10 \\
(\mathrm{~Pa} \cdot \mathrm{s})\end{array}$ & $\begin{array}{l}\mathrm{T} 11 \\
\left({ }^{\circ} \mathrm{C}\right) \\
\end{array}$ & $\begin{array}{c}\text { V11 } \\
(\mathrm{Pa} \cdot \mathrm{s})\end{array}$ & $\begin{array}{l}\mathrm{T} 12 \\
\left({ }^{\circ} \mathrm{C}\right) \\
\end{array}$ & $\begin{array}{c}\mathrm{V} 12 \\
(\mathrm{~Pa} \cdot \mathrm{s})\end{array}$ \\
\hline \multicolumn{22}{|l|}{ HLW98-41 } \\
\hline HLW98-42 & 5.42 & 1100 & 3.502 & 1150 & 2.363 & 1200 & 1.656 & & & & & & & & & & & & & & \\
\hline \multicolumn{22}{|l|}{ HLW98-43 } \\
\hline HLW98-44 & 5.42 & 1100 & 3.502 & 1150 & 2.363 & 1200 & 1.656 & & & & & & & & & & & & & & \\
\hline \multicolumn{22}{|l|}{ HLW98-45 } \\
\hline \multicolumn{22}{|l|}{ HLW98-46 } \\
\hline HLW98-47 & 2.558 & 1100 & 1.624 & 1150 & 1.079 & 1200 & 0.745 & & & & & & & & & & & & & & \\
\hline \multicolumn{22}{|l|}{ HLW98-48 } \\
\hline \multicolumn{22}{|l|}{ HLW98-49 } \\
\hline \multicolumn{22}{|l|}{ HLW98-50 } \\
\hline HLW98-51R & 9.85 & 1100 & 5.916 & 1150 & 3.78 & 1200 & 2.542 & & & & & & & & & & & & & & \\
\hline \multicolumn{22}{|l|}{ HLW98-52 } \\
\hline \multicolumn{22}{|l|}{ HLW98-53A } \\
\hline \multicolumn{22}{|l|}{ HLW98-54 } \\
\hline \multicolumn{22}{|l|}{ HLW98-55 } \\
\hline \multicolumn{22}{|l|}{ HLW98-56 } \\
\hline \multicolumn{22}{|l|}{ HLW98-57 } \\
\hline \multicolumn{22}{|l|}{ HLW98-58 } \\
\hline \multicolumn{22}{|l|}{ HLW98-59 } \\
\hline \multicolumn{22}{|l|}{ HLW98-60 } \\
\hline HLW98-61 & 34.638 & 1100 & 20.384 & 1150 & 12.557 & 1200 & 8.051 & & & & & & & & & & & & & & \\
\hline \multicolumn{22}{|l|}{ HLW98-62 } \\
\hline \multicolumn{22}{|l|}{ HLW98-63 } \\
\hline HLW98-64 & & & & & & & & & & & & & & & & & & & & & \\
\hline
\end{tabular}


Appendix A. Database - mass fraction

RPP-WTP HLW Formulation (Kot and Pegg 2001)

\begin{tabular}{|c|c|c|c|c|c|c|c|c|c|c|c|c|c|c|c|c|}
\hline Glass ID & $\begin{array}{l}\mathrm{T} 13 \\
\left({ }^{\circ} \mathrm{C}\right) \\
\end{array}$ & $\begin{array}{c}\mathrm{V} 13 \\
(\mathrm{~Pa} \cdot \mathrm{s}) \\
\end{array}$ & $\begin{array}{l}\mathrm{T} 14 \\
\left({ }^{\circ} \mathrm{C}\right) \\
\end{array}$ & $\begin{array}{l}\text { V14 } \\
(\mathrm{Pa} \cdot \mathrm{s}) \\
\end{array}$ & $\begin{array}{c}\text { Q PCT } \\
\mathrm{B}\left(\mathrm{g} / \mathrm{m}^{2}\right)\end{array}$ & $\begin{array}{c}\text { Q PCT } \\
\mathrm{Li}\left(\mathrm{g} / \mathrm{m}^{2}\right)\end{array}$ & $\begin{array}{c}\text { Q PCT } \\
\mathrm{Na}\left(\mathrm{g} / \mathrm{m}^{2}\right)\end{array}$ & $\begin{array}{c}\text { Q PCT } \\
\mathrm{Si}\left(\mathrm{g} / \mathrm{m}^{2}\right)\end{array}$ & $\begin{array}{c}\text { Q PCT } \\
\mathrm{pH} \\
\end{array}$ & $\begin{array}{c}\mathrm{CCC} \mathrm{PCT} \\
\mathrm{B}\left(\mathrm{g} / \mathrm{m}^{2}\right)\end{array}$ & $\begin{array}{l}\mathrm{CCC} \mathrm{PCT} \\
\mathrm{Li}\left(\mathrm{g} / \mathrm{m}^{2}\right)\end{array}$ & $\begin{array}{l}\text { CCC PCT } \\
\mathrm{Na}\left(\mathrm{g} / \mathrm{m}^{2}\right)\end{array}$ & $\begin{array}{l}\text { CCC PCT } \\
\mathrm{Si}\left(\mathrm{g} / \mathrm{m}^{2}\right)\end{array}$ & $\begin{array}{c}\text { CCC PCT } \\
\mathrm{pH}\end{array}$ & $\begin{array}{l}\text { Q PCT B at } \\
20^{\circ} \mathrm{C}\left(\mathrm{g} / \mathrm{m}^{2}\right)\end{array}$ & $\begin{array}{l}\text { Q PCT } \mathrm{Li} \text { at } \\
20^{\circ} \mathrm{C}\left(\mathrm{g} / \mathrm{m}^{2}\right)\end{array}$ \\
\hline \multicolumn{17}{|l|}{ HLW98-41 } \\
\hline \multicolumn{17}{|l|}{ HLW98-42 } \\
\hline \multicolumn{17}{|l|}{ HLW98-43 } \\
\hline \multicolumn{17}{|l|}{ HLW98-44 } \\
\hline \multicolumn{17}{|l|}{ HLW98-45 } \\
\hline \multicolumn{17}{|l|}{ HLW98-46 } \\
\hline \multicolumn{17}{|l|}{ HLW98-47 } \\
\hline \multicolumn{17}{|l|}{ HLW98-48 } \\
\hline \multicolumn{17}{|l|}{ HLW98-49 } \\
\hline HLW98-50 & & & & & 1.765 & 1.265 & 1.095 & 0.45 & 10.51 & & & & & & & \\
\hline HLW98-51R & & & & & 1.355 & 1.08 & 0.915 & 0.405 & 10.55 & & & & & & & \\
\hline \multicolumn{17}{|l|}{ HLW98-52 } \\
\hline HLW98-53A & & & & & 0.425 & 0.405 & 0.355 & 0.225 & 10.43 & & & & & & & \\
\hline \multicolumn{17}{|l|}{ HLW98-54 } \\
\hline \multicolumn{17}{|l|}{ HLW98-55 } \\
\hline HLW98-56 & & & & & 0.695 & 0.445 & 0.455 & 0.265 & 10.61 & & & & & & & \\
\hline \multicolumn{17}{|l|}{ HLW98-57 } \\
\hline HLW98-58 & & & & & 0.475 & 0.55 & 0.67 & 0.33 & 11.32 & & & & & & & \\
\hline HLW98-59 & & & & & 0.695 & 0.445 & 0.455 & 0.265 & 10.61 & & & & & & & \\
\hline HLW98-60 & & & & & 0.485 & 0.505 & 0.59 & 0.295 & 11.38 & & & & & & & \\
\hline HLW98-61 & & & & & 0.23 & 0.33 & 0.345 & 0.275 & 11.01 & & & & & & & \\
\hline HLW98-62 & & & & & 0.285 & 0.305 & 0.23 & 0.135 & 10.16 & & & & & & & \\
\hline \multicolumn{17}{|l|}{ HLW98-63 } \\
\hline HLW98-64 & & & & & & & & & & & & & & & & \\
\hline
\end{tabular}


Appendix A. Database - mass fraction

RPP-WTP HLW Formulation (Kot and Pegg 2001)

\begin{tabular}{|c|c|c|c|c|c|c|c|c|c|c|c|c|}
\hline Glass ID & $\begin{array}{l}\text { Q PCT Na at } \\
20^{\circ} \mathrm{C}\left(\mathrm{g} / \mathrm{m}^{2}\right)\end{array}$ & $\begin{array}{l}\text { Q PCT Si at } \\
20^{\circ} \mathrm{C}\left(\mathrm{g} / \mathrm{m}^{2}\right)\end{array}$ & $\begin{array}{c}\mathrm{QpH} \\
\text { at } 20^{\circ} \mathrm{C}\end{array}$ & $\begin{array}{c}\text { TCLP Ag } \\
\text { (ppm) }\end{array}$ & $\begin{array}{c}\text { TCLP As } \\
\text { (ppm) }\end{array}$ & $\begin{array}{c}\text { TCLP Ba } \\
\text { (ppm) }\end{array}$ & $\begin{array}{c}\text { TCLP Cd } \\
(\mathrm{ppm})\end{array}$ & $\begin{array}{c}\text { TCLP Cr } \\
(\mathrm{ppm})\end{array}$ & $\begin{array}{c}\text { TCLP Ni } \\
(\mathrm{ppm})\end{array}$ & $\begin{array}{c}\text { TCLP Pb } \\
\text { (ppm) }\end{array}$ & $\begin{array}{c}\text { TCLP Se } \\
(\mathrm{ppm})\end{array}$ & $\begin{array}{c}\text { TCLP Zn } \\
(\mathrm{ppm})\end{array}$ \\
\hline HLW98-41 & & & & 0.02 & 0.05 & 0.126 & 0.082 & 0.024 & 0.291 & 0.162 & 0.0532 & 1.475 \\
\hline HLW98-42 & & & & 0.02 & NA & 0.09 & 0.484 & 0.011 & 0.179 & 0.635 & NA & 0.692 \\
\hline HLW98-43 & & & & 0.06 & NA & 0.122 & 0.077 & 0.017 & 0.213 & 0.242 & NA & 1.581 \\
\hline HLW98-44 & & & & 0.065 & NA & 0.106 & 0.072 & 0.013 & 0.201 & 0.249 & NA & 1.449 \\
\hline \multicolumn{13}{|l|}{ HLW98-45 } \\
\hline \multicolumn{13}{|l|}{ HLW98-46 } \\
\hline HLW98-47 & & & & 0.061 & NA & 0.106 & 0.066 & 0.011 & 0.183 & 0.232 & NA & 1.636 \\
\hline \multicolumn{13}{|l|}{ HLW98-48 } \\
\hline \multicolumn{13}{|l|}{ HLW98-49 } \\
\hline \multicolumn{13}{|l|}{ HLW98-50 } \\
\hline HLW98-51R & & & & 0.025 & 0.05 & 0.038 & 0.029 & 0.02 & 0.247 & 0.101 & 0.068 & 3.23 \\
\hline \multicolumn{13}{|l|}{ HLW98-52 } \\
\hline \multicolumn{13}{|l|}{ HLW98-53A } \\
\hline HLW98-54 & & & & 0.014 & 0.05 & 0.041 & 0.203 & 0.014 & 0.187 & 0.06 & 0.062 & 1.479 \\
\hline \multicolumn{13}{|l|}{ HLW98-55 } \\
\hline \multicolumn{13}{|l|}{ HLW98-56 } \\
\hline \multicolumn{13}{|l|}{ HLW98-57 } \\
\hline HLW98-58 & & & & $<0.0031$ & 0.05 & 0.465 & 0.268 & $<0.0055$ & 0.11 & 0.081 & 0.0532 & 0.13 \\
\hline HLW98-59 & & & & 0.007 & 0.118 & 0.469 & 0.157 & $<0.0055$ & 0.095 & $<0.0243$ & 0.0532 & 0.422 \\
\hline \multicolumn{13}{|l|}{ HLW98-60 } \\
\hline HLW98-61 & & & & $<0.0031$ & $<0.049$ & 0.994 & 0.19 & $<0.0055$ & 0.079 & 0.034 & $<0.0532$ & 0.207 \\
\hline \multicolumn{13}{|l|}{ HLW98-62 } \\
\hline \multicolumn{13}{|l|}{ HLW98-63 } \\
\hline HLW98-64 & & & & & & & & & & & & \\
\hline
\end{tabular}


Appendix A. Database - mass fraction

RPP-WTP HLW Formulation (Kot and Pegg 2001)

\begin{tabular}{|c|c|c|c|c|c|c|c|c|c|c|c|c|c|c|c|c|c|c|c|c|c|}
\hline Glass ID & $\begin{array}{c}\mathrm{A} 12 \mathrm{O} 3 \\
-\mathrm{t}\end{array}$ & $\begin{array}{c}\mathrm{B} 2 \mathrm{O} 3 \\
-\mathrm{t}\end{array}$ & $\begin{array}{c}\mathrm{CaO} \\
-\mathrm{t} \\
\end{array}$ & $\begin{array}{c}\mathrm{Fe} 2 \mathrm{O} 3 \\
-\mathrm{t} \\
\end{array}$ & $\begin{array}{c}\mathrm{FeO} \\
-\mathrm{t}\end{array}$ & $\begin{array}{c}\mathrm{K} 2 \mathrm{O} \\
-\mathrm{t}\end{array}$ & $\begin{array}{c}\mathrm{Li} 2 \mathrm{O} \\
-\mathrm{t}\end{array}$ & $\begin{array}{c}\mathrm{MgO} \\
-\mathrm{t} \\
\end{array}$ & $\begin{array}{c}\mathrm{Na} 2 \mathrm{O} \\
-\mathrm{t}\end{array}$ & $\begin{array}{c}\mathrm{NiO} \\
-\mathrm{t}\end{array}$ & $\begin{array}{c}\mathrm{P} 2 \mathrm{O} 5 \\
-\mathrm{t}\end{array}$ & $\begin{array}{c}\mathrm{SiO} 2 \\
-\mathrm{t}\end{array}$ & $\begin{array}{c}\mathrm{ZrO} 2 \\
-\mathrm{t}\end{array}$ & $\begin{array}{c}\mathrm{Ag} 2 \mathrm{O} \\
-\mathrm{t}\end{array}$ & $\begin{array}{c}\mathrm{As} 2 \mathrm{O} 3 \\
-\mathrm{t}\end{array}$ & $\begin{array}{c}\mathrm{BaO} \\
-\mathrm{t}\end{array}$ & $\begin{array}{c}\mathrm{Bi} 2 \mathrm{O} 3 \\
-\mathrm{t}\end{array}$ & $\begin{array}{l}\mathrm{Br} \\
-\mathrm{t}\end{array}$ & $\begin{array}{c}\mathrm{CdO} \\
-\mathrm{t}\end{array}$ & $\begin{array}{c}\mathrm{Ce} 2 \mathrm{O} 3 \\
-\mathrm{t}\end{array}$ & $\begin{array}{c}\mathrm{CeO} 2 \\
-\mathrm{t}\end{array}$ \\
\hline HLWMS-01 & 0.0500 & 0.0600 & & & & & 0.0600 & & 0.0260 & & 0.0100 & 0.3800 & & & & & & & & & \\
\hline HLWMS-02 & & 0.1100 & & & & & 0.0600 & & 0.0340 & & & 0.3500 & & & & & & & & & \\
\hline HLWMS-03 & 0.0100 & 0.0560 & & & & & 0.0600 & & 0.0280 & & & 0.3800 & & & & & & & & & \\
\hline HLWMS-04 & 0.0400 & 0.0500 & & & & & 0.0500 & & 0.0200 & & 0.0050 & 0.3310 & & & & & & & & & \\
\hline HLWMS-05 & & 0.0600 & & & & & 0.0450 & & 0.0200 & & & 0.3390 & & & & & & & & & \\
\hline HLWMS-06 & 0.0100 & 0.0400 & & & & & 0.0450 & & 0.0200 & & & 0.3340 & & & & & & & & & \\
\hline HLWMS-07 & 0.0300 & 0.1300 & & & & & 0.0600 & & 0.0300 & & & 0.2500 & 0.0200 & & & & & & & & \\
\hline HLWMS-08 & & 0.1200 & & & & & 0.0600 & & 0.0300 & & & 0.3350 & 0.0200 & & & & & & & & \\
\hline HLWMS-09 & & 0.1100 & & & & & 0.0450 & & 0.0150 & & & 0.3350 & 0.0600 & & & & & & & & \\
\hline HLWMS-10 & 0.0300 & 0.1100 & & & & & 0.0400 & & 0.0150 & & & 0.3100 & 0.0600 & & & & & & & & \\
\hline HLWMS-11 & 0.0500 & 0.1000 & & & & & 0.0350 & & 0.0100 & & & 0.3000 & 0.0700 & & & & & & & & \\
\hline HLWMS-12 & 0.1120 & 0.1000 & & & & & 0.0350 & & 0.0100 & & & 0.3660 & 0.0700 & & & & & & & & \\
\hline HLWMS-13 & 0.1120 & 0.1000 & & & & & 0.0350 & & 0.0100 & & & 0.3340 & 0.0700 & & & & & & & & \\
\hline HLWMS-14 & 0.1120 & 0.1000 & & & & & 0.0350 & & 0.0100 & & & 0.3960 & 0.0400 & & & & & & & & \\
\hline HLWMS-15 & 0.1320 & 0.1000 & & & & & 0.0350 & & 0.0100 & & & 0.3760 & 0.0400 & & & & & & & & \\
\hline HLW99-01 & 0.0000 & 0.0500 & 0.0700 & 0.0200 & & 0.0100 & 0.0350 & 0.0200 & 0.0500 & 0.0300 & 0.0000 & 0.5500 & 0.0000 & 0.0025 & 0.0020 & 0.0400 & 0.0200 & & 0.0300 & & 0.0020 \\
\hline HLW99-02 & 0.0000 & 0.2000 & 0.0000 & 0.1250 & & 0.0100 & 0.0000 & 0.0000 & 0.2000 & 0.0000 & 0.0270 & 0.3000 & 0.0000 & 0.0025 & 0.0020 & 0.0000 & 0.0000 & & 0.0000 & & 0.0020 \\
\hline HLW99-03 & 0.1100 & 0.2000 & 0.0000 & 0.0200 & & 0.0100 & 0.0650 & 0.0200 & 0.0500 & 0.0300 & 0.0000 & 0.3000 & 0.0300 & 0.0025 & 0.0020 & 0.0400 & 0.0200 & & 0.0300 & & 0.0020 \\
\hline HLW99-04 & 0.1100 & 0.0500 & 0.0000 & 0.0200 & & 0.0100 & 0.0700 & 0.0000 & 0.0500 & 0.0000 & 0.0300 & 0.4900 & 0.0230 & 0.0025 & 0.0020 & 0.0000 & 0.0000 & & 0.0000 & & 0.0020 \\
\hline HLW99-05 & 0.0000 & 0.0600 & 0.0000 & 0.0200 & & 0.0100 & 0.0100 & 0.0200 & 0.2000 & 0.0300 & 0.0184 & 0.3100 & 0.1000 & 0.0025 & 0.0020 & 0.0400 & 0.0200 & & 0.0300 & & 0.0020 \\
\hline HLW99-06 & 0.1100 & 0.0500 & 0.0700 & 0.0200 & & 0.0100 & 0.0000 & 0.0000 & 0.2000 & 0.0000 & 0.0000 & 0.4800 & 0.0050 & 0.0025 & 0.0020 & 0.0000 & 0.0000 & & 0.0000 & & 0.0020 \\
\hline HLW99-07 & 0.0000 & 0.2000 & 0.0700 & 0.0200 & & 0.0100 & 0.0350 & 0.0000 & 0.1500 & 0.0000 & 0.0037 & 0.3450 & 0.1000 & 0.0025 & 0.0020 & 0.0000 & 0.0000 & & 0.0000 & & 0.0020 \\
\hline HLW99-08 & 0.0000 & 0.0500 & 0.0700 & 0.1250 & & 0.0100 & 0.0700 & 0.0000 & 0.0800 & 0.0000 & 0.0300 & 0.3800 & 0.0380 & 0.0025 & 0.0020 & 0.0000 & 0.0000 & & 0.0000 & & 0.0020 \\
\hline HLW99-09 & 0.0000 & 0.2000 & 0.0700 & 0.0200 & & 0.0100 & 0.0350 & 0.0173 & 0.0500 & 0.0259 & 0.0300 & 0.3000 & 0.0000 & 0.0025 & 0.0020 & 0.0345 & 0.0173 & & 0.0259 & & 0.0020 \\
\hline HLW99-10 & 0.0000 & 0.0500 & 0.0630 & 0.0200 & & 0.0100 & 0.0000 & 0.0000 & 0.1300 & 0.0000 & 0.0300 & 0.5500 & 0.0000 & 0.0025 & 0.0020 & 0.0000 & 0.0000 & & 0.0000 & & 0.0020 \\
\hline HLW99-11 & 0.1100 & 0.2000 & 0.0700 & 0.0200 & & 0.0100 & 0.0070 & 0.0000 & 0.1060 & 0.0000 & 0.0300 & 0.3000 & 0.0000 & 0.0025 & 0.0020 & 0.0000 & 0.0000 & & 0.0000 & & 0.0020 \\
\hline HLW99-12 & 0.0100 & 0.2000 & 0.0700 & 0.1250 & & 0.0100 & 0.0350 & 0.0000 & 0.0500 & 0.0000 & 0.0000 & 0.4450 & 0.0000 & 0.0025 & 0.0020 & 0.0000 & 0.0000 & & 0.0000 & & 0.0020 \\
\hline
\end{tabular}


Appendix A. Database - mass fraction

RPP-WTP HLW Formulation (Kot and Pegg 2001)

\begin{tabular}{|c|c|c|c|c|c|c|c|c|c|c|c|c|c|c|c|c|c|c|c|c|c|}
\hline Glass ID & $\begin{array}{l}\mathrm{Cl} \\
-\mathrm{t}\end{array}$ & $\begin{array}{c}\mathrm{CoO} \\
-\mathrm{t}\end{array}$ & $\begin{array}{c}\mathrm{Co} 2 \mathrm{O} 3 \\
-\mathrm{t}\end{array}$ & $\begin{array}{c}\mathrm{Cr} 2 \mathrm{O} 3 \\
-\mathrm{t}\end{array}$ & $\begin{array}{c}\mathrm{Cs} 2 \mathrm{O} \\
-\mathrm{t}\end{array}$ & $\begin{array}{c}\mathrm{CuO} \\
-\mathrm{t} \\
\end{array}$ & $\begin{array}{c}\mathrm{Eu} 2 \mathrm{O} 3 \\
-\mathrm{t}\end{array}$ & $\begin{array}{l}F \\
-t\end{array}$ & $\begin{array}{c}\mathrm{Ga} 2 \mathrm{O} 3 \\
-\mathrm{t}\end{array}$ & $\begin{array}{c}\mathrm{Gd} 2 \mathrm{O} 3 \\
-\mathrm{t}\end{array}$ & $\begin{array}{c}\mathrm{HgO} \\
-\mathrm{t}\end{array}$ & $\begin{array}{l}I \\
-t\end{array}$ & $\begin{array}{c}\mathrm{La} 2 \mathrm{O} 3 \\
-\mathrm{t}\end{array}$ & $\begin{array}{c}\mathrm{MnO} 2 \\
-\mathrm{t}\end{array}$ & $\begin{array}{c}\mathrm{MnO} \\
-\mathrm{t}\end{array}$ & $\begin{array}{c}\mathrm{MoO} \\
-\mathrm{t}\end{array}$ & $\begin{array}{c}\mathrm{MoO} 3 \\
-\mathrm{t}\end{array}$ & $\begin{array}{c}\mathrm{Nb} 2 \mathrm{O} 5 \\
-\mathrm{t}\end{array}$ & $\begin{array}{c}\mathrm{Nd} 2 \mathrm{O} 3 \\
-\mathrm{t}\end{array}$ & $\begin{array}{c}\mathrm{PbO} \\
-\mathrm{t}\end{array}$ & $\begin{array}{c}\mathrm{PdO} 2 \\
-\mathrm{t}\end{array}$ \\
\hline HLWMS-01 & & & & & & & & & & & & & & & 0.1200 & & & & & & \\
\hline HLWMS-02 & & & & & & & & & & & & & & & 0.1300 & & & & & & \\
\hline HLWMS-03 & & & & & & & & 0.0200 & & & & & & & 0.1300 & & & & & & \\
\hline HLWMS-04 & & & & & & & & & & & & & & & 0.1510 & & & & & & \\
\hline HLWMS-05 & & & & & & & & & & & & & & & 0.1610 & & & & & & \\
\hline HLWMS-06 & & & & & & & & 0.0150 & & & & & & & 0.1610 & & & & & & \\
\hline HLWMS-07 & & & & & & & & & & & & & & & 0.1500 & & & & & & \\
\hline HLWMS-08 & & & & & & & & & & & & & & & 0.1360 & & & & & & \\
\hline HLWMS-09 & & & & & & & & & & & & & & & 0.1360 & & & & & & \\
\hline HLWMS-10 & & & & & & & & & & & & & & & 0.1360 & & & & & & \\
\hline HLWMS-11 & & & & & & & & & & & & & & & 0.1360 & & & & & & \\
\hline HLWMS-12 & & & & & & & & & & & & & & & 0.0960 & & & & & & \\
\hline HLWMS-13 & & & & & & & & & & & & & & & 0.1060 & & & & & & \\
\hline HLWMS-14 & & & & & & & & & & & & & & & 0.0960 & & & & & & \\
\hline HLWMS-15 & & & & & & & & & & & & & & & 0.0960 & & & & & & \\
\hline HLW99-01 & 0.0020 & 0.0020 & & 0.0000 & 0.0020 & 0.0020 & & 0.0020 & & & & & 0.0020 & 0.0020 & & & & & & 0.0100 & \\
\hline HLW99-02 & 0.0020 & 0.0020 & & 0.0045 & 0.0020 & 0.0020 & & 0.0020 & & & & & 0.0020 & 0.0020 & & & & & & 0.0000 & \\
\hline HLW99-03 & 0.0020 & 0.0020 & & 0.0000 & 0.0020 & 0.0020 & & 0.0020 & & & & & 0.0020 & 0.0020 & & & & & & 0.0100 & \\
\hline HLW99-04 & 0.0020 & 0.0020 & & 0.0050 & 0.0020 & 0.0020 & & 0.0020 & & & & & 0.0020 & 0.0020 & & & & & & 0.0000 & \\
\hline HLW99-05 & 0.0020 & 0.0020 & & 0.0031 & 0.0020 & 0.0020 & & 0.0020 & & & & & 0.0020 & 0.0020 & & & & & & 0.0100 & \\
\hline HLW99-06 & 0.0020 & 0.0020 & & 0.0000 & 0.0020 & 0.0020 & & 0.0020 & & & & & 0.0020 & 0.0020 & & & & & & 0.0000 & \\
\hline HLW99-07 & 0.0020 & 0.0020 & & 0.0006 & 0.0020 & 0.0020 & & 0.0020 & & & & & 0.0020 & 0.0020 & & & & & & 0.0000 & \\
\hline HLW99-08 & 0.0020 & 0.0020 & & 0.0050 & 0.0020 & 0.0020 & & 0.0020 & & & & & 0.0020 & 0.0020 & & & & & & 0.0000 & \\
\hline HLW99-09 & 0.0020 & 0.0020 & & 0.0050 & 0.0020 & 0.0020 & & 0.0020 & & & & & 0.0020 & 0.0020 & & & & & & 0.0086 & \\
\hline HLW99-10 & 0.0020 & 0.0020 & & 0.0050 & 0.0020 & 0.0020 & & 0.0020 & & & & & 0.0020 & 0.0020 & & & & & & 0.0000 & \\
\hline HLW99-11 & 0.0020 & 0.0020 & & 0.0050 & 0.0020 & 0.0020 & & 0.0020 & & & & & 0.0020 & 0.0020 & & & & & & 0.0000 & \\
\hline HLW99-12 & 0.0020 & 0.0020 & & 0.0000 & 0.0020 & 0.0020 & & 0.0020 & & & & & 0.0020 & 0.0020 & & & & & & 0.0000 & \\
\hline
\end{tabular}


Appendix A. Database - mass fraction

RPP-WTP HLW Formulation (Kot and Pegg 2001)

\begin{tabular}{|c|c|c|c|c|c|c|c|c|c|c|c|c|c|c|c|c|c|c|c|c|c|}
\hline Glass ID & $\begin{array}{c}\mathrm{PdO} \\
-\mathrm{t} \\
\end{array}$ & $\begin{array}{c}\mathrm{Pr} 2 \mathrm{O} 3 \\
-\mathrm{t}\end{array}$ & $\begin{array}{c}\text { Pr6O11 } \\
-t\end{array}$ & $\begin{array}{c}\mathrm{Rb} 2 \mathrm{O} \\
-\mathrm{t}\end{array}$ & $\begin{array}{c}\mathrm{ReO} \\
-\mathrm{t}\end{array}$ & $\begin{array}{c}\mathrm{ReO} 2 \\
-\mathrm{t}\end{array}$ & $\begin{array}{c}\mathrm{Rh} 2 \mathrm{O} 3 \\
-\mathrm{t}\end{array}$ & $\begin{array}{c}\mathrm{RhO} 2 \\
-\mathrm{t}\end{array}$ & $\begin{array}{c}\mathrm{RuO} 2 \\
-\mathrm{t}\end{array}$ & $\begin{array}{c}\mathrm{Sb} 2 \mathrm{O} 3 \\
-\mathrm{t}\end{array}$ & $\begin{array}{c}\mathrm{Sb} 2 \mathrm{O} 5 \\
-\mathrm{t}\end{array}$ & $\begin{array}{c}\mathrm{SeO} 2 \\
-\mathrm{t}\end{array}$ & $\begin{array}{c}\mathrm{Sm} 2 \mathrm{O} 3 \\
-\mathrm{t}\end{array}$ & $\begin{array}{c}\mathrm{SnO} \\
-\mathrm{t} \\
\end{array}$ & $\begin{array}{c}\mathrm{SnO} 2 \\
-\mathrm{t}\end{array}$ & $\begin{array}{c}\mathrm{SO} 3 \\
-\mathrm{t} \\
\end{array}$ & $\begin{array}{c}\mathrm{SrO} \\
-\mathrm{t}\end{array}$ & $\begin{array}{c}\mathrm{Tc} 2 \mathrm{O} 7 \\
-\mathrm{t}\end{array}$ & $\begin{array}{c}\mathrm{TeO} 2 \\
-\mathrm{t}\end{array}$ & $\begin{array}{c}\mathrm{ThO} 2 \\
-\mathrm{t}\end{array}$ & $\begin{array}{c}\mathrm{TiO} 2 \\
-\mathrm{t}\end{array}$ \\
\hline HLWMS-01 & & & & & & & & & & & & & & & & & 0.2640 & & & & \\
\hline HLWMS-02 & & & & & & & & & & & & & & & & & 0.2860 & & & & \\
\hline HLWMS-03 & & & & & & & & & & & & & & & & & 0.2860 & & & & \\
\hline HLWMS-04 & & & & & & & & & & & & & & & & & 0.3330 & & & & \\
\hline HLWMS-05 & & & & & & & & & & & & & & & & & 0.3550 & & & & \\
\hline HLWMS-06 & & & & & & & & & & & & & & & & & 0.3550 & & & & \\
\hline HLWMS-07 & & & & & & & & & & & & & & & & & 0.3300 & & & & \\
\hline HLWMS-08 & & & & & & & & & & & & & & & & & 0.2990 & & & & \\
\hline HLWMS-09 & & & & & & & & & & & & & & & & & 0.2990 & & & & \\
\hline HLWMS-10 & & & & & & & & & & & & & & & & & 0.2990 & & & & \\
\hline HLWMS-11 & & & & & & & & & & & & & & & & & 0.2990 & & & & \\
\hline HLWMS-12 & & & & & & & & & & & & & & & & & 0.2110 & & & & \\
\hline HLWMS-13 & & & & & & & & & & & & & & & & & 0.2330 & & & & \\
\hline HLWMS-14 & & & & & & & & & & & & & & & & & 0.2110 & & & & \\
\hline HLWMS-15 & & & & & & & & & & & & & & & & & 0.2110 & & & & \\
\hline HLW99-01 & 0.0004 & & & & & & 0.0008 & & 0.0013 & & 0.0020 & 0.0020 & & & & 0.0000 & 0.0020 & & 0.0020 & 0.0000 & 0.0100 \\
\hline HLW99-02 & 0.0004 & & & & & & 0.0008 & & 0.0013 & & 0.0020 & 0.0020 & & & & 0.0045 & 0.0020 & & 0.0020 & 0.0018 & 0.0000 \\
\hline HLW99-03 & 0.0004 & & & & & & 0.0008 & & 0.0013 & & 0.0020 & 0.0020 & & & & 0.0000 & 0.0020 & & 0.0020 & 0.0000 & 0.0100 \\
\hline HLW99-04 & 0.0004 & & & & & & 0.0008 & & 0.0013 & & 0.0020 & 0.0020 & & & & 0.0050 & 0.0020 & & 0.0020 & 0.0020 & 0.0000 \\
\hline HLW99-05 & 0.0004 & & & & & & 0.0008 & & 0.0013 & & 0.0020 & 0.0020 & & & & 0.0031 & 0.0020 & & 0.0020 & 0.0012 & 0.0100 \\
\hline HLW99-06 & 0.0004 & & & & & & 0.0008 & & 0.0013 & & 0.0020 & 0.0020 & & & & 0.0000 & 0.0020 & & 0.0020 & 0.0000 & 0.0000 \\
\hline HLW99-07 & 0.0004 & & & & & & 0.0008 & & 0.0013 & & 0.0020 & 0.0020 & & & & 0.0006 & 0.0020 & & 0.0020 & 0.0002 & 0.0000 \\
\hline HLW99-08 & 0.0004 & & & & & & 0.0008 & & 0.0013 & & 0.0020 & 0.0020 & & & & 0.0050 & 0.0020 & & 0.0020 & 0.0020 & 0.0000 \\
\hline HLW99-09 & 0.0004 & & & & & & 0.0008 & & 0.0013 & & 0.0020 & 0.0020 & & & & 0.0050 & 0.0020 & & 0.0020 & 0.0020 & 0.0086 \\
\hline HLW99-10 & 0.0004 & & & & & & 0.0008 & & 0.0013 & & 0.0020 & 0.0020 & & & & 0.0050 & 0.0020 & & 0.0020 & 0.0020 & 0.0000 \\
\hline HLW99-11 & 0.0004 & & & & & & 0.0008 & & 0.0013 & & 0.0020 & 0.0020 & & & & 0.0050 & 0.0020 & & 0.0020 & 0.0020 & 0.0000 \\
\hline HLW99-12 & 0.0004 & & & & & & 0.0008 & & 0.0013 & & 0.0020 & 0.0020 & & & & 0.0000 & 0.0020 & & 0.0020 & 0.0000 & 0.0000 \\
\hline
\end{tabular}


Appendix A. Database - mass fraction

RPP-WTP HLW Formulation (Kot and Pegg 2001)

\begin{tabular}{|c|c|c|c|c|c|c|c|c|c|c|c|c|c|c|c|c|c|c|c|c|c|}
\hline Glass ID & $\begin{array}{c}\mathrm{T} 12 \mathrm{O} 3 \\
-\mathrm{t}\end{array}$ & $\begin{array}{c}\mathrm{U} 3 \mathrm{O} 8 \\
-\mathrm{t}\end{array}$ & $\begin{array}{c}\mathrm{UO} 2 \\
-\mathrm{t}\end{array}$ & $\begin{array}{c}\mathrm{UO3} 3 \\
-\mathrm{t}\end{array}$ & $\begin{array}{c}\mathrm{V} 2 \mathrm{O} 5 \\
-\mathrm{t}\end{array}$ & $\begin{array}{c}\text { WO3 } \\
-t\end{array}$ & $\begin{array}{c}\mathrm{Y} 2 \mathrm{O} 3 \\
-\mathrm{t}\end{array}$ & $\begin{array}{c}\mathrm{ZnO} \\
-\mathrm{t}\end{array}$ & $\begin{array}{c}\text { Others } \\
-t\end{array}$ & $\begin{array}{c}\text { Sum } \\
-t\end{array}$ & $\begin{array}{c}\mathrm{A} 12 \mathrm{O} 3 \\
-\mathrm{a}\end{array}$ & $\begin{array}{c}\mathrm{B} 2 \mathrm{O} 3 \\
-\mathrm{a}\end{array}$ & $\begin{array}{c}\mathrm{CaO} \\
-\mathrm{a} \\
\end{array}$ & $\begin{array}{c}\mathrm{Fe} 2 \mathrm{O} 3 \\
-\mathrm{a}\end{array}$ & $\begin{array}{c}\mathrm{FeO} \\
-\mathrm{a}\end{array}$ & $\begin{array}{c}\mathrm{K} 2 \mathrm{O} \\
-\mathrm{a}\end{array}$ & $\begin{array}{c}\mathrm{Li} 2 \mathrm{O} \\
-\mathrm{a}\end{array}$ & $\begin{array}{c}\mathrm{MgO} \\
-\mathrm{a}\end{array}$ & $\begin{array}{c}\mathrm{Na} 2 \mathrm{O} \\
-\mathrm{a}\end{array}$ & $\begin{array}{c}\mathrm{NiO} \\
-\mathrm{a} \\
\end{array}$ & $\begin{array}{c}\mathrm{P} 2 \mathrm{O} 5 \\
-\mathrm{a}\end{array}$ \\
\hline HLWMS-01 & & & & & & & & 0.0300 & & 1.0000 & & & & & & & & & & & \\
\hline HLWMS-02 & & & & & & & & 0.0300 & & 1.0000 & & & & & & & & & & & \\
\hline HLWMS-03 & & & & & & & & 0.0300 & & 1.0000 & & & & & & & & & & & \\
\hline HLWMS-04 & & & & & & & & 0.0200 & & 1.0000 & & & & & & & & & & & \\
\hline HLWMS-05 & & & & & & & & 0.0200 & & 1.0000 & & & & & & & & & & & \\
\hline HLWMS-06 & & & & & & & & 0.0200 & & 1.0000 & & & & & & & & & & & \\
\hline HLWMS-07 & & & & & & & & & & 1.0000 & & & & & & & & & & & \\
\hline HLWMS-08 & & & & & & & & & & 1.0000 & & & & & & & & & & & \\
\hline HLWMS-09 & & & & & & & & & & 1.0000 & & & & & & & & & & & \\
\hline HLWMS-10 & & & & & & & & & & 1.0000 & & & & & & & & & & & \\
\hline HLWMS-11 & & & & & & & & & & 1.0000 & & & & & & & & & & & \\
\hline HLWMS-12 & & & & & & & & & & 1.0000 & & & & & & & & & & & \\
\hline HLWMS-13 & & & & & & & & & & 1.0000 & & & & & & & & & & & \\
\hline HLWMS-14 & & & & & & & & & & 1.0000 & & & & & & & & & & & \\
\hline HLWMS-15 & & & & & & & & & & 1.0000 & & & & & & & & & & & \\
\hline HLW99-01 & 0.0020 & & 0.0000 & & & 0.0020 & & 0.0200 & & 1.0000 & 0.0182 & 0.0488 & 0.0633 & 0.0206 & & 0.0114 & 0.0340 & 0.0185 & 0.0475 & 0.0277 & 0.0012 \\
\hline HLW99-02 & 0.0020 & & 0.0721 & & & 0.0020 & & 0.0200 & & 0.9999 & 0.0157 & 0.1860 & 0.0012 & 0.1101 & & 0.0115 & 0.0003 & 0.0004 & 0.1742 & 0.0002 & 0.0280 \\
\hline HLW99-03 & 0.0020 & & 0.0000 & & & 0.0020 & & 0.0200 & & 1.0000 & 0.1052 & 0.2003 & 0.0013 & 0.0201 & & 0.0109 & 0.0576 & 0.0192 & 0.0471 & 0.0284 & 0.0013 \\
\hline HLW99-04 & 0.0020 & & 0.0800 & & & 0.0020 & & 0.0200 & & 1.0000 & 0.1040 & 0.0529 & 0.0008 & 0.0241 & & 0.0114 & 0.0604 & 0.0003 & 0.0507 & 0.0003 & 0.0309 \\
\hline HLW99-05 & 0.0020 & & 0.0492 & & & 0.0020 & & 0.0200 & & 1.0000 & 0.0103 & 0.0564 & 0.0010 & 0.0200 & & 0.0111 & 0.0114 & 0.0178 & 0.1807 & 0.0278 & 0.0196 \\
\hline HLW99-06 & 0.0020 & & 0.0000 & & & 0.0020 & & 0.0200 & & 1.0000 & 0.1037 & 0.0504 & 0.0658 & 0.0203 & & 0.0116 & 0.0009 & 0.0003 & 0.1748 & 0.0001 & 0.0013 \\
\hline HLW99-07 & 0.0020 & & 0.0098 & & & 0.0020 & & 0.0200 & & 0.9999 & 0.0178 & 0.1947 & 0.0652 & 0.0209 & & 0.0106 & 0.0368 & 0.0006 & 0.1338 & 0.0006 & 0.0056 \\
\hline HLW99-08 & 0.0020 & & 0.0800 & & & 0.0020 & & 0.0200 & & 1.0000 & 0.0106 & 0.0487 & 0.0643 & 0.1131 & & 0.0111 & 0.0618 & 0.0003 & 0.0734 & 0.0004 & 0.0346 \\
\hline HLW99-09 & 0.0020 & & 0.0800 & & & 0.0020 & & 0.0200 & & 1.0001 & 0.0229 & 0.1771 & 0.0610 & 0.0194 & & 0.0106 & 0.0322 & 0.0154 & 0.0449 & 0.0229 & 0.0293 \\
\hline HLW99-10 & 0.0020 & & 0.0800 & & & 0.0020 & & 0.0200 & & 1.0000 & 0.0081 & 0.0506 & 0.0620 & 0.0206 & & 0.0109 & 0.0012 & 0.0003 & 0.1140 & 0.0004 & 0.0351 \\
\hline HLW99-11 & 0.0020 & & 0.0800 & & & 0.0020 & & 0.0200 & & 1.0000 & 0.1037 & 0.1838 & 0.0644 & 0.0218 & & 0.0109 & 0.0099 & 0.0002 & 0.0924 & 0.0003 & 0.0330 \\
\hline HLW99-12 & 0.0020 & & 0.0000 & & & 0.0020 & & 0.0200 & & 1.0000 & 0.0248 & 0.1858 & 0.0618 & 0.1136 & & 0.0105 & 0.0324 & 0.0003 & 0.0444 & 0.0001 & 0.0015 \\
\hline
\end{tabular}


Appendix A. Database - mass fraction

RPP-WTP HLW Formulation (Kot and Pegg 2001)

\begin{tabular}{|c|c|c|c|c|c|c|c|c|c|c|c|c|c|c|c|c|c|c|c|c|c|}
\hline Glass ID & $\begin{array}{c}\mathrm{SiO} 2 \\
-\mathrm{a}\end{array}$ & $\begin{array}{c}\mathrm{ZrO} 2 \\
-\mathrm{a}\end{array}$ & $\begin{array}{c}\mathrm{Ag} 2 \mathrm{O} \\
-\mathrm{a}\end{array}$ & $\begin{array}{c}\mathrm{As} 2 \mathrm{O} 3 \\
-\mathrm{a} \\
\end{array}$ & $\begin{array}{c}\mathrm{BaO} \\
-\mathrm{a}\end{array}$ & $\begin{array}{c}\mathrm{Bi} 2 \mathrm{O} 3 \\
-\mathrm{a}\end{array}$ & $\begin{array}{l}\mathrm{Br} \\
-\mathrm{a}\end{array}$ & $\begin{array}{c}\mathrm{CdO} \\
-\mathrm{a}\end{array}$ & $\begin{array}{c}\mathrm{Ce} 2 \mathrm{O} 3 \\
-\mathrm{a}\end{array}$ & $\begin{array}{c}\mathrm{CeO} 2 \\
-\mathrm{a}\end{array}$ & $\begin{array}{l}\mathrm{Cl} \\
-\mathrm{a}\end{array}$ & $\begin{array}{c}\mathrm{CoO} \\
-\mathrm{a}\end{array}$ & $\begin{array}{c}\mathrm{Co} 2 \mathrm{O} 3 \\
-\mathrm{a}\end{array}$ & $\begin{array}{c}\mathrm{Cr} 2 \mathrm{O} 3 \\
-\mathrm{a} \\
\end{array}$ & $\begin{array}{c}\mathrm{Cs} 2 \mathrm{O} \\
-\mathrm{a}\end{array}$ & $\begin{array}{c}\mathrm{CuO} \\
-\mathrm{a} \\
\end{array}$ & $\begin{array}{c}\mathrm{Eu} 2 \mathrm{O} 3 \\
-\mathrm{a}\end{array}$ & $\begin{array}{c}\mathrm{F} \\
-\mathrm{a}\end{array}$ & $\begin{array}{c}\mathrm{Ga} 2 \mathrm{O} 3 \\
-\mathrm{a}\end{array}$ & $\begin{array}{c}\mathrm{Gd} 2 \mathrm{O} 3 \\
-\mathrm{a}\end{array}$ & $\begin{array}{c}\mathrm{HgO} \\
-\mathrm{a}\end{array}$ \\
\hline HLWMS-01 & & & & & & & & & & & & & & & & & & & & & \\
\hline HLWMS-02 & & & & & & & & & & & & & & & & & & & & & \\
\hline HLWMS-03 & & & & & & & & & & & & & & & & & & & & & \\
\hline HLWMS-04 & & & & & & & & & & & & & & & & & & & & & \\
\hline HLWMS-05 & & & & & & & & & & & & & & & & & & & & & \\
\hline HLWMS-06 & & & & & & & & & & & & & & & & & & & & & \\
\hline HLWMS-07 & & & & & & & & & & & & & & & & & & & & & \\
\hline HLWMS-08 & & & & & & & & & & & & & & & & & & & & & \\
\hline HLWMS-09 & & & & & & & & & & & & & & & & & & & & & \\
\hline HLWMS-10 & & & & & & & & & & & & & & & & & & & & & \\
\hline HLWMS-11 & & & & & & & & & & & & & & & & & & & & & \\
\hline HLWMS-12 & & & & & & & & & & & & & & & & & & & & & \\
\hline HLWMS-13 & & & & & & & & & & & & & & & & & & & & & \\
\hline HLWMS-14 & & & & & & & & & & & & & & & & & & & & & \\
\hline HLWMS-15 & & & & & & & & & & & & & & & & & & & & & \\
\hline HLW99-01 & 0.5228 & 0.0004 & 0.0023 & 0.0019 & 0.0366 & 0.0200 & & 0.0276 & & & & 0.0020 & & 0.0000 & & 0.0020 & & & & & \\
\hline HLW99-02 & 0.3053 & 0.0001 & 0.0023 & 0.0018 & 0.0001 & 0.0004 & & 0.0001 & & & & 0.0023 & & 0.0038 & & 0.0023 & & & & & \\
\hline HLW99-03 & 0.3035 & 0.0253 & 0.0025 & 0.0019 & 0.0373 & 0.0201 & & 0.0283 & & & & 0.0022 & & 0.0000 & & 0.0021 & & & & & \\
\hline HLW99-04 & 0.4705 & 0.0153 & 0.0025 & 0.0020 & 0.0001 & 0.0003 & & 0.0000 & & & & 0.0027 & & 0.0031 & & 0.0023 & & & & & \\
\hline HLW99-05 & 0.3185 & 0.0821 & 0.0024 & 0.0020 & 0.0372 & 0.0192 & & 0.0268 & & & & 0.0025 & & 0.0027 & & 0.0025 & & & & & \\
\hline HLW99-06 & 0.4527 & 0.0050 & 0.0017 & 0.0019 & 0.0002 & 0.0000 & & 0.0000 & & & & 0.0021 & & 0.0000 & & 0.0022 & & & & & \\
\hline HLW99-07 & 0.3588 & 0.0862 & 0.0020 & 0.0019 & 0.0008 & 0.0005 & & 0.0005 & & & & 0.0023 & & 0.0007 & & 0.0023 & & & & & \\
\hline HLW99-08 & 0.3771 & 0.0325 & 0.0027 & 0.0019 & 0.0004 & 0.0005 & & 0.0002 & & & & 0.0027 & & 0.0043 & & 0.0025 & & & & & \\
\hline HLW99-09 & 0.3122 & 0.0020 & 0.0021 & 0.0014 & 0.0302 & 0.0149 & & 0.0225 & & & & 0.0025 & & 0.0039 & & 0.0021 & & & & & \\
\hline HLW99-10 & 0.5336 & 0.0023 & 0.0026 & 0.0021 & 0.0003 & 0.0006 & & 0.0002 & & & & 0.0026 & & 0.0043 & & 0.0024 & & & & & \\
\hline HLW99-11 & 0.2956 & 0.0011 & 0.0026 & 0.0019 & 0.0001 & 0.0003 & & 0.0000 & & & & 0.0028 & & 0.0030 & & 0.0024 & & & & & \\
\hline HLW99-12 & 0.4305 & 0.0001 & 0.0023 & 0.0019 & 0.0002 & 0.0002 & & 0.0001 & & & & 0.0019 & & 0.0000 & & 0.0021 & & & & & \\
\hline
\end{tabular}


Appendix A. Database - mass fraction

RPP-WTP HLW Formulation (Kot and Pegg 2001)

\begin{tabular}{|c|c|c|c|c|c|c|c|c|c|c|c|c|c|c|c|c|c|c|c|c|c|}
\hline Glass ID & $\begin{array}{c}\mathrm{I} \\
-\mathrm{a}\end{array}$ & $\begin{array}{c}\mathrm{La} 2 \mathrm{O} 3 \\
-\mathrm{a}\end{array}$ & $\begin{array}{c}\mathrm{MnO} 2 \\
-\mathrm{a}\end{array}$ & $\begin{array}{c}\mathrm{MnO} \\
-\mathrm{a}\end{array}$ & $\begin{array}{c}\mathrm{MoO} \\
-\mathrm{a}\end{array}$ & $\begin{array}{c}\mathrm{MoO} 3 \\
-\mathrm{a}\end{array}$ & $\begin{array}{c}\mathrm{Nb} 2 \mathrm{O} 5 \\
-\mathrm{a}\end{array}$ & $\begin{array}{c}\mathrm{Nd} 2 \mathrm{O} 3 \\
-\mathrm{a}\end{array}$ & $\begin{array}{c}\mathrm{PbO} \\
-\mathrm{a}\end{array}$ & $\begin{array}{c}\mathrm{PdO} 2 \\
-\mathrm{a}\end{array}$ & $\begin{array}{c}\mathrm{PdO} \\
-\mathrm{a}\end{array}$ & $\begin{array}{c}\mathrm{Pr} 2 \mathrm{O} 3 \\
-\mathrm{a}\end{array}$ & $\begin{array}{c}\text { Pr6O11 } \\
-\mathrm{a}\end{array}$ & $\begin{array}{c}\mathrm{Rb} 2 \mathrm{O} \\
-\mathrm{a}\end{array}$ & $\begin{array}{c}\mathrm{ReO} \\
-\mathrm{a}\end{array}$ & $\begin{array}{c}\mathrm{ReO} 2 \\
-\mathrm{a}\end{array}$ & $\begin{array}{c}\mathrm{Rh} 2 \mathrm{O} 3 \\
-\mathrm{a}\end{array}$ & $\begin{array}{c}\mathrm{RhO} 2 \\
-\mathrm{a}\end{array}$ & $\begin{array}{c}\mathrm{RuO}^{2} \\
-\mathrm{a}\end{array}$ & $\begin{array}{c}\mathrm{Sb} 2 \mathrm{O} 3 \\
-\mathrm{a}\end{array}$ & $\begin{array}{c}\mathrm{Sb} 2 \mathrm{O} 5 \\
-\mathrm{a}\end{array}$ \\
\hline HLWMS-01 & & & & & & & & & & & & & & & & & & & & & \\
\hline HLWMS-02 & & & & & & & & & & & & & & & & & & & & & \\
\hline HLWMS-03 & & & & & & & & & & & & & & & & & & & & & \\
\hline HLWMS-04 & & & & & & & & & & & & & & & & & & & & & \\
\hline HLWMS-05 & & & & & & & & & & & & & & & & & & & & & \\
\hline HLWMS-06 & & & & & & & & & & & & & & & & & & & & & \\
\hline HLWMS-07 & & & & & & & & & & & & & & & & & & & & & \\
\hline HLWMS-08 & & & & & & & & & & & & & & & & & & & & & \\
\hline HLWMS-09 & & & & & & & & & & & & & & & & & & & & & \\
\hline HLWMS-10 & & & & & & & & & & & & & & & & & & & & & \\
\hline HLWMS-11 & & & & & & & & & & & & & & & & & & & & & \\
\hline HLWMS-12 & & & & & & & & & & & & & & & & & & & & & \\
\hline HLWMS-13 & & & & & & & & & & & & & & & & & & & & & \\
\hline HLWMS-14 & & & & & & & & & & & & & & & & & & & & & \\
\hline HLWMS-15 & & & & & & & & & & & & & & & & & & & & & \\
\hline HLW99-01 & & & 0.0024 & & & & & & 0.0033 & & & & & & & & & & & & 0.0022 \\
\hline HLW99-02 & & & 0.0023 & & & & & & 0.0010 & & & & & & & & & & & & 0.0020 \\
\hline HLW99-03 & & & 0.0025 & & & & & & 0.0032 & & & & & & & & & & & & 0.0020 \\
\hline HLW99-04 & & & 0.0023 & & & & & & 0.0007 & & & & & & & & & & & & 0.0021 \\
\hline HLW99-05 & & & 0.0024 & & & & & & 0.0108 & & & & & & & & & & & & 0.0023 \\
\hline HLW99-06 & & & 0.0024 & & & & & & 0.0001 & & & & & & & & & & & & 0.0020 \\
\hline HLW99-07 & & & 0.0025 & & & & & & 0.0008 & & & & & & & & & & & & 0.0022 \\
\hline HLW99-08 & & & 0.0023 & & & & & & 0.0008 & & & & & & & & & & & & 0.0020 \\
\hline HLW99-09 & & & 0.0023 & & & & & & 0.0089 & & & & & & & & & & & & 0.0024 \\
\hline HLW99-10 & & & 0.0023 & & & & & & 0.0008 & & & & & & & & & & & & 0.0023 \\
\hline HLW99-11 & & & 0.0023 & & & & & & 0.0008 & & & & & & & & & & & & 0.0019 \\
\hline HLW99-12 & & & 0.0023 & & & & & & 0.0002 & & & & & & & & & & & & 0.0019 \\
\hline
\end{tabular}


Appendix A. Database - mass fraction

RPP-WTP HLW Formulation (Kot and Pegg 2001)

\begin{tabular}{|c|c|c|c|c|c|c|c|c|c|c|c|c|c|c|c|c|c|c|c|c|}
\hline Glass ID & $\begin{array}{c}\mathrm{SeO} 2 \\
-\mathrm{a} \\
\end{array}$ & $\begin{array}{c}\mathrm{Sm} 2 \mathrm{O} 3 \\
-\mathrm{a} \\
\end{array}$ & $\begin{array}{c}\mathrm{SnO} \\
-\mathrm{a} \\
\end{array}$ & $\begin{array}{c}\mathrm{SnO} 2 \\
-\mathrm{a} \\
\end{array}$ & $\begin{array}{c}\mathrm{SO} 3 \\
-\mathrm{a} \\
\end{array}$ & $\begin{array}{c}\mathrm{SrO} \\
-\mathrm{a} \\
\end{array}$ & $\begin{array}{c}\mathrm{Tc} 2 \mathrm{O} 7 \\
-\mathrm{a}\end{array}$ & $\begin{array}{c}\mathrm{TeO} 2 \\
-\mathrm{a}\end{array}$ & $\begin{array}{c}\mathrm{ThO} 2 \\
-\mathrm{a}\end{array}$ & $\begin{array}{c}\mathrm{TiO} 2 \\
-\mathrm{a} \\
\end{array}$ & \begin{tabular}{|c|}
$\mathrm{T} 2 \mathrm{O} 3$ \\
$-\mathrm{a}$
\end{tabular} & $\begin{array}{c}\mathrm{U3O} 8 \\
-\mathrm{a} \\
\end{array}$ & $\begin{array}{c}\mathrm{UO} 2 \\
-\mathrm{a} \\
\end{array}$ & $\begin{array}{c}\text { UO3 } \\
-\mathrm{a} \\
\end{array}$ & $\begin{array}{c}\mathrm{V} 2 \mathrm{O} 5 \\
-\mathrm{a} \\
\end{array}$ & $\begin{array}{c}\mathrm{WO} 3 \\
-\mathrm{a}\end{array}$ & $\begin{array}{c}\mathrm{Y} 2 \mathrm{O} 3 \\
-\mathrm{a}\end{array}$ & $\begin{array}{c}\mathrm{ZnO} \\
-\mathrm{a} \\
\end{array}$ & $\begin{array}{c}\text { Others } \\
-\mathrm{a}\end{array}$ & $\begin{array}{c}\text { Sum } \\
-\mathrm{a}\end{array}$ \\
\hline HLWMS-01 & & & & & & & & & & & & & & & & & & & & \\
\hline HLWMS-02 & & & & & & & & & & & & & & & & & & & & \\
\hline HLWMS-03 & & & & & & & & & & & & & & & & & & & & \\
\hline HLWMS-04 & & & & & & & & & & & & & & & & & & & & \\
\hline HLWMS-05 & & & & & & & & & & & & & & & & & & & & \\
\hline HLWMS-06 & & & & & & & & & & & & & & & & & & & & \\
\hline HLWMS-07 & & & & & & & & & & & & & & & & & & & & \\
\hline HLWMS-08 & & & & & & & & & & & & & & & & & & & & \\
\hline HLWMS-09 & & & & & & & & & & & & & & & & & & & & \\
\hline HLWMS-10 & & & & & & & & & & & & & & & & & & & & \\
\hline HLWMS-11 & & & & & & & & & & & & & & & & & & & & \\
\hline HLWMS-12 & & & & & & & & & & & & & & & & & & & & \\
\hline HLWMS-13 & & & & & & & & & & & & & & & & & & & & \\
\hline HLWMS-14 & & & & & & & & & & & & & & & & & & & & \\
\hline HLWMS-15 & & & & & & & & & & & & & & & & & & & & \\
\hline HLW99-01 & 0.0007 & & & & & 0.0026 & & 0.0021 & & 0.0101 & 0.0007 & & 0.0002 & & & 0.0026 & & 0.0196 & & 0.9533 \\
\hline HLW99-02 & 0.0006 & & & & & 0.0019 & & 0.0019 & & 0.0006 & 0.0009 & & 0.0486 & & & 0.0023 & & 0.0192 & & 0.9274 \\
\hline HLW99-03 & 0.0007 & & & & & 0.0025 & & 0.0023 & & 0.0109 & 0.0010 & & 0.0004 & & & 0.0026 & & 0.0198 & & 0.9625 \\
\hline HLW99-04 & 0.0009 & & & & & 0.0019 & & 0.0021 & & 0.0006 & 0.0013 & & 0.0693 & & & 0.0025 & & 0.0189 & & 0.9372 \\
\hline HLW99-05 & 0.0013 & & & & & 0.0024 & & 0.0024 & & 0.0106 & 0.0018 & & 0.0412 & & & 0.0028 & & 0.0200 & & 0.9500 \\
\hline HLW99-06 & 0.0013 & & & & & 0.0024 & & 0.0022 & & 0.0003 & 0.0007 & & 0.0004 & & & 0.0022 & & 0.0193 & & 0.9283 \\
\hline HLW99-07 & 0.0008 & & & & & 0.0023 & & 0.0021 & & 0.0010 & 0.0018 & & 0.0107 & & & 0.0025 & & 0.0204 & & 0.9897 \\
\hline HLW99-08 & 0.0011 & & & & & 0.0021 & & 0.0020 & & 0.0006 & 0.0017 & & 0.0617 & & & 0.0027 & & 0.0192 & & 0.9393 \\
\hline HLW99-09 & 0.0011 & & & & & 0.0023 & & 0.0019 & & 0.0090 & 0.0014 & & 0.0556 & & & 0.0028 & & 0.0184 & & 0.9356 \\
\hline HLW99-10 & 0.0009 & & & & & 0.0022 & & 0.0021 & & 0.0004 & 0.0014 & & 0.0753 & & & 0.0031 & & 0.0203 & & 0.9653 \\
\hline HLW99-11 & 0.0010 & & & & & 0.0020 & & 0.0022 & & 0.0004 & 0.0019 & & 0.0695 & & & 0.0027 & & 0.0191 & & 0.9340 \\
\hline HLW99-12 & 0.0002 & & & & & 0.0021 & & 0.0020 & & 0.0009 & 0.0009 & & 0.0006 & & & 0.0023 & & 0.0188 & & 0.9467 \\
\hline
\end{tabular}


Appendix A. Database - mass fraction

\begin{tabular}{|c|c|c|c|c|c|c|c|c|}
\hline -WTP HLW & la & n (Kot an & Pegg 2001) & & & & & \\
\hline Glass ID & $\begin{array}{l}\mathrm{TM} \\
\left({ }^{\circ} \mathrm{C}\right)\end{array}$ & $\begin{array}{l}\text { Gradient } \\
\text { TL }\left({ }^{\circ} \mathrm{C}\right)\end{array}$ & $\begin{array}{l}\text { Uniform } \\
\text { TL }\left({ }^{\circ} \mathrm{C}\right)\end{array}$ & Primary Phase & $\begin{array}{l}\text { Quenched } \\
\text { Visual/OM }\end{array}$ & $\begin{array}{c}\text { Quenched } \\
\text { SEM/EDS or TEM }\end{array}$ & $\begin{array}{l}\text { Quenched } \\
\text { XRD }\end{array}$ & $\begin{array}{c}\text { Quenched } \\
\text { Homogeneous? }\end{array}$ \\
\hline HLWMS-01 & 1150 & & $<950^{\circ} \mathrm{C}$ & & Homogeneous dark brown glass & & & \\
\hline HLWMS-02 & 1150 & & $<950^{\circ} \mathrm{C}$ & & Homogeneous dark brown glass & & & \\
\hline HLWMS-03 & 1150 & & $>950^{\circ} \mathrm{C}$ & & $\begin{array}{l}\text { Brown glass with metallic blue appear-ance. } \\
\text { Trace amount of secondary phase }\end{array}$ & & & \\
\hline HLWMS-04 & 1150 & & $>950^{\circ} \mathrm{C}$ & & Homogeneous dark glass with metallic sheen & & & \\
\hline HLWMS-05 & 1150 & & $>950^{\circ} \mathrm{C}$ & & Homogeneous glass with metallic appearance & & & \\
\hline HLWMS-06 & 1150 & & $>950^{\circ} \mathrm{C}$ & & $\begin{array}{l}\text { Metallic blue glass with extensive surface } \\
\text { crystallization }\end{array}$ & & & \\
\hline HLWMS-07 & 1150 & & $<950^{\circ} \mathrm{C}$ & & $\begin{array}{l}\text { Mostly homogeneous with some metallic } \\
\text { swirls }\end{array}$ & & & \\
\hline HLWMS-08 & 1150 & & $<950^{\circ} \mathrm{C}$ & & Homogeneous glass with metallic appearance & & & \\
\hline HLWMS-09 & 1200 & & $<950^{\circ} \mathrm{C}$ & & Homogeneous dark glass & & & \\
\hline HLWMS-10 & 1200 & & $<950{ }^{\circ} \mathrm{C}$ & & Homogeneous dark glass & & & \\
\hline HLWMS-11 & 1200 & & $<950^{\circ} \mathrm{C}$ & & Homogeneous dark brown glass & & & \\
\hline HLWMS-12 & 1200 & & $>950^{\circ} \mathrm{C}$ & & Homogeneous dark brown glass & & & \\
\hline HLWMS-13 & 1200 & & $>950^{\circ} \mathrm{C}$ & & Homogeneous dark brown glass & & & \\
\hline HLWMS-14 & 1200 & & $<950^{\circ} \mathrm{C}$ & & Homogeneous reddish brown glass & & & \\
\hline HLWMS-15 & 1200 & & $<950^{\circ} \mathrm{C}$ & & Homogeneous dark brown glass & & & \\
\hline HLW99-01 & 1150 & & 1075 & & $\begin{array}{l}\text { Homogeneous dark brown glass with a tinge } \\
\text { of blue. Undis-solved materials left in } \\
\text { crucible }\end{array}$ & & & \\
\hline HLW99-02 & & & & & & & & \\
\hline HLW99-03 & 1150 & & 1075 & & $\begin{array}{l}\text { Mostly homogeneous dark brown glass with } \\
\text { some cloudiness on surface }\end{array}$ & & & \\
\hline HLW99-04 & & & & & & & & \\
\hline HLW99-05 & & & & & & & & \\
\hline HLW99-06 & 1150 & & 950 & & $\begin{array}{l}\text { Homogeneous deep blue glass. Silver patches } \\
\text { on blue glass in crucible }\end{array}$ & & & \\
\hline HLW99-07 & & & & & & & & \\
\hline HLW99-08 & & & & & & & & \\
\hline HLW99-09 & & & & & & & & \\
\hline HLW99-10 & 1150 & & $>1056^{\circ} \mathrm{C}$ & & $\begin{array}{l}\text { Blue glass with metallic like fragments and } \\
\text { brown regions }\end{array}$ & & & \\
\hline HLW99-11 & 1150 & & $>978^{\circ} \mathrm{C}$ & & $\begin{array}{l}\text { Mostly clear brown/blue glass with dull gray } \\
\text { lumps on surface. Some gray-colored swirls }\end{array}$ & & & \\
\hline HLW99-12 & 1150 & & 950 & & $\begin{array}{l}\text { Inhomogeneous olive green glass with blue } \\
\text { patches. Traces of silvery clusters on crucible } \\
\text { wall }\end{array}$ & & & \\
\hline
\end{tabular}


Appendix A. Database - mass fraction

RPP-WTP HLW Formulation (Kot and Pegg 2001)

\begin{tabular}{|c|c|c|c|c|c|}
\hline Glass ID & $\begin{array}{c}\mathrm{CCC} \\
\text { Visual/OM }\end{array}$ & $\begin{array}{c}\text { CCC } \\
\text { SEM/EDS or TEM }\end{array}$ & $\begin{array}{l}\mathrm{CCC} \\
\mathrm{XRD}\end{array}$ & $\begin{array}{c}\text { Heat Treated } \\
\text { Visual/OM }\end{array}$ & $\begin{array}{c}\text { Heat Treated } \\
\text { SEM/EDS or TEM }\end{array}$ \\
\hline HLWMS-01 & & & & & $\begin{array}{l}\text { After } 20 \text { hours, no crystallization observed except } \\
\text { two dark crystals }\end{array}$ \\
\hline HLWMS-02 & & & & & After 20 hours, no crystallization observed \\
\hline HLWMS-03 & & & & & $\begin{array}{l}\text { After } 20 \text { hours, } 5-10 \text { vol } \% \text { of large clear light blue } \\
\text { Sr-silicate with oxides of } \mathrm{Na}, \mathrm{Al}, \mathrm{Mn} \text { and } \mathrm{Zn}\end{array}$ \\
\hline HLWMS-04 & & & & & $\begin{array}{l}\text { After } 20 \text { hours, } 0.5 \text { vol } \% \text { of Mn oxide and } 23 \\
\text { vol\% of clear Sr-silicate with } \mathrm{Al}, \mathrm{Mn} \text { and } \mathrm{Zn}\end{array}$ \\
\hline HLWMS-05 & & & & & $\begin{array}{l}\text { After } 20 \text { hours, } 34 \text { vol } \% \text { of clear leaf-shaped Sr- } \\
\text { silicate with } \mathrm{Mn} \text { and } \mathrm{Zn}\end{array}$ \\
\hline HLWMS-06 & & & & & $\begin{array}{l}\text { After } 20 \text { hours, } 35 \text { vol\% of leaf-shaped Sr-silicate } \\
\text { with Al, Mn and } \mathrm{Zn} ; 5 \text { vol\% of Mn oxide with } \mathrm{Zn}\end{array}$ \\
\hline HLWMS-07 & & & & & $\begin{array}{l}\text { After } 20 \text { hours, } 0.2-0.4 \mathrm{vol} \% \text { of dark spinel-like } \\
\text { crystal }\end{array}$ \\
\hline HLWMS-08 & & & & & After 20 hours, no crystallization observed \\
\hline HLWMS-09 & & & & & After 20 hours, no crystallization \\
\hline HLWMS-10 & & & & & After 20 hours, no crystallization observed \\
\hline HLWMS-11 & & & & & $\begin{array}{l}\text { After } 20 \text { hours, a few light color crystals at the } \\
\text { glass/crucible interface }\end{array}$ \\
\hline HLWMS-12 & & & & & $\begin{array}{l}\text { After } 20 \text { hours, } 2.3 \text { vol } \% \text { of cubic or acicular } \\
\text { ZrO2 }\end{array}$ \\
\hline HLWMS-13 & & & & & $\begin{array}{l}\text { After } 20 \text { hours, } 3.6 \text { vol } \% \text { of } \mathrm{ZrO} 2,3-5 \mathrm{vol} \% \text { of } \\
\text { SrA12Si2O8, and small amount of Mn3O4 }\end{array}$ \\
\hline HLWMS-14 & & & & & After 20 hours, no crystallization observed \\
\hline HLWMS-15 & & & & & After 20 hours, no crystallization observed \\
\hline HLW99-01 & & & & & $\begin{array}{l}10 \mathrm{vol} \% \text { of clinopy-roxene (calcium) crystal. } \\
\text { Traces of } \mathrm{RuO} 2\end{array}$ \\
\hline \multicolumn{6}{|l|}{ HLW99-02 } \\
\hline HLW99-03 & & & & & $\begin{array}{l}\text { Up to } 2 \text { vol } \% \text { rod-shaped crystals. No spinel phase } \\
\text { is apparent }\end{array}$ \\
\hline \multicolumn{6}{|l|}{ HLW99-04 } \\
\hline HLW99-05 & & & & & \\
\hline HLW99-06 & & & & & $\begin{array}{l}\text { About } 0.2 \text { vol } \% \text { of rhodium metal and } \mathrm{RuO} 2 \text {. No } \\
\text { spinel found }\end{array}$ \\
\hline \multicolumn{6}{|l|}{ HLW99-07 } \\
\hline \multicolumn{6}{|l|}{ HLW99-08 } \\
\hline HLW99-09 & & & & & \\
\hline HLW99-10 & & & & & $\begin{array}{l}\text { At } 1056^{\circ} \mathrm{C}, 4 \mathrm{vol} \% \text { of clear/white crystal of } \\
\text { calcium phosphate (with some Na). Rhodium } \\
\text { metal and RuO2 are also present }\end{array}$ \\
\hline HLW99-11 & & & & & $\begin{array}{l}\text { At } 978^{\circ} \mathrm{C}, 2 \text { vol } \% \text { of calcium phosphate, } 0.5 \\
\text { vol } \% \text { of spinel }(\mathrm{Cr}, \mathrm{Zn}) \text { and } 0.1 \mathrm{vol} \% \text { of } \mathrm{RuO} 2\end{array}$ \\
\hline HLW99-12 & & & & & $\begin{array}{l}\text { Equiaxed crystals consisting of rhodium } \\
\text { incorporating Fe and } \mathrm{Zn}(0.5-4 \mathrm{~mm}) \text { and } \mathrm{RuO} 2 \text {. } \\
\text { Total } 0.6 \mathrm{vol} \%\end{array}$ \\
\hline
\end{tabular}


Appendix A. Database - mass fraction

RPP-WTP HLW Formulation (Kot and Pegg 2001)

\begin{tabular}{|c|c|c|c|c|c|c|c|c|c|c|c|c|c|c|c|}
\hline Glass ID & $\begin{array}{c}\text { Heat Treated } \\
\text { XRD } \\
\end{array}$ & $\begin{array}{l}\text { Density } \\
\left(\mathrm{g} / \mathrm{cm}^{3}\right)\end{array}$ & $\begin{array}{c}\text { Fulc Visc } \\
\mathrm{A}\end{array}$ & $\begin{array}{c}\text { Fulc Visc } \\
\text { B }\end{array}$ & $\begin{array}{c}\text { Fulc Visc } \\
\text { To }\end{array}$ & $\begin{array}{c}\mathrm{FV} 1150^{\circ} \mathrm{C} \\
(\mathrm{Pa} \cdot \mathrm{s})\end{array}$ & $\begin{array}{c}\text { Arrh Visc } \\
\text { A }\end{array}$ & $\begin{array}{c}\text { Arrh Visc } \\
\mathrm{B}\end{array}$ & $\begin{array}{l}1150^{\circ} \mathrm{C} \\
\left(\mathrm{D}_{0,0}\right)\end{array}$ & $\begin{array}{c}\mathrm{T}\left({ }^{\circ} \mathrm{C}\right) \text { at } \\
2 \mathrm{~Pa} \cdot \mathrm{s}\end{array}$ & $\begin{array}{c}\mathrm{T}\left({ }^{\circ} \mathrm{C}\right) \text { at } \\
5 \mathrm{~Pa} \cdot \mathrm{s}\end{array}$ & $\begin{array}{c}\mathrm{T}\left({ }^{\circ} \mathrm{C}\right) \text { at } \\
10 \mathrm{~Pa} \cdot \mathrm{s}\end{array}$ & $\begin{array}{l}\mathrm{T} 1 \\
\left({ }^{\circ} \mathrm{C}\right)\end{array}$ & $\begin{array}{c}\mathrm{V} 1 \\
(\mathrm{~Pa} \cdot \mathrm{s})\end{array}$ & $\begin{array}{l}\mathrm{T} 2 \\
\left({ }^{\circ} \mathrm{C}\right) \\
\end{array}$ \\
\hline \multicolumn{16}{|l|}{ HLWMS-01 } \\
\hline \multicolumn{16}{|l|}{ HLWMS-02 } \\
\hline \multicolumn{16}{|l|}{ HLWMS-03 } \\
\hline \multicolumn{16}{|l|}{ HLWMS-04 } \\
\hline \multicolumn{16}{|l|}{ HLWMS-05 } \\
\hline \multicolumn{16}{|l|}{ HLWMS-06 } \\
\hline \multicolumn{16}{|l|}{ HLWMS-07 } \\
\hline HLWMS-08 & & & & & & & -11.569 & 14636.0 & 0.28 & & & & 1000 & 0.937 & 1050 \\
\hline \multirow{2}{*}{\multicolumn{16}{|c|}{$\begin{array}{l}\text { HLWMS-09 } \\
\text { HLWMS-10 }\end{array}$}} \\
\hline & & & & & & & & & & & & & & & \\
\hline HLWMS-11 & & 3.37 & & & & & -14.364 & 20240.2 & 0.87 & & & & 1000 & 4.801 & 1050 \\
\hline \multicolumn{16}{|l|}{ HLWMS-12 } \\
\hline \multicolumn{16}{|l|}{ HLWMS-13 } \\
\hline \multicolumn{16}{|l|}{ HLWMS-14 } \\
\hline HLWMS-15 & & & & & & & -13.883 & 22359.8 & 6.23 & & & & 1000 & 41.183 & 1050 \\
\hline \multicolumn{16}{|l|}{ HLW99-01 } \\
\hline \multicolumn{16}{|l|}{ HLW99-02 } \\
\hline HLW99-03 & & 2.8 & & & & & & & & & & & & & \\
\hline HLW99-04 & & & & & & & & & & & & & & & \\
\hline HLW99-05 & & 3.15 & & & & & & & & & & & & & \\
\hline HLW99-06 & & 2.64 & & & & & -11.857 & 20819.7 & 16.02 & & & & 1000 & 91.212 & 1050 \\
\hline HLW99-07 & & 2.78 & & & & & & & & & & & & & \\
\hline HLW99-08 & & 2.94 & & & & & & & & & & & & & \\
\hline HLW99-09 & & 2.99 & & & & & & & & & & & & & \\
\hline HLW99-10 & & 2.76 & & & & & & & & & & & & & \\
\hline HLW99-11 & & 2.72 & & & & & & & & & & & & & \\
\hline HLW99-12 & & 2.67 & & & & & & & & & & & & & \\
\hline
\end{tabular}


Appendix A. Database - mass fraction

\begin{tabular}{|c|c|c|c|c|c|c|c|c|c|c|c|c|c|c|c|c|c|c|c|c|c|}
\hline Glass ID & $\begin{array}{c}\mathrm{V} 2 \\
(\mathrm{~Pa} \cdot \mathrm{s})\end{array}$ & $\begin{array}{c}\mathrm{T} 3 \\
\left({ }^{\circ} \mathrm{C}\right) \\
\end{array}$ & $\begin{array}{c}\mathrm{V} 3 \\
(\mathrm{~Pa} \cdot \mathrm{s})\end{array}$ & $\begin{array}{c}\mathrm{T} 4 \\
\left({ }^{\circ} \mathrm{C}\right) \\
\end{array}$ & $\begin{array}{c}\mathrm{V} 4 \\
(\mathrm{~Pa} \cdot \mathrm{s}) \\
\end{array}$ & $\begin{array}{c}\mathrm{T} 5 \\
\left({ }^{\circ} \mathrm{C}\right) \\
\end{array}$ & $\begin{array}{c}\text { V5 } \\
(\mathrm{Pa} \cdot \mathrm{s}) \\
\end{array}$ & $\begin{array}{c}\mathrm{T} 6 \\
\left({ }^{\circ} \mathrm{C}\right) \\
\end{array}$ & $\begin{array}{c}\mathrm{V} 6 \\
(\mathrm{~Pa} \cdot \mathrm{s})\end{array}$ & $\begin{array}{c}\mathrm{T} 7 \\
\left({ }^{\circ} \mathrm{C}\right) \\
\end{array}$ & $\begin{array}{c}\text { V7 } \\
(\mathrm{Pa} \cdot \mathrm{s}) \\
\end{array}$ & $\begin{array}{c}\mathrm{T} 8 \\
\left({ }^{\circ} \mathrm{C}\right) \\
\end{array}$ & $\begin{array}{c}\mathrm{V} 8 \\
(\mathrm{~Pa} \cdot \mathrm{s})\end{array}$ & $\begin{array}{c}\mathrm{T} 9 \\
\left({ }^{\circ} \mathrm{C}\right) \\
\end{array}$ & $\begin{array}{c}\mathrm{V} 9 \\
(\mathrm{~Pa} \cdot \mathrm{s})\end{array}$ & $\begin{array}{l}\mathrm{T} 10 \\
\left({ }^{\circ} \mathrm{C}\right) \\
\end{array}$ & $\begin{array}{c}\mathrm{V} 10 \\
(\mathrm{~Pa} \cdot \mathrm{s}) \\
\end{array}$ & $\begin{array}{l}\mathrm{T} 11 \\
\left({ }^{\circ} \mathrm{C}\right) \\
\end{array}$ & $\begin{array}{c}\text { V11 } \\
(\mathrm{Pa} \cdot \mathrm{s})\end{array}$ & $\begin{array}{l}\mathrm{T} 12 \\
\left({ }^{\circ} \mathrm{C}\right) \\
\end{array}$ & $\begin{array}{c}\mathrm{V} 12 \\
(\mathrm{~Pa} \cdot \mathrm{s})\end{array}$ \\
\hline \multicolumn{22}{|l|}{ HLWMS-01 } \\
\hline \multicolumn{22}{|l|}{ HLWMS-02 } \\
\hline \multicolumn{22}{|l|}{ HLWMS-03 } \\
\hline \multicolumn{22}{|l|}{ HLWMS-04 } \\
\hline \multicolumn{22}{|l|}{ HLWMS-05 } \\
\hline \multicolumn{22}{|l|}{ HLWMS-06 } \\
\hline \multicolumn{22}{|l|}{ HLWMS-07 } \\
\hline HLWMS-08 & 0.598 & 1100 & 0.4 & 1150 & 0.279 & & & & & & & & & & & & & & & & \\
\hline \multicolumn{22}{|l|}{ HLWMS-09 } \\
\hline \multicolumn{22}{|l|}{ HLWMS-10 } \\
\hline HLWMS-11 & 2.493 & 1100 & 1.412 & 1150 & 0.859 & 1200 & 0.554 & & & & & & & & & & & & & & \\
\hline \multicolumn{22}{|l|}{ HLWMS-12 } \\
\hline \multicolumn{22}{|l|}{ HLWMS-13 } \\
\hline \multicolumn{22}{|l|}{ HLWMS-14 } \\
\hline HLWMS-15 & 19.961 & 1100 & 10.656 & 1150 & 6.153 & 1200 & 3.79 & & & & & & & & & & & & & & \\
\hline \multicolumn{22}{|l|}{ HLW99-01 } \\
\hline \multicolumn{22}{|l|}{ HLW99-02 } \\
\hline \multicolumn{22}{|l|}{ HLW99-03 } \\
\hline \multicolumn{22}{|l|}{ HLW99-04 } \\
\hline \multicolumn{22}{|l|}{ HLW99-05 } \\
\hline HLW99-06 & 47.915 & 1100 & 26.862 & 1150 & 15.921 & 1200 & 9.9 & & & & & & & & & & & & & & \\
\hline \multicolumn{22}{|l|}{ HLW99-07 } \\
\hline \multicolumn{22}{|l|}{ HLW99-08 } \\
\hline \multicolumn{22}{|l|}{ HLW99-09 } \\
\hline \multicolumn{22}{|l|}{ HLW99-10 } \\
\hline \multicolumn{22}{|l|}{ HLW99-11 } \\
\hline HLW99-12 & & & & & & & & & & & & & & & & & & & & & \\
\hline
\end{tabular}


Appendix A. Database - mass fraction

RPP-WTP HLW Formulation (Kot and Pegg 2001)

\begin{tabular}{|c|c|c|c|c|c|c|c|c|c|c|c|c|c|c|c|c|}
\hline Glass ID & $\begin{array}{l}\mathrm{T} 13 \\
\left({ }^{\circ} \mathrm{C}\right) \\
\end{array}$ & $\begin{array}{c}\mathrm{V} 13 \\
(\mathrm{~Pa} \cdot \mathrm{s}) \\
\end{array}$ & $\begin{array}{l}\mathrm{T} 14 \\
\left({ }^{\circ} \mathrm{C}\right) \\
\end{array}$ & $\begin{array}{l}\text { V14 } \\
(\mathrm{Pa} \cdot \mathrm{s}) \\
\end{array}$ & $\begin{array}{c}\text { Q PCT } \\
\mathrm{B}\left(\mathrm{g} / \mathrm{m}^{2}\right)\end{array}$ & $\begin{array}{c}\text { Q PCT } \\
\mathrm{Li}\left(\mathrm{g} / \mathrm{m}^{2}\right)\end{array}$ & $\begin{array}{c}\text { Q PCT } \\
\mathrm{Na}\left(\mathrm{g} / \mathrm{m}^{2}\right)\end{array}$ & $\begin{array}{c}\text { Q PCT } \\
\mathrm{Si}\left(\mathrm{g} / \mathrm{m}^{2}\right)\end{array}$ & $\begin{array}{l}\text { Q PCT } \\
\mathrm{pH} \\
\end{array}$ & $\begin{array}{c}\mathrm{CCC} \mathrm{PCT} \\
\mathrm{B}\left(\mathrm{g} / \mathrm{m}^{2}\right)\end{array}$ & $\begin{array}{l}\mathrm{CCC} \mathrm{PCT} \\
\mathrm{Li}\left(\mathrm{g} / \mathrm{m}^{2}\right)\end{array}$ & $\begin{array}{l}\text { CCC PCT } \\
\mathrm{Na}\left(\mathrm{g} / \mathrm{m}^{2}\right)\end{array}$ & $\begin{array}{l}\mathrm{CCC} \mathrm{PCT} \\
\mathrm{Si}\left(\mathrm{g} / \mathrm{m}^{2}\right)\end{array}$ & $\begin{array}{c}\text { CCC PCT } \\
\mathrm{pH} \\
\end{array}$ & $\begin{array}{l}\text { Q PCT B at } \\
20^{\circ} \mathrm{C}\left(\mathrm{g} / \mathrm{m}^{2}\right)\end{array}$ & $\begin{array}{l}\text { Q PCT Li at } \\
20^{\circ} \mathrm{C}\left(\mathrm{g} / \mathrm{m}^{2}\right)\end{array}$ \\
\hline \multicolumn{17}{|l|}{ HLWMS-01 } \\
\hline \multicolumn{17}{|l|}{ HLWMS-02 } \\
\hline \multicolumn{17}{|l|}{ HLWMS-03 } \\
\hline \multicolumn{17}{|l|}{ HLWMS-04 } \\
\hline \multicolumn{17}{|l|}{ HLWMS-05 } \\
\hline \multicolumn{17}{|l|}{ HLWMS-06 } \\
\hline \multicolumn{17}{|l|}{ HLWMS-07 } \\
\hline HLWMS-08 & & & & & 1.205 & 1.455 & 1.41 & 0.465 & 11.43 & & & & & & & \\
\hline HLWMS-09 & & & & & 0.71 & 0.745 & 0.945 & 0.26 & 10.53 & & & & & & & \\
\hline HLWMS-10 & & & & & 0.56 & 0.575 & 0.64 & 0.18 & 10.43 & & & & & & & \\
\hline \multicolumn{17}{|l|}{ HLWMS-11 } \\
\hline \multicolumn{17}{|l|}{ HLWMS-12 } \\
\hline \multicolumn{17}{|l|}{ HLWMS-13 } \\
\hline \multicolumn{17}{|l|}{ HLWMS-14 } \\
\hline \multicolumn{17}{|l|}{ HLWMS-15 } \\
\hline \multicolumn{17}{|l|}{ HLW99-01 } \\
\hline \multicolumn{17}{|l|}{ HLW99-02 } \\
\hline \multicolumn{17}{|l|}{ HLW99-03 } \\
\hline \multicolumn{17}{|l|}{ HLW99-04 } \\
\hline \multicolumn{17}{|l|}{ HLW99-05 } \\
\hline \multicolumn{17}{|l|}{ HLW99-06 } \\
\hline \multicolumn{17}{|l|}{ HLW99-07 } \\
\hline \multicolumn{17}{|l|}{ HLW99-08 } \\
\hline \multicolumn{17}{|l|}{ HLW99-09 } \\
\hline \multicolumn{17}{|l|}{ HLW99-10 } \\
\hline \multicolumn{17}{|l|}{ HLW99-11 } \\
\hline HLW99-12 & & & & & & & & & & & & & & & & \\
\hline
\end{tabular}


Appendix A. Database - mass fraction

RPP-WTP HLW Formulation (Kot and Pegg 2001)

\begin{tabular}{|c|c|c|c|c|c|c|c|c|c|c|c|c|}
\hline Glass ID & $\begin{array}{l}\text { Q PCT Na at } \\
20^{\circ} \mathrm{C}\left(\mathrm{g} / \mathrm{m}^{2}\right)\end{array}$ & $\begin{array}{l}\text { Q PCT Si at } \\
20^{\circ} \mathrm{C}\left(\mathrm{g} / \mathrm{m}^{2}\right)\end{array}$ & $\begin{array}{c}\mathrm{QpH} \\
\text { at } 20^{\circ} \mathrm{C}\end{array}$ & $\begin{array}{c}\text { TCLP Ag } \\
\text { (ppm) }\end{array}$ & $\begin{array}{c}\text { TCLP As } \\
\text { (ppm) }\end{array}$ & $\begin{array}{c}\text { TCLP Ba } \\
\text { (ppm) }\end{array}$ & $\begin{array}{c}\text { TCLP Cd } \\
(\mathrm{ppm})\end{array}$ & $\begin{array}{c}\text { TCLP Cr } \\
(\mathrm{ppm})\end{array}$ & $\begin{array}{c}\text { TCLP Ni } \\
(\mathrm{ppm})\end{array}$ & $\begin{array}{c}\text { TCLP Pb } \\
\text { (ppm) }\end{array}$ & $\begin{array}{c}\text { TCLP Se } \\
\text { (ppm) }\end{array}$ & $\begin{array}{c}\text { TCLP Zn } \\
(\mathrm{ppm})\end{array}$ \\
\hline \multicolumn{13}{|l|}{ HLWMS-01 } \\
\hline \multicolumn{13}{|l|}{ HLWMS-02 } \\
\hline \multicolumn{13}{|l|}{ HLWMS-03 } \\
\hline \multicolumn{13}{|l|}{ HLWMS-04 } \\
\hline \multicolumn{13}{|l|}{ HLWMS-05 } \\
\hline \multicolumn{13}{|l|}{ HLWMS-06 } \\
\hline \multicolumn{13}{|l|}{ HLWMS-07 } \\
\hline \multicolumn{13}{|l|}{ HLWMS-08 } \\
\hline \multicolumn{13}{|l|}{ HLWMS-09 } \\
\hline \multicolumn{13}{|l|}{ HLWMS-10 } \\
\hline \multicolumn{13}{|l|}{ HLWMS-11 } \\
\hline \multicolumn{13}{|l|}{ HLWMS-12 } \\
\hline \multicolumn{13}{|l|}{ HLWMS-13 } \\
\hline \multicolumn{13}{|l|}{ HLWMS-14 } \\
\hline \multicolumn{13}{|l|}{ HLWMS-15 } \\
\hline HLW99-01 & & & & 0.068 & 0.049 & 1.283 & 0.875 & $<0.0055$ & 0.66 & 0.111 & 0.0532 & 0.598 \\
\hline HLW99-02 & & & & 0.079 & 0.049 & 0.053 & 0.171 & 0.573 & 0.357 & 0.4 & 0.108 & 38.928 \\
\hline HLW99-03 & & & & 0.363 & 0.055 & 12.084 & 8.776 & $<0.0055$ & 7.382 & 0.727 & 0.0532 & 5.47 \\
\hline HLW99-04 & & & & 0.029 & 0.148 & 0.13 & 0.075 & 0.008 & 0.064 & 0.171 & 0.0532 & 0.942 \\
\hline HLW99-05 & & & & 0.085 & 0.25 & 35.742 & 33.345 & 1.165 & 29.936 & 1.552 & 0.355 & 20.134 \\
\hline HLW99-06 & & & & 0.09 & 0.049 & 0.013 & 0.006 & $<0.0055$ & $<0.0079$ & $<0.0243$ & 0.0532 & 0.813 \\
\hline HLW99-07 & & & & 0.088 & 0.406 & 0.139 & 0.18 & 0.056 & 0.275 & 0.502 & 0.708 & 40.201 \\
\hline HLW99-08 & & & & 0.169 & 0.089 & 0.408 & 0.269 & 0.022 & 0.252 & 0.12 & 0.121 & 1.547 \\
\hline HLW99-09 & & & & 0.023 & 0.205 & 26.083 & 53.865 & 0.089 & 49.261 & 1.991 & 0.402 & 39.512 \\
\hline HLW99-10 & & & & 0.087 & 1.487 & 0.134 & 0.076 & 0.041 & 0.063 & 0.153 & 0.081 & 0.764 \\
\hline HLW99-11 & & & & 0.256 & 0.049 & 0.099 & 0.026 & 0.073 & 0.071 & 0.178 & 0.164 & 3.577 \\
\hline HLW99-12 & & & & 0.1 & 0.074 & 0.197 & 0.12 & $<0.0055$ & 0.097 & $<0.0243$ & 0.0532 & 1.084 \\
\hline
\end{tabular}


RPP-WTP HLW Formulation (Kot and Pegg 2001)

\begin{tabular}{|c|c|c|c|c|c|c|c|c|c|c|c|c|c|c|c|c|c|c|c|c|c|}
\hline Glass ID & $\begin{array}{c}\mathrm{A} 12 \mathrm{O} 3 \\
-\mathrm{t}\end{array}$ & $\begin{array}{c}\mathrm{B} 2 \mathrm{O} 3 \\
-\mathrm{t}\end{array}$ & $\begin{array}{c}\mathrm{CaO} \\
-\mathrm{t}\end{array}$ & $\begin{array}{c}\mathrm{Fe} 2 \mathrm{O} 3 \\
-\mathrm{t}\end{array}$ & $\begin{array}{c}\mathrm{FeO} \\
-\mathrm{t}\end{array}$ & $\begin{array}{c}\mathrm{K} 2 \mathrm{O} \\
-\mathrm{t}\end{array}$ & $\begin{array}{c}\mathrm{Li} 2 \mathrm{O} \\
-\mathrm{t}\end{array}$ & $\begin{array}{c}\mathrm{MgO} \\
-\mathrm{t}\end{array}$ & $\begin{array}{c}\mathrm{Na} 2 \mathrm{O} \\
-\mathrm{t}\end{array}$ & $\begin{array}{c}\mathrm{NiO} \\
-\mathrm{t}\end{array}$ & $\begin{array}{c}\mathrm{P} 2 \mathrm{O} 5 \\
-\mathrm{t}\end{array}$ & $\begin{array}{c}\mathrm{SiO} 2 \\
-\mathrm{t}\end{array}$ & $\begin{array}{c}\mathrm{ZrO} 2 \\
-\mathrm{t}\end{array}$ & $\begin{array}{c}\mathrm{Ag} 2 \mathrm{O} \\
-\mathrm{t}\end{array}$ & $\begin{array}{c}\mathrm{As} 2 \mathrm{O} 3 \\
-\mathrm{t}\end{array}$ & $\begin{array}{c}\mathrm{BaO} \\
-\mathrm{t}\end{array}$ & $\begin{array}{c}\mathrm{Bi} 2 \mathrm{O} 3 \\
-\mathrm{t}\end{array}$ & $\begin{array}{l}\mathrm{Br} \\
-\mathrm{t}\end{array}$ & $\begin{array}{c}\mathrm{CdO} \\
-\mathrm{t}\end{array}$ & $\begin{array}{c}\mathrm{Ce} 2 \mathrm{O} 3 \\
-\mathrm{t}\end{array}$ & $\begin{array}{c}\mathrm{CeO} 2 \\
-\mathrm{t}\end{array}$ \\
\hline HLW99-13 & 0.0000 & 0.0500 & 0.0000 & 0.0200 & & 0.0100 & 0.0000 & 0.0200 & 0.2000 & 0.0300 & 0.0061 & 0.4800 & 0.0000 & 0.0025 & 0.0020 & 0.0400 & 0.0200 & & 0.0300 & & 0.0020 \\
\hline HLW99-14 & 0.0000 & 0.2000 & 0.0000 & 0.1250 & & 0.0100 & 0.0350 & 0.0200 & 0.0500 & 0.0300 & 0.0000 & 0.3317 & 0.0333 & 0.0025 & 0.0020 & 0.0400 & 0.0200 & & 0.0300 & & 0.0020 \\
\hline HLW99-15 & 0.0000 & 0.2000 & 0.0350 & 0.0200 & & 0.0100 & 0.0100 & 0.0200 & 0.2000 & 0.0300 & 0.0000 & 0.3100 & 0.0000 & 0.0025 & 0.0020 & 0.0400 & 0.0200 & & 0.0300 & & 0.0020 \\
\hline HLW99-16 & 0.0000 & 0.0500 & 0.0000 & 0.1250 & & 0.0100 & 0.0700 & 0.0113 & 0.0500 & 0.0169 & 0.0000 & 0.5500 & 0.0000 & 0.0025 & 0.0020 & 0.0225 & 0.0113 & & 0.0169 & & 0.0020 \\
\hline HLW99-17 & 0.0000 & 0.0500 & 0.0700 & 0.0200 & & 0.0100 & 0.0100 & 0.0000 & 0.2000 & 0.0000 & 0.0300 & 0.3630 & 0.1000 & 0.0025 & 0.0020 & 0.0000 & 0.0000 & & 0.0000 & & 0.0020 \\
\hline HLW99-18 & 0.1100 & 0.0700 & 0.0700 & 0.0200 & & 0.0100 & 0.0100 & 0.0156 & 0.2000 & 0.0234 & 0.0000 & 0.3000 & 0.0300 & 0.0025 & 0.0020 & 0.0313 & 0.0156 & & 0.0234 & & 0.0020 \\
\hline HLW99-19 & 0.0850 & 0.2000 & 0.0000 & 0.1250 & & 0.0100 & 0.0530 & 0.0000 & 0.0500 & 0.0000 & 0.0300 & 0.3000 & 0.0000 & 0.0025 & 0.0020 & 0.0000 & 0.0000 & & 0.0000 & & 0.0020 \\
\hline HLW99-20 & 0.0530 & 0.0900 & 0.0000 & 0.0200 & & 0.0100 & 0.0700 & 0.0200 & 0.0800 & 0.0300 & 0.0300 & 0.3400 & 0.0000 & 0.0025 & 0.0020 & 0.0400 & 0.0200 & & 0.0300 & & 0.0020 \\
\hline HLW99-21 & 0.0650 & 0.0500 & 0.0700 & 0.0200 & & 0.0100 & 0.0700 & 0.0000 & 0.0750 & 0.0000 & 0.0000 & 0.5350 & 0.0500 & 0.0025 & 0.0020 & 0.0000 & 0.0000 & & 0.0000 & & 0.0020 \\
\hline HLW99-22 & 0.0400 & 0.2000 & 0.0000 & 0.0230 & & 0.0100 & 0.0000 & 0.0000 & 0.1500 & 0.0000 & 0.0300 & 0.3000 & 0.1000 & 0.0025 & 0.0020 & 0.0000 & 0.0000 & & 0.0000 & & 0.0020 \\
\hline HLW99-23 & 0.0000 & 0.0500 & 0.0700 & 0.0200 & & 0.0100 & 0.0000 & 0.0179 & 0.2000 & 0.0268 & 0.0300 & 0.3300 & 0.0000 & 0.0025 & 0.0020 & 0.0358 & 0.0179 & & 0.0268 & & 0.0020 \\
\hline HLW99-24R & 0.0000 & 0.1550 & 0.0700 & 0.1100 & & 0.0100 & 0.0000 & 0.0000 & 0.2000 & 0.0000 & 0.0000 & 0.3000 & 0.1000 & 0.0025 & 0.0020 & 0.0000 & 0.0000 & & 0.0000 & & 0.0020 \\
\hline HLW99-25 & 0.1100 & 0.0900 & 0.0700 & 0.1000 & & 0.0100 & 0.0700 & 0.0094 & 0.0800 & 0.0141 & 0.0000 & 0.3400 & 0.0000 & 0.0025 & 0.0020 & 0.0188 & 0.0094 & & 0.0141 & & 0.0020 \\
\hline HLW99-26 & 0.0000 & 0.0500 & 0.0000 & 0.0730 & & 0.0100 & 0.0350 & 0.0200 & 0.0500 & 0.0300 & 0.0300 & 0.4450 & 0.0000 & 0.0025 & 0.0020 & 0.0400 & 0.0200 & & 0.0300 & & 0.0020 \\
\hline HLW99-27 & 0.0000 & 0.1600 & 0.0700 & 0.1250 & & 0.0100 & 0.0700 & 0.0200 & 0.0500 & 0.0300 & 0.0000 & 0.3000 & 0.0000 & 0.0025 & 0.0020 & 0.0400 & 0.0200 & & 0.0300 & & 0.0020 \\
\hline HLW99-28 & 0.0250 & 0.1600 & 0.0700 & 0.0200 & & 0.0100 & 0.0000 & 0.0200 & 0.1200 & 0.0300 & 0.0000 & 0.3000 & 0.0800 & 0.0025 & 0.0020 & 0.0400 & 0.0200 & & 0.0300 & & 0.0020 \\
\hline HLW99-29 & 0.0000 & 0.0500 & 0.0000 & 0.0200 & & 0.0100 & 0.0300 & 0.0123 & 0.1300 & 0.0184 & 0.0000 & 0.5200 & 0.0867 & 0.0025 & 0.0020 & 0.0246 & 0.0123 & & 0.0184 & & 0.0020 \\
\hline HLW99-30 & 0.0400 & 0.1250 & 0.0000 & 0.0200 & & 0.0100 & 0.0100 & 0.0056 & 0.2000 & 0.0084 & 0.0000 & 0.3950 & 0.1000 & 0.0025 & 0.0020 & 0.0113 & 0.0056 & & 0.0084 & & 0.0020 \\
\hline HLW99-31 & 0.1100 & 0.1830 & 0.0000 & 0.0200 & & 0.0100 & 0.0000 & 0.0000 & 0.2000 & 0.0000 & 0.0300 & 0.3000 & 0.0000 & 0.0025 & 0.0020 & 0.0000 & 0.0000 & & 0.0000 & & 0.0020 \\
\hline HLW99-32 & 0.0000 & 0.0500 & 0.0000 & 0.1250 & & 0.0100 & 0.0463 & 0.0029 & 0.1275 & 0.0043 & 0.0300 & 0.3563 & 0.0850 & 0.0025 & 0.0020 & 0.0058 & 0.0029 & & 0.0043 & & 0.0020 \\
\hline
\end{tabular}


Appendix A. Database - mass fraction

RPP-WTP HLW Formulation (Kot and Pegg 2001)

\begin{tabular}{|c|c|c|c|c|c|c|c|c|c|c|c|c|c|c|c|c|c|c|c|c|c|}
\hline Glass ID & $\begin{array}{l}\mathrm{Cl} \\
-\mathrm{t}\end{array}$ & $\begin{array}{c}\mathrm{CoO} \\
-\mathrm{t}\end{array}$ & $\begin{array}{c}\mathrm{Co} 2 \mathrm{O} 3 \\
-\mathrm{t}\end{array}$ & $\begin{array}{c}\mathrm{Cr} 2 \mathrm{O} 3 \\
-\mathrm{t}\end{array}$ & $\begin{array}{c}\mathrm{Cs} 2 \mathrm{O} \\
-\mathrm{t}\end{array}$ & $\begin{array}{c}\mathrm{CuO} \\
-\mathrm{t}\end{array}$ & $\begin{array}{c}\mathrm{Eu} 2 \mathrm{O} 3 \\
-\mathrm{t}\end{array}$ & $\begin{array}{l}F \\
-t\end{array}$ & $\begin{array}{c}\mathrm{Ga} 2 \mathrm{O} 3 \\
-\mathrm{t}\end{array}$ & $\begin{array}{c}\mathrm{Gd} 2 \mathrm{O} 3 \\
-\mathrm{t}\end{array}$ & $\begin{array}{c}\mathrm{HgO} \\
-\mathrm{t}\end{array}$ & $\begin{array}{l}I \\
-t\end{array}$ & $\begin{array}{c}\mathrm{La} 2 \mathrm{O} 3 \\
-\mathrm{t}\end{array}$ & $\begin{array}{c}\mathrm{MnO} 2 \\
-\mathrm{t}\end{array}$ & $\begin{array}{c}\mathrm{MnO} \\
-\mathrm{t}\end{array}$ & $\begin{array}{c}\mathrm{MoO} \\
-\mathrm{t}\end{array}$ & $\begin{array}{c}\mathrm{MoO} 3 \\
-\mathrm{t}\end{array}$ & $\begin{array}{c}\mathrm{Nb} 2 \mathrm{O} 5 \\
-\mathrm{t}\end{array}$ & $\begin{array}{c}\mathrm{Nd} 2 \mathrm{O} 3 \\
-\mathrm{t}\end{array}$ & $\begin{array}{c}\mathrm{PbO} \\
-\mathrm{t}\end{array}$ & $\begin{array}{c}\mathrm{PdO} 2 \\
-\mathrm{t}\end{array}$ \\
\hline HLW99-13 & 0.0020 & 0.0020 & & 0.0010 & 0.0020 & 0.0020 & & 0.0020 & & & & & 0.0020 & 0.0020 & & & & & & 0.0100 & \\
\hline HLW99-14 & 0.0020 & 0.0020 & & 0.0000 & 0.0020 & 0.0020 & & 0.0020 & & & & & 0.0020 & 0.0020 & & & & & & 0.0100 & \\
\hline HLW99-15 & 0.0020 & 0.0020 & & 0.0000 & 0.0020 & 0.0020 & & 0.0020 & & & & & 0.0020 & 0.0020 & & & & & & 0.0100 & \\
\hline HLW99-16 & 0.0020 & 0.0020 & & 0.0000 & 0.0020 & 0.0020 & & 0.0020 & & & & & 0.0020 & 0.0020 & & & & & & 0.0056 & \\
\hline HLW99-17 & 0.0020 & 0.0020 & & 0.0050 & 0.0020 & 0.0020 & & 0.0020 & & & & & 0.0020 & 0.0020 & & & & & & 0.0000 & \\
\hline HLW99-18 & 0.0020 & 0.0020 & & 0.0000 & 0.0020 & 0.0020 & & 0.0020 & & & & & 0.0020 & 0.0020 & & & & & & 0.0078 & \\
\hline HLW99-19 & 0.0020 & 0.0020 & & 0.0050 & 0.0020 & 0.0020 & & 0.0020 & & & & & 0.0020 & 0.0020 & & & & & & 0.0000 & \\
\hline HLW99-20 & 0.0020 & 0.0020 & & 0.0050 & 0.0020 & 0.0020 & & 0.0020 & & & & & 0.0020 & 0.0020 & & & & & & 0.0100 & \\
\hline HLW99-21 & 0.0020 & 0.0020 & & 0.0000 & 0.0020 & 0.0020 & & 0.0020 & & & & & 0.0020 & 0.0020 & & & & & & 0.0000 & \\
\hline HLW99-22 & 0.0020 & 0.0020 & & 0.0050 & 0.0020 & 0.0020 & & 0.0020 & & & & & 0.0020 & 0.0020 & & & & & & 0.0000 & \\
\hline HLW99-23 & 0.0020 & 0.0020 & & 0.0050 & 0.0020 & 0.0020 & & 0.0020 & & & & & 0.0020 & 0.0020 & & & & & & 0.0089 & \\
\hline HLW99-24R & 0.0020 & 0.0020 & & 0.0000 & 0.0020 & 0.0020 & & 0.0020 & & & & & 0.0020 & 0.0020 & & & & & & 0.0000 & \\
\hline HLW99-25 & 0.0020 & 0.0020 & & 0.0000 & 0.0020 & 0.0020 & & 0.0020 & & & & & 0.0020 & 0.0020 & & & & & & 0.0047 & \\
\hline HLW99-26 & 0.0020 & 0.0020 & & 0.0050 & 0.0020 & 0.0020 & & 0.0020 & & & & & 0.0020 & 0.0020 & & & & & & 0.0100 & \\
\hline HLW99-27 & 0.0020 & 0.0020 & & 0.0000 & 0.0020 & 0.0020 & & 0.0020 & & & & & 0.0020 & 0.0020 & & & & & & 0.0100 & \\
\hline HLW99-28 & 0.0020 & 0.0020 & & 0.0000 & 0.0020 & 0.0020 & & 0.0020 & & & & & 0.0020 & 0.0020 & & & & & & 0.0100 & \\
\hline HLW99-29 & 0.0020 & 0.0020 & & 0.0000 & 0.0020 & 0.0020 & & 0.0020 & & & & & 0.0020 & 0.0020 & & & & & & 0.0061 & \\
\hline HLW99-30 & 0.0020 & 0.0020 & & 0.0000 & 0.0020 & 0.0020 & & 0.0020 & & & & & 0.0020 & 0.0020 & & & & & & 0.0028 & \\
\hline HLW99-31 & 0.0020 & 0.0020 & & 0.0050 & 0.0020 & 0.0020 & & 0.0020 & & & & & 0.0020 & 0.0020 & & & & & & 0.0000 & \\
\hline HLW99-32 & 0.0020 & 0.0020 & & 0.0050 & 0.0020 & 0.0020 & & 0.0020 & & & & & 0.0020 & 0.0020 & & & & & & 0.0014 & \\
\hline
\end{tabular}


Appendix A. Database - mass fraction

RPP-WTP HLW Formulation (Kot and Pegg 2001)

\begin{tabular}{|c|c|c|c|c|c|c|c|c|c|c|c|c|c|c|c|c|c|c|c|c|c|}
\hline Glass ID & $\begin{array}{c}\mathrm{PdO} \\
-\mathrm{t}\end{array}$ & $\begin{array}{c}\mathrm{Pr} 2 \mathrm{O} 3 \\
-\mathrm{t}\end{array}$ & $\begin{array}{c}\text { Pr6O11 } \\
-t\end{array}$ & $\begin{array}{c}\mathrm{Rb} 2 \mathrm{O} \\
-\mathrm{t}\end{array}$ & $\begin{array}{c}\mathrm{ReO} \\
-\mathrm{t}\end{array}$ & $\begin{array}{c}\mathrm{ReO} 2 \\
-\mathrm{t}\end{array}$ & $\begin{array}{c}\mathrm{Rh} 2 \mathrm{O} 3 \\
-\mathrm{t}\end{array}$ & $\begin{array}{c}\mathrm{RhO} 2 \\
-\mathrm{t}\end{array}$ & $\begin{array}{c}\mathrm{RuO} 2 \\
-\mathrm{t}\end{array}$ & $\begin{array}{c}\mathrm{Sb} 2 \mathrm{O} 3 \\
-\mathrm{t}\end{array}$ & $\begin{array}{c}\mathrm{Sb} 2 \mathrm{O} 5 \\
-\mathrm{t}\end{array}$ & $\begin{array}{c}\mathrm{SeO} 2 \\
-\mathrm{t}\end{array}$ & $\begin{array}{c}\mathrm{Sm} 2 \mathrm{O} 3 \\
-\mathrm{t}\end{array}$ & $\begin{array}{c}\mathrm{SnO} \\
-\mathrm{t}\end{array}$ & $\begin{array}{c}\mathrm{SnO} 2 \\
-t\end{array}$ & $\begin{array}{c}\mathrm{SO} 3 \\
-\mathrm{t}\end{array}$ & $\begin{array}{c}\mathrm{SrO} \\
-\mathrm{t}\end{array}$ & $\begin{array}{c}\mathrm{Tc} 2 \mathrm{O} 7 \\
-\mathrm{t}\end{array}$ & $\begin{array}{c}\mathrm{TeO} 2 \\
-\mathrm{t}\end{array}$ & $\begin{array}{c}\mathrm{ThO} 2 \\
-\mathrm{t}\end{array}$ & $\begin{array}{c}\mathrm{TiO} 2 \\
-\mathrm{t}\end{array}$ \\
\hline HLW99-13 & 0.0004 & & & & & & 0.0008 & & 0.0013 & & 0.0020 & 0.0020 & & & & 0.0010 & 0.0020 & & 0.0020 & 0.0004 & 0.0100 \\
\hline HLW99-14 & 0.0004 & & & & & & 0.0008 & & 0.0013 & & 0.0020 & 0.0020 & & & & 0.0000 & 0.0020 & & 0.0020 & 0.0000 & 0.0100 \\
\hline HLW99-15 & 0.0004 & & & & & & 0.0008 & & 0.0013 & & 0.0020 & 0.0020 & & & & 0.0000 & 0.0020 & & 0.0020 & 0.0000 & 0.0100 \\
\hline HLW99-16 & 0.0004 & & & & & & 0.0008 & & 0.0013 & & 0.0020 & 0.0020 & & & & 0.0000 & 0.0020 & & 0.0020 & 0.0000 & 0.0056 \\
\hline HLW99-17 & 0.0004 & & & & & & 0.0008 & & 0.0013 & & 0.0020 & 0.0020 & & & & 0.0050 & 0.0020 & & 0.0020 & 0.0020 & 0.0000 \\
\hline HLW99-18 & 0.0004 & & & & & & 0.0008 & & 0.0013 & & 0.0020 & 0.0020 & & & & 0.0000 & 0.0020 & & 0.0020 & 0.0000 & 0.0078 \\
\hline HLW99-19 & 0.0004 & & & & & & 0.0008 & & 0.0013 & & 0.0020 & 0.0020 & & & & 0.0050 & 0.0020 & & 0.0020 & 0.0020 & 0.0000 \\
\hline HLW99-20 & 0.0004 & & & & & & 0.0008 & & 0.0013 & & 0.0020 & 0.0020 & & & & 0.0050 & 0.0020 & & 0.0020 & 0.0020 & 0.0100 \\
\hline HLW99-21 & 0.0004 & & & & & & 0.0008 & & 0.0013 & & 0.0020 & 0.0020 & & & & 0.0000 & 0.0020 & & 0.0020 & 0.0000 & 0.0000 \\
\hline HLW99-22 & 0.0004 & & & & & & 0.0008 & & 0.0013 & & 0.0020 & 0.0020 & & & & 0.0050 & 0.0020 & & 0.0020 & 0.0020 & 0.0000 \\
\hline HLW99-23 & 0.0004 & & & & & & 0.0008 & & 0.0013 & & 0.0020 & 0.0020 & & & & 0.0050 & 0.0020 & & 0.0020 & 0.0020 & 0.0089 \\
\hline HLW99-24R & 0.0004 & & & & & & 0.0008 & & 0.0013 & & 0.0020 & 0.0020 & & & & 0.0000 & 0.0020 & & 0.0020 & 0.0000 & 0.0000 \\
\hline HLW99-25 & 0.0004 & & & & & & 0.0008 & & 0.0013 & & 0.0020 & 0.0020 & & & & 0.0000 & 0.0020 & & 0.0020 & 0.0000 & 0.0047 \\
\hline HLW99-26 & 0.0004 & & & & & & 0.0008 & & 0.0013 & & 0.0020 & 0.0020 & & & & 0.0050 & 0.0020 & & 0.0020 & 0.0020 & 0.0100 \\
\hline HLW99-27 & 0.0004 & & & & & & 0.0008 & & 0.0013 & & 0.0020 & 0.0020 & & & & 0.0000 & 0.0020 & & 0.0020 & 0.0000 & 0.0100 \\
\hline HLW99-28 & 0.0004 & & & & & & 0.0008 & & 0.0013 & & 0.0020 & 0.0020 & & & & 0.0000 & 0.0020 & & 0.0020 & 0.0000 & 0.0100 \\
\hline HLW99-29 & 0.0004 & & & & & & 0.0008 & & 0.0013 & & 0.0020 & 0.0020 & & & & 0.0000 & 0.0020 & & 0.0020 & 0.0000 & 0.0061 \\
\hline HLW99-30 & 0.0004 & & & & & & 0.0008 & & 0.0013 & & 0.0020 & 0.0020 & & & & 0.0000 & 0.0020 & & 0.0020 & 0.0000 & 0.0028 \\
\hline HLW99-31 & 0.0004 & & & & & & 0.0008 & & 0.0013 & & 0.0020 & 0.0020 & & & & 0.0050 & 0.0020 & & 0.0020 & 0.0020 & 0.0000 \\
\hline HLW99-32 & 0.0004 & & & & & & 0.0008 & & 0.0013 & & 0.0020 & 0.0020 & & & & 0.0050 & 0.0020 & & 0.0020 & 0.0020 & 0.0014 \\
\hline
\end{tabular}


Appendix A. Database - mass fraction

RPP-WTP HLW Formulation (Kot and Pegg 2001)

\begin{tabular}{|c|c|c|c|c|c|c|c|c|c|c|c|c|c|c|c|c|c|c|c|c|c|}
\hline Glass ID & $\begin{array}{c}\mathrm{T} 12 \mathrm{O} 3 \\
-\mathrm{t}\end{array}$ & $\begin{array}{c}\text { U3O8 } \\
-t\end{array}$ & $\begin{array}{c}\mathrm{UO} 2 \\
-\mathrm{t}\end{array}$ & $\begin{array}{c}\text { UO3 } \\
-t\end{array}$ & $\begin{array}{c}\mathrm{V} 2 \mathrm{O} 5 \\
-\mathrm{t}\end{array}$ & $\begin{array}{c}\text { WO3 } \\
-t\end{array}$ & $\begin{array}{c}\mathrm{Y} 2 \mathrm{O} 3 \\
-\mathrm{t}\end{array}$ & $\begin{array}{c}\mathrm{ZnO} \\
-\mathrm{t}\end{array}$ & $\begin{array}{c}\text { Others } \\
-t\end{array}$ & $\begin{array}{c}\text { Sum } \\
-t\end{array}$ & $\begin{array}{c}\mathrm{A} 12 \mathrm{O} 3 \\
-\mathrm{a}\end{array}$ & $\begin{array}{c}\mathrm{B} 2 \mathrm{O} 3 \\
-\mathrm{a}\end{array}$ & $\begin{array}{c}\mathrm{CaO} \\
-\mathrm{a} \\
\end{array}$ & $\begin{array}{c}\mathrm{Fe} 2 \mathrm{O} 3 \\
-\mathrm{a}\end{array}$ & $\begin{array}{c}\mathrm{FeO} \\
-\mathrm{a} \\
\end{array}$ & $\begin{array}{c}\mathrm{K} 2 \mathrm{O} \\
-\mathrm{a}\end{array}$ & $\begin{array}{c}\mathrm{Li} 2 \mathrm{O} \\
-\mathrm{a}\end{array}$ & $\begin{array}{c}\mathrm{MgO} \\
-\mathrm{a}\end{array}$ & $\begin{array}{c}\mathrm{Na} 2 \mathrm{O} \\
-\mathrm{a}\end{array}$ & $\begin{array}{c}\mathrm{NiO} \\
-\mathrm{a}\end{array}$ & $\begin{array}{c}\mathrm{P} 2 \mathrm{O} 5 \\
-\mathrm{a}\end{array}$ \\
\hline HLW99-13 & 0.0020 & & 0.0164 & & & 0.0020 & & 0.0200 & & 0.9999 & 0.0065 & 0.0511 & 0.0030 & 0.0199 & & 0.0109 & 0.0004 & 0.0177 & 0.1879 & 0.0276 & 0.0080 \\
\hline HLW99-14 & 0.0020 & & 0.0000 & & & 0.0020 & & 0.0200 & & 1.0000 & 0.0249 & 0.1885 & 0.0008 & 0.1138 & & 0.0198 & 0.0328 & 0.0177 & 0.0487 & 0.0253 & 0.0010 \\
\hline HLW99-15 & 0.0020 & & 0.0000 & & & 0.0020 & & 0.0200 & & 1.0000 & 0.0173 & 0.1924 & 0.0353 & 0.0214 & & 0.0116 & 0.0116 & 0.0195 & 0.1808 & 0.0272 & 0.0004 \\
\hline HLW99-16 & 0.0020 & & 0.0000 & & & 0.0020 & & 0.0200 & & 1.0001 & 0.0137 & 0.0516 & 0.0025 & 0.1228 & & 0.0112 & 0.0666 & 0.0112 & 0.0503 & 0.0163 & 0.0016 \\
\hline HLW99-17 & 0.0020 & & 0.0800 & & & 0.0020 & & 0.0200 & & 1.0000 & 0.0080 & 0.0488 & 0.0641 & 0.0197 & & 0.0106 & 0.0104 & 0.0005 & 0.1829 & 0.0009 & 0.0258 \\
\hline HLW99-18 & 0.0020 & & 0.0000 & & & 0.0020 & & 0.0200 & & 0.9999 & 0.1074 & 0.0708 & 0.0698 & 0.0214 & & 0.0111 & 0.0123 & 0.0157 & 0.1859 & 0.0234 & 0.0012 \\
\hline HLW99-19 & 0.0020 & & 0.0800 & & & 0.0020 & & 0.0200 & & 1.0000 & 0.0797 & 0.1924 & 0.0028 & 0.1127 & & 0.0111 & 0.0462 & 0.0002 & 0.0485 & 0.0004 & 0.0354 \\
\hline HLW99-20 & 0.0020 & & 0.0800 & & & 0.0020 & & 0.0200 & & 1.0000 & 0.0551 & 0.0970 & 0.0014 & 0.0227 & & 0.0102 & 0.0635 & 0.0176 & 0.0737 & 0.0276 & 0.0354 \\
\hline HLW99-21 & 0.0020 & & 0.0000 & & & 0.0020 & & 0.0200 & & 1.0000 & 0.0680 & 0.0501 & 0.0667 & 0.0202 & & 0.0107 & 0.0648 & 0.0004 & 0.0757 & 0.0002 & 0.0012 \\
\hline HLW99-22 & 0.0020 & & 0.0800 & & & 0.0020 & & 0.0200 & & 1.0000 & 0.0407 & 0.1856 & 0.0006 & 0.0229 & & 0.0111 & 0.0026 & 0.0007 & 0.1355 & 0.0011 & 0.0337 \\
\hline HLW99-23 & 0.0020 & & 0.0800 & & & 0.0020 & & 0.0200 & & 1.0000 & 0.0086 & 0.0615 & 0.0637 & 0.0201 & & 0.0116 & 0.0014 & 0.0157 & 0.1754 & 0.0247 & 0.0327 \\
\hline HLW99-24R & 0.0020 & & 0.0000 & & & 0.0020 & & 0.0200 & & 1.0000 & 0.0072 & 0.1502 & 0.0676 & 0.1022 & & 0.0112 & 0.0008 & 0.0002 & 0.1726 & 0.0003 & 0.0028 \\
\hline HLW99-25 & 0.0020 & & 0.0000 & & & 0.0020 & & 0.0200 & & 1.0002 & 0.1126 & 0.0899 & 0.0686 & 0.0943 & & 0.0114 & 0.0659 & 0.0100 & 0.0797 & 0.0132 & 0.0005 \\
\hline HLW99-26 & 0.0020 & & 0.0800 & & & 0.0020 & & 0.0200 & & 1.0000 & 0.0051 & 0.0559 & 0.0041 & 0.0628 & & 0.0106 & 0.0301 & 0.0171 & 0.0530 & 0.0260 & 0.0329 \\
\hline HLW99-27 & 0.0020 & & 0.0000 & & & 0.0020 & & 0.0200 & & 1.0000 & 0.0297 & 0.1457 & 0.0642 & 0.1096 & & 0.0109 & 0.0616 & 0.0182 & 0.0464 & 0.0263 & 0.0010 \\
\hline HLW99-28 & 0.0020 & & 0.0000 & & & 0.0020 & & 0.0200 & & 1.0000 & 0.0397 & 0.1490 & 0.0630 & 0.0205 & & 0.0106 & 0.0016 & 0.0185 & 0.1065 & 0.0268 & 0.0017 \\
\hline HLW99-29 & 0.0020 & & 0.0000 & & & 0.0020 & & 0.0200 & & 0.9999 & 0.0046 & 0.0532 & 0.0012 & 0.0210 & & 0.0099 & 0.0315 & 0.0122 & 0.1156 & 0.0187 & 0.0017 \\
\hline HLW99-30 & 0.0020 & & 0.0000 & & & 0.0020 & & 0.0200 & & 0.9999 & 0.0437 & 0.1218 & 0.0009 & 0.0204 & & 0.0117 & 0.0115 & 0.0059 & 0.1840 & 0.0078 & 0.0012 \\
\hline HLW99-31 & 0.0020 & & 0.0800 & & & 0.0020 & & 0.0200 & & 1.0000 & 0.1018 & 0.1756 & 0.0011 & 0.0223 & & 0.0113 & 0.0022 & 0.0012 & 0.1708 & 0.0017 & 0.0329 \\
\hline HLW99-32 & 0.0020 & & 0.0800 & & & 0.0020 & & 0.0200 & & 1.0001 & 0.0116 & 0.0641 & 0.0008 & 0.1156 & & 0.0115 & 0.0420 & 0.0031 & 0.1213 & 0.0044 & 0.0362 \\
\hline
\end{tabular}


Appendix A. Database - mass fraction

RPP-WTP HLW Formulation (Kot and Pegg 2001)

\begin{tabular}{|c|c|c|c|c|c|c|c|c|c|c|c|c|c|c|c|c|c|c|c|c|c|}
\hline Glass ID & $\begin{array}{c}\mathrm{SiO} 2 \\
-\mathrm{a}\end{array}$ & $\begin{array}{c}\mathrm{ZrO} 2 \\
-\mathrm{a}\end{array}$ & $\begin{array}{c}\mathrm{Ag} 2 \mathrm{O} \\
-\mathrm{a}\end{array}$ & $\begin{array}{c}\mathrm{As} 2 \mathrm{O} 3 \\
-\mathrm{a}\end{array}$ & $\begin{array}{c}\mathrm{BaO} \\
-\mathrm{a} \\
\end{array}$ & $\begin{array}{c}\mathrm{Bi} 2 \mathrm{O} 3 \\
-\mathrm{a}\end{array}$ & $\begin{array}{l}\mathrm{Br} \\
-\mathrm{a}\end{array}$ & $\begin{array}{c}\mathrm{CdO} \\
-\mathrm{a}\end{array}$ & $\begin{array}{c}\mathrm{Ce} 2 \mathrm{O} 3 \\
-\mathrm{a}\end{array}$ & $\begin{array}{c}\mathrm{CeO} 2 \\
-\mathrm{a}\end{array}$ & $\begin{array}{l}\mathrm{Cl} \\
-\mathrm{a} \\
\end{array}$ & $\begin{array}{c}\mathrm{CoO} \\
-\mathrm{a} \\
\end{array}$ & $\begin{array}{c}\mathrm{Co} 2 \mathrm{O} 3 \\
-\mathrm{a}\end{array}$ & $\begin{array}{c}\mathrm{Cr} 2 \mathrm{O} 3 \\
-\mathrm{a}\end{array}$ & $\begin{array}{c}\mathrm{Cs} 2 \mathrm{O} \\
-\mathrm{a}\end{array}$ & $\begin{array}{c}\mathrm{CuO} \\
-\mathrm{a}\end{array}$ & $\begin{array}{c}\mathrm{Eu} 2 \mathrm{O} 3 \\
-\mathrm{a}\end{array}$ & $\begin{array}{l}\mathrm{F} \\
-\mathrm{a}\end{array}$ & $\begin{array}{c}\mathrm{Ga} 2 \mathrm{O} 3 \\
-\mathrm{a}\end{array}$ & $\begin{array}{c}\mathrm{Gd} 2 \mathrm{O} 3 \\
-\mathrm{a}\end{array}$ & $\begin{array}{c}\mathrm{HgO} \\
-\mathrm{a}\end{array}$ \\
\hline HLW99-13 & 0.4713 & 0.0004 & 0.0020 & 0.0020 & 0.0386 & 0.0190 & & 0.0274 & & & & 0.0021 & & 0.0010 & & 0.0023 & & & & & \\
\hline HLW99-14 & 0.3466 & 0.0312 & 0.0022 & 0.0019 & 0.0378 & 0.0186 & & 0.0268 & & & & 0.0017 & & 0.0000 & & 0.0021 & & & & & \\
\hline HLW99-15 & 0.3256 & 0.0004 & 0.0017 & 0.0018 & 0.0404 & 0.0196 & & 0.0278 & & & & 0.0018 & & 0.0000 & & 0.0021 & & & & & \\
\hline HLW99-16 & 0.5360 & 0.0001 & 0.0024 & 0.0019 & 0.0230 & 0.0115 & & 0.0162 & & & & 0.0018 & & 0.0000 & & 0.0022 & & & & & \\
\hline HLW99-17 & 0.3647 & 0.1009 & 0.0028 & 0.0019 & 0.0010 & 0.0006 & & 0.0005 & & & & 0.0029 & & 0.0042 & & 0.0021 & & & & & \\
\hline HLW99-18 & 0.3083 & 0.0285 & 0.0018 & 0.0020 & 0.0322 & 0.0159 & & 0.0229 & & & & 0.0023 & & 0.0000 & & 0.0023 & & & & & \\
\hline HLW99-19 & 0.3177 & 0.0032 & 0.0025 & 0.0020 & 0.0002 & 0.0002 & & 0.0001 & & & & 0.0027 & & 0.0033 & & 0.0023 & & & & & \\
\hline HLW99-20 & 0.3387 & 0.0007 & 0.0024 & 0.0021 & 0.0368 & 0.0199 & & 0.0278 & & & & 0.0027 & & 0.0038 & & 0.0024 & & & & & \\
\hline HLW99-21 & 0.4989 & 0.0483 & 0.0021 & 0.0020 & 0.0002 & 0.0000 & & 0.0001 & & & & 0.0023 & & 0.0001 & & 0.0023 & & & & & \\
\hline HLW99-22 & 0.3043 & 0.0878 & 0.0023 & 0.0023 & 0.0013 & 0.0009 & & 0.0008 & & & & 0.0027 & & 0.0044 & & 0.0023 & & & & & \\
\hline HLW99-23 & 0.3248 & 0.0045 & 0.0018 & 0.0020 & 0.0334 & 0.0183 & & 0.0245 & & & & 0.0028 & & 0.0046 & & 0.0023 & & & & & \\
\hline HLW99-24R & 0.3042 & 0.0871 & 0.0028 & 0.0020 & 0.0003 & 0.0000 & & 0.0001 & & & & 0.0022 & & 0.0001 & & 0.0023 & & & & & \\
\hline HLW99-25 & 0.3496 & 0.0020 & 0.0022 & 0.0019 & 0.0188 & 0.0093 & & 0.0135 & & & & 0.0023 & & 0.0000 & & 0.0022 & & & & & \\
\hline HLW99-26 & 0.4072 & 0.0008 & 0.0025 & 0.0019 & 0.0373 & 0.0200 & & 0.0279 & & & & 0.0027 & & 0.0032 & & 0.0022 & & & & & \\
\hline HLW99-27 & 0.3299 & 0.0004 & 0.0021 & 0.0016 & 0.0372 & 0.0189 & & 0.0270 & & & & 0.0021 & & 0.0001 & & 0.0021 & & & & & \\
\hline HLW99-28 & 0.3204 & 0.0696 & 0.0024 & 0.0019 & 0.0371 & 0.0198 & & 0.0276 & & & & 0.0023 & & 0.0001 & & 0.0022 & & & & & \\
\hline HLW99-29 & 0.5209 & 0.0858 & 0.0026 & 0.0021 & 0.0254 & 0.0124 & & 0.0182 & & & & 0.0023 & & 0.0002 & & 0.0020 & & & & & \\
\hline HLW99-30 & 0.3965 & 0.0898 & 0.0024 & 0.0022 & 0.0113 & 0.0056 & & 0.0080 & & & & 0.0021 & & 0.0000 & & 0.0022 & & & & & \\
\hline HLW99-31 & 0.3014 & 0.0009 & 0.0020 & 0.0019 & 0.0025 & 0.0014 & & 0.0016 & & & & 0.0026 & & 0.0046 & & 0.0024 & & & & & \\
\hline HLW99-32 & 0.3593 & 0.0699 & 0.0023 & 0.0021 & 0.0065 & 0.0034 & & 0.0044 & & & & 0.0029 & & 0.0045 & & 0.0023 & & & & & \\
\hline
\end{tabular}


Appendix A. Database - mass fraction

RPP-WTP HLW Formulation (Kot and Pegg 2001)

\begin{tabular}{|c|c|c|c|c|c|c|c|c|c|c|c|c|c|c|c|c|c|c|c|c|c|}
\hline Glass ID & $\begin{array}{c}\mathrm{I} \\
-\mathrm{a} \\
\end{array}$ & $\begin{array}{c}\mathrm{La} 2 \mathrm{O} 3 \\
-\mathrm{a}\end{array}$ & $\begin{array}{c}\mathrm{MnO} 2 \\
-\mathrm{a} \\
\end{array}$ & $\begin{array}{c}\mathrm{MnO} \\
-\mathrm{a} \\
\end{array}$ & $\begin{array}{c}\mathrm{MoO} \\
-\mathrm{a} \\
\end{array}$ & $\begin{array}{c}\mathrm{MoO} 3 \\
-\mathrm{a}\end{array}$ & $\begin{array}{c}\mathrm{Nb} 2 \mathrm{O} 5 \\
-\mathrm{a} \\
\end{array}$ & $\begin{array}{c}\mathrm{Nd} 2 \mathrm{O} 3 \\
-\mathrm{a}\end{array}$ & $\begin{array}{c}\mathrm{PbO} \\
-\mathrm{a} \\
\end{array}$ & $\begin{array}{c}\mathrm{PdO} 2 \\
-\mathrm{a}\end{array}$ & $\begin{array}{c}\mathrm{PdO} \\
-\mathrm{a} \\
\end{array}$ & $\begin{array}{c}\mathrm{Pr} 2 \mathrm{O} 3 \\
-\mathrm{a} \\
\end{array}$ & $\begin{array}{c}\text { Pr6O11 } \\
-\mathrm{a} \\
\end{array}$ & $\begin{array}{c}\mathrm{Rb} 2 \mathrm{O} \\
-\mathrm{a}\end{array}$ & $\begin{array}{c}\mathrm{ReO} \\
-\mathrm{a} \\
\end{array}$ & $\begin{array}{c}\mathrm{ReO} 2 \\
-\mathrm{a}\end{array}$ & $\begin{array}{c}\mathrm{Rh} 2 \mathrm{O} 3 \\
-\mathrm{a} \\
\end{array}$ & $\begin{array}{c}\mathrm{RhO} 2 \\
-\mathrm{a}\end{array}$ & $\begin{array}{c}\mathrm{RuO2} \\
-\mathrm{a}\end{array}$ & $\begin{array}{c}\mathrm{Sb} 2 \mathrm{O} 3 \\
-\mathrm{a} \\
\end{array}$ & $\begin{array}{c}\mathrm{Sb} 2 \mathrm{O} 5 \\
-\mathrm{a} \\
\end{array}$ \\
\hline HLW99-13 & & & 0.0024 & & & & & & 0.0109 & & & & & & & & & & & & 0.0023 \\
\hline HLW99-14 & & & 0.0023 & & & & & & 0.0032 & & & & & & & & & & & & 0.0019 \\
\hline HLW99-15 & & & 0.0021 & & & & & & 0.0035 & & & & & & & & & & & & 0.0019 \\
\hline HLW99-16 & & & 0.0022 & & & & & & 0.0019 & & & & & & & & & & & & 0.0021 \\
\hline HLW99-17 & & & 0.0023 & & & & & & 0.0011 & & & & & & & & & & & & 0.0023 \\
\hline HLW99-18 & & & 0.0023 & & & & & & 0.0030 & & & & & & & & & & & & 0.0021 \\
\hline HLW99-19 & & & 0.0024 & & & & & & 0.0007 & & & & & & & & & & & & 0.0021 \\
\hline HLW99-20 & & & 0.0025 & & & & & & 0.0112 & & & & & & & & & & & & 0.0023 \\
\hline HLW99-21 & & & 0.0022 & & & & & & 0.0002 & & & & & & & & & & & & 0.0019 \\
\hline HLW99-22 & & & 0.0024 & & & & & & 0.0010 & & & & & & & & & & & & 0.0023 \\
\hline HLW99-23 & & & 0.0024 & & & & & & 0.0099 & & & & & & & & & & & & 0.0022 \\
\hline HLW99-24R & & & 0.0025 & & & & & & 0.0003 & & & & & & & & & & & & 0.0018 \\
\hline HLW99-25 & & & 0.0024 & & & & & & 0.0017 & & & & & & & & & & & & 0.0020 \\
\hline HLW99-26 & & & 0.0023 & & & & & & 0.0109 & & & & & & & & & & & & 0.0023 \\
\hline HLW99-27 & & & 0.0022 & & & & & & 0.0034 & & & & & & & & & & & & 0.0020 \\
\hline HLW99-28 & & & 0.0022 & & & & & & 0.0034 & & & & & & & & & & & & 0.0020 \\
\hline HLW99-29 & & & 0.0021 & & & & & & 0.0022 & & & & & & & & & & & & 0.0022 \\
\hline HLW99-30 & & & 0.0023 & & & & & & 0.0011 & & & & & & & & & & & & 0.0022 \\
\hline HLW99-31 & & & 0.0025 & & & & & & 0.0011 & & & & & & & & & & & & 0.0024 \\
\hline HLW99-32 & & & 0.0025 & & & & & & 0.0023 & & & & & & & & & & & & 0.0025 \\
\hline
\end{tabular}


Appendix A. Database - mass fraction

RPP-WTP HLW Formulation (Kot and Pegg 2001)

\begin{tabular}{|c|c|c|c|c|c|c|c|c|c|c|c|c|c|c|c|c|c|c|c|c|}
\hline Glass ID & $\begin{array}{c}\mathrm{SeO} 2 \\
-\mathrm{a}\end{array}$ & $\begin{array}{c}\mathrm{Sm} 2 \mathrm{O} 3 \\
-\mathrm{a}\end{array}$ & $\begin{array}{c}\mathrm{SnO} \\
-\mathrm{a}\end{array}$ & $\begin{array}{c}\mathrm{SnO} 2 \\
-\mathrm{a}\end{array}$ & $\begin{array}{c}\mathrm{SO} 3 \\
-\mathrm{a}\end{array}$ & $\begin{array}{c}\mathrm{SrO} \\
-\mathrm{a}\end{array}$ & $\begin{array}{c}\mathrm{Tc} 2 \mathrm{O} 7 \\
-\mathrm{a}\end{array}$ & $\begin{array}{c}\mathrm{TeO} 2 \\
-\mathrm{a}\end{array}$ & $\begin{array}{c}\mathrm{ThO} 2 \\
-\mathrm{a}\end{array}$ & $\begin{array}{c}\mathrm{TiO} 2 \\
-\mathrm{a}\end{array}$ & $\begin{array}{c}\mathrm{T} 12 \mathrm{O} 3 \\
-\mathrm{a}\end{array}$ & $\begin{array}{c}\mathrm{U} 3 \mathrm{O} 8 \\
-\mathrm{a}\end{array}$ & $\begin{array}{c}\mathrm{UO} 2 \\
-\mathrm{a}\end{array}$ & $\begin{array}{c}\mathrm{UO} 3 \\
-\mathrm{a}\end{array}$ & $\begin{array}{c}\mathrm{V} 2 \mathrm{O} 5 \\
-\mathrm{a}\end{array}$ & $\begin{array}{c}\text { WO3 } \\
-\mathrm{a}\end{array}$ & $\begin{array}{c}\mathrm{Y} 2 \mathrm{O} 3 \\
-\mathrm{a}\end{array}$ & $\begin{array}{c}\mathrm{ZnO} \\
-\mathrm{a}\end{array}$ & $\begin{array}{c}\text { Others } \\
-\mathrm{a}\end{array}$ & $\begin{array}{l}\text { Sum } \\
-\mathrm{a}\end{array}$ \\
\hline HLW99-13 & 0.0011 & & & & & 0.0024 & & 0.0020 & & 0.0101 & 0.0010 & & 0.0167 & & & 0.0030 & & 0.0196 & & 0.9706 \\
\hline HLW99-14 & 0.0006 & & & & & 0.0027 & & 0.0021 & & 0.0110 & 0.0013 & & 0.0001 & & & 0.0024 & & 0.0186 & & 0.9884 \\
\hline HLW99-15 & 0.0006 & & & & & 0.0025 & & 0.0020 & & 0.0107 & 0.0005 & & 0.0001 & & & 0.0025 & & 0.0190 & & 0.9841 \\
\hline HLW99-16 & 0.0010 & & & & & 0.0023 & & 0.0020 & & 0.0061 & 0.0009 & & 0.0001 & & & 0.0024 & & 0.0199 & & 0.9838 \\
\hline HLW99-17 & 0.0011 & & & & & 0.0021 & & 0.0020 & & 0.0008 & 0.0029 & & 0.0853 & & & 0.0033 & & 0.0192 & & 0.9757 \\
\hline HLW99-18 & 0.0017 & & & & & 0.0024 & & 0.0020 & & 0.0085 & 0.0008 & & 0.0003 & & & 0.0027 & & 0.0199 & & 0.9809 \\
\hline HLW99-19 & 0.0005 & & & & & 0.0021 & & 0.0018 & & 0.0005 & 0.0012 & & 0.0680 & & & 0.0026 & & 0.0182 & & 0.9637 \\
\hline HLW99-20 & 0.0014 & & & & & 0.0025 & & 0.0022 & & 0.0103 & 0.0015 & & 0.0634 & & & 0.0029 & & 0.0202 & & 0.9619 \\
\hline HLW99-21 & 0.0013 & & & & & 0.0022 & & 0.0021 & & 0.0006 & 0.0010 & & 0.0006 & & & 0.0022 & & 0.0195 & & 0.9481 \\
\hline HLW99-22 & 0.0008 & & & & & 0.0021 & & 0.0020 & & 0.0007 & 0.0021 & & 0.0565 & & & 0.0027 & & 0.0211 & & 0.9373 \\
\hline HLW99-23 & 0.0013 & & & & & 0.0025 & & 0.0020 & & 0.0089 & 0.0009 & & 0.0632 & & & 0.0031 & & 0.0198 & & 0.9506 \\
\hline HLW99-24R & 0.0006 & & & & & 0.0024 & & 0.0022 & & 0.0006 & 0.0015 & & 0.0008 & & & 0.0024 & & 0.0200 & & 0.9513 \\
\hline HLW99-25 & 0.0013 & & & & & 0.0025 & & 0.0017 & & 0.0056 & 0.0006 & & 0.0003 & & & 0.0024 & & 0.0200 & & 0.9884 \\
\hline HLW99-26 & 0.0007 & & & & & 0.0023 & & 0.0021 & & 0.0099 & 0.0015 & & 0.0712 & & & 0.0029 & & 0.0190 & & 0.9284 \\
\hline HLW99-27 & 0.0009 & & & & & 0.0024 & & 0.0019 & & 0.0115 & 0.0006 & & 0.0004 & & & 0.0024 & & 0.0193 & & 0.9820 \\
\hline HLW99-28 & 0.0012 & & & & & 0.0023 & & 0.0020 & & 0.0110 & 0.0016 & & 0.0006 & & & 0.0026 & & 0.0190 & & 0.9692 \\
\hline HLW99-29 & 0.0010 & & & & & 0.0022 & & 0.0020 & & 0.0066 & 0.0013 & & 0.0006 & & & 0.0023 & & 0.0235 & & 0.9875 \\
\hline HLW99-30 & 0.0012 & & & & & 0.0022 & & 0.0045 & & 0.0031 & 0.0015 & & 0.0003 & & & 0.0019 & & 0.0199 & & 0.9692 \\
\hline HLW99-31 & 0.0013 & & & & & 0.0022 & & 0.0020 & & 0.0009 & 0.0012 & & 0.0454 & & & 0.0026 & & 0.0203 & & 0.9241 \\
\hline HLW99-32 & 0.0015 & & & & & 0.0022 & & 0.0020 & & 0.0020 & 0.0021 & & 0.0686 & & & 0.0027 & & 0.0198 & & 0.9764 \\
\hline
\end{tabular}


Appendix A. Database - mass fraction

RPP-WTP HLW Formulation (Kot and Pegg 2001)

\begin{tabular}{|c|c|c|c|c|c|c|c|c|}
\hline Glass ID & $\begin{array}{l}\mathrm{TM} \\
\left({ }^{\circ} \mathrm{C}\right)\end{array}$ & $\begin{array}{l}\text { Gradient } \\
\text { TL }\left({ }^{\circ} \mathrm{C}\right)\end{array}$ & $\begin{array}{l}\text { Uniform } \\
\text { TL }\left({ }^{\circ} \mathrm{C}\right)\end{array}$ & Primary Phase & $\begin{array}{l}\text { Quenched } \\
\text { Visual/OM }\end{array}$ & $\begin{array}{c}\text { Quenched } \\
\text { SEM/EDS or TEM }\end{array}$ & $\begin{array}{l}\text { Quenched } \\
\text { XRD }\end{array}$ & $\begin{array}{c}\text { Quenched } \\
\text { Homogeneous? }\end{array}$ \\
\hline \multicolumn{9}{|l|}{ HLW99-13 } \\
\hline HLW99-14 & 1150 & & $>1150$ & & $\begin{array}{l}\text { Dark brown glass with green swirls and } \\
\text { streaks on the surface. Cross section appears } \\
\text { uniform }\end{array}$ & & & \\
\hline HLW99-15 & 1150 & & 850 & & $\begin{array}{l}\text { Dark green glass with black patches on top } \\
\text { and bottom. Cross section appears uniform }\end{array}$ & & & \\
\hline HLW99-16 & 1150 & & 1100 & & $\begin{array}{l}\text { Brown glass with brownish green streaks on } \\
\text { top and bottom. Cross section appears } \\
\text { uniform }\end{array}$ & & & \\
\hline \multicolumn{9}{|l|}{ HLW99-17 } \\
\hline HLW99-18 & 1150 & & 1075 & & $\begin{array}{l}\text { Homogeneous black glass with silvery } \\
\text { patches left in crucible }\end{array}$ & & & \\
\hline HLW99-19 & 1150 & & $>950^{\circ} \mathrm{C}$ & & $\begin{array}{l}\text { Brown glass with blue and green regions } \\
\text { across cross sections. Crystals found in glass } \\
\text { shards }\end{array}$ & & & \\
\hline HLW99-20 & 1150 & & $>950^{\circ} \mathrm{C}$ & & $\begin{array}{l}\text { Dark brown glass with gray swirls on surface } \\
\text { and silvery patches. Some undissolved } \\
\text { crystals }\end{array}$ & & & \\
\hline HLW99-21 & 1150 & & 975 & & $\begin{array}{l}\text { Blue glass with green regions on surface and } \\
\text { cross sections }\end{array}$ & & & \\
\hline HLW99-22 & 1150 & & $>950^{\circ} \mathrm{C}$ & & $\begin{array}{l}\text { Mostly uniform dark brown glass with gray } \\
\text { patches on surface and small amount of } \\
\text { undissolved crystals }\end{array}$ & & & \\
\hline \multicolumn{9}{|l|}{ HLW99-23 } \\
\hline HLW99-24R & 1200 & & 925 & & $\begin{array}{l}\text { Brown glass with multicolored regions and } \\
\text { undissolved lumps. Glass cloudy after remelt } \\
\text { at } 1300^{\circ} \mathrm{C}\end{array}$ & & & \\
\hline HLW99-25 & 1150 & & 1125 & & $\begin{array}{l}\text { Brown glass with streaks and silver patches } \\
\text { on surface }\end{array}$ & & & \\
\hline HLW99-26 & 1150 & & $>950^{\circ} \mathrm{C}$ & & $\begin{array}{l}\text { Brown glass with green areas in cross section } \\
\text { Glass shards with crystals }\end{array}$ & & & \\
\hline HLW99-27 & 1150 & & 1075 & & $\begin{array}{l}\text { Brown glass with swirls on surface. } \\
\text { Inhomogeneous cross section with } \\
\text { undissolved material }\end{array}$ & & & \\
\hline HLW99-28 & 1150 & & $>950^{\circ} \mathrm{C}$ & & $\begin{array}{l}\text { Mostly homogeneous black glass with red } \\
\text { tinge. Trace amount of undissolved material }\end{array}$ & & & \\
\hline HLW99-29 & 1250 & & 1150 & & $\begin{array}{l}\text { Inhomogeneous glass with green and gray } \\
\text { regions and silver patches. Trace amount of } \\
\text { undissolved material }\end{array}$ & & & \\
\hline HLW99-30 & 1150 & & 925 & & $\begin{array}{l}\text { Mostly homogeneous black glass with brown } \\
\text { streaks on surface }\end{array}$ & & & \\
\hline \multicolumn{9}{|l|}{ HLW99-31 } \\
\hline HLW99-32 & & & & & & & & \\
\hline
\end{tabular}


Appendix A. Database - mass fraction

RPP-WTP HLW Formulation (Kot and Pegg 2001)

\begin{tabular}{|c|c|c|c|c|c|}
\hline Glass ID & $\begin{array}{c}\mathrm{CCC} \\
\text { Visual/OM }\end{array}$ & $\begin{array}{c}\text { CCC } \\
\text { SEM/EDS or TEM }\end{array}$ & $\begin{array}{l}\mathrm{CCC} \\
\mathrm{XRD}\end{array}$ & $\begin{array}{l}\text { Heat Treated } \\
\text { Visual/OM }\end{array}$ & $\begin{array}{c}\text { Heat Treated } \\
\text { SEM/EDS or TEM }\end{array}$ \\
\hline \multicolumn{6}{|l|}{ HLW99-13 } \\
\hline HLW99-14 & & & & & $\begin{array}{l}\text { Up to } 2.5 \mathrm{vol} \% \text { of magnetic spinel crystals, mostly } \\
\text { settle to the bottom }\end{array}$ \\
\hline HLW99-15 & & & & & $\begin{array}{l}\text { Homogeneous glass with trace } \mathrm{RuO} 2\left(20^{\prime} 70\right. \\
\mathrm{mm})\end{array}$ \\
\hline HLW99-16 & & & & & $\begin{array}{l}\text { About } 2 \mathrm{vol} \% \text { of magnetic spinel throughout glas } \\
\text { but concentrate at bottom }\end{array}$ \\
\hline \multicolumn{6}{|l|}{ HLW99-17 } \\
\hline HLW99-18 & & & & & $\begin{array}{l}\text { 1) Up to } 1.5 \mathrm{vol} \% \text { of rod shaped } \mathrm{NiO} 2) 0.2 \mathrm{vol} \% \\
\text { of } \mathrm{ZrO} 2(\mathrm{w} / \mathrm{Ce}, \mathrm{Sb} \mathrm{Ca}) 3)>0.1 \mathrm{vol} \% \text { of } \mathrm{RuO} 24) \\
\text { Trace amount of } \mathrm{Pd} / \mathrm{Rh} \text { alloy }\end{array}$ \\
\hline HLW99-19 & & & & & $\begin{array}{l}\text { Up to } 15 \mathrm{vol} \% \text { of magnetic spinel crystals with } \\
\text { heavy coverage at the glass/air interface }\end{array}$ \\
\hline HLW99-20 & & & & & $\begin{array}{l}\text { Up to } 0.6 \mathrm{vol} \% \text { of rectangular prismatic crystals o } \\
\mathrm{NiO}\end{array}$ \\
\hline HLW99-21 & & & & & $\begin{array}{l}\text { Dark crystals of RuO2 and Rh throughout the } \\
\text { bulk of glass. Total } 0.2 \mathrm{vol} \%\end{array}$ \\
\hline HLW99-22 & & & & & $\begin{array}{l}\text { Clusters of white zirconium containing crystals. } \\
\text { Dark RuO2 crystals. Total } 2.5 \text { vol } \% \text {. } \\
\text { Inhomogeneous possibly due to silver }\end{array}$ \\
\hline \multicolumn{6}{|l|}{ HLW99-23 } \\
\hline HLW99-24R & & & & & $\begin{array}{l}\text { About } 5 \text { vol } \% \text { of white zirconium containing } \\
\text { crystals, mostly settle to the bottom }\end{array}$ \\
\hline HLW99-25 & & & & & $\begin{array}{l}5 \mathrm{vol} \% \text { of spinel (trevorite) with minor } \\
\text { incorporation (Al, Zn, Rh). About } 0.2 \text { vol\% of } \\
\text { RuO2 }\end{array}$ \\
\hline HLW99-26 & & & & & $\begin{array}{l}\text { Heavy crystallization with over } 1 \mathrm{vol} \% \text { of } \\
\text { magnetic spinel and over } 10 \mathrm{vol} \% \text { of clino- } \\
\text { pyroxene }\end{array}$ \\
\hline HLW99-27 & & & & & $\begin{array}{l}\text { About } 3 \mathrm{vol} \% \text { of spinel (trevorite) with minor } \\
\text { incorporation of other elements, centered around } \\
\mathrm{Rh} / \mathrm{Pd} \text { metal }\end{array}$ \\
\hline HLW99-28 & & & & & $\begin{array}{l}\text { About } 1.5 \mathrm{vol} \% \text { of } \mathrm{NiO} \text { with incorpo-ration of } \mathrm{Fe} \\
\text { and } \mathrm{Sb} \text {. Trace amount of } \mathrm{Rh} \text { and } \mathrm{RuO} 2\end{array}$ \\
\hline HLW99-29 & & & & & $1 \mathrm{vol} \%$ of small dark non-magnetic crystals \\
\hline HLW99-30 & & & & & $\begin{array}{l}0.5 \mathrm{vol} \% \text { of RuO2 About } 0.5 \mathrm{vol} \% \text { of non- } \\
\text { magnetic rod shaped crystals }\end{array}$ \\
\hline HLW99-31 & & & & & \\
\hline HLW99-32 & & & & & \\
\hline
\end{tabular}


Appendix A. Database - mass fraction

RPP-WTP HLW Formulation (Kot and Pegg 2001)

\begin{tabular}{|c|c|c|c|c|c|c|c|c|c|c|c|c|c|c|c|}
\hline Glass ID & $\begin{array}{c}\text { Heat Treated } \\
\text { XRD } \\
\end{array}$ & $\begin{array}{l}\text { Density } \\
\left(\mathrm{g} / \mathrm{cm}^{3}\right)\end{array}$ & $\begin{array}{c}\text { Fulc Visc } \\
\mathrm{A}\end{array}$ & $\begin{array}{c}\text { Fulc Visc } \\
\text { B } \\
\end{array}$ & $\begin{array}{c}\text { Fulc Visc } \\
\text { To } \\
\end{array}$ & $\begin{array}{c}\mathrm{FV} 1150^{\circ} \mathrm{C} \\
(\mathrm{Pa} \cdot \mathrm{s})\end{array}$ & \begin{tabular}{|c|} 
Arrh Visc \\
A
\end{tabular} & $\begin{array}{c}\text { Arrh Visc } \\
\text { B } \\
\end{array}$ & $\begin{array}{c}1150^{\circ} \mathrm{C} \\
\left(\mathrm{D}_{0.0}\right)\end{array}$ & $\begin{array}{c}\mathrm{T}\left({ }^{\circ} \mathrm{C}\right) \text { at } \\
2 \mathrm{~Pa} \cdot \mathrm{s}\end{array}$ & $\begin{array}{c}\mathrm{T}\left({ }^{\circ} \mathrm{C}\right) \text { at } \\
5 \mathrm{~Pa} \cdot \mathrm{s}\end{array}$ & $\begin{array}{c}\mathrm{T}\left({ }^{\circ} \mathrm{C}\right) \text { at } \\
10 \mathrm{~Pa} \cdot \mathrm{s}\end{array}$ & $\begin{array}{l}\mathrm{T} 1 \\
\left({ }^{\circ} \mathrm{C}\right) \\
\end{array}$ & $\begin{array}{c}\mathrm{V} 1 \\
(\mathrm{~Pa} \cdot \mathrm{s}) \\
\end{array}$ & $\begin{array}{l}\mathrm{T} 2 \\
\left({ }^{\circ} \mathrm{C}\right) \\
\end{array}$ \\
\hline HLW99-13 & & 2.98 & & & & & & & & & & & & & \\
\hline HLW99-14 & & 2.93 & & & & & & & & & & & & & \\
\hline HLW99-15 & & 2.86 & & & & & -10.171 & 12934.2 & 0.34 & & & & 1000 & 1.018 & 1050 \\
\hline \multicolumn{16}{|l|}{ HLW99-16 } \\
\hline \multicolumn{16}{|l|}{ HLW99-17 } \\
\hline \multicolumn{16}{|l|}{ HLW99-18 } \\
\hline \multicolumn{16}{|l|}{ HLW99-19 } \\
\hline \multicolumn{16}{|l|}{ HLW99-20 } \\
\hline \multicolumn{16}{|l|}{ HLW99-21 } \\
\hline \multicolumn{16}{|l|}{ HLW99-22 } \\
\hline \multicolumn{16}{|l|}{ HLW99-23 } \\
\hline \multicolumn{16}{|l|}{ HLW99-24R } \\
\hline \multicolumn{16}{|l|}{ HLW99-25 } \\
\hline \multicolumn{16}{|l|}{ HLW99-26 } \\
\hline \multicolumn{16}{|l|}{ HLW99-27 } \\
\hline \multicolumn{16}{|l|}{ HLW99-28 } \\
\hline \multicolumn{16}{|l|}{ HLW99-29 } \\
\hline HLW99-30 & & & & & & & -13.007 & 19960.0 & 2.77 & & & & 1000 & 15.036 & 1050 \\
\hline HLW99-31 & & & & & & & & & & & & & & & \\
\hline HLW99-32 & & & & & & & & & & & & & & & \\
\hline
\end{tabular}


Appendix A. Database - mass fraction

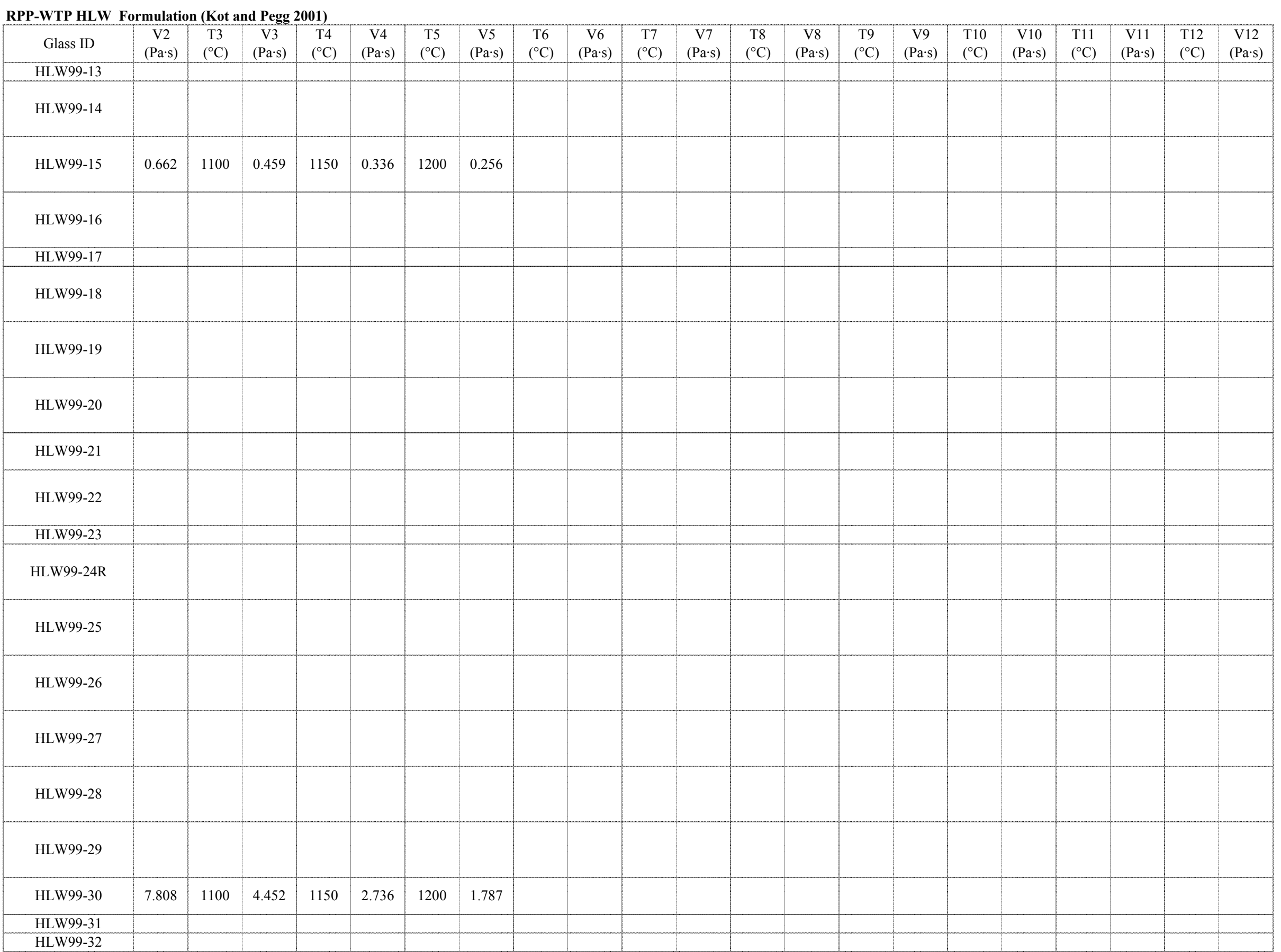


Appendix A. Database - mass fraction

RPP-WTP HLW Formulation (Kot and Pegg 2001)

\begin{tabular}{|c|c|c|c|c|c|c|c|c|c|c|c|c|c|c|c|c|}
\hline Glass ID & $\begin{array}{l}\mathrm{T} 13 \\
\left({ }^{\circ} \mathrm{C}\right) \\
\end{array}$ & $\begin{array}{c}\mathrm{V} 13 \\
\mathrm{(Pa} \cdot \mathrm{s}) \\
\end{array}$ & $\begin{array}{l}\mathrm{T} 14 \\
\left({ }^{\circ} \mathrm{C}\right) \\
\end{array}$ & $\begin{array}{c}\mathrm{V} 14 \\
(\mathrm{~Pa} \cdot \mathrm{s})\end{array}$ & $\begin{array}{c}\text { Q PCT } \\
\text { B }\left(\mathrm{g} / \mathrm{m}^{2}\right)\end{array}$ & $\begin{array}{c}\text { Q PCT } \\
\mathrm{Li}\left(\mathrm{g} / \mathrm{m}^{2}\right)\end{array}$ & $\begin{array}{c}\text { Q PCT } \\
\mathrm{Na}\left(\mathrm{g} / \mathrm{m}^{2}\right) \\
\end{array}$ & $\begin{array}{c}\text { Q PCT } \\
\text { Si }\left(\mathrm{g} / \mathrm{m}^{2}\right)\end{array}$ & $\begin{array}{c}\text { Q PCT } \\
\mathrm{pH}\end{array}$ & $\begin{array}{c}\text { CCC PCT } \\
\mathrm{B}\left(\mathrm{g} / \mathrm{m}^{2}\right) \\
\end{array}$ & $\begin{array}{l}\text { CCC PCT } \\
\mathrm{Li}\left(\mathrm{g} / \mathrm{m}^{2}\right) \\
\end{array}$ & $\begin{array}{l}\mathrm{CCC} \text { PCT } \\
\mathrm{Na}\left(\mathrm{g} / \mathrm{m}^{2}\right) \\
\end{array}$ & $\begin{array}{c}\text { CCC PCT } \\
\mathrm{Si}\left(\mathrm{g} / \mathrm{m}^{2}\right) \\
\end{array}$ & $\begin{array}{c}\text { CCC PCT } \\
\mathrm{pH}\end{array}$ & $\begin{array}{l}\text { Q PCT B at } \\
20^{\circ} \mathrm{C}\left(\mathrm{g} / \mathrm{m}^{2}\right)\end{array}$ & $\begin{array}{l}\text { Q PCT Li at } \\
20^{\circ} \mathrm{C}\left(\mathrm{g} / \mathrm{m}^{2}\right)\end{array}$ \\
\hline HLW99-13 & & & & & & & & & & & & & & & & \\
\hline HLW99-14 & & & & & & & & & & & & & & & & \\
\hline HLW99-15 & & & & & 13.27 & 12.93 & 11.83 & 1.385 & 11.35 & & & & & & & \\
\hline HLW99-16 & & & & & & & & & & & & & & & & \\
\hline HLW99-17 & & & & & & & & & & & & & & & & \\
\hline HLW99-18 & & & & & & & & & & & & & & & & \\
\hline HLW99-19 & & & & & & & & & & & & & & & & \\
\hline HLW99-20 & & & & & & & & & & & & & & & & \\
\hline HLW99-21 & & & & & & & & & & & & & & & & \\
\hline HLW99-22 & & & & & & & & & & & & & & & & \\
\hline HLW99-23 & & & & & & & & & & & & & & & & \\
\hline HLW99-24R & & & & & & & & & & & & & & & & \\
\hline HLW99-25 & & & & & & & & & & & & & & & & \\
\hline HLW99-26 & & & & & & & & & & & & & & & & \\
\hline HLW99-27 & & & & & 0.555 & 0.615 & 0.61 & 0.18 & 10.71 & & & & & & & \\
\hline HLW99-28 & & & & & & & & & & & & & & & & \\
\hline HLW99-29 & & & & & & & & & & & & & & & & \\
\hline HLW99-30 & & & & & & & & & & & & & & & & \\
\hline HLW99-31 & & & & & & & & & & & & & & & & \\
\hline HLW99-32 & & & & & & & & & & & & & & & & \\
\hline
\end{tabular}


Appendix A. Database - mass fraction

RPP-WTP HLW Formulation (Kot and Pegg 2001)

\begin{tabular}{|c|c|c|c|c|c|c|c|c|c|c|c|c|}
\hline Glass ID & $\begin{array}{l}\text { Q PCT Na at } \\
20^{\circ} \mathrm{C}\left(\mathrm{g} / \mathrm{m}^{2}\right)\end{array}$ & $\begin{array}{l}\text { Q PCT Si at } \\
20^{\circ} \mathrm{C}\left(\mathrm{g} / \mathrm{m}^{2}\right)\end{array}$ & $\begin{array}{c}\mathrm{QpH} \\
\text { at } 20^{\circ} \mathrm{C}\end{array}$ & $\begin{array}{c}\text { TCLP Ag } \\
\text { (ppm) }\end{array}$ & $\begin{array}{c}\text { TCLP As } \\
\text { (ppm) }\end{array}$ & $\begin{array}{c}\text { TCLP Ba } \\
\text { (ppm) }\end{array}$ & $\begin{array}{l}\text { TCLP Cd } \\
\text { (ppm) }\end{array}$ & $\begin{array}{c}\text { TCLP Cr } \\
\text { (ppm) }\end{array}$ & $\begin{array}{c}\text { TCLP Ni } \\
(\mathrm{ppm})\end{array}$ & $\begin{array}{c}\text { TCLP Pb } \\
\text { (ppm) }\end{array}$ & $\begin{array}{c}\text { TCLP Se } \\
(\mathrm{ppm})\end{array}$ & $\begin{array}{c}\text { TCLP Zn } \\
(\mathrm{ppm})\end{array}$ \\
\hline HLW99-13 & & & & 0.388 & 0.049 & 8.764 & 6.645 & 0.026 & 5.047 & 2.024 & 0.266 & 4.531 \\
\hline HLW99-14 & & & & 0.079 & 0.049 & 3.804 & 2.648 & 0.008 & 1.945 & 0.211 & 0.075 & 1.63 \\
\hline HLW99-15 & & & & 0.577 & 2.768 & 99.555 & 47.236 & 0.073 & 65.141 & 4.868 & 0.806 & 12.33 \\
\hline HLW99-16 & & & & 0.064 & 0.049 & 0.404 & 0.276 & $<0.0055$ & 0.207 & $<0.0243$ & 0.0532 & 0.309 \\
\hline HLW99-17 & & & & 0.028 & 0.219 & 0.053 & 0.057 & 1.43 & 0.102 & 0.185 & 0.719 & 16.94 \\
\hline HLW99-18 & & & & 0.736 & 0.053 & 17.703 & 11.891 & 0.02 & 8.929 & 0.912 & 0.195 & 9.45 \\
\hline HLW99-19 & & & & 0.07 & 0.049 & 0.048 & 0.011 & 0.021 & 0.022 & 0.082 & 0.138 & 0.96 \\
\hline HLW99-20 & & & & 0.128 & 0.277 & 17.5 & 17.884 & 0.085 & 14.79 & 1.998 & 0.423 & 10.72 \\
\hline HLW99-21 & & & & 0.026 & 0.049 & 0.089 & 0.048 & $<.0055$ & 0.023 & 0.049 & 0.056 & 0.625 \\
\hline HLW99-22 & & & & 0.392 & 0.049 & 0.29 & 0.188 & 0.085 & 0.2 & 0.105 & 0.185 & 4.481 \\
\hline HLW99-23 & & & & 0.079 & 0.627 & 28.944 & 43.541 & 4.222 & 53.192 & 0.35 & 1.333 & 13.138 \\
\hline HLW99-24R & & & & 0.125 & 0.06 & 0.27 & 0.088 & 0.021 & 0.141 & 0.22 & 0.106 & 22.953 \\
\hline HLW99-25 & & & & 0.072 & 0.049 & 2.239 & 1.626 & 0.011 & 0.967 & 0.156 & 0.0532 & 2.102 \\
\hline HLW99-26 & & & & 0.033 & 0.049 & 2.344 & 1.521 & 0.037 & 1.24 & 0.492 & 0.138 & 1.102 \\
\hline HLW99-27 & & & & 0.025 & 0.063 & 41.818 & 30.943 & 0.006 & 23.934 & 0.781 & 0.0532 & 18.43 \\
\hline HLW99-28 & & & & 0.179 & 0.103 & 20.759 & 16.978 & 0.009 & 14.359 & 0.888 & 0.085 & 9.841 \\
\hline HLW99-29 & & & & 0.086 & 0.049 & 0.719 & 0.482 & $<.0055$ & 0.389 & 0.114 & 0.0532 & 0.611 \\
\hline HLW99-30 & & & & 0.333 & 0.049 & 1.832 & 1.444 & $<0.0055$ & 0.962 & 0.072 & 0.0532 & 3.187 \\
\hline HLW99-31 & & & & 0.254 & 0.087 & 0.111 & 0.082 & 0.582 & 0.203 & 0.362 & 0.419 & 13.152 \\
\hline HLW99-32 & & & & 0.088 & 0.049 & 0.303 & 0.185 & 0.033 & 0.133 & 0.079 & 0.116 & 1.024 \\
\hline
\end{tabular}


RPP-WTP HLW Formulation (Kot and Pegg 2001)

\begin{tabular}{|c|c|c|c|c|c|c|c|c|c|c|c|c|c|c|c|c|c|c|c|c|c|}
\hline Glass ID & $\begin{array}{c}\mathrm{A} 12 \mathrm{O} 3 \\
-\mathrm{t}\end{array}$ & $\begin{array}{c}\mathrm{B} 2 \mathrm{O} 3 \\
-\mathrm{t}\end{array}$ & $\begin{array}{c}\mathrm{CaO} \\
-\mathrm{t} \\
\end{array}$ & $\begin{array}{c}\mathrm{Fe} 2 \mathrm{O} 3 \\
-\mathrm{t}\end{array}$ & $\begin{array}{c}\mathrm{FeO} \\
-\mathrm{t}\end{array}$ & $\begin{array}{c}\mathrm{K} 2 \mathrm{O} \\
-\mathrm{t}\end{array}$ & $\begin{array}{c}\mathrm{Li} 2 \mathrm{O} \\
-\mathrm{t}\end{array}$ & $\begin{array}{c}\mathrm{MgO} \\
-\mathrm{t}\end{array}$ & $\begin{array}{c}\mathrm{Na} 2 \mathrm{O} \\
-\mathrm{t}\end{array}$ & $\begin{array}{c}\mathrm{NiO} \\
-\mathrm{t}\end{array}$ & $\begin{array}{c}\mathrm{P} 2 \mathrm{O} 5 \\
-\mathrm{t}\end{array}$ & $\begin{array}{c}\mathrm{SiO} 2 \\
-\mathrm{t}\end{array}$ & $\begin{array}{c}\mathrm{ZrO} 2 \\
-\mathrm{t}\end{array}$ & $\begin{array}{c}\mathrm{Ag} 2 \mathrm{O} \\
-\mathrm{t}\end{array}$ & $\begin{array}{c}\mathrm{As} 2 \mathrm{O} 3 \\
-\mathrm{t}\end{array}$ & $\begin{array}{c}\mathrm{BaO} \\
-\mathrm{t}\end{array}$ & $\begin{array}{c}\mathrm{Bi} 2 \mathrm{O} 3 \\
-\mathrm{t} \\
\end{array}$ & $\begin{array}{l}\mathrm{Br} \\
-\mathrm{t}\end{array}$ & $\begin{array}{c}\mathrm{CdO} \\
-\mathrm{t}\end{array}$ & $\begin{array}{c}\mathrm{Ce} 2 \mathrm{O} 3 \\
-\mathrm{t} \\
\end{array}$ & $\begin{array}{c}\mathrm{CeO} 2 \\
-\mathrm{t}\end{array}$ \\
\hline HLW99-33 & 0.0000 & 0.2000 & 0.0700 & 0.0200 & & 0.0100 & 0.0700 & 0.0000 & 0.0800 & 0.0000 & 0.0300 & 0.3730 & 0.0000 & 0.0025 & 0.0020 & 0.0000 & 0.0000 & & 0.0000 & & 0.0020 \\
\hline HLW99-34 & 0.0000 & 0.0500 & 0.0000 & 0.0297 & & 0.0100 & 0.0700 & 0.0000 & 0.0800 & 0.0000 & 0.0300 & 0.5300 & 0.0533 & 0.0025 & 0.0020 & 0.0000 & 0.0000 & & 0.0000 & & 0.0020 \\
\hline HLW99-35 & 0.0914 & 0.0500 & 0.0400 & 0.0700 & & 0.0100 & 0.0536 & 0.0000 & 0.0729 & 0.0000 & 0.0000 & 0.5086 & 0.0486 & 0.0025 & 0.0020 & 0.0000 & 0.0000 & & 0.0000 & & 0.0020 \\
\hline HLW99-36 & 0.0000 & 0.2000 & 0.0133 & 0.1250 & & 0.0100 & 0.0700 & 0.0017 & 0.0800 & 0.0025 & 0.0000 & 0.3800 & 0.0533 & 0.0025 & 0.0020 & 0.0033 & 0.0017 & & 0.0025 & & 0.0020 \\
\hline HLW99-37 & 0.1100 & 0.0700 & 0.0000 & 0.0200 & & 0.0100 & 0.0500 & 0.0094 & 0.1200 & 0.0141 & 0.0000 & 0.4900 & 0.0000 & 0.0025 & 0.0020 & 0.0188 & 0.0094 & & 0.0141 & & 0.0020 \\
\hline HLW99-38 & 0.0000 & 0.0500 & 0.0700 & 0.0200 & & 0.0100 & 0.0700 & 0.0137 & 0.0500 & 0.0206 & 0.0300 & 0.4100 & 0.0333 & 0.0025 & 0.0020 & 0.0274 & 0.0137 & & 0.0206 & & 0.0020 \\
\hline HLW99-39 & 0.0000 & 0.1250 & 0.0700 & 0.1250 & & 0.0100 & 0.0400 & 0.0013 & 0.1400 & 0.0019 & 0.0000 & 0.4250 & 0.0000 & 0.0025 & 0.0020 & 0.0025 & 0.0013 & & 0.0019 & & 0.0020 \\
\hline HLW99-40 & 0.1100 & 0.2000 & 0.0000 & 0.0675 & & 0.0100 & 0.0000 & 0.0000 & 0.1200 & 0.0000 & 0.0000 & 0.4075 & 0.0300 & 0.0025 & 0.0020 & 0.0000 & 0.0000 & & 0.0000 & & 0.0020 \\
\hline HLW99-41 & 0.0516 & 0.1171 & 0.0303 & 0.0614 & & 0.0100 & 0.0317 & 0.0062 & 0.1129 & 0.0094 & 0.0124 & 0.3973 & 0.0322 & 0.0025 & 0.0020 & 0.0125 & 0.0062 & & 0.0094 & & 0.0020 \\
\hline HLW99-42 & 0.1100 & 0.1775 & 0.0450 & 0.0200 & & 0.0100 & 0.0350 & 0.0000 & 0.0500 & 0.0000 & 0.0000 & 0.4675 & 0.0300 & 0.0025 & 0.0020 & 0.0000 & 0.0000 & & 0.0000 & & 0.0020 \\
\hline HLW99-43 & 0.0850 & 0.2000 & 0.0700 & 0.1250 & & 0.0100 & 0.0675 & 0.0000 & 0.0850 & 0.0000 & 0.0000 & 0.3025 & 0.0000 & 0.0025 & 0.0020 & 0.0000 & 0.0000 & & 0.0000 & & 0.0020 \\
\hline HLW99-44 & 0.0850 & 0.0700 & 0.0700 & 0.1250 & & 0.0100 & 0.0100 & 0.0000 & 0.2000 & 0.0000 & 0.0184 & 0.3000 & 0.0000 & 0.0025 & 0.0020 & 0.0000 & 0.0000 & & 0.0000 & & 0.0020 \\
\hline HLW99-45 & 0.1067 & 0.1600 & 0.0700 & 0.0200 & & 0.0100 & 0.0700 & 0.0004 & 0.0500 & 0.0006 & 0.0300 & 0.3000 & 0.0333 & 0.0025 & 0.0020 & 0.0008 & 0.0004 & & 0.0006 & & 0.0020 \\
\hline HLW99-46 & 0.0000 & 0.1158 & 0.0700 & 0.0200 & & 0.0100 & 0.0700 & 0.0200 & 0.0800 & 0.0300 & 0.0000 & 0.3658 & 0.0533 & 0.0025 & 0.0020 & 0.0400 & 0.0200 & & 0.0300 & & 0.0020 \\
\hline HLW99-47 & 0.0500 & 0.0500 & 0.0350 & 0.1250 & & 0.0100 & 0.0300 & 0.0000 & 0.0950 & 0.0000 & 0.0000 & 0.5500 & 0.0000 & 0.0025 & 0.0020 & 0.0000 & 0.0000 & & 0.0000 & & 0.0020 \\
\hline HLW99-48 & 0.1100 & 0.0650 & 0.0000 & 0.0850 & & 0.0100 & 0.0000 & 0.0200 & 0.2000 & 0.0300 & 0.0000 & 0.3150 & 0.0000 & 0.0025 & 0.0020 & 0.0400 & 0.0200 & & 0.0300 & & 0.0020 \\
\hline HLW99-49 & 0.0425 & 0.0650 & 0.0000 & 0.1250 & & 0.0100 & 0.0000 & 0.0029 & 0.2000 & 0.0043 & 0.0300 & 0.3150 & 0.0425 & 0.0025 & 0.0020 & 0.0058 & 0.0029 & & 0.0043 & & 0.0020 \\
\hline HLW99-50 & 0.0500 & 0.0600 & 0.0000 & 0.0200 & & 0.0100 & 0.0700 & 0.0000 & 0.0500 & 0.0000 & 0.0250 & 0.5500 & 0.0333 & 0.0025 & 0.0020 & 0.0000 & 0.0000 & & 0.0000 & & 0.0020 \\
\hline HLW99-51R & 0.0000 & 0.2000 & 0.0700 & 0.0200 & & 0.0100 & 0.0000 & 0.0000 & 0.2000 & 0.0000 & 0.0300 & 0.3000 & 0.0230 & 0.0025 & 0.0020 & 0.0000 & 0.0000 & & 0.0000 & & 0.0020 \\
\hline HLW99-52 & 0.0250 & 0.2000 & 0.0000 & 0.0200 & & 0.0100 & 0.0000 & 0.0200 & 0.1600 & 0.0300 & 0.0000 & 0.3700 & 0.0000 & 0.0025 & 0.0020 & 0.0400 & 0.0200 & & 0.0300 & & 0.0020 \\
\hline HLW99-53 & 0.0000 & 0.0500 & 0.0700 & 0.1250 & & 0.0100 & 0.0000 & 0.0124 & 0.1200 & 0.0186 & 0.0150 & 0.4100 & 0.0000 & 0.0025 & 0.0020 & 0.0248 & 0.0124 & & 0.0186 & & 0.0020 \\
\hline HLW99-54 & 0.1100 & 0.0500 & 0.0000 & 0.0200 & & 0.0100 & 0.0350 & 0.0138 & 0.1200 & 0.0206 & 0.0000 & 0.4900 & 0.0000 & 0.0025 & 0.0020 & 0.0275 & 0.0138 & & 0.0206 & & 0.0020 \\
\hline HLW99-55 & 0.0400 & 0.0500 & 0.0700 & 0.0700 & & 0.0100 & 0.0100 & 0.0094 & 0.2000 & 0.0141 & 0.0000 & 0.3200 & 0.1000 & 0.0025 & 0.0020 & 0.0188 & 0.0094 & & 0.0141 & & 0.0020 \\
\hline HLW99-56R & 0.0367 & 0.0500 & 0.0000 & 0.1250 & & 0.0100 & 0.0700 & 0.0200 & 0.0500 & 0.0300 & 0.0000 & 0.4100 & 0.0333 & 0.0025 & 0.0020 & 0.0400 & 0.0200 & & 0.0300 & & 0.0020 \\
\hline HLW99-57 & 0.0000 & 0.0500 & 0.0000 & 0.1250 & & 0.0100 & 0.0100 & 0.0000 & 0.2000 & 0.0000 & 0.0000 & 0.4700 & 0.0800 & 0.0025 & 0.0020 & 0.0000 & 0.0000 & & 0.0000 & & 0.0020 \\
\hline HLW99-58 & 0.1100 & 0.2000 & 0.0700 & 0.0200 & & 0.0100 & 0.0000 & 0.0044 & 0.2000 & 0.0066 & 0.0000 & 0.3000 & 0.0000 & 0.0025 & 0.0020 & 0.0088 & 0.0044 & & 0.0066 & & 0.0020 \\
\hline HLW99-59 & 0.1100 & 0.0500 & 0.0650 & 0.0200 & & 0.0100 & 0.0700 & 0.0200 & 0.0500 & 0.0300 & 0.0000 & 0.4100 & 0.0000 & 0.0025 & 0.0020 & 0.0400 & 0.0200 & & 0.0300 & & 0.0020 \\
\hline
\end{tabular}


Appendix A. Database - mass fraction

RPP-WTP HLW Formulation (Kot and Pegg 2001)

\begin{tabular}{|c|c|c|c|c|c|c|c|c|c|c|c|c|c|c|c|c|c|c|c|c|c|}
\hline Glass ID & $\begin{array}{l}\mathrm{Cl} \\
-\mathrm{t}\end{array}$ & $\begin{array}{c}\mathrm{CoO} \\
-\mathrm{t}\end{array}$ & $\begin{array}{c}\mathrm{Co} 2 \mathrm{O} 3 \\
-\mathrm{t}\end{array}$ & $\begin{array}{c}\mathrm{Cr} 2 \mathrm{O} 3 \\
-\mathrm{t}\end{array}$ & $\begin{array}{c}\mathrm{Cs} 2 \mathrm{O} \\
-\mathrm{t}\end{array}$ & $\begin{array}{c}\mathrm{CuO} \\
-\mathrm{t}\end{array}$ & $\begin{array}{c}\mathrm{Eu} 2 \mathrm{O} 3 \\
-\mathrm{t}\end{array}$ & $\begin{array}{l}F \\
-t\end{array}$ & $\begin{array}{c}\mathrm{Ga} 2 \mathrm{O} 3 \\
-\mathrm{t}\end{array}$ & $\begin{array}{c}\mathrm{Gd} 2 \mathrm{O} 3 \\
-\mathrm{t}\end{array}$ & $\begin{array}{c}\mathrm{HgO} \\
-\mathrm{t}\end{array}$ & $\begin{array}{l}\mathrm{I} \\
-\mathrm{t}\end{array}$ & $\begin{array}{c}\mathrm{La} 2 \mathrm{O} 3 \\
-\mathrm{t}\end{array}$ & $\begin{array}{c}\mathrm{MnO} 2 \\
-\mathrm{t}\end{array}$ & $\begin{array}{c}\mathrm{MnO} \\
-\mathrm{t}\end{array}$ & $\begin{array}{c}\mathrm{MoO} \\
-\mathrm{t}\end{array}$ & $\begin{array}{c}\mathrm{MoO} 3 \\
-\mathrm{t}\end{array}$ & $\begin{array}{c}\mathrm{Nb} 2 \mathrm{O} 5 \\
-\mathrm{t}\end{array}$ & $\begin{array}{c}\mathrm{Nd} 2 \mathrm{O} 3 \\
-\mathrm{t}\end{array}$ & $\begin{array}{c}\mathrm{PbO} \\
-\mathrm{t}\end{array}$ & $\begin{array}{c}\mathrm{PdO} 2 \\
-\mathrm{t}\end{array}$ \\
\hline HLW99-33 & 0.0020 & 0.0020 & & 0.0050 & 0.0020 & 0.0020 & & 0.0020 & & & & & 0.0020 & 0.0020 & & & & & & 0.0000 & \\
\hline HLW99-34 & 0.0020 & 0.0020 & & 0.0050 & 0.0020 & 0.0020 & & 0.0020 & & & & & 0.0020 & 0.0020 & & & & & & 0.0000 & \\
\hline HLW99-35 & 0.0020 & 0.0020 & & 0.0000 & 0.0020 & 0.0020 & & 0.0020 & & & & & 0.0020 & 0.0020 & & & & & & 0.0000 & \\
\hline HLW99-36 & 0.0020 & 0.0020 & & 0.0000 & 0.0020 & 0.0020 & & 0.0020 & & & & & 0.0020 & 0.0020 & & & & & & 0.0008 & \\
\hline HLW99-37 & 0.0020 & 0.0020 & & 0.0000 & 0.0020 & 0.0020 & & 0.0020 & & & & & 0.0020 & 0.0020 & & & & & & 0.0047 & \\
\hline HLW99-38 & 0.0020 & 0.0020 & & 0.0050 & 0.0020 & 0.0020 & & 0.0020 & & & & & 0.0020 & 0.0020 & & & & & & 0.0069 & \\
\hline HLW99-39 & 0.0020 & 0.0020 & & 0.0000 & 0.0020 & 0.0020 & & 0.0020 & & & & & 0.0020 & 0.0020 & & & & & & 0.0006 & \\
\hline HLW99-40 & 0.0020 & 0.0020 & & 0.0000 & 0.0020 & 0.0020 & & 0.0020 & & & & & 0.0020 & 0.0020 & & & & & & 0.0000 & \\
\hline HLW99-41 & 0.0020 & 0.0020 & & 0.0021 & 0.0020 & 0.0020 & & 0.0020 & & & & & 0.0020 & 0.0020 & & & & & & 0.0031 & \\
\hline HLW99-42 & 0.0020 & 0.0020 & & 0.0000 & 0.0020 & 0.0020 & & 0.0020 & & & & & 0.0020 & 0.0020 & & & & & & 0.0000 & \\
\hline HLW99-43 & 0.0020 & 0.0020 & & 0.0000 & 0.0020 & 0.0020 & & 0.0020 & & & & & 0.0020 & 0.0020 & & & & & & 0.0000 & \\
\hline HLW99-44 & 0.0020 & 0.0020 & & 0.0031 & 0.0020 & 0.0020 & & 0.0020 & & & & & 0.0020 & 0.0020 & & & & & & 0.0000 & \\
\hline HLW99-45 & 0.0020 & 0.0020 & & 0.0050 & 0.0020 & 0.0020 & & 0.0020 & & & & & 0.0020 & 0.0020 & & & & & & 0.0002 & \\
\hline HLW99-46 & 0.0020 & 0.0020 & & 0.0000 & 0.0020 & 0.0020 & & 0.0020 & & & & & 0.0020 & 0.0020 & & & & & & 0.0100 & \\
\hline HLW99-47 & 0.0020 & 0.0020 & & 0.0000 & 0.0020 & 0.0020 & & 0.0020 & & & & & 0.0020 & 0.0020 & & & & & & 0.0000 & \\
\hline HLW99-48 & 0.0020 & 0.0020 & & 0.0000 & 0.0020 & 0.0020 & & 0.0020 & & & & & 0.0020 & 0.0020 & & & & & & 0.0100 & \\
\hline HLW99-49 & 0.0020 & 0.0020 & & 0.0050 & 0.0020 & 0.0020 & & 0.0020 & & & & & 0.0020 & 0.0020 & & & & & & 0.0014 & \\
\hline HLW99-50 & 0.0020 & 0.0020 & & 0.0042 & 0.0020 & 0.0020 & & 0.0020 & & & & & 0.0020 & 0.0020 & & & & & & 0.0000 & \\
\hline HLW99-51R & 0.0020 & 0.0020 & & 0.0050 & 0.0020 & 0.0020 & & 0.0020 & & & & & 0.0020 & 0.0020 & & & & & & 0.0000 & \\
\hline HLW99-52 & 0.0020 & 0.0020 & & 0.0000 & 0.0020 & 0.0020 & & 0.0020 & & & & & 0.0020 & 0.0020 & & & & & & 0.0100 & \\
\hline HLW99-53 & 0.0020 & 0.0020 & & 0.0025 & 0.0020 & 0.0020 & & 0.0020 & & & & & 0.0020 & 0.0020 & & & & & & 0.0062 & \\
\hline HLW99-54 & 0.0020 & 0.0020 & & 0.0000 & 0.0020 & 0.0020 & & 0.0020 & & & & & 0.0020 & 0.0020 & & & & & & 0.0069 & \\
\hline HLW99-55 & 0.0020 & 0.0020 & & 0.0000 & 0.0020 & 0.0020 & & 0.0020 & & & & & 0.0020 & 0.0020 & & & & & & 0.0047 & \\
\hline HLW99-56R & 0.0020 & 0.0020 & & 0.0000 & 0.0020 & 0.0020 & & 0.0020 & & & & & 0.0020 & 0.0020 & & & & & & 0.0100 & \\
\hline HLW99-57 & 0.0020 & 0.0020 & & 0.0000 & 0.0020 & 0.0020 & & 0.0020 & & & & & 0.0020 & 0.0020 & & & & & & 0.0000 & \\
\hline HLW99-58 & 0.0020 & 0.0020 & & 0.0000 & 0.0020 & 0.0020 & & 0.0020 & & & & & 0.0020 & 0.0020 & & & & & & 0.0022 & \\
\hline HLW99-59 & 0.0020 & 0.0020 & & 0.0000 & 0.0020 & 0.0020 & & 0.0020 & & & & & 0.0020 & 0.0020 & & & & & & 0.0100 & \\
\hline
\end{tabular}


Appendix A. Database - mass fraction

RPP-WTP HLW Formulation (Kot and Pegg 2001)

\begin{tabular}{|c|c|c|c|c|c|c|c|c|c|c|c|c|c|c|c|c|c|c|c|c|c|}
\hline Glass ID & $\begin{array}{c}\mathrm{PdO} \\
-\mathrm{t}\end{array}$ & $\begin{array}{c}\mathrm{Pr} 2 \mathrm{O} 3 \\
-\mathrm{t}\end{array}$ & $\begin{array}{c}\text { Pr6O11 } \\
-t\end{array}$ & $\begin{array}{c}\mathrm{Rb} 2 \mathrm{O} \\
-\mathrm{t}\end{array}$ & $\begin{array}{c}\mathrm{ReO} \\
-\mathrm{t}\end{array}$ & $\begin{array}{c}\mathrm{ReO} 2 \\
-\mathrm{t}\end{array}$ & $\begin{array}{c}\mathrm{Rh} 2 \mathrm{O} 3 \\
-\mathrm{t}\end{array}$ & $\begin{array}{c}\mathrm{RhO}^{2} \\
-\mathrm{t}\end{array}$ & $\begin{array}{c}\mathrm{RuO} 2 \\
-\mathrm{t}\end{array}$ & $\begin{array}{c}\mathrm{Sb} 2 \mathrm{O} 3 \\
-\mathrm{t}\end{array}$ & $\begin{array}{c}\mathrm{Sb} 2 \mathrm{O} 5 \\
-\mathrm{t}\end{array}$ & $\begin{array}{c}\mathrm{SeO} 2 \\
-\mathrm{t}\end{array}$ & $\begin{array}{c}\mathrm{Sm} 2 \mathrm{O} 3 \\
-\mathrm{t} \\
\end{array}$ & $\begin{array}{c}\mathrm{SnO} \\
-\mathrm{t}\end{array}$ & $\begin{array}{c}\mathrm{SnO} 2 \\
-\mathrm{t}\end{array}$ & $\begin{array}{c}\mathrm{SO} 3 \\
-t\end{array}$ & $\begin{array}{c}\mathrm{SrO} \\
-\mathrm{t}\end{array}$ & $\begin{array}{c}\mathrm{Tc} 2 \mathrm{O} 7 \\
-\mathrm{t}\end{array}$ & $\begin{array}{c}\mathrm{TeO} 2 \\
-\mathrm{t}\end{array}$ & $\begin{array}{c}\mathrm{ThO} 2 \\
-\mathrm{t}\end{array}$ & $\begin{array}{c}\mathrm{TiO} 2 \\
-\mathrm{t}\end{array}$ \\
\hline HLW99-33 & 0.0004 & & & & & & 0.0008 & & 0.0013 & & 0.0020 & 0.0020 & & & & 0.0050 & 0.0020 & & 0.0020 & 0.0020 & 0.0000 \\
\hline HLW99-34 & 0.0004 & & & & & & 0.0008 & & 0.0013 & & 0.0020 & 0.0020 & & & & 0.0050 & 0.0020 & & 0.0020 & 0.0020 & 0.0000 \\
\hline HLW99-35 & 0.0004 & & & & & & 0.0008 & & 0.0013 & & 0.0020 & 0.0020 & & & & 0.0000 & 0.0020 & & 0.0020 & 0.0000 & 0.0000 \\
\hline HLW99-36 & 0.0004 & & & & & & 0.0008 & & 0.0013 & & 0.0020 & 0.0020 & & & & 0.0000 & 0.0020 & & 0.0020 & 0.0000 & 0.0008 \\
\hline HLW99-37 & 0.0004 & & & & & & 0.0008 & & 0.0013 & & 0.0020 & 0.0020 & & & & 0.0000 & 0.0020 & & 0.0020 & 0.0000 & 0.0047 \\
\hline HLW99-38 & 0.0004 & & & & & & 0.0008 & & 0.0013 & & 0.0020 & 0.0020 & & & & 0.0050 & 0.0020 & & 0.0020 & 0.0020 & 0.0069 \\
\hline HLW99-39 & 0.0004 & & & & & & 0.0008 & & 0.0013 & & 0.0020 & 0.0020 & & & & 0.0000 & 0.0020 & & 0.0020 & 0.0000 & 0.0006 \\
\hline HLW99-40 & 0.0004 & & & & & & 0.0008 & & 0.0013 & & 0.0020 & 0.0020 & & & & 0.0000 & 0.0020 & & 0.0020 & 0.0000 & 0.0000 \\
\hline HLW99-41 & 0.0004 & & & & & & 0.0008 & & 0.0013 & & 0.0020 & 0.0020 & & & & 0.0021 & 0.0020 & & 0.0020 & 0.0008 & 0.0031 \\
\hline HLW99-42 & 0.0004 & & & & & & 0.0008 & & 0.0013 & & 0.0020 & 0.0020 & & & & 0.0000 & 0.0020 & & 0.0020 & 0.0000 & 0.0000 \\
\hline HLW99-43 & 0.0004 & & & & & & 0.0008 & & 0.0013 & & 0.0020 & 0.0020 & & & & 0.0000 & 0.0020 & & 0.0020 & 0.0000 & 0.0000 \\
\hline HLW99-44 & 0.0004 & & & & & & 0.0008 & & 0.0013 & & 0.0020 & 0.0020 & & & & 0.0031 & 0.0020 & & 0.0020 & 0.0012 & 0.0000 \\
\hline HLW99-45 & 0.0004 & & & & & & 0.0008 & & 0.0013 & & 0.0020 & 0.0020 & & & & 0.0050 & 0.0020 & & 0.0020 & 0.0020 & 0.0002 \\
\hline HLW99-46 & 0.0004 & & & & & & 0.0008 & & 0.0013 & & 0.0020 & 0.0020 & & & & 0.0000 & 0.0020 & & 0.0020 & 0.0000 & 0.0100 \\
\hline HLW99-47 & 0.0004 & & & & & & 0.0008 & & 0.0013 & & 0.0020 & 0.0020 & & & & 0.0000 & 0.0020 & & 0.0020 & 0.0000 & 0.0000 \\
\hline HLW99-48 & 0.0004 & & & & & & 0.0008 & & 0.0013 & & 0.0020 & 0.0020 & & & & 0.0000 & 0.0020 & & 0.0020 & 0.0000 & 0.0100 \\
\hline HLW99-49 & 0.0004 & & & & & & 0.0008 & & 0.0013 & & 0.0020 & 0.0020 & & & & 0.0050 & 0.0020 & & 0.0020 & 0.0020 & 0.0014 \\
\hline HLW99-50 & 0.0004 & & & & & & 0.0008 & & 0.0013 & & 0.0020 & 0.0020 & & & & 0.0042 & 0.0020 & & 0.0020 & 0.0017 & 0.0000 \\
\hline HLW99-51R & 0.0004 & & & & & & 0.0008 & & 0.0013 & & 0.0020 & 0.0020 & & & & 0.0050 & 0.0020 & & 0.0020 & 0.0020 & 0.0000 \\
\hline HLW99-52 & 0.0004 & & & & & & 0.0008 & & 0.0013 & & 0.0020 & 0.0020 & & & & 0.0000 & 0.0020 & & 0.0020 & 0.0000 & 0.0100 \\
\hline HLW99-53 & 0.0004 & & & & & & 0.0008 & & 0.0013 & & 0.0020 & 0.0020 & & & & 0.0025 & 0.0020 & & 0.0020 & 0.0010 & 0.0062 \\
\hline HLW99-54 & 0.0004 & & & & & & 0.0008 & & 0.0013 & & 0.0020 & 0.0020 & & & & 0.0000 & 0.0020 & & 0.0020 & 0.0000 & 0.0069 \\
\hline HLW99-55 & 0.0004 & & & & & & 0.0008 & & 0.0013 & & 0.0020 & 0.0020 & & & & 0.0000 & 0.0020 & & 0.0020 & 0.0000 & 0.0047 \\
\hline HLW99-56R & 0.0004 & & & & & & 0.0008 & & 0.0013 & & 0.0020 & 0.0020 & & & & 0.0000 & 0.0020 & & 0.0020 & 0.0000 & 0.0100 \\
\hline HLW99-57 & 0.0004 & & & & & & 0.0008 & & 0.0013 & & 0.0020 & 0.0020 & & & & 0.0000 & 0.0020 & & 0.0020 & 0.0000 & 0.0000 \\
\hline HLW99-58 & 0.0004 & & & & & & 0.0008 & & 0.0013 & & 0.0020 & 0.0020 & & & & 0.0000 & 0.0020 & & 0.0020 & 0.0000 & 0.0022 \\
\hline HLW99-59 & 0.0004 & & & & & & 0.0008 & & 0.0013 & & 0.0020 & 0.0020 & & & & 0.0000 & 0.0020 & & 0.0020 & 0.0000 & 0.0100 \\
\hline
\end{tabular}


Appendix A. Database - mass fraction

RPP-WTP HLW Formulation (Kot and Pegg 2001)

\begin{tabular}{|c|c|c|c|c|c|c|c|c|c|c|c|c|c|c|c|c|c|c|c|c|c|}
\hline Glass ID & $\begin{array}{c}\mathrm{T} 12 \mathrm{O} 3 \\
-\mathrm{t}\end{array}$ & $\begin{array}{c}\mathrm{U} 308 \\
-\mathrm{t}\end{array}$ & $\begin{array}{c}\mathrm{UO} 2 \\
-\mathrm{t}\end{array}$ & $\begin{array}{c}\text { UO3 } \\
-t\end{array}$ & $\begin{array}{c}\mathrm{V} 2 \mathrm{O} 5 \\
-\mathrm{t}\end{array}$ & $\begin{array}{c}\text { WO3 } \\
-t\end{array}$ & $\begin{array}{c}\mathrm{Y} 2 \mathrm{O} 3 \\
-\mathrm{t}\end{array}$ & $\begin{array}{c}\mathrm{ZnO} \\
-\mathrm{t}\end{array}$ & $\begin{array}{c}\text { Others } \\
-t\end{array}$ & $\begin{array}{c}\text { Sum } \\
-t\end{array}$ & $\begin{array}{c}\mathrm{A} 12 \mathrm{O} 3 \\
-\mathrm{a}\end{array}$ & $\begin{array}{c}\mathrm{B} 2 \mathrm{O} 3 \\
-\mathrm{a}\end{array}$ & $\begin{array}{c}\mathrm{CaO} \\
-\mathrm{a} \\
\end{array}$ & $\begin{array}{c}\mathrm{Fe} 2 \mathrm{O} 3 \\
-\mathrm{a}\end{array}$ & $\begin{array}{c}\mathrm{FeO} \\
-\mathrm{a} \\
\end{array}$ & $\begin{array}{c}\mathrm{K} 2 \mathrm{O} \\
-\mathrm{a}\end{array}$ & $\begin{array}{c}\mathrm{Li} 2 \mathrm{O} \\
-\mathrm{a}\end{array}$ & $\begin{array}{c}\mathrm{MgO} \\
-\mathrm{a}\end{array}$ & $\begin{array}{c}\mathrm{Na} 2 \mathrm{O} \\
-\mathrm{a}\end{array}$ & $\begin{array}{c}\mathrm{NiO} \\
-\mathrm{a} \\
\end{array}$ & $\begin{array}{c}\mathrm{P} 2 \mathrm{O} 5 \\
-\mathrm{a}\end{array}$ \\
\hline HLW99-33 & 0.0020 & & 0.0800 & & & 0.0020 & & 0.0200 & & 1.0000 & 0.0118 & 0.1903 & 0.0631 & 0.0259 & & 0.0117 & 0.0645 & 0.0005 & 0.0761 & 0.0007 & 0.0325 \\
\hline HLW99-34 & 0.0020 & & 0.0800 & & & 0.0020 & & 0.0200 & & 1.0000 & 0.0060 & 0.0622 & 0.0038 & 0.0297 & & 0.0113 & 0.0652 & 0.0003 & 0.0763 & 0.0004 & 0.0368 \\
\hline HLW99-35 & 0.0020 & & 0.0000 & & & 0.0020 & & 0.0200 & & 1.0001 & 0.0895 & 0.0500 & 0.0387 & 0.0686 & & 0.0102 & 0.0500 & 0.0004 & 0.0699 & 0.0004 & 0.0013 \\
\hline HLW99-36 & 0.0020 & & 0.0000 & & & 0.0020 & & 0.0200 & & 0.9999 & 0.0140 & 0.1927 & 0.0137 & 0.1170 & & 0.0101 & 0.0646 & 0.0020 & 0.0759 & 0.0023 & 0.0009 \\
\hline HLW99-37 & 0.0020 & & 0.0000 & & & 0.0020 & & 0.0200 & & 1.0002 & 0.1076 & 0.0707 & 0.0009 & 0.0198 & & 0.0101 & 0.0488 & 0.0096 & 0.1158 & 0.0134 & 0.0017 \\
\hline HLW99-38 & 0.0020 & & 0.0800 & & & 0.0020 & & 0.0200 & & 1.0001 & 0.0086 & 0.0513 & 0.0651 & 0.0207 & & 0.0115 & 0.0611 & 0.0133 & 0.0463 & 0.0189 & 0.0317 \\
\hline HLW99-39 & 0.0020 & & 0.0000 & & & 0.0020 & & 0.0200 & & 1.0001 & 0.0098 & 0.1233 & 0.0680 & 0.1179 & & 0.0115 & 0.0404 & 0.0015 & 0.1268 & 0.0018 & 0.0010 \\
\hline HLW99-40 & 0.0020 & & 0.0000 & & & 0.0020 & & 0.0200 & & 1.0000 & 0.1037 & 0.1944 & 0.0003 & 0.0645 & & 0.0117 & 0.0003 & 0.0001 & 0.1123 & 0.0001 & 0.0012 \\
\hline HLW99-41 & 0.0020 & & 0.0332 & & & 0.0020 & & 0.0200 & & 1.0000 & 0.0521 & 0.1124 & 0.0292 & 0.0587 & & 0.0105 & 0.0297 & 0.0060 & 0.0986 & 0.0087 & 0.0151 \\
\hline HLW99-42 & 0.0020 & & 0.0000 & & & 0.0020 & & 0.0200 & & 1.0000 & 0.1060 & 0.1631 & 0.0426 & 0.0195 & & 0.0109 & 0.0328 & 0.0003 & 0.0461 & 0.0001 & 0.0013 \\
\hline HLW99-43 & 0.0020 & & 0.0000 & & & 0.0020 & & 0.0200 & & 1.0000 & 0.0913 & 0.1901 & 0.0666 & 0.1153 & & 0.0112 & 0.0617 & 0.0003 & 0.0789 & 0.0001 & 0.0010 \\
\hline HLW99-44 & 0.0020 & & 0.0492 & & & 0.0020 & & 0.0200 & & 1.0000 & 0.0835 & 0.0693 & 0.0658 & 0.1169 & & 0.0114 & 0.0121 & 0.0004 & 0.1792 & 0.0003 & 0.0202 \\
\hline HLW99-45 & 0.0020 & & 0.0800 & & & 0.0020 & & 0.0200 & & 1.0002 & 0.1003 & 0.1584 & 0.0664 & 0.0208 & & 0.0103 & 0.0666 & 0.0007 & 0.0490 & 0.0010 & 0.0312 \\
\hline HLW99-46 & 0.0020 & & 0.0000 & & & 0.0020 & & 0.0200 & & 0.9999 & 0.0152 & 0.1132 & 0.0657 & 0.0206 & & 0.0111 & 0.0639 & 0.0188 & 0.0724 & 0.0284 & 0.0010 \\
\hline HLW99-47 & 0.0020 & & 0.0000 & & & 0.0020 & & 0.0200 & & 1.0000 & 0.0481 & 0.0466 & 0.0338 & 0.1127 & & 0.0111 & 0.0272 & 0.0003 & 0.0834 & 0.0003 & 0.0008 \\
\hline HLW99-48 & 0.0020 & & 0.0000 & & & 0.0020 & & 0.0200 & & 1.0000 & 0.1050 & 0.0624 & 0.0008 & 0.0808 & & 0.0118 & 0.0007 & 0.0187 & 0.1799 & 0.0281 & 0.0012 \\
\hline HLW99-49 & 0.0020 & & 0.0800 & & & 0.0020 & & 0.0200 & & 1.0000 & 0.0404 & 0.0628 & 0.0002 & 0.1126 & & 0.0110 & & 0.0029 & 0.1765 & 0.0044 & 0.0313 \\
\hline HLW99-50 & 0.0020 & & 0.0667 & & & 0.0020 & & 0.0200 & & 1.0001 & 0.0486 & 0.0603 & 0.0007 & 0.0208 & & 0.0116 & 0.0607 & 0.0002 & 0.0484 & 0.0005 & 0.0280 \\
\hline HLW99-51R & 0.0020 & & 0.0800 & & & 0.0020 & & 0.0200 & & 1.0000 & 0.0098 & 0.1876 & 0.0644 & 0.0205 & & 0.0118 & 0.0009 & 0.0004 & 0.1626 & 0.0004 & 0.0329 \\
\hline HLW99-52 & 0.0020 & & 0.0000 & & & 0.0020 & & 0.0200 & & 1.0000 & 0.0301 & 0.1852 & 0.0008 & 0.0193 & & 0.0105 & 0.0005 & 0.0182 & 0.1471 & 0.0276 & 0.0012 \\
\hline HLW99-53 & 0.0020 & & 0.0400 & & & 0.0020 & & 0.0200 & & 1.0002 & 0.0077 & 0.0526 & 0.0598 & 0.1037 & & 0.0093 & 0.0045 & 0.0112 & 0.0973 & 0.0157 & 0.0161 \\
\hline HLW99-54 & 0.0020 & & 0.0000 & & & 0.0020 & & 0.0200 & & 1.0001 & 0.1043 & 0.0491 & 0.0010 & 0.0187 & & 0.0106 & 0.0330 & 0.0133 & 0.1100 & 0.0195 & 0.0004 \\
\hline HLW99-55 & 0.0020 & & 0.0000 & & & 0.0020 & & 0.0200 & & 1.0002 & 0.0443 & 0.0523 & 0.0666 & 0.0675 & & 0.0117 & 0.0129 & 0.0095 & 0.1612 & 0.0129 & 0.0009 \\
\hline HLW99-56R & 0.0020 & & 0.0000 & & & 0.0020 & & 0.0200 & & 1.0000 & 0.0415 & 0.0531 & 0.0012 & 0.1153 & & 0.0105 & 0.0638 & 0.0183 & 0.0470 & 0.0275 & 0.0015 \\
\hline HLW99-57 & 0.0020 & & 0.0000 & & & 0.0020 & & 0.0200 & & 1.0000 & 0.0060 & 0.0516 & 0.0006 & 0.1184 & & 0.0118 & 0.0138 & 0.0007 & 0.1743 & 0.0009 & 0.0017 \\
\hline HLW99-58 & 0.0020 & & 0.0000 & & & 0.0020 & & 0.0200 & & 1.0002 & 0.1043 & 0.1881 & 0.0654 & 0.0230 & & 0.0114 & 0.0011 & 0.0044 & 0.1708 & 0.0062 & 0.0011 \\
\hline HLW99-59 & 0.0020 & & 0.0000 & & & 0.0020 & & 0.0200 & & 1.0000 & 0.1073 & 0.0533 & 0.0617 & 0.0209 & & 0.0112 & 0.0623 & 0.0190 & 0.0456 & 0.0284 & 0.0020 \\
\hline
\end{tabular}


Appendix A. Database - mass fraction

RPP-WTP HLW Formulation (Kot and Pegg 2001)

\begin{tabular}{|c|c|c|c|c|c|c|c|c|c|c|c|c|c|c|c|c|c|c|c|c|c|}
\hline Glass ID & $\begin{array}{c}\mathrm{SiO} 2 \\
-\mathrm{a}\end{array}$ & $\begin{array}{c}\mathrm{ZrO} 2 \\
-\mathrm{a}\end{array}$ & $\begin{array}{c}\mathrm{Ag} 2 \mathrm{O} \\
-\mathrm{a}\end{array}$ & $\begin{array}{c}\mathrm{As} 2 \mathrm{O} 3 \\
-\mathrm{a} \\
\end{array}$ & $\begin{array}{c}\mathrm{BaO} \\
-\mathrm{a} \\
\end{array}$ & $\begin{array}{c}\mathrm{Bi} 2 \mathrm{O} 3 \\
-\mathrm{a} \\
\end{array}$ & $\begin{array}{l}\mathrm{Br} \\
-\mathrm{a}\end{array}$ & $\begin{array}{c}\mathrm{CdO} \\
-\mathrm{a} \\
\end{array}$ & $\begin{array}{c}\mathrm{Ce} 2 \mathrm{O} 3 \\
-\mathrm{a}\end{array}$ & $\begin{array}{c}\mathrm{CeO} 2 \\
-\mathrm{a}\end{array}$ & $\begin{array}{l}\mathrm{Cl} \\
-\mathrm{a}\end{array}$ & $\begin{array}{c}\mathrm{CoO} \\
-\mathrm{a} \\
\end{array}$ & $\begin{array}{c}\mathrm{Co} 2 \mathrm{O} 3 \\
-\mathrm{a} \\
\end{array}$ & $\begin{array}{c}\mathrm{Cr} 2 \mathrm{O} 3 \\
-\mathrm{a}\end{array}$ & $\begin{array}{c}\mathrm{Cs} 2 \mathrm{O} \\
-\mathrm{a} \\
\end{array}$ & $\begin{array}{c}\mathrm{CuO} \\
-\mathrm{a} \\
\end{array}$ & $\begin{array}{c}\mathrm{Eu} 2 \mathrm{O} 3 \\
-\mathrm{a}\end{array}$ & $\begin{array}{c}\mathrm{F} \\
-\mathrm{a} \\
\end{array}$ & $\begin{array}{c}\mathrm{Ga} 2 \mathrm{O} 3 \\
-\mathrm{a} \\
\end{array}$ & $\begin{array}{c}\mathrm{Gd} 2 \mathrm{O} 3 \\
-\mathrm{a} \\
\end{array}$ & $\begin{array}{c}\mathrm{HgO} \\
-\mathrm{a} \\
\end{array}$ \\
\hline HLW99-33 & 0.3727 & 0.0048 & 0.0024 & 0.0022 & 0.0008 & 0.0006 & & 0.0005 & & & & 0.0028 & & 0.0045 & & 0.0022 & & & & & \\
\hline HLW99-34 & 0.4976 & 0.0432 & 0.0026 & 0.0022 & 0.0003 & 0.0004 & & 0.0002 & & & & 0.0028 & & 0.0044 & & 0.0023 & & & & & \\
\hline HLW99-35 & 0.5114 & 0.0462 & 0.0024 & 0.0020 & 0.0005 & 0.0003 & & 0.0002 & & & & 0.0023 & & 0.0001 & & 0.0021 & & & & & \\
\hline HLW99-36 & 0.3884 & 0.0439 & 0.0024 & 0.0020 & 0.0036 & 0.0016 & & 0.0023 & & & & 0.0021 & & 0.0001 & & 0.0019 & & & & & \\
\hline HLW99-37 & 0.4988 & 0.0004 & 0.0022 & 0.0018 & 0.0191 & 0.0093 & & 0.0137 & & & & 0.0022 & & 0.0001 & & 0.0021 & & & & & \\
\hline HLW99-38 & 0.4222 & 0.0276 & 0.0025 & & 0.0258 & & & 0.0180 & & & & 0.0033 & & 0.0047 & & & & & & & \\
\hline HLW99-39 & 0.4181 & 0.0005 & 0.0019 & 0.0021 & 0.0028 & 0.0013 & & 0.0018 & & & & 0.0020 & & 0.0001 & & 0.0020 & & & & & \\
\hline HLW99-40 & 0.4087 & 0.0228 & 0.0023 & 0.0018 & 0.0001 & 0.0002 & & 0.0000 & & & & 0.0021 & & 0.0000 & & 0.0020 & & & & & \\
\hline HLW99-41 & 0.3904 & 0.0301 & 0.0024 & 0.0016 & 0.0120 & 0.0065 & & 0.0089 & & & & 0.0022 & & 0.0018 & & 0.0021 & & & & & \\
\hline HLW99-42 & 0.4549 & 0.0230 & 0.0025 & 0.0020 & 0.0001 & 0.0003 & & 0.0000 & & & & 0.0022 & & 0.0000 & & 0.0021 & & & & & \\
\hline HLW99-43 & 0.3117 & 0.0002 & 0.0023 & 0.0022 & 0.0002 & 0.0006 & & 0.0000 & & & & 0.0022 & & 0.0001 & & 0.0023 & & & & & \\
\hline HLW99-44 & 0.3103 & 0.0007 & 0.0018 & 0.0023 & 0.0001 & 0.0003 & & 0.0001 & & & & 0.0026 & & 0.0029 & & 0.0022 & & & & & \\
\hline HLW99-45 & 0.3252 & 0.0275 & 0.0022 & 0.0018 & 0.0010 & 0.0008 & & 0.0006 & & & & 0.0031 & & 0.0043 & & 0.0023 & & & & & \\
\hline HLW99-46 & 0.3672 & 0.0438 & 0.0023 & 0.0020 & 0.0385 & 0.0216 & & 0.0301 & & & & 0.0022 & & 0.0001 & & 0.0020 & & & & & \\
\hline HLW99-47 & 0.5352 & 0.0005 & 0.0022 & 0.0021 & 0.0004 & 0.0010 & & 0.0002 & & & & 0.0021 & & 0.0000 & & 0.0018 & & & & & \\
\hline HLW99-48 & 0.3166 & 0.0175 & 0.0017 & 0.0020 & 0.0392 & 0.0204 & & 0.0290 & & & & 0.0023 & & 0.0000 & & 0.0021 & & & & & \\
\hline HLW99-49 & 0.3077 & 0.0394 & 0.0019 & 0.0013 & 0.0058 & 0.0031 & & 0.0041 & & & & 0.0026 & & 0.0047 & & 0.0022 & & & & & \\
\hline HLW99-50 & 0.5334 & 0.0262 & 0.0025 & & 0.0001 & & & 0.0001 & & & & 0.0030 & & 0.0040 & & & & & & & \\
\hline HLW99-51R & 0.2896 & 0.0209 & 0.0026 & 0.0019 & 0.0004 & 0.0006 & & 0.0003 & & & & 0.0025 & & 0.0044 & & 0.0031 & & & & & \\
\hline HLW99-52 & 0.3728 & 0.0001 & 0.0024 & 0.0018 & 0.0393 & 0.0195 & & 0.0282 & & & & 0.0019 & & 0.0000 & & 0.0022 & & & & & \\
\hline HLW99-53 & 0.3849 & 0.0037 & 0.0024 & 0.0020 & 0.0194 & 0.0110 & & 0.0165 & & & & 0.0019 & & 0.0020 & & 0.0021 & & & & & \\
\hline HLW99-54 & 0.4768 & 0.0003 & 0.0027 & 0.0015 & 0.0269 & 0.0133 & & 0.0194 & & & & 0.0020 & & 0.0000 & & 0.0023 & & & & & \\
\hline HLW99-55 & 0.3245 & 0.0808 & 0.0020 & & 0.0186 & & & 0.0126 & & & & 0.0024 & & 0.0002 & & & & & & & \\
\hline HLW99-56R & 0.4093 & 0.0292 & 0.0024 & 0.0020 & 0.0362 & 0.0192 & & 0.0268 & & & & 0.0020 & & 0.0001 & & 0.0021 & & & & & \\
\hline HLW99-57 & 0.4646 & 0.0654 & 0.0027 & 0.0020 & 0.0014 & 0.0006 & & 0.0008 & & & & 0.0020 & & 0.0001 & & 0.0022 & & & & & \\
\hline HLW99-58 & 0.3063 & 0.0019 & 0.0023 & 0.0020 & 0.0098 & 0.0044 & & 0.0061 & & & & 0.0021 & & 0.0001 & & 0.0022 & & & & & \\
\hline HLW99-59 & 0.4121 & 0.0003 & 0.0024 & 0.0021 & 0.0381 & 0.0199 & & 0.0279 & & & & 0.0022 & & 0.0001 & & 0.0022 & & & & & \\
\hline
\end{tabular}


Appendix A. Database - mass fraction

RPP-WTP HLW Formulation (Kot and Pegg 2001)

\begin{tabular}{|c|c|c|c|c|c|c|c|c|c|c|c|c|c|c|c|c|c|c|c|c|c|}
\hline Glass ID & $\begin{array}{l}\mathrm{I} \\
-\mathrm{a}\end{array}$ & $\begin{array}{c}\mathrm{La} 2 \mathrm{O} 3 \\
-\mathrm{a}\end{array}$ & $\begin{array}{c}\mathrm{MnO} 2 \\
-\mathrm{a}\end{array}$ & $\begin{array}{c}\mathrm{MnO} \\
-\mathrm{a}\end{array}$ & $\begin{array}{c}\mathrm{MoO} \\
-\mathrm{a} \\
\end{array}$ & $\begin{array}{c}\mathrm{MoO} 3 \\
-\mathrm{a} \\
\end{array}$ & $\begin{array}{c}\mathrm{Nb} 2 \mathrm{O} 5 \\
-\mathrm{a}\end{array}$ & $\begin{array}{c}\mathrm{Nd} 2 \mathrm{O} 3 \\
-\mathrm{a} \\
\end{array}$ & $\begin{array}{c}\mathrm{PbO} \\
-\mathrm{a}\end{array}$ & $\begin{array}{c}\mathrm{PdO} 2 \\
-\mathrm{a} \\
\end{array}$ & $\begin{array}{c}\mathrm{PdO} \\
-\mathrm{a} \\
\end{array}$ & $\begin{array}{c}\mathrm{Pr} 2 \mathrm{O} 3 \\
-\mathrm{a} \\
\end{array}$ & $\begin{array}{c}\text { Pr6O11 } \\
-\mathrm{a}\end{array}$ & $\begin{array}{c}\mathrm{Rb} 2 \mathrm{O} \\
-\mathrm{a} \\
\end{array}$ & $\begin{array}{c}\mathrm{ReO} \\
-\mathrm{a}\end{array}$ & $\begin{array}{c}\mathrm{ReO} 2 \\
-\mathrm{a} \\
\end{array}$ & $\begin{array}{c}\mathrm{Rh} 2 \mathrm{O} 3 \\
-\mathrm{a} \\
\end{array}$ & $\begin{array}{c}\mathrm{RhO}_{2} \\
-\mathrm{a}\end{array}$ & $\begin{array}{c}\mathrm{RuO} 2 \\
-\mathrm{a}\end{array}$ & $\begin{array}{c}\mathrm{Sb} 2 \mathrm{O} 3 \\
-\mathrm{a}\end{array}$ & $\begin{array}{c}\mathrm{Sb} 2 \mathrm{O} 5 \\
-\mathrm{a}\end{array}$ \\
\hline HLW99-33 & & & 0.0023 & & & & & & 0.0009 & & & & & & & & & & & & 0.0023 \\
\hline HLW99-34 & & & 0.0023 & & & & & & 0.0008 & & & & & & & & & & & & 0.0023 \\
\hline HLW99-35 & & & 0.0023 & & & & & & 0.0002 & & & & & & & & & & & & 0.0020 \\
\hline HLW99-36 & & & 0.0022 & & & & & & 0.0005 & & & & & & & & & & & & 0.0019 \\
\hline HLW99-37 & & & 0.0023 & & & & & & 0.0017 & & & & & & & & & & & & 0.0019 \\
\hline HLW99-38 & & & 0.0024 & & & & & & 0.0065 & & 0.0003 & & & & & & 0.0003 & & 0.0003 & & 0.0004 \\
\hline HLW99-39 & & & 0.0023 & & & & & & 0.0003 & & & & & & & & & & & & 0.0019 \\
\hline HLW99-40 & & & 0.0023 & & & & & & 0.0002 & & & & & & & & & & & & 0.0020 \\
\hline HLW99-41 & & & 0.0032 & & & & & & 0.0035 & & 0.0003 & & & & & & 0.0003 & & 0.0002 & & 0.0030 \\
\hline HLW99-42 & & & 0.0025 & & & & & & 0.0002 & & & & & & & & & & & & 0.0020 \\
\hline HLW99-43 & & & 0.0026 & & & & & & 0.0002 & & & & & & & & & & & & 0.0018 \\
\hline HLW99-44 & & & 0.0025 & & & & & & 0.0005 & & 0.0003 & & & & & & 0.0003 & & 0.0004 & & 0.0035 \\
\hline HLW99-45 & & & 0.0027 & & & & & & 0.0009 & & 0.0004 & & & & & & 0.0005 & & 0.0003 & & 0.0039 \\
\hline HLW99-46 & & & 0.0025 & & & & & & 0.0035 & & & & & & & & & & & & 0.0020 \\
\hline HLW99-47 & & & 0.0024 & & & & & & 0.0001 & & & & & & & & & & & & 0.0020 \\
\hline HLW99-48 & & & 0.0026 & & & & & & 0.0034 & & & & & & & & & & & & 0.0019 \\
\hline HLW99-49 & & & 0.0024 & & & & & & 0.0021 & & 0.0004 & & & & & & 0.0003 & & 0.0003 & & 0.0030 \\
\hline HLW99-50 & & & 0.0029 & & & & & & 0.0005 & & 0.0003 & & & & & & 0.0002 & & 0.0002 & & 0.0026 \\
\hline HLW99-51R & & & 0.0024 & & & & & & 0.0006 & & 0.0003 & & & & & & 0.0003 & & 0.0004 & & 0.0022 \\
\hline HLW99-52 & & & 0.0024 & & & & & & 0.0102 & & & & & & & & & & & & 0.0022 \\
\hline HLW99-53 & & & 0.0039 & & & & & & 0.0067 & & 0.0003 & & & & & & 0.0003 & & 0.0002 & & 0.0022 \\
\hline HLW99-54 & & & 0.0026 & & & & & & 0.0074 & & & & & & & & & & & & 0.0022 \\
\hline HLW99-55 & & & 0.0026 & & & & & & 0.0045 & & 0.0005 & & & & & & 0.0004 & & 0.0001 & & 0.0025 \\
\hline HLW99-56R & & & 0.0039 & & & & & & 0.0101 & & & & & & & & & & & & 0.0020 \\
\hline HLW99-57 & & & 0.0025 & & & & & & 0.0005 & & & & & & & & & & & & 0.0022 \\
\hline HLW99-58 & & & 0.0023 & & & & & & 0.0026 & & & & & & & & & & & & 0.0019 \\
\hline HLW99-59 & & & 0.0024 & & & & & & 0.0109 & & & & & & & & & & & & 0.0022 \\
\hline
\end{tabular}


Appendix A. Database - mass fraction

RPP-WTP HLW Formulation (Kot and Pegg 2001)

\begin{tabular}{|c|c|c|c|c|c|c|c|c|c|c|c|c|c|c|c|c|c|c|c|c|}
\hline Glass ID & $\begin{array}{c}\mathrm{SeO} 2 \\
-\mathrm{a} \\
\end{array}$ & $\begin{array}{c}\mathrm{Sm} 2 \mathrm{O} 3 \\
-\mathrm{a}\end{array}$ & $\begin{array}{c}\mathrm{SnO} \\
-\mathrm{a} \\
\end{array}$ & $\begin{array}{c}\mathrm{SnO} 2 \\
-\mathrm{a}\end{array}$ & $\begin{array}{c}\mathrm{SO} 3 \\
-\mathrm{a} \\
\end{array}$ & $\begin{array}{c}\mathrm{SrO} \\
-\mathrm{a} \\
\end{array}$ & $\begin{array}{c}\mathrm{Tc} 2 \mathrm{O} 7 \\
-\mathrm{a} \\
\end{array}$ & $\begin{array}{c}\mathrm{TeO} 2 \\
-\mathrm{a}\end{array}$ & $\begin{array}{c}\mathrm{ThO} 2 \\
-\mathrm{a} \\
\end{array}$ & $\begin{array}{c}\mathrm{TiO} 2 \\
-\mathrm{a} \\
\end{array}$ & $\begin{array}{c}\mathrm{T} 12 \mathrm{O} 3 \\
-\mathrm{a} \\
\end{array}$ & $\begin{array}{c}\mathrm{U} 3 \mathrm{O} 8 \\
-\mathrm{a}\end{array}$ & $\begin{array}{c}\mathrm{UO} 2 \\
-\mathrm{a}\end{array}$ & $\begin{array}{c}\text { UO3 } \\
-\mathrm{a}\end{array}$ & $\begin{array}{c}\mathrm{V} 2 \mathrm{O} 5 \\
-\mathrm{a}\end{array}$ & $\begin{array}{c}\text { WO3 } \\
-\mathrm{a}\end{array}$ & $\begin{array}{c}\mathrm{Y} 2 \mathrm{O} 3 \\
-\mathrm{a}\end{array}$ & $\begin{array}{c}\mathrm{ZnO} \\
-\mathrm{a}\end{array}$ & $\begin{array}{c}\text { Others } \\
-\mathrm{a}\end{array}$ & $\begin{array}{c}\text { Sum } \\
-\mathrm{a}\end{array}$ \\
\hline HLW99-33 & 0.0010 & & & & & 0.0022 & & 0.0020 & & 0.0007 & 0.0012 & & 0.0680 & & & 0.0028 & & 0.0199 & & 0.9739 \\
\hline HLW99-34 & 0.0011 & & & & & 0.0021 & & 0.0020 & & 0.0005 & 0.0018 & & 0.0714 & & & 0.0026 & & 0.0193 & & 0.9542 \\
\hline HLW99-35 & 0.0010 & & & & & 0.0021 & & 0.0018 & & 0.0005 & 0.0012 & & 0.0006 & & & 0.0019 & & 0.0281 & & 0.9882 \\
\hline HLW99-36 & 0.0006 & & & & & 0.0020 & & 0.0017 & & 0.0016 & 0.0009 & & 0.0005 & & & 0.0020 & & 0.0218 & & 0.9772 \\
\hline HLW99-37 & 0.0014 & & & & & 0.0022 & & 0.0021 & & 0.0049 & 0.0010 & & 0.0004 & & & 0.0020 & & 0.0229 & & 0.9909 \\
\hline HLW99-38 & 0.0014 & & & & & 0.0024 & & 0.0024 & & 0.0072 & & & 0.0668 & & & & & 0.0191 & & 0.9421 \\
\hline HLW99-39 & 0.0011 & & & & & 0.0023 & & 0.0021 & & 0.0011 & 0.0005 & & 0.0004 & & & 0.0020 & & 0.0203 & & 0.9689 \\
\hline HLW99-40 & 0.0006 & & & & & 0.0022 & & 0.0020 & & 0.0003 & 0.0015 & & 0.0002 & & & 0.0020 & & 0.0194 & & 0.9613 \\
\hline HLW99-41 & 0.0010 & & & & & 0.0030 & & 0.0026 & & 0.0037 & 0.0015 & & 0.0294 & & & 0.0024 & & 0.0191 & & 0.9522 \\
\hline HLW99-42 & 0.0006 & & & & & 0.0021 & & 0.0017 & & 0.0004 & 0.0013 & & 0.0004 & & & 0.0021 & & 0.0204 & & 0.9435 \\
\hline HLW99-43 & 0.0006 & & & & & 0.0022 & & 0.0019 & & 0.0008 & 0.0006 & & 0.0005 & & & 0.0023 & & 0.0207 & & 0.9725 \\
\hline HLW99-44 & 0.0010 & & & & & 0.0023 & & 0.0024 & & 0.0005 & 0.0009 & & 0.0422 & & & 0.0024 & & 0.0193 & & 0.9609 \\
\hline HLW99-45 & 0.0011 & & & & & 0.0025 & & 0.0021 & & 0.0007 & 0.0018 & & 0.0698 & & & 0.0027 & & 0.0199 & & 0.9828 \\
\hline HLW99-46 & 0.0006 & & & & & 0.0027 & & 0.0021 & & 0.0112 & 0.0008 & & 0.0005 & & & 0.0026 & & 0.0203 & & 0.9689 \\
\hline HLW99-47 & 0.0007 & & & & & 0.0022 & & 0.0020 & & 0.0003 & 0.0007 & & 0.0003 & & & 0.0020 & & 0.0210 & & 0.9435 \\
\hline HLW99-48 & 0.0016 & & & & & 0.0026 & & 0.0022 & & 0.0112 & 0.0012 & & 0.0002 & & & 0.0022 & & 0.0003 & & 0.9496 \\
\hline HLW99-49 & 0.0017 & & & & & 0.0021 & & 0.0024 & & 0.0018 & 0.0017 & & 0.0611 & & & 0.0028 & & 0.0188 & & 0.9158 \\
\hline HLW99-50 & 0.0012 & & & & & 0.0027 & & 0.0022 & & 0.0004 & & & 0.0576 & & & & & 0.0194 & & 0.9393 \\
\hline HLW99-51R & 0.0006 & & & & & 0.0023 & & 0.0017 & & 0.0006 & 0.0012 & & 0.0658 & & & 0.0030 & & 0.0184 & & 0.9174 \\
\hline HLW99-52 & 0.0004 & & & & & 0.0024 & & 0.0021 & & 0.0104 & 0.0008 & & 0.0002 & & & 0.0023 & & 0.0194 & & 0.9615 \\
\hline HLW99-53 & 0.0011 & & & & & 0.0034 & & 0.0025 & & 0.0057 & 0.0010 & & 0.0344 & & & 0.0026 & & 0.0189 & & 0.9070 \\
\hline HLW99-54 & 0.0012 & & & & & 0.0022 & & 0.0021 & & 0.0071 & 0.0011 & & 0.0001 & & & 0.0022 & & 0.0197 & & 0.9530 \\
\hline HLW99-55 & 0.0017 & & & & & 0.0026 & & 0.0021 & & 0.0055 & & & 0.0010 & & & & & 0.0193 & & 0.9237 \\
\hline HLW99-56R & 0.0007 & & & & & 0.0034 & & 0.0018 & & 0.0105 & 0.0013 & & 0.0003 & & & 0.0023 & & 0.0201 & & 0.9654 \\
\hline HLW99-57 & 0.0007 & & & & & 0.0024 & & 0.0019 & & 0.0007 & 0.0013 & & 0.0003 & & & 0.0023 & & 0.0207 & & 0.9571 \\
\hline HLW99-58 & 0.0005 & & & & & 0.0024 & & 0.0020 & & 0.0026 & 0.0007 & & 0.0005 & & & 0.0023 & & 0.0198 & & 0.9506 \\
\hline HLW99-59 & 0.0010 & & & & & 0.0028 & & 0.0021 & & 0.0110 & 0.0005 & & 0.0004 & & & 0.0025 & & 0.0197 & & 0.9745 \\
\hline
\end{tabular}


Appendix A. Database - mass fraction

\begin{tabular}{|c|c|c|c|c|c|c|c|c|}
\hline Glass ID & $\begin{array}{l}\mathrm{TM} \\
\left({ }^{\circ} \mathrm{C}\right) \\
\end{array}$ & $\begin{array}{l}\text { Gradient } \\
\text { TL }\left({ }^{\circ} \mathrm{C}\right)\end{array}$ & $\begin{array}{l}\text { Uniform } \\
\text { TL }\left({ }^{\circ} \mathrm{C}\right)\end{array}$ & Primary Phase & $\begin{array}{l}\text { Quenched } \\
\text { Visual/OM }\end{array}$ & $\begin{array}{c}\text { Quenched } \\
\text { SEM/EDS or TEM }\end{array}$ & $\begin{array}{l}\text { Quenched } \\
\text { XRD }\end{array}$ & $\begin{array}{c}\text { Quenched } \\
\text { Homogeneous? }\end{array}$ \\
\hline HLW99-33 & 1150 & & $<950^{\circ} \mathrm{C}$ & & $\begin{array}{l}\text { Dark green glass with cross section fragment } \\
\text { of white and green }\end{array}$ & & & \\
\hline \multicolumn{9}{|l|}{ HLW99-34 } \\
\hline HLW99-35 & 1150 & & 1025 & & $\begin{array}{l}\text { Inhomogeneous glass with green and black } \\
\text { regions. Silvery crystals and undis-solved } \\
\text { material }\end{array}$ & & & \\
\hline HLW99-36 & 1150 & & 910 & & $\begin{array}{l}\text { Mostly uniform black glass with green and } \\
\text { silver patches }\end{array}$ & & & \\
\hline HLW99-37 & 1150 & & 1000 & & $\begin{array}{l}\text { Mostly homogeneous black glass with } \\
\text { grayish cross sections }\end{array}$ & & & \\
\hline \multicolumn{9}{|l|}{ HLW99-38 } \\
\hline HLW99-39 & 1150 & & 875 & & $\begin{array}{l}\text { Brownish green glass with multicolored } \\
\text { regions. Cross section appears uniform }\end{array}$ & & & \\
\hline HLW99-40 & 1200 & & 1150 & & $\begin{array}{l}\text { Homogeneous black glass with a reddish } \\
\text { yellow tinge }\end{array}$ & & & \\
\hline \multicolumn{9}{|l|}{ HLW99-41 } \\
\hline HLW99-42 & 1150 & & 1100 & & Homogeneous black glass & & & \\
\hline HLW99-43 & 1150 & & 950 & & $\begin{array}{l}\text { Mostly homogeneous brown glass with small } \\
\text { multicolored regions }\end{array}$ & & & \\
\hline \multicolumn{9}{|l|}{ HLW99-44 } \\
\hline \multicolumn{9}{|l|}{ HLW99-45 } \\
\hline HLW99-46 & 1150 & & 925 & & $\begin{array}{l}\text { Green glass with multicolored patches on } \\
\text { surface. Uniform cross section }\end{array}$ & & & \\
\hline HLW99-47 & & & 1050 & & & & & \\
\hline HLW99-48 & & & $>1150$ & & & & & \\
\hline HLW99-49 & 1150 & & $>950^{\circ} \mathrm{C}$ & & $\begin{array}{l}\text { Dark brown glass with small amount of a } \\
\text { yellow non-glassy phase. Yellow phase } \\
\text { deposit on crucible }\end{array}$ & & & \\
\hline \multicolumn{9}{|l|}{ HLW99-50 } \\
\hline \multicolumn{9}{|l|}{ HLW99-51R } \\
\hline HLW99-52 & 1150 & & 1000 & & $\begin{array}{l}\text { Homogeneous black glass with trace amount } \\
\text { of gray precipitate }\end{array}$ & & & \\
\hline \multicolumn{9}{|l|}{ HLW99-53 } \\
\hline HLW99-54 & & & 1150 & & & & & \\
\hline HLW99-55 & 1150 & & 1100 & & $\begin{array}{l}\text { Dark brown glass with patches of metallic } \\
\text { looking precipitates on surface. Crystal- } \\
\text { lization throughout bulk }\end{array}$ & & & \\
\hline HLW99-56R & 1150 & & 1100 & & $\begin{array}{l}\text { Cloudy brown glass with traces of metallic } \\
\text { precipitate on bottom }\end{array}$ & & & \\
\hline HLW99-57 & 1150 & & 1050 & & Cloudy green glass with multicolored bottom & & & \\
\hline HLW99-58 & 1150 & & 1000 & & Dark brown glass with light brown regions & & & \\
\hline HLW99-59 & 1150 & & 1100 & & $\begin{array}{l}\text { Mostly uniform brown glass with small areas } \\
\text { of light brown swirls }\end{array}$ & & & \\
\hline
\end{tabular}


Appendix A. Database - mass fraction

RPP-WTP HLW Formulation (Kot and Pegg 2001)

\begin{tabular}{|c|c|c|c|c|c|}
\hline Glass ID & $\begin{array}{c}\mathrm{CCC} \\
\text { Visual/OM }\end{array}$ & $\begin{array}{c}\text { CCC } \\
\text { SEM/EDS or TEM }\end{array}$ & $\begin{array}{l}\text { CCC } \\
\text { XRD }\end{array}$ & $\begin{array}{l}\text { Heat Treated } \\
\text { Visual/OM }\end{array}$ & $\begin{array}{c}\text { Heat Treated } \\
\text { SEM/EDS or TEM }\end{array}$ \\
\hline HLW99-33 & & & & & Trace amount of dark crystals, likely RuO2 \\
\hline \multicolumn{6}{|l|}{ HLW99-34 } \\
\hline HLW99-35 & & & & & $\begin{array}{l}0.15 \mathrm{vol} \% \text { of zircon and } 0.1 \mathrm{vol} \% \text { of } \mathrm{ZrO} 2.0 .1 \\
\mathrm{vol} \% \text { of spinel with cores of } \mathrm{Rh} / \mathrm{Pd} .0 .05 \mathrm{vol} \% \text { of } \\
\mathrm{RuO} 2\end{array}$ \\
\hline HLW99-36 & & & & & Trace amount of dark crystals, likely RuO2 \\
\hline HLW99-37 & & & & & $>0.5 \mathrm{vol} \%$ of $\mathrm{RuO} 2$ and $\mathrm{Rh}$ metal \\
\hline \multicolumn{6}{|l|}{ HLW99-38 } \\
\hline HLW99-39 & & & & & $\begin{array}{l}0.1 \mathrm{vol} \% \text { of spinel with Rh incorporation } 0.1 \mathrm{vol} \% \\
\text { of } \mathrm{Rh} \text { and } \mathrm{RuO} 2\end{array}$ \\
\hline HLW99-40 & & & & & $\begin{array}{l}\text { About } 0.2 \mathrm{vol} \% \text { of } \mathrm{ZrO} 2 \text { and up to } 1 \mathrm{vol} \% \text { of } \\
\text { spinel }(\mathrm{Fe}, \mathrm{Zn})\left(\text { Premelt } 1300^{\circ} \mathrm{C}\right)\end{array}$ \\
\hline \multicolumn{6}{|l|}{ HLW99-41 } \\
\hline HLW99-42 & & & & & $\begin{array}{l}\text { About } 0.3 \mathrm{vol} \% \text { of RuO2 and }<0.01 \mathrm{vol} \% \text { of Rh } \\
\text { (Premelt } 1300^{\circ} \mathrm{C} \text { ) }\end{array}$ \\
\hline HLW99-43 & & & & & $\begin{array}{l}\text { Up to } 0.5 \mathrm{vol} \% \text { of spinel }(\mathrm{Fe}, \mathrm{Zn}, \mathrm{Rh}) \text { and } 0.1 \\
\text { vol\% of } \mathrm{RuO} 2\end{array}$ \\
\hline \multicolumn{6}{|l|}{ HLW99-44 } \\
\hline \multicolumn{6}{|l|}{ HLW99-45 } \\
\hline HLW99-46 & & & & & $\begin{array}{l}\text { About } 0.1 \text { vol } \% \text { of Ni-based spinel }(<5 \mathrm{~mm}) \text { with } \\
\text { incorporation of } \mathrm{Zn}, \mathrm{Rh}, \mathrm{Sb} \text { and small amount of } \\
\text { Fe }\end{array}$ \\
\hline \multicolumn{6}{|l|}{ HLW99-47 } \\
\hline \multicolumn{6}{|l|}{ HLW99-48 } \\
\hline HLW99-49 & & & & & $\begin{array}{l}\text { About } 0.5 \mathrm{vol} \% \text { of an unidentified phase that } \\
\text { contains } \mathrm{Na}, \mathrm{S}, \mathrm{P}, \mathrm{W}, \mathrm{Cr} \text { and } \mathrm{O} \text {. Brownish white } \\
\text { cluster of } \mathrm{ZrO} 2 \text { with trace amount of } \mathrm{Fe}, \mathrm{Na}\end{array}$ \\
\hline \multicolumn{6}{|l|}{ HLW99-50 } \\
\hline \multicolumn{6}{|l|}{ HLW99-51R } \\
\hline HLW99-52 & & & & & $\begin{array}{l}\text { OM study shows } 2 \text { vol\% of rod shaped non- } \\
\text { magnetic crystals in star like clusters }\end{array}$ \\
\hline \multicolumn{6}{|l|}{ HLW99-53 } \\
\hline HLW99-54 & & & & & \\
\hline HLW99-55 & & & & & $\begin{array}{l}\text { About } 15 \text { vol } \% \text { of zircon (with } \mathrm{Ca}, \mathrm{Ce} \text { and } \mathrm{Na} \text { ), } 1 \\
\text { vol } \% \text { of spinel (trevorite) with Rh cores, and } 0.5 \\
\text { vol } \% \text { of } \mathrm{ZrO} 2\end{array}$ \\
\hline HLW99-56R & & & & & $\begin{array}{l}\text { About } 7 \text { vol\% of spinel (trevorite with } \mathrm{Zn}, \mathrm{Ti}, \mathrm{Sb} \\
\text { and Al) with little to no Rh. } 0.1 \mathrm{vol} \% \text { of RuO2 }\end{array}$ \\
\hline HLW99-57 & & & & & $\begin{array}{l}0.1 \mathrm{vol} \% \text { of rhodium spinel }(\mathrm{Fe}, \mathrm{Zn}) \text {. No evidence } \\
\text { of settling }\end{array}$ \\
\hline HLW99-58 & & & & & $\begin{array}{l}0.2 \mathrm{vol} \% \text { of } \mathrm{RuO} 2 \text { and } 0.1 \mathrm{vol} \% \text { of } \mathrm{Rh} \text { spinel }(\mathrm{Ni}, \\
\mathrm{Zn}, \mathrm{Al} \text { and } \mathrm{Fe})\end{array}$ \\
\hline HLW99-59 & & & & & $\begin{array}{l}1 \mathrm{vol} \% \text { of } \mathrm{NiO} \text { with some incorporation of } \mathrm{Fe}, \mathrm{Mg} \\
\text { and } \mathrm{Zn} .0 .1 \mathrm{vol} \% \text { of spinel rich in } \mathrm{Ni}, \mathrm{Fe} \text { and } \mathrm{Sb}\end{array}$ \\
\hline
\end{tabular}


Appendix A. Database - mass fraction

RPP-WTP HLW Formulation (Kot and Pegg 2001)

\begin{tabular}{|c|c|c|c|c|c|c|c|c|c|c|c|c|c|c|c|}
\hline Glass ID & $\begin{array}{c}\text { Heat Treated } \\
\text { XRD }\end{array}$ & $\begin{array}{l}\text { Density } \\
\left(\mathrm{g} / \mathrm{cm}^{3}\right)\end{array}$ & $\begin{array}{c}\text { Fulc Visc } \\
\mathrm{A}\end{array}$ & $\begin{array}{c}\text { Fulc Visc } \\
\text { B }\end{array}$ & $\begin{array}{c}\text { Fulc Visc } \\
\text { To }\end{array}$ & $\begin{array}{c}\mathrm{FV} 1150^{\circ} \mathrm{C} \\
(\mathrm{Pa} \cdot \mathrm{s})\end{array}$ & \begin{tabular}{|c|} 
Arrh Visc \\
$\mathrm{A}$
\end{tabular} & $\begin{array}{c}\text { Arrh Visc } \\
\text { B }\end{array}$ & $\begin{array}{c}\text { Av } \\
1150^{\circ} \mathrm{C} \\
\left(\mathrm{D}_{0}, \mathrm{C}^{\prime}\right.\end{array}$ & $\begin{array}{c}\mathrm{T}\left({ }^{\circ} \mathrm{C}\right) \text { at } \\
2 \mathrm{~Pa} \cdot \mathrm{s}\end{array}$ & $\begin{array}{c}\mathrm{T}\left({ }^{\circ} \mathrm{C}\right) \text { at } \\
5 \mathrm{~Pa} \cdot \mathrm{s}\end{array}$ & $\begin{array}{l}\mathrm{T}\left({ }^{\circ} \mathrm{C}\right) \text { at } \\
10 \mathrm{~Pa} \cdot \mathrm{s}\end{array}$ & $\begin{array}{l}\mathrm{T} 1 \\
\left({ }^{\circ} \mathrm{C}\right) \\
\end{array}$ & $\begin{array}{c}\mathrm{V} 1 \\
(\mathrm{~Pa} \cdot \mathrm{s})\end{array}$ & $\begin{array}{l}\mathrm{T} 2 \\
\left({ }^{\circ} \mathrm{C}\right) \\
\end{array}$ \\
\hline \multicolumn{16}{|l|}{ HLW99-33 } \\
\hline \multicolumn{16}{|l|}{ HLW99-34 } \\
\hline \multicolumn{16}{|l|}{ HLW99-35 } \\
\hline \multicolumn{16}{|l|}{ HLW99-36 } \\
\hline \multicolumn{16}{|l|}{ HLW99-37 } \\
\hline \multicolumn{16}{|l|}{ HLW99-38 } \\
\hline \multicolumn{16}{|l|}{ HLW99-39 } \\
\hline \multicolumn{16}{|l|}{ HLW99-40 } \\
\hline \multicolumn{16}{|l|}{ HLW99-41 } \\
\hline \multicolumn{16}{|l|}{ HLW99-42 } \\
\hline \multicolumn{16}{|l|}{ HLW99-43 } \\
\hline \multicolumn{16}{|l|}{ HLW99-44 } \\
\hline \multicolumn{16}{|l|}{ HLW99-45 } \\
\hline \multicolumn{16}{|l|}{ HLW99-46 } \\
\hline \multicolumn{16}{|l|}{ HLW99-47 } \\
\hline \multicolumn{16}{|l|}{ HLW99-48 } \\
\hline \multicolumn{16}{|l|}{ HLW99-49 } \\
\hline \multicolumn{16}{|l|}{ HLW99-50 } \\
\hline \multicolumn{16}{|l|}{ HLW99-51R } \\
\hline \multicolumn{16}{|l|}{ HLW99-52 } \\
\hline \multicolumn{16}{|l|}{ HLW99-53 } \\
\hline \multicolumn{16}{|l|}{ HLW99-54 } \\
\hline \multicolumn{16}{|l|}{ HLW99-55 } \\
\hline \multicolumn{16}{|l|}{ HLW99-56R } \\
\hline \multicolumn{16}{|l|}{ HLW99-57 } \\
\hline \multicolumn{16}{|l|}{ HLW99-58 } \\
\hline HLW99-59 & & & & & & & & & & & & & & & \\
\hline
\end{tabular}


Appendix A. Database - mass fraction

\begin{tabular}{|c|c|c|c|c|c|c|c|c|c|c|c|c|c|c|c|c|c|c|c|c|c|}
\hline Glass ID & $\begin{array}{c}\mathrm{V} 2 \\
(\mathrm{~Pa} \cdot \mathrm{s})\end{array}$ & $\begin{array}{c}\mathrm{T} 3 \\
\left({ }^{\circ} \mathrm{C}\right) \\
\end{array}$ & $\begin{array}{c}\mathrm{V} 3 \\
(\mathrm{~Pa} \cdot \mathrm{s}) \\
\end{array}$ & $\begin{array}{c}\mathrm{T} 4 \\
\left({ }^{\circ} \mathrm{C}\right) \\
\end{array}$ & $\begin{array}{c}\mathrm{V} 4 \\
(\mathrm{~Pa} \cdot \mathrm{s})\end{array}$ & $\begin{array}{c}\mathrm{T} 5 \\
\left({ }^{\circ} \mathrm{C}\right) \\
\end{array}$ & $\begin{array}{c}\text { V5 } \\
(\mathrm{Pa} \cdot \mathrm{s})\end{array}$ & $\begin{array}{c}\mathrm{T} 6 \\
\left({ }^{\circ} \mathrm{C}\right) \\
\end{array}$ & $\begin{array}{c}\mathrm{V} 6 \\
(\mathrm{~Pa} \cdot \mathrm{s})\end{array}$ & $\begin{array}{c}\mathrm{T} 7 \\
\left({ }^{\circ} \mathrm{C}\right) \\
\end{array}$ & $\begin{array}{c}\text { V7 } \\
(\mathrm{Pa} \cdot \mathrm{s}) \\
\end{array}$ & $\begin{array}{c}\mathrm{T} 8 \\
\left({ }^{\circ} \mathrm{C}\right) \\
\end{array}$ & $\begin{array}{c}\mathrm{V} 8 \\
(\mathrm{~Pa} \cdot \mathrm{s})\end{array}$ & $\begin{array}{c}\mathrm{T} 9 \\
\left({ }^{\circ} \mathrm{C}\right) \\
\end{array}$ & $\begin{array}{c}\mathrm{V} 9 \\
(\mathrm{~Pa} \cdot \mathrm{s}) \\
\end{array}$ & $\begin{array}{l}\mathrm{T} 10 \\
\left({ }^{\circ} \mathrm{C}\right) \\
\end{array}$ & $\begin{array}{c}\mathrm{V} 10 \\
(\mathrm{~Pa} \cdot \mathrm{s})\end{array}$ & $\begin{array}{l}\mathrm{T} 11 \\
\left({ }^{\circ} \mathrm{C}\right) \\
\end{array}$ & $\begin{array}{c}\text { V11 } \\
(\mathrm{Pa} \cdot \mathrm{s})\end{array}$ & $\begin{array}{l}\mathrm{T} 12 \\
\left({ }^{\circ} \mathrm{C}\right) \\
\end{array}$ & $\begin{array}{c}\mathrm{V} 12 \\
(\mathrm{~Pa} \cdot \mathrm{s})\end{array}$ \\
\hline HLW99-33 & & & & & & & & & & & & & & & & & & & & & \\
\hline HLW99-34 & & & & & & & & & & & & & & & & & & & & & \\
\hline HLW99-35 & & & & & & & & & & & & & & & & & & & & & \\
\hline HLW99-36 & & & & & & & & & & & & & & & & & & & & & \\
\hline HLW99-37 & & & & & & & & & & & & & & & & & & & & & \\
\hline HLW99-38 & & & & & & & & & & & & & & & & & & & & & \\
\hline HLW99-39 & & & & & & & & & & & & & & & & & & & & & \\
\hline HLW99-40 & & & & & & & & & & & & & & & & & & & & & \\
\hline HLW99-41 & & & & & & & & & & & & & & & & & & & & & \\
\hline HLW99-42 & & & & & & & & & & & & & & & & & & & & & \\
\hline HLW99-43 & & & & & & & & & & & & & & & & & & & & & \\
\hline HLW99-44 & & & & & & & & & & & & & & & & & & & & & \\
\hline HLW99-45 & & & & & & & & & & & & & & & & & & & & & \\
\hline HLW99-46 & & & & & & & & & & & & & & & & & & & & & \\
\hline HLW99-47 & & & & & & & & & & & & & & & & & & & & & \\
\hline HLW99-48 & & & & & & & & & & & & & & & & & & & & & \\
\hline HLW99-49 & & & & & & & & & & & & & & & & & & & & & \\
\hline HLW99-50 & & & & & & & & & & & & & & & & & & & & & \\
\hline HLW99-51R & & & & & & & & & & & & & & & & & & & & & \\
\hline HLW99-52 & & & & & & & & & & & & & & & & & & & & & \\
\hline HLW99-53 & & & & & & & & & & & & & & & & & & & & & \\
\hline HLW99-54 & & & & & & & & & & & & & & & & & & & & & \\
\hline HLW99-55 & & & & & & & & & & & & & & & & & & & & & \\
\hline HLW99-56R & & & & & & & & & & & & & & & & & & & & & \\
\hline HLW99-57 & & & & & & & & & & & & & & & & & & & & & \\
\hline HLW99-58 & & & & & & & & & & & & & & & & & & & & & \\
\hline HLW99-59 & & & & & & & & & & & & & & & & & & & & & \\
\hline
\end{tabular}


Appendix A. Database - mass fraction

RPP-WTP HLW Formulation (Kot and Pegg 2001)

\begin{tabular}{|c|c|c|c|c|c|c|c|c|c|c|c|c|c|c|c|c|}
\hline Glass ID & $\begin{array}{l}\mathrm{T} 13 \\
\left({ }^{\circ} \mathrm{C}\right) \\
\end{array}$ & $\begin{array}{c}\mathrm{V} 13 \\
(\mathrm{~Pa} \cdot \mathrm{s}) \\
\end{array}$ & $\begin{array}{l}\mathrm{T} 14 \\
\left({ }^{\circ} \mathrm{C}\right) \\
\end{array}$ & $\begin{array}{c}\mathrm{V} 14 \\
(\mathrm{~Pa} \cdot \mathrm{s}) \\
\end{array}$ & $\begin{array}{c}\text { Q PCT } \\
\mathrm{B}\left(\mathrm{g} / \mathrm{m}^{2}\right)\end{array}$ & $\begin{array}{c}\text { Q PCT } \\
\mathrm{Li}\left(\mathrm{g} / \mathrm{m}^{2}\right)\end{array}$ & $\begin{array}{c}\text { Q PCT } \\
\mathrm{Na}\left(\mathrm{g} / \mathrm{m}^{2}\right)\end{array}$ & $\begin{array}{c}\text { Q PCT } \\
\mathrm{Si}\left(\mathrm{g} / \mathrm{m}^{2}\right)\end{array}$ & $\begin{array}{l}\text { Q PCT } \\
\mathrm{pH}\end{array}$ & $\begin{array}{c}\mathrm{CCC} \mathrm{PCT} \\
\mathrm{B}\left(\mathrm{g} / \mathrm{m}^{2}\right)\end{array}$ & $\begin{array}{c}\mathrm{CCC} \mathrm{PCT} \\
\mathrm{Li}\left(\mathrm{g} / \mathrm{m}^{2}\right)\end{array}$ & $\begin{array}{l}\mathrm{CCC} \text { PCT } \\
\mathrm{Na}\left(\mathrm{g} / \mathrm{m}^{2}\right)\end{array}$ & $\begin{array}{l}\mathrm{CCC} \mathrm{PCT} \\
\mathrm{Si}\left(\mathrm{g} / \mathrm{m}^{2}\right)\end{array}$ & $\begin{array}{c}\text { CCC PCT } \\
\mathrm{pH}\end{array}$ & $\begin{array}{l}\text { Q PCT B at } \\
20^{\circ} \mathrm{C}\left(\mathrm{g} / \mathrm{m}^{2}\right)\end{array}$ & $\begin{array}{l}\text { Q PCT Li at } \\
20^{\circ} \mathrm{C}\left(\mathrm{g} / \mathrm{m}^{2}\right)\end{array}$ \\
\hline \multicolumn{17}{|l|}{ HLW99-33 } \\
\hline \multicolumn{17}{|l|}{ HLW99-34 } \\
\hline \multicolumn{17}{|l|}{ HLW99-35 } \\
\hline \multicolumn{17}{|l|}{ HLW99-36 } \\
\hline \multicolumn{17}{|l|}{ HLW99-37 } \\
\hline \multicolumn{17}{|l|}{ HLW99-38 } \\
\hline \multicolumn{17}{|l|}{ HLW99-39 } \\
\hline \multicolumn{17}{|l|}{ HLW99-40 } \\
\hline \multicolumn{17}{|l|}{ HLW99-41 } \\
\hline \multicolumn{17}{|l|}{ HLW99-42 } \\
\hline \multicolumn{17}{|l|}{ HLW99-43 } \\
\hline HLW99-44 & & & & & & & & & & & & & & & & \\
\hline \multicolumn{17}{|l|}{ HLW99-45 } \\
\hline \multicolumn{17}{|l|}{ HLW99-46 } \\
\hline \multicolumn{17}{|l|}{ HLW99-47 } \\
\hline \multicolumn{17}{|l|}{ HLW99-48 } \\
\hline \multicolumn{17}{|l|}{ HLW99-49 } \\
\hline \multicolumn{17}{|l|}{ HLW99-50 } \\
\hline \multicolumn{17}{|l|}{ HLW99-51R } \\
\hline HLW99-52 & & & & & 9.775 & 1.735 & 7.545 & 0.57 & 9.75 & & & & & & & \\
\hline \multicolumn{17}{|l|}{ HLW99-53 } \\
\hline \multicolumn{17}{|l|}{ HLW99-54 } \\
\hline \multicolumn{17}{|l|}{ HLW99-55 } \\
\hline \multicolumn{17}{|l|}{ HLW99-56R } \\
\hline \multicolumn{17}{|l|}{ HLW99-57 } \\
\hline \multicolumn{17}{|l|}{ HLW99-58 } \\
\hline HLW99-59 & & & & & & & & & & & & & & & & \\
\hline
\end{tabular}


Appendix A. Database - mass fraction

RPP-WTP HLW Formulation (Kot and Pegg 2001)

\begin{tabular}{|c|c|c|c|c|c|c|c|c|c|c|c|c|}
\hline Glass ID & $\begin{array}{l}\text { Q PCT Na at } \\
20^{\circ} \mathrm{C}\left(\mathrm{g} / \mathrm{m}^{2}\right)\end{array}$ & $\begin{array}{l}\text { Q PCT Si at } \\
20^{\circ} \mathrm{C}\left(\mathrm{g} / \mathrm{m}^{2}\right)\end{array}$ & $\begin{array}{c}\mathrm{QpH} \\
\text { at } 20^{\circ} \mathrm{C}\end{array}$ & $\begin{array}{c}\text { TCLP Ag } \\
\text { (ppm) }\end{array}$ & $\begin{array}{c}\text { TCLP As } \\
\text { (ppm) }\end{array}$ & $\begin{array}{c}\text { TCLP Ba } \\
\text { (ppm) }\end{array}$ & $\begin{array}{c}\text { TCLP Cd } \\
(\mathrm{ppm})\end{array}$ & $\begin{array}{c}\text { TCLP Cr } \\
(\mathrm{ppm})\end{array}$ & $\begin{array}{c}\text { TCLP Ni } \\
(\mathrm{ppm})\end{array}$ & $\begin{array}{c}\text { TCLP Pb } \\
(\mathrm{ppm})\end{array}$ & $\begin{array}{c}\text { TCLP Se } \\
(\mathrm{ppm})\end{array}$ & $\begin{array}{c}\text { TCLP Zn } \\
(\mathrm{ppm})\end{array}$ \\
\hline HLW99-33 & & & & 0.109 & 2.427 & 0.025 & 0.007 & 0.085 & 0.072 & 0.564 & 1.917 & 9.783 \\
\hline HLW99-34 & & & & 0.057 & NA & 0.041 & 0.019 & 0.025 & 0.011 & 0.168 & NA & 0.812 \\
\hline HLW99-35 & & & & 0.057 & 0.049 & 0.026 & 0.007 & $<.0055$ & $<.0079$ & 0.086 & 0.0532 & 0.435 \\
\hline HLW99-36 & & & & 0.059 & 0.049 & 0.666 & 0.423 & $<.0055$ & 0.308 & 0.118 & 0.0532 & 3.867 \\
\hline HLW99-37 & & & & 0.049 & 0.049 & 0.538 & 0.362 & $<.0055$ & 0.297 & 0.126 & 0.0532 & 0.596 \\
\hline HLW99-38 & & & & 0.678 & 0.398 & 8.047 & 6.407 & 0.104 & 5.539 & 1.188 & 0.198 & 5.482 \\
\hline HLW99-39 & & & & 0.03 & 0.157 & 3.292 & 2.498 & 0.014 & 2.135 & 0.164 & 0.095 & 23.696 \\
\hline HLW99-40 & & & & 0.073 & 0.064 & 0.011 & $<0.0027$ & $<0.0055$ & $<0.0079$ & $<0.0243$ & 0.0532 & 0.607 \\
\hline HLW99-41 & & & & 0.194 & 0.049 & 1.051 & 0.759 & 0.014 & 0.647 & 0.162 & 0.0532 & 1.475 \\
\hline HLW99-42 & & & & 0.05 & 0.086 & 0.082 & 0.045 & $<0.0055$ & 0.025 & $<0.0243$ & 0.0532 & 0.504 \\
\hline HLW99-43 & & & & 0.109 & 0.071 & 0.025 & $<0.0027$ & $<0.0055$ & $<0.0079$ & 0.033 & 0.0532 & 4.6 \\
\hline HLW99-44 & & & & 0.492 & 0.049 & 0.031 & 0.007 & 0.157 & 0.038 & 0.101 & 0.068 & 3.237 \\
\hline HLW99-45 & & & & 0.209 & NA & 0.116 & 0.067 & 0.076 & 0.079 & 0.09 & NA & 2.138 \\
\hline HLW99-46 & & & & 0.372 & 0.425 & 58.931 & 56.468 & 0.038 & 53.415 & 0.397 & 0.563 & 34.913 \\
\hline HLW99-47 & & & & 0.058 & 0.049 & 0.005 & $<0.0027$ & $<0.0055$ & $<0.0079$ & $<0.0243$ & 0.079 & 0.273 \\
\hline HLW99-48 & & & & 0.216 & 0.049 & 4.356 & 3.162 & $<0.0055$ & 1.456 & 0.297 & 0.072 & 0.016 \\
\hline HLW99-49 & & & & 0.197 & 0.375 & 1.213 & 0.408 & 5.363 & 0.334 & 0.06 & 0.062 & 1.479 \\
\hline HLW99-50 & & & & 0.045 & NA & 0.031 & 0.013 & 0.016 & 0.014 & 1.497 & NA & 0.843 \\
\hline HLW99-51R & & & & 0.174 & 2.363 & 0.19 & $<0.0027$ & 4.729 & 0.234 & 0.777 & 1.851 & 8.813 \\
\hline HLW99-52 & & & & 0.206 & 0.499 & 76.9 & 68.73 & 0.022 & 62.646 & 4.361 & 0.789 & 43.693 \\
\hline HLW99-53 & & & & 0.205 & 0.049 & 1.943 & 1.146 & 0.017 & 0.778 & 0.284 & 0.125 & 1.214 \\
\hline HLW99-54 & & & & 0.047 & 0.098 & 0.742 & 0.484 & $<0.0055$ & 0.39 & 0.148 & 0.084 & 0.436 \\
\hline HLW99-55 & & & & 0.227 & 0.094 & 5.618 & 4.049 & 0.019 & 2.2 & 0.482 & 0.056 & 5.131 \\
\hline HLW99-56R & & & & 0.109 & 0.051 & 1.488 & 0.987 & $<0.0055$ & 0.466 & 0.282 & 0.0532 & 0.624 \\
\hline HLW99-57 & & & & 0.046 & 0.049 & 0.031 & 0.004 & $<0.0055$ & $<0.0079$ & $<0.0243$ & 0.071 & 0.55 \\
\hline HLW99-58 & & & & 0.054 & 0.189 & 7.817 & 13.979 & 0.05 & 12.915 & 0.681 & 0.958 & 37.847 \\
\hline HLW99-59 & & & & 0.128 & 0.049 & 2.346 & 1.678 & $<0.0055$ & 1.296 & 0.506 & 0.088 & 1.022 \\
\hline
\end{tabular}


RPP-WTP HLW Formulation (Kot and Pegg 2001)

\begin{tabular}{|c|c|c|c|c|c|c|c|c|c|c|c|c|c|c|c|c|c|c|c|c|c|}
\hline Glass ID & $\begin{array}{c}\mathrm{A} 12 \mathrm{O} 3 \\
-\mathrm{t}\end{array}$ & $\begin{array}{c}\mathrm{B} 2 \mathrm{O} 3 \\
-\mathrm{t}\end{array}$ & $\begin{array}{c}\mathrm{CaO} \\
-\mathrm{t}\end{array}$ & $\begin{array}{c}\mathrm{Fe} 2 \mathrm{O} 3 \\
-\mathrm{t}\end{array}$ & $\begin{array}{c}\mathrm{FeO} \\
-\mathrm{t}\end{array}$ & $\begin{array}{c}\mathrm{K} 2 \mathrm{O} \\
-\mathrm{t}\end{array}$ & $\begin{array}{c}\mathrm{Li} 2 \mathrm{O} \\
-\mathrm{t}\end{array}$ & $\begin{array}{c}\mathrm{MgO} \\
-\mathrm{t}\end{array}$ & $\begin{array}{c}\mathrm{Na} 2 \mathrm{O} \\
-\mathrm{t}\end{array}$ & $\begin{array}{c}\mathrm{NiO} \\
-\mathrm{t}\end{array}$ & $\begin{array}{c}\mathrm{P} 2 \mathrm{O} 5 \\
-\mathrm{t}\end{array}$ & $\begin{array}{c}\mathrm{SiO} 2 \\
-\mathrm{t}\end{array}$ & $\begin{array}{c}\mathrm{ZrO} 2 \\
-\mathrm{t}\end{array}$ & $\begin{array}{c}\mathrm{Ag} 2 \mathrm{O} \\
-\mathrm{t}\end{array}$ & $\begin{array}{c}\mathrm{As} 2 \mathrm{O} 3 \\
-\mathrm{t}\end{array}$ & $\begin{array}{c}\mathrm{BaO} \\
-\mathrm{t}\end{array}$ & $\begin{array}{c}\mathrm{Bi} 2 \mathrm{O} 3 \\
-\mathrm{t}\end{array}$ & $\begin{array}{l}\mathrm{Br} \\
-\mathrm{t}\end{array}$ & $\begin{array}{c}\mathrm{CdO} \\
-\mathrm{t}\end{array}$ & $\begin{array}{c}\mathrm{Ce} 2 \mathrm{O} 3 \\
-\mathrm{t}\end{array}$ & $\begin{array}{c}\mathrm{CeO} 2 \\
-\mathrm{t}\end{array}$ \\
\hline HLW99-60 & 0.0000 & 0.1350 & 0.0000 & 0.1250 & & 0.0100 & 0.0233 & 0.0135 & 0.0733 & 0.0203 & 0.0300 & 0.3483 & 0.0000 & 0.0025 & 0.0020 & 0.0270 & 0.0135 & & 0.0203 & & 0.0020 \\
\hline HLW99-61 & 0250 & 0.2000 & 0.0000 & 0200 & & 0.0100 & 0000 & 0.0200 & 0.1600 & 0300 & 0.0000 & 0.3700 & 0.0000 & 0.0025 & 0.0020 & 0.0400 & 0.0200 & & 0.0300 & & 0.0020 \\
\hline HLW99-62 & 0.0000 & 0.0500 & 0.0000 & 0.0200 & & 0.0100 & 0.0300 & 0.0123 & 0.1300 & 0.0184 & 0.0000 & 0.5200 & 0.0867 & 0.0025 & 0.0020 & 0.0246 & 0.0123 & & 0.0184 & & 0.0020 \\
\hline HLW99-63 & 0.0516 & 0.1171 & .0303 & 0.0614 & & 0.0100 & 0.0317 & 0.0062 & 0.1129 & 0.0094 & 0.0124 & 0.3973 & 0.0322 & 0.0025 & 0.0020 & 0.0125 & 0.0062 & & 0.0094 & & 0.0020 \\
\hline HLW99-64 & 0.0530 & 0.0900 & 0.0000 & 0.0200 & & 0.0100 & 0.0700 & 0.0200 & 0.0800 & 0.0300 & 0.0300 & 0.3400 & 0.0000 & 0.0025 & 0.0020 & 0.0400 & 0.0200 & & 0.0300 & & 0.0020 \\
\hline HLW99-65 & 0.1100 & 0.1830 & 0.0000 & 0.0200 & & 0.0100 & 0.0000 & 0.0000 & 0.2000 & 0.0000 & 0.0300 & 0.3000 & 0.0000 & 0.0025 & 0.0020 & 0.0000 & 0.0000 & & 0.0000 & & 0.0020 \\
\hline HLW99-66 & 0.0735 & 0.0525 & 0.0210 & 0.0210 & & 0.0105 & 0.0315 & 0.0000 & 0.0992 & 0.0000 & 0.0013 & 0.5008 & 0.0315 & 0.0026 & 0.0021 & 0.0000 & 0.0000 & & 0.0000 & & 0.0021 \\
\hline HLW99-67 & 0.0735 & 0.0525 & 0.0210 & 0.1176 & & 0.0105 & 0.0315 & 0.0000 & 0.0525 & 0.0000 & 0.0013 & 0.3146 & 0.0315 & 0.0026 & 0.0021 & 0.0000 & 0.0000 & & 0.0000 & & 0.0021 \\
\hline HLW99-68 & 0.0735 & 0.0525 & 0.0210 & 0.1313 & & 0.0105 & 0.0315 & 0.0131 & 0.0704 & 0.0197 & 0.0013 & 0.3145 & 0.0315 & 0.0026 & 0.0021 & 0.0263 & 0.0131 & & 0.0197 & & 0.0021 \\
\hline HLW99-69 & 0.0735 & 0.0525 & 0.0210 & 0.0210 & & 0.0105 & 0.0315 & 0.0131 & 0.0525 & 0.0197 & 0.0013 & 0.3146 & 0.0315 & 0.0026 & 0.0021 & 0.0263 & 0.0131 & & 0.0197 & & 0.0021 \\
\hline HLW99-70 & 0.0735 & 0.0525 & 0.0210 & 0.0210 & & 0.0105 & 0.0315 & 0.0000 & 0.1139 & 0.0000 & 0.0013 & 0.3495 & 0.0315 & 0.0026 & 0.0021 & 0.0000 & 0.0000 & & 0.0000 & & 0.0021 \\
\hline HLW99-71 & 0.0735 & 0.0525 & 0.0210 & 0.1313 & & 0.0105 & 0.0315 & 0.0000 & 0.0771 & 0.0000 & 0.0013 & 0.4127 & 0.0315 & 0.0026 & 0.0021 & 0.0000 & 0.0000 & & 0.0000 & & 0.0021 \\
\hline HLW99-72 & 0.0735 & 0.0525 & 0.0210 & 0.0210 & & 0.0105 & 0.0315 & 0.0131 & 0.0781 & 0.0197 & 0.0013 & 0.4169 & 0.0315 & 0.0026 & 0.0021 & 0.0263 & 0.0131 & & 0.0197 & & 0.0021 \\
\hline HLW99-73 & 0.0736 & 0.0525 & 0.0210 & 0.0210 & & 0.0105 & 0.0315 & 0.0000 & 0.1412 & 0.0000 & 0.0013 & 0.4586 & 0.0315 & 0.0026 & 0.0021 & 0.0000 & 0.0000 & & 0.0000 & & 0.0021 \\
\hline HLW99-74 & 0.0735 & 0.0525 & 0.0210 & 0.1313 & & 0.0105 & 0.0315 & 0.0066 & 0.0525 & 0.0098 & 0.0013 & 0.3146 & 0.0315 & 0.0026 & 0.0021 & 0.0131 & 0.0066 & & 0.0098 & & 0.0021 \\
\hline HLW99-75 & 0.0735 & 0.0525 & 0.0210 & 0.1313 & & 0.0105 & 0.0315 & 0.0088 & 0.1051 & 0.0132 & 0.0013 & 0.3142 & 0.0315 & 0.0026 & 0.0021 & 0.0176 & 0.0088 & & 0.0132 & & 0.0021 \\
\hline HLW99-76 & 0.0735 & 0.0525 & 0.0210 & 0.0210 & & 0.0105 & 0.0315 & 0.0131 & 0.1051 & 0.0197 & 0.0013 & 0.3142 & 0.0315 & 0.0026 & 0.0021 & 0.0263 & 0.0131 & & 0.0197 & & 0.0021 \\
\hline HLW99-77 & 0.0735 & 0.0525 & 0.0210 & 0.1313 & & 0.0105 & 0.0315 & 0.0000 & 0.1051 & 0.0000 & 0.0013 & 0.3142 & 0.0315 & 0.0026 & 0.0021 & 0.0000 & 0.0000 & & 0.0000 & & 0.0021 \\
\hline HLW99-78 & 0.0735 & 0.0525 & 0.0210 & 0.0562 & & 0.0105 & 0.0315 & 0.0044 & 0.0788 & 0.0066 & 0.0013 & 0.3144 & 0.0315 & 0.0026 & 0.0021 & 0.0088 & 0.0044 & & 0.0066 & & 0.0021 \\
\hline HLW99-79 & 0.0735 & 0.0525 & 0.0210 & 0.0210 & & 0.0105 & 0.0315 & 0.0000 & 0.0718 & 0.0000 & 0.0013 & 0.3917 & 0.0315 & 0.0026 & 0.0021 & 0.0000 & 0.0000 & & 0.0000 & & 0.0021 \\
\hline HLW99-80 & 0.0700 & 0.1000 & 0.0200 & 0.0725 & & 0.0100 & 0.0300 & 0.0125 & 0.0500 & 0.0188 & 0.0025 & 0.3000 & 0.0300 & 0.0025 & 0.0020 & 0.0250 & 0.0125 & & 0.0188 & & 0.0020 \\
\hline HLW99-81 & 0.0736 & 0.0525 & 0.0210 & 0.0210 & & 0.0105 & 0.0315 & 0.0000 & 0.1276 & 0.0000 & 0.0013 & 0.4040 & 0.0315 & 0.0026 & 0.0021 & 0.0000 & 0.0000 & & 0.0000 & & 0.0021 \\
\hline HLW99-82 & 0.0700 & 0.1000 & 0.0200 & 0.0560 & & 0.0100 & 0.0300 & 0.0125 & 0.1072 & 0.0188 & 0.0025 & 0.3288 & 0.0300 & 0.0025 & 0.0020 & 0.0250 & 0.0125 & & 0.0188 & & 0.0020 \\
\hline HLW99-83 & 0.0700 & 0.1000 & 0.0200 & 0.0804 & & 0.0100 & 0.0300 & 0.0000 & 0.0672 & 0.0000 & 0.0025 & 0.3690 & 0.0300 & 0.0025 & 0.0020 & 0.0000 & 0.0000 & & 0.0000 & & 0.0020 \\
\hline HLW99-84 & 0.0700 & 0.1000 & 0.0200 & 0.0200 & & 0.0100 & 0.0300 & 0.0073 & 0.0674 & 0.0110 & 0.0025 & 0.3698 & 0.0300 & 0.0025 & 0.0020 & 0.0146 & 0.0073 & & 0.0110 & & 0.0020 \\
\hline HLW99-85 & 0.0700 & 0.1000 & 0.0200 & 0.0725 & & 0.0100 & 0.0300 & 0.0063 & 0.0739 & 0.0094 & 0.0025 & 0.3956 & 0.0300 & 0.0025 & 0.0020 & 0.0125 & 0.0063 & & 0.0094 & & 0.0020 \\
\hline HLW99-86 & 0.0700 & 0.1000 & 0.0200 & 0.0200 & & 0.0100 & 0.0300 & 0.0000 & 0.0944 & 0.0000 & 0.0025 & 0.4776 & 0.0300 & 0.0025 & 0.0020 & 0.0000 & 0.0000 & & 0.0000 & & 0.0020 \\
\hline HLW99-87 & 0.0700 & 0.1000 & 0.0200 & 0.0535 & & 0.0100 & 0.0300 & 0.0042 & 0.0750 & 0.0063 & 0.0025 & 0.3000 & 0.0300 & 0.0025 & 0.0020 & 0.0084 & 0.0042 & & 0.0063 & & 0.0020 \\
\hline HLW99-88 & 0.0700 & 0.1000 & 0.0200 & 0.1250 & & 0.0100 & 0.0300 & 0.0125 & 0.0670 & 0.0188 & 0.0025 & 0.3000 & 0.0300 & 0.0025 & 0.0020 & 0.0250 & 0.0125 & & 0.0188 & & 0.0020 \\
\hline HLW99-89 & 0.0700 & 0.1000 & 0.0200 & 0.1250 & & 0.0100 & 0.0300 & 0.0000 & 0.0734 & 0.0000 & 0.0025 & 0.3936 & 0.0300 & 0.0025 & 0.0020 & 0.0000 & 0.0000 & & 0.0000 & & 0.0020 \\
\hline HLW99-90R & 0.0700 & 0.1000 & 0.0200 & 0.1250 & & 0.0100 & 0.0300 & 0.0000 & 0.1000 & 0.0000 & 0.0025 & 0.3000 & 0.0300 & 0.0025 & 0.0020 & 0.0000 & 0.0000 & & 0.0000 & & 0.0020 \\
\hline HLW99-91 & 0.0700 & 0.1000 & 0.0200 & 0.1120 & & 0.0100 & 0.0300 & 0.0000 & 0.0500 & 0.0000 & 0.0025 & 0.3000 & 0.0300 & 0.0025 & 0.0020 & 0.0000 & 0.0000 & & 0.0000 & & 0.0020 \\
\hline HLW99-92 & 0.0700 & 0.1000 & 0.0200 & 0.0200 & & 0.0100 & 0.0300 & 0.0125 & 0.0500 & 0.0188 & 0.0025 & 0.3000 & 0.0300 & 0.0025 & 0.0020 & 0.0250 & 0.0125 & & 0.0188 & & 0.0020 \\
\hline HLW99-93R2 & 0.0700 & 0.1000 & 0.0200 & 0.0200 & & 0.0100 & 0.0300 & 0.0000 & 0.1084 & 0.0000 & 0.0025 & 0.3336 & 0.0300 & 0.0025 & 0.0020 & 0.0000 & 0.0000 & & 0.0000 & & 0.0020 \\
\hline HLW99-94 & 0.0700 & 0.1000 & 0.0200 & 0.0200 & & 0.0100 & 0.0300 & 0.0000 & 0.1344 & 0.0000 & 0.0025 & 0.4376 & 0.0300 & 0.0025 & 0.0020 & 0.0000 & 0.0000 & & 0.0000 & & 0.0020 \\
\hline HLW99-95 & 0.0700 & 0.1000 & 0.0200 & 0.0200 & & 0.0100 & 0.0300 & 0.0000 & 0.1344 & 0.0000 & 0.0025 & 0.4376 & 0.0300 & 0.0025 & 0.0020 & 0.0000 & 0.0000 & & 0.0000 & & 0.0020 \\
\hline HLW99-96 & 0.0700 & 0.1000 & 0.0200 & 0.1250 & & 0.0100 & 0.0300 & 0.0063 & 0.0500 & 0.0094 & 0.0025 & 0.3000 & 0.0300 & 0.0025 & 0.0020 & 0.0125 & 0.0063 & & 0.0094 & & 0.0020 \\
\hline HLW99-97 & 0.0700 & 0.1000 & 0.0200 & 0.1250 & & 0.0100 & 0.0300 & 0.0084 & 0.1000 & 0.0126 & 0.0025 & 0.3000 & 0.0300 & 0.0025 & 0.0020 & 0.0168 & 0.0084 & & 0.0126 & & 0.0020 \\
\hline HLW99-98R & 0.0700 & 0.1000 & 0.0200 & 0.0200 & & 0.0100 & 0.0300 & 0.0125 & 0.1000 & 0.0188 & 0.0025 & 0.3000 & 0.0300 & 0.0025 & 0.0020 & 0.0250 & 0.0125 & & 0.0188 & & 0.0020 \\
\hline HLW99-99 & 0.0700 & 0.1000 & 0.0200 & 0.0200 & & 0.0100 & 0.0300 & 0.0000 & 0.0684 & 0.0000 & 0.0025 & 0.3736 & 0.0300 & 0.0025 & 0.0020 & 0.0000 & 0.0000 & & 0.0000 & & 0.0020 \\
\hline HLW99-100 & 0.0700 & 0.1000 & 0.0200 & 0.0200 & & 0.0100 & 0.0300 & 0.0000 & 0.1214 & 0.0000 & 0.0025 & 0.3856 & 0.0300 & 0.0025 & 0.0020 & 0.0000 & 0.0000 & & 0.0000 & & 0.0020 \\
\hline
\end{tabular}


RPP-WTP HLW Formulation (Kot and Pegg 2001)

\begin{tabular}{|c|c|c|c|c|c|c|c|c|c|c|c|c|c|c|c|c|c|c|c|c|c|}
\hline Glass ID & $\begin{array}{c}\mathrm{Cl} \\
-\mathrm{t}\end{array}$ & $\begin{array}{c}\mathrm{CoO} \\
-\mathrm{t}\end{array}$ & $\begin{array}{c}\mathrm{Co} 2 \mathrm{O} 3 \\
-\mathrm{t}\end{array}$ & $\begin{array}{c}\mathrm{Cr} 2 \mathrm{O} 3 \\
-\mathrm{t}\end{array}$ & $\begin{array}{c}\mathrm{Cs} 2 \mathrm{O} \\
-\mathrm{t}\end{array}$ & $\begin{array}{c}\mathrm{CuO} \\
-\mathrm{t}\end{array}$ & $\begin{array}{c}\mathrm{Eu} 2 \mathrm{O} 3 \\
-\mathrm{t}\end{array}$ & $\begin{array}{l}F \\
-t\end{array}$ & $\begin{array}{c}\mathrm{Ga} 2 \mathrm{O} 3 \\
-\mathrm{t}\end{array}$ & $\begin{array}{c}\mathrm{Gd} 2 \mathrm{O} 3 \\
-\mathrm{t}\end{array}$ & $\begin{array}{c}\mathrm{HgO} \\
-\mathrm{t}\end{array}$ & $\begin{array}{l}I \\
-t\end{array}$ & $\begin{array}{c}\mathrm{La} 2 \mathrm{O} 3 \\
-\mathrm{t}\end{array}$ & $\begin{array}{c}\mathrm{MnO} 2 \\
-\mathrm{t}\end{array}$ & $\begin{array}{c}\mathrm{MnO} \\
-\mathrm{t}\end{array}$ & $\begin{array}{c}\mathrm{MoO} \\
-\mathrm{t}\end{array}$ & $\begin{array}{c}\mathrm{MoO} 3 \\
-\mathrm{t}\end{array}$ & $\begin{array}{c}\mathrm{Nb} 2 \mathrm{O} 5 \\
-\mathrm{t}\end{array}$ & $\begin{array}{c}\mathrm{Nd} 2 \mathrm{O} 3 \\
-\mathrm{t}\end{array}$ & $\begin{array}{c}\mathrm{PbO} \\
-\mathrm{t}\end{array}$ & $\begin{array}{c}\mathrm{PdO} 2 \\
-\mathrm{t}\end{array}$ \\
\hline HLW99-60 & 0.0020 & 0.0020 & & 0.0050 & 0.0020 & 0.0020 & & 0.0020 & & & & & 0.0020 & 0.0020 & & & & & & 0.0068 & \\
\hline HLW99-61 & 0.0020 & 0.0020 & & 0.0000 & 0.0020 & 0.0020 & & 0.0020 & & & & & 0.0020 & 0.0020 & & & & & & 0.0100 & \\
\hline HLW99-62 & 0.0020 & 0.0020 & & 0.0000 & 0.0020 & 0.0020 & & 0.0020 & & & & & 0.0020 & 0.0020 & & & & & & 0.0061 & \\
\hline HLW99-63 & 0.0020 & 0.0020 & & 0.0021 & 0.0020 & 0.0020 & & 0.0020 & & & & & 0.0020 & 0.0020 & & & & & & 0.0031 & \\
\hline HLW99-64 & 0.0020 & 0.0020 & & 0.0050 & 0.0020 & 0.0020 & & 0.0020 & & & & & 0.0020 & 0.0020 & & & & & & 0.0100 & \\
\hline HLW99-65 & 0.0020 & 0.0020 & & 0.0050 & 0.0020 & 0.0020 & & 0.0020 & & & & & 0.0020 & 0.0020 & & & & & & 0.0000 & \\
\hline HLW99-66 & 0.0021 & 0.0063 & & 0.0021 & 0.0021 & 0.0021 & & 0.0021 & & & & & 0.0021 & 0.0373 & & & & & & 0.0000 & \\
\hline HLW99-67 & 0.0021 & 0.0063 & & 0.0021 & 0.0021 & 0.0021 & & 0.0021 & & & & & 0.0021 & 0.0912 & & & & & & 0.0000 & \\
\hline HLW99-68 & 0.0021 & 0.0063 & & 0.0021 & 0.0021 & 0.0021 & & 0.0021 & & & & & 0.0021 & 0.0373 & & & & & & 0.0066 & \\
\hline HLW99-69 & 0.0021 & 0.0063 & & 0.0021 & 0.0021 & 0.0021 & & 0.0021 & & & & & 0.0021 & 0.0878 & & & & & & 0.0066 & \\
\hline HLW99-70 & 0.0021 & 0.0063 & & 0.0021 & 0.0021 & 0.0021 & & 0.0021 & & & & & 0.0021 & 0.0912 & & & & & & 0.0000 & \\
\hline HLW99-71 & 0.0021 & 0.0063 & & 0.0021 & 0.0021 & 0.0021 & & 0.0021 & & & & & 0.0021 & 0.0373 & & & & & & 0.0000 & \\
\hline HLW99-72 & 0.0021 & 0.0063 & & 0.0021 & 0.0021 & 0.0021 & & 0.0021 & & & & & 0.0021 & 0.0373 & & & & & & 0.0066 & \\
\hline HLW99-73 & 0.0021 & 0.0063 & & 0.0021 & 0.0021 & 0.0021 & & 0.0021 & & & & & 0.0021 & 0.0373 & & & & & & 0.0000 & \\
\hline HLW99-74 & 0.0021 & 0.0063 & & 0.0021 & 0.0021 & 0.0021 & & 0.0021 & & & & & 0.0021 & 0.0651 & & & & & & 0.0033 & \\
\hline HLW99-75 & 0.0021 & 0.0063 & & 0.0021 & 0.0021 & 0.0021 & & 0.0021 & & & & & 0.0021 & 0.0373 & & & & & & 0.0044 & \\
\hline HLW99-76 & 0.0021 & 0.0063 & & 0.0021 & 0.0021 & 0.0021 & & 0.0021 & & & & & 0.0021 & 0.0672 & & & & & & 0.0066 & \\
\hline HLW99-77 & 0.0021 & 0.0063 & & 0.0021 & 0.0021 & 0.0021 & & 0.0021 & & & & & 0.0021 & 0.0651 & & & & & & 0.0000 & \\
\hline HLW99-78 & 0.0021 & 0.0063 & & 0.0021 & 0.0021 & 0.0021 & & 0.0021 & & & & & 0.0021 & 0.0912 & & & & & & 0.0022 & \\
\hline HLW99-79 & 0.0021 & 0.0063 & & 0.0021 & 0.0021 & 0.0021 & & 0.0021 & & & & & 0.0021 & 0.0912 & & & & & & 0.0000 & \\
\hline HLW99-80 & 0.0020 & 0.0020 & & 0.0020 & 0.0020 & 0.0020 & & 0.0020 & & & & & 0.0020 & 0.0629 & & & & & & 0.0063 & \\
\hline HLW99-81 & 0.0021 & 0.0063 & & 0.0021 & 0.0021 & 0.0021 & & 0.0021 & & & & & 0.0021 & 0.0643 & & & & & & 0.0000 & \\
\hline HLW99-82 & 0.0020 & 0.0020 & & 0.0020 & 0.0020 & 0.0020 & & 0.0020 & & & & & 0.0020 & 0.0355 & & & & & & 0.0063 & \\
\hline HLW99-83 & 0.0020 & 0.0020 & & 0.0020 & 0.0020 & 0.0020 & & 0.0020 & & & & & 0.0020 & 0.0653 & & & & & & 0.0000 & \\
\hline HLW99-84 & 0.0020 & 0.0020 & & 0.0020 & 0.0020 & 0.0020 & & 0.0020 & & & & & 0.0020 & 0.0657 & & & & & & 0.0037 & \\
\hline HLW99-85 & 0.0020 & 0.0020 & & 0.0020 & 0.0020 & 0.0020 & & 0.0020 & & & & & 0.0020 & 0.0355 & & & & & & 0.0031 & \\
\hline HLW99-86 & 0.0020 & 0.0020 & & 0.0020 & 0.0020 & 0.0020 & & 0.0020 & & & & & 0.0020 & 0.0355 & & & & & & 0.0000 & \\
\hline HLW99-87 & 0.0020 & 0.0020 & & 0.0020 & 0.0020 & 0.0020 & & 0.0020 & & & & & 0.0020 & 0.0868 & & & & & & 0.0021 & \\
\hline HLW99-88 & 0.0020 & 0.0020 & & 0.0020 & 0.0020 & 0.0020 & & 0.0020 & & & & & 0.0020 & 0.0355 & & & & & & 0.0063 & \\
\hline HLW99-89 & 0.0020 & 0.0020 & & 0.0020 & 0.0020 & 0.0020 & & 0.0020 & & & & & 0.0020 & 0.0355 & & & & & & 0.0000 & \\
\hline HLW99-90R & 0.0020 & 0.0020 & & 0.0020 & 0.0020 & 0.0020 & & 0.0020 & & & & & 0.0020 & 0.0620 & & & & & & 0.0000 & \\
\hline HLW99-91 & 0.0020 & 0.0020 & & 0.0020 & 0.0020 & 0.0020 & & 0.0020 & & & & & 0.0020 & 0.0868 & & & & & & 0.0000 & \\
\hline HLW99-92 & 0.0020 & 0.0020 & & 0.0020 & 0.0020 & 0.0020 & & 0.0020 & & & & & 0.0020 & 0.0837 & & & & & & 0.0063 & \\
\hline HLW99-93R2 & 0.0020 & 0.0020 & & 0.0020 & 0.0020 & 0.0020 & & 0.0020 & & & & & 0.0020 & 0.0868 & & & & & & 0.0000 & \\
\hline HLW99-94 & 0.0020 & 0.0020 & & 0.0020 & 0.0020 & 0.0020 & & 0.0020 & & & & & 0.0020 & 0.0355 & & & & & & 0.0000 & \\
\hline HLW99-95 & 0.0020 & 0.0020 & & 0.0020 & 0.0020 & 0.0020 & & 0.0020 & & & & & 0.0020 & 0.0355 & & & & & & 0.0000 & \\
\hline HLW99-96 & 0.0020 & 0.0020 & & 0.0020 & 0.0020 & 0.0020 & & 0.0020 & & & & & 0.0020 & 0.0620 & & & & & & 0.0031 & \\
\hline HLW99-97 & 0.0020 & 0.0020 & & 0.0020 & 0.0020 & 0.0020 & & 0.0020 & & & & & 0.0020 & 0.0355 & & & & & & 0.0042 & \\
\hline HLW99-98R & 0.0020 & 0.0020 & & 0.0020 & 0.0020 & 0.0020 & & 0.0020 & & & & & 0.0020 & 0.0639 & & & & & & 0.0063 & \\
\hline HLW99-99 & 0.0020 & 0.0020 & & 0.0020 & 0.0020 & 0.0020 & & 0.0020 & & & & & 0.0020 & 0.0868 & & & & & & 0.0000 & \\
\hline HLW99-100 & 0.0020 & 0.0020 & & 0.0020 & 0.0020 & 0.0020 & & 0.0020 & & & & & 0.0020 & 0.0612 & & & & & & 0.0000 & \\
\hline
\end{tabular}


RPP-WTP HLW Formulation (Kot and Pegg 2001)

\begin{tabular}{|c|c|c|c|c|c|c|c|c|c|c|c|c|c|c|c|c|c|c|c|c|c|}
\hline Glass ID & $\begin{array}{c}\mathrm{PdO} \\
-\mathrm{t}\end{array}$ & $\begin{array}{c}\mathrm{Pr} 2 \mathrm{O} 3 \\
-\mathrm{t}\end{array}$ & $\begin{array}{c}\text { Pr6O11 } \\
-t\end{array}$ & $\begin{array}{c}\mathrm{Rb} 2 \mathrm{O} \\
-\mathrm{t}\end{array}$ & $\begin{array}{c}\mathrm{ReO} \\
-\mathrm{t}\end{array}$ & $\begin{array}{c}\mathrm{ReO} 2 \\
-\mathrm{t}\end{array}$ & $\begin{array}{c}\mathrm{Rh} 2 \mathrm{O} 3 \\
-\mathrm{t}\end{array}$ & $\begin{array}{c}\mathrm{RhO} 2 \\
-\mathrm{t}\end{array}$ & $\begin{array}{c}\mathrm{RuO} 2 \\
-\mathrm{t}\end{array}$ & $\begin{array}{c}\mathrm{Sb} 2 \mathrm{O} 3 \\
-\mathrm{t}\end{array}$ & $\begin{array}{c}\mathrm{Sb} 2 \mathrm{O} 5 \\
-\mathrm{t}\end{array}$ & $\begin{array}{c}\mathrm{SeO} 2 \\
-\mathrm{t}\end{array}$ & $\begin{array}{c}\mathrm{Sm} 2 \mathrm{O} 3 \\
-\mathrm{t}\end{array}$ & $\begin{array}{c}\mathrm{SnO} \\
-\mathrm{t}\end{array}$ & $\begin{array}{c}\mathrm{SnO} 2 \\
-\mathrm{t}\end{array}$ & $\begin{array}{c}\mathrm{SO} 3 \\
-\mathrm{t}\end{array}$ & $\begin{array}{c}\mathrm{SrO} \\
-\mathrm{t}\end{array}$ & $\begin{array}{c}\mathrm{Tc} 2 \mathrm{O} 7 \\
-\mathrm{t}\end{array}$ & $\begin{array}{c}\mathrm{TeO} 2 \\
-\mathrm{t}\end{array}$ & $\begin{array}{c}\mathrm{ThO} 2 \\
-\mathrm{t}\end{array}$ & $\begin{array}{c}\mathrm{TiO}^{2} \\
-\mathrm{t}\end{array}$ \\
\hline HLW99-60 & 0.0004 & & & & & & 0.0008 & & 0.0013 & & 0.0020 & 0.0020 & & & & 0.0050 & 0.0020 & & 0.0020 & 0.0020 & 0.0068 \\
\hline HLW99-61 & 0.0004 & & & & & & 0.0008 & & 0.0013 & & 0.0020 & 0.0020 & & & & 0.0000 & 0.0020 & & 0.0020 & 0.0000 & 0.0100 \\
\hline HLW99-62 & 0.0004 & & & & & & 0.0008 & & 0.0013 & & 0.0020 & 0.0020 & & & & 0.0000 & 0.0020 & & 0.0020 & 0.0000 & 0.0061 \\
\hline HLW99-63 & 0.0004 & & & & & & 0.0008 & & 0.0013 & & 0.0020 & 0.0020 & & & & 0.0021 & 0.0020 & & 0.0020 & 0.0008 & 0.0031 \\
\hline HLW99-64 & 0.0004 & & & & & & 0.0008 & & 0.0013 & & 0.0020 & 0.0020 & & & & 0.0050 & 0.0020 & & 0.0020 & 0.0020 & 0.0100 \\
\hline HLW99-65 & 0.0004 & & & & & & 0.0008 & & 0.0013 & & 0.0020 & 0.0020 & & & & 0.0050 & 0.0020 & & 0.0020 & 0.0020 & 0.0000 \\
\hline HLW99-66 & 0.0004 & & & & & & 0.0008 & & 0.0014 & & 0.0021 & 0.0021 & & & & 0.0026 & 0.0572 & & 0.0021 & 0.0000 & 0.0000 \\
\hline HLW99-67 & \begin{tabular}{|l|}
0.0004 \\
\end{tabular} & & & & & & 0.0008 & & 0.0014 & & 0.0021 & 0.0021 & & & & 0.0026 & 0.1398 & & 0.0021 & 0.0000 & 0.0000 \\
\hline HLW99-68 & 0.0004 & & & & & & 0.0008 & & 0.0014 & & 0.0021 & 0.0021 & & & & 0.0026 & 0.0572 & & 0.0021 & 0.0000 & 0.0066 \\
\hline HLW99-69 & 0.0004 & & & & & & 0.0008 & & 0.0014 & & 0.0021 & 0.0021 & & & & 0.0026 & 0.1348 & & 0.0021 & 0.0000 & 0.0066 \\
\hline HLW99-70 & 0.0004 & & & & & & 0.0008 & & 0.0014 & & 0.0021 & 0.0021 & & & & 0.0026 & 0.1399 & & 0.0021 & 0.0000 & 0.0000 \\
\hline HLW99-71 & 0.0004 & & & & & & 0.0008 & & 0.0014 & & 0.0021 & 0.0021 & & & & 0.0026 & 0.0572 & & 0.0021 & 0.0000 & 0.0000 \\
\hline HLW99-72 & 0.0004 & & & & & & 0.0008 & & 0.0014 & & 0.0021 & 0.0021 & & & & 0.0026 & 0.0572 & & 0.0021 & 0.0000 & 0.0066 \\
\hline HLW99-73 & 0.0004 & & & & & & 0.0008 & & 0.0014 & & 0.0021 & 0.0021 & & & & 0.0026 & 0.0573 & & 0.0021 & 0.0000 & 0.0000 \\
\hline HLW99-74 & 0.0004 & & & & & & 0.0008 & & 0.0014 & & 0.0021 & 0.0021 & & & & 0.0026 & 0.0998 & & 0.0021 & 0.0000 & 0.0033 \\
\hline HLW99-75 & \begin{tabular}{|l|}
0.0004 \\
\end{tabular} & & & & & & 0.0008 & & 0.0014 & & 0.0021 & 0.0021 & & & & 0.0026 & 0.0572 & & 0.0021 & 0.0000 & 0.0044 \\
\hline HLW99-76 & \begin{tabular}{|l|}
0.0004 \\
\end{tabular} & & & & & & 0.0008 & & 0.0014 & & 0.0021 & 0.0021 & & & & 0.0026 & 0.1030 & & 0.0021 & 0.0000 & 0.0066 \\
\hline HLW99-77 & 0.0004 & & & & & & 0.0008 & & 0.0014 & & 0.0021 & 0.0021 & & & & 0.0026 & 0.0999 & & 0.0021 & 0.0000 & 0.0000 \\
\hline HLW99-78 & 0.0004 & & & & & & 0.0008 & & 0.0014 & & 0.0021 & 0.0021 & & & & 0.0026 & 0.1399 & & 0.0021 & 0.0000 & 0.0022 \\
\hline HLW99-79 & 0.0004 & & & & & & 0.0008 & & 0.0014 & & 0.0021 & 0.0021 & & & & 0.0026 & 0.1399 & & 0.0021 & 0.0000 & 0.0000 \\
\hline HLW99-80 & 0.0004 & & & & & & 0.0008 & & 0.0013 & & 0.0020 & 0.0020 & & & & 0.0025 & 0.0966 & & 0.0020 & 0.0000 & 0.0063 \\
\hline HLW99-81 & 0.0004 & & & & & & 0.0008 & & 0.0014 & & 0.0021 & 0.0021 & & & & 0.0026 & 0.0986 & & 0.0021 & 0.0000 & 0.0000 \\
\hline HLW99-82 & 0.0004 & & & & & & 0.0008 & & 0.0013 & & 0.0020 & 0.0020 & & & & 0.0025 & 0.0545 & & 0.0020 & 0.0000 & 0.0063 \\
\hline HLW99-83 & 0.0004 & & & & & & 0.0008 & & 0.0013 & & 0.0020 & 0.0020 & & & & 0.0025 & 0.1001 & & 0.0020 & 0.0000 & 0.0000 \\
\hline HLW99-84 & 0.0004 & & & & & & 0.0008 & & 0.0013 & & 0.0020 & 0.0020 & & & & 0.0025 & 0.1007 & & 0.0020 & 0.0000 & 0.0037 \\
\hline HLW99-85 & 0.0004 & & & & & & 0.0008 & & 0.0013 & & 0.0020 & 0.0020 & & & & 0.0025 & 0.0545 & & 0.0020 & 0.0000 & 0.0031 \\
\hline HLW99-86 & 0.0004 & & & & & & 0.0008 & & 0.0013 & & 0.0020 & 0.0020 & & & & 0.0025 & 0.0545 & & 0.0020 & 0.0000 & 0.0000 \\
\hline HLW99-87 & 0.0004 & & & & & & 0.0008 & & 0.0013 & & 0.0020 & 0.0020 & & & & 0.0025 & 0.1332 & & 0.0020 & 0.0000 & 0.0021 \\
\hline HLW99-88 & 0.0004 & & & & & & 0.0008 & & 0.0013 & & 0.0020 & 0.0020 & & & & 0.0025 & 0.0545 & & 0.0020 & 0.0000 & 0.0063 \\
\hline HLW99-89 & 0.0004 & & & & & & 0.0008 & & 0.0013 & & 0.0020 & 0.0020 & & & & 0.0025 & 0.0545 & & 0.0020 & 0.0000 & 0.0000 \\
\hline HLW99-90R & 0.0004 & & & & & & 0.0008 & & 0.0013 & & 0.0020 & 0.0020 & & & & 0.0025 & 0.0950 & & 0.0020 & 0.0000 & 0.0000 \\
\hline HLW99-91 & 0.0004 & & & & & & 0.0008 & & 0.0013 & & 0.0020 & 0.0020 & & & & 0.0025 & 0.1332 & & 0.0020 & 0.0000 & 0.0000 \\
\hline HLW99-92 & 0.0004 & & & & & & 0.0008 & & 0.0013 & & 0.0020 & 0.0020 & & & & 0.0025 & 0.1283 & & 0.0020 & 0.0000 & 0.0063 \\
\hline HLW99-93R2 & 0.0004 & & & & & & 0.0008 & & 0.0013 & & 0.0020 & 0.0020 & & & & 0.0025 & 0.1332 & & 0.0020 & 0.0000 & 0.0000 \\
\hline HLW99-94 & 0.0004 & & & & & & 0.0008 & & 0.0013 & & 0.0020 & 0.0020 & & & & 0.0025 & 0.0545 & & 0.0020 & 0.0000 & 0.0000 \\
\hline HLW99-95 & 0.0004 & & & & & & 0.0008 & & 0.0013 & & 0.0020 & 0.0020 & & & & 0.0025 & 0.0545 & & 0.0020 & 0.0000 & 0.0000 \\
\hline HLW99-96 & 0.0004 & & & & & & 0.0008 & & 0.0013 & & 0.0020 & 0.0020 & & & & 0.0025 & 0.0950 & & 0.0020 & 0.0000 & 0.0031 \\
\hline HLW99-97 & 0.0004 & & & & & & 0.0008 & & 0.0013 & & 0.0020 & 0.0020 & & & & 0.0025 & 0.0545 & & 0.0020 & 0.0000 & 0.0042 \\
\hline HLW99-98R & 0.0004 & & & & & & 0.0008 & & 0.0013 & & 0.0020 & 0.0020 & & & & 0.0025 & 0.0981 & & 0.0020 & 0.0000 & 0.0063 \\
\hline HLW99-99 & 0.0004 & & & & & & 0.0008 & & 0.0013 & & 0.0020 & 0.0020 & & & & 0.0025 & 0.1332 & & 0.0020 & 0.0000 & 0.0000 \\
\hline HLW99-100 & 0.0004 & & & & & & 0.0008 & & 0.0013 & & 0.0020 & 0.0020 & & & & 0.0025 & 0.0938 & & 0.0020 & 0.0000 & 0.0000 \\
\hline
\end{tabular}


RPP-WTP HLW Formulation (Kot and Pegg 2001)

\begin{tabular}{|c|c|c|c|c|c|c|c|c|c|c|c|c|c|c|c|c|c|c|c|c|c|}
\hline Glass ID & $\begin{array}{c}\mathrm{T} 12 \mathrm{O} 3 \\
-\mathrm{t}\end{array}$ & $\begin{array}{c}\text { U3O8 } \\
-t\end{array}$ & $\begin{array}{c}\mathrm{UO} 2 \\
-\mathrm{t}\end{array}$ & $\begin{array}{c}\text { UO3 } \\
-t\end{array}$ & $\begin{array}{c}\mathrm{V} 2 \mathrm{O} 5 \\
-\mathrm{t}\end{array}$ & $\begin{array}{c}\text { WO3 } \\
-t\end{array}$ & $\begin{array}{c}\mathrm{Y} 2 \mathrm{O} 3 \\
-\mathrm{t}\end{array}$ & $\begin{array}{c}\mathrm{ZnO} \\
-\mathrm{t}\end{array}$ & $\begin{array}{c}\text { Others } \\
-t\end{array}$ & $\begin{array}{c}\text { Sum } \\
-t\end{array}$ & $\begin{array}{c}\mathrm{A} 12 \mathrm{O} 3 \\
-\mathrm{a}\end{array}$ & $\begin{array}{c}\mathrm{B} 2 \mathrm{O} 3 \\
-\mathrm{a}\end{array}$ & $\begin{array}{c}\mathrm{CaO} \\
-\mathrm{a}\end{array}$ & $\begin{array}{c}\mathrm{Fe} 2 \mathrm{O} 3 \\
-\mathrm{a}\end{array}$ & $\begin{array}{c}\mathrm{FeO} \\
-\mathrm{a}\end{array}$ & $\begin{array}{c}\mathrm{K} 2 \mathrm{O} \\
-\mathrm{a}\end{array}$ & $\begin{array}{c}\mathrm{Li} 2 \mathrm{O} \\
-\mathrm{a}\end{array}$ & $\begin{array}{c}\mathrm{MgO} \\
-\mathrm{a}\end{array}$ & $\begin{array}{c}\mathrm{Na} 2 \mathrm{O} \\
-\mathrm{a}\end{array}$ & $\begin{array}{c}\mathrm{NiO} \\
-\mathrm{a}\end{array}$ & $\begin{array}{c}\mathrm{P} 2 \mathrm{O} 5 \\
-\mathrm{a}\end{array}$ \\
\hline HLW99-60 & 0.0020 & & 0.0800 & & & 0.0020 & & 0.0200 & & 1.0001 & 0.0115 & 0.1289 & 0.0012 & 0.1029 & & 0.0106 & 0.0209 & 0.0129 & 0.0640 & 0.0154 & 0.0333 \\
\hline HLW99-61 & 0.0020 & & 0.0000 & & & 0.0020 & & 0.0200 & & 1.0000 & 0.0285 & 0.1873 & 0.0008 & 0.0191 & & 0.0103 & 0.0003 & 0.0182 & 0.1456 & 0.0277 & 0.0002 \\
\hline HLW99-62 & 0.0020 & & 0.0000 & & & 0.0020 & & 0.0200 & & 0.9999 & 0.0062 & 0.0521 & 0.0014 & 0.0207 & & 0.0116 & 0.0318 & 0.0128 & 0.1112 & 0.0183 & 0.0013 \\
\hline HLW99-63 & 0.0020 & & 0.0332 & & & 0.0020 & & 0.0200 & & 1.0000 & 0.0499 & 0.1151 & 0.0279 & 0.0583 & & 0.0105 & 0.0281 & 0.0068 & 0.0875 & 0.0089 & 0.0151 \\
\hline HLW99-64 & 0.0020 & & 0.0800 & & & 0.0020 & & 0.0200 & & 1.0000 & 0.0541 & 0.0890 & 0.0017 & 0.0211 & & 0.0111 & 0.0605 & \begin{tabular}{|l|}
0.0186 \\
\end{tabular} & 0.0721 & 0.0273 & 0.0333 \\
\hline HLW99-65 & 0.0020 & & 0.0800 & & & 0.0020 & & 0.0200 & & 1.0000 & 0.1006 & 0.1821 & 0.0006 & 0.0194 & & 0.0120 & 0.0020 & 0.0006 & 0.1653 & 0.0008 & 0.0365 \\
\hline HLW99-66 & 0.0021 & & 0.0000 & & & 0.0021 & & 0.0210 & & 0.9997 & 0.0703 & 0.0541 & 0.0215 & 0.0205 & & 0.0107 & 0.0303 & 0.0002 & 0.0874 & 0.0001 & 0.0021 \\
\hline HLW99-67 & 0.0021 & & 0.0000 & & & 0.0021 & & 0.0210 & & 0.9999 & 0.0728 & 0.0542 & 0.0225 & 0.1062 & & 0.0104 & 0.0293 & 0.0003 & 0.0541 & 0.0001 & 0.0028 \\
\hline HLW99-68 & 0.0021 & & 0.0000 & & & 0.0021 & & 0.0210 & & 1.0000 & 0.0742 & 0.0553 & 0.0209 & 0.1172 & & 0.0107 & 0.0285 & 0.0128 & 0.0634 & 0.0177 & 0.0027 \\
\hline HLW99-69 & 0.0021 & & 0.0000 & & & 0.0021 & & 0.0210 & & 1.0000 & 0.0746 & 0.0568 & 0.0217 & 0.0209 & & 0.0101 & 0.0293 & 0.0133 & 0.0491 & 0.0188 & 0.0032 \\
\hline HLW99-70 & 0.0021 & & 0.0000 & & & 0.0021 & & 0.0210 & & 0.9997 & 0.0813 & 0.0533 & 0.0219 & 0.0264 & & 0.0100 & 0.0312 & \begin{tabular}{|l|}
0.0003 \\
\end{tabular} & 0.0988 & 0.0008 & 0.0008 \\
\hline HLW99-71 & 0.0021 & & 0.0000 & & & 0.0021 & & 0.0210 & & 0.9998 & 0.0758 & 0.0547 & 0.0226 & 0.1180 & & 0.0105 & 0.0307 & 0.0002 & 0.0754 & 0.0001 & 0.0025 \\
\hline HLW99-72 & 0.0021 & & 0.0000 & & & 0.0021 & & 0.0210 & & 0.9998 & 0.0741 & 0.0567 & 0.0240 & 0.0232 & & 0.0111 & 0.0335 & 0.0133 & 0.0772 & 0.0205 & 0.0020 \\
\hline HLW99-73 & 0.0021 & & 0.0000 & & & 0.0021 & & 0.0210 & & 0.9997 & 0.0599 & 0.0575 & 0.0235 & 0.0233 & & 0.0112 & 0.0383 & 0.0003 & 0.1209 & 0.0002 & 0.0024 \\
\hline HLW99-74 & 0.0021 & & 0.0000 & & & 0.0021 & & 0.0210 & & 1.0000 & 0.0661 & 0.0543 & 0.0228 & 0.1191 & & 0.0106 & 0.0364 & 0.0068 & 0.0521 & 0.0094 & 0.0020 \\
\hline HLW99-75 & 0.0021 & & 0.0000 & & & 0.0021 & & 0.0210 & & 0.9997 & 0.0599 & 0.0548 & 0.0291 & 0.1220 & & 0.0115 & 0.0398 & 0.0094 & 0.0909 & 0.0131 & 0.0038 \\
\hline HLW99-76 & 0.0021 & & 0.0000 & & & 0.0021 & & 0.0210 & & 0.9998 & 0.0692 & 0.0541 & 0.0222 & 0.0212 & & 0.0115 & 0.0366 & 0.0140 & 0.0898 & 0.0194 & 0.0024 \\
\hline HLW99-77 & 0.0021 & & 0.0000 & & & 0.0021 & & 0.0210 & & 0.9998 & 0.0679 & 0.0558 & 0.0235 & 0.1161 & & 0.0109 & 0.0381 & 0.0003 & 0.0849 & 0.0001 & 0.0027 \\
\hline HLW99-78 & 0.0021 & & 0.0000 & & & 0.0021 & & 0.0210 & & 0.9999 & 0.0718 & 0.0544 & 0.0223 & 0.0546 & & 0.0115 & 0.0352 & 0.0048 & 0.0768 & 0.0069 & 0.0029 \\
\hline HLW99-79 & 0.0021 & & 0.0000 & & & 0.0021 & & 0.0210 & & 0.9998 & 0.0726 & 0.0553 & 0.0224 & 0.0194 & & 0.0113 & 0.0299 & 0.0003 & 0.0697 & 0.0001 & 0.0024 \\
\hline HLW99-80 & 0.0020 & & 0.0000 & & & 0.0020 & & 0.0200 & & 1.0002 & 0.0756 & 0.1035 & 0.0216 & 0.0677 & & 0.0102 & 0.0281 & 0.0121 & 0.0483 & 0.0179 & 0.0030 \\
\hline HLW99-81 & 0.0021 & & 0.0000 & & & 0.0021 & & 0.0210 & & 0.9998 & 0.0712 & 0.0543 & 0.0212 & 0.0213 & & 0.0116 & 0.0305 & 0.0002 & 0.1116 & 0.0001 & 0.0028 \\
\hline HLW99-82 & 0.0020 & & 0.0000 & & & 0.0020 & & 0.0200 & & 1.0002 & 0.0681 & 0.1034 & 0.0207 & 0.0553 & & 0.0107 & 0.0290 & \begin{tabular}{|l|}
0.0128 \\
\end{tabular} & 0.0926 & 0.0178 & 0.0043 \\
\hline HLW99-83 & 0.0020 & & 0.0000 & & & 0.0020 & & 0.0200 & & 1.0000 & 0.0707 & 0.1054 & 0.0210 & 0.0777 & & 0.0109 & 0.0289 & 0.0003 & 0.0637 & 0.0001 & 0.0037 \\
\hline HLW99-84 & 0.0020 & & 0.0000 & & & 0.0020 & & 0.0200 & & 1.0002 & 0.0654 & 0.1007 & 0.0198 & 0.0189 & & 0.0108 & 0.0273 & 0.0077 & 0.0591 & 0.0110 & 0.0034 \\
\hline HLW99-85 & 0.0020 & & 0.0000 & & & 0.0020 & & 0.0200 & & 1.0001 & 0.0737 & 0.1125 & 0.0219 & 0.0655 & & 0.0104 & 0.0292 & 0.0062 & 0.0715 & 0.0090 & 0.0041 \\
\hline HLW99-86 & 0.0020 & & 0.0000 & & & 0.0020 & & 0.0200 & & 1.0000 & 0.0635 & 0.1000 & 0.0195 & 0.0190 & & 0.0111 & 0.0270 & 0.0002 & 0.0808 & 0.0001 & 0.0035 \\
\hline HLW99-87 & 0.0020 & & 0.0000 & & & 0.0020 & & 0.0200 & & 1.0001 & 0.0784 & 0.1047 & 0.0225 & 0.0504 & & 0.0100 & 0.0301 & 0.0041 & 0.0752 & 0.0060 & 0.0034 \\
\hline HLW99-88 & 0.0020 & & 0.0000 & & & 0.0020 & & 0.0200 & & 1.0002 & 0.0674 & 0.1038 & 0.0199 & 0.1124 & & 0.0110 & 0.0280 & 0.0121 & 0.0600 & 0.0171 & 0.0028 \\
\hline HLW99-89 & 0.0020 & & 0.0000 & & & 0.0020 & & 0.0200 & & 1.0000 & 0.0717 & 0.1036 & 0.0213 & 0.1090 & & 0.0107 & 0.0289 & 0.0001 & 0.0714 & 0.0001 & 0.0040 \\
\hline HLW99-90R & 0.0020 & & 0.0000 & & & 0.0020 & & 0.0200 & & 1.0000 & 0.0680 & 0.0985 & 0.0193 & 0.1129 & & 0.0109 & 0.0269 & 0.0002 & 0.0838 & 0.0001 & 0.0031 \\
\hline HLW99-91 & 0.0020 & & 0.0000 & & & 0.0020 & & 0.0200 & & 1.0000 & 0.0712 & 0.0991 & 0.0206 & 0.1022 & & 0.0100 & 0.0295 & 0.0002 & 0.0435 & 0.0001 & 0.0037 \\
\hline HLW99-92 & 0.0020 & & 0.0000 & & & 0.0020 & & 0.0200 & & 1.0002 & 0.0742 & 0.1000 & 0.0195 & 0.0201 & & 0.0105 & 0.0290 & $\mid 0.0117$ & 0.0459 & 0.0171 & 0.0037 \\
\hline HLW99-93R2 & 0.0020 & & 0.0000 & & & 0.0020 & & 0.0200 & & 1.0000 & 0.0795 & 0.1032 & 0.0215 & 0.0201 & & 0.0112 & 0.0290 & 0.0004 & 0.0926 & 0.0001 & 0.0032 \\
\hline HLW99-94 & 0.0020 & & 0.0000 & & & 0.0020 & & 0.0200 & & 1.0000 & 0.0687 & 0.1022 & 0.0197 & 0.0198 & & 0.0114 & 0.0300 & 0.0002 & 0.1173 & 0.0001 & 0.0033 \\
\hline HLW99-95 & 0.0020 & & 0.0000 & & & 0.0020 & & 0.0200 & & 1.0000 & 0.0680 & 0.0996 & 0.0198 & 0.0199 & & 0.0116 & 0.0289 & 0.0002 & 0.1166 & 0.0001 & 0.0044 \\
\hline HLW99-96 & 0.0020 & & 0.0000 & & & 0.0020 & & 0.0200 & & 1.0001 & 0.0751 & 0.1030 & 0.0209 & 0.1169 & & 0.0109 & 0.0284 & 0.0064 & 0.0479 & 0.0094 & 0.0033 \\
\hline HLW99-97 & 0.0020 & & 0.0000 & & & 0.0020 & & 0.0200 & & 1.0002 & 0.0714 & 0.1026 & 0.0214 & 0.1151 & & 0.0109 & 0.0287 & 0.0082 & 0.0851 & 0.0124 & 0.0031 \\
\hline HLW99-98R & 0.0020 & & 0.0000 & & & 0.0020 & & 0.0200 & & 1.0002 & 0.0702 & 0.0992 & 0.0209 & 0.0202 & & 0.0104 & 0.0274 & 0.0111 & 0.0858 & 0.0172 & 0.0033 \\
\hline HLW99-99 & 0.0020 & & 0.0000 & & & 0.0020 & & 0.0200 & & 1.0000 & 0.0731 & 0.1032 & 0.0219 & 0.0208 & & 0.0106 & 0.0321 & 0.0003 & 0.0654 & 0.0001 & 0.0013 \\
\hline HLW99-100 & 0.0020 & & 0.0000 & & & 0.0020 & & 0.0200 & & 1.0000 & 0.0675 & 0.1002 & 0.0195 & 0.0193 & & 0.0112 & 0.0286 & 0.0002 & 0.1063 & 0.0002 & 0.0042 \\
\hline
\end{tabular}


RPP-WTP HLW Formulation (Kot and Pegg 2001)

\begin{tabular}{|c|c|c|c|c|c|c|c|c|c|c|c|c|c|c|c|c|c|c|c|c|c|}
\hline Glass ID & $\begin{array}{c}\mathrm{SiO} 2 \\
-\mathrm{a}\end{array}$ & $\begin{array}{c}\mathrm{ZrO} 2 \\
-\mathrm{a}\end{array}$ & $\begin{array}{c}\mathrm{Ag} 2 \mathrm{O} \\
-\mathrm{a}\end{array}$ & $\begin{array}{c}\mathrm{As} 2 \mathrm{O} 3 \\
-\mathrm{a}\end{array}$ & $\begin{array}{c}\mathrm{BaO} \\
-\mathrm{a}\end{array}$ & $\begin{array}{c}\mathrm{Bi} 2 \mathrm{O} 3 \\
-\mathrm{a}\end{array}$ & $\begin{array}{l}\mathrm{Br} \\
-\mathrm{a}\end{array}$ & $\begin{array}{c}\mathrm{CdO} \\
-\mathrm{a}\end{array}$ & $\begin{array}{c}\mathrm{Ce} 2 \mathrm{O} 3 \\
-\mathrm{a}\end{array}$ & $\begin{array}{c}\mathrm{CeO} 2 \\
-\mathrm{a}\end{array}$ & $\begin{array}{l}\mathrm{Cl} \\
-\mathrm{a}\end{array}$ & $\begin{array}{c}\mathrm{CoO} \\
-\mathrm{a}\end{array}$ & $\begin{array}{c}\mathrm{Co} 2 \mathrm{O} 3 \\
-\mathrm{a}\end{array}$ & $\begin{array}{c}\mathrm{Cr} 2 \mathrm{O} 3 \\
-\mathrm{a}\end{array}$ & $\begin{array}{c}\mathrm{Cs} 2 \mathrm{O} \\
-\mathrm{a}\end{array}$ & $\begin{array}{c}\mathrm{CuO} \\
-\mathrm{a}\end{array}$ & $\begin{array}{c}\mathrm{Eu} 2 \mathrm{O} 3 \\
-\mathrm{a}\end{array}$ & $\begin{array}{l}\mathrm{F} \\
-\mathrm{a}\end{array}$ & $\begin{array}{c}\mathrm{Ga} 2 \mathrm{O} 3 \\
-\mathrm{a}\end{array}$ & $\begin{array}{c}\mathrm{Gd} 2 \mathrm{O} 3 \\
-\mathrm{a}\end{array}$ & $\begin{array}{c}\mathrm{HgO} \\
-\mathrm{a}\end{array}$ \\
\hline HLW99-60 & 0.3278 & 0.0004 & 0.0026 & 0.0020 & 0.0218 & 0.0127 & & 0.0181 & & & & 0.0021 & & 0.0021 & & 0.0042 & & & & & \\
\hline HLW99-61 & 0.3710 & 0.0002 & 0.0024 & 0.0020 & 0.0384 & 0.0197 & & 0.0269 & & & & 0.0019 & & 0.0001 & & 0.0022 & & & & & \\
\hline HLW99-62 & 0.4978 & 0.0779 & 0.0026 & 0.0020 & 0.0243 & 0.0124 & & 0.0176 & & & & 0.0021 & & 0.0000 & & 0.0023 & & & & & \\
\hline HLW99-63 & 0.3686 & 0.0293 & 0.0027 & 0.0025 & 0.0113 & 0.0068 & & 0.0095 & & & & 0.0022 & & 0.0018 & & 0.0023 & & & & & \\
\hline HLW99-64 & 0.3541 & 0.0007 & 0.0026 & 0.0017 & 0.0330 & 0.0196 & & 0.0275 & & & & 0.0022 & & 0.0035 & & 0.0021 & & & & & \\
\hline HLW99-65 & 0.2851 & 0.0002 & 0.0025 & 0.0020 & 0.0010 & 0.0007 & & 0.0006 & & & & 0.0026 & & 0.0046 & & 0.0019 & & & & & \\
\hline HLW99-66 & 0.4843 & 0.0256 & 0.0022 & 0.0019 & 0.0012 & 0.0001 & & 0.0000 & & & & 0.0065 & & 0.0020 & & 0.0021 & & & & & \\
\hline HLW99-67 & 0.3144 & 0.0283 & 0.0026 & 0.0016 & 0.0024 & 0.0000 & & & & & & 0.0060 & & 0.0019 & & 0.0024 & & & & & \\
\hline HLW99-68 & 0.3254 & 0.0297 & 0.0028 & 0.0018 & 0.0225 & 0.0135 & & 0.0190 & & & & 0.0058 & & 0.0017 & & 0.0022 & & & & & \\
\hline HLW99-69 & 0.3218 & 0.0276 & 0.0027 & 0.0015 & 0.0268 & 0.0139 & & 0.0190 & & & & 0.0061 & & 0.0018 & & 0.0020 & & & & & \\
\hline HLW99-70 & 0.3586 & 0.0238 & 0.0026 & 0.0022 & 0.0026 & 0.0000 & & 0.0002 & & & & 0.0058 & & 0.0031 & & 0.0025 & & & & & \\
\hline HLW99-71 & 0.4012 & 0.0313 & 0.0028 & 0.0022 & 0.0011 & 0.0007 & & 0.0000 & & & & 0.0060 & & 0.0018 & & 0.0023 & & & & & \\
\hline HLW99-72 & 0.4250 & 0.0298 & 0.0030 & 0.0020 & 0.0270 & 0.0143 & & 0.0199 & & & & 0.0065 & & 0.0018 & & 0.0023 & & & & & \\
\hline HLW99-73 & 0.4465 & 0.0345 & 0.0029 & 0.0020 & 0.0012 & 0.0003 & & 0.0001 & & & & 0.0062 & & 0.0019 & & 0.0023 & & & & & \\
\hline HLW99-74 & 0.3167 & 0.0332 & 0.0029 & 0.0021 & 0.0151 & 0.0074 & & 0.0099 & & & & 0.0059 & & 0.0019 & & 0.0024 & & & & & \\
\hline HLW99-75 & 0.3214 & 0.0277 & 0.0026 & 0.0021 & 0.0189 & 0.0096 & & 0.0131 & & & & 0.0064 & & 0.0019 & & 0.0023 & & & & & \\
\hline HLW99-76 & 0.3295 & 0.0271 & 0.0029 & 0.0019 & 0.0273 & 0.0138 & & 0.0197 & & & & 0.0064 & & 0.0020 & & 0.0022 & & & & & \\
\hline HLW99-77 & 0.3115 & 0.0300 & 0.0029 & 0.0021 & 0.0019 & 0.0007 & & 0.0001 & & & & 0.0061 & & 0.0019 & & 0.0025 & & & & & \\
\hline HLW99-78 & 0.3260 & 0.0279 & 0.0029 & 0.0021 & 0.0115 & 0.0053 & & 0.0066 & & & & 0.0064 & & 0.0019 & & 0.0021 & & & & & \\
\hline HLW99-79 & 0.3910 & 0.0227 & 0.0029 & 0.0023 & 0.0026 & 0.0015 & & 0.0000 & & & & 0.0064 & & 0.0019 & & 0.0024 & & & & & \\
\hline HLW99-80 & 0.3021 & 0.0256 & 0.0025 & 0.0015 & 0.0247 & 0.0131 & & 0.0191 & & & & 0.0020 & & 0.0018 & & 0.0022 & & & & & \\
\hline HLW99-81 & 0.4005 & 0.0274 & 0.0027 & 0.0022 & 0.0001 & 0.0014 & & 0.0000 & & & & 0.0064 & & 0.0020 & & 0.0031 & & & & & \\
\hline HLW99-82 & 0.3303 & 0.0274 & 0.0026 & 0.0021 & 0.0246 & 0.0131 & & 0.0190 & & & & 0.0021 & & 0.0019 & & 0.0021 & & & & & \\
\hline HLW99-83 & 0.3673 & 0.0244 & 0.0025 & 0.0023 & 0.0001 & 0.0012 & & 0.0000 & & & & 0.0022 & & 0.0018 & & 0.0022 & & & & & \\
\hline HLW99-84 & 0.3612 & 0.0249 & 0.0026 & 0.0018 & 0.0144 & 0.0073 & & 0.0115 & & & & 0.0021 & & 0.0017 & & 0.0026 & & & & & \\
\hline HLW99-85 & 0.3868 & 0.0278 & 0.0027 & 0.0016 & 0.0135 & 0.0067 & & 0.0092 & & & & 0.0020 & & 0.0018 & & 0.0036 & & & & & \\
\hline HLW99-86 & 0.4739 & 0.0285 & 0.0026 & 0.0021 & 0.0001 & 0.0003 & & 0.0000 & & & & 0.0022 & & 0.0018 & & 0.0019 & & & & & \\
\hline HLW99-87 & 0.3013 & 0.0277 & 0.0025 & 0.0017 & 0.0085 & 0.0045 & & 0.0062 & & & & 0.0020 & & 0.0017 & & 0.0022 & & & & & \\
\hline HLW99-88 & 0.3030 & 0.0261 & 0.0024 & 0.0020 & 0.0238 & 0.0127 & & 0.0197 & & & & 0.0020 & & 0.0017 & & 0.0022 & & & & & \\
\hline HLW99-89 & 0.3889 & 0.0252 & 0.0025 & 0.0020 & 0.0001 & 0.0004 & & 0.0000 & & & & 0.0021 & & 0.0016 & & 0.0023 & & & & & \\
\hline HLW99-90R & 0.2948 & 0.0246 & 0.0027 & 0.0019 & 0.0001 & 0.0002 & & 0.0000 & & & & 0.0020 & & 0.0018 & & 0.0021 & & & & & \\
\hline HLW99-91 & 0.3069 & 0.0229 & 0.0025 & 0.0016 & 0.0001 & 0.0006 & & 0.0000 & & & & 0.0020 & & 0.0017 & & 0.0022 & & & & & \\
\hline HLW99-92 & 0.3067 & 0.0278 & 0.0023 & 0.0021 & 0.0246 & 0.0123 & & 0.0179 & & & & 0.0021 & & 0.0019 & & 0.0020 & & & & & \\
\hline HLW99-93R2 & 0.3465 & 0.0280 & 0.0026 & 0.0022 & 0.0024 & 0.0001 & & 0.0000 & & & & 0.0020 & & 0.0018 & & 0.0021 & & & & & \\
\hline HLW99-94 & 0.4262 & 0.0240 & 0.0020 & 0.0017 & 0.0001 & 0.0002 & & 0.0001 & & & & 0.0021 & & 0.0021 & & 0.0022 & & & & & \\
\hline HLW99-95 & 0.4293 & 0.0236 & 0.0021 & 0.0019 & 0.0010 & 0.0002 & & 0.0001 & & & & 0.0023 & & 0.0020 & & 0.0021 & & & & & \\
\hline HLW99-96 & 0.3099 & 0.0298 & 0.0027 & 0.0021 & 0.0142 & 0.0067 & & 0.0102 & & & & 0.0022 & & 0.0018 & & 0.0024 & & & & & \\
\hline HLW99-97 & 0.3048 & 0.0271 & 0.0024 & 0.0022 & 0.0177 & 0.0086 & & 0.0134 & & & & 0.0021 & & 0.0018 & & 0.0022 & & & & & \\
\hline HLW99-98R & 0.3359 & 0.0257 & 0.0025 & 0.0023 & 0.0258 & 0.0126 & & 0.0181 & & & & 0.0020 & & 0.0019 & & 0.0023 & & & & & \\
\hline HLW99-99 & 0.3880 & 0.0240 & 0.0026 & 0.0022 & 0.0024 & 0.0003 & & 0.0001 & & & & 0.0019 & & 0.0016 & & 0.0026 & & & & & \\
\hline HLW99-100 & 0.3935 & 0.0217 & 0.0021 & 0.0021 & 0.0016 & 0.0002 & & 0.0000 & & & & 0.0021 & & 0.0020 & & 0.0021 & & & & & \\
\hline
\end{tabular}


RPP-WTP HLW Formulation (Kot and Pegg 2001)

\begin{tabular}{|c|c|c|c|c|c|c|c|c|c|c|c|c|c|c|c|c|c|c|c|c|c|}
\hline Glass ID & $\begin{array}{c} \\
-a\end{array}$ & $\begin{array}{c}\mathrm{La} 2 \mathrm{O} 3 \\
-\mathrm{a}\end{array}$ & $\begin{array}{c}\mathrm{MnO} 2 \\
-\mathrm{a}\end{array}$ & $\begin{array}{c}\mathrm{MnO} \\
-\mathrm{a}\end{array}$ & $\begin{array}{c}\mathrm{MoO} \\
-\mathrm{a}\end{array}$ & $\begin{array}{c}\mathrm{MoO} 3 \\
-\mathrm{a}\end{array}$ & $\begin{array}{c}\mathrm{Nb} 2 \mathrm{O} 5 \\
-\mathrm{a}\end{array}$ & $\begin{array}{c}\mathrm{Nd} 2 \mathrm{O} 3 \\
-\mathrm{a}\end{array}$ & $\begin{array}{c}\mathrm{PbO} \\
-\mathrm{a}\end{array}$ & $\begin{array}{c}\mathrm{PdO} 2 \\
-\mathrm{a}\end{array}$ & $\begin{array}{c}\mathrm{PdO} \\
-\mathrm{a}\end{array}$ & $\begin{array}{c}\mathrm{Pr} 2 \mathrm{O} 3 \\
-\mathrm{a}\end{array}$ & $\begin{array}{c}\operatorname{Pr} 6011 \\
-\mathrm{a}\end{array}$ & $\begin{array}{c}\mathrm{Rb} 2 \mathrm{O} \\
-\mathrm{a}\end{array}$ & $\begin{array}{c}\mathrm{ReO} \\
-\mathrm{a}\end{array}$ & $\begin{array}{c}\mathrm{ReO} 2 \\
-\mathrm{a}\end{array}$ & $\begin{array}{c}\mathrm{Rh} 2 \mathrm{O} 3 \\
-\mathrm{a}\end{array}$ & $\begin{array}{c}\mathrm{RhO} 2 \\
-\mathrm{a}\end{array}$ & $\begin{array}{c}\mathrm{RuO} 2 \\
-\mathrm{a}\end{array}$ & $\begin{array}{c}\mathrm{Sb} 2 \mathrm{O} 3 \\
-\mathrm{a}\end{array}$ & $\begin{array}{c}\mathrm{Sb} 2 \mathrm{O} 5 \\
-\mathrm{a}\end{array}$ \\
\hline HLW99-60 & & & 0.0024 & & & & & & 0.0074 & & 0.0003 & & & & & & 0.0003 & & 0.0002 & & 0.0022 \\
\hline HLW99-61 & & & 0.0023 & & & & & & 0.0104 & & & & & & & & & & & & 0.0021 \\
\hline HLW99-62 & & & 0.0023 & & & & & & 0.0006 & & & & & & & & & & & & 0.0021 \\
\hline HLW99-63 & & & 0.0024 & & & & & & 0.0040 & & 0.0002 & & & & & & 0.0003 & & 0.0002 & & 0.0023 \\
\hline HLW99-64 & & & 0.0023 & & & & & & 0.0105 & & 0.0003 & & & & & & 0.0002 & & 0.0002 & & 0.0021 \\
\hline HLW99-65 & & & 0.0024 & & & & & & 0.0007 & & 0.0002 & & & & & & 0.0001 & & 0.0004 & & 0.0022 \\
\hline HLW99-66 & & & 0.0376 & & & & & & 0.0005 & & 0.0003 & & & & & & 0.0002 & & 0.0001 & & 0.0048 \\
\hline HLW99-67 & & & 0.0861 & & & & & & 0.0009 & & 0.0005 & & & & & & 0.0009 & & 0.0004 & & 0.0042 \\
\hline HLW99-68 & & & 0.0364 & & & & & & 0.0071 & & 0.0005 & & & & & & 0.0009 & & 0.0002 & & 0.0049 \\
\hline HLW99-69 & & & 0.0859 & & & & & & 0.0078 & & 0.0005 & & & & & & 0.0008 & & 0.0002 & & 0.0044 \\
\hline HLW99-70 & & & 0.0908 & & & & & & 0.0008 & & 0.0004 & & & & & & 0.0002 & & 0.0002 & & 0.0025 \\
\hline HLW99-71 & & & 0.0392 & & & & & & 0.0005 & & 0.0003 & & & & & & 0.0008 & & 0.0002 & & 0.0028 \\
\hline HLW99-72 & & & 0.0400 & & & & & & 0.0076 & & 0.0003 & & & & & & 0.0002 & & 0.0002 & & 0.0027 \\
\hline HLW99-73 & & & 0.0401 & & & & & & 0.0005 & & 0.0003 & & & & & & 0.0003 & & 0.0002 & & 0.0030 \\
\hline HLW99-74 & & & 0.0653 & & & & & & 0.0042 & & 0.0004 & & & & & & 0.0009 & & 0.0002 & & 0.0029 \\
\hline HLW99-75 & & & 0.0389 & & & & & & 0.0053 & & 0.0004 & & & & & & 0.0009 & & 0.0004 & & 0.0023 \\
\hline HLW99-76 & & & 0.0669 & & & & & & 0.0078 & & 0.0005 & & & & & & 0.0007 & & 0.0001 & & 0.0025 \\
\hline HLW99-77 & & & 0.0675 & & & & & & 0.0007 & & 0.0004 & & & & & & 0.0009 & & 0.0002 & & 0.0029 \\
\hline HLW99-78 & & & 0.0900 & & & & & & 0.0032 & & 0.0004 & & & & & & 0.0008 & & 0.0002 & & 0.0024 \\
\hline HLW99-79 & & & 0.0913 & & & & & & 0.0008 & & 0.0003 & & & & & & 0.0002 & & 0.0001 & & 0.0025 \\
\hline HLW99-80 & & & 0.0633 & & & & & & 0.0075 & & 0.0003 & & & & & & 0.0008 & & 0.0002 & & 0.0019 \\
\hline HLW99-81 & & & 0.0645 & & & & & & 0.0007 & & 0.0003 & & & & & & 0.0003 & & 0.0002 & & 0.0025 \\
\hline HLW99-82 & & & 0.0362 & & & & & & 0.0074 & & 0.0004 & & & & & & 0.0004 & & 0.0002 & & 0.0022 \\
\hline HLW99-83 & & & 0.0669 & & & & & & 0.0007 & & 0.0005 & & & & & & 0.0002 & & 0.0001 & & 0.0024 \\
\hline HLW99-84 & & & 0.0624 & & & & & & 0.0043 & & 0.0003 & & & & & & 0.0002 & & 0.0001 & & 0.0021 \\
\hline HLW99-85 & & & 0.0380 & & & & & & 0.0039 & & 0.0004 & & & & & & 0.0003 & & 0.0002 & & 0.0019 \\
\hline HLW99-86 & & & 0.0352 & & & & & & 0.0004 & & 0.0003 & & & & & & 0.0003 & & 0.0001 & & 0.0022 \\
\hline HLW99-87 & & & 0.0889 & & & & & & 0.0030 & & 0.0003 & & & & & & 0.0006 & & 0.0002 & & 0.0020 \\
\hline HLW99-88 & & & 0.0364 & & & & & & 0.0072 & & 0.0004 & & & & & & 0.0010 & & 0.0003 & & 0.0025 \\
\hline HLW99-89 & & & 0.0375 & & & & & & 0.0004 & & 0.0003 & & & & & & 0.0005 & & 0.0002 & & 0.0024 \\
\hline HLW99-90R & & & 0.0589 & & & & & & 0.0007 & & 0.0003 & & & & & & 0.0009 & & 0.0002 & & 0.0022 \\
\hline HLW99-91 & & & 0.0829 & & & & & & 0.0008 & & 0.0004 & & & & & & 0.0006 & & 0.0002 & & 0.0026 \\
\hline HLW99-92 & & & 0.0816 & & & & & & 0.0082 & & 0.0004 & & & & & & 0.0004 & & 0.0002 & & 0.0022 \\
\hline HLW99-93R2 & & & 0.0853 & & & & & & 0.0008 & & 0.0004 & & & & & & 0.0003 & & 0.0001 & & 0.0022 \\
\hline HLW99-94 & & & 0.0363 & & & & & & 0.0008 & & 0.0003 & & & & & & 0.0004 & & 0.0002 & & 0.0021 \\
\hline HLW99-95 & & & 0.0359 & & & & & & 0.0008 & & 0.0004 & & & & & & 0.0004 & & 0.0002 & & 0.0020 \\
\hline HLW99-96 & & & 0.0619 & & & & & & 0.0038 & & 0.0004 & & & & & & 0.0009 & & 0.0003 & & 0.0023 \\
\hline HLW99-97 & & & 0.0368 & & & & & & 0.0046 & & 0.0008 & & & & & & 0.0008 & & 0.0003 & & 0.0023 \\
\hline HLW99-98R & & & 0.0613 & & & & & & 0.0072 & & 0.0005 & & & & & & 0.0002 & & 0.0001 & & 0.0021 \\
\hline HLW99-99 & & & 0.0864 & & & & & & 0.0008 & & 0.0003 & & & & & & 0.0003 & & 0.0001 & & 0.0024 \\
\hline HLW99-100 & & & 0.0599 & & & & & & 0.0013 & & 0.0003 & & & & & & 0.0004 & & 0.0001 & & 0.0021 \\
\hline
\end{tabular}




\section{RPP-WTP HLW Formulation (Kot and Pegg 2001)}

\begin{tabular}{|c|c|c|c|c|c|c|c|c|c|c|c|c|c|c|c|c|c|c|c|c|}
\hline Glass ID & $\begin{array}{c}\mathrm{SeO} 2 \\
-\mathrm{a}\end{array}$ & $\begin{array}{c}\mathrm{Sm} 2 \mathrm{O} 3 \\
-\mathrm{a}\end{array}$ & $\begin{array}{c}\mathrm{SnO} \\
-\mathrm{a}\end{array}$ & $\begin{array}{c}\mathrm{SnO} 2 \\
-\mathrm{a}\end{array}$ & $\begin{array}{c}\mathrm{SO} 3 \\
-\mathrm{a}\end{array}$ & $\begin{array}{c}\mathrm{SrO} \\
-\mathrm{a}\end{array}$ & $\begin{array}{c}\mathrm{Tc} 2 \mathrm{O} 7 \\
-\mathrm{a}\end{array}$ & $\begin{array}{c}\mathrm{TeO} 2 \\
-\mathrm{a}\end{array}$ & $\begin{array}{c}\mathrm{ThO} 2 \\
-\mathrm{a}\end{array}$ & $\begin{array}{c}\mathrm{TiO} 2 \\
-\mathrm{a}\end{array}$ & \begin{tabular}{|c|}
$\mathrm{T} 12 \mathrm{O} 3$ \\
$-\mathrm{a}$
\end{tabular} & $\begin{array}{c}\mathrm{U} 3 \mathrm{O} 8 \\
-\mathrm{a}\end{array}$ & $\begin{array}{c}\mathrm{UO} 2 \\
-\mathrm{a}\end{array}$ & $\begin{array}{c}\mathrm{UO} 3 \\
-\mathrm{a}\end{array}$ & $\begin{array}{c}\mathrm{V} 2 \mathrm{O} 5 \\
-\mathrm{a}\end{array}$ & $\begin{array}{c}\text { WO3 } \\
-\mathrm{a}\end{array}$ & $\begin{array}{c}\mathrm{Y} 2 \mathrm{O} 3 \\
-\mathrm{a}\end{array}$ & $\begin{array}{c}\mathrm{ZnO} \\
-\mathrm{a}\end{array}$ & $\begin{array}{c}\text { Others } \\
-\mathrm{a}\end{array}$ & $\begin{array}{c}\text { Sum } \\
-\mathrm{a}\end{array}$ \\
\hline HLW99-60 & 0.0010 & & & & & 0.0023 & & 0.0026 & & 0.0069 & 0.0012 & & 0.0683 & & & 0.0026 & & 0.0180 & & 0.9111 \\
\hline HLW99-61 & 0.0007 & & & & & 0.0023 & & 0.0023 & & 0.0100 & 0.0008 & & 0.0004 & & & 0.0024 & & 0.0203 & & 0.9568 \\
\hline HLW99-62 & 0.0009 & & & & & 0.0025 & & 0.0019 & & 0.0071 & 0.0018 & & 0.0003 & & & 0.0023 & & 0.0204 & & 0.9486 \\
\hline HLW99-63 & 0.0008 & & & & & 0.0024 & & \begin{tabular}{|l|}
0.0025 \\
\end{tabular} & & 0.0035 & 0.0012 & & 0.0304 & & & 0.0025 & & 0.0184 & & 0.9162 \\
\hline HLW99-64 & 0.0011 & & & & & 0.0024 & & 0.0042 & & 0.0102 & 0.0010 & & 0.0687 & & & 0.0029 & & 0.0188 & & 0.9607 \\
\hline HLW99-65 & 0.0012 & & & & & 0.0023 & & 0.0020 & & 0.0005 & 0.0009 & & 0.0670 & & & 0.0026 & & 0.0192 & & 0.9228 \\
\hline HLW99-66 & 0.0009 & & & & & 0.0534 & & 0.0022 & & 0.0002 & 0.0013 & & 0.0014 & & & 0.0025 & & 0.0211 & & 0.9496 \\
\hline HLW99-67 & 0.0005 & & & & & 0.1239 & & 0.0006 & & 0.0006 & 0.0013 & & 0.0030 & & & 0.0025 & & 0.0204 & & 0.9581 \\
\hline HLW99-68 & 0.0011 & & & & & 0.0522 & & 0.0029 & & 0.0069 & 0.0014 & & 0.0015 & & & 0.0027 & & 0.0201 & & 0.9666 \\
\hline HLW99-69 & 0.0005 & & & & & 0.1229 & & 0.0030 & & 0.0068 & 0.0013 & & 0.0029 & & & 0.0031 & & 0.0207 & & 0.9818 \\
\hline HLW99-70 & 0.0012 & & & & & 0.1217 & & 0.0017 & & 0.0006 & 0.0007 & & 0.0042 & & & 0.0033 & & 0.0212 & & 0.9757 \\
\hline HLW99-71 & 0.0011 & & & & & 0.0562 & & 0.0023 & & 0.0003 & 0.0011 & & 0.0023 & & & 0.0027 & & 0.0218 & & 0.9715 \\
\hline HLW99-72 & 0.0006 & & & & & 0.0561 & & 0.0028 & & 0.0071 & 0.0010 & & 0.0015 & & & 0.0037 & & 0.0222 & & 1.0132 \\
\hline HLW99-73 & 0.0015 & & & & & 0.0584 & & 0.0023 & & 0.0004 & 0.0010 & & 0.0023 & & & 0.0028 & & 0.0216 & & 0.9701 \\
\hline HLW99-74 & 0.0003 & & & & & 0.0824 & & 0.0022 & & 0.0037 & 0.0009 & & 0.0030 & & & 0.0032 & & 0.0213 & & 0.9680 \\
\hline HLW99-75 & 0.0007 & & & & & 0.0556 & & 0.0025 & & 0.0052 & 0.0007 & & 0.0018 & & & 0.0032 & & 0.0216 & & 0.9798 \\
\hline HLW99-76 & 0.0009 & & & & & 0.0889 & & 0.0026 & & 0.0075 & 0.0007 & & 0.0028 & & & 0.0036 & & 0.0210 & & 0.9797 \\
\hline HLW99-77 & 0.0003 & & & & & 0.0831 & & 0.0024 & & 0.0006 & 0.0007 & & 0.0033 & & & 0.0029 & & 0.0215 & & 0.9474 \\
\hline HLW99-78 & 0.0007 & & & & & 0.1177 & & 0.0025 & & 0.0032 & 0.0007 & & 0.0035 & & & 0.0033 & & 0.0209 & & 0.9834 \\
\hline HLW99-79 & 0.0006 & & & & & 0.1218 & & 0.0024 & & 0.0002 & 0.0009 & & 0.0036 & & & 0.0031 & & 0.0214 & & 0.9663 \\
\hline HLW99-80 & 0.0003 & & & & & 0.0833 & & 0.0021 & & 0.0066 & 0.0007 & & 0.0031 & & & 0.0035 & & 0.0202 & & 0.9764 \\
\hline HLW99-81 & 0.0011 & & & & & 0.0898 & & 0.0025 & & 0.0004 & 0.0008 & & 0.0029 & & & 0.0028 & & 0.0217 & & 0.9611 \\
\hline HLW99-82 & 0.0010 & & & & & 0.0529 & & 0.0026 & & 0.0069 & 0.0009 & & 0.0019 & & & 0.0032 & & 0.0201 & & 0.9762 \\
\hline HLW99-83 & 0.0005 & & & & & 0.0895 & & 0.0022 & & 0.0004 & 0.0010 & & 0.0028 & & & 0.0028 & & 0.0206 & & 0.9770 \\
\hline HLW99-84 & 0.0007 & & & & & 0.0942 & & 0.0025 & & 0.0040 & 0.0008 & & 0.0013 & & & 0.0028 & & 0.0201 & & 0.9500 \\
\hline HLW99-85 & 0.0008 & & & & & 0.0559 & & 0.0022 & & 0.0035 & 0.0010 & & 0.0022 & & & 0.0030 & & 0.0210 & & 0.9940 \\
\hline HLW99-86 & 0.0005 & & & & & 0.0509 & & 0.0017 & & 0.0003 & 0.0010 & & 0.0009 & & & 0.0024 & & 0.0202 & & 0.9545 \\
\hline HLW99-87 & 0.0010 & & & & & 0.1189 & & 0.0021 & & 0.0024 & 0.0007 & & 0.0040 & & & 0.0032 & & 0.0199 & & 0.9903 \\
\hline HLW99-88 & 0.0008 & & & & & 0.0522 & & 0.0021 & & 0.0068 & 0.0010 & & 0.0023 & & & 0.0036 & & 0.0200 & & 0.9667 \\
\hline HLW99-89 & 0.0006 & & & & & 0.0554 & & 0.0017 & & 0.0003 & 0.0009 & & 0.0023 & & & 0.0027 & & 0.0200 & & 0.9711 \\
\hline HLW99-90R & 0.0002 & & & & & 0.0869 & & 0.0018 & & 0.0005 & 0.0007 & & 0.0014 & & & 0.0026 & & 0.0198 & & 0.9310 \\
\hline HLW99-91 & 0.0001 & & & & & 0.1237 & & 0.0021 & & 0.0006 & 0.0006 & & 0.0014 & & & 0.0030 & & 0.0196 & & 0.9592 \\
\hline HLW99-92 & 0.0000 & & & & & 0.1237 & & 0.0032 & & 0.0067 & 0.0007 & & 0.0069 & & & 0.0027 & & 0.0181 & & 0.9864 \\
\hline HLW99-93R2 & 0.0004 & & & & & 0.1168 & & 0.0020 & & 0.0009 & 0.0007 & & 0.0049 & & & 0.0029 & & 0.0204 & & 0.9866 \\
\hline HLW99-94 & 0.0003 & & & & & 0.0553 & & 0.0025 & & 0.0003 & 0.0009 & & 0.0039 & & & 0.0025 & & 0.0182 & & 0.9574 \\
\hline HLW99-95 & 0.0008 & & & & & 0.0535 & & 0.0028 & & 0.0002 & 0.0009 & & 0.0040 & & & 0.0025 & & 0.0187 & & 0.9568 \\
\hline HLW99-96 & 0.0005 & & & & & 0.0827 & & 0.0020 & & 0.0039 & 0.0009 & & 0.0041 & & & 0.0033 & & 0.0203 & & 0.9915 \\
\hline HLW99-97 & 0.0008 & & & & & 0.0525 & & 0.0021 & & 0.0046 & 0.0007 & & 0.0028 & & & 0.0031 & & 0.0205 & & 0.9739 \\
\hline HLW99-98R & 0.0010 & & & & & 0.0858 & & 0.0023 & & 0.0063 & 0.0007 & & 0.0044 & & & 0.0036 & & 0.0200 & & 0.9903 \\
\hline HLW99-99 & 0.0003 & & & & & 0.1216 & & 0.0022 & & 0.0004 & 0.0007 & & 0.0045 & & & 0.0029 & & 0.0204 & & 0.9978 \\
\hline HLW99-100 & 0.0007 & & & & & 0.0917 & & 0.0025 & & 0.0002 & 0.0008 & & 0.0058 & & & 0.0025 & & 0.0186 & & 0.9715 \\
\hline
\end{tabular}


Appendix A. Database - mass fraction

RPP-WTP HLW Formulation (Kot and Pegg 2001)

\begin{tabular}{|c|c|c|c|c|c|c|c|c|}
\hline Glass ID & $\begin{array}{l}\mathrm{TM} \\
\left({ }^{\circ} \mathrm{C}\right) \\
\end{array}$ & $\begin{array}{l}\text { Gradient } \\
\text { TL }\left({ }^{\circ} \mathrm{C}\right) \\
\end{array}$ & $\begin{array}{l}\text { Uniform } \\
\text { TL }\left({ }^{\circ} \mathrm{C}\right) \\
\end{array}$ & Primary Phase & $\begin{array}{l}\text { Quenched } \\
\text { Visual/OM }\end{array}$ & $\begin{array}{c}\text { Quenched } \\
\text { SEM/EDS or TEM }\end{array}$ & $\begin{array}{l}\text { Quenched } \\
\text { XRD }\end{array}$ & $\begin{array}{c}\text { Quenched } \\
\text { Homogeneous? }\end{array}$ \\
\hline \multicolumn{9}{|l|}{ HLW99-60 } \\
\hline HLW99-61 & 1150 & & 950 & & Homogeneous red glass & & & \\
\hline HLW99-62 & 1150 & & 1075 & & Mostly homogeneous greenish blue glass & & & \\
\hline \multicolumn{9}{|l|}{ HLW99-63 } \\
\hline \multicolumn{9}{|l|}{ HLW99-64 } \\
\hline \multicolumn{9}{|l|}{ HLW99-65 } \\
\hline HLW99-66 & & & $>1150$ & & & & & \\
\hline HLW99-67 & & & $>1150$ & & & & & \\
\hline \multicolumn{9}{|l|}{ HLW99-68 } \\
\hline HLW99-69 & & & 1150 & & & & & \\
\hline HLW99-70 & & & 1050 & & & & & \\
\hline HLW99-71 & & & $>1150$ & & & & & \\
\hline HLW99-72 & & & 1150 & & & & & \\
\hline HLW99-73 & & & 1050 & & & & & \\
\hline HLW99-74 & & & $>1150$ & & & & & \\
\hline HLW99-75 & & & $>1150$ & & & & & \\
\hline HLW99-76 & & & 1125 & & & & & \\
\hline HLW99-77 & & & 1125 & & & & & \\
\hline HLW99-78 & & & 1125 & & & & & \\
\hline HLW99-79 & & & 1050 & & & & & \\
\hline HLW99-80 & & & 1160 & & & & & \\
\hline HLW99-81 & & & 1050 & & & & & \\
\hline HLW99-82 & & & $>1160$ & & & & & \\
\hline HLW99-83 & & & 1100 & & & & & \\
\hline HLW99-84 & & & 1050 & & & & & \\
\hline HLW99-85 & & & 1175 & & & & & \\
\hline HLW99-86 & & & 1050 & & & & & \\
\hline HLW99-87 & & & 1050 & & & & & \\
\hline HLW99-88 & & & $>1160$ & & & & & \\
\hline HLW99-89 & & & 1150 & & & & & \\
\hline HLW99-90R & & & 1125 & & & & & \\
\hline \multicolumn{9}{|l|}{ HLW99-91 } \\
\hline \multicolumn{9}{|l|}{ HLW99-92 } \\
\hline \multicolumn{9}{|l|}{ HLW99-93R2 } \\
\hline \multicolumn{9}{|l|}{ HLW99-94 } \\
\hline \multicolumn{9}{|l|}{ HLW99-95 } \\
\hline \multicolumn{9}{|l|}{ HLW99-96 } \\
\hline \multicolumn{9}{|l|}{ HLW99-97 } \\
\hline \multicolumn{9}{|l|}{ HLW99-98R } \\
\hline \multicolumn{9}{|l|}{ HLW99-99 } \\
\hline HLW99-100 & & & & & & & & \\
\hline
\end{tabular}


Appendix A. Database - mass fraction

\section{RPP-WTP HLW Formulation (Kot and Pegg 2001)}

\begin{tabular}{|c|c|c|c|c|c|}
\hline Glass ID & $\begin{array}{c}\mathrm{CCC} \\
\text { Visual/OM }\end{array}$ & $\begin{array}{c}\text { CCC } \\
\text { SEM/EDS or TEM }\end{array}$ & $\begin{array}{l}\text { CCC } \\
\text { XRD }\end{array}$ & $\begin{array}{c}\text { Heat Treated } \\
\text { Visual/OM }\end{array}$ & $\begin{array}{c}\text { Heat Treated } \\
\text { SEM/EDS or TEM }\end{array}$ \\
\hline \multicolumn{6}{|l|}{ HLW99-60 } \\
\hline HLW99-61 & & & & & $\begin{array}{l}\text { Less than } 0.5 \mathrm{vol} \% \text { of } \mathrm{NiO} \text { (rod shaped or } \\
\text { rectangular prismatic) with Rh cores. } 0.1 \text { vol } \% \text { of } \\
\mathrm{RuO} 2\end{array}$ \\
\hline HLW99-62 & & & & & $\begin{array}{l}\text { Less than } 0.1 \mathrm{vol} \% \text { of small clusters of Rh spinel } \\
(\mathrm{Ni}, \mathrm{Zn}, \mathrm{Fe}) \text { Trace amount of } \mathrm{Rh} / \mathrm{Ru}\end{array}$ \\
\hline \multicolumn{6}{|l|}{ HLW99-63 } \\
\hline \multicolumn{6}{|l|}{ HLW99-64 } \\
\hline \multicolumn{6}{|l|}{ HLW99-65 } \\
\hline \multicolumn{6}{|l|}{ HLW99-66 } \\
\hline \multicolumn{6}{|l|}{ HLW99-67 } \\
\hline \multicolumn{6}{|l|}{ HLW99-68 } \\
\hline \multicolumn{6}{|l|}{ HLW99-69 } \\
\hline \multicolumn{6}{|l|}{ HLW99-70 } \\
\hline \multicolumn{6}{|l|}{ HLW99-71 } \\
\hline \multicolumn{6}{|l|}{ HLW99-72 } \\
\hline \multicolumn{6}{|l|}{ HLW99-73 } \\
\hline \multicolumn{6}{|l|}{ HLW99-74 } \\
\hline \multicolumn{6}{|l|}{ HLW99-75 } \\
\hline \multicolumn{6}{|l|}{ HLW99-76 } \\
\hline \multicolumn{6}{|l|}{ HLW99-77 } \\
\hline \multicolumn{6}{|l|}{ HLW99-78 } \\
\hline \multicolumn{6}{|l|}{ HLW99-79 } \\
\hline \multicolumn{6}{|l|}{ HLW99-80 } \\
\hline \multicolumn{6}{|l|}{ HLW99-81 } \\
\hline \multicolumn{6}{|l|}{ HLW99-82 } \\
\hline \multicolumn{6}{|l|}{ HLW99-83 } \\
\hline \multicolumn{6}{|l|}{ HLW99-84 } \\
\hline \multicolumn{6}{|l|}{ HLW99-85 } \\
\hline \multicolumn{6}{|l|}{ HLW99-86 } \\
\hline HLW99-87 & & & & & \\
\hline HLW99-88 & & & & & \\
\hline HLW99-89 & & & & & \\
\hline HLW99-90R & & & & & \\
\hline HLW99-91 & & & & & \\
\hline HLW99-92 & & & & & \\
\hline HLW99-93R2 & & & & & \\
\hline HLW99-94 & & & & & \\
\hline HLW99-95 & & & & & \\
\hline HLW99-96 & & & & & \\
\hline HLW99-97 & & & & & \\
\hline HLW99-98R & & & & & \\
\hline HLW99-99 & & & & & \\
\hline HLW99-100 & & & & & \\
\hline
\end{tabular}


Appendix A. Database - mass fraction

RPP-WTP HLW Formulation (Kot and Pegg 2001)

\begin{tabular}{|c|c|c|c|c|c|c|c|c|c|c|c|c|c|c|c|}
\hline Glass ID & $\begin{array}{c}\text { Heat Treated } \\
\text { XRD }\end{array}$ & $\begin{array}{l}\text { Density } \\
\left(\mathrm{g} / \mathrm{cm}^{3}\right)\end{array}$ & $\begin{array}{c}\text { Fulc Visc } \\
\mathrm{A}\end{array}$ & $\begin{array}{c}\text { Fulc Visc } \\
\text { B } \\
\end{array}$ & \begin{tabular}{c|} 
Fulc Visc \\
To \\
\end{tabular} & $\begin{array}{c}\mathrm{FV} 1150^{\circ} \mathrm{C} \\
(\mathrm{Pa} \cdot \mathrm{s})\end{array}$ & \begin{tabular}{|c|} 
Arrh Visc \\
$\mathrm{A}$
\end{tabular} & \begin{tabular}{|c|} 
Arrh Visc \\
B \\
\end{tabular} & $\begin{array}{l}\text { Av } \\
1150^{\circ} \mathrm{C} \\
(\mathrm{D} a, 0)\end{array}$ & $\begin{array}{c}\mathrm{T}\left({ }^{\circ} \mathrm{C}\right) \text { at } \\
2 \mathrm{~Pa} \cdot \mathrm{s}\end{array}$ & $\begin{array}{c}\mathrm{T}\left({ }^{\circ} \mathrm{C}\right) \text { at } \\
5 \mathrm{~Pa} \cdot \mathrm{s}\end{array}$ & \begin{tabular}{|c|}
$\mathrm{T}\left({ }^{\circ} \mathrm{C}\right)$ at \\
$10 \mathrm{~Pa} \cdot \mathrm{s}$ \\
\end{tabular} & $\begin{array}{l}\mathrm{T} 1 \\
\left({ }^{\circ} \mathrm{C}\right) \\
\end{array}$ & $\begin{array}{c}\mathrm{V} 1 \\
(\mathrm{~Pa} \cdot \mathrm{s})\end{array}$ & $\begin{array}{l}\mathrm{T} 2 \\
\left({ }^{\circ} \mathrm{C}\right) \\
\end{array}$ \\
\hline \multicolumn{16}{|c|}{ (D) } \\
\hline \multicolumn{16}{|l|}{ HLW99-61 } \\
\hline \multicolumn{16}{|l|}{ HLW99-62 } \\
\hline \multicolumn{16}{|l|}{ HLW99-63 } \\
\hline \multicolumn{16}{|l|}{ HLW99-64 } \\
\hline \multicolumn{16}{|l|}{ HLW99-65 } \\
\hline \multicolumn{16}{|l|}{ HLW99-66 } \\
\hline \multicolumn{16}{|l|}{ HLW99-67 } \\
\hline \multicolumn{16}{|l|}{ HLW99-68 } \\
\hline \multicolumn{16}{|l|}{ HLW99-69 } \\
\hline \multicolumn{16}{|l|}{ HLW99-70 } \\
\hline \multicolumn{16}{|l|}{ HLW99-71 } \\
\hline \multicolumn{16}{|l|}{ HLW99-72 } \\
\hline \multicolumn{16}{|l|}{ HLW99-73 } \\
\hline \multicolumn{16}{|l|}{ HLW99-74 } \\
\hline \multicolumn{16}{|l|}{ HLW99-75 } \\
\hline \multicolumn{16}{|l|}{ HLW99-76 } \\
\hline \multicolumn{16}{|l|}{ HLW99-77 } \\
\hline \multicolumn{16}{|l|}{ HLW99-78 } \\
\hline \multicolumn{16}{|l|}{ HLW99-79 } \\
\hline HLW99-80 & & & & & & & & & & & & & & & \\
\hline HLW99-81 & & & & & & & & & & & & & & & \\
\hline HLW99-82 & & & & & & & & & & & & & & & \\
\hline HLW99-83 & & & & & & & & & & & & & & & \\
\hline HLW99-84 & & & & & & & & & & & & & & & \\
\hline HLW99-85 & & & & & & & & & & & & & & & \\
\hline HLW99-86 & & & & & & & & & & & & & & & \\
\hline HLW99-87 & & & & & & & & & & & & & & & \\
\hline HLW99-88 & & & & & & & & & & & & & & & \\
\hline HLW99-89 & & & & & & & & & & & & & & & \\
\hline HLW99-90R & & & & & & & & & & & & & & & \\
\hline HLW99-91 & & & & & & & & & & & & & & & \\
\hline HLW99-92 & & & & & & & & & & & & & & & \\
\hline HLW99-93R2 & & & & & & & & & & & & & & & \\
\hline HLW99-94 & & & & & & & & & & & & & & & \\
\hline HLW99-95 & & & & & & & & & & & & & & & \\
\hline HLW99-96 & & & & & & & & & & & & & & & \\
\hline HLW99-97 & & & & & & & & & & & & & & & \\
\hline HLW99-98R & & & & & & & & & & & & & & & \\
\hline HLW99-99 & & & & & & & & & & & & & & & \\
\hline HLW99-100 & & & & & & & & & & & & & & & \\
\hline
\end{tabular}


RPP-WTP HLW Formulation (Kot and Pegg 2001)

\begin{tabular}{|c|c|c|c|c|c|c|c|c|c|c|c|c|c|c|c|c|c|c|c|c|c|}
\hline Glass ID & $\begin{array}{c}\mathrm{V} 2 \\
(\mathrm{~Pa} \cdot \mathrm{s})\end{array}$ & $\begin{array}{c}\mathrm{T} 3 \\
\left({ }^{\circ} \mathrm{C}\right) \\
\end{array}$ & $\begin{array}{c}\mathrm{V} 3 \\
(\mathrm{~Pa} \cdot \mathrm{s})\end{array}$ & $\begin{array}{c}\mathrm{T} 4 \\
\left({ }^{\circ} \mathrm{C}\right) \\
\end{array}$ & $\begin{array}{c}\mathrm{V} 4 \\
(\mathrm{~Pa} \cdot \mathrm{s})\end{array}$ & $\begin{array}{c}\mathrm{T} 5 \\
\left({ }^{\circ} \mathrm{C}\right) \\
\end{array}$ & $\begin{array}{c}\mathrm{V} 5 \\
(\mathrm{~Pa} \cdot \mathrm{s})\end{array}$ & $\begin{array}{c}\mathrm{T} 6 \\
\left({ }^{\circ} \mathrm{C}\right) \\
\end{array}$ & $\begin{array}{c}\mathrm{V} 6 \\
(\mathrm{~Pa} \cdot \mathrm{s})\end{array}$ & $\begin{array}{c}\mathrm{T} 7 \\
\left({ }^{\circ} \mathrm{C}\right) \\
\end{array}$ & $\begin{array}{c}\mathrm{V} 7 \\
(\mathrm{~Pa} \cdot \mathrm{s})\end{array}$ & $\begin{array}{c}\mathrm{T} 8 \\
\left({ }^{\circ} \mathrm{C}\right) \\
\end{array}$ & $\begin{array}{c}\mathrm{V} 8 \\
(\mathrm{~Pa} \cdot \mathrm{s})\end{array}$ & $\begin{array}{c}\mathrm{T} 9 \\
\left({ }^{\circ} \mathrm{C}\right) \\
\end{array}$ & $\begin{array}{c}\mathrm{V} 9 \\
(\mathrm{~Pa} \cdot \mathrm{s})\end{array}$ & $\begin{array}{l}\mathrm{T} 10 \\
\left({ }^{\circ} \mathrm{C}\right) \\
\end{array}$ & $\begin{array}{c}\mathrm{V} 10 \\
(\mathrm{~Pa} \cdot \mathrm{s})\end{array}$ & $\begin{array}{l}\mathrm{T} 11 \\
\left({ }^{\circ} \mathrm{C}\right) \\
\end{array}$ & $\begin{array}{c}\mathrm{V} 11 \\
(\mathrm{~Pa} \cdot \mathrm{s})\end{array}$ & $\begin{array}{l}\mathrm{T} 12 \\
\left({ }^{\circ} \mathrm{C}\right) \\
\end{array}$ & $\begin{array}{c}\mathrm{V} 12 \\
(\mathrm{~Pa} \cdot \mathrm{s})\end{array}$ \\
\hline HLW99-60 & & & & & & & & & & & & & & & & & & & & & \\
\hline HLW99-61 & & & & & & & & & & & & & & & & & & & & & \\
\hline HLW99-62 & & & & & & & & & & & & & & & & & & & & & \\
\hline HLW99-63 & & & & & & & & & & & & & & & & & & & & & \\
\hline HLW99-64 & & & & & & & & & & & & & & & & & & & & & \\
\hline HLW99-65 & & & & & & & & & & & & & & & & & & & & & \\
\hline HLW99-66 & & & & & & & & & & & & & & & & & & & & & \\
\hline HLW99-67 & & & & & & & & & & & & & & & & & & & & & \\
\hline HLW99-68 & & & & & & & & & & & & & & & & & & & & & \\
\hline HLW99-69 & & & & & & & & & & & & & & & & & & & & & \\
\hline HLW99-70 & & & & & & & & & & & & & & & & & & & & & \\
\hline HLW99-71 & & & & & & & & & & & & & & & & & & & & & \\
\hline HLW99-72 & & & & & & & & & & & & & & & & & & & & & \\
\hline HLW99-73 & & & & & & & & & & & & & & & & & & & & & \\
\hline HLW99-74 & & & & & & & & & & & & & & & & & & & & & \\
\hline HLW99-75 & & & & & & & & & & & & & & & & & & & & & \\
\hline HLW99-76 & & & & & & & & & & & & & & & & & & & & & \\
\hline HLW99-77 & & & & & & & & & & & & & & & & & & & & & \\
\hline HLW99-78 & & & & & & & & & & & & & & & & & & & & & \\
\hline HLW99-79 & & & & & & & & & & & & & & & & & & & & & \\
\hline HLW99-80 & & & & & & & & & & & & & & & & & & & & & \\
\hline HLW99-81 & & & & & & & & & & & & & & & & & & & & & \\
\hline HLW99-82 & & & & & & & & & & & & & & & & & & & & & \\
\hline HLW99-83 & & & & & & & & & & & & & & & & & & & & & \\
\hline HLW99-84 & & & & & & & & & & & & & & & & & & & & & \\
\hline HLW99-85 & & & & & & & & & & & & & & & & & & & & & \\
\hline HLW99-86 & & & & & & & & & & & & & & & & & & & & & \\
\hline HLW99-87 & & & & & & & & & & & & & & & & & & & & & \\
\hline HLW99-88 & & & & & & & & & & & & & & & & & & & & & \\
\hline HLW99-89 & & & & & & & & & & & & & & & & & & & & & \\
\hline HLW99-90R & & & & & & & & & & & & & & & & & & & & & \\
\hline HLW99-91 & & & & & & & & & & & & & & & & & & & & & \\
\hline HLW99-92 & & & & & & & & & & & & & & & & & & & & & \\
\hline HLW99-93R2 & & & & & & & & & & & & & & & & & & & & & \\
\hline HLW99-94 & & & & & & & & & & & & & & & & & & & & & \\
\hline HLW99-95 & & & & & & & & & & & & & & & & & & & & & \\
\hline HLW99-96 & & & & & & & & & & & & & & & & & & & & & \\
\hline HLW99-97 & & & & & & & & & & & & & & & & & & & & & \\
\hline HLW99-98R & & & & & & & & & & & & & & & & & & & & & \\
\hline HLW99-99 & & & & & & & & & & & & & & & & & & & & & \\
\hline HLW99-100 & & & & & & & & & & & & & & & & & & & & & \\
\hline
\end{tabular}


Appendix A. Database - mass fraction

RPP-WTP HLW Formulation (Kot and Pegg 2001)

\begin{tabular}{|c|c|c|c|c|c|c|c|c|c|c|c|c|c|c|c|c|}
\hline Glass ID & $\begin{array}{l}\mathrm{T} 13 \\
\left({ }^{\circ} \mathrm{C}\right)\end{array}$ & $\begin{array}{c}\mathrm{V} 13 \\
(\mathrm{~Pa} \cdot \mathrm{s})\end{array}$ & $\begin{array}{l}\mathrm{T} 14 \\
\left({ }^{\circ} \mathrm{C}\right)\end{array}$ & $\begin{array}{c}\mathrm{V} 14 \\
(\mathrm{~Pa} \cdot \mathrm{s})\end{array}$ & $\begin{array}{c}\text { Q PCT } \\
\mathrm{B}\left(\mathrm{g} / \mathrm{m}^{2}\right)\end{array}$ & $\begin{array}{c}\text { Q PCT } \\
\mathrm{Li}\left(\mathrm{g} / \mathrm{m}^{2}\right)\end{array}$ & $\begin{array}{c}\text { Q PCT } \\
\mathrm{Na}\left(\mathrm{g} / \mathrm{m}^{2}\right)\end{array}$ & $\begin{array}{c}\text { Q PCT } \\
\mathrm{Si}\left(\mathrm{g} / \mathrm{m}^{2}\right)\end{array}$ & $\begin{array}{c}\text { Q PCT } \\
\mathrm{pH}\end{array}$ & $\begin{array}{c}\text { CCC PCT } \\
\mathrm{B}\left(\mathrm{g} / \mathrm{m}^{2}\right)\end{array}$ & $\begin{array}{l}\text { CCC PCT } \\
\mathrm{Li}\left(\mathrm{g} / \mathrm{m}^{2}\right)\end{array}$ & $\begin{array}{l}\mathrm{CCC} \text { PCT } \\
\mathrm{Na}\left(\mathrm{g} / \mathrm{m}^{2}\right) \\
\end{array}$ & $\begin{array}{c}\text { CCC PCT } \\
\mathrm{Si}\left(\mathrm{g} / \mathrm{m}^{2}\right)\end{array}$ & $\begin{array}{c}\text { CCC PCT } \\
\text { pH }\end{array}$ & $\begin{array}{l}\text { Q PCT B at } \\
20^{\circ} \mathrm{C}\left(\mathrm{g} / \mathrm{m}^{2}\right)\end{array}$ & $\begin{array}{l}\text { Q PCT Li at } \\
20^{\circ} \mathrm{C}\left(\mathrm{g} / \mathrm{m}^{2}\right)\end{array}$ \\
\hline HLW99-60 & & & & & & & & & & & & & & & & \\
\hline HLW99-61 & & & & & & & & & & & & & & & & \\
\hline HLW99-62 & & & & & & & & & & & & & & & & \\
\hline HLW99-63 & & & & & & & & & & & & & & & & \\
\hline HLW99-64 & & & & & & & & & & & & & & & & \\
\hline HLW99-65 & & & & & & & & & & & & & & & & \\
\hline HLW99-66 & & & & & & & & & & & & & & & & \\
\hline HLW99-67 & & & & & & & & & & & & & & & & \\
\hline HLW99-68 & & & & & & & & & & & & & & & & \\
\hline HLW99-69 & & & & & & & & & & & & & & & & \\
\hline HLW99-70 & & & & & & & & & & & & & & & & \\
\hline HLW99-71 & & & & & & & & & & & & & & & & \\
\hline HLW99-72 & & & & & & & & & & & & & & & & \\
\hline HLW99-73 & & & & & & & & & & & & & & & & \\
\hline HLW99-74 & & & & & & & & & & & & & & & & \\
\hline HLW99-75 & & & & & & & & & & & & & & & & \\
\hline HLW99-76 & & & & & & & & & & & & & & & & \\
\hline HLW99-77 & & & & & & & & & & & & & & & & \\
\hline HLW99-78 & & & & & & & & & & & & & & & & \\
\hline HLW99-79 & & & & & & & & & & & & & & & & \\
\hline HLW99-80 & & & & & & & & & & & & & & & & \\
\hline HLW99-81 & & & & & & & & & & & & & & & & \\
\hline HLW99-82 & & & & & & & & & & & & & & & & \\
\hline HLW99-83 & & & & & & & & & & & & & & & & \\
\hline HLW99-84 & & & & & & & & & & & & & & & & \\
\hline HLW99-85 & & & & & & & & & & & & & & & & \\
\hline HLW99-86 & & & & & & & & & & & & & & & & \\
\hline HLW99-87 & & & & & & & & & & & & & & & & \\
\hline HLW99-88 & & & & & & & & & & & & & & & & \\
\hline HLW99-89 & & & & & & & & & & & & & & & & \\
\hline HLW99-90R & & & & & & & & & & & & & & & & \\
\hline HLW99-91 & & & & & & & & & & & & & & & & \\
\hline HLW99-92 & & & & & & & & & & & & & & & & \\
\hline HLW99-93R2 & & & & & & & & & & & & & & & & \\
\hline HLW99-94 & & & & & & & & & & & & & & & & \\
\hline HLW99-95 & & & & & & & & & & & & & & & & \\
\hline HLW99-96 & & & & & & & & & & & & & & & & \\
\hline HLW99-97 & & & & & & & & & & & & & & & & \\
\hline HLW99-98R & & & & & & & & & & & & & & & & \\
\hline HLW99-99 & & & & & & & & & & & & & & & & \\
\hline HLW99-100 & & & & & & & & & & & & & & & & \\
\hline
\end{tabular}


RPP-WTP HLW Formulation (Kot and Pegg 2001)

\begin{tabular}{|c|c|c|c|c|c|c|c|c|c|c|c|c|}
\hline Glass ID & $\begin{array}{l}\text { Q PCT Na at } \\
20^{\circ} \mathrm{C}\left(\mathrm{g} / \mathrm{m}^{2}\right)\end{array}$ & $\begin{array}{l}\text { Q PCT Si at } \\
20^{\circ} \mathrm{C}\left(\mathrm{g} / \mathrm{m}^{2}\right)\end{array}$ & $\begin{array}{c}\mathrm{QpH} \\
\text { at } 20^{\circ} \mathrm{C}\end{array}$ & $\begin{array}{c}\text { TCLP Ag } \\
\text { (ppm) }\end{array}$ & $\begin{array}{c}\text { TCLP As } \\
(\mathrm{ppm})\end{array}$ & $\begin{array}{c}\text { TCLP Ba } \\
(\mathrm{ppm})\end{array}$ & $\begin{array}{c}\text { TCLP Cd } \\
(\mathrm{ppm})\end{array}$ & $\begin{array}{c}\text { TCLP Cr } \\
(\mathrm{ppm})\end{array}$ & $\begin{array}{c}\text { TCLP Ni } \\
(\mathrm{ppm})\end{array}$ & $\begin{array}{c}\text { TCLP Pb } \\
(\mathrm{ppm})\end{array}$ & $\begin{array}{l}\text { TCLP Se } \\
(\mathrm{ppm})\end{array}$ & $\begin{array}{c}\text { TCLP Zn } \\
(\mathrm{ppm})\end{array}$ \\
\hline HLW99-60 & & & & 0.056 & 0.074 & 2.242 & 1.553 & 0.019 & 0.862 & 0.369 & 0.072 & 1.489 \\
\hline HLW99-61 & & & & 0.072 & 0.291 & 77.997 & 67.006 & 0.019 & 63.71 & 3.922 & 0.951 & 44.108 \\
\hline HLW99-62 & & & & 0.054 & 0.056 & 0.699 & 0.451 & $<0.0055$ & 0.352 & 0.148 & 0.0532 & 0.459 \\
\hline HLW99-63 & & & & 0.129 & 0.049 & 1.238 & 0.524 & 0.017 & 0.381 & 0.143 & 0.17 & 1.109 \\
\hline HLW99-64 & & & & 0.448 & 0.201 & 10.856 & 11.492 & 0.123 & 9.62 & 1.352 & 0.338 & 6.956 \\
\hline HLW99-65 & & & & 0.368 & 0.054 & 0.833 & 0.151 & 0.318 & 0.255 & 0.166 & 0.27 & 8.745 \\
\hline HLW99-66 & & & & 0.064 & 0.065 & 0.48 & $<0.0027$ & $<0.0055$ & $<0.0079$ & $<0.0243$ & 0.105 & 0.614 \\
\hline HLW99-67 & & & & 0.15 & 0.049 & 0.575 & $<0.0027$ & 0.01 & 0.01 & 0.078 & 0.111 & 1.145 \\
\hline HLW99-68 & & & & 0.137 & 0.049 & 1.571 & 0.956 & $<0.0055$ & 0.147 & 0.311 & 0.0532 & 1.01 \\
\hline HLW99-69 & & & & 0.309 & 0.049 & 3.725 & 2.592 & 0.037 & 1.738 & 0.828 & 0.094 & 2.521 \\
\hline HLW99-70 & & & & 0.519 & 0.099 & 1.097 & 0.006 & 0.102 & 0.026 & 0.416 & 0.124 & 5.859 \\
\hline HLW99-71 & & & & 0.069 & 0.061 & 0.46 & $<0.0027$ & $<0.0055$ & 0.008 & $<0.0243$ & 0.065 & 0.591 \\
\hline HLW99-72 & & & & 0.105 & 0.11 & 1.511 & 0.848 & 0.015 & 0.584 & 0.268 & 0.0532 & 0.871 \\
\hline HLW99-73 & & & & 0.187 & 0.063 & 0.883 & $<0.0027$ & 0.041 & 0.016 & 0.042 & 0.0532 & 1.622 \\
\hline HLW99-74 & & & & 0.148 & 0.063 & 1.64 & 0.562 & 0.028 & 0.134 & 0.203 & 0.062 & 1.105 \\
\hline HLW99-75 & & & & 0.208 & 0.212 & 2.195 & 1.102 & 0.032 & 0.374 & 0.29 & 0.057 & 1.606 \\
\hline HLW99-76 & & & & 1.097 & 0.149 & 11.832 & 10.328 & 0.127 & 7.274 & 1.554 & 0.248 & 11.355 \\
\hline HLW99-77 & & & & 0.213 & 0.164 & 1.022 & 0.029 & 0.018 & 0.022 & 0.039 & 0.105 & 1.473 \\
\hline HLW99-78 & & & & 0.326 & $<0.049$ & 2.29 & 0.918 & 0.024 & 0.579 & 0.246 & $<0.0532$ & 2.427 \\
\hline HLW99-79 & & & & 0.203 & 0.214 & 1.2 & 0.006 & 0.034 & $<0.0079$ & 0.124 & 0.144 & 1.774 \\
\hline HLW99-80 & & & & 0.249 & 0.128 & 3.673 & 2.343 & 0.027 & 1.287 & 0.641 & 0.172 & 2.127 \\
\hline HLW99-81 & & & & 0.254 & 0.049 & 1.169 & 0.101 & 0.019 & 0.039 & 0.124 & 0.072 & 2.155 \\
\hline HLW99-82 & & & & 0.334 & 0.049 & 4.458 & 2.875 & 0.02 & 2.089 & 0.69 & 0.0532 & 2.823 \\
\hline HLW99-83 & & & & 0.111 & 0.105 & 1.019 & 0.016 & $<0.0055$ & $<0.0079$ & 0.044 & 0.0532 & 1.097 \\
\hline HLW99-84 & & & & 0.231 & 0.172 & 2.512 & 1.2 & $<0.0055$ & 0.937 & 0.388 & 0.055 & 2.171 \\
\hline HLW99-85 & & & & 0.081 & 0.238 & 1.373 & 0.337 & 0.036 & 0.27 & 0.115 & 0.0532 & 0.0779 \\
\hline HLW99-86 & & & & 0.07 & 0.049 & 0.816 & 0.011 & 0.035 & 0.039 & $<0.0243$ & 0.121 & 0.73 \\
\hline HLW99-87 & & & & 0.559 & 0.122 & 2.737 & 1.64 & 0.073 & 1.171 & 0.583 & 0.0532 & 4.627 \\
\hline HLW99-88 & & & & 0.233 & 0.055 & 2.659 & 1.508 & $<0.0055$ & 0.399 & 0.416 & 0.0532 & 1.376 \\
\hline HLW99-89 & & & & 0.105 & 0.049 & 0.807 & 0.017 & 0.015 & $<0.0079$ & $<0.0243$ & 0.0532 & 0.649 \\
\hline HLW99-90R & & & & 0.201 & 0.049 & 0.767 & $<0.0027$ & 0.02 & 0.015 & 0.085 & 0.0532 & 1.714 \\
\hline HLW99-91 & & & & 0.18 & $<0.049$ & 0.819 & 0.011 & $<0.0055$ & $<0.0079$ & 0.066 & $<0.0532$ & 1.627 \\
\hline HLW99-92 & & & & 0.527 & 0.063 & 6.268 & 4.021 & 0.04 & 3.913 & 1.016 & $<0.0532$ & 4.434 \\
\hline HLW99-93R2 & & & & 0.739 & 0.224 & 1.415 & $<0.0027$ & 0.081 & 0.019 & 0.233 & 0.081 & 8.326 \\
\hline HLW99-94 & & & & 0.253 & 0.083 & 1.206 & 0.072 & 0.019 & 0.061 & 0.055 & $<0.0532$ & 2.307 \\
\hline HLW99-95 & & & & 0.195 & 0.083 & 0.974 & $<0.0027$ & 0.018 & $<0.0079$ & $<0.0243$ & 0.064 & 1.812 \\
\hline HLW99-96 & & & & 0.164 & $<0.0490$ & 1.605 & 0.616 & $<0.0055$ & 0.212 & 0.147 & 0.075 & 1.151 \\
\hline HLW99-97 & & & & 0.225 & $<0.0490$ & 2.453 & 1.151 & $<0.0055$ & 0.523 & 0.275 & $<0.0532$ & 1.781 \\
\hline HLW99-98R & & & & 0.605 & 0.072 & 10.716 & 8.643 & 0.081 & 7.903 & 1.292 & 0.081 & 9.125 \\
\hline HLW99-99 & & & & 0.267 & $<0.0490$ & 1.262 & 0.018 & 0.024 & 0.009 & 0.077 & 0.125 & 2.486 \\
\hline HLW99-100 & & & & 0.795 & 0.186 & 1.627 & 0.02 & 0.072 & 0.022 & 0.227 & 0.12 & 8.829 \\
\hline
\end{tabular}


Appendix A. Database - mass fraction

RPP-WTP LAW Formulation (Muller et al. 2001)

\begin{tabular}{|c|c|c|c|c|c|c|c|c|c|c|c|c|c|c|c|c|c|c|c|c|c|}
\hline Glass ID & $\begin{array}{c}\mathrm{A} 12 \mathrm{O} 3 \\
-\mathrm{t}\end{array}$ & $\begin{array}{c}\mathrm{B} 2 \mathrm{O} 3 \\
-\mathrm{t}\end{array}$ & $\begin{array}{c}\mathrm{CaO} \\
-\mathrm{t}\end{array}$ & $\begin{array}{c}\mathrm{Fe} 2 \mathrm{O} 3 \\
-\mathrm{t} \\
\end{array}$ & $\begin{array}{c}\mathrm{FeO} \\
-\mathrm{t}\end{array}$ & $\begin{array}{c}\mathrm{K} 2 \mathrm{O} \\
-\mathrm{t}\end{array}$ & $\begin{array}{c}\mathrm{Li} 2 \mathrm{O} \\
-\mathrm{t}\end{array}$ & $\begin{array}{c}\mathrm{MgO} \\
-\mathrm{t}\end{array}$ & $\begin{array}{c}\mathrm{Na} 2 \mathrm{O} \\
-\mathrm{t}\end{array}$ & $\begin{array}{c}\mathrm{NiO} \\
-\mathrm{t}\end{array}$ & $\begin{array}{c}\mathrm{P} 2 \mathrm{O} 5 \\
-\mathrm{t}\end{array}$ & $\begin{array}{c}\mathrm{SiO} 2 \\
-\mathrm{t}\end{array}$ & $\begin{array}{c}\mathrm{ZrO} 2 \\
-\mathrm{t}\end{array}$ & $\begin{array}{c}\mathrm{Ag} 2 \mathrm{O} \\
-\mathrm{t}\end{array}$ & $\begin{array}{c}\mathrm{As} 2 \mathrm{O} 3 \\
-\mathrm{t}\end{array}$ & $\begin{array}{c}\mathrm{BaO} \\
-\mathrm{t}\end{array}$ & $\begin{array}{c}\mathrm{Bi} 2 \mathrm{O} 3 \\
-\mathrm{t}\end{array}$ & $\begin{array}{l}\mathrm{Br} \\
-\mathrm{t}\end{array}$ & $\begin{array}{c}\mathrm{CdO} \\
-\mathrm{t}\end{array}$ & $\begin{array}{c}\mathrm{Ce} 2 \mathrm{O} 3 \\
-\mathrm{t}\end{array}$ & $\begin{array}{c}\mathrm{CeO} 2 \\
-\mathrm{t}\end{array}$ \\
\hline LAWA41 & 0.0620 & 0.0750 & 0.0200 & 0.0698 & & 0.0310 & & 0.0199 & 0.2000 & & 0.0008 & 0.4341 & 0.0300 & & & & & & & & \\
\hline LAWA42 & 0.0620 & 0.0903 & 0.0240 & 0.0841 & & 0.0310 & 0.0000 & 0.0240 & 0.2000 & 0.0000 & 0.0008 & 0.3800 & 0.0361 & 0.0000 & & 0.0000 & & & & & \\
\hline LAWA43 & 0.1200 & 0.0739 & 0.0197 & 0.0688 & & 0.0310 & 0.0000 & 0.0197 & 0.2000 & 0.0000 & 0.0008 & 0.3800 & 0.0295 & 0.0000 & & 0.0000 & & & & & \\
\hline LAWA44 & 0.0620 & 0.0890 & 0.0199 & 0.0698 & & 0.0050 & & 0.0199 & 0.2000 & & 0.0003 & 0.4455 & 0.0299 & 0.0000 & & & & & & & \\
\hline LAWA45 & 0.0620 & 0.1190 & & 0.0698 & & 0.0050 & & 0.0148 & 0.2000 & & 0.0003 & 0.4455 & 0.0299 & 0.0000 & & & & & & & \\
\hline LAWA46 & 0.0620 & 0.0890 & & 0.0698 & & 0.0050 & & 0.0148 & 0.2000 & & 0.0003 & 0.4455 & 0.0299 & 0.0000 & & & & & & & \\
\hline LAWA47 & 0.0620 & 0.0890 & & 0.0698 & & 0.0050 & & 0.0148 & 0.2000 & & 0.0003 & 0.4455 & 0.0299 & 0.0000 & & & & & & & \\
\hline LAWA48 & 0.0620 & 0.0890 & & 0.0698 & & 0.0300 & & 0.0148 & 0.2000 & & 0.0003 & 0.4455 & 0.0299 & 0.0000 & & & & & & & \\
\hline LAWA49 & 0.0620 & 0.0890 & & 0.0998 & & & & 0.0148 & 0.2000 & & 0.0003 & 0.4455 & 0.0299 & 0.0000 & & & & & & & \\
\hline LAWA50 & 0.0620 & 0.0890 & & 0.1198 & & & & 0.0148 & 0.2000 & & 0.0003 & 0.4255 & 0.0299 & 0.0000 & & & & & & & \\
\hline LAWA51 & 0.0620 & 0.1197 & & 0.0700 & & & & 0.0148 & 0.1800 & & 0.0003 & 0.4657 & 0.0300 & 0.0000 & & & & & & & \\
\hline LAWA52 & 0.0618 & 0.0619 & 0.0788 & 0.0751 & & 0.0050 & & 0.0148 & 0.2000 & & 0.0003 & 0.4225 & 0.0299 & 0.0000 & & & & & & & \\
\hline LAWA53 & 0.0609 & 0.0611 & 0.0777 & 0.0740 & & 0.0049 & & 0.0146 & 0.1972 & & 0.0003 & 0.4166 & 0.0295 & & & & & & & & \\
\hline LAWA54 & 0.0609 & 0.0611 & & 0.0740 & & 0.0049 & & 0.0146 & 0.1972 & & 0.0003 & 0.4166 & 0.0295 & & & & & & & & \\
\hline LAWA55 & 0.0609 & 0.0611 & & 0.0740 & & 0.0049 & & 0.0146 & 0.1972 & & 0.0003 & 0.4166 & 0.0295 & & & 0.0777 & & & & & \\
\hline LAWA56 & 0.0609 & 0.1193 & 0.0195 & 0.0740 & & 0.0049 & & 0.0146 & 0.1972 & & 0.0003 & 0.4166 & 0.0295 & & & & & & & & \\
\hline LAWA57 & 0.0609 & 0.0611 & 0.0284 & 0.0740 & & 0.0049 & & 0.0146 & 0.1972 & & 0.0003 & 0.4166 & 0.0295 & & & & & & & & \\
\hline LAWA58 & 0.0609 & 0.0611 & 0.0284 & 0.0740 & & 0.0049 & & 0.0146 & 0.1972 & & 0.0003 & 0.4166 & 0.0295 & & & & 0.0493 & & & & \\
\hline LAWA59 & 0.0609 & 0.0611 & 0.0482 & 0.0740 & & 0.0049 & & 0.0146 & 0.1972 & & 0.0003 & 0.4166 & 0.0295 & & & & & & & & \\
\hline LAWA60 & 0.0853 & 0.1123 & 0.0432 & & & 0.0050 & & 0.0199 & 0.2000 & & 0.0003 & 0.4455 & 0.0299 & 0.0000 & & & & & & & \\
\hline LAWA61 & 0.0609 & 0.0611 & 0.0477 & 0.0740 & & 0.0049 & & 0.0146 & 0.1972 & & 0.0003 & 0.4166 & 0.0295 & & & & & & & & \\
\hline LAWA62 & 0.0609 & 0.0611 & 0.0477 & 0.0740 & & 0.0049 & & 0.0146 & 0.1972 & & 0.0003 & 0.4166 & 0.0295 & & & & & & & & \\
\hline LAWA63 & 0.0609 & 0.0611 & 0.0477 & 0.0740 & & 0.0049 & & 0.0146 & 0.1972 & & 0.0003 & 0.4166 & 0.0295 & & & & & & & & \\
\hline LAWA64 & 0.0618 & 0.0619 & & 0.0751 & & 0.0050 & & 0.0148 & 0.2000 & & 0.0003 & 0.4225 & 0.0299 & 0.0000 & & & & & & & \\
\hline LAWA65 & 0.0609 & 0.0611 & 0.0326 & 0.0740 & & 0.0049 & & 0.0597 & 0.1972 & & 0.0003 & 0.4166 & 0.0295 & & & & & & & & \\
\hline LAWA66 & 0.0609 & 0.0611 & 0.0477 & 0.0740 & & 0.0049 & & 0.0146 & 0.1972 & & 0.0303 & 0.4166 & 0.0295 & & & & & & & & \\
\hline LAWA67 & \begin{tabular}{|l|}
0.0609 \\
\end{tabular} & 0.0611 & 0.0477 & 0.0740 & & 0.0049 & & 0.0146 & 0.1972 & & 0.0003 & 0.4166 & 0.0295 & & & & & & & & \\
\hline LAWA68 & 0.0609 & 0.0611 & 0.0316 & 0.0740 & & 0.0049 & & 0.0146 & 0.1972 & & 0.0003 & 0.4327 & 0.0295 & & & & & & & & \\
\hline LAWA69 & 0.0609 & 0.1088 & & 0.0740 & & 0.0049 & & 0.0146 & 0.1972 & & 0.0303 & 0.4166 & 0.0295 & & & & & & & & \\
\hline LAWA70 & 0.0609 & 0.0611 & 0.0577 & 0.0740 & & 0.0049 & & 0.0146 & 0.1972 & & 0.0003 & 0.4166 & 0.0295 & & & & & & & & \\
\hline LAWA71 & 0.0609 & 0.1085 & 0.0777 & 0.0740 & & 0.0049 & & 0.0146 & 0.1499 & & 0.0003 & 0.4166 & 0.0295 & & & & & & & & \\
\hline LAWA72 & 0.0609 & 0.1085 & & 0.0740 & & 0.0049 & & 0.0146 & 0.1499 & & 0.0003 & 0.4166 & 0.0295 & & & & & & & & \\
\hline LAWA73 & 0.0609 & 0.0611 & 0.0777 & 0.0740 & & 0.0049 & & 0.0146 & 0.1972 & & 0.0003 & 0.4166 & 0.0295 & & & & & & & & \\
\hline
\end{tabular}


Appendix A. Database - mass fraction

RPP-WTP LAW Formulation (Muller et al. 2001)

\begin{tabular}{|c|c|c|c|c|c|c|c|c|c|c|c|c|c|c|c|c|c|c|c|c|c|}
\hline Glass ID & $\begin{array}{l}\mathrm{Cl} \\
-\mathrm{t} \\
\end{array}$ & $\begin{array}{c}\mathrm{CoO} \\
-\mathrm{t}\end{array}$ & $\begin{array}{c}\mathrm{Co} 2 \mathrm{O} 3 \\
-\mathrm{t}\end{array}$ & $\begin{array}{c}\mathrm{Cr} 2 \mathrm{O} 3 \\
-\mathrm{t} \\
\end{array}$ & $\begin{array}{c}\mathrm{Cs} 2 \mathrm{O} \\
-\mathrm{t}\end{array}$ & $\begin{array}{c}\mathrm{CuO} \\
-\mathrm{t}\end{array}$ & $\begin{array}{c}\mathrm{Eu} 2 \mathrm{O} 3 \\
-\mathrm{t}\end{array}$ & $\begin{array}{l}F \\
-t\end{array}$ & $\begin{array}{c}\mathrm{Ga} 2 \mathrm{O} 3 \\
-\mathrm{t}\end{array}$ & $\begin{array}{c}\mathrm{Gd} 2 \mathrm{O} 3 \\
-\mathrm{t}\end{array}$ & $\begin{array}{c}\mathrm{HgO} \\
-\mathrm{t}\end{array}$ & $\begin{array}{l}I \\
-t\end{array}$ & $\begin{array}{c}\mathrm{La} 2 \mathrm{O} 3 \\
-\mathrm{t}\end{array}$ & $\begin{array}{c}\mathrm{MnO} 2 \\
-\mathrm{t}\end{array}$ & $\begin{array}{c}\mathrm{MnO} \\
-\mathrm{t}\end{array}$ & $\begin{array}{c}\mathrm{MoO} \\
-\mathrm{t}\end{array}$ & $\begin{array}{c}\mathrm{MoO} 3 \\
-\mathrm{t} \\
\end{array}$ & $\begin{array}{c}\mathrm{Nb} 2 \mathrm{O} 5 \\
-\mathrm{t}\end{array}$ & $\begin{array}{c}\mathrm{Nd} 2 \mathrm{O} 3 \\
-\mathrm{t}\end{array}$ & $\begin{array}{c}\mathrm{PbO} \\
-\mathrm{t} \\
\end{array}$ & $\begin{array}{c}\mathrm{PdO} 2 \\
-\mathrm{t}\end{array}$ \\
\hline LAWA41 & 0.0058 & & & 0.0002 & & & & 0.0004 & & & & & & & & & & & & 0.0000 & \\
\hline LAWA42 & 0.0058 & & & 0.0002 & 0.0000 & & & 0.0004 & & & & & & 0.0000 & & & & & & 0.0000 & \\
\hline LAWA43 & 0.0058 & & & 0.0002 & 0.0000 & & & 0.0004 & & & & & & 0.0000 & & & & & & 0.0000 & \\
\hline LAWA44 & 0.0065 & & & 0.0002 & 0.0000 & & & 0.0001 & & & & & & & & & & & & 0.0000 & \\
\hline LAWA45 & 0.0065 & & & 0.0002 & 0.0000 & & & 0.0001 & & & & & & & & & & & & 0.0000 & \\
\hline LAWA46 & 0.0065 & & & 0.0002 & 0.0000 & & & 0.0001 & 0.0300 & & & & & & & & & & & 0.0000 & \\
\hline LAWA47 & 0.0065 & & & 0.0002 & 0.0000 & & & 0.0001 & & & & & & & & & & & & 0.0000 & \\
\hline LAWA48 & 0.0065 & & & 0.0002 & 0.0000 & & & 0.0001 & 0.0050 & & & & & & & & & & & 0.0000 & \\
\hline LAWA49 & 0.0065 & & & 0.0002 & 0.0000 & & & 0.0001 & 0.0050 & & & & & & & & & & & 0.0000 & \\
\hline LAWA50 & 0.0065 & & & 0.0002 & 0.0000 & & & 0.0001 & 0.0050 & & & & & & & & & & & 0.0000 & \\
\hline LAWA51 & 0.0059 & & & 0.0002 & 0.0000 & & & 0.0001 & 0.0045 & & & & & & & & & & & 0.0000 & \\
\hline LAWA52 & 0.0065 & & & 0.0002 & 0.0000 & & & 0.0001 & & & & & & & & & & & & & \\
\hline LAWA53 & 0.0064 & & & 0.0002 & & & & 0.0001 & & & & & & & & & 0.0001 & & & & \\
\hline LAWA54 & 0.0064 & & & 0.0002 & & & & 0.0001 & & & & & & & & & 0.0001 & & & & \\
\hline LAWA55 & 0.0064 & & & 0.0002 & & & & 0.0001 & & & & & & & & & 0.0001 & & & & \\
\hline LAWA56 & 0.0064 & & & 0.0002 & & & & 0.0001 & & & & & & & & & 0.0001 & & & & \\
\hline LAWA57 & 0.0064 & & & 0.0002 & & & & 0.0001 & & & & & & & & & 0.0001 & & & & \\
\hline LAWA58 & 0.0064 & & & 0.0002 & & & & 0.0001 & & & & & & & & & 0.0001 & & & & \\
\hline LAWA59 & 0.0064 & & & 0.0002 & & & & 0.0001 & & & & & & & & & 0.0001 & & & & \\
\hline LAWA60 & 0.0065 & & & 0.0002 & 0.0000 & & & 0.0001 & & & & & & & & & & & & & \\
\hline LAWA61 & 0.0064 & & & 0.0002 & & & & 0.0001 & & & & & & 0.0300 & & & 0.0001 & & & & \\
\hline LAWA62 & 0.0064 & 0.0300 & & 0.0002 & & & & 0.0001 & & & & & & & & & 0.0001 & & & & \\
\hline LAWA63 & 0.0064 & & & 0.0002 & & 0.0300 & & 0.0001 & & & & & & & & & 0.0001 & & & & \\
\hline LAWA64 & 0.0065 & & & 0.0002 & 0.0000 & & & 0.0001 & & & & & & & & & & & & & \\
\hline LAWA65 & 0.0064 & & & 0.0002 & & & & 0.0001 & & & & & & & & & 0.0001 & & & & \\
\hline LAWA66 & 0.0064 & & & 0.0002 & & & & 0.0001 & & & & & & & & & 0.0001 & & & & \\
\hline LAWA67 & 0.0064 & & & 0.0002 & & & & 0.0001 & & & & & & & & & 0.0001 & & & & \\
\hline LAWA68 & 0.0064 & & & 0.0002 & & & & 0.0301 & & & & & & & & & 0.0001 & & & & \\
\hline LAWA69 & 0.0064 & & & 0.0002 & & & & 0.0001 & & & & & & & & & 0.0001 & & & & \\
\hline LAWA70 & 0.0064 & & & 0.0002 & & & & 0.0201 & & & & & & & & & 0.0001 & & & & \\
\hline LAWA71 & 0.0064 & & & 0.0002 & & & & 0.0001 & & & & & & & & & 0.0001 & & & & \\
\hline LAWA72 & 0.0064 & & & 0.0002 & & & & 0.0001 & & & & & & & & & 0.0001 & & & & \\
\hline LAWA73 & 0.0064 & & & 0.0002 & & & & 0.0001 & & & & & & & & & 0.0001 & & & & \\
\hline
\end{tabular}


RPP-WTP LAW Formulation (Muller et al. 2001)

\begin{tabular}{|c|c|c|c|c|c|c|c|c|c|c|c|c|c|c|c|c|c|c|c|c|c|}
\hline Glass ID & $\begin{array}{c}\mathrm{PdO} \\
-\mathrm{t} \\
\end{array}$ & $\begin{array}{c}\mathrm{Pr} 2 \mathrm{O} 3 \\
-\mathrm{t}\end{array}$ & $\begin{array}{c}\text { Pr6O11 } \\
-t\end{array}$ & $\begin{array}{c}\mathrm{Rb} 2 \mathrm{O} \\
-\mathrm{t}\end{array}$ & $\begin{array}{c}\mathrm{ReO} \\
-\mathrm{t}\end{array}$ & $\begin{array}{c}\mathrm{ReO} 2 \\
-\mathrm{t}\end{array}$ & $\begin{array}{c}\mathrm{Rh} 2 \mathrm{O} 3 \\
-\mathrm{t}\end{array}$ & $\begin{array}{c}\mathrm{RhO} 2 \\
-\mathrm{t}\end{array}$ & $\begin{array}{c}\mathrm{RuO} 2 \\
-\mathrm{t}\end{array}$ & $\begin{array}{c}\mathrm{Sb} 2 \mathrm{O} 3 \\
-\mathrm{t}\end{array}$ & $\begin{array}{c}\mathrm{Sb} 2 \mathrm{O} 5 \\
-\mathrm{t}\end{array}$ & $\begin{array}{c}\mathrm{SeO} 2 \\
-\mathrm{t} \\
\end{array}$ & $\begin{array}{c}\mathrm{Sm} 2 \mathrm{O} 3 \\
-\mathrm{t}\end{array}$ & $\begin{array}{c}\mathrm{SnO} \\
-\mathrm{t} \\
\end{array}$ & $\begin{array}{c}\mathrm{SnO} 2 \\
-\mathrm{t} \\
\end{array}$ & $\begin{array}{c}\mathrm{SO} 3 \\
-\mathrm{t} \\
\end{array}$ & $\begin{array}{c}\mathrm{SrO} \\
-\mathrm{t}\end{array}$ & $\begin{array}{c}\mathrm{Tc} 2 \mathrm{O} 7 \\
-\mathrm{t}\end{array}$ & $\begin{array}{c}\mathrm{TeO} 2 \\
-\mathrm{t}\end{array}$ & $\begin{array}{c}\mathrm{ThO} 2 \\
-\mathrm{t}\end{array}$ & $\begin{array}{c}\mathrm{TiO} 2 \\
-\mathrm{t}\end{array}$ \\
\hline LAWA41 & & & & & & & & & & & & 0.0001 & & & & 0.0010 & & & & & 0.0200 \\
\hline LAWA42 & & & & & & & & & & & & 0.0001 & & & & 0.0010 & & & & & 0.0240 \\
\hline LAWA43 & & & & & & & & & & & & 0.0001 & & & & 0.0010 & & & & & 0.0197 \\
\hline LAWA44 & & & & & & 0.0010 & & & & & & & & & & 0.0010 & & & & & 0.0199 \\
\hline LAWA45 & & & & & & 0.0010 & & & & & & & & & & 0.0010 & & & & & 0.0199 \\
\hline LAWA46 & & & & & & 0.0010 & & & & & & & & & & 0.0010 & & & & & 0.0199 \\
\hline LAWA47 & & & & & & 0.0010 & & & & & & & & & & 0.0010 & & & & & 0.0199 \\
\hline LAWA48 & & & & & & 0.0010 & & & & & & & & & & 0.0010 & & & & & 0.0199 \\
\hline LAWA49 & & & & & & 0.0010 & & & & & & & & & & 0.0010 & & & & & 0.0199 \\
\hline LAWA50 & & & & & & 0.0010 & & & & & & & & & & 0.0010 & & & & & 0.0199 \\
\hline LAWA51 & & & & & & 0.0010 & & & & & & & & & & 0.0009 & & & & & 0.0200 \\
\hline LAWA52 & & & & & & 0.0010 & & & & & & & & & & 0.0010 & & & & & 0.0111 \\
\hline LAWA53 & & & & & & & & & & & & & & & & 0.0148 & & & & & 0.0109 \\
\hline LAWA54 & & & & & & & & & & & & & & & & 0.0148 & 0.0777 & & & & 0.0109 \\
\hline LAWA55 & & & & & & & & & & & & & & & & 0.0148 & & & & & 0.0109 \\
\hline LAWA56 & & & & & & & & & & & & & & & & 0.0148 & & & & & 0.0109 \\
\hline LAWA57 & & & & & & & & & & & & & & & 0.0493 & 0.0148 & & & & & 0.0109 \\
\hline LAWA58 & & & & & & & & & & & & & & & & 0.0148 & & & & & 0.0109 \\
\hline LAWA59 & & & & & & & & & & 0.0295 & & & & & & 0.0148 & & & & & 0.0109 \\
\hline LAWA60 & & & & & & 0.0010 & & & & & & & & & & 0.0010 & & & & & 0.0199 \\
\hline LAWA61 & & & & & & & & & & & & & & & & 0.0148 & & & & & 0.0109 \\
\hline LAWA62 & & & & & & & & & & & & & & & & 0.0148 & & & & & 0.0109 \\
\hline LAWA63 & & & & & & & & & & & & & & & & 0.0148 & & & & & 0.0109 \\
\hline LAWA64 & & & & & & 0.0010 & & & & & & & & & & 0.0010 & 0.0788 & & & & 0.0111 \\
\hline LAWA65 & & & & & & & & & & & & & & & & 0.0148 & & & & & 0.0109 \\
\hline LAWA66 & & & & & & & & & & & & & & & & 0.0148 & & & & & 0.0109 \\
\hline LAWA67 & & & & & & & & & & & & & & & & 0.0148 & & & & & 0.0109 \\
\hline LAWA68 & & & & & & & & & & & & & & & & 0.0148 & & & & & 0.0109 \\
\hline LAWA69 & & & & & & & & & & & & & & & & 0.0148 & & & & & 0.0109 \\
\hline LAWA70 & & & & & & & & & & & & & & & & 0.0148 & & & & & 0.0109 \\
\hline LAWA71 & & & & & & & & & & & & & & & & 0.0148 & & & & & 0.0109 \\
\hline LAWA72 & & & & & & & & & & & & & & & & 0.0148 & 0.0777 & & & & 0.0109 \\
\hline LAWA73 & & & & & & & & & & & & & & & & 0.0148 & & & & & 0.0404 \\
\hline
\end{tabular}


Appendix A. Database - mass fraction

RPP-WTP LAW Formulation (Muller et al. 2001)

\begin{tabular}{|c|c|c|c|c|c|c|c|c|c|c|c|c|c|c|c|c|c|c|c|c|c|}
\hline Glass ID & $\begin{array}{c}\mathrm{T} 12 \mathrm{O} 3 \\
-\mathrm{t}\end{array}$ & $\begin{array}{c}\mathrm{U} 3 \mathrm{O} 8 \\
-\mathrm{t}\end{array}$ & $\begin{array}{c}\mathrm{UO} 2 \\
-\mathrm{t}\end{array}$ & $\begin{array}{c}\text { UO3 } \\
-t\end{array}$ & $\begin{array}{c}\mathrm{V} 2 \mathrm{O} 5 \\
-\mathrm{t}\end{array}$ & $\begin{array}{c}\text { WO3 } \\
-t\end{array}$ & $\begin{array}{c}\mathrm{Y} 2 \mathrm{O} 3 \\
-\mathrm{t}\end{array}$ & $\begin{array}{c}\mathrm{ZnO} \\
-\mathrm{t}\end{array}$ & $\begin{array}{c}\text { Others } \\
-t\end{array}$ & $\begin{array}{c}\text { Sum } \\
-t\end{array}$ & $\begin{array}{c}\mathrm{Al} 2 \mathrm{O} 3 \\
-\mathrm{a}\end{array}$ & $\begin{array}{c}\mathrm{B} 2 \mathrm{O} 3 \\
-\mathrm{a}\end{array}$ & $\begin{array}{c}\mathrm{CaO} \\
-\mathrm{a} \\
\end{array}$ & $\begin{array}{c}\mathrm{Fe} 2 \mathrm{O} 3 \\
-\mathrm{a}\end{array}$ & $\begin{array}{c}\mathrm{FeO} \\
-\mathrm{a}\end{array}$ & $\begin{array}{c}\mathrm{K} 2 \mathrm{O} \\
-\mathrm{a}\end{array}$ & $\begin{array}{c}\mathrm{Li} 2 \mathrm{O} \\
-\mathrm{a}\end{array}$ & $\begin{array}{c}\mathrm{MgO} \\
-\mathrm{a}\end{array}$ & $\begin{array}{c}\mathrm{Na} 2 \mathrm{O} \\
-\mathrm{a}\end{array}$ & $\begin{array}{c}\mathrm{NiO} \\
-\mathrm{a}\end{array}$ & $\begin{array}{c}\mathrm{P} 2 \mathrm{O} 5 \\
-\mathrm{a}\end{array}$ \\
\hline LAWA41 & & & & & & & & 0.0299 & & 0.9999 & 0.0592 & 0.0735 & 0.0218 & 0.0650 & & 0.0283 & 0.0003 & 0.0195 & 0.1698 & 0.0001 & 0.0025 \\
\hline LAWA42 & & & & & & & & 0.0360 & & 1.0000 & 0.0582 & 0.0849 & 0.0248 & 0.0783 & & 0.0279 & 0.0003 & 0.0227 & 0.1694 & 0.0001 & 0.0025 \\
\hline LAWA43 & & & & & & & & 0.0295 & & 1.0000 & 0.1118 & 0.0733 & 0.0208 & 0.0642 & & 0.0291 & 0.0003 & 0.0190 & 0.1778 & 0.0001 & 0.0020 \\
\hline LAWA44 & & & & & & & & 0.0297 & & 0.9999 & 0.0586 & 0.0881 & 0.0203 & 0.0659 & & 0.0061 & 0.0003 & 0.0185 & 0.1792 & 0.0001 & 0.0017 \\
\hline LAWA45 & & & & & & & & 0.0248 & & 0.9999 & 0.0578 & 0.1183 & 0.0006 & 0.0661 & & 0.0060 & 0.0002 & 0.0151 & 0.1803 & 0.0001 & 0.0020 \\
\hline LAWA46 & & & & & & & & 0.0248 & & 0.9999 & 0.0590 & 0.0914 & 0.0009 & 0.0664 & & 0.0061 & 0.0002 & 0.0157 & 0.1878 & 0.0000 & 0.0016 \\
\hline LAWA47 & & & & & & & 0.0300 & 0.0248 & & 0.9999 & 0.0557 & 0.0944 & 0.0006 & 0.0653 & & 0.0060 & 0.0003 & 0.0145 & 0.1799 & 0.0001 & 0.0017 \\
\hline LAWA48 & & & & & & & & 0.0248 & & 0.9999 & 0.0621 & 0.0935 & 0.0007 & 0.0663 & & 0.0036 & 0.0002 & 0.0160 & 0.1858 & 0.0001 & 0.0017 \\
\hline LAWA49 & & & & & & & & 0.0248 & & 0.9999 & 0.0537 & 0.0854 & 0.0006 & 0.0868 & & & 0.0002 & 0.0145 & 0.1772 & 0.0001 & 0.0014 \\
\hline LAWA50 & & & & & & & & 0.0248 & & 0.9999 & 0.0588 & 0.0886 & 0.0006 & 0.1091 & & & 0.0002 & 0.0151 & 0.1832 & 0.0001 & 0.0019 \\
\hline LAWA51 & & & & & & & & 0.0249 & & 0.9999 & 0.0592 & 0.1179 & 0.0006 & 0.0660 & & & 0.0002 & 0.0149 & 0.1675 & 0.0001 & 0.0015 \\
\hline LAWA52 & & & & & & & & 0.0299 & & 0.9999 & 0.0595 & 0.0640 & 0.0769 & 0.0774 & & 0.0053 & 0.0006 & 0.0144 & 0.1900 & 0.0004 & 0.0007 \\
\hline LAWA53 & & & & & & & & 0.0295 & & 0.9988 & & & & & & & & & & & \\
\hline LAWA54 & & & & & & & & 0.0295 & & 0.9988 & & & & & & & & & & & \\
\hline LAWA55 & & & & & & & & 0.0295 & & 0.9988 & & & & & & & & & & & \\
\hline LAWA56 & & & & & & & & 0.0295 & & 0.9988 & & & & & & & & & & & \\
\hline LAWA57 & & & & & & & & 0.0295 & & 0.9988 & & & & & & & & & & & \\
\hline LAWA58 & & & & & & & & 0.0295 & & 0.9988 & & & & & & & & & & & \\
\hline LAWA59 & & & & & & & & 0.0295 & & 0.9988 & & & & & & & & & & & \\
\hline LAWA60 & & & & & & & & 0.0297 & & 0.9999 & 0.0824 & 0.1172 & 0.0432 & 0.0011 & & 0.0054 & 0.0005 & 0.0193 & 0.1823 & 0.0000 & 0.0017 \\
\hline LAWA61 & & & & & & & & 0.0295 & & 0.9988 & & & & & & & & & & & \\
\hline LAWA62 & & & & & & & & 0.0295 & & 0.9988 & & & & & & & & & & & \\
\hline LAWA63 & & & & & & & & 0.0295 & & 0.9988 & & & & & & & & & & & \\
\hline LAWA64 & & & & & & & & 0.0299 & & 0.9999 & 0.0576 & 0.0593 & 0.0009 & 0.0751 & & 0.0049 & 0.0008 & 0.0138 & 0.1747 & 0.0002 & 0.0007 \\
\hline LAWA65 & & & & & & & & 0.0295 & & 0.9988 & & & & & & & & & & & \\
\hline LAWA66 & & & & & & & & 0.0295 & & 0.9988 & & & & & & & & & & & \\
\hline LAWA67 & & & & & 0.0300 & & & 0.0295 & & 0.9988 & & & & & & & & & & & \\
\hline LAWA68 & & & & & & & & 0.0295 & & 0.9988 & & & & & & & & & & & \\
\hline LAWA69 & & & & & & & & 0.0295 & & 0.9988 & & & & & & & & & & & \\
\hline LAWA70 & & & & & & & & 0.0295 & & 0.9988 & & & & & & & & & & & \\
\hline LAWA71 & & & & & & & & 0.0295 & & 0.9989 & & & & & & & & & & & \\
\hline LAWA72 & & & & & & & & 0.0295 & & 0.9989 & & & & & & & & & & & \\
\hline LAWA73 & & & & & & & & & & 0.9988 & & & & & & & & & & & \\
\hline
\end{tabular}


Appendix A. Database - mass fraction

RPP-WTP LAW Formulation (Muller et al. 2001)

\begin{tabular}{|c|c|c|c|c|c|c|c|c|c|c|c|c|c|c|c|c|c|c|c|c|c|}
\hline Glass ID & $\begin{array}{c}\mathrm{SiO} 2 \\
-\mathrm{a}\end{array}$ & $\begin{array}{c}\mathrm{ZrO} 2 \\
-\mathrm{a}\end{array}$ & $\begin{array}{c}\mathrm{Ag} 2 \mathrm{O} \\
-\mathrm{a}\end{array}$ & $\begin{array}{c}\mathrm{As} 2 \mathrm{O} 3 \\
-\mathrm{a} \\
\end{array}$ & $\begin{array}{c}\mathrm{BaO} \\
-\mathrm{a} \\
\end{array}$ & $\begin{array}{c}\mathrm{Bi} 2 \mathrm{O} 3 \\
-\mathrm{a}\end{array}$ & $\begin{array}{l}\mathrm{Br} \\
-\mathrm{a}\end{array}$ & $\begin{array}{c}\mathrm{CdO} \\
-\mathrm{a}\end{array}$ & $\begin{array}{c}\mathrm{Ce} 2 \mathrm{O} 3 \\
-\mathrm{a}\end{array}$ & $\begin{array}{c}\mathrm{CeO} 2 \\
-\mathrm{a}\end{array}$ & $\begin{array}{l}\mathrm{Cl} \\
-\mathrm{a} \\
\end{array}$ & $\begin{array}{c}\mathrm{CoO} \\
-\mathrm{a} \\
\end{array}$ & $\begin{array}{c}\mathrm{Co} 2 \mathrm{O} 3 \\
-\mathrm{a}\end{array}$ & $\begin{array}{c}\mathrm{Cr} 2 \mathrm{O} 3 \\
-\mathrm{a} \\
\end{array}$ & $\begin{array}{c}\mathrm{Cs} 2 \mathrm{O} \\
-\mathrm{a}\end{array}$ & $\begin{array}{c}\mathrm{CuO} \\
-\mathrm{a}\end{array}$ & $\begin{array}{c}\mathrm{Eu} 2 \mathrm{O} 3 \\
-\mathrm{a}\end{array}$ & $\begin{array}{c}\mathrm{F} \\
-\mathrm{a}\end{array}$ & $\begin{array}{c}\mathrm{Ga} 2 \mathrm{O} 3 \\
-\mathrm{a}\end{array}$ & $\begin{array}{c}\mathrm{Gd} 2 \mathrm{O} 3 \\
-\mathrm{a}\end{array}$ & $\begin{array}{c}\mathrm{HgO} \\
-\mathrm{a}\end{array}$ \\
\hline LAWA41 & 0.4160 & 0.0258 & 0.0001 & & 0.0000 & & & & & & & & & 0.0002 & & & & & & & \\
\hline LAWA42 & 0.3584 & 0.0324 & 0.0001 & & 0.0000 & & & & & & & & & 0.0002 & & & & & & & \\
\hline LAWA43 & 0.3576 & 0.0260 & 0.0001 & & 0.0000 & & & 0.0002 & & & & & & 0.0002 & & & & & & & \\
\hline LAWA44 & 0.4202 & 0.0254 & 0.0001 & & 0.0000 & & & 0.0000 & & & & & & 0.0003 & & & & & & & \\
\hline LAWA45 & 0.4274 & 0.0266 & 0.0001 & & 0.0000 & & & 0.0000 & & & & & & 0.0002 & & & & & & & \\
\hline LAWA46 & 0.4183 & 0.0262 & 0.0001 & & 0.0000 & & & 0.0000 & & & & & & 0.0001 & & & & & 0.0321 & & \\
\hline LAWA47 & 0.4256 & 0.0261 & 0.0001 & & 0.0000 & & & 0.0000 & & & & & & 0.0002 & & & & & & & \\
\hline LAWA48 & 0.4280 & 0.0256 & 0.0001 & & 0.0001 & & & 0.0000 & & & & & & 0.0002 & & & & & 0.0062 & & \\
\hline LAWA49 & 0.4124 & 0.0251 & 0.0001 & & 0.0000 & & & 0.0000 & & & & & & 0.0001 & & & & & 0.0056 & & \\
\hline LAWA50 & 0.4161 & 0.0254 & 0.0001 & & 0.0001 & & & 0.0000 & & & & & & 0.0001 & & & & & 0.0059 & & \\
\hline LAWA51 & 0.4315 & 0.0272 & 0.0001 & & 0.0000 & & & 0.0000 & & & & & & 0.0002 & & & & & 0.0052 & & \\
\hline LAWA52 & 0.4188 & 0.0284 & & & 0.0000 & & & & & & & & & 0.0014 & & & & & & & \\
\hline LAWA53 & & & & & & & & & & & & & & & & & & & & & \\
\hline LAWA54 & & & & & & & & & & & & & & & & & & & & & \\
\hline LAWA55 & & & & & & & & & & & & & & & & & & & & & \\
\hline LAWA56 & & & & & & & & & & & & & & & & & & & & & \\
\hline LAWA57 & & & & & & & & & & & & & & & & & & & & & \\
\hline LAWA58 & & & & & & & & & & & & & & & & & & & & & \\
\hline LAWA59 & & & & & & & & & & & & & & & & & & & & & \\
\hline LAWA60 & 0.4322 & 0.0283 & & & 0.0001 & & & & & & & & & 0.0001 & & & & & & & \\
\hline LAWA61 & & & & & & & & & & & & & & & & & & & & & \\
\hline LAWA62 & & & & & & & & & & & & & & & & & & & & & \\
\hline LAWA63 & & & & & & & & & & & & & & & & & & & & & \\
\hline LAWA64 & 0.4080 & 0.0306 & & & 0.0006 & & & & & & & & & 0.0003 & & & & & & & \\
\hline LAWA65 & & & & & & & & & & & & & & & & & & & & & \\
\hline LAWA66 & & & & & & & & & & & & & & & & & & & & & \\
\hline LAWA67 & & & & & & & & & & & & & & & & & & & & & \\
\hline LAWA68 & & & & & & & & & & & & & & & & & & & & & \\
\hline LAWA69 & & & & & & & & & & & & & & & & & & & & & \\
\hline LAWA70 & & & & & & & & & & & & & & & & & & & & & \\
\hline LAWA71 & & & & & & & & & & & & & & & & & & & & & \\
\hline LAWA72 & & & & & & & & & & & & & & & & & & & & & \\
\hline LAWA73 & & & & & & & & & & & & & & & & & & & & & \\
\hline
\end{tabular}


Appendix A. Database - mass fraction

RPP-WTP LAW Formulation (Muller et al. 2001)

\begin{tabular}{|c|c|c|c|c|c|c|c|c|c|c|c|c|c|c|c|c|c|c|c|c|c|}
\hline Glass ID & $\begin{array}{c}\text { I } \\
-\mathrm{a} \\
\end{array}$ & $\begin{array}{c}\mathrm{La} 2 \mathrm{O} 3 \\
-\mathrm{a} \\
\end{array}$ & $\begin{array}{c}\mathrm{MnO} 2 \\
-\mathrm{a}\end{array}$ & $\begin{array}{c}\mathrm{MnO} \\
-\mathrm{a}\end{array}$ & $\begin{array}{c}\mathrm{MoO} \\
-\mathrm{a}\end{array}$ & $\begin{array}{c}\mathrm{MoO} 3 \\
-\mathrm{a} \\
\end{array}$ & $\begin{array}{c}\mathrm{Nb} 2 \mathrm{O} 5 \\
-\mathrm{a} \\
\end{array}$ & $\begin{array}{c}\mathrm{Nd} 2 \mathrm{O} 3 \\
-\mathrm{a}\end{array}$ & $\begin{array}{c}\mathrm{PbO} \\
-\mathrm{a}\end{array}$ & $\begin{array}{c}\mathrm{PdO} 2 \\
-\mathrm{a}\end{array}$ & $\begin{array}{c}\mathrm{PdO} \\
-\mathrm{a}\end{array}$ & $\begin{array}{c}\mathrm{Pr} 2 \mathrm{O} 3 \\
-\mathrm{a}\end{array}$ & $\begin{array}{c}\text { Pr6O11 } \\
-\mathrm{a}\end{array}$ & $\begin{array}{c}\mathrm{Rb} 2 \mathrm{O} \\
-\mathrm{a}\end{array}$ & $\begin{array}{c}\mathrm{ReO} \\
-\mathrm{a}\end{array}$ & $\begin{array}{c}\mathrm{ReO} 2 \\
-\mathrm{a}\end{array}$ & $\begin{array}{c}\mathrm{Rh} 2 \mathrm{O} 3 \\
-\mathrm{a} \\
\end{array}$ & $\begin{array}{c}\mathrm{RhO} 2 \\
-\mathrm{a} \\
\end{array}$ & $\begin{array}{c}\mathrm{RuO} 2 \\
-\mathrm{a} \\
\end{array}$ & $\begin{array}{c}\mathrm{Sb} 2 \mathrm{O} 3 \\
-\mathrm{a}\end{array}$ & $\begin{array}{c}\mathrm{Sb} 2 \mathrm{O} \\
-\mathrm{a}\end{array}$ \\
\hline LAWA41 & & & 0.0001 & & & & & & 0.0007 & & & & & & & & & & & & \\
\hline LAWA42 & & & 0.0001 & & & & & & 0.0002 & & & & & & & & & & & & \\
\hline LAWA43 & & & 0.0001 & & & & & & 0.0003 & & & & & & & & & & & & \\
\hline LAWA44 & & & 0.0001 & & & & & & 0.0002 & & & & & & & & & & & & \\
\hline LAWA45 & & & 0.0000 & & & & & & 0.0002 & & & & & & & & & & & & \\
\hline LAWA46 & & & 0.0000 & & & & & & 0.0002 & & & & & & & & & & & & \\
\hline LAWA47 & & & 0.0001 & & & & & & 0.0002 & & & & & & & & & & & & \\
\hline LAWA48 & & & 0.0000 & & & & & & 0.0002 & & & & & & & & & & & & \\
\hline LAWA49 & & & 0.0000 & & & & & & 0.0003 & & & & & & & & & & & & \\
\hline LAWA50 & & & 0.0001 & & & & & & 0.0002 & & & & & & & & & & & & \\
\hline LAWA51 & & & 0.0001 & & & & & & 0.0002 & & & & & & & & & & & & \\
\hline LAWA52 & & & 0.0001 & & & & & & & & & & & & & & & & & & \\
\hline LAWA53 & & & & & & & & & & & & & & & & & & & & & \\
\hline LAWA54 & & & & & & & & & & & & & & & & & & & & & \\
\hline LAWA55 & & & & & & & & & & & & & & & & & & & & & \\
\hline LAWA56 & & & & & & & & & & & & & & & & & & & & & \\
\hline LAWA57 & & & & & & & & & & & & & & & & & & & & & \\
\hline LAWA58 & & & & & & & & & & & & & & & & & & & & & \\
\hline LAWA59 & & & & & & & & & & & & & & & & & & & & & \\
\hline LAWA60 & & & 0.0001 & & & & & & & & & & & & & & & & & & \\
\hline LAWA61 & & & & & & & & & & & & & & & & & & & & & \\
\hline LAWA62 & & & & & & & & & & & & & & & & & & & & & \\
\hline LAWA63 & & & & & & & & & & & & & & & & & & & & & \\
\hline LAWA64 & & & 0.0003 & & & & & & & & & & & & & & & & & & \\
\hline LAWA65 & & & & & & & & & & & & & & & & & & & & & \\
\hline LAWA66 & & & & & & & & & & & & & & & & & & & & & \\
\hline LAWA67 & & & & & & & & & & & & & & & & & & & & & \\
\hline LAWA68 & & & & & & & & & & & & & & & & & & & & & \\
\hline LAWA69 & & & & & & & & & & & & & & & & & & & & & \\
\hline LAWA70 & & & & & & & & & & & & & & & & & & & & & \\
\hline LAWA71 & & & & & & & & & & & & & & & & & & & & & \\
\hline LAWA72 & & & & & & & & & & & & & & & & & & & & & \\
\hline LAWA73 & & & & & & & & & & & & & & & & & & & & & \\
\hline
\end{tabular}


Appendix A. Database - mass fraction

RPP-WTP LAW Formulation (Muller et al. 2001)

\begin{tabular}{|c|c|c|c|c|c|c|c|c|c|c|c|c|c|c|c|c|c|c|c|c|}
\hline Glass ID & $\begin{array}{c}\mathrm{SeO} 2 \\
-\mathrm{a}\end{array}$ & $\begin{array}{c}\mathrm{Sm} 2 \mathrm{O} 3 \\
-\mathrm{a}\end{array}$ & $\begin{array}{c}\mathrm{SnO} \\
-\mathrm{a} \\
\end{array}$ & $\begin{array}{c}\mathrm{SnO} 2 \\
-\mathrm{a}\end{array}$ & $\begin{array}{c}\mathrm{SO} 3 \\
-\mathrm{a}\end{array}$ & $\begin{array}{c}\mathrm{SrO} \\
-\mathrm{a}\end{array}$ & $\begin{array}{c}\mathrm{Tc} 2 \mathrm{O} 7 \\
-\mathrm{a}\end{array}$ & $\begin{array}{c}\mathrm{TeO} 2 \\
-\mathrm{a}\end{array}$ & $\begin{array}{c}\mathrm{ThO} 2 \\
-\mathrm{a}\end{array}$ & $\begin{array}{c}\mathrm{TiO} 2 \\
-\mathrm{a} \\
\end{array}$ & $\begin{array}{c}\mathrm{T} 12 \mathrm{O} 3 \\
-\mathrm{a} \\
\end{array}$ & $\begin{array}{c}\mathrm{U} 3 \mathrm{O} 8 \\
-\mathrm{a}\end{array}$ & $\begin{array}{c}\mathrm{UO} 2 \\
-\mathrm{a}\end{array}$ & $\begin{array}{c}\text { UO3 } \\
-\mathrm{a}\end{array}$ & $\begin{array}{c}\mathrm{V} 2 \mathrm{O} 5 \\
-\mathrm{a}\end{array}$ & $\begin{array}{c}\text { WO3 } \\
-\mathrm{a} \\
\end{array}$ & $\begin{array}{c}\mathrm{Y} 2 \mathrm{O} 3 \\
-\mathrm{a}\end{array}$ & $\begin{array}{c}\mathrm{ZnO} \\
-\mathrm{a}\end{array}$ & $\begin{array}{c}\text { Others } \\
-\mathrm{a}\end{array}$ & $\begin{array}{c}\text { Sum } \\
-\mathrm{a}\end{array}$ \\
\hline LAWA41 & 0.0001 & & & & & 0.0001 & & & & 0.0209 & & & & & & & & 0.0283 & & 0.9320 \\
\hline LAWA42 & 0.0003 & & & & & 0.0001 & & & & 0.0242 & & & & & & & & 0.0338 & & 0.9189 \\
\hline LAWA43 & 0.0002 & & & & & 0.0001 & & & & 0.0202 & & & & & & & & 0.0275 & & 0.9310 \\
\hline LAWA44 & 0.0002 & & & & & & & & & 0.0199 & & & & & & & & 0.0293 & & 0.9342 \\
\hline LAWA45 & 0.0002 & & & & & & & & & 0.0197 & & & & & & & & 0.0243 & & 0.9452 \\
\hline LAWA46 & & & & & & & & & & 0.0211 & & & & & & & & 0.0247 & & 0.9518 \\
\hline LAWA47 & 0.0002 & & & & & & & & & 0.0192 & & & & & & & 0.0012 & 0.0240 & & 0.9153 \\
\hline LAWA48 & & & & & & & & & & 0.0211 & & & & & & & & 0.0247 & & 0.9361 \\
\hline LAWA49 & 0.0001 & & & & & & & & & 0.0198 & & & & & & & & 0.0229 & & 0.9061 \\
\hline LAWA50 & & & & & & & & & & 0.0207 & & & & & & & & 0.0242 & & 0.9504 \\
\hline LAWA51 & & & & & & & & & & 0.0205 & & & & & & & & 0.0243 & & 0.9371 \\
\hline LAWA52 & & & & & & 0.0001 & & & & 0.0116 & & & & & & & & 0.0291 & & 0.9788 \\
\hline LAWA53 & & & & & & & & & & & & & & & & & & & & \\
\hline LAWA54 & & & & & & & & & & & & & & & & & & & & \\
\hline LAWA55 & & & & & & & & & & & & & & & & & & & & \\
\hline LAWA56 & & & & & & & & & & & & & & & & & & & & \\
\hline LAWA57 & & & & & & & & & & & & & & & & & & & & \\
\hline LAWA58 & & & & & & & & & & & & & & & & & & & & \\
\hline LAWA59 & & & & & & & & & & & & & & & & & & & & \\
\hline LAWA60 & & & & & & 0.0000 & & & & 0.0199 & & & & & & & & 0.0289 & & 0.9629 \\
\hline LAWA61 & & & & & & & & & & & & & & & & & & & & \\
\hline LAWA62 & & & & & & & & & & & & & & & & & & & & \\
\hline LAWA63 & & & & & & & & & & & & & & & & & & & & \\
\hline LAWA64 & & & & & & 0.0727 & & & & 0.0110 & & & & & & & & 0.0303 & & 0.9417 \\
\hline LAWA65 & & & & & & & & & & & & & & & & & & & & \\
\hline LAWA66 & & & & & & & & & & & & & & & & & & & & \\
\hline LAWA67 & & & & & & & & & & & & & & & & & & & & \\
\hline LAWA68 & & & & & & & & & & & & & & & & & & & & \\
\hline LAWA69 & & & & & & & & & & & & & & & & & & & & \\
\hline LAWA70 & & & & & & & & & & & & & & & & & & & & \\
\hline LAWA71 & & & & & & & & & & & & & & & & & & & & \\
\hline LAWA72 & & & & & & & & & & & & & & & & & & & & \\
\hline LAWA73 & & & & & & & & & & & & & & & & & & & & \\
\hline
\end{tabular}


Appendix A. Database - mass fraction

RPP-WTP LAW Formulation (Muller et al. 2001)

\begin{tabular}{|c|c|c|c|c|c|c|c|c|}
\hline Glass ID & $\begin{array}{l}\mathrm{TM} \\
\left({ }^{\circ} \mathrm{C}\right) \\
\end{array}$ & $\begin{array}{l}\text { Gradient } \\
\text { TL }\left({ }^{\circ} \mathrm{C}\right) \\
\end{array}$ & $\begin{array}{l}\text { Uniform } \\
\text { TL }\left({ }^{\circ} \mathrm{C}\right)\end{array}$ & Primary Phase & $\begin{array}{l}\text { Quenched } \\
\text { Visual/OM }\end{array}$ & $\begin{array}{c}\text { Quenched } \\
\text { SEM/EDS or TEM }\end{array}$ & $\begin{array}{l}\text { Quenched } \\
\text { XRD }\end{array}$ & $\begin{array}{c}\text { Quenched } \\
\text { Homogeneous? }\end{array}$ \\
\hline \multicolumn{9}{|l|}{ LAWA41 } \\
\hline \multicolumn{9}{|l|}{ LAWA42 } \\
\hline \multicolumn{9}{|l|}{ LAWA43 } \\
\hline \multicolumn{9}{|l|}{ LAWA44 } \\
\hline \multicolumn{9}{|l|}{ LAWA45 } \\
\hline \multicolumn{9}{|l|}{ LAWA46 } \\
\hline \multicolumn{9}{|l|}{ LAWA47 } \\
\hline \multicolumn{9}{|l|}{ LAWA48 } \\
\hline \multicolumn{9}{|l|}{ LAWA49 } \\
\hline \multicolumn{9}{|l|}{ LAWA50 } \\
\hline \multicolumn{9}{|l|}{ LAWA51 } \\
\hline \multicolumn{9}{|l|}{ LAWA52 } \\
\hline \multicolumn{9}{|l|}{ LAWA53 } \\
\hline \multicolumn{9}{|l|}{ LAWA54 } \\
\hline \multirow{2}{*}{\multicolumn{9}{|c|}{$\begin{array}{l}\text { LAWA55 } \\
\text { LAWA56 }\end{array}$}} \\
\hline LAWA56 & & & & & & & & \\
\hline LAWA57 & & & & & & & & \\
\hline \multicolumn{9}{|l|}{ LAWA58 } \\
\hline \multicolumn{9}{|l|}{ LAWA59 } \\
\hline \multicolumn{9}{|l|}{ LAWA60 } \\
\hline \multicolumn{9}{|l|}{ LAWA61 } \\
\hline \multirow{2}{*}{\multicolumn{9}{|c|}{$\begin{array}{l}\text { LAWA62 } \\
\text { LAWA63 }\end{array}$}} \\
\hline LAWA63 & & & & & & & & \\
\hline \multicolumn{9}{|l|}{ LAWA64 } \\
\hline \multicolumn{9}{|l|}{ LAWA65 } \\
\hline \multicolumn{9}{|l|}{ LAWA66 } \\
\hline \multicolumn{9}{|l|}{ LAWA67 } \\
\hline \multicolumn{9}{|l|}{ LAWA68 } \\
\hline \multicolumn{9}{|l|}{ LAWA69 } \\
\hline \multicolumn{9}{|l|}{ LAWA70 } \\
\hline LAWA71 & & & & & & & & \\
\hline LAWA72 & & & & & & & & \\
\hline LAWA73 & & & & & & & & \\
\hline
\end{tabular}


Appendix A. Database - mass fraction

RPP-WTP LAW Formulation (Muller et al. 2001)

\begin{tabular}{|c|c|c|c|c|c|}
\hline Glass ID & $\begin{array}{c}\mathrm{CCC} \\
\text { Visual/OM }\end{array}$ & $\begin{array}{c}\text { CCC } \\
\text { SEM/EDS or TEM }\end{array}$ & $\begin{array}{l}\text { CCC } \\
\text { XRD }\end{array}$ & $\begin{array}{l}\text { Heat Treated } \\
\text { Visual/OM }\end{array}$ & $\begin{array}{c}\text { Heat Treated } \\
\text { SEM/EDS or TEM }\end{array}$ \\
\hline LAWA41 & & & & & $\begin{array}{l}\text { After } 1200^{\circ} \mathrm{C} 1 \mathrm{hr} \text { and } 950^{\circ} \mathrm{C} 20 \mathrm{hr}-\text { Clear } \\
\text { homogeneous glass }\end{array}$ \\
\hline LAWA42 & & & & & $\begin{array}{l}\text { After } 1200^{\circ} \mathrm{C} 1 \mathrm{hr} \text { and } 950^{\circ} \mathrm{C} 20 \mathrm{hr}-\text { Clear } \\
\text { homogeneous glass }\end{array}$ \\
\hline LAWA43 & & & & & $\begin{array}{l}\text { After } 1200^{\circ} \mathrm{C} 1 \mathrm{hr} \text { and } 950^{\circ} \mathrm{C} 20 \mathrm{hr} \text { - Trace } \\
\text { amount }(\sim 0.01 \mathrm{vol} \%) \mathrm{ZrO} 2\end{array}$ \\
\hline LAWA44 & & & & & $\begin{array}{l}\text { After } 1200^{\circ} \mathrm{C} 1 \mathrm{hr} \text { and } 950^{\circ} \mathrm{C} 20 \mathrm{hr}-\text { Clear } \\
\text { homogeneous glass; After } 1200^{\circ} \mathrm{C} 1 \mathrm{hr} \text { and } 850^{\circ} \mathrm{C} \\
20 \mathrm{hr} \text { - Clear homogeneous glass }\end{array}$ \\
\hline LAWA45 & & & & & $\begin{array}{l}\text { After } 1200^{\circ} \mathrm{C} 1 \mathrm{hr} \text { and } 950^{\circ} \mathrm{C} 20 \mathrm{hr}-\text { Clear } \\
\text { homogeneous glass }\end{array}$ \\
\hline LAWA46 & & & & & $\begin{array}{l}\text { After } 1200^{\circ} \mathrm{C} 1 \mathrm{hr} \text { and } 950^{\circ} \mathrm{C} 20 \mathrm{hr}-\text { Clear } \\
\text { homogeneous glass }\end{array}$ \\
\hline LAWA47 & & & & & $\begin{array}{l}\text { After } 1200^{\circ} \mathrm{C} 1 \mathrm{hr} \text { and } 950^{\circ} \mathrm{C} 20 \mathrm{hr}-\text { Clear } \\
\text { homogeneous glass }\end{array}$ \\
\hline LAWA48 & & & & & $\begin{array}{l}\text { After } 1200^{\circ} \mathrm{C} 1 \mathrm{hr} \text { and } 950^{\circ} \mathrm{C} 20 \mathrm{hr}-\text { Clear } \\
\text { homogeneous glass }\end{array}$ \\
\hline LAWA49 & & & & & $\begin{array}{l}\text { After } 1200^{\circ} \mathrm{C} 1 \mathrm{hr} \text { and } 950^{\circ} \mathrm{C} 20 \mathrm{hr}-\text { Clear } \\
\text { homogeneous glass }\end{array}$ \\
\hline LAWA50 & & & & & $\begin{array}{l}\text { After } 1200^{\circ} \mathrm{C} 1 \mathrm{hr} \text { and } 950^{\circ} \mathrm{C} 20 \mathrm{hr}-\text { Clear } \\
\text { homogeneous glass }\end{array}$ \\
\hline LAWA51 & & & & & $\begin{array}{l}\text { After } 1200^{\circ} \mathrm{C} 1 \mathrm{hr} \text { and } 950^{\circ} \mathrm{C} 20 \mathrm{hr}-\text { Clear } \\
\text { homogeneous glass }\end{array}$ \\
\hline LAWA52 & & & & & $\begin{array}{l}\text { After } 1200^{\circ} \mathrm{C} 1 \mathrm{hr} \text { and } 950^{\circ} \mathrm{C} 20 \mathrm{hr} \text { - Clear } \\
\text { homogeneous glass }\end{array}$ \\
\hline \multicolumn{6}{|l|}{ LAWA53 } \\
\hline \multicolumn{6}{|l|}{ LAWA54 } \\
\hline \multicolumn{6}{|l|}{ LAWA55 } \\
\hline \multicolumn{6}{|l|}{ LAWA56 } \\
\hline \multicolumn{6}{|l|}{ LAWA57 } \\
\hline \multicolumn{6}{|l|}{ LAWA58 } \\
\hline \multicolumn{6}{|l|}{ LAWA59 } \\
\hline LAWA60 & & & & & $\begin{array}{l}\text { After } 1200^{\circ} \mathrm{C} 1 \mathrm{hr} \text { and } 950^{\circ} \mathrm{C} 20 \mathrm{hr}-\text { Clear } \\
\text { homogeneous glass }\end{array}$ \\
\hline \multicolumn{6}{|l|}{ LAWA61 } \\
\hline \multicolumn{6}{|l|}{ LAWA62 } \\
\hline \multicolumn{6}{|l|}{ LAWA63 } \\
\hline LAWA64 & & & & & $\begin{array}{l}\text { After } 1200^{\circ} \mathrm{C} 1 \mathrm{hr} \text { and } 950^{\circ} \mathrm{C} 20 \mathrm{hr}-\text { Clear } \\
\text { homogeneous glass }\end{array}$ \\
\hline \multicolumn{6}{|l|}{ LAWA65 } \\
\hline LAWA66 & & & & & $\begin{array}{l}\text { After } 1200^{\circ} \mathrm{C} 1 \mathrm{hr} \text { and } 950^{\circ} \mathrm{C} 20 \mathrm{hr}-\text { Clear } \\
\text { homogeneous glass }\end{array}$ \\
\hline \multicolumn{6}{|l|}{ LAWA67 } \\
\hline \multicolumn{6}{|l|}{ LAWA68 } \\
\hline \multicolumn{6}{|l|}{ LAWA69 } \\
\hline \multicolumn{6}{|l|}{ LAWA70 } \\
\hline \multicolumn{6}{|l|}{ LAWA71 } \\
\hline \multicolumn{6}{|l|}{ LAWA72 } \\
\hline LAWA73 & & & & & \\
\hline
\end{tabular}


Appendix A. Database - mass fraction

RPP-WTP LAW Formulation (Muller et al. 2001)

\begin{tabular}{|c|c|c|c|c|c|c|c|c|c|c|c|c|c|c|c|}
\hline Glass ID & $\begin{array}{l}\text { Heat Treated } \\
\text { XRD } \\
\end{array}$ & $\begin{array}{l}\text { Density } \\
\left(\mathrm{g} / \mathrm{cm}^{3}\right)\end{array}$ & $\begin{array}{c}\text { Fulc Visc } \\
\text { A }\end{array}$ & $\begin{array}{c}\text { Fulc Visc } \\
\text { B }\end{array}$ & $\begin{array}{c}\text { Fulc Visc } \\
\text { To } \\
\end{array}$ & $\begin{array}{c}\text { FV } 1150^{\circ} \mathrm{C} \\
(\mathrm{Pa} \cdot \mathrm{s})\end{array}$ & $\begin{array}{c}\text { Arrh Visc } \\
\text { A }\end{array}$ & $\begin{array}{c}\text { Arrh Visc } \\
\mathrm{B}\end{array}$ & $1150^{\circ} \mathrm{C}$ & $\begin{array}{c}\mathrm{T}\left({ }^{\circ} \mathrm{C}\right) \text { at } \\
2 \mathrm{~Pa} \cdot \mathrm{s}\end{array}$ & $\begin{array}{c}\mathrm{T}\left({ }^{\circ} \mathrm{C}\right) \text { at } \\
5 \mathrm{~Pa} \cdot \mathrm{s}\end{array}$ & $\begin{array}{c}\mathrm{T}\left({ }^{\circ} \mathrm{C}\right) \text { at } \\
10 \mathrm{~Pa} \cdot \mathrm{s}\end{array}$ & $\begin{array}{c}\mathrm{T} 1 \\
\left({ }^{\circ} \mathrm{C}\right) \\
\end{array}$ & $\begin{array}{c}\mathrm{V} 1 \\
(\mathrm{~Pa} \cdot \mathrm{s}) \\
\end{array}$ & $\begin{array}{l}\mathrm{T} 2 \\
\left({ }^{\circ} \mathrm{C}\right) \\
\end{array}$ \\
\hline LAWA41 & & 2.68 & & & & & -12.546 & 20602.7 & 6.91 & & & & 900 & 159.5 & 950 \\
\hline LAWA42 & & & & & & & -12.723 & 19749.5 & 3.18 & & & & 900 & 66.9 & 950 \\
\hline LAWA43 & & & & & & & -13.231 & 21937.8 & 8.90 & & & & 900 & 260.4 & 950 \\
\hline LAWA44 & & 2.67 & & & & & -12.592 & 20696.1 & 7.04 & & & & 900 & 168.7 & 950 \\
\hline LAWA45 & & & & & & & -12.440 & 20387.4 & 6.60 & & & & 900 & 154.3 & 950 \\
\hline LAWA46 & & & & & & & -12.402 & 20780.7 & 9.04 & & & & 900 & 218.9 & 950 \\
\hline LAWA47 & & & & & & & -13.309 & 22023.0 & 8.74 & & & & 900 & 265.3 & 950 \\
\hline LAWA48 & & & & & & & -12.563 & 20880.7 & 8.25 & & & & 900 & 205 & 950 \\
\hline LAWA49 & & 2.64 & & & & & -12.532 & 20937.5 & 8.86 & & & & 900 & 220.4 & 950 \\
\hline LAWA50 & & & & & & & -12.477 & 20604.5 & 7.41 & & & & 900 & 175.8 & 950 \\
\hline LAWA51 & & & & & & & -12.672 & 21443.3 & 10.99 & & & & 900 & 304.8 & 950 \\
\hline LAWA52 & & & & & & & -13.542 & 21659.8 & 5.36 & & & & 900 & 155.4 & 950 \\
\hline LAWA53 & & & & & & & & & & & & & & & \\
\hline LAWA54 & & & & & & & & & & & & & & & \\
\hline LAWA55 & & & & & & & & & & & & & & & \\
\hline LAWA56 & & & & & & & & & & & & & & & \\
\hline LAWA57 & & & & & & & & & & & & & & & \\
\hline LAWA58 & & & & & & & & & & & & & & & \\
\hline LAWA59 & & & & & & & & & & & & & & & \\
\hline LAWA60 & & 2.64 & & & & & -11.417 & 18952.1 & 6.70 & & & & (900) & (245.6) & (950) \\
\hline LAWA61 & & & & & & & & & & & & & & & \\
\hline LAWA62 & & & & & & & & & & & & & & & \\
\hline LAWA63 & & & & & & & & & & & & & & & \\
\hline LAWA64 & & & & & & & -12.688 & 20668.1 & 6.27 & & & & 900 & 149.6 & 950 \\
\hline LAWA65 & & & & & & & & & & & & & & & \\
\hline LAWA66 & & & & & & & & & & & & & & & \\
\hline LAWA67 & & & & & & & & & & & & & & & \\
\hline LAWA68 & & & & & & & & & & & & & & & \\
\hline LAWA69 & & & & & & & & & & & & & & & \\
\hline LAWA70 & & & & & & & & & & & & & & & \\
\hline LAWA71 & & & & & & & & & & & & & & & \\
\hline LAWA72 & & & & & & & & & & & & & & & \\
\hline LAWA73 & & & & & & & & & & & & & & & \\
\hline
\end{tabular}


Appendix A. Database - mass fraction

RPP-WTP LAW Formulation (Muller et al. 2001)

\begin{tabular}{|c|c|c|c|c|c|c|c|c|c|c|c|c|c|c|c|c|c|c|c|c|c|}
\hline Glass ID & $\begin{array}{c}\mathrm{V} 2 \\
(\mathrm{~Pa} \cdot \mathrm{s}) \\
\end{array}$ & $\begin{array}{c}\mathrm{T} 3 \\
\left({ }^{\circ} \mathrm{C}\right) \\
\end{array}$ & $\begin{array}{c}\mathrm{V} 3 \\
(\mathrm{~Pa} \cdot \mathrm{s})\end{array}$ & $\begin{array}{c}\mathrm{T} 4 \\
\left({ }^{\circ} \mathrm{C}\right)\end{array}$ & $\begin{array}{c}\mathrm{V} 4 \\
(\mathrm{~Pa} \cdot \mathrm{s})\end{array}$ & $\begin{array}{c}\mathrm{T} 5 \\
\left({ }^{\circ} \mathrm{C}\right) \\
\end{array}$ & $\begin{array}{c}\mathrm{V} 5 \\
(\mathrm{~Pa} \cdot \mathrm{s})\end{array}$ & $\begin{array}{c}\mathrm{T} 6 \\
\left({ }^{\circ} \mathrm{C}\right) \\
\end{array}$ & $\begin{array}{c}\mathrm{V} 6 \\
(\mathrm{~Pa} \cdot \mathrm{s})\end{array}$ & $\begin{array}{c}\mathrm{T} 7 \\
\left({ }^{\circ} \mathrm{C}\right) \\
\end{array}$ & $\begin{array}{c}\mathrm{V} 7 \\
(\mathrm{~Pa} \cdot \mathrm{s}) \\
\end{array}$ & $\begin{array}{c}\mathrm{T} 8 \\
\left({ }^{\circ} \mathrm{C}\right) \\
\end{array}$ & $\begin{array}{c}\mathrm{V} 8 \\
(\mathrm{~Pa} \cdot \mathrm{s}) \\
\end{array}$ & $\begin{array}{c}\mathrm{T} 9 \\
\left({ }^{\circ} \mathrm{C}\right) \\
\end{array}$ & $\begin{array}{c}\mathrm{V} 9 \\
(\mathrm{~Pa} \cdot \mathrm{s})\end{array}$ & $\begin{array}{l}\mathrm{T} 10 \\
\left({ }^{\circ} \mathrm{C}\right) \\
\end{array}$ & $\begin{array}{c}\mathrm{V} 10 \\
(\mathrm{~Pa} \cdot \mathrm{s})\end{array}$ & $\begin{array}{l}\mathrm{T} 11 \\
\left({ }^{\circ} \mathrm{C}\right) \\
\end{array}$ & $\begin{array}{c}\mathrm{V} 11 \\
(\mathrm{~Pa} \cdot \mathrm{s})\end{array}$ & $\begin{array}{l}\mathrm{T} 12 \\
\left({ }^{\circ} \mathrm{C}\right) \\
\end{array}$ & $\begin{array}{c}\mathrm{V} 12 \\
(\mathrm{~Pa} \cdot \mathrm{s})\end{array}$ \\
\hline LAWA41 & 73.7 & 1000 & 36.9 & 1050 & 19.9 & 1100 & 11.3 & 1150 & 6.8 & 1200 & 4.3 & 1250 & 2.8 & & & & & & & & \\
\hline LAWA42 & 30.7 & 1000 & 15.5 & 1050 & 8.5 & 1100 & 5 & 1150 & 3.1 & 1200 & 2 & 1250 & 1.4 & & & & & & & & \\
\hline LAWA43 & 110.4 & 1000 & 52 & 1050 & 26.7 & 1100 & 14.8 & 1150 & 8.7 & 1200 & 5.4 & 1250 & 3.5 & & & & & & & & \\
\hline LAWA44 & 75.9 & 1000 & 37.5 & 1050 & 20 & 1100 & 11.4 & 1150 & 6.9 & 1200 & 4.4 & 1250 & 2.9 & & & & & & & & \\
\hline LAWA45 & 68.5 & 1000 & 33.8 & 1050 & 18.2 & 1100 & 10.5 & 1150 & 6.4 & 1200 & 4.2 & 1250 & 2.8 & & & & & & & & \\
\hline LAWA46 & 98.2 & 1000 & 48.4 & 1050 & 25.8 & 1100 & 14.7 & 1150 & 8.9 & 1200 & 5.6 & 1250 & 3.7 & & & & & & & & \\
\hline LAWA47 & 109.2 & 1000 & 50.7 & 1050 & 25.9 & 1100 & 14.4 & 1150 & 8.5 & 1200 & 5.3 & 1250 & 3.5 & & & & & & & & \\
\hline LAWA48 & 90.6 & 1000 & 44.3 & 1050 & 23.6 & 1100 & 13.4 & 1150 & 8.1 & 1200 & 5.1 & 1250 & 3.4 & & & & & & & & \\
\hline LAWA49 & 98 & 1000 & 48 & 1050 & 25.5 & 1100 & 14.5 & 1150 & 8.7 & 1200 & 5.5 & 1250 & 3.6 & & & & & & & & \\
\hline LAWA50 & 78.9 & 1000 & 39 & 1050 & 20.9 & 1100 & 12 & 1150 & 7.2 & 1200 & 4.6 & 1250 & 3.1 & & & & & & & & \\
\hline LAWA51 & 128.8 & 1000 & 61 & 1050 & 31.8 & 1100 & 17.8 & 1150 & 10.7 & 1200 & 6.8 & 1250 & 4.5 & & & & & & & & \\
\hline LAWA52 & 64.4 & 1000 & 30.1 & 1050 & 15.5 & 1100 & 8.7 & 1150 & 5.2 & 1200 & 3.3 & 1250 & 2.2 & & & & & & & & \\
\hline \multicolumn{22}{|l|}{ LAWA53 } \\
\hline LAWA54 & & & & & & & & & & & & & & & & & & & & & \\
\hline LAWA55 & & & & & & & & & & & & & & & & & & & & & \\
\hline \multicolumn{22}{|l|}{ LAWA56 } \\
\hline \multicolumn{22}{|l|}{ LAWA57 } \\
\hline \multicolumn{22}{|l|}{ LAWA58 } \\
\hline \multicolumn{22}{|l|}{ LAWA59 } \\
\hline LAWA60 & $(82.3)$ & 1000 & 35.1 & 1050 & 17.7 & 1100 & 10.1 & 1150 & 6.3 & 1200 & 4.3 & 1250 & 3 & & & & & & & & \\
\hline \multicolumn{22}{|l|}{ LAWA61 } \\
\hline \multirow{2}{*}{\multicolumn{22}{|c|}{$\begin{array}{l}\text { LAWA62 } \\
\text { LAWA63 }\end{array}$}} \\
\hline & & & & & & & & & & & & & & & & & & & & & \\
\hline LAWA64 & 67.3 & 1000 & 33.3 & 1050 & 17.8 & 1100 & 10.2 & 1150 & 6.1 & 1200 & 3.9 & 1250 & 2.6 & & & & & & & & \\
\hline \multicolumn{22}{|l|}{ LAWA65 } \\
\hline \multicolumn{22}{|l|}{ LAWA66 } \\
\hline \multicolumn{22}{|l|}{ LAWA67 } \\
\hline \multirow{2}{*}{\multicolumn{22}{|c|}{$\begin{array}{l}\text { LAWA68 } \\
\text { LAWA69 }\end{array}$}} \\
\hline & & & & & & & & & & & & & & & & & & & & & \\
\hline \multicolumn{22}{|l|}{ LAWA70 } \\
\hline \multicolumn{22}{|l|}{ LAWA71 } \\
\hline LAWA72 & & & & & & & & & & & & & & & & & & & & & \\
\hline LAWA73 & & & & & & & & & & & & & & & & & & & & & \\
\hline
\end{tabular}


Appendix A. Database - mass fraction

RPP-WTP LAW Formulation (Muller et al. 2001)

\begin{tabular}{|c|c|c|c|c|c|c|c|c|c|c|c|c|c|c|c|c|}
\hline Glass ID & $\begin{array}{l}\mathrm{T} 13 \\
\left({ }^{\circ} \mathrm{C}\right) \\
\end{array}$ & $\begin{array}{c}\mathrm{V} 13 \\
(\mathrm{~Pa} \cdot \mathrm{s})\end{array}$ & $\begin{array}{l}\mathrm{T} 14 \\
\left({ }^{\circ} \mathrm{C}\right) \\
\end{array}$ & $\begin{array}{c}\text { V14 } \\
(\mathrm{Pa} \cdot \mathrm{s})\end{array}$ & $\begin{array}{c}\text { Q PCT } \\
\text { B }\left(\mathrm{g} / \mathrm{m}^{2}\right)\end{array}$ & $\begin{array}{c}\text { Q PCT } \\
\mathrm{Li}\left(\mathrm{g} / \mathrm{m}^{2}\right)\end{array}$ & $\begin{array}{c}\text { Q PCT } \\
\mathrm{Na}\left(\mathrm{g} / \mathrm{m}^{2}\right)\end{array}$ & $\begin{array}{c}\text { Q PCT } \\
\mathrm{Si}\left(\mathrm{g} / \mathrm{m}^{2}\right)\end{array}$ & $\begin{array}{c}\text { Q PCT } \\
\mathrm{pH}\end{array}$ & $\begin{array}{c}\text { CCC PCT } \\
\text { B }\left(\mathrm{g} / \mathrm{m}^{2}\right)\end{array}$ & $\begin{array}{l}\text { CCC PCT } \\
\mathrm{Li}\left(\mathrm{g} / \mathrm{m}^{2}\right)\end{array}$ & $\begin{array}{l}\text { CCC PCT } \\
\mathrm{Na}\left(\mathrm{g} / \mathrm{m}^{2}\right)\end{array}$ & $\begin{array}{c}\text { CCC PCT } \\
\mathrm{Si}\left(\mathrm{g} / \mathrm{m}^{2}\right)\end{array}$ & $\begin{array}{c}\text { CCC PCT } \\
\mathrm{pH}\end{array}$ & $\begin{array}{l}\text { Q PCT B at } \\
20^{\circ} \mathrm{C}\left(\mathrm{g} / \mathrm{m}^{2}\right)\end{array}$ & $\begin{array}{l}\text { Q PCT Li at } \\
20^{\circ} \mathrm{C}\left(\mathrm{g} / \mathrm{m}^{2}\right)\end{array}$ \\
\hline LAWA41 & & & & & 0.47 & & 0.52 & 0.2 & 11.11 & & & & & & & \\
\hline LAWA42 & & & & & 0.78 & & 0.7 & 0.22 & 11.27 & & & & & & & \\
\hline LAWA43 & & & & & 0.38 & & 0.43 & 0.16 & 11.21 & & & & & & & \\
\hline LAWA44 & & & & & 0.37 & & 0.36 & 0.16 & 10.27 & & & & & & & \\
\hline LAWA45 & & & & & 0.77 & & 0.51 & 0.15 & 10.17 & & & & & & & \\
\hline LAWA46 & & & & & 0.43 & & 0.35 & 0.16 & 10.33 & & & & & & & \\
\hline LAWA47 & & & & & 0.38 & & 0.33 & 0.15 & 10.55 & & & & & & & \\
\hline LAWA48 & & & & & 0.39 & & 0.33 & 0.15 & 10.55 & & & & & & & \\
\hline LAWA49 & & & & & 0.31 & & 0.29 & 0.15 & 10.51 & & & & & & & \\
\hline LAWA50 & & & & & 0.31 & & 0.3 & 0.15 & 10.62 & & & & & & & \\
\hline LAWA51 & & & & & 0.35 & & 0.26 & 0.12 & 10.03 & & & & & & & \\
\hline LAWA52 & & & & & 0.43 & & 0.55 & 0.17 & 10.82 & & & & & & & \\
\hline LAWA53 & & & & & & & & & & & & & & & & \\
\hline LAWA54 & & & & & & & & & & & & & & & & \\
\hline LAWA55 & & & & & & & & & & & & & & & & \\
\hline LAWA56 & & & & & & & & & & & & & & & & \\
\hline LAWA57 & & & & & & & & & & & & & & & & \\
\hline LAWA58 & & & & & & & & & & & & & & & & \\
\hline LAWA59 & & & & & & & & & & & & & & & & \\
\hline LAWA60 & & & & & 0.29 & & 0.31 & 0.11 & 10.21 & & & & & & & \\
\hline LAWA61 & & & & & & & & & & & & & & & & \\
\hline LAWA62 & & & & & & & & & & & & & & & & \\
\hline LAWA63 & & & & & & & & & & & & & & & & \\
\hline LAWA64 & & & & & 0.38 & & 0.5 & 0.18 & 10.94 & & & & & & & \\
\hline LAWA65 & & & & & & & & & & & & & & & & \\
\hline LAWA66 & & & & & & & & & & & & & & & & \\
\hline LAWA67 & & & & & & & & & & & & & & & & \\
\hline LAWA68 & & & & & & & & & & & & & & & & \\
\hline LAWA69 & & & & & & & & & & & & & & & & \\
\hline LAWA70 & & & & & & & & & & & & & & & & \\
\hline LAWA71 & & & & & & & & & & & & & & & & \\
\hline LAWA72 & & & & & & & & & & & & & & & & \\
\hline LAWA73 & & & & & & & & & & & & & & & & \\
\hline
\end{tabular}


Appendix A. Database - mass fraction

RPP-WTP LAW Formulation (Muller et al. 2001)

\begin{tabular}{|c|c|c|c|c|c|c|c|c|c|c|c|c|}
\hline Glass ID & $\begin{array}{l}\text { Q PCT Na at } \\
20^{\circ} \mathrm{C}\left(\mathrm{g} / \mathrm{m}^{2}\right)\end{array}$ & $\begin{array}{l}\text { Q PCT Si at } \\
20^{\circ} \mathrm{C}\left(\mathrm{g} / \mathrm{m}^{2}\right)\end{array}$ & $\begin{array}{c}\mathrm{QpH} \\
\text { at } 20^{\circ} \mathrm{C}\end{array}$ & $\begin{array}{c}\text { TCLP Ag } \\
(\mathrm{ppm})\end{array}$ & $\begin{array}{c}\text { TCLP As } \\
\text { (ppm) }\end{array}$ & $\begin{array}{c}\text { TCLP Ba } \\
\text { (ppm) }\end{array}$ & $\begin{array}{c}\text { TCLP Cd } \\
(\mathrm{ppm})\end{array}$ & $\begin{array}{c}\text { TCLP Cr } \\
(\mathrm{ppm})\end{array}$ & $\begin{array}{c}\text { TCLP Ni } \\
(\mathrm{ppm})\end{array}$ & $\begin{array}{c}\text { TCLP Pb } \\
\text { (ppm) }\end{array}$ & $\begin{array}{c}\text { TCLP Se } \\
(\mathrm{ppm})\end{array}$ & $\begin{array}{c}\text { TCLP Zn } \\
(\mathrm{ppm})\end{array}$ \\
\hline \multicolumn{13}{|l|}{ LAWA41 } \\
\hline \multicolumn{13}{|l|}{ LAWA42 } \\
\hline \multicolumn{13}{|l|}{ LAWA43 } \\
\hline LAWA44 & & & & $<0.0031$ & $<0.0490$ & 0.005 & $<0.0027$ & $<0.0055$ & $<0.0079$ & $<0.0243$ & $<0.0532$ & 1.121 \\
\hline \multicolumn{13}{|l|}{ LAWA45 } \\
\hline \multicolumn{13}{|l|}{ LAWA46 } \\
\hline \multicolumn{13}{|l|}{ LAWA47 } \\
\hline \multicolumn{13}{|l|}{ LAWA48 } \\
\hline \multicolumn{13}{|l|}{ LAWA49 } \\
\hline \multicolumn{13}{|l|}{ LAWA50 } \\
\hline \multicolumn{13}{|l|}{ LAWA51 } \\
\hline \multicolumn{13}{|l|}{ LAWA52 } \\
\hline \multicolumn{13}{|l|}{ LAWA53 } \\
\hline \multicolumn{13}{|l|}{ LAWA54 } \\
\hline \multicolumn{13}{|l|}{ LAWA55 } \\
\hline \multicolumn{13}{|l|}{ LAWA56 } \\
\hline \multicolumn{13}{|l|}{ LAWA57 } \\
\hline \multicolumn{13}{|l|}{ LAWA58 } \\
\hline \multicolumn{13}{|l|}{ LAWA59 } \\
\hline \multicolumn{13}{|l|}{ LAWA60 } \\
\hline \multicolumn{13}{|l|}{ LAWA61 } \\
\hline \multicolumn{13}{|l|}{ LAWA62 } \\
\hline \multicolumn{13}{|l|}{ LAWA63 } \\
\hline \multicolumn{13}{|l|}{ LAWA64 } \\
\hline \multicolumn{13}{|l|}{ LAWA65 } \\
\hline \multicolumn{13}{|l|}{ LAWA66 } \\
\hline LAWA67 & & & & & & & & & & & & \\
\hline LAWA68 & & & & & & & & & & & & \\
\hline LAWA69 & & & & & & & & & & & & \\
\hline LAWA70 & & & & & & & & & & & & \\
\hline LAWA71 & & & & & & & & & & & & \\
\hline LAWA72 & & & & & & & & & & & & \\
\hline LAWA73 & & & & & & & & & & & & \\
\hline
\end{tabular}


Appendix A. Database - mass fraction

RPP-WTP LAW Formulation (Muller et al. 2001)

\begin{tabular}{|c|c|c|c|c|c|c|c|c|c|c|c|c|c|c|c|c|c|c|c|c|c|}
\hline Glass ID & $\begin{array}{c}\mathrm{A} 12 \mathrm{O} 3 \\
-\mathrm{t}\end{array}$ & $\begin{array}{c}\mathrm{B} 2 \mathrm{O} 3 \\
-\mathrm{t}\end{array}$ & $\begin{array}{c}\mathrm{CaO} \\
-\mathrm{t}\end{array}$ & $\begin{array}{c}\mathrm{Fe} 2 \mathrm{O} 3 \\
-\mathrm{t}\end{array}$ & $\begin{array}{c}\mathrm{FeO} \\
-\mathrm{t}\end{array}$ & $\begin{array}{c}\mathrm{K} 2 \mathrm{O} \\
-\mathrm{t}\end{array}$ & $\begin{array}{c}\mathrm{Li} 2 \mathrm{O} \\
-\mathrm{t}\end{array}$ & $\begin{array}{c}\mathrm{MgO} \\
-\mathrm{t}\end{array}$ & $\begin{array}{c}\mathrm{Na} 2 \mathrm{O} \\
-\mathrm{t}\end{array}$ & $\begin{array}{c}\mathrm{NiO} \\
-\mathrm{t}\end{array}$ & $\begin{array}{c}\mathrm{P} 2 \mathrm{O} 5 \\
-\mathrm{t}\end{array}$ & $\begin{array}{c}\mathrm{SiO} 2 \\
-\mathrm{t}\end{array}$ & $\begin{array}{c}\mathrm{ZrO} 2 \\
-\mathrm{t}\end{array}$ & $\begin{array}{c}\mathrm{Ag} 2 \mathrm{O} \\
-\mathrm{t}\end{array}$ & $\begin{array}{c}\mathrm{As} 2 \mathrm{O} 3 \\
-\mathrm{t}\end{array}$ & $\begin{array}{c}\mathrm{BaO} \\
-\mathrm{t}\end{array}$ & $\begin{array}{c}\mathrm{Bi} 2 \mathrm{O} 3 \\
-\mathrm{t}\end{array}$ & $\begin{array}{c}\mathrm{Br} \\
-\mathrm{t}\end{array}$ & $\begin{array}{c}\mathrm{CdO} \\
-\mathrm{t}\end{array}$ & $\begin{array}{c}\mathrm{Ce} 2 \mathrm{O} 3 \\
-\mathrm{t}\end{array}$ & $\begin{array}{c}\mathrm{CeO} 2 \\
-\mathrm{t}\end{array}$ \\
\hline LAWA74 & 0.0609 & 0.0688 & 0.0777 & 0.0813 & & 0.0049 & & 0.0219 & 0.1972 & & 0.0003 & 0.4166 & 0.0295 & & & & & & & & \\
\hline LAWA75 & 0.0609 & 0.1580 & 0.0777 & 0.0740 & & 0.0049 & & 0.0146 & 0.1003 & & 0.0003 & 0.4166 & 0.0295 & & & & & & & & \\
\hline LAWA76 & 0.0609 & 0.1085 & 0.0777 & 0.0740 & & 0.0049 & 0.0495 & 0.0146 & 0.1003 & & 0.0003 & 0.4166 & 0.0295 & & & & & & & & \\
\hline LAWA77 & 0.1105 & 0.1085 & 0.0777 & 0.0740 & & 0.0049 & & 0.0146 & 0.1003 & & 0.0003 & 0.4166 & 0.0295 & & & & & & & & \\
\hline LAWA78 & 0.0609 & 0.1085 & 0.1273 & 0.0740 & & 0.0049 & & 0.0146 & 0.1003 & & 0.0003 & 0.4166 & 0.0295 & & & & & & & & \\
\hline LAWA79 & 0.1105 & 0.0611 & 0.0777 & 0.0740 & & 0.0049 & & 0.0146 & 0.1972 & & 0.0003 & 0.3670 & 0.0295 & & & & & & & & \\
\hline LAWA80 & 0.1105 & 0.0611 & 0.0777 & 0.0245 & & 0.0049 & & 0.0146 & 0.1972 & & 0.0003 & 0.4166 & 0.0295 & & & & & & & & \\
\hline LAWA81 & 0.0620 & 0.0890 & 0.0399 & 0.0698 & & 0.0050 & & 0.0199 & 0.2000 & & 0.0003 & 0.4455 & 0.0299 & 0.0000 & & & & & & & \\
\hline LAWA82 & 0.0620 & 0.0890 & & 0.0698 & & 0.0050 & & 0.0199 & 0.2000 & & 0.0003 & 0.4455 & 0.0299 & 0.0000 & & & & & & & \\
\hline LAWA83 & 0.0620 & 0.0890 & 0.0199 & 0.0499 & & 0.0050 & & 0.0199 & 0.2000 & & 0.0203 & 0.4455 & 0.0299 & 0.0000 & & & & & & & \\
\hline LAWA84 & 0.0620 & 0.0890 & 0.0199 & 0.0299 & & 0.0050 & & 0.0199 & 0.2000 & & 0.0402 & 0.4455 & 0.0299 & 0.0000 & & & & & & & \\
\hline LAWA85 & 0.0620 & 0.0890 & & 0.0499 & & 0.0050 & & 0.0199 & 0.2000 & & 0.0203 & 0.4455 & 0.0299 & 0.0000 & & & & & & & \\
\hline LAWA86 & 0.0620 & 0.0890 & & 0.0299 & & 0.0050 & & 0.0199 & 0.2000 & & 0.0402 & 0.4455 & 0.0299 & 0.0000 & & & & & & & \\
\hline LAWA87 & 0.0448 & 0.0887 & 0.0199 & 0.0697 & & 0.0258 & & 0.0199 & 0.2000 & & 0.0007 & 0.4446 & 0.0299 & & & & & & & & \\
\hline LAWA88 & 0.0608 & 0.0970 & 0.0199 & 0.0553 & & 0.0258 & & 0.0148 & 0.2000 & & 0.0007 & 0.4399 & 0.0299 & & & & & & & & \\
\hline LAWA89 & 0.0608 & 0.0970 & & 0.0553 & & 0.0258 & & 0.0148 & 0.2000 & & 0.0007 & 0.4399 & 0.0299 & & & & & & & & \\
\hline LAWA90 & 0.0608 & 0.0970 & 0.0398 & 0.0553 & & 0.0258 & & 0.0148 & 0.2000 & & 0.0007 & 0.4399 & 0.0299 & & & & & & & & \\
\hline LAWA91 & 0.0609 & 0.0611 & & 0.0740 & & 0.0049 & & 0.0146 & 0.1972 & & 0.0003 & 0.4166 & 0.0295 & & & & & & & & \\
\hline LAWA92 & 0.0609 & 0.0611 & & 0.0740 & & 0.0049 & & 0.0146 & 0.1972 & & 0.0003 & 0.4166 & 0.0295 & & & & & & & & \\
\hline LAWA93 & 0.0618 & 0.1110 & 0.0788 & 0.0751 & & 0.0050 & 0.0507 & 0.0148 & 0.1003 & & 0.0003 & 0.4225 & 0.0299 & 0.0000 & & & & & & & \\
\hline LAWA94 & 0.0609 & 0.0611 & 0.0632 & 0.0740 & & 0.0049 & & 0.0146 & 0.1972 & & 0.0003 & 0.4166 & 0.0441 & & & & & & & & \\
\hline LAWA95 & 0.0609 & 0.0611 & 0.0559 & 0.0376 & & 0.0049 & & 0.0146 & 0.1972 & & 0.0295 & 0.4166 & 0.0295 & & & & & & & & \\
\hline LAWA96 & 0.0620 & 0.0791 & 0.0399 & 0.0299 & & 0.0050 & & 0.0199 & 0.2000 & & 0.0402 & 0.4356 & 0.0299 & 0.0000 & & & & & & & \\
\hline LAWA97S & 0.0612 & 0.0878 & 0.0197 & 0.0397 & & 0.0049 & & 0.0197 & 0.1972 & & 0.0003 & 0.4394 & 0.0292 & & & & & & & & \\
\hline LAWA98S & 0.0603 & 0.1074 & 0.0508 & 0.0750 & & 0.0037 & 0.0306 & 0.0149 & 0.1480 & & 0.0003 & 0.4300 & 0.0302 & 0.0000 & & & & & & & \\
\hline LAWA99S & 0.0603 & 0.1074 & 0.0613 & 0.0452 & & 0.0037 & 0.0202 & 0.0149 & 0.1480 & & 0.0003 & 0.4300 & 0.0302 & 0.0000 & & & & & & & \\
\hline LAWA100S & 0.0603 & 0.1074 & 0.0613 & 0.0750 & & 0.0037 & 0.0202 & 0.0149 & 0.1480 & & 0.0003 & 0.4300 & 0.0302 & 0.0000 & & & & & & & \\
\hline LAWA101S & 0.0603 & 0.1074 & 0.0710 & 0.0750 & & 0.0037 & 0.0105 & 0.0149 & 0.1480 & & 0.0003 & 0.4300 & 0.0302 & 0.0000 & & & & & & & \\
\hline
\end{tabular}


Appendix A. Database - mass fraction

RPP-WTP LAW Formulation (Muller et al. 2001)

\begin{tabular}{|c|c|c|c|c|c|c|c|c|c|c|c|c|c|c|c|c|c|c|c|c|c|}
\hline Glass ID & $\begin{array}{l}\mathrm{Cl} \\
-\mathrm{t}\end{array}$ & $\begin{array}{c}\mathrm{CoO} \\
-\mathrm{t}\end{array}$ & $\begin{array}{c}\mathrm{Co} 2 \mathrm{O} 3 \\
-\mathrm{t}\end{array}$ & $\begin{array}{c}\mathrm{Cr} 2 \mathrm{O} 3 \\
-\mathrm{t}\end{array}$ & $\begin{array}{c}\mathrm{Cs} 2 \mathrm{O} \\
-\mathrm{t}\end{array}$ & $\begin{array}{c}\mathrm{CuO} \\
-\mathrm{t}\end{array}$ & $\begin{array}{c}\mathrm{Eu} 2 \mathrm{O} 3 \\
-\mathrm{t}\end{array}$ & $\begin{array}{l}F \\
-t\end{array}$ & $\begin{array}{c}\mathrm{Ga} 2 \mathrm{O} 3 \\
-\mathrm{t}\end{array}$ & $\begin{array}{c}\mathrm{Gd} 2 \mathrm{O} 3 \\
-\mathrm{t}\end{array}$ & $\begin{array}{c}\mathrm{HgO} \\
-\mathrm{t}\end{array}$ & $\begin{array}{l}I \\
-t\end{array}$ & $\begin{array}{c}\mathrm{La} 2 \mathrm{O} 3 \\
-\mathrm{t}\end{array}$ & $\begin{array}{c}\mathrm{MnO} 2 \\
-\mathrm{t}\end{array}$ & $\begin{array}{c}\mathrm{MnO} \\
-\mathrm{t}\end{array}$ & $\begin{array}{c}\mathrm{MoO} \\
-\mathrm{t}\end{array}$ & $\begin{array}{c}\mathrm{MoO} 3 \\
-\mathrm{t} \\
\end{array}$ & $\begin{array}{c}\mathrm{Nb} 2 \mathrm{O} 5 \\
-\mathrm{t} \\
\end{array}$ & $\begin{array}{c}\mathrm{Nd} 2 \mathrm{O} 3 \\
-\mathrm{t}\end{array}$ & $\begin{array}{c}\mathrm{PbO} \\
-\mathrm{t}\end{array}$ & $\begin{array}{c}\mathrm{PdO} 2 \\
-\mathrm{t} \\
\end{array}$ \\
\hline LAWA74 & 0.0064 & & & 0.0002 & & & & 0.0001 & & & & & & & & & 0.0001 & & & & \\
\hline LAWA75 & 0.0064 & & & 0.0002 & & & & 0.0001 & & & & & & & & & 0.0001 & & & & \\
\hline LAWA76 & 0.0064 & & & 0.0002 & & & & 0.0001 & & & & & & & & & 0.0001 & & & & \\
\hline LAWA77 & 0.0064 & & & 0.0002 & & & & 0.0001 & & & & & & & & & 0.0001 & & & & \\
\hline LAWA78 & 0.0064 & & & 0.0002 & & & & 0.0001 & & & & & & & & & 0.0001 & & & & \\
\hline LAWA79 & 0.0064 & & & 0.0002 & & & & 0.0001 & & & & & & & & & 0.0001 & & & & \\
\hline LAWA80 & 0.0064 & & & 0.0002 & & & & 0.0001 & & & & & & & & & 0.0001 & & & & \\
\hline LAWA81 & 0.0065 & & & 0.0002 & 0.0000 & & & 0.0001 & & & & & & & & & & & & & \\
\hline LAWA82 & 0.0065 & & & 0.0002 & 0.0000 & & & 0.0001 & & & & & & & & & & & & & \\
\hline LAWA83 & 0.0065 & & & 0.0002 & 0.0000 & & & 0.0001 & & & & & & & & & & & & & \\
\hline LAWA84 & 0.0065 & & & 0.0002 & 0.0000 & & & 0.0001 & & & & & & & & & & & & & \\
\hline LAWA85 & 0.0065 & & & 0.0002 & 0.0000 & & & 0.0001 & & & & & & & & & & & & & \\
\hline LAWA86 & 0.0065 & & & 0.0002 & 0.0000 & & & 0.0001 & & & & & & & & & & & & & \\
\hline LAWA87 & 0.0033 & & & 0.0001 & 0.0000 & & & & & & & & & & & & & & & & \\
\hline LAWA88 & 0.0033 & & & 0.0001 & 0.0000 & & & & & & & & & & & & & & & & \\
\hline LAWA89 & 0.0033 & & & 0.0001 & 0.0000 & & & & & & & & & & & & & & & & \\
\hline LAWA90 & 0.0033 & & & 0.0001 & 0.0000 & & & & & & & & & & & & & & & & \\
\hline LAWA91 & 0.0064 & & & 0.0002 & & & & 0.0001 & & & & & 0.0777 & & & & 0.0001 & & & & \\
\hline LAWA92 & 0.0064 & & & 0.0002 & & & & 0.0001 & & 0.0777 & & & & & & & 0.0001 & & & & \\
\hline LAWA93 & 0.0065 & & & 0.0002 & 0.0000 & & & 0.0001 & & & & & & & & & & & & & \\
\hline LAWA94 & 0.0064 & & & 0.0002 & & & & 0.0001 & & & & & & & & & 0.0001 & & & & \\
\hline LAWA95 & 0.0064 & & & 0.0002 & & & & 0.0001 & & & & & & & & & 0.0001 & & & & \\
\hline LAWA96 & 0.0065 & & & 0.0002 & 0.0000 & & & 0.0001 & & & & & & & & & & & & & \\
\hline LAWA97S & 0.0064 & & & 0.0002 & & & & 0.0001 & & & & & & & & & 0.0001 & & & & \\
\hline LAWA98S & 0.0048 & & & 0.0002 & 0.0000 & & & 0.0001 & & & & & & & & & & & & & \\
\hline LAWA99S & 0.0048 & & & 0.0002 & 0.0000 & & & 0.0001 & & & & & & & & & & & & 0.0000 & \\
\hline LAWA100S & 0.0048 & & & 0.0002 & 0.0000 & & & 0.0001 & & & & & & & & & & & & 0.0000 & \\
\hline LAWA101S & 0.0048 & & & 0.0002 & 0.0000 & & & 0.0001 & & & & & & & & & & & & 0.0000 & \\
\hline
\end{tabular}


RPP-WTP LAW Formulation (Muller et al. 2001)

\begin{tabular}{|c|c|c|c|c|c|c|c|c|c|c|c|c|c|c|c|c|c|c|c|c|c|}
\hline Glass ID & $\begin{array}{c}\mathrm{PdO} \\
-\mathrm{t}\end{array}$ & $\begin{array}{c}\mathrm{Pr} 2 \mathrm{O} 3 \\
-\mathrm{t} \\
\end{array}$ & $\begin{array}{c}\text { Pr6O11 } \\
-t\end{array}$ & $\begin{array}{c}\mathrm{Rb} 2 \mathrm{O} \\
-\mathrm{t} \\
\end{array}$ & $\begin{array}{c}\mathrm{ReO} \\
-\mathrm{t} \\
\end{array}$ & $\begin{array}{c}\mathrm{ReO} 2 \\
-\mathrm{t}\end{array}$ & $\begin{array}{c}\mathrm{Rh} 2 \mathrm{O} 3 \\
-\mathrm{t}\end{array}$ & $\begin{array}{c}\mathrm{RhO} 2 \\
-\mathrm{t}\end{array}$ & $\begin{array}{c}\mathrm{RuO} 2 \\
-\mathrm{t}\end{array}$ & $\begin{array}{c}\mathrm{Sb} 2 \mathrm{O} 3 \\
-\mathrm{t} \\
\end{array}$ & $\begin{array}{c}\mathrm{Sb} 2 \mathrm{O} 5 \\
-\mathrm{t}\end{array}$ & $\begin{array}{c}\mathrm{SeO} 2 \\
-\mathrm{t}\end{array}$ & $\begin{array}{c}\mathrm{Sm} 2 \mathrm{O} 3 \\
-\mathrm{t} \\
\end{array}$ & $\begin{array}{c}\mathrm{SnO} \\
-\mathrm{t} \\
\end{array}$ & $\begin{array}{c}\mathrm{SnO} 2 \\
-\mathrm{t}\end{array}$ & $\begin{array}{c}\mathrm{SO} 3 \\
-\mathrm{t}\end{array}$ & $\begin{array}{c}\mathrm{SrO} \\
-\mathrm{t}\end{array}$ & $\begin{array}{c}\mathrm{Tc} 2 \mathrm{O} 7 \\
-\mathrm{t} \\
\end{array}$ & $\begin{array}{c}\mathrm{TeO} 2 \\
-\mathrm{t}\end{array}$ & $\begin{array}{c}\mathrm{ThO} 2 \\
-\mathrm{t}\end{array}$ & $\begin{array}{c}\mathrm{TiO} 2 \\
-\mathrm{t} \\
\end{array}$ \\
\hline LAWA74 & & & & & & & & & & & & & & & & 0.0148 & & & & & 0.0182 \\
\hline LAWA75 & & & & & & & & & & & & & & & & 0.0148 & & & & & 0.0109 \\
\hline LAWA76 & & & & & & & & & & & & & & & & 0.0148 & & & & & 0.0109 \\
\hline LAWA77 & & & & & & & & & & & & & & & & 0.0148 & & & & & 0.0109 \\
\hline LAWA78 & & & & & & & & & & & & & & & & 0.0148 & & & & & 0.0109 \\
\hline LAWA79 & & & & & & & & & & & & & & & & 0.0148 & & & & & 0.0109 \\
\hline LAWA80 & & & & & & & & & & & & & & & & 0.0148 & & & & & 0.0109 \\
\hline LAWA81 & & & & & & 0.0010 & & & & & & & & & & 0.0010 & & & & & \\
\hline LAWA82 & & & & & & 0.0010 & & & & & & & & & & 0.0010 & & & & & 0.0399 \\
\hline LAWA83 & & & & & & 0.0010 & & & & & & & & & & 0.0010 & & & & & 0.0199 \\
\hline LAWA84 & & & & & & 0.0010 & & & & & & & & & & 0.0010 & & & & & 0.0199 \\
\hline LAWA85 & & & & & & 0.0010 & & & & & & & & & & 0.0010 & 0.0199 & & & & 0.0199 \\
\hline LAWA86 & & & & & & 0.0010 & & & & & & & & & & 0.0010 & 0.0199 & & & & 0.0199 \\
\hline LAWA87 & & & & & & 0.0010 & & & & & & & & & & 0.0021 & & & & & 0.0199 \\
\hline LAWA88 & & & & & & 0.0010 & & & & & & & & & & 0.0021 & & & & & 0.0199 \\
\hline LAWA89 & & & & & & 0.0010 & & & & & & & & & & 0.0021 & & & & & 0.0398 \\
\hline LAWA90 & & & & & & 0.0010 & & & & & & & & & & 0.0021 & & & & & \\
\hline LAWA91 & & & & & & & & & & & & & & & & 0.0148 & & & & & 0.0109 \\
\hline LAWA92 & & & & & & & & & & & & & & & & 0.0148 & & & & & 0.0109 \\
\hline LAWA93 & & & & & & 0.0010 & & & & & & & & & & 0.0010 & & & & & 0.0111 \\
\hline LAWA94 & & & & & & & & & & & & & & & & 0.0148 & & & & & 0.0109 \\
\hline LAWA95 & & & & & & & & & & & & & & & & 0.0148 & & & & & 0.0109 \\
\hline LAWA96 & & & & & & 0.0010 & & & & & & & & & & 0.0010 & & & & & 0.0199 \\
\hline LAWA97S & & & & & & & & & & & & & & & & 0.0148 & & & & & 0.0197 \\
\hline LAWA98S & & & & & & 0.0010 & & & & & & & & & & 0.0071 & & & & & 0.0113 \\
\hline LAWA99S & & & & & & 0.0010 & & & & & & & & & & 0.0084 & & & & & 0.0113 \\
\hline LAWA100S & & & & & & 0.0010 & & & & & & & & & & 0.0061 & & & & & 0.0113 \\
\hline LAWA101S & & & & & & 0.0010 & & & & & & & & & & 0.0062 & & & & & 0.0113 \\
\hline
\end{tabular}


Appendix A. Database - mass fraction

RPP-WTP LAW Formulation (Muller et al. 2001)

\begin{tabular}{|c|c|c|c|c|c|c|c|c|c|c|c|c|c|c|c|c|c|c|c|c|c|}
\hline Glass ID & $\begin{array}{c}\mathrm{T} 12 \mathrm{O} 3 \\
-\mathrm{t}\end{array}$ & $\begin{array}{c}\mathrm{U} 3 \mathrm{O} 8 \\
-\mathrm{t}\end{array}$ & $\begin{array}{c}\mathrm{UO} 2 \\
-\mathrm{t}\end{array}$ & $\begin{array}{c}\mathrm{UO} 3 \\
-\mathrm{t}\end{array}$ & {$\left[\begin{array}{c}\mathrm{V} 2 \mathrm{O} 5 \\
-\mathrm{t}\end{array}\right.$} & $\begin{array}{c}\text { WO3 } \\
-t\end{array}$ & $\begin{array}{c}\mathrm{Y} 2 \mathrm{O} 3 \\
-\mathrm{t}\end{array}$ & $\begin{array}{c}\mathrm{ZnO} \\
-\mathrm{t}\end{array}$ & $\begin{array}{c}\text { Others } \\
-t\end{array}$ & $\begin{array}{c}\text { Sum } \\
-t\end{array}$ & $\begin{array}{c}\mathrm{A} 12 \mathrm{O} 3 \\
-\mathrm{a}\end{array}$ & $\begin{array}{c}\mathrm{B} 2 \mathrm{O} 3 \\
-\mathrm{a}\end{array}$ & $\begin{array}{c}\mathrm{CaO} \\
-\mathrm{a}\end{array}$ & $\begin{array}{c}\mathrm{Fe} 2 \mathrm{O} 3 \\
-\mathrm{a}\end{array}$ & $\begin{array}{c}\mathrm{FeO} \\
-\mathrm{a}\end{array}$ & $\begin{array}{c}\mathrm{K} 2 \mathrm{O} \\
-\mathrm{a}\end{array}$ & $\begin{array}{c}\mathrm{Li} 2 \mathrm{O} \\
-\mathrm{a}\end{array}$ & $\begin{array}{c}\mathrm{MgO} \\
-\mathrm{a}\end{array}$ & $\begin{array}{c}\mathrm{Na} 2 \mathrm{O} \\
-\mathrm{a}\end{array}$ & $\begin{array}{c}\mathrm{NiO} \\
-\mathrm{a}\end{array}$ & $\begin{array}{c}\mathrm{P} 2 \mathrm{O} 5 \\
-\mathrm{a}\end{array}$ \\
\hline LAWA74 & & & & & & & & & & 0.9989 & & & & & & & & & & & \\
\hline LAWA75 & & & & & & & & 0.0295 & & 0.9988 & & & & & & & & & & & \\
\hline LAWA76 & & & & & & & & 0.0295 & & 0.9988 & & & & & & & & & & & \\
\hline LAWA77 & & & & & & & & 0.0295 & & 0.9989 & & & & & & & & & & & \\
\hline LAWA78 & & & & & & & & 0.0295 & & 0.9989 & & & & & & & & & & & \\
\hline LAWA79 & & & & & & & & 0.0295 & & 0.9988 & & & & & & & & & & & \\
\hline LAWA80 & & & & & & & & 0.0295 & & 0.9989 & & & & & & & & & & & \\
\hline LAWA81 & & & & & & & & 0.0297 & & 0.9999 & 0.0619 & 0.0883 & 0.0409 & 0.0704 & & 0.0059 & 0.0011 & 0.0190 & 0.1982 & 0.0000 & 0.0004 \\
\hline LAWA82 & & & & & & & & 0.0297 & & 0.9999 & 0.0614 & 0.0878 & 0.0013 & 0.0663 & & 0.0059 & 0.0002 & 0.0188 & 0.1925 & 0.0000 & 0.0015 \\
\hline LAWA83 & & & & & & & & 0.0297 & & 0.9999 & 0.0633 & 0.0911 & 0.0209 & 0.0502 & & 0.0058 & 0.0002 & 0.0189 & 0.1897 & 0.0001 & 0.0210 \\
\hline LAWA84 & & & & & & & & 0.0297 & & 0.9999 & 0.0613 & 0.0901 & 0.0210 & 0.0299 & & 0.0059 & 0.0002 & 0.0189 & 0.1860 & 0.0000 & 0.0373 \\
\hline LAWA85 & & & & & & & & 0.0297 & & 0.9999 & 0.0553 & 0.0862 & 0.0015 & 0.0467 & & 0.0054 & 0.0002 & 0.0176 & 0.1728 & 0.0001 & 0.0209 \\
\hline LAWA86 & & & & & & & & 0.0297 & & 0.9999 & 0.0571 & 0.0862 & 0.0008 & 0.0286 & & 0.0053 & 0.0002 & 0.0178 & 0.1711 & 0.0001 & 0.0400 \\
\hline LAWA87 & & & & & & & & 0.0296 & & 1.0000 & 0.0424 & 0.0870 & 0.0203 & 0.0653 & & 0.0225 & 0.0003 & 0.0180 & 0.1840 & 0.0001 & 0.0022 \\
\hline LAWA88 & & & & & & & & 0.0295 & & 1.0000 & 0.0611 & 0.0966 & 0.0205 & 0.0546 & & 0.0246 & 0.0004 & 0.0153 & 0.1693 & 0.0001 & 0.0023 \\
\hline LAWA89 & & & & & & & & 0.0295 & & 1.0000 & 0.0593 & 0.0940 & 0.0007 & 0.0487 & & 0.0235 & 0.0004 & 0.0143 & 0.1735 & 0.0002 & 0.0026 \\
\hline LAWA90 & & & & & & & & 0.0295 & & 1.0000 & 0.0578 & 0.0948 & 0.0380 & 0.0516 & & 0.0230 & 0.0004 & 0.0150 & 0.1691 & 0.0001 & 0.0016 \\
\hline LAWA91 & & & & & & & & 0.0295 & & 0.9988 & & & & & & & & & & & \\
\hline LAWA92 & & & & & & & & 0.0295 & & 0.9988 & & & & & & & & & & & \\
\hline LAWA93 & & & & & & & & 0.0299 & & 0.9999 & 0.0578 & 0.1087 & 0.0757 & 0.0710 & & 0.0061 & 0.0465 & 0.0153 & 0.0864 & 0.0001 & 0.0017 \\
\hline LAWA94 & & & & & & & & 0.0295 & & 0.9989 & & & & & & & & & & & \\
\hline LAWA95 & & & & & 0.0291 & & & 0.0295 & & 0.9990 & & & & & & & & & & & \\
\hline LAWA96 & & & & & & & & 0.0297 & & 0.9999 & 0.0610 & 0.0773 & 0.0394 & 0.0288 & & 0.0055 & 0.0006 & 0.0182 & 0.1921 & 0.0001 & 0.0403 \\
\hline LAWA97S & & & & & 0.0294 & & & 0.0292 & & 0.9990 & & & & & & & & & & & \\
\hline LAWA98S & & & & & & & & 0.0307 & & 1.0063 & 0.0574 & 0.1080 & 0.0482 & 0.0790 & & 0.0039 & 0.0282 & 0.0157 & 0.1094 & 0.0013 & 0.0023 \\
\hline LAWA99S & & & & & 0.0298 & & & 0.0307 & & 1.0076 & & & & & & & & & & & \\
\hline LAWA100S & & & & & & & & 0.0307 & & 1.0053 & & & & & & & & & & & \\
\hline LAWA101S & & & & & & & & 0.0307 & & 1.0054 & & & & & & & & & & & \\
\hline
\end{tabular}


Appendix A. Database - mass fraction

RPP-WTP LAW Formulation (Muller et al. 2001)

\begin{tabular}{|c|c|c|c|c|c|c|c|c|c|c|c|c|c|c|c|c|c|c|c|c|c|}
\hline Glass ID & $\begin{array}{c}\mathrm{SiO} 2 \\
-\mathrm{a} \\
\end{array}$ & $\begin{array}{c}\mathrm{ZrO} 2 \\
-\mathrm{a}\end{array}$ & $\begin{array}{c}\mathrm{Ag} 2 \mathrm{O} \\
-\mathrm{a}\end{array}$ & $\begin{array}{c}\mathrm{As} 2 \mathrm{O} 3 \\
-\mathrm{a}\end{array}$ & $\begin{array}{c}\mathrm{BaO} \\
-\mathrm{a}\end{array}$ & $\begin{array}{c}\mathrm{Bi} 2 \mathrm{O} 3 \\
-\mathrm{a} \\
\end{array}$ & $\begin{array}{l}\mathrm{Br} \\
-\mathrm{a}\end{array}$ & $\begin{array}{c}\mathrm{CdO} \\
-\mathrm{a}\end{array}$ & $\begin{array}{c}\mathrm{Ce} 2 \mathrm{O} 3 \\
-\mathrm{a}\end{array}$ & $\begin{array}{c}\mathrm{CeO} 2 \\
-\mathrm{a}\end{array}$ & $\begin{array}{l}\mathrm{Cl} \\
-\mathrm{a}\end{array}$ & $\begin{array}{c}\mathrm{CoO} \\
-\mathrm{a}\end{array}$ & $\begin{array}{c}\mathrm{Co} 2 \mathrm{O} 3 \\
-\mathrm{a}\end{array}$ & $\begin{array}{c}\mathrm{Cr} 2 \mathrm{O} 3 \\
-\mathrm{a}\end{array}$ & $\begin{array}{c}\mathrm{Cs} 2 \mathrm{O} \\
-\mathrm{a}\end{array}$ & $\begin{array}{c}\mathrm{CuO} \\
-\mathrm{a}\end{array}$ & $\begin{array}{c}\text { Eu2O3 } \\
-\mathrm{a}\end{array}$ & $\begin{array}{l}\mathrm{F} \\
-\mathrm{a}\end{array}$ & $\begin{array}{c}\mathrm{Ga} 2 \mathrm{O} 3 \\
-\mathrm{a}\end{array}$ & $\begin{array}{c}\mathrm{Gd} 2 \mathrm{O} 3 \\
-\mathrm{a}\end{array}$ & $\begin{array}{c}\mathrm{HgO} \\
-\mathrm{a}\end{array}$ \\
\hline LAWA74 & & & & & & & & & & & & & & & & & & & & & \\
\hline LAWA75 & & & & & & & & & & & & & & & & & & & & & \\
\hline LAWA76 & & & & & & & & & & & & & & & & & & & & & \\
\hline LAWA77 & & & & & & & & & & & & & & & & & & & & & \\
\hline LAWA78 & & & & & & & & & & & & & & & & & & & & & \\
\hline LAWA79 & & & & & & & & & & & & & & & & & & & & & \\
\hline LAWA80 & & & & & & & & & & & & & & & & & & & & & \\
\hline LAWA81 & 0.4507 & 0.0272 & & & 0.0001 & & & & & & & & & 0.0002 & & & & & & & \\
\hline LAWA82 & 0.4563 & 0.0271 & & & 0.0000 & & & & & & & & & 0.0002 & & & & & & & \\
\hline LAWA83 & 0.4450 & 0.0298 & & & 0.0000 & & & & & & & & & 0.0002 & & & & & & & \\
\hline LAWA84 & 0.4403 & 0.0271 & & & 0.0000 & & & & & & & & & 0.0002 & & & & & & & \\
\hline LAWA85 & 0.4364 & 0.0258 & & & 0.0002 & & & & & & & & & 0.0002 & & & & & & & \\
\hline LAWA86 & 0.4287 & 0.0230 & & & 0.0002 & & & & & & & & & 0.0002 & & & & & & & \\
\hline LAWA87 & 0.4385 & 0.0253 & & & 0.0000 & & & & & & & & & 0.0002 & & & & & & & \\
\hline LAWA88 & 0.4272 & 0.0279 & & & 0.0000 & & & & & & & & & 0.0002 & & & & & & & \\
\hline LAWA89 & 0.4164 & 0.0275 & & & 0.0000 & & & & & & & & & 0.0002 & & & & & & & \\
\hline LAWA90 & 0.4217 & 0.0261 & & & 0.0000 & & & & & & & & & 0.0001 & & & & & & & \\
\hline LAWA91 & & & & & & & & & & & & & & & & & & & & & \\
\hline LAWA92 & & & & & & & & & & & & & & & & & & & & & \\
\hline LAWA93 & 0.4022 & 0.0278 & & & 0.0000 & & & & & & & & & 0.0003 & & & & & & & \\
\hline LAWA94 & & & & & & & & & & & & & & & & & & & & & \\
\hline LAWA95 & & & & & & & & & & & & & & & & & & & & & \\
\hline LAWA96 & 0.4038 & 0.0280 & & & 0.0000 & & & & & & & & & 0.0002 & & & & & & & \\
\hline LAWA97S & & & & & & & & & & & & & & & & & & & & & \\
\hline LAWA98S & 0.4194 & 0.0298 & & & 0.0001 & & & & & & & & & 0.0027 & & & & & & & \\
\hline LAWA99S & & & & & & & & & & & & & & & & & & & & & \\
\hline LAWA100S & & & & & & & & & & & & & & & & & & & & & \\
\hline LAWA101S & & & & & & & & & & & & & & & & & & & & & \\
\hline
\end{tabular}


Appendix A. Database - mass fraction

RPP-WTP LAW Formulation (Muller et al. 2001)

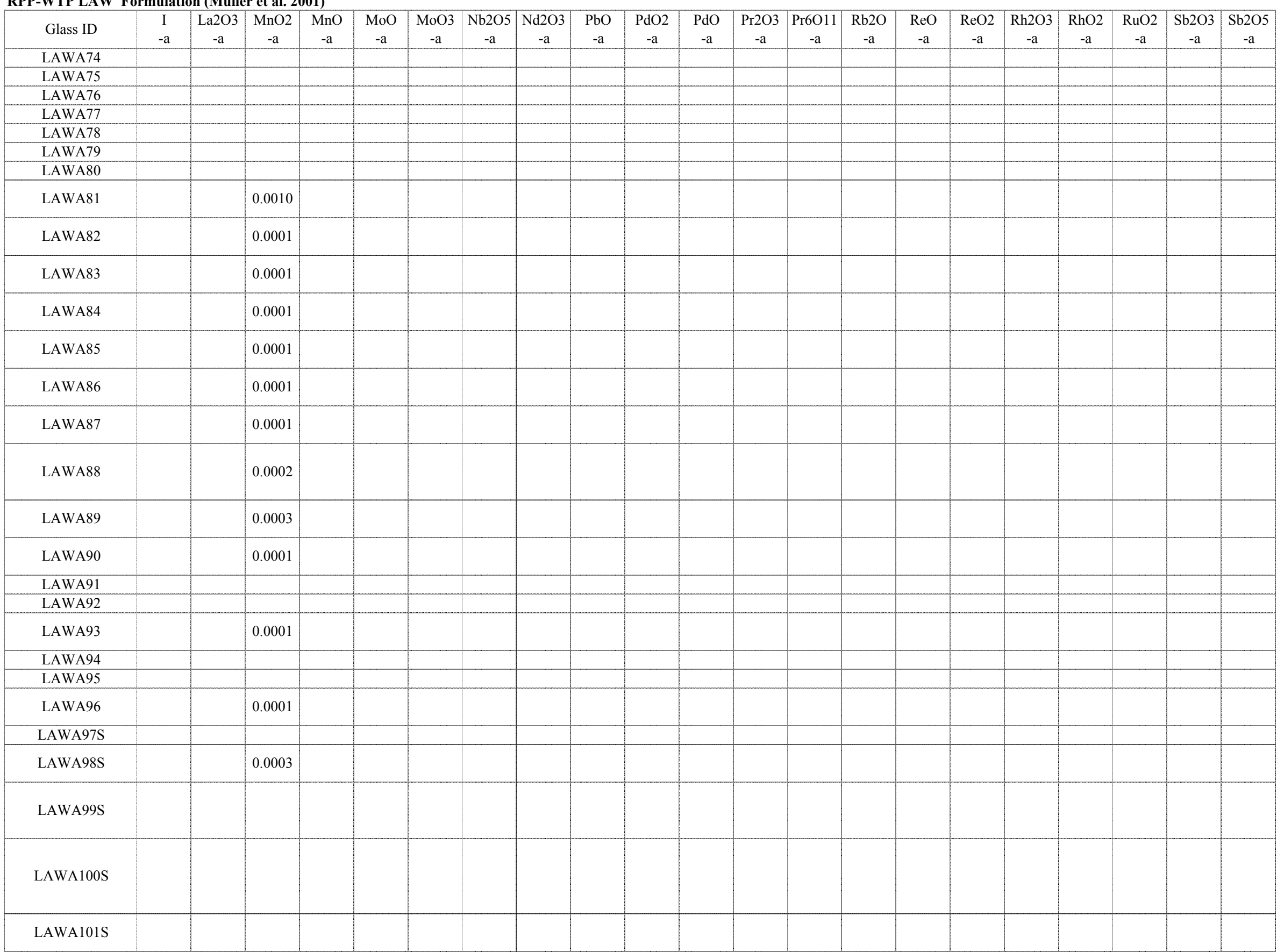


Appendix A. Database - mass fraction

RPP-WTP LAW Formulation (Muller et al. 2001)

\begin{tabular}{|c|c|c|c|c|c|c|c|c|c|c|c|c|c|c|c|c|c|c|c|c|}
\hline Glass ID & $\begin{array}{c}\mathrm{SeO} 2 \\
-\mathrm{a}\end{array}$ & $\begin{array}{c}\mathrm{Sm} 2 \mathrm{O} 3 \\
-\mathrm{a}\end{array}$ & $\begin{array}{c}\mathrm{SnO} \\
-\mathrm{a}\end{array}$ & $\begin{array}{c}\mathrm{SnO} 2 \\
-\mathrm{a}\end{array}$ & $\begin{array}{c}\mathrm{SO} 3 \\
-\mathrm{a}\end{array}$ & $\begin{array}{c}\mathrm{SrO} \\
-\mathrm{a}\end{array}$ & $\begin{array}{c}\mathrm{Tc} 2 \mathrm{O} 7 \\
-\mathrm{a}\end{array}$ & $\begin{array}{c}\mathrm{TeO} 2 \\
-\mathrm{a}\end{array}$ & $\begin{array}{c}\mathrm{ThO} 2 \\
-\mathrm{a}\end{array}$ & $\begin{array}{c}\mathrm{TiO} 2 \\
-\mathrm{a}\end{array}$ & $\begin{array}{c}\mathrm{T} 12 \mathrm{O} 3 \\
-\mathrm{a}\end{array}$ & $\begin{array}{c}\mathrm{U} 3 \mathrm{O} 8 \\
-\mathrm{a}\end{array}$ & $\begin{array}{c}\mathrm{UO} 2 \\
-\mathrm{a}\end{array}$ & $\begin{array}{c}\mathrm{UO} 3 \\
-\mathrm{a}\end{array}$ & $\begin{array}{c}\mathrm{V} 2 \mathrm{O} 5 \\
-\mathrm{a}\end{array}$ & $\begin{array}{c}\text { WO3 } \\
-\mathrm{a}\end{array}$ & $\begin{array}{c}\mathrm{Y} 2 \mathrm{O} 3 \\
-\mathrm{a}\end{array}$ & $\begin{array}{c}\mathrm{ZnO} \\
-\mathrm{a}\end{array}$ & $\begin{array}{c}\text { Others } \\
-\mathrm{a}\end{array}$ & $\begin{array}{c}\text { Sum } \\
-\mathrm{a}\end{array}$ \\
\hline LAWA74 & & & & & & & & & & & & & & & & & & & & \\
\hline LAWA75 & & & & & & & & & & & & & & & & & & & & \\
\hline LAWA76 & & & & & & & & & & & & & & & & & & & & \\
\hline LAWA77 & & & & & & & & & & & & & & & & & & & & \\
\hline LAWA78 & & & & & & & & & & & & & & & & & & & & \\
\hline LAWA79 & & & & & & & & & & & & & & & & & & & & \\
\hline LAWA80 & & & & & & & & & & & & & & & & & & & & \\
\hline LAWA81 & & & & & & 0.0013 & & & & 0.0003 & & & & & & & & 0.0286 & & 0.9952 \\
\hline LAWA82 & & & & & & 0.0001 & & & & 0.0401 & & & & & & & & 0.0283 & & 0.9881 \\
\hline LAWA83 & & & & & & 0.0001 & & & & 0.0207 & & & & & & & & 0.0284 & & 0.9852 \\
\hline LAWA84 & & & & & & 0.0000 & & & & 0.0199 & & & & & & & & 0.0281 & & 0.9663 \\
\hline LAWA85 & & & & & & 0.0166 & & & & 0.0189 & & & & & & & & 0.0293 & & 0.9342 \\
\hline LAWA86 & & & & & & 0.0178 & & & & 0.0190 & & & & & & & & 0.0255 & & 0.9217 \\
\hline LAWA87 & & & & & & 0.0001 & & & & 0.0182 & & & & & & & & 0.0291 & & 0.9535 \\
\hline LAWA88 & & & & & & 0.0001 & & & & 0.0192 & & & & & & & & 0.0279 & & 0.9476 \\
\hline LAWA89 & & & & & & 0.0002 & & & & 0.0365 & & & & & & & & 0.0270 & & 0.9252 \\
\hline LAWA90 & & & & & & 0.0001 & & & & 0.0005 & & & & & & & & 0.0287 & & 0.9286 \\
\hline LAWA91 & & & & & & & & & & & & & & & & & & & & \\
\hline LAWA92 & & & & & & & & & & & & & & & & & & & & \\
\hline LAWA93 & & & & & & 0.0001 & & & & 0.0121 & & & & & & & & 0.0294 & & 0.9414 \\
\hline LAWA94 & & & & & & & & & & & & & & & & & & & & \\
\hline LAWA95 & & & & & & & & & & & & & & & & & & & & \\
\hline LAWA96 & & & & & 0.0004 & 0.0000 & & & & 0.0193 & & & & & & & & 0.0294 & & 0.9444 \\
\hline LAWA97S & & & & & & & & & & & & & & & & & & & & \\
\hline LAWA98S & & & & & 0.0089 & 0.0001 & & & & 0.0120 & & & & & & & & 0.0285 & & 0.9552 \\
\hline LAWA99S & & & & & & & & & & & & & & & & & & & & \\
\hline LAWA100S & & & & & & & & & & & & & & & & & & & & \\
\hline LAWA101S & & & & & & & & & & & & & & & & & & & & \\
\hline
\end{tabular}


Appendix A. Database - mass fraction

\section{RPP-WTP LAW Formulation (Muller et al. 2001)}

\begin{tabular}{|c|c|c|c|c|c|c|c|c|}
\hline Glass ID & $\begin{array}{l}\mathrm{TM} \\
\left({ }^{\circ} \mathrm{C}\right)\end{array}$ & $\begin{array}{l}\text { Gradient } \\
\text { TL }\left({ }^{\circ} \mathrm{C}\right)\end{array}$ & $\begin{array}{l}\text { Uniform } \\
\text { TL }\left({ }^{\circ} \mathrm{C}\right)\end{array}$ & Primary Phase & $\begin{array}{l}\text { Quenched } \\
\text { Visual/OM }\end{array}$ & $\begin{array}{c}\text { Quenched } \\
\text { SEM/EDS or TEM }\end{array}$ & $\begin{array}{l}\text { Quenched } \\
\text { XRD }\end{array}$ & $\begin{array}{c}\text { Quenched } \\
\text { Homogeneous? }\end{array}$ \\
\hline LAWA74 & & & & & & & & \\
\hline LAWA75 & & & & & & & & \\
\hline LAWA76 & & & & & & & & \\
\hline LAWA77 & & & & & & & & \\
\hline LAWA78 & & & & & & & & \\
\hline LAWA79 & & & & & & & & \\
\hline LAWA80 & & & & & & & & \\
\hline LAWA81 & & & & & & & & \\
\hline LAWA82 & & & & & & & & \\
\hline LAWA83 & & & & & & & & \\
\hline LAWA84 & & & & & & & & \\
\hline LAWA85 & & & & & & & & \\
\hline LAWA86 & & & & & & & & \\
\hline LAWA87 & & & & & & & & \\
\hline LAWA88 & & & & & & & & \\
\hline LAWA89 & & & & & & & & \\
\hline LAWA90 & & & & & & & & \\
\hline LAWA91 & & & & & & & & \\
\hline LAWA92 & & & & & & & & \\
\hline LAWA93 & & & & & & & & \\
\hline LAWA94 & & & & & & & & \\
\hline LAWA95 & & & & & & & & \\
\hline LAWA96 & & & & & & & & \\
\hline LAWA97S & & & & & & & & \\
\hline LAWA98S & & & & & & & & \\
\hline LAWA99S & & & & & & & & \\
\hline LAWA100S & & & & & & & & \\
\hline LAWA101S & & & & & & & & \\
\hline
\end{tabular}


Appendix A. Database - mass fraction

RPP-WTP LAW Formulation (Muller et al. 2001)

\begin{tabular}{|c|c|c|c|c|c|}
\hline Glass ID & $\begin{array}{c}\mathrm{CCC} \\
\text { Visual/OM }\end{array}$ & $\begin{array}{c}\text { CCC } \\
\text { SEM/EDS or TEM }\end{array}$ & $\begin{array}{l}\mathrm{CCC} \\
\mathrm{XRD}\end{array}$ & $\begin{array}{l}\text { Heat Treated } \\
\text { Visual/OM }\end{array}$ & $\begin{array}{c}\text { Heat Treated } \\
\text { SEM/EDS or TEM }\end{array}$ \\
\hline \multicolumn{6}{|l|}{ LAWA74 } \\
\hline \multicolumn{6}{|l|}{ LAWA75 } \\
\hline \multicolumn{6}{|l|}{ LAWA76 } \\
\hline \multicolumn{6}{|l|}{ LAWA77 } \\
\hline \multicolumn{6}{|l|}{ LAWA78 } \\
\hline \multicolumn{6}{|l|}{ LAWA79 } \\
\hline \multicolumn{6}{|l|}{ LAWA80 } \\
\hline LAWA81 & & & & & $\begin{array}{l}\text { After } 1200^{\circ} \mathrm{C} 1 \mathrm{hr} \text { and } 950^{\circ} \mathrm{C} 20 \mathrm{hr} \text { - Clear } \\
\text { homogeneous glass }\end{array}$ \\
\hline LAWA82 & & & & & $\begin{array}{l}\text { After } 1200^{\circ} \mathrm{C} 1 \mathrm{hr} \text { and } 950^{\circ} \mathrm{C} 20 \mathrm{hr}-\text { Clear } \\
\text { homogeneous glass }\end{array}$ \\
\hline LAWA83 & & & & & $\begin{array}{l}\text { After } 1200^{\circ} \mathrm{C} 1 \mathrm{hr} \text { and } 950^{\circ} \mathrm{C} 20 \mathrm{hr}-\text { Clear } \\
\text { homogeneous glass }\end{array}$ \\
\hline LAWA84 & & & & & $\begin{array}{l}\text { After } 1200^{\circ} \mathrm{C} 1 \mathrm{hr} \text { and } 950^{\circ} \mathrm{C} 20 \mathrm{hr} \text { - Clear } \\
\text { homogeneous glass }\end{array}$ \\
\hline LAWA85 & & & & & $\begin{array}{l}\text { After } 1200^{\circ} \mathrm{C} 1 \mathrm{hr} \text { and } 950^{\circ} \mathrm{C} 20 \mathrm{hr}-\text { Clear } \\
\text { homogeneous glass }\end{array}$ \\
\hline LAWA86 & & & & & $\begin{array}{l}\text { After } 1200^{\circ} \mathrm{C} 1 \mathrm{hr} \text { and } 950^{\circ} \mathrm{C} 20 \mathrm{hr} \text { - Clear } \\
\text { homogeneous glass }\end{array}$ \\
\hline LAWA87 & & & & & $\begin{array}{l}\text { After } 1200^{\circ} \mathrm{C} 1 \mathrm{hr} \text { and } 950^{\circ} \mathrm{C} 20 \mathrm{hr} \text { - Clear } \\
\text { homogeneous glass }\end{array}$ \\
\hline LAWA88 & & & & & $\begin{array}{l}\text { After } 1200^{\circ} \mathrm{C} 1 \mathrm{hr} \text { and } 950^{\circ} \mathrm{C} 20 \mathrm{hr}-\text { Clear } \\
\text { homogeneous glass; After } 1200^{\circ} \mathrm{C} 1 \mathrm{hr} \text { and } 850^{\circ} \mathrm{C} \\
20 \mathrm{hr} \text { - Clear homogeneous glass }\end{array}$ \\
\hline LAWA89 & & & & & $\begin{array}{l}\text { After } 1200^{\circ} \mathrm{C} 1 \mathrm{hr} \text { and } 950^{\circ} \mathrm{C} 20 \mathrm{hr}-\text { Clear } \\
\text { homogeneous glass }\end{array}$ \\
\hline LAWA90 & & & & & $\begin{array}{l}\text { After } 1200^{\circ} \mathrm{C} 1 \mathrm{hr} \text { and } 950^{\circ} \mathrm{C} 20 \mathrm{hr} \text { - Clear } \\
\text { homogeneous glass }\end{array}$ \\
\hline \multicolumn{6}{|l|}{ LAWA91 } \\
\hline \multicolumn{6}{|l|}{ LAWA92 } \\
\hline LAWA93 & & & & & $\begin{array}{l}\text { After } 1200^{\circ} \mathrm{C} 1 \mathrm{hr} \text { and } 950^{\circ} \mathrm{C} 20 \mathrm{hr}-\text { Clear } \\
\text { homogeneous glass }\end{array}$ \\
\hline \multicolumn{6}{|l|}{ LAWA94 } \\
\hline \multicolumn{6}{|l|}{ LAWA95 } \\
\hline LAWA96 & & & & & $\begin{array}{l}\text { After } 1200^{\circ} \mathrm{C} 1 \mathrm{hr} \text { and } 950^{\circ} \mathrm{C} 20 \mathrm{hr} \text { - Less than } \\
0.1 \mathrm{vol} \% \text { of white, dendritic crystals }\end{array}$ \\
\hline \multicolumn{6}{|l|}{ LAWA97S } \\
\hline LAWA98S & & & & & $\begin{array}{l}\text { After } 1200^{\circ} \mathrm{C} 1 \mathrm{hr} \text { and } 950^{\circ} \mathrm{C} 20 \mathrm{hr} \text { - Clear } \\
\text { homogeneous glass }\end{array}$ \\
\hline LAWA99S & & & & & $\begin{array}{l}\text { After } 1200^{\circ} \mathrm{C} 1 \mathrm{hr} \text { and } 950^{\circ} \mathrm{C} 20 \mathrm{hr} \text { - Clear } \\
\text { homogeneous glass for the most part with one } \\
\text { black, irregular nodule } \sim 75 \mathrm{~mm} \text { dia. }\end{array}$ \\
\hline LAWA100S & & & & & $\begin{array}{l}\text { After } 1200^{\circ} \mathrm{C} 1 \mathrm{hr} \text { and } 950^{\circ} \mathrm{C} 20 \mathrm{hr}-\text { Clear } \\
\text { homogeneous glass for the most part, with one } \\
\text { white, cloudy nodule } 300 \mathrm{~mm} \text { dia. at the crucible } \\
\text { contact surface }\end{array}$ \\
\hline LAWA101S & & & & & $\begin{array}{l}\text { After } 1200^{\circ} \mathrm{C} 1 \mathrm{hr} \text { and } 950^{\circ} \mathrm{C} 20 \mathrm{hr} \text { - Clear } \\
\text { homogeneous glass }\end{array}$ \\
\hline
\end{tabular}


Appendix A. Database - mass fraction

RPP-WTP LAW Formulation (Muller et al. 2001)

\begin{tabular}{|c|c|c|c|c|c|c|c|c|c|c|c|c|c|c|c|}
\hline Glass ID & $\begin{array}{l}\text { Heat Treated } \\
\text { XRD }\end{array}$ & $\begin{array}{l}\text { Density } \\
\left(\mathrm{g} / \mathrm{cm}^{3}\right)\end{array}$ & $\begin{array}{c}\text { Fulc Visc } \\
\text { A }\end{array}$ & \begin{tabular}{|c|} 
Fulc Visc \\
B
\end{tabular} & $\begin{array}{c}\text { Fulc Visc } \\
\text { To }\end{array}$ & $\begin{array}{c}\mathrm{FV} 1150^{\circ} \mathrm{C} \\
(\mathrm{Pa} \cdot \mathrm{s})\end{array}$ & $\begin{array}{c}\text { Arrh Visc } \\
\text { A }\end{array}$ & $\begin{array}{c}\text { Arrh Visc } \\
\text { B }\end{array}$ & $1150^{\circ} \mathrm{C}$ & $\begin{array}{c}\mathrm{T}\left({ }^{\circ} \mathrm{C}\right) \text { at } \\
2 \mathrm{~Pa} \cdot \mathrm{s}\end{array}$ & $\begin{array}{c}\mathrm{T}\left({ }^{\circ} \mathrm{C}\right) \text { at } \\
5 \mathrm{~Pa} \cdot \mathrm{s}\end{array}$ & $\begin{array}{l}\mathrm{T}\left({ }^{\circ} \mathrm{C}\right) \text { at } \\
10 \mathrm{~Pa} \cdot \mathrm{s}\end{array}$ & $\begin{array}{l}\mathrm{T} 1 \\
\left({ }^{\circ} \mathrm{C}\right)\end{array}$ & $\begin{array}{c}\mathrm{V} 1 \\
(\mathrm{~Pa} \cdot \mathrm{s})\end{array}$ & $\begin{array}{c}\mathrm{T} 2 \\
\left({ }^{\circ} \mathrm{C}\right) \\
\end{array}$ \\
\hline LAWA74 & & & & & & & & & & & & & & & \\
\hline LAWA75 & & & & & & & & & & & & & & & \\
\hline \multicolumn{16}{|l|}{ LAWA76 } \\
\hline \multicolumn{16}{|l|}{ LAWA77 } \\
\hline \multicolumn{16}{|l|}{ LAWA78 } \\
\hline LAWA79 & & & & & & & & & & & & & & & \\
\hline \multicolumn{16}{|l|}{ LAWA80 } \\
\hline LAWA81 & & & & & & & -13.382 & 21825.3 & 7.07 & & & & 900 & 197.8 & 950 \\
\hline LAWA82 & & & & & & & -12.527 & 21089.4 & 9.91 & & & & 900 & 250.3 & 950 \\
\hline LAWA83 & & & & & & & -12.882 & 21519.6 & 9.40 & & & & 900 & 254.8 & 950 \\
\hline LAWA84 & & & & & & & -12.603 & 21112.7 & 9.33 & & & & 900 & 238.4 & 950 \\
\hline LAWA85 & & & & & & & -12.525 & 20923.7 & 8.84 & & & & 900 & 225.5 & 950 \\
\hline \multicolumn{16}{|l|}{ LAWA86 } \\
\hline LAWA87 & & & & & & & -11.869 & 19176.9 & 4.99 & & & & 900 & 94.5 & 950 \\
\hline LAWA88 & & 2.67 & & & & & -11.891 & 19487.1 & 6.07 & & & & 900 & 119.9 & 950 \\
\hline LAWA89 & & & & & & & -12.550 & 20570.0 & 6.72 & & & & 900 & 163.9 & 950 \\
\hline LAWA90 & & & & & & & -12.201 & 20053.1 & 6.63 & & & & 900 & 143.9 & 950 \\
\hline \multirow{2}{*}{\multicolumn{16}{|c|}{$\begin{array}{l}\text { LAWA91 } \\
\text { LAWA92 }\end{array}$}} \\
\hline & & & & & & & & & & & & & & & \\
\hline LAWA93 & & & & & & & -11.341 & 16831.1 & 1.63 & & & & 900 & 21.6 & 950 \\
\hline \multicolumn{16}{|l|}{ LAWA94 } \\
\hline LAWA95 & & & & & & & & & & & & & & & \\
\hline LAWA96 & & 2.67 & & & & & -12.791 & 21146.3 & 7.92 & & & & 900 & 210.4 & 950 \\
\hline LAWA97S & & & & & & & -12.661 & 20939.9 & 7.80 & & & & 900 & 203.6 & 950 \\
\hline LAWA98S & & & & & & & -10.909 & 17329.9 & 3.56 & & & & 900 & 51 & 950 \\
\hline LAWA99S & & & & & & & -12.393 & 19647.8 & 4.11 & & & & 900 & 84.1 & 950 \\
\hline LAWA100S & & & & & & & -12.265 & 19509.2 & 4.24 & & & & 900 & 83.1 & 950 \\
\hline LAWA101S & & & & & & & -12.888 & 20915.2 & 6.11 & & & & 900 & 151.8 & 950 \\
\hline
\end{tabular}


Appendix A. Database - mass fraction

\begin{tabular}{|c|c|c|c|c|c|c|c|c|c|c|c|c|c|c|c|c|c|c|c|c|c|}
\hline Glass ID & $\begin{array}{c}\mathrm{V} 2 \\
(\mathrm{~Pa} \cdot \mathrm{s})\end{array}$ & $\begin{array}{c}\mathrm{T} 3 \\
\left({ }^{\circ} \mathrm{C}\right)\end{array}$ & $\begin{array}{c}\mathrm{V} 3 \\
(\mathrm{~Pa} \cdot \mathrm{s})\end{array}$ & $\begin{array}{c}\mathrm{T} 4 \\
\left({ }^{\circ} \mathrm{C}\right)\end{array}$ & $\begin{array}{c}\mathrm{V} 4 \\
(\mathrm{~Pa} \cdot \mathrm{s})\end{array}$ & $\begin{array}{c}\mathrm{T} 5 \\
\left({ }^{\circ} \mathrm{C}\right)\end{array}$ & $\begin{array}{c}\mathrm{V} 5 \\
(\mathrm{~Pa} \cdot \mathrm{s})\end{array}$ & $\begin{array}{c}\mathrm{T} 6 \\
\left({ }^{\circ} \mathrm{C}\right)\end{array}$ & $\begin{array}{c}\mathrm{V} 6 \\
(\mathrm{~Pa} \cdot \mathrm{s})\end{array}$ & $\begin{array}{c}\mathrm{T} 7 \\
\left({ }^{\circ} \mathrm{C}\right) \\
\end{array}$ & $\begin{array}{c}\mathrm{V} 7 \\
(\mathrm{~Pa} \cdot \mathrm{s})\end{array}$ & $\begin{array}{c}\mathrm{T} 8 \\
\left({ }^{\circ} \mathrm{C}\right)\end{array}$ & $\begin{array}{c}\mathrm{V} 8 \\
(\mathrm{~Pa} \cdot \mathrm{s})\end{array}$ & $\begin{array}{c}\mathrm{T} 9 \\
\left({ }^{\circ} \mathrm{C}\right)\end{array}$ & $\begin{array}{c}\mathrm{V} 9 \\
(\mathrm{~Pa} \cdot \mathrm{s})\end{array}$ & $\begin{array}{l}\mathrm{T} 10 \\
\left({ }^{\circ} \mathrm{C}\right) \\
\end{array}$ & $\begin{array}{c}\mathrm{V} 10 \\
(\mathrm{~Pa} \cdot \mathrm{s})\end{array}$ & $\begin{array}{l}\mathrm{T} 11 \\
\left({ }^{\circ} \mathrm{C}\right)\end{array}$ & $\begin{array}{c}\mathrm{V} 11 \\
(\mathrm{~Pa} \cdot \mathrm{s})\end{array}$ & $\begin{array}{l}\mathrm{T} 12 \\
\left({ }^{\circ} \mathrm{C}\right)\end{array}$ & $\begin{array}{c}\mathrm{V} 12 \\
(\mathrm{~Pa} \cdot \mathrm{s})\end{array}$ \\
\hline \multicolumn{22}{|l|}{ LAWA74 } \\
\hline \multicolumn{22}{|l|}{ LAWA75 } \\
\hline \multicolumn{22}{|l|}{ LAWA76 } \\
\hline \multicolumn{22}{|l|}{ LAWA77 } \\
\hline \multicolumn{22}{|l|}{ LAWA78 } \\
\hline \multicolumn{22}{|l|}{ LAWA79 } \\
\hline \multicolumn{22}{|l|}{ LAWA80 } \\
\hline LAWA81 & 86.5 & 1000 & 41.5 & 1050 & 21.5 & 1100 & 11.9 & 1150 & 7 & 1200 & 4.3 & 1250 & 2.7 & & & & & & & & \\
\hline LAWA82 & 111.7 & 1000 & 54.7 & 1050 & 28.9 & 1100 & 16.3 & 1150 & 9.7 & 1200 & 6.1 & 1250 & 4 & & & & & & & & \\
\hline LAWA83 & 111.2 & 1000 & 53.5 & 1050 & 27.9 & 1100 & 15.6 & 1150 & 9.2 & 1200 & 5.8 & 1250 & 3.7 & & & & & & & & \\
\hline LAWA84 & 105.5 & 1000 & 51.4 & 1050 & 27.1 & 1100 & 15.3 & 1150 & 9.2 & 1200 & 5.7 & 1250 & 3.8 & & & & & & & & \\
\hline LAWA85 & 97.5 & 1000 & 47.1 & 1050 & 24.9 & 1100 & 14.2 & 1150 & 8.6 & 1200 & 5.5 & 1250 & 3.7 & & & & & & & & \\
\hline \multicolumn{22}{|l|}{ LAWA86 } \\
\hline LAWA87 & 45.1 & 1000 & 23.5 & 1050 & 13.2 & 1100 & 7.8 & 1150 & 4.9 & 1200 & 3.2 & 1250 & 2.2 & & & & & & & & \\
\hline LAWA88 & 56.9 & 1000 & 29.4 & 1050 & 16.3 & 1100 & 9.6 & 1150 & 6 & 1200 & 3.9 & 1250 & 2.6 & & & & & & & & \\
\hline LAWA89 & 71.2 & 1000 & 34.7 & 1050 & 18.5 & 1100 & 10.7 & 1150 & 6.5 & 1200 & 4.2 & 1250 & 2.9 & & & & & & & & \\
\hline LAWA90 & 66.3 & 1000 & 33.4 & 1050 & 18.2 & 1100 & 10.6 & 1150 & 6.5 & 1200 & 4.2 & 1250 & 2.8 & & & & & & & & \\
\hline \multicolumn{22}{|l|}{ LAWA91 } \\
\hline \multicolumn{22}{|l|}{ LAWA92 } \\
\hline LAWA93 & 11.3 & 1000 & 6.3 & 1050 & 3.8 & 1100 & 2.4 & 1150 & 1.6 & 1200 & 1.1 & 1250 & 0.8 & & & & & & & & \\
\hline \multicolumn{22}{|l|}{ LAWA94 } \\
\hline \multicolumn{22}{|l|}{ LAWA95 } \\
\hline LAWA96 & 89.5 & 1000 & 42.8 & 1050 & 22.5 & 1100 & 12.8 & 1150 & 7.7 & 1200 & 4.9 & 1250 & 3.3 & & & & & & & & \\
\hline LAWA97S & 86.1 & 1000 & 41.2 & 1050 & 21.7 & 1100 & 12.4 & 1150 & 7.6 & 1200 & 4.9 & 1250 & 3.3 & & & & & & & & \\
\hline LAWA98S & 26.1 & 1000 & 14.4 & 1050 & 8.5 & 1100 & 5.3 & 1150 & 3.5 & 1200 & 2.4 & 1250 & 1.7 & & & & & & & & \\
\hline LAWA99S & 39.3 & 1000 & 20.1 & 1050 & 11.1 & 1100 & 6.5 & 1150 & 4 & 1200 & 2.6 & 1250 & 1.8 & & & & & & & & \\
\hline LAWA100S & 39.8 & 1000 & 20.7 & 1050 & 11.5 & 1100 & 6.8 & 1150 & 4.2 & 1200 & 2.7 & 1250 & 1.8 & & & & & & & & \\
\hline LAWA101S & 67.6 & 1000 & 33.1 & 1050 & 17.5 & 1100 & 9.9 & 1150 & 6 & 1200 & 3.8 & 1250 & 2.5 & & & & & & & & \\
\hline
\end{tabular}


Appendix A. Database - mass fraction

RPP-WTP LAW Formulation (Muller et al. 2001)

\begin{tabular}{|c|c|c|c|c|c|c|c|c|c|c|c|c|c|c|c|c|}
\hline Glass ID & $\begin{array}{l}\mathrm{T} 13 \\
\left({ }^{\circ} \mathrm{C}\right) \\
\end{array}$ & $\begin{array}{l}\mathrm{V} 13 \\
(\mathrm{~Pa} \cdot \mathrm{s})\end{array}$ & $\begin{array}{l}\mathrm{T} 14 \\
\left({ }^{\circ} \mathrm{C}\right) \\
\end{array}$ & $\begin{array}{c}\mathrm{V} 14 \\
(\mathrm{~Pa} \cdot \mathrm{s})\end{array}$ & $\begin{array}{c}\text { Q PCT } \\
\mathrm{B}\left(\mathrm{g} / \mathrm{m}^{2}\right)\end{array}$ & $\begin{array}{c}\text { Q PCT } \\
\mathrm{Li}\left(\mathrm{g} / \mathrm{m}^{2}\right)\end{array}$ & $\begin{array}{c}\text { Q PCT } \\
\mathrm{Na}\left(\mathrm{g} / \mathrm{m}^{2}\right)\end{array}$ & $\begin{array}{c}\text { Q PCT } \\
\mathrm{Si}\left(\mathrm{g} / \mathrm{m}^{2}\right)\end{array}$ & $\begin{array}{c}\text { Q PCT } \\
\text { pH }\end{array}$ & $\begin{array}{c}\text { CCC PCT } \\
\mathrm{B}\left(\mathrm{g} / \mathrm{m}^{2}\right)\end{array}$ & \begin{tabular}{|l} 
CCC PCT \\
$\mathrm{Li}\left(\mathrm{g} / \mathrm{m}^{2}\right)$ \\
\end{tabular} & $\begin{array}{l}\text { CCC PCT } \\
\mathrm{Na}\left(\mathrm{g} / \mathrm{m}^{2}\right)\end{array}$ & $\begin{array}{l}\text { CCC PCT } \\
\mathrm{Si}\left(\mathrm{g} / \mathrm{m}^{2}\right) \\
\end{array}$ & $\begin{array}{c}\text { CCC PCT } \\
\text { pH }\end{array}$ & $\begin{array}{l}\text { Q PCT B at } \\
20^{\circ} \mathrm{C}\left(\mathrm{g} / \mathrm{m}^{2}\right)\end{array}$ & $\begin{array}{l}\text { Q PCT Li at } \\
20^{\circ} \mathrm{C}\left(\mathrm{g} / \mathrm{m}^{2}\right)\end{array}$ \\
\hline \multicolumn{17}{|c|}{ P } \\
\hline LAWA75 & & & & & & & & & & & & & & & & \\
\hline LAWA76 & & & & & 0.71 & & 0.66 & 0.2 & 10.82 & & & & & & & \\
\hline \multicolumn{17}{|l|}{ LAWA77 } \\
\hline \multicolumn{17}{|l|}{ LAWA78 } \\
\hline \multicolumn{17}{|l|}{ LAWA79 } \\
\hline \multicolumn{17}{|l|}{ LAWA80 } \\
\hline LAWA81 & & & & & 0.39 & & 0.42 & 0.15 & 10.88 & & & & & & & \\
\hline LAWA82 & & & & & 0.34 & & 0.33 & 0.17 & 10.51 & & & & & & & \\
\hline LAWA83 & & & & & 0.31 & & 0.34 & 0.16 & 10.57 & & & & & & & \\
\hline LAWA84 & & & & & 0.3 & & 0.33 & 0.16 & 10.41 & & & & & & & \\
\hline LAWA85 & & & & & 0.34 & & 0.35 & 0.17 & 10.35 & & & & & & & \\
\hline LAWA86 & & & & & 0.39 & & 0.39 & 0.18 & 10.31 & & & & & & & \\
\hline LAWA87 & & & & & 0.6 & & 0.55 & 0.25 & 10.97 & & & & & & & \\
\hline LAWA88 & & & & & 0.43 & & 0.43 & 0.17 & 10.91 & & & & & & & \\
\hline LAWA89 & & & & & 0.58 & & 0.47 & 0.18 & 10.81 & & & & & & & \\
\hline LAWA90 & & & & & 0.49 & & 0.49 & 0.18 & 10.98 & & & & & & & \\
\hline \multicolumn{17}{|l|}{ LAWA91 } \\
\hline \multicolumn{17}{|l|}{ LAWA92 } \\
\hline LAWA93 & & & & & 0.53 & & 0.54 & 0.17 & 10.86 & & & & & & & \\
\hline \multicolumn{17}{|l|}{ LAWA94 } \\
\hline \multicolumn{17}{|l|}{ LAWA95 } \\
\hline LAWA96 & & & & & 0.31 & & 0.38 & 0.17 & 10.63 & & & & & & & \\
\hline \multicolumn{17}{|l|}{ LAWA97S } \\
\hline LAWA98S & & & & & 0.36 & & 0.28 & 0.12 & 10.36 & & & & & & & \\
\hline LAWA99S & & & & & 0.74 & & 0.54 & 0.19 & 10.96 & & & & & & & \\
\hline LAWA100S & & & & & 0.54 & & 0.38 & 0.16 & 9.86 & & & & & & & \\
\hline LAWA101S & & & & & 0.43 & & 0.32 & 0.14 & 9.87 & & & & & & & \\
\hline
\end{tabular}


Appendix A. Database - mass fraction

RPP-WTP LAW Formulation (Muller et al. 2001)

\begin{tabular}{|c|c|c|c|c|c|c|c|c|c|c|c|c|}
\hline Glass ID & $\begin{array}{l}\text { Q PCT Na at } \\
20^{\circ} \mathrm{C}\left(\mathrm{g} / \mathrm{m}^{2}\right)\end{array}$ & $\begin{array}{l}\text { Q PCT Si at } \\
20^{\circ} \mathrm{C}\left(\mathrm{g} / \mathrm{m}^{2}\right)\end{array}$ & $\begin{array}{c}\mathrm{QpH} \\
\text { at } 20^{\circ} \mathrm{C}\end{array}$ & $\begin{array}{c}\text { TCLP Ag } \\
(\mathrm{ppm})\end{array}$ & $\begin{array}{c}\text { TCLP As } \\
(\mathrm{ppm})\end{array}$ & $\begin{array}{c}\text { TCLP Ba } \\
(\mathrm{ppm})\end{array}$ & $\begin{array}{c}\text { TCLP Cd } \\
(\mathrm{ppm})\end{array}$ & $\begin{array}{c}\text { TCLP Cr } \\
(\mathrm{ppm})\end{array}$ & $\begin{array}{c}\text { TCLP Ni } \\
(\mathrm{ppm})\end{array}$ & $\begin{array}{c}\text { TCLP Pb } \\
(\mathrm{ppm})\end{array}$ & $\begin{array}{c}\text { TCLP Se } \\
(\mathrm{ppm})\end{array}$ & $\begin{array}{c}\text { TCLP Zn } \\
(\mathrm{ppm})\end{array}$ \\
\hline LAWA74 & & & & & & & & & & & & \\
\hline LAWA75 & & & & & & & & & & & & \\
\hline LAWA76 & & & & & & & & & & & & \\
\hline LAWA77 & & & & & & & & & & & & \\
\hline LAWA78 & & & & & & & & & & & & \\
\hline LAWA79 & & & & & & & & & & & & \\
\hline LAWA80 & & & & & & & & & & & & \\
\hline LAWA81 & & & & & & & & & & & & \\
\hline LAWA82 & & & & & & & & & & & & \\
\hline LAWA83 & & & & & & & & & & & & \\
\hline LAWA84 & & & & & & & & & & & & \\
\hline LAWA85 & & & & & & & & & & & & \\
\hline LAWA86 & & & & & & & & & & & & \\
\hline LAWA87 & & & & & & & & & & & & \\
\hline LAWA88 & & & & & & & & & & & & \\
\hline LAWA89 & & & & & & & & & & & & \\
\hline LAWA90 & & & & & & & & & & & & \\
\hline LAWA91 & & & & & & & & & & & & \\
\hline LAWA92 & & & & & & & & & & & & \\
\hline LAWA93 & & & & & & & & & & & & \\
\hline LAWA94 & & & & & & & & & & & & \\
\hline LAWA95 & & & & & & & & & & & & \\
\hline LAWA96 & & & & & & & & & & & & \\
\hline LAWA97S & & & & & & & & & & & & \\
\hline LAWA98S & & & & & & & & & & & & \\
\hline LAWA99S & & & & & & & & & & & & \\
\hline LAWA100S & & & & & & & & & & & & \\
\hline LAWA101S & & & & & & & & & & & & \\
\hline
\end{tabular}


Appendix A. Database - mass fraction

RPP-WTP LAW Formulation (Muller et al. 2001)

\begin{tabular}{|c|c|c|c|c|c|c|c|c|c|c|c|c|c|c|c|c|c|c|c|c|c|}
\hline Glass ID & $\begin{array}{c}\mathrm{Al} 2 \mathrm{O} 3 \\
-\mathrm{t}\end{array}$ & $\begin{array}{c}\mathrm{B} 2 \mathrm{O} 3 \\
-\mathrm{t}\end{array}$ & $\begin{array}{c}\mathrm{CaO} \\
-\mathrm{t}\end{array}$ & $\begin{array}{c}\mathrm{Fe} 2 \mathrm{O} 3 \\
-\mathrm{t} \\
\end{array}$ & $\begin{array}{c}\mathrm{FeO} \\
-\mathrm{t}\end{array}$ & $\begin{array}{c}\mathrm{K} 2 \mathrm{O} \\
-\mathrm{t}\end{array}$ & $\begin{array}{c}\mathrm{Li} 2 \mathrm{O} \\
-\mathrm{t}\end{array}$ & $\begin{array}{c}\mathrm{MgO} \\
-\mathrm{t}\end{array}$ & $\begin{array}{c}\mathrm{Na} 2 \mathrm{O} \\
-\mathrm{t}\end{array}$ & $\begin{array}{c}\mathrm{NiO} \\
-\mathrm{t}\end{array}$ & $\begin{array}{c}\mathrm{P} 2 \mathrm{O} 5 \\
-\mathrm{t}\end{array}$ & $\begin{array}{c}\mathrm{SiO} 2 \\
-\mathrm{t}\end{array}$ & $\begin{array}{c}\mathrm{ZrO} 2 \\
-\mathrm{t}\end{array}$ & $\begin{array}{c}\mathrm{Ag} 2 \mathrm{O} \\
-\mathrm{t}\end{array}$ & $\begin{array}{c}\mathrm{As} 2 \mathrm{O} 3 \\
-\mathrm{t}\end{array}$ & $\begin{array}{c}\mathrm{BaO} \\
-\mathrm{t}\end{array}$ & $\begin{array}{c}\mathrm{Bi} 2 \mathrm{O} 3 \\
-\mathrm{t}\end{array}$ & $\begin{array}{l}\mathrm{Br} \\
-\mathrm{t}\end{array}$ & $\begin{array}{c}\mathrm{CdO} \\
-\mathrm{t} \\
\end{array}$ & $\begin{array}{c}\mathrm{Ce} 2 \mathrm{O} 3 \\
-\mathrm{t}\end{array}$ & $\begin{array}{c}\mathrm{CeO} 2 \\
-\mathrm{t}\end{array}$ \\
\hline LAWA102S & 0.0606 & 0.1000 & 0.0507 & 0.0541 & & 0.0026 & 0.0250 & 0.0150 & 0.1449 & & 0.0013 & 0.4660 & 0.0302 & & & & & & & & \\
\hline LAWA103S & 0.0606 & 0.1000 & 0.0507 & 0.0500 & & 0.0026 & 0.0250 & 0.0150 & 0.1449 & & 0.0013 & 0.4551 & 0.0302 & & & 0.0000 & & & & & \\
\hline LAWA104 & 0.0661 & 0.0859 & 0.0192 & 0.0674 & & 0.0055 & & 0.0192 & 0.2200 & & 0.0004 & 0.4299 & 0.0289 & 0.0000 & & & & & & & \\
\hline LAWA105 & 0.0703 & 0.0828 & 0.0185 & 0.0649 & & 0.0060 & & 0.0185 & 0.2400 & & 0.0004 & 0.4142 & 0.0278 & 0.0000 & & & & & & & \\
\hline LAWB29* & 0.0803 & 0.0807 & 0.0701 & 0.0802 & & 0.0038 & 0.0408 & 0.0298 & 0.1000 & & 0.0008 & 0.4383 & 0.0303 & & & & & & & & \\
\hline LAWB30 & 0.0860 & 0.1004 & 0.0723 & 0.0827 & & 0.0032 & 0.0407 & 0.0307 & 0.0790 & & 0.0004 & 0.4272 & 0.0312 & & & & & & & & \\
\hline LAWB31 & 0.0616 & 0.1209 & 0.0403 & 0.0717 & & 0.0032 & 0.0296 & 0.0224 & 0.0790 & & 0.0272 & 0.4691 & 0.0309 & & & & & & & & \\
\hline LAWB32 & 0.0616 & 0.1509 & 0.0403 & 0.0417 & & 0.0032 & 0.0296 & 0.0224 & 0.0790 & & 0.0272 & 0.4691 & 0.0309 & & & & & & & & \\
\hline LAWB33 & 0.0616 & 0.1209 & 0.0403 & 0.0515 & & 0.0032 & 0.0296 & 0.0224 & 0.0790 & & 0.0474 & 0.4691 & 0.0309 & & & & & & & & \\
\hline LAWB34 & 0.0616 & 0.1209 & 0.0605 & 0.0515 & & 0.0032 & 0.0296 & 0.0224 & 0.0790 & & 0.0272 & 0.4691 & 0.0309 & & & & & & & & \\
\hline LAWB35 & 0.0616 & 0.1209 & 0.0403 & 0.0515 & & 0.0032 & 0.0296 & 0.0426 & 0.0790 & & 0.0272 & 0.4691 & 0.0309 & & & & & & & & \\
\hline LAWB36S & 0.0616 & 0.1209 & 0.0403 & 0.0515 & & 0.0032 & 0.0385 & 0.0224 & 0.0790 & & 0.0474 & 0.4333 & 0.0309 & & & & & & & & \\
\hline LAWB37 & 0.0616 & 0.1209 & 0.0470 & 0.0515 & & 0.0032 & 0.0296 & 0.0291 & 0.0790 & & 0.0340 & 0.4691 & 0.0309 & & & & & & & & \\
\hline LAWB38 & 0.0616 & 0.1209 & 0.0475 & 0.0515 & & 0.0032 & 0.0381 & 0.0224 & 0.0790 & & 0.0317 & 0.4691 & 0.0309 & & & & & & & & \\
\hline LAWB39 & 0.0616 & 0.1209 & 0.0470 & 0.0515 & & 0.0032 & 0.0296 & 0.0291 & 0.0790 & & 0.0004 & 0.4691 & 0.0309 & & & & & & & & \\
\hline LAWB40 & 0.0616 & 0.1209 & 0.0470 & 0.0515 & & 0.0032 & 0.0632 & 0.0291 & 0.0790 & & 0.0004 & 0.4691 & 0.0309 & & & & & & & & \\
\hline LAWB41 & 0.0616 & 0.1209 & 0.0650 & 0.0515 & & 0.0032 & 0.0452 & 0.0291 & 0.0790 & & 0.0004 & 0.4692 & 0.0309 & & & & & & & & \\
\hline LAWB42S & 0.0607 & 0.1192 & 0.0463 & 0.0605 & & 0.0031 & & 0.0287 & 0.0779 & & 0.0004 & 0.4623 & 0.0305 & & & & & & & & \\
\hline LAWB43S & 0.0607 & 0.1192 & 0.0463 & 0.1033 & & 0.0031 & & 0.0287 & 0.0779 & & 0.0004 & 0.4623 & 0.0305 & & & & & & & & \\
\hline LAWB44S & 0.0607 & 0.1192 & 0.0463 & 0.1320 & & 0.0031 & & & 0.0779 & & 0.0004 & 0.4623 & 0.0305 & & & & & & & & \\
\hline LAWB45 & 0.0613 & 0.1234 & 0.0663 & 0.0526 & & 0.0026 & 0.0462 & 0.0297 & 0.0650 & & 0.0003 & 0.4786 & 0.0315 & & & & & & & & \\
\hline LAWB47 & 0.0613 & 0.1234 & 0.0601 & 0.0526 & & 0.0026 & 0.0409 & 0.0297 & 0.0650 & & 0.0003 & 0.4786 & 0.0315 & & & & & & & & \\
\hline LAWB48 & 0.0613 & 0.1234 & 0.0540 & 0.0526 & & 0.0026 & 0.0356 & 0.0297 & 0.0650 & & 0.0003 & 0.4786 & 0.0315 & & & & & & & & \\
\hline LAWB49 & 0.0613 & 0.1234 & 0.0480 & 0.0526 & & 0.0026 & 0.0302 & 0.0297 & 0.0650 & & 0.0003 & 0.4786 & 0.0315 & & & & & & & & \\
\hline LAWB50 & 0.0616 & 0.1209 & 0.0649 & 0.0515 & & 0.0032 & 0.0452 & 0.0291 & 0.0790 & & 0.0004 & 0.4691 & 0.0309 & & & & & & & & \\
\hline
\end{tabular}


Appendix A. Database - mass fraction

RPP-WTP LAW Formulation (Muller et al. 2001)

\begin{tabular}{|c|c|c|c|c|c|c|c|c|c|c|c|c|c|c|c|c|c|c|c|c|c|}
\hline Glass ID & $\begin{array}{l}\mathrm{Cl} \\
-\mathrm{t}\end{array}$ & $\begin{array}{c}\mathrm{CoO} \\
-\mathrm{t}\end{array}$ & $\begin{array}{c}\mathrm{Co} 2 \mathrm{O} 3 \\
-\mathrm{t}\end{array}$ & $\begin{array}{c}\mathrm{Cr} 2 \mathrm{O} 3 \\
-\mathrm{t}\end{array}$ & $\begin{array}{c}\mathrm{Cs} 2 \mathrm{O} \\
-\mathrm{t} \\
\end{array}$ & $\begin{array}{c}\mathrm{CuO} \\
-\mathrm{t} \\
\end{array}$ & $\begin{array}{c}\mathrm{Eu} 2 \mathrm{O} 3 \\
-\mathrm{t} \\
\end{array}$ & $\begin{array}{l}F \\
-t\end{array}$ & $\begin{array}{c}\mathrm{Ga} 2 \mathrm{O} 3 \\
-\mathrm{t}\end{array}$ & $\begin{array}{c}\mathrm{Gd} 2 \mathrm{O} 3 \\
-\mathrm{t} \\
\end{array}$ & $\begin{array}{c}\mathrm{HgO} \\
-\mathrm{t}\end{array}$ & $\begin{array}{l}I \\
-t\end{array}$ & $\begin{array}{c}\mathrm{La} 2 \mathrm{O} 3 \\
-\mathrm{t}\end{array}$ & $\begin{array}{c}\mathrm{MnO} 2 \\
-\mathrm{t}\end{array}$ & $\begin{array}{c}\mathrm{MnO} \\
-\mathrm{t} \\
\end{array}$ & $\begin{array}{c}\mathrm{MoO} \\
-\mathrm{t}\end{array}$ & $\begin{array}{c}\mathrm{MoO} 3 \\
-t\end{array}$ & $\begin{array}{c}\mathrm{Nb} 2 \mathrm{O} 5 \\
-\mathrm{t}\end{array}$ & $\begin{array}{c}\mathrm{Nd} 2 \mathrm{O} 3 \\
-\mathrm{t}\end{array}$ & $\begin{array}{c}\mathrm{PbO} \\
-\mathrm{t} \\
\end{array}$ & $\begin{array}{c}\mathrm{PdO} 2 \\
-\mathrm{t}\end{array}$ \\
\hline LAWA102S & 0.0033 & & & 0.0002 & 0.0000 & & & 0.0003 & & & & & & & & & & & & & \\
\hline LAWA103S & 0.0033 & & & 0.0002 & 0.0000 & & & 0.0003 & & & & & & & & & & & & & \\
\hline LAWA104 & 0.0072 & & & 0.0002 & 0.0000 & & & 0.0001 & & & & & & & & & & & & 0.0000 & \\
\hline LAWA105 & 0.0078 & & & 0.0002 & 0.0000 & & & 0.0001 & & & & & & & & & & & & 0.0000 & \\
\hline LAWB29* & 0.0001 & & & 0.0007 & 0.0000 & & & 0.0012 & & & & & & & & & & & & & \\
\hline LAWB30 & 0.0001 & & & 0.0009 & 0.0000 & & & 0.0010 & & & & & & & & & & & & & \\
\hline LAWB31 & 0.0001 & & & 0.0009 & 0.0000 & & & 0.0010 & & & & & & & & & & & & & \\
\hline LAWB32 & 0.0001 & & & 0.0009 & 0.0000 & & & 0.0010 & & & & & & & & & & & & & \\
\hline LAWB33 & 0.0001 & & & 0.0009 & 0.0000 & & & 0.0010 & & & & & & & & & & & & & \\
\hline LAWB34 & 0.0001 & & & 0.0009 & 0.0000 & & & 0.0010 & & & & & & & & & & & & & \\
\hline LAWB35 & 0.0001 & & & 0.0009 & 0.0000 & & & 0.0010 & & & & & & & & & & & & & \\
\hline LAWB36S & 0.0001 & & & 0.0009 & 0.0000 & & & 0.0010 & & & & & & & & & & & & & \\
\hline LAWB37 & 0.0001 & & & 0.0009 & 0.0000 & & & 0.0010 & & & & & & & & & & & & & \\
\hline LAWB38 & 0.0001 & & & 0.0009 & 0.0000 & & & 0.0010 & & & & & & & & & & & & & \\
\hline LAWB39 & 0.0001 & & & 0.0009 & 0.0000 & & & 0.0010 & & & & & & & & & & & & & \\
\hline LAWB40 & 0.0001 & & & 0.0009 & 0.0000 & & & 0.0010 & & & & & & & & & & & & & \\
\hline LAWB41 & 0.0001 & & & 0.0009 & 0.0000 & & & 0.0010 & & & & & & & & & & & & & \\
\hline LAWB42S & 0.0001 & & & 0.0009 & 0.0000 & & & 0.0010 & & & & & & & & & & & & & \\
\hline LAWB43S & 0.0001 & & & 0.0009 & 0.0000 & & & 0.0010 & & & & & & & & & & & & & \\
\hline LAWB44S & 0.0001 & & & 0.0009 & 0.0000 & & & 0.0010 & & & & & & & & & & & & & \\
\hline LAWB45 & 0.0001 & & & 0.0007 & 0.0000 & & & 0.0008 & & & & & & & & & & & & & \\
\hline LAWB47 & 0.0001 & & & 0.0007 & 0.0000 & & & 0.0008 & & & & & & & & & & & & & \\
\hline LAWB48 & 0.0001 & & & 0.0007 & 0.0000 & & & 0.0008 & & & & & & & & & & & & & \\
\hline LAWB49 & 0.0001 & & & 0.0007 & 0.0000 & & & 0.0008 & & & & & & & & & & & & & \\
\hline LAWB50 & 0.0050 & & & 0.0009 & 0.0000 & & & 0.0010 & & & & & & & & & & & & & \\
\hline
\end{tabular}


Appendix A. Database - mass fraction

RPP-WTP LAW Formulation (Muller et al. 2001)

\begin{tabular}{|c|c|c|c|c|c|c|c|c|c|c|c|c|c|c|c|c|c|c|c|c|c|}
\hline Glass ID & $\begin{array}{c}\mathrm{PdO} \\
-\mathrm{t}\end{array}$ & $\begin{array}{c}\mathrm{Pr} 2 \mathrm{O} 3 \\
-\mathrm{t}\end{array}$ & $\begin{array}{c}\text { Pr6O11 } \\
-t\end{array}$ & $\begin{array}{c}\mathrm{Rb} 2 \mathrm{O} \\
-\mathrm{t}\end{array}$ & $\begin{array}{c}\mathrm{ReO} \\
-\mathrm{t}\end{array}$ & $\begin{array}{c}\mathrm{ReO} 2 \\
-\mathrm{t}\end{array}$ & $\begin{array}{c}\mathrm{Rh} 2 \mathrm{O} 3 \\
-\mathrm{t}\end{array}$ & $\begin{array}{c}\mathrm{RhO} 2 \\
-\mathrm{t}\end{array}$ & $\begin{array}{c}\mathrm{RuO} 2 \\
-\mathrm{t}\end{array}$ & $\begin{array}{c}\mathrm{Sb} 2 \mathrm{O} 3 \\
-\mathrm{t}\end{array}$ & $\begin{array}{c}\mathrm{Sb} 2 \mathrm{O} 5 \\
-\mathrm{t}\end{array}$ & $\begin{array}{c}\mathrm{SeO} 2 \\
-\mathrm{t}\end{array}$ & $\begin{array}{c}\mathrm{Sm} 2 \mathrm{O} 3 \\
-\mathrm{t}\end{array}$ & $\begin{array}{c}\mathrm{SnO} \\
-\mathrm{t}\end{array}$ & $\begin{array}{c}\mathrm{SnO} 2 \\
-\mathrm{t}\end{array}$ & $\begin{array}{c}\mathrm{SO} 3 \\
-\mathrm{t}\end{array}$ & $\begin{array}{c}\mathrm{SrO} \\
-\mathrm{t} \\
\end{array}$ & $\begin{array}{c}\mathrm{Tc} 2 \mathrm{O} 7 \\
-\mathrm{t}\end{array}$ & $\begin{array}{c}\mathrm{TeO} 2 \\
-\mathrm{t}\end{array}$ & $\begin{array}{c}\text { ThO2 } \\
-t\end{array}$ & $\begin{array}{c}\mathrm{TiO} 2 \\
-\mathrm{t}\end{array}$ \\
\hline LAWA102S & & & & & & 0.0010 & & & & & & & & & & 0.0068 & & & & & 0.0114 \\
\hline LAWA103S & & & & & & 0.0010 & & & & & & & & & & 0.0055 & & & & & 0.0114 \\
\hline LAWA104 & & & & & & 0.0010 & & & & & & & & & & 0.0011 & & & & & 0.0192 \\
\hline LAWA105 & & & & & & 0.0010 & & & & & & & & & & 0.0011 & & & & & 0.0185 \\
\hline LAWB29* & & & & & & 0.0010 & & & & & & & & & & 0.0020 & & & & & \\
\hline LAWB30 & & & & & & 0.0010 & & & & & & & & & & 0.0020 & & & & & \\
\hline LAWB31 & & & & & & 0.0010 & & & & & & & & & & 0.0103 & & & & & \\
\hline LAWB32 & & & & & & 0.0010 & & & & & & & & & & 0.0103 & & & & & \\
\hline LAWB33 & & & & & & 0.0010 & & & & & & & & & & 0.0103 & & & & & \\
\hline LAWB34 & & & & & & 0.0010 & & & & & & & & & & 0.0103 & & & & & \\
\hline LAWB35 & & & & & & 0.0010 & & & & & & & & & & 0.0103 & & & & & \\
\hline LAWB36S & & & & & & 0.0010 & & & & & & & & & & 0.0087 & & & & & \\
\hline LAWB37 & & & & & & 0.0010 & & & & & & & & & & 0.0103 & & & & & \\
\hline LAWB38 & & & & & & 0.0010 & & & & & & & & & & 0.0103 & & & & & \\
\hline LAWB39 & & & & & & 0.0010 & & & & & & & & & & 0.0103 & & & & & \\
\hline LAWB40 & & & & & & 0.0010 & & & & & & & & & & 0.0103 & & & & & \\
\hline LAWB41 & & & & & & 0.0010 & & & & & & & & & & 0.0102 & & & & & \\
\hline LAWB42S & & & & & & & & & & & & & & & & 0.0061 & & & & & \\
\hline LAWB43S & & & & & & & & & & & & & & & & 0.0062 & & & & & \\
\hline LAWB44S & & & & & & & & & & & & & & & & 0.0052 & & & & & \\
\hline LAWB45 & & & & & & 0.0010 & & & & & & & & & & 0.0084 & & & & & \\
\hline LAWB47 & & & & & & 0.0010 & & & & & & & & & & 0.0084 & & & & & \\
\hline LAWB48 & & & & & & 0.0010 & & & & & & & & & & 0.0084 & & & & & \\
\hline LAWB49 & & & & & & 0.0010 & & & & & & & & & & 0.0084 & & & & & \\
\hline LAWB50 & & & & & & 0.0010 & & & & & & & & & & & & & & & \\
\hline
\end{tabular}


Appendix A. Database - mass fraction

RPP-WTP LAW Formulation (Muller et al. 2001)

\begin{tabular}{|c|c|c|c|c|c|c|c|c|c|c|c|c|c|c|c|c|c|c|c|c|c|}
\hline Glass ID & $\begin{array}{c}\mathrm{T} 12 \mathrm{O} 3 \\
-\mathrm{t}\end{array}$ & $\begin{array}{c}\mathrm{U} 3 \mathrm{O} 8 \\
-\mathrm{t}\end{array}$ & $\begin{array}{c}\mathrm{UO} 2 \\
-\mathrm{t}\end{array}$ & $\begin{array}{c}\mathrm{UO} 3 \\
-\mathrm{t}\end{array}$ & $\begin{array}{c}\mathrm{V} 2 \mathrm{O} 5 \\
-\mathrm{t}\end{array}$ & $\begin{array}{c}\text { WO3 } \\
-t\end{array}$ & $\begin{array}{c}\mathrm{Y} 2 \mathrm{O} 3 \\
-\mathrm{t}\end{array}$ & $\begin{array}{c}\mathrm{ZnO} \\
-\mathrm{t}\end{array}$ & $\begin{array}{c}\text { Others } \\
-t\end{array}$ & $\begin{array}{c}\text { Sum } \\
-t\end{array}$ & $\begin{array}{c}\mathrm{A} 12 \mathrm{O} 3 \\
-\mathrm{a}\end{array}$ & $\begin{array}{c}\mathrm{B} 2 \mathrm{O} 3 \\
-\mathrm{a}\end{array}$ & $\begin{array}{c}\mathrm{CaO} \\
-\mathrm{a}\end{array}$ & $\begin{array}{c}\mathrm{Fe} 2 \mathrm{O} 3 \\
-\mathrm{a}\end{array}$ & $\begin{array}{c}\mathrm{FeO} \\
-\mathrm{a}\end{array}$ & $\begin{array}{c}\mathrm{K} 2 \mathrm{O} \\
-\mathrm{a}\end{array}$ & $\begin{array}{c}\mathrm{Li} 2 \mathrm{O} \\
-\mathrm{a}\end{array}$ & $\begin{array}{c}\mathrm{MgO} \\
-\mathrm{a}\end{array}$ & $\begin{array}{c}\mathrm{Na} 2 \mathrm{O} \\
-\mathrm{a}\end{array}$ & $\begin{array}{c}\mathrm{NiO} \\
-\mathrm{a}\end{array}$ & $\begin{array}{c}\mathrm{P} 2 \mathrm{O} 5 \\
-\mathrm{a}\end{array}$ \\
\hline LAWA102S & & & & & & & & 0.0306 & & 1.0041 & & & & & & & & & & & \\
\hline LAWA103S & & & & & 0.0150 & & & 0.0306 & & 1.0028 & & & & & & & & & & & \\
\hline LAWA104 & & & & & & & & 0.0286 & & 0.9999 & & & & & & & & & & & \\
\hline LAWA105 & & & & & & & & 0.0276 & & 0.9999 & & & & & & & & & & & \\
\hline LAWB29* & & & & & & & & 0.0399 & & 1.0000 & 0.0648 & 0.0778 & 0.0700 & 0.0813 & & 0.0050 & 0.0391 & 0.0275 & 0.0869 & & 0.0021 \\
\hline LAWB30 & & & & & & & & 0.0411 & & 1.0000 & 0.0840 & 0.0986 & 0.0700 & 0.0793 & & 0.0033 & & 0.0283 & 0.0750 & & 0.0019 \\
\hline LAWB31 & & & & & & & & 0.0309 & & 1.0000 & 0.0569 & 0.1113 & 0.0380 & 0.0657 & & 0.0035 & 0.0262 & 0.0191 & 0.0724 & & 0.0270 \\
\hline LAWB32 & & & & & & & & 0.0309 & & 1.0000 & 0.0599 & 0.1500 & 0.0395 & 0.0399 & & 0.0036 & 0.0294 & 0.0202 & 0.0727 & & 0.0282 \\
\hline LAWB33 & & & & & & & & 0.0309 & & 1.0000 & 0.0558 & 0.1174 & 0.0371 & 0.0469 & & 0.0035 & 0.0263 & 0.0195 & 0.0696 & & 0.0438 \\
\hline LAWB34 & & & & & & & & 0.0309 & & 1.0000 & 0.0553 & 0.1143 & 0.0550 & 0.0476 & & 0.0035 & 0.0274 & 0.0195 & 0.0704 & & 0.0269 \\
\hline LAWB35 & & & & & & & & 0.0309 & & 1.0000 & 0.0584 & 0.1196 & 0.0387 & 0.0493 & & 0.0039 & 0.0270 & 0.0369 & 0.0732 & & 0.0270 \\
\hline LAWB36S & & & & & 0.0269 & & & 0.0309 & & 0.9984 & & & & & & & & & & & \\
\hline LAWB37 & & & & & & & & 0.0309 & & 1.0000 & 0.0625 & 0.1145 & 0.0451 & 0.0470 & & 0.0035 & 0.0285 & 0.0253 & 0.0764 & & 0.0340 \\
\hline LAWB38 & & & & & & & & 0.0309 & & 1.0000 & & & & & & & & & & & \\
\hline LAWB39 & & & & & 0.0336 & & & 0.0309 & & 1.0000 & 0.0601 & 0.1163 & 0.0446 & 0.0487 & & 0.0035 & 0.0287 & 0.0255 & 0.0752 & & 0.0014 \\
\hline LAWB40 & & & & & & & & 0.0309 & & 1.0000 & & & & & & & & & & & \\
\hline LAWB41 & & & & & & & & 0.0309 & & 1.0000 & & & & & & & & & & & \\
\hline LAWB42S & & & & & 0.0428 & & & 0.0402 & & 0.9805 & & & & & & & & & & & \\
\hline LAWB43S & & & & & & & & 0.0402 & & 0.9806 & & & & & & & & & & & \\
\hline LAWB44S & & & & & & & & 0.0402 & & 0.9796 & & & & & & & & & & & \\
\hline LAWB45 & & & & & & & & 0.0315 & & 1.0000 & 0.0586 & 0.1296 & 0.0620 & 0.0500 & & 0.0023 & 0.0426 & 0.0263 & 0.0541 & & 0.0009 \\
\hline LAWB47 & & & & & 0.0114 & & & 0.0315 & & 1.0000 & & & & & & & & & & & \\
\hline LAWB48 & & & & & 0.0229 & & & 0.0315 & & 1.0000 & & & & & & & & & & & \\
\hline LAWB49 & & & & & 0.0343 & & & 0.0315 & & 1.0000 & & & & & & & & & & & \\
\hline LAWB50 & & & & & & & & 0.0309 & & 0.9947 & & & & & & & & & & & \\
\hline
\end{tabular}


Appendix A. Database - mass fraction

RPP-WTP LAW Formulation (Muller et al. 2001)

\begin{tabular}{|c|c|c|c|c|c|c|c|c|c|c|c|c|c|c|c|c|c|c|c|c|c|}
\hline Glass ID & $\begin{array}{c}\mathrm{SiO} 2 \\
-\mathrm{a}\end{array}$ & $\begin{array}{c}\mathrm{ZrO} 2 \\
-\mathrm{a}\end{array}$ & $\begin{array}{c}\mathrm{Ag} 2 \mathrm{O} \\
-\mathrm{a}\end{array}$ & $\begin{array}{c}\mathrm{As} 2 \mathrm{O} 3 \\
-\mathrm{a} \\
\end{array}$ & $\begin{array}{c}\mathrm{BaO} \\
-\mathrm{a}\end{array}$ & $\begin{array}{c}\mathrm{Bi} 2 \mathrm{O} 3 \\
-\mathrm{a}\end{array}$ & $\begin{array}{l}\mathrm{Br} \\
-\mathrm{a}\end{array}$ & $\begin{array}{c}\mathrm{CdO} \\
-\mathrm{a} \\
\end{array}$ & $\begin{array}{c}\mathrm{Ce} 2 \mathrm{O} 3 \\
-\mathrm{a}\end{array}$ & $\begin{array}{c}\mathrm{CeO} 2 \\
-\mathrm{a} \\
\end{array}$ & $\begin{array}{l}\mathrm{Cl} \\
-\mathrm{a}\end{array}$ & $\begin{array}{c}\mathrm{CoO} \\
-\mathrm{a} \\
\end{array}$ & $\begin{array}{c}\mathrm{Co} 2 \mathrm{O} 3 \\
-\mathrm{a} \\
\end{array}$ & $\begin{array}{c}\mathrm{Cr} 2 \mathrm{O} 3 \\
-\mathrm{a} \\
\end{array}$ & $\begin{array}{c}\mathrm{Cs} 2 \mathrm{O} \\
-\mathrm{a} \\
\end{array}$ & $\begin{array}{c}\mathrm{CuO} \\
-\mathrm{a} \\
\end{array}$ & $\begin{array}{c}\mathrm{Eu} 2 \mathrm{O} 3 \\
-\mathrm{a}\end{array}$ & $\begin{array}{c}\mathrm{F} \\
-\mathrm{a}\end{array}$ & $\begin{array}{c}\mathrm{Ga} 2 \mathrm{O} 3 \\
-\mathrm{a} \\
\end{array}$ & $\begin{array}{c}\mathrm{Gd} 2 \mathrm{O} 3 \\
-\mathrm{a}\end{array}$ & $\begin{array}{c}\mathrm{HgO} \\
-\mathrm{a} \\
\end{array}$ \\
\hline \multicolumn{22}{|c|}{ LAWA102S } \\
\hline \multicolumn{22}{|c|}{ LAWA103S } \\
\hline \multicolumn{22}{|l|}{ LAWA104 } \\
\hline \multicolumn{22}{|l|}{ LAWA105 } \\
\hline LAWB29* & 0.4540 & 0.0276 & & & & & & & & & & & & 0.0035 & & & & & & & \\
\hline LAWB30 & 0.4092 & 0.0309 & & & & & & & & & & & & 0.0007 & & & & & & & \\
\hline LAWB31 & 0.4322 & 0.0275 & & & & & & & & & & & & 0.0010 & & & & & & & \\
\hline LAWB32 & 0.4584 & 0.0250 & & & & & & & & & & & & 0.0009 & & & & & & & \\
\hline LAWB33 & 0.4383 & 0.0246 & & & & & & & & & & & & 0.0008 & & & & & & & \\
\hline LAWB34 & 0.4552 & 0.0247 & & & & & & & & & & & & 0.0008 & & & & & & & \\
\hline LAWB35 & 0.4540 & 0.0281 & & & & & & & & & & & & 0.0009 & & & & & & & \\
\hline \multicolumn{22}{|l|}{ LAWB36S } \\
\hline LAWB37 & 0.4728 & 0.0265 & & & & & & & & & & & & 0.0008 & & & & & & & \\
\hline \multicolumn{22}{|l|}{ LAWB38 } \\
\hline LAWB39 & 0.4670 & 0.0276 & & & & & & & & & & & & 0.0008 & & & & & & & \\
\hline \multicolumn{22}{|l|}{ LAWB40 } \\
\hline \multicolumn{22}{|l|}{ LAWB41 } \\
\hline \multicolumn{22}{|l|}{ LAWB42S } \\
\hline \multicolumn{22}{|l|}{ LAWB43S } \\
\hline \multicolumn{22}{|l|}{ LAWB44S } \\
\hline LAWB45 & 0.4624 & 0.0304 & & & & & & & & & & & & 0.0005 & & & & & & & \\
\hline \multicolumn{22}{|l|}{ LAWB47 } \\
\hline \multicolumn{22}{|l|}{ LAWB48 } \\
\hline LAWB49 & & & & & & & & & & & & & & & & & & & & & \\
\hline LAWB50 & & & & & & & & & & & & & & & & & & & & & \\
\hline
\end{tabular}


Appendix A. Database - mass fraction

RPP-WTP LAW Formulation (Muller et al. 2001)

\begin{tabular}{|c|c|c|c|c|c|c|c|c|c|c|c|c|c|c|c|c|c|c|c|c|c|}
\hline Glass ID & $\begin{array}{c}\mathrm{I} \\
-\mathrm{a} \\
\end{array}$ & $\begin{array}{c}\mathrm{La} 2 \mathrm{O} 3 \\
-\mathrm{a}\end{array}$ & $\begin{array}{c}\mathrm{MnO} 2 \\
-\mathrm{a}\end{array}$ & $\begin{array}{c}\mathrm{MnO} \\
-\mathrm{a}\end{array}$ & $\begin{array}{c}\mathrm{MoO} \\
-\mathrm{a}\end{array}$ & $\begin{array}{c}\mathrm{MoO} 3 \\
-\mathrm{a}\end{array}$ & $\begin{array}{c}\mathrm{Nb} 2 \mathrm{O} 5 \\
-\mathrm{a}\end{array}$ & $\begin{array}{c}\mathrm{Nd} 2 \mathrm{O} 3 \\
-\mathrm{a}\end{array}$ & $\begin{array}{c}\mathrm{PbO} \\
-\mathrm{a} \\
\end{array}$ & $\begin{array}{c}\mathrm{PdO} 2 \\
-\mathrm{a}\end{array}$ & $\begin{array}{c}\mathrm{PdO} \\
-\mathrm{a} \\
\end{array}$ & $\begin{array}{c}\mathrm{Pr} 2 \mathrm{O} 3 \\
-\mathrm{a} \\
\end{array}$ & $\begin{array}{c}\text { Pr6O11 } \\
-\mathrm{a}\end{array}$ & $\begin{array}{c}\mathrm{Rb} 2 \mathrm{O} \\
-\mathrm{a}\end{array}$ & $\begin{array}{c}\mathrm{ReO} \\
-\mathrm{a}\end{array}$ & $\begin{array}{c}\mathrm{ReO} 2 \\
-\mathrm{a}\end{array}$ & $\begin{array}{c}\mathrm{Rh} 2 \mathrm{O} 3 \\
-\mathrm{a}\end{array}$ & $\begin{array}{c}\mathrm{RhO}_{2} \\
-\mathrm{a}\end{array}$ & $\begin{array}{c}\mathrm{RuO} 2 \\
-\mathrm{a}\end{array}$ & $\begin{array}{c}\mathrm{Sb} 2 \mathrm{O} 3 \\
-\mathrm{a}\end{array}$ & $\begin{array}{c}\mathrm{Sb} 2 \mathrm{O} 5 \\
-\mathrm{a}\end{array}$ \\
\hline LAWA102S & & & & & & & & & & & & & & & & & & & & & \\
\hline LAWA103S & & & & & & & & & & & & & & & & & & & & & \\
\hline LAWA104 & & & & & & & & & & & & & & & & & & & & & \\
\hline LAWA105 & & & & & & & & & & & & & & & & & & & & & \\
\hline LAWB29* & & & & & & & & & & & & & & & & & & & & & \\
\hline LAWB30 & & & & & & & & & & & & & & & & & & & & & \\
\hline LAWB31 & & & & & & & & & & & & & & & & & & & & & \\
\hline LAWB32 & & & & & & & & & & & & & & & & & & & & & \\
\hline LAWB33 & & & & & & & & & & & & & & & & & & & & & \\
\hline LAWB34 & & & & & & & & & & & & & & & & & & & & & \\
\hline LAWB35 & & & & & & & & & & & & & & & & & & & & & \\
\hline LAWB36S & & & & & & & & & & & & & & & & & & & & & \\
\hline LAWB37 & & & & & & & & & & & & & & & & & & & & & \\
\hline LAWB38 & & & & & & & & & & & & & & & & & & & & & \\
\hline LAWB39 & & & & & & & & & & & & & & & & & & & & & \\
\hline LAWB40 & & & & & & & & & & & & & & & & & & & & & \\
\hline LAWB41 & & & & & & & & & & & & & & & & & & & & & \\
\hline LAWB42S & & & & & & & & & & & & & & & & & & & & & \\
\hline LAWB43S & & & & & & & & & & & & & & & & & & & & & \\
\hline LAWB44S & & & & & & & & & & & & & & & & & & & & & \\
\hline LAWB45 & & & & & & & & & & & & & & & & & & & & & \\
\hline LAWB47 & & & & & & & & & & & & & & & & & & & & & \\
\hline LAWB48 & & & & & & & & & & & & & & & & & & & & & \\
\hline LAWB49 & & & & & & & & & & & & & & & & & & & & & \\
\hline LAWB50 & & & & & & & & & & & & & & & & & & & & & \\
\hline
\end{tabular}


Appendix A. Database - mass fraction

RPP-WTP LAW Formulation (Muller et al. 2001)

\begin{tabular}{|c|c|c|c|c|c|c|c|c|c|c|c|c|c|c|c|c|c|c|c|c|}
\hline Glass ID & $\begin{array}{c}\mathrm{SeO} 2 \\
-\mathrm{a}\end{array}$ & $\begin{array}{c}\mathrm{Sm} 2 \mathrm{O} 3 \\
-\mathrm{a}\end{array}$ & $\begin{array}{c}\mathrm{SnO} \\
-\mathrm{a}\end{array}$ & $\begin{array}{c}\mathrm{SnO} 2 \\
-\mathrm{a}\end{array}$ & $\begin{array}{c}\mathrm{SO} 3 \\
-\mathrm{a}\end{array}$ & $\begin{array}{c}\mathrm{SrO} \\
-\mathrm{a}\end{array}$ & $\begin{array}{c}\mathrm{Tc} 2 \mathrm{O} 7 \\
-\mathrm{a}\end{array}$ & $\begin{array}{c}\mathrm{TeO} 2 \\
-\mathrm{a}\end{array}$ & $\begin{array}{c}\mathrm{ThO} 2 \\
-\mathrm{a}\end{array}$ & $\begin{array}{c}\mathrm{TiO} 2 \\
-\mathrm{a}\end{array}$ & $\begin{array}{c}\mathrm{T} 12 \mathrm{O} 3 \\
-\mathrm{a}\end{array}$ & $\begin{array}{c}\text { U3O8 } \\
-\mathrm{a}\end{array}$ & $\begin{array}{c}\mathrm{UO} 2 \\
-\mathrm{a}\end{array}$ & $\begin{array}{c}\mathrm{UO3} \\
-\mathrm{a}\end{array}$ & $\begin{array}{c}\mathrm{V} 2 \mathrm{O} 5 \\
-\mathrm{a}\end{array}$ & $\begin{array}{c}\text { WO3 } \\
-\mathrm{a}\end{array}$ & $\begin{array}{c}\mathrm{Y} 2 \mathrm{O} 3 \\
-\mathrm{a}\end{array}$ & $\begin{array}{c}\mathrm{ZnO} \\
-\mathrm{a}\end{array}$ & $\begin{array}{c}\text { Others } \\
-\mathrm{a}\end{array}$ & $\begin{array}{c}\text { Sum } \\
-\mathrm{a}\end{array}$ \\
\hline \multicolumn{21}{|l|}{ LAWA102S } \\
\hline \multicolumn{21}{|c|}{ LAWA103S } \\
\hline \multicolumn{21}{|l|}{ LAWA104 } \\
\hline \multicolumn{21}{|l|}{ LAWA105 } \\
\hline LAWB29* & & & & & & & & & & 0.0005 & & & & & & & & 0.0370 & & 0.9770 \\
\hline LAWB30 & & & & & & & & & & 0.0004 & & & & & & & & 0.0402 & & 0.9217 \\
\hline LAWB31 & & & & & 0.0062 & & & & & 0.0002 & & & & & & & & 0.0290 & & 0.9163 \\
\hline LAWB32 & & & & & 0.0067 & & & & & 0.0002 & & & & & & & & 0.0285 & & 0.9630 \\
\hline LAWB33 & & & & & 0.0075 & & & & & 0.0002 & & & & & & & & 0.0285 & & 0.9198 \\
\hline LAWB34 & & & & & 0.0068 & & & & & 0.0002 & & & & & & & & 0.0295 & & 0.9370 \\
\hline LAWB35 & & & & & 0.0073 & & & & & 0.0002 & & & & & & & & 0.0308 & & 0.9551 \\
\hline LAWB36S & & & & & & & & & & & & & & & & & & & & \\
\hline LAWB37 & & & & & 0.0067 & & & & & 0.0003 & & & & & & & & 0.0312 & & 0.9751 \\
\hline LAWB38 & & & & & & & & & & & & & & & & & & & & \\
\hline LAWB39 & & & & & 0.0077 & & & & & 0.0002 & & & & & 0.0339 & & & 0.0310 & & 0.9719 \\
\hline \multicolumn{21}{|l|}{ LAWB40 } \\
\hline \multicolumn{21}{|l|}{ LAWB41 } \\
\hline \multicolumn{21}{|l|}{ LAWB42S } \\
\hline \multicolumn{21}{|l|}{ LAWB43S } \\
\hline \multicolumn{21}{|l|}{ LAWB44S } \\
\hline LAWB45 & & & & & 0.0058 & & & & & 0.0003 & & & & & & & & 0.0305 & & 0.9563 \\
\hline \multicolumn{21}{|l|}{ LAWB47 } \\
\hline \multicolumn{21}{|l|}{ LAWB48 } \\
\hline LAWB49 & & & & & & & & & & & & & & & & & & & & \\
\hline LAWB50 & & & & & & & & & & & & & & & & & & & & \\
\hline
\end{tabular}


Appendix A. Database - mass fraction

\section{RPP-WTP LAW Formulation (Muller et al. 2001)}

\begin{tabular}{|c|c|c|c|c|c|c|c|c|}
\hline Glass ID & $\begin{array}{l}\mathrm{TM} \\
\left({ }^{\circ} \mathrm{C}\right) \\
\end{array}$ & $\begin{array}{l}\text { Gradient } \\
\text { TL }\left({ }^{\circ} \mathrm{C}\right)\end{array}$ & $\begin{array}{l}\text { Uniform } \\
\text { TL }\left({ }^{\circ} \mathrm{C}\right)\end{array}$ & Primary Phase & $\begin{array}{l}\text { Quenched } \\
\text { Visual/OM }\end{array}$ & $\begin{array}{c}\text { Quenched } \\
\text { SEM/EDS or TEM }\end{array}$ & $\begin{array}{l}\text { Quenched } \\
\text { XRD } \\
\end{array}$ & $\begin{array}{c}\text { Quenched } \\
\text { Homogeneous? }\end{array}$ \\
\hline \multicolumn{9}{|c|}{ LAWA102S } \\
\hline \multicolumn{9}{|c|}{ LAWA103S } \\
\hline \multicolumn{9}{|l|}{ LAWA104 } \\
\hline \multicolumn{9}{|l|}{ LAWA105 } \\
\hline \multicolumn{9}{|l|}{ LAWB29* } \\
\hline \multicolumn{9}{|l|}{ LAWB30 } \\
\hline \multicolumn{9}{|l|}{ LAWB31 } \\
\hline \multicolumn{9}{|l|}{ LAWB32 } \\
\hline \multicolumn{9}{|l|}{ LAWB33 } \\
\hline \multicolumn{9}{|l|}{ LAWB34 } \\
\hline \multicolumn{9}{|l|}{ LAWB35 } \\
\hline \multicolumn{9}{|l|}{ LAWB36S } \\
\hline \multirow{2}{*}{\multicolumn{9}{|c|}{$\begin{array}{l}\text { LAWB37 } \\
\text { LAWB38 }\end{array}$}} \\
\hline & & & & & & & & \\
\hline \multicolumn{9}{|l|}{ LAWB39 } \\
\hline \multicolumn{9}{|l|}{ LAWB40 } \\
\hline \multicolumn{9}{|l|}{ LAWB41 } \\
\hline \multicolumn{9}{|l|}{ LAWB42S } \\
\hline \multicolumn{9}{|l|}{ LAWB43S } \\
\hline \multicolumn{9}{|l|}{ LAWB44S } \\
\hline \multicolumn{9}{|l|}{ LAWB45 } \\
\hline \multicolumn{9}{|l|}{ LAWB47 } \\
\hline \multicolumn{9}{|l|}{ LAWB48 } \\
\hline LAWB49 & & & & & & & & \\
\hline LAWB50 & & & & & & & & \\
\hline
\end{tabular}


Appendix A. Database - mass fraction

RPP-WTP LAW Formulation (Muller et al. 2001)

\begin{tabular}{|c|c|c|c|c|c|}
\hline Glass ID & $\begin{array}{c}\mathrm{CCC} \\
\text { Visual/OM }\end{array}$ & $\begin{array}{c}\text { CCC } \\
\text { SEM/EDS or TEM }\end{array}$ & $\begin{array}{l}\mathrm{CCC} \\
\mathrm{XRD}\end{array}$ & $\begin{array}{l}\text { Heat Treated } \\
\text { Visual/OM }\end{array}$ & $\begin{array}{c}\text { Heat Treated } \\
\text { SEM/EDS or TEM }\end{array}$ \\
\hline LAWA102S & & & & & $\begin{array}{l}\text { After } 1200^{\circ} \mathrm{C} 1 \mathrm{hr} \text { and } 950^{\circ} \mathrm{C} 20 \mathrm{hr} \text { - Clear } \\
\text { homogeneous glass }\end{array}$ \\
\hline LAWA103S & & & & & $\begin{array}{l}\text { After } 1200^{\circ} \mathrm{C} 1 \mathrm{hr} \text { and } 850^{\circ} \mathrm{C} 20 \mathrm{hr} \text { - Clear } \\
\text { homogeneous glass }\end{array}$ \\
\hline LAWA104 & & & & & $\begin{array}{l}\text { After } 1200^{\circ} \mathrm{C} 1 \mathrm{hr} \text { and } 850^{\circ} \mathrm{C} 20 \mathrm{hr} \text { - Clear } \\
\text { homogeneous glass }\end{array}$ \\
\hline LAWA105 & & & & & $\begin{array}{l}\text { After } 1200^{\circ} \mathrm{C} 1 \mathrm{hr} \text { and } 850^{\circ} \mathrm{C} 20 \mathrm{hr} \text { - Clear } \\
\text { homogeneous glass }\end{array}$ \\
\hline \multicolumn{6}{|l|}{ LAWB29* } \\
\hline LAWB30 & & & & & $\begin{array}{l}\text { After } 1200^{\circ} \mathrm{C} 1 \mathrm{hr} \text { and } 950^{\circ} \mathrm{C} 20 \mathrm{hr} \text { - trace } \\
\text { amount of high Fe spinel. }\end{array}$ \\
\hline LAWB31 & & & & & After $1200^{\circ} \mathrm{C} 1 \mathrm{hr}$ and $850^{\circ} \mathrm{C} 20 \mathrm{hr}-\sim 2.4 \% *$ \\
\hline LAWB32 & & & & & After $1200^{\circ} \mathrm{C} 1 \mathrm{hr}$ and $850^{\circ} \mathrm{C} 20 \mathrm{hr}-\sim 2.9 \mathrm{vol} \%{ }^{*}$ \\
\hline LAWB33 & & & & & $\begin{array}{l}\text { After } 1200^{\circ} \mathrm{C} 1 \mathrm{hr} \text { and } 950^{\circ} \mathrm{C} 20 \mathrm{hr}-2.9 \% \\
(\mathrm{Ca} 3(\mathrm{PO} 4) 2) \text {; After } 1200^{\circ} \mathrm{C} 1 \mathrm{hr} \text { and } 850^{\circ} \mathrm{C} 20 \mathrm{hr} \\
\text { 6.2 vol\%* }\end{array}$ \\
\hline LAWB34 & & & & & $\begin{array}{l}\text { After } 1200^{\circ} \mathrm{C} 1 \mathrm{hr} \text { and } 950^{\circ} \mathrm{C} 20 \mathrm{hr} \text { - Trace } \\
\text { amount of crystals; After } 1200^{\circ} \mathrm{C} 1 \mathrm{hr} \text { and } 850^{\circ} \mathrm{C} \\
20 \mathrm{hr}-\sim 4.8 \text { vol\%* }\end{array}$ \\
\hline LAWB35 & & & & & After $1200^{\circ} \mathrm{C} 1 \mathrm{hr}$ and $850^{\circ} \mathrm{C} 20 \mathrm{hr}-0.26 \% *$ \\
\hline LAWB36S & & & & & After $1200^{\circ} \mathrm{C} 1 \mathrm{hr}$ and $850^{\circ} \mathrm{C} 20 \mathrm{hr}-4.16 \%$ * \\
\hline LAWB37 & & & & & After $1200^{\circ} \mathrm{C} 1 \mathrm{hr}$ and $850^{\circ} \mathrm{C} 20 \mathrm{hr}-4.8 \% *$ \\
\hline LAWB38 & & & & & After $1200^{\circ} \mathrm{C} 1 \mathrm{hr}$ and $850^{\circ} \mathrm{C} 20 \mathrm{hr}-4.0 \% *$ \\
\hline LAWB39 & & & & & $\begin{array}{l}\text { After } 1200^{\circ} \mathrm{C} 1 \mathrm{hr} \text { and } 850^{\circ} \mathrm{C} 20 \mathrm{hr} \text { - Less than } 0.1 \\
\text { vol\% of clear cubic-shaped crystal }\end{array}$ \\
\hline LAWB40 & & & & & $\begin{array}{l}\text { After } 1200^{\circ} \mathrm{C} 1 \mathrm{hr} \text { and } 850^{\circ} \mathrm{C} 20 \mathrm{hr} \text { - One small } \\
\text { submicron crystal }\end{array}$ \\
\hline LAWB41 & & & & & $\begin{array}{l}\text { After } 1200^{\circ} \mathrm{C} 1 \mathrm{hr} \text { and } 850^{\circ} \mathrm{C} 20 \mathrm{hr} \text { - Clear } \\
\text { homogeneous glass }\end{array}$ \\
\hline LAWB42S & & & & & $\begin{array}{l}\text { After } 1200^{\circ} \mathrm{C} 1 \mathrm{hr} \text { and } 850^{\circ} \mathrm{C} 20 \mathrm{hr} \text { - Trace } \\
\text { amount of } 1 \text { to } 10 \mathrm{~mm} \text { zircon crystals and Fe-Zn } \\
\text { spinel; } \sim 1 \text { vol. } \%\end{array}$ \\
\hline LAWB43S & & & & & $\begin{array}{l}\text { After } 1200^{\circ} \mathrm{C} 1 \mathrm{hr} \text { and } 850^{\circ} \mathrm{C} 20 \mathrm{hr} \text { - Presence of } \\
\text { spinel, Ca-Mg-Fe silicate (augite) and zircon. In } \\
\text { total } \sim 14.9 \mathrm{vol} \%\end{array}$ \\
\hline LAWB44S & & & & & $\begin{array}{l}\text { After } 1200^{\circ} \mathrm{C} 1 \mathrm{hr} \text { and } 850^{\circ} \mathrm{C} 20 \mathrm{hr} \text { - Presence of } \\
\text { spinel, Ca-Mg-Fe silicate (augite) and zircon. In } \\
\text { total } \sim 12.3 \mathrm{vol} \%\end{array}$ \\
\hline LAWB45 & & & & & $\begin{array}{l}\text { After } 1200^{\circ} \mathrm{C} 1 \mathrm{hr} \text { and } 850^{\circ} \mathrm{C} 20 \mathrm{hr} \text { - Clear } \\
\text { homogeneous glass }\end{array}$ \\
\hline LAWB47 & & & & & $\begin{array}{l}\text { After } 1200^{\circ} \mathrm{C} 1 \mathrm{hr} \text { and } 850^{\circ} \mathrm{C} 20 \mathrm{hr} \text { - Clear } \\
\text { homogeneous glass }\end{array}$ \\
\hline LAWB48 & & & & & $\begin{array}{l}\text { After } 1200^{\circ} \mathrm{C} 1 \mathrm{hr} \text { and } 850^{\circ} \mathrm{C} 20 \mathrm{hr} \text { - Clear } \\
\text { homogeneous glass for the most part, with one } \\
\text { black irregular particle of } \sim 5 \mathrm{~mm} \text { dia. possibly } \\
\text { spinel. }\end{array}$ \\
\hline LAWB49 & & & & & After $1200^{\circ} \mathrm{C} 1 \mathrm{hr}$ and $850^{\circ} \mathrm{C} 20 \mathrm{hr}$ - trace \\
\hline LAWB50 & & & & & \\
\hline
\end{tabular}


Appendix A. Database - mass fraction

RPP-WTP LAW Formulation (Muller et al. 2001)

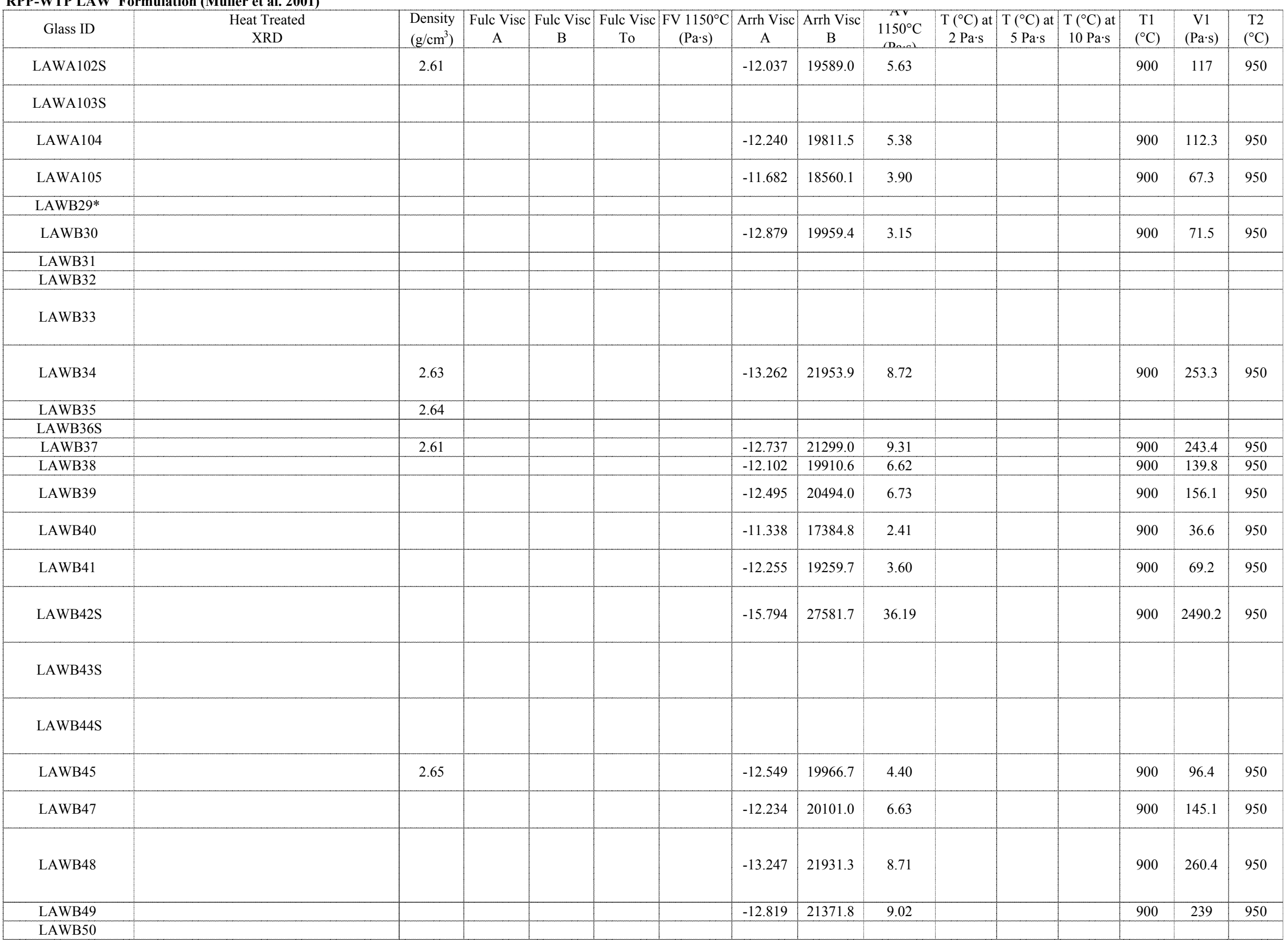


Appendix A. Database - mass fraction

RPP-WTP LAW Formulation (Muller et al. 2001)

\begin{tabular}{|c|c|c|c|c|c|c|c|c|c|c|c|c|c|c|c|c|c|c|c|c|c|}
\hline Glass ID & $\begin{array}{c}\mathrm{V} 2 \\
(\mathrm{~Pa} \cdot \mathrm{s})\end{array}$ & $\begin{array}{c}\mathrm{T} 3 \\
\left({ }^{\circ} \mathrm{C}\right) \\
\end{array}$ & $\begin{array}{c}\mathrm{V} 3 \\
(\mathrm{~Pa} \cdot \mathrm{s})\end{array}$ & $\begin{array}{c}\mathrm{T} 4 \\
\left({ }^{\circ} \mathrm{C}\right) \\
\end{array}$ & $\begin{array}{c}\mathrm{V} 4 \\
(\mathrm{~Pa} \cdot \mathrm{s})\end{array}$ & $\begin{array}{c}\mathrm{T} 5 \\
\left({ }^{\circ} \mathrm{C}\right) \\
\end{array}$ & $\begin{array}{c}\text { V5 } \\
(\mathrm{Pa} \cdot \mathrm{s})\end{array}$ & $\begin{array}{c}\mathrm{T} 6 \\
\left({ }^{\circ} \mathrm{C}\right) \\
\end{array}$ & $\begin{array}{c}\mathrm{V} 6 \\
(\mathrm{~Pa} \cdot \mathrm{s})\end{array}$ & $\begin{array}{c}\mathrm{T} 7 \\
\left({ }^{\circ} \mathrm{C}\right) \\
\end{array}$ & $\begin{array}{c}\mathrm{V} 7 \\
(\mathrm{~Pa} \cdot \mathrm{s})\end{array}$ & $\begin{array}{c}\mathrm{T} 8 \\
\left({ }^{\circ} \mathrm{C}\right) \\
\end{array}$ & $\begin{array}{c}\mathrm{V} 8 \\
(\mathrm{~Pa} \cdot \mathrm{s})\end{array}$ & $\begin{array}{c}\mathrm{T} 9 \\
\left({ }^{\circ} \mathrm{C}\right) \\
\end{array}$ & $\begin{array}{c}\mathrm{V} 9 \\
(\mathrm{~Pa} \cdot \mathrm{s})\end{array}$ & $\begin{array}{l}\mathrm{T} 10 \\
\left({ }^{\circ} \mathrm{C}\right)\end{array}$ & $\begin{array}{c}\mathrm{V} 10 \\
(\mathrm{~Pa} \cdot \mathrm{s})\end{array}$ & $\begin{array}{l}\mathrm{T} 11 \\
\left({ }^{\circ} \mathrm{C}\right)\end{array}$ & $\begin{array}{c}\mathrm{V} 11 \\
(\mathrm{~Pa} \cdot \mathrm{s})\end{array}$ & $\begin{array}{l}\mathrm{T} 12 \\
\left({ }^{\circ} \mathrm{C}\right)\end{array}$ & $\begin{array}{c}\mathrm{V} 12 \\
(\mathrm{~Pa} \cdot \mathrm{s})\end{array}$ \\
\hline LAWA102S & 53.3 & 1000 & 27 & 1050 & 14.9 & 1100 & 8.8 & 1150 & 5.5 & 1200 & 3.6 & 1250 & 2.5 & & & & & & & & \\
\hline \multicolumn{22}{|l|}{ LAWA103S } \\
\hline LAWA104 & 52.3 & 1000 & 26.7 & 1050 & 14.6 & 1100 & 8.6 & 1150 & 5.3 & 1200 & 3.4 & 1250 & 2.3 & & & & & & & & \\
\hline LAWA105 & 32.9 & 1000 & 17.5 & 1050 & 10 & 1100 & 6 & 1150 & 3.8 & 1200 & 2.5 & 1250 & 1.8 & & & & & & & & \\
\hline \multicolumn{22}{|l|}{ LAWB29* } \\
\hline LAWB30 & 30.9 & 1000 & 15.2 & 1050 & 8.3 & 1100 & 4.9 & 1150 & 3.1 & 1200 & 2 & 1250 & 1.4 & & & & & & & & \\
\hline \multicolumn{22}{|l|}{ LAWB31 } \\
\hline LAWB32 & & & & & & & & & & & & & & & & & & & & & \\
\hline \multicolumn{22}{|l|}{ LAWB33 } \\
\hline LAWB34 & 108.5 & 1000 & 51.4 & 1050 & 26.4 & 1100 & 14.6 & 1150 & 8.5 & 1200 & 5.3 & 1250 & 3.4 & & & & & & & & \\
\hline \multicolumn{22}{|l|}{ LAWB35 } \\
\hline \multicolumn{22}{|l|}{ LAWB36S } \\
\hline LAWB37 & 107.2 & 1000 & 52 & 1050 & 27.3 & 1100 & 15.4 & 1150 & 9.2 & 1200 & 5.7 & 1250 & 3.7 & & & & & & & & \\
\hline LAWB38 & 65.1 & 1000 & 33.1 & 1050 & 18.2 & 1100 & 10.6 & 1150 & 6.5 & 1200 & 4.2 & 1250 & 2.8 & & & & & & & & \\
\hline LAWB39 & 70.8 & 1000 & 35.3 & 1050 & 19 & 1100 & 10.9 & 1150 & 6.6 & 1200 & 4.2 & 1250 & 2.8 & & & & & & & & \\
\hline LAWB40 & 17.6 & 1000 & 9.5 & 1050 & 5.6 & 1100 & 3.5 & 1150 & 2.4 & 1200 & 1.6 & 1250 & 1.2 & & & & & & & & \\
\hline LAWB41 & 32.9 & 1000 & 17.1 & 1050 & 9.5 & 1100 & 5.6 & 1150 & 3.5 & 1200 & 2.3 & 1250 & 1.6 & & & & & & & & \\
\hline LAWB42S & 859.3 & 1000 & 336 & 1050 & 145.9 & 1100 & 69.1 & 1150 & 35.3 & 1200 & 19.2 & 1250 & 11.1 & & & & & & & & \\
\hline \multicolumn{22}{|l|}{ LAWB43S } \\
\hline \multicolumn{22}{|l|}{ LAWB44S } \\
\hline LAWB45 & 43.4 & 1000 & 21.8 & 1050 & 11.9 & 1100 & 7 & 1150 & 4.3 & 1200 & 2.8 & 1250 & 1.9 & & & & & & & & \\
\hline LAWB47 & 66.7 & 1000 & 33.6 & 1050 & 18.3 & 1100 & 10.6 & 1150 & 6.5 & 1200 & 4.2 & 1250 & 2.8 & & & & & & & & \\
\hline LAWB48 & 107.9 & 1000 & 50.3 & 1050 & 25.8 & 1100 & 14.3 & 1150 & 8.5 & 1200 & 5.3 & 1250 & 3.5 & & & & & & & & \\
\hline LAWB49 & 104.9 & 1000 & 50.7 & 1050 & 26.6 & 1100 & 14.9 & 1150 & 8.9 & 1200 & 5.5 & 1250 & 3.6 & & & & & & & & \\
\hline LAWB50 & & & & & & & & & & & & & & & & & & & & & \\
\hline
\end{tabular}


Appendix A. Database - mass fraction

RPP-WTP LAW Formulation (Muller et al. 2001)

\begin{tabular}{|c|c|c|c|c|c|c|c|c|c|c|c|c|c|c|c|c|}
\hline Glass ID & $\begin{array}{l}\mathrm{T} 13 \\
\left({ }^{\circ} \mathrm{C}\right) \\
\end{array}$ & $\begin{array}{c}\mathrm{V} 13 \\
(\mathrm{~Pa} \cdot \mathrm{s}) \\
\end{array}$ & $\begin{array}{l}\mathrm{T} 14 \\
\left({ }^{\circ} \mathrm{C}\right) \\
\end{array}$ & $\begin{array}{c}\mathrm{V} 14 \\
(\mathrm{~Pa} \cdot \mathrm{s}) \\
\end{array}$ & $\begin{array}{c}\text { Q PCT } \\
\text { B }\left(\mathrm{g} / \mathrm{m}^{2}\right)\end{array}$ & $\begin{array}{c}\text { Q PCT } \\
\mathrm{Li}\left(\mathrm{g} / \mathrm{m}^{2}\right)\end{array}$ & $\begin{array}{c}\text { Q PCT } \\
\mathrm{Na}\left(\mathrm{g} / \mathrm{m}^{2}\right)\end{array}$ & $\begin{array}{c}\text { Q PCT } \\
\mathrm{Si}\left(\mathrm{g} / \mathrm{m}^{2}\right)\end{array}$ & $\begin{array}{c}\text { Q PCT } \\
\mathrm{pH}\end{array}$ & $\begin{array}{c}\text { CCC PCT } \\
\text { B }\left(\mathrm{g} / \mathrm{m}^{2}\right)\end{array}$ & $\begin{array}{l}\text { CCC PCT } \\
\mathrm{Li}\left(\mathrm{g} / \mathrm{m}^{2}\right)\end{array}$ & $\begin{array}{l}\text { CCC PCT } \\
\mathrm{Na}\left(\mathrm{g} / \mathrm{m}^{2}\right)\end{array}$ & $\begin{array}{l}\text { CCC PCT } \\
\mathrm{Si}\left(\mathrm{g} / \mathrm{m}^{2}\right)\end{array}$ & $\begin{array}{c}\begin{array}{c}\text { CCC PCT } \\
\mathrm{pH}\end{array} \\
\end{array}$ & $\begin{array}{l}\text { Q PCT B at } \\
20^{\circ} \mathrm{C}\left(\mathrm{g} / \mathrm{m}^{2}\right)\end{array}$ & $\begin{array}{l}\text { Q PCT Li at } \\
20^{\circ} \mathrm{C}\left(\mathrm{g} / \mathrm{m}^{2}\right)\end{array}$ \\
\hline LAWA102S & & & & & 0.27 & & 0.22 & 0.16 & 9.84 & & & & & & & \\
\hline \multicolumn{17}{|l|}{ LAWA103S } \\
\hline LAWA104 & & & & & 0.58 & & 0.53 & 0.21 & 11.23 & & & & & & & \\
\hline LAWA105 & & & & & 0.96 & & 0.79 & 0.28 & 11.35 & & & & & & & \\
\hline \multicolumn{17}{|l|}{ LAWB29* } \\
\hline LAWB30 & & & & & 0.24 & & 0.24 & 0.09 & 10.11 & & & & & & & \\
\hline LAWB31 & & & & & 0.21 & & 0.11 & 0.1 & 9.41 & & & & & & & \\
\hline LAWB32 & & & & & 0.25 & & 0.14 & 0.11 & 9.33 & & & & & & & \\
\hline LAWB33 & & & & & 0.19 & & 0.12 & 0.1 & 9.18 & & & & & & & \\
\hline LAWB34 & & & & & 0.22 & & 0.13 & 0.1 & 9.51 & & & & & & & \\
\hline LAWB35 & & & & & 0.5 & & 0.34 & 0.14 & 9.56 & & & & & & & \\
\hline \multicolumn{17}{|l|}{ LAWB36S } \\
\hline LAWB37 & & & & & 0.26 & & 0.18 & 0.11 & 9.43 & & & & & & & \\
\hline LAWB38 & & & & & 0.25 & & 0.18 & 0.12 & 9.56 & & & & & & & \\
\hline LAWB39 & & & & & 0.5 & & 0.4 & 0.13 & 9.43 & & & & & & & \\
\hline LAWB40 & & & & & 1.59 & & 1.17 & 0.32 & 10.41 & & & & & & & \\
\hline LAWB41 & & & & & 0.85 & & 0.7 & 0.19 & 10.17 & & & & & & & \\
\hline \multicolumn{17}{|l|}{ LAWB42S } \\
\hline \multicolumn{17}{|l|}{ LAWB43S } \\
\hline \multicolumn{17}{|l|}{ LAWB44S } \\
\hline LAWB45 & & & & & 0.53 & & 0.44 & 0.14 & 10.13 & & & & & & & \\
\hline \multicolumn{17}{|l|}{ LAWB47 } \\
\hline \multicolumn{17}{|l|}{ LAWB48 } \\
\hline LAWB49 & & & & & & & & & & & & & & & & \\
\hline LAWB50 & & & & & & & & & & & & & & & & \\
\hline
\end{tabular}


Appendix A. Database - mass fraction

RPP-WTP LAW Formulation (Muller et al. 2001)

\begin{tabular}{|c|c|c|c|c|c|c|c|c|c|c|c|c|}
\hline Glass ID & $\begin{array}{l}\text { Q PCT Na at } \\
20^{\circ} \mathrm{C}\left(\mathrm{g} / \mathrm{m}^{2}\right)\end{array}$ & $\begin{array}{l}\text { Q PCT Si at } \\
20^{\circ} \mathrm{C}\left(\mathrm{g} / \mathrm{m}^{2}\right)\end{array}$ & $\begin{array}{c}\mathrm{QpH} \\
\text { at } 20^{\circ} \mathrm{C}\end{array}$ & $\begin{array}{c}\text { TCLP Ag } \\
\text { (ppm) }\end{array}$ & $\begin{array}{c}\text { TCLP As } \\
\text { (ppm) }\end{array}$ & $\begin{array}{c}\text { TCLP Ba } \\
\text { (ppm) }\end{array}$ & $\begin{array}{c}\text { TCLP Cd } \\
(\mathrm{ppm})\end{array}$ & $\begin{array}{c}\text { TCLP Cr } \\
(\mathrm{ppm})\end{array}$ & $\begin{array}{c}\text { TCLP Ni } \\
(\mathrm{ppm})\end{array}$ & $\begin{array}{c}\text { TCLP Pb } \\
\text { (ppm) }\end{array}$ & $\begin{array}{c}\text { TCLP Se } \\
(\mathrm{ppm})\end{array}$ & $\begin{array}{c}\text { TCLP Zn } \\
(\mathrm{ppm})\end{array}$ \\
\hline LAWA102S & & & & $<0.0031$ & $<0.0490$ & 0.003 & $<0.0027$ & 0.016 & 0.017 & $<0.0243$ & $<0.0532$ & 1.327 \\
\hline \multicolumn{13}{|l|}{ LAWA103S } \\
\hline \multicolumn{13}{|l|}{ LAWA104 } \\
\hline \multicolumn{13}{|l|}{ LAWA105 } \\
\hline \multicolumn{13}{|l|}{ LAWB29* } \\
\hline \multicolumn{13}{|l|}{ LAWB30 } \\
\hline \multicolumn{13}{|l|}{ LAWB31 } \\
\hline \multicolumn{13}{|l|}{ LAWB32 } \\
\hline \multicolumn{13}{|l|}{ LAWB33 } \\
\hline \multicolumn{13}{|l|}{ LAWB34 } \\
\hline \multicolumn{13}{|l|}{ LAWB35 } \\
\hline \multicolumn{13}{|l|}{$\begin{array}{c}\text { LAWB36S } \\
\text { LAWB37 }\end{array}$} \\
\hline LAWB37 & & & & & & & & & & & & \\
\hline \multicolumn{13}{|l|}{ LAWB38 } \\
\hline \multicolumn{13}{|l|}{ LAWB39 } \\
\hline \multicolumn{13}{|l|}{ LAWB40 } \\
\hline \multicolumn{13}{|l|}{ LAWB41 } \\
\hline \multicolumn{13}{|l|}{ LAWB42S } \\
\hline \multicolumn{13}{|l|}{ LAWB43S } \\
\hline \multicolumn{13}{|l|}{ LAWB44S } \\
\hline LAWB45 & & & & $<0.0031$ & 0.051 & 0.004 & $<0.0027$ & 0.015 & 0.016 & $<0.0243$ & $<0.0532$ & 1.014 \\
\hline \multicolumn{13}{|l|}{ LAWB47 } \\
\hline \multicolumn{13}{|l|}{ LAWB48 } \\
\hline LAWB49 & & & & & & & & & & & & \\
\hline LAWB50 & & & & & & & & & & & & \\
\hline
\end{tabular}


Appendix A. Database - mass fraction

RPP-WTP LAW Formulation (Muller et al. 2001)

\begin{tabular}{|c|c|c|c|c|c|c|c|c|c|c|c|c|c|c|c|c|c|c|c|c|c|}
\hline Glass ID & $\begin{array}{c}\mathrm{Al} 2 \mathrm{O} 3 \\
-\mathrm{t}\end{array}$ & $\begin{array}{c}\mathrm{B} 2 \mathrm{O} 3 \\
-\mathrm{t}\end{array}$ & $\begin{array}{c}\mathrm{CaO} \\
-\mathrm{t}\end{array}$ & $\begin{array}{c}\mathrm{Fe} 2 \mathrm{O} 3 \\
-\mathrm{t}\end{array}$ & $\begin{array}{c}\mathrm{FeO} \\
-\mathrm{t}\end{array}$ & $\begin{array}{c}\mathrm{K} 2 \mathrm{O} \\
-\mathrm{t}\end{array}$ & $\begin{array}{c}\mathrm{Li} 2 \mathrm{O} \\
-\mathrm{t}\end{array}$ & $\begin{array}{c}\mathrm{MgO} \\
-\mathrm{t}\end{array}$ & $\begin{array}{c}\mathrm{Na} 2 \mathrm{O} \\
-\mathrm{t}\end{array}$ & $\begin{array}{c}\mathrm{NiO} \\
-\mathrm{t} \\
\end{array}$ & $\begin{array}{c}\mathrm{P} 2 \mathrm{O} 5 \\
-\mathrm{t}\end{array}$ & $\begin{array}{c}\mathrm{SiO} 2 \\
-\mathrm{t}\end{array}$ & $\begin{array}{c}\mathrm{ZrO} 2 \\
-\mathrm{t}\end{array}$ & $\begin{array}{c}\mathrm{Ag} 2 \mathrm{O} \\
-\mathrm{t}\end{array}$ & $\begin{array}{c}\mathrm{As} 2 \mathrm{O} 3 \\
-\mathrm{t}\end{array}$ & $\begin{array}{c}\mathrm{BaO} \\
-\mathrm{t}\end{array}$ & $\begin{array}{c}\mathrm{Bi} 2 \mathrm{O} 3 \\
-\mathrm{t}\end{array}$ & $\begin{array}{l}\mathrm{Br} \\
-\mathrm{t}\end{array}$ & $\begin{array}{c}\mathrm{CdO} \\
-\mathrm{t} \\
\end{array}$ & $\begin{array}{c}\mathrm{Ce} 2 \mathrm{O} 3 \\
-\mathrm{t}\end{array}$ & $\begin{array}{c}\mathrm{CeO} 2 \\
-\mathrm{t}\end{array}$ \\
\hline LAWB51S & 0.0609 & 0.1254 & 0.0674 & 0.0534 & & 0.0023 & 0.0473 & 0.0300 & 0.0500 & & 0.0001 & 0.4888 & 0.0318 & & & & & & & & \\
\hline LAWB52S & 0.0609 & 0.1002 & 0.0674 & 0.0674 & & 0.0023 & 0.0585 & 0.0300 & 0.0500 & & 0.0001 & 0.4888 & 0.0318 & & & & & & & & \\
\hline LAWB53S & 0.0609 & 0.1002 & 0.0674 & 0.0534 & & 0.0023 & 0.0585 & 0.0300 & 0.0500 & & 0.0001 & 0.4888 & 0.0318 & & & & & & & & \\
\hline LAWC11 for AN107 & 0.1197 & 0.0862 & 0.0160 & 0.0571 & & 0.0016 & & 0.0139 & 0.2200 & 0.0005 & 0.0003 & 0.3830 & 0.0247 & 0.0000 & & 0.0000 & & & 0.0001 & & \\
\hline LAWC12 for AN107 & 0.1197 & 0.0913 & 0.0159 & 0.0571 & & 0.0014 & & 0.0139 & 0.2000 & 0.0004 & 0.0003 & 0.3933 & 0.0246 & 0.0000 & & 0.0000 & & & 0.0001 & & \\
\hline LAWC13 & 0.0612 & 0.0616 & 0.0550 & 0.0347 & & 0.0025 & & 0.0154 & 0.2000 & 0.0003 & 0.0296 & 0.4234 & 0.0308 & & & & & & & & \\
\hline LAWC14 & 0.0612 & 0.0616 & 0.0550 & 0.0347 & & 0.0025 & & 0.0154 & 0.2000 & 0.0003 & 0.0019 & 0.4234 & 0.0308 & & & & & & & & \\
\hline LAWC15* & 0.0623 & 0.0895 & 0.0201 & 0.0702 & & 0.0014 & & 0.0201 & 0.2000 & 0.0004 & 0.0002 & 0.4480 & 0.0301 & & & & & 0.0008 & 0.0000 & & \\
\hline LAWC16S & 0.0613 & 0.1010 & 0.0637 & 0.0752 & & 0.0015 & 0.0397 & 0.0151 & 0.1180 & & 0.0012 & 0.4454 & 0.0302 & & & & & & & & \\
\hline LAWC17S & 0.0613 & 0.1010 & 0.0736 & 0.0450 & & 0.0015 & 0.0298 & 0.0151 & 0.1180 & & 0.0012 & 0.4454 & 0.0302 & & & & & & & & \\
\hline LAWC18S & 0.0613 & 0.1010 & 0.0736 & 0.0752 & & 0.0015 & 0.0298 & 0.0151 & 0.1180 & & 0.0012 & 0.4454 & 0.0302 & & & & & & & & \\
\hline LAWC19S & 0.0613 & 0.1010 & 0.0836 & 0.0752 & & 0.0015 & 0.0199 & 0.0151 & 0.1180 & & 0.0012 & 0.4454 & 0.0302 & & & & & & & & \\
\hline LAWC20S & 0.0613 & 0.1010 & 0.0736 & 0.0748 & & 0.0015 & & 0.0151 & 0.1180 & & 0.0012 & 0.4454 & 0.0302 & & & & & & & & \\
\hline LAWC21S & 0.0613 & 0.1010 & 0.0641 & 0.0648 & & 0.0015 & 0.0274 & 0.0151 & 0.1188 & & 0.0012 & 0.4677 & 0.0302 & & & & & & & & \\
\hline LAWC $22 * *$ & 0.0607 & 0.1005 & 0.0511 & 0.0542 & & 0.0008 & 0.0251 & 0.0151 & 0.1440 & 0.0003 & 0.0007 & 0.4662 & 0.0303 & & & & & 0.0005 & 0.0000 & & \\
\hline LAWC23 & 0.0612 & 0.1008 & 0.0640 & 0.0647 & & 0.0288 & & 0.0151 & 0.1186 & & 0.0012 & 0.4677 & 0.0302 & & & & & & & & \\
\hline LAWC24 & 0.0595 & 0.0980 & 0.0623 & 0.0629 & & 0.0555 & & 0.0147 & 0.1153 & & 0.0012 & 0.4539 & 0.0294 & & & & & & & & \\
\hline LAWC25 & 0.0579 & 0.0954 & 0.0606 & 0.0612 & & 0.0809 & & 0.0143 & 0.1122 & & 0.0011 & 0.4418 & 0.0286 & & & & & & & & \\
\hline LAWABP1 & 0.1000 & 0.0925 & & 0.0250 & & 0.0220 & & 0.0100 & 0.2000 & & 0.0008 & 0.4189 & 0.0525 & & & & & & & & \\
\hline PNLREF (LD6-5412) & 0.1200 & 0.0500 & 0.0400 & 0.0000 & & 0.0146 & & 0.0000 & 0.2000 & & 0.0019 & 0.5591 & & & & & & & & & \\
\hline TFA-BASE (HLP-01) & 0.0700 & 0.1000 & & 0.0550 & & 0.0041 & & 0.0150 & 0.2000 & & 0.0006 & 0.4907 & 0.0150 & & & & & & & & \\
\hline
\end{tabular}


Appendix A. Database - mass fraction

RPP-WTP LAW Formulation (Muller et al. 2001)

\begin{tabular}{|c|c|c|c|c|c|c|c|c|c|c|c|c|c|c|c|c|c|c|c|c|c|}
\hline Glass ID & $\begin{array}{l}\mathrm{Cl} \\
-\mathrm{t}\end{array}$ & $\begin{array}{c}\mathrm{CoO} \\
-\mathrm{t}\end{array}$ & $\begin{array}{c}\mathrm{Co} 2 \mathrm{O} 3 \\
-\mathrm{t}\end{array}$ & $\begin{array}{c}\mathrm{Cr} 2 \mathrm{O} 3 \\
-\mathrm{t}\end{array}$ & $\begin{array}{c}\mathrm{Cs} 2 \mathrm{O} \\
-\mathrm{t}\end{array}$ & $\begin{array}{c}\mathrm{CuO} \\
-\mathrm{t} \\
\end{array}$ & $\begin{array}{c}\mathrm{Eu} 2 \mathrm{O} 3 \\
-\mathrm{t}\end{array}$ & $\begin{array}{l}F \\
-t\end{array}$ & $\begin{array}{c}\mathrm{Ga} 2 \mathrm{O} 3 \\
-\mathrm{t}\end{array}$ & $\begin{array}{c}\mathrm{Gd} 2 \mathrm{O} 3 \\
-\mathrm{t}\end{array}$ & $\begin{array}{c}\mathrm{HgO} \\
-\mathrm{t}\end{array}$ & $\begin{array}{c}\text { I } \\
-t \\
\end{array}$ & $\begin{array}{c}\mathrm{La} 2 \mathrm{O} 3 \\
-\mathrm{t}\end{array}$ & $\begin{array}{c}\mathrm{MnO} 2 \\
-\mathrm{t}\end{array}$ & $\begin{array}{c}\mathrm{MnO} \\
-\mathrm{t}\end{array}$ & $\begin{array}{c}\mathrm{MoO} \\
-\mathrm{t}\end{array}$ & $\begin{array}{c}\mathrm{MoO} 3 \\
-\mathrm{t}\end{array}$ & $\begin{array}{c}\mathrm{Nb} 2 \mathrm{O} 5 \\
-\mathrm{t}\end{array}$ & $\begin{array}{c}\mathrm{Nd} 2 \mathrm{O} 3 \\
-\mathrm{t}\end{array}$ & $\begin{array}{c}\mathrm{PbO} \\
-\mathrm{t}\end{array}$ & $\begin{array}{c}\mathrm{PdO} 2 \\
-\mathrm{t}\end{array}$ \\
\hline LAWB51S & 0.0001 & & & 0.0011 & & & & 0.0006 & & & & & & & & & & & & & \\
\hline LAWB52S & 0.0001 & & & 0.0011 & & & & 0.0006 & & & & & & & & & & & & & \\
\hline LAWB53S & 0.0001 & & & 0.0011 & & & & 0.0006 & & & & & & & & & & & & & \\
\hline LAWC11 for AN107 & 0.0013 & & & 0.0002 & & & & 0.0001 & & & & & & 0.0006 & & & & & & 0.0003 & \\
\hline LAWC12 for AN107 & 0.0012 & & & 0.0002 & & & & 0.0001 & & & & & & 0.0006 & & & & & & 0.0003 & \\
\hline LAWC13 & 0.0020 & & & 0.0002 & 0.0000 & & & 0.0010 & & & & & & 0.0000 & & & & & & & \\
\hline LAWC14 & 0.0020 & & & 0.0002 & 0.0000 & & & 0.0010 & & & & & & 0.0000 & & & & & & & \\
\hline LAWC15* & 0.0008 & & & 0.0000 & 0.0000 & & & 0.0047 & & & & & & & & & 0.0000 & & & 0.0000 & \\
\hline LAWC16S & 0.0012 & & & 0.0002 & & & & 0.0006 & & & & & & 0.0000 & & & & & & & \\
\hline LAWC17S & 0.0012 & & & 0.0002 & & & & 0.0006 & & & & & & 0.0000 & & & & & & & \\
\hline LAWC18S & 0.0012 & & & 0.0002 & & & & 0.0006 & & & & & & 0.0000 & & & & & & & \\
\hline LAWC19S & 0.0012 & & & 0.0002 & & & & 0.0006 & & & & & & 0.0000 & & & & & & & \\
\hline LAWC20S & 0.0012 & & & 0.0002 & & & & 0.0006 & & & & & & 0.0000 & & & & & & & \\
\hline LAWC21S & 0.0012 & & & 0.0002 & & & & 0.0006 & & & & & & 0.0000 & & & & & & & \\
\hline LAWC $22 * *$ & 0.0005 & & & 0.0001 & & & & 0.0034 & & & & & & & & & 0.0000 & & & 0.0001 & \\
\hline LAWC23 & 0.0012 & & & 0.0002 & & & & 0.0006 & & & & & & 0.0000 & & & & & & & \\
\hline LAWC24 & 0.0012 & & & 0.0002 & & & & 0.0006 & & & & & & 0.0000 & & & & & & & \\
\hline LAWC25 & 0.0012 & & & 0.0002 & & & & 0.0006 & & & & & & 0.0000 & & & & & & & \\
\hline LAWABP1 & 0.0058 & & & 0.0002 & & & & 0.0004 & & & & & 0.0200 & & & & & & & & \\
\hline PNLREF (LD6-5412) & 0.0035 & & & 0.0004 & 0.0015 & & & 0.0029 & & & & 0.0013 & & & 0.0000 & & 0.0015 & & & & \\
\hline TFA-BASE (HLP-01) & 0.0028 & & & 0.0008 & & & & & & & & & & & & & & & & & \\
\hline
\end{tabular}


Appendix A. Database - mass fraction

RPP-WTP LAW Formulation (Muller et al. 2001)

\begin{tabular}{|c|c|c|c|c|c|c|c|c|c|c|c|c|c|c|c|c|c|c|c|c|c|}
\hline Glass ID & $\begin{array}{c}\mathrm{PdO} \\
-\mathrm{t}\end{array}$ & $\begin{array}{c}\mathrm{Pr} 2 \mathrm{O} 3 \\
-\mathrm{t}\end{array}$ & $\begin{array}{c}\text { Pr6O11 } \\
-t\end{array}$ & $\begin{array}{c}\mathrm{Rb} 2 \mathrm{O} \\
-\mathrm{t}\end{array}$ & $\begin{array}{c}\mathrm{ReO} \\
-\mathrm{t}\end{array}$ & $\begin{array}{c}\mathrm{ReO} 2 \\
-\mathrm{t}\end{array}$ & $\begin{array}{c}\mathrm{Rh} 2 \mathrm{O} 3 \\
-\mathrm{t}\end{array}$ & $\begin{array}{c}\mathrm{RhO} 2 \\
-\mathrm{t}\end{array}$ & $\begin{array}{c}\mathrm{RuO} 2 \\
-\mathrm{t}\end{array}$ & $\begin{array}{c}\mathrm{Sb} 2 \mathrm{O} 3 \\
-\mathrm{t}\end{array}$ & $\begin{array}{c}\mathrm{Sb} 2 \mathrm{O} 5 \\
-\mathrm{t}\end{array}$ & $\begin{array}{c}\mathrm{SeO} 2 \\
-\mathrm{t}\end{array}$ & $\begin{array}{c}\mathrm{Sm} 2 \mathrm{O} 3 \\
-\mathrm{t}\end{array}$ & $\begin{array}{c}\mathrm{SnO} \\
-\mathrm{t}\end{array}$ & $\begin{array}{c}\mathrm{SnO} 2 \\
-\mathrm{t}\end{array}$ & $\begin{array}{c}\text { SO3 } \\
-t\end{array}$ & $\begin{array}{c}\mathrm{SrO} \\
-\mathrm{t} \\
\end{array}$ & $\begin{array}{c}\mathrm{Tc} 2 \mathrm{O} 7 \\
-\mathrm{t}\end{array}$ & $\begin{array}{c}\mathrm{TeO} 2 \\
-\mathrm{t}\end{array}$ & $\begin{array}{c}\text { ThO2 } \\
-t\end{array}$ & $\begin{array}{c}\mathrm{TiO} 2 \\
-\mathrm{t}\end{array}$ \\
\hline LAWB51S & & & & & & 0.0010 & & & & & & & & & & 0.0106 & & & & & \\
\hline LAWB52S & & & & & & 0.0010 & & & & & & & & & & 0.0106 & & & & & \\
\hline LAWB53S & & & & & & 0.0010 & & & & & & & & & & 0.0110 & & & & & 0.0140 \\
\hline LAWC11 for AN107 & & & & & & & & & & & & & & & & 0.0020 & & & & & 0.0292 \\
\hline LAWC12 for AN107 & & & & & & & & & & & & & & & & 0.0020 & & & & & 0.0341 \\
\hline LAWC13 & & & & & & 0.0010 & & & & & & & & & & 0.0060 & & & & & 0.0154 \\
\hline LAWC14 & & & & & & 0.0010 & & & & & & & & & & 0.0060 & & & & & 0.0154 \\
\hline LAWC15* & & & & & & 0.0010 & & & & & & & & & & 0.0013 & 0.0000 & & & & 0.0200 \\
\hline LAWC16S & & & & & & 0.0010 & & & & & & & & & & 0.0071 & & & & & 0.0112 \\
\hline LAWC17S & & & & & & 0.0010 & & & & & & & & & & 0.0066 & & & & & 0.0112 \\
\hline LAWC18S & & & & & & 0.0010 & & & & & & & & & & 0.0075 & & & & & 0.0112 \\
\hline LAWC19S & & & & & & 0.0010 & & & & & & & & & & 0.0073 & & & & & 0.0112 \\
\hline LAWC20S & & & & & & 0.0010 & & & & & & & & & & 0.0055 & & & & & 0.0112 \\
\hline LAWC21S & & & & & & 0.0010 & & & & & & & & & & 0.0070 & & & & & 0.0112 \\
\hline LAWC22** & & & & & & 0.0010 & & & & & & & & & & 0.0032 & 0.0002 & & & & 0.0114 \\
\hline LAWC23 & & & & & & 0.0010 & & & & & & & & & & 0.0044 & & & & & 0.0112 \\
\hline LAWC24 & & & & & & 0.0010 & & & & & & & & & & 0.0042 & & & & & 0.0109 \\
\hline LAWC25 & & & & & & 0.0009 & & & & & & & & & & 0.0041 & & & & & 0.0106 \\
\hline LAWABP1 & & & & & & & & & & & & & & & & 0.0010 & & & & & 0.0249 \\
\hline PNLREF (LD6-5412) & & & & & & & & & & & & & & & & 0.0021 & 0.0011 & & & & \\
\hline TFA-BASE (HLP-01) & & & & & & & & & & & & & & & & & & & & & 0.0300 \\
\hline
\end{tabular}


Appendix A. Database - mass fraction

RPP-WTP LAW Formulation (Muller et al. 2001)

\begin{tabular}{|c|c|c|c|c|c|c|c|c|c|c|c|c|c|c|c|c|c|c|c|c|c|}
\hline Glass ID & $\begin{array}{c}\mathrm{T} 12 \mathrm{O} 3 \\
-\mathrm{t}\end{array}$ & $\begin{array}{c}\text { U3O8 } \\
-t\end{array}$ & $\begin{array}{c}\mathrm{UO} 2 \\
-\mathrm{t}\end{array}$ & $\begin{array}{c}\text { UO3 } \\
-t\end{array}$ & $\begin{array}{c}\mathrm{V} 2 \mathrm{O} 5 \\
-\mathrm{t}\end{array}$ & $\begin{array}{c}\text { WO3 } \\
-t\end{array}$ & $\begin{array}{c}\mathrm{Y} 2 \mathrm{O} 3 \\
-\mathrm{t}\end{array}$ & $\begin{array}{c}\mathrm{ZnO} \\
-\mathrm{t}\end{array}$ & $\begin{array}{c}\text { Others } \\
-t\end{array}$ & $\begin{array}{c}\text { Sum } \\
-t\end{array}$ & $\begin{array}{c}\mathrm{A} 12 \mathrm{O} 3 \\
-\mathrm{a}\end{array}$ & $\begin{array}{c}\mathrm{B} 2 \mathrm{O} 3 \\
-\mathrm{a}\end{array}$ & $\begin{array}{c}\mathrm{CaO} \\
-\mathrm{a}\end{array}$ & $\begin{array}{c}\mathrm{Fe} 2 \mathrm{O} 3 \\
-\mathrm{a}\end{array}$ & $\begin{array}{c}\mathrm{FeO} \\
-\mathrm{a}\end{array}$ & $\begin{array}{c}\mathrm{K} 2 \mathrm{O} \\
-\mathrm{a}\end{array}$ & $\begin{array}{c}\mathrm{Li} 2 \mathrm{O} \\
-\mathrm{a}\end{array}$ & $\begin{array}{c}\mathrm{MgO} \\
-\mathrm{a}\end{array}$ & $\begin{array}{c}\mathrm{Na} 2 \mathrm{O} \\
-\mathrm{a}\end{array}$ & $\begin{array}{c}\mathrm{NiO} \\
-\mathrm{a}\end{array}$ & $\begin{array}{c}\mathrm{P} 2 \mathrm{O} 5 \\
-\mathrm{a}\end{array}$ \\
\hline LAWB51S & & & & & & & & 0.0318 & & 1.0025 & & & & & & & & & & & \\
\hline LAWB52S & & & & & & & & 0.0318 & & 1.0025 & & & & & & & & & & & \\
\hline LAWB53S & & & & & & & & 0.0318 & & 1.0029 & & & & & & & & & & & \\
\hline LAWC11 for AN107 & & & & & & & & 0.0423 & & 0.9990 & 0.1139 & 0.0882 & 0.0185 & 0.0525 & & 0.0023 & & 0.0131 & 0.1954 & 0.0005 & 0.0019 \\
\hline LAWC12 for AN107 & & & & & & & & 0.0427 & & 0.9991 & 0.0846 & 0.0972 & 0.0192 & 0.0546 & & 0.0022 & & 0.0133 & 0.1863 & 0.0004 & 0.0022 \\
\hline LAWC13 & & & & & 0.0293 & & & 0.0308 & & 1.0000 & 0.0602 & 0.0626 & 0.0529 & 0.0340 & & 0.0027 & 0.0008 & 0.0154 & 0.1679 & 0.0006 & 0.0297 \\
\hline LAWC14 & & & & & 0.0570 & & & 0.0308 & & 1.0000 & 0.0648 & 0.0583 & 0.0530 & 0.0354 & & 0.0026 & 0.0005 & 0.0160 & 0.1814 & 0.0012 & 0.0021 \\
\hline LAWC15* & & & & & & 0.0001 & & 0.0300 & & 1.0010 & 0.0568 & 0.0917 & 0.0201 & 0.0725 & & 0.0018 & 0.0004 & 0.0187 & 0.1789 & 0.0012 & 0.0024 \\
\hline LAWC16S & & & & & & & & 0.0302 & & 1.0039 & & & & & & & & & & & \\
\hline LAWC17S & & & & & 0.0302 & & & 0.0302 & & 1.0033 & & & & & & & & & & & \\
\hline LAWC18S & & & & & & & & 0.0302 & & 1.0042 & & & & & & & & & & & \\
\hline LAWC19S & & & & & & & & 0.0302 & & 1.0041 & & & & & & & & & & & \\
\hline LAWC20S & & & & & 0.0302 & & & 0.0302 & & 1.0022 & & & & & & & & & & & \\
\hline LAWC21S & & & & & & & & 0.0302 & & 1.0046 & & & & & & & & & & & \\
\hline LAWC $22 * *$ & & & & & & 0.0001 & & 0.0307 & & 1.0000 & & & & & & & & & & & \\
\hline LAWC23 & & & & & & & & 0.0301 & & 1.0009 & & & & & & & & & & & \\
\hline LAWC24 & & & & & & & & 0.0293 & & 1.0000 & & & & & & & & & & & \\
\hline LAWC25 & & & & & & & & 0.0285 & & 1.0000 & & & & & & & & & & & \\
\hline LAWABP1 & & & & & & & & 0.0260 & & 1.0000 & & & & & & & & & & & \\
\hline PNLREF (LD6-5412) & & & & & & & & & & 1.0000 & & & & & & & & & & & \\
\hline TFA-BASE (HLP-01) & & & & & & & & 0.0150 & & 0.9990 & & & & & & & & & & & \\
\hline
\end{tabular}


Appendix A. Database - mass fraction

RPP-WTP LAW Formulation (Muller et al. 2001)

\begin{tabular}{|c|c|c|c|c|c|c|c|c|c|c|c|c|c|c|c|c|c|c|c|c|c|}
\hline Glass ID & $\begin{array}{c}\mathrm{SiO} 2 \\
-\mathrm{a}\end{array}$ & $\begin{array}{c}\mathrm{ZrO} 2 \\
-\mathrm{a}\end{array}$ & $\begin{array}{c}\mathrm{Ag} 2 \mathrm{O} \\
-\mathrm{a}\end{array}$ & $\begin{array}{c}\text { As2 } 203 \\
-a\end{array}$ & $\begin{array}{c}\mathrm{BaO} \\
-\mathrm{a}\end{array}$ & $\begin{array}{c}\mathrm{Bi} 2 \mathrm{O} 3 \\
-\mathrm{a}\end{array}$ & $\begin{array}{l}\mathrm{Br} \\
-\mathrm{a}\end{array}$ & $\begin{array}{c}\mathrm{CdO} \\
-\mathrm{a} \\
\end{array}$ & $\begin{array}{c}\mathrm{Ce} 2 \mathrm{O} 3 \\
-\mathrm{a}\end{array}$ & $\begin{array}{c}\mathrm{CeO} 2 \\
-\mathrm{a} \\
\end{array}$ & $\begin{array}{l}\mathrm{Cl} \\
-\mathrm{a} \\
\end{array}$ & $\begin{array}{c}\mathrm{CoO} \\
-\mathrm{a} \\
\end{array}$ & $\begin{array}{c}\mathrm{Co} 2 \mathrm{O} 3 \\
-\mathrm{a} \\
\end{array}$ & $\begin{array}{c}\mathrm{Cr} 2 \mathrm{O} 3 \\
-\mathrm{a} \\
\end{array}$ & $\begin{array}{c}\mathrm{Cs} 2 \mathrm{O} \\
-\mathrm{a} \\
\end{array}$ & $\begin{array}{c}\mathrm{CuO} \\
-\mathrm{a} \\
\end{array}$ & $\begin{array}{c}\mathrm{Eu} 2 \mathrm{O} 3 \\
-\mathrm{a}\end{array}$ & $\begin{array}{c}\mathrm{F} \\
-\mathrm{a} \\
\end{array}$ & $\begin{array}{c}\mathrm{Ga} 2 \mathrm{O} 3 \\
-\mathrm{a} \\
\end{array}$ & $\begin{array}{c}\mathrm{Gd} 2 \mathrm{O} 3 \\
-\mathrm{a}\end{array}$ & $\begin{array}{c}\mathrm{HgO} \\
-\mathrm{a} \\
\end{array}$ \\
\hline \multicolumn{22}{|l|}{ LAWB51S } \\
\hline \multicolumn{22}{|l|}{ LAWB52S } \\
\hline \multicolumn{22}{|l|}{ LAWB53S } \\
\hline LAWC11 for AN107 & 0.3756 & 0.0196 & 0.0001 & & 0.0000 & & & 0.0002 & & & & & & 0.0004 & & & & & & & \\
\hline LAWC12 for AN107 & 0.3885 & 0.0219 & 0.0001 & & 0.0000 & & & 0.0001 & & & & & & 0.0002 & & & & & & & \\
\hline LAWC13 & 0.4124 & 0.0303 & & & 0.0001 & & & & & & & & & 0.0002 & & & & & & & \\
\hline LAWC14 & 0.4032 & 0.0289 & & & 0.0001 & & & & & & & & & 0.0010 & & & & & & & \\
\hline LAWC15* & 0.4382 & 0.0281 & & & 0.0000 & & & & & & & 0.0003 & & 0.0018 & & & & & & & \\
\hline \multicolumn{22}{|l|}{ LAWC16S } \\
\hline \multicolumn{22}{|l|}{ LAWC17S } \\
\hline \multicolumn{22}{|l|}{ LAWC18S } \\
\hline \multicolumn{22}{|l|}{ LAWC19S } \\
\hline \multicolumn{22}{|l|}{ LAWC20S } \\
\hline \multicolumn{22}{|l|}{ LAWC21S } \\
\hline \multicolumn{22}{|l|}{ LAWC22** } \\
\hline \multicolumn{22}{|l|}{ LAWC23 } \\
\hline \multicolumn{22}{|l|}{ LAWC24 } \\
\hline \multicolumn{22}{|l|}{ LAWC25 } \\
\hline \multicolumn{22}{|l|}{ LAWABP1 } \\
\hline PNLREF (LD6-5412) & & & & & & & & & & & & & & & & & & & & & \\
\hline TFA-BASE (HLP-01) & & & & & & & & & & & & & & & & & & & & & \\
\hline
\end{tabular}


Appendix A. Database - mass fraction

RPP-WTP LAW Formulation (Muller et al. 2001)

\begin{tabular}{|c|c|c|c|c|c|c|c|c|c|c|c|c|c|c|c|c|c|c|c|c|c|}
\hline Glass ID & $\begin{array}{c}\mathrm{I} \\
-\mathrm{a}\end{array}$ & $\begin{array}{c}\mathrm{La} 2 \mathrm{O} 3 \\
-\mathrm{a}\end{array}$ & $\begin{array}{c}\mathrm{MnO} 2 \\
-\mathrm{a}\end{array}$ & $\begin{array}{c}\mathrm{MnO} \\
-\mathrm{a}\end{array}$ & $\begin{array}{c}\mathrm{MoO} \\
-\mathrm{a}\end{array}$ & $\begin{array}{c}\mathrm{MoO} 3 \\
-\mathrm{a}\end{array}$ & $\begin{array}{c}\mathrm{Nb} 2 \mathrm{O} 5 \\
-\mathrm{a}\end{array}$ & $\begin{array}{c}\mathrm{Nd} 2 \mathrm{O} 3 \\
-\mathrm{a}\end{array}$ & $\begin{array}{c}\mathrm{PbO} \\
-\mathrm{a}\end{array}$ & $\begin{array}{c}\mathrm{PdO} 2 \\
-\mathrm{a}\end{array}$ & $\begin{array}{c}\mathrm{PdO} \\
-\mathrm{a}\end{array}$ & $\begin{array}{c}\mathrm{Pr} 2 \mathrm{O} 3 \\
-\mathrm{a}\end{array}$ & $\begin{array}{c}\text { Pr6O11 } \\
-\mathrm{a}\end{array}$ & $\begin{array}{c}\mathrm{Rb} 2 \mathrm{O} \\
-\mathrm{a}\end{array}$ & $\begin{array}{c}\mathrm{ReO} \\
-\mathrm{a}\end{array}$ & $\begin{array}{c}\mathrm{ReO} 2 \\
-\mathrm{a}\end{array}$ & $\begin{array}{c}\mathrm{Rh} 2 \mathrm{O} 3 \\
-\mathrm{a}\end{array}$ & $\begin{array}{c}\mathrm{RhO} 2 \\
-\mathrm{a}\end{array}$ & $\begin{array}{c}\mathrm{RuO} 2 \\
-\mathrm{a}\end{array}$ & $\begin{array}{c}\mathrm{Sb} 2 \mathrm{O} 3 \\
-\mathrm{a}\end{array}$ & $\begin{array}{c}\mathrm{Sb} 2 \mathrm{O} 5 \\
-\mathrm{a}\end{array}$ \\
\hline \multicolumn{22}{|l|}{ LAWB51S } \\
\hline \multicolumn{22}{|l|}{ LAWB52S } \\
\hline \multicolumn{22}{|l|}{ LAWB53S } \\
\hline LAWC11 for AN107 & & & 0.0008 & & & & & & 0.0005 & & & & & & & & & & & & \\
\hline LAWC12 for AN107 & & & 0.0005 & & & & & & 0.0005 & & & & & & & & & & & & \\
\hline LAWC13 & & & 0.0000 & & & & & & & & & & & & & & & & & & \\
\hline LAWC14 & & & 0.0002 & & & & & & & & & & & & & & & & & & \\
\hline \multicolumn{22}{|l|}{ LAWC15* } \\
\hline \multicolumn{22}{|l|}{ LAWC16S } \\
\hline \multicolumn{22}{|l|}{ LAWC17S } \\
\hline \multicolumn{22}{|l|}{ LAWC18S } \\
\hline \multicolumn{22}{|l|}{ LAWC19S } \\
\hline \multicolumn{22}{|l|}{ LAWC20S } \\
\hline \multicolumn{22}{|l|}{ LAWC21S } \\
\hline \multicolumn{22}{|l|}{ LAWC $22 * *$} \\
\hline \multicolumn{22}{|l|}{ LAWC23 } \\
\hline \multicolumn{22}{|l|}{ LAWC24 } \\
\hline \multicolumn{22}{|l|}{ LAWC25 } \\
\hline \multicolumn{22}{|l|}{ LAWABP1 } \\
\hline PNLREF (LD6-5412) & & & & & & & & & & & & & & & & & & & & & \\
\hline TFA-BASE (HLP-01) & & & & & & & & & & & & & & & & & & & & & \\
\hline
\end{tabular}


Appendix A. Database - mass fraction

RPP-WTP LAW Formulation (Muller et al. 2001)

\begin{tabular}{|c|c|c|c|c|c|c|c|c|c|c|c|c|c|c|c|c|c|c|c|c|}
\hline Glass ID & $\begin{array}{c}\mathrm{SeO} 2 \\
-\mathrm{a}\end{array}$ & $\begin{array}{c}\mathrm{Sm} 2 \mathrm{O} 3 \\
-\mathrm{a}\end{array}$ & $\begin{array}{c}\mathrm{SnO} \\
-\mathrm{a}\end{array}$ & $\begin{array}{c}\mathrm{SnO} 2 \\
-\mathrm{a}\end{array}$ & $\begin{array}{c}\mathrm{SO} 3 \\
-\mathrm{a}\end{array}$ & $\begin{array}{c}\mathrm{SrO} \\
-\mathrm{a}\end{array}$ & $\begin{array}{c}\mathrm{Tc} 2 \mathrm{O} 7 \\
-\mathrm{a}\end{array}$ & $\begin{array}{c}\mathrm{TeO} 2 \\
-\mathrm{a}\end{array}$ & $\begin{array}{c}\mathrm{ThO} 2 \\
-\mathrm{a}\end{array}$ & $\begin{array}{c}\mathrm{TiO} 2 \\
-\mathrm{a}\end{array}$ & $\begin{array}{c}\mathrm{T} 12 \mathrm{O} 3 \\
-\mathrm{a}\end{array}$ & $\begin{array}{c}\text { U3O8 } \\
-\mathrm{a}\end{array}$ & $\begin{array}{c}\mathrm{UO} 2 \\
-\mathrm{a}\end{array}$ & $\begin{array}{c}\mathrm{UO3} \\
-\mathrm{a}\end{array}$ & $\begin{array}{c}\mathrm{V} 2 \mathrm{O} 5 \\
-\mathrm{a}\end{array}$ & $\begin{array}{c}\text { WO3 } \\
-\mathrm{a}\end{array}$ & $\begin{array}{c}\mathrm{Y} 2 \mathrm{O} 3 \\
-\mathrm{a}\end{array}$ & $\begin{array}{c}\mathrm{ZnO} \\
-\mathrm{a}\end{array}$ & $\begin{array}{c}\text { Others } \\
-\mathrm{a}\end{array}$ & $\begin{array}{c}\text { Sum } \\
-\mathrm{a}\end{array}$ \\
\hline \multicolumn{21}{|l|}{ LAWB51S } \\
\hline \multicolumn{21}{|l|}{ LAWB52S } \\
\hline \multicolumn{21}{|l|}{ LAWB53S } \\
\hline LAWC11 for AN107 & & & & & 0.0018 & & & & & 0.0290 & & & & & & & & 0.0405 & & 0.9546 \\
\hline LAWC12 for AN107 & & & & & 0.0018 & & & & & 0.0336 & & & & & & & & 0.0425 & & 0.9497 \\
\hline LAWC13 & & & & & 0.0018 & & & & & 0.0165 & & & & & 0.0298 & & & 0.0322 & & 0.9500 \\
\hline LAWC14 & & & & & 0.0033 & & & & & 0.0157 & & & & & 0.0550 & & & 0.0309 & & 0.9535 \\
\hline LAWC15* & & & & & 0.0011 & 0.0001 & & & & 0.0190 & & & & & & & & 0.0295 & & 0.9624 \\
\hline \multicolumn{21}{|l|}{ LAWC16S } \\
\hline \multicolumn{21}{|l|}{ LAWC17S } \\
\hline \multicolumn{21}{|l|}{ LAWC18S } \\
\hline \multicolumn{21}{|l|}{ LAWC19S } \\
\hline \multicolumn{21}{|l|}{ LAWC20S } \\
\hline \multicolumn{21}{|l|}{ LAWC21S } \\
\hline \multicolumn{21}{|l|}{ LAWC $22 * *$} \\
\hline \multicolumn{21}{|l|}{ LAWC23 } \\
\hline \multicolumn{21}{|l|}{ LAWC24 } \\
\hline \multicolumn{21}{|l|}{ LAWC25 } \\
\hline \multicolumn{21}{|l|}{ LAWABP1 } \\
\hline PNLREF (LD6-5412) & & & & & & & & & & & & & & & & & & & & \\
\hline TFA-BASE (HLP-01) & & & & & & & & & & & & & & & & & & & & \\
\hline
\end{tabular}


Appendix A. Database - mass fraction

RPP-WTP LAW Formulation (Muller et al. 2001)

\begin{tabular}{|c|c|c|c|c|c|c|c|c|}
\hline Glass ID & $\begin{array}{l}\mathrm{TM} \\
\left({ }^{\circ} \mathrm{C}\right) \\
\end{array}$ & $\begin{array}{l}\text { Gradient } \\
\text { TL }\left({ }^{\circ} \mathrm{C}\right)\end{array}$ & $\begin{array}{l}\text { Uniform } \\
\text { TL }\left({ }^{\circ} \mathrm{C}\right) \\
\end{array}$ & Primary Phase & $\begin{array}{l}\text { Quenched } \\
\text { Visual/OM }\end{array}$ & $\begin{array}{c}\text { Quenched } \\
\text { SEM/EDS or TEM }\end{array}$ & $\begin{array}{l}\text { Quenched } \\
\text { XRD } \\
\end{array}$ & $\begin{array}{c}\text { Quenched } \\
\text { Homogeneous? }\end{array}$ \\
\hline LAWB51S & & & & & & & & \\
\hline LAWB52S & & & & & & & & \\
\hline LAWB53S & & & & & & & & \\
\hline LAWC11 for AN107 & & & & & & & & \\
\hline LAWC12 for AN107 & & & & & & & & \\
\hline LAWC13 & & & & & & & & \\
\hline LAWC14 & & & & & & & & \\
\hline LAWC15* & & & & & & & & \\
\hline LAWC16S & & & & & & & & \\
\hline LAWC17S & & & & & & & & \\
\hline LAWC18S & & & & & & & & \\
\hline LAWC19S & & & & & & & & \\
\hline LAWC20S & & & & & & & & \\
\hline LAWC21S & & & & & & & & \\
\hline LAWC $22 * *$ & & & & & & & & \\
\hline LAWC23 & & & & & & & & \\
\hline LAWC24 & & & & & & & & \\
\hline LAWC25 & & & & & & & & \\
\hline LAWABP1 & & & & & & & & \\
\hline PNLREF (LD6-5412) & & & & & & & & \\
\hline TFA-BASE (HLP-01) & & & & & & & & \\
\hline
\end{tabular}


Appendix A. Database - mass fraction

RPP-WTP LAW Formulation (Muller et al. 2001)

\begin{tabular}{|c|c|c|c|c|c|}
\hline Glass ID & $\begin{array}{c}\mathrm{CCC} \\
\mathrm{Visual} / \mathrm{OM}\end{array}$ & $\begin{array}{c}\text { CCC } \\
\text { SEM/EDS or TEM }\end{array}$ & $\begin{array}{l}\text { CCC } \\
\text { XRD }\end{array}$ & $\begin{array}{l}\text { Heat Treated } \\
\text { Visual/OM }\end{array}$ & $\begin{array}{c}\text { Heat Treated } \\
\text { SEM/EDS or TEM }\end{array}$ \\
\hline LAWB51S & & & & & $\begin{array}{l}\text { After } 1200^{\circ} \mathrm{C} 1 \mathrm{hr} \text { and } 850^{\circ} \mathrm{C} 20 \mathrm{hr}-\sim 0.2 \text { vol. } \% \\
\text { of a Ca-Fe-Mg Silicate }\end{array}$ \\
\hline LAWB52S & & & & & $\begin{array}{l}\text { After } 1200^{\circ} \mathrm{C} 1 \mathrm{hr} \text { and } 850^{\circ} \mathrm{C} 20 \mathrm{hr}-\sim 0.3 \text { vol. } \% \\
\text { of a Ca-Fe-Mg Silicate }\end{array}$ \\
\hline LAWB53S & & & & & $\begin{array}{l}\text { After } 1200^{\circ} \mathrm{C} 1 \mathrm{hr} \text { and } 850^{\circ} \mathrm{C} 20 \mathrm{hr}-\sim 0.3 \text { vol. } \% \\
\text { of a Ca-Fe-Mg Silicate }\end{array}$ \\
\hline \multicolumn{6}{|l|}{ LAWC11 for AN107 } \\
\hline \multicolumn{2}{|l|}{ LAWC12 for AN107 } & & & & $\begin{array}{l}\text { After } 1200^{\circ} \mathrm{C} 1 \mathrm{hr} \text { and } 850^{\circ} \mathrm{C} 20 \mathrm{hr} \text { - Clear } \\
\text { homogeneous glass }\end{array}$ \\
\hline LAWC13 & & & & & $\begin{array}{l}\text { After } 1200^{\circ} \mathrm{C} 1 \mathrm{hr} \text { and } 950^{\circ} \mathrm{C} 20 \mathrm{hr} \text { - Less than } \\
0.1 \text { vol } \% \text { of white clear dendritic crystals }\end{array}$ \\
\hline LAWC14 & & & & & $\begin{array}{l}\text { After } 1200^{\circ} \mathrm{C} 1 \mathrm{hr} \text { and } 950^{\circ} \mathrm{C} 20 \mathrm{hr} \text { - Clear } \\
\text { homogeneous glass }\end{array}$ \\
\hline LAWC15* & & & & & $\begin{array}{l}\text { After } 1200^{\circ} \mathrm{C} 1 \mathrm{hr} \text { and } 950^{\circ} \mathrm{C} 20 \mathrm{hr} \text { - Not Heat } \\
\text { Treated }\end{array}$ \\
\hline LAWC16S & & & & & $\begin{array}{l}\text { After } 1200^{\circ} \mathrm{C} 1 \mathrm{hr} \text { and } 850^{\circ} \mathrm{C} 20 \mathrm{hr}-\text { Clear } \\
\text { homogeneous glass }\end{array}$ \\
\hline LAWC17S & & & & & $\begin{array}{l}\text { After } 1200^{\circ} \mathrm{C} 1 \mathrm{hr} \text { and } 850^{\circ} \mathrm{C} 20 \mathrm{hr} \text { - Clear } \\
\text { homogeneous glass }\end{array}$ \\
\hline LAWC18S & & & & & $\begin{array}{l}\text { After } 1200^{\circ} \mathrm{C} 1 \mathrm{hr} \text { and } 850^{\circ} \mathrm{C} 20 \mathrm{hr} \text { - Clear } \\
\text { homogeneous glass }\end{array}$ \\
\hline LAWC19S & & & & & $\begin{array}{l}\text { After } 1200^{\circ} \mathrm{C} 1 \mathrm{hr} \text { and } 850^{\circ} \mathrm{C} 20 \mathrm{hr} \text { - Clear } \\
\text { homogeneous glass }\end{array}$ \\
\hline LAWC20S & & & & & $\begin{array}{l}\text { After } 1200^{\circ} \mathrm{C} 1 \mathrm{hr} \text { and } 850^{\circ} \mathrm{C} 20 \mathrm{hr} \text { - Clear } \\
\text { homogeneous glass }\end{array}$ \\
\hline LAWC21S & & & & & $\begin{array}{l}\text { After } 1200^{\circ} \mathrm{C} 1 \mathrm{hr} \text { and } 950^{\circ} \mathrm{C} 20 \mathrm{hr}-\text { Clear } \\
\text { homogeneous glass }\end{array}$ \\
\hline LAWC $22 * *$ & & & & & $\begin{array}{l}\text { After } 1200^{\circ} \mathrm{C} 1 \mathrm{hr} \text { and } 950^{\circ} \mathrm{C} 20 \mathrm{hr}-\text { Clear } \\
\text { homogeneous glass; After } 1200^{\circ} \mathrm{C} 1 \mathrm{hr} \text { and } 850^{\circ} \mathrm{C} \\
20 \mathrm{hr} \text { - Clear homogeneous glass }\end{array}$ \\
\hline LAWC23 & & & & & $\begin{array}{l}\text { After } 1200^{\circ} \mathrm{C} 1 \mathrm{hr} \text { and } 950^{\circ} \mathrm{C} 20 \mathrm{hr} \text { - Clear } \\
\text { homogeneous glass }\end{array}$ \\
\hline LAWC24 & & & & & $\begin{array}{l}\text { After } 1200^{\circ} \mathrm{C} 1 \mathrm{hr} \text { and } 950^{\circ} \mathrm{C} 20 \mathrm{hr}-\text { Clear } \\
\text { homogeneous glass for the most part, with white } \\
\text { granular nodule at the crucible contact surface }\end{array}$ \\
\hline LAWC25 & & & & & $\begin{array}{l}\text { After } 1200^{\circ} \mathrm{C} 1 \mathrm{hr} \text { and } 950^{\circ} \mathrm{C} 20 \mathrm{hr} \text { - Clear } \\
\text { homogeneous glass for the most part, with one on } \\
\sim 200 \mathrm{~mm} \text { dia. white granular nodule at the } \\
\text { crucible contact surface }\end{array}$ \\
\hline LAWABP1 & & & & & $\begin{array}{l}\text { After } 1200^{\circ} \mathrm{C} 1 \mathrm{hr} \text { and } 950^{\circ} \mathrm{C} 20 \mathrm{hr} \text { - Clear } \\
\text { homogeneous glass }\end{array}$ \\
\hline PNLREF (LD6-5412) & & & & & \\
\hline TFA-BASE (HLP-01) & & & & & \\
\hline
\end{tabular}


Appendix A. Database - mass fraction

RPP-WTP LAW Formulation (Muller et al. 2001)

\begin{tabular}{|c|c|c|c|c|c|c|c|c|c|c|c|c|c|c|c|}
\hline Glass ID & $\begin{array}{c}\text { Heat Treated } \\
\text { XRD } \\
\end{array}$ & $\begin{array}{l}\text { Density } \\
\left(\mathrm{g} / \mathrm{cm}^{3}\right)\end{array}$ & $\begin{array}{c}\text { Fulc Visc } \\
\text { A }\end{array}$ & $\begin{array}{c}\text { Fulc Visc } \\
\text { B }\end{array}$ & $\begin{array}{c}\text { Fulc Visc } \\
\text { To }\end{array}$ & $\begin{array}{c}\mathrm{FV} 1150^{\circ} \mathrm{C} \\
(\mathrm{Pa} \cdot \mathrm{s})\end{array}$ & $\begin{array}{c}\text { Arrh Visc } \\
\text { A }\end{array}$ & $\begin{array}{c}\text { Arrh Visc } \\
\text { B }\end{array}$ & $\begin{array}{c}1150^{\circ} \mathrm{C} \\
(\mathrm{Daw})\end{array}$ & $\begin{array}{c}\mathrm{T}\left({ }^{\circ} \mathrm{C}\right) \text { at } \\
2 \mathrm{~Pa} \cdot \mathrm{s} \\
\end{array}$ & $\begin{array}{c}\mathrm{T}\left({ }^{\circ} \mathrm{C}\right) \text { at } \\
5 \mathrm{~Pa} \cdot \mathrm{s}\end{array}$ & $\begin{array}{c}\mathrm{T}\left({ }^{\circ} \mathrm{C}\right) \mathrm{at} \\
10 \mathrm{~Pa} \cdot \mathrm{s}\end{array}$ & $\begin{array}{c}\mathrm{T} 1 \\
\left({ }^{\circ} \mathrm{C}\right) \\
\end{array}$ & $\begin{array}{c}\mathrm{V} 1 \\
(\mathrm{~Pa} \cdot \mathrm{s}) \\
\end{array}$ & $\begin{array}{c}\mathrm{T} 2 \\
\left({ }^{\circ} \mathrm{C}\right) \\
\end{array}$ \\
\hline LAWB51S & & & & & & & -13.034 & 21298.8 & 6.91 & & & & 900 & 191.5 & 950 \\
\hline LAWB52S & & & & & & & -12.114 & 19560.8 & 5.11 & & & & 900 & 102.9 & 950 \\
\hline LAWB53S & & 2.67 & & & & & -12.528 & 20045.7 & 4.75 & & & & 900 & 102.8 & 950 \\
\hline LAWC11 for AN107 & & & & & & & -12.483 & 20316.5 & 6.01 & & & & 900 & 135.5 & 950 \\
\hline LAWC12 for AN107 & & 2.69 & & & & & -12.560 & 20343.6 & 5.68 & & & & 900 & 131.9 & 950 \\
\hline LAWC13 & & 2.69 & & & & & -13.823 & 22785.2 & 8.93 & & & & 900 & 321.2 & 950 \\
\hline LAWC14 & & 2.67 & & & & & -13.517 & 21544.8 & 5.07 & & & & 900 & 143.2 & 950 \\
\hline LAWC15* & & 2.68 & & & & & -12.432 & 20561.6 & 7.52 & & & & 900 & 176.1 & 950 \\
\hline LAWC16S & & & & & & & -11.926 & 18581.6 & 3.10 & & & & 900 & 53.7 & 950 \\
\hline \multicolumn{16}{|l|}{ LAWC17S } \\
\hline LAWC18S & & & & & & & -12.437 & 19884.8 & 4.65 & & & & 900 & 98.4 & 950 \\
\hline LAWC19S & & & & & & & -12.822 & 20494.0 & 4.85 & & & & 900 & 111.1 & 950 \\
\hline \multicolumn{16}{|l|}{ LAWC20S } \\
\hline LAWC21S & & 2.66 & & & & & -12.398 & 20130.8 & 5.75 & & & & 900 & 129.3 & 950 \\
\hline LAWC $22 * *$ & & 2.67 & & & & & -11.375 & 18136.1 & 3.94 & & & & 900 & 63.6 & 950 \\
\hline LAWC23 & & & & & & & -13.499 & 23080.7 & 15.19 & & & & 900 & 523.1 & 950 \\
\hline LAWC24 & & & & & & & -13.494 & 22857.8 & 13.05 & & & & 900 & 434.6 & 950 \\
\hline LAWC25 & & & & & & & -13.437 & 22364.5 & 9.77 & & & & 900 & 301.2 & 950 \\
\hline LAWABP1 & & & & & & & -14.107 & 23857.5 & 14.28 & & & & 900 & 554.3 & 950 \\
\hline \multicolumn{16}{|l|}{ PNLREF (LD6-5412) } \\
\hline TFA-BASE (HLP-01) & & & & & & & & & & & & & & & \\
\hline
\end{tabular}


Appendix A. Database - mass fraction

\begin{tabular}{|c|c|c|c|c|c|c|c|c|c|c|c|c|c|c|c|c|c|c|c|c|c|}
\hline \multicolumn{22}{|c|}{ RPP-WTP LAW Formulation (Muller et al. 2001) } \\
\hline Glass ID & $\begin{array}{c}\mathrm{V} 2 \\
(\mathrm{~Pa} \cdot \mathrm{s})\end{array}$ & $\begin{array}{c}\mathrm{T} 3 \\
\left({ }^{\circ} \mathrm{C}\right) \\
\end{array}$ & $\begin{array}{c}\mathrm{V} 3 \\
(\mathrm{~Pa} \cdot \mathrm{s})\end{array}$ & $\begin{array}{c}\mathrm{T} 4 \\
\left({ }^{\circ} \mathrm{C}\right) \\
\end{array}$ & $\begin{array}{c}\mathrm{V} 4 \\
(\mathrm{~Pa} \cdot \mathrm{s})\end{array}$ & $\begin{array}{c}\mathrm{T} 5 \\
\left({ }^{\circ} \mathrm{C}\right) \\
\end{array}$ & $\begin{array}{c}\mathrm{V} 5 \\
(\mathrm{~Pa} \cdot \mathrm{s})\end{array}$ & $\begin{array}{c}\mathrm{T} 6 \\
\left({ }^{\circ} \mathrm{C}\right) \\
\end{array}$ & $\begin{array}{c}\mathrm{V} 6 \\
(\mathrm{~Pa} \cdot \mathrm{s})\end{array}$ & $\begin{array}{c}\mathrm{T} 7 \\
\left({ }^{\circ} \mathrm{C}\right) \\
\end{array}$ & $\begin{array}{c}\mathrm{V} 7 \\
(\mathrm{~Pa} \cdot \mathrm{s})\end{array}$ & $\begin{array}{c}\mathrm{T} 8 \\
\left({ }^{\circ} \mathrm{C}\right) \\
\end{array}$ & $\begin{array}{c}\mathrm{V} 8 \\
(\mathrm{~Pa} \cdot \mathrm{s})\end{array}$ & $\begin{array}{c}\mathrm{T} 9 \\
\left({ }^{\circ} \mathrm{C}\right) \\
\end{array}$ & $\begin{array}{c}\mathrm{V} 9 \\
(\mathrm{~Pa} \cdot \mathrm{s})\end{array}$ & $\begin{array}{l}\mathrm{T} 10 \\
\left({ }^{\circ} \mathrm{C}\right) \\
\end{array}$ & $\begin{array}{c}\mathrm{V} 10 \\
(\mathrm{~Pa} \cdot \mathrm{s})\end{array}$ & $\begin{array}{l}\mathrm{T} 11 \\
\left({ }^{\circ} \mathrm{C}\right) \\
\end{array}$ & $\begin{array}{c}\mathrm{V} 11 \\
(\mathrm{~Pa} \cdot \mathrm{s})\end{array}$ & $\begin{array}{l}\mathrm{T} 12 \\
\left({ }^{\circ} \mathrm{C}\right) \\
\end{array}$ & $\begin{array}{c}\mathrm{V} 12 \\
(\mathrm{~Pa} \cdot \mathrm{s})\end{array}$ \\
\hline LAWB51S & 79.5 & 1000 & 37.5 & 1050 & 19.5 & 1100 & 11.1 & 1150 & 6.7 & 1200 & 4.3 & 1250 & 2.9 & & & & & & & & \\
\hline LAWB52S & 48.4 & 1000 & 24.8 & 1050 & 13.7 & 1100 & 8.1 & 1150 & 5 & 1200 & 3.3 & 1250 & 2.2 & & & & & & & & \\
\hline LAWB53S & 47.4 & 1000 & 24 & 1050 & 13.1 & 1100 & 7.6 & 1150 & 4.7 & 1200 & 3 & 1250 & 2 & & & & & & & & \\
\hline LAWC11 for AN107 & 62 & 1000 & 31.1 & 1050 & 16.8 & 1100 & 9.7 & 1150 & 5.9 & 1200 & 3.8 & 1250 & 2.5 & & & & & & & & \\
\hline LAWC12 for AN107 & 58.6 & 1000 & 28.9 & 1050 & 15.6 & 1100 & 9 & 1150 & 5.6 & 1200 & 3.6 & 1250 & 2.4 & & & & & & & & \\
\hline LAWC13 & 121.1 & 1000 & 53.4 & 1050 & 26.6 & 1100 & 14.6 & 1150 & 8.6 & 1200 & 5.4 & 1250 & 3.6 & & & & & & & & \\
\hline LAWC14 & 60.2 & 1000 & 28.4 & 1050 & 14.7 & 1100 & 8.2 & 1150 & 4.9 & 1200 & 3.1 & 1250 & 2.1 & & & & & & & & \\
\hline LAWC15* & 79.7 & 1000 & 39.6 & 1050 & 21.2 & 1100 & 12.2 & 1150 & 7.4 & 1200 & 4.7 & 1250 & 3.1 & & & & & & & & \\
\hline LAWC16S & 26.2 & 1000 & 13.9 & 1050 & 7.9 & 1100 & 4.8 & 1150 & 3.1 & 1200 & 2 & 1250 & 1.4 & & & & & & & & \\
\hline \multicolumn{22}{|l|}{ LAWC17S } \\
\hline LAWC18S & 45.6 & 1000 & 23.1 & 1050 & 12.7 & 1100 & 7.4 & 1150 & 4.6 & 1200 & 2.9 & 1250 & 2 & & & & & & & & \\
\hline LAWC19S & 51.1 & 1000 & 25.6 & 1050 & 13.8 & 1100 & 7.9 & 1150 & 4.8 & 1200 & 3 & 1250 & 2 & & & & & & & & \\
\hline \multicolumn{22}{|l|}{ LAWC20S } \\
\hline LAWC21S & 57.9 & 1000 & 28.8 & 1050 & 15.6 & 1100 & 9.1 & 1150 & 5.6 & 1200 & 3.6 & 1250 & 2.5 & & & & & & & & \\
\hline LAWC $22 * *$ & 31.6 & 1000 & 17 & 1050 & 9.8 & 1100 & 6 & 1150 & 3.9 & 1200 & 2.6 & 1250 & 1.8 & & & & & & & & \\
\hline LAWC23 & 214.9 & 1000 & 98 & 1050 & 48.8 & 1100 & 26.1 & 1150 & 14.9 & 1200 & 9 & 1250 & 5.6 & & & & & & & & \\
\hline LAWC24 & 180.1 & 1000 & 82.7 & 1050 & 41.5 & 1100 & 22.3 & 1150 & 12.8 & 1200 & 7.7 & 1250 & 4.9 & & & & & & & & \\
\hline LAWC25 & 127.2 & 1000 & 59.5 & 1050 & 30.3 & 1100 & 16.5 & 1150 & 9.6 & 1200 & 5.9 & 1250 & 3.7 & & & & & & & & \\
\hline LAWABP1 & 220.9 & 1000 & 98.1 & 1050 & 47.7 & 1100 & 25 & 1150 & 14 & 1200 & 8.3 & 1250 & 5.1 & & & & & & & & \\
\hline PNLREF (LD6-5412) & & & & & & & & & & & & & & & & & & & & & \\
\hline TFA-BASE (HLP-01) & & & & & & & & & & & & & & & & & & & & & \\
\hline
\end{tabular}


Appendix A. Database - mass fraction

RPP-WTP LAW Formulation (Muller et al. 2001)

\begin{tabular}{|c|c|c|c|c|c|c|c|c|c|c|c|c|c|c|c|c|}
\hline Glass ID & $\begin{array}{l}\mathrm{T} 13 \\
\left({ }^{\circ} \mathrm{C}\right) \\
\end{array}$ & $\begin{array}{c}\mathrm{V} 13 \\
(\mathrm{~Pa} \cdot \mathrm{s}) \\
\end{array}$ & $\begin{array}{l}\mathrm{T} 14 \\
\left({ }^{\circ} \mathrm{C}\right) \\
\end{array}$ & $\begin{array}{c}\mathrm{V} 14 \\
(\mathrm{~Pa} \cdot \mathrm{s})\end{array}$ & $\begin{array}{c}\text { Q PCT } \\
\text { B }\left(\mathrm{g} / \mathrm{m}^{2}\right)\end{array}$ & $\begin{array}{c}\text { Q PCT } \\
\mathrm{Li}\left(\mathrm{g} / \mathrm{m}^{2}\right)\end{array}$ & $\begin{array}{c}\text { Q PCT } \\
\mathrm{Na}\left(\mathrm{g} / \mathrm{m}^{2}\right) \\
\end{array}$ & $\begin{array}{c}\text { Q PCT } \\
\mathrm{Si}\left(\mathrm{g} / \mathrm{m}^{2}\right)\end{array}$ & $\begin{array}{c}\text { Q PCT } \\
\text { pH }\end{array}$ & $\begin{array}{c}\text { CCC PCT } \\
\mathrm{B}\left(\mathrm{g} / \mathrm{m}^{2}\right) \\
\end{array}$ & $\begin{array}{l}\text { CCC PCT } \\
\mathrm{Li}\left(\mathrm{g} / \mathrm{m}^{2}\right) \\
\end{array}$ & $\begin{array}{l}\text { CCC PCT } \\
\mathrm{Na}\left(\mathrm{g} / \mathrm{m}^{2}\right) \\
\end{array}$ & $\begin{array}{c}\text { CCC PCT } \\
\mathrm{Si}\left(\mathrm{g} / \mathrm{m}^{2}\right) \\
\end{array}$ & $\begin{array}{c}\text { CCC PCT } \\
\mathrm{pH}\end{array}$ & $\begin{array}{l}\text { Q PCT B at } \\
20^{\circ} \mathrm{C}\left(\mathrm{g} / \mathrm{m}^{2}\right)\end{array}$ & $\begin{array}{l}\text { Q PCT Li at } \\
20^{\circ} \mathrm{C}\left(\mathrm{g} / \mathrm{m}^{2}\right)\end{array}$ \\
\hline LAWB51S & & & & & 0.48 & & 0.33 & 0.14 & 9.61 & & & & & & & \\
\hline LAWB52S & & & & & 0.49 & & 0.34 & 0.16 & 9.74 & & & & & & & \\
\hline LAWB53S & & & & & 0.42 & & 0.27 & 0.14 & 10.08 & & & & & & & \\
\hline LAWC11 for AN107 & & & & & & & & & & & & & & & & \\
\hline LAWC12 for AN107 & & & & & 0.42 & & 0.409 & 0.184 & 10.81 & & & & & & & \\
\hline LAWC13 & & & & & 0.354 & & 0.37 & 0.172 & 10.43 & & & & & & & \\
\hline LAWC14 & & & & & 0.54 & & 3.609 & 0.208 & 11.22 & & & & & & & \\
\hline LAWC15* & & & & & 0.329 & & 0.335 & 0.161 & 10.45 & & & & & & & \\
\hline LAWC16S & & & & & 0.584 & & 0.408 & 0.178 & 10.12 & & & & & & & \\
\hline LAWC17S & & & & & 0.399 & & 0.294 & 0.137 & 10.02 & & & & & & & \\
\hline LAWC18S & & & & & 0.413 & & 0.295 & 0.136 & 10.22 & & & & & & & \\
\hline LAWC19S & & & & & 0.232 & & 0.225 & 0.088 & 9.84 & & & & & & & \\
\hline LAWC20S & & & & & 0.244 & & 0.189 & 0.094 & 9.53 & & & & & & & \\
\hline LAWC21S & & & & & 0.15 & & 0.172 & 0.08 & 10.02 & & & & & & & \\
\hline LAWC $22 * *$ & & & & & 0.518 & & 0.469 & 0.181 & 10.53 & & & & & & & \\
\hline LAWC23 & & & & & 0.239 & & 0.273 & 0.096 & 9.74 & & & & & & & \\
\hline LAWC24 & & & & & 0.221 & & 0.282 & 0.092 & 9.62 & & & & & & & \\
\hline LAWC25 & & & & & 0.32 & & 0.385 & 0.109 & 9.76 & & & & & & & \\
\hline LAWABP1 & & & & & 0.29 & & 0.31 & 0.13 & 10.43 & & & & & & & \\
\hline PNLREF (LD6-5412) & & & & & 0.1 & & 0.27 & 0.09 & 10.61 & & & & & & & \\
\hline TFA-BASE (HLP-01) & & & & & 0.39 & & 0.33 & 0.16 & 10.21 & & & & & & & \\
\hline
\end{tabular}


Appendix A. Database - mass fraction

RPP-WTP LAW Formulation (Muller et al. 2001)

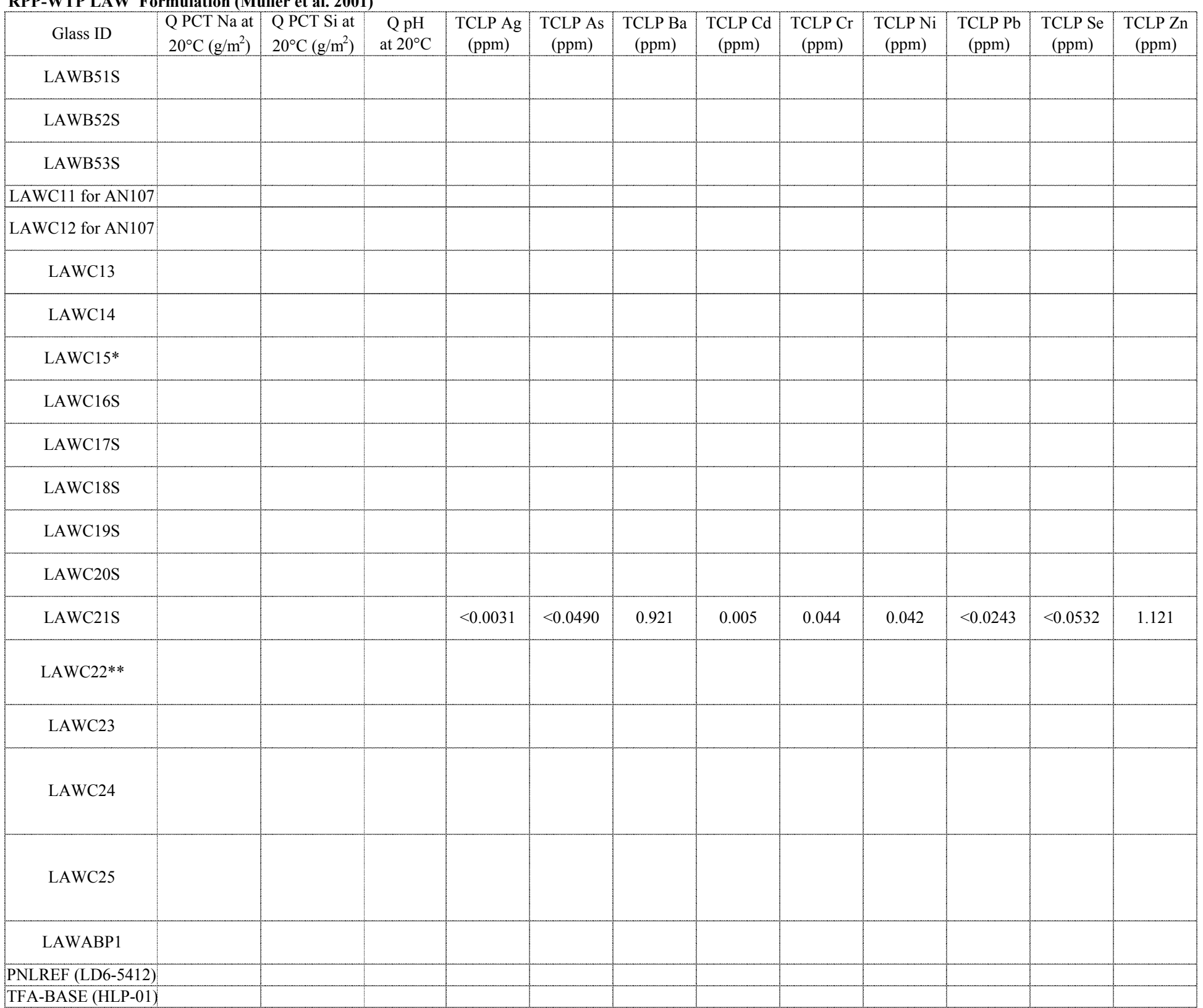


Appendix A. Database - mass fraction

DP Glasses for INEEL HLW (Pittman et al. 2001)

\begin{tabular}{|c|c|c|c|c|c|c|c|c|c|c|c|c|c|c|c|c|c|c|c|c|c|}
\hline Glass ID & $\begin{array}{c}\mathrm{A} 12 \mathrm{O} 3 \\
-\mathrm{t}\end{array}$ & $\begin{array}{c}\mathrm{B} 2 \mathrm{O} 3 \\
-\mathrm{t}\end{array}$ & $\begin{array}{c}\mathrm{CaO} \\
-\mathrm{t}\end{array}$ & $\begin{array}{c}\mathrm{Fe} 2 \mathrm{O} 3 \\
-\mathrm{t}\end{array}$ & $\begin{array}{c}\mathrm{FeO} \\
-\mathrm{t}\end{array}$ & $\begin{array}{c}\mathrm{K} 2 \mathrm{O} \\
-\mathrm{t}\end{array}$ & $\begin{array}{c}\mathrm{Li} 2 \mathrm{O} \\
-\mathrm{t}\end{array}$ & $\begin{array}{c}\mathrm{MgO} \\
-\mathrm{t}\end{array}$ & $\begin{array}{c}\mathrm{Na} 2 \mathrm{O} \\
-\mathrm{t}\end{array}$ & $\begin{array}{c}\mathrm{NiO} \\
-\mathrm{t} \\
\end{array}$ & $\begin{array}{c}\mathrm{P} 2 \mathrm{O} 5 \\
-\mathrm{t}\end{array}$ & $\begin{array}{c}\mathrm{SiO} 2 \\
-\mathrm{t} \\
\end{array}$ & $\begin{array}{c}\mathrm{ZrO} 2 \\
-\mathrm{t}\end{array}$ & $\begin{array}{c}\mathrm{Ag} 2 \mathrm{O} \\
-\mathrm{t}\end{array}$ & $\begin{array}{c}\mathrm{As} 2 \mathrm{O} 3 \\
-\mathrm{t}\end{array}$ & $\begin{array}{c}\mathrm{BaO} \\
-\mathrm{t}\end{array}$ & $\begin{array}{c}\mathrm{Bi} 2 \mathrm{O} 3 \\
-\mathrm{t}\end{array}$ & $\begin{array}{l}\mathrm{Br} \\
-\mathrm{t}\end{array}$ & $\begin{array}{c}\mathrm{CdO} \\
-\mathrm{t} \\
\end{array}$ & $\begin{array}{c}\mathrm{Ce} 2 \mathrm{O} 3 \\
-\mathrm{t}\end{array}$ & $\begin{array}{c}\mathrm{CeO} 2 \\
-t\end{array}$ \\
\hline DP-1 & 0.1250 & 0.0600 & 0.1500 & 0.0000 & & & 0.0700 & & 0.0800 & & 0.0000 & 0.3650 & 0.0800 & & & & & & & & \\
\hline DP-2 & 0.1250 & 0.0600 & 0.1500 & 0.0100 & & & 0.0450 & & 0.1300 & & 0.0000 & 0.3600 & 0.0400 & & & & & & & & \\
\hline DP-3 & 0.1250 & 0.0600 & 0.0950 & 0.0500 & & & 0.0700 & & 0.0800 & & 0.0000 & 0.3750 & 0.0400 & & & & & & & & \\
\hline DP-4 & 0.1250 & 0.0600 & 0.0950 & 0.0000 & & & 0.0450 & & 0.0800 & & 0.0250 & 0.3600 & 0.0800 & & & & & & & & \\
\hline DP-5 & 0.1250 & 0.0600 & 0.0950 & 0.0500 & & & 0.0450 & & 0.0800 & & 0.0250 & 0.3600 & 0.0800 & & & & & & & & \\
\hline DP-6 & 0.1250 & 0.0600 & 0.0950 & 0.0000 & & & 0.0700 & & 0.1300 & & 0.0250 & 0.3600 & 0.0400 & & & & & & & & \\
\hline DP-7 & 0.1250 & 0.1350 & 0.0950 & 0.0000 & & & 0.0700 & & 0.0800 & & 0.0000 & 0.3600 & 0.0400 & & & & & & & & \\
\hline DP-8 & 0.0700 & 0.1500 & 0.0950 & 0.0000 & & & 0.0450 & & 0.0800 & & 0.0250 & 0.3600 & 0.0800 & & & & & & & & \\
\hline DP-9 & 0.0700 & 0.1500 & 0.0950 & 0.0000 & & & 0.0450 & & 0.1300 & & 0.0250 & 0.3650 & 0.0400 & & & & & & & & \\
\hline DP-10 & 0.0700 & 0.1500 & 0.1000 & 0.0000 & & & 0.0700 & & 0.0800 & & 0.0000 & 0.3600 & 0.0400 & & & & & & & & \\
\hline DP-11 & 0.0700 & 0.1500 & 0.1000 & 0.0500 & & & 0.0700 & & 0.0800 & & 0.0000 & 0.3600 & 0.0400 & & & & & & & & \\
\hline DP-12 & 0.0700 & 0.0600 & 0.1500 & 0.0500 & & & 0.0450 & & 0.0800 & & 0.0250 & 0.3600 & 0.0400 & & & & & & & & \\
\hline DP-13 & 0.0700 & 0.0600 & 0.1500 & 0.0000 & & & 0.0700 & & 0.0800 & & 0.0250 & 0.3600 & 0.0800 & & & & & & & & \\
\hline DP-14 & 0.0700 & 0.0600 & 0.1500 & 0.0500 & & & 0.0700 & & 0.0800 & & 0.0250 & 0.3600 & 0.0400 & & & & & & & & \\
\hline DP-15 & 0.0700 & 0.0600 & 0.1500 & 0.0000 & & & 0.0450 & & 0.0800 & & 0.0000 & 0.4500 & 0.0400 & & & & & & & & \\
\hline DP-16 & 0.0700 & 0.0600 & 0.0950 & 0.0500 & & & 0.0450 & & 0.0800 & & 0.0000 & 0.4650 & 0.0400 & & & & & & & & \\
\hline DP-17 & 0.0700 & 0.0600 & 0.0950 & 0.0000 & & & 0.0700 & & 0.1300 & & 0.0250 & 0.4150 & 0.0400 & & & & & & & & \\
\hline DP-18 & 0.0700 & 0.0600 & 0.0950 & 0.0500 & & & 0.0450 & & 0.1300 & & 0.0000 & 0.3650 & 0.0800 & & & & & & & & \\
\hline DP-19 & 0.0700 & 0.0600 & 0.0950 & 0.0500 & & & 0.0700 & & 0.1300 & & 0.0000 & 0.3750 & 0.0800 & & & & & & & & \\
\hline DP-20 & 0.0700 & 0.0600 & 0.1000 & 0.0000 & & & 0.0450 & & 0.0800 & & 0.0000 & 0.4700 & 0.0800 & & & & & & & & \\
\hline DP-21 & 0.0700 & 0.1450 & 0.1500 & 0.0000 & & & 0.0450 & & 0.0800 & & 0.0000 & 0.3600 & 0.0800 & & & & & & & & \\
\hline DP-22 & 0.0700 & 0.0650 & 0.0950 & 0.0000 & & & 0.0700 & & 0.1300 & & 0.0000 & 0.3600 & 0.0800 & & & & & & & & \\
\hline DP-23 & 0.1200 & 0.0600 & 0.1500 & 0.0000 & & & 0.0450 & & 0.1300 & & 0.0000 & 0.3600 & 0.0400 & & & & & & & & \\
\hline DP-24 & 0.0800 & 0.0600 & 0.0950 & 0.0000 & & & 0.0700 & & 0.0800 & & 0.0250 & 0.4700 & 0.0400 & & & & & & & & \\
\hline DP-BL1 & 0.0900 & 0.0900 & 0.1200 & 0.0250 & & & 0.0500 & & 0.1050 & & 0.0150 & 0.3875 & 0.0500 & & & & & & & & \\
\hline DP-BL2 & 0.0859 & 0.0509 & 0.1132 & 0.0300 & & & 0.0600 & & 0.1137 & & 0.0117 & 0.4228 & 0.0471 & & & & & & & & \\
\hline DP-centroid & 0.0890 & 0.0819 & 0.1140 & 0.0183 & & & 0.0558 & & 0.0983 & & 0.0108 & 0.3802 & 0.0555 & & & & & & & & \\
\hline
\end{tabular}

\section{INEEL DZr-CV Glasses (Riley et al. 2001)}

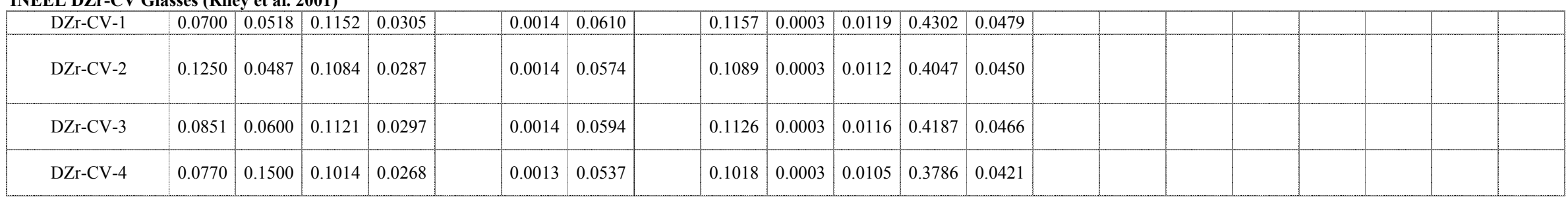


Appendix A. Database - mass fraction

DP Glasses for INEEL HLW (Pittman et al. 2001)

\begin{tabular}{|c|c|c|c|c|c|c|c|c|c|c|c|c|c|c|c|c|c|c|c|c|c|}
\hline Glass ID & $\begin{array}{l}\mathrm{Cl} \\
-\mathrm{t}\end{array}$ & $\begin{array}{c}\mathrm{CoO} \\
-\mathrm{t}\end{array}$ & $\begin{array}{c}\mathrm{Co} 2 \mathrm{O} 3 \\
-\mathrm{t}\end{array}$ & $\begin{array}{c}\mathrm{Cr} 2 \mathrm{O} 3 \\
-\mathrm{t}\end{array}$ & $\begin{array}{c}\mathrm{Cs} 2 \mathrm{O} \\
-\mathrm{t}\end{array}$ & $\begin{array}{c}\mathrm{CuO} \\
-\mathrm{t}\end{array}$ & $\begin{array}{c}\mathrm{Eu} 2 \mathrm{O} 3 \\
-\mathrm{t}\end{array}$ & $\begin{array}{l}F \\
-t\end{array}$ & $\begin{array}{c}\mathrm{Ga} 2 \mathrm{O} 3 \\
-\mathrm{t}\end{array}$ & $\begin{array}{c}\mathrm{Gd} 2 \mathrm{O} 3 \\
-\mathrm{t}\end{array}$ & $\begin{array}{c}\mathrm{HgO} \\
-\mathrm{t}\end{array}$ & $\begin{array}{l}I \\
-t\end{array}$ & $\begin{array}{c}\mathrm{La} 2 \mathrm{O} 3 \\
-\mathrm{t}\end{array}$ & $\begin{array}{c}\mathrm{MnO} 2 \\
-\mathrm{t}\end{array}$ & $\begin{array}{c}\mathrm{MnO} \\
-\mathrm{t}\end{array}$ & $\begin{array}{c}\mathrm{MoO} \\
-\mathrm{t}\end{array}$ & $\begin{array}{c}\mathrm{MoO} 3 \\
-\mathrm{t}\end{array}$ & $\begin{array}{c}\mathrm{Nb} 2 \mathrm{O} 5 \\
-\mathrm{t}\end{array}$ & $\begin{array}{c}\mathrm{Nd} 2 \mathrm{O} 3 \\
-\mathrm{t}\end{array}$ & $\begin{array}{c}\mathrm{PbO} \\
-\mathrm{t}\end{array}$ & $\begin{array}{c}\mathrm{PdO} 2 \\
-\mathrm{t}\end{array}$ \\
\hline DP-1 & & & & & & & & 0.0400 & & & & & 0.0250 & & & & & & & & \\
\hline DP-2 & & & & & & & & 0.0400 & & & & & 0.0250 & & & & & & & & \\
\hline DP-3 & & & & & & & & 0.0650 & & & & & 0.0250 & & & & & & & & \\
\hline DP-4 & & & & & & & & 0.0650 & & & & & 0.0500 & & & & & & & & \\
\hline DP-5 & & & & & & & & 0.0400 & & & & & 0.0250 & & & & & & & & \\
\hline DP-6 & & & & & & & & 0.0400 & & & & & 0.0500 & & & & & & & & \\
\hline DP-7 & & & & & & & & 0.0400 & & & & & 0.0500 & & & & & & & & \\
\hline DP-8 & & & & & & & & 0.0650 & & & & & 0.0250 & & & & & & & & \\
\hline DP-9 & & & & & & & & 0.0400 & & & & & 0.0250 & & & & & & & & \\
\hline DP-10 & & & & & & & & 0.0650 & & & & & 0.0500 & & & & & & & & \\
\hline DP-11 & & & & & & & & 0.0400 & & & & & 0.0250 & & & & & & & & \\
\hline DP-12 & & & & & & & & 0.0650 & & & & & 0.0500 & & & & & & & & \\
\hline DP-13 & & & & & & & & 0.0650 & & & & & 0.0250 & & & & & & & & \\
\hline DP-14 & & & & & & & & 0.0400 & & & & & 0.0500 & & & & & & & & \\
\hline DP-15 & & & & & & & & 0.0400 & & & & & 0.0500 & & & & & & & & \\
\hline DP-16 & & & & & & & & 0.0650 & & & & & 0.0250 & & & & & & & & \\
\hline DP-17 & & & & & & & & 0.0650 & & & & & 0.0250 & & & & & & & & \\
\hline DP-18 & & & & & & & & 0.0400 & & & & & 0.0500 & & & & & & & & \\
\hline DP-19 & & & & & & & & 0.0400 & & & & & 0.0250 & & & & & & & & \\
\hline DP-20 & & & & & & & & 0.0400 & & & & & 0.0500 & & & & & & & & \\
\hline DP-21 & & & & & & & & 0.0400 & & & & & 0.0250 & & & & & & & & \\
\hline DP-22 & & & & & & & & 0.0650 & & & & & 0.0500 & & & & & & & & \\
\hline DP-23 & & & & & & & & 0.0650 & & & & & 0.0250 & & & & & & & & \\
\hline DP-24 & & & & & & & & 0.0400 & & & & & 0.0250 & & & & & & & & \\
\hline DP-BL1 & & & & & & & & 0.0500 & & & & & 0.0000 & & & & & & & & \\
\hline DP-BL2 & & & & & & & & 0.0470 & & & & & 0.0000 & & & & & & & & \\
\hline DP-centroid & & & & & & & & 0.0508 & & & & & 0.0358 & & & & & & & & \\
\hline
\end{tabular}

INEEL DZr-CV Glasses (Riley et al. 2001)

\begin{tabular}{|c|c|c|c|c|c|c|}
\hline $\mathrm{DZr}-\mathrm{CV}-1$ & 0.0003 & 0.0014 & 0.0478 & 0.0000 & 0.0013 & 0.0002 \\
\hline DZr-CV-2 & 0.0003 & 0.0014 & 0.0450 & 0.0000 & 0.0012 & 0.0002 \\
\hline DZr-CV-3 & 0.0003 & 0.0014 & 0.0466 & 0.0000 & 0.0012 & 0.0002 \\
\hline DZr-CV-4 & 0.0003 & 0.0014 & 0.0421 & 0.0000 & 0.0011 & 0.0001 \\
\hline
\end{tabular}


DP Glasses for INEEL HLW (Pittman et al. 2001)

\begin{tabular}{|c|c|c|c|c|c|c|c|c|c|c|c|c|c|c|c|c|c|c|c|c|c|}
\hline Glass ID & $\begin{array}{c}\mathrm{PdO} \\
-\mathrm{t}\end{array}$ & $\begin{array}{c}\mathrm{Pr} 2 \mathrm{O} 3 \\
-\mathrm{t}\end{array}$ & $\begin{array}{c}\text { Pr6O11 } \\
-t\end{array}$ & $\begin{array}{c}\mathrm{Rb} 2 \mathrm{O} \\
-\mathrm{t}\end{array}$ & $\begin{array}{c}\mathrm{ReO} \\
-\mathrm{t}\end{array}$ & $\begin{array}{c}\mathrm{ReO} 2 \\
-\mathrm{t}\end{array}$ & $\begin{array}{c}\mathrm{Rh} 2 \mathrm{O} 3 \\
-\mathrm{t} \\
\end{array}$ & $\begin{array}{c}\mathrm{RhO} 2 \\
-\mathrm{t}\end{array}$ & $\begin{array}{c}\mathrm{RuO} 2 \\
-\mathrm{t}\end{array}$ & $\begin{array}{c}\mathrm{Sb} 2 \mathrm{O} 3 \\
-\mathrm{t} \\
\end{array}$ & $\begin{array}{c}\mathrm{Sb} 2 \mathrm{O} 5 \\
-\mathrm{t}\end{array}$ & $\begin{array}{c}\mathrm{SeO} 2 \\
-\mathrm{t}\end{array}$ & $\begin{array}{c}\mathrm{Sm} 2 \mathrm{O} 3 \\
-\mathrm{t} \\
\end{array}$ & $\begin{array}{c}\mathrm{SnO} \\
-\mathrm{t} \\
\end{array}$ & $\begin{array}{c}\mathrm{SnO} 2 \\
-\mathrm{t}\end{array}$ & $\begin{array}{c}\mathrm{SO} 3 \\
-\mathrm{t} \\
\end{array}$ & $\begin{array}{c}\mathrm{SrO} \\
-\mathrm{t} \\
\end{array}$ & $\begin{array}{c}\mathrm{Tc} 2 \mathrm{O} 7 \\
-\mathrm{t} \\
\end{array}$ & $\begin{array}{c}\mathrm{TeO} 2 \\
-\mathrm{t}\end{array}$ & $\begin{array}{c}\mathrm{ThO} 2 \\
-\mathrm{t}\end{array}$ & $\begin{array}{c}\mathrm{TiO} 2 \\
-\mathrm{t} \\
\end{array}$ \\
\hline DP-1 & & & & & & & & & & & & & & & & 0.0050 & & & & & \\
\hline DP-2 & & & & & & & & & & & & & & & & 0.0150 & & & & & \\
\hline DP-3 & & & & & & & & & & & & & & & & 0.0150 & & & & & \\
\hline DP-4 & & & & & & & & & & & & & & & & 0.0150 & & & & & \\
\hline DP-5 & & & & & & & & & & & & & & & & 0.0150 & & & & & \\
\hline DP-6 & & & & & & & & & & & & & & & & 0.0050 & & & & & \\
\hline DP-7 & & & & & & & & & & & & & & & & 0.0050 & & & & & \\
\hline DP-8 & & & & & & & & & & & & & & & & 0.0050 & & & & & \\
\hline DP-9 & & & & & & & & & & & & & & & & 0.0150 & & & & & \\
\hline DP-10 & & & & & & & & & & & & & & & & 0.0150 & & & & & \\
\hline DP-11 & & & & & & & & & & & & & & & & 0.0150 & & & & & \\
\hline DP-12 & & & & & & & & & & & & & & & & 0.0050 & & & & & \\
\hline DP-13 & & & & & & & & & & & & & & & & 0.0150 & & & & & \\
\hline DP-14 & & & & & & & & & & & & & & & & 0.0050 & & & & & \\
\hline DP-15 & & & & & & & & & & & & & & & & 0.0150 & & & & & \\
\hline DP-16 & & & & & & & & & & & & & & & & 0.0050 & & & & & \\
\hline DP-17 & & & & & & & & & & & & & & & & 0.0050 & & & & & \\
\hline DP-18 & & & & & & & & & & & & & & & & 0.0150 & & & & & \\
\hline DP-19 & & & & & & & & & & & & & & & & 0.0050 & & & & & \\
\hline DP-20 & & & & & & & & & & & & & & & & 0.0050 & & & & & \\
\hline DP-21 & & & & & & & & & & & & & & & & 0.0050 & & & & & \\
\hline DP-22 & & & & & & & & & & & & & & & & 0.0150 & & & & & \\
\hline DP-23 & & & & & & & & & & & & & & & & 0.0050 & & & & & \\
\hline DP-24 & & & & & & & & & & & & & & & & 0.0150 & & & & & \\
\hline DP-BL1 & & & & & & & & & & & & & & & & 0.0100 & & & & & \\
\hline DP-BL2 & & & & & & & & & & & & & & & & 0.0105 & & & & & \\
\hline DP-centroid & & & & & & & & & & & & & & & & 0.0098 & & & & & \\
\hline
\end{tabular}

\section{INEEL DZr-CV Glasses (Riley et al. 2001)}

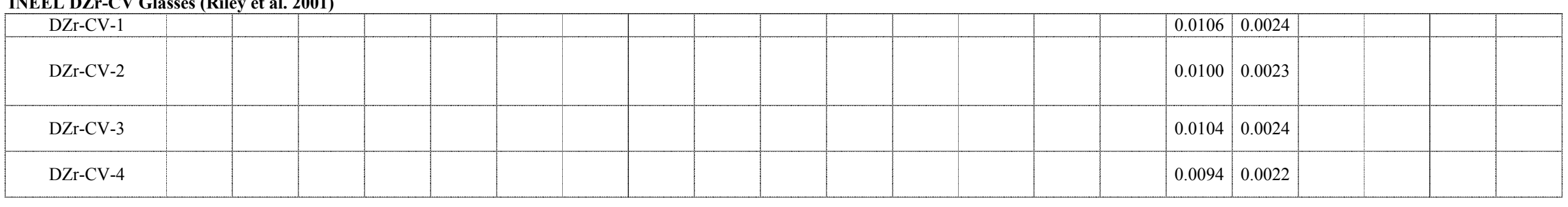


DP Glasses for INEEL HLW (Pittman et al. 2001)

\begin{tabular}{|c|c|c|c|c|c|c|c|c|c|c|c|c|c|c|c|c|c|c|c|c|c|}
\hline Glass ID & $\begin{array}{c}\mathrm{T} 12 \mathrm{O} 3 \\
-\mathrm{t}\end{array}$ & $\begin{array}{c}\text { U3O8 } \\
-t\end{array}$ & $\begin{array}{c}\mathrm{UO} 2 \\
-\mathrm{t}\end{array}$ & $\begin{array}{c}\mathrm{UO} 3 \\
-\mathrm{t}\end{array}$ & $\begin{array}{c}\mathrm{V} 2 \mathrm{O} 5 \\
-\mathrm{t}\end{array}$ & $\begin{array}{c}\text { WO3 } \\
-t\end{array}$ & $\begin{array}{c}\mathrm{Y} 2 \mathrm{O} 3 \\
-\mathrm{t}\end{array}$ & $\begin{array}{c}\mathrm{ZnO} \\
-\mathrm{t}\end{array}$ & $\begin{array}{c}\text { Others } \\
-t\end{array}$ & $\begin{array}{c}\text { Sum } \\
-t\end{array}$ & $\begin{array}{c}\mathrm{A} 12 \mathrm{O} 3 \\
-\mathrm{a}\end{array}$ & $\begin{array}{c}\mathrm{B} 2 \mathrm{O} 3 \\
-\mathrm{a}\end{array}$ & $\begin{array}{c}\mathrm{CaO} \\
-\mathrm{a} \\
\end{array}$ & $\begin{array}{c}\mathrm{Fe} 2 \mathrm{O} 3 \\
-\mathrm{a} \\
\end{array}$ & $\begin{array}{c}\mathrm{FeO} \\
-\mathrm{a}\end{array}$ & $\begin{array}{c}\mathrm{K} 2 \mathrm{O} \\
-\mathrm{a}\end{array}$ & $\begin{array}{c}\mathrm{Li} 2 \mathrm{O} \\
-\mathrm{a}\end{array}$ & $\begin{array}{c}\mathrm{MgO} \\
-\mathrm{a}\end{array}$ & $\begin{array}{c}\mathrm{Na} 2 \mathrm{O} \\
-\mathrm{a}\end{array}$ & $\begin{array}{c}\mathrm{NiO} \\
-\mathrm{a} \\
\end{array}$ & $\begin{array}{c}\mathrm{P} 2 \mathrm{O} 5 \\
-\mathrm{a}\end{array}$ \\
\hline DP-1 & & & & & & & & & & 1.0000 & 0.1270 & 0.0583 & 0.1440 & 0.0002 & & & 0.0703 & & 0.0857 & & \\
\hline DP-2 & & & & & & & & & & 1.0000 & 0.1149 & 0.0554 & 0.1345 & 0.0176 & & & 0.0507 & & 0.1291 & & 0.0039 \\
\hline DP-3 & & & & & & & & & & 1.0000 & 0.1304 & 0.0602 & 0.1015 & 0.0523 & & & 0.0714 & & 0.0815 & & \\
\hline DP-4 & & & & & & & & & & 1.0000 & 0.1272 & 0.0576 & 0.0970 & 0.0001 & & & 0.0445 & & 0.0770 & & 0.0234 \\
\hline DP-5 & & & & & & & & & & 1.0000 & 0.1312 & 0.0596 & 0.1000 & 0.0513 & & & 0.0460 & & 0.0805 & & 0.0170 \\
\hline DP-6 & & & & & & & & & & 1.0000 & 0.1266 & 0.0586 & 0.0990 & 0.0001 & & & 0.0710 & & 0.1316 & & 0.0259 \\
\hline DP-7 & & & & & & & & & & 1.0000 & 0.1240 & 0.1301 & 0.0966 & 0.0005 & & & 0.0688 & & 0.0857 & & \\
\hline DP-8 & & & & & & & & & & 1.0000 & 0.0709 & 0.1462 & 0.0980 & 0.0003 & & & 0.0454 & & 0.0698 & & 0.0243 \\
\hline DP-9 & & & & & & & & & & 1.0000 & 0.0709 & 0.1465 & 0.0988 & 0.0000 & & & 0.0449 & & 0.1238 & & 0.0252 \\
\hline DP-10 & & & & & & & & & & 1.0000 & 0.0811 & 0.1658 & 0.1078 & 0.0003 & & & 0.0011 & & 0.0868 & & \\
\hline DP-11 & & & & & & & & & & 1.0000 & 0.0726 & 0.1517 & 0.1046 & 0.0509 & & & 0.0718 & & 0.0844 & & \\
\hline DP-12 & & & & & & & & & & 1.0000 & 0.0720 & 0.0663 & 0.1442 & 0.0499 & & & 0.0447 & & 0.0744 & & 0.0245 \\
\hline DP-13 & & & & & & & & & & 1.0000 & 0.0716 & 0.0605 & 0.1442 & 0.0001 & & & 0.0710 & & 0.0853 & & 0.0259 \\
\hline DP-14 & & & & & & & & & & 1.0000 & 0.0718 & 0.0592 & 0.1456 & 0.0513 & & & 0.0707 & & 0.0840 & & 0.0252 \\
\hline DP-15 & & & & & & & & & & 0.9700 & 0.0722 & 0.0583 & 0.1442 & 0.0000 & & & 0.0449 & & 0.0726 & & \\
\hline DP-16 & & & & & & & & & & 1.0000 & 0.0716 & 0.0583 & 0.0998 & 0.0511 & & & 0.0456 & & 0.0830 & & \\
\hline DP-17 & & & & & & & & & & 1.0000 & 0.0720 & 0.0592 & 0.1009 & 0.0004 & & & 0.0718 & & 0.1314 & & 0.0254 \\
\hline DP-18 & & & & & & & & & & 1.0000 & 0.0737 & 0.0615 & 0.0998 & 0.0521 & & & 0.0458 & & 0.1288 & & \\
\hline DP-19 & & & & & & & & & & 1.0000 & 0.0748 & 0.0586 & 0.1019 & 0.0518 & & & 0.0716 & & 0.1246 & & \\
\hline DP-20 & & & & & & & & & & 1.0000 & 0.0743 & 0.0599 & 0.1043 & 0.0017 & & & 0.0454 & & 0.0803 & & \\
\hline DP-21 & & & & & & & & & & 1.0000 & 0.0718 & 0.1417 & 0.1442 & 0.0000 & & & 0.0452 & & 0.0822 & & \\
\hline DP-22 & & & & & & & & & & 1.0000 & 0.0726 & 0.0657 & 0.1008 & 0.0004 & & & 0.0710 & & 0.1418 & & \\
\hline DP-23 & & & & & & & & & & 1.0000 & 0.1223 & 0.0596 & 0.1414 & 0.0017 & & & 0.0464 & & 0.1364 & & \\
\hline DP-24 & & & & & & & & & & 1.0000 & 0.0818 & 0.0592 & 0.1004 & 0.0003 & & & 0.0720 & & 0.0929 & & 0.0247 \\
\hline DP-BL1 & & & & & & & & & & 0.9925 & 0.0932 & 0.1001 & 0.1224 & 0.0256 & & & 0.0503 & & 0.1040 & & 0.0152 \\
\hline DP-BL2 & & & & & & & & & & 0.9928 & 0.0886 & 0.0538 & 0.1133 & 0.0330 & & & 0.0613 & & 0.1131 & & 0.0116 \\
\hline DP-centroid & & & & & & & & & & 1.0002 & 0.0903 & 0.0802 & 0.1165 & 0.0192 & & & 0.0568 & & 0.0967 & & 0.0112 \\
\hline
\end{tabular}

INEEL DZr-CV Glasses (Riley et al. 2001)

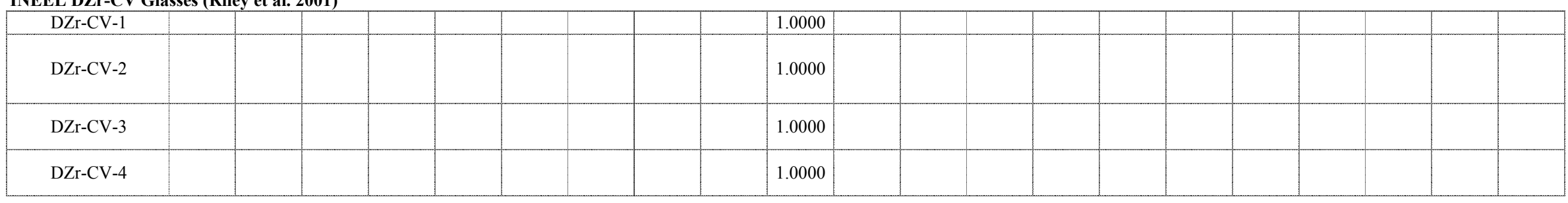


DP Glasses for INEEL HLW (Pittman et al. 2001)

\begin{tabular}{|c|c|c|c|c|c|c|c|c|c|c|c|c|c|c|c|c|c|c|c|c|c|}
\hline Glass ID & $\begin{array}{c}\mathrm{SiO} 2 \\
-\mathrm{a}\end{array}$ & $\begin{array}{c}\mathrm{ZrO} 2 \\
-\mathrm{a}\end{array}$ & $\begin{array}{c}\mathrm{Ag} 2 \mathrm{O} \\
-\mathrm{a}\end{array}$ & $\begin{array}{c}\mathrm{As} 2 \mathrm{O} 3 \\
-\mathrm{a}\end{array}$ & $\begin{array}{c}\mathrm{BaO} \\
-\mathrm{a} \\
\end{array}$ & $\begin{array}{c}\mathrm{Bi} 2 \mathrm{O} 3 \\
-\mathrm{a} \\
\end{array}$ & $\begin{array}{l}\mathrm{Br} \\
-\mathrm{a}\end{array}$ & $\begin{array}{c}\mathrm{CdO} \\
-\mathrm{a} \\
\end{array}$ & $\begin{array}{c}\mathrm{Ce} 2 \mathrm{O} 3 \\
-\mathrm{a} \\
\end{array}$ & $\begin{array}{c}\mathrm{CeO} 2 \\
-\mathrm{a}\end{array}$ & $\begin{array}{l}\mathrm{Cl} \\
-\mathrm{a}\end{array}$ & $\begin{array}{c}\mathrm{CoO} \\
-\mathrm{a} \\
\end{array}$ & $\begin{array}{c}\mathrm{Co} 2 \mathrm{O} 3 \\
-\mathrm{a} \\
\end{array}$ & $\begin{array}{c}\mathrm{Cr} 2 \mathrm{O} 3 \\
-\mathrm{a} \\
\end{array}$ & $\begin{array}{c}\mathrm{Cs} 2 \mathrm{O} \\
-\mathrm{a} \\
\end{array}$ & $\begin{array}{c}\mathrm{CuO} \\
-\mathrm{a} \\
\end{array}$ & $\begin{array}{c}\mathrm{Eu} 2 \mathrm{O} 3 \\
-\mathrm{a} \\
\end{array}$ & $\begin{array}{l}\mathrm{F} \\
-\mathrm{a}\end{array}$ & $\begin{array}{c}\mathrm{Ga} 2 \mathrm{O} 3 \\
-\mathrm{a}\end{array}$ & $\begin{array}{c}\mathrm{Gd} 2 \mathrm{O} 3 \\
-\mathrm{a}\end{array}$ & $\begin{array}{c}\mathrm{HgO} \\
-\mathrm{a} \\
\end{array}$ \\
\hline DP-1 & 0.3890 & 0.0797 & & & & & & & & & & & & & & & & 0.0366 & & & \\
\hline DP-2 & 0.3980 & 0.0427 & & & & & & & & & & & & & & & & 0.0385 & & & \\
\hline DP-3 & 0.4109 & 0.0412 & & & & & & & & & & & & & & & & 0.0599 & & & \\
\hline DP-4 & 0.3831 & 0.0774 & & & & & & & & & & & & & & & & 0.0597 & & & \\
\hline DP-5 & 0.3938 & 0.0707 & & & & & & & & & & & & & & & & 0.0362 & & & \\
\hline DP-6 & 0.3852 & 0.0401 & & & & & & & & & & & & & & & & 0.0375 & & & \\
\hline DP-7 & 0.3788 & 0.0408 & & & & & & & & & & & & & & & & 0.0369 & & & \\
\hline DP-8 & 0.3724 & 0.0791 & & & & & & & & & & & & & & & & 0.0613 & & & \\
\hline DP-9 & 0.3831 & 0.0401 & & & & & & & & & & & & & & & & 0.0368 & & & \\
\hline DP-10 & 0.4259 & 0.0446 & & & & & & & & & & & & & & & & 0.0649 & & & \\
\hline DP-11 & 0.3766 & 0.0401 & & & & & & & & & & & & & & & & 0.0368 & & & \\
\hline DP-12 & 0.3702 & 0.0401 & & & & & & & & & & & & & & & & 0.0564 & & & \\
\hline DP-13 & 0.3681 & 0.0791 & & & & & & & & & & & & & & & & 0.0587 & & & \\
\hline DP-14 & 0.3638 & 0.0408 & & & & & & & & & & & & & & & & 0.0380 & & & \\
\hline DP-15 & 0.4622 & 0.0409 & & & & & & & & & & & & & & & & 0.0350 & & & \\
\hline DP-16 & 0.4858 & 0.0405 & & & & & & & & & & & & & & & & 0.0612 & & & \\
\hline DP-17 & 0.4066 & 0.0402 & & & & & & & & & & & & & & & & 0.0602 & & & \\
\hline DP-18 & 0.3980 & 0.0776 & & & & & & & & & & & & & & & & 0.0362 & & & \\
\hline DP-19 & 0.4002 & 0.0798 & & & & & & & & & & & & & & & & 0.0338 & & & \\
\hline DP-20 & 0.4986 & 0.0784 & & & & & & & & & & & & & & & & 0.0363 & & & \\
\hline DP-21 & 0.3831 & 0.0803 & & & & & & & & & & & & & & & & 0.0367 & & & \\
\hline DP-22 & 0.3702 & 0.0805 & & & & & & & & & & & & & & & & 0.0638 & & & \\
\hline DP-23 & 0.3766 & 0.0409 & & & & & & & & & & & & & & & & 0.0615 & & & \\
\hline DP-24 & 0.5008 & 0.0401 & & & & & & & & & & & & & & & & 0.0376 & & & \\
\hline DP-BL1 & 0.4066 & 0.0509 & & & & & & & & & & & & & & & & 0.0424 & & & \\
\hline DP-BL2 & 0.4558 & 0.0471 & & & & & & & & & & & & & & & & 0.0448 & & & \\
\hline DP-centroid & 0.4002 & 0.0560 & & & & & & & & & & & & & & & & 0.0468 & & & \\
\hline
\end{tabular}

INEEL DZr-CV Glasses (Riley et al. 2001)

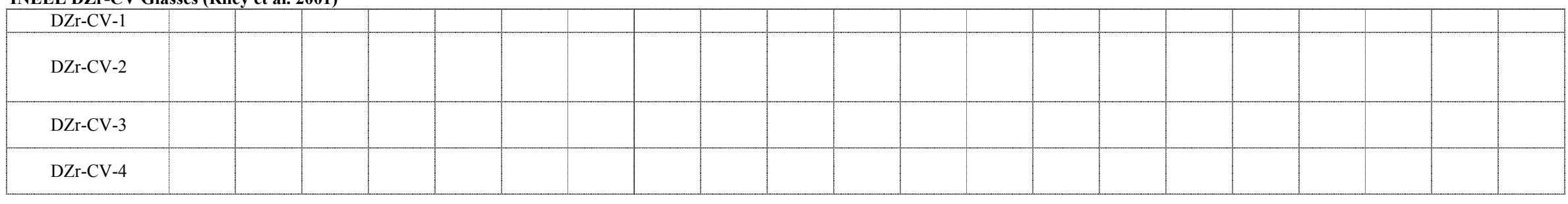


DP Glasses for INEEL HLW (Pittman et al. 2001)

\begin{tabular}{|c|c|c|c|c|c|c|c|c|c|c|c|c|c|c|c|c|c|c|c|c|c|}
\hline Glass ID & $\begin{array}{c}\mathrm{I} \\
-\mathrm{a}\end{array}$ & $\begin{array}{c}\mathrm{La} 2 \mathrm{O} 3 \\
-\mathrm{a}\end{array}$ & $\begin{array}{c}\mathrm{MnO} 2 \\
-\mathrm{a}\end{array}$ & $\begin{array}{c}\mathrm{MnO} \\
-\mathrm{a}\end{array}$ & $\begin{array}{c}\mathrm{MoO} \\
-\mathrm{a}\end{array}$ & $\begin{array}{c}\mathrm{MoO} 3 \\
-\mathrm{a}\end{array}$ & $\begin{array}{c}\mathrm{Nb} 2 \mathrm{O} 5 \\
-\mathrm{a}\end{array}$ & $\begin{array}{c}\mathrm{Nd} 2 \mathrm{O} 3 \\
-\mathrm{a}\end{array}$ & $\begin{array}{c}\mathrm{PbO} \\
-\mathrm{a}\end{array}$ & $\begin{array}{c}\mathrm{PdO} 2 \\
-\mathrm{a}\end{array}$ & $\begin{array}{c}\mathrm{PdO} \\
-\mathrm{a}\end{array}$ & $\begin{array}{c}\mathrm{Pr} 2 \mathrm{O} 3 \\
-\mathrm{a}\end{array}$ & $\begin{array}{c}\text { Pr6O11 } \\
-\mathrm{a}\end{array}$ & $\begin{array}{c}\mathrm{Rb} 2 \mathrm{O} \\
-\mathrm{a}\end{array}$ & $\begin{array}{c}\mathrm{ReO} \\
-\mathrm{a}\end{array}$ & $\begin{array}{c}\mathrm{ReO} 2 \\
-\mathrm{a}\end{array}$ & $\begin{array}{c}\mathrm{Rh} 2 \mathrm{O} 3 \\
-\mathrm{a}\end{array}$ & $\begin{array}{c}\mathrm{RhO} 2 \\
-\mathrm{a}\end{array}$ & $\begin{array}{c}\mathrm{RuO} 2 \\
-\mathrm{a}\end{array}$ & $\begin{array}{c}\mathrm{Sb} 2 \mathrm{O} 3 \\
-\mathrm{a}\end{array}$ & $\begin{array}{c}\mathrm{Sb} 2 \mathrm{O} 5 \\
-\mathrm{a}\end{array}$ \\
\hline DP-1 & & 0.0250 & & & & & & & & & & & & & & & & & & & \\
\hline DP-2 & & 0.0166 & & & & & & & & & & & & & & & & & & & \\
\hline DP-3 & & 0.0257 & & & & & & & & & & & & & & & & & & & \\
\hline DP-4 & & 0.0490 & & & & & & & & & & & & & & & & & & & \\
\hline DP-5 & & 0.0256 & & & & & & & & & & & & & & & & & & & \\
\hline DP-6 & & 0.0500 & & & & & & & & & & & & & & & & & & & \\
\hline DP-7 & & 0.0489 & & & & & & & & & & & & & & & & & & & \\
\hline DP-8 & & 0.0249 & & & & & & & & & & & & & & & & & & & \\
\hline DP-9 & & 0.0250 & & & & & & & & & & & & & & & & & & & \\
\hline DP-10 & & 0.0536 & & & & & & & & & & & & & & & & & & & \\
\hline DP-11 & & 0.0256 & & & & & & & & & & & & & & & & & & & \\
\hline DP-12 & & 0.0498 & & & & & & & & & & & & & & & & & & & \\
\hline DP-13 & & 0.0252 & & & & & & & & & & & & & & & & & & & \\
\hline DP-14 & & 0.0491 & & & & & & & & & & & & & & & & & & & \\
\hline DP-15 & & 0.0500 & & & & & & & & & & & & & & & & & & & \\
\hline DP-16 & & 0.0250 & & & & & & & & & & & & & & & & & & & \\
\hline DP-17 & & 0.0255 & & & & & & & & & & & & & & & & & & & \\
\hline DP-18 & & 0.0488 & & & & & & & & & & & & & & & & & & & \\
\hline DP-19 & & 0.0254 & & & & & & & & & & & & & & & & & & & \\
\hline DP-20 & & 0.0515 & & & & & & & & & & & & & & & & & & & \\
\hline DP-21 & & 0.0252 & & & & & & & & & & & & & & & & & & & \\
\hline DP-22 & & 0.0500 & & & & & & & & & & & & & & & & & & & \\
\hline DP-23 & & 0.0256 & & & & & & & & & & & & & & & & & & & \\
\hline DP-24 & & 0.0255 & & & & & & & & & & & & & & & & & & & \\
\hline DP-BL1 & & & & & & & & & & & & & & & & & & & & & \\
\hline DP-BL2 & & 0.0012 & & & & & & & & & & & & & & & & & & & \\
\hline DP-centroid & & 0.0360 & & & & & & & & & & & & & & & & & & & \\
\hline
\end{tabular}

\section{INEEL DZr-CV Glasses (Riley et al. 2001)}

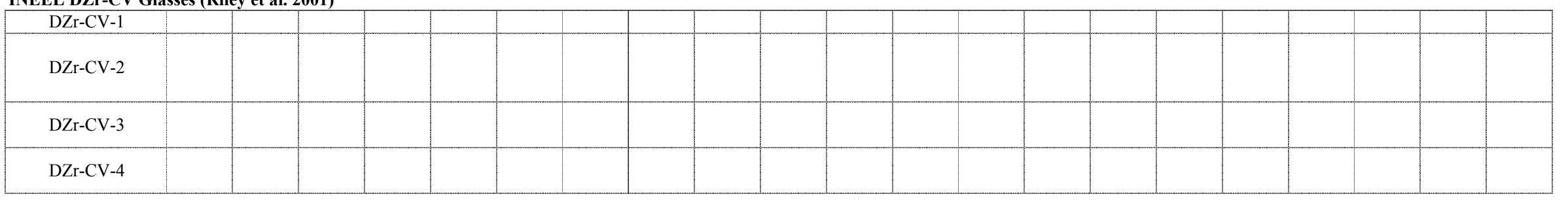


Appendix A. Database - mass fraction

DP Glasses for INEEL HLW (Pittman et al. 2001)

\begin{tabular}{|c|c|c|c|c|c|c|c|c|c|c|c|c|c|c|c|c|c|c|c|c|}
\hline Glass ID & $\begin{array}{c}\mathrm{SeO} 2 \\
-\mathrm{a} \\
\end{array}$ & $\begin{array}{c}\mathrm{Sm} 2 \mathrm{O} 3 \\
-\mathrm{a}\end{array}$ & $\begin{array}{c}\mathrm{SnO} \\
-\mathrm{a}\end{array}$ & $\begin{array}{c}\mathrm{SnO} 2 \\
-\mathrm{a} \\
\end{array}$ & $\begin{array}{c}\mathrm{SO} 3 \\
-\mathrm{a}\end{array}$ & $\begin{array}{c}\mathrm{SrO} \\
-\mathrm{a} \\
\end{array}$ & $\begin{array}{c}\mathrm{Tc} 2 \mathrm{O} 7 \\
-\mathrm{a}\end{array}$ & $\begin{array}{c}\mathrm{TeO} 2 \\
-\mathrm{a} \\
\end{array}$ & $\begin{array}{c}\mathrm{ThO} 2 \\
-\mathrm{a}\end{array}$ & $\begin{array}{c}\mathrm{TiO} 2 \\
-\mathrm{a} \\
\end{array}$ & $\begin{array}{c}\mathrm{T} 12 \mathrm{O} 3 \\
-\mathrm{a} \\
\end{array}$ & $\begin{array}{c}\mathrm{U3O} 8 \\
-\mathrm{a} \\
\end{array}$ & $\begin{array}{c}\mathrm{UO} 2 \\
-\mathrm{a} \\
\end{array}$ & $\begin{array}{c}\text { UO3 } \\
-\mathrm{a} \\
\end{array}$ & $\begin{array}{c}\mathrm{V} 2 \mathrm{O} 5 \\
-\mathrm{a} \\
\end{array}$ & $\begin{array}{c}\text { WO3 } \\
-\mathrm{a} \\
\end{array}$ & $\begin{array}{c}\mathrm{Y} 2 \mathrm{O} 3 \\
-\mathrm{a} \\
\end{array}$ & $\begin{array}{c}\mathrm{ZnO} \\
-\mathrm{a} \\
\end{array}$ & $\begin{array}{c}\text { Others } \\
-\mathrm{a}\end{array}$ & $\begin{array}{c}\text { Sum } \\
-\mathrm{a}\end{array}$ \\
\hline DP-1 & & & & & & & & & & & & & & & & & & & & 1.0158 \\
\hline DP-2 & & & & & & & & & & & & & & & & & & & & 1.0019 \\
\hline DP-3 & & & & & & & & & & & & & & & & & & & & 1.0350 \\
\hline DP-4 & & & & & & & & & & & & & & & & & & & & 0.9960 \\
\hline DP-5 & & & & & & & & & & & & & & & & & & & & 1.0119 \\
\hline DP-6 & & & & & & & & & & & & & & & & & & & & 1.0256 \\
\hline DP-7 & & & & & & & & & & & & & & & & & & & & 1.0111 \\
\hline DP-8 & & & & & & & & & & & & & & & & & & & & 0.9926 \\
\hline DP-9 & & & & & & & & & & & & & & & & & & & & 0.9951 \\
\hline DP-10 & & & & & & & & & & & & & & & & & & & & 1.0319 \\
\hline DP-11 & & & & & & & & & & & & & & & & & & & & 1.0151 \\
\hline DP-12 & & & & & & & & & & & & & & & & & & & & 0.9925 \\
\hline DP-13 & & & & & & & & & & & & & & & & & & & & 0.9897 \\
\hline DP-14 & & & & & & & & & & & & & & & & & & & & 0.9995 \\
\hline DP-15 & & & & & & & & & & & & & & & & & & & & 0.9803 \\
\hline DP-16 & & & & & & & & & & & & & & & & & & & & 1.0219 \\
\hline DP-17 & & & & & & & & & & & & & & & & & & & & 0.9936 \\
\hline DP-18 & & & & & & & & & & & & & & & & & & & & 1.0223 \\
\hline DP-19 & & & & & & & & & & & & & & & & & & & & 1.0225 \\
\hline DP-20 & & & & & & & & & & & & & & & & & & & & 1.0307 \\
\hline DP-21 & & & & & & & & & & & & & & & & & & & & 1.0104 \\
\hline DP-22 & & & & & & & & & & & & & & & & & & & & 1.0168 \\
\hline DP-23 & & & & & & & & & & & & & & & & & & & & 1.0124 \\
\hline DP-24 & & & & & & & & & & & & & & & & & & & & 1.0353 \\
\hline DP-BL1 & & & & & & & & & & & & & & & & & & & & 1.0107 \\
\hline DP-BL2 & & & & & & & & & & & & & & & & & & & & 1.0236 \\
\hline DP-centroid & & & & & & & & & & & & & & & & & & & & 1.0099 \\
\hline
\end{tabular}

INEEL DZr-CV Glasses (Riley et al. 2001)

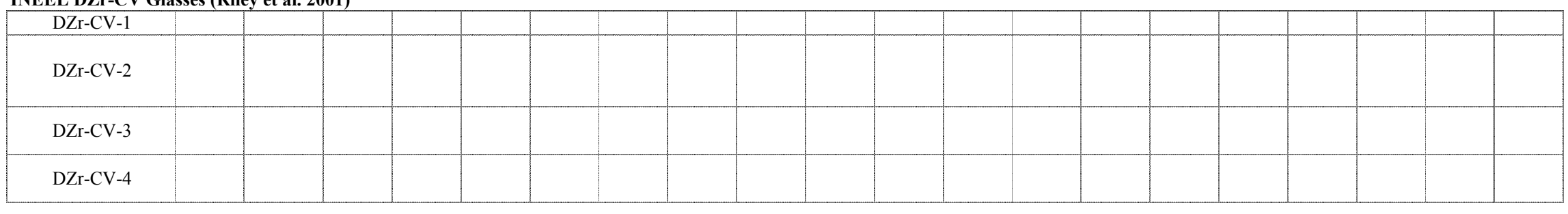


Appendix A. Database - mass fraction

DP Glasses for INEEL HLW (Pittman et al. 2001)

\begin{tabular}{|c|c|c|c|c|c|c|c|c|}
\hline Glass ID & $\begin{array}{l}\mathrm{TM} \\
\left({ }^{\circ} \mathrm{C}\right)\end{array}$ & $\begin{array}{l}\text { Gradient } \\
\text { TL }\left({ }^{\circ} \mathrm{C}\right)\end{array}$ & $\begin{array}{l}\text { Uniform } \\
\text { TL }\left({ }^{\circ} \mathrm{C}\right)\end{array}$ & Primary Phase & $\begin{array}{l}\text { Quenched } \\
\text { Visual/OM }\end{array}$ & $\begin{array}{c}\text { Quenched } \\
\text { SEM/EDS or TEM }\end{array}$ & $\begin{array}{l}\text { Quenched } \\
\text { XRD }\end{array}$ & $\begin{array}{c}\text { Quenched } \\
\text { Homogeneous? }\end{array}$ \\
\hline DP-1 & & & & & & & Baddeleyite $^{\mathrm{a}}$ & no \\
\hline DP-2 & & & & & & & Amorphous & yes \\
\hline DP-3 & & & & & & & $\begin{array}{l}\text { Griceite }{ }^{b}, \text { Calcium } \\
\text { Fluoride }^{c}\end{array}$ & no \\
\hline DP-4 & & & & & & & $\begin{array}{l}\text { Fluorellestadite, } \\
\text { Calcium Fluoride }\end{array}$ & no \\
\hline DP-5 & & & & & & & $\begin{array}{l}\text { Fluorellestadite, } \\
\text { Calcium Fluoride, } \\
\text { Baddeleyite }\end{array}$ & no \\
\hline DP-6 & & & & & & & Amorphous & yes \\
\hline DP-7 & & & & & & & Amorphous & yes \\
\hline DP-8 & & & & & & & $\begin{array}{l}\text { Calcium Fluoride, } \\
\text { Fluorapatite }\end{array}$ & no \\
\hline DP-9 & & & & & & & Fluorapatite & no \\
\hline DP-10 & & & & & & & Calcium Fluoride & no \\
\hline DP-11 & & & & & & & Amorphous & yes \\
\hline DP-12 & & & & & & & Calcium Fluoride & no \\
\hline DP-13 & & & & & & & $\begin{array}{l}\text { Calcium Fluoride, } \\
\text { Zircon }^{\mathrm{e}}\end{array}$ & no \\
\hline DP-14 & & & & & & & Amorphous & yes \\
\hline DP-15 & & & & & & & Amorphous & yes \\
\hline DP-16 & & & & & & & Calcium Fluoride & no \\
\hline DP-17 & & & & & & & Amorphous & yes \\
\hline DP-18 & & & & & & & $\begin{array}{l}\text { Baddeleyite, } \\
\text { Fluorellestadite }\end{array}$ & no \\
\hline DP-19 & & & & & & & Baddeleyite & no \\
\hline DP-20 & & & & & & & Zircon & no \\
\hline DP-21 & & & & & & & Amorphous & yes \\
\hline DP-22 & & & & & & & Baddeleyite & no \\
\hline DP-23 & & & & & & & Amorphous & yes \\
\hline DP-24 & & & & & & & Fluorapatite & no \\
\hline DP-BL1 & & & & & & & Calcium Fluoride & no \\
\hline DP-BL2 & & & & & & & Amorphous & yes \\
\hline DP-centroid & & & & & & & Amorphous & yes \\
\hline
\end{tabular}

INEEL DZr-CV Glasses (Riley et al. 2001)

\begin{tabular}{|c|c|c|c|c|c|}
\hline DZr-CV-1 & & & amorphous Homo. \\
\hline DZr-CV-2 & & & & slight phase speration & \\
\hline DZr-CV-3 & & & & amorphous Homo. & \\
\hline DZr-CV-4 & & & & amorphous Homo. & \\
\hline
\end{tabular}


Appendix A. Database - mass fraction

DP Glasses for INEEL HLW (Pittman et al. 2001)

\begin{tabular}{|c|c|c|c|c|c|}
\hline Glass ID & $\begin{array}{c}\mathrm{CCC} \\
\text { Visual/OM }\end{array}$ & $\begin{array}{c}\text { CCC } \\
\text { SEM/EDS or TEM }\end{array}$ & $\begin{array}{l}\text { CCC } \\
\text { XRD } \\
\end{array}$ & $\begin{array}{c}\text { Heat Treated } \\
\text { Visual/OM }\end{array}$ & $\begin{array}{c}\text { Heat Treated } \\
\text { SEM/EDS or TEM }\end{array}$ \\
\hline DP-1 & & & $\begin{array}{l}\text { Zirconium Oxide }{ }^{\mathrm{a}} \text {, Nepheline }{ }^{\mathrm{b}} \text {, Lithium } \\
\text { Silicate }^{\mathrm{c}}\end{array}$ & & \\
\hline DP-2 & & & $\begin{array}{l}\text { Sodium Aluminum Silicated, } 1 \\
\text { unidentifiable }\end{array}$ & & \\
\hline DP-3 & & & $\begin{array}{l}\text { Lazurite }{ }^{\mathrm{e}} \text {, Calcium Fluoride } \mathrm{f}^{\mathrm{f}} \text {, Lithium } \\
\text { Sodium Sulfate }\end{array}$ & & \\
\hline DP-4 & & & $\begin{array}{l}\text { Baddeleyite }{ }^{\mathrm{h}}, \text { Calcium Fluoride, } \\
\text { Brotholite-(Ce) }\end{array}$ & & \\
\hline DP-5 & & & $\begin{array}{l}\text { Hydroxylapatite }{ }^{\mathrm{j}}, \text { Britholite-(Ce), } \\
\text { Fluorite }^{\mathrm{k}}\end{array}$ & & \\
\hline DP-6 & & & Britholite-(Ce), Zirconium Oxide & & \\
\hline DP-7 & & & Amorphous & & \\
\hline DP-8 & & & Fluorite, Fluorellestadite & & \\
\hline DP-9 & & & Hydroxylapatite & & \\
\hline DP-10 & & & Fluorite, Fluorellestadite & & \\
\hline DP-11 & & & Amorphous & & \\
\hline DP-12 & & & $\begin{array}{l}\text { Calcium Fluoride, Britholite-(Y) } \\
\text { Britholite-(Ce) }\end{array}$ & & \\
\hline DP-13 & & & Calcium Fluoride, Zircon, Nosean ${ }^{\circ}$ & & \\
\hline DP-14 & & & Britholite-(Ce) & & \\
\hline DP-15 & & & 1 unidentifiable & & \\
\hline DP-16 & & & Fluorite & & \\
\hline DP-17 & & & Hydroxylapatite & & \\
\hline DP-18 & & & Baddeleyite & & \\
\hline DP-19 & & & Baddeleyite & & \\
\hline DP-20 & & & Zircon & & \\
\hline DP-21 & & & Amorphous & & \\
\hline DP-22 & & & Nosean, Baddeleyite & & \\
\hline DP-23 & & & 3 unidentifiable & & \\
\hline DP-24 & & & Fluorapatite & & \\
\hline DP-BL1 & & & Baddeleyite, Hydroxylapatite & & \\
\hline DP-BL2 & & & Amorphous & & \\
\hline DP-centroid & & & Fluorite, Hydroxylapatite & & \\
\hline
\end{tabular}

INEEL DZr-CV Glasses (Riley et al. 2001)

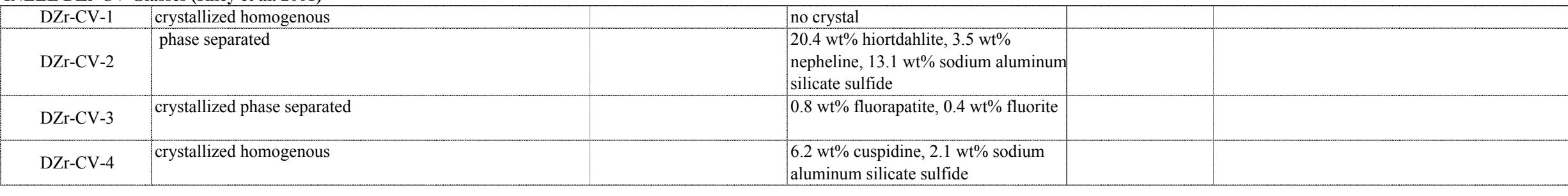


Appendix A. Database - mass fraction

DP Glasses for INEEL HLW (Pittman et al. 2001)

\begin{tabular}{|c|c|c|c|c|c|c|c|c|c|c|c|c|c|c|c|}
\hline Glass ID & $\begin{array}{c}\text { Heat Treated } \\
\text { XRD } \\
\end{array}$ & $\begin{array}{l}\text { Density } \\
\left(\mathrm{g} / \mathrm{cm}^{3}\right)\end{array}$ & $\begin{array}{c}\text { Fulc Visc } \\
\mathrm{A}\end{array}$ & \begin{tabular}{|c|}
$\begin{array}{c}\text { Fulc Visc } \\
\mathrm{B}\end{array}$ \\
\end{tabular} & \begin{tabular}{|c|}
$\begin{array}{c}\text { fulc Visc } \\
\text { To }\end{array}$ \\
\end{tabular} & $\begin{array}{c}\mathrm{FV} 1150^{\circ} \mathrm{C} \\
(\mathrm{Pa} \cdot \mathrm{s})\end{array}$ & $\begin{array}{c}\text { Arrh Visc } \\
\mathrm{A}\end{array}$ & $\begin{array}{c}\text { Arrh Visc } \\
\text { B }\end{array}$ & $\begin{array}{l}1150^{\circ} \mathrm{C} \\
\left(\mathrm{D}_{0,0}\right)\end{array}$ & $\begin{array}{c}\mathrm{T}\left({ }^{\circ} \mathrm{C}\right) \text { at } \\
2 \mathrm{~Pa} \cdot \mathrm{s}\end{array}$ & $\begin{array}{c}\mathrm{T}\left({ }^{\circ} \mathrm{C}\right) \text { at } \\
5 \mathrm{~Pa} \cdot \mathrm{s}\end{array}$ & $\begin{array}{r}\mathrm{T}\left({ }^{\circ} \mathrm{C}\right) \text { at } \\
10 \mathrm{~Pa} \cdot \mathrm{s}\end{array}$ & $\begin{array}{c}\mathrm{T} 1 \\
\left({ }^{\circ} \mathrm{C}\right) \\
\end{array}$ & $\begin{array}{c}\mathrm{V} 1 \\
(\mathrm{~Pa} \cdot \mathrm{s})\end{array}$ & $\begin{array}{l}\mathrm{T} 2 \\
\left({ }^{\circ} \mathrm{C}\right) \\
\end{array}$ \\
\hline \multicolumn{16}{|l|}{ DP-1 } \\
\hline \multicolumn{16}{|l|}{ DP-2 } \\
\hline \multicolumn{16}{|l|}{ DP-3 } \\
\hline \multicolumn{16}{|l|}{ DP-4 } \\
\hline \multicolumn{16}{|l|}{ DP-5 } \\
\hline \multicolumn{16}{|l|}{ DP-6 } \\
\hline \multicolumn{16}{|l|}{ DP-7 } \\
\hline \multicolumn{16}{|l|}{ DP-8 } \\
\hline \multicolumn{16}{|l|}{ DP-9 } \\
\hline \multicolumn{16}{|l|}{ DP-10 } \\
\hline \multicolumn{16}{|l|}{ DP-11 } \\
\hline \multicolumn{16}{|l|}{ DP-12 } \\
\hline \multicolumn{16}{|l|}{ DP-13 } \\
\hline \multicolumn{16}{|l|}{ DP-14 } \\
\hline \multicolumn{16}{|l|}{ DP-15 } \\
\hline \multicolumn{16}{|l|}{ DP-16 } \\
\hline \multicolumn{16}{|l|}{ DP-17 } \\
\hline \multicolumn{16}{|l|}{ DP-18 } \\
\hline \multicolumn{16}{|l|}{ DP-19 } \\
\hline \multicolumn{16}{|l|}{ DP-20 } \\
\hline \multicolumn{16}{|l|}{ DP-21 } \\
\hline \multicolumn{16}{|l|}{ DP-22 } \\
\hline \multicolumn{16}{|l|}{ DP-23 } \\
\hline \multicolumn{16}{|l|}{ DP-24 } \\
\hline \multicolumn{16}{|l|}{ DP-BL1 } \\
\hline \multicolumn{16}{|l|}{ DP-BL2 } \\
\hline DP-centroid & & & & & & & & & & & & & & & \\
\hline
\end{tabular}

\section{INEEL DZr-CV Glasses (Riley et al. 2001)}

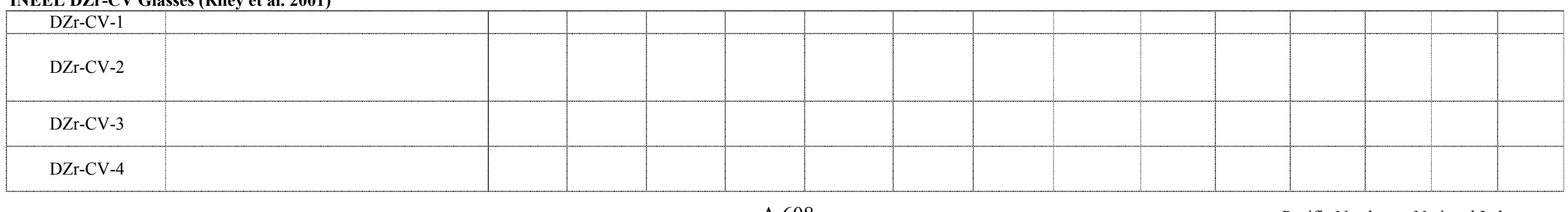


Appendix A. Database - mass fraction

\begin{tabular}{|c|c|c|c|c|c|c|c|c|c|c|c|c|c|c|c|c|c|c|c|c|c|}
\hline \multicolumn{22}{|c|}{ DP Glasses for INEEL HLW (Pittman et al. 2001) } \\
\hline Glass ID & $\begin{array}{c}\mathrm{V} 2 \\
(\mathrm{~Pa} \cdot \mathrm{s})\end{array}$ & $\begin{array}{c}\mathrm{T} 3 \\
\left({ }^{\circ} \mathrm{C}\right) \\
\end{array}$ & $\begin{array}{c}\mathrm{V} 3 \\
(\mathrm{~Pa} \cdot \mathrm{s})\end{array}$ & $\begin{array}{c}\mathrm{T} 4 \\
\left({ }^{\circ} \mathrm{C}\right) \\
\end{array}$ & $\begin{array}{c}\mathrm{V} 4 \\
(\mathrm{~Pa} \cdot \mathrm{s})\end{array}$ & $\begin{array}{c}\mathrm{T} 5 \\
\left({ }^{\circ} \mathrm{C}\right) \\
\end{array}$ & $\begin{array}{c}\mathrm{V} 5 \\
(\mathrm{~Pa} \cdot \mathrm{s})\end{array}$ & $\begin{array}{c}\mathrm{T} 6 \\
\left({ }^{\circ} \mathrm{C}\right) \\
\end{array}$ & $\begin{array}{c}\mathrm{V} 6 \\
(\mathrm{~Pa} \cdot \mathrm{s})\end{array}$ & $\begin{array}{c}\mathrm{T} 7 \\
\left({ }^{\circ} \mathrm{C}\right) \\
\end{array}$ & $\begin{array}{c}\mathrm{V} 7 \\
(\mathrm{~Pa} \cdot \mathrm{s})\end{array}$ & $\begin{array}{c}\mathrm{T} 8 \\
\left({ }^{\circ} \mathrm{C}\right)\end{array}$ & $\begin{array}{c}\mathrm{V} 8 \\
(\mathrm{~Pa} \cdot \mathrm{s})\end{array}$ & $\begin{array}{c}\mathrm{T} 9 \\
\left({ }^{\circ} \mathrm{C}\right)\end{array}$ & $\begin{array}{c}\mathrm{V} 9 \\
(\mathrm{~Pa} \cdot \mathrm{s})\end{array}$ & $\begin{array}{l}\mathrm{T} 10 \\
\left({ }^{\circ} \mathrm{C}\right)\end{array}$ & $\begin{array}{c}\mathrm{V} 10 \\
(\mathrm{~Pa} \cdot \mathrm{s})\end{array}$ & $\begin{array}{l}\mathrm{T} 11 \\
\left({ }^{\circ} \mathrm{C}\right)\end{array}$ & $\begin{array}{c}\text { V11 } \\
(\mathrm{Pa} \cdot \mathrm{s})\end{array}$ & $\begin{array}{l}\mathrm{T} 12 \\
\left({ }^{\circ} \mathrm{C}\right)\end{array}$ & $\begin{array}{c}\mathrm{V} 12 \\
(\mathrm{~Pa} \cdot \mathrm{s})\end{array}$ \\
\hline \multicolumn{22}{|l|}{ DP-1 } \\
\hline \multicolumn{22}{|l|}{ DP-2 } \\
\hline \multicolumn{22}{|l|}{ DP-3 } \\
\hline \multicolumn{22}{|l|}{ DP-4 } \\
\hline \multicolumn{22}{|l|}{ DP-5 } \\
\hline \multicolumn{22}{|l|}{ DP-6 } \\
\hline \multicolumn{22}{|l|}{ DP-7 } \\
\hline \multicolumn{22}{|l|}{ DP-8 } \\
\hline \multicolumn{22}{|l|}{ DP-9 } \\
\hline \multicolumn{22}{|l|}{ DP-10 } \\
\hline \multicolumn{22}{|l|}{ DP-11 } \\
\hline \multicolumn{22}{|l|}{ DP-12 } \\
\hline \multicolumn{22}{|l|}{ DP-13 } \\
\hline \multicolumn{22}{|l|}{ DP-14 } \\
\hline \multicolumn{22}{|l|}{ DP-15 } \\
\hline \multicolumn{22}{|l|}{ DP-16 } \\
\hline \multicolumn{22}{|l|}{ DP-17 } \\
\hline \multicolumn{22}{|l|}{ DP-18 } \\
\hline \multicolumn{22}{|l|}{ DP-19 } \\
\hline \multicolumn{22}{|l|}{ DP-20 } \\
\hline \multicolumn{22}{|l|}{ DP-21 } \\
\hline \multicolumn{22}{|l|}{ DP-22 } \\
\hline \multicolumn{22}{|l|}{ DP-23 } \\
\hline DP-24 & & & & & & & & & & & & & & & & & & & & & \\
\hline DP-BL1 & & & & & & & & & & & & & & & & & & & & & \\
\hline DP-BL2 & & & & & & & & & & & & & & & & & & & & & \\
\hline DP-centroid & & & & & & & & & & & & & & & & & & & & & \\
\hline
\end{tabular}

\section{INEEL DZr-CV Glasses (Riley et al. 2001)}

$$
\text { DZr-CV-1 }
$$

DZr-CV-2

DZr-CV-3

DZr-CV-4 
Appendix A. Database - mass fraction

DP Glasses for INEEL HLW (Pittman et al. 2001)

\begin{tabular}{|c|c|c|c|c|c|c|c|c|c|c|c|c|c|c|c|c|}
\hline Glass ID & $\begin{array}{l}\mathrm{T} 13 \\
\left({ }^{\circ} \mathrm{C}\right) \\
\end{array}$ & $\begin{array}{c}\mathrm{V} 13 \\
(\mathrm{~Pa} \cdot \mathrm{s})\end{array}$ & $\begin{array}{l}\mathrm{T} 14 \\
\left({ }^{\circ} \mathrm{C}\right) \\
\end{array}$ & $\begin{array}{c}\mathrm{V} 14 \\
(\mathrm{~Pa} \cdot \mathrm{s})\end{array}$ & $\begin{array}{c}\text { Q PCT } \\
\text { B }\left(\mathrm{g} / \mathrm{m}^{2}\right)\end{array}$ & $\begin{array}{c}\text { Q PCT } \\
\mathrm{Li}\left(\mathrm{g} / \mathrm{m}^{2}\right)\end{array}$ & $\begin{array}{c}\text { Q PCT } \\
\mathrm{Na}\left(\mathrm{g} / \mathrm{m}^{2}\right)\end{array}$ & $\begin{array}{c}\text { Q PCT } \\
\mathrm{Si}\left(\mathrm{g} / \mathrm{m}^{2}\right)\end{array}$ & $\begin{array}{c}\text { Q PCT } \\
\mathrm{pH}\end{array}$ & $\begin{array}{c}\text { CCC PCT } \\
\mathrm{B}\left(\mathrm{g} / \mathrm{m}^{2}\right)\end{array}$ & $\begin{array}{l}\text { CCC PCT } \\
\mathrm{Li}\left(\mathrm{g} / \mathrm{m}^{2}\right)\end{array}$ & $\begin{array}{l}\text { CCC PCT } \\
\mathrm{Na}\left(\mathrm{g} / \mathrm{m}^{2}\right)\end{array}$ & $\begin{array}{c}\text { CCC PCT } \\
\mathrm{Si}\left(\mathrm{g} / \mathrm{m}^{2}\right)\end{array}$ & $\begin{array}{c}\text { CCC PCT } \\
\mathrm{pH} \\
\end{array}$ & $\begin{array}{l}\text { Q PCT B at } \\
20^{\circ} \mathrm{C}\left(\mathrm{g} / \mathrm{m}^{2}\right)\end{array}$ & $\begin{array}{l}\text { Q PCT Li at } \\
20^{\circ} \mathrm{C}\left(\mathrm{g} / \mathrm{m}^{2}\right)\end{array}$ \\
\hline DP-1 & & & & & 0.0892 & 0.2158 & 0.1778 & & 10.98 & 7.3820 & 5.5727 & 0.9082 & & 11.53 & & \\
\hline DP-2 & & & & & 0.1270 & 0.1934 & 0.2896 & & 11.06 & 2.5972 & 2.1046 & 0.7435 & & 11.42 & & \\
\hline DP-3 & & & & & 0.1024 & 0.2091 & 0.1536 & & 10.56 & 0.3546 & 0.6560 & 0.2695 & & 10.74 & & \\
\hline DP-4 & & & & & 0.2149 & 0.6946 & 0.1471 & & 9.87 & 0.6589 & 4.6297 & 0.5133 & & 10.27 & & \\
\hline DP-5 & & & & & 0.1160 & 0.2526 & 0.1160 & & 10.21 & 0.3079 & 0.3644 & 0.1509 & & 10.42 & & \\
\hline DP-6 & & & & & 0.4131 & 0.4761 & 0.5596 & & 11.33 & 1.0486 & 1.9470 & 0.8039 & & 11.68 & & \\
\hline DP-7 & & & & & 0.2166 & 0.2813 & 0.2173 & & 10.58 & 0.2137 & 0.2463 & 0.1994 & & 10.61 & & \\
\hline DP-8 & & & & & 1.4467 & 1.3760 & 0.8402 & & 9.37 & 1.2929 & 1.7891 & 0.9326 & & 9.53 & & \\
\hline DP-9 & & & & & 2.0334 & 1.9860 & 1.7948 & & 10.37 & 1.9231 & 1.9910 & 1.7661 & & 10.32 & & \\
\hline DP-10 & & & & & 0.5406 & 0.0000 & 0.4089 & & 8.97 & 0.7942 & 0.0000 & 0.5231 & & 9.07 & & \\
\hline DP-11 & & & & & 1.0105 & 1.0939 & 0.9040 & & 10.55 & 0.9915 & 1.0230 & 0.8564 & & 10.68 & & \\
\hline DP-12 & & & & & 0.1277 & 0.2134 & 0.2177 & & 10.63 & 0.2184 & 0.2549 & 0.2282 & & 10.69 & & \\
\hline DP-13 & & & & & 0.2403 & 0.3654 & 0.2958 & & 10.98 & 0.4733 & 2.7189 & 0.6607 & & 11.51 & & \\
\hline DP-14 & & & & & 0.2611 & 0.4345 & 0.3536 & & 11.15 & 0.2449 & 0.3117 & 0.2814 & & 11.21 & & \\
\hline DP-15 & & & & & 0.1036 & 0.1840 & 0.1979 & & 10.74 & NR & 0.1966 & 0.1885 & & 10.69 & & \\
\hline DP-16 & & & & & 0.1644 & 0.2809 & 0.1411 & & 10.23 & 0.1805 & 0.2713 & 0.1409 & & 10.37 & & \\
\hline DP-17 & & & & & 0.7354 & 0.7711 & 0.8544 & & 11.31 & 0.5586 & 0.8699 & 0.7295 & & 11.43 & & \\
\hline DP-18 & & & & & 0.2324 & 0.3078 & 0.3868 & & 11.1 & 0.2062 & 0.2779 & 0.3463 & & 11.09 & & \\
\hline DP-19 & & & & & 0.5251 & 0.5098 & 0.7460 & & 11.42 & 0.6218 & 0.8075 & 0.8065 & & 11.59 & & \\
\hline DP-20 & & & & & 0.1228 & 0.2207 & 0.2034 & & 10.63 & NR & 0.2035 & 0.1660 & & 10.61 & & \\
\hline DP-21 & & & & & 0.2519 & 0.2899 & 0.2763 & & 10.33 & 0.2293 & 0.2649 & 0.2434 & & 10.42 & & \\
\hline DP-22 & & & & & 0.4208 & 0.5245 & 0.6067 & & 11.33 & 0.8330 & 1.8898 & 0.7674 & & 11.61 & & \\
\hline DP-23 & & & & & 0.1277 & 0.1887 & 0.2766 & & 11.05 & 7.9731 & 6.2887 & 2.7311 & & 11.35 & & \\
\hline DP-24 & & & & & 0.1327 & 0.2571 & 0.1559 & & 10.68 & 0.3509 & 0.2700 & 0.1701 & & 10.75 & & \\
\hline DP-BL1 & & & & & 0.1624 & 0.2525 & 0.2461 & & 10.64 & 0.2675 & 0.2235 & 0.2245 & & 10.61 & & \\
\hline DP-BL2 & & & & & 0.1612 & 0.2571 & 0.2744 & & 10.96 & 0.2191 & 0.2360 & 0.2450 & & 11 & & \\
\hline DP-centroid & & & & & 0.1830 & 0.2528 & 0.2498 & & 10.66 & 0.2001 & 0.2273 & 0.2224 & & 10.7 & & \\
\hline
\end{tabular}

INEEL DZr-CV Glasses (Riley et al. 2001)

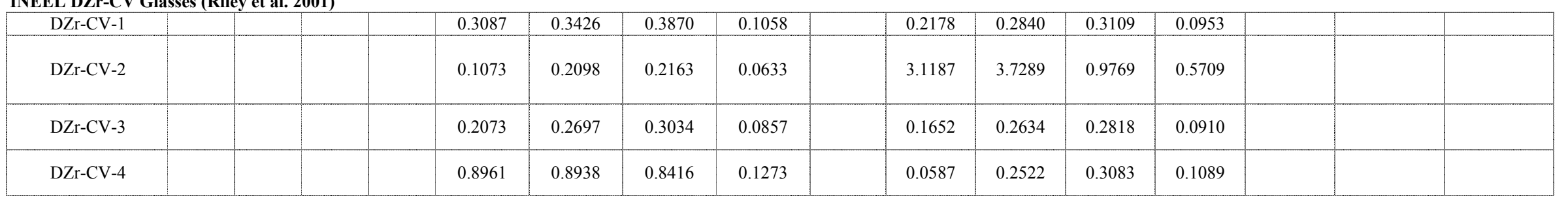


Appendix A. Database - mass fraction

DP Glasses for INEEL HLW (Pittman et al. 2001)

\begin{tabular}{|c|c|c|c|c|c|c|c|c|c|c|c|c|}
\hline Glass ID & $\begin{array}{l}\text { Q PCT Na at } \\
20^{\circ} \mathrm{C}\left(\mathrm{g} / \mathrm{m}^{2}\right)\end{array}$ & $\begin{array}{l}\text { Q PCT Si at } \\
20^{\circ} \mathrm{C}\left(\mathrm{g} / \mathrm{m}^{2}\right)\end{array}$ & $\begin{array}{c}\mathrm{QpH} \\
\text { at } 20^{\circ} \mathrm{C}\end{array}$ & $\begin{array}{c}\text { TCLP Ag } \\
(\mathrm{ppm})\end{array}$ & $\begin{array}{c}\text { TCLP As } \\
\text { (ppm) }\end{array}$ & $\begin{array}{c}\text { TCLP Ba } \\
\text { (ppm) }\end{array}$ & $\begin{array}{c}\text { TCLP Cd } \\
(\mathrm{ppm})\end{array}$ & $\begin{array}{c}\text { TCLP Cr } \\
(\mathrm{ppm})\end{array}$ & $\begin{array}{c}\text { TCLP Ni } \\
(\mathrm{ppm})\end{array}$ & $\begin{array}{c}\text { TCLP Pb } \\
(\mathrm{ppm})\end{array}$ & $\begin{array}{c}\text { TCLP Se } \\
(\mathrm{ppm})\end{array}$ & $\begin{array}{c}\text { TCLP Zn } \\
(\mathrm{ppm})\end{array}$ \\
\hline \multicolumn{13}{|l|}{ DP-1 } \\
\hline \multicolumn{13}{|l|}{ DP-2 } \\
\hline \multicolumn{13}{|l|}{ DP-3 } \\
\hline \multicolumn{13}{|l|}{ DP-4 } \\
\hline \multicolumn{13}{|l|}{ DP-5 } \\
\hline \multicolumn{13}{|l|}{ DP-6 } \\
\hline \multicolumn{13}{|l|}{ DP-7 } \\
\hline \multicolumn{13}{|l|}{ DP-8 } \\
\hline \multicolumn{13}{|l|}{ DP-9 } \\
\hline \multicolumn{13}{|l|}{ DP-10 } \\
\hline \multicolumn{13}{|l|}{ DP-11 } \\
\hline \multicolumn{13}{|l|}{ DP-12 } \\
\hline \multicolumn{13}{|l|}{ DP-13 } \\
\hline \multicolumn{13}{|l|}{ DP-14 } \\
\hline \multicolumn{13}{|l|}{ DP-15 } \\
\hline \multirow{2}{*}{\multicolumn{13}{|c|}{$\frac{\text { DP-16 }}{\text { DP-17 }}$}} \\
\hline & & & & & & & & & & & & \\
\hline \multicolumn{13}{|l|}{ DP-18 } \\
\hline \multicolumn{13}{|l|}{ DP-19 } \\
\hline \multirow{2}{*}{\multicolumn{13}{|c|}{ DP-20 }} \\
\hline \multirow{2}{*}{\multicolumn{13}{|c|}{ DP-21 }} \\
\hline \multirow{2}{*}{\multicolumn{13}{|c|}{ DP-22 }} \\
\hline \multicolumn{4}{|l|}{ DP-23 } & & & & & & & & & \\
\hline \multicolumn{13}{|l|}{ DP-24 } \\
\hline \multicolumn{13}{|l|}{$\begin{array}{l}\text { DP-BL1 } \\
\text { DP-BL2 }\end{array}$} \\
\hline DP-BL2 & & & & & & & & & & & & \\
\hline P-centroid & & & & & & & & & & & & \\
\hline
\end{tabular}

\section{INEEL DZr-CV Glasses (Riley et al. 2001)}

\begin{tabular}{|c|c|c|c|c|c|c|c|c|c|c|c|}
\hline DZr-CV-1 & & & & & & \\
\hline DZr-CV-2 & & & & & & & & \\
\hline DZr-CV-3 & & & & & & & & & \\
\hline DZr-CV-4 & & & & & & & & & \\
\hline
\end{tabular}


INEEL DZr-CV Glasses (Riley et al. 2001)

\begin{tabular}{|c|c|c|c|c|c|c|c|c|c|c|c|c|c|c|c|c|c|c|c|c|c|}
\hline Glass ID & $\begin{array}{c}\mathrm{Al} 2 \mathrm{O} 3 \\
-\mathrm{t}\end{array}$ & $\begin{array}{c}\mathrm{B} 2 \mathrm{O} 3 \\
-\mathrm{t}\end{array}$ & $\begin{array}{c}\mathrm{CaO} \\
-\mathrm{t}\end{array}$ & $\begin{array}{c}\mathrm{Fe} 2 \mathrm{O} 3 \\
-\mathrm{t}\end{array}$ & $\begin{array}{c}\mathrm{FeO} \\
-\mathrm{t}\end{array}$ & $\begin{array}{c}\mathrm{K} 2 \mathrm{O} \\
-\mathrm{t}\end{array}$ & $\begin{array}{c}\mathrm{Li} 2 \mathrm{O} \\
-\mathrm{t}\end{array}$ & $\begin{array}{c}\mathrm{MgO} \\
-\mathrm{t}\end{array}$ & $\begin{array}{c}\mathrm{Na} 2 \mathrm{O} \\
-\mathrm{t}\end{array}$ & $\begin{array}{c}\mathrm{NiO} \\
-\mathrm{t}\end{array}$ & $\begin{array}{c}\mathrm{P} 2 \mathrm{O} 5 \\
-\mathrm{t}\end{array}$ & $\begin{array}{c}\mathrm{SiO} 2 \\
-\mathrm{t}\end{array}$ & $\begin{array}{c}\mathrm{ZrO} 2 \\
-\mathrm{t}\end{array}$ & $\begin{array}{c}\mathrm{Ag} 2 \mathrm{O} \\
-\mathrm{t}\end{array}$ & $\begin{array}{c}\mathrm{As} 2 \mathrm{O} 3 \\
-\mathrm{t}\end{array}$ & $\begin{array}{c}\mathrm{BaO} \\
-\mathrm{t}\end{array}$ & $\begin{array}{c}\mathrm{Bi} 2 \mathrm{O} 3 \\
-\mathrm{t}\end{array}$ & $\begin{array}{c}\mathrm{Br} \\
-\mathrm{t}\end{array}$ & $\begin{array}{c}\mathrm{CdO} \\
-\mathrm{t}\end{array}$ & $\begin{array}{c}\mathrm{Ce} 2 \mathrm{O} 3 \\
-\mathrm{t}\end{array}$ & $\begin{array}{c}\mathrm{CeO} 2 \\
-\mathrm{t}\end{array}$ \\
\hline DZr-CV-5 & 0.0877 & 0.0519 & 0.0950 & 0.0306 & & 0.0015 & 0.0612 & & 0.1161 & 0.0003 & 0.0120 & 0.4315 & 0.0480 & & & & & & & & \\
\hline DZr-CV-6 & 0.0824 & 0.0488 & 0.1500 & 0.0287 & & 0.0014 & 0.0575 & & 0.1090 & 0.0003 & 0.0112 & 0.4052 & 0.0451 & & & & & & & & \\
\hline DZr-CV-7 & 0.0866 & 0.0513 & 0.1140 & 0.0302 & & 0.0014 & 0.0604 & & 0.1146 & 0.0003 & 0.0118 & 0.4259 & 0.0474 & & & & & & & & \\
\hline DZr-CV-8 & 0.0843 & 0.0499 & 0.1111 & 0.0294 & & 0.0014 & 0.0589 & & 0.1116 & 0.0003 & 0.0115 & 0.4148 & 0.0462 & & & & & & & & \\
\hline DZr-CV-9 & 0.0886 & 0.0525 & 0.1167 & 0.0000 & & 0.0015 & 0.0619 & & 0.1173 & 0.0003 & 0.0121 & 0.4359 & 0.0485 & & & & & & & & \\
\hline DZr-CV-10 & 0.0842 & 0.0498 & 0.1109 & 0.0500 & & 0.0014 & 0.0588 & & 0.1114 & 0.0003 & 0.0115 & 0.4141 & 0.0461 & & & & & & & & \\
\hline DZr-CV-11 & 0.0838 & 0.0496 & 0.1104 & 0.0292 & & 0.0014 & 0.0585 & & 0.1109 & 0.0003 & 0.0114 & 0.4122 & 0.0459 & & & & & & & & \\
\hline DZr-CV-12 & 0.0816 & 0.0483 & 0.1075 & 0.0285 & & 0.0014 & 0.0570 & & 0.1080 & 0.0003 & 0.0111 & 0.4016 & 0.0447 & & & & & & & & \\
\hline DZr-CV-13 & 0.0873 & 0.0517 & 0.1150 & 0.0304 & & 0.0014 & 0.0450 & & 0.1156 & 0.0003 & 0.0119 & 0.4296 & 0.0478 & & & & & & & & \\
\hline DZr-CV-14 & 0.0850 & 0.0504 & 0.1120 & 0.0297 & & 0.0014 & 0.0700 & & 0.1125 & 0.0003 & 0.0116 & 0.4183 & 0.0466 & & & & & & & & \\
\hline DZr-CV-15 & 0.0892 & 0.0528 & 0.1175 & 0.0311 & & 0.0015 & 0.0623 & & 0.0800 & 0.0003 & 0.0122 & 0.4389 & 0.0488 & & & & & & & & \\
\hline DZr-CV-16 & 0.0844 & 0.0500 & 0.1111 & 0.0294 & & 0.0014 & 0.0589 & & 0.1300 & 0.0003 & 0.0115 & 0.4150 & 0.0462 & & & & & & & & \\
\hline DZr-CV-17 & 0.0870 & 0.0515 & 0.1146 & 0.0303 & & 0.0014 & 0.0607 & & 0.1151 & 0.0003 & 0.0000 & 0.4278 & 0.0476 & & & & & & & & \\
\hline DZr-CV-18 & 0.0848 & 0.0502 & 0.1117 & 0.0296 & & 0.0014 & 0.0592 & & 0.1122 & 0.0003 & 0.0250 & 0.4171 & 0.0464 & & & & & & & & \\
\hline DZr-CV-19 & 0.0864 & 0.0512 & 0.1138 & 0.0301 & & 0.0014 & 0.0603 & & 0.1144 & 0.0003 & 0.0118 & 0.4251 & 0.0473 & & & & & & & & \\
\hline DZr-CV-20 & 0.0856 & 0.0507 & 0.1127 & 0.0298 & & 0.0014 & 0.0597 & & 0.1132 & 0.0003 & 0.0117 & 0.4209 & 0.0468 & & & & & & & & \\
\hline DZr-CV-21 & 0.0953 & 0.0564 & 0.1256 & 0.0332 & & 0.0016 & 0.0665 & & 0.1261 & 0.0003 & 0.0130 & 0.3600 & 0.0522 & & & & & & & & \\
\hline DZr-CV-22 & 0.0789 & 0.0467 & 0.1039 & 0.0275 & & 0.0013 & 0.0551 & & 0.1044 & 0.0003 & 0.0108 & 0.4699 & 0.0432 & & & & & & & & \\
\hline DZr-CV-23 & 0.0866 & 0.0513 & 0.1140 & 0.0302 & & 0.0014 & 0.0604 & & 0.1146 & 0.0003 & 0.0118 & 0.4259 & 0.0400 & & & & & & & & \\
\hline DZr-CV-24 & 0.0830 & 0.0491 & 0.1093 & 0.0289 & & 0.0014 & 0.0579 & & 0.1098 & 0.0003 & 0.0113 & 0.4082 & 0.0800 & & & & & & & & \\
\hline
\end{tabular}

\section{HLP glasses (Vienna et al. 2001)}

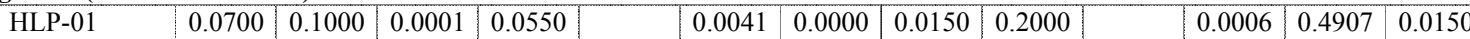

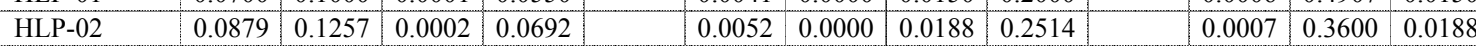

\begin{tabular}{|l|l|l|l|l|l|l|l|l|l|l|l|l|l|}
\hline HLP-03 & 0.0660 & 0.0943 & 0.0001 & 0.0519 & & 0.0039 & 0.0000 & 0.0141 & 0.1885 & & 0.0005 & 0.5200 & 0.0141 \\
\hline
\end{tabular}

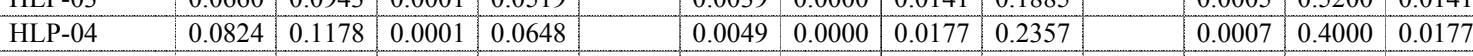

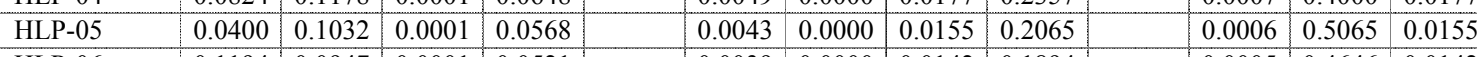

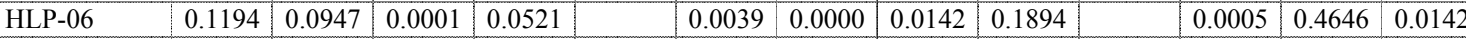

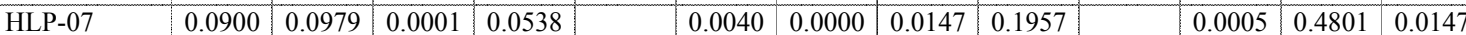

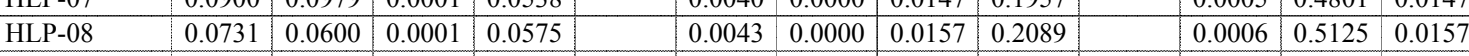

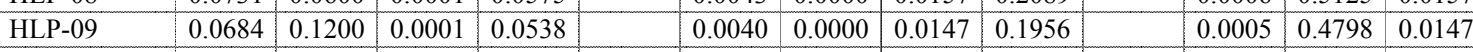

\begin{tabular}{|l|l|l|l|l|l|l|l|l|l|l|l|l|l|l|}
\hline HLP-10 & 0.0715 & 0.0800 & 0.0001 & 0.0563 & & 0.0042 & 0.0000 & 0.0153 & 0.2045 & & 0.0006 & 0.5016 & 0.0153 \\
\hline
\end{tabular}

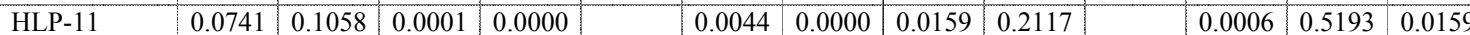

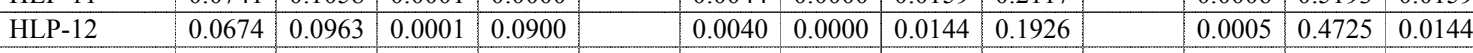

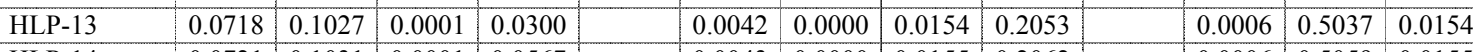

\begin{tabular}{|l|l|l|l|l|l|l|l|l|l|l|l|l|l|l|}
\hline HLP-14 & 0.0721 & 0.1031 & 0.0001 & 0.0567 & & 0.0043 & 0.0000 & 0.0155 & 0.2062 & & 0.0006 & 0.5059 & 0.0155 \\
\hline
\end{tabular}

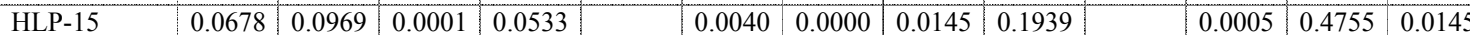

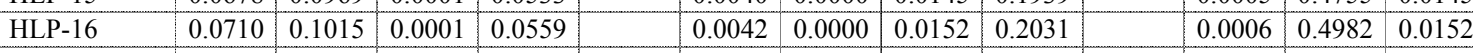

\begin{tabular}{|l|l|l|l|l|l|l|l|l|l|l|l|l|l|}
\hline HLP-17 & 0.0682 & 0.0975 & 0.0001 & 0.0536 & & 0.0040 & 0.0000 & 0.0146 & 0.1950 & & 0.0005 & 0.4782 & 0.0146 \\
\hline
\end{tabular}

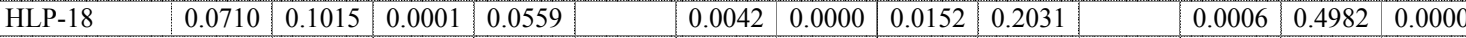

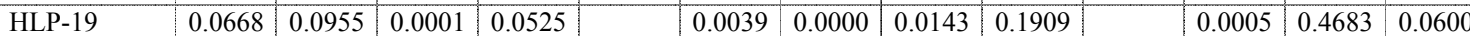

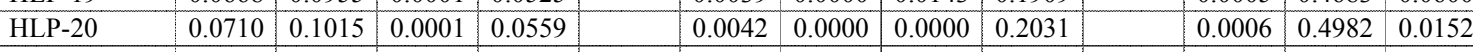

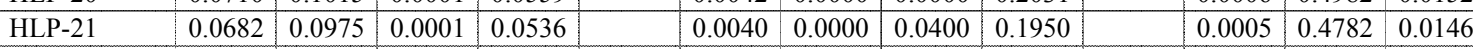

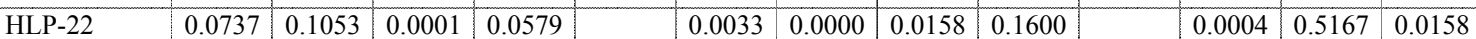


INEEL DZr-CV Glasses (Riley et al. 2001)

\begin{tabular}{|c|c|c|c|c|c|c|c|c|c|c|c|c|c|c|c|c|c|c|c|c|c|}
\hline Glass ID & $\begin{array}{l}\mathrm{Cl} \\
-\mathrm{t}\end{array}$ & $\begin{array}{c}\mathrm{CoO} \\
-\mathrm{t}\end{array}$ & $\begin{array}{c}\mathrm{Co} 2 \mathrm{O} 3 \\
-\mathrm{t}\end{array}$ & $\begin{array}{c}\mathrm{Cr} 2 \mathrm{O} 3 \\
-\mathrm{t}\end{array}$ & $\begin{array}{c}\mathrm{Cs} 2 \mathrm{O} \\
-\mathrm{t}\end{array}$ & $\begin{array}{c}\mathrm{CuO} \\
-\mathrm{t}\end{array}$ & $\begin{array}{c}\mathrm{Eu} 2 \mathrm{O} 3 \\
-\mathrm{t}\end{array}$ & $\begin{array}{l}F \\
-t\end{array}$ & $\begin{array}{c}\mathrm{Ga} 2 \mathrm{O} 3 \\
-\mathrm{t}\end{array}$ & {$\left[\begin{array}{c}\mathrm{Gd} 2 \mathrm{O} 3 \\
-\mathrm{t}\end{array}\right.$} & $\begin{array}{c}\mathrm{HgO} \\
-\mathrm{t}\end{array}$ & $\begin{array}{l}I \\
-t\end{array}$ & $\begin{array}{c}\mathrm{La} 2 \mathrm{O} 3 \\
-\mathrm{t}\end{array}$ & $\begin{array}{c}\mathrm{MnO} 2 \\
-\mathrm{t}\end{array}$ & $\begin{array}{c}\mathrm{MnO} \\
-\mathrm{t}\end{array}$ & $\begin{array}{c}\mathrm{MoO} \\
-\mathrm{t}\end{array}$ & $\begin{array}{c}\mathrm{MoO} 3 \\
-\mathrm{t}\end{array}$ & $\begin{array}{c}\mathrm{Nb} 2 \mathrm{O} 5 \\
-\mathrm{t}\end{array}$ & $\begin{array}{c}\mathrm{Nd} 2 \mathrm{O} 3 \\
-\mathrm{t}\end{array}$ & $\begin{array}{c}\mathrm{PbO} \\
-\mathrm{t}\end{array}$ & $\begin{array}{c}\mathrm{PdO} 2 \\
-\mathrm{t}\end{array}$ \\
\hline DZr-CV-5 & 0.0003 & & & 0.0014 & & & & 0.0480 & & & & & 0.0000 & & 0.0013 & & & & & 0.0002 & \\
\hline DZr-CV-6 & 0.0003 & & & 0.0014 & & & & 0.0451 & & & & & 0.0000 & & 0.0012 & & & & & 0.0002 & \\
\hline DZr-CV-7 & 0.0003 & & & 0.0014 & & & & 0.0400 & & & & & 0.0000 & & 0.0013 & & & & & 0.0002 & \\
\hline DZr-CV-8 & 0.0003 & & & 0.0014 & & & & 0.0650 & & & & & 0.0000 & & 0.0012 & & & & & 0.0002 & \\
\hline DZr-CV-9 & 0.0003 & & & 0.0014 & & & & 0.0485 & & & & & 0.0000 & & 0.0013 & & & & & 0.0002 & \\
\hline DZr-CV-10 & 0.0003 & & & 0.0014 & & & & 0.0460 & & & & & 0.0000 & & 0.0012 & & & & & 0.0002 & \\
\hline DZr-CV-11 & 0.0003 & & & 0.0014 & & & & 0.0458 & & & & & 0.0250 & & 0.0012 & & & & & 0.0002 & \\
\hline DZr-CV-12 & 0.0003 & & & 0.0014 & & & & 0.0447 & & & & & 0.0500 & & 0.0012 & & & & & 0.0001 & \\
\hline DZr-CV-13 & 0.0003 & & & 0.0014 & & & & 0.0478 & & & & & 0.0000 & & 0.0013 & & & & & 0.0002 & \\
\hline DZr-CV-14 & 0.0003 & & & 0.0014 & & & & 0.0465 & & & & & 0.0000 & & 0.0012 & & & & & 0.0002 & \\
\hline DZr-CV-15 & 0.0003 & & & 0.0014 & & & & 0.0488 & & & & & 0.0000 & & 0.0013 & & & & & 0.0002 & \\
\hline DZr-CV-16 & 0.0003 & & & 0.0014 & & & & 0.0461 & & & & & 0.0000 & & 0.0012 & & & & & 0.0002 & \\
\hline DZr-CV-17 & 0.0003 & & & 0.0014 & & & & 0.0476 & & & & & 0.0000 & & 0.0013 & & & & & 0.0002 & \\
\hline DZr-CV-18 & 0.0003 & & & 0.0014 & & & & 0.0464 & & & & & 0.0000 & & 0.0012 & & & & & 0.0002 & \\
\hline DZr-CV-19 & 0.0003 & & & 0.0014 & & & & 0.0473 & & & & & 0.0000 & & 0.0013 & & & & & 0.0002 & \\
\hline DZr-CV-20 & 0.0003 & & & 0.0014 & & & & 0.0468 & & & & & 0.0000 & & 0.0012 & & & & & 0.0002 & \\
\hline DZr-CV-21 & 0.0003 & & & 0.0014 & & & & 0.0521 & & & & & 0.0000 & & 0.0014 & & & & & 0.0002 & \\
\hline DZr-CV-22 & 0.0003 & & & 0.0014 & & & & 0.0432 & & & & & 0.0000 & & 0.0011 & & & & & 0.0001 & \\
\hline DZr-CV-23 & 0.0003 & & & 0.0014 & & & & 0.0474 & & & & & 0.0000 & & 0.0013 & & & & & 0.0002 & \\
\hline DZr-CV-24 & 0.0003 & & & 0.0014 & & & & 0.0454 & & & & & 0.0000 & & 0.0012 & & & & & 0.0002 & \\
\hline
\end{tabular}

HLP glasses (Vienna et al. 2001)

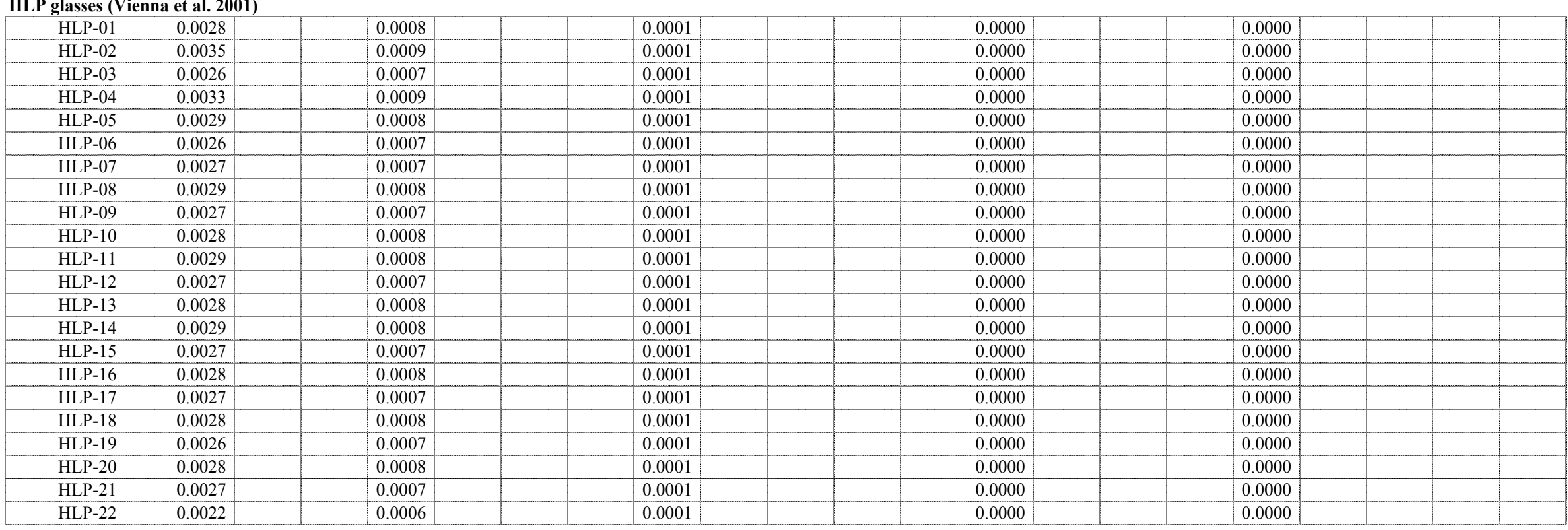


INEEL DZr-CV Glasses (Riley et al. 2001)

\begin{tabular}{|c|c|c|c|c|c|c|c|c|c|c|c|c|c|c|c|c|c|c|c|c|c|}
\hline Glass ID & $\begin{array}{c}\mathrm{PdO} \\
-\mathrm{t}\end{array}$ & $\begin{array}{c}\mathrm{Pr} 2 \mathrm{O} 3 \\
-\mathrm{t}\end{array}$ & $\begin{array}{c}\text { Pr6O11 } \\
-\mathrm{t}\end{array}$ & $\begin{array}{c}\mathrm{Rb} 2 \mathrm{O} \\
-\mathrm{t}\end{array}$ & $\begin{array}{c}\mathrm{ReO} \\
-\mathrm{t}\end{array}$ & $\begin{array}{c}\mathrm{ReO} 2 \\
-\mathrm{t}\end{array}$ & $\begin{array}{c}\mathrm{Rh} 2 \mathrm{O} 3 \\
-\mathrm{t}\end{array}$ & $\begin{array}{c}\mathrm{RhO}^{2} \\
-\mathrm{t}\end{array}$ & $\begin{array}{c}\mathrm{RuO} 2 \\
-\mathrm{t}\end{array}$ & $\begin{array}{c}\mathrm{Sb} 2 \mathrm{O} 3 \\
-\mathrm{t}\end{array}$ & $\begin{array}{c}\mathrm{Sb} 2 \mathrm{O} 5 \\
-\mathrm{t}\end{array}$ & $\begin{array}{c}\mathrm{SeO} 2 \\
-\mathrm{t}\end{array}$ & $\begin{array}{c}\mathrm{Sm} 2 \mathrm{O} 3 \\
-\mathrm{t}\end{array}$ & $\begin{array}{c}\mathrm{SnO} \\
-\mathrm{t}\end{array}$ & $\begin{array}{c}\mathrm{SnO} 2 \\
-\mathrm{t}\end{array}$ & $\begin{array}{c}\mathrm{SO} 3 \\
-\mathrm{t}\end{array}$ & $\begin{array}{c}\mathrm{SrO} \\
-\mathrm{t}\end{array}$ & $\begin{array}{c}\mathrm{Tc} 2 \mathrm{O} 7 \\
-\mathrm{t}\end{array}$ & $\begin{array}{c}\mathrm{TeO} 2 \\
-\mathrm{t}\end{array}$ & $\begin{array}{c}\mathrm{ThO} 2 \\
-\mathrm{t}\end{array}$ & $\begin{array}{c}\mathrm{TiO} 2 \\
-\mathrm{t}\end{array}$ \\
\hline DZr-CV-5 & & & & & & & & & & & & & & & & 0.0107 & 0.0025 & & & & \\
\hline DZr-CV-6 & & & & & & & & & & & & & & & & 0.0100 & 0.0023 & & & & \\
\hline DZr-CV-7 & & & & & & & & & & & & & & & & 0.0105 & 0.0024 & & & & \\
\hline DZr-CV-8 & & & & & & & & & & & & & & & & 0.0103 & 0.0024 & & & & \\
\hline DZr-CV-9 & & & & & & & & & & & & & & & & 0.0108 & 0.0025 & & & & \\
\hline DZr-CV-10 & & & & & & & & & & & & & & & & 0.0102 & 0.0024 & & & & \\
\hline DZr-CV-11 & & & & & & & & & & & & & & & & 0.0102 & 0.0023 & & & & \\
\hline DZr-CV-12 & & & & & & & & & & & & & & & & 0.0099 & 0.0023 & & & & \\
\hline DZr-CV-13 & & & & & & & & & & & & & & & & 0.0106 & 0.0024 & & & & \\
\hline DZr-CV-14 & & & & & & & & & & & & & & & & 0.0104 & 0.0024 & & & & \\
\hline DZr-CV-15 & & & & & & & & & & & & & & & & 0.0109 & 0.0025 & & & & \\
\hline DZr-CV-16 & & & & & & & & & & & & & & & & 0.0103 & 0.0024 & & & & \\
\hline DZr-CV-17 & & & & & & & & & & & & & & & & 0.0106 & 0.0024 & & & & \\
\hline DZr-CV-18 & & & & & & & & & & & & & & & & 0.0103 & 0.0024 & & & & \\
\hline DZr-CV-19 & & & & & & & & & & & & & & & & 0.0050 & 0.0024 & & & & \\
\hline DZr-CV-20 & & & & & & & & & & & & & & & & 0.0150 & 0.0024 & & & & \\
\hline DZr-CV-21 & & & & & & & & & & & & & & & & 0.0116 & 0.0027 & & & & \\
\hline DZr-CV-22 & & & & & & & & & & & & & & & & 0.0096 & 0.0022 & & & & \\
\hline DZr-CV-23 & & & & & & & & & & & & & & & & 0.0105 & 0.0024 & & & & \\
\hline DZr-CV-24 & & & & & & & & & & & & & & & & 0.0101 & 0.0023 & & & & \\
\hline
\end{tabular}

HLP glasses (Vienna et al. 2001)

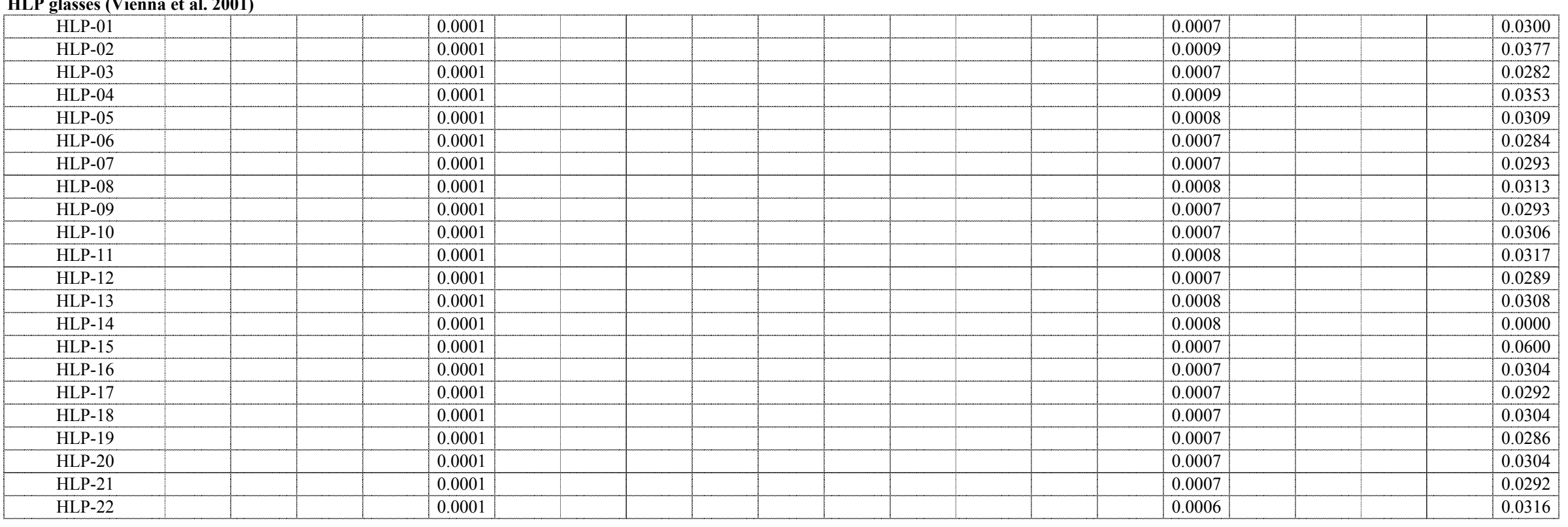


Appendix A. Database - mass fraction

INEEL DZr-CV Glasses (Riley et al. 2001)

\begin{tabular}{|c|c|c|c|c|c|c|c|c|c|c|c|c|c|c|c|c|c|c|c|c|c|}
\hline Glass ID & $\begin{array}{c}\mathrm{T} 12 \mathrm{O} 3 \\
-\mathrm{t}\end{array}$ & $\begin{array}{c}\mathrm{U} 3 \mathrm{O} 8 \\
-\mathrm{t}\end{array}$ & $\begin{array}{c}\mathrm{UO} 2 \\
-\mathrm{t}\end{array}$ & $\begin{array}{c}\mathrm{UO} 3 \\
-\mathrm{t}\end{array}$ & $\begin{array}{c}\mathrm{V} 2 \mathrm{O} 5 \\
-\mathrm{t}\end{array}$ & $\begin{array}{c}\text { WO3 } \\
-t\end{array}$ & $\begin{array}{c}\mathrm{Y} 2 \mathrm{O} 3 \\
-\mathrm{t}\end{array}$ & $\begin{array}{c}\mathrm{ZnO} \\
-\mathrm{t}\end{array}$ & $\begin{array}{c}\text { Others } \\
-t\end{array}$ & $\begin{array}{c}\text { Sum } \\
-t\end{array}$ & $\begin{array}{c}\mathrm{A} 12 \mathrm{O} 3 \\
-\mathrm{a}\end{array}$ & $\begin{array}{c}\mathrm{B} 2 \mathrm{O} 3 \\
-\mathrm{a}\end{array}$ & $\begin{array}{c}\mathrm{CaO} \\
-\mathrm{a}\end{array}$ & $\begin{array}{c}\mathrm{Fe} 2 \mathrm{O} 3 \\
-\mathrm{a}\end{array}$ & $\begin{array}{c}\mathrm{FeO} \\
-\mathrm{a}\end{array}$ & $\begin{array}{c}\mathrm{K} 2 \mathrm{O} \\
-\mathrm{a}\end{array}$ & $\begin{array}{c}\mathrm{Li} 2 \mathrm{O} \\
-\mathrm{a}\end{array}$ & $\begin{array}{c}\mathrm{MgO} \\
-\mathrm{a}\end{array}$ & $\begin{array}{c}\mathrm{Na} 2 \mathrm{O} \\
-\mathrm{a}\end{array}$ & $\begin{array}{c}\mathrm{NiO} \\
-\mathrm{a}\end{array}$ & $\begin{array}{c}\mathrm{P} 2 \mathrm{O} 5 \\
-\mathrm{a}\end{array}$ \\
\hline DZr-CV-5 & & & & & & & & & & 1.0000 & & & & & & & & & & & \\
\hline DZr-CV-6 & & & & & & & & & & 1.0000 & & & & & & & & & & & \\
\hline DZr-CV-7 & & & & & & & & & & 1.0000 & & & & & & & & & & & \\
\hline DZr-CV-8 & & & & & & & & & & 1.0000 & & & & & & & & & & & \\
\hline DZr-CV-9 & & & & & & & & & & 1.0000 & & & & & & & & & & & \\
\hline DZr-CV-10 & & & & & & & & & & 1.0000 & & & & & & & & & & & \\
\hline DZr-CV-11 & & & & & & & & & & 1.0000 & & & & & & & & & & & \\
\hline DZr-CV-12 & & & & & & & & & & 1.0000 & & & & & & & & & & & \\
\hline DZr-CV-13 & & & & & & & & & & 1.0000 & & & & & & & & & & & \\
\hline DZr-CV-14 & & & & & & & & & & 1.0000 & & & & & & & & & & & \\
\hline DZr-CV-15 & & & & & & & & & & 1.0000 & & & & & & & & & & & \\
\hline DZr-CV-16 & & & & & & & & & & 1.0000 & & & & & & & & & & & \\
\hline DZr-CV-17 & & & & & & & & & & 1.0000 & & & & & & & & & & & \\
\hline DZr-CV-18 & & & & & & & & & & 1.0000 & & & & & & & & & & & \\
\hline DZr-CV-19 & & & & & & & & & & 1.0000 & & & & & & & & & & & \\
\hline DZr-CV-20 & & & & & & & & & & 1.0000 & & & & & & & & & & & \\
\hline DZr-CV-21 & & & & & & & & & & 1.0000 & & & & & & & & & & & \\
\hline DZr-CV-22 & & & & & & & & & & 1.0000 & & & & & & & & & & & \\
\hline DZr-CV-23 & & & & & & & & & & 1.0000 & & & & & & & & & & & \\
\hline DZr-CV-24 & & & & & & & & & & 1.0000 & & & & & & & & & & & \\
\hline
\end{tabular}

HLP glasses (Vienna et al. 2001)

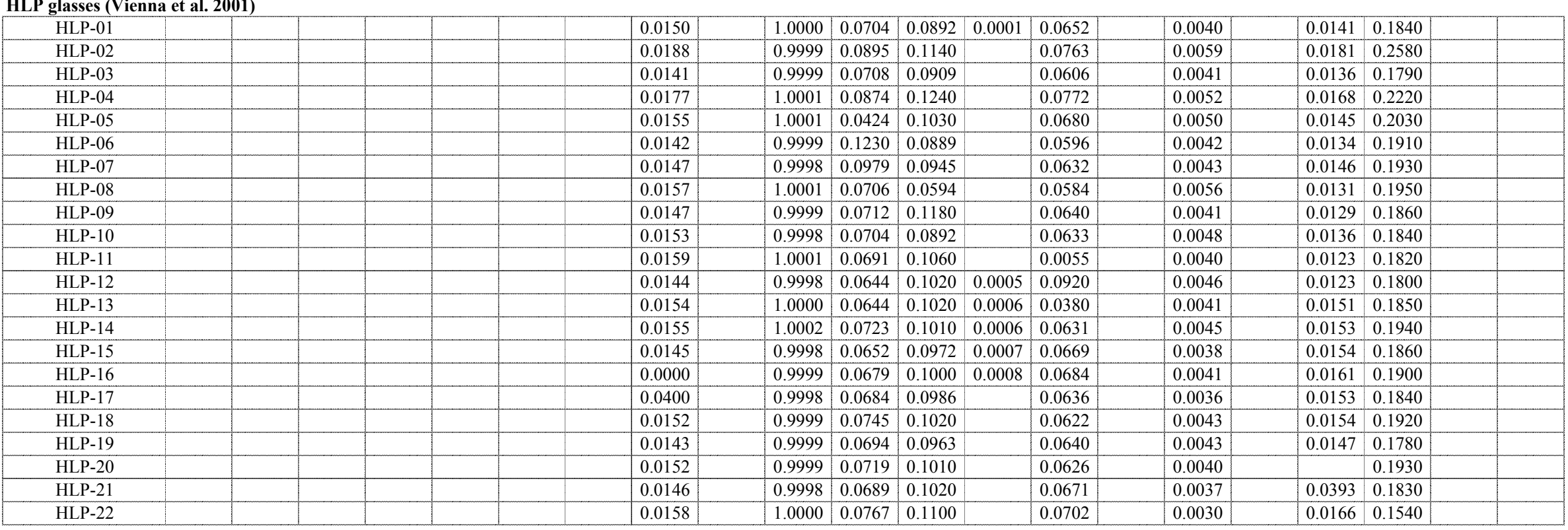


INEEL DZr-CV Glasses (Riley et al. 2001)

\begin{tabular}{|c|c|c|c|c|c|c|c|c|c|c|c|c|c|c|c|c|c|c|c|c|c|}
\hline Glass ID & $\begin{array}{c}\mathrm{SiO} 2 \\
-\mathrm{a}\end{array}$ & $\begin{array}{c}\mathrm{ZrO} 2 \\
-\mathrm{a}\end{array}$ & $\begin{array}{c}\mathrm{Ag} 2 \mathrm{O} \\
-\mathrm{a}\end{array}$ & $\begin{array}{c}\mathrm{As} 2 \mathrm{O} 3 \\
-\mathrm{a}\end{array}$ & $\begin{array}{c}\mathrm{BaO} \\
-\mathrm{a}\end{array}$ & $\begin{array}{c}\mathrm{Bi} 2 \mathrm{O} 3 \\
-\mathrm{a}\end{array}$ & $\begin{array}{l}\mathrm{Br} \\
-\mathrm{a}\end{array}$ & $\begin{array}{c}\mathrm{CdO} \\
-\mathrm{a}\end{array}$ & $\begin{array}{c}\mathrm{Ce} 2 \mathrm{O} 3 \\
-\mathrm{a}\end{array}$ & $\begin{array}{c}\mathrm{CeO} 2 \\
-\mathrm{a}\end{array}$ & $\begin{array}{l}\mathrm{Cl} \\
-\mathrm{a}\end{array}$ & $\begin{array}{c}\mathrm{CoO} \\
-\mathrm{a}\end{array}$ & $\begin{array}{c}\mathrm{Co} 2 \mathrm{O} 3 \\
-\mathrm{a}\end{array}$ & $\begin{array}{c}\mathrm{Cr} 2 \mathrm{O} 3 \\
-\mathrm{a}\end{array}$ & $\begin{array}{c}\mathrm{Cs} 2 \mathrm{O} \\
-\mathrm{a}\end{array}$ & $\begin{array}{c}\mathrm{CuO} \\
-\mathrm{a}\end{array}$ & $\begin{array}{c}\mathrm{Eu} 2 \mathrm{O} 3 \\
-\mathrm{a}\end{array}$ & $\begin{array}{l}\mathrm{F} \\
-\mathrm{a}\end{array}$ & $\begin{array}{c}\mathrm{Ga} 2 \mathrm{O} 3 \\
-\mathrm{a}\end{array}$ & $\begin{array}{c}\mathrm{Gd} 2 \mathrm{O} 3 \\
-\mathrm{a}\end{array}$ & $\begin{array}{c}\mathrm{HgO} \\
-\mathrm{a}\end{array}$ \\
\hline DZr-CV-5 & & & & & & & & & & & & & & & & & & & & & \\
\hline DZr-CV-6 & & & & & & & & & & & & & & & & & & & & & \\
\hline DZr-CV-7 & & & & & & & & & & & & & & & & & & & & & \\
\hline DZr-CV-8 & & & & & & & & & & & & & & & & & & & & & \\
\hline DZr-CV-9 & & & & & & & & & & & & & & & & & & & & & \\
\hline DZr-CV-10 & & & & & & & & & & & & & & & & & & & & & \\
\hline DZr-CV-11 & & & & & & & & & & & & & & & & & & & & & \\
\hline DZr-CV-12 & & & & & & & & & & & & & & & & & & & & & \\
\hline DZr-CV-13 & & & & & & & & & & & & & & & & & & & & & \\
\hline DZr-CV-14 & & & & & & & & & & & & & & & & & & & & & \\
\hline DZr-CV-15 & & & & & & & & & & & & & & & & & & & & & \\
\hline DZr-CV-16 & & & & & & & & & & & & & & & & & & & & & \\
\hline DZr-CV-17 & & & & & & & & & & & & & & & & & & & & & \\
\hline DZr-CV-18 & & & & & & & & & & & & & & & & & & & & & \\
\hline DZr-CV-19 & & & & & & & & & & & & & & & & & & & & & \\
\hline DZr-CV-20 & & & & & & & & & & & & & & & & & & & & & \\
\hline DZr-CV-21 & & & & & & & & & & & & & & & & & & & & & \\
\hline DZr-CV-22 & & & & & & & & & & & & & & & & & & & & & \\
\hline DZr-CV-23 & & & & & & & & & & & & & & & & & & & & & \\
\hline DZr-CV-24 & & & & & & & & & & & & & & & & & & & & & \\
\hline
\end{tabular}

\section{HLP glasses (Vienna et al. 2001)}

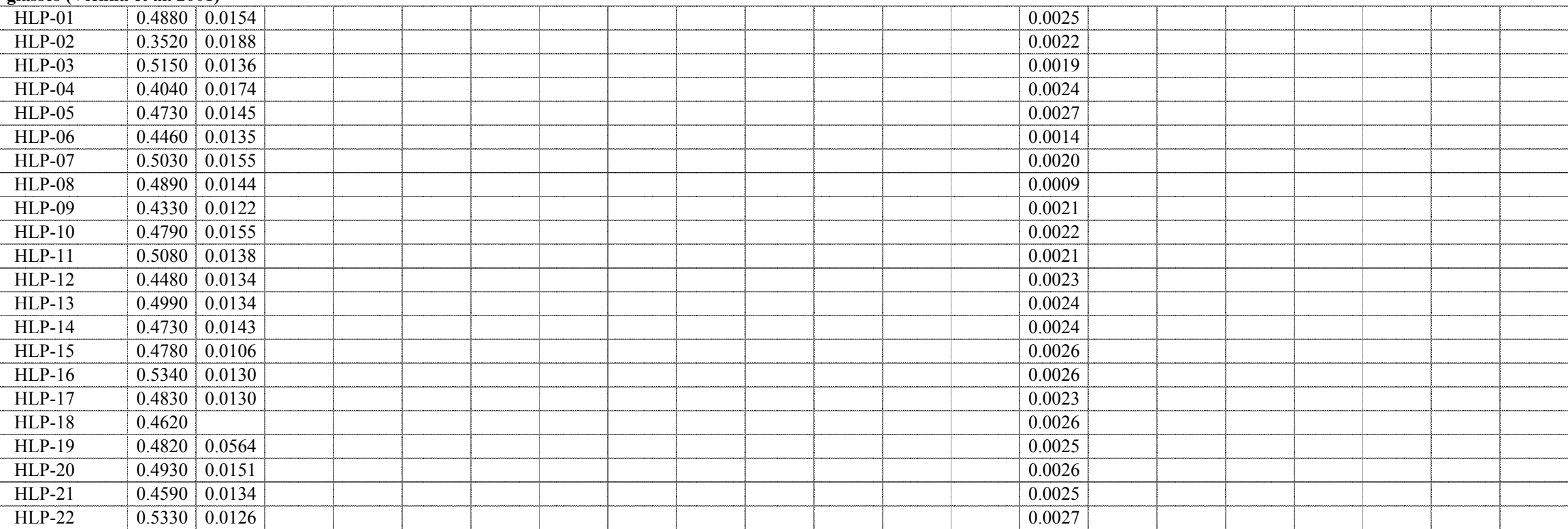


INEEL DZr-CV Glasses (Riley et al. 2001)

\begin{tabular}{|c|c|c|c|c|c|c|c|c|c|c|c|c|c|c|c|c|c|c|c|c|c|}
\hline Glass ID & $\begin{array}{c}I \\
-a\end{array}$ & $\begin{array}{c}\mathrm{La} 2 \mathrm{O} 3 \\
-\mathrm{a}\end{array}$ & $\begin{array}{c}\mathrm{MnO} 2 \\
-\mathrm{a}\end{array}$ & $\begin{array}{c}\mathrm{MnO} \\
-\mathrm{a}\end{array}$ & $\begin{array}{c}\mathrm{MoO} \\
-\mathrm{a}\end{array}$ & $\begin{array}{c}\mathrm{MoO} 3 \\
-\mathrm{a}\end{array}$ & $\begin{array}{c}\mathrm{Nb} 2 \mathrm{O} 5 \\
-\mathrm{a}\end{array}$ & $\begin{array}{c}\mathrm{Nd} 2 \mathrm{O} 3 \\
-\mathrm{a}\end{array}$ & $\begin{array}{c}\mathrm{PbO} \\
-\mathrm{a}\end{array}$ & $\begin{array}{c}\mathrm{PdO} 2 \\
-\mathrm{a}\end{array}$ & $\begin{array}{c}\mathrm{PdO} \\
-\mathrm{a}\end{array}$ & $\begin{array}{c}\mathrm{Pr} 2 \mathrm{O} 3 \\
-\mathrm{a}\end{array}$ & $\begin{array}{c}\text { Pr6O11 } \\
-\mathrm{a}\end{array}$ & $\begin{array}{c}\mathrm{Rb} 2 \mathrm{O} \\
-\mathrm{a}\end{array}$ & $\begin{array}{c}\mathrm{ReO} \\
-\mathrm{a}\end{array}$ & $\begin{array}{c}\mathrm{ReO} 2 \\
-\mathrm{a}\end{array}$ & $\begin{array}{c}\mathrm{Rh} 2 \mathrm{O} 3 \\
-\mathrm{a}\end{array}$ & $\begin{array}{c}\mathrm{RhO} 2 \\
-\mathrm{a}\end{array}$ & $\begin{array}{c}\mathrm{RuO} 2 \\
-\mathrm{a}\end{array}$ & $\begin{array}{c}\mathrm{Sb} 2 \mathrm{O} 3 \\
-\mathrm{a}\end{array}$ & $\begin{array}{c}\mathrm{Sb} 2 \mathrm{O} 5 \\
-\mathrm{a}\end{array}$ \\
\hline DZr-CV-5 & & & & & & & & & & & & & & & & & & & & & \\
\hline DZr-CV-6 & & & & & & & & & & & & & & & & & & & & & \\
\hline DZr-CV-7 & & & & & & & & & & & & & & & & & & & & & \\
\hline DZr-CV-8 & & & & & & & & & & & & & & & & & & & & & \\
\hline DZr-CV-9 & & & & & & & & & & & & & & & & & & & & & \\
\hline DZr-CV-10 & & & & & & & & & & & & & & & & & & & & & \\
\hline DZr-CV-11 & & & & & & & & & & & & & & & & & & & & & \\
\hline DZr-CV-12 & & & & & & & & & & & & & & & & & & & & & \\
\hline DZr-CV-13 & & & & & & & & & & & & & & & & & & & & & \\
\hline DZr-CV-14 & & & & & & & & & & & & & & & & & & & & & \\
\hline DZr-CV-15 & & & & & & & & & & & & & & & & & & & & & \\
\hline DZr-CV-16 & & & & & & & & & & & & & & & & & & & & & \\
\hline DZr-CV-17 & & & & & & & & & & & & & & & & & & & & & \\
\hline DZr-CV-18 & & & & & & & & & & & & & & & & & & & & & \\
\hline DZr-CV-19 & & & & & & & & & & & & & & & & & & & & & \\
\hline DZr-CV-20 & & & & & & & & & & & & & & & & & & & & & \\
\hline DZr-CV-21 & & & & & & & & & & & & & & & & & & & & & \\
\hline DZr-CV-22 & & & & & & & & & & & & & & & & & & & & & \\
\hline DZr-CV-23 & & & & & & & & & & & & & & & & & & & & & \\
\hline DZr-CV-24 & & & & & & & & & & & & & & & & & & & & & \\
\hline
\end{tabular}

HLP glasses (Vienna et al. 2001)

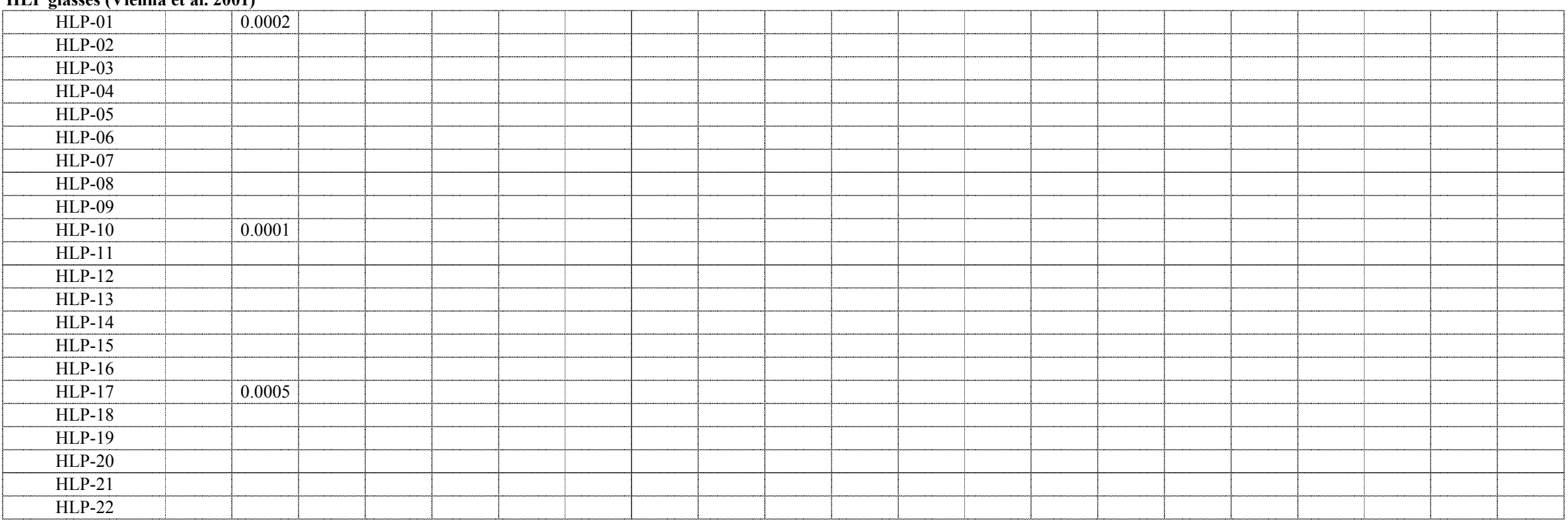


Appendix A. Database - mass fraction

INEEL DZr-CV Glasses (Riley et al. 2001)

\begin{tabular}{|c|c|c|c|c|c|c|c|c|c|c|c|c|c|c|c|c|c|c|c|c|}
\hline Glass ID & $\begin{array}{c}\mathrm{SeO} 2 \\
-\mathrm{a}\end{array}$ & $\begin{array}{c}\mathrm{Sm} 2 \mathrm{O} 3 \\
-\mathrm{a}\end{array}$ & $\begin{array}{c}\mathrm{SnO} \\
-\mathrm{a} \\
\end{array}$ & $\begin{array}{c}\mathrm{SnO} 2 \\
-\mathrm{a}\end{array}$ & $\begin{array}{c}\mathrm{SO} 3 \\
-\mathrm{a}\end{array}$ & $\begin{array}{c}\mathrm{SrO} \\
-\mathrm{a}\end{array}$ & $\begin{array}{c}\mathrm{Tc} 2 \mathrm{O} 7 \\
-\mathrm{a}\end{array}$ & $\begin{array}{c}\mathrm{TeO} 2 \\
-\mathrm{a}\end{array}$ & $\begin{array}{c}\mathrm{ThO} 2 \\
-\mathrm{a}\end{array}$ & $\begin{array}{c}\mathrm{TiO} 2 \\
-\mathrm{a}\end{array}$ & $\begin{array}{c}\mathrm{T} 12 \mathrm{O} 3 \\
-\mathrm{a}\end{array}$ & $\begin{array}{c}\mathrm{U} 3 \mathrm{O} 8 \\
-\mathrm{a}\end{array}$ & $\begin{array}{c}\mathrm{UO} 2 \\
-\mathrm{a}\end{array}$ & $\begin{array}{c}\mathrm{UO} 3 \\
-\mathrm{a}\end{array}$ & $\begin{array}{c}\mathrm{V} 2 \mathrm{O} 5 \\
-\mathrm{a}\end{array}$ & $\begin{array}{c}\text { WO3 } \\
-\mathrm{a}\end{array}$ & $\begin{array}{c}\mathrm{Y} 2 \mathrm{O} 3 \\
-\mathrm{a}\end{array}$ & $\begin{array}{c}\mathrm{ZnO} \\
-\mathrm{a}\end{array}$ & $\begin{array}{c}\text { Others } \\
-\mathrm{a}\end{array}$ & $\begin{array}{c}\text { Sum } \\
-\mathrm{a}\end{array}$ \\
\hline DZr-CV-5 & & & & & & & & & & & & & & & & & & & & \\
\hline DZr-CV-6 & & & & & & & & & & & & & & & & & & & & \\
\hline DZr-CV-7 & & & & & & & & & & & & & & & & & & & & \\
\hline DZr-CV-8 & & & & & & & & & & & & & & & & & & & & \\
\hline DZr-CV-9 & & & & & & & & & & & & & & & & & & & & \\
\hline DZr-CV-10 & & & & & & & & & & & & & & & & & & & & \\
\hline DZr-CV-11 & & & & & & & & & & & & & & & & & & & & \\
\hline DZr-CV-12 & & & & & & & & & & & & & & & & & & & & \\
\hline DZr-CV-13 & & & & & & & & & & & & & & & & & & & & \\
\hline DZr-CV-14 & & & & & & & & & & & & & & & & & & & & \\
\hline DZr-CV-15 & & & & & & & & & & & & & & & & & & & & \\
\hline DZr-CV-16 & & & & & & & & & & & & & & & & & & & & \\
\hline DZr-CV-17 & & & & & & & & & & & & & & & & & & & & \\
\hline DZr-CV-18 & & & & & & & & & & & & & & & & & & & & \\
\hline DZr-CV-19 & & & & & & & & & & & & & & & & & & & & \\
\hline DZr-CV-20 & & & & & & & & & & & & & & & & & & & & \\
\hline DZr-CV-21 & & & & & & & & & & & & & & & & & & & & \\
\hline DZr-CV-22 & & & & & & & & & & & & & & & & & & & & \\
\hline DZr-CV-23 & & & & & & & & & & & & & & & & & & & & \\
\hline DZr-CV-24 & & & & & & & & & & & & & & & & & & & & \\
\hline
\end{tabular}

\section{HLP glasses (Vienna et al. 2001)}

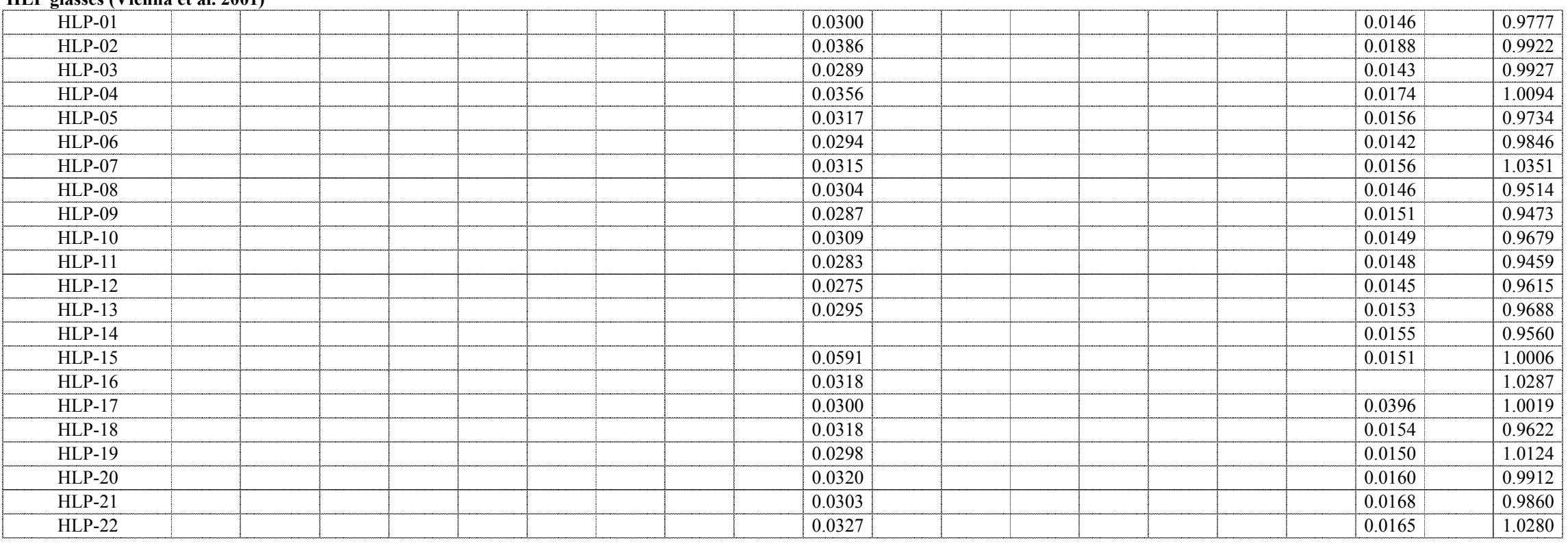


Appendix A. Database - mass fraction

INEEL DZr-CV Glasses (Riley et al. 2001)

\begin{tabular}{|c|c|c|c|c|c|c|c|c|}
\hline Glass ID & $\begin{array}{l}\mathrm{TM} \\
\left({ }^{\circ} \mathrm{C}\right)\end{array}$ & $\begin{array}{l}\text { Gradient } \\
\text { TL }\left({ }^{\circ} \mathrm{C}\right)\end{array}$ & $\begin{array}{l}\text { Uniform } \\
\text { TL }\left({ }^{\circ} \mathrm{C}\right)\end{array}$ & Primary Phase & $\begin{array}{l}\text { Quenched } \\
\text { Visual/OM }\end{array}$ & $\begin{array}{c}\text { Quenched } \\
\text { SEM/EDS or TEM }\end{array}$ & $\begin{array}{l}\text { Quenched } \\
\text { XRD }\end{array}$ & $\begin{array}{c}\text { Quenched } \\
\text { Homogeneous? }\end{array}$ \\
\hline DZr-CV-5 & & & & & slight phase separation & & & \\
\hline DZr-CV-6 & & & & & amorphous Homo. & & & \\
\hline $\mathrm{DZr}-\mathrm{CV}-7$ & & & & & amorphous Homo. & & & \\
\hline DZr-CV-8 & & & & & massive phase separation & & & \\
\hline DZr-CV-9 & & & & & amorphous Homo. & & & \\
\hline DZr-CV-10 & & & & & amorphous Homo. & & & \\
\hline DZr-CV-11 & & & & & amorphous Homo. & & & \\
\hline DZr-CV-12 & & & & & amorphous Homo. & & & \\
\hline DZr-CV-13 & & & & & slight phase separation & & & \\
\hline DZr-CV-14 & & & & & amorphous Homo. & & & \\
\hline DZr-CV-15 & & & & & slight phase separation & & & \\
\hline DZr-CV-16 & & & & & amorphous Homo. & & & \\
\hline DZr-CV-17 & & & & & amorphous Homo. & & & \\
\hline DZr-CV-18 & & & & & moderate phase separation & & & \\
\hline DZr-CV-19 & & & & & amorphous Homo. & & & \\
\hline DZr-CV-20 & & & & & moderate phase separation & & & \\
\hline DZr-CV-21 & & & & & amorphous Homo. & & & \\
\hline DZr-CV-22 & & & & & amorphous Homo. & & & \\
\hline DZr-CV-23 & & & & & amorphous Homo. & & & \\
\hline DZr-CV-24 & & & & & amorphous Homo. & & & \\
\hline
\end{tabular}

HLP glasses (Vienna et al. 2001)

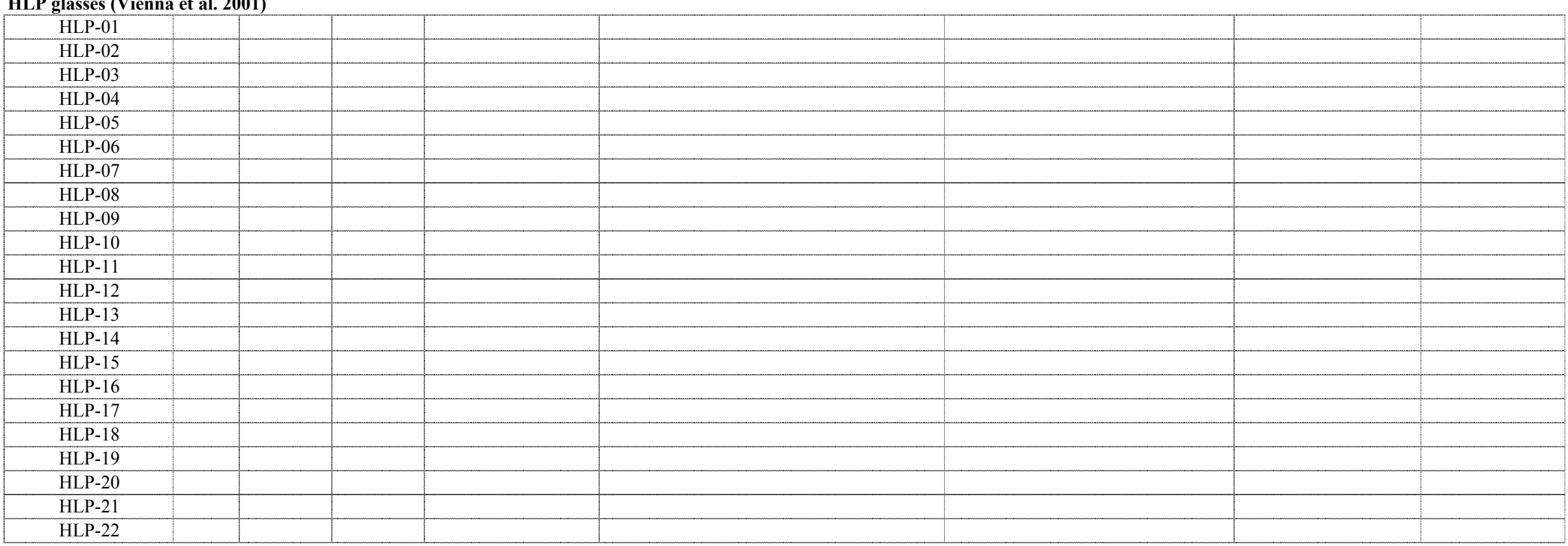


Appendix A. Database - mass fraction

\begin{tabular}{|c|c|c|c|c|c|}
\hline Glass ID & $\begin{array}{c}\mathrm{CCC} \\
\text { Visual/OM }\end{array}$ & $\begin{array}{c}\text { CCC } \\
\text { SEM/EDS or TEM }\end{array}$ & $\begin{array}{l}\text { CCC } \\
\text { XRD }\end{array}$ & $\begin{array}{l}\text { Heat Treated } \\
\text { Visual/OM }\end{array}$ & $\begin{array}{c}\text { Heat Treated } \\
\text { SEM/EDS or TEM }\end{array}$ \\
\hline DZr-CV-5 & crystallized phase separated & & $1.7 \mathrm{wt} \%$ fluorapatite & & \\
\hline DZr-CV-6 & crystallized homogenous & & no crystal & & \\
\hline DZr-CV-7 & crystallized homogenous & & no crystal & & \\
\hline DZr-CV-8 & crystallized phase separated & & $0.3 \mathrm{wt} \%$ fluorapatite, $3.8 \mathrm{wt} \%$ fluorite & & \\
\hline DZr-CV-9 & crystallized phase separated & & $2.1 \mathrm{wt} \%$ fluorapatite & & \\
\hline DZr-CV-10 & crystallized homogenous & & $3.0 \mathrm{wt} \%$ cuspidine & & \\
\hline DZr-CV-11 & crystallized homogenous & & $1.6 \mathrm{wt} \%$ cuspidine & & \\
\hline DZr-CV-12 & crystallized phase separated & & $5.5 \mathrm{wt} \%$ apatite & & \\
\hline DZr-CV-13 & crystallized phase separated & & $\begin{array}{l}1.4 \mathrm{wt} \% \text { fluorite, } 6.2 \mathrm{wt} \% \text { sodium } \\
\text { calcium oxide fluoride phosphate }\end{array}$ & & \\
\hline DZr-CV-14 & crystallized homogenous & & no crystal & & \\
\hline DZr-CV-15 & crystallized phase separated & & $2.4 \mathrm{wt} \%$ fluorite & & \\
\hline DZr-CV-16 & crystallized phase separated & & no crystal & & \\
\hline DZr-CV-17 & crystallized homogenous & & $1.3 \mathrm{wt} \%$ cuspidine & & \\
\hline DZr-CV-18 & crystallized phase separated & & $4.8 \mathrm{wt} \%$ fluorapatite & & \\
\hline DZr-CV-19 & amorphous Homo. & & no crystal & & \\
\hline DZr-CV-20 & crystallized phase separated & & $8.7 \mathrm{wt} \%$ sodalite & & \\
\hline DZr-CV-21 & crystallized phase separated & & $3.7 \mathrm{wt} \%$ cuspidine, $12.1 \mathrm{wt} \%$ nosean & & \\
\hline DZr-CV-22 & crystallized phase separated & & $0.6 \mathrm{wt} \%$ fluorapatite, $0.6 \mathrm{wt} \%$ fluorite & & \\
\hline DZr-CV-23 & crystallized phase separated & & $1.0 \mathrm{wt} \%$ cuspidine & & \\
\hline DZr-CV-24 & crystallized phase separated & & $0.6 \mathrm{wt} \%$ fluorite, $15.3 \mathrm{wt} \%$ hiortdahlite & & \\
\hline
\end{tabular}

\section{HLP glasses (Vienna et al. 2001)}

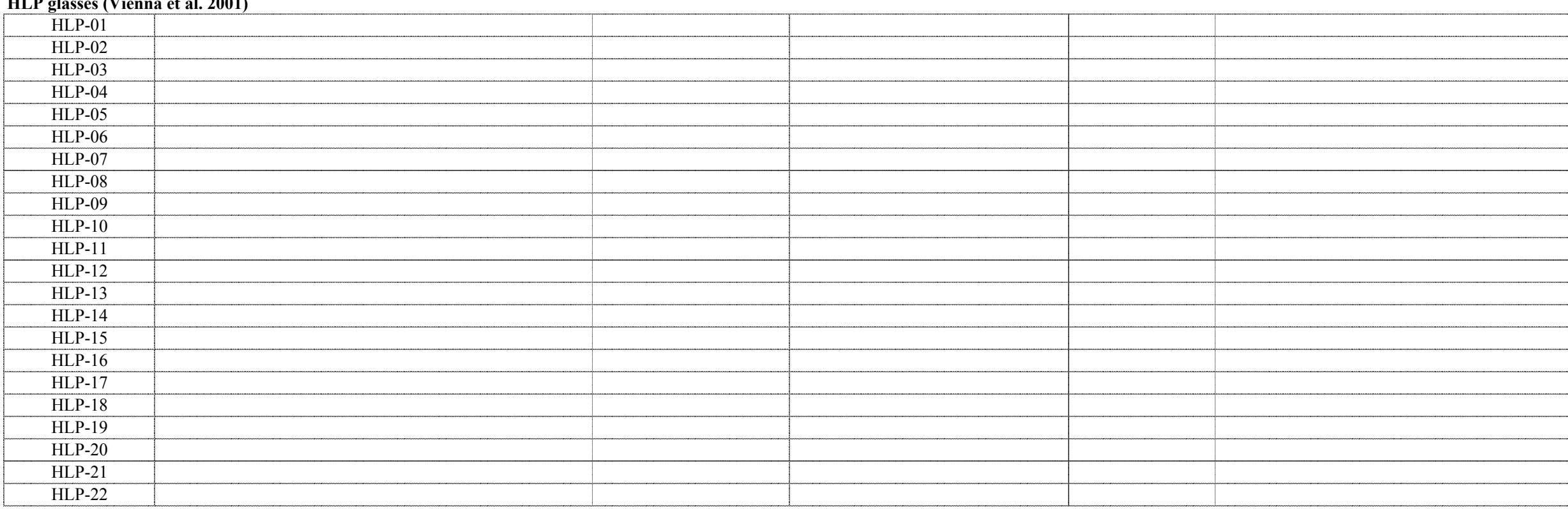


Appendix A. Database - mass fraction

INEEL DZr-CV Glasses (Riley et al. 2001)

\begin{tabular}{|c|c|c|c|c|c|c|c|c|c|c|c|c|c|c|c|}
\hline Glass ID & $\begin{array}{c}\text { Heat Treated } \\
\text { XRD } \\
\end{array}$ & $\begin{array}{l}\text { Density } \\
\left(\mathrm{g} / \mathrm{cm}^{3}\right)\end{array}$ & $\begin{array}{c}\text { Fulc Visc } \\
\mathrm{A}\end{array}$ & $\begin{array}{c}\text { Fulc Visc } \\
\text { B }\end{array}$ & $\begin{array}{c}\text { Fulc Visc } \\
\text { To }\end{array}$ & $\begin{array}{c}\mathrm{FV} 1150^{\circ} \mathrm{C} \\
(\mathrm{Pa} \cdot \mathrm{s})\end{array}$ & \begin{tabular}{|c|} 
Arrh Visc \\
$\mathrm{A}$
\end{tabular} & $\begin{array}{c}\text { Arrh Visc } \\
\text { B } \\
\end{array}$ & $\begin{array}{c}\text { Av } \\
1150^{\circ} \mathrm{C} \\
\left(\mathrm{D}_{0}, \mathrm{C}^{\prime}\right.\end{array}$ & $\begin{array}{c}\mathrm{T}\left({ }^{\circ} \mathrm{C}\right) \text { at } \\
2 \mathrm{~Pa} \cdot \mathrm{s}\end{array}$ & $\begin{array}{c}\mathrm{T}\left({ }^{\circ} \mathrm{C}\right) \text { at } \\
5 \mathrm{~Pa} \cdot \mathrm{s}\end{array}$ & $\begin{array}{l}\mathrm{T}\left({ }^{\circ} \mathrm{C}\right) \text { at } \\
10 \mathrm{~Pa} \cdot \mathrm{s}\end{array}$ & $\begin{array}{l}\mathrm{T} 1 \\
\left({ }^{\circ} \mathrm{C}\right) \\
\end{array}$ & $\begin{array}{c}\mathrm{V} 1 \\
(\mathrm{~Pa} \cdot \mathrm{s}) \\
\end{array}$ & $\begin{array}{c}\mathrm{T} 2 \\
\left({ }^{\circ} \mathrm{C}\right) \\
\end{array}$ \\
\hline DZr-CV-5 & & & & & & & & & & & & & & & \\
\hline DZr-CV-6 & & & & & & & & & & & & & & & \\
\hline DZr-CV-7 & & & & & & & & & & & & & & & \\
\hline DZr-CV-8 & & & & & & & & & & & & & & & \\
\hline DZr-CV-9 & & & & & & & & & & & & & & & \\
\hline DZr-CV-10 & & & & & & & & & & & & & & & \\
\hline DZr-CV-11 & & & & & & & & & & & & & & & \\
\hline DZr-CV-12 & & & & & & & & & & & & & & & \\
\hline DZr-CV-13 & & & & & & & & & & & & & & & \\
\hline DZr-CV-14 & & & & & & & & & & & & & & & \\
\hline DZr-CV-15 & & & & & & & & & & & & & & & \\
\hline DZr-CV-16 & & & & & & & & & & & & & & & \\
\hline DZr-CV-17 & & & & & & & & & & & & & & & \\
\hline DZr-CV-18 & & & & & & & & & & & & & & & \\
\hline DZr-CV-19 & & & & & & & & & & & & & & & \\
\hline DZr-CV-20 & & & & & & & & & & & & & & & \\
\hline DZr-CV-21 & & & & & & & & & & & & & & & \\
\hline DZr-CV-22 & & & & & & & & & & & & & & & \\
\hline DZr-CV-23 & & & & & & & & & & & & & & & \\
\hline DZr-CV-24 & & & & & & & & & & & & & & & \\
\hline
\end{tabular}

\section{HLP glasses (Vienna et al. 2001)}

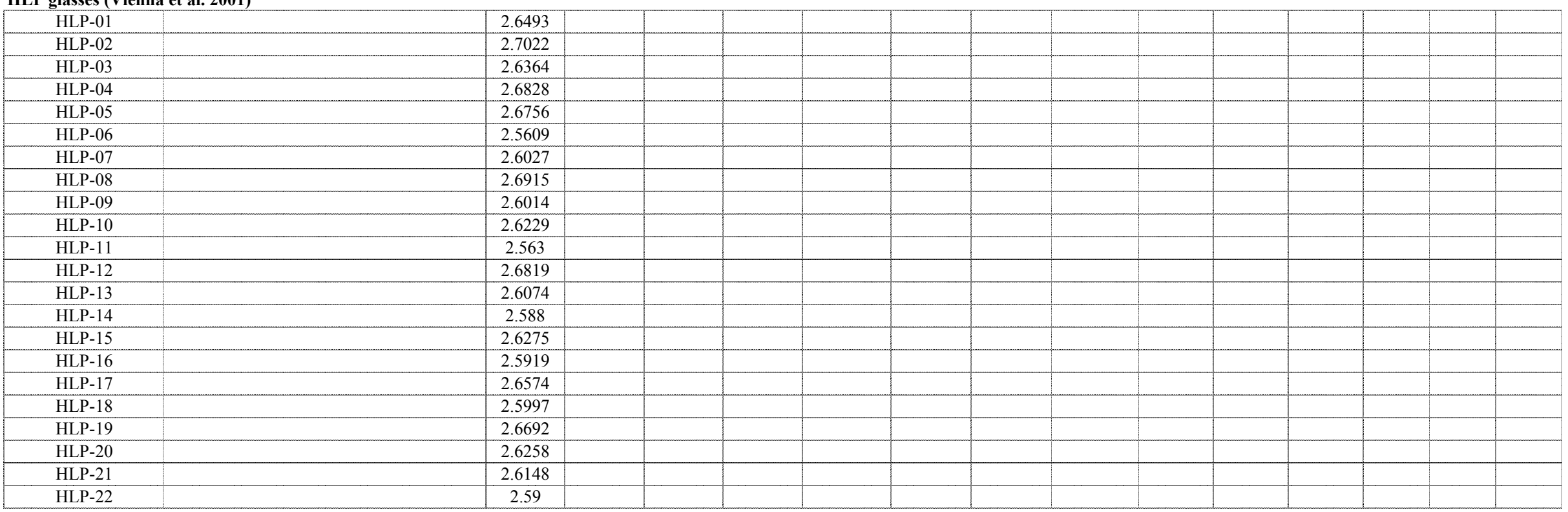


Appendix A. Database - mass fraction

INEEL DZr-CV Glasses (Riley et al. 2001)

\begin{tabular}{|c|c|c|c|c|c|c|c|c|c|c|c|c|c|c|c|c|c|c|c|c|c|}
\hline Glass ID & $\begin{array}{c}\mathrm{V} 2 \\
(\mathrm{~Pa} \cdot \mathrm{s})\end{array}$ & $\begin{array}{c}\text { T3 } \\
\left({ }^{\circ} \mathrm{C}\right)\end{array}$ & $\begin{array}{c}\mathrm{V} 3 \\
(\mathrm{~Pa} \cdot \mathrm{s})\end{array}$ & $\begin{array}{c}\mathrm{T} 4 \\
\left({ }^{\circ} \mathrm{C}\right)\end{array}$ & $\begin{array}{c}\mathrm{V} 4 \\
(\mathrm{~Pa} \cdot \mathrm{s})\end{array}$ & $\begin{array}{c}\mathrm{T} 5 \\
\left({ }^{\circ} \mathrm{C}\right)\end{array}$ & $\begin{array}{c}\text { V5 } \\
(\mathrm{Pa} \cdot \mathrm{s})\end{array}$ & $\begin{array}{l}\mathrm{T} 6 \\
\left({ }^{\circ} \mathrm{C}\right)\end{array}$ & $\begin{array}{c}\mathrm{V} 6 \\
(\mathrm{~Pa} \cdot \mathrm{s})\end{array}$ & $\begin{array}{c}\mathrm{T} 7 \\
\left({ }^{\circ} \mathrm{C}\right)\end{array}$ & $\begin{array}{c}\text { V7 } \\
(\mathrm{Pa} \cdot \mathrm{s})\end{array}$ & $\begin{array}{c}\mathrm{T} 8 \\
\left({ }^{\circ} \mathrm{C}\right)\end{array}$ & $\begin{array}{c}\mathrm{V} 8 \\
(\mathrm{~Pa} \cdot \mathrm{s})\end{array}$ & $\begin{array}{c}\mathrm{T} 9 \\
\left({ }^{\circ} \mathrm{C}\right)\end{array}$ & $\begin{array}{c}\text { V9 } \\
(\mathrm{Pa} \cdot \mathrm{s})\end{array}$ & $\begin{array}{l}\mathrm{T} 10 \\
\left({ }^{\circ} \mathrm{C}\right)\end{array}$ & $\begin{array}{c}\mathrm{V} 10 \\
(\mathrm{~Pa} \cdot \mathrm{s})\end{array}$ & $\begin{array}{l}\mathrm{T} 11 \\
\left({ }^{\circ} \mathrm{C}\right)\end{array}$ & $\begin{array}{c}\mathrm{V} 11 \\
(\mathrm{~Pa} \cdot \mathrm{s})\end{array}$ & $\begin{array}{l}\mathrm{T} 12 \\
\left({ }^{\circ} \mathrm{C}\right)\end{array}$ & $\begin{array}{c}\mathrm{V} 12 \\
(\mathrm{~Pa} \cdot \mathrm{s})\end{array}$ \\
\hline DZr-CV-5 & & & & & & & & & & & & & & & & & & & & & \\
\hline DZr-CV-6 & & & & & & & & & & & & & & & & & & & & & \\
\hline DZr-CV-7 & & & & & & & & & & & & & & & & & & & & & \\
\hline DZr-CV-8 & & & & & & & & & & & & & & & & & & & & & \\
\hline DZr-CV-9 & & & & & & & & & & & & & & & & & & & & & \\
\hline DZr-CV-10 & & & & & & & & & & & & & & & & & & & & & \\
\hline DZr-CV-11 & & & & & & & & & & & & & & & & & & & & & \\
\hline DZr-CV-12 & & & & & & & & & & & & & & & & & & & & & \\
\hline DZr-CV-13 & & & & & & & & & & & & & & & & & & & & & \\
\hline DZr-CV-14 & & & & & & & & & & & & & & & & & & & & & \\
\hline DZr-CV-15 & & & & & & & & & & & & & & & & & & & & & \\
\hline DZr-CV-16 & & & & & & & & & & & & & & & & & & & & & \\
\hline DZr-CV-17 & & & & & & & & & & & & & & & & & & & & & \\
\hline DZr-CV-18 & & & & & & & & & & & & & & & & & & & & & \\
\hline DZr-CV-19 & & & & & & & & & & & & & & & & & & & & & \\
\hline DZr-CV-20 & & & & & & & & & & & & & & & & & & & & & \\
\hline DZr-CV-21 & & & & & & & & & & & & & & & & & & & & & \\
\hline DZr-CV-22 & & & & & & & & & & & & & & & & & & & & & \\
\hline DZr-CV-23 & & & & & & & & & & & & & & & & & & & & & \\
\hline DZr-CV-24 & & & & & & & & & & & & & & & & & & & & & \\
\hline
\end{tabular}

\section{HLP glasses (Vienna et al. 2001)}

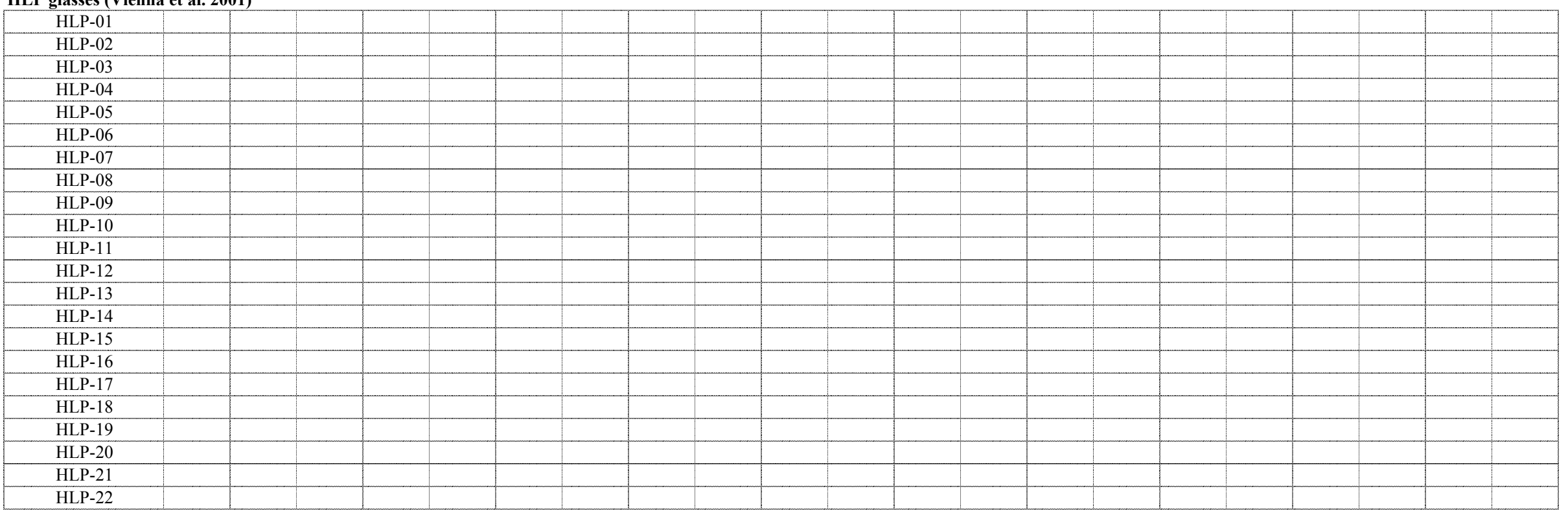


Appendix A. Database - mass fraction

INEEL DZr-CV Glasses (Riley et al. 2001)

\begin{tabular}{|c|c|c|c|c|c|c|c|c|c|c|c|c|c|c|c|c|}
\hline Glass ID & $\begin{array}{l}\mathrm{T} 13 \\
\left({ }^{\circ} \mathrm{C}\right)\end{array}$ & $\begin{array}{c}\mathrm{V} 13 \\
(\mathrm{~Pa} \cdot \mathrm{s})\end{array}$ & $\begin{array}{l}\mathrm{T} 14 \\
\left({ }^{\circ} \mathrm{C}\right)\end{array}$ & $\begin{array}{c}\mathrm{V} 14 \\
(\mathrm{~Pa} \cdot \mathrm{s})\end{array}$ & $\begin{array}{c}\text { Q PCT } \\
\mathrm{B}\left(\mathrm{g} / \mathrm{m}^{2}\right)\end{array}$ & $\begin{array}{c}\text { Q PCT } \\
\mathrm{Li}\left(\mathrm{g} / \mathrm{m}^{2}\right)\end{array}$ & $\begin{array}{c}\text { Q PCT } \\
\mathrm{Na}\left(\mathrm{g} / \mathrm{m}^{2}\right)\end{array}$ & $\begin{array}{c}\text { Q PCT } \\
\mathrm{Si}\left(\mathrm{g} / \mathrm{m}^{2}\right)\end{array}$ & $\begin{array}{c}\text { Q PCT } \\
\text { pH }\end{array}$ & $\begin{array}{l}\text { CCC PCT } \\
\text { B }\left(\mathrm{g} / \mathrm{m}^{2}\right)\end{array}$ & $\begin{array}{l}\text { CCC PCT } \\
\mathrm{Li}\left(\mathrm{g} / \mathrm{m}^{2}\right)\end{array}$ & $\begin{array}{l}\text { CCC PCT } \\
\mathrm{Na}\left(\mathrm{g} / \mathrm{m}^{2}\right)\end{array}$ & $\begin{array}{l}\text { CCC PCT } \\
\mathrm{Si}\left(\mathrm{g} / \mathrm{m}^{2}\right)\end{array}$ & $\begin{array}{c}\text { CCC PCT } \\
\text { pH }\end{array}$ & $\begin{array}{l}\text { Q PCT B at } \\
20^{\circ} \mathrm{C}\left(\mathrm{g} / \mathrm{m}^{2}\right)\end{array}$ & $\begin{array}{l}\text { Q PCT Li at } \\
20^{\circ} \mathrm{C}\left(\mathrm{g} / \mathrm{m}^{2}\right)\end{array}$ \\
\hline DZr-CV-5 & & & & & 0.2097 & 0.2926 & 0.3211 & 0.0955 & & 2.2434 & 0.6887 & 0.6005 & 0.1025 & & & \\
\hline DZr-CV-6 & & & & & 0.1305 & 0.2631 & 0.2634 & 0.0683 & & 0.1766 & 0.2340 & 0.2425 & 0.0776 & & & \\
\hline DZr-CV-7 & & & & & 0.1723 & 0.2881 & 0.3004 & 0.0886 & & 0.1253 & 0.2342 & 0.2373 & 0.0794 & & & \\
\hline DZr-CV-8 & & & & & 0.1834 & 0.2740 & 0.2939 & 0.0897 & & 0.2330 & 0.4462 & 0.3178 & 0.1516 & & & \\
\hline DZr-CV-9 & & & & & 6.5610 & 0.2841 & 0.3376 & 0.0909 & & 0.1636 & 0.2482 & 0.2855 & 0.0840 & & & \\
\hline DZr-CV-10 & & & & & 0.2151 & 0.3022 & 0.3367 & 0.0858 & & 0.1558 & 0.2259 & 0.2634 & 0.0974 & & & \\
\hline DZr-CV-11 & & & & & 0.2062 & 0.2987 & 0.3366 & 0.0868 & & 0.1345 & 0.0653 & 0.2512 & 0.0815 & & & \\
\hline DZr-CV-12 & & & & & 0.2062 & 0.3166 & 0.3194 & 0.0916 & & 0.1056 & 0.2141 & 0.2435 & 0.0768 & & & \\
\hline $\mathrm{DZr}-\mathrm{CV}-13$ & & & & & 0.0978 & 0.2027 & 0.2134 & 0.0635 & & 0.1178 & 0.2138 & 0.2157 & 0.0982 & & & \\
\hline DZr-CV-14 & & & & & 0.2368 & 0.3501 & 0.3963 & 0.1053 & & 0.1818 & 0.3026 & 0.3274 & 0.0989 & & & \\
\hline DZr-CV-15 & & & & & 0.1022 & 0.1952 & 0.1640 & 0.0563 & & 0.0959 & 0.1861 & 0.1430 & 0.0603 & & & \\
\hline DZr-CV-16 & & & & & 0.2170 & 0.3110 & 0.3982 & 0.0986 & & 0.1514 & 0.2511 & 0.3221 & 0.0885 & & & \\
\hline DZr-CV-17 & & & & & 0.1099 & 0.2394 & 0.2611 & 0.0696 & & 0.0766 & 0.1894 & 0.2281 & 0.0732 & & & \\
\hline DZr-CV-18 & & & & & 0.2360 & 0.3502 & 0.3750 & 0.1157 & & 0.1777 & 0.2635 & 0.2692 & 0.0848 & & & \\
\hline DZr-CV-19 & & & & & 0.1249 & 0.2500 & 0.2657 & 0.0776 & & 0.0959 & 0.2086 & 0.2189 & 0.0731 & & & \\
\hline DZr-CV-20 & & & & & 0.1529 & 0.2582 & 0.2897 & 0.0767 & & 0.0568 & 0.1918 & 0.2391 & 0.0710 & & & \\
\hline DZr-CV-21 & & & & & 0.3099 & 0.4298 & 0.4835 & 0.1114 & & 0.6589 & 0.2665 & 0.5741 & 0.2151 & & & \\
\hline DZr-CV-22 & & & & & 0.1180 & 0.2275 & 0.2340 & 0.0699 & & 0.1048 & 0.2115 & 0.2039 & 0.0665 & & & \\
\hline DZr-CV-23 & & & & & 0.1396 & 0.2475 & 0.2883 & 0.0765 & & 0.1260 & 0.2215 & 0.2550 & 0.0786 & & & \\
\hline $\mathrm{DZr}-\mathrm{CV}-24$ & & & & & 0.1415 & 0.2494 & 0.2509 & 0.0722 & & 0.1303 & 0.2144 & 0.2182 & 0.1127 & & & \\
\hline
\end{tabular}

HLP glasses (Vienna et al. 2001)

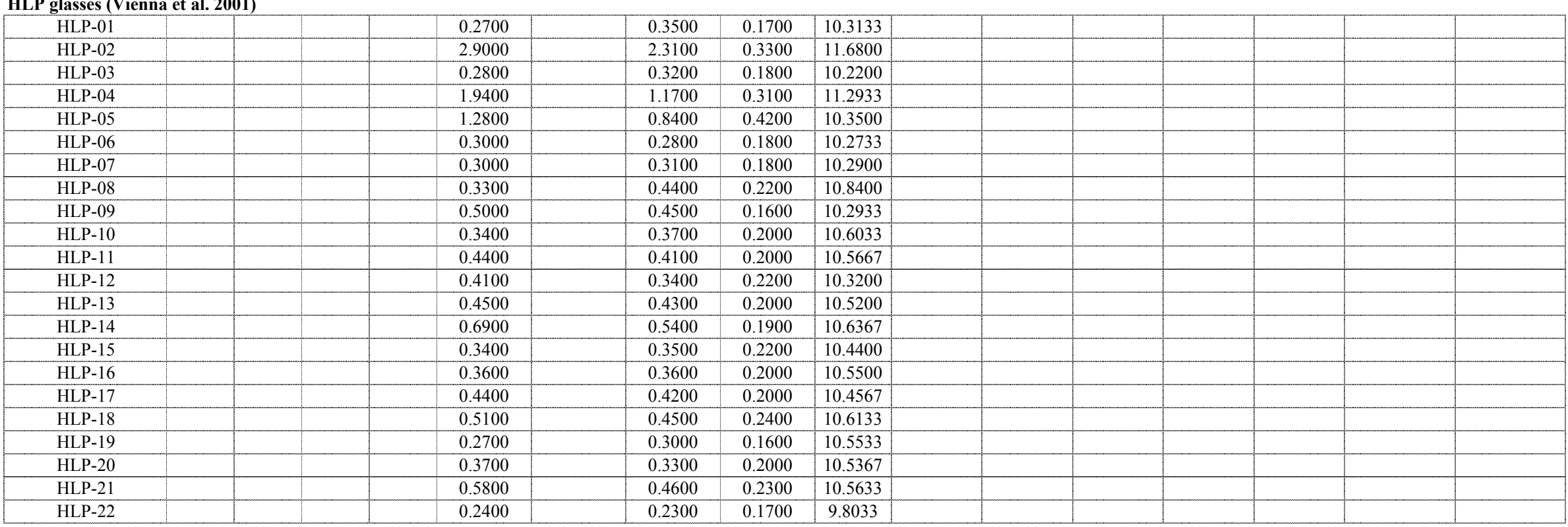


Appendix A. Database - mass fraction

INEEL DZr-CV Glasses (Riley et al. 2001)

\begin{tabular}{|c|c|c|c|c|c|c|c|c|c|c|c|c|}
\hline Glass ID & $\begin{array}{l}\text { Q PCT Na at } \\
20^{\circ} \mathrm{C}\left(\mathrm{g} / \mathrm{m}^{2}\right)\end{array}$ & $\begin{array}{l}\text { Q PCT Si at } \\
20^{\circ} \mathrm{C}\left(\mathrm{g} / \mathrm{m}^{2}\right)\end{array}$ & $\begin{array}{c}\mathrm{QpH} \\
\text { at } 20^{\circ} \mathrm{C}\end{array}$ & $\begin{array}{c}\text { TCLP Ag } \\
\text { (ppm) }\end{array}$ & $\begin{array}{c}\text { TCLP As } \\
(\mathrm{ppm})\end{array}$ & $\begin{array}{c}\text { TCLP Ba } \\
(\mathrm{ppm})\end{array}$ & $\begin{array}{c}\text { TCLP Cd } \\
(\mathrm{ppm})\end{array}$ & $\begin{array}{c}\text { TCLP Cr } \\
(\mathrm{ppm})\end{array}$ & $\begin{array}{c}\text { TCLP Ni } \\
(\mathrm{ppm})\end{array}$ & $\begin{array}{c}\text { TCLP Pb } \\
(\mathrm{ppm})\end{array}$ & $\begin{array}{l}\text { TCLP Se } \\
(\mathrm{ppm})\end{array}$ & $\begin{array}{c}\text { TCLP Zn } \\
\text { (ppm) }\end{array}$ \\
\hline DZr-CV-5 & & & & & & & & & & & & \\
\hline DZr-CV-6 & & & & & & & & & & & & \\
\hline DZr-CV-7 & & & & & & & & & & & & \\
\hline DZr-CV-8 & & & & & & & & & & & & \\
\hline DZr-CV-9 & & & & & & & & & & & & \\
\hline DZr-CV-10 & & & & & & & & & & & & \\
\hline DZr-CV-11 & & & & & & & & & & & & \\
\hline DZr-CV-12 & & & & & & & & & & & & \\
\hline DZr-CV-13 & & & & & & & & & & & & \\
\hline DZr-CV-14 & & & & & & & & & & & & \\
\hline DZr-CV-15 & & & & & & & & & & & & \\
\hline DZr-CV-16 & & & & & & & & & & & & \\
\hline DZr-CV-17 & & & & & & & & & & & & \\
\hline DZr-CV-18 & & & & & & & & & & & & \\
\hline DZr-CV-19 & & & & & & & & & & & & \\
\hline DZr-CV-20 & & & & & & & & & & & & \\
\hline DZr-CV-21 & & & & & & & & & & & & \\
\hline DZr-CV-22 & & & & & & & & & & & & \\
\hline DZr-CV-23 & & & & & & & & & & & & \\
\hline DZr-CV-24 & & & & & & & & & & & & \\
\hline
\end{tabular}

\section{HLP glasses (Vienna et al. 2001)}

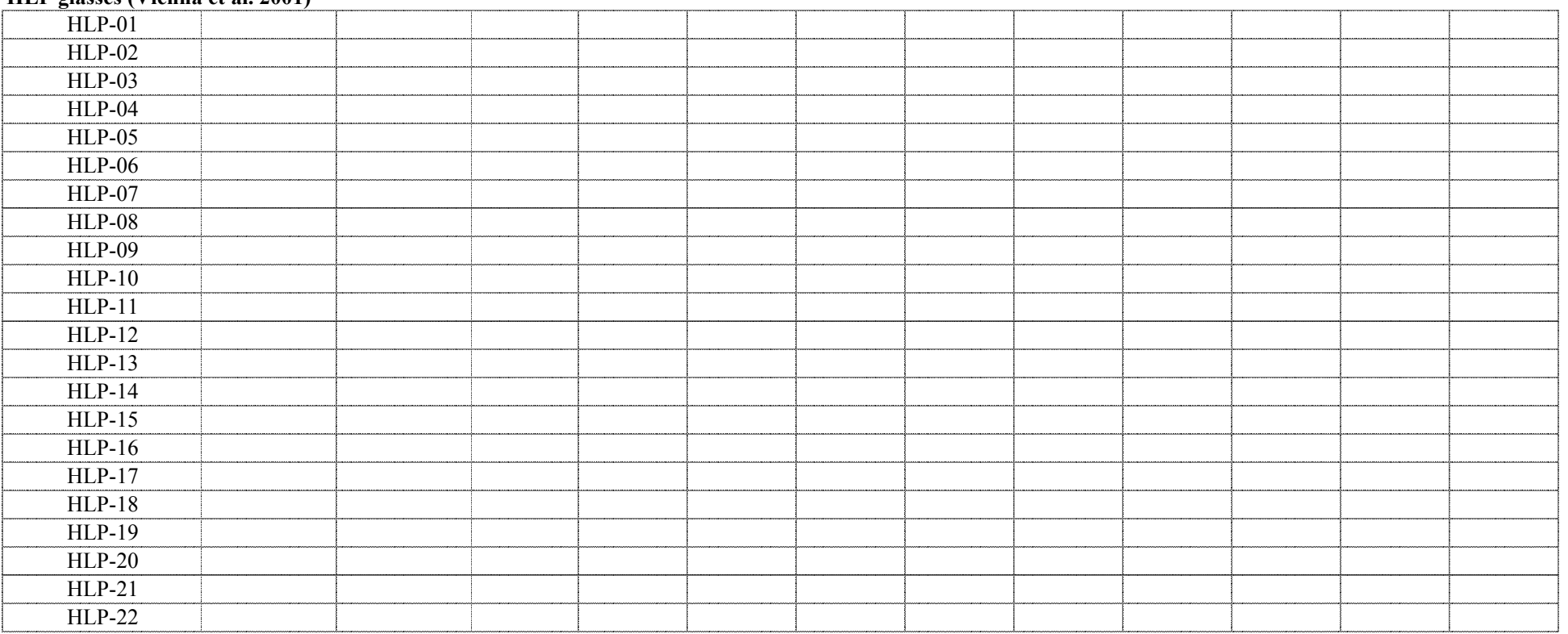


HLP glasses (Vienna et al. 2001)

\begin{tabular}{|c|c|c|c|c|c|c|c|c|c|c|c|c|c|c|c|c|c|c|c|c|c|}
\hline Glass ID & $\begin{array}{c}\mathrm{A} 12 \mathrm{O} 3 \\
-\mathrm{t}\end{array}$ & $\begin{array}{c}\mathrm{B} 2 \mathrm{O} 3 \\
-\mathrm{t}\end{array}$ & $\begin{array}{c}\mathrm{CaO} \\
-\mathrm{t} \\
\end{array}$ & $\begin{array}{c}\mathrm{Fe} 2 \mathrm{O} 3 \\
-\mathrm{t}\end{array}$ & $\begin{array}{c}\mathrm{FeO} \\
-\mathrm{t}\end{array}$ & $\begin{array}{c}\mathrm{K} 2 \mathrm{O} \\
-\mathrm{t}\end{array}$ & $\begin{array}{c}\mathrm{Li} 2 \mathrm{O} \\
-\mathrm{t}\end{array}$ & $\begin{array}{c}\mathrm{MgO} \\
-\mathrm{t}\end{array}$ & $\begin{array}{c}\mathrm{Na} 2 \mathrm{O} \\
-\mathrm{t}\end{array}$ & $\begin{array}{c}\mathrm{NiO} \\
-\mathrm{t}\end{array}$ & $\begin{array}{c}\mathrm{P} 2 \mathrm{O} 5 \\
-\mathrm{t}\end{array}$ & $\begin{array}{c}\mathrm{SiO} 2 \\
-\mathrm{t}\end{array}$ & $\begin{array}{c}\mathrm{ZrO} 2 \\
-\mathrm{t}\end{array}$ & $\begin{array}{c}\mathrm{Ag} 2 \mathrm{O} \\
-\mathrm{t}\end{array}$ & $\begin{array}{c}\mathrm{As} 2 \mathrm{O} 3 \\
-\mathrm{t}\end{array}$ & $\begin{array}{c}\mathrm{BaO} \\
-\mathrm{t}\end{array}$ & $\begin{array}{c}\mathrm{Bi} 2 \mathrm{O} 3 \\
-\mathrm{t}\end{array}$ & $\begin{array}{l}\mathrm{Br} \\
-\mathrm{t}\end{array}$ & $\begin{array}{c}\mathrm{CdO} \\
-\mathrm{t}\end{array}$ & $\begin{array}{c}\mathrm{Ce} 2 \mathrm{O} 3 \\
-\mathrm{t}\end{array}$ & $\begin{array}{c}\mathrm{CeO} 2 \\
-\mathrm{t}\end{array}$ \\
\hline HLP-23 & 0.0672 & 0.0961 & 0.0001 & 0.0529 & & 0.0047 & 0.0000 & 0.0144 & 0.2300 & & 0.0006 & 0.4713 & 0.0144 & & & & & & & & \\
\hline HLP-24 & 0.0718 & 0.1027 & 0.0001 & 0.0565 & & 0.0037 & 0.0000 & 0.0154 & 0.1800 & & 0.0005 & 0.5037 & 0.0154 & & & & & & & & \\
\hline HLP-25 & 0.0700 & 0.1000 & 0.0001 & 0.0550 & & 0.0041 & 0.0000 & 0.0150 & 0.2000 & & 0.0006 & 0.4907 & 0.0150 & & & & & & & & \\
\hline HLP-26 & 0.0700 & 0.1000 & 0.0001 & 0.0550 & & 0.0041 & 0.0000 & 0.0150 & 0.2000 & & 0.0006 & 0.4907 & 0.0150 & & & & & & & & \\
\hline HLP-27 & 0.1194 & 0.1200 & 0.0001 & 0.0000 & & 0.0047 & 0.0000 & 0.0000 & 0.2300 & & 0.0006 & 0.5200 & 0.0000 & & & & & & & & \\
\hline HLP-28 & 0.1194 & 0.1200 & 0.0001 & 0.0310 & & 0.0033 & 0.0000 & 0.0084 & 0.1600 & & 0.0004 & 0.5200 & 0.0084 & & & & & & & & \\
\hline HLP-29 & 0.1194 & 0.0600 & 0.0001 & 0.0254 & & 0.0047 & 0.0000 & 0.0069 & 0.2300 & & 0.0006 & 0.5200 & 0.0069 & & & & & & & & \\
\hline HLP-30 & 0.1194 & 0.0600 & 0.0001 & 0.0564 & & 0.0033 & 0.0000 & 0.0154 & 0.1600 & & 0.0004 & 0.5200 & 0.0154 & & & & & & & & \\
\hline HLP-31 & 0.0400 & 0.1200 & 0.0001 & 0.0336 & & 0.0047 & 0.0000 & 0.0092 & 0.2300 & & 0.0006 & 0.5200 & 0.0092 & & & & & & & & \\
\hline HLP-32 & 0.0400 & 0.1200 & 0.0001 & 0.0646 & & 0.0033 & 0.0000 & 0.0176 & 0.1600 & & 0.0004 & 0.5200 & 0.0176 & & & & & & & & \\
\hline HLP-33 & 0.0400 & 0.0600 & 0.0001 & 0.0590 & & 0.0047 & 0.0000 & 0.0161 & 0.2300 & & 0.0006 & 0.5200 & 0.0161 & & & & & & & & \\
\hline HLP-34 & 0.0400 & 0.0600 & 0.0001 & 0.0900 & & 0.0033 & 0.0000 & 0.0245 & 0.1600 & & 0.0004 & 0.5200 & 0.0245 & & & & & & & & \\
\hline HLP-35 & 0.1194 & 0.1200 & 0.0001 & 0.0677 & & 0.0047 & 0.0000 & 0.0185 & 0.2300 & & 0.0006 & 0.3600 & 0.0185 & & & & & & & & \\
\hline HLP-36 & 0.1194 & 0.1200 & 0.0001 & 0.0987 & & 0.0033 & 0.0000 & 0.0269 & 0.1600 & & 0.0004 & 0.3600 & 0.0269 & & & & & & & & \\
\hline HLP-37 & 0.1194 & 0.0600 & 0.0001 & 0.0931 & & 0.0047 & 0.0000 & 0.0254 & 0.2300 & & 0.0006 & 0.3600 & 0.0254 & & & & & & & & \\
\hline HLP-38 & 0.1194 & 0.0600 & 0.0001 & 0.1241 & & 0.0033 & 0.0000 & 0.0338 & 0.1600 & & 0.0004 & 0.3600 & 0.0338 & & & & & & & & \\
\hline HLP-39 & 0.0400 & 0.1200 & 0.0001 & 0.1013 & & 0.0047 & 0.0000 & 0.0276 & 0.2300 & & 0.0006 & 0.3600 & 0.0276 & & & & & & & & \\
\hline HLP-40 & 0.0400 & 0.1200 & 0.0001 & 0.1323 & & 0.0033 & 0.0000 & 0.0361 & 0.1600 & & 0.0004 & 0.3600 & 0.0361 & & & & & & & & \\
\hline HLP-40Q & 0.0400 & 0.1200 & 0.0001 & 0.1323 & & 0.0033 & 0.0000 & 0.0361 & 0.1600 & & 0.0004 & 0.3600 & 0.0361 & & & & & & & & \\
\hline HLP-41 & 0.0400 & 0.0600 & 0.0001 & 0.1267 & & 0.0047 & 0.0000 & 0.0345 & 0.2300 & & 0.0006 & 0.3600 & 0.0345 & & & & & & & & \\
\hline HLP-42 & 0.0400 & 0.0600 & 0.0001 & 0.1577 & & 0.0033 & 0.0000 & 0.0430 & 0.1600 & & 0.0004 & 0.3600 & 0.0430 & & & & & & & & \\
\hline HLP-42Q & 0.0400 & 0.0600 & 0.0001 & 0.1577 & & 0.0033 & 0.0000 & 0.0430 & 0.1600 & & 0.0004 & 0.3600 & 0.0430 & & & & & & & & \\
\hline HLP-43 & 0.0700 & 0.1000 & 0.0001 & 0.0550 & & 0.0041 & 0.0000 & 0.0150 & 0.2000 & & 0.0006 & 0.4907 & 0.0150 & & & & & & & & \\
\hline HLP-44 & 0.0700 & 0.1000 & 0.0001 & 0.0550 & & 0.0041 & 0.0000 & 0.0150 & 0.2000 & & 0.0006 & 0.4907 & 0.0150 & & & & & & & & \\
\hline HLP-45 & 0.0700 & 0.1000 & 0.0001 & 0.0550 & & 0.0041 & 0.0000 & 0.0150 & 0.2000 & & 0.0006 & 0.4907 & 0.0150 & & & & & & & & \\
\hline HLP-46 & 0.1200 & 0.0500 & 0.0400 & 0.0000 & & 0.0146 & & 0.0000 & 0.2000 & & 0.0019 & 0.5591 & & & & & & & & & \\
\hline HLP-47 & 0.1000 & 0.0800 & 0.0050 & 0.0100 & & 0.0150 & 0.0010 & 0.0010 & 0.2000 & 0.0010 & 0.0050 & 0.5437 & 0.0100 & & & 0.0001 & & & 0.0020 & & \\
\hline HLP-48 & 0.1197 & 0.0885 & 0.0000 & 0.0577 & & 0.0310 & 0.0000 & 0.0199 & 0.2000 & & 0.0008 & 0.3825 & 0.0249 & & & & & & & & \\
\hline HLP-49 & 0.0803 & 0.0807 & 0.0703 & 0.0803 & & 0.0036 & 0.0408 & 0.0300 & 0.1000 & & 0.0001 & 0.4393 & 0.0304 & & & & & & & & \\
\hline HLP-51 & 0.1000 & 0.0925 & 0.0000 & 0.0250 & & 0.0220 & 0.0000 & 0.0100 & 0.2000 & & 0.0008 & 0.4189 & 0.0525 & & & & & & & & \\
\hline HLP-52 & 0.1019 & 0.0000 & 0.0260 & 0.0254 & & 0.0197 & 0.0000 & 0.0118 & 0.2874 & & 0.0191 & 0.4464 & 0.0502 & & & & & & & & \\
\hline HLP-53 & 0.0986 & 0.0423 & 0.0438 & 0.0730 & & 0.0310 & 0.0204 & 0.0204 & 0.2000 & & 0.0008 & 0.4015 & 0.0299 & & & & & & & & \\
\hline HLP-53Q & 0.0986 & 0.0423 & 0.0438 & 0.0730 & & 0.0310 & 0.0204 & 0.0204 & 0.2000 & & 0.0008 & 0.4015 & 0.0299 & & & & & & & & \\
\hline HLP-54 & 0.1200 & 0.0900 & 0.0000 & 0.0000 & & 0.0033 & 0.0000 & 0.0000 & 0.2000 & & 0.0119 & 0.5678 & 0.0000 & & & & & & & & \\
\hline HLP-55 & 0.0900 & 0.0900 & 0.0000 & 0.0000 & & 0.0033 & 0.0000 & 0.0000 & 0.2000 & & 0.0119 & 0.5978 & 0.0000 & & & & & & & & \\
\hline HLP-56 & 0.0620 & 0.0890 & 0.0199 & 0.0698 & & 0.0050 & 0.0000 & 0.0199 & 0.2000 & & 0.0003 & 0.4455 & 0.0299 & & & & & & & & \\
\hline HLP-58 & 0.0686 & 0.0980 & 0.0200 & 0.0539 & & 0.0040 & 0.0000 & 0.0147 & 0.1960 & & 0.0006 & 0.4809 & 0.0147 & & & & & & & & \\
\hline HLP-59 & 0.0665 & 0.0950 & 0.0500 & 0.0523 & & 0.0039 & 0.0000 & 0.0143 & 0.1900 & & 0.0006 & 0.4662 & 0.0143 & & & & & & & & \\
\hline HLP-60 & 0.1194 & 0.1257 & 0.0001 & 0.0171 & & 0.0033 & 0.0000 & 0.0430 & 0.1600 & & 0.0200 & 0.3600 & 0.0600 & & & & & & & & \\
\hline HLP-61 & 0.0400 & 0.1257 & 0.0500 & 0.0000 & & 0.0500 & 0.0000 & 0.0430 & 0.1600 & & 0.0004 & 0.3965 & 0.0000 & & & & & & & & \\
\hline HLP-62 & 0.0400 & 0.1257 & 0.0500 & 0.0000 & & 0.0033 & 0.0000 & 0.0000 & 0.1600 & & 0.0004 & 0.4692 & 0.0600 & & & & & & & & \\
\hline HLP-63 & 0.0400 & 0.0600 & 0.0001 & 0.1504 & & 0.0033 & 0.0000 & 0.0430 & 0.2514 & & 0.0004 & 0.3600 & 0.0000 & & & & & & & & \\
\hline HLP-64 & 0.0400 & 0.0600 & 0.0001 & 0.0000 & & 0.0500 & 0.0000 & 0.0430 & 0.1600 & & 0.0200 & 0.5200 & 0.0600 & & & & & & & & \\
\hline HLP-65 & 0.0400 & 0.0600 & 0.0500 & 0.0982 & & 0.0033 & 0.0000 & 0.0430 & 0.1600 & & 0.0200 & 0.5200 & 0.0000 & & & & & & & & \\
\hline HLP-66 & 0.0400 & 0.1257 & 0.0001 & 0.0536 & & 0.0033 & 0.0000 & 0.0000 & 0.2514 & & 0.0004 & 0.5200 & 0.0000 & & & & & & & & \\
\hline HLP-67 & 0.0400 & 0.1257 & 0.0001 & 0.0443 & & 0.0500 & 0.0000 & 0.0000 & 0.2514 & & 0.0200 & 0.3600 & 0.0600 & & & & & & & & \\
\hline HLP-68 & 0.0964 & 0.0600 & 0.0500 & 0.1577 & & 0.0500 & 0.0000 & 0.0000 & 0.1600 & & 0.0004 & 0.3600 & 0.0600 & & & & & & & & \\
\hline HLP-69 & 0.1194 & 0.0600 & 0.0001 & 0.0024 & & 0.0033 & 0.0000 & 0.0000 & 0.1600 & & 0.0004 & 0.5200 & 0.0000 & & & & & & & & \\
\hline HLP-70 & 0.1194 & 0.0600 & 0.0478 & 0.0000 & & 0.0500 & 0.0000 & 0.0000 & 0.2514 & & 0.0200 & 0.3600 & 0.0000 & & & & & & & & \\
\hline
\end{tabular}


Appendix A. Database - mass fraction

HLP glasses (Vienna et al. 2001)

\begin{tabular}{|c|c|c|c|c|c|c|c|c|c|c|c|c|c|c|c|c|c|c|c|c|c|}
\hline Glass ID & $\begin{array}{l}\mathrm{Cl} \\
-\mathrm{t}\end{array}$ & $\begin{array}{c}\mathrm{CoO} \\
-\mathrm{t}\end{array}$ & $\begin{array}{c}\mathrm{Co} 2 \mathrm{O} 3 \\
-\mathrm{t}\end{array}$ & $\begin{array}{c}\mathrm{Cr} 2 \mathrm{O} 3 \\
-\mathrm{t}\end{array}$ & $\begin{array}{c}\mathrm{Cs} 2 \mathrm{O} \\
-\mathrm{t}\end{array}$ & $\begin{array}{c}\mathrm{CuO} \\
-\mathrm{t}\end{array}$ & $\begin{array}{c}\mathrm{Eu} 2 \mathrm{O} 3 \\
-\mathrm{t}\end{array}$ & $\begin{array}{l}F \\
-t\end{array}$ & $\begin{array}{c}\mathrm{Ga} 2 \mathrm{O} 3 \\
-\mathrm{t}\end{array}$ & $\begin{array}{c}\mathrm{Gd} 2 \mathrm{O} 3 \\
-\mathrm{t}\end{array}$ & $\begin{array}{c}\mathrm{HgO} \\
-\mathrm{t}\end{array}$ & $\begin{array}{l}I \\
-t\end{array}$ & $\begin{array}{c}\mathrm{La} 2 \mathrm{O} 3 \\
-\mathrm{t}\end{array}$ & $\begin{array}{c}\mathrm{MnO} 2 \\
-\mathrm{t}\end{array}$ & $\begin{array}{c}\mathrm{MnO} \\
-\mathrm{t}\end{array}$ & $\begin{array}{c}\mathrm{MoO} \\
-\mathrm{t}\end{array}$ & $\begin{array}{c}\mathrm{MoO} 3 \\
-\mathrm{t}\end{array}$ & $\begin{array}{c}\mathrm{Nb} 2 \mathrm{O} 5 \\
-\mathrm{t}\end{array}$ & $\begin{array}{c}\mathrm{Nd} 2 \mathrm{O} 3 \\
-\mathrm{t}\end{array}$ & $\begin{array}{c}\mathrm{PbO} \\
-\mathrm{t}\end{array}$ & $\begin{array}{c}\mathrm{PdO} 2 \\
-\mathrm{t}\end{array}$ \\
\hline HLP-23 & 0.0032 & & & 0.0009 & & & & 0.0001 & & & & & 0.0000 & & & & 0.0000 & & & & \\
\hline HLP-24 & 0.0025 & & & 0.0007 & & & & 0.0001 & & & & & 0.0000 & & & & 0.0000 & & & & \\
\hline HLP-25 & 0.0028 & & & 0.0008 & & & & 0.0001 & & & & & 0.0000 & & & & 0.0000 & & & & \\
\hline HLP-26 & 0.0028 & & & 0.0008 & & & & 0.0001 & & & & & 0.0000 & & & & 0.0000 & & & & \\
\hline HLP-27 & 0.0032 & & & 0.0009 & & & & 0.0001 & & & & & 0.0000 & & & & 0.0000 & & & & \\
\hline HLP-28 & 0.0022 & & & 0.0006 & & & & 0.0001 & & & & & 0.0000 & & & & 0.0000 & & & & \\
\hline HLP-29 & 0.0032 & & & 0.0009 & & & & 0.0001 & & & & & 0.0000 & & & & 0.0000 & & & & \\
\hline HLP-30 & 0.0022 & & & 0.0006 & & & & 0.0001 & & & & & 0.0000 & & & & 0.0000 & & & & \\
\hline HLP-31 & 0.0032 & & & 0.0009 & & & & 0.0001 & & & & & 0.0000 & & & & 0.0000 & & & & \\
\hline HLP-32 & 0.0022 & & & 0.0006 & & & & 0.0001 & & & & & 0.0000 & & & & 0.0000 & & & & \\
\hline HLP-33 & 0.0032 & & & 0.0009 & & & & 0.0001 & & & & & 0.0000 & & & & 0.0000 & & & & \\
\hline HLP-34 & 0.0022 & & & 0.0006 & & & & 0.0001 & & & & & 0.0000 & & & & 0.0000 & & & & \\
\hline HLP-35 & 0.0032 & & & 0.0009 & & & & 0.0001 & & & & & 0.0000 & & & & 0.0000 & & & & \\
\hline HLP-36 & 0.0022 & & & 0.0006 & & & & 0.0001 & & & & & 0.0000 & & & & 0.0000 & & & & \\
\hline HLP-37 & 0.0032 & & & 0.0009 & & & & 0.0001 & & & & & 0.0000 & & & & 0.0000 & & & & \\
\hline HLP-38 & 0.0022 & & & 0.0006 & & & & 0.0001 & & & & & 0.0000 & & & & 0.0000 & & & & \\
\hline HLP-39 & 0.0032 & & & 0.0009 & & & & 0.0001 & & & & & 0.0000 & & & & 0.0000 & & & & \\
\hline HLP-40 & 0.0022 & & & 0.0006 & & & & 0.0001 & & & & & 0.0000 & & & & 0.0000 & & & & \\
\hline HLP-40Q & 0.0022 & & & 0.0006 & & & & 0.0001 & & & & & 0.0000 & & & & 0.0000 & & & & \\
\hline HLP-41 & 0.0032 & & & 0.0009 & & & & 0.0001 & & & & & 0.0000 & & & & 0.0000 & & & & \\
\hline HLP-42 & 0.0022 & & & 0.0006 & & & & 0.0001 & & & & & 0.0000 & & & & 0.0000 & & & & \\
\hline HLP-42Q & 0.0022 & & & 0.0006 & & & & 0.0001 & & & & & 0.0000 & & & & 0.0000 & & & & \\
\hline HLP-43 & 0.0028 & & & 0.0008 & & & & 0.0001 & & & & & 0.0000 & & & & 0.0000 & & & & \\
\hline HLP-44 & 0.0028 & & & 0.0008 & & & & 0.0001 & & & & & 0.0000 & & & & 0.0000 & & & & \\
\hline HLP-45 & 0.0028 & & & 0.0008 & & & & 0.0001 & & & & & 0.0000 & & & & 0.0000 & & & & \\
\hline HLP-46 & 0.0035 & & & 0.0004 & 0.0015 & & & 0.0029 & & & & 0.0013 & & & 0.0000 & & 0.0015 & & & & \\
\hline HLP-47 & 0.0080 & & & 0.0020 & & & & 0.0100 & & & & 0.0000 & 0.0001 & & 0.0010 & & 0.0000 & & & 0.0010 & \\
\hline HLP-48 & 0.0058 & & & 0.0002 & & & & 0.0004 & & & & & 0.0000 & & & & 0.0000 & & & & \\
\hline HLP-49 & 0.0029 & & & 0.0010 & & & & 0.0000 & & & & & 0.0000 & & & & 0.0000 & & & & \\
\hline HLP-51 & 0.0058 & & & 0.0002 & & & & 0.0004 & & & & & 0.0200 & & & & 0.0000 & & & & \\
\hline HLP-52 & 0.0013 & & & 0.0008 & & & & 0.0031 & & & & & 0.0000 & & & & 0.0000 & & & & \\
\hline HLP-53 & 0.0036 & & & 0.0001 & & & & 0.0012 & & & & & 0.0000 & & & & 0.0000 & & & & \\
\hline HLP-53Q & 0.0036 & & & 0.0001 & & & & 0.0012 & & & & & 0.0000 & & & & 0.0000 & & & & \\
\hline HLP-54 & 0.0009 & & & 0.0004 & & & & 0.0021 & & & & & 0.0001 & & & & 0.0000 & & & & \\
\hline HLP-55 & 0.0009 & & & 0.0004 & & & & 0.0021 & & & & & 0.0001 & & & & 0.0000 & & & & \\
\hline HLP-56 & 0.0065 & & & 0.0002 & & & & 0.0001 & & & & & 0.0000 & & & & 0.0001 & & & & \\
\hline HLP-58 & 0.0027 & & & 0.0008 & & & & 0.0001 & & & & & 0.0000 & & & & 0.0000 & & & & \\
\hline HLP-59 & 0.0027 & & & 0.0008 & & & & 0.0001 & & & & & 0.0000 & & & & 0.0000 & & & & \\
\hline HLP-60 & 0.0035 & & & 0.0009 & & & & 0.0001 & & & & & 0.0000 & & & & 0.0000 & & & & \\
\hline HLP-61 & 0.0035 & & & 0.0009 & & & & 0.0001 & & & & & 0.0000 & & & & 0.0000 & & & & \\
\hline HLP-62 & 0.0035 & & & 0.0009 & & & & 0.0001 & & & & & 0.0000 & & & & 0.0000 & & & & \\
\hline HLP-63 & 0.0035 & & & 0.0009 & & & & 0.0001 & & & & & 0.0000 & & & & 0.0000 & & & & \\
\hline HLP-64 & 0.0035 & & & 0.0009 & & & & 0.0001 & & & & & 0.0000 & & & & 0.0000 & & & & \\
\hline HLP-65 & 0.0035 & & & 0.0009 & & & & 0.0001 & & & & & 0.0000 & & & & 0.0000 & & & & \\
\hline HLP-66 & 0.0035 & & & 0.0009 & & & & 0.0001 & & & & & 0.0000 & & & & 0.0000 & & & & \\
\hline HLP-67 & 0.0035 & & & 0.0009 & & & & 0.0001 & & & & & 0.0000 & & & & 0.0000 & & & & \\
\hline HLP-68 & 0.0035 & & & 0.0009 & & & & 0.0001 & & & & & 0.0000 & & & & 0.0000 & & & & \\
\hline HLP-69 & 0.0035 & & & 0.0009 & & & & 0.0001 & & & & & 0.0000 & & & & 0.0000 & & & & \\
\hline HLP-70 & 0.0035 & & & 0.0009 & & & & 0.0001 & & & & & 0.0000 & & & & 0.0000 & & & & \\
\hline
\end{tabular}




\section{HLP glasses (Vienna et al. 2001)}

\begin{tabular}{|c|c|c|c|c|c|c|c|c|c|c|c|c|c|c|c|c|c|c|c|c|c|}
\hline Glass ID & $\begin{array}{c}\mathrm{PdO} \\
-\mathrm{t}\end{array}$ & $\begin{array}{c}\mathrm{Pr} 2 \mathrm{O} 3 \\
-\mathrm{t}\end{array}$ & {$\left[\begin{array}{c}\operatorname{Pr6O11} \\
-\mathrm{t}\end{array}\right.$} & $\begin{array}{c}\mathrm{Rb} 2 \mathrm{O} \\
-\mathrm{t}\end{array}$ & $\begin{array}{c}\mathrm{ReO} \\
-\mathrm{t}\end{array}$ & $\begin{array}{c}\mathrm{ReO} 2 \\
-\mathrm{t}\end{array}$ & $\begin{array}{c}\mathrm{Rh} 2 \mathrm{O} 3 \\
-\mathrm{t}\end{array}$ & $\begin{array}{c}\mathrm{RhO}^{2} \\
-\mathrm{t}\end{array}$ & $\begin{array}{c}\mathrm{RuO}_{2} \\
-\mathrm{t}\end{array}$ & $\begin{array}{c}\mathrm{Sb} 2 \mathrm{O} 3 \\
-\mathrm{t}\end{array}$ & $\begin{array}{c}\mathrm{Sb} 2 \mathrm{O} 5 \\
-\mathrm{t}\end{array}$ & $\begin{array}{c}\mathrm{SeO} 2 \\
-\mathrm{t}\end{array}$ & $\begin{array}{c}\mathrm{Sm} 2 \mathrm{O} 3 \\
-\mathrm{t}\end{array}$ & $\begin{array}{c}\mathrm{SnO} \\
-\mathrm{t}\end{array}$ & $\begin{array}{c}\mathrm{SnO} 2 \\
-\mathrm{t}\end{array}$ & $\begin{array}{c}\mathrm{SO} 3 \\
-\mathrm{t}\end{array}$ & $\begin{array}{c}\mathrm{SrO} \\
-\mathrm{t}\end{array}$ & $\begin{array}{c}\mathrm{Tc} 2 \mathrm{O} 7 \\
-\mathrm{t}\end{array}$ & $\begin{array}{c}\mathrm{TeO} 2 \\
-\mathrm{t}\end{array}$ & $\begin{array}{c}\mathrm{ThO} 2 \\
-\mathrm{t}\end{array}$ & $\begin{array}{c}\mathrm{TiO} 2 \\
-\mathrm{t}\end{array}$ \\
\hline HLP-23 & & & & & 0.0001 & & & & & & & & & & & 0.0008 & & & & & 0.0288 \\
\hline HLP-24 & & & & & 0.0001 & & & & & & & & & & & 0.0007 & & & & & 0.0308 \\
\hline HLP-25 & & & & & 0.0001 & & & & & & & & & & & 0.0007 & & & & & 0.0300 \\
\hline HLP-26 & & & & & 0.0001 & & & & & & & & & & & 0.0007 & & & & & 0.0300 \\
\hline HLP-27 & & & & & 0.0001 & & & & & & & & & & & 0.0008 & & & & & 0.0000 \\
\hline HLP-28 & & & & & 0.0001 & & & & & & & & & & & 0.0006 & & & & & 0.0169 \\
\hline HLP-29 & & & & & 0.0001 & & & & & & & & & & & 0.0008 & & & & & 0.0138 \\
\hline HLP-30 & & & & & 0.0001 & & & & & & & & & & & 0.0006 & & & & & 0.0307 \\
\hline HLP-31 & & & & & 0.0001 & & & & & & & & & & & 0.0008 & & & & & 0.0183 \\
\hline HLP-32 & & & & & 0.0001 & & & & & & & & & & & 0.0006 & & & & & 0.0352 \\
\hline HLP-33 & & & & & 0.0001 & & & & & & & & & & & 0.0008 & & & & & 0.0321 \\
\hline HLP-34 & & & & & 0.0001 & & & & & & & & & & & 0.0006 & & & & & 0.0490 \\
\hline HLP-35 & & & & & 0.0001 & & & & & & & & & & & 0.0008 & & & & & 0.0369 \\
\hline HLP-36 & & & & & 0.0001 & & & & & & & & & & & 0.0006 & & & & & 0.0538 \\
\hline HLP-37 & & & & & 0.0001 & & & & & & & & & & & 0.0008 & & & & & 0.0507 \\
\hline HLP-38 & & & & & 0.0001 & & & & & & & & & & & 0.0006 & & & & & 0.0676 \\
\hline HLP-39 & & & & & 0.0001 & & & & & & & & & & & 0.0008 & & & & & 0.0552 \\
\hline HLP-40 & & & & & 0.0001 & & & & & & & & & & & 0.0006 & & & & & 0.0721 \\
\hline HLP-40Q & & & & & 0.0001 & & & & & & & & & & & 0.0006 & & & & & 0.0721 \\
\hline HLP-41 & & & & & 0.0001 & & & & & & & & & & & 0.0008 & & & & & 0.0690 \\
\hline HLP-42 & & & & & 0.0001 & & & & & & & & & & & 0.0006 & & & & & 0.0859 \\
\hline HLP-42Q & & & & & 0.0001 & & & & & & & & & & & 0.0006 & & & & & 0.0859 \\
\hline HLP-43 & & & & & 0.0001 & & & & & & & & & & & 0.0007 & & & & & 0.0300 \\
\hline HLP-44 & & & & & 0.0001 & & & & & & & & & & & 0.0007 & & & & & 0.0300 \\
\hline HLP-45 & & & & & 0.0001 & & & & & & & & & & & 0.0007 & & & & & 0.0300 \\
\hline HLP-46 & & & & & & & & & & & & & & & & 0.0022 & 0.0011 & & & & \\
\hline HLP-47 & & & & & 0.0000 & & & & & & & & & & & 0.0020 & & & & & 0.0010 \\
\hline HLP-48 & & & & & 0.0000 & & & & & & & & & & & 0.0010 & & & & & 0.0249 \\
\hline HLP-49 & & & & & 0.0000 & & & & & & & & & & & 0.0002 & & & & & 0.0000 \\
\hline HLP-51 & & & & & 0.0000 & & & & & & & & & & & 0.0010 & & & & & 0.0249 \\
\hline HLP-52 & & & & & 0.0000 & & & & & & & & & & & 0.0030 & & & & & 0.0038 \\
\hline HLP-53 & & & & & 0.0000 & & & & & & & & & & & 0.0004 & & & & & 0.0000 \\
\hline HLP-53Q & & & & & 0.0000 & & & & & & & & & & & 0.0004 & & & & & 0.0000 \\
\hline HLP-54 & & & & & 0.0000 & & & & & & & & & & & 0.0032 & & & & & 0.0000 \\
\hline HLP-55 & & & & & 0.0000 & & & & & & & & & & & 0.0032 & & & & & 0.0000 \\
\hline HLP-56 & & & & & 0.0010 & & & & & & & & & & & 0.0010 & & & & & 0.0199 \\
\hline HLP-58 & & & & & 0.0001 & & & & & & & & & & & 0.0007 & & & & & 0.0294 \\
\hline HLP-59 & & & & & 0.0001 & & & & & & & & & & & 0.0007 & & & & & 0.0285 \\
\hline HLP-60 & & & & & 0.0001 & & & & & & & & & & & 0.0009 & & & & & 0.0859 \\
\hline HLP-61 & & & & & 0.0001 & & & & & & & & & & & 0.0009 & & & & & 0.0859 \\
\hline HLP-62 & & & & & 0.0001 & & & & & & & & & & & 0.0009 & & & & & 0.0859 \\
\hline HLP-63 & & & & & 0.0001 & & & & & & & & & & & 0.0009 & & & & & 0.0859 \\
\hline HLP-64 & & & & & 0.0001 & & & & & & & & & & & 0.0009 & & & & & 0.0000 \\
\hline HLP-65 & & & & & 0.0001 & & & & & & & & & & & 0.0009 & & & & & 0.0000 \\
\hline HLP-66 & & & & & 0.0001 & & & & & & & & & & & 0.0009 & & & & & 0.0000 \\
\hline HLP-67 & & & & & 0.0001 & & & & & & & & & & & 0.0009 & & & & & 0.0000 \\
\hline HLP-68 & & & & & 0.0001 & & & & & & & & & & & 0.0009 & & & & & 0.0000 \\
\hline HLP-69 & & & & & 0.0001 & & & & & & & & & & & 0.0009 & & & & & 0.0859 \\
\hline HLP-70 & & & & & 0.0001 & & & & & & & & & & & 0.0009 & & & & & 0.0859 \\
\hline
\end{tabular}


Appendix A. Database - mass fraction

HLP glasses (Vienna et al. 2001)

\begin{tabular}{|c|c|c|c|c|c|c|c|c|c|c|c|c|c|c|c|c|c|c|c|c|c|}
\hline Glass ID & $\begin{array}{c}\mathrm{T} 12 \mathrm{O} 3 \\
-\mathrm{t}\end{array}$ & $\begin{array}{c}\text { U3O8 } \\
-t\end{array}$ & $\begin{array}{c}\mathrm{UO} 2 \\
-\mathrm{t}\end{array}$ & $\begin{array}{c}\text { UO3 } \\
-t\end{array}$ & $\begin{array}{c}\mathrm{V} 2 \mathrm{O} 5 \\
-\mathrm{t}\end{array}$ & $\begin{array}{c}\text { WO3 } \\
-t\end{array}$ & $\begin{array}{c}\mathrm{Y} 2 \mathrm{O} 3 \\
-\mathrm{t}\end{array}$ & $\begin{array}{c}\mathrm{ZnO} \\
-\mathrm{t}\end{array}$ & $\begin{array}{c}\text { Others } \\
-t\end{array}$ & $\begin{array}{c}\text { Sum } \\
-t\end{array}$ & $\begin{array}{c}\mathrm{A} 12 \mathrm{O} 3 \\
-\mathrm{a}\end{array}$ & $\begin{array}{c}\mathrm{B} 2 \mathrm{O} 3 \\
-\mathrm{a}\end{array}$ & $\begin{array}{c}\mathrm{CaO} \\
-\mathrm{a}\end{array}$ & $\begin{array}{c}\mathrm{Fe} 2 \mathrm{O} 3 \\
-\mathrm{a}\end{array}$ & $\begin{array}{c}\mathrm{FeO} \\
-\mathrm{a}\end{array}$ & $\begin{array}{c}\mathrm{K} 2 \mathrm{O} \\
-\mathrm{a}\end{array}$ & $\begin{array}{c}\mathrm{Li} 2 \mathrm{O} \\
-\mathrm{a}\end{array}$ & $\begin{array}{c}\mathrm{MgO} \\
-\mathrm{a}\end{array}$ & $\begin{array}{c}\mathrm{Na} 2 \mathrm{O} \\
-\mathrm{a}\end{array}$ & $\begin{array}{c}\mathrm{NiO} \\
-\mathrm{a}\end{array}$ & $\begin{array}{c}\mathrm{P} 2 \mathrm{O} 5 \\
-\mathrm{a}\end{array}$ \\
\hline HLP-23 & & & & & & & & 0.0144 & & 1.0000 & 0.0692 & 0.0965 & & 0.0591 & & 0.0042 & & 0.0147 & 0.2150 & & \\
\hline HLP-24 & & & & & & & & 0.0154 & & 1.0001 & 0.0744 & 0.1000 & & 0.0659 & & 0.0040 & & 0.0163 & 0.1720 & & \\
\hline HLP-25 & & & & & & & & 0.0150 & & 1.0000 & 0.0676 & 0.1010 & 0.0001 & 0.0627 & & 0.0041 & & 0.0154 & 0.1850 & & \\
\hline HLP-26 & & & & & & & & 0.0150 & & 1.0000 & 0.0668 & 0.0943 & & 0.0597 & & 0.0043 & & 0.0145 & 0.1820 & & \\
\hline HLP-27 & & & & & & & & 0.0000 & & 0.9999 & 0.1200 & 0.1230 & & 0.0060 & & 0.0044 & & & 0.2170 & & \\
\hline HLP-28 & & & & & & & & 0.0084 & & 0.9999 & 0.1210 & 0.1300 & & 0.0382 & & & & 0.0080 & 0.1510 & & \\
\hline HLP-29 & & & & & & & & 0.0069 & & 0.9998 & 0.1180 & 0.0651 & & 0.0312 & & 0.0042 & & 0.0069 & 0.2150 & & \\
\hline HLP-30 & & & & & & & & 0.0154 & & 1.0001 & 0.1220 & 0.0656 & & 0.0638 & & 0.0029 & & 0.0150 & 0.1540 & & \\
\hline HLP-31 & & & & & & & & 0.0092 & & 1.0000 & 0.0420 & 0.1230 & & 0.0430 & & 0.0040 & & 0.0084 & 0.2200 & & \\
\hline HLP-32 & & & & & & & & 0.0176 & & 1.0000 & 0.0421 & 0.1290 & & 0.0705 & & 0.0031 & & 0.0166 & 0.1570 & & \\
\hline HLP-33 & & & & & & & & 0.0161 & & 0.9999 & 0.0416 & 0.0644 & & 0.0692 & & 0.0045 & & 0.0157 & 0.2200 & & \\
\hline HLP-34 & & & & & & & & 0.0245 & & 0.9999 & 0.0397 & 0.0624 & & 0.1020 & & 0.0030 & & 0.0210 & 0.1430 & & \\
\hline HLP-35 & & & & & & & & 0.0185 & & 1.0000 & 0.1150 & 0.1210 & & 0.0747 & & 0.0050 & & 0.0168 & 0.2160 & & \\
\hline HLP-36 & & & & & & & & 0.0269 & & 1.0000 & 0.1200 & 0.1260 & & 0.1030 & & 0.0027 & & 0.0241 & 0.1550 & & \\
\hline HLP-37 & & & & & & & & 0.0254 & & 0.9999 & 0.1160 & 0.0678 & & 0.0971 & & 0.0040 & & 0.0219 & 0.2180 & & \\
\hline HLP-38 & & & & & & & & 0.0338 & & 0.9999 & 0.1200 & 0.0609 & & 0.1260 & & 0.0030 & & 0.0315 & 0.1530 & & \\
\hline HLP-39 & & & & & & & & 0.0276 & & 0.9998 & 0.0399 & 0.1260 & & 0.1040 & & 0.0045 & & 0.0259 & 0.2150 & & \\
\hline HLP-40 & & & & & & & & 0.0361 & & 1.0001 & 0.0426 & 0.1240 & & 0.1340 & & 0.0031 & & 0.0365 & 0.1630 & & \\
\hline HLP-40Q & & & & & & & & 0.0361 & & 1.0001 & 0.0401 & 0.1270 & & 0.1340 & & 0.0031 & & 0.0346 & 0.1540 & & \\
\hline HLP-41 & & & & & & & & 0.0345 & & 0.9997 & 0.0391 & 0.0647 & & 0.1300 & & 0.0043 & & 0.0320 & 0.2160 & & \\
\hline HLP-42 & & & & & & & & 0.0430 & & 1.0000 & 0.0413 & 0.0646 & & 0.1580 & & 0.0042 & & 0.0409 & 0.1560 & & \\
\hline HLP-42Q & & & & & & & & 0.0430 & & 1.0000 & 0.0409 & 0.0632 & & 0.1680 & & 0.0037 & & 0.0405 & 0.1550 & & \\
\hline HLP-43 & & & & & & & & 0.0150 & & 1.0000 & 0.0669 & 0.0985 & 0.0001 & 0.0623 & & 0.0047 & & 0.0133 & 0.1850 & & \\
\hline HLP-44 & & & & & & & & 0.0150 & & 1.0000 & 0.0693 & 0.0997 & & 0.0607 & & 0.0035 & & 0.0146 & 0.1860 & & \\
\hline HLP-45 & & & & & & & & 0.0150 & & 1.0000 & 0.0710 & 0.1010 & & 0.0606 & & 0.0034 & & 0.0155 & 0.1850 & & \\
\hline HLP-46 & & & & & & & & & & 1.0000 & 0.1170 & 0.0550 & 0.0392 & 0.0049 & & 0.0028 & & & 0.1800 & & \\
\hline HLP-47 & & & & & & & & 0.0000 & & 0.9989 & 0.0942 & 0.0764 & 0.0052 & 0.0204 & & 0.0140 & 0.0012 & 0.0011 & 0.1890 & & \\
\hline HLP-48 & & & & & & & & 0.0427 & & 1.0000 & 0.1150 & 0.0893 & & 0.0637 & & 0.0275 & & 0.0183 & 0.1850 & & \\
\hline HLP-49 & & & & & & & & 0.0399 & & 0.9998 & 0.0776 & 0.0874 & 0.0696 & 0.0871 & & 0.0036 & 0.0411 & 0.0281 & 0.0965 & & \\
\hline HLP-51 & & & & & & & & 0.0260 & & 1.0000 & 0.0968 & 0.0926 & & 0.0294 & & 0.0207 & & 0.0092 & 0.1880 & & \\
\hline HLP-52 & & & & & & & & 0.0000 & & 1.0000 & 0.0963 & 0.0010 & 0.0251 & 0.0300 & & 0.0180 & & 0.0108 & 0.2750 & & \\
\hline HLP-53 & & & & & & & & 0.0328 & & 0.9998 & 0.0931 & 0.0546 & 0.0421 & 0.0767 & & 0.0275 & 0.0229 & 0.0193 & 0.1860 & & \\
\hline HLP-53Q & & & & & & & & 0.0328 & & 0.9998 & 0.0979 & 0.0454 & 0.0433 & 0.0752 & & 0.0308 & 0.0213 & 0.0184 & 0.2040 & & \\
\hline HLP-54 & & & & & & & & 0.0000 & & 0.9997 & 0.1170 & 0.0876 & & 0.0056 & & 0.0028 & & & 0.1780 & & \\
\hline HLP-55 & & & & & & & & 0.0000 & & 0.9997 & 0.0887 & 0.0892 & & 0.0058 & & 0.0032 & & & 0.1770 & & \\
\hline HLP-56 & & & & & & & & 0.0296 & & 0.9997 & 0.0633 & 0.0931 & 0.0206 & 0.0705 & & 0.0212 & & 0.0204 & 0.1810 & & \\
\hline HLP-58 & & & & & & & & 0.0147 & & 0.9999 & 0.0663 & 0.0998 & 0.0202 & 0.0545 & & 0.0045 & 0.0013 & 0.0143 & 0.1903 & & 0.0008 \\
\hline HLP-59 & & & & & & & & 0.0143 & & 1.0003 & 0.0659 & 0.0932 & 0.0468 & 0.0540 & & 0.0045 & 0.0013 & 0.0138 & 0.1837 & & 0.0005 \\
\hline HLP-60 & & & & & & & & 0.0000 & & 1.0000 & 0.1181 & 0.1225 & 0.0002 & 0.0185 & & 0.0038 & 0.0013 & 0.0390 & 0.1569 & & 0.0068 \\
\hline HLP-61 & & & & & & & & 0.0430 & & 1.0000 & 0.0408 & 0.1252 & 0.0474 & 0.0013 & & 0.0478 & 0.0014 & 0.0387 & 0.1592 & & 0.0007 \\
\hline HLP-62 & & & & & & & & 0.0000 & & 1.0000 & 0.0403 & 0.1278 & 0.0467 & 0.0019 & & 0.0037 & 0.0012 & & 0.1641 & & 0.0007 \\
\hline HLP-63 & & & & & & & & 0.0000 & & 1.0000 & 0.0390 & 0.0600 & 0.0001 & 0.1452 & & 0.0038 & 0.0011 & 0.0391 & 0.2565 & & 0.0008 \\
\hline HLP-64 & & & & & & & & 0.0414 & & 1.0000 & 0.0404 & 0.0609 & & 0.0016 & & 0.0460 & 0.0011 & 0.0392 & 0.1599 & & 0.0106 \\
\hline HLP-65 & & & & & & & & 0.0000 & & 1.0000 & 0.0399 & 0.0602 & 0.0466 & 0.0990 & & 0.0037 & 0.0011 & 0.0398 & 0.1688 & & 0.0198 \\
\hline HLP-66 & & & & & & & & 0.0000 & & 1.0000 & 0.0418 & 0.1257 & 0.0004 & 0.0544 & & 0.0041 & 0.0013 & & 0.2349 & & 0.0004 \\
\hline HLP-67 & & & & & & & & 0.0430 & & 1.0000 & 0.0410 & 0.1232 & 0.0002 & 0.0443 & & 0.0473 & 0.0013 & & 0.2372 & & 0.0055 \\
\hline HLP-68 & & & & & & & & 0.0000 & & 1.0000 & 0.0941 & 0.0624 & 0.0470 & 0.1590 & & 0.0464 & 0.0012 & & 0.1631 & & 0.0006 \\
\hline HLP-69 & & & & & & & & 0.0430 & & 1.0000 & 0.1131 & 0.0611 & & 0.0038 & & 0.0038 & 0.0011 & & 0.1694 & & 0.0009 \\
\hline HLP-70 & & & & & & & & 0.0000 & & 1.0000 & 0.1179 & 0.0616 & 0.0456 & 0.0023 & & 0.0497 & 0.0012 & & 0.2431 & & 0.0193 \\
\hline
\end{tabular}


Appendix A. Database - mass fraction

HLP glasses (Vienna et al. 2001)

\begin{tabular}{|c|c|c|c|c|c|c|c|c|c|c|c|c|c|c|c|c|c|c|c|c|c|}
\hline Glass ID & $\begin{array}{c}\mathrm{SiO} 2 \\
-\mathrm{a}\end{array}$ & $\begin{array}{c}\mathrm{ZrO} 2 \\
-\mathrm{a}\end{array}$ & $\begin{array}{c}\mathrm{Ag} 2 \mathrm{O} \\
-\mathrm{a}\end{array}$ & $\begin{array}{c}\mathrm{As} 2 \mathrm{O} 3 \\
-\mathrm{a}\end{array}$ & $\begin{array}{c}\mathrm{BaO} \\
-\mathrm{a}\end{array}$ & $\begin{array}{c}\mathrm{Bi} 2 \mathrm{O} 3 \\
-\mathrm{a}\end{array}$ & $\begin{array}{l}\mathrm{Br} \\
-\mathrm{a}\end{array}$ & $\begin{array}{c}\mathrm{CdO} \\
-\mathrm{a}\end{array}$ & $\begin{array}{c}\mathrm{Ce} 2 \mathrm{O} 3 \\
-\mathrm{a}\end{array}$ & $\begin{array}{c}\mathrm{CeO} 2 \\
-\mathrm{a}\end{array}$ & $\begin{array}{l}\mathrm{Cl} \\
-\mathrm{a}\end{array}$ & $\begin{array}{c}\mathrm{CoO} \\
-\mathrm{a}\end{array}$ & $\begin{array}{c}\mathrm{Co} 2 \mathrm{O} 3 \\
-\mathrm{a}\end{array}$ & $\begin{array}{c}\mathrm{Cr} 2 \mathrm{O} 3 \\
-\mathrm{a}\end{array}$ & $\begin{array}{c}\mathrm{Cs} 2 \mathrm{O} \\
-\mathrm{a}\end{array}$ & $\begin{array}{c}\mathrm{CuO} \\
-\mathrm{a}\end{array}$ & $\begin{array}{c}\mathrm{Eu} 2 \mathrm{O} 3 \\
-\mathrm{a}\end{array}$ & $\begin{array}{l}\mathrm{F} \\
-\mathrm{a}\end{array}$ & $\begin{array}{c}\mathrm{Ga} 2 \mathrm{O} 3 \\
-\mathrm{a}\end{array}$ & $\begin{array}{c}\mathrm{Gd} 2 \mathrm{O} 3 \\
-\mathrm{a}\end{array}$ & $\begin{array}{c}\mathrm{HgO} \\
-\mathrm{a}\end{array}$ \\
\hline HLP-23 & 0.4410 & 0.0138 & & & & & & & & & & & & 0.0027 & & & & & & & \\
\hline HLP-24 & 0.4890 & 0.0151 & & & & & & & & & & & & 0.0024 & & & & & & & \\
\hline HLP-25 & 0.4720 & 0.0148 & & & & & & & & & & & & 0.0021 & & & & & & & \\
\hline HLP-26 & 0.4680 & 0.0150 & & & & & & & & & & & & 0.0021 & & & & & & & \\
\hline HLP-27 & 0.5040 & & & & & & & & & & & & & 0.0026 & & & & & & & \\
\hline HLP-28 & 0.5180 & 0.0075 & & & & & & & & & & & & 0.0022 & & & & & & & \\
\hline HLP-29 & 0.4950 & 0.0064 & & & & & & & & & & & & 0.0025 & & & & & & & \\
\hline HLP-30 & 0.4730 & 0.0143 & & & & & & & & & & & & 0.0023 & & & & & & & \\
\hline HLP-31 & 0.4890 & 0.0070 & & & & & & & & & & & & 0.0024 & & & & & & & \\
\hline HLP-32 & 0.4600 & 0.0160 & & & & & & & & & & & & 0.0025 & & & & & & & \\
\hline HLP-33 & 0.5230 & 0.0131 & & & & & & & & & & & & 0.0030 & & & & & & & \\
\hline HLP-34 & 0.5100 & 0.0200 & & & & & & & & & & & & 0.0020 & & & & & & & \\
\hline HLP-35 & 0.3600 & 0.0173 & & & & & & & & & & & & 0.0022 & & & & & & & \\
\hline HLP-36 & 0.3560 & 0.0244 & & & & & & & & & & & & 0.0017 & & & & & & & \\
\hline HLP-37 & 0.3560 & 0.0228 & & & & & & & & & & & & 0.0019 & & & & & & & \\
\hline HLP-38 & 0.3540 & 0.0338 & & & & & & & & & & & & 0.0017 & & & & & & & \\
\hline HLP-39 & 0.3440 & 0.0262 & & & & & & & & & & & & 0.0023 & & & & & & & \\
\hline HLP-40 & 0.3510 & 0.0344 & & & & & & & & & & & & 0.0018 & & & & & & & \\
\hline HLP-40Q & 0.3410 & 0.0316 & & & & & & & & & & & & 0.0020 & & & & & & & \\
\hline HLP-41 & 0.3500 & 0.0319 & & & & & & & & & & & & 0.0022 & & & & & & & \\
\hline HLP-42 & 0.3420 & 0.0378 & & & & & & & & & & & & 0.0017 & & & & & & & \\
\hline HLP-42Q & 0.3510 & 0.0327 & & & & & & & & & & & & 0.0019 & & & & & & & \\
\hline HLP-43 & 0.4840 & 0.0150 & & & & & & & & & & & & 0.0023 & & & & & & & \\
\hline HLP-44 & 0.4830 & 0.0122 & & & & & & & & & & & & 0.0021 & & & & & & & \\
\hline HLP-45 & 0.4860 & 0.0134 & & & & & & & & & & & & 0.0022 & & & & & & & \\
\hline HLP-46 & 0.5770 & & & & & & & & & & & & & 0.0016 & & & & & & & \\
\hline HLP-47 & 0.5510 & 0.0094 & & & & & & & & & & & & 0.0032 & & & & & & & \\
\hline HLP-48 & 0.3640 & 0.0204 & & & & & & & & & & & & 0.0014 & & & & & & & \\
\hline HLP-49 & 0.4110 & 0.0273 & & & & & & & & & & & & 0.0024 & & & & & & & \\
\hline HLP-51 & 0.4120 & 0.0390 & & & & & & & & & & & & 0.0014 & & & & & & & \\
\hline HLP-52 & 0.4580 & 0.0254 & & & & & & & & & & & & 0.0021 & & & & & & & \\
\hline HLP-53 & 0.3950 & 0.0276 & & & & & & & & & & & & 0.0014 & & & & & & & \\
\hline HLP-53Q & 0.4080 & 0.0282 & & & & & & & & & & & & & & & & & & & \\
\hline HLP-54 & 0.5640 & 0.0007 & & & & & & & & & & & & 0.0016 & & & & & & & \\
\hline HLP-55 & 0.6060 & & & & & & & & & & & & & 0.0017 & & & & & & & \\
\hline HLP-56 & 0.4690 & 0.0135 & & & & & & & & & & & & 0.0002 & & & & & & & \\
\hline HLP-58 & 0.4822 & 0.0146 & & & & & & & & & 0.0027 & & & 0.0008 & & & & 0.0005 & & & \\
\hline HLP-59 & 0.4482 & 0.0152 & & & & & & & & & 0.0031 & & & 0.0008 & & & & 0.0005 & & & \\
\hline HLP-60 & 0.3531 & 0.0414 & & & & & & & & & 0.0031 & & & 0.0010 & & & & 0.0011 & & & \\
\hline HLP-61 & 0.3945 & 0.0007 & & & & & & & & & 0.0032 & & & 0.0010 & & & & 0.0005 & & & \\
\hline HLP-62 & 0.4839 & 0.0552 & & & & & & & & & 0.0032 & & & 0.0007 & & & & 0.0015 & & & \\
\hline HLP-63 & 0.3696 & 0.0006 & & & & & & & & & 0.0036 & & & 0.0008 & & & & 0.0005 & & & \\
\hline HLP-64 & 0.5296 & 0.0424 & & & & & & & & & 0.0033 & & & 0.0007 & & & & 0.0005 & & & \\
\hline HLP-65 & 0.5340 & 0.0004 & & & & & & & & & 0.0032 & & & 0.0008 & & & & 0.0005 & & & \\
\hline HLP-66 & 0.5151 & 0.0002 & & & & & & & & & 0.0034 & & & 0.0009 & & & & 0.0005 & & & \\
\hline HLP-67 & 0.3526 & 0.0379 & & & & & & & & & 0.0035 & & & 0.0009 & & & & 0.0005 & & & \\
\hline HLP-68 & 0.3778 & 0.0563 & & & & & & & & & 0.0029 & & & 0.0008 & & & & 0.0005 & & & \\
\hline HLP-69 & 0.5389 & 0.0007 & & & & & & & & & 0.0030 & & & 0.0007 & & & & 0.0005 & & & \\
\hline HLP-70 & 0.3799 & 0.0005 & & & & & & & & & 0.0037 & & & 0.0007 & & & & 0.0005 & & & \\
\hline
\end{tabular}


Appendix A. Database - mass fraction

HLP glasses (Vienna et al. 2001)

\begin{tabular}{|c|c|c|c|c|c|c|c|c|c|c|c|c|c|c|c|c|c|c|c|c|c|}
\hline Glass ID & $\begin{array}{c}I \\
-a\end{array}$ & $\begin{array}{c}\mathrm{La} 2 \mathrm{O} 3 \\
-\mathrm{a}\end{array}$ & $\begin{array}{c}\mathrm{MnO} 2 \\
-\mathrm{a}\end{array}$ & $\begin{array}{c}\mathrm{MnO} \\
-\mathrm{a}\end{array}$ & $\begin{array}{c}\mathrm{MoO} \\
-\mathrm{a}\end{array}$ & $\begin{array}{c}\mathrm{MoO} 3 \\
-\mathrm{a}\end{array}$ & $\begin{array}{c}\mathrm{Nb} 2 \mathrm{O} 5 \\
-\mathrm{a}\end{array}$ & $\begin{array}{c}\mathrm{Nd} 2 \mathrm{O} 3 \\
-\mathrm{a}\end{array}$ & $\begin{array}{c}\mathrm{PbO} \\
-\mathrm{a}\end{array}$ & $\begin{array}{c}\mathrm{PdO} 2 \\
-\mathrm{a}\end{array}$ & $\begin{array}{c}\mathrm{PdO} \\
-\mathrm{a}\end{array}$ & $\begin{array}{c}\mathrm{Pr} 2 \mathrm{O} 3 \\
-\mathrm{a}\end{array}$ & $\begin{array}{c}\operatorname{Pr} 6011 \\
-\mathrm{a}\end{array}$ & $\begin{array}{c}\mathrm{Rb} 2 \mathrm{O} \\
-\mathrm{a}\end{array}$ & $\begin{array}{c}\mathrm{ReO} \\
-\mathrm{a}\end{array}$ & $\begin{array}{c}\mathrm{ReO} 2 \\
-\mathrm{a}\end{array}$ & $\begin{array}{c}\mathrm{Rh} 2 \mathrm{O} 3 \\
-\mathrm{a}\end{array}$ & $\begin{array}{c}\mathrm{RhO} 2 \\
-\mathrm{a}\end{array}$ & $\begin{array}{c}\mathrm{RuO} 2 \\
-\mathrm{a}\end{array}$ & $\begin{array}{c}\mathrm{Sb} 2 \mathrm{O} 3 \\
-\mathrm{a}\end{array}$ & $\begin{array}{c}\mathrm{Sb} 2 \mathrm{O} 5 \\
-\mathrm{a}\end{array}$ \\
\hline \multicolumn{22}{|l|}{ HLP-23 } \\
\hline \multicolumn{22}{|l|}{ HLP-24 } \\
\hline HLP-25 & & 0.0002 & & & & & & & & & & & & & & & & & & & \\
\hline \multicolumn{22}{|l|}{ HLP-26 } \\
\hline \multicolumn{22}{|l|}{ HLP-27 } \\
\hline \multicolumn{22}{|l|}{ HLP-28 } \\
\hline \multicolumn{22}{|l|}{ HLP-29 } \\
\hline \multicolumn{22}{|l|}{ HLP-30 } \\
\hline \multicolumn{22}{|l|}{ HLP-31 } \\
\hline \multicolumn{22}{|l|}{ HLP-32 } \\
\hline \multicolumn{22}{|l|}{ HLP-33 } \\
\hline \multicolumn{22}{|l|}{ HLP-34 } \\
\hline \multicolumn{22}{|l|}{ HLP-35 } \\
\hline \multicolumn{22}{|l|}{ HLP-36 } \\
\hline HLP-37 & & & & & & & & & & & & & & & & & & & & & \\
\hline HLP-38 & & & & & & & & & & & & & & & & & & & & & \\
\hline HLP-39 & & & & & & & & & & & & & & & & & & & & & \\
\hline HLP-40 & & & & & & & & & & & & & & & & & & & & & \\
\hline HLP-40Q & & & & & & & & & & & & & & & & & & & & & \\
\hline HLP-41 & & & & & & & & & & & & & & & & & & & & & \\
\hline HLP-42 & & & & & & & & & & & & & & & & & & & & & \\
\hline HLP-42Q & & 0.0010 & & & & & & & & & & & & & & & & & & & \\
\hline HLP-43 & & & & & & & & & & & & & & & & & & & & & \\
\hline HLP-44 & & & & & & & & & & & & & & & & & & & & & \\
\hline HLP-45 & & & & & & & & & & & & & & & & & & & & & \\
\hline HLP-46 & & & & & & & & & & & & & & & & & & & & & \\
\hline HLP-47 & & & & & & & & & & & & & & & & & & & & & \\
\hline HLP-48 & & & & & & & & & & & & & & & & & & & & & \\
\hline HLP-49 & & & & & & & & & & & & & & & & & & & & & \\
\hline HLP-51 & & 0.0194 & & & & & & & & & & & & & & & & & & & \\
\hline HLP-52 & & & & & & & & & & & & & & & & & & & & & \\
\hline HLP-53 & & & & & & & & & & & & & & & & & & & & & \\
\hline HLP-53Q & & & & & & & & & & & & & & & & & & & & & \\
\hline HLP-54 & & & & & & & & & & & & & & & & & & & & & \\
\hline HLP-55 & & & & & & & & & & & & & & & & & & & & & \\
\hline HLP-56 & & & & & & & & & & & & & & & & & & & & & \\
\hline HLP-58 & & 0.0001 & & & & 0.0002 & & & & & & & & & & & & & & & \\
\hline HLP-59 & & 0.0001 & & & & 0.0002 & & & & & & & & & & & & & & & \\
\hline HLP-60 & & 0.0001 & & & & 0.0002 & & & & & & & & & & & & & & & \\
\hline HLP-61 & & 0.0001 & & & & 0.0002 & & & & & & & & & & & & & & & \\
\hline HLP-62 & & 0.0001 & & & & 0.0002 & & & & & & & & & & & & & & & \\
\hline HLP-63 & & 0.0001 & & & & 0.0002 & & & & & & & & & & & & & & & \\
\hline HLP-64 & & 0.0001 & & & & 0.0002 & & & & & & & & & & & & & & & \\
\hline HLP-65 & & 0.0001 & & & & 0.0002 & & & & & & & & & & & & & & & \\
\hline HLP-66 & & 0.0001 & & & & 0.0002 & & & & & & & & & & & & & & & \\
\hline HLP-67 & & 0.0001 & & & & 0.0002 & & & & & & & & & & & & & & & \\
\hline HLP-68 & & 0.0001 & & & & 0.0002 & & & & & & & & & & & & & & & \\
\hline HLP-69 & & 0.0001 & & & & 0.0002 & & & & & & & & & & & & & & & \\
\hline HLP-70 & & 0.0001 & & & & 0.0002 & & & & & & & & & & & & & & & \\
\hline
\end{tabular}


Appendix A. Database - mass fraction

\section{HLP glasses (Vienna et al. 2001)}

\begin{tabular}{|c|c|c|c|c|c|c|c|c|c|c|c|c|c|c|c|c|c|c|c|c|}
\hline Glass ID & $\begin{array}{c}\mathrm{SeO} 2 \\
-\mathrm{a}\end{array}$ & $\begin{array}{c}\mathrm{Sm} 2 \mathrm{O} 3 \\
-\mathrm{a}\end{array}$ & $\begin{array}{c}\mathrm{SnO} \\
-\mathrm{a} \\
\end{array}$ & $\begin{array}{c}\mathrm{SnO} 2 \\
-\mathrm{a} \\
\end{array}$ & $\begin{array}{c}\mathrm{SO} 3 \\
-\mathrm{a}\end{array}$ & $\begin{array}{c}\mathrm{SrO} \\
-\mathrm{a} \\
\end{array}$ & $\begin{array}{c}\mathrm{Tc} 2 \mathrm{O} 7 \\
-\mathrm{a}\end{array}$ & $\begin{array}{c}\mathrm{TeO} 2 \\
-\mathrm{a}\end{array}$ & $\begin{array}{c}\mathrm{ThO} 2 \\
-\mathrm{a}\end{array}$ & $\begin{array}{c}\mathrm{TiO} 2 \\
-\mathrm{a} \\
\end{array}$ & $\begin{array}{c}\mathrm{T} 12 \mathrm{O} 3 \\
-\mathrm{a} \\
\end{array}$ & $\begin{array}{c}\text { U3O8 } \\
-\mathrm{a} \\
\end{array}$ & $\begin{array}{c}\mathrm{UO} 2 \\
-\mathrm{a} \\
\end{array}$ & $\begin{array}{c}\text { UO3 } \\
-\mathrm{a} \\
\end{array}$ & $\begin{array}{c}\mathrm{V} 2 \mathrm{O} 5 \\
-\mathrm{a} \\
\end{array}$ & $\begin{array}{c}\text { WO3 } \\
-\mathrm{a}\end{array}$ & $\begin{array}{c}\mathrm{Y} 2 \mathrm{O} 3 \\
-\mathrm{a}\end{array}$ & $\begin{array}{c}\mathrm{ZnO} \\
-\mathrm{a} \\
\end{array}$ & $\begin{array}{c}\text { Others } \\
-\mathrm{a}\end{array}$ & $\begin{array}{c}\text { Sum } \\
-\mathrm{a}\end{array}$ \\
\hline HLP-23 & & & & & & & & & & 0.0290 & & & & & & & & 0.0147 & & 0.9599 \\
\hline HLP-24 & & & & & & & & & & 0.0324 & & & & & & & & 0.0160 & & 0.9875 \\
\hline HLP-25 & & & & & & & & & & 0.0266 & & & & & & & & 0.0149 & & 0.9665 \\
\hline HLP-26 & & & & & & & & & & 0.0286 & & & & & & & & 0.0140 & & 0.9493 \\
\hline HLP-27 & & & & & & & & & & & & & & & & & & & & 0.9770 \\
\hline HLP-28 & & & & & & & & & & 0.0174 & & & & & & & & 0.0092 & & 1.0025 \\
\hline HLP-29 & & & & & & & & & & 0.0141 & & & & & & & & 0.0073 & & 0.9657 \\
\hline HLP-30 & & & & & & & & & & 0.0320 & & & & & & & & 0.0155 & & 0.9604 \\
\hline HLP-31 & & & & & & & & & & 0.0178 & & & & & & & & 0.0094 & & 0.9660 \\
\hline HLP-32 & & & & & & & & & & 0.0357 & & & & & & & & 0.0186 & & 0.9511 \\
\hline HLP-33 & & & & & & & & & & 0.0323 & & & & & & & & 0.0167 & & 1.0035 \\
\hline HLP-34 & & & & & & & & & & 0.0470 & & & & & & & & 0.0232 & & 0.9733 \\
\hline HLP-35 & & & & & & & & & & 0.0361 & & & & & & & & 0.0183 & & 0.9824 \\
\hline HLP-36 & & & & & & & & & & 0.0549 & & & & & & & & 0.0269 & & 0.9947 \\
\hline HLP-37 & & & & & & & & & & 0.0504 & & & & & & & & 0.0246 & & 0.9805 \\
\hline HLP-38 & & & & & & & & & & 0.0688 & & & & & & & & 0.0333 & & 0.9860 \\
\hline HLP-39 & & & & & & & & & & 0.0550 & & & & & & & & 0.0272 & & 0.9700 \\
\hline HLP-40 & & & & & & & & & & 0.0765 & & & & & & & & 0.0373 & & 1.0042 \\
\hline HLP-40Q & & & & & & & & & & 0.0728 & & & & & & & & 0.0362 & & 0.9764 \\
\hline HLP-41 & & & & & & & & & & 0.0678 & & & & & & & & 0.0348 & & 0.9728 \\
\hline HLP-42 & & & & & & & & & & 0.0866 & & & & & & & & 0.0417 & & 0.9748 \\
\hline HLP-42Q & & & & & & & & & & 0.0831 & & & & & & & & 0.0429 & & 0.9839 \\
\hline HLP-43 & & & & & & & & & & 0.0286 & & & & & & & & 0.0140 & & 0.9747 \\
\hline HLP-44 & & & & & & & & & & 0.0312 & & & & & & & & 0.0151 & & 0.9774 \\
\hline HLP-45 & & & & & & & & & & 0.0293 & & & & & & & & 0.0146 & & 0.9820 \\
\hline HLP-46 & & & & & & & & & & & & & & & & & & & & 0.9775 \\
\hline HLP-47 & & & & & & & & & & 0.0009 & & & & & & & & & & 0.9660 \\
\hline HLP-48 & & & & & & & & & & 0.0245 & & & & & & & & 0.0410 & & 0.9501 \\
\hline HLP-49 & & & & & & & & & & & & & & & & & & 0.0393 & & 0.9710 \\
\hline HLP-51 & & & & & & & & & & 0.0237 & & & & & & & & 0.0251 & & 0.9573 \\
\hline HLP-52 & & & & & & & & & & 0.0037 & & & & & & & & & & 0.9454 \\
\hline HLP-53 & & & & & & & & & & & & & & & & & & 0.0309 & & 0.9771 \\
\hline HLP-53Q & & & & & & & & & & & & & & & & & & 0.0324 & & 1.0049 \\
\hline HLP-54 & & & & & & & & & & & & & & & & & & & & 0.9573 \\
\hline HLP-55 & & & & & & & & & & & & & & & & & & & & 0.9716 \\
\hline HLP-56 & & & & & & & & & & & & & & & & & & 0.0409 & & 0.9937 \\
\hline HLP-58 & & & & & 0.0009 & & & & & 0.0301 & & & & & & & & 0.0143 & & 0.9984 \\
\hline HLP-59 & & & & & 0.0009 & & & & & 0.0288 & & & & & & & & 0.0143 & & 0.9758 \\
\hline HLP-60 & & & & & 0.0009 & & & & & 0.0857 & & & & & & & & 0.0002 & & 0.9539 \\
\hline HLP-61 & & & & & 0.0013 & & & & & 0.0846 & & & & & & & & 0.0399 & & 0.9885 \\
\hline HLP-62 & & & & & 0.0013 & & & & & 0.0856 & & & & & & & & & & 1.0181 \\
\hline HLP-63 & & & & & 0.0014 & & & & & 0.0883 & & & & & & & & & & 1.0107 \\
\hline HLP-64 & & & & & 0.0004 & & & & & & & & & & & & & 0.0373 & & 0.9742 \\
\hline HLP-65 & & & & & 0.0011 & & & & & & & & & & & & & & & 1.0192 \\
\hline HLP-66 & & & & & 0.0011 & & & & & 0.0004 & & & & & & & & 0.0001 & & 0.9850 \\
\hline HLP-67 & & & & & 0.0011 & & & & & 0.0001 & & & & & & & & 0.0395 & & 0.9364 \\
\hline HLP-68 & & & & & 0.0014 & & & & & & & & & & & & & & & 1.0138 \\
\hline HLP-69 & & & & & 0.0004 & & & & & 0.0879 & & & & & & & & 0.0394 & & 1.0250 \\
\hline HLP-70 & & & & & 0.0009 & & & & & 0.0843 & & & & & & & & & & 1.0115 \\
\hline
\end{tabular}


Appendix A. Database - mass fraction

HLP glasses (Vienna et al. 2001)

\begin{tabular}{|c|c|c|c|c|c|c|c|c|}
\hline Glass ID & $\begin{array}{l}\mathrm{TM} \\
\left({ }^{\circ} \mathrm{C}\right)\end{array}$ & $\begin{array}{l}\text { Gradient } \\
\text { TL }\left({ }^{\circ} \mathrm{C}\right)\end{array}$ & $\begin{array}{l}\text { Uniform } \\
\text { TL }\left({ }^{\circ} \mathrm{C}\right)\end{array}$ & Primary Phase & $\begin{array}{l}\text { Quenched } \\
\text { Visual/OM }\end{array}$ & $\begin{array}{c}\text { Quenched } \\
\text { SEM/EDS or TEM }\end{array}$ & $\begin{array}{l}\text { Quenched } \\
\text { XRD }\end{array}$ & $\begin{array}{c}\text { Quenched } \\
\text { Homogeneous? }\end{array}$ \\
\hline \multicolumn{9}{|l|}{ HLP-23 } \\
\hline \multicolumn{9}{|l|}{ HLP-24 } \\
\hline \multicolumn{9}{|l|}{ HLP-25 } \\
\hline \multicolumn{9}{|l|}{ HLP-26 } \\
\hline \multicolumn{9}{|l|}{ HLP-27 } \\
\hline \multicolumn{9}{|l|}{ HLP-28 } \\
\hline \multicolumn{9}{|l|}{ HLP-29 } \\
\hline \multicolumn{9}{|l|}{ HLP-30 } \\
\hline \multicolumn{9}{|l|}{ HLP-31 } \\
\hline \multicolumn{9}{|l|}{ HLP-32 } \\
\hline \multicolumn{9}{|l|}{ HLP-33 } \\
\hline \multicolumn{9}{|l|}{ HLP-34 } \\
\hline \multicolumn{9}{|l|}{ HLP-35 } \\
\hline \multicolumn{9}{|l|}{ HLP-36 } \\
\hline \multicolumn{9}{|l|}{ HLP-37 } \\
\hline \multicolumn{9}{|l|}{ HLP-38 } \\
\hline \multicolumn{9}{|l|}{ HLP-39 } \\
\hline \multicolumn{9}{|l|}{ HLP-40 } \\
\hline \multicolumn{9}{|l|}{ HLP-40Q } \\
\hline \multicolumn{9}{|l|}{ HLP-41 } \\
\hline HLP-42 & & & & & & & & \\
\hline HLP-42Q & & & & & & & & \\
\hline HLP-43 & & & & & & & & \\
\hline HLP-44 & & & & & & & & \\
\hline HLP-45 & & & & & & & & \\
\hline HLP-46 & & & & & & & & \\
\hline HLP-47 & & & & & & & & \\
\hline HLP-48 & & & & & & & & \\
\hline HLP-49 & & & & & & & & \\
\hline HLP-51 & & & & & & & & \\
\hline HLP-52 & & & & & & & & \\
\hline HLP-53 & & & & & & & & \\
\hline HLP-53Q & & & & & & & & \\
\hline HLP-54 & & & & & & & & \\
\hline HLP-55 & & & & & & & & \\
\hline HLP-56 & & & & & & & & \\
\hline HLP-58 & & & & & & & & \\
\hline HLP-59 & & & & & & & & \\
\hline HLP-60 & & & & & & & & \\
\hline HLP-61 & & & & & & & & \\
\hline HLP-62 & & & & & & & & \\
\hline HLP-63 & & & & & & & & \\
\hline HLP-64 & & & & & & & & \\
\hline HLP-65 & & & & & & & & \\
\hline HLP-66 & & & & & & & & \\
\hline HLP-67 & & & & & & & & \\
\hline HLP-68 & & & & & & & & \\
\hline HLP-69 & & & & & & & & \\
\hline HLP-70 & & & & & & & & \\
\hline
\end{tabular}


Appendix A. Database - mass fraction

HLP glasses (Vienna et al. 2001)

\begin{tabular}{|c|c|c|c|c|c|}
\hline Glass ID & $\begin{array}{c}\mathrm{CCC} \\
\text { Visual/OM }\end{array}$ & $\begin{array}{c}\text { CCC } \\
\text { SEM/EDS or TEM }\end{array}$ & $\begin{array}{l}\mathrm{CCC} \\
\mathrm{XRD}\end{array}$ & $\begin{array}{l}\text { Heat Treated } \\
\text { Visual/OM }\end{array}$ & $\begin{array}{c}\text { Heat Treated } \\
\text { SEM/EDS or TEM }\end{array}$ \\
\hline \multicolumn{6}{|l|}{ HLP-23 } \\
\hline \multicolumn{6}{|l|}{ HLP-24 } \\
\hline \multicolumn{6}{|l|}{ HLP-25 } \\
\hline \multicolumn{6}{|l|}{ HLP-26 } \\
\hline \multicolumn{6}{|l|}{ HLP-27 } \\
\hline \multicolumn{6}{|l|}{ HLP-28 } \\
\hline \multicolumn{6}{|l|}{ HLP-29 } \\
\hline \multicolumn{6}{|l|}{ HLP-30 } \\
\hline \multicolumn{6}{|l|}{ HLP-31 } \\
\hline \multicolumn{6}{|l|}{ HLP-32 } \\
\hline \multicolumn{6}{|l|}{ HLP-33 } \\
\hline \multicolumn{6}{|l|}{ HLP-34 } \\
\hline \multicolumn{6}{|l|}{ HLP-35 } \\
\hline \multicolumn{6}{|l|}{ HLP-36 } \\
\hline \multicolumn{6}{|l|}{ HLP-37 } \\
\hline \multicolumn{6}{|l|}{ HLP-38 } \\
\hline \multicolumn{6}{|l|}{ HLP-39 } \\
\hline \multicolumn{6}{|l|}{ HLP-40 } \\
\hline \multicolumn{6}{|l|}{ HLP-40Q } \\
\hline \multicolumn{6}{|l|}{ HLP-41 } \\
\hline \multicolumn{6}{|l|}{ HLP-42 } \\
\hline HLP-42Q & & & & & \\
\hline HLP-43 & & & & & \\
\hline HLP-44 & & & & & \\
\hline HLP-45 & & & & & \\
\hline HLP-46 & & & & & \\
\hline HLP-47 & & & & & \\
\hline HLP-48 & & & & & \\
\hline HLP-49 & & & & & \\
\hline HLP-51 & & & & & \\
\hline HLP-52 & & & & & \\
\hline HLP-53 & & & & & \\
\hline HLP-53Q & & & & & \\
\hline HLP-54 & & & & & \\
\hline HLP-55 & & & & & \\
\hline HLP-56 & & & & & \\
\hline HLP-58 & & & & & \\
\hline HLP-59 & & & & & \\
\hline HLP-60 & & & & & \\
\hline HLP-61 & & & & & \\
\hline HLP-62 & & & & & \\
\hline HLP-63 & & & & & \\
\hline HLP-64 & & & & & \\
\hline HLP-65 & & & & & \\
\hline HLP-66 & & & & & \\
\hline HLP-67 & & & & & \\
\hline HLP-68 & & & & & \\
\hline HLP-69 & & & & & \\
\hline HLP-70 & & & & & \\
\hline
\end{tabular}


Appendix A. Database - mass fraction

HLP glasses (Vienna et al. 2001)

\begin{tabular}{|c|c|c|c|c|c|c|c|c|c|c|c|c|c|c|c|}
\hline Glass ID & $\begin{array}{c}\text { Heat Treated } \\
\text { XRD }\end{array}$ & $\begin{array}{l}\text { Density } \\
\left(\mathrm{g} / \mathrm{cm}^{3}\right)\end{array}$ & \begin{tabular}{|c|} 
Fulc Visc \\
$\mathrm{A}$
\end{tabular} & \begin{tabular}{|c|} 
Fulc Visc \\
B
\end{tabular} & \begin{tabular}{|c|} 
Fulc Visc \\
To \\
\end{tabular} & $\begin{array}{c}\mathrm{FV} 1150^{\circ} \mathrm{C} \\
(\mathrm{Pa} \cdot \mathrm{s})\end{array}$ & $\begin{array}{c}\text { Arrh Visc } \\
\text { A }\end{array}$ & $\begin{array}{c}\text { Arrh Visc } \\
\text { B }\end{array}$ & $1150^{\circ} \mathrm{C}$ & $\begin{array}{l}\mathrm{T}\left({ }^{\circ} \mathrm{C}\right) \text { at } \\
2 \mathrm{~Pa} \cdot \mathrm{s}\end{array}$ & \begin{tabular}{|c|}
$\mathrm{T}\left({ }^{\circ} \mathrm{C}\right)$ at \\
$5 \mathrm{~Pa} \cdot \mathrm{s}$
\end{tabular} & \begin{tabular}{|l|}
$\mathrm{T}\left({ }^{\circ} \mathrm{C}\right)$ at \\
$10 \mathrm{~Pa} \cdot \mathrm{s}$
\end{tabular} & $\begin{array}{c}\mathrm{T} 1 \\
\left({ }^{\circ} \mathrm{C}\right)\end{array}$ & $\begin{array}{c}\mathrm{V} 1 \\
(\mathrm{~Pa} \cdot \mathrm{s})\end{array}$ & $\begin{array}{l}\mathrm{T} 2 \\
\left({ }^{\circ} \mathrm{C}\right)\end{array}$ \\
\hline HLP-23 & & 2.6285 & & & & & & & & & & & & & \\
\hline HLP-24 & & 2.6087 & & & & & & & & & & & & & \\
\hline HLP-25 & & 2.6409 & & & & & & & & & & & & & \\
\hline HLP-26 & & 2.6418 & & & & & & & & & & & & & \\
\hline HLP-27 & & 2.5048 & & & & & & & & & & & & & \\
\hline HLP-28 & & 2.5165 & & & & & & & & & & & & & \\
\hline HLP-29 & & 2.5691 & & & & & & & & & & & & & \\
\hline HLP-30 & & 2.599 & & & & & & & & & & & & & \\
\hline HLP-31 & & 2.6244 & & & & & & & & & & & & & \\
\hline HLP-32 & & 2.6249 & & & & & & & & & & & & & \\
\hline HLP-33 & & 2.6351 & & & & & & & & & & & & & \\
\hline HLP-34 & & 2.6729 & & & & & & & & & & & & & \\
\hline HLP-35 & & 2.6437 & & & & & & & & & & & & & \\
\hline HLP-36 & $\mathrm{X}$ & & & & & & & & & & & & & & \\
\hline HLP-37 & X & & & & & & & & & & & & & & \\
\hline HLP-38 & X & & & & & & & & & & & & & & \\
\hline HLP-39 & & 2.7304 & & & & & & & & & & & & & \\
\hline HLP-40 & $\mathrm{X}$ & & & & & & & & & & & & & & \\
\hline \multicolumn{16}{|l|}{ HLP-40Q } \\
\hline HLP-41 & $\mathrm{X}$ & & & & & & & & & & & & & & \\
\hline HLP-42 & $\mathrm{X}$ & & & & & & & & & & & & & & \\
\hline \multicolumn{16}{|l|}{ HLP-42Q } \\
\hline HLP-43 & & 2.6354 & & & & & & & & & & & & & \\
\hline HLP-44 & & 2.6391 & & & & & & & & & & & & & \\
\hline HLP-45 & & 2.6572 & & & & & & & & & & & & & \\
\hline HLP-46 & & 2.5389 & & & & & & & & & & & & & \\
\hline HLP-47 & & 2.5233 & & & & & & & & & & & & & \\
\hline HLP-48 & & 2.6872 & & & & & & & & & & & & & \\
\hline HLP-49 & $\mathrm{X}$ & & & & & & & & & & & & & & \\
\hline HLP-51 & & 2.6751 & & & & & & & & & & & & & \\
\hline HLP-52 & & 2.6539 & & & & & & & & & & & & & \\
\hline HLP-53 & $\mathrm{X}$ & & & & & & & & & & & & & & \\
\hline HLP-53Q & & 2.7258 & & & & & & & & & & & & & \\
\hline HLP-54 & & 2.4897 & & & & & & & & & & & & & \\
\hline HLP-55 & & 2.4545 & & & & & & & & & & & & & \\
\hline HLP-56 & & 2.6804 & & & & & & & & & & & & & \\
\hline HLP-58 & & 2.6217 & & & & & & & & & & & & & \\
\hline HLP-59 & & 2.6452 & & & & & & & & & & & & & \\
\hline HLP-60 & $\mathrm{X}$ & & & & & & & & & & & & & & \\
\hline HLP-61 & & 2.6494 & & & & & & & & & & & & & \\
\hline HLP-62 & & 2.6795 & & & & & & & & & & & & & \\
\hline HLP-63 & & 2.7808 & & & & & & & & & & & & & \\
\hline HLP-64 & & 2.6331 & & & & & & & & & & & & & \\
\hline HLP-65 & & 2.6494 & & & & & & & & & & & & & \\
\hline HLP-66 & & 2.5834 & & & & & & & & & & & & & \\
\hline HLP-67 & & 2.7122 & & & & & & & & & & & & & \\
\hline HLP-68 & $\mathrm{X}$ & 2.7948 & & & & & & & & & & & & & \\
\hline HLP-69 & & 2.6048 & & & & & & & & & & & & & \\
\hline HLP-70 & $\mathrm{X}$ & & & & & & & & & & & & & & \\
\hline
\end{tabular}


Appendix A. Database - mass fraction

\begin{tabular}{|c|c|c|c|c|c|c|c|c|c|c|c|c|c|c|c|c|c|c|c|c|c|}
\hline \multicolumn{22}{|c|}{ HLP glasses (Vienna et al. 2001) } \\
\hline Glass ID & $\begin{array}{c}\mathrm{V} 2 \\
(\mathrm{~Pa} \cdot \mathrm{s})\end{array}$ & $\begin{array}{c}\mathrm{T} 3 \\
\left({ }^{\circ} \mathrm{C}\right)\end{array}$ & $\begin{array}{c}\mathrm{V} 3 \\
(\mathrm{~Pa} \cdot \mathrm{s})\end{array}$ & $\begin{array}{c}\mathrm{T} 4 \\
\left({ }^{\circ} \mathrm{C}\right)\end{array}$ & $\begin{array}{c}\mathrm{V} 4 \\
(\mathrm{~Pa} \cdot \mathrm{s})\end{array}$ & $\begin{array}{c}\mathrm{T} 5 \\
\left({ }^{\circ} \mathrm{C}\right)\end{array}$ & $\begin{array}{c}\text { V5 } \\
(\mathrm{Pa} \cdot \mathrm{s})\end{array}$ & $\begin{array}{c}\mathrm{T} 6 \\
\left({ }^{\circ} \mathrm{C}\right)\end{array}$ & $\begin{array}{c}\mathrm{V} 6 \\
(\mathrm{~Pa} \cdot \mathrm{s})\end{array}$ & $\begin{array}{c}\mathrm{T} 7 \\
\left({ }^{\circ} \mathrm{C}\right)\end{array}$ & $\begin{array}{c}\text { V7 } \\
(\mathrm{Pa} \cdot \mathrm{s})\end{array}$ & $\begin{array}{c}\mathrm{T} 8 \\
\left({ }^{\circ} \mathrm{C}\right)\end{array}$ & $\begin{array}{c}\mathrm{V} 8 \\
(\mathrm{~Pa} \cdot \mathrm{s})\end{array}$ & $\begin{array}{c}\text { T9 } \\
\left({ }^{\circ} \mathrm{C}\right)\end{array}$ & $\begin{array}{c}\mathrm{V} 9 \\
(\mathrm{~Pa} \cdot \mathrm{s})\end{array}$ & $\begin{array}{l}\mathrm{T} 10 \\
\left({ }^{\circ} \mathrm{C}\right)\end{array}$ & $\begin{array}{c}\mathrm{V} 10 \\
(\mathrm{~Pa} \cdot \mathrm{s})\end{array}$ & $\begin{array}{l}\mathrm{T} 11 \\
\left({ }^{\circ} \mathrm{C}\right)\end{array}$ & $\begin{array}{c}\text { V11 } \\
(\mathrm{Pa} \cdot \mathrm{s})\end{array}$ & $\begin{array}{l}\mathrm{T} 12 \\
\left({ }^{\circ} \mathrm{C}\right)\end{array}$ & $\begin{array}{c}\text { V12 } \\
(\mathrm{Pa} \cdot \mathrm{s})\end{array}$ \\
\hline \multicolumn{22}{|l|}{ HLP-23 } \\
\hline \multicolumn{22}{|l|}{ HLP-24 } \\
\hline \multicolumn{22}{|l|}{ HLP-25 } \\
\hline \multicolumn{22}{|l|}{ HLP-26 } \\
\hline \multicolumn{22}{|l|}{ HLP-27 } \\
\hline \multicolumn{22}{|l|}{ HLP-28 } \\
\hline \multicolumn{22}{|l|}{ HLP-29 } \\
\hline \multicolumn{22}{|l|}{ HLP-30 } \\
\hline \multicolumn{22}{|l|}{ HLP-31 } \\
\hline \multicolumn{22}{|l|}{ HLP-32 } \\
\hline \multicolumn{22}{|l|}{ HLP-33 } \\
\hline \multicolumn{22}{|l|}{ HLP-34 } \\
\hline HLP-35 & & & & & & & & & & & & & & & & & & & & & \\
\hline HLP-36 & & & & & & & & & & & & & & & & & & & & & \\
\hline HLP-37 & & & & & & & & & & & & & & & & & & & & & \\
\hline HLP-38 & & & & & & & & & & & & & & & & & & & & & \\
\hline HLP-39 & & & & & & & & & & & & & & & & & & & & & \\
\hline HLP-40 & & & & & & & & & & & & & & & & & & & & & \\
\hline HLP-40Q & & & & & & & & & & & & & & & & & & & & & \\
\hline HLP-41 & & & & & & & & & & & & & & & & & & & & & \\
\hline HLP-42 & & & & & & & & & & & & & & & & & & & & & \\
\hline HLP-42Q & & & & & & & & & & & & & & & & & & & & & \\
\hline HLP-43 & & & & & & & & & & & & & & & & & & & & & \\
\hline HLP-44 & & & & & & & & & & & & & & & & & & & & & \\
\hline HLP-45 & & & & & & & & & & & & & & & & & & & & & \\
\hline HLP-46 & & & & & & & & & & & & & & & & & & & & & \\
\hline HLP-47 & & & & & & & & & & & & & & & & & & & & & \\
\hline HLP-48 & & & & & & & & & & & & & & & & & & & & & \\
\hline HLP-49 & & & & & & & & & & & & & & & & & & & & & \\
\hline HLP-51 & & & & & & & & & & & & & & & & & & & & & \\
\hline HLP-52 & & & & & & & & & & & & & & & & & & & & & \\
\hline HLP-53 & & & & & & & & & & & & & & & & & & & & & \\
\hline HLP-53Q & & & & & & & & & & & & & & & & & & & & & \\
\hline HLP-54 & & & & & & & & & & & & & & & & & & & & & \\
\hline HLP-55 & & & & & & & & & & & & & & & & & & & & & \\
\hline HLP-56 & & & & & & & & & & & & & & & & & & & & & \\
\hline HLP-58 & & & & & & & & & & & & & & & & & & & & & \\
\hline HLP-59 & & & & & & & & & & & & & & & & & & & & & \\
\hline HLP-60 & & & & & & & & & & & & & & & & & & & & & \\
\hline HLP-61 & & & & & & & & & & & & & & & & & & & & & \\
\hline HLP-62 & & & & & & & & & & & & & & & & & & & & & \\
\hline HLP-63 & & & & & & & & & & & & & & & & & & & & & \\
\hline HLP-64 & & & & & & & & & & & & & & & & & & & & & \\
\hline HLP-65 & & & & & & & & & & & & & & & & & & & & & \\
\hline HLP-66 & & & & & & & & & & & & & & & & & & & & & \\
\hline HLP-67 & & & & & & & & & & & & & & & & & & & & & \\
\hline HLP-68 & & & & & & & & & & & & & & & & & & & & & \\
\hline HLP-69 & & & & & & & & & & & & & & & & & & & & & \\
\hline HLP-70 & & & & & & & & & & & & & & & & & & & & & \\
\hline
\end{tabular}


Appendix A. Database - mass fraction

HLP glasses (Vienna et al. 2001)

\begin{tabular}{|c|c|c|c|c|c|c|c|c|c|c|c|c|c|c|c|c|}
\hline Glass ID & $\begin{array}{l}\mathrm{T} 13 \\
\left({ }^{\circ} \mathrm{C}\right) \\
\end{array}$ & $\begin{array}{c}\mathrm{V} 13 \\
(\mathrm{~Pa} \cdot \mathrm{s})\end{array}$ & $\begin{array}{l}\mathrm{T} 14 \\
\left({ }^{\circ} \mathrm{C}\right) \\
\end{array}$ & $\begin{array}{c}\mathrm{V} 14 \\
(\mathrm{~Pa} \cdot \mathrm{s})\end{array}$ & $\begin{array}{c}\text { Q PCT } \\
\mathrm{B}\left(\mathrm{g} / \mathrm{m}^{2}\right)\end{array}$ & $\begin{array}{c}\text { Q PCT } \\
\mathrm{Li}\left(\mathrm{g} / \mathrm{m}^{2}\right)\end{array}$ & $\begin{array}{c}\text { Q PCT } \\
\mathrm{Na}\left(\mathrm{g} / \mathrm{m}^{2}\right)\end{array}$ & $\begin{array}{c}\text { Q PCT } \\
\mathrm{Si}\left(\mathrm{g} / \mathrm{m}^{2}\right)\end{array}$ & $\begin{array}{c}\text { Q PCT } \\
\text { pH }\end{array}$ & $\begin{array}{c}\text { CCC PCT } \\
\mathrm{B}\left(\mathrm{g} / \mathrm{m}^{2}\right)\end{array}$ & $\begin{array}{l}\text { CCC PCT } \\
\mathrm{Li}\left(\mathrm{g} / \mathrm{m}^{2}\right)\end{array}$ & $\begin{array}{l}\text { CCC PCT } \\
\mathrm{Na}\left(\mathrm{g} / \mathrm{m}^{2}\right)\end{array}$ & $\begin{array}{l}\text { CCC PCT } \\
\mathrm{Si}\left(\mathrm{g} / \mathrm{m}^{2}\right)\end{array}$ & $\begin{array}{c}\text { CCC PCT } \\
\mathrm{pH}\end{array}$ & $\begin{array}{l}\text { Q PCT B at } \\
20^{\circ} \mathrm{C}\left(\mathrm{g} / \mathrm{m}^{2}\right)\end{array}$ & $\begin{array}{l}\text { Q PCT Li at } \\
20^{\circ} \mathrm{C}\left(\mathrm{g} / \mathrm{m}^{2}\right)\end{array}$ \\
\hline HLP-23 & & & & & 0.9600 & & 0.7600 & 0.3000 & 11.1667 & & & & & & & \\
\hline HLP-24 & & & & & 0.3300 & & 0.3500 & 0.1900 & 10.1367 & & & & & & & \\
\hline HLP-25 & & & & & 0.4300 & & 0.4200 & 0.2000 & 10.5033 & & & & & & & \\
\hline HLP-26 & & & & & 0.5200 & & 0.3800 & 0.2200 & 10.4533 & & & & & & & \\
\hline HLP-27 & & & & & 2.1700 & & 1.5200 & 0.2300 & 11.3567 & & & & & & & \\
\hline HLP-28 & & & & & 0.2500 & & 0.1900 & 0.1800 & 9.5867 & & & & & & & \\
\hline HLP-29 & & & & & 0.3800 & & 0.5300 & 0.2600 & 11.0667 & & & & & & & \\
\hline HLP-30 & & & & & 0.1500 & & 0.2400 & 0.1500 & 10.1733 & & & & & & & \\
\hline HLP-31 & & & & & 6.5400 & & 4.6700 & 1.4800 & 11.4000 & & & & & & & \\
\hline HLP-32 & & & & & 0.4600 & & 0.3400 & 0.1800 & 9.8400 & & & & & & & \\
\hline HLP-33 & & & & & 1.9200 & & 1.6100 & 0.8100 & 11.3500 & & & & & & & \\
\hline HLP-34 & & & & & 0.3800 & & 0.3600 & 0.1900 & 10.2033 & & & & & & & \\
\hline HLP-35 & & & & & 1.3200 & & 1.0000 & 0.2100 & 11.1567 & & & & & & & \\
\hline HLP-36 & & & & & & & & & & 0.4300 & & 0.3500 & 0.1600 & 9.8700 & & \\
\hline HLP-37 & & & & & 0.6300 & & 0.7500 & 0.3000 & 11.4467 & & & & & & & \\
\hline HLP-38 & & & & & 0.4000 & & 0.3700 & 0.1800 & 10.5833 & & & & & & & \\
\hline HLP-39 & & & & & 2.3100 & & 1.4900 & 0.4000 & 11.4567 & & & & & & & \\
\hline HLP-40 & & & & & & & & & & 0.9400 & & 0.7300 & 0.2100 & 10.0533 & & \\
\hline HLP-40Q & & & & & 0.8100 & & 0.6400 & 0.2100 & 10.0300 & & & & & & & \\
\hline HLP-41 & & & & & & & & & & 6.9400 & & 4.6600 & 1.1100 & 12.2033 & & \\
\hline HLP-42 & & & & & & & & & & 0.7400 & & 0.5900 & 0.2600 & 10.8667 & & \\
\hline HLP-42Q & & & & & 0.9700 & & 0.7100 & 0.2700 & 10.9900 & & & & & & & \\
\hline HLP-43 & & & & & 0.4000 & & 0.3300 & 0.1800 & 10.4933 & & & & & & & \\
\hline HLP-44 & & & & & 0.4500 & & 0.4000 & 0.1700 & 10.4800 & & & & & & & \\
\hline HLP-45 & & & & & 0.4200 & & 0.3800 & 0.1700 & 10.4633 & & & & & & & \\
\hline HLP-46 & & & & & 0.0500 & & 0.4700 & 0.1100 & 10.9033 & & & & & & & \\
\hline HLP-47 & & & & & 0.4900 & & 0.4900 & 0.1400 & 10.9300 & & & & & & & \\
\hline HLP-48 & & & & & 0.6100 & & 0.5200 & 0.1700 & 10.5667 & & & & & & & \\
\hline HLP-49 & & & & & & & & & & 0.3100 & & 0.2900 & 0.0900 & 10.8700 & & \\
\hline HLP-51 & & & & & 0.3400 & & 0.3800 & 0.1500 & 12.6800 & & & & & & & \\
\hline HLP-52 & & & & & & & 5.5700 & 0.6500 & 10.3300 & & & & & & & \\
\hline \multicolumn{17}{|l|}{ HLP-53 } \\
\hline \multicolumn{17}{|l|}{ HLP-53Q } \\
\hline HLP-54 & & & & & 0.4300 & & 0.4500 & 0.1900 & 10.3233 & & & & & & & \\
\hline HLP-55 & & & & & 0.1400 & & 0.2500 & 0.1100 & 11.3633 & & & & & & & \\
\hline \multicolumn{17}{|l|}{ HLP-56 } \\
\hline HLP-58 & & & & & 0.2600 & & 0.3100 & 0.1400 & 10.4633 & & & & & & & \\
\hline HLP-59 & & & & & 0.2900 & & 0.3700 & 0.1500 & 10.7367 & & & & & & & \\
\hline HLP-60 & & & & & & & & & & 0.0300 & & 0.0400 & 0.0200 & & & \\
\hline HLP-61 & & & & & 0.9600 & & 0.3300 & 0.2400 & 10.5733 & & & & & & & \\
\hline HLP-62 & & & & & 0.2500 & & 0.2700 & 0.1200 & 9.9700 & & & & & & & \\
\hline HLP-63 & & & & & 1.2700 & & 3.7300 & 0.9900 & 11.8600 & & & & & & & \\
\hline HLP-64 & & & & & 3.2000 & & 2.4000 & 0.5800 & 11.1933 & & & & & & & \\
\hline HLP-65 & & & & & 0.4600 & & 0.4900 & 0.2300 & 10.5700 & & & & & & & \\
\hline HLP-66 & & & & & 8.9200 & & 6.5000 & 2.3400 & 11.5400 & & & & & & & \\
\hline HLP-67 & & & & & 7.6600 & & 5.6200 & 0.9100 & 12.1967 & & & & & & & \\
\hline HLP-68 & & & & & & & & & & 0.3200 & & 0.4300 & 0.1100 & 11.1867 & & \\
\hline HLP-69 & & & & & 0.2000 & & 0.2100 & 0.1400 & 10.2967 & & & & & & & \\
\hline HLP-70 & & & & & & & & & & 0.3100 & & 0.1400 & 0.1300 & 12.2767 & & \\
\hline
\end{tabular}


Appendix A. Database - mass fraction

HLP glasses (Vienna et al. 2001)

\begin{tabular}{|c|c|c|c|c|c|c|c|c|c|c|c|c|}
\hline Glass ID & $\begin{array}{l}\text { Q PCT Na at } \\
20^{\circ} \mathrm{C}\left(\mathrm{g} / \mathrm{m}^{2}\right)\end{array}$ & $\begin{array}{l}\text { Q PCT Si at } \\
20^{\circ} \mathrm{C}\left(\mathrm{g} / \mathrm{m}^{2}\right)\end{array}$ & $\begin{array}{c}\mathrm{QpH} \\
\text { at } 20^{\circ} \mathrm{C}\end{array}$ & $\begin{array}{l}\text { TCLP Ag } \\
(\mathrm{ppm})\end{array}$ & $\begin{array}{c}\text { TCLP As } \\
(\mathrm{ppm})\end{array}$ & $\begin{array}{c}\text { TCLP Ba } \\
(\mathrm{ppm})\end{array}$ & $\begin{array}{c}\text { TCLP Cd } \\
(\mathrm{ppm})\end{array}$ & $\begin{array}{c}\text { TCLP Cr } \\
(\mathrm{ppm})\end{array}$ & $\begin{array}{l}\text { TCLP Ni } \\
(\mathrm{ppm})\end{array}$ & $\begin{array}{c}\text { TCLP Pb } \\
\text { (ppm) }\end{array}$ & $\begin{array}{c}\text { TCLP Se } \\
(\mathrm{ppm})\end{array}$ & $\begin{array}{c}\text { TCLP Zn } \\
(\mathrm{ppm})\end{array}$ \\
\hline \multicolumn{13}{|l|}{ HLP-23 } \\
\hline \multicolumn{13}{|l|}{ HLP-24 } \\
\hline \multicolumn{13}{|l|}{ HLP-25 } \\
\hline \multicolumn{13}{|l|}{ HLP-26 } \\
\hline \multicolumn{13}{|l|}{ HLP-27 } \\
\hline \multicolumn{13}{|l|}{ HLP-28 } \\
\hline \multicolumn{13}{|l|}{ HLP-29 } \\
\hline \multicolumn{13}{|l|}{ HLP-30 } \\
\hline \multicolumn{13}{|l|}{ HLP-31 } \\
\hline \multicolumn{13}{|l|}{ HLP-32 } \\
\hline \multicolumn{13}{|l|}{ HLP-33 } \\
\hline \multicolumn{13}{|l|}{ HLP-34 } \\
\hline \multicolumn{13}{|l|}{ HLP-35 } \\
\hline \multicolumn{13}{|l|}{ HLP-36 } \\
\hline \multirow{2}{*}{\multicolumn{13}{|c|}{$\begin{array}{l}\text { HLP-37 } \\
\text { HLP-38 }\end{array}$}} \\
\hline \multicolumn{8}{|l|}{ HLP-38 } & & & & & \\
\hline \multicolumn{13}{|l|}{ HLP-39 } \\
\hline \multicolumn{13}{|l|}{ HLP-40 } \\
\hline HLP-40Q & & & & & & & & & & & & \\
\hline HLP-41 & & & & & & & & & & & & \\
\hline HLP-42 & & & & & & & & & & & & \\
\hline HLP-42Q & & & & & & & & & & & & \\
\hline HLP-43 & & & & & & & & & & & & \\
\hline HLP-44 & & & & & & & & & & & & \\
\hline HLP-45 & & & & & & & & & & & & \\
\hline HLP-46 & & & & & & & & & & & & \\
\hline HLP-47 & & & & & & & & & & & & \\
\hline HLP-48 & & & & & & & & & & & & \\
\hline HLP-49 & & & & & & & & & & & & \\
\hline HLP-51 & & & & & & & & & & & & \\
\hline HLP-52 & & & & & & & & & & & & \\
\hline HLP-53 & & & & & & & & & & & & \\
\hline HLP-53Q & & & & & & & & & & & & \\
\hline HLP-54 & & & & & & & & & & & & \\
\hline HLP-55 & & & & & & & & & & & & \\
\hline HLP-56 & & & & & & & & & & & & \\
\hline HLP-58 & & & & & & & & & & & & \\
\hline HLP-59 & & & & & & & & & & & & \\
\hline HLP-60 & & & & & & & & & & & & \\
\hline HLP-61 & & & & & & & & & & & & \\
\hline HLP-62 & & & & & & & & & & & & \\
\hline HLP-63 & & & & & & & & & & & & \\
\hline HLP-64 & & & & & & & & & & & & \\
\hline HLP-65 & & & & & & & & & & & & \\
\hline HLP-66 & & & & & & & & & & & & \\
\hline HLP-67 & & & & & & & & & & & & \\
\hline HLP-68 & & & & & & & & & & & & \\
\hline HLP-69 & & & & & & & & & & & & \\
\hline HLP-70 & & & & & & & & & & & & \\
\hline
\end{tabular}


HLP glasses (Vienna et al. 2001)

\begin{tabular}{|c|c|c|c|c|c|c|c|c|c|c|c|c|c|c|c|c|c|c|c|c|c|}
\hline Glass ID & $\begin{array}{c}\mathrm{A} 12 \mathrm{O} 3 \\
-\mathrm{t}\end{array}$ & $\begin{array}{c}\mathrm{B} 2 \mathrm{O} 3 \\
-\mathrm{t}\end{array}$ & $\begin{array}{c}\mathrm{CaO} \\
-\mathrm{t}\end{array}$ & $\begin{array}{c}\mathrm{Fe} 2 \mathrm{O} 3 \\
-\mathrm{t}\end{array}$ & $\begin{array}{c}\mathrm{FeO} \\
-\mathrm{t}\end{array}$ & $\begin{array}{c}\mathrm{K} 2 \mathrm{O} \\
-\mathrm{t}\end{array}$ & $\begin{array}{c}\mathrm{Li} 2 \mathrm{O} \\
-\mathrm{t}\end{array}$ & $\begin{array}{c}\mathrm{MgO} \\
-\mathrm{t}\end{array}$ & $\begin{array}{c}\mathrm{Na} 2 \mathrm{O} \\
-\mathrm{t}\end{array}$ & $\begin{array}{c}\mathrm{NiO} \\
-\mathrm{t}\end{array}$ & $\begin{array}{c}\mathrm{P} 2 \mathrm{O} 5 \\
-\mathrm{t}\end{array}$ & $\begin{array}{c}\mathrm{SiO} 2 \\
-\mathrm{t}\end{array}$ & $\begin{array}{c}\mathrm{ZrO} 2 \\
-\mathrm{t}\end{array}$ & $\begin{array}{c}\mathrm{Ag} 2 \mathrm{O} \\
-\mathrm{t}\end{array}$ & $\begin{array}{c}\mathrm{As} 2 \mathrm{O} 3 \\
-\mathrm{t}\end{array}$ & $\begin{array}{c}\mathrm{BaO} \\
-\mathrm{t}\end{array}$ & $\begin{array}{c}\mathrm{Bi} 2 \mathrm{O} 3 \\
-\mathrm{t}\end{array}$ & $\begin{array}{l}\mathrm{Br} \\
-\mathrm{t}\end{array}$ & $\begin{array}{c}\mathrm{CdO} \\
-\mathrm{t}\end{array}$ & {$\left[\begin{array}{c}\mathrm{Ce} 2 \mathrm{O} 3 \\
-\mathrm{t}\end{array}\right.$} & $\begin{array}{c}\mathrm{CeO} 2 \\
-\mathrm{t}\end{array}$ \\
\hline HLP-71 & 0.1194 & 0.0600 & 0.0500 & 0.0040 & & 0.0033 & 0.0000 & 0.0430 & 0.2514 & & 0.0004 & 0.3600 & 0.0600 & & & & & & & & \\
\hline HLP-72 & 0.0400 & 0.0600 & 0.0001 & 0.1577 & & 0.0033 & 0.0000 & 0.0000 & 0.1600 & & 0.0200 & 0.3645 & 0.0600 & & & & & & & & \\
\hline HLP-73 & 0.1194 & 0.1257 & 0.0001 & 0.1359 & & 0.0500 & 0.0000 & 0.0430 & 0.1600 & & 0.0004 & 0.3600 & 0.0000 & & & & & & & & \\
\hline HLP-74 & 0.1194 & 0.1257 & 0.0500 & 0.1131 & & 0.0033 & 0.0000 & 0.0000 & 0.1600 & & 0.0200 & 0.3600 & 0.0000 & & & & & & & & \\
\hline HLP-75 & 0.0743 & 0.0893 & 0.0231 & 0.0554 & & 0.0249 & 0.0000 & 0.0200 & 0.1984 & & 0.0099 & 0.4155 & 0.0270 & & & & & & & & \\
\hline HLP-76 & 0.1000 & 0.0800 & 0.0050 & 0.0100 & & 0.0150 & 0.0010 & 0.0010 & 0.2000 & 0.0010 & 0.0050 & 0.5437 & 0.0100 & & & 0.0001 & & & 0.0020 & & \\
\hline HLP-77 & 0.1000 & 0.0800 & 0.0050 & 0.0100 & & 0.0150 & 0.0010 & 0.0010 & 0.2000 & 0.0010 & 0.0050 & 0.5437 & 0.0100 & & & 0.0001 & & & 0.0020 & & \\
\hline
\end{tabular}

Hanford LLW Glass Formulation (Feng et at. 1996)

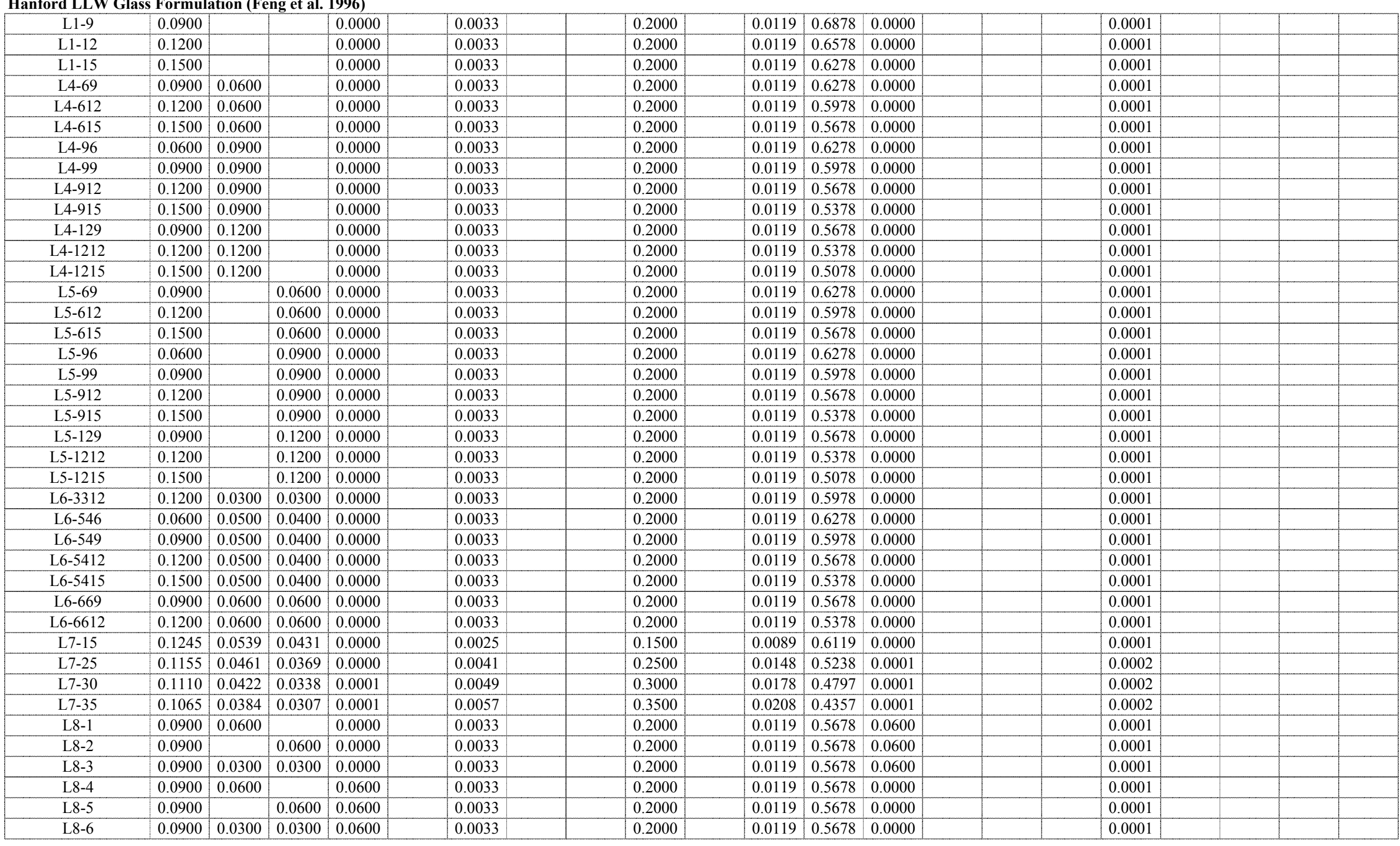


HLP glasses (Vienna et al. 2001)

\begin{tabular}{|c|c|c|c|c|c|c|c|c|c|c|c|c|c|c|c|c|c|c|c|c|c|}
\hline Glass ID & $\begin{array}{l}\mathrm{Cl} \\
-\mathrm{t}\end{array}$ & $\begin{array}{c}\mathrm{CoO} \\
-\mathrm{t}\end{array}$ & $\begin{array}{c}\mathrm{Co} 2 \mathrm{O} 3 \\
-\mathrm{t}\end{array}$ & $\begin{array}{c}\mathrm{Cr} 2 \mathrm{O} 3 \\
-\mathrm{t}\end{array}$ & $\begin{array}{c}\mathrm{Cs} 2 \mathrm{O} \\
-\mathrm{t}\end{array}$ & $\begin{array}{c}\mathrm{CuO} \\
-\mathrm{t}\end{array}$ & $\begin{array}{c}\mathrm{Eu} 2 \mathrm{O} 3 \\
-\mathrm{t}\end{array}$ & $\begin{array}{l}F \\
-t\end{array}$ & $\begin{array}{c}\mathrm{Ga} 2 \mathrm{O} 3 \\
-\mathrm{t}\end{array}$ & {$\left[\begin{array}{c}\mathrm{Gd} 2 \mathrm{O} 3 \\
-\mathrm{t}\end{array}\right.$} & $\begin{array}{c}\mathrm{HgO} \\
-\mathrm{t}\end{array}$ & $\begin{array}{l}I \\
-t\end{array}$ & $\begin{array}{c}\mathrm{La} 2 \mathrm{O} 3 \\
-\mathrm{t}\end{array}$ & $\begin{array}{c}\mathrm{MnO} 2 \\
-\mathrm{t}\end{array}$ & $\begin{array}{c}\mathrm{MnO} \\
-\mathrm{t}\end{array}$ & $\begin{array}{c}\mathrm{MoO} \\
-\mathrm{t}\end{array}$ & $\begin{array}{c}\mathrm{MoO} 3 \\
-\mathrm{t}\end{array}$ & $\begin{array}{c}\mathrm{Nb} 2 \mathrm{O} 5 \\
-\mathrm{t}\end{array}$ & $\begin{array}{c}\mathrm{Nd} 2 \mathrm{O} 3 \\
-\mathrm{t}\end{array}$ & $\begin{array}{c}\mathrm{PbO} \\
-\mathrm{t}\end{array}$ & $\begin{array}{c}\mathrm{PdO} 2 \\
-\mathrm{t}\end{array}$ \\
\hline HLP-71 & 0.0035 & & & 0.0009 & & & & 0.0001 & & & & & 0.0000 & & & & 0.0000 & & & & \\
\hline HLP-72 & 0.0035 & & & 0.0009 & & & & 0.0001 & & & & & 0.0000 & & & & 0.0000 & & & & \\
\hline HLP-73 & 0.0035 & & & 0.0009 & & & & 0.0001 & & & & & 0.0000 & & & & 0.0000 & & & & \\
\hline HLP-74 & 0.0035 & & & 0.0009 & & & & 0.0001 & & & & & 0.0000 & & & & 0.0000 & & & & \\
\hline HLP-75 & 0.0035 & & & 0.0009 & & & & 0.0001 & & & & & 0.0000 & & & & 0.0000 & & & & \\
\hline HLP-76 & 0.0080 & & & 0.0020 & & & & 0.0100 & & & & 0.0000 & 0.0001 & & 0.0010 & & 0.0000 & & & 0.0010 & \\
\hline HLP-77 & 0.0080 & & & 0.0020 & & & & 0.0100 & & & & 0.0000 & 0.0001 & & 0.0010 & & 0.0000 & & & 0.0010 & \\
\hline
\end{tabular}

Hanford LLW Glass Formulation (Feng et al. 1996)

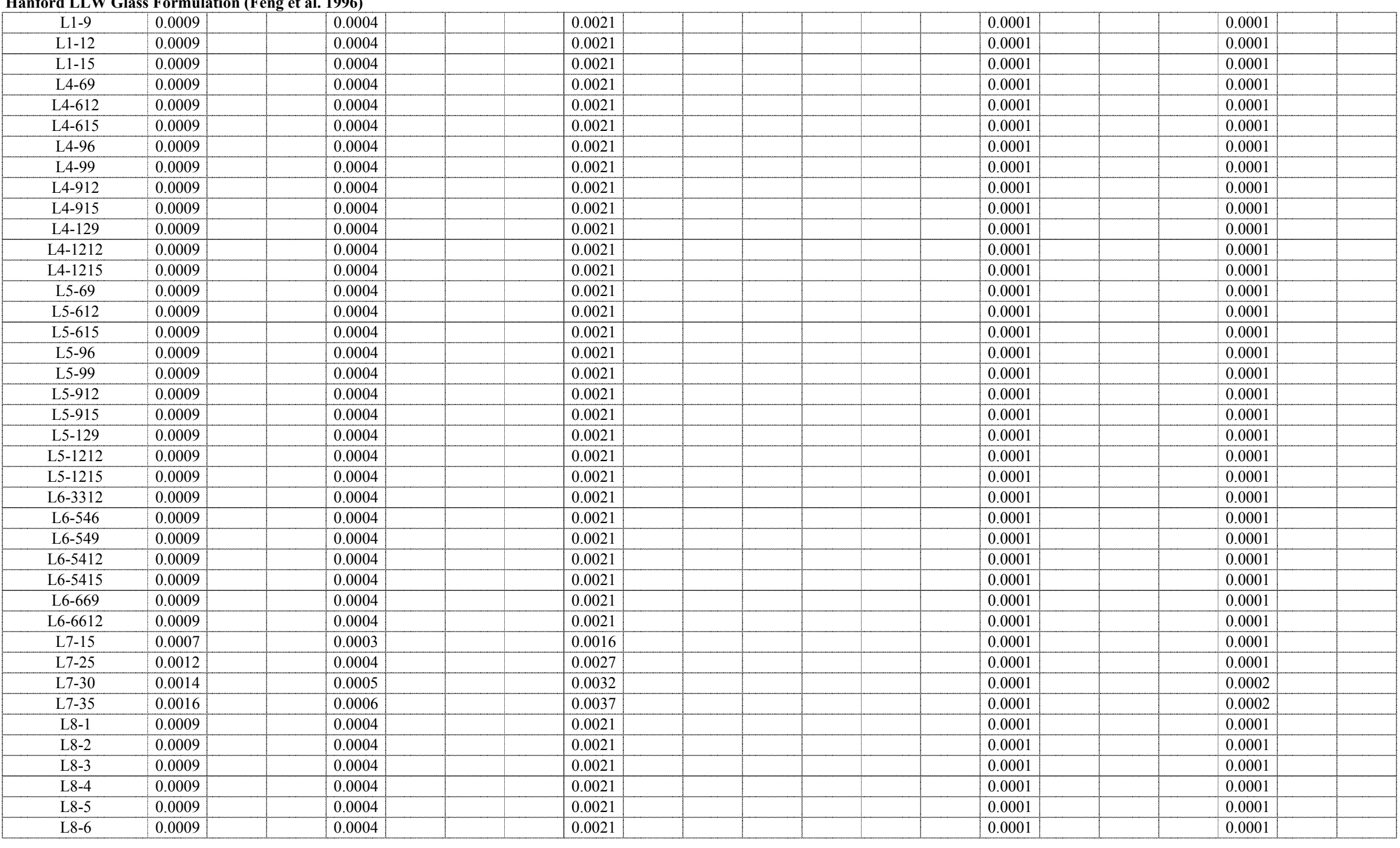


HLP glasses (Vienna et al. 2001)

\begin{tabular}{|c|c|c|c|c|c|c|c|c|c|c|c|c|c|c|c|c|c|c|c|c|c|}
\hline Glass ID & $\begin{array}{c}\mathrm{PdO} \\
-\mathrm{t}\end{array}$ & $\begin{array}{c}\mathrm{Pr} 2 \mathrm{O} 3 \\
-\mathrm{t}\end{array}$ & $\begin{array}{c}\operatorname{Pr} 6 \mathrm{O} 11 \\
-\mathrm{t}\end{array}$ & $\begin{array}{c}\mathrm{Rb} 2 \mathrm{O} \\
-\mathrm{t}\end{array}$ & $\begin{array}{c}\mathrm{ReO} \\
-\mathrm{t}\end{array}$ & $\begin{array}{c}\mathrm{ReO} 2 \\
-\mathrm{t}\end{array}$ & $\begin{array}{c}\mathrm{Rh} 2 \mathrm{O} 3 \\
-\mathrm{t}\end{array}$ & $\begin{array}{c}\mathrm{RhO} 2 \\
-\mathrm{t}\end{array}$ & $\begin{array}{c}\mathrm{RuO} 2 \\
-\mathrm{t}\end{array}$ & $\begin{array}{c}\mathrm{Sb} 2 \mathrm{O} 3 \\
-\mathrm{t}\end{array}$ & $\begin{array}{c}\mathrm{Sb} 2 \mathrm{O} 5 \\
-\mathrm{t}\end{array}$ & $\begin{array}{c}\mathrm{SeO} 2 \\
-\mathrm{t}\end{array}$ & $\begin{array}{c}\mathrm{Sm} 2 \mathrm{O} 3 \\
-\mathrm{t}\end{array}$ & $\begin{array}{c}\mathrm{SnO} \\
-\mathrm{t}\end{array}$ & $\begin{array}{c}\mathrm{SnO} 2 \\
-t\end{array}$ & $\begin{array}{c}\mathrm{SO} 3 \\
-\mathrm{t}\end{array}$ & $\begin{array}{c}\mathrm{SrO} \\
-\mathrm{t}\end{array}$ & $\begin{array}{c}\mathrm{Tc} 2 \mathrm{O} 7 \\
-\mathrm{t}\end{array}$ & $\begin{array}{c}\mathrm{TeO} 2 \\
-\mathrm{t}\end{array}$ & $\begin{array}{c}\mathrm{ThO} 2 \\
-\mathrm{t}\end{array}$ & $\begin{array}{c}\mathrm{TiO} 2 \\
-\mathrm{t}\end{array}$ \\
\hline HLP-71 & & & & & 0.0001 & & & & & & & & & & & 0.0009 & & & & & 0.0000 \\
\hline HLP-72 & & & & & 0.0001 & & & & & & & & & & & 0.0009 & & & & & 0.0859 \\
\hline HLP-73 & & & & & 0.0001 & & & & & & & & & & & 0.0009 & & & & & 0.0000 \\
\hline HLP-74 & & & & & 0.0001 & & & & & & & & & & & 0.0009 & & & & & 0.0000 \\
\hline HLP-75 & & & & & 0.0001 & & & & & & & & & & & 0.0009 & & & & & 0.0368 \\
\hline HLP-76 & & & & & 0.0000 & & & & & & & & & & & 0.0020 & & & & & 0.0010 \\
\hline HLP-77 & & & & & 0.0000 & & & & & & & & & & & 0.0020 & & & & & 0.0010 \\
\hline
\end{tabular}

\section{Hanford LLW Glass Formulation (Feng et al. 1996)}

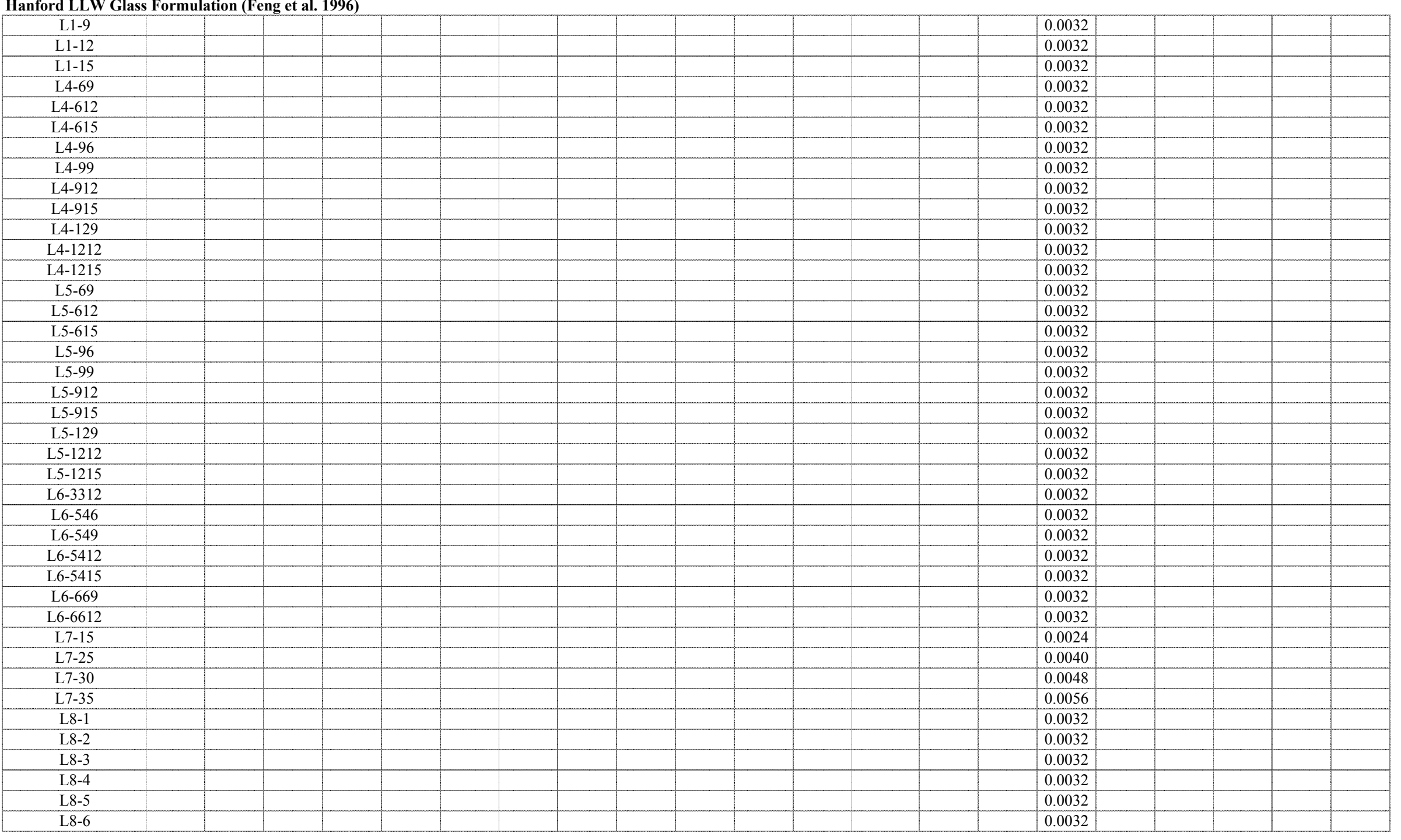


HLP glasses (Vienna et al. 2001)

\begin{tabular}{|c|c|c|c|c|c|c|c|c|c|c|c|c|c|c|c|c|c|c|c|c|c|}
\hline Glass ID & $\begin{array}{c}\mathrm{T} 12 \mathrm{O} 3 \\
-\mathrm{t} \\
\end{array}$ & $\begin{array}{c}\mathrm{U} 3 \mathrm{O} 8 \\
-\mathrm{t}\end{array}$ & $\begin{array}{c}\mathrm{UO} 2 \\
-\mathrm{t} \\
\end{array}$ & $\begin{array}{c}\mathrm{UO} 3 \\
-\mathrm{t} \\
\end{array}$ & $\begin{array}{c}\mathrm{V} 2 \mathrm{O} 5 \\
-\mathrm{t} \\
\end{array}$ & $\begin{array}{c}\text { WO3 } \\
-t\end{array}$ & $\begin{array}{c}\mathrm{Y} 2 \mathrm{O} 3 \\
-\mathrm{t}\end{array}$ & $\begin{array}{c}\mathrm{ZnO} \\
-\mathrm{t} \\
\end{array}$ & $\begin{array}{c}\text { Others } \\
-t\end{array}$ & $\begin{array}{c}\text { Sum } \\
-t\end{array}$ & $\begin{array}{c}\mathrm{A} 12 \mathrm{O} 3 \\
-\mathrm{a} \\
\end{array}$ & $\begin{array}{c}\mathrm{B} 2 \mathrm{O} 3 \\
-\mathrm{a} \\
\end{array}$ & $\begin{array}{c}\mathrm{CaO} \\
-\mathrm{a}\end{array}$ & $\begin{array}{c}\mathrm{Fe} 2 \mathrm{O} 3 \\
-\mathrm{a}\end{array}$ & $\begin{array}{c}\mathrm{FeO} \\
-\mathrm{a}\end{array}$ & $\begin{array}{c}\mathrm{K} 2 \mathrm{O} \\
-\mathrm{a}\end{array}$ & $\begin{array}{c}\mathrm{Li} 2 \mathrm{O} \\
-\mathrm{a}\end{array}$ & $\begin{array}{c}\mathrm{MgO} \\
-\mathrm{a} \\
\end{array}$ & $\begin{array}{c}\mathrm{Na} 2 \mathrm{O} \\
-\mathrm{a} \\
\end{array}$ & $\begin{array}{c}\mathrm{NiO} \\
-\mathrm{a} \\
\end{array}$ & $\begin{array}{c}\mathrm{P} 2 \mathrm{O} 5 \\
-\mathrm{a} \\
\end{array}$ \\
\hline HLP-71 & & & & & & & & 0.0430 & & 1.0000 & 0.1184 & 0.0590 & 0.0465 & 0.0045 & & 0.0037 & 0.0013 & 0.0391 & 0.2447 & & 0.0004 \\
\hline HLP-72 & & & & & & & & 0.0430 & & 1.0000 & 0.0401 & 0.0615 & 0.0017 & 0.1533 & & 0.0037 & 0.0015 & 0.0004 & 0.1642 & & 0.0087 \\
\hline HLP-73 & & & & & & & & 0.0000 & & 1.0000 & 0.1181 & 0.1220 & 0.0004 & 0.1349 & & 0.0478 & 0.0013 & 0.0391 & 0.1556 & & 0.0005 \\
\hline HLP-74 & & & & & & & & 0.0430 & & 1.0000 & 0.1193 & 0.1245 & 0.0473 & 0.1108 & & 0.0042 & 0.0014 & & 0.1573 & & 0.0201 \\
\hline HLP-75 & & & & & & & & 0.0200 & & 1.0001 & 0.0762 & 0.0943 & 0.0238 & 0.0585 & & 0.0264 & 0.0028 & 0.0195 & 0.1925 & & 0.0080 \\
\hline HLP-76 & & & & & & & & 0.0000 & & 0.9989 & 0.0997 & 0.0785 & 0.0054 & 0.0157 & & 0.0164 & 0.0018 & 0.0009 & 0.1938 & & 0.0052 \\
\hline HLP-77 & & & & & & & & 0.0000 & & 0.9989 & 0.0958 & 0.0790 & 0.0049 & 0.0167 & & 0.0156 & 0.0019 & 0.0006 & 0.2029 & & 0.0052 \\
\hline
\end{tabular}

Hanford LLW Glass Formulation (Feng et al. 1996)

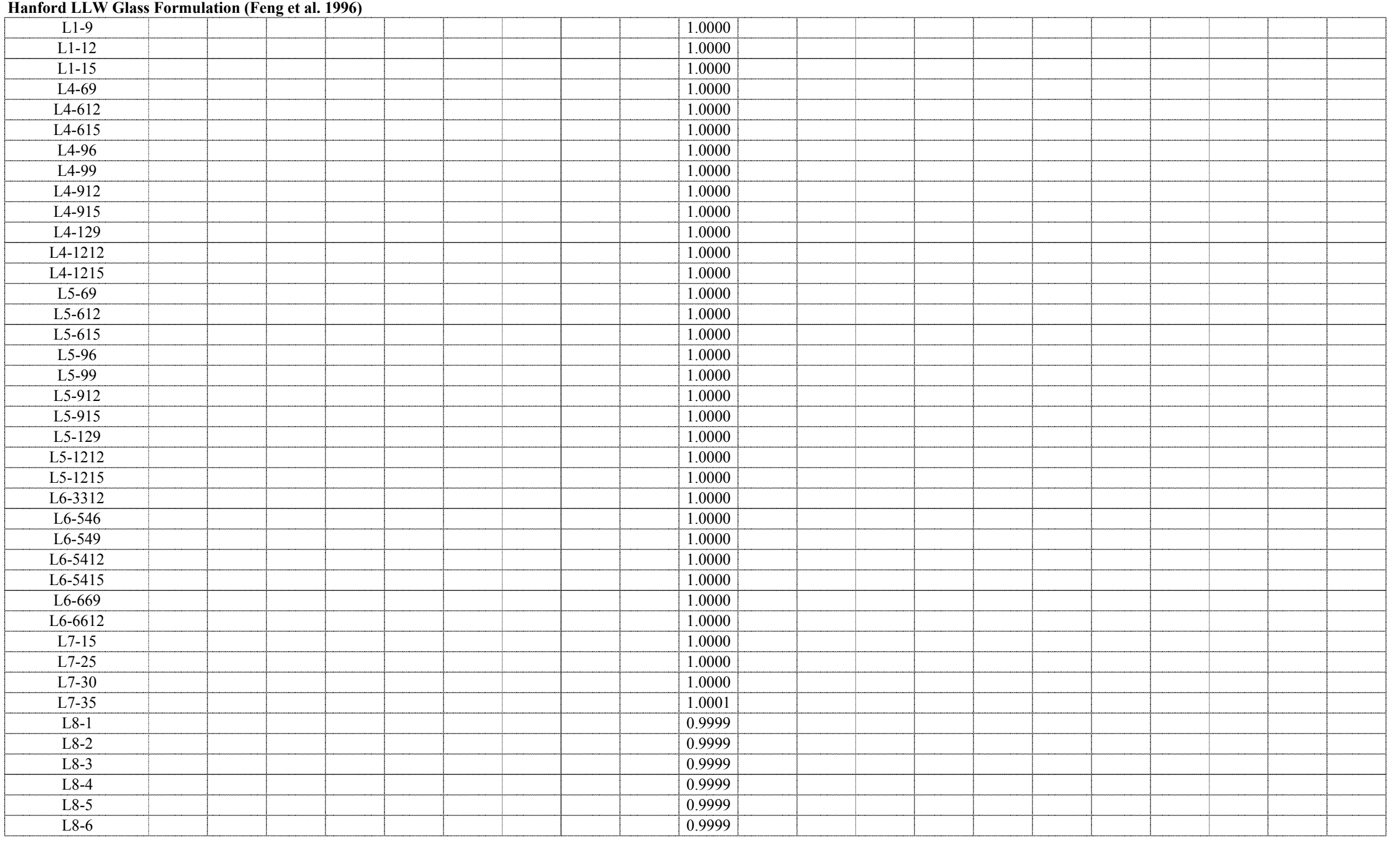


Appendix A. Database - mass fraction

HLP glasses (Vienna et al. 2001)

\begin{tabular}{|c|c|c|c|c|c|c|c|c|c|c|c|c|c|c|c|c|c|c|c|c|c|}
\hline Glass ID & $\begin{array}{c}\mathrm{SiO} 2 \\
-\mathrm{a}\end{array}$ & $\begin{array}{c}\mathrm{ZrO} 2 \\
-\mathrm{a}\end{array}$ & $\begin{array}{c}\mathrm{Ag} 2 \mathrm{O} \\
-\mathrm{a}\end{array}$ & $\begin{array}{c}\mathrm{As} 2 \mathrm{O} 3 \\
-\mathrm{a}\end{array}$ & $\begin{array}{c}\mathrm{BaO} \\
-\mathrm{a}\end{array}$ & $\begin{array}{c}\mathrm{Bi} 2 \mathrm{O} 3 \\
-\mathrm{a}\end{array}$ & $\begin{array}{l}\mathrm{Br} \\
-\mathrm{a}\end{array}$ & $\begin{array}{c}\mathrm{CdO} \\
-\mathrm{a}\end{array}$ & $\begin{array}{c}\mathrm{Ce} 2 \mathrm{O} 3 \\
-\mathrm{a}\end{array}$ & $\begin{array}{c}\mathrm{CeO} 2 \\
-\mathrm{a}\end{array}$ & $\begin{array}{l}\mathrm{Cl} \\
-\mathrm{a}\end{array}$ & $\begin{array}{c}\mathrm{CoO} \\
-\mathrm{a}\end{array}$ & $\begin{array}{c}\mathrm{Co} 2 \mathrm{O} 3 \\
-\mathrm{a}\end{array}$ & $\begin{array}{c}\mathrm{Cr} 2 \mathrm{O} 3 \\
-\mathrm{a}\end{array}$ & $\begin{array}{c}\mathrm{Cs} 2 \mathrm{O} \\
-\mathrm{a}\end{array}$ & $\begin{array}{c}\mathrm{CuO} \\
-\mathrm{a}\end{array}$ & $\begin{array}{c}\mathrm{Eu} 2 \mathrm{O} 3 \\
-\mathrm{a}\end{array}$ & $\begin{array}{l}\mathrm{F} \\
-\mathrm{a}\end{array}$ & $\begin{array}{c}\mathrm{Ga} 2 \mathrm{O} 3 \\
-\mathrm{a}\end{array}$ & $\begin{array}{c}\mathrm{Gd} 2 \mathrm{O} 3 \\
-\mathrm{a}\end{array}$ & $\begin{array}{c}\mathrm{HgO} \\
-\mathrm{a}\end{array}$ \\
\hline HLP-71 & 0.3579 & 0.0612 & & & & & & & & & 0.0036 & & & 0.0009 & & & & 0.0005 & & & \\
\hline HLP-72 & 0.3701 & 0.0385 & & & & & & & & & 0.0033 & & & 0.0008 & & & & 0.0005 & & & \\
\hline HLP-73 & 0.3532 & 0.0001 & & & & & & & & & 0.0032 & & & 0.0009 & & & & 0.0005 & & & \\
\hline HLP-74 & 0.3584 & & & & & & & & & & 0.0034 & & & 0.0008 & & & & 0.0005 & & & \\
\hline HLP-75 & 0.4225 & 0.0253 & & & & & & & & & 0.0028 & & & 0.0009 & & & & 0.0005 & & & \\
\hline HLP-76 & 0.5546 & 0.0111 & & & & & & & & & 0.0011 & & & 0.0019 & & & & 0.0086 & & & \\
\hline HLP-77 & 0.5612 & 0.0101 & & & & & & & & & 0.0010 & & & 0.0018 & & & & 0.0086 & & & \\
\hline
\end{tabular}

Hanford LLW Glass Formulation (Feng et al. 1996)

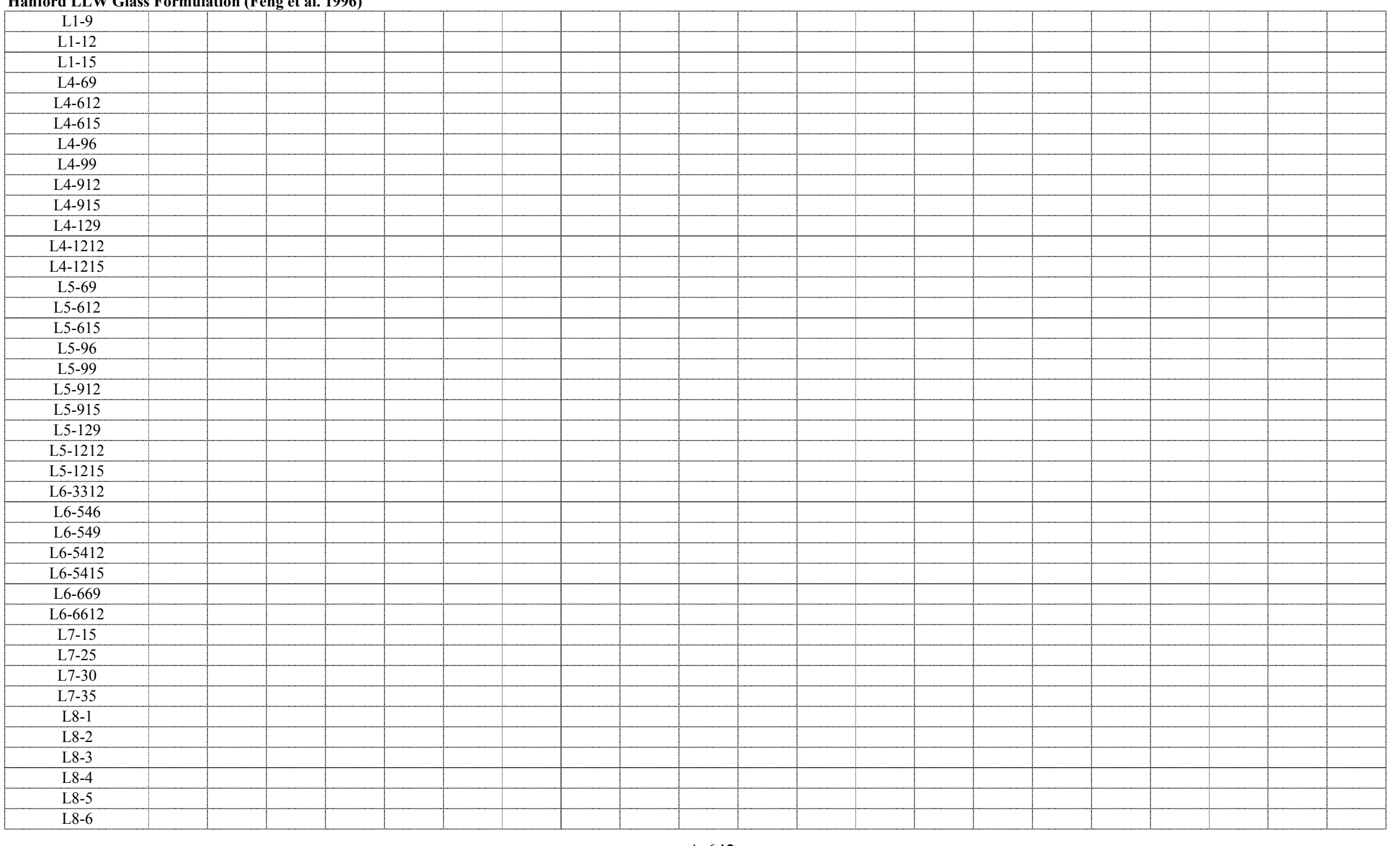


HLP glasses (Vienna et al. 2001)

\begin{tabular}{|c|c|c|c|c|c|c|c|c|c|c|c|c|c|c|c|c|c|c|c|c|c|}
\hline Glass ID & $\begin{array}{c}\mathrm{I} \\
-\mathrm{a}\end{array}$ & $\begin{array}{c}\mathrm{La} 2 \mathrm{O} 3 \\
-\mathrm{a}\end{array}$ & $\begin{array}{c}\mathrm{MnO} 2 \\
-\mathrm{a}\end{array}$ & $\begin{array}{c}\mathrm{MnO} \\
-\mathrm{a}\end{array}$ & $\begin{array}{c}\mathrm{MoO} \\
-\mathrm{a}\end{array}$ & $\begin{array}{c}\mathrm{MoO} 3 \\
-\mathrm{a}\end{array}$ & $\begin{array}{c}\mathrm{Nb} 2 \mathrm{O} 5 \\
-\mathrm{a}\end{array}$ & $\begin{array}{c}\mathrm{Nd} 2 \mathrm{O} 3 \\
-\mathrm{a}\end{array}$ & $\begin{array}{c}\mathrm{PbO} \\
-\mathrm{a}\end{array}$ & $\begin{array}{c}\mathrm{PdO} 2 \\
-\mathrm{a}\end{array}$ & $\begin{array}{c}\mathrm{PdO} \\
-\mathrm{a}\end{array}$ & $\begin{array}{c}\mathrm{Pr} 2 \mathrm{O} 3 \\
-\mathrm{a}\end{array}$ & $\begin{array}{c}\text { Pr6O11 } \\
-\mathrm{a}\end{array}$ & $\begin{array}{c}\mathrm{Rb} 2 \mathrm{O} \\
-\mathrm{a}\end{array}$ & $\begin{array}{c}\mathrm{ReO} \\
-\mathrm{a}\end{array}$ & $\begin{array}{c}\mathrm{ReO} 2 \\
-\mathrm{a}\end{array}$ & $\begin{array}{c}\mathrm{Rh} 2 \mathrm{O} 3 \\
-\mathrm{a}\end{array}$ & $\begin{array}{c}\mathrm{RhO} 2 \\
-\mathrm{a}\end{array}$ & $\begin{array}{c}\mathrm{RuO} 2 \\
-\mathrm{a}\end{array}$ & $\begin{array}{c}\mathrm{Sb} 2 \mathrm{O} 3 \\
-\mathrm{a}\end{array}$ & $\begin{array}{c}\mathrm{Sb} 2 \mathrm{O} 5 \\
-\mathrm{a}\end{array}$ \\
\hline HLP-71 & & 0.0001 & & & & 0.0002 & & & & & & & & & & & & & & & \\
\hline HLP-72 & & 0.0001 & & & & 0.0002 & & & & & & & & & & & & & & & \\
\hline HLP-73 & & 0.0001 & & & & 0.0002 & & & & & & & & & & & & & & & \\
\hline HLP-74 & & 0.0001 & & & & 0.0002 & & & & & & & & & & & & & & & \\
\hline HLP-75 & & 0.0001 & & & & 0.0002 & & & & & & & & & & & & & & & \\
\hline HLP-76 & & 0.0001 & & & & 0.0009 & & & & & & & & & & & & & & & \\
\hline HLP-77 & & 0.0001 & & & & 0.0008 & & & & & & & & & & & & & & & \\
\hline
\end{tabular}

Hanford LLW Glass Formulation (Feng et al. 1996)

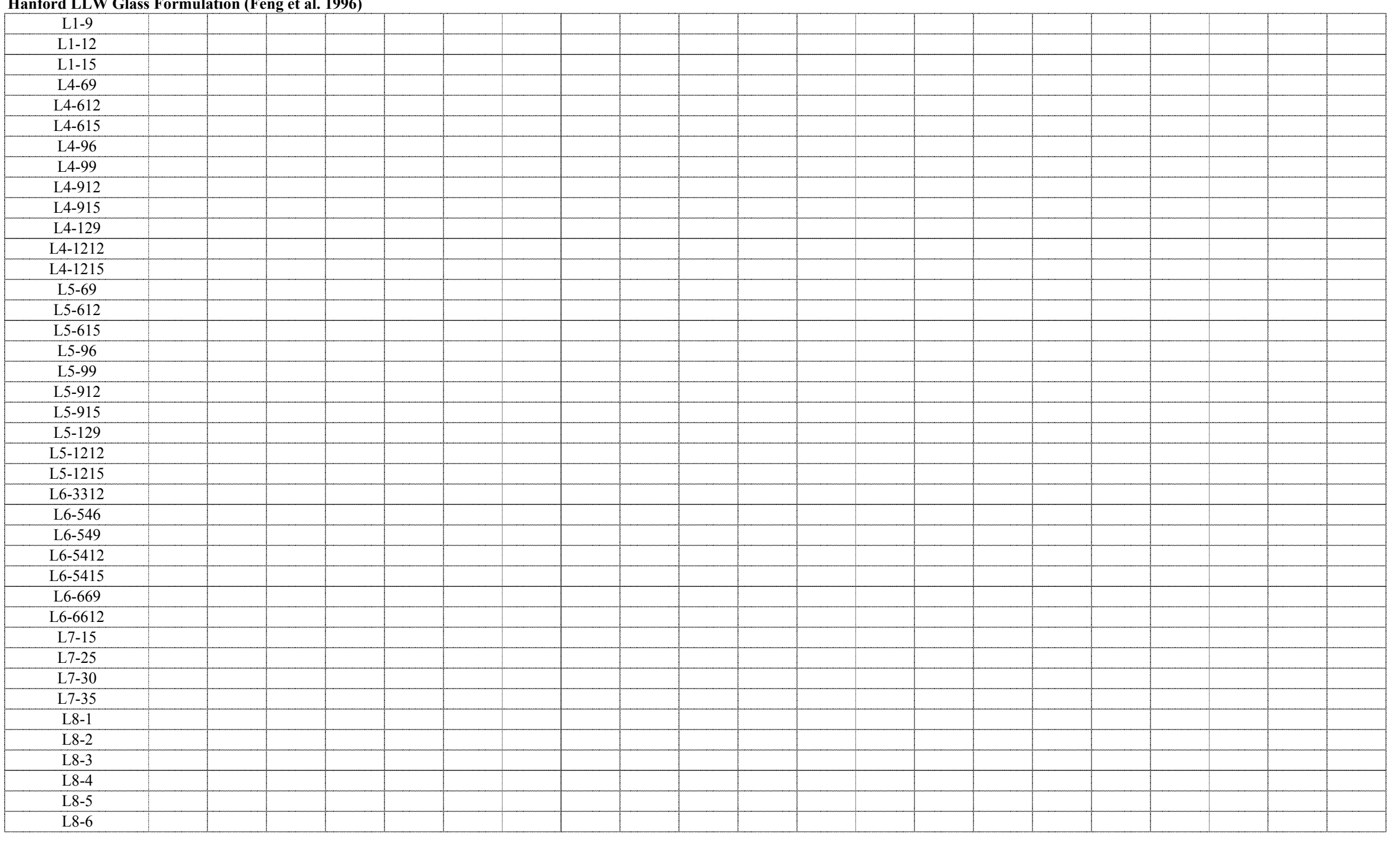


Appendix A. Database - mass fraction

HLP glasses (Vienna et al. 2001)

\begin{tabular}{|c|c|c|c|c|c|c|c|c|c|c|c|c|c|c|c|c|c|c|c|c|}
\hline Glass ID & $\begin{array}{c}\mathrm{SeO} 2 \\
-\mathrm{a}\end{array}$ & $\begin{array}{c}\mathrm{Sm} 2 \mathrm{O} 3 \\
-\mathrm{a}\end{array}$ & $\begin{array}{c}\mathrm{SnO} \\
-\mathrm{a}\end{array}$ & $\begin{array}{c}\mathrm{SnO} 2 \\
-\mathrm{a}\end{array}$ & $\begin{array}{c}\mathrm{SO} 3 \\
-\mathrm{a}\end{array}$ & $\begin{array}{c}\mathrm{SrO} \\
-\mathrm{a}\end{array}$ & $\begin{array}{c}\mathrm{Tc} 2 \mathrm{O} 7 \\
-\mathrm{a}\end{array}$ & $\begin{array}{c}\mathrm{TeO} 2 \\
-\mathrm{a}\end{array}$ & $\begin{array}{c}\text { ThO2 } \\
-\mathrm{a}\end{array}$ & $\begin{array}{c}\mathrm{TiO} 2 \\
-\mathrm{a}\end{array}$ & $\begin{array}{c}\mathrm{T} 12 \mathrm{O} 3 \\
-\mathrm{a}\end{array}$ & $\begin{array}{c}\mathrm{U} 3 \mathrm{O} 8 \\
-\mathrm{a}\end{array}$ & $\begin{array}{c}\mathrm{UO} 2 \\
-\mathrm{a}\end{array}$ & $\begin{array}{c}\mathrm{UO3} \\
-\mathrm{a}\end{array}$ & $\begin{array}{c}\mathrm{V} 2 \mathrm{O} 5 \\
-\mathrm{a}\end{array}$ & $\begin{array}{c}\text { WO3 } \\
-\mathrm{a}\end{array}$ & $\begin{array}{c}\mathrm{Y} 2 \mathrm{O} 3 \\
-\mathrm{a}\end{array}$ & $\begin{array}{c}\mathrm{ZnO} \\
-\mathrm{a}\end{array}$ & $\begin{array}{c}\text { Others } \\
-\mathrm{a}\end{array}$ & $\begin{array}{l}\text { Sum } \\
-\mathrm{a}\end{array}$ \\
\hline HLP-71 & & & & & 0.0012 & & & & & 0.0001 & & & & & & & & 0.0393 & & 0.9826 \\
\hline HLP-72 & & & & & 0.0014 & & & & & 0.0856 & & & & & & & & 0.0386 & & 0.9742 \\
\hline HLP-73 & & & & & 0.0014 & & & & & 0.0002 & & & & & & & & 0.0001 & & 0.9796 \\
\hline HLP-74 & & & & & 0.0010 & & & & & & & & & & & & & 0.0401 & & 0.9894 \\
\hline HLP-75 & & & & & 0.0010 & & & & & 0.0366 & & & & & & & & 0.0197 & & 1.0116 \\
\hline HLP-76 & & & & & 0.0020 & & & & & 0.0013 & & & & & & & & 0.0001 & & 0.9991 \\
\hline HLP-77 & & & & & 0.0019 & & & & & 0.0008 & & & & & & & & & & 1.0089 \\
\hline
\end{tabular}

Hanford LLW Glass Formulation (Feng et al. 1996)

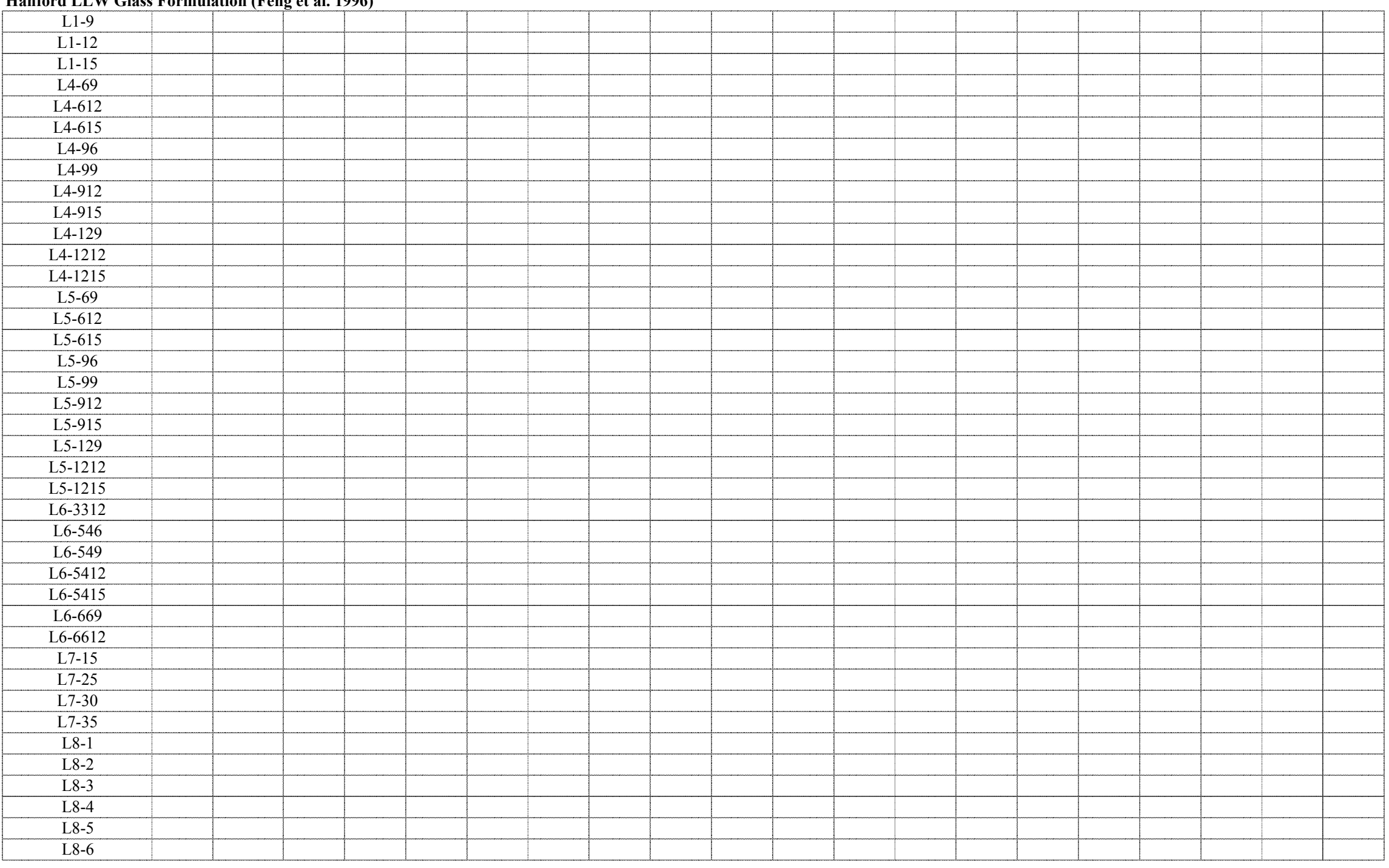


Appendix A. Database - mass fraction

HLP glasses (Vienna et al. 2001)

\begin{tabular}{|c|c|c|c|c|c|c|c|}
\hline Glass ID & $\begin{array}{c}\text { TM } \\
\left({ }^{\circ} \mathrm{C}\right)\end{array}$ & $\begin{array}{c}\text { Gradient } \\
\text { TL }\left({ }^{\circ} \mathrm{C}\right)\end{array}$ & $\begin{array}{c}\text { Uniform } \\
\text { TL }\left({ }^{\circ} \mathrm{C}\right)\end{array}$ & Primary Phase & $\begin{array}{c}\text { Quenched } \\
\text { Visual/OM }\end{array}$ & $\begin{array}{c}\text { Quenched } \\
\text { SEM/EDS or TEM }\end{array}$ & $\begin{array}{c}\text { Quenched } \\
\text { XRD }\end{array}$ \\
\hline HLP-71 & & & & & & \\
\hline HLP-72 & & & & & & \\
\hline HLP-73 & & & & & & \\
\hline HLP-74 & & & & & \\
\hline HLP-75 & & & & \\
\hline HLP-76 & & & & \\
\hline
\end{tabular}

Hanford LLW Glass Formulation (Feng et al. 1996)

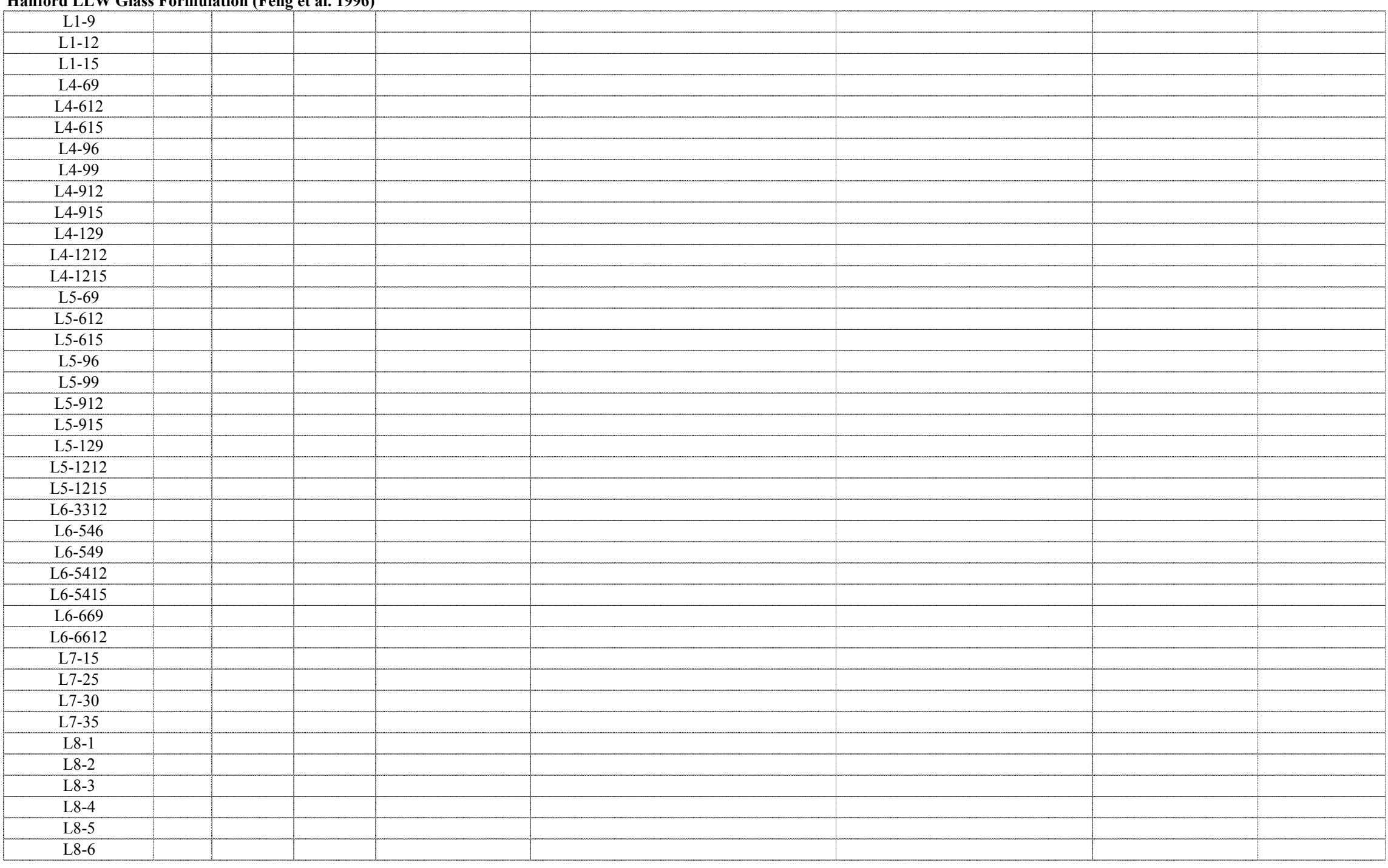


Appendix A. Database - mass fraction

HLP glasses (Vienna et al. 2001)

\begin{tabular}{|c|c|c|c|c|c|}
\hline Glass ID & $\begin{array}{c}\mathrm{CCC} \\
\text { Visual/OM }\end{array}$ & $\begin{array}{c}\text { CCC } \\
\text { SEM/EDS or TEM }\end{array}$ & $\begin{array}{l}\text { CCC } \\
\text { XRD }\end{array}$ & $\begin{array}{c}\text { Heat Treated } \\
\text { Visual/OM }\end{array}$ & $\begin{array}{c}\text { Heat Treated } \\
\text { SEM/EDS or TEM }\end{array}$ \\
\hline \multicolumn{6}{|l|}{ HLP-71 } \\
\hline \multicolumn{6}{|l|}{ HLP-72 } \\
\hline \multicolumn{6}{|l|}{ HLP-73 } \\
\hline \multicolumn{6}{|l|}{ HLP-74 } \\
\hline \multicolumn{6}{|l|}{ HLP-75 } \\
\hline HLP-76 & & & & & \\
\hline HLP-77 & & & & & \\
\hline
\end{tabular}

Hanford LLW Glass Formulation (Feng et al. 1996)

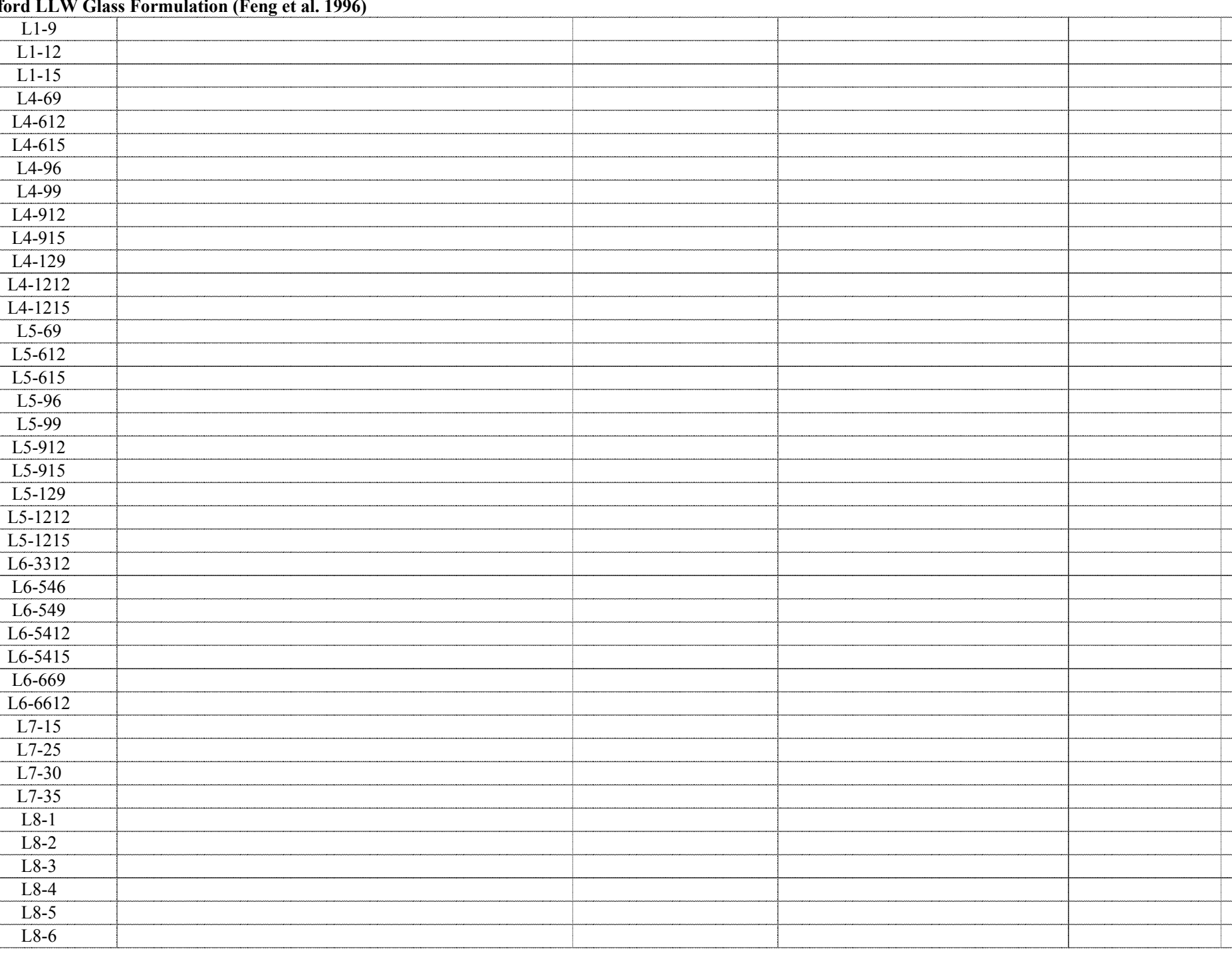


HLP glasses (Vienna et al. 2001)

\begin{tabular}{|c|c|c|c|c|c|c|c|c|c|c|c|c|c|c|c|}
\hline Glass ID & $\begin{array}{c}\text { Heat Treated } \\
\text { XRD }\end{array}$ & $\begin{array}{l}\text { Density } \\
\left(\mathrm{g} / \mathrm{cm}^{3}\right)\end{array}$ & $\begin{array}{c}\text { Fulc Visc } \\
\text { A } \\
\end{array}$ & $\begin{array}{c}\text { Fulc Visc } \\
\text { B } \\
\end{array}$ & $\begin{array}{c}\text { Fulc Visc } \\
\text { To } \\
\end{array}$ & $\begin{array}{c}\mathrm{FV} 1150^{\circ} \mathrm{C} \\
(\mathrm{Pa} \cdot \mathrm{s})\end{array}$ & $\begin{array}{c}\text { Arrh Visc } \\
\text { A }\end{array}$ & $\begin{array}{c}\text { Arrh Visc } \\
\text { B } \\
\end{array}$ & $1150^{\circ} \mathrm{C}$ & $\begin{array}{l}\mathrm{T}\left({ }^{\circ} \mathrm{C}\right) \text { at } \\
2 \mathrm{~Pa} \cdot \mathrm{s}\end{array}$ & $\begin{array}{c}\mathrm{T}\left({ }^{\circ} \mathrm{C}\right) \text { at } \\
5 \mathrm{~Pa} \cdot \mathrm{s}\end{array}$ & $\begin{array}{l}\mathrm{T}\left({ }^{\circ} \mathrm{C}\right) \text { at } \\
10 \mathrm{~Pa} \cdot \mathrm{s}\end{array}$ & $\begin{array}{c}\mathrm{T} 1 \\
\left({ }^{\circ} \mathrm{C}\right) \\
\end{array}$ & $\begin{array}{c}\mathrm{V} 1 \\
(\mathrm{~Pa} \cdot \mathrm{s})\end{array}$ & $\begin{array}{c}\mathrm{T} 2 \\
\left({ }^{\circ} \mathrm{C}\right) \\
\end{array}$ \\
\hline HLP-71 & $\mathrm{X}$ & 2.7159 & & & & & & & & & & & & & \\
\hline HLP-72 & $\mathrm{X}$ & & & & & & & & & & & & & & \\
\hline HLP-73 & $\mathrm{X}$ & & & & & & & & & & & & & & \\
\hline HLP-74 & $\mathrm{X}$ & & & & & & & & & & & & & & \\
\hline HLP-75 & & 2.6806 & & & & & & & & & & & & & \\
\hline HLP-76 & & 2.5537 & & & & & & & & & & & & & \\
\hline HLP-77 & & 2.5613 & & & & & & & & & & & & & \\
\hline
\end{tabular}

\section{Hanford LLW Glass Formulation (Feng et al. 1996)}

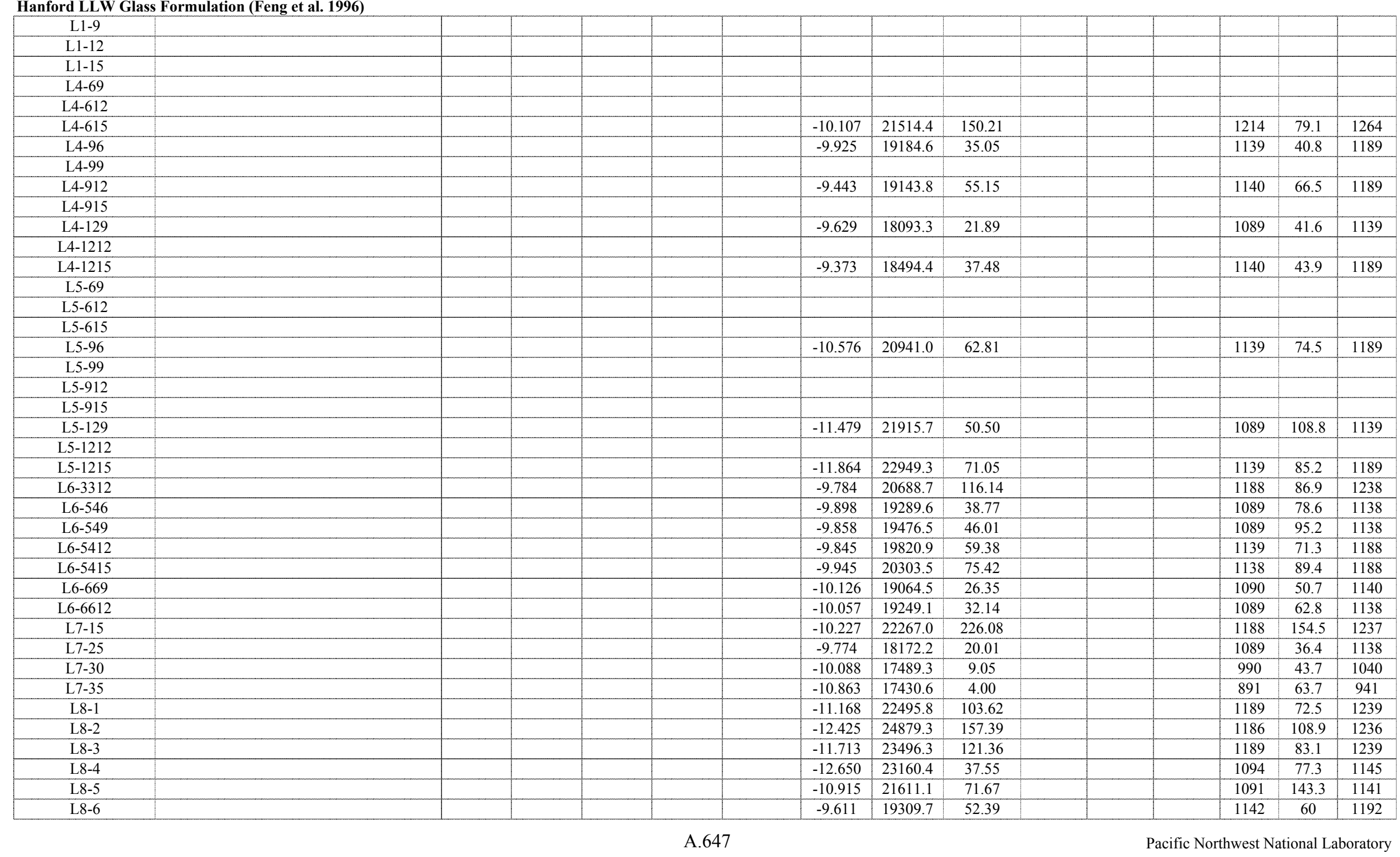




\section{HLP glasses (Vienna et al. 2001)}

\begin{tabular}{|c|c|c|c|c|c|c|c|c|c|c|c|c|c|c|c|c|c|c|c|c|c|}
\hline Glass ID & $\begin{array}{c}\mathrm{V} 2 \\
(\mathrm{~Pa} \cdot \mathrm{s})\end{array}$ & $\begin{array}{c}\mathrm{T} 3 \\
\left({ }^{\circ} \mathrm{C}\right) \\
\end{array}$ & $\begin{array}{c}\mathrm{V} 3 \\
(\mathrm{~Pa} \cdot \mathrm{s})\end{array}$ & $\begin{array}{c}\mathrm{T} 4 \\
\left({ }^{\circ} \mathrm{C}\right) \\
\end{array}$ & $\begin{array}{c}\mathrm{V} 4 \\
(\mathrm{~Pa} \cdot \mathrm{s}) \\
\end{array}$ & $\begin{array}{c}\mathrm{T} 5 \\
\left({ }^{\circ} \mathrm{C}\right) \\
\end{array}$ & $\begin{array}{c}\text { V5 } \\
(\mathrm{Pa} \cdot \mathrm{s}) \\
\end{array}$ & $\begin{array}{c}\mathrm{T} 6 \\
\left({ }^{\circ} \mathrm{C}\right) \\
\end{array}$ & $\begin{array}{c}\mathrm{V} 6 \\
(\mathrm{~Pa} \cdot \mathrm{s})\end{array}$ & $\begin{array}{c}\mathrm{T} 7 \\
\left({ }^{\circ} \mathrm{C}\right) \\
\end{array}$ & $\begin{array}{c}\mathrm{V} 7 \\
(\mathrm{~Pa} \cdot \mathrm{s})\end{array}$ & $\begin{array}{c}\mathrm{T} 8 \\
\left({ }^{\circ} \mathrm{C}\right) \\
\end{array}$ & $\begin{array}{c}\mathrm{V} 8 \\
(\mathrm{~Pa} \cdot \mathrm{s})\end{array}$ & $\begin{array}{c}\mathrm{T} 9 \\
\left({ }^{\circ} \mathrm{C}\right) \\
\end{array}$ & $\begin{array}{c}\mathrm{V} 9 \\
(\mathrm{~Pa} \cdot \mathrm{s})\end{array}$ & $\begin{array}{l}\mathrm{T} 10 \\
\left({ }^{\circ} \mathrm{C}\right) \\
\end{array}$ & $\begin{array}{c}\mathrm{V} 10 \\
(\mathrm{~Pa} \cdot \mathrm{s})\end{array}$ & $\begin{array}{l}\mathrm{T} 11 \\
\left({ }^{\circ} \mathrm{C}\right) \\
\end{array}$ & $\begin{array}{c}\text { V11 } \\
\text { (Pa.s) } \\
\end{array}$ & $\begin{array}{l}\mathrm{T} 12 \\
\left({ }^{\circ} \mathrm{C}\right)\end{array}$ & $\begin{array}{c}\mathrm{V} 12 \\
(\mathrm{~Pa} \cdot \mathrm{s})\end{array}$ \\
\hline \multicolumn{22}{|l|}{ HLP-71 } \\
\hline \multicolumn{22}{|l|}{ HLP-72 } \\
\hline \multicolumn{22}{|l|}{ HLP-73 } \\
\hline \multicolumn{22}{|l|}{ HLP-74 } \\
\hline \multicolumn{22}{|l|}{ HLP-75 } \\
\hline HLP-76 & & & & & & & & & & & & & & & & & & & & & \\
\hline
\end{tabular}

\section{Hanford LLW Glass Formulation (Feng et al. 1996)}

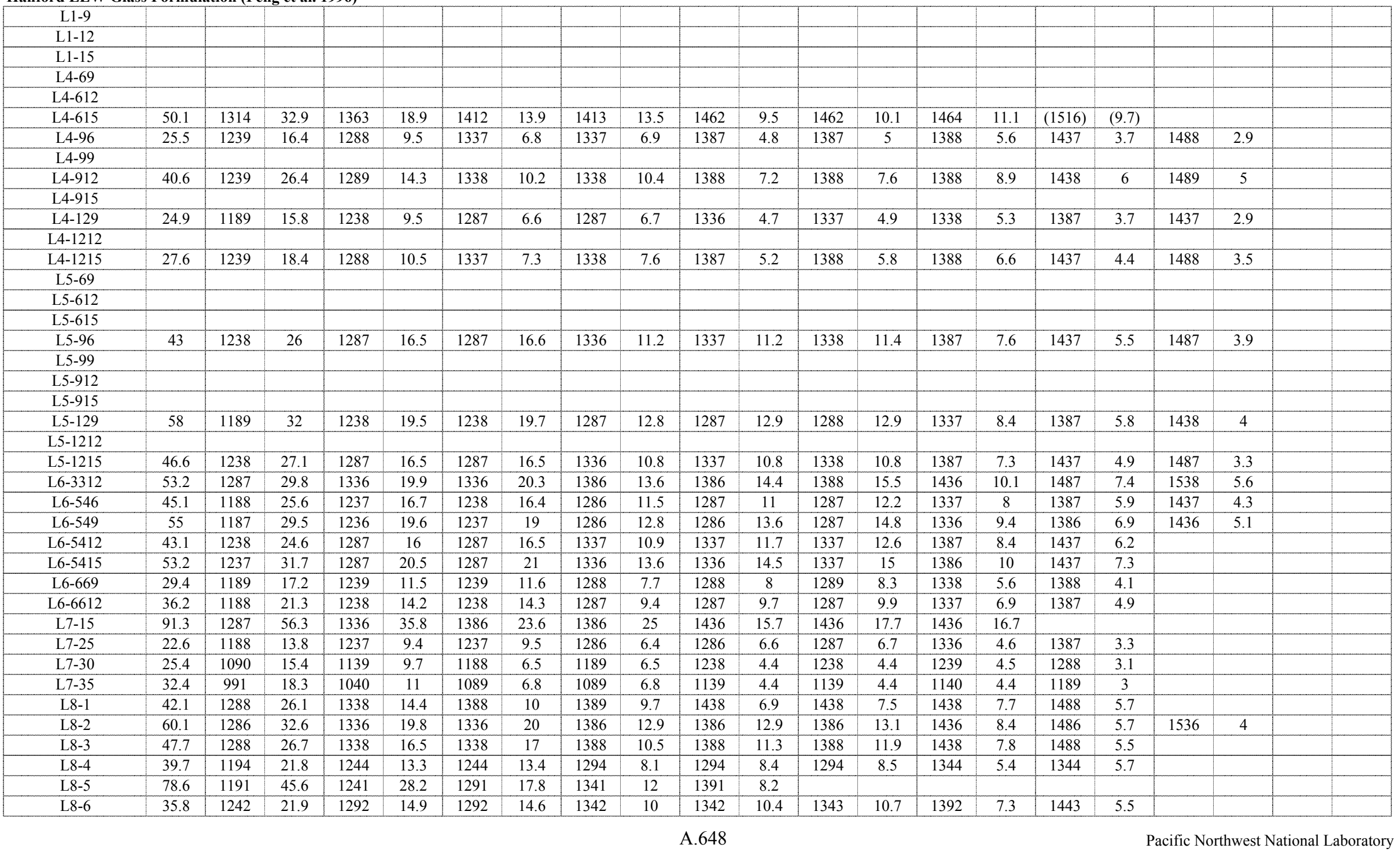


Appendix A. Database - mass fraction

HLP glasses (Vienna et al. 2001)

\begin{tabular}{|c|c|c|c|c|c|c|c|c|c|c|c|c|c|c|c|c|}
\hline Glass ID & $\begin{array}{l}\mathrm{T} 13 \\
\left({ }^{\circ} \mathrm{C}\right)\end{array}$ & $\begin{array}{c}\mathrm{V} 13 \\
(\mathrm{~Pa} \cdot \mathrm{s})\end{array}$ & $\begin{array}{l}\mathrm{T} 14 \\
\left({ }^{\circ} \mathrm{C}\right)\end{array}$ & $\begin{array}{c}\text { V14 } \\
(\mathrm{Pa} \cdot \mathrm{s})\end{array}$ & $\begin{array}{c}\text { Q PCT } \\
\text { B }\left(\mathrm{g} / \mathrm{m}^{2}\right)\end{array}$ & $\begin{array}{c}\text { Q PCT } \\
\mathrm{Li}\left(\mathrm{g} / \mathrm{m}^{2}\right)\end{array}$ & $\begin{array}{c}\text { Q PCT } \\
\mathrm{Na}\left(\mathrm{g} / \mathrm{m}^{2}\right)\end{array}$ & $\begin{array}{c}\text { Q PCT } \\
\mathrm{Si}\left(\mathrm{g} / \mathrm{m}^{2}\right)\end{array}$ & $\begin{array}{c}\text { Q PCT } \\
\text { pH }\end{array}$ & $\begin{array}{l}\text { CCC PCT } \\
\text { B }\left(\mathrm{g} / \mathrm{m}^{2}\right)\end{array}$ & \begin{tabular}{|c|} 
CCC PCT \\
$\mathrm{Li}\left(\mathrm{g} / \mathrm{m}^{2}\right)$ \\
\end{tabular} & $\begin{array}{l}\text { CCC PCT } \\
\mathrm{Na}\left(\mathrm{g} / \mathrm{m}^{2}\right)\end{array}$ & $\begin{array}{l}\text { CCC PCT } \\
\mathrm{Si}\left(\mathrm{g} / \mathrm{m}^{2}\right)\end{array}$ & $\begin{array}{c}\text { CCC PCT } \\
\mathrm{pH}\end{array}$ & $\begin{array}{l}\text { Q PCT B at } \\
20^{\circ} \mathrm{C}\left(\mathrm{g} / \mathrm{m}^{2}\right)\end{array}$ & $\begin{array}{l}\text { Q PCT Li at } \\
20^{\circ} \mathrm{C}\left(\mathrm{g} / \mathrm{m}^{2}\right)\end{array}$ \\
\hline HLP-71 & & & & & & & & & & 42.6800 & & 11.0100 & 0.4300 & 12.4667 & & \\
\hline HLP-72 & & & & & & & & & & 0.2900 & & 0.3000 & 0.1200 & 9.8933 & & \\
\hline HLP-73 & & & & & & & & & & 2.0600 & & 1.2400 & 0.1400 & 10.2667 & & \\
\hline HLP-74 & & & & & & & & & & 0.2500 & & 0.2900 & 0.1000 & 10.1833 & & \\
\hline HLP-75 & & & & & 0.3300 & & 0.4300 & 0.1700 & 10.9667 & & & & & & & \\
\hline HLP-76 & & & & & 0.2300 & & 0.3000 & 0.1300 & 10.7967 & & & & & & & \\
\hline HLP-77 & & & & & 0.2200 & & 0.3000 & 0.1300 & 10.7600 & & & & & & & \\
\hline
\end{tabular}

Hanford LLW Glass Formulation (Feng et al. 1996)

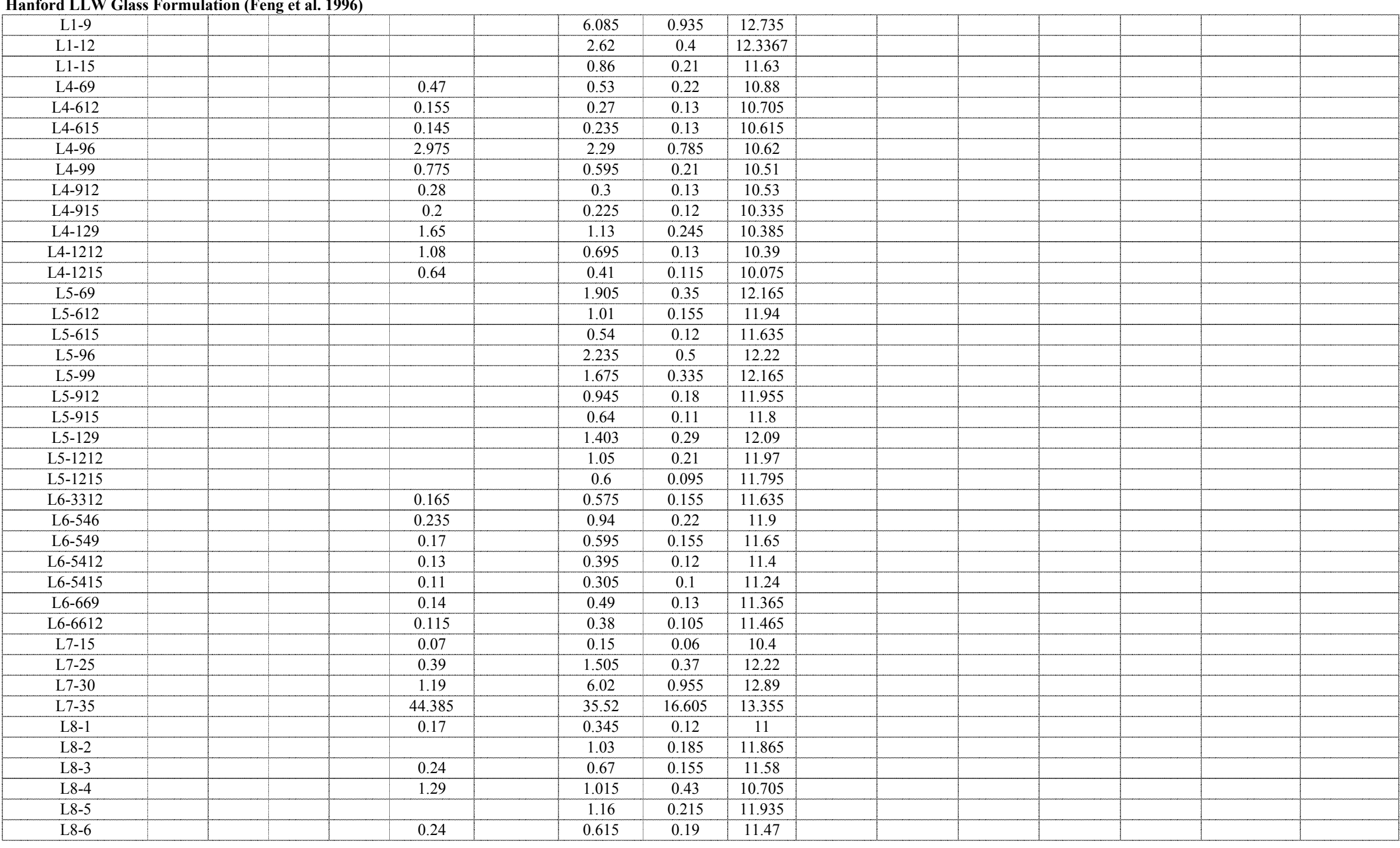


Appendix A. Database - mass fraction

HLP glasses (Vienna et al. 2001)

\begin{tabular}{|c|c|c|c|c|c|c|c|c|c|c|c|c|}
\hline Glass ID & $\begin{array}{l}\text { Q PCT Na at } \\
20^{\circ} \mathrm{C}\left(\mathrm{g} / \mathrm{m}^{2}\right)\end{array}$ & $\begin{array}{l}\text { Q PCT Si at } \\
20^{\circ} \mathrm{C}\left(\mathrm{g} / \mathrm{m}^{2}\right)\end{array}$ & $\begin{array}{c}\mathrm{QpH} \\
\text { at } 20^{\circ} \mathrm{C}\end{array}$ & $\begin{array}{c}\text { TCLP Ag } \\
(\mathrm{ppm})\end{array}$ & $\begin{array}{c}\text { TCLP As } \\
(\mathrm{ppm})\end{array}$ & $\begin{array}{c}\text { TCLP Ba } \\
(\mathrm{ppm})\end{array}$ & $\begin{array}{c}\text { TCLP Cd } \\
(\mathrm{ppm})\end{array}$ & $\begin{array}{c}\text { TCLP Cr } \\
(\mathrm{ppm})\end{array}$ & $\begin{array}{c}\text { TCLP Ni } \\
(\mathrm{ppm})\end{array}$ & $\begin{array}{c}\text { TCLP Pb } \\
(\mathrm{ppm})\end{array}$ & $\begin{array}{c}\text { TCLP Se } \\
(\mathrm{ppm})\end{array}$ & $\begin{array}{c}\text { TCLP Zn } \\
(\mathrm{ppm})\end{array}$ \\
\hline \multicolumn{13}{|c|}{ (1) } \\
\hline \multicolumn{13}{|l|}{ HLP-72 } \\
\hline \multicolumn{13}{|l|}{ HLP-73 } \\
\hline \multicolumn{13}{|l|}{ HLP-74 } \\
\hline \multicolumn{13}{|l|}{ HLP-75 } \\
\hline HLP-76 & & & & & & & & & & & & \\
\hline HLP-77 & & & & & & & & & & & & \\
\hline
\end{tabular}

Hanford LLW Glass Formulation (Feng et al. 1996)

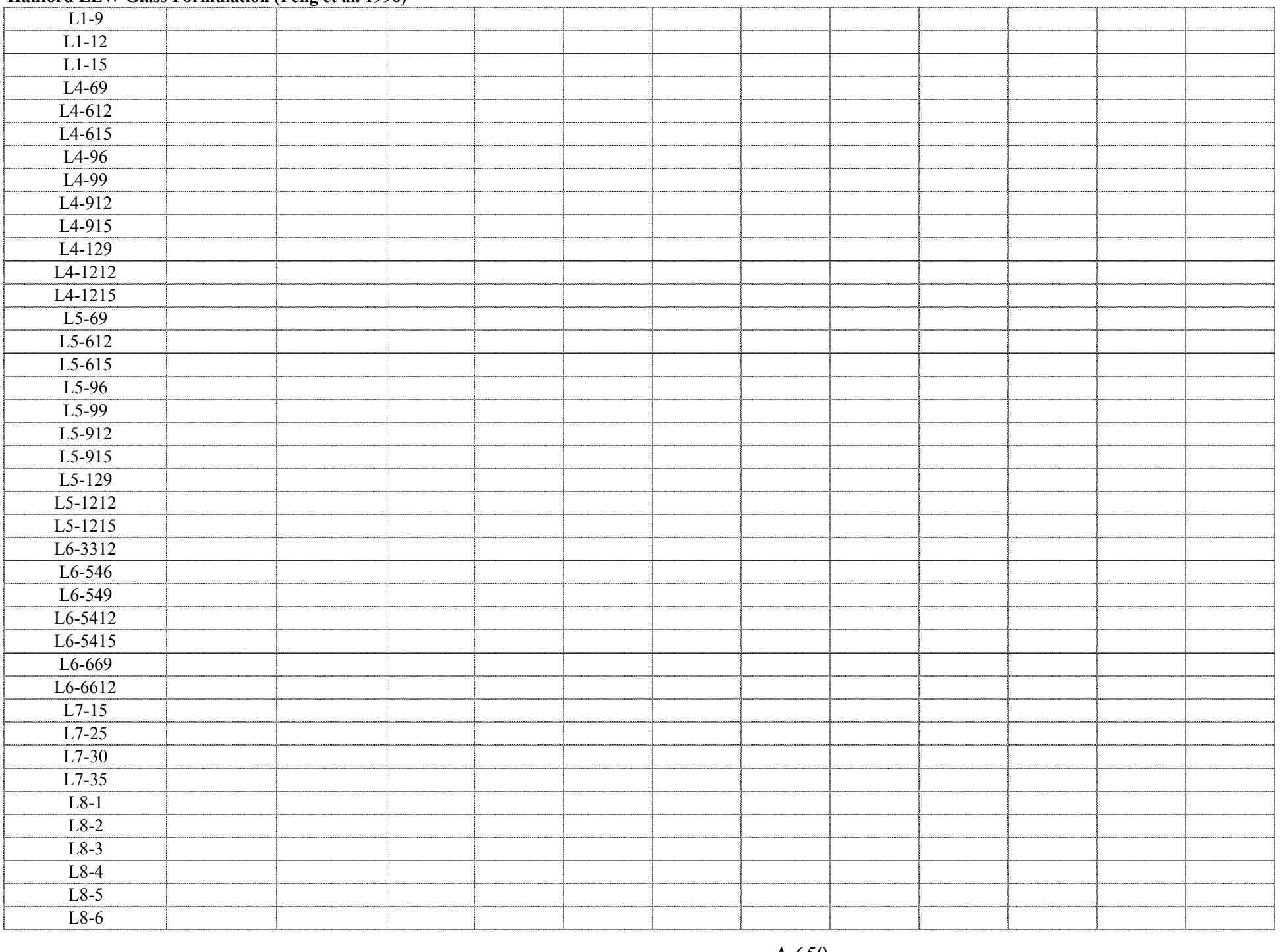


Hanford LLW Glass Formulation (Feng et al. 1996)

\begin{tabular}{|c|c|c|c|c|c|c|c|c|c|c|c|c|c|c|c|c|c|c|c|c|c|}
\hline Glass ID & $\begin{array}{c}\mathrm{A} 12 \mathrm{O} 3 \\
-\mathrm{t}\end{array}$ & $\begin{array}{c}\mathrm{B} 2 \mathrm{O} 3 \\
-\mathrm{t}\end{array}$ & $\begin{array}{c}\mathrm{CaO} \\
-\mathrm{t}\end{array}$ & $\begin{array}{c}\mathrm{Fe} 2 \mathrm{O} 3 \\
-\mathrm{t}\end{array}$ & $\begin{array}{c}\mathrm{FeO} \\
-\mathrm{t}\end{array}$ & $\begin{array}{c}\mathrm{K} 2 \mathrm{O} \\
-\mathrm{t}\end{array}$ & $\begin{array}{c}\mathrm{Li} 2 \mathrm{O} \\
-\mathrm{t}\end{array}$ & $\begin{array}{c}\mathrm{MgO} \\
-\mathrm{t}\end{array}$ & $\begin{array}{c}\mathrm{Na} 2 \mathrm{O} \\
-\mathrm{t}\end{array}$ & $\begin{array}{c}\mathrm{NiO} \\
-\mathrm{t}\end{array}$ & $\begin{array}{c}\mathrm{P} 2 \mathrm{O} 5 \\
-\mathrm{t}\end{array}$ & $\begin{array}{c}\mathrm{SiO} 2 \\
-\mathrm{t}\end{array}$ & $\begin{array}{c}\mathrm{ZrO} 2 \\
-\mathrm{t}\end{array}$ & $\begin{array}{c}\mathrm{Ag} 2 \mathrm{O} \\
-\mathrm{t}\end{array}$ & $\begin{array}{c}\mathrm{As} 2 \mathrm{O} 3 \\
-\mathrm{t}\end{array}$ & $\begin{array}{c}\mathrm{BaO} \\
-\mathrm{t}\end{array}$ & $\begin{array}{c}\mathrm{Bi} 2 \mathrm{O} 3 \\
-\mathrm{t}\end{array}$ & $\begin{array}{c}\mathrm{Br} \\
-\mathrm{t}\end{array}$ & $\begin{array}{c}\mathrm{CdO} \\
-\mathrm{t}\end{array}$ & $\begin{array}{c}\mathrm{Ce} 2 \mathrm{O} 3 \\
-\mathrm{t}\end{array}$ & $\begin{array}{c}\mathrm{CeO} 2 \\
-\mathrm{t}\end{array}$ \\
\hline L8-7 & 0.0900 & 0.0600 & & 0.0300 & & 0.0033 & & & 0.2000 & & 0.0119 & 0.5678 & 0.0300 & & & & 0.0001 & & & & \\
\hline L8-8 & 0.0900 & 0.0500 & & 0.0000 & & 0.0033 & & 0.0400 & 0.2000 & & 0.0119 & 0.5979 & 0.0000 & & & & 0.0001 & & & & \\
\hline LD4-912 & 0.1200 & 0.0900 & & 0.0000 & & 0.0146 & & 0.0000 & 0.2000 & & 0.0019 & 0.5591 & & & & & & & & & \\
\hline LD5-912 & 0.1200 & & 0.0900 & 0.0000 & & 0.0146 & & 0.0000 & 0.2000 & & 0.0019 & 0.5591 & & & & & & & & & \\
\hline LD6-5314 & 0.1400 & 0.0500 & 0.0300 & 0.0000 & & 0.0146 & & 0.0000 & 0.2000 & & 0.0019 & 0.5491 & & & & & & & & & \\
\hline LD6-5412 & 0.1200 & 0.0500 & 0.0400 & 0.0000 & & 0.0146 & & 0.0000 & 0.2000 & & 0.0019 & 0.5591 & & & & & & & & & \\
\hline LD6-5510 & 0.1000 & 0.0500 & 0.0500 & 0.0000 & & 0.0146 & & 0.0000 & 0.2000 & & 0.0019 & 0.5691 & & & & & & & & & \\
\hline LDM-912 & 0.1200 & & 0.0900 & & & 0.0143 & & & 0.2000 & & 0.0019 & 0.5514 & & & & & & & & & \\
\hline LDM-1 & 0.1200 & 0.0200 & 0.0200 & 0.0600 & & 0.0143 & & & 0.2000 & & 0.0019 & 0.5014 & 0.0400 & & & & & & & & \\
\hline LDM-2 & 0.1200 & & 0.0600 & 0.0600 & & 0.0143 & & & 0.2000 & & 0.0019 & 0.5214 & & & & & & & & & \\
\hline LDM-3 & 0.1200 & 0.0600 & & & & 0.0143 & & & 0.2000 & & 0.0019 & 0.5214 & 0.0600 & & & & & & & & \\
\hline LDM-4 & 0.1000 & 0.0600 & 0.0600 & 0.0600 & & 0.0143 & & & 0.2000 & & 0.0019 & 0.4414 & 0.0400 & & & & & & & & \\
\hline LDM-5412 & 0.1200 & 0.0500 & 0.0400 & & & 0.0143 & & & 0.2000 & & 0.0019 & 0.5514 & & & & & & & & & \\
\hline LDMS-1 & 0.1200 & 0.0200 & 0.0200 & 0.0600 & & 0.0143 & & & 0.2000 & & 0.0019 & 0.5014 & 0.0400 & & & & & & & & \\
\hline LRM-912 & 0.1200 & & 0.0900 & & & 0.0003 & & & 0.2000 & & 0.0252 & 0.5454 & & & & & & & & & \\
\hline LRM-1 & 0.1200 & 0.0200 & 0.0200 & 0.0600 & & 0.0003 & 0.0100 & & 0.2000 & & 0.0252 & 0.4854 & 0.0400 & & & & & & & & \\
\hline LRM-2 & 0.1200 & & 0.0600 & 0.0600 & & 0.0003 & & & 0.2000 & & 0.0252 & 0.5154 & & & & & & & & & \\
\hline LRM-3 & 0.1200 & 0.0600 & & & & 0.0003 & & & 0.2000 & & 0.0252 & 0.5154 & 0.0600 & & & & & & & & \\
\hline LRM-4 & 0.1000 & 0.0600 & 0.0600 & 0.0600 & & 0.0003 & 0.0050 & & 0.2000 & & 0.0252 & 0.4304 & 0.0400 & & & & & & & & \\
\hline LRM-5412 & 0.1200 & 0.0500 & 0.0400 & & & 0.0003 & & & 0.2000 & & 0.0252 & 0.5454 & & & & & & & & & \\
\hline LRMS-1 & 0.1200 & 0.0200 & 0.0200 & 0.0600 & & 0.0003 & 0.0100 & & 0.2000 & & 0.0252 & 0.4854 & 0.0400 & & & & & & & & \\
\hline SSHTM-3 & 0.1200 & 0.0500 & 0.0400 & 0.0000 & & 0.0146 & & 0.0000 & 0.2000 & & 0.0019 & 0.5591 & & & & & & & & & \\
\hline B1G9-0IIC4 & 0.1000 & 0.0500 & 0.0500 & 0.0000 & & 0.0146 & & 0.0000 & 0.2000 & & 0.0019 & 0.5691 & & & & & & & & & \\
\hline B1G9-013C5 & 0.1000 & 0.0500 & 0.0500 & 0.0000 & & 0.0146 & & 0.0000 & 0.2000 & & 0.0019 & 0.5691 & & & & & & & & & \\
\hline B1G9-014C & 0.1000 & 0.0500 & 0.0500 & 0.0000 & & 0.0146 & & 0.0000 & 0.2000 & & 0.0019 & 0.5691 & & & & & & & & & \\
\hline D1G4-022P2 & 0.0614 & 0.0615 & 0.0780 & 0.0750 & & 0.0368 & & 0.0000 & 0.1882 & & 0.0018 & 0.4223 & 0.0509 & & & & & & & & \\
\hline D1G4-023P3 & 0.0614 & 0.0615 & 0.0780 & 0.0750 & & 0.0368 & & 0.0000 & 0.1882 & & 0.0018 & 0.4223 & 0.0509 & & & & & & & & \\
\hline Duratek & 0.0614 & 0.0615 & 0.0780 & 0.0750 & & 0.0368 & & 0.0000 & 0.1882 & & 0.0018 & 0.4223 & 0.0509 & & & & & & & & \\
\hline PEI & 0.0600 & & 0.0973 & 0.0100 & & 0.0150 & & 0.0013 & 0.1882 & & 0.0019 & 0.5922 & 0.0200 & & & & & & & & \\
\hline M1G1-008P & 0.1000 & 0.0500 & 0.0500 & 0.0000 & & 0.0146 & & 0.0000 & 0.2000 & & 0.0019 & 0.5691 & & & & & & & & & \\
\hline M1G1-011P & 0.1000 & 0.0500 & 0.0500 & 0.0000 & & 0.0146 & & 0.0000 & 0.2000 & & 0.0019 & 0.5691 & & & & & & & & & \\
\hline V1M2 632011 P1 & 0.1000 & 0.0800 & 0.0290 & 0.0100 & & 0.0146 & & 0.0210 & 0.2000 & & 0.0019 & 0.5290 & & & & & & & & & \\
\hline V1M2 632040 P2 & 0.1000 & 0.0800 & 0.0290 & 0.0100 & & 0.0146 & & 0.0210 & 0.2000 & & 0.0019 & 0.5290 & & & & & & & & & \\
\hline V1M3 632059 P1 & 0.1000 & 0.0800 & 0.0290 & 0.0100 & & 0.0146 & & 0.0210 & 0.2000 & & 0.0019 & 0.5290 & & & & & & & & & \\
\hline V1M3 632075 P2 & 0.1000 & 0.0800 & 0.0290 & 0.0100 & & 0.0146 & & 0.0210 & 0.2000 & & 0.0019 & 0.5290 & & & & & & & & & \\
\hline V1M4 632088 P1 & 0.1000 & 0.0800 & 0.0290 & 0.0100 & & 0.0146 & & 0.0210 & 0.2000 & & 0.0019 & 0.5290 & & & & & & & & & \\
\hline V1M4 632096 P2 & 0.1000 & 0.0800 & 0.0290 & 0.0100 & & 0.0146 & & 0.0210 & 0.2000 & & 0.0019 & 0.5290 & & & & & & & & & \\
\hline Vectra & 0.1000 & 0.0800 & 0.0290 & 0.0100 & & 0.0146 & & 0.0210 & 0.2000 & & 0.0019 & 0.5290 & & & & & & & & & \\
\hline WSTC & 0.1822 & 0.0945 & 0.0465 & 0.0000 & & 0.0144 & 0.0083 & 0.0000 & 0.1882 & & 0.0019 & 0.4290 & 0.0210 & & & & & & & & \\
\hline
\end{tabular}

\section{SBW CVS Phase 1 (Scholes et al. 2002)}

\begin{tabular}{|c|c|c|c|c|c|c|c|c|c|c|c|c|c|c|}
\hline SBW1-01 & 0.1003 & 0.0900 & 0.0218 & 0.0412 & 0.0278 & 0.0200 & 0.0000 & 0.1772 & 0.0008 & 0.0122 & 0.4548 & 0.0120 & 0.0200 & 0.0020 \\
\hline SBW1-02 & 0.0980 & 0.0900 & 0.0218 & 0.0608 & 0.0278 & 0.0300 & 0.0000 & 0.1408 & 0.0008 & 0.0084 & 0.4650 & 0.0120 & 0.0200 & 0.0030 \\
\hline SBW1-03 & 0.0980 & 0.0900 & 0.0218 & 0.0412 & 0.0278 & 0.0200 & 0.0000 & 0.1408 & 0.0008 & 0.0084 & 0.5029 & 0.0080 & 0.0200 & 0.0020 \\
\hline SBW1-04 & 0.0980 & 0.1100 & 0.0218 & 0.0412 & 0.0362 & 0.0300 & 0.0000 & 0.1450 & 0.0008 & 0.0122 & 0.4548 & 0.0080 & 0.0200 & 0.0020 \\
\hline
\end{tabular}


Hanford LLW Glass Formulation (Feng et al. 1996)

\begin{tabular}{|c|c|c|c|c|c|c|c|c|c|c|c|c|c|c|c|c|c|c|c|c|c|}
\hline Glass ID & $\begin{array}{c}\mathrm{Cl} \\
-\mathrm{t}\end{array}$ & $\begin{array}{c}\mathrm{CoO} \\
-\mathrm{t}\end{array}$ & $\begin{array}{c}\mathrm{Co} 2 \mathrm{O} 3 \\
-\mathrm{t}\end{array}$ & $\begin{array}{c}\mathrm{Cr} 2 \mathrm{O} 3 \\
-\mathrm{t}\end{array}$ & $\begin{array}{c}\mathrm{Cs} 2 \mathrm{O} \\
-\mathrm{t}\end{array}$ & $\begin{array}{c}\mathrm{CuO} \\
-\mathrm{t} \\
\end{array}$ & $\begin{array}{c}\mathrm{Eu} 2 \mathrm{O} 3 \\
-\mathrm{t}\end{array}$ & $\begin{array}{l}F \\
-t\end{array}$ & $\begin{array}{c}\mathrm{Ga} 2 \mathrm{O} 3 \\
-\mathrm{t}\end{array}$ & $\begin{array}{c}\mathrm{Gd} 2 \mathrm{O} 3 \\
-\mathrm{t}\end{array}$ & $\begin{array}{c}\mathrm{HgO} \\
-\mathrm{t}\end{array}$ & $\begin{array}{l}I \\
-t\end{array}$ & $\begin{array}{c}\mathrm{La} 2 \mathrm{O} 3 \\
-\mathrm{t}\end{array}$ & $\begin{array}{c}\mathrm{MnO} 2 \\
-\mathrm{t} \\
\end{array}$ & $\begin{array}{c}\mathrm{MnO} \\
-\mathrm{t}\end{array}$ & $\begin{array}{c}\mathrm{MoO} \\
-\mathrm{t} \\
\end{array}$ & $\begin{array}{c}\mathrm{MoO} 3 \\
-\mathrm{t}\end{array}$ & $\begin{array}{c}\mathrm{Nb} 2 \mathrm{O} 5 \\
-\mathrm{t}\end{array}$ & $\begin{array}{c}\mathrm{Nd} 2 \mathrm{O} 3 \\
-\mathrm{t}\end{array}$ & $\begin{array}{c}\mathrm{PbO} \\
-\mathrm{t}\end{array}$ & $\begin{array}{c}\mathrm{PdO} 2 \\
-\mathrm{t}\end{array}$ \\
\hline L8-7 & 0.0009 & & & 0.0004 & & & & 0.0021 & & & & & & & 0.0001 & & & & 0.0001 & & \\
\hline L8-8 & 0.0009 & & & 0.0004 & & & & 0.0021 & & & & & & & 0.0001 & & & & 0.0001 & & \\
\hline LD4-912 & 0.0035 & & & 0.0004 & 0.0015 & & & 0.0029 & & & & 0.0013 & & & 0.0000 & & 0.0015 & & & & \\
\hline LD5-912 & 0.0035 & & & 0.0004 & 0.0015 & & & 0.0029 & & & & 0.0013 & & & 0.0000 & & 0.0015 & & & & \\
\hline LD6-5314 & 0.0035 & & & 0.0004 & 0.0015 & & & 0.0029 & & & & 0.0013 & & & 0.0000 & & 0.0015 & & & & \\
\hline LD6-5412 & 0.0035 & & & 0.0004 & 0.0015 & & & 0.0029 & & & & 0.0013 & & & 0.0000 & & 0.0015 & & & & \\
\hline LD6-5510 & 0.0035 & & & 0.0004 & 0.0015 & & & 0.0029 & & & & 0.0013 & & & 0.0000 & & 0.0015 & & & & \\
\hline LDM-912 & 0.0064 & & & 0.0004 & 0.0014 & & & 0.0082 & & & & 0.0013 & & & & & 0.0015 & & & & \\
\hline LDM-1 & 0.0064 & & & 0.0004 & 0.0014 & & & 0.0082 & & & & 0.0013 & & & & & 0.0015 & & & & \\
\hline LDM-2 & 0.0064 & & & 0.0004 & 0.0014 & & & 0.0082 & & & & 0.0013 & & & & & 0.0015 & & & & \\
\hline LDM-3 & 0.0064 & & & 0.0004 & 0.0014 & & & 0.0082 & & & & 0.0013 & & & & & 0.0015 & & & & \\
\hline LDM-4 & 0.0064 & & & 0.0004 & 0.0014 & & & 0.0082 & & & & 0.0013 & & & & & 0.0015 & & & & \\
\hline LDM-5412 & 0.0064 & & & 0.0004 & 0.0014 & & & 0.0082 & & & & 0.0013 & & & & & 0.0015 & & & & \\
\hline LDMS-1 & 0.0064 & & & 0.0004 & 0.0014 & & & 0.0082 & & & & 0.0013 & & & & & 0.0014 & & & & \\
\hline LRM-912 & 0.0004 & & & 0.0004 & 0.0015 & & & 0.0026 & & & & 0.0014 & & & 0.0001 & & 0.0015 & & & & \\
\hline LRM-1 & 0.0004 & & & 0.0004 & 0.0015 & & & 0.0026 & & & & 0.0014 & & & 0.0001 & & 0.0015 & & & & \\
\hline LRM-2 & 0.0004 & & & 0.0004 & 0.0015 & & & 0.0026 & & & & 0.0014 & & & 0.0001 & & 0.0015 & & & & \\
\hline LRM-3 & 0.0004 & & & 0.0004 & 0.0015 & & & 0.0026 & & & & 0.0014 & & & 0.0001 & & 0.0015 & & & & \\
\hline LRM-4 & 0.0004 & & & 0.0004 & 0.0015 & & & 0.0026 & & & & 0.0014 & & & 0.0001 & & 0.0015 & & & & \\
\hline LRM-5412 & 0.0004 & & & 0.0004 & 0.0015 & & & 0.0026 & & & & 0.0014 & & & 0.0001 & & 0.0015 & & & & \\
\hline LRMS-1 & 0.0004 & & & 0.0004 & 0.0015 & & & 0.0026 & & & & 0.0014 & & & 0.0001 & & 0.0015 & & & & \\
\hline SSHTM-3 & 0.0035 & & & 0.0004 & 0.0015 & & & 0.0029 & & & & 0.0013 & & & 0.0000 & & 0.0015 & & & & \\
\hline B1G9-0IIC4 & 0.0035 & & & 0.0004 & 0.0015 & & & 0.0029 & & & & 0.0013 & & & 0.0000 & & 0.0015 & & & & \\
\hline B1G9-013C5 & 0.0035 & & & 0.0004 & 0.0015 & & & 0.0029 & & & & 0.0013 & & & 0.0000 & & 0.0015 & & & & \\
\hline B1G9-014C & 0.0035 & & & 0.0004 & 0.0015 & & & 0.0029 & & & & 0.0013 & & & 0.0000 & & 0.0015 & & & & \\
\hline D1G4-022P2 & 0.0033 & & & 0.0004 & 0.0014 & & & 0.0028 & & & & 0.0012 & & & 0.0000 & & 0.0014 & & & & \\
\hline D1G4-023P3 & 0.0033 & & & 0.0004 & 0.0014 & & & 0.0028 & & & & 0.0012 & & & 0.0000 & & 0.0014 & & & & \\
\hline Duratek & 0.0033 & & & 0.0004 & 0.0014 & & & 0.0028 & & & & 0.0012 & & & 0.0000 & & 0.0014 & & & & \\
\hline PEI & 0.0035 & & & 0.0004 & 0.0015 & & & 0.0029 & & & & 0.0013 & & & 0.0000 & & 0.0015 & & & & \\
\hline M1G1-008P & 0.0035 & & & 0.0004 & 0.0015 & & & 0.0029 & & & & 0.0013 & & & 0.0000 & & 0.0015 & & & & \\
\hline M1G1-011P & 0.0035 & & & 0.0004 & 0.0015 & & & 0.0029 & & & & 0.0013 & & & 0.0000 & & 0.0015 & & & & \\
\hline V1M2 632011 P1 & 0.0035 & & & 0.0004 & 0.0015 & & & 0.0029 & & & & 0.0013 & & & 0.0000 & & 0.0015 & & & & \\
\hline V1M2 632040 P2 & 0.0035 & & & 0.0004 & 0.0015 & & & 0.0029 & & & & 0.0013 & & & 0.0000 & & 0.0015 & & & & \\
\hline V1M3 632059 P1 & 0.0035 & & & 0.0004 & 0.0015 & & & 0.0029 & & & & 0.0013 & & & 0.0000 & & 0.0015 & & & & \\
\hline V1M3 632075 P2 & 0.0035 & & & 0.0004 & 0.0015 & & & 0.0029 & & & & 0.0013 & & & 0.0000 & & 0.0015 & & & & \\
\hline V1M4 632088 P1 & 0.0035 & & & 0.0004 & 0.0015 & & & 0.0029 & & & & 0.0013 & & & 0.0000 & & 0.0015 & & & & \\
\hline V1M4 632096 P2 & 0.0035 & & & 0.0004 & 0.0015 & & & 0.0029 & & & & 0.0013 & & & 0.0000 & & 0.0015 & & & & \\
\hline Vectra & 0.0035 & & & 0.0004 & 0.0015 & & & 0.0029 & & & & 0.0013 & & & 0.0000 & & 0.0015 & & & & \\
\hline WSTC & 0.0035 & & & 0.0004 & 0.0015 & & & 0.0029 & & & & 0.0013 & & & 0.0000 & & 0.0015 & & & & \\
\hline
\end{tabular}

SBW CVS Phase 1 (Scholes et al. 2002)

\begin{tabular}{|c|c|c|c|c|c|c|c|c|}
\hline SBW1-01 & 0.0015 & 0.0013 & 0.0058 & 0.0001 & 0.0003 & 0.0049 & 0.0002 & 0.0007 \\
\hline SBW1-02 & 0.0015 & 0.0013 & 0.0058 & 0.0001 & 0.0003 & 0.0066 & 0.0002 & 0.0007 \\
\hline SBW1-03 & 0.0015 & 0.0013 & 0.0042 & 0.0001 & 0.0003 & 0.0049 & 0.0002 & 0.0007 \\
\hline SBW1-04 & 0.0015 & 0.0013 & 0.0042 & 0.0001 & 0.0003 & 0.0066 & 0.0002 & 0.0007 \\
\hline
\end{tabular}




\section{Hanford LLW Glass Formulation (Feng et al. 1996)}

\begin{tabular}{|c|c|c|c|c|c|c|c|c|c|c|c|c|c|c|c|c|c|c|c|c|c|}
\hline Glass ID & $\begin{array}{c}\mathrm{PdO} \\
-\mathrm{t}\end{array}$ & $\mathrm{Pr} 2 \mathrm{O} 3$ & $\mathrm{Pr}_{-\mathrm{t}}$ & $\begin{array}{c}\mathrm{Rb} 2 \mathrm{O} \\
-\mathrm{t}\end{array}$ & $\begin{array}{c}\mathrm{ReO} \\
-\mathrm{t}\end{array}$ & $\begin{array}{c}\mathrm{ReO} 2 \\
-\mathrm{t}\end{array}$ & $\begin{array}{c}\mathrm{Rh} 2 \mathrm{O} 3 \\
-\mathrm{t}\end{array}$ & $\begin{array}{c}\mathrm{RhO} 2 \\
-\mathrm{t}\end{array}$ & $\begin{array}{c}\mathrm{RuO} 2 \\
-\mathrm{t}\end{array}$ & $\begin{array}{c}\mathrm{Sb} 2 \mathrm{O} 3 \\
-\mathrm{t}\end{array}$ & $\begin{array}{c}\mathrm{Sb} 2 \mathrm{O} 5 \\
-\mathrm{t}\end{array}$ & $\begin{array}{c}\mathrm{SeO} 2 \\
-t\end{array}$ & $\begin{array}{c}\mathrm{Sm} 2 \mathrm{O} 3 \\
-\mathrm{t}\end{array}$ & $\begin{array}{c}\mathrm{SnO} \\
-\mathrm{t}\end{array}$ & $\begin{array}{c}\mathrm{SnO} 2 \\
-t\end{array}$ & $\begin{array}{c}\mathrm{SO} 3 \\
-\mathrm{t}\end{array}$ & $\begin{array}{c}\mathrm{SrO} \\
-\mathrm{t}\end{array}$ & $\begin{array}{c}\mathrm{Tc} 2 \mathrm{O} 7 \\
-\mathrm{t}\end{array}$ & $\begin{array}{c}\mathrm{TeO} 2 \\
-\mathrm{t}\end{array}$ & $\begin{array}{c}\mathrm{ThO} 2 \\
-\mathrm{t}\end{array}$ & $\begin{array}{c}\mathrm{TiO} 2 \\
-\mathrm{t}\end{array}$ \\
\hline L8-7 & & & & & & & & & & & & & & & & 0.0032 & & & & & \\
\hline L8-8 & & & & & & & & & & & & & & & & 0.0032 & & & & & \\
\hline LD4-912 & & & & & & & & & & & & & & & & 0.0022 & 0.0011 & & & & \\
\hline LD5-912 & & & & & & & & & & & & & & & & 0.0022 & 0.0011 & & & & \\
\hline LD6-5314 & & & & & & & & & & & & & & & & 0.0022 & 0.0011 & & & & \\
\hline LD6-5412 & & & & & & & & & & & & & & & & 0.0022 & 0.0011 & & & & \\
\hline LD6-5510 & & & & & & & & & & & & & & & & 0.0022 & 0.0011 & & & & \\
\hline LDM-912 & & & & & & & & & & & & & & & & 0.0021 & 0.0010 & & & & \\
\hline LDM-1 & & & & & & & & & & & & & & & & 0.0021 & 0.0010 & & & & \\
\hline LDM-2 & & & & & & & & & & & & & & & & 0.0021 & 0.0010 & & & & \\
\hline LDM-3 & & & & & & & & & & & & & & & & 0.0021 & 0.0010 & & & & \\
\hline LDM-4 & & & & & & & & & & & & & & & & 0.0021 & 0.0010 & & & & \\
\hline LDM-5412 & & & & & & & & & & & & & & & & 0.0021 & 0.0010 & & & & \\
\hline LDMS-1 & & & & & & & & & & & & & & & & 0.0021 & 0.0010 & & & & \\
\hline LRM-912 & & & & & & & & & & & & & & & & 0.0101 & 0.0011 & & & & \\
\hline LRM-1 & & & & & & & & & & & & & & & & 0.0101 & 0.0011 & & & & \\
\hline LRM-2 & & & & & & & & & & & & & & & & 0.0101 & 0.0011 & & & & \\
\hline LRM-3 & & & & & & & & & & & & & & & & 0.0101 & 0.0011 & & & & \\
\hline LRM-4 & & & & & & & & & & & & & & & & 0.0101 & 0.0011 & & & & \\
\hline LRM-5412 & & & & & & & & & & & & & & & & 0.0101 & 0.0011 & & & & \\
\hline LRMS-1 & & & & & & & & & & & & & & & & 0.0101 & 0.0011 & & & & \\
\hline SSHTM-3 & & & & & & & & & & & & & & & & 0.0022 & 0.0011 & & & & \\
\hline B1G9-0IIC4 & & & & & & & & & & & & & & & & 0.0022 & 0.0011 & & & & \\
\hline B1G9-013C5 & & & & & & & & & & & & & & & & 0.0022 & 0.0011 & & & & \\
\hline B1G9-014C & & & & & & & & & & & & & & & & 0.0022 & 0.0011 & & & & \\
\hline D1G4-022P2 & & & & & & & & & & & & & & & & 0.0020 & 0.0010 & & & & 0.0100 \\
\hline D1G4-023P3 & & & & & & & & & & & & & & & & 0.0020 & 0.0010 & & & & 0.0100 \\
\hline Duratek & & & & & & & & & & & & & & & & 0.0020 & 0.0010 & & & & 0.0100 \\
\hline PEI & & & & & & & & & & & & & & & & 0.0021 & 0.0010 & & & & \\
\hline M1G1-008P & & & & & & & & & & & & & & & & 0.0022 & 0.0011 & & & & \\
\hline M1G1-011P & & & & & & & & & & & & & & & & 0.0022 & 0.0011 & & & & \\
\hline V1M2 $632011 \mathrm{P} 1$ & & & & & & & & & & & & & & & & 0.0022 & 0.0011 & & & & \\
\hline V1M2 632040 P2 & & & & & & & & & & & & & & & & 0.0022 & 0.0011 & & & & \\
\hline V1M3 632059 P1 & & & & & & & & & & & & & & & & 0.0022 & 0.0011 & & & & \\
\hline V1M3 632075 P2 & & & & & & & & & & & & & & & & 0.0022 & 0.0011 & & & & \\
\hline V1M4 632088 P1 & & & & & & & & & & & & & & & & 0.0022 & 0.0011 & & & & \\
\hline V1M4 632096 P2 & & & & & & & & & & & & & & & & 0.0022 & 0.0011 & & & & \\
\hline Vectra & & & & & & & & & & & & & & & & 0.0022 & 0.0011 & & & & \\
\hline WSTC & & & & & & & & & & & & & & & & 0.0022 & 0.0011 & & & & \\
\hline
\end{tabular}

SBW CVS Phase 1 (Scholes et al. 2002)

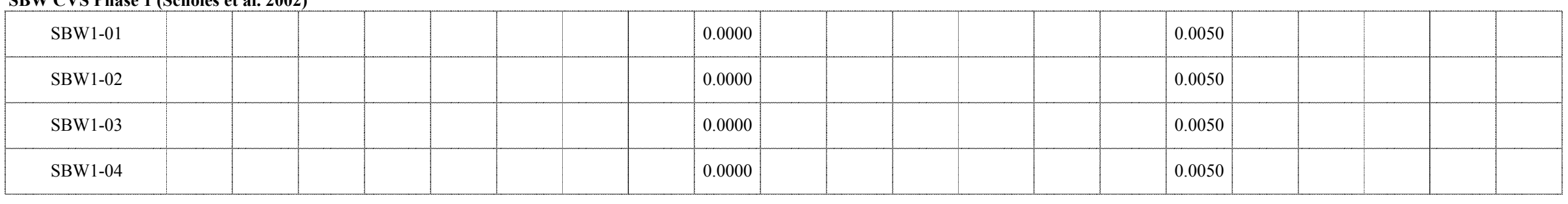


Hanford LLW Glass Formulation (Feng et al. 1996)

\begin{tabular}{|c|c|c|c|c|c|c|c|c|c|c|c|c|c|c|c|c|c|c|c|c|c|}
\hline Glass ID & $\begin{array}{c}\mathrm{T} 12 \mathrm{O} 3 \\
-\mathrm{t}\end{array}$ & $\begin{array}{c}\mathrm{U} 3 \mathrm{O} 8 \\
-\mathrm{t}\end{array}$ & $\begin{array}{c}\mathrm{UO} 2 \\
-\mathrm{t}\end{array}$ & $\begin{array}{c}\mathrm{UO} 3 \\
-\mathrm{t}\end{array}$ & $\begin{array}{c}\mathrm{V} 2 \mathrm{O} 5 \\
-\mathrm{t}\end{array}$ & $\begin{array}{c}\text { WO3 } \\
-t\end{array}$ & $\begin{array}{c}\mathrm{Y} 2 \mathrm{O} 3 \\
-\mathrm{t}\end{array}$ & $\begin{array}{c}\mathrm{ZnO} \\
-\mathrm{t}\end{array}$ & $\begin{array}{c}\text { Others } \\
-t\end{array}$ & $\begin{array}{c}\text { Sum } \\
-t\end{array}$ & $\begin{array}{c}\mathrm{A} 12 \mathrm{O} 3 \\
-\mathrm{a}\end{array}$ & $\begin{array}{c}\mathrm{B} 2 \mathrm{O} 3 \\
-\mathrm{a}\end{array}$ & $\begin{array}{c}\mathrm{CaO} \\
-\mathrm{a}\end{array}$ & $\begin{array}{c}\mathrm{Fe} 2 \mathrm{O} 3 \\
-\mathrm{a}\end{array}$ & $\begin{array}{c}\mathrm{FeO} \\
-\mathrm{a}\end{array}$ & $\begin{array}{c}\mathrm{K} 2 \mathrm{O} \\
-\mathrm{a}\end{array}$ & $\begin{array}{c}\mathrm{Li} 2 \mathrm{O} \\
-\mathrm{a}\end{array}$ & $\begin{array}{c}\mathrm{MgO} \\
-\mathrm{a}\end{array}$ & $\begin{array}{c}\mathrm{Na} 2 \mathrm{O} \\
-\mathrm{a}\end{array}$ & $\begin{array}{c}\mathrm{NiO} \\
-\mathrm{a}\end{array}$ & $\begin{array}{c}\mathrm{P} 2 \mathrm{O} 5 \\
-\mathrm{a}\end{array}$ \\
\hline L8-7 & & & & & & & & & & 0.9999 & & & & & & & & & & & \\
\hline L8-8 & & & & & & & & & & 1.0001 & & & & & & & & & & & \\
\hline LD4-912 & & & & & & & & & & 1.0000 & & & & & & & & & & & \\
\hline LD5-912 & & & & & & & & & & 1.0000 & & & & & & & & & & & \\
\hline LD6-5314 & & & & & & & & & & 1.0000 & & & & & & & & & & & \\
\hline LD6-5412 & & & & & & & & & & 1.0000 & 0.1217 & 0.0505 & 0.0412 & 0.0011 & & 0.0166 & 0.0001 & & 0.2041 & 0.0003 & 0.0022 \\
\hline LD6-5510 & & & & & & & & & & 1.0000 & & & & & & & & & & & \\
\hline LDM-912 & & & & & & & & & & 0.9999 & 0.1222 & & 0.0960 & 0.0006 & & 0.0167 & & 0.0010 & 0.1992 & & 0.0024 \\
\hline LDM-1 & & & & & & & & & & 0.9999 & 0.1217 & 0.0201 & 0.0178 & 0.0621 & & 0.0146 & & & 0.2105 & & 0.0046 \\
\hline LDM-2 & & & & & & & & & & 0.9999 & 0.1208 & & 0.0624 & 0.0619 & & 0.0179 & & & 0.2030 & & 0.0028 \\
\hline LDM-3 & & & & & & & & & & 0.9999 & 0.1244 & 0.0631 & 0.0012 & 0.0005 & & 0.0230 & & & 0.1885 & 0.0005 & 0.0028 \\
\hline LDM-4 & & & & & & & & & & 0.9999 & 0.1006 & 0.0625 & 0.0648 & 0.0614 & & 0.0202 & & & 0.2036 & 0.0003 & 0.0027 \\
\hline LDM-5412 & & & & & & & & & & 0.9999 & 0.1205 & 0.0501 & 0.0394 & 0.0006 & & 0.0213 & & 0.0015 & 0.2122 & & 0.0062 \\
\hline LDMS-1 & & & & & & & & & & 0.9998 & 0.1213 & 0.0203 & 0.0205 & 0.0607 & & 0.0250 & 0.0001 & 0.0011 & 0.2083 & 0.0002 & 0.0028 \\
\hline LRM-912 & & & & & & & & & & 1.0000 & 0.1179 & 0.0003 & 0.0941 & 0.0005 & & 0.0070 & & 0.0012 & 0.2042 & & 0.0267 \\
\hline LRM-1 & & & & & & & & & & 1.0000 & 0.1219 & 0.0202 & 0.0182 & 0.0624 & & & 0.0102 & & 0.2123 & & 0.0272 \\
\hline LRM-2 & & & & & & & & & & 1.0000 & 0.1141 & & 0.0613 & 0.0587 & & & & 0.0011 & 0.2213 & & 0.0252 \\
\hline LRM-3 & & & & & & & & & & 1.0000 & 0.1262 & 0.0638 & 0.0019 & 0.0005 & & 0.0044 & & & 0.1811 & & 0.0282 \\
\hline LRM-4 & & & & & & & & & & 1.0000 & 0.0977 & 0.0601 & 0.0619 & 0.0598 & & 0.0146 & 0.0049 & 0.0011 & 0.2022 & 0.0002 & 0.0262 \\
\hline LRM-5412 & & & & & & & & & & 1.0000 & 0.1186 & 0.0509 & 0.0423 & 0.0005 & & 0.0092 & & & 0.2027 & 0.0003 & 0.0267 \\
\hline LRMS-1 & & & & & & & & & & 1.0000 & 0.1191 & 0.0201 & 0.0220 & 0.0598 & & 0.0176 & 0.0101 & 0.0011 & & 0.0002 & 0.0257 \\
\hline SSHTM-3 & & & & & & & & & & 1.0000 & 0.1163 & 0.0609 & 0.0414 & 0.0002 & & 0.0104 & 0.0002 & & 0.2255 & 0.0003 & 0.0031 \\
\hline B1G9-0IIC4 & & & & & & & & & & 1.0000 & 0.1215 & 0.0141 & 0.0516 & 0.0044 & & 0.0371 & & 0.0025 & 0.1205 & 0.0004 & \\
\hline B1G9-013C5 & & & & & & & & & & 1.0000 & 0.1294 & 0.0139 & 0.0514 & 0.0066 & & 0.0315 & & 0.0023 & 0.1250 & 0.0004 & \\
\hline B1G9-014C & & & & & & & & & & 1.0000 & 0.1260 & 0.0162 & 0.0522 & 0.0058 & & 0.0284 & & 0.0024 & 0.1337 & 0.0004 & \\
\hline $\mathrm{D} 1 \mathrm{G} 4-022 \mathrm{P} 2$ & & & & & & & & & & 0.9994 & 0.0670 & 0.0736 & 0.0869 & 0.0777 & & 0.0246 & & 0.0061 & 0.1800 & 0.0006 & 0.0006 \\
\hline D1G4-023P3 & & & & & & & & & & 0.9994 & 0.0641 & 0.0710 & 0.0857 & 0.0776 & & 0.0302 & & 0.0053 & 0.1824 & 0.0007 & 0.0004 \\
\hline Duratek & & & & & & & & & & 0.9994 & & & & & & & & & & & \\
\hline PEI & & & & & & & & & & 1.0002 & & & & & & & & & & & \\
\hline M1G1-008P & & & & & & & & & & 1.0000 & 0.1498 & 0.0181 & 0.0950 & 0.0106 & & & & 0.0039 & 0.1321 & & \\
\hline M1G1-011P & & & & & & & & & & 1.0000 & 0.1335 & 0.0253 & 0.0675 & 0.0116 & & & & 0.0030 & 0.1524 & & \\
\hline V1M2 632011 P1 & & & & & & & & & & 0.9999 & 0.1028 & 0.0701 & 0.0299 & 0.0104 & & 0.0123 & & 0.0214 & 0.1622 & 0.0004 & \\
\hline V1M2 632040 P2 & & & & & & & & & & 0.9999 & 0.1042 & 0.0718 & 0.0306 & 0.0102 & & 0.0226 & & 0.0221 & 0.1553 & 0.0004 & \\
\hline V1M3 632059 P1 & & & & & & & & & & 0.9999 & 0.1062 & 0.0729 & 0.0333 & 0.0104 & & 0.0200 & & 0.0221 & 0.1597 & 0.0004 & \\
\hline V1M3 632075 P2 & & & & & & & & & & 0.9999 & 0.1098 & 0.0761 & 0.0351 & 0.0114 & & 0.0181 & 0.0001 & 0.0228 & 0.1267 & 0.0004 & \\
\hline V1M4 632088 P1 & & & & & & & & & & 0.9999 & 0.1059 & 0.0733 & 0.0335 & 0.0120 & & 0.0202 & 0.0003 & 0.0213 & 0.1680 & 0.0004 & \\
\hline V1M4 632096 P2 & & & & & & & & & & 0.9999 & 0.1087 & 0.0752 & 0.0299 & 0.0099 & & 0.0212 & & 0.0215 & 0.1654 & 0.0003 & \\
\hline Vectra & & & & & & & & & & 0.9999 & 0.0945 & 0.0820 & 0.0290 & 0.0110 & & 0.0091 & & 0.0187 & 0.2082 & 0.0003 & 0.0040 \\
\hline WSTC & & & & & & & & & & 1.0004 & & & & & & & & & & & \\
\hline
\end{tabular}

SBW CVS Phase 1 (Scholes et al. 2002)

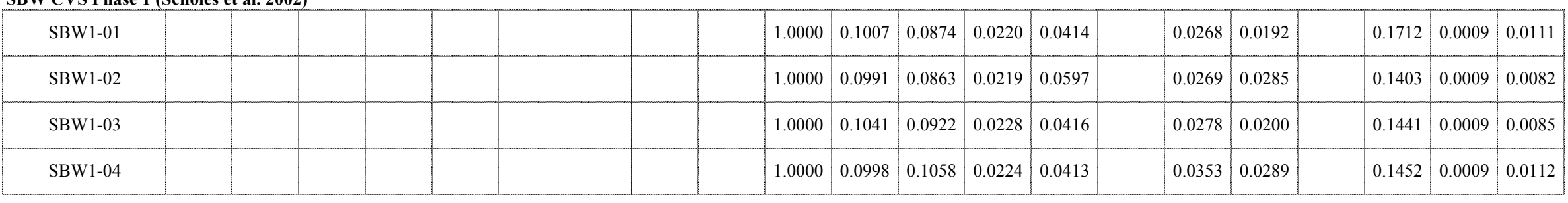


Hanford LLW Glass Formulation (Feng et al. 1996)

\begin{tabular}{|c|c|c|c|c|c|c|c|c|c|c|c|c|c|c|c|c|c|c|c|c|c|}
\hline Glass ID & $\begin{array}{c}\mathrm{SiO} 2 \\
-\mathrm{a}\end{array}$ & $\begin{array}{c}\mathrm{ZrO} 2 \\
-\mathrm{a}\end{array}$ & $\begin{array}{c}\mathrm{Ag} 2 \mathrm{O} \\
-\mathrm{a}\end{array}$ & $\begin{array}{c}\mathrm{As} 2 \mathrm{O} 3 \\
-\mathrm{a} \\
\end{array}$ & $\begin{array}{c}\mathrm{BaO} \\
-\mathrm{a}\end{array}$ & $\begin{array}{c}\mathrm{Bi} 2 \mathrm{O} 3 \\
-\mathrm{a}\end{array}$ & $\begin{array}{l}\mathrm{Br} \\
-\mathrm{a}\end{array}$ & $\begin{array}{c}\mathrm{CdO} \\
-\mathrm{a}\end{array}$ & $\begin{array}{c}\mathrm{Ce} 2 \mathrm{O} 3 \\
-\mathrm{a}\end{array}$ & $\begin{array}{c}\mathrm{CeO} 2 \\
-\mathrm{a}\end{array}$ & $\begin{array}{l}\mathrm{Cl} \\
-\mathrm{a} \\
\end{array}$ & $\begin{array}{c}\mathrm{CoO} \\
-\mathrm{a}\end{array}$ & $\begin{array}{c}\mathrm{Co} 2 \mathrm{O} 3 \\
-\mathrm{a}\end{array}$ & $\begin{array}{c}\mathrm{Cr} 2 \mathrm{O} 3 \\
-\mathrm{a}\end{array}$ & $\begin{array}{c}\mathrm{Cs} 2 \mathrm{O} \\
-\mathrm{a}\end{array}$ & $\begin{array}{c}\mathrm{CuO} \\
-\mathrm{a}\end{array}$ & $\begin{array}{c}\mathrm{Eu} 2 \mathrm{O} 3 \\
-\mathrm{a}\end{array}$ & $\begin{array}{l}\mathrm{F} \\
-\mathrm{a}\end{array}$ & $\begin{array}{c}\mathrm{Ga} 2 \mathrm{O} 3 \\
-\mathrm{a}\end{array}$ & $\begin{array}{c}\mathrm{Gd} 2 \mathrm{O} 3 \\
-\mathrm{a}\end{array}$ & $\begin{array}{c}\mathrm{HgO} \\
-\mathrm{a}\end{array}$ \\
\hline \multicolumn{22}{|l|}{ L8-7 } \\
\hline \multicolumn{22}{|l|}{ L8-8 } \\
\hline \multicolumn{22}{|l|}{ LD4-912 } \\
\hline \multicolumn{22}{|l|}{ LD5-912 } \\
\hline \multicolumn{22}{|l|}{ LD6-5314 } \\
\hline LD6-5412 & 0.5544 & 0.0008 & & & & & & & & & & & & 0.0005 & & & & & & & \\
\hline \multicolumn{22}{|l|}{ LD6-5510 } \\
\hline LDM-912 & 0.5530 & & & & & & & & & 0.0006 & & & & & & & & & & & \\
\hline LDM-1 & 0.5006 & 0.0402 & & & & & & & & 0.0005 & & & & 0.0005 & & & & & & & \\
\hline LDM-2 & 0.5187 & 0.0001 & & & & & & & & & & & & 0.0005 & & & & & & & \\
\hline LDM-3 & 0.5350 & 0.0527 & & & & & & & & 0.0008 & & & & 0.0005 & & & & & & & \\
\hline LDM-4 & 0.4370 & 0.0382 & & & & & & & & & & & & 0.0005 & & & & & & & \\
\hline LDM-5412 & 0.5356 & & & & & & & & & & & & & 0.0005 & & & & & & & \\
\hline LDMS-1 & 0.4803 & 0.0405 & & & & & & & & 0.0005 & & & & 0.0003 & & & & & & & \\
\hline LRM-912 & 0.5319 & & & & & & & & & 0.0005 & & & & 0.0004 & & & & & & & \\
\hline LRM-1 & 0.4742 & 0.0399 & & & & & & & & & & & & 0.0004 & & & & & & & \\
\hline LRM-2 & 0.4820 & & & & & & & & & & & & & 0.0004 & & & & & & & \\
\hline LRM-3 & 0.5529 & 0.0507 & & & & & & & & & & & & 0.0004 & & & & & & & \\
\hline LRM-4 & 0.4174 & 0.0382 & & & & & & & & 0.0006 & & & & 0.0004 & & & & & & & \\
\hline LRM-5412 & 0.5341 & & & & & & & & & 0.0005 & & & & 0.0004 & & & & & & & \\
\hline LRMS-1 & 0.4717 & & & & & & & & & 0.0005 & & & & 0.0003 & & & & & & & \\
\hline SSHTM-3 & 0.5281 & 0.0034 & & & & & & & & & & & & 0.0016 & & & & & & & \\
\hline B1G9-0IIC4 & 0.6387 & 0.0008 & & & 0.0001 & & & & & & & & & 0.0005 & & & & & & & \\
\hline $\mathrm{B} 1 \mathrm{G} 9-013 \mathrm{C} 5$ & 0.6300 & 0.0006 & & & 0.0001 & & & & & & & & & 0.0005 & & & & & & & \\
\hline B1G9-014C & 0.6256 & 0.0006 & & & 0.0001 & & & & & & & & & 0.0005 & & & & & & & \\
\hline D1G4-022P2 & 0.4247 & 0.0362 & & & 0.0004 & & & & & 0.0007 & & & & 0.0021 & & & & & & & \\
\hline D1G4-023P3 & 0.4227 & 0.0382 & & & 0.0004 & & & & & 0.0004 & & & & 0.0019 & & & & & & & \\
\hline \multicolumn{22}{|l|}{ Duratek } \\
\hline \multicolumn{22}{|l|}{ PEI } \\
\hline M1G1-008P & 0.4782 & & & & & & & & & & & & & & & & & & & & \\
\hline M1G1-011P & 0.5053 & & & & & & & & & & & & & & & & & & & & \\
\hline V1M2 $632011 \mathrm{P} 1$ & 0.5809 & 0.0006 & & & & & & & & & & & & 0.0031 & & & & & & & \\
\hline V1M2 632040 P2 & 0.5735 & 0.0004 & & & & & & & & 0.0001 & & & & 0.0029 & & & & & & & \\
\hline V1M3 632059 P1 & 0.5669 & 0.0005 & & & & & & & & 0.0006 & & & & 0.0027 & & & & & & & \\
\hline V1M3 632075 P2 & 0.5908 & 0.0003 & & & 0.0001 & & & & & 0.0006 & & & & 0.0029 & & & & & & & \\
\hline V1M4 $632088 \mathrm{P} 1$ & 0.5563 & 0.0004 & & & 0.0001 & & & & & 0.0005 & & & & 0.0031 & & & & & & & \\
\hline V1M4 632096 P2 & 0.5597 & 0.0004 & & & & & & & & & & & & 0.0030 & & & & & & & \\
\hline Vectra & 0.5331 & 0.0002 & & & & & & & & & & & & 0.0004 & & & & & & & \\
\hline WSTC & & & & & & & & & & & & & & & & & & & & & \\
\hline
\end{tabular}

\section{SBW CVS Phase 1 (Scholes et al. 2002)}

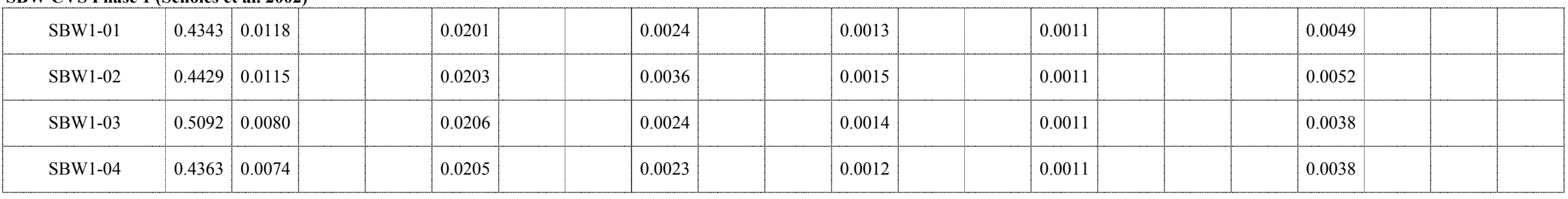


Appendix A. Database - mass fraction

Hanford LLW Glass Formulation (Feng et al. 1996)

\begin{tabular}{|c|c|c|c|c|c|c|c|c|c|c|c|c|c|c|c|c|c|c|c|c|c|}
\hline Glass ID & $\begin{array}{c}\mathrm{I} \\
-\mathrm{a} \\
\end{array}$ & $\begin{array}{c}\mathrm{La} 2 \mathrm{O} 3 \\
-\mathrm{a} \\
\end{array}$ & $\begin{array}{c}\mathrm{MnO} 2 \\
-\mathrm{a}\end{array}$ & $\begin{array}{c}\mathrm{MnO} \\
-\mathrm{a}\end{array}$ & $\begin{array}{c}\mathrm{MoO} \\
-\mathrm{a}\end{array}$ & $\begin{array}{c}\mathrm{MoO} 3 \\
-\mathrm{a} \\
\end{array}$ & $\begin{array}{c}\mathrm{Nb} 2 \mathrm{O} 5 \\
-\mathrm{a}\end{array}$ & $\begin{array}{c}\mathrm{Nd} 2 \mathrm{O} 3 \\
-\mathrm{a}\end{array}$ & $\begin{array}{c}\mathrm{PbO} \\
-\mathrm{a}\end{array}$ & $\begin{array}{c}\mathrm{PdO} 2 \\
-\mathrm{a}\end{array}$ & $\begin{array}{c}\mathrm{PdO} \\
-\mathrm{a}\end{array}$ & $\begin{array}{c}\mathrm{Pr} 2 \mathrm{O} 3 \\
-\mathrm{a}\end{array}$ & $\begin{array}{c}\operatorname{Pr} 6 \mathrm{O} 11 \\
-\mathrm{a}\end{array}$ & $\begin{array}{c}\mathrm{Rb} 2 \mathrm{O} \\
-\mathrm{a}\end{array}$ & $\begin{array}{c}\mathrm{ReO} \\
-\mathrm{a}\end{array}$ & $\begin{array}{c}\mathrm{ReO} 2 \\
-\mathrm{a}\end{array}$ & $\begin{array}{c}\mathrm{Rh} 2 \mathrm{O} 3 \\
-\mathrm{a}\end{array}$ & $\begin{array}{c}\mathrm{RhO} 2 \\
-\mathrm{a}\end{array}$ & $\begin{array}{c}\mathrm{RuO} 2 \\
-\mathrm{a}\end{array}$ & $\begin{array}{c}\mathrm{Sb} 2 \mathrm{O} 3 \\
-\mathrm{a}\end{array}$ & $\begin{array}{c}\mathrm{Sb} 2 \mathrm{O} 5 \\
-\mathrm{a}\end{array}$ \\
\hline \multicolumn{22}{|l|}{ L8-7 } \\
\hline \multicolumn{22}{|l|}{ L8-8 } \\
\hline \multicolumn{22}{|l|}{ LD4-912 } \\
\hline \multicolumn{22}{|l|}{ LD5-912 } \\
\hline \multicolumn{22}{|l|}{ LD6-5314 } \\
\hline LD6-5412 & & & & 0.0001 & & 0.0013 & & 0.0006 & & & & & & & & & & & & & \\
\hline \multicolumn{22}{|l|}{ LD6-5510 } \\
\hline LDM-912 & & & & & & 0.0016 & & & & & & & & & & & & & & & \\
\hline LDM-1 & & & & 0.0001 & & 0.0015 & & 0.0005 & & & & & & & & & & & & & \\
\hline LDM-2 & & & & & & 0.0015 & & 0.0006 & & & & & & & & & & & & & \\
\hline LDM-3 & & & & & & 0.0016 & & 0.0007 & & & & & & & & & & & & & \\
\hline LDM-4 & & & & 0.0001 & & 0.0015 & & 0.0008 & & & & & & & & & & & & & \\
\hline LDM-5412 & & & & & & 0.0016 & & 0.0006 & & & & & & & & & & & & & \\
\hline LDMS-1 & & & & 0.0001 & & 0.0013 & & 0.0008 & & & & & & & & & & & & & \\
\hline LRM-912 & & & & 0.0001 & & 0.0016 & & 0.0009 & & & & & & & & & & & & & \\
\hline LRM-1 & & & & 0.0002 & & 0.0015 & & & & & & & & & & & & & & & \\
\hline LRM-2 & & & & 0.0002 & & 0.0016 & & 0.0003 & & & & & & & & & & & & & \\
\hline LRM-3 & & & & 0.0001 & & 0.0017 & & 0.0004 & & & & & & & & & & & & & \\
\hline LRM-4 & & & & 0.0002 & & 0.0015 & & 0.0009 & & & & & & & & & & & & & \\
\hline LRM-5412 & & & & 0.0001 & & 0.0016 & & 0.0008 & & & & & & & & & & & & & \\
\hline LRMS-1 & & & & 0.0001 & & 0.0015 & & 0.0010 & & & & & & & & & & & & & \\
\hline SSHTM-3 & & & & 0.0001 & & 0.0014 & & 0.0002 & & & & & & & & & & & & & \\
\hline B1G9-0IIC4 & & & & 0.0002 & & 0.0006 & & 0.0008 & & & & & & & & & & & & & \\
\hline B1G9-013C5 & & & & 0.0002 & & 0.0006 & & 0.0011 & & & & & & & & & & & & & \\
\hline B1G9-014C & & & & 0.0002 & & 0.0007 & & 0.0013 & & & & & & & & & & & & & \\
\hline D1G4-022P2 & & & & 0.0002 & & 0.0015 & & 0.0005 & & & & & & & & & & & & & \\
\hline $\mathrm{D} 1 \mathrm{G} 4-023 \mathrm{P} 3$ & & & & 0.0002 & & 0.0015 & & 0.0003 & & & & & & & & & & & & & \\
\hline \multicolumn{22}{|l|}{ Duratek } \\
\hline \multicolumn{22}{|l|}{ PEI } \\
\hline M1G1-008P & & & & & & 0.0007 & & & & & & & & & & & & & & & \\
\hline M1G1-011P & & & & & & 0.0009 & & & & & & & & & & & & & & & \\
\hline V1M2 632011 P1 & & & & 0.0001 & & 0.0022 & & 0.0002 & & & & & & & & & & & & & \\
\hline V1M2 632040 P2 & & & & 0.0001 & & 0.0019 & & 0.0004 & & & & & & & & & & & & & \\
\hline V1M3 632059 P1 & & & & 0.0001 & & 0.0016 & & 0.0010 & & & & & & & & & & & & & \\
\hline V1M3 632075 P2 & & & & 0.0002 & & 0.0016 & & 0.0009 & & & & & & & & & & & & & \\
\hline V1M4 632088 P1 & & & & 0.0003 & & 0.0013 & & 0.0009 & & & & & & & & & & & & & \\
\hline V1M4 632096 P2 & & & & 0.0001 & & 0.0013 & & 0.0006 & & & & & & & & & & & & & \\
\hline Vectra & & & & & & 0.0015 & & 0.0003 & & & & & & & & & & & & & \\
\hline WSTC & & & & & & & & & & & & & & & & & & & & & \\
\hline
\end{tabular}

\section{SBW CVS Phase 1 (Scholes et al. 2002)}

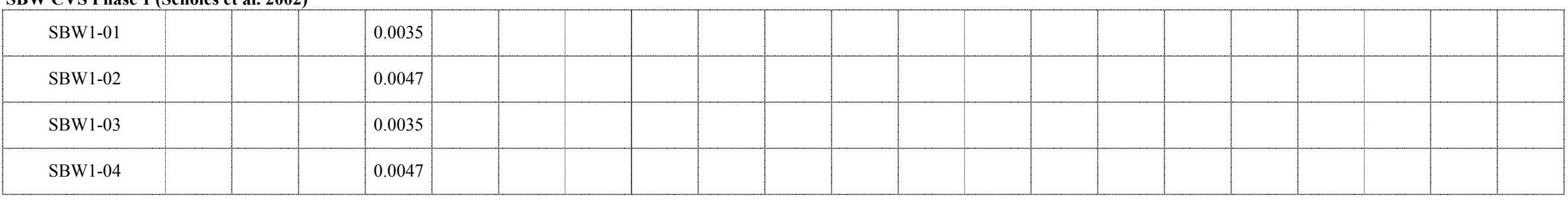


Appendix A. Database - mass fraction

\section{Hanford LLW Glass Formulation (Feng et al. 1996)}

\begin{tabular}{|c|c|c|c|c|c|c|c|c|c|c|c|c|c|c|c|c|c|c|c|c|}
\hline Glass ID & $\begin{array}{c}\mathrm{SeO} 2 \\
-\mathrm{a}\end{array}$ & $\begin{array}{c}\mathrm{Sm} 2 \mathrm{O} 3 \\
-\mathrm{a}\end{array}$ & $\begin{array}{c}\mathrm{SnO} \\
-\mathrm{a}\end{array}$ & $\begin{array}{c}\mathrm{SnO} 2 \\
-\mathrm{a}\end{array}$ & $\begin{array}{c}\mathrm{SO} 3 \\
-\mathrm{a}\end{array}$ & $\begin{array}{c}\mathrm{SrO} \\
-\mathrm{a}\end{array}$ & $\begin{array}{c}\mathrm{Tc} 2 \mathrm{O} 7 \\
-\mathrm{a}\end{array}$ & $\begin{array}{c}\mathrm{TeO} 2 \\
-\mathrm{a}\end{array}$ & $\begin{array}{c}\mathrm{ThO} 2 \\
-\mathrm{a}\end{array}$ & $\begin{array}{c}\mathrm{TiO} 2 \\
-\mathrm{a}\end{array}$ & $\begin{array}{c}\mathrm{T} 12 \mathrm{O} 3 \\
-\mathrm{a}\end{array}$ & $\begin{array}{c}\mathrm{U} 3 \mathrm{O} 8 \\
-\mathrm{a}\end{array}$ & $\begin{array}{c}\mathrm{UO} 2 \\
-\mathrm{a}\end{array}$ & $\begin{array}{c}\mathrm{UO} 3 \\
-\mathrm{a}\end{array}$ & $\begin{array}{c}\mathrm{V} 2 \mathrm{O} 5 \\
-\mathrm{a}\end{array}$ & $\begin{array}{c}\text { WO3 } \\
-\mathrm{a}\end{array}$ & $\begin{array}{c}\mathrm{Y} 2 \mathrm{O} 3 \\
-\mathrm{a}\end{array}$ & $\begin{array}{c}\mathrm{ZnO} \\
-\mathrm{a}\end{array}$ & $\begin{array}{c}\text { Others } \\
-\mathrm{a}\end{array}$ & $\begin{array}{l}\text { Sum } \\
-\mathrm{a}\end{array}$ \\
\hline \multicolumn{21}{|l|}{ L8-7 } \\
\hline \multicolumn{21}{|l|}{ L8-8 } \\
\hline \multicolumn{21}{|l|}{ LD4-912 } \\
\hline \multicolumn{21}{|l|}{ LD5-912 } \\
\hline \multicolumn{21}{|l|}{ LD6-5314 } \\
\hline LD6-5412 & & & & & 0.0018 & 0.0009 & & & & 0.0002 & & & & & & & & 0.0014 & & 0.9998 \\
\hline \multicolumn{21}{|l|}{ LD6-5510 } \\
\hline LDM-912 & & & & & 0.0026 & 0.0012 & & & & & & & & & & & & & & 0.9971 \\
\hline LDM-1 & & & & & 0.0022 & 0.0010 & & & & 0.0001 & & & & & & & & 0.0011 & & 0.9997 \\
\hline LDM-2 & & & & & 0.0023 & 0.0011 & & & & & & & & & & & & 0.0006 & & 0.9942 \\
\hline LDM-3 & & & & & 0.0021 & 0.0012 & & & & 0.0001 & & & & & & & & 0.0008 & & 0.9995 \\
\hline LDM-4 & & & & & 0.0026 & 0.0012 & & & & & & & & & & & & 0.0014 & & 0.9994 \\
\hline LDM-5412 & & & & & 0.0069 & 0.0011 & & & & 0.0001 & & & & & & & & 0.0016 & & 0.9998 \\
\hline LDMS-1 & & & & & 0.0026 & 0.0009 & & & & 0.0002 & & & & & & & & 0.0006 & & 0.9884 \\
\hline LRM-912 & & & & & 0.0101 & 0.0013 & & & & & & & & & & & & 0.0010 & & 0.9997 \\
\hline LRM-1 & & & & & 0.0076 & 0.0012 & & & & 0.0002 & & & & & & & & 0.0021 & & 0.9997 \\
\hline LRM-2 & & & & & 0.0074 & 0.0012 & & & & 0.0001 & & & & & & & & 0.0018 & & 0.9767 \\
\hline LRM-3 & & & & & 0.0059 & 0.0013 & & & & 0.0001 & & & & & & & & 0.0024 & & 1.0220 \\
\hline LRM-4 & & & & & 0.0093 & 0.0012 & & & & 0.0001 & & & & & & & & 0.0009 & & 0.9994 \\
\hline LRM-5412 & & & & & 0.0087 & 0.0013 & & & & & & & & & & & & 0.0011 & & 0.9998 \\
\hline LRMS-1 & & & & & 0.0101 & 0.0011 & & & & 0.0002 & & & & & & & & 0.0006 & & 0.7628 \\
\hline SSHTM-3 & & & & & 0.0029 & 0.0007 & & & & 0.0007 & & & & & & & & 0.0026 & & 1.0000 \\
\hline B1G9-0IIC4 & & & & & & 0.0010 & & & & 0.0019 & & & & & & & & 0.0014 & & 0.9981 \\
\hline B1G9-013C5 & & & & & & 0.0010 & & & & 0.0017 & & & & & & & & 0.0012 & & 0.9975 \\
\hline B1G9-014C & & & & & & 0.0010 & & & & 0.0017 & & & & & & & & 0.0019 & & 0.9987 \\
\hline $\mathrm{D} 1 \mathrm{G} 4-022 \mathrm{P} 2$ & & & & & 0.0021 & 0.0010 & & & & 0.0089 & & & & & & & & 0.0021 & & 0.9975 \\
\hline D1G4-023P3 & & & & & 0.0021 & 0.0010 & & & & 0.0091 & & & & & & & & 0.0027 & & 0.9979 \\
\hline \multicolumn{21}{|l|}{ Duratek } \\
\hline \multicolumn{21}{|l|}{ PEI } \\
\hline M1G1-008P & & & & & & & & & & 0.0068 & & & & & & & & & & 0.8952 \\
\hline M1G1-011P & & & & & & & & & & 0.0035 & & & & & & & & & & 0.9030 \\
\hline V1M2 632011 P1 & & & & & & 0.0006 & & & & 0.0001 & & & & & & & & 0.0008 & & 0.9981 \\
\hline V1M2 632040 P2 & & & & & & 0.0006 & & & & 0.0001 & & & & & & & & 0.0006 & & 0.9978 \\
\hline V1M3 632059 P1 & & & & & & 0.0008 & & & & 0.0001 & & & & & & & & 0.0007 & & 1.0000 \\
\hline V1M3 632075 P2 & & & & & & 0.0008 & & & & 0.0002 & & & & & & & & 0.0008 & & 0.9997 \\
\hline V1M4 632088 P1 & & & & & & 0.0009 & & & & 0.0002 & & & & & & & & 0.0008 & & 0.9997 \\
\hline V1M4 632096 P2 & & & & & & 0.0008 & & & & 0.0001 & & & & & & & & & & 0.9981 \\
\hline Vectra & & & & & 0.0022 & 0.0011 & & & & & & & & & & & & 0.0028 & & 0.9984 \\
\hline WSTC & & & & & & & & & & & & & & & & & & & & \\
\hline
\end{tabular}

SBW CVS Phase 1 (Scholes et al. 2002)

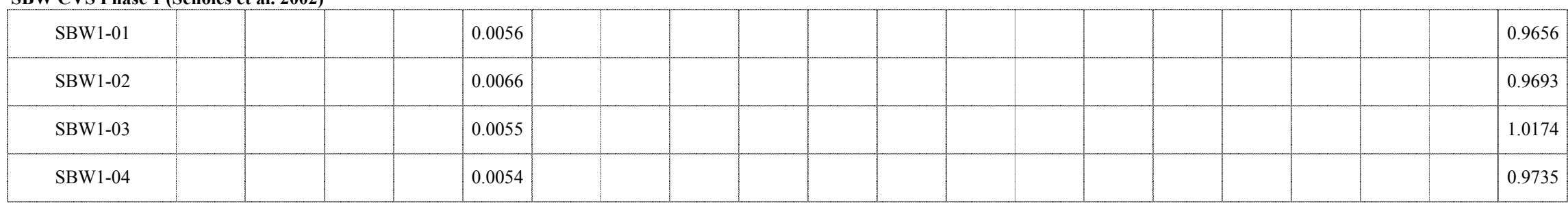


Appendix A. Database - mass fraction

Hanford LLW Glass Formulation (Feng et al. 1996)

\begin{tabular}{|c|c|c|c|c|c|c|c|c|}
\hline Glass ID & $\begin{array}{l}\mathrm{TM} \\
\left({ }^{\circ} \mathrm{C}\right)\end{array}$ & $\begin{array}{l}\text { Gradient } \\
\text { TL }\left({ }^{\circ} \mathrm{C}\right) \\
\end{array}$ & $\begin{array}{l}\text { Uniform } \\
\text { TL }\left({ }^{\circ} \mathrm{C}\right) \\
\end{array}$ & Primary Phase & $\begin{array}{l}\text { Quenched } \\
\text { Visual/OM }\end{array}$ & $\begin{array}{c}\text { Quenched } \\
\text { SEM/EDS or TEM }\end{array}$ & $\begin{array}{l}\text { Quenched } \\
\text { XRD }\end{array}$ & $\begin{array}{c}\text { Quenched } \\
\text { Homogeneous? }\end{array}$ \\
\hline \multicolumn{9}{|l|}{ L8-7 } \\
\hline \multicolumn{9}{|l|}{ L8-8 } \\
\hline \multirow{2}{*}{\multicolumn{9}{|c|}{$\begin{array}{l}\text { LD4-912 } \\
\text { LD5-912 }\end{array}$}} \\
\hline & & & & & & & & \\
\hline \multicolumn{9}{|l|}{ LD6-5314 } \\
\hline \multicolumn{9}{|l|}{ LD6-5412 } \\
\hline \multicolumn{9}{|l|}{ LD6-5510 } \\
\hline \multirow{2}{*}{\multicolumn{9}{|c|}{$\begin{array}{l}\text { LDM-912 } \\
\text { LDM-1 }\end{array}$}} \\
\hline LDM-1 & & & & & & & & \\
\hline \multicolumn{9}{|l|}{ LDM-2 } \\
\hline \multicolumn{9}{|l|}{ LDM-3 } \\
\hline \multicolumn{9}{|l|}{ LDM-4 } \\
\hline \multicolumn{9}{|l|}{ LDM-5412 } \\
\hline \multicolumn{9}{|l|}{ LDMS-1 } \\
\hline \multicolumn{9}{|l|}{ LRM-912 } \\
\hline \multicolumn{9}{|l|}{ LRM-1 } \\
\hline \multicolumn{9}{|l|}{ LRM-2 } \\
\hline \multicolumn{9}{|l|}{ LRM-3 } \\
\hline \multicolumn{9}{|l|}{ LRM-4 } \\
\hline \multicolumn{9}{|l|}{ LRM-5412 } \\
\hline \multicolumn{9}{|l|}{ LRMS-1 } \\
\hline \multicolumn{9}{|l|}{ SSHTM-3 } \\
\hline \multicolumn{9}{|l|}{ B1G9-0IIC4 } \\
\hline \multicolumn{9}{|l|}{ B1G9-013C5 } \\
\hline B1G9-014C & & & & & & & & \\
\hline D1G4-022P2 & & & & & & & & \\
\hline D1G4-023P3 & & & & & & & & \\
\hline Duratek & & & & & & & & \\
\hline PEI & & & & & & & & \\
\hline M1G1-008P & & & & & & & & \\
\hline M1G1-011P & & & & & & & & \\
\hline V1M2 632011 & & & & & & & & \\
\hline V1M2 632040 & & & & & & & & \\
\hline V1M3 632059 & & & & & & & & \\
\hline V1M3 632075 & & & & & & & & \\
\hline V1M4 632088 & & & & & & & & \\
\hline V1M4 632096 & & & & & & & & \\
\hline Vectra & & & & & & & & \\
\hline WSTC & & & & & & & & \\
\hline SBW CVS Phas & choles $\mathrm{C}$ & al. 2002) & & & & & & \\
\hline SBW1-01 & 1150 & & 793 & $\mathrm{Ca} 5(\mathrm{PO} 4) 3 \mathrm{~F}$ & $\begin{array}{l}\text { Clear dark brown glass with black specks anc } \\
\text { air bubbles, low viscosity }\end{array}$ & & Amorphous & Single-phase \\
\hline SBW1-02 & 1150 & & 783 & $\mathrm{Ca} 5(\mathrm{PO} 4) 3 \mathrm{~F}$ & $\begin{array}{l}\text { Clear dark brown glass with black specks anc } \\
\text { air bubbles, low viscosity }\end{array}$ & & Amorphous & Single-phase \\
\hline SBW1-03 & 1150 & & 848 & $(\mathrm{Na}, \mathrm{K})(\mathrm{Si} 3 \mathrm{Al}) \mathrm{O} 8$ & $\begin{array}{l}\text { Clear dark brown glass with black specks anc } \\
\text { air bubbles, avg. viscosity }\end{array}$ & & Amorphous & Single-phase \\
\hline SBW1-04 & 1150 & & 783 & $\mathrm{Ca} 5(\mathrm{PO} 4) 3 \mathrm{~F}$ & $\begin{array}{l}\text { Clear dark brown glass with black specks anc } \\
\text { air bubbles, low viscosity }\end{array}$ & & Amorphous & Single-phase \\
\hline
\end{tabular}


Appendix A. Database - mass fraction

\section{Hanford LLW Glass Formulation (Feng et al. 1996)}

\begin{tabular}{|c|c|c|c|c|c|}
\hline Glass ID & $\begin{array}{c}\text { CCC } \\
\text { Visual/OM }\end{array}$ & $\begin{array}{c}\text { CCC } \\
\text { SEM/EDS or TEM }\end{array}$ & $\begin{array}{l}\text { CCC } \\
\text { XRD }\end{array}$ & $\begin{array}{c}\text { Heat Treated } \\
\text { Visual/OM }\end{array}$ & $\begin{array}{c}\text { Heat Treated } \\
\text { SEM/EDS or TEM }\end{array}$ \\
\hline \multicolumn{6}{|l|}{ L8-7 } \\
\hline \multicolumn{6}{|l|}{ L8-8 } \\
\hline \multicolumn{6}{|l|}{ LD4-912 } \\
\hline \multicolumn{6}{|l|}{ LD5-912 } \\
\hline \multicolumn{6}{|l|}{ LD6-5314 } \\
\hline \multicolumn{6}{|l|}{ LD6-5412 } \\
\hline \multicolumn{6}{|l|}{ LD6-5510 } \\
\hline \multicolumn{6}{|l|}{ LDM-912 } \\
\hline \multicolumn{6}{|l|}{ LDM-1 } \\
\hline \multicolumn{6}{|l|}{ LDM-2 } \\
\hline \multicolumn{6}{|l|}{ LDM-3 } \\
\hline \multicolumn{6}{|l|}{ LDM-4 } \\
\hline \multicolumn{6}{|l|}{ LDM-5412 } \\
\hline \multicolumn{6}{|l|}{ LDMS-1 } \\
\hline \multicolumn{6}{|l|}{ LRM-912 } \\
\hline \multicolumn{6}{|l|}{ LRM-1 } \\
\hline \multicolumn{6}{|l|}{ LRM-2 } \\
\hline \multicolumn{6}{|l|}{ LRM-3 } \\
\hline \multicolumn{6}{|l|}{ LRM-4 } \\
\hline \multicolumn{6}{|l|}{ LRM-5412 } \\
\hline \multicolumn{6}{|l|}{ LRMS-1 } \\
\hline \multicolumn{6}{|l|}{ SSHTM-3 } \\
\hline \multicolumn{6}{|l|}{ B1G9-0IIC4 } \\
\hline \multicolumn{6}{|l|}{ B1G9-013C5 } \\
\hline \multicolumn{6}{|l|}{ B1G9-014C } \\
\hline \multicolumn{6}{|l|}{$\mathrm{D} 1 \mathrm{G} 4-022 \mathrm{P} 2$} \\
\hline D1G4-023P3 & & & & & \\
\hline Duratek & & & & & \\
\hline PEI & & & & & \\
\hline M1G1-008P & & & & & \\
\hline M1G1-011P & & & & & \\
\hline V1M2 $632011 \mathrm{P} 1$ & & & & & \\
\hline V1M2 $632040 \mathrm{P} 2$ & & & & & \\
\hline V1M3 632059 P1 & & & & & \\
\hline V1M3 632075 P2 & & & & & \\
\hline V1M4 632088 P1 & & & & & \\
\hline V1M4 632096 P2 & & & & & \\
\hline Vectra & & & & & \\
\hline WSTC & & & & & \\
\hline
\end{tabular}

SBW1-01 Clear dark brown glass, with few air bubbles and black specks (expected to be Ru).

SBW1-02 Clear dark brown glass, air bubbles and black specks

(expected to be Ru).

SBW1-03 Clear dark reddish brown glass, air bubbles and black specks (expected to be $\mathrm{Ru}$ ).

SBW1-04 Clear dark brown glass, few air bubbles and black specks (expected to be $\mathrm{Ru}$ ).

\section{Amorphous \\ Amorphous \\ Amorphous \\ Amorphous}


Hanford LLW Glass Formulation (Feng et al. 1996)

\begin{tabular}{|c|c|c|c|c|c|c|c|c|c|c|c|c|c|c|c|}
\hline Glass ID & $\begin{array}{c}\text { Heat Treated } \\
\text { XRD } \\
\end{array}$ & $\begin{array}{l}\text { Density } \\
\left(\mathrm{g} / \mathrm{cm}^{3}\right)\end{array}$ & $\begin{array}{c}\text { Fulc Visc } \\
\mathrm{A}\end{array}$ & $\begin{array}{c}\text { Fulc Visc } \\
\text { B }\end{array}$ & $\begin{array}{c}\text { Fulc Visc } \\
\text { To } \\
\end{array}$ & $\begin{array}{c}\mathrm{FV} 1150^{\circ} \mathrm{C} \\
(\mathrm{Pa} \cdot \mathrm{s})\end{array}$ & \begin{tabular}{|c} 
Arrh Visc \\
A
\end{tabular} & \begin{tabular}{|c|} 
Arrh Visc \\
B
\end{tabular} & $1150^{\circ} \mathrm{C}$ & $\begin{array}{l}\mathrm{T}\left({ }^{\circ} \mathrm{C}\right) \text { at } \\
2 \text { Pa.s }\end{array}$ & \begin{tabular}{|c|}
$\mathrm{T}\left({ }^{\circ} \mathrm{C}\right)$ at \\
$5 \mathrm{~Pa} \cdot \mathrm{s}$ \\
\end{tabular} & \begin{tabular}{|c|}
$\mathrm{T}\left({ }^{\circ} \mathrm{C}\right)$ at \\
$10 \mathrm{~Pa} \cdot \mathrm{s}$ \\
\end{tabular} & $\begin{array}{c}\mathrm{T} 1 \\
\left({ }^{\circ} \mathrm{C}\right) \\
\end{array}$ & $\begin{array}{c}\mathrm{V} 1 \\
(\mathrm{~Pa} \cdot \mathrm{s})\end{array}$ & $\begin{array}{c}\mathrm{T} 2 \\
\left({ }^{\circ} \mathrm{C}\right) \\
\end{array}$ \\
\hline L8-7 & & & & & & & -10.390 & 20691.6 & 63.49 & & & & 1141 & 76.3 & 1191 \\
\hline L8-8 & & & & & & & -9.780 & 20032.8 & 73.54 & & & & 1190 & 54.5 & 1239 \\
\hline LD4-912 & & & & & & & -10.096 & 19838.1 & 46.76 & & & & 1045 & 153.4 & 1144 \\
\hline LD5-912 & & & & & & & -11.888 & 23324.3 & 90.28 & & & & 1095 & 179.9 & 1145 \\
\hline LD6-5314 & & & & & & & -10.564 & 21253.2 & 79.16 & & & & 1070 & 198.3 & 1119 \\
\hline LD6-5412 & & & & & & & -10.879 & 21013.7 & 48.82 & & & & 1046 & 161 & 1145 \\
\hline LD6-5510 & & & & & & & -11.161 & 21110.3 & 39.41 & & & & 997 & 244.2 & 1096 \\
\hline LDM-912 & & & & & & & -10.759 & 21165.8 & 61.26 & & & & 1138 & 76.8 & 1186 \\
\hline LDM-1 & & & & & & & -11.786 & 23083.2 & 84.40 & & & & 1092 & 179.3 & 1142 \\
\hline LDM-2 & & & & & & & -11.119 & 21720.8 & 63.12 & & & & 1091 & 133.5 & 1141 \\
\hline LDM-3 & & & & & & & -11.395 & 22585.1 & 87.92 & & & & 1138 & 111.1 & 1187 \\
\hline LDM-4 & & & & & & & -12.435 & 21132.1 & 11.19 & & & & 941 & 158.7 & 992 \\
\hline LDM-5412 & & & & & & & -8.601 & 17292.2 & 34.85 & & & & (1088) & (78.7) & 1138 \\
\hline LDMS-1 & & & & & & & -11.648 & 22995.7 & 91.10 & & & & 1143 & 108.1 & 1193 \\
\hline LRM-912 & & & & & & & -10.848 & 21757.9 & 84.96 & & & & 1187 & 59.9 & 1237 \\
\hline LRM-1 & & & & & & & -11.491 & 22167.2 & 59.55 & & & & 1142 & 67.3 & 1192 \\
\hline LRM-2 & & & & & & & -10.836 & 21715.1 & 83.43 & & & & 1191 & 54.9 & 1241 \\
\hline LRM-3 & & & & & & & -11.854 & 23833.9 & 133.62 & & & & 1187 & 90.3 & 1237 \\
\hline LRM-4 & & & & & & & -13.327 & 22476.2 & 11.80 & & & & 940 & 203.9 & 990 \\
\hline LRM-5412 & & & & & & & -8.839 & 18144.8 & 50.00 & & & & 1188 & 40.8 & 1237 \\
\hline LRMS-1 & & & & & & & -11.130 & 21635.2 & 58.79 & & & & 1142 & 67.3 & 1192 \\
\hline SSHTM-3 & & & & & & & -12.900 & 24419.0 & 70.83 & & & & 1195 & 46.9 & 1245 \\
\hline \multicolumn{16}{|l|}{ B1G9-0IIC4 } \\
\hline \multicolumn{16}{|l|}{ B1G9-013C5 } \\
\hline \multirow{2}{*}{\multicolumn{16}{|c|}{$\begin{array}{l}\mathrm{B} 1 \mathrm{G} 9-014 \mathrm{C} \\
\mathrm{D} 1 \mathrm{G} 4-022 \mathrm{P} 2\end{array}$}} \\
\hline & & & & & & & & & & & & & & & \\
\hline \multicolumn{16}{|l|}{ D1G4-023P3 } \\
\hline Duratek & & & & & & & -12.584 & 20386.2 & 5.71 & & & & 941 & 76.5 & 992 \\
\hline PEI & & & & & & & -11.457 & 22008.1 & 55.09 & & & & 1090 & 116.8 & 1140 \\
\hline \multicolumn{16}{|l|}{ M1G1-008P } \\
\hline \multicolumn{16}{|l|}{ M1G1-011P } \\
\hline \multicolumn{16}{|l|}{ V1M2 632011 P1 } \\
\hline \multicolumn{16}{|l|}{ V1M2 632040 P2 } \\
\hline \multicolumn{16}{|l|}{ V1M3 632059 P1 } \\
\hline \multicolumn{16}{|l|}{ V1M3 632075 P2 } \\
\hline \multicolumn{16}{|l|}{ V1M4 632088 P1 } \\
\hline \multicolumn{16}{|l|}{ V1M4 632096 P2 } \\
\hline Vectra & & & & & & & -9.500 & 17677.5 & 18.59 & & & & 1090 & 33.4 & 1144 \\
\hline WSTC & & & & & & & -9.980 & 18288.5 & 17.67 & & & & 1040 & 54.7 & 1090 \\
\hline
\end{tabular}

\section{SBW CVS Phase 1 (Scholes et al. 2002)}

\begin{tabular}{|c|c|c|c|c|c|c|c|c|c|}
\hline SBW1-01 & 2.6045 & -9.866 & 16504.0 & 5.65 & 1290 & 1083 & 1155.4 & 5.2593 & 1079.5 \\
\hline SBW1-02 & 2.6102 & -10.105 & 16909.0 & 5.92 & 1293 & 1090 & 1157.1 & 5.5161 & 1081.3 \\
\hline SBW1-03 & 2.5754 & -9.996 & 17892.0 & 13.16 & 1401 & 1182 & 1155.8 & 12.149 & 1080 \\
\hline SBW1-04 & 2.5891 & -9.904 & 16145.0 & 4.23 & 1250 & 1050 & 1157 & 3.9018 & 1081.6 \\
\hline
\end{tabular}




\section{Hanford LLW Glass Formulation (Feng et al. 1996)}

\begin{tabular}{|c|c|c|c|c|c|c|c|c|c|c|c|c|c|c|c|c|c|c|c|c|c|}
\hline Glass ID & $\begin{array}{c}\mathrm{V} 2 \\
(\mathrm{~Pa} \cdot \mathrm{s})\end{array}$ & $\begin{array}{c}\mathrm{T} 3 \\
\left({ }^{\circ} \mathrm{C}\right)\end{array}$ & $\begin{array}{c}\mathrm{V} 3 \\
(\mathrm{~Pa} \cdot \mathrm{s})\end{array}$ & $\begin{array}{c}\mathrm{T} 4 \\
\left({ }^{\circ} \mathrm{C}\right)\end{array}$ & $\begin{array}{c}\mathrm{V} 4 \\
(\mathrm{~Pa} \cdot \mathrm{s})\end{array}$ & $\begin{array}{c}\mathrm{T} 5 \\
\left({ }^{\circ} \mathrm{C}\right)\end{array}$ & $\begin{array}{c}\text { V5 } \\
(\mathrm{Pa} \cdot \mathrm{s})\end{array}$ & $\begin{array}{c}\mathrm{T} 6 \\
\left({ }^{\circ} \mathrm{C}\right)\end{array}$ & $\begin{array}{c}\mathrm{V} 6 \\
(\mathrm{~Pa} \cdot \mathrm{s})\end{array}$ & $\begin{array}{c}\mathrm{T} 7 \\
\left({ }^{\circ} \mathrm{C}\right)\end{array}$ & $\begin{array}{c}\text { V7 } \\
(\mathrm{Pa} \cdot \mathrm{s})\end{array}$ & $\begin{array}{c}\mathrm{T} 8 \\
\left({ }^{\circ} \mathrm{C}\right)\end{array}$ & $\begin{array}{c}\mathrm{V} 8 \\
(\mathrm{~Pa} \cdot \mathrm{s})\end{array}$ & $\begin{array}{c}\mathrm{T} 9 \\
\left({ }^{\circ} \mathrm{C}\right)\end{array}$ & $\begin{array}{c}\text { V9 } \\
(\mathrm{Pa} \cdot \mathrm{s})\end{array}$ & $\begin{array}{l}\mathrm{T} 10 \\
\left({ }^{\circ} \mathrm{C}\right)\end{array}$ & $\begin{array}{c}\text { V10 } \\
(\mathrm{Pa} \cdot \mathrm{s})\end{array}$ & $\begin{array}{l}\text { T11 } \\
\left({ }^{\circ} \mathrm{C}\right)\end{array}$ & $\begin{array}{c}\text { V11 } \\
(\mathrm{Pa} \cdot \mathrm{s})\end{array}$ & $\begin{array}{l}\mathrm{T} 12 \\
\left({ }^{\circ} \mathrm{C}\right)\end{array}$ & $\begin{array}{c}\mathrm{V} 12 \\
(\mathrm{~Pa} \cdot \mathrm{s})\end{array}$ \\
\hline L8-7 & 45.3 & 1240 & 24.1 & 1290 & 15.7 & 1290 & 15.9 & 1340 & 10.6 & 1340 & 11.2 & 1341 & 12.4 & 1390 & 7.9 & 1442 & 6 & & & & \\
\hline L8-8 & 33.1 & 1289 & 19.6 & 1339 & 13.3 & 1339 & 13.4 & 1388 & 9.3 & 1389 & 9 & 1390 & 10.2 & 1439 & 7.1 & 1489 & 5.4 & & & & \\
\hline LD4-912 & 48.5 & 1243 & 19.4 & 1342 & 7.4 & 1343 & 9.2 & 1392 & 5.8 & 1442 & 4.4 & 1492 & 3.7 & & & & & & & & \\
\hline LD5-912 & 94.6 & 1244 & 32.2 & 1343 & 12.3 & 1343 & 12.6 & 1393 & 8.2 & 1443 & 5.8 & & & & & & & & & & \\
\hline LD6-5314 & 109.4 & 1220 & 39.6 & 1318 & 14.4 & 1319 & 16.9 & 1368 & 10.6 & 1418 & 8.1 & & & & & & & & & & \\
\hline LD6-5412 & 49.7 & 1244 & 19.1 & 1342 & 8.7 & & & & & & & & & & & & & & & & \\
\hline LD6-5510 & 69.4 & 1195 & 23.7 & 1294 & 9.6 & 1294 & 10.3 & 1344 & 7.1 & & & & & & & & & & & & \\
\hline LDM-912 & 40.9 & 1236 & 24.8 & 1236 & 25.9 & 1285 & 15.6 & 1285 & 16.5 & 1286 & 17.1 & 1336 & 11.2 & 1385 & 7.6 & 1436 & 5.3 & & & & \\
\hline LDM-1 & 96.1 & 1191 & 50.8 & 1241 & 29.8 & 1241 & 31.1 & 1290 & 17.9 & 1290 & 19.3 & 1292 & 19.8 & 1343 & 13 & 1392 & 8.6 & & & & \\
\hline LDM-2 & 72.7 & 1190 & 38.6 & 1239 & 24.7 & 1240 & 23.7 & 1289 & 15.7 & 1290 & 14.9 & 1290 & 16.1 & 1340 & 10.8 & 1392 & 7.8 & & & & \\
\hline LDM-3 & 62.8 & 1236 & 31.8 & 1286 & 20 & 1286 & 20.8 & 1336 & 12.5 & 1336 & 13.9 & 1336 & 15 & 1386 & 9.7 & 1436 & 6.9 & & & & \\
\hline LDM-4 & 72.6 & 1041 & 35.9 & 1090 & 20.1 & 1091 & 20.1 & 1140 & 12.2 & 1141 & 11.9 & 1141 & 12.4 & 1190 & 7.5 & 1240 & 5.2 & & & & \\
\hline LDM-5412 & 40 & 1187 & 24.9 & 1187 & 25.9 & 1236 & 15.8 & 1237 & 17 & 1237 & 18.8 & 1286 & 12 & 1336 & 8.6 & 1386 & 6.3 & & & & \\
\hline LDMS-1 & 60.3 & 1243 & 30.1 & 1292 & 19.9 & 1293 & 18.8 & 1342 & 12.2 & 1342 & 13.4 & 1344 & 14.4 & 1393 & 8.8 & 1444 & 6.3 & & & & \\
\hline LRM-912 & 35.3 & 1286 & 21.8 & 1336 & 14 & 1336 & 14.2 & 1386 & 9.6 & 1386 & 9.7 & 1386 & 9.7 & 1436 & 6.6 & 1487 & 4.7 & & & & \\
\hline LRM-1 & 38.3 & 1242 & 22.6 & 1291 & 14.2 & 1292 & 14 & 1341 & 9.2 & 1341 & 9.5 & 1342 & 9.5 & 1392 & 6.3 & 1442 & 4.3 & & & & \\
\hline LRM-2 & 33 & 1291 & 20.9 & 1291 & 21 & 1340 & 13.7 & 1340 & 13.9 & 1342 & 13.6 & 1392 & 9.4 & 1441 & 6.2 & 1492 & 4.3 & & & & \\
\hline LRM-3 & 51.9 & 1286 & 31.3 & 1336 & 17.9 & 1386 & 11.7 & 1386 & 12.2 & 1435 & 7.6 & 1436 & 8.2 & 1436 & 8.5 & 1485 & 6 & & & & \\
\hline LRM-4 & 89.4 & 1040 & 41.9 & 1089 & 22.4 & 1090 & 21.3 & 1139 & 12.7 & 1140 & 12.8 & 1140 & 13.1 & 1189 & 7.9 & 1240 & 5.4 & & & & \\
\hline LRM-5412 & 23.6 & 1286 & 15.8 & 1287 & 15.3 & 1336 & 10.3 & 1336 & 11.1 & 1337 & 11.9 & 1386 & 8 & 1437 & 5.9 & 1488 & 4.8 & & & & \\
\hline LRMS-1 & 38.5 & 1243 & 22.5 & 1292 & 14.1 & 1293 & 14 & 1341 & 9.4 & 1342 & 9.6 & 1343 & 9.8 & 1392 & 6.6 & 1443 & 4.6 & & & & \\
\hline SSHTM-3 & 22.4 & 1294 & 14.2 & 1295 & 13.7 & 1344 & 8.6 & 1345 & 9.1 & 1345 & 9 & 1395 & 5.8 & 1446 & 3.9 & & & & & & \\
\hline \multicolumn{22}{|l|}{ B1G9-0IIC4 } \\
\hline \multicolumn{22}{|l|}{ B1G9-013C5 } \\
\hline \multicolumn{22}{|l|}{ B1G9-014C } \\
\hline \multicolumn{22}{|l|}{$\begin{array}{l}\mathrm{D} 1 \mathrm{G} 4-022 \mathrm{P} 2 \\
\mathrm{D} 1 \mathrm{G} 4-023 \mathrm{P}\end{array}$} \\
\hline \multicolumn{22}{|l|}{ D1G4-023P3 } \\
\hline Duratek & 35 & 1041 & 17.3 & 1091 & 9.8 & 1091 & 9.9 & 1140 & 6 & 1141 & 5.9 & 1141 & 6.2 & 1190 & 3.9 & 1241 & 2.9 & & & & \\
\hline PEI & 64.7 & 1188 & 35 & 1238 & 21.3 & 1238 & 21.4 & 1287 & 13.6 & 1287 & 13.7 & 1288 & 13.9 & 1337 & 9.1 & 1387 & 6.3 & 1438 & 4.4 & & \\
\hline \multicolumn{22}{|l|}{ M1G1-008P } \\
\hline \multicolumn{22}{|l|}{ M1G1-011P } \\
\hline \multicolumn{22}{|l|}{ V1M2 632011 P1 } \\
\hline \multicolumn{22}{|l|}{ V1M2 632040 P2 } \\
\hline \multicolumn{22}{|l|}{ V1M3 $632059 \mathrm{P} 1$} \\
\hline \multicolumn{22}{|l|}{ V1M3 632075 P2 } \\
\hline \multicolumn{22}{|l|}{ V1M4 632088 P1 } \\
\hline \multicolumn{22}{|l|}{ V1M4 632096 P2 } \\
\hline Vectra & 20.6 & 1190 & 12.6 & 1240 & 8.4 & 1240 & 8.4 & 1289 & 5.9 & 1290 & 6 & 1291 & 6.4 & 1344 & 4.4 & 1390 & 3.2 & & & & \\
\hline WSTC & 30.8 & 1090 & 31.3 & 1139 & 18.6 & 1139 & 18.8 & 1140 & 20.1 & 1188 & 12.1 & 1237 & 8.2 & 1238 & 8.6 & 1287 & 5.8 & 1337 & 4.1 & & \\
\hline
\end{tabular}

\section{SBW CVS Phase 1 (Scholes et al. 2002)}

\begin{tabular}{|l|l|l|l|l|l|l|l|l|l|}
\hline SBW1-01 & 9.9147 & 1004.2 & 20.96 & 954.51 & 37.227 & 1252.6 & 2.6951 & 1153.2 & 5.528 \\
\hline
\end{tabular}

\begin{tabular}{|l|l|l|l|l|l|l|l|l|l|}
\hline SBW1-02 & 10.474 & 1006.4 & 22.059 & 956.85 & 39.293 & 1255 & 2.6643 & 1155.9 & 5.6532 \\
\hline
\end{tabular}

\begin{tabular}{l|l|l|l|l|l|l|l|l|l} 
SBW1-03 & 24.098 & 1004.6 & 54.205 & 954.81 & 101.35 & 1253.7 & 5.8842 & 1153.9 & 12.574
\end{tabular}

\begin{tabular}{l|l|l|l|l|l|l|l|l|l|}
\hline SBW1-04 & 7.1934 & 1006.8 & 14.83 & 957.17 & 25.998 & 1255.3 & 2.012 & 1156.1 & 4.0156
\end{tabular} 
Appendix A. Database - mass fraction

Hanford LLW Glass Formulation (Feng et al. 1996)

\begin{tabular}{|c|c|c|c|c|c|c|c|c|c|c|c|c|c|c|c|c|}
\hline Glass ID & $\begin{array}{l}\mathrm{T} 13 \\
\left({ }^{\circ} \mathrm{C}\right)\end{array}$ & $\begin{array}{c}\text { V13 } \\
(\mathrm{Pa} \cdot \mathrm{s})\end{array}$ & $\begin{array}{l}\text { T14 } \\
\left({ }^{\circ} \mathrm{C}\right)\end{array}$ & $\begin{array}{c}\mathrm{V} 14 \\
(\mathrm{~Pa} \cdot \mathrm{s})\end{array}$ & $\begin{array}{c}\text { Q PCT } \\
\mathrm{B}\left(\mathrm{g} / \mathrm{m}^{2}\right)\end{array}$ & $\begin{array}{c}\text { Q PCT } \\
\mathrm{Li}\left(\mathrm{g} / \mathrm{m}^{2}\right)\end{array}$ & $\begin{array}{c}\text { Q PCT } \\
\mathrm{Na}\left(\mathrm{g} / \mathrm{m}^{2}\right)\end{array}$ & $\begin{array}{c}\text { Q PCT } \\
\mathrm{Si}\left(\mathrm{g} / \mathrm{m}^{2}\right)\end{array}$ & $\begin{array}{c}\text { Q PCT } \\
\text { pH }\end{array}$ & $\begin{array}{c}\text { CCC PCT } \\
\text { B }\left(\mathrm{g} / \mathrm{m}^{2}\right)\end{array}$ & $\begin{array}{l}\text { CCC PCT } \\
\mathrm{Li}\left(\mathrm{g} / \mathrm{m}^{2}\right)\end{array}$ & $\begin{array}{l}\text { CCC PCT } \\
\mathrm{Na}\left(\mathrm{g} / \mathrm{m}^{2}\right)\end{array}$ & $\begin{array}{l}\text { CCC PCT } \\
\mathrm{Si}\left(\mathrm{g} / \mathrm{m}^{2}\right)\end{array}$ & $\begin{array}{c}\text { CCC PCT } \\
\text { pH }\end{array}$ & $\begin{array}{l}\text { Q PCT B at } \\
20^{\circ} \mathrm{C}\left(\mathrm{g} / \mathrm{m}^{2}\right)\end{array}$ & $\begin{array}{l}\text { Q PCT Li at } \\
20^{\circ} \mathrm{C}\left(\mathrm{g} / \mathrm{m}^{2}\right)\end{array}$ \\
\hline L8-7 & & & & & 0.185 & & 0.35 & 0.14 & 10.985 & & & & & & & \\
\hline L8-8 & & & & & 1.435 & & 1.285 & 0.4 & 11.285 & & & & & & & \\
\hline LD4-912 & & & & & 0.3515 & & 0.3155 & 0.127 & 10.545 & & & & & & & \\
\hline LD5-912 & & & & & & & 1.2285 & 0.224 & 12.03 & & & & & & & \\
\hline LD6-5314 & & & & & 0.0985 & & 0.2565 & 0.09 & 11.05 & & & & & & & \\
\hline LD6-5412 & & & & & 0.112 & & 0.376 & 0.1045 & 11.385 & & & & & & & \\
\hline LD6-5510 & & & & & 0.1323333 & & 0.5176667 & 0.1196667 & 11.475 & & & & & & & \\
\hline LDM-912 & & & & & & & 1.197 & 0.2185 & 11.815 & & & & & & & \\
\hline LDM-1 & & & & & 0.1725 & & 0.365 & 0.131 & 11.12 & & & & & & & \\
\hline LDM-2 & & & & & & & 0.8445 & 0.1425 & 10.665 & & & & & & & \\
\hline LDM-3 & & & & & 0.1655 & & 0.2875 & 0.111 & 10.665 & & & & & & & \\
\hline LDM-4 & & & & & 0.2815 & & 0.463 & 0.129 & 10.945 & & & & & & & \\
\hline LDM-5412 & & & & & 0.126 & & 0.4045 & 0.109 & 10.945 & & & & & & & \\
\hline LDMS-1 & & & & & 0.154 & & 0.3055 & 0.1255 & 10.905 & & & & & & & \\
\hline LRM-912 & & & & & & & 1.0295 & 0.1845 & 11.73 & & & & & & & \\
\hline LRM-1 & & & & & 0.2045 & 0.105 & 0.462 & 0.154 & 11.225 & & & & & & & \\
\hline LRM-2 & & & & & & & 0.7615 & 0.185 & 11.495 & & & & & & & \\
\hline LRM-3 & & & & & 0.1635 & & 0.3585 & 0.118 & 10.855 & & & & & & & \\
\hline LRM-4 & & & & & 0.2865 & 0.134 & 0.47 & 0.1485 & 11.19 & & & & & & & \\
\hline LRM-5412 & & & & & 0.1535 & & 0.3905 & 0.1335 & 10.975 & & & & & & & \\
\hline LRMS-1 & & & & & 0.205 & 0.096 & 0.4445 & 0.159 & 11.23 & & & & & & & \\
\hline SSHTM-3 & & & & & 0.1735 & & 0.4385 & 0.1185 & 11.325 & & & & & & & \\
\hline B1G9-0IIC4 & & & & & 0.049 & & 0.125 & 0.05 & 10.365 & & & & & & & \\
\hline B1G9-013C5 & & & & & 0.0645 & & 0.1575 & 0.0515 & 10.62 & & & & & & & \\
\hline B1G9-014C & & & & & 0.045 & & 0.124 & 0.049 & 10.445 & & & & & & & \\
\hline D1G4-022P2 & & & & & 0.538 & & 0.62 & 0.1675 & 11.455 & & & & & & & \\
\hline D1G4-023P3 & & & & & 0.501 & & 0.608 & 0.1665 & 11.455 & & & & & & & \\
\hline \multicolumn{17}{|l|}{ Duratek } \\
\hline PEI & & & & & & & 1.696 & 0.2575 & 12.14 & & & & & & & \\
\hline M1G1-008P & & & & & 0.08 & & 0.22 & 0.06 & 10.535 & & & & & & & \\
\hline M1G1-011P & & & & & 0.1 & & 0.32 & 0.08 & 11.06 & & & & & & & \\
\hline V1M2 $632011 \mathrm{P} 1$ & & & & & 0.1335 & & 0.213 & 0.094 & 10.295 & & & & & & & \\
\hline V1M2 632040 P2 & & & & & 0.129 & & 0.1895 & 0.087 & 10.215 & & & & & & & \\
\hline V1M3 632059 P1 & & & & & 0.1465 & & 0.223 & 0.096 & 10.34 & & & & & & & \\
\hline V1M3 632075 P2 & & & & & 0.13 & & 0.262 & 0.0875 & 10.315 & & & & & & & \\
\hline V1M4 632088 P1 & & & & & 0.1415 & & 0.2115 & 0.0935 & 10.375 & & & & & & & \\
\hline V1M4 632096 P2 & & & & & 0.148 & & 0.2185 & 0.096 & 10.37 & & & & & & & \\
\hline Vectra & & & & & 0.4605 & & 0.5485 & 0.1845 & 11.115 & & & & & & & \\
\hline WSTC & & & & & 0.1513333 & 0.0793333 & 0.244 & 0.0846667 & 10.815 & & & & & & & \\
\hline
\end{tabular}

SBW CVS Phase 1 (Scholes et al. 2002)

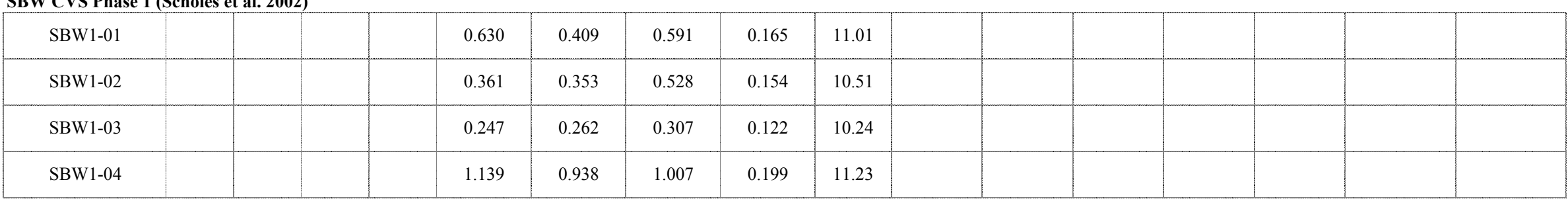


Appendix A. Database - mass fraction

Hanford LLW Glass Formulation (Feng et al. 1996)

\begin{tabular}{|c|c|c|c|c|c|c|c|c|c|c|c|c|}
\hline Glass ID & $\begin{array}{l}\text { Q PCT Na at } \\
20^{\circ} \mathrm{C}\left(\mathrm{g} / \mathrm{m}^{2}\right)\end{array}$ & $\begin{array}{l}\text { Q PCT Si at } \\
20^{\circ} \mathrm{C}\left(\mathrm{g} / \mathrm{m}^{2}\right)\end{array}$ & $\begin{array}{c}\mathrm{QpH} \\
\text { at } 20^{\circ} \mathrm{C}\end{array}$ & $\begin{array}{c}\text { TCLP Ag } \\
(\mathrm{ppm})\end{array}$ & $\begin{array}{l}\text { TCLP As } \\
\text { (ppm) }\end{array}$ & $\begin{array}{c}\text { TCLP Ba } \\
(\mathrm{ppm})\end{array}$ & $\begin{array}{c}\text { TCLP Cd } \\
\text { (ppm) }\end{array}$ & $\begin{array}{c}\text { TCLP Cr } \\
(\mathrm{ppm})\end{array}$ & $\begin{array}{c}\text { TCLP Ni } \\
(\mathrm{ppm})\end{array}$ & $\begin{array}{c}\text { TCLP Pb } \\
(\mathrm{ppm})\end{array}$ & $\begin{array}{c}\text { TCLP Se } \\
(\mathrm{ppm})\end{array}$ & $\begin{array}{c}\text { TCLP Zn } \\
\text { (ppm) }\end{array}$ \\
\hline \multicolumn{13}{|c|}{ 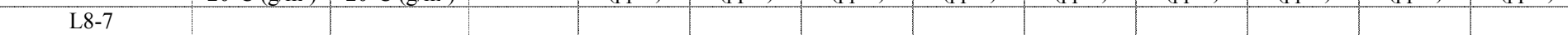 } \\
\hline \multicolumn{13}{|l|}{ L8-8 } \\
\hline \multicolumn{13}{|l|}{ LD4-912 } \\
\hline \multicolumn{13}{|l|}{ LD5-912 } \\
\hline \multicolumn{13}{|l|}{ LD6-5314 } \\
\hline \multicolumn{13}{|l|}{ LD6-5412 } \\
\hline \multicolumn{13}{|l|}{ LD6-5510 } \\
\hline \multicolumn{13}{|l|}{ LDM-912 } \\
\hline \multicolumn{13}{|l|}{ LDM-1 } \\
\hline \multicolumn{13}{|l|}{ LDM-2 } \\
\hline \multicolumn{13}{|l|}{ LDM-3 } \\
\hline \multicolumn{13}{|l|}{ LDM-4 } \\
\hline \multicolumn{13}{|l|}{ LDM-5412 } \\
\hline \multicolumn{13}{|l|}{ LDMS-1 } \\
\hline \multicolumn{13}{|l|}{ LRM-912 } \\
\hline \multicolumn{13}{|l|}{ LRM-1 } \\
\hline \multicolumn{13}{|l|}{ LRM-2 } \\
\hline \multicolumn{13}{|l|}{ LRM-3 } \\
\hline \multicolumn{13}{|l|}{ LRM-4 } \\
\hline \multicolumn{13}{|l|}{ LRM-5412 } \\
\hline \multicolumn{13}{|l|}{ LRMS-1 } \\
\hline \multicolumn{13}{|l|}{ SSHTM-3 } \\
\hline \multicolumn{13}{|l|}{ B1G9-0IIC4 } \\
\hline B1G9-013C5 & & & & & & & & & & & & \\
\hline B1G9-014C & & & & & & & & & & & & \\
\hline $\mathrm{D} 1 \mathrm{G} 4-022 \mathrm{P} 2$ & & & & & & & & & & & & \\
\hline $\mathrm{D} 1 \mathrm{G} 4-023 \mathrm{P} 3$ & & & & & & & & & & & & \\
\hline Duratek & & & & & & & & & & & & \\
\hline PEI & & & & & & & & & & & & \\
\hline M1G1-008P & & & & & & & & & & & & \\
\hline M1G1-011P & & & & & & & & & & & & \\
\hline V1M2 632011 P1 & & & & & & & & & & & & \\
\hline V1M2 $632040 \mathrm{P} 2$ & & & & & & & & & & & & \\
\hline V1M3 $632059 \mathrm{P1}$ & & & & & & & & & & & & \\
\hline V1M3 632075 P2 & & & & & & & & & & & & \\
\hline V1M4 632088 P1 & & & & & & & & & & & & \\
\hline V1M4 632096 P2 & & & & & & & & & & & & \\
\hline Vectra & & & & & & & & & & & & \\
\hline WSTC & & & & & & & & & & & & \\
\hline
\end{tabular}

SBW CVS Phase 1 (Scholes et al. 2002)

\begin{tabular}{|c|c|c|c|c|c|c|c|c|c|}
\hline SBW1-01 & & & & & & & & & \\
\hline SBW1-02 & & & & & & & & \\
\hline SBW1-03 & & & & & & & & & \\
\hline SBW1-04 & & & & & & & & \\
\hline
\end{tabular}


Appendix A. Database - mass fraction

SBW CVS Phase 1 (Scholes et al. 2002)

\begin{tabular}{|c|c|c|c|c|c|c|c|c|c|c|c|c|c|c|c|c|c|c|c|c|c|}
\hline Glass ID & $\begin{array}{c}\mathrm{A} 12 \mathrm{O} 3 \\
-\mathrm{t}\end{array}$ & $\begin{array}{c}\mathrm{B} 2 \mathrm{O} 3 \\
-\mathrm{t}\end{array}$ & $\begin{array}{c}\mathrm{CaO} \\
-\mathrm{t}\end{array}$ & $\begin{array}{c}\mathrm{Fe} 2 \mathrm{O} 3 \\
-\mathrm{t}\end{array}$ & $\begin{array}{c}\mathrm{FeO} \\
-\mathrm{t}\end{array}$ & $\begin{array}{c}\mathrm{K} 2 \mathrm{O} \\
-\mathrm{t}\end{array}$ & $\begin{array}{c}\mathrm{Li} 2 \mathrm{O} \\
-\mathrm{t}\end{array}$ & $\begin{array}{c}\mathrm{MgO} \\
-\mathrm{t}\end{array}$ & $\begin{array}{c}\mathrm{Na} 2 \mathrm{O} \\
-\mathrm{t}\end{array}$ & $\begin{array}{c}\mathrm{NiO} \\
-\mathrm{t}\end{array}$ & $\begin{array}{c}\mathrm{P} 2 \mathrm{O} 5 \\
-\mathrm{t}\end{array}$ & $\begin{array}{c}\mathrm{SiO} 2 \\
-\mathrm{t}\end{array}$ & $\begin{array}{c}\mathrm{ZrO} 2 \\
-\mathrm{t}\end{array}$ & $\begin{array}{c}\mathrm{Ag} 2 \mathrm{O} \\
-\mathrm{t}\end{array}$ & $\begin{array}{c}\mathrm{As} 2 \mathrm{O} 3 \\
-\mathrm{t}\end{array}$ & $\begin{array}{c}\mathrm{BaO} \\
-\mathrm{t}\end{array}$ & $\begin{array}{c}\mathrm{Bi} 2 \mathrm{O} 3 \\
-\mathrm{t}\end{array}$ & $\begin{array}{l}\mathrm{Br} \\
-\mathrm{t}\end{array}$ & $\begin{array}{c}\mathrm{CdO} \\
-\mathrm{t}\end{array}$ & $\begin{array}{c}\mathrm{Ce} 2 \mathrm{O} 3 \\
-\mathrm{t}\end{array}$ & $\begin{array}{c}\mathrm{CeO} 2 \\
-\mathrm{t}\end{array}$ \\
\hline SBW1-05 & 0.1270 & 0.0900 & 0.0218 & 0.0412 & & 0.0278 & 0.0300 & 0.0000 & 0.1408 & 0.0008 & 0.0084 & 0.4548 & 0.0080 & & & 0.0258 & & & 0.0020 & & \\
\hline SBW1-06 & 0.1171 & 0.0900 & 0.0218 & 0.0608 & & 0.0362 & 0.0200 & 0.0000 & 0.1408 & 0.0008 & 0.0084 & 0.4548 & 0.0080 & & & 0.0200 & & & 0.0030 & & \\
\hline SBW1-07 & 0.0980 & 0.0900 & 0.0218 & 0.0608 & & 0.0362 & 0.0200 & 0.0000 & 0.1432 & 0.0008 & 0.0122 & 0.4548 & 0.0120 & & & 0.0282 & & & 0.0020 & & \\
\hline SBW1-08 & 0.1270 & 0.0904 & 0.0300 & 0.0412 & & 0.0278 & 0.0200 & 0.0000 & 0.1408 & 0.0008 & 0.0122 & 0.4548 & 0.0120 & & & 0.0200 & & & 0.0030 & & \\
\hline SBW1-09 & 0.0980 & 0.1100 & 0.0218 & 0.0412 & & 0.0278 & 0.0300 & 0.0000 & 0.1408 & 0.0008 & 0.0122 & 0.4559 & 0.0120 & & & 0.0282 & & & 0.0030 & & \\
\hline SBW1-10 & 0.0980 & 0.0900 & 0.0300 & 0.0412 & & 0.0362 & 0.0300 & 0.0000 & 0.1583 & 0.0008 & 0.0084 & 0.4548 & 0.0120 & & & 0.0200 & & & 0.0020 & & \\
\hline SBW1-11 & 0.1029 & 0.1100 & 0.0218 & 0.0412 & & 0.0362 & 0.0200 & 0.0000 & 0.1408 & 0.0008 & 0.0084 & 0.4548 & 0.0120 & & & 0.0282 & & & 0.0030 & & \\
\hline SBW1-12 & 0.0980 & 0.0900 & 0.0300 & 0.0412 & & 0.0362 & 0.0200 & 0.0000 & 0.1408 & 0.0008 & 0.0122 & 0.4782 & 0.0080 & & & 0.0200 & & & 0.0030 & & \\
\hline SBW1-13 & 0.0980 & 0.0900 & 0.0218 & 0.0608 & & 0.0278 & 0.0300 & 0.0000 & 0.1447 & 0.0008 & 0.0122 & 0.4548 & 0.0080 & & & 0.0282 & & & 0.0030 & & \\
\hline SBW1-14 & 0.0980 & 0.0908 & 0.0218 & 0.0412 & & 0.0278 & 0.0200 & 0.0000 & 0.1772 & 0.0008 & 0.0084 & 0.4548 & 0.0080 & & & 0.0282 & & & 0.0030 & & \\
\hline SBW1-15 & 0.0980 & 0.1100 & 0.0300 & 0.0595 & & 0.0278 & 0.0200 & 0.0000 & 0.1408 & 0.0008 & 0.0084 & 0.4548 & 0.0080 & & & 0.0200 & & & 0.0020 & & \\
\hline SBW1-16 & 0.0690 & 0.1300 & 0.0124 & 0.0216 & & 0.0194 & 0.0400 & 0.0000 & 0.1044 & 0.0008 & 0.0045 & 0.5550 & 0.0040 & & & 0.0100 & & & 0.0040 & & \\
\hline SBW1-17 & 0.0690 & 0.0700 & 0.0400 & 0.0804 & & 0.0194 & 0.0400 & 0.0000 & 0.1044 & 0.0008 & 0.0161 & 0.5299 & 0.0040 & & & 0.0100 & & & 0.0010 & & \\
\hline SBW1-18 & 0.1560 & 0.0700 & 0.0400 & 0.0804 & & 0.0446 & 0.0400 & 0.0000 & 0.1044 & 0.0008 & 0.0045 & 0.4215 & 0.0040 & & & 0.0100 & & & 0.0040 & & \\
\hline SBW1-19 & 0.0690 & 0.0700 & 0.0124 & 0.0216 & & 0.0446 & 0.0100 & 0.0000 & 0.1954 & 0.0008 & 0.0161 & 0.5253 & 0.0040 & & & 0.0100 & & & 0.0010 & & \\
\hline SBW1-20 & 0.0690 & 0.0700 & 0.0400 & 0.0804 & & 0.0194 & 0.0170 & 0.0000 & 0.2136 & 0.0008 & 0.0045 & 0.4304 & 0.0160 & & & 0.0100 & & & 0.0040 & & \\
\hline SBW1-21 & 0.1560 & 0.0700 & 0.0124 & 0.0804 & & 0.0194 & 0.0400 & 0.0000 & 0.1276 & 0.0008 & 0.0161 & 0.4254 & 0.0160 & & & 0.0100 & & & 0.0010 & & \\
\hline SBW1-22 & 0.1560 & 0.0700 & 0.0124 & 0.0216 & & 0.0446 & 0.0400 & 0.0000 & 0.1484 & 0.0008 & 0.0045 & 0.4567 & 0.0160 & & & 0.0100 & & & 0.0040 & & \\
\hline SBW1-23 & 0.1110 & 0.0700 & 0.0124 & 0.0216 & & 0.0194 & 0.0400 & 0.0000 & 0.1906 & 0.0008 & 0.0161 & 0.4524 & 0.0040 & & & 0.0376 & & & 0.0040 & & \\
\hline
\end{tabular}


Appendix A. Database - mass fraction

SBW CVS Phase 1 (Scholes et al. 2002)

\begin{tabular}{|c|c|c|c|c|c|c|c|c|c|c|c|c|c|c|c|c|c|c|c|c|c|}
\hline Glass ID & $\begin{array}{l}\mathrm{Cl} \\
-\mathrm{t} \\
\end{array}$ & $\begin{array}{c}\mathrm{CoO} \\
-\mathrm{t} \\
\end{array}$ & $\begin{array}{c}\mathrm{Co} 2 \mathrm{O} 3 \\
-\mathrm{t}\end{array}$ & $\begin{array}{c}\mathrm{Cr} 2 \mathrm{O} 3 \\
-\mathrm{t} \\
\end{array}$ & $\begin{array}{c}\mathrm{Cs} 2 \mathrm{O} \\
-\mathrm{t}\end{array}$ & $\begin{array}{c}\mathrm{CuO} \\
-\mathrm{t}\end{array}$ & $\begin{array}{c}\mathrm{Eu} 2 \mathrm{O} 3 \\
-\mathrm{t}\end{array}$ & $\begin{array}{l}F \\
-t\end{array}$ & $\begin{array}{c}\mathrm{Ga} 2 \mathrm{O} 3 \\
-\mathrm{t}\end{array}$ & $\begin{array}{c}\mathrm{Gd} 2 \mathrm{O} 3 \\
-\mathrm{t}\end{array}$ & $\begin{array}{c}\mathrm{HgO} \\
-\mathrm{t}\end{array}$ & $\begin{array}{l}I \\
-t\end{array}$ & $\begin{array}{c}\mathrm{La} 2 \mathrm{O} 3 \\
-\mathrm{t}\end{array}$ & $\begin{array}{c}\mathrm{MnO} 2 \\
-\mathrm{t}\end{array}$ & $\begin{array}{c}\mathrm{MnO} \\
-\mathrm{t}\end{array}$ & $\begin{array}{c}\mathrm{MoO} \\
-\mathrm{t}\end{array}$ & $\begin{array}{c}\mathrm{MoO} 3 \\
-\mathrm{t}\end{array}$ & $\begin{array}{c}\mathrm{Nb} 2 \mathrm{O} 5 \\
-\mathrm{t}\end{array}$ & $\begin{array}{c}\mathrm{Nd} 2 \mathrm{O} 3 \\
-\mathrm{t}\end{array}$ & $\begin{array}{c}\mathrm{PbO} \\
-\mathrm{t}\end{array}$ & $\begin{array}{c}\mathrm{PdO} 2 \\
-\mathrm{t}\end{array}$ \\
\hline SBW1-05 & 0.0015 & & & 0.0013 & & & & 0.0058 & & & & 0.0001 & 0.0003 & & 0.0066 & & 0.0002 & & & 0.0007 & \\
\hline SBW1-06 & 0.0015 & & & 0.0013 & & & & 0.0042 & & & & 0.0001 & 0.0003 & & 0.0049 & & 0.0002 & & & 0.0007 & \\
\hline SBW1-07 & 0.0015 & & & 0.0013 & & & & 0.0042 & & & & 0.0001 & 0.0003 & & 0.0066 & & 0.0002 & & & 0.0007 & \\
\hline SBW1-08 & 0.0015 & & & 0.0013 & & & & 0.0042 & & & & 0.0001 & 0.0003 & & 0.0066 & & 0.0002 & & & 0.0007 & \\
\hline SBW1-09 & 0.0015 & & & 0.0013 & & & & 0.0042 & & & & 0.0001 & 0.0003 & & 0.0049 & & 0.0002 & & & 0.0007 & \\
\hline SBW1-10 & 0.0015 & & & 0.0013 & & & & 0.0042 & & & & 0.0001 & 0.0003 & & 0.0049 & & 0.0002 & & & 0.0007 & \\
\hline SBW1-11 & 0.0015 & & & 0.0013 & & & & 0.0058 & & & & 0.0001 & 0.0003 & & 0.0049 & & 0.0002 & & & 0.0007 & \\
\hline SBW1-12 & 0.0015 & & & 0.0013 & & & & 0.0058 & & & & 0.0001 & 0.0003 & & 0.0066 & & 0.0002 & & & 0.0007 & \\
\hline SBW1-13 & 0.0015 & & & 0.0013 & & & & 0.0058 & & & & 0.0001 & 0.0003 & & 0.0049 & & 0.0002 & & & 0.0007 & \\
\hline SBW1-14 & 0.0015 & & & 0.0013 & & & & 0.0042 & & & & 0.0001 & 0.0003 & & 0.0066 & & 0.0002 & & & 0.0007 & \\
\hline SBW1-15 & 0.0015 & & & 0.0013 & & & & 0.0058 & & & & 0.0001 & 0.0003 & & 0.0049 & & 0.0002 & & & 0.0007 & \\
\hline SBW1-16 & 0.0015 & & & 0.0013 & & & & 0.0074 & & & & 0.0001 & 0.0003 & & 0.0083 & & 0.0002 & & & 0.0007 & \\
\hline SBW1-17 & 0.0015 & & & 0.0013 & & & & 0.0026 & & & & 0.0001 & 0.0003 & & 0.0032 & & 0.0002 & & & 0.0007 & \\
\hline SBW1-18 & 0.0015 & & & 0.0013 & & & & 0.0074 & & & & 0.0001 & 0.0003 & & 0.0032 & & 0.0002 & & & 0.0007 & \\
\hline SBW1-19 & 0.0015 & & & 0.0013 & & & & 0.0074 & & & & 0.0001 & 0.0003 & & 0.0032 & & 0.0002 & & & 0.0007 & \\
\hline SBW1-20 & 0.0015 & & & 0.0013 & & & & 0.0074 & & & & 0.0001 & 0.0003 & & 0.0083 & & 0.0002 & & & 0.0007 & \\
\hline SBW1-21 & 0.0015 & & & 0.0013 & & & & 0.0074 & & & & 0.0001 & 0.0003 & & 0.0083 & & 0.0002 & & & 0.0007 & \\
\hline SBW1-22 & 0.0015 & & & 0.0013 & & & & 0.0026 & & & & 0.0001 & 0.0003 & & 0.0032 & & 0.0002 & & & 0.0007 & \\
\hline SBW1-23 & 0.0015 & & & 0.0013 & & & & 0.0026 & & & & 0.0001 & 0.0003 & & 0.0083 & & 0.0002 & & & 0.0007 & \\
\hline
\end{tabular}


Appendix A. Database - mass fraction

SBW CVS Phase 1 (Scholes et al. 2002)

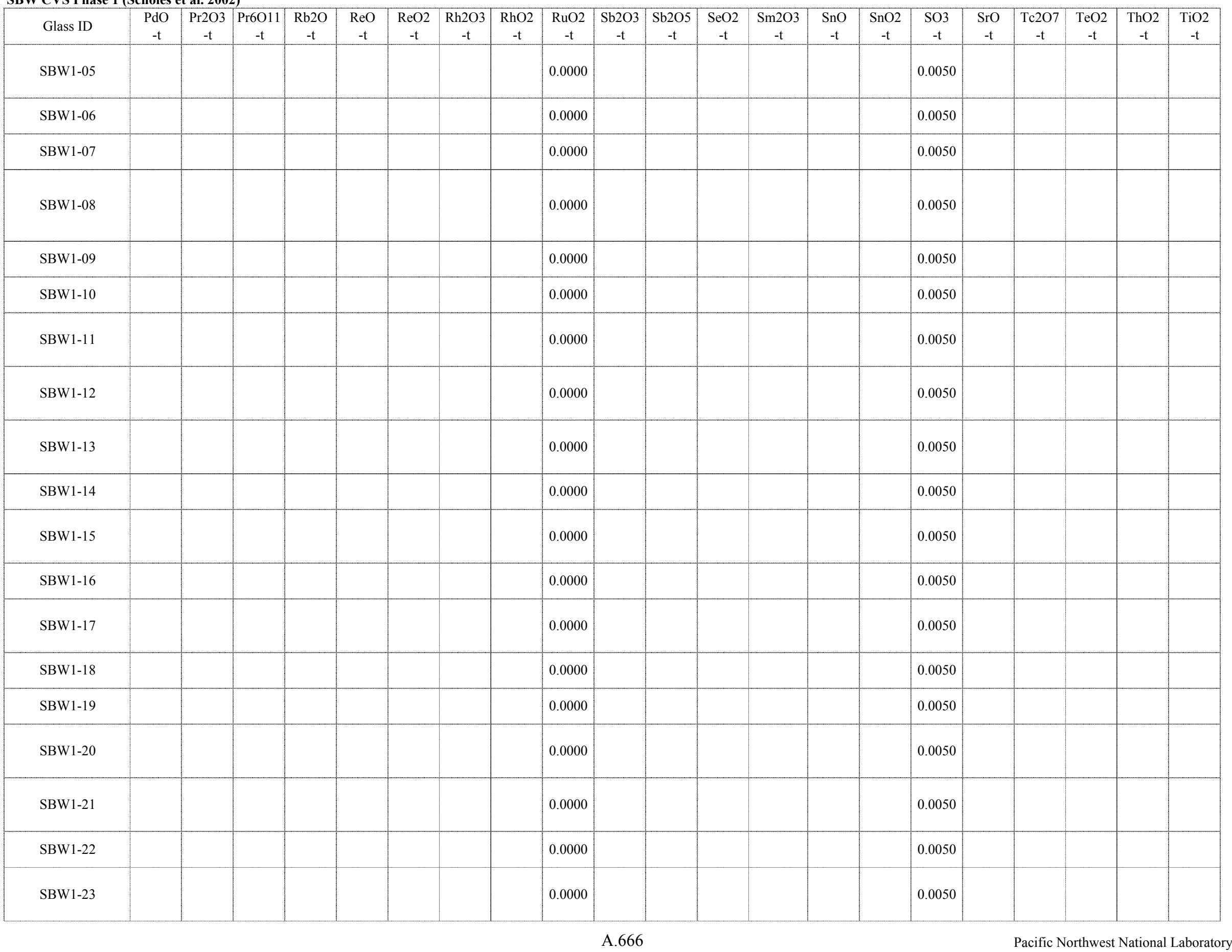


Appendix A. Database - mass fraction

SBW CVS Phase 1 (Scholes et al. 2002)

\begin{tabular}{|c|c|c|c|c|c|c|c|c|c|c|c|c|c|c|c|c|c|c|c|c|c|}
\hline Glass ID & $\begin{array}{c}\mathrm{T} 12 \mathrm{O} 3 \\
-\mathrm{t} \\
\end{array}$ & $\begin{array}{c}\mathrm{U} 3 \mathrm{O} 8 \\
-\mathrm{t}\end{array}$ & $\begin{array}{c}\mathrm{UO} 2 \\
-\mathrm{t} \\
\end{array}$ & $\begin{array}{c}\text { UO3 } \\
-t \\
\end{array}$ & $\begin{array}{c}\mathrm{V} 2 \mathrm{O} 5 \\
-\mathrm{t}\end{array}$ & $\begin{array}{c}\text { WO3 } \\
-t \\
\end{array}$ & $\begin{array}{c}\mathrm{Y} 2 \mathrm{O} 3 \\
-\mathrm{t}\end{array}$ & $\begin{array}{c}\mathrm{ZnO} \\
-\mathrm{t}\end{array}$ & $\begin{array}{c}\text { Others } \\
-t\end{array}$ & $\begin{array}{c}\text { Sum } \\
-t\end{array}$ & $\begin{array}{c}\mathrm{A} 12 \mathrm{O} 3 \\
-\mathrm{a} \\
\end{array}$ & $\begin{array}{c}\mathrm{B} 2 \mathrm{O} 3 \\
-\mathrm{a} \\
\end{array}$ & $\begin{array}{c}\mathrm{CaO} \\
-\mathrm{a} \\
\end{array}$ & $\begin{array}{c}\mathrm{Fe} 2 \mathrm{O} 3 \\
-\mathrm{a} \\
\end{array}$ & $\begin{array}{c}\mathrm{FeO} \\
-\mathrm{a} \\
\end{array}$ & $\begin{array}{c}\mathrm{K} 2 \mathrm{O} \\
-\mathrm{a} \\
\end{array}$ & $\begin{array}{c}\mathrm{Li} 2 \mathrm{O} \\
-\mathrm{a} \\
\end{array}$ & $\begin{array}{c}\mathrm{MgO} \\
-\mathrm{a} \\
\end{array}$ & $\begin{array}{c}\mathrm{Na} 2 \mathrm{O} \\
-\mathrm{a} \\
\end{array}$ & $\begin{array}{c}\mathrm{NiO} \\
-\mathrm{a}\end{array}$ & $\begin{array}{c}\mathrm{P} 2 \mathrm{O} 5 \\
-\mathrm{a} \\
\end{array}$ \\
\hline SBW1-05 & & & & & & & & & & 1.0000 & 0.1293 & 0.0868 & 0.0226 & 0.0420 & & 0.0277 & 0.0289 & & 0.1421 & 0.0009 & 0.0071 \\
\hline SBW1-06 & & & & & & & & & & 1.0000 & 0.1181 & 0.0895 & 0.0220 & 0.0617 & & 0.0357 & 0.0193 & & 0.1409 & 0.0009 & 0.0079 \\
\hline SBW1-07 & & & & & & & & & & 1.0000 & 0.0976 & 0.0906 & 0.0219 & 0.0602 & & 0.0353 & 0.0194 & & 0.1449 & 0.0009 & 0.0108 \\
\hline SBW1-08 & & & & & & & & & & 1.0000 & 0.1299 & 0.0873 & 0.0306 & 0.0415 & & 0.0273 & 0.0190 & & 0.1439 & 0.0011 & 0.0103 \\
\hline SBW1-09 & & & & & & & & & & 1.0000 & 0.1013 & 0.1092 & 0.0222 & 0.0418 & & 0.0281 & 0.0295 & & 0.1432 & 0.0009 & 0.0112 \\
\hline SBW1-10 & & & & & & & & & & 1.0000 & 0.0991 & 0.0878 & 0.0297 & 0.0411 & & 0.0349 & 0.0287 & & 0.1555 & 0.0009 & 0.0081 \\
\hline SBW1-11 & & & & & & & & & & 1.0000 & 0.1093 & 0.1120 & 0.0230 & 0.0426 & & 0.0366 & 0.0202 & & 0.1440 & 0.0009 & 0.0082 \\
\hline SBW1-12 & & & & & & & & & & 1.0000 & 0.0993 & 0.0901 & 0.0300 & 0.0415 & & 0.0351 & 0.0196 & & 0.1400 & 0.0009 & 0.0099 \\
\hline SBW1-13 & & & & & & & & & & 1.0000 & 0.1018 & 0.0905 & 0.0222 & 0.0611 & & 0.0273 & 0.0293 & & 0.1467 & 0.0009 & 0.0108 \\
\hline SBW1-14 & & & & & & & & & & 1.0000 & 0.1004 & 0.0887 & 0.0222 & 0.0422 & & 0.0272 & 0.0194 & & 0.1760 & 0.0009 & 0.0081 \\
\hline SBW1-15 & & & & & & & & & & 1.0000 & 0.0986 & 0.1087 & 0.0300 & 0.0591 & & 0.0266 & 0.0194 & & 0.1383 & 0.0009 & 0.0082 \\
\hline SBW1-16 & & & & & & & & & & 1.0000 & 0.0719 & 0.1262 & 0.0128 & 0.0218 & & 0.0191 & 0.0387 & & 0.1052 & 0.0009 & 0.0046 \\
\hline SBW1-17 & & & & & & & & & & 1.0000 & 0.0725 & 0.0688 & 0.0407 & 0.0796 & & 0.0191 & 0.0392 & & 0.1060 & 0.0009 & 0.0163 \\
\hline SBW1-18 & & & & & & & & & & 1.0000 & 0.1602 & 0.0712 & 0.0396 & 0.0792 & & 0.0437 & 0.0391 & & 0.1055 & 0.0008 & 0.0044 \\
\hline SBW1-19 & & & & & & & & & & 1.0000 & 0.0703 & 0.0695 & 0.0124 & 0.0219 & & 0.0435 & 0.0096 & & 0.1927 & 0.0008 & 0.0146 \\
\hline SBW1-20 & & & & & & & & & & 1.0000 & 0.0727 & 0.0696 & 0.0402 & 0.0795 & & 0.0191 & 0.0166 & & 0.2186 & 0.0010 & 0.0048 \\
\hline SBW1-21 & & & & & & & & & & 1.0000 & 0.1579 & 0.0691 & 0.0129 & 0.0793 & & 0.0188 & 0.0386 & & 0.1259 & 0.0011 & 0.0143 \\
\hline SBW1-22 & & & & & & & & & & 1.0000 & 0.1593 & 0.0682 & 0.0130 & 0.0219 & & 0.0433 & 0.0376 & & 0.1491 & 0.0009 & 0.0048 \\
\hline SBW1-23 & & & & & & & & & & 1.0000 & 0.1139 & 0.0717 & 0.0128 & 0.0221 & & 0.0193 & 0.0396 & & 0.1923 & 0.0009 & 0.0151 \\
\hline
\end{tabular}


Appendix A. Database - mass fraction

SBW CVS Phase 1 (Scholes et al. 2002)

\begin{tabular}{|c|c|c|c|c|c|c|c|c|c|c|c|c|c|c|c|c|c|c|c|c|c|}
\hline Glass ID & $\begin{array}{c}\mathrm{SiO} 2 \\
-\mathrm{a} \\
\end{array}$ & $\begin{array}{c}\mathrm{ZrO} 2 \\
-\mathrm{a}\end{array}$ & $\begin{array}{c}\mathrm{Ag} 2 \mathrm{O} \\
-\mathrm{a}\end{array}$ & $\begin{array}{c}\mathrm{As} 2 \mathrm{O} 3 \\
-\mathrm{a} \\
\end{array}$ & $\begin{array}{c}\mathrm{BaO} \\
-\mathrm{a}\end{array}$ & $\begin{array}{c}\mathrm{Bi} 2 \mathrm{O} 3 \\
-\mathrm{a} \\
\end{array}$ & $\begin{array}{l}\mathrm{Br} \\
-\mathrm{a}\end{array}$ & $\begin{array}{c}\mathrm{CdO} \\
-\mathrm{a}\end{array}$ & $\begin{array}{c}\mathrm{Ce} 2 \mathrm{O} 3 \\
-\mathrm{a} \\
\end{array}$ & $\begin{array}{c}\mathrm{CeO} 2 \\
-\mathrm{a}\end{array}$ & $\begin{array}{l}\mathrm{Cl} \\
-\mathrm{a} \\
\end{array}$ & $\begin{array}{c}\mathrm{CoO} \\
-\mathrm{a} \\
\end{array}$ & $\begin{array}{c}\mathrm{Co} 2 \mathrm{O} 3 \\
-\mathrm{a} \\
\end{array}$ & $\begin{array}{c}\mathrm{Cr} 2 \mathrm{O} 3 \\
-\mathrm{a}\end{array}$ & $\begin{array}{c}\mathrm{Cs} 2 \mathrm{O} \\
-\mathrm{a} \\
\end{array}$ & $\begin{array}{c}\mathrm{CuO} \\
-\mathrm{a} \\
\end{array}$ & $\begin{array}{c}\mathrm{Eu} 2 \mathrm{O} 3 \\
-\mathrm{a} \\
\end{array}$ & $\begin{array}{c}\mathrm{F} \\
-\mathrm{a} \\
\end{array}$ & $\begin{array}{c}\mathrm{Ga} 2 \mathrm{O} 3 \\
-\mathrm{a}\end{array}$ & $\begin{array}{c}\mathrm{Gd} 2 \mathrm{O} 3 \\
-\mathrm{a} \\
\end{array}$ & $\begin{array}{c}\mathrm{HgO} \\
-\mathrm{a}\end{array}$ \\
\hline SBW1-05 & 0.4427 & 0.0082 & & & 0.0268 & & & 0.0024 & & & 0.0013 & & & 0.0011 & & & & 0.0048 & & & \\
\hline SBW1-06 & 0.4515 & 0.0073 & & & 0.0206 & & & 0.0035 & & & 0.0012 & & & 0.0011 & & & & 0.0038 & & & \\
\hline SBW1-07 & 0.4867 & 0.0112 & & & 0.0285 & & & 0.0023 & & & 0.0014 & & & 0.0011 & & & & 0.0036 & & & \\
\hline SBW1-08 & 0.4340 & 0.0112 & & & 0.0203 & & & 0.0036 & & & 0.0014 & & & 0.0011 & & & & 0.0036 & & & \\
\hline SBW1-09 & 0.4512 & 0.0112 & & & 0.0286 & & & 0.0035 & & & 0.0014 & & & 0.0011 & & & & 0.0037 & & & \\
\hline SBW1-10 & 0.4396 & 0.0116 & & & 0.0199 & & & 0.0023 & & & 0.0012 & & & 0.0011 & & & & 0.0037 & & & \\
\hline SBW1-11 & 0.4621 & 0.0116 & & & 0.0293 & & & 0.0035 & & & 0.0013 & & & 0.0011 & & & & 0.0051 & & & \\
\hline SBW1-12 & 0.4672 & 0.0082 & & & 0.0202 & & & 0.0035 & & & 0.0007 & & & 0.0011 & & & & 0.0065 & & & \\
\hline SBW1-13 & 0.4479 & 0.0061 & & & 0.0287 & & & 0.0036 & & & 0.0013 & & & 0.0011 & & & & 0.0050 & & & \\
\hline SBW1-14 & 0.4406 & 0.0072 & & & 0.0293 & & & 0.0036 & & & 0.0012 & & & 0.0011 & & & & 0.0038 & & & \\
\hline SBW1-15 & 0.4444 & 0.0082 & & & 0.0200 & & & 0.0024 & & & 0.0013 & & & 0.0011 & & & & 0.0048 & & & \\
\hline SBW1-16 & 0.5334 & 0.0041 & & & 0.0104 & & & 0.0047 & & & 0.0015 & & & 0.0011 & & & & 0.0062 & & & \\
\hline SBW1-17 & 0.5132 & 0.0038 & & & 0.0104 & & & 0.0012 & & & 0.0014 & & & 0.0011 & & & & 0.0029 & & & \\
\hline SBW1-18 & 0.4206 & 0.0038 & & & 0.0104 & & & 0.0046 & & & 0.0012 & & & 0.0011 & & & & 0.0063 & & & \\
\hline SBW1-19 & 0.5199 & 0.0037 & & & 0.0102 & & & 0.0012 & & & 0.0012 & & & 0.0010 & & & & 0.0064 & & & \\
\hline SBW1-20 & 0.4305 & 0.0166 & & & 0.0102 & & & 0.0047 & & & 0.0012 & & & 0.0011 & & & & 0.0062 & & & \\
\hline SBW1-21 & 0.4137 & 0.0156 & & & 0.0105 & & & 0.0013 & & & 0.0011 & & & 0.0011 & & & & 0.0061 & & & \\
\hline SBW1-22 & 0.4305 & 0.0163 & & & 0.0102 & & & 0.0048 & & & 0.0011 & & & 0.0011 & & & & 0.0025 & & & \\
\hline SBW1-23 & 0.4576 & 0.0039 & & & 0.0387 & & & 0.0047 & & & 0.0013 & & & 0.0010 & & & & 0.0026 & & & \\
\hline
\end{tabular}


Appendix A. Database - mass fraction

SBW CVS Phase 1 (Scholes et al. 2002)

\begin{tabular}{|c|c|c|c|c|c|c|c|c|c|c|c|c|c|c|c|c|c|c|c|c|c|}
\hline Glass ID & $\begin{array}{c}\text { I } \\
-\mathrm{a}\end{array}$ & $\begin{array}{c}\mathrm{La} 2 \mathrm{O} 3 \\
-\mathrm{a}\end{array}$ & $\begin{array}{c}\mathrm{MnO} 2 \\
-\mathrm{a}\end{array}$ & $\begin{array}{c}\mathrm{MnO} \\
-\mathrm{a}\end{array}$ & $\begin{array}{c}\mathrm{MoO} \\
-\mathrm{a}\end{array}$ & $\begin{array}{c}\mathrm{MoO} 3 \\
-\mathrm{a}\end{array}$ & $\begin{array}{c}\mathrm{Nb} 2 \mathrm{O} 5 \\
-\mathrm{a}\end{array}$ & $\begin{array}{c}\mathrm{Nd} 2 \mathrm{O} 3 \\
-\mathrm{a}\end{array}$ & $\begin{array}{c}\mathrm{PbO} \\
-\mathrm{a}\end{array}$ & $\begin{array}{c}\mathrm{PdO} 2 \\
-\mathrm{a}\end{array}$ & $\begin{array}{c}\mathrm{PdO} \\
-\mathrm{a}\end{array}$ & $\begin{array}{c}\mathrm{Pr} 2 \mathrm{O} 3 \\
-\mathrm{a}\end{array}$ & \begin{tabular}{|c|} 
Pr6O11 \\
$-\mathrm{a}$
\end{tabular} & $\begin{array}{c}\mathrm{Rb} 2 \mathrm{O} \\
-\mathrm{a}\end{array}$ & $\begin{array}{c}\mathrm{ReO} \\
-\mathrm{a}\end{array}$ & $\begin{array}{c}\mathrm{ReO} 2 \\
-\mathrm{a}\end{array}$ & $\begin{array}{c}\mathrm{Rh} 2 \mathrm{O} 3 \\
-\mathrm{a}\end{array}$ & $\begin{array}{c}\mathrm{RhO} 2 \\
-\mathrm{a}\end{array}$ & $\begin{array}{c}\mathrm{RuO} 2 \\
-\mathrm{a}\end{array}$ & $\begin{array}{c}\mathrm{Sb} 2 \mathrm{O} 3 \\
-\mathrm{a}\end{array}$ & $\begin{array}{c}\mathrm{Sb} 2 \mathrm{O} 5 \\
-\mathrm{a}\end{array}$ \\
\hline SBW1-05 & & & & 0.0047 & & & & & & & & & & & & & & & & & \\
\hline SBW1-06 & & & & 0.0035 & & & & & & & & & & & & & & & & & \\
\hline SBW1-07 & & & & 0.0046 & & & & & & & & & & & & & & & & & \\
\hline SBW1-08 & & & & 0.0047 & & & & & & & & & & & & & & & & & \\
\hline SBW1-09 & & & & 0.0035 & & & & & & & & & & & & & & & & & \\
\hline SBW1-10 & & & & 0.0035 & & & & & & & & & & & & & & & & & \\
\hline SBW1-11 & & & & 0.0036 & & & & & & & & & & & & & & & & & \\
\hline SBW1-12 & & & & 0.0048 & & & & & & & & & & & & & & & & & \\
\hline SBW1-13 & & & & 0.0036 & & & & & & & & & & & & & & & & & \\
\hline SBW1-14 & & & & 0.0047 & & & & & & & & & & & & & & & & & \\
\hline SBW1-15 & & & & 0.0035 & & & & & & & & & & & & & & & & & \\
\hline SBW1-16 & & & & 0.0059 & & & & & & & & & & & & & & & & & \\
\hline SBW1-17 & & & & 0.0023 & & & & & & & & & & & & & & & & & \\
\hline SBW1-18 & & & & 0.0022 & & & & & & & & & & & & & & & & & \\
\hline SBW1-19 & & & & 0.0023 & & & & & & & & & & & & & & & & & \\
\hline SBW1-20 & & & & 0.0061 & & & & & & & & & & & & & & & & & \\
\hline SBW1-21 & & & & 0.0059 & & & & & & & & & & & & & & & & & \\
\hline SBW1-22 & & & & 0.0023 & & & & & & & & & & & & & & & & & \\
\hline SBW1-23 & & & & 0.0061 & & & & & & & & & & & & & & & & & \\
\hline
\end{tabular}


Appendix A. Database - mass fraction

SBW CVS Phase 1 (Scholes et al. 2002)

\begin{tabular}{|c|c|c|c|c|c|c|c|c|c|c|c|c|c|c|c|c|c|c|c|c|}
\hline Glass ID & $\begin{array}{c}\mathrm{SeO} 2 \\
-\mathrm{a}\end{array}$ & $\begin{array}{c}\mathrm{Sm} 2 \mathrm{O} 3 \\
-\mathrm{a}\end{array}$ & $\begin{array}{c}\mathrm{SnO} \\
-\mathrm{a}\end{array}$ & $\begin{array}{c}\mathrm{SnO} 2 \\
-\mathrm{a}\end{array}$ & $\begin{array}{c}\mathrm{SO} 3 \\
-\mathrm{a} \\
\end{array}$ & $\begin{array}{c}\mathrm{SrO} \\
-\mathrm{a}\end{array}$ & $\begin{array}{c}\mathrm{Tc} 2 \mathrm{O} 7 \\
-\mathrm{a}\end{array}$ & $\begin{array}{c}\mathrm{TeO} 2 \\
-\mathrm{a}\end{array}$ & $\begin{array}{c}\mathrm{ThO} 2 \\
-\mathrm{a}\end{array}$ & $\begin{array}{c}\mathrm{TiO} 2 \\
-\mathrm{a}\end{array}$ & $\begin{array}{c}\mathrm{T} 12 \mathrm{O} 3 \\
-\mathrm{a}\end{array}$ & $\begin{array}{c}\mathrm{U} 3 \mathrm{O} 8 \\
-\mathrm{a}\end{array}$ & $\begin{array}{c}\mathrm{UO} 2 \\
-\mathrm{a}\end{array}$ & $\begin{array}{c}\text { UO3 } \\
-\mathrm{a}\end{array}$ & $\begin{array}{c}\mathrm{V} 2 \mathrm{O} 5 \\
-\mathrm{a}\end{array}$ & $\begin{array}{c}\text { WO3 } \\
-\mathrm{a}\end{array}$ & $\begin{array}{c}\mathrm{Y} 2 \mathrm{O} 3 \\
-\mathrm{a}\end{array}$ & $\begin{array}{c}\mathrm{ZnO} \\
-\mathrm{a}\end{array}$ & $\begin{array}{c}\text { Others } \\
-\mathrm{a}\end{array}$ & $\begin{array}{c}\text { Sum } \\
-\mathrm{a}\end{array}$ \\
\hline SBW1-05 & & & & & 0.0055 & & & & & & & & & & & & & & & 0.9849 \\
\hline SBW1-06 & & & & & 0.0054 & & & & & & & & & & & & & & & 0.9939 \\
\hline SBW1-07 & & & & & 0.0054 & & & & & & & & & & & & & & & 1.0261 \\
\hline SBW1-08 & & & & & 0.0051 & & & & & & & & & & & & & & & 0.9759 \\
\hline SBW1-09 & & & & & 0.0056 & & & & & & & & & & & & & & & 0.9971 \\
\hline SBW1-10 & & & & & 0.0056 & & & & & & & & & & & & & & & 0.9744 \\
\hline SBW1-11 & & & & & 0.0054 & & & & & & & & & & & & & & & 1.0198 \\
\hline SBW1-12 & & & & & 0.0049 & & & & & & & & & & & & & & & 0.9833 \\
\hline SBW1-13 & & & & & 0.0055 & & & & & & & & & & & & & & & 0.9935 \\
\hline SBW1-14 & & & & & 0.0056 & & & & & & & & & & & & & & & 0.9823 \\
\hline SBW1-15 & & & & & 0.0055 & & & & & & & & & & & & & & & 0.9809 \\
\hline SBW1-16 & & & & & 0.0053 & & & & & & & & & & & & & & & 0.9740 \\
\hline SBW1-17 & & & & & 0.0057 & & & & & & & & & & & & & & & 0.9851 \\
\hline SBW1-18 & & & & & 0.0053 & & & & & & & & & & & & & & & 0.9992 \\
\hline SBW1-19 & & & & & 0.0054 & & & & & & & & & & & & & & & 0.9865 \\
\hline SBW1-20 & & & & & 0.0057 & & & & & & & & & & & & & & & 1.0044 \\
\hline SBW1-21 & & & & & 0.0053 & & & & & & & & & & & & & & & 0.9785 \\
\hline SBW1-22 & & & & & 0.0054 & & & & & & & & & & & & & & & 0.9724 \\
\hline SBW1-23 & & & & & 0.0055 & & & & & & & & & & & & & & & 1.0091 \\
\hline
\end{tabular}


Appendix A. Database - mass fraction

SBW CVS Phase 1 (Scholes et al. 2002)

\begin{tabular}{|c|c|c|c|c|c|c|c|c|}
\hline Glass ID & $\begin{array}{l}\mathrm{TM} \\
\left({ }^{\circ} \mathrm{C}\right)\end{array}$ & $\begin{array}{l}\text { Gradient } \\
\text { TL }\left({ }^{\circ} \mathrm{C}\right)\end{array}$ & $\begin{array}{l}\text { Uniform } \\
\text { TL }\left({ }^{\circ} \mathrm{C}\right)\end{array}$ & Primary Phase & $\begin{array}{l}\text { Quenched } \\
\text { Visual/OM }\end{array}$ & $\begin{array}{c}\text { Quenched } \\
\text { SEM/EDS or TEM }\end{array}$ & $\begin{array}{l}\text { Quenched } \\
\text { XRD }\end{array}$ & $\begin{array}{c}\text { Quenched } \\
\text { Homogeneous? }\end{array}$ \\
\hline SBW1-05 & 1150 & & 808 & $\mathrm{Na6}(\mathrm{AlSiO} 4) 6$ & $\begin{array}{l}\text { Clear dark reddish brown glass with a few } \\
\text { black specks and air bubbles, low viscosity }\end{array}$ & & Amorphous & Single-phase \\
\hline SBW1-06 & 1150 & & 848 & $\mathrm{Ca} 5(\mathrm{PO} 4) 3 \mathrm{~F}$ & $\begin{array}{l}\text { Clear dark brown glass with black specks anc } \\
\text { air bubbles, low viscosity }\end{array}$ & & Amorphous & Single-phase \\
\hline SBW1-07 & 1150 & & 828 & $\mathrm{Ca} 5(\mathrm{PO} 4) 3 \mathrm{~F}$ & $\begin{array}{l}\text { Clear dark brown glass with black specks anc } \\
\text { air bubbles, low viscosity }\end{array}$ & & Amorphous & Single-phase \\
\hline SBW1-08 & 1150 & & 908 & $\mathrm{Ca} 5(\mathrm{PO} 4) 3 \mathrm{~F}$ & $\begin{array}{l}\text { Clear very dark brown glass with a few black } \\
\text { specks and lots of air bubbles, avg. viscosity }\end{array}$ & & Amorphous & Single-phase \\
\hline SBW1-09 & 1150 & & 793 & $\mathrm{Ca} 5(\mathrm{PO} 4) 3 \mathrm{~F}$ & $\begin{array}{l}\text { Clear dark brown glass with a few black } \\
\text { specks and air bubbles, low viscosity }\end{array}$ & & Amorphous & Single-phase \\
\hline SBW1-10 & 1150 & & 743 & Na6(AlSiO4)6 & $\begin{array}{l}\text { Clear dark brown glass with black specks anc } \\
\text { air bubbles, low viscosity }\end{array}$ & & Amorphous & Single-phase \\
\hline SBW1-11 & 1150 & & 828 & $\mathrm{~K}(\mathrm{Si} 3 \mathrm{Al}) \mathrm{O} 8$ & $\begin{array}{l}\text { Clear dark brown glass with a few black } \\
\text { specks and lots of air bubbles, low viscosity }\end{array}$ & & Amorphous & Single-phase \\
\hline SBW1-12 & 1150 & & 878 & $\mathrm{Ca} 5(\mathrm{PO} 4) 3 \mathrm{~F}$ & $\begin{array}{l}\text { Clear dark brown glass with a few black } \\
\text { specks and air bubbles, low viscosity }\end{array}$ & & Amorphous & Single-phase \\
\hline SBW1-13 & 1150 & & 783 & $\mathrm{Ca} 5(\mathrm{PO} 4) 3 \mathrm{~F}$ & $\begin{array}{l}\text { Clear dark brown glass with a few black } \\
\text { specks and lots of air bubbles, low viscosity }\end{array}$ & & Amorphous & Single-phase \\
\hline SBW1-14 & 1150 & & 773 & $\mathrm{Na} 6(\mathrm{AlSiO} 4) 6$ & $\begin{array}{l}\text { Clear dark brown glass with black specks anc } \\
\text { air bubbles, low viscosity }\end{array}$ & & Amorphous & Single-phase \\
\hline SBW1-15 & 1150 & & 866 & $\mathrm{Ca} 5(\mathrm{PO} 4) 3 \mathrm{~F}$ & $\begin{array}{l}\text { Clear dark brown glass with black specks anc } \\
\text { a few air bubbles, low viscosity }\end{array}$ & & Amorphous & Single-phase \\
\hline SBW1-16 & 1150 & & 783 & $\mathrm{NaAlSi3O} 8$ & $\begin{array}{l}\text { Clear dark brown glass with black specks anc } \\
\text { air bubbles, low viscosity }\end{array}$ & & Amorphous & Single-phase \\
\hline SBW1-17 & 1150 & & 931 & $\mathrm{Ca} 5(\mathrm{PO} 4) 3 \mathrm{~F}$ & $\begin{array}{l}\text { Clear brown glass with black specks and air } \\
\text { bubbles, avg. viscosity }\end{array}$ & & Amorphous & Single-phase \\
\hline SBW1-18 & 1150 & & 948 & NaAlSiO4 & $\begin{array}{l}\text { Clear reddish brown glass with black specks } \\
\text { and air bubbles, low viscosity }\end{array}$ & & Amorphous & Single-phase \\
\hline SBW1-19 & 1150 & & 801 & Na3PO4 & $\begin{array}{l}\text { Clear brown glass with few black specks and } \\
\text { lots of air bubbles, low viscosity }\end{array}$ & & Amorphous & Single-phase \\
\hline SBW1-20 & 1150 & & 801 & Na6(AlSiO4)6 & $\begin{array}{l}\text { Clear dark reddish brown glass with a few } \\
\text { black specks and very few air bubbles, low } \\
\text { viscosity }\end{array}$ & & Amorphous & Single-phase \\
\hline SBW1-21 & 1150 & & 938 & $\mathrm{Fe} 2 \mathrm{O} 3$ & $\begin{array}{l}\text { Clear dark brown glass with a few black } \\
\text { specks and lots of air bubbles, avg. viscosity }\end{array}$ & & Amorphous & Single-phase \\
\hline SBW1-22 & 1150 & & 908 & $\mathrm{NaAlSiO} 4$ & $\begin{array}{l}\text { Clear brown glass with a few black specks } \\
\text { and lots of air bubbles, avg. viscosity }\end{array}$ & & Amorphous & Single-phase \\
\hline SBW1-23 & 1150 & & 828 & $\mathrm{Na} 6(\mathrm{AlSiO} 4) 6$ & $\begin{array}{l}\text { Clear dark reddish brown glass with a few } \\
\text { black specks and a few air bubbles, low } \\
\text { viscosity }\end{array}$ & & Amorphous & Single-phase \\
\hline
\end{tabular}


Appendix A. Database - mass fraction

\begin{tabular}{|c|c|c|c|c|c|}
\hline Glass ID & $\begin{array}{c}\text { CCC } \\
\text { Visual/OM }\end{array}$ & $\begin{array}{c}\text { CCC } \\
\text { SEM/EDS or TEM }\end{array}$ & $\begin{array}{l}\mathrm{CCC} \\
\mathrm{XRD}\end{array}$ & $\begin{array}{l}\text { Heat Treated } \\
\text { Visual/OM }\end{array}$ & $\begin{array}{c}\text { Heat Treated } \\
\text { SEM/EDS or TEM }\end{array}$ \\
\hline SBW1-05 & $\begin{array}{l}\text { Clear very dark brown glass, with air bubbles and black } \\
\text { specks (expected to be Ru). }\end{array}$ & & Amorphous & & \\
\hline SBW1-06 & $\begin{array}{l}\text { Clear brown glass, air bubbles and black specks (expected to } \\
\text { be Ru). }\end{array}$ & & Amorphous & & \\
\hline SBW1-07 & $\begin{array}{l}\text { Clear dark brown glass, air bubbles and black specks } \\
\text { (expected to be Ru). }\end{array}$ & & Amorphous & & \\
\hline SBW1-08 & $\begin{array}{l}\text { Clear dark brown glass with clear needles around outside } \\
\text { edges, some sheet-type crystals on top surface, some dark } \\
\text { cubic clusters, and other clear crystal clusters around the blact } \\
\text { specks (expected to be } \mathrm{Ru} \text { ). }\end{array}$ & & $\begin{array}{l}\mathrm{Fe} 2 \mathrm{O} 3, \mathrm{LiOH}, \mathrm{NaHF} 2 \text {, and } \\
\mathrm{Na} 2 \mathrm{Ca}(\mathrm{SiO} 4)(0.19 \text { vol} \% \\
\text { crystallinity) }\end{array}$ & & \\
\hline SBW1-09 & $\begin{array}{l}\text { Clear dark brown glass, with few air bubbles and black specks } \\
\text { (expected to be Ru). }\end{array}$ & & Amorphous & & \\
\hline SBW1-10 & $\begin{array}{l}\text { Clear dark brown glass, air bubbles and black specks } \\
\text { (expected to be Ru). }\end{array}$ & & Amorphous & & \\
\hline SBW1-11 & $\begin{array}{l}\text { Clear dark brown glass, air bubbles and black specks } \\
\text { (expected to be Ru). }\end{array}$ & & $\begin{array}{l}\text { NaMn3F7 and } \mathrm{ZrO} 2(0.00 \mathrm{vol} \% \\
\text { crystallinity) }\end{array}$ & & \\
\hline SBW1-12 & $\begin{array}{l}\text { Clear dark brown glass with some sheet-type crystals on top } \\
\text { surface, air bubbles and black specks (expected to be Ru). }\end{array}$ & & NaMn3F7 (0.05 vol\% crystallinity) & & \\
\hline SBW1-13 & $\begin{array}{l}\text { Clear brown glass, with few air bubbles and black specks } \\
\text { (expected to be Ru). }\end{array}$ & & Amorphous & & \\
\hline SBW1-14 & $\begin{array}{l}\text { Clear dark brown glass, air bubbles and black specks } \\
\text { (expected to be Ru). }\end{array}$ & & $\begin{array}{l}\text { Ca5Si6O16 }(\mathrm{OH}) 2(0.03 \mathrm{vol} \% \\
\text { crystallinity })\end{array}$ & & \\
\hline SBW1-15 & $\begin{array}{l}\text { Clear brown glass with one surface crystal and some dark } \\
\text { crystal clusters, air bubbles and black specks (expected to be } \\
\mathrm{Ru} \text { ). }\end{array}$ & & $\begin{array}{l}\text { Ca5Si6O16(OH)2 (0.11 vol\% } \\
\text { crystallinity) }\end{array}$ & & \\
\hline SBW1-16 & $\begin{array}{l}\text { Clear dark purplish glass, few air bubbles and a few black } \\
\text { specks (expected to be } \mathrm{Ru} \text { ). }\end{array}$ & & Amorphous & & \\
\hline SBW1-17 & $\begin{array}{l}\text { Clear greenish brown glass with clusters of thin rod-type } \\
\text { crystals forming on top and along the crucible walls, air } \\
\text { bubbles and black specks (expected to be Ru). }\end{array}$ & & Ca5(PO4)3F (0.20 vol $\%$ crystallinity) & & \\
\hline SBW1-18 & $\begin{array}{l}\text { Clear brown glass, few air bubbles and clusters of crystals } \\
\text { around each black speck (expected to be Ru). }\end{array}$ & & Amorphous & & \\
\hline SBW1-19 & $\begin{array}{l}\text { Clear dark purplish glass, air bubbles and black specks } \\
\text { (expected to be } \mathrm{Ru} \text { ). }\end{array}$ & & Amorphous & & \\
\hline SBW1-20 & $\begin{array}{l}\text { Clear dark reddish brown glass, a few black specks (expected } \\
\text { to be } \mathrm{Ru} \text { ) but no air bubbles. }\end{array}$ & & Amorphous & & \\
\hline SBW1-21 & $\begin{array}{l}\text { Clear dark brown glass, with clusters of fuzzy sphere crystals } \\
\text { throughout. }\end{array}$ & & LiAlO2 (0.15 vol\% crystallinity) & & \\
\hline SBW1-22 & $\begin{array}{l}\text { Clear dark brown glass, air bubbles and black specks } \\
\text { (expected to be Ru). }\end{array}$ & & Amorphous & & \\
\hline SBW1-23 & $\begin{array}{l}\text { Clear dark brown glass, air bubbles and black specks } \\
\text { (expected to be Ru). }\end{array}$ & & Amorphous & & \\
\hline
\end{tabular}


Appendix A. Database - mass fraction

SBW CVS Phase 1 (Scholes et al. 2002)

\begin{tabular}{|c|c|c|c|c|c|c|c|c|c|c|c|c|c|c|c|}
\hline Glass ID & $\begin{array}{l}\text { Heat Treated } \\
\text { XRD }\end{array}$ & $\begin{array}{l}\text { Density } \\
\left(\mathrm{g} / \mathrm{cm}^{3}\right)\end{array}$ & $\begin{array}{c}\text { Fulc Visc } \\
\mathrm{A}\end{array}$ & $\begin{array}{c}\text { Fulc Visc } \\
\text { B }\end{array}$ & $\begin{array}{c}\text { Fulc Visc } \\
\text { To } \\
\end{array}$ & $\begin{array}{c}\mathrm{FV} 1150^{\circ} \mathrm{C} \\
(\mathrm{Pa} \cdot \mathrm{s})\end{array}$ & \begin{tabular}{|c|} 
Arrh Visc \\
A \\
\end{tabular} & \begin{tabular}{|c|} 
Arrh Visc \\
B
\end{tabular} & $1150^{\circ} \mathrm{C}$ & $\begin{array}{c}\mathrm{T}\left({ }^{\circ} \mathrm{C}\right) \text { at } \\
2 \mathrm{~Pa} \cdot \mathrm{s}\end{array}$ & $\begin{array}{c}\mathrm{T}\left({ }^{\circ} \mathrm{C}\right) \text { at } \\
5 \mathrm{~Pa} \cdot \mathrm{s} \\
\end{array}$ & $\begin{array}{l}\mathrm{T}\left({ }^{\circ} \mathrm{C}\right) \text { at } \\
10 \mathrm{~Pa} \cdot \mathrm{s}\end{array}$ & $\begin{array}{c}\mathrm{T} 1 \\
\left({ }^{\circ} \mathrm{C}\right) \\
\end{array}$ & $\begin{array}{c}\mathrm{V} 1 \\
(\mathrm{~Pa} \cdot \mathrm{s})\end{array}$ & $\begin{array}{c}\mathrm{T} 2 \\
\left({ }^{\circ} \mathrm{C}\right) \\
\end{array}$ \\
\hline SBW1-05 & & 2.5925 & & & & & -9.649 & 16730.0 & 8.23 & 1344 & & 1127 & 1155.7 & 7.5633 & 1079.9 \\
\hline SBW1-06 & & 2.5997 & & & & & -9.904 & 17477.0 & 10.78 & 1376 & & 1159 & 1155.8 & 10.009 & 1080.3 \\
\hline SBW1-07 & & 2.6258 & & & & & -10.333 & 17591.0 & 7.61 & 1322 & & 1119 & 1160.2 & 6.961 & 1083.1 \\
\hline SBW1-08 & & 2.5841 & & & & & -10.365 & 18249.0 & 11.70 & 1377 & & 1167 & 1156.5 & 10.768 & 1081.1 \\
\hline SBW1-09 & & 2.6066 & & & & & -9.967 & 16451.0 & 4.92 & 1270 & & 1068 & 1153.7 & 4.661 & 1079.3 \\
\hline SBW1-10 & & 2.6066 & & & & & -9.985 & 16250.0 & 4.20 & 1249 & & 1049 & 1156.6 & 3.9068 & 1080.9 \\
\hline SBW1-11 & & 2.6016 & & & & & -10.200 & 17229.0 & 6.74 & 1308 & & 1105 & 1156.7 & 6.1792 & 1081.1 \\
\hline SBW1-12 & & 2.5945 & & & & & -10.243 & 17669.0 & 8.79 & 1343 & & 1135 & 1159.8 & 7.8889 & 1082.8 \\
\hline SBW1-13 & & 2.6261 & & & & & -9.824 & 16351.0 & 5.29 & 1282 & & 1075 & 1154.1 & 5.0109 & 1078.8 \\
\hline SBW1-14 & & 2.6178 & & & & & -9.899 & 16323.0 & 4.82 & 1268 & & 1065 & 1155.1 & 4.5201 & 1079.7 \\
\hline SBW1-15 & & 2.6103 & & & & & -10.262 & 17277.0 & 6.55 & 1304 & & 1102 & 1156 & 6.0766 & 1079.7 \\
\hline SBW1-16 & & 2.5127 & & & & & -10.055 & 17309.0 & 8.24 & 1337 & & 1128 & 1155.3 & 7.488 & 1079.6 \\
\hline SBW1-17 & & 2.5929 & & & & & -10.418 & 18108.0 & 10.05 & 1357 & & 1150 & 1156.2 & 9.6228 & 1080.5 \\
\hline SBW1-18 & & 2.6045 & & & & & -10.585 & 18071.0 & 8.28 & 1329 & & 1129 & 1155.1 & 7.9986 & 1079.6 \\
\hline SBW1-19 & & 2.5464 & & & & & -9.707 & 16981.0 & 9.26 & 1360 & & 1141 & 1155.8 & 8.4691 & 1079.9 \\
\hline SBW1-20 & & 2.6735 & & & & & -10.229 & 15879.0 & 2.53 & 1181 & & 994 & 1156.2 & 2.3822 & 1080.4 \\
\hline SBW1-21 & & 2.5936 & & & & & -10.728 & 18683.0 & 11.04 & 1363 & & 1161 & 1153.6 & 10.838 & 1078.3 \\
\hline SBW1-22 & & 2.5479 & & & & & -9.799 & 17272.0 & 10.37 & 1373 & & 1154 & 1156.5 & 9.64 & 1080.6 \\
\hline SBW1-23 & & 2.6065 & & & & & -9.222 & 15119.0 & 4.06 & 1252 & & 1039 & 1155.3 & 3.8327 & 1080 \\
\hline
\end{tabular}


Appendix A. Database - mass fraction

\begin{tabular}{|c|c|c|c|c|c|c|c|c|c|c|c|c|c|c|c|c|c|c|c|c|c|}
\hline \multicolumn{22}{|c|}{ SBW CVS Phase 1 (Scholes et al. 2002) } \\
\hline Glass ID & $\begin{array}{c}\mathrm{V} 2 \\
(\mathrm{~Pa} \cdot \mathrm{s})\end{array}$ & $\begin{array}{l}\mathrm{T} 3 \\
\left({ }^{\circ} \mathrm{C}\right)\end{array}$ & $\begin{array}{c}\mathrm{V} 3 \\
(\mathrm{~Pa} \cdot \mathrm{s})\end{array}$ & $\begin{array}{l}\mathrm{T} 4 \\
\left({ }^{\circ} \mathrm{C}\right)\end{array}$ & $\begin{array}{c}\mathrm{V} 4 \\
(\mathrm{~Pa} \cdot \mathrm{s})\end{array}$ & $\begin{array}{c}\text { T5 } \\
\left({ }^{\circ} \mathrm{C}\right)\end{array}$ & $\begin{array}{c}\text { V5 } \\
(\mathrm{Pa} \cdot \mathrm{s})\end{array}$ & $\begin{array}{l}\text { T6 } \\
\left({ }^{\circ} \mathrm{C}\right)\end{array}$ & $\begin{array}{c}\mathrm{V} 6 \\
(\mathrm{~Pa} \cdot \mathrm{s})\end{array}$ & $\begin{array}{c}\mathrm{T} 7 \\
\left({ }^{\circ} \mathrm{C}\right)\end{array}$ & $\begin{array}{c}\text { V7 } \\
(\mathrm{Pa} \cdot \mathrm{s})\end{array}$ & $\begin{array}{c}\mathrm{T} 8 \\
\left({ }^{\circ} \mathrm{C}\right)\end{array}$ & $\begin{array}{c}\mathrm{V} 8 \\
(\mathrm{~Pa} \cdot \mathrm{s})\end{array}$ & $\begin{array}{l}\mathrm{T} 9 \\
\left({ }^{\circ} \mathrm{C}\right)\end{array}$ & $\begin{array}{c}\mathrm{V} 9 \\
(\mathrm{~Pa} \cdot \mathrm{s})\end{array}$ & $\begin{array}{l}\mathrm{T} 10 \\
\left({ }^{\circ} \mathrm{C}\right)\end{array}$ & $\begin{array}{c}\mathrm{V} 10 \\
(\mathrm{~Pa} \cdot \mathrm{s})\end{array}$ & $\begin{array}{l}\mathrm{T} 11 \\
\left({ }^{\circ} \mathrm{C}\right)\end{array}$ & $\begin{array}{c}\mathrm{V} 11 \\
(\mathrm{~Pa} \cdot \mathrm{s})\end{array}$ & $\begin{array}{l}\mathrm{T} 12 \\
\left({ }^{\circ} \mathrm{C}\right)\end{array}$ & $\begin{array}{c}\mathrm{V} 12 \\
(\mathrm{~Pa} \cdot \mathrm{s})\end{array}$ \\
\hline SBW1-05 & 14.474 & 1004.6 & 30.953 & 954.96 & 55.569 & 1252.9 & 3.9229 & 1153.6 & 7.9369 & & & & & & & & & & & & \\
\hline SBW1-06 & 19.455 & 1005.3 & 42.664 & 955.53 & 78.332 & 1253.6 & 4.9056 & 1154.2 & 10.225 & & & & & & & & & & & & \\
\hline SBW1-07 & 13.227 & 1007.4 & 29.087 & 960.75 & 53.407 & 1254.6 & 3.3452 & 1158.4 & 7.1193 & & & & & & & & & & & & \\
\hline SBW1-08 & 21.495 & 1006.3 & 48.807 & 956.58 & 91.116 & 1255.2 & 5.0299 & 1155.5 & 11.07 & & & & & & & & & & & & \\
\hline SBW1-09 & 8.6702 & 1003.7 & 18.265 & 954.07 & 32.449 & 1252 & 2.378 & 1152.6 & 4.7617 & & & & & & & & & & & & \\
\hline SBW1-10 & 7.2541 & 1006.1 & 14.942 & 956.48 & 26.176 & 1253.3 & 1.9994 & 1154.3 & 4.0525 & & & & & & & & & & & & \\
\hline SBW1-11 & 11.872 & 1006 & 25.908 & 956.18 & 47.447 & 1255.2 & 3.0595 & 1156.3 & 6.387 & & & & & & & & & & & & \\
\hline SBW1-12 & 15.535 & 1007.8 & 34.267 & 958.22 & 63.416 & 1255.9 & 3.8793 & 1156.7 & 8.2315 & & & & & & & & & & & & \\
\hline SBW1-13 & 9.3391 & 1004.1 & 19.445 & 954.5 & 34.062 & 1252.6 & 2.528 & 1153.3 & 5.1395 & & & & & & & & & & & & \\
\hline SBW1-14 & 8.3932 & 1005.4 & 17.439 & 955.29 & 30.69 & 1253.6 & 2.2901 & 1154.2 & 4.6615 & & & & & & & & & & & & \\
\hline SBW1-15 & 11.736 & 1004.9 & 25.575 & 955.26 & 46.874 & 1253.7 & 2.9998 & 1154.2 & 6.2825 & & & & & & & & & & & & \\
\hline SBW1-16 & 14.619 & 1004.4 & 32.419 & 954.57 & 60.602 & 1253.2 & 3.9059 & 1153.8 & 7.8332 & & & & & & & & & & & & \\
\hline SBW1-17 & 18.767 & 1005.3 & 41.6 & 955.61 & 76.681 & 1154.8 & 9.6888 & & & & & & & & & & & & & & \\
\hline SBW1-18 & 15.742 & 1004.7 & 34.64 & 954.91 & 63.118 & 1154.2 & 7.995 & & & & & & & & & & & & & & \\
\hline SBW1-19 & 16.231 & 1004.6 & 35.452 & 954.91 & 64.729 & 1252.8 & 4.3656 & 1153.6 & 9.033 & & & & & & & & & & & & \\
\hline SBW1-20 & 4.3905 & 1005.4 & 8.8737 & 955.83 & 15.098 & 1253 & 1.2207 & 1153.5 & 2.4559 & & & & & & & & & & & & \\
\hline SBW1-21 & 21.817 & 1003.5 & 49.043 & 953.91 & 91.267 & 1202.7 & 6.9506 & 1152.9 & 10.587 & & & & & & & & & & & & \\
\hline SBW1-22 & 18.791 & 1005.5 & 40.469 & 955.87 & 72.09 & 1254 & 4.6425 & 1154.5 & 9.9693 & & & & & & & & & & & & \\
\hline SBW1-23 & 6.8778 & 1005 & 13.455 & 955.25 & 22.393 & 1253.7 & 2.0227 & 1154.2 & 3.9427 & & & & & & & & & & & & \\
\hline
\end{tabular}


Appendix A. Database - mass fraction

SBW CVS Phase 1 (Scholes et al. 2002)

\begin{tabular}{|c|c|c|c|c|c|c|c|c|c|c|c|c|c|c|c|c|}
\hline Glass ID & $\begin{array}{l}\mathrm{T} 13 \\
\left({ }^{\circ} \mathrm{C}\right) \\
\end{array}$ & $\begin{array}{c}\mathrm{V} 13 \\
(\mathrm{~Pa} \cdot \mathrm{s}) \\
\end{array}$ & $\begin{array}{l}\mathrm{T} 14 \\
\left({ }^{\circ} \mathrm{C}\right) \\
\end{array}$ & $\begin{array}{c}\mathrm{V} 14 \\
(\mathrm{~Pa} \cdot \mathrm{s}) \\
\end{array}$ & $\begin{array}{c}\text { Q PCT } \\
\text { B }\left(\mathrm{g} / \mathrm{m}^{2}\right)\end{array}$ & $\begin{array}{c}\text { Q PCT } \\
\mathrm{Li}\left(\mathrm{g} / \mathrm{m}^{2}\right)\end{array}$ & $\begin{array}{c}\text { Q PCT } \\
\mathrm{Na}\left(\mathrm{g} / \mathrm{m}^{2}\right)\end{array}$ & $\begin{array}{c}\text { Q PCT } \\
\mathrm{Si}\left(\mathrm{g} / \mathrm{m}^{2}\right) \\
\end{array}$ & $\begin{array}{c}\text { Q PCT } \\
\mathrm{pH}\end{array}$ & $\begin{array}{c}\text { CCC PCT } \\
\text { B }\left(\mathrm{g} / \mathrm{m}^{2}\right)\end{array}$ & $\begin{array}{l}\text { CCC PCT } \\
\mathrm{Li}\left(\mathrm{g} / \mathrm{m}^{2}\right)\end{array}$ & $\begin{array}{l}\text { CCC PCT } \\
\mathrm{Na}\left(\mathrm{g} / \mathrm{m}^{2}\right)\end{array}$ & $\begin{array}{l}\text { CCC PCT } \\
\mathrm{Si}\left(\mathrm{g} / \mathrm{m}^{2}\right)\end{array}$ & $\begin{array}{c}\begin{array}{c}\text { CCC PCT } \\
\mathrm{pH}\end{array} \\
\end{array}$ & $\begin{array}{l}\text { Q PCT B at } \\
20^{\circ} \mathrm{C}\left(\mathrm{g} / \mathrm{m}^{2}\right)\end{array}$ & $\begin{array}{l}\text { Q PCT Li at } \\
20^{\circ} \mathrm{C}\left(\mathrm{g} / \mathrm{m}^{2}\right)\end{array}$ \\
\hline SBW1-05 & & & & & 0.292 & 0.309 & 0.330 & 0.142 & 10.44 & & & & & & & \\
\hline SBW1-06 & & & & & 0.238 & 0.257 & 0.254 & 0.111 & 10.285 & & & & & & & \\
\hline SBW1-07 & & & & & 0.338 & 0.324 & 0.383 & 0.125 & 10.63 & & & & & & & \\
\hline SBW1-08 & & & & & 0.182 & 0.209 & 0.325 & 0.100 & 10.005 & & & & & & & \\
\hline SBW1-09 & & & & & 0.738 & 0.622 & 0.598 & 0.164 & 10.67 & & & & & & & \\
\hline SBW1-10 & & & & & 0.663 & 0.567 & 0.770 & 0.187 & 11.22 & & & & & & & \\
\hline SBW1-11 & & & & & 0.667 & 0.526 & 0.665 & 0.133 & 10.22 & & & & & & & \\
\hline SBW1-12 & & & & & 0.315 & 0.316 & 0.469 & 0.135 & 10.57 & & & & & & & \\
\hline SBW1-13 & & & & & 0.374 & 0.353 & 0.411 & 0.156 & 10.635 & & & & & & & \\
\hline SBW1-14 & & & & & 0.852 & 0.578 & 0.902 & 0.207 & 11.37 & & & & & & & \\
\hline SBW1-15 & & & & & 0.442 & 0.379 & 0.380 & 0.138 & 10.19 & & & & & & & \\
\hline SBW1-16 & & & & & 1.490 & 1.355 & 0.950 & 0.267 & 9.905 & & & & & & & \\
\hline SBW1-17 & & & & & 0.243 & 0.389 & 0.277 & 0.136 & 10.165 & & & & & & & \\
\hline SBW1-18 & & & & & 0.180 & 0.290 & 0.175 & 0.099 & 10.25 & & & & & & & \\
\hline SBW1-19 & & & & & 3.514 & 2.529 & 2.952 & 0.957 & 11.595 & & & & & & & \\
\hline SBW1-20 & & & & & 1.197 & 0.804 & 1.424 & 0.370 & 11.695 & & & & & & & \\
\hline SBW1-21 & & & & & 0.229 & 0.309 & 0.215 & 0.161 & 10.215 & & & & & & & \\
\hline SBW1-22 & & & & & 0.357 & 0.368 & 0.613 & 0.173 & 10.77 & & & & & & & \\
\hline SBW1-23 & & & & & 1.139 & 0.832 & 1.372 & 0.372 & 11.575 & & & & & & & \\
\hline
\end{tabular}


Appendix A. Database - mass fraction

\begin{tabular}{|c|c|c|c|c|c|c|c|c|c|c|c|c|}
\hline \multicolumn{13}{|c|}{ SBW CVS Phase 1 (Scholes et al. 2002) } \\
\hline Glass ID & $\begin{array}{l}\text { Q PCT Na at } \\
20^{\circ} \mathrm{C}\left(\mathrm{g} / \mathrm{m}^{2}\right)\end{array}$ & $\begin{array}{l}\text { Q PCT Si at } \\
20^{\circ} \mathrm{C}\left(\mathrm{g} / \mathrm{m}^{2}\right)\end{array}$ & $\begin{array}{c}\mathrm{QpH} \\
\text { at } 20^{\circ} \mathrm{C}\end{array}$ & $\begin{array}{c}\text { TCLP Ag } \\
(\mathrm{ppm})\end{array}$ & $\begin{array}{c}\text { TCLP As } \\
\text { (ppm) }\end{array}$ & $\begin{array}{c}\text { TCLP Ba } \\
(\mathrm{ppm})\end{array}$ & $\begin{array}{c}\text { TCLP Cd } \\
(\mathrm{ppm})\end{array}$ & $\begin{array}{c}\text { TCLP Cr } \\
(\mathrm{ppm})\end{array}$ & $\begin{array}{c}\text { TCLP Ni } \\
(\mathrm{ppm})\end{array}$ & $\begin{array}{c}\text { TCLP Pb } \\
\text { (ppm) }\end{array}$ & $\begin{array}{c}\text { TCLP Se } \\
(\mathrm{ppm})\end{array}$ & $\begin{array}{c}\text { TCLP Zn } \\
(\mathrm{ppm})\end{array}$ \\
\hline \multicolumn{13}{|l|}{ SBW1-05 } \\
\hline \multicolumn{13}{|l|}{ SBW1-06 } \\
\hline \multicolumn{13}{|l|}{ SBW1-07 } \\
\hline \multicolumn{13}{|l|}{ SBW1-08 } \\
\hline \multicolumn{13}{|l|}{ SBW1-09 } \\
\hline \multicolumn{13}{|l|}{ SBW1-10 } \\
\hline \multicolumn{13}{|l|}{ SBW1-11 } \\
\hline \multicolumn{13}{|l|}{ SBW1-12 } \\
\hline \multicolumn{13}{|l|}{ SBW1-13 } \\
\hline \multicolumn{13}{|l|}{ SBW1-14 } \\
\hline \multicolumn{13}{|l|}{ SBW1-15 } \\
\hline \multicolumn{13}{|l|}{ SBW1-16 } \\
\hline \multicolumn{13}{|l|}{ SBW1-17 } \\
\hline \multicolumn{13}{|l|}{ SBW1-18 } \\
\hline \multicolumn{13}{|l|}{ SBW1-19 } \\
\hline \multicolumn{13}{|l|}{ SBW1-20 } \\
\hline \multicolumn{13}{|l|}{ SBW1-21 } \\
\hline \multicolumn{13}{|l|}{ SBW1-22 } \\
\hline SBW1-23 & & & & & & & & & & & & \\
\hline
\end{tabular}


Appendix A. Database - mass fraction

\begin{tabular}{|c|c|c|c|c|c|c|c|c|c|c|c|c|c|c|c|c|c|c|c|c|c|}
\hline Glass ID & $\begin{array}{c}\mathrm{A} 12 \mathrm{O} 3 \\
-\mathrm{t}\end{array}$ & $\begin{array}{c}\mathrm{B} 2 \mathrm{O} 3 \\
-\mathrm{t}\end{array}$ & $\begin{array}{c}\mathrm{CaO} \\
-\mathrm{t}\end{array}$ & $\begin{array}{c}\mathrm{Fe} 2 \mathrm{O} 3 \\
-\mathrm{t}\end{array}$ & $\begin{array}{c}\mathrm{FeO} \\
-\mathrm{t}\end{array}$ & $\begin{array}{c}\mathrm{K} 2 \mathrm{O} \\
-\mathrm{t}\end{array}$ & $\begin{array}{c}\mathrm{Li} 2 \mathrm{O} \\
-\mathrm{t}\end{array}$ & $\begin{array}{c}\mathrm{MgO} \\
-\mathrm{t}\end{array}$ & $\begin{array}{c}\mathrm{Na} 2 \mathrm{O} \\
-\mathrm{t}\end{array}$ & $\begin{array}{c}\mathrm{NiO} \\
-\mathrm{t}\end{array}$ & $\begin{array}{c}\mathrm{P} 2 \mathrm{O} 5 \\
-\mathrm{t}\end{array}$ & $\begin{array}{c}\mathrm{SiO} 2 \\
-\mathrm{t}\end{array}$ & $\begin{array}{c}\mathrm{ZrO} 2 \\
-\mathrm{t}\end{array}$ & $\begin{array}{c}\mathrm{Ag} 2 \mathrm{O} \\
-\mathrm{t}\end{array}$ & $\begin{array}{c}\mathrm{As} 2 \mathrm{O} 3 \\
-\mathrm{t}\end{array}$ & $\begin{array}{c}\mathrm{BaO} \\
-\mathrm{t}\end{array}$ & $\begin{array}{c}\mathrm{Bi} 2 \mathrm{O} 3 \\
-\mathrm{t}\end{array}$ & $\begin{array}{l}\mathrm{Br} \\
-\mathrm{t}\end{array}$ & $\begin{array}{c}\mathrm{CdO} \\
-\mathrm{t}\end{array}$ & $\begin{array}{c}\mathrm{Ce} 2 \mathrm{O} 3 \\
-\mathrm{t}\end{array}$ & $\begin{array}{c}\mathrm{CeO} 2 \\
-\mathrm{t}\end{array}$ \\
\hline SBW1-24 & 0.1560 & 0.1300 & 0.0124 & 0.0804 & & 0.0446 & 0.0100 & 0.0000 & 0.1059 & 0.0008 & 0.0161 & 0.4057 & 0.0040 & & & 0.0100 & & & 0.0040 & & \\
\hline SBW1-25 & 0.0690 & 0.1300 & 0.0400 & 0.0216 & & 0.0194 & 0.0100 & 0.0000 & 0.1293 & 0.0008 & 0.0161 & 0.5188 & 0.0160 & & & 0.0100 & & & 0.0040 & & \\
\hline SBW1-26 & 0.0690 & 0.0700 & 0.0124 & 0.0804 & & 0.0446 & 0.0244 & 0.0000 & 0.1044 & 0.0008 & 0.0045 & 0.5148 & 0.0160 & & & 0.0376 & & & 0.0010 & & \\
\hline SBW1-27 & 0.1560 & 0.1300 & 0.0124 & 0.0216 & & 0.0194 & 0.0100 & 0.0000 & 0.1301 & 0.0008 & 0.0045 & 0.4408 & 0.0160 & & & 0.0376 & & & 0.0010 & & \\
\hline SBW1-28 & 0.0938 & 0.1300 & 0.0124 & 0.0804 & & 0.0446 & 0.0400 & 0.0000 & 0.1044 & 0.0008 & 0.0161 & 0.4024 & 0.0160 & & & 0.0353 & & & 0.0040 & & \\
\hline SBW1-29 & 0.1155 & 0.1300 & 0.0400 & 0.0216 & & 0.0446 & 0.0400 & 0.0000 & 0.1419 & 0.0008 & 0.0161 & 0.4024 & 0.0160 & & & 0.0100 & & & 0.0010 & & \\
\hline SBW1-30 & 0.0727 & 0.1300 & 0.0124 & 0.0804 & & 0.0194 & 0.0100 & 0.0000 & 0.2123 & 0.0008 & 0.0045 & 0.4275 & 0.0040 & & & 0.0100 & & & 0.0010 & & \\
\hline SBW1-31D & 0.1617 & 0.1354 & 0.0085 & 0.0854 & & 0.0173 & 0.0428 & 0.0000 & 0.0917 & 0.0008 & 0.0171 & 0.4066 & 0.0030 & & & 0.0058 & & & 0.0043 & & \\
\hline SBW1-32 & 0.0400 & 0.1500 & 0.0030 & 0.0020 & & 0.0530 & 0.0500 & 0.0000 & 0.0680 & 0.0008 & 0.0006 & 0.6044 & & & & & & & & & \\
\hline SBW1-33A & 0.0400 & 0.1500 & 0.0500 & 0.1000 & & 0.0530 & & 0.0000 & 0.0680 & 0.0008 & 0.0200 & 0.4780 & 0.0200 & & & & & & & & \\
\hline SBW1-34 & 0.0400 & 0.1289 & 0.0030 & 0.0020 & & 0.0110 & 0.0500 & 0.0000 & 0.0680 & 0.0008 & 0.0006 & 0.6120 & 0.0200 & & & 0.0470 & & & 0.0050 & & \\
\hline SBW1-35 & 0.1799 & 0.1500 & 0.0500 & 0.0020 & & 0.0530 & & 0.0000 & 0.1970 & 0.0008 & 0.0006 & 0.3500 & & & & & & & 0.0050 & & \\
\hline SBW1-36 & 0.1850 & 0.0500 & 0.0030 & 0.0020 & & 0.0110 & & 0.0000 & 0.2390 & 0.0008 & 0.0006 & 0.4334 & & & & 0.0470 & & & & & \\
\hline SBW1-37 & 0.0400 & 0.0500 & 0.0030 & 0.1000 & & 0.0110 & & 0.0000 & 0.2163 & 0.0008 & 0.0200 & 0.5142 & 0.0200 & & & & & & 0.0050 & & \\
\hline SBW1-38B & 0.1617 & 0.1354 & 0.0085 & 0.0854 & & 0.0173 & 0.0078 & 0.0000 & 0.1127 & 0.0008 & 0.0035 & 0.4010 & 0.0030 & & & 0.0387 & & & 0.0043 & & \\
\hline SBW1-39B & 0.1592 & 0.0586 & 0.0048 & 0.0951 & & 0.0509 & 0.0026 & 0.0000 & 0.1920 & 0.0008 & 0.0016 & 0.3886 & 0.0190 & & & 0.0019 & & & 0.0048 & & \\
\hline SBW1-40 & 0.1523 & 0.1356 & 0.0030 & 0.1000 & & 0.0530 & 0.0500 & 0.0000 & 0.0680 & 0.0008 & 0.0006 & 0.3500 & 0.0200 & & & 0.0470 & & & & & \\
\hline SBW1-41 & 0.0400 & 0.1500 & 0.0030 & 0.1000 & & 0.0110 & & 0.0000 & 0.2280 & 0.0008 & 0.0006 & 0.4549 & & & & & & & & & \\
\hline SBW1-42 & 0.0400 & 0.1500 & 0.0500 & 0.1000 & & 0.0110 & 0.0500 & 0.0000 & 0.0798 & 0.0008 & 0.0006 & 0.4646 & 0.0200 & & & & & & 0.0050 & & \\
\hline SBW1-43 & 0.1650 & 0.1500 & 0.0030 & 0.0020 & & 0.0110 & 0.0500 & 0.0000 & 0.1781 & 0.0008 & 0.0200 & 0.3799 & 0.0200 & & & & & & & & \\
\hline
\end{tabular}


Appendix A. Database - mass fraction

SBW CVS Phase 1 (Scholes et al. 2002)

\begin{tabular}{|c|c|c|c|c|c|c|c|c|c|c|c|c|c|c|c|c|c|c|c|c|c|}
\hline Glass ID & $\begin{array}{l}\mathrm{Cl} \\
-\mathrm{t} \\
\end{array}$ & $\begin{array}{c}\mathrm{CoO} \\
-\mathrm{t}\end{array}$ & $\begin{array}{c}\mathrm{Co} 2 \mathrm{O} 3 \\
-\mathrm{t}\end{array}$ & $\begin{array}{c}\mathrm{Cr} 2 \mathrm{O} 3 \\
-\mathrm{t} \\
\end{array}$ & $\begin{array}{c}\mathrm{Cs} 2 \mathrm{O} \\
-\mathrm{t}\end{array}$ & $\begin{array}{c}\mathrm{CuO} \\
-\mathrm{t}\end{array}$ & $\begin{array}{c}\mathrm{Eu} 2 \mathrm{O} 3 \\
-\mathrm{t}\end{array}$ & $\begin{array}{l}F \\
-t\end{array}$ & $\begin{array}{c}\mathrm{Ga} 2 \mathrm{O} 3 \\
-\mathrm{t}\end{array}$ & $\begin{array}{c}\mathrm{Gd} 2 \mathrm{O} 3 \\
-\mathrm{t}\end{array}$ & $\begin{array}{c}\mathrm{HgO} \\
-\mathrm{t}\end{array}$ & $\begin{array}{l}I \\
-t\end{array}$ & $\begin{array}{c}\mathrm{La} 2 \mathrm{O} 3 \\
-\mathrm{t}\end{array}$ & $\begin{array}{c}\mathrm{MnO} 2 \\
-\mathrm{t}\end{array}$ & $\begin{array}{c}\mathrm{MnO} \\
-\mathrm{t}\end{array}$ & $\begin{array}{c}\mathrm{MoO} \\
-\mathrm{t}\end{array}$ & $\begin{array}{c}\mathrm{MoO} 3 \\
-\mathrm{t}\end{array}$ & $\begin{array}{c}\mathrm{Nb} 2 \mathrm{O} 5 \\
-\mathrm{t}\end{array}$ & $\begin{array}{c}\mathrm{Nd} 2 \mathrm{O} 3 \\
-\mathrm{t}\end{array}$ & $\begin{array}{c}\mathrm{PbO} \\
-\mathrm{t}\end{array}$ & $\begin{array}{c}\mathrm{PdO} 2 \\
-\mathrm{t}\end{array}$ \\
\hline SBW1-24 & 0.0015 & & & 0.0013 & & & & 0.0026 & & & & 0.0001 & 0.0003 & & 0.0083 & & 0.0002 & & & 0.0007 & \\
\hline SBW1-25 & 0.0015 & & & 0.0013 & & & & 0.0026 & & & & 0.0001 & 0.0003 & & 0.0032 & & 0.0002 & & & 0.0007 & \\
\hline SBW1-26 & 0.0015 & & & 0.0013 & & & & 0.0026 & & & & 0.0001 & 0.0003 & & 0.0083 & & 0.0002 & & & 0.0007 & \\
\hline SBW1-27 & 0.0015 & & & 0.0013 & & & & 0.0074 & & & & 0.0001 & 0.0003 & & 0.0032 & & 0.0002 & & & 0.0007 & \\
\hline SBW1-28 & 0.0015 & & & 0.0013 & & & & 0.0074 & & & & 0.0001 & 0.0003 & & 0.0032 & & 0.0002 & & & 0.0007 & \\
\hline SBW1-29 & 0.0015 & & & 0.0013 & & & & 0.0026 & & & & 0.0001 & 0.0003 & & 0.0083 & & 0.0002 & & & 0.0007 & \\
\hline SBW1-30 & 0.0015 & & & 0.0013 & & & & 0.0026 & & & & 0.0001 & 0.0003 & & 0.0032 & & 0.0002 & & & 0.0007 & \\
\hline SBW1-31D & 0.0015 & & & 0.0013 & & & & 0.0078 & & & & 0.0001 & 0.0003 & & 0.0028 & & 0.0002 & & & 0.0007 & \\
\hline SBW1-32 & 0.0015 & & & 0.0013 & & & & 0.0090 & & & & 0.0001 & 0.0003 & & 0.0100 & & 0.0002 & & & 0.0007 & \\
\hline SBW1-33A & 0.0015 & & & 0.0013 & & & & 0.0010 & & & & 0.0001 & 0.0003 & & 0.0100 & & 0.0002 & & & 0.0007 & \\
\hline SBW1-34 & 0.0015 & & & 0.0013 & & & & 0.0010 & & & & 0.0001 & 0.0003 & & 0.0015 & & 0.0002 & & & 0.0007 & \\
\hline SBW1-35 & 0.0015 & & & 0.0013 & & & & 0.0010 & & & & 0.0001 & 0.0003 & & 0.0015 & & 0.0002 & & & 0.0007 & \\
\hline SBW1-36 & 0.0015 & & & 0.0013 & & & & 0.0090 & & & & 0.0001 & 0.0003 & & 0.0100 & & 0.0002 & & & 0.0007 & \\
\hline SBW1-37 & 0.0015 & & & 0.0013 & & & & 0.0090 & & & & 0.0001 & 0.0003 & & 0.0015 & & 0.0002 & & & 0.0007 & \\
\hline SBW1-38B & 0.0015 & & & 0.0013 & & & & 0.0022 & & & & 0.0001 & 0.0003 & & 0.0087 & & 0.0002 & & & 0.0007 & \\
\hline SBW1-39B & 0.0015 & & & 0.0013 & & & & 0.0014 & & & & 0.0001 & 0.0003 & & 0.0096 & & 0.0002 & & & 0.0007 & \\
\hline SBW1-40 & 0.0015 & & & 0.0013 & & & & 0.0090 & & & & 0.0001 & 0.0003 & & 0.0015 & & 0.0002 & & & 0.0007 & \\
\hline SBW1-41 & 0.0015 & & & 0.0013 & & & & 0.0010 & & & & 0.0001 & 0.0003 & & 0.0015 & & 0.0002 & & & 0.0007 & \\
\hline SBW1-42 & 0.0015 & & & 0.0013 & & & & 0.0090 & & & & 0.0001 & 0.0003 & & 0.0100 & & 0.0002 & & & 0.0007 & \\
\hline SBW1-43 & 0.0015 & & & 0.0013 & & & & 0.0010 & & & & 0.0001 & 0.0003 & & 0.0100 & & 0.0002 & & & 0.0007 & \\
\hline
\end{tabular}


Appendix A. Database - mass fraction

SBW CVS Phase 1 (Scholes et al. 2002)

\begin{tabular}{|c|c|c|c|c|c|c|c|c|c|c|c|c|c|c|c|c|c|c|c|c|c|}
\hline Glass ID & $\begin{array}{c}\mathrm{PdO} \\
-\mathrm{t}\end{array}$ & $\begin{array}{c}\mathrm{Pr} 2 \mathrm{O} 3 \\
-\mathrm{t}\end{array}$ & $\begin{array}{c}\text { Pr6O11 } \\
-t\end{array}$ & $\begin{array}{c}\mathrm{Rb} 2 \mathrm{O} \\
-\mathrm{t}\end{array}$ & $\begin{array}{c}\mathrm{ReO} \\
-\mathrm{t}\end{array}$ & $\begin{array}{c}\mathrm{ReO} 2 \\
-\mathrm{t}\end{array}$ & $\begin{array}{c}\mathrm{Rh} 2 \mathrm{O} 3 \\
-\mathrm{t}\end{array}$ & $\begin{array}{c}\mathrm{RhO} 2 \\
-\mathrm{t}\end{array}$ & $\begin{array}{c}\mathrm{RuO} 2 \\
-\mathrm{t}\end{array}$ & $\begin{array}{c}\mathrm{Sb} 2 \mathrm{O} 3 \\
-\mathrm{t} \\
\end{array}$ & $\begin{array}{c}\mathrm{Sb} 2 \mathrm{O} 5 \\
-\mathrm{t}\end{array}$ & $\begin{array}{c}\mathrm{SeO} 2 \\
-\mathrm{t}\end{array}$ & $\begin{array}{c}\mathrm{Sm} 2 \mathrm{O} 3 \\
-\mathrm{t}\end{array}$ & $\begin{array}{c}\mathrm{SnO} \\
-\mathrm{t} \\
\end{array}$ & $\begin{array}{c}\mathrm{SnO} 2 \\
-\mathrm{t}\end{array}$ & $\begin{array}{c}\mathrm{SO} 3 \\
-\mathrm{t}\end{array}$ & $\begin{array}{c}\mathrm{SrO} \\
-\mathrm{t} \\
\end{array}$ & $\begin{array}{c}\mathrm{Tc} 2 \mathrm{O} 7 \\
-\mathrm{t}\end{array}$ & $\begin{array}{c}\mathrm{TeO} 2 \\
-\mathrm{t}\end{array}$ & $\begin{array}{c}\mathrm{ThO} 2 \\
-\mathrm{t}\end{array}$ & $\begin{array}{c}\mathrm{TiO} 2 \\
-\mathrm{t}\end{array}$ \\
\hline SBW1-24 & & & & & & & & & 0.0000 & & & & & & & 0.0050 & & & & & \\
\hline SBW1-25 & & & & & & & & & 0.0000 & & & & & & & 0.0050 & & & & & \\
\hline SBW1-26 & & & & & & & & & 0.0000 & & & & & & & 0.0050 & & & & & \\
\hline SBW1-27 & & & & & & & & & 0.0000 & & & & & & & 0.0050 & & & & & \\
\hline SBW1-28 & & & & & & & & & 0.0000 & & & & & & & 0.0050 & & & & & \\
\hline SBW1-29 & & & & & & & & & 0.0000 & & & & & & & 0.0050 & & & & & \\
\hline SBW1-30 & & & & & & & & & 0.0000 & & & & & & & 0.0050 & & & & & \\
\hline SBW1-31D & & & & & & & & & 0.0000 & & & & & & & 0.0050 & & & & & \\
\hline SBW1-32 & & & & & & & & & 0.0000 & & & & & & & 0.0050 & & & & & \\
\hline SBW1-33A & & & & & & & & & 0.0000 & & & & & & & 0.0050 & & & & & \\
\hline SBW1-34 & & & & & & & & & 0.0000 & & & & & & & 0.0050 & & & & & \\
\hline SBW1-35 & & & & & & & & & 0.0000 & & & & & & & 0.0050 & & & & & \\
\hline SBW1-36 & & & & & & & & & 0.0000 & & & & & & & 0.0050 & & & & & \\
\hline SBW1-37 & & & & & & & & & 0.0000 & & & & & & & 0.0050 & & & & & \\
\hline SBW1-38B & & & & & & & & & 0.0000 & & & & & & & 0.0050 & & & & & \\
\hline SBW1-39B & & & & & & & & & 0.0000 & & & & & & & 0.0050 & & & & & \\
\hline SBW1-40 & & & & & & & & & 0.0000 & & & & & & & 0.0050 & & & & & \\
\hline SBW1-41 & & & & & & & & & 0.0000 & & & & & & & 0.0050 & & & & & \\
\hline SBW1-42 & & & & & & & & & 0.0000 & & & & & & & 0.0050 & & & & & \\
\hline SBW1-43 & & & & & & & & & 0.0000 & & & & & & & 0.0050 & & & & & \\
\hline
\end{tabular}


Appendix A. Database - mass fraction

\begin{tabular}{|c|c|c|c|c|c|c|c|c|c|c|c|c|c|c|c|c|c|c|c|c|c|}
\hline Glass ID & $\begin{array}{c}\mathrm{T} 12 \mathrm{O} 3 \\
-\mathrm{t} \\
\end{array}$ & $\begin{array}{c}\text { U3O8 } \\
-t\end{array}$ & $\begin{array}{c}\mathrm{UO} 2 \\
-\mathrm{t}\end{array}$ & $\begin{array}{c}\mathrm{UO} 3 \\
-\mathrm{t}\end{array}$ & $\begin{array}{c}\mathrm{V} 2 \mathrm{O} 5 \\
-\mathrm{t}\end{array}$ & $\begin{array}{c}\text { WO3 } \\
-t\end{array}$ & $\begin{array}{c}\mathrm{Y} 2 \mathrm{O} 3 \\
-\mathrm{t}\end{array}$ & $\begin{array}{c}\mathrm{ZnO} \\
-\mathrm{t}\end{array}$ & $\begin{array}{c}\text { Others } \\
-t\end{array}$ & $\begin{array}{c}\text { Sum } \\
-\mathrm{t}\end{array}$ & $\begin{array}{c}\mathrm{A} 12 \mathrm{O} 3 \\
-\mathrm{a}\end{array}$ & $\begin{array}{c}\mathrm{B} 2 \mathrm{O} 3 \\
-\mathrm{a}\end{array}$ & $\begin{array}{c}\mathrm{CaO} \\
-\mathrm{a} \\
\end{array}$ & $\begin{array}{c}\mathrm{Fe} 2 \mathrm{O} 3 \\
-\mathrm{a} \\
\end{array}$ & $\begin{array}{c}\mathrm{FeO} \\
-\mathrm{a} \\
\end{array}$ & $\begin{array}{c}\mathrm{K} 2 \mathrm{O} \\
-\mathrm{a}\end{array}$ & $\begin{array}{c}\mathrm{Li} 2 \mathrm{O} \\
-\mathrm{a}\end{array}$ & $\begin{array}{c}\mathrm{MgO} \\
-\mathrm{a}\end{array}$ & $\begin{array}{c}\mathrm{Na} 2 \mathrm{O} \\
-\mathrm{a}\end{array}$ & $\begin{array}{c}\mathrm{NiO} \\
-\mathrm{a} \\
\end{array}$ & $\begin{array}{c}\mathrm{P} 2 \mathrm{O} 5 \\
-\mathrm{a}\end{array}$ \\
\hline SBW1-24 & & & & & & & & & & 1.0000 & 0.1589 & 0.1251 & 0.0129 & 0.0802 & & 0.0428 & 0.0096 & & 0.1058 & 0.0009 & 0.0136 \\
\hline SBW1-25 & & & & & & & & & & 1.0000 & 0.0628 & 0.1266 & 0.0406 & 0.0224 & & 0.0188 & 0.0097 & & 0.1277 & 0.0009 & 0.0140 \\
\hline SBW1-26 & & & & & & & & & & 1.0000 & 0.0699 & 0.0720 & 0.0125 & 0.0797 & & 0.0430 & 0.0244 & & 0.1042 & 0.0009 & 0.0049 \\
\hline SBW1-27 & & & & & & & & & & 1.0000 & 0.1565 & 0.1282 & 0.0125 & 0.0215 & & 0.0188 & 0.0098 & & 0.1288 & 0.0009 & 0.0047 \\
\hline SBW1-28 & & & & & & & & & & 1.0000 & 0.0955 & 0.1281 & 0.0127 & 0.0804 & & 0.0441 & 0.0385 & & 0.1063 & 0.0009 & 0.0141 \\
\hline SBW1-29 & & & & & & & & & & 1.0000 & 0.1161 & 0.1313 & 0.0396 & 0.0218 & & 0.0431 & 0.0397 & & 0.1401 & 0.0009 & 0.0140 \\
\hline SBW1-30 & & & & & & & & & & 1.0000 & 0.0731 & 0.1259 & 0.0129 & 0.0798 & & 0.0191 & 0.0096 & & 0.2107 & 0.0009 & 0.0046 \\
\hline SBW1-31D & & & & & & & & & & 1.0000 & 0.1690 & 0.1386 & 0.0090 & 0.0854 & & 0.0177 & 0.0421 & & 0.0942 & 0.0010 & 0.0161 \\
\hline SBW1-32 & & & & & & & & & & 1.0000 & 0.0369 & 0.1494 & 0.0032 & 0.0026 & & 0.0506 & 0.0491 & & 0.0675 & 0.0011 & 0.0003 \\
\hline SBW1-33A & & & & & & & & & & 1.0000 & 0.0387 & 0.1502 & 0.0473 & 0.0993 & & 0.0514 & 0.0000 & & 0.0681 & 0.0009 & 0.0158 \\
\hline SBW1-34 & & & & & & & & & & 1.0000 & 0.0008 & 0.1481 & 0.0032 & 0.0021 & & 0.0111 & 0.0488 & & 0.0693 & 0.0009 & 0.0003 \\
\hline SBW1-35 & & & & & & & & & & 1.0000 & 0.1648 & 0.1483 & 0.0506 & 0.0026 & & 0.0518 & 0.0000 & & 0.1980 & 0.0009 & 0.0004 \\
\hline SBW1-36 & & & & & & & & & & 1.0000 & 0.1732 & 0.0492 & 0.0033 & 0.0024 & & 0.0103 & 0.0000 & & 0.2341 & 0.0008 & 0.0004 \\
\hline SBW1-37 & & & & & & & & & & 1.0000 & 0.0428 & 0.0510 & 0.0034 & 0.1004 & & 0.0112 & 0.0000 & & 0.2196 & 0.0009 & 0.0195 \\
\hline SBW1-38B & & & & & & & & & & 1.0000 & 0.1664 & 0.1392 & 0.0090 & 0.0866 & & 0.0173 & 0.0077 & & 0.1151 & 0.0009 & 0.0038 \\
\hline SBW1-39B & & & & & & & & & & 1.0000 & 0.1610 & 0.0596 & 0.0051 & 0.0957 & & 0.0485 & 0.0026 & & 0.1928 & 0.0008 & 0.0028 \\
\hline SBW1-40 & & & & & & & & & & 1.0000 & 0.1528 & 0.1381 & 0.0034 & 0.0995 & & 0.0534 & 0.0488 & & 0.0701 & 0.0010 & 0.0004 \\
\hline SBW1-41 & & & & & & & & & & 1.0000 & 0.0411 & 0.1444 & 0.0031 & 0.0987 & & 0.0109 & 0.0000 & & 0.2232 & 0.0009 & 0.0004 \\
\hline SBW1-42 & & & & & & & & & & 1.0000 & 0.0465 & 0.1451 & 0.0521 & 0.0986 & & 0.0138 & 0.0440 & & 0.0790 & 0.0009 & 0.0041 \\
\hline SBW1-43 & & & & & & & & & & 1.0000 & 0.1586 & 0.1455 & 0.0033 & 0.0024 & & 0.0108 & 0.0476 & & 0.1762 & 0.0009 & 0.0169 \\
\hline
\end{tabular}


Appendix A. Database - mass fraction

SBW CVS Phase 1 (Scholes et al. 2002)

\begin{tabular}{|c|c|c|c|c|c|c|c|c|c|c|c|c|c|c|c|c|c|c|c|c|c|}
\hline Glass ID & $\begin{array}{c}\mathrm{SiO} 2 \\
-\mathrm{a} \\
\end{array}$ & $\begin{array}{c}\mathrm{ZrO} 2 \\
-\mathrm{a} \\
\end{array}$ & $\begin{array}{c}\mathrm{Ag} 2 \mathrm{O} \\
-\mathrm{a}\end{array}$ & $\begin{array}{c}\mathrm{As} 2 \mathrm{O} 3 \\
-\mathrm{a} \\
\end{array}$ & $\begin{array}{c}\mathrm{BaO} \\
-\mathrm{a} \\
\end{array}$ & $\begin{array}{c}\mathrm{Bi} 2 \mathrm{O} 3 \\
-\mathrm{a} \\
\end{array}$ & $\begin{array}{l}\mathrm{Br} \\
-\mathrm{a}\end{array}$ & $\begin{array}{c}\mathrm{CdO} \\
-\mathrm{a} \\
\end{array}$ & $\begin{array}{c}\mathrm{Ce} 2 \mathrm{O} 3 \\
-\mathrm{a}\end{array}$ & $\begin{array}{c}\mathrm{CeO} 2 \\
-\mathrm{a} \\
\end{array}$ & $\begin{array}{l}\mathrm{Cl} \\
-\mathrm{a} \\
\end{array}$ & $\begin{array}{c}\mathrm{CoO} \\
-\mathrm{a} \\
\end{array}$ & $\begin{array}{c}\mathrm{Co} 2 \mathrm{O} 3 \\
-\mathrm{a} \\
\end{array}$ & $\begin{array}{c}\mathrm{Cr} 2 \mathrm{O} 3 \\
-\mathrm{a} \\
\end{array}$ & $\begin{array}{c}\mathrm{Cs} 2 \mathrm{O} \\
-\mathrm{a} \\
\end{array}$ & $\begin{array}{c}\mathrm{CuO} \\
-\mathrm{a} \\
\end{array}$ & $\begin{array}{c}\mathrm{Eu} 2 \mathrm{O} 3 \\
-\mathrm{a}\end{array}$ & $\begin{array}{c}\mathrm{F} \\
-\mathrm{a} \\
\end{array}$ & $\begin{array}{c}\mathrm{Ga} 2 \mathrm{O} 3 \\
-\mathrm{a} \\
\end{array}$ & $\begin{array}{c}\mathrm{Gd} 2 \mathrm{O} 3 \\
-\mathrm{a} \\
\end{array}$ & $\begin{array}{c}\mathrm{HgO} \\
-\mathrm{a} \\
\end{array}$ \\
\hline SBW1-24 & 0.3878 & 0.0039 & & & 0.0103 & & & 0.0047 & & & 0.0014 & & & 0.0011 & & & & 0.0018 & & & \\
\hline SBW1-25 & 0.5032 & 0.0132 & & & 0.0105 & & & 0.0046 & & & 0.0013 & & & 0.0011 & & & & 0.0024 & & & \\
\hline SBW1-26 & 0.5208 & 0.0164 & & & 0.0396 & & & 0.0012 & & & 0.0014 & & & 0.0011 & & & & 0.0026 & & & \\
\hline SBW1-27 & 0.4298 & 0.0164 & & & 0.0367 & & & 0.0012 & & & 0.0012 & & & 0.0011 & & & & 0.0060 & & & \\
\hline SBW1-28 & 0.3937 & 0.0146 & & & 0.0362 & & & 0.0048 & & & 0.0013 & & & 0.0011 & & & & 0.0047 & & & \\
\hline SBW1-29 & 0.3987 & 0.0129 & & & 0.0103 & & & 0.0012 & & & 0.0013 & & & 0.0010 & & & & 0.0026 & & & \\
\hline SBW1-30 & 0.4211 & 0.0041 & & & 0.0101 & & & 0.0012 & & & 0.0013 & & & 0.0011 & & & & 0.0023 & & & \\
\hline SBW1-31D & 0.4084 & 0.0029 & & & 0.0062 & & & 0.0050 & & & 0.0013 & & & 0.0011 & & & & 0.0063 & & & \\
\hline SBW1-32 & 0.5946 & 0.0001 & & & 0.0003 & & & 0.0000 & & & 0.0013 & & & 0.0011 & & & & 0.0075 & & & \\
\hline SBW1-33A & 0.4721 & 0.0174 & & & 0.0003 & & & 0.0000 & & & 0.0013 & & & 0.0011 & & & & 0.0011 & & & \\
\hline SBW1-34 & 0.5930 & 0.0393 & & & 0.0480 & & & 0.0060 & & & 0.0013 & & & 0.0010 & & & & 0.0015 & & & \\
\hline SBW1-35 & 0.3409 & 0.0000 & & & 0.0000 & & & 0.0058 & & & 0.0020 & & & 0.0011 & & & & 0.0009 & & & \\
\hline SBW1-36 & 0.4202 & 0.0000 & & & 0.0460 & & & 0.0000 & & & 0.0012 & & & 0.0010 & & & & 0.0076 & & & \\
\hline SBW1-37 & 0.5158 & 0.0194 & & & 0.0001 & & & 0.0059 & & & 0.0014 & & & 0.0011 & & & & 0.0081 & & & \\
\hline SBW1-38B & 0.4073 & 0.0028 & & & 0.0391 & & & 0.0050 & & & 0.0010 & & & 0.0011 & & & & 0.0020 & & & \\
\hline SBW1-39B & 0.3842 & 0.0180 & & & 0.0023 & & & 0.0053 & & & 0.0007 & & & 0.0010 & & & & 0.0014 & & & \\
\hline SBW1-40 & 0.3653 & 0.0209 & & & 0.0467 & & & 0.0000 & & & 0.0011 & & & 0.0011 & & & & 0.0056 & & & \\
\hline SBW1-41 & 0.4505 & 0.0001 & & & 0.0001 & & & 0.0000 & & & 0.0015 & & & 0.0011 & & & & 0.0012 & & & \\
\hline SBW1-42 & 0.4580 & 0.0209 & & & 0.0003 & & & 0.0054 & & & 0.0012 & & & 0.0011 & & & & 0.0048 & & & \\
\hline SBW1-43 & 0.3704 & 0.0140 & & & 0.0002 & & & 0.0000 & & & 0.0013 & & & 0.0010 & & & & 0.0012 & & & \\
\hline
\end{tabular}


Appendix A. Database - mass fraction

SBW CVS Phase 1 (Scholes et al. 2002)

\begin{tabular}{|c|c|c|c|c|c|c|c|c|c|c|c|c|c|c|c|c|c|c|c|c|c|}
\hline Glass ID & $\begin{array}{c}\mathrm{I} \\
-\mathrm{a} \\
\end{array}$ & $\begin{array}{c}\mathrm{La} 2 \mathrm{O} 3 \\
-\mathrm{a} \\
\end{array}$ & $\begin{array}{c}\mathrm{MnO} 2 \\
-\mathrm{a} \\
\end{array}$ & $\begin{array}{c}\mathrm{MnO} \\
-\mathrm{a} \\
\end{array}$ & $\begin{array}{c}\mathrm{MoO} \\
-\mathrm{a}\end{array}$ & $\begin{array}{c}\mathrm{MoO} 3 \\
-\mathrm{a}\end{array}$ & $\begin{array}{c}\mathrm{Nb} 2 \mathrm{O} 5 \\
-\mathrm{a}\end{array}$ & $\begin{array}{c}\mathrm{Nd} 2 \mathrm{O} 3 \\
-\mathrm{a} \\
\end{array}$ & $\begin{array}{c}\mathrm{PbO} \\
-\mathrm{a} \\
\end{array}$ & $\begin{array}{c}\mathrm{PdO} 2 \\
-\mathrm{a}\end{array}$ & $\begin{array}{c}\mathrm{PdO} \\
-\mathrm{a} \\
\end{array}$ & $\begin{array}{c}\mathrm{Pr} 2 \mathrm{O} 3 \\
-\mathrm{a} \\
\end{array}$ & $\begin{array}{c}\text { Pr6O11 } \\
-\mathrm{a}\end{array}$ & $\begin{array}{c}\mathrm{Rb} 2 \mathrm{O} \\
-\mathrm{a}\end{array}$ & $\begin{array}{c}\mathrm{ReO} \\
-\mathrm{a}\end{array}$ & $\begin{array}{c}\mathrm{ReO} 2 \\
-\mathrm{a} \\
\end{array}$ & $\begin{array}{c}\mathrm{Rh} 2 \mathrm{O} 3 \\
-\mathrm{a}\end{array}$ & $\begin{array}{c}\mathrm{RhO}_{2} \\
-\mathrm{a}\end{array}$ & $\begin{array}{c}\mathrm{RuO} 2 \\
-\mathrm{a}\end{array}$ & $\begin{array}{c}\mathrm{Sb} 2 \mathrm{O} 3 \\
-\mathrm{a} \\
\end{array}$ & $\begin{array}{c}\mathrm{Sb} 2 \mathrm{O} 5 \\
-\mathrm{a}\end{array}$ \\
\hline SBW1-24 & & & & 0.0060 & & & & & & & & & & & & & & & & & \\
\hline SBW1-25 & & & & 0.0022 & & & & & & & & & & & & & & & & & \\
\hline SBW1-26 & & & & 0.0060 & & & & & & & & & & & & & & & & & \\
\hline SBW1-27 & & & & 0.0023 & & & & & & & & & & & & & & & & & \\
\hline SBW1-28 & & & & 0.0024 & & & & & & & & & & & & & & & & & \\
\hline SBW1-29 & & & & 0.0059 & & & & & & & & & & & & & & & & & \\
\hline SBW1-30 & & & & 0.0023 & & & & & & & & & & & & & & & & & \\
\hline SBW1-31D & & & & 0.0021 & & & & & & & & & & & & & & & & & \\
\hline SBW1-32 & & & & 0.0071 & & & & & & & & & & & & & & & & & \\
\hline SBW1-33A & & & & 0.0071 & & & & & & & & & & & & & & & & & \\
\hline SBW1-34 & & & & 0.0011 & & & & & & & & & & & & & & & & & \\
\hline SBW1-35 & & & & 0.0011 & & & & & & & & & & & & & & & & & \\
\hline SBW1-36 & & & & 0.0070 & & & & & & & & & & & & & & & & & \\
\hline SBW1-37 & & & & 0.0012 & & & & & & & & & & & & & & & & & \\
\hline SBW1-38B & & & & 0.0065 & & & & & & & & & & & & & & & & & \\
\hline SBW1-39B & & & & 0.0069 & & & & & & & & & & & & & & & & & \\
\hline SBW1-40 & & & & 0.0011 & & & & & & & & & & & & & & & & & \\
\hline SBW1-41 & & & & 0.0011 & & & & & & & & & & & & & & & & & \\
\hline SBW1-42 & & & & 0.0072 & & & & & & & & & & & & & & & & & \\
\hline SBW1-43 & & & & 0.0071 & & & & & & & & & & & & & & & & & \\
\hline
\end{tabular}


Appendix A. Database - mass fraction

SBW CVS Phase 1 (Scholes et al. 2002)

\begin{tabular}{|c|c|c|c|c|c|c|c|c|c|c|c|c|c|c|c|c|c|c|c|c|}
\hline Glass ID & $\begin{array}{c}\mathrm{SeO} 2 \\
-\mathrm{a}\end{array}$ & $\begin{array}{c}\mathrm{Sm} 2 \mathrm{O} 3 \\
-\mathrm{a}\end{array}$ & $\begin{array}{c}\mathrm{SnO} \\
-\mathrm{a} \\
\end{array}$ & $\begin{array}{c}\mathrm{SnO} 2 \\
-\mathrm{a}\end{array}$ & $\begin{array}{c}\mathrm{SO} 3 \\
-\mathrm{a} \\
\end{array}$ & $\begin{array}{c}\mathrm{SrO} \\
-\mathrm{a} \\
\end{array}$ & $\begin{array}{c}\mathrm{Tc} 2 \mathrm{O} 7 \\
-\mathrm{a}\end{array}$ & $\begin{array}{c}\mathrm{TeO} 2 \\
-\mathrm{a}\end{array}$ & $\begin{array}{c}\mathrm{ThO} 2 \\
-\mathrm{a}\end{array}$ & $\begin{array}{c}\mathrm{TiO} 2 \\
-\mathrm{a} \\
\end{array}$ & $\begin{array}{c}\mathrm{T} 12 \mathrm{O} 3 \\
-\mathrm{a}\end{array}$ & $\begin{array}{c}\mathrm{U} 3 \mathrm{O} 8 \\
-\mathrm{a}\end{array}$ & $\begin{array}{c}\mathrm{UO} 2 \\
-\mathrm{a}\end{array}$ & $\begin{array}{c}\mathrm{UO} 3 \\
-\mathrm{a}\end{array}$ & $\begin{array}{c}\mathrm{V} 2 \mathrm{O} 5 \\
-\mathrm{a}\end{array}$ & $\begin{array}{c}\text { WO3 } \\
-\mathrm{a}\end{array}$ & $\begin{array}{c}\mathrm{Y} 2 \mathrm{O} 3 \\
-\mathrm{a}\end{array}$ & $\begin{array}{c}\mathrm{ZnO} \\
-\mathrm{a} \\
\end{array}$ & $\begin{array}{c}\text { Others } \\
-\mathrm{a}\end{array}$ & $\begin{array}{c}\text { Sum } \\
-\mathrm{a}\end{array}$ \\
\hline SBW1-24 & & & & & 0.0037 & & & & & & & & & & & & & & & 0.9705 \\
\hline SBW1-25 & & & & & 0.0052 & & & & & & & & & & & & & & & 0.9673 \\
\hline SBW1-26 & & & & & 0.0054 & & & & & & & & & & & & & & & 1.0059 \\
\hline SBW1-27 & & & & & 0.0048 & & & & & & & & & & & & & & & 0.9812 \\
\hline SBW1-28 & & & & & 0.0056 & & & & & & & & & & & & & & & 0.9848 \\
\hline SBW1-29 & & & & & 0.0055 & & & & & & & & & & & & & & & 0.9861 \\
\hline SBW1-30 & & & & & 0.0058 & & & & & & & & & & & & & & & 0.9857 \\
\hline SBW1-31D & & & & & 0.0050 & & & & & & & & & & & & & & & 1.0115 \\
\hline SBW1-32 & & & & & 0.0051 & & & & & & & & & & & & & & & 0.9778 \\
\hline SBW1-33A & & & & & 0.0048 & & & & & & & & & & & & & & & 0.9767 \\
\hline SBW1-34 & & & & & 0.0049 & & & & & & & & & & & & & & & 0.9807 \\
\hline SBW1-35 & & & & & 0.0053 & & & & & & & & & & & & & & & 0.9747 \\
\hline SBW1-36 & & & & & 0.0054 & & & & & & & & & & & & & & & 0.9621 \\
\hline SBW1-37 & & & & & 0.0059 & & & & & & & & & & & & & & & 1.0077 \\
\hline SBW1-38B & & & & & 0.0028 & & & & & & & & & & & & & & & 1.0137 \\
\hline SBW1-39B & & & & & 0.0043 & & & & & & & & & & & & & & & 0.9928 \\
\hline SBW1-40 & & & & & 0.0053 & & & & & & & & & & & & & & & 1.0144 \\
\hline SBW1-41 & & & & & 0.0057 & & & & & & & & & & & & & & & 0.9839 \\
\hline SBW1-42 & & & & & 0.0050 & & & & & & & & & & & & & & & 0.9880 \\
\hline SBW1-43 & & & & & 0.0055 & & & & & & & & & & & & & & & 0.9628 \\
\hline
\end{tabular}


Appendix A. Database - mass fraction

SBW CVS Phase 1 (Scholes et al. 2002)

\begin{tabular}{|c|c|c|c|c|c|c|c|c|}
\hline Glass ID & $\begin{array}{l}\mathrm{TM} \\
\left({ }^{\circ} \mathrm{C}\right)\end{array}$ & $\begin{array}{l}\text { Gradient } \\
\text { TL }\left({ }^{\circ} \mathrm{C}\right)\end{array}$ & $\begin{array}{l}\text { Uniform } \\
\text { TL }\left({ }^{\circ} \mathrm{C}\right)\end{array}$ & Primary Phase & $\begin{array}{l}\text { Quenched } \\
\text { Visual/OM }\end{array}$ & $\begin{array}{c}\text { Quenched } \\
\text { SEM/EDS or TEM }\end{array}$ & $\begin{array}{l}\text { Quenched } \\
\text { XRD }\end{array}$ & $\begin{array}{c}\text { Quenched } \\
\text { Homogeneous? }\end{array}$ \\
\hline SBW1-24 & 1150 & & 1038 & $\mathrm{Ca} 4 \mathrm{P} 2 \mathrm{O} 9$ & $\begin{array}{l}\text { Clear dark brown glass with black specks anc } \\
\text { air bubbles, high viscosity }\end{array}$ & & $\mathrm{Ca} 5(\mathrm{PO} 4) 3(\mathrm{OH})$ & Single-phase \\
\hline SBW1-25 & 1150 & & 1031 & $\mathrm{Ca} 5(\mathrm{PO} 4) 3 \mathrm{~F}$ & $\begin{array}{l}\text { Clear dark brown glass with black specks anc } \\
\text { air bubbles, avg. viscosity }\end{array}$ & & Na3Ca6(PO4)5 & Single-phase \\
\hline SBW1-26 & 1150 & & 866 & $\mathrm{~K}(\mathrm{Si} 3 \mathrm{Al}) \mathrm{O} 8$ & $\begin{array}{l}\text { Clear dark brown glass with black specks anc } \\
\text { air bubbles, avg. viscosity }\end{array}$ & & Amorphous & Single-phase \\
\hline SBW1-27 & 1150 & & 943 & $\mathrm{Ca} 5(\mathrm{PO} 4) 3 \mathrm{~F}$ & $\begin{array}{l}\text { Clear dark brown glass with black specks anc } \\
\text { lots of air bubbles, high viscosity }\end{array}$ & & Amorphous & Single-phase \\
\hline SBW1-28 & 1150 & & 878 & NaAlSiO4 & $\begin{array}{l}\text { Clear brown glass with black specks and air } \\
\text { bubbles, low viscosity }\end{array}$ & & Amorphous & Single-phase \\
\hline SBW1-29 & 1150 & & 793 & $\mathrm{Ca} 5(\mathrm{PO} 4) 3 \mathrm{~F}$ & $\begin{array}{l}\text { Clear very dark brown glass with black } \\
\text { specks and few air bubbles, low viscosity }\end{array}$ & & Amorphous & Single-phase \\
\hline SBW1-30 & 1150 & & 756 & $\mathrm{NaAlSiO} 4$ & $\begin{array}{l}\text { Clear dark brown glass with few black speck } \\
\text { and air bubbles, low viscosity }\end{array}$ & & Amorphous & Single-phase \\
\hline SBW1-31D & 1150 & & 1028 & $\mathrm{Fe} 2 \mathrm{O} 3$ & $\begin{array}{l}\text { Clear dark brown glass with black specks anc } \\
\text { air bubbles, low viscosity }\end{array}$ & & Amorphous & Single-phase \\
\hline SBW1-32 & 1150 & & 773 & $\mathrm{SiO} 2$ & $\begin{array}{l}\text { Clear very dark reddish brown glass with } \\
\text { black specks and air bubbles, low viscosity }\end{array}$ & & Amorphous & Single-phase \\
\hline SBW1-33A & 1250 & & & & $\begin{array}{l}\text { Opaque brownish green glass with multi- } \\
\text { phases and thin clear layer of glass on } \\
\text { bottom, avg. viscosity }\end{array}$ & & $\mathrm{Ca} 5(\mathrm{PO} 4) 3 \mathrm{~F}$ & $\begin{array}{l}\text { Multi-phase, } \\
\text { opaque }\end{array}$ \\
\hline SBW1-34 & 1150 & & 978 & $\mathrm{SiO} 2$ & $\begin{array}{l}\text { Clear brown glass with some black specks } \\
\text { and lots of air bubbles, low viscosity }\end{array}$ & & Amorphous & Single-phase \\
\hline SBW1-35 & 1150 & & 908 & Na6(AlSiO4)6 & $\begin{array}{l}\text { Clear light brown glass with few black } \\
\text { specks and lots of air bubbles, low viscosity }\end{array}$ & & Amorphous & Single-phase \\
\hline SBW1-36 & 1150 & & 1123 & $\mathrm{Na} 6(\mathrm{AlSiO} 4) 6$ & $\begin{array}{l}\text { Clear dark reddish brown glass with air } \\
\text { bubbles mostly on top of melt, high viscosity }\end{array}$ & & Amorphous & Single-phase \\
\hline SBW1-37 & 1150 & & 916 & $\mathrm{Na} 2 \mathrm{CaSiO} 4$ & $\begin{array}{l}\text { Clear brown glass with black specks and air } \\
\text { bubbles, avg. viscosity }\end{array}$ & & Amorphous & Single-phase \\
\hline SBW1-38B & 1350 & & 1131 & $\mathrm{Fe} 3 \mathrm{O} 4$ & $\begin{array}{l}\text { Clear very dark black shiny glass foamed out } \\
\text { of crucible, avg. viscosity }\end{array}$ & & Amorphous & \begin{tabular}{|l} 
Single-phase \\
\end{tabular} \\
\hline SBW1-39B & 1350 & & 1073 & $\mathrm{NaAlSiO} 4$ & $\begin{array}{l}\text { Clear dark brown glass with black specks ane } \\
\text { air bubbles, low viscosity }\end{array}$ & & Amorphous & Single-phase \\
\hline SBW1-40 & 1150 & & 1038 & $\mathrm{LiFe} 2 \mathrm{O} 3 \mathrm{~F}$ & $\begin{array}{l}\text { Clear dark brown glass with air bubbles, low } \\
\text { viscosity }\end{array}$ & & Amorphous & Single-phase \\
\hline SBW1-41 & 1150 & & 736 & $(\mathrm{~K}, \mathrm{Na})(\mathrm{Si} 3 \mathrm{Al}) \mathrm{O} 8$ & $\begin{array}{l}\text { Clear brown glass with a few black specks } \\
\text { and some air bubbles, low viscosity }\end{array}$ & & Amorphous & Single-phase \\
\hline SBW1-42 & 1150 & & 838 & $(\mathrm{Na}, \mathrm{Ca}) \mathrm{FeSi} 2 \mathrm{O} 6$ & $\begin{array}{l}\text { Clear dark brown glass with black specks anc } \\
\text { few air bubbles, low viscosity }\end{array}$ & & Amorphous & Single-phase \\
\hline SBW1-43 & 1150 & & 858 & Na6(AlSiO4)6 & $\begin{array}{l}\text { Clear dark black glass with some black } \\
\text { specks and very few air bubbles, low } \\
\text { viscosity }\end{array}$ & & Amorphous & Single-phase \\
\hline
\end{tabular}


Appendix A. Database - mass fraction

\begin{tabular}{|c|c|c|c|c|c|}
\hline Glass ID & $\begin{array}{c}\mathrm{CCC} \\
\text { Visual/OM }\end{array}$ & $\begin{array}{c}\text { CCC } \\
\text { SEM/EDS or TEM }\end{array}$ & $\begin{array}{l}\text { CCC } \\
\text { XRD }\end{array}$ & $\begin{array}{l}\text { Heat Treated } \\
\text { Visual/OM }\end{array}$ & $\begin{array}{c}\text { Heat Treated } \\
\text { SEM/EDS or TEM }\end{array}$ \\
\hline SBW1-24 & $\begin{array}{l}\text { Reddish brown and completely devitrified with very small } \\
\text { crystalline specks with some clusters of dark red crystals. }\end{array}$ & & $\begin{array}{l}\mathrm{Ca} 5(\mathrm{PO} 4) 3 \mathrm{~F} \text { and } \mathrm{Fe} 3 \mathrm{O} 4(0.76 \mathrm{vol} \% \\
\text { crystallinity) }\end{array}$ & & \\
\hline SBW1-25 & $\begin{array}{l}\text { Light reddish brown and phase-separated making the glass } \\
\text { cloudy also some long flaky crystals near the surface. }\end{array}$ & & $\begin{array}{l}\mathrm{Ca} 5(\mathrm{PO} 4) 3 \mathrm{~F} \text { and } \mathrm{CaF} 2(0.42 \mathrm{vol} \% \\
\text { crystallinity) }\end{array}$ & & \\
\hline SBW1-26 & $\begin{array}{l}\text { Clear dark brown glass, air bubbles and black specks } \\
\text { (expected to be Ru). }\end{array}$ & & Amorphous & & \\
\hline SBW1-27 & $\begin{array}{l}\text { Clear dark brown glass, air bubbles and black specks } \\
\text { (expected to be Ru). }\end{array}$ & & Amorphous & & \\
\hline SBW1-28 & $\begin{array}{l}\text { Clear brown glass with some clear crystilline needle clusters, } \\
\text { air bubbles and a few black specks (expected to be Ru). }\end{array}$ & & $\begin{array}{l}\mathrm{BaZr}(\mathrm{BO} 3) 2 \text { and } \mathrm{AlFe}(\mathrm{PO} 3) 6(0.04 \\
\text { vol\% crystallinity })\end{array}$ & & \\
\hline SBW1-29 & $\begin{array}{l}\text { Clear dark brown glass, with few air bubbles and black specks } \\
\text { (expected to be Ru). }\end{array}$ & & Amorphous & & \\
\hline SBW1-30 & $\begin{array}{l}\text { Clear brown glass, air bubbles and black specks (expected to } \\
\text { be Ru). }\end{array}$ & & Amorphous & & \\
\hline SBW1-31D & $\begin{array}{l}\text { Reddish brown and completely devitrified with small red and } \\
\text { white clusters of crystals. }\end{array}$ & & Fe2O3 ( 0.88 vol $\%$ crystallinity) & & \\
\hline SBW1-32 & $\begin{array}{l}\text { Clear dark purplish glass, air bubbles and black specks } \\
\text { (expected to be Ru). }\end{array}$ & & Amorphous & & \\
\hline SBW1-33A & $\begin{array}{l}\text { Very dark reddish brown and phase separated with a few } \\
\text { clusters of clear crystals. }\end{array}$ & & $\begin{array}{l}\mathrm{Ca} 5(\mathrm{PO} 4) 3(\mathrm{OH})(0.40 \mathrm{vol} \% \\
\text { crystallinity })\end{array}$ & & \\
\hline SBW1-34 & $\begin{array}{l}\text { Clear reddish brown glass with clusters of white crystals } \\
\text { scattered throughout and completely covering the walls of the } \\
\text { crucible. }\end{array}$ & & SiO2 (0.13 vol\% crystallinity) & & \\
\hline SBW1-35 & $\begin{array}{l}\text { Clear brown glass, air bubbles and black specks (expected to } \\
\text { be Ru). }\end{array}$ & & Amorphous & & \\
\hline SBW1-36 & $\begin{array}{l}\text { Clear purplish brown glass, few small clusters of very small } \\
\text { crystals near edges, also air bubbles and black specks } \\
\text { (expected to be } \mathrm{Ru} \text { ). }\end{array}$ & & Amorphous & & \\
\hline SBW1-37 & $\begin{array}{l}\text { Clear brown glass with clear cubic crystals and a slight cloudy } \\
\text { or opaque appearance, air bubbles and black specks (expected } \\
\text { to be } \mathrm{Ru} \text { ). }\end{array}$ & & $\mathrm{Ca} 2 \mathrm{~A} 1 \mathrm{SiO} 7$ & & \\
\hline SBW1-38B & $\begin{array}{l}\text { Opaque dark brown and phase separated with very small } \\
\text { crystals. }\end{array}$ & & $\begin{array}{l}\mathrm{Fe} 3 \mathrm{O} 4, \mathrm{KFeO} 2, \mathrm{Ba} 2 \mathrm{FeF} 6 \text {, and } \mathrm{Mn} 3 \mathrm{O} 4 \\
(0.34 \text { vol } \% \text { crystallinity })\end{array}$ & & \\
\hline SBW1-39B & $\begin{array}{l}\text { Clear brown glass with some spots that appear cloudy, also } \\
\text { some clear cubic crystals and flaky crystals. }\end{array}$ & & Amorphous & & \\
\hline SBW1-40 & $\begin{array}{l}\text { Clear reddish brown glass with clusters of white crystals } \\
\text { throughout. }\end{array}$ & & $\mathrm{Fe} 2 \mathrm{O} 3$ ( 0.53 vol $\%$ crystallinity) & & \\
\hline SBW1-41 & $\begin{array}{l}\text { Clear brown glass, few air bubbles and a few black specks } \\
\text { (expected to be Ru). }\end{array}$ & & Amorphous & & \\
\hline SBW1-42 & $\begin{array}{l}\text { Clear dark reddish brown glass, air bubbles and black specks } \\
\text { (expected to be Ru). }\end{array}$ & & Amorphous & & \\
\hline SBW1-43 & $\begin{array}{l}\text { Clear dark reddish brown glass, air bubbles and black specks } \\
\text { (expected to be Ru). }\end{array}$ & & Amorphous & & \\
\hline
\end{tabular}


Appendix A. Database - mass fraction

SBW CVS Phase 1 (Scholes et al. 2002)

\begin{tabular}{|c|c|c|c|c|c|c|c|c|c|c|c|c|c|c|c|}
\hline Glass ID & $\begin{array}{c}\text { Heat Treated } \\
\text { XRD }\end{array}$ & $\begin{array}{l}\text { Density } \\
\left(\mathrm{g} / \mathrm{cm}^{3}\right)\end{array}$ & $\begin{array}{c}\text { Fulc Visc } \\
\text { A }\end{array}$ & $\begin{array}{c}\text { Fulc Visc } \\
\text { B }\end{array}$ & $\begin{array}{c}\text { Fulc Visc } \\
\text { To } \\
\end{array}$ & $\begin{array}{c}\mathrm{FV} 1150^{\circ} \mathrm{C} \\
(\mathrm{Pa} \cdot \mathrm{s})\end{array}$ & $\begin{array}{c}\text { Arrh Visc } \\
\text { A }\end{array}$ & $\begin{array}{c}\text { Arrh Visc } \\
\text { B }\end{array}$ & 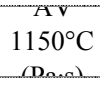 & $\begin{array}{c}\mathrm{T}\left({ }^{\circ} \mathrm{C}\right) \text { at } \\
2 \mathrm{~Pa} \cdot \mathrm{s}\end{array}$ & $\begin{array}{c}\mathrm{T}\left({ }^{\circ} \mathrm{C}\right) \text { at } \\
5 \mathrm{~Pa} \cdot \mathrm{s}\end{array}$ & $\begin{array}{l}\mathrm{T}\left({ }^{\circ} \mathrm{C}\right) \text { at } \\
10 \mathrm{~Pa} \cdot \mathrm{s}\end{array}$ & $\begin{array}{c}\mathrm{T} 1 \\
\left({ }^{\circ} \mathrm{C}\right) \\
\end{array}$ & $\begin{array}{c}\mathrm{V} 1 \\
(\mathrm{~Pa} \cdot \mathrm{s}) \\
\end{array}$ & $\begin{array}{c}\mathrm{T} 2 \\
\left({ }^{\circ} \mathrm{C}\right) \\
\end{array}$ \\
\hline SBW1-24 & & 2.5222 & & & & & -11.127 & 20624.0 & 28.97 & 1472 & & 1263 & 1154.2 & 28.332 & 1079.4 \\
\hline SBW1-25 & & 2.557 & & & & & -11.565 & 20246.0 & 14.33 & 1378 & & 1187 & 1156.9 & 12.822 & 1081 \\
\hline SBW1-26 & & 2.6375 & & & & & -10.730 & 19202.0 & 15.86 & 1408 & & 1200 & 1160.1 & 14.707 & 1084.3 \\
\hline SBW1-27 & & 2.5432 & & & & & -10.614 & 19604.0 & 23.63 & 1461 & & 1245 & 1157 & 21.673 & 1081.8 \\
\hline SBW1-28 & & 2.6379 & & & & & -10.100 & 15749.0 & 2.63 & 1186 & & 997 & 1159.7 & 2.3927 & 1083.6 \\
\hline SBW1-29 & & 2.5757 & & & & & -9.941 & 15261.0 & 2.19 & 1162 & & 973 & 1154.7 & 2.0715 & 1078.8 \\
\hline SBW1-30 & & 2.6163 & & & & & -9.866 & 15126.0 & 2.15 & 1159 & & 970 & 1156.8 & 1.9862 & 1081.9 \\
\hline SBW1-31D & & 2.5317 & & & & & -10.249 & 17603.0 & 8.34 & 1336 & & 1129 & 1155.4 & 7.8874 & 1080.1 \\
\hline SBW1-32 & & 2.4424 & & & & & -10.601 & 17980.0 & 7.65 & 1319 & & 1120 & 1155.3 & 6.9677 & 1079.8 \\
\hline SBW1-33A & & 2.5511 & & & & & & & & & & & & & \\
\hline \multicolumn{16}{|l|}{ SBW1-34 } \\
\hline SBW1-35 & & 2.516 & & & & & -9.882 & 16043.0 & 4.02 & 1244 & & 1044 & 1156.6 & 3.7113 & 1080.3 \\
\hline SBW1-36 & & 2.4982 & & & & & -9.945 & 18745.0 & 25.23 & 1489 & & 1257 & 1157 & 23.67 & 1081.2 \\
\hline SBW1-37 & & 2.6297 & & & & & -10.942 & 19060.0 & 11.61 & 1365 & & 1166 & 1161.7 & 10.183 & 1085.8 \\
\hline SBW1-38B & & 2.5806 & & & & & -10.922 & 20392.0 & 30.21 & 1482 & & 1269 & 1254.8 & 11.102 & 1180.3 \\
\hline SBW1-39B & & 2.6269 & & & & & -11.160 & 20554.0 & 26.69 & 1461 & & 1254 & 1260 & 9.1749 & 1184.3 \\
\hline SBW1-40 & & 2.6405 & & & & & -10.230 & 16274.0 & 3.34 & 1217 & & 1025 & 1157.4 & 3.0624 & 1082.5 \\
\hline SBW1-41 & & 2.6055 & & & & & -10.452 & 15888.0 & 2.04 & 1152 & & 973 & 1158.9 & 1.8486 & 1083.2 \\
\hline SBW1-42 & & 2.6517 & & & & & -10.780 & 16302.0 & 1.97 & 1148 & & 973 & 1155.9 & 1.8362 & 1079.7 \\
\hline SBW1-43 & & 2.5045 & & & & & -9.563 & 14877.0 & 2.44 & 1177 & & 981 & 1157.3 & 2.2767 & 1082.6 \\
\hline
\end{tabular}


Appendix A. Database - mass fraction

\begin{tabular}{|c|c|c|c|c|c|c|c|c|c|c|c|c|c|c|c|c|c|c|c|c|c|}
\hline \multicolumn{22}{|c|}{ SBW CVS Phase 1 (Scholes et al. 2002) } \\
\hline Glass ID & $\begin{array}{c}\mathrm{V} 2 \\
(\mathrm{~Pa} \cdot \mathrm{s})\end{array}$ & $\begin{array}{c}\mathrm{T} 3 \\
\left({ }^{\circ} \mathrm{C}\right) \\
\end{array}$ & $\begin{array}{c}\mathrm{V} 3 \\
(\mathrm{~Pa} \cdot \mathrm{s})\end{array}$ & $\begin{array}{c}\mathrm{T} 4 \\
\left({ }^{\circ} \mathrm{C}\right) \\
\end{array}$ & $\begin{array}{c}\mathrm{V} 4 \\
(\mathrm{~Pa} \cdot \mathrm{s})\end{array}$ & $\begin{array}{c}\mathrm{T} 5 \\
\left({ }^{\circ} \mathrm{C}\right) \\
\end{array}$ & $\begin{array}{c}\mathrm{V} 5 \\
(\mathrm{~Pa} \cdot \mathrm{s})\end{array}$ & $\begin{array}{c}\text { T6 } \\
\left({ }^{\circ} \mathrm{C}\right) \\
\end{array}$ & $\begin{array}{c}\mathrm{V} 6 \\
(\mathrm{~Pa} \cdot \mathrm{s}) \\
\end{array}$ & $\begin{array}{c}\mathrm{T} 7 \\
\left({ }^{\circ} \mathrm{C}\right) \\
\end{array}$ & $\begin{array}{c}\mathrm{V} 7 \\
(\mathrm{~Pa} \cdot \mathrm{s})\end{array}$ & $\begin{array}{c}\mathrm{T} 8 \\
\left({ }^{\circ} \mathrm{C}\right) \\
\end{array}$ & $\begin{array}{c}\mathrm{V} 8 \\
(\mathrm{~Pa} \cdot \mathrm{s}) \\
\end{array}$ & $\begin{array}{c}\mathrm{T} 9 \\
\left({ }^{\circ} \mathrm{C}\right) \\
\end{array}$ & $\begin{array}{c}\mathrm{V} 9 \\
(\mathrm{~Pa} \cdot \mathrm{s})\end{array}$ & $\begin{array}{l}\mathrm{T} 10 \\
\left({ }^{\circ} \mathrm{C}\right)\end{array}$ & $\begin{array}{c}\mathrm{V} 10 \\
(\mathrm{~Pa} \cdot \mathrm{s})\end{array}$ & $\begin{array}{l}\mathrm{T} 11 \\
\left({ }^{\circ} \mathrm{C}\right) \\
\end{array}$ & $\begin{array}{c}\mathrm{V} 11 \\
(\mathrm{~Pa} \cdot \mathrm{s})\end{array}$ & $\begin{array}{l}\mathrm{T} 12 \\
\left({ }^{\circ} \mathrm{C}\right) \\
\end{array}$ & $\begin{array}{c}\mathrm{V} 12 \\
(\mathrm{~Pa} \cdot \mathrm{s})\end{array}$ \\
\hline SBW1-24 & 60.128 & 1004.7 & 147.76 & 954.81 & 295.87 & & & & & & & & & & & & & & & & \\
\hline SBW1-25 & 27.433 & 1005.8 & 69.184 & 955.95 & 146.33 & 1254.4 & 5.896 & 1154.7 & 13.347 & & & & & & & & & & & & \\
\hline SBW1-26 & 29.521 & 1009.2 & 68.16 & 959.4 & 130.85 & 1157.7 & 14.777 & & & & & & & & & & & & & & \\
\hline SBW1-27 & 45.233 & 1005.9 & 110.3 & 956.34 & 215.53 & 1254.9 & 9.665 & 1155.3 & 21.849 & & & & & & & & & & & & \\
\hline SBW1-28 & 4.3675 & 1008.4 & 8.7955 & 958.76 & 15.138 & 1255.4 & 1.2615 & 1157.6 & 2.4861 & & & & & & & & & & & & \\
\hline SBW1-29 & 3.7258 & 1003.4 & 7.4194 & 953.65 & 12.537 & 1251.9 & 1.102 & 1152.8 & 2.1406 & & & & & & & & & & & & \\
\hline SBW1-30 & 3.508 & 1007.2 & 6.9017 & 957.55 & 11.77 & 1255.8 & 1.0706 & 1156.9 & 2.0318 & & & & & & & & & & & & \\
\hline SBW1-31D & 15.421 & 1004.9 & 33.644 & 955.2 & 60.362 & 1204.5 & 5.4093 & 1154.1 & 8.005 & & & & & & & & & & & & \\
\hline SBW1-32 & 13.866 & 1004.7 & 31.626 & 954.97 & 60.165 & 1253.7 & 3.4653 & 1154.4 & 7.2826 & & & & & & & & & & & & \\
\hline \multicolumn{22}{|l|}{ SBW1-33A } \\
\hline \multicolumn{22}{|l|}{ SBW1-34 } \\
\hline SBW1-35 & 6.8909 & 1004.8 & 14.234 & 955.04 & 25.045 & 1253.2 & 1.9498 & 1153.9 & 3.9126 & & & & & & & & & & & & \\
\hline SBW1-36 & 47.48 & 1006.3 & 108.7 & 956.63 & 206.68 & 1254.7 & 10.423 & 1155.4 & 23.974 & & & & & & & & & & & & \\
\hline SBW1-37 & 20.74 & 1010.8 & 48.913 & 961.18 & 93.577 & 1258.1 & 4.6471 & 1159.9 & 10.734 & & & & & & & & & & & & \\
\hline SBW1-38B & 22.073 & 1106 & 47.537 & 1055.7 & 85.116 & 1351.9 & 5.2433 & 1253 & 11.325 & & & & & & & & & & & & \\
\hline SBW1-39B & 18.575 & 1109.9 & 40.422 & 1060.3 & 71.429 & 1356.9 & 4.3304 & 1258 & 9.8112 & & & & & & & & & & & & \\
\hline SBW1-40 & 5.7409 & 1007.4 & 11.826 & 957.69 & 20.491 & 1256.5 & 1.5553 & 1157.2 & 3.1584 & & & & & & & & & & & & \\
\hline SBW1-41 & 3.3358 & 1007.8 & 6.8682 & 958.01 & 12.332 & 1255.6 & 0.9989 & 1156.9 & 1.9118 & & & & & & & & & & & & \\
\hline SBW1-42 & 3.3996 & 1004.9 & 7.099 & 954.76 & 12.75 & 1254.2 & 0.9471 & 1153.8 & 1.8764 & & & & & & & & & & & & \\
\hline SBW1-43 & 4.001 & 1007.6 & 7.7022 & 957.99 & 12.768 & 1255.4 & 1.2152 & 1157.1 & 2.3107 & & & & & & & & & & & & \\
\hline
\end{tabular}


Appendix A. Database - mass fraction

SBW CVS Phase 1 (Scholes et al. 2002)

\begin{tabular}{|c|c|c|c|c|c|c|c|c|c|c|c|c|c|c|c|c|}
\hline Glass ID & $\begin{array}{l}\mathrm{T} 13 \\
\left({ }^{\circ} \mathrm{C}\right) \\
\end{array}$ & $\begin{array}{c}\mathrm{V} 13 \\
(\mathrm{~Pa} \cdot \mathrm{s})\end{array}$ & $\begin{array}{l}\mathrm{T} 14 \\
\left({ }^{\circ} \mathrm{C}\right)\end{array}$ & $\begin{array}{c}\mathrm{V} 14 \\
(\mathrm{~Pa} \cdot \mathrm{s})\end{array}$ & $\begin{array}{c}\text { Q PCT } \\
\text { B }\left(\mathrm{g} / \mathrm{m}^{2}\right)\end{array}$ & $\begin{array}{c}\text { Q PCT } \\
\mathrm{Li}\left(\mathrm{g} / \mathrm{m}^{2}\right)\end{array}$ & $\begin{array}{c}\text { Q PCT } \\
\mathrm{Na}\left(\mathrm{g} / \mathrm{m}^{2}\right) \\
\end{array}$ & $\begin{array}{c}\text { Q PCT } \\
\mathrm{Si}\left(\mathrm{g} / \mathrm{m}^{2}\right)\end{array}$ & $\begin{array}{c}\text { Q PCT } \\
\text { pH }\end{array}$ & $\begin{array}{c}\text { CCC PCT } \\
\text { B }\left(\mathrm{g} / \mathrm{m}^{2}\right)\end{array}$ & $\begin{array}{c}\text { CCC PCT } \\
\mathrm{Li}\left(\mathrm{g} / \mathrm{m}^{2}\right) \\
\end{array}$ & $\begin{array}{l}\text { CCC PCT } \\
\mathrm{Na}\left(\mathrm{g} / \mathrm{m}^{2}\right)\end{array}$ & $\begin{array}{c}\text { CCC PCT } \\
\mathrm{Si}\left(\mathrm{g} / \mathrm{m}^{2}\right) \\
\end{array}$ & $\begin{array}{c}\text { CCC PCT } \\
\mathrm{pH}\end{array}$ & $\begin{array}{l}\text { Q PCT B at } \\
20^{\circ} \mathrm{C}\left(\mathrm{g} / \mathrm{m}^{2}\right)\end{array}$ & $\begin{array}{l}\text { Q PCT Li at } \\
20^{\circ} \mathrm{C}\left(\mathrm{g} / \mathrm{m}^{2}\right)\end{array}$ \\
\hline SBW1-24 & & & & & 0.409 & 0.451 & 0.395 & 0.120 & 9.2 & & & & & & & \\
\hline SBW1-25 & & & & & 0.907 & 0.791 & 0.815 & 0.160 & 9.63 & & & & & & & \\
\hline SBW1-26 & & & & & 0.224 & 0.333 & 0.251 & 0.114 & 10.33 & & & & & & & \\
\hline SBW1-27 & & & & & 0.339 & 0.333 & 0.377 & 0.099 & 8.89 & & & & & & & \\
\hline SBW1-28 & & & & & 1.445 & 1.218 & 0.949 & 0.146 & 10.605 & & & & & & & \\
\hline SBW1-29 & & & & & 1.643 & 1.428 & 1.275 & 0.220 & 11.235 & & & & & & & \\
\hline SBW1-30 & & & & & 4.192 & 2.990 & 3.040 & 0.412 & 11.795 & & & & & & & \\
\hline SBW1-31D & & & & & 0.392 & 0.514 & 0.073 & 0.186 & 9.34 & & & & & & & \\
\hline SBW1-32 & & & & & 4.141 & 3.722 & 3.342 & 0.695 & 10.045 & & & & & & & \\
\hline SBW1-33A & & & & & 1.143 & & 0.761 & 0.147 & 8.885 & & & & & & & \\
\hline SBW1-34 & & & & & & & & & & & & & & & & \\
\hline SBW1-35 & & & & & 1.386 & & 1.256 & 0.173 & 11.09 & & & & & & & \\
\hline SBW1-36 & & & & & 0.413 & & 0.674 & 0.215 & 11.42 & & & & & & & \\
\hline SBW1-37 & & & & & 1.466 & & 1.366 & 0.505 & 11.305 & & & & & & & \\
\hline SBW1-38B & & & & & 0.116 & 0.241 & 0.093 & 0.044 & 8.795 & & & & & & & \\
\hline SBW1-39B & & & & & 0.412 & 0.172 & 0.631 & 0.217 & 10.945 & & & & & & & \\
\hline SBW1-40 & & & & & 0.799 & 0.761 & 0.372 & 0.122 & 9.99 & & & & & & & \\
\hline SBW1-41 & & & & & 6.724 & & 5.099 & 0.917 & 11.295 & & & & & & & \\
\hline SBW1-42 & & & & & 1.478 & 1.398 & 1.066 & 0.263 & 9.88 & & & & & & & \\
\hline SBW1-43 & & & & & 3.631 & 2.568 & 2.192 & 0.239 & 11.715 & & & & & & & \\
\hline
\end{tabular}


Appendix A. Database - mass fraction

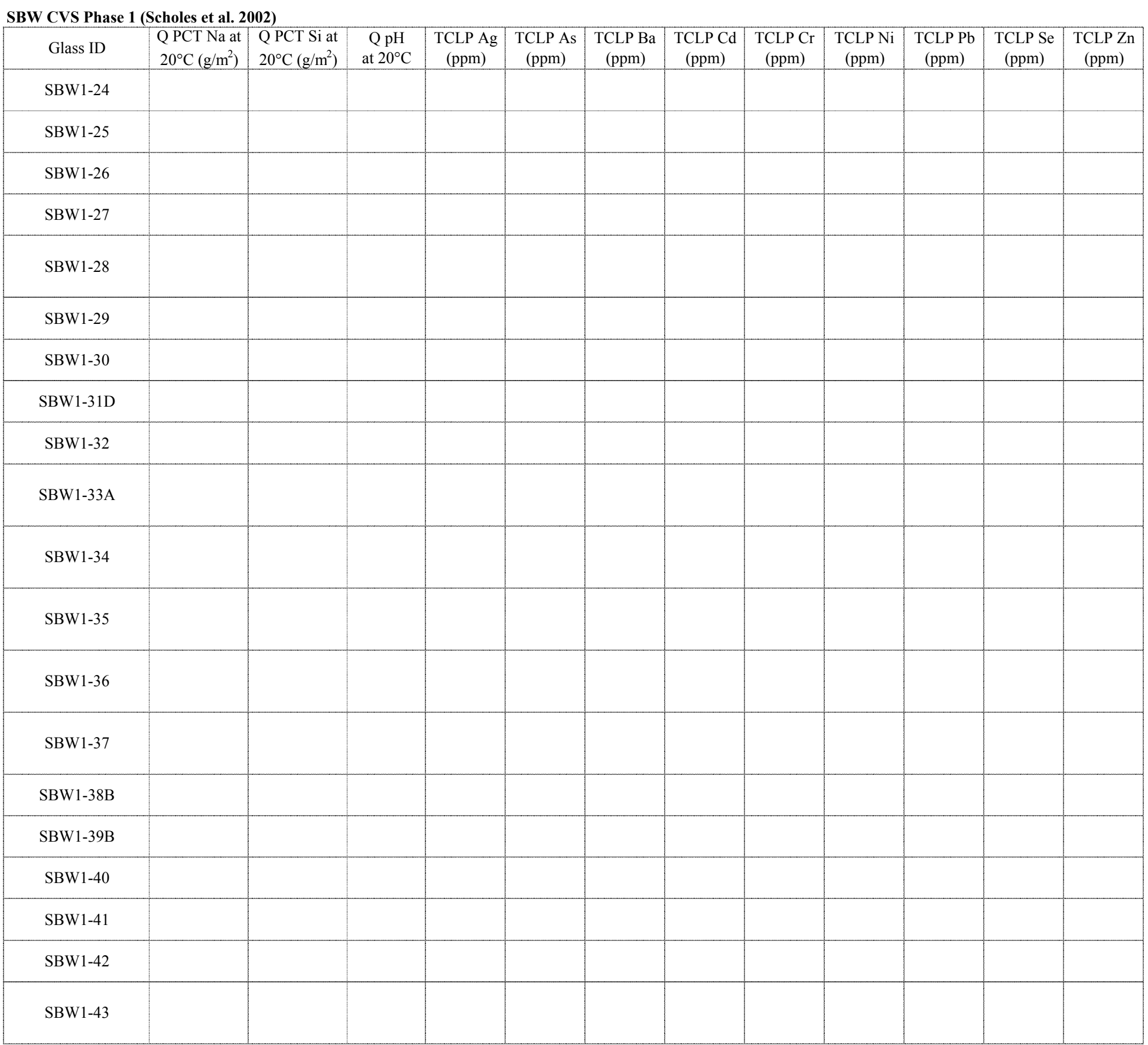


Appendix A. Database - mass fraction

SBW CVS Phase 1 (Scholes et al. 2002)

\begin{tabular}{|c|c|c|c|c|c|c|c|c|c|c|c|c|c|c|c|c|c|c|c|c|c|}
\hline Glass ID & $\begin{array}{c}\mathrm{A} 12 \mathrm{O} 3 \\
-\mathrm{t}\end{array}$ & $\begin{array}{c}\mathrm{B} 2 \mathrm{O} 3 \\
-\mathrm{t}\end{array}$ & $\begin{array}{c}\mathrm{CaO} \\
-\mathrm{t}\end{array}$ & $\begin{array}{c}\mathrm{Fe} 2 \mathrm{O} 3 \\
-\mathrm{t}\end{array}$ & $\begin{array}{c}\mathrm{FeO} \\
-\mathrm{t}\end{array}$ & $\begin{array}{c}\mathrm{K} 2 \mathrm{O} \\
-\mathrm{t}\end{array}$ & $\begin{array}{c}\mathrm{Li} 2 \mathrm{O} \\
-\mathrm{t}\end{array}$ & $\begin{array}{c}\mathrm{MgO} \\
-\mathrm{t}\end{array}$ & $\begin{array}{c}\mathrm{Na} 2 \mathrm{O} \\
-\mathrm{t}\end{array}$ & $\begin{array}{c}\mathrm{NiO} \\
-\mathrm{t}\end{array}$ & $\begin{array}{c}\mathrm{P} 2 \mathrm{O} 5 \\
-\mathrm{t}\end{array}$ & $\begin{array}{c}\mathrm{SiO} 2 \\
-\mathrm{t}\end{array}$ & $\begin{array}{c}\mathrm{ZrO} 2 \\
-\mathrm{t}\end{array}$ & $\begin{array}{c}\mathrm{Ag} 2 \mathrm{O} \\
-\mathrm{t}\end{array}$ & $\begin{array}{c}\mathrm{As} 2 \mathrm{O} 3 \\
-\mathrm{t}\end{array}$ & $\begin{array}{c}\mathrm{BaO} \\
-\mathrm{t}\end{array}$ & $\begin{array}{c}\mathrm{Bi} 2 \mathrm{O} 3 \\
-\mathrm{t}\end{array}$ & $\begin{array}{c}\mathrm{Br} \\
-\mathrm{t}\end{array}$ & $\begin{array}{c}\mathrm{CdO} \\
-\mathrm{t}\end{array}$ & $\begin{array}{c}\mathrm{Ce} 2 \mathrm{O} 3 \\
-\mathrm{t}\end{array}$ & $\begin{array}{c}\mathrm{CeO} 2 \\
-\mathrm{t}\end{array}$ \\
\hline SBW1-44B & 0.0602 & 0.0654 & 0.0085 & 0.0854 & & 0.0467 & 0.0428 & 0.0000 & 0.1400 & 0.0008 & 0.0171 & 0.4672 & 0.0030 & & & 0.0387 & & & 0.0043 & & \\
\hline SBW1-45 & 0.0400 & 0.1500 & 0.0030 & 0.0020 & & 0.0530 & & 0.0000 & 0.1867 & 0.0008 & 0.0200 & 0.4443 & 0.0200 & & & 0.0470 & & & 0.0050 & & \\
\hline SBW1-46 & 0.1072 & 0.1014 & 0.0214 & 0.0513 & & 0.0320 & 0.0259 & 0.0000 & 0.1471 & 0.0008 & 0.0102 & 0.4512 & 0.0099 & & & 0.0192 & & & 0.0025 & & \\
\hline SBW1-47 & 0.1072 & 0.1014 & 0.0214 & 0.0513 & & 0.0320 & 0.0259 & 0.0000 & 0.1471 & 0.0008 & 0.0102 & 0.4512 & 0.0099 & & & 0.0192 & & & 0.0025 & & \\
\hline SBW1-48 & 0.0904 & 0.1236 & 0.0103 & 0.0894 & & 0.0270 & 0.0057 & 0.0000 & 0.1422 & 0.0005 & 0.0064 & 0.4866 & 0.0029 & & & & & & 0.0015 & & \\
\hline SBW1-49 & 0.0904 & 0.1236 & 0.0103 & 0.0054 & & 0.0270 & 0.0412 & 0.0000 & 0.1422 & 0.0005 & 0.0064 & 0.5001 & 0.0029 & & & 0.0350 & & & 0.0015 & & \\
\hline SBW1-50 & 0.0904 & 0.1198 & 0.0453 & 0.0894 & & 0.0270 & 0.0412 & 0.0000 & 0.1422 & 0.0005 & 0.0064 & 0.4199 & 0.0029 & & & & & & 0.0015 & & \\
\hline SBW1-51 & 0.0904 & 0.0700 & 0.0103 & 0.0894 & & 0.0270 & 0.0136 & 0.0000 & 0.1422 & 0.0005 & 0.0064 & 0.4973 & 0.0029 & & & 0.0350 & & & 0.0015 & & \\
\hline SBW1-52 & 0.0904 & 0.1236 & 0.0453 & 0.0367 & & 0.0270 & 0.0061 & 0.0000 & 0.1422 & 0.0005 & 0.0064 & 0.5039 & 0.0029 & & & & & & 0.0015 & & \\
\hline SBW1-53 & 0.0904 & 0.0700 & 0.0112 & 0.0894 & & 0.0270 & 0.0412 & 0.0000 & 0.1422 & 0.0005 & 0.0064 & 0.5038 & 0.0029 & & & & & & 0.0015 & & \\
\hline SBW1-54 & 0.0725 & 0.1125 & 0.0461 & 0.0795 & & 0.0224 & 0.0375 & 0.0000 & 0.1169 & 0.0006 & 0.0052 & 0.4873 & 0.0047 & & & & & & 0.0012 & & \\
\hline SBW1-55 & 0.0870 & 0.1050 & 0.0453 & 0.0754 & & 0.0269 & 0.0350 & 0.0000 & 0.1403 & 0.0007 & 0.0062 & 0.4549 & 0.0056 & & & & & & 0.0014 & & \\
\hline SBW1-56 & 0.1014 & 0.0975 & 0.0445 & 0.0713 & & 0.0314 & 0.0325 & 0.0000 & 0.1637 & 0.0009 & 0.0073 & 0.4224 & 0.0065 & & & & & & 0.0017 & & \\
\hline SBW1-57 & 0.0725 & 0.1125 & 0.0086 & 0.0795 & & 0.0224 & 0.0375 & 0.0000 & 0.1169 & 0.0006 & 0.0052 & 0.4873 & 0.0047 & & & 0.0375 & & & 0.0012 & & \\
\hline SBW1-58 & 0.0870 & 0.1050 & 0.0103 & 0.0754 & & 0.0269 & 0.0350 & 0.0000 & 0.1403 & 0.0007 & 0.0062 & 0.4549 & 0.0056 & & & 0.0350 & & & 0.0014 & & \\
\hline SBW1-59 & 0.1014 & 0.0975 & 0.0120 & 0.0713 & & 0.0314 & 0.0325 & 0.0000 & 0.1637 & 0.0009 & 0.0073 & 0.4224 & 0.0065 & & & 0.0325 & & & 0.0017 & & \\
\hline SBW1-60 & 0.0725 & 0.1125 & 0.0273 & 0.0795 & & 0.0224 & 0.0375 & 0.0000 & 0.1169 & 0.0006 & 0.0052 & 0.4873 & 0.0047 & & & 0.0188 & & & 0.0012 & & \\
\hline SBW1-61 & 0.0870 & 0.1050 & 0.0278 & 0.0754 & & 0.0269 & 0.0350 & 0.0000 & 0.1403 & 0.0007 & 0.0062 & 0.4549 & 0.0056 & & & 0.0175 & & & 0.0014 & & \\
\hline SBW1-62 & 0.1014 & 0.0975 & 0.0282 & 0.0713 & & 0.0314 & 0.0325 & 0.0000 & 0.1637 & 0.0009 & 0.0073 & 0.4224 & 0.0065 & & & 0.0163 & & & 0.0017 & & \\
\hline SBW1-63 & 0.1501 & 0.1501 & 0.0008 & 0.0005 & & & 0.0845 & & 0.0500 & & & 0.5307 & 0.0301 & & & 0.0000 & & & & & \\
\hline SBW1-64 & 0.0375 & 0.1250 & 0.0009 & 0.0005 & & 0.0250 & 0.0631 & & 0.0875 & & 0.0125 & 0.5391 & 0.1050 & & & 0.0000 & & & & & \\
\hline
\end{tabular}


Appendix A. Database - mass fraction

SBW CVS Phase 1 (Scholes et al. 2002)

\begin{tabular}{|c|c|c|c|c|c|c|c|c|c|c|c|c|c|c|c|c|c|c|c|c|c|}
\hline Glass ID & $\begin{array}{l}\mathrm{Cl} \\
-\mathrm{t} \\
\end{array}$ & $\begin{array}{c}\mathrm{CoO} \\
-\mathrm{t}\end{array}$ & $\begin{array}{c}\mathrm{Co} 2 \mathrm{O} 3 \\
-\mathrm{t}\end{array}$ & $\begin{array}{c}\mathrm{Cr} 2 \mathrm{O} 3 \\
-\mathrm{t} \\
\end{array}$ & $\begin{array}{c}\mathrm{Cs} 2 \mathrm{O} \\
-\mathrm{t}\end{array}$ & $\begin{array}{c}\mathrm{CuO} \\
-\mathrm{t}\end{array}$ & $\begin{array}{c}\mathrm{Eu} 2 \mathrm{O} 3 \\
-\mathrm{t}\end{array}$ & $\begin{array}{l}F \\
-t\end{array}$ & $\begin{array}{c}\mathrm{Ga} 2 \mathrm{O} 3 \\
-\mathrm{t}\end{array}$ & $\begin{array}{c}\mathrm{Gd} 2 \mathrm{O} 3 \\
-\mathrm{t}\end{array}$ & $\begin{array}{c}\mathrm{HgO} \\
-\mathrm{t}\end{array}$ & $\begin{array}{l}I \\
-t\end{array}$ & $\begin{array}{c}\mathrm{La} 2 \mathrm{O} 3 \\
-\mathrm{t}\end{array}$ & $\begin{array}{c}\mathrm{MnO} 2 \\
-\mathrm{t}\end{array}$ & $\begin{array}{c}\mathrm{MnO} \\
-\mathrm{t}\end{array}$ & $\begin{array}{c}\mathrm{MoO} \\
-\mathrm{t}\end{array}$ & $\begin{array}{c}\mathrm{MoO} 3 \\
-\mathrm{t}\end{array}$ & $\begin{array}{c}\mathrm{Nb} 2 \mathrm{O} 5 \\
-\mathrm{t}\end{array}$ & $\begin{array}{c}\mathrm{Nd} 2 \mathrm{O} 3 \\
-\mathrm{t}\end{array}$ & $\begin{array}{c}\mathrm{PbO} \\
-\mathrm{t}\end{array}$ & $\begin{array}{c}\mathrm{PdO} 2 \\
-\mathrm{t}\end{array}$ \\
\hline SBW1-44B & 0.0015 & & & 0.0013 & & & & 0.0022 & & & & 0.0001 & 0.0003 & & 0.0087 & & 0.0002 & & & 0.0007 & \\
\hline SBW1-45 & 0.0015 & & & 0.0013 & & & & 0.0090 & & & & 0.0001 & 0.0003 & & 0.0100 & & 0.0002 & & & 0.0007 & \\
\hline SBW1-46 & 0.0015 & & & 0.0013 & & & & 0.0050 & & & & 0.0001 & 0.0003 & & 0.0057 & & 0.0002 & & & 0.0007 & \\
\hline SBW1-47 & 0.0015 & & & 0.0013 & & & & 0.0050 & & & & 0.0001 & 0.0003 & & 0.0057 & & 0.0002 & & & 0.0007 & \\
\hline SBW1-48 & 0.0009 & & & 0.0008 & & & & 0.0037 & & & & 0.0001 & 0.0002 & & 0.0044 & & 0.0001 & & & 0.0004 & \\
\hline SBW1-49 & 0.0009 & & & 0.0008 & & & & 0.0037 & & & & 0.0001 & 0.0002 & & 0.0044 & & 0.0001 & & & 0.0004 & \\
\hline SBW1-50 & 0.0009 & & & 0.0008 & & & & 0.0037 & & & & 0.0001 & 0.0002 & & 0.0044 & & 0.0001 & & & 0.0004 & \\
\hline SBW1-51 & 0.0009 & & & 0.0008 & & & & 0.0037 & & & & 0.0001 & 0.0002 & & 0.0044 & & 0.0001 & & & 0.0004 & \\
\hline SBW1-52 & 0.0009 & & & 0.0008 & & & & 0.0037 & & & & 0.0001 & 0.0002 & & 0.0044 & & 0.0001 & & & 0.0004 & \\
\hline SBW1-53 & 0.0009 & & & 0.0008 & & & & 0.0037 & & & & 0.0001 & 0.0002 & & 0.0044 & & 0.0001 & & & 0.0004 & \\
\hline SBW1-54 & 0.0011 & & & 0.0010 & & & & 0.0031 & & & & 0.0001 & 0.0002 & & 0.0036 & & 0.0001 & & & 0.0005 & \\
\hline SBW1-55 & 0.0014 & & & 0.0012 & & & & 0.0037 & & & & 0.0001 & 0.0003 & & 0.0043 & & 0.0002 & & & 0.0006 & \\
\hline SBW1-56 & 0.0016 & & & 0.0014 & & & & 0.0043 & & & & 0.0001 & 0.0003 & & 0.0050 & & 0.0002 & & & 0.0007 & \\
\hline SBW1-57 & 0.0011 & & & 0.0010 & & & & 0.0031 & & & & 0.0001 & 0.0002 & & 0.0036 & & 0.0001 & & & 0.0005 & \\
\hline SBW1-58 & 0.0014 & & & 0.0012 & & & & 0.0037 & & & & 0.0001 & 0.0003 & & 0.0043 & & 0.0002 & & & 0.0006 & \\
\hline SBW1-59 & 0.0016 & & & 0.0014 & & & & 0.0043 & & & & 0.0001 & 0.0003 & & 0.0050 & & 0.0002 & & & 0.0007 & \\
\hline SBW1-60 & 0.0011 & & & 0.0010 & & & & 0.0031 & & & & 0.0001 & 0.0002 & & 0.0036 & & 0.0001 & & & 0.0005 & \\
\hline SBW1-61 & 0.0014 & & & 0.0012 & & & & 0.0037 & & & & 0.0001 & 0.0003 & & 0.0043 & & 0.0002 & & & 0.0006 & \\
\hline SBW1-62 & 0.0016 & & & 0.0014 & & & & 0.0043 & & & & 0.0001 & 0.0003 & & 0.0050 & & 0.0002 & & & 0.0007 & \\
\hline SBW1-63 & & & & & 0.0009 & 0.0007 & & & & 0.0007 & & & & & & & 0.0001 & & & 0.0000 & \\
\hline SBW1-64 & & & & & 0.0010 & 0.0007 & & 0.0005 & & 0.0007 & & & & & & & 0.0001 & & & 0.0000 & \\
\hline
\end{tabular}


Appendix A. Database - mass fraction

SBW CVS Phase 1 (Scholes et al. 2002)

\begin{tabular}{|c|c|c|c|c|c|c|c|c|c|c|c|c|c|c|c|c|c|c|c|c|c|}
\hline Glass ID & $\begin{array}{c}\mathrm{PdO} \\
-\mathrm{t} \\
\end{array}$ & $\begin{array}{c}\mathrm{Pr} 2 \mathrm{O} 3 \\
-\mathrm{t}\end{array}$ & $\begin{array}{c}\text { Pr6O11 } \\
-t\end{array}$ & $\begin{array}{c}\mathrm{Rb} 2 \mathrm{O} \\
-\mathrm{t}\end{array}$ & $\begin{array}{c}\mathrm{ReO} \\
-\mathrm{t} \\
\end{array}$ & $\begin{array}{c}\mathrm{ReO} 2 \\
-\mathrm{t}\end{array}$ & $\begin{array}{c}\mathrm{Rh} 2 \mathrm{O} 3 \\
-\mathrm{t}\end{array}$ & $\begin{array}{c}\mathrm{RhO} 2 \\
-\mathrm{t}\end{array}$ & $\begin{array}{c}\mathrm{RuO} 2 \\
-\mathrm{t}\end{array}$ & $\begin{array}{c}\mathrm{Sb} 2 \mathrm{O} 3 \\
-\mathrm{t}\end{array}$ & $\begin{array}{c}\mathrm{Sb} 2 \mathrm{O} 5 \\
-\mathrm{t}\end{array}$ & $\begin{array}{c}\mathrm{SeO} 2 \\
-\mathrm{t}\end{array}$ & $\begin{array}{c}\mathrm{Sm} 2 \mathrm{O} 3 \\
-\mathrm{t}\end{array}$ & $\begin{array}{c}\mathrm{SnO} \\
-\mathrm{t} \\
\end{array}$ & $\begin{array}{c}\mathrm{SnO} 2 \\
-\mathrm{t}\end{array}$ & $\begin{array}{c}\mathrm{SO} 3 \\
-\mathrm{t}\end{array}$ & $\begin{array}{c}\mathrm{SrO} \\
-\mathrm{t}\end{array}$ & $\begin{array}{c}\mathrm{Tc} 2 \mathrm{O} 7 \\
-\mathrm{t}\end{array}$ & $\begin{array}{c}\mathrm{TeO} 2 \\
-\mathrm{t}\end{array}$ & $\begin{array}{c}\mathrm{ThO} 2 \\
-\mathrm{t}\end{array}$ & $\begin{array}{c}\mathrm{TiO} 2 \\
-\mathrm{t} \\
\end{array}$ \\
\hline SBW1-44B & & & & & & & & & 0.0000 & & & & & & & 0.0050 & & & & & \\
\hline SBW1-45 & & & & & & & & & 0.0000 & & & & & & & 0.0050 & & & & & \\
\hline SBW1-46 & & & & & & & & & 0.0000 & & & & & & & 0.0050 & & & & & \\
\hline SBW1-47 & & & & & & & & & 0.0000 & & & & & & & 0.0050 & & & & & \\
\hline SBW1-48 & & & & & & & & & 0.0000 & & & & & & & 0.0030 & & & & & \\
\hline SBW1-49 & & & & & & & & & 0.0000 & & & & & & & 0.0030 & & & & & \\
\hline SBW1-50 & & & & & & & & & 0.0000 & & & & & & & 0.0030 & & & & & \\
\hline SBW1-51 & & & & & & & & & 0.0000 & & & & & & & 0.0030 & & & & & \\
\hline SBW1-52 & & & & & & & & & 0.0000 & & & & & & & 0.0030 & & & & & \\
\hline SBW1-53 & & & & & & & & & 0.0000 & & & & & & & 0.0030 & & & & & \\
\hline SBW1-54 & & & & & & & & & 0.0000 & & & & & & & 0.0038 & & & & & \\
\hline SBW1-55 & & & & & & & & & 0.0000 & & & & & & & 0.0045 & & & & & \\
\hline SBW1-56 & & & & & & & & & 0.0000 & & & & & & & 0.0053 & & & & & \\
\hline SBW1-57 & & & & & & & & & 0.0000 & & & & & & & 0.0038 & & & & & \\
\hline SBW1-58 & & & & & & & & & 0.0000 & & & & & & & 0.0045 & & & & & \\
\hline SBW1-59 & & & & & & & & & 0.0000 & & & & & & & 0.0053 & & & & & \\
\hline SBW1-60 & & & & & & & & & 0.0000 & & & & & & & 0.0038 & & & & & \\
\hline SBW1-61 & & & & & & & & & 0.0000 & & & & & & & 0.0045 & & & & & \\
\hline SBW1-62 & & & & & & & & & 0.0000 & & & & & & & 0.0053 & & & & & \\
\hline SBW1-63 & & & & & & & & & & & & & & & & & 0.0002 & & & & \\
\hline SBW1-64 & & & & & & & & & & & & & & & & & 0.0002 & & & & \\
\hline
\end{tabular}


Appendix A. Database - mass fraction

SBW CVS Phase 1 (Scholes et al. 2002)

\begin{tabular}{|c|c|c|c|c|c|c|c|c|c|c|c|c|c|c|c|c|c|c|c|c|c|}
\hline Glass ID & $\begin{array}{c}\mathrm{T} 12 \mathrm{O} 3 \\
-\mathrm{t}\end{array}$ & $\begin{array}{c}\mathrm{U} 3 \mathrm{O} 8 \\
-\mathrm{t}\end{array}$ & $\begin{array}{c}\mathrm{UO} 2 \\
-\mathrm{t}\end{array}$ & $\begin{array}{c}\text { UO3 } \\
-t\end{array}$ & $\begin{array}{c}\mathrm{V} 2 \mathrm{O} 5 \\
-\mathrm{t}\end{array}$ & $\begin{array}{c}\text { WO3 } \\
-t\end{array}$ & $\begin{array}{c}\mathrm{Y} 2 \mathrm{O} 3 \\
-\mathrm{t}\end{array}$ & $\begin{array}{c}\mathrm{ZnO} \\
-\mathrm{t} \\
\end{array}$ & $\begin{array}{c}\text { Others } \\
-t\end{array}$ & $\begin{array}{c}\text { Sum } \\
-t\end{array}$ & $\begin{array}{c}\mathrm{Al} 2 \mathrm{O} 3 \\
-\mathrm{a}\end{array}$ & $\begin{array}{c}\mathrm{B} 2 \mathrm{O} 3 \\
-\mathrm{a}\end{array}$ & $\begin{array}{c}\mathrm{CaO} \\
-\mathrm{a} \\
\end{array}$ & $\begin{array}{c}\mathrm{Fe} 2 \mathrm{O} 3 \\
-\mathrm{a} \\
\end{array}$ & $\begin{array}{c}\mathrm{FeO} \\
-\mathrm{a} \\
\end{array}$ & $\begin{array}{c}\mathrm{K} 2 \mathrm{O} \\
-\mathrm{a} \\
\end{array}$ & $\begin{array}{c}\mathrm{Li} 2 \mathrm{O} \\
-\mathrm{a}\end{array}$ & $\begin{array}{c}\mathrm{MgO} \\
-\mathrm{a} \\
\end{array}$ & $\begin{array}{c}\mathrm{Na} 2 \mathrm{O} \\
-\mathrm{a}\end{array}$ & $\begin{array}{c}\mathrm{NiO} \\
-\mathrm{a} \\
\end{array}$ & $\begin{array}{c}\mathrm{P} 2 \mathrm{O} 5 \\
-\mathrm{a}\end{array}$ \\
\hline SBW1-44B & & & & & & & & & & 1.0000 & 0.0593 & 0.0653 & 0.0087 & 0.0854 & & 0.0451 & 0.0411 & & 0.1404 & 0.0009 & 0.0161 \\
\hline SBW1-45 & & & & & & & & & & 1.0000 & 0.0434 & 0.1483 & 0.0033 & 0.0025 & & 0.0536 & 0.0001 & & 0.1923 & 0.0009 & 0.0177 \\
\hline SBW1-46 & & & & & & & & & & 1.0000 & 0.1082 & 0.1009 & 0.0216 & 0.0506 & & 0.0311 & 0.0251 & & 0.1455 & 0.0010 & 0.0093 \\
\hline SBW1-47 & & & & & & & & & & 1.0000 & 0.1087 & 0.1015 & 0.0213 & 0.0509 & & 0.0314 & 0.0255 & & 0.1464 & 0.0009 & 0.0095 \\
\hline SBW1-48 & & & & & & & & & & 1.0000 & 0.0869 & 0.1196 & 0.0105 & 0.0884 & & 0.0261 & 0.0055 & & 0.1406 & 0.0006 & 0.0066 \\
\hline SBW1-49 & & & & & & & & & & 1.0000 & 0.0926 & 0.1214 & 0.0107 & 0.0057 & & 0.0262 & 0.0398 & & 0.1415 & 0.0006 & 0.0066 \\
\hline SBW1-50 & & & & & & & & & & 1.0000 & 0.0810 & 0.1189 & 0.0461 & 0.0886 & & 0.0259 & 0.0395 & & 0.1407 & 0.0006 & 0.0064 \\
\hline SBW1-51 & & & & & & & & & & 1.0000 & 0.0922 & 0.0702 & 0.0106 & 0.0912 & & 0.0261 & 0.0131 & & 0.1391 & 0.0005 & 0.0063 \\
\hline SBW1-52 & & & & & & & & & & 1.0000 & 0.0922 & 0.1220 & 0.0458 & 0.0368 & & 0.0262 & 0.0060 & & 0.1400 & 0.0006 & 0.0064 \\
\hline SBW1-53 & & & & & & & & & & 1.0000 & 0.0852 & 0.0706 & 0.0115 & 0.0899 & & 0.0265 & 0.0399 & & 0.1416 & 0.0006 & 0.0062 \\
\hline SBW1-54 & & & & & & & & & & 1.0000 & 0.0742 & 0.1074 & 0.0465 & 0.0783 & & 0.0217 & 0.0357 & & 0.1162 & 0.0007 & 0.0055 \\
\hline SBW1-55 & & & & & & & & & & 1.0000 & 0.0923 & 0.1083 & 0.0481 & 0.0764 & & 0.0268 & 0.0356 & & 0.1434 & 0.0008 & 0.0064 \\
\hline SBW1-56 & & & & & & & & & & 1.0000 & 0.1057 & 0.0979 & 0.0462 & 0.0726 & & 0.0311 & 0.0324 & & 0.1660 & 0.0010 & 0.0070 \\
\hline SBW1-57 & & & & & & & & & & 1.0000 & 0.0746 & 0.1085 & 0.0088 & 0.0791 & & 0.0217 & 0.0359 & & 0.1150 & 0.0007 & 0.0059 \\
\hline SBW1-58 & & & & & & & & & & 1.0000 & 0.0832 & 0.1031 & 0.0108 & 0.0761 & & 0.0256 & 0.0340 & & 0.1379 & 0.0008 & 0.0062 \\
\hline SBW1-59 & & & & & & & & & & 1.0000 & 0.1056 & 0.0986 & 0.0127 & 0.0709 & & 0.0313 & 0.0326 & & 0.1665 & 0.0009 & 0.0075 \\
\hline SBW1-60 & & & & & & & & & & 1.0000 & 0.0744 & 0.1108 & 0.0274 & 0.0797 & & 0.0224 & 0.0366 & & 0.1191 & 0.0007 & 0.0054 \\
\hline SBW1-61 & & & & & & & & & & 1.0000 & 0.0896 & 0.1026 & 0.0282 & 0.0744 & & 0.0259 & 0.0339 & & 0.1399 & 0.0008 & 0.0063 \\
\hline SBW1-62 & & & & & & & & & & 1.0000 & 0.1067 & 0.0979 & 0.0300 & 0.0718 & & 0.0306 & 0.0315 & & 0.1695 & 0.0009 & 0.0074 \\
\hline SBW1-63 & & & 0.0006 & & & & & & & 1.0000 & 0.1496 & 0.1474 & 0.0010 & 0.0009 & & 0.0002 & 0.0811 & & 0.0503 & 0.0003 & 0.0001 \\
\hline SBW1-64 & & & 0.0007 & & & & & & & 1.0000 & 0.0389 & 0.1260 & 0.0012 & 0.0008 & & 0.0241 & 0.0619 & & 0.0881 & 0.0001 & 0.0107 \\
\hline
\end{tabular}


Appendix A. Database - mass fraction

SBW CVS Phase 1 (Scholes et al. 2002)

\begin{tabular}{|c|c|c|c|c|c|c|c|c|c|c|c|c|c|c|c|c|c|c|c|c|c|}
\hline Glass ID & $\begin{array}{c}\mathrm{SiO} 2 \\
-\mathrm{a}\end{array}$ & $\begin{array}{c}\mathrm{ZrO} 2 \\
-\mathrm{a}\end{array}$ & $\begin{array}{c}\mathrm{Ag} 2 \mathrm{O} \\
-\mathrm{a}\end{array}$ & $\begin{array}{c}\mathrm{As} 2 \mathrm{O} 3 \\
-\mathrm{a}\end{array}$ & $\begin{array}{c}\mathrm{BaO} \\
-\mathrm{a} \\
\end{array}$ & $\begin{array}{c}\mathrm{Bi} 2 \mathrm{O} 3 \\
-\mathrm{a}\end{array}$ & $\begin{array}{l}\mathrm{Br} \\
-\mathrm{a}\end{array}$ & $\begin{array}{c}\mathrm{CdO} \\
-\mathrm{a}\end{array}$ & $\begin{array}{c}\mathrm{Ce} 2 \mathrm{O} 3 \\
-\mathrm{a}\end{array}$ & $\begin{array}{c}\mathrm{CeO} 2 \\
-\mathrm{a}\end{array}$ & $\begin{array}{l}\mathrm{Cl} \\
-\mathrm{a}\end{array}$ & $\begin{array}{c}\mathrm{CoO} \\
-\mathrm{a}\end{array}$ & $\begin{array}{c}\mathrm{Co} 2 \mathrm{O} 3 \\
-\mathrm{a}\end{array}$ & $\begin{array}{c}\mathrm{Cr} 2 \mathrm{O} 3 \\
-\mathrm{a} \\
\end{array}$ & $\begin{array}{c}\mathrm{Cs} 2 \mathrm{O} \\
-\mathrm{a}\end{array}$ & $\begin{array}{c}\mathrm{CuO} \\
-\mathrm{a}\end{array}$ & $\begin{array}{c}\mathrm{Eu} 2 \mathrm{O} 3 \\
-\mathrm{a}\end{array}$ & $\begin{array}{l}\mathrm{F} \\
-\mathrm{a}\end{array}$ & $\begin{array}{c}\mathrm{Ga} 2 \mathrm{O} 3 \\
-\mathrm{a}\end{array}$ & $\begin{array}{c}\mathrm{Gd} 2 \mathrm{O} 3 \\
-\mathrm{a}\end{array}$ & $\begin{array}{c}\mathrm{HgO} \\
-\mathrm{a}\end{array}$ \\
\hline SBW1-44B & 0.4546 & 0.0028 & & & 0.0398 & & & 0.0050 & & & 0.0013 & & & 0.0011 & & & & 0.0021 & & & \\
\hline SBW1-45 & 0.4332 & 0.0191 & & & 0.0488 & & & 0.0060 & & & 0.0015 & & & 0.0011 & & & & 0.0075 & & & \\
\hline SBW1-46 & 0.4455 & 0.0087 & & & 0.0194 & & & 0.0030 & & & 0.0011 & & & 0.0011 & & & & 0.0042 & & & \\
\hline SBW1-47 & 0.4417 & 0.0096 & & & 0.0192 & & & 0.0030 & & & 0.0011 & & & 0.0011 & & & & 0.0042 & & & \\
\hline SBW1-48 & 0.4738 & 0.0030 & & & 0.0001 & & & 0.0018 & & & 0.0009 & & & 0.0007 & & & & 0.0033 & & & \\
\hline SBW1-49 & 0.4843 & 0.0029 & & & 0.0353 & & & 0.0018 & & & 0.0009 & & & 0.0007 & & & & 0.0038 & & & \\
\hline SBW1-50 & 0.4119 & 0.0029 & & & 0.0001 & & & 0.0017 & & & 0.0012 & & & 0.0006 & & & & 0.0033 & & & \\
\hline SBW1-51 & 0.4945 & 0.0028 & & & 0.0359 & & & 0.0018 & & & 0.0008 & & & 0.0007 & & & & 0.0033 & & & \\
\hline SBW1-52 & 0.4903 & 0.0030 & & & 0.0001 & & & 0.0018 & & & 0.0008 & & & 0.0007 & & & & 0.0032 & & & \\
\hline SBW1-53 & 0.4945 & 0.0028 & & & 0.0001 & & & 0.0018 & & & 0.0011 & & & 0.0007 & & & & 0.0034 & & & \\
\hline SBW1-54 & 0.4600 & 0.0047 & & & 0.0001 & & & 0.0014 & & & 0.0010 & & & 0.0008 & & & & 0.0030 & & & \\
\hline SBW1-55 & 0.4622 & 0.0057 & & & 0.0001 & & & 0.0017 & & & 0.0009 & & & 0.0010 & & & & 0.0033 & & & \\
\hline SBW1-56 & 0.4220 & 0.0067 & & & 0.0002 & & & 0.0021 & & & 0.0014 & & & 0.0011 & & & & 0.0036 & & & \\
\hline SBW1-57 & 0.4716 & 0.0049 & & & 0.0376 & & & 0.0014 & & & 0.0009 & & & 0.0009 & & & & 0.0032 & & & \\
\hline SBW1-58 & 0.4444 & 0.0057 & & & 0.0360 & & & 0.0017 & & & 0.0012 & & & 0.0010 & & & & 0.0032 & & & \\
\hline SBW1-59 & 0.4248 & 0.0066 & & & 0.0335 & & & 0.0020 & & & 0.0014 & & & 0.0011 & & & & 0.0040 & & & \\
\hline SBW1-60 & 0.4794 & 0.0048 & & & 0.0194 & & & 0.0014 & & & 0.0009 & & & 0.0008 & & & & 0.0021 & & & \\
\hline SBW1-61 & 0.4371 & 0.0055 & & & 0.0174 & & & 0.0016 & & & 0.0013 & & & 0.0010 & & & & 0.0035 & & & \\
\hline SBW1-62 & 0.4290 & 0.0069 & & & 0.0167 & & & 0.0021 & & & 0.0012 & & & 0.0011 & & & & 0.0039 & & & \\
\hline SBW1-63 & 0.5181 & 0.0315 & & & 0.0001 & & & 0.0000 & & & 0.0002 & & & 0.0001 & & & & 0.0003 & & & \\
\hline SBW1-64 & 0.5323 & 0.0911 & & & 0.0001 & & & 0.0000 & & & 0.0001 & & & 0.0001 & & & & 0.0006 & & & \\
\hline
\end{tabular}


Appendix A. Database - mass fraction

SBW CVS Phase 1 (Scholes et al. 2002)

\begin{tabular}{|c|c|c|c|c|c|c|c|c|c|c|c|c|c|c|c|c|c|c|c|c|c|}
\hline Glass ID & $\begin{array}{c}\mathrm{I} \\
-\mathrm{a} \\
\end{array}$ & $\begin{array}{c}\mathrm{La} 2 \mathrm{O} 3 \\
-\mathrm{a}\end{array}$ & $\begin{array}{c}\mathrm{MnO} 2 \\
-\mathrm{a}\end{array}$ & $\begin{array}{c}\mathrm{MnO} \\
-\mathrm{a}\end{array}$ & $\begin{array}{c}\mathrm{MoO} \\
-\mathrm{a}\end{array}$ & $\begin{array}{c}\mathrm{MoO} 3 \\
-\mathrm{a}\end{array}$ & $\begin{array}{c}\mathrm{Nb} 2 \mathrm{O} 5 \\
-\mathrm{a}\end{array}$ & $\begin{array}{c}\mathrm{Nd} 2 \mathrm{O} 3 \\
-\mathrm{a}\end{array}$ & $\begin{array}{c}\mathrm{PbO} \\
-\mathrm{a}\end{array}$ & $\begin{array}{c}\mathrm{PdO} 2 \\
-\mathrm{a}\end{array}$ & $\begin{array}{c}\mathrm{PdO} \\
-\mathrm{a}\end{array}$ & $\begin{array}{c}\mathrm{Pr} 2 \mathrm{O} 3 \\
-\mathrm{a}\end{array}$ & $\begin{array}{c}\text { Pr6O11 } \\
-\mathrm{a}\end{array}$ & $\begin{array}{c}\mathrm{Rb} 2 \mathrm{O} \\
-\mathrm{a}\end{array}$ & $\begin{array}{c}\mathrm{ReO} \\
-\mathrm{a}\end{array}$ & $\begin{array}{c}\mathrm{ReO} 2 \\
-\mathrm{a}\end{array}$ & $\begin{array}{c}\mathrm{Rh} 2 \mathrm{O} 3 \\
-\mathrm{a}\end{array}$ & $\begin{array}{c}\mathrm{RhO} 2 \\
-\mathrm{a}\end{array}$ & $\begin{array}{c}\mathrm{RuO} 2 \\
-\mathrm{a}\end{array}$ & $\begin{array}{c}\mathrm{Sb} 2 \mathrm{O} 3 \\
-\mathrm{a}\end{array}$ & $\begin{array}{c}\mathrm{Sb} 2 \mathrm{O} 5 \\
-\mathrm{a}\end{array}$ \\
\hline SBW1-44B & & & & 0.0062 & & & & & & & & & & & & & & & & & \\
\hline SBW1-45 & & & & 0.0072 & & & & & & & & & & & & & & & & & \\
\hline SBW1-46 & & & & 0.0041 & & & & & & & & & & & & & & & & & \\
\hline SBW1-47 & & & & 0.0041 & & & & & & & & & & & & & & & & & \\
\hline SBW1-48 & & & & 0.0031 & & & & & & & & & & & & & & & & & \\
\hline SBW1-49 & & & & 0.0032 & & & & & & & & & & & & & & & & & \\
\hline SBW1-50 & & & & 0.0031 & & & & & & & & & & & & & & & & & \\
\hline SBW1-51 & & & & 0.0032 & & & & & & & & & & & & & & & & & \\
\hline SBW1-52 & & & & 0.0032 & & & & & & & & & & & & & & & & & \\
\hline SBW1-53 & & & & 0.0032 & & & & & & & & & & & & & & & & & \\
\hline SBW1-54 & & & & 0.0026 & & & & & & & & & & & & & & & & & \\
\hline SBW1-55 & & & & 0.0032 & & & & & & & & & & & & & & & & & \\
\hline SBW1-56 & & & & 0.0037 & & & & & & & & & & & & & & & & & \\
\hline SBW1-57 & & & & 0.0026 & & & & & & & & & & & & & & & & & \\
\hline SBW1-58 & & & & 0.0031 & & & & & & & & & & & & & & & & & \\
\hline SBW1-59 & & & & 0.0037 & & & & & & & & & & & & & & & & & \\
\hline SBW1-60 & & & & 0.0026 & & & & & & & & & & & & & & & & & \\
\hline SBW1-61 & & & & 0.0031 & & & & & & & & & & & & & & & & & \\
\hline SBW1-62 & & & & 0.0038 & & & & & & & & & & & & & & & & & \\
\hline SBW1-63 & & & & 0.0000 & & & & & & & & & & & & & & & & & \\
\hline SBW1-64 & & & & 0.0000 & & & & & & & & & & & & & & & & & \\
\hline
\end{tabular}


Appendix A. Database - mass fraction

SBW CVS Phase 1 (Scholes et al. 2002)

\begin{tabular}{|c|c|c|c|c|c|c|c|c|c|c|c|c|c|c|c|c|c|c|c|c|}
\hline Glass ID & $\begin{array}{c}\mathrm{SeO} 2 \\
-\mathrm{a}\end{array}$ & $\begin{array}{c}\mathrm{Sm} 2 \mathrm{O} 3 \\
-\mathrm{a}\end{array}$ & $\begin{array}{c}\mathrm{SnO} \\
-\mathrm{a} \\
\end{array}$ & $\begin{array}{c}\mathrm{SnO} 2 \\
-\mathrm{a}\end{array}$ & $\begin{array}{c}\mathrm{SO} 3 \\
-\mathrm{a}\end{array}$ & $\begin{array}{c}\mathrm{SrO} \\
-\mathrm{a} \\
\end{array}$ & $\begin{array}{c}\mathrm{Tc} 2 \mathrm{O} 7 \\
-\mathrm{a}\end{array}$ & $\begin{array}{c}\mathrm{TeO} 2 \\
-\mathrm{a}\end{array}$ & $\begin{array}{c}\mathrm{ThO} 2 \\
-\mathrm{a}\end{array}$ & $\begin{array}{c}\mathrm{TiO} 2 \\
-\mathrm{a}\end{array}$ & $\begin{array}{c}\mathrm{T} 12 \mathrm{O} 3 \\
-\mathrm{a}\end{array}$ & $\begin{array}{c}\mathrm{U} 3 \mathrm{O} 8 \\
-\mathrm{a}\end{array}$ & $\begin{array}{c}\mathrm{UO} 2 \\
-\mathrm{a}\end{array}$ & $\begin{array}{c}\text { UO3 } \\
-\mathrm{a}\end{array}$ & $\begin{array}{c}\mathrm{V} 2 \mathrm{O} 5 \\
-\mathrm{a}\end{array}$ & $\begin{array}{c}\text { WO3 } \\
-\mathrm{a}\end{array}$ & $\begin{array}{c}\mathrm{Y} 2 \mathrm{O} 3 \\
-\mathrm{a}\end{array}$ & $\begin{array}{c}\mathrm{ZnO} \\
-\mathrm{a}\end{array}$ & $\begin{array}{c}\text { Others } \\
-\mathrm{a}\end{array}$ & $\begin{array}{c}\text { Sum } \\
-\mathrm{a}\end{array}$ \\
\hline SBW1-44B & & & & & 0.0056 & & & & & & & & & & & & & & & 0.9806 \\
\hline SBW1-45 & & & & & 0.0057 & & & & & & & & & & & & & & & 0.9919 \\
\hline SBW1-46 & & & & & 0.0054 & & & & & & & & & & & & & & & 0.9858 \\
\hline SBW1-47 & & & & & 0.0055 & & & & & & & & & & & & & & & 0.9855 \\
\hline SBW1-48 & & & & & 0.0034 & & & & & & & & & & & & & & & 0.9748 \\
\hline SBW1-49 & & & & & 0.0035 & & & & & & & & & & & & & & & 0.9815 \\
\hline SBW1-50 & & & & & 0.0037 & & & & & & & & & & & & & & & 0.9762 \\
\hline SBW1-51 & & & & & 0.0037 & & & & & & & & & & & & & & & 0.9957 \\
\hline SBW1-52 & & & & & 0.0037 & & & & & & & & & & & & & & & 0.9827 \\
\hline SBW1-53 & & & & & 0.0037 & & & & & & & & & & & & & & & 0.9834 \\
\hline SBW1-54 & & & & & 0.0038 & & & & & & & & & & & & & & & 0.9638 \\
\hline SBW1-55 & & & & & 0.0051 & & & & & & & & & & & & & & & 1.0215 \\
\hline SBW1-56 & & & & & 0.0060 & & & & & & & & & & & & & & & 1.0068 \\
\hline SBW1-57 & & & & & 0.0047 & & & & & & & & & & & & & & & 0.9779 \\
\hline SBW1-58 & & & & & 0.0049 & & & & & & & & & & & & & & & 0.9789 \\
\hline SBW1-59 & & & & & 0.0059 & & & & & & & & & & & & & & & 1.0096 \\
\hline SBW1-60 & & & & & 0.0047 & & & & & & & & & & & & & & & 0.9927 \\
\hline SBW1-61 & & & & & 0.0050 & & & & & & & & & & & & & & & 0.9772 \\
\hline SBW1-62 & & & & & 0.0058 & & & & & & & & & & & & & & & 1.0171 \\
\hline SBW1-63 & & & & & 0.0001 & & & & & & & & & & & & & & & 0.9813 \\
\hline SBW1-64 & & & & & 0.0001 & & & & & & & & & & & & & & & 0.9763 \\
\hline
\end{tabular}


Appendix A. Database - mass fraction

SBW CVS Phase 1 (Scholes et al. 2002)

\begin{tabular}{|c|c|c|c|c|c|c|c|c|}
\hline Glass ID & $\begin{array}{l}\mathrm{TM} \\
\left({ }^{\circ} \mathrm{C}\right)\end{array}$ & $\begin{array}{l}\text { Gradient } \\
\text { TL }\left({ }^{\circ} \mathrm{C}\right)\end{array}$ & $\begin{array}{l}\text { Uniform } \\
\text { TL }\left({ }^{\circ} \mathrm{C}\right)\end{array}$ & Primary Phase & $\begin{array}{l}\text { Quenched } \\
\text { Visual/OM }\end{array}$ & $\begin{array}{c}\text { Quenched } \\
\text { SEM/EDS or TEM }\end{array}$ & $\begin{array}{l}\text { Quenched } \\
\text { XRD }\end{array}$ & $\begin{array}{c}\text { Quenched } \\
\text { Homogeneous? }\end{array}$ \\
\hline SBW1-44B & 1150 & & 736 & $\mathrm{~K}(\mathrm{Si} 3 \mathrm{Al}) \mathrm{O} 8$ & $\begin{array}{l}\text { Clear dark brown glass with black specks anc } \\
\text { few air bubbles, low viscosity }\end{array}$ & & Amorphous & Single-phase \\
\hline SBW1-45 & 1150 & & 801 & $\mathrm{NaBaPO} 4$ & $\begin{array}{l}\text { Clear very dark purplish brown glass with } \\
\text { black specks and a few air bubbles, low } \\
\text { viscosity }\end{array}$ & & Amorphous & Single-phase \\
\hline SBW1-46 & 1150 & & 808 & $\mathrm{Ca} 5(\mathrm{PO} 4) 3 \mathrm{~F}$ & $\begin{array}{l}\text { Clear dark brown glass with black specks anc } \\
\text { air bubbles, low viscosity }\end{array}$ & & Amorphous & Single-phase \\
\hline SBW1-47 & 1150 & & 798 & $\mathrm{Ca} 5(\mathrm{PO} 4) 3 \mathrm{~F}$ & $\begin{array}{l}\text { Clear dark brown glass with few black speck } \\
\text { and lots of air bubbles, low viscosity }\end{array}$ & & Amorphous & Single-phase \\
\hline SBW1-48 & 1150 & & 868 & $\mathrm{Ca} 5(\mathrm{PO} 4) 3 \mathrm{~F}$ & $\begin{array}{l}\text { Clear brown glass with few black specks and } \\
\text { lots of air bubbles, avg. viscosity }\end{array}$ & & Amorphous & Single-phase \\
\hline SBW1-49 & 1150 & & 766 & $(\mathrm{~K}, \mathrm{Na})(\mathrm{Si} 3 \mathrm{Al}) \mathrm{O} 8$ & $\begin{array}{l}\text { Clear reddish brown glass with black specks } \\
\text { and air bubbles, low viscosity }\end{array}$ & & Amorphous & Single-phase \\
\hline SBW1-50 & 1150 & & & & $\begin{array}{l}\text { Clear brown glass with black specks and a } \\
\text { few air bubbles, low viscosity }\end{array}$ & & Amorphous & Single-phase \\
\hline SBW1-51 & 1151 & & 788 & $(\mathrm{~K}, \mathrm{Na})(\mathrm{Si} 3 \mathrm{Al}) \mathrm{O} 8$ & $\begin{array}{l}\text { Clear brown glass with black specks and a } \\
\text { few air bubbles, avg. viscosity }\end{array}$ & & Amorphous & Single-phase \\
\hline SBW1-52 & 1150 & & 971 & $\mathrm{Ca} 5(\mathrm{PO} 4) 3 \mathrm{~F}$ & $\begin{array}{l}\text { Clear brown glass with black specks and lots } \\
\text { of tiny air bubbles, avg. viscosity }\end{array}$ & & Amorphous & Single-phase \\
\hline SBW1-53 & 1150 & & 783 & $(\mathrm{~K}, \mathrm{Ba}, \mathrm{Na})(\mathrm{Si}, \mathrm{Al}) 4 \mathrm{O} 8$ & $\begin{array}{l}\text { Clear brown glass with black specks and air } \\
\text { bubbles, low viscosity }\end{array}$ & & Amorphous & Single-phase \\
\hline SBW1-54 & 1150 & & 828 & $\mathrm{Ca} 5(\mathrm{PO} 4) 3 \mathrm{~F}$ & $\begin{array}{l}\text { Clear light brown glass with very few black } \\
\text { specks and lots of air bubbles, low viscosity }\end{array}$ & & Amorphous & Single-phase \\
\hline SBW1-55 & 1150 & & 828 & $\mathrm{NaAlSiO4}$ & $\begin{array}{l}\text { Clear brown glass with black specks and air } \\
\text { bubbles, low viscosity }\end{array}$ & & Amorphous & Single-phase \\
\hline SBW1-56 & 1150 & & 828 & $\mathrm{Na} 6(\mathrm{AlSiO} 4) 6$ & $\begin{array}{l}\text { Clear dark brown glass with black specks anc } \\
\text { a few air bubbles, low viscosity }\end{array}$ & & Amorphous & Single-phase \\
\hline SBW1-57 & 1150 & & 756 & $(\mathrm{Na}, \mathrm{K})(\mathrm{Si} 3 \mathrm{Al}) \mathrm{O} 8$ & $\begin{array}{l}\text { Clear light brown glass with black specks anc } \\
\text { air bubbles, low viscosity }\end{array}$ & & Amorphous & Single-phase \\
\hline SBW1-58 & 1150 & & 748 & $\mathrm{~K}(\mathrm{Si} 3 \mathrm{Al}) \mathrm{O} 8$ & $\begin{array}{l}\text { Clear brown glass with black specks and air } \\
\text { bubbles, low viscosity }\end{array}$ & & Amorphous & Single-phase \\
\hline SBW1-59 & 1150 & & 793 & Na6(A1SiO4)6 & $\begin{array}{l}\text { Clear dark brown glass with black specks anc } \\
\text { air bubbles, low viscosity }\end{array}$ & & Amorphous & Single-phase \\
\hline SBW1-60 & 1150 & & 773 & $(\mathrm{Na}, \mathrm{K})(\mathrm{Si} 3 \mathrm{Al}) \mathrm{O} 8$ & $\begin{array}{l}\text { Clear brown glass with black specks and air } \\
\text { bubbles, low viscosity }\end{array}$ & & Amorphous & Single-phase \\
\hline SBW1-61 & 1150 & & 766 & $\mathrm{~K}(\mathrm{Si} 3 \mathrm{Al}) \mathrm{O} 8$ & $\begin{array}{l}\text { Clear brown glass with black specks and air } \\
\text { bubbles, low viscosity }\end{array}$ & & Amorphous & Single-phase \\
\hline SBW1-62 & 1150 & & 828 & $\mathrm{Na} 6(\mathrm{AlSiO} 4) 6$ & $\begin{array}{l}\text { Clear dark brown glass with black specks anc } \\
\text { a few air bubbles, low viscosity }\end{array}$ & & Amorphous & Single-phase \\
\hline SBW1-63 & 1150 & & 878 & LiAlSi2O6 & $\begin{array}{l}\text { Clear blue glass with lots of air bubbles, } \\
\text { avg.viscosity }\end{array}$ & & Amorphous & Single-phase \\
\hline SBW1-64 & 1150 & & 888 & NaLiZrSi6O15 & $\begin{array}{l}\text { Clear blue glass with lots of air bubbles, high } \\
\text { viscosity }\end{array}$ & & Amorphous & Single-phase \\
\hline
\end{tabular}


Appendix A. Database - mass fraction

\begin{tabular}{|c|c|c|c|c|c|}
\hline Glass ID & $\begin{array}{c}\mathrm{CCC} \\
\text { Visual/OM }\end{array}$ & $\begin{array}{c}\text { CCC } \\
\text { SEM/EDS or TEM }\end{array}$ & $\begin{array}{l}\mathrm{CCC} \\
\mathrm{XRD}\end{array}$ & $\begin{array}{c}\text { Heat Treated } \\
\text { Visual/OM }\end{array}$ & $\begin{array}{c}\text { Heat Treated } \\
\text { SEM/EDS or TEM }\end{array}$ \\
\hline SBW1-44B & $\begin{array}{l}\text { Clear dark brown glass, with very few air bubbles and black } \\
\text { specks (expected to be } \mathrm{Ru} \text { ). }\end{array}$ & & Amorphous & & \\
\hline SBW1-45 & $\begin{array}{l}\text { Clear dark purplish glass, a few black specks (expected to be } \\
\text { Ru) and no air bubbles. }\end{array}$ & & Amorphous & & \\
\hline SBW1-46 & $\begin{array}{l}\text { Clear dark brown glass, air bubbles and black specks } \\
\text { (expected to be } \mathrm{Ru} \text { ). }\end{array}$ & & Amorphous & & \\
\hline SBW1-47 & $\begin{array}{l}\text { Clear dark brown glass, air bubbles and black specks } \\
\text { (expected to be } \mathrm{Ru} \text { ). }\end{array}$ & & Amorphous & & \\
\hline SBW1-48 & $\begin{array}{l}\text { Clear brown glass, with air bubbles and black specks } \\
\text { (expected to be Ru). }\end{array}$ & & Amorphous & & \\
\hline SBW1-49 & $\begin{array}{l}\text { Clear dark brown glass, air bubbles and black specks } \\
\text { (expected to be Ru). }\end{array}$ & & Amorphous & & \\
\hline SBW1-50 & $\begin{array}{l}\text { Clear brown glass, with black specks (expected to be Ru) but } \\
\text { no air bubbles . }\end{array}$ & & Amorphous & & \\
\hline SBW1-51 & $\begin{array}{l}\text { Clear brown glass, with black specks (expected to be Ru) but } \\
\text { no air bubbles . }\end{array}$ & & Amorphous & & \\
\hline SBW1-52 & $\begin{array}{l}\text { Clear brown glass, air bubbles and black specks (expected to } \\
\text { be Ru). }\end{array}$ & & Amorphous & & \\
\hline SBW1-53 & $\begin{array}{l}\text { Clear brown glass, air bubbles and black specks (expected to } \\
\text { be Ru). }\end{array}$ & & Amorphous & & \\
\hline SBW1-54 & $\begin{array}{l}\text { Clear brown glass, with air bubbles and black specks } \\
\text { (expected to be Ru). }\end{array}$ & & Amorphous & & \\
\hline SBW1-55 & $\begin{array}{l}\text { Clear brown glass, air bubbles and black specks (expected to } \\
\text { be Ru). }\end{array}$ & & Amorphous & & \\
\hline SBW1-56 & $\begin{array}{l}\text { Clear dark brown glass, with very few air bubbles and black } \\
\text { specks (expected to be Ru). }\end{array}$ & & Amorphous & & \\
\hline SBW1-57 & $\begin{array}{l}\text { Clear brown glass, with few air bubbles and black specks } \\
\text { (expected to be Ru). }\end{array}$ & & Amorphous & & \\
\hline SBW1-58 & $\begin{array}{l}\text { Clear brown glass, air bubbles and black specks (expected to } \\
\text { be Ru). }\end{array}$ & & Amorphous & & \\
\hline SBW1-59 & $\begin{array}{l}\text { Clear dark brown glass, air bubbles and black specks } \\
\text { (expected to be Ru). }\end{array}$ & & Amorphous & & \\
\hline SBW1-60 & $\begin{array}{l}\text { Clear brown glass, few air bubbles and black specks (expected } \\
\text { to be } \mathrm{Ru} \text { ). }\end{array}$ & & Amorphous & & \\
\hline SBW1-61 & $\begin{array}{l}\text { Clear dark brown glass, air bubbles and black specks } \\
\text { (expected to be Ru). }\end{array}$ & & Amorphous & & \\
\hline SBW1-62 & $\begin{array}{l}\text { Clear brown glass with few black specks (expected to be Ru), } \\
\text { no air bubbles. }\end{array}$ & & Amorphous & & \\
\hline SBW1-63 & Clear blue glass, lots air bubbles. & & Amorphous & & \\
\hline SBW1-64 & Clear blue glass, lots air bubbles. & & Amorphous & & \\
\hline
\end{tabular}


Appendix A. Database - mass fraction

SBW CVS Phase 1 (Scholes et al. 2002)

\begin{tabular}{|c|c|c|c|c|c|c|c|c|c|c|c|c|c|c|c|}
\hline Glass ID & $\begin{array}{c}\text { Heat Treated } \\
\text { XRD }\end{array}$ & $\begin{array}{l}\text { Density } \\
\left(\mathrm{g} / \mathrm{cm}^{3}\right)\end{array}$ & $\begin{array}{c}\text { Fulc Visc } \\
\mathrm{A}\end{array}$ & $\begin{array}{c}\text { Fulc Visc } \\
\text { B }\end{array}$ & \begin{tabular}{c|} 
Fulc Visc \\
To \\
\end{tabular} & $\begin{array}{c}\mathrm{FV} 1150^{\circ} \mathrm{C} \\
(\mathrm{Pa} \cdot \mathrm{s})\end{array}$ & \begin{tabular}{|c} 
Arrh Visc \\
A \\
\end{tabular} & \begin{tabular}{|c|} 
Arrh Visc \\
B
\end{tabular} & $1150^{\circ} \mathrm{C}$ & $\begin{array}{c}\mathrm{T}\left({ }^{\circ} \mathrm{C}\right) \text { at } \\
2 \mathrm{~Pa} \cdot \mathrm{s}\end{array}$ & $\begin{array}{c}\mathrm{T}\left({ }^{\circ} \mathrm{C}\right) \text { at } \\
5 \mathrm{~Pa} \cdot \mathrm{s} \\
\end{array}$ & $\begin{array}{l}\mathrm{T}\left({ }^{\circ} \mathrm{C}\right) \text { at } \\
10 \mathrm{~Pa} \cdot \mathrm{s}\end{array}$ & $\begin{array}{c}\mathrm{T} 1 \\
\left({ }^{\circ} \mathrm{C}\right) \\
\end{array}$ & $\begin{array}{c}\mathrm{V} 1 \\
(\mathrm{~Pa} \cdot \mathrm{s})\end{array}$ & $\begin{array}{c}\mathrm{T} 2 \\
\left({ }^{\circ} \mathrm{C}\right) \\
\end{array}$ \\
\hline SBW1-44B & & 2.6652 & & & & & -9.389 & 15058.0 & 3.30 & 1220 & & 1015 & 1154.7 & 3.1362 & 1079.3 \\
\hline SBW1-45 & & 2.6397 & & & & & -11.520 & 17589.0 & 2.32 & 1167 & & 999 & 1154 & 2.1289 & 1078.9 \\
\hline SBW1-46 & & 2.6003 & & & & & -10.014 & 16789.0 & 5.96 & 1295 & & 1090 & 1156.1 & 5.5615 & 1080.2 \\
\hline SBW1-47 & & 2.5972 & & & & & -10.041 & 16785.0 & 5.78 & 1291 & & 1087 & 1159.1 & 5.2659 & 1083.4 \\
\hline SBW1-48 & & 2.5422 & & & & & -10.838 & 19308.0 & 15.34 & 1401 & & 1196 & 1154.6 & 14.841 & 1078.8 \\
\hline SBW1-49 & & 2.5573 & & & & & -9.582 & 15609.0 & 4.00 & 1246 & & 1040 & 1155.5 & 3.7271 & 1080.1 \\
\hline SBW1-50 & & 2.6139 & & & & & -9.684 & 14557.0 & 1.73 & 1130 & & 941 & 1155.9 & 1.6515 & 1079.8 \\
\hline SBW1-51 & & 2.6386 & & & & & -10.371 & 18767.0 & 16.73 & 1423 & & 1208 & 1155.6 & 16.027 & 1079.6 \\
\hline SBW1-52 & & 2.5406 & & & & & -11.090 & 19320.0 & 12.02 & 1366 & & 1169 & 1157.9 & 10.749 & 1082.1 \\
\hline SBW1-53 & & 2.583 & & & & & -9.384 & 16003.0 & 6.43 & 1315 & & 1096 & 1154.5 & 6.1183 & 1079 \\
\hline SBW1-54 & & 2.5967 & & & & & -10.144 & 16445.0 & 4.11 & 1244 & & 1048 & 1155.6 & 3.8889 & 1079.8 \\
\hline SBW1-55 & & 2.6049 & & & & & -10.031 & 15968.0 & 3.29 & 1216 & & 1022 & 1157.5 & 3.0212 & 1082.8 \\
\hline SBW1-56 & & 2.609 & & & & & -9.767 & 15266.0 & 2.61 & 1186 & & 992 & 1154 & 2.5424 & 1078 \\
\hline SBW1-57 & & 2.6291 & & & & & -9.859 & 16260.0 & 4.80 & 1268 & & 1064 & 1156.5 & 4.4991 & 1080.8 \\
\hline SBW1-58 & & 2.6365 & & & & & -9.647 & 15607.0 & 3.75 & 1236 & & 1033 & 1156.8 & 3.4718 & 1081.2 \\
\hline SBW1-59 & & 2.6385 & & & & & -9.504 & 15071.0 & 2.97 & 1205 & & 1003 & 1155.4 & 2.7929 & 1080.5 \\
\hline SBW1-60 & & 2.6101 & & & & & -10.036 & 16410.0 & 4.46 & 1256 & & 1057 & 1157.5 & 4.1374 & 1082.4 \\
\hline SBW1-61 & & 2.6179 & & & & & -9.804 & 15716.0 & 3.46 & 1224 & & 1025 & 1155.4 & 3.2467 & 1080.1 \\
\hline SBW1-62 & & 2.6238 & & & & & -9.638 & 15230.0 & 2.90 & 1201 & & 1002 & 1155.2 & 2.777 & 1079.4 \\
\hline SBW1-63 & & 2.4303 & & & & & -10.113 & 17680.0 & 10.09 & 1363 & & 1151 & 1157.6 & 9.1859 & 1082.6 \\
\hline SBW1-64 & & 2.5697 & & & & & -12.395 & 20569.0 & 7.84 & 1298 & & 1126 & 1157.5 & 7.0288 & 1082.6 \\
\hline
\end{tabular}




\begin{tabular}{|c|c|c|c|c|c|c|c|c|c|c|c|c|c|c|c|c|c|c|c|c|c|}
\hline Glass ID & $\begin{array}{c}\mathrm{V} 2 \\
(\mathrm{~Pa} \cdot \mathrm{s})\end{array}$ & $\begin{array}{c}\mathrm{T} 3 \\
\left({ }^{\circ} \mathrm{C}\right)\end{array}$ & $\begin{array}{c}\mathrm{V} 3 \\
(\mathrm{~Pa} \cdot \mathrm{s})\end{array}$ & $\begin{array}{c}\mathrm{T} 4 \\
\left({ }^{\circ} \mathrm{C}\right)\end{array}$ & $\begin{array}{c}\mathrm{V} 4 \\
(\mathrm{~Pa} \cdot \mathrm{s})\end{array}$ & $\begin{array}{c}\mathrm{T} 5 \\
\left({ }^{\circ} \mathrm{C}\right) \\
\end{array}$ & $\begin{array}{c}\mathrm{V} 5 \\
(\mathrm{~Pa} \cdot \mathrm{s})\end{array}$ & $\begin{array}{c}\mathrm{T} 6 \\
\left({ }^{\circ} \mathrm{C}\right) \\
\end{array}$ & $\begin{array}{c}\mathrm{V} 6 \\
(\mathrm{~Pa} \cdot \mathrm{s})\end{array}$ & $\begin{array}{c}\mathrm{T} 7 \\
\left({ }^{\circ} \mathrm{C}\right)\end{array}$ & $\begin{array}{c}\text { V7 } \\
(\mathrm{Pa} \cdot \mathrm{s})\end{array}$ & $\begin{array}{c}\mathrm{T} 8 \\
\left({ }^{\circ} \mathrm{C}\right)\end{array}$ & $\begin{array}{c}\mathrm{V} 8 \\
(\mathrm{~Pa} \cdot \mathrm{s})\end{array}$ & $\begin{array}{c}\mathrm{T} 9 \\
\left({ }^{\circ} \mathrm{C}\right)\end{array}$ & $\begin{array}{c}\mathrm{V} 9 \\
(\mathrm{~Pa} \cdot \mathrm{s})\end{array}$ & $\begin{array}{l}\mathrm{T} 10 \\
\left({ }^{\circ} \mathrm{C}\right)\end{array}$ & $\begin{array}{c}\mathrm{V} 10 \\
(\mathrm{~Pa} \cdot \mathrm{s})\end{array}$ & $\begin{array}{l}\mathrm{T} 11 \\
\left({ }^{\circ} \mathrm{C}\right)\end{array}$ & $\begin{array}{c}\mathrm{V} 11 \\
(\mathrm{~Pa} \cdot \mathrm{s})\end{array}$ & $\begin{array}{l}\mathrm{T} 12 \\
\left({ }^{\circ} \mathrm{C}\right)\end{array}$ & $\begin{array}{c}\mathrm{V} 12 \\
(\mathrm{~Pa} \cdot \mathrm{s})\end{array}$ \\
\hline SBW1-44B & 5.6044 & 1004.3 & 10.914 & 954.63 & 18.154 & 1252.8 & 1.6543 & 1153.1 & 3.2108 & & & & & & & & & & & & \\
\hline SBW1-45 & 4.1231 & 1004.3 & 9.271 & 954.75 & 17.731 & 1252.2 & 1.0906 & 1152.9 & 2.2532 & & & & & & & & & & & & \\
\hline SBW1-46 & 10.54 & 1004.7 & 22.376 & 954.82 & 40.335 & 1253.3 & 2.7835 & 1154 & 5.7004 & & & & & & & & & & & & \\
\hline SBW1-47 & 9.9564 & 1008.2 & 20.983 & 958.65 & 37.278 & 1254.7 & 2.6491 & 1156.9 & 5.465 & & & & & & & & & & & & \\
\hline SBW1-48 & 30.158 & 1004 & 70.681 & 954.3 & 137.76 & 1202.4 & 9.7138 & 1152.9 & 14.683 & & & & & & & & & & & & \\
\hline SBW1-49 & 6.7468 & 1005.5 & 13.589 & 955.79 & 23.674 & 1254.1 & 1.9868 & 1154.6 & 3.8339 & & & & & & & & & & & & \\
\hline SBW1-50 & 2.8677 & 1004.7 & 5.4465 & 954.64 & 9.022 & 1252.9 & 0.8853 & 1153.5 & 1.6652 & & & & & & & & & & & & \\
\hline SBW1-51 & 32.506 & 1004.5 & 73.526 & 954.49 & 139.39 & 1154 & 16.241 & & & & & & & & & & & & & & \\
\hline SBW1-52 & 22.229 & 1007 & 53.628 & 957.28 & 107.86 & 1255.4 & 5.1018 & 1155.5 & 11.092 & & & & & & & & & & & & \\
\hline SBW1-53 & 11.287 & 1004.1 & 23.071 & 954.55 & 39.494 & 1252.8 & 3.0958 & 1153.7 & 6.2195 & & & & & & & & & & & & \\
\hline SBW1-54 & 7.2362 & 1004.8 & 15.102 & 955.18 & 26.445 & 1253.6 & 1.937 & 1154 & 3.905 & & & & & & & & & & & & \\
\hline SBW1-55 & 5.5325 & 1008 & 11.238 & 958.67 & 19.487 & 1256.4 & 1.5659 & 1157.2 & 3.0906 & & & & & & & & & & & & \\
\hline SBW1-56 & 4.5076 & 1003.1 & 8.8429 & 953.45 & 14.944 & 1251.9 & 1.2959 & 1152.7 & 2.5423 & & & & & & & & & & & & \\
\hline SBW1-57 & 8.3111 & 1005.2 & 17.207 & 955.67 & 30.249 & 1253.4 & 2.2878 & 1153.9 & 4.5871 & & & & & & & & & & & & \\
\hline SBW1-58 & 6.3131 & 1006.4 & 12.649 & 956.73 & 21.739 & 1254.6 & 1.8362 & 1155.3 & 3.569 & & & & & & & & & & & & \\
\hline SBW1-59 & 4.9721 & 1005.9 & 9.6696 & 956.29 & 16.142 & 1254.3 & 1.4747 & 1155.2 & 2.8609 & & & & & & & & & & & & \\
\hline SBW1-60 & 7.6618 & 1007.5 & 15.92 & 957.82 & 27.952 & 1255.9 & 2.0912 & 1156.5 & 4.1465 & & & & & & & & & & & & \\
\hline SBW1-61 & 5.893 & 1005.1 & 11.93 & 955.45 & 20.586 & 1254.4 & 1.6884 & 1155.5 & 3.2839 & & & & & & & & & & & & \\
\hline SBW1-62 & 4.9358 & 1004.5 & 9.7052 & 954.89 & 16.273 & 1252.8 & 1.4419 & 1153.4 & 2.8005 & & & & & & & & & & & & \\
\hline SBW1-63 & 17.959 & 1007.7 & 39.496 & 958.04 & 72.978 & 1256.1 & 4.4612 & 1157.1 & 9.3947 & & & & & & & & & & & & \\
\hline SBW1-64 & 15.251 & 1007.7 & 38.414 & 958.09 & 78.728 & 1256 & 3.0691 & 1156.4 & 7.2017 & & & & & & & & & & & & \\
\hline
\end{tabular}


Appendix A. Database - mass fraction

SBW CVS Phase 1 (Scholes et al. 2002)

\begin{tabular}{|c|c|c|c|c|c|c|c|c|c|c|c|c|c|c|c|c|}
\hline Glass ID & $\begin{array}{l}\mathrm{T} 13 \\
\left({ }^{\circ} \mathrm{C}\right) \\
\end{array}$ & $\begin{array}{c}\text { V13 } \\
(\mathrm{Pa} \cdot \mathrm{s}) \\
\end{array}$ & $\begin{array}{l}\mathrm{T} 14 \\
\left({ }^{\circ} \mathrm{C}\right) \\
\end{array}$ & $\begin{array}{c}\text { V14 } \\
(\mathrm{Pa} \cdot \mathrm{s}) \\
\end{array}$ & $\begin{array}{c}\text { Q PCT } \\
\text { B }\left(\mathrm{g} / \mathrm{m}^{2}\right)\end{array}$ & $\begin{array}{c}\text { Q PCT } \\
\mathrm{Li}\left(\mathrm{g} / \mathrm{m}^{2}\right)\end{array}$ & $\begin{array}{c}\text { Q PCT } \\
\mathrm{Na}\left(\mathrm{g} / \mathrm{m}^{2}\right)\end{array}$ & $\begin{array}{c}\text { Q PCT } \\
\mathrm{Si}\left(\mathrm{g} / \mathrm{m}^{2}\right)\end{array}$ & $\begin{array}{c}\text { Q PCT } \\
\mathrm{pH}\end{array}$ & $\begin{array}{l}\text { CCC PCT } \\
\mathrm{B}\left(\mathrm{g} / \mathrm{m}^{2}\right)\end{array}$ & $\begin{array}{l}\text { CCC PCT } \\
\mathrm{Li}\left(\mathrm{g} / \mathrm{m}^{2}\right)\end{array}$ & $\begin{array}{l}\text { CCC PCT } \\
\mathrm{Na}\left(\mathrm{g} / \mathrm{m}^{2}\right)\end{array}$ & $\begin{array}{l}\text { CCC PCT } \\
\mathrm{Si}\left(\mathrm{g} / \mathrm{m}^{2}\right)\end{array}$ & $\begin{array}{c}\text { CCC PCT } \\
\mathrm{pH}\end{array}$ & $\begin{array}{l}\text { Q PCT B at } \\
20^{\circ} \mathrm{C}\left(\mathrm{g} / \mathrm{m}^{2}\right)\end{array}$ & $\begin{array}{l}\text { Q PCT Li at } \\
20^{\circ} \mathrm{C}\left(\mathrm{g} / \mathrm{m}^{2}\right)\end{array}$ \\
\hline SBW1-44B & & & & & 0.908 & 0.998 & 1.078 & 0.360 & 11.39 & & & & & & & \\
\hline SBW1-45 & & & & & 15.272 & & 14.267 & 1.701 & 10.71 & & & & & & & \\
\hline SBW1-46 & & & & & 0.520 & 0.422 & 0.461 & 0.162 & 10.525 & & & & & & & \\
\hline SBW1-47 & & & & & 0.516 & 0.435 & 0.478 & 0.131 & 10.765 & & & & & & & \\
\hline SBW1-48 & & & & & 0.602 & 0.462 & 0.392 & 0.131 & 9.33 & & & & & & & \\
\hline SBW1-49 & & & & & 2.587 & 2.233 & 2.028 & 0.418 & 11.36 & & & & & & & \\
\hline SBW1-50 & & & & & 0.961 & 0.899 & 0.893 & 0.240 & 10.935 & & & & & & & \\
\hline SBW1-51 & & & & & 0.191 & 0.234 & 0.289 & 0.114 & 10.23 & & & & & & & \\
\hline SBW1-52 & & & & & 0.461 & 0.508 & 0.325 & 0.105 & 9.89 & & & & & & & \\
\hline SBW1-53 & & & & & 0.402 & 0.435 & 0.469 & 0.222 & 10.78 & & & & & & & \\
\hline SBW1-54 & & & & & 0.449 & 0.491 & 0.389 & 0.166 & 10.34 & & & & & & & \\
\hline SBW1-55 & & & & & 0.455 & 0.461 & 0.583 & 0.168 & 10.88 & & & & & & & \\
\hline SBW1-56 & & & & & 0.569 & 0.492 & 0.597 & 0.203 & 10.905 & & & & & & & \\
\hline SBW1-57 & & & & & 0.661 & 0.616 & 0.458 & 0.173 & 10.385 & & & & & & & \\
\hline SBW1-58 & & & & & 0.859 & 0.715 & 0.810 & 0.203 & 10.99 & & & & & & & \\
\hline SBW1-59 & & & & & 1.295 & 0.983 & 1.158 & 0.263 & 11.48 & & & & & & & \\
\hline SBW1-60 & & & & & 0.415 & 0.434 & 0.319 & 0.149 & 10.335 & & & & & & & \\
\hline SBW1-61 & & & & & 0.604 & 0.538 & 0.660 & 0.184 & 10.56 & & & & & & & \\
\hline SBW1-62 & & & & & 1.033 & 0.768 & 1.019 & 0.253 & 11.24 & & & & & & & \\
\hline SBW1-63 & & & & & 0.576 & 0.646 & 0.165 & 0.355 & 9.845 & & & & & & & \\
\hline SBW1-64 & & & & & 1.868 & 1.618 & 0.891 & 0.340 & 10.395 & & & & & & & \\
\hline
\end{tabular}


Appendix A. Database - mass fraction

SBW CVS Phase 1 (Scholes et al. 2002)

\begin{tabular}{|c|c|c|c|c|c|c|c|c|c|c|c|c|}
\hline Glass ID & $\begin{array}{l}\text { Q PCT Na at } \\
20^{\circ} \mathrm{C}\left(\mathrm{g} / \mathrm{m}^{2}\right)\end{array}$ & $\begin{array}{l}\text { Q PCT Si at } \\
20^{\circ} \mathrm{C}\left(\mathrm{g} / \mathrm{m}^{2}\right)\end{array}$ & $\begin{array}{c}\mathrm{QpH} \\
\text { at } 20^{\circ} \mathrm{C}\end{array}$ & $\begin{array}{c}\text { TCLP Ag } \\
\text { (ppm) }\end{array}$ & $\begin{array}{c}\text { TCLP As } \\
\text { (ppm) }\end{array}$ & $\begin{array}{c}\text { TCLP Ba } \\
\text { (ppm) }\end{array}$ & $\begin{array}{c}\text { TCLP Cd } \\
(\mathrm{ppm})\end{array}$ & $\begin{array}{c}\text { TCLP Cr } \\
(\mathrm{ppm})\end{array}$ & $\begin{array}{c}\text { TCLP Ni } \\
(\mathrm{ppm})\end{array}$ & $\begin{array}{c}\text { TCLP Pb } \\
(\mathrm{ppm})\end{array}$ & $\begin{array}{c}\text { TCLP Se } \\
(\mathrm{ppm})\end{array}$ & $\begin{array}{c}\text { TCLP Zn } \\
\text { (ppm) }\end{array}$ \\
\hline SBW1-44B & & & & & & & & & & & & \\
\hline SBW1-45 & & & & & & & & & & & & \\
\hline SBW1-46 & & & & & & & & & & & & \\
\hline SBW1-47 & & & & & & & & & & & & \\
\hline SBW1-48 & & & & & & & & & & & & \\
\hline SBW1-49 & & & & & & & & & & & & \\
\hline SBW1-50 & & & & & & & & & & & & \\
\hline SBW1-51 & & & & & & & & & & & & \\
\hline SBW1-52 & & & & & & & & & & & & \\
\hline SBW1-53 & & & & & & & & & & & & \\
\hline SBW1-54 & & & & & & & & & & & & \\
\hline SBW1-55 & & & & & & & & & & & & \\
\hline SBW1-56 & & & & & & & & & & & & \\
\hline SBW1-57 & & & & & & & & & & & & \\
\hline SBW1-58 & & & & & & & & & & & & \\
\hline SBW1-59 & & & & & & & & & & & & \\
\hline SBW1-60 & & & & & & & & & & & & \\
\hline SBW1-61 & & & & & & & & & & & & \\
\hline SBW1-62 & & & & & & & & & & & & \\
\hline SBW1-63 & & & & & & & & & & & & \\
\hline SBW1-64 & & & & & & & & & & & & \\
\hline
\end{tabular}


SBW Melter Glass Formulation (Vienna et al. 2002)

\begin{tabular}{|c|c|c|c|c|c|c|c|c|c|c|c|c|c|c|c|c|c|c|c|c|c|}
\hline Glass ID & $\begin{array}{c}\mathrm{A} 12 \mathrm{O} 3 \\
-\mathrm{t}\end{array}$ & $\begin{array}{c}\mathrm{B} 2 \mathrm{O} 3 \\
-\mathrm{t}\end{array}$ & $\begin{array}{c}\mathrm{CaO} \\
-\mathrm{t}\end{array}$ & $\begin{array}{c}\mathrm{Fe} 2 \mathrm{O} 3 \\
-\mathrm{t}\end{array}$ & $\begin{array}{c}\mathrm{FeO} \\
-\mathrm{t}\end{array}$ & $\begin{array}{c}\mathrm{K} 2 \mathrm{O} \\
-\mathrm{t}\end{array}$ & $\begin{array}{c}\mathrm{Li} 2 \mathrm{O} \\
-\mathrm{t}\end{array}$ & $\begin{array}{c}\mathrm{MgO} \\
-\mathrm{t}\end{array}$ & $\begin{array}{c}\mathrm{Na} 2 \mathrm{O} \\
-\mathrm{t}\end{array}$ & $\begin{array}{c}\mathrm{NiO} \\
-\mathrm{t}\end{array}$ & $\begin{array}{c}\mathrm{P} 2 \mathrm{O} 5 \\
-\mathrm{t}\end{array}$ & $\begin{array}{c}\mathrm{SiO} 2 \\
-\mathrm{t}\end{array}$ & $\begin{array}{c}\mathrm{ZrO} 2 \\
-\mathrm{t}\end{array}$ & $\begin{array}{c}\mathrm{Ag} 2 \mathrm{O} \\
-\mathrm{t}\end{array}$ & $\begin{array}{c}\mathrm{As} 2 \mathrm{O} 3 \\
-\mathrm{t}\end{array}$ & $\begin{array}{c}\mathrm{BaO} \\
-\mathrm{t}\end{array}$ & $\begin{array}{c}\mathrm{Bi} 2 \mathrm{O} 3 \\
-\mathrm{t}\end{array}$ & $\begin{array}{l}\mathrm{Br} \\
-\mathrm{t}\end{array}$ & $\begin{array}{c}\mathrm{CdO} \\
-\mathrm{t}\end{array}$ & $\begin{array}{c}\mathrm{Ce} 2 \mathrm{O} 3 \\
-\mathrm{t}\end{array}$ & $\begin{array}{c}\mathrm{CeO} 2 \\
-\mathrm{t}\end{array}$ \\
\hline SBW-11-18.5 & 0.0510 & 0.0997 & 0.0449 & 0.0150 & & 0.0140 & 0.0498 & 0.0150 & 0.1117 & 0.0002 & 0.0015 & 0.5235 & 0.0199 & & & 0.0000 & & & 0.0001 & & \\
\hline SBW-11-30 & 0.0827 & 0.0861 & 0.0416 & 0.0149 & & 0.0226 & 0.0428 & 0.0134 & 0.1693 & 0.0003 & 0.0024 & 0.4496 & 0.0171 & & & 0.0000 & & & 0.0002 & & \\
\hline SBW-12-18.5 & 0.0510 & 0.0498 & 0.0449 & 0.0150 & & 0.0140 & 0.0498 & 0.0150 & 0.1531 & 0.0002 & 0.0015 & 0.5320 & 0.0199 & & & 0.0000 & & & 0.0001 & & \\
\hline SBW-13-18.5 & 0.0510 & 0.0997 & 0.0040 & 0.0150 & & 0.0804 & 0.0498 & 0.0007 & 0.1117 & 0.0002 & 0.0015 & 0.5122 & 0.0199 & & & 0.0000 & & & 0.0001 & & \\
\hline SBW-14-18.5 & 0.0510 & 0.1184 & 0.0449 & 0.0150 & & 0.0140 & 0.0498 & 0.0150 & 0.1117 & 0.0002 & 0.0015 & 0.5445 & 0.0199 & & & 0.0000 & & & 0.0001 & & \\
\hline SBW-15-18.5 & 0.0510 & 0.0577 & 0.0692 & 0.0678 & & 0.0140 & 0.0489 & 0.0007 & 0.1532 & 0.0002 & 0.0015 & 0.5216 & 0.0000 & & & 0.0000 & & & 0.0001 & & \\
\hline SBW-16-18.5 & 0.0510 & 0.0498 & 0.0449 & 0.0150 & & 0.0140 & 0.0498 & 0.0150 & 0.1312 & 0.0002 & 0.0015 & 0.5538 & 0.0199 & & & 0.0000 & & & 0.0001 & & \\
\hline SBW-17-18.5 & 0.0510 & 0.0984 & 0.0203 & 0.1004 & & 0.0140 & 0.0326 & 0.0007 & 0.0962 & 0.0002 & 0.0015 & 0.5705 & 0.0000 & & & 0.0000 & & & 0.0001 & & \\
\hline SBW-18-18.5 & 0.0510 & 0.0984 & 0.0203 & 0.1004 & & 0.0140 & 0.0245 & 0.0007 & 0.1288 & 0.0002 & 0.0015 & 0.5216 & 0.0082 & & & 0.0000 & & & 0.0001 & & \\
\hline SBW-19-18.5 & 0.0510 & 0.0984 & 0.0203 & 0.1004 & & 0.0140 & 0.0245 & 0.0007 & 0.1288 & 0.0002 & 0.0015 & 0.5053 & 0.0082 & & & 0.0000 & & & 0.0001 & & \\
\hline SBW-20-18.5 & 0.0510 & 0.0984 & 0.0366 & 0.1004 & & 0.0140 & 0.0245 & 0.0007 & 0.1288 & 0.0002 & 0.0015 & 0.4890 & 0.0082 & & & 0.0000 & & & 0.0001 & & \\
\hline SBW-21-18.5 & 0.0510 & 0.0821 & 0.0366 & 0.1004 & & 0.0140 & 0.0245 & 0.0007 & 0.1288 & 0.0002 & 0.0015 & 0.5053 & 0.0082 & & & 0.0000 & & & 0.0001 & & \\
\hline SBW-22-15 & 0.0414 & 0.0518 & 0.0459 & 0.0150 & & 0.0113 & 0.0519 & 0.0155 & 0.1145 & 0.0001 & 0.0012 & 0.5776 & 0.0208 & & & 0.0000 & & & 0.0001 & & \\
\hline SBW-22-18.5 & 0.0510 & 0.0498 & 0.0449 & 0.0150 & & 0.0140 & 0.0498 & 0.0150 & 0.1312 & 0.0002 & 0.0015 & 0.5538 & 0.0199 & & & 0.0000 & & & 0.0001 & & \\
\hline SBW-22-20 & 0.0551 & 0.0490 & 0.0445 & 0.0150 & & 0.0151 & 0.0489 & 0.0148 & 0.1384 & 0.0002 & 0.0016 & 0.5436 & 0.0195 & & & 0.0000 & & & 0.0002 & & \\
\hline SBW-22-25 & 0.0689 & 0.0461 & 0.0430 & 0.0149 & & 0.0189 & 0.0458 & 0.0141 & 0.1622 & 0.0002 & 0.0020 & 0.5096 & 0.0183 & & & 0.0000 & & & 0.0002 & & \\
\hline SBW-23-15 & 0.0414 & 0.1038 & 0.0032 & 0.0150 & & 0.0480 & 0.0519 & 0.0006 & 0.0941 & 0.0001 & 0.0012 & 0.5668 & 0.0208 & & & 0.0000 & & & 0.0001 & & \\
\hline SBW-23-18.5 & 0.0510 & 0.0997 & 0.0040 & 0.0150 & & 0.0492 & 0.0498 & 0.0007 & 0.1117 & 0.0002 & 0.0015 & 0.5435 & 0.0199 & & & 0.0000 & & & 0.0001 & & \\
\hline SBW-23-20 & 0.0551 & 0.0979 & 0.0043 & 0.0150 & & 0.0496 & 0.0489 & 0.0008 & 0.1192 & 0.0002 & 0.0016 & 0.5335 & 0.0195 & & & 0.0000 & & & 0.0002 & & \\
\hline SBW-23-25 & 0.0689 & 0.0920 & 0.0054 & 0.0149 & & 0.0512 & 0.0458 & 0.0010 & 0.1442 & 0.0002 & 0.0020 & 0.5001 & 0.0183 & & & 0.0000 & & & 0.0002 & & \\
\hline SBW-24-18.5 & 0.0510 & 0.0911 & 0.0449 & 0.0150 & & 0.0140 & 0.0498 & 0.0150 & 0.1117 & 0.0002 & 0.0015 & 0.5718 & 0.0199 & & & 0.0000 & & & 0.0001 & & \\
\hline SBW-25-15 & 0.0414 & 0.1038 & 0.0459 & 0.1041 & & 0.0113 & 0.0292 & 0.0155 & 0.0780 & 0.0001 & 0.0012 & 0.4958 & 0.0208 & & & 0.0000 & & & 0.0001 & & \\
\hline SBW-25-18.5 & 0.0510 & 0.0997 & 0.0449 & 0.1004 & & 0.0140 & 0.0280 & 0.0150 & 0.0962 & 0.0002 & 0.0015 & 0.4753 & 0.0199 & & & 0.0000 & & & 0.0001 & & \\
\hline SBW-25-20 & 0.0551 & 0.0979 & 0.0445 & 0.0988 & & 0.0151 & 0.0275 & 0.0148 & 0.1040 & 0.0002 & 0.0016 & 0.4666 & 0.0195 & & & 0.0000 & & & 0.0002 & & \\
\hline SBW-25-25 & 0.0689 & 0.0920 & 0.0430 & 0.0935 & & 0.0189 & 0.0258 & 0.0141 & 0.1300 & 0.0002 & 0.0020 & 0.4374 & 0.0183 & & & 0.0000 & & & 0.0002 & & \\
\hline SBW-26-18.5 & 0.0510 & 0.0498 & 0.1196 & 0.0150 & & 0.0140 & 0.0287 & 0.0150 & 0.1117 & 0.0002 & 0.0015 & 0.5197 & 0.0199 & & & 0.0000 & & & 0.0001 & & \\
\hline SBW-27-18.5 & 0.0510 & 0.0498 & 0.0997 & 0.0150 & & 0.0140 & 0.0333 & 0.0150 & 0.1117 & 0.0002 & 0.0015 & 0.5152 & 0.0199 & & & 0.0000 & & & 0.0001 & & \\
\hline
\end{tabular}


Appendix A. Database - mass fraction

SBW Melter Glass Formulation (Vienna et al. 2002)

\begin{tabular}{|c|c|c|c|c|c|c|c|c|c|c|c|c|c|c|c|c|c|c|c|c|c|}
\hline Glass ID & $\begin{array}{l}\mathrm{Cl} \\
-\mathrm{t}\end{array}$ & $\begin{array}{c}\mathrm{CoO} \\
-\mathrm{t}\end{array}$ & $\begin{array}{c}\mathrm{Co} 2 \mathrm{O} 3 \\
-\mathrm{t}\end{array}$ & $\begin{array}{c}\mathrm{Cr} 2 \mathrm{O} 3 \\
-\mathrm{t}\end{array}$ & $\begin{array}{c}\mathrm{Cs} 2 \mathrm{O} \\
-\mathrm{t}\end{array}$ & $\begin{array}{c}\mathrm{CuO} \\
-\mathrm{t}\end{array}$ & $\begin{array}{c}\mathrm{Eu} 2 \mathrm{O} 3 \\
-\mathrm{t}\end{array}$ & $\begin{array}{l}F \\
-t\end{array}$ & $\begin{array}{c}\mathrm{Ga} 2 \mathrm{O} 3 \\
-\mathrm{t}\end{array}$ & {$\left[\begin{array}{c}\mathrm{Gd} 2 \mathrm{O} 3 \\
-\mathrm{t}\end{array}\right.$} & $\begin{array}{c}\mathrm{HgO} \\
-\mathrm{t}\end{array}$ & $\begin{array}{l}I \\
-t\end{array}$ & $\begin{array}{c}\mathrm{La} 2 \mathrm{O} 3 \\
-\mathrm{t}\end{array}$ & $\begin{array}{c}\mathrm{MnO} 2 \\
-\mathrm{t}\end{array}$ & $\begin{array}{c}\mathrm{MnO} \\
-\mathrm{t}\end{array}$ & $\begin{array}{c}\mathrm{MoO} \\
-\mathrm{t}\end{array}$ & $\begin{array}{c}\mathrm{MoO} 3 \\
-\mathrm{t}\end{array}$ & $\begin{array}{c}\mathrm{Nb} 2 \mathrm{O} 5 \\
-\mathrm{t}\end{array}$ & $\begin{array}{c}\mathrm{Nd} 2 \mathrm{O} 3 \\
-\mathrm{t}\end{array}$ & $\begin{array}{c}\mathrm{PbO} \\
-\mathrm{t}\end{array}$ & $\begin{array}{c}\mathrm{PdO} 2 \\
-\mathrm{t}\end{array}$ \\
\hline SBW-11-18.5 & 0.0016 & & & 0.0004 & & 0.0001 & & 0.0014 & & 0.0001 & & & & & 0.0015 & & 0.0000 & & & 0.0004 & \\
\hline SBW-11-30 & 0.0026 & & & 0.0006 & & 0.0002 & & 0.0022 & & 0.0001 & & & & & 0.0024 & & 0.0001 & & & 0.0007 & \\
\hline SBW-12-18.5 & 0.0016 & & & 0.0004 & & 0.0001 & & 0.0014 & & 0.0001 & & & & & 0.0015 & & 0.0000 & & & 0.0004 & \\
\hline SBW-13-18.5 & 0.0016 & & & 0.0004 & & 0.0001 & & 0.0014 & & 0.0001 & & & & & 0.0015 & & 0.0000 & & & 0.0004 & \\
\hline SBW-14-18.5 & 0.0016 & & & 0.0004 & & 0.0001 & & 0.0014 & & 0.0001 & & & & & 0.0015 & & 0.0000 & & & 0.0004 & \\
\hline SBW-15-18.5 & 0.0016 & & & 0.0004 & & 0.0001 & & 0.0014 & & 0.0001 & & & & & 0.0015 & & 0.0000 & & & 0.0004 & \\
\hline SBW-16-18.5 & 0.0016 & & & 0.0004 & & 0.0001 & & 0.0014 & & 0.0001 & & & & & 0.0015 & & 0.0000 & & & 0.0004 & \\
\hline SBW-17-18.5 & 0.0016 & & & 0.0004 & & 0.0001 & & 0.0014 & & 0.0001 & & & & & 0.0015 & & 0.0000 & & & 0.0004 & \\
\hline SBW-18-18.5 & 0.0016 & & & 0.0004 & & 0.0001 & & 0.0014 & & 0.0001 & & & & & 0.0015 & & 0.0000 & & & 0.0004 & \\
\hline SBW-19-18.5 & 0.0016 & & & 0.0004 & & 0.0001 & & 0.0014 & & 0.0001 & & & & & 0.0015 & & 0.0000 & & & 0.0004 & \\
\hline SBW-20-18.5 & 0.0016 & & & 0.0004 & & 0.0001 & & 0.0014 & & 0.0001 & & & & & 0.0015 & & 0.0000 & & & 0.0004 & \\
\hline SBW-21-18.5 & 0.0016 & & & 0.0004 & & 0.0001 & & 0.0014 & & 0.0001 & & & & & 0.0015 & & 0.0000 & & & 0.0004 & \\
\hline SBW-22-15 & 0.0013 & & & 0.0003 & & 0.0001 & & 0.0011 & & 0.0000 & & & & & 0.0012 & & 0.0000 & & & 0.0004 & \\
\hline SBW-22-18.5 & 0.0016 & & & 0.0004 & & 0.0001 & & 0.0014 & & 0.0001 & & & & & 0.0015 & & 0.0000 & & & 0.0004 & \\
\hline SBW-22-20 & 0.0017 & & & 0.0004 & & 0.0001 & & 0.0015 & & 0.0001 & & & & & 0.0016 & & 0.0000 & & & 0.0005 & \\
\hline SBW-22-25 & 0.0022 & & & 0.0005 & & 0.0001 & & 0.0018 & & 0.0001 & & & & & 0.0020 & & 0.0001 & & & 0.0006 & \\
\hline SBW-23-15 & 0.0013 & & & 0.0003 & & 0.0001 & & 0.0011 & & 0.0000 & & & & & 0.0012 & & 0.0000 & & & 0.0004 & \\
\hline SBW-23-18.5 & 0.0016 & & & 0.0004 & & 0.0001 & & 0.0014 & & 0.0001 & & & & & 0.0015 & & 0.0000 & & & 0.0004 & \\
\hline SBW-23-20 & 0.0017 & & & 0.0004 & & 0.0001 & & 0.0015 & & 0.0001 & & & & & 0.0016 & & 0.0000 & & & 0.0005 & \\
\hline SBW-23-25 & 0.0022 & & & 0.0005 & & 0.0001 & & 0.0018 & & 0.0001 & & & & & 0.0020 & & 0.0001 & & & 0.0006 & \\
\hline SBW-24-18.5 & 0.0016 & & & 0.0004 & & 0.0001 & & 0.0014 & & 0.0001 & & & & & 0.0015 & & 0.0000 & & & 0.0004 & \\
\hline SBW-25-15 & 0.0013 & & & 0.0003 & & 0.0001 & & 0.0011 & & 0.0000 & & & & & 0.0012 & & 0.0000 & & & 0.0004 & \\
\hline SBW-25-18.5 & 0.0016 & & & 0.0004 & & 0.0001 & & 0.0014 & & 0.0001 & & & & & 0.0015 & & 0.0000 & & & 0.0004 & \\
\hline SBW-25-20 & 0.0017 & & & 0.0004 & & 0.0001 & & 0.0015 & & 0.0001 & & & & & 0.0016 & & 0.0000 & & & 0.0005 & \\
\hline SBW-25-25 & 0.0022 & & & 0.0005 & & 0.0001 & & 0.0018 & & 0.0001 & & & & & 0.0020 & & 0.0001 & & & 0.0006 & \\
\hline SBW-26-18.5 & 0.0016 & & & 0.0004 & & 0.0001 & & 0.0014 & & 0.0001 & & & & & 0.0015 & & 0.0000 & & & 0.0004 & \\
\hline SBW-27-18.5 & 0.0016 & & & 0.0004 & & 0.0001 & & 0.0014 & & 0.0001 & & & & & 0.0015 & & 0.0000 & & & 0.0004 & \\
\hline
\end{tabular}


Appendix A. Database - mass fraction

SBW Melter Glass Formulation (Vienna et al. 2002)

\begin{tabular}{|c|c|c|c|c|c|c|c|c|c|c|c|c|c|c|c|c|c|c|c|c|c|}
\hline Glass ID & $\begin{array}{c}\mathrm{PdO} \\
-\mathrm{t}\end{array}$ & $\begin{array}{c}\mathrm{Pr} 2 \mathrm{O} 3 \\
-\mathrm{t}\end{array}$ & $\begin{array}{c}\text { Pr6O11 } \\
-t\end{array}$ & $\begin{array}{c}\mathrm{Rb} 2 \mathrm{O} \\
-\mathrm{t}\end{array}$ & $\begin{array}{c}\mathrm{ReO} \\
-\mathrm{t}\end{array}$ & $\begin{array}{c}\mathrm{ReO} 2 \\
-\mathrm{t}\end{array}$ & $\begin{array}{c}\mathrm{Rh} 2 \mathrm{O} 3 \\
-\mathrm{t}\end{array}$ & $\begin{array}{c}\mathrm{RhO} 2 \\
-\mathrm{t}\end{array}$ & $\begin{array}{c}\mathrm{RuO} 2 \\
-\mathrm{t}\end{array}$ & $\begin{array}{c}\mathrm{Sb} 2 \mathrm{O} 3 \\
-\mathrm{t}\end{array}$ & $\begin{array}{c}\mathrm{Sb} 2 \mathrm{O} 5 \\
-\mathrm{t}\end{array}$ & $\begin{array}{c}\mathrm{SeO} 2 \\
-\mathrm{t}\end{array}$ & $\begin{array}{c}\mathrm{Sm} 2 \mathrm{O} 3 \\
-\mathrm{t}\end{array}$ & $\begin{array}{c}\mathrm{SnO} \\
-\mathrm{t}\end{array}$ & $\begin{array}{c}\mathrm{SnO} 2 \\
-\mathrm{t}\end{array}$ & $\begin{array}{c}\mathrm{SO} 3 \\
-\mathrm{t}\end{array}$ & $\begin{array}{c}\mathrm{SrO} \\
-\mathrm{t}\end{array}$ & $\begin{array}{c}\mathrm{Tc} 2 \mathrm{O} 7 \\
-\mathrm{t}\end{array}$ & $\begin{array}{c}\mathrm{TeO} 2 \\
-\mathrm{t}\end{array}$ & $\begin{array}{c}\mathrm{ThO} 2 \\
-\mathrm{t}\end{array}$ & $\begin{array}{c}\mathrm{TiO} 2 \\
-\mathrm{t}\end{array}$ \\
\hline SBW-11-18.5 & & & & & & & & & & & & & & & & 0.0084 & & & & & \\
\hline SBW-11-30 & & & & & & & & & & & & & & & & 0.0137 & & & & & \\
\hline SBW-12-18.5 & & & & & & & & & & & & & & & & 0.0084 & & & & & \\
\hline SBW-13-18.5 & & & & & & & & & & & & & & & & 0.0084 & & & & & \\
\hline SBW-14-18.5 & & & & & & & & & & & & & & & & 0.0084 & & & & & \\
\hline SBW-15-18.5 & & & & & & & & & & & & & & & & 0.0084 & & & & & \\
\hline SBW-16-18.5 & & & & & & & & & & & & & & & & 0.0084 & & & & & \\
\hline SBW-17-18.5 & & & & & & & & & & & & & & & & 0.0084 & & & & & \\
\hline SBW-18-18.5 & & & & & & & & & & & & & & & & 0.0084 & & & & & \\
\hline SBW-19-18.5 & & & & & & & & & & & & & & & & 0.0084 & & & & & \\
\hline SBW-20-18.5 & & & & & & & & & & & & & & & & 0.0084 & & & & & \\
\hline SBW-21-18.5 & & & & & & & & & & & & & & & & 0.0084 & & & & & \\
\hline SBW-22-15 & & & & & & & & & & & & & & & & 0.0068 & & & & & \\
\hline SBW-22-18.5 & & & & & & & & & & & & & & & & 0.0084 & & & & & \\
\hline SBW-22-20 & & & & & & & & & & & & & & & & 0.0091 & & & & & \\
\hline SBW-22-25 & & & & & & & & & & & & & & & & 0.0114 & & & & & \\
\hline SBW-23-15 & & & & & & & & & & & & & & & & 0.0068 & & & & & \\
\hline SBW-23-18.5 & & & & & & & & & & & & & & & & 0.0084 & & & & & \\
\hline SBW-23-20 & & & & & & & & & & & & & & & & 0.0091 & & & & & \\
\hline SBW-23-25 & & & & & & & & & & & & & & & & 0.0114 & & & & & \\
\hline SBW-24-18.5 & & & & & & & & & & & & & & & & 0.0084 & & & & & \\
\hline SBW-25-15 & & & & & & & & & & & & & & & & 0.0068 & & & & & \\
\hline SBW-25-18.5 & & & & & & & & & & & & & & & & 0.0084 & & & & & \\
\hline SBW-25-20 & & & & & & & & & & & & & & & & 0.0091 & & & & & \\
\hline SBW-25-25 & & & & & & & & & & & & & & & & 0.0114 & & & & & \\
\hline SBW-26-18.5 & & & & & & & & & & & & & & & & 0.0084 & & & & & \\
\hline SBW-27-18.5 & & & & & & & & & & & & & & & & 0.0084 & & & & & \\
\hline
\end{tabular}


SBW Melter Glass Formulation (Vienna et al. 2002)

\begin{tabular}{|c|c|c|c|c|c|c|c|c|c|c|c|c|c|c|c|c|c|c|c|c|c|}
\hline Glass ID & $\begin{array}{c}\mathrm{T} 12 \mathrm{O} 3 \\
-\mathrm{t}\end{array}$ & $\begin{array}{c}\mathrm{U} 3 \mathrm{O} 8 \\
-\mathrm{t}\end{array}$ & $\begin{array}{c}\mathrm{UO} 2 \\
-\mathrm{t}\end{array}$ & $\begin{array}{c}\mathrm{UO} 3 \\
-\mathrm{t}\end{array}$ & $\begin{array}{c}\mathrm{V} 2 \mathrm{O} 5 \\
-\mathrm{t}\end{array}$ & $\begin{array}{c}\text { WO3 } \\
-t\end{array}$ & $\begin{array}{c}\mathrm{Y} 2 \mathrm{O} 3 \\
-\mathrm{t}\end{array}$ & $\begin{array}{c}\mathrm{ZnO} \\
-\mathrm{t}\end{array}$ & $\begin{array}{c}\text { Others } \\
-t\end{array}$ & $\begin{array}{c}\text { Sum } \\
-t\end{array}$ & $\begin{array}{c}\mathrm{A} 12 \mathrm{O} 3 \\
-\mathrm{a}\end{array}$ & $\begin{array}{c}\mathrm{B} 2 \mathrm{O} 3 \\
-\mathrm{a}\end{array}$ & $\begin{array}{c}\mathrm{CaO} \\
-\mathrm{a}\end{array}$ & $\begin{array}{c}\mathrm{Fe} 2 \mathrm{O} 3 \\
-\mathrm{a}\end{array}$ & $\begin{array}{c}\mathrm{FeO} \\
-\mathrm{a}\end{array}$ & $\begin{array}{c}\mathrm{K} 2 \mathrm{O} \\
-\mathrm{a}\end{array}$ & $\begin{array}{c}\mathrm{Li} 2 \mathrm{O} \\
-\mathrm{a}\end{array}$ & $\begin{array}{c}\mathrm{MgO} \\
-\mathrm{a}\end{array}$ & $\begin{array}{c}\mathrm{Na} 2 \mathrm{O} \\
-\mathrm{a}\end{array}$ & $\begin{array}{c}\mathrm{NiO} \\
-\mathrm{a}\end{array}$ & $\begin{array}{c}\mathrm{P} 2 \mathrm{O} 5 \\
-\mathrm{a}\end{array}$ \\
\hline SBW-11-18.5 & & & & & 0.0399 & & & 0.0000 & & 1.0000 & 0.0533 & 0.1060 & 0.0465 & 0.0163 & & 0.0121 & 0.0527 & 0.0159 & 0.1100 & & \\
\hline SBW-11-30 & & & & & 0.0344 & & & 0.0000 & & 1.0000 & & & & & & & & & & & \\
\hline SBW-12-18.5 & & & & & 0.0399 & & & 0.0000 & & 1.0000 & 0.0516 & 0.0486 & 0.0456 & 0.0150 & & 0.0148 & 0.0488 & 0.0150 & 0.1540 & 0.0003 & \\
\hline SBW-13-18.5 & & & & & 0.0399 & & & 0.0000 & & 1.0000 & 0.0505 & 0.0921 & 0.0039 & 0.0144 & & 0.0822 & 0.0475 & 0.0005 & 0.1110 & 0.0002 & \\
\hline SBW-14-18.5 & & & & & 0.0001 & & & 0.0000 & & 1.0000 & 0.0512 & 0.1130 & 0.0456 & 0.0150 & & 0.0149 & 0.0486 & 0.0150 & 0.1120 & 0.0002 & \\
\hline SBW-15-18.5 & & & & & 0.0001 & & & 0.0000 & & 1.0000 & 0.0508 & 0.0554 & 0.0701 & 0.0683 & & 0.0145 & 0.0477 & 0.0045 & 0.1490 & 0.0002 & \\
\hline SBW-16-18.5 & & & & & 0.0399 & & & 0.0000 & & 1.0000 & 0.0512 & 0.1080 & 0.0619 & 0.0031 & & 0.0145 & 0.0473 & 0.0005 & 0.1130 & 0.0003 & \\
\hline SBW-17-18.5 & & & & & 0.0001 & & & 0.0000 & & 1.0000 & 0.0523 & 0.1000 & 0.0180 & 0.0959 & & 0.0124 & 0.0321 & & 0.0987 & & \\
\hline SBW-18-18.5 & & & & & 0.0164 & & & 0.0000 & & 1.0000 & 0.0524 & 0.0989 & 0.0177 & 0.0969 & & 0.0127 & 0.0218 & & 0.1240 & & \\
\hline SBW-19-18.5 & & & & & 0.0327 & & & 0.0000 & & 1.0000 & 0.0533 & 0.0986 & 0.0180 & 0.0989 & & 0.0128 & 0.0214 & & 0.1260 & & \\
\hline SBW-20-18.5 & & & & & 0.0327 & & & 0.0000 & & 1.0000 & 0.0530 & 0.0985 & 0.0371 & 0.1000 & & 0.0127 & 0.0213 & & 0.1260 & & \\
\hline SBW-21-18.5 & & & & & 0.0327 & & & 0.0000 & & 1.0000 & 0.0542 & 0.0829 & 0.0382 & 0.1010 & & 0.0127 & 0.0210 & & 0.1260 & & \\
\hline SBW-22-15 & & & & & 0.0416 & & & 0.0000 & & 1.0000 & 0.0427 & 0.0528 & 0.0423 & 0.0176 & & 0.0125 & 0.0497 & 0.0161 & 0.1129 & 0.0002 & 0.0013 \\
\hline SBW-22-18.5 & & & & & 0.0399 & & & 0.0000 & & 1.0000 & 0.0518 & 0.0489 & 0.0406 & 0.0150 & & 0.0149 & 0.0479 & 0.0152 & 0.1281 & 0.0002 & 0.0015 \\
\hline SBW-22-20 & & & & & 0.0392 & & & 0.0000 & & 1.0000 & 0.0563 & 0.0467 & 0.0407 & 0.0147 & & 0.0166 & 0.0462 & 0.0151 & 0.1350 & 0.0002 & 0.0017 \\
\hline SBW-22-25 & & & & & 0.0368 & & & 0.0000 & & 1.0000 & 0.0703 & 0.0435 & 0.0400 & 0.0149 & & 0.0210 & 0.0430 & 0.0146 & 0.1620 & 0.0002 & 0.0021 \\
\hline SBW-23-15 & & & & & 0.0416 & & & 0.0000 & & 1.0000 & 0.0412 & 0.1020 & & 0.0167 & & 0.0427 & 0.0544 & 0.0004 & 0.0932 & 0.0002 & 0.0017 \\
\hline SBW-23-18.5 & & & & & 0.0399 & & & 0.0000 & & 1.0000 & 0.0516 & 0.0972 & 0.0017 & 0.0163 & & 0.0438 & 0.0528 & 0.0006 & 0.1110 & 0.0002 & 0.0020 \\
\hline SBW-23-20 & & & & & 0.0392 & & & 0.0000 & & 1.0000 & 0.0554 & 0.0955 & 0.0015 & 0.0167 & & 0.0450 & 0.0522 & \begin{tabular}{|l|}
0.0006 \\
\end{tabular} & 0.1180 & 0.0002 & 0.0021 \\
\hline SBW-23-25 & & & & & 0.0368 & & & 0.0000 & & 1.0000 & 0.0675 & 0.0884 & 0.0025 & 0.0166 & & 0.0446 & 0.0485 & 0.0009 & 0.1410 & 0.0003 & 0.0024 \\
\hline SBW-24-18.5 & & & & & 0.0001 & & & 0.0000 & & 1.0000 & & & & & & & & & & & \\
\hline SBW-25-15 & & & & & 0.0416 & & & 0.0000 & & 1.0000 & 0.0418 & 0.1010 & 0.0494 & 0.1030 & & 0.0112 & 0.0295 & 0.0155 & 0.0760 & 0.0001 & 0.0017 \\
\hline SBW-25-18.5 & & & & & 0.0399 & & & 0.0000 & & 1.0000 & 0.0516 & 0.0959 & 0.0473 & 0.0998 & & 0.0145 & 0.0277 & 0.0150 & 0.0952 & 0.0003 & 0.0019 \\
\hline SBW-25-20 & & & & & 0.0392 & & & 0.0000 & & 1.0000 & 0.0559 & 0.0966 & 0.0484 & 0.1000 & & 0.0145 & 0.0275 & 0.0149 & 0.1060 & 0.0002 & 0.0021 \\
\hline SBW-25-25 & & & & & 0.0368 & & & 0.0000 & & 1.0000 & 0.0686 & 0.0911 & 0.0473 & 0.0978 & & 0.0184 & 0.0258 & 0.0144 & 0.1240 & 0.0002 & 0.0025 \\
\hline SBW-26-18.5 & & & & & 0.0399 & & & 0.0000 & & 1.0000 & & & & & & & & & & & \\
\hline SBW-27-18.5 & & & & & 0.0598 & & & 0.0000 & & 1.0000 & & & & & & & & & & & \\
\hline
\end{tabular}


Appendix A. Database - mass fraction

SBW Melter Glass Formulation (Vienna et al. 2002)

\begin{tabular}{|c|c|c|c|c|c|c|c|c|c|c|c|c|c|c|c|c|c|c|c|c|c|}
\hline Glass ID & $\begin{array}{c}\mathrm{SiO} 2 \\
-\mathrm{a}\end{array}$ & $\begin{array}{c}\mathrm{ZrO} 2 \\
-\mathrm{a}\end{array}$ & $\begin{array}{c}\mathrm{Ag} 2 \mathrm{O} \\
-\mathrm{a}\end{array}$ & $\begin{array}{c}\mathrm{As} 2 \mathrm{O} 3 \\
-\mathrm{a}\end{array}$ & $\begin{array}{c}\mathrm{BaO} \\
-\mathrm{a}\end{array}$ & $\begin{array}{c}\mathrm{Bi} 2 \mathrm{O} 3 \\
-\mathrm{a}\end{array}$ & $\begin{array}{l}\mathrm{Br} \\
-\mathrm{a}\end{array}$ & $\begin{array}{c}\mathrm{CdO} \\
-\mathrm{a}\end{array}$ & $\begin{array}{c}\mathrm{Ce} 2 \mathrm{O} 3 \\
-\mathrm{a}\end{array}$ & $\begin{array}{c}\mathrm{CeO} 2 \\
-\mathrm{a}\end{array}$ & $\begin{array}{l}\mathrm{Cl} \\
-\mathrm{a}\end{array}$ & $\begin{array}{c}\mathrm{CoO} \\
-\mathrm{a}\end{array}$ & $\begin{array}{c}\mathrm{Co} 2 \mathrm{O} 3 \\
-\mathrm{a}\end{array}$ & $\begin{array}{c}\mathrm{Cr} 2 \mathrm{O} 3 \\
-\mathrm{a}\end{array}$ & $\begin{array}{c}\mathrm{Cs} 2 \mathrm{O} \\
-\mathrm{a}\end{array}$ & $\begin{array}{c}\mathrm{CuO} \\
-\mathrm{a}\end{array}$ & $\begin{array}{c}\mathrm{Eu} 2 \mathrm{O} 3 \\
-\mathrm{a}\end{array}$ & $\begin{array}{c}\mathrm{F} \\
-\mathrm{a}\end{array}$ & $\begin{array}{c}\mathrm{Ga} 2 \mathrm{O} 3 \\
-\mathrm{a}\end{array}$ & $\begin{array}{c}\mathrm{Gd} 2 \mathrm{O} 3 \\
-\mathrm{a}\end{array}$ & $\begin{array}{c}\mathrm{HgO} \\
-\mathrm{a}\end{array}$ \\
\hline SBW-11-18.5 & 0.5410 & 0.0189 & & & & & & & & & & & & 0.0004 & & 0.0001 & & & & 0.0001 & \\
\hline SBW-11-30 & & & & & & & & & & & & & & & & & & & & & \\
\hline SBW-12-18.5 & 0.5410 & 0.0197 & & & & & & & & & & & & 0.0004 & & 0.0001 & & & & & \\
\hline SBW-13-18.5 & 0.5240 & 0.0196 & & & & & & & & & & & & 0.0004 & & 0.0001 & & & & & \\
\hline SBW-14-18.5 & 0.5430 & 0.0194 & & & & & & & & & & & & 0.0004 & & 0.0001 & & & & & \\
\hline SBW-15-18.5 & 0.5240 & 0.0002 & & & & & & & & & & & & 0.0004 & & 0.0001 & & & & & \\
\hline SBW-16-18.5 & 0.5710 & 0.0001 & & & & & & & & & & & & 0.0006 & & 0.0001 & & & & & \\
\hline SBW-17-18.5 & 0.5360 & 0.0000 & & & & & & & & & & & & 0.0003 & & 0.0001 & & & & & \\
\hline SBW-18-18.5 & 0.5140 & 0.0074 & & & & & & & & & & & & 0.0003 & & 0.0001 & & & & & \\
\hline SBW-19-18.5 & 0.5090 & 0.0076 & & & & & & & & & & & & 0.0003 & & 0.0001 & & & & & \\
\hline SBW-20-18.5 & 0.4990 & 0.0078 & & & & & & & & & & & & 0.0004 & & 0.0001 & & & & & \\
\hline SBW-21-18.5 & 0.5170 & 0.0076 & & & & & & & & & & & & 0.0005 & & 0.0001 & & & & & \\
\hline SBW-22-15 & 0.5842 & 0.0196 & & & & & & & & & & & & 0.0003 & & 0.0000 & & & & 0.0001 & \\
\hline SBW-22-18.5 & 0.5393 & 0.0182 & & & & & & & & & & & & 0.0007 & & 0.0001 & & & & 0.0001 & \\
\hline SBW-22-20 & 0.5500 & 0.0182 & & & & & & & & & & & & 0.0006 & & 0.0001 & & & & 0.0001 & \\
\hline SBW-22-25 & 0.5222 & 0.0176 & & & & & & & & & & & & 0.0006 & & 0.0001 & & & & 0.0001 & \\
\hline SBW-23-15 & 0.5630 & 0.0201 & & & & & & & & & & & & 0.0003 & & 0.0003 & & & & 0.0001 & \\
\hline SBW-23-18.5 & 0.5240 & 0.0192 & & & & & & & & & & & & 0.0004 & & 0.0003 & & & & 0.0001 & \\
\hline SBW-23-20 & 0.5240 & 0.0190 & & & & & & & & & & & & 0.0005 & & 0.0003 & & & & 0.0001 & \\
\hline SBW-23-25 & 0.4990 & 0.0182 & & & & & & & & & & & & 0.0005 & & 0.0003 & & & & 0.0001 & \\
\hline SBW-24-18.5 & & & & & & & & & & & & & & & & & & & & & \\
\hline SBW-25-15 & 0.4860 & 0.0201 & & & & & & & & & & & & 0.0003 & & 0.0003 & & & & & \\
\hline SBW-25-18.5 & 0.4580 & 0.0190 & & & & & & & & & & & & 0.0005 & & 0.0010 & & & & 0.0001 & \\
\hline SBW-25-20 & 0.4240 & 0.0200 & & & & & & & & & & & & 0.0014 & & 0.0003 & & & & & \\
\hline SBW-25-25 & 0.4560 & 0.0178 & & & & & & & & & & & & 0.0007 & & 0.0004 & & & & & \\
\hline SBW-26-18.5 & & & & & & & & & & & & & & & & & & & & & \\
\hline SBW-27-18.5 & & & & & & & & & & & & & & & & & & & & & \\
\hline
\end{tabular}


Appendix A. Database - mass fraction

SBW Melter Glass Formulation (Vienna et al. 2002)

\begin{tabular}{|c|c|c|c|c|c|c|c|c|c|c|c|c|c|c|c|c|c|c|c|c|c|}
\hline Glass ID & $\begin{array}{c}\mathrm{I} \\
-\mathrm{a}\end{array}$ & $\begin{array}{c}\mathrm{La} 2 \mathrm{O} 3 \\
-\mathrm{a}\end{array}$ & $\begin{array}{c}\mathrm{MnO} 2 \\
-\mathrm{a}\end{array}$ & $\begin{array}{c}\mathrm{MnO} \\
-\mathrm{a}\end{array}$ & $\begin{array}{c}\mathrm{MoO} \\
-\mathrm{a}\end{array}$ & $\begin{array}{c}\mathrm{MoO} 3 \\
-\mathrm{a}\end{array}$ & $\begin{array}{c}\mathrm{Nb} 2 \mathrm{O} 5 \\
-\mathrm{a}\end{array}$ & $\begin{array}{c}\mathrm{Nd} 2 \mathrm{O} 3 \\
-\mathrm{a}\end{array}$ & $\begin{array}{c}\mathrm{PbO} \\
-\mathrm{a}\end{array}$ & $\begin{array}{c}\mathrm{PdO} 2 \\
-\mathrm{a}\end{array}$ & $\begin{array}{c}\mathrm{PdO} \\
-\mathrm{a}\end{array}$ & $\begin{array}{c}\mathrm{Pr} 2 \mathrm{O} 3 \\
-\mathrm{a}\end{array}$ & $\begin{array}{c}\operatorname{Pr} 6 \mathrm{O} 11 \\
-\mathrm{a}\end{array}$ & $\begin{array}{c}\mathrm{Rb} 2 \mathrm{O} \\
-\mathrm{a}\end{array}$ & $\begin{array}{c}\mathrm{ReO} \\
-\mathrm{a}\end{array}$ & $\begin{array}{c}\mathrm{ReO} 2 \\
-\mathrm{a}\end{array}$ & $\begin{array}{c}\mathrm{Rh} 2 \mathrm{O} 3 \\
-\mathrm{a}\end{array}$ & $\begin{array}{c}\mathrm{RhO} 2 \\
-\mathrm{a}\end{array}$ & $\begin{array}{c}\mathrm{RuO} 2 \\
-\mathrm{a}\end{array}$ & $\begin{array}{c}\mathrm{Sb} 2 \mathrm{O} 3 \\
-\mathrm{a}\end{array}$ & $\begin{array}{c}\mathrm{Sb} 2 \mathrm{O} 5 \\
-\mathrm{a}\end{array}$ \\
\hline SBW-11-18.5 & & & & 0.0014 & & & & & & & & & & & & & & & & & \\
\hline \multicolumn{22}{|l|}{ SBW-11-30 } \\
\hline SBW-12-18.5 & & & & 0.0015 & & & & & 0.0005 & & & & & & & & & & & & \\
\hline SBW-13-18.5 & & & & 0.0015 & & & & & 0.0004 & & & & & & & & & & & & \\
\hline SBW-14-18.5 & & & & 0.0015 & & & & & 0.0004 & & & & & & & & & & & & \\
\hline SBW-15-18.5 & & & & & & & & & 0.0005 & & & & & & & & & & & & \\
\hline SBW-16-18.5 & & & & 0.0015 & & & & & 0.0004 & & & & & & & & & & & & \\
\hline SBW-17-18.5 & & & & 0.0014 & & & & & 0.0004 & & & & & & & & & & & & \\
\hline SBW-18-18.5 & & & & 0.0014 & & & & & 0.0004 & & & & & & & & & & & & \\
\hline SBW-19-18.5 & & & & 0.0014 & & & & & 0.0004 & & & & & & & & & & & & \\
\hline SBW-20-18.5 & & & & 0.0014 & & & & & 0.0004 & & & & & & & & & & & & \\
\hline SBW-21-18.5 & & & & 0.0014 & & & & & 0.0004 & & & & & & & & & & & & \\
\hline SBW-22-15 & & & & 0.0012 & & 0.0010 & & & 0.0004 & & & & & & & & & & & & \\
\hline SBW-22-18.5 & & & & 0.0014 & & 0.0010 & & & 0.0004 & & & & & & & & & & & & \\
\hline SBW-22-20 & & & & 0.0015 & & 0.0010 & & & 0.0005 & & & & & & & & & & & & \\
\hline SBW-22-25 & & & & 0.0020 & & 0.0009 & & & 0.0006 & & & & & & & & & & & & \\
\hline SBW-23-15 & & & & 0.0012 & & & & & 0.0003 & & & & & & & & & & & & \\
\hline SBW-23-18.5 & & & & 0.0015 & & & & & 0.0004 & & & & & & & & & & & & \\
\hline SBW-23-20 & & & & 0.0016 & & & & & 0.0005 & & & & & & & & & & & & \\
\hline SBW-23-25 & & & & 0.0020 & & 0.0001 & & & 0.0006 & & & & & & & & & & & & \\
\hline \multicolumn{22}{|l|}{ SBW-24-18.5 } \\
\hline SBW-25-15 & & & & 0.0012 & & & & & 0.0004 & & & & & & & & & & & & \\
\hline SBW-25-18.5 & & & & 0.0015 & & 0.0001 & & & 0.0007 & & & & & & & & & & & & \\
\hline SBW-25-20 & & & & 0.0016 & & 0.0001 & & & 0.0005 & & & & & & & & & & & & \\
\hline SBW-25-25 & & & & 0.0021 & & 0.0001 & & & 0.0006 & & & & & & & & & & & & \\
\hline \multicolumn{22}{|l|}{ SBW-26-18.5 } \\
\hline SBW-27-18.5 & & & & & & & & & & & & & & & & & & & & & \\
\hline
\end{tabular}


Appendix A. Database - mass fraction

SBW Melter Glass Formulation (Vienna et al. 2002)

\begin{tabular}{|c|c|c|c|c|c|c|c|c|c|c|c|c|c|c|c|c|c|c|c|c|}
\hline Glass ID & $\begin{array}{c}\mathrm{SeO} 2 \\
-\mathrm{a}\end{array}$ & $\begin{array}{c}\mathrm{Sm} 2 \mathrm{O} 3 \\
-\mathrm{a} \\
\end{array}$ & $\begin{array}{c}\mathrm{SnO} \\
-\mathrm{a} \\
\end{array}$ & $\begin{array}{c}\mathrm{SnO} 2 \\
-\mathrm{a} \\
\end{array}$ & $\begin{array}{c}\mathrm{SO} 3 \\
-\mathrm{a}\end{array}$ & $\begin{array}{c}\mathrm{SrO} \\
-\mathrm{a} \\
\end{array}$ & $\begin{array}{c}\mathrm{Tc} 2 \mathrm{O} 7 \\
-\mathrm{a} \\
\end{array}$ & $\begin{array}{c}\mathrm{TeO} 2 \\
-\mathrm{a}\end{array}$ & $\begin{array}{c}\mathrm{ThO}_{2} \\
-\mathrm{a}\end{array}$ & $\begin{array}{c}\mathrm{TiO} 2 \\
-\mathrm{a} \\
\end{array}$ & $\begin{array}{c}\mathrm{T} 12 \mathrm{O} 3 \\
-\mathrm{a} \\
\end{array}$ & $\begin{array}{c}\mathrm{U} 3 \mathrm{O} 8 \\
-\mathrm{a}\end{array}$ & $\begin{array}{c}\text { UO2 } \\
-\mathrm{a}\end{array}$ & $\begin{array}{c}\text { UO3 } \\
-\mathrm{a} \\
\end{array}$ & $\begin{array}{c}\mathrm{V} 2 \mathrm{O} 5 \\
-\mathrm{a} \\
\end{array}$ & $\begin{array}{c}\text { WO3 } \\
-\mathrm{a}\end{array}$ & $\begin{array}{c}\mathrm{Y} 2 \mathrm{O} 3 \\
-\mathrm{a}\end{array}$ & $\begin{array}{c}\mathrm{ZnO} \\
-\mathrm{a} \\
\end{array}$ & $\begin{array}{c}\text { Others } \\
-\mathrm{a} \\
\end{array}$ & $\begin{array}{c}\text { Sum } \\
-\mathrm{a}\end{array}$ \\
\hline SBW-11-18.5 & & & & & 0.0075 & & & & & & & & & & 0.0396 & & & & & 1.0217 \\
\hline SBW-11-30 & & & & & & & & & & & & & & & & & & & & 0.0000 \\
\hline SBW-12-18.5 & & & & & 0.0082 & & & & & & & & & & & & & & & 0.9651 \\
\hline SBW-13-18.5 & & & & & 0.0082 & & & & & & & & & & & & & & & 0.9565 \\
\hline SBW-14-18.5 & & & & & 0.0072 & & & & & & & & & & & & & & & 0.9876 \\
\hline SBW-15-18.5 & & & & & 0.0084 & & & & & & & & & & & & & & & 0.9941 \\
\hline SBW-16-18.5 & & & & & 0.0074 & & & & & & & & & & & & & & & 0.9808 \\
\hline SBW-17-18.5 & & & & & 0.0048 & & & & & & & & & & 0.0009 & & & & & 0.9532 \\
\hline SBW-18-18.5 & & & & & 0.0060 & & & & & & & & & & 0.0164 & & & & & 0.9704 \\
\hline SBW-19-18.5 & & & & & 0.0057 & & & & & & & & & & 0.0324 & & & & & 0.9860 \\
\hline SBW-20-18.5 & & & & & 0.0059 & & & & & & & & & & 0.0331 & & & & & 0.9966 \\
\hline SBW-21-18.5 & & & & & 0.0058 & & & & & & & & & & 0.0329 & & & & & 1.0017 \\
\hline SBW-22-15 & & & & & 0.0060 & & & & & & & & & & 0.0401 & & & 0.0000 & & 1.0009 \\
\hline SBW-22-18.5 & & & & & 0.0074 & & & & & & & & & & 0.0374 & & & 0.0000 & & 0.9701 \\
\hline SBW-22-20 & & & & & 0.0081 & & & & & & & & & & 0.0369 & & & 0.0000 & & 0.9901 \\
\hline SBW-22-25 & & & & & 0.0107 & & & & & & & & & & 0.0349 & & & 0.0000 & & 1.0012 \\
\hline SBW-23-15 & & & & & 0.0061 & & & & & & & & & & 0.0377 & & & 0.0002 & & 0.9818 \\
\hline SBW-23-18.5 & & & & & 0.0078 & & & & & & & & & & 0.0357 & & & 0.0001 & & 0.9668 \\
\hline SBW-23-20 & & & & & 0.0084 & & & & & & & & & & 0.0357 & & & 0.0001 & & 0.9774 \\
\hline SBW-23-25 & & & & & 0.0112 & & & & & & & & & & 0.0325 & & & 0.0001 & & 0.9773 \\
\hline SBW-24-18.5 & & & & & & & & & & & & & & & & & & & & 0.0000 \\
\hline SBW-25-15 & & & & & 0.0051 & & & & & & & & & & 0.0381 & & & 0.0002 & & 0.9809 \\
\hline SBW-25-18.5 & & & & & 0.0058 & & & & & & & & & & 0.0357 & & & 0.0004 & & 0.9720 \\
\hline SBW-25-20 & & & & & 0.0059 & & & & & & & & & & 0.0359 & & & 0.0001 & & 0.9560 \\
\hline SBW-25-25 & & & & & 0.0085 & & & & & & & & & & 0.0340 & & & 0.0002 & & 1.0104 \\
\hline SBW-26-18.5 & & & & & & & & & & & & & & & & & & & & 0.0000 \\
\hline SBW-27-18.5 & & & & & & & & & & & & & & & & & & & & 0.0000 \\
\hline
\end{tabular}


Appendix A. Database - mass fraction

SBW Melter Glass Formulation (Vienna et al. 2002)

\begin{tabular}{|c|c|c|c|c|c|c|c|c|}
\hline Glass ID & $\begin{array}{l}\mathrm{TM} \\
\left({ }^{\circ} \mathrm{C}\right)\end{array}$ & $\begin{array}{l}\text { Gradient } \\
\mathrm{TL}\left({ }^{\circ} \mathrm{C}\right)\end{array}$ & $\begin{array}{l}\text { Uniform } \\
\text { TL }\left({ }^{\circ} \mathrm{C}\right)\end{array}$ & Primary Phase & $\begin{array}{l}\text { Quenched } \\
\text { Visual/OM }\end{array}$ & $\begin{array}{c}\text { Quenched } \\
\text { SEM/EDS or TEM }\end{array}$ & $\begin{array}{l}\text { Quenched } \\
\text { XRD }\end{array}$ & $\begin{array}{c}\text { Quenched } \\
\text { Homogeneous? }\end{array}$ \\
\hline \multicolumn{9}{|l|}{ SBW-11-18.5 } \\
\hline \multicolumn{9}{|l|}{ SBW-11-30 } \\
\hline \multicolumn{9}{|l|}{ SBW-12-18.5 } \\
\hline \multicolumn{9}{|l|}{ SBW-13-18.5 } \\
\hline \multicolumn{9}{|l|}{ SBW-14-18.5 } \\
\hline \multicolumn{9}{|l|}{ SBW-15-18.5 } \\
\hline \multicolumn{9}{|l|}{ SBW-16-18.5 } \\
\hline \multicolumn{9}{|l|}{ SBW-17-18.5 } \\
\hline \multicolumn{9}{|l|}{ SBW-18-18.5 } \\
\hline \multicolumn{9}{|l|}{ SBW-19-18.5 } \\
\hline \multicolumn{9}{|l|}{ SBW-20-18.5 } \\
\hline \multicolumn{9}{|l|}{ SBW-21-18.5 } \\
\hline \multicolumn{9}{|l|}{ SBW-22-15 } \\
\hline \multicolumn{9}{|l|}{ SBW-22-18.5 } \\
\hline \multicolumn{9}{|l|}{ SBW-22-20 } \\
\hline \multicolumn{9}{|l|}{ SBW-22-25 } \\
\hline \multicolumn{9}{|l|}{ SBW-23-15 } \\
\hline \multicolumn{9}{|l|}{ SBW-23-18.5 } \\
\hline \multicolumn{9}{|l|}{ SBW-23-20 } \\
\hline \multicolumn{9}{|l|}{ SBW-23-25 } \\
\hline \multicolumn{9}{|l|}{ SBW-24-18.5 } \\
\hline \multicolumn{9}{|l|}{ SBW-25-15 } \\
\hline \multicolumn{9}{|l|}{ SBW-25-18.5 } \\
\hline \multicolumn{9}{|l|}{ SBW-25-20 } \\
\hline \multicolumn{9}{|l|}{ SBW-25-25 } \\
\hline \multicolumn{9}{|l|}{ SBW-26-18.5 } \\
\hline SBW-27-18.5 & & & & & & & & \\
\hline
\end{tabular}


Appendix A. Database - mass fraction

SBW Melter Glass Formulation (Vienna et al. 2002)

\begin{tabular}{|c|c|c|c|c|c|}
\hline Glass ID & $\begin{array}{c}\mathrm{CCC} \\
\text { Visual/OM }\end{array}$ & $\begin{array}{c}\text { CCC } \\
\text { SEM/EDS or TEM }\end{array}$ & $\begin{array}{l}\text { CCC } \\
\text { XRD }\end{array}$ & $\begin{array}{c}\text { Heat Treated } \\
\text { Visual/OM }\end{array}$ & $\begin{array}{c}\text { Heat Treated } \\
\text { SEM/EDS or TEM }\end{array}$ \\
\hline \multicolumn{6}{|l|}{ SBW-11-18.5 } \\
\hline \multicolumn{6}{|l|}{ SBW-11-30 } \\
\hline \multicolumn{6}{|l|}{ SBW-12-18.5 } \\
\hline \multicolumn{6}{|l|}{ SBW-13-18.5 } \\
\hline \multicolumn{6}{|l|}{ SBW-14-18.5 } \\
\hline \multicolumn{6}{|l|}{ SBW-15-18.5 } \\
\hline \multicolumn{6}{|l|}{ SBW-16-18.5 } \\
\hline \multicolumn{6}{|l|}{ SBW-17-18.5 } \\
\hline \multicolumn{6}{|l|}{ SBW-18-18.5 } \\
\hline \multicolumn{6}{|l|}{ SBW-19-18.5 } \\
\hline \multicolumn{6}{|l|}{ SBW-20-18.5 } \\
\hline \multicolumn{6}{|l|}{ SBW-21-18.5 } \\
\hline \multicolumn{6}{|l|}{ SBW-22-15 } \\
\hline \multicolumn{6}{|l|}{ SBW-22-18.5 } \\
\hline \multicolumn{6}{|l|}{ SBW-22-20 } \\
\hline \multicolumn{6}{|l|}{ SBW-22-25 } \\
\hline \multicolumn{6}{|l|}{ SBW-23-15 } \\
\hline \multicolumn{6}{|l|}{ SBW-23-18.5 } \\
\hline \multicolumn{6}{|l|}{ SBW-23-20 } \\
\hline \multicolumn{6}{|l|}{ SBW-23-25 } \\
\hline \multicolumn{6}{|l|}{ SBW-24-18.5 } \\
\hline \multicolumn{6}{|l|}{ SBW-25-15 } \\
\hline \multicolumn{6}{|l|}{ SBW-25-18.5 } \\
\hline \multicolumn{6}{|l|}{ SBW-25-20 } \\
\hline \multicolumn{6}{|l|}{ SBW-25-25 } \\
\hline \multicolumn{6}{|l|}{ SBW-26-18.5 } \\
\hline SBW-27-18.5 & & & & & \\
\hline
\end{tabular}


Appendix A. Database - mass fraction

SBW Melter Glass Formulation (Vienna et al. 2002)

\begin{tabular}{|c|c|c|c|c|c|c|c|c|c|c|c|c|c|c|c|}
\hline Glass ID & $\begin{array}{l}\text { Heat Treated } \\
\text { XRD }\end{array}$ & $\begin{array}{l}\text { Density } \\
\left(\mathrm{g} / \mathrm{cm}^{3}\right)\end{array}$ & \begin{tabular}{|c|} 
Fulc Visc \\
A \\
\end{tabular} & \begin{tabular}{|c|} 
Fulc Visc \\
B \\
\end{tabular} & \begin{tabular}{|c|} 
Fulc Visc \\
To \\
\end{tabular} & $\begin{array}{c}\mathrm{FV} 1150^{\circ} \mathrm{C} \\
(\mathrm{Pa} \cdot \mathrm{s})\end{array}$ & \begin{tabular}{|c|} 
Arrh Visc \\
A
\end{tabular} & \begin{tabular}{|c|} 
Arrh Visc \\
B
\end{tabular} & $1150^{\circ} \mathrm{C}$ & $\begin{array}{c}\mathrm{T}\left({ }^{\circ} \mathrm{C}\right) \text { at } \\
2 \mathrm{~Pa} \cdot \mathrm{s}\end{array}$ & $\begin{array}{c}\mathrm{T}\left({ }^{\circ} \mathrm{C}\right) \text { at } \\
5 \mathrm{~Pa} \cdot \mathrm{s}\end{array}$ & \begin{tabular}{|c|}
$\mathrm{T}\left({ }^{\circ} \mathrm{C}\right)$ at \\
$10 \mathrm{~Pa} \cdot \mathrm{s}$
\end{tabular} & $\begin{array}{l}\mathrm{T} 1 \\
\left({ }^{\circ} \mathrm{C}\right)\end{array}$ & $\begin{array}{c}\mathrm{V} 1 \\
(\mathrm{~Pa} \cdot \mathrm{s})\end{array}$ & $\begin{array}{l}\mathrm{T} 2 \\
\left({ }^{\circ} \mathrm{C}\right) \\
\end{array}$ \\
\hline SBW-11-18.5 & & & & & & & -8.682 & 14283.8 & 3.88 & & & & 1150 & 3.9202 & 1095.5 \\
\hline \multicolumn{16}{|l|}{ SBW-11-30 } \\
\hline \multicolumn{16}{|l|}{ SBW-12-18.5 } \\
\hline \multicolumn{16}{|l|}{ SBW-13-18.5 } \\
\hline \multicolumn{16}{|l|}{ SBW-14-18.5 } \\
\hline \multicolumn{16}{|l|}{ SBW-15-18.5 } \\
\hline \multicolumn{16}{|l|}{ SBW-16-18.5 } \\
\hline SBW-17-18.5 & & & & & & & -8.353 & 15801.1 & 15.66 & & & & 1150 & 16.392 & 1089 \\
\hline \multicolumn{16}{|l|}{ SBW-18-18.5 } \\
\hline \multicolumn{16}{|l|}{ SBW-19-18.5 } \\
\hline SBW-20-18.5 & & & & & & & -8.934 & 15147.9 & 5.53 & & & & 1154.5 & 5.3519 & 1100.5 \\
\hline SBW-21-18.5 & & & & & & & -8.488 & 14810.3 & 6.82 & & & & 1148.5 & 6.9857 & 1090 \\
\hline SBW-22-15 & & & & & & & -10.123 & 17398.0 & 8.20 & & & & 1150 & 8.2552 & 1094.5 \\
\hline SBW-22-18.5 & & & & & & & -10.122 & \begin{tabular}{|l|}
17137.9 \\
\end{tabular} & 6.83 & & & & 1151.5 & 6.7105 & 1098.5 \\
\hline SBW-22-20 & & & & & & & -9.986 & 16871.6 & 6.49 & & & & 1154 & 6.2008 & 1098 \\
\hline SBW-22-25 & & & & & & & -9.954 & 16499.6 & 5.16 & & & & 1145.5 & 5.3027 & 1091.5 \\
\hline \multicolumn{16}{|l|}{ SBW-23-15 } \\
\hline SBW-23-18.5 & & & & & & & -9.350 & 15767.2 & 5.64 & & & & 1150 & 5.6384 & 1099 \\
\hline \multicolumn{16}{|l|}{ SBW-23-20 } \\
\hline \multicolumn{16}{|l|}{ SBW-23-25 } \\
\hline \multicolumn{16}{|l|}{ SBW-24-18.5 } \\
\hline \multicolumn{16}{|l|}{ SBW-25-15 } \\
\hline SBW-25-18.5 & & & & & & & -10.796 & 17984.2 & 6.31 & & & & 1145 & 6.5209 & 1094.5 \\
\hline SBW-25-20 & & & & & & & -10.215 & 17032.8 & 5.78 & & & & 1151.1 & 5.5963 & 1094.5 \\
\hline SBW-25-25 & & & & & & & -9.426 & 15478.7 & 4.27 & & & & 1150.5 & 4.2057 & 1097 \\
\hline SBW-26-18.5 & & & & & & & -11.308 & 18781.8 & 6.63 & & & & 1149.5 & 6.5778 & 1082 \\
\hline SBW-27-18.5 & & & & & & & -10.962 & 18222.3 & 6.32 & & & & 1151 & 6.3862 & 1096 \\
\hline
\end{tabular}


Appendix A. Database - mass fraction

SBW Melter Glass Formulation (Vienna et al. 2002)

\begin{tabular}{|c|c|c|c|c|c|c|c|c|c|c|c|c|c|c|c|c|c|c|c|c|c|}
\hline Glass ID & $\begin{array}{c}\mathrm{V} 2 \\
(\mathrm{~Pa} \cdot \mathrm{s})\end{array}$ & $\begin{array}{c}\mathrm{T} 3 \\
\left({ }^{\circ} \mathrm{C}\right)\end{array}$ & $\begin{array}{c}\mathrm{V} 3 \\
(\mathrm{~Pa} \cdot \mathrm{s})\end{array}$ & $\begin{array}{c}\mathrm{T} 4 \\
\left({ }^{\circ} \mathrm{C}\right)\end{array}$ & $\begin{array}{c}\mathrm{V} 4 \\
(\mathrm{~Pa} \cdot \mathrm{s})\end{array}$ & $\begin{array}{c}\text { T5 } \\
\left({ }^{\circ} \mathrm{C}\right)\end{array}$ & $\begin{array}{c}\text { V5 } \\
(\mathrm{Pa} \cdot \mathrm{s})\end{array}$ & $\begin{array}{l}\text { T6 } \\
\left({ }^{\circ} \mathrm{C}\right)\end{array}$ & $\begin{array}{c}\mathrm{V} 6 \\
(\mathrm{~Pa} \cdot \mathrm{s})\end{array}$ & $\begin{array}{c}\mathrm{T} 7 \\
\left({ }^{\circ} \mathrm{C}\right)\end{array}$ & $\begin{array}{c}\mathrm{V} 7 \\
(\mathrm{~Pa} \cdot \mathrm{s})\end{array}$ & $\begin{array}{c}\mathrm{T} 8 \\
\left({ }^{\circ} \mathrm{C}\right)\end{array}$ & $\begin{array}{c}\mathrm{V} 8 \\
(\mathrm{~Pa} \cdot \mathrm{s})\end{array}$ & $\begin{array}{c}\mathrm{T} 9 \\
\left({ }^{\circ} \mathrm{C}\right)\end{array}$ & $\begin{array}{c}\mathrm{V} 9 \\
(\mathrm{~Pa} \cdot \mathrm{s})\end{array}$ & $\begin{array}{l}\text { T10 } \\
\left({ }^{\circ} \mathrm{C}\right)\end{array}$ & $\begin{array}{c}\mathrm{V} 10 \\
(\mathrm{~Pa} \cdot \mathrm{s})\end{array}$ & $\begin{array}{l}\mathrm{T} 11 \\
\left({ }^{\circ} \mathrm{C}\right)\end{array}$ & $\begin{array}{c}\mathrm{V} 11 \\
(\mathrm{~Pa} \cdot \mathrm{s})\end{array}$ & $\begin{array}{l}\mathrm{T} 12 \\
\left({ }^{\circ} \mathrm{C}\right)\end{array}$ & $\begin{array}{c}\mathrm{V} 12 \\
(\mathrm{~Pa} \cdot \mathrm{s})\end{array}$ \\
\hline SBW-11-18.5 & 5.7763 & 1043 & 8.7052 & 1150.5 & 3.8799 & 1206 & 2.604 & 1151.1 & 3.8987 & & & & & & & & & & & & \\
\hline \multicolumn{22}{|l|}{ SBW-11-30 } \\
\hline \multicolumn{22}{|l|}{ SBW-12-18.5 } \\
\hline \multicolumn{22}{|l|}{ SBW-13-18.5 } \\
\hline \multicolumn{22}{|l|}{ SBW-14-18.5 } \\
\hline \multicolumn{22}{|l|}{ SBW-15-18.5 } \\
\hline \multicolumn{22}{|l|}{ SBW-16-18.5 } \\
\hline SBW-17-18.5 & 25.515 & 1046 & 37.396 & 1147 & 15.839 & 1199.5 & 10.522 & 1150 & 15.72 & & & & & & & & & & & & \\
\hline \multicolumn{22}{|l|}{ SBW-18-18.5 } \\
\hline \multicolumn{22}{|l|}{ SBW-19-18.5 } \\
\hline SBW-20-18.5 & 8.4352 & 1046 & 12.476 & 1150.5 & 5.5187 & 1201 & 3.75 & 1150 & 5.5791 & & & & & & & & & & & & \\
\hline SBW-21-18.5 & 11.477 & 1052.5 & 13.943 & 1147 & 6.9505 & 1201 & 4.6296 & 1152 & 6.7486 & & & & & & & & & & & & \\
\hline SBW-22-15 & 13.366 & 1048 & 21.184 & 1152 & 8.0016 & 1200.5 & 5.4136 & 1151 & 8.1037 & & & & & & & & & & & & \\
\hline SBW-22-18.5 & 10.681 & 1050 & 17.074 & 1152 & 6.719 & 1201 & 4.5329 & 1150 & 6.8233 & & & & & & & & & & & & \\
\hline SBW-22-20 & 10.098 & 1052 & 15.724 & 1151.5 & 6.4145 & 1207 & 4.1454 & 1150 & 6.5172 & & & & & & & & & & & & \\
\hline SBW-22-25 & 8.3954 & 1044.5 & 13.153 & 1151 & 5.103 & 1201 & 3.4698 & 1150 & 5.2039 & & & & & & & & & & & & \\
\hline \multicolumn{22}{|l|}{ SBW-23-15 } \\
\hline SBW-23-18.5 & 8.6219 & 1043 & 13.79 & 1149.5 & 5.6198 & 1204 & 3.7453 & 1151 & 5.6558 & & & & & & & & & & & & \\
\hline \multicolumn{22}{|l|}{ SBW-23-20 } \\
\hline \multicolumn{22}{|l|}{ SBW-23-25 } \\
\hline \multicolumn{22}{|l|}{ SBW-24-18.5 } \\
\hline \multicolumn{22}{|l|}{ SBW-25-15 } \\
\hline SBW-25-18.5 & 10.507 & 1045.5 & 17.341 & 1150 & 6.2901 & 1207 & 3.9333 & 1151.5 & 6.2072 & & & & & & & & & & & & \\
\hline SBW-25-20 & 9.3449 & 1043.5 & 15.489 & 1150 & 5.6779 & 1216 & 3.4956 & & & & & & & & & & & & & & \\
\hline SBW-25-25 & 6.3985 & 1037 & 10.918 & 1152 & 4.2623 & 1208 & 2.7463 & 1149.5 & 4.4149 & & & & & & & & & & & & \\
\hline SBW-26-18.5 & 12.804 & 1047.5 & 18.738 & 1151 & 6.5046 & 1202.5 & 4.2596 & 1150 & 6.5074 & & & & & & & & & & & & \\
\hline SBW-27-18.5 & 10.561 & 1047.5 & 17.05 & 1149 & 6.273 & 1200 & 4.1094 & 1148 & 6.3546 & & & & & & & & & & & & \\
\hline
\end{tabular}


Appendix A. Database - mass fraction

SBW Melter Glass Formulation (Vienna et al. 2002)

\begin{tabular}{|c|c|c|c|c|c|c|c|c|c|c|c|c|c|c|c|c|}
\hline Glass ID & $\begin{array}{l}\mathrm{T} 13 \\
\left({ }^{\circ} \mathrm{C}\right)\end{array}$ & $\begin{array}{c}\mathrm{V} 13 \\
(\mathrm{~Pa} \cdot \mathrm{s})\end{array}$ & $\begin{array}{l}\mathrm{T} 14 \\
\left({ }^{\circ} \mathrm{C}\right)\end{array}$ & $\begin{array}{c}\text { V14 } \\
(\mathrm{Pa} \cdot \mathrm{s})\end{array}$ & $\begin{array}{c}\text { Q PCT } \\
\mathrm{B}\left(\mathrm{g} / \mathrm{m}^{2}\right)\end{array}$ & $\begin{array}{c}\text { Q PCT } \\
\mathrm{Li}\left(\mathrm{g} / \mathrm{m}^{2}\right)\end{array}$ & $\begin{array}{c}\text { Q PCT } \\
\mathrm{Na}\left(\mathrm{g} / \mathrm{m}^{2}\right)\end{array}$ & $\begin{array}{c}\text { Q PCT } \\
\mathrm{Si}\left(\mathrm{g} / \mathrm{m}^{2}\right)\end{array}$ & $\begin{array}{c}\text { Q PCT } \\
\text { pH }\end{array}$ & $\begin{array}{c}\text { CCC PCT } \\
\text { B }\left(\mathrm{g} / \mathrm{m}^{2}\right)\end{array}$ & $\begin{array}{l}\text { CCC PCT } \\
\mathrm{Li}\left(\mathrm{g} / \mathrm{m}^{2}\right)\end{array}$ & $\begin{array}{l}\text { CCC PCT } \\
\mathrm{Na}\left(\mathrm{g} / \mathrm{m}^{2}\right)\end{array}$ & $\begin{array}{l}\text { CCC PCT } \\
\mathrm{Si}\left(\mathrm{g} / \mathrm{m}^{2}\right)\end{array}$ & $\begin{array}{c}\text { CCC PCT } \\
\text { pH }\end{array}$ & $\begin{array}{l}\text { Q PCT B at } \\
20^{\circ} \mathrm{C}\left(\mathrm{g} / \mathrm{m}^{2}\right)\end{array}$ & $\begin{array}{l}\text { Q PCT Li at } \\
20^{\circ} \mathrm{C}\left(\mathrm{g} / \mathrm{m}^{2}\right)\end{array}$ \\
\hline SBW-11-18.5 & & & & & 1.428 & 1.468 & 1.307 & 0.496 & & 1.257 & 1.305 & 1.177 & 0.465 & & & \\
\hline SBW-11-30 & & & & & 0.598 & 0.558 & 0.720 & 0.251 & & 0.494 & 0.514 & 0.604 & 0.226 & & & \\
\hline SBW-12-18.5 & & & & & 0.541 & 0.836 & 0.911 & 0.357 & & 0.526 & 0.804 & 0.830 & 0.344 & & & \\
\hline SBW-13-18.5 & & & & & 14.883 & 13.479 & 13.341 & 4.165 & & 14.928 & 13.467 & 13.334 & 4.177 & & & \\
\hline SBW-14-18.5 & & & & & 1.051 & 1.009 & 0.948 & 0.397 & & 0.913 & 0.902 & 0.844 & 0.369 & & & \\
\hline SBW-15-18.5 & & & & & 0.298 & 0.614 & 0.691 & 0.165 & & 0.232 & 0.546 & 0.564 & 0.142 & & & \\
\hline SBW-16-18.5 & & & & & 0.186 & 0.306 & 0.293 & 0.126 & & 0.135 & 0.248 & 0.226 & 0.105 & & & \\
\hline SBW-17-18.5 & & & & & 0.176 & 0.299 & 0.194 & 0.136 & & 0.124 & 0.212 & 0.143 & 0.109 & & & \\
\hline SBW-18-18.5 & & & & & 0.191 & 0.275 & 0.223 & 0.127 & & 0.290 & 0.359 & 0.310 & 0.161 & & & \\
\hline SBW-19-18.5 & & & & & 0.454 & 0.509 & 0.421 & 0.186 & & 0.437 & 0.499 & 0.406 & 0.187 & & & \\
\hline SBW-20-18.5 & & & & & 0.341 & 0.417 & 0.367 & 0.162 & & 0.290 & 0.378 & 0.325 & 0.149 & & & \\
\hline SBW-21-18.5 & & & & & 0.230 & 0.344 & 0.301 & 0.144 & & 0.240 & 0.349 & 0.315 & 0.149 & & & \\
\hline SBW-22-15 & & & & & 0.824 & 0.939 & 0.858 & 0.427 & & 0.569 & 0.724 & 0.649 & 0.343 & & & \\
\hline SBW-22-18.5 & & & & & 0.350 & 0.578 & 0.558 & 0.258 & & 0.365 & 0.596 & 0.548 & 0.265 & & & \\
\hline SBW-22-20 & & & & & 0.388 & 0.617 & 0.611 & 0.267 & & 0.299 & 0.547 & 0.516 & 0.236 & & & \\
\hline SBW-22-25 & & & & & 0.376 & 0.721 & 0.803 & 0.302 & & 0.341 & 0.694 & 0.714 & 0.285 & & & \\
\hline SBW-23-15 & & & & & 7.049 & 7.020 & 4.338 & 1.677 & & 6.754 & 6.647 & 4.160 & 1.629 & & & \\
\hline SBW-23-18.5 & & & & & 7.500 & 6.998 & 6.318 & 1.894 & & 7.013 & 7.080 & 5.234 & 1.894 & & & \\
\hline SBW-23-20 & & & & & 8.285 & 7.128 & 6.893 & 2.076 & & 7.694 & 6.671 & 6.440 & 1.980 & & & \\
\hline SBW-23-25 & & & & & 8.841 & 7.368 & 9.441 & 2.298 & & 7.730 & 6.503 & 8.155 & 2.067 & & & \\
\hline SBW-24-18.5 & & & & & 0.722 & 0.760 & 0.719 & 0.328 & & 0.664 & 0.668 & 0.625 & 0.311 & & & \\
\hline SBW-25-15 & & & & & 0.750 & 0.654 & 0.429 & 0.200 & & 0.516 & 0.472 & 0.303 & 0.169 & & & \\
\hline SBW-25-18.5 & & & & & 0.363 & 0.450 & 0.371 & 0.150 & & 0.329 & & 0.309 & 0.142 & & & \\
\hline SBW-25-20 & & & & & 0.389 & 0.367 & 0.351 & 0.139 & & 0.374 & 0.356 & 0.351 & 0.139 & & & \\
\hline SBW-25-25 & & & & & 0.357 & 0.337 & 0.489 & 0.135 & & 0.339 & 0.335 & 0.467 & 0.135 & & & \\
\hline \multicolumn{17}{|l|}{ SBW-26-18.5 } \\
\hline SBW-27-18.5 & & & & & & & & & & & & & & & & \\
\hline
\end{tabular}


Appendix A. Database - mass fraction

SBW Melter Glass Formulation (Vienna et al. 2002)

\begin{tabular}{|c|c|c|c|c|c|c|c|c|c|c|c|c|}
\hline Glass ID & $\begin{array}{l}\text { Q PCT Na at } \\
20^{\circ} \mathrm{C}\left(\mathrm{g} / \mathrm{m}^{2}\right)\end{array}$ & $\begin{array}{l}\text { Q PCT Si at } \\
20^{\circ} \mathrm{C}\left(\mathrm{g} / \mathrm{m}^{2}\right)\end{array}$ & $\begin{array}{c}\mathrm{Q} \mathrm{pH} \\
\text { at } 20^{\circ} \mathrm{C}\end{array}$ & $\begin{array}{c}\text { TCLP Ag } \\
\text { (ppm) }\end{array}$ & $\begin{array}{c}\text { TCLP As } \\
(\mathrm{ppm})\end{array}$ & $\begin{array}{c}\text { TCLP Ba } \\
\text { (ppm) }\end{array}$ & $\begin{array}{c}\text { TCLP Cd } \\
(\mathrm{ppm})\end{array}$ & $\begin{array}{c}\text { TCLP Cr } \\
\text { (ppm) }\end{array}$ & $\begin{array}{c}\text { TCLP Ni } \\
(\mathrm{ppm})\end{array}$ & $\begin{array}{c}\text { TCLP Pb } \\
\text { (ppm) }\end{array}$ & $\begin{array}{c}\text { TCLP Se } \\
(\mathrm{ppm})\end{array}$ & $\begin{array}{c}\text { TCLP Zn } \\
(\mathrm{ppm})\end{array}$ \\
\hline \multicolumn{13}{|l|}{ SBW-11-18.5 } \\
\hline \multicolumn{13}{|l|}{ SBW-11-30 } \\
\hline \multicolumn{13}{|l|}{ SBW-12-18.5 } \\
\hline \multicolumn{13}{|l|}{ SBW-13-18.5 } \\
\hline \multicolumn{13}{|l|}{ SBW-14-18.5 } \\
\hline \multicolumn{13}{|l|}{ SBW-15-18.5 } \\
\hline \multicolumn{13}{|l|}{ SBW-16-18.5 } \\
\hline \multicolumn{13}{|l|}{ SBW-17-18.5 } \\
\hline \multicolumn{13}{|l|}{ SBW-18-18.5 } \\
\hline \multicolumn{13}{|l|}{ SBW-19-18.5 } \\
\hline \multicolumn{13}{|l|}{ SBW-20-18.5 } \\
\hline \multicolumn{13}{|l|}{ SBW-21-18.5 } \\
\hline \multicolumn{13}{|l|}{ SBW-22-15 } \\
\hline \multicolumn{13}{|l|}{ SBW-22-18.5 } \\
\hline \multicolumn{13}{|l|}{ SBW-22-20 } \\
\hline \multicolumn{13}{|l|}{ SBW-22-25 } \\
\hline \multicolumn{13}{|l|}{ SBW-23-15 } \\
\hline \multicolumn{13}{|l|}{ SBW-23-18.5 } \\
\hline \multicolumn{13}{|l|}{ SBW-23-20 } \\
\hline \multicolumn{13}{|l|}{ SBW-23-25 } \\
\hline \multicolumn{13}{|l|}{ SBW-24-18.5 } \\
\hline \multicolumn{13}{|l|}{ SBW-25-15 } \\
\hline \multicolumn{13}{|l|}{ SBW-25-18.5 } \\
\hline \multicolumn{13}{|l|}{ SBW-25-20 } \\
\hline \multicolumn{13}{|l|}{ SBW-25-25 } \\
\hline \multicolumn{13}{|l|}{ SBW-26-18.5 } \\
\hline SBW-27-18.5 & & & & & & & & & & & & \\
\hline
\end{tabular}




\section{Distribution}

No. of

\section{Copies}

\section{OFFSITE}

$4 \quad$ Catholic University of America

Vitreous State Laboratory

620 Michigan Ave., N. E.

Washington, D. C. 20064, Attn:

H. Gan

W. K. Kot

I. S. Muller

I. L. Pegg (w/ appendix)

4 Westinghouse Savannah River Company

Aiken, SC 29808, Attn:
E. W. Holtzscheiter
773-A
C. M. Jantzen
$773-\mathrm{A}$
S. L. Marra
D. K. Peeler (w/appendix) 999-W

1 CEA-Valrho / Marcoule

SCD/LEBM

Bâtiment 208, BP 171

F-30207 Bagnols- Sur-Ceze Cedex, France, Attn:

C. Fillet

1 A. Aloy

V. G. Khlopin Radium Institute

28, $2^{\text {nd }}$ Murinsky Pr.

RF-194021 St. Petersburg, Russia

1 S. I. A. Radon

2/14 $8^{\text {th }}$ Rostovsky Line

RF-119121 Moscow, Russia, Attn:

S. V. Stefanovsky

1 B. Varshal (w/appendix)

Northern Technologies International Corp.

25 Cherry Street, \#3

Lynn, MA 01902
No. of

Copies

\section{ONSITE}

3 DOE/Office of River Protection

R. Carreon H6-60

E. J. Cruz H6-60

B. Mauss H6-60

1 DOE/Richland Operations Office

T. E. Pietrok K8-50

2 CH2M Hill Hanford Group

T. W. Crawford R3-73

K. A. Gasper L4-07

2 GTS Duratek

C. C. Chapman

R. D. Peters

8 Numatec Hanford Company

$\begin{array}{ll}\text { G. K. Allen R3-73 } & \text { R2-50 }\end{array}$

A-M. F. Choho R2-50

B. A. Higley R3-73

S. L. Lambert (3) R3-75

R. S. Wittman R3-73

5 Bechtel National Inc./Washington Group

$\begin{array}{ll}\text { S. M. Barnes } & \mathrm{H} 4-02 \\ \text { C. M. Musick } & \mathrm{H} 4-02 \\ \text { J. M. Perez } & \mathrm{H} 4-02 \\ \text { K. J. Rueter } & \mathrm{H} 4-02 \\ \text { J. G. Reynolds } & \mathrm{H} 4-02 \\ \text { D. J. Swanberg } & \mathrm{H} 4-02\end{array}$

S. M. Barnes

$\mathrm{H} 4-02$

H4-02

H4-02

H4-02

Distr. 1 
No. of

Copies

ONSITE
No. of

\section{Copies}

\section{ONSITE}

28 Pacific Northwest National Laboratory

B. R. Allen

K6-24

G. H. Beeman

K9-18

W. F. Bonner

K9-14

S. K. Cooley

K5-12

J. V. Crum

K6-24

P. R. Hrma (w/appendix)

K6-24

D-S. Kim (w/appendix)

K6-24

D. E. Kurath

P7-28

L. K. Holton

H6-61

E. V. Morrey

P7-28

L. M. Peurrung

K6-24

G. F. Piepel (w/appendix)

K5-12

B. A. Pulsipher

K5-12

M. J. Schweiger

K6-24

G. L. Smith

K6-24

H. D. Smith

K6-24

J. D. Vienna (10) (3 w/appendix)

K6-24

J. H. Westsik

K9-91

B. J. Williams - TFA (w/appendix)

K9-69

Total copies: 28,9 w/appendix

Distr. 2 